A \102 142094

NATL INST OF STANDARDS \& TECH A.I.C.

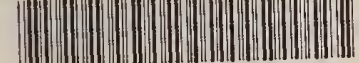

A11102142094

/Miscellaneous publlication - Natlonal Bu 1934

\title{
STANDARDS AND SPECIFICATIONS FOR NONMETALLIC MINERALS AND THEIR PRODUCTS
}

1930

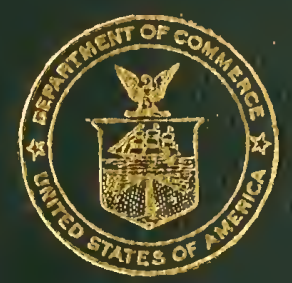





U. S. DEPARTMENT OF COMMERCE

R. P. LAMONT, Secretary

NATIONAL BUREAU OF STANDARDS

GEORGE K. BURGESS, Director

\section{STANDARDS AND SPECIFICATIONS FOR NONMETALLIC MINERALS AND THEIR PRODUCTS}

MISCELLANEOUS PUBLICATION No. 110

APRIL, 1930

PREPARED BY JOHN Q. CANNON, Jr., UNDER THE DIRECTION OF

A. S. MCALLISTER, CHIEF OF THE DIVISION OF SPECIFICATIONS

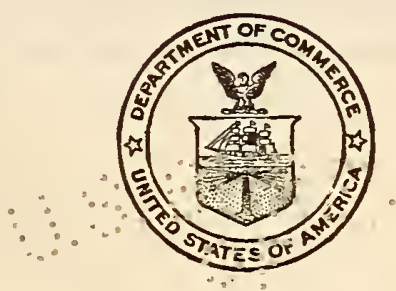

UNITED STATES

GOVERNMENT PRINTING OFFICE

WASHINGTON : 1930 
7 HIS volume represents an attempt on the part of the Department of Commerce to collect and publish the substance of the standards and specifications relating to nonmetallic minerals and manufactures thereof, formulated by the national technical societies, the trade associations having national recognition, or other organizations which speak for industry or with the authority of the Federal Government as a whole.

Criticisms and suggestions are desired from all interested persons, so that the presentation of the information concerning the nationally recognized specifications may be made most nearly universally satisfactory and that the collection of existing specifications and classification thereof may be kept as complete, accurate, and up to date as possible. All recommendations for improvement received will be given careful consideration when future editions are issued. 


\title{
LETTER OF SUBMITTAL
}

\author{
Department of Commerce, \\ National Bureau of Standards, \\ Washington, March 6, 1930.
}

SIR: I have the honor to submit herewith for publication the manuscript for a compilation relating to "Standards and Specifications for Nonmetallic Minerals and Their Products."

In conformity to plans outlined by the Secretary of Commerce in 1923, there has been made under the joint auspices of the Bureau of Standards and the Bureau of Foreign and Domestic Commerce a review of the standards, specifications, simplifications, and testing methods in use in this country. The material obtained as a result of this review, following recommendations of an advisory board composed of official representatives of 14 national organizations interested in the utilization of specifications, was divided into two distinct parts. One part, in the nature of an index, was issued in 1925 as the National Directory of Commodity Specifications. The second part, under the recommendations, was to contain copies of actual specifications (or abstracts) instead of merely titles. The first step in accomplishment of this second part was achieved in 1927, in the publication of the volume Standards and Specifications in the Wood Using Industries.

The present compilation represents a further step in fulfillment of the second part of the program as outlined, being the second of the series of publications dealing with standards and specifications in various industries, to be issued as rapidly as conditions permit.

The material in this volume has been arranged and distributed to conform with the decimal system of indexing made use of in the National Directory of Commodity Specifications. In certain cases, due to the expansion of standardization, it has been necessary to enlarge upon the system laid out in the volume referred to.

Respectfully,

Hon. R. P. LAMont,

George K. Burgess, Director, Bureau of Standards.

Secretary of Commerce. 



\section{CONTENTS}

Page

Introduction

IX

500

GENERAL ITEMS RELATING TO NONMETALLIC MINERALS

Class No

Subject

Page $\mid$ Class No.

Subject

Page

500. 1

501-509

501.

501. 0

501.1

501. 2

501. 3

501. 4

501. 41

501. 42

501. 5

501. 6

502.

502 . 0

502. 1

502. 2

503.

503. 0

503. 1

503. 2

503. 3

503. 1

503. 5

503. 6

503. 7

504.

504.0

504.1

504. 11

504. 12

504. 13

504. 14

504. 15

504. 16

504. 19

504. 2

504. 21

504. 22

504. 23

504. 24

504. 3

504. 31

504. 32

504. 33

504. 34

504. 35

504. 4

504.40

504. 41
COAL, PETROLEOM, ASPHALT, AND MINERAI WAX

Coal and coke

General items....

Anthracite coal (see also 501.0) -

Bituminous coal (see also 501.0) --

Semibituminous coal (see 501.0) _.

Coal-tar products and bituminous materials _._._._.

Coal-tar products

Bituminous materials (see 505).

Coke (see also 501.0)

Charcoal

Petroleum . .

General items.........

Crude petroleum

Petroleum products (see also 503).

Fuel and illuminating oils.......

General items . . . . . . . . . .

Benzine.........................

Distillates....................

Gasoline and naphtha........

Fuel and gas oils

Kerosene and similar illuminating oils

Mineral seal oil

Long-time burning (signal) oil -.-

Lubricating and insulating oils (including paraffin oil) and greases.

General items _..............

Special lubricating oils(see 504.19) Asphaltic oils (see 504.19) ......

Axle generator oils .........

Journal box oils ............

Mineral oils (see 504.19)

Black oil_._._.

Blending oils

Miscellaneous specifications for lubricating oils and greases..

Cylinder oils _. . . . . .

Compressor oil (including air, ammonia ice machine) -.----

Engine oil, steam (including noncondensing, high-speed, and turbine)

Engine oil, internal combustion.

Valve oils ....................

Machine oils .................

Dynamos, spindles, pumps, etc

Machine-gun oil..........-

Recoil and recuperator oils...-

Transmission oil (including crank case) -.......

Typewriter, sewing machine, and clock oils

Lubricating greases. .

General items.

Cup grease.

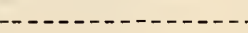

108

108

505. 20

505. 21

505. 22

505. 23

108

108

108

108

108

108

108

108

108

108

108
505. 29

505. 3

505. 30

505. 31

505. 32

505. 33

505. 34

505. 35

505. 36

505. 37

505. 4 road oils

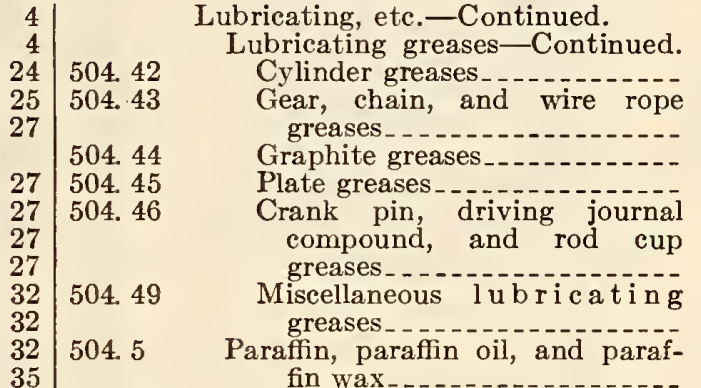

108

108

109

109

109

109

Candles...............

Illuminating wax..........

Paraffin oils_...............

Paraffin wax

Petrolatum

Cutting oils

Transformer and switch oils

Asphalt and other bituminous materials _....................

General items......

Asphalts

Native asphalt._..........

Asphalt pavements (see 518.37)

Asphalt cement.............

Oil asphalt

nous fillers
Asphalt for roofing and waterproofing (see also 505.36) _...

Asphalt mastic flooring-.-.---

Miscellaneous specifications for asphalt and other bituminous materials ............

Road oils ...................

General items _.............

Oil for cold application.......

Oil for hot application.......

Oil asphalt (see 505.14) .......

Miscellaneous specifications for

Tar and tar pitch......

General items _..............

Tars for cold application to roads _....................

Tars for hot application to roads .........

Tars for road construction...-

Tars for repair work..........

Tar cement ..............

Tar for roofing and waterproofing (see also 505.16)

Tar oils

Bituminous surface treatments (see also 518.34) 


\begin{tabular}{|c|c|c|c|c|}
\hline $\begin{array}{l}\text { Class No. } \\
506 .\end{array}$ & $\begin{array}{l}\text { Subject } \\
\text { Ozokerite and other mineral waxes }\end{array}$ & $\begin{array}{l}\text { Page } \\
181\end{array}$ & Class No. & Subject \\
\hline $\begin{array}{l}506 . \\
500\end{array}$ & and other mineral waxes_ & & & iscellaneous oils-Continued. \\
\hline $\begin{array}{l}509 . \\
509 .\end{array}$ & laneous olls & 18 & 509. & Well oil_-_--------- \\
\hline 509.2 & 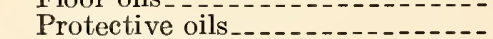 & 18 & 509.6 & ng oil___.. \\
\hline 09.3 & Hydroline oils _ & 18 & 509. 7 & Washing oil _... \\
\hline
\end{tabular}

510 .

511.

511. 1

511. 2

511. 3

511. 4

511. 5

511. 50

511. 51

511. 52

511. 53

511. 6

511. 7

511. 70

511. 71

511. 72

511. 73

511. 9

512.

512. 1

512. 10

512. 11

512. 12

512. 13

512. 14

512. 15

512. 16

512. 2

513.

514.

514. 0

514. 1

514. 2

514. 3

514. 4

514.5

514. 6

514. 61

514. 62

514. 63

515.

516.

516. 0

516. 1

516. 11

516. 12

516. 13

516. 19

516. 2

516.3

516. 4

516. 5

516. 9

517.

517. 0

517. 1

517. 2

518.

518. 0

518. 1

518. 10

518. 11

518. 12

518. 13

518. 14
General items.

Stone and stone manufactures...-

Granite.......

Limestone.........

Marble

Slate.

General items

Blackboard slate

Building slate (see also 505.16)

Slate for electrical purposes _. -

Roapstone and stone for road building _.... General items. Stone and granite blocks....Riprap.- Ballast

Miscellaneous specifications for stone and stone manufactures -

Sand, gravel, and slag.-.....-.

Sand and gravel...........

General items............

Gravel _.........

Sand

Fine aggregate.

Coarse aggregate.......

Broken stone..._._._._._._.

Fillers.

Slag-_._.

Sand-lime brick
Gypsum and other plasters

General items.....

Gypsum Calcined gypsum (plaster of Paris) _.

Gypsum plaster....

Lime plaster.

Portland cement plaster........

Plaster manufactures......... Gypsum plaster board.......Gypsum tiles and blocks.....Wall plasters............

Magnesite

Cement and concrete.-.... General items_.

Portland cement_..........

Natural cement.

Masonry cement_..._._._._._-

Miscellaneous cements.......

Stucco_..._._._._._._._._.

Concrete and mortar._.......

Concrete bricks and blocks

Sidewalks and driveways

Miscellaneous specifications for cement and concrete.........

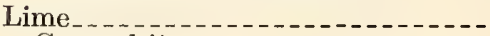

General items.....

Lime.....

Hydrated lime or quicklime....

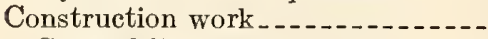

General items.........

Grading for roads

General items . .........

Roadway

Excavation and embankment.

Slopes and shoulders.........

Subbases for roads. \begin{tabular}{l|l}
182 & \\
187 & 518.2
\end{tabular}

187 518. 20

190 518. 21

192 518. 22

194 518. 23

194 518. 24

194 518. 25

196

197

204

206

206

206

207

208

212

212

212

212
217

217
220

235

242

244

253
254

257

258
258

262

263

265
271

271
275

275

275

278

280

281

281

287

287

295

295

297

297

301

323

327

347

347
348

349

364

364

365

365
365

366

367

367

518. 26

518. 27

518. 28

518. 29

518. 3

518. 30

518. 31

518. 32

518. 33

518. 34

518. 35

518. 36

518. 37

518. 38

518. 39

518. 4

518. 40

518. 41

518. 42

518. 43

518. 44

518. 45

518. 49

518. 5

518. 50

518. 51

518. 52

518. 53

518. 54

518. 55

518. 56

518. 57

518. 59

518. 6

518. 60

518. 61

518. 62

518. 63

518. 65

518. 66

518. 67 .

518. 7

518. 71

518. 72
Construction work-Continued.

Foundations for roads_._._... 367

General items_............. 367

Cement concrete foundation -.- 368

Telford foundation.........- 370

Macadam foundation

Broken stone foundation _..._. 371

Bituminous macadam foundation

518. 46

518. 64

tion_.....................

Asphaltic foundation.......

Slag foundation

Miscellaneous specifications for foundation for roads _._._._.

Paving surfaces.

General items............

Cement concrete pavements.--

Gravel pavements.........--

Macadam pavements.......-

Bituminous macadam and concrete pavements (see also

505. 34)

Stone block pavements

Sand clay or topsoil pavements

Miscellaneous pavement specifications . . . .

Bridge construction

General items. . .

Bridges _..._...........

Piling - .

Parapets and railings.......

Slabs and girders............

Floors and wearing surfaces.--

Arches_....................

Miscellaneous items under bridge construction .........

Building construction and materials

General items (see also 505.16 and 505.36)

Buildings and dwellings ...-.-

Foundations for building construction......................

Walls and partitions.....-

Inclosures _..................

Chimneys...

Ceilings and roofing (see also 505.16 and 505.36) -

Miscellaneous specifications for building construction and materials _. . .

Drainage structures (see also $5 \overline{3}$ and 534.21) 


\section{CONTENTS}

Class No.

518. 73

518. 74

518. 75

518. 76

518. 77

518. 8

518. 80

518. 81

520-529

520.

521.

521. 0

521.1

521. 2

521. 3

521.4

521.5

521. 6

521.7

522 .

523.

523. 1

523. 2

523. 3

523. 4

523. 9

$530-539$

531.

531. 0

531. 1

531. 2

531. 3

531. 4

531. 5

532.

532. 0

532.1

532. 2

532. 21

532. 22

532. 23

533.

533. 1

533. 2

533. 3

540-549

541.

541. 0

541.1

541. 2

541.3

541. 4

541.5

545.

545. 1

550-559

551. 560-569

560.

561 .
Subject

Construction work-Continued.

Reservoir, tanks, pools, etc.-Con. Swimming pools...........

Watering troughs..........

Reservoirs . . . . . .

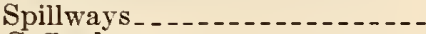

Cofferdams

Masonry

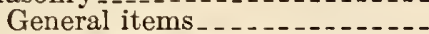

Dry rubble masonry
Page | Class No.

471

472

472

472

472

472

473

473

518. 82

518. 83

518. 84

518. 85

518. 89

518. 9
Subject

Construction work-Continued.

Masonry-Continued.
Cement rubble masonry _. . . _ $\quad 473$

Brick masonry ............... 475

Arches

Monuments_._._._._._._. 478

Miscellaneous specifications for masonry _... _.............

Miscellaneous specifications for construction work ...........

478

481
Page

498

502

502

502

502

503

504

504

504

504

504

505

Tubes and tubing

Water gauges

Miscellaneous specifications for glass_._._._._.

Bottles (vinegar)

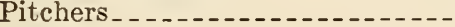

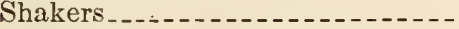

Tumblers.............

Miscellaneous table glassware...
496 526. 9

497

497527.

497

497
506

506

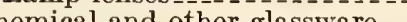

Mirrors
Clay

General items...

Ball clay

China clay

Fire clay

Kaolin

Clay products

China and porcelain ware

General items_........-......

Table chinaware....

Porcelain .......... Crucibles _. . _.............. Porcelain for electrical purposes Tubs, sinks, and lavatories...-

Earthenware and stoneware.....Bowls..................... Crockery . . .

Pots_

ABRASIVE MATE

5
8
8

78

\section{AND CLAY PRODUCTS}

\begin{tabular}{l|l}
507 & 534. \\
507 & 534.1 \\
512 & 534.10 \\
512 & 534.11 \\
512 & \\
514 & 534.12 \\
515 & 534.19 \\
518 & \\
518 & 534.2 \\
519 & 534.20 \\
523 & 534.21 \\
523 & 534.22 \\
525 & 534.23 \\
535 & 534.24 \\
559 & 534.29 \\
559 & \\
559 & \\
559 &
\end{tabular}

Bricks and tiles........

559

559

559

General items.

Face, common, and vitrified brick - - - -

Fire brick and fire-clay brick --

Miscellaneous specifications for brick

577

586

593

General items 593

Drainage tiles (see also 518.62)_ $\quad 606$

Hollow building tile........- 614

Roof tiles................. 619

Terra cotta_..... 620

Miscellaneous specifications for tile.......................

622

Abrasives, natural and artificial_-.-

General items...........-

623

\section{3}

Flint_..................... 623

Hones, whetstones, and oilstones

Abrasive wheels

Pumice.-.

Asbestos...

Asbestos, unmanufactured....

\section{3}

623

\section{1}

639

639

639546.
MICA, RARE MINERALS

Asbestos-Continued.

545. 2 Asbestos paper, millboard, and roll board 545. 4 Textiles, yarns, and packing Miscellaneous manufactures of asbestos _...
639

640

640

645

645

648

Crude mica_................. 648 | 552. Manufactured mica_............

\section{PRECIOUS STONES AND IMITATIONS}

\begin{tabular}{ll|l} 
General items & \\
Diamonds and pearls & $562 . \quad$ Sapphires_.
\end{tabular} 


\section{CONTENTS}

570-579

Class No.

571.

572.

573.

574.

590-599
SULPHUR, MAGNESIA, SALT, AND GRAPHITE

Subject
Magnesia_.
Salt
Graphite and manufactures thereof

Page Class No.

650575.

$650 \quad 575$.

658 575. 2

Subject

Prge

658

Carbons and electrodes

658

658

658

\section{OTHER NONMETALIIC MINERALS}

591.

Fluorspar and cryolite

$659 \mid 593$.

659599.

Silica

Miscellaneous nonmetallic minerals_

659

592.

\section{SOCIETIES AND ASSOCIATIONS}

Technical societies, trade associations, and other organizations issuing standards and specifications dealing with nonmetallic minerals

\section{INDEX}

Alphabetical list of commodities covered by standards and specifications, and methods of inspection

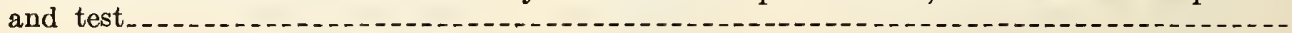




\section{INTRODUCTION}

In general terms this volume can be described briefly as an editorial compendium of standards and specifications relating to nonmetallic minerals and manufactures thereof.

In it are included in full, or by means of adequate abstracts, tabulations, or cross references, the substance of every available nationally recognized specification for such commodities as are embraced within the numerical classification designations 500 to 599 , in the National Directory of Commodity Specifications.

Neither in the selection of the specifications for inclusion in the volume, nor in the arrangement and thesubstance of the specifications included, has any preference been exerted. An effort has been made to include all standards and specifications properly belonging within the scope of nonmetallic minerals, which have been promulgated or adopted by technical societies, trade associations, or other organizations qualified to speak authoritatively for industry, and nationally recognized to that extent; and by agencies authorized to represent the Federal Government as a whole. It has been found that for many of the commodities there exist several specifications, and naturally many of these specifications overlap, to an extent, in their substance. In such cases free use has been made of a system of reference, with no idea of indicating preference, simply to avoid unnecessary duplication in presentation.

This volume furnishes a fair portrayal of the extent of standardization. It discloses the fact that simplification of commercial practices, or, in other words, standardization of standards, is in certain fields an accomplished fact, while in others little progress has thus far been made. Under the auspices of the commercial standardization group of the Bureau of Standards, and the American Standards Association, formerly known as the American Engineering Standards Committee, simplification and standardization on a national scale are being carried forward at an ever-increasing speed. However, much yet remains to be done in the unification of standards and specifications.

With more than threescore agencies contributing the material utilized in the present volume, variances in texts are plentiful. It is for the user of this volume, where options in specifications are offered, to make his own selections, and to determine whether or not his needs are to be met by the standards and specifications presented here.

Use has been made of the decimal system of classification of nonmetallic minerals which was employed in the National Directory of Commodity Specifications. In utilizing this system advantage has been taken of the opportunity to insert under the proper classification numerals, certain important commodities or groups of commodities for which there might well be, but are not as yet, specifications that can be referred to as nationally recognized or sponsored by nationally recognized organizations. 



\section{STANDARDS AND SPECIFICATIONS RELATING TO NON- METALLIC MINERALS}

\section{GENERAL ITEMS RELATING TO NON- METALIIC MINERALS}

\subsection{METHODS OF TEST.}

(Test methods for the various items in the nonmetallic mineral group are presented under commodity designations corresponding with the subject matter of the tests.)

\subsection{TESTING EQUIPMENT.}

American Society for Testing Materials, tentative definition of the term screen (sieve), serial designation E13-28T, 1928.

Screen (sieve).-A plate or sheet or a woven cloth, or other device, with regularly spaced apertures of uniform size, mounted in a suitable frame or holder, for use in separating material according to size.

Note 1.-The shape and spacing of apertures, size of wires or threads, thickness of plate or sheet, allowable variations and similar properties should be taken care of in specifications.

Note 2-In mechanical analysis testing work, when not otherwise specified, the term "sieve" shall apply to an apparatus in which the apertures are rectangular, and the term "screen" shall apply to an apparatus in which the apertures are circular.

American Society for Testing Materials, standard specifications for sieves for testing purposes, serial designation E11-26, 1926.

1. These specifications cover sieves for use in the usual cases of precision testing for the fineness of materials (classification of materials according to size). The sieves have been designated according to the size of openings in terms of microns, the corresponding United States Standard Sieve Series designation in arbitrary numbers being given for the convenience of those familiar with the latter scheme of designation.

\section{PHYSICAL REQUIREMENTS}

2. Wire cloth for standard sieves shall be woven (not twilled, except that the cloth of 44,53 , and 62 micron sieves may be twilled until further notice) from brass, bronze, or other suitable wire and mounted on the frames without distortion. To prevent the material being sieved from catching in the joint betreen the cloth and the frame, the joint shall be smoothly filled with solder, or so made that the material will not catch. The sieve frames should be circular, about $20 \mathrm{~cm}$ ( 8 inches) in diameter and about $5 \mathrm{~cm}$ (2 inches) or $2.5 \mathrm{~cm}$ ( 1 inch) between the top of the frame and the cloth.

Note.-The size specified above is for the regular standard testing sieve, but for special purposes other sizes may be used, as, for ezample the 3 -inch sieve, which is designed especially for use on laboratory balances as in the testing of pigments.

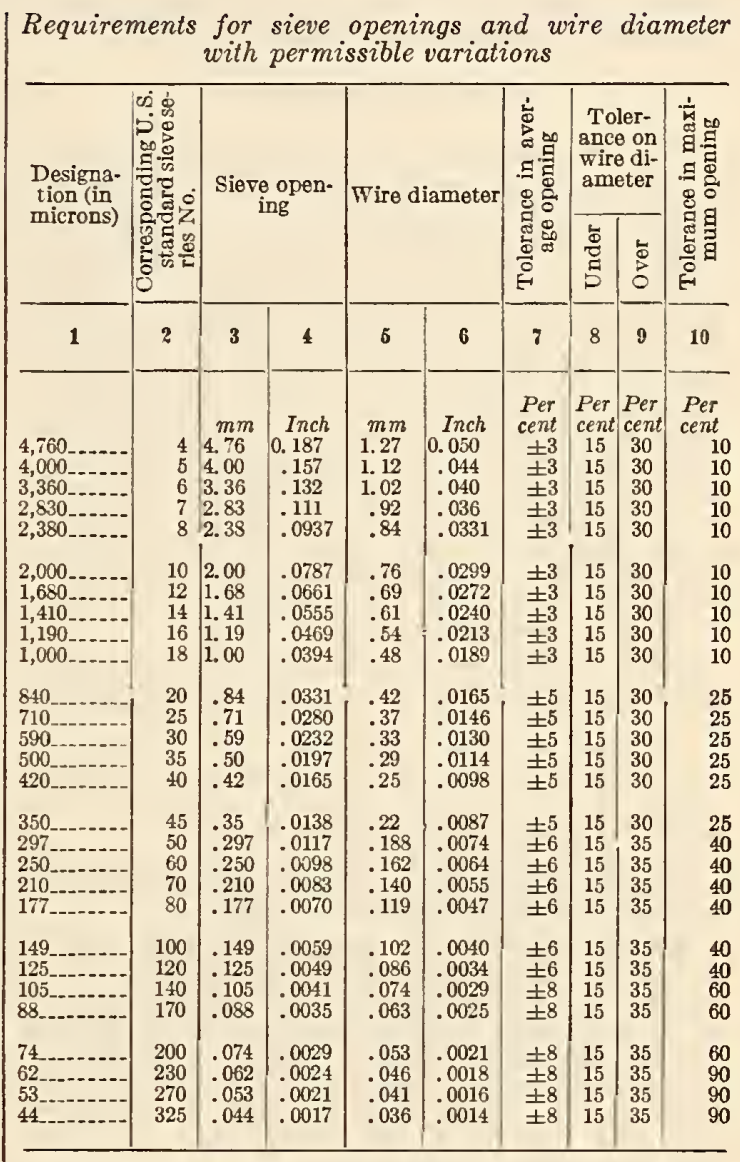

Note.-Screens having larger openings than those included in the table may be specified, but in specifying screens of larger openings the actual size of the opening (in millimeters or in inches) shall be stated. A tolerance of \pm 3 per cent on average openings and of 10 per cent in maximum openings shall be permitted.

3. The average opening between the adjacent warp and the adjacent shoot wires, taken separately, shall be that given in column 3 (or 4) of the table, within the "tolerance in average opening" given in column 7. The average diameter of the warp and of the shoot wires, taken separately, of the cloth of any given sieve shall be that given in column 5 (or 6 ) of the table, within the "tolerance on wire diameter" given in columns 8 and 9 . The maximum opening between adjacent parallel wires shall not exceed the nominal width of opening for that sieve by more than the "tolerance in maximum opening" given in column 10 of the table, 


\section{WORKMANSHIP}

4. The sieves shall be free from imperfections in the sieve cloth or its mounting, as, for example, punctured, loose or wavy cloth, imperfections in soldering, etc.

\section{MARKING}

5. The sieves shall bear a label upon which the manufacturer's nominal opening in metric units (preferably in microns) with the equivalent in inches, together with the size of wire, should be given.

Note.-It is recommended that, until the new micron designation shall have been thoroughly established, in addition the corresponding United States Standard Sieve Series number he given.

\section{METHODS OF TISTING}

6. (a) To determine whether the cloth conforms to the requirements of these specifications, the wire diameter shall be measured in each direction by means of a micrometer-microscope or projection apparatus or other suitable apparatus of requisite precision, care being taken in all cases to avoid diffraction effects in measuring to the edge of a wire. Micrometer calipers and steel scales should not be used for this purpose. The mesh shall be determined by counting the number of openings for a distance of at least 6 inches in both directions of the sieve, using a scale, either glass or steel, and a microscope. The use of scales with special rulings for obtaining mesh by counting "interference bands" is recommended in cases where large numbers of any of the sieves with the smaller openings are to be measured. From the wire diameter and the mesh the average opening shall be computed.

Note.-No general rule can be given for the number of observations to be taken, except that they be sufficient to cause no change in the result if, say, three or four additional measurements are made.

(b) Measurements of large openings shall be made by means of the micrometer-microscope or the projection lantern or other suitable apparatus in the same way as wire diameters are measured.

Note.-In order to insure the securing of standard sieves it is possible to ohtain from the manufacturer or dealer sieves which have been certified by the National Bureau of Standards.

American Society for Testing Materials, specifications for thermometers to be used in tests.

(This association has prepared specifications for 17 thermometers to be used in standard and tentative test methods which are presented in this volume. The reference where a specific type of thermometer is required in a method, is by a number, to correspond with the individual numbering given the thermometers in the table below.)

Thermometers shall be graduated in either centigrade or Fahrenheit degrees, as specified.

\section{GENERAL REQUIREMENTS, APPLYNG TO ALL THERMIOMETERS LISTED}

Type.-Etched stem, glass.

Liquid.-Mercury, except for No. 5, which requires toluene or other suitable liquid colored red with a permanent dye.

Stem.-Plain front, enamel back, suitable thermometer tubing.

Bulb.-Corning normal or equally suitable thermometric glass. Bulb for No. 16 shall have a heavy wall.

Contraction chamber.-Required for thermometers Nos. 4, 9, 15, and 17.

No. 4 to be a long narrow type, top to be not more than $65 \mathrm{~mm}$ (2.56 inches) above bottom of bulb.
No. 9, top to be not more than $41 \mathrm{~mm}$ (1.61 inches) above bottom of bulb.

Nos. 15 and 17, to be long narrow type, top to be not more than $60 \mathrm{~mm}$ (2.36 inches) above bottom of bulb, mercury to stand in contraction chamber at $32^{\circ} \mathrm{F} .\left(0^{\circ} \mathrm{C}\right.$. $)$.

Filling above liquid.-Nitrogen gas, except for No. 5, which requires gas under pressure.

Graduation.-All lines, figures, and letters clear cut and distinct.

Immersion.- On partial immersion thermometers, the immersion, in inches for Fahrenheit and millimeters for centigrade thermometers, together with a line around the stem at height indicated in the table, shall be etched on the thermometer. This last is not required for No. 17.

Special marking. - The name, as given in table in special marking, a serial number, and the manufacturer's name or trade-mark shall be etched on the stem.

Scale error.-Scale error is measured at any point on the scale, with the exceptions of Nos. 2,8 , and 12. See notes below table.

Test for permanency of range.-After being subjected for 24 hours to temperature given in the table, accuracy shall be within limit specified.

Standardization.-The thermometers shall be standardized as follows:

No. 1 , at the ice point and at intervals of approximately $20^{\circ} \mathrm{C}$. or $40^{\circ} \mathrm{F}$. for $108 \mathrm{~mm}$ or $4 \frac{1}{4}$ inches immersion, and for an average temperature of $21^{\circ} \mathrm{C}$. or $70^{\circ} \mathrm{F}$. for the emergent mercury column. No. 2, immersed in the testing bath to the top of the mercury column, at the ice point and at temperature intervals of approximately $50^{\circ} \mathrm{C}$. or $100^{\circ} \mathrm{F}$. up to $370^{\circ} \mathrm{C}$. or $700^{\circ} \mathrm{F}$.

No. 3, immersed in the testing bath to the top of the mercury column, at intervals of approximately $40^{\circ} \mathrm{C}$. or $70^{\circ} \mathrm{F}$.

No. 4, at three points, including $163^{\circ} \mathrm{C}$. for total immersion.

No. 5 , at the ice point and at intervals of approximately $20^{\circ} \mathrm{C}$. or $35^{\circ} \mathrm{F}$. for $108 \mathrm{~mm}$ or $4 \frac{1}{4}$ inches immersion, and for an average temperature of $21^{\circ} \mathrm{C}$. or $70^{\circ} \mathrm{F}$. for the emergent liquid column.

No. 6, immersed in the testing bath to the top of the mercury column, at the ice point and at temperature intervals of approximately $50^{\circ} \mathrm{C}$. or $100^{\circ} \mathrm{F}$. up to $300^{\circ}$ C. or $572^{\circ} \mathrm{F}$.

No. 7, immersed in the testing bath to the top of the mercury column, at the ice point and at temperature intervals of approximately $20^{\circ} \mathrm{C}$. or $40^{\circ} \mathrm{F}$.

No. 8 , at the ice point and at intervals of approximately $100^{\circ} \mathrm{F}$. for $25 \mathrm{~mm}$ or 1 inch immersion, and for the following temperatures of the emergent mercury column:

\begin{tabular}{|c|c|}
\hline $\begin{array}{l}\text { Thermometer } \\
\text { reading }\end{array}$ & $\begin{array}{l}\text { A verage temper- } \\
\text { ature of emergent } \\
\text { mercury column }\end{array}$ \\
\hline $\begin{array}{cc}{ }^{\circ} C . & { }^{\circ} F . \\
100 & 200 \\
150 & 300 \\
200 & 400 \\
250 & 500 \\
300 & 600 \\
350 & 700\end{array}$ & $\begin{array}{rr}{ }^{\circ} C . & { }^{\circ} F \\
44 & 110 \\
54 & 129 \\
64 & 150 \\
77 & 175 \\
91 & 205 \\
108 & 240\end{array}$ \\
\hline
\end{tabular}

No. 9, at intervals of approximately $10^{\circ} \mathrm{C}$. or $20^{\circ} \mathrm{F}$. for $79 \mathrm{~mm}$ or $3 \frac{1}{8}$ inches immersion and for an average temperature of the emergent mercury column of $25^{\circ} \mathrm{C}$. or $77^{\circ} \mathrm{F}$.

No. 10 , for $76 \mathrm{~mm}$ (3 inches) immersion and for the following temperatures of the emergent mercury 


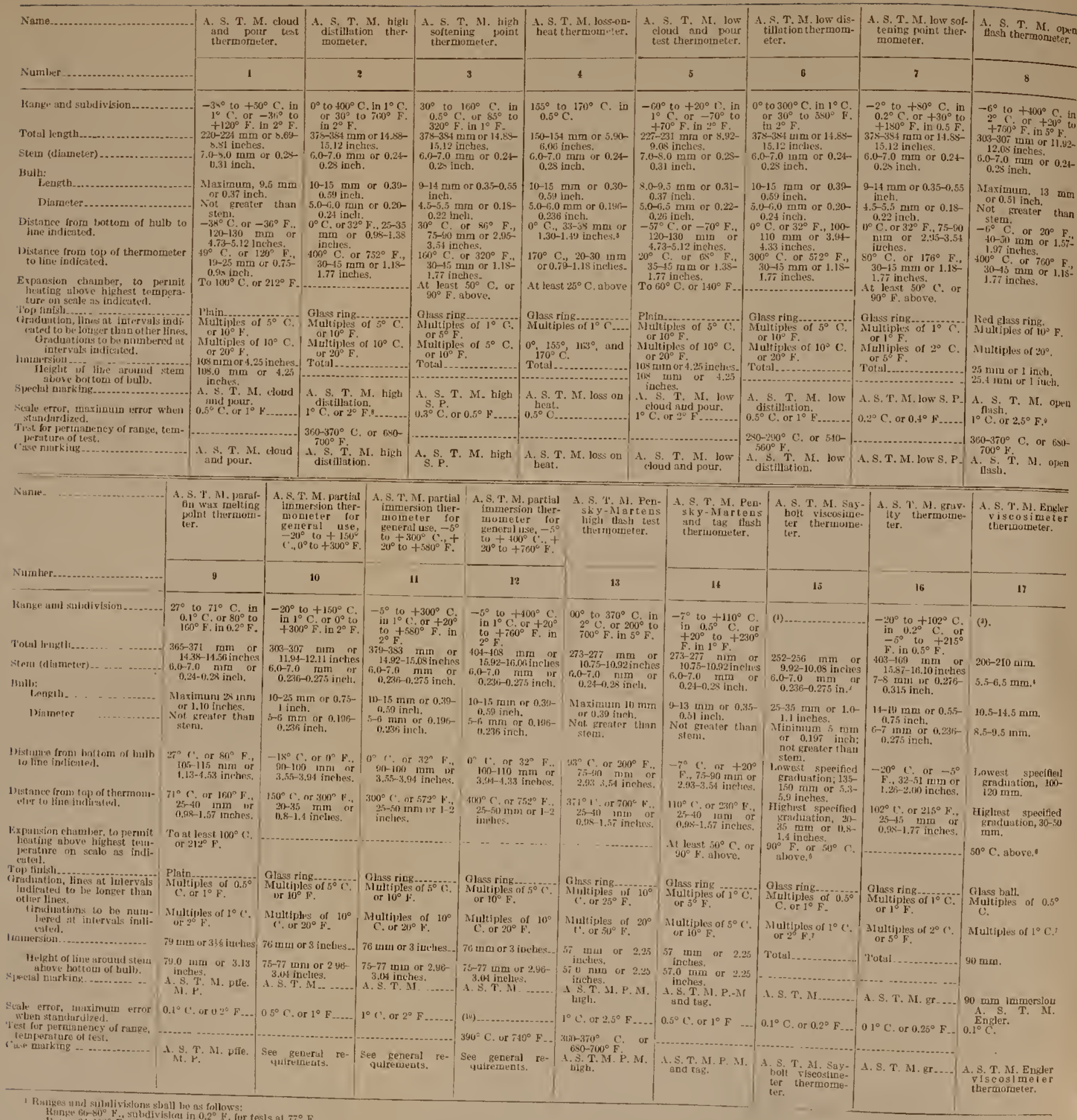

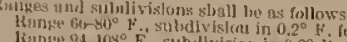

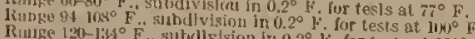

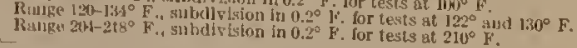

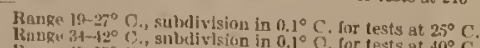

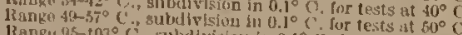

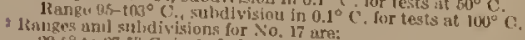

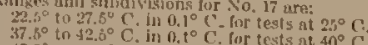

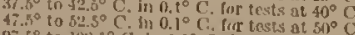

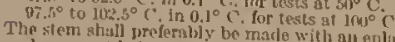

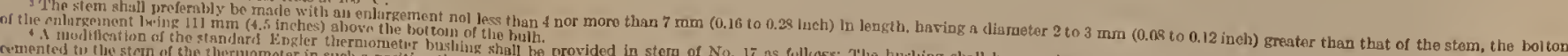

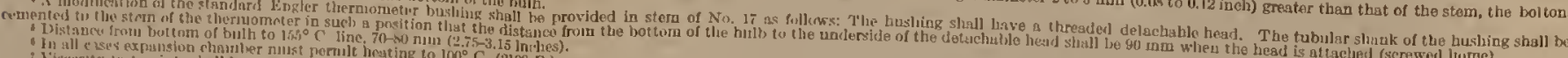

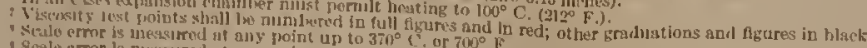

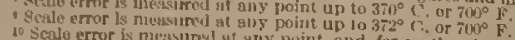

10 scalo error is newasured ut ariy point, aud, for centlgracle ther

(1111325:-30. (Face p. 3.1 


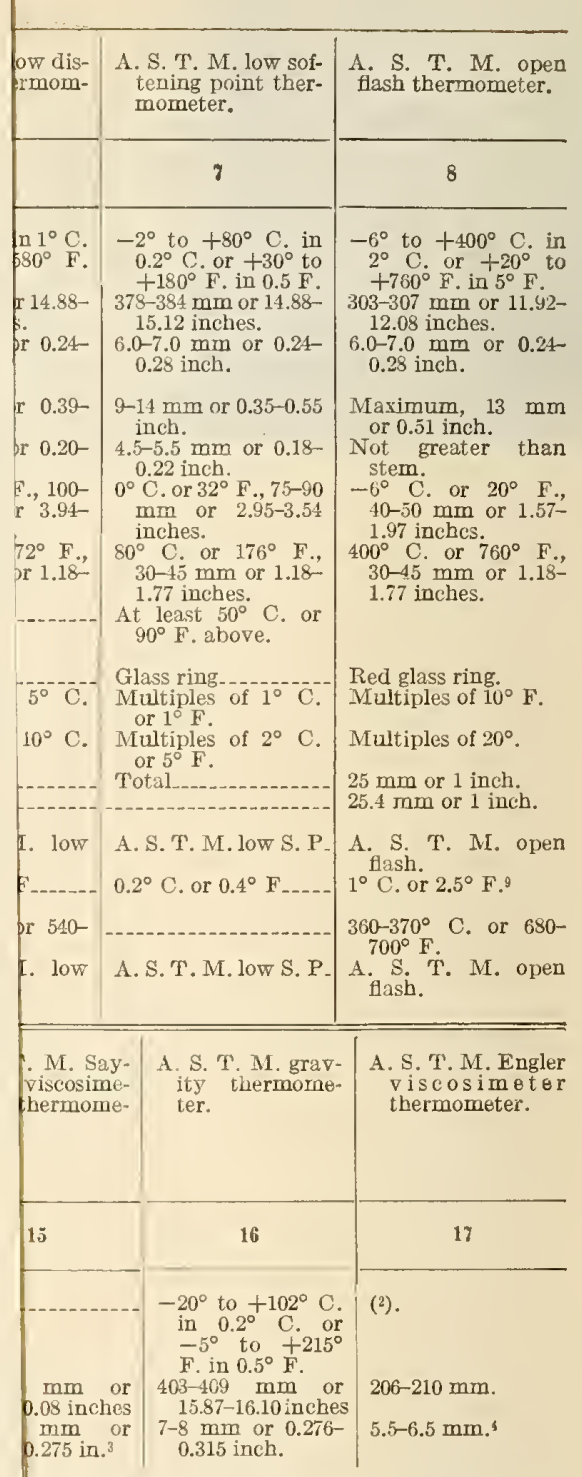


column. These stem temperatures have been chosen as corresponding, on the average, to those likely to occur in the use of the thermometer.

\begin{tabular}{|c|c|}
\hline $\begin{array}{l}\text { Thermometer } \\
\text { reading }\end{array}$ & $\begin{array}{l}\text { A verage temper- } \\
\text { ature of emergent } \\
\text { mercury column }\end{array}$ \\
\hline $\begin{array}{rl}{ }^{\circ} C . & { }^{\circ} F . \\
50 & 122 \\
100 & 212 \\
150 & 302\end{array}$ & $\begin{array}{rr}{ }^{\circ} \mathrm{C} . & { }^{\circ} \mathrm{F} . \\
35 & 94 \\
48 & 118 \\
55 & 131\end{array}$ \\
\hline
\end{tabular}

No. 11, for $76 \mathrm{~mm}$ (3 inches) immersion and for the following temperatures of the emergent mercury column. These stem temperatures have been chosen as corresponding, on the average, to those likely to occur in the use of the thermometer:

\begin{tabular}{|c|c|}
\hline $\begin{array}{l}\text { Thermometer } \\
\text { reading }\end{array}$ & $\begin{array}{l}\text { A verage temper- } \\
\text { ature of emergent } \\
\text { mercury co.umn }\end{array}$ \\
\hline $\begin{array}{rr}{ }^{\circ} C . & { }^{\circ} F . \\
50 & 122 \\
100 & 212 \\
150 & 302 \\
200 & 392 \\
250 & 482 \\
300 & 572\end{array}$ & $\begin{array}{rr}{ }^{\circ} \mathrm{C} . & { }^{\circ} \mathrm{F} . \\
35 & 94 \\
49 & 120 \\
61 & 142 \\
70 & 158 \\
76 & 169 \\
80 & 176\end{array}$ \\
\hline
\end{tabular}

No. 12 , for $76 \mathrm{~mm}$ ( 3 inches) immersion and for the following temperatures of the emergent mercury column. These stem temperatures have been chosen as corresponding, on the average, to those likely to occur in the use of the thermometer:

\begin{tabular}{|c|c|}
\hline $\begin{array}{l}\text { Thermometer } \\
\text { reading }\end{array}$ & $\begin{array}{l}\text { A verage temper- } \\
\text { ature of emergent } \\
\text { mercury column }\end{array}$ \\
\hline $\begin{array}{ll}{ }^{\circ} \mathrm{C} . & { }^{\circ} \mathrm{F} . \\
100 & 212 \\
200 & 392 \\
300 & 482 \\
400 & 572\end{array}$ & $\begin{array}{rr}{ }^{\circ} \mathrm{C} . & { }^{\circ} \mathrm{F} . \\
50 & 122 \\
75 & 167 \\
89 & 192 \\
94 & 201\end{array}$ \\
\hline
\end{tabular}

No. 13 , at intervals of approximately $50^{\circ} \mathrm{C}$. or $100^{\circ} \mathrm{F}$. for $57 \mathrm{~mm}$ or $21 / 4$ inches immersion, and for the following temperatures of the emergent mercury column:

\begin{tabular}{|c|c|}
\hline $\begin{array}{l}\text { Thermometer } \\
\text { reading }\end{array}$ & $\begin{array}{l}\text { A verage temper- } \\
\text { ature of emergent } \\
\text { mercury column }\end{array}$ \\
\hline & ${ }^{\circ} \mathrm{C} . \quad{ }^{\circ} \mathrm{F}$. \\
\hline $100200^{\circ}$ & $61 \quad 140^{\circ}$ \\
\hline & $\begin{array}{ll}65 & 149\end{array}$ \\
\hline 200400 & $71 \quad 160$ \\
\hline $250 \quad 500$ & $\begin{array}{ll}78 & 175\end{array}$ \\
\hline $300 \quad 600$ & $\begin{array}{ll}87 & 195\end{array}$ \\
\hline $350 \quad 700$ & $99 \quad 220$ \\
\hline
\end{tabular}

No. 14, at the ice point and at intervals of approximately $30^{\circ} \mathrm{C}$. or $50^{\circ} \mathrm{F}$. for $57 \mathrm{~mm}$ or $2 \frac{1}{4}$ inches immersion and for the following temperatures of the emergent mercury column:

\begin{tabular}{|c|c|}
\hline $\begin{array}{l}\text { Thermometer } \\
\text { reading }\end{array}$ & $\begin{array}{l}\text { Average temper- } \\
\text { ature of emergent } \\
\text { mercury column }\end{array}$ \\
\hline $\begin{array}{rr}{ }^{\circ} \mathrm{C} . & { }^{\circ} \mathrm{F} . \\
20 & 70 \\
40 & 100 \\
70 & 150 \\
100 & 212\end{array}$ & $\begin{array}{rr}{ }^{\circ} \mathrm{C} . & { }^{\circ} \mathrm{F} . \\
20 & 70 \\
31 & 86 \\
40 & 104 \\
48 & 118\end{array}$ \\
\hline
\end{tabular}

No. 15, for the condition of total immersion. Correction for emergent stem shall not be applied.

No. 16, immersed in the testing bath to the top of the mercury column, at the ice point and at temperature intervals of from $15^{\circ}$ to $20^{\circ} \mathrm{C}$. or $30^{\circ}$ to $40^{\circ} \mathrm{F}$

No. 17 , for the condition of $90 \mathrm{~mm}$ immersion.

Case.-The thermometer shall be supplied in a suitable case which shall be marked as indicated in table in case marking, together with the range in ${ }^{\circ} \mathrm{F}$. or ${ }^{\circ} \mathrm{C}$., according to the type of thermometer. Cases for Nos. 10, 11 , and 12 shall be marked "A. S. T. M., $76 \mathrm{~mm}$ immersion," with the range in "C., or "A. S. T. M., 3-inch. immersion," with the range in ${ }^{\circ} \mathrm{F}$., according to the type of thermometer.

Note. -For the purpose of interpreting these specifications the following definitions apply:

The total length is the over-all length of the finished instrument. The diameter is that measured with a ring gage.

The length of the bulb is the distance from the bottom of the bulb to the beginning of the enamel backing.

The top of the thermometer is the top of the finished instrument. 


\section{1-509 COAL, PETROLEUM, ASPHALT, AND MINERAL WAX}

\section{COAL AND COKE 501.0 GENERAL ITEMS.}

American Chemical Society, coal analysis. (This society has specifications for coal analysis identical with those of the American Society for Testing Materials, serial designation D271. See p. 8.)

American Klectric Railway Association, specification and contract for the purchase of fuel (recommended practice), serial No. G200-15, 1915.

(This association has formulated a specification and contract for the purchase of coal, setting out in detail, conditions of purchase, delivery, test, and acceptance to control the transaction, and indorsing the standard of analysis of coal adopted by the American Chemical Society. See A. S. T. M.: D271, p. 8.)

American Society for Testing Materials, standard method of sampling coal, serial designation D21-16, 1916.

Approved as "Tentative American Standard," by the American Engineering Standards Committee, now the American Standards Association.

It is imperative that every sample be collected and prepared carefully and conscientiously and in strict accordance with the standard methods described herein, for if the sampling is improperly done the sample will be in error, and it may be impossible or impracticable to take another sample; but if an analysis is in error another analysis can easily be made of the original sample.

Gross samples of the quantities designated herein must be taken whether the coal to be sampled consists of a few tons or several hundred tons, because of the following cardinal principle in sampling coal that must be recognized and understood; that is, the effect of the chance inclusion or exclusion of too many or too few pieces of slate or other impurities in what, or from what, would otherwise have been a representative sample will cause the analysis to be in error accordingly, regardless of the tonnage sampled. For example, the chance inclusion or exclusion of 10 pounds too much or too little of impurities in or from an otherwise representative sample of 100 pounds would cause the analysis to show an error in ash content and in heat units of approximately 10 per cent, whereas for a $1,000-$ pound sample, the effect would be approximately only 1 per cent, being the same whether the sample is collected from a 1-ton lot or from a lot consisting of several hundred tons.

When this method of sampling is to be employed as a part of any contract or agreement, the following provisions shall be specifically agreed to by the parties to such contract or agreement:

(a) The place at which the coal is to be sampled (see sec. 1).

(b) The approximate sizc of the sample required when the standard conditions do not apply (see sec. 3).

(c) The number of samples to be taken or the amount of coal to be represented by each sample when the standard conditions do not apply (see sec. 4).

\section{FOR ALI DETERMINATIONS EXCEPT TOTAI MOISTURE}

1. The coal shall be sampled when it is being loaded into or unloaded from railroad cars, ships, barges, or wagons, or when discharged from supply bins, or from industrial railway cars, or grab buckets, or from any coalaconveying equipment, as the case may be. If the coal is crushed as received, samples usually can be taken advantageously after the coal has passed through the crusher. Samples collected from the surface of coal in piles or bins, or in cars, ships, or barges are generally unreliable.

2 . To collect samples, a shovel or specially designed tool, or mechanical means shall be used for taking equal portions or increments. For slack or small sizes of anthracite, increments as small as 5 to 10 pounds may be taken, but for run-of-mine or lump coal, increments should be at least 10 to 30 pounds.

3 . The increments shall be regularly and systematically collected, so that the entire quantity of coal sampled will be represented proportionately in the gross sample, and with such frequency that a gross sample of the required amount shall be collected. The standard gross sample shall not be less than 1,000 pounds, except that for siack coal and small sizes of anthracite in which the impurities do not exist in abnormal quantities or in pieces larger than three-fourths inch, a gross sample of approximately 500 pounds shall be considered sufficient. If the coal contains an unusual amount of impurities, such as slate, and if the pieces of such impurities are very large, a gross sample of 1,500 pounds or more shall be collected. The gross sample should contain the same proportion of lump coal, fine coal, and impurities as is contained in the coal sampled. When coal is extremely lumpy, it is best to break a proportional amount of the lumps before taking the various increments of a sample. Provision should be made for the preservation of the integrity of the sample.

4. A gross sample shall be taken for each 500 tons or less, or in case of larger tonnages, for such quantities as may be agreed upon.

5. After the gross sample has been collected, it shall be systematically crushed, mixed, and reduced in quantity to convenient size for transmittal to the laboratory. The sample may bo crushed by hand or by any mechanical means, but under such conditions as shall prevent loss or the accidental admixture of foreign matter. Sampies of the quantities indicatcd in the table shall be crushed so that no pieces of coal and impurities will be greater in any dimension, as judged by eye, than specified for the sample before division into two approximately equal parts.

The method of reducing by hand the quantity of coal in a gross sample shall be carried out as prescribed in section 6, even should the initial size of coal and impurities be less than indicated in the table.

Samples and quantities

\begin{tabular}{|c|c|}
\hline Weight of sample to be divided (pounds) & $\begin{array}{c}\text { Largest } \\
\text { size of coal } \\
\text { and impuri- } \\
\text { ties allow- } \\
\text { able in } \\
\text { sample be- } \\
\text { fore divi- } \\
\text { sion }\end{array}$ \\
\hline $\begin{array}{l}1,000 \text { or over } \\
6000 \\
60 \\
60\end{array}$ & $\begin{array}{ll}\text { Inch } & \\
& 1 \\
& 3 / 4 \\
& 1 / 3 \\
3 / 8 & 3 / 4 \\
1 / 46 & \end{array}$ \\
\hline
\end{tabular}

1 Or to pass a 4,760-micron (No. 4) sieve. 
6. The progressire reduction in the meight of lated by placing in a waterproof receptacle with the sample to the quantities indicated in the table a tight-fitting and waterproof lid small equal shall be done $b^{\circ}$ the methods illustrated in Figure 1. parts of freshly taken increments of the standard NorE. - The full tert of the specification gires in detail a descrip- gross sample. The accumulated moisture sample tion of the method illustrated.

7. Only such mechanical means as will give or by hand to about a 5-pound quantity, which equally representative samples shall be used in shall be immediately placed in a container and
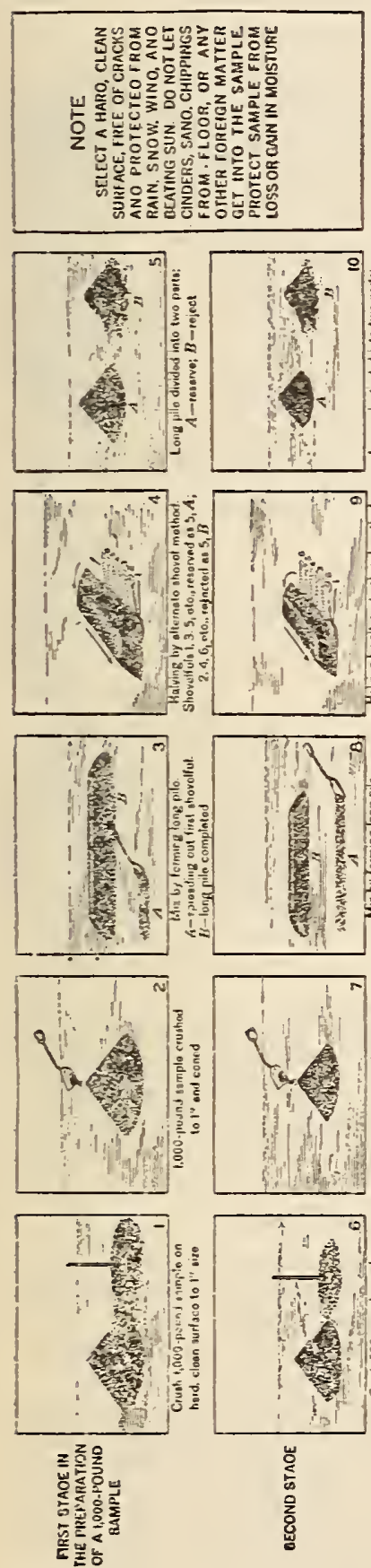
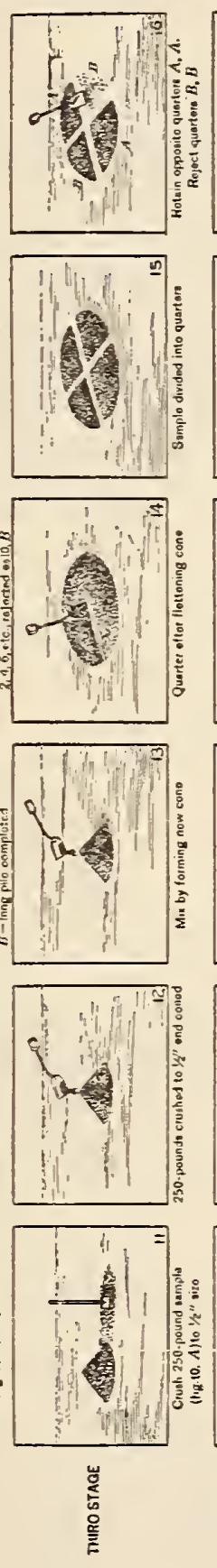
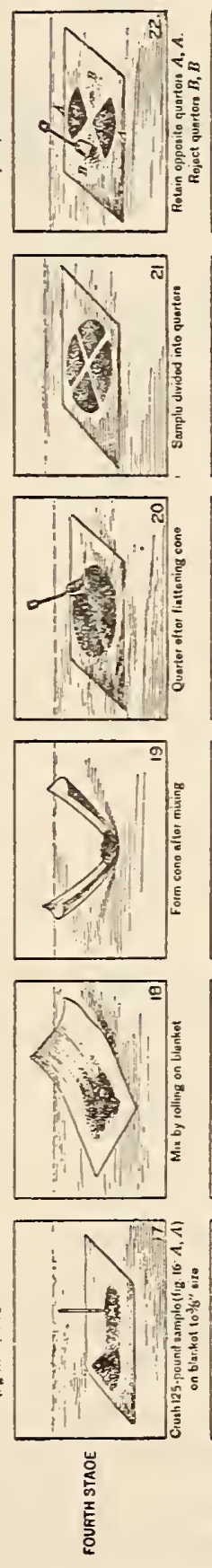
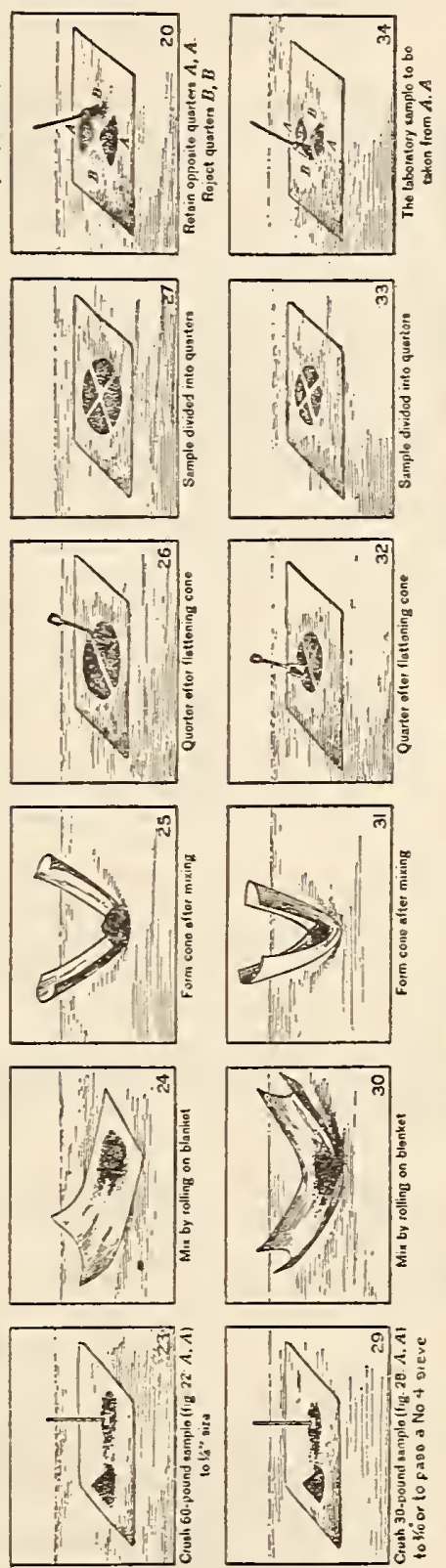

崖
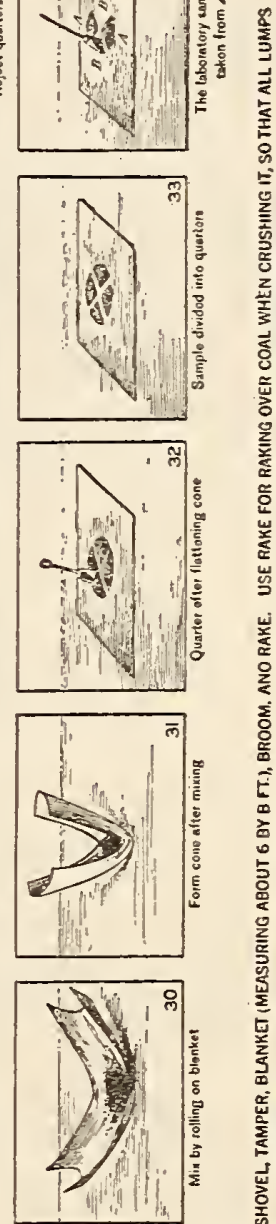

Eే

突

응

논인

홍 존

㟧
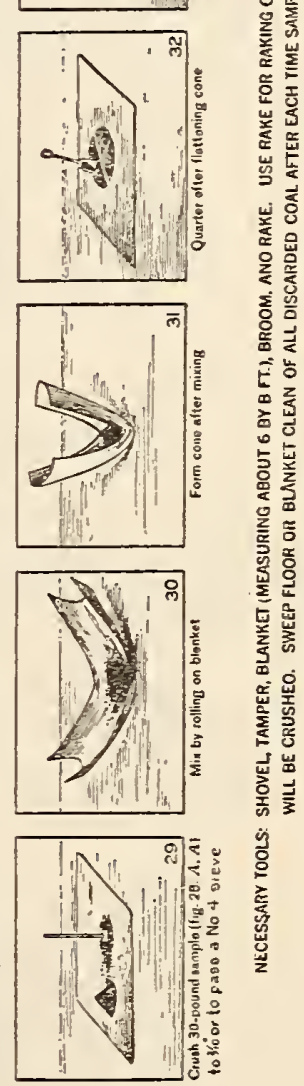

容 substitution for the hand method of preparation herein standardized.

\section{FOR THE DETERMINATION OF TOTAL MOISTURE}

8. The special moisture sample shall weigh approximately 100 pounds, and shall be accumu-

sealed airtight and forwarded to the laboratory without delay.

9. Only when equally representative results will be obtained shall the standard gross sample be used instead of the special moisture sample for the determination of total moisture. 
American Society for Testing Materials, standard definitions of terms relating to coal and coke, serial designation D121-27, 1927.

Proximate analysis.-In the case of coal and coke, the determination, by prescribed methods, of moisture, volatile matter, fixed carbon (by difference), and ash.

Note.-Unless otherwise spccified, the terin "proximate analysis" does not included determinations of sulphur or phosphorus or any determinations other than those named.

Ultimate analysis.- In the case of coal and coke, the determination of carbon and hydrogen in the material, as found in the gaseous products of its complete combustion, the determination of sulphur nitrogen, and ash in the material as a whole, and the estimation of oxygen by difference.

Note 1.-The determination of phosphorus is not by definition a part of the ultimate analysis of coal or coke, but may be specified when desired.

Note 2 -When the analysis is made on an undried sample, part of the hydrogen and oxygen as determincd is present in the free moisture accompanying the coal. Therefore, in comparing coals on the basis of their ultimate analysis, it is advisable always to state the analysis of their ultimate analysis, it is advisable al ways to state the analysis

(as some coals contain mineral carhonates, and practically all contain clay or shale containing com hined water a part of the carhon, hydrogen, and oxygen found in the products o comhustion may arise from these mineral components.

Moisture.-Essentially water, quantitatively determined by definite prescribed methods which may vary according to the nature of the material.

NoTE 1.-Such methods may not determine all of the water present NOTE 2.-In the case of coal and coke the methods employed shal be those preseribed in A.S.T. M. D271, see 501.0, page 8.

Ash.-Inorganic residue remaining after ignition of combustible substances, determined by definite prescribed methods.

Note 1.-Ash may not he identical, in composition or quantity, with the inorganic suhstances present in the material before ignition Note 2.-In the case of coal and coke the methods employed shal be those prescrihed in A.S. T. M. D271.

Volatile matter.-Those products, exclusive of moisture, given off by a material as gas or vapor, determined by definite prescribed methods which may vary according to the nature of the material.

Note. - In the casc of coal and coke the methods employed shall he those prescrihed in A. S. T. M. D271.

Fixed carbon.-In the case of coal, coke, and bituminous materials, the solid residue other than ash, obtained by destructive distillation, determined by definite prescribed methods.

Note 1.-It is made up principally of carbon, hut may contain appreciable amounts of sulphur, hydrogen, nitrogen, and oxygen.

NoTE 2.- In the case of coal and coke the methods employed shall be those prescribed in A, S. T, M. D271.

American Society for Testing Materials, tentative definitions of terms relating to coal and coke, serial designation: D121-27T, 1927.

Coke.-The infusible, cellular, coherent solid material obtained from coal as the rcsidue from its destructive distillation.

Note 1.-Such material ohtained from the destructive distillation Note 1.- Such material ohtained from the destructive distillat

Note 2. - Such material obtained from the destructive distillation of coal-tar pitch is known as "pitch coke."

Beehive coke.-Coke manufactured in beehive, rectangular, or similar forms of ovens in a horizontal bed, where heat for the coking process is secured by combustion within the oven chamber.

By-product coke.-Coke manufactured with attendant recovery of by-products, in ovens that are heated externally, and having properties making it available for metallurgical purposes.

Coke breeze.-The fine screenings from crushed coke or from coke as taken from the ovens, of a size varied in local practice, but usually passing a one-half or threc-fourths inch screen opening.

Dry coke.-Coke which has been dried to constant weight at temperatures not less than $104^{\circ} \mathrm{C}$. nor more than $200^{\circ} \mathrm{C}$. in the case of lump coke, and between $104^{\circ}$ and $110^{\circ} \mathrm{C}$. in the case of coke passing a No. 60 sieve, in accordance with methods of determining moisture prescribed in A. S. T. M. D271. (See p. 8.)

American Society for Testing Materials, tentative method of test for fineness of powdered coal, serial designation D197-26T, 1926.

\section{APPARATUS}

1. Sieves Nos. $16,30,50,100$, and 200 of the U.S. Standard Sieve Series, each with a close-fitting pan and cover, shall be used. Wire cloth for the sieves shall be woven (not twilled) from brass, bronzc, or other suitable wire and mounted on the frames without distortion. To prevent the material being sieved from catching in the joint between the cloth and the frame, the joint shall be smoothly filled with solder, or so made that the material will not catch. The sieve frames shall be circular, about $20 \mathrm{~cm}$ ( 8 inches) in diameter and about $5 \mathrm{~cm}$ (2 inches) or $2.5 \mathrm{~cm}$ ( $1 \mathrm{inch}$ ) between the top of the frame and the cloth. The sieve openings and wire diameters shall conform to the requirements as given in the table.

Requirements for sieve openings and wire diameters, with permissible variations

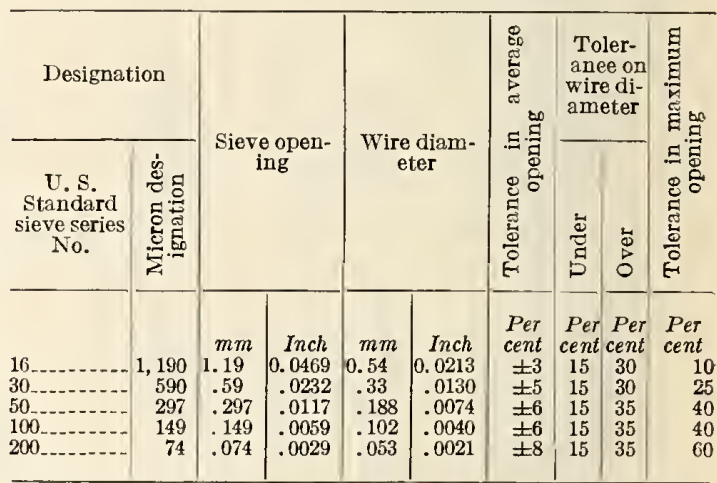

NotE.-These sieve specifications conform to A. S. T. M. E11. (See 500.2, p. 1.)

2. (a) For collecting gross sample.-The containers shall be of metal of about 8 to 10 liter capacity, provided with tightly fitting covers.

NoтE.-Two-gallon milk cans are suitahle for this purpose.

(b) For collecting laboratory sample.-The containers may be of metal or glass of about 1-liter capacity, provided with tightly fitting covers.

\section{SAMPLING}

3. The coal pulverized, per mill, during the period of one hour shall be considered a unit for sampling.

\section{A. MULTIPLE SYSTEM}

In the multiple powdered coal system, the eoal after pulverization is eonveyed into hins.

4. Not less than $2 \mathrm{~kg}$ of representative powdered coal shall be collccted as it is being discharged from the pulverizer. This is best accomplished by collecting increments of not more than $200 \mathrm{~g}$ at regular intervals by means of a scoop. The increments shall be placed into a can of about 8 to 10 liter capacity, provided with a tightly fitting cover.

5 . The gross sample shall be placed on a sheet of rubber or oilcloth, and thoroughly mixed by raising first one corner of the cloth and then the other so as to roll the coal over and over at least 100 times. After mixing, the sample shall be coned and quartered. The operations of mixing, coning, and quartering shall be continued if necessary until the 
sample is reduced sufficiently so that all of one of the quarters weighs about $500 \mathrm{~g}$, which coal shall constitute the laboratory sample. The laboratory sample shall be transferred to a metal or glass container of about 1-liter capacity, provided with a tightly fitting cover.

NoTE.-The operations of mixing, coning and quartering are described and illustrated in A. S. T. M. D21. (See 501.0, p. 4.)

\section{B. ONIT SYSTEM}

In the unit powdered coal system, the coal during and aftcr being pulverized is at once mixed with air and delivered to the furnace. It is difficult to obtain representative samples, as it is necessary to sample the coal from a moving stream of coal and air inside the pipe between the pulverizer and the furnace. It is preferable to collect such samples from vertical pipes if possible, as in horizontal pipes considerable segregation takes place, the coarser particles tending to flow along the bottom of the pipes.

6. (a) The sampling device shall consist of a 1-inch thin-walled brass tube, with one end bent at a right angle or provided with a $90^{\circ}$ elbow and short length of tube. At the other end of the sampling tube a vacuum-sweeper bag shall be tightly attached. The inside of the sampling tube shall have no projections to interfere with the flow of coal.

(b) The sampling tube shall be inserted into the powdered coal pipe through a stuffing box in such a manner that the opening of the sampling tube shall be directed against the stream of coal and air, and permit moving back and forth across the entire diameter of the coal pipe. If the sample must be taken from a horizontal pipe, the sampling tube shall be inserted through the top of the coal pipe, in such a manner as will permit moving the tube back and forth from top to bottom along the vertical diameter of the pipe.

7. In collecting the sample, the sampling tube shall be moved uniformly back and forth along the diameter of the pipe. In order to permit rady elimination of air from the bag, the vacuum-cleaner bag shall not be filled more than about one-fifth full at any time. Sampling shall be continued at regular intervals until a gross sample of not less than $5 \mathrm{~kg}$ of coal is collected.

8. The gross sample shall be mixed, coned, and quartered, and reduced in the same manner as described for the multiplc powdered coal system.

\section{PROCEDURE}

9. The entire laboratory sample shall be air dried in a drying oven at $10^{\circ}$ to $15^{\circ} \mathrm{C}$. above room temperature. The drying shall be continued until the loss in weight is not more than 0.1 per cent per hour.

10. After air drying, the sample shall be thoroughly mixed by rolling over and over at least 100 times on a sheet of rubber or oilcloth, as described for the mixing of the gross sample. The sample, after mixing, shall be spread out to a depth of about onehalf inch and portions, representing a section from the top of the coal to the mixing cloth, shall be taken with a spatula at regular intervals.

11. A $50 \mathrm{~g}$ sample of the air-dried coal shall be placed on the No. 200 sieve, to which a pan and cover shall be attached. The sieve shall be held in one hand in a slightly inclined position, so that the sample will be well distributed over the sieve, while at the same time the operator shall gently strike the side about 150 times per minute against the palm of the other hand on the upstroke. Every 25 strokes the sieve shall be turned about one-sixth of a revolution in the same direction. The sieve shall be held over a sheet of glazed white paper during the sieving operation, so as to detect any loss that might result from accidental spilling of a portion of the sample, in which case another sample shall be taken.
After sieving for one minute, the sieve pan shall be removed, and the material which has passed through the sieve discarded. The side of the sieve shall be tapped with a brush liandle, and the coal adhering to the underside of the wire cloth brushed off. During the operation of tapping and brushing the sieve cover shall remain on the sieve so as to prevent any loss of material over the side of the sieve.

NoTE. - A 1-inch bristle brush is satisfactory for brushing the underside of the wire cloth.

The sieve pan shall be replaced, and the sieving continued for two minutes; then the tapping and brushing shall be repeated, and again repeated after two minutes of additional sieving. The operation shall then be continued in the usual manner, omitting the tapping and brushing, until the amount passing through in one minute of continuous sieving is about $0.1 \mathrm{~g}$. Each time, before weighing the material passing through the sieve, the side of the sieve shall be tapped with the brush handle in order to remove any material adhering to the underside of the wire cloth. The cover of the sieve shall then be removed, and the residue rcmaining on the sieve carefully removed. The sieve shall be inverted over a piece of glazed white paper, and the wire cloth cleaned by carefully brushing the under side. The material thus removed from the wire cloth shall be added to the residue removed from the sieve.

After cleaning the sieve, the residue shall be placed thereupon, the sieve pan and cover attached, and the sieving resumed in the usual manner, omitting the operations of tapping and brushing, until not more than $0.05 \mathrm{~g}$ passes through in one minute of continuous sieving, when the sieving shall be considered to be finished. The sieve shall be inverted and cleaned as before, and the residue weighed.

12. The residue shall then be successively sieved on the Nos. $100,50,30$, and 16 sieves. Sieving shall be continued in the usual manner, omitting the tapping and brushing, until not more than $0.05 \mathrm{~g}$ passes through in one minute of continuous sieving, when the sieving shall be considered finished. The sieve shall then be inverted, cleaned, and the residue weighed as described for the No. 200 sicve.

NotE.-As the fine material which tends to clog the meshes of the sieves has been removed by sieving through the No. 200 sieve, it is not necessary to brush the underside of the wire cloth or clean the sieves during the sieving operation, as was done for the No. 200 sieve.

13. The fineness shall be calculated from the weights of the residues on the sieves, and shall be expressed as percentages of the weight of the original sample. Sieving value corrections for the No. 200 sieves, as determined by the National Bureau of Standards, shall be applied to the material sieved on the No. 200 sieves. The fineness test shall be reported as follows:

Per cent

Retained on No. 16 sieve

Retained on No. 30 sieve, passing No. 16 sieve

Retained on No. 00 sieve, passing No. 30 sieve

Retained on No. 50 sieve, passing N. 30 sieve

Retained on No. 200 sieve, passing No. 100 sieve

Passing No 200 sieve sieve, passing No.

Note.-In applying the sieving value corrections, the graphical or analytical methods for obtaining such corrections for sieves which have been tested with a single standard sample shall be used. These methods are described in National Bureau of Standards Technologic Paper No. 42, pages 45-47; 1914.

14. Duplicate determinations by the same operator, using the same sieves, shall check within 1 per cent on all sizes. Different operators, using different sieves, shall check within 3 per cent on the material sieved on the No. 200 sieves.

NoTE.-Until such time as standard materials are available for determining sieving values of Nos. $100,50,30$, and 16 sieves, it is not feasible to specify check limits for the sizes as determined with these sieves, when different sieves are used. 


\section{RAPID ROUTINE METHOD BY MACHINE SIEVING}

For routine testing, mechanical sieving devices, by which a number of sieves are shaken at the same time, afford a convenient method of making fineness tests. The hand-sieving method shall, however, be the standard method of test. The results obtained by machine sieving should be compared to those obtained by hand sieving, to determine whether the sieving action of the machine used is practically the

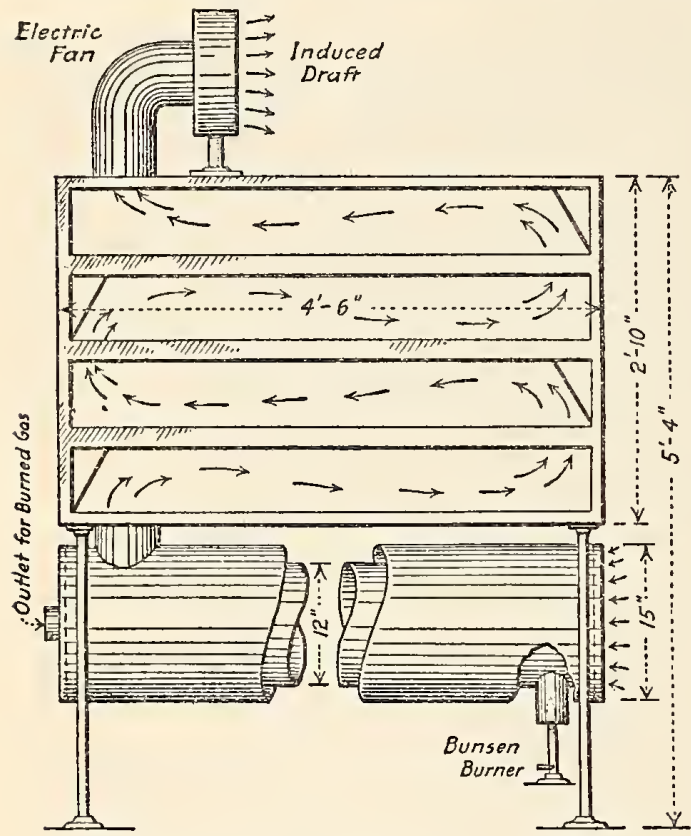

FiguRE 2.-Drier for coarse samples

The outlet for air at the top may be connected with a chimnes or any other device which will furnish a suitable draft. (Bulletin No. 9, Geological Survey of Ohio, p. 312,

samc as by hand sieving, for the coal being sieved.

The same sieves should be used as specified for hand sieving, and each sieve should be provided with a close-fitting pan and cover to prevent possible loss of material during sieving. A $50 \mathrm{~g}$ sample of air-dried coal should be placed on each sieve(previously tared), and the sieves shaken until not more than about $0.1 \mathrm{~g}$ passes through the finest sieve of the series in the machine in one minute of continuous sieving. The sieves should then be removed from the machine and the undersurface of each sieve brushed clean. Sieving should then be resumed until not more than 0.1 g passes through the finest sieve of the series in the machine in one minute of continuous sieving, when the test shall be considered finished, after which the sieves should be cleaned as before and weighed on a balance sensitive to $0.1 \mathrm{~g}$. The fineness should be calculated and reported as in the hand-sieving method.

American Society for Testing Materials, standard methods of laboratory sampling and analysis of coal and coke, serial designation, D271-29, 1929.

(These methods agree with those of the American Chemical Society and were prepared jointly by that organization and the A. S. T. M.)

\section{PREPARATION OF LABORATORY SAMPLES}

(A) For coal: Apparatus

1. The apparatus used for sampling coal shall consist of the following: (a) Air drying oven.-An oven for air-drying wet samples. A suggested form is shown in Figure 2. Such an oven is not essential, but is economical where many samples are to be dried.

(b) Pans for air-drying wet samples.-Galvanized iron pans 18 by 18 inches by 1.5 inches in depth.

(c) Balance or solution scale.-A balance or scale having a capacity of $5 \mathrm{~kg}$ sensitive to $0.5 \mathrm{~g}$ for weighing the galvanized iron pans with samples.

(d) Crusher.-A jaw crusher suitable for crushing coarse samples to pass a 4760 -micron (No. 4) sieve.

(e) Grinder.-A roll crusher or coffee-mill type of grinder suitable for reducing the material passing a 4,760-micron (No. 4) sieve to pass an 840-micron (No. 20) sieve. To reduce the moisture loss while crushing, a coffce-mill type of grinder should be entirely inclosed and have an inclosed hopper and receptacle capable of holding 10 pounds of coal.

(f) Pulverizer.-An Abbé ball mill, planetary disk crusher, chrome-steel bucking board, or any satisfactory form of pulverizer for reducing the material passing an 840-micron (No. 20) sieve to pass a 250 micron (No. 60) sieve. The porcelain jars for the ball mill should be approximately 9 inches in diameter and 10 inches high. The flint pebbles should be smooth, hard, and well rounded.

(g) Large riffle sampler.-A large riffle sampler with one-half or five-eighth inch divisions for reducing the coal passing the 4,760-micron (No. 4) sieve to 10 pounds (see fig. 3 ).

(h) Small riffe sampler.-A small riffle sampler with one-fourth or three-eighths inch divisions for reducing the material passing the 840 -micron (No.

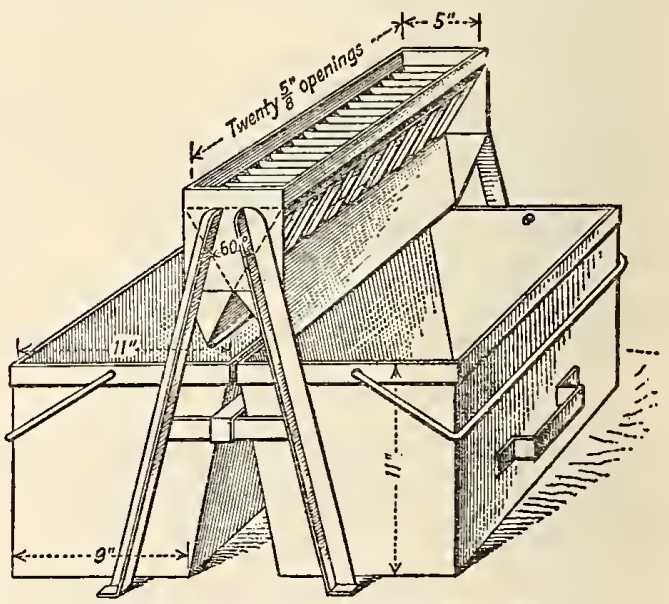

Figure 3.-Large rifle sampler

20) and the 250-micron (No. 60) sieves to a laboratory sample (see fig. 4).

(i) Sieve.-An 8-inch 250-micron (No. 60) sieve with cover and receiver.

NotE. - The sieve designations employed are those of the Standard pecifications for Sieves for Testing Purposes (serial designation: E 11, see 500.2, p. 1), of the American Society for Testing Materials, and all sieves shall conform to the detailed requirements of these specifications.

(j) Containers.-Samples in which the moisture content is important should always be shipped in moisture-tight containers. A galvanized-iron or tin can with an air-tight friction top or a screw top which is sealed with a rubber gasket and adhesive

1 For details of air-drying oven, see F. M. Stanton and A. C. Field. Methods of Analyzing Coal and Coke, Tech. Paper No. 8 , Bureau of Mines, p. $2 ; 1926$. 
tape is best adapted to this purpose. Glass fruit jars sealed with rubber gaskets may be used, but require very careful packing to avoid breakage in transit. Samples in which the moisture content is of no importance need no special protection from loss of moisture.

\section{(B) For coke :}

2. The apparatus used for sampling coke shall consist of the following:

(a) Pans for total moisture determination.-Galvanized iron pans 24 by 24 inches by 4 inches in depth.

(b) Balance or solution scale.-A balance or scale having a capacity of $10 \mathrm{~kg}$ sensitive to $1 \mathrm{~g}$ for weighing the galvanized-iron pans with samples.

(c) Crusher.-The same as section $1(d)$.

(d) Roll crusher.-A hard-steel roll crusher suitable for reducing the material passing a 4,760 micron (No. 4) sieve to pass an 840-micron (No. 20) sieve.

(e) Pulverizer.-An Abbé ball mill, hard-steel roll crusher or hard-steel diamond mortar for reducing the product passing an 840 -micron (No. 20) sieve to pass a 250-micron (No. 60) sieve. The porcelain jars for the ball mill should be approximately 9 inches in diameter and 10 inches high. The flint pebbles should be smooth, hard, and well rounded. The rolls of the hard-steel roll crusher should revolve at the same speed.

$(f)$ Large rifle sampler. - Same as in section $1(g)$ for reducing coke passing the 4,760-micron (No. 4) sieve to 10 pounds. (See fig. 3.)

(g) Small riffe sampler.-The same as section 1 (h). (See fig. 4.)

(h) Sieve. -The same as section 1 (i).

(i) Containers. - The same as section 1 (j).

(j) Oven, stove, or hot plate.-An oven, stove, or hot plate for drying coke samples in the determination of total moisture. If an oven is used it should have openings provided for natural ventilation and should be capable of being regulated between $104^{\circ}$ and $200^{\circ} \mathrm{C}$. If the coke is dried on a stove or hot plate a thermometer should be placed in it, and care exercised that the temperature does not exceed $200^{\circ} \mathrm{C}$. at any point in the pan of coke.

\section{METHOD OF SAMIPLING}

(A) For coal appearing dry:

3. (a) If the sample is coarser than will pass completely a 4,760-micron (No. 4) sieve and larger in amount than 10 pounds, quickly crush it with the jaw crusher to pass a 4,760-micron (No. 4) sieve and reduce it on the large riffle sampler to 10 pounds $; 2$ then crush it at once to pass an 840-micron (No. 20) sieve by passing through rolls or an inclosed grinder, and take, without sieving a $50 \mathrm{~g}$ total moisture sample, immediately after the material has passed through the crushing apparatus. This sample should be taken with a spoon from various parts of the product passing an 840-micron (No. 20) sieve, and should be placed directly in a rubber-stoppered bottle.

(b) Thoroughly mix the main portion of the sample, reduce on the small riffle sampler to about $200 \mathrm{~g}$, and pulverize to pass a 250 -micron (No. 60) sieve by any suitable apparatus without regard to loss of moisture. After all the material has been passed through the 250-micron (No. 60) sieve, mix and divide it on the small riffle sampler to about $50 \mathrm{~g}$. Transfer the final sample to a 4-ounce rubber-stoppered bottle. Determine moisture in both the 250-micron (No. 60) sieve sample, and the 840-micron (No. 20) sieve sample, in accordance

If the sample is crushed to pass a 3,360 micron (No. 6) sieve it may be reduced to 5 pounds. with sections 6,7 , and 8 under the determination of moisture.

(c) Calculation.-Calculate the analysis of the coal passing the 250-micron (No.60) sieve which has become partly air-dried during sampling, to the dry-coal basis, by dividing each result by 1 minus its content of moisture. Compute the analysis of the coal "as received" from the dry-coal analysis by multiplying by 1 minus the total moisture found in the sample passing an 840-micron (No. 20) sieve.

\section{(B) For coal appearing wet:}

4. (a) Spread the sample on tared pans, weigh, and air dry at room temperature, or in the special drying oven, shown in Figure 2 , at $10^{\circ}$ to $15^{\circ} \mathrm{C}$. above room temperature, and weigh again. The drying should be continued until the loss in meight is not more than 0.1 per cent per hour. Complete the sampling as described in section 3 for dry coal.

(b) Calculation.-Correct the moisture found in

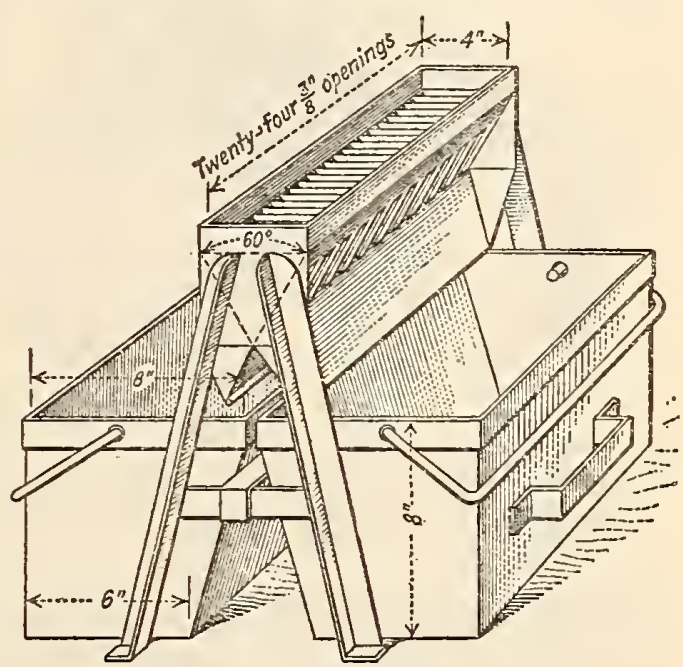

Figure 4.-Small riffle sampler

the air-dried sample passing an 840-micron (No. 20) sieve to total moisture "as received," as follows:

$\frac{100-\text { percentage of air-drying loss }}{100} \times$ (percentage of moisture in air-dried sample passing an 840-micron (No. 20) sieve) + (percentage of air-drying loss $)=($ total moisture "as received" $)$

(c) Calculate the analysis to "dry-coal" and "as-received" bases as described in section 3 for dry coal, using for the "as-received" caluclation the total moisture as found by the formula in paragraph (b) in place of the moisture found in the coal passing an 840-micron (No. 20) sieve.

Notes.-Freshly mined or wet coal loses moisture rapidly on exposure to the air of the laboratory, hence the sampling operations between opening the container and taking the total-moisture sample passing an 840-micron (No. 20) sieve must be conducted with the utmost dispatch and with minimum exposure to air.

The accuracy of the method of preparing laboratory samples should be checked frequently by resampling the rejected portions and preparing a duplicate sample. The ash in the two samples should not differ more than the following limits:

No carbonates present

Per cent

Considerable carbonate and pyrite present

Coals with more than 12 per cent ash, containing considerable

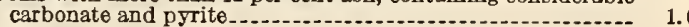

\section{(C) For coke:}

5. (a) Total moisture determination.-Dry the entire sample received at the laboratory, without any preliminary crushing, to constant weight at a 
temperature of not less than $104^{\circ}$ nor more than $200^{\circ} \mathrm{C}^{3}{ }^{3}$

Calculate the loss in weight to percentage of moisture, which shall constitute the total moisture in the coke as received at the laboratory.

The allowable difference in duplicate determinations by the same analyst is 0.5 per cent.

(b) Reduction of sample.-Crush the dried sample mechanically with a jaw or roll crusher, or by hand on a chilled iron or hard-steel plate by impact of a hard bar or sledge, avoiding all rubbing action, as otherwise the ash content will be materially increased by the addition of iron from the sampling apparatus,

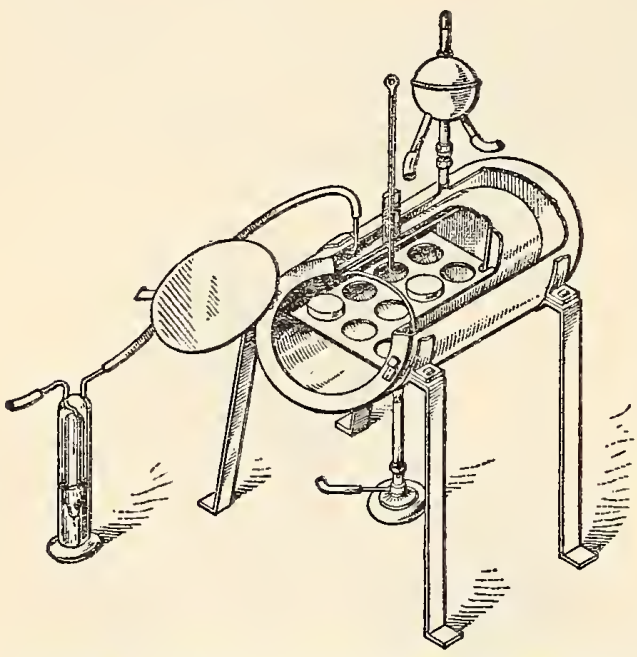

FIGURE 5.-Toluene or glycerin and water oven for determining moisture (Technical Paper No. 8, Bureau of Mines, p. 8; 1926)

even though hardened iron or steel is used. Continue the crushing until all the sample passes through a 4,760-micron (No. 4) sieve, mix and reduce on the large riffle sampler to not less than 5 pounds; again crush the 5-pound sample sufficiently fine to pass an 840-micron (No. 20) sieve; mix and reduce on the small riffle sampler to $200 \mathrm{~g}$. Transfer this $200 \mathrm{~g}$ portion to the porcelain jar of an Abbé ball mill and pulverize to pass a 250 -micron (No. 60) sieve. When pulverization is complete, pour the contents of the jar on a 1/2-inch screen and separate the sample from the pebbles by shaking the screen. Pass all the material through a 250-micron (No. 60) sieve, pulverizing any coarse particles by impact in a hard-steel diamond mortar, and mix with the remainder of the sample. Reduce the sample through the small riffle sampler to about $50 \mathrm{~g}$ and transfer to a rubber-stoppered glass bottle.

(c) In case a ball mill is not available for fine grinding, quarter the 5-pound sample passing an 840 -micron (No. 20) sieve to $200 \mathrm{~g}$ and pulverize to pass a 250-micron (No. 60) sieve by means of a hardsteel roll crusher in which the rolls revolve at the same speed or by impact in a hard-steel diamond mortar. The use of rubbing surfaces, such as a disk pulverizer or a bucking board, is never permissible for grinding coke.

NotE.-The accuracy of the method of preparing laboratory samples should be checked frequently by resampling the rejected portions and preparing a duplicate sample. The ash in the two samples should not differ more than 0.4 per cent.

Fxperiments made at the U.S. Bureau of Mines have shown that results checking within 0.5 per cent are obtained between these
temperature limits. See A. C. Fieldner and W. A. Selvig, The temperature limits. See A. C. Fieldner and W. A. Selvig, The cal Paper No. 148; 1917.

\section{METHODS OF ANALYSIS}

\section{DETERAINATION OF MOISTORE (FOR COAL AND COKE)}

Apparatus

6. (a) Moisiure oven (for coal).-For determining the moisture of coal, the oven shall be so constructed as to have a uniform temperature in all parts and a minimum of air space. It may be of the form shown in Figure 5. Provision shall be made for renewing the air in the oven at the rate of two to four times a minute, with the air dried by passing it through concentrated $\mathrm{H}_{2} \mathrm{SO}_{4}$.

(For coke).-For determining the moisture of coke, an ordinary drying oven with openings for natural air circulation and capable of temperature regulation between limits of $104^{\circ}$ and $110^{\circ} \mathrm{C}$. may be used.

(b) Capsules with covers.-A convenient form, which allows the ash determination to be made on the same sample, is a porcelain capsule, seveneighths inch deep and $13 / 4$ inches in diameter; or a fused silica capsule of similar shape. This shall be used with a well-fitting flat aluminum cover, illustrated in Figure 6.

Platinum crucibles or glass capsules with groundglass caps may also be used. They should be as shallow as possible, consistent with convenient handling.

\section{Method}

(A) For coal or coke passing 250-micron (No. 60) sieve:

7. (a) Heat the empty capsules under the conditions at which the sample is to be dried, place stopper or cover on capsule, cool over concentrated $\mathrm{H}_{2} \mathrm{SO}_{4}$ (sp. gr. 1.84) for 30 minutes, and weigh. Dip out with a spoon or spatula from the sample bottle approximately $1 \mathrm{~g}$ of the sample; put this quickly into the capsule, close, and weigh at once.

(b) An alternate procedure (more open to error) after transferring an amount slightly in excess of $1 \mathrm{~g}$, is to bring to exactly $1 \mathrm{~g}$ in weight $( \pm 0.5 \mathrm{mg}$ ) by quickly removing the

excess weight of the sample with a spatula. The utmost dispatch must be used in order to minimize the exposure of the sample until the weight is found.

After removing the covers, quickly place the capsules in a preheated oven (at $104^{\circ}$ to $110^{\circ} \mathrm{C}$.) through which passes a current of air dried by concentrated $\mathrm{H}_{2} \mathrm{SO}_{4}$ (the current of dry air is not necessary for coke).

Close the oven at once and heat for one hour. Then open the oven, cover the capsules quickly and place them in a desiccator over concentrated $\mathrm{H}_{2} \mathrm{SO}_{4}$. When cool, weigh.

The percentage of moisture in the sample passing a 250-micron (No.60) sieve shall be used to calculate the other results to a dry basis.

(B) For coal passing 840-micron (No. 20) sieve:

8. Use $5 \mathrm{~g}$ samples, weighed with an accuracy of $2 \mathrm{mg}$, and heat for one and one-half hours; the procedure is otherwise the same as with the sample passing a 250-micron (No. 60) sieve as described in section 7 .

Note.-For methods of greater accuracy see Report of Subcommittee II on Moisture, of the Joint Committee on Analysis of Coal, Proceedings, Am. Soc. Testing Mats., Vol. XIV, Part I, pp. 419423; 1914. 
DETERMTNATION OF ASH (FOR COAL AND COKE)

Apparatus

9. (a) Gas or electric muffle furnace (for coal).-For determination of ash of coal, the muffle shall have good air circulation and be capable of having its temperature regulated between $700^{\circ}$ and $750^{\circ} \mathrm{C}$.

(b) Gas or electric muffle furnace or Meker burner (for coke).-For determination of ash of coke, the muffle shall have good air circulation, and be capable of having its temperature regulated to not exceed $950^{\circ} \mathrm{C}$.

(c) Porcelain capsules.-Porcelain capsules, seveneighths inch deep and $13 / 4$ inches in diameter, or similar shallow dishes or platimun crucibles.

\section{(A) For coal: \\ Method}

10. Place the porcelain capsules containing the dried coal from the moisture determination in a cold muffle furnace, or on the hearth at a low temperature, and gradually heat to redness at such a rate as to avoid mechanical loss from too rapid expulsion of volatile matter. Finish the ignition to constant weight $( \pm 0.001 \mathrm{~g})$ at a temperature between $700^{\circ}$ and $750^{\circ} \mathrm{C}$. Cool in a desiccator, and weigh as soon as cold.

Notes.-Before replacing. the capsules in the muffe for ignition to constant weight, the ash should be stirred with a platinum or to constant weight, the ash should be stirred with a platinum or hastens complete ignition.

The result obtained by this method is "uncorrected" ash. For "corrected" ash see the preliminary report." The actual mineral matters in the original coal are usually very different in weight and composition from the weight of the "uncorrected " ash.

(B) For coke :

11. Place the capsules containing the dried coke from the moisture determination in a muffle furnace or over a burner, and heat to redness at such a rate as to avoid mechanical loss. Finish the ignition to constant weight $( \pm 0.001 \mathrm{~g})$ at a temperature not exceeding $950^{\circ} \mathrm{C}$. Cool in a desiccator and weigh.

Notes.-Before replacing the capsules in the muffe for ignition to constant weight the ash should be stirred with a platinum or nichrome wire. Stirring once or twice before the first weighing hastens complete ignition.

Test the ash for unburned carbon by moistening it with alcohol; any carbon remaining will show as black particles.

\section{DETERMINATION OF VOLATILE MATTER (FOR COAL AND COKE)}

\section{Apparatus}

12. (a) Platinum crucible with closely fitting cover (for coal). -The crucible shall be of not less than 10 nor more than 20 cc capacity; of not less than 25 nor more than $35 \mathrm{~mm}$ in diameter; of not less than 30 nor more than $35 \mathrm{~mm}$ in height.

(b) Platinum crucible with closely fitting cover (for coke). - The crucible shall be of 10 cc capacity, with capsule cover having thin fiexible sides fitting down into crucible. Or the double-crucible method may be used, in which the sample is placed in a 10 or $20 \mathrm{cc}$ platinum crucible, which is then covered with another crucible of such a size that it will fit closely to the sides of the outer crucible, and its bottom will rest one-third to one-half inch above the bottom of the outer crucible.

(c) Vertical electric tube furnace; or a gas or electrically heated mufle furnace (for coal or coke).-The furnace may be of the form as shown in Figure 7. It shall be regulated to maintain a temperature of $950^{\circ} \mathrm{C} . \pm 20^{\circ} \mathrm{C}$. in the crucible, as shown by a thermocouple kept in the furnace. If the determination of volatile matter is not an essential feature of the

4 Report on Fired Carbon and Ash, Proc., Am. Soc. Testing Mats., specifications under which the coal or coke is bought, a Meker burner may be used.

\section{Method}

\section{(A) For coal and coke, usual method:}

13. Weigh $1 \mathrm{~g}$ of the sample in a weighed platinum crucible, closed with a cover, and place on platinum or nichrome-wire supports in the furnace chamber, which shall be at a temperature of $950^{\circ} \mathrm{C} . \pm 20^{\circ} \mathrm{C}$. After the more rapid discharge of volatile matter has subsided, as shown by the disappearance of the luminous flame, or in the case of coke after heating two or three minutes, tap the cover lightly to more

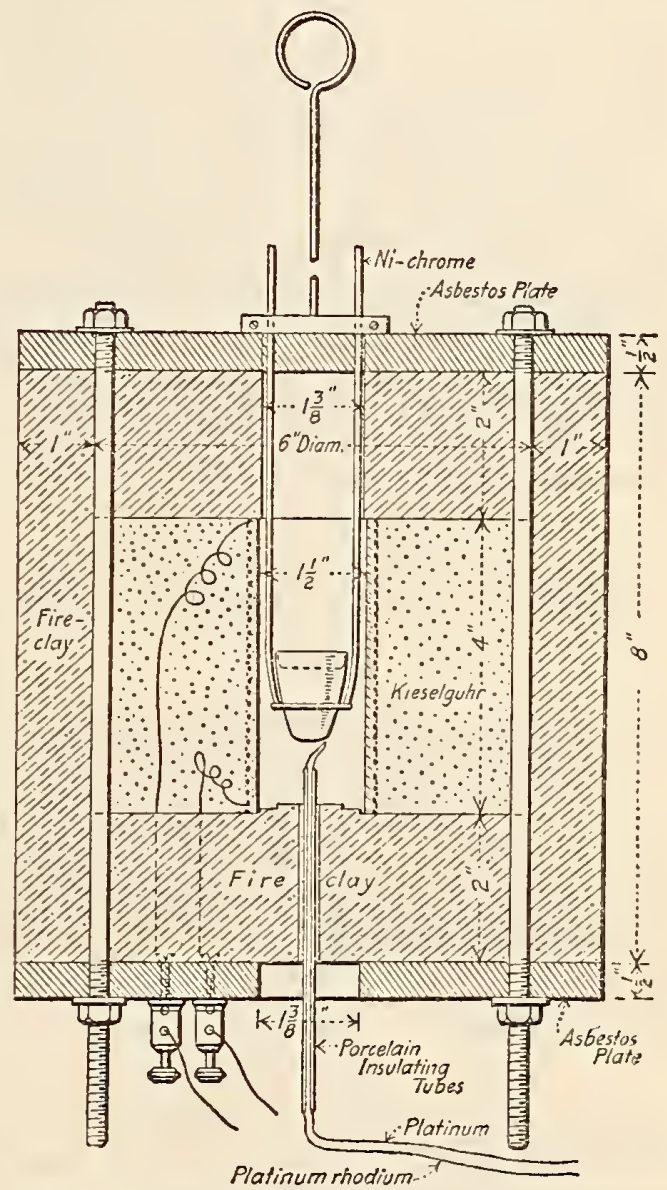

Figure 7.-Electric tube furnace for determining volatile matter

For 110-volt alternating current, 60 feet of nichrome wire, No. 17 B. \& S. gage will give the required temperature. The temperature must be controlled by an external resistance. (Technical Paper No. 8, Bureau of Mines, p. 11; 1926.)

perfectly seal the crucible and thus guard against the admission of air. After heating for exactly seven minutes, remove the crucible from the furnace and, without disturbing the cover, allow it to cool. Coke should be cooled in a desiccator. Weigh as soon as cold. The loss of weight minus moisture equals the volatile matter.

14. Modification for subbituminous coal, lignite, and peat.-Mechanical losses are incurred on suddenly heating peat, subbituminous coal, and lignite; therefore, they must be subjected to a preliminary gradual heating for five minutes; this is best done by playing the flame of a burner upon the bottom of 
the crucible in such a manner as to bring about the discharge of volatile matter at a rate not sufficient to cause sparking. After the preliminary heating, transfer the crucible to the volatile-matter furnace and heat for six minutes at $950^{\circ} \mathrm{C}$. as in the regular method described in Section 13.

Notes.-The cover should fit closely enough so that the carbon deposit from bituminous and lignite coals does not burn away from the under side.

Regulation of temperature to within the preseribed limit is impor. tant.

(B) For coal and coke, using Meker burner:

15. Weigh $1 \mathrm{~g}$ of the sample in a weighed platinum crucible and close with a cover or, in the case of coke, another crucible as described in section $12(b)$. Place in the flame of a No. 4 Meker burner, having approximately an outside diameter at the top of 25 $\mathrm{mm}$ and giving a flame not less than $15 \mathrm{~cm}$ high. The temperature should be $950^{\circ}$ C. $\pm 20^{\circ}$ C., as determined by placing a thermocouple through the perforated cover, which for this purpose may be of nickel or asbestos. The junction of the couple should be placed in contact with the center of the bottom of the crucible; or the temperature may be indicated by the fusion of pure $\mathrm{K}_{2} \mathrm{CrO}_{4}$ in the covered crucible (fusion of $\mathrm{K}_{2} \mathrm{CrO}_{4}, 940^{\circ}$ C.). The crucible shall be placed in the flame about $1 \mathrm{~cm}$ above the top of the burner and the heating continued seven minutes. Where the gas pressure is variable it is well to use a $U$ tube attachment to the burner.

\section{DETERIIIMATION OF FIXED CARBON (FOR COAI AND COKE)}

16. Caltulate fixed carbon as follows:

$100-($ moisture $+a s h+$ volatile matter $)=$ percentage of fixed carbon

\section{DETERMINATION OF SULPHOR (FOR COAL AND COEE)}

(A) Eschka method:

\section{Apparatus}

17. (a) Gas or electric muffe furnace, or burners.For igniting the sample with the Eschka mixture and for igniting the $\mathrm{BaSO}_{4}$.

(b) Porcelain or platinum crucibles or capsules.Porcelain capsules, seven-eighths inch deep and $13 / 4$ inches in diameter, or porcelain crucibles of $30 \mathrm{cc}$ capacity, high or low form, or platinum crucibles of similar size shall be used for igniting the sample with the Eschka mixture.

(c) Porcelain, platinum, alundum, or silica crucibles of 10 to 15 cc capacity, shall be used for igniting the $\mathrm{BaSO}_{4}$.

\section{Solutions and Reagents}

18. (a) Barium chloride.-Dissolve $100 \mathrm{~g}$ of $\mathrm{BaCl}_{2} \cdot 2 \mathrm{H}_{2} \mathrm{O}$ in $1,000 \mathrm{cc}$ of distilled water.

(b) Saturated bromine water.-Add an excess of bromine to $1,000 \mathrm{cc}$ of distilled water.

(c) Eschlia mixture.-Thoroughly mix 2 parts (by weight) of light calcined $\mathrm{MgO}$ and 1 part of anhydrous $\mathrm{Na}_{2} \mathrm{CO}_{2}$. Both materials should be as free as possible from sulphur.

(d) Methyl orange.-Dissolve $0.02 \mathrm{~g}$ in $100 \mathrm{cc}$ of hot distilled water and filter.

(e) Hydrochloric acid.-Mix $500 \mathrm{ce}$ of $\mathrm{HCl}$ (sp. gr. 1.19), and $500 \mathrm{ce}$ of distilled water.

(f) Normal hydrochloric acid.-Dilute $80 \mathrm{cc}$ of $\mathrm{HCl}$ (sp. gr. 1.19), to 1 liter with distilled water.

(g) Sodium carbonate.-A saturated solution, approximately $60 \mathrm{~g}$ of crystallized or $22 \mathrm{~g}$ of anhydrous $\mathrm{Na}_{2} \mathrm{CO}_{3}$ in 100 ce of distilled water.

(h) Sodium-hydroxide solution.-Dissolve $100 \mathrm{~g}$ in 1 liter of distilled water. This solution may be used in place of the $\mathrm{Na}_{2} \mathrm{CO}_{3}$ solution.

\section{Method}

19. Preparation of sample and mixture.-Thoroughly mix on glazed paper $1 \mathrm{~g}$ of the sample and
$3 \mathrm{~g}$ of Eschka mixture. Transfer to a porcelain capsule, seven-eighths inch deep and $1 \frac{3 / 4}{4}$ inches in diameter, or a porcelain crucible of 30 cc capacity, high or low form, or platinum crucible of similar size, and cover with about $1 \mathrm{~g}$ of Eschka mixture.

20. (a) Ignition.-On account of the amount of sulphur contained in artificial gas, the crucible shall be heated over an alcohol, gasoline, or natural-gas flame as described in paragraph (b), or in a gas or electrically heated muffle, as described in paragraph (c) for coal and in paragraph $(d)$ for coke. The use of artificial gas for heating the sample and Eschka mixture is permissible only when the crucibles are heated in a muffle.

(b) Heat the crucible, placed in a slanting position on a triangle, over a very low flame to avoid rapid explusion of the volatile matter, which tends to prevent complete absorption of the products of combustion of the sulphur. Heat the crucible slowly for 30 minutes, gradually increasing the temperature and stirring after all black particles have disappeared, which is an indication of the completeness of the procedure.

(c) (For coal).-Place the crucible in a cold muffle and gradually raise the temperature to $870^{\circ}$ to $925^{\circ} \mathrm{C}$. (cherry-red heat) in about one hour. Maintain the maximum temperature for about one and one-half hours.and then allow the crucible to cool in the muffe.

(d) (For coke).-Place the crucible in a warm muffle and gradually raise the temperature to $870^{\circ}$ to $925^{\circ} \mathrm{C}$. (cherry-red heat) in about 30 minutes. Maintain this maximum temperature until on stirring all black particles have disappeared.

(e) Subsequent treatment.-Remove and empty the contents into a $200 \mathrm{cc}$ beaker and digest with $100 \mathrm{cc}$ of hot water for one-half to three-fourths hour, with occasional stirring. Filter and wash the insoluble matter by decantation. After several washings in this manner, transfer the insoluble matter to the filter and wash five times, keeping the mixture well agitated. Treat the filtrate, amounting to about $250 \mathrm{cc}$, with 10 to $20 \mathrm{cc}$ of saturated bromine water, make slightly acid with $\mathrm{HCl}$ and boil to expel the liberated bromine. Make just neutral to methyl orange with $\mathrm{NaOH}$ or $\mathrm{Na}_{2} \mathrm{CO}_{3}$ solution, then add 1 ce of $N \mathrm{HCl}$. Boil again and add slowly from a pipette, with constant stirring, 10 cc of a 10 per cent solution of $\mathrm{BaCl}_{2} \cdot 2 \mathrm{H}_{2} \mathrm{O}$. Continue boiling for 15 minutes and allow to stand for at least two hours, or preferably over night, at a temperature just below boiling. Filter through an ashless filter paper and wash with hot distilled water until a $\mathrm{AgNO}_{3}$ solution shows no precipitate with a drop of the filtrate. Place the wet filter containing the precipitate of $\mathrm{BaSO}_{4}$ in a weighed platinum, porcelain, silica, or alundum crucible, allowing a free access of air by folding the paper over the precipitate loosely to prevent spattering. Smoke the paper off gradually and at no time allow it to burn with flame. After the paper is practically consumed, raise the temperature to approximately $925^{\circ} \mathrm{C}$. and heat to constant weight.

The residue of $\mathrm{MgO}$, etc., after leaching, should be dissolved in $\mathrm{HCl}$ and tested with great care for sulphur. When an appreciable amount is found this should be determined quantitatively. The amount of sulphur retained is by no means a negligible quantity.

$(f)$ Blanks and corrections.-In all cases a correction must be applied either (1) by running a blank exactly as described above, using the same amount of all reagents that were employed in the regular determination, or more surely (2) by determining a known amount of sulphate added to a solution of the reagents after these have been put 
through the preseribed series of operations. If this latter procedure is adopted and carried out, say, once a week or whenever a new supply of a reagent must be used, and for a series of solutions covering the range of sulphur content likely to be met with in the samples, it is only necessary to add to or subtract from the weight of $\mathrm{BaSO}_{4}$ obtained from a sample, whatever deficiency or excess may have been found in the appropriate "check" in order to obtain a result that is more certain to be correct than if a "blank" correction as determined by the former procedure is applied. This is due to the fact that the solubility error for $\mathrm{BaSO}_{4}$, for the amounts of sulphur in question and the conditions of precipitation prescribed, is probably the largest one to be considered. $\mathrm{BaSO}_{4}$ is soluble in acids and even in pure water, and the solubility limit is reached almost immediately on contact with the solvent. Hence, in the event of using reagents of very superior quality or of exercising more than ordinary precautions, there may be no apparent "blank," because the solubility limit of the solution for $\mathrm{BaSO}_{4}$ has not been reached or at any rate not exceeded.

(g) Calculate sulphur content as follows:

Percentage of sulphur $=\frac{\left(\text { Weight of } \mathrm{BaSO}_{1} \text { - blank }\right) \times 13.74}{\text { Tight of }}$

Note.-As shown in the preliminary report, the Atkinson and sodium-peroxide methods give results in close agreement with the Eschka method. Register has shown that if 5 per cent of nitrogen is present in the gases contained in the homh calorimeter the sulphur of a sample is almost completely oxidized to $\mathrm{H}_{2} \mathrm{SO}_{4}$ and the washings of the calorimeter may he used for the determination of sulphur.

(B) Bomb-washing method:

\section{Method}

21. (a) Ignition.-Sulphur is determined in the washings from the oxygen-bomb calorimeter following the calorimetric determination. The type of bomb, amount of water in the bomb, oxygen pressure, and amount of sample taken shall be the same as specified under the calorimetric determination. The bomb shall stand in the calorimeter water for not less than five minutes after firing.

(b) Subsequent treatment.-Remove the bomb from the calorimeter water and open the valve carefully so as to allow the gases to escape at an approximately even rate so the pressure is reduced to atmospheric in not less than one minute. Bombs equipped with valves other than needle valves, such as compression valves, shall be provided with a device so the valve can be controlled to permit a slow and uniform release of the gases. Open the bomb and examine the inside for traces of unburned material or sooty deposit. If these are found, the determination shall be discarded. Wash carefully all parts of the interior of the bomb, including the tray, with a fine jet of distilled water containing $1 \mathrm{cc}$ per liter of a saturated solution of methyl orange, until no acid reaction is observed. It is essential to wash through the valve opening in the case of bombs equipped with compression valves, or other types of valves with large openings, as considerable spray may collect in such valve openings.

Collect the washings in a 250 cc beaker and titrate with standard alkali solution to obtain the "acid correction" for the heating value, as specified under the calorimetric determination. Add 1 cc of $\mathrm{NH}_{4} \mathrm{OH}$ (sp. gr. 0.90) heat the solution to boiling, and filter through qualitative filter paper. Wash the residue and filter paper thoroughly five or six times with hot distilled water. To the filtrate and washings, amounting to about $250 \mathrm{cc}$, add $1 \mathrm{cc}$ of saturated bromine water and sufficient $\mathrm{HCl}$ to make it slightly acid. Boil the solution to expel the excess bromine. Adjust the acidity and precipitate and determine the sulphur, as specified under the Eschka method, section 19.

\section{(C) Sodium peroxide fusion method:}

\section{Apparatus}

22. (a) Combustion bomb.-The Parr coal sulphur bomb, or its equivalent, shall be used. The bomb shall have an inner surface which is not attacked by the chemicals on ignition of the charge.

(b) Reagents. - Sulphur-free powdered sodium peroxide $\left(\mathrm{Na}_{2} \mathrm{O}_{2}\right)$ powdered c. p. potassium perchlorate $\left(\mathrm{KClO}_{4}\right)$ or potassium chlorate $\left(\mathrm{KClO}_{3}\right)$ and powdered c. p. benzoic acid shall be used.

\section{Method}

23. (a) Place $1 \mathrm{~g}$ of $\mathrm{KClO}_{4}$ or $\mathrm{KClO}_{3}$ in a dry sulphur bomb and break up any lumps that occur. Add a $0.5 \mathrm{~g}$ sample and mix thoroughly with a glass rod. Then add one measure (about $15 \mathrm{~g}$ ) sodium peroxide, close the bomb and mix thoroughly by shaking. In the case of cokes or anthracites, or coals excessively high in ash which fail to ignite or fuse properly (as indicated by the fusion being honeycombed in appearance) and $0.3 \mathrm{~g}$ of benzoic acid to the bomb at the time the chlorate and sample are added. It should be noted that a mixture of potassium chlorate and organic matter alone produces a mixture of extremely explosive properties. One of the important functions of the sodium peroxide is to provide a diluent, thus slowing down the reaction, so care should be taken that it is not omitted in the charge. Potassium perchlorate is fully equal if not superior to the $\mathrm{KClO}_{3}$, and is without turbulence in its reaction.

Fasten the cover securely to the bomb and ignite the charge by applying a sharply pointed flame from a blast lamp to the bottom of the bomb for a brief period, or by electric ignition, according to the type of bomb used. Place the bomb inside a piece of steel pipe when the charge is ignited to prevent possible injury to the operator if the bomb should burst. Allow one minute for complete combustion to take place after ignition, then cool under the tap or in a vessel of water.

Remove the cover from the bomb, place the bomb on its side in a 400 ce beaker and wash off the cover with a fine jet of hot distilled water. Place a watch glass over the beaker and cautiously add about $100 \mathrm{cc}$ of hot distilled water. After the contents of the bomb have dissolved, remove and rinse it carefully with the distilled water. Add slowly, concentrated $\mathrm{HCl}$ to the neutral point, then add 1 to $2 \mathrm{cc}$ of the acid in excess. Filter through qualitative filter paper into a $600 \mathrm{ec}$ beaker and wash thoroughly five or six times with distilled water. Dilute the filtrate to approximately 400 ce and precipitate the sulphur with barium chloride and determine as specified under the Eschka method, section 19.

(b) Blank correction.-A blank correction shall be applied by running a blank on the reagents used.

\section{DETERMINATION OF PHOSPHORUS IN ASE (FOR COAL AND}

\section{Solution Required}

24. Molybdate solution.-Dissolve $65 \mathrm{~g}$ of molybdic acid (85 per cent) in a mixture of $143 \mathrm{cc}$ of $\mathrm{NH}_{4} \mathrm{OH}$ (sp. gr. 0.90 ) and $142 \mathrm{ec}$ of water. Add this solution slowly, with constant stirring, to $715 \mathrm{cc}$ of $\mathrm{HNO}_{3}$ (sp. gr. 1.20). If the solution is cloudy, add two drops of a solution of ammonium phosphate (20 per cent) and allow the precipitate which forms to settle. Filter the solution into bottles, and if necessary refilter just before using. If the molybdic acid used is 100 per cent, $56 \mathrm{~g}$ should be taken instead of $65 \mathrm{~g}$. 


\begin{abstract}
Method
Method No. 1, for all cases:

25 . Add to the ash from a $5 \mathrm{~g}$ sample, in a platinum crucible, $10 \mathrm{cc}$ of concentrated $\mathrm{HNO}_{3}$ and 3 to 5 cc of HF. Evaporate the liquid and fuse the residue with $3 \mathrm{~g}$ of $\mathrm{Na}_{2} \mathrm{CO}_{3}$. If unburned carbon is present, mix $0.2 \mathrm{~g}$ of $\mathrm{NaNO}_{3}$ with the carbonate. Leach the melt with water and filter the solution. Ignite the residue, fuse with $\mathrm{Na}_{2} \mathrm{CO}_{3}$ alone, leach the melt with water and filter the solution. Just acidify with $\mathrm{HNO}_{3}$ the combined filtrate lield in a flask, add 3 to $5 \mathrm{cc}$ of concentrated $\mathrm{HNO}_{3}$ in excess, and concentrate to a volume of $100 \mathrm{cc}$. Add $6 \mathrm{~g}$ of $\mathrm{NH}_{4} \mathrm{NO}_{3}$, bring the temperature of the solution to $80^{\circ} \mathrm{C}$., add $50 \mathrm{cc}$ of molybdate solution and shake the flask for 10 minutes. When the precipitate has settled, filter and wash the precipitate, until free from acid, with a $\mathrm{KNO}_{3}$ solution (2 per cent). Place the filter paper with the precipitate in the flask, add 25 cc of recently boiled distilled water, and macerate the filter paper with a glass stirring rod. Add a measured excess of standard $\mathrm{NaOH}$ solution and agitate the solution to completely dissolve the precipitate. Add three drops of phenolphthalein solution as an indicator and titrate the excess $\mathrm{NaOH}$ with a standard $\mathrm{HNO}_{3}$ solution. The alkali solution may well be made equal to 0.00025 $\mathrm{g}$ of phosphorus per cubic centimeter, or 0.005 per cent for a $5 \mathrm{~g}$ samplc. Such a solution would be 0.926 of $0.2 N .^{5}$
\end{abstract}

Note. - The advantage of the use of $\mathrm{HF}$ in the initial attack of the ash lies in the resulting removal of silica. Fusion with alkali carbonate is necessary for the elimination of titanium, which if present and not removed, will contaminate the phosphomolybdate and is said to removed, will contaminate the ph
sometimes retard its precipitation.

Method No. 2, when titanium is low:

26. When titanium is so low as to offer no objection, decompose the ash as described in section 25 , method No. 1, but carry the evaporation only to a volume of about $5 \mathrm{cc}$. Dilute the solution with water to $30 \mathrm{cc}$, boil and filter into a flask. If the washings are turbid pass them again through the filter. Ignite the residue in as platinum crucible, fuse with a little $\mathrm{Na}_{2} \mathrm{CO}_{3}$, dissolve the melt in $\mathrm{HNO}_{3}$ and add the solution, if clear, to the main one. If not clcar, filter. The fusion of the residue may be dispensed with in routine work on a given coal or coke if it is certain that the residue is free from phosphorus. Add $\mathrm{NH}_{4} \mathrm{OH}$ until a slight precipitate ensues. Add concentrated $\mathrm{HNO}_{3}$ to just dissolve the precipitate, then add 3 to 5 cc of the acid in excess. Heat the solution, which should have a volume of about $100 \mathrm{cc}$, to $80^{\circ} \mathrm{C}$., and add $50 \mathrm{cc}$ of molybdate solution. Shake the flask for 10 minutes, filter, and determine the phosphorus as described in scetion 25 , method No. 1 .

\section{DETERMUNATION OF FUSIBIIITY OF ASH (FOR COAL AND COEE)}

\section{Apparatus}

27. (a) Standard furnace-Gas-fired furnaces, such as the coal-ash fusion furnace of the Denver Fire Clay Co., the No. 3 Melter's furnace of the American Gas Furnace Co., or their equivalent shall be used.

These gas-fired furnaces are especially suitable for fusion detcrmination in that the burners are arranged on a tangent near the base of the furnace, thus producing a rotary flame which completely surrounds the crucible in which the ash cones are placed. The whirling flame heats the crucible uniformly, and when the furnaces are operated with an excess of gas over air a reducing atmosphere is maintained in the crucible, which condition gives the lowest point at which the ash fuses. Either natural or

- Ulmann and Buch, Chemical Engineer, 10, p. 130; 1909. artificial gas may be used. Air should be supplied at uniform pressure.

The stock design of the No. 3 Melter's furnace should be modified by providing the upper cylinder with two holes in the side; a 2 -inch observation hole with its center 4 inches from the top of the cylinder (excluding cover plate), and a 1-inch thermocouple hole $90^{\circ}$ to the right of the obscrvation hole; the bottoms of these holes being in the same horizontal plane. The coal-ash fusion furnace as furnished by the Denver Fire Clay Co. is provided with suitable observation and thermocouple holes. The interiors of the furnaces are cylindrical and approximately 7 inches in diameter. Counterbalanced sheet-iron canopies connected with telescopic flues to an exhaust systcm are placed over the furnaces for conducting the hot gases out of the room.

(b) Refractory crucibles.-The interior of the fusion furnace as arranged for making a test is shown in Figurc 8. Crucible $a$ is a corundite crucible about 3 inches in inside diamcter and about $4 \frac{1}{2} 2$ inches in

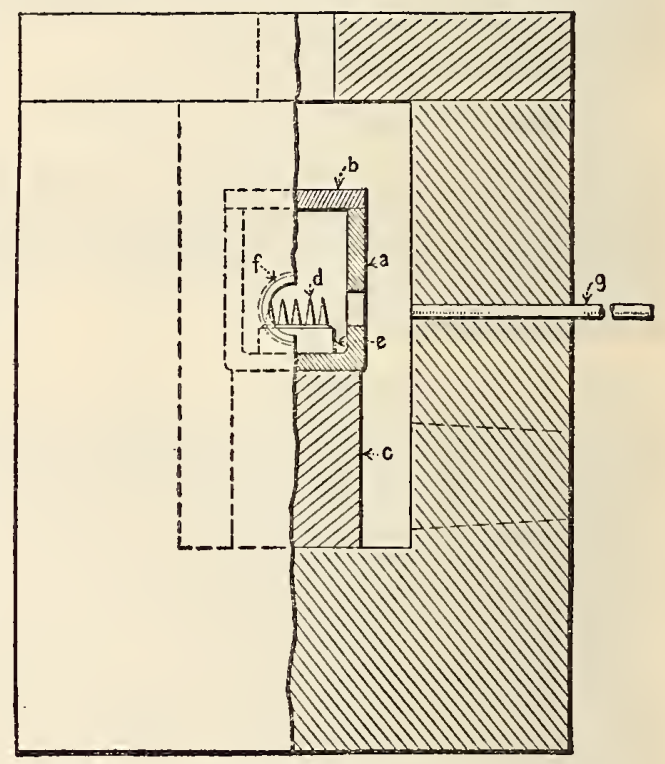

Figure 8.- Section of furnace arranged for fusion tests

height outside, with a wall thickness of about onehalf inch. The crucible is provided with a cover, $b$, and shall have two holes in the side, an observation hole 2 inches in diametcr and a thermocouple hole 1 inch in diameter, $90^{\circ}$ to the right of the observation hole. The bottoms of the two holes shall be in the same horizontal plane. The crucible is supported by a corundite support, $c$, so that the two holes in the crucible are in line with the corresponding holes in the furnace wall. The ash cones, $d$, are supported on corundite disk $e$ so that they are visible through the observation hole of the crucible and furnace wall.

(c) Observation hole- $\mathrm{A}$ fused silica, alundum, or refractory porcelain tube, $f, 178$ inches in external diameter and 6 to 7 inches long, is cemented in the 2-inch observation hole of the furnace, the inner end being flush with the insidc furnace wall and the other end projecting out of the furnace. A brass sleeve carrying a single thin glass window, made from ordinary soda glass, shall be slipped on the outer end of the observation tube to prevent the escape of burning gas, which would interfere with a convenient observation of the cones. When observations at 
high temperatures are made, the cones should be viewed through a piece of colored glass.

(d) Blowing tube.-At furnace temperatures above $1,000^{\circ} \mathrm{C}$. it is very difficult to observe the ash cones. An open refractory porcelain blowing tube $(g$, fig. 8) about $1 / 4$ inch in inside diameter shall be inserted in the thermocouple hole, the inner end being flush with the inside of the furnace wall. The blowing tube shall be connected to the compressed air line by means of rubber tubing and the air shall be let into the tube at the time of observation by means of a pinchcock, thus momentarily cooling the ash cones and rendering them visible.

(e) Brass cone mold.-A brass cone mold for making ash cones, three-fourths inch high and one-fourth inch at each side of the base, which is an equilateral triangle, is illustrated in Figure 9.

(f) Pyrometer. ${ }^{\text {- }}$-Temperature measurements sha!l be made with a thermocouple of platinum and platinum-rhodium used in conjunction with a highresistance millivoltmeter or potentiometer; or with an optical pyrometer of the risappearing filament type, which has been calibrated to be accurate to $10^{\circ} \mathrm{C}$. up to $1,400^{\circ} \mathrm{C}$. and to $15^{\circ} \mathrm{C}$. from $1,400^{\circ}$ to $1,600^{\circ} \mathrm{C}$. If a thermocouple is used it should be protected from the furnace gases by a glazed porcelain tube and so placed in the furnacc that the hot junction is in the immediate vicinity of the cones. The pyrometer equipment shall be checked frequently by mounting small pieces of pure gold and nickel in the same manner as the cones. With a strong reducing atmosphere, $1,452^{\circ} \mathrm{C}$. should be obtained for the melting point of nickel and $1,063^{\circ} \mathrm{C}$. for the gold. The pyrometer equipment should also be standardized from time to time through the temperature range for which it is used, by a suilabiy equipped standardiying iaboratory, such as that of the National Bureau of Standards.

(g) If temperature measurements are made by sighting an optical pyrometer through the glass window of the observation tube the following corrections shall be added to the observed temperatures to correct absorption of light by the glass window:

Correction to observed temperature for absorption of light by a single clean gla ss window ${ }^{1}$

\begin{tabular}{|c|c|}
\hline $\begin{array}{c}\text { Observed } \\
\text { tempera- } \\
\text { ture }\end{array}$ & $\begin{array}{c}\text { Correction } \\
\text { to be } \\
\text { added }\end{array}$ \\
\hline${ }^{\circ} C$. & ${ }^{\circ} C$. \\
800 & 5 \\
1,000 & 8 \\
1,200 & 10 \\
1,400 & 13 \\
1,600 & 16 \\
\hline
\end{tabular}

1 B. S. Tech. Paper No. 170, p. 117; 1921.

\section{Preparation of Ash}

28. Spread out 50 to $100 \mathrm{~g}$ of the sample passing a 250-micron (No.60) sieve on a 6-inch fire-clay roasting dish, and completely convert to ash in a muffle furnace at a temperature of $800^{\circ}$ to $900^{\circ} \mathrm{C}$. Transfer 5 to $10 \mathrm{~g}$ of this ash to an agate mortar ${ }^{7}$ and grind to pass a 74-micron (No. 200) sieve. Then place the ash in a silica or porcelain capsule, $5 / 8$-inch deep and $13 / 4$ inches in diameter, and ignite for a period of two hours in a current of oxygen, at a tcmperature of $800^{\circ}$ to $850^{\circ} \mathrm{C}$. This ignition is made to insure complete and uniform oxidation of the ash.

- For detailed information concerning the use of pyrometers see Pyrometric Practice, B.S. Tech. Paper No. 170; 1921.

7 A mechanical agate-mortar grinder will save time where many determinations are made.

\section{Preparation of Cones}

29. Moisten the ignited ash with a 10 per cent dextrin solution and work into a plastic mass with a spatula. Mold the plastic material into small triangular pyramids $3 / 4$-inch ligh and 1/4-inch wide at the side of the base. The pyramids are made by firmly pressing the plastic material with a steel spatula into a brass mold of the dimensions mentioned, the mold being similar to that shown in Figure 9 . Strike off

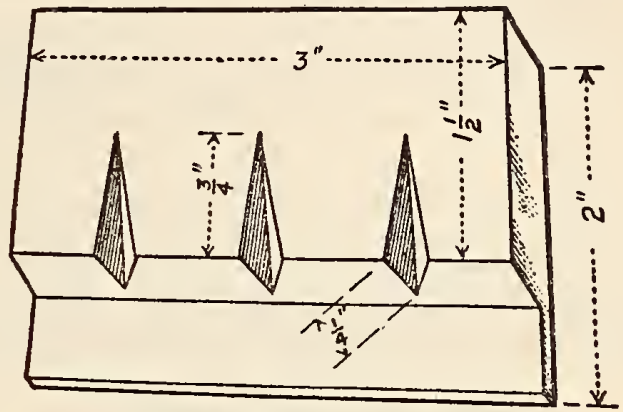

Figure 9.-Brass cone mold

the surface smooth and remove the cone from the mold by applying a small knife blade at the base. Mount the cones when dry in a refractory base composed of a mixture of equal parts of kaolin and calcined alumina. Moisten the base mixture to make it workable, and spread a part of it out on a sheetiron plate. Then mount the cone in a vertical position in a small hole made in the base, and put base material into the hole around the bottom of the cone to fill the crevices and make the cone stand firmly. Usually five cones are mounted in one base in the manner shown in Figure 10. Dry the sheet-iron plate with the test piece on a hot plate. Ignite the

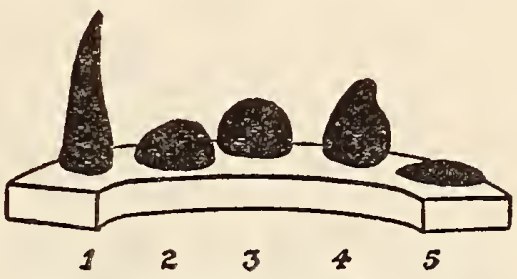

Figure 10.-Typical forms of cones fused in the No. 3 melter's furnace

cones at a dull red heat for 30 minutes in an open muffle to remove the carbonaceous material.

\section{Method of Heating}

30. Place the test piece in the muffle crucible of the furnace in the position shown in Figure 8 , place the loosely fitting cover $b$, on the crucible, and ignite the gas. It is necessary to let the gas burn about 10 minutes to heat the furnace parts before the large cover plate of the furnace is replaced; otherwise the flame is apt to blow out. During this time increase the flow of gas and air sufficiently to cause the combustion to take place just above the tuyeres and yet maintain a yellowish flame at least 6 inches above the opening in the furnace cover plate. While such a flame is maintained above the furnace, gradually increase the temperature by a suitable adjustment of gas and air to $800^{\circ} \mathrm{C}$., then reduce the rate of heat increase to not less than $5^{\circ} \mathrm{C}$. and not more than $10^{\circ} \mathrm{C}$. per minute. Maintain this rate until the end 
of the test. It is also important that the 6-inch reducing flame be maintained at the furnace vent throughout the test, if possible, and at all events up to a temperature of $1,450^{\circ} \mathrm{C}$. Temperatures above $1,450^{\circ} \mathrm{C}$. require larger proportions of air to gas; however, a strongly reducing atmosphere is not so essential at the higher temperatures, as refractory ashes, owing to their low iron-oxide content, are only slightly affected by oxidizing or reducing atmospheres. After a test has been completed turn the supply of gas and air off gradually to avoid cracking the muffle crucible.

31. The softening temperature is defined as the temperature at which the cone has fused down to a spherical lump, as shown in cones 2 and 3 of Figure 10. Cone 4 has almost reached the softening temperature.

Notes.-Critical points other than the softening temperature, that may be observed during the test, and which may be of value are as foliows:

The initial deformation temperature.-The temperature at which the first rounding or bending of the apex of the cone takes place, as shown in cone 1 of Figure 10 . Such bending must not be coníused with a shrinking or warping of the cone.

with a shrinking or warping of the cone.
The iuid temperature.-The temperature at which the cone has spread out over the base in a flat layer, as represented by cone 5 of Figure 10.

\section{ULTIMATE ANALYSIS (FOR COAL AND COKE)}

\section{Carbon and Hydrogen}

\section{Method with gas-heated combustion furnace:}

32. The determination of carbon and of hydrogen shall be made with a weighed quantity of the sample in a 25-burner combustion furnace of the Glaser type. The products of combustion shall be thoroughly oxidized by passing them over red-hot $\mathrm{CuO}$ and $\mathrm{PbCrO}_{4}$, and shall be fixed by absorbing the water in a weighed Marchand tube filled with granular $\mathrm{CaCl}_{2}$ and by absorbing the $\mathrm{CO}_{2}$ in a Liebig bulb containing a 30 per cent solution of $\mathrm{KOH}$.

33. A pparatus. - The apparatus used shall consist of a purifying train in duplicate, a combustion tube in the furnace, and an absorption train.

(a) The purifying train shall consist of the following purifying rcagents arranged in order of passage of air and oxygen through them: $\mathrm{H}_{2} \mathrm{SO}_{4}, \mathrm{KOH}$ solution, soda lime, and granular $\mathrm{CaCl}_{2}$. One of the trains is for air and one for oxygen. In the $\mathrm{H}_{2} \mathrm{SO}_{4}$ and $\mathrm{KOH}$ scrubbing bottles the air and the oxygen shall be made to bubble through about $5 \mathrm{~mm}$ of the purifying reagent. Both purifying trains shall be connected to the combustion tube by a $Y$ tube, the joint being made tight by a rubber stopper.

(b) The combustion tube shall be made of hard Jena glass, or its equivalent. Its external diameter shall be about $21 \mathrm{~mm}$, and its total length $1 \mathrm{~m}$. The first $30 \mathrm{~cm}$ of the tube shall be empty; following this empty space is an asbestos plug (acidwashed and ignited) or in its place a roll of oxidized copper gauze may be used; the next $40 \mathrm{~cm}$ shall be filled with "wire" $\mathrm{CuO}$; a second asbestos plug separates the copper oxide from $10 \mathrm{~cm}$ of fused $\mathrm{PbCrO}_{4}$, which shall be held in place by another asbestos plug $20 \mathrm{~cm}$ from the end of the tube. The end of the tube shall be drawn out for rubber-tubing connection with the absorption train.

(c) The absorption train shall consist of a Marchand tube filled with granular $\mathrm{CaCl}_{2}$ to absorb moisture. The $\mathrm{CaCl}_{2}$ should be saturated with $\mathrm{CO}_{2}$ before using. The Marchand tube shall be followed by a Liebig bulb containing a 30 per cent $\mathrm{KOH}$ solution, in which any possible impurities, as ferrous iron or nitrates, have been oxidized by a little $\mathrm{KMnO}_{4}$. A guard tube, containing granular $\mathrm{CaCl}_{2}$ and soda lime, shall be attached to the Liebig bulb to absorb any $\mathrm{CO}_{2}$ escaping the $\mathrm{KOH}$ solution and any water evaporating from that solution.

34. Procedure.-Connect the train to an aspirator which draws the products of combustion through the entire train. A guard tube of $\mathrm{CaCl}_{2}$ prevents moisture from running back into the absorption train. Maintain the suction constant by a Mariotte flask. The advantage of aspirating the gases through the train rather than forcing them through by pressure is that the pressure on the rubber connections is from the outside, so that gas-tight connections are more easily maintained than if the pressure is on the inside of the tube. Make the connections as tight as possible. The usual test for tightness is to start aspiration at the rate of about three bubbles of air per second through the potash bulb, and then to close the inlet for air and oxygen at the opposite end of the train; if there is no more than one bubble per minute in the potash bulb, the apparatus shall be considered tight.

Before starting a determination when the train has been idle some hours, or after any changes in chemicals or connections, run a blank by aspirating about 1 liter of ail through the train, which is heated in the same manner as if a determination on the sample were being made. If the Liebig bulb and the tube containing calcium chloride show a change in weight of less than $0.5 \mathrm{mg}$ each, the apparatus is in proper condition for use.

Use a porcelain or platinum boat provided with a glass weighing tube of suitable size, which is fitted with an accurately ground glass stopper. Weigh the tube and empty boat. Quickly place in the boat approximately $0.2 \mathrm{~g}$ of the air-dry sample (passing a 250-micron (No.60) sieve and finer, or better, passing a 149-micron (No. 100) sieve if much free impurity is present). Place the boat at once in the weighing tube, which is quickly stoppered to prevent moisture change in the sample while weighing, and transfer to the furnace. Connect the absorption tubes and transfer the boat and sample from the weighing tube to the combustion tube; which should be cool for the first $30 \mathrm{~cm}$. The $\mathrm{CuO}$ should be red hot and the $\mathrm{PbCrO}_{4}$ at a dull-red heat. Transfer the boat from the weighing tube to the combustion tube as rapidly as possible.

As soon as the boat is in place near the asbestos plug at the beginning of the copper oxide, insert the stopper connecting with the purifying train and start the aspiration with pure oxygen gas at the rate of three bubbles per second. Turn on one burner about $10 \mathrm{~cm}$ back from the boat, and continue the aspiration carefully until practically all the moisture is expelled from the sample. Then increase the heat very gradually until all the volatile matter has been driven off. In driving off the volatile matter the heat must be applied gradually in order to prevent a too rapid evolution of gas and tar, which may cither escape complete combustion or may be driven back into the purifying train. Increase the heat slowly by turning on more burners under the open part of the tube until the sample is ignited; then the temperature may be increased rapidly, but care should be taken not to melt the combustion tube.

Any moisture collecting in the end of the combustion tube or in the rubber connection joining it to the $\mathrm{CaCl}_{2}$ tube may be driven over into the $\mathrm{CaCl}_{2}$ tube by carefully warming with a piece of hot tile. Continue the aspiration with oxygen for two minutes after the sample ceases to glow, then turn off the heat and aspirate about $1,200 \mathrm{cc}$ of air. Disconnect the absorption bulbs, wipe with a clean cloth, and allow to cool to the balance-room temperature before weighing. 
Calculate the percentages of hydrogen and carbon as follows:

Percentage of hydrogen $=\frac{11.19 \times \text { (increase in weight of } \mathrm{CaCl}_{2} \text { tube) }}{\text { Weight of sample }}$

Percentage of carbon $=\frac{27.27 \times \text { (increase in weight of } \mathrm{KOH} \mathrm{bulb)}}{}$

Weigh the ash in the boat and carefully inspect it for any unburned carbon, which would destroy the value of the determination.

\section{Method with electrically-heated combustion furnace:}

35. (a) Apparatus.-An electrically heated combustion furnace of the Heraeus type is used by the Bureau of Mines. ${ }^{8}$ It consists of three independent heaters, two of which are provided with sheave wheels, and are mounted on a track so that they are movable along the tube; the third heater which surrounds the $\mathrm{PbCrO}_{4}$, is stationary.

(b) The furnace as provided by the manufacturer does not include the small stationary heater. This may be made in the laboratory by winding an alundum tube $12 \mathrm{~cm}$ in length with No. 20 nichrome II wire and inclosing it in a cylinder packed with magnesia-asbestos. The movable heaters have very thin platinum foil, weighing about $9 \mathrm{~g}$ in all, wound on a porcelain tube of $30 \mathrm{~mm}$ internal diameter. The larger one, which heats the $\mathrm{CuO}$, is $350 \mathrm{~mm}$ in length, and the smaller one, which heats the sample in the boat, is $200 \mathrm{~mm}$ in length.

(c) The Jena glass or fused silica combustion tube, of about $21 \mathrm{~mm}$ external diameter and 900 $\mathrm{mm}$ in length, is supported by an asbestos-lined nickel trough. The current through each heater shall be regulated independently by separate rheostats, mounted on the frame of the furnace. The two platinum-wound heaters require an average current of about 4.5 amperes at a pressure of 220 volts, although for heating rapidly a larger amperage is necessary.

36. Procedure.-Purify the oxygen or air entering the combustion tube by passing through a Tauber's drying apparatus, which contains the following reagents arranged in order of the passage of air or oxygen through them: $\mathrm{H}_{2} \mathrm{SO}_{4}$, for removing possible traces of ammonia, 30 per cent $\mathrm{KOH}$ solution, granular soda-lime, and granular $\mathrm{CaCl}_{2}$. Connect one side of the train directly to a Linde oxygen tank provided with a reducing valve for regulating the oxygen pressure; use the other side of the train for purifying the air supply.

The absorption train shall consist of a 5-inch $U$ tube, filled with granular $\mathrm{CaCl}_{2}$ to absorb moisture. Before using, saturate the $\mathrm{CaCl}_{2}$ with $\mathrm{CO}_{2}$ to avoid possible absorption of $\mathrm{CO}_{2}$ during a determination by any traces of $\mathrm{CaO}$ that may be present. This saturating is done most conveniently by placing a quantity of $\mathrm{CaCl}_{2}$ in a large drying jar, and filling the jar with $\mathrm{CO}_{2}$. After standing over night, draw dry air through the jar to remove the $\mathrm{CO}_{2}$. Keep the treated $\mathrm{CaCl}_{2}$ in well-stoppered bottles.

Connect the $\mathrm{CaCl}_{2}$ tube to a Vanier potash bulb containing a 30 per cent $\mathrm{KOH}$ solution and granular $\mathrm{CaCl}_{2}$. Six to eight determinations may be made without recharging this bulb. Connect the potash bulb to an aspirator through a guard tube containing granular $\mathrm{CaCl}_{2}$ and soda lime, and a Mariotte flask. The Mariotte flask keeps the pressure constant.

In general, the method of determination is the same as the one used with the gas furnace as described in sections 33 and 34 . By moving the heaters toward the end of the tube where the gases enter, and cutting in the electric current, the air may be warmed enough to thoroughly dry the tube and its contents. Cut off the current from the small heater, and move the large heater over the

8 United States Bureau of Mines, Tech. Paper No. 8, p. 18, 1926.
$\mathrm{CuO}$; keep exposed about $250 \mathrm{~mm}$ of that part of the combustion tube between the two heaters where the boat containing the sample is to be placed. Turn the full current on the large heater to bring the $\mathrm{CuO}$ to a red heat. When this temperature is reached it is necessary to reduce the current with the rheostat to avoid melting the tube. In the meantime, weigh and connect the absorption train and place the boat containing the sample in the exposed and cooler part of the tube between the two heaters.

Pass the current through the shorter heater. By manipulating the rheostat and by gradually pushing this heater toward the boat, the rate of evaporation of moisture and evolution of volatile matter may be readily controlled.

After combustion is complete, turn the electric current off the smaller heater and move this heater back, to allow the tube to cool for the next determination. Conduct the final aspiration of air and the weighing of the absorption train as described in sections 33 and 34 under the gas-furnace method.

Note, - In place of granulated $\mathrm{CaCl}_{2}$, concentrated $\mathrm{H}_{2} \mathrm{SO}_{4}$ may be used for collecting the water formed by combustion. In such cases the air and oxygen entering the combustion tube and the gas leaving the potash bulb must also be dried by $\mathrm{H}_{2} \mathrm{SO}_{4}$.

Other suitable forms of absorption vessels than those indicated in the above procedure may be used.

\section{Nitrogen}

37. The Kjeldahl-Gunning method is recommended for the determination of nitrogen. This method has the advantage over either the simple Kjeldahl or the Gunning method, in requiring less time for the complete oxidation of the organic matter and in giving the most uniform results.

The Kjeldahl-Gunning method.-Boil $1 \mathrm{~g}$ of the sample with $30 \mathrm{cc}$ of concentrated $\mathrm{H}_{2} \mathrm{SO}_{4}, 7$ to $10 \mathrm{~g}$ of $\mathrm{K}_{2} \mathrm{SO}_{4}$, and 0.6 to $0.8 \mathrm{~g}$ of metallic mercury in a $500 \mathrm{cc}$ Kjeldahl flask until all particles of the sample are oxidized and the solution nearly colorless. Continue the boiling at least two hours after the solution has reached the straw-colored stage. The total time of digestion will be from three to four hours except in the case of coke and anthracite coal which require much longer digestion. The addition of a few crystals of $\mathrm{KMnO}_{4}$, after the solution has cooled enough to avoid violent reaction, tends to insure complete oxidation. Coke and anthracite coal should be ground to an impalpable powder, as they are very difficult to oxidize. Even if this is done the digestion may require 12 to 16 hours.

After cooling, dilute the solution to about $200 \mathrm{cc}$ with cold water. If the dilution with water has warmed the solution, cool it again and add the following reagents: 25 cc $K_{2} S$ solution (40 g $K_{2} \mathrm{~S}$ per liter) to precipitate the mercury, 1 to $2 \mathrm{~g}$ of granular zinc to prevent bumping, and finally enough strong $\mathrm{NaOH}$ solution (usually 80 to $100 \mathrm{cc}$ ) to make the solution distinctly alkaline. The danger of loss of $\mathrm{NH}_{3}$ may be minimized by holding the flask in an inclined position while the $\mathrm{NaOH}$ solution is being added. The alkaline solution runs down the side of the flask and forms a layer below the lighter acid solution. After adding the alkaline solution, connect the flask at once to the condensing apparatus and mix the solution by gently shaking the flask.

Distill the $\mathrm{NH}_{3}$ over into a measured amount (10 cc) of standard $\mathrm{H}_{2} \mathrm{SO}_{4}$ solution, to which has been added sufficient cochineal indicator for titration. Care should be taken that the glass connecting tube on the end of the condenser dips under the surface of the standard acid. Distill the solution slowly until 150 to $200 \mathrm{cc}$ of distillate has passed over. To avoid mechanically entrained alkali passing over into the condenser, the rate of distillation should not exceed $100 \mathrm{cc}$ per hour. Titrate 
the distillate with standard $\mathrm{NH}_{3}$ solution $(20 \mathrm{cc}$ $\mathrm{NH}_{4} \mathrm{OH}$ solution $=10 \mathrm{cc} \mathrm{H}_{2} \mathrm{SO}_{4}$ solution $=0.05 \mathrm{~g}$ nitrogen). Standard $\mathrm{NaOH}$ or $\mathrm{KOH}$ solution with methyl orange or methyl red as indicator may be used instead of $\mathrm{NH}_{3}$ and cochineal.

A blank determination shall be made in exactly the same manner as described above, except that $1 \mathrm{~g}$ of pure sucrose (cane sugar) shall be substituted in place of the sample. The nitrogen found in this blank determination shall be deducted from the result obtained with the sample.

The $\mathrm{K}_{2} \mathrm{~S}$ and $\mathrm{NaOH}$ may be dissolved in a single stock solution. Dissolve sufficient $\mathrm{K}_{2} \mathrm{~S}$ in the water before adding the $\mathrm{NaOH}$, to make a solution in which the quantity necessary for a nitrogen determination $\left(80\right.$ to $100 \mathrm{cc}$ ) contains $1 \mathrm{~g}$ of $K_{2} \mathrm{~S}$. Twelve grams of $\mathrm{K}_{2} \mathrm{~S}$ and $500 \mathrm{~g}$ of $\mathrm{NaOH}$ in 1 liter of water, are required for the above proportions.

\section{Oxygen}

38. There being no satisfactory direct method of determining oxygen, it shall be computed by subtracting the sum of the percentages of hydrogen, carbon, nitrogen, sulphur, water, and ash from 100 . The result so obtained is affected by all the errors incurred in the other determinations and especially by the change in weight of the ash-forming constituents on ignition. In the case of coal, iron pyrite changes to ferric oxide, increasing the ash and causing a negative error in the oxygen equivalent to three-eighths of the pyritic sulphur. On the other hand, there is always a loss on ignition, of water of composition from the clayey and shaley constituents, $\mathrm{CO}_{2}$ from carbonates, etc., which tends to compensate the absorption of oxygen.

39. Correcled oxygen (for coal).-When a more correct oxygen value is desired, it may be obtained by making the corrections indicated in the following formula:

Corrected oxygen $=100-\left[\left(C-C^{\prime}\right)+\left(H-H^{\prime}\right)+N+H_{2} O+S^{\prime}+\right.$ corrected where

$C=$ total carbon.

$C^{\prime}=$ carbon of carbonates.

$H$ = total hydrogen less hydrogen of water.

$H^{\prime}=$ hydrogen from water of composition in clay, shale, etc

$N=$ nitrogen.

$\mathrm{H}^{2} \mathrm{O}=$ moisture as found at $105^{\circ} \mathrm{C}$.

${\underset{\mathrm{S}}{\mathrm{S}^{\prime}}}^{\mathrm{H}} \mathrm{O}=$ moisture as found at $105^{\circ} \mathrm{C}$. small In many types of coal it may be disregarded.

Corrected ash $=$ mineral constitucnts originally present in the coal. For most purposes this can be determined with sufficient
For accuracy by adding to the ash, as found, five-eighths of the weight of pyritic sulphur, the $\mathrm{CO}_{2}$ of carbonates and the water of composition of clay, shale, etc. See also Determinaticn of ash, sections 9 and 10 .

\section{CALORTMETRIC DETERMINATION (FOR COAL AND COKE)}

Apparatus

40. (a) Combustion bombs.-The Atwater, Davis, Emerson, Mahler, Parr, Peters, Williams, or similar bombs may be used. The bomb shall have an inner surface of platinum, gold, porcelain enamel, or other material which is not attacked by $\mathrm{HNO}_{3}$ and $\mathrm{H}_{2} \mathrm{SO}_{4}$, or other products of combustion.

(b) Calorimeter jacket.-The calorimeter shall be provided with a water jacket having a cover to protect the calorimeter from air currents. The jacket shall be kept filled with water within $2^{\circ}$ or $3^{\circ} \mathrm{C}$. of the temperature of the room (except in calorimeters which are totally submerged, where the jacket temperature is controlled by a thermostat) and should be stirred continuously by some mechanical stirring device.

(c) Stirring of the calorimeter water.-The water in the calorimeter shall be stirred sufficiently well to give consistent thermometer readings while the temperature is rising rapidly. The speed of stirring should be kept constant. A motor-driven screw or turbine stirrer is recommended, and the speed should not be excessive. This may be determined by adjusting the temperature of the calorimeter to equality with that of the jacket and allowing the stirrer to run continuously for 10 minutes. If the temperature of the calorimeter rises more than about $0.01^{\circ} \mathrm{C}$. in this length of time, the rate of stirring is excessive. Accurate results can not be obtained when too much energy is supplied by the stirring device or when the rate of stirring is irregular. The portion of the stirring device immersed in the calorimeter should be separated from that outside by nonconducting material, such as hard rubber, to prevent conduction of heat from the motor or outside air.

(d) Thermometers. - Thermometers used shall have been certified by a Government testing bureau and shall be used with the corrections given on the certificate. This shall also apply to electrical resistance or thermoelectric thermometers. Correction shall also be made for the temperature of the emergent stem of all mercurial thermometers, and for the "setting" of Beckmann thermometers. For accurate work, either Beckmann or special calorimetric thermometers graduated to $0.01^{\circ}$ or or $0.02^{\circ}$ C. are required. Such thermometers should be tapped lightly just before each reading to avoid errors caused by the sticking of the mercury meniscus, particularly when the temperature is falling. A convenient method is to mount a small electric buzzer directly on the top of the thermometer and connect it with a dry cell and a push button. The button should be pressed for a few seconds immediately before each reading.

(e) Oxygen.-The oxygen used for combustions shall be free from combustible material. The bomb when filled should contain at least 5 per cent of nitrogen to inusre complete oxidation of the sulphur. ${ }^{9}$ The total amount of oxygen contained in the bomb for combustion shall not be less than $5 \mathrm{~g}$ per gram of the sample. But the combustion must be complete, as shown by the absence of any sooty deposit on opcning the bomb after firing.

(f) Firing wire.-The sample in the bomb may be ignited by means of either iron or platinum wire. If iron wire is used, it should be of about No. 34 B. \& S. gage and not more than $10 \mathrm{~cm}$ (preferably 5 $\mathrm{cm}$ ) should be uscd at a time. A correction of 1,600 calories per gram weight of iron wire burned shall be subtracted from the observed number of calories.

(g) Standardization.-The water equivalent of a calorimeter can best be determined by the use of the standard combustion samples supplied by the National Bureau of Standards. The required water equivalent is equal to the weight of the sample multiplied by its heat of combustion per gram and divided by the corrected rise in temperature.

The calorimeter shall be standardized by the combustion of standard samples supplied by the Bureau of Standards, and used according to the directions given in the certificates which accompany them. A standardization shall consist of a series of not less than five combustions of either the same or different standard materials. The conditions as to the amount of water, oxygen, firing wire, method of correcting for radiation, etc., under which these combustions are made shall be the same as for combustions of the sample. In the case of any disagreement between contracting parties a check standardization may consist of two or more combustions of standardizing samples.

\section{Procedure}

41. (a) Preparation of sample.-The ground sample shall be thoroughly mixed in the bottle and an

- Register, J. Ind. and Eng. Chem., 16, p. 812; 1914. 
amount, approximately $1 \mathrm{~g}$, shall be taken out and weighed in the crucible in which it is to be burned. Samples which are likely to be blown out of the crucible should be briqueted. After weighing, the sample should preferably be immediately placed in the bomb and this closed. This procedure is necessary to avoid sublimation in the use of naphthalene for standardization.

(b) Preparation of the bomb.-The firing wire, if iron, shall be measured and coiled in a small spiral and connected between the platinum terminals, using, if necessary, a piece of platinum wire somewhat heavier than the iron wire, to make the connection. The platinum and the iron shall both be clean. About $0.5 \mathrm{cc}$ of water should be placed in the bottom of the bomb to saturate with moisture the oxygen used for combustion. When the crucible is put in place in the bomb, the firing wire should touch the sample or briquet of standard material. For the combustion of standardizing samples iron wire is preferable to platinum.

(c) Filling the bomb with oxygen.-Oxygen from the supply tank shall be admitted slowly to avoid blowing the sample from the crucible, and the pressure allowed to reach 20 atmospheres for the larger bombs or about 30 atmospheres for the smaller bombs, so that the bomb shall contain an amount of oxygen sufficient for complete combustion, namely, at least $5 \mathrm{~g}$ per gram of the sample. This method of filling should insure 4 per cent of nitrogen in the larger bombs, irrespective of the nitrogen contained in the oxygen.

(d) Calorimeter water.-The calorimeter shall be filled with the required amount of distilled water, depending upon the type of calorimeter. The amount may be determined either by measurement in a standardized flask or by weighing. The amount shall be kept the same as that used in the standardization of the apparatus.

(e) Temperature adjustments.-The initial temperature in the calorimeter shall be so adjusted that the final temperature, after the combustion, will not be more than $1^{\circ} \mathrm{C}$, , preferably about $0.5^{\circ} \mathrm{C}$., above that of the jacket, under which conditions the total correction for heat gained from or lost to the surroundings will be small when the rise of temperature is $2^{\circ}$ or $3^{\circ} \mathrm{C}$., and the effect of evaporation will also be small.

(f) Firing current.-The electric current used for firing the charge shall be obtained from storage or dry cells having an electromotive force of not more than 12 volts. A higher voltage is liable to cause an arc between the firing terminals, introducing additional heat, which can not be measured with certainty. The circuit should be closed by means of a switch which should remain closed for not more than two seconds. When possible, it is recommended that an ammeter be used in the firing circuit to indicate when the firing wire has burned out.

(g) Method of making an observation.-The bomb when ready for firing, shall be placed in the calorimeter, the firing wires connected, the cover put in place, and the stirrer and thermometer so placed as not to be in contact with the bomb or container. The stirrer shall then be started and after the thermometer reading has become steady, not less than 2 minutes after the stirrer is started, temperatures shall be read at 1 -minute intervals for 5 minutes and the charge then fired, the exact time of firing being noted. Observations of temperature shall then be made at intervals depending upon the method to be used for computing the cooling cor- rection. When the temperature has reached its maximum and is falling uniformly, a series of thermometer readings shall be taken at 1-minute intervals for $\mathbf{5}$ minutes to determine the final cooling rate.

(h) Titration.-After a combustion and after allowing the gas to escape the bomb shall be opened and the inside examined for traces of unburned material or sooty deposit. If these are found, the observations shall be discarded. If the combustion appears complete, the bomb shall be rinsed out thoroughly and the washings titrated with a standard alkali solution ( $1 \mathrm{cc}=0.02173 \mathrm{~g} \mathrm{HNO}=5$ calories) using methyl-orange or methyl-red indicator, to determine the amount of acid formed. A correction of 230 calories per gram of $\mathrm{HNO}_{3}$ should be subtracted from the total heat observed. An additional correction of 1,300 calories per gram of sulphur in the sample should be made for the excess of difference in heats of formation of $\mathrm{SO}_{2}$ and aqueous $\mathrm{H}_{2} \mathrm{SO}_{4}$ over the heat of formation of aqueous $\mathrm{HNO}_{3}$.

\section{Calculation of Results}

42. The following method of calculation is recommended to take the place of the Pfaundler or other similar formulas for calculating the cooling correction (radiation correction):

Observe (1) the rate of rise, $r_{1}$, of the calorimeter temperature in degrees per minute for five minutes before firing; (2) the time, $a$, at which the last temperature reading is made immediately before firing; (3) the time, $b$, when the rise of temperature has reached six-tenths of its total amount (this point can generally be determined by adding to the temperature observed before firing, 60 per cent of the expected 10 temperature rise, and noting the time at which this point is reached); (4) the time, c, of a thermometer reading taken when the temperature change has become uniform some five minutes after firing; (5) the final rate of cooling, $r_{2}$, in degrees per minute for five minutes.

Multiply the rate $r_{1}$ by the time $b-a$ in minutes and tenths of a minute, and add to this product (subtracted if the temperature was falling at the time a) to the thermometer reading taken at the time $a$. Multiply the rate $r_{2}$ by the time $c-b$ and add this product (subtracted if the temperature was rising at the time $c$ and later) to the thermometer reading taken at the time $c$. The difference of the two thermometer readings thus corrected, provided the corrections from the certificate have already been applied, gives the total rise of temperature due to the combustion. This multiplied by the water equivalent of the calorimeter gives the total amount of heat liberated. Divide this result, corrected for the heats of formation of $\mathrm{HNO}_{3}$ and $\mathrm{H}_{2} \mathrm{SO}_{4}$ observed and for the heat of combustion of the firing wire, when that is included, by the weight of the charge to find the heat of combustion in calories per gram.

10 When the temperature rise is not approximately known bcforehand, it is only necessary to take thermometer readings at 40,50 , 60 seconds (and possibly 70 seconds with some calorimeters) after firing, and from these observations to find when the temperature rise had reached 60 per cent of the total. Thus, if the temperature at firing was $2.135^{\circ} \mathrm{C}$., at 40 seconds $3.05^{\circ}$, at 50 seconds $3.92^{\circ}$, at 60 at firing was $2.135^{\circ}$ C., at 40 seconds $3.05^{\circ}$, at 50 seconds $3.92^{\circ}$, at 60 seconds $4.16^{\circ}$, and the final tem perature was $4.200^{\circ}$, the total rise was $2.07^{\circ} ; 60$ per cent of it was $1.24^{\circ}$. The temperature to be observed was then $2.14^{\circ}+1.24^{\circ}=3.38^{\circ}$. Referring to the observations at 40 and 50 seconds, the temperatures were, respectively, $3.05^{\circ}$ and $3.92^{\circ}$. The time corresponding to the temperature of $3.38^{\circ}$ was, therefore:

$$
40+\frac{3.38-3.05}{3.92-3.05} \times 10=44 \text { seconds }
$$


Calories per gram multiplied by 1.8 give the British thermal units per pound. (See example.)

NoтE-In practice, the time $b-a$ will be found so nearly constant for a given calorimeter with the usual amounts of fuel tbat $b$ need be determined only occasionally.

The results shall be reduced to calories per gram or Britisb tbermal units per pound of dry sample, the moisture being determined upon a sample taken from the bottle at about tbe same time as tbe combustion sample is taken.

Example:

\section{Observations}

Water equivalent $=2,550 \mathrm{~g}$

Weight of charge $=1.0535$

Approximate rise of temperature expected $=3.2^{\circ} \mathrm{O}$ 60 per cent of approximate rise $=1.9^{\circ} \mathrm{C}$.

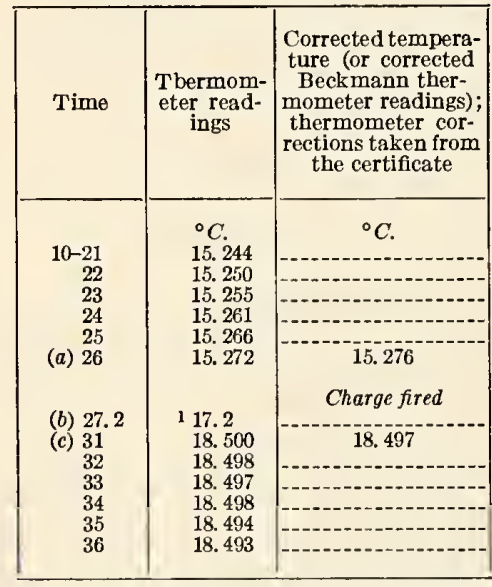

1 Tbc initial temperature is $15.27^{\circ} \mathrm{C}$; 60 per cent of the expected risc is $1.9^{\circ} \mathrm{C}$. The reading to observe is then $17.2^{\circ}$.

\section{Calculation}

$r_{1}=0.028^{\circ} \div 5=0.0056^{\circ}$ per minute; $b-a=1.2$ minutes.

The corrected initial temperature is $15.276^{\circ}+0.0056^{\circ} \times 1.2_{-.}=15.283^{\circ}$ $r_{2}=0.007^{\circ} \div 5=0.0014^{\circ}$ per minute; $c-b=3.8$ minutes.

The corrected final temperature is $18.497^{\circ}+0.0014^{\circ} \times 3.8_{-} . .-=18.502^{\circ}$ Total rise $18.502^{\circ}-15.283^{\circ}$

Total calories $2,550 \times 3.219$
Titration, etc

Calories from $1.0535 \mathrm{~g}$ sample $\ldots . . . . . .8,202$

$\begin{array}{lr}\text { Calories per gram } & 7,785 \\ \text { or Britisb tbermal units per pound. } & 14,013\end{array}$

The result obtained by tbe above method of computation and determination is the total beat of combustion at constant volume with the water in the products of combustion condensed to liquid at tbe temperature of tbe calorimeter; that is, about $20^{\circ}$ to $35^{\circ} \mathrm{C}$.

Net heat of combustion at $20^{\circ}$ shall refer to results corrected for latent heat of vaporization, as follows:

Total beat of combustion in B. t. u. $-1,040$ (total bydrogen $\times 9$ ) $=$ net beat of combustion in B. $t$. $u$. per pound.

Also: Total beat of combustion in calories-580 (total bydrogen $\times 9)=$ nct beat of combustion in calories per gram.

Nores.-For anthracite, coke, and coal of higb asb content, which do not readily burn completely, tbe following procedure is recommended:

Tbe inside of the crucible is lined completely witb ignited asbestos in a tbin layer pressed well down into tbe angles. The sample is then sprinkled evenly over the surface of tbe asbestos. Otherwise the procedure is as previously described.

procedure is as previously described. United States Bureau of Mines, Technical Paper No. 8, pp. 24-28 (1026), may also be used.

\section{Tolerances}

The permissible differences between two or more determinations shall not exceed the values specified.

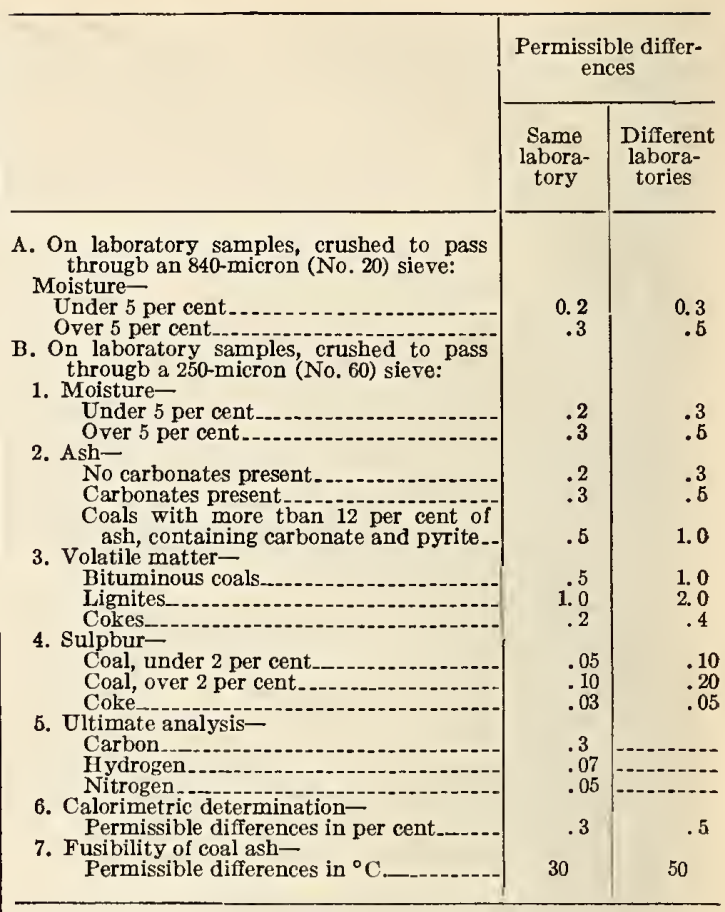

American Society of Mechanical Engineers, test code for solid fuels, March 31, 1927.

\section{INTRODUCTION}

1. The test code for solid fuels is intended primarily to specify standard methods for a determination of those ascertainable chemical and physical properties which serve as indicators of the value of extensively used solid fuels when burned in boiler, industrial, and domestic furnaces. As a secondary part of the code are included methods for determining the efficiency of the combustion process itself. In working out such a code it has been found advisable to adopt, with a few modifications, codes already in use by other societies, such as the American Society for Testing Materials and the American Chemical Society. These modified codes as adopted are to be included in their proper places with this code.

\section{OBJECTS}

2. The objects of testing solid fuels may be any one or more of the following:

(a) A determination of the composition of the fuel.

(b) A determination of the heating value of the fuel.

(c) A determination of the size of the fuel (solid fuels).

(d) Classification of fuel.

(e) A determination of the character of the combustion by an analysis of the products of combustion.

$(f)$ A determination of certain ash constituents.

(g) A determination of the fusing point of the ash.

3 . Under the term "solid fuels" are included the ignites, bituminous coals, anthracite coals, and coke. 


\section{COLLECTING THE SAMPLE}

4. (a) For a standard test the fuel shall be collected and prepared substantially in accordance with the method worked out jointly by the American Chemical Society and the American Society for Testing Materials and described in A. S. T. M. D21-16, standard method of sampling of coal. (See 501.0, p. 4.)

(b) As an alternative method for cases in which less accurate results are permissible, a smaller total weight of fuel sample than is specified in above code may be collected, it being, of course, understood that the probable error in sampling varies inversely with the weight of the sample collected. If not otherwise agreed to, solid fuel samples shall, however, be collected and prepared in accordance with the rules set forth in said code.

\section{FUEL SLZING}

5. In determining the fuel size (for bituminous coal) the gross samples collected as explained in A. S. T. M. D21, or an equivalent sample collected in a similar manner and at least equal in weight to the sample referred to above, will be used. Such samples before they have been broken up will be passed over and through the standard screens such as are more completely described in the Instruments and Apparatus (A. S. M. E. series), and in Bulletin No. 101, engineering experiment station, University of Illinois, and the percentage by weight remaining over each screen will be determined.

The first screen in the series shall be large enough so that not more than 5 per cent by weight of total sample will remain on the screen. The last screen size (smallest opening) in the scale will be such that not more than 20 per cent by weight of the total sample will pass through the screen.

Over 5 inches

Per cent

On 216 inches and through 5 inches

On $11 / 4$ inches and through $21 / 2$ inches

On $5 / 8$ inch and through $11 / 4$ inches.

6. In designating the size of anthracite fuel used for test purposes (particularly for power boilers using steam sizes of anthracite) the use of trade names should be discouraged. Where, however, it is desired to refer to fuel by trade size designation the size of the perforations in the screens through and over which the coal must pass should be definitely stated. Furthermore, limits should be set to the permissible percentages of coal as shown by the sample that will not go through the larger size screen and that will go through the smaller size screen. The foregoing is of importance when the size of coal is to be specified in advance of the conduct of the test and particularly where guarantees are to be made when using a certain size of coal.

In determining the fuel size (for anthracite samples) where no trade size is designated, the gross samples collected as explained in A. S. T. M. D21, or an equivalent sample collected in a similar manner and at least equal in weight to the sample referred to above, will be used. Such samples before they have been broken up will be passed over and through screens having the following sizes of round-hole perforations: Screen No. 1, $41 / 2$ inches; No. 2, $3 \frac{1 / 4}{4}$ inches; No. $3,23 / 8$ inches; No. 4 , $1 \frac{5}{8}$ inches; No. 5, $7 / 8$ inch; No. 6, $\% / 16$ inch; No. 7, $5 / 10$ inch; No. $8,3 / 16$ inch; No. $9,3 / 32$ inch.

In the process of shaking the fuel through the screens care shall be taken to prevent breaking up the coal. The shaking process must, however, be continued a sufficient length of time so that a complete separation results.

\section{LABORATORY SAMPLING AND ANALYSIS}

7. In calorific determinations the high heating value shall be used for solid fuels. This shall be stated in terms of standard B. t. u. per pound of fuel on "as-received" or "as-used" basis.

(a) The proximate analysis shall contain the percentages by weight of the following constituents and shall likewise be stated on the "as-received" or "as-used" basis: Moisture, volatile matter, fixed carbon, and ash.

(b) The ultimate analysis shall contain the percentages by weight of the following constituents which shall be reported on the "as-received" or "as-used" basis with moisture and ash content expressed as separate items; and a second column shall give analysis on dry basis.

Example

\begin{tabular}{|c|c|c|}
\hline & As received & Dry basis 1 \\
\hline Moisture & Per cent & Per cent \\
\hline Carhon.-- & 76.80 & 79.58 \\
\hline Hydrogen................. & 4. 45 & 4.62 \\
\hline Oxygen & 4.00 & 4.14 \\
\hline Nitrogen. & 1.40 & 1.45 \\
\hline $\begin{array}{l}\text { Sulphur. } \\
\text { A sh }\end{array}$ & $\begin{array}{l}1.65 \\
8.20\end{array}$ & $\begin{array}{l}1.71 \\
8.50\end{array}$ \\
\hline & 100.00 & 100.00 \\
\hline
\end{tabular}

I In Bureau of Mines Reports one statement of ultimate analysis is given on dry hasis.

8. The total moisture, the proximate and ultimate analyses, the heating value and the fusion point of ash shall be determined under the direction of a compctent chemist engaged in fuel-testing work and shall be carried out in substantially the form prescribed in A. S. T. M. D271. (See 501.0, p. 8.)

(NotE.-The full text of this code includes reproduction, with slight modification, of A. S. T. M. method D22-24, standard method of lahoratory sampling and analysis of coal. This method has heen of lahoratory sampling and analysis of coal. This method has heen p. 8. The method D271, which is formed to conform with D22 and the modifications added in the original text of this code, may he referred to in connection with this code.)

\section{CLASSIFICATION OF SOLID FUEIS}

9. Any of the commercially adaptable systems of classifying solid fuels give only very limited information concerning its behavior characteristics. In other words, a knowledge of the class to which a fuel belongs can be used only in the most general way in determining where and how it shall be burned and what results may be expected.

For such purposes the classifications based on fixed carbon volatile and moisture are just as good as those requiring an ultimate analysis for proper classification. With this in view the Frazer classifications given in Appendix A may be used.

\section{FLUE GAS}

10. (a) Sampling.-The standard flue-gas sampler shall consist of single open-ended iron, copper, or glass exploring tubes of one-fourth to threefourths inch diameter and of which the open ends are placed at such positions that the collected sample has a composition representative of the average composition of the gas being sampled. A determination of such positions will require preliminary exploration and judgment. Pockets back of dampers and at bends shall be avoided.

(b) Temperature. - The thermometer bulb or hot. junction or other device used for temperature measurements shall be placed as near the open end 
of the sampling tube as possible and in such a position that the stream flow impinges freely on the temperature-responsive elements, but, of course, not so as to interfere seriously with gas flow into sampling tube.

The thermometer or hot junction must be protected from radiation to colder or hotter surfaces which may be in the vicinity, by providing metal, asbestos, or other shields so placed in the gas stream that no direct radiation can take place between the bulb or hot junction and the hot or cold surfaces.

11. Collecting flue-gas sample.-The gas sample shall be carried from the sampling pipe (fig. 11) in a single tube to the bottle $A$ for the separation of condensed moisture and tars, past the $T$ (the tube should be capillary bore) to which the collecting bottle $B$ is connected, through the suction bottle $C$ half filled with water, which indicates that a sample is being drawn and the rate, and thence to the aspirator. The aspirating process shall be strong, eontinuous, and at a uniform rate. The collecting bottle is a standard 2-liter aspirating bottle fitted with a 3-way stopcock, having capillary bore in the rubber stopper at the top. Any vessel $D$, having a latter case the average analysis of the gases must be arrived at by weighing the separated determinations proportionally to the weights of fuel burned during the times of collecting the samples from which the analyses are made. Whenever possible the analysis shall be made immediately after collection of the sample.

13. Flue-gas analysis.-The gas samples collected in the manner described above shall be analyzed for $\mathrm{CO}_{2}, \mathrm{CO}$, and $\mathrm{O}_{2}$ by means of an Orsat apparatus. When more than 0.5 per cent of $\mathrm{CO}$ is present the carbon monoxide shall be removed with two cuprous chloride pipes in the Orsat apparatus. If hydrogen is present in the products, an apparatus such as the Mercury, Orsat, Hempel, or other suitable apparatus should be used.

\section{SMOKD DETERMINATIONS}

14. Qualitative tests.-See Instruments and Apparatus, section on Exhaust Gas Analysis (A. S. M. E. Power Test Codes).

For quantitative tests of solids in gases see Appendix B.

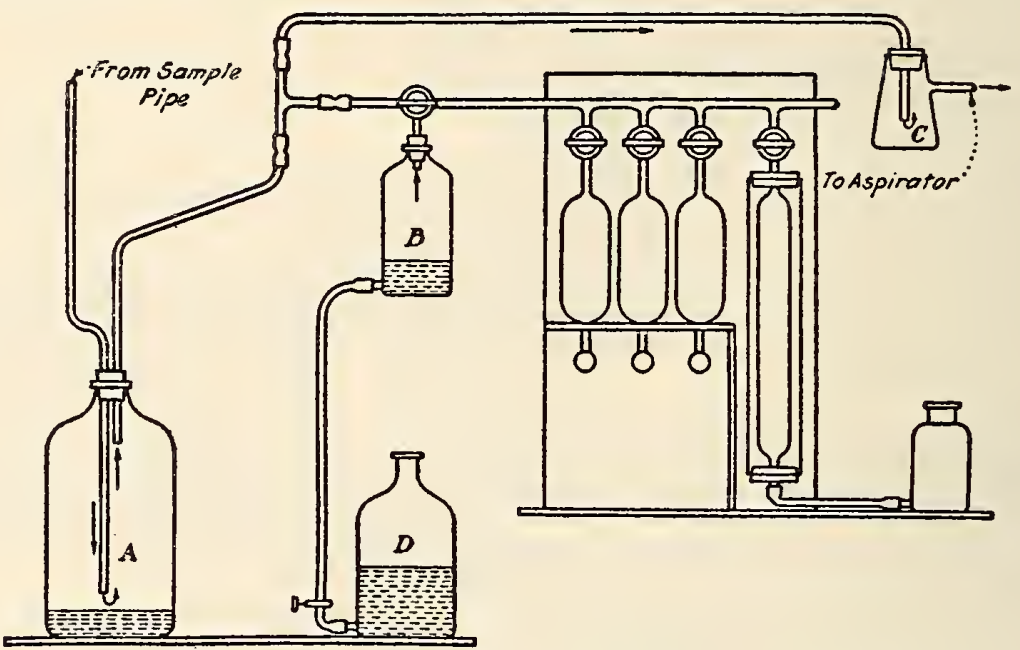

FIGURE 11.-A pparatus for collecting and storing gas samples

capacity somewhat greater than the bottle, may be used to contain the water used for displacement. It is connected to the bottle by a rubber tubing fitted with a pincheock for regulating the flow of water out of and into the bottle. With this apparatus a continuous sample is drawn from the main stream of gases, a small part of this sample is drawn off continuously for 15 or 30 minutes or other suitable period, and a portion of this sample is then analyzed in the Orsat. As only two to three minutes are required to fill the Orsat, and empty the the gas from the bottle, there is practically a continuous sample and analysis of the stream of gas aspirated from the breeching. If water is used in the bottle $B$ and vessel $D$, it shall be saturated with gas being analyzed; or concentrated brine may be used.

12. It is preferable to have the flue-gas sample drawn directly into the Orsat apparatus. When this is not feasible the gas sample shall be collected at the aspirator in standard collecting bottles such as shown in Figure 11 or in a tube of tin or glass such as illustrated by Figure 12. The gas samples may be collceted in quantities proportioned to the rate of burning fuel, or they may be eollected in equal quantities at equal intervals of time. In the
ASH

15. Sampling ash.-Immediately after removal of ash from the ash pit, quench with water to stop the burning of any combustible carried over. The method of collecting gross samples for eombustible and the moisture determination will depend upon whether or not the total ash can be crushed.

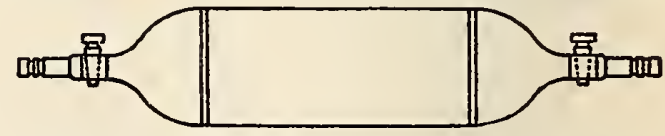

FIGURE 12.- Sheet-metal or glass container for collecting gas samples

When the gas is at a pressure below normal it may be drawn in a when however, it is under pressure above normal it will readily displace the water in the container.

16. Standard method of collecting gross sample.If the total ash ean be erushed down to maximum pieces not over three-fourths inch diameter, then the gross sample shall be collected in increments having a proportion of about 50 pounds for each ton of ash weighed out, unless the total gross sample 
collected in this manner weighs less than 1.000 pounds. In the latter case the increments shall be large enough to result in a gross sample weighing at least 1,000 pounds. In cases where the total weight of ash is less than 1,000 pounds the total ash will be treated as the gross sample. The increments or samples taken as above directed shall be collected in galvanized-iron cans provided with tightly fitting covers to prevent evaporation of moisture from the sample. The cover shall of course only be removed to add the successive increments.

17. Alternate method of collecting gross sample.-If it is not feasible to crush the fine refuse and clinker in one operation, pass the total through a 2 -inch-mesh wire screen to separate the clinker from the finer material. If any unburnt combustible remains over the screen, separate by hand picking, crush, and throw in with fine ash. Both clinker material and the screened ash and combustible must bc weighed and the weight so found will be known as "weight of wet clinker from ash pit" and "weight of wet ash plus combustible from ash pit."

The separated clinker shall now be broken up, crushed, and throughly mixed. The wct ash plus combustible shall likewise be throughly mixed.

To obtain the gross sample of the refuse, mix the crushed clinker and ash plus combustible in proportion by weight to the ratio between "weight of wet clinker from ash pit" and "weight of wet ash plus combustible from ash pit." The wcight increments in the mixing process for the gross sample shall be taken according to the foregoing proportions; that is, 50 pounds per ton of screened ash, unless the total sample so collected is less than 1,000 pounds, in which case the increments shall be so proportioned that the gross sample is at least equal to 1,000 pounds; and in cases where the total weight of screened ash is less than 1,000 pounds the total ash so collected will be treated as the gross sample. The increments taken as above directed shall be collected in galvanized-iron cans provided with tightly fitting covers.

18. Laboratory sample for determination of combustible.-(a) When gross sample has been collected by standard method. - The gross sample so collected shall be systematically crushed, mixed, and reduced in quantity according to Table 1 of A. S. T. M. specification D2l.

(b) When gross sample has been collected according to alternate method.-For this case the gross sample of ash and combustible which has been collected from the screened portion shall be systematically crushed, mixed, and reduced in guantity according to Table 1 of A. S. T. M. specification D2l.

19. Moisture sample.-The moisture sample shall be taken from the collected gross sample before the latter has been crushed for subdivision in prcparation for the laboratory sample. A grab sample of from 10 to 20 pounds will serve as the moisture sample. Before taking this, mix the gross sample by turning over several times with a shovel.

In taking this sample the least possible time should be used in order to reduce the moisture loss by evaporation to a minimum. This moisture sample shall then be conveyed in a sealed container to the laboratory or place in which the moisture determination is to be made.

20. Moisture determination.-The ash sample as received from the test shall be quickly crushed down in a jaw crusher to pass through a 4 -mesh sieve. It shall now be reduced to approximately a 5-pound sample, weighed quickly, spread over galvanizediron pans, such as are described for air drying in A. S. T. M. specification D271. These pans containing the sample shall now be placed in an air-drying oven such as shown in Figure 2, page 8 , and shall have air passed over them at a temperature not exceeding $200^{\circ} \mathrm{F}$. The process shall be continued until the weight loss is not more than 0.1 per cent per hour.

21. Determination of combustible matter.-(a) Combustible in refuse shall be computed by means of difference in weight of total coal, computed weight of total dry ash from the ultimate analysis, and weight of boiler refuse. The heating value of the combustible is to be taken as that of pure carbon.

(b) For determination of the combustible in fuel siftings through the grate the sifting sample shall be treated according to the method described under proximate analyses in A. S. T. M. specification D271 for the determination of ash in coal. The heating value of the combustible in the siftings shall be determined by the calorimetric method.

\section{LOCOMOTIVES}

22. Gross sample from pan.-Collect the gross ash sample from the pan as outlined in paragraph 6 or 7, being careful to prevent loss of ashes.

23. Stack sample of cinders.-For an accurate determination of the cinder content in gases exhausting from locomotive stacks, all known methods which may lead to accuracy under actual running conditions are impracticable. It is therefore necessary to make these determinations in locomotive-testing plants, such as those of the Pennsylvania Railroad at Altoona, $\mathrm{Pa}$. , and of the departments of railroad engineering of the Universities of Illinois and Purdue.

24. Laboratory sample for determination of combustible in solids.-By quartering and reducing according to the approved method a sample of from 2 to 4 pounds may be taken from the gross sample. This should be placed in a tightly scaled jar for delivery to the laboratory.

25. Analysis of solid samples.-The cinder sample shall be analyzed for combustible according to the method outlined in section 4 of A. S. T. M. D271.

\section{POWDERED COAI}

26. Gross ash sample from furnace.-This shall be collected and weighed at convenient intervals, care being taken that none of the fine ash deposit is lost. If there are large slag formations, these should be broken down and weighed separately.

27. Gross ash sample from stack.-In collecting this sample the rules outlined in Appendix B may be used.

28. Preparing laboratory samples.-Laboratory samples from both the gross stack-ash sample and the gross furnace-ash sample shall be prepared from said gross samples by the standard process of reducing, accompanied by quartering and mixing. The laboratory samples shall weigh from 2 to 5 pounds. Scparate samples shall be taken for the furnace and stack. If the slag is to be analyzed the sample shall be prepared by crushing all of the slag so that it will pass through a $3 / 16$-inch screen. The laboratory sample shall be prepared from the total crushed slag by a reducing process, accompanied by quartering and mixing according to the standard method.

29. Analyzing laboratory samples.-The laboratory samples above prepared shall be analyzed for combustible according to the standard method.

\section{COOPERATION FROM THE BUREAU OF MINES}

30. In cases of important acceptance tests, exportation of coal, etc., it is often imperative to have the analysis performed by such an organization as the Government laboratories afford. The conditions under which the Bureau of Mines will analyze and test coal are stated in Appendix C. 
APPENDIX A

Frazer classification based on volatile matter

Anthracites
Semianthracites.
Semibituminous.
Bituminous $\left\{\begin{array}{l}\text { Eastern } \\ \text { Midcontinental }\end{array}\right.$
Black lignites or subbituminous
Brown lignites .

Frazer Classification Based on Fuel Ratio

$$
\text { Fucl ratio }=\frac{\text { Fixed carhon }}{\text { Volatile matter }}
$$

Anthracite.

Semianthracite

Semihituminous.

Bituminous

The first of the above classifications is strongly influenced by the quantity of ash present when a coal falls near one of the lines of demarcation; that is, for the same actual volatile content a low as compared to a high ash content may throw the coal into the next higher rank.

The second systcm of classification is not suitcd for western coals, although it may be advantageously used in ranking eastern coal.

Other methods of classification are in use but all are set up on a more or less arbitrary basis, and are therefore subject to criticism of one kind or another.

\section{APPENDIX B}

\section{Quantitative Test for Solids in Flue Gases}

For the determination of solids, such as cinders, an cfficient cinder catcher through which the total gases are passed will lead to more accurate results than any sampling device which takes only a very small fraction of the total gases. Furthermore, due to the relatively slow movement of the gases in many natural-draft installations, it is impossible to obtain a representative sample by simple means through a small sampling tubc. (There is insufficient kinetic energy in the particles to carry them through such tubes.) It is often impracticable to have the total gases passed through a cinder catcher and for such cases no generally recognized method is in use. The methods used $(a)$ by the Chicago department of smoke inspection 11 and $(b)$ those reported in Bureau of Mines Bulletin 223, entitled An Investigation of Powdered Coal as Fuel for Power Plant Boilers, by Kreisinger, Blizard, Augustine, and Cross, have been used with success.

The latter method is particularly to be recommended when it is desired to obtain in addition to the cinder content also a measurement of the fine dust such as may be present in gases escaping from powdered-coal furnaces.

\section{APPENDIX C}

\section{Fuel Testing and Analysis by Bureau of Mines}

In the bureaus' Schedule 3-A, the fees for analyzing coal are announced, and the conditions under which analyses and tests will be made are stated as follows:

Tbe conditions under whicb the Bureau of Mines will, for a fee, analyze samples of coal and determine the softening temperature of coal ash are set forth below.

(1) Application for the making of such analyses and tests must be made to the Director of the Burcau of Mines, Washington, D. C.

(2) The Bureau of Mines can not undertake to make analyses of coals for private parties except in cases where good reason exists why such analyses should be made in a Government laboratory, as in

11 Sce report of 1915 of Cbicago Association of Commerce Committee of Investigation on Smoke A batement and Electrification of Railway Terminals, pp, 150-151. cases where the coals are to be exported for sale in otber countries, or in cases involving arbitration, etc.

(3) In order tbat the analyses and tests made by the hureau may be fairly representative of the coal to be analyzed, it is essential that the samples of such coal be collected and shipped in the manner approved hy the Bureau of Mines. 12 (See method described in Technical Paper 1. The Sampling of Coal in the Mine, and Bulletin 110 , nical Paper 1, The Sampling of Coal in the Mine, and Bulletin 116 , Sampling Coal Deliveries, With each sample of coal sbould be furnisbed tbe name of the coal bed and of the mine from which the sample came. Tbe location of the mine with respect to the State, county, and tbe shipping point or nearest town should also be given.

American Standards Association, classification of coals.

(Under the procedure of the American Standards Association, standardization work is under way for the classification of all coals from anthracite to lignite, to be based upon such chemical and physical characteristics as will make the plan most readily adaptable to industrial and commercial use on a national scale.)

National Association of Purchasing Agents, standard coal contract. (In cooperation with National Coal Association).

Form A (with analysis clause).

Form B (without analysis clause).

(The above-entitled forms, to be used in preparing contracts for the purchase of coal, have been adopted as standard by the association.)

501.1 ANTHRACITE COAL. (See also 501.0.)

American Society for Testing IMaterials, tentative method of test for size of anthracite, serial designation D310-29T, 1929.

\section{Scope}

1. This method of test covers the screen tests of anthracite to determine the percentage of undersize or oversize in any given commercial size.

\section{Apparatus}

2. The screens for testing the various sizes of anthracite shall consist of No. 16 U. S. gage metal plates with staggered round openings. Screens mounted in hardwood box frames 16 to 20 inches square are satisfactory for testing chestnut, pca, and buckwheat sizes of allthracite. For broken, egg, and stove sizes it is more convenient to use scrcens square or rectangular in shape having an ares of 4 to 6 square feet. The screens may conveniently be mounted on a rack, so as to slide like a drawer, with a pan underneath to catch the undersize.

3. The screen openings for testing the different commercial sizes of anthracite ${ }^{13}$ shall be as follows:

\begin{tabular}{|c|c|}
\hline Size & $\begin{array}{l}\text { Size of round-hole openings in testing } \\
\text { screens }\end{array}$ \\
\hline Broken. & $\begin{array}{l}\text { Passing a } 47 / 16 \text { inch screen, retained on a } \\
37 / 16 \text { inch screen. }\end{array}$ \\
\hline Egg- & $\begin{array}{l}\text { Passing a } 37 / 16 \text { inch screen, retained on } \\
\text { a } 28 / 16 \text { inch screen. }\end{array}$ \\
\hline Stove & Passing a 2816 incb screen, retained on \\
\hline Chestnut. & Passing a $19 / 16$ inch screen, retained on \\
\hline Pea & Passing a 1316 incb screen, retained on \\
\hline No. I buckwheat & Passing a $9 / 16$ inch screen, retained on \\
\hline No. 2 buckwheat (r & Passing a $5 / 16$ inch screen, retained on \\
\hline No. 3 buckwheat (barley). - & $\begin{array}{l}\text { Passing a } 3 / 10 \text { inch screen, retained on } \\
\text { a } 3 / 32 \text { inch screen. }\end{array}$ \\
\hline
\end{tabular}

Tolerances of \pm 3 per cent on average openings and of 10 per cent in maximum openings are permissible.

12 Conditions here referred to for sampling are practically identical to those specified by this code as the standard method.

13 The 13 The names of anthracite sizes and the size of screen openings for broken, egg, stove, chestnut, and pea sizes, are in conformity with those recommended by the antbracite operators' conference of June
8,1928 . 


\section{Sampling}

4. Each carload, or its equivalent, shall be considered as a unit for sampling.

5. The gross sample shall not be less than 200 pounds for broken and egg sizes, and not less than 100 pounds for stove, chestnut, pea, and the buckwheat sizes.

6. (a) The gross sample shall preferably be collected when the coal is being loaded or unloaded in accordance with the procedure described in sections 1 and 2 of A. S. T. M. D21. (See 501.0, p. 4.) The increments shall be regularly and systematically collected, so that the entire quantity of coal sampled will be represented proportionately in the gross sample, and with such frequency that a gross sample of the required amount shall be collected.

(b) In case it is necessary to collect the sample from the exposed surface of the car, at least nine equal increments shall be taken about 1 foot below the surface at regular intervals along three parallel lines running from one end of the car to the other. One of these lines shall pass through the center of the car and each of the other lines shall be about two-thirds the distance from the center line to the side of the car.

7. For broken, egg, stove, and chestnut sizes the gross sample shall be screened without being mixed or reduced. For pea and the buckwheat sizes the gross sample shall be thoroughly mixed and reduced by quartering, without erushing, in accordance with sections 6 and 7 of A. S. T. M. D21 to a laboratory sample of the following approximate minimum amounts:

\begin{tabular}{l|r}
\hline Size & $\begin{array}{c}\text { Laboratory } \\
\text { sample } \\
\text { approxi- } \\
\text { matc mini- } \\
\text { mum } \\
\text { weight }\end{array}$ \\
\hline Pea & Pounds \\
No. 1 buckwheat (rice) & 50 \\
No. 2 buckwheat 3 buckwheat (barley) & 25 \\
\end{tabular}

Procedure

8. In case the coal is wet it shall be air-dried before screening. The undersize shall be determined first. For broken, egg, and stove sizes, each piece shall be upended on the screen, that is, tested to see if it will in any position pass through the screen. For chestnut, pea, and the buckwheat sizes, the screens shall be shaken gently, so as to avoid breakage of the coal, with a reciprocating horizontal motion until practically no more coal will pass through the openings. The coal shall be screened in such increments as will allow the pieces to be in direct contact with the screen openings after the completion of the shaking of each increment.

9. The undersize and oversize shall be reported to the nearest 1 per cent.

501.2 BITUMINOUS COAL. (See also 501.0.)

American Gas Association, standard specifications for gas and coking coals.

(These specifications agree with those of the American Society for Testing Materials (given below) and were prepared jointly by that organization and the American Gas Association)

American Society for Testing Materials, standard specifications for gas and coking coals; serial designation: D166-24; 1924.
(These specifications agree with those of the American Gas Association and were prepared jointly by that organization and the American Society for Testing Materials.)

1. Gas and coking coals must yield both merchantable gas and coke when distilled in a retort or oven by commercial methods. The type of coals may vary within rather wide limits according to the treatment in the retort and the market for the products. These specifications, therefore, merely give the limits within which gas and coking coals will usually fall, and indicate the circumstances under which further restrictive conditions should be imposed.

\section{SAMPLING AND ANALYSIS}

2. The coal shall be sampled in accordance with A. S. T. M. D21. (See 501.0, p. 4.)

3. Analyses of the coal, when required, shall be made in accordance with A. S. T. M. D271. (Sec 501.0, p. 8.)

\section{CHEMICAL AND PHYSICAI PROPERTIES}

The carbon ratio; that is, the ratio of fixed carbon to volatile matter while not entirely reliable, is the best simple index to the behavior of the coal when carbonized. The carbon ratio in the case of gas coals will vary from 1.4 to 2.0 and for coking coals from 1.4 to 5.0 The latter includes a wide range of coals varying from high volatile gas coal to low volatile or "smokeless" coal.

4. (a) The percentage of moisture in the coal as mined shall be subject to agreement by the purchaser and the seller.

(b) In the absence of a definite agreement bctween the purchaser and the seller, the mine moisture in the coal as mined shall not exceed 4.0 per cent. The moisture shall be determined by the general average composition of coal from the mine in question and an analysis of each shipment shall not be required.

5. The fusion temperature of ash of coal, the coke from which is intended for domestic and industrial use, shall not be below $2,200^{\circ} \mathrm{F}$. In the case of coke for use in the manufacture of water gas, the fusion temperature of the ash of the coal shall preferably be highcr than $2,300^{\circ} \mathrm{F}$. The fusion temperature of the ash shall be determined in accordance with the method for determination of fusibility of coaI ash appcaring in A. S. T. M. D271.

NotE.-The fusion point of ash is not usually important for metallurgical work. It is important, however, in the case of coke for domestic and industrial furnace use and for the manufacture of water gas.

\section{(a) Special Requirements for Gas Coals}

6. Gas coal shall contain not less than 35.0 per cent of volatile matter when determined on the moisture and ash-free basis.

Note.-This is equivalent to 30.8 per cent volatile matter for a coal containing 12.0 per cent of combined ash and moisture.

7. In the case of gas coals, the ash in the dry coal shall be not over 9 per cent.

8. The composition of gas coal shall be such that the dry coke produced therefrom will contain not over 1.5 per cent of sulphur and the resultant gas will contain not more than 30 grains of sulphur, in the form of compounds other than hydrogen sulphide, per 100 cubic feet of gas.

9. Gas coal shall be such that the coke produced thereform will be of sufficient size and strength to be suitable for domestic use or for the manufacture of water gas.

NotE.-These physical characteristics of coke are not amenable to simple explicit definition, and must necessarily be left to the judgment of experienced operators.

(b) Special Requirements for Coking Coals

10. In the case of coking coals, the ash in the dry coal shall be not over 9 per cent.

11. (a) If metallurgical coke is to be produced, the composition of the coking coal shall be such 
that the dry coke produced therefrom will not contain more than 1.0 per cent of sulphur in the case of foundry coke, and 1.3 per cent of sulphur in the ease of blast-furnace coke.

(b) If gas is to be sold for domestic use, the composition of the coking coal shall be such that the resultant gas will contain not more than 30 grains of sulphur, in the form of compounds other than hydrogen sulphide, per 100 cubic feet of gas.

12. A limitation as to phosphorus, which may be required when coke is used for metallurgical purposes, shall be subject to agreement between the purchaser and the seller.

13. The composition of coking coal which is to be charged into a by-product oven without admixture shall be such that the coke produced thercfrom will shrink sufficiently to permit of its being discharged from the oven without difficulty.

NOTE.-The mixing of coals for hy-product coke-oven use is widely practiced, and such mixtures usually contain, as a very important component, low volatile or "smokeless" coking coals, which when carhonized alone would not give the requisite shrinkage.

14. The composition of the coking coal shall be such that the coke produced therefrom will meet such requirements as to size, strength, and structure as are necessary for good practice in the industries using the coke.

Note.-These physical characteristies of coke are not amenable to simple explicit definition and must necessarily be left to the judgment of experienced operators.

American Society for Testing Materials, standard method of test for cubic foot weight of crushed bituminous coal, serial designation: D291-29, 1929.

\section{APPARATOS}

1. This method is intended for the rather coarsely crushed coal less than $1 \frac{1}{2} 2$ inches in size, sucl as is charged into coke ovens. It does not apply to powdered coal as used in boiler plants.

2. A box 12 by 12 by 12 inches in inside dimensions shall be used. A box made of one-eighth inch sheet steel welded together and provided with handles, makes a very suitable cubic foot box and would weigh approximately 35 pounds.

\section{SAMPLING}

3. For collecting gross samples of crushed bituminous coal, the procedure deseribed in sections 1 to 4 , inclusive, and the directions preceding section 1 of A. S. T. M. D21 (see 501.0, p. 4) shall apply. During the period of collecting the gross sample, the increments of the sample shall be stored in a waterproof container with a tightly fitting cover in order to prevent the loss of moisture.

4. The gross sample of coal shall, as quickly as possible to avoid loss of moisturc, be thoroughly mixed and reduced by quartering, without crushing, to a laboratory sample of approximately 60 pounds in accordance with sections 6 and 7 of D21. The cubic-foot weight should be determined immediately. If the determination of the cubic-foot weight is not to be made immediately, the 60-pound laboratory sample slould be kept in a waterproof container with a tightly fitting cover until the time for making the determination.

\section{PROCEDTRD}

5. The cubic-foot weight box shall be placed on a suitable platform scale, weighed empty and then filled with crushed bituminous eoal from the laboratory sample while the box is on the scale in order to avoid handling the heavy box of coal. The box shall be filled by means of a scoop or small shovel by allowing the coal to slide off the scoop or shovel from a height of 2 inches above the top of the box until the coal overflows on all sides of the box. Pre- cautions should be taken that none of the coal is dropped into the box so as to cause packing and that the cubic-foot box is not shaken or tapped during filling. The excess coal shall be struck off by means of a straightedge without shaking or tapping the box. The box filled with coal shall then be weighed. The difference between the two weights to the nearest 0.1 pound shall be reported as the weight of 1 cubic foot of the crushed bituminous coal.

NOTE.-Aside from the character of the coal itself, moisture content and size of the coal are the two main factors which affect the cubic foot weight. A moisture determination and a sieve analysis of the coal should he reported along with the cubic foot weight for proper interpretation of the cuhic foot weight.

For directions for making the moisture determination see A. S. T. M. D271. (See 501.0, p. 8.)

American Society for Testing Materials, tentative method of test for sieve analysis of crushed bituminous coal, serial designation: D311-29T, 1929.

\section{SCOPE}

1. This method is intended for the rather coarsely crushed coal, less than $1 \frac{1}{2}$ inches in size, such as is charged into coke ovens. It does not apply to powdered coal as used in boiler plants.

\section{APPARATUS}

2. The following square-mesh sieves, so selected that the actual openings between the wires of the succeeding sizes of sieves shall have a constant ratio of 1.414 (the square root of 2) shall be used: 0.131 , $0.185,0.263,0.371,0.525,0.742$, and 1.050 inches. Tolerances of \pm 5 per cent on a verage openings and of 15 per cent in maximum openings are permissible. ${ }^{14}$ Sieves of double-crimped wire with circular frames about 18 inches in diameter are satisfactory.

\section{SAMPLING}

3. For collecting gross samples of crushed bituminous coal, the procedure described in sections 1 to 4 , inclusive, and the directions preceding section 1 of A. S. T. M. D21 (see 501.0, p. 4) shall apply.

4. The gross sample of coal shall be thoroughly mixed and reduced by quartering, without crushing, to a laboratory sample of approximately 60 pounds in accordance with $\mathrm{A}$. S. T. M. D21. In case the coal is wet, the gross sample shall be air-dried before reduction to the 60 -pound laboratory sample.

\section{PROCEDURE}

5. The laboratory sample (approximately 60 pounds) shall be accurately weighed. Starting with the sieve having the largest opening, the laboratory sample of coal shall be sieved in such increments as will allow the pieces to be in direct contact with the ineshes after the completion of the shaking of each increment. In sieving, the coal shall be shaken rather vigorously, in order to upend the pieces, until practically no more coal will pass through the openings. The coal retained on each sieve and that which passes the smallest sieve shall be weighed separately.

6. The sieve analysis of the coal shall be reported in percentage to the nearest 0.1 per cent as follows:

Retained on a 1.050-inch sieve. Per cent

Passing a 1.050-inch sieve, retained on a 0.742 -inch sievo Passing a 0.742 -inch sieve, retained on a 0.525 -inch sieve.......... Passing a 0.525 -inch sieve, retained on a 0.371 -inch sieve Passing a 0.371-inch sieve, retained on a 0.263-inch sieve Passing a 0.263 -inch sieve, retained on a 0.185 -inch sieve Passing a 0.185-inch sieve retained on a 0.131-inch sieve

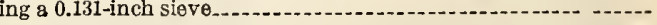

Total

14 For methods of testing sieves see A. S.T.M. E 11 . (See 500.2, p. 1.) 
In case the sum of the percentages does not total 100.0 , correction shall be made on the quantity passing through the smallest sieve so that the total will be 100.0. However, if the sum of the weights retained on each sieve and that which passes the smallest sieve shows a loss of over 0.5 per cent, the analysis shall be rejected and another test made.

501.3 SENIBITUMINOUS COAL. (See 501.0.)

\subsection{COAL-TAR PRODUCTS AND BITU. MINOUS MATERIAIS.}

501.41 Coal-tar products.

American Society for Testing Materials, analysis and tests for creosote oil.

(This society has formulated the following methods: D38-27, 1927, standard method of sampling and analysis of creosote oil; D246-28, 1928, standard method of test for distillation of creosote oil.)

\subsection{Bituminous materials. (See 505.)}

\subsection{COKE. (See also 501.0.)}

American Society for Testing Niaterials, standard definitions of terms relating to coal and coke. (See D121-, 501.0 p. 6.)

American Society for Testing Materials, tentative definitions of terms relating to coal and coke.

D121-27T, 501.0, p. 6.)

American Society for Testing Materials, standard method of laboratory sampling and analysis of coal and coke. (See D271-, 501.0, p. 8.)

American Society for Testing Materials, tentative methods for the determination of sulphur in coal and coke by the bomb washing and sodium peroxide fusion methods. (See D271-, 501.0, p. 8.)

American Society for Testing Materials, standard specifications for gas and coking coals. (See D166-, 501.2, p. 25.)

American Society for Testing Materials, standard specifications for foundry coke, serial designation: D17-16, 1916.

\section{CHEMICAL PROPERTIES AND TESTS}

\section{(a) Sampling}

1. Each carload, or its equivalent, shall be considered as a unit.

2. (a) The sample shall be taken from the exposed surface of the car, by knocking off with a hammer a piece approximately the size of a walnut, at regular intervais of 18 inches along three limes running from one end of the car to the other. One of these lines shall pass through the center of the car and the other two lines shall be 2 feet from the respective sides of the car.

(b) The intervals of sampling along the three lines may be measured by using a hammer with a handle 18 inches long, breaking off a piece of coke the size of a walnut at each point where the head of the hammer rests, regardless of the appearance of the particular piece that happens to occur under the head of the hammer.

3. The total quantity of sample collected in the above manner shall not be less than 1 cubic foot.

4. When the total moisture content is not to be determined, the entire gross sample shall be crushed to pass through a 4,760-micron (No. 4) sieve, under such conditions as shall prevent loss or the accidental admixture of foreign matter. The crushing shall be done mechanically with a jaw crusher, or by hand on a chilled iron or hard steel plate by impact of a chilled iron or hard steel tamping bar, hammer, or sledge, avoiding all rubbing action, otherwise the ash content of the sample will be materially increased by the addition of iron from the crushing apparatus, even though hardened steel or chilled iron is used.
After all the gross sample has been passed through the 4,760-micron (No. 4) sieve, it shall be mixed on a strong, closely woven cloth about 5 feet square by raising successively the four sides of the cloth, thus rolling the sample about until thoroughly mixed. The four corners of the cloth shall then be gathered up, and the sample shall be formed in a conical pile and reduced in quantity by quartering as follows:

The cone shall be flattened, its apex being pressed down so that eacli quarter contains the material originally in it. The flattened mass shall then be divided into four equal quarters. The diagonally opposite quarters shall then be removed and discarded and the space that they occupied brushed clean. The two remaining quarters shall be successively mixed, coned, and quartered on the cloth as before, until two opposite quarters shall weigh not less than 5 pounds, which shall then be placed in a suitable container for transportation to the laboratory. In case duplicate laboratory samples are desired, the rejected portions of the original sample passing the 4,760-micron (No. 4) sieve shall be combined, mixed, and quartered down to a similar 5 -pound sample.

5. The sample prepared by the above method may, at the option of the purchaser, be used for an approximate inoisture determination. In such cases the gross sample shall be immediately crushed and reduced to the 5-pound laboratory sample as rapidly as possible, to minimize the loss of moisture. The container for shipment to the laboratory shall be moisture tight. Since the sample obtained by this method will usually show less than the true moisture content of the gross sample, the purchaser shall have the privilege of a special moisture sample as hereinafter provided, if the standard sample shows more then 3 per cent moisture.

6. The special moisture sample shall consist of not less than 1 cubic foot of walnut size. It shall be taken in the manner described in section 2 , and shall be placed, immediately after collection, in a moisture-tight container for transportation to the laboratory. The car shall be weighed at the time the special moisture sample is collected.

7. In case of disagreement between buyer and seller, an independent chemist, mutually agreed upon shall be employed to sample and analyze the coke, the cost to be borne by the party at fault.

The resample shall be taken and prepared as prescribed in the foregoing sections, except that the minimum quantity of gross sample shall be not less than 1 bushel in volume, taken at intervals of 18 inches on six equidistant lines parallel to the side of the car.

\section{(b) Chemical Analysis}

8. The sample received at the laboratory shall be prepared for analysis, and the percentage of moisture, volatile matter, fixed carbon, ash, and sulphur shall be determined as specified in A. S. T. M. D271. (See 501.0, p. 8.)

9. The dry coke shall not exceed the following limits in chemical composition:

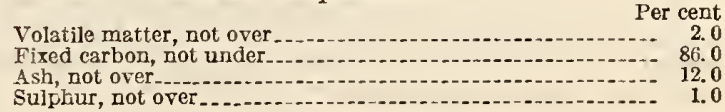

\section{REJECTION}

10. (a) In case the original standard sample was taken in accordance with section 5 and showed more than 3 per cent moisture, the purchaser shall have the option of taking a special moisture sample according to section 6 , and of deducting the moisture found in excess of 3 per cent from the weight of coke found on reweighing the car at the time the special moisture sample was taken. 
(b) In case the original standard sample was taken with special regard to moisture in accordance with section 6 , the purchaser shall have the option of deducting the moisture in excess of 3 per cent from the wcight of coke, provided that the car was weighed at the time of sampling.

11. Coke which fails to conform to the limits of chemical composition given in section 9 will be rejected, and the seller shall be notified within five working days from the date of sampling.

American Society for Testing Materials, standard method of test for sieve analysis of coke, serial designation: D293-29; 1929.

\section{APPARATUS}

1. The following square-mesh sieves, so selected that the actual openings between the wires of the succeeding sizes shall have a constant ratio of 1.414 (the square root of 2), in so far as this sicve scale has becomc cstablished by use, shall be used: 0.131 , $0.185,0.263,0.371,0.525,0.742,1.050,1.5,2.0,3.0$, and 4.0 inches. Tolerances of \pm 5 per cent on average openings and of 15 per cent in maximum openings are permissible. (For sieve tests see A. S. T. M. E $11,500.2$, p. 1.) For sizes of coke smaller than 2 inches, sieves of double-crimped wire with circular frame about 18 inches in diameter are satisfactory. For coke 2 inches and larger in size, it is more convenient to use sieves square or rectangular in shape, of heavy, double-crimped wire having an area of 6 to 9 square fect. These larger sieves may be mounted in a rack so as to slide like a drawer with a pan at the bottom.

\section{SAMPLING}

2. For collecting samples of coke, the procedure described in sections 1 to 4 , inclusive, and the directions preceding section 1 of A. S. T. M. D21 (see 501.0, p. 4), shall apply in general. The weight of the sample selected for the sieve-analysis determination depends upon the size and character of the coke and shall conform to the following:

For run-of-oven coke and coke 1 inch and larger in size containing breeze, not less than

For coke 1 inch and larcer in size free from breeze, not less than

For coke smaller than 1 inch in size, not less than,

It is extremely difficult to obtain truly representative samples of colse having a range of sizes. When the coke is on a belt conveyor the sample shall be selected by stopping the belt at regular intervals and selecting increments in sections about 3 feet in length and the entire width of the belt, or when the coke is going over a pulley or coming down a chute the sample shall be selected by inserting a container or scoop into the stream of coke at regular intcrvals so as to takc increments of the full width and thickness. The increments shall be regularly and systematically collected, so that the entire quantity of coke sampled will be represented proportionately in the sample, and with such frequency that a sample of the required amount shall be collected. It is not feasible to collect representative samples of coke from loaded cars or bins for sieve analyses. Samples of coke in cars or bins should be taken as the cars or bins are being filled or emptied.

\section{PROCEDURE}

4. The sample shall be accurately weighed. Starting with the sieve having the largest opening, the sample of coke shall be sieved in such increments as will allow the pieces to be in dircet contact with the meshes after the completion of the shaking of each incrcment. For coke 1 inch and larger in size the following square-mesh sieves are usually used: 4.0, $3.0,2.0,1.5,1.050$, and 0.525 inches. For coke smaller than 1 inch in size the square-mesh sieves usually used are: $0.742,0.525, \quad 0.371, \quad 0.263$ 0.185 , and 0.131 inch. Each piece of coke retained on the 2-inch or larger sieve shall be upended on the sieve; that is, tested to see if it will in any position pass through the sieve. Coke pieces passing the 2-inch sieve shall be shaken rather vigorously on each succeeding sieve in order to upend the picces until practically no more coke will pass through the openings. The coke retained on each sieve and that which passes the smallest sieve used shall be weighed separately. In case the coke is wet, the coke shall be dried before making the sieve analysis. However, usually any errol due to moisture content would be very small and may be neglected except in the case of coke smaller than 1 inch in size, in which case the coke shall be dried before making the sieve test.

5 . The sieve analysis of the coke shall be reported in percentagc to the nearest 0.1 per cent as follows:

Per cent

Retained on a 4.0-inch sieve

Passing a 40 -inch sieve, retained on a 30 -inch sievo

Passing a 4.0-inch sieve, retained on a 3.0 -inch sieve Passing a 2.0 -inch sieve, retained on a 1.5 -inch sieve

Passing a 1.5-inch sieve, retained on a 1.050 -inch sieve

Passing a 0.185 -inch sieve, retained on a $0.131-i n c h$ sieve

Passing a 0.131-inch sieve.

Total.

100.0

In case the sum of the percentages do not total 100.0 , correction shall be made on the quantity passing through the smallest sieve so that the total will be 100.0. However, if the sum of the weights retained on each sieve and that which passes the smallest sieve shows a loss of over 0.5 per cent, the analysis shall be rejected and another test made.

In view of the difficulty of obtaining representative samples of coke with regard to the size of the pieces, even when 500-pound samples are used for the sieve analysis, it is desirable to take several samples for sieve tests and average the figures for the several samples.

American Society for Testing Materials, standard method of test for cubic foot weight of coke, serial designation: D292-29; 1929.

1. This method is intended for coke 5 inches and smaller; that is, coke which would in any position pass through a 5-inch square-mesh sieve.

2 . A box 24 by 24 by 24 inches in inside dimensions shall be used. In order to keep the weight of the empty box as light as possible, the box may be made of wood, but it must be rigid. Two strips of wood may be fastened to the sides of the box to form "sedan-chair" handles for convenience in handling.

NorE.-For determining the cubic foot weight of coke smaller than 1 inch in size, a cubic foot weight box measuring 12 by 12 by 12 inches in inside dimensions may be used.

\section{SAMPLING}

3. For collecting samples of coke, the procedure described in sections 1 to 4 , inclusive, and the directions preceding section 1 of A. S. T. M. D21 (see 501.0, p. 4) shall apply in general. The weight of the sample selected for the determination of the cubic foot weight depends upon the size and character of the coke and shall conform to the following:

For run-of-oven coke and coke 1 inch and larger in size not less than 300 pounds.

For coke smaller than 1 inch in size not less than 50 pounds.

It is extremely difficult to obtain truly representative samples of coke having a range of sizes. When the coke is on a belt conveyor the sample shall be selected by stopping the belt at regular intervals and selecting increments in sections about 3 feet 
in length and the entire width of the belt or when the coke is going over a pulley or coming down a chute the sample shall be selected by inserting a container or scoop into the stream of coke at regular intervals so as to take increments of the full width and thickness. The increments shall be regularly and systematically collected, so that the entire quantity of coke sampled will be represented proportionately in the sample, and with such frequency that a sample of the required amount shall be collected. It is not feasible to collect representative samples of coke from loaded cars or bins for cubic foot weight determinations. Samples of coke in cars or bins should be taken as the cars or bins are being filled or emptied.

\section{PROCEDURE}

4. The cubic foot weight box shall be placed on a suitable platform scale, weighed empty and then filled with coke from the sample while the box is on the scale in order to avoid handling the heavy box of coke. The box shall be filled by means of a shovel or other suitable container by allowing the coke to slide out of the shovel or container from a height of 2 inches above the top of the box, with no attempt to spread or arrange the coke. Because of the physical character of coke, it is not practical to strike off the exccss coke by means of a straightedge, so it is necessary to do this largely by eye, with the assistance of a straightedge to check obscrvations. The box shall not be shaken, tapped, or dropped during filling or leveling off. The box filled with coke shall then be weighed. The difference between the two weights divided by the number of cubic feet in the box will give the weight per cubic foot of coke.

NotE.-For proper interpretation of the cuhic foot weight of coke, a moisture determination and a sieve analysis of the coke should he reported along with the cuhic foot weight. For directions for making these determinations see the following methods of the American Society for Testing Materials:

In the case of a moisture determination, D271 (see 501.0, p. 8)

In the case of a sieve analysis, D293-28T (given ahove).

American Society for Testing Materials, standard method of tumbler test for coke, serial designation: D294-29, 1929.

\section{APPARATUS}

1. The tumbler machine shall consist of a circular steel drum 36 inches in inside diameter and 18 inches in inside length made of plate at least onc-fourth inch in thickness. Two equally spaced 2 by 2 by $1 / 4$ inch angles shall be riveted longitudinally inside the drum. These angles shall be riveted to the shell so that the attached legs point away from the direction of rotation, thus giving a clear unobstructed shelf for lifting the coke. The drum shall be mounted on a horizontal shaft to provide for rotating the drum. An opening shall be provided, preferably in the shell, for introducing and removing the sample. During the test, the cover shall be rigidly fastened to the shell and shall be so constructed as to fit into the shell in order to have a smooth inner surface.

2. For sizing the sample for test, square-mesh sieves having 2 -inch and 3 -inch actual openings between the wires shall be used. For sieving the coke after the tumbler test, square-mesh sieves having 2 -inch, 1.5 -inch, 1.050 -inch, 0.525 -inch, and 0.263 -inch actual openings between the wires shall be used. In accordance with A. S. T. M. E11 (see 500.2, p. 1) a tolerance of \pm 3 per cent on average openings and of 10 per cent in maximum openings are permissible. Sieves of heavy double-crimped wire with circular frames about 24 inches in diameter are satisfactory.

\section{SAMPLING}

3. The quantity of coke collected for sizing shall be sufficient to obtain approximately 25 pounds of coke which will pass a 3 -inch and be retained on a 2-inch square-mesh sieve. This may best be acconıplished by placing a container or scoop in the stream of coke and collecting small increments at regular intervals in order to obtain a representative sample of the entire quantity of coke under consideration.

4. The coke shall be sized by sieving on a 3-inch and a 2-inch square-mesh sieve, without crushing the larger pieces, in order to obtain a sample that will pass the 3 -inch and be retained on the 2-inch sieve. In sizing the sample, each piecc of coke shall be upended on the sieve; that is, tested to see if it will in any position pass the sieve. If a large proportion of the pieces arc larger than 3 inches, it will be necessary to break out representative smaller pieces of the desired size. This shall be accomplished without shattering the coke picces and may often be done with a heavy screw driver by prying apart at fracture cracks.

\section{PROCEDURE}

5. Approximately 22 pounds $(10 \mathrm{~kg}$ ) of the coke sample which has been sized in accordance with section 4 , and previously dricd at $104^{\circ}$ to $200^{\circ} \mathrm{C}$. shall be accurately weighed and placed in the drum of the tumbler machine. The cover shall be rigidly fastened and the drum rotated at 24 r. p. m. \pm 1 r. p. $m$. for a total of 1,400 revolutions. All of the coke shall then be removed from the drum and sieved, using the following square-mesh sieves: 2-inch, 1.5-inch, 1.050-inch, 0.525-inch, and 0.263 -inch. Each piece of coke retained on the 2 -inch sievc shall be upended to determine whether in any position it passes the 2 -inch sieve, while the coke passing the 2 -inch sieve shall be shaken rather vigorously on each succeeding sieve in order to upend the pieces until practically no more coke will pass through the openings. The coke retained on each sieve and that which passes through sliall be weighed separately.

\section{REPORT}

6. The sieve analysis after the tumbler test shall be reported in cumulative percentages to the nearest 0.1 per cent, as follows:

Total retained on the 2-inch sieve

Per cont cumulative

Total retained on the 1.5 -inch sieve

Total retained on the 1.050 -inch sieve.

Total retained on the 0.525 -inch sieve

Total retained on the 0.263 -inch sieve

Stability factor) (Hardness factor)

Names have been assigned to two values in the sieve test following the tumbler test. The percentage of coke retained on the 1.050 -inch sieve has been designated the stability factor, and the percentage retained on the 0.263 -inch sieve as the hardness factor as indicated in the report of the sieve test in the above table.

American Society for Testing Materials, standard method of shatter test for coke, serial designation: D141-23; 1923.

1. The shatter-test machine shall consist of a box 18 inches in width, 28 inches in length, and approximately 15 inches in depth, supported above a rigidly mounted cast iron or steel plate, not less than one-half inch in thickness, 38 inches in width, and 48 inches in length. The inside of the bottom of the box shall be 6 feet above the plate. The bottom of the box shall consist of two doors hinged lengthwise and latched so that they will swing open freely and not impede the fall of the coke. Boards about 8 inches in height should be placed around the plate so that no coke is lost. To prevent the breakage of coke, which may occur while placing the sample in the box, the box shall be constructed so that it can be lowered to a convenient level, which is 
best done by means of a pulley and counterweight. A convenient form of shatter-test machine is shown in Figure 13.

2. For determining the breakage of coke, squaremesh screcns with the following openings shall be used: 2 inches, $1 \frac{1}{2}$ inches, 1 inch, and $1 / 2$ inch. A circular screen, 2 feet in diameter, is satisfactory.

3 . Each carload, or its equivalent, shall be considered a unit for sampling.

\section{SAMPINNG}

4. (a) If the shatter test is to be used to indicate the probable breakage of coke on handling, the sample should be taken before the coke is subjected to possible breakage resulting from screening and loading into cars. In the case of by-product coke, the sample should be collected from the coke wharf; while samples of beehive coke should be collected as it is delivered from the ovens.

(b) If the shatter test is to be used to indicate the fitness of the coke for furnace or cupola use, the sample should be collected at the point of delivery.
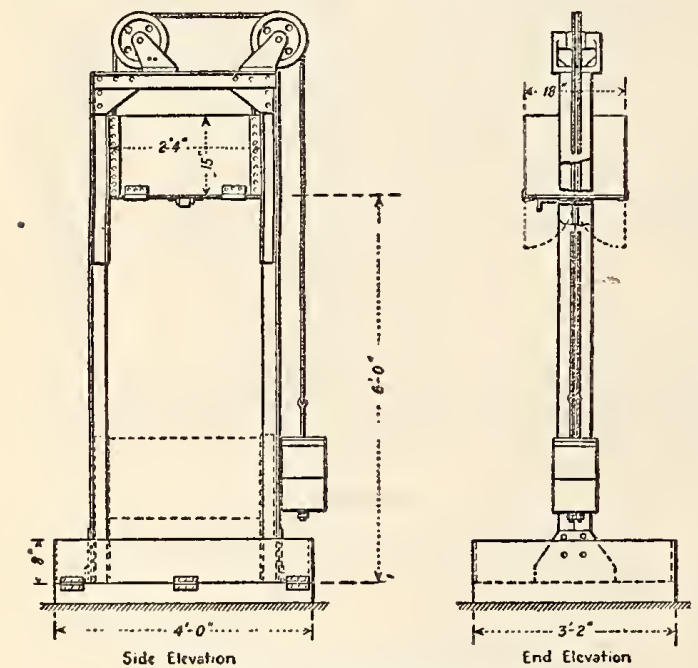

FIGUR丨 13.- Shatter test machine

5. (a) By-product coke.-About 75 pounds of representative pieces of coke, none of which would in any position pass through a 2-inch square-mesh screen, shall be selected from the coke wharf for each test. This is best accomplished by dividing the coke on the wharf into approximately equal areas, and selecting an equal number of pieces from each area. Each piece selected shall be approximately equal in length to one-half the wiath of the coke ovens, and should show a "cauliflower" end produced at the walls of the ovens, and an "inner" end produced at the center of the ovens.

(b) Beehive coke.-About 75 pounds of representative pieces of coke shall be selected for each test as the coke is drawn from the ovens. This is best done by selecting full-length pieces, or their equivalent, as the coke is being drawn from previously determined points in the oven, so that they include pieces from the front, sides, center, and back. If the individual pieces as taken from the ovens are too large and bulky, the 75-pound sample shall be collected by removing three small pieces from each large piece-one from the top, the middle, and the bottom. The sample finally collected should be composed of an equal number of pieces showing top, middle, and bottom. None of the pieces comprising the sample shall in any position pass through the 2-inch square-mesh screen.
6. By-product and beehive coke.-The sample is best collected as the coke is delivered from the railroad cars into the bins. This may be accomplished by inserting a scoop of 10 to 15 pounds capacity in the coke stream at regular intervals during the period of unloading. The sample collected shall be large enough to give about 75 pounds of coke pieces, none of which would in any position pass through a 2-inch square-mesh screen.

As it is very difficult to collect a representative sample from coke exposed in bins and cars, care should be taken to take pieces representing the entire exposed area, if sampling must be done in this manner. This is best accomplished by dividing the exposed surface to be sampled into approximately equal areas, and selecting an equal number of pieces from each area. Approximately 75 pounds of representative pieces, none of which would in any position pass through a 2 -inch square-mesh sieve, shall be collected.

\section{PROCEDURE}

7. About 50 pounds of the sample shall be placed in the box of the coke shatter-test machine, the coke leveled, the box raised, and the coke dropped four times on the plate, the small material produced being returned each time to the box with the large coke. To prevent breakage of the coke, the box should be lowered to a convenient height when transferring the sample into it. After the fourth drop the material shall be successively run through the screens mentioned below. The coke should be screened in such increments as will allow all pieces to be in direct contact with the screen openings.

In screening, care should be taken to prevent breakage of the coke pieces. The screen shall be shaken gently until all of the pieces are in direct contact with the meshes. The coke remaining on each screen, and that which passes through, shall be weighed separately. If the sum of these weights shows a loss of over 1 per cent, the test shall be rejected and another made.

The results of the shatter test shall be reported as follows:

Retained on 2-inch screen

Per cont

Passing 2-inch screen, retained on 11/2-inch Passing 11 in

Passing 1 Passing

8. Since the average probable error of a single shatter test determination is approximately 2 per cent, it is advisable to make several tests and report the average result.

American Society for Testing Materials, standard method of test for volume of cell space of lump coke, serial designation: D167-24; 1924.

1. The percentage by volume of cell space of lump coke shall be calculated from the apparent specific gravity of the moisture-free lump coke and the true specific gravity of moisture-free coke passing a 74micron (No. 200) sieve as follows:

Percentage of cell space $=100-100 \frac{\text { Apparent specific gravity }}{\text { True specific gravity }}$

\section{A. DETERIMUATION OF APPARENT SPECIFIC GRAVITY}

\section{Apparatus}

2. The apparatus for the determination of the apparent specific gravity of coke shall consist of the following:

(a) A suitable container not smaller than approximately 13 inches in height, 22 inches in length and 11 inches in width, of or equivalent size, provided with a spout consisting of a short one-half inch nipple extending horizontally from the container about $2 \frac{1}{2} 2$ inches below the top.

Note.-A wash boiler of suitable size to which a spout has been soldered answers the above description. 
(b) A wire cage or basket of about one-half inch square-mesh screen wire cloth provided with a cover and two long handles, suitable for holding the entire sample of coke and so made as to fit inside the container below the spout.

(c) A 3-gallon bucket or other vessel suitable for receiving the displaced water.

(d) A pan about 15 inches square and 3 inches in height or the equivalent for containing the coke during the determination of its weight.

(e) A scale sensitive to one-half ounce.

Sampling

3. Each carload, or its equivalent, shall be considered a unit for sampling.

4. (a) If the porosity test is desired on run-ofoven coke, the sample should be collected from the coke wharf in the case of by-product coke, while samples of beehive coke should be collected as it is delivered from the ovens.

(b) If the porosity test is desired on coke for furnace or cupola use, the sample should be representative of the material in question and collected at the point of delivery.

5. (a) By-product coke.-About 50 pounds of representative picces of coke shall be selected from the coke wharf for each test. This is best accomplished by dividing the coke on the wharf into approximately equal areas, and selecting an equal number of pieces from each area. Each piece selected shall be approximately equal in length to one-half of the width of the coke ovens, and shall show a "cauliflower" end produced at the walls of the ovens, and an "inner" end produced at the center of the ovens.

(b) Beehive coke.-About 50 pounds of representative pieces of coke shall be selected for each test as the coke is drawn from the ovens. This is best done by selecting full-length pieces, or their equivalent, as the coke is being drawn from previously determined points in the oven, so that they include pieces from the front, sides, center, and back. If the individual pieces as taken from the ovens are too large and bulky, the 50-pound sample shall be collected by removing three small pieces from each large piece-one from the top, the middle, and the bottom. The sample finally collected should be composed of an equal number of pieces showing top, middle, and bottom.

6. By-product and beehive coke.-The sample is best collected as the coke is delivered from the railroad cars into the bins. This may be accomplished by inserting a scoop of 10 to 15 pounds capacity in the coke stream at regular intervals during the period of unloading. The sample collected shall be large enough to give about 50 pounds of coke pieces, none of which would in any position pass through a 1-inch square-mesh screen.

Note.-Since the eage or basket is of 1/2-inch square-mesh screen-wire cloth, it is necessary to bave coke pieces that will remain in the cage when the the cage is removed from the water.

As it is very difficult to collect a representative sample from coke exposed in bins and cars, care should be taken to take pieces representing the entire exposed area, if sampling must be done in this manner. This is best accomplished by dividing the exposed surface to be sampled into approximately equal areas, and selecting an equal number of pieces from each area. A 50-pound sample of representative pieces shall be collected, none of which should in any position pass through a 1 -inch square-mesh screen.

\section{Procedure}

7. About 25 pounds of coke from the sample shall be selected so as to be representative of the material under consideration with regard to size, shape, and general appearance. The coke shall be dried to constant weight at a temperature of from $105^{\circ}$ to $200^{\circ} \mathrm{C}$. The coke shall be weighed when cool, after shaking and brushing off any adhering dust.

A cork shall be placed in the spout of the container which has been placed on a level and rigid base or floor. After the empty cage has been placed into the container, water at room temperature shall be poured into the container until the water level is above the spout. After the water has come to rest, the cork shall be removed from the spout and the excess water be permitted to drain out for one minute after the overflow-stream starts to discharge drop by drop. The cork shall then be replaced and the cage removed from the water, care being taken to shake all adhering water back into the container. The weighed dried cobe sample shall then be placed into the cage and after fastening the cover, the cage containing the coke shall be lowered into the water.

Note.-If there is not sufficient capacity in the container above the spout to retain the displaced water, some of the water may be drawn off into a weighed bucket, or other suitable ressel, by removing the cork from the spout while the coke is being lowered.

The cage shall be permitted to remain in the water for 15 minutes with occasional shaking to detach any air bubbles adhering to the surface of the coke, care being taken not to disturb the position of the container. At the end of the 15-minute period, during which the coke shall have been completely submerged at all times, after the water has come to rest, the cork shall be removed and the displaced water permitted to drain into a weighed bucket or other suitable vessel for one minute after the overflow stream starts to discharge drop by drop. The cork shall then be replaced, the cage containing the coke removed from the water and permitted to drain for one minute. The wet coke shall then be removed from the cage and weighed.

The weight of the displaced water, which has been caught in the bucket, shall then be determined.

8. The apparent specific gravity shall be calculated from the formula:

$$
\text { A pparent specific gravity }=\frac{A}{B+(C-A)}
$$

in which

$A=$ weight of dry coke;

$\mathrm{B}=$ weight of water displaced by wet coke; and

$\mathrm{C}=$ weight of wet $\operatorname{cok} \theta$.

\section{B. DETERMINATION OF TRUE SPECIFIC GRAVITT}

\section{Apparatus}

9. The apparatus for the determination of the true specific gravity of coke passing a 74-micron (No. 200) sieve shall consist of a Hogarth's specific gravity bottle with side tubulure, having a capacity of about $100 \mathrm{cc}$. The bottle shall be accurately calibrated so that a table may be constructed, giving the contents of the bottle at the room temperatures likely to occur in the laboratory.

Note.-This may be done conveniently from data in tables of corrections for determining the true capacities of glass vessels from the weight of water in air, as given in National Bureau of Standards Circular No. 19, pages 52 to 56; 1916.

\section{Procedure}

10. A $10 \mathrm{~g}$ portion of 200 -mesh coke, which has been previously dried for one hour at $105^{\circ} \mathrm{C}$., shall be carefully introduced into the specific-gravity bottle with a sufficient quantity of distilled water to fill the bottle about one-half full. The bottle shall then be placed on a hot plate and the contents kept boiling for one hour, the specific-gravity bottle being shaken frequently so as to wash down any coke adhering to the sides. After boiling for one hour, the bottle shall be removed from the plate, filled to the tubulure with recently boiled and cooled distilled water, and the stopper inserted. The bottle shall be per- 
mitted to stand until the contents have cooled to room temperature.

NotE.-Cooling may be hastened by placing the bottle in water.

After the contents of the bottle have cooled to room temperature, the bottle shall be filled to slightly above the mark on the capillary of the stopper with recently boiled distilled water which has been cooled to room temperature.

NOTE.- This is conveniently done by inserting the end of the tubulure in a small beaker of the distilled water and applying a slight suction on the stopper.

The water level shall be adjusted to the mark on the capillary by touching a piece of filter paper to the end of the tubulure. The bottle shall then be wiped dry and weighed immediately. Immediately after the weighing, the stopper shall be removed and the temperature of the contents taken.

11. The true specific gravity shall be calculated from the formula:

$$
\text { True specific gravity }=\frac{W}{W-\left(W^{\prime}-\bar{P}\right)}
$$

in which $W=$ weight in gxams of dry coke

$W^{\prime}=$ weight in grams of bottle + dry coke + water required to fill the bottle;

$P=$ weight in grams of bottle + water required to fill the bottle.

12. The differences in duplicate determinations shall not be more than the following:

Same analyst

Different analyst

\subsection{CHARCOAL.}

(No nationally recognized specifications available.) 502.

\section{PETROLEUM}

\subsection{GENERAL ITEMS.}

American Society for Testing Materials, tentative definitions of terms relating to petroleum, serial designation: D288-28T; 1928.

Crude petroleum.-A naturally occurring mixture, consisting predominantly of hydrocarbons and/or of sulphur, nitrogen, and/or oyxgen derivatives of hydrocarbons, which is removed from the earth in liquid state or is capable of being so removed.

Note.-Crude petroleum is commonly accompanied by varying quantities of extraneous substances, such as water, inorganic matter, and gas. The removal of such extraneous substances alone does not change the status of the mixture as crude petroleum. If such removal appreciably affects the composition of the oil mixture, then the rcsult ing product is no longer crude petroleum.

Topped crude petroleum.-A residual product remaining after the removal, by distillation, or other artificial means, of an appreciable quantity of the more volatile components of crude petroleum.

Weathered crude petroleum.-The product resulting from crude petroleum through loss, due to natural causes, during storage and handling of an appreciable quantity of the more volatile components.

Benzine.-This term is archaic and misleading and should not be used.

Petroleum naphtha.-A generic term applied to refined, partly refined, or unrefined petroleum products and liquid products of natural gas, not less than 10 per cent of which distills below $347^{\circ} \mathrm{F}$ $\left(175^{\circ}\right.$ C. $)$ and not less than 95 per cent of which distills below $464^{\circ} \mathrm{F}$. ( $240^{\circ} \mathrm{C}$.), when subjected to distillation in accordance with the current method of test of the American Society for Testing Materials.

NoтE.-The "naphthas" used for specific purposes such as cleaning, manufacture of rubber, manu facture of paints and varnishes, etc., are made to conform to specifications which may require products of considerably greater volatility than that set by the limits of this generic definition.

Gasoline.-A refined petroleum naphtha which by its composition is suitable for use as a carburant in internal-combustion engines.
Petroleum spirits (white spirits).-A refined petroleum distillate with a minimum flasl point of $70^{\circ} \mathrm{F}$. $\left(21^{\circ}\right.$ C. $)$ determined by the tag closed tester in accordance with A. S. T. M. D56 (see 503.0, p. 43) or by the Abel tester, with volatility and other properties making it suitable as a thinner and solvent in paints, varnishes, and similar products.

NoTE.-The term "turpentine substitute" as applied to petroleum spirits is to be condemned as false and misleading. The term "mineral spirits," which is frequently used in the paint and varnish industry, is a misnomcr, as it includes within its scope not only petroleum products, but other hydrocarbon mixtures, such as coal-tar distillates. In Great Britain the term "pctroleum spirits" is applied to a very light hydrocarbon mixture having a flash point below $32^{\circ} \mathrm{F}$. $\left(\mathrm{O}^{\circ} \mathrm{C}.\right)$.

Kerosene.-A refined petroleum distillate having a flash point not below $73^{\circ} \mathrm{F}$. $\left(23^{\circ} \mathrm{C}\right.$.), as determined by the Abel tester (which is approximately equivalent to $73^{\circ}$ F. $\left(23^{\circ}\right.$ C.) as determined by the tag closed tester (see A. S. T. M. D56) and suitable for use as an illuminant when burned in a wick lamp.

Note.-In the United States of America local ordinances or insurance regulations require at times flash points higher than $73^{\circ} \mathrm{F}$ $\left(23^{\circ}\right.$ C. $)$, tag closed tester.

Gas oil.-A liquid petroleum distillate having a viscosity intermediate between that of kerosene and lubricating oil.

NotE.-It should be understood that oils, other than gas oil as defined above, may be and are used in the manufacture of gas.

Fuel oil.-Any liquid or liquefiable petroleum product used for the generation of heat in a furnace or firebox, the controlling factors being cost and adaptability. Fuel oils in common use fall into one of four classes: (a) Residual fuel oils, which are topped crude petroleums or viscous residiums obtained in refinery operations; $(b)$ distillate fuel oils, which are distillates derived directly or indirectly from crude petroleum; (c) crude petroleums and weathered crude petroleums of relatively low commercial value; and $(d)$ blended fuels, which are mixtures of two or more of the three classes.

Tar, pitch.- These terms shall not be applied to petroleum products.

Petroleum lubricating grease.-A combination of a petroleum product and a soap or a mixture of soaps, suitable for certain types of lubrication.

Oil shale.-A compact rock of sedimentary origin, with an ash content of more than 33 per cent and containing organic matter that yields oil when destructively distilled, but not appreciably when extracted with the ordinary solvents for petroleum.

Crude shale oil. - The oil obtained as a distillate by the destructive distillation of oil shale.

End point.-The highest temperature reading observed on the distillation thermometer during the distillation procedure conducted in accordance with A. S. T. M. D86. (See 503.0, p. 44.)

Engine distillate. - A refined or unrefined petroleum distillate similar to naphtha, but often of higher distillation range.

Tops.-The unrefined distillate obtained in topping a crude petroleum.

American Society for Testing Materials, tentative method of test for gravity of petroleum and petroleum products by means of the hydrometer, serial designation: D287-28T; 1928.

1. This method covers the determination of the specific gravity or the A. P. I. gravity of crude petroleum and petroleum products normally handled as liquids. The determination of the gravity of mixtures of petroleum products with other substances is treated as a special case owing to the fact that the coefficients of expansion of such mixtures may not be the same as those of petroleum or its products. The determination of the specific gravity of road oils, road tars, asphalt cements, and soft tar pitches is covered by A. S. T. M. D70 (see $505.0, \mathrm{p} .125$.), and the testing of these products is 
therefore specifically cxcluded from the scope of this method.

\section{DEFINITIONS}

2. (a) Specific gravity.-The specific gravity of a petroleum oil and of mixtures of petroleum products with other substances is the ratio of the weight of a given volume of the material at a temperature of $60^{\circ} \mathrm{F} .\left(15.56^{\circ} \mathrm{C}\right.$.) to the weight of an equal volume of distilled water at the same temperature, both weights being correctcd for the buoyancy of air.

(b) A. P. I. gravity-The A. P. I. gravity scale is an arbitrary one which is related to the specific gravity of a petroleum oil in accordance with the formula:

$$
\text { Degrees A. P. I. }=\frac{141.5}{\text { sp. gr. } 60^{\circ} / 60^{\circ} \mathrm{F}} .-131.5
$$

A table showing the equivalent specific gravity, pounds per gallon and gallons per pound at $60^{\circ} \mathrm{F}$. for each degree A. P. I. from $0^{\circ}$ to $100^{\circ}$ A. P. I. is given in the appendix to this method.

\section{APPARATUS}

There are two kinds of hydrometers in common use, the plain type ter and hydrometer, kwown as a thermohydrometer. Although the thermohydrometer type of instrument is sufticiently satisfactory for routine use, the thermomoter incorroutine dae, the thesm wich inend porated in it possesses certain ioherent delects of design which rende tested must be determined by means of a separate thermometer.

3. Hydrometer calibrations.-The hydrometer may be calibrated either in terms of specific gravity or degrees A. P. I. It shall be of glass of the conventional constant mass and variable displacement type, with a paper hand-written scale. The range may be any suitable portion of the interval between $0^{\circ}$ and $100^{\circ}$ A. P. I. (1.0760 to $0.6112 \mathrm{sp}$. gr.) and it shall be so divided that $0.1^{\circ}$ A. P. I. or 0.0005 of a unit of specific gravity shall not be less than $1 \mathrm{~mm}$ (0.039 inch) in width. The value of the smallest subdivisions shall be $0.1^{\circ} \mathrm{A}$. P. I. when the instrument is calibrated in degrees A. P. I. or 0.0005 of a unit of specific gravity when it is calibrated in the specific gravity scale. The accuracy of calibration of the hydrometer shall be such that the error at any point of the scale shall not exceed the value of one smallest scale division. Each fifth smallest subdivision on the scale shall be denoted by a longer line. The graduations shall be numbered at each multiple of $1^{\circ}$ A. P. I. or at each multiple of 0.01 of a unit of specific gravity, in accordance with the type of calibration used. The paper scale, or an extension thereof, of the hydrometer shall have plainly printed on it the name of the manufacturer, a serial number, and the definition of the scale.

Nore.-In the case of A. P. I. hydrometers the scale is defined by the expression:

$$
\text { Degrees A. P. I. }=\frac{141.5}{\text { sp. gr. } 60^{\circ} / 60^{\circ} \mathrm{F} .}-131.5
$$

In the case of specific gravity hydrometers the inscription "sp. gr. $60^{\circ} / 60^{\circ} \mathrm{F}$., petroleum," is sufficient to denote that the hydrometer is intended to determine specific gravities at $60^{\circ} \mathrm{F}$. relative to water at $\epsilon 0^{\circ} \mathrm{F}$. when the readings are taken at $60^{\circ} \mathrm{F}$.

The long axis of the hydrometer shall assume a vertical position when the instrument is floating freely in the sample. Any solid material, such as shot, paper, etc., used in the weighting of the hydrometer shall be firmly secured in place by the use of a suitable cement, the softening point of which lies well above $195^{\circ} \mathrm{F}$. When mercury is used for weighting, it shall be placed in a small bulb below the main bulb of the hydrometer and completely separated from the bulb by means of a glass partition.

4. (a) Separate thermometer--No. 16. (Sce A. S. T. M. table of thermometers, 500.2, p. 2). (b) Thermometer for thermohydrometer.-The thermometer incorporated in the thermohydrometer type of instrument shall be of the paper-scale type (scale to be placed in either the bulb of stem of the hydrometer) having a range of from $20^{\circ}$ to $130^{\circ} \mathrm{F}$. or $60^{\circ}$ to $220^{\circ} \mathrm{F}$., depending upon the range of the hydrometer. The paper scale shall be graduated in $2^{\circ} \mathrm{F}$. divisions accura te to $\pm 1^{\circ} \mathrm{F}$. (note) and shall be printed in red ink to avoid any possibliity of its being confused with the black gravity scale.

Note.-This approximates the limit of accuracy which it is possible to obtain in this type of thermometer. It becomes at once obvious, therefore, in view of the following facts, that a high degree of accuracy in the detormination of the gravity of an oil can not be expected when this type of thermometer is used for the determination expected when this type of thermometer is used for

Between $10^{\circ}$ and $11^{\circ} \mathrm{A}$. P.I., $1^{\circ}$ A.P. I. is equivalent to 0.0070 of a Between $10^{\circ}$ and $11^{\circ} \Lambda$. P. I., $1^{\circ}$ A.P. . is equivalent to 0.0070 of unit of specific gravity. Between $90^{\circ}$ and $91^{\circ}$ A. P. $1 ., 1^{\circ} \mathrm{A}$. P. 1 . is equivalent to only 0.0028 of a unit of spccific gravity. In correcting the observed gravity of an oil of $10^{\circ} \mathrm{A}$. P. I. for temperature, an erro of $\pm 2^{\circ} \mathrm{F}$. iu the determination of the temperature of the oil will introduce maximum error of only $0.1^{\circ} \mathrm{A}$.P. I. (0.0007 sp. gr.) in the gravity. In correcting the observed gravity of an oil of $90^{\circ} \mathbf{A}$. P. I. for temperature, an error of $1^{\circ} \mathrm{F}$. in the determination of the temperature of the oil will introduce a maximum error of $0.3^{\circ} \mathrm{A}$. P. I. $(0.00084 \mathrm{sp}$. gr.) in the gravity. For convenience, the following table is given which shows the errors at each tenth degree A. P. I. from $10^{\circ}$ to $100^{\circ} \mathrm{A}$. P. I which may be introduced into a corrected gravity as the result of an error of $+1^{\circ}$. in the determiution of the observed tomperature:

\begin{tabular}{|c|c|}
\hline $\begin{array}{c}\text { Observed } \\
\text { gravity de- } \\
\text { grees A. P. I. }\end{array}$ & $\begin{array}{c}\text { Resultant } \\
\text { inaximum } \\
\text { error in cor- } \\
\text { rected grav- } \\
\text { ity, degrees } \\
\text { A. P. I. per } \\
\pm 1^{\circ} \text {. . }\end{array}$ \\
\hline $10-11$ & 0.1 \\
$20-21$ & .1 \\
$30-31$ & .1 \\
$40-41$ & .1 \\
$50-51$ & .2 \\
$60-61$ & .2 \\
$70-71$ & .2 \\
$80-81$ & .2 \\
$90-91$ & .3 \\
At 99 & .3 \\
\hline
\end{tabular}

5. IIydrometer cylinder.-The hydrometer cylinder in which the sample for the gravity test is confined shall be made of clear glass and shall be cylindrical in shape. For convenience in pouring it may have a lip on the rim. The inside diameter of the cylinder shall be at least $2.54 \mathrm{~cm}(1.0 \mathrm{inch})$ greater than the outside diameter of the hydrometer used in it. The height of the cylinder shall be such that the length of the column of sample it contains is greater by at least $2.54 \mathrm{~cm}(1.0$ inch) than the portion of the hydrometer which is immersed beneath the surface of the sample after a state of equilibrium has been reached.

\section{PROCEDURE}

6. (a) The determination of the gravity of crude petroleum or its products may be made at any suitable temperature between $0^{\circ}$ and $195^{\circ} \mathrm{F}$. in accordance with the procedure described in section 7. The observed gravity at the observed temperature shall be corrected to the gravity at $60^{\circ} \mathrm{F}$. as described in section 8 .

(b) The gravity of mixtures of petroleum products with other substances shall be determined at a temperature of $60^{\circ} \mathrm{F}$. in accordance with the procedure described in section 7 , since there are no nationally recognized tables giving the coefficients of expansion of such mixtures.

7. The sample to be tested for gravity shall be poured into the clean hydrometer cylinder without splashing, so as to avoid the formation of air bubbles and to reduce to a minimum the evaporation of the lower boiling constituents of the lighter oils. If air bubbles are formed they shall be removed after they 
have collected on the surface, by touching them with a piece of clean blotting paper or filter paper before the hydrometer is placed in the semple. If the sample to be tested is easily volatile both the hydrometer and hydrometer cylinder shall be cooled before use to a temperature below that of the sample.

The cylinder containing the prepared sample shall be placed in a vertical position in a place free from air currents. The hydrometer shall be carefully lowered into the sample to a level two smallest scale divisions below that at which it will fioat and shal then be released. When the hydrometer has come to rest, floating freely away from the walls of the cylinder, the gravity shall be read as the point at which the surface of the sample apparently cuts the hydrometer scale. In the case of samples sufficiently transparent this point shall be determined by placing the eye slightly below the level of the liquid and slowly raising it until the surface of the sample first seen as a distorted ellipse seems to become a straight line cutting the hydrometer scale. In the case of nontransparent samples where this procedure can not be followed, the point on the hydrometer scale to which the sample rises above the main surface of the liquid shall be read with the eye placed slightly above the plane of the surface of the sample. This reading shall then be corrected by subtracting in the case of A. P. I. hydrometers or adding in the case of specific-gravity hydrometers, an amount equal to the height which the sample rises on the hydrometer stem above the main liquid surface. This height will vary for different oils and different hydrometers, and the amount of correction necessary will depend upon the width of the hydrometer scale graduations. The necessary correction factor shall be determined, therefore, for the particular hydrometer in use by observing the height above the main surface of the liquid to which the oil rises on the hydrometer scale when the hydrometer in question is immersed in a transparent oil having a surface tension similar to that of the sample under test.

The temperature of the sample shall be determined from the reading of the separate thermometer placed in the sample or from that of the thermometer contained in the hydrometer when the thermometerhydrometer type of instrument is used. (See note.)

Note.-Precautions sbould, of course, be taken to prevent tbe temperature of the sample from changing appreciably during tbe time necessary to complete tbe test. Tbis may be done when testing nonviscous oils by cboosing a temperature of test only slightly different from the room tcmperature. Wben testing viscous oils which must be heated hefore testing it will be necessary to surround the test cylinder with a batb maintained at a constantdesired temperature during the time of test.

Although it is usually satisfactory to determine the temperature of the sample immediately after reading the hydrometer, it is recommended that in all referee tests this temperature be determined both before and after the bydrometer is read.

\section{CaLCULATION}

8. In the case of crude petrolcum and petroleum products the, determinations made in section 7 are commonly referred to as "observed gravity and observed temperature." These observations shall be corrected to the gravity at $60^{\circ} \mathrm{F}$. by the use of Tables 1 and 3 of the "National Standard Petroleum Oil Tables" as published in National Bureau of Standards Circular No. 154. Table 1 shall be employed if the observed gravity is in degrees A. P. I. and Tablc 3 if expressed as specific gravity.

Interpolations in Table 1 shall be made as follows: When the observed gravity ends in $1,2,3,4$, or 5 tenths of a degree A.P. I., find the degrees A. P. I. at $60^{\circ} \mathrm{F}$. corresponding to the observed gravity, disregarding the tenths of a degree. To this value add tenths of a degree dropped from the obscrved gravity to obtain the corrected value of the gravity in dcgrees A, P. I, at $60^{\circ} \mathrm{F}$. When the observed gravity ends in $6,7,8$, or 9 tenths find the degrees A. P. I. at $60^{\circ} \mathrm{F}$. corresponding to the next larger value of observed gravity in the table. Correct this figure by subtracting a number of tenths equal to the difference between the observed value and the next larger value.

\section{ACCORACY}

9. Duplicate determinations shall agree within $0.1^{\circ}$ A. P. I. or the equivalent thereof in units of specific gravity at the particular gravity being determined when the same hydrometer and thermometer are used in the tests.

Separate laboratories using different hydrometers and thermorneters should obtain duplicate results agreeing within $0.5^{\circ} \mathrm{A}$. P. I. or the equivalent thereof in units of specific gravity at the particular gravity being determined.

\section{APPENDIX}

The following table is primarily an abridgement of Table 5 of the "National Standard Petroleum Oil Tables" as published in the United States Bureau of Standards Circular No. 154, which contains similar data for each $0.1^{\circ}$ A. P. I. from $10^{\circ}$ to $100^{\circ}$ A. P. I., inclusive. The data for gravities from $0^{\circ}$ to $9^{\circ}$ A. P. I., inclusive, have been added in order to extend the use of the A. P. I. gravity scale to petroleum oils having a specific gravity slightly greater than unity.

Table showing specific gravities, pounds per gallon, and gallons per pound at $60^{\circ} \mathrm{F}$., corresponding to degrees A. P. I.

\begin{tabular}{|c|c|c|c|c|c|c|c|}
\hline $\begin{array}{l}\text { Degrees } \\
\text { A. P. I. }\end{array}$ & $\begin{array}{c}\text { Specific } \\
\text { gravity } \\
\text { at } 60^{\circ} \\
/ 60^{\circ} \mathrm{F} \text {. }\end{array}$ & $\begin{array}{l}\text { Pounds } \\
\text { per } \\
\text { gallon } \\
\text { at. } 60^{\circ} \\
\mathrm{F} .1\end{array}$ & $\begin{array}{c}\text { Gallons } \\
\text { per } \\
\text { pound } \\
\text { at } 60^{\circ} \\
\text { F.2 }\end{array}$ & $\begin{array}{l}\text { Degr } \\
\text { A. } P\end{array}$ & $\begin{array}{l}\text { Specific } \\
\text { gravity } \\
\text { at } 60^{\circ} \\
160^{\circ} \mathrm{F} \text {. }\end{array}$ & $\begin{array}{c}\text { Pounds } \\
\text { per } \\
\text { gallon } \\
\text { at } 60^{\circ} \\
\text { F. } 1\end{array}$ & $\begin{array}{l}\text { Gal- } \\
\text { lons } \\
\text { per } \\
\text { pound } \\
\text { at } 60^{\circ} \\
\text { F.1 }\end{array}$ \\
\hline $\begin{array}{l}0 . \\
1 . \\
2 \\
3 \\
4\end{array}$ & $\begin{array}{l}1.0760 \\
1.0679 \\
1.0599 \\
1.0520 \\
1.0443\end{array}$ & $\begin{array}{l}8.962 \\
8.895 \\
8.828 \\
8.762 \\
8.698\end{array}$ & $\begin{array}{r}0.1116 \\
.1124 \\
.1133 \\
.1141 \\
.1150\end{array}$ & $\begin{array}{l}3 \\
3 \\
3\end{array}$ & $\begin{array}{r}0.8498 \\
.8448 \\
.8398 \\
.8348 \\
.8299\end{array}$ & $\begin{array}{l}7.076 \\
7.034 \\
6.993 \\
6.951 \\
6.910\end{array}$ & $\begin{array}{l}0.1413 \\
.1422 \\
.1430 \\
.1439 \\
.1447\end{array}$ \\
\hline $\begin{array}{l}5 \\
6 \\
7 \\
8 \\
9\end{array}$ & $\begin{array}{l}\text { 1. } 0366 \\
1.0291 \\
1.0217 \\
1.0143 \\
1.0071\end{array}$ & $\begin{array}{l}8.634 \\
8.571 \\
8.509 \\
8.448 \\
8.388\end{array}$ & $\begin{array}{l}.1158 \\
.1167 \\
.1175 \\
.1184 \\
.1192\end{array}$ & $\begin{array}{l}41 . \\
42- \\
43 . \\
44 .\end{array}$ & $\begin{array}{l}.8251 \\
.8203 \\
.8155 \\
.8109 \\
.8063\end{array}$ & $\begin{array}{l}6.870 \\
6.830 \\
6.790 \\
6.752 \\
6.713\end{array}$ & $\begin{array}{l}.1456 \\
.1464 \\
.1473 \\
.1481 \\
.1490\end{array}$ \\
\hline $\begin{array}{l}10 \\
11 \\
12 \\
13 \\
14\end{array}$ & $\begin{array}{r}1.0000 \\
.9030 \\
.9861 \\
.9792 \\
.9725\end{array}$ & $\begin{array}{l}8.328 \\
8.270 \\
8.212 \\
8.155 \\
8.099\end{array}$ & $\begin{array}{l}.1201 \\
.1209 \\
.1218 \\
.1226 \\
.1235\end{array}$ & 4 & $\begin{array}{l}.8017 \\
.7972 \\
.7927 \\
.7883 \\
.7839\end{array}$ & $\begin{array}{l}6.675 \\
6.637 \\
6.600 \\
6.563 \\
6.526\end{array}$ & $\begin{array}{l}.1507 \\
.1515 \\
.1524 \\
.1532\end{array}$ \\
\hline $\begin{array}{l}15 \\
16 \\
17 \\
18 \\
19\end{array}$ & $\begin{array}{l}.9659 \\
.9593 \\
.9529 \\
.9465\end{array}$ & $\begin{array}{l}8.044 \\
7.989 \\
7.935 \\
7.882 \\
7.830\end{array}$ & $\begin{array}{l}.1243 \\
.1252 \\
.1260 \\
.1269 \\
.1277\end{array}$ & 5 & $\begin{array}{l}.7796 \\
.7753 \\
.7711 \\
.7669 \\
.7628\end{array}$ & & $\begin{array}{l}.1521 \\
.1549 \\
.1558 \\
.1566 \\
.1575\end{array}$ \\
\hline $\begin{array}{l}20 \\
21 \\
22 \\
23 \\
24\end{array}$ & $\begin{array}{l}.9340 \\
.9279 \\
.9218 \\
.9159 \\
.9100\end{array}$ & $\begin{array}{l}7.778 \\
7.727 \\
7.676 \\
7.627 \\
7.578\end{array}$ & $\begin{array}{l}.1286 \\
.1294 \\
.1303 \\
.1311 \\
.1320\end{array}$ & $\begin{array}{l}56 \\
57 \\
58 \\
59\end{array}$ & $\begin{array}{l}.7587 \\
.7547 \\
.7507 \\
.7467 \\
.7428\end{array}$ & $\begin{array}{l}6.316 \\
6.283 \\
6.249 \\
6.216 \\
6.184\end{array}$ & $\begin{array}{l}.1592 \\
.1600 \\
.1609 \\
.1617\end{array}$ \\
\hline $\begin{array}{l}25 \\
26 \\
27 \\
28\end{array}$ & $\begin{array}{l}.9042 \\
.8984 \\
.8927 \\
.8871 \\
.8816\end{array}$ & $\begin{array}{l}7.529 \\
7.481 \\
7.434 \\
7.387 \\
7.341\end{array}$ & $\begin{array}{l}.1328 \\
.1337 \\
.1345 \\
.1354 \\
.1362\end{array}$ & & $\begin{array}{l}.7389 \\
.7351 \\
.7313 \\
.7275 \\
.7238\end{array}$ & $\begin{array}{l}6.151 \\
6.119 \\
6.087 \\
6.056 \\
6.025\end{array}$ & $\begin{array}{l}.1626 \\
.1634 \\
.1643 \\
.1651 \\
.1660\end{array}$ \\
\hline 3 & $\begin{array}{l}.8762 \\
.8708 \\
.8654 \\
.8602 \\
.8550\end{array}$ & $\begin{array}{l}7.296 \\
7.251 \\
7.206 \\
7.163 \\
7.119\end{array}$ & $\begin{array}{l}.1371 \\
.1379 \\
.1388 \\
.1396 \\
.1405\end{array}$ & $\begin{array}{l}66 \\
67 \\
68 \\
69\end{array}$ & $\begin{array}{l}.7201 \\
.7165 \\
.7128 \\
.7093 \\
.7057\end{array}$ & $\begin{array}{l}5.994 \\
5.964 \\
5.934 \\
5.904 \\
5.874\end{array}$ & $\begin{array}{l}.1668 \\
.1677 \\
.1685 \\
.1694 \\
.1702\end{array}$ \\
\hline
\end{tabular}

1 The data in this column are based apon apparent weigbts in air tbe weight of 1 gallon of water at $60^{\circ} \mathrm{F}$. in air heing assumed to be 8.32828 pounds. Consequently, a correction for the buoyaney of air is required in computing these figures from the specific gravities in the second column. 
Table showing specific gravities, pounds per gallon, and gallons per pound at $60^{\circ} \mathrm{F}$., corresponding to degrees A. P. I.-Continued

\begin{tabular}{|c|c|c|c|c|c|c|c|}
\hline $\begin{array}{c}\text { Degrees } \\
\text { A.P.I. }\end{array}$ & $\begin{array}{c}\text { Specific } \\
\text { gravity } \\
\text { 8t } 60^{\circ} \\
160^{\circ} \mathrm{F} .\end{array}$ & $\begin{array}{c}\text { Pounds } \\
\text { per } \\
\text { gallon } \\
\text { at } 60^{\circ} \\
\text { F.1 }\end{array}$ & $\begin{array}{c}\text { Gallons } \\
\text { per } \\
\text { pound } \\
\text { at } 69^{\circ} \\
\text { F.1 }\end{array}$ & $\begin{array}{l}\text { Degree } \\
\text { A. P. I. }\end{array}$ & $\begin{array}{c}\text { Specific } \\
\text { gravity } \\
\text { at } 60^{\circ} \\
160 \mathrm{~F}\end{array}$ & $\begin{array}{c}\text { Pounds } \\
\text { per } \\
\text { gallon } \\
\text { at } 60^{\circ} \\
\mathrm{F} .1\end{array}$ & $\begin{array}{l}\text { Gal- } \\
\text { lons } \\
\text { per } \\
\text { pound } \\
\text { at } 60^{\circ} \\
\text { F.1 }\end{array}$ \\
\hline $\begin{array}{l}70 \ldots \\
71 \ldots \\
72 \ldots \\
73- \\
74 \\
7\end{array}$ & $\begin{array}{r}0.7022 \\
.6988 \\
.6953 \\
.6919 \\
.6886\end{array}$ & $\begin{array}{l}\text { 5. } 845 \\
5.817 \\
5.788 \\
5.759 \\
5.731\end{array}$ & $\begin{array}{r}0.1711 \\
: 1719 \\
: 1728 \\
.1736 \\
.1745\end{array}$ & $\begin{array}{l}85-- \\
86-- \\
87- \\
88 .- \\
89-\end{array}$ & $\begin{array}{r}0.6536 \\
.6506 \\
.6476 \\
.6446 \\
.6417\end{array}$ & $\begin{array}{l}5.440 \\
5.415 \\
5.390 \\
5.365 \\
5.341\end{array}$ & $\begin{array}{r}0.1838 \\
.1847 \\
.1855 \\
.1864 \\
.1872\end{array}$ \\
\hline $\begin{array}{l}75 \\
76 \\
77 \\
78 \\
79 \\
79\end{array}$ & $\begin{array}{l}.6852 \\
.6819 \\
.6787 \\
.6754 \\
.6722\end{array}$ & $\begin{array}{l}5.703 \\
5.676 \\
5.649 \\
5.622 \\
5.595\end{array}$ & $\begin{array}{l}.1753 \\
.1762 \\
.1770 \\
.1779 \\
.1787\end{array}$ & $\begin{array}{l}90 \ldots \ldots \\
91 \ldots \ldots \\
92 \ldots- \\
93 \ldots \\
94 \ldots\end{array}$ & $\begin{array}{l}.6388 \\
.6360 \\
.6331 \\
.6303 \\
.6275\end{array}$ & $\begin{array}{l}5.316 \\
5.293 \\
5.269 \\
5.246 \\
5.222\end{array}$ & $\begin{array}{l}.1881 \\
.1889 \\
.1898 \\
.1906 \\
.1915\end{array}$ \\
\hline $\begin{array}{l}80 . \\
81 . \\
82 \\
83 . \\
81 .\end{array}$ & $\begin{array}{r}.6690 \\
.6659 \\
.6628 \\
.6597 \\
.6566\end{array}$ & $\begin{array}{l}5.568 \\
5.542 \\
5.516 \\
5.491 \\
5.465\end{array}$ & $\begin{array}{l}.1796 \\
.1804 \\
.1813 \\
.1821 \\
.1830\end{array}$ & $\begin{array}{l}95 \\
96-- \\
97-- \\
98= \\
99 .- \\
100 .\end{array}$ & $\begin{array}{l}.6247 \\
.6220 \\
.6193 \\
.6166 \\
.6139 \\
.6112\end{array}$ & $\begin{array}{l}5.199 \\
5.176 \\
5.154 \\
5.131 \\
5.109 \\
5.086\end{array}$ & $\begin{array}{l}.1924 \\
.1932 \\
.1940 \\
.1949 \\
.1957 \\
.1966\end{array}$ \\
\hline
\end{tabular}

See footnote 1 on p. 34

\subsection{CRUDE PETROLEUII}

American Society for Testing Materials, tentative method of test for distillation of crude petroleum, serial designation D285-29T; 1929.

1. This method is intended for determinirg the percentages and distillation range of the naphtha in any crude oil of the class commercially known as refinable crudes. This method does not attempt to specify what quality of product shall be defined as naphtha nor can it be expected to duplicate the results of commercial refining operations. It defines apparatus and procedure, leaving selection of numerical limits and interpretation of results to be agreed upon by the interested parties.

\section{APPARATUS}

2. Flask.-The distillation fiask shall be a $300 \mathrm{cc}$ Hempel distilling flask (fig. 14), the dimensions and allowable tolerances being as follows:

\begin{tabular}{|c|c|c|c|c|}
\hline & \multicolumn{4}{|c|}{ Tolerances } \\
\hline & $\begin{array}{l}\text { Centi- } \\
\text { meters }\end{array}$ & Inches & $\begin{array}{l}\text { Centi- } \\
\text { meters }\end{array}$ & Inches \\
\hline $\begin{array}{l}\text { Diameter of bulb, outside } \\
\text { Diameter of neck, outside. } \\
\text { Length of neck } \\
\text { Distance, top of neck to center of } \\
\text { vapos tube } \\
\text { Length of vapor tube } \\
\text { Diameter of vapor tube, outside.- }\end{array}$ & $\begin{array}{r}10.2 \\
2.9 \\
25.4 \\
6.4 \\
17.2 \\
.8\end{array}$ & $\begin{array}{l}\text { 4. } 0 \\
1.125 \\
10.0 \\
\\
2.5 \\
6.75 \\
.3125\end{array}$ & $\begin{array}{l} \pm 0.2 \\
\pm .1 \\
\pm .4 \\
\pm .3 \\
\pm .5 \\
\pm .1\end{array}$ & $\begin{array}{l} \pm 0.08 \\
\pm .04 \\
\pm .16 \\
\pm .12 \\
\pm .20 \\
\pm .04\end{array}$ \\
\hline
\end{tabular}

The vapor tube shall be set at an angle of $75^{\circ}$ with the neck of the flask, subject to a permissible variation of $\pm 5^{\circ}$.

3. Fractionating column.-The fractionating column (see (a), fig. 12) shall be made of a length of No. 18 iron jack chain, enough to pack under its own weight into a column $2.54 \mathrm{~cm}$ (1 inch) in diameter and $17.51 \mathrm{~cm}$ (61/2 inches) in length. It may conveniently be "strung" on a loop of wire so that it hangs in loops about 2 feet in length, which makes the chain more convenient to handle than when it is not looped together. The device used for supporting the column (see (b), fig. 14) shall be made of a suitable length of wire, preferably, though not necessarily of nickel-chromium, about No. 18 gage. One end shall be wound in a spiral a little less than 1 inch in diameter, and the remaining wire shall be bent at right angles to the plane of the spiral and cut off at a length of about $91 / 2$ inches. A small loop shall be bent into the end away from the spiral and put through a hole drilled in a strip of spring steel (see (c), fig. 12) or other suitable material about 0.015 inch in thickness, $3 / 8$ inch in width, and 3 inches in length, bent around a cylinder 1 inch in diameter. When allowed to expand this spring strip should hold firmly on the inside of the neck of the flask and provide a secure support for the column of chain.

4. Thermometer. - The A. S. T. M. low-distillation thermometer, conforming to the requirements

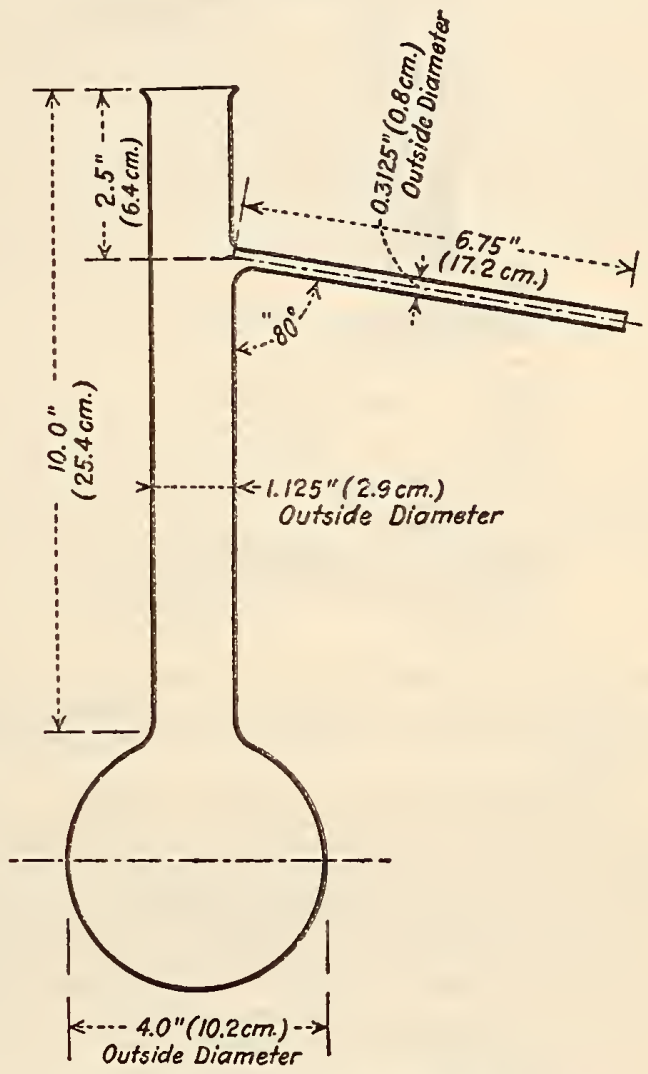

Figure 14.-Dimensions of a $300 \mathrm{cc} \mathrm{Hempel}$ flask

specified for thermometer No. 6 , table of A. S. T.M. thermometers (see 500.2, p. 2) shall be used.

5. Condenser. - The condenser shall consist of a $9 / 16$-inch $(14.29 \mathrm{~mm})$ OD No. 20 Stubbs gage seamless brass tube, 22 inches $(55.88 \mathrm{~cm})$ in length. It shall be set at an angle of $75^{\circ}$ from the perpendicular and surrounded with a cooling bath 15 inches $(38.1 \mathrm{~cm})$ in length, approximately 4 inches $(10.16$ cm) in width, by 6 inches $(15.24 \mathrm{~cm})$ in height. The lower end of the condenser tube shall be cut off at an acute angle, and curved downward for a length of 3 inches $(7.62 \mathrm{~cm})$ and slightly backward to insure contact with the wall of the graduate 1 to $1 \frac{1}{4}$ inches $(2.54$ to $3.18 \mathrm{~cm})$ below the top of the graduate when it is in position to receive the distillate.

6. Shield and support.- The shield shall be of any convenient type having a minimum horizontal dimension of 6 inches. The flask shall rest on a board of one-fourth inch $(0,64 \mathrm{~cm})$ transite or hard 
asbestos, in the center of which is cut a hole $3 \frac{1}{2}$ inches $(8.9 \mathrm{~cm})$ in diameter. The board shall not be less than 6 inches $(15.24 \mathrm{~cm})$ in width and shall be supported by an ordinary ring stand and ring.

7. Gas burner or electric healer.-(a) Gas burner.The burner shall be so constructed that sufficient heat can be obtained to distill the product at the uniform rate specified below. The flame should never be so large that it spreads over a circle of a diameter

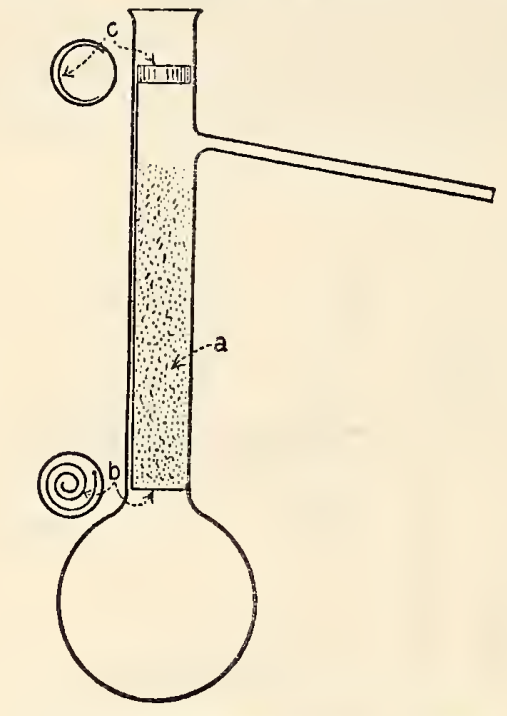

FIgURE 15.-Fractionating column and supporting device in a Hempel flask

greater than 5 inches $(12.7 \mathrm{~cm})$ on the undersurface of the asbestos board. A sensitive regulating valve is a necessary adjunct, as it gives complete control of heating.

(b) Electric heater.-The electric heater, which may be used in place of the gas flame, shall be capable of maintaining the distillation at the rate specified in section 9 (i). The electric heater shall be fitted with an asbestos board top one-eighth to one-fourth inch (3.18 to $6.35 \mathrm{~mm}$ ) in thickness having a hole $3 \frac{1}{2}$ inches $(8.9 \mathrm{~cm})$ in diameter in the center. When an electric heater is employed the portion of the shield above the asbestos board shall be the same as with the gas burner, but the part below the board may be omitted.

8. Measuring graduates and pipettes.-Filling charges and distillation fractions shall be measured with suitable accurate graduated cylinders or other volumetric glassware. A special $300-c c$ pipette, (fig. 16), is a convenient device for measuring charges, but an accurate graduate may be used if desired. The regular 100-ce graduate used in gasoline distillations as specified in section 7 of A. S. T. M. D86 (see 503.0, p. 44) is reasonably satisfactory for measuring distillation fractions, but a graduate of similar dimensions that can be tightly stoppered is preferable. One can be made by cutting down a standard graduate to remove the lip and then fire polishing.

\section{PROCEDURE}

9. (a) The condenser bath shall be filled with cracked ice and sodium chloride or calcium chloride to form a freezing mixture that will cover the condenser tube and maintain a temperature between $0^{\circ}$ and $10^{\circ} \mathrm{F}$. (b) The condenser tube shall be swabbed or cleaned inside to remove any liquid remaining from a previous test.

(c) The specific gravity of the oil to be tested shall first be determined in accordance with A. S. T. M. D287-28T. (See 502.0, p. 32.)

(d) A 300 cc sample of the oil ${ }^{15}$ shall be measured into the Hempel flask by any suitable means. For less viscous oils a pipette or graduate may be used. If the oil is decidedly viscous a sample equivalent to $300 \mathrm{ce}$ at $60^{\circ} \mathrm{F}$. shall be weighed into the flask. None of the liquid shall be permitted to flow into the vapor tube.

The weight in grams of the $300-c c$ sample shall then be calculated. (The weight in grams of $300 \mathrm{cc}$ of oil is equivalent to three hundred times its specific gravity determined in accordance with paragraph (c).)

(e) The supporting device for the fractionating column (fig. 15) shall be put in place and the proper quantity of iron chain dropped in carefully so that it fills the space uniformly and without channels. Tapping the flask while the iron chain is being added is helpful, but the column shall not be compressed after all the chain is in place.

(f) The thermometer, provided with a cork, shall be fitted tightly into the flask, so that it will be in the middle of the neck and so that the lower end of the capillary tube is about one-sixteenth inch below the level of the inside of the bottom of the vapor tube at its junction with the neck of the flask.

(g) The charged flask shall be placed in position on the hard asbestos board used with the gas burner or the electric heater and connected to the condenser with a carefully fitted cork through which the vapor tube passes. The position of the flask shall be adjusted so that the vapor tube extends into the condenser tube not less than 1 inch $(2.54 \mathrm{~cm})$ nor more than 2 inches $(5.08 \mathrm{~cm})$.

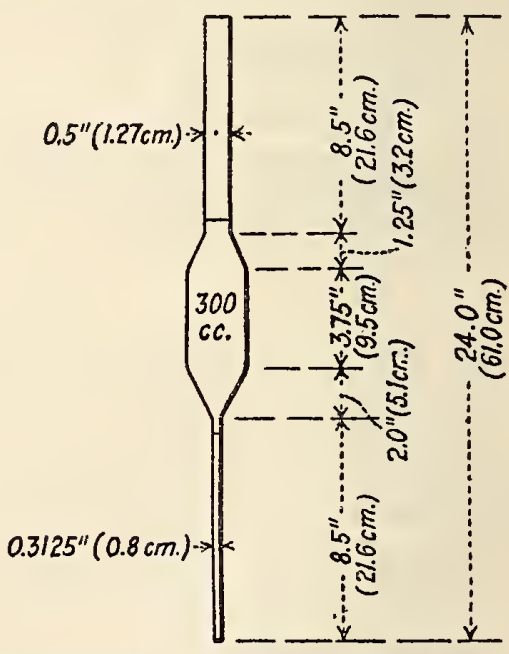

Figure 16.-Measuring pipetie

(h) A clean, dry, graduated cylinder shall be placed at the outlet of the condenser tube in such a position that the condenser tube shall extend at least 1 inch into the graduate. The graduate shall be immersed up to the level of about 1 inch below the top of the condenser tube in a transparent container of water maintained at a temperature of from $32^{\circ}$ to $40^{\circ} \mathrm{F}$. $\left(0^{\circ}\right.$ to $4.4^{\circ} \mathrm{C}$. $)$. During the distillation the

15 In case water in the crude oil causes bumping the sample shall be dehydrated by some suitable method, without loss of naphtha, before making the determination. 
top of the graduate shall be covered closely with a piece of blotting paper or its equivalent, cut to fit the condenser tube tightly.

(i) When everthing is in readiness, heat shall be applied. The fractionating column causes some little delay between the time when the liquid starts to boil and the time the vapor enters the condenser. It is, therefore, permissible to apply heat vigorously until the liquid begins to boil, then to slow down the application of heat so that the distillate begins to come over at a moderate rate. For the first 5 to $10 \mathrm{cc}$ distilled the rate shall be from 2 to 3 ce per minute; thereafter the rate of distillation shall be increased to from 4 to $5 \mathrm{cc}$ per minute.

(j) When the thermometer reads ..... ${ }^{16}$ the graduate shall be withdrawn from beneath the condenser and the distillation discontinued. The graduate shall be tightly stoppered and allowed to stand until all sediment and moisture have settled out and until its contents have reached a temperature between $55^{\circ}$ and $65^{\circ} \mathrm{F}$. The total volume in the graduate shall then be read and recorded as $D$. The volume of water, if any, shall be read and recorded as $W$.

The percentage of naphtha in the crude oil shall be calculated from the following formula:

$$
\text { Percentage of naphtha }=\frac{D-W}{300-W} \times 100
$$

The whole procedure shall be repeated enough times to yield a total volume of distillate of not less than $100 \mathrm{cc}$.

10. Naphtha distillation.-The distillates shall be poured together (taking care to avoid losses by evaporation and rejecting the layers of water, if present) and shall be mixed throughly by shaking. A distillation test of the combined distillates shall be made in accordance with A. S. T. M. D86 (see 503.0, p. 44), except that the initial boiling point shall be recorded and the volume of distillate collected in the cylinder shall be observed and recorded to the nearest $0.5 \mathrm{cc}$ when the mercury of the thermometer reaches $212^{\circ} \mathrm{F}$. $\left(100^{\circ} \mathrm{C}\right.$. $), 221^{\circ} \mathrm{F}$. $\left(105^{\circ} \mathrm{C}\right.$. $), 284^{\circ} \mathrm{F}$. $\left(140^{\circ}\right.$ C. $), 392^{\circ}$ F. $\left(200^{\circ}\right.$ C. $)$ and the end point.

\section{ACCURACY}

11. Results of duplicate tests in the crude-oil distiliation should not differ from each other by more than 0.5 per cent. In the naphtha distillation duplicate results obtained for initial boiling point and maximum temperature, respectivcly, shall not differ from each other by more than $6^{\circ} \mathrm{F}$. $\left(3.33^{\circ} \mathrm{C}\right.$.). Duplicate readings of the volume of distillate collected in the cylinder when each of the prescribed temperature points is reached should not differ by more than 2 cc.

\section{CORRECTION FOR BAROMETRIC PRESSURE}

12. The actual barometric pressure shall be ascertained and recorded, but no correction shall be made except in case of dispute, when the temperature points shall be corrected to $760 \mathrm{~mm}$ (29.92 inches) by the use of the Sydney Young equation, as follows:

For centigrade readings:

For Fahrenheit $\begin{aligned} C_{c}=0 \\ =0\end{aligned}$

$$
0.00012(760-P)\left(273+t_{c}\right)
$$$$
C_{f}=0.00012(760-P)\left(460+t_{f}\right)
$$

in which $C_{c}$ and $C_{f}$ are, respectively, corrections to be added to the observed temperaturc $t_{c}$ or $t_{f}$ and $P$ is the barometric pressure in millimeters of mercury.

New York Produce Exchange, rules regulating the petroleum trade, 1920.

16 The temperature at which the distillation shall be stopped can best he decided hy mutual agreement between the parties concerned in the evaluation of a given crude petroleum.

\section{CRUDE PETROLEUM}

Crude petroleum shall be understood to be pure natural mincral oil, neither steamed nor treated, free from water, sediment, or any adulteration.

When crude petroleum is sold in bulk, the quantity shall be ascertained by tank measurement, at the time of delivery.

Crude petroleum in bulk, barrels, and cases, shall be sold per measured gallon at $60^{\circ} \mathrm{F}$. It may, however, be sold by weight if specially agreed upon by scller and buyer.

In the absence of any stipulation, crude petroleum, when sold in barrels, shall be understood to mean, so far as regards packages, such packages as were originally refined petroleum barrels, whose last contents was crude petroleum, refined petroleum, or naphtha.

When contracts for crude petroleum call for second-hand refined petroleum barrels (that is, barrels whose last contents have been refined petroleum or naphtha) the seller shall have the privilege of substituting new barrels, but they shall be glued.

The measuring and verification of crude petroleum shall be governed by the rules applicable thereto under the head of refined petroleum. (See 502.2 , p. 43.)

\subsection{PETROLEUM PRODUCTS. (See also}

\section{3.)}

American Petroleum Institute, methods of testing petroleum produets.

(The above association, on February 12, 1929, formally approved as standard 21 American Society for Testing Materials methods of testing petroleum products. The approved standards, with their classifications and page numbers as placed within

\begin{tabular}{|c|c|c|c|}
\hline $\begin{array}{l}\text { A. S. T. M. } \\
\text { serial No. }\end{array}$ & Title & $\begin{array}{l}\text { Com- } \\
\text { mod- } \\
\text { ity } \\
\text { classif- } \\
\text { cation } \\
\text { No. }\end{array}$ & Page \\
\hline $\mathrm{D} 206-25$ & Abridged volume correction table for & 503.0 & 56 \\
\hline D12 & Analysis of grease. & 504.0 & 93 \\
\hline & Test for burning quality of kerosene oils & 503. 5 & 85 \\
\hline D21 & $\begin{array}{l}\text { Test for burning quality of long time } \\
\text { burning oil for railway use. }\end{array}$ & 503.7 & 89 \\
\hline D239-27 & Test for burning quality of mineral seal & 503.6 & 87 \\
\hline D $189-2$ & Test for carbon residue in petroleum & 504.0 & 100 \\
\hline D97-28. & $\begin{array}{l}\text { products (Conradson carhon residue). } \\
\text { Test for cloud and pour points of petro- }\end{array}$ & 502.2 & 40 \\
\hline D216 & $\begin{array}{l}\text { leum products. } \\
\text { Test for distillation of natural-gas gaso- }\end{array}$ & 503.3 & 72 \\
\hline D 86 & $\begin{array}{l}\text { line. } \\
\text { Test for distillation of gasoline, naph- } \\
\text { tha, kerosene, and similar petroleum } \\
\text { products. }\end{array}$ & 503.0 & 44 \\
\hline D $56-2$ & $\begin{array}{l}\text { Test for flash point of volatile flamma- } \\
\text { ble liquids. }\end{array}$ & 503.0 & 43 \\
\hline D93- & Test for flash point by means of the & 503.0 & 49 \\
\hline $\mathrm{D} 92$ & Test for flash and fire points hy means & 504.0 & 90 \\
\hline D158-28. & $\begin{array}{l}\text { Testing gas oils (gravity, distillation, } \\
\text { sulphur, carbon residue, pour point, } \\
\text { viscosity, water). }\end{array}$ & 503.0 & 54 \\
\hline D87 & Test for melting point of paraffin wax & 504.54 & 110 \\
\hline & Test for saponification number & 504.0 & 92 \\
\hline D1s & $\begin{array}{l}\text { Test for steam emulsion of lubricating } \\
\text { oils. }\end{array}$ & 504.0 & 98 \\
\hline D $129-2$ & $\begin{array}{l}\text { Test for sulphur in petroleum oils heav- } \\
\text { ier than illuminating oil. }\end{array}$ & 503.0 & 53 \\
\hline $\mathrm{D} 2$ & Test for thermal value of fuel oil .. & 503.0 & 58 \\
\hline & $\begin{array}{l}\text { Test for viscosity of petroleum products } \\
\text { and lubricants. }\end{array}$ & 503.0 & 47 \\
\hline D95- & Test for water in petroleum products & 502.2 & 38 \\
\hline D96-28. & $\begin{array}{l}\text { Test for water and sediment in petro- } \\
\text { leum products by means of centrifuge }\end{array}$ & 502.2 & 39 \\
\hline
\end{tabular}
this volume, follow.) 
Federal Specifications Board, specification No. 310, United States Government master specifications for petroleum ether, July 6, 1925 .

\section{GRADE}

Petroleum ether shall be of one grade only as hereinafter described.

\section{GENERAL REQUIREMENTS}

Petroleum ether shall be composed solely of petroleum distillates distilling between $30^{\circ}$ and $80^{\circ} \mathrm{C}$.

\section{DETAIL REQOIREMENTS}

Failure to meet any of the following requirements will be cause for rejection.

1. Color.-The color shall not be darker than No. 25 Saybolt. Federal Specifications Board specification No. 2d, test method No. 1. (See 503.0, p. 66, Bureau of Mines Technical Paper 323B.)

2. Sediment.-None.

3. Boiling point range.-It shall distill completely between $30^{\circ}$ and $80^{\circ} \mathrm{C}$. Not less than 90 per cent of the petroleum ether taken for distillation shall be collected from a condenser as directed.

4. Limit of residue.-The residue remaining from the evaporation of $50 \mathrm{cc}$ at the temperature of boiling water shall not exceed $0.0005 \mathrm{~g}$.

5. Spot test.-Negative.

6. Acidity.-Negative.

7. Benzene hydrocarbons.-None.

\section{METHOD OF INSPECTION AND TEST}

1. Sampling.-(a) A 500 cc sample shall be taken from each drum or tank. If delivered in bottles, a single bottle taken at random shall constitute a sample.

2. Methods of testing.-(a) Distillation.-The distillation shall be performed as directed in A. S. T. M. D86. (See 503.0, p. 44.)

(b) Spot test.-Ten cubic centimeters evaporated at room temperature from a piece of clean filter paper shall leave no greasy stain after 30 minutes, and the odor of the residue shall not be disagreeable nor sulphureted.

(c) Acidity.-This test shall be made as directed in Federal Specifications Board specification No. 2d, test method No. 30. (See 503.0, p. 66.)

(d) Benzene hydrocarbons. - The test shall be made according to the requirements of the United States Pharmacopoia that is official at the time of examining the sample.

\section{ADDITIONAI INFORMATION}

Basis of purchase.-The material will be purchased by convenient units of volume.

American Society for Testing Materials, standard method of test for water in petroleum products and other bituminous materials, serial designation D95-28; 1928.

Approved as American Standard by the American Standards Association.

1. This method of test determines water existing in a sample of bituminous material by distilling the sample with a violatile solvent. This method is suitable for a variety of materials, but is especially applicable to petroleum, fuel oil, road oil, coal tar, water-gas tar, coke-oven tar, and other petroleum products or bituminous materials.

\section{APPARATUS}

2. The apparatus shall consist of a metal still or glass flask, heated by suitable means and provided with a reflux condenser discharging into a trap con- nected to the still or flask. The trap serves to collect and measure the condensed water and to return the solvent to the still.

The type of distilling apparatus used is not an essential feature of this method, but glass has been generally used for petroleum products and the metal still for road materials and tars.

3. (a) The metal still (fig. $17(a)$ ) shall be a vertical cylindrical vessel, preferably of copper, having a faced flange at the top to which the head is tightly attached by means of a clamp. The head shall be of metal, preferably of brass or copper, and be provided with a tubulation 1 inch inside diameter.

(b) The glass fiask (fig. $17(b)$ ) shall be of the short neck, round-bottom type, made of well-annealed glass, having an approximate capacity of $500 \mathrm{cc}$.

4. The burner used with the metal still shall be a ring gas burner 4 inches $(100 \mathrm{~mm})$ in inside diameter. With the glass flask, an ordinary gas burner or electric heater may be used as the source of heat.

5. The condenser shall be of the water-cooled, reflux, glass-tube type, having a condenser jacket not less than $400 \mathrm{~mm}$ ( $15 \%$ inches) in length with an inner tube 9.5 to $12.7 \mathrm{~mm}$ (three-eighths to onehalf inch) in outside diameter. The end of the condenser to be inserted in the trap shall be ground off at an angle of $60^{\circ}$.

6. The trap shall be made of well-annealed glass constructed in accordance with Figure $17(\mathrm{c})$ and shall be graduated as shown from 0 to $10 \mathrm{cc}$ in $0.1 \mathrm{cc}$ divisions. The error of any indicated capacity shall not be greater than $0.05 \mathrm{cc}$.

The outside diameters should be preferably 2.5 to $3.5 \mathrm{~mm}$ (three thirty-seconds to one-eighth inch) greater than the inside diameters specified.

7. (a) The solvent used when testing petroleum products or bituminous materials derived from petroleum shall be gasoline free from water and shall conform to the following distillation requirements, determined in accordance with A. S. T. M. D86.. (See 503.0, p. 44.)

5 per ccnt shall distill at a temperature not below $194^{\circ} \mathrm{F} .\left(90^{\circ} \mathrm{C}\right.$.) nor above $212^{\circ} \mathrm{F}$. $\left(100^{\circ} \mathrm{C}\right.$.)

90 per cent shall distill bclow $410^{\circ} \mathrm{F} .\left(210^{\circ} \mathrm{C}\right.$.)

(b) The solvent used when testing bituminous materials derived from coal tar, water-gas tar, etc., shall be a coal-tar naphtha or a light oil and shall conform to the following distillation requirements, determined in accordance with A. S. T. M. D86:

98 per cent shall distill between $248^{\circ} \mathrm{F} .\left(120^{\circ} \mathrm{C}\right.$. $)$ and $482^{\circ} \mathrm{F}$. $\left(250^{\circ} \mathrm{C}\right.$.)

\section{SAMPLE}

8. The sample shall be thoroughly representative of the material to be tested, and the portion of the sample used for the test shall be thoroughly representative of the sample itself. Deviation from this requirement shall not be permitted.

Note.-The difficulties in obtaining proper representative samples for this determination are unusually great so that the importance of sampling can not be too strongly emphasizcd.

\section{PROCEDURE}

9. When the sample to be tested contains less than 10 per cent of water, exactly $100 \mathrm{cc}$ of the material to be tested shall be placed into the still or flask and thoroughly mixed with an equal volume of solvent by swirling, proper care being taken to avoid any loss of material. If the material is measured by volume, an accurate $100 \mathrm{cc}$ graduated cylinder shall be used and the contents transferred to the still by rinsing with one $50 \mathrm{cc}$ portion of solvent followed by two successive $25 \mathrm{cc}$ portions of solvent, the cylinder being allowed to drain each time. When the sample to be tested contains more than 10 per cent of water, the volume of material 
used shall be decreased to that which will yield Eomewhat less than $10 \mathrm{cc}$ of water.

Note.-In special cases where the water content exceeds 10 per cent and it is not desirable to reduce the size of the sample to that which will yield somewhat less than $10 \mathrm{cc}$ of water, a distilling tube receiver graduated from 0 to $25 \mathrm{cc}$ may be used. This tuhe shall be graduated from 0 to $2 \mathrm{cc}$ in $0.1 \mathrm{cc}$, from 2 to $5 \mathrm{cc}$ in $0.2 \mathrm{cc}$, and from 5 to $25 \mathrm{cc}$ in $0.5 \mathrm{cc}$.

The connections between the still or flask, trap, and condenser shall be made by means of tightfitting corks as shown in Figure 17 (a) and (b). When the metal still is used, a heavy paper gasket moistened with the solvent shall be inserted between the lid and flange before attaching the clamp. A loose cotton plug shall be inserted in the top of the be the percentage of water and shall be reported as "...., . per cent water by volume, A. S. T. M. method."

11. The accuracy to be expected with this method is that duplicate determinations of water should not differ from each other by more than one division on the trap.

American Society for Testing Materials, standard method of test for water and sediment in petroleum products, by means of centrifuge, serial designation D96-28; 1928. tion.
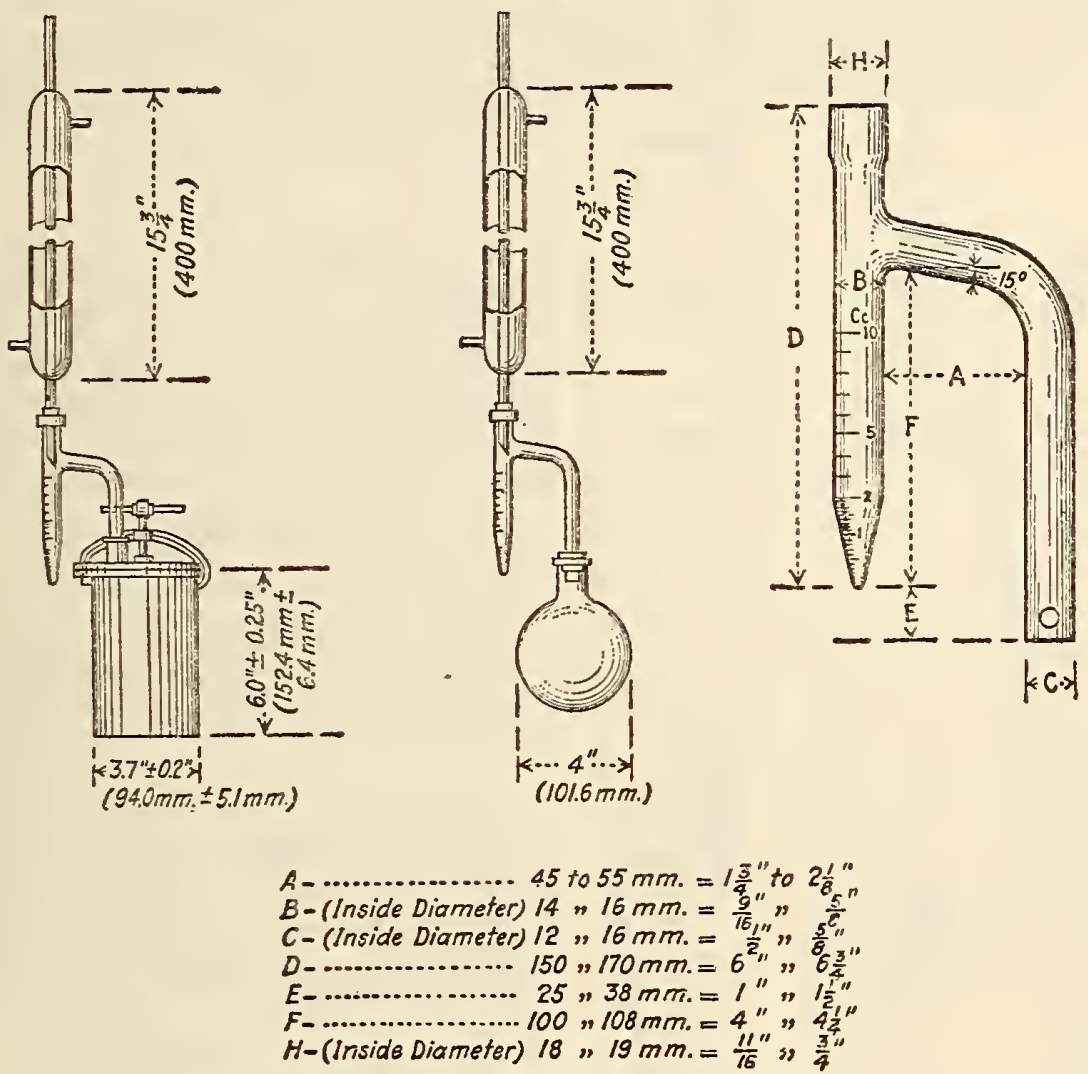

(b)

Figure 17. Metal still, glass flask and trap

condenser tube to prevent condensation of atmospheric moisture in the condenser tube.

Heat shall then be applied and so regulated that the condensed distillate falls from the end of the condenser at the rate of from two to five drops per second. The ring burner used with the metal still should be placed about 3 inches above the bottom of the still at the beginning of the distillation and gradually lowered as the distillation proceeds.

The distillation shall be continued at the specified rate until no water is visible on any part of the apparatus except at the bottom of the trap. This operation usually requires less than an hour. A persistent ring of condensed water in the condenser tube shall be removed by increasing the rate of distillation for a few minutes.

10. The volume of condensed water measured in the trap at room temperature multiplied by 100 and divided by the volume of the sample used shall
1. This method may be used for crude mineral oils and fuel oils. A centrifuge method for "water and sediment" is not entirely satisfactory because the amount of water obtained is nearly always lower than the actual water content. Nevertheless, on account of the wide use of the centrifuge for this purpose, it is desirable that the method of making the determination be standardized as far as possible. It must be clearly understood that the reading of the centrifuge tube includes both the sediment and the precipitated water. Accurate determination of water content, if desired, should be made in accordance with A. S. T. M. D95 (given above).

2. The sample shall be thoroughly representative of the material in question and the portion used for the test shall be thoroughly representative of the sample itself. Deviation from this rule shall not be permitted, The difficulties in obtaining 
proper representative samples for this determination are unusually great, hence the importance of sampling can not be too strongly emphasized.

\section{APPARATUS}

3. The centrifuge shall be capable of whirling at the required speed at least two 100 cc centrifuge tubes filled with water. It shall be of sound design and rugged construction so that it may be operated without danger. The tube carriers shall be so designed that the glass centrifuge tubes may be cushioned with water, rubber, or other suitable material. The tube holders shall be surrounded during the operation by a suitable metal shield or case strong enough to eliminate danger if any breakage occurs.

The preferred form of centrifuge shall have a diameter of swing (tip to tip of whirling tubes) of 15 to 17 inches and a speed of at least 1,500
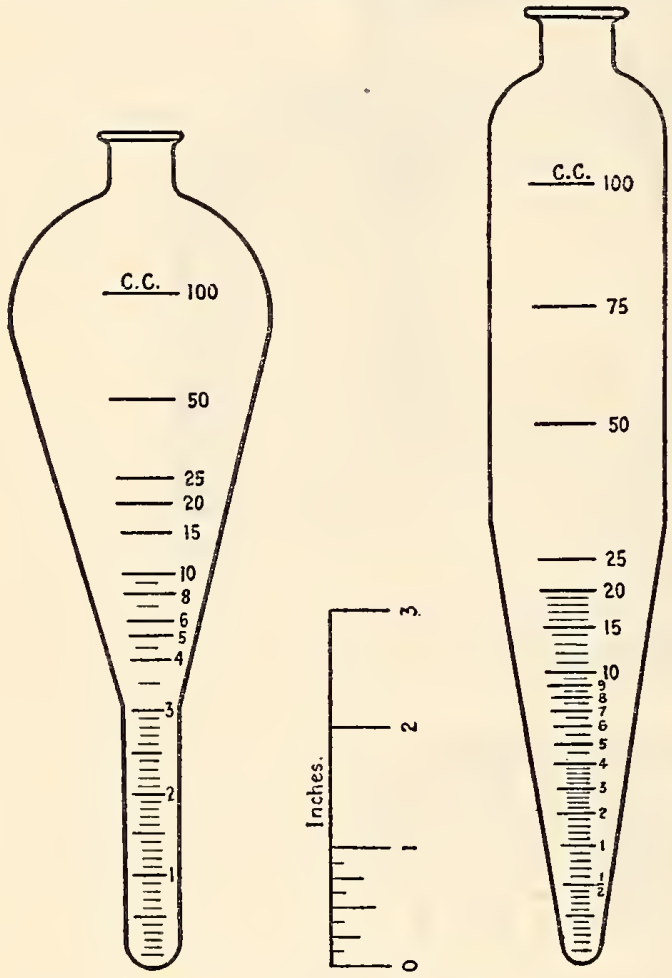

Figure 18.-Centrifuge tubes

r. p. m. or the equivalent. If the available centrifuge has a diameter of swing varying from these limits, it shall be run at the proper speed to give the same centrifugal force at the tips of the tubes as that obtained with the preferred form of centrifuge. The proper speed shall be calculated from the following formula in which $d$ represents diameter of swing (tip to tip of whirling tubes) of the centrifuge used:

$$
\text { R. p. m. }=1,500 \sqrt{\frac{16}{d}}
$$

4. The centrifuge tubes, A. S. T. M. type, shall be made of suitable glass and thoroughly annealed. The total capacity sliall be about $125 \mathrm{ec}$ and the mouth shall be suitably constricted for closing with a cork. The graduations shall be clear and distinct, reading upward from the bottom of the tube. The value of the divisions for each range shall not be greater than shown in the following tabulation:

\begin{tabular}{c|c|c|l|}
\hline Range & $\begin{array}{c}\text { Scale } \\
\text { divisions }\end{array}$ & $\begin{array}{c}\text { Limit of } \\
\text { error }\end{array}$ & Numbered \\
\cline { 1 - 1 }$c c$ & $c c$ & $c c$ & \multicolumn{1}{cc}{$c$} \\
$0-3$ & 0.1 & 0.05 & $1,2,3$ \\
$3-5$ & .5 & .2 & 4,5 \\
$5-10$ & 1.0 & .5 & $6,8,10$ \\
$10-25$ & 5.0 & 1.0 & $15,20,25$ \\
$50-100$ & 50.0 & 1.0 & 50,100 \\
\hline
\end{tabular}

The shape is optional provided it does not conflict with the other requirements. Satisfactory types are shown in Figure 18.

5. The water or oil bath shall be of sufficient depth for immersing the centrifuge tubes in a vertical position to the $100 \mathrm{cc}$ mark. Means shall be provided for heating this bath to $120^{\circ} \mathrm{F}$.

\section{PROCEDURE}

6. (a) Exactly $50 \mathrm{ec}$ of 90 per cent benzol shall be measured into each of two centrifuge tubes and exactly $50 \mathrm{cc}$ of the oil to be tested shall then be added to each. The centrifuge tubes shall be tightly stoppered and shall be shaken vigorously until the contents are thoroughly mixed. The temperature of the bath shall be maintained at $120^{\circ} \mathrm{F}$. and the centrifuge tubes shall be immersed therein to the $100 \mathrm{cc}$ mark for 10 minutes.

(b) The two centrifuge tubes shall then be placed in the centrifuge on opposite sides and shall be whirled at a rate of 1,400 to $1,500 \mathrm{r}$. p. m. or the equivalent for 10 minutes. The combined volume of water and sediment at the bottom of each tube shall be read and recorded, estimating to $0.1 \mathrm{cc}$ if necessary. The centrifuge tubes shall then be replaced in the centrifuge, again whirled for $10 \mathrm{~min}$ utes and removed for reading the volume of water and sediment as before. This operation shall be repeated until the combined volume of water and sediment in each tube remains constant for three consecutive readings. In general, not more than four whirlings will be required.

7. The combined total volume of water and sediment shall be read on each tube, estimating to $0.1 \mathrm{cc}$ if necessary. The sum of the two readings shall be recorded as percentage of water and sediment, centrifuge method.

\section{ACCURACY}

8. With care and proper attention to details, duplicate determinations of water and sediment by this method should not differ by more than $0.2 \mathrm{cc}$ provided the centrifuge tubes are accurate and readable to this degree.

American Society for Testing Materials, standard method of test for cloud and pour points of petroleum products, serial designation D97-28; 1928.

Approved as Tentative American Standard by the American Engineering Standards Committee, now the American Standards Association.

1. (a) The cloud point of a petroleum oil is that temperature at which paraffin wax or other solid substances begin to crystallize out or separate from solution when the oil is chilled under certain definite specified conditions.

(b) The pour point of a petroleum oil is the lowest temperature at which this oil will pour or flow when it is chilled without disturbance under certain definite specified conditions.

2. (a) The test for cloud point shall be used only for oils which are transparent in layers $1 \frac{1}{2}$ inches thick. 
(b) The test for pour point shall be used for all other petroleum oils and may be used for oils on which the test for cloud point is permitted.

\section{APPARATUS \\ (See fig. 19)}

3. The test jar, $a$, shall be of clear glass, cylindrical form, flat bottom, approximately $1 \% / 16$ to $1 \% 16$ inches in inside diameter and $4 \frac{1}{2}$ to 5 inches high. An ordinary 4-ounce oil sample bottle may be used if it is within the above specifications and no test jar is available.

4. The thermometer, $b$, shall conform to the requirements of either thermometer No. 1 or No. 5. (See table of A. S. T. M. thermometers, 500.2 , p. 2.)

5. The cork, $c$, shall fit the test jar, and shall be bored centrally to take the test thermometer.

6. The jacket, $d$, shall be of glass or metal, shall be water-tight, of cylindrical form, flat bottom, about $4 \frac{1}{2}$ inches deep, with inside diameter threeeighths to one-half inch greater than outside diameter of the test jar.

7. A disk of cork or felt, $e$, one-fourth inch thick and of the same diameter as the inside of the jacket will be required.

8. The ring gasket, $f$, shall be about threesixteenths inch thick and made to fit snugly around the outside of the test jar and loosely inside the jacket. This gasket may be made of cork, felt, or other suitable material, elastic enough to cling to the test jar and hard enough to hold its shape. The purpose of the ring gasket is to prevent the test jar from touching the jacket.

9. The cooling bath, $g$, shall be of a type suitable for obtaining the required temperatures. The size and shape of the bath are optional, but a support suitable for holding the jacket firmly in a vertical position is essential. For determination of very low pour points, a smaller insulated cooling bath may be used and the test jar placed directly in it. The required bath temperatures may be maintained by refrigeration if available, otherwise by suitable freezing mixtures.

Note.-The freezing mixtures commonly used are as follows: For temperatures down to $50^{\circ} \mathrm{F}$., ice and water.

For temperatures down to $10^{\circ} \mathrm{F}$., crushed ice and sodium chloride. For temperatures down to $-15^{\circ} \mathrm{F}$., crushed ice and calcium chloride crystals.

For temperatures down to $-70^{\circ} \mathrm{F}$, solid carbon dioxide and acetone or gasoline.

The last-named mixture may be made as follows: In a covered metal beaker chill a suitable amount of acetone or gasoline to $10^{\circ} \mathrm{F}$ metal beaker chill a suitable amount of acetone or gasoline to $10^{\circ} \mathrm{F}$, or lower, by means of an ice-salt mixture. Invert a cylinder of liquiri carbon dioxide and draw off carefully into a chamois skin bag the
desired amount of carbon dioxide, which through rapid evaporation desired amount of carbon dioxide, which through rapid evaporation
will quickly become solid. Then add to the chilled acetone or gasoline enough of the solid carbon dioxide togive the desired temperature.

\section{PROCEDURE}

10. The oil to be tested shall be brought to a temperature at least $25^{\circ} \mathrm{F}$. above the approximate cloud point. Moisture, if present, shall be removed by any suitable method, as by filtration through dry filter paper until the oil is perfectly clear, but such filtration shall be made at a temperature at least $25^{\circ} \mathrm{F}$. above the approximate cloud point.

The clear oil shall be poured into the test jar, $a$, to a height of not less than 2 nor more than $21 / 4$ inches. The test jar may be marked to indicate the proper level.

The test jar shall be tightly closed by the cork, $c$, carrying the test thermometer, $b$, in a vertical position in the center of the jar with the thermometer bulb resting on the bottom of the jar.

The disk, $e$, shall be placed in the bottom of the jacket, $d$, and the test jar with the ring gasket, $f, 1$ inch above the bottom, shall be inserted into the jacket. The disk, jacket and inside of jacket shall be clean and dry.
The temperature of the cooling bath, $g$, shall be adjusted so that it is below the cloud point of the oil by not less than $15^{\circ}$ nor more than $30^{\circ} \mathrm{F}$. and this temperature shall be maintained throughout the test. The jacket, containing the test jar, shall be supported firmly in a vertical position in the cooling bath so that not more than 1 inch of the jacket projects out of the cooling medium.

At each test thermometer reading which is a multiple of $2^{\circ} \mathrm{F}$., the test jar shall be removed from the jacket quickly, but without disturbing the oil, inspected for cloud, and replaced in the jacket.

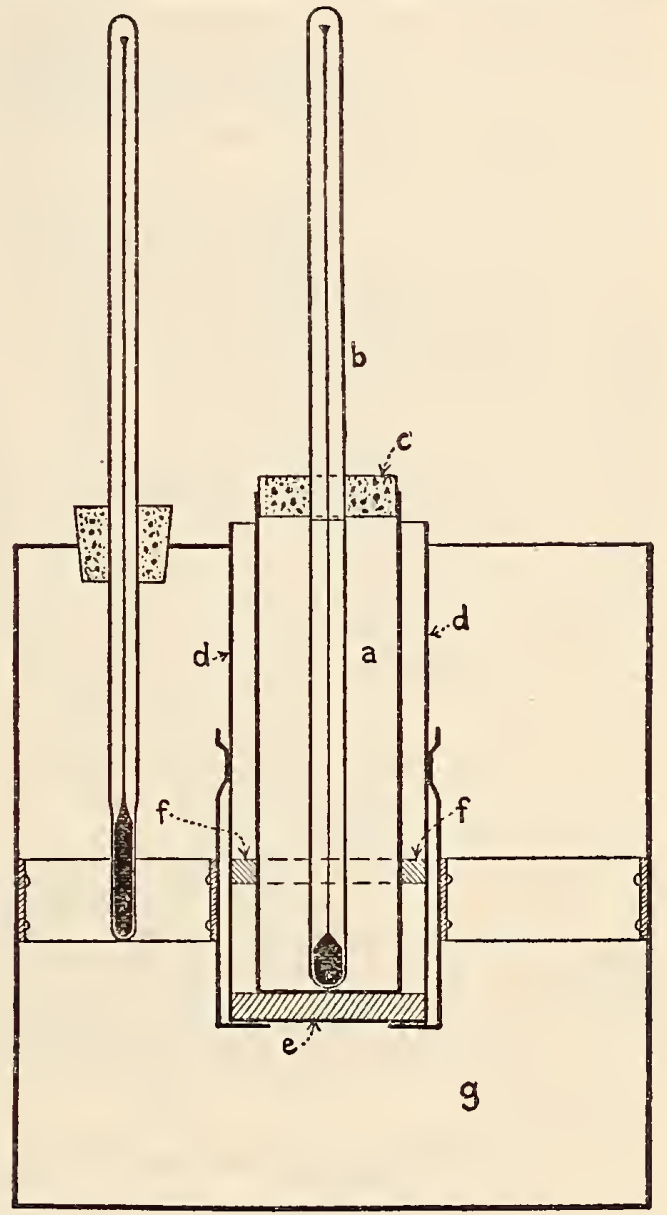

Figure 19.-A pparatus for cloud and pour test (as assembled for cloud test)

This complete operation shall require not more than three seconds.

When such inspection first reveals a distinct cloudiness or haze in the oil at the bottom of the test jar, the reading of the test thermometer, corrected for error if necessary, shall be recorded as the cloud point.

11. The oil shall be poured into the test jar, $a$, to a height of not less than 2 nor more than $2 \frac{1}{4}$ inches. The jar may be marked to indicate the proper level. When necessary, the oil shall be heated in a water bath just sufficiently for pouring into the test jar.

The test jar shall be tightly closed by the cork, $c$, carrying the test thermometer, $b$, in a vertical position in the center of the jar with the thermometer bulb immersed so that the beginning of the capillary shall be one-eighth inch below the surface of the oil. 
Heat without stirring to a temperature of $115^{\circ} \mathrm{F}$. in a bath maintained at not higher than $118^{\circ} \mathrm{F}$. The oil shall then be cooled to $90^{\circ} \mathrm{F}$. in air or in a water bath approximately $77^{\circ} \mathrm{F}$. in temperature. Oils with which the low cloud and pour test thermometer can be used from the beginning of the test shall be cooled to $60^{\circ} \mathrm{F}$. in any convenient manner before the thermometer is placed in position.

The disk, $e$, shall be placed in the bottom of the jacket, $d$, and the test jar, with the ring gasket, $f$, 1 inch above the bottom, shall be inserted into the jacket. The disk, gasket, and inside of jacket shall be clean and dry.

After the oil has cooled enough to allow the formation of paraffin wax crystals, great care shall be taken not to disturb the mass of the oil nor to permit the thermometer to shift in the oil. Any disturbance of the spongy network of wax crystals will lead to low and fictitious results.

The temperature of the cooling bath, $g$, shall be adjusted so that it is below the pour point of the oil by not less than $15^{\circ}$ nor more than $30^{\circ} \mathrm{F}$., and this temperature shall be maintained throughout the test. The jacket, containing the test jar, shall be supported firmly in a vertical position in the cooling bath so that not more than 1 inch of the jacket projects out of the cooling medium.

Beginning at a temperature $20^{\circ} \mathrm{F}$. before the expected pour point, at each test thermometer reading which is a multiple of $5^{\circ} \mathrm{F}$., the test jar shall be removed from the jacket carefully and shall be tilted just sufficiently to ascertain whether there is a movement of the oil in the test jar. The complete operation of removal and replacement shall require not more than three seconds. As soon as the oil in the test jar does not flow when the jar is tilted, the test jar shall be held in a horizontal position for exactly five seconds, as noted by a stop watch or other accurate timing device and observed carefully. If the oil shows any movement under these conditions, the test jar shall be immediately replaced in the jacket and the same procedure repeated at the next temperature reading $5^{\circ} \mathrm{F}$. lower.

The test shall be continued in this manner until a point is reached at which the oil in the test jar shows no movement when the test jar is held in a horizontal position for exactly five seconds. Certain lubricating oils tend to move as a whole and should be very closely observed. The reading of the test thermometer at this temperature, corrected for error if necessary, shall be recorded. The pour point shall be taken as the temperature $5^{\circ} \mathrm{F}$. above this solid point.

\section{SPECTAL PROCEDURE FOR BLACK OULS AND CXLINDER
STOCKS}

12. (a) In those cases where it is known that a sample has been subjected to some temperature higher than $115^{\circ} \mathrm{F}$. during the preceding 24 hours, or where the history of the sample in this respect is not known, the sample shall be held in the laboratory 24 hours before testing, unless three consecutive tests of the same sample in the same test jar check results. For these particular oils this shall be called the upper (maximum) pour point.

(b) The lower (minimum) pour point shall be determined by heating a sample with stirring to $220^{\circ} \mathrm{F}$. The oil shall then be poured in the test jar, cooled to $90^{\circ} \mathrm{F}$. as before, and the pour point determined as described in section 11 .

(c) The upper and lower pour points shall be reported separately.

American Society for Testing Materials, proposed tentative method of test for the determination of autogenous ignition temperatures, serial designation D286-28T; 1928.

\section{APFARATUS}

1. Scope.-This method is applicable to liquid and semiliquid petroleum products.

2. (a) Solder bath.-The solder bath shall consist of a round-bottomed iron pot about $4 \frac{1}{2}$ inches in diameter and $3 \frac{1}{2}$ inches in height, and filled with alloy to within about 1 inch of the top.

Nore.-The alloy should have a melting point below the ignition temperature of the product being tested. For products with relatively low ignition temperature, an alloy of lead, tin, and cadmium is advisable; for medium temperature, a 50 per cent tin-lead alloy; for high temperatures, lead.

(b) Conical flask.-The flask shall be of Pyrex glass in conical form with flat bottom, and shall be about $41 / 4$ inches $(11.4 \mathrm{~cm})$ in height, $23 \% 8$ inches $(6.0 \mathrm{~cm})$ in diameter at the bottom and $11 / 8$ inch $(2.85 \mathrm{~cm})$ in diameter at the top. The capacity for these dimensions is about $160 \mathrm{cc}$, and the ratio of surface area to volume is about 1.1 .

(c) Gas burner.-The gas burner for heating the bath may be of any ordinary form and shall be large enough to be capable of heating the bath to the desired temperature.

(d) Thermocouple or thermometer.-The temperature-measuring device shall be a calibrated thermocouple with a quartz protecting tube. A calibrated, high-temperature, nitrogen-filled thermometer may be substituted for the thermocouple, in which case appropriate corrections shall be applied.

(e) Pipette.--The pipette shall be of small capacity readily adaptable to the delivery of drops.

\section{PROCEDORE}

3. The alloy in the solder bath shall be melted and the flask partially submerged in the molten alloy to a depth equal to two-thirds its height. It should be centered in the bath, and shall not touch the walls of the iron pot at any point. The thermocouple (or thermometer) shall be placed and held firmly by suitable clips in the solder bath in such a position that its end shall be about one-fourth inch from the bottom of the bath and one-fourth inch from the side of the flask. The temperature of the bath shall be brought to and held at a temperature near the probable ignition temperature of the sample under test. Various amounts of the sample $(1,2,3,4$, etc., drops from the pipette) shall be added successively to the flask, at least one minute being allowed to elapse after each addition and the gases and vapors completely displaced after each addition of the sample by the aid of a slow stream of air or a hand bulb with a bent (right angle) delivery tube. If ignition takes place when one of the charges is added, the temperature should be lowered about $5^{\circ} \mathrm{C}$. and the process repeated, starting with the number of drops that ignited in the first instance, and using other volumes if necessary. The operator should proceed, in general, in this manner, raising or lowering the temperature by approximately $5^{\circ} \mathrm{C}$. intervals, according as ignition does not or does not take place, holding each temperature approximately constant during all trials at that temperature, until an indicated temperature is found at which ignition invariably takes place, but at $5^{\circ} \mathrm{C}$. below which ignition fails to take place. The minimum indicated ignition temperature may then be determined by repeated trials between the indicated temperature and a temperature $5^{\circ} \mathrm{C}$. less. Results should be duplicable to within $\pm 2^{\circ} \mathrm{C}$. of the indicated temperature. It is advisable in some of the various trials, especially where the large numbers of drops of the sample are added, to introduce a little additional air into the flask by means of the rubber bulb and bent delivery tube. 


\section{REFINED PETROLEUN}

New York Produce Exchange, rules regulating the petroleum trade, 1920.

Refined petroleum shall be of good quality, having a burning test of $110^{\circ} \mathrm{F}$. or upward, and of a gravity not below $40^{\circ}$ Baumé at $60^{\circ} \mathrm{F}$.

The burning test of refined petroleum shall be determined by the use of the Saybolt electric instrument, or other instrument in general use as agreed upon by buyer and seller, and shall be operated in arriving at a result as follows:

In $110^{\circ}$ and upward, the flashing points, after the first flash (which will generally occur between $90^{\circ}$ and $95^{\circ}$ ) shall be taken at $95^{\circ}, 100^{\circ}, 104^{\circ}, 108^{\circ}$, $110^{\circ}, 112^{\circ}$, and $115^{\circ}$.

In $120^{\circ}$ and upward, after first flash, at $100^{\circ}, 105^{\circ}$, $110^{\circ}, 115^{\circ}, 118^{\circ}, 120^{\circ}, 122^{\circ}$, and $125^{\circ}$.

In $130^{\circ}$ and upward, every $5^{\circ}$ until burning point is reached.

The gravity of refined petroleum shall be determined as per the "Tagliabue" Manual for Inspectors of Petroleum as adopted by the United States Petroleum Association and now in use generally by the petroleum trade.

When refined petroleum is sold in bulk, the quantity shall be ascertained by tank measurement at the time of delivery.

Refined petroleum in barrels shall be delivered in blue, well-painted barrels, with white heads. Barrels shall be well glued and filled within 1 or 2 inches of the bung.

Refined petroleum in bulk, barrels, and cases shall be sold per measured gallon at $60^{\circ} \mathrm{F}$. It may, however, be sold by weight if specially agreed upon by seller and buyer.

Barrels shall be made of well-seasoned timber, and shall be hooped not lighter than as follows: Either with six steel or iron hoops, the head hoop $13 / 4$ inches wide, No. 17 gage U. S. Standard, the quarter hoop $1 \frac{1}{2}$ inches wide, No. 18 gage, and the bilge hoop $13 / 4$ inches wide, No. 17 gage; or with 8 steel or iron hoops, the head hoop $13 / 4$ inches wide, No. 17 gage, the collar hoop $1 \frac{1}{2}$ inches wide No. 18 gage, the quarter hoop $1 \frac{112}{2}$ inches wide No. 18 gage, and the bilge hoop $1 \frac{3}{4}$ inches wide No. 17 gage.

The actual gross weight or gage shall be plainly marked on the barrel. Buyers may check, at their own expense, the correctness of the gross weight or gage of the whole or part of any lot delivered, and the average shortage found on a portion of not less than 10 per cent shall be taken as the average amount to be deducted from the lot.

The tare shall be plainly marked upon each barrel before it is filled. Buyers may check the accuracy of the tare so marked to the extent of 5 per cent of the lot, and the average difference between the tare thus ascertained and the marked tare on the barrels tested shall be accepted as the average difference on the entire lot. Any excess of tare so discovered shall be allowed the buyer.

Naphtha and/or gasoline shall be of good merchantable quality and water white in color.

When naphtha and/or gasoline is sold in bulk, the quantity shall be ascertained by tank measurement at the time of delivery.

\section{FUEL AND ILLUMINATING OILS}

\subsection{GENERAL ITEIMS.}

American Society for Testing Materials, standard method of test for flash point of volatile flammable liquids, serial designation D56-21; 1921.

Approved as Tentative American Standard by

the American Engineering Standards Committee now the American Standards Association.

1. (a) The Tag closed tester shall be used for all volatile flammable liquids flashing below $175^{\circ} \mathrm{F}$. with the exception of products classed as fuel oil. Determination of the flash point of fuel oil by the Tag tester is permissible, but the Pensky Martens tester, as described in A. S. T. M. D93 (see 503.0, p. 49), is to be preferred.

(b) The Tag closed tester shall conform to the following dimensions within the limits of tolerances given:

\begin{tabular}{|c|c|c|}
\hline Dimension & Normal & Tolerance \\
\hline $\begin{array}{l}\text { Depth of water surface below top } \\
\text { of cup (inches). }\end{array}$ & $13 / 32(27.8 \mathrm{~mm})$ & $\pm 1 / 64(0.4 \mathrm{~mm})$ \\
\hline $\begin{array}{l}\text { Depth of oil surface below top of } \\
\text { cup (inches). }\end{array}$ & $15 \% 32(29.4 \mathrm{~mm})$ & $\pm 164(0.4 \mathrm{~mm})$ \\
\hline $\begin{array}{l}\text { Depth of top of bulb of oil ther- } \\
\text { mometer when in place below } \\
\text { top of cup, (inches). }\end{array}$ & $15 / 16(33.3 \mathrm{~mm})$ & $\pm 1 / 32(0.8 \mathrm{~mm})$ \\
\hline $\begin{array}{l}\text { Inside diameter of oil cup at top } \\
\text { (inch). }\end{array}$ & $218(54.0 \mathrm{~mm})_{-}$ & $\pm 0.005(0.1 \mathrm{~mm})$ \\
\hline $\begin{array}{l}\text { Weight of oil cup (grams) } \\
\text { Diameter of bead on top of cover } 1 \\
\text { (inches). }\end{array}$ & $\frac{68}{5 / 32}(4.0 \mathrm{~mm})$ & $\begin{array}{l} \pm 1 . \\
\pm 1 / 64(0.4 \mathrm{~mm})\end{array}$ \\
\hline
\end{tabular}

1 The plane of underside of cover to be between the top and bottom of the burner tip when the latter is fully depressed.

(c) The thermometer shall conform to the requirements of thermometer No. 14. (See A. S. T. M. table of thermometers, 500.2 , p. 2.)

2. (a) If gas is available, connect a $1 / 8$-inch rubber tube to the corrugated gas connection on the oil cup cover. If no gas is available, unserew the test flame burner tip from the oil chamber on the cover and insert a wick of cotton cord in the burner tip and replace it. Put a small quantity of cotton waste in the oil chambor, and insert a small quantity of signal, sperm, or lard oil in the chamber. light the wick and adjust the flame, so that it is exactly the size of the small white bead mounted on the top of the tester.

(b) The test shall be performed in a dim light so as to sec the flash plainly.

(c) Surround the tester on three sides with an inclusure to keep away draughts.

A shield about 18 inches square and 2 feet high, open in front, is satisfactory, but any safe precaution against all possible room draughts is acceptable. Tests made in a lahoratory hood or near ventilators will give unreliable results.

(d) See that the tester sets firm and level.

(e) For accuracy, the flash-point thermometers which are especially designed for the instrument should be used, as the position of the bulb of the thermometer in the oil cup is essential.

3. Put the water-bath thermometer in place and place a receptacle under the overflow spout to catch the overflow. Fill the water bath with water at such a temperature that, when testing is started, the temperature of the water bath will be at least $20^{\circ} \mathrm{F}$. $\left(11^{\circ} \mathrm{C}\right.$.) below the probable flash point of the oil to be tested.

4. Put the oil cup in place in the water bath. Measure $50 \mathrm{cc}$ of the oil to be tested in a pipette or a graduate, and place in the oil cup. The temperature of the oil shall be at least $20^{\circ} \mathrm{F}$. ( $11^{\circ} \mathrm{C}$.) below its probable flash point when testing is started. Destroy any bubbles on the surface of the oil. Put on the cover, with flash-point thermometer in place and gas tube attached. Light the pilot light on the cover and adjust the flame to the size of the small white bead on the cover.

5. Light and place the heating lamp, filled with alcohol, in the base of the tester and see that it is centrally located. Adjust the flame of the alcohol lamp so that the temperature of the oil in the cup rises at the rate of about $1.8^{\circ} \mathrm{F} .\left(1^{\circ} \mathrm{C}\right.$.) per minute, not faster than $2^{\circ} \mathrm{F}$. $\left(1.1^{\circ} \mathrm{C}\right.$.) nor slower than $1.6^{\circ} \mathrm{F}$. $\left(0.9^{\circ} \mathrm{C}\right.$. $)$ per minute.

6. (a) Record the barometric pressure which, in the absence of a laboratory instrument, may be obtained from the nearest Weather Bureau station. 
(b) Record the temperature of the oil sample at start.

(c) When the temperature of the oil reaches $9^{\circ} \mathrm{F}$. $\left(5^{\circ} \mathrm{C}\right.$.) below the probable flash point of the oil, turn the knob on the cover so as to introduce the test flame into the cup, and turn it promptly back again. Do not let it snap back. The time consumed in turning the knob down and back should be about onc full second, or the time required to pronounce distinctly the words "one-thousand-andone."

(d) Record the time of making the first introduction of the test flame.

(e) Record the tempcrature of the oil sample at the time of first test.

(f) Repeat the application of the test flame at every $1^{\circ} \mathrm{F} .\left(0.5^{\circ} \mathrm{C}\right.$.) rise in temperature of the oil until there is a flash of the oil within the cup.

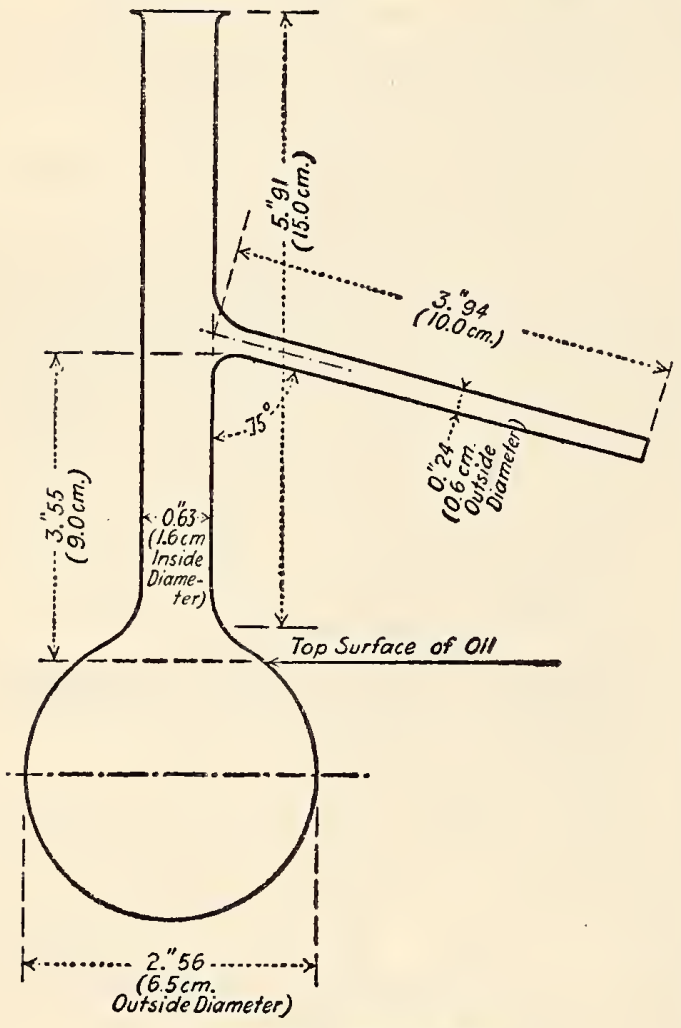

FigURE 20.-Engler flask

Do not be misled by an enlargement of the test flame or halo around it when entered into the cup, or by slight flickering of the flame; the true flash consumes the gas in the top of the cup and causes a very slight explosion.

(g) Record the time at which the flash point is reached.

(h) Record the flash point.

(i) If the rise in temperature of the oil, from the "time of making the first introduction of the test flame" to the "time at which the flash point is reached" was faster than $2^{\circ} \mathrm{F}$. $\left(1.1^{\circ}\right.$ C. $)$ or slower than $1.6^{\circ} \mathrm{F}$. $\left(0.9^{\circ} \mathrm{C}\right.$.) per minute, the test should be questioned, and the alcohol heating lamp adjusted so as to correct the rate of heating. It will be found that the wick of this lamp can be so accurately adjusted as to give a uniform rate of rise in temperature within the above limits and remain so.

7. (a) It is not necessary to turn off the test flame with the small regulating valve on the cover; leave it adjusted to give the proper size of flame. (b) Having completed the preliminary test, remove the heating lamp, lift up the oil cup cover, and wipe off the thermometer bulb. Lift out the oil cup, and empty and carefully wipe it. Throw away all oil samples after once used in making a test.

(c) Pour cold water into the water bath, allowing it to overflow into a receptacle, until the temperature of the water in the bath is lowered to $15^{\circ} \mathrm{F}$. $\left(8^{\circ} \mathrm{C}\right.$.) below the flash point of the oil, as shown by the previous test.

With cold water of nearly constant temperature, it will be found that a uniform amount will be required to reduce the temperature of the water bath to the required point.

(d) Place the oil cup back in the bath and measure into it a 50-cc charge of fresh oil. Destroy any bubbles on the surface of the oil, put on the cover with its thermometer, put in the heating lamp, record the temperature of the oil, and proceed to repeat the test as described above in sections 4 to 6 , inclusive. Introduce the test flame for first time at a tempcrature of $10^{\circ} \mathrm{F} .\left(5.5^{\circ} \mathrm{C}\right.$.) below the flash point obtained on the previous test.

8. If two or more determinations agree within $1^{\circ} \mathrm{F}$. $\left(0.5^{\circ} \mathrm{C}.\right)$, the average of these results, corrected for baromctric pressure, shall be considered the flash point. If two determinations do not check within $1^{\circ} \mathrm{F} .\left(0.5^{\circ} \mathrm{C}.\right)$, a third determination shall be made, and, if the maximum variation of the three tests is not greater than $2^{\circ} \mathrm{F} .\left(1^{\circ} \mathrm{C}\right.$.), their a verage, after correcting for barometric pressure, shall be considered the flash point.

9. Correction for barometric pressure shall only be made in cases of dispute or when the barometer reading varies more than one-half inch $(13 \mathrm{~mm})$ from the standard pressure of 29.92 inches $(760$. $\mathrm{mm}$ ). When the barometer reading is below this standard pressurc, add to the thermometcr reading $1.6^{\circ} \mathrm{F}$. $\left(0.9^{\circ} \mathrm{C}\right.$.) for each inch $(25 \mathrm{~mm})$ of barometer difference to obtain the true flash point. When the barometer rcading is above the standard pressure, deduct $1.6^{\circ} \mathrm{F}$. $\left(0.9^{\circ} \mathrm{C}\right.$.) for each inch $(25 \mathrm{~mm})$ of barometer difference to obtain the true flash point.

American Society for Testing Materials, standard method of test for distillation of gasoline, naphtha, kerosene, and similar petroleum products, serial designation D86-27, 1927.

Approved as American Standard by the American Engineering Standards Committee, now the American Standards Association.

\section{APPARATUS}

\section{Flask}

The standard 100-cc Engler flask is shown in Figure 20, the dimensions and allowable tolerance being as follows:

\begin{tabular}{|c|c|c|c|}
\hline & $\begin{array}{l}\text { Centi- } \\
\text { meters }\end{array}$ & Inches & $\begin{array}{l}\text { Toler- } \\
\text { ances }\end{array}$ \\
\hline $\begin{array}{l}\text { Viameter of bulb, outside. } \\
\text { Diameter of neck, inside. } \\
\text { Length of neck } \\
\text { Length of vapor tube } \\
\text { Diameter of vapor tube, outside. } \\
\text { Diameter of vapor tube, inside } \\
\text { Thickness of vapor tube wall. }\end{array}$ & $\begin{array}{r}6.5 \\
1.6 \\
15.0 \\
10.0 \\
.6 \\
.4 \\
.1\end{array}$ & $\begin{array}{r}2.56 \\
.63 \\
5.91 \\
3.94 \\
.24 \\
.16 \\
.04\end{array}$ & $\begin{array}{c}c m \\
0.2 \\
.1 \\
.4 \\
.3 \\
.05 \\
.05 \\
.05\end{array}$ \\
\hline
\end{tabular}

The position of the vapor tube shall be $9 \mathrm{~cm}$ (3.55 inches) $\pm 3 \mathrm{~mm}$ above the surface of the liquid when the flask contains its charge of $100 \mathrm{cc}$. The tube is approximately in the middle of the neck and set at an angle of $75^{\circ}$ (tolerance $\pm 3^{\circ}$ ) with the vertical.

\section{Condenser}

The condenser (fig. 21) consists of a nine-sixteenths-inch $(14.29 \mathrm{~mm})$ OD No. 20 Stubbs gage 
seamless brass tube, 22 inches $(55.88 \mathrm{~cm})$ long. It is set at an angle of $75^{\circ}$ from the perpendicular and is surrounded with a cooling bath 15 inches long $(38.1 \mathrm{~cm})$, approximately 4 inches $(10.16 \mathrm{~cm})$ wide by 6 inches $(15.24 \mathrm{~cm})$ high. The lower end of the condenser tube is cut off at an acute angle, and curved downward for a length of 3 inches $(7.62 \mathrm{~cm})$ and slightly backward so as to insure contact with the wall of the graduate at a point 1 to $1 \frac{1}{4}$ inches $(2.54$ to $3.8 \mathrm{~cm}$ ) below the top of the graduate when it is in position to receive the distillate.

\section{Shield}

The shield (fig. 21) is made of approximately 22 gage sheet metal and is 19 inches $(48.26 \mathrm{~cm})$ high, 11 inches $(27.94 \mathrm{~cm})$ long and 8 inches $(20.32 \mathrm{~cm})$ wide, with a door on one narrow side, with two openings, 1 inch $(2.54 \mathrm{~cm})$ in diameter, equally spaced, in each of the two narrow sides, and with a slot cut in one side for the vapor tube. The centers of these four openings are $8 \frac{1}{2}$ inches $(21.59 \mathrm{~cm})$ below the top of the shield. There are also three
$1 \frac{1 / 4}{-1}$ ch $(3.175 \mathrm{~cm})$ opening in the first asbestos board.

5. Gas Burner or Electric Heater

(a) Gas burner.-The burner is so constructed that sufficient heat can be obtained to distill the product at the uniform rate specified below. The flame should never be so large that it spreads over a circle of diameter greater than $3 \frac{1}{2}$ inches $(8.89 \mathrm{~cm})$ on the under surface of the asbestos board. A sensitive regulating valve is a necessary adjunct, as it gives complete control of heating.

(b) Electric heater.-The electric heater, which may be used in place of the gas flame, shall be capable of bringing over the first drop within the time specified below when started cold, and of continuing the distillation at the uniform rate. The electric heater shall be fitted with an asbestos board top one-eighth to one-fourth inch ( 3.175 to $6.35 \mathrm{~mm})$ thick, having a hole $1 \frac{1}{4}$ inches $(3.175 \mathrm{~cm})$ in diameter in the center. When an electric heater is employed, the portion of the shield above the asbestos board

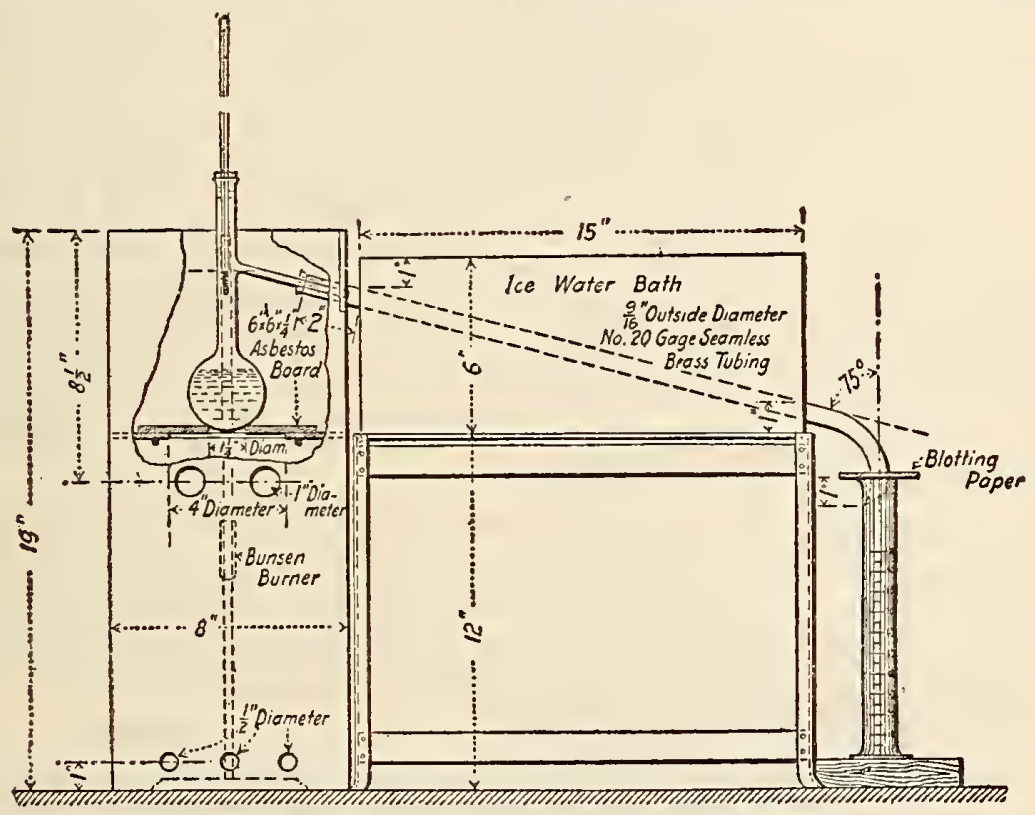

Figure 21.-Distillation assembly

one-half-inch $(1.27 \mathrm{~cm})$ holes in each of the four sides with their centers 1 inch $(2.54 \mathrm{~cm})$ above the base of the shield.

\section{Ring Support and Hard Asbestos Boards}

The ring support is of the ordinary laboratory type, 4 inches $(10.16 \mathrm{~cm})$ or larger in diameter, and is supported on a stand inside the shield. There are two hard asbestos boards: One 6 by 6 by onefourth inch ( 15.24 by 15.24 by $6.35 \mathrm{~mm}$ ) with a hole $11 / 4$ inches $(3.175 \mathrm{~cm})^{17}$ in diameter in its center, the sides of which shall be perpendicular to the surface; the other, an asbestos board to fit tightly inside the shield, with an opening 4 inches $(10.16 \mathrm{~cm})$ in diameter concentric with the ring support. These are arranged as follows: The second asbestos board is placed on the ring and the first or smaller asbestos board on top so that it may be moved in accordance with the directions for placing the distilling flask. Direct heat is applied to the flask only through the

17 When distilling petroleum products having an end point above $470^{\circ} \mathrm{F}$. $\left(243.34^{\circ} \mathrm{C}\right.$.) the hole in the asbestos board shall be $11 / 2$ inches $(3.81 \mathrm{~cm})$ in diameter. shall be the same as with the gas burner, but the part below the board may be omitted.

\section{B. Thermometers}

Nos. 2 and 6. (See A. S. T. M. table of thermometers, 500.2 , p. 2.)

\section{Graduate}

The graduate shall be of the cylindrical type, of uniform diameter, with a pressed or molded base and a lipped top. The cylinder shall be graduated to contain $100 \mathrm{cc}$, and the graduated portion shall be not less than 7 inches $(17.78 \mathrm{~cm})$ nor more than 8 inches $(20.32 \mathrm{~cm})$ long. It shall be graduated in single cubic centimeters, and each fifth mark shall be distinguished by a longer line. It shall be numbered from the bottom up at intervals of $10 \mathrm{cc}$ The over-all height of the graduate shall be not less than $93 / 4$ inches $(24.8 \mathrm{~cm})$, nor more than $10^{1 / 4}$ inches $(26.0 \mathrm{~cm})$. The graduations shall not be in error by more than 1 ce at any point on the scale. 


\section{PROCEDURE}

8. (a) The condenser bath shall be filled with cracked ice, ${ }^{18}$ and enough water added to cover the condenser tube. The temperature shall be maintained between $32^{\circ}$ and $40^{\circ} \mathrm{F}$. $\left(0^{\circ}\right.$ and $4.45^{\circ} \mathrm{C}$. $)$.

(b) The condenser tube shall be swabbed to remove any liquid remaining from the previous test. A piece of soft cloth attached to a cord or copper wire may be used for this purpose.

(c) One hundred cubic centimeters of the product shall be measured in the $100 \mathrm{ce}$ graduated cylinder at $55^{\circ}$ to $65^{\circ} \mathrm{F}$. $\left(12.78^{\circ}\right.$ to $18.33^{\circ} \mathrm{C}$. $)$ and transferred directly to the Engler flask. None of the liquid shall be permitted to flow into the vapor tube.

(d) The thermometer ${ }^{19}$ provided with a cork shall be fitted tightly into the flask so that it will be in the middle of the neck and so that the lower end of the capillary tube is on a level with the inside of the bottom of the vapor outlet tube at its junction with the ncck of the flask. The thermometer shall be approximately at room temperature when placed in the flask.

(e) The charged flask shall be placed in the $1 \frac{1}{4}$ inch $(3.175 \mathrm{~cm})$ opening in the 6 by 6 inch $(15.24$ by $15.24 \mathrm{~cm}$ ) asbestos board with the vapor outlet tube inserted into the condenser tube. A tight connection may be made by means of a cork through which the vapor tube passes. The position of the flask shall be so adjusted that the vapor tube "extends into the condenser tube not less than 1 inch $(2.54 \mathrm{~cm})$ nor more than 2 inches $(5.08 \mathrm{~cm})$.

(f) The graduated cylinder used in measuring the charge shall be placed, without drying, at the outlet of the condenser tube in such a position that the condenser tube shall extend into the graduate at least 1 inch $(2.54 \mathrm{~cm})$ but not below the $100-\mathrm{cc}$ mark. Unless the temperature is between $55^{\circ}$ and $65^{\circ} \mathrm{F}$. $\left(12.78^{\circ}\right.$ and $18.33^{\circ} \mathrm{C}$.) the receiving graduate shall be immersed up to the 100-ce mark in a transparent bath maintained between these temperatures. The top of the graduate shall be covered closely during the distillation with a piece of blotting paper or its equivalent, cut so as to fit the condenser tube tightly.

9. When everything is in readiness, heat shall be applied at a uniform rate, so regulated that the first drop of condensate falls from the condenser in not less than 5 nor more than 10 minutes. The distillation thermometer shall be read two minutes after heat is applied and the indication recorded as the "correction temperature." This figure is of significance only in cases when there is a question as to the accuracy ${ }^{20}$ of the initial boiling point,

${ }^{39}$ Any otber convenient cooling medium may be used

19 An boiling point of $212^{\circ} \mathrm{F} .\left(100^{\circ} \mathrm{C}\right.$.) or higher, the higb-distillation thermometer shall be used; for all other higher, the higb-distillation thermometer shall be used; for all other $500.2,0.2$.)

30 The initial boiling point is one of the observations wbicb it is customary to report as indicative of the quality of gasoline. It is a point which is very difficult to cbeck, especially when duplicate tests are made at different room temperatures, and it has not been found practical to devise an accurate metbod of correcting for tbis unavoidable variation in operating conditions. The practical significance of initial hoiling point is not regarded as sufficicnt to warrant requiring that tests be conducted under regulated conditions of room temperature. The following directions are included as an exnedicnt intended to obviate some of the difficulties that develop on account of the practice of inciuding initial hoiling point limits in specifications for the purchase and sale of gasoline

specifications for the purchase and sale of gasoline: $80^{\circ} \mathrm{F}\left(27^{\circ} \mathrm{C}\right.$ ) the temperature" is below $70^{\circ} \mathrm{F}$. $\left(21^{\circ} \mathrm{C}\right.$.) or ahovo $\left(66^{\circ} \mathrm{C}\right)$, may be considered incorrect by an amount not less tban one-third the difference hetween the "correction temperature" and one-third the difference hetween the "correction temperature" and the ohserved initial boiling point figures obtained by all parties sball be revised according to the following formula; as subsequently determined. When the first drop falls from the end of the condenser the reading of the distillation thermometer shall be recorded as the initial boiling point. The receiving cylinder shall then be moved so that the end of the condenser tube shall touch the side of the cylinder. The heat shall then be so regulated that the distillation will proceed at a uniform rate of not less than 4 nor more than 5 cc per minute. The volume of distillate collected in the cylinder shall be observed and recorded, to the nearest $0.5 \mathrm{cc}$, when the mercury of the thermometer reaches each point that is an even multiple of $25^{\circ} \mathrm{C}$. or the Fahrenheit equivalent of this point $\left(50^{\circ}, 75^{\circ}, 100^{\circ}, 125^{\circ} \mathrm{C}\right.$., etc., or $122^{\circ}$, $167^{\circ}, 212^{\circ}, 257^{\circ} \mathrm{F}$., etc.). 'If preferred, the reading of the distillation thermometer may be observed and recorded when the level of the distillate reaches each 10-cc mark on the graduate. In case a product is being tested to ascertain whether or not it conforms with a given specification, all necessary observations shall be made and recorded, whether or not they are included in the series ordinarily employed by the laboratory making the test.

When the liquid residue in the distillation flask is approximately 5 cc the heat may be increased because of the presence of heavy ends which have relatively high boiling points. However, no further increase of heat shall be applied after this adjustment. The 4 to 5 cc rate can rarely be maintained from this point to the end of the distillation, but in no case shall the period between the point when approximately $5 \mathrm{cc}$ of liquid remains in the flask and the end point be more than five minutes.

The heating shall be continued until the mercury reaches a maximum and starts to fall consistently. The highest temperature observed on the distillation thermometer shall be recorded as the maximum temperature or end point. Usually this point will be reached after the bottom of the flask has become dry.

The total volume of the distillate collected in the receiving graduate shall be recorded as the recovery.

The cooled residue shall be poured from the flask into a small cylinder graduated in $0.1 \mathrm{cc}$, measured when cool and the volume recorded as residue.

The difference between $100 \mathrm{cc}$ and the sum of the recovery and the residue shall be calculated and recorded as distillation loss.

\section{ACCDRACY}

10. With proper care and attention to detail, duplicate results obtained for initial boiling point and maximum temperature, respectively, should not

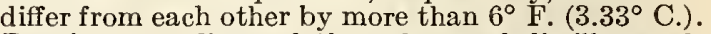
Duplicate readings of the volume of distillate collected in the cylinder when each of the prescribed temperature points is reached should not differ from each other by more than $2 \mathrm{cc}$. In case observations are made on the basis of prescribed percentage points, the differences in temperature readings should not exceed the amounts equivalent to $2 \mathrm{cc}$ of distillate at each point in question.

\section{CORRECTION FOR BAROMETRIC PRESSURE}

11. The actual barometric pressure shall be ascertained and recorded, but no correction shall be

$\left.\begin{array}{c}\text { Revised ini- } \\ \text { tial boiling } \\ \text { point }\end{array}\right\}=\left\{\begin{array}{c}\text { observcd ini- } \\ \text { tial boiling } \\ \text { point }\end{array}\right\}-\frac{\text { correction temperature }\left({ }^{\circ} \text { F. }\right)-75}{3}$
point $\}=\{$ point

If all of the revised figures fall within the specification limit the gasoline in question sball be considered as passing; otherwise arrangements shall, if possihle, he made to conduct a distillation test witb the room temperature maintained between the limits of $70^{\circ}$ and $80^{\circ} \mathrm{F}$. $\left(21^{\circ}\right.$ and $27^{\circ}$ C.), inclusive, 
made except in case of dispute. In such cases the temperature points shall be corrected to $760 \mathrm{~mm}$ (29.92 inches) by the use of the Sydney Young equation, as follows:

For centigrade readings:

For Fahrenheit readings:

$$
C_{c}=0.00012(760-P)\left(273+t_{c}\right)
$$

$$
C_{f}+0.00012(760-P)\left(460+t_{f}\right)
$$

in which $C_{c}$ and $C_{f}$ are, respectively, corrections to be added to the observed temperature $t_{c}$ or $t_{f}$, and

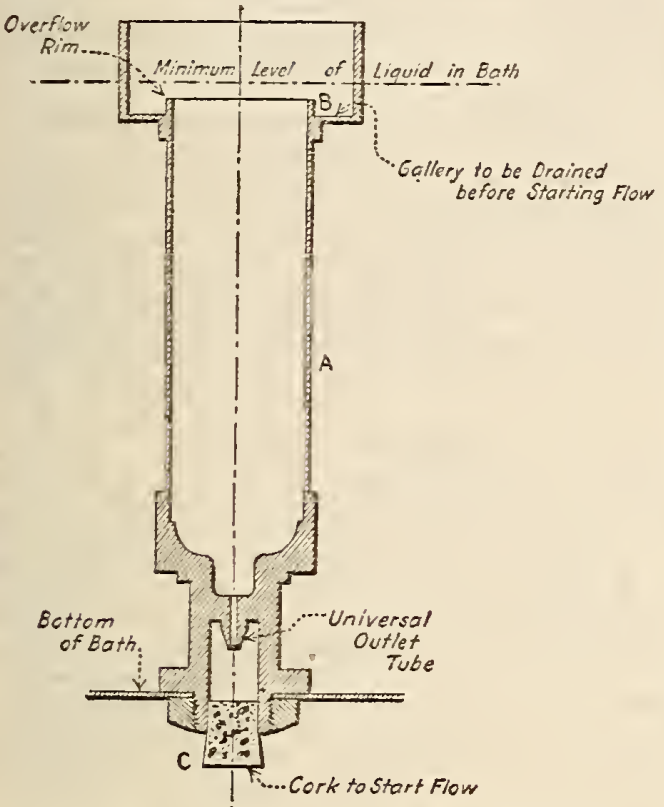

Figure 22.-Sectional view of standard oil tube

$P$ is the actual barometric pressure in millimeters of mercury.

The following table is a convenient approximation of the corrections as calculated by the above equation:

\begin{tabular}{|c|c|c|c|}
\hline \multicolumn{2}{|c|}{} & \multicolumn{2}{c|}{$\begin{array}{c}\text { Correction }{ }^{1} \text { per } \\
10 \text { mm (difier- } \\
\text { ence in pres* } \\
\text { sure) }\end{array}$} \\
\hline${ }^{\circ} \mathrm{C}$. & ${ }^{\circ} \mathrm{F}$. & ${ }^{\circ} \mathrm{C}$. & ${ }^{\circ} \mathrm{F}$ \\
$10-30$ & $50-86$ & 0.35 & 0.63 \\
$30-50$ & $86-122$ & .38 & .68 \\
$50-70$ & $122-158$ & .40 & .72 \\
$70-90$ & $158-194$ & .42 & .76 \\
$90-110$ & $194-230$ & .45 & .81 \\
$110-130$ & $230-266$ & .47 & .85 \\
$130-150$ & $266-302$ & .50 & .89 \\
$150-170$ & $302-338$ & .52 & .94 \\
$170-190$ & $338-374$ & .54 & .98 \\
$190-210$ & $374-410$ & .57 & 1.02 \\
$210-230$ & $410-446$ & .59 & 1.06 \\
$230-250$ & $446-482$ & .62 & 1.11 \\
$250-270$ & $482-518$ & .64 & 1.15 \\
$270-290$ & $518-554$ & .66 & 1.19 \\
$290-310$ & $554-590$ & .69 & 1.24 \\
$310-330$ & $590-626$ & .71 & 1.28 \\
$330-350$ & $626-662$ & .74 & 1.32 \\
$350-370$ & $662-698$ & .76 & 1.37 \\
$370-390$ & $698-734$ & .78 & 1.41 \\
$390-410$ & $734-770$ & .81 & 1.45 \\
\hline
\end{tabular}

1 To be added in case barometric pressure is below $760 \mathrm{~mm}$; to be subtracted in case barometric pressure is above $760 \mathrm{~mm}$.

American Society for Testing Materials, standard methods of test for viscosity of petroleum products and lubricants, serial designation D88-26; 1926.
Approved as American Standard by the American Engineering Standards Committee, now the American Standards Association.

1. (a) Viscosity shall be determined by means of the Saybolt universal or Saybolt Furol, viscosimeter.

(b) In general, the Saybolt universal viscosimeter shall be used for lubricants and the Saybolt Furol viscosimeter for fuel oils and other oils of similar viscosity.

\begin{tabular}{|c|c|c|c|c|c|c|}
\hline \multirow{2}{*}{ Dimension } & \multicolumn{3}{|c|}{$\begin{array}{l}\text { Saybolt universal } \\
\text { riscosimeter }\end{array}$} & \multicolumn{3}{|c|}{$\begin{array}{l}\text { Saybolt Furol } \\
\text { viscosimeter }\end{array}$} \\
\hline & $\begin{array}{l}\text { Min- } \\
\text { imum }\end{array}$ & $\begin{array}{c}\text { Nor- } \\
\text { mal }\end{array}$ & Max- & imum & $\begin{array}{l}\text { Nor- } \\
\text { mal }\end{array}$ & $\begin{array}{l}\text { Max- } \\
\text { imum }\end{array}$ \\
\hline $\begin{array}{l}\text { Insicie diameter of outlet } \\
\text { tube }\end{array}$ & $\begin{array}{l}c m \\
0.175\end{array}$ & $\begin{array}{c}c m \\
0.1705\end{array}$ & $\begin{array}{c}c m \\
0.178\end{array}$ & $\begin{array}{l}c m \\
0.313\end{array}$ & $\begin{array}{c}c m \\
0.315\end{array}$ & $\begin{array}{l}\mathrm{cm} \\
0.317\end{array}$ \\
\hline $\begin{array}{l}\text { Outside diameter of outlet } \\
\text { at the lower end }\end{array}$ & .28 & .30 & .32 & .40 & .43 & .46 \\
\hline Lengtb of outlet tube 1 & 1. 215 & 1.225 & 1. 235 & 1. 215 & 1. 225 & 1. 235 \\
\hline $\begin{array}{l}\text { bottom of outlct tube } \\
\text { Outside diamerer of over- } \\
\text { flow rim at the top }\end{array}$ & 12.40 & 12.50 & $\begin{array}{r}12.60 \\
3.30\end{array}$ & 12. 40 & 12.50 & 12. 60 \\
\hline $\begin{array}{l}\text { Diameter of container } \\
\text { Deptb of cylindrical part of } \\
\text { container }\end{array}$ & $\begin{array}{l}2.955 \\
8.8\end{array}$ & 2.975 & 2.995 & $\begin{array}{l}2.955 \\
8.8\end{array}$ & 2.975 & 2.995 \\
\hline $\begin{array}{l}\text { Diameter of container be- } \\
\text { tween bottom of crlindri- } \\
\text { cal part of container and } \\
\text { top of outlet tube }\end{array}$ & .9 & & & 9 & & \\
\hline
\end{tabular}

Dimensions of oil tubes

1 This dimension is identical in the Saybolt universal and the Saybolt Furol instruments.

2 Tbe section of overfow rim shall be bounded by straigbt lines, except tbat a fillet is permissible at tbe junction witb tbe bottom of tho gallery.

? The minimum value shall preferably be not less than $3.2 \mathrm{~cm}$.

(c) The Saybolt universal viscosimeter shall not be used for times of flow less than 32 seconds.

\section{APPARATUS}

2. (a) The Saybołt viscosimeters (see fig. 22) are made entirely of metal. The oil tube, $A$, is fitted at the top with an overflow cup, $B$, and the tube is surrounded by a bath. At the bottom of the oil tube is a small outlet tube through which the oil to be tested flows into a receiving fiask (fig. 23), whose capacity at $20^{\circ} \mathrm{C}$. $\left(68^{\circ} \mathrm{F}\right.$.) to a mark on its neck is 60 , +0.15 , cc. The outlet tube is of hard and corrosive resistant metal, such as stainless steel, Monel metal, etc. The inside diameter of neck of flask, at the level where the graduation mark is placed, is 0.8 to $1.1 \mathrm{~cm}$. The lower end of the outlet tube is inclosed by a larger tube, which when stoppered by a cork, $C$, acts as a closed air chamber and prevents the flow of oil through the outlet tube until the cork is removed and the test started. A looped string may be at-

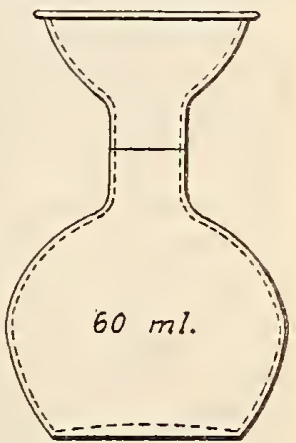

Elevation of Flask.

Figure 23. - Receiving flask

Tbis type of flask is recommended as convenient and durable. tached to the lower end of the cork as an aid to its rapid removal. The temperatures in the oil tube and in the bath are shown by thermometers. The bath may be heated by any suitable means. The oil tube shall be 
thoroughly cleaned, and all oil entering the oil tube shall be strained through a 100-mesh wire strainer. A stop watch shall be used for taking the time of flow of the oil and a pipette shall be used for draining the overflow cup.

(b) The oil tubes, which may be standardized by the National Bureau of Standards, shall conform to the dimensions given in the table. The time of flow shall be within \pm 1 per cent of the time as obtained with the Bureau of Standards' master tube. ${ }^{21}$

(c) The bath and oil tube thermometers shall conform to the requirements of thermometer No. 15, see A. S. T. M. table of thermometers, 500.2 , page 2 . They cover two sets of 4 thermometers each, one set being graduated in Fahrenheit degrees and the other set in centigrade degrees, the ranges being chosen to include the temperatures commonly used in testing.

\section{TEMPERATURE OF TESTUNG}

3. (a) With the Saybolt universal viscosimeter, determinations shall be made at $100^{\circ} \mathrm{F}$. $\left(37.8^{\circ} \mathrm{C}\right.$.), $130^{\circ} \mathrm{F}$. $\left(54.4^{\circ} \mathrm{C}\right.$. $)$ or $210^{\circ} \mathrm{F} .\left(98.9^{\circ} \mathrm{C}\right.$. $)$.

(b) With the Saybolt Furol viscosimeter, determinations shall be made at $122^{\circ} \mathrm{F}$. $\left(50^{\circ} \mathrm{C}\right.$.).

(c) Viscosities shall be expressed as seconds, Saybolt universal (or Saybolt Furol), being the time in seconds for the delivery of $60 \mathrm{cc}$ of oil.

(d) Fuel oils and other oils of similar viscosity showing a time of less than 25 seconds, Saybolt Furol, at $122^{\circ}$ F., shall be tested on the Saybolt universal at $122^{\circ} \mathrm{F}$. Oil showing a time of less than 32 seconds, Saybolt universal, at $122^{\circ} \mathrm{F}$., shall be measured in the Saybolt universal at $100^{\circ} \mathrm{F}$. $\left(37.8^{\circ} \mathrm{C}\right.$.). These methods of test do not apply to fuels having a viscosity at $100^{\circ} \mathrm{F}$. of less than 32 seconds Saybolt universal, which are not considered to be fuel oils.

\section{PROCEDURE}

4. In tests at $100^{\circ}$ and $130^{\circ} \mathrm{F} .\left(37.8^{\circ}\right.$ and $54.4^{\circ} \mathrm{C}$. $)$ the bath temperature throughout the test shall not vary more than $\pm 0.1^{\circ} \mathrm{F}$. $\left(0.06^{\circ} \mathrm{C}\right.$.) from the predetermined temperature which will maintain thermal equilibrium as long as the oil is well stirred with the thermometer. In tests at $210^{\circ} \mathrm{F} .\left(98.9^{\circ} \mathrm{C}\right.$. $)$ a variation of $\pm 0.2^{\circ} \mathrm{F}$. $\left(0.11^{\circ} \mathrm{C}\right.$. $)$ is permitted.

Any construction of bath may be employed provided the bath tcmperature necessary to maintain thermal equilibrium (while the oil in the tube is well stirred by the oil tube thermometer) is not in excess of $100.25^{\circ}, 122.35^{\circ}, 130.50^{\circ}$, and $212.00^{\circ} \mathrm{F}$. $\left(37.9^{\circ}, 50.2^{\circ}, 54.7^{\circ}\right.$, and $100.00^{\circ}$ C. $)$, respectively, for the standard temperatures previously mentioned. The level of the bath liquid shall not be lower than $0.5 \mathrm{~cm}$ above the overflow rim of the oil tube. In tests at $210^{\circ} \mathrm{F}$. $\left(98.9^{\circ} \mathrm{C}\right.$.) the water bath shall be vigorously stirred and may be heated by the direct injection of steam near the bottom of the bath. A bath of other suitablc liquid may be used provided it is properly heated and stirred.22 The heating and stirring of the bath may be accomplished by any suitable means, provided the source of heat is not less than the following distances from any part of the oil tube: 2 inches $(5 \mathrm{~cm})$ with

21 This tube represents the average mechanically perfect tube, based on the A.S.T.M. specifications, and has been approved by the American Petroleum Institute Committee on Viscosity

Standards.

22 For example, the bath liquid may be water, to which glycerin or salt has been added. In ordinary routine testing it is frequently desirable to employ oil as a bath medium. This is allowable, provided the temperature of the oil bath is adjusted so that the necessary condition of thermal equilibrium is maintained. It is usually necessary to maintain the oil bath at slightly higher temperatures than are nccessary when water is the bath medium. Oil-bath temperatures will need to be from $0.1^{\circ}$ to $0.2^{\circ} \mathrm{F}$. $\left(0.06^{\circ}\right.$ to $\left.0.11^{\circ} \mathrm{C}\right)$ higher for tests at $100^{\circ} \mathrm{F}$. $\left(37.8^{\circ} \mathrm{C}\right)$ and $1.5^{\circ}$ to $20^{\circ} \mathrm{F}\left(0.85^{\circ}\right.$ to $1.11^{\circ}$ C.) higher for tests at $210^{\circ} \mathrm{F},\left(98,8^{\circ} \mathrm{C}\right.$.) than the corresponding water-bath temperatures. an external heater, $1 \frac{1}{4}$ inches $(3 \mathrm{~cm})$ with an immersion heater. Viscosity determinations shall be made in a room free from draughts and from rapid changes in temperature. The room temperature shall be between $68^{\circ}$ and $86^{\circ} \mathrm{F}$. $\left(20^{\circ}\right.$ and $30^{\circ}$ C. $) .^{23}$

All oil introduced into the oil tube, either for cleaning or for test, shall first be passed through the strainer.

To make the test, heat the oil to the necessary temperature and clean out the oil tube. Pour some of the oil to be tested through the cleaned tube. Insert the cork stopper into the lower end of the air chamber at the bottom of the oil tube sufficiently to prevent the escape of air, but not to touch the small outlet tube.

Heat the oil to be tested, outside the viscosimeter, to slightly but not more than $3^{\circ} \mathrm{F}$. (1.7 ${ }^{\circ} \mathrm{C}$.) above the tempcrature at which the viscosity is to be determincd and pour it into the oil tube until it ceases to overflow into the overflow cup. By means of the oil tube thermometer, keep the oil in the oil tube well stirred and also stir well the liquid in the bath. It is extremely important that the temperature of the bath be maintained constant during the entire time consumed in making the test. After the temperature of the bath and of the oil in the oil tube have been constant for one minute at the desired temperature, withdraw the oil tube thermometer, quickly remove the surplus oil from the overflow cup by means of a pipette ${ }^{2 s}$ so that the level of the oil in the overflow cup is below the level of the oil in the oil tube proper; place the 60 cc flask (fig. 23) in position so that the stream of oil from the outlet tube will strike the neck of the flask so as to avoid foam. Snap the cork from its position and at the same instant start the stop watch. Stir the liquid in the bath during the run, and carefully maintain it at the previously determined proper temperature. Stop the watch wher the bottom of the meniscus of the oil reaches the mark on the neck of the receiving flask.

The time in seconds for the delivery of $60 \mathrm{cc}$ of oil is the Saybolt universal (or Saybolt Furol) viscosity of the oil at the temperature at which the test is made.

With proper attention to details of methods of procedure, duplicate results should not differ from each other by more than 1 per cent.

American Society for Testing Materials, tentative method of test for sulphur in motor fuels, naphthas, and illuminating oils (lamp method), serial designation D90-29T, 1929.

\section{SCOPE}

1. This method is intended to cover the determination of sulphur in gasolines and other volatile motor fuels, in illuminating oils, and in petroleum naphthas. It is not strictly applicable to mixtures containing carbon bisulfide.

Note.-When this method is applied to mixtures containing carbon bisulfide approximately 85 per cent of the sulphur present as carbon bisulfide is usually determined.

\section{APPARATUS}

2. The apparatus shall consist of the following:

(a) Absorber.- An absorber of chemically resistant glass conforming to the dimensions shown in Figure 24. The larger of the two bulbs of the absorber shall be filled at least two-thirds full with pieces of chemically resistant glass rod from 8 to 10 $\mathrm{mm}$ in length and from 5 to $6 \mathrm{~mm}$ in diameter, or

23 These limits are necessary for extreme accuracy in standardization and referee tests, but for routine purposes the use of higher temperatures up to $100^{\circ} \mathrm{F}$. $\left(37.8^{\circ} \mathrm{C}\right.$.) will not ordinarily cause an error of more than 1 per cent.

${ }_{24}$ It is recommended that the tip of the pipette should be about $2 \mathrm{~mm}$ inside and about $3 \mathrm{~mm}$ outside diameter, and great care should be exercised to avoid touching the overflow rim while draining the overflow cup. 
with perforated beads of chemically resistant glass 5 to $8 \mathrm{~mm}$ in diameter.

(b) Chimney. - A chimney of chemically resistant glass conforming to the dimensions shown in Figure 24 , and connected with the absorber by a cork stopper.

(c) Spray trap.-A spray trap of chemically resistant glass conforming to the dimensions shown in Figure 24, and connected with the absorber by a cork or by a rubber stopper.

(d) Lamp. - A small lamp of about 25 cc capacity. The lamp may conveniently consist of a 25 to $35 \mathrm{cc}$ Erlenmeyer flask, preferably of the dimensions shown in Figure 24, and a cork carrying a piece of glass tubing $40 \mathrm{~mm}$ in length, from 6 to $7 \mathrm{~mm}$ in outside diameter, and $3 \mathrm{~mm}$ in inside diameter, with ends ground or fused level. The wick tube should project above the cork at least $10 \mathrm{~mm}$, and the cork shall have a vertical groove in its side so that air may enter the flask to replace the oil as it is consumed.

(e) Cotton wicking. - Two strands of clean, unused cotton wicking, 10 to $12 \mathrm{~cm}$ in length and weighing about 5 to $6 \mathrm{mg}$ per $\mathrm{cm}$ per strand.

(f) Filter pump.-A filter pump or other means for continuous suction.

\section{SOLUTIONS REQUIRED}

3. (a) Hydrochloric acid.-Prepare a solution of $\mathrm{HCl}$ in distilled water which will contain $2.275 \mathrm{~g}$ of $\mathrm{HCl}$ per liter and check carefully for accuracy.

(b) Sodium carbonate.-Dissolve $3.306 \mathrm{~g}$ of $\mathrm{Na}_{2} \mathrm{CO}_{3}$ in 1 liter of distilled water. Ten cubic centimeters of this solution should neutralize exactly $10 \mathrm{cc}$ of the $\mathrm{HCl}$ solution.

(c) Methyl orange.-Dissolve $0.004 \mathrm{~g}$ of methyl orange in one liter of distilled water.

\section{PROCEDURE}

4. The sulphur shall be determined as follows:

(a) Pass two strands of the new cotton wicking through the wick tube so that they are not twisted, but are parallel in the wick tube. Pour into the clean, dry lamp about $15 \mathrm{cc}$ of the oil to be tested, and insert the cork containing the wick tube. After the wick has become saturated with the oil, trin it off as nearly as possible to the top of the wick tube with a pair of sharp scissors, finally adjusting the wick height by touching the top of it with the tip of a finger. Weigh the oil and lamp assembly to the nearest $0.001 \mathrm{~g}$. Run a blank determination at the same time and under the same conditions by burning sulphur-free alcohol in a similar lamp. Thoroughly rinse out the absorber with distilled water, and put into it exactly $10 \mathrm{cc}$ of the sodium carbonate solution from an accurately calibrated pipette, and then dilute the solution with $10 \mathrm{cc}$ of distilled water. Rinse out also the chimney and the spray trap with distilled water, dry the chimney, and connect both to the absorber as shown in Figure 24. Prepare the apparatus for the blank determination in the same manner, putting into it exactly $10 \mathrm{cc}$ of the sodium carbonate solution and $10 \mathrm{cc}$ of distilled water. Apply gentle suction to both absorbers; light both the oil lamp and the alcohol lamp and then place them in position under the chimneys so that the top of each wick tube extends into the chimney not more than 1 or $2 \mathrm{~mm}$ above the edge. Use a sulphur-free flame, such as an alcohol lamp, for lighting the lamps; do not use matches. Adjust the suction so that a steady flame 12 to $18 \mathrm{~mm}$ in height and free from smoking is obtained (note 1 ). The suction shall be adjusted so that air is drawn through both absorbers at approximately the same rate. The maintenance of proper flame height usually requires that the wick be flush with the top of the wick tube for naphthas or motor fuels and a little higher for illuminating oils. 'The room shall be free from draughts. Continue burning for from one to one and one-half hours, or less if the sulphur content of the oil is high. During this time the oil should be consumed at the rate of about 2 to 2.5 $\mathrm{g}$ per hour.

(b) Extinguish the flames and stop the suction on both absorbers. Weigh the oil lamp immediately to the nearest $0.001 \mathrm{~g}$, and determine by difference the weight of oil consumed. Working with the blank first, disconnect the spray trap and chimney and wash them thoroughly with the methyl orange solution, using a wash bottle with a very fine jet and collecting the washings in the absorber. Use approximately $35 \mathrm{cc}$ of the solution for washing. Carefully titrate the faintly yellow solution with the $\mathrm{HCl}$ solution. During titration, carefully agitate the contents of the absorber either by alternate sucking and blowing through a rubber tube held between the lips, or else by the use of a suitable rubber-syringe bulb. As the end point is approached, draw the liquid back and forth between the bulbs after each addition of acid, agitating as before. When the first permanent pink color appears, the end point has been reached. Read and record the volume of $\mathrm{HCl}$ solution used (note 2).

(c) Rinse the chimney and the spray trap used in the actual determination into the absorber to which they were connected, exactly as has been prescribed for the blank. If at this point the solution in the absorber should have a pink color, too much oil has been burned and the determination shall be repeated, but burning for a shorter time. The temporary pink color that sometimes appears in the chimney when rinsing it out should be disregarded. Titrate just as in the blank, continuing to add the $\mathrm{HCl}$ solution until the color matches that obtained in the blank. Read and record the volume of $\mathrm{HCl}$ solution used.

Note 1.-If it is not possible to obtain a good flame free from smoking, as sometimes happens if the oil contains considerahle percentages of benzol, dilute the oil with an equal weight of sulphur-free anhydrous alcohol before putting the sample into the lamp. If, while maintaining the specified flame height, the flame still smokes, a smaller flame is permissible. In order to get the cor rect value when smaller flame is permissible. In order to get the correct value when calculating the percentage of sulphur present in this case, allowance shall be made for the fact that the sample as burned in the lamp is one-half alcohol. This shall be done by doubling the percentage of sulphur obtained in computation by the formula given in section 5 . NoTE 2.-For accurate work, conditions shall be such that a minimum or zero blank is obtained. If the titration of the blank requires more than $0.1 \mathrm{cc}$ of the $\mathrm{HCl}$ solution per gram of sample burned, which corresponds to 0.01 per cent of sulphur, a high degree of accu-

5. Calculate the sulphur content of the oil by substituting the proper values in the following formula:

\section{Percentage of sulphur $=\frac{\left.\begin{array}{c}\text { Cubic centimeters } \\ \text { of } \mathrm{HCl} \text { for blank }\end{array}\right\}\left\{\begin{array}{l}\text { Cubic centimeters } \\ \text { of } \mathrm{HCl} \text { for sample }\end{array}\right.}{\text { Grams of oil burned } \times 10}$}

This formula is correct only for the standard solutions specified in section 3,1 cc of each being equivalent to $0.001 \mathrm{~g}$ of sulphur. The use of solutions of any other strength, such as $0.1 \mathrm{~N}$, involves more complicated calculation and is not advisable.

6. As a check on the determination, a sample of known sulphur content shall be analyzed. Such a reference sample may be made by adding a pure volatile sulphur compound, such as carbon bisulphide, to sulphur-free anhydrous alcohol. This check shall indicate from 85 to 100 per cent of the sulphur present.

American Society for Testing Materials, standard method of test for flash point by means of the Pensky-Martens closed tester, serial designation: D93-22; 1922.

Approved as American Standard by the American Engineering Standards Committee, now the American Standards Association. 
The A. S. T. M. standard Pensky-Martens closed tester shall be used for determining the flash point of fuel oil unless the use of the tag closed tester is specified.

\section{APPARATUS}

The Pensky-Martens tester, a diagram of which appears in Figure 25, shall include the following major parts:

\section{Cup}

The cup of the A. S. T. M. Pensky-Martens flash tester shall be made of brass and shall satisfy the following dimensional specifications:

\begin{tabular}{|c|c|c|c|c|c|c|}
\hline Dimensions & $\begin{array}{l}\text { Mini- } \\
\text { mum }\end{array}$ & $\begin{array}{l}\text { Nor- } \\
\text { mal }\end{array}$ & $\begin{array}{l}\text { Maxi- } \\
\text { mum }\end{array}$ & $\begin{array}{l}\text { Mini- } \\
\text { mum }\end{array}$ & $\begin{array}{l}\text { Nor- } \\
\text { mal }\end{array}$ & $\begin{array}{l}\text { Maxi- } \\
\text { mum }\end{array}$ \\
\hline $\begin{array}{l}\text { Inside diameter below fill- } \\
\text { ing mark }\end{array}$ & $\begin{array}{r}\text { Inches } \\
1.950\end{array}$ & $\begin{array}{r}\text { Inches } \\
2.000\end{array}$ & $\begin{array}{r}\text { Inches } \\
2.050\end{array}$ & $\begin{array}{l}\mathrm{cm} \\
4.953\end{array}$ & $\begin{array}{l}c m \\
5.080\end{array}$ & $\begin{array}{l}c m \\
5.207\end{array}$ \\
\hline $\begin{array}{l}\text { Difference, inside and out- } \\
\text { side diameters below fill- }\end{array}$ & 1.500 & & & & & \\
\hline Inside height & 2.150 & 2.200 & 2.250 & $5.46 \mathrm{I}$ & 5.588 & $\begin{array}{r}.330 \\
5.715\end{array}$ \\
\hline Thickness o & .070 & .095 & .120 & .178 & .241 & .305 \\
\hline $\begin{array}{l}\text { Distance from rim to filling } \\
\text { mark }\end{array}$ & 845 & .860 & .875 & 2. 146 & 2.184 & 2. 223 \\
\hline $\begin{array}{l}\text { Distance Jower surface flange } \\
\text { to bottom of cup. }\end{array}$ & 1.780 & 1. 795 & 1.810 & 4. 521 & 4. 559 & 4.597 \\
\hline
\end{tabular}

The inside of the cup may be turned to a slightly larger diameter above the filling mark and the outside may be tapered above the flange, but the wall thickness at the upper edge shall be not less than 0.04 inch $(0.102 \mathrm{~cm})$. The flange should be approximately 0.5 inch $(1.27 \mathrm{~cm})$ wide and approximately 0.125 inch $(0.318 \mathrm{~cm})$ thick. It shall be equipped with devices for locating the position of the lid on the cup and the cup in the stove. A handle, attached permanently to the fiange of the cup, is a desirable accessory.

\section{Iid}

(a) Stirring device.-The lid shall be equipped with a stirring device consisting of a vertical steel shaft, not less than 0.1 inch $(0.254 \mathrm{~cm})$ nor more than 0.125 inch $(0.318 \mathrm{~cm})$ in diameter, mounted in the center of the cup, and carrying two 2-bladed brass propellers. The blades of both propellers shall be approximately 0.313 inch $(0.795 \mathrm{~cm})$ wide and shall be set at an angle of approximately $45^{\circ}$. The smaller (upper) propeller shall have an over-all diameter of approximately 0.75 inch $(1.905 \mathrm{~cm})$. The larger (lower) propeller shall have an over-all diameter between 1.25 and 1.75 inches $(3.175$ and 4.445 $\mathrm{cm})$. The thickness of the propeller blades shall be not less than 0.057 inch $(0.145 \mathrm{~cm})$ nor more than 0.081 inch $(0.206 \mathrm{~cm})$, which limits correspond, respectively, to No. 15 and No. 12 B. \& S. gage sheet brass. The collars on which the propeller blades are mounted shall have horizontal and vertical dimensions not greater than 0.4 inch $(1.016 \mathrm{~cm})$.

The plane of the center of the upper propeller shall be 0.4 inch $(1.016 \mathrm{~cm})$ below the level of the rim of the cup. The plane of the center of the lower propeller shall be 2.0 inches $(5.08 \mathrm{~cm})$ below the level of the rim of the cup. The level of the rim of the cup is in effect the level of the plant part of the portion of the lower surface of the lid inside the rim.

(b) Cover proper.-The cover proper shall be of brass and shall have a rim projecting downward almost to the flange of the cup and fitting the outside of the cup closely. The thickness of the cover, measured just inside the rim shall be not less than 0.031 inch $(0.079 \mathrm{~cm})$ nor more than 0.078 inch $(0.198 \mathrm{~cm})$. There shall be a proper locating device engaging with a corresponding locating device on the flange of the cup.
There shall be four openings in the cover, as indicated in Figure 26.

Opening $A$ is an area defined by arcs of two concentric circles and the intersected lengths of two radii. The radius of the outer circle shall be not less than 0.938 inch $(2.383 \mathrm{~cm})$ nor more than 0.969 inch $(2.461 \mathrm{~cm})$. The chord of the arc of the outer circle shall be not less than 0.500 inch $(1.270 \mathrm{~cm})$ nor more than 0.540 inch $(1.372 \mathrm{~cm})$.

Openings $B$ and $C$ are equal areas, each of the same general form as opening $A$, but of approximatcly half the (angular) width. The radii of the defining inner and outer circles shall be within the limits specified for the radii of the two circles, ares of which partially define opening $A$. The chord of the outer arc for opening $B$ or opening $C$ shall be not less than 0.187 inch $(0.475 \mathrm{~cm})$ nor more than 0.219 inch $(0.556 \mathrm{~cm})$. The sum of the areas of openings $B$ and $C$ shall be not less than 75 per cent nor more than 100 per cent of the area of opening $A$. Openings $B$ and $C$ shall be equally distant from opening $A$ and radii drawn through each of their centers shall be at an angle of not less than $135^{\circ}$ nor more than $140^{\circ}$.

Openings $A, B$, and $C$ need not conform exactly to the shape of geometrical figures bounded by arcs of two concentric circles and intersected lengths of radii. Their boundaries must, however, fall on or between the lines indicated by the limiting values of the dimensional specification of the preceding text and of Figure 26.

Opening $D$ is for a thermometer collar. Its center is approximately $0.75 \mathrm{inch}(1.905 \mathrm{~cm})$ from the center of the lid and on a radius at an angle of not less than $50^{\circ}$ nor more than $60^{\circ}$ from a radius passing through the center of opening $C$. The thermometer collar shall have an inside diameter of approximately 0.5 inch $(1.27 \mathrm{~cm})$. It shall be set at an angle of not less than $10^{\circ}$ nor more than $15^{\circ}$ from the perpendicular.

(c) Shutter.-The lid shall be equipped with a brass shutter, approximately 0.094 inch $(0.239 \mathrm{~cm})$ thick operating on the plane of the upper surface of the lid. The shutter shall be so shaped and mounted that it rotates on the axis of the horizontal center of the lid between two stops so placed that when in one extreme position the opcnings $A, B$, and $C$ of the lid are completely closed and when in the other extremc position these orifices are completely opened.

(d) Flame-exposure device.-The flame-exposure device shall have a tip with an opening 0.027 inch $(0.069 \mathrm{~cm})$ to 0.031 inch $(0.079 \mathrm{~cm})$ in diameter. The flame-exposure device shall be equipped with an operating mechanism which, when the shutter is in the "open" position, depresses the tip so that the center of the orifice is between the planes of the under and upper surfaces of the lid proper at a point on a radius passing through the center of the larger opening $A$ and approximately 0.1 inch $(0.254$ $\mathrm{cm})$ from the outer edge of the opening.

NoTE. - A pilot flame for automatic relighting of the exposure flame should be provided.

A bead 0.156 inch $(0.396 \mathrm{~cm})$ in diameter, of some suitable material, may be mounted on the lid so that the size of the test flame can be regulated by comparison.

The mechanism operating the shutter should be of the spring type and constructed so that when at rest the shutter shall exactly close the three openings. When operated to the other extreme the three openings in the lid shall be exactly open and the tip of the exposure tube shall be fuily depressed.

\section{Stove}

Heat shall be supplied to the cup by means of a properly designed stove which is equivalent to an air bath. This stove shall consist of (1) an air bath 
and (2) a top plate on which the flange of the cup rests.

(a) A ir bath. - The air bath shall have a cylindrical interior 1.625 inches $(4.128 \mathrm{~cm})$ to 1.656 inches $(4.206 \mathrm{~cm})$ deep and a diameter not less than 0.125 inch $(0.317 \mathrm{~cm})$ nor more than 0.156 inch $(0.396 \mathrm{~cm})$ greater than the outside diameter of the cup. The air bath may be either a flame-heated metal casting or an electric-resistance element. (b) Top plate.-The top plate shall be of metal. The total distance from the upper surface of the plate to the bottorn of the air bath shall exceed the distance from the under surface of the flange to the bottom of the cup by not less than 0.063 inch $(0.160$ $\mathrm{cm})$ nor more than 0.125 inch $(0.317 \mathrm{~cm})$.

The top plate shall be mounted with an air gap between it and the air bath. The top plate may be attached to the air bath by means of three screws

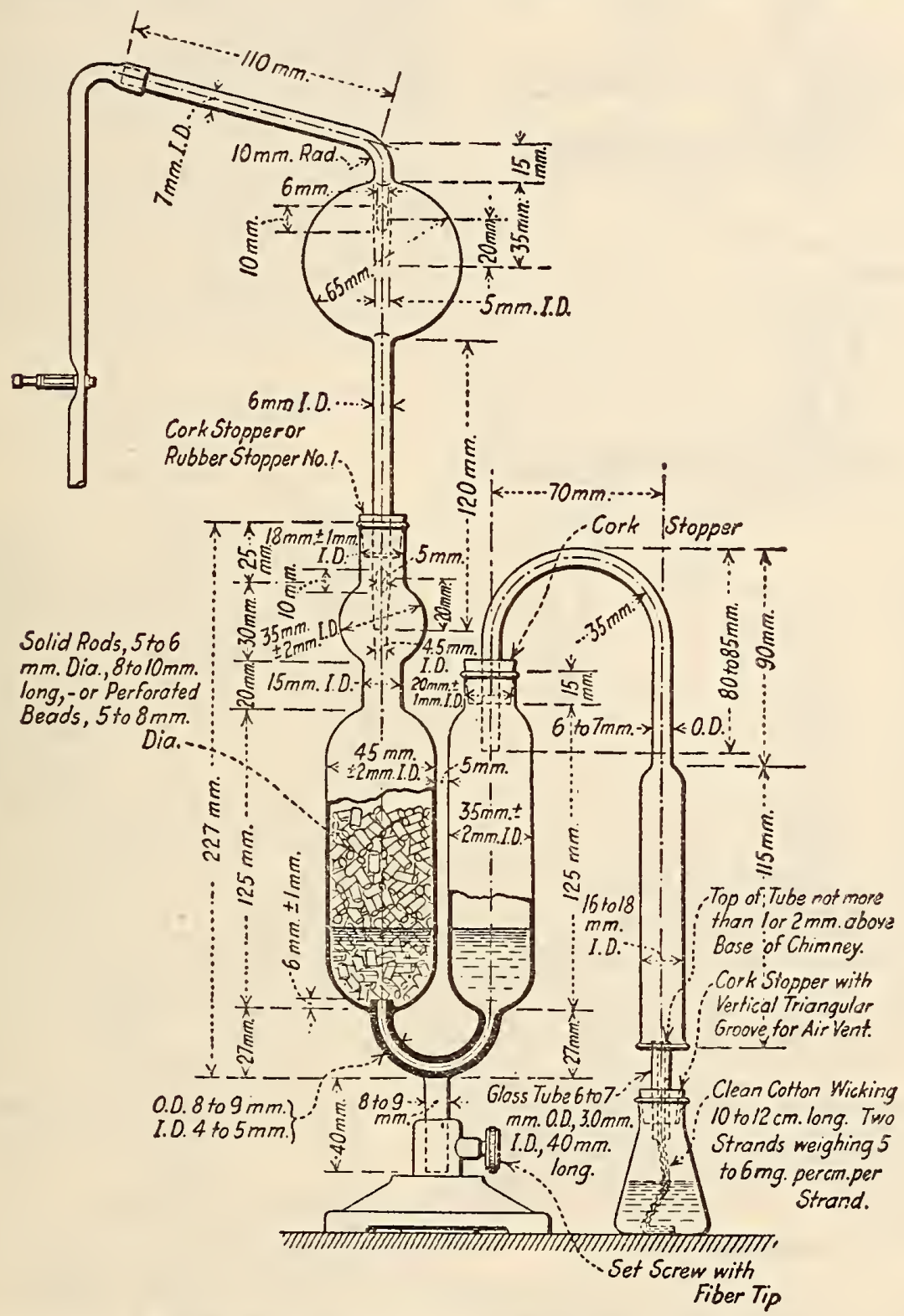

FIGURE 24.-Apparatus for determination of sulphur in oils

Nors.- If the heating element is a flame-heated metal casting it shall be so designed and used that the temperature of bottom and walls is approximately the same. On this account it should he not less than 0.25 inch $(0.635 \mathrm{~cm})$ thick. The casting shall be designed so that products of combustion of the flame can not pass up and in contact with the cup.

If the air bath is of the electric-resistance type it shall be con. structed so that all parts of the interior suriace are heated equally. This necessitates an even distrihution of resistance wire over bottom and walls and a method of construction such that heat is given out from the whole core of the resistance element rather than directly from the wire. and spacing bushings. The spacing bushings should be of proper thickness to define the air gap which shall be not less than 0.125 inch $(0.317 \mathrm{~cm})$ nor more than 0.187 inch $(0.475 \mathrm{~cm})$. The spacing bushings shall be not more than 0.375 inch $(0.952$ $\mathrm{cm}$ ) in diameter.

\section{Thermometer}

Two standard thermometers shall be used with the A. S. T. M. Pensky-Martens tester. The low 
range, "P. M. and tag" thermometer shall be used for tests when the indicated reading falls within the limits $20^{\circ}$ to $200^{\circ} \mathrm{F}$. The "P. M. high" thermometer shall be used for tests when the indicated reading falls within the limits $230^{\circ}$ to $700^{\circ} \mathrm{F}$. For
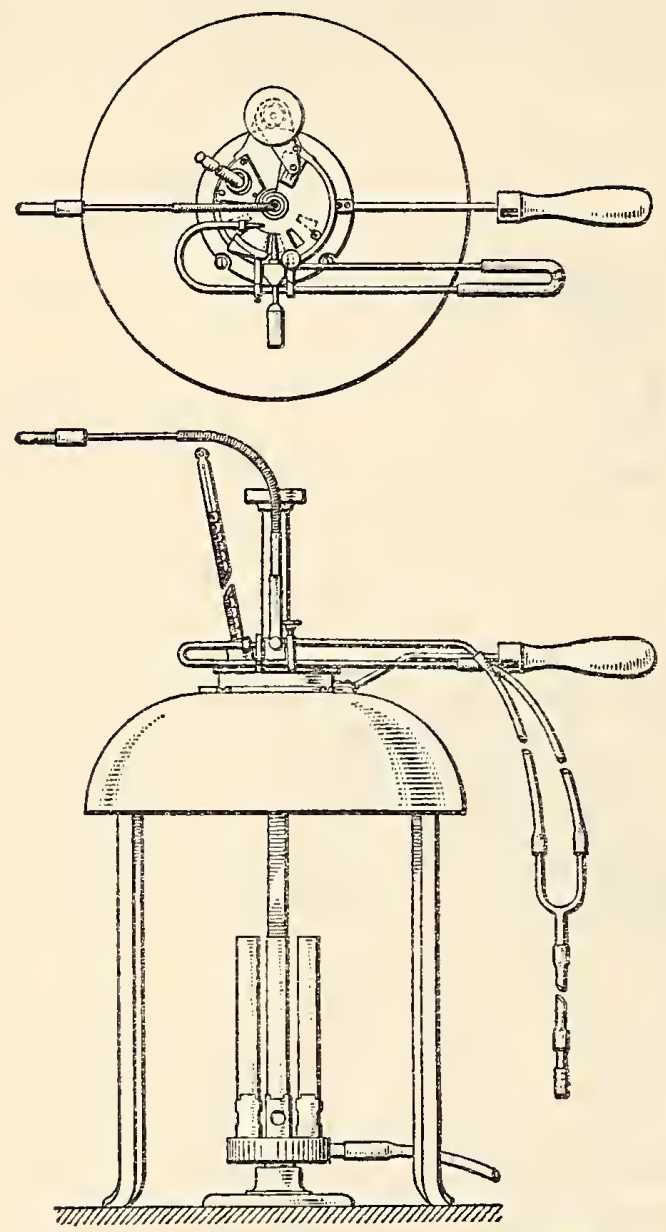

Figure 25.-The Pensky-Marlens tester

the range $200^{\circ}$ to $230^{\circ} \mathrm{F}$. either thermometer may be employed, depending on the convenience of the operator. The thermometers shall comply with the requirements of Nos. 13 and 14 . (See table of A. S. T. M. thermometers, 500.2, p. 2.)

Thermometers shall be mounted so that the bottom of the bulb is 1.75 inches $(4.445 \mathrm{~cm})$ below the level of the rim of the cup (which corresponds to the level of the lower surface of the portion of the lid inside the rim).

\section{PROCEDURE}

(a) All parts of the cup and its accessories shall be thoroughly clean and dry before starting the test. Particular care shall be taken to avoid the presence of any gasoline or naphtha used to clean the apparatus after a previous test.

(b) The cup shall be filled with the oil to be tested up to the level indicated by the filling mark.

(c) The lid shall be placed on the cup and the latter set in the stove. Care shall be taken to have the locating devices properly engaged. The thermometer shall be inserted. If it is known that the oil will flash above $220^{\circ} \mathrm{F}$. the "P. M. high" thermometer may be selected; otherwise, it is preferable to start with the "P. M. and tag" thermometer and change in case a temperature of $220^{\circ}$ to $230^{\circ} \mathrm{F}$. is reached.

(d) The test flame shall be lighted and adjusted so that it is of the size of a bead $5 / 32$ inch $(3.97 \mathrm{~mm})$ in diameter.

(e) Heat shall be supplied at such a rate that the temperature read on the thermometer increases not less than $9^{\circ}$ nor more than $11^{\circ} \mathrm{F}$. per minute. The stirrer shall be turned at a rate of from one to two revolutions per second.

(f) Application of the test flame shall be made at each temperature reading which is a multiple of $2^{\circ} \mathrm{F}$. up to $220^{\circ} \mathrm{F}$. For the temperature range above $220^{\circ} \mathrm{F}$., application shall be made at each temperature reading which is a multiple of $5^{\circ} \mathrm{F}$. The first application of the test flame shall be made at a temperature at least $30^{\circ} \mathrm{F}$. below the actual flash point. Application of the test flame shall be made by operating the device controlling the shutter and test-flame burner so that the flame is lowered in one-half second, left in its lowered position for one second, and quickly raised to its high position.
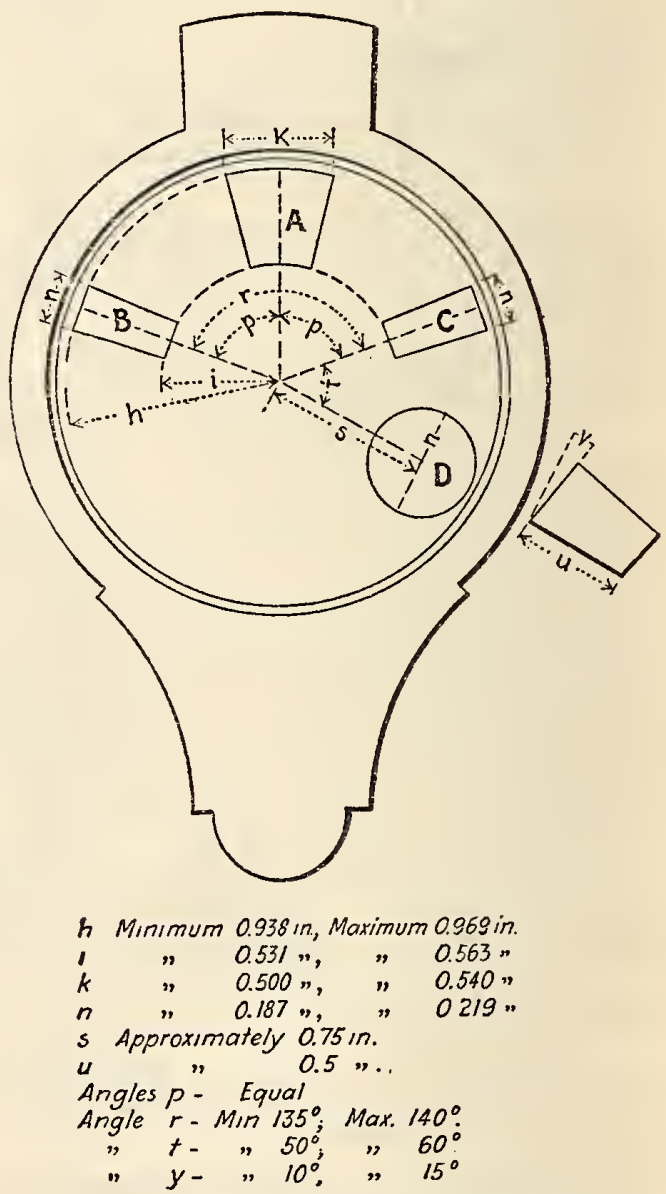

Figure 26.-Cover for Pensky-Martens tester

Stirring shall be discontinued during the application of the test flame.

The flash point is taken as the temperature read on the thermometer at the.time of the flame application that causes a distinct flash in the interior of the cup. The true flash must not be confused with the bluish halo that sometimes surrounds the test 
flame for the applications preceding the one that causes the actual flash.

The barometric pressure shall be observed and recorded. No corrections shall be made except in case of dispute, when the flash point figure shall be corrected according to the following rule:

For each inch $(25 \mathrm{~mm})$ below 29.92 inches $(760$ $\mathrm{mm}$ ) barometric reading add $1.6^{\circ} \mathrm{F}$. to the flash point.

For each inch $(25 \mathrm{~mm})$ above 29.92 inches $(760$ $\mathrm{mm}$ ) barometric reading subtract $1.6^{\circ} \mathrm{F}$. from the flash point.

American Society for Testing Materials, standard method of test for water in petroleum products and other bituminous materials, serial designation D95-28; 1928 .

See 502.2 , page 38 .

American Society for Testing Materials, standard method of test for water and sediment in petroleum products, by means of centrifuge, serial designation D96-28; 1928.

See 502.2 , page 39 .

American Society for Testing Materials, standard method of test for sulphur in petroleum oils heavier than illuminating oil, serial designation D129-27; 1927.

Approved as American Standard by the American Engineering Standards Committee, now the American Standards Association.

\section{APPARATUS}

1. The oxygen bomb shall have a capacity of not less than $300 \mathrm{cc}$, and shall be of a design or construction such that no leaks shall occur at any pressure or temperature generated during the test, and such that when the bomb is open, liquid contents can be easily and completely drained. The inner surfaces shall be of materials that are chemically and physically resistant to the process or products of combustion. The gaskets, insulating materials, etc., shall be, as far as possible, physically and chemically resistant, and in no event shall they undergo any reaction which would increase or decrease the sulphur content of the bomb liquors.

2. The oil cup shall be of platinum, glazed silica, or other suitable material, with a capacity of not less than 2.5 nor more than $5 \mathrm{cc}$.

3. If a platinum oil cup is used, the fuse wire shall be of platinum; if a glazed silica oil cup is used, the fuse wire may be of either platinum or iron. No. 35 B. \& S. gage is a convenient size.

4. (a) The distilled water and all reagents should be sulphur free; but in such cases where it is necessary to employ reagents not sulphur free, blanks shall be run and the figures thus obtained used to correct the results of actual determinations.

(b) The barium chloride solution shall contain $100 \mathrm{~g}$ of $\mathrm{BaCl}_{2} \cdot 2 \mathrm{H}_{2} \mathrm{O}$ per liter.

\section{PROCEDURE}

5. (a) Twenty cubic centimeters of distilled water shall be placed in the bottom of the bomb. From 0.6 to $0.8 \mathrm{~g}$ of the oil to be tested shall be placed in the weighed oil cup and the weight of this charge shall be determined to an accuracy of at least $\pm 0.002 \mathrm{~g}$. The cup shall be placed in the proper position in the bomb, the ignition mechanism arranged, and the bomb closed. Oxygen shall be admitted slowly until a pressure is reached as indicated by the following table:

\begin{tabular}{|c|c|}
\hline Capacity of bomb & $\begin{array}{c}\text { Minimum } \\
\text { gauge pres- } \\
\text { sure }\end{array}$ \\
\hline & \\
\hline & $\begin{array}{c}\text { Atmos- } \\
\text { pheres }\end{array}$ \\
300 to 350 & 40 \\
350 to 400 & 35 \\
400 to 450 & 39 \\
450 to $500 \ldots$ & 27.5 \\
Above $500 \ldots \ldots$ & 25 \\
\hline
\end{tabular}

The lcads from the firing circuit shall be attached, the bomb placed in a bucket of cold water, and ignited. The boinb shall be allowed to stand in the water for 10 minutes and shall then be removed. The valve of the bomb shall be opened, allowing the gas to escape at an approximately even rate so that the pressure is reduced to atmospheric in not less than one minute. The bomb shall be opened, and all parts of its interior, including the oil cup, rinsed with a fine jet of distilled water. All washings, which should not amount to more than $350 \mathrm{cc}$, shall be collected in a beaker. Particular care should be taken not to lose, by splashing or otherwise, any of the liquid contents of the bomb. The washings shall be filtered through a washed "qualitative" filter paper. The filter shall be washed thoroughly. Two cubic centimeters of concentrated $\mathrm{HCl}$ and $10 \mathrm{cc}$ of saturated bromine water shall be added to the filtrate. The solution shall be evaporated to about 75 cc on a steam bath or hot plate. Ten cubic centimeters of hot barium chloride solution shall be added in a fine stream or dropwise to the hot solution, stirring during the addition and for two minutes afterwards. The solution shall be allowed to stand over night, or shall be kept hot for one hour on the steam bath or hot plate, allowing the prccipitate to settle for another hour, while cooling. The supernatant liquid shall be filtered through an "ashless quantitative" filter paper, the precipitate washed with water, first by decantation, then on the filter, till free from chloride. The paper and precipitate shall be transferred to a suitable weighed crucible, dried at low hcat till moisture is evaporated, the paper charred (without flaming), and finally ignited at a good red heat till the precipitate is just burned white. A satisfactory means of accomplishing these operations is to place the crucible containing the wet filter paper in a cold electric muffle furnace and to turn on the current. Drying, charring, and ignition will usually occur at the desired rate.

(b) After ignition is complete the crucible shall be allowed to cool to room temperature and weighed. The use of a desiccator is not recommended.

(c) From the increase in weight of the crucible the percentage of sulphur shall be calculated from the formula:

$$
\text { Percentage of sulphur }=\frac{\text { grams of } \mathrm{BaSO}_{4} \times 13.734}{\text { grams of oil used }}
$$

\section{ACCURACY}

6. Duplicate determinations should agree to \pm 2 per cent on the basis of the sulphur found. 
American Society for Testing Materials, tentative method of test for color of refined petroleum oil by means of Saybolt chromometer, serial designation: D156-23T; 1923.

1. The color of all refined oils (naphthas, kerosenes, etc.) shall be determined by means of the Saybolt chromometer.

\section{APPARATUS}

2. (a) The Saybolt chromometer shall consist of two glass tubes having an internal diameter of not less than $14 \mathrm{~mm}$ nor more than $16 \mathrm{~mm}$. One glass tube, 20 inches in length, shall be pernuanently closed at one end with a colorless, plano glass disk, and be mounted in a suitable metal collar, provided with a small pet cock, for the purpose of draining the tube. This glass tube shall be graduated in $1 / 8$-inch divisions, numbered in inches. The other tube, 19 inches in length, shall be open at both ends, one end being mounted in a suitable metal collar which will hold the color standards and a black metal diaphragm with a circular aperture $12 \mathrm{~mm}$ in diameter. The mounting of this tube shall be such as to give equal length to both tubes. The tubes shall be mounted vertically and shall be covered at their upper end with a removable diaphragmed metal cap about 1 inch in length and of sufficient diameter to easily slip over the end of the tubes. The circular diaphragm aperture shall be $14 \mathrm{~mm}$ in diameter.

(b) The instrument shall be provided with a suitable optical head consisting of prisms and eyepiece. The prisms shall be of a suitable form, matched in their refracting angles and areas, and so mounted as to avoid possioility of disarrangement. They shall be so arranged that the light rays passing through the tubes shall be deflected to a central aperture which is viewed by the eyepiece. The arrangement shall be such as to provide a circular field of vision, the two halves of which are illuminated by the light transmitted by the sample and the color standard, respectively.

3. (a) Light shall be provided by means of a reflecting mirror with a finely frosted surface set at a suitable angle and so arranged that the reflected light shall pass through suitable openings and through the glass tubes in parallel rays; or it shall be provided by projecting a diffused light directly up through the tubes, from the base of the instrument.

(b) The light shall be supplied by an artificial daylight lamp so arranged as to project a diffused light on the reflecting mirror or directly up througb the tubes.

Color standards, corresponding to heights of oil

\begin{tabular}{|c|c|c|c|c|c|}
\hline \multirow{2}{*}{$\begin{array}{c}\text { Number of } \\
\text { disks }\end{array}$} & \multicolumn{2}{|c|}{$\begin{array}{l}\text { Saybolt chro- } \\
\text { mometer }\end{array}$} & \multirow{2}{*}{$\begin{array}{l}\text { Number of } \\
\text { disks }\end{array}$} & \multicolumn{2}{|c|}{$\begin{array}{l}\text { Saybolt chro- } \\
\text { mometer }\end{array}$} \\
\hline & $\begin{array}{l}\text { Height } \\
\text { of oil }\end{array}$ & $\begin{array}{l}\text { Color } \\
\text { shade }\end{array}$ & & $\begin{array}{c}\text { Height } \\
\text { of oil }\end{array}$ & $\begin{array}{l}\text { Color } \\
\text { shada }\end{array}$ \\
\hline $\begin{array}{l}1 \\
1 \\
1 \\
1 \\
1 \\
1\end{array} \ldots$ & $\begin{array}{r}\text { Inches } \\
20.0 \\
18.0 \\
16.0 \\
14.0 \\
12.0 \\
10.75\end{array}$ & $\begin{array}{l}+25 \\
+24 \\
+23 \\
+22 \\
+21 \\
+20\end{array}$ & $\begin{array}{l}2 . \\
2 \\
2 . \\
2 \\
2 . \\
2 .\end{array}$ & $\begin{array}{r}\text { Inches } \\
5.50 \\
5.25 \\
5.00 \\
4.75 \\
4.50 \\
4.25\end{array}$ & $\begin{array}{r}+4 \\
+3 \\
+2 \\
+1 \\
0 \\
-1\end{array}$ \\
\hline $\begin{array}{l}1 \ldots \ldots- \\
1 \\
1 \\
1 \\
2 \ldots \ldots\end{array}$ & $\begin{array}{r}9.50 \\
8.25 \\
7.25 \\
6.25 \\
10.50\end{array}$ & $\begin{array}{l}+19 \\
+18 \\
+17 \\
+16 \\
+15\end{array}$ & & $\begin{array}{l}4.00 \\
3.75 \\
3.625 \\
3.50 \\
3.375\end{array}$ & $\begin{array}{l}-2 \\
-3 \\
-4 \\
-5 \\
-6\end{array}$ \\
\hline $\begin{array}{l}2 \\
2 \\
2 \\
2 \\
2\end{array}$ & $\begin{array}{l}9.75 \\
9.00 \\
8.25 \\
7.75 \\
7.25\end{array}$ & $\begin{array}{l}+14 \\
+13 \\
+12 \\
+11 \\
+10\end{array}$ & $\begin{array}{l}2 \\
2 \\
2 \\
2 \\
2\end{array}$ & $\begin{array}{l}\text { 3. } 25 \\
3.125 \\
3.00 \\
2.875 \\
2.75\end{array}$ & $\begin{array}{l}-7 \\
-8 \\
-9 \\
-10 \\
-11\end{array}$ \\
\hline $\begin{array}{l}2 \\
2 \\
2 \\
2 \\
2\end{array}$ & $\begin{array}{l}6.75 \\
6.50 \\
6.25 \\
6.00 \\
5.75\end{array}$ & $\begin{array}{l}+9 \\
+8 \\
+7 \\
+6 \\
+5\end{array}$ & $\begin{array}{l}2 \\
2 \\
2 \\
2 \\
2\end{array}$ & $\begin{array}{l}2.625 \\
2.50 \\
2.375 \\
2.25 \\
2.125\end{array}$ & $\begin{array}{l}-12 \\
-13 \\
-14 \\
-15 \\
-16\end{array}$ \\
\hline
\end{tabular}

(c) The artificial daylight lamp' shall consist of a C2 national mazda lamp, the rays of light from which shall pass through a glass color screen of such composition and color, as, in conjunction with the C2 national mazda lamp, will yield by spectrum analysis a light closely approximating northern daylight.

In case electric current is not available, the chromometer may be used by exposing the mirror to northern daylight with no colored objects in the immediate foreground, but the artificial daylight lamp shall be required in referee work.

4. The color standards shall consist of suitable glass disks.

5. (a) The Saybolt chromometer shall be assembled and the light source so adjusted that a diffused light, with absence of glare or shadow, shall be projected on the reflecting mirror, or projected directly through the tubes from the base of the instrument, thereby eliminating the mirror. Light from all other sources shall be excluded.

(b) The oil tube shall be cleaned by rinsing with some of the oil to be tested, care being taken to allow the tube to drain thoroughly. The pet cock on the oil tube shall then be closed, and the tube shall be filled with the oil to be tested to a height of 10.5 inches.

(c) The measurement of the color shall be made by using two standard color disks when the oil is darker than the two disks and by using one disk when the oil is lighter than the two disks. After determining the number of disks to be used and with the proper number in place in the color standard, the oil tube shall contain a sufficient height of oil for the color of the oil to be decidedly darker than the color standard, additional oil being added if necessary. The oil shall then be drawn off slowly by means of the pet cock until the oil appears slightly darker than the color standard. The oil shall then be drawn down to the nearest height corresponding to a standard color shade as shown in the table. If the color of the oil observed through the eyepiece is still darker than the color standard, the oil shall be drawn down to the next height given in the table, and examined again. This operation shall be continued until the oil and color standard match or show questionable differences. The column of oil shall be lowered one shade more and if the oil is unmistakably lighter than the color standard, the previous color shade shall be recorded as the Saybolt chromometer color.

\section{APPENDIX}

The following examples of the procedure are given:

\section{Using 1 disk:}

$\begin{array}{ccc}\text { Oil darker at height of } & \\ \text { Do } & & \end{array}$

oil questionable at height of -

Oil lighter at height of

Color is

Color is-

Using 2 disks:

Oil darker at height of

Oil questionable at height of

Oil lighter at lheight of -

Color is

American Society for Testing Materials, standard methods of testing gas oils, serial designation: D158-28; 1928.

1. The gravity shall be determined at $60^{\circ} / 60^{\circ}$ : 9 . $\left(15.6^{\circ} / 15.6^{\circ} \mathrm{C}\right.$.) in a pyknometer, accurately sta? ardized, or by means of a hydrometer or West. balance, the accuracy of which has been verifi The gravity shall be recorded as specific gravity gravity, degrees A, P. I., at $60^{\circ} / 60^{\circ} \mathrm{F}$. (15.6\% $15.6^{\circ} \mathrm{C}$.). 


\section{DISTILLATION}

\section{APPARATUS}

Flask

2. The distillation flask shall be a Saybolt distilling flask, the dimensions and permissible variations being as follows:

\begin{tabular}{|c|c|c|c|}
\hline & $\begin{array}{l}\text { Centi- } \\
\text { meters }\end{array}$ & Inches & $\begin{array}{l}\text { Toler- } \\
\text { ances }\end{array}$ \\
\hline $\begin{array}{l}\text { Diameter of balb, outside } \\
\text { Diameter of neck, inside. } \\
\text { Orer all length, neck plus bulb } \\
\text { Length of vapor tube } \\
\text { Diameter of vapor tube, outside.... } \\
\text { Diameter of vapor tube, inside } \\
\text { Thickness of vapor tube wall }\end{array}$ & $\begin{array}{r}8.57 \\
1.50 \\
21.50 \\
17.50 \\
.60 \\
.40 \\
.10\end{array}$ & $\begin{array}{r}3.37 \\
59 \\
8.16 \\
6.89 \\
.24 \\
.16 \\
.04\end{array}$ & $\begin{array}{c}c m \\
0.3 \\
.1 \\
.3 \\
.5 \\
.05 \\
.05 \\
.02\end{array}$ \\
\hline
\end{tabular}

The center of the vapor tube shall be $6.5 \mathrm{~cm}$ $(2.56$ inches $) \pm 0.3 \mathrm{~cm}$, below the top of the neck. The tube is approximately in the middle of the neck and set at an angle of $75^{\circ}, \pm 3^{\circ}$, with the vertical.

\section{Condenser}

3. The condenser ${ }^{25}$ shall consist of a $\% 16$-inch $(14.29$ $\mathrm{mam}$ ) outside diameter, No. 20 Stubbs gage seamless brass tube, 22 inches $(55.88 \mathrm{~cm})$ in length. It shall be set at an angle of $75^{\circ}$ with the vertical and shall be surrounded with a bath 15 inches $(38.1 \mathrm{~cm})$ in length, approximately 4 inches $(10.16 \mathrm{~cm}$ ) in width by 6 inches $(15.24 \mathrm{~cm})$ in height. The bath shall be provided with suitable connections for circulating water through it. The lower end of the condenser tube shall be cut off at an acute angle, and curved downward for a length of 3 inches $(7.62 \mathrm{~cm})$ and slightly backward so as to insure contact with the wail of the receiving graduate at a point approximately 1 to $1 \frac{1 / 4}{4}$ inches ( 2.54 to $3.18 \mathrm{~cm}$ ) below the top of the graduate when it is in position to receive the distillate.

\section{Shield}

4. The shield ${ }^{26}$ shall be made of approximately No. 22 gage sheet metal and shall be 19 inches $(48.26 \mathrm{~cm})$ in height, 11 inches $(27.94 \mathrm{~cm})$ in length, and 8 inches $(20.32 \mathrm{~cm})$ in width, with a door on one narrow side, with two openings, 1 inch $(2.54 \mathrm{~cm})$ in diameter, equally spaced, in each of the two narrow sides, and with a slot cut in one side for the vapor tube. The centers of these four openings shall be $8 \frac{1}{2}$ inches $(21.59 \mathrm{~cm})$ below the top of the shield. There shall also be three $1 / 2-i n c h(1.27 \mathrm{~cm})$ holes in each of the four sides with their centers 1 inch $(2.54 \mathrm{~cm})$ above the base of the shield.

\section{Ring Support and Hard Asbestos Boards}

5. The ring support may be of the ordinary laboratory type, 4 inches $(10.16 \mathrm{~cm})$ or larger in diameter, and shall be supported on a stand inside the shield. There shall be two hard asbestos boards: One 6 by 6 by $1 / 4$ inch ( 15.24 by $15.24 \mathrm{~cm}$ by $6.35 \mathrm{~mm}$ ) with a hole $2^{3 / 4}$ inches $(6.99 \mathrm{~cm})$ in diameter in its center, the sides of which shall be perpendicular to the surface; the other, an asbestos board to fit tightly inside the shield, with an opening 4 inches $(10.16 \mathrm{~cm})$ in diameter concentric with the ring support. These shall be arranged as follows: The second asbestos $\because$ ooard shall be placed on the ring and the first or maller asbestos board on top so that it may be lesteved in accordance with the directions for placing .bos.

is A condenser conforming to these specifications is illustrated in A. S. T. M. D86. See 503.0, p. 44

${ }^{28} \mathrm{~A}$ shield conforming to these specifications is illustrated in D85. the distilling flask. Direct heat shall be applied to the flask only through the $23 / 4-$ inch $(6.99 \mathrm{~cm})$ opening in the first asbestos board.

\section{Gas Burner}

6. The burner shall be so constructed that sufficient heat can be obtained to distill the product at the uniform rate specified below. The flame should never be so large that it spreads over a circle of diameter greater than $41 / 2$ inches $(11.43 \mathrm{~cm})$ on the under surface of the asbestos board. A sensitive regulating valve and gas pressure governor are desirable adjuncts, as they give complete control of heating.

\section{Thermometer}

7. The A. S. T. M. high distillation thermometer, conforming to the requirements specified for thermometer No. 2, see table of A. S. T. M. thermometers, 500.2 , p. 2, shall be used.

\section{Graduates}

8. (a) For sample.-A graduate or pipette graduated to deliver $200 \mathrm{cc}, \pm 1.0 \mathrm{cc}$, may be used for measuring the sample to be tested.

(b) For fractions. - The graduates used for measuring fractions shall be of the cylindrical type, of uniform diameter, with a pressed or molded base and a lipped top. The cylinder shall be graduated to contain $100 \mathrm{cc}$ and the graduated portion shall be not less than 7 inches $(17.78 \mathrm{~cm})$ nor more than 8 inches $(20.32 \mathrm{~cm})$ in length. It shall be graduated in single cubic centimeters and each fifth mark shall be distinguished by a longer line. It shall be numbered from the bottom at intervals of $10 \mathrm{cc}$. The over-all height of the graduate shall be not less than $93 / 4$ inches $(24.8 \mathrm{~cm})$ nor more than $10^{1 / 4}$ inches $(26.0 \mathrm{~cm})$. The graduations shall not be in error by more than $1 \mathrm{cc}$ at any point on the scale.

\section{PROCEDURE}

9. (a) Provision shall be made for circulating water through the condenser.

(b) The condenser tube shall be swabbed to remove any liquid remaining from the previous test. A piece of soft, lint-free cloth attached to a cord or copper wire may be used for this purpose.

(c) A volume of water-free oil equivalent to 200 cc at $55^{\circ}$ to $65^{\circ} \mathrm{F} .\left(12.8^{\circ}\right.$ to $18.3^{\circ} \mathrm{C}$. $)$ shall be placed in the flask.

(d) The thermometer, provided with a cork, shall be fitted tightly into the flask so that it will be in the middle of the neck and so that the lower end of the capillary tube is on a level with the inside of the bottom of the vapor outlet tube at its junction with the neck of the flask.

(e) The charged flask shall be placed over the $23 / 4^{-}$ inch $(6.99 \mathrm{~cm})$ opening in the asbestos board with the vapor-outlet tube inserted into the condenser tube. A tight connection may be made by means of a cork through which the vapor tube passes. The position of the flask shall be so adjusted that the vapor tube extends into the condenser tube not less than 1 inch $(2.54 \mathrm{~cm})$ nor more than 2 inches $(5.08 \mathrm{~cm})$.

(f) A clean, dry graduated cylinder shall be placed at the outlet of the condenser tube in such position that the condenser tube shall extend into the graduate approximately 1 inch $(2.54 \mathrm{~cm})$ but not below the 100 ce mark.

10. When everything is in readiness, water shall be circulated through the condenser bath at such a rate that the water overflowing the condenser shall be at a temperature of $90^{\circ}$ to $100^{\circ} \mathrm{F}$. $\left(32.2^{\circ}\right.$ to $37.8^{\circ}$ C.). Heat shall be applied to the contents of the flask at a uniform rate, so regulated that the first 
drop of condensate falls from the condenser in not less than 10 nor more than 15 minutes. When the first drop falls from the end of the condenser, the reading of the distillation thermometer shall be recorded as the initial boiling point. The receiving cylinder shall then be moved so that the end of the condenser tube shall touch the side of the cylinder. The heat shall then be so regulated that the distillation will proceed at a uniform rate of not less than 8 cc nor more than 10 cc per minute. A fraction shall be separated at every temperature point above the initial boiling point that is a multiple of $50^{\circ} \mathrm{F} .{ }^{27}$ When the temperature of the distilling vapors reaches $500^{\circ} \mathrm{F}$. $\left(260^{\circ}\right.$ C. $)$, the temperature of the water in the condenser bath shall be adjusted to approximately $140^{\circ} \mathrm{F}$. and the bath maintained between $140^{\circ}$ and $160^{\circ} \mathrm{F}$. $\left(60^{\circ}\right.$ to $71.1^{\circ} \mathrm{C}$.) for the remainder of the test. The distillation shall be continued until a vapor temperature of $700^{\circ} \mathrm{F}$. $\left(371.1^{\circ}\right.$ C. $)$ is reached or until the maximum temperature point is reached. The maximum temperature point may result from complete distillation of the oil below $700^{\circ} \mathrm{F}$. $\left(371.1^{\circ} \mathrm{C}\right.$.), or from cracking. ${ }^{28}$ In case the oil distills completely below $700^{\circ} \mathrm{F}$. $\left(371.1^{\circ}\right.$ C. $)$, the finai adjustment of heat shall be made when a quantity of distillate amounting to 90 per cent of the sample has been collected. The heating shall be continued until the mereury reaches a maximum height and starts to fall consistently; but in no case should the period between the 90 per cent point and the maximum temperature be more than 10 minutes. The highest temperature observed on the distillation thermometer shall be recorded as the maximum temperature.

Sufficient time shall be allowed after distillation is discontinued for the condenser to drain into the final fraction. Each fraction shall be brought to room temperature, the volume read and recorded, and the fraction set aside for the determination of the gravity. The percentage of distillate in each fraction shall be calculated and recorded. The difference between 100 and the sum of the distillates in per cent shall be calculated and recorded as residue and loss.

The gravity of each fraction and of the residue shall be determined at or corrected to $60^{\circ} \mathrm{F}$. $\left(15.6^{\circ}\right.$ C.). The gravity of each fraction shall bc recorded as specific gravity or as gravity, degrees A. P. I.

\section{SULPFIR}

11. The sulphur shall be determined in accordance with A. S. T. M. D129-22T. (See 503.0, p. 53.)

\section{CAREON RESIDUE}

12. The carbon residue shall be determined in accordance with the Conradson method, as deseribed in A. S. T. M. D189. (See 504.0, p. 100.)

${ }^{27}$ When it is agreed to make allowance for the effect of barometric pressure, the observed temperature points at which fractions are to be separated in order to conform to multiples of $50^{\circ} \mathrm{F}$. at $760 \mathrm{~mm}$ shall be calculated by the Sydney Young equation, so expressed as to simplify the application:

$$
C_{f}=0.00012 P\left(460+t_{f}\right)
$$

in which $C_{f}$ is the correction to be added to the observed temperature when the barometer is above $760 \mathrm{~mm}$ and to be subtracted from the observed temperature when the barometer is below $700 \mathrm{~mm}$.

$P$ is the difference in millimeters of mercury (at $32^{\circ} \mathrm{F}$ ) between $760 \mathrm{~mm}$ and the observed barometric pressure, corrceted for instrumental errors.

$t_{f}$ is the temperature point to be corrected.

${ }_{23}$ Cracking will be evidenced by an increase in distilling rate with a thermometer reading that may advance very slowly, remain stationary, or recede, and an effort to adjust the distilling rate will usually result in a decided drop in the temperature reading. When
this condition is observed, it is advisable to discontinue the disthis condition is observed, it is advisable to discontinue the dis-
tillation.

\section{POUR POINT}

13. The pour point shall be determined in accordance with A. S. T. M. D97. (See 502.2, p. 40.)

\section{VISCOSITY}

14. The viscosity shall be determined on the Saybolt universal viscosimeter at $100^{\circ} \mathrm{F}$. in accordance with A. S. T. M. D88. (See 503.0, p. 47.)

\section{WATER}

15. Water shall be determined in accordance with A. S. T. M. D95. (See 502.2 , p. 38.)

American Society for Testing Materials, standard method of test for carbon residue of petroleum products (Conradson carbon residue) serial designation D189-28; 1928.

(This specification furnishes information relative to domestic oil fuels and oils used in the manufacture of gas.)

See 504.0, p. 100

American Society for Testing Materials, standard abridged volume correction table for petroleum oils, serial designation: D206-25; 1925.

Approved as American Standard by the American Engineering Standards Committce, now the American Standards Association.

This abridged table has been prepared by the National Bureau of Standards to meet a demand from the oil industry for a short and convenient table for reducing oil volumes to the basis of $60^{\circ} \mathrm{F}$. $\left(15.5^{\circ} \mathrm{C}\right.$.). It is based on the same data as Table 2 , Bureau of Standards Circular No. 154. The groups, coefficients of expansion, degrees A. P. I. and gravity ranges of the abridged table follow: ${ }^{2 \theta}$

\begin{tabular}{|c|c|c|c|}
\hline Group No. & $\begin{array}{l}\text { Coefficient } \\
\text { of expansion } \\
\text { at } 60^{\circ} \mathrm{F} \text {. }\end{array}$ & $\begin{array}{l}\text { Corre- } \\
\text { sponding } \\
\text { degrees } \\
\text { A. P. I. }\end{array}$ & $\begin{array}{l}\text { Range of group } \\
\text { (degrees A. P. I } \\
\text { at } 60^{\circ} \mathrm{F} . \text { ) }\end{array}$ \\
\hline $\begin{array}{l}\text { I } \\
\text { III } \\
\text { IV } \\
\text { IV } \\
\text { VI } \\
\text { VI }\end{array}$ & $\begin{array}{l}0.0004 \\
.0005 \\
.0006 \\
.0007 \\
.0008 \\
.00085\end{array}$ & $\begin{array}{l}22 \\
44 \\
58 \\
72 \\
86 \\
91\end{array}$ & $\begin{array}{l}\text { Up to } 34.9 \text {. } \\
35 \text { to } 50.9 . \\
51 \text { to } 63.9 . \\
64 \text { to } 78.9 \text {. } \\
79 \text { to } 88.9 \text {. } \\
89 \text { and higher. }\end{array}$ \\
\hline
\end{tabular}

The table shows the volume occupied at $60^{\circ} \mathrm{F}$. by a quantity of oil occupying a unit volume at the indicated temperatures. For example, if at $60^{\circ} \mathrm{F}$. the A. P. I. gravity of the oil is 28 (Group I), 1 gallon of this oil measured at $120^{\circ} \mathrm{F}$. will have a volume of 0.9763 gallon at $60^{\circ} \mathrm{F}$. The values given in the table are in the form of "multipliers"; that is, the volume of oil at the indicated temperature and degrees A. P. I. for each group, multiplied by the corresponding factor in the table, equals the volume at $60^{\circ} \mathrm{F}$. For example, if the A. P. I. gravity of an oil at $60^{\circ} \mathrm{F}$. equals 28 (Group I) and the volume at $120^{\circ} \mathrm{F}$. equals 6,000 gallons, then the volume at $60^{\circ} \mathrm{F}$. equals $6,000 \times 0.9763$, or $5,857.8$ gallons.

20 This table has bcen approved by the Bureau of Standards, the Bureau of Mines, and the American Pctrolcum Institute and is now published as a standard of this society.

This abridged table is not intended to replace the more complete and accurate volume correction table contained in Bureau of Standand accirate volune correction table contalned in Bureau of Standards Circular No. 154, but rather to supplement it and especially to replace the various, abridged tables and aprection factors heretofing accuracy is essential the complete table contained in Circular No. 154 should be used, especially if large volumes or wide temperature ranges are involved. The A. S. T. M. standard (serial designation D20f-25) applies only to the abridged table and should be so understood when written into contracts. 
Standard abridged volume correction table for petroleum oils

\begin{tabular}{|c|c|c|c|c|c|c|c|c|c|c|c|c|c|}
\hline \multirow[b]{2}{*}{$\begin{array}{c}\text { Observed } \\
\text { tempera- } \\
\text { ture } \\
\left(^{\circ} \mathrm{F} .\right)\end{array}$} & \multicolumn{6}{|c|}{$\begin{array}{l}\text { Volume at } 60^{\circ} \mathrm{F} \text {. occupied by unit volume at indicated } \\
\text { temperature }\end{array}$} & \multirow[b]{2}{*}{$\begin{array}{c}\text { Observed } \\
\text { tempera- } \\
\text { ture } \\
\left({ }^{\circ} \mathrm{F} .\right)\end{array}$} & \multicolumn{6}{|c|}{$\begin{array}{c}\text { Volume at } 60^{\circ} \mathrm{F} \text {. occupied by unit volume at indicated } \\
\text { temperature }\end{array}$} \\
\hline & $\begin{array}{c}\text { Group I, } \\
\text { up to } \\
34.9^{\circ} \mathrm{A} . \\
\text { P. I. at } \\
69^{\circ} \mathrm{F} \text {. }\end{array}$ & $\begin{array}{l}\text { Group } \\
\text { II, } 35.0^{\circ} \\
\text { to } 50.9^{\circ} \\
\text { A. P. I. } \\
\text { at } 69^{\circ} \mathrm{F} .\end{array}$ & $\begin{array}{l}\text { Group } \\
\text { III, } 51.0^{\circ} \\
\text { to } 63.9^{\circ} \\
\text { A. P. I. } \\
\text { at } 60^{\circ} \text { F. }\end{array}$ & $\begin{array}{l}\text { Group } \\
\text { IV, } 64.0^{\circ} \\
\text { to } 78.9^{\circ} \\
\text { A. P. I. } \\
\text { at } 60^{\circ} \mathrm{F}\end{array}$ & $\begin{array}{l}\text { Group } \\
\text { V, } 79.0^{\circ} \\
\text { to } 88.9^{\circ} \\
\text { A. P. I. } \\
\text { at } 60^{\circ} \mathrm{F} .\end{array}$ & $\begin{array}{l}\text { Group } \\
\text { VI, } 89.0^{\circ} \\
\text { and } \\
\text { higher, } \\
\text { A. P. I. } \\
\text { at } 60^{\circ} \mathbf{F} \text {. }\end{array}$ & & $\begin{array}{l}\text { Group I, } \\
\text { up to } \\
34.9^{\circ} \mathrm{A} . \\
\mathrm{P} . \mathrm{I} \text {. at } \\
60^{\circ} \mathrm{F} .\end{array}$ & $\begin{array}{l}\text { Group } \\
\text { II, } 35.0^{\circ} \\
\text { to } 50.9^{\circ} \\
\text { A. P. I. } \\
\text { at } 60^{\circ} \mathrm{F} .\end{array}$ & $\begin{array}{l}\text { Group } \\
\text { III, } 51.0^{\circ} \\
\text { to } 63.9^{\circ} \\
\text { A. P. I. } \\
\text { at } 60^{\circ} \mathrm{F} \text {. }\end{array}$ & $\begin{array}{l}\text { Group } \\
\text { IV, } 64.0^{\circ} \\
\text { to } 78.9^{\circ} \\
\text { A. } \mathrm{P} \text { I. } \\
\text { at } 60^{\circ} \mathrm{F} \text {. }\end{array}$ & $\begin{array}{l}\text { Group } \\
\text { V, } 79.0^{\circ} \\
\text { to } 88.9^{\circ} \\
\text { A. P. I. } \\
\text { at } 60^{\circ} \mathrm{F}\end{array}$ & $\begin{array}{l}\text { Group } \\
\text { VI } 89.0^{\circ} \\
\text { and } \\
\text { higher, } \\
\text { A. P. I. } \\
\text { at } 60^{\circ} \mathbf{F} \text {. }\end{array}$ \\
\hline & $\begin{array}{l}1.0212 \\
1.0238 \\
1.0234 \\
1.0230 \\
1.0225\end{array}$ & $\begin{array}{l}1.0297 \\
1.0292 \\
1.0287 \\
1.0283 \\
1.0278\end{array}$ & $\begin{array}{l}1.0361 \\
1.0355 \\
1.0349 \\
1.0343 \\
1.0337\end{array}$ & $\begin{array}{l}1.0419 \\
1.0412 \\
1.0405 \\
1.0398 \\
1.0391\end{array}$ & $\begin{array}{l}1.0476 \\
1.0468 \\
1.0460 \\
1.0453 \\
1.0445\end{array}$ & $\begin{array}{l}1.0501 \\
1.0493 \\
1.0484 \\
1.0476 \\
1.0467\end{array}$ & $\begin{array}{l}60 \\
61 . \\
62 . \\
62 . \\
64\end{array}$ & $\begin{array}{r}1.0000 \\
.9996 \\
.9992 \\
.9988 \\
.9984\end{array}$ & $\begin{array}{r}1.0000 \\
.9995 \\
.9990 \\
.9955 \\
.9980\end{array}$ & $\begin{array}{r}1.0000 \\
.9994 \\
.9988 \\
.9982 \\
.9976\end{array}$ & $\begin{array}{r}1.0000 \\
.9993 \\
.9986 \\
.9979 \\
.9972\end{array}$ & $\begin{array}{r}1.0000 \\
.9992 \\
.9984 \\
.9976 \\
.9968\end{array}$ & $\begin{array}{r}1.0000 \\
.9992 \\
.9983 \\
.9975 \\
.9966\end{array}$ \\
\hline & $\begin{array}{l}1.0222 \\
1.0218 \\
1.0214 \\
1.0210 \\
1.0205\end{array}$ & $\begin{array}{l}1.0273 \\
1.0268 \\
1.0263 \\
1.0258 \\
1.0253\end{array}$ & $\begin{array}{l}1.0331 \\
1.0325 \\
1.0319 \\
1.0313 \\
1.0307\end{array}$ & $\begin{array}{l}1.0384 \\
1.0377 \\
1.0370 \\
1.0364 \\
1.0357\end{array}$ & $\begin{array}{l}1.0437 \\
1.0429 \\
1.0421 \\
1.0414 \\
1.0406\end{array}$ & $\begin{array}{l}1.0459 \\
1.0451 \\
1.0443 \\
1.0434 \\
1.0425\end{array}$ & $\begin{array}{l}65 . \\
66 . \\
67 .\end{array}$ & $\begin{array}{l}.9980 \\
.9976 \\
.9972 \\
.9958 \\
.9964\end{array}$ & $\begin{array}{l}.9975 \\
.9970 \\
.9965 \\
.9951 \\
.9956\end{array}$ & & & $\begin{array}{l}.9960 \\
.9952 \\
.9944 \\
.9935 \\
.9927\end{array}$ & $\begin{array}{l}.9958 \\
.9949 \\
.9941 \\
.9932 \\
.9924\end{array}$ \\
\hline & $\begin{array}{l}1.0202 \\
1.0198 \\
1.0194 \\
1.0189 \\
1.0185\end{array}$ & $\begin{array}{l}1.0248 \\
1.0243 \\
1.0238 \\
1.0233 \\
1.0228\end{array}$ & $\begin{array}{l}1.0301 \\
1.0295 \\
1.0289 \\
1.0283 \\
1.0277\end{array}$ & & $\begin{array}{l}1.0375 \\
1.0367\end{array}$ & 1. 0385 & & & & & $\begin{array}{l}.9929 \\
.9922 \\
.9915 \\
.9908 \\
.9901\end{array}$ & $\begin{array}{l}.9919 \\
.9911 \\
.9903 \\
.9895 \\
.9887\end{array}$ & $\begin{array}{l}.9915 \\
.9907 \\
.9898 \\
.9890 \\
.9881\end{array}$ \\
\hline & $\begin{array}{l}1.0181 \\
1.0177 \\
1.0173 \\
1.0169 \\
1.0165\end{array}$ & $\begin{array}{l}1.0223 \\
1.0218 \\
1.0213 \\
1.0208 \\
1.0203\end{array}$ & $\begin{array}{l}\text { 1. } 0271 \\
1.0265 \\
1.0259 \\
1.0253 \\
1.0247\end{array}$ & $\begin{array}{l}1.0315 \\
1.0308 \\
1.0301 \\
1.0294 \\
1.0287\end{array}$ & & 1.0377 & & $\begin{array}{l}.0940 \\
.9936 \\
.9932 \\
.9929 \\
.9925\end{array}$ & & & & $\begin{array}{l}.9879 \\
.9871 \\
.9863 \\
.9554 \\
.9846\end{array}$ & $\begin{array}{l}.9873 \\
.9864 \\
.9856 \\
.9347 \\
.9820\end{array}$ \\
\hline & $\begin{array}{l}1.0161 \\
1.0157 \\
1.0153 \\
1.0148 \\
1.0144\end{array}$ & $\begin{array}{l}1.0198 \\
1.0193 \\
1.0188 \\
1.0184 \\
1.0179\end{array}$ & $\begin{array}{l}1.0241 \\
1.0235 \\
1.0229 \\
1.0223 \\
1.0217\end{array}$ & & & & & & & $\begin{array}{l}.9879 \\
.9873 \\
.9867 \\
.9860 \\
.9854\end{array}$ & $\begin{array}{l}.9859 \\
.9851 \\
.9344 \\
.9837 \\
.9830\end{array}$ & $\begin{array}{l}.9838 \\
.9830 \\
.9822 \\
.9814 \\
.9806\end{array}$ & $\begin{array}{l}.9830 \\
.9821 \\
.9813 \\
.9804 \\
.9796\end{array}$ \\
\hline & $\begin{array}{l}1.0140 \\
1.0136 \\
1.0132 \\
1.0128 \\
1.0124\end{array}$ & $\begin{array}{l}1.0174 \\
1.0169 \\
1.0164 \\
1.0159 \\
1.0154\end{array}$ & & & & & & & & & $\begin{array}{l}.9823 \\
.9816 \\
.9809 \\
.9802 \\
.9795\end{array}$ & $\begin{array}{l}.9798 \\
.9790 \\
.9782 \\
.9773 \\
.9765\end{array}$ & $\begin{array}{l}.9787 \\
.9779 \\
.9770 \\
.9762 \\
.9753\end{array}$ \\
\hline & $\begin{array}{l}1.0110 \\
1.0112 \\
1.0108 \\
1.0104\end{array}$ & & & & $\begin{array}{l}1.0240 \\
1.0232 \\
1.0224 \\
1.0216 \\
1.0208\end{array}$ & & & .9865 & $\begin{array}{l}.9851 \\
.9846 \\
.9841 \\
.9536 \\
.9831\end{array}$ & $\begin{array}{r}.9818 \\
.9812 \\
.9806 \\
.9800 \\
.9794\end{array}$ & .9788 & .0757 & .9745 \\
\hline & $\begin{array}{l}1.0100 \\
1.0096 \\
1.0092 \\
1.0088 \\
1.0084\end{array}$ & & & & & & & & & $\begin{array}{r}.9788 \\
.9782 \\
.9776 \\
.9769 \\
.9763\end{array}$ & & & \\
\hline & $\begin{array}{l}1.0072 \\
1.0068 \\
1.0064\end{array}$ & $\begin{array}{l}1.0099 \\
1.0094 \\
1.0089 \\
1.0084 \\
1.0079\end{array}$ & 1.0097 & $\begin{array}{l}1.0120 \\
1.0113\end{array}$ & $\begin{array}{l}1.0136 \\
1.0128\end{array}$ & & & & $\begin{array}{r}.9802 \\
.9977 \\
.9792 \\
.9786 \\
.9781\end{array}$ & $\begin{array}{r}.9757 \\
.9751 \\
.9745 \\
.9738 \\
.9732\end{array}$ & & & \\
\hline 西 & $\begin{array}{l}1.0056 \\
1.0052 \\
1.0048 \\
1.0014\end{array}$ & $\begin{array}{l}1.0059 \\
1.0064 \\
1.0059 \\
1.0054\end{array}$ & & $\begin{array}{l}1.0092 \\
1.0084 \\
1.0077\end{array}$ & & & & & $\begin{array}{l}.9 \\
.9 \\
.9 \\
.9 \\
.9\end{array}$ & $\begin{array}{r}.9726 \\
.99720 \\
.9714 \\
.99708 \\
.9702\end{array}$ & & & \\
\hline & $\begin{array}{l}\text { 1. } 0040 \\
1.0036 \\
1.0032 \\
\text { 1. } 0028 \\
1.0024\end{array}$ & $\begin{array}{l}1.0049 \\
1.0044 \\
1.0039 \\
1.0035 \\
1.0030\end{array}$ & 1.0036 & 1.0042 & $\begin{array}{l}1.0080 \\
1.0072 \\
1.0064 \\
1.0056 \\
1.0048\end{array}$ & 1. 6050 & & $\begin{array}{l}.9791 \\
.9787\end{array}$ & $\begin{array}{r}.9752 \\
.9974 \\
.9742 \\
.9737 \\
.9732\end{array}$ & $\begin{array}{l}.9696 \\
.9690 \\
.9684 \\
.9678 \\
.8672\end{array}$ & & & \\
\hline & $\begin{array}{l}1.0016 \\
1.0912 \\
1.0008 \\
1.0004\end{array}$ & $\begin{array}{l}1.0020 \\
1.0015 \\
1.0010 \\
1.0005\end{array}$ & $\begin{array}{l}\text { 1. } 0012 \\
\text { 1. } 0006\end{array}$ & $\begin{array}{l}\text { 1. } 0021 \\
\text { 1. } 0014 \\
1.0007\end{array}$ & $\begin{array}{l}\text { 1. } 0032 \\
\text { 1. } 0024 \\
1.0016 \\
\text { 1. } 0008\end{array}$ & $\begin{array}{l}1.0025 \\
1.0017 \\
1.0008\end{array}$ & & $\begin{array}{l}.9778 \\
.9775 \\
.9771 \\
.9767\end{array}$ & $\begin{array}{l}.972 \\
.972 \\
.971 \\
.971 \\
.970\end{array}$ & $\begin{array}{l}.9666 \\
.9660 \\
.9654 \\
9647\end{array}$ & & & \\
\hline
\end{tabular}

Standard abridged volume correction table for petroleum oils-Continued 
Standard abridged volume correction table for petroleum oils-Continued

\begin{tabular}{|c|c|c|c|c|c|c|c|}
\hline \multirow[t]{2}{*}{$\begin{array}{c}\text { Ob- } \\
\text { served } \\
\text { tern- } \\
\text { pera- } \\
\text { ture } \\
\text { in }{ }^{\circ} \mathrm{F} .\end{array}$} & \multicolumn{3}{|c|}{$\begin{array}{l}\text { Volume at } 60^{\circ} \mathrm{F} \text {. oc- } \\
\text { cupied by unit vol- } \\
\text { ume at indicated } \\
\text { temperature }\end{array}$} & \multirow[t]{2}{*}{$\begin{array}{c}\text { Ob- } \\
\text { served } \\
\text { tem- } \\
\text { pera- } \\
\text { ture } \\
\text { in }{ }^{\circ} F .\end{array}$} & $\begin{array}{l}\text { Vol- } \\
\text { ume at } \\
60^{\circ} \mathrm{F} \text {. } \\
\text { occu- } \\
\text { pied by } \\
\text { unit } \\
\text { volume } \\
\text { at indi- } \\
\text { cated } \\
\text { tem- } \\
\text { pera- } \\
\text { ture }\end{array}$ & \multirow[t]{2}{*}{$\begin{array}{l}\text { Ob- } \\
\text { served } \\
\text { tem- } \\
\text { pera- } \\
\text { ture } \\
\text { in }{ }^{\circ} \mathrm{F} .\end{array}$} & $\begin{array}{l}\text { Vol- } \\
\text { ume at } \\
60^{\circ} \mathrm{F} \text {. } \\
\text { occu- } \\
\text { pied by } \\
\text { unit } \\
\text { volume } \\
\text { at indi- } \\
\text { catad } \\
\text { tem- } \\
\text { pera- } \\
\text { ture }\end{array}$ \\
\hline & $\begin{array}{l}\text { Group } \\
\text { I up to } \\
34.9^{\circ} \\
\text { A. P. I, } \\
\text { at } \\
60^{\circ} \mathrm{F} \text {. }\end{array}$ & $\begin{array}{c}\text { Group } \\
\text { II } 35.0 \\
\text { to } 50.9^{\circ} \\
\text { A. P. I. } \\
\text { at } \\
60^{\circ} \mathrm{F} \text {. }\end{array}$ & $\begin{array}{l}\text { Group } \\
\text { III } 51.0 \\
\text { to } 63.9^{\circ} \\
\text { A. P. I. } \\
\text { at } \\
60^{\circ} \text { F. }\end{array}$ & & $\begin{array}{l}\text { Group } \\
\text { I up to } \\
34.9^{\circ} \\
\text { A. P. I. } \\
\text { at } \\
60^{\circ} \mathrm{F} \text {. }\end{array}$ & & $\begin{array}{l}\text { Group } \\
\text { I up to } \\
34.9^{\circ} \\
\text { A.P. I. } \\
\text { at } \\
60^{\circ} \mathrm{F} .\end{array}$ \\
\hline $\begin{array}{l}20-- \\
21- \\
22-- \\
23- \\
24--\end{array}$ & $\begin{array}{r}0.9763 \\
.9759 \\
.9755 \\
.9752 \\
.9748\end{array}$ & $\begin{array}{r}0.9702 \\
.9697 \\
.9692 \\
.9687 \\
.9682\end{array}$ & $\begin{array}{r}0.9 \\
.9 \\
.9 \\
.9 \\
.9\end{array}$ & $\begin{array}{l}164 . \\
165 \\
166 \\
167 \\
163\end{array}$ & $\begin{array}{l}0.9594 \\
.9590 \\
.9586 \\
.9582 \\
.9578\end{array}$ & $\begin{array}{l}208 \\
209 \\
210- \\
211 \\
212\end{array}$ & $\begin{array}{r}0.9427 \\
.9423 \\
.9419 \\
.9415 \\
.9412\end{array}$ \\
\hline $\begin{array}{l}125 \\
126 \\
127 \\
128 \\
129\end{array}$ & $\begin{array}{l}.9744 \\
.9740 \\
.9736 \\
.9732 \\
.9728\end{array}$ & $\begin{array}{l}.9677 \\
.9672 \\
.9667 \\
.9662 \\
.9657\end{array}$ & .9 & $\begin{array}{l}169 \\
170 \\
171 \\
172 \\
173\end{array}$ & $\begin{array}{l}.9574 \\
.9570 \\
.9566 \\
.9562 \\
.9559\end{array}$ & $\begin{array}{l}213 \\
214 \\
215 \\
216 \\
217\end{array}$ & $\begin{array}{l}.9408 \\
.9405 \\
.9401 \\
.9397 \\
.9393\end{array}$ \\
\hline $\begin{array}{l}130 \ldots \\
131 \ldots \\
132 \ldots \\
133 \ldots \\
134 \ldots\end{array}$ & $\begin{array}{l}.9724 \\
.9720 \\
.9716 \\
.9713 \\
.9709\end{array}$ & $\begin{array}{l}.9652 \\
.9647 \\
.9642 \\
.9637 \\
.9632\end{array}$ & & $\begin{array}{l}174 \\
175 \\
176- \\
177- \\
178\end{array}$ & $\begin{array}{l}.9555 \\
.9551 \\
.9547 \\
.9543 \\
.9540\end{array}$ & $\begin{array}{l}218 \\
219 \\
220 \\
221 \\
222\end{array}$ & $\begin{array}{l}.9390 \\
.9386 \\
.9382 \\
.9378 \\
.9374\end{array}$ \\
\hline 39 & $\begin{array}{l}.9705 \\
.9701 \\
.9697 \\
.9694 \\
.9690\end{array}$ & $\begin{array}{l}.9627 \\
.9622 \\
.9617 \\
.9613 \\
.9608\end{array}$ & & $\begin{array}{l}179 . \\
180 . \\
181 \\
182 . \\
183 .\end{array}$ & $\begin{array}{l}.9536 \\
.9532 \\
.9528 \\
.9524 \\
.9521\end{array}$ & $\begin{array}{l}223 \\
224 \\
225 \\
226 \\
227\end{array}$ & $\begin{array}{l}.9371 \\
.9367 \\
.9363 \\
.9359 \\
.9356\end{array}$ \\
\hline 40. & $\begin{array}{l}.9686 \\
.9682 \\
.9678 \\
.9675 \\
.9671\end{array}$ & $\begin{array}{l}.9603 \\
.9598 \\
.9593 \\
.9588 \\
.9583\end{array}$ & & $\begin{array}{l}184 . \\
185 \\
186 . \\
187 . \\
188 .\end{array}$ & $\begin{array}{l}.9517 \\
.9513 \\
.9509 \\
.9505 \\
.9502\end{array}$ & $\begin{array}{l}228 \\
229 \\
230 \\
231 \\
232\end{array}$ & $\begin{array}{l}.9352 \\
.9349 \\
.9345 \\
.9341 \\
.9337\end{array}$ \\
\hline & $\begin{array}{l}.9667 \\
.9663 \\
.9659 \\
.9655 \\
.9651\end{array}$ & $\begin{array}{l}.9578 \\
.9573 \\
.9568 \\
.9563 \\
.9558\end{array}$ & & $\begin{array}{l}189 \\
190 \\
191 \\
192 \\
193\end{array}$ & $\begin{array}{l}.9498 \\
.9494 \\
.9490 \\
.9487 \\
.9483\end{array}$ & $\begin{array}{l}233 \\
234 \\
235 \\
236 \\
237\end{array}$ & $\begin{array}{l}.9334 \\
.9330 \\
.9326 \\
.9322 \\
.9318\end{array}$ \\
\hline $\begin{array}{l}153 \\
154\end{array}$ & $\begin{array}{l}.9647 \\
.9643 \\
.9639 \\
.9636 \\
.9632\end{array}$ & .9553 & & $\begin{array}{l}194 \\
195- \\
196- \\
197- \\
198\end{array}$ & $\begin{array}{l}.9480 \\
.9476 \\
.9472 \\
.9468 \\
.9465\end{array}$ & $\begin{array}{l}238 . \\
239 \\
240- \\
241 \\
242\end{array}$ & $\begin{array}{l}.9315 \\
.9311 \\
.9307 \\
.9303 \\
.9300\end{array}$ \\
\hline $\begin{array}{l}157 \\
158 \\
159\end{array}$ & $\begin{array}{l}.9628 \\
.9624 \\
.9620 \\
.9616 \\
.9612\end{array}$ & & & $\begin{array}{l}199 .- \\
200- \\
201- \\
202 . \\
203 .\end{array}$ & $\begin{array}{l}.9461 \\
.9457 \\
.9453 \\
.9449 \\
.9446\end{array}$ & $\begin{array}{l}243_{-} \\
244- \\
245_{-} \\
246- \\
247 .\end{array}$ & $\begin{array}{l}.9296 \\
.9293 \\
.9289 \\
.9285 \\
.9281\end{array}$ \\
\hline $\begin{array}{l}160 \\
161- \\
162 \\
163\end{array}$ & $\begin{array}{l}.9608 \\
.9604 \\
.9601 \\
.9597\end{array}$ & & & $\begin{array}{l}204 . \\
205 \\
206 \\
207\end{array}$ & $\begin{array}{l}.9442 \\
.9438 \\
.9434 \\
.9430\end{array}$ & $\begin{array}{l}248 \\
249 \\
250\end{array}$ & $\begin{array}{l}.9278 \\
.9274 \\
.9270\end{array}$ \\
\hline
\end{tabular}

Note.-For temperatures above $203^{\circ} \mathrm{F}$. the tabulated values of the volume at $60^{\circ} \mathrm{F}$. are extrapolated from the experimental results obtained over the temperature range $32^{\circ}$ to $203^{\circ} \mathrm{F}$.

American Society for Testing Materials, standard method of test for thermal value of fuel oil, serial designation D240-27; 1927.

Approved as American Standard by the American Engineering Standards Committee, now the American Standards Association.

\section{APPARATUS}

1. The Atwater, Davis, Emerson, Mahler, Parr, Peters, Williams or similar bombs may be used. The bomb shall have an inner surface of platinum, gold, porcelain enamel, or other material which is not attacked by nitric and sulphuric acids, or other products of combustion.

2. The calorimeter shall be provided with a vacuum jacket or with a water jacket having a cover to protect the calorimeter from air currents. In case a water jacket is used, it shall be kept filled with water within $2^{\circ}$ or $3^{\circ} \mathrm{C}$. of the temperature of the room (except in calorimeters which are totally submerged, where the jacket temperature is controlled by a thermostat) and this water should be stirred continuously by some mechanical stirring device.

3. The water in the calorimeter shall be stirred sufficiently well to give consistent thermometer readings while the temperature is rising rapidly. The speed of stirring should be kept constant. A motor-driven screw or turbine stirrer is recommended and the speed should not be excessive. This may be determined by adjusting the temperature of the calorimeter to equality with that of the jacket and allowing the stirrer to run continuously for 10 minutes. If the temperature of the calorimeter rises more than about $0.01^{\circ} \mathrm{C}$. in this length of time, the rate of stirring is excessive. Accurate results can not be obtained when too much energy is supplied by the stirring device or when the rate of stirring is irregular. The portion of the stirring device immersed in the calorimeter should be separated from that oustide by nonconducting material, such as hard rubber, to prevent conduction of heat from the motor or outside air.

4. The thermometers used shall have been certified by a Government testing bureau and shall be used with the corrections given on the certificate. This shall also apply to electrical resistance or thermoelectric thermometers. Correction shall also be made for the temperature of the emergent stem of all mercurial thermometers, and for the "setting" of Beckmann thermometers. For accurate work, either Beckmann or special calorimetric thermometers, graduated to $0.01^{\circ}$ or $0.02^{\circ} \mathrm{C}$., are required. Such thermometers should be tapped lightly just before each reading to a void errors caused by the sticking of the mercury meniscus, particularly when the temperature is falling. A convenient method is to mount a small electric buzzer directly on the top of the thermometer and connect it with a dry cell and a push button. The button should be pressed for a few seconds immediately before each reading.

5. The oxygen used for combustions shall be free from combustible material. The bomb when filled should contain at least 5 per cent of nitrogen to insure complete oxidation of the sulphur. The total amount of oxygen contained in the bomb for combustion shall be not less than $5 \mathrm{~g}$ per gram of fuel oil. But the combustion must be complete, as shown by the absence of any sooty deposit on opening the bomb after firing.

6. The fuel oil in the bomb may be ignited by means of either iron or platinum wire. If iron wire is used, it should be of about No. 34 B. \& S. gage and not more than $10 \mathrm{~cm}$ (preferably $5 \mathrm{~cm}$ ) should be used at a time. A correction of 1,600 calories per gram weight of iron wire burned shall be subtracted from the observed number of calories.

7. The water equivalent of a calorimeter can best be determined by the use of the standard combustion samples supplied by the National Bureau of Standards. The required water equivalent is equal to the weight of the sample multiplied by its heat of combustion per gram and divided by the corrected rise in temperature.

The calorimeter shall be standardized by the combustion of standard samples supplied by the National Bureau of Standards, and used according to the cirections given in the certificates which accompany them. A standardization shall consist of a series of not less than five combustions of 
each of two of the standard materials, which are sucrose, naphthalene, and benzoic acid. The conditions as to the amount of water, oxygen, firing wire, method of correcting for radiation, etc., under which these combustions are made, shall be the same as for fuel-oil combustions. In the case of any disagreement between contracting parties a check standardization may consist of two or more combustions of standardizing samples.

\section{PROCEDURE}

8. Approximately enough oil shall be taken to give a thermometer rise of from $3^{\circ}$ to $4^{\circ} \mathrm{C}$. After weighing, the sample should preferably be immediately placed in the bomb and this closed. This procedure is necessary to avoid sublimation in the use of naphthalelle for standardization.

9. The firing wire, if iron, shall be measured and coiled in a small spiral and connected between the platinum terminals, using, if necessary, a piece of platinum wire somewhat heavier than the iron wire to make the connection. The platinum and the iron shall both be clean. About $0.5 \mathrm{cc}$ of water should be placed in the bottom of the bomb to saturate with moisture the oxygen used for combustion. When the crucible is put in place in the bomb, the firing wire should touch the fuel oil or briquet of standard material. For the combustion of standardizing samples, iron wire is preferable to platinum.

10. Oxygen from the supply tank shall be admitted slowly, to avoid blowing the oil from the crucible, until a pressure is reached as indicated by the following table:

\begin{tabular}{|c|c|}
\hline Capacity of bomb (cc) & $\begin{array}{l}\text { Minimum } \\
\text { gáuge pres- } \\
\text { sure }\end{array}$ \\
\hline $\begin{array}{l}300 \text { to } 350 \\
400 \text { to } 400 \\
450 \text { to } 50000 \\
\text { Abore } 500\end{array}$ & $\begin{array}{l}\text { Atrinos- } \\
\text { pheres } \\
40 \\
35 \\
30 \\
27.5 \\
25\end{array}$ \\
\hline
\end{tabular}

11. The calorimeter shall be filled with the required amount of distilled water, depending upon the type of calorimeter. The amount may be determined either br measurement in a standardized flask or by weighing. The amount shall be kept the same as that used in the standardization of the apparatus.

12. The initial temperature in the calorimeter shall be so adjusted that the final temperature, after the combustion, will not be more than $1^{\circ} \mathrm{C}$., preferably about $0.5^{\circ} \mathrm{C}$., above that of the jacket. Under these conditions, the total correction for heat gained from or lost to the surroundings will be small when the rise of temperature is $3^{\circ}$ or $4^{\circ} \mathrm{C}$ and the effect of evaporation will also be small.

13. The electric current used for firing the charge shall be obtained from storage or dry cells having an electromotive force of not more than 12 volts. A higher voltage is liable to cause an arc between the firing terminals, introducing additional heat which can not be measured with certainty. The circuit should be closed by means of a switch which should remain closed for not more that two seconds. When possible it is recommended that an ammeter be used in the firing circuit to indicate when the firing wire has burned out.

14. The bomb, when ready for firing, shall be placed in the calorimeter, the firing wires connected, the cover put in place, and the stirrer and thermometer so placed as not to be in contact with the bomb or container. The stirrer shall then be started and after the thermometer reading has become steady, not less than two minutes after the stirrer is started, the temperatures shall be read at 1-minute intervals for five minutes and the charge then fired, the exact time of firing being noted. Observations of temperature shall then be made at intervals, depending upon the method to be used for computing the cooling correction. When the temperature has reached its maximum and is falling uniformly, a series of thermometer readings shall be taken at 1-minute intervals for five minutes to determine the final cooling rate.

15. After a combustion and after allowing the gas to escape, the bomb shall be opened and the inside examined for traces of unburned material or sooty deposit. If these are found, the observations shall be discarced. If the combustion appears complete, the bomb shall be rinsed out thoroughly and the washings titrated with a standard alkali solution (1 cc $=0.02173 \mathrm{~g} \mathrm{HNO}_{3}=5$ calories) using methylorange or methyl-red indicator to determine the amount of acid formed. A correction of 230 calories per gram of $\mathrm{IINO}_{3}$ should be subtracted from the total heat observed. An additional correction of 1,300 calories per gram of sulphur in the oil should be made for the excess of difference in heats of formation of $\mathrm{SO}_{2}$ and aqueous $\mathrm{H}_{2} \mathrm{SO}_{4}$ over the heat of formation of aqueous $\mathrm{HNO}_{3}$.

\section{COMPOTATION OF RESUITS}

16. The following method of computation is recommended to take the place of the Pfaundler or other similar formulas for computing the cooling correction (radiation correction):

Observe (1) the rate of rise, $r_{1}$ of the calorimeter temperature in degrees per minute for five minutes before firing; (2) the time, $a$, at which the last temperature reading is made immediately before firing; (3) the time, $b$, when the rise of temperature has reached six-tenths of its total amount (this point can generally be determined by adding to the temperature observed before firing, 60 per cent of the expected temperature rise, and noting the time at which this point is reached); (4) the time, $c$, of a thermometer reading taken when the temperature change has become uniform some five minutes after firing; (5) the final rate of cooling, $r_{2}$, in degrees per minute for five minutes.

Multiply the rate $r_{1}$ by the time $b-a$ in minutes and tenths of a minute and add to this product (subtracted if the temperature was falling at the time $a$ ) the thermometer reading taken at the time $a$. Multiply the rate $r_{2}$ by the time $c-b$ and add this product (subtracted if the temperature was rising at the time $c$ and later) to the thermometer reading taken at the time $c$. The difference of the two thermometer readings thus corrected, provided the corrections from the certificate have already been applied, gives the total rise of temperature due to the combustion. This, multiplied by the water equivalent of the calorimeter, gives the total amount of heat liberated. Divide this result, corrected for the heats of formation of $\mathrm{HNO}_{3}$ and $\mathrm{H}_{2} \mathrm{SO}_{4}$ oberved and for the heat of combustion of the firing wire, when that is included, by the weight of the charge to find the heat of combustion in calories per gram. Calories per gram multiplied by 1.8 give the British thermal units per pound.

17. The permissible differences in duplicate determinations are as follows:

Same analyst Per cent 0.3

18. In practice, the time $b-a$ will be found so nearly constant for a given calorimeter with the 
usual amounts of fuel that $b$ need be determined only occasionally.

The results shall be reduced to calories per gram or British thermal units per pound of oil.

American Society for Testing Materials, tentative methods of sampling petroleum and petroleum products, serial designation D270-27T; 1927.

\section{PURPOSE AND SCOPE}

1. Samples are taken for the purpose of having representative quantities, of economical and convenient size, from a section, or from the whole of a lot of material, for examination and test, so that an agreement may be reached in regard to the following:

(a) The extent of variation of qualities in different portions of the lot. rial.

(b) The average quality of the whole lot of mate-

2. These methods of sampling are for use in obtaining representative portions of stocks or shipments of petroleum and all petroleum products, except gases.

\section{GENERAI CONSIDERATIONS}

3. No set of directions for sampling, however explicit, can take the place of judgment, skill, and previous experience on the part of the persons actually engaged in the sampling and in the supervision of the sampling. These directions are intended to supplement this experience and particularly to serve as a guide in the selection of the method which is to be used in common by each of two or more contracting parties.

4. The materials sampled may be graded as follows:

(a) Crude or unrefined materials, such as petroleum from wells, pipe lines, and tanks.

(b) Semirefined materials, such as fuel oil, and gas oil.

(c) Refined products, such as gasoline, lubricating oils, and insulating oils.

5. For sampling purposes, material may be classified as follows:

(a) Liquid materials, such as light crude oils, gas oils, lubricating oils, and napthas.

(b) Semiliquid and highly viscous materials, such as cylinder stocks and heavy fuel oils.

(c) Soft solid and semisolid materials, such as asphalts, waxes, petrolatum, and greases.

(d) Lumpy solid materials, such as petroleum coke. 6. These methods apply to material in the following types of containers:

(a) Cans or small containers (up to 10 U. S. gallons, inclusive).

(b) Drums or barrels.

(c) Tank cars or tank trucks.

(d) Shore tanks.

(e) Ship tanks or barge tanks.

(f) Pipe lines.

(g) Bags.

(h) Cakes.

(i) Bulk or loose form.

7. Samples are taken for the following types of tests:

(a) General quality tests.

(b) Definite chemical and physical tests.

(c) Special quality tests.

8. Samples are used for one or more of the following purposes:

(a) Visual examination.

(b) Laboratory test.

(c) Preservation for record.

(d) Check and referee tests.

9. Samples shall be marked, recorded, and handled throughont in accordance with all the usual precautions of the best laboratory technique.

\section{DEFINITIONS}

10. Average sample ${ }^{30}$.-An average sample would be one so taken as to contain parts from all sections of the container or pipe in proportion to the volume of each part.

11. All-levels sample ${ }^{31}$ - An all-levels sample is one obtained by submerging a closed sample container to a point as near as possible to the draw-off level, opening it and raising it at such a rate that it will be nearly but not quite full when withdrawn.

12. Upper sample.-An upper sample is one taken at a point 10 per cent of the depth of a uniform cross-section vessel or 10 per cent of the diameter of a horizontal cylindrical tank below the surface.

13. Middle sample.-A middle sample is one taken at half the depth of the material.

14. Lower sample.-A lower sample is one taken at a point 10 per cent of the depth of a uniform crosssection vessel or 10 per cent of the diameter of a horizontal cylindrical tank above the bottom of the vessel.

15. Composite sample.-(a) A composite sample is a mixture of upper, middle, and lower samples containing, for vessels of different shapes, volume proportions which correspond approximately to the volumes of the material at these levels, as follows:

\begin{tabular}{|c|c|c|}
\hline & \multicolumn{2}{|c|}{$\begin{array}{l}\text { Composite sample pro- } \\
\text { portions }\end{array}$} \\
\hline & $\begin{array}{l}\text { Uniform } \\
\text { cross- } \\
\text { section } \\
\text { vessels }\end{array}$ & $\begin{array}{l}\text { Horizontal } \\
\text { cylindri- } \\
\text { cal tanks } \\
\text { (assumed } \\
\text { full) }\end{array}$ \\
\hline $\begin{array}{l}\text { Upper sample } \\
\text { Middle sample } \\
\text { Lower sample }\end{array}$ & $\begin{array}{r}\text { Parts } \\
1 \\
3 \\
1\end{array}$ & Parts \\
\hline
\end{tabular}

(b) For horizontal cylindrical tanks that are only partially filled, the simple geometrically proportioned sampling levels and composite sample mixtures stated above can not give samples that are substantially proportionate to the volumes at the different depths, unless the tanks are filled to at least 80 per cent of the diameter. Each tank level may be worked out in accordance with the principle stated in section 10 .

(c) In referee tests, a mutuajly satisfactory selection of sampling levels and sample quantities at each level shall be mutually agreed upon if any departure from the methods specified are to be allowed.

(d) The following table gives a set of substantially correct sampling levels and sample quantities for partially filled horizontal cylindrical tanks, which may be used in the absence of other arrangements, employing values in the tabulation that lis nearest to the actual filling depth.

16. Continuous sample.-A continuous sample is one obtained from a pipe or conduit conveying the

${ }^{30} \mathrm{It}$ is practically impossible to obtain an average sample, except perhaps, through a continuous sampling connection from a vertical run in a pipe line with specially constructed draw-off pipes, or by vigorously agitating and stirring the contents of a vessel and drawing igorously agitating and stirring the contents of a vesse and drawing if a sample while the contents are still agitated. by lowering and raising a bottle while allowing it to fill have been considered average samples, but obviously the proportions are not related to the tank volumes at the various levels.

31 The all-levels sampling method is widely used for ship tanks, barges, and large shore tanks. The method can not, however, give an average or a strictly representative sample, not only because the tank volume may not be proportional to the depth and because the operator can not move the sample container with a uniform speed. but also because the rate of filling the sample container is propor tionate to the square root of the depth of immersion of the sample container opening below the surface. 
material, in such a manner as to give at all times a representative average of all the sections and section velocities of the stream throughout the period of transit.

17. Dipper sample.-A dipper sample is one taken by interposing a dipper or collecting vessel into the path of a free-flowing stream, so as to collect a definite volume from the full cross section of the stream at regular time intervals, or at time intervals so varied as to obtain samples in proportion to the amounts being delivered.

18. Mixed sample.-A mixed sample is one obtained by mixing or vigorously stirring the contents of the original container and pouring out or drawing off the quantity desired.

19. Thief sample.-A thief sample is one taken by means of a sainpling tube from a specified point in the containing ressel being sampled.

Sampling levels and sample quantities for partially filled horizontal cylindrical tanks

\begin{tabular}{|c|c|c|c|c|c|c|}
\hline \multirow{2}{*}{$\begin{array}{r}\text { Liquid depth, } \\
\text { percentage } \\
\text { of diameter }\end{array}$} & \multicolumn{3}{|c|}{$\begin{array}{l}\text { Sampling level, per- } \\
\text { centage of diameter } \\
\text { above bottom }\end{array}$} & \multicolumn{3}{|c|}{$\begin{array}{l}\text { Quantity of sample to } \\
\text { be taken at each sam- } \\
\text { pling level }\end{array}$} \\
\hline & Upper & Middle & Lower & Upper & Middle & Lower \\
\hline $\begin{array}{l}10 \ldots \\
20 \ldots \ldots \\
30 \ldots \ldots \\
40 \ldots \ldots\end{array}$ & & $\begin{array}{l}20 \\
25 \\
30\end{array}$ & $\begin{array}{r}5 \\
10 \\
10 \\
10 \\
10\end{array}$ & $\begin{array}{c}\text { Part } \\
\\
\end{array}$ & $\begin{array}{r}\text { Parts } \\
6 \\
8 \\
8\end{array}$ & $\begin{array}{r}\text { Parts } \\
10 \\
10 \\
4 \\
3 \\
2\end{array}$ \\
\hline $\begin{array}{l}60 \\
80 \\
90 \\
100\end{array}$ & $\begin{array}{l}55 \\
65 \\
75 \\
85 \\
90\end{array}$ & $\begin{array}{l}35 \\
40 \\
45 \\
50 \\
50\end{array}$ & $\begin{array}{l}10 \\
10 \\
10 \\
10 \\
10\end{array}$ & $\begin{array}{l}1 \\
1 \\
1 \\
1 \\
1\end{array}$ & $\begin{array}{l}8 \\
8 \\
8 \\
8 \\
8\end{array}$ & $\begin{array}{l}1 \\
1 \\
1 \\
1 \\
1\end{array}$ \\
\hline
\end{tabular}

20. Drain sample.32-A drain sample is one taken by opening the draw-off or the discharge valve and drawing off a suitable portion of the material.

21. Bottom sainple. ${ }^{32}$ - A bottom sample is one obtained by collecting a portion of the material lying on the bottom surface of the tank, container or line at its lowest point.

22. Borings sample. $-\mathrm{A}$ borings sample is one obtained by collecting the chips made by boring holes with a ship auger from top to bottom of the material contained in a barrel, case, bag, or cake.

23. Grab sample.-A grab sample is one obtained by collecting representative portions from loose solids in equal quantities from each part or package of a shipment, in sufficient amount to be representative of all the sizes and components.

24. Official samples shall be taken by or under the immediate supervision of a person of judgment, skill, and previous experience in sampling.

25. The portions taken for samples shall represent the general character and average condition of the lot sampled.

26 . If there is any substantial reason indicating that a sample or set of samples may not be fully representative, another sample or set of samples shall be taken.

27 . Great care and good judgment are necessary in taking and handling samples from crude or unrefined materials not only because of possible segregation, sludging, and volatilization, but because of the bearing upon value, refining problems, and methods of handling.

${ }_{32}$ Occasionally, as in tank cars, the drain sample will also give a bottom sample.

Drain samples and botton samples are usually taken only in order to examine for moisture sludge, scale, etc., and in some cases for the purpose of drawing off any free water or extraneous material.
28. A high degree of care shall be employed in handling samples from semirefined materials upon which definite chemical and physical tests are usually made.

29. The utmost precautions and the most meticulous care shall be exercised in handling samples from refined products, upon which special quality tests are usually made.

30. For all grades of materials, precautions shall be taken to assure that the sampling apparatus and the samples themselves are neither contaminated with nor altered by any material not representative of the lot being sampled.

31. The operator engaged in sampling shall have clean hands, free from any material (unless it be the material being sampled). Cleaned gloves may be worn, but only when absolutely necessary, as in extremely cold weather, or in handling materials at high temperatures, or materials involving some health or other hazard.

32. The sampling apparatus shall be thoroughly clean and dry, and frce from any substance that will dissolve into and/or color water-white gasoline.

Note.-Exception: Crude and dark-colored semirefined materials (such as dark-colored fuel oils) when fluid enough to drain completely from sampling apparatus (Saybolt universal viscosity less than 250 at $100^{\circ} \mathrm{F}$.) may be sampled repeatedly with apparatus that has been hung up and thoroughly drained.

33. Samples shall be permanently marked (prefcrably with a pencil hard enough to dent the paper ${ }^{23}$ immediately after collection with the date, also the hour and minute for continuous and dipper samples, name of the sampler, name or number and owner of vessel, barge, car, or container; material sampled, and a reference symbol or number. Thin aluminum strips stamped with the sample number and other information are acceptable. The reference symbol or number and other markings should be entered in a bound record book.

34. Sample containers shall be closed immediately after collection. Sample containers shall be of such types as will protect the sample from contamination or deterioration. Specific precautions shall be taken as follows:

(a) Corks, for bottles, shall be clean, free from holes and loose bits of cork.

(b) Sealing wax, or paraffin, shall not be poured over corks after filling.

(c) Light-sensitive samples, when placed in bottles shall be wrapped or otherwise covered at once, to protect them from light.

$(d)$ Tin cans with screwed or overlapped and soldered caps are acceptable, but only if the inside has been scrupulously cleaned and known to be free from dirt, water, washing compounds, naphtha or other solvents, soldering flux or acids, corrosion, rust, and pinholes.

(e) Refined materials should be further protected by covers of paper or metal foil over the stopper and top to keep moisture and dust away from the filling and emptying opening, and to protect it from contact with the hands. Covers also prevent sealing substances, such as wax or paraffin, from getting on the lip when the seal is made or later opened, and so getting into the sample when it is poured out.

(f) Samples shipped by mail, express, or messenger shall be well packed to avoid spillage, leakage, breakage, and loss by evaporation or alteration while in transit.

35. Volatile samples shall be protected from evaporation while being collected, pal ticularly in the case of continuous and dipper samples, and at once placed in closed containers.

36. Samples shall (if possible) be taken at the time of loading of a shipment and at the point of

33 Soft pencil and ink markings are liable to obliteration from moisture, handling, or smearing with oil. 
origination of a shipment. When sampling at the point of origin has not been provided for, or is not feasible, then the sampling shall be carried out as soon as possible after receipt of the shipment.

37. Shipments consisting of a number of separate packages, such as cans, drums, barrels, or boxes, shall have a number opened and sampled equal to the cube root (or the next larger whole number) of the total number of packages in the lot.

38. (a) Individual liquid samples.-Individual liquid samples taken with a beaker, bottle or dipper shall be approximately 1 quart or 1 liter.

(b) Composite liquid samples.-Composite liquid samples taken from vessels of all kinds and sizes shall be at least 5 quarts (or 5 liters) for vessels of uniform cross section and 10 quarts (or 10 liters) for horizontal cylindrical tanks.

(c) Gross liquid mixed cargo samples.-Gross liquid mixed cargo samples taken from the various ship tanks shall have a total gross amount corresponding to the number of tanks; that is, multiples of 5 to 10 quarts, depending on the tank shapes.

(d) Liquid samples taken by the continuous method or by the dipper method.-Liquid samples taken by the continuous method, section 50 , or by the dipper method, section 53 , shall be approximately 0.1 per cent of the total quantity shipped, but not less than 5 U. S. gallons (or 20 liters) nor more than 40 U. S. gallons (or 160 liters).

(e) Soft solid samples (waxes, etc.).-Soft solid samples taken by the borings method, section 62 , shall consist of three sets of borings three-f ourths inch in diameter and approximately the depth of the material.

(f) Lumpy solid samples.-Lumpy solid samples taken by the grab method, section 65, shall be equivalent to approximately 0.1 per cent of the lot, but not less than about 50 pounds $(25 \mathrm{~kg})$ nor more than 1,000 pounds $(500 \mathrm{~kg})$.

(g) Subdivision of gross samples.-Subdivision of gross samples shall be carried out in the laboratory and should be performed by someone from the laboratory staff, rather than by the person who originally took the samples. The subdivision shall be carried out in accordance with careful laboratory practice and the detailed directions given as a part of the procedure for each method.

\section{CHOICE OF SAMPLING METHODS}

39. (a) Where standard or tentative standard methods for sampling of the American Society for Testing Materials are already in existence, they shall be followed in detail in addition to the more general requirements appearing in these methods, and shall supersede them if there is a conflict.

Nore.-Specifically this includes the following methods of sampling of the American Society for Testing Materials: D21 (see 501.0, p. 4), D271 (see 501.0, p. 8), D140 (see 505.0, p. 128).

(b) Where special conditions exist in regard to the quality of the material and/or the method of test, and particularly when existing standard or tentative standard methods of tests of the American Society for Testing Materials call for special precautions or methods for sampling, they shall be followed in detail in addition to the requirements appearing in these methods and shall supersede them if there is a conflict.

NOTE.-Specifically this includes the following methods of test of the American Society for Testing Materials: D117 (seo 504.8, p. 115), D217 27 T (see 504.0, p. 101), D128 (see 504.0, p. 93).

40. Alternative methods for sampling shall be employed only after a mutually satisfactory agreement shall have been reached by persons authorized to act on this question. The agreement shall be put in writing and signed by competent officials.
41. Liquid materials may be sampled by the bottle or beaker, thief, continuous, or dipper method, in accordance with the type of container, as described below:

(a) Tank cars, tank trucks, shore tanks, ship tanks, and barge tanks shall be sampled by the bottle or beaker method as described in sections 45 , 46 , and 47 for upper, middle, and lower samples (sees. 12,13, and 14), from which a composite sample for all referee tests shall be made up in accordance with section 15 .

The all-levels sample (sec. 11) is convenient in practice but shall be employed for routine work only.

(b) Pipe lines shall be sampled by the continuous method as described in sections 48,49 , and 50 .

(c) Small pipe lines (approximately 2 inches in diameter or less) ending in an open discharge, or where an open discharge is permissible, may be sampled by the dipper method as described in sections 51,52 , and 53 . In the absence of an open discharge, a larger diameter pipe nipple may be placed in a vertical section of the pipe line and the continuous method used as described in sections 48 , 49 , and 50.

(d) Cans, drums, or barrels shall be sampled by the thief method as described in sections 54 to 59 , inclusive, except that small cans, 1 gallon or less, may be taken complete. Containers shall have their contents thoroughly mixed by being rolled or shaken to allow the taking of a mixed sample (sec. 18) with the thief.

42. Semiliquid materials shall be warmed till they are sufficiently fluid to be sampled as liquid materials as described in section 41 .

43. Soft solid materials shall be sampled by the borings method as described in sections 60,61 , and 62.

44. Loose, lumpy, solid materials shall be sampled by the grab method as described in sections 63,64 , and 65 .

\section{BOTTLE OR BEAKER SAMPLING}

45. The bottle or beaker method of sampling is applicable to the following types of containers: (a) Tank cars and/or tank trucks, (b) shore tanks, and $(c)$ ship tanks and barge tanks.

\section{APPARATUS 4}

46. The sampling bottle and sampling beaker shall be made substantially in accordance with the form and dimensions shown in Figure 27, with the size of opening as follows:

\begin{tabular}{|c|c|c|}
\hline \multirow[b]{2}{*}{ Class of material } & \multicolumn{2}{|c|}{ Size of opening } \\
\hline & $\begin{array}{c}\text { Weighted } \\
\text { sampling } \\
\text { bottle }\end{array}$ & $\begin{array}{l}\text { Weighted } \\
\text { sampling } \\
\text { beaker }\end{array}$ \\
\hline Heavy crudes and fuel oils......... & Inches & Inches \\
\hline $\begin{array}{l}\text { Light crudes and full cargoes of Diesel fuels, } \\
\text { distillates or nontransparent gas oils. }\end{array}$ & & $3 / 4$ \\
\hline Heavy lubricating oils & $13 / 2$ & \\
\hline $\begin{array}{l}\text { Light lubricating oils, kerosene, gasoline, } \\
\text { transparent gas oils, also part cargoes of } \\
\text { Diesel fuel, distillates or nontransparent gas } \\
\text { oils. }\end{array}$ & & \\
\hline
\end{tabular}

\section{PROCEDURE}

47. The following instructions shall apply to a substantially full tank:

(a) An upper sample shall be taken by lowering the weighted stoppered bottle or beaker till its

${ }^{34}$ Adapted from Instructions for Measuring, Sampling and Testing Bulk Oil Cargoes, Standard Oil Co. of New Jersey, pp. 20-22; 1926. 
mouth is 10 per cent of the depth of a tank of uniform cross section or diameter of a horizontal cylindrical tank below the surface, uncorking it by a quick jerk, allowing it to fill completely, as evidenced by cessation of air bubbles, and then immediately withdrawing it.

(b) Three middle samples shall be taken in a similar manner from a tank of uniform cross section ship tank, or barge tank shall be combined into a composite sample.

(e) The composite samples taken with the beaker from the various ship tanks shall be combined into a mixed cargo sample for: All unfinished products, including crude oils and bunker fuel; and full cargoes of Diesel fuel, distillates, and nontransparent gas oils.

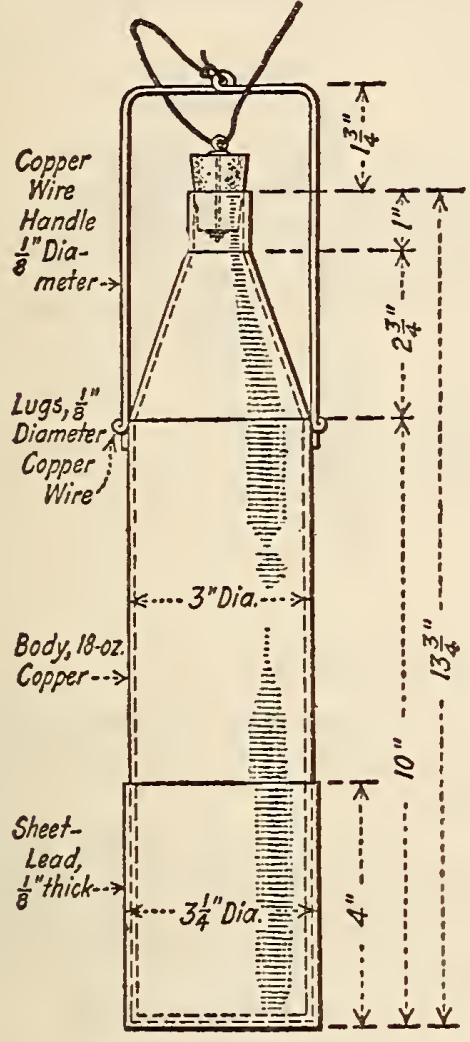

Weighted Beaker.

Note: This Container Should be Used when one Composite Sample is desired for each Grade of Cargo.

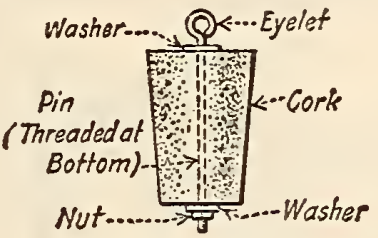

Detail of Cork Arrangement.

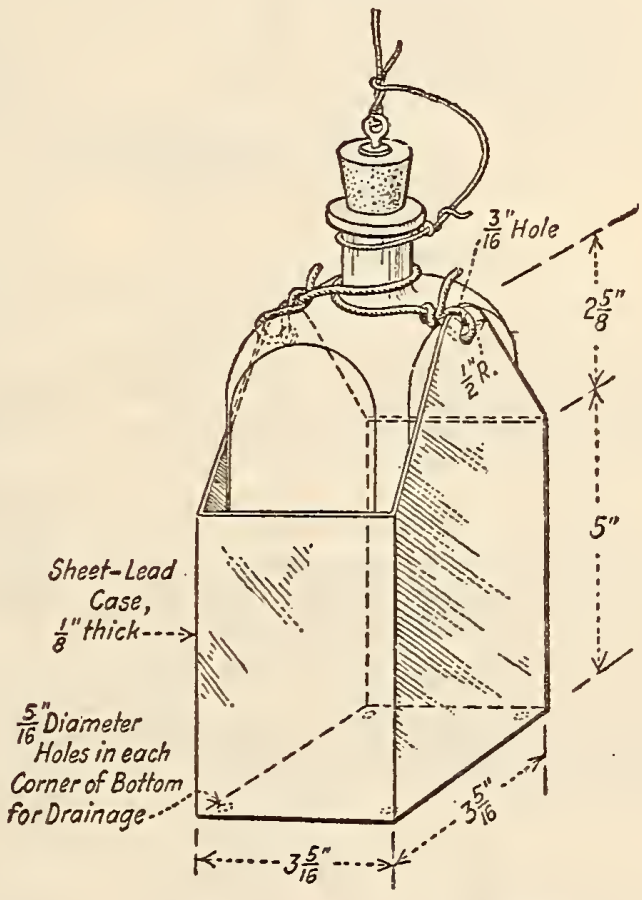

Weighted Bottle.

Note: This. Bottle to be used for Obtaining the Individual Samples when individual Samples are to be kept Separate, that is whenever the Sample must be delivered to the Labora. tory in the same Container in which it was contained.

FIGURE 27.-Standard sampling containers for bottle or beaker sampling

and eight middle samples from a horizontal cylindrical tank with the bottle or beaker mouth lowered to the middle of the tank.

(c) A lower sample shall be taken in a similar manner with the bottle or beaker mouth lowered to a point 10 per cent of the depth of a tank of uniform cross section or diameter of a horizontal cylindrical tank above the bottom of the tank.

(d) The upper, middle, and lower samples from each tank car, tank truck, shore tank, individual (f) The beaker samples shall be poured as taken into a clean 5 or 10 gallon can, or cans, and the cans closed and covered, labeled, and delivered to the laboratory, shaken or mixed with the churn-dasher type of stirrer (sec. $49(b))$, and portions withdrawn by means of a thief for test.

(g) The composite samples taken with the bottle from the various ship tanks shall be kept separate and delivered separately to the laboratory for all finished products, including gasoline, kerosene, 
lubricating oils, and transparent gas oils; and part cargoes of Diesel fuel, distillates, and nontransparent gas oils.

(h) The bottle samples shall all be delivered to the laboratory in the bottle in which they were taken.

(i) When loading or discharging any finished product, samples shall also be taken from the shore tanks and at frequent intervals from the shore pipe line on the dock. These samples shall also be tested whenever it seems advisable. (a) For semiliquid materials, the receiver, or receivers, shall be kept warm by means of steam coils adjusted to keep the material just above the liquefying temperature.

(b) The receiver, or rcceivers, should be kept covered and closed except for their vents and filling stream openings, or pipes.

(c) Screens of larger area than the opcnings may be placed around the $1 / 4$-inch pipes or $1 / 4$-inch screen fittings inserted in the drip lines.

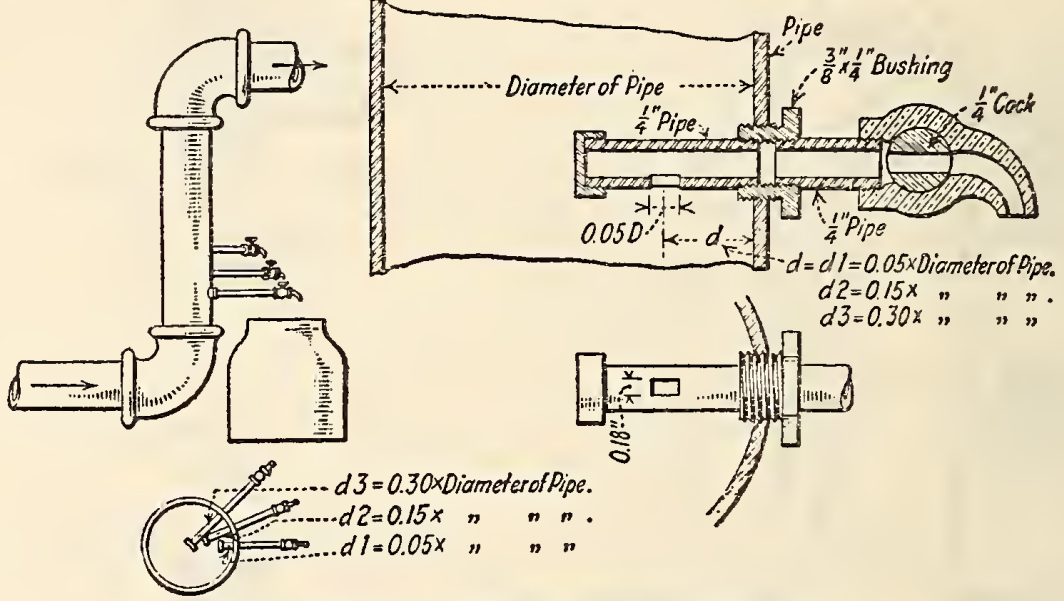

FIgURE 28.-Continuous sampling connection

(j) A portion of all samples from all shipments shall be fully labeled and kept in storage until the final disposition of the material.

(k) Ship and barge tanks should be sampled not only immediately after loading, but also before discharging.

\section{CONTINUOUS SAMPEING}

48. The continuous method of sampling is applicable to the following conditions and types of containers: (a) Pipe lincs, (b) filling lines, and (c) transfer lines.

\section{APPARATUS}

49. (a) The continuous sampling connection shall be constructed and installed in accordance with the form and dimensions shown in Figure 28.

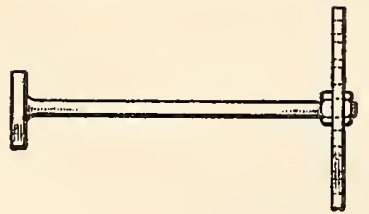

Figure 29.-Churn dasher type of stirrer

(b) The churn-dasher type of stirrer shall conform to the form and dimensions shown in Figure 29.

\section{PROCEDURE}

50. The three plug cocks shall be opened, as nearly as possible, to the same angle of opening, so that a steady stream is drawn off from each cock and at such a rate as will allow about 0.1 per cent of the stream to be diverted during the passage of the lot being sampled, but not more than 40 U. S. gallons. The following precautions should be observed: (d) A pipe cap with a suitable orifice hole may be substituted for the cocks, where the orifice size has been predetermined so as to give the right amount of sample.

(e) The gross continuous sample as collected shall be stirred with the churn-dasher type of stirrer, or mixed by rolling or shaking, and then sampled by means of a thief to get a 1-quart sample, or more where larger quantities may be desired and are called for.

\section{DIPPER SAMPIING}

51. The dipper method of sampling is applicable where a free or open discharge of a stream exists or can be obtained readily, as follows: (a) Small filling and transfer pipe lines (2 inches in diameter or less), and (b) package, can, or barrel filling apparatus.

\section{APPARATUS}

52. The dipper shall have a flared bowl holding approximately 1 quart (or 1 liter) and a handle of any convenient length. Tinned steel is acceptable.

\section{PROCEDURE}

53. (a) The dipper shall be inserted in the free flowing part of the stream, collecting a sample from the full cross section of the stream at regular intervals, and the sample collected poured into a clean can of a convenient size, such as 5 or 10 gallon capacity.

(b) If the pumping or delivery rate varies, the samples shall be taken at time intervals so chosen as to give a sample for equal pumped quantities.

(c) The container into which the individual dipper samples of approximately 1 quart each are poured shall be kept closed and covered except when the close-fitting lid and cover are lifted to pour in the dipper sample.

(d) The gross amount of material collected should be about 0.1 per cent of the quantity trans- 
ferred, and the time intervals should be so chosen as to correspond, but not less than 5 gallons nor more than 40 gallons should be collected.

(e) The 5 or 10 gallon can shall be closed and covered, labeled, and delivered to the laboratory, where it shall be shaken or mixed with the churndasher type of stirrer and portions withdrawn by means of a thief for test.

\section{THIEF SAMPLING}

54. The thief method of sampling is applicable to the following types of containers: (a) Cans, (b) drums or barrels, and (c) tank cars.

\section{APPARATUS}

55. (a) A convenient and simple thief (see fig. 59, p. 115) for use with 50-gallon drum containers may be rade of tinned steel as follows: Length 36 inches and diameter 11/4 inches, with cone-shaped caps over the ends and having openings at the ends three-eighths inch in diameter. Three legs equally spaced around the thief at the bottom, long enough to hold the opening one-eighth inch from the bottom of the container being sampled, aid in securing a good representative sample. Two rings soldered to opposite sides of the tube at the upper end will be found convenient for holding the thief by slipping two fingers through them, leaving the thumb free to close the opening. For tank cars a thief emploring a trap at the bottom may be used.

(b) When sampling a shipment of oil enough thiefs should be provided to insure drainage of each before it is used a second time. For obtaining only a few samples two or three thiefs are enough, but for sampling a car lot of drummed oil six or more are desirable.

56. Tin cans or clear glass cork-stoppered bottles in cartons may be used. Containers shall be thoroughly clean and dry.

NoTE.-The glass bottle offers the adrantage that it may be examined to see that it is clern. It also allows visual inspection of the oil before testing, particularly as regards free water and solid impurities. However, if samples are placed in glass hottles, they shall be kent in the dark until the portions are remored for testing for color and sludge-forming characteristics, as light produces changes in these properties. Ruhber stoppers should nerer he used with the in these properties. Ruhber stoppers should nerer he used with the glass containers. Only good quality cork should be used with a prevented by means of wrapping around the cork before forcin into the moutb of tbe hottle some metal foil or a similar materia not attacked by oil. The tin can fitted with a screw cap protected witb a nonabsorbent gasket will endure harder usage. Tin cans sbould he soldered on the exterior surfaces.

57. All thiefs and sample receptacles shall be thoroughly cleaned before using, special care being taken that no lint or other fibrous material remains in or on them.

Note.-It is suggested that the thiefs be cleaned by rinsing with oil-free gasoline and dried in the hot cahinet (see see. 58 ) before using. Tbe best procedure is to rinse them after the sampling bas been finished and tben place them in the hot cahinet. They will in this way be ready for use witbout further cleaning when tbe next shipway be ready for use $\mathrm{w}$.

All sample contajners before using should be rinsed with oil-free All sample containers before using should be rinsed with oil-free
asoline and dried. They sbould tben he washed with strong soapgasoline and dried. They sbould tben he washed with strong soap-
suds, rinsed thoroughly witb water, and then dried in an oren at 105 to $110^{\circ} \mathrm{C}$. A similar washing will he sufficient for reuse of the con tainers for samples of new oil, hut tbose for used oil should he washed witb both gasoline and henzol before using soapsuds. Container wbicb are not tboroughly elean and dry are likely to contaminat the sample of oil and cause misleading results in some of the tests.

58. When not in use, all sampling apparatus should be kept in a hot, dry, dust-free cabinet or compariment at a temperature of not less than $38^{\circ}$ C. $\left(100^{\circ}\right.$ F.). Containers for samples, after washing and drying as indicated in section 57 , shall be kept unstoppered while in the oven or hot cabinet, but shall be closed immediately after they are taken out of the hot cabinet. However, it is permissible to prepare containers in advance, leaving them in the $105^{\circ}$ to $110^{\circ} \mathrm{C}$. oven until dry, then removing, corking or capping and sealing by dipping the neck into melted paraffin wax. Bottles shall, before dipping, have cork and neck wrapped with paper, securely tied. If this procedure is followed, before removing the cork or cap preparatory to filling the bottle or can with the sample, carc shall be taken to remove the parafin so as to prevent its contaminating the sainple. Thiefs shall be kept at all times in a vertical position in the hot cabinet in a rack having a suitable drainage receptacle at the base.

59. (a) Drums and barrels of oil to be sampled should be lined up preferably on the side and numbered, bungs up. The bungs shall be unsealed and removed and laid with the oily side up beside the bungholes. The unstoppered sample receptacle may be placed on the opposite side of the bungholes. The top hole of the thief shall be left open, and the thief thrust to the lowest point of recess of the container. When the thief is filled, it shall be closed, the thief quickly withdrawn and the contents allowed to flow into the sample receptacle. The lower holes shall not be closed with the fingers of the other hand. The free hand shall be used to guide the stream of oil by touching the thief only as necessary. The oil shall not be allowed to flow over the hand or fingers before it flows into the sample receptacle, nor shall any excess be ailowed to fiow over the drum and back into the drum

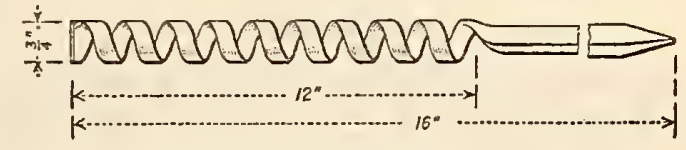

Note : Greater Overall lengths may be obtained when necessary to poss entirely through the Matorial to be sampled.

FIGURE 30.-I Improved ship auger

through the open bunghole. When the sample receptacle is filled, it shall be closed quickly and the bung replaced in the container and tightened. The sample receptacle shall be taken to the testing laboratory as quickly as feasible.

(b) Drums or tank cars, etc., of oil shall not be sampled until the oil is at least as warm as the surrounding air. Electrical insulating oil is not hygroscopic, but cold oil may condense enough moisture on the surface from a humid atmosphere to seriously affect its insulating properties.

(c) Samples shall not be taken in a place exposed to rain and precautions shall be taken during sampling to protect both the material being sampled and the samples themselves from moisture and dust.

(d) Electrical insulating oil samples shall be taken only after the oil in the original container has been allowed to settle undisturbed for eight hours.

(e) The containers for oils other than electrical insulating oil shall have their contents thoroughly mixed by being rolled or shaken, so that a mixed sample may be taken with the thief.

\section{BORINGS SAMPLING}

60. The borings method of sampling is applicable to wax and soft solids in cases, cakes, bags, or harrels when they can not properly or conveniently be melted and sampled as liquids.

\section{APPARATUS}

61. The ship auger shall be three-fourth inch in diameter and shall conform to the form and dimensions shown in Figure 30, and be of such a length as to pass entirely through the material to be sampled. 


\section{PROCEDURE}

62. (a) Opening.-Cases and barrels shall have the covers or heads removed. Bags shall have the mouths opened. Cakes shall have the wrappings, if any, opened.

(b) Foreign matter.-If foreign substances, such as dirt, sticks, string, etc., appear upon the surface, they shall be removed. Foreign matter found in the interior shall be included, as it may come with the borings.

(c) Boring hole location.-Three test holes shall be bored through the body of the material as follows: One at the center, one at onc-quarter of the diameter (or width of the package) from the right side, and one at onc-quarter of the diameter (or width of the package) from the lcft side.

$(d)$ If any visible differences appear in the three sets of borings, they shall be preserved, examined, and tested separately.

(e) If no visible differences appear in the borings they may be combined, preserved, examined, and tested as a single sample, but this shall be done only after the three sets of borings have been delivered to the laboratory and by the laboratory tester.

(f) Subdivision.-If subdivision of the borings is desired, they may be chilled, pulverized if necessary for handling, mixed, and quartered till reduced to the amount desired after their receipt in the laboratory. This subdivision shall not be done in the field by the sampler.

\section{GRAB SAMPLING}

63. The grab method of sampling is applicable to all lumpy materials loose in bins, bunkers, or carried in sacks, barrels, or boxes.

\section{APPARATUS}

64. A shovel if employed for sampling (see A. S. T. M. D140, 505.0, p. 128) may be of any convenient size, but should have a width at least twice as great as the maximum dimension of the largest pieces sampled.

\section{PROCEDURE 35}

65. (a) Solids are almost always heterogeneous in constitution. It is impossible to give absolutely definite and arbitrary methods for obtaining samples. Each problem must be worked out for itself, bearing in mind the particular conditions.

(b) It is usually preferable to take samples during the unloading of cars or during transit of the material in conveyors. In such instances, a number of small samples should be taken at frequent and regular intervals from the material in transit and these samples combined to form a representative combined sample.

(c) Occasionally, solids are tested as received in bags or barrels. In such instances it is desirable to take a small sample from a number of packages selected at random and equivalent in number to the cube root (or the next larger whole number) of the total number of packages in the lot.

(d) Generally, samples taken from the bulk, in piles or cars, are unreliable and not representative. Where it is necessary to take such samples before the unloading of the car, small samples should preferably be taken from at least 12 spots throughout the bulk, and these small samples collected to form the representative combined sample. In taking such small samples, it is desirable to take 8 samples from the corners of the car, 4 near the bottom,

${ }^{36}$ The Standard Method of Sampling Coal, serial designation D21 (see $501.0,0.4$ ), gives very full instructions and may be very helpful in directing the sampling of any loose bulk material and 4 near the top of the material. To those should be added 4 samples from the center of the car, 2 at the top and 2 near the bottom of the material.

(e) The combined sample taken by any of the above methods should be in amount at least 0.1 per cent of the total bulk of material sampled. These combined samples should be carefully mixed and reduced in size to a convenient laboratory sample, by the standard method of quartering. In carrying out this quartering, a hard, clean surface should be selected, free from cracks and protected from rain, snow, wind, and beating sun. Cinders, sand, chips from the floor, or any other material shall be avoided. The sample shall be protected from loss or gain in moisture or dust. The combined sample should be carefully mixed, spread out on the surface into a circular layer, and divided into four equal quadrants. Two opposite quadrants should be combined to form the representative reduced sample. If this sample is still too large for laboratory purposes, the quartering operation shall be repeated. In this manner, the sample shall finally be reduced to a size suitable for handling in the laboratory.

$(f)$ The use of a riffle sampler, as described in A. S. T. M. D271 (see 501.0, p. 8), is also recommended, with proper precautions against loss of dust, for subdivision in the laboratory.

American Society for Testing Materials, proposed tentative method of test for the determination of autogenous ignition temperatures, serial designation D286-28T, 1928. (See 502.2, p. 42.)

American Society for Testing Materials, tentative method of test for gravity of petroleum and petroleum products by means of the hydrometer, serial designation D287-28T, 1928. (See 502.0, p. 32.)

Federal Specifications Board, specification 2d, United States Government master specification for lubricants and liquid fuels.

(This specification, containing detailed requirements, methods of testing and methods of sampling, is published by the United States Government Printing Office as Technical Paper 323B, Department of Commerce, Bureau of Mines. The specification includes test methods, listed numerically, which are given below.)

\section{METHODS OF TEST FOR LUBRICANTS AND LIQUID} FUELS

No. 1. Color of refined petroleum oil by means of Saybolt chromometer; A. S. T. M. method D156-23T. (See 503.0, p. 54.)

No. 2. Color of lubricating oil by means of union colorimeter; A. S. T. M. method D155-23T. (See 504.0, p. 96.)

No. 3. Cloud and pour points; A. S. T. M. method D97-28. (See 502.2, p. 40 , omitting centigrade thermometer specifications.)

No. 4. Viscosity of petroleum products and lubricants; A. S. T. M. method D88-26. (See 503.0, p. 47.)

No. 5. Penetration of greases and petrolatum; A. S. T. M. method D217-27T. (See 504.0, p. 101.)

No. 6. Distillation of gasoline. This method is similar to A. S. T. M. method D86-27. (See 503.0$, p. 44.$)$

No. 7. Distillation of kerosene. This method is similar to A. S. T. M. method D86-27. (See 503.0, p. 44.)

No. 8. Flash point of volatile flammable liquids by the tag closed tester; A. S. T. M. method D56-21. (See 503.0, p. 43, omitting centigrade thermometer specifications.)

No.9. Flash point by means of the PenskyMartens closed tester; A. S. T. M. method D93-22. (See 503.0 , p. 49 , omitting centigrade thermometer specifications.) 
No. 10. Flash and fire points by means of open cup; A. S. T. M. method D92-24. (See 504.0, p.90, omitting centigrade thermometer specifications.)

No. 11. Flock test for kerosene.

\section{Apparatus and Procedure}

Place 300 cc of the sample in a 500 cc Florence or Erlenmeyer flask (after filtering if it contains suspended matter). Suspend a thermometer in the oil by means of a cork slotted on the side, and place the flask containing the oil in a sand bath or on an electric hot plate. Heat at such a rate that the oil will reach a temperature of $240^{\circ} \mathbf{F}$. at the end of one hour. Hold the oil at a temperature of not less than $240^{\circ} \mathrm{F}$. nor more than $250^{\circ} \mathrm{F}$. for six hours. Give the flask a slight rotary motion, and if any flock is seen rising from the bottom the test shall be reported as positive. Discoloration of the oil shall be disregarded.

No. 12. Flock test for mineral seal oil.

\section{Apparatus and Procedure}

Place $300 \mathrm{cc}$ of the sample in a $500 \mathrm{cc}$ Florence or Erlenmeyer flask (after filtering if it contains suspended matter). Suspend a thermometer in the oil by means of a cork slotted on the side, and place the flask containing the oil in a sand bath, or on an electric hot plate. Heat the oil at the rate of $10^{\circ} \mathrm{F}$. per minute to a temperature of $450^{\circ} \mathrm{F}$. and hold at that temperature for 15 minutes. Give the flask a slight rotary motion, and if any flock is seen rising from the bottom the test shall be reported as positive. Allow the flask to cool for one hour and again examine for flock. Discoloration of the oil shall be disregarded.

No. 13. Wick feed test.

\section{Apparatus}

1. An oil container, made of brass, capacity about 1 quart, fitted in the center with a brass tube of onehalf inch internal diameter, which serves as an oilway and which feeds into a graduated glass cylinder. (See fig. 31.)

2. A wick composed of eight strands of worsted zephyr of the best quality, pure, long-fiber, cream white fine wool, thoroughly washed, scoured, and carded; not dyed nor subjected to any chemical process. Strands shall be 4-ply, soft-spun, and twisted. The separate plies shall be of uniform thickness throughout their entire length.

3. A wick support in the form of a hook of copper wire which grips the outlet end of the wick below the level of the oil as is the usual manner in a wick feed.

\section{Procedure}

4. Dip the wick in the oil and place it in position and fill the oil container so that the lift of the wick is one-fourth inch. Maintain the lift at from onehalf to one-fourth inch during the test. Measure the flow of oil at the end of 24 and 48 hours and again at the end of 14 days.

No. 14. Burning test for long-time burning oil for railway use; A. S. T. M. method D219-27. (See 503.7, p. 89.)

No. 15. Burning quality of mineral seal oil. This method conforms in general with A. S. T. M. method D239-27. (See 503.6, p. 87)

No. 16. Burning test for kerosene (16 hours). This method conforms in general with A. S. T. M. standard method D187-27. (See 503.5, p. 85.)

No. 17. Water in petroleum products and other bituminous materials; A. S. T. M. method D95-28. (See 502.2, p. 38.)

No. 18. Sediment.

\section{Apparatus}

Alundum (porous grade) thimbles, ${ }^{30} 1$ inch in diameter by $2 \frac{3}{4}$ inches high, weighing not less than 15 nor more than $17 \mathrm{~g}$.

Extraction apparatus (see fig. 32) of such construction that the thimble is completely surrounded by the vapor of the boiling solvent. Siphon extractors must not be used.

\section{Procedure}

Place approximately $10 \mathrm{~g}$ of the sample in the previously extracted and dried, accurately weighed thimble and weigh to $\pm 0.01 \mathrm{~g}$, place in the extraction apparatus, and extract with 90 per cent benzol until the solvent dropping from the thimble is colorless. Dry the thimble for one hour at $105^{\circ} \mathrm{C}$. and weigh to $\pm 0.0001 \mathrm{~g}$. Repeat the extraction until the weight of the dried thimble and sediment is constant.

Note.-The rate of extraction shall be such that the mixture of oil and benzol in the thimble does not rise to within one-fourth inch of the top.

No. 19. Water and sediment by centrifuge; A. S. T. M. method D96-28. (See 502.2, p. 39.)

No. 20. Water by centrifuge.

\section{Apparatus}

1. The apparatus shall be the same as in No. 19 .

Procedure

2. Exactly 40 cc of 90 per cent benzol shall be measured into each of two centrifuge tubes, and exactly $20 \mathrm{ce}$ of the oil to be tested shall then be added to each. The centrif uge tubes shall be tightly stoppered and shall be shaken vigorously until the contents are thoroughly mixed.

3 . The two centrifuge tubes shall then be placed in the centrifuge on opposite sides and shall be whirled at a rate of 1,400 to $1,500 \mathrm{r}$. p. m., or the equivalent, for 10 minutes. The volume of water at the bottom of each tube shall be read and recorded, estimating to $0.1 \mathrm{cc}$ if necessary. The centrifuge tubes shall then be replaced in the centrifuge, again whirled for 10 minutes as before, and removed for reading the volume of water as before. This operation shall be repeated until the volume of water in each tube remains constant for three consecutive readings.

4. The volume of water shall be read on each tube, estimating to $0.1 \mathrm{cc}$ if necessary, and the average percentage of water in the sample calculated.

No. 21. Precipitation number of lubricating oils; A. S. T. M. method D91-29T. (See 504.0, p. 90.)

No. 22. Emulsion Test at $130^{\circ} \mathrm{F}$.

\section{Apparatus}

1. A $100 \mathrm{ec}$ graduated cylinder, $1 \frac{1 / 16}{16}$ to $1 \frac{1}{8}$ inches inside diameter.

2. An oil or water bath for maintaining the contents of the cylinder at the specified temperature. The line of the bath liquid shall not be lower than the 85-cc mark on the graduated cylinder.

3. A paddle consisting of a copper plate $43 / 4$ inches long, between three-fourths and seven-eighth inch wide, and one-sixteenth inch thick.

4. Means for revolving this paddle about a veltical axis parallel to and midway between its two longer edges and for keeping the speed fairly constant at 1,500 r. p. m.

5. A stop so arranged that when the paddle is lowered into the cylinder the distance from the bottom of the paddle to the bottom of the cylinder will be about one-fourth inch.

"Alundun thimbles listed by the Norton Co., Worcester, Mass., as 5163RA98, have proved satisfactory. 


\section{Procedure}

6. Place $40 \mathrm{cc}$ of the emulsifying liquid in the 100 ce graduated cylinder and add $40 \mathrm{cc}$ of the oil to be tested. (See fig. 33.)

7. Heat the cylinder by means of the bath to $130^{\circ} \mathrm{F}$.

8. Stir by rotating the paddle for five minutes at a speed of 1,500 r. p. m., stop the paddle, withdraw from the cylinder, and wipe clean with the finger
Apparatus and Procedure

The apparatus and procedure are the same as in No. 22 except that paragraphs 7 and 9 shall read:

7. Heat the cylinder by means of the bath to $180^{\circ} \mathrm{F}$.

9. Allow the cylinder to stand at $180^{\circ} \mathrm{F}$. for the specified time and then inspect. No continuous layer of emulsion shall remain.

No. 24. Demulsibility at $130^{\circ} \mathrm{F}$.
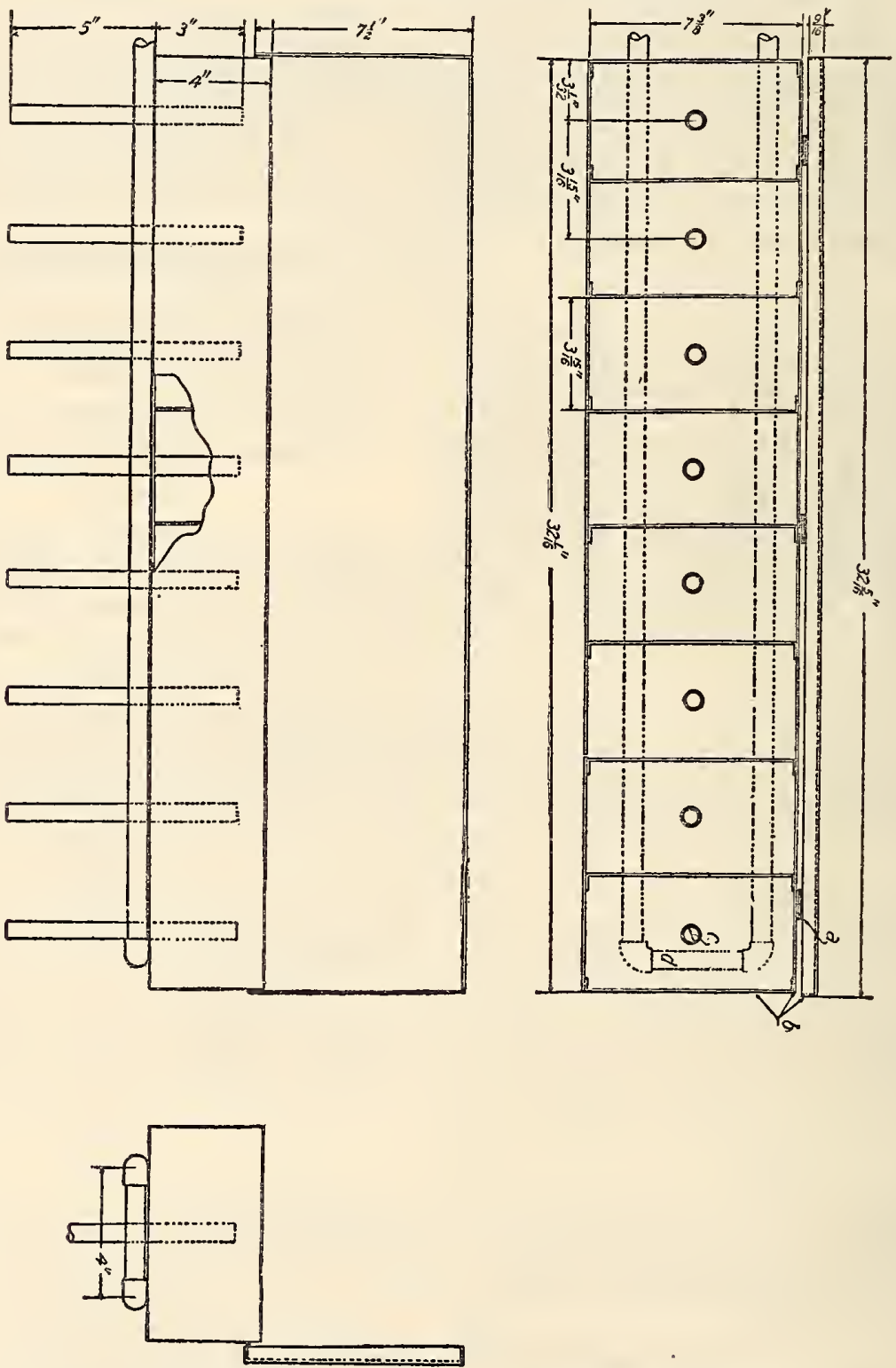

Figure 31.-Wick-feed test apparatus, as used by the United States Naval Engineering Experiment Station, Annapolis, $M d$.

$a, 1$-inch brass hinge; $b, 1 / 16$-inch sheet brass; $c$, 1/2-inch (inside diameter) brass tube; $d, 3 / 6$-inch steam pipe.

or rubber policeman, returning to the cylinder as much of the emulsion as possible.

9. Allow the cylinder to stand at $130^{\circ} \mathrm{F}$. for the specified time and then inspect. No continuous layer of emulsion shall remain.

No. 23. Emulsion test at $180^{\circ} \mathrm{F}$.
Same as in No. 22 .

\section{Apparatus}

\section{Procedure}

6. Place $27 \mathrm{cc}$ of the oil to be tested and $53 \mathrm{cc}$ of distilled water in the cylinder. 
7. Heat the cylinder by means of the bath to $130^{\circ} \mathrm{F}$.

8. Stir by rotating the paddle for five minutes at a speed of 1,500 r. p. m., stop the paddle, withdrawing from the cylinder, and wipe clean with the

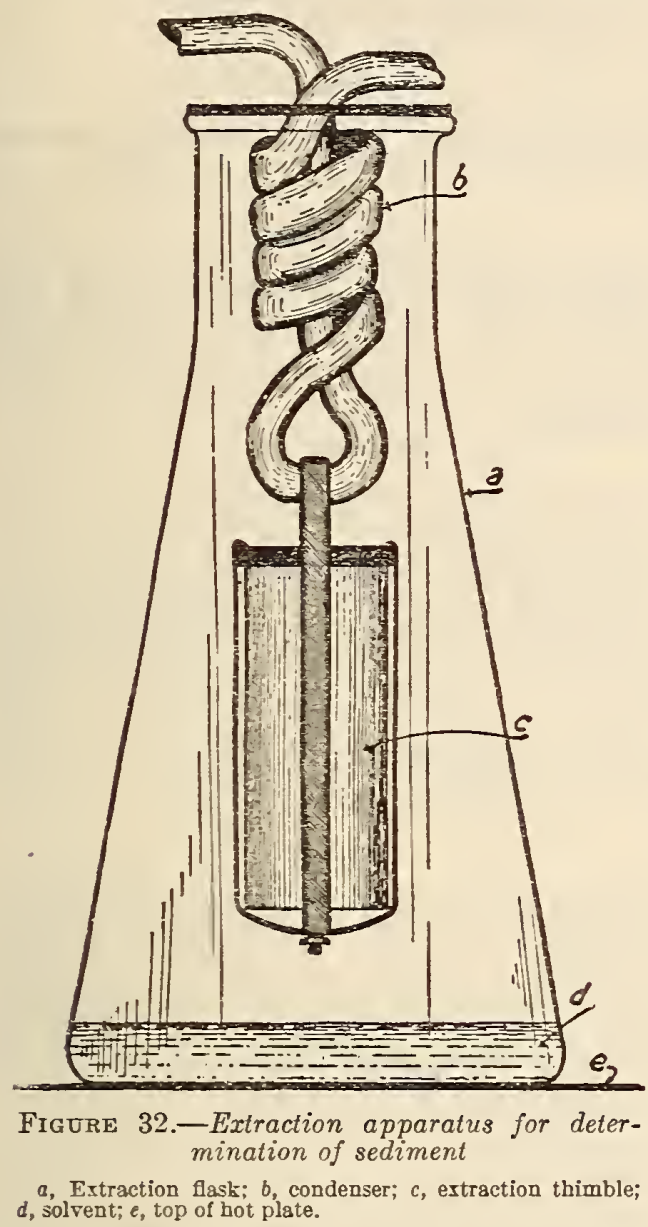

finger or rubber policeman, returning to the cylinder as much of the emulsion as possible.

9. Keep the temperature of the cylinder constant at $130^{\circ} \mathrm{F}$.

10. Take readings every minute of the position of the line of demarcation between the topmost layer of oil and the adjoining emulsion. Take the first reading one minute after stopping the paddle. With oils which act normally the rate of settling out of the oil increases up to a maximum and then decreases, and the maximum value in cubic centimeters per hour is called the "demulsibility" and is recorded as the numerical result of the test. Each rate of settling is the average rate calculated from the time of stopping the paddle to the time of reading, as shown in the following condensed table:
Rate of settling

\begin{tabular}{|r|c|c|c|c|}
\hline Time & $\begin{array}{c}\text { Time } \\
\text { since } \\
\text { stopping } \\
\text { paddle }\end{array}$ & $\begin{array}{c}\text { Reading } \\
\text { at inter- } \\
\text { face } \\
\text { between } \\
\text { oil and } \\
\text { emulsion }\end{array}$ & $\begin{array}{c}\text { Oil } \\
\text { settied } \\
\text { out }\end{array}$ & $\begin{array}{c}\text { Rate of } \\
\text { settling } \\
\text { per hour }\end{array}$ \\
\cline { 1 - 2 } & Minutes & & $c c$ & $c c$ \\
9.50 & 0 & 80 & 0 & 0 \\
9.55 & 5 & 77 & 3 & 36 \\
10.02 & 12 & 67 & 13 & 65 \\
10.05 & 15 & 63 & 17 & 68 \\
10.10 & 20 & 61 & 19 & 57 \\
\hline
\end{tabular}

The demulsibility in this case would be 68 , the highest value in the last column. When the maximum rate of settling has not been reached at the

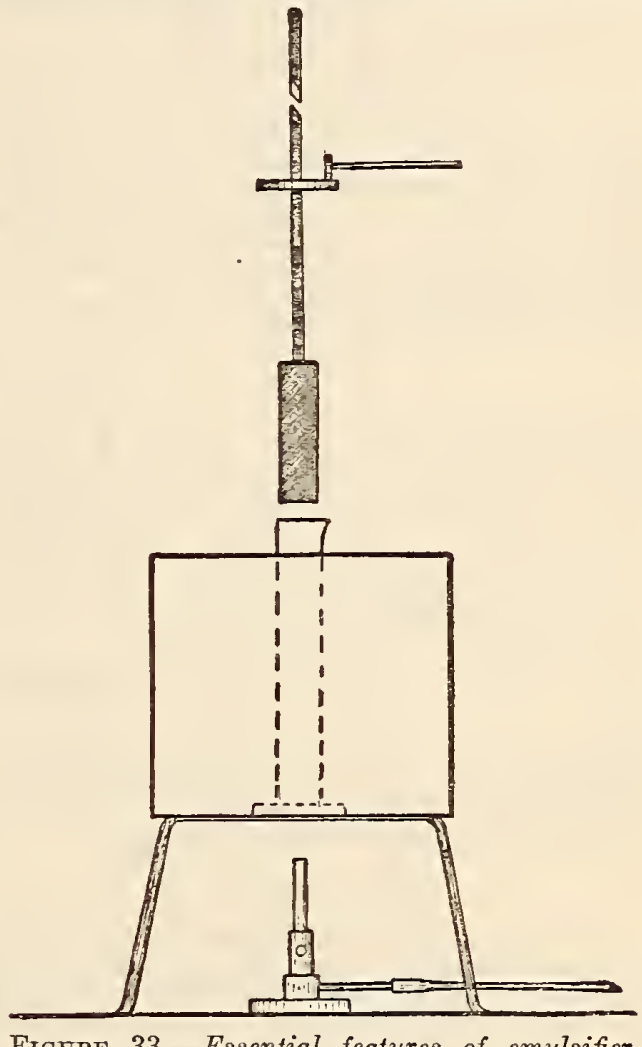

FIgURE 33.-Essential features of emulsifier

end of one hour the test is discontinued and the demulsibility taken as the number of cubic centimeters that settled out in the hour.

No. 25. Demulsibility at $180^{\circ} \mathrm{F}$.

\section{Apparatus and Procedure}

The apparatus and procedure are the same as in No. 24, except that paragraphs 7 and 9 shall read: 7. Heat the cylinder by means of the bath to $180^{\circ} \mathrm{F}$. 
9. Keep the temperature of the cylinder constant at $180^{\circ} \mathrm{F}$.

No. 26. Emulsion test for soluble cutting oils. In a 4-ounce oil sample bottle or similar vessel place $95 \mathrm{cc}$ of distilled water and add thereto $5 \mathrm{ec}$ of the oil. Agitate vigorously for one minute at room temperature $\left(70^{\circ}\right.$ to $90^{\circ}$ F.). After standing for one hour no sevaration whatever shall have occurred.

No. 27. Protection test. Coat a clean polished steel plate 2 inches long, one-half inch wide, and one-eighth inch thick by immersing it in the sample to be tested, which has been heated to a temperature of $212^{\circ} \mathrm{F}$. Remove the plate while it is still hot, allow to cool in a vertical position, and suspend vertically within a 10 per cent salt solution at room temperature for 30 days. At the end of this time rinse with gasoline and examine for corrosion.

No. 28. Carbon residue of petroleum products (Conradson carbon residue, modified); A. S. T. M. method D189. (See 504.0, p. 100.)

No. 29. Reaction test. Place 50.ce of the sample and 15 ec of distilled water in a 150-cc flask. Warm to $150^{\circ} \mathrm{F}$. and shake thoroughly. Allow the mixture to cool and transfer $5 \mathrm{cc}$ of the aqueous layer to each of two test tubes by means of a pipette. Add one drop of 1 per cent solution of methyl orange to the contents of one tube and one drop of a 1 per cent solution of phenolphthalein to the other. No red or pink color shall result in either case.

No. 30 . Acidity in gasoline.

Nore.-This test is to be made immediately after distillation test No. 6.

Collect in a test tube the cooled residue from the distillation flask, add three volumes of distilled water, and shake the tube thoroughly. Allow the mixture to separate and remove the aqueous layer to a clean test tube by means of a pipette. Add one drop of a 1 per cent solution of methyl orange. No pink or red color shall be formed.

No. 31. Neutralization number; A. S. T. M. method D188-27T. (See 504.0, p.99.)

No. 32. Sulphur in naphthas and illuminating oils; A. S. T. M. method D90-29T. (See 503.0, p. 48.)

No. 33. Sulphur in petroleum oils heavier than illuminating oil; A. S. T. M. method D129-27. (See 503.0, p. 53.)

No. 34. Doctor test (sodium plumbite).

\section{Preparation of Reagents}

(1) Sodium plumbite (doctor solution).-Dissolve approximately $125 \mathrm{~g}$ of sodium hydroxide ( $\mathrm{NaOH}$ ) in 1 liter of distilled water. Add $60 \mathrm{~g}$ of litharge $(\mathrm{PbO})$ and shake vigorously for 15 minutes or let stand with occasional shaking for at least a day. Allow to settle and decant or siphon off the clear liquid. Filtration through a mat of asbestos may be employed if the solution does not settle clear. The solution should be kept in a tightly corked bottle and should be refiltered before use if not perfectly clear.

(2) Sulphur.-Pure, dry flowers of sulphur.

\section{Making of Test}

Shake vigorously together in a test tube 10 cc of the sample to be tested and 5 cc of sodium plumbite solution for about 15 seconds. Add a small pinch of flowers of sulphur, again shake for 15 seconds, and allow to settle. The quantity of sulphur used should be such that practically all of it floats on the interface between the sample and the sodium plumbite solution.

\section{Interpretation of Results}

If the sample is discolored, or if the yellow color of the sulphur film is noticeably masked, the test shall be reported as positive and the sample condemned as "sour." If the sample remains unchanged in color, and if the sulphur film is bright yellow or only slightly discolored with gray or flecked with black, the test shall be reported negative and the sample considered "sweet."

No. 35. Corrosion test (copper dish).

\section{Apparatus}

A freshly polished hemispherical dish of spun copper approximately $3 \frac{1}{2}$ inches in diameter.

\section{Making of Test}

Place $100 \mathrm{cc}$ of the gasoline to be examined in the dish and place the dish in an opening of an actively boiling steam bath, so that the steam comes in contact with the outer surface of the dish up to the level of the gasoline. Leave the dish on the steam bath until all volatile material has disappeared.

Interpretation of Results

If the gasoline contains dissolved elementary sulphur or corrosive sulphur compounds, the bottom of the dish will be colored gray or black.

If the gasoline contains undesirable gum-forming constituents, there will be a weighable amount of gum deposited on the dish. Acid residues will show as gum in this test. The gasoline shall cause no gray or black corrosion and the amount of deposit shall not exceed $3 \mathrm{mg}$.

No. 36. Corrosion test at $122^{\circ}$ F.; A. S. T. M. method D130-27T. (See 503.3, p. 71.)

No. 37. Corrosion test at $212^{\circ} \mathrm{F}$.

\section{Copper Strip}

Place a clean strip of mechanically polished pure sheet copper about one-half inch wide and 3 inches long in a clean test tube. Add enough of the sample to be tested completely to cover the strip. Close the tube with a vented stopper and maintain for three hours at $212^{\circ} \mathrm{F}$. Rinse the copper strip with sulphur-free acetone and compare it with a similar strip of freshly polished copper.

Discoloration or pitting indicates corrosion.

No. 38. Corrosion test for solid or semisolid products.

1. This method of test shall be used for the detection of corrosive properties of greases or other solid or semisolid products.

2. Clean, well-polished sheets of copper shall be used for this test. In general, sheets about 1 inch $(25 \mathrm{~mm})$ square will be found most convenient.

3. Place a small portion of the grease to be tested on each of the metal plates, taking care not to cover the whole surface of the metal plates. Cover each plate with an inverted watch glass and let stand at room temperature for 24 hours. There must be no evidence of corrosion on the plate, either when examined with the sample in place or after removing it by washing with benzol. Either the development of a green color in the material on the copper or a brown stain remaining on the copper after washing with benzene which passes the corrosion test No. 36 is evidence of corrosion.

No. 39. Corrosion test at room temperatures.

1. This method of test shall be used for the detection of corrosive compounds in soluble cutting oils.

2. Clean strips of mechanically polished sheet steel and copper about one-half inch wide and 3 inches long shall be placed separately in suitable clean tubes or sample bottles. The cutting oil under test shall be added so that the strips are but partly immersed and the tubes or bottles shall be closed with corks. The container shall then be 
manipulated so as to completely wet the metal strips with the cutting oil. The tubes or bottles shall be set up in an approximately vertical position and allowed to remain in the laboratory at room temperature $\left(70^{\circ}\right.$ to $90^{\circ} \mathrm{F}$.) for 48 hours.

3 . At the end of the 48 -hour period the metal strips shall be removed from the containers and washed with benzol which passes corrosion test No. 36. The presence of corrosive compounds is indicated by corrosion or discoloration of the steel or copper strips when compared with freshly polished ones.

(a) A soluble cutting oil shall be reported as passing the test when the exposed strips show at most an extremely slight discoloration.

(b) A soluble cutting oil shall be reported as not passing the test when the exposed strips of either metal show more than extremely slight discoloration.

No. 40. Saponification number; A. S. T. M. method D94-28. (See 504.0, p. 92.)

No. 41. Fatty oil. The percentage of fatty oil shall be calculated from the saponification number (No. 40) by means of the following formula:

\section{Percentage of fatty oil $=100 \times$ saponification number of sample}

The saponification number of the fatty oil used in compounding shall be assumed to be 195 , unless the manufacturer of the sample under examination submits an affidavit stating the kind of fatty oil used and its saponification number.

No. 42. Analysis of grease; A. S. T. M. method D128-27. (See 504.0, p. 93.)

National Association of Purchasing Agents, standard fuel oil contract guide, May, 1925.

(The above guide has been adopted by the association to be used as a standard for writing contracts for the purchase of fuel.)

United States Department of Agriculture, Bulletin 1216, Method of Test for Percentage of Residue of Desired Penetration, 1928.

1. Fifty grams of the oil are placed on a 3-ounce deep seamless tin box; the box is placed in a sand bath and heated over a Bunsen burner. A thermometer is suspended in the oil, the bulb not touching the bottom of the box. The temperature of the oil is kept at from $249^{\circ}$ to $260^{\circ} \mathrm{C}$. $\left(480^{\circ}\right.$ to $500^{\circ} \mathrm{F}$.), and the oil is stirred from time to time with the thermometer to prevent overheating in any part. Depending upon the nature of the oil, as usually indicated by its flash, consistency at $25^{\circ} \mathrm{C} .\left(77^{\circ} \mathrm{F}\right.$.) and specific gravity, the operator can with experience tell about what percentage it will be necessary to evaporate before cooling and taking a penetration of the residue. It is sometimes necessary to make several trials before the desired result is obtained. When the required penetration is reached, the residue left from evaporation is weighed and its per cent of the original sample taken is computed.

2. Ordinarily a residue shall be considered as satisfactorily obtained on the basis of 100 penetration being specified when its penetration is within 25 points of that desired. When it is necessary to determine more precisely the per cent of residue for a specified penetration, such percentage shall be computed by interpolation between percentages of two residues, one having a penetration greater, and one having a penetration lower than that specified.

\subsection{BENZINE.} able.)

(No nationally recognized specifications avail-

\subsection{DISTILLATES.}

American Society for Testing Materials, tentative specifications for petroleum spirits (mineral spirits) serial designation: D235-26T; 1926.
1. These specifications apply only to petroleurn distillates.

\section{PROPERTTES}

2. The material shall conform to the following requirements:

(a) Appearance.-It shall be clear and free from suspended matter and water.

(b) Color.-The color shall be "water white"; that is, not darker than No. 21 Saybolt chromometer.

(c) Flash point.-The flash point shall not be lower than $86^{\circ} \mathrm{F}$. $\left(30^{\circ} \mathrm{C}\right.$. $)$ when tested in the Tag closed tester.

(d) Blackening.-It shall not blacken or corrode clean metallic copper in 30 minutes at the boiling point of the spirits.

(e) Distillation.-The distillate below $266^{\circ} \mathrm{F}$.

$\left(130^{\circ}\right.$ C.) shall not exceed 5 per cent.

The distillate below $446^{\circ} \mathrm{F}$. $\left(230^{\circ} \mathrm{C}\right.$. $)$ shall not be less than 97 per cent.

(f) Acidity. - The residue after distillation shall be neutral.

\section{METHODS OF TEST}

3. The properties enumerated in these specifications shall be determined in accordance with the following methods of test:

(a) Color. - The test for color shall be in accordance with A.S. T. M. D156-23 T. (See 503.0, p. 54.)

A fresh aqueous solution of potassium dichromate in distilled water, containing $0.0048 \mathrm{~g} \mathrm{~K}_{2} \mathrm{Cr}_{2} \mathrm{O}_{7}$ per liter, is approximately equivalent to No. 21 Saybolt chromometer.

(b) Flash point.-The test shall be made in accordance with A. S. T. M. D56. (See 503.0, p. 43.)

(c) Blackening.-A clean strip of mechanically polished pure sheet copper, about $1 / 2$ inch $(1.3 \mathrm{~cm})$ in width and 3 inches $(7.5 \mathrm{~cm})$ in length, shall be placed in a glass test tube about $3 / 4$ inch $(1.9 \mathrm{~cm})$ in width and 18 inches $(46 \mathrm{~cm})$ in length. Sufficient of the sample to be tested shall be added to completely cover the strip and heated rapidly to boiling. It is most convenient to heat the tube by immersion in an oil bath maintained at a temperature slightly higher than the initial boiling point of the mineral spirits. The sample shall be kept boiling for 30 minutes without any actual distillation taking place and the copper strip then examined for blackening. A slight tarnish shall be disregarded, but any marked blackening shall be cause for rejection.

(d) Distillation.-The test shall be in accordance with A. S. T. M. D86. (See 503.0, p. 44.)

(e) Acidity. - The cooled residue from the distillation flask shall be collected in a test tube, three volumes of distilled water added, and the tube thoroughly shaken. The mixture shall be allowed to separate and the aqueous laver removed to a clean test tube by means of a pipette. One drop of a 1 per cent solution of methyl orange shall be added. No pink or red color should be formed.

\subsection{GASOLINE AND NAPHTHA.}

American Railway Association, signal section, specification 13324, July, 1924, motor gasoline.

(This specification is based on United States Government master specification for motor gasoline. (See F. S. B. No. 2d, p. 72, "Gasoline.")

American Society for Testing Materials, tentative method of test for detection of free sulphur and corrosive sulphur compounds in gasoline, serial designation: D $130-27 \mathrm{~T} ; 1927$.

1. This method of test shall be used for the detection of free sulphur and corrosive sulphur compounds in gasoline.

2. A clean strip of mechanically polished pure sheet copper about $1 / 2$ inch in width and 3 inches in length shall be placed in a suitable clean tube or 
sample bottle. Gasoline under test shall be added so that the copper strip is completely immersed. The test tube or sample bottle shall be closed with a loosely fitting cork and held in a suitable bath at $122^{\circ}$ F. $\left(50^{\circ} \mathrm{C}\right.$. $)$.

At the end of three hours the gasoline exposed strip shall be removed and shall be compared with a similar strip of freshly polished copper.

3. The presence of sulphur or corrosive sulphur compound is indicated by the corrosion or discoloration of the gasoline exposed strip when compared with the fresh copper strip.

4. (a) Gasoline shall be reported as passing the test when on examination the exposed strip shows not more than extremely slight discoloration as compared with the fresh copper strip.

(b) Gasoline shall be reported as not passing the test when on examination the exposed strip shows more than extremely slight discoloration as compared with the fresh copper strip.

American Society for Testing Materials, standard method of test for distillation of natural gas gasoline, serial designation D216-27, 1927.

Approved as American Standard, by the American Engineering Standards Committee, now the American Standards Association.

\section{APPARATUS}

The apparatus to be used in this specification is the same as that specified in A. S. T. M. D86 (see 503.0, p. 44), sections $1,2,3,4,5$, and 7 .

Thermometer. -The A. S. T. M. low-distillation thermometer shall conform to the requirements specified for thermometer No. 6. (See table of A. S. T. M. thermometers, 500.2, p. 2.)

\section{PROCEDURE}

Sampling.-Samples should be collected in a previously cooled bottle, preferably by immersing the bottle in the liquid, where possible, and discarding the first sample. Where immersion is not possible the sample should be drawn off into a previously cooled bottle in such a manner that agitation is kept at a minimum. Immediately close the bottle with a tight-fitting stopper, and place in an ice bath or refrigerator capable of bringing the gasoline to a temperature of not less than $32^{\circ} \mathrm{F}$. $\left(0^{\circ} \mathrm{C}\right.$.), nor more than $40^{\circ} \mathrm{F}$. $\left(4.45^{\circ} \mathrm{C}\right.$.).

The condenser bath shall be filled with cracked ice 37 and enough water added to cover the condenser tube. The temperature shall be maintained between $32^{\circ}$ and $34^{\circ} \mathrm{F}$. $\left(0^{\circ}\right.$ and $1.1^{\circ} \mathrm{C}$.). Agitation with air greatly aids in maintaining the required temperature range.

The condenser tube shall be swabbed to remove any liquid remaining from the previous test. A piece of soft cloth attached to a cord or copper wire may be used for this purpose.

One hundred cubic centimeters of the product shall be measured in the 100-cc graduated cylinder at $32^{\circ}$ to $40^{\circ} \mathrm{F}$. $\left(0^{\circ}\right.$ to $4.45^{\circ} \mathrm{C}$. $)$ and transferred directly to the Engler flask. Both the flask and the graduated cylinder shall have been cooled to a temperature of from $32^{\circ}$ to $40^{\circ} \mathrm{F}$. $\left(0^{\circ}\right.$ to $4.45^{\circ} \mathrm{C}$. $)$ before use. None of the liquid shall be permitted to flow into the vapor tube.

The thermometer provided with a cork shall be fitted tightly into the flask so that it will be in the middle of the neck and so that the lower end of the capillary tube is on a level with the inside of the bottom of the vapor outlet tube at its junction with the neck of the flask.

The charged flask shall be placed in the $11 / 4$ inches $(3.175 \mathrm{~cm})$ opening in the 6 by 6 inch $(15.24$ by by $15.24 \mathrm{~cm}$ ) asbestos board with the vapor-outlet

any other convenient cooling medium may be used. tube inserted into the condenser tube. A tight connection may be made by means of a cork through which the vapor tube passes. The position of the flask shall be so adjusted that the vapor tube extends into the condenser tube not less than 1 inch $(2.54 \mathrm{~cm})$ nor more than 2 inches $(5.08 \mathrm{~cm})$.

The graduated receiver used in measuring the charge shall be placed, without drying, at the outlet of the condenser tube in such a position that the condenser tube shall extend into the graduate at least 1 inch $(2.54 \mathrm{~cm})$, but not below the $100 \mathrm{cc}$ mark. The graduate shail be immersed at least up to the $100 \mathrm{cc}$ mark in a transparent bath maintained between the temperatures of $32^{\circ}$ and $34^{\circ} \mathrm{F}$. $\left(0^{\circ}\right.$ and $1.11^{\circ} \mathrm{C}$.). The top of the graduate shall be covered closcly during the distillation with a piece of blotting paper, or its equivalent, and cut to fit the condenser tube tightly. A lead washer, or other suitable material, resting on the blotting paper is a convenient accessory as it serves to hold the paper tightly against the top of the graduate and also provides the additional weight necessary to overcome the buoyant effect of the liquid in the cooling bath.

When everything is in readiness, heat shall be applied at a uniform rate, so regulated that the first drop of the condensate falls from the condenser in not less than two nor more than five minutes. When the first drop falls from the end of the condenser, the receiving cylinder shall be moved so that the end of the condenser tube shall touch the side of the cylinder. The heat shall then be so regulated that the first $10 \mathrm{cc}$ of distillate shall be recovered in not less than three nor more than four minutes. Thereafter, the rate of distillation shall be maintained uniformly at not less than 4 nor more than 5 cc per minute. The reading of the distillation thermometer shall be recorded when the level of the distillate reaches each 10-cc mark on the graduate.

When the liquid residue in the distillation flask is approximately $5 \mathrm{cc}$, the heat may be increased because of the presence of heavy ends which have relatively high boiling points. However, no further increase of heat shall be applied after this adjustment. The 4 to 5 cc rate can not always be maintained from this point to the end of the distillation, but in no case should the period between the point when approximately $5 \mathrm{cc}$ of the liouid remains in the flask and the end point be more than five minutes

The heating shall be continued until the mercury reaches a maximum and starts to fall consistently. The highest temperature observed on the distillation thermometer shall be recorded as the end point. Usually this point will be reached after the bottom of the flask has become dry. If the bottom of the flask is not dry, the operator should note this on his report.

The total volume of the distillate collected in the receiving graduate shall be recorded as the "recovery.",

The cooled residue shall be poured from the flask into a small cylinder graduated in $0.1 \mathrm{cc}$, measured when cool, and the volume recorded as "residue" at $32^{\circ}$ to $40^{\circ} \mathrm{F}$. $\left(0^{\circ}\right.$ to $4.45^{\circ} \mathrm{C}$.).

The difference between $100 \mathrm{cc}$ and the sum of the recovery and the residue shall be calculated and recorded as "distillation loss."

\section{CORRECTION FOR BAROMETRIC PRESSURE}

Same as section 11, including table and note of A. S. T. M. D86.

The form shown in Figure 34 is suggested for the recording of results of tests of natural gas gasoline.

Federal Specifications Board specification 2d, United States Government master specification for lubricants and liquid fuels. 
(This specification, containing detailed requirements, methods of testing, and methods of sampling is published by the United States Government Printing Office as technical paper 323B, Department of Commerce, Bureau of Mines. The specification includes specifications and tests for gasoline, which are given below.)

\section{GASOLINE}

\section{GENRRAL REQUIRETENTS}

Sampling.-See A. S. T. M. method D270-27T, 503.0 , page 60 .

Testing.-See F. S. B. No. 2d, 503.0, page 66.
NoтE.-For convenience in presentation the test methods have been listed numerically and reference made accordingly in the following table of specifications where tests apply.

\section{DETAIL REQUIREMENTS}

(a) General

1. The gasoline shall be free from water and suspended matter.

2. Uses:

Fighting grade.-Used as a fuel for fighting planes, where the highest efficiency is required.

Domestic grade.-Used for aviation fuel, where the fighting grade is not required.

(b) Properties and tests.

\begin{tabular}{|c|c|c|c|c|c|c|c|c|c|c|c|c|c|c|c|c|c|c|c|}
\hline \multirow[b]{2}{*}{ Name and grade } & \multirow{2}{*}{$\begin{array}{l}\text { Color, } \\
\text { dark- } \\
\text { est al- } \\
\text { lowed } \\
\text { (No. 1) }\end{array}$} & \multirow[b]{2}{*}{$\begin{array}{c}\text { Doctor test } \\
\text { (No. 34) }\end{array}$} & \multirow[b]{2}{*}{$\begin{array}{c}\text { Corrosion } \\
\text { tesi }\end{array}$} & \multicolumn{12}{|c|}{ Distillation range (No. 6) } & \multirow{2}{*}{$\begin{array}{c}\text { Recov- } \\
\text { ery, } \\
\text { mini- } \\
\text { mum }\end{array}$} & \multirow{2}{*}{$\begin{array}{l}\text { Dis- } \\
\text { tilla- } \\
\text { tion } \\
\text { loss, } \\
\text { maxi- } \\
\text { mum }\end{array}$} & \multirow{2}{*}{$\begin{array}{l}\text { Acidity } \\
\text { test } \\
\text { (No. 30) }\end{array}$} & \multirow{2}{*}{$\begin{array}{l}\text { Sul- } \\
\text { phur, } \\
\text { maxi- } \\
\text { mum } \\
\text { (No. } \\
\text { 32) }\end{array}$} \\
\hline & & & & \multicolumn{2}{|c|}{$\begin{array}{l}5 \text { per } \\
\text { cent, } \\
\text { mini- } \\
\text { mum }\end{array}$} & \multicolumn{2}{|c|}{$\begin{array}{l}5 \text { per } \\
\text { cent, } \\
\text { maxi- } \\
\text { mum }\end{array}$} & \multicolumn{2}{|c|}{$\begin{array}{l}50 \text { per } \\
\text { cent, } \\
\text { maxi- } \\
\text { mum }\end{array}$} & \multicolumn{2}{|c|}{$\begin{array}{l}90 \text { per } \\
\text { cent, } \\
\text { maxi- } \\
\text { mum }\end{array}$} & \multicolumn{2}{|c|}{$\begin{array}{l}96 \text { per } \\
\text { cent, } \\
\text { maxi- } \\
\text { mum }\end{array}$} & \multicolumn{2}{|c|}{$\begin{array}{l}\text { End } \\
\text { point, } \\
\text { maxi- } \\
\text { mum }\end{array}$} & & & & \\
\hline $\begin{array}{l}\text { A viation gasoline: } \\
\text { Fighting } \\
\text { Domestic }\end{array}$ & $\begin{array}{r}\text { Saybolt } \\
\text { No. } \\
25 \\
25\end{array}$ & $\begin{array}{l}\text { Negative.- } \\
-\mathrm{do}\end{array}$ & $\begin{array}{r}\text { (No. 35) } \\
-\end{array}$ & $\begin{array}{r}{ }^{\circ} \mathrm{C} . \\
50 \\
50\end{array}$ & $\begin{array}{l}{ }^{\circ} F \\
122 \\
122\end{array}$ & $\begin{array}{r}{ }^{\circ} \mathrm{C} \\
65 \\
75\end{array}$ & $\begin{array}{l}{ }^{\circ} F \\
149 \\
167\end{array}$ & $\begin{array}{r}{ }^{\circ} \mathrm{C} . \\
95 \\
105\end{array}$ & $\begin{array}{l}{ }^{\circ} F \\
203 \\
22 \mathrm{I}\end{array}$ & $\begin{array}{l}{ }^{\circ} C \\
125 \\
155\end{array}$ & $\begin{array}{l}{ }^{\circ} F \\
257 \\
311\end{array}$ & $\begin{array}{l}{ }^{\circ} \mathrm{C} \\
150 \\
175\end{array}$ & $\begin{array}{l}{ }^{\circ} F \\
302 \\
347\end{array}$ & $\begin{array}{l}{ }^{\circ} \mathrm{C} \\
16.5 \\
190\end{array}$ & $\begin{array}{l}{ }^{\circ} F \\
329 \\
374\end{array}$ & $\begin{array}{r}P . c t . \\
96 \\
96\end{array}$ & $\begin{array}{r}P . c t . \\
2 \\
2\end{array}$ & Required_ & $\begin{array}{r}0.10 \\
.10\end{array}$ \\
\hline
\end{tabular}

Distillation Test for

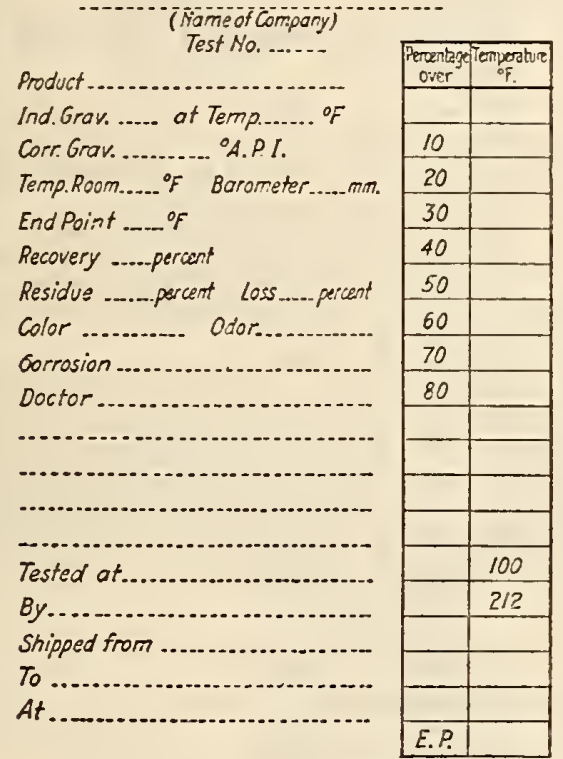

Carlnit..

CarNa.

Date 192.

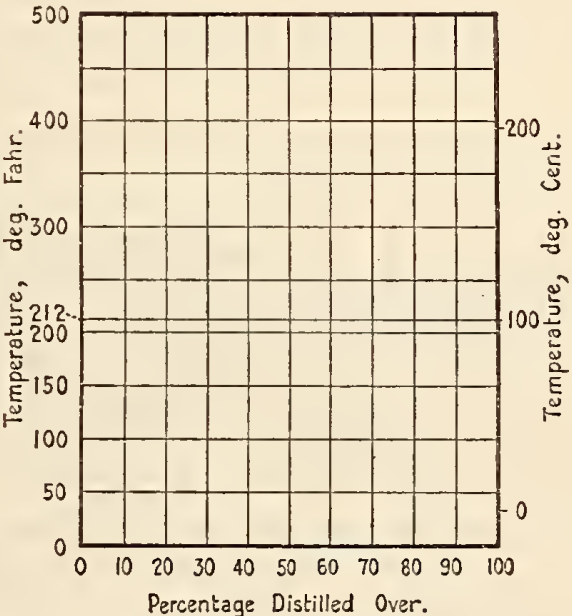

FIGURE 34.-Form for recording tests on natural gas gasoline

Federal Speciflcations Board specification 622a, United States Government master specification for motor gasoline, June 11, 1929.

1. This specification covers the grade of gasoline used by the United States Government and its agencies as a fuel for automobile, truck, tractor, motor boat, and similar engines.
2. Gasoline purchased under this specification shall be described as "U. S. Government motor gasoline" with the understanding that this designation always refers to the current specification of the Federal Specifications Board.

3 . The gasoline shall be free from water and sus. pended matter. 


\section{PROPERTIES AND TESTS}

NotE.-All tests shall be made according to the methods of testing contained in United States Government master specification 2d. (See $503.0, p, 66$.) The numbers given in the tests following refer to those methods.

4. Sampling.-See A. S. T. M. D270, 503.0, page 60.

5. Corrosion test.-No. 36. A clean copper strip shall not show more than extremely slight discoloration when submerged in the gasoline for three hours at $122^{\circ} \mathrm{F}$.

6. Distillation range.-No. 6. When 10 per cent has been recovered in the receiver, the thermometer shall not read more than $80^{\circ} \mathrm{C}$. $\left(176^{\circ} \mathrm{F}\right.$.) nor less than $50^{\circ}$ C. $\left(122^{\circ} \mathrm{F}\right.$.); provided that, for each per cent distillation loss less than 4 per cent, obtained in the A. S. T. M. distillation, the minimum 10 per cent temperature requirements shall be lowered $3^{\circ} \mathrm{C}$. $\left(5.4^{\circ} \mathrm{F}\right.$.). The Government reserves the right by proper provision in the contract or purchase order to lower maximum 10 per cent point either $9^{\circ} \mathrm{F}$. or $18^{\circ} \mathrm{F}$. Administrative action in this respect may be taken by contracting officers to meet the temperature conditions under which the equipment must operate. (See note 1.)

NотЕ 1--Contracting officers are advised that, in general, the expectation of freezing temperatures warrants the lowest maximum 10 per cent temperature.

NOTE 2.- When United States Government motor gasoline is to be used for some special purpose, such as that requiring tropical storage, the Government reserves the right to raise the lower limit at the 10 per cent point to not higher than $60^{\circ} \mathrm{C}$. $\left(140^{\circ} \mathrm{F}\right.$.) with the same corrections for distillation loss as given above. This limit, however, should not be applied unless absolutely necessary, since the gasoline so procured is less satisfactory for general purposes.

When 50 per cent has been recovered in the receiver, the thermometer shall not read more than $140^{\circ} \mathrm{C}$. $\left(284^{\circ} \mathrm{F}\right.$. $)$.

When 90 per cent has been recovered in the receiver, the thermometer shall not read more than $200^{\circ}$ C. $\left(392^{\circ} \mathrm{F}\right.$.).

The end point shall not be higher than $225^{\circ} \mathrm{C}$. $\left(437^{\circ} \mathrm{F}\right.$.).

At least 95 per cent shall be recovered as distillate in the receiver from the distillation.

7. Sulphur.-No. 32. Sulphur shall not exceed 0.10 per cent.

Federal Specifications Board specification 623, United Statcs Government master specification for motor fuel V, Junie 11, 1929.

1. This specification covers a special grade of fuel which may be used by the Government at the discretion of the head of the department or bureau concerned and its agencies, for ambulances, fire engines, emergency vehicles, military and naval equipment, and for other equipment under special conditions.

2. This fuel shall be free from water and suspended matter.

\section{PROPERTIES AND TESTS}

NotE.-All tests shall be made according to the methods of testing contained in United States Government master specification $2 d$. (See 503.0, p. 66.) The numbers given in the tests following refer to those methods.

3. Sampling.-Samples shall be taken according to A. S. T. M. D. 270 . (See 503.0 , p. 60.)

4. Corrosion test.-No. 36. A clean copper strip shall not show more than extremely slight discoloration when submerged in the fuel for three hours at $122^{\circ} \mathrm{F}$.

5. Distillation range.-No. 6. When 10 per cent has been recovered in the receiver, the thermometer shall not read more than $65^{\circ} \mathrm{C}$. $\left(149^{\circ} \mathrm{F}.\right)$, nor less than $50^{\circ}$ C. $\left(122^{\circ}\right.$ F.); provided that, for each per cent distillation loss less than 4 per cent, obtained in the A. S. T. M. distillation, the minimum 10 per cent temperature requirement shall be lowered $3^{\circ} \mathrm{C}$. $\left(5.4^{\circ} \mathbf{F}\right.$.).
When 50 per cent has been recovered in the receiver, the thermometer shall not read more than $125^{\circ}$ C. $\left(257^{\circ}\right.$ F.).

When 90 per cent has been recovered in the receiver, the thermometer shall not read more than $180^{\circ}$ C. $\left(356^{\circ}\right.$ F. $)$.

The end point shall not be higher than $205^{\circ} \mathrm{C}$. $\left(401^{\circ}\right.$ F.).

At least 95 per cent shall be recovered as distillate in the receiver from the distillation.

6. Sulphur.-No. 32. Sulphur shall not exceed 0.10 per cent.

Natural Gasoline Association of America, tentative code for testing natural gas for gasoline content, 1924.

\section{GENERAL INTRODUCTION-PURPOSE}

To establish in the industry definite standardized methods for testing natural gas, so planned that the quantities of condensed fluid extracted from a unit volume of natural gas by these specific tests will constitute a standard measure of reference useful for the following purposes: (1) To determine the comparative richness in gasoline content of the gases from different localities; (2) to afford a basis for estimating the probable yield of gasoline from any given gas; and (3) to form a basic measure for rcference which may be used in comparing the daily performance of commercial gasoline extraction plants.

\section{GENERAL FACTS CONCERNING THE PROBLEM OF TESTING NATURAL GAS FOR GASOLINE CONTENT}

The word "gasoline" is a popular term, used in the trade to describe all of the mixtures of combustible liquids which may be extracted from natural gas.

Further than this, the word "gasoline" is applied to all thosc finished products forming motor fuels.

Thus the word "gasoline" has no specific meaning; that is to say, it does not designate any one combustible fluid.

In general, natural gas is composed of a mixture of fixed gases and the vapor of several substances normally liquids. These liquids are present in the gas mixture in the form of vapors.

The proportion which these vapors form of the whole natural gas mixture varies widely in different sections of the country. In most gases the gases in the mixture are-

Methane $\left(\mathrm{CH}_{4}\right)$.

Ethane $\left(\mathrm{C}_{2} \mathrm{H}_{6}\right)$.

Propane $\left(\mathrm{C}_{3} \mathrm{H}_{8}\right)$.

Butane $\left(\mathrm{C}_{4} \mathrm{H}_{10}\right)$.

The vapors are:

Pentane $\left(\mathrm{C}_{5} \mathrm{H}_{12}\right)$.

Hexane $\left(\mathrm{C}_{6} \mathrm{H}_{14}\right)$.

Heptane $\left(\mathrm{C}_{7} \mathrm{H}_{16}\right)$

Octanc $\left(\mathrm{C}_{8} \mathrm{H}_{18}\right)$.

In the very "dry" natural gas sold in most cities and towns for fuel, the vapors form only a very small part of the natural gas mixture. But in the casc of "wet" gases, such as that known as casinghead gas, the vapors form quite a large percentagc of the gas mixture, in some cases as much as 40 per cent or more by volume.

If any natural gas is subjected to pressure and at the same time is cooled, a certain part of the vapors contained by the gas will be condensed to liquid, and may be thus separated from the gas. The proportion of such liquid separated from the gas will depend upon the pressure, and the degree of cold to which the gas is subjected. Thus with high pressure and intense cold a much larger portion of the vapors will be separated than when the pressure 
is low and the temperature high, but the mixed fluid from the various vapors which is obtained by high pressure and intense cold is much "wilder" and more volatile than the lesser quantity obtained by the low pressure and high temperature.

Now since the word "gasoline" as it is used to-day applies equally to either quality of fluid, it becolnes apparent that when we speak of testing natural gas for "gasoline" content we must at once define what kind of gasoline we refer to.

Furthermore, some commercial gasoline plants are so operated as to obtain very "wild" gasoline. Other plants, because of marketing conditions, only operate at low rates of recovery and produce a more stable product.

In testing natural gas without any reference to the quality of the product obtained in the test, and with varying forms of apparatus, much confusion has arisen in the industry by indefiniteness of all state-
The apparatus for each form of test has been fixed upon after much careful study to meet the following requirements:

(1) Simplicity of design, which makes possible concordant results in the hands of operators, and also tends to economy in costs.

(2) Reliability and performance, so that the working of the apparatus itself will be regular and introduce no errors.

The method prescribed to be followed in operating each form of test was fixed for the purpose of meeting the following requirements:

(1) Duplication of results.-To this end, the pressure, temperature, and rates of operation have been chosen in each case so that the operator can easily obtain the conditions in the test and can continue to hold same throughout the test.

(2) Simplicity of performance.-To this end the methods of manipulation have been made simple

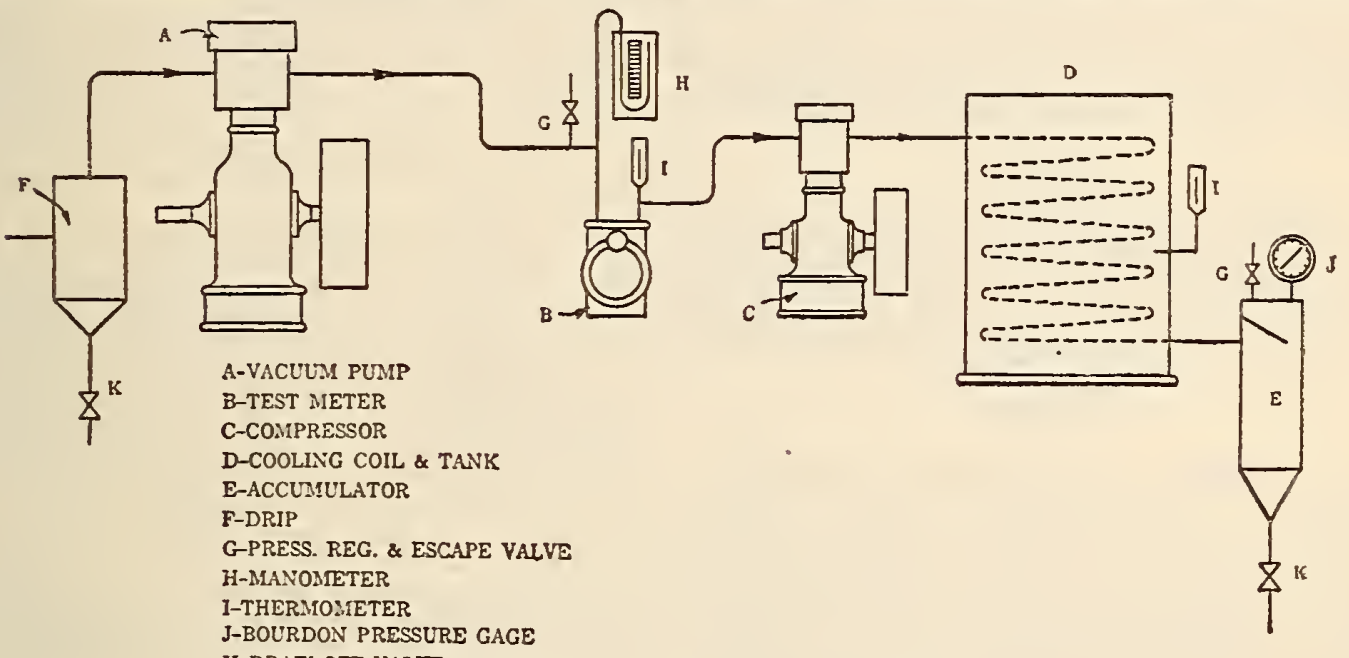

I-DRAW OFF VALVE

FIGURE 35.-Compression and cooling test

ments merely referring to a "gasoline content," which has not been deinitely defined.

From the nature of the problem described in the foregoing paragraph, the associations put forward these three inethods of testing natural gas, to be used in a fixed and definite manner under all circumstances, and when examining all qualities of natural gas.

Each test method consists of a fixed form of apparatus, operated always in one particular manner, the results of which test are stated as a quantity of fluid per 1,000,000 cubic feet of gas. The results are not stated as "gasoline" but as "natural gas condensate." More "gasoline" or varying degrees of volatility might be extracted under certain conditions from the same quantity of gas, but by eventually standardizing the test methods the results obtained by such test methods may always be used as a reference of the comparative richness of the various gases, and experience with a definite test method will also enable the engineer to draw reliable conclusions as to the amount of more volatile fluids which may be extracted from the gas.

\section{TEE THREE TESTING METHODS}

The three tentative testing methods have been adopted because the principles of each method are used in commercial plants extracting "gasoline" fiuids from natural gas. and direct, and the results of the test are measured at the temperatures and pressures set out in the test method.

\section{COMPRESSION AND COOLIAG TEST METHOD}

\section{FOREWORD}

The apparatus and procedure herein described for the compression and cooling test method were adopted after much study and experimentation. Necessarily choice had to be made between various alternate forms of apparatus, and steps in procedure. Should experience in general use throughout the industry develop facts which would make any changes or modifications in apparatus or procedure advisable, such changes will be made before this test method is adopted as a standard.

\section{APPARATUS}

(Refer to fig. 35)

Vacrum Pump

It is very essential that the vacuum pump used for this test shall be absolutely free from leakage in order to prevent air mixing with the gas being tested. In-leakage of air will cause a low test and the error is undeterminable. Consequently, the vacuum pump used shall be of the crosshead type 
with the piston rod passing through a stuffing box which, when packed, will prevent all leakage. The vacuum pump shall be of such a capacity that it will maintain on the meter a pressure of 4 ounces per square inch or any other particular pressure above atmospheric agreed upon by contracting parties with the compressor running at rated speed under any field vacuum condition the operator may encounter.

\section{Test Meter}

The test meter shall be installed at the intake of the compressor. The meter shall be scaled to onehundredths of a cubic foot. At the intake of the meter or tapped into the upper gas chamber of the meter, a $U$-tube pressure gauge shall be connected as a means of determining the pressure on the meter. A thermometer shall be placed as near the outlet of the meter as possible so that the volume can be corrected to $60^{\circ} \mathrm{F}$. or any other temperature agreed upon between contracting parties. The meter shall be within 3 per cent of correct by displacement test. The test meter shall have its bellows suitably treated to prevent the absorption of gasoline vapor from the gas by passing through it.

\section{Compressor}

The compressor shall be of the crosshead type, with piston rod packed to prevent the gas from leaking and escaping to the air. Its capacity shall be $2^{1 / 2}$ cubic feet of gas per minute at atmospheric pressure when run at rated speed with a tolerance of one-half cubic foot per minute.

\section{Cocling Coil}

The cooling coil for each stage of compression shall be built of copper tubing not less than threeeighths inch outside diameter nor larger than fiveeighths inch outside diameter and shall be not less than 30 feet long. The coil shall have a drop of not less than three-fourths inch per foot in order to secure continuous drainage, and shall be rigidly fastened within a water chamber of capacity ample to permit the complete submergence of the coil in a water bath. The water bath to which cracked ice shall be added, shail be held at a temperature not less than $32^{\circ} \mathrm{F}$. nor more than $40^{\circ} \mathrm{F}$., and the water bath shall be provided with an indicating thermometer midway between the top and bottom of the coil.

\section{Accumulater}

The accumulator for each stage of compression shall hold at least 1 quart of condensate below the intake opening. Each accumulator shall have a baffle above the intake opening to prevent the condensate from blowing out through the gas escape connection. The bottom of the accumulator shall be of such shape as to permit complete drainage through the draw-off connection.

\section{Drip}

A drip shall be placed before the vacuum pump, to collect any oil or water that may be in the line, in order to protect the meter and other machinery from being stopped up with foreign substances. The bottom of the drip shall be of such shape as to permit complete drainage through the draw-off connections.

Notes. -1 . Connections between tbe various parts of tbe equipment may be tubing, rubber hose, or pipe.

2. The entire apparatus shall be constructed of tbe best materials, with finest workmanship, sball be maintained in good mccbanical condition, and must be absolutely free from leaks under operating condition,

3 The abov

ove description of the test apparatus sball be construed to apply to both single-stage and two-stage compressing equipment.

\section{PROCEDURE}

Before starting the compression test, let the machine run with the pressure on the meter constant and the pressure on the last stage accumulator at 255 pounds until at least 20 cubic feet of gas shall have passed through the meter.

When at least 20 cubic feet of gas shall have been passed through the apparatus, the condensate therefrom shall be withdrawn completely from the accumulators without relieving the last stage accumulator pressure below 250 pounds gauge pressure, and the test than started by quickly closing the accumulator draw-off valves and simultaneously reading the gas meter.

Either 13.2 cubic feet or 26.4 cubic feet of gas at 4-ounce pressure corrected for the temperature shown by the meter thermometer, to $60^{\circ} \mathrm{F}$., shall be passed through the machine.

NoTE.-Otber temperature and pressure bases may be used by agreement between contracting parties.

A constant pressure of 250 pounds gauge shall be maintained on the last stage accumulator by means of an escape valve provided on the top of the accumulator, and as soon as the required amount of gas has been passed through the apparatus, as stipulated above for the test, the apparatus shall be quickly stopped.

The pressure shall then be relieved from the laststage accumulator through the escape valve provided, at a rate not to exceed 50 pounds per minute to a limit of 25 pounds per square inch. If the gas has been compressed with a single-stage apparatus the condensate shall then be withdrawn from the accumulator into a graduated cylinder, and the quantity and temperature of the contents be immediately determined. The temperature of this condensate shall then be steadily raised to $60^{\circ} \mathrm{F}$., at a rate not to exceed $2^{\circ}$ per minute.

Where 2-stage cempressing equipment is used the contents of each accumulator shall be drawn off in separate graduated cylinders and the quantity and temperature of the contents of each graduated cylinder shall be immediately determined. The contents of the two graduated cylinders shall then be combined in a graduated cylinder or cylinders and the temperature thereof brought to $60^{\circ} \mathrm{F}$., at a rate not to exceed $2^{\circ}$ per minute.

At least two tests shall be made with the given apparatus to determine the gasoline content of the gas.

\section{S. COMFOTATION OF YIELD}

For a run of 13.2 cubic feet, the gallons of gasoline per thousand cubic feet of gas shall be computed by multiplving the cubic centimeters of condensate weathered to $60^{\circ} \mathrm{F}$. by the factor 2, and dividing this product by 100 .

For a run of 26.4 cubic feet, the gallons of gasoline per thousand cubic feet of gas shall be computed by dividing the cubic centimeters of condensate weathered to $60^{\circ} \mathrm{F}$. by 100 .

If the results are desired in gallons of gasoline per million cubic feet of gas, the following formula shall be used:

$$
\frac{264.2 B}{A}=X \text { gallons of fluid per million }
$$

Where $A$ is the total corrected volume of gas used in cubic feet; $B$ is the total gasoline measured in cubic centimeters at $60^{\circ} \mathrm{F}$, and $X$ is gallons of gasoline per million cubic feet of gas.

\section{OIL ABSORPTION TEST METHOD} FOREWORD

The apparatus and procedure herein described for the oil-absorption-test method were adopted 
giter much study and experimentation. Necessarily, choice had to be made between various alternate forms of apparatus and steps in procedure. Should experience in general use throughout the industry develop facts which would make any changes or modifications in apparatus or procedure advisable, such changes will be made before this test method is adopted as a standard by the associations.

\section{MATERTAIS}

Oil. - The oil to be used for the purpose of this test is known commercially as mineral seal oil, or absorbent oil, and shall have the following specifications:

1. Color: Thite to pale yellow.

2. Odor: Sweet.

3. Cold test: Less than $32^{\circ} \mathrm{F}$.

4. Viscosity: 35 to 140 seconds at $100^{\circ} \mathrm{F}$. (Sa vbolt).

5. Emulsifying bodies: Practically none.

6. Fire distillation: Initial boiling point to be not less than $480^{\circ} \mathrm{F}$. and not more than 3 per cent over at $500^{\circ} \mathrm{F}$.

Ice for condenser

\section{ADSORPTIOT APPARATUS}

(Reler to fig. 3s)

A1, A2, AS. Three absorbers, in series, 5-inch diameter, 30 inches long.

$B$. Three-fourths inch nipple, 2 inches long for introduction of oil. Cap on top for closing.

C. Gas inlet pipe extending to within 1 inch of bottom of absorber, fitted with a tee and two short nipples, each nipple being closed at end and perforated with ten $1 / 16$-inch holes on the underside.

$D$. Gas outlet connection flush with underside of the top head of absorber.

$E$. U gauge.-Preferably of the pressure, enclosed type.

M. Meter. - If an orifice is used instead of a direct meter, then for convenience it should be calibrated at a certain U-gauge pressure to deliver the gas at a fised rate.

\section{DISTILIATION APPARATUS}

(Refer to fig. 37 )

A. Flask-Standard 1,000 ce Engler distillation flask. Dimensions, diameter of bulb (outside) 5 inches, ${ }^{1 / 4}$-inch tolerance; dimaeter of column (inside) between $7 / 8$ and 1 inch. Length of column, 6 inches, $1 / 4$-inch tolerance. Vapor tube to be connected in middle of column.

$B$. Condenser.-A. S. T. M. standard gasoline distillation condenser described in A. S. T. M. D86, see page 44 . In addition, a small vapor tube should be soldered on condenser just above the outlet, in such a way that uncondensed vapor may be removed without trapping condensate.

C. Thermometer-A. T. S. M. low distillation. (See table of A. S. T. M. thermometers, 500.2, p. 2, thermometer No. 6.)

$D$. Cones.-To remove mechanically entrained liquids from hot vapor, made of 14 to 20 mesh brass of nichrome gage. Cone dimensions 1 inch diameter, 1 inch long.

$E$. Receiver. - A graduate cylinder shall be used for a condensate receiver. The receiver shall be equipped with a rubber stopper through which the condenser outlet extends. The receiver shall be surrounded with an ice bath.

F. Barometer. - Not shown.

G. Hydrometer.-For determination of A. P. I. gravity of absorbing oil. Not shown.
H. Thermometer.-For determination of gas temperature and for determination of temperature of ice bath. Not shown.

I. Meter.-Not shown.

\section{OPERATIONS OF TEST}

Procedure for absorption.-One and one-half gallons of the absorbent oil are placed in each absorber.

The several parts of the apparatus are set up as shown in Figure 36 , connection being made at valve $F$, with the gas supply to be tested. All connections are made leak-proof and needle valves $G, H, I, J$, $K$, and $L$ are tightly closed. Valve $N$ is opened. Meter reading is taken. Valve $F$ is then opened.

Record the pressure shown on the gauge and the time. Open needle valve $G$ carefully and siowly to its capacity. When the pressure shown on absorber No. 1 equals the well, or line pressure, as shown by no further passage of gas through the oil, needle valve $H$ is opened slowly to its capacity. Needle valve $I$ is next carefully opened until the pressures are equalized, then valve $J$ is opened, and valve $K$ is slowly opened until the pressure in all three absorbers is the same as the line pressure. Needle valve $L$ is opened slightly, attention being given to the level of the mercury in the $U$ gauge. The rate of gas flow must now be so regulated by manipulation of the valves $L$ and $N$ so that: (1) There is a differential of 1 inch of mercury shown on the $U$ gauge, and (2) the rate of flow shall be as per the following schedule, depending upon the pressure under which the test is being conducted.

1. 15 cubic feet per hour at atmospheric pressure.

2. 30 cubic feet per hour at 15 pounds gauge pressure.

3. 45 cubic feet per hour at 50 pounds gauge pressure.

4. 60 cubic feet per hour at 120 pounds gauge pressure or higher.

Place thermometer in the thermometer well and shortly thereafter record the gas temperature. The temperature of both gas and oil at the time of test should be $60^{\circ} \mathrm{F}$., with an allowance of $5^{\circ} \mathrm{F}$. above or below.

Gas temperature should be recorded at one-half hour intevals during the test, and an average of these taken as the gas temperature.

The barometer is read at the same time as the gas temperature is taken. These readings are likewise averaged.

General considerations of the nature of the gas under test will enable the operator to approximate the volume of gas, which should be used as follows: (1) If the gas is residual gas from gasoline plants, 400 cubic feet should be ample for the test, (2) if the gas is untreated gas from wells, 300 cubic feet should be passed, and (3) if the gas is from oil wells or very low-pressure gas wells, 100 cubic feet should be passed.

It is, of course, understood that as the vapor content of the gas increases, no matter what the origin of the gas, the volume of the gas passed in a test should be decreased to an amount which will yield not to exceed 40 cubic centimeters of condensate per liter of oil, distilled from absorber A-1.

When the selected volume of gas has been passed through the apparatus, as shown by the meter, close valve $G$ and then valve $F$. Allow the gas from the absorbers to vent through the meter until the $U$ gauge shows zero pressure. Close valves $H, I, J$, $K$, and $L$, in order given. Read the meter and note the time. Detach absorbers and set in a cool place.

Procedure of distillation.-Pour from the absorber A1, Figure $36,750 \mathrm{cc}$ of oil, Assemble the distilla- 
tion apparatus as shown in Figure 37 and proceed with the distillation as specified below:

1. The condenser and receiver shall be iced so that a temperature of $32^{\circ}$ to $34^{\circ} \mathrm{F}$. may be obtained. 2 . The condenser shall be clean.
8. The distillation shall be carried out by so regulating the flame that the test may be completed in approximately 45 minutes.

The distillation shall be considered complete when the thermometer shows a reading of $350^{\circ} \mathrm{F}$.

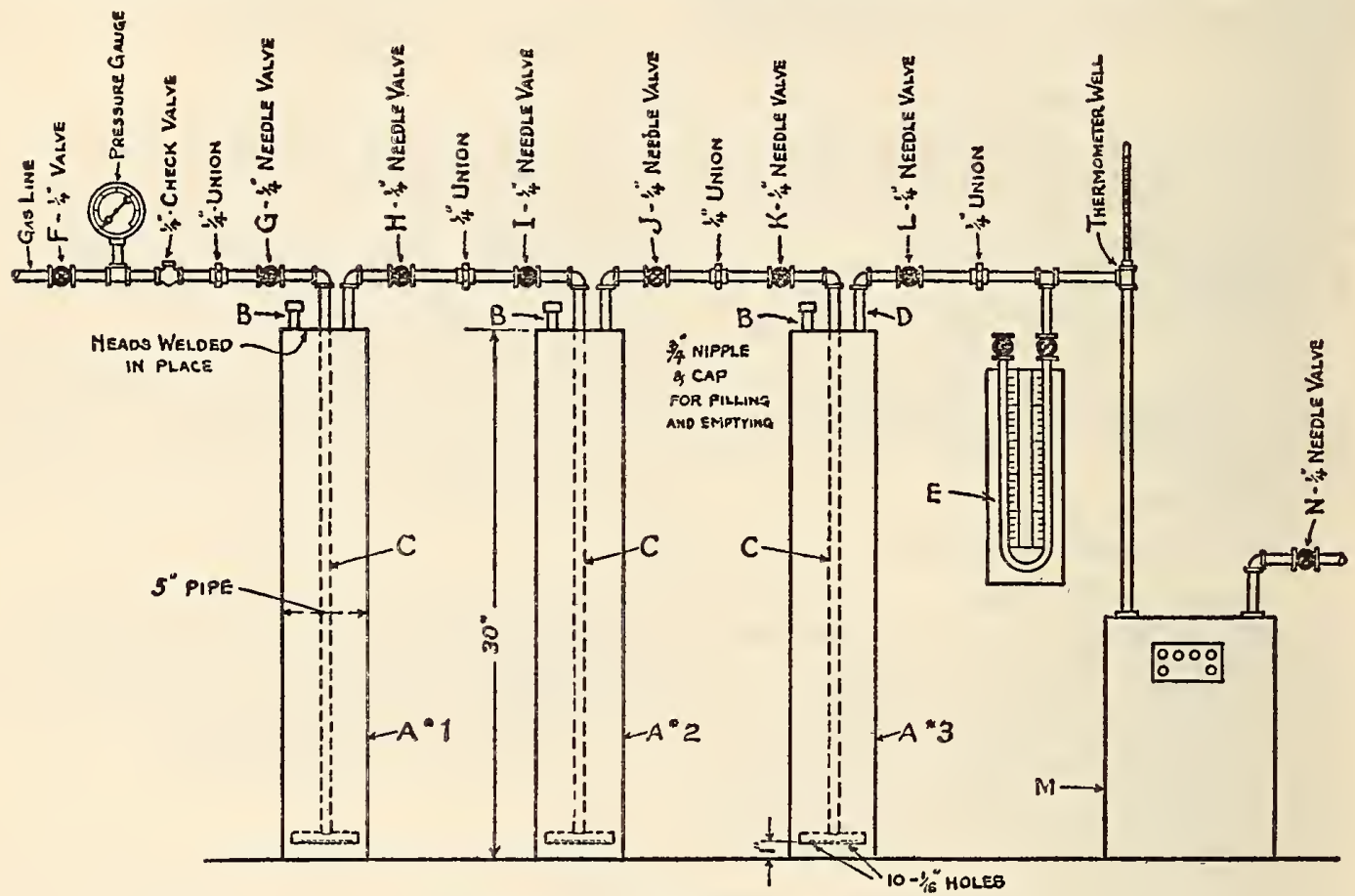

FIGURE 36.-Oil absorption test method

3. $750 \mathrm{cc}$ of the oil shall be introduced into the flask.

4. Two gauze cones shall then be placed in the neck of the flask, the tip of the first at the base of the column, and the top of the second just below the side outlet.

5. The thermometer with its bulb extending just below the side outlet shall be fitted tightly in the flask by means of a cork.

6 . The receiver shall be tightly connected to the discharge of the condenser.

7. The line carrying uncondensed vapors shall be connected to the intake of a suitable meter.

As an additional precaution in control, the condensing ring of oil which appears just before the distillation is complete should be not allowed to rise higher than 1 inch in the column.

After the completion of the distillation the volume of distillate is noted, and the distillate is then run into a cold bottle and stored for subsequent study. A second distillation of another $750 \mathrm{cc}$ sample from the first absorber is now run. If a check result is obtained, the first absorber is emptied, the exact volume of ois is recorded, and the gravity and temperatures taken.

Similar distillations are next carried out on the oil from absorbers 2 and 3.

\section{CALCDLATION OF RESULTS}

Gas volume-1. Correct the gas volume metered at the outlet of the absorbers to $60^{\circ} \mathrm{F}$. and 30 inches of mercury.

2. From the volume of condensate recovered by the distilations and the aliquot parts used, as well as the volume of oil recovered from the absorbers, calculate the total volume of condensate contained in the oil from the three absorbers.
3. Calculate the volume of vapor that this total volume of condensate would make, on the basis that 1 gallon of condensate makes 35 cubic feet of vapor.

4 . Correct the volume of gas measured at the outlet of the condenser during the distillation to $60^{\circ} \mathrm{F}$. and 30 inches mercury. (See 7 , in procedure of distillation.)

From the aliquot parts of oil used for the distillations and the volume of oil recovered from the absorbers calculate the total amount of noncondensable gas contained in the oil of the absorbers.

5. Take as the total gas volume the sum of the items calculated under 1,3 , and 4 , above.

\section{COMPUTATION OF YIELD}

The gallons of fluid per million cubic feet of gas may be computed conveniently according to the following formula:

$$
\frac{264.2 B}{A}=X \text { gallons of fluid per million }
$$

Where $A=$ the total corrected gas volume (item 5 , calculation of results), and $B=$ calculated total condensate contained in the oil in the absorbers (itcm 2, calculation of results).

The result $(X)$ is gallons per million, the volume of liquid being measured at $32^{\circ} \mathrm{F}$. and the volume of the gas being measured at $60^{\circ} \mathrm{F}$. and 30 inches of mercury pressure.

\section{CHARCOAL ADSORPTION TEST METHOD}

\section{FOREWORD}

The apparatus and procedure herein described for the charcoal adsorption test method were adopted after much study and experimentation. Necessarily, 
choice had to be made between various alternate forms of apparatus and steps in procedure. Should experience in general use throughout the industry develop facts which would make any changes or modifications in apparatus or procedure advisable, such change will be made before this test method is adopted as a standard.

\section{MATERIALS}

(a) Charcoal.-The charcoal used for this test shall meet the following specifications:

1. Degree of activity, 40 minutes. (See Appendix No. 1.)

2. Size, 6-14 mesh; not more than 5 per cent material finer than 14 mesh.

3. Moisture content, not more than 4 per cent by weight as determined by the following method: $\dot{A}$ 1 -grain sample heated for one hour at $150^{\circ} \mathrm{C}$. shall not show a loss of weight of more than four onehundredths of 1 gram.

(b) Ice for condenser.

(c) Glycerine, C.P.

\section{ADSORPTION APPARATUS}

Adsorption tubes for which see Figures 38 and 39.

\section{DISTILLATION APPARATUS}

(Refer to fig. 37)

A. Flask.-Standard 1,000 cc Engler distillation flask. Dimensions, diameter of bulb (outside), 5

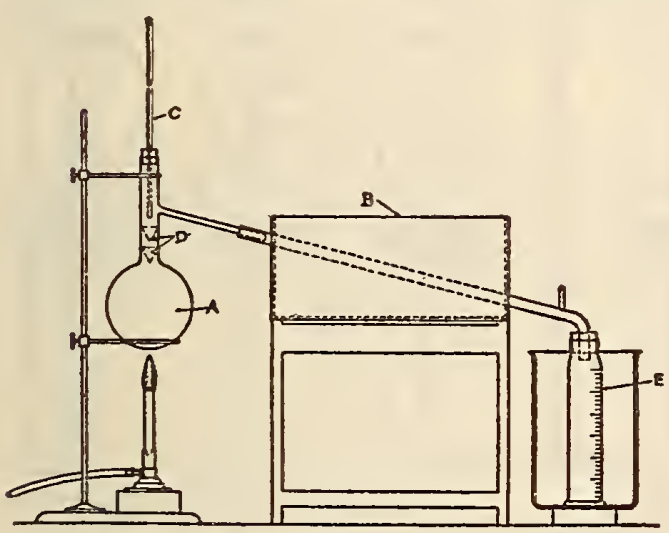

Frgure 37.-Distillation apparatus

inches, 1/4-inch tolerance. Diameter of column (inside), between $7 / 8$ and 1 inch. Length of column $61 / 4$ inches, $1 / 4$-inch tolerance. Length of vapor side tube, approximately 7 inches. Vapor tube to be connected in middle of column.

B. Condenser-A. S. T. M. standard gasoline distillation condenser, described in A. S. T. M. D86, ee page 44 . In addition, a small vapor tube shoulds be soldered in condenser tube just above outlet in such a way that uncondensed vapor may be removed without trapping condensate.

C. Thermometer-A. S. T. M. low distillation, see table of A.S.T.M. thermometers, 500.2 , page 2 ; thermometer No. 6.

D. Meters.-Dry, orifice, or wet, carefully calibrated. See also procedure.

$E$. Receiver. - A graduated glass-stoppered cylinder shall be used as a condensate receiver. The receiver shall be equipped with a rubber stopper through which outlet tube of condenser extends. The receiver shall be surrounded by an ice bath.

$F$. Barometer.-Not shown.
G. Thermometers for taking aas temperature.-Not shown.

\section{OPERATIONS OF TEST}

(a) Procedure for adsorption.-1. Adsorption tube is charged with 150 grams of specified charcoal. Previous to use charcoal shall be heated for one hour at $150^{\circ} \mathrm{C}$.

2. All gas measurements are to be made on inlet side of charcoal tube. The gas is to be dried by

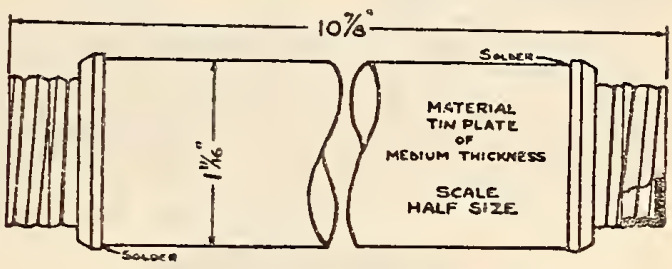

FIgURe 38.-Charcoal adsorption tube

means of calcium chloride, before metering, if a dry or orifice meter is used; and on outlet of meter if a wet meter is used.

If an orifice meter is used for gas measurement, proper correction shall be made for specific gravity of gas flowing into meter.

All gas volumes are to be corrected to $60^{\circ} \mathrm{F}$. and 30 inches of mercury without correction for saturation.

3. Rate of flow of gas through charcoal varies with the vapor content of the gas.

Rate is defined as the number of cubic feet passing through the charcoal tube per hour.

Several preliminary tests should be made to learn approximate gasoline content of the gas to be tested, and with this information available the proper rate should be employed in making a determination. The following rates should be used:

1. When the vapor content of the gas is under 100 gallons per million, 20 cubic feet of gas per hour.

2 . When vapor content is between 100 and 300 gallons per million, 15 cubic feet per hour.

3. When the vapor content is between 300 and 500 gallonsper million, 10 cubic feet per hour.

4. When the vapor content is between 500 and 1,000 gallons per million, 5 cubic feet per hour.

5. When the vapor content is over 1,000 gallons per million, 2 cubic feet per hour.

Nore-The volume of gas passed must be such that there will be a recovery of approximately 25 cubic centimeters of fluid on distillation.

(b) Procedure for distillation. - The apparatus for distillation is assembled in accordance with Figure 37. The distilling operation is conducted in the following manner:

1. One-hundred and fifty cubic centimeters of pure glycerin are poured into distillation flask. Use a funnel for the purpose in order to prevent glycerine adhering to the neck of the flask.

2. Transfer the charcoal used in the test from the adsorbing tube to the flask.

3. A cork carrying the thermometer is inserted in the neck of the flask, the thermometer bulb being located just below the side outlet of flask. 
4. The condenser box is filled with cracked ice and water. The temperature shall be maintained between $32^{\circ}$ and $34^{\circ} \mathrm{F}$. in the condenser box.

5. The beaker or jar in which the condensate receiver is placed is filled with cracked ice and water.

6 . It is very important that the temperature in both the condenser box and the receiver jar be maintained the same. Furthermore, they should be stirred in order that an even temperature be maintained throughout.

7. A low flame is lighted under the flask. During the first few moments of the distillation the burner is offset so that the flame impinges on the side of the flask just below the level of the glycerin. This materially assists in preventing foaming of the glycerin.

8. In case the glycerin should foam to the extent of filling the flask and flowing out the side outlet, the test is lost. When foaming occurs, this contin.
The result $X$ is gallons per million; the volume of liquid is measured at $32^{\circ} \mathrm{F}$; and the volume of the gas is measured at $60^{\circ} \mathrm{F}$. and 30 inches of mercury pressure.

APPENDIX N0. 1

Method for Determination of Degree of Activation of Charcoal

The apparatus for this test is shown in Figure 40. It is mounted on a suitable board frame. Air enters at $A$, passes through drying bottles $B 1, B 2$, and $B 3$, containing sulphuric acid to remove water vapor. Next the air passes through two bottles containing carbon tetrachloride, kept at the temperature of melting ice. (These bottles should be iced for at least 30 minutes before making the test.)

Saturated with carbon tetrachloride vapor at this temperature, the air vapor mixture passes

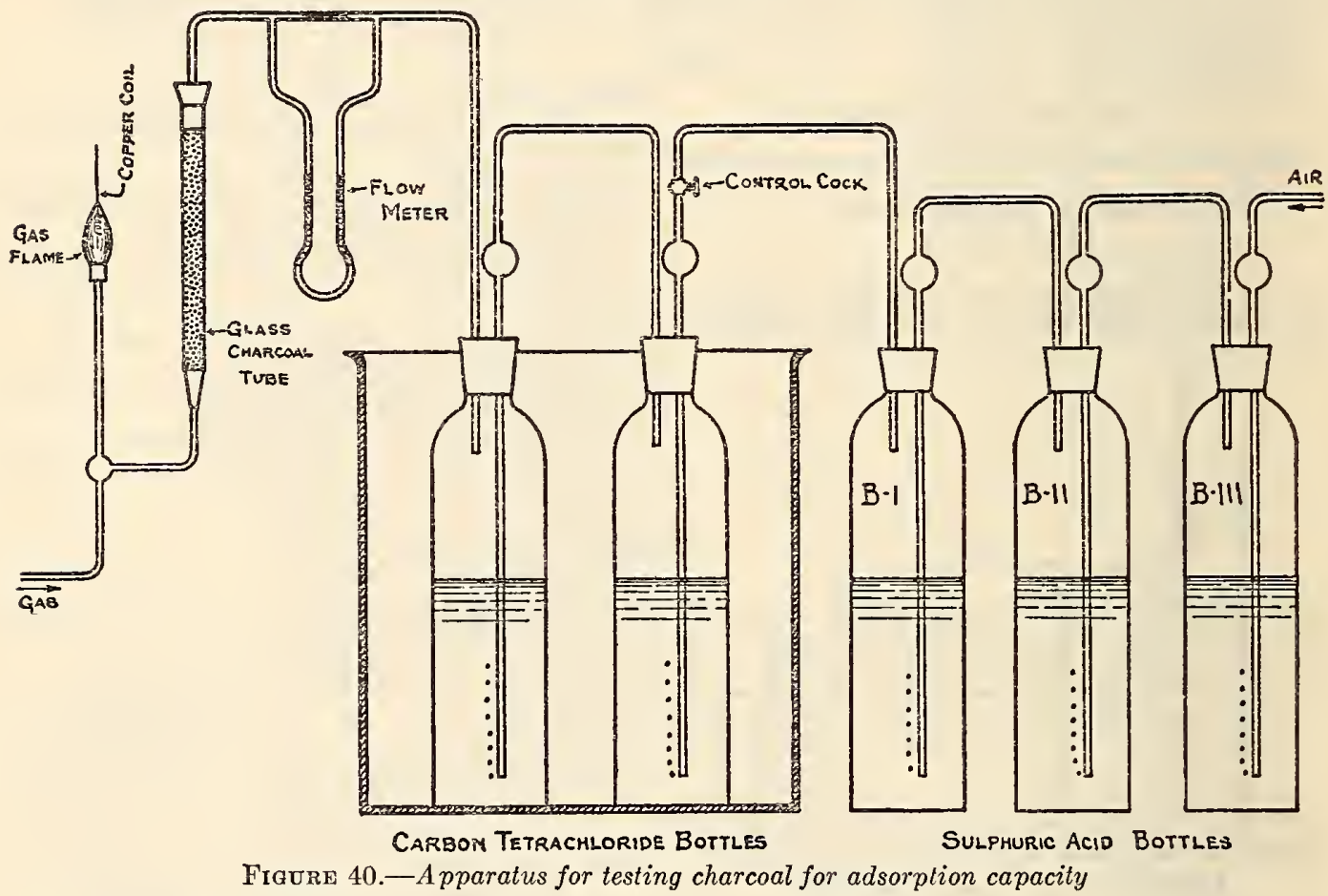

gency can be met by playing a flame cautiously around the upper part of the flask. After the danger point from foaming is passed the flame is adjusted to the center of the flask and is so regulated that the distillation consumes about 45 minutes.

9. When the temperature reaches $350^{\circ} \mathrm{F}$. the flame is cut off and the flask allowed to cool.

10. After the flask has cooled enough to permit handling, disconnect from condenser inlet and flush out condenser with $20 \mathrm{cc}$ water. The volume of condensate in the receiver is read and noted.

\section{COMPUTATION OF RESULTS}

The gallons of fluid per million cubic feet of gas may be computed conveniently according to the following formula:

Where

$$
\frac{264.2 B}{A}=X \text { gallons of fluid per million }
$$

$A=$ corrected gas volume in cubic feet which has passed through the charcoal and

$B=$ the volume in cubic centimeters of the condensate recovered. through the flow meter, then through the charcoal tube, and finally into the gas flame in which the copper coil is suspended.

The glass charcoal tube should be exactly $14 \mathrm{~mm}$ in diameter, and the tube should be exactly $10 \mathrm{~cm}$ long. A perforated porcelain disk in the bottom of the tube holds the charcoal in place. The flow meter is calibrated so it passes exactly $770 \mathrm{cc}$ per minute.

In conducting the test the time is noted, the gas burner lighted to give a flame height of about $11 / 4$ inches and air supplied under positive pressure until the first indication of a green color in the flame is noticed. The time is again taken. The difference gives the carbon tetrachloride time for the charcoal. This time multiplied by three gives the chloropicrin time. The degree of activation referred to in these standards is the chloropicrin time.

It is advised that the charcoal be bought from a manufacturer who can guarantee a product which will meet the above specifications.

Natural Gas Association of America, tentative standard vapor pressure testing method, September 1, 1928. 


\section{APPARATUS}

The apparatus and method of procedure is essentially the same as proposed by Walter Reid, which apparatus consists of a bomb, a pressure gauge, and a water bath.

(a) The Bomb

The bomb consists of two parts. The first, which is the air bomb, is made of a piece of 2 -inch standard pipe 10 inches long, with welded ends having a one-quarter-inch collar welded in the center of one end and a one-quarter-inch nipple 1 inch long welded in the center of the other end. The bomb must drain completely from either end when held in a vertical position.

The second part, the gasoline bomb, consists of a piece of 2 -inch standard line pipe $2 \frac{1}{2}$ inches long with welded ends having a one-quarter-inch collar welded in the center of one end, the other end being completely closed.

Method of testing.-The apparatus is shown in Figure 41.

(b) Pressure Gange

The pressure gauge shall be an approved make of vapor tension or test gauge, with a diameter of 3 or $41 \frac{2}{2}$ inches, and scaled for 30 or 45 pounds pressure. Gauges shall be checked frequently and adjusted if found to be in error.

\section{(c) Water Bath}

The water bath shall be of such dimensions that the bomb may be immersed to the bottom of the pressure gauge. Means for maintaining a constant bath temperature of $90^{\circ} \mathrm{F}$. should be provided.

\section{PROCEDURE}

(a) Purging the Air Bomb

Prior to each test the air bomb must be thoroughly purged of any gasoline or gasoline vapors that may remain from a previous test. This is best accomplished by filling the air bomb, from which the gauge has been removed, with hot water, then allowing the bomb to drain. The procedure should be repeated several times. The air bomb is then suspended in the shade on a rack in a vertical position. The bomb should remain on the rack for at least one hour.

Where a large nimmber of determinations are to be made, several air bombs should be provided for each gasoline bomb.

Just prior to the test the pressure gauge is serewed tightly into the air bomb using a little shellac or powdered lead on the threads of the gauge to make a tight connection.

\section{(b) Filling the Gasoline Bomb}

Before each test the gasoline bomb is rinsed with water. The water is poured out and the bomb allowed to drain for one minute.

The bomb is immersed in a tank of the material to be tested and allowed to fill while being raised or lowered through the liquid. This liquid is used to thoroughly wash the bomb and is then emptied. The bomb is refilled in the same manner, care being taken to see it is completely full of the liquid. The sample is now ready for the test.

Should it be necessary to transfer the sample to the bomb by means of pouring, both the liquid and the bomb must be cold enough so that no appreciable change in composition of the liquid occurs during the operation. The bomb shall be completely full of the liquid when the test is run. The following table gives the temperatures at which gasolines of several vapor pressures should be held when pouring:
Temperature to which gasoline should be cooled before pouring

\begin{tabular}{|c|c|}
$\begin{array}{c}\text { Vapor pressure at } 99^{\circ} \mathrm{F} \text {. (pounds per } \\
\text { square inch absolute) }\end{array}$ & $\begin{array}{c}\text { Temperature } \\
\text { of sample } \\
\text { when pour- } \\
\text { ing }\end{array}$ \\
\hline 9 or under- & $\circ \mathrm{F}$. \\
9 to 12 & $32-40$ \\
12 to $16-20$ \\
$16-15$
\end{tabular}

(c) Conducting Test

The temperature of the air in the air bomb is recorded. The air bomb with the gauge in place

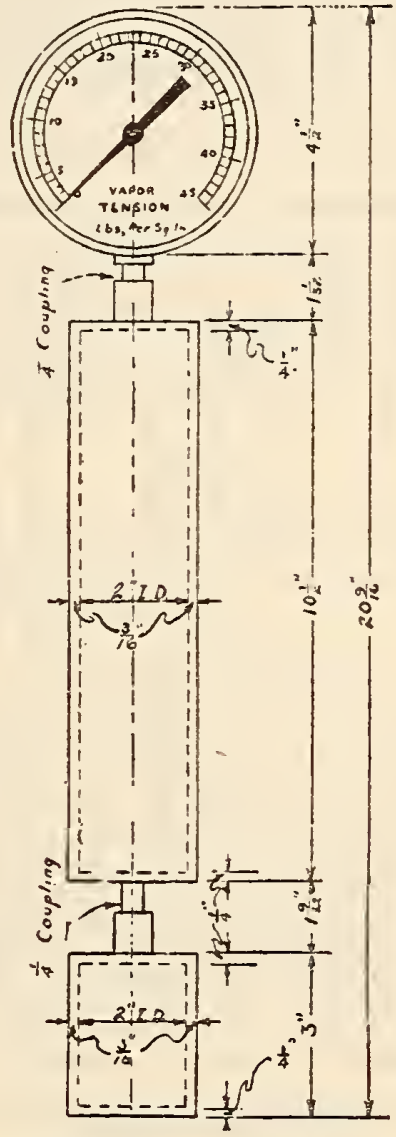

FIgURE $41 .-$ Testing appa-
ratus

is then immediately attached tightly to the gasoline bomb. The apparatus is turned upside down several times to allow gasoline to run into the air chamber. It is then immersed in a water bath held constant at $90^{\circ} \mathrm{F}$. At the end of five minutes the bomb is removed from the bath, inverted several times and shaken vigorously. It is then replaced in the bath, and as soon as the gauge reading becomes constant, the pressure is recorded. This will usually require from 5 to 10 minutes.

\section{(d) Calculating Results}

In order to calculate the vapor pressure of the gasoline, the final pressure recorded above must 
be corrected for the change in the pressure of the air in the bomb due to change in temperature. This is done by adding to or subtracting from the observed pressure the correction given in the following table opposite the air bomb temperature. Since the pressure of the air in the air bomb was zcro pounds gauge when attached to the gasoline bomb, any pressure shown on the gauge at the end of the test must have been due to the pressure of the gasoline vapors and the change in the air pressure. For this reason the gauge pressure when corrected for the change of the air pressure is the vapor pressure of the gasoline in pounds per square inch absolute; for example, the observed gauge pressure is 15 pounds per square inch and the air bomb temperature is $76^{\circ} \mathrm{F}$. Reference to the table will show the correction for this air bomb temperature to be 0.4 pound per square inch. Subtracting this correction from the observed gauge pressure of 15 pounds will give 14.6 pounds per square inch absolute as the vapor pressure of the gasoline.

\section{Correction factors for expansion of air}

[Barometric pressure 14.4 pounds per square inch]

\begin{tabular}{|c|c|c|c|}
\hline \multicolumn{2}{|c|}{ Air bomb } & \multicolumn{2}{|c|}{ Air bomb } \\
\hline $\begin{array}{l}\text { Temper- } \\
\text { ature }{ }^{\circ} \mathrm{F}\end{array}$ & $\begin{array}{c}\text { Correc- } \\
\text { tion }\end{array}$ & $\begin{array}{l}\text { Temper- } \\
\text { ature }{ }^{\circ} \mathrm{F} \text {. }\end{array}$ & $\begin{array}{c}\text { Correc- } \\
\text { tion }\end{array}$ \\
\hline $\begin{array}{l}30 \\
32 \\
34 \\
36 \\
38 \\
40 \\
42 \\
44 \\
46 \\
48\end{array}$ & $\begin{array}{c}\text { Subtract } \\
1.8 \\
1.7 \\
1.6 \\
1.6 \\
1.5 \\
1.4 \\
1.4 \\
1.3 \\
1.3 \\
1.2\end{array}$ & $\begin{array}{l}72 \\
74 \\
76 \\
78 \\
80 \\
82 \\
84 \\
86 \\
88 \\
90\end{array}$ & $\begin{array}{c}\text { Subtract } \\
0.5 \\
.4 \\
.4 \\
.3 \\
.3 \\
.2 \\
.2 \\
.1 \\
.1 \\
.0\end{array}$ \\
\hline $\begin{array}{l}50 \\
52 \\
54 \\
56 \\
58 \\
60 \\
62 \\
64 \\
66 \\
68 \\
70\end{array}$ & $\begin{array}{l}1.1 \\
1.1 \\
1.0 \\
1.0 \\
.9 \\
.8 \\
.8 \\
.7 \\
.7 \\
.6 \\
.5\end{array}$ & $\begin{array}{r}92 \\
94 \\
96 \\
98 \\
100 \\
102 \\
104 \\
106 \\
108 \\
110\end{array}$ & $\begin{array}{r}A d d \\
.1 \\
.1 \\
.2 \\
.2 \\
.3 \\
.3 \\
.4 \\
.4 \\
.5 \\
.5\end{array}$ \\
\hline
\end{tabular}

Figures given in the table to be subtracted from or added to the observed reading to give vapor pressure of gasoline in pounds per square inch absolute at $90^{\circ} \mathrm{F}$.

The above values were calculated from the formula: Increase in air pressure equals

Barometric pressure $\times(90-$ air bomb temp.)

Natural Gasoline Association of America, specifications and test methods for natural gasoline, 1928 .

\begin{tabular}{|c|c|c|c|}
\hline & Gravity & $\begin{array}{l}\text { End point } \\
\text { (maximum) }\end{array}$ & $\begin{array}{c}\text { Recovery } \\
\text { (minimum) }\end{array}$ \\
\hline $\begin{array}{l}\text { Grade "double A" } \\
\text { Grade "A" } \\
\text { Grade "double B" } \\
\text { Grade "B", } \\
\text { Grade "C", }\end{array}$ & $\begin{array}{l}{ }^{\circ} \text { A. P. I. } \\
80.0-87.9 \\
72.0-79.9 \\
84.0-92.0 \\
76.0-83.9 \\
80.0-92.0\end{array}$ & $\begin{array}{r}{ }^{\circ} F . \\
375 \\
375 \\
375 \\
375 \\
375\end{array}$ & $\begin{array}{r}\text { Per cent } \\
90 \\
90 \\
85 \\
85 \\
78\end{array}$ \\
\hline
\end{tabular}

All grades must be noncorrosive.

The doctor test on all grades must be negative ("sweet").

The color of all grades must be not less than pius 25 Saybolt.

\section{TESTS}

1. Doctor test.-Same as method No. 34, Federal Specifications Board specification 2d (see 503.0, p. 66), with the following variations and additions:

Change first sentence under the heading "Making the test," to read: "Shake vigorously together in a 4-ounce sample bottle fitted with a clean glass stopper or a new clean cork, $10 \mathrm{cc}$ of the sample to be tested and $5 \mathrm{cc}$ of sodium plumbite solution for about 15 seconds."

Add the following:

Note. -The reagents used shall be chemically pure. Extreme care must be taken in storing all cherricals and the sodium plumbite solution in strictly clean, tightly closed receptacles.

2. Corrosion test.-Same as A. S. T. M. D130-27T. (See 503.3, p. 71.)

3. Distillation test.-Same as A. S. T. M. D216-27 (see p. 72) with the following variations and additions:

The application of heat, when everything is in readiness, shall be so regulated that the first drop of the condensate falls from the condenser in not less than two nor more than five minutes from the time heat is first applied to the flask.

The heating shall be continued until the mercury reaches a maximum and starts to fall consistently, after the bottom of the flask has become dry. The highest temperature observed on the distillation thermometer shall be recorded as the end point.

Note--Absolute pressure on the distillation apparatus during test must be not less than $735 \mathrm{~mm}$ of mercury.

New York Produce Exchange, rules regulating the petroleum trade, 1920.

\section{NAPHTRA AND GASOLINE}

Naphtha and/or gasoline in bulk, barrels, and cases shall be sold per measured gallon at $60^{\circ} \mathrm{F}$. It may, however, be sold by weight if specially agreed upon by seller and buyer.

Barrels containing naphtha and/or gasoline shall be painted green with white heads and be well glued.

The gross and tare weight or gaugc shall be plainly marked on the barrel and the buyer may check for correctness, the same rules applying as provided in the N. Y. P. E. rules relating to refined petroleum. (See 502.2, p. 43.)

Residuum shall be understood to mean a heavy residue from the distillation of petroleum oil, free from coke, water, and foreign impurities. Fuel oil shall be understood to mean a liquid petroleum oil suitable for use as a liquid fuel and having a pour test not above $55^{\circ} \mathrm{F}$. Gas oil shall be understood to mean a distillate product from petroleum oil of $23^{\circ}$ Beaumé gravity or above.

Residuum, fuel oil, and gas oil shall be sold per measured gallon at $60^{\circ} \mathrm{F}$. It may, however, be sold by weight if specially agreed upon by seller and buyer.

The gross and tare weight or gauge shall be plainly marked on the barrel and the buyer may check for correctness, the same rules applying as provided in the New York Produce Exchange rules relating to refined petroleum. 
503.4 FUEL AND GAS OIIS.

American Oil Burner Association, uniform oil specifications, 1928.

(The uniform oil specifications of the American Oil Burner Association were used as the basis for the formulation of United States Department of Commerce Commercial Standard CS12-29, for domestic and industrial fuel oils. (See below.) Commercial Staudard CS12 has been adopted as a standard by the American Oil Burner Association).

Federal Specifications Board specification 2d, United States Government master specification for lubricants and liquid fuels.

(This publication, containing detailed specifications, methods of testing, and methods of sampling is published by the United States Government Printing Office as Technical Paper 323B, Department of Commerce, Bureau of Mines. The publication includes specifications for fuel oils, which are given below.)

\section{FUEL OILS}

\section{GENERAI REQUIREMENTS .}

Sampling.-See A. S. T. M. method D270-27T, 503.0, page 60 .

Testing.-See F. S. B. No. 2d, 503.0, page 66.

Note.-For conrenience in presentation the test methods hare been listed numerically and reference made accordingly in the following table where tests apply.

\section{DETAR REQUIREMENTS}

(a) General

1. Fuel oil shall be a hydrocarbon oil, free from grit, acid, and fibrous or other foreign matters likely to clog or injure the burners or valves. If required by the purchaser, the oil shall be strained by being drawn through two or more filters of wire gauze of 16 meshes to the linear inch. The clearance through the strainer shall be at least twice the area of the suction pipe and the strainers shall be in series.

2. Uses.-Navy standard.-Used where a highgrade fuel oil is required. This oil may be used in Diesel engines.

Bunker A.-Used where low-viscosity oil is required. This oil may be used in Diesel engines.

Bunker B.-Used where a more viscous oil than bunker $A$ can be used. This oil may be used in Diesel engines of a type adapted to an oil of medium viscosity.

Bunker C.-Used where a high-viscosity oil is satisfactory. This oil may be used in Diesel engines of a type adapted to an oil of high viscosity. (b) Properties and Tests

\begin{tabular}{|c|c|c|c|c|c|c|c|}
\hline Name and grade & 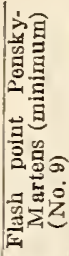 & 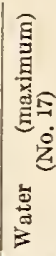 & 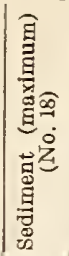 & 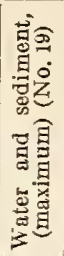 & 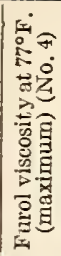 & 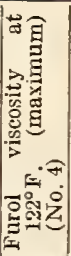 & 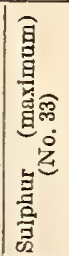 \\
\hline Nav & $\begin{array}{l}{ }^{\circ} \mathrm{F} \\
1150\end{array}$ & $\begin{array}{l}\text { Per } \\
\text { cent }\end{array}$ & $\begin{array}{l}P_{e r} \\
\text { cent }\end{array}$ & $\begin{array}{c}\text { Per } \\
\text { cent } \\
1.0\end{array}$ & $\begin{array}{r}\text { Sec- } \\
\text { onds } \\
100\end{array}$ & $\begin{array}{l}\text { Sec- } \\
\text { onds }\end{array}$ & $\begin{array}{l}P \in T \\
\text { cent } \\
1.5\end{array}$ \\
\hline $\begin{array}{l}\text { Bunker A } \\
\text { Bunker B }\end{array}$ & $\begin{array}{l}1150 \\
150\end{array}$ & & & 1.0 & 100 & 100 & \\
\hline Bunker C. & 150 & 21.75 & 20.25 & & 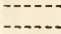 & $\begin{array}{l}1100 \\
300\end{array}$ & \\
\hline
\end{tabular}

1 In case of oils having viscosity greater than 30 seconds at $150^{\circ}$ F. (Saybolt Furol viscosimeter) ( $8^{\circ}$ Engler), the flash point shall not be below the temperature at which the oil has a viscosity of 30 seconds.

$2 \mathrm{~A}$ deduction in quantity will be made for all water plus sediment in excess of 1.0 per cent.

United States Department of Commerce, trade standards unit, Commercial Standard CS12-29, Domestic and Industrial Fuel Oils, January 31, 1929.

(Adopted at a conference of representative manufacturers, distributors, and users, and later accepted and approved by the industry for promulgation by the Department of Commerce.)

\section{SCOPE}

1. These specifications cover light, medium, and heavy domestic fuel oils, and light, medium, and heavy industrial fuel oils for domestic and industrial oil-burning equipment.

\section{GENERAI REQUIREMENTS}

2. Properties.-Grades 1 to 4, inclusive, shall be hydrocarbon oils free from water, acid, grit, and fibrous or other foreign matters likely to clog or injure the burncr or valves. Grades 5 and 6 shall be hydrocarbon oils, free from grit, acid, and fibrous or other foreign matters likely to clog or injure the burner or valves. If required, it shall be strained by being drawn through filters or wire gauze of 16 meshes to the inch. The clearance area through the strainers shall be at least twice the area of the suction pipe and the strainers shall be in duplicate. 
Detailed requirements for domestic fuel oils

[For metbod of test see paragrapb indicated]

\begin{tabular}{|c|c|c|c|c|c|c|c|}
\hline \multirow[b]{2}{*}{ Grade of oil } & \multicolumn{2}{|c|}{ Flash point } & \multirow{2}{*}{$\begin{array}{l}\text { Water and } \\
\text { sediment } \\
\text { (maxi- } \\
\text { mum) }\end{array}$} & \multirow{2}{*}{$\begin{array}{l}\text { Pour } 1 \\
\text { point } \\
\text { (maxi- } \\
\text { mum) }\end{array}$} & \multicolumn{2}{|c|}{ Distillation tast } & \multirow[b]{2}{*}{$\begin{array}{l}\text { Viscosity } \\
\text { (maxi- } \\
\text { mum) }\end{array}$} \\
\hline & Minimum & Maximum & & & $\begin{array}{l}10 \text { per cent } \\
\text { point } \\
\text { (maxi- } \\
\text { mum) }\end{array}$ & $\begin{array}{l}\text { End point } \\
\text { (maxi- } \\
\text { mum) }\end{array}$ & \\
\hline $\begin{array}{l}\text { No. 1. Light domestic fuel oil } \\
\text { A light distillate oil for use in burners requiring a } \\
\text { higb-grade fuel. }\end{array}$ & 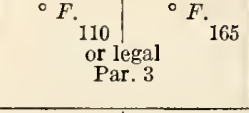 & ${ }^{\circ}{ }^{\circ}{ }_{165}$ & $\begin{array}{l}\text { Per cent } \\
\quad 0.05 \\
\text { Par. } 5\end{array}$ & $\begin{array}{l}{ }^{\circ} F . \\
15 \\
\text { Par. } 7\end{array}$ & $\begin{array}{l}{ }^{\circ} F . \\
\text { Par. } 8\end{array}$ & $\begin{array}{l}\circ F . \\
\text { Par. } 8\end{array}$ & \\
\hline \multirow{3}{*}{$\begin{array}{l}\text { No. 2. Medium domestic fuel oil } \\
\text { A medium distillate oil for use in burners requiring } \\
\text { a higb-grade fuel. }\end{array}$} & \multirow{2}{*}{\multicolumn{2}{|c|}{$\begin{array}{l}125 \\
\text { or legal } \\
\text { Par. } 3\end{array}$}} & & & $\begin{array}{l}10 \text { per cent } \\
\text { point } \\
\text { (maxi- } \\
\text { mum) }\end{array}$ & $\begin{array}{l}90 \text { per cent } \\
\text { point } \\
\text { (maxi- } \\
\text { mum) }\end{array}$ & \\
\hline & & & $\begin{array}{l}\quad 0.05 \\
\text { Par. } 5\end{array}$ & Par. 7 & $\begin{array}{l}{ }^{\circ} F_{440} \\
\text { Par. } 9\end{array}$ & $\begin{array}{l}\circ F . \\
620 \\
\text { Par. } 9\end{array}$ & \\
\hline & & & & & $\begin{array}{l}10 \text { per cent } \\
\text { point } \\
\text { (maxi- } \\
\text { mum) }\end{array}$ & $\begin{array}{l}90 \text { per cent } \\
\text { point } \\
\text { (maxi- } \\
\text { mum) }\end{array}$ & \\
\hline $\begin{array}{l}\text { No. 3. Heavy domestic fuel oil } \\
\text { A distillate fuel cil for use in burners wbere a low } \\
\text { viscosity oil is required. }\end{array}$ & $\begin{array}{c}150 \\
\text { or } 1 \\
\mathrm{~Pa}\end{array}$ & gal & $\begin{array}{l}0.1 \\
\text { Par. } 5\end{array}$ & Par. $7^{15}$ & $\begin{array}{l}\circ . \\
\quad 460 \\
\text { Par. } 9\end{array}$ & $\begin{array}{l}{ }^{\circ} F . \\
675 \\
\text { Par. } 9\end{array}$ & $\begin{array}{c}\text { Saybolt } \\
\text { universal } \\
\text { at } 100^{\circ} F \\
55 \text { seconds. } \\
\text { Par. } 10\end{array}$ \\
\hline
\end{tabular}

1 Lower or higher pour points may be specified whenever required by conditions of storage and use. However, these specifications shall nct require a pour point less than $0^{\circ} \mathrm{F}$. under any conditions.

\section{Detailed requirements for industrial fuel oils}

[For method of test see paragrapb indicated]

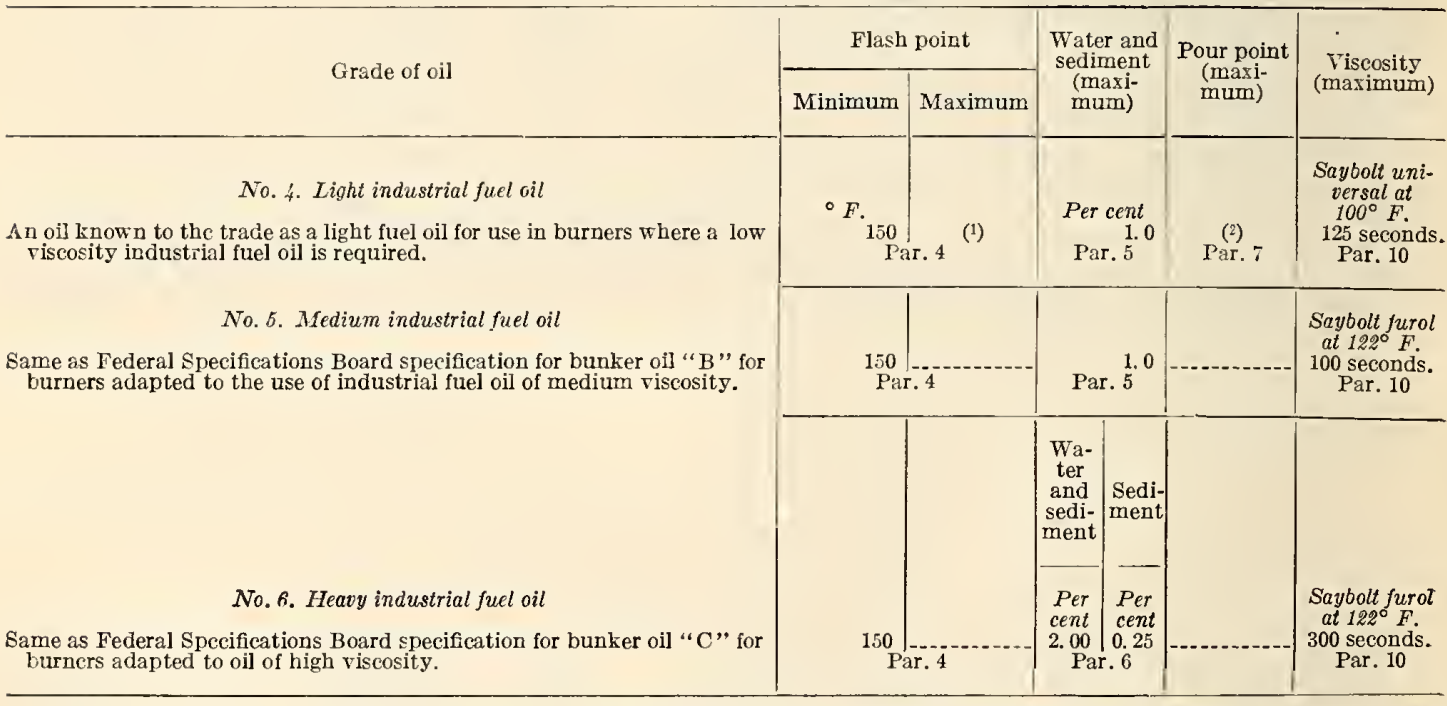

1 Whenever required, as, for example, in burncrs witb automatic ignition, a maximum flash point may be specified. However, these specifications shall not require a flash point less than $250^{\circ} \mathrm{F}$. under any conditions.

2 Pour point may be specified whenever required by conditions of storage and use. However, these specifications sball not require a pour point less tban $15^{\circ} \mathrm{F}$. under any conditions. 


\section{METHODS OF TEST}

\section{FLASE POINT}

3. Minimum.-The flash point, instrument, and method for determining minimum flash point shall be that legal for the locality in which the oil is sold. In absence of legal requirements, the minimum flash point shall be determined in accordance with $\mathrm{A}$. S. T. M. D93. (See 503.0, p. 49.)

4. Maximum.-Standard method of test for flash point by means of the Pensky-Martens closed tester as in A. S. T. M. D93.

\section{WATER AND SEDIMENT}

5. Standard method of test for water and sediment in petroleum products by means of centrifuge as described in A. S. T. M. D96. (See 502.2, p. 39.)

6. Standard method of test for water and sediment in petroleum products by means of distillation and extraction as described in A. S. T. M. D95 (see 502.2 , p. 38) for water, and test method No. 18, Federal Specifications Board specification 2d (see 503.0, p. 66) for sediment. A deduction in quantity will be made for all water plus sediment in excess of 1.0 per cent.

\section{POUR POINT}

7. Tentative method of test for pour points of petroleum products as in A. S. T. M. D97. (See 502.2 , p. 40.$)$

\section{DISTILLATION TEST}

8. Standard method of test for distillation of gasoline, naphtha, kerosene, and similar petroleum products as in A. S. T. M. D86. (See 503.0, p. 44.)

9. Standard method of testing gas oils as in A. S. T. M. D158 (see 503.0 , p. 154), except as to the procedure in taking readings, which shall be taken as required in these specifications.

\section{VISCOSITY}

10. Standard method of test for viscosity of petroleum products and lubricants as in A. S. T. M. D88. (See 503.0, p. 47.)

\section{SIGNIFICANCE OF TESTS PRESCRIBED}

\section{FLASH POINT}

11. The flash point of a product may be defined as the temperature to which it must be heated in order to give off sufficient vapor to form an inflammable mixture with air. This temperature varies with the apparatus and procedure employed and consequently both must be specified when the flash point of an oil is stated.

12. The minimum flash point of oils used for fuel is usally controlled by law. When there are no legal requirements, the minimum values in the table are to be employed. Maximum values are specified in oils Nos. 1, 2, and 3 to insure the required ease of ignition.

\section{WATER AND SEDIMENT}

13. Water and sediment are impurities which are almost entirely excluded in oils Nos. 1, 2, and 3 and which are permitted in somewhat larger quantities in oils Nos. 4,5 , and 6 . It is difficult to eliminate them entirely from this latter group of oils, and the advantage is not sufficient to justify the cost.

14. Water and sediment are determined together by the centrifuge, except with oil No. 6, where water is determined by a distillation method, and sediment by extraction with benzol.

\section{POUR POINT}

15. The "pour point" of an oil is the lowest temperature at which an oil will flow under prescribed conditions. Pour-point specifications are included in order that oil may be secured which will not cause difficulty in handling or in use at the lowest temperatures to which it may normally be subjected.

\section{DISTILLATION}

16. Laboratory distillation of a sample under prescribed conditions gives an index of the volatility of the oil. The 10 and 90 per cent points represent, respectively, the temperatures at which 10 and 90 per cent of the sample are distilled over. The end point is the maximum temperature recorded by the distillation thermometer at the end of the distillation.

17. The 10 per cent point serves as an index of the ease of ignition of the oil and the 90 per cent point and the end point are specified to make sure that the oil will burn completely and produce a minimum amount of carbon.

\section{VISCOSITY}

18. The viscosity of an oil is the measure of its resistance to flow. Maximum limits are placed on this property because of its effect upon the rate at which oil will flow through pipe lines and upon the degree of atomization that may be secured in any given equipment.

19. Viscosity is measured as the seconds required for a definite volume of oil to pass through a small tube of specified dimensions at a definite temperature. Viscosity decreases rapidly as temperature increases and preheating makes possible the use of oils of relatively high viscosity at normal temperatures. The Saybolt universal viscosimeter is used for fuel oils of fairly low viscosity and the Saybolt furol viscosimeter for heavier oils.

NotE.-It will be noted that these specifications make no reference to the gravity of the oil, a quality that has been widely used in the past as an index of volatility. Gravity is of no value as an indicator of the volatility or viscosity of a fuel oil, and consequently no gravity limits are given.

\subsection{KEROSENE AND SIMILAR ILLUMI- NATING OILS.}

American Railway Association, signal section, specification 1911, illuminating oil.

1. Sample must not flash below a temperature of $110^{\circ} \mathrm{F}$. in the "Elliott" closed cup nor burn below $160^{\circ} \mathrm{F}$. in the "Tagliabue" open eup when heated at the rate of $2^{\circ}$ per minute and flame applied every $2^{\circ}$, beginning at $100^{\circ} \mathrm{F}$.

2. Must be not less than 47.3 Baumé, or a specific gravity of 0.7896 .

3. Must be strictly water white in color and remain perfectly clear and transparent when cooled to a temperature of $0^{\circ} \mathrm{F}$. and held there for 10 minutes. Oil must be perfectly neutral, showing neither acid nor alkaline reaction.

4. A sample will be taken from each carload or part carload lot (either in bulk or in packages) and such sample shall be considered representative of the entire shipment.

5. Samples will be taken with a thief.

6. The oil must burn steadily and clearly in a suitable lamp without smoking and with a minimum incrustation of the wick for a period of at least 100 hours.

7. Any sample failing to meet the requirements of this specification will be condemned, and the shipment represented by it will be returned to the manufacturer, who must pay all freight charges.

American Society for Testing Materials, standard method of test for burning quality of kerosene oils, serial designation D187-27; 1927.

Approved as American Standard by the American Engineering Standards Committee, now the American Standards Association. 
1. The burning test for kercsene oil is intended for the determination of the burning quality of ordinary kerosene used for illuminating purposes.

\section{APPARATUS 88}

2. Standard brass Saybolt test lamp (see fig. 42 , facing p. 222). The lamp shall conform to the following require-ments:

\begin{tabular}{|c|c|c|c|}
\hline & Inches & $\begin{array}{l}\text { Centi- } \\
\text { meters }\end{array}$ & $\begin{array}{l}\text { Toler- } \\
\text { ances }\end{array}$ \\
\hline Inside diameter of burner opening & & & $\stackrel{c m}{c m}$ \\
\hline Top of font to top of burner opening.-. & $\begin{array}{r}1.21 \\
.45\end{array}$ & 1. 14 & $\begin{array}{r} \pm .01 \\
.02\end{array}$ \\
\hline Outside diameter of font & 5. 50 & 13.97 & .... \\
\hline Outside depth of font...... & 2.49 & 6.33 & \\
\hline Bottom of font to bottom of base & 5. 50 & 13.97 & \\
\hline Filiing opening: & & & \\
\hline Inside diameter. & .69 & 1.75 & \\
\hline Distance from side of for & .08 & .20 & $\ldots$ \\
\hline Height above font & .33 & .83 & \\
\hline Width of rim at bottom of font. & .65 & 1.65 & \\
\hline Thickness of metal-_................ & .03 & .07 & ... \\
\hline
\end{tabular}

3. Miller No. 2 sun hinge burner (see fig. 43) or any other burner meeting the following:

The burner shall conform to the following requirements:

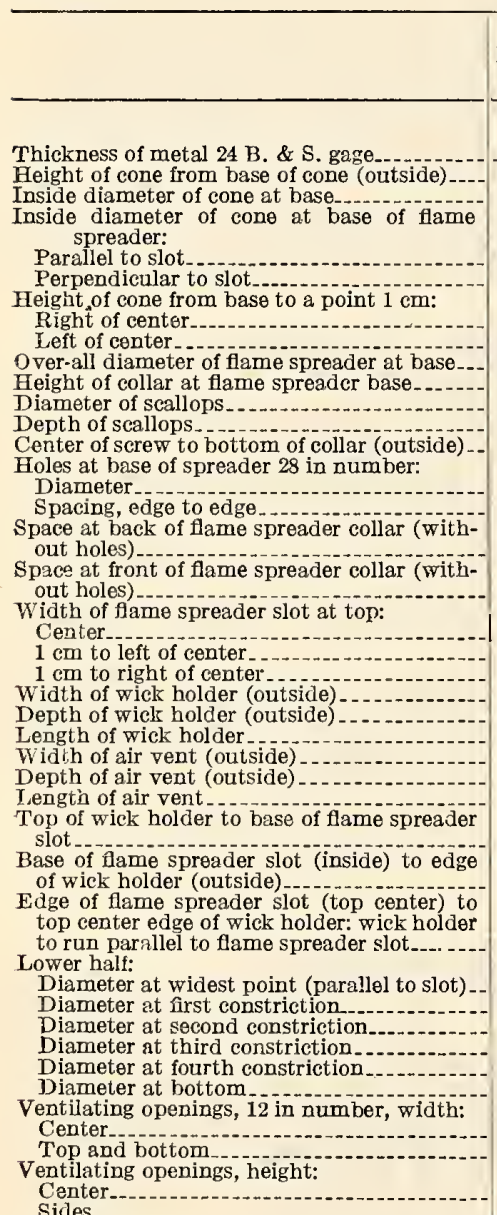

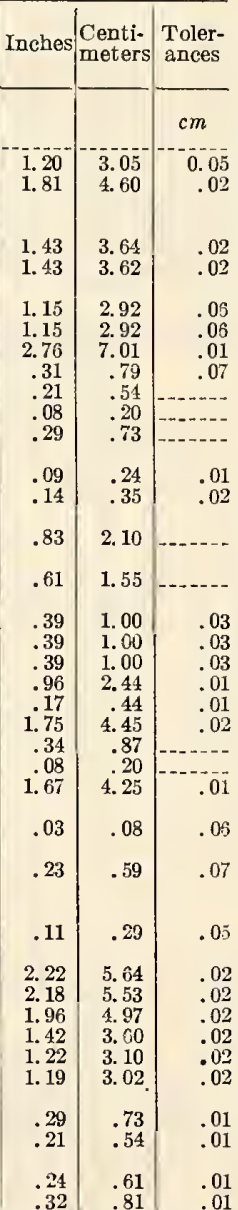

${ }_{38}$ The equipment used in this test is selected to secure results in a reasonably short time, and it should be understood that this does not indicate any preference as to the proper type for general household use.

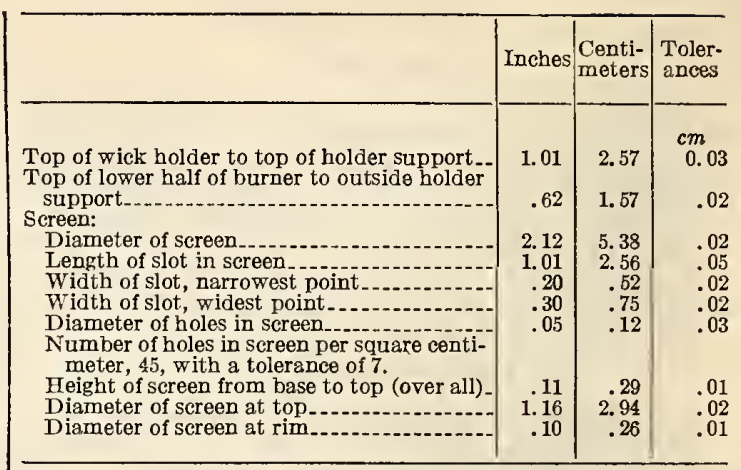

Note.-The burner shall be used as received from the manufacturer provided it meets the above specifications and is free from gross imperfections of manufacture. It shall be clean and in good condition at the start of the test, but emery paper shall not be used in cleaning nor imperfections removed by means of a file or otherwise.

4. Macbeth-Evans No. 514 pearl top chimney (see fig. 42).-The chimney shall conform to the following requirements, all figures being outside measurements:

\begin{tabular}{|c|c|c|c|}
\hline & Inches & $\begin{array}{l}\text { Centi- } \\
\text { meters }\end{array}$ & $\begin{array}{l}\text { Toler- } \\
\text { ances }\end{array}$ \\
\hline , & & & $\mathrm{cm}$ \\
\hline Total height_.. & 8.06 & 20.48 & 0.50 \\
\hline Diameter of constriction: & & & \\
\hline Upper- & 1. 77 & 4. 50 & .08 \\
\hline Lower & 2.27 & 5.77 & .07 \\
\hline Diameter at top. & $\begin{array}{l}3.69 \\
2.83\end{array}$ & $\begin{array}{l}9.36 \\
7.20\end{array}$ & .09 \\
\hline Diameter at bottom & 2.60 & 6.61 & .06 \\
\hline Distance from bottom to narrowest point. & 7. 03 & 17.86 & .55 \\
\hline Distance from bottom to widest point & 2.47 & 6.27 & .55 \\
\hline Distance from bottom to constriction near & & & \\
\hline bottom & .30 & .77 & .19 \\
\hline Top_- & .07 & .19 & .05 \\
\hline Bottom & .05 & .12 & .03 \\
\hline
\end{tabular}

5. American Wicle Co.'s "B" or similar No. Q wick. - The wick shall be used as received from the manufacturer and shall not be extracted with ether or other solvent previous to use. A wick shall be rejected if it nits the wick guide too loosely or too tightly, and a new wick shall be used for each burning test.

6. A sight gage for measuring the flame height and width accurate to one-sixteenth inch shall be used. The sight gage shown in Figure 45, facing page 222, is satisfactory.

7. A special room for conducting the burning test is desirable, but the lamp may be burned in any part of any adequately ventilated room reasonably free from drafts. The temperature of the room is immaterial, but it shall not vary more than $20^{\circ} \mathrm{F}$. during the test.

\section{PROCEDURE}

8. The lamp shall be cleaned and $850 \mathrm{cc}$ of the oil to be tested shall be poured into the font. The wick, wetted with the oil, shall be inserted in the burner, and the burner shall be firmly screwed into the font. The wick shall be trimmed with the scissors so as to produce a flame, which, unless otherwise specified, shall be $1 \frac{3 / 4}{4}$ inches in height measured from the top of the wick guide and $13 / 4$ inches in width at its widest point. (See fig. 43.) The trimming should be done very carefully to produce a smooth symmetrical flame free from peaks or ears, and the lamp should be lit and the flame inspected from time to time during the course of trimming in order to determine whether the trim is satisfactory. 
Proper height can be obtained by turming the wick up or down and the proper width obtained by trimming the corners of the wick. These adjustments should be made to $\pm 1 / 16$ inch from the standard flame dimensions. The lamp shall be allowed to burn for one hour, and the flame then readjusted, if necessary, to the standard dimensions. At the end of the first hour the lamp shall be weighed, while burning, on a platform balance accurate to $1 \mathrm{~g}$, and weighed again after exactly 60 minutes further burning. The oil consumed shall be calculated in cubic centimeters per hour. The initial rate of oil consumption, thus established, shall not vary from $43 \mathrm{cc}$ per hour by more than \pm 2 cc. With proper attention to the flame dimensions this measurement is needed only as a check, since with a given flame size and shape the initial rate of oil consumption will always be within the limit set.

9. The oil shall be allowed to burn continuously, without further adjustment of any kind, for the duration of the test. The lamp shall be refilled to within one-fourth inch of the top of the fount, at intervals of eight hours, without putting out the flame.

10. Unless otherwise specified, the duration of the test shall be 24 hours of continuous burning.

11. At the end of the test the height and width of the flame and the condition of the chimney, flame, and wick shall be noted. The final flame measurement shall be taken without refilling the lamp.

12. With care and proper attention to detail, the average oil consumption per hour should not differ in duplicate determinations by more than 2 ec. With this condition fixed, the other characteristics of the oil will be reproduced invariably, with the exception of initial mushroom formations ("ears," "niggers," "toadstools"), reproducibility of which will vary with the quality of the oil, amounting in the poorer oils to 10 per cent of the burning time.

Federal Specifications Board specification 2d, United States Government master specification for lubricants and liquid fuels.

(This publication, containing detailed specifications, methods of testing, and methods of sampling, is published by the United States Government Printing Office as Technical Paver 323B, Department of Commerce, Bureau of Mines. The publication includes specifications for illuminating oils, which are given below.)

\section{ILLUMINATING OILS}

\section{GENERAI REQDIREMENTS}

Sampling.-See A. S. T. M. method D270-27T, 503.0 , page 60 .

Testing.-See F. S. B. No. 2d, 503.0, page 66.

Noт:.-For convenience in presentation, the test methods have been listed numerically and referenco made accordingly in the following table where tests apply.

\section{DETAII REQUIREMENTS}

(a) General.-1. The oil shall be free from water, glue, and suspended matter.

2. Uses.-Kerosene: Used as an illuminating oil. Long-time burning oil: Used where a long-time burning oil is required. Three-hundred-degree mineral seal oil: Used for lamps in passenger coaches and for illuminating railroad equipment, and where a high-flash illuminant is required. (b) Properties and tests.-

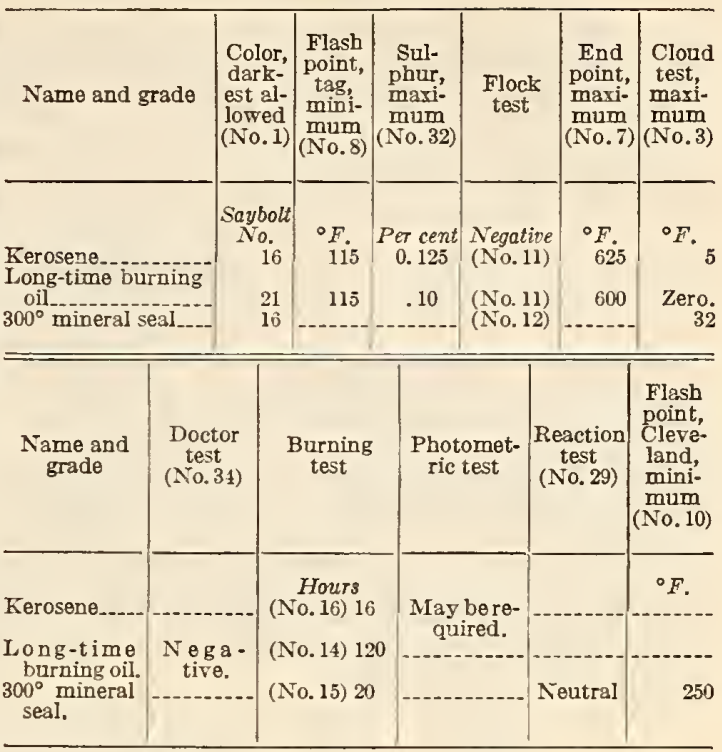

\subsection{MINERAL SEAL OIL.}

American Society for Testing Materials, standard method of test for burning quality of mineral seal oil, serial designation: D239-27, 1927.

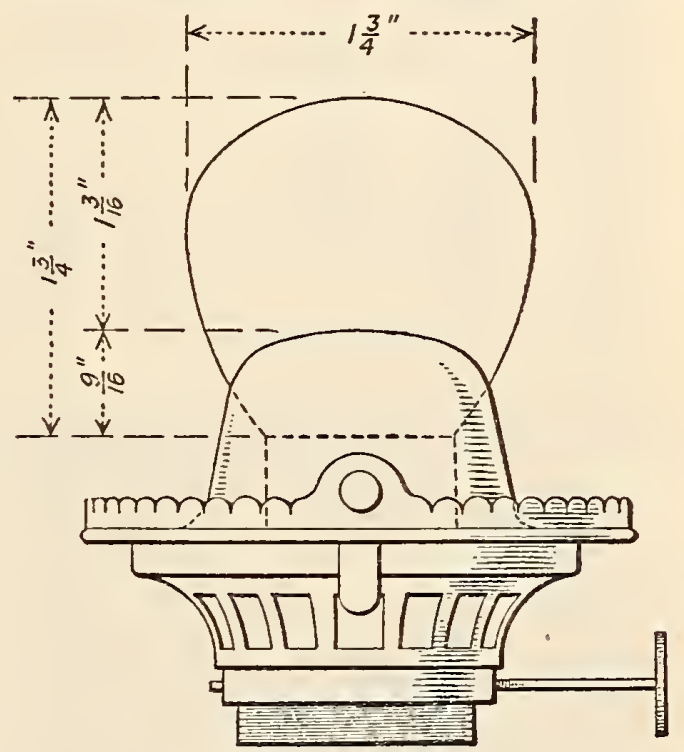

FIGURE 43.-Burner

Approved as American Standard, by the American Engineering Standards Committee, now the American Standards Association.

1. The burning test for mineral colza oil is intended for the determination of the burning quality of the special illuminating oil known to the trade as mineral seal oil, 300 oil, or mineral colza oil, used in railway coach lamps. 


\section{APPARATUS}

2. The lamp shall be the standard Dressel No. 520 side lamp fitted with a No. 3 dual burner without spring chimney holder and without smoke bell.

3. A 24-fluid-ounce font having the following measurements shall be used:

\begin{tabular}{|c|c|c|c|}
\hline & Inches & $\begin{array}{l}\text { Centi- } \\
\text { meters }\end{array}$ & $\begin{array}{l}\text { Toler- } \\
\text { ances }\end{array}$ \\
\hline Inside diameter of burner opening. - & 1.39 & 3.52 & $\begin{aligned} & \mathrm{cm} \\
\pm & 0.01\end{aligned}$ \\
\hline Outside diameter of font at bottom. & 3. 74 & 9.49 & \\
\hline Outside diameter of font above constriction.- & 4. 06 & 10.32 & \\
\hline Outside diameter of font at widest point & 4.50 & 11.43 & \\
\hline Outside diameter of font at neck & 1.85 & 4. 69 & \\
\hline Height of font (to top of collar) & 4. 31 & 10.95 & \\
\hline Height of font (to outside edge top) & 3.65 & 9.27 & .08 \\
\hline Diameter of inside compartment.... & 1.89 & 4.80 & \\
\hline Thickness of metal & .03 & .07 & \\
\hline
\end{tabular}

4. The burner shall be a Dressel "D" or No. 3 dual burner, without skirt, or any other burner meeting the following requirements:

\begin{tabular}{|c|c|c|c|}
\hline & Inches & $\begin{array}{l}\text { Centi- } \\
\text { meters }\end{array}$ & $\begin{array}{l}\text { Toler- } \\
\text { ances }\end{array}$ \\
\hline \multicolumn{4}{|l|}{$\begin{array}{l}\text { Upper part of burner: } \\
\text { Thickness of metal, No. } 24 \text { B. \& S. gage. } \\
\text { Height of cone from base of cone- }\end{array}$} \\
\hline Height of cone from base of cone- & & & $\mathrm{cm}$ \\
\hline & 1.61 & 4.09 & 0.03 \\
\hline $1 \mathrm{~cm}$ to right of center. & 1.57 & 3. 98 & .03 \\
\hline $1 \mathrm{~cm}$ to left of center... & 1.56 & 3.97 & .05 \\
\hline Inside diameter of cone at base & 2.05 & 5.20 & \\
\hline Inside diameter of cone at constriction. & 2.00 & 5.08 & \\
\hline \multicolumn{4}{|l|}{$\begin{array}{l}\text { Inside diameter of cone at base of flame } \\
\text { spreader slot- }\end{array}$} \\
\hline Parallel to slot & 1.49 & 3. 79 & .01 \\
\hline Perpendicular to slot..... & 1. 52 & 3.85 & 01 \\
\hline Height of collar & 1.95 & 4.96 & \\
\hline \multicolumn{4}{|l|}{ Holes near base of cone, 18 in number- } \\
\hline \multicolumn{4}{|l|}{ Diameter } \\
\hline \multicolumn{4}{|l|}{ Spacing edge to edge } \\
\hline Spaces at sides of cone (without holes) & .78 & 1.98 & \\
\hline \multicolumn{4}{|l|}{ Width of flame spreader slot- } \\
\hline Center & .31 & .78 & .02 \\
\hline $1 \mathrm{cr}$ & .31 & .78 & .02 \\
\hline $1 \mathrm{~cm}$ to left of center & .31 & .78 & .02 \\
\hline \multicolumn{4}{|l|}{$\begin{array}{l}\text { Ventilating openings, } 40 \text { in number in each } \\
\text { row- }\end{array}$} \\
\hline \multicolumn{4}{|l|}{ Top row (diamond shaped), height and } \\
\hline & .09 & .22 & \\
\hline \multicolumn{4}{|l|}{ Third row (diamond shaped), height and } \\
\hline \multicolumn{4}{|l|}{ width } \\
\hline \multicolumn{4}{|l|}{ Bottom row (diamond shaped) - } \\
\hline Height & .22 & .55 & \\
\hline \multicolumn{4}{|l|}{ Width. } \\
\hline \multicolumn{4}{|l|}{ Lower part of burner: } \\
\hline \multirow{2}{*}{\multicolumn{4}{|c|}{$\begin{array}{l}\text { Diameter of widest point } \\
\text { Diameter at narrowest point (just below }\end{array}$}} \\
\hline & & & \\
\hline & 1.28 & 3. 26 & \\
\hline \multicolumn{4}{|l|}{ Diameter at bottom (thread) } \\
\hline \multicolumn{4}{|l|}{ Top of screen (outer edge) to bottom of } \\
\hline \multirow{2}{*}{\multicolumn{4}{|c|}{$\begin{array}{l}\text { Top of wick holde } \\
\text { Holes in screen- }\end{array}$}} \\
\hline & & & \\
\hline \multicolumn{4}{|l|}{ Outer circle, 36 in number, diameter } \\
\hline \multirow{2}{*}{\multicolumn{4}{|c|}{$\begin{array}{l}\text { Second circle, } 36 \text { in number, diameter } \\
\text { Inner circle, } 36 \text { in number, diameter }\end{array}$}} \\
\hline & & & \\
\hline Space between wick holders...... & .11 & .27 & .04 \\
\hline \multicolumn{4}{|l|}{$\begin{array}{l}\text { Space between } \\
\text { Wick holders- }\end{array}$} \\
\hline \multicolumn{4}{|l|}{ Width (outside) - } \\
\hline & .19 & .48 & .06 \\
\hline \multirow{2}{*}{\multicolumn{4}{|c|}{$\begin{array}{l}\text { Length } \\
\text { wick holder brace: }\end{array}$}} \\
\hline & & & \\
\hline Width (outside) & 1.02 & 2. 60 & \\
\hline Depth (outside). & .15 & .38 & \\
\hline
\end{tabular}

Following measurements taken with upper part of burner fitted evenly and tightly in lower half:

\begin{tabular}{l|r|r|r}
\hline & Inches $\begin{array}{r}\text { Centi- } \\
\text { meters }\end{array}$ & $\begin{array}{r}\text { Toler- } \\
\text { ances }\end{array}$ \\
\hline Top of wick holder to base of flame spreader & & & \\
$\begin{array}{l}\text { slot. } \\
\text { Base of flame spreader slot (inside) to edge of } \\
\text { wick holder (outside) }\end{array}$ & 0.32 & 0.81 & $\begin{array}{r}\mathrm{cm} \\
\text { Height of burner (over-all) }\end{array}$ \\
\hline
\end{tabular}

5. The chimney shall be a Keystone No. 24 chimney, or any other chimney meeting the following requirements: ${ }^{39}$

$$
\text { . }
$$

Total height

Diameter at top

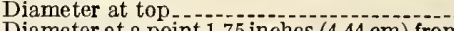

1ameter at a point 1.75 inches $(4.44 \mathrm{~cm})$ from

Diameter at a point 5 inches from top.

Diameter at widest point

Diameter at the bottom.

Distance from bottom to widest point.

Distance from bottom to constriction near bottom

bottom

Thickness of glass at bottom

6. The wicks shall be the American "D" dual wick and shall be used as received from the manufacturer. New wicks shall be used in each test.

7. A platform balance sensitive to $0.1 \mathrm{~g}$ shall be used.

8. The lamp shall be burned in a well-ventilated room, free from draughts, and not subjected to the direct rays of the sun. The lamp shall be mounted on a wall, at a distance of approximately $2 \frac{1}{4}$ inches from the wall, by means of a bracket provided with the lamp.

9. A sight gage, or some other suitable flamemeasuring device, accurate to one-sixteenth inch, shall be used. The gage shown in Figure 45 , facing page 222 , is satisfactory.

\section{PROCEDURE}

10. Place approximately $600 \mathrm{cc}$ of the oil to be tested in a clean font. The wicks, wetted with the oil, shall be inserted in the burner and then assembled with the font and chimney. The wicks shall then be trimmed with scissors so that they are of the same height and so as to produce a flame which, unlcss otherwise specified, shall be $1 \frac{1}{16}$ inches high, measured from the top of the wick guide, and $13 / 8$ inches wide at its widest point. The trimming shall be done very carefully to produce a flame free from peaks or ears and the lamp should be lit and the flame frequently inspected during the course of the trimming in order to determine the conditions of the flame as to size and shape. Proper height can be obtained by turning up or down, and the proper width by trimming the corners of the wick. These adjustments shall be made to $\pm 1 / 16$.

38 All measurements are outside. 
inch from the standard flame. After the proper flame has been obtained, the burner and wicks shall be removed, the wicks dried as thoroughly as possible without removing the wicks from the burner, and the apparatus assembled with a clcan font and chimney. Weigh the assembly and then pour exactly $600 \mathrm{cc}$ of the sample into the font, then light the lamp and weigh again while burning. After one hour, the flame shall be measured and adjusted, if necessary, to the proper height and width. Allow the lamp to burn continuously until $570 \mathrm{cc}$ of oil has been consumed, as determined by weight. In general, $570 \mathrm{cc}$ of oil will bc consumed in not less than 20 hours. At the end of the test, record the number of hours burned, the flame change, and the condition of wick and chimney.

Federal Specifications Board, specification 2d, United States Government master specification for lubricants and liquid fuels. (See 503.5, p. 87.)

\subsection{LONG-TIME BURNING (SIGNAL) OIL}

American Society for Testing Materials, standard method of test for burning quality of long-time burning oil for railway use, serial designation D219-27; 1927.

Approved as American Standard, by the American Engineering Standards Committee, now the American Standards Association.

1. The burning test for long-time burning oil is intended for the determination of the burning quality of special kerosene oil used in railway semaphore signal lamps.

\section{APPARATUS}

2. The lamp shall be the standard American Railway Association, signal section, semaphore lamp.

3. A 31-fluid-ounce font, about 3 inches high, measured from inside the bottom to top of burner collar, and fitting the standard lamp, shall be used.

4. The burner shall be a Dressel No. 23 long-time burner, New York Central typc, or any other burner meeting the following requirements: A nickelplated long-time burner made of suitable weight metal, provided with the conventional wick-adjusting device, with any convenient means of fitting to font, and in other respects corresponding to the following requirements:

Flame spreader.-Cylindrical, five-sixteenths inch in height and one-half inch in inside diameter, soldered to the top of wick tube. Two V-shaped slots, placed opposite to each other, with top of $v$ one-fourth inch wide and bottom ending just above top of wick tube.

Wick tube.-One and fifteen-sixteenths inches in length, one-fourth inch in inside diameter, and extending five-eighths inch above top of wick tube

Chimney-support ring.-One and one-fourth inches in inside diameter, seven-sixteenths inch below top of flame spreader, equipped with four springs to hold chimney in place.

Air vents. - Four 1/16-inch holes placed symmetrically in flame spreader just above the top of the wick tube; four rectangular openings, sevensixteenths inch high, by nine-sixteenths inch wide, placed below the chimney support ring and between the chimney springs.

5. The chimney shall be a No. 55-D chimney, 40 or any other chimney meeting the following requirements: Straight wall glass $2 \frac{1}{2}$ inches high and $1 \frac{1}{8}$ inches in inside diameter, with rim at top and bottom.

6. A new gray round felt wick, approximately $73 / 4$ inches long and three-sixteenths inch in diameter

10 This term, “55-D chimney" is fully descriptive with glass manufacturing companies as the long-time burner chimney covered by these specifications. made $b y$ sewing a strip of fclt one-half inch wide by $73 / 4$ inches long by one-cighth inch thick into proper form is suitable. The wick shall be washed with redistilled ether and dried at room temperature before use.

7. A platform balance sensitive to $1 \mathrm{~g}$ shall be used.

8. The lamp may be burned in any well ventilated room, or out of doors, if not subjected to the direct rays of the sun.

9. A sight gage, or other suitable flame measuring device, accurate to one-sixteenth inch shall be used. The sight gage shown in Figure 45 is satisfactory.

\section{PROCEDURE}

10. Seven hundred and fifty cubic centimeters of the oil to be tested shall be placed in the font. The burner, wick, chimney, and font shall be assembled, the rough edge trimmed from the wick and the wick centered in the flame spreader, lighted and adjusted to give a flame three-fourths inch high, measured from top of wick. This, and all following flame measurements, should be made with the assembly inside of the lamp, with

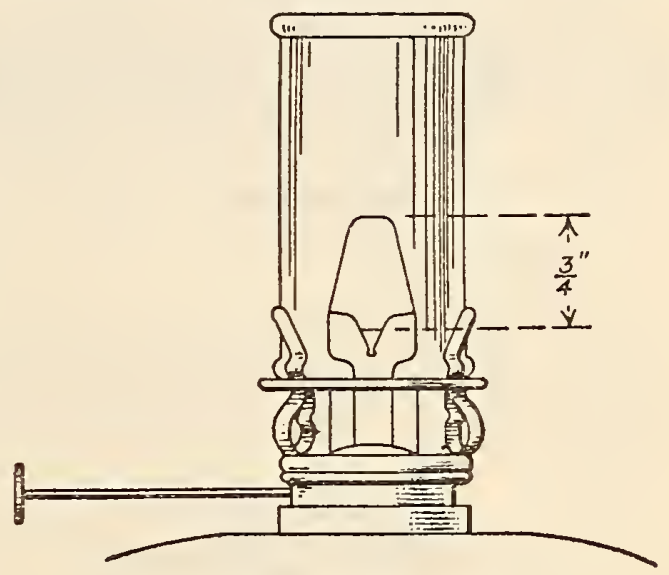

FIGURE 44.-Burner

slide open, and in a room free from drafts. The flame should have a flat top and be of the general shape indicated in Figure 44. If this can not be accomplished by leveling the lamp, the burner should be rejected. Inability to secure the specified flame shape will be due, in such cases, to improper alignment of the sides of the flame spreader. It is essential that the specified flame shape be adhered to, as any deviation from it may influence the rate of oil consumption and the flame drop. The assembly shall be weighed while burning, then placed in the lamp. The wick shall be readjusted, if necessary, without removing the font from the lamp, during the first four hours. The lamp shall be allowed to burn continuously for the duration of the test. After 96 hours, the font assembly shall be removed from the lamp, weighed while burning and then replaced in the lamp. After 120 hours the lamp shall again be weighed and the average rate of oil consumption per hour calculated for the 96 to 120 hour period. From this rate and the original weight of oil in the font, the number of additional hours required to make the total quantity of oil consumed $650 \mathrm{cc}$ shall be calculated and the lamp burned the calculated length of time. The oil consumption shall be verified by reighing at the end of the test. The time required to burn $650 \mathrm{cc}$ will, in general, be not lcss than 120 hours and not more than 144 hours if 
proper attention is paid to initial flame height and shape. At the end of the test, observe and record the change in height of flame, and condition of the wick and chimney.

Federal Specifications Board, specification 2d, United States Government master specification for lubricants and liquid fuels. (See 503.5, p. 87.)

\section{IUBRICATING AND INSULATIYG OILS (INCLUDING PARAFFIN OIL) AND GREASES}

\subsection{GENERAI ITEMS.}

American Society for Testing Materials, standard methods of test for viscosity of petroleum products and lubricants, serial designation D88-26; 1926.

See 503.0, page 47 .

American Society for Testing Materials, tentative method of test for precipitation number of lubricating oils, serial designation D91-29T; 1929.

1. This method is commonly used for steam cylinder stocks and black oils and may be used for other lubricating oils.

\section{DEFINITION}

2. A. S. T. M. precipitation number of lubricating oils. - The number of cubic centimeters of precipitate formed when $10 \mathrm{cc}$ of lubricating oil are mixed with 90 cc of petroleum naphtha as specified in section 5 and centrifuged under prescribed conditions.

\section{APPARATUS}

3. Centrifuge.-Same as section 3 of A. S. T. M. D96. (See 502.2, p. 39.)

4. Centrifuge tubes, A. S. T. M. type.-These tubes shall be made of suitable glass and thoroughly annealed. The total capacity shall be about $125 \mathrm{cc}$ and the mouth shall be suitably constricted for closing with a cork. The graduations shall be clear and distinct, reading upward from the bottom of the tube as follows:

\begin{tabular}{|c|c|c|l|}
\hline Range & $\begin{array}{c}\text { Scale } \\
\text { divisions }\end{array}$ & $\begin{array}{c}\text { Limit of } \\
\text { error }\end{array}$ & Numbered \\
\cline { 1 - 3 }$c c$ & $c c$ & $c c$ & \multicolumn{1}{c|}{$c c$} \\
$0-0.5$ & 0.05 & 0.02 & $1 / 2$. \\
$0.5-3$ & .1 & .05 & $1,2,3$. \\
$3-5$ & .5 & .2 & $4,5$. \\
$5-10$ & 1.0 & .5 & $6,8,10$. \\
$10-25$ & 5.0 & 1.0 & $15,20,25$. \\
$50-100$ & 50.0 & 1.0 & $50,100$. \\
\hline
\end{tabular}

The shape is optional provided it does not conflict with the other requirements.

5. (a) Petroluem naplitha conforming to the following requirements shall be used for the diluent.

Gravity, ${ }^{\circ}$ A. P. I. at $60^{\circ} \mathrm{F} \ldots \ldots 70$ to 73

Anilize point, ${ }^{\circ} \mathrm{C}$

Initial boiling point, A.S.T.M.-. Not less than $122^{\circ} \mathrm{F} .\left(50^{\circ} \mathrm{C}.\right)$

50 per cent point, A.S.T. M ..... $158^{\circ}$ to $176^{\circ} \mathrm{F}$. $\left(70^{\circ}\right.$ to $80^{\circ} \mathrm{C}$.)

End point, A.S. T. M........ Not more than $266^{\circ} \mathrm{F} .\left(130^{\circ} \dot{\mathrm{C}}.\right)$.

(b) The initial boiling point, the 50 per cent point, and the end point of the petroleum naphtha shall be determined in accordance with A. S. T. M. D86. (See 503.0 p. 44.)

(c) The aniline point of the petroleum naphtha shall be determined as follows: Measure $10 \mathrm{cc}$ of the sample and $10 \mathrm{ce}$ of the aniline (freshly distilled, free from aminothiophenes) into a large test tube fitted with a stirrer and a thermometer. Heat the mixture by means of a naked flame until it becomes homogeneous, and then allow to cool until it becomes turbid. Raise the temperature slightly and make a duplicate determination. Disregard any slight initial turbidity, taking the temperature when the solution becomes completely cloudy. If the aniline point is more than $10^{\circ} \mathrm{C}$. above the room temperature, use another larger test tube to form an air jacket.

\section{PROCEDURE}

6. Exactly $10.0 \mathrm{cc}$ of the oil to be tested shall be measured in each of two clean and dry centrifuge tubes at room temperature. Each tube shall be filled to the $100 \mathrm{cc}$ mark with the prescribed diluent and closed tightly with a softened cork (not a rubber stopper). Each tube shall then be inverted at least twenty times, allowing the liquid to drain thoroughly from the tapered tip of the tube each time. The tubes shall then be placed in a water bath at $90^{\circ}$ to $95^{\circ} \mathrm{F}$. for five minutes. The corks shall be momentarily removed to relieve any pressure and each tube shall again be inverted at least twenty times exactly as before. The success of this method depends to a large degree upon having a thoroughly homogeneous mixture which will drain quickly and completely from the tapered tip when the tube is inverted.

7. The two centrifuge tubes shall then be placed in the centrifuge on opposite sides and shall be whirled at a rate of 1,400 to $1,500 \mathrm{r}$. p. m. or equivalent for 10 minutes. The volume of sediment at the bottom of each tube shall be read and recorded, estimating to $0.05 \mathrm{cc}$ if possible. The tubes shall then be replaced in the centrifuge, again whirled for 10 minutes as before, and removed for reading the volume of the sediment as before. This operation shall be repeated until the volume of sediment in each tube remains constant for three consecutive readings. In general, not more than four whirlings are required, for oils having a low precipitation number.

8. The volume of the solid sediment at the bottom of each centrifuge tube shall be read, estimating to $0.1 \mathrm{cc}$ or closer if possible. If the two readings differ by not more than $0.1 \mathrm{cc}$ the mean of the two shall be reported as the "A. S. T. M. precipitation number." If the two readings differ by more than 0.1 cc two more determinations shall be made and the average of the four determinations shall be reported.

\section{ACCURACY}

9. With care and proper attention to details, duplicate determinations of precipitation number by this method should not differ by more than 0.1 , provided the centrifuge tubes are accurate and readable to this degree.

American Society for Testing Materials, standard method of test for flash and fire points by means of open cup, serial designation D92-24; 1924.

Approved as American Standard by the American Engineering Standards Committee, now the American Standards Association.

1. The open cup flash and fire test on all products except fuel oils and those having an open cup flash below $175^{\circ} \mathrm{F}$. shall be determined in the Cleveland open cup.

\section{APPARATUS}

2. The cup shall be supported by a metal plate one-fourth inch $(0.635 \mathrm{~cm})$ in thickness and 6 inches $(15.24 \mathrm{~cm})$ in width. The plate shall be of brass, cast iron, wrought iron, or steel. In the center of the plate there shall be a plane depression onethirty-second inch $(0.079 \mathrm{~cm})$ in depth, and of just sufficient diameter to fit the cup. There shall be a circular opening $23 / 16$ inches $(5.50 \mathrm{~cm})$ in diameter, cut through the plate, centering with the center of the above-mentioned depression. The plate shall be covered with a sheet of hard asbestos board one-fourth inch in thickness, and of the same shape 
as the metal plate. There shall be cut in the center of the asbestos board a circular hole just fitting the cup. Heat may be supplied from any convenient source. The use of a gas burner, electric heater, or alcohol lamp is permitted, but under no circumstances are products of combustion or free flame allowed to come up around the cup. The source of heat shall be centered under the opening in the plate and shall be of a type that will not produce
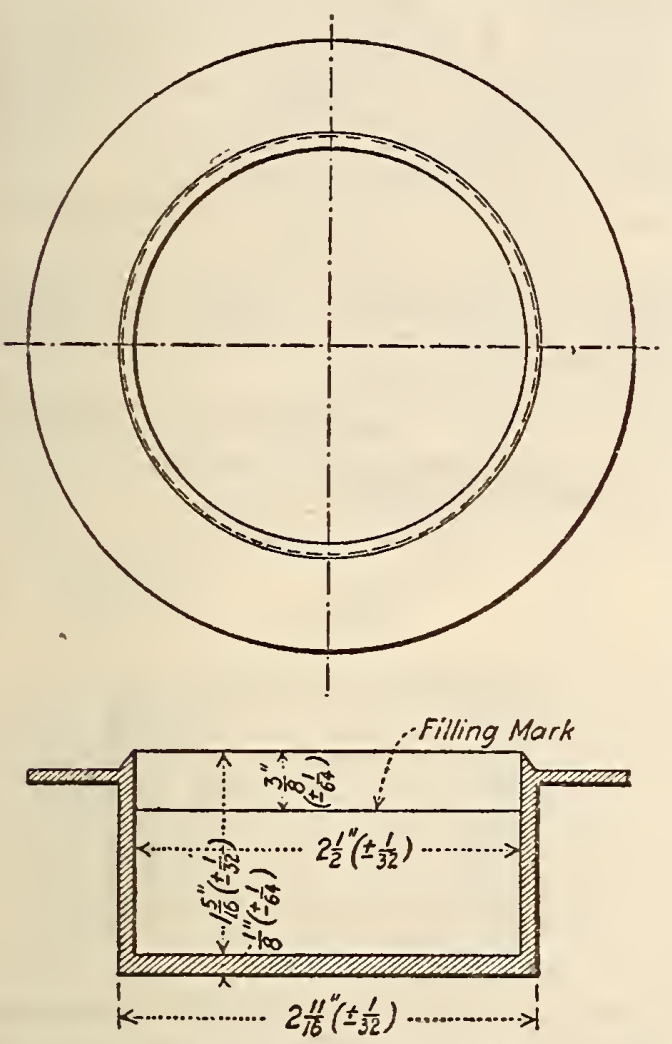

Figdre 46.-Cleveland open cup

local superheating. If a flame heater is used, it may be protected from drafts or excessive radiation by any suitable type of shield, that does not project above the level of the upper surface of the asbestos board.

3 . The thermometer shall conform to the requirements of thermometer No. 8. (See A. S. T. M. table of thermometers, 500.2, p. 2.)

\section{PROCEDURE}

4. (a) The thermometer shall be suspended or held in a vertical position by any suitable device. the bottom of the bulb shall be one-fourth inch $(0.635 \mathrm{~cm})^{41}$ from the bottom of the cup, and above a point half way between the center and back of the cup.

(b) The cup shall be filled with the oil to be tested in such a manner that the top of the meniscus is exactly at the filling line at room temperature. The surface of the oil shall be free from bubbles. There shall be no oil above the filling line or on the outside of the apparatus.

(c) The test flame shall be approximately five thirty-seconds inch $(0.397 \mathrm{~cm})$ in diameter.

41 The immersion line engraved on the thermometer stem will be one-sixteenth inch $(0.159 \mathrm{~cm})$ below the level of the rim of tie cup.
NoTE.-For purposes of comparison it is recommended that a bead of suitable light-colored material be mounted in a convenient position so that the size of the test flame can be determined. The device for applying the flame may be of any suitsble type, but it is suggested that the th o that the tip be approximately one-sixteenth inch $(0.159 \mathrm{~cm})$ in diameter at the end and that the orifice be one thirty-second inch $(0.079$ $\mathrm{cm}$ ) in diameter. If the device for operating the test flame be mounted in such a manner as to permit automatic duplication of the sweep
of the test flame, the radius of swing shall be not less than 6 inches.

(d) The test flame shall be applied as the temperature read on the thermometer reaches each successive $5^{\circ}$ F. mark. The flame shall pass in a straight line (or on the circumference of a circle having a radius of at least 6 inches) across the center of the cup and at right angles to the diameter passing through the thermometer. The test flame shall, while passing across the surface of the oil, be in the plane of the upper edge of the cup. The time for the passage of the test flame across the cup shall be approximately one second.

(e) The oil shall be heated at a rate not exceeding $30^{\circ} \mathrm{F}$. per minute temperature rise till a point is reached approximately $100^{\circ} \mathrm{F}$. below the probable flash point of the oil. Thereafter the rate of heating shall be decreased and for at least the last $50^{\circ} \mathrm{F}$. before the flash point is reached the rate shall be not less than $9^{\circ}$ nor more than $11^{\circ} \mathrm{F}$. per minute.

5. The flash point shall be taken as the temperature read on the thermometer when a flash appears at any point on the surface of the oil. The true flash must not be confused with a bluish halo that sometimes surrounds the test flame.

6. After determining the flash point, the heating shall be continued at the specified rate of $9^{\circ}$ to $11^{\circ}$ F. per minute, and application of the test flame shall be made at the specified intervals until the oil

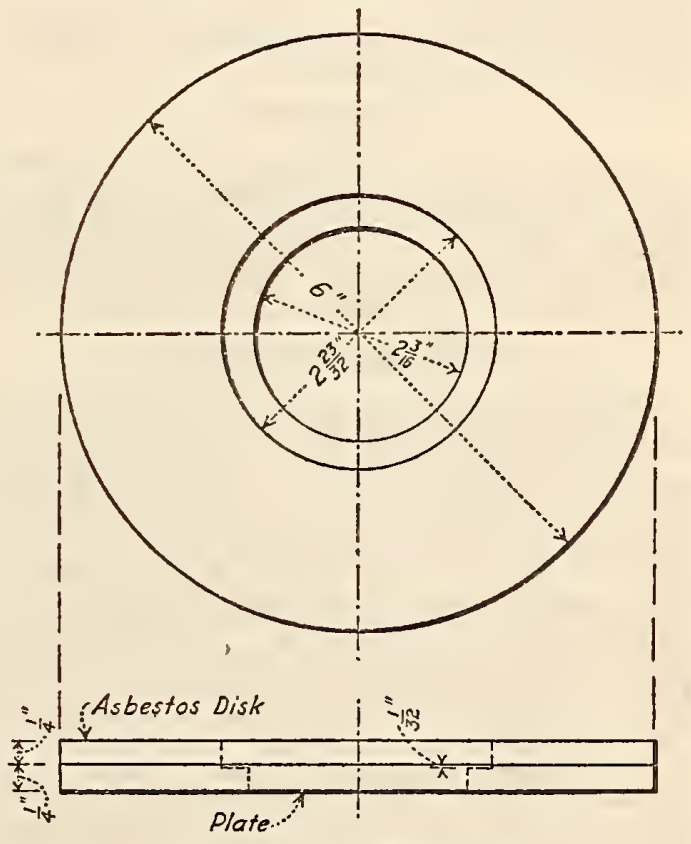

FigURE 47.-Heating plate

ignites and continues to burn for a period of at least five seconds. The method of application of the flame shall be the same as for flash point. The temperature read at the time of the flame application, which causes burning for a period of five seconds or more, shall be recorded as the fire point.

7. The flash-point and fire-point tests shall be made in a room or compartment free from air drafts. The operator shall avoid breathing over the surface 
of the oil. It is desirable that the room or compartment be darkened sufficiently so that the flash may be readily discernible.

American Society for Testing Materials, standard method of test for saponification number, serial designation D94-28, 1928.

\section{SCOPE}

This method is one of the means used for the identification of unmixed animal and vegetable oils and for measuring the quantity of fatty material in compounded products. It may also be used in the study of noncompounded petroleum products.

\section{SOLUTIONS REQUIRED}

Alcoholic solution for saponification.-Dissolve $58 \mathrm{~g}$ of potassium hydrate "purified by alcohol" in 500 ec of 95 per cent purified ethyl alcohol. Allow the solution to settle in a dark place. Draw off the clear solution or filter through an asbestos filter and make up to 1 liter with 95 per cent alcohol. The solution so prepared shall stand at least 20 hours before it is standardized.

Alcohol.-Purify 95 per cent ethyl alcohol with silver oxide in the following manner:

Dissolve $1.5 \mathrm{~g}$ of silver nitrate c. p. in about $3 \mathrm{cc}$ of water and add to 1 liter of alcohol in a glassstoppered bottle and mix thoroughly. Dissolve $3 \mathrm{~g}$ of potassium hydrate (by alcohol) in 10 to $15 \mathrm{cc}$ of warm alcohol. After cooling add slowly to the alcoholic silver nitrate solution, stirring slightly. Allow the precipitated silver oxide to settle, siphon off the clear solution, and distill on a steam bath.

Standard hydrochloric acid solution.-One-half normal solution.

Phenolphthalein solution.-One gram phenolphthalein in $100 \mathrm{cc}$ alcohol and water.

\section{APPARATUS}

The saponification shall be carried out in a widemouthed flat-bottom extraction flask, or Erlenmeyer flask, of chemically resistant glass, of 250 to $300 \mathrm{cc}$ capacity, fitted to a reliable condenser properly connected with a good cork. The boiling shall preferably be carried on by means of an electric hot plate.

\section{BLANK DETERMINATION}

Determination shall be made in a duplicate in the alcoholic potash solution in the following manner:

Measure accurately into the flask $25 \mathrm{cc}$ of alcoholic potash solution from a calibrated pipette. The tip and outside of the pipette shall be wiped off with a clean filter paper before the solution is delivered. Then rinse out with $25 \mathrm{cc}$ of neutral alcohol. If a standard burette is used, allow 60 seconds total time for drawing and draining. Connect the flask to a suitable condenser and boil for three hours. Before disconnecting the flask, wash out the condenser with a few cubic centimeters of neutral alcohol; if a soxhlet is used as the condenser the tip shall be washed off into the flask. Titrate while hot with $N / 2 \mathrm{HCl}$, using three drops phenolphthalein indicator. The total number of cubic centimeters of $N / 2 \mathrm{HCl}$ required for the blank represents the strength of the alcoholic potash solution.

\section{PROCEDURE}

For straight fats or oils use 2 to $3 \mathrm{~g}$ of the material, for oils containing over 30 per cent of fatty oils use about $5 \mathrm{~g}$, and for oils containing less than 30 per cent of fatty oils use about $10 \mathrm{~g}$.

Weigh the oil accurately, by difference, from a small beaker into the saponification flask. Add $25 \mathrm{cc}$ of alcoholic potash solution and $25 \mathrm{cc}$ of neutral alcohol in the same manner as for the blank, connect to the condenser and boil for three hours. Titrate while hot. Calculate the saponification number from the difference between the number of cubic centimeters of $N / 2 \mathrm{HCl}$ required for the determination and the average of the two blanks, using the formula:

Saponfication number $=\frac{\text { Difference, cubic centimeters } \times 28.05}{\text { Weight of oil, grams }}$

Petrolic ether may be used with compounded cylinder oils in the quantity of $50 \mathrm{cc}$ providing a soxhlet extraction flask is used to periodically collect this petrolic ether. The soxhlet flask should be so adjusted, through the addition of glass rodding or beads when necessary, so that it will just overflow with the full quantity of petrolic ether.

The percentage of fatty oil (or fat) in a compounded petroleum product can be calculated from the saponification number of such a product only when the saponification number of the fatty oil is known. If the saponification numbers of both fatty oil and compounded oil are known, the following formula should be used:

Per cent of fatty oil $=\frac{100 \times \text { saponification number of compounded oil }}{\text { Saponification }}$

For this determination the following values of saponification number may be used:

\begin{tabular}{|c|c|}
\hline Fatty oil & $\begin{array}{l}\text { Saponifi- } \\
\text { cation } \\
\text { number }\end{array}$ \\
\hline $\begin{array}{l}\text { Lard oil } \\
\text { Tallow } \\
\text { Neat's-foot } \\
\text { Fish } \\
\text { Sperm } \\
\text { Castor } \\
\text { Rapeseed } \\
\text { Soybean } \\
\text { Peanut } \\
\text { Cottonseed } \\
\text { Blown rapeseed } \\
\text { Blown cottonseed } \\
\text { Degras }\end{array}$ & $\begin{array}{l}192-198 \\
193-198 \\
193-204 \\
140-193 \\
120-140 \\
176-187 \\
170-179 \\
189-197 \\
186-197 \\
191-197 \\
195-216 \\
210-225 \\
110-210\end{array}$ \\
\hline
\end{tabular}

The above calculations and data presume a knowledge of the nature of the fat present. In an unknown sample, and particularly when a low saponification number leaves doubt as to whether fat is actually present, the fatty acid may be extracted (as the soap) and recovered for gravimetric determination. For this purpose the saponified and neutralized solution, obtained as above, shall be handled as in a grease analysis, following the second paragraph of section 19 of A. S. T. M. D128. (See 504.0 , p. 93.) The fatty acid is thus obtained and may then be used for subsequent identification tests.

\section{ACCURACY}

It may be expected that the same operator will find variations of \pm 0.3 saponification number on compounded oils containing less than 30 per cent compounding, \pm 0.5 saponification number on compounded oils containing over 30 per cent compounding, and \pm 1.0 saponification number for unmixed fatty oils. Corresponding permissible variations for different operators will be $\pm 0.5, \pm 1.0$, and \pm 2.0 saponification numbers, respectively. For dark oils, the errors may be double this magnitude.

American Society for Testing Materials, standard method of test for water in petroleum products and other bituminous materials, serial designation D95-28; 1928.

See 502.2 , page 38 .

American Society for Testing Materials, standard method of test for water and sediment in petroleum 
products, by means of centrifuge, serial designation D96-28; 1928.

See 502.2 , page 39 .

American Society for Testing Materials, standard method of analysis of grease, serial designation D128-27, 1927.

Approved as American Standard by the American Engineering Standards Committee, now the American Standards Association.

1. These methods of analysis permit determinations sufficiently accurate for referee purposes of all the constituents of greases likely to be covered by specifications. These constituents are fillers and ash, soap bases, soap, fat, water, excess alkali or acid, petroleum products, and unsaponifiable matter. No quantitative determination of glycerin is described, but a procedure is outlined for its qualitative detection.

\section{SAMPIE}

2. The size of sample for the soap determination (methods (1) and (2) for determination of fillers, soap, fat, petroleum oil, and unsaponifiable matter) shall be from about 8 to $30 \mathrm{~g}$, depending on the conconsistency of the grease, which is chiefly determined by the percentage of soap present. Ten to twenty grams is usually a convenient amount for No. 3 cup grease, while thin transmission and other greases require a larger sample. The original sample should be stirred or mixed until uniform.

3. Samples need not be weighed more closely than $0.1 \mathrm{~g}$.

\section{SOLVENTS}

4. The petroleum ether shall have an end point not higher than $200^{\circ} \mathrm{F}$., and $125 \mathrm{cc}$ of it shall not consume more than $0.2 \mathrm{cc}$ of $0.5 \mathrm{~N}$ potassium hydroxide when a blank test is made as follows:

One hundred and twenty-five cubic centimeters of petroleum ether shall be boiled with $10 \mathrm{cc}$ of $0.5 \mathrm{~N}$ alcoholic potassium hydroxide and $50 \mathrm{cc}$ of neutral 50 per cent alcohol for one and one-half hours on a hot plate, using a glass tube of about $7 \mathrm{~mm}$ internal diameter and $75 \mathrm{~cm}$ long as a reflux condenser. After cooling, the solutions shall be titrated with $0.5 N$ hydrochloric acid and phenolphthalein. Not less than $9.8 \mathrm{cc}$ of $0.5 \mathrm{~N}$ acid shall be required for neutralization. The amount of alkali consumed in this test shall be deducted as a blank correction in the fat determination on solution (E). ${ }^{42}$

5 . The alcohol shall be prepared from commercial 95 per cent grain or denatured grain alcohol by distilling from sodium hydroxide, diluting to 50 per cent (or 70 per cent) by volume with distilled water, and neutralizing exactly with sodium or potassium hydroxide and phenolphthalein.

6. The phenolphthalein solution shall be prepared by dissolving $1.0 \mathrm{~g}$ of phenolphthalein in $50 \mathrm{cc}$ of strong redistilled alcohol, adding $50 \mathrm{cc}$ of water, and neutralizing with sodium or potassium hydroxide.

7. Concentrated c. p. hydrochloric acid shall be used when "concentrated hydrochloric acid" is specified. Hydrochloric acid, 10 per cent, shall contain 10 per cent by weight of absolute $\mathrm{HCl}$, with a permissible variation of \pm 0.5 per cent.

\section{PROCEDURE}

\section{(A) DETERMINATION OF ASH}

Note.-The determination of the total ash should not in general be regarded as of any great importance. It is, however, sometimes required. This determination is often unsatisfactory on account of interaction between sodium carbonate derived from the soap and inorganic fillers. There is always likelihood of reaction with the porcelain crucible itself on account of the long-continued heating necessary to burn out all carbon. Moreover, if much sodium or

12 Solutions designated by capital letters in parentheses refer to the solutions given in Figure 48. potassium carbonate is present, the ash is fusible and often incloses carbon, making complete removal of the latter very difficult sults will always be low in the presence of easily reducible oxides of rolation volatile metals. There is also uncertainty as to when calcium car bonate has been completely ignited to calcium oxide. Ash determinations made on the same sample in different laboratories are likely separate determinations of the percentage of fllers and of soap, from which data a calculation is easily made giving all the information to which data a calculation is easily made giving all th
be gained from a direct determination of total ash.

8. The percentage of ash shall not be included in the total of the analysis.

\section{(1) Rapid Routine Method of Ash Determination}

9. From 2 to $5 \mathrm{~g}$ of grease shall be placed in a weighed porcelain crucible, and the sample weighed to the nearest $0.1 \mathrm{~g}$. If lead or zinc soap is known to be absent, a platinum crucible is more convenient. The combustible matter shall be burned off slowly and the residue finally ignited until the ash is free of carbonaceous matter. The crucible and contents shall then be cooled in a desiccator and weighed, reporting the result as percentage of ash.

\section{(2) Alternative Method of Ash Determination}

10. The sample shall be burned as in method (1) until the ash is nearly free from carbon. The crucible and contents shall then be cooled, the soluble portions dissolved in a little water, and a slight excess of dilute sulphuric acid added, running the acid in carefully from a pipette inserted under a small watch glass covering the crucible. The crucible and contents shall then be warmed on the steam bath until effervescence has ceased. The watch glass shall then be rinsed with water into the crucible. The solution when tested with methyl orange for free acidity shall show free acid present. The contents of the crucible shall be evaporated to dryness, and the whole ignited at a low red heat, adding a few small pieces of dry ammonium carbonate to drive off the excess of sulphur trioxide. After cooling and weighing, the result shall be reported as "percentage of ash as sulphates."

The alternative method gives more concordant results than the first method, but requires more time and manipulation.

\section{(B) QUALITATIVE EXAMINATION OF ASE}

11. An easily fusible ash, dissolving completely in water to give a strongly alkaline solution, indicates a grease containing sodium, potassium, or both. A white infusible ash, practically insoluble in water, but imparting to it an alkaline reaction, may indicate calcium, with or without magnesium or aluminum. Zinc is shown by the yellow color of the ash while hot, and lead may be indicated by the presence of metallic globules, or by the yellow color of the ash when cold.

12. The ash shall be dissolved in dilute nitric or hydrochloric acid, and the presence of the several bases confirmed by suitable chemical tests, any standard scheme of qualitative analysis bcing followed.

\section{(C) QUANTITATIVE EXAMINATION OF ASH}

13. For the quantitative examination of ash any standard analytical procedure shall be used, the choice of methods being based on the information gained from the qualitative tests. If only one base is present, a quantitative determination is in general unnecessary.

\section{(D) DETERMINATION OF FILLERS, SOAP, FAT, PETROLEDM OIL AND UNSAPONIFTABLE MATTER}

14. Cup, fiber, and sponge greases, with or without graphite, and all comparatively light-colored greases, including axle greases, shall be examined by 
method (1). Dark greases containing residuum, asphaltic oils and asphalt, tars, etc., shall be examined by method (2), as these ingredients usually cause stubborn emulsions if a shaking-out process is applied at the start.

\section{(1) Determination of Filler, Soap, Fat, Etc.}

15. If no fillers are present, sections 15 and 16 shall be passed over and the grease sample introduced directly from a weighing bottle into a sepa- leum ether, and the crucible finally washed with strong alcohol, collecting the alcohol washings separately and discarding them. The crucible and contents shall then be dried at $120^{\circ} \mathrm{C}$. and weighed, and the result reported as "percentage of insoluble matter" (graphite, mica, talc, asbestos, gypsum, wood pulp, etc.).

16. If gypsum is present as a filler, the results obtained in the determination described in section 15 will be too low becausc of the solubility of cal-

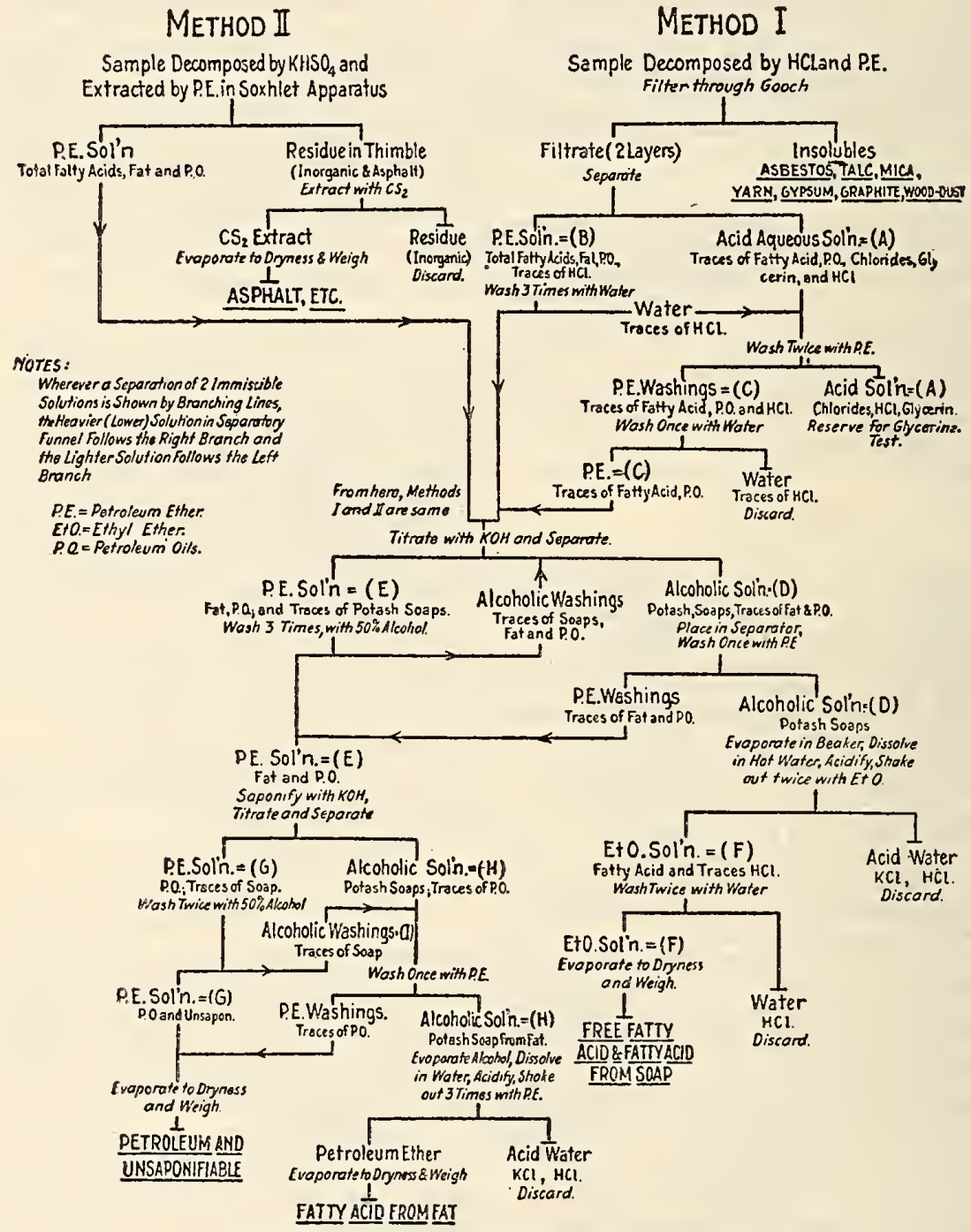

Ficure 48.-Scheme of grease analysis

rator, the weight of the sample being obtained by difference. (See sec. 2.) The sample shall then be shaken with $75 \mathrm{cc}$ of petroleum ether and $50 \mathrm{cc}$ of 10 per cent hydrochloric acid until the grease is completely decomposed, after which section 17 shall be followed. If fillers are present, the sample (see sec. 2) shall be weighed in a small beaker, 50 cc of 10 per cent hydrochloric acid added and the beaker warmed on the steam bath, stirring until all soap lumps have disappeared and the upper layer is clear. If undissolved mineral matter or other fillcr is present, both layers shall be filtered through a Gooch crucible provided with a suitable mat, the beaker and crucible washed with water and petro- cium sulphate in hydrochloric acid. A sample of about $5 \mathrm{~g}$ of grease shall be weighed in a small beaker, and decomposed with 50 cc of petroleum ether and $25 \mathrm{cc}$ of concentrated hydrochloric acid, warming on the steam bath until all gypsum is dissolved. The contents of the beaker shall be cooled and poured into a separatory funnel, the beaker rinsed with water and a little petroleum ether and the solutions in the funnel allowed to separate clearly. The lower layer shall then be drawn into a beaker, and the petroleum ether in the separator washed with two portions of $20 \mathrm{cc}$ each of 10 per cent hydrochloric acid, adding the washes to the strong acid solution in the separator. 
The hydrochloric acid solution in the beaker shall then be evaporated on the steam bath almost to dryness, diluted to $150 \mathrm{cc}$ with water, heated to boiling and treated with $10 \mathrm{cc}$ of 10 per cent barium chloride solution. The barium sulphate shall then be filtered off and ignited in the usual way, and from the weight of the barium sulphate, the percentage of calcium sulphate shall be calculated, reporting it as $\mathrm{CaSO}_{4} \cdot 2 \mathrm{H}_{2} \mathrm{O}$.

Note.-Chalk and other forms of alkaline earth carbonates are not determined by this method, but are considered under the determination of free alkalinity, section 24 .

17. The solution resulting from the decomposition of the filler-free grease or the combined filtrate and washes from the determination of fillers (sec. 15), except the alcohol, which is discarded, shall be placed in a separator, allowed to clear, and the aqueous layer (A), 43 which contains all the bases as well as glycerin, shall be drawn in another separator. The petroleum ether layer (b) shall be washed three times with $25 \mathrm{cc}$ of watcr to remove hydrochloric acid, adding the washings to (A). Solution (B) shall then be run into a 250-cc separator. The aqueous solution (A) shall then be washed twice with 20 cc of petroleum ether (C), whereupon (A) is set aside for examination for glycerin. (See sec. 18.) Solution (C) shall then be washed once with $15 \mathrm{cc}$ of water, which is rejected, and (C) shall be added to (B). If (B) and (C) are comparatively light colored, an approximate determination of free fatty acids and fatty acids from soap may now be made by titrating the solution in the separator with $0.5 \mathrm{~N}$ alcoholic potassium hydroxide and phenolphthalein, using 200 as the average neutralization value of the fatty acid (that is, $1.0 \mathrm{~g}$ of fatty acid requires $200 \mathrm{mg}$ of absolute KOH for neutralization). If solutions (B) and (C) are dark, a few drops of phenolphthalein and suffcient $0.5 \mathrm{~N}$. alcoholic potassium hydroxide shall be added to make the alcoholic layer distinctly alkaline after vigorous shaking. If the solutions have been titrated, a slight excess of alkali shall be added before separating. The conservative addition of strong alcohol at this point may aid in securing rapid and sharp separation. The two solutions shall be allowed to separate sharply and the lower alcoholic layer (D) drawn off into another separator. The upper petroleum ether layer (E) shall be washed three times, with 30,25 , and $20 \mathrm{cc}$ of neutral 50 per cent alcohol, respectively, adding these washes to (D). Solution (D) shall be washed with $25 \mathrm{cc}$ of petroleum ether, after which (D) is drawn off into a beaker, and the petroleum ether added to (E). Solution (D) shall then be evaporated to a small volume to remove alcohol, the residue of potassium soap washed into a separator with hot water, acidified with hydrochloric acid, and shaken out twice with 50 and 25 cc of ethyl ether $\left(F^{\prime}\right)$, respectively. Solution $(F)$ shall be run into another separator and washed twice with $20 \mathrm{cc}$ portions of water, which is discarded. Solution (F) shall then be transferred to a weighed beaker and evaporated to dryness on the steam bath, blowing with air to remove all traces of petroleum ether. The residue consisting of free fatty acid and fatty acid from soap shall be heated for a short time on a steam bath, adding and evaporating $5 \mathrm{cc}$ of absolute alcohol to remove the last traces of water, and weighed. The exact neutralization value shall then be determined on as large a sample of these fatty acids as possible. From the total fatty acid found here the free acid if any (see sec. 23 and 25) shall be deducted and the remainder calculated to percentage of soap, using the ash analysis as a guide

${ }^{43}$ Solutions designated by capital letters in parentheses refer to the solutions given in Figure 48 . in distributing the bases, if more than one are present.

The fatty acids may be identified to some ertent by special tests, such as odor, crystal form, melting point, iodine number, neutralization value, color reactions, etc.

If the grease is appreciably oxidized, the fatty acids obtained by the ethyl ether extraction are likely to be dark in color and hard to identify. For further study and identification, the neutralized acids may be extracted again (qualitatively) with petroleum ether and dilute $\mathrm{HCl}$, thus eliminating the small amount of more darkly colored matter.

18. In case it is desired to determine whether a grease has been made from whole fats or from fatty acids, solution (A) shall be neutralized with dry sodium carbonate and sufficient excess added to precipitate calcium or other metals. The whole mass shall then be evaporated to dryness, the residue extracted several times with strong alcohol, the combined alcoholic extracts filtered, and the alcohol evaporated. The residue will then contain most of the glycerin, with a little sodium chloride. The presence or absence of glycerin in the residue shall be confirmed by suitable qualitative tests.

Since fats on saponification yield between 10 and 11 per cent of glycerin, and this analytical scheme does not include a quantitative determination of glycerin, the total percentages in greases made from fats should always be less than 100.44

19. The petroleum ether solution (E) (sec. 17), containing free fat, petroleum oils, and unsaponifiable matter shall be concentrated to a volume of about $125 \mathrm{cc}$ in a 300-cc Erlenmeyer flask, $10 \mathrm{cc}$ of $0.5 N$ alcoholic potassium hydroxide and $50 \mathrm{cc}$ of strong neutral alcohol added, and the whole boiled on a hot plate with an air condenser for one and onehalf hours. The uncombined alkali shall be titrated with $0.5 \mathrm{~N}$ hydrochloric acid, and from the alkali consumed, corrected for the blank determination as specified in section 4 , the percentage of free fat shall be calculated, using 195 as the average saponification value (that is, $1.0 \mathrm{~g}$ of fat requires $195 \mathrm{mg}$ of absolute IKOH for saponification).

The titrated solution (G) shall be placed in a scparator, the alcoholic lower layer (H) drawn off into another separator, and the remaining traces of soap removed as described for (B) and (C), section 17 , making only two washes with 30 and $20 \mathrm{cc}$ of 50 per cent alcohol (I). Solutions (H) and (I) shall be combined, washed once with a little petroleum ether, which is added to solution $(G)$, and solutions (H) and (I) then evaporated to a small volume and the fatty acid isolated as described for (D), section 17. The percentage of fat may be checked by weighing and titrating the free fatty acid. .5

20 . The petroleum ether solution (G), now containing all the hydrocarbon oils and unsaponifiable matter shall be evaporated to dryness in a weighed beaker as described for (F), section 17 , the residue weighed, and the result reported as "petroleum oils plus unsaponifiable matter."

An approximate determination of the viscosity of the petroleum oil may be made by using a 2 to $5 \mathrm{cc}$ pipette, which has been standardized against oils of known viscosities. If a complete characterization of the petroleum products is required, a new sample of from 150 to $200 \mathrm{~g}$ of grease shall be decomposed as described above, except that all quantitative operations shall be omitted, as well as the isolation of the

1 A close approximation to the amount of glycerin present may be calculated and included in the analysis by taking 11.0 per cent of the weight of the fatty acids from soaps, providing, of course, that the grease was made from neutral fats.

${ }_{45}$ Multiplying the weight of fatty acid by 1.045 gives a very close approximation of the weight of fat from which it was derived. This factor varies very little with the molecular weight of the fat. 
free fatty acids, and the use of standard alkali and acid is not necessary.

If the grease contains rosin oil, beeswax, de gras, spermaceti, spermatin, montan wax, candle pitch, and other materials containing a large amount of unsaponifiable substances, the petroleum oils isolated from solution (G) will contain the unsaponifiable matter, and the constants found will differ from those of the petroleum products used in making the grease. In most cases, no further separation is possible except in the hands of skillful and experienced operators who can devise special methods to suit the individual conditions.

(2) Determination of Filler, Soap, Fat, Etc.

21. The sample shall be weighed in a 3 -inch porcelain dish, $10 \mathrm{~g}$ of granulated acid potassium sulphate added and $10 \mathrm{~g}$ of clean, dry ignited sand. The dish and contents shall be heated on the steam bath with frequent stirring until all water is driven off, two hours usually being sufficient. After cooling and breaking up lumps with a small pestle, the mixture shall be transferred quantitatively to an extraction thimble, using a little petroleum ether to wash the last traces into the thimble, which should be already placed in a soxhlet apparatus. The thimble shall be extracted thoroughly with petroleum ether, the extract concentrated somewhat if necessary, and the free fatty acid and fatty acid from soap titrated with $0.5 \mathrm{~N}$ alcoholic potassium hydroxide as in solutions (B) and (C), in method (1), section 17. From this point on, the procedure is identical with that given in $\operatorname{method}(1)$, sections 17,19 , and 20 .

22. The thimble shall be extracted a second time with carbon disulphide, the extract evaporated to dryness, heated for one hour to $120^{\circ} \mathrm{C}$., and weighed, the results being reported as asphaltic and tarry matter. The residue in the thimble shall be discarded.

\section{(E) DETERMINATION OF FREE ALKAII AND FREE ACID}

23. From 10 to $30 \mathrm{~g}$ of grease shall be weighed in a small beaker, dissolved as completely as possible in $75 \mathrm{cc}$ of petroleum ether by stirring with a spatula, the mixture washed into a 250-cc Erlenmeyer flask with a small amount of petroleum ether, and the beaker rinsed with $50 \mathrm{cc}$ of 50 per cent alcohol, the alcohol being poured into the flask, after which a few drops of phenolphthalein solution shall be added and the whole shaken vigorously. If the alcoholic layer, after settling for a few seconds, is pink, $10 \mathrm{cc}$ of $0.5 \mathrm{~N}$ hydrochloric acid shall be added, the solutions boiled on a hot plate for 10 minutes to expel carbon dioxide, and the excess acid titrated back with $0.5 \mathrm{~N}$ alcoholic potassium hydroxide. The free alkalinity shall be calculated in terms of hydroxide of the predominating base.

24 . If chalk or any other form of alkaline earth carbonate, or lead carbonate, is present as a filler, it will be detected by effervescence on adding the hydrochloric acid. As the amount of such carbonates is likely to be considerable, the volume of $0.5 \mathrm{~N}$ hydrochloric acid added shall be increased sufficiently to dissolve all carbonate and leave a slight excess of acid. The solution shall be boiled for two minutes and the excess acid titrated back with $0.5 \mathrm{~N}$ alcoholic potassium hydroxide, and from the acid consumed, its equivalent in calcium carbonate, etc., shall be calculated, disregarding any other forms of alkalinity which may have been present.

25. If the original alcoholic layer is not pink, the solution shall be titrated carefully in the cold with $0.5 N$ alcoholic potassium hydroxide, shaking well after each addition. The acidity shall be calculated as oleic acid.
26. If soaps of iron, zinc, aluminum, or other weak bases are present, a determination of free acid is not possible, since these metallic soaps react with potassium hydroxide. Up to the present, no means has been devised whereby this determination can be made directly.

\section{(F) DETERMINATION OF WATER}

27. The quantity of water shall be determined in accoldance with A. S. T. M. D95. (See 502.2, p. 38.)

American Society for Testing Materials, tentative method of test for color of lubricating oils by means of union colorimeter, serial designation D155-23T, 1923.

\section{APPARATUS}

1. The color of all lubricating oils shall be determined by means of the union colorimeter.

2. (a) The union colorimeter (fig. 49) shall consist of a box, $a$, glass color standards, a glass jar, a light shield, $b$, and a daylight lamp, $c$.

(b) The box, $a$, (fig. 49), shall have an interior finish of dull black, and may be constructed of metal or wood, approximately 18 inches in length and $11 / 2$ inches in depth in inside dimensions, the observation end being $2 \frac{1}{8}$ inches in width and the end next to the source of light 3 inches in width.

A transverse vertical partition having a circular hole in the end $1 / 2$ inch in diameter shall be placed $1 \frac{1}{8}$ inches from the observation end of the box. In the opposite end shall be mounted a rectangular piece of pure white opal glass, approximately $3 \frac{1}{4}$ by $13 / 4$ by $1 / 16$ inch, which shall be ground or etched on the inner side to diffuse light rays. Approximately $2 \frac{1}{8}$ inches from the light end of the box shall be a second vertical partition 1 inch in thickness having two openings about $3 / 4$ inch in diameter at lightsource side and about $\% 16$ inch in diameter on the opposite side, symmetrically located in regard to the vision line. In case the standards are not mounted, a vertical partition shall be placed approximately $33_{4}^{\prime}$ inches from the light-source end, having a thickness of $1 / 8$ inch, and shall have two circular openings $9 / 16$ inch in diameter and $1 \frac{1}{2}$ inches apart. Extending from the opal glass to this partition along the horizontal axis of the box shall be a vertical partition approximately $1 / 8$ inch in thickness for a distance of $15 / 8$ inches, and then continuing with a thickness of $7 / 8$ inch until abutting against the transverse partition which in conjunction serves to divide the light end of the box into two compartments. In the top of the box, immediately over the centers of these two compartments, there shall be circular holes of such a size to accommodate the standard jar and 4-ounce oil sample bottles. In the top of the box, directly above the $3 / 4$-inch opening in the left side of the second partition, there shall be an opening or slot suitable for placing the glass color standards in a vertical position in the opening in the partition. In case the color standards are not mounted in a magazine, this opening shall be provided with a sliding cover so that all light may be excluded. In case all the glass standards are mounted in a magazine, suitable arrangements shall be made for this magazine to be raised or lowered through the opening provided for the glass standards, and when such a magazine is used a hole may be located in the left side of the box through which the numbers of the mounted glass standards may be read. For convenience, numbers may be stamped on the magazine as mirror images, and a swinging mirror may be provided on the side of the box so that the operator may read the numbers on the magazine from the front of the instrument.

(c) The glass color standards shall be 15 in number as specified in the table. These glasses may be kept separately or for convenience may be mounted in a 
magazine. If so mounted, there shall be a blank opening provided in the magazine so that two bottiles of oil may be compared in the colorimeter as well as a sample of oil and the glass color standards. The glass color standards shall be not less than fiveeighths inch across.

(d) The standard glass jar (fig. 50) shall be a true cylinder of clear colorless glass. The internal diameter shall be not less than $32.5 \mathrm{~mm}$ nor more than $33.5 \mathrm{~mm}$. The thickness of the wall shall be (e) The light proof cover, $b$, shall be of any suitable material. It shall be dull black on the inside and of such size as to completely shield all light from the tops of the cylinders, or oil sample bottles, when they are in place in the colorimeter.

(f) The artificial daylight lamp, $c$ (fig. 49), may for convenience be fastened to the base supporting the box. It shall consist of a 50 -watt $\mathrm{C} 2$ national Mazda lamp, fitting into a socket on a swivel fitted with a parabolic metal reflector, The inside of this

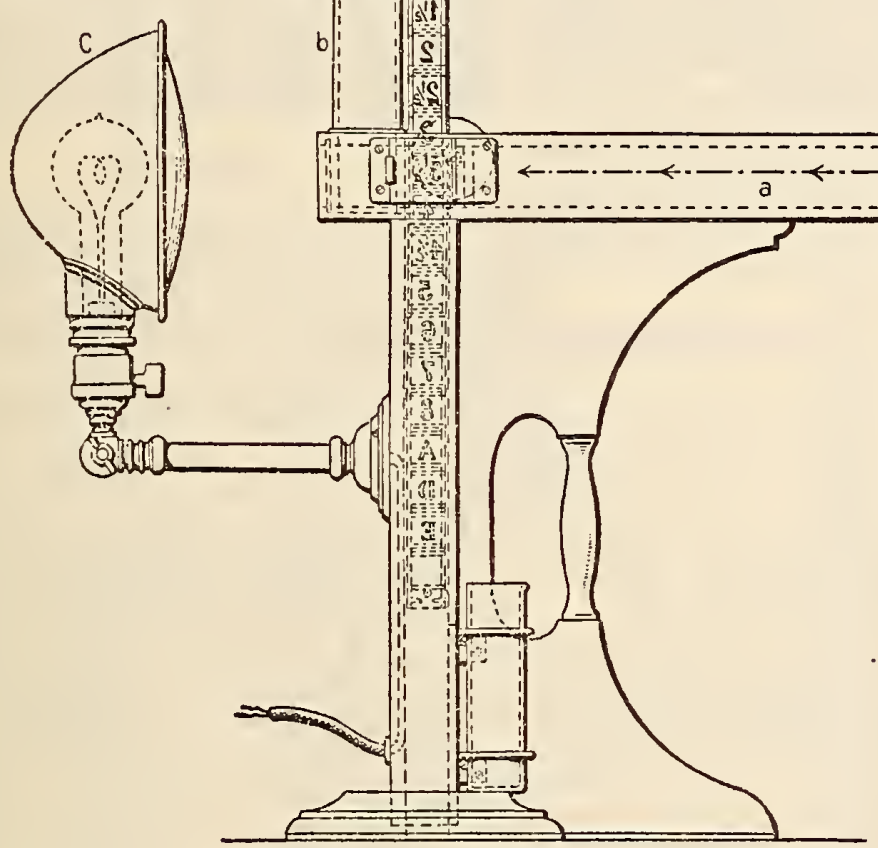

Figure 49.-Union colorimeter

$1.75 \mathrm{~mm} \pm 0.25 \mathrm{~mm}$. The height of the cylinder shall be 5 inches $\pm 1 / 4$ inch.

Glass color standards of union colorimeter

\begin{tabular}{|c|c|c|c|c|c|c|}
\hline \multirow[b]{2}{*}{$\begin{array}{l}\text { A.S.T.M. M. } \\
\text { color } \\
\text { numbers }\end{array}$} & \multirow{2}{*}{$\begin{array}{l}\text { Un- } \\
\text { ion } \\
\text { Petro- } \\
\text { leum } \\
\text { Co. } \\
\text { des- } \\
\text { igna- } \\
\text { tions }\end{array}$} & \multirow[b]{2}{*}{$\begin{array}{l}\text { National Petro- } \\
\text { leum Association } \\
\text { color numbers } \\
\text { (1915) }\end{array}$} & \multirow[b]{2}{*}{$\begin{array}{l}\text { National } \\
\text { Petroleum } \\
\text { Association } \\
\text { names }\end{array}$} & \multicolumn{3}{|c|}{$\begin{array}{l}\text { Lovibond } \\
\text { analysis }\end{array}$} \\
\hline & & & & 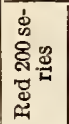 & $\mid \begin{array}{l}0 \\
0 \\
0 \\
0 \\
0 \\
0 \\
0 \\
0\end{array}$ & 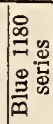 \\
\hline & $\underset{\mathrm{J}}{\mathrm{H}}$ & $\begin{array}{l}\text { No.1 N.P. A } \\
\text { No. } 11 / 2 \text { N.P. } \\
\text { No. } 2 \text { N. P. } \\
\text { No. } 21 / 2 \text { N. P. }\end{array}$ & $\begin{array}{l}\text { Lily White.- } \\
\text { Cream white } \\
\text { Extra pale... } \\
\text { Extra lemon } \\
\text { pale. }\end{array}$ & $\begin{array}{l}0.12 \\
.60 \\
2.5 \\
4.6\end{array}$ & $\begin{array}{r}2.4 \\
8.0 \\
26.0 \\
27.0\end{array}$ & . \\
\hline & $\frac{\mathrm{K}}{\mathrm{L}}$ & No. 3 N. P. A & $\begin{array}{l}\text { Lemon pale } \\
\text { Extra orange }\end{array}$ & $\begin{array}{l}6.9 \\
9.4\end{array}$ & $\begin{array}{l}32.0 \\
15.0\end{array}$ & \\
\hline & $\underset{\mathrm{N}}{\mathrm{M}}$ & $\begin{array}{l}\text { No. } 4 \text { N. P. A } \\
\text { No. } 41 / 2 \text { N. P. }\end{array}$ & $\begin{array}{l}\text { Orange pale. } \\
\text { Pale. }\end{array}$ & $\begin{array}{l}14.0 \\
21.0\end{array}$ & $\begin{array}{l}50.0 \\
56.0\end{array}$ & 0.55 \\
\hline & $\begin{array}{l}\mathbf{O} \\
\mathbf{P} \\
\mathrm{Q} \\
\mathrm{R}\end{array}$ & $\begin{array}{l}\text { No. } 5 \text { N. P. } \\
\text { No. } 6 \text { N. P. } \\
\text { Q. } \\
(2)\end{array}$ & $\begin{array}{l}\text { Light red...- } \\
\text { Dark red-.-- } \\
\text { Claret red.-- }\end{array}$ & $\begin{array}{r}35.0 \\
60.0 \\
60.0 \\
166.0\end{array}$ & $\begin{array}{r}93.0 \\
60.0 \\
106.0 \\
64.0\end{array}$ & 0 \\
\hline & $\begin{array}{l}\mathrm{A} \\
\mathrm{D} \\
\mathbf{E}\end{array}$ & $\begin{array}{l}\text { Cylinder oil, extra } \\
\text { Cylinder oil, ligh } \\
\text { Cylinder oil, med }\end{array}$ & $\begin{array}{l}\text { light filtered } \\
\text { filtered....- } \\
\text { um filtered. }\end{array}$ & $\begin{array}{l}10.2 \\
21.0 \\
89.0\end{array}$ & $\begin{array}{l}29.0 \\
31.0 \\
56.0\end{array}$ & \\
\hline
\end{tabular}

1 N. P. A. color of 1915 changed slightly to make $31 / 2$ more nearly midway between 3 and 4 .

A new color. reflector shall be brightly silvered at all times with bright aluminum paint or brightly polished silver or nickel. In the front of the reflector shall be mounted a dished, but not lenticular, glass-color sereen of such composition and color as, in conjunction with the C2 Mazda lamp, will yield by spectrum analysis a light elosely approximating northern daylight. When in normal position, the color screen shall be 4 inches from the white opal glass.

In case electric current is not available, the coiorimeter may be used by exposing the opal glass to northern daylight with no colored objects in the immediate foreground, but the artificial daylight lamp shall be required in referee work.

\section{PROCEDURE}

3. The oil, when examined, shall be at room temperature unless its cloud point is above the temperature of the room, in which case the oil shall be heated to a temperature of not more than $10^{\circ} \mathrm{F}$. above its cloud point. The oil to be examined may be observed in the ordinary 4-ounce sample bottle, but in referee work a standard jar shall be used. The jar shall be placed in the right-hand compartment while in the other compartment shall be placed a 4-ounce sample bottle of distilled water. The jar and bottle shall be covered with the shield, and the artificial day-light lamp lighted. One at a time, the glass color standards shall be placed between the 
bottle of distilled water and the eye, in the slot provided for them, until the nearest mateh possible is obtained. In case the standards are not mounted in a magazine, the slide on the top of the box shall be pressed against the glass standard to keep out light while making an observation.

4. Filtered cylinder oils and other oils that are darker than No. 8 color (see the table) shall be diluted with water-white kerosene, the color of which is not darker than No. 21 as determined in A. S. T. M. D156-23T (see 503.0, p. 54), a mixture of exactly 15 per cent by volume of oil and 85 per cent by volume of kerosene being used in all cases. This mixture shall then be compared with the three cylinder oil stanuards, or optionally with the standards ranging from Nos. 1 to 8 , inclusive. The daylight lamp may be brought closer to the opal glass in determining the darker colors.

The numbers or letters, designating the color of all oils that have been diluted to make the determination, shall be followed by the designation "Dil."

American Society for Testing Materials, standard

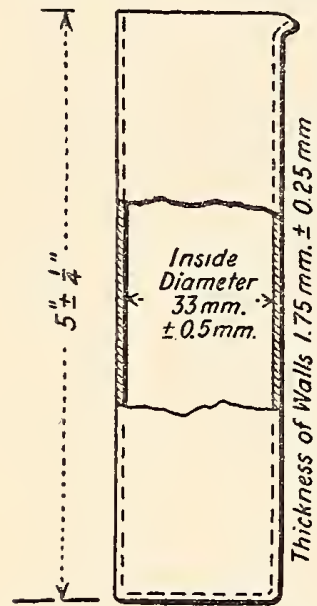

Figure 50.-Standard glass jar for use with union colorimeter method of test for steam emulsion of lubricating oils, serial designation D15728, 1928.

Approved by the American Standards Association.

1. This method shall be used on all oils when an emulsion, demulsibility, or emulsification test is required. This method is commonly used for turbine oils and may be used for other lubricating oils.

NoTE.-Methods for sampling are covered in A. S. T. M. D270 $27 \mathrm{~T}$. (See $503.0 \mathrm{p} .60$.) The following paragraphs, which are particularly important for protection of samples for the steam-emulsion test, are quoted from these meth ods:

" 34 (a) Corks for bottles shall be clean, free from holes and loose bits of cork.

"(b) Sealing wax, or paraffin, shall not be ,poured over corks aiter cooling,"

2. The steam emulsion number (A. S. T. M. S. E.) is the number of seconds required for an oil to separate when emulsified and separated under definitely prescribed conditions.

\section{APPARATUS}

3 . The steam generator shall be made of either metal or glass of at least 1-liter capacity, capable of withstanding the heat necessary for continued use in the production of steam. It shall be fitted with three outlets with suitable connections for rubber tubing. In case of a metal generator, a large opening for filling and a suitable water gauge shall be a necessary part of the apparatus.

4. The baths shall be of glass, with a capacity of 3 to $3 \frac{1}{2}$ liters and $a$ depth of $7 \frac{1}{2}$ to 9 inches. A good quality battery jar or beaker is entirely satisfactory.

5. Heat for the steam generator shall be supplied by a suitable gas burner or electric hot plate. The separating bath may be heated by any convenient means, including an auxiliary steam line as shown in Figure 51.

6 . The oil container shall be a 25 by $200 \mathrm{~mm}$ test tube graduated from zero or from 10 to $50 \mathrm{cc}$ in cubic centimeters, each even 5 -cc line to encircle the tube.
7. The steam piping or the steam delivery tube shall consist of a piece of thin-wall glass tubing, not less than 2.3 nor more than $2.7 \mathrm{~mm}$ in inside diameter and 12 inches in length. The steam pipe shall be cut off diagonally at an angle of $30^{\circ}$ with the axis of the tube at the discharge orifice and shall be bent at right angles 10 inches from the discharge orifice.

8. Accessories shall consist of :

(a) Suitable wooden or metal frames or holders for holding all containers in a vertical position in the baths.

(b) Thermometers for the separating and emulsifying baths (floating type thermometers of suitable range).

(c) Thermometers for the oil container tube (engraved-stem type, of suitable range, graduated in $1^{\circ} \mathrm{F}$., 5 to $7 \mathrm{~mm}$ in diameter).

(d) Corks, rubber tubing, and serew pincheocks.

\section{PROCEDURE}

9. The apparatus shall be assembled as shown in Figure 51. The steam generator shall be filled onehalf full of water and heat applied. The baths shall be filled with 3 liters \pm 60 cc of water. The temperature in the separating bath shall be raised to and maintained at $200^{\circ}$ to $203^{\circ} \mathrm{F}$.

NoтE.-Care must be taken if glass battery jars are used, as direct heating by flame or electric heater may cause breakage.

The temperature of the emulsifying bath shall be brought to not less than $67^{\circ} \mathrm{F}$. nor more than $78^{\circ} \mathrm{F}$. at the start of the test, and is not controlled thereafter. Twenty cubic centimeters of the oil to be tested shall be measured in the oil container at room temperature and the latter placed in the holder of the emulsifying bath. The steam pipe, or delivery tube, shall be connected to the steam generator with suitable rubber tubing, and screw pincheocks placed as shown in Figure 51 .

Care shall be taken to see that the apparatus, particularly the oil container, oil container thermometer, and the steam delivery tube are chemically clean before using. Care shall also be taken to prevent any foreign materials from entering the steam generator as any contamination of the steam renders the test valueless.

10. The steam delivery tube line shall be steamed out until all condensation disappears. A cork having two openings, with the thermometer in one, shall be placed in the mouth of the oil container. The thermometer shall be adjusted so that the bottom of the bulb is $3 / 4$ to 1 inch from the bottom of the oil container. The steam delivery tube shall be inserted through the second opening in the cork (this fitting shall be loose) so that the end of the steam delivery tube shall touch the center of the bottom of the oil container. Steam shall be admitted at a rate that will maintain the temperature of the oil, as shown on the thermometer in the oil container, between $190^{\circ}$ and $195^{\circ} \mathrm{F}$.

Nore -. The usual time necessary for the temperature of the oil to come to this point is 45 to 75 seconds, depending on its character.

This control shall be effected by manipulation of the pinchcocks on the steam delivery line and steam exhaust line from the steam generator. The steam supply shall be sufficient at all times to cause a generous discharge from the exhaust line. Steaming shall be continued until the volume of condensed steam and oil in the oil container tube is $40 \mathrm{cc}+3$ cc. The time required for this operation shall be 4 to 6.5 minutes, depending on the quality of the oil, altitude, etc. If condensed water amounts to $20 \mathrm{cc}$ in less than four minutes, it shall be taken as 
an indication of net steam or incomplete steaming out of the line and the test shall be rerun.

Nore.-The apparent rolume in the tube near the end of the steaming operation is approximately 12 to $15 \mathrm{cc}$ greater than the actual rolume due to displacement caused by steam thermometer and steam delivery tube.

11. The steam delivery tube shall be withdrawn as soon as the required volume is obtained. The oil container shall be transferred immediately to the separating bath which shall be maintained at $200^{\circ}$ to $203^{\circ} \mathrm{F}$. It is extremely important that the temperature of the separating bath be maintained within the given limits. A stop watch shall be started at the instant of withdrawal of the steam delivery tube. The cork containing the thermometer shall be removed after the oil container and contents have been placed in the separating bath. The contents of the oil container shall be examined directly proportional to the resistance to emulsification of the tested oil.

Nore.-The expansion of the oil at the higher temperature is disregarded, so when the separation of the oil is complete as regards the interface between the oil and emulsion layers, the volume of separated oil will always be more than $20 \mathrm{cc}$. This in no way interferes with the reproducibility of results, and the time shall be recorded when 20 cc.- and not necessarily all - of the oil have separated, when this occurs in less than 20 minutes.

13. Results shall be reported, to the nearest second, of the time necessary for 20 cc oil separation. The time in seconds shall be reported as the $\mathrm{S}$. $\mathrm{E}$. number A. S. T. M. method. In cases where the required volume shall not have separated in 20 minutes, the oil shall be reported as having an $\mathrm{S}$. $\mathrm{E}$. number, A. S. T. M. method, of 1,200 plus. If a duplicate determination varies by more than 10 per cent, a third test shall be made and the average of the three tests reported.

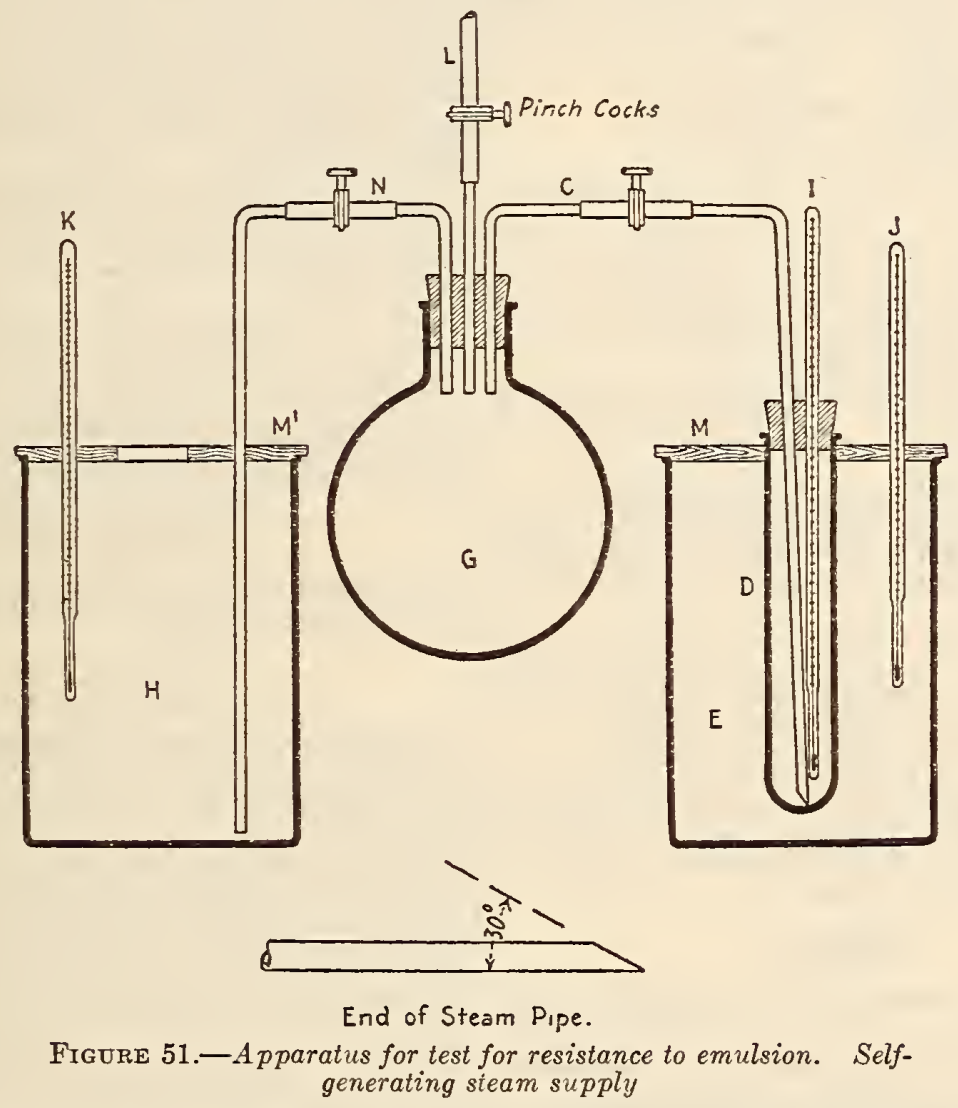

at least every 30 seconds, and the volume of the separated oil layer shall be recorded. When this reading operation has continued until $20 \mathrm{cc}$ of the oil have separated, the elapsed time in seconds shall be reported as the steam emulsion number (S. E. No.). No differentiation shall be made between clear and turbid oil. This examination shall be made without withdrawing the oil container and its contents from the separating bath and reading the volumes.

In cases where the interlace between more or less clear oil and emulsion is not a clear, straight, horizontal line, the position of such a line should be carefully estimated to the nearest $0.5 \mathrm{cc}$.

If 20 cc of oil do not separate, the test shall be discontinued.

12. In interpreting results, the assumption shall be made that the rate of emulsion separation is
NotE.-Oils which emulsify separate into three layers without exception: Top, clear or turbid oil; middle, lacy or creamy emulsion; bottom, clear or milky water. The $\mathrm{S}$. E. number is derived from the top layer.

American Society for Testing Materials, tentative method of test for neutralization number of petroleum products and lubricants, serial designation D188-27T, 1927.

Approved as tentative American Standard by the American Engineering Standards Committee, now the American Standard Association.

1. The methods described herein are designed to indicate in petroleum products and lubricants and compounded products the presence of organic constituents having acid characteristics and the contamination by alkalies and mineral acids.

2. (a) The neutralization number is the weight in milligrams of potassium hydroxide required to neutralize $1 \mathrm{~g}$ of oil. 
NoтE.-The neutralization number expresses the total amount of the mineral acid, and of the organic constituents having acid characteristics.

(b) The alkali neutralization number is defined as the weight of acid required to neutralize $1 \mathrm{~g}$ of oil, expressed in equivalent milligrams of potassium hydroxide.

(c) The mineral acid neutralization number is the number of milligrams of potassium hydroxide required to neutralize the mineral acid content in 1 $\mathrm{g}$ of oil.

\section{SOLUTIONS REQUIRED}

3. (a) Aqueous potassium hydroxide ( 1 cc $=5 \mathrm{mg}$ $K O H$ ).-Dissolve $5.1 \mathrm{~g}$ of potassium hydroxide, c. p., in 1 liter of freshly boiled and cooled distilled water. Add a very small amount of barium hydroxide, sufficient to precipitate any potassium carbonate present. Standardize this solution against National Bureau of Standards certified benzoic acid, using phenolphthalein as an indicator according to the relation:

$$
\begin{gathered}
\frac{5 \mathrm{mg} \mathrm{KOH}}{x \mathrm{mg} \text { benzoic } \mathrm{acid}}=\frac{56.104 \mathrm{~g} \mathrm{KOH}}{122.048 \mathrm{~g} \text { benzoic acid }} \\
1 \mathrm{cc} \text { of } \mathrm{KOH}=10.88 \mathrm{mg} \text { benzoic acid }
\end{gathered}
$$

This weight of benzoic acid is required for standardization.

Make necessary adjustments so that the value of potassium hydroxide equals $5 \mathrm{mg} \mathrm{KOH}$ per 1 cc.

Nore.-Fit the solution bottle with a guard tube of soda lime to prevent access of carbon dioxide. The solution should be standardizod at necessary intervals.

The weight of benzoic acid should be dissolved in 50 ce of 95 per cent alcohol and titrated cold. For blank, use same amount of alcohol and correct the titration.

(b) Sulphuric acid solution ( $1 \mathrm{cc}=5 \mathrm{mg} \mathrm{KOH}$ ).Match a sulphuric acid solution so that $1 \mathrm{cc}$ of the acid will be required to neutralize $1 \mathrm{cc}$ of the $\mathrm{KOH}$, using phenolphthalein as an indicator, titrating in boiling solution.

(c) Neutralized 95 per cent alcohol.-Add a few drops of phenolphthalein and neutralize carefully the alcohol to a very faint pink end point with some of the above prepared alkali solution.

(d) Phenolphthalein indicator.-Dissolve $10 \mathrm{~g}$ of the indicator in 1 liter of 95 per cent alcohol, preferably ethyl. Use 1 cc of this strength for titration.

(e) Methyl orange indicator.-Dissolve $1 \mathrm{~g}$ of the indicator in 1 liter of distilled water. Use $0.1 \mathrm{cc}$ of this strength for titration.

\section{METHODS}

4. Method A.-Neutralization number of petroleum products.

Weight of oil: Approximately $20 \mathrm{~g}$ weighed to $0.1 \mathrm{~g}$.

Volume of solvent: 100 cc of a mixture of $1: 1$ neutralized alcohol and distilled water.

Procedure: Agitate oil and solvent thoroughly and heat to boiling. Add 1 cc of phenolphthalein indicator and titrate rapidly, with vigorous agitation, to a sharp pink end point. The titration must be completed in a hot solution, reheating same if found necessary.

The color change is noted in the alcohol water layer.

Calculation:

$\frac{\text { (Cubic centimeters of } \mathrm{KOH}) \times 5}{\text { Weight of oil taken }}=\mathrm{mg} \mathrm{KOH}$ per $1 \mathrm{~g}$ of oil.

5. Method B.-Neutralization number of compounded Petroleum Products. $0.1 \mathrm{~g}$.

Weight of oil: Approximately $10 \mathrm{~g}$ weighed to Volume of solvent; $50 \mathrm{cc}$ of neutralized alcohol.
Procedure: Agitate oil and solvent thoroughly and heat to boiling. Add 1 cc of phenolphthalein indicator and titrate rapidly, with vigorous agitation, to a sharp pink end point. The titration must be completed in a hot solution, reheating same if found necessary.

The color change is noted in the alcohol layer.

6. Method C.46.-Alkali or mineral acid neutralization number in noncompounded and compounded petroleum products.

Weight of oil: 25 to $50 \mathrm{~g}$ weighed to $0.1 \mathrm{~g}$.

Volume of solvent: Boiling distilled water, $200 \mathrm{cc}$.

Procedure: Introduce into a 250-ce separatory funnel 25 to $50 \mathrm{~g}$ of oil weighed to $0.1 \mathrm{~g}$ and add 100 cc of boiling distilled water. Shake vigorously, and after oil and water have separated, drain the water layer into a 500-cc casserole. Wash the oil in the separatory funnel twice by vigorously shaking with 50-ce portions of boiling distilled water, and after separation, drain into the casserole. To the accumulated 200 cc of water add one drop of 1 per cent phenolphthalein solution. Boil. If solution turns pink add 1 cc of the indicator and titrate with the sulphuric acid solution until extraction is colorless.

If the addition of one drop of phenolphthalein causes no change in color, add $0.1 \mathrm{cc}$ of methyl orange. If color changes to red or pink, titrate with the $\mathrm{KOH}$ solution until the red color is just discharged.

An equal volume of distilled water shall be titrated with the standard solution used, and the amount of alkali or acid required shall be subtracted from the titration for blank correction.

Calculation: If the acid solution was required to discharge the phenolphthalein end point, the alkali neutralization number equals:

\section{(Cubic centimeters of acid) $\times 5$}

If the alkali solution was required to discharge the methyl orange end point, the mineral acid neutralization number equals:

\section{(Cubic centimeters of alkali) $\times 5$ \\ Weight of oil}

American Society for Testing Materials, standard method of test for carbon residue of petroleum products (Conradson carbon residue) serial designation D189-28; 1928.

1. This method of test is a means of determining the amount of carbon residue left on evaporating an oil under specified conditions, and is intended to throw some light on the relative carbon-forming propensity of an oil. The results of the test must be considered in connection with other tests and the use for which the oil is intended. This test furnishes pertinent information relative to lubricants for internal-combustion engines, domestic oil fuels, and oils used in the manufacture of gas.

\section{APPARATUS}

2. The apparatus (see fig. 52) shall consist of the following:

(a) Porcelain crucible, wide form, glazed throughout, or a silica crucible, 25 to $26 \mathrm{ml}(0.8 \mathrm{oz})$ capacity, $46 \mathrm{~mm}$ (17/8 inches) in approximate rim diameter.

(b) Skidmore iron crucible, flanged and rimmed, 60 to $80 \mathrm{ml}$ approximate capacity, $55 \mathrm{~mm}\left(2 \frac{1}{5}\right.$ inches) inside, and $65 \mathrm{~mm}$ ( $25 / 8$ inches) outside diameter at the top, 37 to $39 \mathrm{~mm}$ (about $11 / 2$ to $1 \frac{5}{8}$ inches) in height, supplied with a cover without delivery tubes and having the vertical opening closed. The horizontal opening shall be kept clean.

16 When the oil is acid, the acidity shall not be reported as sulphuric or hydrochloric acid unless the presence of these acids shall have been 
(c) Spun sheet-iron crucible, with cover, about $200 \mathrm{ml}$ ( 7 ounces) capacity, $80 \mathrm{~mm}$ (31/8 inches) in outside diameter at the top, 58 to $60 \mathrm{~mm}$ (about 23/8 inches) in height, approximately $0.8 \mathrm{~mm}$ (1/32 inch) in thickness and weighing, without cover, 70 to $75 \mathrm{~g}$ (about 21/2 ounces). Place at the bottom of this crucible, and level before each test, a layer of about $25 \mathrm{ml}$ of dry sand, or enough to bring the Skidmore crucible with cover on, nearly to the top of the sheet-iron crucible.

(d) Triangle of bare nichrome wire of approximately No. 13 B. \& S. gage, having an opening small enough to support the bottom of the sheetiron crucible at the same level as the bottom of the asbestos block or hollow sheet-metal box, paragraph $(f)$.

(e) Circular sheet-iron hood from 120 to $130 \mathrm{~mm}$ ( $4^{3 / 4}$ to $5 \frac{1}{4}$ inches) in diameter, the height of the lower perpendicular side to be from 50 to $53 \mathrm{~mm}$ ( 2 to $2 \frac{1}{8}$ inches); provided at the top with a chimney 50 to $60 \mathrm{~mm}$ ( 2 to $2 \frac{1}{2}$ inches) in height and from 50 to $56 \mathrm{~mm}$ ( 2 to $2 \frac{1}{4}$ inches) inside diameter, which is attached to the lower part having the perpendicular sides by a cone-shaped member, bringing the total height of the complete hood from 125 to $130 \mathrm{~mm}$ (51/8 inches). The hood may be made from a single piece of metal provided the foregoing dimensions are adhered to. As a guide for the height of the flame above the chimney, a bridge made of approximately $3 \mathrm{~mm}$ (1's inch) iron or nichrome wire, $h$, shall be attached having a height of $50 \mathrm{~mm}$ (2 inches) above the top of the chimney.

(f) Asbestos block or hollow sheet-metal box, 150 to $175 \mathrm{~mm}$ (6 to 7 inches) in diameter if round or on a side if square, 32 to $38 \mathrm{~mm}$ (11/4 to $1 \frac{11}{2}$ inches) in thickness, provided with a metal-lined, inverted cone-shaped opening through the center; $83 \mathrm{~mm}$ ( $3 \frac{1}{4}$ inches) in diameter at the bottom, and $89 \mathrm{~mm}$ (31/2 inches) in diameter at the top.

(g) Burner, Meker type, $24 \mathrm{~mm}$ (1 inch) in diameter by $155 \mathrm{~mm}$ ( 6 inches) in height, suitable for either manufactured or natural gas.

\section{PROCEDURE}

3. The test shall be conducted as follows:

Place two glass beads about 0.1 inch in diameter, in, and include them in the weight of, the tared porcelain or silica crucible in which is accurately weighed $10 \mathrm{~g}$ of the oil to be tested, free from moisture or other suspended matter. Place this crucible in the center of the Skidmore crucible. Level the sand in the large sheet-iron crucible and set the Skidmore crucible on it in the exact center of the iron crucible. Apply covers to both the Skidmore and the iron crucible, the one to the latter fitting loosely to allow free exit to the vapors as formed.

On a suitable stand or ring place the bare nichrome wire triangle and on it the asbestos block or hollow sheet-metal box. Next, center the sheet-iron crucible in the block with its bottom resting on top of the triangle, and cover the whole with the sheetiron hood in order to distribute the heat uniformly during the process. (See fig. 52.)

Apply heat with a high strong flame from the Meker-type gas burner, so that the preignition period will be 10 minutes $\pm 11 / 2$ minutes (a shorter time may start the distillation so rapidly as to cause foaming or too high a flame). When smoke appears above the chimney, immediately move or tilt the burner so that the gas flame plays on the sides of the crucible for the purpose of igniting the vapors. Then remove the heat temporarily, and before replacing adjust by screwing down the pinchcock on the gas tubing so that the ignited vapors burn uniformly with the flame above the chimney but not above the wire bridge. Heat may be increased, if necessary, when the flame does not show above the chimney.

When the vapors cease to burn and no further blue smoke can be observed, readjust the burner and hold the heat as at the beginning so as to make the bottom and lower part of the sheet-iron crucible a cherry red and maintain for exactly seven minutes. The total period of heating shall be 30 minutes \pm 2 minutes. There should be no difficulty in carry ing out the test exactly as directed with the gas burner of the type named, using city gas (about 550 B. t. u.) with the top of the burner about 2 inches below the bottom of the crucible. The time

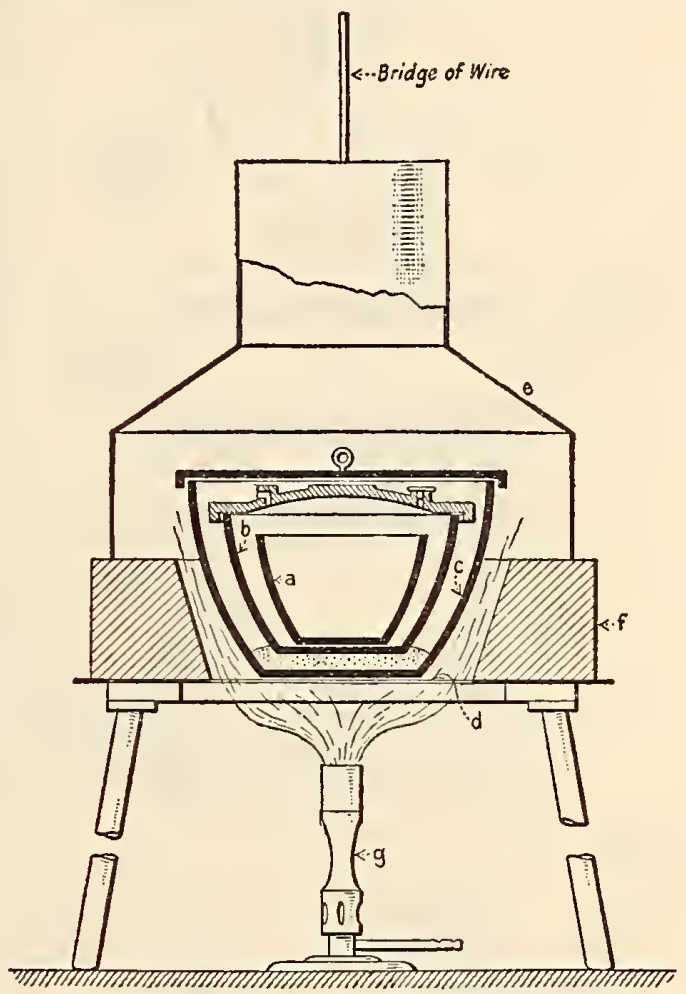

FigURE 52.-A pparatus for determining carbon residue

periods shall be observed with whatever burner and gas is used.

Remove the burner and allow the apparatus to cool until no smoke appears, and then remove the cover of the Skidmore crucible (about 15 minutes). Remove the porcelain or silica crucible with heated tongs, place in the desiccator, cool and weigh. Calculate the percentage of carbon residue on the original sample.

\section{TOLERANCES}

4. Weights of oil samples shall be accurate to within $5 \mathrm{mg}$. Tests shall be run in duplicate and repeated if necessary until the percentages of carbon residue differ by not more than 10 per cent from an average.

American Society for Testing Materials, tentative method of test for penetration of greases and petrolatum, serial designation D217-27T; 1927.

Approved as tentative American Standard by the American Engineering Standards Committee, now the American Standards Association. 


\section{INTERPRETATION OF RESULTS}

It should be understood that the original consistency of grease is determined by a variety of factors which are very difficult to control. The soap content is the most important single factor, but the kind of fats used, the method of mixing, the final water content, and the temperature of pouring are all very important in determining the final consistency, so that it is impracticable to maintain these consistencies within narrow limits. Any working of the sample after it is poured, or any remelting,

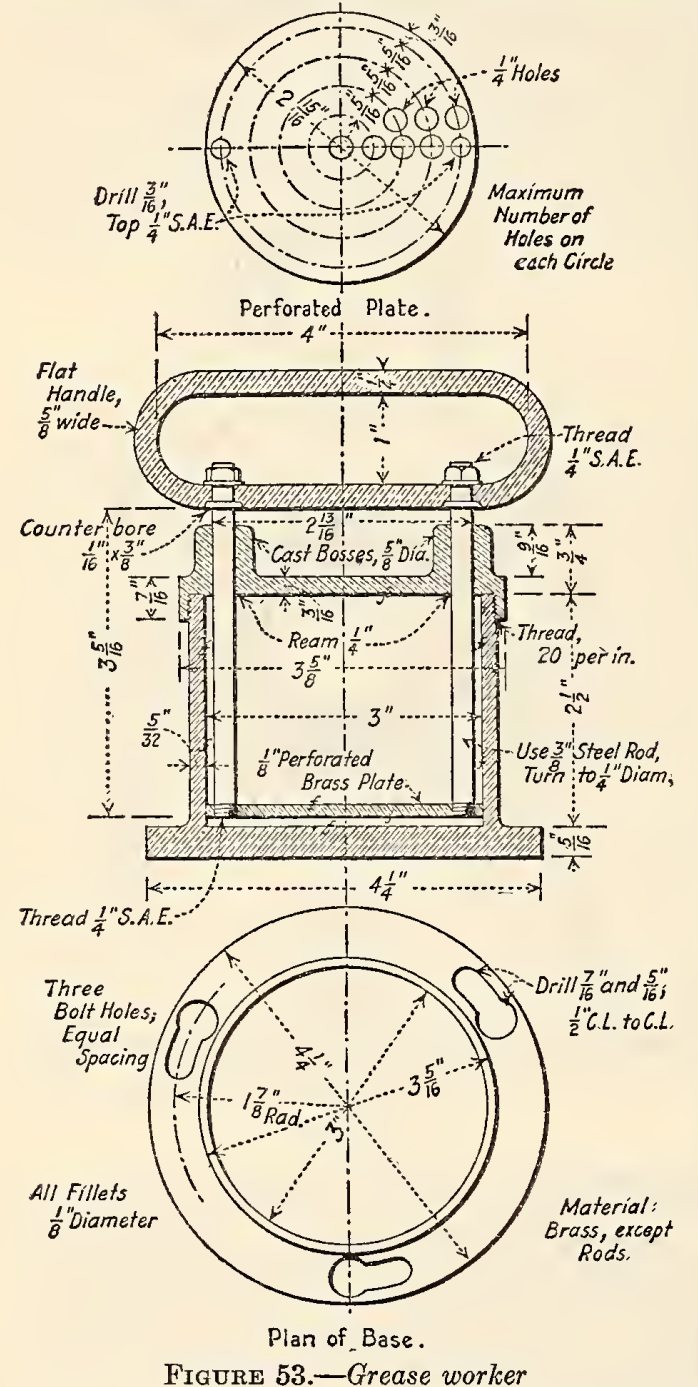

will greatly change the consistency. The original consistency also changes to some extent on standing, changing most rapidly during the first 48 hours after manufacture and more slowly thereafter.

Although most tests in the past have been based on the original consistency of greases, this consistency bears no necessary relationship to the worked consistency which is the factor of practical importance for most uses of cup greases, etc. Tests on such greases should therefore be made on the worked consistency. It is also almost impossible to measure the unworked consistency when samples have to be taken from barrels or other large containers.
On the other hand, for very hard greases, such as railroad greases used in slab form, the original or unworked consistency is generally the property of practical importance and should be the object of the test.

\section{SCOPE}

1. This method shall be used to measure the original consistency or the worked consistency of No. 0 cup grease and all harder greases and of petrolatum (unworked).

2. The method shall not be applied to greases which have been melted after having been poured and cooled during manufacture. Petrolatum, however, should be melted, poured into a suitable container and cooled before testing.

\section{APPARATUS}

3. The consistency shall be determined by measuring the penetration on what is customarily known as an asphalt penetrometer such as that illustrated in Figure 55, facing page 222. It is desirable, however, to modify this penetrometer by placing a flat metal plate or transite board on top of the present grid base to give a flat support for the container. If many tests are to be made, it is preferable to replace the ordinary grid base with a special flat base 6 inches in diameter.

This plate should have a cork insert in the center to prevent dulling the tip of the cone if it should drop.

4. The needle used in the asphalt penetration tests shall be replaced with the cone shown in Figure 54. ${ }^{47}$ The cone shall be constructed of stainless steel or of brass with a detachable hardened steel or stainless steel tip and made in accordance with the outside dimensions and tolerances shown. The interior construction and dimensions without tolerances are intended to be suggestive only. The outside surface of the cone and tip shall be given a very smooth finish.

5. The total moving weight in the test shall be $150 \mathrm{~g}$. If the asphalt plunger weighs $47.5 \mathrm{~g}$ (50 g-2.5 g needle) the cone or the cone plus an attached weight must weight $102.5 \mathrm{~g}$. It is in general desirable to make the cone as light as possible so that it will have a wide range of usefulness, and to add a weight to give the desired total for the grease test. If the conditions of the tests are not specified, the penetration of a grease shall be understood to refer to the results of this test made at $77^{\circ} \mathrm{F}$. $\left(25^{\circ} \mathrm{C}\right.$. $)$.

6. The grease worker shown in Figure 53 shall be used to work the grease to constant consistency in tests where the worked consistency is to be measured.

7. A constant temperature bath regulated to $77^{\circ}$ $\mathrm{F} . \pm 1^{\circ} \mathrm{F} .\left(25^{\circ} \mathrm{C} . \pm 0.5^{\circ}\right.$ C. $)$ is desirable to bring the samples to the temperature of test if many tests are to be made.

\section{SAMPLES}

8. Tests on the unworked consistency of grease shall be made only on grease in the original container (or cake in the case of very hard greases), 1-pound tins being especially convenient for the purpose. If samples are to be taken from large containers, the tests must be made only on a sample worked as specified below. In taking samples from large containers, any discolored layers near the surface shall first be completely cut away and rejected. All

47 The cone may be combined with the asphalt plunger if desired, providing the total weight of cone and plunger and the outside dimensions of the cone are not thereby changed. 
samples of petrolatum shall be tested for original (unworked) consistency after melting and cooling to the temperature of test.

\section{PROCEDURE}

(A) ORIGINAI (UNWOREED) CONSISTENCY

9. The temperature of the sample shall be brought to within $1^{\circ} \mathrm{F} .\left(0.5^{\circ} \mathrm{C}\right.$. $)$ of $77^{\circ} \mathrm{F}$. $\left(25^{\circ} \mathrm{C}\right.$. $)$ before the test. If the sample is initially within $3^{\circ}$ to $4^{\circ} \mathrm{F}$. $\left(1.5^{\circ}\right.$ to $2^{\circ} \mathrm{C}$.) of this temperature, it can be brought to $77^{\circ} \mathrm{F}$. $\left(25^{\circ}\right.$ C.) by placing in a water bath for 30 or 40 minutes, but if the initial temperature is
11. The can of grease or petrolatum shall be placed on the penetrometer table and the cone lowered until the tip just touches the surface. Watching the shadow of the tip aids in accurately setting the cone. The scale shall then be set to zero and the plunger released suddenly and kept released for five seconds. The penetration is read from the scale (the units being tenths of a millimeter) by the same procedure as is customary in measuring asphalt penetration as described in A. S. T. M. D 5. (See 505.0, p. 118.)

12. In making tests, the total surface area disturbed by the test has a diameter about equal to

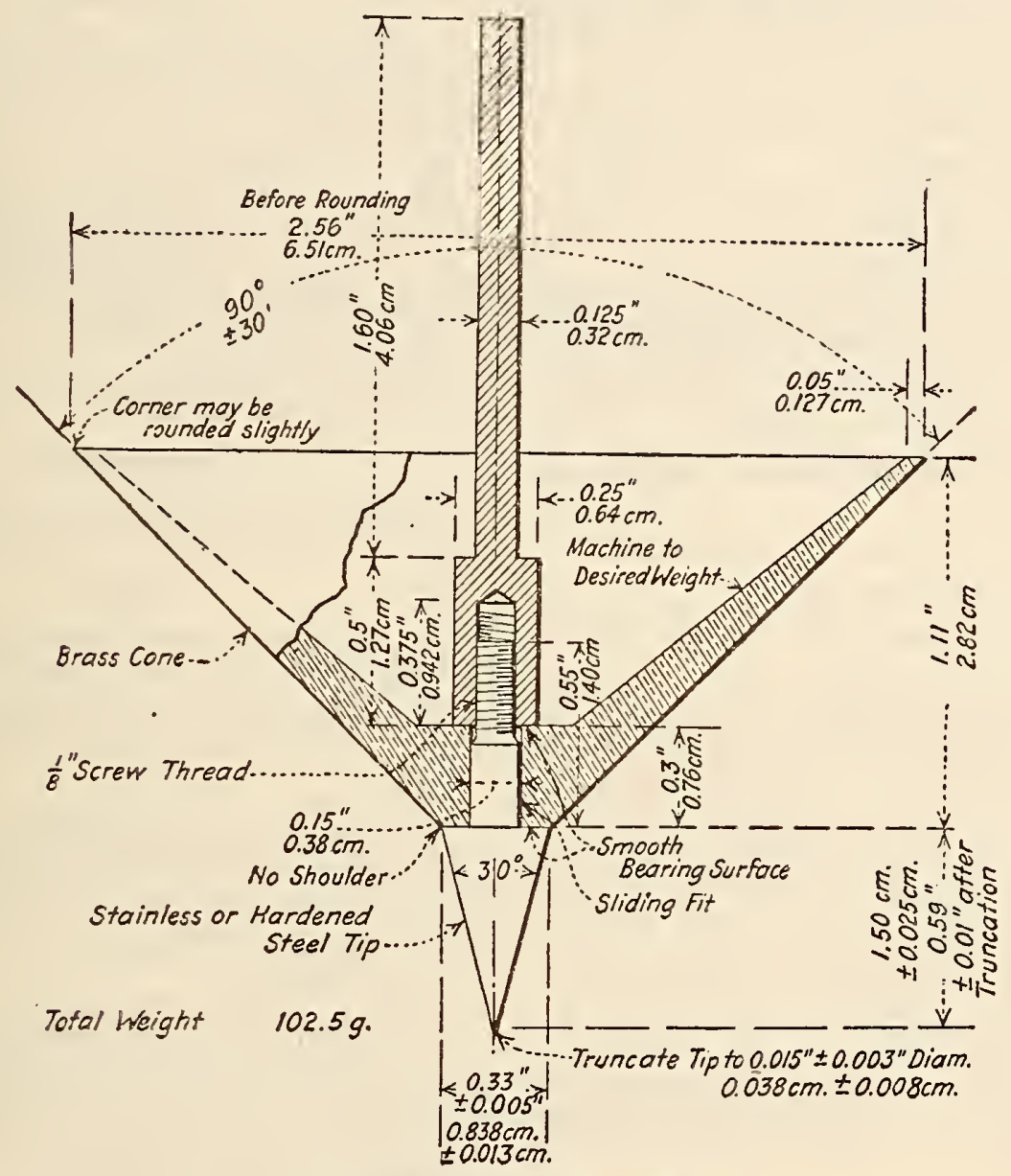

Figure 54.-Penetrometer cone

outside this range it must be placed in the constant temperature bath for one and one-half hours to insure reaching the final constant temperature. If the room temperature is more than $3^{\circ}$ to $4^{\circ} \mathrm{F}$. $\left(1.5^{\circ}\right.$ to $2^{\circ}$ C. $)$ from $77^{\circ}$ F. $\left(25^{\circ}\right.$ C.) a lid should be placed on the can, sealing with grease or petrolatum to prevent the entrance of water and the can immersed in the bath for the required period as, otherwise, the temperature of the surface will be different from that of the main body of the grease or petrolatum. Any holes or seams in the can should also be sealed with grease (petrolatum) or paraffin.

10. The surface of the grease or petrolatum shall be cut level and very smooth with a knife. Care must be taken not to work the surface of the grease or petrolatum. Any crust or discolored layers shall be completely removed before testing. the measured depth of penetration. In order to prevent one test from being affected by another disturbed area or by sides of the can, in starting a test the tip shall never be placed closer to the sides of the can or the edge of a previous hole than the penetration distance of that particular grease or petrolatum. The grease or petrolatum shall not be smoothed over for further tests.

13. Five tests shall be made on each sample and the average reported as the penetration, if the mean deviation of these readings does not exceed 3.0 per cent. If the mean deviation does exceed 3.0 per cent, the average of ten readings shall be reported as the penetration of the sample. More than one 1-pound can will be needed for the required number of tests on the softer greases or petrolatums. 


\section{(B) PROCEDURE FOR WORKED CONSISTENCY}

14. The procedure for worked greases shall be the same as that for unworked consistency except that the grease to be tested shall be transferred to the worker, which shall be filled heaping full with a minimum inclusion of air brought to within $2^{\circ}$ F. $\left(1^{\circ}\right.$ C. $)$ of $75^{\circ}$ F. $\left(24^{\circ}\right.$ C. $)$ and worked with 60 double strokes of the plunger. ${ }^{48}$ The top and plunger can then be removed, the surface smoothed over, and the test made at once if the temperature is within $1^{\circ} \mathrm{F}$. $\left(0.5^{\circ} \mathrm{C}\right.$.) of $77^{\circ} \mathrm{F}$. $\left(25^{\circ} \mathrm{C}\right.$. $)$. As soon as one test is made, the surface may be smoothed over for the next, taking care to avoid creating air pockets. If desired, the grease may be transferred carefully to another container before bringing to $77^{\circ} \mathrm{F}$. $\left(25^{\circ} \mathrm{C}\right.$.) and testing. One 1-pound sample is sufficient for all tests.

Federal Specifications Board, specification 2d, United States Government master specification for lubricants and liquid fuels.

(For methods of test listed in the above specification see 503.0 , page 66. .)

Independent $0 \mathrm{il}$ Men of America, specifications for various grades of motor oils.

(These specifications are compounded to conform with the Society of Automotive Engineers viscosity numbers (see 504.34, p. 108) and use the methods of test of the American Society for Testing Materials and those contained in Federal Specifications Board specification $2 \mathrm{~d}$ (see 503.0 , p. 66).)

\subsection{SPECIAL LUBRICATING OILS. (See} 504.19.)

\subsection{Asphaltic Oils. (See 504.19.) \\ 504.12 Axle Generator Oils.}

(No nationally recognized specifications available.)

\subsection{Journal Box Oils.}

American Railway Association, mechanical division, standard specification for reclaimed oil, 1928.

1. The grade of reclaimed oil to be used for lubricating journals for freight and passenger-train cars, should meet the following specifications:

2. Flash and fire points.-Flash and fire points shall not be lower than the following: Flash point $250^{\circ} \mathrm{F}$., fire point $300^{\circ} \mathrm{F}$.

3. Viscosity. - The viscosity at $210^{\circ} \mathrm{F}$. shall be within the following limits: 50 to 70 seconds, based on A. S. T. M. method D88. (See 503.0, p. 47.)

4. Pour point.-The pour point shall not be above a temperature of $45^{\circ} \mathrm{F}$., based on A. S. T. M. method D97. (See 502.2, p. 40.)

5. Precipitation number of sediment.-The precipitation number of sediment shall not be greater than 0.5 based on A. S. T. M. method D96. (See 502.2, p. 39.)

6. Water.-This oil shall contain not more than 0.5 per cent water by volume, based on A. S. T. M. method D95. (See 502.2, p. 38.)

7. Oil must be bright and clean and free from any extraneous solids that will not pass through a number 325-mesh screen.

\subsection{Mineral Oils. (See 504.19.)}

\subsection{Black Oil.}

(No nationally recognized specifications available.) 504.16 Blending Oils.

(No nationally recognized specifications available.)

${ }^{48}$ In general, quite similar results may be obtained by working the grease thoroughly for five minutes with a spatula on a flat plate, providing care is taken to prevent working in much air. For referee tests, however, the standard worker must be used.

\subsection{Miscellaneous Specifications for Lu. bricating Oils and Greases.}

American Railway Association, signal section, specification 10220, March, 1921, zero Fahrenheit lubricating oil.

\section{PURPOSE}

(a) The purpose of this specification is to provide for a lubricating oil for signal mechanisms and crossing protection apparatus in territories where the temperature does not fall below $0^{\circ} \mathrm{F}$. This oil shall not be used on relay or in oil dashpots.

\section{MATERIAL}

(a) Oil shall be pale in color, neutral, and free from vegetable or animal oils or fats, alkali, or other material which might gum or corrode metals under any condition.

(b) Oil shall conform to the following requirements:

(1) Flash.-Minimum $305^{\circ}$ F. $\left(151.7^{\circ} \mathrm{C}\right.$.).

(2) Fire.-Minimum $345^{\circ}$ F. $\left(173.9^{\circ}\right.$ C.).

(3) Viscosity. -100 seconds minimum to $135 \mathrm{sec}-$ onds maximum at $100^{\circ}$ F. $\left(37.8^{\circ}\right.$ C.) on the Saybolt standard universal viscosimeter.

(4) Pour. $-0^{\circ}$ F. $\left(-17.8^{\circ}\right.$ C. $)$.

(5) Moisture.-None.

(6) Sulphur.-Free sulphur, none.

(7) A cid.- Not more than 0.03 of 1 per cent calculated as $\mathrm{SO}_{3}$.

\section{INSPECTION}

(Inspection requirements same as for A. R. A. signal section specification 11328 . (See 522., p. 491.)

\section{TESTS}

(a) Tests may be made at point of production, or on samples submitted, and may also be made at destination.

(b) Contractor shall give the purchaser sufficient notice of time when material will be ready for testing.

(c) Contractor shall provide at point of production, apparatus and labor for making the required tests under supervision of the purchaser.

(d) Sample for test shall be taken from the bottom of the oil through an opening in the container or by use of a thief.

(e) Tests shall be made in accordance with the following methods:

(1) Flash test.-(a) The flash test shall be made in the Cleveland open cup, consisting of two parts, one the cup, the other a brass metal plate 5 inches in diameter by one-fourth inch thick, slightly recessed in its upper face to receive the base of the cup. The metal plate and oil holder are usually mounted on a tripod to which a wire is attached for supporting the thermometer. The latter may be supported by a clamp on a ring stand or by a chain. The thermometer used shall be of the engraved chemical stem "bulb-immersion" type, corrected for 1-inch immersion and reading to $\begin{array}{lll}\text { approximately } 700^{\circ} & \text { F. }\left(371.1^{\circ}\right. & \text { C. }) \text {. The bulb }\end{array}$ shall be about three-eighths inch long, not exceeding one-half inch. The thermometer shall be suspended in the center of the cup, and free from the bottom from one-fourth to three-eighths inch. Fill cup to within one-half inch from the top. Avoid overheating locally by wiping the flange free of oil and not allowing oil to besspattered up the sides of the cup.

(b) The oil shall be heated at a uniform rate of $10^{\circ} \mathrm{F}$. $\left(5.6^{\circ} \mathrm{C}\right.$.) per minute, and at approximately $25^{\circ} \mathrm{F}$. $\left(13.9^{\circ}\right.$ C. $)$, below the expected flash, the test shall be applied at every rise of $5^{\circ} \mathrm{F} .\left(2.8^{\circ} \mathrm{C}\right.$. $)$ by resting the gas tube on the edge of the cup and 
searching for the flash in the semicircle around the thermometer, midway between it and the inside of the cup, carrying the flame level with the top of the cup. The temperature at which flashes occur on the surface of the oil at two points or one that covers at least half of the surface and immediately goes out shall be recorded as the flash point.

(c) The testing flame is provided by using a slender tube tapered at one end to an opening approximately one-sixteenth inch diameter. The flame should be in the form of a bead and not more than one-eighth inch diameter.

(2) Fire test.-(a) The fire test shall be made by continuing the method of the flash test, the operation being the same except in the application of the flame. The flame in the fire test shall be quickly brought to one-sixteenth inch above the surface of the oil near the center of the cup and as quickly removed, and the temperature to which the oil must be heated to cause it to give off gases which will burn continuously when the flame is so applied shall be recorded as the fire point. The fire test is usually from $40^{\circ} \mathrm{F}$. $\left(22.2^{\circ}\right.$ C.) to $80^{\circ} \mathrm{F}$. $\left(44.4^{\circ}\right.$ C.) higher than the flash point.

(3) Viscosity test.- (a) The viscosity shall be determined by means of the Saybolt standard universal viscosimeter. The oil tube ${ }^{49}$ shall be swabbed with the plunger accompanying the instrument by using oil from sample, making sure the outflow tube is not obstructed. Strain the oil into the oil tube at a temperature not above that of the bath, stir until the oil is of the test temperature throughout, remove the thermometer, remove excess oil from the overflow chamber with the pipette, and proceed with the test. The elapsed time in seconds required for the oil to fill the flask to the $60 \mathrm{cc}$ mark on the neck shall be the viscosity of the oil.

(4) Pour test.- (a) The pour test shall be made by using a glass jar approximately $1 \frac{1}{4}$ inches in diameter and 4 to 5 inches high, provided with a tightly fitting cork. The glass jar shall be incased in a closely fitting metal jacket, which shall be provided at the bottom with a disk of cork or felt one-fourth inch thick.

(b) A bulb immersion type mercury thermometer having a bulb one-fourth to three-eighths inch long shall be fitted securely in the cork so that the shaft will be held centrally in the jar with the tip of the bulb one-half inch from the bottom. A toluol thermometer shall be used for temperatures lower than $-20^{\circ}$ F. $\left(-28.9^{\circ}\right.$ C. $)$.

(c 1) Material used in the freezing mixture varies with the temperature required to cause the oil to solidify. Use cracked ice for a temperature above $35^{\circ} \mathrm{F}$. $\left(1.7^{\circ} \mathrm{C}\right.$. $)$. From $15^{\circ} \mathrm{F}$. $\left(-9.4^{\circ} \mathrm{C}\right.$. $)$ to $-5^{\circ} \mathrm{F}$. $\left(-20.6^{\circ}\right.$ C. $)$ use ice and salt in proportion of one to two. The salt shall be dry and fine enough to pass through a twenty-mesh screen. For temperature lower than $-5^{\circ}$ F. $\left(-20.6^{\circ} \mathrm{C}\right.$. $)$ use a mixture of solid carbon dioxide and acetone.

(c 2) The carbon dioxide-acetone mixture may be made as follows:

Place a sufficient amount of dry acetone in a covered copper or nickel beaker. Place the beaker in an ice-salt mixture and when the acetone reaches $10^{\circ} \mathrm{F}$. $\left(-12.2^{\circ} \mathrm{C}\right.$.) or less, add solid carbon dioxide gently until the desired temperature is reached. To obtain the solid carbon dioxide, invert an ordinarily liquefied carbon dioxide cylinder, open the valve carefully and let the gas flow into a chamois skin bag. Rapid evaporation will cause the carbon dioxide to solidify.

(d) The oil shall be placed in a jar, to a depth of about $1 \frac{1}{4}$ inches, or to a sufficient depth to reach

9 The standard oil tube should be standardized by the National Bureau of Standards, Washington, D, C. about one-fourth inch above bulb of thermometer. Put the thermometer in cork, fit the cork tightly into the jar, making sure that bottom of bulb is about one-half inch from the bottom of the jar. Place jar in metal jacket and place jacket in freezing mixture.

(e) When near the expected pour point, at every drop in temperature of $5^{\circ} \mathrm{F} .\left(2.8^{\circ} \mathrm{C}\right.$. $)$, remove the jar from the jacket and tip just enough to make the oil flow. The last $5^{\circ} \mathrm{F} .\left(2.8^{\circ} \mathrm{C}\right.$.) reading previous to the termperature at which the oil will not flow when jar is tipped to a horizontal position shall be taken as the pour point.

(5) Moisture test.-(a) The moisture test shall be made by taking a sample of oil from the bottom of the container, which, when placed in clean and dry glass or porcelain beaker and stirred without touching the sides or bottom with a one-fourth inch brass or iron rod heated to a cherry red, shall not cause a snapping sound.

(6) Acid test.- (a) The acid test shall be made by agitating thoroughly a solution at a boiling point of $5 \mathrm{~g}$ of the oil with $25 \mathrm{cc}$ of distilled water and 25 ce of 95 per cent neutralized alcohol. A few drops of phenolphthalein shall be added as an indicator and the result, if colorless (indicating acid present) shall be titrated with one-tenth normal alkali, free from carbonate until permanently pink when agitated. The percentage of acid shall be calculated from reading the burette and shown in percentage of $\mathrm{SO}_{3}$ or as an acid number (milligrams of potassium hydroxide required to saturate the free acid in $1 \mathrm{~g}$ of oil). One cc of one-tenth normal alkali solution is equivalent to $4 \mathrm{mg}$ of $\mathrm{SO}_{3}$.

(b) A clean strip of pure copper one-half inch wide and 4 inches long shall be heated to redness and while red hot dipped in 95 per cent neutralized alcohol. The strip shall then be allowed to dry as quickly as possible in the air and dropped into a sample of oil contained in the test tube. About onehalf of the length of the copper strip shall be submerged. The test tube shall then be closed with a cork stopper and left to stand for 24 hours at a temperature of approximately $70^{\circ} \mathrm{F}$. At the end of this time the copper strip shall be removed and cleansed with proper solvents, then compared with a similar strip of copper freshly cleaned in alcohol as previously described. No discoloration of the test strip shall be shown by this test.

\section{MARKING}

(b) Detail list of loose pieces, containers, and their contents shall be furnished for each shipment. Where carload shipments are made, routing and car identification shall be shown.

(d) Each quart container shall be marked with label as follows:

\section{A. R. A. Signal Division Specification 10220}

\section{Zero F. Lubricating Oil}

This oil must not be used for lubricating signal mechanisms or crossing protection apparatus in territories where temperature falls below $0^{\circ} \mathrm{F}$. This oil must not be used on relays or in oil dashpots.

(e) Purchaser's order, requisition and package number, name of consignor, and name and address of consignee shall be plainly marked on outside of each case or drum.

(f) Where carload shipments are made, each case or drum shall be marked with contents and order number, but address may be omitted. 


\section{PACKING}

(b) The oil shall be put up in clean, dry containers of 1-quart size, provided with a screw top and airtight gasket, or in steel drums of approximately 50 gallons capacity, and shall be protected so as to permit convenient handling and to prevent loss or damage during shipment.

American Railway Association, signal section, specification 10320 , March, 1921, $45^{\circ}$ below zero Fahrenheit lubricating oil.

This specification is the same as A. R. A. signal section specification 10220 , given above, with the exception of material under the headings: 1, Purpose; 2, material; and $5(d)$, marking. The substance at variance is as follows:

\section{PURPOSE}

(a) The purpose of this specification is to provide for a lubricating oil for signal mechanisms. This oil shall not be used in oil dash pots.

\section{MATERIAL}

(a) Oil shall be pale in color, neutral, and free from vegetable or animal oils and fats, alkali, or other material which might gum or corrode metals under any condition.

(b) The oil shall conform to the following requirements:

1. Flash-Minimum $290^{\circ} \mathrm{F} .\left(143.3^{\circ} \mathrm{C}.\right)$

2. Fire.-Minimum $325^{\circ} \mathrm{F}$. $\left(162.8^{\circ} \mathrm{C}\right.$. $)$.

3. Viscosity.-Minimum 80 seconds at $100^{\circ} \mathrm{F}$. $\left(37.8^{\circ}\right.$ C.) on the Saybolt standard universal viscosimeter.

4. Pour at $-45^{\circ}$ F. $\left(-42.8^{\circ}\right.$ C. $)$.

5. Moisture.-None.

6. Sulphur.-Free sulphur, none.

7. Acid.- Not more than 0.03 of 1 per cent calculated as $\mathrm{SO}_{3}$.

\section{MARKING}

(d) Each quart container shall be marked with label as follows:

\section{A. R. A. Signal Division Specification $1032045^{\circ}$ Below $0^{\circ} \mathrm{F}$ uil}

This oil must not be used in oil dash pots.

Furnisbed by

Federal Specifications Board, specification 2d, United States Government master specification for lubricants and liquid fuels.

(This publication, containing detailed specifications, methods of testing, and methods of sampling, is published by the United States Government Printing Office as Technical Paper 323B, Department of Commerce, Bureau of Mines. The publication contains specifications for lubricating oils, which are given below.)

\section{IUBRICATING OILS}

\section{GENERAL REQUIREMENTS}

Sampling.-See A. S. T. M. method D270-27T, 503.0 , page 60 .

Testing. - See F. S. B. No. 2d, 503.0, page 66 .

NOTE.-For convenience in presel.tation the test methods have been listed numerically and reference made accordingly in the follow. ing table where tests apply.

\section{DETAII REQUIREMENTS}

(a) General

Class $A$ oils.-Used where highly refined oil is not required and is not to be used for steam-cylinder lubrication. ${ }^{50}$

Class $B$ oils.-Used in turbines, dynamos, and high-speed steam-engines, using circulating and forced-feed systems. 50

Class $C$ oils.- - Used when, and only when, service conditions necessitate an oil suitable for lubricating both turbine and internal combustion engines. ${ }^{50}$

Class $D$ oils.-Used for lubrication of internal combustion machines, except aircraft, and Diesel engines.5

Car and locomotive engine oil.-Used as lubricant on journals of all cars, passenger coaches, and steam and electric locomotives.

Diesel engine oil.-Used for lubrication of Diesel engines. ${ }^{50}$

Gear, chain, and wire rope lubricant.-Used for the lubrication and protection of chains, wire ropes, and gears. ${ }^{50}$

Transmission oil.-Used for the lubrication of transmission gears and bearings, differential gears, worm drives, winch drives, and roller and ball bearings used in connection with such parts of the equipment of motor vehicles. ${ }^{60}$

Compounded marine-engine oil.-Used for reciprocating steam engines in marine service where compounded engine oil is required. These oils must not be used in forced feed or circulating systems. Grade 1 oil shall be compounded from refined petroleum oil and 10 to 20 per cent of blown refined rapeseed oil or blown refined peanut oil, so compounded that it will not separate or break down in any way either before or while it is in service. Grade 2 oil shall be a compounded oil made from refined petroleum oil and 10 to 20 per cent of blown refined rapeseed oil or oil of equivalent tests and shall have an emulsifying value equivalent to that of rapeseed compounded oil.

Marine-engine oil.-Used for reciprocating steam engines in marine service. This oil shall not be used in circulating or forced-feed systems. ${ }^{51}$

Compounded steam-cylinder oil for noncondensing engines.-For steam cylinders of noncondensing engines and pumps where a compounded oil is required.

Mineral steam-cylinder oil for noncondensing engines.-Used for noncondensing steam-engine cylinders where mineral oil is required..$^{50}$

Aircraft machine-gun oil.-Used for machine guns on aircraft, for the cc interrupter gears, and for gun oil for cleaning and oiling machine guns and small arms. ${ }^{50}$

Gun and ice-machine oil.-Used for cleaning and oiling guns and small arms where aircraft machine-gun oil is not required; also for lubi ication of the cylinders of ice machines, for lubrication of pneumatic tools, and for hydraulic systems. ${ }^{50}$

Liberty aero oil.-Used for stationary-cylinder aircraft engines. ${ }^{50}$

Buffer oil.-Used for filling the recoil and recuperator cylinders of all British-type howitzers and gun carriages. ${ }^{50}$

Recoil oil.-Used to fill the recoil cylinders of gun carriages. ${ }^{50}$

so Only refined petroleum oils without the admixture of fatty oils, resins, soap, or other compounds not derived from crude petroleum will be considered.

s1 Marine-engine oil shall be a mineral lubricating oil free from water, mineral acids, alkalies, and asphaltic matter. 
(b) Properties and Tests

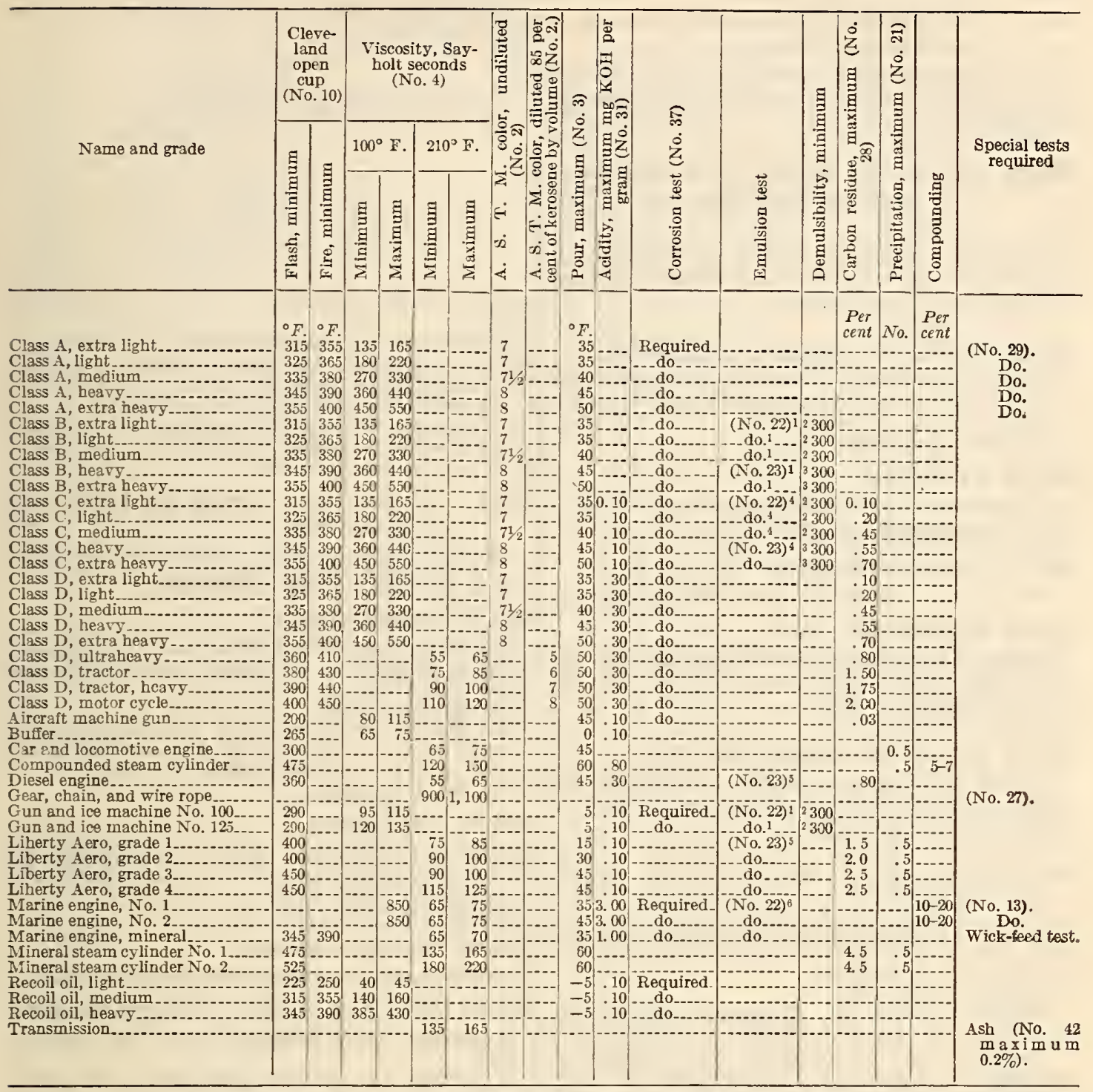

1 The oil shall separate (this means there shall be only a slight cuff between the oil and water) in 30 minutes from an emulsion with normal caustic soda solution, and the upper layer at the end of 30 minutes shall not contain more than 10 per cent of water, as determined by method No. 20.

Method No. 24.

\$The oil shall separate (this means there shall he only a slight cuff hetween the oil and water) in 30 minutes from an emulsion with (1) distilled water, (2) 1 per cent salt solution, and (3) normal caustic solution.

6 The oil shall separate completely in one hour from an emulsion with distilled water at a temperature of $180^{\circ} \mathrm{F}$.

6 The oil shall remain completely emulsified for 1 hour for Nos. 1 and 2, and $1 / 2$ hour for mineral, when the emulsifying liquid is (1) distilled water and (2) 1 per cent salt solution.

NOTE. - For the class B and gun and ice-machine oils under notes (5) and (6) the following requirement is stipulated: The upper layer at the end of 30 minutes shall not contain more than 5 per cent water by method No. 20. 
504.2 CYLINDER OILS.

504.21 Compressor Oil (Including Air, Ammonia Ice IMachine).

Federal Specifications Board, specification 2d, United States Government master specification for lubricants and liquid fuels.

See 504.19, page 106.

504.22 Engine Oil, Steam (Including Noncondensing, High-Speed, and Turbine).

Federal Specifications Board, specification 2d, United States Government master specification for lubricants and liquid fuels.

See 504.19, page 106.

\subsection{Engine Oil, Internal Combustion.}

Federal Specifications Board, specification 2d, United States Government master specification for lubricants and liquid fuels.

See 504.19, page 106 .

\subsection{Valve Oils.}

(No nationally recognized specifications available.)

\subsection{MACHINE OILS.}

504.31 Dynamos, Spindles, Pumps, Etc.

(No nationally recognized specifications available.)

\subsection{Machine-Gun Oil.}

Federal Specifications Board, specification 2d, United States Government master specification for lubricants and liquid fuels.

See 504.19, page 106 .

\subsection{Recoil and Recuperator Oils.}

Federal Specifications Board, specification 2d, United States Government master specification for lubricants and liquid fuels.

See 504.19, page 106.

\subsection{Transmission Oil (Including Crank Case).}

Federal Specifications Board, specification 2d, United States Government master specification for lubricants and liquid fuels.

See 504.19 , page 106 .

Society of Automotive Engineers, transmission lubricating oils (standard, 1924).

General.-These specifications cover grades of petroleum oil suitable for the lubrication of transmission and differential gears, worm drives, and rolker and ball bearings used in connection therewith.

Compounded lubricating oils containing products other than those derived from petroleum are not dealt with in these specifications.

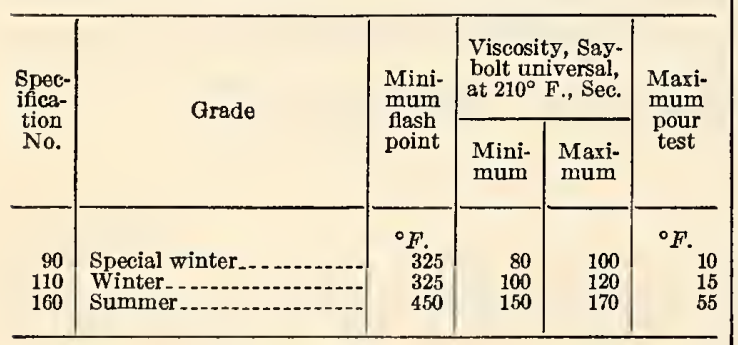

Tests shall be made in accordance with A. S. T. M. specifications.
Society of Automotive Engineers, crank-case lubricating-oil viscosity numbers (recommended practice, 1929).

\begin{tabular}{|c|c|c|}
\hline \multirow{2}{*}{$\begin{array}{l}\text { Viscosity } \\
\text { No. }\end{array}$} & \multicolumn{2}{|c|}{$\begin{array}{l}\text { Viscosity range } 1 \\
\text { Saybolt universal, } \\
\text { Sec. }\end{array}$} \\
\hline & At $130^{\circ} \mathrm{F}$. & At $210^{\circ} \mathrm{F}$. \\
\hline $\begin{array}{l}10 \\
20 \\
30 \\
40 \\
50 \\
60\end{array}$ & $\begin{array}{r}90-115 \\
120-150 \\
185-220 \\
255 \\
\end{array}$ & $\begin{array}{r}70 \\
75-95 \\
105-120\end{array}$ \\
\hline
\end{tabular}

1 Oils with viseosities falling between the ranges specified shall be classified in the next lower grades.

In the case of prediluted oils, S. A. E. viscosity numbers by which the oils are classified shall be determined by the viscosity of the undiluted oils.

Wherever the S. A. E. viscosity numbers are used on prediluted oils, the container labels should show in some suitable manner that the S. A. E. number applies to the undiluted oil.

504.35 Typewriter, Sewing-Machine, and Clock Oils.

(No nationally recognized specifications available.)

\subsection{IUBRICATING GREASES.}

\subsection{General Items.}

American Society for Testing Materials, standard method of analysis of grease, serial designation D128-27, 1927.

See 504.0, page 93

American Society for Testing Materials, tentative method of test for penetration of greases and petrolatum, serial designation D217-27T, 1927.

See 504.0, page 101 .

\subsection{Cup Grease.}

Federal Speciflcations Board, specification 2d, United States Government master specification for lubricants and liquid fuels.

See 504.49, page . 109

\subsection{Cylinder Greases.}

(No nationally recognized specifications available.)

504.43. Gear, Chain, and Wire-Rope Greases. American Mining Congress, 1927, wire rope for mines.

Approved as an American Standard by the American Engineering Standards Committee, now the American Standards Association.

(This standard includes the tollowing requirements for wire-rope lubrication.)

It is advisable that the lubricant recommended by the rope manufacturer be used.

Wire-rope lubricant should be commercially chemically neutral.

It should not harden sufficiently to crack, chip, or peel, nor soften sufficiently to drip off the rope under extreme atmospheric conditions.

It should not dry out rapidly in service.

It should be practically free from moisture, not absorb moisture readily, and be impervious to surface moisture.

It should be of such nature that it can penetrate between the strands to the core of the rope, and should be applied in such a manner as to secure the maximum penetration.

It should possess such adhesive qualities as not to be thrown from the rope by vibration nor rapid motion. 
Its physical and chemical qualities should permit heating to liquid consistency for ease of application. Lubricant should be applied at frequent intervals to protect the wires of the rope from friction and corrosion.

Federal Specifications Board, specification 2d, United States Government master specification for lubricants and liquid fuels.

See 504.49, below.

\subsection{Graphite Greases.}

Federal Specifications Board, specification 2d, United States Government master specification for lubricants and liquid fuels.

See 504.49, below.

\subsection{Plate Greases.}

(No nationally recognized specifications available.)

504.46 Crank Pin, Driving Journal Compound, and Rod Cup Greases.

Federal Specifications Board, specification 2d, United States Government master specification for lubricants and liquid fuels.

See 504.49 , below.

\subsection{Miscellaneous Lubricating Greases.}

Federal Specifications Board, specification 2d, United States Government master specification for lubricants and liquid fuels.

(This publication, containing detailed specifications, methods of testing, and methods of sampling, is published by the United States Government Printing Office as Technical Paper 323B, Department of Commerce, Bureau of Mines. The publication includes specifications for greases, which are given below.)

\section{GREASES}

\section{GENERAL REQUIREMENTS}

Sampling.-See A. S. T. M. method D270-27T, 503.0, page 60 .

Testing.-See F. S. B. No. 2d, 503.0, page 66.

NotE.-For convenience in presentation the test methods have been listed numerically and reference made in the following table where tests apply.

\section{DETAII REQUIREMENTS}

(a) General

1. Recuperator grease shall be a well-manufactured product composed of calcium soap made from high-grade animal or vegetable oils or fatty acids and a highly refined mineral oil. It is used by the United States Government for the recoil mechanism of 75 and $155 \mathrm{~mm}$ French gun carriages.

2. Crank-pin grease shall be composed of soda soap, combined with a well-refined cylinder-stock petroleum oil. It is used for the driving journals on locomotives (provided with grease cellars) and for the lubrication of cranks and rods on locomotives (provided with grease cups). The grease shall be smooth and uniform and must not crumble under pressure.

3. Mineral lubricating grease shall be a smooth, homogeneous mixture of mineral oil and pure odorless lime soap made from animal or vegetable fats or oils, or fatty acids completely saponified with hydrated lime. It is used for the lubrication of such parts of motor equipment and other machinery as are lubricated by means of compression cups, and also for use on ball bearings and roller bearings. The grease shall contain no fillers such as rosin, rosin oils, talc, wax, powdered mica, sulphur, clay, asbestos, or other undesirable or deleterious impurities It shall possess only a slight odor of mineral oil, and may be rejected if it has the odor of any objectionable material.

Mineral lubricating grease shall be furnished in the following grades as specified: Hard, medium, soft. Soft grease is intended for use in ball bearings and roller bearings. Medium grease and hard grease are intended for use in compression grease cups for bearings. For rapid running machines in cool climates medium grease should be ordered. For hot climates or heavy running machinery hard grease should in general be ordered.

4. Graphite lubricating grease.-The requirements, grades, and uses are the same as for mineral lubricating grease, given above, with the variations (1) that the mixture for this product shall contain graphite, in addition to the mineral oil and lime soap, and (2) this specification does not include fatty acids as a source for the grease.

(b) Properties and Tests

\begin{tabular}{|c|c|c|c|c|c|c|c|c|c|c|c|c|c|c|}
\hline \multirow{3}{*}{ Name and grade } & \multirow{3}{*}{ 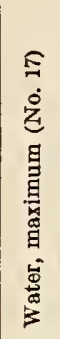 } & \multirow{3}{*}{ 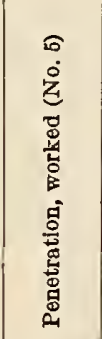 } & \multirow{3}{*}{$\begin{array}{c}\text { Corrosion } \\
\text { test }\end{array}$} & \multirow{3}{*}{ 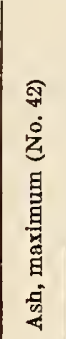 } & \multirow{3}{*}{ 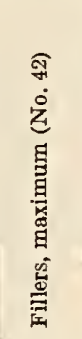 } & \multirow{3}{*}{ 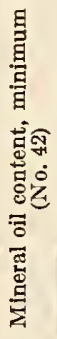 } & \multicolumn{3}{|c|}{$\begin{array}{l}\text { Properties of the min- } \\
\text { eral-oil constituent }\end{array}$} & \multirow{3}{*}{ 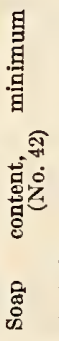 } & \multirow{3}{*}{ 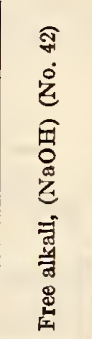 } & \multirow{3}{*}{ Color } & \multirow{3}{*}{ 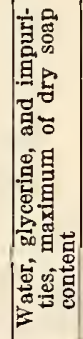 } & \multirow[b]{3}{*}{ 兽 } \\
\hline & & & & & & & \multirow{2}{*}{ 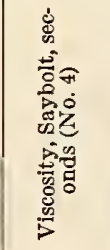 } & \multicolumn{2}{|c|}{$\begin{array}{l}\text { Cleveland } \\
\text { open cup } \\
\text { (No. 10) }\end{array}$} & & & & & \\
\hline & & & & & & & & 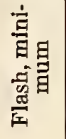 & 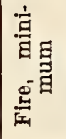 & & & & & \\
\hline Recuperator & $\begin{array}{l}\text { Per } \\
\text { cent } \\
3.0\end{array}$ & $240-290$ & (No.37)_- & $\begin{array}{c}\text { Per } \\
\text { cent } \\
12.3\end{array}$ & $\begin{array}{c}\text { Per } \\
\text { cent } \\
\text { None. }\end{array}$ & $\begin{array}{l}\text { Per } \\
\text { cent }\end{array}$ & $2180 @ 100$ & ${ }^{\circ} F$. & ${ }^{\circ} \mathrm{F}$. & $\begin{array}{r}\text { Per } \\
\text { cent } \\
18\end{array}$ & Per cent & & $\begin{array}{l}\text { Per } \\
\text { cent }\end{array}$ & \\
\hline Crank-pin grease & $\ldots$ & - & 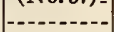 & $\ldots . .$. & 190.- & -1 & 1006 & & & & $0.5-2.5$ & Yellowish & $33 \overline{1} / 3$ & 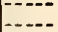 \\
\hline Driving-journal compound. & $-\ldots$ & & & & & & & & & & $.5-2.5$ & Greenish & $331 / 3$ & --- \\
\hline $\begin{array}{l}\text { Rod-cup grease } \\
\text { Mineral lubricating grease: }\end{array}$ & 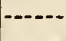 & & & & & & & & & & $.5-2.5$ & Yellowish & $331 / 3$ & $-\cdots$ \\
\hline Hard. & 2.0 & $190-230$ & (No.38) & 38.5 & 0.10 & 75.0 & 125@130 & 340 & 370 & & & & & \\
\hline Medium & 2.0 & $240-290$ & ho do & 3. 6.0 & .10 & 80.0 & 125@130 & 340 & 370 & & & & & \\
\hline Soft & 1.5 & $300-355$ & - do do & 35.0 & .10 & 85.0 & $125 @ 130$ & 340 & 370 & & & & & \\
\hline Hard. & 2.0 & $190-230$ & ...do. & & .10 & 75.0 & 125@130 & 340 & 370 & & & & & 42.0 \\
\hline Medium. & 2.0 & $240-290$ & ...do... & & .10 & 80.0 & 125@130 & 340 & 370 & & & & & 42.0 \\
\hline & 1.5 & & -_-_do_-_ & & .10 & 85.0 & 125@130 & 340 & 370 & & & & & 42 \\
\hline
\end{tabular}

1 Rapid routine method.

Saybolt universal.

- May contain 2 to 3 per cent of flake graphite which is free from grit and which contains at least 82 per cent of graphitic carbon. 


\subsection{PARAFFIN, PARAFFIN OIL, AND PARAFFIN WAX.}

\subsection{Candles.} able.)

(No nationally recognized specifications avail-

\subsection{Iluminating Wax.} able.)

(No nationally recognized specifications avail-

\subsection{Paraffin Oils.}

(No nationally recognized specifications available.)

\subsection{Paraffin Wax.}

American Society for Testing Materials, standard method of test for melting point of paraffin wax, serial designation D87-22, 1922.

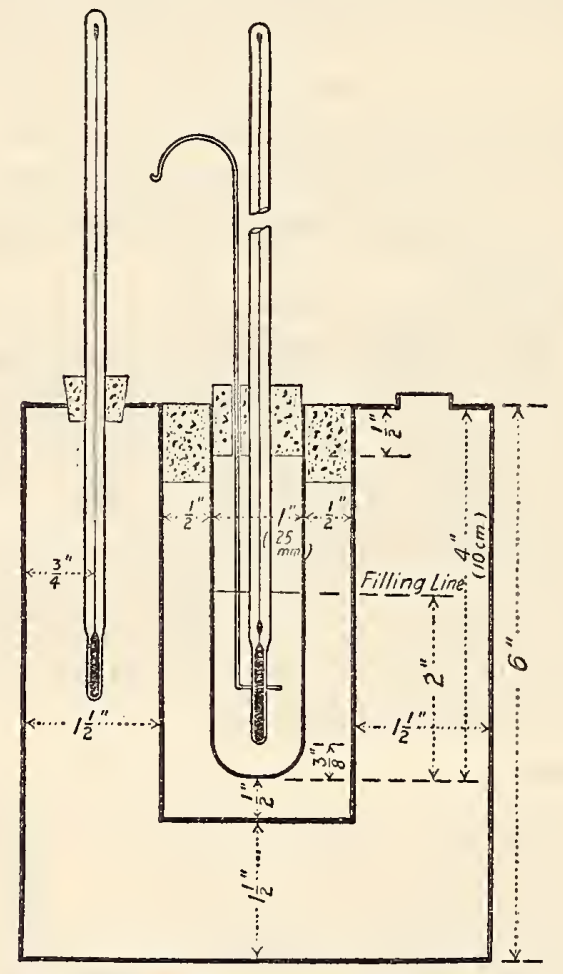

Figure 56.-A pparatus for determination of melting point of paraffin wax

Approved as American Standard by the American Engineering Standards Committee now the American Standards Association.

\section{DEFINITION}

1. A. S. T. M. paraffin wax melting point.-The temperature at which melted paraffin wax, when allowed to cool under definite prescribed conditions, first shows a minimum rate of temperature change.

Note.-The so-called American Melting Point is an arbitrary figure $3^{\circ} \mathrm{F}$. higher than the A.S. T. M. paraffin wax melting point.

\section{APPARATUS}

2. Wax container.-Test tube of standard form, $25 \mathrm{~mm}$ (1 inch) outside diameter and $100 \mathrm{~mm}$ (4 inches) long. It may be marked with a filling line 2 inches above the bottom. This test tube shall be closed in a tightly fitting cork having two openings, one at the center for the melting-point thermometer and the other for a stirrer at one side of the center. The opening for the stirrer may be lined with glass or metal tubing to act as a guide for the stirrer.

3. Air bath.-Suitable water-tight cylinder, 2 inches in inside diameter and $4 \frac{1}{2}$ inches deep. This air bath shall be provided with a tightly fitting cork having a central opening for holding the test tube firmly in a vertical position in the center of the air bath.

4. Water bath.-Suitable cylinder, 51/8 inches in inside diameter and 6 inches deep. This water bath shall be provided with a suitable cover and with the guides and fasteners necessary to hold the air bath firmly in a vertical central position so that the sides and bottom of the air bath shall be surrounded by a layer of water $1 \frac{1}{2}$ inches thick. The water-bath cover shall have a slot for introduction of a suitable stirrer and shall have an opening for the bath thermometer so that the latter may be suspended in a vertical position three-fourths inch from the outside wall of the water bath. Air bath, water bath, and water-bath cover may be conveniently made of metal in one assembly as shown in Figure 56.

5. Stirrer in test tube--Brass or copper wire, one-eighth inch in diameter and about 12 inches long. A circular loop one-half inch in diameter shall be formed at one end of this wire in such a manner that the loop lies in a horizontal plane when the rest of the wire is in a vertical position. The stirrer thus formed shall be passed through the proper opening in the test-tube cork and the upper end may be then bent into a shape convenient for holding.

6. Thermomeier.-The A. S. T. M. paraffin wax melting point thermometer shall conform to the requirements of thermometer No. 9. (See table of A. S. T. M. thermometers, 500.2, p. 2.)

7. Bath thermometer of any suitable type, accurate to $2^{\circ} \mathrm{F}$. throughout the required range.

\section{PROCEDURE}

8. An average sample of the wax to be tested shall be melted in a suitable container in a water bath whose temperature shall be not more than $35^{\circ} \mathrm{F}$. above the approximate melting point of the wax sample. Direct heat, such as a flame or hot plate, shall not be used and the wax sample shall not be held in the melted condition any longer than necessary.

The test tube shall be filled with melted wax to a height of 2 inches. The test-tube cork, carrying the stirrer and the melting-point thermometer with the $3^{1 / 8}$-inch immersion line at the under surface of the cork, shall be inserted into the test tube for a distance of one-half inch. The lower end of the thermometer bulb shall then be three-eighths inch from the bottom of the test tube.

The air bath being in its proper position in the water bath, the latter shall be filled to within onehalf inch of the top with water at a temperature $15^{\circ}$ to $20^{\circ} \mathrm{F}$. below the approximate melting point of the wax sample.

The test tube containing the melted wax, with wax stirrer and thermometer in place, shall be inserted into the air bath in a central vertical position so that the bottom of the test tube is one-half inch from the bottom of the air bath. The temperature of the water bath shall be adjusted by stirring if necessary, so that it shall be lower than the temperature of the wax sample by not more than $30^{\circ} \mathrm{F}$. and not less than $25^{\circ} \mathrm{F}$., when the wax sample lias cooled to a 
temperature $10^{\circ} \mathrm{F}$. above its approximate melting point.

When these conditions have been obtained, temperature adjustment and stirring of the water bath shall be discontinued. The wax shall be stirred continuously during the remainder of the test, the stirring loop being moved up and down throughout the entire length of the test tube in a steady motion at

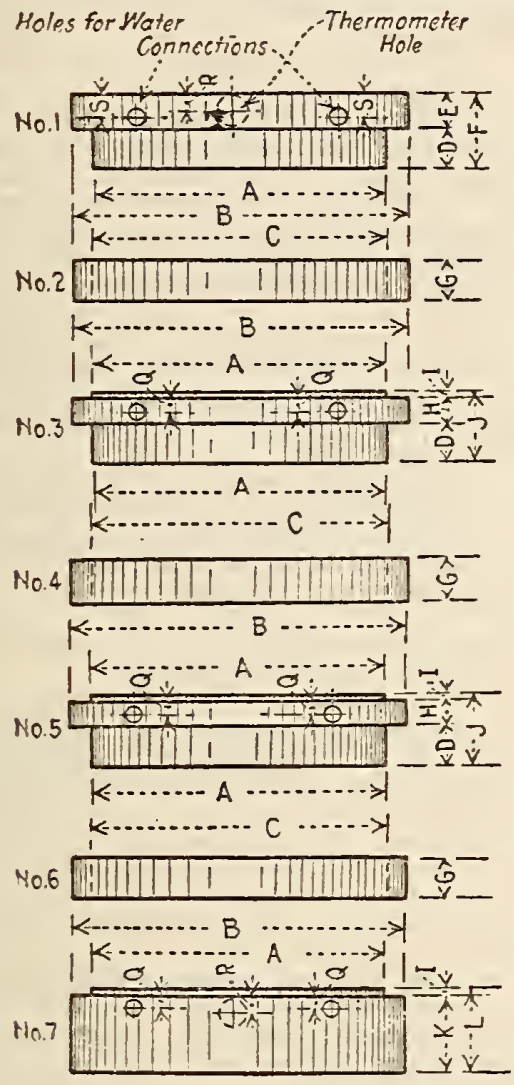

error in the thermometer scale and the corrected temperature shall be reported as the "A. S. T. M. paraffin-wax melting point."

\section{ACCURACY}

9. Duplicate determinations on the same sample should differ by not more than $0.2^{\circ} \mathrm{F}$.

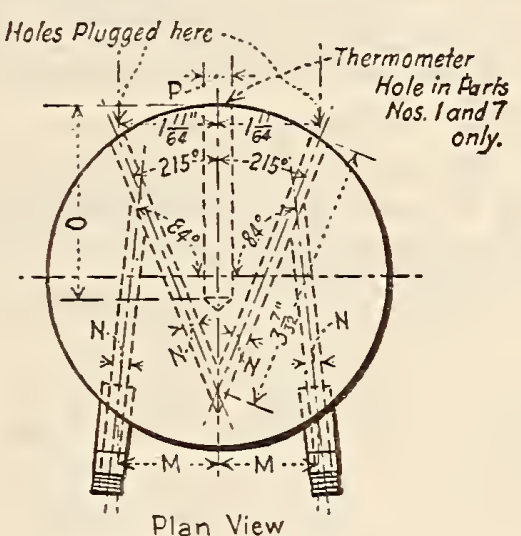

Parts Nos. 1, 3, 5 and 7 .

DIMENSIONS. TOLERANCES.

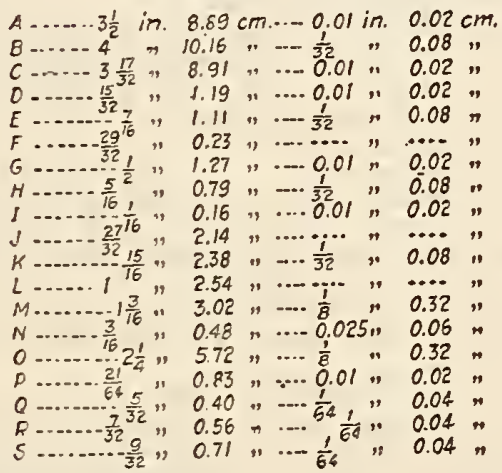

FIGURE 57.-Press-platen assernbly

the rate of 20 complete cycles per minute. The melting point thermometer reading, estimated to $0.1^{\circ} \mathrm{F}$., shall be observed and recorded every 30 seconds. The temperature of the wax will fall gradually at first, will then become almost constant, and will then again fall gradually.

The melting point thermometer reading, estimated to $0.1^{\circ} \mathrm{F}$., shall be observed and recorded
American Society for Testing Materials, tentative method of test for expressible oil and moisture in paraffin waxes, serial designation D308-29T, 1929.

\section{SCOPE}

1. This method is the well known press method for the determination of oil and moisture in paraffin wax.

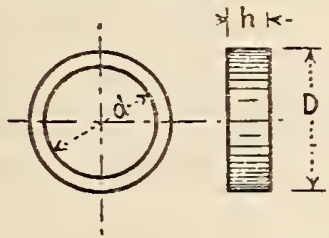

DIMENSIONS. TOLERANCES.

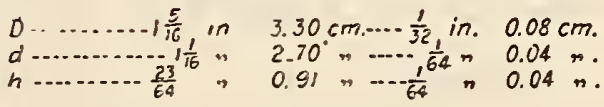

Figore 58.-Ring mald for wax samples

every 30 seconds, for at least three minutes after the It is applicable to crude, semirefined, and refined temperature again begins to fall after remaining almost constant. The record of temperature readings shall then be inspected and the average of the first four readings that lie within a range of $0.2^{\circ} \mathrm{F}$. shall be considered as the uncorrected melting point. This temperature shall be corrected if necessary for paraffin waxes melting at or above $108^{\circ} \mathrm{F}$. $\left(42.2^{\circ} \mathrm{C}\right.$.).

Note,-I thas been established that the expressible oil and moisture content does not bear a direct relation to the actual oil content of a given sample of paraffin wax - However, it is extremely difficult to determine actual oil content by any known method. The expressible oil and moisture method has been in almost universal use for many years and no doubt comes nearer to giving the information desired 


\section{APPARATUS}

2. Press.-The press shall be of any suitable form, and shall be capable of producing and maintaining a pressure of $1,000 \mathrm{lbs} . / \mathrm{in}^{2} \pm 100 \mathrm{lbs} . / \mathrm{in} .^{2}$ over the working surface of the platens, the latter having a diameter of 3.50 inches. This will require a total pressure of 9,620 pounds \pm 962 pounds upon the platen assembly. When presses of either the lever type or hydraulic type are used, it may be necessary to readjust the weights in the case of the former, or the gauge pressure in the case of the latter, to secure the specified pressure.

3. Press platens.-(a) The press platens shall be constructed of steel, properly plated, or of a suitable rust-resisting metal. The working surfaces of the platens shall be so finished as to be completely free from tool marks. The press platens shall conform to the dimensions shown in Figure 57.

(b) When asscmbled for use, the platens shall be connected in series by means of rubber tubes of such length that the platens may be manipulated without interrupting the flow of water through the assembly. The use of the complete platen assembly makes possible the simultaneous pressing of three samples of paraffin wax.

4. Wax sample molds.-The molds for wax samples shall consist of three smoothly turned brass rings conforming to the dimensions shown in Figure 58. When in use the rings shall be supported by a smooth No. 16 B. \& S. gage brass plate, 3 inches $\pm 1 / 8$ inch $(7.62 \mathrm{~cm} \pm 0.3 \mathrm{~cm})$ square

5. Thermometer.-The A. S. T. M. cloud and pour thermometer, conforming to the requirements of thermometer No. 5 (see table of A. S. T. M. thermometers, 500.2, p. 2), shall be used.

6. Graduated cylinder.-An ordinary 25-cc graduated cylinder may be used.

7. Cloth.-The cloth shall be a white or colored "silesia" or "duretta" or similar tightly woven cloth free from nap. The cloth shall be accurately cut in disks 3.50 inches in diameter.

8. Filter paper.-The filter paper used shall be Whatman's No. 1 filter paper, cut in disks 3.50 inches in diameter.

9. Desiccator.-The desiccator may be of any suitable size and design. The desiccator shall have a side opening in which a thermometer can be mounted. Calcium chloride shall be used as the desiccant.

10. Balance.-Any suitable analytical balance sensitive to $0.001 \mathrm{~g}$ may be used.

11. Electric oven.-The electric oven shall be capable of maintaining a temperature of $212^{\circ} \mathrm{F} . \pm 2^{\circ} \mathrm{F}$. $\left(100^{\circ} \mathrm{C} . \pm 1^{\circ} \mathrm{C}\right.$. $)$.

12. Refrigerator.-The refrigerator shall be capable of maintaining a temperature such that the wax samples when contained in the desiccator will be brought in a reasonable time, to a temperature of $60^{\circ} \mathrm{F} . \pm 5^{\circ} \mathrm{F} .\left(15.6^{\circ} \mathrm{C} . \pm 3^{\circ}\right.$ C. $)$.

13. Weighing bottle.-The weighing bottle may be of any suitable dimensions ${ }^{52}$ such that cloth disks may be placed therein without being tightly folded. The weighing bottle shall have a tightly fitting cover.

\section{PROCEDURE}

14. (a) An average sample of the wax shall be melted in a suitable container using a water bath whose temperature shall not be more than $35^{\circ} \mathrm{F}$. $\left(19.5^{\circ}\right.$ C. $)$ above the approximate melting point of the wax sample. Direct heat, such as a flame or hot plate, shall not be used and the wax sample

52 The American Can Co.'s gill style ointment box, deep pattern, 3-ounce capacity, may be used, provided they are selected with closely fitting lids. shall not be held in the melted condition any longer than necessary. The sample mold ring shall be placed upon the brass plate, the latter being accurately supported in a horizontal position. After thorough mixing, a portion of the wax sample shall be poured into the 25-ce graduated cylinder which has previously been heatcd by immersion in the water bath, and 2.5 to $3.0 \mathrm{cc}$ of the melted wax poured from the graduate into the sample mold ring. The wax sample shall be allowed to solidify at room temperature.

(b) Two cloth disks shall be placed in the weighing bottle and the bottle with its cover removed shall be dried for one hour at a tempertaure of $212^{\circ} \mathrm{F} . \pm 2^{\circ} \mathrm{F}$. $\left(100^{\circ} \mathrm{C} . \pm 1^{\circ} \mathrm{C}\right.$.). The cover of the weighing bottle shall be replaced, the bottle cooled in a desiccator and weighed to the nearest milligram. The wax sample shall be removed from the mold and placed within the weighing bottle with the cloth disks and the bottle reweighed to the nearest milligram. After reweighing, the closed weighing bottle shall be returned to the desiccator and the latter placed in the refrigerator, where the wax sample shall be cooled to a temperature of $60^{\circ} \mathrm{F} . \pm 5^{\circ} \mathrm{F} .\left(15.6^{\circ} \mathrm{C}\right.$. $)\left( \pm 3^{\circ} \mathrm{C}.\right)$, as indicated by a thermometer mounted in the side of the desiccator.

(c) The press platens shall be assembled as shown in Figure 57, connected together with rubber tubing, and water passed through the assembly in such a manner that both the upper and lower platens shall be brought to and maintained at a temperature of $60^{\circ} \mathrm{F} . \pm 5^{\circ} \mathrm{F} .\left(15.6^{\circ} \mathrm{C} . \pm 3^{\circ} \mathrm{C}\right.$. $)$ as indicated by thermometers placed in the thermometer holes in the upper and lower platens.

(d) The wax sample and the dry platens being at the required temperature, and the lower platen and ring being in place in the press, three disks of filter paper shall be placed upon the lower platen, making certain that the papers lie smoothly upon the platen. One disk of cloth shall be placed upon the filter papers. The wax sample shall then be placed upon the center of the cloth, and the other cloth disk laid upon the wax sample. Three filter papers shall be laid upon the second cloth disk and thc upper platen, or the next intermediate platen, if more than one sample of wax is to be pressed, shall be placed in position. Under no circumstances shall a pressing be made with other than the lower platen at the bottom of the assembly and the upper platen at the top of the assembly. A pressure of $1,000 \mathrm{lbs} . /$ in. $^{2} \pm 100 \mathrm{lbs}$./in. ${ }^{2}$ of the working surface of the platens, equivalent to a total pressure of 9,620 pounds \pm 962 pounds, shall be applied and maintained for a period of five minutes, after which time the pressure shall be released and the upper platen and ring removed. The two filter papers immediately adjacent to the sandwich of wax and cloth and any others which show traces of oil, when observed by transmitted light, shall be removed and replaced by fresh papers, and the pressing operation repeated. The filter paper shall be changed a second time and a third pressing made. During humid weather, platens may sweat considerably. In this case, care shall be exercised to prevent moisture from getting on the cloth-wax sandwich while changing the filter papers. Immediately before assembling the platens, they shall be wiped as dry as possible.

(e) After the three pressings have been made, the cloth-wax sandwich shall be folded and returned to the weighing bottle, care being taken to avoid loss of wax by crumbling. The weighing bottle witl its cover removed shall be placed in the electric oven and dried for one hour at a temperature of $212^{\circ} \mathrm{F} . \pm 2^{\circ} \mathrm{F} . \quad\left(100^{\circ} \mathrm{C} . \pm 1^{\circ} \mathrm{C}\right.$. $)$. The cover of the weighing bottle shall be replaced, the bottle cooled in a desiccator and weighed to the nearest milligram. 
15. The percentage of expressible oil and moisture shall be calculated as follows:

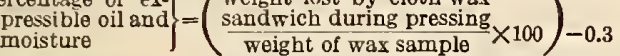

Nore - The correction of 0.3 is applied in order to correct for the lass of paraftin wax expressed along with the oil and moisture

16. With care and proper attention to detail, the amount of expressible oil and moisture determined in duplicate tests should not differ by more than 0.5 per cent.

17. Should a wax show negative content of expressible oil and moisture, the wax shall be reported as containing no expressible oil and moisture.

New York Produce Exchange, annual report, 1921-22, Paraffin Wax.

Paraffin wax shall be sold on color and melting point. White crude wax shall be understood to be on basis of 2 per cent oil and moisture contents; yellow crude wax on basis of 4 per cent oil and moisture contents.

The melting point is to be expressed in Fahrenheit degrees, American method of testing. The melting point is understood to be guaranteed, and buyer may reject the goods outright if not according to representation, and seller shall be required to replace.

Refined paraffin wax shall be sold according to melting point and if not as represented, goods may be rejected by buyer and seller be required to replace.

The price shall be understood for the net weight.

\subsection{PETROLATUM.}

American Railway Association, signal section, specification 7719 , May, 1920 , petrolatum for use in impedance bonds.

Nore. - The full text of this specification includes a requisite sheet, or contract form, and the following specifications for materials:

\section{MATERIAL}

(a) Shall be a pure mineral oil, free from crystalline wax, acid, alkali, or other chemicals.

(b) It shall conform to the following physical characteristics:

1. The specific gravity shall be between $27^{\circ} \mathrm{B}$. 0.891 and $30^{\circ}$ B. 0.875 .

F.)

2. The flash shall be not less than $246^{\circ} \mathrm{C}$. $\left(474^{\circ}\right.$

3. The viscosity at $99^{\circ}$ shall be between 105 and 120 seconds taken in a Saybolt standard universal viscosimeter.

4. The melting point shall be between $49^{\circ}$ and $57^{\circ} \mathrm{C}$. taken by the Saybolt method.

5. The solidifying point shall be between $49^{\circ} \mathrm{C}$. $\left(120^{\circ}\right.$ F.) and $57^{\circ}$ C. $\left(135^{\circ}\right.$ F.).

\section{INSPECTION}

Inspection requirements same as A. R. A. signal section specification 11328 . (See 522, p. 491.)

\section{TESTS}

(a) Tests may be made at point of production, or on samples submitted, and may also be made at destination.

(b) Contractor shall give the purchaser sufficient notice of time when material will be ready for testing.

(c) Contractor shall provide, at point of production, apparatus and labor for making the required tests under supervision of the purchaser.

\section{MARKING}

(a) Purchaser's order, requisition and package number, name of consignor, and name and address of consignee shall be plainly marked on outside of package.

(b) Detail list of loose pieces, containers, and their contents shall be furnished for each shipment. Where carload shipments are made, routing and car identification shall be shown.

(c) Where carload shipments are made, name and address of consignee may be omitted.

\section{PACKING}

(a) Material shall be so prepared as to permit convenient handling and to protect against loss or damage during shipment.

American Society for Testing Materials, tentative method of test for melting point of petrolatum, serial designation D127-28T, 1928.

\section{DEFINITION}

1. A. S. T. M. petrolatum melting point.-The temperature at which petrolatum becomes sufficiently fluid to drop from the thermometer used in making the determination under definite, prescribed conditions.

\section{APPARATUS}

2. (a) The melting point thermometer shall conform to the specifications for the A. S. T. M. paraffin wax melting point thermometer. (See thermometer No. 9, table of A. S. T. M. thermometers, 500.2 , p. 2.)

(b) The bath thermometer shall be of any suitable type accurate throughout the required range to $2^{\circ} \mathrm{F}$.

3. The test tubes shall be 25 by $100 \mathrm{~mm}$ in size, and shall be supplied with corks grooved at sides to permit air circulation and bored at the exact center to receive the thermometer.

4. The beakers shall be of the tall form type and have a capacity of $600 \mathrm{cc}$.

\section{PROCEDURE}

5. (a) Samples of sufficient size that exactly represent the petrolatum under inspection shall be secured. The material shall be melted slowly in a casserole or other suitable dish with stirring until the temperature reaches $180^{\circ} \mathrm{F}$. A fresh portion of the sample shall be used for each determination. The source of heat shall then be removed and the petrolatum allowed to cool to $15^{\circ} \mathrm{F}$. above the temperature that it is anticipated will be its melting point. The thermometer bulb shall be chilled to $40^{\circ} \mathrm{F}$., wiped dry, and while still cold thrust into the melted petrolatum so that approximately the lower half is submerged. It shall be withdrawn immediately, held vertically away from the heat until the surface dulls, and then placed in a water bath having a temperature not over $60^{\circ} \mathbf{F}$. for five minutes.

(b) The thermometer shall be securely fixed in a test tube by means of a cork so that the lowest point is $15 \mathrm{~mm}$ above the bottom of the test tube. The test tube shall be surrounded with a water bath at a temperature of $60^{\circ} \mathrm{F}$. The temperature of the bath shall be raised at a rate of $2^{\circ} \mathrm{F}$. per minute to $100^{\circ} \mathrm{F}$., then at a rate of $1^{\circ} \mathrm{F}$. per minute until the first drop of petrolatum leaves the thermometer. The temperature at this instant shall be recorded.

6 . If the variation of three such determinations does not exceed $2^{\circ}$ F., the average of these three 
determinations shall be reported as the melting point of the sample under test. If the variation of three determinations is greater than $2^{\circ} \mathbf{F}$., two additional determinations shall be made and the average of five determinations reported as the melting point.

American Society for Testing Materials, tentative method of test for color of petrolatum by means of the union colorimeter, serial designation D218-25T.

\section{APPARATUS}

The color of all petrolatums shall be determined by means of the union colorimeter.

(For description of apparatus and table of glass color standards see Sec. I, Apparatus, A. S. T. M. D155, 504.0, p. 96.)

\section{PROCEDURE}

The petrolatum shall be heated to $20^{\circ}$ to $30^{\circ} \mathrm{F}$. $\left(11^{\circ}\right.$ to $17^{\circ} \mathrm{C}$.) above the melting point. If darker than No. 8 color it shall be diluted with water white kerosene, the color of which is not darker than No. 21 as determined in accordance with A. S. T. M. D158 (see 503.0, p. 54), a mixture of exactly 15 per cent by volume of petrolatum and 85 per cent by volume of keroscne being used in all cases.

The water-white kerosene shall be heated to a temperature $20^{\circ}$ to $30^{\circ} \mathrm{F}$. $\left(11^{\circ}\right.$ to $17^{\circ} \mathrm{C}$.) above the melting point of the petrolatum being tested before mixing them together.

This mixture shall then be placed in the standard glass jar and compared with the three cylinder oil standards or optionally with the standards ranging from No. 1 to No. 8 , inclusive.

The daylight lamp may be brought closer to the opal glass in determining the darker colors. The numbers or letters designating the color of all petrolatum that has been diluted to make determinations shall be followed by the designation "Dil."

\subsection{CUTTING OILS.}

Federal Specifications Board, specification 2d, United States Government master specification for lubricants and liquid fuels.

(This publication, containing detailed specifications, methods of testing and methods of sampling, is published by the United States Government Printing Office as technical paper 323B, Department of Commerce, Bureau of Mines. The publication includes specifications for cutting oils which are given below.)

\section{CUTTING OILS}

\section{GENERAL REQUIREMENTS}

Sampling.-See A. S. T. M. method D270-27T, 503.0 , page 60 .

Testing.-See F. S. B. 2d, 503.0, page 66.

NoTE.-For convenience in presentation the test methods have been listed numerically and reference miade accordingly in the following table where tests apply.

\section{DETAIL REQUIREMENTS}

(a) General

Compounded cutting oil.--Shall be a clean and homogeneous solution of well-refined mineral oil and lard oil. It shall be free from disagreeable odors, rancidity, dirt, sediment, or other foreign matter. It shall not deposit gum on machinery or parts with use, nor shall separation of fatty or mineral oils occur at working temperatures. It shall be easily soluble and retain its oily consistence in kerosene, or soda and cold-water mixtures. The concentration of the soda solution which may be mixed with the compounded cutting oil prior to use should not exceed 1 per cent alkalinity calculated as $\mathrm{Na}_{2} \mathrm{CO}_{3}$. Aqueous solutions of this or lower concentrations may be obtained by adding approximately 1 ounce or less (28.5 g or less) of 58 per cent soda ash to 7 pints (3.31 liters) of water.

This oil is used as lubricant and coolant for machine cutting tools where soluble cutting oil is not suitable. It may be used as supplied or after mixing with either mineral oil or soda solution.

Soluble cutting oil.- - Shall be a clean, homogeneous mixture, free from disagreeable odors, mineral acids, sediment, or ingredients injurious to persons using the oil either in its original state or in emulsion. It shall show no segregation of any kind and shall not be rancid either in its original state or in emulsion. The oil shall not interfere with the lubrication of the machinery upon which it is used and shall not clog circulating systems, pumps, or bearings. It shall form stable emulsions with water in any proportion without the addition of any other product, and the emulsion so formed shall be free from froth, such as would be characteristic of soapsuds.

The oil is used as a coolant and lubricant for cutting and threading tools.

Lard oil shall be clean and free from other oil, fat, or grease. It shall be free from dirt, cracklings, and rancidity.

The oil is used for the lubrication of tools for pipe cutting and threading and other purposes.

(b) Properties and Tests

\begin{tabular}{|c|c|c|c|c|c|c|c|c|c|c|c|c|}
\hline Name & $\begin{array}{l}\text { Flash, } \\
\text { mini- } \\
\text { mum } \\
(\mathrm{No} .10)\end{array}$ & $\begin{array}{l}\text { Fire, } \\
\text { mini- } \\
\text { mum }\end{array}$ & $\begin{array}{l}\text { Viscos* } \\
\text { ity } \\
\text { seconds, } \\
\text { (No. 4) }\end{array}$ & $\begin{array}{l}\text { Saybolt } \\
100^{\circ} \mathrm{F}\end{array}$ & $\begin{array}{c}\text { Pour } \\
\text { test, } \\
\text { maxi- } \\
\text { mum } \\
(\text { No. 3) }\end{array}$ & $\begin{array}{l}\text { Corrosion } \\
\text { test (No. } \\
39)\end{array}$ & $\begin{array}{l}\text { Emukion } \\
\text { test (No. } \\
26)\end{array}$ & $\begin{array}{l}\text { Com- } \\
\text { pound- } \\
\text { ing } \\
\text { (Nos. } \\
40,41)\end{array}$ & $\begin{array}{c}\text { Free } \\
\text { fatty } \\
\text { acid } \\
\text { maxi- } \\
\text { mum } \\
\text { (No. 31) }\end{array}$ & $\begin{array}{l}\text { Water } \\
\text { (No. 17) }\end{array}$ & $\begin{array}{c}\text { Sedi- } \\
\text { ment } \\
\text { (No. 18) }\end{array}$ & $\begin{array}{l}\text { Special tests } \\
\text { required }\end{array}$ \\
\hline $\begin{array}{l}\text { Compounded cutting } \\
\text { oil. } \\
\text { Soluble cutting oil_-_- }\end{array}$ & ${ }^{\circ} F$. & ${ }^{\circ} F$ & $\begin{array}{c}\text { Mini- } \\
\text { mum } \\
190\end{array}$ & $\begin{array}{c}\text { Maxi- } \\
m u m \\
220\end{array}$ & ${ }^{\circ} F$ & Required 2 & Required & $\begin{array}{c}\text { Per cent } \\
25-35\end{array}$ & Per cent & \multirow{2}{*}{$\begin{array}{r}\text { Per cent } \\
0.5 \\
10.0 \\
.5\end{array}$} & None & \multirow{2}{*}{$\begin{array}{l}\text { No. } 2.1 \\
\text { No. } 42.8 \\
\text { Specific grav- } \\
\text { ity.4 } \\
\text { No. } 40.5\end{array}$} \\
\hline Lard oil & 440 & - & 190 & 220 & 45 & nequined & & $-\infty-1$ & 5 & & None & \\
\hline
\end{tabular}

1 A solution of the oil containing 50 per cent by volume of kerosene of quality specified in this specification, shall not be darker than No. 6 . 2 Clean, polished steel and polished copper strips shall not show more than extremely slight discoloration when partly immersed in the cutting oil for 24 hours.

The ash content shall not he more than 3.5 per cent.

- The specific gravity at $60^{\circ} \mathrm{F}$. $\left(15.5^{\circ} \mathrm{C}\right.$.) shall be not more than 0.925 or less than 0.912

8 The saponification shall be within the following limits: 190 to 198. 
504.8 TRANSFORMER AND SWITCH OILS.

American Railway Association, signal section, specification 4614, transformer oil.

\section{MATERIAI}

(a) Shall be a pure mineral oil obtained by the fractional distillation of petroleum, unmixed with any other substance. It shall be prepared and refined especially for the purpose and shall be free from moisture, acid, and alkali, and shall not contain sulphur compounds to exceed one-fifth of 1 per cent.

\section{TESTS}

(a) The flash of the oil determined in an open cup shall be not less than $320^{\circ} \mathrm{F} .\left(160^{\circ} \mathrm{C}\right.$. $)$ and on fire test not less than $347^{\circ} \mathrm{F}$. $\left(175^{\circ} \mathrm{C}\right.$.) .

(b) The viscosity shall be not more than 60 seconds at $104^{\circ} \mathrm{F}$. $\left(40^{\circ}\right.$ C.) by the Saybolt universal viscosimeter.

(c) The oil shall withstand a breakdown test of 30,000 volts A. C. between spherical surfaces of 0.5 $\mathrm{cm}$ radius, $0.40 \mathrm{~cm}$ apart.

American Society for Testing Materials, standard methods of testing electrical insulating oils, serial designation D117-27, 1927.

1. These tests are intended for oil used in transformers, oil circuit breakers and other electrical apparatus as an insulating or cooling medium, or both.

\section{SAMPLING}

2. Some of the tests, notably dielectric strength and sludge tests, ${ }^{53}$ of this class of material are very greatly affected by the most minute traces of certain impurities. Moisture, for example, greatly affects the dielectric test. It is exceedingly important that the samples be handled with the utmost care to avoid contamination. Therefore, the following procedure and precautions shall be observed in obtaining and transporting samples of the oil to be tested.

3. (a) Containers for samples.-Tin cans, or clear glass cork-stoppered bottles in cartons may be used. Containers shall be chemically clean and dry.

NotE.-The glass bottle offers the advantage that it may be examined to see that it is clean. It also allows risual inspection of the oil before testing, particularly as regards free water and solid impurities. However, if samples are placed in glass bottles, they sball be kept in tbe dark until the portions are removed for testing for color and sludge forming characteristies, as ligbt produces changes in these properties. Rubber stoppers sbould never be used with the glass containers. Only good quality cork sbould be used with a new cork for eacb samplo cont by by means of wrapping around the cork hefore forcing it into the moutb of the bottle some pure tbin tin foil or similar material not attacked by oil. The tin can fitted witb a screw cap protected witb a nonabsorbent gasket will endure barder usage. Tin cans sbould be soldered on tbe exterior surfaces.

(b) Thiefs.-When sampling a shipment of oil, enough thiefs should be provided to insure drainage of each before it is used a second time. For obtaining only a few samples, two or three thiefs are enough, but for sampling a car lot of drummed oil, six or more are desirable.

Note. $-A$ convenient and simple thief (see fig. 59) is described in par. 55 (a), A. S. T. M. D270-27T. (See 503.0, D. 60.)

4. All thiefs and sample receptacles shall be thoroughly cleaned before using, special care being taken that no lint or other fibrous material remains in or on them.

Note.-It is suggested tbat the thiefs be cleaned by rinsing with oil-free gasoline and dried in tbe hot cabinet (see sec. 5) before using. The best procedure is to rinse tbem after the sampling has heen

63 The great importance of a sludging test is recognized hut none is included in this standard at this time hecause none of the methods in general use are considered sufficiently satisfactory by committee D-9 to warrant consideration for standardization purposes. finished and tben place them in the bot cabinet. They will in tbis way be ready for use without further cleaning wben the next sbipment is to he sampled.

All sample containers before using sbould be rinsed witb oil-free All sample containers before using sbould be rith strong gand suds, rinsed thoroughy witb water and then dried in an oven at tor sam containers for samples or new oil, but those for used oil sbould be wasbed with both gasoline and henzol before using soapsuds. Containers whicb are not chemically clean and dry are likely to contaminate tbe sample of oil and cause misleading results in some of the tests.

5. When not in use all sampling apparatus should be kept in a hot, dry, dust-free cabinet or compartment at a temperature not less than $38^{\circ} \mathrm{C}$. $\left(100^{\circ}\right.$ F.). Containers for samples, after washing and drying as indicated in section 4 , shall be kept unstoppered while in the oven or hot cabinet but shall be closed immediately after they are taken out of the hot cabinet. However, it is permissible to prepare containers in advance, leaving them in the $105^{\circ}$ to $110^{\circ} \mathrm{C}$. oven until dry, then removing, corking, or capping and sealing by dipping the neck into melted paraffin wax. Bottles shall, before dipping, have cork and neck wrapped with paper and securely tied. If this procedure is followed, the paraffin should be wiped off carefully before removing the cork or cap preparatory to filling the bottle with the sample. Thief tubes and cups shall be kept at all times in a vertical position in the hot cabinet in a rack having a suitable drainage receptacle at the base.

6. (a) It is recommended that 1 quart of oil be taken as a sample if complete physical and chemical tests are to be made; at least 1 pint if the dielectric test alone is to be made.

(b) Drums or tank cars, etc., of oil shall not be sampled until the oil is at least as narm as the surrounding air. Electrical insulating oil is not hygroscopic, but cold oil may condense enough

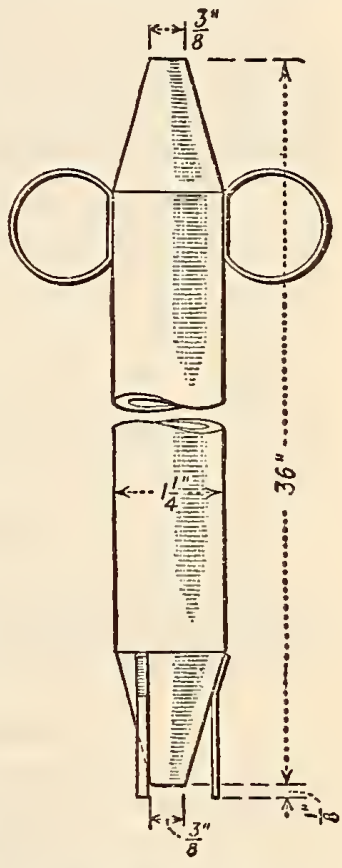

FIgURE 59.-Thief for sampling drums moisture on the surface from a humid atmosphere to seriously affect its insulating propcrties.

(c) Samples of oil shall be taken only after the oil has remained undisturbed and has settled for at least eight hours. Samples must, of course, never be taken in the rain and precautions must be taken during sampling to prevent contamination by windblown dust, etc.

(d) Drums and barrels of oil to be sampled should be lined up preferably on their sides, and numbered, bungs up. The bungs shall be unsealed and removed and laid with the oily side up beside the bungholes. The unstoppered sampling receptacle may be placed on the opposite side of the bungholes. The top hole of the thief tube shall be closed with the thumb, the tube thrust to the bottom of the container and the thumb removed. When the thief tube is filled the thumb shall be replaced, the tube quickly withdrawn and the contents allowed to flow into the sampling receptacle. The lower holes shall not be closed with the fingers of the 
other hand. The free hand shall be used to guide the stream of oil by touching the thief only as necessary. The oil shall not be allowed to flow over the hand or fingers before it flows into the sample receptacle nor shall any excess be allowed to flow over the drum and back into the drum through the open bunghole. When the sampling receptacle is filled it shall be closed quickly and the bung replaced in the container and tightened. The sampling receptacle shall be taken to the testing laboratory as quickly as feasible.

(e) Tank cars of oil may be sampled with the thief, introducing it through the manhole on top of the car, the cover of which shall be removed carefully so as not to introduce any dirt into the oil. The sample shall be taken as near as possible to the bottom of the tank car.

7. (a) When taking samples of oil from transformers, oil circuit breakers, and other apparatus where a thief tube or cup can not be used, the cautions outlined in section 6 shall be observed as far as practicable. In addition, care shall be taken to procure a sample which fairly represents the oil at the bottom of the tank. Transformer and oil circuit-breaker tanks, etc., are usually provided with drain pipes or sampling cocks from which the sample can be allowed to flow into the sample container. Sufficient oil should be allowed to flow to waste before the test sample is taken to insure that the sample will not be that portion which was stored in the drain pipe. For this reason the valve and the drain pipe should be sufficiently small to be emptied with convenience and yet sufficiently large to give an even flow of oil and avoid clogging by sediment. A 1/4-inch pipe and valve are recommended. This, of course, may be separate from the drainage pipe and valve or may be connected to the drainage valve by means of a suitable reducer.

\section{VISCOSITY}

8. The viscosity test shall be made in accordance with A. S. T. M. D88 (see 503.0, p. 47) and unless otherwise directed shall be taken at a temperature of $37.8^{\circ} \mathrm{C}$. $\left(100^{\circ} \mathrm{F}\right.$.).

\section{FLASH POINT}

9. The flash point shall be determined in accordance with A. S. T. M. D92. (See 504.0, p. 90.)

\section{POUR POINT}

10. The pour point shall be determined in accordance with A. S. T. M. D97-28. (See 502.2, p. 40.)

\section{NEUTRALIZATION NUMBER}

11. The neutralization number shall be determined in accordance with A. S. T. M. D188-27T (504.0, p. 99), using method $A$, section 4 .

\section{MINERAL ACIDS (CHLORIDES AND SULPHATES)}

12. The presence of mineral acids ( $\mathrm{Cl}$ or $\mathrm{SO}_{3}$ ) shall be determined by thoroughly shaking in a separatory funnel $100 \mathrm{cc}$ of the oil with 50 to 100 cc of hot $\left(90^{\circ}\right.$ to $100^{\circ} \mathrm{C}$.) distilled water and then separating and drawing off the water layer and testing it qualitatively with the usual procedures for the presence of chlorides or sulphates.

\section{FREE AND CORROSIVE SULPHUR}

14. (a) The apparatus for the determination of free sulphur shall consist of the following:

(a) Electrolytic sheet copper.-Approximate dimensions: Length, 3 inches $(7.62 \mathrm{~cm})$; width, 0.5 inch $(1.27 \mathrm{~cm})$; thickness, 0.031 inch $(0.079 \mathrm{~cm})$. (b) Glass tube.-Acid-resistant glass, 125 cc capacity, fitted with a slotted cork (a test tube of suitable size is satisfactory).

(c) Suitable bath in which a temperature of from $95^{\circ}$ to $110^{\circ} \mathrm{C}$. can be maintained.

15. Reagents. - The following reagents will be required: (a) Fuming nitric acid, c. p.; (b) hydrochloric acid, c. p. concentrated; and (c) barium chloride, 10 per cent solution.

16. The copper strip shall be mechanically polished. It shall be thoroughly washed to remove the polishing compounds and dried between filter paper. The polished copper strip shall be placed in the glass tube and $100 \mathrm{cc}$ of oil added. The strip shall be completely immersed. The tube shall be closed with the vented cork and the oil heated in the bath for not less than five hours, maintaining on the oil sample a temperature between $95^{\circ}$ and $110^{\circ} \mathrm{C}$.

If free sulphur or corrosive sulphur compounds are present, the copper strip will be discolored. This discoloration (a dark characteristic film) is not definite proof of the presence of free sulphur or corrosive sulphur compounds. It shall be tested as follows:

Remove the oil from the copper strip by rinsing with petroleum ether. After the ether has evaporated, completely dissolve the discoloring film with a few drops of fuming nitric acid, catching the solution in a 50-ce beaker. In this manner a minimum of copper is removed. Wash the strip with a minimum of distilled water, catching the washings in the beaker. To the combined solution, add about 5 cc of concentrated hydrochloric acid. Boil the solution to drive off free nitric acid. If necessary, dilute with a small amount of distilled water, and then to the boiling solution add $5 \mathrm{cc}$ of the 10 per cent solution of barium chloride. A white precipitate proves the presence of sulphur. Minute traces of sulphur are not precipitated immediately. If no precipitate forms in six hours, the temperature of the solution having been maintained at akout $95^{\circ} \mathrm{C}$., free sulphur or corrosive-sulphur compounds shall be considered absent.

Note.-All reagents shall be sulphur free. A blank should be run with each batch of determinations, following the above procedure, but using water in equal volume instead of oil. With necessary precautions the volume of solution for proving the presence or absence of sulphur should not exceed 10 to $20 \mathrm{cc}$ throughout the manipulation.

\section{DIELECTRIC STRENGTH}

17. (a) The apparatus shall be as described in section 14 of the Standard Methods of Testing Molded Insulating Materials (serial designation D48-27) of the American Society for Testing Materials. The test cup for holding the sample of oil shall be made of a material having a suitable dielectric strength. It must be insoluble in and unattacked by mineral oil and gasoline and nonabsorbent as far as moisture, mineral oil, and gasoline are concerned. The electrodes in the test cup between which the sample is tested shall be circular disks of polished brass or copper, 1 inch in diameter and having square edges. The electrodes shall be mounted in the test cup having their axes horizontal and coincident, with a gap of 0.1 inch between their adjacent faces, and with top of electrodes about $33 \mathrm{~mm}$ (11/4 inches) below the top of the cup.

18. (a) The electrodes and the test cup shall be wiped clean with dry, calendared tissue paper or with a clean dry chamois skin and thoroughly rinsed with oil-free, dry gasoline until they are entirely free from fibers.

(b) The spacing of electrodes shall be checked with a standard round gage having a diameter of 0.100 inch and the electrodes then locked in position. Care shall be taken not to touch the electrodes with the gage or in any other manner after cleaning the electrodes and cup, so as to avoid any possible contamination. 
(c) The test cup shall be filled with dry gasoline, and voltage applied with uniform increase at the rate of approximately $3 \mathrm{kv}$ (rms.) per second until breakdomn occurs. If the dielectric strength is not less than $25 \mathrm{kv}$ the cup shall be considered in suitable condition for testing the oil. If a lower test value is obtained the cup shall be cleaned with gasoline and the test repeated.

Note.-Evaporation of gasoline from the electrodes may chill them sufficiently to cause moisture to condense on their surface. For this reason, after the final rinsing with gasoline, the test cup should be immediately filled with the oil which is being tested, and the test proceeded with at once, or the electrodes should be thoroughly dried.

(d) The temperature of the oil when tested shall be between $20^{\circ}$ and $30^{\circ} \mathrm{C}$. $\left(68^{\circ}\right.$ and $86^{\circ} \mathrm{F}$.). Testing at lower temperatures is likely to give variable results which may be misleading.

(e) The sample in the container shall be shaken so as to thoroughly mix the oil before filling the test cup. This is even more important with used than with new oil as the impurities may settle to the bottom and the test may be misleading.

(f) The cup shall be filled with oil to a height of not less than $20 \mathrm{~mm}(0.787 \mathrm{inch})$ above the top of the electrodes.

(g) The oil shall be gently agitated by rocking the cup and then the oil shall be allowed to stand in the cup for three minutes before the first and one minute before each succeeding puncture. This will allow air bubbles to escape.

(h) Voltage shall be applied and increased uniformly at a rate of approximately $3 \mathrm{kv}$ (rms.) per second until breakdown occurs as indicated by a continuous discharge across the gap. (Occasional momentary discharges which do not result in a permanent car may occur; these should be disregarded.)

(i) Provision shall be made for opening the circuit as promptly as possible after breakdown has occurred in order to prevent unnecessary carbonization of the oil. After each puncture, the testing vessel shall be jarred to loosen particles of carbon adhering to the electrodes and the oil gently agitated but not with sufficient violence to introduce air bubbles.

(j) Five breakdowns shall be made on each filling after which the ressel shall be emptied and refilled with fresh oil from the original sample. The test shall be continued until the averaged values of at least three fillings do not differ from their mean by more than 10 per cent.

(k) The last filling of the cup should drain the container. If a quart sample be received, allowance shall be made in withdraming portions so that just enough be left for a final filling of the cup with the drainings from the container.

19. The report shall include the following: $(a)$ The volts (rms. value) at each puncture, the average voltage for each of the three or more fillings, and the grand average; $(b)$ the report shall be stated as $\mathrm{kv} 1.0$ in disk 0.1-inch gap; and (c) the approximate temperature of the oil at the time of the test.

NOTE.-A precision of about 3 per cent may reasonably be expected in 15 tests distributed among 3 consistent fillings taken in succession. But if the length of the gap is readjusted and possibilities of contamination exist, the precision may be only 6 or 7 per cent. Differences as great as 10 or 12 per cent may occur between different laboratorie even where the work is carefully done. (See Appendix I to Report of Committee D-9 of the A merican Society for Testing Materials in 1921, Proceedings, A. S. T. M., vol. 21, p. 397 (1921).)

\section{ASPHALT AND OTHER BITUMINOUS MATERIALS}

\subsection{GENERAI ITEMS.}

American Society for Testing Materials, standard method of test for the determination of bitumen, serial designation D4-27, 1927.

Approved by the American Standards Association.
SCOPE

1. Bitumen may usually be expeditiously and accurately determined by method No. 1, section 5 . In the case of certain native asphalts containing finely divided mineral matter, the mineral residue is not easily retained by the filter, and it is necessary to resort to method No. 2 , section 6 , in order to obtain accurate results. Method No. 2 shall not be used unless the filter clogs unduly or unless the mineral matter passing through the filter exceeds 0.5 per cent. In every case the report shall indicate whether method No. 1 or method No. 2 has been employed

\section{APPARATUS}

2. The apparatus shall consist of the following:

(a) Gooch crucible, approximately $4.4 \mathrm{~cm}$ in width at the top, tapering to $3.6 \mathrm{~cm}$ at the bottom, with a depth of $2.5 \mathrm{~cm}$.

(b) Filtering flask.

(c) Rubber stopper

(d) Filter tube.

(e) Section of rubber tubing to hold the Gooch crucible on the filter tube.

(f) Asbestos (amphibole) cut in pieces not exceeding $1 \mathrm{~cm}$ in length, shredded and shaken up with water.

(g) Two 150-ce Erlenmeyer flasks.

(h) Bunsen burner.

(i) Drying oven.

(j) Suction pump.

(k) Rubber tubing.

(l) Analytical balance.

$(m)$ Desiccator.

\section{PREPARATION OF SAMPLE}

3. The sample shall be representative and if it contains more than 2 per cent of water it shall be dehydrated by distillation in a copper still in accordance with A. S. T. M. D20-28T (see 505.0, p. 120), the water-free distillate being returned to the residue. If the material is hard and brittle, it may be ground and dried at a temperature below the temperature of volitilization of the material.

\section{PROCEDURE}

4. The Gooch crucible shall be set in the filter tube inserted in the stopper of the filtering flask. The flask shall be connected with the suction pump. Before suction shall be applied, the crucible shall be filled with asbestos suspended in water which shall be allowed to partly settle in the crucible. A light suction shall be applied to draw off the water, leaving a firm mat of asbestos in the crucible. More suspended asbestos shall be added and the process repeated until a felt is built up that barely transmits light. The felt shall then be thoroughly washed with water, dried in a drying oven, and ignited over a Bunsen burner. The crucible shall then be cooled in a desiccator and weighed.

\section{(a) METHOD NO. 1}

5. An amount of material which shall contain approximately $1 \mathrm{~g}$ of bitumen shall be weighed into a tared Erlenmeyer flask. One hundred cubic centimeters of chemically pure carbon disulphide shall be added to the flask in small portions with continued agitation until all lumps disappear and nothing adheres to the bottom. The flask shall be corked and set aside for 15 minutes.

The Gooch crucible shall be set up again with the suction flask and the carbon disulphide solution carefully decanted through the asbestos felt, with or without suction, as may be necessary. No sediment shall be allowed to go onto the filter. A small 
amount of carbon disulphide shall be used to wash down the sides of the flask and then the precipitate shall be brought onto the felt and the flask scrubbed with a feather if necessary to remove all precipitate. The contents of the crucible shall be washed with carbon disulphide until the washings are colorless. Suction shall be applied to the crucible to remove the carbon disulphide. The crucible shall be dried in the oven at $100^{\circ}$ to $125^{\circ} \mathrm{C}$. for 20 minutes, cooled in the desiccator, and weighed.

In case insoluble matter adheres to the flask, the flask shall be dried and weighed and the increase in weight over the original weight shall be added to the weight of insoluble matter in the crucible.

The crucible shall be ignited at a red heat and, after thorough ignition, cooled and weighed.

The weight of substance taken, minus the total weight of matter insoluble in carbon disulphide, is the total bitumen. The weight of material after ignition is ash.

\section{(b) METHOD NO. 2}

6. Material shall be weighed out in the same way as in (a) method No. 1 (sec. 5) into a tared Erlenmeyer flask and treated with 100 cc of chemically pure carbon disulphide.

The flask shall be loosely corked and shaken at intervals until all large particles of material have been broken down. The flask shall be left undisturbed for 48 hours. The solution shall be decanted into a similar tared flask, care being taken to disturb the material under known conditions of loading, time, and temperature. Where the conditions of test are not specifically mentioned, the load, time, and temperature are understood to be $100 \mathrm{~g}, 5$ seconds, $25^{\circ}$ C. $\left(77^{\circ} \mathrm{F}\right.$.), respectively, and the units of penetration to indicate hundredths of a centimeter.

\section{APPARATUS}

2. The container for holding the material to be tested shall be a fiat-bottom, cylindrical dish, 55 $\mathrm{mm}\left(2 \frac{1}{16}\right.$ inches $)$ in diameter and $35 \mathrm{~mm}\left(1 \frac{3}{8}\right.$ inches) deep. ${ }^{54}$

3. The needle (fig. 60) for this test shall bc made from a cylindrical steel rod approximately $50.8 \mathrm{~mm}$ (2 inches) long, having a diameter of 1.01 to 1.02 $\mathrm{mm}$. This shall be symmetrically tapered at one end to a cone approximately $6.35 \mathrm{~mm}$ ( $1 / 4$ inch) in height and whose angle shall be within the range of $8^{\circ} 40^{\prime}$ and $9^{\circ} 40^{\prime}$. After tapering, the point shall be "blunted" by grinding off to a truncated cone, the smaller base of which shall be from 0.14 to 0.16 $\mathrm{mm}$ in diameter. The finished needle shall be hardened and highly polished.

Note.-The National Bureau of Standards will measure and certify the accuracy of penetration needles in accordance with the tolerances given.

4. The water bath shall be maintained at a temperature not varying more than $0.1^{\circ} \mathrm{C}$. from $25^{\circ} \mathrm{C}$. $\left(77^{\circ} \mathrm{F}.\right)$. The volume of water shall be not less than 10 liters and the sample shall be immersed to

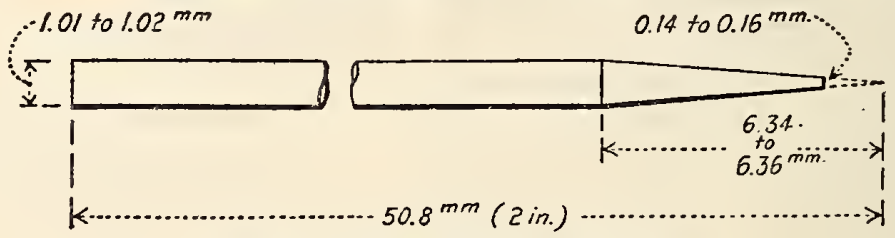

Figure, 60.-Needle for penetration test

as little of the residue as possible. The first flask shall be treated again with fresh carbon disulphide as before and left undisturbed for 48 hours. The solution from the second flask shall then be carefully decanted upon the Gooch crucible without use of vacuum, and this shall be followed by the solution from the first flask. The filter shall be washed with fresh carbon disulphide. The residue remaining in each flask shall be shaken again with fresh carbon disulphide and allowed to settle for 24 hours. The solution from both flasks shall then be decanted through the filter and the residues remaining in the flasks shall be washed again with carbon disulphide, the solution decanted and the process repeated until the washings are practically colorless. The temperature shall be maintained between $20^{\circ}$ and $25^{\circ} \mathrm{C}$.

The crucible and both flasks shall be dried at $100^{\circ}$ to $125^{\circ} \mathrm{C}$. and weighed. The filtrate containing the bitumen shall be evaporated, the bituminous residue burned, and the weight of the ash thus obtained added to that of the residue in the two flasks and the crucible. The sum of these weights deducted from the weight of substance taken is the weight of bitumen.

American Society for Testing Materials, standard method of test for penetration of bituminous materials, serial designation D5-25, 1925.

Approved by the American Standards Association.

\section{DEFINITION}

1. Penetration is defined as the consistency of a bituminous material, expressed as the distance that a standard needle vertically penetrates a sample of a depth of not less than $10 \mathrm{~cm}$ (4 inches) and shall be supported on a perforated shelf not less than 5 $\mathrm{cm}$ ( 2 inches) from the bottom of the bath.

5. Any apparatus which will allow the needle to penetrate without appreciable friction, and which is accurately calibrated to yield results in accordance with the definition of penetration, will be acceptable.

6. The transfer dish for container shall be a small dish or tray of such capacity as will insure complete immersion of the container during the test. It shall be provided with some means which will insure a firm bearing and prevent rocking of the container.

\section{PREPARATION OF SAMPIE}

7. The sample shall be completely melted at the lowest possible temperature and stirred thoroughly until it is homogeneous and free from air bubbles. It shall then be poured into the sample container to a depth of not less than $15 \mathrm{~mm}(5 / 8$ inch). The sample shall be protected from dust and allowed to cool in an atmosphere not lower than $18^{\circ} \mathrm{C}$. $\left(65^{\circ} \mathrm{F}\right.$.) for one hour. It shall then be placed in the water bath along with the transfer dish and allowed to remain one hour.

\section{PROCEDURE}

8. (a) In making the test the sample shall be placed in the transfer dish filled with water from the water bath of sufficient depth to completely cover the container. The transfer dish containing the sample shall then be placed upon the stand of

34 This requirement is fulfilled by the American Can Co.'s Gill style ointment box, deep pattern, 3-ounce capacity. 
the penetration machine. The needle, loaded with specified weight, shall be adjusted to make contact with the surface of the sample. This may be accomplished by making contact of the actual needle point with its image reflected by the surface of the sample from a properly placed source of light. Either the reading of the dial shall then be noted or the needle brought to zero. The needle is then released for the specified period of time, after which the penetration machine is adjusted to measure the distance penetrated.

At least three tests shall be made at points on the surface of the sample not less than $1 \mathrm{~cm}(3 / 8$ inch) from the side of the container and not less than 1 $\mathrm{cm}(3 / 8$ inch) apart. After each test the sample and transfer dish shall be returned to the water bath and the needle shall be carefully wiped toward its point with a clean, dry cloth to remove all adhering bitumen. The reported penetration shall be the average of at least three tests whose values shall not differ more than four points between maximum and minimum.

(b) When desirable to vary the temperature, time and weight. and, in order to provide for a and depth or diameter, at least $5.08 \mathrm{~cm}$ (2 inches) greater than the diameter of the revolving shelf.

It shall be well rentilated and shall be fitted with a window in the upper half of the door, so placed and of sufficient size to permit the accurate reading of the thermometer without opening the door. It shall also be provided with a perforated circular shelf preferably of approximately $24.8 \mathrm{~cm}(9.75$ inches) in diameter. This shelf shall be placed in the center of the oven and shall be suspended by a vertical shaft and provided with mechanical means for rotating it at the rate of five to six revolutions per minute. It shall be provided with recesses equidistant from the central shaft in which the tins containing the samples are to be placed. (A recommended form of aluminum shelf is shown in fig. 61.)

3 . The thermometer shall conform to the requirements for thermometer No. 4. (See table of A. S. T. M. thermometers, 500.2 , p. 2.)

4. The container in which the sample is to be tested shall be of tin, cylindrical in shape, and shall have a flat bottom. Its inside dimensions shall be substantially as follows: Diameter, $55 \mathrm{~mm}(2.17$

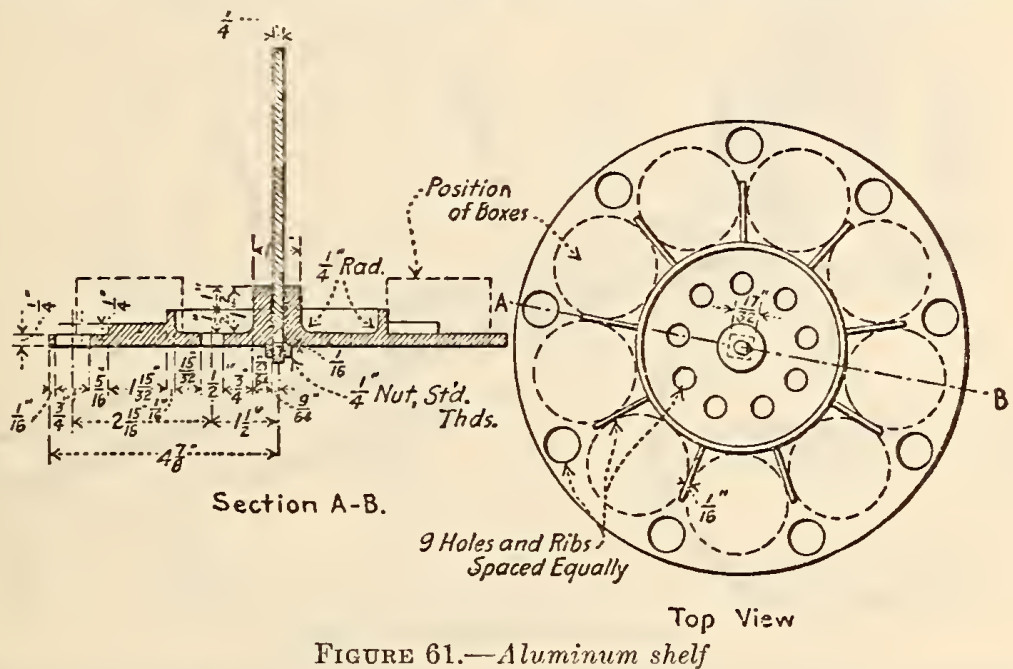

uniform method of reporting results when variations are made, the samples shall be melted and cooled in air as above directed. They shall then be immersed in water or brine, as the case may require, for one hour at the temperature desired. The following combinations are suggested: At $0^{\circ}$ C. $\left(32^{\circ} \mathrm{F}\right.$.) $200 \mathrm{~g}$ weight, 60 seconds. At $46.1^{\circ} \mathrm{C}$. (115 $\mathrm{F}$.) $50 \mathrm{~g}$ weight, 5 seconds.

American Society for Testing Materials, standard method of test for loss on heating of oil and asphaltic compounds, serial designation D6-27, 1927.

1. This test covers the determination of the loss in weight (exclusive of water) of oil and asphaltic compounds when heated as hereinafter prescribed. The material under examination must, therefore, first be tested for water, and if water is found to be present it shall be removed by suitable methods of dehydration before the material is subjected to the loss on heating test; or another sample obtained which is free from water.

\section{APPARATUS}

2. The oven may be either circular or rectangular in form and may be heated by either gas or electricity. Its interior dimensions shall be as follows: Height, not less than $40.64 \mathrm{~cm}$ (16 inches); width inches); depth, $35 \mathrm{~mm}$ (1.38 inches). (A 3-ounce Gill-style ointment box, deep pattern, fulfills these requirements.)

\section{PREPARATION OF SAMPLE}

5. The sample as reccived shall be thoroughly stirred and agitated, warming, if necessary, to insure a complete mixture before the portion for analysis is removed.

\section{PROCEDURE}

6. Weight $50 \mathrm{~g}$ of the water-free material to be tested into a tared container conforming to the requirements of section 4 . The oven shall be brought to a temperature of $163^{\circ}$ C. $\left(325^{\circ}\right.$ F.), and the tin box containing the sample placed in one of the recesses of the revolving shelf. The thermometer shall be immersed for the depth of its bulb in a separate 50-g sample of the material under test, placed in a similar container, and shall be conveniently suspended from the vertical shaft. This sample shall rest in one of the recesses upon the same shelf and revolve with the sample or samples under test. The oven shall then be closed and the shelf rotated five to six revolutions per minute 
during the entire test. The temperature shall be maintained at $163^{\circ} \mathrm{C}$. $\left(325^{\circ} \mathrm{F}\right.$.) for five hours, then the sample shall be removed from the oven, cooled and weighed, and the loss due to volatilization calculated.

7. During the 5-hour period the temperature shall not vary more than $1^{\circ} \mathrm{C}$. All tests showing a greater variation in temperature shall be rejected.

8. Under ordinary circumstances a number of samples having about the same degree of volatility may be tested at the same time. Samples varying greatly in volatility should be tested separately. Where extreme accuracy is required not more than one material should be tested at one time and duplicate samples of it should be placed simultaneously in the oven. Such duplicates shall check within the limits of accuracy given below. Results obtained on samples showing evidences of foaming during the test shall be rejected.

\section{ACCURACY}

9. Up to 5 per cent loss in weight the results obtained may be considered as correct within 0.5 .

\section{APPARATUS}

1. The apparatus consists of a flask, condenser tube, shield, receivers, and thermometers as specified in sections 2 to 6 .

2. Flask.-The distillation flask (fig. 62) shall be a side-neck distilling flask, having the following dimensions:

Diameter of bulb, outside

Diameter of neck, inside

Diameter of tubulature, inside

$86 \mathrm{~mm} \pm 1.5 \mathrm{~mm}$.

Length of neck

Distance, top of neck to tubulature - .......- $25 \mathrm{~mm} \pm 1.0 \mathrm{~mm}$

Length of tubulature

Angle of tubulature

3. Condenser tube.-The condenser tube shall be a suitable form of tapered glass tubing of the following dimensions:

Outside diameter of small end........ $12.5 \mathrm{~mm}$, permissible variation $\pm 1.5 \mathrm{~mm}$.

$\begin{array}{cc}\text { Outside diameter of large end. . ...... } & 28.5 \mathrm{~mm} \text {, permissible varia- } \\ \text { tion } \pm 3.0 \mathrm{~mm} \text {. }\end{array}$

Outside diameter of large end. ....... $28.5 \mathrm{~mm}$, permissible varia-
tion $\pm 3.0 \mathrm{~mm}$. $60.0 \mathrm{~mm}$, permissible variaTength of tapered part tion $\pm 5.0 \mathrm{~mm}$

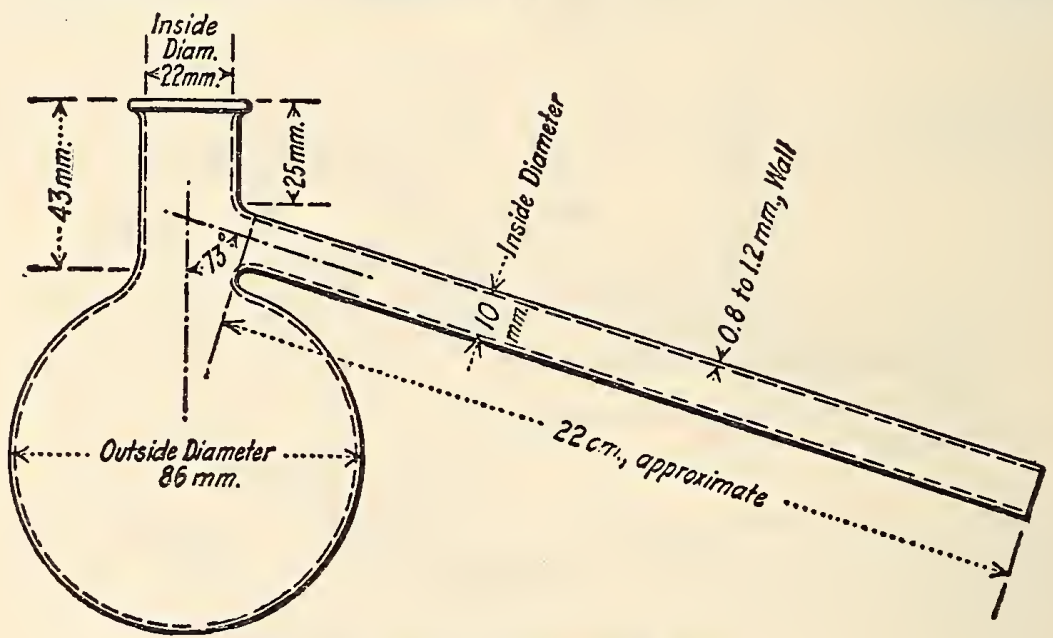

Figdre 62.-Distillation flask

Above 5 per cent loss in weight the numerical limit of error increases 0.01 for every 0.5 per cent increase in loss by volatilization as follows:

\begin{tabular}{|r|r|r|}
$\begin{array}{r}\text { Volatiliza- } \\
\text { tion loss }\end{array}$ & $\begin{array}{c}\text { Numerical } \\
\text { correction }\end{array}$ & $\begin{array}{c}\text { True volatili- } \\
\text { zation loss }\end{array}$ \\
\cline { 1 - 2 } Per cent & & Per cent \\
5.0 & \pm 0.50 & $4.50-5.50$ \\
5.5 & \pm .51 & $4.91-6.01$ \\
6.0 & \pm .52 & $5.48-6.52$ \\
10.0 & \pm .60 & $9.40-10.60$ \\
15.0 & \pm .70 & $14.30-15.70$ \\
25.0 & \pm .90 & $24.10-25.90$ \\
40.0 & \pm 1.20 & $38.80-41.20$ \\
\hline
\end{tabular}

Note.-If additional periods of heating are desired, it is recommended that they be made in successive increments of five hours each.

When the penetration of the sample after heating is required, melt the residue in the container at the lowest possible temperature and thoroughly mix by stirring, taking care to avoid incorporating air bubbles in the mass. Then bring it to the standard temperature and test as prescribed under the directions for A. S. T. M. D5, page 118.

American Society for Testing Materials, standard definitions of terms relating to materials for roads and pavements, serial designation D8-18, 1918. (See 510., p. 182.)

American Society for Testing Materials, tentative method of test for distillation of bituminous materials suitable for road treatment, serial designation $\mathrm{D} 20-28 \mathrm{~T}$.
4. Shield.-A galvanized iron shield, lined with $1 / 8$-inch asbestos, of the form and dimensions shown in Figure 63, shall be used to protect the flask from air currents and to prevent radiation. The cover (top) may be of transit board made in two parts, or it may be of galvanized iron lined with $1 / 8$-inch asbestos.

5. Receiver.-The distillates will be collected in tared Erlenmeyer flasks having a capacity of $\mathbf{5 0}$ to $100 \mathrm{ml}$.

6. Thermometer.-The thermometer shall conform to the requirements of thermometer No. 2. (See table of A. S. T. M. thermometers, 500.2, p. 2.)

\section{PREPARATION OF SAMPLE}

7. The sample, as received, shall be thoroughly stirred and agitated, warming, if necessary, to insure a complete mixture before the portion for analysis is removed.

8. The material may be tested for distillation without dehydration, if water is present not to exceed 2.0 per cent. If water is present in excess of 2.0 per cent, the bituminous material shall be dehydrated before distillation in accordance with the method for dehydration as given in sections 2 to 4 of the Standard Methods of Sampling and Analysis of Creosote Oil (serial designation D 38-27) of the American Society for Testing Materials. 


\section{PROCEDURE}

9. (a) The flask shall be supported on a tripod or rings over two sheets of 20 -mesh gauze, $150 \mathrm{~mm}$ square, as shown in Figure 64 . It shall be connected to the condenser tube by a tight cork joint. The thermometer shall be inserted through a cork in the neck with the top of the bulb level with the lowest point of juncture of the tubulature and neck of the flask.

(b) The axis of the flask through the neck shall be vertical.

(c) The distance from the bulb of the thermometer to the outlet end of the condenser tube be removed and any oil which has condensed in the condenser tube shall be drained into the last fraction.

(b) The residue sliall remain in the flask with the cork and thermometer in position until no vapors are visible, and it sliall then be weighed. If tests of the residue are required, the flask shall then be inclined so that the residue will flow around the sides, thus collecting any condensed vapors that may be on the sides of the flask, after which the residue shall be poured into a suitable receptacle and covered. If the residue becomes so cool that it can not be poured readily from the flask, it shall be reheated to a temperature not exceeding

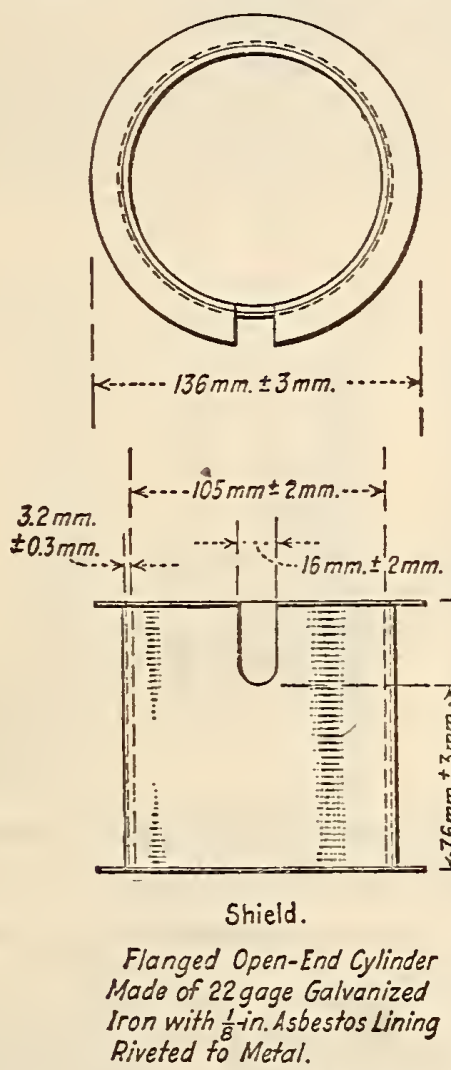

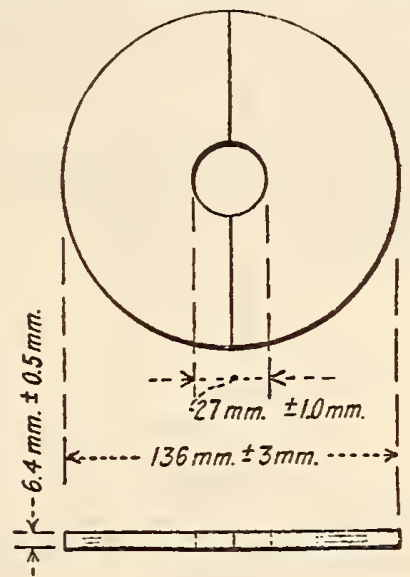

Cover

In Two Parts.

\section{FigdRE 63. - Shield}

shall be not more than 600 nor less than $500 \mathrm{~mm}$. The burner should be protected from draughts by a suitable shield or chimney. (See fig. 64.)

\section{DISTILLATION TEST}

10. (a) One hundred grams (100 $\mathrm{g} \pm 0.1 \mathrm{~g})$ of the sample shall be weighed into the flask, the apparatus assembled, and heat applied so that the first drop comes over in from 5 to 15 minutes. The distillation shall be conducted at the rate of between 50 and 70 drops per minute and the distillate collected in weighed receivers. The condenser tube shall be warmed whenever necessary to prevent accumulation of solid distillates. The fraction shall be collected at the points designated by the specifications. The receivers shall be changed when the thermometer indicates the maximum temperature for each fraction. When the maximum specified temperature of the test is indicated by the thermometer, the flame shall $125^{\circ} \mathrm{C}$. by holding the bulb of the flask in a suitable bath and not by the application of flame.

For weighing the receivers and fractions, a balance accurate to at least $0.05 \mathrm{~g}$ sliall be used.

(c) During the progress of the distillation the thermometer shall remain in its original position. No correction shall be made for the emergent stem of the thermometer.

(d) The results of the distillation test shall be reported in percentages by weight of water-free material. The following fractions are usually reported:

$$
\begin{aligned}
& \text { Tp to } 170^{\circ} \mathrm{C} \text {. } \\
& 170^{\circ} \text { to } 235^{\circ} \mathrm{C} \text {. } \\
& 235^{\circ} \text { to } 270^{\circ} \mathrm{C} \text {. } \\
& 270^{\circ} \text { to } 300^{\circ} \mathrm{C} \text {. } \\
& \text { Residue. }
\end{aligned}
$$

American Society for Testing Materials, standard method of test for softening point of bituminous materials (ring-ball method), serial designation D36-26, 1926.

1. The softening of bituminous materials generally takes place at no definite moment or temperature. 
As the temperature rises, they gradually and imperceptibly change from a brittle or exceedingly thick and slow flowing material to a softer and less viscous liquid. For this reason the determination of the softening point must be made by a fixed, arbitrary, and closely defined method if the results obtained are to be comparable.

\section{APPARATUS}

2. The apparatus shall consist of the following:

(a) A brass ring $15.875 \mathrm{~mm}(5 / 8$ inch) in inside diameter and $6.35 \mathrm{~mm}$ ( $1 / 4$ inch) deep; thickness of wall, $2.38 \mathrm{~mm}(3 / 32$ inch); permissible variation on inside diameter and thickness of ring, $0.25 \mathrm{~mm}$ (0.01 inch). This ring shall be attached in a convenient manner to a No. $13 \mathrm{~B}$. \& S. gage brass wire (diameter $1.83 \mathrm{~mm}=0.072$ inch). (See figure 65.)

(b) A steel ball $9.53 \mathrm{~mm}(3 / 8$ inch) in diameter weighing between 3.45 and $3.55 \mathrm{~g}$.

(c) A glass vessel, capable of being heated, not less than $8.5 \mathrm{~cm}$ (3.34 inches) in diameter and measuring $10.5 \mathrm{~cm}$ ( 4.13 inches) in depth from the bottom of
Suspend the thermometer so that the bottom of the bulb is level with the bottom of the ring and within $0.635 \mathrm{~cm}$ ( $1 / 1$ inch), but not touching the ring. Maintain the temperature of the water at $5^{\circ} \mathrm{C}$. $\left(41^{\circ}\right.$ F.) for 15 minutes. With suitable forceps, place the ball in the center of the upper surface of the bitumen in the ring, thus completing the assembly as in Figure 53.

5. Apply the heat in such a manner that the temperature of the water is raised $5^{\circ} \mathrm{C} .\left(9^{\circ} \mathrm{F}\right.$.) each minute.

6 . The temperature recorded by the thermometer at the instant the bituminous material touches the bottom of the glass vessel shall be reported as the softening point. No correction shall be made for emergent stem.

7. The rate of rise of temperature shall be uniform and shall not be averaged over the period of the test. The maximum permissible variation for any minute period after the first three shail be $\pm 0.5^{\circ} \mathrm{C} .\left(0.9^{\circ} \mathrm{F}\right.$.). All tests in which the rate of rise in temperature exceeds these limits shall be rejected,

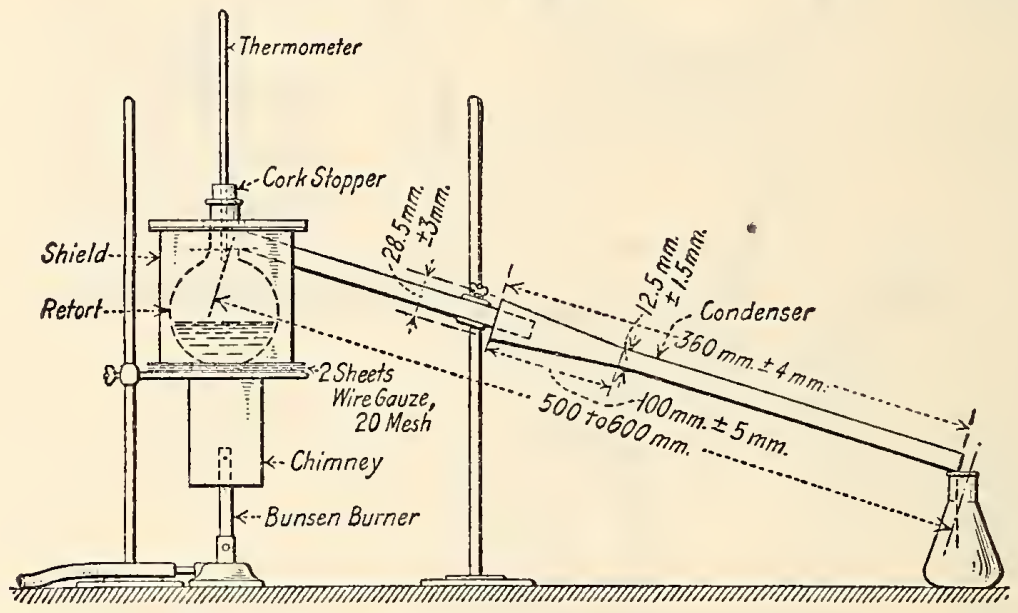

Figure 64.-Distillation apparatus assembly

the flare. (A 600-cc beaker, low form, meets this requirement.)

(d) A thermometer which shall conform to the requirements of thermometel No. 7. (See table of A. S. T. M. thermometers, 500.2, p. 2.)

\section{PREPARATION OF SAMPLE}

3. The sample shall be melted and stirred thoroughly, avoiding incorporating air bubbles in the mass, and then poured into the ring so as to lcave an excess on cooling. The ring, while being filled, should rest on a brass plate which has been amalgamated to prevent the bituminous material from adhering to it. After cooling, the excess material shall be cut off cleanly with a slightly heated knife.

\section{PROCEDURE}

(a) Bituminous Materials Having Soltening Points $80^{\circ} \mathrm{C} .\left(176^{\circ} \mathrm{F}.\right)$ or Belov

4. Fill the glass vesscl to a depth of substantially $8.24 \mathrm{~cm}$ (3.25 inches) with freshly boiled, distilled water at $5^{\circ} \mathrm{C} .\left(41^{\circ}\right.$ F.). Suspend the ring containing the sample in the watcr so that the lower surface of the filled ring is exactly $2.54 \mathrm{~cm}$ (1 inch) above the bottom of the glass vessel and its upper surface is $5.08 \mathrm{~cm}$ ( 2 inches) below the surface of the water. Place the ball in the water, but not on the specimen. (b) Bituminous Materials Having Softening Points Above $80^{\circ} \mathrm{C}$. $\left(178^{\circ} \mathrm{F}.\right)$

8. Use the same method as given under $(a)$, except that U.S. P. glycerin shall be used instead of water, and the starting point of the glycerin bath shall be $32^{\circ}$ C. $\left(89.6^{\circ}\right.$ F.). The bath shall be brought to this temperature and thoroughly agitated, then the apparatus and specimens shall be placed in the bath which shall be maintained under agitation at the starting temperature for 15 minutes, after which the assembly shall be completed by placing the ball on the center of the specimen and the test carried on as in $(a)$. In applying the heat, the ring apparatus shall be placed off the center of the container and the burner placed midway between the center and edge of the beaker away from the specimen.

The thermometer shall conform to the requirements of thermometer No. 3. (See table of A. S. T. M. thermometers, 500.2 , p. 2.)

\section{PRECAUTIONS}

9. The use of freshly boiled distilled water is essential, as otherwise air bubbles may form on the speciinen and afiect the accuracy of the results. Rigid adherence to the prescribed rate of heating is absolutely essential in order to secure accuracy of results.

A sheet of paper placed on the bottom of the glass vessel and conveniently weighted will prevent the bituminous material from sticking to the glass ves- 
sel, thereby saving considerable time and trouble in cleaning.

\section{ACCURACY}

10. The limit of accuracy of the test is $\pm 0.5^{\circ} \mathrm{C}$ $\left(0.9^{\circ} \mathrm{F}\right.$.).

American Society for Testing Materials, standard method of test for softening point of tar products (cube in water method), serial designation D61-24, 1924. (b) An L-shaped right-angled hook made of No. $12 \mathrm{~B}$. \& S. gage copper wire (diameter $2.05 \mathrm{~mm}=$ 0.0808 inch) the foot of which shall be $2.54 \mathrm{~cm}$ (1 inch) long. (See fig. 66.)

(c) A glass ressel, capable of being heated, not less than $8.5 \mathrm{~cm}$ ( 3.34 inches) in diameter and measuring $10.5 \mathrm{~cm}$. (4.13 inches) in depth from the bottom of the fiare. (A 600-ce beaker, Griffin low form, meets this requirement.)

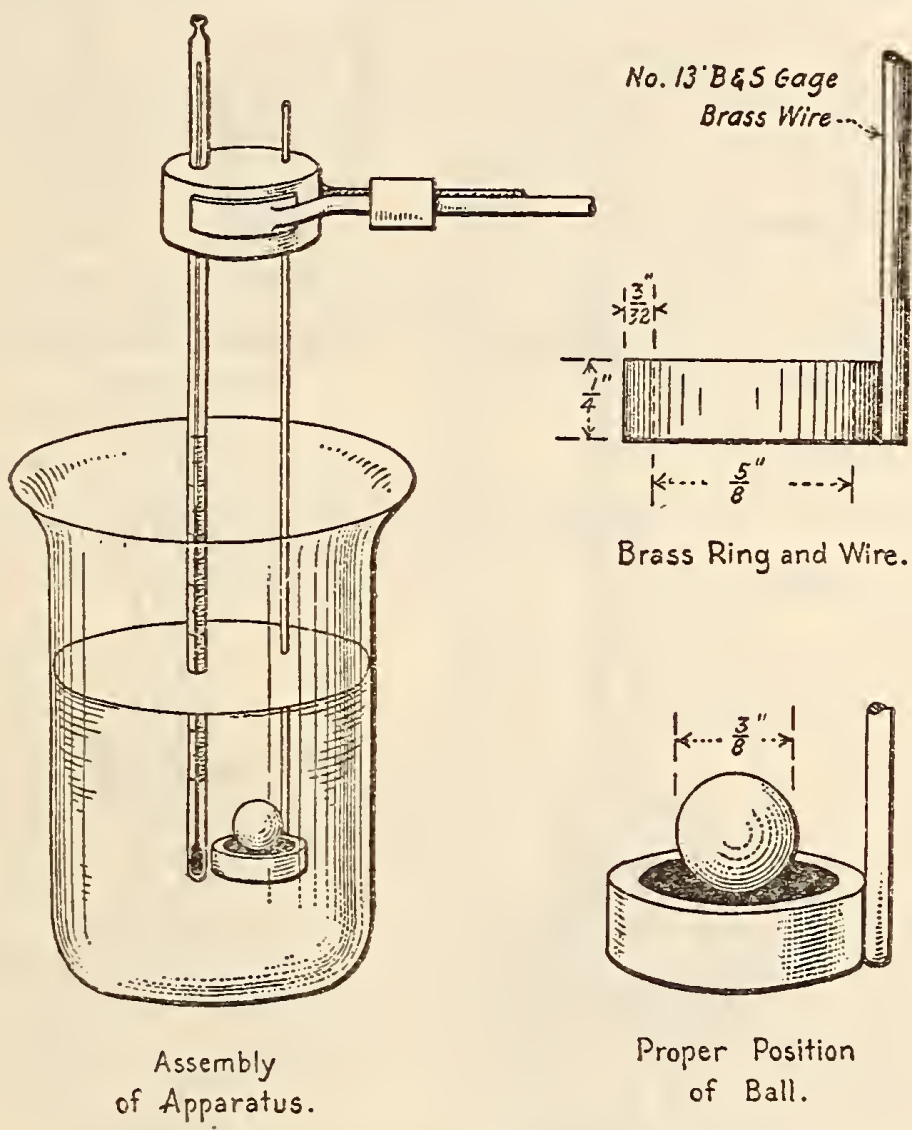

FigURE 65.-Apparatus for ring-and-ball method

1. (a) The softening of pitch takes place at no definite moment or temperature. As the temperature rises, pitch gradually and imperceptibly changes from a brittle or exceedingly thick and slow-flowing material to a softer and less viscous liquid. For this reason the determination of the softening point must be made by a fixed, arbitrary, and closely defined method if the results obtained are to be comparable.

(b) The methods herein described are not applicable to pitches having a melting point above $77^{\circ} \mathrm{C}$. $\left(170.6^{\circ} \mathrm{F}.\right)$.

(c) For the purpose of shortening the time required for testing, hard pitches having a softening point between $43^{\circ}$ and $77^{\circ} \mathrm{C}$. $\left(109.4^{\circ}\right.$ and $\left.170.6^{\circ} \mathrm{F}.\right)$ are cooled at $15.5^{\circ} \mathrm{C}$. $\left(60^{\circ} \mathrm{F}\right.$.) instead of at $4^{\circ} \mathrm{C}$. $\left(39.2^{\circ} \mathrm{F}\right.$.) as prescribed for sof $t$ pitches.

\section{APPARATUS}

2. The apparatus shall consist of the following:

(a) A mold suitable for forming a $12.7 \mathrm{~mm}$ ( $1 / 2$ inch) cube of pitch. (A recommended type is shown in fig, 66.) (d) A thermometer which shall conform to the requirements specified for thermometer No. 7, see table of A. S. T. M. thermometers, 500.2, p. 2 .

\section{PREPARATION OF SAMPIE}

3. The pitch shall be formed into a $12.7-\mathrm{mm}$ (1/2-inch) cube, truly shaped and with sharp edges, either by melting and pouring, or softening and pressing, into a mold. In all cases an excess of pitch shall be used and the surplus material shall be cut off cleanly with a slightly heated knife. The harder pitches specified can ordinarily be molded at room temperature, the softer pitches in water at about $4^{\circ}$ C. $\left(39.2^{\circ}\right.$ F.). If they are melted, they should first be thoroughly stirred, avoiding incorporating air bubbles in the mass, and then poured into the mold so as to leave an excess on cooling. The mold should rest on a brass plate and the surface of the plate and the interior surfaces of the mold should be amalgamated to prevent the pitch from adhering to them. 


\section{PROCEDURE}

(a) Pitches Having Softening Points Between $43^{\circ}$ and $77^{\circ}$ C. $\left(109.4^{\circ}\right.$ and $170.6^{\circ} \mathrm{F}$.)

4. Assemble the apparatus as shown in Figure 66. Fill the glass vessel to a depth of substantially 9.5 cm ( 3.75 inches) with freshly boiled, distilled water at $15.5^{\circ} \mathrm{C}$. $\left(60^{\circ} \mathrm{F}\right.$.). Place the cube of pitch on the wire as shown in Figure 66 and suspend it in the
5. Apply the heat in such a manner that the temperature of the water is raised $5^{\circ} \mathrm{C} .\left(9^{\circ} \mathrm{F}\right.$.) each minute.

6. The temperature recorded by the thermometer at the instant the pitch touches the bottom of the glass vessel shall be reported as the softening point. No correction shall be made for emergent stem.

7. The rate of rise of temperature shall be uniform and shall not be averaged over the period of

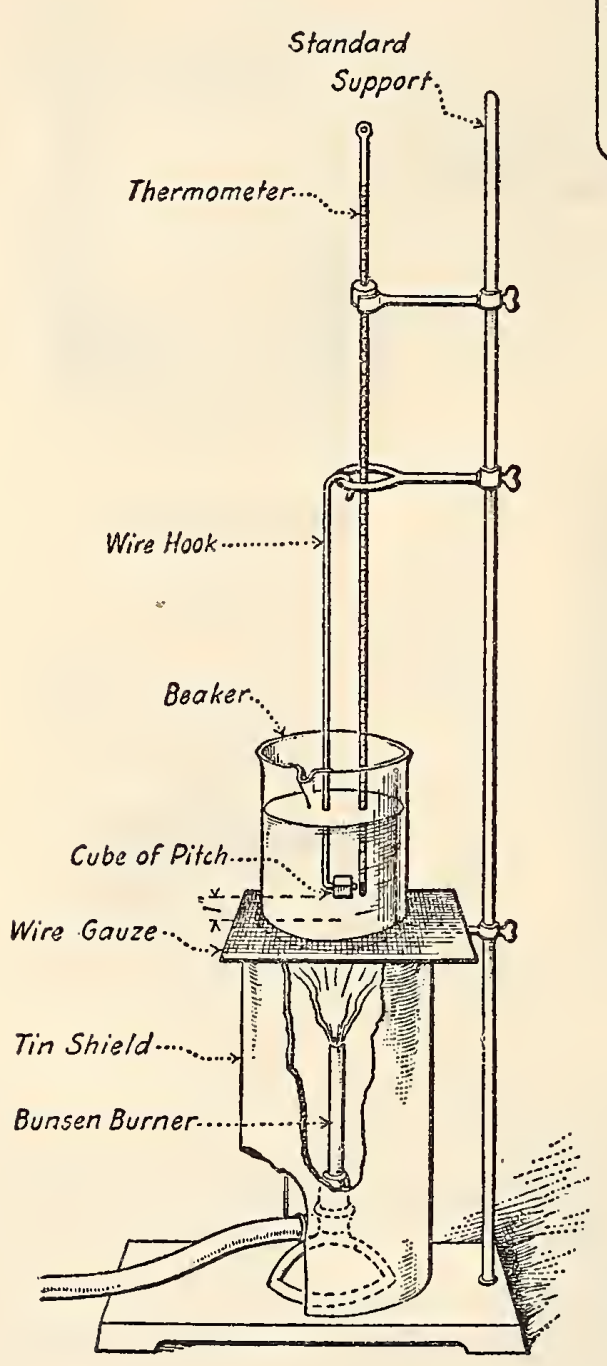

Assembly

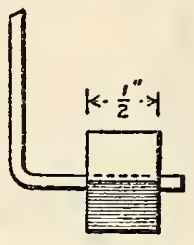

side.

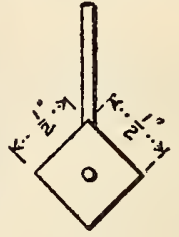

Front

Method of Placing Cube on Wire Hook.

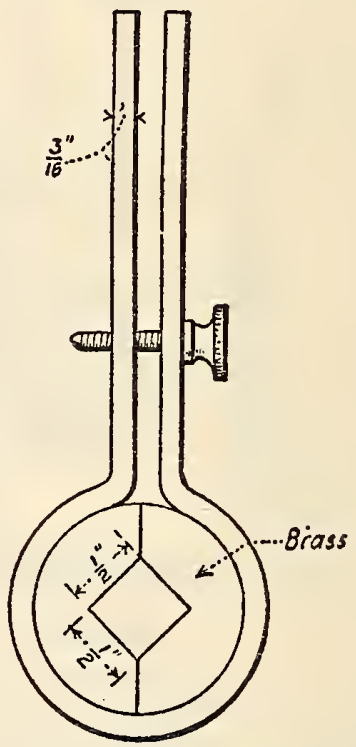

W.I. Pitch Mold (Special)

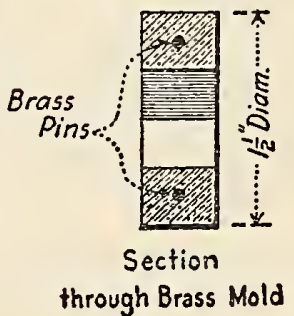

Fiqune 66.-Apparatus for cube-in-water method

water so that its lower edge is exactly $2.54 \mathrm{~cm}$ (1 inch) above the bottom of the glass vessel and its upper edge is $5.08 \mathrm{~cm}$ ( 2 inches) below the surface of the water. Allow it to remain in the water for 15 minutes before applying heat. Suspend the thermometer so that the bottom of the bulb is level with the bottom edge of the cube of pitch and within $0.635 \mathrm{~cm}$ (1/4 inch), but not touching the cube. the test. The maximum permissible variation for any minute period after the first three shall be $\pm 0.5^{\circ}$ C. $\left(0.9^{\circ}\right.$ F.). All tests in which the rate of rise in temperature exceeds these limits shall be rejected.

(b) Pitches Having Softening Points Below $43^{\circ}$ C. $\left(109.4^{\circ}\right.$ F.)

8. Use the same method as given under $(a)$, except that the water when placed in the glass 
vessel shall be at a temperature of $4^{\circ} \mathrm{C} .\left(39.2^{\circ} \mathrm{F}.\right)$. The cube shall be allowed to remain 15 minutes in this water before applying the heat.

\section{PRECAOTIONS}

9. The use of freshly distilled water is essential, as otherwise air bubbles may form on the cube and retard its sinking. Rigid adherence to the prescribed rate of heating is absolutely essential in order to secure accuracy of results.

A sheet of paper placed on the bottom of the glass vessel and conveniently weighted will prevent the pitch from sticking to the glass vessel, thereby saving considerable time and trouble in cleaning.

\section{ACCURACY}

10. The limit of accuracy of the test is $\pm 0.5^{\circ} \mathrm{C}$ $\left(0.9^{\circ}\right.$ F.).

American Society for Testing Materials, standard method of test for specific gravity of road oils, road tars, asphalt cements, and soft tar pitches, serial designation D70-27, 1927.

\section{DEFINITION}

1. The specific gravity of road oils, road tars, asphalt cements, and soft tar pitches shall be expressed as the ratio of the weight of a given volume of the material at $25^{\circ} \mathrm{C}$. $\left(77^{\circ} \mathrm{F}\right.$.) to that of an equal volume of water at the same temperature and shall be expressed thus:

\section{Specific gravity $25^{\circ} \mathrm{C} .\left(77^{\circ} \mathrm{F}.\right),-$}

\section{APPARATUS}

2. The determination of specific gravity shall be made with a pyknometer or weighing bottle (fig. 67), which shall consist of a straight-walled glass tube approximately $70 \mathrm{~mm}$ long and $22 \mathrm{~mm}$ in diameter, carefully ground to receive an accurately fitting solid glass stopper with a hole of 1.5 to $1.7 \mathrm{~mm}$ bore in place of the usual capillary opening. The lower part of the stopper is made concave in order to allow all air bubbles to escape through the bore. The depth of the cup-shape depression "shall be about $4.8 \mathrm{~mm}$ at the center. The stoppered tube should have a capacity of about $24 \mathrm{cc}$ and when empty should weigh not over $35 \mathrm{~g}$.

\section{PROCEDURE}

3. Before making a determination, the pyknometer with stopper shall first be calibrated by weighing it clean and dry upon an analytical balance. This weight is called $a$. It shall then be filled with freshly boiled distilled water at a temperature of $25^{\circ}$ C. $\left(77^{\circ} \mathrm{F}.\right)$, the stopper firmly inserted, all surplus moisture wiped from the surface with a clean, dry cloth and again weighed. This weight is called $b$.

4. When determining the specific gravity of road oils or road tars which flow readily, the material shall be brought to a temperature of $25^{\circ} \mathrm{C}$. $\left(77^{\circ} \mathrm{F}\right.$.) and poured into the pyknometer until it is full, with care to prevent the inclusion of air bubbles. The stopper is then firmly inserted and all excess of material forced through the opening is carefully removed with a clean, dry cloth. The pyknometer and contents are than weighed and this weight is called $c$. The specific gravity of the material shall be calculated from the formula:

$$
\text { Specific gravity }=\frac{c-a}{b-a}
$$

5. When determining the specific gravity of tar and asphalt products which are too viscous for the method described in section 4, a small amount of the material shall be brought to a fluid condition by the gentle application of heat, care being exercised to prevent loss by evaporation. When sufficiently fluid, enough is poured into the clean, dry pyknometer to about half fill it. Precautions shall be taken to keep the material from touching the sides of the tube above the final level and to prevent the inclusion of air bubbles. The tube should be slightly warmed before filling. The pyknometer and contents are then cooled to room temperature and weighed with the stopper. This weight is called $c$. The pyknometer is next removed from the balance, filled with freshly boiled distilled water, and the stopper firmly inserted. It is then completely immersed for not less than 30 minutes in a beakel of distilled water maintained at $25^{\circ} \mathrm{C}$. $\left(77^{\circ} \mathrm{F}\right.$.) after which it is removed, and all surplus water is wiped off with a clean cloth. It is immediately weighed. This weight is called $d$. The specific gravity of the material shall be calculated from the formula

$$
\text { Specific gravity }=\frac{c-a}{(b-a)-(d-c)}
$$

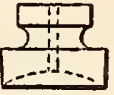

6. When making the specific gravity determination it is important that-

(a) Only freshly boiled distilled water shall be used.

(b) When weighing the pyknometer completely filled, the temperature of its contents shall be within $1^{\circ} \mathrm{C}$. $\left(1.8^{\circ} \mathrm{F}\right.$.) of $25^{\circ} \mathrm{C}$. $\left(77^{\circ} \mathrm{F}\right.$.)

(c) Precautions shall be taken to prevent expansion and overflow of the contents from the heat of the hand when wiping the surface of the pyknometer.

(d) The presence of all air bubbles shall be eliminated in filling the pyknometer and inserting the stopper.

(e) Weighings shall be made quickly after filling the pyknometer and shall be accurate to $1 \mathrm{mg}$. A number of trial fillings and catch weights may be necessary to obtain the desired degree of accuracy.

(f) To prevent breakage of the pyknometer when cleaning it out after a determination has been made upon a very viscous or semisolid material, it will be found advisable to warm it in an oven at not over $100^{\circ} \mathrm{C}$. until most of the material may be poured out and then to swab it with a piece of soft cloth or cotton waste. When cool it may be finally rinsed with carbon disulphide, benzol, or other solvent and wipcd clean.

7. The limit of accuracy of the test is \pm 0.005 specific gravity.

American Society for Testing Materials, standard method of test for specific gravity of asphalts and tar pitches sufficiently solid to be handled in fragments, serial designation D71-27, 1927.

\section{DEFINITION}

1. The specific gravity of asphalts and tar pitches shall be expressed as the ratio of the weight of a given volume of the material at $25^{\circ} \mathrm{C}$. $\left(77^{\circ} \mathrm{F}\right.$.) to that of an equal volume of water at the same temperature and shall be expressed thus: 


\section{APPARATUS}

2. The determination of specific gravity shall be made with an analytical balance equipped with a pan straddle or other stationary support (fig. 68).

\section{TEST SPECIMEN}

3 . The test specimen shall be a cube of the material measuring approximately one-half inch to the edge. It shall be prepared by melting a small sample of the material by the gentle application of heat, care being exercised to prevent loss by evaporation, and pouring when sufficiently fluid into a $1 / 2$-inch brass cubical mold, which has been amalgamated with mercury and which is placed on an amalgamated brass plate. Precautions should be taken to
5. The limit of accuracy of the test is \pm 0.005 specific gravity.

American Society for Testing Materials, standard method of test for water in petroleum products and other bituminous materials, serial designation D95-28, 1928.

See 502.2 , page 38 .

American Society for Testing Materials, tentative method of test for ductility of bituminous materials, serial designation D113-26T, 1926.

1. The ductility of an asphalt cement or semisolid bitumen is measured by the distance to which it will elongate before breaking when two ends of a briguet of the material are pulled apart at a specified rate of speed and at a specified temperature, which for a normal test shall be $5 \mathrm{~cm}$ per minute at $25^{\circ} \mathrm{C}$. $\left(77^{\circ} \mathrm{F}.\right)$.

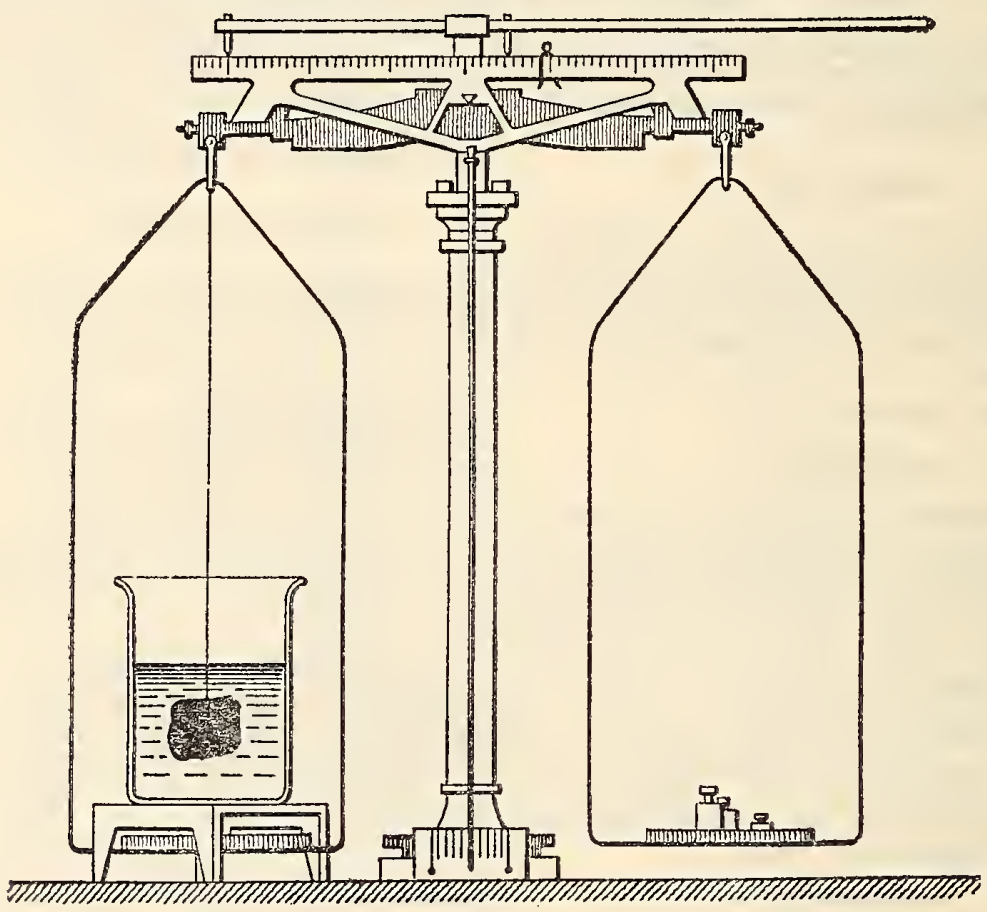

Figure 68.-Analytical balance equipped with pan siraddle

prevent the inclusion of air bubbles. The hot material should slightly more than fill the mold and when cool the excess may be cut off with a hot spatula. The specimen shall be removed from the mold when cooled to room temperature.

\section{PROCEDURE}

4. The balance shall first be tared with a piece of fine waxed silk thread sufficiently long to reach from the hook on one of the pan supports to the straddle or rest. The test specimen shall then be attached to the thread, so as to be suspended about 1 inch above the straddle from the hook on the pan support, and weighed. This weight is called $a$ and shall be accurate to $0.1 \mathrm{mg}$. The specimen, still suspended by the thread, shall then be weighed completely immersed in freshly-boiled distilled water at $25^{\circ}$ C. $\left(77^{\circ} \mathrm{F}\right.$. $) \pm 1^{\circ}$ C. $\left(1.8^{\circ}\right.$ F. $)$, adhering air bubbles being first removed with a fine wire. This weight is called $b$ and shall also be accurate to $0.1 \mathrm{mg}$.

The specific gravity of the material shall be calculated from the formula:

$$
\text { Specific gravity }=\frac{a}{a-b}
$$

\section{APPARATUS}

2. The mold shall be as shown in Figure 69 . It shall be made of blass, the ends, $b$ and $b^{\prime}$, being known as clips, and the parts, $a$ and $a^{\prime}$, as sides of the mold. The dimensions of the mold shall be as follows:

Total length (internal) $\mathrm{cm}$ Distance between clips _........ 2.97-3. 06 Width of clips at mouth Width of briquet at minimum cross section (halfway between clips) Thickness of briquet throughout

3. The water bath shall be maintained at a temperature not varying more than $0.1^{\circ} \mathrm{C}$. from $25^{\circ} \mathrm{C}$. $\left(77^{\circ} \mathrm{F}\right.$.). The volume of water shall be not less than 10 liters and the sample shall be immersed to a depth of not less than $10 \mathrm{~cm}$ and shall be supported on a perforated shelf not less than $5 \mathrm{~cm}$ from the bottom of the bath.

4. Any apparatus may be used for pulling the briquet of bitumen apart that is so constructed that the briquet will be continuously immersed in water and the two clips pulled apart at a uniform rate of speed of $5 \mathrm{~cm}$ per minute. 


\section{PROCEDURE}

5. The asphalt cement or bituminous material to be tested shall be completely melted at such a temperature that it will be thoroughly fluid-with ordinary paving asphalt cement this is about $160^{\circ} \mathrm{C}$. $\left(320^{\circ}\right.$ F.). It shall then be strained through a 50 -mesh sieve and, after a thorough stirring, poured into the mold. The mold shall be assembled on a brass plate and so as to prevent the material under test from sticking, the surface of the plate and the interior surfaces of the side pieces $a a^{\prime}$ of the mold shall be thoroughly amalgamated. In filling the mold care shall be taken not to disarrange the parts and thus distort the briquet. In filling, the material shall be poured in a thin stream back and forth from end to end of the mold until it is more than level full. It shall be left to cool to room temperature and then placed in water maintained at $25^{\circ} \mathrm{C}$. $\left(77^{\circ} \mathrm{F}\right.$.) for 30 minutes, after which the excess of bitumen shall be cut off by means of a hot putty knife or spatula so that the mold shall be just level full.

5. The brass plate and mold, with briquet, shall then be placed in the water bath and kept at a temperature of $25^{\circ} \mathrm{C}$. $\left(77^{\circ} \mathrm{F}\right.$.) for at least one and one-half hours, when the briquet shall be removed from the plate and the side pieces detached.

\begin{tabular}{|c|c|c|c|}
\hline & $\begin{array}{l}\text { Mini- } \\
\text { muma }\end{array}$ & $\begin{array}{l}\text { Nor- } \\
\text { mal }\end{array}$ & $\begin{array}{l}\text { Maxi- } \\
\text { mumm }\end{array}$ \\
\hline $\begin{array}{l}\text { Weight of float } \\
\text { Total height of float } \\
\text { Height of rim above lower side of shoul- } \\
\text { der } \\
\text { Thickness of shoulder-a } \\
\text { Diameter of opening }\end{array}$ & $\begin{array}{l}37.70 \\
34.0 \\
26.5 \\
1.3 \\
11.0\end{array}$ & $\begin{array}{l}37.90 \\
35.0 \\
27.0 \\
1.4 \\
11.1\end{array}$ & $\begin{array}{r}38.10 \\
36.0 \\
27.5 \\
1.5 \\
11.2\end{array}$ \\
\hline
\end{tabular}

2. The collar (fig. 70) shall be made of brass and shall be in accordance with the following requirements:

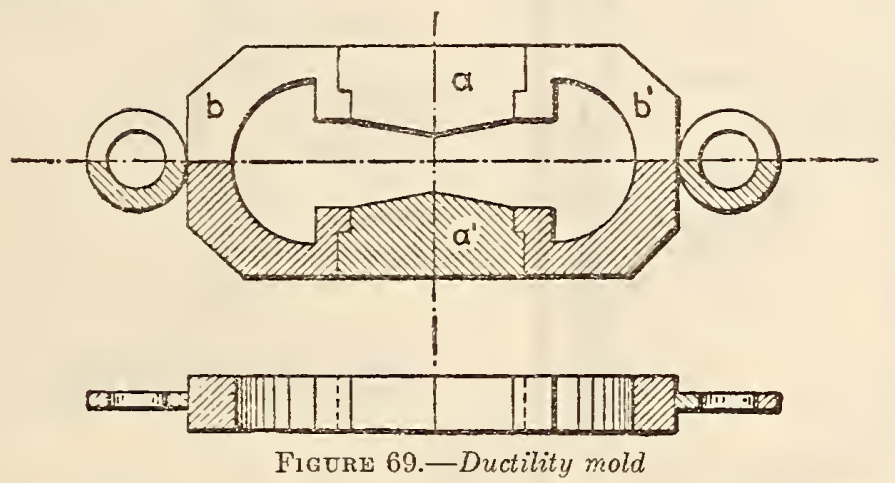

7. The rings at each end of the clip shall then be attached to the pins or hooks in the ductility machine and the two clips pulled apart at a uniform rate of $5 \mathrm{~cm}$ per minute $( \pm 5$ per cent) until the briquet ruptures. The distance through which the clips have been pulled to produce rupture shall then be measured in centimeters. While the test is being made, the water in the tank of the ductility machine shall cover the sample by at least $2.5 \mathrm{~cm}$ and shall be kept continuously at a temperature within $0.5^{\circ} \mathrm{C}$. of $25^{\circ} \mathrm{C}$. $\left(77^{\circ} \mathrm{F}.\right)$.

A normal test is one in which the material between the two clips pulls out to a point or thread until rupture occurs at the point where the thread has practically no cross sectional area. The average of three normal tests shall be taken and reported as the ductility of the sample.

If a normal test is not obtainable on three successive tests, the ductility should be reported as being unobtainable under the conditions of the test.

American Society for Testing MIaterials, standard method of float test for bituminous materials, serial designation D139-27, 1927.

Approved by the American Standards Association.

\section{APPARATUS}

1. The float (fig. 70) shall be made of aluminum or aluminum alloy and shall be in accordance with the following requirements: weight of $53.2 \mathrm{~g}$, shall float upon water with the rim $8.5 \pm 1.5 \mathrm{~mm}$ above the surface of the water. Dimensions of the apparatus additional to those required above are given in Figure 70.

4. The thermometer shall conform to the requirements of thermometer No. 7. (See A. S. T. M. table of thermometers, 500.2, p. 2 .)

5 . The diameter of the bath and the depth of water shall be at least $185 \mathrm{~mm}$.

\section{PREPARATION OF SAMPLE}

6. The brass collar shall be placed with the smaller end on a brass plate which has been previously amalgamated with mercury by first rubbing it with a dilute solution of mercuric chloride or nitrate and then with mercury.

The sample shall be completely melted at the lowest possible temperature that will bring it to a sufficiently fluid condition for pouring, excepting creosote oil residues, which shall be mixed and poured at a temperature of $100^{\circ}$ to $125^{\circ} \mathrm{C}$. It shall be stirred thoroughly until it is homogeneous and free from air bubbles. The sample shall then be poured into the collar in any convenient manner until slightly more than level with the top.

Asphalt and asphalt products.-Asphalt and asphalt products shall be cooled to room temperature, placed in water maintained at $5^{\circ} \mathrm{C}$. for five minutes, after which the surplus material shall be 
removed by means of a spatula, or steel knife, which has been slightly heated. The collar and plate shall then be placed in a tin cup containing ice water maintained at $5^{\circ} \mathrm{C} . \pm 1^{\circ} \mathrm{C}$., and left in this bath for at least 15 minutes.

Tar products.- Tar products shall be immediately immersed in ice water maintained at $5^{\circ} \mathrm{C}$. for five minutes, after which the surplus material shall be removed by means of a spatula or steel knife, which has been slightly heated. The collar and plate shall then be placed in a tin cup containing ice water for one minute. Any water shall then be removed from the inside of the float and the latter immediately floated in the warm bath. As the plug of material becomes warm and fluid it is forced upward and out of the collar until the water gains entrance into the saucer and causes it to sink.

(c) The time in seconds between placing the apparatus on the water and when the water breaks through the material shall be determined by means of a stop watch, and shall be taken as a measure of the consistency of the material under examination.

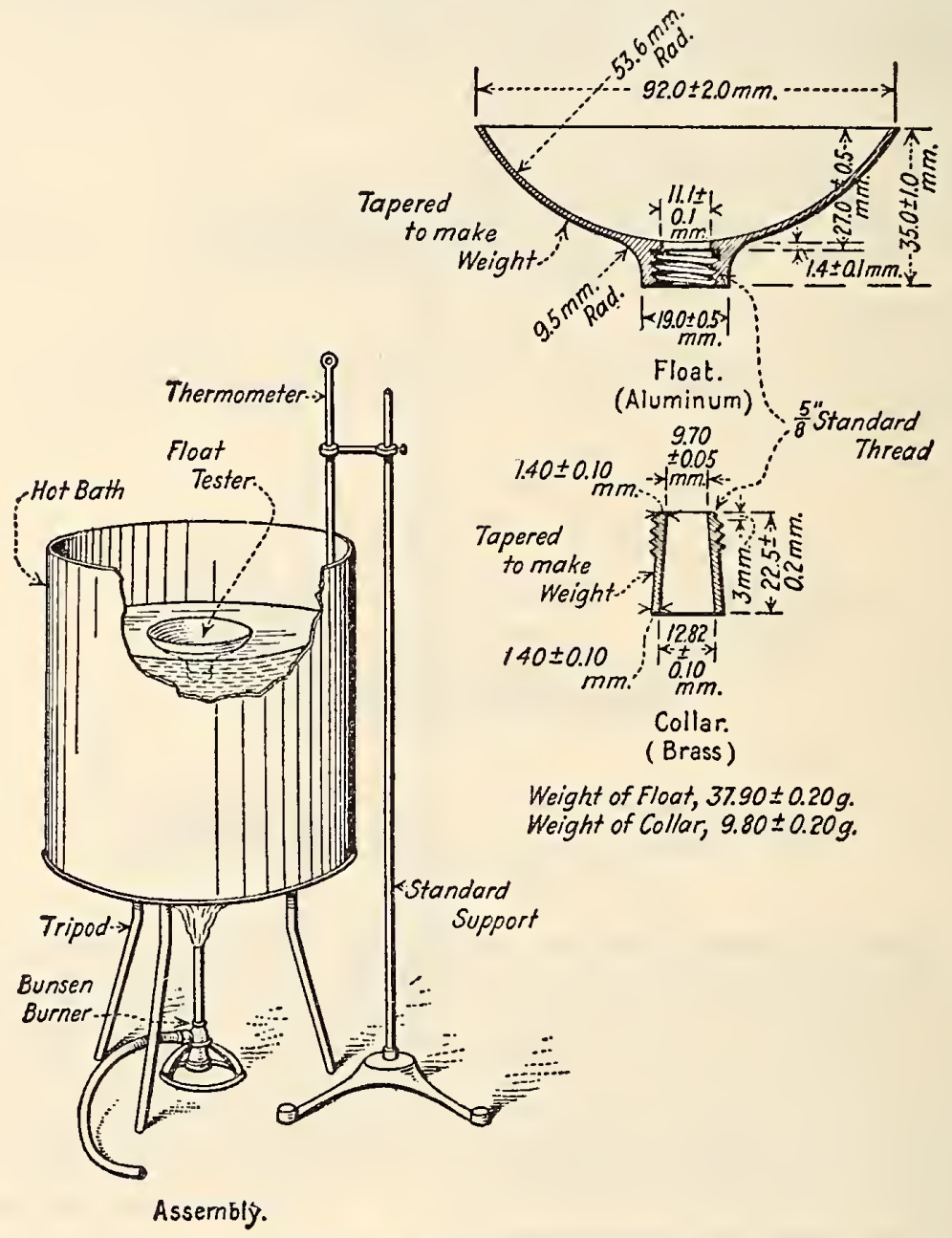

Figere 70.-Float test apparatus

maintained at $5^{\circ}$ C. $\pm 1^{\circ}$ C., and left in this bath for at least 15 minutes.

\section{PROCEDURE}

7. (a) The bath shall be filled with water and the water heated to the temperature at which the test is to be made. This temperature shall be accurately maintained and shall at no time throughout the test be allowed to vary more than $0.5^{\circ} \mathrm{C}$. from the temperature specified.

(b) After the material to be tested has been kept in the ice water for not less than 15 minutes nor more than 30 minutes, the collar with its contents shall be removed from the plate and screwed into the aluminum float and immersed in water at $5^{\circ} \mathrm{C}$.
Note.-Special precaution should be taken to insure the collar fitting tightly into the float and to see that there is no seepage of water between the collar and float during the test.

American Society for Testing Materials, standard methods of sampling bituminous materials, serial designation D140-25, 1925.

1. Samples may be taken for either of two purposes:

(a) To represent as nearly as possible an average of the bulk of the material sampled.

(b) To ascertain the maximum variation in characteristics which the material may possess. In either case they shall be obtained by methods hereinafter described.

2. (a) Care shall be taken that the samples are not contaminated with dirt or any other extraneous 
matter and that the sample containers are perfectly clean and dry before filling.

(b) Immediately after filling, the sample containers shall be tightly closed and properly marked for identification on the container itself or on a linen tag attached to the container.

3. (a) Whenever practical, bituminous materials shall be sampled at the point of manufacture, and at such time as to allow the tests, controlling acceptance or rejection, to be made in advance of shipment.

(b) When impracticable to take samples at the point of manufacture, they should be taken from the shipment immediately upon delivery.

4. For routine laboratory examination of acceptability of a given lot not less than 1 quart of material should be submitted, which should be representative of the average sample collected as hereinafter described.

5. Containers for liquid bituminous materials shall be small-mouth cans with cork-lined screw caps. Containers for semisolid and solid materials shall be friction-top cans.

\section{SAMPLING AT PLACE OF MANUFACTURE}

6. The inlet and outlet to the storage tank shall be sealed and a 1-gallon sample drawn from the top, middle, and bottom contents. The sample may be taken from drain cocks on the side of the tank, if such are available. Enough material should be discarded to insure a representative sample. Otherwise, samples may be taken by lowering weighted bottles or cans into the material. The bottle or can should be fitted with a stopper which can be removed by a string or wire attached to it after it has been lowered to the proper depth. The three samples from bulk storage shall be tested separately for consistency in order to detect stratification. They may then be combined and thoroughly mixed for other tests that may be required.

7. Where tank cars, distributors, or barrels are being filled, samples may conveniently be taken from the pipe line through which the material is flowing, as hereinafter described.

\section{(a) WHEN MATERIAL IS PUMPED ONDER PRESSURE}

8. The sampling pipe shall be inserted into a rising section of the pipe line on the discharge side of the pump. The sampling pipe shall be not more then one-eighth the diameter of the line pipe and its opening should be turned at an angle of $90^{\circ}$ facing the flow of the liquid. This pipe shall be provided with a plug cock and shall discharge into a receiving drum of 50 -gallon capacity. The plug cock shall be so adjusted that there is a steady continuous flow of bituminous material through it and shall be so regulated that the receiver will fill in the same time that is required to make the entire pumping. In the case of semisolid materials, the receiver shall be provided with a steam coil which shall keep the contents at a temperature just above the liquef ying point. At completion of the pumping, the receiver shall be thoroughly agitated, and a 1-quart sample taken therefrom. The sampling shall be so regulated that for each 1,000 gallons of material pumped, at least 1 gallon of sample is taken; but 40 gallons shall be the limit required for any one cargo.

Care should be taken that the drip cock, after once having been set, allows a constant flow during the pumping.

This method is also applicable to gravity flows where the pipe line is completely filled by the outflowing liquid and has a rising section.

\section{(b) WHEN MATERIALS FLOW BY GRAVTTY}

9. Materials flowing by gravity through pipes which are not completely filled may be sampled by taking dipperfuls at the outlet at frequent and regular intervals. These samples shall be combined, and the total sample shall be not less than 0.1 per cent of the whole material. The samples shall be collected in a receiver and resampled as described in section 8

\section{SAMPLING AT POINT OF DELIVERY}

10. Samples may be taken by means of a weighted bottle or can as described in section 6 , or from the unloading pipe line as described in section 7 .

Liquid bituminous materials shall be sampled before heating. Semisolid or solid bituminous materials shall be rendered fluid by heating. Sampling should be so conducted as to eliminate the possibility of adventitious water resulting from leaky steam heating coils, rain, or snow.

11. Samples may be taken from distributors by means of a weighted bottle or can, as described in section 6 . One sample shall be sufficient.

12. (a) Semisolid or solid materials.-Where the lot of material to be sampled is obviously from a single run or batch of the producer, one package or cake shall be selected at random and sampled as described in the following paragraph. Where the lot of material to be sampled is not obviously from a single run or batch of the producer, or where the single sample selected as described above fails on test to conform to the requirements of the specifications, a number of packages or cakes shall be selected at randoin equivalent to the cube root of the total number of packages or cakes in the lot. For convenience the following table is given, showing the number of samples to be selected for shipments of various sizes:

\begin{tabular}{|c|c|c|c|}
\hline $\begin{array}{c}\text { Packages or cakes in } \\
\text { shipment }\end{array}$ & $\begin{array}{l}\text { Packages } \\
\text { or cakes } \\
\text { selected }\end{array}$ & $\begin{array}{c}\text { Packages or cakes in } \\
\text { shipment }\end{array}$ & $\begin{array}{l}\text { Packages } \\
\text { or cakes } \\
\text { selccted }\end{array}$ \\
\hline $\begin{array}{l}2 \text { to } 8 \\
9 \text { to } 27 \\
28 \text { to } 64 \\
65 \text { to } 125 \\
126 \text { to } 216\end{array}$ & $\begin{array}{l}2 \\
3 \\
4 \\
5 \\
6\end{array}$ & $\begin{array}{l}217 \text { to } 343 \\
344 \text { to } 512 \\
513 \text { to } 729 \\
730 \text { to } 1,000 \\
1,001 \text { to } 1,331\end{array}$ & $\begin{array}{r}7 \\
8 \\
9 \\
10 \\
11\end{array}$ \\
\hline
\end{tabular}

Samples shall be taken from at least 3 inches below the surface and at least 3 inches from the side of the container or cake or from the center of a cake. A clean hatchet may be used if the material is hard enough to shatter and a broad, stiff putty knife if the material is soft. An auger, or brace and $3 / 4$-inch bit, or other suitable means may also be used. When more than one package or cake in a lot is sampled, each individual sample shall be not less than one-fourth pound in weight. When the lot of material is obviously from a single run or batch of the producer, all samples from the lot shall be melted and thoroughly mixed, and an average sample taken from the combined material for examination. In case more than a single run or batch of the producer is present and the batches can be clearly differentiated, a composite sample shall be prepared for examination from each batch. Where it is not possible to differentiate between the various batches each sample shall be examined separately.

(b) Liquid materials.-Samples of liquid bituminous materials from barrels or drums shall be secured by taking 1 quart of material from packages selected at random according to the cube-root method described in paragraph $(a)$. When the lot of material is obviously from a single run or batch of the producer the samples shall be combined and thoroughly mixed and an average sample taken from the combined material. In case more than one 
run or batch is present and can be clearly differentiated, a composite sample shall be prepared from each batch. Where it is impossible to differentiate between the various batches or runs, each sample shall be examined separately.

13. Solid bituminous materials in crushed fragments or powder, such as gilsonite, grahamite, etc., shall be sampled in accordance with A. S. T. M. D21. (See 501.0, p. 4.)

American Society for Testing Materials, standard methods of testing felted and woven fabrics saturated with bituminous substances for use in waterproofing and roofing, serial designation D146-27, 1927.

1. These methods cover the examination of felted or woven fabrics saturated (but not coated) with asphalt or coal-tar products, for use in the "membrane" system of waterproofing.

\section{SAMPIING}

2. From each shipment or portion thereof representing a product of the same kind, class, and weight a number of rolls shall be selected at random, equivalent to one-half the cube root of the total number of rolls in the lot, except that in lots of 1,000 or less, 5 rolls shall be taken. If the cube root, as calculated, proves to be a fractional number, it shall be expressed as the next higher whole number. For convenience, the following table is given, showing the number of samples to be selected for shipments of various sizes:

\begin{tabular}{l|r|r|r}
\hline $\begin{array}{c}\text { Packages in ship- } \\
\text { ment }\end{array}$ & $\begin{array}{c}\text { Number of } \\
\text { packages } \\
\text { selected }\end{array}$ & $\begin{array}{c}\text { Packages in ship- } \\
\text { ment }\end{array}$ & $\begin{array}{c}\text { Number of } \\
\text { packages } \\
\text { sclected }\end{array}$ \\
\hline Up to 1,000 & 5 & 5,833 to 8,000 & 10 \\
1,001 to 1,728 & 6 & 11 \\
1,729 to 2,744 & 8,001 to 10,648 \\
2,745 to 4,096 & 10,649 to 13,842 \\
4,097 to 5,832 & 8 & 13,843 to 17,576 & 13 \\
& 9 & 17,577 to 21,952 & 14 \\
\hline
\end{tabular}

\section{EXAMINATION OF FINISHED FABRIC}

3. The width of each roll taken shall be measured to the nearest one thirty-second inch. The minimum and maximum width so obtained shall be reported.

4. Each roll taken shall be weighed intact to the nearest one-fourth pound and the minimum and maximum gross weight shall be reported.

5. Each roll sampled shall be stripped. All the wrappers and packing material shall be weighed together to the ncarest one-fourth pound and the average maximum weight thereof per roll shall be reported.

6. Each roll shall be unwound, observing the workmanship and finish while so doing. The length and width of each roll shall thereupon be measured to the nearest one-fourth inch and the square feet of material contained in each roll calculated.

7. The rolls shall be rewound, fastened with pieces of light string, and then each roll shall be wcighed to the nearest one-fourth pound. In the case of felted fabrics, calculate the weight of each roll in pounds per 100 square feet and report the maximum and minimum, likewise recording the average for the rolls sampled, which shall be regarded as the corresponding weights of the entire shipment. In the case of woven fabrics, calculate the weight of each roll in ounces per square yard, and report the minimum, likewise recording the average for the rolls sampled, which shall be regarded as the corresponding weights of the entire shipment.

8. From the rolls examined select the one whose weight per unit area is nearest the average weight per unit area of the lot. The roll so selected shall be laid flat, the first convolution or two carefully unwound, and with a knife and straightedge the sheet shall be cleanly cut across at right angles to the edges. A section measuring 30 inches in the direction of the roll's length shall be removed. If the material is surfaced with sand or other fine material any detached particles belonging to each 30 -inch section shall be retained. The width of each section shall be measured to the nearest one thirty-second inch. Each section, together with any detached surfacing, shall be weighed to the nearest gram. The weight in pounds per 100 square feet or the weight in ounces per square yard shall be calculated from the formulas:

Pounds per 100 square feet $=1.0582 \times \frac{\text { weight of } 30 \text {-inch section in grams }}{\text { width of } 30 \text {-inch section in inches }}$
Ounces per square yard $=1.5238 \times \frac{\text { weight of } 30 \text {-inch section in grams }}{\text { width of } 30 \text {-inch section in inches }}$

This must check within 1 per cent of the average weight per unit area of shipment (sec. 7). If the sample fails to do this, then additional samples shall be cut from the same roll, until one is obtained which does, which sample shall be kept for further examination.

NoTE.-As a referee method or in case any disputearises regarding the properties as may be ascertained from the particular sample selected, a 30-inch sample shall be taken and examined separately froin each roll sampled in section 2 .

9. If the material is surfaced with sand or other finely comminuted material, the surfacing shall be swept with a moderately stiff brush from all the 30 -inch sections taken. All the material thus removed shall be caught and weighed to the nearest ounce. From the aggregate areas of all the sections taken the average weight of detached comminuted surfacing shall be calculated in pounds per 100 square feet.

10. The thickness of each specimen shall be measured at 10 equally spaced points, 1 inch from a cut edge, with a micrometer gate having flat-bearing surfaces at both contact points of not less than onehalf inch in diameter and preferably of the springand-dial type. The readings for each roll sampled shall be averaged and the minimum, maximum, and average thickness calculated to the nearest 0.001 inch.

11. From each specimen, 2-inch strips shall be cut as shown at $A-1$ and $A-Q$ in Figure 71 . The 2-inch strips shall be cut into 1-inch squares. About $50 \mathrm{~g}$, selected at random, shall be accurately weighed and distilled with 100 cc of a coal-tar distillate of which 5 to 10 per cent boils below $100^{\circ} \mathrm{C}$. $\left(212^{\circ} \mathrm{F}\right.$.) and of which at least 90 per cent distills up to $180^{\circ} \mathrm{C}$. $\left(356^{\circ} \mathrm{F}\right.$.) in an apparatus of the form and under the conditions prescribed in A. S. T. M. D95. (See 502.2 , p. 38.) The flask containing the solvent and sample shall be heated in a paraffin bath at a temperature of $302^{\circ}$ to $338^{\circ} \mathrm{F}$. $\left(150^{\circ}\right.$ to $170^{\circ} \mathrm{C}$.) until no further water passes over. The distillate shall be allowed to stand in the graduated receiver until the water separates, Whereupon the volume of water shall be measured and its weight calculated. From this the average percentage of moisture in the fabric, as received, shall be calculated.

12. (a) Felted fabrics.-With a photographic trimmer having a stop attached exactly 1 inch behind the blade, 10 strips shall be cut with the fiber grain, as shown at $B-1$ to $B-10$ (fig. 71) and 10 strips across the fiber grain, as shown at $C-1$ to $C-10$. Each strip should measure 6 inches and have parallel sides 1 inch apart (within one-thirty-secondinch) with the edges cut straight and clean. Both sets of strips shall be tested at $70^{\circ} \mathrm{F}$. $\left(21.1^{\circ} \mathrm{C}\right.$. $)$, 11sing a tension-testing machine, such as the Scott strength tester (type F, 100-pound capacity), the Perkins strip tester, or equal, in which the clamps 
are attached to swivels free to move in any direction. The test strips shall be gripped $1 \frac{1}{2}$ inches on each end, leaving approximately 3 inches between the clamps. The tension shall be increased by causing the lower clamp of the machine to travel at a uniform speed of 12 inches per minute. If any strip breaks nearer than one-half inch to either clamp, the reading shall be disregarded, and an additional strip shall be tested in its place. The 10 readings, with and across the grain, respectively, shall be averaged for each roll sampled. From these results the average strength with and across the fiber grain, respectively, of the fabric as supplied, shall be calculated.

(b) Woven fabrics. - Woven fabrics shall be tested at $70^{\circ} \mathrm{F} .\left(21.1^{\circ}\right.$ C. $)$ in accordance with the grab test as described in section $12(b)$ of the Standard
(6) Cracks on the $10-\mathrm{mm}$ mandrel.

(7) Cracks on the 5 -mm mandrel.

(8) Cracks when bent through $180^{\circ}$ over a $1 / 16^{-i n c h}$ (1.588-mm) mandrel.

(9) May be bent through $180^{\circ}$ over a 1/16-inch (1.588-mm) mandrel in one direction without cracking, but will crack when bent through $360^{\circ}$ in the opposite direction.

(10) May be bent through $180^{\circ}$ over a 1/10-inch $(1.588-\mathrm{mm})$ mandrel in one direction and then through $360^{\circ}$ over a $1 / 16^{-i n c h}(1.588-\mathrm{mm})$ mandrel in the opposite direction without cracking.

The readings for each roll shall be averaged separately, and from these results the minimum, maximum, and average pliability of the felt shall be calculated.

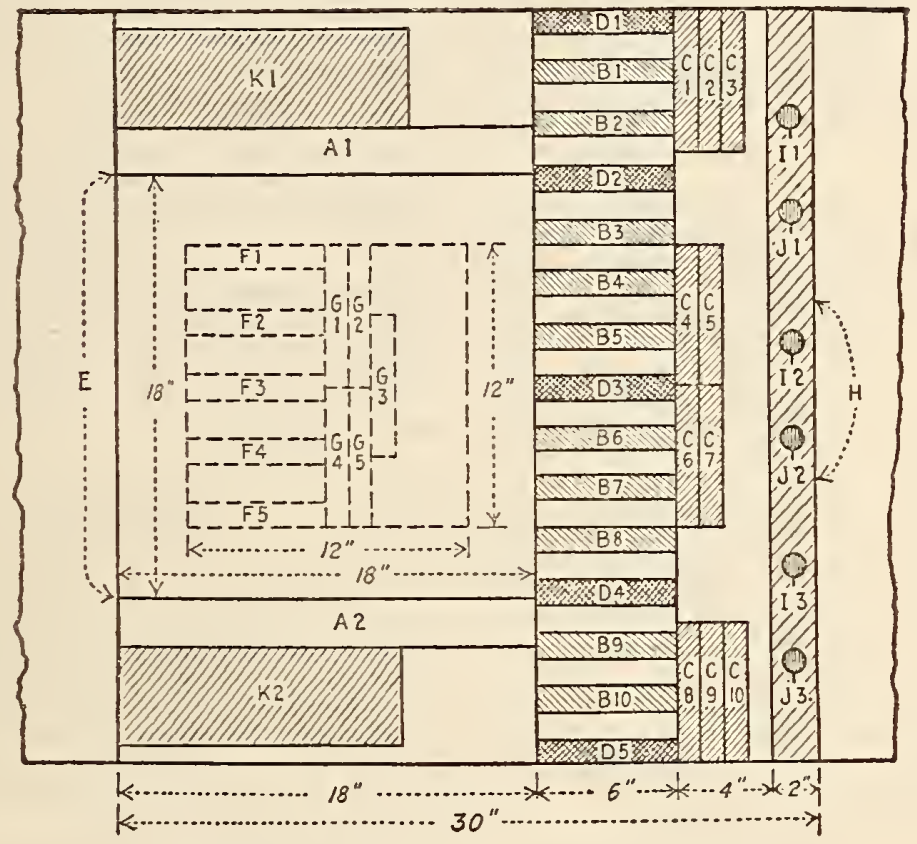

Figure 71.-Cutting of samples from fubric

Methods of Testing Woven Textile Fabrics (serial designation D39) of the American Society for Testing Materials.

Note.-As a referee method, or in case ans dispute arises regarding the strength, the test shall be repeated, with the exception that the fabric before testing shall be exposed at least two hours in an atmos phere of 65 per,cent relative humidity at $70^{\circ} \mathrm{F}$. $\left(21.1^{\circ} \mathrm{C}\right.$.).

13. With the trimmer described in section $12(a)$, five 6 -inch strips shall be cut with the fiber grain, as shown at $D-1$ to $D-5$ (fig. 71) and immersed in water at $77^{\circ} \mathrm{F}$. $\left(25^{\circ} \mathrm{C}\right.$.) for from 10 to 15 minutes. These strips shall be bent through $180^{\circ}$ at a uniform speed, in exactly two seconds, around a mandrel $25 \mathrm{~mm}$ in diameter; the second, around a mandrel $20 \mathrm{~mm}$ in diameter; the third, around a mandrel $15 \mathrm{~mm}$ in diameter; the fourth, around a mandrel $10 \mathrm{~mm}$ in diameter; and the fifth, around a mandrel $5 \mathrm{~mm}$ in diameter. The test may also be made at $32^{\circ} \mathrm{F}$. $\left(0^{\circ} \mathrm{C}\right.$. $)$. The pliability shall be expressed numerically from 1 to 10 as follows:

(1) Cracks entirely through the sheet on the 25mm mandrel.

(2) Cracks part way through the sheet on the 25-mm mandrel.

(3) Cracks on the $25-\mathrm{mm}$ mandrel.

(4) Cracks on the $20-\mathrm{mm}$ mandrel.

(5) Cracks on the $15-\mathrm{mm}$ mandrel.
14. (a) $A$ section 18 inches square (as shown at $E$, fig. 71) shall be cut from each specimen, weighed, and completely immersed in distilled water at $77^{\circ} \mathrm{F}$. $\left(25^{\circ}\right.$ C. $)$ for 24 hours. The specimen shall then be removed and dried superficially by pressing lightly between two towels. As the moisture enters through the edges of the sheet more rapidly than through the surfaces, each specimen shall be trimmed to exactly 12 inches square, representing four-ninths of the original area, and reweighed rapidly. The increase in weight shall be calculated on the basis of the original test specimen by multiplying by $21 / 4$. The percentage increase in weight represents the water absorption. The minimum, maximum, and average for the shipment shall be calcuiated.

(b) Five strips shall be cut with the fiber grain as shown at $F-1$ to $F-5$ (fig. 71 ); five other strips shall be cut across the fiber grain as shown at $G-1$ to G-5 (fig. 71) and the strength shall be redetermined as in section 12. The decrease in strength in percentage shall be calculated after the specimen has been subjected to water.

15. (a) Asphalt-saturated products only.-From each specimen, 12 by 6 inch strips shall be cut at $K-1$ and $K-2$ in Figure 71 , care being taken not to disturb any of the detached surfacing. Each strip shall be weighed and suspended in the center of an 
air oven maintained at $221^{\circ} \mathrm{F} .\left(105^{\circ}\right.$ C. $) \pm 5^{\circ} \mathrm{F}$. $\left(3^{\circ}\right.$ C.) with a thin wire fastened through holes punctured near one edge. The thermometer shall be inserted in the oven to such a depth that its bulb will be in line with the center of the specimens. The strips shall be kept in the oven for exactly five hours, then cooled and removed carefully, and each specimen weighed. The average loss shall be calculated as a percentage and the average percentage of moisture (ascertained in sec. 11) deducted. The final figure shall represent the average loss on heating, exclusive of moisture.

(b) Coal-tar-saturated felt only.-From the rolls making up the sample, strips 6 inches wide shall be cut across the sheet. Sufficient of these shall be taken to make up a sample of $250 \mathrm{~g} \pm 5.0 \mathrm{~g}$. These strips then shall be rolled up and placed in the wire basket of the extraction apparatus described in A. S. T. M. D147 (see 505.0, p. 133), or other extraction apparatus of suitable construction. The sample shall then be covered with a disk of soft filter paper to insure an even distribution of the solvent. The extraction shall be carried out as described in methods D147.

The $\mathrm{CS}_{2}$ extract containing the bitumen shall be transferred to a 500-cc, short-neck, round-bottom flask. The flask shall be equipped with a Hempel column, $16 \mathrm{~cm}$ in length, $15 \mathrm{~mm}$ in inside diameter, and filled to a depth of $5 \mathrm{~cm}$ with glass beads. The Hempel column shall be connected with a watercooled condenser. $A-2$ to $+80^{\circ} \mathrm{C}$. thermometer as described in A. S. T. M. D139 (see 505.0, p. 127), shall be placed in the Hempel column so that the top of the bulb shall be opposite the bottom of the tubulure. Distillation for the removal of $\mathrm{CS}_{2}$ shall be continued by means of a steam bath until drops cease to fall from the condenser. The distillation then shall be carried out over a small flame, heating carefully and observing the thermometer until the distillation ceases and the temperature of the vapor as indicated by the thermometer shows no further rise on slightly increasing the flame.

When the contents of the flask is sufficiently cool to be poured without appreciable vaporizing, $100 \mathrm{~g} \pm 0.1 \mathrm{~g}$ shall be distilled in accordance with the Tentative Method of Test for Distillation of Creosote Oil (serial designation D246-27T) of the American Society for Testing Materials.

Fractions shall be taken at $210^{\circ}$ and $235^{\circ} \mathrm{C}$. and the distillation stopped at that point. The per cent of distillate by weight at each temperature shall be calculated on the basis of the weight of sample taken for distillation.

\section{DESATURATING THE FABRIC}

16. From each roll sampled, a 2-inch strip (within one-thirty-second inch) shall be cut across the specimen as shown at $H$ (fig. 71). Each strip shall be extracted with carbon disulphide ${ }^{55}$ in an extractor described in section 4 of A. S. T. M. D147 (see 505.0, p. 133), the extraction being continued for several hours after the drippings have become colorless. The desaturated fabric shall bc removed and heated in a ventilated oven at $225^{\circ} \mathrm{F} .\left(107.1^{\circ} \mathrm{C}\right.$.), cooled in a desiccator, any adhering comminuted surfacing being brushed off and retained, and wcighed as rapidly as possible. The heating shall be repeated until the weight of the fabric remains constant as determined by two consecutive weighings taken not less than 10 minutes apart which shall show a further loss of not more than 0.1 per cent. This will give the weight of the fabric in the moisture-free state. Where a coal-tar pitch saturant has been used, the moisture-free weight

ss In this test, chloroform may be used as an equivalent solvent. of the desaturated fabric shall be corrected for the carbonaceous matter retained mechanically in its interstices, by means of the method described in section 17 .

17. The following colorimetric method shall be used to ascertain the carbonaceous matter derived from a coal-tar pitch saturant and retained by the desaturated fabric:

(a) About $15 \mathrm{~g}$ of an unsaturated fabric of the same general character as the one under examination, shall be macerated by boiling in water, disintegrating with a rotary egg beater and picking the fibers apart with needles. The fibers shall be filtered through fine cloth and dried at a temperature of $225^{\circ}$ F. $\left(107.1^{\circ} \mathrm{C}\right.$.) to constant weight. One gram of the fibers shall be accurately weighed into a flask and diluted to exactly $100 \mathrm{cc}$ with distilled water at room temperature. About $50 \mathrm{~g}$ of glass beads shall be added and the contents of the flask shaken vigorously until the fibcrs are reduced to a homogeneous pulp in uniform suspension.

(b) A distilled coal tar, having approximately 10 to 25 per cent of insoluble carbonaceous matter, shall be procured and the carbonaceous matter extracted with benzol until it is free from soluble matter; then dried at a temperature of $225^{\circ} \mathrm{F}$. $\left(107.1^{\circ}\right.$ C.) to constant weight. One gram of the purified carbonaceous matter shall be accurately weighed and diluted to exactly $100 \mathrm{cc}$ at room temperature with a starch solution of a consistency sufficient to carry the carbonaceous matter into temporary suspension. (A 12.5 per cent solution is recommended.)

(c) The liquid carrying the fibers, obtained as described in paragraph $(a)$, shall be titrated with the suspension of carbonaceous matter, obtained as described in paragraph $(b)$, and from time to time a field prepared from a drop of the well-agitated mixture shall be examined under a microscope at 100 diameters magnification until the color exactly matches a field prepared from the desaturated fabric under examination (obtained as described in sec. 16), when both are viewed side by side under parallel conditions. The end point is fairly sharply defined. The burette reading gives directly the percentage of carbonaceous matter adhering to the moisture-free fabric under examination.

(d) The weight of moisture-free fabric ascertained in section 16 shall be corrected by deducting the weight of adhering carbonaceous matter.

18. The total comminuted surfacing plus any filler present in the bituminous saturant represents the sum of the detached comminuted surfacing (sec. 9 ), the amount brushed off from the surface of the desaturated fabric (sec. 16), plus the amount recovered upon evaporating and igniting the bituminous extract obtained in accordance with section 16 .

19. The weight of bituminous saturant represents the difference between the weight of the original fabric (sec. 7) and the sum of the weights of the moisture-free desaturated fabric (secs. 16 and 17), plus moisture (sec. 11), plus the total comminuted surfacing and filler present (sec. 18).

\section{EXAMINATION OF THE EXTRACTED FABRIC}

\section{(A) WOVEN FABRICS}

20. Woven fabrics shall be tested for weight and number of threads per inch in accordance with sections 7 to 9 , inclusive, of the Standard Methods of Testing Woven Textile Fabrics (serial designation D39) of the American Society for Testing Materials.

\section{(B) FELTED FABRICS}

21. The weight in pounds per 480 square feet of the desaturated felt (corrected for any carbonaceous 
matter present) for each specimen examined shall be calculated separately from the formulas:

"Number" $=\frac{\text { weight of specimen in grams }}{\text { area of specimen in square centimeters }} \times 983$

Or

$$
\text { "Number" }=\frac{\text { weight of specimen in grams }}{\text { area of specimen in square inches }} \times 152
$$

This represents the "number" of the moisturefree felt on the felt makers' scale. The minimum, maximum, and average "number" of the felt in its moisture-free state shall be calculated. This may be corrected to the condition in which it actually existed in the material under examination, by adding thereto the percentage of moisture as determined in section 11 .

22. A representative sample shall be secured by cutting from each strip of desaturated felt a piece about one-half inch in diameter as shown at $I-1, I-2$, and $1-3$. (Fig. 71.) About 25 g selected at random from all the specimens sampled in this manner shall be accurately weighed, and incinerated in a weighed porcelain or quartz crucible either over an open flame or in a muffle, until all the carbon is consumed. A few drops of ammonium carbonate solution shall be added, the sample ignited gently, and weighed. The percentage of ash shall be calculated on the basis of the moisture-free felt.

Note.-As a referee method, or in case greater accuracy is desired. the three portions taken from each specimen roll shall be weighed and ignited separately. The minimum, maximum, and s rerage of ash present shall be calculated on the basis of the moisture-free felt

American Society for Testing Materials, standard methods of testing bituminous mastics, grouts, and like mixtures, serial designation D147-27, 1927.

\section{DEFINITIONS}

1. These methods apply to bituminous mastics, grouts, and like mixtures, which may be classified and defined as follows:

Bituminous grout.-A mixture of bituminous material as a binder and sandy mineral matter as an aggregate, which when heated to a suitable temperature becomes sufficiently fluid to flow into place without mechanical manipulation, and which on cooling congeals to a compact mass.

Asphalt mastic.-(1) A mixture containing asphaltic material as a binder and graded mineral matter as an aggregate, or (2) pulverized native rock asphalt (to which asphaltic material may bave been added); either of which when heated to a suitable temperature may be poured into place but which requires trowelling to form it into a compact mass.

Asphalt mastic cake.-A mixture containing asphaltic material as a binder and an aggregate consisting chiefly of calcareous or siliceous dust cast into the form of blocks or "cakes" and adapted for use in preparing asphalt mastic.

\section{PREPARATION OF SAMPLES}

2. (a) Bituminous grouts shall be heated in an oven or on a hot plate in a pan or other suitable container at the lowest possible temperature to prevent overheating and volatilization; and when sufficiently fluid, shall be thoroughly stirred to insure a uniform sample, whereupon 10 to $30 \mathrm{~g}$ shall be taken for analysis.

(b) Asphalt mastics or mastic cake shall be warmed on a hot plate or in a hot oven until soft enough to be broken up or stirred so that a representative sample for analysis may be taken. The amount taken for analysis will depend upon the amount of coarse gravel or stone in the mixture. The larger the gravel or stone, the larger will be the

sample required for accuracy. The size of samples to be taken shall be as follows: Where all particles pass a No. 10 sieve, 10 to $30 \mathrm{~g}$; where 25 per cent of the aggregate is retained on a No. 10 sieve, $50 \mathrm{~g}$; where 50 per cent of the aggregate is retained on a No. 10 sieve, $100 \mathrm{~g}$; and where 75 per cent of the aggregate is retained on a No. 10 sieve, $200 \mathrm{~g}$.

NoTE.- Where the properties of the extracted bituminous matter are to be determined, $500 \mathrm{~g}$ shall he taken and extracted with pure henzol as described in sections 4 and 6 and examined as described in section 7 .

\section{APPARATUS}

\section{(A) FOR ANALIYSIS OF 10 TO $30 \mathrm{~g}$ SAMPLES}

3. In cases where a 10 to $30 \mathrm{~g}$ sample is sufficient, the analysis shall be carried out by means of the

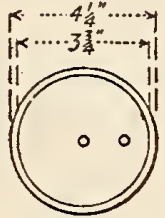

Section D-D.

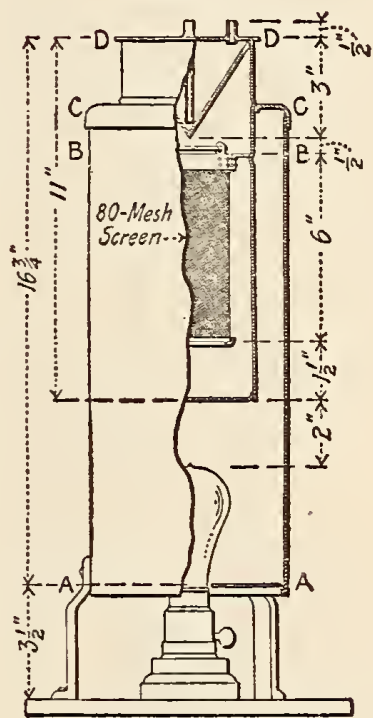

Figdre 72.-Large extractor

glass extractor described in section 8 of the Standard Methods of Testing Rubber Products (serial designation D15) of the American Society for Testing Materials.

\section{(B) FOR ANALISIS OF 50 TO $500 \mathrm{~g}$ SAMPLES}

4. The apparatus for analysis of samples containing coarser aggregate shall be the large extractor shown in Figure 72, consisting of a large brass cylinder, through the bottom of which projects a 16candlepower incandescent carbon filament bulb to supply heat to the extraction apparatus proper, which is held in the upper portion of the cylinder. This apparatus is composed of a cylindrical brass vessel for holding the solvent, a cylindrical wire basket made of 80 -mesh wire cloth suspended in the cylinder, and an inverted conical condenser which serves as a top. 


\section{PROCEDURE}

\section{(A) ANALYSIS OF 10 TO 30 g SAMPLES 60}

5. An ordinary Whatman or S. \& S. filter thimble shall be dried and weighed. The weighed sample shall be pleced in the thimble and 40 to $50 \mathrm{cc}$ of $\mathrm{CS}_{2}$ poured over the sample. The thimble containing the sample shall be suspended under the condenser by a fine wire bail. The flask shall be cautiously heated by a steam bath or electric heater just enough to vaporize the solvent. Cold water is circulated through the condenser. The heat evaporates the carbon disulphide in the flask. This condenses upon the condenser and drops back upon the sample through which it filters, thus dissolving out the bitumen which collects in the bottom of the flask.

The extraction should be discontinued when the $\mathrm{CS}_{2}$ drops colorless from the filter. The time of extraction will depend upon the nature of the bitumen and mineral aggregate in the sample and upon the degree of heat applied, the coldness of the water in the condenser, and other factors. In some cases extraction may be complete in one hour; in others four or five hours may be necessary.

When the solvent comes through clear, the filter shall be removed and washed with a fine jet of $\mathrm{CS}_{2}$ from a washing bottle to wash out any bitumen that may be retained at the top of the paper and to break up any channels that may have been formed by the $\mathrm{CS}_{2}$ passing through. If the washings show any color, the thimble shall be put back and extraction continued until the solvent again becomes colorless. It shall then be removed, dried carefully, at a low temperature at first to prevent ignition of the absorbed $\mathrm{CS}_{2}$, and finally to constant weight at $100^{\circ} \mathrm{C}$. $\left(212^{\circ} \mathrm{F}\right.$.), cooled, and weighed.

The solution in the flask shall be rinsed into a weighed porcelain or silica evaporating dish or crucible and the solvent burned off under a hood. The residue shall be ignited over a flame or in a muffle and the ash weighed and the weight added to that of the mineral matter in the filter paper. This is to correct for the fine mineral matter which will be carried through the paper by the solvent. Should there be a considerable amount of ash recovered in this way, and if it is found that the mineral matter is calcium or other carbonate, it shall be recarbonated by repeated treatment with ammonium carbonate solution and finally ignited at a dull red heat. Ordinarily, however, the mineral matter going through the paper will be so small in amount that the difference caused by ignition may be neglected.

The corrected loss in weight on the original sample represents the percentage of bitumen.

The sieve analysis of the mineral aggregate shall be made in accordance with A. S. T. M. D7. (See 512.12 , p. 234 .) The extracted residue shall now be transferred from the thimble to the No. 200 sieve, the paper being gently rubbed to free adhering particles.

The aggregate on the sieve shall be gently rubbed with the fingers to break up lumps and to free any particles of fine dust that might adhere to larger sand particles. The sieve shall be shaken over a piece of paper from side to side with the right hand, striking it sharply against the palm of the left hand until no appreciable amount of dust comes through the sieve on to the paper. The paper shall be cleared from time to time by raising one side with the left hand and rolling off the siftings, so that it can be seen when the sifting is complete.

The material remaining on the No. 200 sieve shall be weighed and the amount of material which has

${ }^{86}$ This method and the method for analysis of larger samples are especially adapted for asphalt masties, grouts, and mastic cake. I the binder is coal-tar pitch, the "free-carbon" constituent of the binder will remain with the mineral aggregate. passed through the No. 200 sieve shall be determined by difference.

This operation shall be repeated upon the coarser sieves in order and the amount passing each sieve and retained on the next finer recorded as percentage of the original sample.

\section{(B) AINALYSTS OF 50 TO $500 \mathrm{~g}$ SAMPLES}

6. A large filter paper, 12 or 13 inches in diameter, shall be fitted inside the wire basket of the extractor by folding once more than in ordinary filtering, or by wrapping it over a form which fits inside the basket (a cylindrical bottle of proper size makes a good form) and placing it inside the basket.

The basket with contained filter paper shall be dried and weighed. The sample shall be weighed and packed in the filter paper in the basket. Care should be taken not to pack all coarse particles in one place and the fine particles in another but to have them mixed together in uniform proportions.

The sample shall be covered with a disk of felt or wad of absorbent cotton to insure even distribution of the dropping solvent, thus preventing it from forming a channel through the sample. The basket shall be suspended in the extractor and 150 to 200 cc of $\mathrm{CS}_{2}$ poured over the felt or cotton. The condenser shall be placed over the top and water circulated through it. Current shall be started through the electric lamp underneath the Extractor and the extraction carried on exactly as in the smaller glass extractor, but on a larger scale. The time for extraction will vary from 3 to 12 or more hours, depending upon the nature of the sample.

To determine when extraction is complete, the condenser shall be raised and the basket lifted out to observe if drippings are clear. One or two drops caught upon white filter paper should leave but a light stain.

The drying and weighing of the basket, burning off of the solution for correction, and the sifting of the mineral aggregate shall be carried on exactly as in the case of the smaller sample as described in section 5, except that in sifting mineral aggregates containing coarse stone it is well to remove the stone by putting the mineral aggregate first through a No. 10 sieve, as the large stone would injure the No. 200 sieve.

The stone removed by the No. 10 sieve may be screened through 1/4-inch, 1/2-inch, and coarser screens in order, as described in A. S. T. M. D18 (see 512.15 p. 250), and the fine material passing the No. 10 sieve through No. 200 and coarser sieves in order, all amounts passing any one screen or sieve and retained on the next finer one being reported as percentage of the original sample.

Thus the ingredients of the mixture may be roughly separated by the use of the proper sized sieves, and examined for physical and chemical characteristics.

Note.-The siftings passing the No. 200 sieve will consist largely of the dust or pulverized rock used in the mixture. The material hetween the No. 100 and No. 10 will consist largely of the sand used in the mixture, with any particles of fine crushed stone within these limits that existed in the original materials. Gravel or broken stone as a rule will he larger than No. 10 or No. 8 .

Carbon tetrachloride, benzol, or chloroform may be used instead of carbon disulphide, with the only difference that in the case of noninflammable solvents the solvent will have to be evaporated from the solution of bitumen to determine the ash for correction instead of burning off directly. When the solvent is expelled, the bitumen can be ignited for ash.

\section{(C) RECOVERY AND EXAMINATION OF EXTRACTED BITUMEN}

7. The benzol solution containing the asphalt shall be evaporated to a thick viscous liquid on a steam or water bath, then transferred to a steam 
oven at $105^{\circ} \mathrm{C} .\left(221^{\circ} \mathrm{F}\right.$.) until its weight is nearly equal to the percentage of asphalt calculated in accordance with section 5 . It shall then be transferred to a hot plate and heated carefully with stirring at a temperature not exceeding $121^{\circ} \mathrm{C}$. $\left(250^{\circ}\right.$ F.) until its weight is exactly that determined in accordance with section 6 .

\section{(D) SOLUBIITY OF AGGREGATE IN HYDROCHLORIC ACID}

8. Where the mastic is to be used for acid-proof floors, tank linings, etc., pulverized silica and other materials insoluble in acid are used in preparing the mixture. The most important chemical property, therefore, is solubility or insolubility in mineral acids. This determination may be carried out in the following manner:

About $2.5 \mathrm{~g}$ of the dust, or of the coarser material, or stone which has been previously pulverized in a mortar shall be dried to constant weight at $325^{\circ} \mathrm{F}$. The loss will represent any moisture that may have been retained in the material as well as any absorbed solvent that may not have been expelled while drying the sample after extraction.

A $1-g$ sample of the dried material shall be placed in a 250-cc beaker, covered with a watch glass and $10 \mathrm{cc}$ of concentrated c. p. hydrochloric acid added, diluted with $50 \mathrm{cc}$ of distilled water. Any lumps shall be broken up by means of a glass stirring rod. Any effervescence which may occur shall be noted. If considerable effervescing takes place, the sample may be said to be carbonate. If all the sample, or practically all, dissolves, the determination need not be carried farther. If apparently insoluble, it shall be heated carefully, brought to boiling for 15 minutes, and filtered through a tared Gooch filter, washing the insoluble residue with hot water. It shall be ignited and weighed and the loss reported as the percentage soluble in hydrochloric acid.

American Society for Testing Materials, standard method of test for the determination of proportion of bitumen soluble in carbon tetrachloride, serial designation D165-27, 1927.

\section{APPARATUS}

1. The apparatus shall consist of the following:

(a) Gooch crucible, approximately $4.4 \mathrm{~cm}$ in width at the top, tapering to $3.6 \mathrm{~cm}$ at the bottom, with a depth of $2.5 \mathrm{~cm}$.

(b) Filtering flask.

(c) Rubber stopper.

(d) Filter tube.

(e) Section of rubber tubing to hold the Gooch crucible on the filter tube.

(f) Asbestos (amphibole) cut in pieces not exceeding $1 \mathrm{~cm}$ in length, shredded and shaken up with water.

(g) Two 150-ce Erlenmeyer flasks.

(h) Bunsen burner.

(i) Drying oven.

(j) Suction pump.

(k) Rubber tubing.

(l) Analytical balance.

(m) Desiccator.

\section{PREPARATION OF SAMPLE}

2. The sample shall be representative, and if it contains more than 2 per cent of water it shall be dehydrated by distillation in a copper still in accordance with A. S. T. M. D20-28T (see 505.0, p. 120), and water-free distillate returned to the residue. If the material is hard and brittle, it may be ground and dried at a temperature below the temperature of volatilization of the material.

\section{PROCEDURE}

3. The Gooch crucible shall be set in the filter tube inserted in the stopper of the filtering flask. The flask shall be connected with the suction pump. Before suction is applied the crucible shall be filled with asbestos suspended in water which shall be allowed to partly settle in the crucible. A light suction shall be applied to draw off the water, leaving a firm mat of asbestos in the crucible. More suspended asbestos shall be added and the process repeated until a felt is built up that barely transmits light. The felt shall then be thoroughly washed with water, dried in a drying oven, and ignited over a Bunsen burner. The crucible then shall be cooled in a desiccator and weighed.

4. An amount of material which shall contain approximately $1 \mathrm{~g}$ of bitumen shall be weighed into a tared Erlenmeyer flask. One hundred cubic centimeters of chemically pure carbon tetrachloride shall be added to the flask in small portions with continued agitation until all lumps disappear and nothing adheres to the bottom. The flask shall be corked and set aside in subdued light for at least 12 hours.

The Gooch crucible shall be set up again with the suction flask and the carbon tetrachloride solution carefully decanted through the asbestos felt, with or without light suction, as may be found necessary. No sediment shall be allowed to go onto the filter. A small amount of carbon tetrachloride shall be used to wash down the sides of the flask and then the precipitate shall be brought onto the felt and the flask scrubbed with a feather if necessary to remove all precipitate. The contents of the crucible shall be washed with carbon tetrachloride until the washings are colorless. Suction shall be applied to the crucible to remove the carbon tetrachloride. The crucible shall be dried in the oven at $100^{\circ}$ to $125^{\circ} \mathrm{C}$. for 20 minutes, cooled in the desiccator, and weighed.

In case insoluble matter adheres to the flask, the flask shall be dried and weighed and the increase in weight over the original weight shall be added to the weight of insoluble matter in the crucible.

The proportion of bitumen soluble in carbon tetrachloride shall be reported on the basis of total bitumen taken as 100 . $\begin{gathered}\text { Proportion of bitumen } \\ \text { soluble in carbon tetra- } \\ \text { cholride }\end{gathered}$
.

American Society for Testing Materials, tentative method of test for residue of specified penetration, serial designation D243-28T, 1928.

1. This method of test covers the determination of percentage of residue having a specified penetration at $100 \mathrm{~g}$, five seconds, $25^{\circ} \mathrm{C}$. $\left(77^{\circ} \mathrm{F}\right.$.), obtained by heating a road oil or a semisolid asphalt having a penetration of more than 100 , at a temperature of $249^{\circ}$ to $260^{\circ} \mathrm{C}$. $\left(480^{\circ}\right.$ to $500^{\circ} \mathrm{F}$. $)$. When the penetration of the residue is not otherwise stated it shall be understood to be 100 . The residue obtained is available for tests as desired.

\section{APPARATUS}

2. The apparatus shall consist of an iron dish, flask heater, thermometer, Bunsen burner, tripod, and stand with a thermometer clamp. The dish, flask heater, and thermometer shall be as specified in sections 3,4 , and 5 .

3. The container for holding the sample to be tested shall be a hemispherical iron dish having the following dimensions:

Diameter at the top, outside $\mathrm{cm}_{7 \pm 0.3}$

$5.7 \pm 0.2$ Radius of curvature, inside..- 6, $0 \pm 0.1$

Note.-A hemispherical iron sand bath, deep form of 5 inches diameter, should meet these requirements. 
4. The flask heater shall be in the form of a truncated cone, with a diameter at the top of approximately $18.0 \mathrm{~cm}$, a diameter at the bottom of approximately $5.2 \mathrm{~cm}$, and a height of approximately $7.0 \mathrm{~cm}$. It shall be made of sheet iron of about No. 22 gage with asbestos strips riveted to the sides, and with a sheet-iron shield at the bottom.

5 . The thermometer shall conform to the requirements of thermometer No. 8. (See table of A. S. T. M. thermometers, 500.2 , p. 2.)

\section{PREPARATION OF SAMPLE}

6. The sample as received shall be thoroughly stirred and agitated, to insure a complete mixture before the portion for testing is removed.

\section{PROCEDURE}

7. One hundred grams $(100.00 \pm 1.10 \mathrm{~g})$ of the material to be tested shall be weighed into a tared container, which shall then be placed in the flask heater in position to be heated. The thermometer shall be supported in the sample equidistant from the sides of the container and with the bottom of the bulb neither more than one-fourth inch above nor touching the bottom of the container. The bulb shall be completely immersed in the sample throughout the heating. An assembly of the apparatus is shown in Figure 73 facing page 222 .

8. The sample should be heated as rapidly as possible, to prevent foaming, to a temperature of $249^{\circ}$ C. $\left(480^{\circ}\right.$ F.) and during the evaporation, the temperature shall be maintained between $249^{\circ} \mathrm{C}$. $\left(480^{\circ}\right.$ F.) and $260^{\circ}$ C. $\left(500^{\circ}\right.$ F.). The sample shall be stirred with the thermometer from time to time to prevent local overheating and, to maintain a homogeneous sample, all cakes of hardened bitumen which form at the sides of the container shall be fluxed in the sample.

An experienced operator can judge approximately what percentage of residue he should obtain to secure the desired penetration. When it is supposed that the residue will show the required penetration, the bitumen on the thermometer which may be readily scraped off shall be returned to the container, which then shall be removed from the llask heater, cooled, and weighed. The penetration of the residue shall then be determined in accordance with A. S. T. M. D5. (See 505.0, p. 118.)

9. It frequently is necessary to make several trials before a residue of the required penetration is obtained. If it is determined to be greater than that required, all water shall be removed from the container and the surface of the sample, and the beating and determination of penetration may be repeated as before. Ordinarily a residue shall be considered as satisfactorily obtained when its penetration is within 15 of that desired, and its percentage by weight of the original sample shall be calculated. When it is necessary to determine more precisely the percentage of residue having the specified penetration, such a percentage shall be computed by interpolation between percentages of two residues, one having a penetration greater and one having a penetration lower than that specified. The percentage shall be reported as:

Percentage of residue of ....... penetration (determined .........) stating, first, the specified penetration, and second, the penetration actually determined for the sample tested or calculated by interpolation.

\section{PRECAUTIONS}

10. Certain types of road oil will readily form rings of hard asphalt at the side of the container. Great care should be taken that this material be completely fluxed in the sample before the penetration of the residue is determined.

\section{ACCURACY}

11. Duplicate determinations should not differ by more than 1.0 per cent with the same operator nor more than 2.5 per cent between different laboratories.

American Society for Testing Materials, tentative method of testing bituminous emulsions, serial designation D244-28T, 1928.

1. For testing purposes, emulsions shall be classified as follows:

T. Emulsified light oils or liquid petroleum products intended for dust laying purposes.

II. Emulsified asphaltic materials, the asphalt content of which is of a consistency suitable for construction or repair.

(a) Containing little or no mineral matter.

(b) Containing appreciable quantities of mineral matter.

\section{METHOD FOR DETERMINING MISCIBILITY OF} BITUMINOUS EMULSIONS WITH WATER

\section{(Applicable to both Classes I and II)}

2. About $50 \mathrm{cc}$ of the emulsion shall be gradually added to about $150 \mathrm{cc}$ of distilled water, stirring the mixture while adding the water. The temperature is not important, but should be between $21^{\circ}$ and $25^{\circ} \mathrm{C}$. $\left(70^{\circ}\right.$ and $79^{\circ} \mathrm{F}$.). The mixture shall be allowed to stand for two hours and then examined for any appreciable separation.

\section{STONE COATING TEST}

\section{(Applicable only to emulsions of Class II $(a)$ )}

3 . Four hundred and sixty-five grams of clean stone (one-fourth to three-fourths inch in size, not more than 5 per cent passing a $1 / 4$-inch screen and 100 per cent passing a $3 / 4$-inch screen), (see A.S. T. M. D63-23T, 511.71, p. 207) of either trap or hard limestone shall be placed in a No. 10 sieve, drenched and washed with distilled water, allowed to drain for three minutes and transferred to a suitable pan in which the mixture is to be made. (A 6-inch hemispherical iron dish has been found suitable and satisfactory for this purpose.) Thirty-five grams of the emulsion shall then be added to and mixed vigorously with the stone for three minutes. (A steel spatula is satisfactory for mixing.) There shall be no signs of appreciable separation of the asphalt contained in the emulsion during the 3-minute mixing period.

\section{METHOD FOR DETERMINATION OF WATER, OII DISTILLED WITH WATER, AND PREPARATION OF ASPHALTIC BASE FOR FURTHER TESTS}

\section{(A) METHOD APPLICABLE TO EMULSIONS OF CLASS II $(a)$}

4. The apparatus shall consist of an iron still, 6 by $3 \frac{1}{2}$ inches in inside diameter with an air adjustable ring burner to fit the still. The still shall be provided with a connecting tube of approximately $12-\mathrm{mm}$ diameter glass tubing, a tin shield, a condenser trough, a condenser tube, and a suitable graduated cylinder. A thermometer $0^{\circ}$ to $400^{\circ} \mathrm{C}$. shall be provided conforming to the requirements for thermometer No. 2. (See table of A. S. T. M. thermometers, 500.2 , p. 2.) The apparatus shall be set up as shown in Figure 74. The still shall be charged with $200 \mathrm{~g}$ of the well-mixed sample. An oiled paper gasket shall be applied to the flange at 
the.top of the still and the cover clamped on securely. The thermometer shall be placed in position so that the tip of the bulb is one-half inch from the inside bottom of the still. A gentle heat shall be applied to the top of the still by the ring burner. Just sufficient heat shall be applied to the connecting tube to prevent condensation of water therein.

The heat shall be applied to the extreme upper part of the still, until practically all of the water has distilled over, or until a temperature of $121^{\circ} \mathrm{C}$. $\left(250^{\circ} \mathrm{F}\right.$.) has been reached. The ring burner shall then be lowered very gradually to a position midway between the top and bottom of the still and held there until the temperature is about $176^{\circ} \mathrm{C}$. $\left(349^{\circ}\right.$ F.) The ring burner shall then be lowered more rapidly to within one-fourth inch of the bottom of the still and the temperature then raised to $260^{\circ} \mathrm{C}$. $\left\langle 500^{\circ}\right.$ F.) and maintained for 15 minutes. This
The weight of residue remaining in the still divided by two is the percentage of nonvolatile matter under the conditions of this test.

The sum of the percentage of water and nonvolatile matter deducted from 100 represents distilled oils.

\section{(B) METHOD APPLICABLE TO EMULSIONS OF CLASS II (b)}

Water.-The water shall be deternined by the distillation method above described.

Oils.-Two 50-g samples of well-mixed emulsion shall be placed in a standard 3-ounce tin box, in an oven at $163^{\circ} \mathrm{C}$. $\left(325^{\circ} \mathrm{F}\right.$.) for five hours, and the loss determined. If the loss is greater than the percentage of water (see preceding paragraph) it would indicate the presence of volatile oils and the difference in the two determinations would closely approximate the percentage of volatile oil.

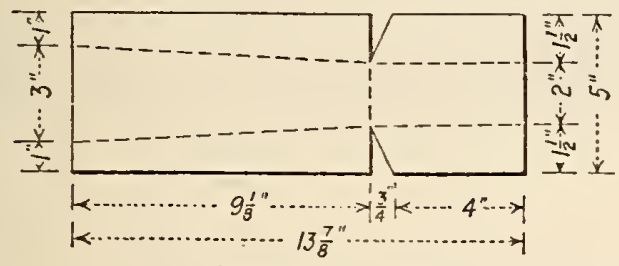

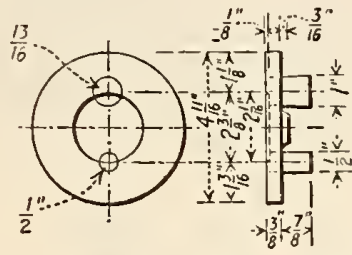

Detail of Lid on Still.
Thermomeier

Detail of Tin Shield (I).

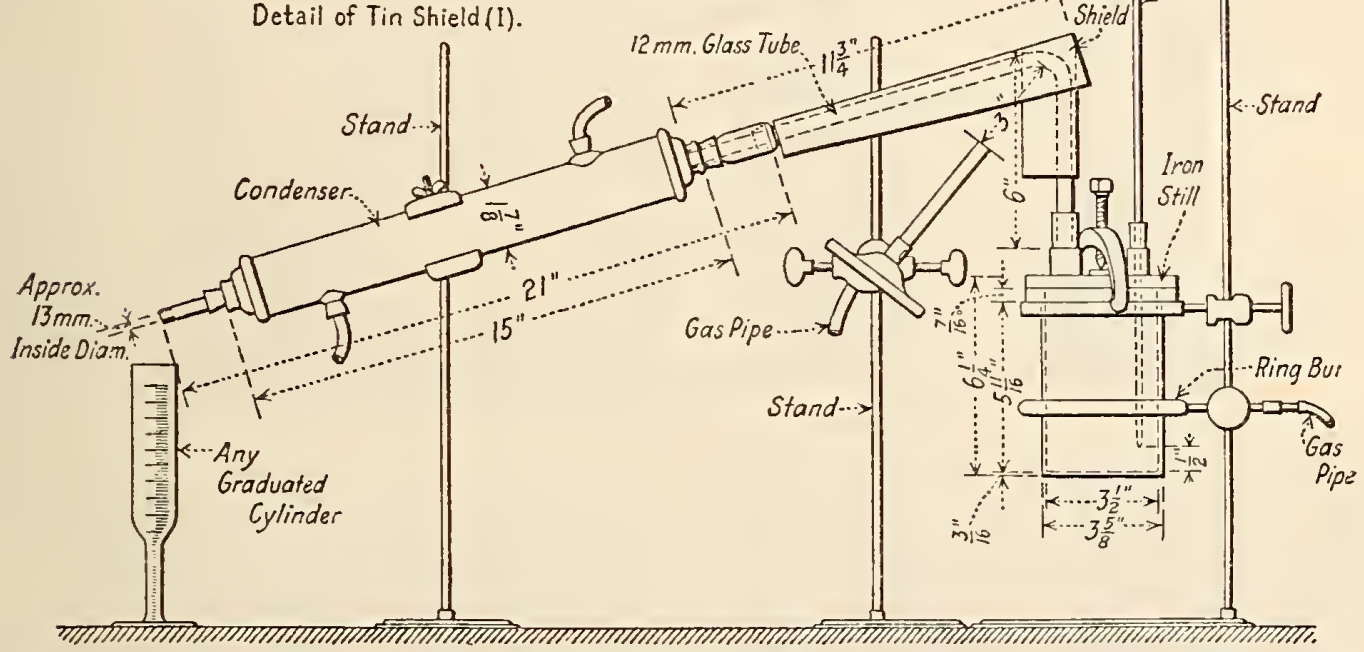

FiguRe 74.- Assembly for distillation test for bituminous emulsions

period of heating is important to insure a smooth homogeneous residue in the still.

The cover shall then be removed from the still and the hot residue poured into a ductility mold and into a standard 3-ounce tin box for the penetration test, the receptacles being left uncovered. While cooling, any excess shall be poured into suitable containers for any additional tests desired.

Any water or oil remaining in the condenser tube shall be forced out carefully into the graduated cylinder, by means of a rubber stopper fixed on the end of a long rod.

The distillation should be completed in not less than one hour and in not more than one and onefourth hours.

If the residue is not homogeneous and appears granular, it shall be rejected and the distillation repeated upon a fresh sample, maintaining the temperature at $260^{\circ} \mathrm{C}$. $\left(500^{\circ} \mathrm{F}\right.$.) for slightly more than 15 minutes.

The number of cubic centimeters of water divided by two is the per cent by weight of water.
The residue of the $50-\mathrm{g}$ sample shall be dissolved in benzol, filtered, and the filtrate evaporated on a water bath until practically all the benzol is evaporated, then placed in an oven at $105^{\circ} \mathrm{C}$. $\left(221^{\circ} \mathrm{F}\right.$.) for 30 minutes and finally heated on a hot plate to $121^{\circ}$ C. $\left(250^{\circ}\right.$ F.) with constant stirring. The consistency, ductility, or other tests desired are made upon this residue.

Mineral matter.- The mineral matter on the filter paper from the extraction shall be dried and weighed or a separate determination on a smaller sample of the original emulsion may be made.

American Society for Testing Materials, standard method of test for steam distillation of bituminous protective coatings, serial designation D255-28, 1928.

1. This is a general method for the separation and recovery of the solvent and the base in bituminous mixtures.

2. The bituminous mixture is distilled in a current of steam, the solvent is condensed and separated from the water. 


\section{APPARATUS}

3. The steam generator shall be made of either metal or glass, with a capacity of from 2 to 4 liters, suitable for continued use in the production of steam. If of glass, it shall be fitted with two outlets with suitable connections for rubber tubing. In the case of a metal generator, a large opening for filling and a water gage shall be additional parts of the apparatus. The generator shall be supplied with suitable pinchcocks or valves so that steam may be blown off to the atmosphere until the test is ready.

4. The bath shall be of metal of sufficient capacity to permit immersion of the distilling flask to a depth of not less than $10 \mathrm{~cm}$.

5. Heat for the steam generator shall be supplied by a suitable gas generator or electric hot plate. The bath may be heated by any convenient means.

6. The distilling flask shall be a short ring neck, round-bottom flask of 1,000 cc capacity. It shall be fitted with a 3-hole rubber stopper; with a steam distilling tube which will reach to within one-half inch $(12.7 \mathrm{~mm})$ of the bottom of the flask and project ments specified for thermometer No. 6. (See table of A. S. T. M. thermometers, 500.2, p. 2.)

\section{PROCEDURE}

10. The apparatus shall be assembled as shown in Figure 75. The steam generator shall be filled with water, and heat applied. The bath shall be filled with a high-flash-point oil and raised to approximately $140^{\circ}$ C. $\left(284^{\circ}\right.$ F.). Five hundred cubic centimeters of the sample shall be weighed into the round-bottom flask. The connection shall be made from the steam distillate to the steam delivery tube, the end of which shall be within one-half inch of the bottom of the distilling flask. The outlet from the distilling flask shall be connected to the condenser and the separatory funnel placed in position at the outlet of the condenser to receive the distillate. The end of the bulb of the thermometer in the steam distilling flask shall be placed within one-half inch $(12.7 \mathrm{~mm})$ of the bottom of the distilling flask.

When the temperature of the sample in the distilling flask reaches $130^{\circ} \mathrm{C}$, the outlet of the steam

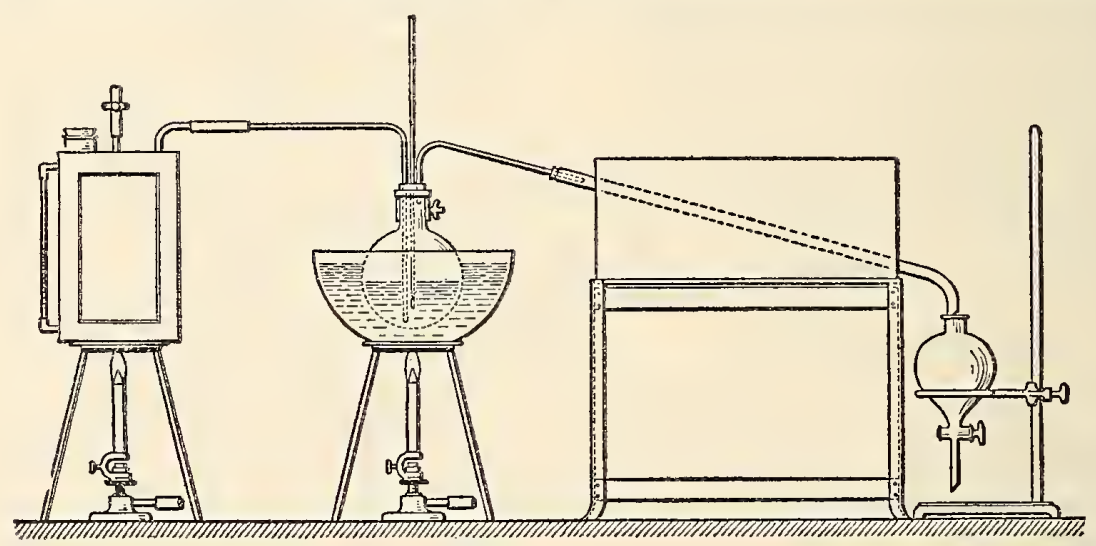

Frgdre 75.-Assemb?y of apparatus

from the top at a convenient distance for connection to the generator; a vapor outlet tube which extends from beneath the rubber stopper to a point sufficiently above the distilling flask that will permit convenient connection to the condenser; and with a thermometer. The steam tubing should be not less than 2 nor more than $4 \mathrm{~mm}$ in internal diameter and the vapor outlet tube should be not less than $5 \mathrm{~mm}$ in internal diameter.

7. The condenser shall consist of a nine-sixteenths inch $(14.29 \mathrm{~mm})$ outside diameter No. 20 Stubbs gage seamless brass tube, 22 inches $(55.88 \mathrm{~cm})$ long. It shall be set at an angle of $75^{\circ}$ from the perpendicular and be surrounded with a cooling bath 15 inches $(38.1 \mathrm{~cm})$ long, approximately 4 inches $(10.16 \mathrm{~cm})$ wide by 6 inches $(15.24 \mathrm{~cm})$ high. The lower end of the condenser tube shall be cut off at an acute angle, and curved downward for a length of 3 inches $(7.62 \mathrm{~cm})$ and slightly backward so as to insure contact with the wall of the graduate at a point 1 to $1 \frac{1}{4}$ inches $(2.54$ to $3.18 \mathrm{~cm}$ ) below the top of the graduate when it is in position to receive the distillate.

8. A separatory funnel having a capacity of not less than $500 \mathrm{cc}$, as shown in Figure 75 , should be provided.

9. (a) Accessories consist of suitable ring stands for supporting the stcam generator, distilling flask, bath for distilling flask and separatory funnel.

(b) The A. S. T. M. low-distillation thermometer for the distillation flask, conforming to the require- generator shall be closed, thus forcing the steam to pass through the sample. The flow of steam shall be adjusted so that the distillate is collected at the rate of approximately 6 to $10 \mathrm{cc}$ per minute.

The distillation shall be stopped when $100 \mathrm{cc}$ of the distillate contains not more than $0.5 \mathrm{cc}$ of solvent as determined by measuring the amount of oil in $100 \mathrm{cc}$ of distillate.

When the distillation is finished, the water shall be separated from the distillate and the distillate measured and retained for further tests if required by the specifications.

In some cases, the distillate does not separate readily from the water, and this separation can be facilitated by the addition of sodium chloride which will result in a sufficient difference in gravity to produce a clear separation of the two layers.

The results shall be reported in per cent by weight or volume as required by the specifications, based on the weight of the sample taken.

American Society for Testing Materials, tentative recommended practice for bituminous paving plant inspection, serial designation D290-28T; 1928.

(This recommended practice defines the authority and duties of the inspector at a bituminous paving plant.)

American Society for Testing Materials, tentative method of test for coarse particles in bituminous materials by means of elutriation, serial designation D313-29T, 1929. 


\section{SCOPE}

1. These methods cover the determination of the amount of relatively coarse particles of mineral or other insoluble matter in bituminous materials, which settle under the conditions prescribed.

\section{SAMPLING}

2. The sampling shall be performed in accordance with A.S. T. M. D140. (See p. 128.)

\section{TREATMENT OF SAMPIE}

3. The sample as received shall be completely melted at the lowest possible temperature and stirred thoroughly while cooling to insure homogeneity.

\section{PROCEDURE}

4. (a) Ten grams of the sample shall be dissolved in $100 \mathrm{cc}$ of carbon disulfide in a 150-ce tall-form beaker. The solution shall bc stirred thoroughly, allowed to stand for 15 minutes and approximately 90 per cent of the solution decanted. The sediment in the beaker shall then be treated in a similar manner three successive times with benzol at room ternperature, using $90 \mathrm{cc}$ of benzol each time. Drying of the residue should be avoided until it is finally dried as described in paragraph $(b)$.

(b) The sediment and remaining benzol shall be washed into a 400-ce tall-form beaker with waterwhite keroscne (having a flash point of $120^{\circ} \mathrm{F}$. at room temperature, and sufficient kerosene added to bring the total volume to $300 \mathrm{cc}$. This sediment and liquid shall then be agitated with an air blast until thoroughly mixed, avoiding the formation of cyclonic currents. The mixture shall then be allowed to settle for 10 seconds and about 90 per cent of the solution decanted. This operation shall be repcated in a similar manner two additional times with 300 cc of kerosene. After final decantation, the kerosene shall be removed from the remaining sediment by washing with carbon disulfide on to a tared filter paper, or a Gooch, or an alundum crucible and dried to constant weight at from $200^{\circ}$ to $220^{\circ} \mathrm{F}$. $\left(95^{\circ}\right.$ to $105^{\circ} \mathrm{C}$.).

\section{PERCENTAGE OF COARSE PARTICLES}

5 . The percentage of coarse particles of mineral or other insoluble matter shall be calculated from the formula:

$$
\text { Percentage of coarse particles }=\frac{\text { Weight of final sediment }}{\text { Original weight of sample }} \times 100
$$

Asphalt Shingle and Roofing Association (now Institute), mill analysis, of bituminous roofings (recommended) 1922 .

\section{OBJECT OF THE TESTS}

The following methods are designed to ascertain:

1. Weight and caliper of original felt as received.

2. Weight and caliper of original felt, moisturefree.

3. Moisture per cent in original felt as received.

4. Ash per cent in original felt, moisture-free.

5. Per cent saturation in moisture-free felt.

6. Pounds dry felt in 100 square feet roofing.

7. Pounds saturation in 100 square feet roofing.

8. Pounds pure coating in $\mathbf{1 0 0}$ square feet roofing.

9. Pounds mineral filler in coating in 100 square feet roofing.

10. Pounds slate or other granular surfacing in 100 square feet roofing.

11. Pounds dust on surface in 100 square feet roofing.

12. Pounds total weight in 100 square feet roofing.
For comparative purposes in mill records all results are expressed in terms of 100 square feet, surface (equivalent "square"). Such results may be converted directly into values per 108, 216, 250, or 300 square feet by simple mutliplication, using the appropriate factor indicated. (Pounds per 100 square feet $\times 2.5=$ pounds per 250 square feet.)

The information desired is calculated chiefly from a series of weights of mill samples on a paper-makers' scale and a few laboratory determinations of ash, depending upon the type of roofing employed.

\section{SAMPLES REQUIRED AND SCHEME FOR TAKING THEM}

1. Original felt.-Size 12 by 13 inches. Cut from the last end of roll when making splice.

2. Saturated felt.-Size 7 by 25 inches. Cut from edge of sheet while passing from saturator to coating rolls, just ahead of splice.

3. Saturated and coated felt.-Size 13 by 13 inches. As the roofing leaves the coating rolls, place a 13 by 13 inch piece of kraft paper directly on top of the sheet so it will stick to the hot coating. This should be located just ahead of the splice beside the opening left by cutting out the sample of saturated felt (item 2). Recover this sample at the winder or cutter when it reaches that point.

4. Finished roofing. Size 13 by 13 inches. Take a sample of this just ahead of the splice when it reaches the winder or cutter.

It is the intention to take the above samples from locations on the roofing as nearly adjacent as possible.

\section{ANALYTICAT OPERATIONS}

Note. The paper-makers' scale is used for all detorminations, except ash. As the ordinary paper-makers' scale when testing square foot of roofing shows pounds per 480 square feet, such reading should be divided by 4.8 in each instance to show pounds per 100 square feet. A direct reading in pounds per 100 square feet may he ohtained on this scale hy testing 30 square inches of roofing strip 3 hy 10 on this scale hy testing 30 square inches of roofing (a converted into pounds per 480 square feet hy multiplying it by 4.8 .

\section{SAMPLE OF ORIGINAI FEIT}

First. Trim the exact size ( 12 by 12 inches or 3 by 10 inches). Caliper and weigh on P. M. scale.

Result.-Pounds original felt per 100 square feet $=a$.

Second. After drying this same piece of felt at $220^{\circ} \mathrm{F}$. to constant weight, weigh it on $\mathrm{P}$. M. scale. Result.-Pounds dry felt per 100 square feet $=b$.

Third. Cut out a piece of felt 3 by 3 inches from this dried sample or a similar one, weigh it in grams on analytical balance, place it in a weighed quartz dish and burn to constant weight in 3 mufle or over a Bunsen burner. Deduct weight of dish.

$$
\text { Result.-Ash, per cent }\left(\frac{\text { grams ash }}{\text { grams felt }} \times \mathbf{1 0 0}\right)=c \text {. }
$$

Result.-Ash, pounds per 100 square feet (grams $\operatorname{ash} \times 3.527)=d$.

\section{SAIIPLE SATURATED FELT}

First. Trim to exact size (two pieces 6 by 12 inches or one piece 3 by 10 inches). Caliper and weigh on $\mathrm{P}$. M. scale.

Result.-Pounds saturated felt 100 square feet $=e$.

NoTE.-If saturated felt is final sample and is sanded, brush off sand and weigh again. Difference $=$ pounds sand per 100 square feet.

\section{SAMPLE SATURATED AND COATED FELT WITH KRAFT PAPER ON ONE SIDE}

First. Trim to exact size, and weigh on P. M. scale.

Result.-Pounds roofing plus kraft paper per 100 square feet $=f$. 
Second. Trim a piece of kraft paper to the same size as that included in the weight $(f)$. Weigh this on P. M. scale.

Result.-Pounds kraft paper per 100 square feet $=g$.

Third. Cut out a piece of this sample of roofing exactly 3 by 3 inches, weigh and burn to ash as in dry felt.

Result.-(Grams ash $\times 3.527)=$ pounds ash in roofing per 100 square feet $=h$.

Fourth. Cut out a piece of kraft paper exactly 3 by 3 inches, weigh and burn to ash as in dry felt. Result.-(Grams ash $\times 3.527)=$ pounds ash in $\mathrm{kraft}$ paper per 100 square feet $=m$.

Note.-If the dust or filler in use loses weight when ignited, a loss factor must be determincd and added to weight of ash $h$.

\section{SAMPLE FINISHED SMOOTH-SURFACED ROOFING}

First. Trim to exact size and weigh on P. M. scale.

Result.-Total weight smooth-surfaced roofing 100 square feet $=o$.

\section{SAMPLE FINISFED GRANULAR-SURFACED ROOFING}

First. Trim to exact size and weigh on P. M. scale.

Result.-Total weight granular-surfaced roofing per 100 square feet $=p$.

\section{CALCULATIONS}

1. Weight of original felt as received: $a=$ pounds per 100 square feet.

$(a \times 4.8)=$ pounds per 480 square feet.

2. Weight of original felt, moisture-free:

$b=$ pounds per 100 square feet.

$(b \times 4.8)=$ pounds per 480 square feet.

3. Per cent moisture in original felt:

$\frac{a-b}{a} \times 100=$ per cent moisture in felt.

4. Ash in moisture-free felt:

$c=$ per cent ash in moisture-free felt.

$d=$ pounds ash per 100 square feet felt.

5. Per cent saturation in moisture-free felt: $\frac{e-b}{b} \times 100=$ parts saturation per 100 parts dry

felt.

6. Pounds dry felt per 100 square feet roofing:

$a=$ pounds dry felt per 100 square feet roofing.

7. Pounds saturation per 100 square feet roofing: $e-b=$ pounds saturation per 100 square feet roofing.

8. Pounds pure coating per 100 square feet roofing.

NOTE.-The dusting on one or both sides of roofing is calculated as described under 11. This amount runs sufficiently constant on one type of roofing to be accepted as a standard factor when once determined and checked and may be deducted at once in routine work without individual checking each time.

Similarly, a supply of kraft paper sheets may be accumulated and factor weights for the sizes employed, established both under $g$ and $m$, which may be used thereafter without rechecking.

Smooth-surfaced roofing:

$2[o-(f-g)]=(1)$ Total dust on roofing (pounds per 100 square feet).

$a=$ (2) Total dry felt (pounds per 100 square feet).

$e-b=(3)$ Total saturation (pounds per 100 square feet)

$h-(d+m+$ dust on roofing $)=(4)$ Total mineral

filler in coating.

Thercfore $o-$ (sum of above 4 items) $=$ Pure coating (pounds per 100 square feet).
Slate or other granular roofing:

$p-(f-g)=(1)$ 'Tutal slate or other granules.

$1 / 2$ dust factor $=(2)$ Total dust on one side.

$a=(3)$ Total dry felt.

$e-b=(4)$ Total saturation.

$h-(d+m+$ dust on roofing $)=(5)$ Total mineral

filler in

coating.

Therefore $p-$ (sum of above 5 items) $=$ Pure coat

ing (pounds per 100 square feet).

9. Mineral filler in coating:

$h-(d+m+$ dust on roofing $)=$ Mineral filler in coating (pounds per 100 square feet).

10. Slate or other granular surfacing:

$p-(f-g)=$ Mineral surfacing (pounds per 100 square feet)

11. Dust on surface:

$f-g=$ Saturated and coated roofing with dust on one side only (pounds per 100 square feet).

$o-(f-g)=$ Dust on one side (pounds per 100 square feet)

$2[o-(f-g)]=$ Dust on two sides (pounds per 100 square feet)

12. Total weight of roofing:

$o=$ Pounds per 100 square feet smooth surface roofing.

$p=$ Pounds per 100 square feet slate or other granular roofing.

Asphalt Shingle and Roofing Association (now Institute), test for discoloration of coatings.

The following test is adapted for ascertaining in a brief period of time whether or not a coating will discolor the talc surfacing on aging:

The asphalt is melted at the lowest possible temperature, to prevent loss of volatile constituents, and by means of a small spatula spread uniformly on one side of a 1 by 3 inch microscope slide glass, in a layer one-sixteenth to one-eighteenth inch thick. Before the asphalt coating has set it is sprinkled liberally with pure calcium asphalt coating has set it is sprinkled liberally with pure calcium carbonate (prepared from a saturated solution of pure calcium hydroxide, which is precipitated with an excess of pure ammonium carbonate, then boiled until all the ammonia is driven off, filtered,
and dried on a steam bath). When cool, the calcium carbonate is and dried on a steam bath). When cool, the calcium carbonate is distributes the powder and removes the excess. Support the slide horizontally on an asbestos pad and heat for five hours in an oven, the air temperature of which is maintained at $150^{\circ} \mathrm{F}$. Remove from the oven at the end of the test and observe the color of the surfacing, recording your observations in the following terms: "no discolora

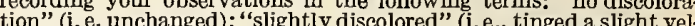
low); "moderately discolored" (i. e., cream color); "discolored" (i.e. yellow); "badly discolored" (i. e., yellowish brown).

Asphalt Shingle and Roofing Association (now Institute), rccommends the standard method of test for soluble bitumen of the American Society for Testing Materials, serial designation D4. (See p. 117.)

This association has a "short method" for the above test, where a moderate degree of accuracy is required, which is as follows:

Weigh 1 to $2 \mathrm{~g}$ of the bituminous material free from moisture into a $150 \mathrm{cc}$ tared flask, $50 \mathrm{cc}$ of c. p. carbon disulphide are added, and the contents agitated until all the lumps disappear and nothing adheres to the bottom. The flask is then loosely corked and set aside for 15 minutes, whereupon it is filtered through a weighed Gooch crucible measuring $4 \mathrm{~cm}$ wide at the top, tapering to $3.2 \mathrm{~cm}$ at the bottom, and $2.5 \mathrm{~cm}$. deep, carrying freshly ignited long-fibered asbestos compacted in a layer not over one-eighth inch deep. Wash the sides of the llask with a small amount of carbon disulphide and catch the sediment on the crucible. Then wash the residue with carbon disulphide, using suction until the washings are colorless and continue the suction until the odor of the carbon disulphide disappears. Heat the crucible for one-half hour at $220^{\circ} \mathrm{F}$., cool in a desiccator, and weigh. The weight of the rcsidue on the crucible added to the residue obtained on evaporating and igniting the carbon-disulphide extract represents the insoluble 
matter. The portion soluble in carbon disulphide is calculated by difference.

Asphalt Shingle and Roofing Association (now Institute), standard method of testing the ductility of coatings composed of bituminous materials.

This method is substantially the same as A. S. T. M. D113. (See p. 126.)

Asphalt Shingle and Roofing Association (now Institute), standard methods of testing the viscosity of saturation. This method is the same as that of the United States Department of Agriculture, Bulletin 1216. (See 505.20, p. 172.)

Federal Specifications Board, specification No. 157, United States Government master specification for the installation of plastic flashings with bituminous built-up roofing, and specification No. 156, United States Government master specification for the installation of metal flashings with bituminous built-up roofing, June 1, 1924.

These specifications cover the installation of plastic basc flashings with or without appropriate cap or counter flashings and all metal flashings, and other metal fittings, in connection with built-up bituminous roofing in the angles formed where the roof surface abuts walls and similar vertical surfaces and whele required to make the roof covering watertight. They shall be installed by the roofing contractor or he shall at lcast be responsible for their proper installation.

Plastic flashings shall be composed of either asphalt-saturated felt or coal-tar saturated rag felt cemented together, respectively, with asphalt plastic roofing cement or coal-tar plastic roofing cement.

Note-Detailed requirements for installation of the flashings are included in the full text of the above specifications.

United States Department of Agriculture, Bulletin 1216 , method for examination of bituminous mixtures, 1928.

\section{CENTRIFUGAL METHOD}

1. The aggregate is prepared for analysis by heating it in an enamel-ware pan on the hot plate until it is sufficiently soft to be thoroughly disintegrated by means of a large spoon. Care must be taken, however, that the individual particles are not crushed. If a section of pavement is under examination, a piece weighing somewhat over $1 \mathrm{~kg}$ may be cut off with hammer and chisel. The disintegrated aggregate is then allowed to cool. Not less than $500 \mathrm{~g}$ of aggregate containing particles larger than three-quarter inch in diameter or $200 \mathrm{~g}$ of aggregate with all particles smaller than threequarter inch are placed in the bowl of the centrifuge extractor, and a ring three-quarters of an inch wide, cut from felt paper, is fitted on the rim, after which the cover plate is placed in position and drawn down tightly by means of the milled nut. If the bitumen is to be recovered and extracted, the felt ring should be treated previously in the empty extractor with a couple of charges of carbon disulphide in order to remove any small amount of grease or resin that may be present, although a proper grade of felt should be practically free from such products. The bowl is then placed on the motor shaft of the extractor and the slot and pin are carefully locked. An empty bottle is placed under the spout and $150 \mathrm{cc}$. of carbon disulphide (carbon tetrachloride, benzol, or chloroform may also be used as solvents) is poured into the bowl through the small holes. After allowing the material to digest for a few minutes, the motor is started, slowly at first, in order to permit the aggregate to distributc uniformly.

The speed should then be increased sufficiently by means of the regulator to cause the dissolved bitumen to flow from the spout in a thin stream. When the first charge has drained, the motor is stopped and a fresh portion of disulphide is added. This operation is repeated from four to six times with $150 \mathrm{cc}$ of disulphide. With a little experience the operator can soon gauge exactly what treatment is necessary for any given material. When the last addition of solvent lias drained off, the bowl is removed and placed with the cover plate uppermost on a sheet of manila paper. The cover plate and felt ring are carefully laid aside on the paper and, when the aggregatc is thoroughly dry, it can be brushed on a pan of the rough balance and weighed. The difference between this weight and the original weight taken shows the amount of bitumen extracted. The aggregate may then be tested as occasion requires.

2. When it is desired to examine the bitumen, or recover the solvent, a suitable distillation apparatus may be used, taking necessary precautions when handling inflammable solvents.

3 . The solution of bitumen should be allowed to stand overnight in order to permit the settling of any fine mineral matter that is sometimes carried through the felt ring in the extractor. The solution is then dccantcd into the flask, and the solvent is driven off by means of heat from an incandescent lamp until the residue is of a thick sirupy consistency. Meanwhile the solvent is condensed and recovered in the flask. The residue is poured into an $11-\mathrm{cm}$ porcelain evaporating dish and evaporated on a steam bath. The most scrupulous care must be taken at all times that no flames are in its immediate vicinity. Evaporation is carried on at a gentle heat, with continual stirring, until foaming practically ceases. It is advisable to have a large watch glass at hand to smother the flames quickly should the material ignite. As the foaming subsides, the heat of the steam bath may be gradually raised, and evaporation continued until the bubbles beaten or stirred to the surface of the bitumen fail to give a blue flame or odor of sulphur dioxide when ignited by a small gas jet. The dish of bitumen should then be set in a hot-air oven maintained at $105^{\circ} \mathrm{C}$. for about an hour, after which it should be allowed to cool. Its general character is noted and any tests for bitumen that are necessary are then made upon it.

4. The difference between the final aggregate and the original amount taken gives the amount of bitumen cxtracted, which is subject to correction, dependent on the amount of ash determined from the washings.

5. Ash correction shall be made in the following manner: The total solution of bitumen, well stirred, is rapidly measured and an aliquot portion taken, usually $100 \mathrm{cc}$, and poured into a previously weighcd suitable flat-bottom dish, preferably quartz. The solvent is evaporated over a very low flame and the residual coke is then ignited with a burner capable of furnishing high temperature, such as a Meker. (Caution: When an inflammable solvent is used evaporation should be conducted on a steam bath and care should be taken that no flames are in the immediate vicinity.) The dish and contents are then cooled in a desiccator and the percentage of ash calculated.

\section{HOT EXTRACTION METHOD}

6. The New York testing laboratory extractor consists of a large brass cylinder, through the bottom of which projects a 16-candlepower incandescent carbon-filament bulb to supply heat to the extraction apparatus proper, which is held in the upper portion of the cylinder. This apparatus is composed of a cylindrical brass vessel for holding the solvent, a cylindrical wire basket made of 
80-mesh wire cloth, suspended in the cylinder, and an inverted conical condenser which serves as a top. 7. The aggregate is prepared for analysis by heating it in a tin dish on the hot plate until it is sufficiently soft to be disintegrated by means of a large spoon. The disintegrated aggregate is then allowed to cool. Not less than 500 grams of aggregates containing particles larger than three-quarters inch in diameter or $200 \mathrm{~g}$ of aggregates with all particles smaller than three-quarters inch are then closely packed in the wire basket or suitably sized extraction thimbles and covered with a disk or wad of absorbent cotton or felt. From 175 to $200 \mathrm{cc}$ of carbon disulphide (carbon tetrachloride, benzol, or chloroform may be used) are next placed in the inside vessel, in which the wire basket should be suspended. The top is then placed in position and cooling water circulated through it. Heat is applied by means of the electric-light bulb. The solvent is boiled in the lower part of the extractor and condenses on the undersurface of the top, from which it drips upon the wad of absorbent cotton and then percolates through the sample. A complete extraction may be made in three hours. At the end of this time the apparatus is allowed to cool and the basket containing the extracted aggregate carefully removed. After thoroughly drying the aggregate is placed upon a pan of the rough balance and weighed. The difference between this weight and the original weight taken shows the amount of bitumen extracted, which is calculated upon a percentage basis of the original. This figure should be corrected for fine mineral matter which passes through the meshes of the wire basket, as follows: The solution of extracted bitumen is thoroughly agitated and measured in a glass graduate. Five or ten cubic centimeters are then poured into a weighed platinum crucible or dish, burned, and ignited to ash. The amount of mineral matter in the entire solution may then be calculated from the amount of ash produced from that portion ignited. The total percentage of such ash is then deducted from the percentage of bitumen already calculated in order to obtain the true percentage of bitumen. The amount of this correction will ordinarily vary from 0.1 per cent in uniformly coarse aggregates to 1 or 2 per cent in the analysis of aggregates containing a considerable amount of very fine mineral matter.

\section{SUGGESTED HETHOD FOR EXAMINATION OF BITUMINOUS MORTARS}

8. Bituminous mortars may be extracted by the use of bronze tubes which are capable of being whirled in the type of centrifuge similar to the Babcock milk tester. This method is based on decantation of the supernatant solvent. (The sample shall consist of not less than $25 \mathrm{~g}$.) The difference between the amount of final aggregate and the original amount taken gives the amount of bitumen extracted, which is subject to correction by deducting the amount of ash determined from the washings. The ash correction shall be made as given under the centrifugal method.

9. Mechanical analysis of the extracted aggregate shall be conducted in accordance with United States Department of Agriculture, Bulletin 1216, Method of Mechanical Analysis of Extracted Aggregates. (See 512.13, p. 241.)

United States Department of Agriculture, Bulletin 1216, Methods of Sampling Bituminous Mixtures, 1928.

A daily sample shall be taken by any of the following methods:

(a) Samples shall be cut from the finished pavement about 6 to 8 inches square and extending full depth of pavement before application of any sealing coat which may be used.

(b) Samples of sheet asphalt mixtures shall be taken during preparation of the paving mixture from trucks or wagons at the asphalt plant and combined into composite samples of not less than 1 pound each in the following manner: These composite samples shall be taken by collecting small quantities at different times from not less than 10 and preferably more separate batches, molding the portions into the container provided for the purpose.

In the sampling of bituminous concrete either of the two methods given below shall be used in order to obtain a composite sample of not less than 5 pounds, the amount being dependent on, the maximum size of the coarse aggregate. Small portions from a number of batches are taken and stored on a metal plate until ncar the end of the day, when the entire mass is reheated-care being exercised not to apply too great heat-mixed thoroughly, and the day's sample selected in small portions from this mass. In case of dense bituminous-concrete mixtures the samples may be taken by collecting portions from one or more batches on a large shovel, thoroughly mixing while still warm, and taking a small scoopful which is placed directly in the carton. This operation is to be repeated as often as possible, thus representing a maximum number of batches. In either of these methods care must be taken to avoid segregation of either stone or bituminous mortar. In selecting the portions of the sample care should be taken to scrape away the top material in the load, so as to avoid dust or any segregated unrepresentative mixture.

(c) Samples shall be taken either at the road or asphalt plant of any bituminous mixture which appears defective.

United States Department of Agriculture, Bulletin 1216 , methods of test for specific gravity of bituminous materials, 1928.

1. The specific gravity of bituminous materials shall be expressed preferably as the ratio of the weight of a given volume of the material at $25^{\circ}$ C. $\left(77^{\circ}\right.$ F.) to that of an equal volume of water at the same temperature, and shall be expressed thus:

Specific gravity $25^{\circ} / 25^{\circ} \mathrm{C}$. $\left(77^{\circ} / 77^{\circ} \mathrm{F}\right.$.)

\section{HYDROMETER METHOD}

\section{(Used for thin fluid bitumens)}

2. The specific gravity of thin fluid bituminous road materials may be determined with the abovementioned apparatus by first pouring a sufficient quantity of the material into a tin cup which is then placed in a large dish containing cold or warm water, as occasion may require. The material in the cup should be stirred with the thermometer until it is brought to a temperature of $25^{\circ} \mathrm{C}$., after which it should be immediately poured into the hydrometer jar and its gravity determined by means of the proper hydrometer. In case the hydrometer sinks slowly, owing to the viscosity of the material, it should be given sufficient time to come to a definite resting point, and this point should be checked by raising the hydrometer and allowing it to sink a second time. The hydrometer should never be pushed below the point at which it naturally comes to rest until the last reading has been made. It may then be pushed below the reading for a distance of three or four of the small divisions on the scale, whereupon it should immediately begin to rise. If it fails to do so, the material is too viscous for the hydrometer method, and the pycnometer method should be employed. 
3. The direct specific gravity reading obtained by the foregoing method is based upon water at $15.5^{\circ} \mathrm{C}$. taken as unity. For all practical purposes this reading may be corrected to water at $25^{\circ} \mathrm{C}$. considered as unity, by multiplying it by 1.002. Thus:

Specific gravity $25^{\circ} / 25^{\circ}$ C. $=$ specific gravity $25^{\circ} / 15.5^{\circ} \mathrm{C} . \times 1.002$

\section{PYCNOMETER METHOD}

(Used for viscous fluid and semisolid bitumens and emulsions)

4. The determination of specific gravity shall be made with a pycnometer (Hubbard-Carmick type recommended) or weighing bottle, which shall consist of a conical or Erlenmeyer-shaped flask approximately $45 \mathrm{~mm}$ high, $40 \mathrm{~mm}$ in diameter at the bottom, and $25 \mathrm{~mm}$ in diameter at the mouth, carefully ground to receive an accurately fitting solidglass stopper with a hole of about $1 \mathrm{~mm}$ bore in place of the usual capillary opening. The lower surface of the stopper is made concave in order to allow all air bubbles to escape through the bore. The depth of the cup-shaped depression shall be about $4.8 \mathrm{~mm}$ at the center. The stoppered flask has a capacity of about $25 \mathrm{cc}$ and when empty weighs about $25 \mathrm{~g}$.

5. Before making a determination, the pycnometer with stopper shall first be calibrated by weighing it clean and dry upon an analytical balance. This weight is called $a$. It shall then be filled with freshly boiled distilled water at a temperature of $25^{\circ} \mathrm{C}$. $\left(77^{\circ} \mathrm{F}\right.$.), the stopper firmly inserted, all surplus moisture wiped from the surface with a clean, dry cloth, and again weighed. This weight is called $b$.

6 . When determining the specific gravity of road oils or road tars which flow readily, the material shall be brought to a temperature of $25^{\circ} \mathrm{C}$. $\left(77^{\circ} \mathrm{F}\right.$.) and poured into the pycnometer until it is full, with care to prevent the inclusion of air bubbles. The stopper is then firmly inserted and all excess of material forced through the opening is carefully removed with a clean, dry cloth. The pycnometer and contents are then weighed and this weight is called $c$. The specific gravity of the material shall be calculated from the formula:

$$
\text { Specific gravity }=\frac{c-a}{b-a}
$$

7. When determining the specific gravity of tar and asphalt products which are too viscous for the method described in section 4, a small amount of the material shall be brought to a fluid condition by the gentle application of heat, care being exercised to prevent loss by evaporation. When sufficiently fluid, enough is poured into the clean, dry pycnometer to about half fill it. Precautions shall be taken to keep the material from touching the sides of the tube above the final level and to prevent the inclusion of air bubbles. The tube should be slightly warmed before filling. The pycnometer and contents are then cooled to room temperature and weighed with the stopper. This weight is called $c$. The pycnometer is next removed from the balance, filled with freshly boiled distilled water, and the stopper firmly inserted. It is then completely immersed for not less than 30 minutes in a beaker of distilled water maintained at $25^{\circ} \mathrm{C}$. $\left(77^{\circ} \mathrm{F}\right.$. $)$, after which it is removed, and all surplus water is wiped off with a clean cloth. It is immediately weighed. This weight is called $d$. The specific gravity of the material shall be calculated from the formula:

$$
\text { Specific gravity }=\frac{c-a}{(b-a)-(d-c)}
$$

8. When making the specific-gravity determination it is important that:

(a) Only freshly boiled distilled water shall be used.

(b) When weighing the pycnometer completely filled, the temperature of its contents shall be within $1^{\circ} \mathrm{C} .\left(1.8^{\circ} \mathrm{F}\right.$.) of $25^{\circ} \mathrm{C} .\left(77^{\circ} \mathrm{F}\right.$.).

(c) Precautions shall be taken to prevent expansion and overflow of the contents from the heat of the hand when wiping the surface of the pycnometer.

(d) The presence of all air bubbles shall be eliminated in filling the pycnometer and inserting the stopper.

(e) Weighings shall be made quickly after filling the pycnometer and shall be accurate to $1 \mathrm{mg}$. A number of trial fillings and catchweights may be necessary to obtain the desired degree of accuracy.

$(f)$ To prevent breakage of the pycnometer when cleaning it out after a determination has been made upon a very viscous or semisolid matelial, it will be found advisable to warm it in an oven at not over $100^{\circ} \mathrm{C}$. until most of the material may be poured out and then to swab it with a piece of soft cloth or cotton waste. When cool, it may be finally rinsed with carbon disulphide, benzol, or other solvent and wiped clean.

9. The linit of accuracy of the test is \pm 0.005 specific gravity.

\section{DISPLACEMENT METHOD}

(Used for hard, solid bitumens)

10. For materials which are hard enough to be broken and handled in fragments at room temperature the following method will prove convenient: A small fragment of the bitumen (about $1 \mathrm{cc}$ ) is suspended by means of a silk thread from the hook on one of the pan supports, about $1 \frac{1}{2}$ inches above the pan and weighed. This weight is called $a$. It is then weighed immersed in water at $25^{\circ} \mathrm{C}$. and this weight is called $b$. The specific gravity may then be calculated by means of the following formula:

$$
\text { Specific gravity }=\frac{a}{a-b}
$$

\section{DISPLACEMENT METHOD}

\section{(Used for semisolids)}

11. The specific gravity of semisolid bituminous materials may be determined by the displacement method. Weigh a silica crucible suspended from the beam of the balance in air and call the weight $a$, and in water and call the weight $b$. Fill the crucible approximately two-thirds full with the material under examination. Free from bubbles by heating on a hot plate, cool and weigh, calling this weight $c$. Immerse the filled crucible in water at $25^{\circ} \mathrm{C}$. for one-half hour, then suspend by a wire from the beam of the balance and weigh it immersed in water at $25^{\circ}$ C.; call this weight $d$. The specific gravity is then calculated by means of the following formula:

$$
\text { Specific gravity }=\frac{c-a}{d-b}
$$

United States Department of Agriculture, Bulletin 1216, method of test for percentage of bitumen soluble in carbon disulphide, 1928.

1. This test consists in dissolving the bitumen in carbon disulphide and recovering any insoluble matter by filtering the solution through an asbestos felt. The form of Gooch crucible best adapted for the determination is $4.4 \mathrm{~cm}$ wide at the top, tapering to $3.6 \mathrm{~cm}$ at the bottom, and is $2.5 \mathrm{~cm}$ deep.

2. For preparing the felt, the asbestos is cut with scissors into pieces not exceeding $1 \mathrm{~cm}$ in length, after which it is shaken up with just sufficient water 
to pour easily. The Gooch crucible is filled with the suspended asbestos, which is allowed to settle for a few moments. A light suction is then applied to draw off all the water and leave a firm mat of asbestos in the crucible. More of the suspended material is added, and the operation is repeated until the felt is so dense that it scarcely transmits light when held so that the bottom of the crucible is between the eye and the source of light. The felt should then be washed several times with water and drawn firmly against the bottom of the crucible by an increased suction. The crucible is removed to a drying oven for a few minutes, after which it is ignited at red heat over a Bunsen burner, cooled in a desiccator, and weighed.

3. From 1 to $2 \mathrm{~g}$ of bitumen or about $10 \mathrm{~g}$ of an asphalt topping or rock asphalt are now placed in the Erlenmeyer flask, which has been previously weighed, and the accuratc weight of the sample is obtained. One hundred cubic centimeters of chemically pure carbon disulphide are poured into the flask in small portions, with continual agitation, until all lumps disappear and nothing adheres to the bottom. The flask is then corked and set aside for 15 minutes.

4. After being weighed, the Gooch crucible containing the felt is set up over the dry-suction flask, and the solution of bitumen in carbon disulphide is decanted through the felt with light suction or without suction by gradually tilting the flask, with care not to stir up any precipitate that may have settled out. At the first sign of any sediment coming out, the decantation is stopped and the filter allowed to drain. A small amount of carbon disulphide is then washed down the sides of the flask, after which the precipitate is brought upon the felt and the flask scrubbed, if necessary, with a feather or "policeman" to remove all adhering material. The contents of the crucible are washed with carbon disulphide, until the washings run colorless. Suction is then applied until there is practically no odor of carbon disulphide in the crucible, after which the outside of the crucible is cleaned with a cloth moistened with a small amount of solvent. The crucible and contents are allowed to dry over the top of the oven until no odor of carbon disulphide is detected and then dried in the hot-air oven at $100^{\circ} \mathrm{C}$. for about 20 minutes, cooled in a desiccator, and weighed. If any appreciable amount of insoluble matter adheres to the flask, it should also be dried and weighed, and any increase over the original weight of the flask should be added to the weight of insoluble matter in the crucible. The total weight of insoluble material may include both organic and mineral matter. The former, if present, is burned off by ignition at a red heat until no incandescent particles remain, thus leaving the mineral matter or ash, which can be weighed on cooling. The difference between the total weight of material insoluble in carbon disulphide and the weight of substance taken equals the total bitumen, and the percentage weights are calculated and reported as total bitumen, and organic and inorganic matter insoluble, on the basis of the weight of material taken for analysis.

5. This method is quite satisfactory for straight oil and tar products, but where certain natural asphalts are present it will be found practically impossible to retain all of the finely divided mineral matter on an asbestos felt. It is therefore generally more accurate to obtain the results for total mineral matter by direct ignition of a l-g sample in a platinum crucible. The total bitumen is then determined by deducting from 100 per cent the sum of the percentage of total mineral matter and organic matter insoluble. If the presence of a carbonate mineral is suspected, the percentage of mineral matter may be most accurately obtained by treating the ash with a few drops of ammonium carbonate solution, drying at $100^{\circ} \mathrm{C}$., then heating for a few minutes at a dull red heat, cooling, and weighing again.

6 . When difficulty in filtering is experiencedfor instance, when Trinidad asphalt is present in any amount-a period of longer subsidence than 15 minutes is necessary, and A. S. T. M. test method D4, method No. 2, is recommended. (See 505.0, p. 117.)

United States Department of Agriculture, Bulletin 1216, calcium chloride method for determination of percentage of water in bituminous emulsions, 1928.

\section{(Not applicable to clay emulsions)}

Approximately $10 \mathrm{~g}$ of the emulsion is accurately weighed in an Erlenmeyer flask and exactly $25 \mathrm{cc}$ of a 10 per cent solution of calcium chloride added and thoroughly agitated by shaking. The liquid content of the flask is then poured into a graduated cylinder. Carbon disulphide is then added to the Erlenmeyer flask and the separated asphalt dissolved and washed with carbon disulphide into the graduated cylinder above referred to containing the decanted liquid. The total contents of the cylinder are then thoroughly shaken and after standing the quantity of separated supernatant water read.

$$
\text { Calculations: Per cent water }=\frac{A-B}{C} \times 100
$$

$A=$ volume of supernatant water.

$B=$ volume of 10 per cent caleium chloridc, solution originally used. $C=$ weight of emulsion taken for water determination.

United States Department of Agriculture, Bulletin 1216 , method of test for percentage of bitumen insoluble in paraffin naphtha, 1928.

1. This determination is made in the same general manner as the total bitumen determination, except that $100 \mathrm{~cm}^{3}$ of $86^{\circ}$ to $88^{\circ}$ Bé. paraffin naphtha, at least 85 per cent distilling between $35^{\circ}$ and $65^{\circ} \mathrm{C}$., is employed as a solvent instead of carbon disulphide. Considerable difficulty is sometimes experienced in breaking up some of the heavy semisolid bitumens; the surface of the material is attacked, but it is necessary to remove some of the insoluble matter in order to expose fresh material to the action of the solvent. It is, therefore, advisable to heat the sample after it is weighed, allowing it to cool in a thin layer around the lower part of the flask. If difficulty is still experienced in dissolving the material, a rounded glass rod will be found convenient for brcaking up the undissolved particles. Not more than one-half of the total amount of naphtha required should be used until the sample is entirely broken up. The balance of the $100 \mathrm{cc}$ is then added, and the flask is twirled a moment in order to mix the contents thoroughly, after which it is corked and set aside for 30 minutes.

2. In making the filtration the utmost care should be exercised to avoid stirring up any of the precipitate, in order that the filter may not be clogged and that the first decantation may be as complete as possible. The sides of the flask should then be quickly washed down with naphtha and, when the crucible has drained, the bulk of insoluble matter is brought upon the felt. Suction may be applied when the filtration by gravity almost ceases, but should be used sparingly, as it tends to clog the filter by packing the precipitate too tightly. The material on the felt should never be allowed to run entirely dry until the washing is completed, as shown by the colorless filtrate. When considerable insoluble matter adheres to the flask no attempt should be made to remove it completely. In such cases the adhering material is merely washed until free from soluble matter and the flask is dried with the cruciblc at $100^{\circ} \mathrm{C}$. for about one hour, after which it is cooled and weighed. The percentage of 
bitumen insoluble is reported upon the basis of total bitumen taken as 100 .

3. The difference between the material insoluble in carbon disulphide and in the naphtha is the bitumen insoluble in the latter. Thus, if in a certain instance it is found that the material insoluble ir. carbon disulphide amounts to 1 per cent and that 10.9 per cent is insoluble in naphtha, the percentage of bitumen insoluble would be calculated as follows:

$$
\frac{\text { Bitumen insoluble in naphtha }}{\text { Total hitumen }}=\frac{10.9-1}{109-1}=\frac{9.9}{99}=10 \text { per cent }
$$

United States Department of Asriculture, Bulletin 1216, method of test for percentage of bitumen insoluble in carbon tetrachloride, 1928.

1. This determination is conducted in exactly the same manner as described under "Determination of bitumen soluble in carbon disulphide" (see p. 143), using $100 \mathrm{~cm}^{3}$ of chemically pure carbon tetrachloride as a solvent in place of carbon disulphide.

2. The percentage of bitumen insoluble is reported upon the basis of total bitumen taken as 100, as described under "Method of test for percentage of bitumen insoluble in paraffin naptha" given above.

United States Department of Commerce, Bureau of Standards, simplified practice recommendation No. 4, for asphalt (penetration limits), January. 1, 1924.

In accordance with unanimous action of a joint conference of representatives of manufacturers, distributors, and users of asphalt for paving purposes and reaffirmed by the standing committee on June 8 , 1926, the United States Department of Commerce, through the Bureau of Standards, recommends that recognized varieties of paving asphalt be reduced to those shown in the following tables.

I. For construction of sheet ashpalt, asphaltic concrete, and asphalt macadam pavements, and also for surface treatment.

\section{Penetration limits}

$\begin{array}{lll}25 \text { to } 30 & 50 \text { to } 60 & 100 \text { to } 120 \\ 30 \text { to } 40 & 60 \text { to } 70 & 120 \text { to } 150 \\ 40 \text { to } 50 & 85 \text { to } 100 & 150 \text { to } 200\end{array}$

II. For joint filler for various types of construction:

Penetration limits
30 to 50
60 to 70
50 to 60
85 to 100

The first is used primarily for brick pavements, and does not require the admixture of sand, whereas the latter three, which are identical with three of the grades adopted for asphalt pavement construction, are those which would ordinarly be used in admix ture with sand to produce an asphalt grout.

III. In adopting these limits, it is understood that the producer will furnish asphalts with penetration equal to the mid-point in each range, a plus and minus tolerance from that mid-point being acceptable to all parties, but in no case shall the deviation exceed the limits of the grade specified.

\subsection{ASPHAITS.}

\subsection{Native Asphalt.}

Federal Specifications Board, specification No. 276a, United States Government master specification for asphalt for use in road and pavement construction, May 8, 1925.

\section{PURPOSE AND INTENT OF THIS SPECIFICA. TION}

1. It is the purpose of this specification to describe asphalts suitable for ordinary types of bituminous construction by physical and chemical requirements without prejudice as to source.

2 . The bidder shall state in his bid whether he proposes to furnish asphalt of a petroleum type, a fluxed native type, or both, and he shall also state the source or sources, from which the material is derived.

3. Bids shall be solicited on all types of asphalt of particular grade (or grades) desired.

\section{TYPES AND GRADES}

This specification covers the types, grades, and intended uses of materials shown in the following table. The material shall be supplied in the particular type (or types) and grade (or grades) ordered.

\begin{tabular}{|c|c|c|c|}
\hline $\begin{array}{l}\text { Grade (pene- } \\
\text { tration) }\end{array}$ & Type of material & $\begin{array}{c}\text { Designa- } \\
\text { tion }\end{array}$ & Intended use \\
\hline 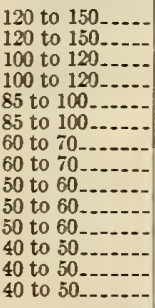 & 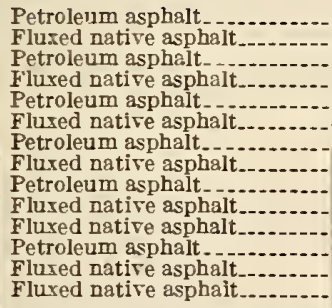 & $\begin{array}{l}\text { AP-1-25 } \\
\text { A }-1-25 \\
\text { AP-2-25 } \\
\text { A }-2-25 \\
\text { AP-3-25 } \\
\text { AB-3-25 } \\
\text { AP-5-25 } \\
\text { A }-5-25 \\
\text { AP-6-25 } \\
\text { A }-6-25 \\
\text { AT }-6-25 \\
\text { AP-7-25 } \\
\text { AB-7-25 } \\
\text { AT }-7-25\end{array}$ & $\begin{array}{l}\text { Bituminous macadam, northern United States, comparatively light traffic. } \\
\text { Bituminous macadam, middle United States or northern United States, com. } \\
\text { paratively heavy traffic. } \\
\text { Bituminous macadam, southern United States. } \\
\text { Graded hituminous concrete, northern United States, light or moderate traffic. } \\
\text { Graded bituminous concrete, southern United States or northern United States, } \\
\text { heavy traffic. } \\
\text { Sheet asphalt, northern United States, light or moderate traffic. } \\
\text { Sheet asphalt, southern United States or northern United States, heavy traffic. }\end{array}$ \\
\hline
\end{tabular}

NotE.-The information as to intended use shown above is necessarily general in character and should not be interpreted too strictly. Varying combinations of climate, traffic, and nature of the nonbituminous portion of the road or pavement will often influence the selection of the bituminous material and its method of use.

The right is reserved to make award for that type (or types) of asphalt, and from that source (or sources) which in the judgment of the purchaser is best suited for his particular requirements.

\section{MATERIAL AND WORKMANSHIP}

1. The materials supplied under this specification shall be asphalts prepared by the distillation of asphaltic petroleum or by the fluxing of hard native asphalts with a suitable petroleum flux.

2 . Those materials only which have been demonstrated by service tests as satisfactory for intended use will be acceptable under this specification.

3. No mineral matter other than that naturally contained in the asphalt shall be present.

\section{GENERAL REQUIREMENTS}

The asphalt shall be homogeneous, free from water and shall not foam when heated to $175^{\circ} \mathrm{C}$. $\left(347^{\circ} \mathrm{F}\right.$.).

\section{DETAIL REQUIREMENTS}

1. Physical and chemical properties.-The respective types and grades shall meet the requirements shown in the following table. 
2. Uniformity.-The material furnished under this specification for a given contract, type and grade shall be uniform in character and shall not vary more than $10^{\circ} \mathrm{C}$. in softening point from the test limits specified in the following table, nor more than 0.020 in specific gravity where no maximum limit is specified.

\section{METHODS OF TESTING}

Tests of the physical and chemical properties of the asphalt shall be made in accordance with the following methods:
1. Specific gravity.-A. S. T. M. D70, see 505.0, page 125.

2. Flash point.-A. S. T. M. D92, see 504.0, page 90.

3. Softening point.-A. S. T. M. D36, see 505.0, page 121.

4. Penetration--A. S. T. M. D5, see 505.0, page 118.

5. Ductility.-A. S. T. M. D113-26T, see 505.0, page 126.

6. Loss on heating.-A. S. T. M. D6, see 505.0, page 119.

7. Bitumen (soluble in carbon disulphide).A. S. T. M. D4, see 505.0, page 117, (Pt. I),.

Physical and chemical properties

\begin{tabular}{|c|c|c|c|c|c|c|c|}
\hline Designation & AP-1-25 & $A B-1-25$ & $A P-2-25$ & AB-2-25 & $A P-3-25$ & AB-3-25 & $A P-5-25$ \\
\hline 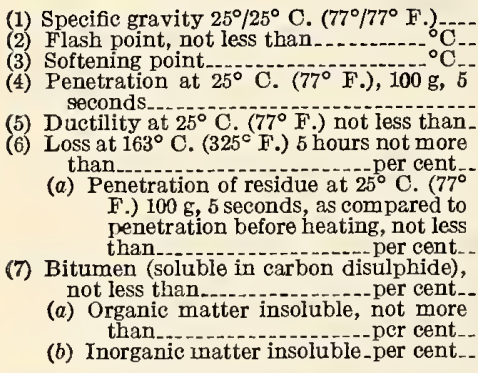 & 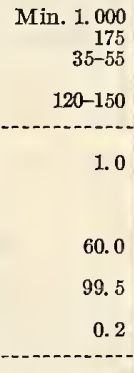 & $\begin{array}{r}1.025-1.050 \\
175 \\
35-45 \\
120-150\end{array}$ & $\begin{array}{r}\text { Min. } 1.000 \\
175 \\
35-55 \\
\\
100-120\end{array}$ & $\begin{array}{r}1.040-1.060 \\
175 \\
40-50 \\
100-120\end{array}$ & $\begin{array}{r}\text { Min. } 1.000 \\
175 \\
40-60 \\
85-100\end{array}$ & $\begin{array}{r}\text { 1. } 050-1.070 \\
175 \\
40-50 \\
85-100\end{array}$ & $\begin{array}{r}\text { Min. } 1.010 \\
175 \\
40-60 \\
60-70 \\
40 \\
1.0\end{array}$ \\
\hline Designation & $A B-5-25$ & AP-6-25 & $A B-6-25$ & AT-6-25 & AP-7-25 & A B $-7-25$ & AT-7-25 \\
\hline 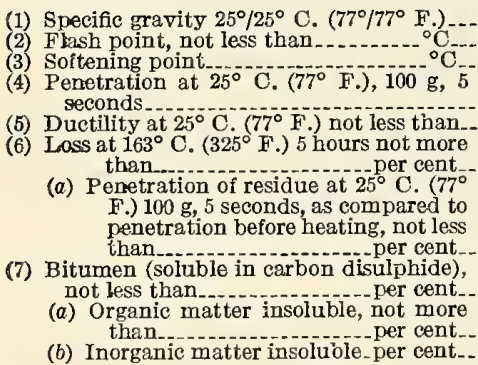 & $\begin{array}{r}1.050-1.070 \\
175 \\
45-55 \\
60-70 \\
40 \\
3.0\end{array}$ & $\begin{array}{r}\text { Min. } 1.010 \\
175 \\
40-60 \\
50-60 \\
40 \\
1.0\end{array}$ & $\begin{array}{r}1.050-1.070 \\
175 \\
45-55 \\
50-60 \\
40 \\
3.0\end{array}$ & $\begin{array}{r}\text { 1. } 200-1.250 \\
175 \\
45-55 \\
50-60 \\
40 \\
3.0\end{array}$ & $\begin{array}{r}\text { Min. } 1.010 \\
175 \\
45-64 \\
40-50 \\
40 \\
1.0\end{array}$ & $\begin{array}{r}1.055-1.075 \\
175 \\
45-55 \\
40-50 \\
40 \\
3.0\end{array}$ & $\begin{array}{r}1.210-1.270 \\
175 \\
50-60 \\
40-50 \\
40 \\
3.0\end{array}$ \\
\hline
\end{tabular}

\subsection{Asphalt Pavements. (See 518.37.)}

\subsection{Asphait Cement.}

American Association of State Highway Officials, tentative standard specifications for highway materials, 1927.

No. 18. Oil asphalt filler (type A).

No. 19. Asphalt filler (type B).

No. 20. Asphalt cement (prepared from petroleum), penetration grades 30 to 40,40 to 50,50 to 60 , 60 to 70 .

No. 21. Asphalt cement (prepared from petroleum), penetration grades 85 to 100,100 to 120 , 120 to 150.

No. 22. Asphalt cement (prepared from native asphait), penetration grades 30 to 40,40 to 50,50 to 60,60 to 70 .

No. 23. Asphalt cement (prepared from native asphalt), penetration grades 85 to 100,100 to 120 , 120 to 150 .

1. The product shall be homogeneous, free from water, and shall not foam when heated to a temperature of $177^{\circ} \mathrm{C}$., for Nos. 18 and 19 , and $175^{\circ} \mathrm{C}$. for Nos. $20,21,22$, and 23 .
2. The produet shall conform to the following requirements:

\begin{tabular}{|c|c|c|c|}
\hline & No. 18 & No. 19 & No. 20 \\
\hline 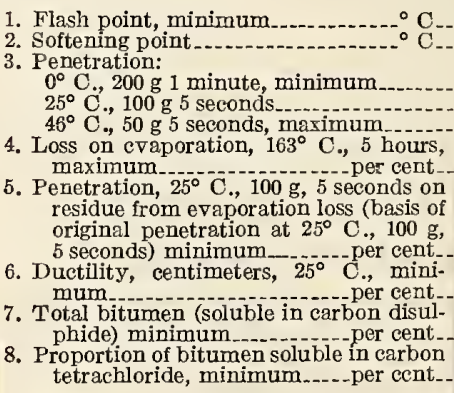 & $\begin{array}{r}200 \\
65-110 \\
\\
10 \\
30-50 \\
110 \\
1.0\end{array}$ & $\begin{array}{r}200 \\
60-75 \\
\\
20 \\
50-60 \\
160 \\
1.0\end{array}$ & $\begin{array}{r}60.0 \\
30\end{array}$ \\
\hline
\end{tabular}

See footnotes at end of table. 


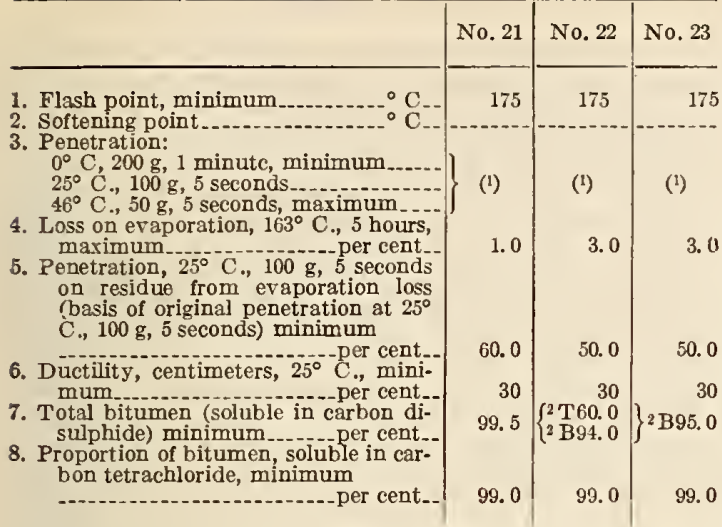

1 Insert penetration limits for grade desired. The exact penetration grade to he used depends on the type of road, climatic condition grade to ha used depends on the

tions, and kind and nature of traffic.
${ }^{\mathrm{T}}$ for Trinidad asphaltic cement; $\mathrm{B}$ for Bermudez asphaltic cement.

SSampling and testing: Test methods listed below in table of A. S. T. M. asphalt cement specifications. Also U.S. Department of Agriculture, Bull. 1216, method of test for proportion of bitumen soluble in carbon disulphide, 505.0, p. 143 .

American Association of State Highway Officials, standard specifications for highway bridges and incidental structures, 1928.

\section{BITUMNOUS MATERIALS}

\section{ASPHAIT FOR BITUMINOUS CARPETS}

The asphalt for either mixed or penetration asphalt bituminous carpets on concrete or wood bridge floors shall be an asphaltic cement conforming to either (A) or (B) as follows:

(A) Specification No. 21, American Association of State Highway Officials (given above), with the following exceptions:

1. The penetration grade requirement must be within the limits 85 to 100.

2. Add to No. 21 a requirement for specific gravity at $25^{\circ} / 25^{\circ} \mathrm{C}$., not less than 1.0 .

3. Ductility, centimeters, $25^{\circ}$ C. minimum 75 .

Note. - The ductility test shall be made at the penetration specified for use.

(B) Specification No. 23 (above) with the following exceptions:
1. The penetration grade requirement must be withing the limits 85 to 100 .

2. Add to No. 23 a requirement for specific gravity at $25^{\circ} / 25^{\circ} \mathrm{C}$., not less than 1.03 .

3. Ductility, centimeters, $25^{\circ}$ C. minimum 50 .

NorE.-The ductility test shall be made at the penetration specified for use.

American Society for Municipal Improvements, specifications for cut-back asphalt cement, optional with high and low carbon tars for road surface treatment, 1922.

See A. S. M. I. specifications for broken stone and gravel roads, 512.15 , page 245 .

American Society for Testing Materials, asphalt cement.

The society has prepared 10 tentative specifications for asphalt cement according to grades of penetration, as follows:

Serial designation: D99-26T, 1926, asphalt cement, 40 to 50 penetration, for use in sheet asphalt and asphaltic-concrete pavements and as filler for brick and block pavements. ${ }^{57}$

D100-26T, 1926, penetration 50 to 60 , for use in sheet asphalt and asphaltic-concrete pavements and as filler for block pavements. ${ }^{68}$

D101-26T, 1926, penetration 60 to 70 , for use in sheet asphalt, asphaltic-concrete, and asphaltmacadam pavements and as filler for block pavements..$^{58}$

D102-24T, 1924, penetration 85 to 100 , for 'use in asphalt-macadam pavements.

D103-24T, 1924, penetration 100 to 120 , for use in asphalt-macadam pavements.

D133-23T, 1923, penetration 10 to 15 , for the manufacture of asphalt block.

D134-23T, 1923, penetration 15 to 25, for the manufacture of asphalt block.

D135-23T, 1923, penetration 120 to 150 , for use in asphalt-macadam pavements.

D163-23T, 1923, penetration 25 to 30 , for use in sheet asphalt and asphaltic-concrete pavements.

D164-23T, 1923, penetration 30 to 40 , for use in sheet asphalt and asphaltic-concrete pavements.

The asphalt shall be homogeneous and free from water. It shall conform to requirements as listed in the accompanying table.

${ }^{67}$ As a filler the asphalt cement is not to be used in admixtures with sand.

ss For use as filler for block pavements the asphalt should be mixed with a suitable proportion of sand.

\begin{tabular}{|c|c|c|c|c|c|c|c|c|c|c|}
\hline & D99-26T & D $100-26 \mathrm{~T}$ & D 101-26T & D $102-24 T$ & $\mathrm{D} 103-24 \mathrm{~T}$ & D $133-23 T$ & D134-23T & D135-23T & D163-23T & $\begin{array}{c}\text { D164- } \\
23 T\end{array}$ \\
\hline 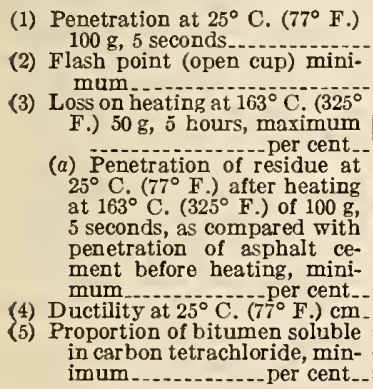 & $\left\{\begin{array}{r}40-50 \\
175^{\circ} \mathrm{C} . \\
\left(347^{\circ} \mathrm{F} .\right)\end{array}\right.$ & $\begin{array}{r}50-60 \\
175^{\circ} \mathrm{C} \\
\left(347^{\circ} \mathrm{F} .\right)\end{array}$ & $\begin{array}{r}60-70 \\
175^{\circ} \mathrm{C} . \\
\left(347^{\circ} \mathrm{F} .\right)\end{array}$ & $\begin{array}{r}85-100 \\
175^{\circ} \mathrm{C} . \\
\left(347^{\circ} \mathrm{F} .\right)\end{array}$ & $\begin{array}{l}100-120 \\
175^{\circ} \mathrm{C} . \\
\left(347^{\circ} \mathrm{F} .\right)\end{array}$ & $\mid \begin{array}{r}10-15 \\
200^{\circ} \mathrm{C} . \\
\left(392^{\circ} \mathrm{F} .\right)\end{array}$ & $\begin{array}{r}15-25 \\
200^{\circ} \mathrm{C} \\
\left(392^{\circ} \mathrm{F}\right)\end{array}$ & $\begin{array}{r}120-150 \\
175^{\circ} \mathrm{C} . \\
\left(347^{\circ} \mathrm{F} .\right)\end{array}$ & $\begin{array}{r}25-30 \\
175^{\circ} \mathrm{C} . \\
\left(347^{\circ} \mathrm{Fj}\right)\end{array}$ & $\begin{array}{c}30-40 \\
175^{\circ} \mathrm{C} . \\
\left(347^{\circ} \mathrm{F} .\right)\end{array}$ \\
\hline
\end{tabular}

The properties enumerated in the accompanying table shall be determined in accordance with the following methods of test of the American Society for Testing Materials:

(a) Penetration. - Standard method of test for penetration of bituminous materials (serial designation: D5, see 505.0, p. 118).

(b) Flash point.- Standard method of test for flash and fire points by means of open cup (serial designation: D92, see 504.0, p. 90).

(c) Loss on hea:ing. - Standard method of test for loss on heating of oil and asphaltic compounds (serial designation: D 6, see 505.0, p. 119).

(d) Drecility.-Tentative method of test for ductility of hituminous materials (serial designation: D 113-26T, see 505.0, p. 126).

(e) Proportion of bitumen soluble in caroon tetrachloride. - Tentative method of test for the determination of proportion of bitumen soluble in carbon tetrachloride (serial designation: D 165, see 505.0, p. 135).

No'te.-When less than 99 per cent of the asphalt cement is soluble in carbon tetrachloride, the per centage of bitumen (solubility in carbon disulphide) shall be reported. 
American Society for Testing Materials, tentative specifications for acid-resisting asphalt mastic, serial designation D223-25T, 1925.

This specification is the same as A. S. T. M. D169 (see 505.16, p. 152), with the following exceptions:

The grade of asphalt mastic covered by these specifications is suitable for waterproofing, tank linings, floors, etc., where dilute mineral acids at temperatures below $100^{\circ} \mathrm{F}$. (38 $8^{\circ}$ C.) are encountered.

The matter insoluble in pure benzol shall contain not more than 5 per cent by weight of material soluble in hydrochloric acid.

The mineral aggregate shall contain not more than 5 per cent by weight of material soluble in hydrochloric acid and shall be free from carbonates.

The weight per mastic cake for this specification shall be not more than 80 pounds.

Federal Specifications Board, specification No. 380 , United States Government master specification for asphalt plastic cement.

\section{GRADE}

Asphalt plastic cement shall be of one grade only, as hereinafter described.

\section{MATERIAI}

The cement shall be composed entirely of an asphaltic base, asbestos fiber, and a suitable solvent and shall be of such a consistency that it can be applied easily with a trowel.

\section{DETAIL REQUIREMENTS}

1. Condition in container.-The plastic cement shall be a smooth homogeneous mixture and shall not separate, thicken, or liver in the container.

2. Nonvolatile matter. - The plastic cement shall yield not less than 70 per cent of nonvolatile matter when $10 \mathrm{~g}$ are heated in an oven at $105^{\circ} \mathrm{C}$. $\left(221^{\circ} \mathrm{F}\right.$.) for 24 hours.

3. Asbestos fiber.-The plastic cement shall contain not less than 15 per cent and not more than 25 per cent of asbestos fiber. The asbestos fiber shall be not less than one-fourth inch in length and shall be free from coarse particles.

4. Workability - The plastic cement shall spread readily without drawing or pulling when applied with a trowel to prepared roofing, asphalt saturated rag felt, and to metal and concrete surfaces.

5. Time of setting.- When applied in a layer of from one-sixteenth to one-eighth inch, the cement shall set throughout within 24 hours to a tough plastic coating free from blisters.

6. Behavior at $60^{\circ} \mathrm{C}$. $\left(140^{\circ} \mathrm{F}\right.$. $)$-A patch of the cement one-sixteenth to one-eighth of an inch in thickness applied to the material upon which it is to be used, after drying for 72 hours, on exposure to a temperature of $60^{\circ} \mathrm{C}$. $\left(140^{\circ} \mathrm{F}\right.$.) for five hours shall not sag or slip more than one-fourth of an inch and shall not blister. In addition, in the case of prepared roofing or saturated felt, it shall not be absorbed into the roofing or felt.

7. Behavior at $0^{\circ} C$. $\left(32^{\circ} F\right.$.). - A patch of the cement one-sixteenth to one-eighth of an inch in thickness after drying for 72 hours and after exposure to a temperature of $60^{\circ} \mathrm{C}$. $\left(140^{\circ} \mathrm{F}\right.$.) for five hours shall be plastic and adhere well to prepared roofing, saturated felt, metal, and concrete after exposure at $0^{\circ} \mathrm{C}$. $\left(32^{\circ} \mathrm{F}\right.$. $)$ for one hour.

8. Toughness. - A patch of the cement one-sixteenth to one-eighth of an inch in thickness after drying for 24 hours and after exposure to a temperature of $60^{\circ}$ C. $\left(140^{\circ}\right.$ F.) for 24 hours and cooling to $21^{\circ}$ to $25^{\circ} \mathrm{C}$. $\left(70^{\circ}\right.$ to $77^{\circ} \mathrm{F}$.) shall not crack nor break from the roofing or metal when bent quickly over a mandrel 1 inch in diameter.

\section{METHODS OF SAMPLING AND TESTS}

1. Sampling.-From each shipment the inspector shall take at random a number of packages equivalent to one-half the cube root of the total number of packages in the lot. If this proves to be a fractional number it shall be expressed as the next ligher whole number. The contents of each package selected shall be thoroughly stirred until a homogeneous mixture is obtained. One pint of the cement shall then be immediately drawn from each package and transferred to a clean receptacle of a suitable size which shall be kept tightly covered except while the cement is being introduced. After all the pint samples have been entered the contents of this container shall be thoroughly stirred and two 1-quart samples of the composite sample shall be transferred to clean dry containers, shall immediately be stoppered with new clean corks or well fitting cover's or cans, sealed, and distinctly labeled. One sample shall be transmitted to the testing laboratory and the other retained for check analysis in case of dispute.

2. Laboratory examination.- (a) Condition in container.-Open the container when received at the laboratory and examine its contents. The cement shall be a smooth, homogeneous mixture and shall not show any separation, thickening, or livering.

(b) Nonvolatile matter. - Weight about $10 \mathrm{~g}$ of the sample in a weighed flat-bottomed metal dish about 5 to $8 \mathrm{~cm}$ in dianneter or a friction top can plug. Heat the dish with its contents in an oven maintained at $105^{\circ} \mathrm{C}$. $\left(221^{\circ} \mathrm{F}\right.$. $) \pm 3^{\circ} \mathrm{C}$. $\left(5^{\circ} \mathrm{F}\right.$. $)$ for 24 hours, cool, and weight. From the weight of the residue left in the dish and from the weight of the original sample taken compute the percentage of nonvolatile matter.

(c) Asbestos fiber.-Weigh from 2 to $5 \mathrm{~g}$ of the cement into a small beaker add 25 to $50 \mathrm{cc}$ of carbon bisulphide, stir thoroughly until mixed and allow to stand for 15 minutes. Filter the solution through a weighed Gooch crucible prepared with a medium thick mat of asbestos, using suction if necessary to aid filtration. Wash the residue in the crucible with cold carbon bisulphide until the washings are colorless, dry the crucible with its contents in air at room temperature until the odor of carbon bisulphide has almost disappeared and then for 1 hour in an oven at $100^{\circ}$ to $105^{\circ} \mathrm{C}$. $\left(212^{\circ}\right.$ to $221^{\circ} \mathrm{F}$.), cool, and weigh. From the weight of the insoluble matter in the crucible and the weight of the sample taken, compute the percentage of matter insoluble in carbon bisulphide and consider it as asbestos. Separate the layer of insouble matter obtained from the cement from the asbestos mat in the crucible and examine portions of it under the microscope in order to determine the length of fiber and the presence of materials other than asbestos fiber. Ignite also a weighed portion of the insobule matter to a red heat for one-half hour, cool, and weigh. The residue obtained shall not be less than 80 per cent of the sample taken.

(d) Workability.-Spread the cement with a trowel to a thickness of one-sixteenth to one-eighth of an inch on asphalt prepared roofing, asphalt saturated felt, a clean piece of metal, and to a concrete surface free from dust. The cement shall spread easily without drawing or pulling.

(e) Time of setting.-Apply the cement to a thickness of one-sixteenth to one-eighth of an inch as above to prepared roofing, asphalt saturáted felt, metal, and concrete. Place the test pieces so prepared in a horizontal position in a well-ventilated room, but not in the direct rays of the sun. Test the coating at points not less than $2^{\frac{1}{2}} / \mathrm{cm}$ ( 1 inch) from the edges by touching lightly with the finger. The cement is considered to have set throughout to a tough plastic mass when gentle pressure of the finger 
does not more the coating and none of the coating adheres to the finger.

(f) Behavior at $60^{\circ} \mathrm{C} .\left(140^{\circ} \mathrm{F}\right.$.).-Prepare test pieces as in IV $2(d)$ and allow to dry for 72 hours in a rell-ventilated room. Now suspend the test pieces vertically for five hours in an oven maintained at $60^{\circ} \mathrm{C} .\left(140^{\circ} \mathrm{F}.\right)$. On examination at the end of this period the coating shall not show blistering, sagging, or slipping of more than one-quarter of an inch and in the cases of test pieces prepared on roofing and saturated felt there shall be no absorption of the cement into the roofing or felt.

(g) Behavior at $0^{\circ} \mathrm{C} .\left(32^{\circ} F^{\circ}\right.$.).-Prepare test pieces as in IV $2(d)$ and allow to stand in a rellventilated room for 72 hours. Now expose these test pieces for five hours to a temperature of $60^{\circ}$ C. $\left(140^{\circ} \mathrm{F}\right.$.) as in IV $2(e)$, and at the end of this period cool to room temperature and then immerse in a water bath at a temperature of $0^{\circ} \mathrm{C}$. $\left(32^{\circ} \mathrm{F}\right.$.) for one hour. On examination at the end of this one-hour period the patch shall be plastic and adhere well to the surface upon which it has been applied.

(h) Toughness.-Prepare test pieces on metal and saturated felt as in IV $2(d)$ and allow to stand in a well-ventilated room for 72 hours. Expose these pieces as in IV 2 (e) to a temperature of $60^{\circ} \mathrm{C}$. $\left(140^{\circ} \mathrm{F}\right.$.) for five hours. At the end of this period remove from the oven and cool to a temperature of $21^{\circ}$ to $25^{\circ} \mathrm{C}$. $\left(70^{\circ}\right.$ to $77^{\circ} \mathrm{F}$.) and bend quickly over a mandrel 1 inch in diameter. In bending, the prepared roofing and metal should be next to the mandrel.

\section{NOTES}

Asphalc prastic cement is intended for use in the construction of plastic flashings in conjunction with bituminous roofing, for the repair of asphalt and metal roofing and as an expansion joint material for concrete and masonry.

\subsection{Oil Asphalt.}

American Association of State Highway Officials tentative standard specifications for highway materials, 1927 .

See 505.13, page 146 .

American Railway Association, signal section, specification 6117, 1917, petroleum asphaltum, to protect insulated wires in trunking.

\section{MATERIAL}

(a) The asphaltum shall be homogeneous and free from granular constituents.

(b) It shall be free from creosote, alkali, acid, free sulphur, and water.

(c) It shall be insulating, moisture proof, and insoluble in water.

(d) The melting point shall be not under $71.1^{\circ} \mathrm{C}$ $\left(160^{\circ} \mathrm{F}\right.$ ) nor over $85^{\circ} \mathrm{C}$. $\left(185^{\circ} \mathrm{F}\right.$.)

(e) It shall not flash below $176.6^{\circ} \mathrm{C}$. $\left(350^{\circ} \mathrm{F}\right.$.).

(f) It shall be soluble in pure carbon bisulphide to the extent of 98 per cent.

(g) The penetration shall conform to the requirements of the following table:

\begin{tabular}{|c|c|c|c|}
\hline $\begin{array}{l}\text { Tempera- } \\
\text { ture }\end{array}$ & Weight & Time & $\begin{array}{l}\text { Tenths of milli- } \\
\text { meters }\end{array}$ \\
\hline${ }^{\circ} C_{0} \quad{ }^{\circ} F$. & ${ }_{200}$ & $\begin{array}{l}\text { Seconds } \\
60\end{array}$ & 25 minimum. \\
\hline 25 & 100 & 5 & $\left\{\begin{array}{l}40 \text { minimum. } \\
80 \text { maximuym. }\end{array}\right.$ \\
\hline $46.1 \quad 115$ & 50 & 5 & 130 maximum. \\
\hline
\end{tabular}

\section{INSPECTION}

Inspection requirements same as inspection items $(a),(b)$, and $(c)$ of A. R. A. signal section, specification 11328. (See 522, p. 491.)

\section{TESTS}

(a) When required, manufacturer shall provide at point of production, apparatus and labor for making tests.

(b) In testing for free sulphur, a copper band $1 / 2$ by 6 inches, after being polished clean and then immersed one-third its length in a sample of asphaltum at a temperature of $121.1^{\circ}$ C. $\left(250^{\circ}\right.$ F.) for five minutes when cleaned with kerosene or benzol, shall not show a discoloration on the surface that came in contact with the asphaltum.

Melting point.-This test is similar to A. S. T. M. D36. (See 505.0 , p. 121.)

Peneiration.-This test is similar to A. S. T. M. D5. (See 505.0, p. 118.)

\section{MARKING}

(a) Purchaser's order and requisition number, name of consignor, and name and address of consignee, also detail of contents, shall be plainly marked on outside of package.

(b) Shipping memoranda shall be furnished. It shall show, in detail, the contents of boxes, barrels, or packages, also all loose pieces.

\section{PACKING}

(a) Material shall be protected so as to permit convenient handling and to prevent loss or damage during shipment.

(b) Material shall be placed in $\left\{\begin{array}{c}\text { steel drums } \\ \text { wooden barrels }\end{array}\right\}$ holding approximately 450 pounds each.

American Society for Municipal Improvements, specification for asphaltic oil for hot-surface treatment, optional with refined tar A or refined tar B.

See specification for broken stone or gravel roads, 512.15 , page 245 .

505.15 Asphalt and Other Bituminous Fillers.

American Association of State Highway Officials, standard specifications for bridges and incidental structures, 1928.

\section{BITUMINOUS MATERIALS}

\section{FILIERS}

1. Asphalt filler for brick-wearing surfaces, woodblock floors, and poured-expansion joints shall be an asphalt oil conforming to the requirements of either specification No. 18 or No. 19, American Association of State Highway Officials, tentative standard specifications for highway materials. (See 505.13,p. 146.)

2. Pitch filler for wood-block floors shall be a straight-run residue obtained entirely from the distillation of coal tar and shall conform to the following requirements:

(1) Specific gravity, $25^{\circ} \mathrm{C} . / 25^{\circ} \mathrm{C}$. $\left(77^{\circ} \mathrm{F} . / 77^{\circ} \mathrm{F}\right.$ ) 1.24 to 1.32 .

(2) Softening point, $50^{\circ}$ C. $\left(122^{\circ}\right.$ F.) to $56^{\circ} \mathrm{C}$ $\left(133^{\circ} \mathrm{F}.\right)$.

(3) Bitumen (soluble in carbon disulphide), 63 to 78 per cent.

(4) Specific gravity of distillate up to $355^{\circ} \mathrm{C}$., not less than 1.07 at $38^{\circ} \mathrm{C}$. compared with water at $15.5 \mathrm{C}$.

3. Premolded expansion joint fillers, same as specification No. 33, American Association of State High- 
way Officials, tentative standard specifications for highway materials. (See below.)

American Association ô State Highway Officials, tentative standard specifications for highway materials, 1927.

Bituminous sand. (See 512.12, p. 220.)

American Association of State Highway Officials, tentative standard specifications for highway materials, 1927.

See 505.13, page 146 .

American Association of State Highway Officials, tentative standard specifications for highway materials, 1927.

\section{NO. 33. PREMOLDED EXPANSION JOINTS}

1. The bituminous premolded joint shall be of the dimensions shown on the plans or in the estimate and shall be of asphaltic or tar composition of approved quality. The joint shall be of such character that it will not be deformed by ordinary handling during the hot summer months or become hard and brittle in cold weather. Thin strips of stiffener will be allowed. The bitumen shall be uniformly impregnated with suitable filler, to reduce its brittleness at low temperatures to a minimum.

\section{PHYSICAL PROPERTIES}

(a) Absorption.-When a specimen 2 by 6 inches cut from the joint material is immersed in water for 24 hours it shall absorb not more than 5 per cent by weight.

(b) Brittleness.-The bituminous premolded joint shall not crack or shatter when subjected to the following tests: The sample to be tested, 2 by 6 inches, is clamped between two boards so that the expansion joint cantilevers $3 \frac{1}{2}$ inches, same being held in any suitable support. A cast-iron ball weighing 0.95 pound and having a diameter of 1.875 inches is suspended by a cord which is tied to an eyelet soldered to the ball. For samples having a thickness of one-half inch and less, the ball is suspended 1 foot above the center of the projected portion of the specimen. For samples over one-half inch in thickncss the ball is suspended 2 feet above the specimen. (The ball is released by burning the string above the eyelet.) The test is made on the sample after it has been maintained at a temperature of from $4^{\circ}$ to $6^{\circ} \mathrm{C}$. for at least two hours prior to testing.

(c) Distortions.-The sample shall not show a deflection from horizontal of more than 1 inch when subjected to the following test: The sample 2 by 6 inches absolutely flat and straight is clamped between two blocks so that the expansion joint cantilevers 3.5 inches. The clamp, with the expansion joint, is then placed in an oven at $125^{\circ} \mathrm{F}$. and left thercin for two hours.

American Society for Testing Materials, tentative specifications for asphalt filler for brick pavements (blown type). Serial designation D241-26T, 1926.

1. The asphalt filler shall be homogeneous and free from water, and shall not foam when heated to $200^{\circ}$ C. $\left(392^{\circ} \mathrm{F}\right.$.). It shall conform to the following requirements:

(a) Flash point (open cup) not less than $200^{\circ} \mathrm{C}$. ( $392^{\circ}$ F.).

(b) Softening point (ring and ball method), $65^{\circ}$ C. $\left(149^{\circ} \mathrm{F}\right.$. $)$ to $110^{\circ} \mathrm{C}$. $\left(230^{\circ} \mathrm{F}\right.$. $)$.

(c) Penetration at $0^{\circ}$ C. ( $32^{\circ}$ F.), $200 \mathrm{~g}, 1 \mathrm{~min}-$ ute, not less than 10 . Penetration at $25^{\circ} \mathrm{C}$. $\left(77^{\circ}\right.$ F.) $, 100 \mathrm{~g}, 5$ seconds, 30 to 50 . Penetration at $46.1^{\circ} \mathrm{C}$. $\left(115^{\circ} \mathrm{F}.\right), 50 \mathrm{~g}, 5$ scconds, not more than 110.

(d) Loss on heating at $163^{\circ}$ C. $\left(325^{\circ}\right.$ F.), $50 \mathrm{~g}$, 5 hours, less than 1.0 per cent. Penetration at $25^{\circ}$ C. $\left(77^{\circ} \mathrm{F}.\right), 100 \mathrm{~g}, 5$ seconds, of residue after heating at $163^{\circ}$ C. $\left(325^{\circ} \mathrm{F}\right.$.) as compared with penetration of asphalt filler before heating, not less than 60 per cent.

(e) Ductility at $25^{\circ} \mathrm{C}$. ( $77^{\circ} \mathrm{F}$.), not less than $3 \mathrm{~cm}$.

$(f)$ Proportion of bitumen soluble in carbon tetrachloride, not less than 99 per cent.

Nore.- When less than 99 per cent of the asphalt filler is soluble in carbon tetrachloride, the percentage of bitumen (solubility in carbon disulfide) shall be reported.

2. The properties enumerated in these specifications shall be determined in accordance with the following methods of test of the American Society for Testing Materials:

(a) Flash point.-D92. (See 504.0, p. 90.)

(b) Softenting point.-D36. (See 505.0, p. 131.)

(c) Penetration.-D5. (See 505.0, p. 118.)

(d) Loss on heating.-D6. (See 505.0 p. 119.)

(e) Ductility.-D113-26T. (See 505.0, p. 126.)

(f) Proportion of bitumen soluble in carbon tetrachloride.-D165. (See 505.0, p. 135.)

American Society for Testing Materials, tentative specifications for mineral filler for sheet asphalt and bituminous concrete pavements, serial designation D242-26T, 1926.

1. The mincral filler shall consist of limestone dust, Portland cement, or other mineral matter from sources agreed upon between the purchaser and seller.

2. Material shall be thoroughly dry and free from lumps consisting of aggregations of fine particlcs. It shall meet the following requirements for mechanical analysis:

Passing No. 200 sieve, not less than

Per cent

Total passing No. 80 sieve, not less than

Total passing No. 30 sieve

3. The mechanical analysis shall be determined in accordance with $\mathrm{A}$. S. T. M. D7 (see 512.12, p. 234), using only the Nos. 30,80 , and 200 sieves. Federal Specifications Board, specification No. 277, United States Government master specification for petroleum asphalt for joint filler, May 8, 1925 (squeegee or pouring method) (type $\mathrm{PAF}-1-25)$.

(This specification is the same as American Society for Testing Materials, serial designation D241-26T (sec above), with the exception of the ductility requirement, which for this specification is $2 \mathrm{~cm}$.)

Federal Specifications Board, specification No. 463, United States Government master specification for mineral asphalt filler for sheet asphalt or asphaltic concrete pavements (Portland cement, limestone dust, and dolomite dust), January 25, 1927.

(This specification is similar to American Society for Testing Materials, serial designation D242-26T, 1926, omitting the 80 -mesh sieve test.)

National Paving Brick Manufacturers' Association, recommended specifications for oil asphalt filler, squeegee method.

(These spccifications are substantially the same as American Society for Testing Materials, serial designation D241-26T, with the exception that this specification requires not less than 99 per cent total bitumen (soluble in carbon disulphide). This organization also has rules for applying the filler.)

National Paving Brick Manufacturers' Association, 1924, recommended specifications for premolded expansion joint.

\section{QDALITY}

Expansion joint shall be a bituminous material unaffected by the action of water or street liquids and shall be of such consistency that the penctration registered by a Roberts sharp No. 2 needle under a weight of $200 \mathrm{~g}$ for one minute at a temperaturc of $32^{\circ} \mathrm{F}$. shall be not less than 20 and when penetrated by a No. 2 needle under $50 \mathrm{~g}$ for five seconds at a temperature of $115^{\circ} \mathrm{F}$., shall be not over 100 . 


\section{DIMENSIONS}

Joint shall be not less than three-fourths inch in thickness and not less than depth of the brick in width.

\section{PIACING}

Expansion joint shall be placed immediately prior to laying the brick-wearing surface. It shall be placed with ends of strips closely joined and in such manner that when brick are rolled the top of joint shall be flush with the top of pavement.

\subsection{Asphalt for Roofing and Water- proofing. (See also 505.36.)}

American Association of State Highway Officials, standard specifications for highway bridges and incidental structures, 1928.

\section{WATERPROOFING}

(This specification contains construction directions for asphalt, fabric, and joint filler waterproofing.)

American Association of State Highway Officials, standard specifications for highway bridges and incidental structures, 1928.

\section{BITUMINOUS MATERIALS}

\section{WATERPROOFING MATERIALS}

1. Waterproofing asphalt shall be the product of the distillation and refining of crude asphaltic petroleum. It shall be free from coal-tar pitch or any of its derivatives.

2. Horizontal joint filler shall be a straight refined oil asphalt.

The materials shall conform to the following requirements:

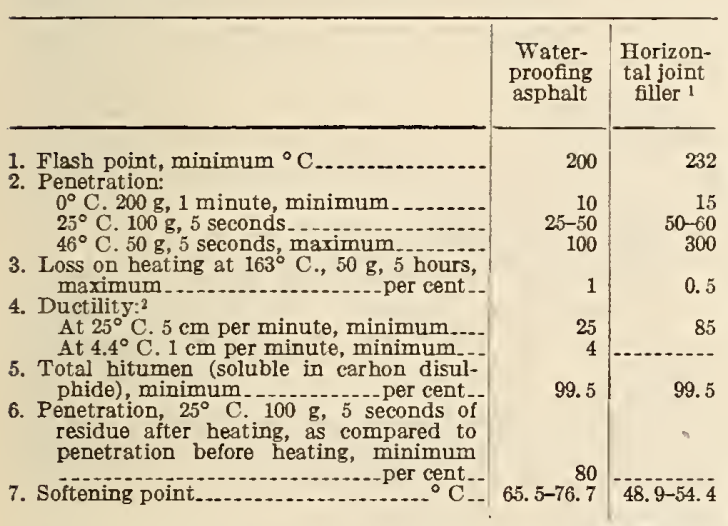

1 Vertical joint filler, for use in vertical joints in waterproofing I Vertical joint filler, for use in vertical joints in waterproofing
work shall he an asphalt conforming to the requirements specified above for horizontal joint filler, to which has heen added 20 per cent hy weight of ashestos fiher. The incorporation of the ashestos fiher with the asphalt shall be done at the factory of the manufacturer to insure a uniform distrihution of the fiher throughout the mix.

2 A higher ductility than that specified is desirahle, provided that the material conforms in all other respects to the specified requirements.

\section{INSPECTION AND DELIVERY}

All waterproofing materials shall be tested before shipment. Unless otherwise ordered by the engineer, they shall be tested at the place of manufacture and when so tested a copy of the test results shall be sent to the engineer by the chemist or inspection bureau which has been designated to make the tests, and each package shall have affixed to it a label, seal, or other mark of identification showing that it has been tested and found accept- able, and identifying the package with the laboratory tests.

Factory inspection is preferred, but, in lieu thereof, the engineer may order that representative samples, properly identified, be sent him for test prior to shipment of the materials. After delivery of the materials, representative check samples shall be taken which shall determine the acceptability of the materials.

All materials shall be delivered on the work in the original containers, plainly marked with the manufacturer's brand or label.

\section{SAMPLING AND TESTING}

See 505.31, page 174, test methods listed following table of A. S. T. M. asphalt cement specifications, also United States Department of Agriculture Bulletin 1216, method of test for proportion of bitumen soluble in carbon disulphide, 505.0, page 143 .

Note.-The full text of this specification includes requirements and tests for water proofing fahric.

American Railway Engineering Association, specifications for buildings for railway purposes, 1929 .

\section{BUILT-UP ROOFING}

See 518.57 , page 427 .

American Society for Testing Materials, standard specifications for (1) asphalt for use in damp proofing and waterproofing below ground level, serial designation D40-25, 1925, and (2) standard specifications for asphalt for use in damp proofing and waterproofing above ground level, serial designation D144-25, 1925.

The asphalts shall be homogeneous and free from water. They shall conform to the following requirements:

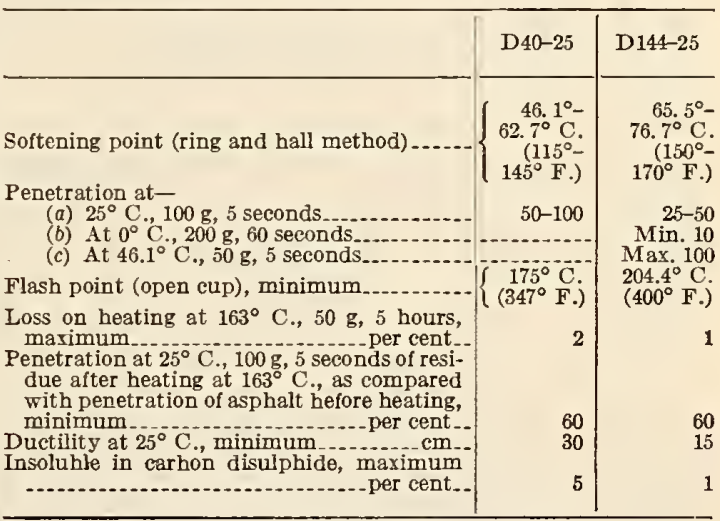

\section{SAMPIING}

The sampling shall be performed in accordance with A. S. T. M. D140. (See 505.0, p. 128.)

\section{ADDITIONAL INFORMATION}

D40-25.-1. These specifications cover asphalt suitable for use as a mopping coat in damp proofing, or as a plying cement in the construction of a membrane system of waterproofing below ground level, under uniformly moderate temperature conditions both during the process of installation and during service. Such asphalt is not intended to be heated above $400^{\circ}$ F. $\left(204.4^{\circ}\right.$ C.) during its application.

2 . The grade of asphalt covered in these specifications is suitable for damp proofing and waterproofing foundations, tunnels, subways, etc. 
D144-25.-1. These specifications cover asphalt suitable for use as a mopping coat in damp proofing or as a plying cement in the construction of a membrane system of waterproofing above ground level, where not exposed to a temperature exceeding $46^{\circ}$ C. $\left(115^{\circ} \mathrm{F}\right.$.). It may be used below ground level. Such asphalt is not intended to be heated above $450^{\circ} \mathrm{F}$. $\left(232.2^{\circ}\right.$ C.) during its application.

2 . The grade of asphalt covered in these specifications is suitable for damp proofing and waterproofing railroad bridges, tanks, retaining walls, culverts, dams, conduits, etc.

The properties enumerated in these specifications shall be determined in accordance with the following methods of test of the American Society for Testing Materials:

(a) Softening point.-D36. (See 505.0, p. 131.)

(b) Penetration.-D5. (See 505.0, p. 118.)

(c) Flash point.-D92. (See 504.0, p. 90.)

(d) Lass on heating.-D6. (See 505.0, p. 119.)

(e) Ductility.-D113. (See 505.0, p. 126.)

(f) Insoluble in carbon disulphide.-D4.

(See 505.0, p. 117 .)

American Society for Testing Materials, standard specifications for primer for use with asphalt in damp proofing and waterproofing below and above ground level, serial designation D41-26, 1926.

1. These specifications cover asphaltic primer for use, when specified, with asphalt in damp proofing and waterproofing below or above ground level, for application to concrete and masonry surfaces.

\section{SANPLING}

2. From each shipment or portion thereof representing primer of the same kind, a number of packages shall be selected at random equivalent to the cube root of the total number of packages in the lot. If the cube root as calculated proves to be a fractional number, it shall be expressed as the next higher whole number. For convenience, the following table is given, showing the number of samples to be selected for shipments of various sizes:

\begin{tabular}{|c|c|c|c|}
\hline Packages in shipment & $\begin{array}{c}\text { Packages } \\
\text { seleeted }\end{array}$ & Packages in shipment & $\begin{array}{l}\text { Packages } \\
\text { selected }\end{array}$ \\
\hline $\begin{array}{l}2 \text { to } 8 \\
9 \text { to } 27 \\
28 \text { to } 64 \\
65 \text { to } 125 \\
126 \text { to } 216\end{array}$ & $\begin{array}{l}2 \\
3 \\
4 \\
5 \\
6\end{array}$ & \begin{tabular}{|l}
217 to 343 \\
344 to 512 \\
513 to $729-$ \\
730 to 1,000 \\
1,001 to 1,331
\end{tabular} & $\begin{array}{r}7 \\
8 \\
9 \\
10 \\
11\end{array}$ \\
\hline
\end{tabular}

3. By means of a paddle, the contents of each package selected shall be thoroughly stirred so as to bring all portions thereof into uniform distribution. With an appropriate sampler, not less than 1 pint of the primer shall be immediately drawn from the center of each package and transferred to a clean receptacle of suitable size, which in turn shall be kept tightly covered, prior and immediately after said samples are being introduced. After all the pint samples have been added, the contents of the container shall be thoroughly agitated, whereupon 1 quart of the mixture shall immediately be transferred to a clean quart can provided with a tightly fitting screw cap, which shall be immediately fastened in place. This sample shall be considered as representative of the shipment and shall be transmitted to the laboratory for examination, bearing a suitable tag or other means of identification.

\section{PROPERTTES}

4. The asphaltic base used in preparing the primer shall meet the requirements specified in A. S. T. M. D40. (See 505.16, p. 151.)
5. The solvent used in preparing the primer shall be a hydrocarbon distillate having an end point on distillation of not above $250^{\circ} \mathrm{C}$. $\left(500^{\circ} \mathrm{F}\right.$.), of which not more than 20 per cent shall distill under $120^{\circ} \mathrm{C}$. $\left(248^{\circ}\right.$ F. $)$.

6. The primer shall be free from water and shall conform to the following requirements:

(a) Sediment, not more than 1 per cent; (b) asphaltic base by weight, 25 to 35 per cent.

\section{METHODS OF TESTING}

7. The properties enumerated in these specifications shall be determined in accordance with the following methods of test of the American Society for Testing Materials except as specified in paragraph $(c)$ :

(a) Distillation.-D86. (See 503.0, p. 44.)

(b) Sediment.-D96. (See 502.2, p. 39.)

(c) Weight of asphaltic base present.-D255.

(See 505.0, p. -.)

American Society for Testing Materials, standard specifications for asphalt mastic for use in waterproofing, serial designation D169-25, 1925.

1. (a) These specifications cover the materials for asphalt mastic suitable for use in waterproofing, consisting of mastic cake, asphalt, cement, and mineral aggregate.

(b) The grade of asphalt mastic covered by these specifications is suitable for waterproofing bridges, floors, reservoirs, subways, ete.

2. This asphalt mastic is a mixture of mastic cake, asphalt cement, and mineral aggregate, which at a temperature of approximately $205^{\circ} \mathrm{C} .\left(400^{\circ} \mathrm{F}\right.$.) is sufficiently plastic to be poured into place and compressed with a wooden trowel or equivalent tool into a compact mass.

As used for strictly waterproofing purposes the mastic cake, asphalt cement, and mineral aggregate are combined under heat and manual stirring in substantially the following proportions by weight:

Mastic cake.

Per cent

A sphalt comen

Mineral aggregate

3. (a) The mastic cake shall conform to the following requirements:

Weight per cake, not more than 75 pounds.

Soluble in pure benzol, 14 to 18 per cent by weight.

Insoluble in pure benzol, 82 to 86 per cent by weight.

(b) The matter soluble in pure benzol shall be asphalt cement, which after recovery from the mastic cake shall conform to A. S. T. M. D163-23T. (See 505.13 , p. 147.)

. (c) The matter insoluble in pure benzol shall be granular mineral matter which after recovery from the mastic cake shall conform to the following requirements as to grading:

Passing No. 200 sieve, not less than 25 per cent.

Passing No. 10 sieve, retained on No. 50 sieve, not more than 25 per cent.

Retained on No. 10 sieve, not morc than 1 per cent.

4. The asphalt cement shall conform to the requirements of A. S. T. M. D163-23T.

5. The mineral aggregate shall be gravel or crushed stone and sand conforming to the following requirements as to grading:

Passing No. 200 sieve, none.

Passing No. 50 sieve, retained on 200 -mesh sieve, not more than 25 per cent.

Passing No, 10 sieve, retained on No. 50 sieve, not less than 25 per cent.

Passing No.4 sieve, retained on No. 10 sieve, not less than 50 per cent.

Retained on No. 4 sieve, not more than 10 per cent.

6. The properties enumerated in these specifications shall be determined in accordance with A. S. T. M. D147. (See 505.0, p. 133.) 
American Society for Testing ITaterials, standard specifications for bituminous grout for use in waterproofing above ground level, serial designation D170-25, 1925.

1. (a) These specifications cover the materials for bituminous grout suitable for use in waterproofing above ground level, either as a protective covering of membrane systems of waterproofing or for bedding brick or filling the joints or flooding the surface of a brick protective covering.

(b) The grade of bituminous grout covered by these specifications is suitable for waterproofing railroad bridges, culverts, subways, etc.

2. This bituminous grout is a mixture of substantially 45 parts by weight of bituminous binder and 55 parts by weight of mineral aggregate as coarse as sand, which becomes sufficiently fluid when heated to approximately $205^{\circ} \mathrm{C}$. $\left(400^{\circ} \mathrm{F}\right.$.) to flow without mechanical manipulation, and which on cooling congeals to a compact mass.

3. The bituminous binder shall consist of either asphalt binder or coal-tar pitch as follows:

(a) The asphalt binder shall conform to the requirements of A.S. T. M. D144. (See 505.16, p. 151.)

(b) The coal-tar pitch shall conform to either of the following standard specifications of the American Society for Testing Materials.

(1) D42. (See 505.36, p. 178.)

(2) D200. (See 505.36 , p. 178.)

4. The mineral aggregate shall consist of silicious sand, all of which will pass a No. 20 sieve, and not more than 5 per cent of which will pass a No. 200 sieve.

5. The properties enumerated in sections 2 and 4 shall be determined in accordance with A. S. T. M. D147. (See 505.0, p. 133.)

American Society for Testing Materials, standard specifications for bituminous grout for use in waterproofing below ground level, scrial designation D171-25, 1925.

(This specification is the same as A. S. T. M. D170-25, with the exception of paragraphs numbered 1,2 , and $3(a)$, which for this specification are as follows:)

1. (a) These specifications cover the materials for bituminous grout suitable for use in waterproofing below ground level, either as a protective covering of membrane systems of waterproofing or for bedding brick or filling the joints or flooding the surface of a brick protective covering.

(b) The grade of bituminous grout covered by these specifications is suitable for waterproofing tunnels, subways, etc.

2. This bituminous grout is a mixture of substantially 45 parts by weight of bituminous binder and 55 parts by weight of mineral aggregate as coarse as sand, which becomes sufficiently fluid when heated to approximately $149^{\circ} \mathrm{C}$. $\left(300^{\circ} \mathrm{F}\right.$.), to flow without mechanical manipulation, and which on cooling congeals to a compact mass.

3. (a) The asphalt binder shall confor'm to the requirements of A. S. T. M. D40. (See 505.16, p. 151.)

American Society for Testing Materials, standard specifications for woven cotton fabrics saturated with bituminous substances for use in waterproofing, serial designation D173-27, 1927.

1. These specifications cover bituminized cotton fabric, composed of woven cotton cloth waterproofed with either asphalt or coal-tar pitch, as specified by the purchaser, for use in the membrane system of waterproofing.

2 . In the process of manufacture, the dry cotton fabric shall be thoroughly and uniformly waterproofed with an asphaltic or coal-tar pitch saturant, at a temperature and speed which will not injure the fabric. This shall be accomplished by passing the fabric through the saturant and then calendering it in the presence of heat, whereupon it shall be cooled and wound into rolls.

3 . The material shall conform to the requirements listed in the following table, which also gives requirements for A. S. T. M. serial designation D174-25:

\begin{tabular}{|c|c|c|}
\hline Physical properties & & 84 \\
\hline 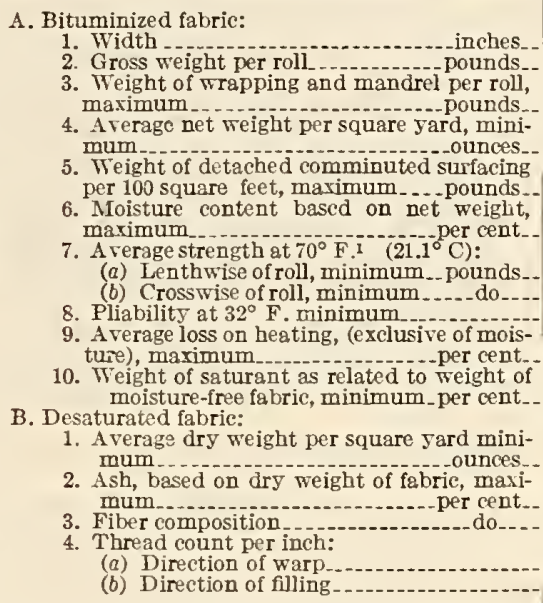 & $\begin{array}{r}30-38 \\
35-80 \\
2.5 \\
11 \\
1\end{array}$ & $\begin{array}{l}30-2 \\
35-8\end{array}$ \\
\hline
\end{tabular}

1 The committee recognizes the advisability of including a requirement relative to the percentage elongation before rupture, but in the absence of any reliablc method of test, such requirement can not be stated at the present time.

With asphalt saturated fabrics only.

3 Cotton. This shall not exclude the presence of the customary sizing materials in amounts not exceeding 8 per cent by weight of the moisture-free cotton.

Sute and manila.

\section{WORKMANSHIP AND FINISH}

4. The finished material shall be free from visible external defects, such as ragged or untrue edges, breaks, rents, or eracks.

5. The meshes of the fabric shall not be completely closed or sealed by the process of saturation, but there shall be sufficient porosity maintained to allow successive moppings of the plying cement to scep through.

6 . The selvage shall not measure over threesixteenths inch.

7. The roll shall be capable of being unrolled easily at atmospheric temperatures above $50^{\circ} \mathrm{F}$. $\left(10^{\circ} \mathrm{C}\right.$.) without sticking together in such a manner as to injure the fabric.

8. The surface of the fabric shall not be coated or covered with talc or other substances which would tend to interfere with the adhesion between the fabric and the plying cement. The use of silica or wood flour will be permitted.

9. The surface shall be uniformly smooth and free from irregularities, folds, or knots.

\section{PACKING}

10. The rolls shall be wound on wooden 2 by 2 inch mandrils extending 2 to 4 inches beyond the ends of the roll. They shall be securely tied or wrapped in a manner which will prevent unrolling in transit.

\section{SAMPLING AND TESTING}

11. The material shall be sampled and the properties enumerated in these specifications shall be determined in accordance with A. S. T. M. D146. (See 505.0, p. 130.) 


\section{INSPECTION}

12. Inspection of material shall be made as agreed upon by the purchaser and seller as part of the purchase contract.

American Society for Testing Materials, standard specifications for burlap saturated with bituminous substances for use in waterproofing, serial designation D174-25, 1925.

1. These specifications cover bituminized jute fabric, composed of burlap waterproofed with either asphalt or coal-tar pitch, as specified by the purchaser, for use in the membrane system of waterproofing.

2 . In the process of manufacture the dry burlap shall be thoroughly and uniformly waterproofed with an asphaltic or coal-tar piteh saturant at a temperature and speed which will not injure the fabric. This shall be accomplished by passing the fabric through the saturant and then calendering it in the presence of heat, whereupon it shall be cooled and wound into rolls.

For (1) physical properties, (2) workmanship and finish, (3) packing, (4) sampling and testing, and (5) inspection, see A. S. T. M. serial designation D173-27, given above.

American Society for Testing Materials, roofing materials.

1. Standard specifications for asphalt roll-roofing surfaced with powdered talc, serial designation D224-27.

2. Standard specifications for slate-surfaced asphalt roll-roofing and slate-surfaced asphalt shingles, serial designation D225-29.

3 . Standard specifications for asphalt roll-roofing surfaced with granular talc, serial designation D248-27.

4. Standard specifications for heavy weight slatesurfaced asphalt roll-roofing and heavy weight slate surfaced asphalt shingles; serial designation D249-27.

The above specifications are uniform as to the following:

\section{MANUFACTURE}

The felt shall be a roofing felt produced by "felting," vegetable, and animal fibers. The surface of the felt shall be uniformly smooth, and upon splitting or tearing on the bias shall appear reasonably free from lumps of underbeaten stock (i. e., stock which has not been beaten or shredded into fiber in the process of manufacture), and particles of foreign substances (i. e., fragments of stone, metal, leather, rubber, straw, wood, etc.).

The saturant and coatings shall be composed principally of asphaltic materials, and the presence of saponifiable constituents shall be permitted.

The felt shall be thoroughly and uniformly saturated and shall show no unsaturated spots at any point upon cutting 2 -inch strips at random across the entire sheet and splitting them open for their f ull length.

\section{WORKMANSHIP AND FINISH}

The finished product shall be free from visible external defects, such as holes, ragged, or untrue edges, breaks, cracks, tears, protuberances, and indentations.

For roll roofing the coating and surfacings shall be of such a nature that the finished product will not crack or stick together so as to cause tearing or damage upon being unrolled at atmospheric temperatures above $50^{\circ} \mathrm{F}$. $\left(10^{\circ} \mathrm{C}\right.$. $)$. For shingles, the coating and surfacings shall be of such a nature that the n̂nished products will not adhere together in the bundles so as to tear off or loosen the coating when unpacked.

\section{PACKING}

(a) Slate-surfaced asphalt roll roofing shall be put up in rolls of 108 square feet. Asphalt roll roofing shall be put up in rolls of 108 square feet or 216 square feet, as specified in the order. No rolls shall contain more than two pieces nor shall there be more than 3 per cent of rolls containing two pieces in any shipment. The rolls shall be tightly wound and securely wrapped in a substantial grade of paper. The wrapper shall completely encircle the roll and shall be pasted at the overlap in a manner which will prevent it shifting from position. The ends of the roll shall be protected with metal caps, cloth, or other suitable means. The nails and lap-cement shall be furnished either in each roll or in bulk, as specified in the order. When furnished in bulk, the cloth or metal ends may be omitted. Rolls for export shipment shall be packed as agreed upon by the purchaser and seller.

Shingles shall be packed in bundles weighing not more than 135 pounds gross. The bundles shall either be packed in cardboard cartons or between wooden or cardboard ends and tied with rope, wire, or steel bands so as to make a substantial package, as may be agreed upon by the purchaser and seller, and shall contain printed directions for laying.

(b) Nails. - The gage of the wire shall not be greater than "A. S. \& W." No. 10 (0.135 inch) nor thinner than No. 12 ( 0.105 inch); the head shall be not less than three-eighths inch in diameter, and not less than 0.025 inch thick; the shank shall be between $3 / 4$ and 1 inch long for slate-roll roofing, and for shingles 1 inch long when laid on solid sheathing or $1 \frac{1}{2}$ to $13 \frac{1}{4}$ inches when laid over old shingles. The lower end of the shank shall be pointed, and either smooth or barbed, and if the latter, then the barbings shall not increase the diameter of the lower two-thirds more than 0.01 inch. The nails shall be zinc coated with not less than $1 / 2$ ounce nor more than $11 / 4$ ounce per square foot of nail surface. Hotgalvanized, electrogalvanized, or sherardized coatings will be accepted. There shall be furnished per "square" of slate-roll roofing not less than an average of 252 nails for 36 inch, and not less than an average of 275 nails for 32 -inch roofing. For shingles, the nails shall be purchased separately, and the number required per "square" will depend upon the size and form of the shingles and shall be agreed upon by the purchaser and seller.

(c) Lap-cement (for slate roll).-The cement shall be composed of bituminous materials dissolved in a volatile solvent, and shall be of such a nature as to firmly bind the laps without injurious effect upon the roofing. The cement shall have a minimum flash point of $71^{\circ} \mathrm{F} .\left(21.5^{\circ} \mathrm{C}\right.$.) when tested by the Tagliabue closed-cup tester. There shall be furnished for each square of 36 -inch roofing not less than $3 / 4$ pint and for 32-inch roofing not less than 1 pint. The addition of 5 to 20 per cent by weight of short-fibered asbestos shall be permitted, in which case the quantity of cement shall be increased 1/4 pint per "square."

\section{SAMPLING AND TESTING}

14. The material shall be sampled and the properties enumerated in these specifications shall be determined in accordance with A. S. T. M. D228. (See 505.16, p. 156.)

\section{INSPECTION}

15. Inspection of material shall be made as agreed upon by the purchaser and seller as part of the purchase contract.

The specifications are individually distinct as follows: 
Serial designation D224-27.-These specifications cover smooth-surfaced roll-roofing (medium and heavy weights), either 36 or 32 inches in width, coinposed of roofing felt saturated and coated on both sides with asphalt, and surfaced top and bottom with powdered talc or mica. The medium-weight roofing is intended for use on semipermanent structures and the heavy-weight roofing for use on permanent structures.

\section{MANUFACTURE}

In the process of manufacture, a single thickness of dry roofing felt shall be impregnated with an asphaltic saturant, then coated on both sides with an asphaltic coating, and finally surfaced on both sides with powdered talc or mica.

The surfacing shall be noncombustible material, such as powdered talc or mica.

\section{PHYSICAL PROPERTIES}

The fabricated product shall conform to the following requirements:

(a) Width of roll.-Either 36 or 32 inches, as may be specified, $\pm 1 / 4$ inch.

(b) A rea of roll.- The average area of the rolls examined shall contain as specified not less than either 108 or 216 square feet, which shall be sufficient to cover one or two "squares," respectively.

(c) Weight of wrapping, packing, nails, and cement per "square."-Maximum, 4 pounds.

NotE.-Special fixtures may be furnished in lieu of nails and cement, if agreed upon by tbe purchaser and seller, in wich erent the above weigbt may be increased.

(d) Weight of material per 108 square feet exclusive of wrapping, packing, loose mineral surfacing, nails, and cement.-Medium weight, average, not less than 42 pounds; minimum, 40 pounds. Heavy weight, average, not less than 52 pounds; minimum, 49 pounds.

(e) Pliability at $77^{\circ} \mathrm{F}$. (25 $\mathrm{C}$.). - At least four strips out of five shall not crack on $10-\mathrm{mm}$ mandrel.

( $f$ ) Behavior on heating to $176^{\circ} \mathrm{F}$. $\left(80^{\circ} \mathrm{C}\right.$.) for two hours.-Volatile matter, maximum, 1.5 per cent. There shall be no flowing, sagging, blistering, or absorption of the surface coatings.

(g) Saturant in moisture-free felt.-Medium and heavy weights, minimum, 140 per cent by weight.

(h) Weight of moisture-free desaturated felt per 108 square feet.-Medium weight, minimum, 10.8 pounds (No. 48). Heavy weight, minimum, 13.5 pounds (No. 60).

(i) Ash in moisture-free desaturated felt.-Maximum, 10 per cent.

(j) Weight of mineral surfacings and mineral matter in coatings per 108 square feet.-Medium and heavy weights, maximum, 2 pounds.

Note.-Should tbe weigbt exceed 2 pounds tbe material sball nerertheless be acceptable, provided the finisbed weights per 108 square feet are increased by a like amount over tbe figures specified in paragrapb (d).

\section{WORKMANSHIP AND FINISH}

The surfaces shall be smooth or veined. Deeply ribbed surfaces shall not be acceptable.

The coating and mineral surfacing shall be applied uniformly up to the edges of the sheet.

The mineral surfacing shall uniformly cover both sides of the sheet.

Serial designation D225-2\%,-1. These specifications cover slate-surfaced roll roofing, either 36 or 32 inches in width, and slate-surfaced composition shingles, composed of roofing felt saturated and coated on both sides with asphalt and surfaced on the weather side with granulated slate (green, red, black, or such color or combinations of color as may be agreed upon between the purchaser and seller) or similar mineral material to be specified by the purchaser, and on the reverse side with powdered talc or mica to prevent the roofing or shingles adhering together in the package. Such roofing and shingles are intended for use on semipermanent and permanent buildings.

\section{MANUFACTURE}

In the process of manufacture, a single thickness of dry roofing felt shall be impregnated with an asphaltic saturant, then coated on both sides with an asphaltic coating, and finally surfaced on the weather side with granulated slate or similar mineral material to be specified by the purchaser (in either green, red, blue-black, or such other color or combinations of color as may be agreed upon between the purchaser and seller), and shall be sufficiently free from fines or dust to permit the firm adhesion of the larger particles of the surfacing and provide a uniform distribution. The under side of the sheet shall be surfaced with a noncombustible material such as powdered mica or talc.

\section{PHYSICAL PROPERTIES}

The fabricated product shall conform to the following requirements:

(a) Width of roll; size and form of shingles.- Slate roll-roofing shall be either 36 or 32 inches in width, as may be specified, $\pm 1 \frac{1}{4}$-inch.

Shingles shall be of a size and form as may be agreed upon between the purchaser and seller.

(b) Area per package; slate roll.-The average area of the rolls examined shall contain not less than 108 square feet, which shall be sufficient to cover one "square."

Shingles.-Sufficient material shall be furnished per "square" as may be agreed upon between the purchaser and seller to adequately waterproof, when laid, the surface to which the shingles are applied.

(c) Weight of wrapping and packing material per "square."-Slate roll including nails and lap cement, maximum 4 pounds.

NOTE.-Special fixtures may be furnished in lieu of nails and cement, if agreed upon by the purchaser and seller, in which event tbe above weights may be increased.

Shingles (packing only) maximum 12 pounds.

(d) Weight of material per 108 square feet exclusive of wrapping, packing, loose mineral surfacing, nails, and cement.- Slate roll and shingles, average not less than 82 pounds, minimum 80 pounds.

(e) Pliability at $\gamma \gamma^{\circ} F$. (25 C.); slate roll.- Shall not crack on $25 \mathrm{~mm}$ mandril. Shingles.-No requirements.

(f) Behaviour on heating to $176^{\circ} \mathrm{F}$. ( $80^{\circ} \mathrm{C}$.) for two hours.-Volatile inatter, maximum 1.5 per cent. There shall be no flowing, sagging, blistering, or absorption of the surface coatings. Granular surfacings shall not slide more than one-sixteenth inch when suspended vertically.

(g) Saturant in moisture-free felt.-Slate roll, minimum 140 per cent by weight. Shingles, minimum 150 per cent by weight.

(h) Weight of moisture-free, desaturated felt per 108 square feet.- Slate roll and shingles, minimum 10.8 pounds (No. 48).

(i) Ash in moisture-free desaturated felt.-Maximum, 10 per cent.

(j) Weight of mineral surfacings and mineral matter in coating per 108 square feet. - Slate roll and shingles, maximum 35 pounds.

NOTE.-Should the weigbt exceed tbe above amount, the material shall nevertheless be acceptable provided the finished weight per 108 square feet is increased by a lite specified in paragrapb $(d)$. 


\section{WORKMANSHIP AND FINISH}

The weather surface shall be uniform in finish and texture.

The coating and granules shall be applied in a uniformly smooth layer over the entire weather surface, except that slate-roll roofing may be provided with a bare lapping edge, 2 inches wide, along one edge of the sheet, where either the slate alone, or both the slate and coating may be omitted. The under surface of the sheet shall have the asphalt coating and mineral surfacing applied uniformly to the edges.

The granular surfacing shall be embedded in the ooating in such. a manner that when rubbed vigorously with the hand, sufficient surfacing will remain embedded to completely cover the coating.

Serial designation D248-27.-These specifications cover roll-roofing (medium and heavy weights) either 36 or 32 inches in width, composed of roofing felt saturated and coated on both sides with asphalt, and surfaced on the face with granular talc, and on the back with powdered talc or mica. The medium weight is intended for use on semipermanent structures, and the heavy weight on permanent structures.

\section{MANUFACTURE}

In the process of manufacture, a single thickness of dry roofing felt shall be impregnated with an asphaltic saturant, then coated on both sides with an asphaltic coating, and finally surfaced on the weather side with granular talc, and on the reverse side with powdered talc or mica. The surfacing on the weather side of the sheet shall be coarsely crushed talc and of a color as may be agreed upon between the purchaser and seller. It shall be of such a character as to permit the firm adhesion of the larger particles of surfacing and provide a uniform distribution. The under side of the sheet shall be surfaced with a noncombustible material, such as powdered mica or talc.

\section{PHYSICAL PROPERTIES}

The physical properties in this standard are identical with those of A. S. T. M. D224-27 (see p. 154) with the following variances:

1. Weight of material per 108 square feet, exclusive of wrapping, packing, loose mineral surfacing, nails, and cement.

Medium weight, average 52 pounds; minimum, 50 pounds.

Heavy weight, average 62 pounds; minimum, 59 pounds.

2. Pliability at $77^{\circ} \mathrm{F}$. $\left(25^{\circ} \mathrm{C}\right.$.) at least three strips out of five shall not crack on $10 \mathrm{~mm}$ mandrel.

3 . Weight of mineral surfacings and mineral matter in coating per 108 square feet.

Medium and heavy weights, maximum, 11 pounds.

Medium and heavy weights, passing a No. 65 sieve, maximum, 3 pounds.

\section{WORKMANSHIP AND FINISH}

(Same as A. S. T. M. D224-27. See p. 154.)

Serial designation D249-27.- This specification is identical with A. S. T. M. serial designation D225-27 (see p. 154), standard specifications for slate-surfaced asphalt roll-roofilg and slate-surfaced asphalt shingles, with the following variance in physical properties:

1. Weight of material per 108 square feet, exclusive of wrapping, packing, loose mineral surfacing, nails, and cement.- Slate roll and shingles, average 105 pounds, minimum, 100 pounds.
2. Pliability at $77^{\circ} \mathrm{F}$. $\left(25^{\circ} \mathrm{C}\right.$.) slate roll and shingles.- No requirements.

3. Saturant in moisture-free felt; slate roll and shingles.-Minimum, 170 per cent by weight.

4. Weight of moisture-free desaturated felt per 108 square feet; slate roll and shingles.-Minimum, 15.75 pounds (No. 70).

5. Weight of mineral surfacing and mineral maiter in coating per 108 square feet.- Slate roll and shingles, maximum, 40 pounds.

American Society for Testing Materials, standard specifications for asphalt-saturated roofing felt for use in waterproofing and in constructing built-up roofs, serial designation D226-27, 1927.

These specifications cover asphalt-saturated felt, either 36 or 32 inches in width, composed of roofing felt saturated, but not coated, with asphalt for use in the membrane system of waterproofing and in the construction of built-up roofs.

\section{MANUFACTURE}

2. In the process of manufacture, a single thickness of dry roofing felt shall be saturated with an asphaltic saturant.

3. The felt shall be a roofing-felt produced by "felting" vegetable and animal fibers. The surface of the felt shall be uniformly smooth and, upon splitting or tearing on the bias, shall appear reasonably free from lumps of underbeaten stock (i. e., stock which has not been beaten or shredded into fiber in the process of manufacture) and particles of foreign substances (i. e., fragments of stone, metal, leather, rubber, straw, wood, etc.).

4. The saturant shall be composed of asphaltie materials.

5. The felt shall be thoroughly and uniformly saturated and shall show no unsaturated spots at any point upon cutting 2 -inch strips at random across the entire sheet and splitting them open for their full length.

6. The surface of the felt shall not be coated or covered with talc or other substance which would tend to interfere with the adhesion between the felt and the plying cement.

\section{PHYSICAL PROPERTIES}

7. The fabricated bituminized felt shall conform to the following requirements:

(a) Width of roll.-Either 36 or 32 inches, as may be specified, $\pm 1 / 4$ in.

(b) Gross weight per roll.-40 to 80 pounds.

(c) Weight of wrapping and packing material per roll.- Maximum one-half pound.

(d) Weight of material per 100 square feet exclusive of packing and wrapping.-14 pounds \pm 1 pound.

(e) Loss on heating at $105^{\circ} \mathrm{C}$. (221 ${ }^{\circ} \mathrm{F}$.) for five hours.-Maximum, 4 per cent.

(f) Pliability at $7 \gamma^{\circ} F$. (25 $5^{\circ}$ C.).-At least four strips out of five shall not crack when bent through $180^{\circ}$ over a $1 / 16$-inch mandrel.

(g) Saturant in moisture-free felt.-Minimum 140 per cent by weight.

8. The desaturated felt shall conform to the following requirements:

(a) Weight of moisture-free desaturated felt.Minimum, 5.2 pounds per 100 square feet (No. 25).

(b) Ash in moisture-free desaturated felt.-Maximum, 10 per cent.

For (1) workmanship and finish (2) packing, (3) sampling and testing and (4) inspection. (See 505.16, A. S. T. M. D250, p. 158.)

American Society for Testing Materials, standard methods of testing smooth-surfaced asphalt roll roofing, slate-surfaced asphalt roll roofing and slate- 
surfaced asphalt shingles, serial designation D228$27,1927$.

1. These methods cover the examination of roofings and shingles composed of roofing felt, saturated and coated on both sides with asphalt, and surfaced on the top with either powdered or granulated mineral matter, and on the underside with powdered mineral matter.

\section{SAMPLING}

2. From each shipment or portion thereof representing a product of the same kind, class, and weight, a number of rolls or bundles shall be selected at random, equivalent to one-half the cube root of the total number of rolls or bundles included in the lot, except that in lots of 1,000 or less, five rolls or bundles shall be taken. If the cube root, as calculated, proves to be a fractional number, it shall be expressed as the nearest higher whole number. For convenience, the following table is given, showing the number of samples to be selected from shipments of various sizes.

\begin{tabular}{|c|c|c|c|}
\hline $\begin{array}{l}\text { Packages in ship. } \\
\text { ment }\end{array}$ & $\begin{array}{l}\text { Number of } \\
\text { packages } \\
\text { selected }\end{array}$ & $\begin{array}{l}\text { Packages in ship- } \\
\text { ment }\end{array}$ & $\begin{array}{l}\text { Number of } \\
\text { packages } \\
\text { selected }\end{array}$ \\
\hline $\begin{array}{l}\text { Up to } 1,000 \\
1,001 \text { to } 1,728 \\
1,729 \text { to } 2,744 \\
2,745 \text { to } 4,096 \\
4,097 \text { to } 5,832 \ldots\end{array}$ & $\begin{array}{l}5 \\
6 \\
7 \\
8 \\
9\end{array}$ & $\begin{array}{l}5,833 \text { to } 8,000 \\
8,001 \text { to } 10,648 \\
10,649 \text { to } 13,824 \\
13,825 \text { to } 17,576 \ldots \\
17,577 \text { to } 21,952 \ldots\end{array}$ & $\begin{array}{l}11 \\
11 \\
12 \\
13 \\
14\end{array}$ \\
\hline
\end{tabular}

\section{DETERMINATION OF SIZE AND WEIGHT}

\section{(A) SMOOTH-ROLL AND SLATE-ROLL ROOFING}

3. Each roll selected shall be stripped. The wrappers, packing material, nails, and cement taken from each roll shall be weighed together to the nearest one-fourth pound and the maximum weight thereof per roll shall be recorded. The quantity and character of nails and ccment shall be recorded.

4. Each roll shall be unwound, discarding any detached surfacing that may fall off in so doing, and the workmanship and finish of the roofing observed.

5. The length and width of each roll shall be measured to the nearest one-fourth inch, and the square feet of material contained in each roll calculated. From this the average area of the rolls examined shall be calculated.

6 . The rolls shall be rewound, fastened with pieces of light string and then each roll shall be weighed to the nearest one-fourth pound. The weight of roofing per 108 square feet contained in each roll shall be calculated and the minimum weight per 108 square feet recorded. This shall be regarded as the minimum weight per 108 square feet of the entire shipment.

7. The average weight per 108 square feet for the rolls examined shall be calculated. This shall be regarded as the average weight per 108 square feet of the entire shipment.

8. From the rolls examined, the one whose weight is nearest the average weight of the lot shall be selected. The first convolution or two shall be unrolled, and with a knife and straight edge, the sheet shall be cut across at right angles to the edges. A section measuring exactly 30 inches in the direction of the roll's length shall then be removed. The width of this section shall be measured to the nearest one thirty-second inch and weighed with an accuracy of one-fourth ounce.
The weight in pounds per 108 square feet shall then be calculated from the following formula:

Pounds per 108 square feet $=\frac{\text { Weight of } 30 \text {-inch section in ounces }}{\text { Width of } 30 \text {-inch section in inches }} \times 32.4$

This must check within 1 per cent of the average weight per 108 square feet of the shipment. (Sec. 7.) If this sample fails to do this then additional samples shall be cut from the same roll, until one is obtained which does, which sample shall be kept for further examination.

NotE_-As a referee method, or in case any dispute arises regarding the properties as may he ascertained from the particular sample selected, a 30-inch sample shall be taken and examined separately from each roll sampled in section 2

\section{(B) SLATE-SURFACED SHIFGLES}

9. Fach bundle selected shall be stripped. The packing material shall be weighed to the nearest one-fourth pound, recording the weight separately for each bundle. The maximum weight of packing material per "square" shall be recorded.

10. The shingles in each bundle, $a$, shall be counted, discarding any detached surfacing that may fall off in so doing. The workmanship and finish of the shingles shall be observed.

11. The shingles contained in each bundle shall be weighed to the nearest one-fourth pound $(b)$. The dimensions of the shingles shall be accurately measured and the net area of material in each shingle calculated in square feet $(c)$.

12. The area in square feet of material contained in each bundle $(a \times c)$ shall be calculated. The weight of roofing material per 108 square feet contained in each bundle $\left(\frac{b}{a \times c} \times 108\right)$ shall be calculated, and the minimum weight per 108 square feet recorded. This shall be regarded as the minimum weight per 108 square feet of the entire shipment.

13. The average weight $(d)$ per 108 square feet for the bundles examined shall be calculated. This shall be regarded as the average weight per 108 square feet of the entire shipment. Also, the a verage weight per shingle $\left(\frac{c \times d}{108}\right)$ shall be calculated.

14. From the bundles selected, a definite number of representative shingles, whose weight shall fall within 1 per cent of the average weight ascertained in section 13 shall be taken for further consideration. The number of shingles so selected shall represent as closely as possible 6 square feet of material, and shall be selected as far as possible from different bundles.

NotE.-As a referee method, or in case any dispute arises regarding the properties as may be ascertained from the particular sample selected, then a similar sample shall he taken and examined sepa. rately from each hundle of shingles sampled in section 2.

\section{DETERMINATION OF PLIABILITY OF SMOOTH- ROLL ROOFING AND SLATE-ROLL ROOFING}

15. With a photographic trimmer five strips shall be cut from the sample of roofing, each 1 inch in width and 6 inches in length, in the direction of the fiber grain, and immersed in water at $77^{\circ} \mathrm{F} .\left(25^{\circ} \mathrm{C}\right.$.) for 10 to 15 minutes. These strips shall be removed from the water and immediately bent through $180^{\circ}$ at uniform speed, in exactly two seconds, over a mandril of the following size:

Smooth-roll roofing.-At least three of the five strips shall not crack over a mandril $10 \mathrm{~mm}$ in diameter.

Slate-roll roofing.-At least three of the five strips shall not crack on a mandril $25 \mathrm{~mm}$ in diameter. 


\section{DETERMINATION OF BEHAVIOR ON HEATING}

16. Two strips shall be cut from the sample of roofing or shingles, each approximately 8 by 8 inches. They shall be weighed and suspended vertically in the center of an air oven maintained at $176^{\circ} \mathrm{F}$. $\left(80^{\circ} \mathrm{C}\right.$. $) \pm 5^{\circ} \mathrm{F}$. $\left(3^{\circ} \mathrm{C}\right.$. $)$ with a thin wire fastened through holes punctured near one edge. The thermometer shall be inserted in the oven to such a depth that its bulb will be in line with the center of the specimens. The strips shall be maintained at the prescribed temperature for exactly two hours, then cooled, removed carefully and each specimen weighed. The average loss shall be calculated as a percentage. Any change in appearance of the specimen shall be recorded, such as flowing, sagging, blistering, or absorption of the surface coatings; also sliding of granular surfacing.

\section{DETERMINATION OF SATURANT IN MOISTURE- FREE FELT}

17. Two strips shall be cut from the sample of roofing or shingles, each approximately 2 inches in width and 8 inches in length. They shall be warmed in an oven at a temperature of approximately $176^{\circ}$ F. $\left(80^{\circ}\right.$ C. $)$. Then with a knife, the front and back coatings shall be peeled off, care being taken to remove as little as possible of the saturated felt, and to assure that all of the coatings and surfacing are stripped from the felt. The saturated felt so obtained shall be weighed. It shall be extracted with benzol, chloroform, carbon tetrachloride, or carbon disulphide, in a suitable extractor and the extraction continued until the drippings have become colorless. The desaturated felt shall be removed from the extractor, placed in a tared vessel, heated in a ventilated oven at $225^{\circ} \mathrm{F}$. $\left(107^{\circ} \mathrm{C}\right.$.) for 30 minutes, cooled in a desiccator, and the felt finally weighed as rapidly as possible. The heating shall be repeated until the weight of the felt remains constant as determined by two consecutive weighings taken not less than 10 minutes apart, which shall show a further loss of not more than 0.1 per cent. From the weight so obtained and the original weight, the percentage of saturation carried by the moisture-free felt shall be computed.

\section{DETERMINATION OF WEIGHT OF MOISTURE- FREE FELT}

18. Two strips shall be cut from the sample of roofing or shingles, each approximately 2 inches in width and 8 inches in length. Each of these shall be accurately weighed in grams and the length and width measured to within $1 \mathrm{~mm}$ or one thirty-second inch. It shall be extracted with carbon disulphide, ${ }^{50}$ in a suitable extractor and the extraction continued until the drippings have become colorless. The desaturated felt shall be removed from the extractor, being careful not to lose any adhering mineral matter, placed in a tared vessel, heated in a ventilated oven at $225^{\circ} \mathrm{F}$. $\left(107^{\circ} \mathrm{C}\right.$.) for 30 minutes, cooled in a desiccator, any adhering surfacing material brushed off and retained and the felt finally weighed as rapidly as possible. The heating shall be repeated until the weight of the felt remains constant as determined by two consecutive weighings taken not less than 10 minutes apart, which shall show a further loss of not more than 0.1 per cent. The benzol extract and accompanying mineral matter shall be retained for further examination. From the weights so obtained and the respective areas of the strips of desaturated felt, the weight of moisture-

${ }^{60}$ In this test, chloroform may be used as an equivalent solvent. free felt shall be computed in pounds per 108 square feet, from the following formulas and the results averaged:

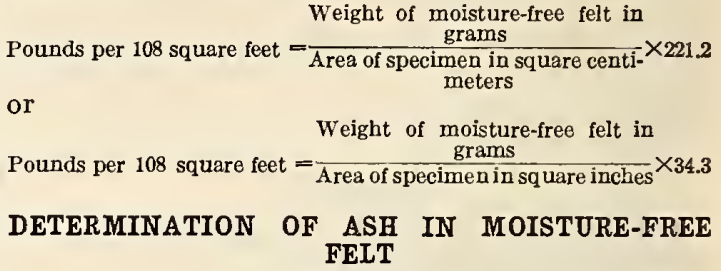

19. The strips of desaturated felt obtained as described in section 18 shall be cut into squares about $2 \mathrm{~cm}$ on each side and thoroughly mixed. Pieces amounting to about $10 \mathrm{~g}$ shall be selected at random and dried at $225^{\circ} \mathrm{F}$. $\left(107^{\circ}\right.$ C.) for 30 minutes. They shall be cooled in a desiccator, accurately weighed, and then incinerated in a weighed crucible either over an open flame or in a muffle until all the carbon is consumed. A few drops of ammonium carbonate solution shall then be added, the sample ignited gently, and weighed. The percentage of ash shall be calculated on the basis of the moisturefree felt.

\section{DETERMINATION OF MINERAL SURFACING AND MINERAL MATTER IN COATINGS}

20. (a) The slate and other mineral matter recovered from the extraction process described in section 18 shall be combined with the mineral matter removed from the surface of the desaturated felt, and that resulting from the evaporation and ignition of the carbon disulphide extract. The mineral matter shall be dried in air free from the odor of carbon disulphide, then heated in an oven for 30 minutes at $225^{\circ} \mathrm{F}$. $\left(107^{\circ} \mathrm{C}\right.$.), and finally cooled in a desiccator and weighed. From the combined weight of the specimens taken in section 18, the total "mineral surfacing and mineral matter in the coatings" shall be calculated in pounds per 108 square feet.

(b) For asphalt roll-roofing surfaced with granular talc, the mineral matter recovered as described in paragraph (a) shall be washed through a No. 65 copper-wire sieve with three washings of water of $100 \mathrm{cc}$ each. The total aqueous suspension of mineral matter which passes through the sieve shall then be filtered through a weighed Gooch crucible. It shall then be dried at $225^{\circ}$ F. $\left(107^{\circ}\right.$ C.) and rewcighed. The difference in weight represents the mineral matter passing a No. 65 sieve which shall be converted into pounds per 108 square feet.

American Society for Testing Materials, standard specifications for asphalt-saturated asbestos felt for use in constructing built-up roofs, serial designation D250-27, 1927.

1. These specifications cover asphalt-saturated asbestos felt, either 32 or 36 inches in width, composed of asbestos felt saturated, but not coated, with asphalt, for use in the construction of built-up roofs.

\section{MANUFACTURE}

2 . In the process of manufacture, a single thickness of asbestos felt shall be saturated with an asphaltic saturant.

3. The felt shall be an asbestos felt produced by "felting" at least 85 per cent by weight of asbestos fiber. The surface of the felt shall be uniformly smooth and, upon splitting or tearing on the bias, shall appear reasonably free from lumps or particles of foreign substances.

4. The saturant shall be composed of asphaltic materials. 


\section{PHYSICAL PROPERTIES}

5. The fabricated bituminized felt shall conform to the following requirements:

(a) Width of roll.-Either 36 or 32 inches, as may be specified, $\pm 1 / 4$ inch.

(b) Gross weight per roll. -40 to 80 pounds.

(c) Weight of wrapping and packing material per roll.- Maximum one-half pound.

(d) Weight of material per 100 square feet exclusive of packing and wrapping. -14 pounds \pm 1 pound.

(e) Loss on heating at $105^{\circ} \mathrm{C}$. (221 $1^{\circ} \mathrm{F}$.) for five hours.-Maximum, 2 per cent.

(f) Pliability at $77^{\circ} F$. $\left(25^{\circ} \mathrm{C}\right.$.). - Shall not crack when bent through $180^{\circ}$ over a one-sixteenth-inch mandrel.

(g) Saturant in moisture-free felt.-Minimum 50 per cent by weight.

6. The desaturated felt shall conform to the following requirements:

(a) Weight of moisture-free desaturated felt.-The minimum weight per 100 square feet shall be 9 pounds.

(b) The ash in moisture-free desaturated felt.Minimum, 73 per cent.

Note.-This presupposes the presence of at least 85 per cent of asbestos fiber carrying 14 per cent water of hydration.

\section{WORKMANSHIP AND FINISH}

7. The felt shall be thoroughly and uniformly saturated and shall show no unsaturated spots at any point upon cutting 2-inch strips at random across the entire sheet and splitting them open for their full length.

8. The surface of the felt shall not be coated or covered with talc or other substance which would tend to interfere with the adhesion between the felt and the plying cement.

9. The finished product shall be free from visible external defects, such as holes, ragged or untrue edges, breaks, cracks, tears, protuberances, and indentations.

10. The rolls shall not crack, or be sticky enough to cause tearing or material damage upon being unrolled at atmospheric temperatures above $50^{\circ} \mathrm{F}$. $\left(10^{\circ}\right.$ C. $)$

\section{PACKING}

11. The rolls of saturated felt need not be wound on cores, but they shall be securely wrapped in a substantial grade of paper of the same width as the fabric. The wrapper shall completely encircle the roll and shall be pasted at the overlap in a manner which will prevent it shifting from position. The ends of the rolls need not be covered. As an alternative, the rolls of saturated felt may either be tied with strings or simply pasted at the ends, as may be agreed upon by the purchaser and seller. No roll shall contain more than two pieces and there shall not be more than 3 per cent of rolls containing two pieces in any shipment. Each roll shall be plainly marked with the manufacturer's name and brand or as agreed by the purchaser and seller and shall bear a notation showing the type of saturant.

\section{SAMPLING AND TESTING}

12. The material shall be sampled, and the properties enumerated in these specifications shall be determined in accordance with A. S. T. M. D146. (See 505.0, p. 130.)

\section{INSPECTION}

13. Inspection of material shall be made as agreed upon by the purchaser and seller as part of the purchase contract.
American Society for Testing Materials, tentative specifications for asphalt for use in constructing built-up roof coverings, serial designation D312-29T, 1929.

\section{SCOPE}

1. These specifications cover asphalt intended for use as hot cement and mopping coat in the construction of built-up roof coverings for roofs surfaced in various manners, laid either over boards or concrete on various inclines, in connection with the Standard Specifications for Asphalt-Saturated Roofing Felt for Use in Waterproofing and in Constructing Built-up Roofs (A. S. T. M. D226, p. 156.) or the Standard Specifications for Asphalt-Saturated Asbestos Felt for Use in Constructing Built-up Roofs (A. S. T. M. D250, p. 156).

\section{TYPES}

2. The asphalts covered by these specifications include steam-distilled and blown-petroleum asphalts and also include mixtures of native asphalts with petroleum asphalts, likewise steam-distilled and blown-petroleum asphalts or combinations thereof mixed with mineral matter. The purchaser shall reserve the right to select that type of asphalt which in his judgment is best suited for his particular requirements.

\section{SAMPLING}

3. The sampling shall be performed in accordance with A. S. T. M. D140. (See 505.0, p. 128.)

\section{PROPERTIES}

4. The asphalt shall be homogeneous and free from water. It shall conform to the following requirements:

(a) Softening point (ring-and-ball method):

For mineral-surfaced roofs with inclines up to 2 inches per foot, $57^{\circ}$ to $74^{\circ} \mathrm{C}$. $\left(135^{\circ}\right.$ to $165^{\circ} \mathrm{F}$.).

For unsurfaced roofs with inclines up to 3 inches per foot, $66^{\circ}$ to $93^{\circ} \mathrm{C}$. $\left(150^{\circ}\right.$ to $200^{\circ} \mathrm{F}$.).

For unsurfaced roofs with inclines up to 6 inches per foot, $74^{\circ}$ to $93^{\circ} \mathrm{C}$. $\left(165^{\circ}\right.$ to $200^{\circ} \mathrm{F}$.).

(b) Penetration at $0^{\circ} \mathrm{C} .\left(32^{\circ} \mathrm{F}\right.$. $), 200 \mathrm{~g}_{2} 60$ seconds, not less than 5.

(c) Penetration at $25^{\circ} \mathrm{C}$. ( $\left(77^{\circ}\right.$ F.), $100 \mathrm{~g}, 5$ seconds, 20 to 50.

(d) Loss on heating at $163^{\circ}$ C. $\left(325^{\circ}\right.$ F.), $50 \mathrm{~g}$, 5 hours, not more than 1.5 per cent.

(e) Penetration at $25^{\circ} \mathrm{C}$. $\left(77^{\circ}\right.$ F.) $, 100 \mathrm{~g}, 5$ seconds, of residue after heating at $163^{\circ}$ C. $\left(325^{\circ}\right.$ F.), as compared with penetration of asphalt before heating, not less than 60 per cent.

(f) Ductility at $25^{\circ} \mathrm{C} .\left(77^{\circ} \mathrm{F}\right.$.), not less than $3 \mathrm{~cm}$.

(g) Soluble in carbon disulphide, not less than 65 per cent.

(h) Ash on incineration, either 0 to 1 or 10 to 28 per cent, inclusive.

(i) Soluble in carbon tetrachloride, not less than 99 per cent.

(j) Elutriation test (sediment), not more than 7 per cent.

\section{METHODS OF TESTING}

5. The properties enumerated in these specifications shall be determined in accordance with the following methods of test of the American Society for Testing Materials:

(a) Softening point.-A. S. T. M. D36. (See 505.0, p. 131.)

(b) Penetration.-A. S. T. M. D5. (See 505.0, p. 118.)

(c) Loss on heating.-A. S. T. M. D6. (See 505.0, p. 119.)

(d) Ductility.-A. S. T. M. D113-26T. (See $505.0, \mathrm{p}, 126$. 
(e) Soluble in carbon disulphide.-A. S. T. M. D4. (See 505.0, p. 117.)

(f) Ash.-A. S. T. M. D271. (See 501.0, p. 8.)

(g) Soluble in carbon tetrachloride.-A. S. T. M. D165. (See 505.0, p. 135.)

(h) Elutriation test (sediment).-A. S. T. M. D313-29T. (See 505.0, p. 138.)

Asphalt Shingle and Roofing Association (now Institute), standards for the laying of roofing. This association has adopted detailed methods for the laying of roofing, as follows:

1. Directions for laying roll roofings, adopted December 8, 1920.

2. Directions for laying shingles, adopted March $9,1921$.

Asphalt Shingle and Roofing Association (now Institute). This organization has the following standards:

1. Dry felt tests, adopted 1921.

2. Methods of testing the thoroughness with which felt will saturate (recommended 1922).

3. Methods of testing the speed with which felt will saturate (recommended 1923).
Federal Specifications Board. The board has prepared specifications for saturated felt as follows: 1. Specification No. 295, United States Government master specification for asphalt saturated rag felt for flashings, May 20, 1925.

2. Specification No. 86, United States Government specification for asphalt saturated rag felt for roofing and waterproofing, December 29, 1923.

3. Specification No. 81, United States Government specification for coal-tar saturated rag felt for roofing and waterproofing, December 29, 1923.

The requirements of the specifications are as follows:

\section{GENERAL}

A ppearance.-It shall be free from visible external defects and uniform throughout. When unrolled at temperatures between $50^{\circ}$ and $90^{\circ} \mathrm{F}$. it shall not stick to such an extent as to cause tearing.

Packing. - The felt shall be properly wrapped and labeled with the manufacturer's name, the brand, grade, weight, area of roll, and type of saturant.

\begin{tabular}{|c|c|c|c|}
\hline Specific properties & Specification No. 295 & Specification No. 86 & Specification No. 81 \\
\hline $\begin{array}{l}\text { 1. Width } \\
\text { 2. Gross weight of roll } \\
\text { 3. Weight of wrapping, packing, etc, maximum } \\
\text { 4. Weight per } 100 \text { square feet, exclusive of packing, etc--pounds } \\
\text { 5. Pliability (at } 25^{\circ} \mathrm{C} \text {.) } \\
\text { 6. Average breaking strength: } \\
\text { (a) With fiber grain, minimum } \\
\text { (b) Across fiber grain, minimum } \\
\text { 7. Volatile matter, maximum (at } 105^{\circ} \mathrm{C} \text { ) } \\
\text { 8. Weight per } 100 \text { square fcet of moisture-free, desaturated felt, } \\
\text { minimum } \\
\text { 9. Weight per } 100 \text { square feet of saturant, as related to weight of } \\
\text { moisture-free felt per } 100 \text { square feet, minimum } \\
\text { 10. Weight of comminuted surfacing, per } 100 \text { square feet, maxi- } \\
\text { mum - } \\
\text { 11. Ash of desaturated felt, maximum } \\
\text { Thickness of desaturated felt, minimum }\end{array}$ & $\begin{array}{l}32 \text { or } 36 \text { in. } \pm 1 / 4 \\
50 \text { to } 80 \\
1 / 2 \\
29 \pm 1 / 2 / 2 \\
\text { No cracking on bend- } \\
\text { ing over } 1 / 16 \text {-inch } \\
\text { mandrel. } \\
40 \\
10.4\end{array}$ & $\begin{array}{l}32 \text { or } 36 \text { in. } \pm 1 / 4 \\
50 \text { to } 80 \\
14 \pm 1 \\
\quad \text { ing cracking on bend- } \\
\text { ing on itself. }\end{array}$ & $\begin{array}{l}32 \text { or } 36 \text { in. } \pm 1 / 4 . \\
50 \text { to } 80 . \\
1 / 2 . \\
14 \pm 1 \text {. } \\
\text { No cracking on bend- } \\
\text { ing flat on itself. } \\
30 . \\
15 .\end{array}$ \\
\hline
\end{tabular}

1 Equivalent to No. 50 feit.

\section{METHODS OF TESTING}

To determine the properties listed in the above table, the following methods of examination shall be used if required, but the purchaser reserves the right to use any additional available information to determine whether the material meets the specification.

(a) Appearance.-Examine both sides of the sheet of felt and note the uniformity of color. The felt shall be thoroughly and uniformly impregnated and shall show no unsaturated spots at any point upon cutting 2 -inch strips at random across the entire sheet and splitting them open for their full length.

(b) Weight.-Trim the sample so that it is 36 inches long and the full width of the roll. Measure accurately and weigh to the nearest gram (15 grains). From the measurements and weight so obtained compute the weight per 100 square feet.

(c) Pliability. - With a photographic print trimmer or by other suitable means cut five strips 1 inch wide and 6 inches long in the direction of the fiber grain and immerse in water at $25^{\circ} \mathrm{C}$. $\left(77^{\circ} \mathrm{F}\right.$.) for 10 to 15 minutes. Remove the strips from the water and immediately bend each strip flat over a 1/16-inch mandrel through an arc of $180^{\circ}$ at a uniform rate in approximately two seconds time.

(d) Brcaking strength.-Cut 10 strips of the felt 1 by 6 inches with the fiber grain and 10 strips of the same size across the fiber grain. Test both sets of strips at $21^{\circ} \mathrm{C}$. $\left(70^{\circ} \mathrm{F}\right.$.) in a Scott or similar testing machine of the inclination balance type having a capacity of 100 to 150 pounds. The test strips shall be gripped $1 \frac{1}{2}$ inches on each end, leaving 3 inches between the clamps and the lower jaw shall travel at the rate of 12 inches per minute. If any strip breaks nearer than one-half inch to either clamp, the reading shall be disregarded, and an additional strip tested in its place. The average of the 10 readings from strips cut in one direction shall be taken as the breaking strength of the sample in that direction.

(e) Volatile matter. - Cut two strips of the asphalt saturated felt 6 inches wide and 12 inches long; weigh each strip and suspend it for five hours in an oven maintained at $105^{\circ} \mathrm{C} .\left(221^{\circ} \mathrm{F}\right.$. $) \pm 3^{\circ} \mathrm{C}$. $\left(5^{\circ} \mathrm{F}\right.$. $)$. Remove from the oven, cool in a desiccator, and weigh. Compute the loss in weight and average the results on the two samples.

(f) Weight and thickness of dcsaturated felt.-Cut a 2-inch strip the full width of the sample, measure accurately, weigh and extract with benzol, carbon tetrachloride, or carbon bisulphide, in a suitable extraction apparatus, until the solvent runs through colorless. Remove the desaturated felt from the extractor and allow to remain in air until the solvent has evaporated. Brush off any adhering particles of foreign matter. In making the test for specification No. 86 , retain any adhering particles of surfacing. In making the test for specification No. 81 this strip shall be cut into four equal portions, and each piece calipered at 10 equally spaced points, at least threefourths of an inch from a cut edge, with a micrometer having a flat bearing surface of at least one-half inch in diameter at each contact point. Average these readings to get the thickness of the desaturated felt. In the tests for specifications Nos. 86 and 295 
the desaturated feit strip shall be placed in a tared weighing bottle, dried at $105^{\circ}$ to $110^{\circ} \mathrm{C}$. $\left(221^{\circ}\right.$ to $230^{\circ}$ F.) for one-half hour, cooled and weighed. Compute the weight per 100 square feet on the desaturated felt.

(g) Comminuted surfacing.-(This test used for specification No. 86 only.) Filter the extract (obtained above in section $(f)$ ) through a tared Gooch crucible prepared with a mat of asbestos fiber. Wash all sediment from the extraction apparatus into the Gooch crucible with carbon tetrachloride or benzol and transfer also all surfacing brushed from the felt (in section $(f)$ ) to the Gooch crucible. Wash the insoluble matter on the filter with carbon tetrachloride or benzol until the washings run through colorless. Suction may be used to aid in filtering. Place in an oven at $105^{\circ}$ to $110^{\circ} \mathrm{C}$. $\left(221^{\circ}\right.$ to $230^{\circ} \mathrm{F}$.) for one-half hour. Cool in a desiccator, weigh, and from the weights of the sample taken (in section $(f)$ ) and the weight of surfacing obtained, compute the weight of comminuted surfacing per 100 square feet.

(h) Saturation of felt. - The weight of asphalt in the saturated felt is the difference in weight between the original sample taken for extraction (see $(f)$ ) and the weight of the desaturated felt obtained in $(f)$. (In testing for specification No. 86 the weights of the comminuted surfacing $(g)$ shall be added to the weight of the desaturated felt and subtracted from the weight of the original sample.) The percentage saturation is obtained from the following formula:

Teight of asphalt in saturated felt

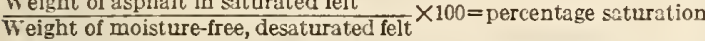

(i) Ash.-Cut the strip of desaturated felt obtained in $(f)$ into squares about $1 \mathrm{~cm}$ on each side, and thoroughly mix. Select at random pieces amounting to about $10 \mathrm{~g}$ and, after drying at $105^{\circ}$ to $111^{\circ} \mathrm{C}$. $\left(100^{\circ}\right.$ to $105^{\circ} \mathrm{C}$. for No. 295) for onehalf hour and cooling, weigh accurately and incinerate in a weighed crucible in a muffle or over an open flame until all the carbon is consumed. Cool, weigh, and compute the percentage of ash in the moisture-free, desaturated felt.

Notes.-Carbon tetrachloride or carbon bisulphide will usually give the sime results, but benzol shall be used in umpire tests in case of dispute.

The specifications are indiridually distinct as follows:

Specification No. 295:

\section{ADDITIONAL REQUIREMENTS}

The asplalt saturated rag felt covered by this specification is intended for use in the construction of flashings in connection with built-up roofing and when it is desired to use a sheet heavier than 14 pounds for unsurfaced built-up roofing. The material shall be composed of rag roofing felt impregnated with asphaltic saturating materials.

Sampling and determination of weight and width.The weight per 100 square feet of the material and its variation in weight can be most accurately determined by the inspector at the time he takes a sample for transmittal to the testing laboratory.

(a) From each shipment of more than 1,000 rolls take at random a number of rolls of felt equivalent to one-half the cube root of the total included in the lot. If this proves to be a fractional number express it as the next higher digit. From each shipment of 1,000 rolls or less take at random five rolls in each case.

(b) Remove wrappers, cores, and other packing material from each roll taken and weigh the roll and the packing material, etc., separately to the nearest one-fourth pound. Then unwind each roll and measure the length and the width of each end of the roll to the nearest one-fourth inch.
Compute the weight per 100 square feet of each roll examined. At approximately 0.25 feet from the end of each roll taken cut two samples the full width of the roll and approximately 40 inches long, label, carefully wrap, and transmit one to the testing laboratory. Retain the other sample for check analysis in case of dispute.

Note.-For reference to specification for the installation of plastic and metal flashings, see F.S. B. specificaticn No. 156, 565.0, page 141 .

Specification No. 86 :

\section{ADDITIONAL REQUIREMENTS}

\section{GENERAL}

This specification applies to asphalt-saturated rag felt intended for use with asphalts for mineral surfaced roofing (F. S. B. specification No. 84; see 505.16 , p. 162), and asphalt for unsurfaced built-up roofing (F. S. B. specification No. 88; see 505.16, p. 163), and asphalt for waterproofing and damp proofing (F. S. B. specification No. 85; see 505.16, p. 163), in the construction of built-up roofing and membrane waterproofing, respectively.

It shall be composed of rag roofing felt impregnated with an asphaltic saturant.

\section{SAMPIING AND DETERMINATION OF WEIGHT AND WIDTH}

The weight per 100 square feet of the material and its variation in weight can be most accurately determined by the inspector at the time he takes a sample for transmittal to the testing laboratory.

(a) From each shipment select at random a number of rolls of felt equivalent to the cube root of the total included in the lot. If the cube root as calculated proves to be a fractional number, express it as the next higher digit.

(b) Remove wrappers, cores, and other packing material from each roll selected and weigh the roll and the packing material, etc., separately to the nearest one-fourth pound. Then unwind each roll, and with a steel tape measure the length and the width of each end of the roll to the nearest one-fourth inch and reroll. Observe the appearance of the material while unrolling and rerolling.

The finished material shall be free from visible external defects, such as holes, ragged or untrue edges, breaks, rents, cracks, or indentations. The rolls shall be capable of being unrolled easily at temperatures between $50^{\circ}$ and $90^{\circ} \mathrm{F}$. without sticking in a manner that will injure the felt. The surface of the felt shall not be coated or covered with talc, or other substances which would tend to interfere with the adhesion between the felt and the plying cement. The use of silica or wood flour will be permitted. The surface shall be uniformly smooth and shall be free from areas or patches of unabsorbed saturant and superficial dry spots.

Compute the weight per 100 square feet of each roll examined, and from these weights compute the average weight of the shipment. In no case shall this be less than 13 pounds per 100 square feet, and any roll examined whose weight is less than 13 pounds per 100 square feet shall be cause for the rejection of the whole shipment. If the average weight is above 15 pounds per 100 square feet, the material shall be paid for on the basis of 15 pounds per 100 square feet.

(c) From the rolls examined for sampling select one, the weight of which is near the average weight of the whole lot. Unroll and at approximately 25 feet from the end cut two samples the full width of the roll and approximately 40 inches long, label, carefully wrap, and transmit one to the testing laboratory. Retain the other sample for check analysis in case of dispute. 
Specification No. 81:

\section{ADDITIONAL REQUIREMENTS}

\section{GENERAL}

This specification applies to coal-tar saturated rag felt intended for use with coal-tar pitch for roofing (F. S. B. specification. No 80 ; see 505.36 , p. 179), and coal-tar pitch for waterproofing and damp proofing (F.S. B. specification No. 83 ; see 505.36 , p. 179), in the construction of built-up roofing and membrane waterproofing, respectively.

It shall be composed of rag roofing felt impregnated with a coal-tar saturant.

\section{SAMPLING AND DETERMINATION OF WEIGHT AND WIDTH}

Same as for F. S. B. specification No. 86, given herewith.

Federal Specifications Board, specification No. 82, United States Government master specification for surfacing materials for bituminous built-up roofing December 29, 1923.

\section{GENERAL}

These specifications apply to surfacing materials intended for surfacing built-up bituminous roofing and may be used interchangeably, but must be applied in the manner described in the specification for application of the particular surfacing material. Surfacing materials shall consist of either roofing gravel, slag, crushed hard stone, promenade tile, or slate slabs.

(a) Roofing gravel.--Roofing gravel shall be hard, durable, waterworn, dry, and free from clay, loam, sand, and other foreign substances. It shall be preferably within the following limits with respect to size:

Per cent

Passing a $3 / 4$-inch sieve

Passing a 5/rinch sieve and retained on a Passing a 8 inch

Retained on a $1 / 6$ inch sieve

(b) Roofing slag.-Roofing slag shall be crushed hard blast-furnace slag, dry, and free from sand, dirt, clay, or other foreign substances. It shall be preferably within the following limits with respect to size:

Passing a $3 / 4$-inch sieve.

Per cent

Passing a $5 / 8$-inch sieve and retained on a $3 / 4$-inch sieve, not less

than -

Retained on a $1 / 8$-inch sieve

(c) Crushed stone.-Crushed stone for roofing shall be any hard, durable rock other than limestone which will crush to particles of a fairly cubical shape instead of long, thin, flat splinters. It shall be dry, free from sand, dirt, clay, or other foreign substances and shall be preferably within the following limits with respect to size:

Passing a $3 / 4$-inch sieve Per cent Passing a $5 / 8$-inch sieve and retained on a 14 -inch sieve, not less than a 8 -inch sleve and retained on a y4-inch sieve, not less 80 Retained on a $1 / 8$-inch sieve ... 100

(d) Promenade tile.-Promenade tile shall be first quality vitrified shale tile, square edged, sound, unwarped, and free from blisters, chipped edges, or other surface defects. The tile shall be grooved on the back, so as to afford an ample bond, and of the size and color specified in the contract. No dimension shall be more than 2 per cent above or below the size specified.

(e) Slate--Slate shall be of the quality, width, length, and color specified for the particular job. It shall be clear and shall have a minimum thickness of three-sixteenths inch with sides cut true.

\section{SAMPLING}

Roofing gravel, slag, and crushed stone shall be sampled in accordance with A. S. T. M. D 75. (See 512.10 , p. 216.)

\section{LABORATORY EXAMINATION}

(a) Sieve analysis of roofing gravel, slag, and crushed stone.-A representative test sample of the surfacing material, which in an air-dry condition weighs about 5 pounds, shall be selected from the sample submitted to the laboratory by quartering or by the use of a sampler.

The sieves shall be of square mesh wire cloth in the following sizes: One-eighth inch, one-fourth inch, five-eighths inch, and three-fourths inch, these being the widths of the clear openings.

The sample shall be separated by means of the sieves, sifting being continued until not more than 1 per cent by weight of the sample passes any sieve during one minute. Each size shall be weighed on a balance or scale which is sensitive to 0.1 ounce (approximately $3 \mathrm{~g}$ ).

The percentage by weight of the total sample passing each of the sieves shall be determined and the percentage reported to the nearest whole number.

(b) Promenade tile and slate.-No laboratory examination will be made.

Federal Specifications Board, specification No. 84, United States Government master specification for asphalt for mineral surfaced roofing, December 29, 1923.

\section{GENERAL}

This specification applies to asphalt intended for use with asphalt-saturated rag felt for roofing and waterproofing (F. S. B. specification No. 86; see p. 160), in the construction of mineral-surfaced built-up roofing in inclines not exceeding 3 inches to the foot. Either petroleum asphalt, or mixtures of refined Trinidad asphalt with petroleum asphalt, or asphalt fluxing materials will be acceptable under these specifications, but the contract must state the kind to be furnished.

The asphalt shall conform to the requirements listed in the following table, which also gives requirements for additional specifications, as follows:

Asphalt for waterproofing and damp proofing, Federal Specifications Board, specification No. 85. (See 505.16, p. 163.)

Asphalt for unsurfaced built-up roofing, Federal Specifications Board, specification No. 88. (See 505.16, p. 163.)

NoтE.--If required, deliveries will, in general, he sampled and tested hy the following methods, hut the purchaser reserves the right to use any additional information to ascertain whether the material meets the specification.

\begin{tabular}{|c|c|c|c|}
\hline Properties & $\begin{array}{l}\text { Specifi- } \\
\text { cation } \\
\text { No. } 84\end{array}$ & \begin{tabular}{|l|} 
Specifi- \\
cation \\
No. 85
\end{tabular} & $\begin{array}{l}\text { Specifi- } \\
\text { cation } \\
\text { No. } 88\end{array}$ \\
\hline Melting point.-- & $\{140-165$ & $140-170$ & $\begin{array}{l}1150-165 \\
2165-190\end{array}$ \\
\hline $\begin{array}{l}\text { Penetration: } \\
\text { (a) At } 77^{\circ} \mathrm{F} \\
\text { (b) At } 32^{\circ} \mathrm{F} \text {, minimum } \\
\text { (c) At } 115^{\circ} \mathrm{F} \text {., maximum }\end{array}$ & $\begin{array}{r}25-50 \\
10\end{array}$ & $\begin{array}{r}25-50 \\
10 \\
100\end{array}$ & $\begin{array}{r}20-50 \\
10 \\
100\end{array}$ \\
\hline $\begin{array}{l}\text { Ductility at } 77^{\circ} \mathrm{F} \text {, minimum } \\
\text { Volatile matter at } 325^{\circ} \mathrm{F} \text {., maximum }\end{array}$ & 5 & 15 & 5 \\
\hline $\begin{array}{l}\text { Decrease in penetration at } 77^{\circ} \mathrm{F} \text {. } \\
\text { after heating, maximum - per cent }\end{array}$ & 40 & $\begin{array}{r}1 \\
40\end{array}$ & 40 \\
\hline $\begin{array}{l}\text { Soluhle in carhon disulphide, mini- } \\
\text { mum }\end{array}$ & $\begin{array}{r}499 \\
8 \min .20\end{array}$ & 99 & $\begin{array}{r}399 \\
810-20\end{array}$ \\
\hline
\end{tabular}

1 Grade 1

2 Grade 2.

a Other considerations being equal, preference will he given to asphalt having a minimum ductility at $77^{\circ} \mathrm{F}$. of $20 \mathrm{~cm}$.

${ }_{4}^{4}$ In the case of petroleum asphalt only.

$\checkmark$ This requirement applies in the case of mixtures of refined Trinidad asphalt with petroleum asphalt or asphalt in fluxing materials. The ash shall have the characteristies of the ash ohtained from refined Trinidad asphalt. 
The following tests shall be used to determine the properties of asphalt for mineral-surfaced roofing, for water proofing and damp proofing, and for unsurfaced built-up roofing covered by Federal Specifications Board, specifications Nos. 84, 85, and 88 .

(a) Appearance.-(See below "Appearance.")

(b) Melting point.-A. S. T. M. D36. (See 505.0, p. 131.)

(c) Penetration at $77^{\circ}$ F.-A. S. T. M. D5.

505.0, p. 118.)

(d) Penetration at $.22^{\circ}$ F.-This shall be determined as in (c) at a temperature of $0^{\circ} \mathrm{C}$. $\left(32^{\circ} \mathrm{F}\right.$.), under a $200 \mathrm{~g}$ load, and for a period of 60 seconds instead of a temperature of $25^{\circ} \mathrm{C}$. $\left(77^{\circ} \mathrm{F}\right.$.), a load of $100 \mathrm{~g}$ and a period of five seconds.

(e) Penetration at $115^{\circ} \mathrm{F}$.- This shall be determined as in (c) using a temperature of $46^{\circ} \mathrm{C}$. $\left(115^{\circ}\right.$ F.) a $50 \mathrm{~g}$ load, and a period of five seconds instead of a temperature of $25^{\circ} \mathrm{C}$. $\left(77^{\circ} \mathrm{F}\right.$.) a load of $100 \mathrm{~g}$, and a period of five seconds.

(f) Ductility.-A. S. T. M. D113. (See 505.0, p. 126.)

(g) Volatile matter.-A. S. T. M. D6. (Sce 505.0, p. 119.)

(h) Penetration at $7 \gamma^{\circ} F$. after heating.-The sample from test $(g)$ shall be melted at a temperature not exceeding $200^{\circ} \mathrm{C}$. $\left(392^{\circ} \mathrm{F}\right.$.) until fluid and thoroughly mixed. It shall then be allowed to cool to room temperature and tested for penetration as in $3(\mathrm{c})$ at a temperature of $25^{\circ} \mathrm{C}$. $\left(77^{\circ} \mathrm{F}\right.$.) under a load of $100 \mathrm{~g}$, and for a period of five seconds.

(i) (1) Soluble in carbon bisulphide.-One $\mathrm{g}$ of the asphalt shall be placed in a beaker of 100 to 150 ce capacity, covered with cold carbon bisulphide, and set aside for about 15 minutes, the liquid being stirred every few minutes until the lumps break up and the asphalt goes into solution. The solid particles shall be allowed to settle and the carbonbisulphide solution of the asphalt decanted through a weighed Gooch crucible, prepared with a mat of asbestos fiber of a thickness that will scarcely transmit light.

The sediment remaining in the beaker shall be crushed with a stirring rod, and a little more carbon bisulphide added. The sides of the beaker also shall be washed down with carbon bisulphide. The contents of the beaker shall be poured into the crucible and all sediment washed from the beaker into the crucible with carbon bisulphide. Suction may be used to aid in filtering. The sediment on the filter shall be washed with carbon bisulphide until the washings run through colorless. The crucible shall be set aside in a place free from dust until the carbon bisulphide evaporates and then placed in an oven at $105^{\circ}$ C. $\left(221^{\circ}\right.$ F. $),\left(100^{\circ}\right.$ C. $\left(212^{\circ}\right.$ F. $)$ for No. 85$)$ for one hour. It shall then be cooled and weighed and the soluble matter computed.

(2) Ash.-About $1 \mathrm{~g}$ of asphalt shall be placed in a crucible of about 30 cc capacity and incinerated until the carbon is consumed and only mineral matter remains. Cool in a desiccator, and weigh. From the weights of the original sample and the ash obtained compute the percentage of ash.

Federal Specifications Board specifications Nos. 84,85 , and 88 are uniform as to the following:

\section{APPEARANCE}

Freshly melted material shall be uniformly glossy, and on aging one week its surface shall not become dull or show any separation of oil, grease, paraffin scale, or similar material.

\section{SAMPLING}

From each shipment select at random a number of packages equivalent to the cube root of the total number of packages included in the lot. If the cube root as calculated proves to be a fractional number, express it as the next higher digit.

Samples shall be taken at least 3 inches below the surface and at least 3 inches from the side of each container selected. A clean hatchet may be used if the material is hard enough to shatter, and a broad, stiff putty knife if the material is soft. An auger, or brace and $3 / 4$-inch bit, or other suitable means may also be used. Avoid contamination with superficial water, wooden slivers, or foreign matter.

The samples so taken shall be combined by melting together, with constant stirring to prevent overheating, but the temperature must not exceed $200^{\circ} \mathrm{C}$. $\left(392^{\circ} \mathrm{F}\right.$.). When thoroughly melted, two 1-quart friction-top cans shall be filled with the well-mixed material, allowed to cool, tightly sealed, marked for identification with the manufacturer's name and brand, etc., and one transmitted to the testing laboratory. The other shall be retained for use in cáse of dispute.

\section{IABORATORY EXAMINATION}

(a) Appearance.-Carefully melt about $10 \mathrm{~g}$ of the asphalt at a temperature not exceeding $200^{\circ} \mathrm{C}$. $\left(392^{\circ}\right.$ F.) and pour it into a small tin box or other suitable container provided with a cover. At least one-half the surface shall be free from froth or bubbles. Allow the asphalt to cool in a place free from drafts and dust. When cool, examine the surface, then cover with the lid and set aside for one week, after which period examine again. Immediately after cooling the surface shall be glossy black, and after standing one week the surface shall not show any dulling or the separation of paraffin scale or greasy or oily material.

\section{PACKING}

This material shall be put up in steel drums or other suitable containers and delivered at the site of the job in original sealed packages as put up by the manufacturer. They shall be marked with the manufacturer's name and brand.

Federal Specifications Board, specification No. 85 United States Government specification for asphalt for waterproofing and damp proofing, December $29,1923$.

This specification applies to asphalt intended for use with asphalt saturated rag felt for roofing and waterproofing (F. S. B. specification No. 86 ; see p. 160) as a plying cement in the construction of membrane waterproofing, or alone as a dampproof coating.

This asphalt is suitable for damp proofing and waterproofing railroad bridges, tanks, retaining walls, embankments, dams, conduits, foundations of buildings, tunnels, subways, pools, reservoirs, ete.

For properties, methods of testing, appearance, sampling, laboratory examination, and packing, see 505.16 (F. S. B. No. 84), page 162

Federal Specifications Board, specification No. 88, United States Government master specification for asphalt for unsurfaced built-up roofing, June 1, 1924.

\section{GRADES}

This specification applies to asphalt intended for use with asphalt-saturated rag felt for roofing and waterproofing (F. S. B. Spcc. No. 86; see p. 160,) or asphalt-saturated asbestos felt, in the construction of unsurfaced built-up roofing. Either petroleum asphalt or mixtures of refined Trinidad asphalt with petroleum asphalt or asphaltic fluxing materials will be acceptable under these specifications; but the contract shall state the kind to be furnished. 
It shall be furnished in one of two grades as spccified in the contract, as follows: (1) For use on inclines not exceeding 3 inches to the foot over boards, and $1 \frac{1}{2}$ inches to the foot over concrete; (2) for use on inclines not exceeding 6 inches to the foot over boards, and 3 inches to the foot over concrete.

(For properties, methods of testing, appearance, sampling, laboratory examination and packing, see 505.16 , F. S. B. No. 84 , p. 162.)

Federal Specifications Board, spccification No. 87, United States Government master specification for asphalt primer for roofing and waterprooîng.

\section{GENERAL}

This specification applies to asphalt primer intended for use, when specified, as a priming coat for concrete, gypsum, and other masonry surfaces over which asphalt built-up roofing, waterproofing, or damp proofing are to be applied. It shall consist of an asphaltic base thinned to suitable brushing consistency with a volatile solvent, and when tested by the methods contained in this specification shall meet the following requirements:

(a) The asphaltic base shall be not less than 35 per cent by weight.

(b) The asphaltic base separated as under $3(b)$ shall have the following characteristics:

1. Melting point, $131^{\circ}$ to $170^{\circ} \mathrm{F}$.

2. Penetration, 15 to 50 .

(c) The solvent used shall be a hydrocarbon distillate having an end point on distillation not exceeding $500^{\circ} \mathrm{F}$., of which not more than 20 per cent shall distill under $248^{\circ} \mathrm{F}$. The solvent shall be completely removed by the method given under $3(b)$.

If required, deliveries will, in gencral, be sampled and tested by the following methods, but the purchaser rescrves the right to use any additional information to ascertain whether the material meets the specification:

\section{SAMPLING}

See "Sampling," American Society for Testing Materials, serial designation D41. (See p. 152.)

\section{IABORATORY EXAMINATION}

(a) Weight of asphaltic base.-A portion of the sample shall be placed in a stoppered bottle or weighing pipette and weighed. About $1.5 \mathrm{~g}$ of the sample shall be transferred to a flat-bottomed metal dish about $8 \mathrm{~cm}$ in diameter (a friction-top can plug). The container shall be weighed again and the exact weight of the portion of the sample transferred to the weighed dish shall be computed by difference. The dish with its contents shall be heated in an oven maintained at $105^{\circ}$ to $110^{\circ} \mathrm{C}$. $\left(221^{\circ}\right.$ to $230^{\circ} \mathrm{F}$.) for three hours, cooled, and weighed. From the weight of the residue left in the dish and the weight of the sample taken, the percentage of nonvolatile residue (asphaltic base) shall be computed.

(b) Separation of asphaltic base for examination.One hundred grams of the primer shall be distilled with steam at a temperature of $130^{\circ}$ to $140^{\circ} \mathrm{C}$. $\left(266^{\circ}\right.$ to $284^{\circ} \mathrm{F}$.) and the distillate collected in a separatory funnel. The distillation shall be stopped when $400 \mathrm{cc}$ of water has distilled over. The residue from the distillation shall be dried at $105^{\circ}$ to $110^{\circ} \mathrm{C}$. $\left(221^{\circ}\right.$ to $230^{\circ} \mathrm{F}$.) for five hours, and then gradually heated until all the moisture is expelled, but the temperature must not exceed $150^{\circ} \mathrm{C}$. $\left(300^{\circ} \mathrm{F}\right.$.).

(c) Examination of asphaltic base.-The asphaltic base separated in $3(b)$ shall be tested for melting point and penetration as follows:
1. Melting point.-The melting point shall be determined by A. S. T. M. D36. (See 505.0, p. 131.)

2. Penetration at $77^{\circ} F$.-The penetration shall be determined by A. S. T. M. D5. (See 505.0, p. 118.)

Federal Specifications Board, United States Government master specifications for the construction of built-up roofing of the following types:

Specification No. 146, for type 4 AWS, having four layers or plies of felt.

Specification No. 147, for type 5 AWS, having five layers or plies of felt.

Specification No. 148, for type 3 ACS, having three layers or plies of felt.

Specification No. 149 , for type 4 ACS, having four layers or plies of felr.

Specification No. 150, for type 5 ACS, having five layers or plies of felt.

\section{GENERAL REQUIREMENTS}

The roofing shall consist of layers or plies of asphalt saturated rag felt cemented together with asphalt and surfaced with slag, gravel, or similar surfacing material as called for in the proposal for the work.

Flashings of the type required under the specifications for the building shall be included and shall conform to Government specification for flashings No. 156 or No. 157 . (See 505.0, p. 141.)

\section{DETAIL REQUIREMENTS}

The roofing shall contain not less than the following quantities of materials per 100 square feet:

\begin{tabular}{|c|c|c|c|c|c|c|c|c|c|c|}
\hline \multirow[t]{2}{*}{ Туре } & \multicolumn{2}{|c|}{$\begin{array}{c}\text { Layers of } \\
\text { asphalt } \\
\text { saturated } \\
\text { rag felt } \\
(\text { F. S. B. } \\
\text { No. } 86 \text {, } \\
\text { p. } 160 \text { ) }\end{array}$} & \multicolumn{2}{|c|}{$\begin{array}{c}\text { Asphalt } \\
\text { primer } \\
\text { (F. S. B. } \\
\text { No. } 87 \text {, } \\
\text { see } \\
\text { above) }\end{array}$} & \multicolumn{2}{|c|}{$\begin{array}{c}\text { Asphalt } \\
\text { for } \\
\text { mineral } \\
\text { surfaced } \\
\text { roofing } \\
\text { (F. S. B. } \\
\text { No. 84, } \\
\text { p. 162) }\end{array}$} & \multicolumn{2}{|c|}{$\begin{array}{c}\text { Roofing } \\
\text { gravel } \\
\left(\begin{array}{l}\text { F. S. B. } \\
\text { No 82, } \\
\text { p. 1o2) }\end{array}\right.\end{array}$} & \multicolumn{2}{|c|}{$\begin{array}{c}\begin{array}{c}\text { Roofing } \\
\text { slag }\end{array} \\
\qquad \begin{array}{c}\text { (F. S. B, } \\
\text { No. 82. } \\
\text { p. 162) }\end{array}\end{array}$} \\
\hline & \multicolumn{2}{|c|}{$\begin{array}{l}\text { Over } \\
\text { wood }\end{array}$} & \multicolumn{2}{|c|}{$\begin{array}{l}\text { Over } \\
\text { wood }\end{array}$} & \multicolumn{2}{|c|}{$\begin{array}{l}\text { Over } \\
\text { wood }\end{array}$} & \multicolumn{2}{|c|}{$\begin{array}{l}\text { Over } \\
\text { wood }\end{array}$} & \multicolumn{2}{|c|}{$\begin{array}{l}\text { Over } \\
\text { wood }\end{array}$} \\
\hline \multirow[t]{2}{*}{$\begin{array}{l}4 \text { AWS } \\
5 \text { AWS }\end{array}$} & \multicolumn{2}{|c|}{$\begin{array}{c}\text { Pounds } \\
60 \\
76\end{array}$} & \multicolumn{2}{|c|}{$\begin{array}{l}\text { None. } \\
\text { None. }\end{array}$} & \multicolumn{2}{|c|}{$\begin{array}{c}\text { Pcunds } \\
105 \\
125\end{array}$} & \multicolumn{2}{|c|}{$\begin{array}{c}\text { Pounds } \\
400 \\
400\end{array}$} & \multicolumn{2}{|c|}{$\begin{array}{c}\text { Pounds } \\
300 \\
300\end{array}$} \\
\hline & $\begin{array}{l}\text { Over } \\
\text { con- } \\
\text { crete }\end{array}$ & $\begin{array}{l}\text { Over } \\
\text { gyp- } \\
\text { sum }\end{array}$ & $\begin{array}{l}\text { Over } \\
\text { con- } \\
\text { crete }\end{array}$ & $\begin{array}{l}\text { Over } \\
\text { gyp- } \\
\text { sum }\end{array}$ & $\begin{array}{l}\text { Over } \\
\text { con- } \\
\text { crete }\end{array}$ & $\begin{array}{l}\text { Over } \\
\text { gyp- } \\
\text { sum }\end{array}$ & $\begin{array}{l}\text { Over } \\
\text { con- } \\
\text { crete }\end{array}$ & $\begin{array}{l}\text { Over } \\
\text { gyp- } \\
\text { sum }\end{array}$ & $\begin{array}{l}\text { Over } \\
\text { con- } \\
\text { crete }\end{array}$ & $\begin{array}{l}\text { Over } \\
\text { gyp- } \\
\text { sum }\end{array}$ \\
\hline $\begin{array}{l}3 \mathrm{ACS} \\
4 \mathrm{ACS}- \\
5 \mathrm{ACS}\end{array}$ & $\begin{array}{r}L b s . \\
45 \\
60 \\
76\end{array}$ & $\begin{array}{r}L b s . \\
45 \\
60 \\
76\end{array}$ & $\begin{array}{l}\text { Lbs. } \\
7.5 \\
7.5 \\
7.5\end{array}$ & $\begin{array}{r}\text { Lbs. } \\
15 \\
15 \\
15\end{array}$ & $\begin{array}{c}\text { Lbs. } \\
130 \\
150 \\
170\end{array}$ & $\begin{array}{c}\text { Lbs. } \\
130 \\
150 \\
170\end{array}$ & $\begin{array}{c}\text { Lbs. } \\
400 \\
400 \\
400\end{array}$ & $\begin{array}{l}L b s . \\
400 \\
400 \\
400\end{array}$ & $\begin{array}{c}\text { Lbs. } \\
300 \\
300 \\
300\end{array}$ & $\begin{array}{r}L b s . \\
300 \\
300 \\
300\end{array}$ \\
\hline
\end{tabular}

In laying the roofing, the following general requirements shall apply:

The asphalt shall not be heated above $400^{\circ} \mathrm{F}$. and shall be hot when the felts are laid.

The layers of felt shall be laid so as to be free from wrinkles and buckles.

The surfacing material shall always be dry when applied and in addition shall be heated in cold weather.

\section{ADDITIONAI INFORMATION}

Roofing of types 4 AWS and 5 AWS is adapted to board sheathing with inclines not exceeding 3 inches to the foot or precase gypsum slabs with inclines not exceeding 1 inch to the foot and shall not be used on inclines steeper than these. 
Roofing of the type $4 \mathrm{AWS}$ should not require repairs or renewals on account of the action of the elements for a period of 10 years. and roofing of type 5 A WS should so endure for 20 years.

Roofing of types $3 \mathrm{ACS}, 4 \mathrm{ACS}$, and $5 \mathrm{ACS}$ is adapted to concrete and poured gypsum surfaces with inclines not exceeding 3 inches to the foot and shall not be used on inclines greater than this.

Such roofing is durable and should not require repairs or renewals on account of the action of the elements, in case of type 3 ACS, for 10 years, and in the case of types $4 \mathrm{ACS}$ and $5 \mathrm{ACS}$ for 20 years.

While the surface of roofing of type $5 \mathrm{ACS}$ will require some attention in about the same time as roofing of type $4 \mathrm{ACS}$, if the roofing is allowed to stand until complete failure, longer life can be expected from type $5 \mathrm{ACS}$ than from type $4 \mathrm{ACS}$.

(The full text of these specifications includes specific requirements to be met and various methods to be followed in the laying of the various types of roofing.)

Federal Specifications Board.

1. Specification No. 214, United States Government master specification for asphalt prepared roofing, September 30, 1924.

2. Specification No. 423, United States Government master specification for roofing, prepared, asphalt and asbestos, slate surfaced, July 9, 1926.

3. Specification No. 296, United States Government master specification for slate surfaced, asphalt prepared roofing and shingles, May 20, 1925.

(The above specifications are uniform as to the following:)

\section{METHODS OF SAMPIING AND TESTING}

1. The methods of sampling and testing of these specifications conform in substance with A. S. T. M. D228, with additions and variations as indicated under the individual specifications.

\section{PACKING AND MARKING}

This roofing shall be put up in rolls of 108 or 216 square feet, as specified in the order. (Rolls for No. 296 shall be put up in the quantity of 108 square feet only.) No roll shall contain more than two pieces of roofing nor shall there be more than 3 per cent of such rolls in a shipment. The rolls shall be tightly wound and their ends shall be protected with metal caps, scrap roofing cloth, or other suitable means. Each roll shall be wrapped with paper and shall be plainly marked with the manufacturer's name and brand and shall contain printed directions for laying. Nails and lap cement shall be furuished either in each roll or in bulk, as specified in the contract. Roofing for export shipment shall be packed as mutually agreed upon by purchaser and seller.

The specifications are individually distinct, as follows:

Specification No. 214:

\section{CLASSES}

This asphalt-prepared roofing shall be furnished in two weights, medium and heavy, as specified in the order, proposal, or contract.

\section{MATERIAL AND WORKMANSHIP}

This asphalt-prepared roofing shall be composed of rag-roofing felt saturated and coated on both sides with asphaltic compounds and surfaced with finely powdered talc or mica, which must prevent sticking in the rolls.

\section{DETAII REQUIREMENTS}

(a) Appearance.-The surface shall be smooth or finely veined in appearance. Deeply ribbed roofing will not be acceptable. The coating shall be uniformly applied on both sides and up to the edge of the sheet, and no uncoated spots or defects such as blisters, or large lumps of coating shall be perceptible.

(b) Width and area of roll.-

Width. -32 or 36 inches plus or minus one-quarter inch

Area.-Not less than 108 square feet or 216 square feet as specified in order.

(c) Average gross weight per 108 square feet.-

Medium. -45 pounds.

Heavy -55 pounds.

(d) Weight per roll of wrapping, packing, nails, and cement.-Maximum, 3 pounds.

(e) Weight per 108 square feet of roofing exclusive of wrappings, packings, nails, and cement.-

\begin{tabular}{|c|c|c|}
\hline & Medium & Heavy \\
\hline $\begin{array}{l}\text { A rerage weight per_108 square feet } \\
\text { Minimum weight per } 108 \text { square feet }\end{array}$ & $\begin{array}{c}\text { Pounds } \\
42 \\
39.0\end{array}$ & $\begin{array}{c}\text { Pounds } \\
52 \\
48.3\end{array}$ \\
\hline
\end{tabular}

(f) Pliability at $\gamma \gamma^{\circ} F$.-No cracking shall occur on bending through an are of $180^{\circ}$ over a $2 / 5$-inch mandrel

(g) Behavior on heating at $176^{\circ} \mathrm{F}$.-No slidiug, blistering, or absorption of the surface coating shall occur.

(h) Minimum weight of moisture-free, desaturated felt per 108 square feet.-

Medium. -10.8 pounds.

Heavy.-13.5 pounds.

(i) Thickness of desaturated felt, minimum.-

Medium. -0.048 inch.

Heavy. -0.060 inch.

(j) Ash of desaturated felt.-Maximum 10 per cent.

(k) Saturation of felt, minimum.-

Medium.-130 per cent.

Heavy. -140 per cent.

The sheet of felt shall be thoroughly and uniformly saturated.

(l) Weight of mineral surfacing and mineral matter in coating 108 square feet.-Maximum, 3 pounds.

(m) Nails.- The gage of the wire shall be 10 to 12 , inclusive; the heads shall be not less than three-eighths inch in diameter and not less than 0.025 inch thick. The shank shall be between three-fourths and 1 inch long, and its lower end pointed. The nails shall be zine coated.

Not less than an average of 250 nails shall be furnished for each 108 square feet of 36 -inch roofing, and not less than an average of 275 nails per 108 square feet of 32-inch roofing.

(n) Lap cement.-The lap cement shall be of such nature as to firmly bind the laps without injurious effect upon the prepared roofing. It shall consist of asphalt or coal tar pitch base dissolved in a volatile solvent. Three-fourths of a pint per 108 square feet shall be furnished for 36-inch roofing and 1 pint per 108 square feet for 32-inch roofing.

\section{METHODS OF SAMPIING AND TESTING}

\section{(Additional)}

The roofing shall not stick and shall be free from visible external defects, such as holes, ragged or 
untrue edges, breaks, rents, cracks, indentations, or lumps of coating. The coating shall be applied on both sides and shall extend to the edge of the sheet.

Compute the area and weight per 108 square feet of each roll selected and from these weights compute the average weight of the shipment. Should the weight of any 108 square feet be less than the minimum weight specified, 39.0 pounds for medium and 48.3 pounds for heavy, it shall be cause for the rejection of the whole shipment. Compute also the average weight of the packing materials, nails, and cement.

Pliability.-Cut five strips 1 inch wide and 6 inches long in the direction of the fiber grain and immerse them in water at $25^{\circ} \mathrm{C}$. $\left(77^{\circ} \mathrm{F}\right.$.) for 10 to 15 minutes.

Remove the strips from the water and immediately bend each strip over a mandrel two-fifths-inch in diameter through an arc of $180^{\circ}$ at a uniform speed in approximately two seconds time. The roofing shall not crack when so bent.

Specification No. 423:

\section{GRADE}

Slate-surfaced asphalt and asbestos prepared roofing shall be of one grade only, as hereinafter described.

\section{MATERIAL AND WORKIMANSHIP}

This prepared roofing shall be composed of asbestos roofing felt containing at least 85 per cent of asbestos fiber, saturated and coated with asphaltic compounds. One side shall be surfaced with granulated slate. The other side may be dusted with talc or mica to prevent sticking in the rolls.

\section{DETAIL REQUIREMENTS}

1. Color and appearance.-The color and distribution of the slate shall be uniform throughout and when so specified shall substantially match a sample mutually agreed upon by buyer and seller. The coating and slate surfacing must be continuous and must extend to the edge of the sheet on one side and may have an unsurfaced lapping edge approximately 2 inches wide on the other side. The edges shall not be ragged.

2. Width and area of roll.-Width, 32 or 36 inches; plus or minus one-fourth inch; area, 108 square feet.

3. Weight per 108 square feet exclusive of wrapping, packing, nails, and cement.-Minimum, 80 pounds.

Notw.-Should the weight of any roll per 108 square feet be less than the mininum weight specified ( 80 pounds), it shall be cause for the rejection of the whole shipment.

4. Volatile matter at $80^{\circ} \mathrm{C}$. $\left(176^{\circ} \mathrm{F}\right.$.) for two hours.-Maximum, 11/2 per cent.

5. Behavior on heating to $80^{\circ} \mathrm{C}$. $\left(1 \pi 6^{\circ} \mathrm{F}\right.$.) for two hours.-There shall be no flowing, sagging, blistering, or absorption of the surface coating. The particles of slate shall not become detached and fall off.

6. Weight of moisture-free, desaturated felt per 108 square feet.-Minimum, 18 pounds.

7. Thickness of desaturated felt.-Minimum, 0.040 inch.

8. Ash of desaturated felt.-Minimum, 70 per cent.

9. Weight of saturant per 108 square feet.-Minimum, 0.60 times the weight of the moisture-f ree felt.

10. Weight of granulated slate per 108 square feet.-Not more than 35 pounds.

11. Composition of felt.-The felt shall contain at least 85 per cent of asbestos fiber. The remainder may be hair, cotton rags or linters, starch, or other suitable binding materials.
Nails and lap cement requirements are the same as given in F. S. B. specification No. 214, page 165. Specification No. 296:

This specification is substantially the same as that of the American Society for Testing Materials, serial designation D225 (see p. 154), standard specifications for slate-surfaced asphalt roll roofing and slate-surfaced asphalt shingles, with the exception of the following:

\section{PHYSICAL PROPERTIES}

(Additions and variances)

1. Thickness of moisture-free desaturated felt.Minimum, 0.050 inch.

2. Trimmings.-If desired, trimmings shall be as specified in order.

3. Weight per roll of wrapping, packing, etc.Maximum, 5 pounds.

Note.-The two most common varieties of shingles are the single or individual shingle, 8 by $12^{3} \%$ inches, and the strip or four-in-one shingle, 10 by $321 / 8$ inches, with four cut-outs $1 / 2$ by 4 inches.

\section{METHODS OF SAMPLING AND TESTING}

(Additions and variances)

\section{DETERMINATION OF WEIGHT}

(1) Slate-surfaced asphalt shingles.-Remove the wrappers and other packing material from each package of shingles selected and weigh the shingles and also the packing material to the nearest quarter pound. Count the number of shingles or strips in each package taken, compute the area, and from these results and the weight compute the weight per 108 square feet. A minimum weight of less than 80 pounds shall be cause for rejection of the whole shipment. Observe the color and general appearance of the shingles while counting them and compare with the mutually agreed upon sample. The color and distribution of the slate shall be uniform over the entire surface of the shingles. The coating and surfacing must extend to the edge of each shingle. The edges shall not be ragged. No patches of coating shall show through the slate surfacing nor shall the slate and coating be broken from the edges; the shingles shall be of the specified shape and shall not stick together in the package to such an extent as to tear off or loosen the coating when unpacked.

\section{SAMPLING FOR LABORATORY EXAMINATION}

(2) Slaie-surfaced asphalt shingles.-For sampling, take in the case of individual shingles, two shingles from each package examined, or in the case of strip shingles, one strip from each two packages examined. Caref ully wrap, label, and transmit to the testing laboratory. The sample submitted to the laboratory shall contain at least six individual shingles or two strips. Samples similar to those transmitted to the testing laboratory should be taken and retained for use in case of dispute.

\section{LABORATORY EXAMUNATION-WEIGHT}

Weigh each shingle or strip submitted to the nearest gram (15 grains); measure to the nearest one-sixteenth inch and from the weights and area so obtained compute the weight per 108 square feet of the roofing fabric represented by each shingle or strip.

\section{PACKING AND MARKING}

Slate-surfaced asphalt shingles shall be packed in one-fourth or one-half square cartons or, as an 
alternative, shall be wrapped in beavy paper or board ends and tied with substantial rope, wire, or steel bands. The packages shall be plainly labeled with the manufacturer's name and brand and shall contain printed directions for laying. Shingles for export shipment shall be packed as mutually agreed upon by the purchaser and seller.

Federal Specifications Board, specification No. 294, United States Government master specification for asphalt-saturated woven cotton fabric for waterproofing, May 20, 1925.

(This specification is practically identical with A. S. T. M. serial designation D173-27 (see above) as to (1) physical properties, (2) workmanship and finish, and (3) packing. The following requirements for inspection of the material shall apply:)

When required, deliveries of asphalt-saturated cotton fabric will be sampled and tested by the following methods, but the purchaser reserves the right to use any additional information to ascertain whether the requirements of the specification have been met.

1. Sampling and determination of weight and width.-The weight per square yard and the width of the material can be most accurately determined by the inspector at the time he takes the samples for transmittal to the testing laboratory.

(a) From each shipment of more than 1,000 rolls take at random a number of rolls of fabric equivalent to the one-half cube root of the total number of rolls included in the lot. If the cube root as calculated proves to be a fractional number, express it as the next higher digit. From each shipment of 1,000 rolls or less take at random five rolls in each case.

(b) Remove wrappers and other packing materials from each roll selected and weigh the roll and the packing materials, etc., separately to the nearest quarter pound, then unwind each roll and measure the length and width to the nearest quarter inch and observe the appearance of the material. Compute the area of each roll and the weights per square yard of each roll of fabric examined.

At approximately 25 feet from the end of each roll cut two samples the full width of the roll and 40 inches long, label carefully, wrap, and transmit one to the testing laboratory. Retain the other sample for use in case of dispute.

2. Laboratory examination.-(a) Appearance.Examine both sides of the sheet of saturated fabric and note the color and general appearance.

(b) Weight.-Trim the sample so that it is 36 inches long and the full width of the roll, measure accurately to one-sixteenth inch and weigh to the nearest gram (15 grains). From the measurements and weight so obtained compute the weight per square yard.

(c) Pliability.-Cut five strips 1 inch wide and 6 inches long in the direction of the warp and immerse in a cooling mixture of ice and water at $0^{\circ} \mathrm{C}$. $\left(32^{\circ}\right.$ F.) for 10 to 15 minutes. The strips shall then be removed from the water and each strip bent flat upon itself through an arc of $180^{\circ}$ at a uniform speed in approximately two seconds and then through $360^{\circ}$ flat on itself in the opposite direction.

(d) Breaking strength.-Cut 10 strips of the fabric 6 inches long by 4 inches wide, taking five in the direction of the warp and five in the direction of the filling, respectively. Care shall be taken that no two strips include the same threads. No sample for testing shall be taken at less than 8 inches from either selvage. If the width of the goods does not admit of cutting pieces as stated, they shall be taken as near the center as possible. Test both sets of strips at $21^{\circ} \mathrm{C} .\left(70^{\circ} \mathrm{F}\right.$.) in a Scott or similar testing machine of the inclination balance type, having a capacity of from 100 to 150 pounds. The 1 by 1 by 3 inch grab method of testing shall be used and is defined as follows: The lower half of each pair of jaws of the machine shall be 2 inches or more in width and the upper half shall be 1 inch in width. The jaws shall be plain, smooth, and flat, with edges slightly rounded to prevent cutting. The initial length of the test pieces between the jaws of the testing machine shall be 3 inches and the pulling jaw snall travel at a uniform rate of 12 inches per minute. In making the test if any strip breaks nearer than one-half inch to either jaw the reading shall be disregarded and an additional strip on the same threads, if possible, shall be tested in its place. The average of the five readings from strips cut in one direction shall be taken as the breaking strength of the sample in that direction.

(e) Volatile matter.-Cut two strips of asphaltsaturated fabric 6 inches wide and 12 inches long. Weigh each strip and suspend it for five hours in an oven maintained at $105^{\circ} \mathrm{C}$. $\left(221^{\circ} \mathrm{F}\right.$.) plus or minus $3^{\circ} \mathrm{C}$. $\left(5^{\circ} \mathrm{F}\right.$.), remove from the oven, cool in a desiccator, and weigh. Compute the loss in weight and average the two results.

(f) Weight of desaturated fabric.-Cut a 2-inch strip the full width of the sample, measure accurately, weigh, and extract with benzol 60 in a suitable extraction apparatus until the solvent runs through colorless. Remove the desaturated fabric from the extractor and allow to remain in air until the solvent has evaporated; brush off any adhering particles of surfacing and retain. Then place the desaturated fabric in a tared weighing bottle, dry at $100^{\circ} \mathrm{C}$. $\left(212^{\circ} \mathrm{F}\right.$.) for one-half hour, cool, and weigh. Compute the weight per square yard of the desaturated fabric. The desaturated fabric so obtained is in a moisture-f ree state.

(g) Surfacing material.-Filter the extract obtained in V, $2(f)$ through a tared Gooch crucible prepared with a mat of asbestos fiber, wash all sediment from the extraction apparatus into the Gooch crucible with benzol ${ }^{60}$ (see note above), and also transfer all surfacing brushed from the fabric above in V, $2(f)$ into the Gooch crucible, wash the insoluble matter on the filter with benzol ${ }^{80}$ (see note above) until the washings run through colorless. Place the crucible with the contents in an oven at $100^{\circ}$ C. $\left(212^{\circ}\right.$ F.) for one-half hour, cool in a desiccator, weigh, and from the weights of the sample taken in V, $2(f)$, and the weight of surfacing obtained compute the weight of surfacing per square yard.

(h) Saturation of fabric.-The saturation or weight of asphalt in the saturated fabric is the difference in weight between the original sample taken for extraction in $V, 2(f)$, and the sum of the weights of desaturated fabric obtained in $V, 2(f)$, plus the weights of the surfacing material obtained in V, 2 $(f)$. The percentage saturation is obtained from the following formula:

Weight of asphalt in the saturated fabric percentage of sat-

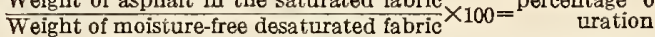

(i) Thread count.-Count the number of threads to the inch in both the warp and filling at five different places. The average of these shall be taken as the threads per inch or thread count.

(j) Character of fiber.- Select 10 threads at random from the desaturated fabrics and separate the fibers in water on a microscope slide so that the fibers are uniformly distributed. Now dry the slides so prepared until all the water is removed, cool, and add a drop of zinc-chloride solution (Herzberg solution).

60 Carbon tetrachloride or carbon bisulphide will usually give the same results, but bezzol shall be used in umpire tests in case of dispute. 
The Herzberg stain is made according to the following formula:

Solution A $\{20 \mathrm{~g}$ zinc chloride.

$$
\text { Solution } \mathrm{B}\left\{\begin{array}{l}
2.1 \mathrm{~g} \text { potassium iodide. } \\
0.1 \mathrm{~g} \text { iodine. } \\
5.0 \mathrm{cc} \text { distilled water. }
\end{array}\right.
$$

Dissolve solutions A and B separately; then mix and allow to stand several hours or until all sediment has settled out. The clear liquid is then decanted into a dark-colored bottle and is ready for use. The stain shall be kept in the dark and should be tested before use on known fibers.

Cover the stained fibers with a cover glass, draw off the excess stain, and examine under a microscope with a magnification of about 100 diameters. The fibers should all be stained a wine-red color.

Federal Specifications Board, specification No. 424, United States Government master specification for roof coating, asphalt fibrous, July 9, 1926.

\section{GRADT}

Asphalt fibrous roof coating shall be of one grade only, as hereinafter described.

\section{MATERIAL AND WORKMANSHTP}

The coating shall consist of asphaltic materials, with or without fatty oils, thinned with a volatile solvent to a heavy brushing consistency, and asbestos fiber.

\section{DETAIL REQUIREMENTS}

1. Condition in container.- The roof coating must be a smooth, homogeneous mixture. It must not separate or liver in the container.

2. Consistency.-It shall be of a heavy brushing consistency to insure a film of appreciable thickness and yet not so stiff as to prevent easy application with a 3-knot roof brush at the rate of approximately 1 gallon per 50 square feet over asphalt roofing.

3. Nonvolatile matter.-The roof coating shall yield not less than 60 per cent nonvolatile matter when $10 \mathrm{~g}$ are heated in an oven at $105^{\circ} \mathrm{C}$. $\left(221^{\circ} \mathrm{F}\right.$.) for 24 hours.

4. Asbestos fiber.-The roof coating shall contain not less than 5 per cent and not more than 15 per cent of asbestos fiber. None of the asbestos fiber shall be less than one-eighth inch in length.

5. Time of setting.-When brushed on prepared roofing and metal at the rate of 1 gallon per 50 square feet and exposed in the laboratory, the coating shall set to a tough plastic coating free from stickiness within 24 hours.

6. Behavior at $60^{\circ} \mathrm{C}$. (140 $0^{\circ}$.).-When brushed on prepared roofing at the rate of 1 gallon per 50 square feet and dried for 72 hours and then exposed to a temperature of $60^{\circ} \mathrm{C}$. $\left(140^{\circ} \mathrm{F}\right.$.) for five hours the coating shall not slip or sag and shall not be absorbed into the roofing.

7. Toughness.-When the test piece from III 6 has cooled to $25^{\circ} \mathrm{C}$. bend quickly over a mandrel $10 \mathrm{~mm}$ (two-fifths inch) in diameter. The coating shall not crack or flake from the roofing.

\section{METHODS OF SAMPLING AND TESTING}

1. Sampling.-From each shipment the inspector shall take at random a number of packages equivalent to the one-half cube root of the total number of packages in the lot. If this proves to be a fractional number, it shall be expressed as the next higher whole number. The contents of each package taken shall be thoroughly stirred until a homogeneous mixture is obtained. One pint of the coating shall then be immediately drawn from each package and transferred to a clean receptacle of suitable size, which shall be kept tightly covered except while the coating is being introduced. After all the pint samples have been taken, the contents of this container shall be thoroughly stirred and two 1-quart samples of the composite sample shall be transferred to a clean, dry container, which shall be immediately stoppered, sealed, and distinctly labeled. One sample shall be transmitted to the testing laboratory and the other shall be retained for check analysis in case of dispute.

2. Laboratory examination.- $(A)$ Condition in container.-Open the container when received at the laboratory and examine its contents. The material must be a smooth, homogeneous mixture, and there must not be any separation, caking, or livering.

(B) Nonvolatile matter.-Weigh about $10 \mathrm{~g}$ of the sample in a weighed flat-bottom metal dish about 8 $\mathrm{cm}$ in diameter or in a friction top can plug. Heat the dish with its contents in an oven maintained at $105^{\circ}$ C. $\left(221^{\circ}\right.$ F.) $\pm 3^{\circ}$ C. $\left(5^{\circ}\right.$ F.) for 24 hours, cool, and weigh. From the weight of the residue left in the dish and from the weight of the original sample taken compute the percentage of nonvolatile inatter.

(C) Asbestos fiber.-Weigh from 2 to $5 \mathrm{~g}$ of the coating and transfer to a small beaker. Add 25 to $50 \mathrm{cc}$ of carbon bisulphide, stir until thoroughly mixed and allow to stand for 15 minutes. Filter the solution through a weighed Gooch crucible prepared with a medium thick mat of paper pulp or a paper disk, using suction, if necessary, to aid filtration. Wash the residue in the crucible with cold carbon bisulphide until the washings are colorless, dry the crucible with its contents in air at room temperature until the odor of carbon bisulphide has almost disappeared, and then for one hour in an oven at $100^{\circ}$ to $105^{\circ} \mathrm{C} .\left(212^{\circ}\right.$ to $221^{\circ} \mathrm{F}$.), cool and weigh. From the weight of the insoluble matter in the crucible and the weight of the sample taken compute the percentage of the matter insoluble in carbon bisulphide and consider it as asbestos. Separate the layer of insoluble matter obtained from the mat or disk in the crucible and examine portions of it under the microscope in order to determine the length of fiber and the presence of material other than fibrous asbestos. Ignite also a weighed portion of the insoluble matter to a red heat for one-half hour, cool, and weigh. The residue obtained shall be not less than 80 per cent.

(D) Time of setting.-Brush the coating at the rate of approximately 1 gallon per 50 square feet on asphalt saturated felt and clean metal and allow to dry in the laboratory. Test the coating at points not less than $2 \frac{1}{2} \mathrm{~cm}$ ( 1 inch) from the top by touching lightly with the finger. The coating is considered to have set throughout to a tough plastic mass free from stickiness when gentle pressure of the finger does not move the coating and none of it adheres to the finger.

(E) Behavior at $60^{\circ} \mathrm{C} .\left(140^{\circ} \mathrm{F}\right.$.)-Prepare test pieces on asphalt saturated felt as in IV, $2,(D)$ and allow to dry for 72 hours in a well-ventilated room. Suspend the test pieces vertically for five hours in an oven maintained at $60^{\circ}$ C. $\left(140^{\circ}\right.$ F.). On examination at the end of this period the coating shall not show blistering, sagging, or slipping of more than one-quarter of an inch and shall not be absorbed into the felt.

(F) Toughness.-Cool the test pieces from IV, 2, $(E)$ to room temperature and bend quickly over a mandrel $10 \mathrm{~mm}$ (two-fifths of an inch) in diameter. In bending, the felt should be next to the mandrel. The coating shall not crack or flake from the roofing.

\section{NOTES}

Asphalt fibrous roof coating is intended for use in the repair and coating of asphalt and metal 
roofing and for application to concrete, masonry, and steel structures as a damp-proofing and protective coating. In the application of this material our concrete and masonry, it is advisable to apply a priming coat of asphalt primer described in United States Government master specification No. 87. (See p. 164.)

Jnderwriters' Laboratories, specifications for class $\mathrm{C}$ asphalt rag-felt sheet roofing and shingles, March, 1929.

\section{SCOPE}

These specifications cover sheet roofing and shingles composed of rag-felt saturated and coated with asphalt and surfaced with mineral surfacing material.

\section{MATERIALS}

\section{FELT}

Character.-This shall be a roofing felt produced by the "felting" vegetable and animal fibers.

Quality. - Felt of acceptable quality shall be uniformly smooth, and upon splitting or tearing on the bias, shall appear reasonably free from knots or lumps of stock which have not been beaten or shredded into fiber in the process of manufacture. It shall also be reasonably free from foreign substances, such as fragments of stone, metal, leather, rubber, straw, wood, etc.

$A s h$.-The ash in the moisture-free felt shall not exceed 10 per cent by weight.

Weight.-The "number" (that is, weight in pounds per 480 square feet) of the moisture-free (desaturated when determined from a sample of finished product) felt shall be not less than 48 for regular weight sheet roofing and shingles and 70 for heavy weight shingles.

\section{SATURANT AND COATING}

Character.-The saturating and coating compounds shall be composed principally of asphaltic materials. The presence of saponifiable constituents shall be permitted.

Flash point.-The saturating and coating compounds shall each have a flash-point of not less than $410^{\circ} \mathrm{F} .\left(210^{\circ} \mathrm{C}\right.$.) Pensky-Martens closed-cup method.

Where the Pensky-Martens closed-cup apparatus is not available for use at a factory, a modified Elliot cup having the dimensions shown in Figure 25, p. 52 and supplied with a glass cover may be used provided the minimum requirement is considered as $419^{\circ} \mathrm{F}$. $\left(215^{\circ} \mathrm{C}\right.$.) or a Cleveland open-cup apparatus (A. S. T. M. D92 (see 504.0, p. 90) may be used provided the minimum requirement is considered as $437^{\circ} \mathrm{F}$. $\left(225^{\circ}\right.$ C.).

\section{SURFACING MATERIALS}

Character.-The surfacing material for sheet roofing shall be of a noncombustible material, such as powdered talc, mica, or granulated slate. The surfacing on the weather side of shingles shall be granular crushed slate or similar material.

Granular surfacing shall be sufficiently free from fines or dust to permit the firm adhesion of the larger particles of the surfacing and provide a uniform distribution. The underside of the sheet shall be surfaced with noncombustible material, such as powered talc or mica.

Weight of mineral surfacings and mineral in coating.-Talc and mica surfaced, maximum, 2 pounds per 108 square feet; granular surfaced, maximum, 35 pounds per 108 square feet.

Note.-Should the weight exceed the above amounts, the materials shall nevertheless be acceptable, provided the finished weights per 108 square feet are increased by a like amount over the figures stipulated under "Finished weight."
FELT AFTER SATURATION

Character.-The felt shall be thoroughly and uniformly saturated and show no unsaturated spots at any point upon cutting 2 -inch strips at random across the entire sheet and splitting them open for their full length.

Weight of saturant.-The weight of the saturant introduced into sheet roofing shall be not less than 1.4 times, and in shingles not less than 1.5 times, the weight of the moisture-free felt in the same respective area.

\section{FINISHED PRODUCT}

Character.-The finished product shall be composed of a single thickness of rag-felt saturated and coated on both sides with asphaltic compound and finished with a mineral surfacing.

The finished product shall be free from visible external defects, such as holes, ragged or untrue edges and breaks, tears, cracks, protuberances, and indentations.

\section{FELT AFTER SATURATING, COATIYG, AND SURFACLNG}

Distribution of coating.-The coating shall uniformly and completely cover both sides of the material.

Pliability. - The coating of talc and granular surfaced sheet roofing shall be of such a nature that, when a strip of roofing maintained at $77^{\circ} \mathrm{F}$. $\left(25^{\circ} \mathrm{C}\right.$.) is bent through $180^{\circ}$ at a uniform speed in exactly two seconds time, four out of five samples shall not crack over a mandrel of the following size:

Smooth sheet roofing. -0.4 inch $(10 \mathrm{~mm})$ in diameter.

Granular sheet roofing.-1.0 inch $(25 \mathrm{~mm})$ in diameter.

Distribution of surfacing.-The surfacing shall be uniformly distributed over the coated felt. It shall be so distributed that any coating which may have been forced up between particles will not give rise to a discolored or mottled appearance.

Granular surfacing and coating nay be omitted for a strip along one edge of sheet roofing to provide a bare lapping edge.

Adhesion of surfacing.-The granular surfacing shall be embedded in the coating compound in such a manner that when rubbed vigorously with the hand sufficient surfacing will remain embedded to completely cover the coating.

Bchavior on heating. - When suspended vertically in an oven heated to $176^{\circ} \mathrm{F}$. $\left(80^{\circ} \mathrm{C}\right.$.) for two hours there shall be no flowing, sagging, blistering, or absorption of the surface coatings. Granular surfacings shall not slide more than orre sixteenth of an inch.

The loss in weight (volatile matter) shall not exceed 1.5 per cent.

Stickiness.-The coating and surfacing shall be of such a nature that sheet roofing will not crack or stick together so as to cause tearing or damage upon being unrolled at atmospheric temperatures above $50^{\circ} \mathrm{F}$. ( $\left(10^{\circ}\right.$ C. $)$, and that shingles will not adhere together in the bundles so as to tear off or loosen the coating when unpacked.

\section{DIMENSIONS}

Sheet roofing.-Sheet roofing may be made in 36inch, 32-inch, or smaller widths.

Individual shingles.-Individual shingles shall average not less than $12 \frac{1}{2}$ inches long by 8 or 9 inches wide.

The variation in the dimensions of the shingles shall not exceed one-fourth inch under those specified.

The use of labels on shingles of other than the individual rectangular type (laid not more than 4 
inches to the weather) is not authorized for any manufacturer unless such authorization is contained in supplement to this standard.

Special shingles.-Each special shingle must be submitted to the laboratories for investigation, and the use of labels is permitted only when authorized in the form of a supplement to this standard.

\section{WEIGH'T}

The weight of the finished product, exclusive of wrapping, packing, loose mineral surfacing, nails, and cement, shall not be less than the following minimum for individual packages and average for the lot:

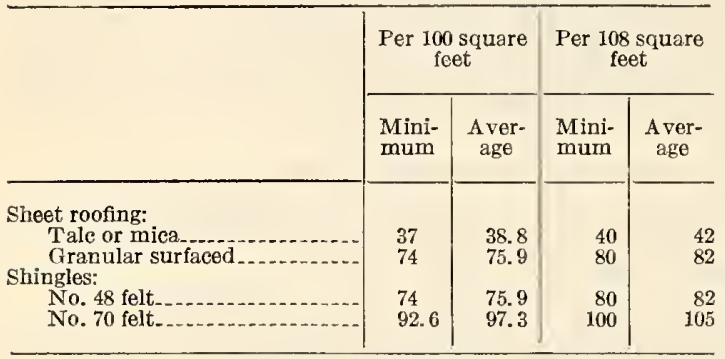

\section{LAP CEMENT}

Character.-The lap cement shall be composed of bituminous materials dissolved in a volatile solvent and shall be of such a nature as to firmly bind the laps without injurious effect upon the roofing.

Flash point.-The cement shall have a minimum flash point of $71^{\circ} \mathrm{F}$. $\left(21.5^{\circ} \mathrm{C}\right.$.) (Tagliabue closed cup tester).

Quantity. - Sheet roofing in 36-inch width shall be supplied with not less than three-fourths pint of lap cement per "square," 32 -inch width with 1 pint, and in 16 and 18 inch widths with $1 \frac{1 / 2}{2}$ to 2 pints of lap cement. Roofer's rolls shipped in carload lots need not contain lap cement.

\section{NAILS FOR SHEET ROOFING}

Dimensions. - The diameter of wire shall not be greater than No. 10 (0.135 inch) nor thinner than No. $12(0.105$ inch); the heads shall not be less than one-eighth inch in diameter nor less than 0.025 inch thick; the shank shall be not less than three-fourths inch long and shall be either smooth or barbed, and if the latter, then the barbings shall not increase the diameter of the lower two-thirds more than 0.01 inch; the lower end of the shank shall be pointed.

Protective coating. - Nails shall be zinc coated with not less than one-half ounce nor more than $1 \frac{1}{4}$ ounces per square foot of nail surface. Hot galvanized, electro-galvanized, or sherardized coating will be accepted.

Quantity.-There shall be furnished per "square" an average of not less than 252 nails for 36-inch, 275 for 32 -inch, 504 for 18 -inch, and 550 for 16 inch roll roofings, respectively. "Roofer's rolls" shipped in carload lots need not contain nails.

\section{PACKING}

Sheet roofing shall be wound tightly and wrapped in a substantial package, except that the wrappers and heads may be omitted on "roofers' rolls" when shipped in carload lots. These may be tied with a stout cord in at least two places or secured by 3-inch gummed paper tape on each end.

Shingles shall be packed in cardboard or wooden containers, or otherwise wrapped to make a substantial package.

\section{INSTRUCTIONS FOR LAYING}

Instructions for laying shall be supplied with each package of sheet roofing and with each package of shingles, except as noted below.

Instructions for laying and trimmings may be omitted from "roofers' rolls" when shipped in carload lots.

The instructions should cover explicitly the manner of preparing the deck; laying the sheets or shingles; forming the joints between the sheets or shingles; nailing; applying the lap cement; flashing at walls, chimneys, vent pipes, hips, ridges, eaves, and gutters; forming valleys; and suggestions for care and maintenance of the roof covering.

Instruction sheets for laying sheet roofing and shingles shall be subject to review and acceptance by the laboratories.

\section{MARKING}

In addition to Underwriters' Laboratories inspection label as evidence of compliance with these specifications, the manufacturer's name or trade-mark or identification mark shall be on each package. Where the product is made in several plants of a given manufacturer, each package shall be marked to indicate the plant of origin.

Markings may be in code when registered with Underwriters' Laboratories. Such code markings, if used, will be embodied in a supplement to this standard.

\subsection{Asphaltic Mastic Flooring.}

(No nationally recognized specifications available.)

\subsection{Miscellaneous Specifications for As- phalt and Other Bituminous Mate- rials.}

Federal Specifications Board, specification No. 19, United States Government specification for asphalt varnish, January 2, 1923.

\section{GENERAL}

This varnish shall be composed of a high grade of asphalt fluxed and blended with properly treated drying oil and thinned to the proper consistency with a volatile solvent. It must be resistant to air, light, lubricating oil, water, and, when the contract so specifies, to mineral acids of the concentration hereinafter specified. It must meet the following requirements:

A ppearance.-Smooth and homogeneous; no livering or stringiness.

Color.-Jet black.

Flash point (closed-cup).-Not below $30^{\circ}$ C. $\left(86^{\circ} \mathrm{F}.\right)$.

Action with linseed oil.- -Varnish must mix readily to a homogeneous mixture with an equal volume of raw linseed oil.

Matter insoluble in carbon bisulphide.-Not more than 1 per cent.

Nonvolatile matter. - Not less than 40 per cent by weight.

Fatty matter.-Not less than 20 per cent of the nonvolatile. Must be liquid and not show a violet coloration by the Liebermann-Storch test.

Set to touch.-Within five hours.

Dry hard and tough.-Within 24 hours.

Toughness.-Film on metal must withstand rapid bending over a rod $3 \mathrm{~mm}$ (1/8 inch) in diameter.

Working properties.-Varnish must have good brushing, flowing, covering, and leveling properties.

Resistance to water.-Dried film must withstand cold water for 18 hours. 
Resistance to oil.-Dried film must withstand lubricating oil for six hours.

Resistance to mineral acids. ${ }^{61}$-Dried film must withstand action of the following acids for six hours: Sulphuric acid, specific gravity 1.25 (about 33 per cent); nitric acid, specific gravity 1.12 (about 20 per cent); hydrochloric acid, specific gravity 1.09 (about 18 per cent).

Note.-Deliveries will, in general, be sampled and tested by the following methods, hut the purchaser reserves the right to use an additional availahle information to ascertain whether the material meets the specification.

\section{SAMPLING}

It is mutually agreed by buyer and seller that a single package out of each lot of not more than 1,000 packages be taken as representative of the whole Whenever possible an original unopened container shall be sent to the laboratory. When for any reason this can not be done, the inspector shall select a package and thoroughly mix its contents. He shall fill a 1-quart, clean, dry container from this package, securely stopper it with a new clean cork, or well-fitting cover or cap, seal, and distinctly label it. The inspector shall take a duplicate from the container sampled to be held for check in case of dispute, and, when requested, shall take a sample for the seller.

\section{IABORATORY EXAMINATION}

(a) Appearance and color.-Pour some of the thoroughly mixed sample on a clean, clear glass plate and stand in a vertical position until the excess varnish has drained off. Examine the film by transmitted light. The varnish must be smooth and homogeneous and must not show any separation or segregation of the constituents. Examine the film by reflected light. The film must be jet black in color.

(b) Flash point.-Determine with either the "tag" or Elliott closed-cup tester. The former is preferred. ${ }^{62}$

(c) Action with linseed oil.-Pour $10 \mathrm{cc}$ of the varnish into a test tube and add an equal volume of raw linseed oil conforming to Bureau of Standards Circular No. 82. Stopper the test tube and shake vigorously for several minutes. Then pour some of the mixture on a clear glass plate and stand in a vertical position. After the excess varnish has drained off examine the film by transmitted light. There shall be no separation of the oil and varnish.

(d) Matter insoluble in carbon bisulphide.-Weigh about $5 \mathrm{~g}$ of the varnish into a small beaker, add 25 cc of carbon bisulphide, and allow to stand for 15 minutes. Filter through a weighed Gooch crucible, prepared with a medium thick mat of asbestos, using suction if necessary to aid in filtration. Wash the residue in the crucible with carbon bisulphide until the washings are colorless. Dry in air at room temperature until the odor of carbon bisulphide has almost disappeared, and then for one hour in an oven at $110^{\circ} \mathrm{C}$. Cool and weigh. From the weight of the insoluble left in the crucible and the weight of sample taken calculate the percentage of insoluble in carbon bisulphide.

(e) Nonvolatile matter.-Place a portion of the sample in a stoppered bottle or weighing pipette. Weigh the container and sample. Transfer about $1.5 \mathrm{~g}$ of the sample to a weighed flat-bottomed metal dish about $8 \mathrm{~cm}$ in diameter (a friction-top can plug). Weigh the container again and by difference calculate the exact weight of the portion of sample trans-

61 Only required when the contract specifically demands asphalt

varnish that is resistant to mineral acids.
62 Directions for using the "tag" tester may be found in A. S. T. M. D56. (See 503.0, 0.43.) ferred to the weighed dish. Heat the dish with its contents in an oven maintained at $105^{\circ}$ to $110^{\circ} \mathrm{C}$. for three hours. Cool and weigh. From the weight of the residue left in the dish and the weight of the sample taken calculate the percentage of nonvolatile residue.

(f) Fatty matter.-Weigh about $5 \mathrm{~g}$ of the varnish into a wide-mouthed flask, add $50 \mathrm{cc}$ of benzol and $5 \mathrm{~g}$ of clean, fine silica sand and heat under a reflux condenser on a steam bath until the varnish is entirely dissolved. Add 25 ce of ethyl alcohol denatured with methyl alcohol and $25 \mathrm{cc}$ of a $0.5 \%$ alcoholic caustic soda solution and continue boiling under the reflux condenser for one-half hour. Remove the condenser and evaporate the solution to dryness. Add to the residue in the flask $50 \mathrm{cc}$ of distilled water and heat until the residue is disintegrated. Filter the water solution of the soaps. Repeat this operation with $25 \mathrm{cc}$ portions of water until the residue is completely disintegrated and the wash water is clear and colorless.

Combine the filtrates (the soap solution and washings), acidify with hydrochloric acid, and heat until the fatty acids and any emulsified asphalt separate and rise to the top and the water below is clear. Cool, transfer to a separatory funnel, and extract three times with $25 \mathrm{cc}$ portions of ether. Combine the etler extracts and wash with water until free from acid. Filter the ether extracts through paper into a beaker and wash the residue on the paper with ether until the washings run through colorless. Evaporate the ether solutions to dryness.

Add 10 to $15 \mathrm{cc}$ of 95 per cent ethyl alcohol to the residue in the beaker and warm on the steam bath. Cool to room temperature and filter through paper into a tared flask or dish. Repeat this operation with 5 ec portions of 95 per cent ethyl alcohol until the alcohol remains colorless. Finally wash the residue on the paper with 95 per cent ethyl alcohol until the washings run through colorless. Evaporate the alcoholic solution to dryness on a steam bath and heat for an hour in an oven at $105^{\circ} \mathrm{C} .\left(221^{\circ} \mathrm{F}\right.$.). Cool and weigh. From the weight of the residue in the flask and the weight of the original sample calculate the percentage of fatty matter.

(Sometimes the residue obtained after saponification and the evaporation of the benzol and alcohol from the saponifying mixture is not completely disintegrated by boiling with water. In that case extract with water until nothing further dissolves and then dry. Dissolve in benzol, using heat if necessary, and wash the benzol solution several times with water. Heat the washings until the odor of benzol has disappeared and add to the soap solution before acidifying.)

The fatty matter obtained above must be a clear, amber-colored liquid. A fugitive violet color shall not be obtained when the fatty matter is subjected to the following test: Dissolve a small amount of the fatty matter in 5 ce of acetic anhydride, warming if necessary to aid solution. Cool, draw off the acetic anhydride solution, and add a drop of sulphuric acid, 1.53 specific gravity.

(g) Drying time.-Pour the varnish on a clean glass plate not less than $15 \mathrm{~cm}$ (6 inches) long and $10 \mathrm{~cm}$ (4 inches) wide. Place the plate in a nearly vertical position in a well-ventilated room, but not in the direct rays of the sun. The temperature of the room should be from $21^{\circ}$ to $32^{\circ} \mathrm{C}$. $\left(70^{\circ}\right.$ to $90^{\circ} \mathrm{F}$.). Test the film at points at not less than $2.5 \mathrm{~cm}$ ( 1 inch) from the edges of the film by touching lightly with the finger. The varnish is considered to have set to touch when gentle pressure of the finger shows a tacky condition, but none of the varnish adheres to the finger. The varnish is considered to have dried hard when the pressure that can be exerted between the thumb and finger does not move the film or leave a mark which remains noticeable after the spot 
is lightly polished. If rapid light rubbing breaks the surface, the sample is not considered to have satisfactorily dried hard. In case the test shows time of setting to touch or drying hard more than 5 and 24 hours, respectively, two additional tests shal!? be run on different days, and if the varnish does not meet the above drying and hardening requirements on both of these additional tests it shall be considered unsatisfactory. In cases where different laboratories fail to agree on the drying test, due to different atmospheric conditions, and umpire tests are necessary, such tests shall be made in a well-ventilated room maintained at a temperature of $70^{\circ} \mathrm{F}$. and relative humidity of 65 per cent saturation.

(h) Toughness.-Flow the varnish on one side of a dry steel plate that has previously been cleaned of all scale, rust, and grease. This plate should be about $0.4 \mathrm{~mm}(0.016 \mathrm{inch})$ thick, and 10 by $15 \mathrm{~cm}$ (4 by 6 inches) will be found of convenient size. Let the test piece dry in a vertical position, not in direet rays of the sun, in a well-ventilated room at a temperature not below $21^{\circ} \mathrm{C}$. $\left(70^{\circ} \mathrm{F}\right.$.) for a period of not less than six days, Now bring the

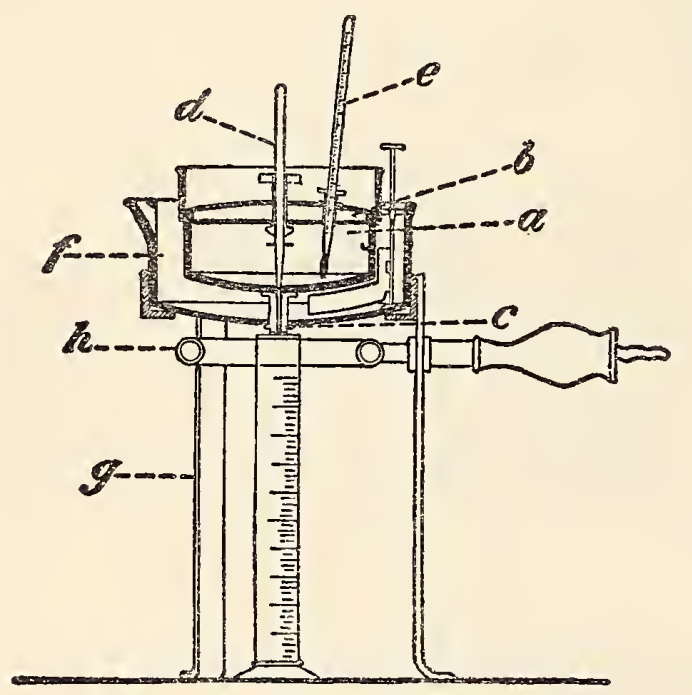

Figure 76.-Engler viscosimeter

test piece to a temperature between $21^{\circ}$ and $24^{\circ} \mathrm{C}$. $\left(70^{\circ}\right.$ to $75^{\circ} \mathrm{F}$.) and, with the varnish film on the outside, bend rapidly over a rod $3 \mathrm{~mm}$ (1/8 inch) in diameter. The film must show no evidence of cracking or flaking.

(i) Working properties.-A clean piece of steel plate similar to that used for testing the toughness of the varnish shall be used for determining the working properties. The plate shall be thoroughly cleaned of all grease and rust and dried. It shal then be laid in a horizontal position and one coat of the varnish applied by brushing. The varnish shall work easily under the brush, showing no tendency to draw or pull, and shall flow out to a smooth, glossy, jet-black film, free from brush marks, blisters, pinholes, or other defects. Test pieces prepared in the above manner and allowed to dry in a horizontal position, not in the direct rays of the sun, in a wellventilated room, at a temperature not below $21^{\circ} \mathrm{C}$. $\left(70^{\circ} \mathrm{F}\right.$.) for a period of not less than six days, shall be used for testing the resistance of the varnish to cold water and lubricating oil.

(j) Resistance to water.-A test piece, prepared and dried as under (i), shall be inclined at an angle of $45^{\circ}$ to the vertical, and a gentle stream of cold tap water with a temperature of about $25^{\circ} \mathrm{C} .\left(77^{\circ} \mathrm{F}\right.$.) allowed to flow for 18 hours down the middle of the varnished surface. After wiping off with a soft cloth or chamois skin any deposit due to the tap water the varnish must show no whitening, dulling, softening, or other visible defects.

(k) Resistance to lubricating oil.-A test piece prepared and dried as under (i) shall be laid flat and in at least two different places several drops of locomotive engine lubricating oil ${ }^{63}$ allowed to stand in contact with the film for six hours. During the test the spots of oil shall be covered with small watch glasses. After wiping off the oil with cotton waste no softening or other deterioration of the film due to the lubricating oil shall be perceptible.

(l) Resistance to mineral acid.-A piece of dry steel plate free from scale, rust, and grease shall be used for this test. It shall be laid in a horizontal position and one coat of the varnish applied by brushing. This coat of varnish shall then be allowed to dry for 24 to 48 hours, when a second coat shall be applied. The second coat shall be allowed to dry, as in (i), for a period of not less than six days before testing. The test pieces shall then be laid flat and in different places several drops each of sulphuric acid, specific gravity 1.25 , nitric acid, specific gravity 1.12 , and hydrochloric acid, specific gravity 1.09 , shall be allowed to remain on the surface of the film for six hours at room tempcrature, about $21^{\circ} \mathrm{C}$. $\left(70^{\circ} \mathrm{F}\right.$.). During the test the drops of acid shall be covered with small watch glasses to prevent evaporation. After six hours the acid shall be washed off with tap water, and after drying for one hour the spots previously in contact with the acid examined. They shall show no appreciable change in-hardness. The film shall then be allowed to dry for 12 hours, when it shall again be examined. The spots exposed to the acid shall show no disintegration or browning, and their luster shall not be impaired. A slight bloom around the area of the spot exposed to the acid shall not be considered an indication of failure. The film shall then bc removed with earbon tetrachloride, chloroform, or carbon bisulphide and the metal examined. It shall show no corrosion.

\section{BASIS OF PURCHAST}

Asphalt varnish shall be purchased by volume, the unit being a gallon of 231 cubic inches at $15.5^{\circ} \mathrm{C}$. $\left(60^{\circ}\right.$ F. $)$. The volume may be determined by measure or, in case of large deliveries, it may be easier to determine the net weight and specific gravity at $15.5 / 15.5^{\circ} \mathrm{C}$. $\left(60 / 60^{\circ} \mathrm{F}\right.$.) of the delivery. The weight per gallon in pounds can then be determined by multiplying the specific gravity by 8.33 . The net weight in pounds divided by the weight per gallon gives the number of gallons.

\subsection{ROAD OILS.}

\subsection{General Items.}

United States Department of Agriculture, Bulletin 1216 , method of test for specific viscosity, 1928 .

1. The viscosity of fluid bituminous road materials may be determined at any suitable temperature by means of the Engler viscosimeter. This apparatus is shown in Figure 76, and may be described as follows: $a$ is a brass vessel for holding the material to be tested, and may be closed by the cover $b$. To the conical bottom of $a$ is fitted a conical outfiow tube, $c$, exactly $20 \mathrm{~mm}$ long, with a diameter at the top of $2.9 \mathrm{~mm}$ and at the bottom of $2.8 \mathrm{~mm}$. This tube can be closed and opened by the pointed hardwood stopper $d$. Pointed metal projections are placed on the inside of $a$ at equal distances from the bottom, and serve for measuring the charge of

$63 \mathrm{~A}$ straight mineral oil having a viscosity (Saybolt universal) of about 75 seconds at $210^{\circ} \mathrm{F}$. 
material, which is 240 cc. The thermometer $e$ is used to ascertain the temperature of the material to be tested. The vessel $a$ is surrounded by a brass jacket, $f$, which holds the material used as a heating bath, either water or cottonseed oil, according to the temperature at which the test is to be made. A tripod, $g$, serves as a support for the apparatus and also carries a ring burner, $h$, by means of which the bath is directly heated. The measuring cylinder of 50 ce capacity, which is sufficiently accurate for work with road materials, is placed directly under the outflow tube.

2. As all viscosity determinations should be compared with that of water at $25^{\circ} \mathrm{C}$., the apparatus should be previously calibrated as follows: The cup and outlet tube should first be scrupulously cleaned. A piece of soft tissue paper is convenient for cleaning the latter. The stopper is then inserted in the tube and the cup filled with water at $25^{\circ}$ C. to the top of the projections. The measuring cylinder should be placed directly under the outfiow tube, so that the material, upon flowing out, will not touch the sides, and the stopper may then be removed. The time required for $50 \mathrm{cc}$ to run out should be ascertained by means of a stop watch, and the results so obtained slould be checked a number of times. The time required for $50 \mathrm{cc}$ of water should be about 11 seconds.

3. Bituminous road materials are tested in the same manner as water, and the temperature at which the test is made is controlled by the bath. The material should be brought to the desired temperature and maintained there for at least three minutes before making the test. The results are expressed as specific viscosity compared with water at $25^{\circ} \mathrm{C}$, as follows:

Specific viscosity at $A^{\circ} \mathrm{C}=\frac{\text { Seconds for passage of } 50 \mathrm{cc} \text { at } \mathrm{A}^{\circ} \mathrm{C} \text {. }}{\text { Seconds for passage of } 50 \mathrm{cc} \text { of water at }}$ 505.21 Oil for Cold Application. able.

(No nationally recognized specifications avail-

\subsection{Oil for Hot Application.}

Federal Specifications Board, specification No. 275, United States Government master specification for road oil for hot application, type $\mathrm{OH}-1-25$, May 8, 1925.

\section{GRADE}

One grade only is covered by this specification.

\section{MATERIAL AND WORKMANSHIP}

The material supplied under this specification shall be a road oil prepared from suitable asphaltic petroleum.

\section{GENERAL REQUIREMENTS}

The road oil shall be homogeneous, free from water, and shall not foam when heated to $120^{\circ} \mathrm{C}$. $\left(248^{\circ} \mathrm{F}\right.$.).

\section{DETAIL REQUIREMENTS}

\section{PHYSICAL AND CHEMHCAL PROPERTIES}

The road oil shall meet the following requirements:

1. Specific gravity $25^{\circ} / 25^{\circ}$ C. $\left(77^{\circ} / 77^{\circ}\right.$ F.), not less than 0.970 .

2. Flash point, not less than $80^{\circ}$ C. $\left(176^{\circ} \mathrm{F}\right.$.).

3. Specific viscosity $100^{\circ} \mathrm{C}$. $\left(212^{\circ} \mathrm{F}\right.$.), not more than 60 .

4. Float test at $32^{\circ}$ C. $\left(89.6^{\circ}\right.$ F.), not less than 60 seconds.

5. Loss at $163^{\circ} \mathrm{C}$. $\left(325^{\circ} \mathrm{F}\right.$.) $50 \mathrm{~g}$, five hours, not more than 10 per cent. (a) Float test of residue $50^{\circ}$ C. $\left(122^{\circ} \mathrm{F}\right.$.), not less than 60 seconds.

6. Bitumen (soluble in carbon disulphide), not less than 99.5 per cent.

7. Per cent of bitumen insoluble in $86^{\circ} \mathrm{B}$. naphtha, not less than 6 per cent.

\section{METHODS OF TESTING}

Tests of the physical and chemical products of the road oil shall be made in accordance with the following methods:

1. Specific gravity, A. S. T. M. D70. (See 505.0, p. 125.)

2. Flash point, A. S. T. M. D92. (See 504.0, p. 90.$)$

3. Specific viscosity (on first $50 \mathrm{cc}$ ), United States Department of Agriculture Bulletin 1216. (See 505.20 , p. 172.)

4. Float test, A. S. T. M. D139. (See 505.0, 7. 127.)

5. Loss on heating, A. S. T. M. D6. (See 505.0, p. 119.)

6. Bitumen (soluble in carbon disulphide), A. S. T. M. D4. (See 505.0, p. 117.)

7. Bitumen insoluble in naphtha, United States Department of Agriculture Bulletin 1216. (See 505.0, p. 144.)

\section{ADDITIONAL INFORMATION}

1. The material covered by this specification is intended for use primarily in the hot surface treatment of macadam, gravel, or shell roads. It may also be used in the maintenance of bituminous macadam roads.

2. In connection with this specification, see United States Government master specification, F. S. B. No. 280 for Refined Tar for Hot Application. (See 505.32, p. 177.)

\subsection{Oil Asphalt. (See 505.14.)}

\subsection{Miscellaneous Specifications for Road Oils.}

American Society for Municipal Improvements, temporary substitute for road oil, 1922.

See A. S. M. I. specifications for broken stone and gravel roads, section $31,512.15$, page 249 .

American Society for Municipal Improvements, specifications for broken stone and gravel roads, 1922.

See 512.15 , page 245 .

(This specification contains requirements for certain types of oils and tars for road construction.)

American Society for Testing Materials, tentative specifications for calcium chloride for dust prevention, serial designation D98-22T, 1922 .

1. These specifications cover calcium chloride to be applied to the surface of highways as a dust preventive.

\section{PROPERTIES AND TESTS}

2. The calcium chloride shall be in the form of loose dry lumps or flakes, and fine enough to feed readily through the common forms of spreaders used in road work, and when tested by means of laboratory screens and sieves shall meet the following requirements:

Passing 3/8-inch screen

Retained on $1 / 2$-inch screen Passing No. 20 sieve...

3. Ten grams of the material dissolved in $100 \mathrm{cc}$ of boiling water shall show less than 1.0 per cent insoluble residue. Not more than $1.0 \mathrm{cc}$ of normal acid shall be required to neutralize the alkalinity of the filtrate, using methyl orange. The chemical 
composition shall conform to the following requirements.

$\mathrm{CaCl}_{2}$ (anhydrous) -...-... Not less than 73 per cent. $\mathrm{MgCl}_{2}$

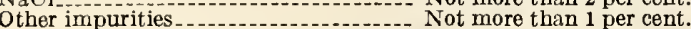

The percentage of calcium chloride shall be calculated from the percentage of calcium in the filtrate above referred to, after making a deduction for the $\mathrm{SO}_{4}$, which shall be assumed to be in combination as $\mathrm{CaSO}_{4}$.

\section{PACKAGES}

4. The calcium chloride shall be delivered in airtight moisture-proof bags or sacks containing approximately 100 pounds each, or in air-tight steel drums weighing not more than 350 pounds each. The name of the manufacturer, the lot number, the approximate net weight, and the percentages of calcium chloride guaranteed by the manufacturer shall be plainly marked on each container.

\section{INSPECTION}

5. Every facility shall be provided the purchaser should he elect to have his own representative sample the material at the plant. If the purchaser decides to sample the material after delivery in the field it is understood that a 3 per cent variation in content of $\mathrm{CaCl}_{2}$ from the chemical composition stated in section 3 shall be permissible.

6. Calcium chloride shall be rejected if it fails to pass any of the requirements of these specifications and if it has become caked or sticky in the containers before opening.

\section{BASIS OF PAYMENT}

7. Payment will be made on the basis of total number of pounds of 100 per cent calcium chloride delivered.

\subsection{TAR AND TAR PITCH.}

\subsection{General Items.}

American Wood-Preservers' Association, Manual of Recommended Practice, standard definition of tar, 1921.

Tar, in the scientific sense, may be properly defined as a nonaqueous liquid product obtained in the destructive distillation of complex organic matter. Tars vary greatly in character, both chemically and physically. They may be roughly divided into three classes:

Class A.-Tars consisting principally of compounds belonging to the aromatic series, and containing well-defined amounts of phenoloids.

Class B.-Tars consisting principally of compounds belonging to the aromatie series, but lacking phenoloids.

Class C.-Tars consisting principally of compounds belonging to the aliphatic series.

505.31 Tars for Cold Application to Roads.

American Association of State Highway Officials, tentative standard specifications for highway materials, 1929.

No. 24. Tar for surface treatment (cold application).

No. 25. Tar for surface treatment (hot application).

No. 26. Tar for use in the maintenance of eracks and for sealing joints in concrete roads.

No. 27. Tar cement.

No. 28. Tar cement for repair work (cut-back product),
1. The tar cement shall conform to the following requirements:

\begin{tabular}{|c|c|c|c|c|c|}
\hline & No.241 & No. 25 & No. 26 & No. 27 & No. 28 \\
\hline Water, maximum & 2.0 & 0.0 & 0.0 & 0.0 & 2.0 \\
\hline $\begin{array}{l}\text { Specific viscosity Engler } \\
50 \text { cc at } 40^{\circ} \mathrm{C}\end{array}$ & $25-35$ & & & & $235-80$ \\
\hline $\begin{array}{l}\text { Total bitumen, soluhle } \\
\text { in carhon disulphide, } \\
\text { minimum }\end{array}$ & 88 & 80 & 80 & 78 & $235-80$ \\
\hline $\begin{array}{l}\text { Distillation test of water- } \\
\text { free material, total dis- }\end{array}$ & 88 & & & & \\
\hline $\begin{array}{l}0^{\circ} \text { to } 170^{\circ} \mathrm{C}_{-} \text {per cent } \\
0^{\circ} \text { to } 235^{\circ} \mathrm{C}_{-} \text {. do }\end{array}$ & 3.0 & ${ }^{3} 1.0$ & ${ }^{3} 1.0$ & 31.0 & $\begin{array}{l}2-10 \\
8-20\end{array}$ \\
\hline $0^{\circ}$ to $270^{\circ} \mathrm{C}$ & 37.0 & 15.0 & 15.0 & 10.0 & $18-30$ \\
\hline $0^{\circ}$ to $300^{\circ} \mathrm{C}$ & 45.0 & 25.0 & 325.0 & 320.0 & 38 \\
\hline due from distillation & 360.0 & 365.0 & 370.0 & 370.0 & 370.0 \\
\hline Float test, $50^{\circ} \mathrm{C}$. & & $60-150$ & $75-100$ & $4100-220$ & \\
\hline
\end{tabular}

1 It is suggested that the maximum requirements for percentages of distillate for tars having a specific viscosity of 18 to 35 be as follows: 0 to $170^{\circ}$ C., 5 per cent; 0 to $270^{\circ}$ C., 30 per cent; 0 to $300^{\circ}$ C., 40 per

2 The viscosity of the material will be subject to variation within the limits designated, as may he directed by prior instructions furnished the producer, in order to meet the local and varying conditions of temperature and pavement. It is suggested that the following ranges of specific viscosity he used: For No. 24,5 to 8,8 to 13,13 to 18 , 18 to 25,25 to 35 . For No. 28,35 to 60,60 to 80 .

3 Maximum.

4 The consistency of the tar cement will he subject to variation between a 60 -second range, within the limits designated hy prior instructions furnished the producer in order to meet the local climatic conditions. It is suggested that the following ranges of float test he used: 100 to $160 ; 160$ to 220 .

2. Sampling and testing.-See 505.31, (see below) test methods listed following table of A.S. T. M. tar specifications, and for the float test, see A. S. T. M. D139,505.0, page 127 .

American Association of State Highway Officials, standard specifications for highway bridges and incidental structures, 1928.

\section{BITUMINOUS MATERIALS}

\section{BITUMUNOUS CARPETS}

Tar for first or prime coat.-The first or prime coat (cold application) for tar bituminous carpets on concrete or wood bridge floors, shall be a homogeneous tar which conforms in substance with specification No. 24, American Association of State Highway Officials (given above), except as to the specific viscosity requirement, which for this specification must be within the limits 8 to 13 .

Tar for second coat. - The second coat (hot application) for tar bituminous carpets on concrete or wood bridge floors shall conform to the requirements of specification No. 25, American Association of State Highway Officials (above).

American Society for Testing Materials, tentative specifications for high and low carbon tars for surface treatment, cold application, serial designations D104-27T and 105-27T, 1927.

The tars shall conform to the requircments listed in the accompanying table, which also gives specific requirements for 13 additional specifications for tars, classified with regard to uses and methods of application, as follows:

Tar for hot application to roads (see 505.32, p. 177), serial designations D108-27T and D109-27T.

Tar for repair work (see 505.31, p.175), serial designation D112-27T.

Tar cement (see 505.31, p. 175), serial designations D106-28T, D107-27T, D110-27T, andD111-27T.

Tar for roofing and waterproofing (see 505.36, p. 178), serial designations D42, D145, D200, D201, D251, and D252. 


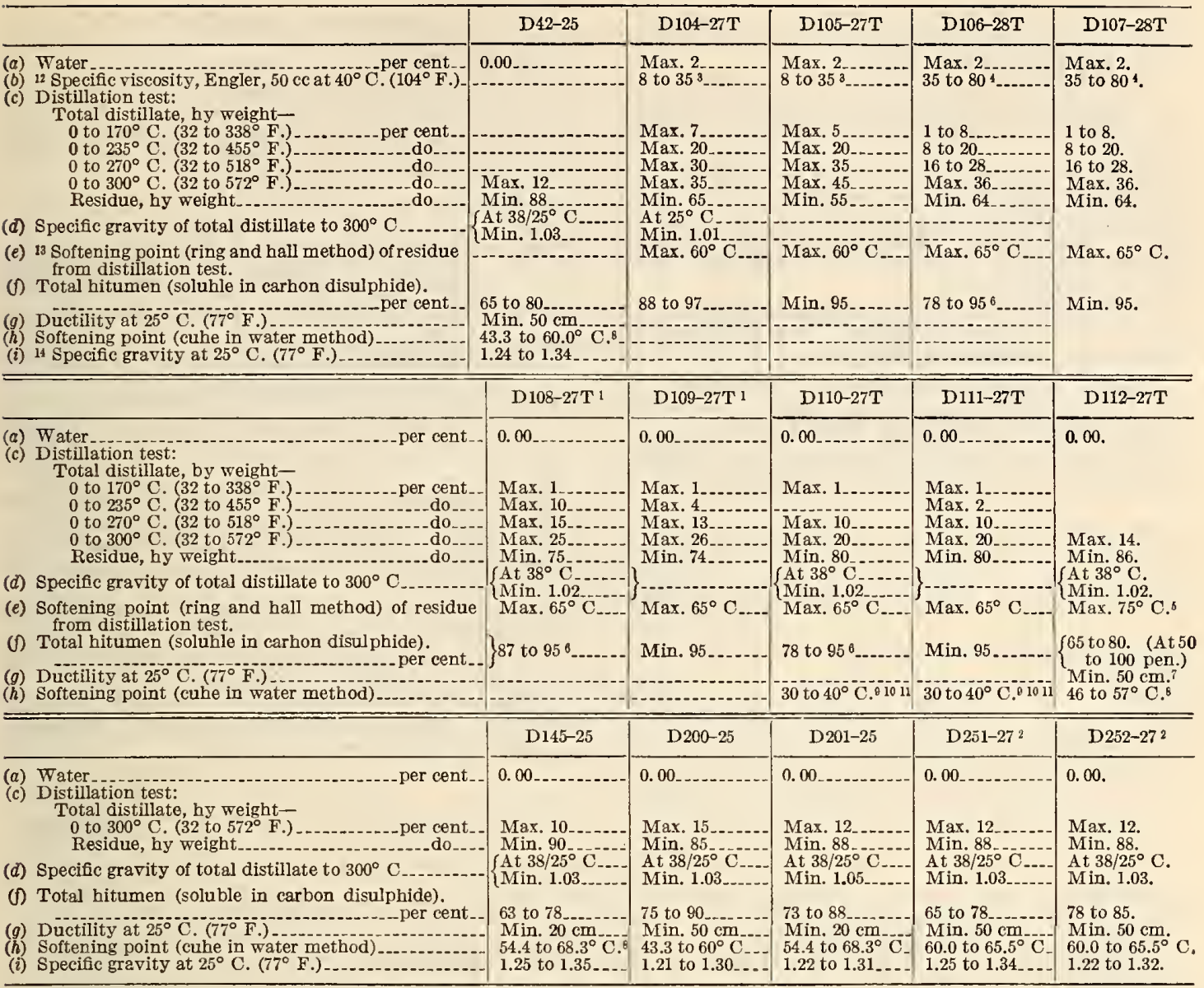

${ }^{1}$ D 103-27T and D109-27T, in addition to the requirements specified, must he subjected at $32^{\circ} \mathrm{C}$. to the A. S. T. M. standard method of float test for hituminous materials, serial designation D 139 (505.0, see p. 127), and the result must fall within the range of 60 to 150 seconds.

${ }^{2}$ D251-27 and D252-27, in addition to the requirements specified, shall have not more than 0.5 per cent of ash on incineration, after being tested in accordance with the A. S. T. M. standard method of lahoratory sampling and analysis of coal and coke, serial designation D271. (See 501.2, p. 8.)

Within the viscosity limits designated a material should he chosen to meet the local conditions of temperature, road conditions, and climate. It is recommended that materials be called for under the following range and headings: Light 8 to 13 , medium 13 to 18 , heavy 18 to 25 , extra heavy 25 to 35 .

4 It is recommended that materials be called for under the following range and headings: Light 35 to 60 , heavy 60 to 80 . The heavy material may require heating hefore use, and care should be taken to avoid foaming on account of tho possible water content.

Cube in water method.

- The specification range for bitumen covers a wide range of materials. If products from vertical retort or low-carhon coke-oven tars are desired, a range of 88 to 95 per cent should he specified. If high-carbon coke-oven or mixtures of coke-oven and gas-house tars are desired, a range of 78 to 88 per cent should be specified.

7 The penetration of the pitch shall be brought within the range of 50 to 100 penetration hy heating in an open vessel with frequent stirrings at a temperature of not over $350^{\circ} \mathrm{F}$.

8 The softening point (cube in water method) specified should have a range of not over $5.5^{\circ} \mathrm{C}$. (10 $\mathrm{F}$.) within the ahove limits. For D112, the range, within the limits of $115^{\circ}$ to $135^{\circ} \mathrm{F}$. should vary with the use of the material; for example, if used in admixture with sand, in a northern locality or a southern locality. The softening point range, within the ahove limits, should also vary according to the character of the paving.

$\theta$ Ring and hall method.

10 If desired, a float test may he substituted for the softening point test, in which case the requirements shall be as follows: Float test at $50^{\circ}$ C., 100 to 220 seconds. (A. S. T. M. serial designation D 139 , see 505.0, p. 127 .)

11 The specification range for softening point, ring and ball method, within the limits given, should he $5^{\circ} \mathrm{C}$. for any given locality, for example, $30^{\circ}$ to $35^{\circ} \mathrm{C}$. for cold climates, equivalent to a float test at $50^{\circ} \mathrm{C}$. of 100 to 160 seconds, $35^{\circ}$ to $40^{\circ} \mathrm{C}$. for warm elimates, equivalent to a float test at $50^{\circ} \mathrm{C}$. of 160 to 220 seconds.

12 No values for specific viscosity in sections 2 and 3 of tahle.

13 No values for softening point in section 3 of tahle.

14 No values for specific gravity at $25^{\circ} \mathrm{C}$. in section 2 of tahle.

The properties enumeraetd in the accompanying table shall be determined in accordance with the following methods of test of the American Society for Testing Materials, except as specified in paragraph (b).

(a) Percentage of water. - Standard method of test for water in petroleum products and other bituminous materials. (Serial designation D95; see $505.2, \mathrm{p} .38$.)

(b) Specific viscosity. ${ }^{6}$-United States Depart-

64 The Engler test has not heen standardized by the American Society for Testing Materials. Committees of the society have under consideration the Saybolt-Furol apparatus, which may be adopted later. ment of Agriculture, methods of examination of bituminous road materials (see $505.0, \mathrm{p}$. 141), the instrument being standardized by the Bureau of Standards. The results shall be reported as specific viscosity compared with water at $25^{\circ} \mathrm{C}$.

(c) Distillation.-Tentative method of test for distillation of bituminous materials suitable for road treatment. (Serial designation D20-28T; see 505.0, p. 120.)

(d) Specific gravity.-Standard method of test for specific gravity of road oils, road tars, asphalt cements, and soft tar pitches. (Serial designation D70; see $505.0, \mathrm{p}, 125$.) This method is given for 
specifications D42, D145, D200, D201, D251, and D252, but no method is prescribed for specifications D104, D108, D110, and D112.

(e) Softening point.-(a) Standard method of test for softening point of bituminous materials (ring and ball method). (Serial designation D36; see 505.0, p. 121.)

(b) Standard method of test for softening point of tar products (cube in water method). (Serial designation D61; see 505.0 , p. 123.)

(f) Total bitumen.-Standard method of test for the determination of bitumen. (Serial designation D4; see 505.0 , p. 117 .)

(g) Ductility.-Tentative method of test for ductility of bituminous materials. (Serial designation D113-26T; see 505.0, p. 126.)

Federal Specifications Board, specification No. 279 , United States Government master specification for tars for cold application, May 8, 1925.

The materials covered in this specification shall be supplied in one or more of the following grades:

\begin{tabular}{|c|c|}
\hline Grade & $\begin{array}{l}\text { Specific } \\
\text { viscosity, } \\
\text { Engler, at } \\
40^{\circ} \mathrm{C} \text {. } \\
\left(104^{\circ} \mathrm{F} .\right)\end{array}$ \\
\hline $\begin{array}{l}\text { TC-1-25 } \\
\text { TC-2-25 } \\
\text { TC-3-25 } \\
\text { TC-4-25 }\end{array}$ & $\begin{array}{r}\text { 8. } 0-13.0 \\
13.0-18.0 \\
18.0-25.0 \\
25.0-35.0\end{array}$ \\
\hline
\end{tabular}

The materials shall be produced from gas-house, coke-oven, water gas, and/or similar tars, and shall be homogeneous.

The materials covered by this specification are intended for use in the surface treatment of macadam, gravel, or shell roads, and in the maintenance of bituminous macadam roads by surface treatment methods, as follows:

\begin{tabular}{|c|c|}
\hline Grade & Intended use \\
\hline $\mathrm{TC}-1$ & $\begin{array}{l}\text { Priming application to be followed by bot-surface treat- } \\
\text { ment, or single application when a very fluid material } \\
\text { is required. }\end{array}$ \\
\hline $\mathrm{TC}-2-$ & $\begin{array}{l}\text { More viscous material than TC-1-25 for surface treat- } \\
\text { ment. }\end{array}$ \\
\hline $\mathrm{TC}-3$ & $\begin{array}{l}\text { More viscous tban TC-2-25. Under general conditions } \\
\text { tbe beavier grades are better adapted for surface treat- } \\
\text { ment as giving a more lasting surface. }\end{array}$ \\
\hline TC-4-25 & $\begin{array}{l}\text { More viscous than TC-3-25. Application may usually } \\
\text { be made witbout beating except wben cold weather } \\
\text { renders the tar too viscous to distribute properly. }\end{array}$ \\
\hline
\end{tabular}

The tars shall conform to the requirements listed in the accompanying table, which also gives specific requirements for nine additional varieties of tars, classified with regard to uses and methods of application as follows:

Refined tar for hot application, type TH-1-25 (see 505.32), specification No. 280.

Tars for use in repair work, types TR-1-25 and TR-2-25, (see 505.34), specification No. 281.

Refined tar for construction, types TP-1-25, TP-2-25, TP-3-25, TP-4-25, TP-5-25, TP-6-25 (see 505.33), specification No, 282 .

\begin{tabular}{|c|c|c|c|c|c|}
\hline \multirow[b]{2}{*}{ 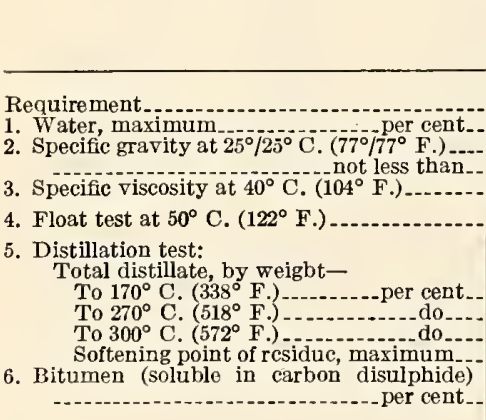 } & \multicolumn{4}{|c|}{ Specification No. 279} & \multirow{2}{*}{ 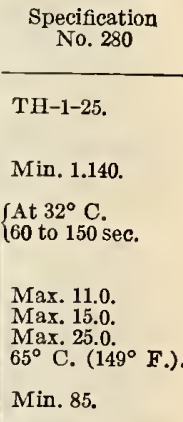 } \\
\hline & $\begin{array}{l}\text { Max. } 7.0 \\
\text { Max. } 32.0 \\
\text { Max. } 42.0 \\
60^{\circ} \text { C. }\left(140^{\circ} \text { F.). }\right. \\
90.0\end{array}$ & $\begin{array}{l}\text { Max. } 5.0 \\
\operatorname{Max} .30 .0 \\
\operatorname{Max} .40 .0 \\
60^{\circ} \mathrm{C} .\left(140^{\circ} \mathrm{F} .\right) \\
90.0\end{array}$ & $\begin{array}{l}\text { Max. } 5.0 \\
\text { Max. } 30.0 \\
\text { Max, } 40.0 \\
60^{\circ} \text { C. }\left(140^{\circ} \mathrm{F} .\right) \\
90.0\end{array}$ & $\begin{array}{l}\text { Max. } 3.0 \\
\operatorname{Max} 30.0 \\
\operatorname{Max} 40.0 \\
60^{\circ} \mathrm{C} .\left(140^{\circ} \mathrm{F} .\right) \\
90.0\end{array}$ & \\
\hline & & \multicolumn{2}{|c|}{ Specification No. 281} & \multicolumn{2}{|c|}{ Specification No. 282} \\
\hline 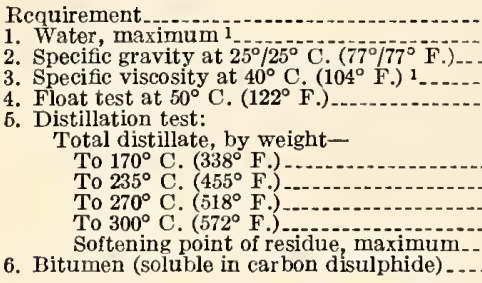 & per cent._ & $\begin{array}{l}2.0 \text { to } 8.0 \\
10.0 \text { to } 20.0 \\
18.0 \text { to } 30.0 \\
\text { Max. } 38.0 \\
65^{\circ} \text { C. }\left(149^{\circ} \text { F.) }\right. \\
80.0\end{array}$ & $\begin{array}{l}2.0 \text { to } 8.0 \\
8.0 \text { to } 16.0 \\
15.0 \text { to } 30.0 \\
\text { Max. } 35.0 \\
65^{\circ} \mathrm{C} .\left(149^{\circ} \mathrm{F} .\right) \\
80.0\end{array}$ & $\begin{array}{l}\text { TP-1-25 } \\
100 \text { to } 160 \text { sec. } \\
\text { Max. } 1.0 \\
\text { Max. } 10.0 \\
65^{\circ} \text { C. } 20.0 \\
\text { Min. } 95.0 \\
\text { Ta. F. }\end{array}$ & $\begin{array}{l}\text { TP-2-25. } \\
1.200 \text { to } 1.260 \text {. } \\
100 \text { to } 160 \text { sec. } \\
\text { Max. } 1.0 \text {. } \\
\text { Max. } 10.0 \text {. } \\
\text { Max. } 20.0 . \\
65^{\circ} \text { C. }\left(149^{\circ} \text { F.). }\right. \\
80.0 \text { to } 95.0 \text {. }\end{array}$ \\
\hline & & \multicolumn{4}{|c|}{ Spccification No. 282} \\
\hline $\begin{array}{l}\text { Requirement } \\
\text { 2. Specific gravity at } 25^{\circ} / 25^{\circ} \mathrm{C} .\left(77^{\circ} / 77^{\circ} \mathrm{F} .\right) \\
\text { 4. Float test at } 50^{\circ} \text { C. }\left(122^{\circ} \text { F.) }\right. \\
\text { 5. Distillation test: } \\
\text { Total distillate, by weigbt- } \\
\text { To } 170^{\circ} \text { C. }\left(338^{\circ} \mathrm{F} .\right) \\
\text { To } 270^{\circ} \text { C. }\left(518^{\circ} \text { F.) }\right. \\
\text { To } 300^{\circ} \text { C. ( }\left(572^{\circ} \text { F.) }\right. \\
\text { Softening point of residue, maximum } \\
\text { 6. Bitumen (soluble in carbon disulphide) }\end{array}$ & not less than & $\begin{array}{l}\text { TP-3 } 25 \\
1.150 \text { to } 1.200 \\
130 \text { to } 190 \mathrm{sec} \\
\text { Max. } 1.0 \\
\text { Max. } 10.0 \\
\text { Max. } 20.0 \\
65^{\circ} \mathrm{C} .\left(149^{\circ} \mathrm{F} .\right) \\
\text { Min. } 95.0\end{array}$ & $\begin{array}{l}\text { TP-4-25 } \\
1.200 \text { to } 1.260 \\
130 \text { to } 190 \text { sec...- } \\
\text { Max. } 1.0 \\
\text { Max. } 10.0 \\
\text { Max. } 20.0 \\
65^{\circ} \mathrm{C} .\left(149^{\circ} \mathrm{F} .\right)_{--} \\
80.0 \text { to } 95.0\end{array}$ & $\begin{array}{l}\text { TP-5 } 25 \\
1.150 \text { to } 1.200 \\
160 \text { to } 220 \text { sec.-.- } \\
\text { Max. } 1.0 \\
\text { Max. } 10.0 \\
\text { Max. } 20.0 \\
65^{\circ} \text { C. }\left(149^{\circ} \mathrm{F} .\right) \\
\text { Min. } 95.0\end{array}$ & $\begin{array}{l}\text { TP-6-25. } \\
1.200 \text { to } 1.260 \text {. } \\
160 \text { to } 220 \text { sec. } \\
\text { Max. } 1.0 \text {. } \\
\text { Max. } 10.0 \text {. } \\
\text { Max. } 20.0 . \\
65^{\circ} \text { C. }\left(149^{\circ} \text { F.). }\right. \\
80.0 \text { to } 95.0 .\end{array}$ \\
\hline
\end{tabular}

1 No values for water and specific viscosity given in Specification No. 282 TP-3-25, TP-4-25, TP-5-25, and TP-6-25. 
Tests of the physical and chemical properties of the tars enumerated in the accompanying table shall be determined in accordance with the methods listed under 505.31, American Society for Testing Materials, serial designations D104 and D105 (see p. 175), with the following modifications of the A. S. T. M. method D20-27T:

For use in the distillation test the thermometer shall be calibrated by distilling $(a)$ water, $(b)$ chemically pure naphthalene, and (c) chemically pure diphenylamine in the apparatus as assembled for a test. When the pure material is distilling at the specified rate, and not less than 15 cc have been condensed, the thermometer reading and a barometric reading are taken. The actual temperatures are calculated from the equations-for water, temperature $=100+0.037$ (mm barometer -760$)$; for naphthalene, temperature $=218+0.058(\mathrm{~mm}$ barometer760 ); for diphenylamine, temperature $=302+0.055$ ( $\mathrm{mm}$ barometer-760). From the observed thermometer readings and corresponding actual temperatures as thus calculated, thermometer readings corresponding to specified fractionating temperatures shall be calculated and used in distillation tests of bituminous materials. (Par. 10 of A. S. T. M., method D20-27T.)

The test for softening point shall be by the ring and ball method in all cases, A. S. T. M. standard method D36-26, 1926. (See 505.0, p. 131.)

505.32 Tars for Hot Application to Roads.

American Society for Testing Materials, tentative specifications for high-carbon tar for suriace treatment, hot application, serial designation D108-27'T, 1927.

(For properties and tests, see 505.31, p. 175.)

Tentative specifications for low-carbon tar for surface treatment, hot application, serial designation D109-27T, 1927.

(For properties and tests, see 505.31, p. 175.)

Federal Specifications Board, specification No. 280, United States Government master specification for refined tar for hot application, type TH-1-25, May 8, 1925.

Material supplied under this specification shall be produced from gas-house, coke-oven, water-gas and/or similar tars. The refined tar shall be homogeneous and free from water.

The material covered by this specification is intended for use in the hot-surface treatment of macaadam, gravel, or shell roads, either with or without a primer coat to form a wearing mat or carpet. It may be used in the maintenance of bituminous macadam roads.

In connection with this specification, see United States Government master specification, F. S. B. No. 275, (505.22, p. 173) for road oil for hot application.

For detail requirements and tests, see 505.31, page 176.

\subsection{Tars for Road Construction.}

American Society for Municipal Improvements, specifications for broken stone and gravel roads, 1922. (See 512.15 , p. 245.)

(This specification contains requirements for certain types of tars for road construction.)

Federal Specifications Board, specification No.

282, United States Government master specification for refined tar for construction, May 8, 1925 .

The materials covered by this specification shall be supplied in the particular grade or grades ordered by the purchasing department:

\begin{tabular}{|c|c|c|c|}
\hline Grade & Material & $\begin{array}{l}\text { Float } \\
\text { test at } \\
\text { ä } 0^{\circ} \mathrm{C} . \\
\left(122^{\circ} \mathrm{F} .\right)\end{array}$ & Intended use \\
\hline $\begin{array}{l}\text { TP-1-25.. } \\
\text { TP-2-25.. }\end{array}$ & $\begin{array}{l}\text { Refined water-gas } \\
\text { tar. } \\
\text { Refined coal tar.... }\end{array}$ & $\begin{array}{l}\text { Seconds } \\
100-160 \\
100-160\end{array}$ & $\begin{array}{l}\text { Bituminous macadam in } \\
\text { the northern United } \\
\text { States, light or moder- } \\
\text { ate traffic. }\end{array}$ \\
\hline $\begin{array}{l}\mathrm{TP}-3-25 . . \\
\mathrm{TP}-4-25 \ldots\end{array}$ & $\begin{array}{l}\text { Refined water-gas } \\
\text { tar. } \\
\text { Refined coal tar.-.- }\end{array}$ & $\begin{array}{l}130-190 \\
130-190\end{array}$ & $\begin{array}{l}\text { Bituminous macadam in } \\
\text { the middle United } \\
\text { States or in the north- } \\
\text { ern United States, } \\
\text { heavy traffic. }\end{array}$ \\
\hline $\begin{array}{l}\text { TP-5-25.. } \\
\text { TP-6-25.. }\end{array}$ & $\begin{array}{l}\text { Refined water.gas } \\
\text { tar. } \\
\text { Refined coal tar.-. }\end{array}$ & $\begin{array}{l}160-220 \\
160-220\end{array}$ & $\begin{array}{l}\text { Bituminous macadam in } \\
\text { the southern United } \\
\text { States. }\end{array}$ \\
\hline
\end{tabular}

Material furnished under this specification shall be prepared from suitable water-gas tars, or from suitable gas-house and/or coke-oven tars. The refined tar shall be homogeneous and free from water.

In connection with this specification, see United States Government master specification for asphalt for use in road and pavement construction, Federal Specifications Board specification No. 276a. (See 505.11, p. 145.)

(For detail requirements and tests, see 505.31, p. 176.$)$

\subsection{Tars for Repair Work.}

Federal Specifications Board, specification No. 281, United States Government master spccification for tar for use in repair work, May 8, 1925.

The materials covered by this specification shall be supplied in either or both of the following grades, as ordered:

\begin{tabular}{|c|c|}
\hline Grade & $\begin{array}{c}\text { Specific } \\
\text { viscosity, } \\
\text { Engler, at } \\
40^{\circ} \mathrm{C} \text {. } \\
\left(104^{\circ} \mathrm{F} .\right)\end{array}$ \\
\hline TR-1-25 & $\begin{array}{l}35-60 \\
60-80\end{array}$ \\
\hline
\end{tabular}

Material supplied under this specification shall be prepared from refined gas-house, coke-oven, and/or water-gas tars fluxed with suitable distillates. The tar shall be homogeneous.

The materials are intended for use primarily in the repair of surface breaks, holes, and depressions in bituminous macadam and surface-treated roads, by surface application or by mixing with broken stone (and sand). The lighter material, TR-1-25, may be used without heating, while the heavier will require slight heating, except under the most favorable temperature conditions.

(For detail requirements and tests, see 505.31, p. 176.)

American Society for Testing Materials, tentative specifications for coal-tar pitch for stone-block filler, serial designation D112-27T, 1927.

(For properties and tests, see 505.31, p. 175.)

\subsection{Tar Cement.}

American Society for Testing Materials, tentative specifications for high-carbon tar cement for use cold in repair work (cut-back product), serial designation D106-28T, 1928. 
Tentative specifications for low-carbon tar cement for use cold in repair work (cut-back product) serial designation D107-28T, 1928.

Tentative specifications for high-carbon tar cement, serial designation D110-27T, 1927.

Tentative specifications for low-carbon tar cement, serial designation D111-27T, 1927.

For properties and tests of the materials covered by the above specifications, see 505.31, page 175 .

\subsection{Tar for Roofing and Waterproofing.} (See also 505.16.)

American Railway Engineering Association, specifications for buildings for railway purposes, 1929

\section{BUILT-UP ROOFING}

See 518.57 , page 427 .

American Society for Testing Materials, standard specifications for creosote oil for priming coat with coal-tar pitch in damp proofing and waterproofing below and above ground level, serial designation D43-25, 1925.

1. These specifications cover creosote oil primer for use, when specified, with coal-tar pitch in damp proofing and waterproofing below or above ground level, for application to concrete and masonry surfaces.

\section{SAMPIING}

2. From each shipment or portion thereof representing creosote oil of the same kind, a number of packages shall be selected at random equivalent to the cube root of the total number of packages in the lot. If the cube root as calculated proves to be a fractional number, it shall be expressed as the next highest whole number. For convenience, the following table is given, showing the number of samples to be selected for shipments of various sizes:

\begin{tabular}{|c|c|c|c|}
\hline Packages in shipment & $\begin{array}{l}\text { Packages } \\
\text { selected }\end{array}$ & Packages in shipment & $\begin{array}{l}\text { Packages } \\
\text { selected }\end{array}$ \\
\hline $\begin{array}{l}2 \text { to } 8 \\
9 \text { to } 27 \\
28 \text { to } 64 \\
65 \text { to } 125 \\
126 \text { to } 216\end{array}$ & $\begin{array}{l}2 \\
3 \\
4 \\
5 \\
6\end{array}$ & $\begin{array}{l}217 \text { to } 343 \\
344 \text { to } 512 \\
513 \text { to } 729 \\
730 \text { to } 1,000 \\
1,001 \text { to } 1,331\end{array}$ & $\begin{array}{r}7 \\
8 \\
8 \\
10 \\
11\end{array}$ \\
\hline
\end{tabular}

3. By means of a paddle, the contents of each package selected shall be thoroughly stirred so as to bring all portions thereof into uniform distribution. With an appropriate sampler, not less than 1 pint of the creosote oil shall be immediately drawn out from the center of each package and transferred to a clean receptacle of suitable size, which in turn shall be kept tightly covered, prior and immediately after said samples are being introduced. After all the pint samples have been added, the contents of the container shall be thoroughly agitated, whereupon 1 quart of the mixture shall immediately be transferred to a clean quart can provided with a tightly fitting screw cap, which shall be immediately fastened in place. This sample shall be considered as representative of the shipment and shall be transmitted to the laboratory for examination, bearing a suitable tag or other means of identification.

\section{PROPERTIES}

4. The creosote oil primer shall meet the following requirements:

(a) Water, not more than 2 per cent.

(b) Consistency at $38^{\circ} \mathrm{C}$. (100 $\mathrm{F}$.), entirely fluid.

(c) Specific gravity at $38^{\circ} / 25^{\circ} \mathrm{C}$. $\left(100^{\circ} / 77^{\circ} \mathrm{F}\right.$ ), 1.00 to 1.06

(d) Insoluble in benzol, not more than 1 per cent. (e) Distillation test:

Total distillate, by weight, under $200^{\circ} \mathrm{C}$. $\left(392^{\circ} \mathrm{F}\right.$.), not more than 5 per cent.

than 5 per cent.

Total distillate, by weight, under 235

50 per cent, ninimum 30 per cent Residue, by weight, above $355^{\circ} \mathrm{C} .\left(671^{\circ} \mathrm{F}\right.$.), maximum 15 per

(f) Specific gravity at $38^{\circ} / 25^{\circ}$ C. $\left(100^{\circ} / 77^{\circ}\right.$ F.) of the fraction distilling between $235^{\circ}$ and $315^{\circ} \mathrm{C}$. ( $\left(455^{\circ}\right.$ and $599^{\circ} \mathrm{F}$.), not less than 1.00 .

(g) Consistency at $25^{\circ} \mathrm{C}$. $\left(77^{\circ} \mathrm{F}\right.$.) of residue, soft.

\section{Methods of Testing}

5. The properties enumerated in these specifications shall be determined in accordance with the Standard Methods of Sampling and Analysis of Creosote Oil (serial designation D38-27) of the American Society for Testing Materials.

American Society for Testing Materials. The society has specifications for tars for roofing and waterproofing, as presented below. In all cases the coal-tar pitch shall be homogeneous and shall be sampled in accordance with the standard method of sampling bituminous materials, American Society for Testing Materials (serial designation D140, see 505.0, p. 128.)

Standard specifications for high-carbon coal-tar pitch for use in damp proofing and waterproofing below ground level, serial designation D42-25, 1925.

1. These specifications cover high-carbon coal-tar pitch suitable for use as a mopping coat in damp proofing or as a plying cement in the construction of a membrane system of waterproofing below ground level, under uniformly moderate temperature conditions both during the process of installation and during service. Such coal-tar pitch is not intended to be heated above $300^{\circ} \mathrm{F}$. (148.8 $\mathrm{C}$.) during its application.

2. The grade of high-carbon coal-tar pitch covered by these specifications is suitable for damp proofing and water-proofing foundations, tunnels, subways, etc.

\section{PROPERTIES AND TESTS}

(See 505.31, p. 175.)

Standard specifications for high-carbon coal-tar pitch for use in damp proofing and waterproofing above ground level, serial designation D145-25, 1925 .

1. These specifications cover high-carbon coaltar pitch suitable for use as a mopping coat in damp proofing, or as a plying-cement in the construction of a membrane system of waterproofing above ground level, when not exposed to a temperature exceeding $46^{\circ}$ C. (115 $\circ$ F.) Such coal-tar pitch is not intended to be heated above $350^{\circ} \mathrm{F} .\left(176.6^{\circ} \mathrm{C}\right.$.) during its application.

2. The grade of high-carbon coal-tar pitch covered in these specifications is suitable for damp proofing and waterproofing railroad bridges, tanks, retaining walls, culverts, dams, conduits, etc.

\section{PROPERTIES AND TESTS}

(See 505.31, p. 175.)

Standard specifications for high-bitumen coal-tar pitch for use in damp proofing and waterproofing below ground level, serial designation D200-25, 1295.

1. These specifications cover high-bitumen coaltar pitch suitable for use as a mopping coat in damp proofing or as a plying cement in the construction of a membrane system of waterproofing below ground level, under uniformly moderate temperature conditions both during the process of installation and during service.

2. The grade of high-bitumen coal-tar pitch covered by these specifications is suitable for damp proofing and waterproofing foundations, tunnels, subways, etc. 


\section{PROPERTIES AND TESTS}

(See 505.31, p. 175 .)

Standard specifications for high-bitumen coal-tar pitch for use in damp proofing and waterproofing above ground level, serial designation D201-25, 1925.

1. These specifications cover high-bitumen coaltar pitch suitable for use as a mopping coat in damp proofing, or as a plying cement in the construction of a membrane system of waterproofing above ground level, when not exposed to a temperature exceeding $46^{\circ} \mathrm{C}$. $\left(115^{\circ} \mathrm{F}\right.$.).

2. The grade of high-bitumen coal-tar pitch covered in these specifications is suitable for damp proofing and waterproofing railroad bridges, tanks, retaining walls, culverts, dams, conduits, etc.

\section{PROPERTLES AND TESTS}

(See 505.31, p. 175.)

Standard specifications for high-carbon coal-tar pitch for use in constructing built-up roofs surfaced with slag or gravel, serial designation D251-27, 1927.

1. These specifications cover high-carbon coal-tar pitch intended for use as a mopping coat in the construction of built-up roofs surfaced with slag or gravel on inclines not exceeding 3 inches, to the foot over boards and 1 inch to the foot over concrete, in connection with the Standard Specifications for Coal-Tar Saturated Roofing Felt for Use in Waterproofing and in Constructing Built-Up Roofs (serial designation D227) of the American Society for Testing Materials. (See below.)

\section{PROPERTIES AND TESTS}

(See 505.31, p. 175 .)

Standard specifications for high-bitumen coal-tar pitch for use in constructing built-up roofs surfaced with slag or gravel, serial designation D252-27, 1927.

1. These specifications cover high-bitumen coaltar pitch intended for use as a mopping coat in the construction of built-up roofs surfaced with slag or gravel on inclines not exceeding 3 inches to the foot over boards and 1 inch to the foot over concrete, in connection with the Standard Specifications for Coal-Tar Saturated Roofing Felt for Use in Waterproofing and in Constructing Built-Up Roofs (serial designation D227) of the American Society for Testing Materials. (See below.)

\section{PROPERTIES AND TESTS}

(See 505.31, p. 175.)

American Society for Testing Materials, standard specifications for woven cotton fabrics saturated with bituminous substances for use in waterproofing, serial designation D173-27, 1927.

(See 505.16, p. 153.)

American Society for Testing Materials, standard specifications for burlap saturated with bituminous substances for use in waterproofing, serial designation D174-25, 1925.

(See 505.16, p. 154.)

American Society for Testing Materials, standard specifications for coal-tar saturated roofing felt for use in waterproofing and in constructing built-up roofs, serial designation D227-27, 1927.

These specifications cover coal-tar saturated felt, either 36 or 32 inches in width, composed of roofing felt saturated, but not coated, with refined coal tar, for use in the membrane system of waterproofing and in the construction of built-up roofs.

This specification is the same as A. S. T. M. serial designation D226 (see 505.16, p. 156), with the following exceptions:

1. The saturant for this specification is coal tar, from which all the highly volatile constituents have been removed, in place of asphalt as used in the specification referred to.

2 . In the specification referred to, the item "loss on heating" is replaced in this specification by tests for distillate, with the following requirements:

(a) Distillate, per cent by weight to $210^{\circ} \mathrm{C}$. calculated on extracted saturant, not more than 2 .

(b) Distillate, per cent by weight to $235^{\circ} \mathrm{C}$. calculated on extracted saturant, not more than 5 .

This specification contains the following additional requirement: Moisture-Maximum, 2.5 per cent of the net weight.

Federal Specifications Board, specification No. 80 , United States Government master specification for coal-tar pitch for roofing, December 29, 1923.

This specification applies to coal-tar pitch intended for use with coal-tar saturated rag felt for roofing and waterproofing (F. S. B. specification No. 81, see p. 160), in the construction of built-up roofing over board sheathing with inclines not exceeding 3 inches to the foot and over concrete surfaces with inclines not exceeding 1 inch per foot. .

The requirements for this specification are identical with those for F. S. B. specification No. 83 (see below) with the following exception: 1. Melting point $140^{\circ}$ to $150^{\circ} \mathrm{F}$.

Federal Specifications Board, specification No. 81, United States Government master specification for coal-tar saturated rag felt for roofing and waterproofing.

(This specification is given in full in connection with F. S. B. specifications Nos. 295 and 86 . See 505.16 , p. 160.)

Federal Specifications Board, specification No. 83, United States Government master specification for coal-tar pitch for waterproofing and damp proofing, December 29, 1923.

\section{GENERAL}

This specification applies to coal-tar pitch intended for use with coal-tar saturated rag felt for roofing and waterproofing (F. S. B. specification No. 81, see p. 160), as a plying cement in the construction of membrane waterproofing or alone as a damp proofing coating.

This coal-tar pitch is suitable for damp proofing and waterproofing tanks, retaining walls, dams, conduits, foundations of buildings, tunnels, subways, pools, reservoirs, etc., where not exposed except during installation to temperatures exceeding $38^{\circ} \mathrm{C}$. $\left(100^{\circ} \mathrm{F}\right.$.), and where not subject to vibration.

\section{APPEARANCE}

Freshly melted material shall be uniformly glossy, and on aging one week its surface shall not become dull or show any separation of oil, grease, paraffin scale, or similar material.

\section{PROPERTIES}

When tested by the methods contained in this specification it shall conform to the following requirements:

(a) Melting point. $-125^{\circ}$ to $150^{\circ} \mathrm{F}$.

(b) Ductility.-Minimum, $50 \mathrm{~cm}$.

(c) Specific gravity. -1.22 to 1.34 .

(d) Free carbon. -15 to 30 per cent.

(e) Distillation test.-Not more than 12 per cent by weight shall distill below $572^{\circ} \mathrm{F}$. The specific gravity of the distillate shall be not less than 1.03.

\section{ADDITIONAL REQUIREMENTS}

1. Sampling.-The sampling requirements are the same as for F. S. B. specification No. 84 (see 505.16, p. 162), with the exception that the melting of the 
samples shall be at a temperature not exceeding $121^{\circ}$ C. $\left(250^{\circ}\right.$ F.).

2. Appearance.-(The requirements under "Appearance" are the same as for F. S. B. specification No. 84 (see 505.16, p. 162), with the exception that the melting of the material shall be at a temperature not exceeding $121^{\circ} \mathrm{C}$. $\left(250^{\circ} \mathrm{F}.\right)$.)

3. Packing.-(Identical with F. S. B. specification No. 84. See 505.16, p. 162.)

NoтE.-When required, deliveries will, in general, be sampled and tested by the methods here given or referred to, but the purchaser reserves the right to use any additional information torascerAdin whether the material meets the specification.

The following methods of tests shall be used to determine the properties listed above:

(a) Melting point.-Standard method of test for softening point of tar products (cube in water method), American Society for Testing Materials, serial designation D61. (See 505.0, p. 123.)

(b) Ductility. - Tentative method of test for ductility of bituminous materials, A. S. T. M. D113-26T. (See 505.0, p. 126.)

(c) Specific gravity. - The specific gravity may be determined by any approved method, but some method similar to the following is recommended:

A small platinum pan or crucible supported in a wire sling shall be accurately weighed in air and the weight designated by $(a)$. It shall then be weighed in a similar manner suspended in freshly distilled water at $25^{\circ}$ C., but immersed except for the fine platinum wire used for suspension and this weight designated by $(b)$. The crucible shall then be dried and poured almost full of coal-tar pitch which has been melted at the lowest possible temperature. It shall then be allowed to cool to $25^{\circ} \mathrm{C}$. $\left(77^{\circ} \mathrm{F}\right.$. and weighed in the sling in air. This weight shall be designated as $(c)$. Then it shall be weighed in water and the weight shall be designated as $(d)$. From these weights the specific gravity shall be computed according to the following formula:

$$
\text { Sp. gr. }=\frac{c-a}{(c-a)-(d-b)}
$$

(d) Free carbon.-Free carbon, as defined by the American Society for Testing Materials, is that matter in tars minus the ash which is insoluble in cold carbon bisulphide.

Method No. 1.-One gram of coal-tar pitch shall be placed in a beaker of 100 to 150 cc capacity, covered with cold carbon bisulphide and set aside for about 15 minutes, the liquid being stirred every few minutes until the lumps break up and the bitumen goes into solution. The solid particles shall be allowed to settle, and the carbon bisulphide solution of the bitumen shall be decanted through a weighed Gooch crucible prepared with a mat of asbestos fiber of a thickness that will scarcely transmit light.

The sediment remaining in the beaker shall be crushed with a stirring rod and a little more carbon bisulphide added. The sides of the beaker also shall be washed down with carbon bisulphide The contents of the beaker shall then be poured into the crucible and all sediment washed from the beaker into the crucible with carbon bisulphide. Suction may be used to aid in filtering. The sediment on the filter shall be washed with carbon bisulphide until the washings run through colorless. The crucible shall then be set in a place free from dust until the carbon bisulphide evaporates and then placed in an oven at $105^{\circ} \mathrm{C}$. $\left(221^{\circ} \mathrm{F}\right.$.) for one hour. It shall then be cooled and weighed, the increase giving the total insoluble matter.

To determine the ash, $1 \mathrm{~g}$ of the pitch shall be weighed in a crucible of about $30 \mathrm{cc}$ capacity and incinerated until the carbon is consumed and only mineral matter remains. Cool and weigh and compute the ash.
Subtract the amount of ash from the total insoluble matter and consider the difierence as free carbon. Should the ash exceed 1 per cent, it is an indication that mineral matter has been added to the pitch and the material should be rejected.

Comparative results may be obtained by the following method, but in case of dispute the method given above will be used:

Method No. 2.-A $1 \mathrm{~g}$ sample of the coal-tar pitch shall be placed in a 100 cc beaker and digested on a steam bath with about 50 ce of c. p. toluol for not over 20 minutes, the liquid being stirred occasionally to break up lumps and solid particles. An extraction thimble or filter cup shall be prepared by washing with hot benzol and drying at $105^{\circ} \mathrm{C}$. $\left(221^{\circ} \mathrm{F}\right.$.) for one hour. It shall be cooled in a desiccator and weighed in a weighing bottle. The extraction thimble in a glass filter cup shall be held over a beaker and wet with c. p. toluol, then the toluol-pitch solution shall be decanted through it. Both it and the beaker shall be washed with hot c. p. toluol until clean, particles adhering to the beaker being detached with a camel's hair brush. The extraction thimble shall then be washed with c. p. benzol, allowed to drain, covered with a cap of filter paper, placed in the extraction apparatus, and extracted with benzol until the descending benzol runs through colorless. The thimble shall then be removed, the cap taken off, and the thimble dried at $105^{\circ} \mathrm{C} .\left(221^{\circ} \mathrm{F}\right.$.) for one-half hour. It shall then be placed in the weighing bottle, cooled in a desiccator, and weighed. The increase in weight is total insoluble matter.

Determine the ash as in method No. 1 and substract it from the total insoluble matter to obtain the amount of free carbon.

(e) Distillation test.-A. S. T. M. D20-28T. (See 505.0, p. 120.)

(f) Specific gravity of distillate-A specific gravity bottle or pyknometer shall be cleaned, dried a $105^{\circ}$ C. $\left(221^{\circ}\right.$ F.), cooled, and weighed. It shall then be filled with freshly boiled distilled water at $38^{\circ}$ C. $\left(100^{\circ}\right.$ F. $)$ and weighed. The bottle shall then be dried and filled at $38^{\circ}$ C. $\left(100^{\circ}\right.$ F.) with distillate obtained in $(f)$ and weighed. From these weights the specific gravity can be computed according to the following formula:

$$
\text { Sp. gr. at } 38^{\circ} / 15.5^{\circ} \text { C. }\left(100^{\circ} / 60^{\circ} \text { F. }\right)=\frac{c-a}{b-a} \times \frac{0.99299}{0.99905}
$$

$o=$ weight of bottle

$b=$ weight of bottle and distilled water

$c=$ weight of bottle and distillate

$0.99299=$ density of water at $38^{\circ} \mathrm{C}$.

$0.99905=$ density of water at $15.5^{\circ} \mathrm{C}$.

Federal Specifications Board, United States Government master specifications for the construction of built-up roofing of the following types:

1. Specification No. 151, for type 3 TCS, having three layers or plies of felt.

2. Specification No. 152, for type 4 TCS, having four layers or plies of felt.

3. Specification No. 153, for type 5 TCS, having five layers or plies of felt.

4. Specification No. 154, for type 4 TWS, having four layers or plies of felt.

5. Specification No. 155, for type 5 TWS, having five layers or plies of felt.

\section{GENERAL REQUIREMENTS}

The roofing shall consist of layers or plies of coaltar saturated rag felt cemented together with coaltar pitch and surfaced with slag, gravel, or similar surfacing material, as called for in the proposal for the work.

Flashings of the type required under the specification for the building shall be included and shall 
conform to Federal Specifications Board specification for flashings No. 156 or No. 157. (See 505.0, p. 141.)

\section{DETAIL REQUIREMENTS}

The roofing shall contain not less than the following quantities of materials per 100 square feet.

\begin{tabular}{|c|c|c|c|c|c|}
\hline Type & $\begin{array}{c}\text { Coal- } \\
\text { tar sat- } \\
\text { urated } \\
\text { rag felt } \\
\text { (F.S.B. } \\
\text { No.81) } \\
(\text { See } \\
505.16, \\
\text { p. } 160)\end{array}$ & $\begin{array}{c}\text { Coal-tar } \\
\text { pitch } \\
\text { (F.S. B. } \\
\text { No. 80) } \\
\text { (See } \\
505.36, \\
\text { p. 162) }\end{array}$ & $\begin{array}{c}\text { Roofing } \\
\text { gravel } \\
\text { (F.S. B. } \\
\text { No.82) o } \\
\text { (See } \\
505.16 \\
\text { p. 179) }\end{array}$ & $\begin{array}{l}\text { Roofing } \\
\text { slag } \\
\text { (F.S.B. } \\
\text { No.82) }\end{array}$ & $\begin{array}{l}\text { Sheathing paper or } \\
\text { unimpregnated felt }\end{array}$ \\
\hline $\begin{array}{l}3 \mathrm{TCS}- \\
4 \mathrm{TCS} . \\
5 \mathrm{TCS} . \\
4 \mathrm{TWS} \\
5 \mathrm{TWS}\end{array}$ & \begin{tabular}{|r} 
Pounds \\
45 \\
60 \\
76 \\
60 \\
76
\end{tabular} & $\begin{array}{r}\text { Pounds } \\
160 \\
185 \\
210 \\
120 \\
145\end{array}$ & \begin{tabular}{|c|} 
Pounds \\
400 \\
400 \\
400 \\
400 \\
400
\end{tabular} & $\begin{array}{r}\text { Pounds } \\
300 \\
300 \\
300 \\
300 \\
300\end{array}$ & $\begin{array}{l}\text { None. } \\
\text { Do. } \\
\text { Do. } \\
5 \text { pounds (one layer). } \\
\text { Do. }\end{array}$ \\
\hline
\end{tabular}

I. In laying the roofing, the following general requirements shall apply:

The coal-tar pitch shall not be heated above $375^{\circ} \mathrm{F}$. and shall be hot when the felts are laid.

The surfacing material shall always be dry when applied and, in addition, shall be heated in cold weather.

The layers of felt shall be laid so as to be free from wrinkles and buckles, and so that pronounced ridges are not formed at the laps.

II. Specific requirements for the laying of types $3 \mathrm{TCS}, 4 \mathrm{TCS}$, and $5 \mathrm{TCS}$ are as follows:

When the roof surface is of gypsum, each sheet of felt shall be nailed at approximately 6 inches from its upper edge with nails driven through flat tin disks.

Concrete and gypsum roof surfaces installed by other contractors before being considered satisfactory for laying the roof covering, shall be smooth, firm, dry, and free from high spots, depressions, and all loose and foreign material. Precast concrete slabs shall be firmly set and well pointed.

For roofing of types 4TWS and 5TWS the following additional requirements shall apply:

When the roof surface is of gypsum block, the sheathing paper may be omitted.

The board roof surface installed by other contractors before being considered satisfactory for laying the roof covering shall be smooth and free from loose boards, large cracks, or projecting ends which might injure the roofing. All loose and foreign material shall be removed. Precast gypsum slabs shall be firmly set, well pointed, and free from any unevenness which might injure the roofing.

NotE.-The full terts of the fire specifications given includes, in each case, directions for application of the roofing.

\section{ADDITIONAL INFORMATION}

Roofing of types $3 \mathrm{TCS}, 4 \mathrm{TCS}$, and $5 \mathrm{TCS}$ is adapted to concrete and poured gypsum surfaces with inclines not exceeding 1 inch to the foot and shall not be used on inclines greater than this.

Such roofing is durable and should not require repairs or renewals on account of the action of the elements, in the case of type 3TCS, for 10 years, and in the case of types 4TCS and 5TCS for 20 years.

While the surface of roofing of type 5TCS will require some attention in about the same time as roofing of type 4TCS. if the roofing is allowed to stand without attention until complete failure, longer life can be expected of type 5TCS than from type 4TCS.

Roofing of types 4TWS and 5TWS is adapted to board sheathing with inclines not exceeding 3 inches to the foot or precast gypsum slabs or blocks with inclines not exceeding 1 inch to the foot and shall not be used on inclines greater than these.
Roofing constructed in the manner specified is durable and should not require repairs or renewals on account of the action of the elements for a period of 10 years for type $4 \mathrm{TWS}$ and 20 years for type 5TWS.

\subsection{Tar Oils.}

American Wood-Preservers' Association, Manual of Recommended Practice. Standard specification for coal-tar oil for flooring and paving blocks, 1922.

The oil shall be a coal-tar product, of which at least 65 per cent shall be a distillate of coal-gas tar or coke-oven tar, and the remainder shall be refined or filtered coal-gas tar or coke-oven tar. It shall comply with the following requirements:

1. It shall not contain more than 3 per cent of water.

2 . It shall not contain more than 3 per cent of matter insoluble in benzol.

3. The specific gravity of the oil at $38^{\circ} \mathrm{C}$. compared with water at $15.5^{\circ} \mathrm{C}$. shall be not less than 1.07 nor more than 1.14 .

4. The distillate, based on water-free oil, shall be within the following limits:

Up to $210^{\circ}$ C., not more than 5 per cent.

Up to $235^{\circ} \mathrm{C}$., not more than 25 per cent.

5. The specific gravity of the fraction between $235^{\circ}$ and $315^{\circ} \mathrm{C}$. shall be not less than 1.03 at $38^{\circ} \mathrm{C}$. compound with water at $15.5^{\circ} \mathrm{C}$.

The specific gravity of the fraction between $315^{\circ}$ and $355^{\circ} \mathrm{C}$. shall be not less than 1.10 at $38^{\circ} \mathrm{C}$. compared with water at $15.5^{\circ} \mathrm{C}$.

6. The residue above $355^{\circ} \mathrm{C}$., if it exceeds 35 per cent, shall have a float test of not more than 80 seconds at $70^{\circ} \mathrm{C}$.

7. The oil shall yield not more than 10 per cent coke residue.

\subsection{BITUMINOUS SURFACE TREAT-}

\section{MENTS. (See also 518.34.)}

American Association of State Highway Officials, tentative standard specifications for highway materials, 1927.

(See 505.13, p. 146.)

\section{OZOKERITE AND OTHER MINERAL WAXES}

(No nationally recognized specifications available.)

509. MISCELLANEOUS OILS

509.1 FLOOR OILS.

(No nationally recognized specifications available.)

509.2 PROTECTIVE OILS.

(No nationally recognized specifications available.)

509.3 HYDROLINE OILS.

(No nationally recognized specifications available.)

509.4 WELI OIL.

(No nationally recognized specifications available.)

509.5 SPECIAI OIL.

(No nationally recognized specifications available.)

509.6 TEIMPERING OIL.

(No nationally recognized specifications available.)

\subsection{WASHING OIL.} able.)

No nationally recognized specifications avail- 


\section{0-519 STONE, SAND, AND CEMENTITIOUS MATERIALS}

\section{GENERAI ITEMS.}

American Society for Testing Materials, standard method of test for abrasion of rock, serial designation D2-26, 1926.

This well-known method of test is similar in almost all respects to the Deval abrasion test of the French School of Roads and Bridges. It has been used since 1878 , and is entirely satisfactory for the purpose for which it was designed.

1. The machine shall consist of one or more hollow iron cylinders; closed at one end and furnished with a tightly fitting iron cover at the other; the cylinders to be $20 \mathrm{~cm}$ in diameter and $34 \mathrm{~cm}$ in depth inside. These cylinders are to be mounted on a shaft at an angle of $30^{\circ}$ with the axis of rotation of the shaft.

2. At least 30 pounds of coarsely broken stone shall be available for a test. The rock to be tested shall be broken in pieces as nearly uniform in size as possible, and as nearly 50 pieces as possible shall constitute a test sample.

3. The total weight of rock in a test shall be within $10 \mathrm{~g}$ of $5 \mathrm{~kg}$. All test pieces shall be washed and thoroughly dried before weighing. Ten thousand revolutions, at a rate of between 30 and 33 per minute, shall constitute a test. Only the percentage of material worn off which will pass through a $0.16-\mathrm{cm}$ (1/16-inch) mesh sieve shall be considered in determining the amount of wear. This may be expressed either as the percentage of the $5 \mathrm{~kg}$ used in the test, or the French coefficient, which is in more general use, may be given; that is, coefficient of wear $=20 \times \frac{20}{w}=\frac{400}{w}$, where $w$ is the weight in grams of the detritus under $0.16 \mathrm{~cm}$ ( $1 / 16$ inch) in size per kilogram of rock used.

American Society for Testing Materials, standard method of test for toughness of rock, serial designation D3-18, 1918.

Approved as Tentative American Standard, by the American Engineering Standards Committee now the American Standards Association.

1. Toughness, as applied to rock, is the resistance offered to fracture under impact, expressed as the final height of blow required of a standard hammer to cause fracture of a cylindrical test specimen of given dimensions.

2. Any form of impact machine which will comply with the following essentials may be used in making the test:

(a) A cast-iron anvil weighing not less than $50 \mathrm{~kg}$, firmly fixed upon a solid foundation.

(b) A hammer weighing $2 \mathrm{~kg}$, arranged so as to fall freely between suitable guides.

(c) A plunger made of hardened steel and weighing $1 \mathrm{~kg}$, arranged to slide freely in a vertical direction in a sleeve, the lower end of the plunger being spherical in shape with a radius of $1 \mathrm{~cm}$.

(d) Means for raising the hammer and for dropping it upon the plunger from any specified height from 1 to not less than $75 \mathrm{~cm}$, and means for determining the height of fall to approximately $1 \mathrm{~mm}$.

(e) Means for holding the cylindrical test specimen securely on the anvil without rigid lateral support, and under the plunger in such a way that the center of its upper surface shall, throughout the test, be tangent to the spherical end of the plunger at its lowest point,
3. Quarry samples of rock from which test specimens are to be prepared shall measure at least 6 inches on a side and at least 4 inches in thickness, and when possible shall have the plane of structural weakness of the rock plainly marked thereon. Samples should be taken from freshly quarried material, and only from pieces which show no evidences of incipient fracture due to blasting or other causes. The samples should preferably be split from large pieces by the use of plugs and feathers and not by sledging. Commercial stone-block samples from which test specimens are to be prepared shall measure at least 3 inches on each edge.

4. Specimens for test shall be cylinders prepared as described in section $5,25 \mathrm{~mm}$ in height and from 24 to $25 \mathrm{~mm}$ in diameter. Three test specimens shall constitute a test set. The ends of the specimen shall be plane surfaces at right angles to the axis of the cylinder.

5 . One set of specimens shall be drilled perpendicular and another parallel to the plane of structural weakness of the rock, if such plane is apparent. If a plane of structural weakness is not apparent, one set of specimens shall be drilled at random. Specimens shall be drilled in a manner which will not subject the material to undue stresses and which will insure the specified dimensions. The ends of the cylinders may be sawed by means of a band or diamond saw ${ }^{1}$ or in any other way which will not induce incipient fracture, but shall not be chipped or broken off with a hammer. After sawing, the ends of the specimen shall be ground plane with water and carborundum or emery on a cast-iron lap until the cylinders are $25 \mathrm{~mm}$ in length.

6 . The test shall consist of a $1 \mathrm{~cm}$ fall of the hammer for the first blow, a $2 \mathrm{~cm}$ fall for the second blow, and an increase of $1 \mathrm{~cm}$ fall for each succeeding blow until failure of the test specimen occurs.

7. The height of the blow in centimeter at failures shall be the toughness of the test specimen. The individual and the average toughness of three test specimens shall be reported when no plane of structural weakness is apparent. In cases where a plane of structural weakness is apparent, the individual and average toughness of the three test specimens in each set shall be reported and identified. Any peculiar condition of a test specimen which might affect the result, such as the presence of seams, fissures, etc., shall be noted and recorded with the test result.

American Society for Testing Materials, standard definitions of terms relating to materials for roads and pavements, serial designation D8-18, 1918.

\section{BITUMINOUS MATERIALS}

\section{RELATING IN GENERAI TO BITUMINOUS MATERIALS}

Bitumens.-Mixtures of native or pyrogenous hydrocarbons and their nonmetallic derivatives, which may be gases, liquids, viscous liquids, or solids, and which are soluble in carbon disulfide

Bituminous.-Containing bitumen or constituting the source of bitumen.

1 The form of diamond drill and the form of diamond saw as described in U.S. Department of Agriculture methods for the determination of physical properties of road-huilding rock is recommended and should prove satisfactory if instructions are followed. 
Bituminous emulsion.-A liquid mixture in which minute globules of bitumen are held in suspension in water or a watery solution.

Cut-back products.-Petroleum or tar residuums which have been filised with distillates.

Flux.-Bitumens, generally liquid, used in combination with harder bitumens for the purpose of softening the latter.

Liquid bituminous materials.-Those haring a penetration at $25^{\circ} \mathrm{C}$. $\left(77^{\circ} \mathrm{F}\right.$.), under a load of $50 \mathrm{~g}$ applied for one second of more than 350 .

Semisolid bituminous materials.-Those having a penetration at $25^{\circ} \mathrm{C} .\left(77^{\circ} \mathrm{F}\right.$.), under a load of $100 \mathrm{~g}$ applied for five seconcs, of more than 10 , and a penetration at $25^{\circ} \mathrm{C}$. $\left(77^{\circ} \mathrm{F}.\right)$, under a load of $50 \mathrm{~g}$ applied for one second, of not more than 350 .

Solid bituminous materials.-Those having a penetration at $25^{\circ} \mathrm{C}$. $\left(77^{\circ} \mathrm{F}\right.$.), under a load of $100 \mathrm{~g}$ applied for five seconds, of not more than 10 .

\section{RELATING SPECIFICALIY TO PETROLEOM OR ASPHALTS}

Asphalts.-Solid or semisolid native bitumens, solid or semisolid bitumens obtained by refining petroleum, or solid or semisolid bitumens which are combinations of the bitumens mentioned with petroleums or derivatives thereof, which melt upon the application of heat and which consist of a mixture of hydrocarbons and their derivatives of complex structure, largely cyclic and bridge compounds.

Artificial asphalt.-Recommended that the use of term be discontinued.

Asphalt cement.-A fluxed or unfluxed asphalt specially prepared as to quality and consistency for direct use in the manufacture of bituminous parements, and having a penetration at $25^{\circ} \mathrm{C}$. $\left(77^{\circ} \mathrm{F}\right.$.) of between 5 and 250 , under a load of $100 \mathrm{~g}$ applied for five seconds.

Liquid asphalt.-This is a trade term not subject to definition.

Native asphalt.-Asphalt occurring as such in nature.

Road asphalt.-This is a trade term not subject to definition.

Rock asphalt.- Sandstone or limestone naturally impregnated with asphalt.

Petroleum.- - Liquid bitumen occurring as such in nature.

Blown petroleums.-Semisolid or solid products produced primarily by the action of air upon liquid native bitumens which are heated during the blowing process.

Topped petroleum.-Petroleum deprived of its more volatile constituents.

Asphaltenes.- The components of the bitumen in petroleums, petroleum products, malthas, asphalt cements, and solid native bitumens, which are soluble in carbon disulphide but insoluble in paraffin naphthas.

Carbenes.-The components of the bitumen in petroleums, petroleum products, malthas, asphalt cements, and solid native bitumens, which are soluble in carbon disulphide but insoluble in carbon tetrachloride.

\section{RELATING SPECIFICAIIT TO TARS AND PITCHES}

Tars.-Bitumens which yield pitches upon fractional distillation and which are produced as distillates by the destructive distillation of bitumens, pyrobitumens, or organis materials.

Coal tar.-The mixture of hydrocarbon distillates, mostly unsaturated ring compounds, produced in the destructive distillation of coal.

Gas-house coal tar.-Coal tar produced in gashouse retorts in the manufacture of illuminating gas from bituminous coal.
Coke-oven tar-Coal tar produced in by-product coke ovens in the manufacture of coke from bituminous coal.

Oil-gas tars.-Tars produced by cracking oil rapors at high temperatures in the manufacture of oil gas.

Water-gas tars.-Tars produced by cracking oil vapors at high temperatures in the manufacture of carburetted water gas.

Dehydrated tars.- Tars from which all water has been removed.

Refined tar.-Tar freed from water by evaporation or distillation which is continued until the residue is of desired consistency; or a product produced by fluxing tar residuum with tar distillate.

Dcad oils.-Oils with a density greater than water which are distilled from tars.

$P$ itches.- Solid residues produced in the evaporation or distillation of bitumens, the term being usually applied to residues obtained from tars.

Straight-run pitch.-A piteh run to the consistency desired, in the initial process of distillation, without subsequent fluxing.

Free carbon in tars.-Organic matter which is insoluble in carbon disulphide.

\section{RELATING SPECIFICALIY TO TESTS}

Normal temperature.-As applied to laboratory observations of the physical characteristics of bituminous materials, is $25^{\circ} \mathrm{C}$. $\left(77^{\circ} \mathrm{F}\right.$.).

Consistency.-The degree of solidity or fluidity of bituminous materials.

Fixed carbon.-The organic matter of the residual coke obtained upon burning hydrocarbon products in a covered vessel in the absence of free oxygen.

Penetration.-The consistency of a bituminous material expressed as the distance that a standard needle vertically penetrates a sample of the material under known conditions of loading, time, and temperature. Where the conditions of test are not specifically mentioned, the load, time, and temperature are understood to be $100 \mathrm{~g}$, five seconds, and $25^{\circ} \mathrm{C}$. $\left(77^{\circ} \mathrm{F}\right.$. $)$, respectively, and the units of penetration to indicate hundredths of a centimeter.

$V$ iscosity.-The measure of the resistance to flow of a bituminous material, usually stated as the time of flow of a given amount of the material through a given orifice.

\section{NONBITUMINOUS MATERIALS}

\section{RELATING IN GENERAL TO NONBITUMINOUS MATERIALS}

Aggregate.-The inert material, such as sand, gravel, shell, slag or broken stone, or combinations thereof, with which the cementing material is mixed to form a mortar or concrete.

Matrix.-The binding material or mixture of binding material and fine aggregate in which the large aggregate is imbedded or held in place.

Crusher-run.-The total unscreened product of a stone crusher.

Stone chips.- Small angular fragments of stone containing no dust.

Tailings.-Stones which after going through the crusher do not pass through the largest openings of the screen.

Rubble.-Rough stones of irregular shapes and sizes, broken from laiger masses either naturally or artificially, as by geological action, in quarrying, or in stone cutting or blasting.

\section{RELATING SPECIFICALIY TO MATERIALS}

Bank gravel.-Gravel found in natural deposits, usually more of less intermixed with fine material, such as sand or clay, or combinations thereof; gravelly clay, gravelly sand, clayey gravel, and sandy 
gravel indicate the varying proportions of the materials in the mixture.

Clinker.-Generally a fused or partly fused byproduct of the combustion of coal, but also including lava and Portland-cement clinker and partly vitrified slag and brick.

Chert.-Compact siliceous rock formed of calcedonic or opaline silica, or both.

Granite.-A granitoid igneous rock consisting of quartz, orthoclase, more or less oligoclase, biotite, and muscovite.

Granitoid.-A textural term to describe those igneous rocks which are entirely composed of recognizable minerals.

Slag.- Fused or partly fused compounds of silica in combination with lime or other bases, resulting in secondary products from the reduction of metallic ores.

Soil.-A mixture of fine earthy material with more or less organic matter resulting from the growth and decomposition of vegetation or animal matter.

\section{RELATING SPECIFICALIY TO TESTS}

Mesh.-The square opening of a sieve.

Screen.-In laboratory work an apparatus, in which the apertures are circular, for separating sizes of material.

Sieve.-In laboratory work an apparatus, in which the apertures are square, for separating sizes of material.

American Society for Testing Materials, standard methods of sampling stone, slag, gravel, sand, and stone block for use as highway materials, including some material survey methods, serial designation D75-22; 1922 .

Approved as tentative American standard by the American Engineering Standards Committee, now the American Standards Association.

1. Samples of all materials for test upon which is to be based the acceptance or rejection of the supply shall be taken by the engineer or his authorized representative. Samples for inspection or preliminary test may be submitted by a producer or owner of the supply.

\section{SAMPLING OF STOIE}

\section{A. SAMPIITG OF STON FROM LEDEES OR QUARRISS FOR}

2. The ledge or quarry face shall be inspected closely to determine any variation in different layers. Any difference in color or structure shall be observed, and if necessary to secure unweathered specimens, pieces broken from different layers.

$3(a)$. For standard stone test separate samples shall be taken of at least 30 pounds each of fresh unweathered specimens from all layers that appear to vary in color or structure. When more than one piece is taken, the minimum size shall be 2 inches, except that there shall be one piece of each sample of a minimum size of 4 by 5 by 3 inches on which the bedding plane is marked and which shall be free from seams or fractures to be used in the toughness or compression test.

(b) The sample for concrete test will depend on the kind of tests to be made and the number of specimens necessary.

4. In addition to the general information accompanying all samples, samples from local ledges not commercial sources shall contain the following: Name of owner, approximate quantity available (if quantity is very large this can be recorded as practically unlimited), amount and character of overburden or stripping, haul to nearest point on road where the material is to be used, character of haul (kind of road and grade); also some detailed record of the extent and location of the material represented by each sample. For this purpose a sketch, plan, and elevation showing the thickness and location of the different layers is recommended.

\section{B. SAILPLING OF STONE FRONI COMBIERCLAL QUARRIES}

5. Where practical, samples from commercial quarries shall be taken from the ledge or quarry face and the same procedure shall be followed as when sampling local ledges.

6. At the plant, the following factors affect the size of broken stone: Size and shape of screen openings, length of screen sections, the angle of screen with horizontal, the speed of screen rotation, and the rate at which the screen is fed. A general inspection and record of these conditions shall be made. The sample shall be taken preferably from cars or boats while loading from stock piles or bins. It is recommended, in order that the sample may be representative, that separate samples be taken at different times while the material is being loaded. If the sample has to be taken from the bin or stock pile, several samples shall be taken from different parts of the stock pile and from the top of the bin and the loading chute. These separate samples shall be well mixed in a composite sample and the sample for test obtained by the quartering method.

7. (a) Where it is not practical to visit the plant, samples for both quality and size shall be taken from different parts of the car or boat during unloading. It is recommended that separate samples be taken from the top, middle, and bottom of car or boat. These separate samples shall be well mixed in a composite sample and the sample for test obtained by the quartering method. The results of tests on crushed stone for quality are not considered comparable with results from the samples specially broken for test in the laboratory, but the general quality of stone can be ascertained and check tests on various shipments should indicate any change in quality. In this case, tests for both quality and size can be made on the same sample.

(b) Where test is to be made for size only, it is recommended that a small set of screens and a pair of scales or some unit measure receptacle for measuring volume be used for field testing in order not to delay decision on the use of the material. Occasional check tests can be obtained from the laboratory to assure a fair degree of accuracy in field testing.

8. (a) The sample of crushed stone for mechanical analysis shall weigh at least fifty times the weight of the largest piece therein.

(b) The sample for concrete test will depend on the kind of tests to be made and the number of specimens necessary.

\section{SAMPLING OF FIELD STOEE ATD BOWLDERS}

9. A detailed inspection of the deposits of field stone and bowlders, over the area where the supply is to be obtained, shall be made. The different kinds of stone and its state of preservation in the various deposits shall be recorded.

10. Separate samples shall be taken of all stone of different classes that a visual inspection indicates, from state of preservation and degrees of lamination, would be considered for use in construction.

11. Records accompanying samples of field stone and bowlders, in addition to general information, shall contain the following:

(a) Location of supply. - The plotting of the fieldstone and bowlder area on a United States topographic or a similar map is recommended for this purpose.

(b) A pproximate quantity available.-A fairly accurate estimate of amount of stone in fences can be 
made by measuring a cross section and pacing the length. It is more difficult to estimate the amount of stone scattered on the ground, and ordinarily such deposits do not pay to work unless quite congested.

(c) Information regarding the percentage of different classes of stone which were sampled and the percentages of material which can be rejected by visual examination and may have to be handled and spoiled.-This information regarding the percentages of different kinds of material can only be estimated and the degree of accuracy attained will depend almost entirely upon the experience and skill of the individual.

\section{SAMPLING OF BLAST-FURNACE SLAG}

12. It is recommended that blast-furnace slag be sampled for size and quality by the method specified for broken stone.

\section{SAMPLING OF SAND AND GRAVEL}

\section{A. NONCOMMERCIAL DEPOSITS}

13. Noncommercial as used here includes all undereloped sand and gravel deposits and all developed deposits where the material is not washed or screened.

14. (a) The investigator should realize that few, if any, natural sand and gravel deposits are uniform and when a sample is taken the quantity of material in the deposit similar to the sample shall be ascertained as nearly as possible.

(b) Where possible, samples of sand shall be taken when it is in a damp condition.

(c) If the deposit is worked as a bank or pit and has an open face, the sample shall be taken by channeling the open face so as to represent material that visual inspection indicates could be used. Care shall be taken to eliminate any overburden or stripping at the top or any that has fallen along the face from the top. It is necessary, especially in small deposits, to excarate test pits some distance back of and parallel to the face to determine the extent of the supply. The number and depth of these pits depend on the quantity of material that is to be taken from the deposit. Separate samples shall be taken from the face of the bank and from test pits. These shall be well mixed in a composite sample and the sample for test obtained by the quartering method.

(d) Deposits that have no open face shall be sampled by means of test pits. The number and depth of these will depend on local conditions and the amount of material to be used from the source. A separate sample shall be taken from each pit and where visual examination indicates no radical difference in size of grain, color, etc., these shall be well mixed in a composite sample and the sample for test obtained by the quartering method. Where visual inspection indicates a distinct difference in material from different pits, separate samples shall be taken for test.

(e) It is suggested that the colorimetric test be used for determining the percentage of organic material. ${ }^{2}$

15. In addition to the general information accompanying all samples from sand and gravel deposits not commercial sources, the same detailed information shall be supplied as with samples of stone taken from local ledges.

\section{B. COMMERCIAL SAND AND GRAVEL PLANTS}

16. Samples of both sand and gravel from commercial screening or washing plants shall be taken from the bins or storage piles preferably while the

3 For a description of this test see A. S. T. M. C40, 512.10, p. 213 material is being loaded from these supplies. The sand and gravel shall be sainpled as separate units and shipped as such. It is difficult to secure a representative sample from stock piles and bins and, if conditions require sampling from these sources, the following is recommended: Separate samples shall be taken from different parts of the stock pile care being taken to avoid sampling a segregated area of coarse-grained material which is likely to exist at the base of the pile. In sampling from a bin, separate samples shall be taken from the top and the loading chute. At the latter place at least one-half eubic yard of material shall be run off and representative samples taken from it. These separate samples shall be well mixed in a composite sample and the sample for test obtained by the quartering method.

17. The size of sand and gravel will be affected by the same factors as broken stone and the same inspection is necessary. In addition, the relative amount of water used in screening must be taken into consideration. The same procedure for taking a sample of broken stone for size is recommended for sampling sand and gravel for size, both at plant and at delivery.

18. Where it is not practical to visit the plant, samples for both quality and size shall be taken from different parts of the car or boat during unloading. It is recommended that separate samples be taken from the top, middle, and bottom of car or boat. These separate samples shall be well mixed in a composite sample and the sample for test obtained by the quartering method.

\section{QDANTITY OF SAND AND GRAVEL SAMPLES}

19. Samples of run of bank (where the sand and gravel are combined) shall consist of at least 100 pounds of material where the gravel content is 50 per cent or more of the whole. If the gravel is less in percentage, the sample shall be increased in proportion. For example, where the gravel percentage is 25 per cent of the whole the sample should contain 200 pounds.

20. Samples of sand shall contain at least 20 pounds of material.

21. Samples of gravel shall contain at least 50 pounds of material.

22. The sainple for concrete test will depend on the kind of tests to be made and the number of specimens necessary.

\section{SAMPIING OF MISCELLANEOUS MATERIALS}

23. Samples of slag sand, screenings, mine tailings, and all other materials used as a substitute for sand and gravel or broken stone, shall be inspected in the same manner and samples taken in the same way as the materials for which they are substituted.

\section{SAMPLING OF STONE BLOCK}

24. Samples shall be taken either at the quarry or from cars or boats as directed by the engineer. They shall be representative of the block which it is proposed to use and no sample shall include blocks that would be rejected by visual inspection.

25. The sample shall consist of at least six blocks and the bedding plane shall be marked on at least two of these.

\section{GENERAL DIRECTIONS FOR SHIPPIHG AND MARKING SAMPLES}

26. Samples of ledge stone, crushed stone, and slag shall be shipped in a secure box or bag.

27. Samples of stone block shall be securely crated. 
28. Samples of run of bank gravel, sand screenings, and other fine material shall be shipped in a tight box or closely woven bag so there shall be no loss of the finer particles.

29. Each sample or separate container shall be accompanied by a card or regular form, preferably in the container, giving the following information: By whom taken, official title or rank of the sampler; by whom submitted; source of supply; proposed use for the material; and in case of commercial supplies, daily production; geographic location; shipping facilities (name of railroad, canal or river, or other common carrier); and price of the material.

Asphalt Shingle and Roofing Association (now Institute), tests for granular surfacing materials, 1922.

The following tests are adapted for examining crushed slate, or other mineral granules, for use in surfacing composition roll roofings and shingles:

1. Sampling.-Each carload of granular surfacing material shall be sampled as follows: By means of a

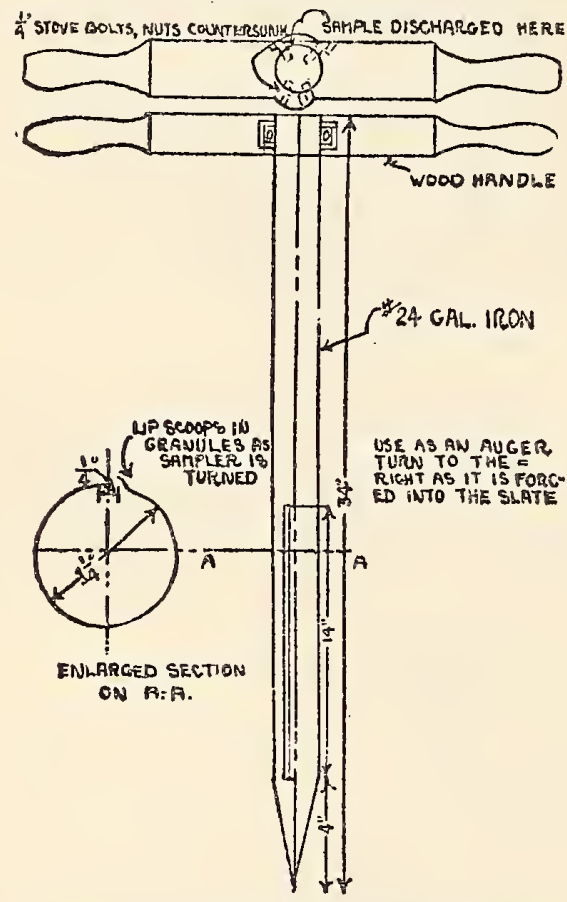

FigURE 77.-Sampler for testing granular surfacing materials

sampler of the form illustrated (see fig. 77) or any other equivalent type of sampler, procure at least 12 samples throughout the car ranging from 1 to 2 pounds each, including one near the top and one near the bottom of the load, respectively, from at least six different places, each near the center of an imaginary square dividing the car horizontally into a corresponding number of areas, approximateiy equal in magnitude. The samples are then combined, carefully mixed together and by the standard method of quartering, which may either be performed by hand, or by a Jones, or other equivalent type of riffler, reduced in bulk to obtain a representative sample of $500 \mathrm{~g}$.

2. Moisture.-Weigh out $50 \mathrm{~g}$ of the well-mixed sample and heat in an oven to constant weight at a temperature of $225^{\circ} \mathrm{F}$. The loss in weight represents "moisture," which is calculated in percentage based on the material as received.
3. Permanency in color.-Fill two flat pint flasks of clear white glass, with the granules, and pack tightly with cotton wadding before inserting the corks. One of the flasks is kept indoors in a dark place and the other exposed flat on its side out of doors, in a sunny spot. The colors of the granules in the two flasks are then compared at the end of one, two, three, and four weeks, respectively. Any fading or alteration in the color of the granules in the flask exposed out of doors is recorded.

4. Granularmetric test.-Standard 8-inch sieves of the following mesh shall be used:

\begin{tabular}{|c|c|c|}
\hline Sieve & Opening & $\begin{array}{c}\text { Wire } \\
\text { diameter }\end{array}$ \\
\hline $\begin{array}{l}\text { 8-mesh } \\
\text { 10-mesh } \\
\text { 14-mesh } \\
\text { 35-mesh }\end{array}$ & $\begin{array}{l}\text { Inch } \\
0.093 \\
.065 \\
.046 \\
.0164\end{array}$ & $\begin{array}{l}\text { Inch } \\
0.032 \\
.035 \\
.025 \\
.0122\end{array}$ \\
\hline
\end{tabular}

The sieves must be thoroughly clean and dry. The sieving operation may either be performed by a mechanical shaker, where an apparatus of this type is available, or else by hand. Due to the inaccuracy of the finer mesh sieves after continued use, it is desirable to have on hand a set of new sieves against which the service set may be checked at intervals. Assemble the various sieves in the sequence named, with the coarsest on top and the dustpan on the bottom. Weigh $100 \mathrm{~g}$ of the wellmixed, moisture-free granules on the 8-mesh sieve. Attach the cover and shake the sieves in the machine (or else vigorously by hand) for five minutes. Each sieve (as well as the pan) plus the material retained shall then be weighed, and after deducting the weight of the sieve (or pan) the percentage retained shall be calculated on the weight of the moisturefree sample. A soft bristle brush is recommended for removing the particles which may have become lodged in the meshes of the sieves. Tapping the sieves after the brushing will help to free the particles. Express the results as follows:

Percentage by weight of the moisture-free granules retained on the 8 -mesh sieve.

Percentage by weight of the moisture-frce granules retained on the 10 -mesh sieve.

Percentage by weight of the moisture-free granules retained on the 14-mesh sieve.

Percentage by weight of the moisture-free granules retained on the 35-mesh sieve.

Percentage by weight of the moisture-free granules (that is, "fines") passing through the 35-mesh sieve.

5. Dust.-Weigh $50 \mathrm{~g}$ of the well-mixed, moisturefree granules on the 200-mesh sieve and treat as in section 4. After the shaking operation, weigh the material retained on the sieve. Then wash under a tap of water until the washings no longer appear turbid. The residue in the sieve is dried to constant weight at $225^{\circ} \mathrm{F}$. and reweighed. The loss in weight represents the powder which passes a 200mesh sieve plus any "dust" adhering to the coarscr granules. These combined weights are calculated in percentage against the moisture-free sample. Such powder and dust would have a tendency to inhibit the granules from adhering to the bituminous coating in manufacturing composition roofings and shingles.

Asphalt Shingle and Roofing Association (now Institute), tests for powdered surfacing materials (recommended), 1923.

The following test are adapted for examining talc, sand, mica, and other powdered materials used for surfacing composition roofing, as well as "fillers," including powdered material or dust of any kind 
intended to be incorporated in the bituminous surface coating of roll roofings or shingles.

\section{SAMPLING}

(a) When shipped loose in bulk, follow the method outhed in section 1 of "Tests for Granular Surfacing Materials," given above.

(b) When shipped in bags or other containers, select at random from each shipment or portion thereof representing a specific grade, a number of packages equivalent to the cube root of the total number included in the lot. If the cube root as calculated proves to be a fractional number, express it as the next higher digit in the following manner:

\begin{tabular}{|c|c|c|c|}
\hline If - & Take- & If- & Take- \\
\hline $\begin{array}{l}2-8 \\
9-27 \\
28-64 \\
65-125 \\
126-216 \\
217-343 \\
344-512 \\
513-729 \\
730-1,000 \\
1,001-1,331\end{array}$ & $\begin{array}{r}2 \\
3 \\
4 \\
5 \\
6 \\
7 \\
8 \\
9 \\
10 \\
11\end{array}$ & $\begin{array}{l}1,332-1,728 \\
1,729-2,197 \\
2,198-2,744 \\
2,745-3,375 \\
3,376-4,096 \\
4,097-4,913 \\
4,914-5,832 \\
5,833-6,859 \\
6,860-8,000 \\
8,001-9,261\end{array}$ & $\begin{array}{l}12 \\
13 \\
14 \\
15 \\
16 \\
17 \\
18 \\
19 \\
20 \\
21\end{array}$ \\
\hline
\end{tabular}

Remore approximately one-fourth pound from each package, then combine the samples, thoroughly mix them together and by the standard method of quartering, which may be performed by a Jones or other equivalent type of riffler, reduce the bulk of the material to obtain a representative sample of $500 \mathrm{~g}$.

\section{MOISTURE}

This shall be ascertained as in section 2 of tests for Granular Surfacing Materials.

\section{GRANULARMETRIC TEST}

This shall be ascertained as in section 4 of Tests for Granular Surfacing Materials, with the exception that the 48 and 200 mesh sieves shall be used in addition. The material which is retained on the 200-mesh sieve shall be treated as in section 5 of Tests for Granular Surfacing Materials, the loss in weight being reported as "dust." Should it be desired to classify the "dust" passing a 200mesh sieve, then the following method shall be used:

Place $5 \mathrm{~g}$ of the surfacing material in a beaker about $120 \mathrm{~mm}$ high holding $600 \mathrm{cc}$ and fill almost to the top with distilled water at exactly $70^{\circ} \mathrm{F}$. Agitate with compressed air until the mineral particles are brought into suspension, and in such a manner that no whirling results. Stop the blast and allow the liquid to stand exactly 20 seconds, whereupon the water above the sediment is immediately decanted through a 200-mesh sieve without, however, pouring off any of the sediment. The operations of agitation, sedimentation, and decantation are repeated with fresh water three times. The particles caught on the 200-mesh sieve are washed back into the sample remaining in the beaker, which is dried to constant weight and weighed. The difference represents the amount removed by elutrition, which should be expressed in percentage.

\section{SPECIFIC GRAVITY}

This shall be ascertained as in A. S. T. M. D55. (See 512.10, p. 214.)

United States Department of Agriculture, Bulletin 1216 , method of test for abrasion of broken stone and broken slag, 1928 .
This specification is based on A. S. T. M. D2 (See p. 182.)

1. The machine shall consist of one or more hollow iron cylinders, closed at one end and furnished with a tightly fitting iron cover at the other; the cylinders to be $20 \mathrm{~cm}$ in diameter and $34 \mathrm{~cm}$ in depth inside. These cylinders shall be mounted on a shaft at an angle of $30^{\circ}$ with the axis of rotation of the shaft.

2 . The rock to be tested shall be broken in the laboratory from pieces at least $3 \frac{1}{2}$ inches in size to as nearly uniform size and shape as possible, and as near to 50 pieces as possible shall constitute a test sample. No pieces with edges or faces that have been rounded by wear shall be included. The total weight of rock in a test shall be within $10 \mathrm{~g}$ of $5 \mathrm{~kg}$. All test pieces shall be washed and thoroughly dried before weighing. Ten thousand revolutions, at a rate of between 30 and 33 per minute, shall constitute a test. Only the percentage of material worn off which will pass through an American Society for Testing Materials standard No. 12 sieve (see 500.2, p. 1) shall be considered in determining the amount of wear. This shall be expressed as a percentage of the $5 \mathrm{~kg}$ used in the test.

Nоте.--In the case of broken stone, samples should, wherever possible, be selected from the quarry face.

3. When the material has a specific gravity below 2.20 the quantity used for the test shall be adjusted on a weight basis, retaining the specified number of pieces. For such materials a weight of $4,000 \mathrm{~g}$ of the broken stone or broken slag shall be used.

4. The results shall be reported as percentage of wear.

5. The operator is cautioned that drying some varities of stone at high temperatures has a deleterious effect upon the abrasion loss, and therefore care should be taken that the temperature be low enough to prevent injury to the stone through heat.

\section{STONE AND STONE MANUFACTURES}

\subsection{GRANITE.}

American Society for Municipal Improvements, specifications for stone block pavements, 1927.

See 518.36, p. 393 .

(This specification includes requirements for granite blocks.)

American Society for Testing Materials, block for granite pavements.

(This society has the following standards, which ar'e presented under 511.71 , p. 208.)

1. Block for granite block pavements, serial designation D59-26, 1926.

2. Block for recut granite pavements, serial designation D131-23, 1923.

3. Block for durax granite pavements, serial designation D132-23, 1923.

Federal Specifications Board, specification No. 510, United States Government master specification for block for granite, recut granite and durax granite pavements, August 12, 1927.

See 511.71 , p. 208.

National Builaing Granite Quarries Association, granite specifications.

Section 1. General conditions.-All work included under the specifications for granite is to be subject to the general conditions which govern the entire work.

Sec. 2. Material.-All granite shall be of compact structure, hard and practically nonabsorbent, and equal in durability and strength to the best granite of the kind required. Granite shall be (mention color, tore, and grain) and of the kind designated as (mention name) granite from quarries at (mention 
location). Granites designated as (mention names and quarries), will also be considered. In submitting estimates, the contractor shall state the name of granite, and quarry, upon which his proposal is based.

Sec. 3. Quarry and plant.-Granite must be obtained from approved, well-known quarries having capacity and facilities for furnishing the quantity sizes, and quality of granite required, and the cutting and finishing must be done by firms properly equipped to produce the finished material without causing delay in the progress of the work. Evidence to this effect must be submitted if required by the architect.

Sec. 4. Quality.-All the granite shall be selected to meet the requirements of these specifications, and shall be absolutely sound and free from seams or other defacts which would impair its strength. Exposed surfaces shall be free from spots, stain, discoloration, knot formations, spalls, chips, or other defects which would impair the appearance of the work, except that in inconspicuous places a reasonable number of knot spots or texture variations inherent to the particular granite proposed may be permissible if samples showing the maximum of such characteristics be submitted to, and approved by, the architect.

In quarrying the granite the blocks shall be so selected that any variations in color permitted by the architect will be uniformly distributed throughout the exposed surfaces of the walls and other portions of the work. If granites from different quarries are used such granites shall be similar in texture and shall satisfactorily match in color and tone throughout the work.

Sec. 5. Work included.-The work to be done by the contractor under the heading of "granite" shall include the furnishing, delivery, and setting in place and completion of all granite work as required by the drawings and herein specified.

The work generally shall include (state portions of work to be of granite).

Note.-State any portions or special features which are not to be of granite or not to be included in this contract.

Sec. 6. Shop drawings.-The granite contractor shall prepare all necessary shop drawings, showing the bedding, bonding, and jointing of all the granite work and typical and special anchoring of same. The dimensions and setting number of each granite stone shall be indicated upon the drawing which shall be submitted and approved by the architect as required under general conditions. No cutting shall be done or work completed except from shop drawings which have been approved by the architect. Sec. 7. Corner stone. ${ }^{3}$-The granite contractor shall furnish and set where indicated on the drawing (or as directed) a corner stone of the requircd dimensions, having an inscription cut thereon in accordance with the drawings and recessed to receive the copper box to be furnished by the (general contractor).

Sec. 8. Samples.-After the award of the contract two samples of each kind of granite required 8 by 8 by 2 inches, showing the extreme variation in quality, color, and texture that will occur in any granite which will be used, shall be submitted to the architect. Upon approval of these samples, one of each shall be returned to the granite contractor for use at the quarry or plant and the other retained by the architect for comparison with work at the building. Samples shall be dressed on the face and one edge to show finish required by the specification; opposite face may be split or dressed to the approximate size called for or may be dressed to another of the specified finishes called for.
Sec. 9. Finishes.-The exposed surfaces of the granite shall be dressed as indicated on the drawings or specified herein. In general, surface finishes shall be as follows: From 2 inches below grade to level (state level or course) shall be dressed with best (state finish as polished, 6-cut, 8-cut) work; from level (state level) to level (state level) shall be dressed with best (state finish) work; and from level (state level) to top of parapet shall be dressed with best (state finish) work.

NotE.-State here any exceptions to tbe above such as "treads of steps or platforms shall be ...... finisb;" "back of parapet and coping courses shall be ...... finish," etc.

The cut marks of all bush hammer work shall be vertical except as noted.

Soffits shall be bushed at right angles to the face.

Faces of key blocks and voussoirs shall be bushed (state whether vertical or radial).

Top surfaces of window and door sills, steps, copings, washes, and projecting courses shall be bushed at right angles to the nosing.

Molded surfaces shall be bushed parallel to the direction of the moldings.

NotE.-Mention otber specific instructions on direction of bushing.

Sec. 10. Cutting-All exposed surfaces must be out of wind, free from waves, projections or depressions and faces of granite in the same plane must be absolutely flush at joints. Arrises must be cut sharp and true to square or pattern and continuous with adjoining arrises.

Slight inequalities which may occur in setting shall be trimmed to the proper surfaces and refinished equal to the original finish.

Sec. 11. Beds and joints.-Beds shall be horizontal and shall be cut full and square for a distance of at least 2 inches back from the face, from which point they may fall off not to exceed 1 inch in 12 inches; and shall be reasonably free from large depressions and cuppings, which might impair the stability of the work.

Joints shall be dressed at right angles to the face for at least $1 \frac{1}{2}$ inches back from which point they may fall away, not to exceed $1 \frac{1}{1} 2$ inches in 12 inches.

Backs of granite stones shall be scabbled or split to approximate vertical surfaces which shall not vary more than 1 inch in 12 inches from the true vertical, nor vary more than 1 inch either way from the thickness called for on the drawings.

Sec. 12. Jointing.-The jointing of the granite work shall be as shown on the drawings, and no additional joints will be permitted except upon written consent of the architect. The joints shall be uniformly three-sixteenth inch (or one-fourth inch) in thickness.

Sec. 13. Bonding.-The bonding of various portions of the work shall be as shown on drawings. Alternate courses of granite shall bond at least 4 inches with the backing except where otherwise shown. No granite stone shall have less than 4inch bed; projecting courses shall have beds equal to the projections unless otherwise shown. Where brick backing is required the granite shall not go closer than $4 \frac{1}{2}$ inches to the inside of the brick wall. Where granite facing occurs at grades it shall extend nowhere less than 4 inches below grade unless otherwise shown.

Sec. 14. Reveals and returns.-Reveals of all openings, unless otherwise shown shall be cut solid without vertical joints. Returns shall be not less than indicated on the drawings. Mitering of granite stones at corners will not be permitted.

Scc. 15. Moldings.-Granite stones forming continuous molded courses shall be of uniform profile on the face with continuous unbroken lines absolutely flush at the joints and with the surfaces free from projections or depressions and out of wind. 
Sec. 16. Tashes and drips.-All exterior projecting granite stones and all exterior sills, steps, platforms, coping, and other stones with exposed top surfaces, shall be cut with a wash on top. Where other work is built upon such granite stones, they shall be cut with raised seats and lugs to form level beds for work built upon them.

All projecting granite stone, such as sills, cornices, copings, etc., shall have a groove drip cut on the underside unless otherwise detailed.

All exterior door sills shall be cut with raised thresholds unless otherwise shown.

Sec. 17. Miscellaneous.-Moldings, and projections must not be subjected to pressure; and granite stones having projecting members which have weight of any kind bearing upon the upper surface shall have seats cut to bear such weights; and in all cases the edges of moldings or projections must be kept free from pressure.

Reglets shall be cut for flashing and counter flashing as required.

Sec. 18. Models.-Full-size plaster models of all ornamental and carved work, shall be furnished to the granite contractor as hereinbefore specified.

Where necessary for the proper execution of the work, models will be delivered at the plant of the granite contractor free of expense to him, to be used by him for the purpose of roughing and such carving as may be done at the plant, the granite contractor to carefully preserve these models, for reshipment to the building if required. The expense of handling and recrating for shipment at the plant to be borne by the granite contractor.

Sec. 19. Roughing for carving.-No roughing for carved work is to be done from drawings, but from approved models only. Sufficient stock in all cases shall be left for the carving and the granite shall be roughed to suitable form and condition for the carver. The cutter and carver shall cooperate in the method of securing the proper roughing for ornamental rork.

Sec. 20. Carving.-Carving may be done at the site either before or after the granite is set, or the mork may be delivered at the site already carved. In case the carving is done at the plant or at the site before being set in place, this contractor shall do all necessary refinishing or retouching to make the carving conform to the models and to the satisfaction of the architect. All carved ornament shall be executed by hand by skilled carvers in a spirited and artistic manner and in strict accordance with the approved models.

Where carving is done after the work is set, all necessary staging and protection shall be furnished by the general contractor, and if required the models shall be hoisted into position and properly secured to the scaffolding for the convenient use of the carvers by the general contractor.

Inscriptions, lettering, or numerals, if required, shall be clean cut and in accordance with the models, if provided, or otherwise with the full-sized details of same.4 The incised surfaces of lettering shall be cut smooth and accurately to the full depth and section shown on the models or drawings.

Sec. 21. Crating and shipping.-This contractor shall properly crate the finished granite for shipment, the crating being so constructed as to properly protect the edges and surfaces of the exposed portions of the work during shipment and handling prior to setting same. Due precaution shall be taken to use crating material which will not stain or discolor the exposed surfaces of granite; and especial care shall be used to protect and suitably note any delicate portion where extra care should be observed in handling.

4 If carving is to be done by others than the granite contractor, same should he noted here and this specification modified to suit conditions.
The finished granite properly crated shall be carefully loaded for shipment by this contractor who shall exercise all necessary precautions in loading to withstand the usual hazards in transit.

Sec. 22. Precaution against stain.-Special precaution shall be taken in the setting to guard against possible seepage through the joints of moisture from the mortar or material used in backing up the granite work, which will cause discoloration around the face joints or surface of the granite.

At least 12 hours before the granite is set, all surfaces not exposed shall be thoroughly coated with an approved damp proof compound to within 1 inch of the exposed face. After the granite is set, and before backing up, another coat of the same damp proofing compound shall be applied to the back for the special purpose of covering the backs of the mortar joints.

The painting of the granite may be omitted with the approval of the architect when it is definitely known that the setting mortar will not stain the granite, but the backs of the mortar joints should be damp proofed in any event to guard against seepage.

If the first coat of damp proofing is applied at the mill, the setting numbers must be painted conspicuously over the damp proofing.

The granite shall at all times be protected from stain and upon delivery at the site shall be kept stacked on timber or platforms at least 4 inches above the ground, until set in place in the wall.

Under no circumstances shall salt be used for thawing out Lewis holes or otherwise in connection with the granite work.

Sec. 23. Setting.-Each granite stone shall be brushed clean and drenched immediately before being set. Each piece shall be carefully bedded in a full bed of nonstaining mortar and tapped home with a wooden mallet to a full and solid bearing.

The face of the granite work shall be kept free from mortar at all times.

Granite facing shall not in any case be built up more than two courses ahead of the backing and no stone having a greater width of bed then the one below it shall be set until the lower course is backed up.

All surplus mortar shall be immediately raked out to a depth of at least 1 inch and every precaution taken to prevent stones bearing upon the edges.

Sills, etc., subject to pressure, shall be bedded only at the ends.

The cement in the mortar used for setting all granite work where the joint is exposed to the weather shall be made waterproof with a satisfactory waterproofing compound, mixed with the mortar.

The sand used in all setting mortar shall be such as to cause no stain or chemical action with the cement.

Sec. 24. Anchors, dowels, etc.-All bolts, expansion bolts, anchors, ties, etc., required in the setting of the granite work, will be furnished to the granite contractor. Al! ashlar shall be anchored to the backing with heavily galvanized wrought-iron anchors one-fourth by $1 \frac{1}{4}$ inches turned down into the granite $1 \frac{1}{4}$ inches and extending into the backing 8 inches, if the thickness of wall permits; the end to be turned up $1 \frac{1}{2}$ inches into the backing. There shall be at least two anchors to every stone whose length exceed its height, and, in general, there shall be not less than two anchors to each superficial square yard of ashlar.

NOTE.-Special anchoring for heavy cornices and overhanging courses, cramps, dowels, etc., for parapets, balustrades, pilasters, and columns, etc., should he suitahly descrihed or shown according to the requirements of the work.

Sec. 25. Boxing and protection.-All granite work must be protected from damage during the progress of the work and until the completion of the building.

The general contractor shall provide the necessary protections, covering all projections, top surfaces, 
angles, etc., protective boxing to be securely fastened in position and securely nailed throughout with galvanized iron nails. No lumber or material to be used which would in any way stain or deface the granite work.

All necessary forms, centers, scaffolding, etc., required by the setter or carver to be furnished by the general contractor.

Sec. 26. Pointing and cleaning.-After the completion of the granite work or at such time thereafter as all liability from stain of other operations on the building is passed, and when there is no danger therefrom the whole of the granite work shall be carefully cleaned down, removing all dirt, mortar, stains, and other defacements.

The use of wire brushes, acids, or solutions which might cause discoloration will not be permitted.

All face joints shall be raked out to a depth of not less than 1 inch, brushed clean, thoroughly wetted, and filled with pointing mortar and then carefully jointed. The pointing mortar must be packed solidly into all joints, completely filling the same; and the form of joint shall be as directed by the architect.

Vertical joints in the top courses of uncovered cornices having a projection of 8 inches or more shall be filled with mortar by grouting to within 3 inches of the top of the granite, then calked with picked oakum and filled with molten lead, calked against the edges and slightly convex at the top, taking care that the oakum is kept at least 2 inches away from the face and top of granite.

Joints in the upper surfaces of projecting stones which are not so protected and in all platforms, steps, and coping, shall be raked out at least 2 inches deep and thoroughly grouted flush with the surface of the granite.

Pointing and cleaning shall start at the top and be continued until such work is completed.

Sec. 27. Defective work.-No patching or hiding of defects will be permitted. Defective granite stones shall be replaced with perfect ones except in extreme cases where a stone has been damaged through no fault of the granite contractor, and where it is possible and practicable to remedy the defect without in any way impairing the appearance, strength, or durability of the work-and then only with the approval and under the supervision of the architect-and where a satisfactory allowance has been agreed upon which shall be deducted from the contract price.

Note.-A short form of granite specifications has been prepared by the association for use where a comparatively small amount of granite is required, but in which the stendards of workmanship and other essentials should conform to requirements set forth in detail in the above complete form. Copies may be ohtained from the secretary of the National Building Granite Quarries Association.

National Paving Brick Manufacturers Association, recommended specifications, stone curb and header, 1924.

(See 518.63, p. 448.)

(This specification includes requirements for granitc, to be used as material.)

\subsection{LIMESTONE.}

Indiana Limestone Ins titute, uniform classification of Indiana limestone, September 26, 1928.

\section{CLASSIFICATION}

Buff statuary stock.

Select buff.

Standard buff.

Rustic buff.

Select gray.

Standard gray.

Rustic gray.

Variegated.

Old gothic.
Special hard buff (same as "select").

Special hard gray (same as "select").

NoтE.-This classification and designation of the grades is based on the assumption that in the submitting of samples of any particular grade for approval, the well-estahlished practice of two samples show. ing the maximum and minimum or fineness and coarseness of texture, thus showing the range of variation from uniformity, will be submitted, instead of only one sample intended to indicate the average run of the particular grade.

Standard practice-Samples.-The submitting of two samples has therefore been adopted as "standard practice"; except in the case of "statuary buff," where a single sample will usually suffice, and in the case of old gothic, the characteristic range of which can not be appropriately shown by only two samples, and for which grade there is no fixed limit as to variation in texture and formation or as to markings that are characteristic of the material.

\section{STATUARY BUFF}

This grade covers only the very fine uniformgrained buff stock, sold principally for sculpture and carved doorways, tracery, sculptured panels, memorials, and elaborate interior work.

In texture the grain shall not exceed $1 \mathrm{~mm}$ (about $1 / 25$ inch) in cross section, visibly uniform and well cemented in structure and entirely free from seams, streaks, and pit-holes, and without calcite crystals larger than $4 \mathrm{~mm}$ (about $1 / 6$ inch) in diameter.

\section{SELECT BUFF}

"Select buff" stock is sound and durable and shall embrace the average finer-grained stone, in texture ranging from statuary stock in which minor surface markings occur to stone having an average grain as shown by the samples and photo reproductions thereof designating the range of this grade. It shall include stone in which any openness of grain does not show pore spaces exceeding $1 \mathrm{~mm}$ (about $1 / 25$ inch) in diameter and in which tight fine opaque white or glasslike calcite streaks or veins not exceeding $1 \mathrm{~mm}$ (about $1 / 2.5 \mathrm{inch}$ ) in width may occur, and will include stonc having occasional solid fossils and grains, crystals, or spots of calcite. Pit-holes not exceeding $6 \mathrm{~mm}$ (about $1 / 4 \mathrm{inch}$ ) in diameter may occur, providing that there are not more than the equivalent of one of these to any 10 square fect of area of slabs nor more than 15 per cent of the cube of blocks so affected and in which any of these allowed minor variations in the formation and texture do not constitute a defect that will impair the strength and durability or appearance of the fabricated and finished stonework produced therefrom, and when appreciably affecting the appearance are such that can be eliminated readily from appearing on the face of stonework in the finished building.

\section{STANDARD BUFF}

"Standard buff" stock is sound and durable and is the average of the moderately large-grained buff product of the quarries that constitutes the bulk of the total output of buff stone and is therefore classed as "standard." This grade has certain variations in density, color tone, and texture not allowed in "select" and may contain streaks of calcite or moderately coarse-grained or reedy formation and small pit-holes, providing such variations in texture and color tone do not exceed the following limits or impair the strength or durability of the stone.

Texture or grain ranging from "select" to a somewhat shelly appearing formation having an average grain as shown by samples and photo reproductions thereof designating the range of this grade.

White calcite or crystalline streaks not over $4 \mathrm{~mm}$ (about $1 \%$ inch) in width nor more than one such marking in any 30 square feet of stone so affected. 
Glasslike calcite streaks or veins not over $2 \mathrm{~mm}$ (about $1 / 12$ inch) in width nor more tlian one such marking in any 30 square feet of stone so affected.

Coarse or open texture bands averaging not over 1 inch in width nor shall not aggregate over 6 per cent of the measurement across grain.

Pit or shell holes aggregating not over 1 square inch in area to any 30 square feet of stone.

Stock to be well cemented and, with the exception of the pit-holes and coarse, somewhat open-textured streaks allowed, shall not be visibly open or porous in general structure.

\section{RUSTIC BUFF}

"Rustic buff" stock is sound and durable and shall embrace the coarser and irregular, more or less open shelly grained stone, having a varying amount of crystalline calcite intermixed, including stone having a decidedly coarse, somewhat honeycomb or reedy formation, as shown by the samples and photo reproductions thereof designating the range of this grade. It may include some stone which is appreciably darker in color tone, some stone with streaks of fine texture, and some stone that is quite hard, due to the size and amount of crystalline calcite that it contains. This grade is therefore distinctly coarser and more varying in texture, showing a wider range of granular formation and more variation in color tone than any other grade, except "old gothic."

\section{SPECIAL HARD BUFF}

"Special hard buff" for the purpose of grading is classed as "select buff." It is the fine-grained, hard, dense stone similar to the regular "select buff", grade in texture and may be included as such, but as a grade by itself, shall be specially selected for its hard, dense, usually somewhat crystalline texture, making it particularly suitable for grade or base courses, steps, platforms, floor tiling, paving, and other walkway surfaces.

\section{SELECT GRAY}

"Select gray" stock is sound and durable and shall embrace the average finer-grained gray stone, in texture ranging from the finest, most uniform grained stock to stone having an average grain, as shown by the samples and photo reproductions thereof designating the range of this grade. It shall include stone in which any openness of grain does not show pore spaces exceeding about $1 \mathrm{~mm}(1 / 25$ inch) in diameter and in which tight, fine opaque white or glasslike calcite streaks or veins not exceeding $1 \mathrm{~mm}$ (about $1 / 25$ inch) in width may occur and will include stone having occasional solid fossils and grains, crystals or spots of calcite. Pit-holes not exceeding $6 \mathrm{~mm}$ (about $1 / 4$ inch) in diameter may occur, providing that there are not more than the equivalent of one of these to any 10 square feet of area of slabs, nor more than 15 per cent of the cube of blocks so affected, and in which any of these allowed minor variations in the formation and texture do not constitute a defect that impairs the strength and durability or appearance of the fabricated and finished stonework produced therefrom, and when appreciably affecting the appearance are such that can be eliminated readily from appearing on the face of stonework in the finished building.

\section{STANDARD GRAY}

"Standard gray" stock is sound and durable and is the average of the moderately large-grained gray product of the quarries that constitutes the bulk of the total output of gray stone and is therefore classed as "standard." This grade has certain variations in density, color tone, and texture not allowed in "select" and may contain streaks of calcite or moderately coarse grained or reedy formation and small pit-holes, providing such variations in texture and color tone do not exceed the following limits or impair the strength or durability of the stone:

Texture or grain ranging from select to a somewhat shelly appearing formation having an average grain, as shown by samples and photo reproductions thereof designating the range of this grade.

White calcite or crystalline streaks not over $4 \mathrm{~mm}$ (about $1 / 6$ inch) in width nor more than one such marking in any 30 square feet of stonc so affected.

Glasslike calcite streaks or veins not over $2 \mathrm{~mm}$ (about $1 / 12$ inch) in width nor more than one such defect in any 30 squarc feet of stone so affected.

Coarse or open texture bands averaging not over 1 inch in width nor shall not aggregate over 6 per cent of the measurement across grain.

Pit or shell holes aggregating not over 1 square inch in area to any 30 square feet of stone.

Stock to be well cemented and with the exception of the pit-holes and coarse somewhat open-textured streaks allowed shall not be visibly open or porous in general structure.

\section{RUSTIC GRAY}

"Rustic gray" stock is sound and durable and shall embrace the coarser, more or less open shelly grained gray stone, having a varying amount of crystalline calcite intermixed, including stone having a decidedly coarse structure with some honeycomb formation, as shown by the samples and photo reproductions thereof designating the range of this grade. Rustic gray, by the nature of the deposit, will generally be somewhat denser in formation than rustic buff, and regardless of its coarse shelly texture will usually be practically free of any very open-grain stone of more or less cellular formation like travertine, such as is characteristic of and will occur in the rustic buff grade, but may include some stone which is darker in color tone, some stone with streaks of fine texture and some stone that is quite hard, due to the size and amount of crystalline calcite that it contains. This grade, like rustic buff, is coarser and more varying in texture and shows a wider range of granular formation and variation in color tone than any of the other grades, excepting "rustic buff" and "old gothic."

\section{SPECIAL HARD GRAY}

"Special hard gray" for the purpose of grading is classed as "select gray." It is the fine-grained, hard, dense stone, similar to the regular select gray grade in texture and may be included as such, but as a grade by itself, shall be specially selected for its hard, dense, usually somewhat crystalline texture, making it particularly suitable for grade or base courses, steps, platforms, floor tiling, paving, and other walkway surfaces.

\section{VARIEGATED}

"Variegated" stock is sound and durable and shall embrace the irregular mixture of the buff and gray color tones which occur in the blocks that are quarried where the buff and gray color tones adjoin in the quarry ledges and includes stone showing variation in texture as well as the variation in color tone, as shown by the samples and photo reproductions thereof designating the range of this grade.

"Variegated" stock includes stone that may vary in texture from the lower range of "standard" up through "select" as herein graded by texture, and consequently from moderately large to quite finegrained stone, and will include some stone containing streaks or veins of crystalline calcite and bands or areas of shelly formation that do not affect the 
structural soundness of the stone or make the stone stock too coarse or variable to be used for the average run of "cut stone" work, trim, etc.

The coarser-grained and more variable of the varibuff-gray color toned stone that is equivalent to rustic buff or rustic gray in texture, shall be included in the "old gothic" classification.

It is understood that while the quarry blocks shall contain stone of both color tones, that variegated stock when cut up for building purposes, will in the finished work produce pieces of solid buff and solid gray and only a certain percentage of stone with the two color tones in one piece.

\section{OLD GOTHIC}

This grade of stock is sound and durable and embraces gray, buff, and variegated. It includes stone of pronounced texture, unselected as to color, ranging from a fine to a distinctly coarse grain, and may contain shelly, honeycomb formations with both white and dark crystalline streaks or streaks of glasslike calcite and tight crow feet, none of which, however, affect the structural soundness and weathering properties of the stone. For this grade of stone there are no fixed limits as to variation in texture, general formation, and surface markings, and when the material is fabricated some pieces will be produced in solid buff and in solid gray.

\section{ADDITIONAL}

\section{MILI BLOCKS}

It is understood that the term "mill blocks" applies to sound block stone in promiscuous sizes, or in blocks from 6 feet 6 inches to 12 feet 0 inches in length; that is, stone having a measured length at no point less than 6 feet 6 inches and generally averaging more than 8 feet 0 inches in length in any carload shipment.

\section{PROMISCUOUS SIZES}

Promiscuous size blocks cover block stone 6 feet 6 inches to 12 feet 0 inches in length, 2 feet 6 inches to 5 feet 0 inches in width between channel cuts and up to 4 feet 6 inches across the grain or to sawed stone produced therefrom.

\section{DIIENSION STONE}

Where order requires that any stone is to be furnished in any specified length, width, or height the stone so ordered is classed as "dimension stone" and is subject to such grade and size rules as may be established to cover that classification.

All blocks having length specified greater than 12 feet 0 inch are classed as "dimension stone."

\section{SPECIAL SIZE STONE}

All blocks or slabs having a specified width greater than 5 feet 0 inch between channel cuts and a height more than 4 feet 6 inches across grain and all slabs having a length greater than 12 feet 0 inch are classed as "special-size" stone and are subject to such grade and size rules as may be established to cover that classification.

\section{MONOLITH BLOCKS}

All stone having a specified length greater than 24 feet 0 inch are classed as "monolith blocks" and is subject to such grade and size rules as may be established to cover that classification.

\section{SHORT-LENGTH BLOCKS}

Under this classification stone can be supplied in the regular grades, segregated as to texture and color tone, the same as in foregoing classification, covering mill blocks, dimension stone, etc. Blocks can be furnished in promiscuous lengths $3-6$ to $6-5$ and in promiscuous widths and thicknesses.

Slabs can be furnished, sawed to the required thickness, in promiscuous lengths $3-6$ to $6-5$ and in promiscuous widths.

Sawed 4-side strips can also be furnished in these promiscuous lengths.

\section{DEFINITIONS}

"Promiscuous length" in both blocks and sawed stone shall imply stone ranging from 6-6 to $12-0$ in length.

"Promiscuous sizes," therefore, shall imply blocks or slabs ranging from 6-6 to $12-0$ in length, $2-6$ to 5-0 in width between channel cuts, and not over 4-6 across grain.

National Paving Brick Manufacturers Association, recommended specifications, stone curb and header, 1924. (Limestone.)

See 518.63 , page 448 .

\subsection{MARBLE.}

American Railway Engineering Association, specifications for buildings for railway purposes, 1926 .

\section{MARBLE AND TILE WORK}

\section{GENERAI}

The contractor shall furnish all labor, materials, tools, scaffolding, and equipment, except as otherwise noted, necessary to entirely complete any or all classes of marble or tile work herein specified, according to the class of building, and as shown or implied on the drawings.

\section{DESCRIPTION OF MARBLE}

All marble specified or shown on drawings shall be the best of their several kinds, carefully selected for color, marking, and location in the work, and each piece shall be subject to the engineer's approval or rejection.

Samples 8 by 12 inches in size of each kind of marble specified shall be submitted for the engineer's approval. These samples shall be finished as specified for the work and there shall be separate samples for each finish.

The completed work shall be equal to the approved samples as to quality, color, markings, and finish.

\section{THICKNESS}

All marble shall be thick enough to be amply strong for its size and location, and no slabs shall be less than seven-eighths ineh in thickness.

All returns of 8 inches or less projection shall be from stock of sufficient thickness to form solid angles without vertical joints; angle pieces may be cut with handsaw, provided the position of joints is not altered from that shown on drawings. All molded or ornamented members shall be from stock sufficiently thick to permit of the finished work being an exact reproduction of the models or drawings without flattening. Projecting angles throughout shall be slighly rounded to prevent the edges chipping.

\section{SETTING $\triangle N D$ ANCHORING}

All slabs shall be set free from backing surfaces using brick furring on vertical surfaces and other approved materials elsewhere. All bearing edges shall be bedded solidly and continuously their entire length, and no material other than pure plaster of Paris and pure nonstaining Portland cement shall be used in setting. Each piece of marble throughout the entire work shall be securely fastened in place with brass or bronze dowels, clamps, and tees, which 
must be provided and used in ample numbers to make a rigid and permanent job. No tees shall be fastened to the marble or walls by cement or plaster only, but each must fit into a properly drilled seat, shaped to retain the setting mortar.

In no case shall any metal fastening show on the exposed faces unless specially mentioned herein or shown on the drawings. Specially made metal fastenings shall be used where necessary or as may be directed.

\section{FINISH}

The finishes to be given marble are as follows:

(a) All floors and floor borders, stair treads, risers, and landings and door saddles are to be honed.

(b) All marble, except as noted in (a) above, to be highly polished.

\section{JOINTS}

All joints shall be close, showing only a hair line, and each piece of marble shall be worked to absolutely perfect edges. The exposed surfaces of all marble shall be worked to true planes, so that abutting edges can not be felt. Any suiface dressing necessary to obtain these results shall be continued the full length and width of the piece affected, so that the dressing can not be felt or seen, and this shall include any dressing required after the marble is set in place.

\section{SUPPORTS FOR MARBLE WORK}

The marble contractor shall provide and set all steel supports of every description required specially for the proper setting of his work. Wherever this special steel framing is supported on the framing furnished and set by others, the contractor for the marble shall furnish to such other contractors, within a reasonable time, carefully prepared drawings showing all required framing connections in detail so that provisions may be made for the proper construction of the work, without unnecessary cutting and drilling but if such framing is already in place the contractor for the marble work shall do all cutting, fitting, and drilling required to properly connect his work to such framing.

All steel supports furnished by this contractor shall be given three coats, one shop coat and two field coats, of approved paint.

\section{FLOORS ARD FIOOR BORDERS}

Wherever floors, floor borders, or panel divisions are specified or shown they shall be not less than seven-eighths inch in thickness and at all openings shall be increased in width to meet the door saddles or filling pieces which take the place of saddles.

\section{TERRAZZO (MONOLITHIC)}

Floors are to be divided into panels, as indicated on the drawings. Wherever shown, borders of different colored terrazzo are to be run, and panels are to be formed by strips of colored terrazzo.

Floors shall be composed of Portland cement and selected first quality chips of marble. Chips shall be of a reasonably uniform size, perfectly clean and uniformly distributed over the surface, and showing the greatest possible proportion of marble in the finished state.

Wherever terrazzo base is indicated it shall be of the height required above the floor, finished with a 1 -inch radius cove at the intersection with the floor.

Contractor shall submit finished samples not less than 6 inches square showing the color and finish for the engineer's approval before work is started.

\section{TERRAZZO (TILE)}

All rooms so indicated on drawings shall have terrazzo tile floors and base as manufactured by _._-_._._. or equal. The pressure used in the manufacture of all tile for the work shall not be less than 2,500 pounds to the square inch. All tile shall be 12 by 12 inches for the field and have a double border. All tile to be not less than 1 inch in thickness. All rooms having terrazzo tile floors shall have a 6-inch terrazzo cove base as detailed. All doors shall have terrazzo plinths in connection with terrazzo base. Tile must be so made that their structure will be free from air bubbles, and when rubbed to a finished surface must present a solid body. No tile, the surface of which has been filled or otherwise trcated after rubbing, shall be used. The tile shall be laid in a true and level plane at elevations shown on drawings, in a first-class and workmanlike manner. Great care shall be taken to have all lines and spacings true and straight and all joints of even width, not exceeding one-sixteenth inch. Provide terrazzo thresholds at all doors in connection with terrazzo tile floors, unless thresholds of other materials are called for.

Tile shall be set on a bed of mortar composed of 2 parts Portland cement and 3 parts sand. Tile shall be rammed to a solid and even bed, grouted and rubbed.

Before depositing bedding mortar, the entire surface shall be cleaned and well saturated with water to prevent too rapid absorption of water from bedding mortar.

After tile is laid and properly grouted, all tile floors shall be holystoned to reduce inequalities of surface. All tile for floors and base shall be made with marble chips of kind and color selected by the engineer. The contractor shall submit samples of tile and setting plan for approval before getting out any work.

The contractor shall guarantee all terrazzo work for a period of one year after completion against defects of workmanship or material.

\section{MARELE FOR FLOORS}

Marble floors and floor borders shall be composed of marble tile not less than seven-eighths inch in thickness, cut with full faces, sawn bed, and all edges rubbed to the exact size required.

Each piece of marble shall be set in full bed of Portland cement mortar, and when set all joints shall butt and match perfectly and closely and shall be perfectly flush. All pieces shall be selected and located in the floor according to character of veinings and color.

\section{VITRIFIED TILE}

(a) On walls.-Tile for walls shall be salt-glazed white tile laid in cement with hair-line joints absolutely plumb and true without waves. Where trim of other material is not specified the tile shall be returned into all reveals and soffits and the angle shall be formed of angle tile with corner rounded to a radius of about 1 inch. Where tile wainscot is called for there will also be required a sanitary tile base 8 inches high.

(b) Floor tile.-Shall be hexagonaı or other approved shapes of vitrified tile, set in cement mortar. Border of 2-inch square tile shall be laid at the intersection of floor with base.

\section{BEDS}

The distance from the finished floor to the rough floor will not be less than 2 inches, and this contractor shall fill in on top of the rough floors to the desired line with concrete composed of 1 part Portland cement, 2 parts sand, and 4 parts crushed stone or gravel to form bed for flooring material.

\section{GENERAL CONDITIONS}

All materials entering into the work and all methods used by the contractor shall be subject to 
the approval of the engineer, and no part of the work will be considered as finally accepted until all the work is completed and accepted.

The general conditions as given in 518.50 , page 416 . shall be considered as to apply to these specifications.

Federal Specifications Board, specification No. 448, United States Government master specifications for plumbing fixtures, November 22, 1926.

(The specification is published in full as Bureau of Standards Circular No. 310 and gives general and detail requirements for 22 plumbing-fixture items, including marble and glass for use in plumbing, general requirements for which are as follows:)

Quality of these materials and finish of same shall be described in the specification for the work.

National Association of Marble Dealers, standard specifications for interior marble.

(This specification is issued as an explanatory measure for composing a specification for interior marble. The full text of the specification includes material relative to construction and includes 42 plates containing drawings for different treatments of marblc.)

(The following classifications and specifications are included:)

\section{CLASSIFICATION}

\section{MARBLE}

Due to the diversity of composition, texture, color, and characteristics peculiar to the various marbles used throughout the world, the National Association of Marble Dealers has classified marbles into four groups, the basis of classification being the characteristics presented in the work required in finishing.

Group A.-Sound marbles and stones which require no "sticking," "waxing," or "filling," characteristically uniform, and favorable working qualities.

Group B.-Marbles and stones similar in character to the preceding group, but somewhat less favorable working qualities; occasional natural faults; limited amount of waxing and sticking necessary.

Group C.- Marbles of uncertain variation in working qualities; geological flaws, voids, veins, and lines of separation common; standard shop practice to repair nature's shortcomings by sticking, waxing, and filling; "liners" and other forms of reinforcement freely employed when necessary.

Group D.- Marbles and stones similar to the preceding group and subject to the same methods of finishing and manufacture, but embracing those materials which contain a larger proportion of natural faults and a maximum variation in working qualities, etc.; this group comprises many of the highly colored marbles prized for their decorative qualities.

There are three distinct classifications for the finishing of marbles; namely, sand rubbed, hone finish, and high polish.

Sand-rubbed finish is commonly used for walk-way surfaces such as floors, thresholds, stair treads, and platforms. Hone as well as high-polish finish is generally used for vertical standing material, and is a matter of selection by the architect.

Unless otherwise shown on drawings or specified, the standard thickness for marble used for wall wainscot or other purposes where one face is finished only is accepted by the trade as being seven-eighths inch; that is, a slab which is seven-eighths inch thick as it comes from the saws; finishing processes usually reduce this by about one-sixteenth inch.

\section{FLOOR THLI}

Inasmuch as floor material is a by-product of the marble trade, no specific thickness shall be called for, but it is understood that the minimum is to be three fourths inch and the maximum $1 \frac{1}{2}$ inches in order to allow the proper bed between the rough concrete slab and the floor tile.

\section{EXTERNAL CORNERS}

Unless otherwise specified or shown on the drawings, it is accepted by the trade that butt joints shall be used; that is, the exposed return of the member will not exceed the accepted or specified thickness of the member.

Another method of treating external corners, especially where only the minimum thickness of marble is required, is what is called by the trade, the "quirk miter." This quirk miter is cut on a $45^{\circ}$ angle from the finish face toward the back face of the member. This sharp edge formed by this method, is taken off so it will show a finished edge of $1 / 8$ to $1 / 4$ inch. This method is used to more or less indicate a solid corner. This method involves more labor, and it should, therefore, be distinctly specified when desired. The quirk miter is not recommendable for base, as the construction forms insanitary internal corner at floor.

\section{SETTIING}

The setting space, a term used by the trade indicates the distance from the rough construction receiving the marble to the finished face of the latter.

For standing marbles, classified under letters A and $B, 1 \frac{1}{2}$ inches is the minimum setting space. For $\mathrm{C}$ and $\mathrm{D}$ marbles, some of which require reinforcing liners as mentioned in this classification, 21/2 inches should be used for setting space.

All standing marble should be set in what the trade call molding plaster of Paris, this to be a quick-setting variety.

Vertical joints should be buttered. It is essential that the beds as well as the vertical joints should be properly buttered with plaster of Paris; the pointing of joints after marble has been set, will not be permanent.

Plaster of Paris "spots," a term used in the trade, are used as backing for standing marble. For ashlar work and smaller pieces these spots are usually sufficient to be applied around the locations of the anchors only. For larger pieces, such as wall panels, spots are usually applied between the top and bottom joint in addition to those around the anchors. No fixed rule can be given as to the number of spots, and this should be left to the discretion of the marble contractor.

The association also has a "Setting Manual," giving information as to the setting of interior marble.

\subsection{SANDSTONE.}

American Society for Municipal Improvements, specifications for stone block pavements, 1927.

See 518.36, page 393.

(This specification includes specifications for new sandstone blocks.)

National Paving Brick Manufacturers Association, recommended specifications, stone curb and header, 1924 (sandstone).

See 518.63, page 448 .

\subsection{SLATE.}

\subsection{General Items.}

American Society for Testing Materials, tentative method of test for water absorption of slate, serial designation D221-27T, 1927.

It is oiten desirable to gain some idea of the porosity of a materia in question. Actual determinations of the pore space require the use of rather elaborate and refined equipment as well as considerabie precision in carrying out the tests. For comparative purposes the precision in carrying out tho tosts. For conparativo purposes the obtaining the desired information. As applied to slate this test re- 
quires somewhat more care and precision than many other materials because of its dense nature, and consequently the small quantitie to be dealt with. Furthermore, the cleavage of slate-that property which permits it to be split into thin sheets of uniform thicknessmust be taken into consideration when this test is made. On this must be takis into consteration whon this to is mical specin account misleading results are often obtained on cubical specimen due to accidental cleavage cracks in the specimens. The shapes of ing procedure are intended to eliminate to a large extent the inconsisting procedure are intended to eliminate to a large extent

\section{TEST SPECIMENS}

1. Each specimen shall be 6 by 6 inches measured in the direction of cleavage and one-fourth inch in thickness.

2. Preferably the specimens should be split to a thickness somewhat greater than one-fourth inch sawed into 6-inch squares and finished on the faces and edges with a hone or fine abrasive to plane surfaces. Where this is not practical the slate may be split as nearly as possible to the desired thickness sawed into 6-inch squares, and tested in this form.

3. The number of specimens shall depend on the manner of preparation, as follows:

Hone finished on faces and edges

Cleavage faces and sawed edge.

\section{PROCEDURE}

4. The specimens shall be dried in an oven at a temperature between $110^{\circ}$ and $120^{\circ} \mathrm{C}$. for a period of 24 hours.

5. After drying, the specimens may be cooled in the room for 15 minutes and then weighed. In cases where it is not possible to weigh the specimens immediately upon cooling they shall be placed in a desiccator. The weights shall be determined to the nearest $0.05 \mathrm{~g}$.

6. After the initial weighing the specimens shall be wholly immersed in water at approximately $20^{\circ} \mathrm{C}$. for 48 hours. At the end of this period all faces and edges shall be thoroughly wiped off with a dry cloth, and the specimens immediately weighed to the same precision as employed for the initial weights.

7. The percentage of absorption shall be calculated from the formula:

$$
\text { Percentage of absorption }=\frac{(B-A) 100}{A}
$$

where $A=$ dry weight of specimen and $B=$ weight of specimen after immersion.

8. In general, the average of all the tests shall be reported as the absorption of the slate. Ir dividual determinations which differ by more than 25 per cent from the average of all the determinations may be disregarded when there is evidence that such results are of uncertain value. The absorption shall then be reported as the average of the remaining determinations, but all determinations shall be reported as information.

\section{ALTERNATIVE METHOD}

Occasionally it is desirable to obtain results in a shorter period than that required by the above procedure. In such cases the 48-hour immersion period may be supplanted by an 8-hour boiling period. In this procedure the different steps are the same as the foregoing method up to that of immersion. Instead of allowing the specimen. to soak for 48 hours they may be placed in an enameled pan or other suitable vessel, covered with water, and boiled for eight hours. Before the final weighing the specimens shall be cooled by allowing tap water to flow over them for at least 30 minutes. The computation and reporting of results are as outlined in the above procedure. hours' boiling has been found to give practically the same saturation as 48 hours' soaking.

American Society for Testing Materials, tentative methods of flexure testing of slate (determination of modulus of rupture and modulus of elasticity) serial designation D222-27T, 1927.

Due to the unique properties of slate the flexure test is better adapted to use for strength and elasticity determinations than either

In testing slate shingles which have sheared edges this type of rimes the type of type in which the blade can be set at right angles to the frame. compression or tension tests. Furthermore, several uses of slate are such that these determinations are of special interest and value, besides furnishing comparative data.

The property of slate termed "grain" causes a slab of the material to break transversely in one direction somewhat more readily than th right angles to this direction. For this reason it is desirable to tran to this direction. the strength and elasticity both parallel and perpendicular to

In the quarrying of slate, blasting is frequently resorted to, and for this reason certain portions of the material may have been unduly strained. Low or erratic strength results on some of the test specimens should be regarded in the light of defective material.

It is sometimes desirable to determine the modulus of rupture and modulus of ebsticity of slate in one operation. The shape of specimens recommended for modulus of rupture under method $\mathrm{A}$ is not well adapted to elesticity meesurements. However, a shape may be chosen which is adepted to both tests. That recommended under method B will be found more convenient for use on roofing slates.

\section{A. MODULUS OF RUPTURE}

\section{TEST SPECTMENS}

1. Six representative specimens, 12 by $1 \frac{1}{2}$ by 1 inch in size, of the particular slate under consideration shall be submitted for this test.

2. The slate for test shall have been split to a thickness of approximately $1 \frac{1 / 4}{4}$ inches and then

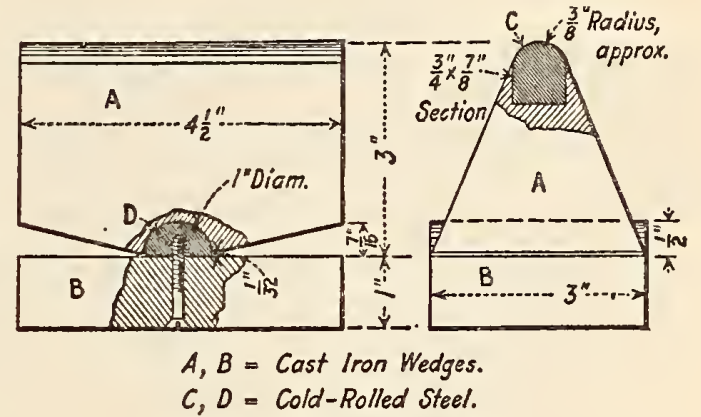

Figure 78.-Knife-edge

sawed into strips 12 inches in length by $1 \frac{1}{2}$ inches in width. Half of these shall be cut with the length parallel to the grain and the other half with the length perpendicular to the grain. The 12 by $1 \frac{1}{2}$ inch faces shall then be planed to smooth surfaces and rubbed down to a thickness of approximately 1 inch, care being taken to have the finished faces as nearly parallel as practical.

NoTE.-The shapes of specimens recommended for modulus of rupture and elasticity tests were determined partly by consideration of the proportions best adapted to the respective tests and partly on account of the convenience of preparing them from stock material. The small sizes recommended were first for economy of material, and The small sizes recommended were frst for economy of material, and, second, to permit the use of lighter testing equipment. Where only the large machines are available it is desirable, in order to obtain consistent results, to use larger specimens. In such cases approximatel the same proportionate dimensions should be adhered to for th respective tests.

\section{PROCED URE}

3. Before testing, the specimens shall be dried in an oven at a temperature of $110^{\circ}$ to $120^{\circ} \mathrm{C}$. for 24 hours.

4. The thickness and the width of the specimen at the middle shall be measured to the nearest 0.01 inch.

5. The testing machine shall be sensitive to 5 pounds when loaded to 500 pounds. The specimen shall be supported flatwise on knife-edges of the type shown in Figure 78 , spaced 10 inches on centers. The load shall be applied by a third knife-edge at the middle of the span. The rate of loading shall be approximately 100 pounds per minute. The breaking load shall be recorded to the nearest 5 pounds.

6. The modulus of rupture shall be calculated from the formula:

$$
R=\frac{3 w l}{2 b d^{2}}
$$


where $w=$ breaking load in pounds, $l=$ length of span in inches, $b=$ width of specimen in inches, and $d=$ thickness of specimen in inches.

7. The average value of the three specimens cut parallel to the grain shall be reported as the modulus of rupture parallel to the grain, and the average value of the other three, as the modulus of rupture perpendicular to the grain. In case any specimen shows a value 25 per cent lower than the average of its group, it shall be examined for defects, and if the low value appears to be due to an imperfection the average of the other two shall be reported as the modulus of rupture of the group. All determinations shall be reported as information.

NoTE.-The following method of marking the specimens before testing is recommended:

By means of a try-square the middle section and two end sections, 5 inches distant from the center, may be circumscribed. These marks serve as a guide to the operator in properly centering and aligning the specimen in the testing machine.

\section{B. MODULUS OF RUPTURE AND MODULUS OF ELASTICITY}

\section{TEST SPECIMENS}

8. The specimens shall be 12 by 4 inches in the direction of cleavage and $3 / 8$ inch in thickness.

9. The slate should preferably be split to a thickness somewhat greater than $3 / 8$ inch and sawed into specimens 12 by 4 inches. These should be finished on the faccs with a hone or fine abrasive to plane parallel surfaces. Where this is not practical the slate may be split to as near the desired thickness as possible, and the specimens sawed therefrom. ${ }^{6}$ Onehalf the specimens from each sample shall be cut with the length parallel to the grain and the other half with the length perpendicular to the grain.

10. The number of specimens shall depend on the method of preparation as follows:

Hone-finished faccs and edges, 3 cach way of grain

Cleavage faces and sawed edges, 4 each way of grain

\section{PROCEDUPE}

11. The specimens shall be dried, measured, and placed in a testing machine of the same type as deseribed in method $\mathrm{A}$, on modulus of rupture. A deflcctometer, capable of measuring accurately to 0.001 inch, shall be used to indicate the deflections at the center of the span. The load shall then be applicd at the rate of about 100 pounds per minute and deflection readings recorded for cach 50-pound increment. ${ }^{7}$ The load $w$ at which the specimens break shall be recorded to the nearest 5 pounds.

12. The moduius of rupture $R$, shall be calculated from the breaking load $w$ from the formula:

$$
R=\frac{3 w l}{2 b d^{2}}
$$

wherc the symbols have the same meaning as in method A.

The load-deflection readings shall be plotted on cross-section paper to a convenient seale and a straight line drawn to represent as nearly as possible

${ }^{6}$ In testing slate shingles which have sheared edges, this type of "In testing slate shingles which have sheared edges, this type of specimen ean be readily cut from the shingle with a hack saw of

type in which the blade can be set at right angles to the frame. when there is no load on the specimen. The best practice is to put a small initial load on the specimen, as 10 pounds and set the deflector to zero for this load. Since it is only the slope of the stress-strain curve that is desired, this initial load does not affect the final result. the average of the points plottcd. ${ }^{8}$ If this line does not pass through the zero point a corrected line shall be drawn through the zero point parallel to stressstrain curve. The modulus of elasticity shall be computed from the coordinates of some convenient point on the corrected curve from the formula:

$$
E=\frac{W^{\prime} L^{3}}{4 \overline{b \Delta d^{3}}}
$$

where $w^{\prime}=$ the load ordinate of this point, $\Delta=$ deformation ordinate of same, $l=$ the length of span in inches, $b=$ the width of the specimen in inches, and $d=$ thickness of the specimen in inches.

13. In general, the average of results obtained for specimens prepared with the length parallel to the grain shall be reported as the modulus of rupture and modulus of elasticity parallel to the grain. Likewise the average results obtained on the specimens cut with the length perpendicular to the grain shall be reported as the modulus of rupture and the modulus of elasticity perpendicular to the grain. In case an individual determination differs by more than 25 per cent from the average of its group it may be disregarded in computing the average if there is evidence that the determination was of uncertain value, but all determinations shall be reported as information.

American Society for Testing Materials, standard definition of the term slate, serial designation D247-27, 1927.

Slate.-A microgranular crystallin stone derived from argillaceous sediments by regional metamorphism, and characterized by a perfect cleavage entirely independent of original bedding, which cleavage has been induced by pressure within the earth.

Note 1.-The essential mineral constituents are white mica (chiefly sericite) and quartz. Prominent accessory constituents are black mica (biotite), chlorite, and hematite. Minor accessory constituents are carbonates, magnetite, apatite clay, andalusite, barite, rutile, purite, graphite, feldspar, zircon, tourmaline, and carbonaccous matter.

carbonaccous matter. significant commercial importance are not covered in this defnition.

Federal Specifications Board, specification No. 448, United States Government master specification for plumbing fixtures, November 22, 1926.

(The specification is published in full as Bureau of Standards Circular No. 310 , and gives general and detail requirements for 22 plumbing fixture items, including slate for use in plumbing, general requirements for which are as follows:)

Slate shall be sound, hard, nonabsorbent, the exposed surfaces finished a smooth sand-ribbed finish, with exposed edges rubbed smooth. The slabs shall be of full thickness called for, with true surfaces and edges, and free from defects in manufacture, and the abutting edges shall be close jointed. Commercial ribbon stock slate meeting the above requirements will be acceptable.

\subsection{Blackboard Slate.}

United States Department of Commerce, Bureau of Standards, Simplified Practice Recommendation No. 15, for blackboard slate, July 1, 1924.

In accordance with unanimous action of a joint conference of representatives of producers, distributors, and users of blackboard slate, the United States Department of Commerce, through the $\mathrm{Bu}-$ reau of Standards, lecommends that recognized sizes be reduced to the following:

s Slate does not ordinarily show a yield point in the stress-strain curve. 
Dimensions of blachboard slabs

\begin{tabular}{|c|c|c|}
\hline \multirow[b]{2}{*}{ Heights of slabs 1} & \multicolumn{2}{|c|}{ Number of slabs to be used in wall spaces } \\
\hline & $\begin{array}{l}\text { Mari- } \\
\text { mum } \\
\text { num- } \\
\text { ber of } \\
\text { slabs }\end{array}$ & Length of wall space \\
\hline $\begin{array}{l}3 \text { feet, } 3 \text { feet } 6 \text { inches, or } \\
4 \text { feet. } \\
\text { Do } \\
\text { Do } \\
\text { Do } \\
\text { Do } \\
\text { Do }\end{array}$ & $\begin{array}{l}4 \\
5 \\
6\end{array}$ & $\begin{array}{l}\text { Up to } 5 \text { feet. } \\
\text { Orer } 5 \text { feet but not exceeding } 9 \text { feet. } \\
\text { Orer } 9 \text { feet but not exceeding } 13 \text { feet } \\
6 \text { inches. } \\
\text { Over } 13 \text { feet } 6 \text { inches but not ex- } \\
\text { ceeding } 18 \text { feet. } \\
\text { Over } 18 \text { feet but not exceeding } 22 \\
\text { feet } 6 \text { inches. } \\
\text { Orer } 22 \text { feet } 6 \text { inches but not ex- } \\
\text { ceeding } 27 \text { feet. }\end{array}$ \\
\hline
\end{tabular}

1 The majority of locations for slate blackboards and the regulations of some State or municipal boards of education require slab heights of 3 feet 6 inches and 4 feet. The 3 -foot height is included in this recommendation for the purpose cf filling a need when the use of the other two heights would prove impracticable.

Location of slab joints in covering wall spaces

\begin{tabular}{c|c}
\hline \multicolumn{1}{c|}{ Number of slabs } & \multicolumn{1}{c}{ Location of slab joints } \\
\cline { 2 - 3 } & $\begin{array}{l}\text { At or near } 1 \text { the middle of the space. } \\
\text { At or near one-third of the space. } \\
\text { At or near one-fourth of the space. } \\
\text { At or near one-6ith of the space. } \\
\text { At or near one-sixth of the space. }\end{array}$ \\
\hline
\end{tabular}

$1 \mathrm{By}$ "at or near" is meant that any slab may be used which places the joint within 3 inches in either direction of what would be exact spacing of the units stated.

In order to utilize available blackboard slate to the fullest possible extent, the National Slate Association recommends that architects, school authorities, contractors, and others adopt the growing practice of specifying "sufficient blackboard slate to fill the lengths of wall spaces," and avoid calling for individual sizes of slabs. This will make it possible to place orders in advance and to prevent delays in waiting for final measurements. Such practice will make it unnecessary for quarries to wait for an exact number of equal-length slabs, or those which would place all joints within 3 inches (in either direction) of what would be exact spacing. The number of slabs, however, should not exceed the maximum number shown in this simplified practice recommendation.

PORTABLE, OR SMALL BIACKBOARDS-BULLETIN BOARDS

Dimensions ${ }^{1}$ of clear writing surface

\begin{tabular}{|c|c|}
\hline Inches & Inches \\
\hline 10 by 12 & 18 by 24 \\
\hline 12 by 18 & 20 by 30 \\
\hline 14 by 14 & 24 by 36 \\
\hline 14 by 16 & 27 by 40 \\
\hline 14 by 20 & 36 by 48 \\
\hline 16 by 16 & $42 \mathrm{by} 60$ \\
\hline $18 \mathrm{by} 18$ & 48 by 72 \\
\hline
\end{tabular}

1 When ordering slate slabs that are to be set in to frames, due allowance must be made for the margin that will be covered by the frames, in order to provide clear writing surfaces of the aborespecified dimensions. When ordering slabs for wall mountings, the dimensions of both slate and frame must be considered. SCHOOL SLATES, KITCHEN SLATES, SCORE-CARD SLATES, AND
MEMORANDUM SLATES

Dimensions ${ }^{1}$ of clear writing surface

\begin{tabular}{|c||c|}
\hline Inches & Inches \\
\hline 4 by 6 & 7 by 10 \\
5 by 7 & 7 by 11 \\
6 by 8 & 8 by 12 \\
6 by 9 & 9 by 13 \\
\hline
\end{tabular}

1 Then ordering slate slabs that are to be set into frames, due allowance must be made for the margin that will be covered by the frames, in order to provide clear writing surfaces of the abore-specified iimensions. When ordering slabs for wall mountings, the dimensions of both slate and frame must be considered.

Thickness.-The thickness of all finished blackboards shall be not less than one-fourth inch or more than three-eighth inch. Since it is not a simple matter to precisely control uniformity in the splitting of this natural rock, a maximum deviation of one-sixteenth inch from the standard limits is permissible when an average thickness of at least one-fourth inch is maintained.

Finish.-The writing face of each blackboard shall be finished to a true, even, uniform, and finely smoothed surface.

Upon request of the slate interests in the State of Maine, notice is called to the fact that Maine slate for blackboard purposes is limited, and for that reason blackboards of the heights specified in this recommendation may be furnished in lengths 3 feet or more, at the option of the manufacturer.

Note.-United States Department of Commerce, Bureau of Standards, simplified practice recommendation 75 (see 545.9 , p. 645) recommends dimensions for composition blackboard.

511.52 Building Slate. (See also 505.16.)

American Railway Engineering Association, specifications for buildings for railway purposes, section I0-C, slate roofing, 1927.

\section{GENERAL}

The contractor shall furnish all labor, tools, material, equipment, and everything necessary to entirely complete the slate roofing shown on the drawings, together with all slate covering on sides of dormers and other vertical surfaces where so indicated.

\section{MATERIALS} (a) Slate.-The slate shall be No.-, - - - inches
They shall be - inches by - inches in size, thick, and - in color.

All slate shall be hard, dense, sound rock, machine punched for two nails each, for nailing above top edge of second course below.

Exposed corners shall be practically full. No broken corners on covered ends, which sacrifice nailing strength or the laying of a water-tight roof will be allowed. No broken or cracked slates shall be used. Samples of slate shall be submitted for approval before ordering, and all slate shall conform with the approved samples.

(b) Roofing felt.- The felt to be used with slate roofs shall be asphalt saturated rag felt, weighing not less than 14 pounds per 108 square feet. It shall be sufficiently pliable to meet the requirements of application, and samples taken 5 feet from the 
outside ends of the rolls shall bend flat without cracking through $180^{\circ}$ at a temperature of $60^{\circ}$.

(c) Nails.-All nails shall be large-headed solid copper or yellow metal slaters' nails, $1 \frac{1 / 4}{4}$ inches for slates 18 inches or less in length and $1 \frac{1}{2}$ inches for slates longer than 18 inches.

\section{APPIICATION}

The roof deck shall be clean, smooth, thoroughly dry and free from projections which would injure
Hips shall have a concealed sheet-metal flashing laid with each slate. Slate at valleys and hips shall be laid in elastic cement of the same color as the slate.

Slate overlapping sheet-metal shall have the nails so placed as to avoid puncturing the sheet metal.

All valleys, roof edges, and hips shall be carefully fitted and lined. Slate shall be carefuily fitted to all wall flastings and at ridges to receive ridge roll or cresting. Small pieces of slate at valleys and hips will not be allowed. Slate should project 1 inch
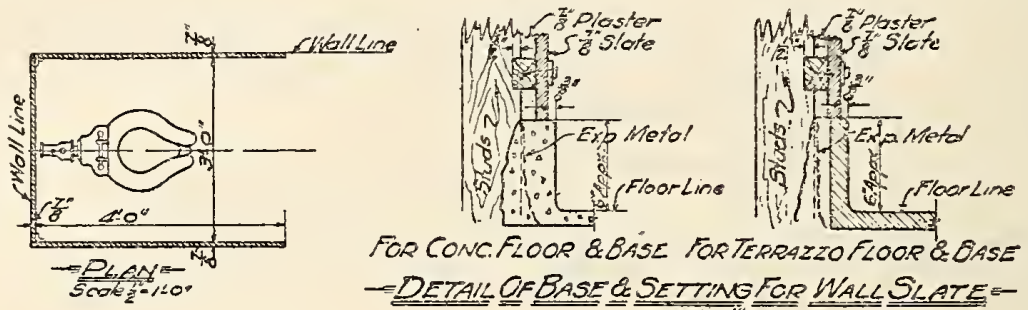

FOR CONC.FLOOR \&BASE FORTERRAZZO FLOOR \& OASE -DETALL OFBASEE SETTING FOR WALLSLATE $=-$
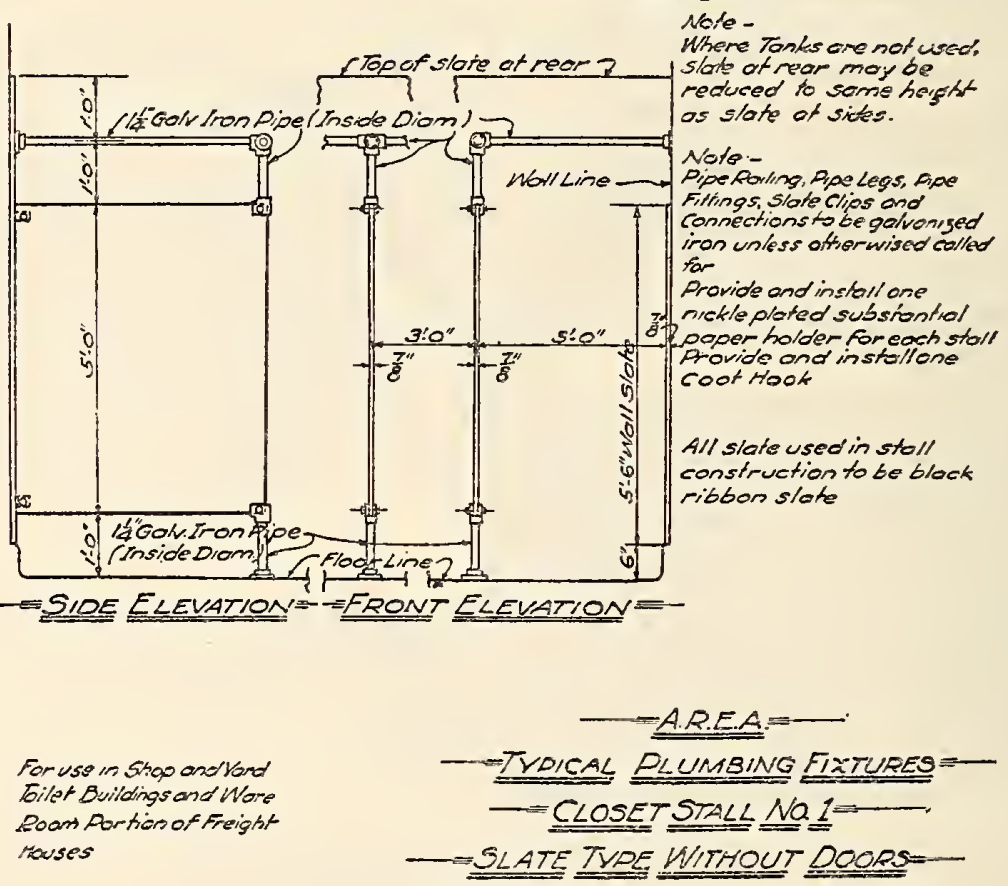

Figure 79

the roof covering. The roofing felt shall be laid smoothly, without wrinkles or buckles, and finished surfaces shall be free from eracks and bubbles.

Before applying the roofing felt, all loose knots and other flaws shali be removed, and knot holes and large eracks shall be covered with tin or other sheet metal, nailed in place.

The roofing felt shall first be applied to the roof sheathing, starting at the eaves. All joints shall be lapped 2 inches, and the felt shall be securely tacked to the sheathing.

The slate shall be laid in horizontal courses, with 3-inch head lap over the second course below and each course shall break joints with the preceding one. Each slate shall be fastened with two nails, driven so that the next course will not ride the nail heads. Slates at eaves or cornice line shall be doubled and canted one-fourth inch by a wooden cant strip, over edge at gable ends or eaves where gutter is omitted.

Under eaves and top courses shall be of same width and with grain of slate vertical. No stretchers or slate with grain running horizontal will be permitted.

Slate shall be neatly fitted around all pipes, ventilators, ete. Hips, ridges, and valleys shall be built as called for on the drawings.

The slating contractor shall build in and place all flashing pieces furnished by the sheet-metal contractor and cooperate with him in doing the work of flashing.

On completion, all slate must be sound, whole, and clean, and the roof shall be water-tight.

\section{SHEET-METAL WORK}

Flashings and other sheet-metal work in connection with the slate roofing shall be furnished and 
installed in accordance with the specifications for sheet-metal work of the Amerlcan Railway Engineering Association.

\section{GUARANTY}

Upon completion of the work the contractor shall furnish a written guarant $y$ covering the maintenance of the slate roofing in a water-tight condition for a
The general conditions as given in 518.50 , p. 416 , shall be considered to apply to the above specifications.

American Railway Engineering Association, specifications for buildings for railway purposes.

PLUMBING (1926)

(This specification includes requirements for the installation of plumbing fixtures and typical types

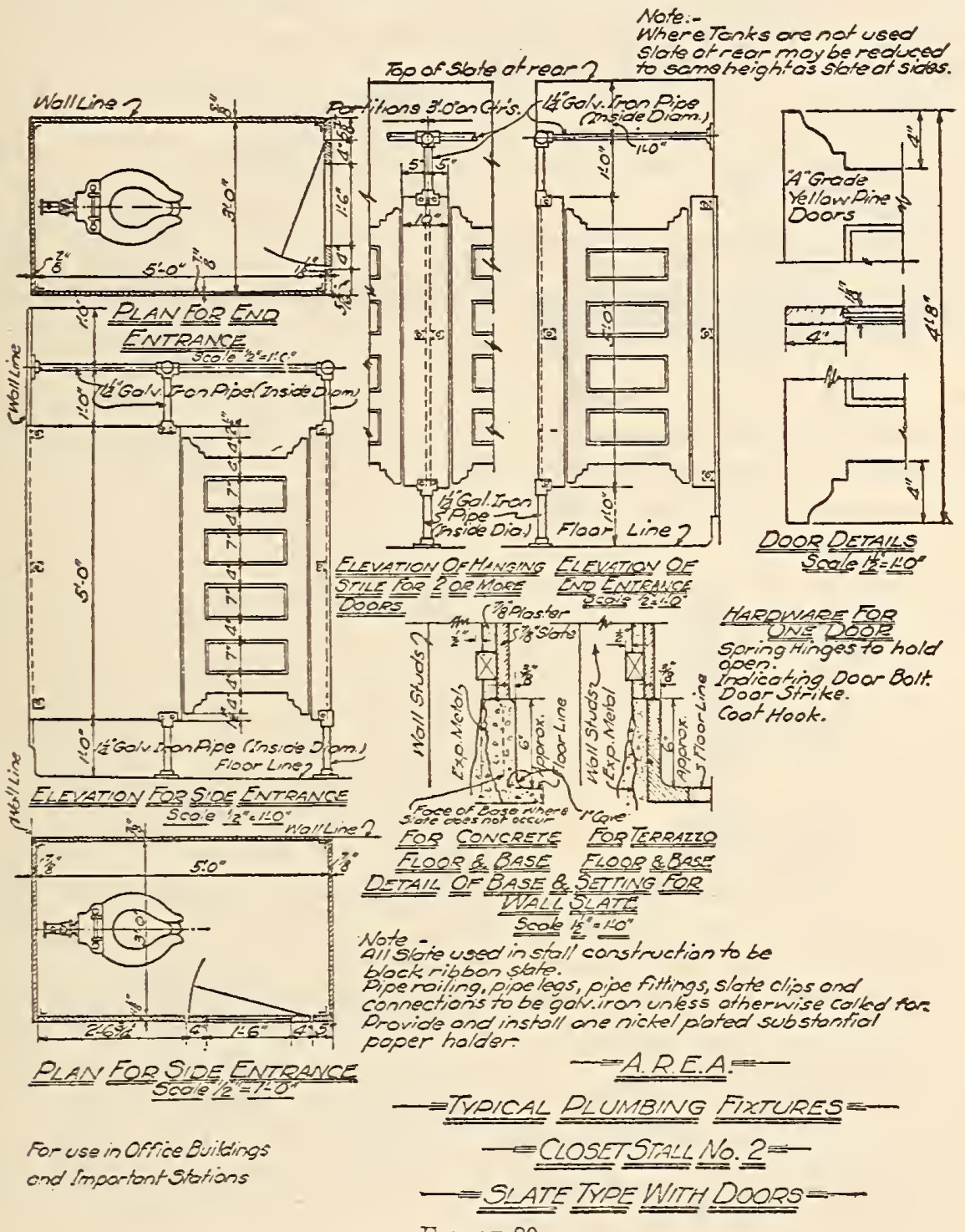

FIGURE 80

period of one year from date of acceptance, replacing or repairing any broken slates or leaks, promptly as notified, until the end of the guaranty period.

\section{GENERAI CONDITIONS}

All materials entering into the work and all methods used by the contractor shall be subject to the approval of the engineer, and no part of the work will be considered as finally accepted until all of the work is cornpleted and accepted. for guidance in use of plumbing specifications, including slate type closet stalls, as shown in figs. 79 and 80.)

Asphalt Shingle and Roofing Institute, granular metric specifications for slates.

The granules desired shall be produced from a natural nonfading slate; are to be as free from dust as possible; to all pass an $\delta$-mesh screen; be retained on a 35 -mesh screen; and, in addition, shall grade within the following percentage limits when tested 
by means of standard Tyler screens at destination:

\begin{tabular}{|c|c|c|c|}
\hline & Red slate & Green slate & $\begin{array}{c}\text { Blue-black } \\
\text { slate }\end{array}$ \\
\hline $\begin{array}{l}\text { Passing 65-mesh screen (maxi- } \\
\text { mum }\end{array}$ & \multirow[t]{2}{*}{0.5} & \multirow[t]{2}{*}{0.5} & \multirow[t]{2}{*}{0.5} \\
\hline Passing 35-mesh screen (less & & & \\
\hline $\begin{array}{l}\text { than) } \\
\text { Retained on } 35 \text {-mesh sereen }\end{array}$ & $\begin{array}{r}2.0 \\
5.0-13.0\end{array}$ & $\begin{array}{r}2.0 \\
5.0-13.0\end{array}$ & 1. $2-12$. \\
\hline Retained on 28 -mesh screes. & 10. $0-25.0$ & $10.0-25.0$ & 9. $0-20.0$ \\
\hline Retained on 20 -mesh screen & $28.0-40.0$ & $28.0-40.0$ & $25.0-38.0$ \\
\hline Retained on 14-mesh screen... & $25.0-40.0$ & $25.0-40.0$ & $25.0-40.0$ \\
\hline Retained on 10-mesh screen... & $.0-5.0$ & $.0-5.0$ & $5.0-15.0$ \\
\hline Retained on 8-mesh sereen.... & & & 0 \\
\hline
\end{tabular}

National Slate Association, specifications for slate floors, terraces, and walks, 1925 .

See 518.56, page 425 .

NOTE.-In this specification, the association indorses the same color nomenclatures for slates as is contained in United States
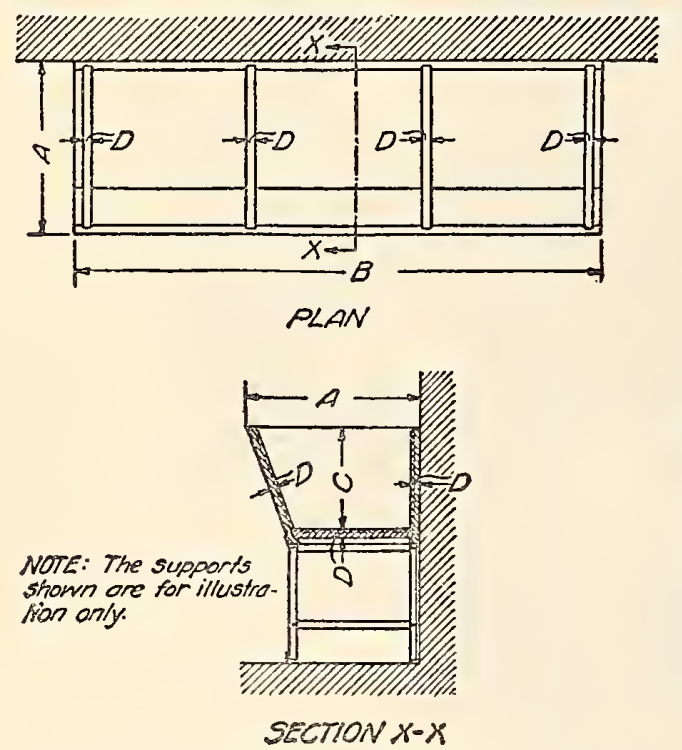

Figure 81.-Slate laundry tubs-with or without integral backs. (See Table 1)

Department of Commerce Division of Simplified Practice Recommendation No. 14, page 203.

National Slate Association, slate roofs, 1926.

See 518.57 , page 430 .

Pennsylvania Slare Institute, standard for roofing slate, 1929.

(The requirements of the above-entitled standard, as set out in a certificate-guaranty carrying the name of the Pennsylvania Institute, are as follows:)

Modulus of rupture.-Over 7,000 pounds per square inch. (A. S. T. M. D222-27T; see 511.50, p. 195.)

Porosity.-Not less than one half of one per cent, A. S. T. M. D221. (See 511.50, p. 194.)

Free from longitudinal curvature to the extent of less than one-fourth of an inch variation in 16-inch slates or three-eighths of an inch in 24-incl slates.

Of a thickness to average 22 to 24 inches to the hundred slate.

United States Department of Commerce, Simplified Practice Recommendation No. 13, structural slate (for plumbing and sanitary purposes), 1928.

In accordance with the unanimous action of a general conference of representative manufacturers, distributors, and users of structural slate, the industry has adopted, and approved for promulgation by the United States Department of Commerce, a simplified practice recommendation establishing the following schedule of dimensions, sizes, and nomenclature of structural slate for plumbing and sanitary purposes:

TABLE 1.-Slate laundry tubs-with or without integral backs. (See fig. 81)

\begin{tabular}{|c|c|c|c|c|}
\hline \multirow{2}{*}{$\begin{array}{l}\text { Number of } \\
\text { equal-sized com- } \\
\text { partments }\end{array}$} & A & B & C & D \\
\hline & $\begin{array}{c}\text { Com- } \\
\text { partment } \\
\text { width }\end{array}$ & Length & $\begin{array}{l}\text { Inside } \\
\text { depth }\end{array}$ & $\begin{array}{l}\text { Slate } \\
\text { thick- } \\
\text { ness }\end{array}$ \\
\hline $\begin{array}{l}1-\ldots- \\
2-\ldots \\
3-\ldots\end{array}$ & $\begin{array}{r}\text { Inches } \\
24 \\
24 \\
24\end{array}$ & $\begin{array}{l}\text { Inches } \\
24,30 \text {, and } 36 \\
48,54 \text {, and } 60 \\
72,78 \text {, and } 84\end{array}$ & $\begin{array}{l}\text { Inches } \\
12 \text { or } 14 \\
12 \text { or } 14 \\
12 \text { or } 14\end{array}$ & $\begin{array}{c}\text { Inches } \\
11 / 4 \\
114 \\
11 / 4\end{array}$ \\
\hline
\end{tabular}

TABLE 2.-Sink and 1-tub combination-with integral back. (See fig. 82)

\begin{tabular}{|c|c|c|c|c|c|c|c|}
\hline \multicolumn{3}{|c|}{ Sink dimensions } & \multicolumn{3}{|c|}{ Tub dimensions } & \multirow{2}{*}{$\begin{array}{c}\begin{array}{c}\text { Over-all } \\
\text { dimen- } \\
\text { sions }\end{array} \\
\mathrm{G}\end{array}$} & \multirow{2}{*}{$\begin{array}{c}\text { Slate } \\
\text { thick- } \\
\text { ness } \\
\end{array}$} \\
\hline$A$ & B & C & $\mathrm{D}$ & $E$ & $\mathrm{~F}$ & & \\
\hline $\begin{array}{c}\text { Out- } \\
\text { side } \\
\text { width }\end{array}$ & $\begin{array}{l}\text { Outside } \\
\text { length }\end{array}$ & $\begin{array}{l}\text { Inside } \\
\text { depth }\end{array}$ & $\begin{array}{l}\text { Out- } \\
\text { side } \\
\text { width }\end{array}$ & $\begin{array}{c}\text { Out- } \\
\text { side } \\
\text { length }\end{array}$ & $\begin{array}{l}\text { Inside } \\
\text { depth }\end{array}$ & $\begin{array}{l}\text { Outside } \\
\text { Iength }\end{array}$ & II \\
\hline $\begin{array}{c}\text { Inches } \\
24 \\
24 \\
24\end{array}$ & $\begin{array}{c}\text { Inches } \\
251 / 4 \\
311 / 4 \\
371 / 4\end{array}$ & $\begin{array}{c}\text { Inches } \\
8 \\
8 \\
8\end{array}$ & $\begin{array}{c}\text { Inches } \\
21 \\
24 \\
24\end{array}$ & $\begin{array}{c}\text { Inches } \\
24 \\
24 \\
24\end{array}$ & $\begin{array}{l}\text { Inches } \\
12 \text { or } 14 \\
12 \text { or } 14 \\
12 \text { or } 14\end{array}$ & $\begin{array}{c}\text { Inches } \\
48 \\
54 \\
60\end{array}$ & $\begin{array}{c}\text { Inches } \\
11 / 4 \\
11 / 4 \\
11 / 4\end{array}$ \\
\hline
\end{tabular}

TABLE 3.-Sink and 2-tub combination-with integral back

\begin{tabular}{|c|c|c|c|c|c|c|c|}
\hline \multicolumn{3}{|c|}{ Sink dimensions } & \multicolumn{3}{|c|}{ Tub dimensions } & \multirow{2}{*}{$\begin{array}{c}\begin{array}{c}\text { Over-all } \\
\text { dimen- } \\
\text { sions }\end{array} \\
\begin{array}{l}\text { Outside } \\
\text { length }\end{array}\end{array}$} & \multirow{2}{*}{$\begin{array}{c}\text { Slate } \\
\text { thick- } \\
\text { ness }\end{array}$} \\
\hline $\begin{array}{l}\text { Out- } \\
\text { side } \\
\text { width }\end{array}$ & $\begin{array}{l}\text { Outside } \\
\text { length }\end{array}$ & $\begin{array}{l}\text { Inside } \\
\text { depth }\end{array}$ & $\begin{array}{l}\text { Out- } \\
\text { side } \\
\text { width }\end{array}$ & $\begin{array}{c}\text { Over- } \\
\text { all } \\
\text { length } \\
\text { (two } \\
\text { tubs) }\end{array}$ & $\begin{array}{l}\text { Inside } \\
\text { depth }\end{array}$ & & \\
\hline $\begin{array}{c}\text { Inches } \\
24 \\
24 \\
24 \\
24 \\
24\end{array}$ & $\begin{array}{c}\text { Inches } \\
261 / 2 \\
321 / 2 \\
321 / 2 \\
381 / 2 \\
381 / 2\end{array}$ & $\begin{array}{c}\text { Inches } \\
8 \\
8 \\
8 \\
8 \\
8\end{array}$ & $\begin{array}{c}\text { Inches } \\
24 \\
24 \\
24 \\
24 \\
24\end{array}$ & $\begin{array}{c}\text { Inches } \\
48 \\
48 \\
54 \\
48 \\
54\end{array}$ & $\begin{array}{l}\text { Inches } \\
12 \text { or } 14 \\
12 \text { or } 14 \\
12 \text { or } 14 \\
12 \text { or } 14 \\
12 \text { or } 14\end{array}$ & $\begin{array}{c}\text { Inches } \\
72 \\
78 \\
84 \\
84 \\
90\end{array}$ & $\begin{array}{c}\text { Inches } \\
11 / 4 \\
11 / 4 \\
11 / 4 \\
11 / 4 \\
11 / 4\end{array}$ \\
\hline
\end{tabular}

The outside length of the sink is determined by subtracting the outside length of the tub as given (and which is kept as a basic standard throughout) from the over-all length and adding the thickness of the partition (Table 2), or the thicknesses of the two partitions (Table 3 ).

It should be noted in Tables 2 and 3 that the overall dimension is for the outside of the tub and sink combined. Some manufacturers extend the front, bottom, and back beyond the over-all length given here. The amount of this extension should be ascertained and taken into consideration when ordering this combination to fill a definite space. 
TABLE 4.-Sinks-with or without integral backs. (See fig. 83)

\begin{tabular}{|c|c|c|c|c|}
\hline A & B & $\mathrm{C}$ & & $\mathrm{D}$ \\
\hline $\begin{array}{l}\text { Over-all } \\
\text { width }\end{array}$ & $\begin{array}{l}\text { Orer-all } \\
\text { length }\end{array}$ & $\begin{array}{l}\text { Inside } \\
\text { depth }\end{array}$ & Style of front & $\begin{array}{l}\text { Slate } \\
\text { thick- } \\
\text { ness }\end{array}$ \\
\hline $\begin{array}{c}\text { Inches } \\
12 \\
18 \\
20 \\
20 \\
22\end{array}$ & $\begin{array}{c}\text { Inches } \\
18 \\
24 \\
30 \\
36 \\
30\end{array}$ & $\begin{array}{c}\text { Inches } \\
6 \\
6 \\
6 \\
6 \\
6\end{array}$ & $\begin{array}{l}\text { Straight or sloping } \\
\text { do do } \\
\end{array}$ & $\begin{array}{c}\text { Inches } \\
11 / 4 \\
11 / 4 \\
11 / 4 \\
11 / 4 \\
11 / 4\end{array}$ \\
\hline $\begin{array}{l}22 \\
22 \\
24 \\
24 \\
24\end{array}$ & $\begin{array}{l}36 \\
42 \\
30 \\
36 \\
48\end{array}$ & $\begin{array}{l}6 \\
6 \\
6 \\
6 \\
6\end{array}$ & 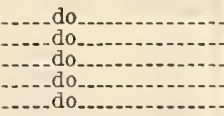 & $\begin{array}{l}11 / 4 \\
11 / 4 \\
11 / 4 \\
11 / 4 \\
11 / 4\end{array}$ \\
\hline
\end{tabular}

TABLE 5.-Sinks 1-with or without integral backswith one or two drain boards. (See fig. 84)

\begin{tabular}{|c|c|c|c|c|c|}
\hline A & B & C & $\mathrm{D}$ & $\mathrm{E}$ & $\mathrm{F}$ \\
\hline $\begin{array}{l}\text { Orer-all } \\
\text { width }\end{array}$ & $\begin{array}{l}\text { Over-all } \\
\text { length }\end{array}$ & $\begin{array}{l}\text { Inside } \\
\text { depth }\end{array}$ & $\begin{array}{l}\text { One drain } \\
\text { board } 2 \text { on } \\
\text { right or } \\
\text { Ieft (over- } \\
\text { alllength) }\end{array}$ & $\begin{array}{l}\text { Two } \\
\text { drain } \\
\text { boards }{ }^{2} \\
\text { (o ver-all } \\
\text { length) }\end{array}$ & $\begin{array}{l}\text { Slate } \\
\text { thick- } \\
\text { ness }\end{array}$ \\
\hline $\begin{array}{c}\text { Inches } \\
12 \\
18 \\
20 \\
20 \\
22\end{array}$ & $\begin{array}{c}\text { Inches } \\
18 \\
24 \\
30 \\
36 \\
30\end{array}$ & $\begin{array}{c}\text { Inches } \\
6 \\
6 \\
6 \\
6 \\
6\end{array}$ & $\begin{array}{c}\text { Inches } \\
42 \\
48 \\
54 \\
60 \\
54\end{array}$ & $\begin{array}{c}\text { Inches } \\
54 \\
60 \\
66 \\
72 \\
66\end{array}$ & $\begin{array}{c}\text { Inches } \\
11 / 4 \\
11 / 4 \\
11 / 4 \\
11 / 4 \\
11 / 4\end{array}$ \\
\hline $\begin{array}{l}22 \\
22 \\
24 \\
24 \\
24\end{array}$ & $\begin{array}{l}36 \\
42 \\
30 \\
36 \\
48\end{array}$ & $\begin{array}{l}6 \\
6 \\
6 \\
6 \\
6\end{array}$ & $\begin{array}{l}60 \\
56 \\
54 \\
60 \\
72\end{array}$ & $\begin{array}{l}72 \\
78 \\
66 \\
72 \\
84\end{array}$ & $\begin{array}{l}11 / 4 \\
11 / 4 \\
11 / 4 \\
11 / 4 \\
11 / 4\end{array}$ \\
\hline
\end{tabular}

1 Extra apron, or ends, to be 1 inch in thichness-other sizes to fit. 2 Regular type drain board, countersunk and groored, or countersunt only.

The regular type of top, grooved and countersunk (or countersunk only), surrounds the sink with an integral slate rim. The over-all dimensions of the top depend upon the size of sink opening and on whether there is a single or double drain board. The width of the top must be sufficient to provide a $23 / 2$-inch slate rim at both the back and the front of the sink opening. The over-all length of top, with single drain board end must provide $2 \frac{1}{2}$ inches of slate on remaining three sides of sink. Single drain boards, measuring from sink opening, should be 24 inches in length and double drain boards 18 inches in length.

Slate tops can also be furnished with separate aprons or separate backs.

TABLE 6.-Slate sink tops oniy.

(See fig. 85)

\begin{tabular}{|c|c|c|}
\hline $\mathrm{A}$ & $\mathrm{B}$ & $\mathrm{C}$ \\
\cline { 1 - 2 } $\begin{array}{c}\text { Width of } \\
\text { tops }\end{array}$ & $\begin{array}{c}\text { Length of } \\
\text { tops }\end{array}$ & $\begin{array}{c}\text { Thickness } \\
\text { of slate }\end{array}$ \\
\hline $\begin{array}{c}\text { Inches } \\
18 \text { to 22 } \\
24 \text { to 30 }\end{array}$ & $\begin{array}{c}\text { Inches } \\
\text { Up to 78 } \\
\text { Above 78 }\end{array}$ & $\begin{array}{c}\text { Inches } \\
11 / 4 \\
11 / 2\end{array}$ \\
\hline
\end{tabular}

TABLE 7.-Slop hoppers-with or without integral backs

\begin{tabular}{|c|c|c|c|c|}
\hline $\begin{array}{l}\text { Over-all } \\
\text { width }\end{array}$ & $\begin{array}{l}\text { Over-all } \\
\text { length }\end{array}$ & Inside depth & Style of front & $\begin{array}{l}\text { Slate } \\
\text { thickness }\end{array}$ \\
\hline $\begin{array}{c}\text { Inches } \\
24 \\
24 \\
24\end{array}$ & $\begin{array}{c}\text { Inches } \\
24 \\
30 \\
36\end{array}$ & $\begin{array}{c}\text { Inches } \\
12 \text { or } 14 \\
12 \text { or } 14 \\
12 \text { or } 14\end{array}$ & Sloping & $\begin{array}{r}\text { Inches } \\
11 / 4 \\
11 / 4 \\
11 / 4\end{array}$ \\
\hline
\end{tabular}

TABLE 8. - Shower stalls. (See fig. 86)

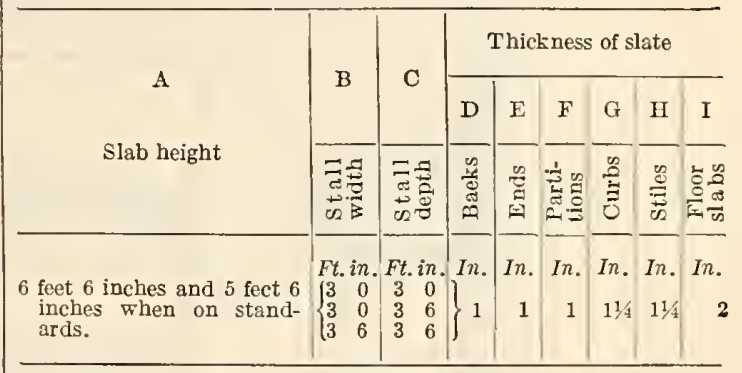

Where one floor drain serves several showers, the partitions are 5 feet 6 inches high and are supported by legs 1 foot in length.

Floor slabs 2 inches thick, sloping to drain outlet in center.

In types where slate dressing rooms are placed in front of shower stalls separate slabs are extended to line with partitions and ends, forming compartments 6 inches less in depth than shower stalls. Shower stalls are moved to front of dressing compartments and 14-inch covering separations are placed between them and stalls from top of curbs to top of shower stalls. A 14-inch diagonal slate seat is fastened to wing and end, or partition.

TaBLE 9.-Toilet inclosures

\begin{tabular}{|c|c|c|c|c|c|c|c|}
\hline \multirow[b]{2}{*}{ Slab height } & \multirow{2}{*}{ 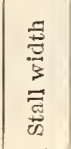 } & \multirow{2}{*}{ 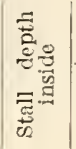 } & \multicolumn{5}{|c|}{ Thickness of slate } \\
\hline & & & 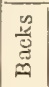 & 窇 & 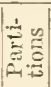 & $\stackrel{\mathscr{c}}{\vec{Z}}$ & $\frac{1}{20}$ \\
\hline $\begin{array}{l}\text { Backs and ends } 6 \text { and } 7 \text { feet; } \\
\text { partitions } 5 \text { and } 6 \text { feet. }\end{array}$ & 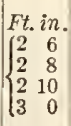 & 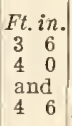 & \}$_{1}^{I n .}$ & $\begin{array}{c}\text { In. } \\
1\end{array}$ & $\begin{array}{c}\text { In. } \\
1\end{array}$ & $\left\{\begin{array}{l}\text { In. } \\
1 \text { 1 }\end{array}\right.$ & $\begin{array}{l}\text { In. } \\
11 / 4\end{array}$ \\
\hline
\end{tabular}

TABLE 10.-Urinals. (See fig. 8\%)

\begin{tabular}{|c|c|c|c|c|c|c|c|c|}
\hline \multicolumn{3}{|c|}{ With partitions } & \multicolumn{3}{|c|}{ Without partitions } & \multicolumn{3}{|c|}{ Thickness of slate } \\
\hline A & $\mathrm{B}$ & $\mathrm{C}$ & & & & $\mathrm{D}$ & $\mathbf{E}$ & $\mathrm{F}$ \\
\hline Height & Width & $\begin{array}{l}\text { Depth } \\
\text { inside }\end{array}$ & Helgnt & inside & $\begin{array}{l}\text { depth } \\
\text { of ends }\end{array}$ & Backs & Ends & $\begin{array}{l}\text { Parti- } \\
\text { tions }\end{array}$ \\
\hline Ft.in. & Ft. in. & Ft. in. & Ft. in. & Ft. in. & Ft.in. & Inch & Inch & Inch \\
\hline $\begin{array}{ll}3 & 6 \\
4 & 0 \\
4 & 6 \\
5 & 0\end{array}$ & $\begin{array}{ll}1 & 8 \\
1 & 8 \\
2 & 0 \\
2 & 0\end{array}$ & $\begin{array}{ll}1 & 6 \\
1 & 8 \\
1 & 2 \\
1 & 6\end{array}$ & $\begin{array}{cc}4 & 0 \\
\text { and } \\
4 \quad 6\end{array}$ & $\left\{\begin{array}{rr}4 & 0 \\
6 & 0 \\
8 & 0 \\
10 & 0 \\
12 & 0 \\
14 & 0\end{array}\right.$ & 18 & 1 & 1 & 1 \\
\hline
\end{tabular}


The top front edges of all urinal stall partitions A corner urinal stall is made by forming an angle and ends are to be rounded to a 6 -inch radius or cut to a slope 6 inches back and 12 inches down. with two slate ends and setting the same snug]y into the corner of the wall, upon a countersunk
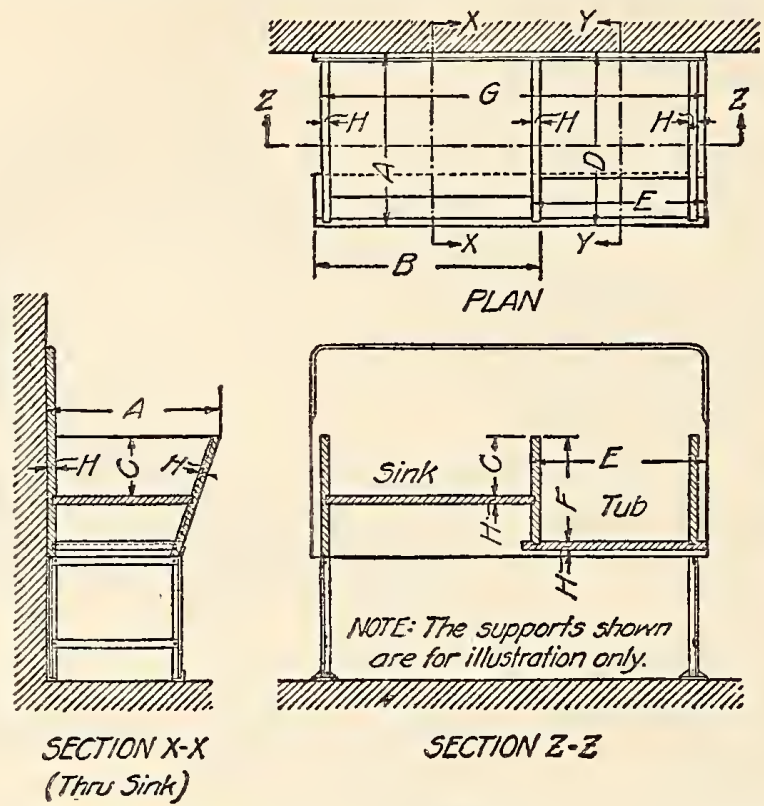

FIgURE 82.-Sink and 1-tub combination-with integral back. (See Table 2)

Backs that measure from 6 to 9 feet in length may be made up of two pieces, while those measuring over 9 feet in length may be made up of three pieces.
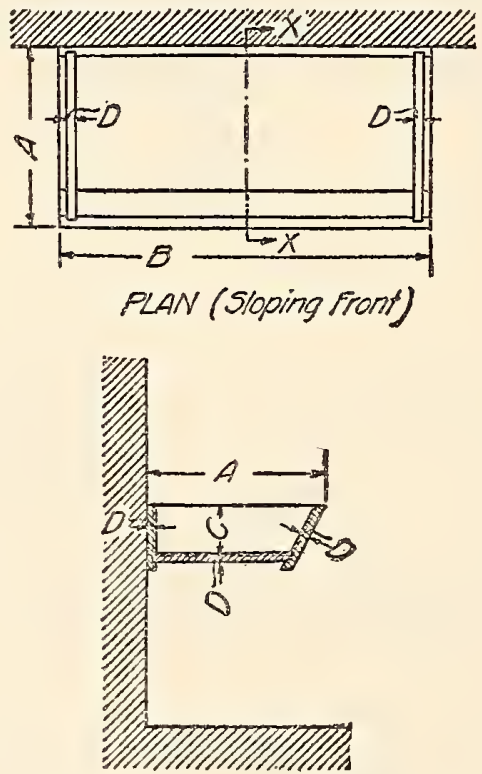

\section{SECTIONXXX}

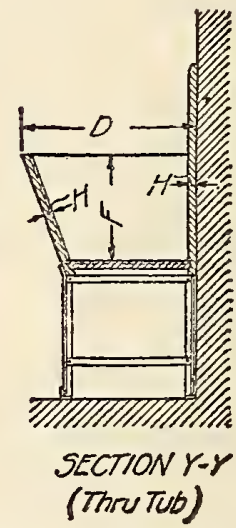

oor slab $1 \frac{1}{2}$ inches in thickness to the outer edge of backs. The angle is formed by placing a 21 or a 24 inch slate end against a 22 or a 25 inch slate
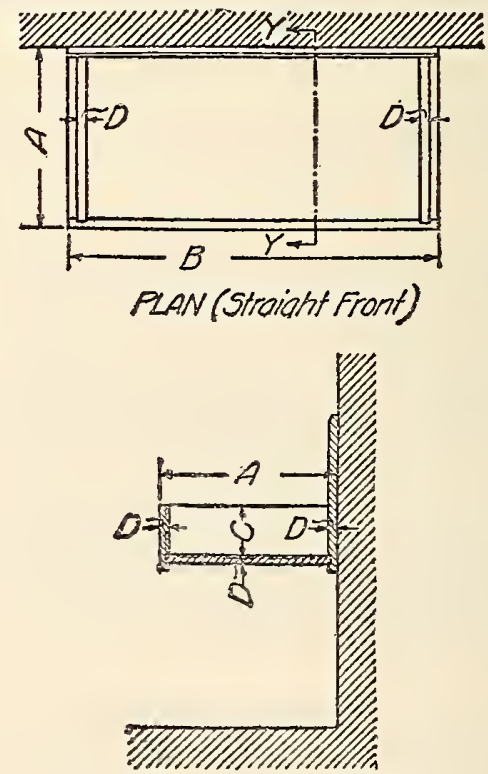

SECTION Y.Y

FIGURE 83.-Sinks with or without integral backs. (See Table f)

Gutters (where used) are hollowed out of solid slate end, so that the outside face of the former is in and two or more pieces may be used in constructing total lengths of 6 and 9 feet.

Floor slabs are $1 \frac{1 / 2}{2}$ or 2 inches in thickness and should line up with face of ends.

line with the thickness of the latter.

The 4 or 7 inch spaces to be covered at top with 1-inch slate, to fit. The exposed ends to be covered by increasing the depth of the end slabs. For 
the back-to-back type, the end slabs to be made in one piece for the purpose of covering both sets of stalls and the vent spaces.

All partitions (see Tables 8, 9, and 10) and ends are placed against backs so that the depth of stall or inclosure and the inside dimension of the same
Floor slabs with płain or beveled edges, flat or countersunk surfaces, are made in sizes to fit the above standard. The size and location of all pipe openings should be mentioned in all specifications.

Unless otherwise specified, all exposed surfaces of slate, including both sides of all end and partition
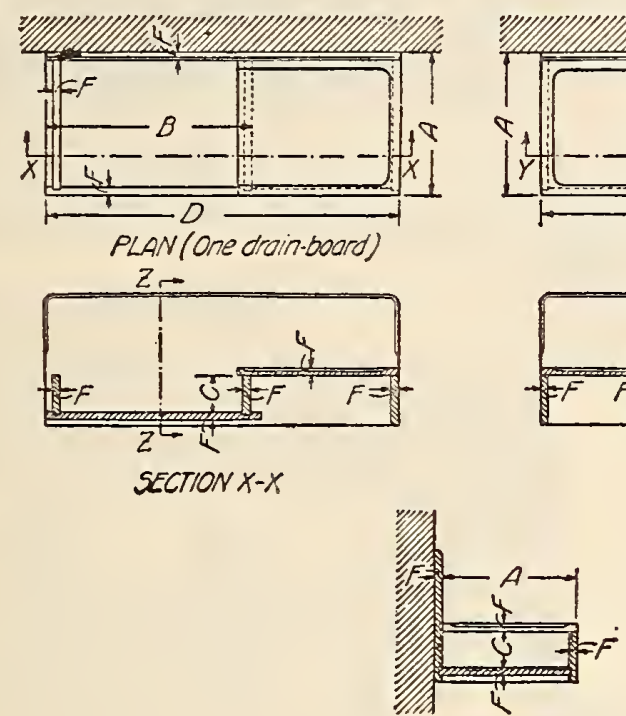

SECTION Z-Z

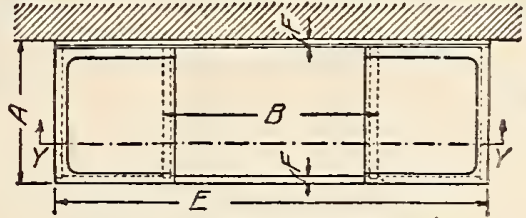

PLAN (Two drain-boords)

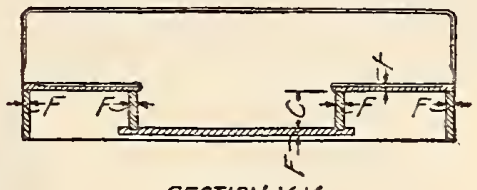

SECTION $Y-Y$

NOTE: Oroin-boards for both types counter sunk oniy, or countersunk and grooved

FIGURE 84.- Sinks, with or without integral backs, with one or two drain boards. (See Table 5)

are identical. But for batteries of two or more the widths of all except the end stalls or inclosures are taken as being the distance between the center lines of the partitions. In order that all back slabs may be of the same dimensions, the width of the end stalls or inclosures is the distance between the center line of the partition and the outside face of slabs (whether against the wall or not), are to be furnished with the standard sand-rubbed finish.

It is recommended that hone finish be specified for work in connection with urinals.

It is believed that the above sizes of slate will contribute to the convenience of architects and builders in preparing plans and specifications and
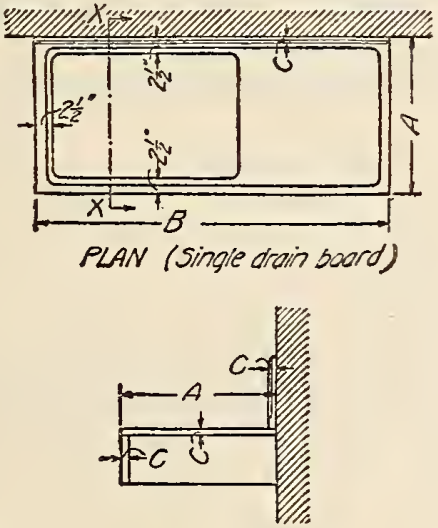

END VIEW
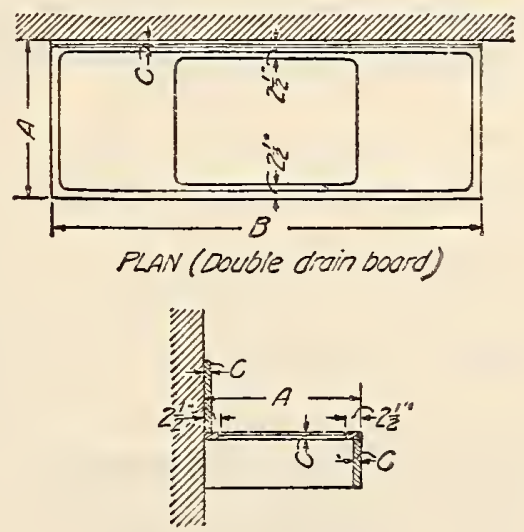

SECTION $X-X$

Figure 85.-Slate sink tops only. (See Table 6)

the end slabs. Vent spaces 7 inches in width to be provided.

Working spaces 18 inches wide are covered and closed with slate 1 inch in thickness at free standing ends.

In back-to-back batteries, working space is 30 inches, and where neither vent nor working space is required one back slab is sufficient. will be helpful to owners and buyers in buying units of slate.

This recommendation is effective from February 1 , 1928, subject to annual revision by a standing committee of the industry.

United States Department of Commerce, Bureau of Standards, Simplified Practice Recommendation No. 14, for roofing slate, February, 1928. 
In accordance with unanimous action of a general conference of representative manufacturers, distributors, and users of roofing slate and of a revision conference, the industry has adopted and approved for promulgation by the United States Department of Commerce a simplified practice recommendation establishing the following schedule of stock sizes and dimensions:

TABLE 1.-Dimensions of slate shingles for sloping roofs; minimum to a square

(Eacb size split 1 to thickness of $3 / 16,1 / 4,3 / 8,1 / 2,3 / 4,1,11 / 4,11 / 2,1 \frac{1}{4}$, and 2 inches ${ }^{2}$ )

\begin{tabular}{|c|c|c|c|}
\hline $\begin{array}{l}\text { Face dimensions, } \\
\text { in incbes }\end{array}$ & $\begin{array}{l}\text { Minimum } \\
\text { number to } \\
\text { square } \\
\text { (3-inch lap) }\end{array}$ & $\begin{array}{c}\text { Face dimenstions, } \\
\text { in inches }{ }^{3}\end{array}$ & $\begin{array}{l}\text { Minimum } \\
\text { number to } \\
\text { square } \\
\text { (3-inch lap) }\end{array}$ \\
\hline $\begin{array}{l}10 \text { by } 6 \\
10 \text { by } 7 \\
10 \text { by } 8 \\
12 \text { by } 6 \\
12 \text { by } 7 \\
12 \text { by } 8 \\
12 \text { by } 9 \\
12 \text { by } 10 \\
14 \text { by } 7 \\
14 \text { by } 8 \\
14 \text { by } 9 \\
14 \text { by } 10 \\
14 \text { by } 12 \\
16 \text { by } 8 \\
16 \text { by } 9\end{array}$ & $\begin{array}{l}686 \\
588 \\
515 \\
533 \\
457 \\
400 \\
355 \\
320 \\
374 \\
327 \\
290 \\
261 \\
218 \\
277 \\
246\end{array}$ & $\begin{array}{l}16 \text { by } 10 \\
16 \text { by } 12 \\
18 \text { by } 9 \\
18 \text { by } 10 \\
18 \text { by } 11 \\
18 \text { by } 12 \\
20 \text { by } 10 \\
20 \text { by } 11 \\
20 \text { by } 12 \\
20 \text { by } 14 \\
22 \text { by } 11 \\
22 \text { by } 12 \\
22 \text { by } 14 \\
24 \text { by } 12 \\
24 \text { by } 14\end{array}$ & $\begin{array}{r}221 \\
185 \\
213 \\
192 \\
175 \\
160 \\
169 \\
154 \\
114 \\
121 \\
138 \\
126 \\
109 \\
115 \\
98\end{array}$ \\
\hline
\end{tabular}

1 The art of splitting slate blocks consists in progressively reducing resultant halves until the desired roofing slate thickness has been reached or approximated. Tbis band-wrought characteristic appeals to architects and owners. It is not a simple matter to precisely con-
trol the splitting of this natural rock, not can a uniformity of tbickness throughout be assured. The recommended range of thicknesses to be aimed at by operative splitters will meet all normal requirements and will insure the maximum of economy in tbe utilization of tbe many sizes of quarried blocks.

2 It is customary to regard a thickness falling between two standard thicknesses as a "special," and it is the practice to base tbe price of the "special" upon the greater of tbe two standard tbicknesses.

the "special" upon the greater of tbe two standard tbicknesses. ${ }^{3}$ For thicknesses one-half incb and more it is not generally con-
sidered practicable to use lengths that are less than 16 inches, althougb for roofs of special treatment it may be done in small quantities. In for roofs of special treatment it may be done in small quantities. In carrying out a desired design on special roofs it is sometimes necessary slates are used.

Note. - Wbere large quantities of tbick slates are required of tbe shortest practical lengtb one or more of the next longer slates should be permitted for most economical utilization of material from the quarry.

TABLE 2.-Dimensions of slate shingles for flat roofs

(Each size split to following thicknesses: For ordinary service, 3/16 inch; for promenade and extraordinary service, $1 / 4$ inch and $3 / 5$ inch)

\begin{tabular}{|l|l|l|}
\hline \multicolumn{3}{|c|}{ Face dimensions, in inches } \\
\hline 6 by 6 & 10 by 6 & 12 by 6 \\
6 by 8 & 10 by 7 & 12 by 7 \\
6 by 9 & 10 by 8 & 12 by 8 \\
\hline
\end{tabular}

\section{SIZES OF SLATE FOR MISCELLANEOUS PURPOSES}

It is recommended that smaller slate, such as 12 or 14 inch lengths, be used in covering pents, porch, and dormer roofs and sides, and garage or other lowroofed buildings. This practice is also recommended even in situations where the main roof is of larger slate.

Owing to the fact that certain sizes of slate may be more available than the size called for in the specification or order, it is recommended that architects, builders, engineers, and contractors provide for alternate selection on usual slate-roofing installations In this connection particular attention is also called to the increasing use of random widths of the desired lengths, and to the fact that architects are adopting this practice wherever possible. While slate is plentiful, such practices will bring about the elimination of waste of an important natural resource and will obviate the necessity of waiting for specified sizes while an accumulated finished stock of other usable sizes is available and accessible.

\section{NAIL HOLES}

The standard practice is to punch two nail holes in all slates.

\section{COLOR NOMENCLATURE}

For the purpose of utilizing the basic natural colors of roofing slate available in large quantities for general usage, it is recommended that the following color nomenclature be used by architects, contractors, engineers, and others in their specifications.

$\begin{array}{lll}\text { Black. } & \text { Gray. } & \text { Purple variegated. } \\ \text { Blue black. } & \text { Green. } & \text { Mottled purple and green. } \\ \text { Blue gray. } & \text { Purple. } & \text { Red. }\end{array}$

These color designations shall be preceded by the word "unfading" or "weathering," according to the ultimate color effect that may be desired.

For roofs of special treatment certain quarries supply colors and combinations of colors other than those mentioned in the above list and these should be regarded as "specials."

\section{DIMENSION NOMENCLATURE}

Commercial standard thickness (that is, average or basic).-The terms " $3 / 16$-inch slate," "full $3 / 16$-inch slate," or "not less than 3/16-inch slate" indicate a desire for a hand-picked selection, regardless of the added labor and cost. "Commercial standard" is the quarry run of production, and shows tolerable variations above or below $3 / 16$ inch. For the thicker slates, however, reasonable plus tolerances only are permissible; thus, a $1 / 4$-inch slate must be a full $1 / 4$ inch or thicker.

A square of roofing slate.-A square of roofing slate means a sufficient number of slate shingles of any size to cover 100 square feet of plain roofing surface when laid with approved or customary standard lap of 3 inches. Slates for surfacing flat roofs are usually laid tile fashion, without lap, in which case a square of slate would cover an area greater than 100 square feet.

Estimates.-For the purpose of figuring roofing surface it is the practice to add to the net surface of the roof one additional square foot for each linear foot of hips and valleys for loss in cutting and fitting. To provide for this and other details, the practice is to add from 5 to 10 per cent to the net roofing surface when estimating the total quantity of slate for any roof.

\subsection{Slate for Electrical Purposes.}

American Society for Testing Materials, tentative method of test for determining the insulating qualities of slate, serial designation D273-27T, 1927.

Wben it is desired to select slate for specific applications or when a routine factory or shop test is desired, either metbod $(a)$ or $(b)$ may be used, the testing applied before the slate is drilled. These tests are of high sensitivity and are applicable to laboratory or routine testing at tbe plant using tbe slate for electrical purposes. 


\section{FACTORY OR LABORATORY TEST ${ }^{\theta}$}

\section{METHOD A}

1. Method $(a)$ covers the determination of the impedance leakage current flowing in the high-voltage winding of the transformer by means of an ammeter in the low-voltage winding.

2. The apparatus shall consist of the following:

(a) Transformer.-A standard 10,000-volt portable testing transformer of $5 \mathrm{kva}$. rating.

(b) Ammeter.-An ammeter having a full scale reading of 5 amperes.

(c) Current source.-A source of 115 to 125 volt alternating current having a frequency of from 25 to 60 cycles and preferably of approximately sinewave form.

(d) Electrodes.-Two electrodes of 4/0 copper wire with rounded ends and provided with suitable insulation for the protection of the operator. (b) The slate shall be stood on edge on a sheet of metal. One electrode shall be placed in contact with the metal sheet and the other electrode moved over the surfaces and edges of the slate.

(c) The slate shall be laid on a sheet of metal with the surface making good contact. One electrode shall be placed in contact with the metal sheet and the other electrode moved over the upper surface of the slate.

\section{METHOD B}

6. Method (b) covers the determination of the impedance leakage current flowing in the highvoltage winding of the transformer or the specific ohmic impedance of the slate by reading two voltmeters in the low-voltage circuit.

7. The apparatus shall be connected as shown in Figure 88 and shall consist of the following:

(a) Transformer-A standard $30: 1$ potential transformer PT of $200 \mathrm{~V}$. A. capacity,

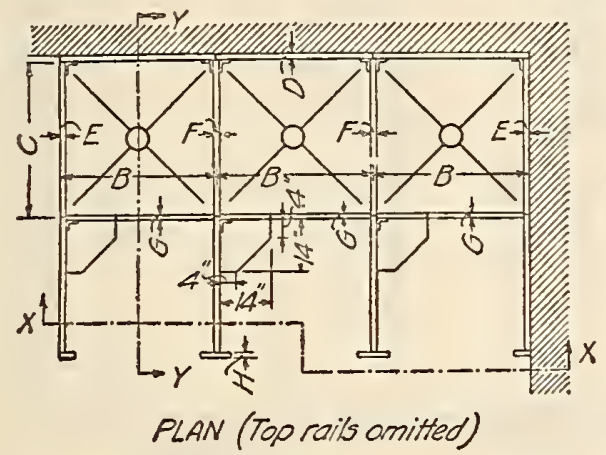

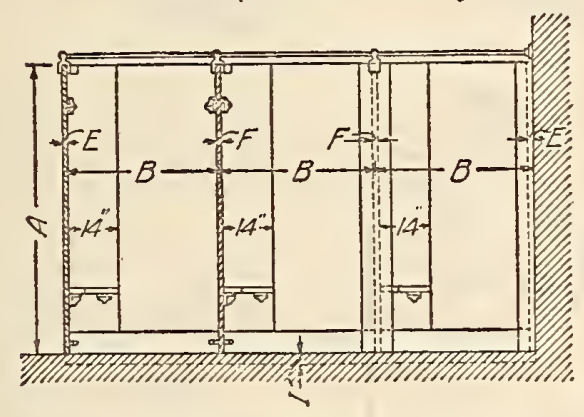

SECTION $X-X$

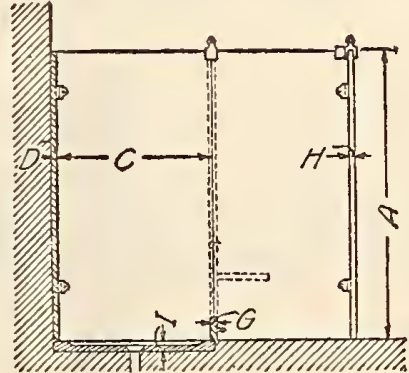

SECTION $Y-\gamma$

Figure 86.-Shower stalls. (See Table 8)

3. The test specimens may be of any size and shall have all surfaces, including edges, sand rubbed or hone finished.

4. (a) The ammeter shall be connected in series with the low-voltage side of the testing transformer and the exciting current noted. (This should be approximately 3 amperes for 25 cycles and 1 ampere for 60 cycles.)

(b) The high-voltage side shall be connected for 5,000 volts.

5. The leakage current; that is, the increase in current above the no-load exciting current of the transformer, shall be noted in the ammeter for the three following tests:

(a) The surfaces and edges of the slate shall be gone over with the electrodes not less than 2 inches apart. ${ }^{9}$ The A.S.T. M. committee has been at work on the development
of a quarry test, designated to make possible the selection at the quarry of slate suitable for electrical purposes, which it plans to include ultimately with the present methods. (b) Voltmeter-Two 150-volt alternating-current voltmeters $V_{1}$ and $V_{2}$.

(c) Current source.-A source of 115 to 125 volt alternating current having a frequency of from 25 to 60 cycles and preferably of approximately sine-wave form.

(d) Electrodes.-Two padded electrodes 4 inches square so arranged as to elamp down and make intimate contact with directly opposite sides of the slate and provided with insulated handles for the protection of the operator.

(e) Rheostat.-A variable rheostat, $R_{1}$, for controlling the voltage across the voltmeter, $V_{1}$.

$(f)$ Resistance.-A fixed resistance, $R_{2}$, of $14 \mathrm{ohms}$.

8. The test specimens may be of any size or thickness which would be used for electrical purposes. They shall have the face and back sand rubbed or hone finished.

9. The electrodes shall be clamped in place so that they come exactly opposite each other and exert a considerable pressure so as to reduce the 
contact resistance between the electrodes and the slate.

10. (a) With a reading of 110 volts held on voltmeter $V_{1}$, the voltage reading on voltmeter $V_{2}$, due to the voltage-drop through the fixed resistance, $R_{1}$, indicates the insulation value of the slate.

(b) As soon as a reading of voltmeter $V_{2}$ is obtained the electrodes shall be moved to another section of the slate. This shall be repeated until the entire surface of the slate has been covered.

(c) The reading of voltmeter $V_{2}$ shows great variation for low values of impedance and very little variation for high values.

Note.-This testing device can be safely used by an unskilled operator. The control circuit may be such that the placing of the electrodes on the slate is done by long insulating handles and the readings of voltmeter $V_{2}$. It is necessary to test a considerable number of samples of slate of different insulation values in order to obtain a satisfactory calibration curve. Aftel the apparatus is once calibrated a very accurate measurement of the ohmic impedance of the slate may be obtained.

\subsection{SOAPSTONE.}

Federal Specifications Board, specification No. 448, United States Government master specification for plumbing fixtures, November 22, 1926.

(The specification is published in full as Bureau of Standards Circular No. 310, and gives general and detail requirements for 22 plumbing fixture items, including soapstone for use in plumbing, general requirements for which are as follows:)
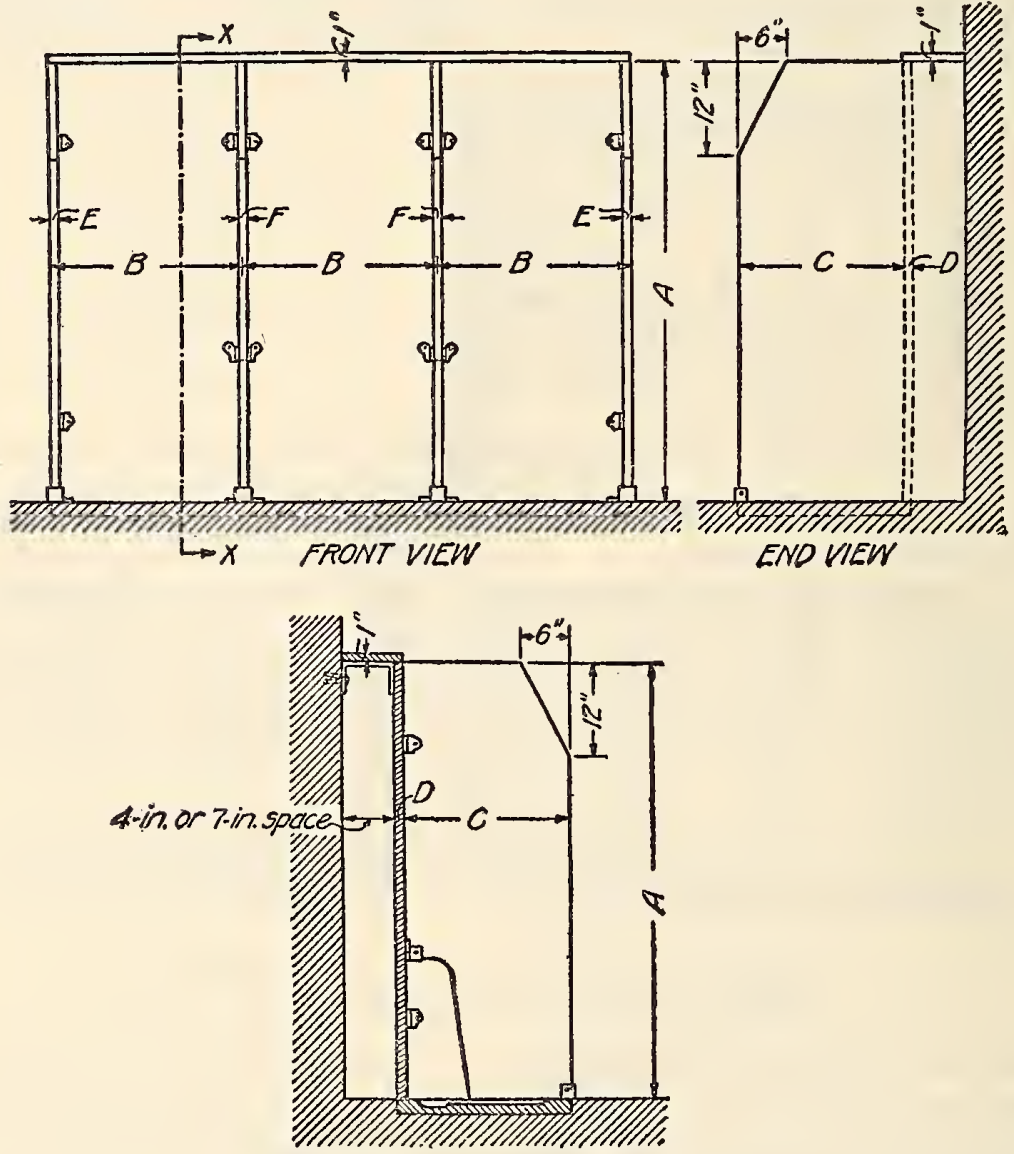

SECTION $X-X$

Figure 87.-Urinals. (See Table 10)

primary circuit of the transformer is open when the location of the electrodes is being changed. As the reading of voltmeter $V_{1}$ is held at a constant value, the scale of voltmeter $V_{2}$ may be marked in red for voltage values below the point predetermined as acceptable for electrical slate. If the needle is on the red portion of the scale when any section is being tested the slate shall be rejected.

11. For calibrating, a milliammeter or voltmeter of high resistance shall be inserted in series with the high voltage side of the transformer and the current flowing through the slate and the voltage across the slate determined for various values of readings on voltmeter $V_{2}$. The ohmic impedance shall be calculated from this data. A calibration curve for the apparatus shall be made by plotting ohmic impedance per square inch of electrode surface against
Soapstone shall be first quality, gray or bluish gray in color, close grained, nonporous, of uniform density and hardness and free from stratification; shall have a smooth finish, free from defects, with surfaces true and abutting edges close jointed.

\subsection{ROCK AND STONE FOR ROAD BUILD.} ING.

\subsection{General Items.}

American Society for Testing Materials, standard form of specifications for certain commercial grades of broken stone, serial designation: D35-18,1918. 
The broken stone shall consist of one product of the operation of a stone-crushing and screening plant without recombining or mixing, and shall conform to the following mechanical analysis, using laboratory screens:

Passing screen having smallest holes selected_- From _ _to _ - per cent. Passing screen having next to largest holes

Pelected Passing screen having largest holes selected.-_ From _. to _. per cent. Total passing screcn having largcst holes selected......... 100 per cent.

Example.-The broken stone shall consist of one product of the operation of a stone-crushing and screening plant without recombining or mixing, and shall conform to the following mechanical analysis using laboratory screens:

Passing $1 / 4$-inch screen Passing 1 -inch screen and retained on $1 / 4$-inch

Passing 1 -inch screen and retained on $1 / 4$-inch

Passing $11 / 4$-inch screen and retained on 1 -inch

screen $\quad$ Total passing $13 / 4$-inch screen.......... 3 to 10 per cent. 80 to 95 per cent. 2 to 10 per cent. 100 per cent.

or the analysis may be expressed as follows:

Total passing 1/1-inch screen.............. 3 to 10 per cent. Total passing 1-inch screen........ 90 to 98 per cent. Total passing $1 \frac{1}{4}$-inch screen. 100 per cent.

\section{PERMISSIBLE VARIATIONS IN MECHANICAL ANALYSIS}

4. The designated size for each grade together with the corresponding maximum permissible variations in mechanical analyses as determined by the use of laboratory screens, are given in the following table:

Maximum permissible range in mechanical analysis for each size

[Pcrcentage by weight passing laboratory screens]

\begin{tabular}{|c|c|c|c|c|c|c|c|}
\hline \multirow{2}{*}{$\begin{array}{l}\text { Designated } \\
\text { size (inch) }\end{array}$} & \multicolumn{7}{|c|}{ Diameter of screen openings (inch) } \\
\hline & $1 / 4$ & $1 / 2$ & $3 / 4$ & 1 & $11 / 4$ & $21 / 2$ & $31 / 2$ \\
\hline & $85-100$ & & & & & & \\
\hline & $\begin{array}{r}0-75 \\
40-80\end{array}$ & $95-100$ & 95 & & & & \\
\hline & $0-15$ & $25-75$ & $95-$ & & & & \\
\hline $1 / 4-$ & $\begin{array}{l}0-5 \\
0-5\end{array}$ & & $25-75$ & & $95-100$ & $05-100$ & \\
\hline & & & $0-15$ & $25-75$ & $95-100$ & & \\
\hline & & & $\ldots$ & - - - - & $0-15$ & $95-100$ & \\
\hline 372 & & & & & & $0-15$ & $95-100$ \\
\hline
\end{tabular}

1 In the case of ligl.t or porous slags a 4-inch maximum size may be specified instcad of $3 \frac{1}{2}$ inches.

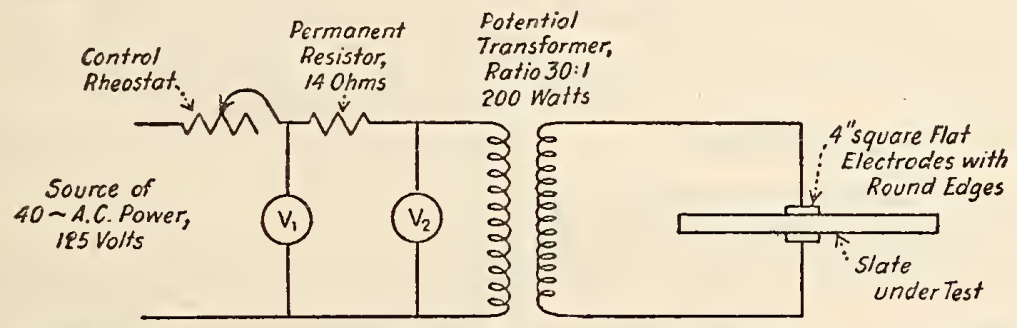

FiguRE 88.-Diagram of connections for measuring leakage current through slate

\subsection{Stone and Granite Blocks.}

American Society for HIunicipal Improvements, specifications for stone block pavements, 1927.

See 518.36, page 393 .

(This specification includes requirements for new granite blocks, resurfacing blocks, new sandstone blocks, and durax paving blocks.)

American Society for Testing Materials, tentative specifications for commercial sizes of broken stone and broken slag for highway construction, serial designation: D63-23T, 1923.

1. These specifications cover the standard size designations and maximum permissible range in mechanical analyses for nine commercial grades of broken stone and broken slag, when used in the construction of plain or bituminous macadam, bituminous concrete, sheet asphalt, and cementconcrete roads and pavements.

\section{MANUFACTURE}

2. The standard sizes herein specified may be produced from the run of the crusher by means of any screening process in which the factors influencing the efficiency of the screening operation are so adjusted that the designated sizes will conform to the requirements for mechanical analysis specified in section 4.

\section{STANDARD SIZES}

3. Each of the standard sizes shall be designated by reference to both the upper and lower nominal limiting size of the product: Thus, " $1 / 4$ to $3 / 4$ inch size" indicating that portion of the product of a crusher, all of which is nominally between $1 / 4$ and $3 / 4$ inch in size.

\section{SIZE OF SAMPLES}

5. Samples of broken stone or broken slag which are to be examined to deternine conformity with these specifications shall weigh at least 50 times the weight of the largest piece therein and be strictly representative of the product sampled.

\section{EXPLANATORY STATEMENT}

The commercial sizes designated in the accompanying proposed specifications provide primarily for the separation of the run of crusher into five sizes not including the 0 to $1 / 2$ inch size. Although the limiting sizes were determined after carefully considering the size requirements of the various types of roads using broken stone or slag, no definite requirements regarding use have been inserted, as such requirements will be included in specifications which are to be prepared by A. S. T. M. Committee D-4. It will be noted that a general tolerance of 15 per cent on the lower limiting size and 5 per cent on the upper limiting size for each grade is specified. It is felt that this will cover all unavoidable variations in grading of a given designated product. An intermediate size requirement of from 25 to 75 per cent is also given for the $1 / 4$ to $3 / 4$ inch and the $3 / 4$ to $1 \frac{1}{4}$ inch sizes. It is felt that for the two larger sizes an intermediate size requirement is not necessary. For the combination sizes which will ordinarily be used in cement-concrete work, a lower tolerance of 5 per cent is specified. In Table 1 various uses to which the different grades are adapted are indicated. 
TABLE 1.-Various uses for which the different grades of stone are adapted

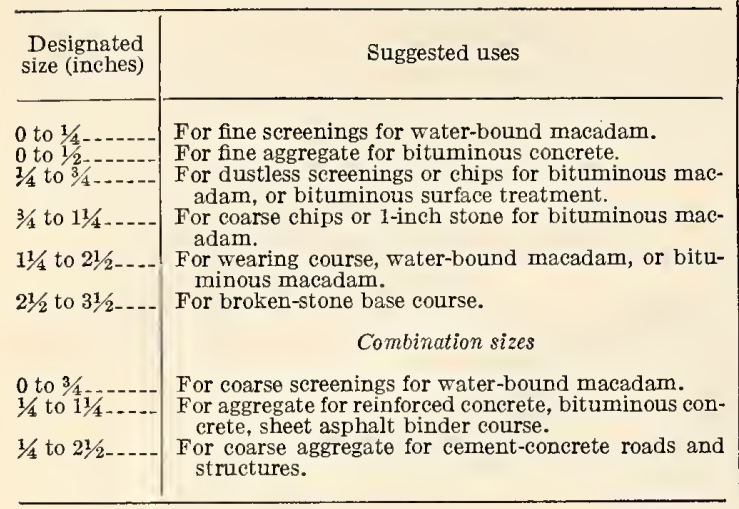

American Society for Testing Materials, standard specifications for:

1. Block for granite block pavements, serial designation D59-26, 1926.

2. Block for recut granite block pavements, serial designation D131-23, 1923.

3. Block for durax-granite pavements, serial designation D132-23, 1923.

\section{GENERAI REQUIREMENTS}

1. The blocks shall be of granite of medium-size grain, showing an even distribution of constituent minerals. They shall be of uniform quality and texture throughout, and free from seams or disintegrated materials.

\section{PHYSICAL PROPERTIES AND TESTS}

2 . The average of three tests on sample blocks shall conform to the following requirements as to physicial properties:

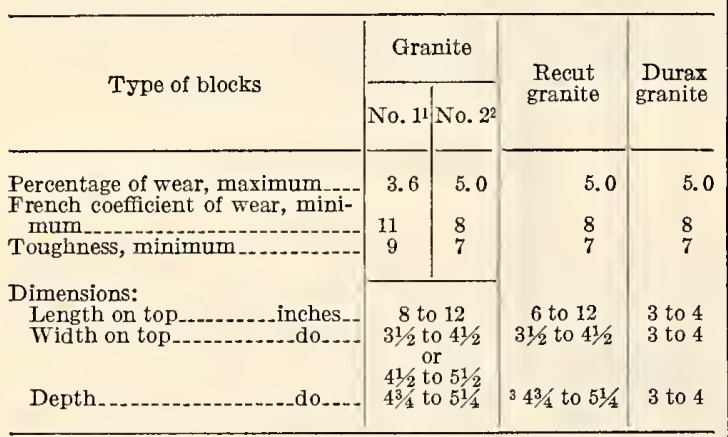

1 For heavy traffic.

2 For moderately heavy traffic.

8 The depth may be varied by the engineer between 4 and 5 inches, depth of blocks to suit the old material, provided that the blocks used on one contract shall not vary more than $1 / 4$ inch above or below the depth specified.

3. (a) The percentage of wear and the French coefficient of wear shall be determined in accordance with A. S. T. M. D2, see 510 , p. 183 .

(b) The toughness shall be determined in accordance with A. S. T. M. D3. (See 510., p. 183.)

\section{DRESSING}

5. (a) The blocks shall be so dressed that the faces will be approximately rectangular in shape, and the ends and sides sufficiently smooth to permit the blocks to be laid with joints not exceeding $1 / 2$ inch in width at the top, and for 1 inch downward therefrom, and not exceeding 1 inch in width at any other part of the joint.

(b) The wearing surface of the blocks shall show no depressions more than $3 / 8$ inch in depth, and the edges and corners shall be unchipped and unbroken.

\section{INSPECTION}

6. (a) At least 6 blocks for D59, 8 blocks for D131, and 25 blocks for D132 shall be selected for physical tests by the engineer or his authorized representative, subsequent to delivery at the place of use, so as to fairly represent actual deliveries. No sample shall include blocks that would be rejected by a visual examination. The bedding plane shall be marked on at least two of the blocks selected.

(b) All deliveries shall be subjected to further inspection at the place of use, prior to and during laying. All blocks which fail to conform to the requirements given under "general requirements," "dimensions," and "dressing" shall be rejected.

Federal Specifications Board, specification No. 510, United States Government master specification for block for granite, recut granite and durax granite pavements, August 12, 1927.

(This specification is the same as American Society for Testing Materials, serial designations D59, D131, and D132 (given above) with the following exceptions):

1. A No. 3 granite block having percentage of wear, maximum 6 , and a toughness, minimum 6 , has been provided for use under heavy traffic for the specific purpose of utilizing the products of certain quarries which may not meet the requirements for grade No. 2, but can meet the requirements for grade No. 3 , and have proved entirely satisfactory in service.

2 . This specification includes in addition to the A. S. T. M. specification for durax granite, a durax grade for extra heavy traffic having a percentage of wear, maximum 4, and a toughness, minimum 9 .

National Paving Brick Manufacturers' Association. recommended specifications, stone curb and header, 1924.

See 518.63 , p. 448.

\subsection{Riprap.}

American Association of State Highway Officials, standard specifications for bridges and incidental structures, 1928.

\section{RIP-RAP}

(The full text of this specification includes material relative to construction methods, and measurement and payment.)

Materials.- When stone is used for rip-rap it shall conform in quality to the requirements specified in "Stone for Masonry." (See 511.9, p. 212, American Association of State Highway Officials.)

Dry rip-rap, class 1.-Unless otherwise specified, all stones used in this class of rip-rap shall weigh between 50 and 150 pounds each and at least 60 per cent of them shall weigh more than 100 pounds each.

Dry rip-rap, class 2.- Stones having one broad flat surface shall be used when possible. Fifty per cent of the mass shall be of stones having a volume of 2 cubic feet or more.

Mortar rip-rap.-Stone for this purpose shall, as far as practicable, be selected as to size and shape in order' to secure fairly large, flat-surfaced stone which will lay up with a true and even surface and a minimum of voids. Fifty per cent of the mass shall be of broad flat stones of a volume of 2 cubic feet or more, laid with the flat surface uppermost and parallel to the slope. Warped and curved surfaces shall have the same general degree of accuracy as specified above for plane surfaces. 
Grouted rip-rap.-Grout for grouted rip-rap shall consist of 1 part of Portland cement and 3 parts of sand, thoroughly mixed with water to produce grout having a thick, creamy consistency.

The stones shall be of the same sizes and placed in the same manner as specified above for dry rip-rap, class 1.

Stone rip-rap for foundation protection.-Stone rip-rap for pier and abutment protection shall be of sizes ranging from derrick stone down and shall be graded from coarse to fine in such manner as to produce a minimum of roids. It shall be deposited where directed. Stone deposited contrary to directions will be considered wasted and will not be paid for.

Concrete rip-rap in bags. - Concrete rip-rap in bags shall consist of class C concrete. (See 516.3, p. 301, American Association of State Highway Officials, specification for "concrete masonry," in cement sacks or suitable burlap bags.) The bags shall be about two-thirds filled with concrete, securely tied, and immediately placed in the work.

\subsection{Ballast.}

American Railway Engineering Association, specifications for burnt clay ballast, 1921 .

\section{KIND OF MATERIAL}

1. Good ballast clay is heary and plastic, free from sand, gypsum, or other impurities. It must not crumble when exposed to air or when brought in contact with heat.

\section{IOCATION}

2. The pit should be located on level or moderately sloping ground, not subject to overflow. A water supply is desirable and it should be borne in mind that the sulphurous and carbonaceous gases liberated during the burning period, damage the surrounding vegetation and make habitation in the near vicinity very disagreeable.

\section{TEST}

3. The location site should be thoroughly tested to determine quality of clay, depth and uniform consistency of deposit, and small quantities should be burned in test kilns to show the quality of ballast to be secured.

\section{BURNING}

4. Fuel should be fresh, clean slack, and arrangements should be made to secure constant supply. One ton of slack coal is generally sufficient for the perfect burning of 4 cubic yards of acceptable ballast. From 1 to $1 \frac{1}{2}$ inch layer of slack is alternated with from 10 to 12 inch layer of clay, a new layer of slack and clay being applied to the fire every five or six dars.

Fires once started must be kept steadily and uniformly burning.

To insure thorough and proper burning of the clay, the top and face of the fire should be frequently raked down to avoid clinker or black spots, caused by too much or too little air.

When fully burnt a proper ballast clay becomes red in color, when the clay contains iron; when underburnt the clay will show a yellow color.

\section{SIZE}

5. Burnt clay ballast should be crushed or broken, if necessary, so that the largest piece will pass through a 4 -inch ring.

\section{DENSITY}

6. The finished product should absorb not to exceed 15 per cent of moisture by weight.

American Railway Engineering Association, specifications for pit-run gravel ballast, 1922.

\section{FOR CIASS A RAILWAYS}

Bank gravel, which contains more than 2 per cent dust or 40 per cent sand, should be washed or screened.

\section{FOR CLASS B RAILWAYS}

Bank gravel, which contains more than 3 per cent dust or 60 per cent sand, should be screened or washed. Screened gravel should not contain less than 25 per cent nor more than 50 per cent sand.

\section{FOR CIASS C RAIIWAYS}

Any material which makes better track than the natural roadbed may be economically used.

\section{METHOD OF TESTING QUALITY OF PIT RUN GRAVEL FOR BALLAST}

1. The size of the sample to be tested should be approximately 1 cubic foot.

2. Five average samples of about 1 cubic foot each should be selected from various parts of the pit which is to be tested. The five samples sbould then be thoroughly mixed and about 1 cubic foot of the mixture selected for testing.

3. To separate the gravel from the sand and dust, use a No. 10 screen, 10 meshes to the inch, made of No. 24 wire, B. \& S. gage. To separate the sand from the dust, use a No. 50 screen, 50 meshes to the inch, made of No. 31 wire, B. \& S. gage.

4. Measure the percentage of gravel, sand, and dust taken from the sample by volume, giving the percentage of each ingredient compared to the volume of the sum of the ingredients, as follows:

Where

$$
\text { Per cent of sand }=\frac{S}{G+S+D}
$$

$S=$ volume of sand.

$G=$ volume of gravel.

$D=$ volume of dust.

5. When sample is shipped for test it should be carefully and securely marked with name and location of the pit from which it was taken.

American Railway Engineering Association, specifications for washed gravel ballast, 1926 .

1. Gravel for ballast shall be so prepared that all dust, dirt, and loam are removed; that all aggregates that will not in every position pass through a $1 \frac{1}{2}$-inch ring are either rejected or crushed and returned to the ballast and that the resultant product conform to the following:

2. Where the percentages of crushed material run between nothing and 20 , the ratios of various sizes of aggregates to the whole shall be as follows:

\begin{tabular}{|c|c|}
\hline & $\begin{array}{c}\text { Per cent } \\
\text { fminimum } 25\end{array}$ \\
\hline $1 / 10$ to $1 / 4$ in & maximum 40 \\
\hline $1 / 4$ to $1 / 2$ inc & $\left\{\begin{array}{l}\text { minimum } 20 \\
\text { maximum } 30\end{array}\right.$ \\
\hline $1 / 2$ to 1 inch & minimum \\
\hline to $1 \frac{1}{2}$ inch & aimum \\
\hline
\end{tabular}

3. Where the percentage of crushed material runs more than 20 and less than 40 , the ratios of 
various sizes of aggregates to the whole shall be as follows

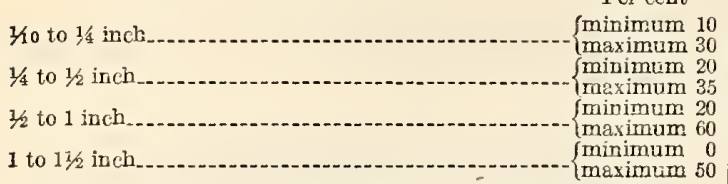

4. Where the percentage of crushed material is more than 40 , the ratios of the various sizes of aggregates to the whole shall be as follows:

Per cent

\begin{tabular}{|c|c|}
\hline \multirow{2}{*}{\multicolumn{2}{|c|}{$\begin{array}{l}1 / 4 \text { to } 1 / 2 \text { inch } \\
1 / 2 \text { to } 1 \text { inch }\end{array}$}} \\
\hline & $\begin{array}{l}1 / 2 \text { to } 1 \text { inch } \\
\text { maximum }\end{array}$ \\
\hline & \\
\hline & \\
\hline
\end{tabular}

5. Test No. 1, dust, dirt or loam.-A sample of the prepared ballast containing onc-eighth cubic foot shall be placed in a watcr-tight receptacie having a capacity of not less than 1 cubic foot. Into this receptacle shall then be placed 2 quarts of clear water, after which the receptaele shall be agitated until the gravel is thoroughly washed. The water shall be drained off immediately and plaeed in a glass jar and allowed to settle. If the sediment deposited in the bottom of the jar is more than onehalf of 1 per cent of the sample, as determined by weight, the output of the plant shall be rejected until the fault has been corrected.

6. Test No. 2, large aggregate.-A sample weighing not less than 150 pounds shall be placed in or on a screen having round holes $11 / 2$ inches in diametcr. If a thorough agitation of the sereen fails to pass through the screen 98 per cent of the material, as determined by weight, the output of the plant shall be rejected until the fault has been corrected.

7. Test No. 8, sand.--One cubic foot of the prepared ballast shall be thoroughly dried, weighed, and placed in a screen having 10 meshes to the inch and the screen agitated till all particles which will pass have passed through the screen. If the material which passes through the screen excecds 3 per cent of the original sample, as determined by weight, the output shall be rejected until the fault has been corrected.

8. Test No. 4, proportion of aggregaies in washed, crushed and screened gravel.- A sample of the prepared ballast, weighing not less than 150 pounds, shall be thoroughly dried, weighcd, and placed on a screen having holes $1 \frac{1}{2}$ inches in diameter; all material which can be passed through this screen shall be placed upon a scrcen having holes 1 inch in diameter; all material which can be passed through this sereen shall be placed upon a scrcen having holes one-half inch in diameter; all material which can be passed through this sercen shall be placed upon a screen having meshes of one-fourth inch, and all material which can be passed through this screen shall be placed upon a No. 10 screen, and all material which can be, shall be passed through it.

9 . If the ratio of the amount of material retained on the $1 \frac{1}{2}$ inch screen to the amount of the sample as a whole exceeds two per cent, the product shall be rejected until the fault has been corrected.

10. If the ratio of the amount of the material passing the No. 10 screen to the amount of the sample as a whole excecds 3 per cent, the product shall be rejected until the fault has been corrected.

11. If the ratio of the amount of the material retained on each of the screens to the amount of the sample as a whole does not come within the tolerances given above, the material shall be rejected until the fault has bcen corrected.

\section{INSPECTION}

12. In case inspection develops the fact that the material which has been or is being loaded is not in accordance with these specifications, the inspector shall notify the manufacturer to stop further loading until the fault has been corrected, and to dispose of all defective material that had been loaded in cars, which shall be done at the expense of the contractor.

\section{MEASUREMEMTS}

13. When ballast is being paid for by the ton, and it is impracticable to weigh each car, the wcight per yard shall be obtained by weighing at frequent intervals not less than five cars loaded with ballast, the contents of which have been carefully measured. The weight per yard obtained by such a test shall be used in figuring the weight per car until another test is made.

14. When ballast is paid for by the yard, the amount shall be determined by weighing each car, wherc practicable, and applying the weight per yard as determined by frequent tests. When impracticable to weigh each car, the contents of each car will be carefully estimated by comparison with cars, the contents of which have been actually measured.

American Railway Engineering Association, specification for stone ballast, 1925.

\section{GENERAL}

1. Stone for use in the manufacture of ballast shall break into angular fragments which range with fair uniformity between the maximum and minimum size specified herein; it shall test high in weight, toughness, wear, and soundness, but low in cementing qualities.

\section{TESTS}

\section{Tests shall be made as follows:}

\section{WEIGHT}

2. Not less than one-half cubic foot of solid stone accurately measured and dried for not less than 12 hours in dry air at a temperature of between $125^{\circ}$ and $140^{\circ} \mathrm{F}$. $\left(51.7^{\circ}\right.$ and $60^{\circ} \mathrm{C}$.) shall be weighed. The weight shail bc not less than — pounds per cubic foot.

\section{TOUGHNESS}

3. A piece of solid rock from which a cylindrical core perpendicular to the bedding plane of the rock, 0.98 inch by 0.98 inch ( 25 by $25 \mathrm{~mm}$ ), can be cut with a diamond core drill, and the ends ground plane shall, after drying, be held on an anvil, weighing not less than 110.23 pounds $(50 \mathrm{~kg})$ in the Page impact machine, like a miniature pile driver, under a plunger with sphere-shaped striking surface of 0.39 -inch $(1 \mathrm{~cm})$ radius, which is struck by a hammer when released weighing 4.4 pounds $(2 \mathrm{~kg})$. The test begins with a 0.39 inch $(1 \mathrm{~cm})$ fall of the hammer for the first blow, and continues with an increased fall of 0.39 inch $(1 \mathrm{~cm})$ for each succecding blow until the test piece fails, the number of blows and height being the same and representing the toughness of the rock. Rock that shows a toughness of less than - shall be rejected. (A. S. T. M., D3, see 510, p. 182.)

\section{SOUNDNESS}

4. Ten small pieces (total weight about $1,000 \mathrm{~g}$ ( 2 pounds)) of the rock shall be immersed in a saturated solution at $70^{\circ} \mathrm{F} .\left(21.1^{\circ} \mathrm{C}\right.$. $)$ of sodium sulphate $\left(\mathrm{Na}_{2} \mathrm{SO}_{4}\right)$ for 20 hours, after which they shall be placed for 4 hours in a drying oven maintained at 
$212^{\circ} \mathrm{F} .\left(100^{\circ} \mathrm{C}\right.$.). The treatment shall be repeated fre times. Rock which exhibits checking, cracking, or disintegration shall be rejected.

\section{WEAR}

5. Eleven pounds $(5 \mathrm{~kg})$ of freshly broken fragments of stone as nearly alike as possible, shall, after thorough drring, be placed in hollow-iron abrasion cylinders, $7 \%$ inches $(20 \mathrm{cin})$ in diameter and 13.4 inches $(34 \mathrm{~cm})$ in depth, which are revolved at the rate of 30 revolutions per minute for 10,000 revolutions for each test, after which the pieces shall be thoroughly washed, dried, and weighed, and the percentage of dust or detritus by weight that will pass through a screen with $1 / 16$-inch $(0.16$ $\mathrm{cm}$ ) mesh shall be considered the percentage of wear. Rock that has a greater percentage of wear than - for trap rock and - for limestone shall be rejected. (A. S. T. MI., D2, see 510, p. 182.)

\section{CEMENTING QUALITY}

6. One and one-tenth pounds (one-half $\mathrm{kg}$ ) of stone which can be crushed to pea size shall be placed (dry) in a ball inill which contains two steel shot weighing 20 pounds $(9.07 \mathrm{~kg}$ ) each, given 5,000 revolutions at the rate of 30 revolutions per minute, and the dough resulting from a mixture of the dust screened through a 100-mesh sieve, and water placed in an air-tight vessel for three hours, and then rekneaded, shall be made into six cylindrical briquettes 0.98 inch $(25 \mathrm{~mm})$ in diameter and $25 \mathrm{~mm}$ in height formed under a pressure of $1,877.5 \mathrm{lbs} . / \mathrm{in}^{2}$ $\left(132 \mathrm{~kg} / \mathrm{cm}^{2}\right)$, after which they shall be allowed to dry 20 hours in air, 4 hours in a hot-air bath at $212^{\circ} \mathrm{F}$. $\left(100^{\circ}\right.$ C.) and then cooled for 20 minutes in a desiccator, and immediately tested in a machine for ascertaining the crushing strength in pounds per square inch, which is the measure of the cementing value of the rock, the average of five determinations being taken. Rock which has a greater cementing value than $\longrightarrow$ shall be rejected.

\section{FREQUENCY}

7. Tests may be made from time to time at the option of the purchaser, and especially when new strata are being opened up for crushing into ballast.

\section{SELECTION OF SAMILES}

8. Each stratum of a quarry shall be tested separately and not averaged with any other stratum. (A. S. T. M., D75, see 510. p. 184.)

\section{AVERAGING}

9. For obtaining the values for physical tests, the average results of the numbers of specimens stated in the following table shall be taken:

\begin{tabular}{c|r|r|r|r|r}
\hline Find of tests & Weight & $\begin{array}{c}\text { Percent- } \\
\text { age of } \\
\text { wear }\end{array}$ & $\begin{array}{c}\text { Tough- } \\
\text { ness }\end{array}$ & $\begin{array}{c}\text { Cement- } \\
\text { ing } \\
\text { value }\end{array}$ & $\begin{array}{c}\text { Sound- } \\
\text { ness }\end{array}$ \\
\hline Number of tests_----- & 5 & 5 & 5 & 5 & 5 \\
\hline
\end{tabular}

PIACE FOR TESTS

10. Such tests as are deemed necessary shall be made at a testing laboratory selected by the purchaser, but visual inspection and other tests shall be made at the place of manufacture prior to shipments as often as considered necessary.

\section{PRODJCTION REQUIREMENTS}

\section{BREARING}

11. Stone for ballast shall be broken into fragments which range with fair uniformity between the size which will in any position pass through a $2 \frac{1}{2}$-inch ring, and the size wbich will not pass through a $3 / 4$-inch ring.

\section{TEST FOR STZE}

12. Maximum.-A sample weighing not less than 150 pounds shall be taken from the ballast as loaded in the cars and placed in or on a screen having round holes $23 / 4$ inches in diameter. If thorough agitation of the screen fails to pass through the screen 95 per cent of the fragments, as determined by weight, the output from the plant shall be rejected until the fault has been corrected.

Minimum.-A sample weighing not less than 150 pounds shall be taken from the ballast as loaded in the cars, weighed carefully and placed in or on a suitable screen having round holes three-fourths inch in diameter. The screen shall then be agitated until all fragments which will pass through the screen have been eliminated. The fragments retained in the screen shall then be weighed; and if the weight is less than 95 per cent of the original weight of the sample, the output of the plant shall be rejected until the fault is corrected.

\section{HANDLING}

13. Broken stone for ballast must be delivered from the screens directly to the cars or to clean bins provided for the storage of the output of the crusher.

Ballast must be loaded into cars which are in good order and tight enough to prevent leakage and waste of material and are clean and free from sand, dirt, rubbish, or any other substance which would foul or damage the ballast material.

\section{CLEANING}

14. The ballast shall be free from dirt loam, dust, or rubbish. When the rock is of such a nature that it does not become clean without preliminary scrubbing, a scrubbing machine shall be provided at the quarry.

\section{DEFECT FOUND AFTER DELIVERY}

15. As it is impracticable to inspect all the ballast loaded in cars, carloads of defective material arriving at the site for unloading and not previously inspected shall be rejected and returned to the manufacturer, who must pay the freight charges both ways. If unloaded prior to discovery of defectiveness, payment without return of the rejected ballast shall be refused to the manufacturer.

\section{INSPECTION}

16. Inspectors representing the purchaser shall have free entry to the work of the manufacturer at all times while the contract is being executed, and shall have all reasonable facilities afforded them by the manufacturer to satisfy them that the ballast is prepared and loaded in accordance with the specifications and contracts.

In case the inspection develops that the material which has been or is being loaded is not according to specifications, the inspector shall notify the manufacturer to stop further loading and to dispose of all cars under load with defective material. 


\section{MEASUREMENT}

17. Ballast material may be reckoned in cubic yards or by tons, as expedient. Where ballast material is handled in cars, the yardage may be determined by weight, after ascertaining the weight per cubic yard of the particular stone in question by careful measurement and weighing of not less than five cars filled with the material, or the tonnage may be determined for subsequent cars by measurement and coverting the yardage into tonnage by the use of the weight per yard as determined above.

Nore.-High quality stone will have the following values:

\begin{tabular}{|c|c|c|}
\hline & $\begin{array}{l}\text { Lime- } \\
\text { stone }\end{array}$ & Trap \\
\hline $\begin{array}{l}\text { Weight per cubic loot_- } \\
\text { Toughness }\end{array}$ & $\begin{array}{r}168 \\
10\end{array}$ & $\begin{array}{r}175 \\
15\end{array}$ \\
\hline 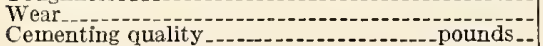 & $\begin{array}{l}5 \\
4\end{array}$ & $\begin{array}{l}3 \\
1\end{array}$ \\
\hline
\end{tabular}

National Sand and Gravel Association, 1928, specifications for washed, screened, and graded ballast.

(This organization cooperated with the American Railway Engineering Association in the preparation of the specifications for these commodities, given above.)

\subsection{MISCELIANEOUS SPECIFICATIONS FOR STONE AND STONE IIANU. FACTURES.}

American Association of State Highway Officials, standard specifications for bridges and incidental structures, 1928.

\section{STOHE FOR MASONRY}

1. Ashlar stone.-Stone for ashlar masonry shall be of the kind specified on the plans or elsewhere in the contract. The stone shall be tough, dense, sound, and durable, resistant to weathering action, reasonably fine grained, uniform in color, and free from seams, cracks, pyrite inclusions, or other structural defects. Preferably stone shall be from a quarry the product of which is known to be of satisiactory quality. All stones shall be quarried in sufficient time so that they may be thoroughly seasoned before being incorporated in the work. Stone shall be of such character that it can be truly wrought to such lines and surfaces, whether curved or plane, as may be required. Any stone having defects which have been repaired with cement or other materials shall be rejected.

Each bidder shall submit with his bid a 6-inch cubical block of the stone he proposes to furnish and shall state the quarry from which it is obtained. The quality of the stone furnished shall be at least equal to that of the sample. The sample shall be squared and dressed on three sides; one side shall be smooth-finished, one side fine-finished, and one side shall be given the finish indicated on the plans for exposed surfaees of face stone. The remaining sides shall be left with quarry face. (For definitions of surface finishes of stone, see "Ashlar masonry," 518.82$, p. 473.$)$

When permitted by the engineer, bidders may submit bids, accompanied by samples as specified above, on kinds of stone other than that specified.

2. Rubblestone.-Stone for mortar rubble or dry rubble masonry shall be of approved quality, sound, and durable, and free from segregations, seams, cracks, and other structural defects or imperfections tending to destroy its resistance to the weather. It shall be free from rounded, worn, or weathered surfaces. All weathered stone shall be rejected.

3. Riprap.-Stone for riprap shall consist of field stone or rough unhewn quarry stone as nearly rectangular in section as is practicable. The stone shall be resistant to the action of air and water; it shall be of sufficient density and suitable in all other respects for the purpose intended.

American Railway Engineering Association, stone masonry.

1. Spccifications for buildings for railway purposes (stone masonry and cut-stone work, 1926). (See 518.89 , p. 478.)

2. Specifications for stone masonry. (See 518.89, p. 478.)

\section{SAND, GRAVEL, AND SLAG}

\subsection{SAND AND GRAVEL.}

\subsection{General Items.}

American Society for Testing Raterials, standard method of test for unit weight of aggregate for concrete, serial designation $\mathrm{C} 29-27,1927$.

1. This method is intended to cover the determination of unit weight of fine, coarse, or mixed aggregate for concrete.

2. (a) The apparatus required consists of a cylindrical metal measure, a tamping rod, and a scale or balance, sensitive to 0.5 per cent of the weight of the sample to be weighed.

(b) Measures.-The measure shall be of metal, preferably machined to accurate dimensions on the inside, cylindrical in form, water-tight, and of sufficient rigidity to retain its form under rough usage, with top and bottom true and even, and preferably provided with handles.

The measure shall be of $1 / 10,1 / 2$, or 1 cubic foot capacity, depending on the maximum diameter of the coarsest particles in the aggregate, and shall be of the following dimensions:

\begin{tabular}{|c|c|c|c|c|}
\hline Capacity & $\begin{array}{l}\text { Inside } \\
\text { diametcr }\end{array}$ & $\begin{array}{l}\text { Inside } \\
\text { beight }\end{array}$ & $\begin{array}{c}\text { Minj- } \\
\text { mum } \\
\text { thickness } \\
\text { of metal } \\
\text { U. S. gage }\end{array}$ & $\begin{array}{c}\text { Diameter of } \\
\text { largest par- } \\
\text { ticles of } \\
\text { aggregate }\end{array}$ \\
\hline $\begin{array}{c}C u . \text { foot } \\
1 / 10 \\
1^{1 / 2}\end{array}$ & $\begin{array}{r}\text { Inches } \\
6.00 \\
10.00 \\
14.00\end{array}$ & $\begin{array}{r}\text { Inches } \\
6.10 \\
11.00 \\
11.23\end{array}$ & $\begin{array}{r}\text { Number } \\
11 \\
8 \\
5\end{array}$ & $\begin{array}{l}\quad \text { Inches } \\
\text { Under } 1 / 2 . \\
\text { Under } 11 / 2 . \\
\text { Over } 11 / 2 .\end{array}$ \\
\hline
\end{tabular}

(c) Tamping rod.-The tamping rod shall be a straight metal rod five-eighths inch in diameter and 24 inches long, with one end tapered for a distance of 1 inch to a blunt bullet-shaped point.

3. The measure shall be calibrated by accurately determining the weight of water at $16.7^{\circ} \mathrm{C}$. $\left(62^{\circ} \mathrm{F}\right.$.) required to fill it. The factor for any unit shall be obtained by dividing the unit weight of water at $16.7^{\circ} \mathrm{C}$. $\left(62^{\circ} \mathrm{F}.\right)(62.355$ pounds per cubic foot) by the weight of water at $16.7^{\circ} \mathrm{C}$. $\left(62^{\circ} \mathrm{F}\right.$.) required to fill the measure.

4. The sample of aggregate shall be room dry and thoroughly mixed.

5. (a) The measure shall be filled one-third full and the top leveled off with the fingers. The mass shall be puddled with the tamping rod with 25 strokes, evenly distributed over the surface. The measure shall be filled two-thirds full and again puddled with 25 strokes as before. The measure shall then be filled to overflowing, puddled 25 times, and the surplus aggregate struck off, using the tamping rod as a straight edge.

In puddling the first layer the rod should not be permitted to forcibly strike the bottom of the meas- 
ure; in puddling the second and final layers, only enough force to cause the tamping rod to penetrate the last layer of aggregate placed in the measure should be used.

(b) The net weight of the aggregate in the measure shall be determined. The unit weight of the aggregate shall then be obtained by multiplying the net weight of the aggregate by the factor found as described in section 3 .

6. Results with the same sample should check within 1 per cent.

American Society for Testing Materials, standard method of test for determination of voids in fine aggregate for concrete, serial designation C30-22, 1922.

Approved as tentative American standard by the American Engineering Standards Committee, now the American Standards Association.

1. The voids in fine aggregate for cement concrete shall be determined by the formula:

$$
\text { Percentage of roids }=\frac{(\text { Sp. gr. } \times 62.355)-\text { Wt. }}{\text { Sp. gr. } \times 62.355} \times 100
$$

2. The symbol "Sp. gr." represents the apparent specific gravity of the fine aggregate as determined by A. S. T. M. D55. (See p. 214.)

3 . The quantity 62.355 is the weight in pounds of 1 cubic foot of water at the standard temperature of $16.7^{\circ} \mathrm{C}$. $\left(62^{\circ} \mathrm{F}\right.$.).

4. The symbol "Wt." represents the weight in pounds per cubic foot of fine aggregate as determined by A. S. T. M. C29. (See p. 212.)

American Society for Testing Materials, standard method of test for organic impurities in sands for concrete, serial designation $\mathrm{C} 40-27,1927$.

1. The method of test is an approximate method of determining the prescnce of injurious organic compounds in natural sands which are to be used in cement mortar or concrete. The principal value of the test is to furnish a warning that further tests of the sands are necessary before they are approved for use.

2. A representative test sample of sand weighing about 1 pound shall be obtained by quartering or by the use of a sampler.

3. (a) A 12-ounce graduated clear-glass bottle shall be filled to the $4 \frac{1}{2} 2$-ounce mark with the sand to be tested.

(b) A 3 per cent solution of sodium hydroxide ( $\mathrm{NaOH}$ ) in water shall be added until the volume of sand and liquid indicated after shaking is 7 liquid ounces.

(c) The bottle shall be stoppered, shaken vigorously, and then allowed to stand for 24 hours.

4. A standard color solution shall be prepared by adding $2.5 \mathrm{cc}$ of a 2 per cent solution of tannic acid in 10 per cent alcohol to $97.5 \mathrm{cc}$ of a 3 per cent sodium hydroxide solution. This shall be placed in a 12-ounce bottle, stoppered, shaken vigorously, and then allowed to stand for 24 hours.

5. (a) After standing 24 hours the color of the clear liquid above the sand shall be compared with the color of the standard color solution prepared at the same time and in accordance with the method described in section 4 or with a glass of color similar to the color of the standard solution.

(b) Solutions darker in color than the standard color have a "color value" higher than 250 parts per million in terms of tannic acid.

American Society for Testing Materials, standard method of test for sieve analysis of aggregates for concrete, serial designation C41-24, 1924.

1. A representative test sample of the aggregate shall be selected by quartering or by use of a sampler, which after drying will give not less than the following :

(a) Fine aggregate, $500 \mathrm{~g}$. (b) Coarse aggregate or a mixture of fine and coarse aggregates, weight in grams, three thousand times size of largest sieve required, measured in inches.

TABLE 1

\begin{tabular}{|c|c|c|c|c|c|c|c|c|}
\hline \multirow{3}{*}{$\begin{array}{l}\text { Sieve No.I } \\
\text { of size } \\
\text { (in inches) }\end{array}$} & \multicolumn{2}{|c|}{ Sieve opening } & \multicolumn{2}{|c|}{ Wire diameter } & \multicolumn{4}{|c|}{ Tolerance } \\
\hline & \multirow{2}{*}{$\begin{array}{l}\text { Milli- } \\
\text { meters }\end{array}$} & \multirow{2}{*}{ Inches } & \multirow{2}{*}{$\begin{array}{l}\text { Milli- } \\
\text { meters }\end{array}$} & \multirow{2}{*}{ Inches } & \multirow{2}{*}{$\begin{array}{l}\text { Aver- } \\
\text { age } \\
\text { open- } \\
\text { ing }\end{array}$} & \multicolumn{2}{|c|}{$\begin{array}{l}\text { Wire diam- } \\
\text { eter }\end{array}$} & \multirow{2}{*}{$\begin{array}{l}\text { Maxi- } \\
\text { mum } \\
\text { open- } \\
\text { ing }\end{array}$} \\
\hline & & & & & & Under & Over & \\
\hline $\begin{array}{l}\text { No. } 100 \\
\text { No. } 50 \\
\text { No. } 30 \\
\text { No. } 16\end{array}$ & $\begin{array}{r}0.149 \\
.297 \\
.59 \\
1.19\end{array}$ & $\begin{array}{r}0.0059 \\
.0117 \\
.0232 \\
.0459\end{array}$ & $\begin{array}{l}0.102 \\
.188 \\
.33 \\
.54\end{array}$ & $\begin{array}{r}0.0040 \\
.0074 \\
.0130 \\
.0213\end{array}$ & $\begin{array}{r}\text { Per } \\
\text { cent } \\
6 \\
6 \\
5 \\
3\end{array}$ & $\begin{array}{r}\text { Per } \\
\text { cent } \\
15 \\
15 \\
15 \\
15\end{array}$ & $\begin{array}{r}\text { Per } \\
\text { cent } \\
35 \\
35 \\
30 \\
30\end{array}$ & $\begin{array}{r}\text { Per } \\
\text { cent } \\
40 \\
40 \\
25 \\
10\end{array}$ \\
\hline $\begin{array}{l}\text { No. } 8 \\
\text { No. } 4 \\
3 / 8 \text { inch.- } \\
3 / 4 \text { inch. } \\
1 \text { inch... }\end{array}$ & $\begin{array}{l}2.38 \\
4.76 \\
9.5 \\
19.0 \\
25.4\end{array}$ & $\begin{array}{l}.0937 \\
.187 \\
.375 \\
.75 \\
1.00\end{array}$ & $\begin{array}{l}.84 \\
1.27 \\
2.33 \\
3.42 \\
4.12\end{array}$ & $\begin{array}{l}.0331 \\
.050 \\
.092 \\
.135 \\
.162\end{array}$ & $\begin{array}{l}3 \\
3 \\
3 \\
3 \\
3\end{array}$ & $\begin{array}{l}15 \\
15 \\
10 \\
10 \\
10\end{array}$ & $\begin{array}{l}30 \\
30 \\
10 \\
10 \\
10\end{array}$ & $\begin{array}{l}10 \\
10 \\
10 \\
10 \\
10\end{array}$ \\
\hline $\begin{array}{l}11 / 2 \text { inches. } \\
2 \text { inches... } \\
3 \text { inches... }\end{array}$ & $\begin{array}{l}38.0 \\
50.8 \\
76.0\end{array}$ & $\begin{array}{l}1.50 \\
2.00 \\
3.00\end{array}$ & $\begin{array}{l}4.50 \\
4.88 \\
6.3\end{array}$ & $\begin{array}{l}.177 \\
.192 \\
.25\end{array}$ & $\begin{array}{l}3 \\
3 \\
3\end{array}$ & $\begin{array}{l}10 \\
10 \\
10\end{array}$ & $\begin{array}{l}10 \\
10 \\
10\end{array}$ & $\begin{array}{l}10 \\
10 \\
10\end{array}$ \\
\hline
\end{tabular}

1 The dimensions and tolerances for these sieves conform to the requirements of A. S. T. M. E 11 . (See 500.2 , p. 1.)

2. The sample shall be dried at not over $110^{\circ} \mathrm{C}$ $\left(230^{\circ} \mathrm{F}\right.$.) to constant weight.

3. (a) The sieves shali be of square-mesh wire cloth and shall be mounted on substantial frames constructed in a manner that will prevent loss of material during sieving.

(b) The size of wire and sieve openings shall be as given in Table 1.

4. (a) The sample shall be separated into a series of sizes by means of the sieves specified in section 3 . Sieving shall be continued until not more than 1 per cent by weight of the sample passes any sieve during one minute.

(b) Each size shall be weighed on a balance or scale which is sensitive to $1 / 1000$ of the weight of the test sample.

(c) The percentage by weight of the total sample which is finer than each of the sieves shall be computed.

5. (a) The percentages in sieve analysis shall be reported to the nearest whole number.

(b) If more than 15 per cent of a fine aggregate is coarser than the No. 4 (4,760-micron) sieve, or more than 15 per cent of a coarse aggregate is finer than the No. 4 (4,760-mieron) sieve, the sieve analysis of the portions finer and coarser than this sieve shall be reported separately.

American Society for Mesting Materials, standard definitions of the term "sand," serial designation C58-28, 1928.

Sand.-The fine granular material (usually less than one-fourth inch in diameter) resulting from the natural disintegration of rock or from the crushing of friable sandstone rocks.

Note.-When used without a qualifying adjective, the term "sand" is generally understood to mean the product of the natural disintegration of siliceous or calcareous rock. Sand should be distinguished from screenings, gravel, etc. The size of particle and other physical characteristics should be taken care of in specifications. The fine material resulting from the crushing of blast-furnace slag is known as slag sand.

American Society for Testing Materials, tentative definition for the term "aggregate," serial designation C58-28T, 1928.

Aggregate.-In the case of materials of construction, designated "inert" material, which when 
bound together into a conglomerated mass by a matrix forms concretes, mastic, mortar, plaster, etc.

American Society for Testing Materials, standard method of mechanical analysis of mixtures of sand or other fine material with broken stone or broken slag, except aggregates used in cement concrete, serial designation D19-16, 1916.

The method shall consist of (1) drying at not over $110^{\circ} \mathrm{C}$. $\left(230^{\circ} \mathrm{F}\right.$.) to a constant weight a sample

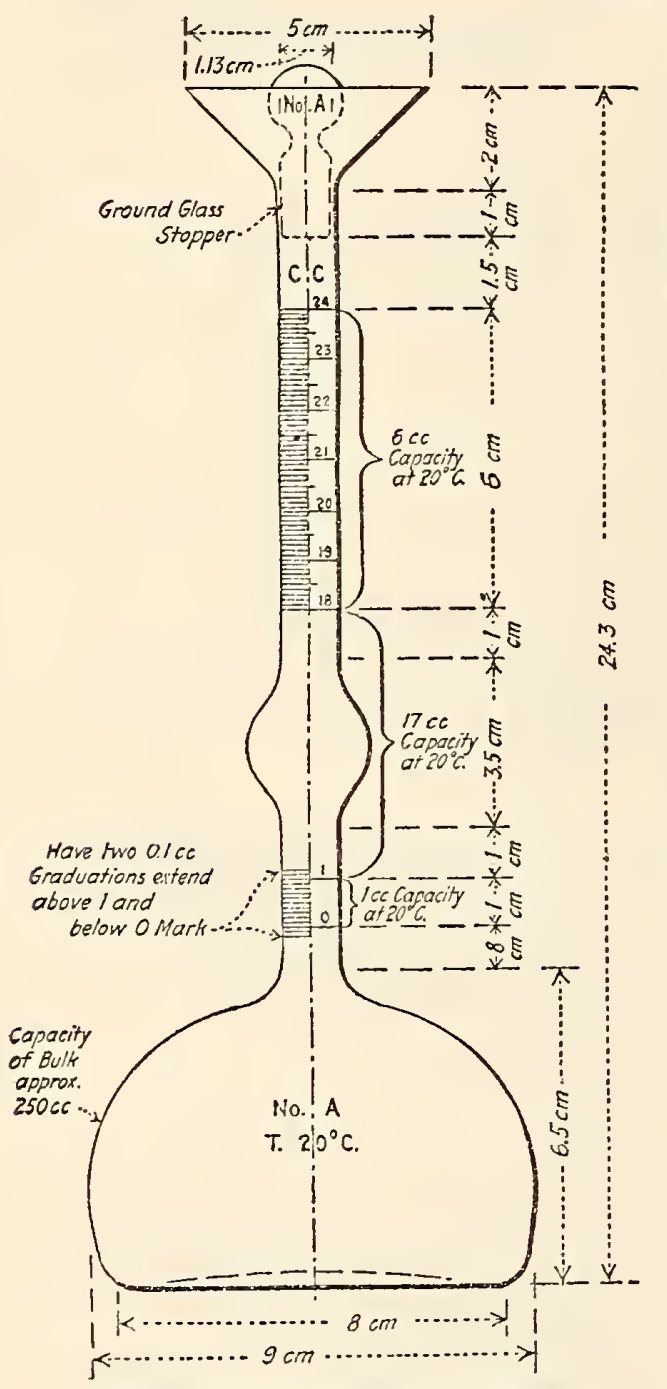

FIGURE 89.-Le Chatelier apparatus

weighing in pounds six times the diameter in inches of the largest holes required; (2) separating the sample by the use of a screen having circular openings $0.61 \mathrm{~cm}$ (one-fourth inch) in diameter; (3) examining the portion retained on the screen in accordance with A. S. T. M. D18 (see 512.15, p. 250); (4) examining the portion passing this screen in accordance with A. S. T. M. D7 (see 512.12, p. 234); and (5) recording the mechanical aralysis in the following manner:
Per cent

Passing No. 200 sieve Passing No. 80 sieve and retained on a No. 100 sieve Passing No. 10 sieve and retained on a No. 20 sieve Passing 0.64-cm (1/4-inch) screen and retained on a No. 10 sieve

Passing $1.27-\mathrm{cm}(1 / 2-\mathrm{inch})$ screen and retained on a $0.64-\mathrm{cm}$

(1/4-inch) screen Passing 1.90-cm (3/4-inch) screen and retained on a $1.27-\mathrm{cm}$ (1/2-inch) screen

American Society for Testing Materials, standard method of test for apparent specific gravity of coarse aggregates, serial designation D30-18, 1918.

Approved as tentative American standard by the American Engineering Standards Committee, now the American Standards Association.

The apparent specific gravity shall be determined in the following manner:

1. The sample, weighing $1,000 \mathrm{~g}$ and composed of pieces approximately cubical or spherical in shape and retained on a screen having $1.27-\mathrm{cm}$ ( $1 / 2$ inch) circular openings, shall be dried to constant weight at a temperature between $100^{\circ}$ and $110^{\circ} \mathrm{C}$. $\left(212^{\circ}\right.$ and $230^{\circ} \mathrm{F}$.), cooled, and weighed to the nearest $0.5 \mathrm{~g}$. Record this weight as weight $A$. In the case of homogeneous material, the smallest particles in the sample may be retained on a screen having $1 \frac{1}{1} 4^{\prime}-$ inch circular openings.

2. Immerse the sample in water for 24 hours, surface-dry individual pieces with aid of a towel or blotting paper, and weigh. Record this weight as weight $\mathrm{B}$.

3 . Place the sample in a wire basket of approximately 1/4-inch mesh, and about $12.7 \mathrm{~cm}$ (5 inches) square and $10.3 \mathrm{~cm}$ (4 inches) deep, suspend in water ${ }^{30}$ from center of scale pan, and weigh. Record the difference between this weight and the weight of the empty basket suspended in water as weight $\mathrm{C}$. (Weight of saturated sample immersed in watcr.)

4. The apparent specific gravity shall be calculated by dividing the weight of the dry sample (A) by the difference between the weights of the saturated sample in air (B) and in water (C), as follows:

$$
\text { Apparent specific gravity }=\frac{A}{B-C}
$$

5. Attention is called to the distinction between apparent specific gravity and true specific gravity. Apparent specific gravity includes the voids in the specimen, and is therefore always less than or equal to but never greater than the true specific gravity of the material.

American Society for Testing Materials, standard method of test for apparent specific gravity of sand, stone, and slag screenings and other fine nonbituminous highway materials, serial designation D55-25, 1925.

1. The following tests, "Le Chatelier" and "Jackson," are equally suited for use in determining the apparent specific gravity of sand, stone, and slag screenings and other fine nonbituminous highway materials, and may be considered as alternates:

\section{IE CHATELIER TEST}

2. The determination of specific gravity shall be made with a standardized Le Chatelier apparatus which conforms to the requirements illustrated in Figure 89. This apparatus is standardized by the

10 The basket may be conveniently suspended by means of a fine wire hung from a hook shaped in the form of a question mark with the top end resting on the center of the scale pan. 
National Bureau of Standards. Kerosene or water may be used in malking this determination.

3. (a) The flask shall be filled with either of these liquids to a point on the stem between 0 and 1 cc and a reading taken. From 50 to $64 \mathrm{~g}$ (depending upon its approximate specific gravity and to bring the reading on the scale) of sand or other fine nonbituminous highway material of the same temperature as the liquid shall be slowly introduced, taking care that the material does not adhere to the inside of the flask above the liquid and to free the material from air by rolling the flask in an inclined position. After all material is introduced, the level of the liquid in the graduated stem shall be read and the difference between readings is the volume displaced by the known weight of the material.

(b) The specific gravity shall then be calculated from the formula:

$$
\text { Specific gravity }=\frac{\text { Weight of material }(\mathrm{g})}{\text { Displaced volume }(\mathrm{cc})}
$$

(c) The flask, during the operation, shall be kept immersed in water, in order to avoid variations in the temperature of the liquid in the flask, which shall not exceed $0.5^{\circ} \mathrm{C}$. The results of reveated tests should agree within 0.01 .

\section{JACKSON TEST}

4. The determination shall be made with the Jackson specific gravity apparatus (illustrated in fig. 90) which shall consist of a burette, with graduations reading to 0.02 in specific gravity, about $28 \mathrm{~cm}$ (11 inches) in length and with an inside diameter of about $0.7 \mathrm{~cm}(0.3 \mathrm{inch})$, which shall be connected with a glass bulb approximately $13 \mathrm{~cm}$ (51/8 inches) in length and $4.5 \mathrm{~cm}$ (1.75 inches) in diameter, the glass bulb being of such size that from a mark on the neck above the bulb to the top graduation on the burette, the capacity shall be such that the graduations shall read directly the specific gravity when $50 \mathrm{~g}$ of materials are used; and an Erlenmeyer flask, which shall contain a hollow ground-glass stopper having a neck of approximately 6 to $8 \mathrm{~mm}$ bore and which shall have a capacity of exactly $200 \mathrm{cc}$ (6.76 ounces) up to the graduation on the neck of the stopper.

5. The method is as follows: (1) Dry at not more than $110^{\circ} \mathrm{C} .\left(230^{\circ} \mathrm{F}\right.$.) to a constant weight a sample weighing about $55 \mathrm{~g}$; (2) weigh $50 \mathrm{~g}$ of the dry sample to $0.1 \mathrm{~g}$ and pour it into the unstoppered Erlenmeyer fiask, which shall be cleaned and dried before each determination; (3) fill the bulb and burette with kerosene, leaving just space enough to take the temperature by introducing a thermometer through the neck; (4) remove the thermometer and add sufficient kerosene to fill exactly to the mark on the neck, drawing off any excess with the burette; (5) run into the flask about one-half of the kerosene in the bulb to remove air bubbles and then run in more kerosene, removing any material adhering to the neck of the flask, until the kerosene is just below the ground glass; (6) place the hollow ground-glass stopper in position and turn it to fit tightly, and then run in kerosene exactly to the $200 \mathrm{cc}$ (6.76 ounce) graduation on the neck, care being taken to remove all air bubbles in the flask; (7) read the specific gravity from the graduation on the burette, and the temperature of the oil in the flask, noting the difference between the temperature of the oil in the bulb before the determination and that of the oil in the flask after the determination; (8) make a temperature correction to the reading of the specific gravity in accordance with the table furnished by the manufacturer of the apparatus, adding the correction if the temperature of the kerosene has increased and subtracting it if the temperature of the kerosene has decreased.

American Society for Testing Materials, tentative specifications for commercial sizes of sand and gravel for highway construction, serial designation D64-20T.

1. These specifications cover the standard size designations and maximum permissible range in mechanical analyses for nine commercial grades of sand and gravel, when used in the constructlon of

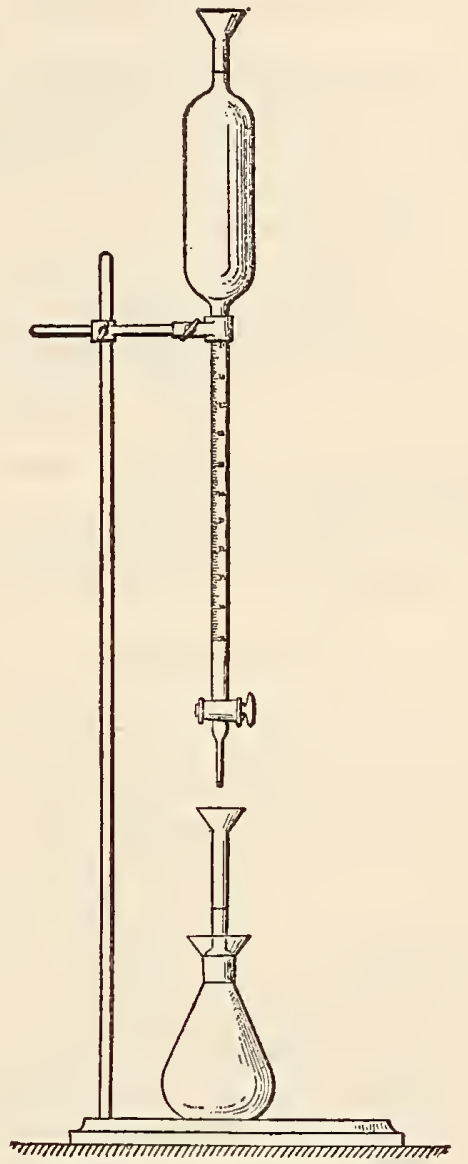

Figure 90.-Jackson apparatus

water-bound gravel and cement-concrete roads and pavements.

\section{MANUFACTURE}

2. The standard sizes herein specified may be produced from any screening process in which the factors influencing the efficiency of the screening operation are so adjusted that the designated sizes will conform to the requirements for mechanical analysis specified in section 4.

\section{STANDARD SIZES}

3. Each of the standard sizes shall be designated by reference to both the upper and lower nominal limiting size of the product: Thus, "one-fourth to three-fourths inch size" indicating that portion of the product of a sand and gravel screening plant, all of which is nominally between one-fourth and threefourths inch in size. 


\section{PERTIISSIBLE VARIATIONS IN JECHANICAL ANATYSES}

4. The designated size for each grade, together with the corresponding maximum permissible variations in mechanical analyses as determined by the use of laboratory screens and with sieves as specified in A. S. 'T. M. D7 (see 512.12, p. 234), are given in the following table:

Maximum permissible range in mechanical analysis for each size

[Percentage by weight passing each screen]

\begin{tabular}{|c|c|c|c|c|c|c|c|c|}
\hline \multirow{2}{*}{$\begin{array}{l}\text { Designated size } \\
\text { (inches) }\end{array}$} & \multicolumn{8}{|c|}{ Diameter of screen openings (inches) } \\
\hline & $1 / 4$ & $1 / 2$ & $3 / 4$ & 1 & $11 / 2$ & 2 & $21 / 2$ & $3 / 2$ \\
\hline & $\begin{array}{l}95-100 \\
85-100\end{array}$ & & & & & & & \\
\hline & $(3)$ & $95-100$ & & & & & & \\
\hline $\begin{array}{l}1 / 4-3 \\
1 / 4-1\end{array}$ & $\begin{array}{l}(3) \\
(3)\end{array}$ & $\overline{25-75}$ & $95-100$ & $95-100$ & & & & \\
\hline & (3) & 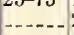 & $2 \overrightarrow{5}-\overrightarrow{75}$ & & $\overrightarrow{95}-100$ & & & \\
\hline $1 / 3-2$ & $\begin{array}{l}\text { (3) } \\
(3)\end{array}$ & -- & - & $25-75$ & $-\overline{25-75}$ & $95-100$ & & \\
\hline-2 & (न) & & & $0-15$ & 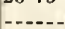 & $85-100$ & & \\
\hline & & & & & & $0-15$ & & $85-$ \\
\hline
\end{tabular}

1 Additional requirements for grading shall be as follows: Passing No. 20 sieve, 25 to 75 per cent.

Passing No. 50 sieve, not over 25 per cent.

Passing No 100 sieve, not over 5 per cent.

2 Limits for silt and clay content may be inserted if desired.

3 Any percentage from 0 to 10 per cent may be designated, with a maximum permissible variation therefrom of not more than $2 \frac{1}{2}$ per cent.

\section{SIZE OF SAMPLES}

5. Samples of sand shall weigh at least 10 pounds Samples of gravel and samples composed of mixtures of sand and gravel shall weigh at least fifty times the weight of the largest piece therein.

American Society for Testing Materials, standard methods of sampling stone, slag, gravel, sand, and stone block for use as highway materials, including some material survey methods, serial designation D75-22, 1922

(See 510, p. 184.)

United States Department of Agriculture, bulletin 1216, method of proportioning natural sand and gravel for concrete construction, 1928.

For determining the amount of cement to make concrete equivalent to any base mixture, when the percentage of sand in the total aggregate varies from the base mixture.

Rule.-Decrease or increase the ratio by weight of total aggregate to cement by 0.05 times the increase, or decrease in the percentage of sand in the total aggregates by weight. This involves the following steps in the process:

1. If the base mixture is stated as a relation by volume, it will be necessary to transfer the proportion to a statement of relation by weight. This requires that the weights per unit of loose volume of the various materials be known or assumed.

2. Determine the percentage of fine aggregate in the total aggregate in the base mixture and in the new mixture to be used, using the weight proportions.

3. Multiply the difference between these two percentages by 0.05 and subtract the result from the sum of the parts of fine and coarse aggregates in the weight proportion for the base mixture if the percentage of fine aggregate is greater in the new mixture. If the percentage of fine aggregate is less in the new mixture, add instead of subtracting.

4. If screened materials are to be used, divide the parts of total aggregate determined above into fine and coarse aggregates according to the percentage of fine aggregate in the new mixture.
5. Transfer the resulting weight proportion into a statement of relation by loose volumes.

Example:

Assume, base $\operatorname{mix}, 1: 2: 3 \frac{1}{2}$ by volume.

Unit weights-

Fine aggregate, 95 pounds per cubic foot.

Coarse aggregate, 110 pounds per cubic foot.

Cement, 94 pounds per cubic foot.

Percentage of fine aggregate in new mixture $=60$.

(1) $1: 2: 3^{1} \frac{1}{2}=94: 2$ times $95: 3^{1 / 2}$ times 110 . $=94: 190: 385$ proportion by weight. $=1: 2.02: 4.10=1: 6.12$.

(2) Percentage of sand in base mixture aggregate $=\frac{190}{190+385} \times 100=33$.

Percentage of sand in new mixture $=60$

(3) $60-33=27$.

27 times $0.05=1.35$.

$6.12-1.35=4.77$, therefore, the new proportion by weight is $1: 4.77$.

(4) Since 60 per cent of the total aggregate is to be fine aggregate

$$
1: 4.77=1: 2.86: 1.91
$$

(5) $1: 2.86: 1.91=94$ pounds : 269 pounds: 180 pounds

Dividing by the unit weights of materials. $\frac{94}{94}: \frac{269}{95}: \frac{180}{110}=1$ cubic foot: 2.83 cubic feet: 1.64 cubic feet.

Or if unscreened gravel is to be used, determine the unit weight of the gravel. Assume for this case 115 pounds per cubic foot.

Then 1:4.77 $=94$ pounds to 449 pounds Dividing by unit weights,

$$
\frac{94}{94}: \frac{449}{115}=1 \text { cubic foot: } 3.90 \text { cubic feet }
$$

This method applies to either screened or unscreened mixtures of sand and gravel in which the specific gravities of the fine and coarse aggregate are approximately equal, and to percentages of fine aggregate greater than 33.11

Recommended practice in the use of unscreened gravel:

1. The fine aggregate portion must pass the user's specification for fine aggregate.

2. The coarse aggregate portion must pass the user's specification for coarse aggregate.

3. Unscreened gravel should not be used, unless, in the opinion of the engineer in charge, the amount of sand in the aggregate will not vary more than 10 per cent.

4. Unscreened gravel should only be used from stock piles containing the largest practicable amount of material. ${ }^{12}$

United States Department of Agriculture, Bulletin 1216 , method of test for apparent specific gravity of sand, stone, and slag screenings, and other fine nonbituminous highway materials, 1928 .

(This method is the same as the Le Chatelier test of A. S. T. M. D55, see 512.10 , p. 214. The Jackson test is deleted. This method is not accurate when sand containing a relatively large proportion of absorptive grains is tested.)

United States Department of Agriculture, Bulletin 1216, method of test for apparent specific gravity and absorption or free moisture content for sand gravel, stone, or other nonbituminous highway materials, 1928 .

11 The method can be applied to mixtures of materials of different in specific gravities, such as sand and crushed limestone by reducing the proportions to relations

adustment upon that basis.

${ }^{12}$ For a theoretical discussion of the method of proportioning see Bulletin No. 60, Engineering Experiment Station, Iowa State College (6), and also a paper by R. W. Crum, Proc. Am. Soc.Testing Materials, 7; 1922 . 


\section{PYCNOMETER METHOD}

1. This method is designed primarily for the purpose of determining the apparent specific gravity of saturated surface-dry materials and the absorption or the free moisture content of materials as used in the field. While this method has been adapted for field use, it is suggested as a convenient method for making these determinations in the laboratory.

2. The special apparatus used consists of a pycnometer which shall conform to the following conditions:

(a) The opening at the top shall be approximately three-eighths inch in diameter.

(b) The shape of the apparatus shall be such as will facilitate the escape of air bubbles.

(c) It shall have an opening for the introduction of samples which will admit particles as large as 2 inches.

(d) It shall be capable of being cleaned quickly and easily.

\section{APPARENT SPECIFIC GRAVITY}

3. (a) Coarse material.-The apparent specific gravity of coarse material shall be determined upon material that has been immersed in water at $70^{\circ} \mathrm{F}$. for 24 hours. The pieces shall be individually surface-dried with a towel. Two thousand grams of this surface-dried material shall be weighed out to the nearest $0.5 \mathrm{~g}$. This sample shall be placed in the pycnometer and the pycnometer partly filled with water at $70^{\circ} \mathrm{F}$. The pycnometer shall be gently shaken and rolled to remove entrained air. The pycnometer shall then be exactly filled with water, and its outside surface dried. The pycnometer and its contents shall then be weighed to the nearest $0.5 \mathrm{~g}$. The apparent specific gravity shall be computed from the formula:

$$
\text { Specific gravity }=\frac{S}{P+S-W}
$$

where

$S=$ the weight of the sample in grams.

$P=$ the weight in grams of the pycnometer full of water.

$W=$ the weight in grams of the pycnometer plus the sample plus sufficient water to fill the pycnometer.

(b) Fine material.-The apparent specific gravity of fine material shall be determined in the same way as for coarse material except that the sample shail consist of air-dried material and shall consist of $1,000 \mathrm{~g}$.

\section{ABSORPTION OR FREE WATER CONTENT}

4. The absorption or the free-water content of material shall be determined upon a sample consisting of material in the condition in which it is to be used.

5. Two thousand grams of the sample shall be reighed out to the nearest $0.5 \mathrm{~g}$. This sample shall be placed in the pycnometer and the pycnometer partly filled with water at $70^{\circ} \mathrm{F}$. The pycnometer shall be gently shaken and rolled to remove entrained air. The pycnometer shall then be exactly filled with water and its outside surface dried. The py cnometer and its contents shall then be weighed to the nearest $0.5 \mathrm{~g}$.

6 . The absorption or the free-moisture content of the sample shall be computed from the following formula:

Percentage of moisture or absorption ${ }^{13}$

$$
=\frac{100}{S}\left(W-W_{1}\right) \frac{G_{\mathrm{s}}}{G_{\mathrm{s}}-1}
$$

13 If the algehraic sign of the result is positive, the material contains ree water. If the sign is negative, the material absorhs water. where $S=$ the weight of the sample in grams.

$W=P+S-\frac{S}{G_{s}}$

$P=$ the weight in grams of the pycnometer filled with water.

$W_{1}=$ the weight of the pycnometer containing the sample and sufficient water to fill the remaining space.

$G_{\mathrm{s}}=$ the specific gravity of the saturated surface dry material.

7. A pycnometer that has been used successfully by the Iowa Highway Commission consists of an ordinary glass Mason fruit jar with a special cap. This cap is the frustrum of a cone superimposed upon a ring which will screw upon the top of the jar. This cap may be made from the screw ring from a Kerr fruit-jar cap to which is soldered a tin cone with a slope of $60^{\circ}$ and $a \frac{3}{8}$-inch hole at the top. A more satisfactory cap could be cast from brass or some other metal that does not corrode. In using a pycnometer of this kind the cap and the jar should be marked so that the cap will be screwed on to the same position each time so that the volume of the apparatus will not be varied.

\subsection{Gravel.}

American Association of State Highway Officials, tentative standard specifications for highway materials, 1929 .

\section{GRAVEL FOR PORTLAND CEMENT CONCRETE}

1. This specification covers the quality and size of gravel for use as coarse aggregate in concrete.

2 . The gravel shall be composed of hard, durable particles of stone, thoroughly clean, and shall be free from an excess of thin or elongated pieces, frozen lumps, vegetable, or other deleterious matter. (Note 1.)

3. (a) Per cent of wear for uncrushed material $(\operatorname{method} \mathrm{A})$.

When grading A (see methods of testing) is unt When grading A (see methods of testing) is used, not over

, not over

When grading D (see methods of testing) is used, not over

(b) For gravel containing angular pieces, the "test for gravel containing crushed pieces" (method B) shall govern and the per cent of wear allowed on the angular portion shall be not less than - per cent.

4. When tested by means of laboratory screens the gravel shall meet the following requirements:

Passing Per cent Passing ..._inch sereen (maximum size) note 2 . ........... 95-100 Passing

5. Sampling and testing of the gravel shall be in accordance with the method of test for abrasion of gravel, United States Department of Agriculture Bulletin 1216 (given below).

Note 1.-Where it is desired to specify definite maximum amounts of deleterious substances, the following form of specification may he employed:

Per cent

Removed by decantation, not more than

Shale, not more than

Clay lumps, not more than

Soft fragments, not more than

(n) ther local deleterious substances (such as alkali, friable,

thin, elongated or laminated pieces), not more than

Note 2.-Either round or square mesh sieves should he specified as required.

American Railway Engineering Association, specification for pit-run gravel ballast, 1922 .

(See 511.73, p. 209.) 
American Railway Engineering Association, specification for washed gravel ballast, 1927 .

(See 511.73, p. 209.)

American Society for Municipal Improvements, specifications for broken stone and gravel roads, 1922.

(Sce 512.15 , p. 245.)

American Society for Testing Materials, standard method of test for quantity of clay and silt in gravel for highway construction, serial designation D72-21, 1921.

1. This test covers the determination of the quantity of clay and silt in natural gravel to be used in highway construction.

2. The samples as received shall be moistened and thoroughly mixed, then dried to constant weight at a temperature between $100^{\circ}$ and $110^{\circ} \mathrm{C}$. $\left(212^{\circ}\right.$ and $230^{\circ}$ F.).

3 . The pan or vessel to be used in the determination shall be 12 inches $(30.5 \mathrm{~cm})$ in diameter by not less than 4 inches $(10.2 \mathrm{~cm})$ deep, as nearly as may be obtaincd.

4. (a) A representative portion of the dry material weighing not less than fifty times the weight of the largest stone in the sample shall be selected from the sample and placed in the pan which has been dried and accurately weighed.

(b) Sufficient watcr shall be poured into the pan to cover the gravel. The gravel shall be agitated vigorously for 15 seconds with a trowel or stirring rod. After it has settled for 15 seconds the water shall be poured off into a tared evaporating dish, care being taken not to pour off any gravel. This is repeated until the wasl water is clear. The washed material shall be dried to constant weight in an oven at a temperature between $100^{\circ}$ and $110^{\circ}$ C. $\left(212^{\circ}\right.$ and $230^{\circ}$ F.), weighed, and the net weight of gravcl determined.

5 . The percentage of clay and silt shall be calculated from the formula:

Percentage of $=\frac{\text { Original weight }- \text { weight aftor washing }}{} \times 100$

6. For a check on the results, the wash water shall be evaporated to dryness and the residue weighed:

$$
\text { Percentago of clay and silt }=\frac{\text { Weight of residue }}{\text { Original weight }} \times 100
$$

American Society for Testing Materials, tentative method of test for abrasion of gravel, serial designation D289-28T, 1928.

(This specification is practically identical with the method of test for the abrasion of gravel of the United States Department of Agriculture, Bulletin 1216, sec p. 219.

American Society for Testing Materials, tentative spccifications for gravel for bituminous concrete base, scrial designation D309-29T, 1929.

1. These specifications cover $1 / 4$ to $1 \frac{1}{4}$ inch and $1 / 4$ to $2 \frac{1}{2}$ inch size gravel, either of which size is suitable for use in the construction of a bituminous concrcte base prepared by the mixing method and upon which may be placed any type of surface course.

2. The gravel shall consist of hard, sound, tough, durable stone particles, either crushed or uncrushed, and shall be free from injurious amounts of adherent coatings and soft, thin, elongated or laminated picces, or other delcterious substances.

3. Gravel shall show a satisfactory resistance to abrasion as measured by an acceptable abrasion test for gravel.

Note.-No test limits for abrasion are specified due to the status of knowledge concerning suitable specification limits for this test. Many engineers focl that the quality of gravel may be indicated by the results of an abrasion test, and, accordingly, the tentative method of test for abrasion of gravel (A.S. T. M. D289-28T) is recommended. specification D289 is practically identical with the method of test or the abrasion of gravel of the United States Department of Agriculture, Bulletin 1216. See p. 219.)

4. The amount of deleterious substances shall not exceed the following maximum requirements:

\section{Per cent} by weight

Removed by decantation 1

Shate

Clay lumps

Clay lumps

Total shale, coal, clay lumps, and soft fragments

ther local deleterious substances (such as alkali, friable, thin,

or elongated or laminated pieces)

Note,-It is recognized that under certain conditions percentages of deleterious substances less than those shown in the table should be specified as a maximum.

5. The gravel when tested by means of laboratory screens shall conform to the following rcquirements:

$1 / 4$ to $11 / 4$ inch size

Passing 11/2-inch screen.

Passing 114-inch screen 100 Passing 3/4-inch screen Passing 1/4-inch screen

$1 / 1$ to $21 / 2$ inch size

Passing 3-inch screen Passing 21/2-inch screen Passing 11/4-inch sereen.-_-_.-. 40 to 75 Passing 3/4-inch screen Passing 1/4-inch screen

6. The method of sampling shall conform to A. S. T. M. D75. (See 510, p. 184.)

Federal Specifications Board, specification No. 453, United States Government master specification for gravel for gravel roads, January 25, 1927.

\section{TYPES AND SIZES}

The materials covered by this specification shall be supplied in the particular type(s) and size(s) ordered. (See note 2.)

\begin{tabular}{|c|c|}
\hline Type of material & Size \\
\hline Sand-clay gravel_............ & $\left\{\begin{array}{r}\text { Inch } \\
0-3 \\
0-1 \\
0-3 \\
0-1 \\
1 / 4-3 / 4\end{array}\right.$ \\
\hline
\end{tabular}

\section{GENERAL REQUIREMENTS}

Gravel shall be composed of hard, durable stone particles of high resistance to abrasion, free from an excess of thin or elongated pieces, together with sand and clay or other binding material as required in this specification.

\section{DETAIL REQUIREMENTS}

1. Sand-clay gravel for use or size designated shall mect the following requirements:

(a) Base course gravel, o to s-inch size:

Per cent

Clay and silt

Passing No. 8 sieve

Passing 0.8 sieve 3 -

烈

Cementing value of material passing $\mathrm{No} .8$ sieve, not less than

(b) Surface course gravel, 0 to 1 inch size:

Passing No. 8 sieve.

Passing 1-inch screen

Cementing value of material passing No. 8 sieve, not less

than

2. Sand gravel for use or size as designated shall meet the following requirements: 
(a) Base course gravel, 0 to s-inch size:

Clay and silt. Passing No. 8 sieve Passing 3-inch screen

(b) Surface course gravel, 0 to 1 -inch size: Clay and silt........... Passing No. 8 sieve.-.

Passing No. 8 sieve

3. Maintenance gravel, $1 / 4$ to $3 / 4$ inch size, shall meet the following requirements:

Per cent

assing $1 / 4$-inch screen

Passing 8 -inch screen

\section{METHODS OF SAMPLING AND TESTING}

1. Methods of sampling shall be in accordance with A. S. T. Mi. D75. (See 510, p. 184.)

2. Tests of gravel shall be made in accordance with the following methods:

(a) Clay and silt: A. S. T. M. D72. (See p. 218.)

(b) Mechanical analysis: A. S. T. M. D18. (See 512.15 , p. 250 ), using a No. 8 sieve as required in addition to the screens specified in Method D18. The No. 8 sieve shall be a United States standard sieve, meeting the requirements of A. S. T. M. E11. (See 500.2 , p. 1.)

(c) Cementing value: United States Department of Agriculture, test. (See 510, p. 187.)

\section{NOTES}

1. In economical construction of gravel roads, it is necessary to use the best available local materials. In promulgating this master specification, the Federal Specifications Board does not intend, therefore, that the use of its requirements shall be strictly mandatory; where material conforming to the requirements is not econmically available the master specification should serve as a guide in the selection of material.

2. In the Northern States it is the current practice to use sand gravel on clay subgrades, and sand-clay gravel on sandy subgrades. In the Southern States the practice is to use either the sand-clay gravel or a washed gravel bound together with added clay or sand clay.

Federal Specifications Board, specification No. 455, United States Government master specification for broken stone, broken slag, or gravel for bituminous surface treatment, January 25, 1927.

(See 512.13, p. 240.)

National Sand and Gravel Association, Bulletin 3, representative specifications for different uses of sand and gravel, November, 1928.

(In this bulletin representative specifications for sand and gravel, many of which are presented in this volume, have been summarized.)

National Sand and Gravel Association, suggested commercial sizes of sand and gravel (tentative).

\section{Gravel}

\begin{tabular}{|c|c|}
\hline Size & Common uses \\
\hline No. $4,1 / 2$ i & $\left\{\begin{array}{l}\text { Gravel for bituminous macadam or bituminous } \\
\text { surface treatment. } \\
\text { Coarse aggregate for thin concrete sections; as, } \\
\text { for example, curb and gutter work, building } \\
\text { block, etc. }\end{array}\right.$ \\
\hline No. $4,3 / 4$ inch. & $\left\{\begin{array}{l}\text { Gravel for maintenance of gravel roads. } \\
\text { Gravel for light reinforced concrete construction. }\end{array}\right.$ \\
\hline No. 4,1 inch & $\left\{\begin{array}{l}\text { Gravel for light reinforced concrete construction. } \\
\text { Gravel for concrete highway construction. }\end{array}\right.$ \\
\hline No. $4,1 \frac{1}{2}$ inches. & $\left\{\begin{array}{l}\text { Gravel for concrete highway construction. } \\
\text { Gravel for railroad bellast. }\end{array}\right.$ \\
\hline inches. & $\{$ Gravel for concretc highway construction. \\
\hline $3 / 4,21 / 2$ inches & $\begin{array}{l}\text { Gravel for foundation course of gravel or water- } \\
\text { bound macadam roads. }\end{array}$ \\
\hline
\end{tabular}
p. 1 .

NOTE.-In connection with sieve sizes, see A. S. T. M. E11, 500.2
(For commercial sizes of sand, see 512.12, p. 235).

United States Department of Agriculture, Bulletin 1216 , method of test for abrasion of gravel, 1928.

\section{A. TEST FOR GRAVEL CONTAINING NO CRUSHED PIECES}

1. The sample shall consist entirely of uncrushed fragments of gravel, and the test shall be made using one of the four gradings $(A, B, C$, or D) given in paragraph 3 . The grading most nearly representing that of the material furnished for the work shall be selected for the test.

2 . The aggregate shall first be screened into the different sizes required for the test, and the material of these sizes shall be washed and dried.

3 . The sample shall consist of $5,000 \mathrm{~g}$ of the dry gravel, with the different sizes combined to conform to one of the following four gradings:

TABLE 2.-Gradings of gravel

\begin{tabular}{|c|c|c|c|}
\hline \multirow{2}{*}{ Grading } & \multicolumn{2}{|c|}{$\begin{array}{c}\text { Size of screens (circular } \\
\text { openings) }\end{array}$} & \multirow{2}{*}{$\begin{array}{l}\text { Percent- } \\
\text { age }\end{array}$} \\
\hline & Passing- & $\begin{array}{l}\text { Retained } \\
\text { on }-\end{array}$ & \\
\hline & Inch & Inch & \\
\hline$A_{-.-}$ & & $1^{3 / 4}$ & 25 \\
\hline B & & $\begin{array}{l}7 / 2 \\
3 / 4\end{array}$ & $\begin{array}{l}25 \\
25\end{array}$ \\
\hline C......... & & $1 / 2$ & 50 \\
\hline$D_{-. .}$ & & $\begin{array}{l}1 / 4 \\
1 / 2\end{array}$ & $\begin{array}{l}50 \\
50\end{array}$ \\
\hline
\end{tabular}

4. The sample for the test shall be placed in the cast-iron cylinder of the Deval abrasion-testing machine as specified for the abrasion test for stone. (See 510,p. 187.) Six cast-iron spheres 1.875 inches in diameter and weighing approximately 0.95 pound $(0.45 \mathrm{~kg})$ each shall be placed in the cylinder as an abrasive charge. ${ }^{15}$

5 . The duration of the test and the rate of rotation shall be the same as specified for the standard test for stone; namely, 10,000 levolutions, at a rate of 30 to 33 revolutions per minute. At the completion of the test the material shall be taken out and screened over a No. 12 sieve, conforming to the requirements of the standard A.S. T. M. specifications for sieves, E11 (500.2, p. 1.) The material retained upon the sieve shall be washed, dried, and weighed. The difference between this weight and the weight of the original sample, expressed as a percentage of the original weight, shall be considered as the loss by abrasion.

6 . When the gravel has a specific gravity below 2.20 , a sample of $4,000 \mathrm{~g}$, instead of $5,000 \mathrm{~g}$, shall be used for the test. The testing procedure shall be the same in all other respects.

7. When the gravel, as used in the work, contains as much as 25 per cent of material finer than onehalf inch, but is of such size that either grading $A$, $\mathrm{B}$, or $\mathrm{C}$ would be used for the abrasion test, a second abrasion test shall be made, using grading $\mathrm{D}$, if, in the opinion of the engineer, the particles finer than one-half inch are not at least equal in hardness to those coarser than one-half inch.

\section{B. TEST FOR GRAVEL CONTAINING CRUSHED PIECES 16}

8. Gravel containing more than about 10 per cent of crushed pieces shall, for the purpose of this test,

15 These spheres are the same as those used in the standard rattler test for paving brick.

16 For the purpose of this test, a crushed gravel fragment may be consiảered as a fragment of gravel having at least one fractured face. 
be considered as crushed gravel. In such cases the abrasion test shall be made on a representative sample of the wholc, including the crushed pieces, following the procedure described in paragraphs 1 to 7 . The percentage, by weight, of crushed pieces shall be determined and the permissible percentage of wear which shall govern for any given sample shall be calculated from the following formula:

$$
W=\frac{A L+(100-A) L^{\prime}}{100}
$$

in which

$A=$ percentage of uncrushed pieces.

$100-A=$ percentage of crushed pieces.

$L=$ maximum percentage of wear permitted by the specifications for gravel containing no crushed pieces.

$L^{\prime}=$ maximum percentage of wear permitted by the specifications for gravel consisting entirely of crushed pieces.

$W=$ permissible percentage of wear.

United States Department of Agriculture, Bulletin 1216, method of test for quantity of soft pebbles in gravel, 1928.

1. The method consists in determining the resistance of the pebbles to uniform static pressure.

2. A sample of at least $1,000 \mathrm{~g}$ shall be separated into three sizes and subjected to uniform pressure without impact, as follows:

0.185 inch to 0.375 inch

Pounds

.375 inch to .750 inch

60

3. Pebbles crushing under these loads shall be classed as soft pebbles, and shall be reported as a percentage of the original sample by weight.

4. Disintegrated rocks or other rocks which will obviously break up into fine material during the screening or mixing operation shall not be considered in this test.

Note.-A satisfactory instrument for classifying soft stone by this method was devised hy the late W. H. Douglass, of the Iowa highway commission. A description of this apparatus follows:

The Douglass stone meter consists of a jack acting through tho specimen to compress a calibrated spring. The jack, which is of the specinen to com rack and pinion tye, is located in the base of the machine and is To the base are attached two vertical standards which are attached to each other by two cross memhers, one at the top and one near the center. The coil-compression spring is located between these two cross members. The top of the spring bears against the top cross har and the bottom against a collar on the movable plunger which passes through hoth cross members and the spring. To the lower end of the plunger is serewed a mechanic's jack which allows adjustment for the various sizes of stones and also carries a ball and socket head. The top end of the plinger is graduated to read the desired pressurcs as it passes through the top cross momber.

United States Department of Agriculture, Bulletin 1216 , method of test for the toughness of gravel, 1928.

The material to be tested is accurately quartered until a sample is obtained which contains at least 50 pieces, ranging in size from three-quarters inch to 2 inches in diameter. Care should be taken to see that the various sizes appear in about the same proportion as in the original sample. The pieces thus obtained are then tested individually by first determining the average diametcr across the shortest dimension and then subjecting each to a single blow of the hammer, using the machine described in a paper printed in the Proceedings of the American Society for Testing Materials for 1922 entitled "An Impact Test for Gravel," by F. H. Jackson, Bureau of Public Roads.

NoTE.-It is suggested that the height of fall shall be that indicated in the following table:

\begin{tabular}{|c|c|c|}
\hline & \multicolumn{2}{|c|}{ Height of fall } \\
\cline { 3 - 3 } $\begin{array}{c}\text { Diameter } \\
\text { of } \\
\text { particle }\end{array}$ & $\begin{array}{c}\text { Sedi- } \\
\text { mentary } \\
\text { origin }\end{array}$ & $\begin{array}{c}\text { Igneous } \\
\text { or meta- } \\
\text { morphic } \\
\text { origin }\end{array}$ \\
Inches & Inches & Inches \\
$3 / 4$ & 2 & 3 \\
$1^{1 / 4}$ & 3 & 4 \\
$11 / 2$ & 4 & 5 \\
\hline
\end{tabular}

Failure in this test shall be construed as the actual crushing or splitting of a fragment under a single blow of the hammer.

United States Department of Agriculture, Bulletin 1216, method of determining quality of glacial gravels by lithological count of particles, 1928.

From 100 to 200 pieces of gravel stones, selected at random, ranging in size from one-half inch to 2 inches, shall be separated into the various rock types and the percentage composition noted.

Note.-This is a simple test which, especially in dealing with the northern glacial gravels, will obviate considerable laboratory work. There is no single laboratory test for this type of material which will give such conclusive results as to quality of the material as this when the adjacent hedrock and direction of the glaciation are known.

\subsection{Sand.}

American Association of State Highway Officials, tentative standard spccifications for highway materials, 1927.

\section{BITUMINOUS SAND}

1. This specification covers the general characteristics and grading of sand for use in bituminous pavements, bituminous mortars, bituminous filler or mastics.

2. The sand shall consist of grains of quartz or other hard, durable rock, moderately sharp and free from a coating of any injurious material. The sand shall be free from Jumps of clay, loam, organic matter, or other foreign matter.

3. When tested by means of laboratory screens, the sand shall conform to the following requirements:

\begin{tabular}{|c|c|c|c|}
\hline Passing- & Retained on- & Minimum & Maximum \\
\cline { 1 - 2 } & & Per cent & Per cent \\
4-mesh & 10-mesh & 0 & 5 \\
10-mesh & 40 -mesh & 10 & 40 \\
40-mesh & 80 -mesh & 25 & 60 \\
80-mesh & 200-mesh & 12 & 45 \\
200-mesh & & 0 & 6 \\
\hline
\end{tabular}

1 For sheet asphalt the minimum requirement shall be 16 .

4. Sampling and testing, A. S. T. M. method D75. (See 510., p. 184.)

American Ceramic Society, tentative method for complete sand analysis, 1928.

\section{(A) PREPARATION OF THE SAMPLE}

The sample may be a well-mixed unground portion or it may be ground in an agate mortar.

\section{(B) SOLUTION OF THE SAMPLE}

Weigh out a $10 \mathrm{~g}$ sample and place it in a platinum dish of suitable size; 250 cc is recommended. Moisten the sample with water and add 2 cc concentrated sulphuric acid. Fill the platinum dish two- 
thirds full with concentrated hydrofluoric acid and evaporate on the sand bath, or if so desired on the hot plate, with an asbestos board beneath, to a volume of about $50 \mathrm{cc}$. Care must be taken to aroid mechanical losses. Examine the contents of the dish, and if complete solution of the sample has not taken place, add more hydrofluoric acid and evaporate again. When complete solution of the sample has been accomplished evaporate to copious fumes of $\mathrm{SO}_{3}$, add one or two drops of fuming nitric acid to oxidize any organic matter. Cool the solution, dilute to about $100 \mathrm{cc}$, filter, and transfer any residue left in the platinum dish to the filter. Wash the filter five times with hot water, place in a covered platinum crucible, dry, and ignite until the filter paper has entirely burned off. Cool the crucible, add about $2 \mathrm{~g}$ of sodium carbonate and fuse over a Meker burner or its equivalent. Cool the crucible, dissolve the melt with dilute hydrochloric acid and hot water in a small covered beaker. If a residue still remains after this treatment, filter into the main solution and wash four times with hot water. Dry and ignite the filter in a covered platinum crucible and fuse with $1 \mathrm{~g}$ of potassium pyrosulphate. Dissolve the melt in dilute hydrochloric acid and hot water and add to the main solution.

Evaporate the total solution to a small volume and transfer to a platinum dish. Add 5 ec of concentrated hydrofluoric acid, evaporate to fumes of sulphuric acid, cool, wash down the sides of the dish with hot water, and again evaporate. Cool, add $50 \mathrm{cc}$ of water and allow to stand five minutes. If the solution is not clear, filter and wash the residue six times with hot water.

\section{(C) $\mathrm{R}_{2} \mathrm{O}_{3}$}

Heat the filtrate from "Solution of sample" to boiling; add two or three drops of methyl red indicator, and then dilute ammonium hydroxide drop by drop to a yellow end point. Boil from one to two minutes and filter immediately. Transfer the contents of the dish or beaker to the filter with hot two per cent neutral ammonium chloride and wash five times with this solution. Dissolve the precipitate in the minimum amount of hot hydrochloric acid (1 to 10 ) receiving the washings in the original beaker in which the precipitation was made. Wash the filter thoroughly with hot water and reserve. Precipitate again as before with the aid of macerated filter paper if desired to aid in filtering. Transfer the precipitate quantitatively to a filter with hot neutral two per cent ammonium chloride or ammonium nitrate and wash six times with either of these solutions.

Place the reserved paper and the precipitate and paper in a previously ignited platinum crucible weighed with cover; dry, and ignite under good oxidizing conditions at approximately $1,100^{\circ} \mathrm{C}$. finally covered. Cool in a desiccator over a good desiccant, never calcium chloride, and quickly weigh the covered crucible. Repeat the ignition until constant weight of " $\mathrm{R}_{2} \mathrm{O}_{3}$ " is obtained.

\section{(D) $\mathrm{CaO}$}

Combine the filtrates from the two $\mathrm{R}_{2} \mathrm{O}_{3}$ precipitations, make slightly acid with dilute hydrochloric acid and evaporate to a volume of about $150 \mathrm{cc}$. Add a slight excess of dilute ammonium hydroxide and heat to boiling. Add slowly, with constant stirring, $10 \mathrm{cc}$ of a 4 per cent solution of ammonium oxalate and boil five minutes. Let stand on steam bath for at least four hours or preferably overnight.

Transfer the precipitate to a small filter and wash with ten 5 cc portions of cool 1 per cent ammonium oxalate solution. Place in an ignited platinum crucible weighed with cover, dry, and ignite at approximately $1,000^{\circ}$ C. Cool over a good desiccant, never over calcium chloride, and quickly weigh the covered crucible. Repeat the ignition until constant weight is obtained.

\section{(E) $\mathrm{MgO}$}

Make the filtrate from the oxalate precipitation distinctly acid with $\mathrm{HCl}$, and add one-half gram of diammonium phosphate $\left(\mathrm{NH}_{4}\right) 2 \mathrm{HPO}_{4}$; evaporate to $150 \mathrm{cc}$ and cool. Add ammonium hydroxide slowly to a slight excess. Stir vigorously until precipitation begins. After several minutes add ammonium hydroxide (specific gravity 0.90 ) to make one-ninth by volume. Let stand overnight. Filter on a previously ignited and weighed Gooch crucible or on filter paper if so desired. Wash with 5 per cent by volume ammonium hydroxide five times, dry and ignite gently. Finally ignite to constant weight at $1,100^{\circ}$ C. as $\mathrm{Mg}_{2} \mathrm{P}_{2} \mathrm{O}_{7}$.

\section{(F) IGNITION LOSS}

Ignite $5 \mathrm{~g}$ of sample at approximately $1,100^{\circ} \mathrm{C}$. in an ignited and weighed platinum crucible to constant weight. Report the loss in weight of the sample as per cent ignition loss.

American Foundrymen's Association, standard and tentatively adopted methods of testing and grading foundry sands, 1928.

\section{STANDARD TESTS}

\section{DETERMLNATION OF MOISTURE}

1. The moisture content of a sand sample is to be determined as follows: Dry $100 \mathrm{~g}$ of tempered sand for one hour between $105^{\circ}$ and $110^{\circ} \mathrm{C}$. When dry reweigh. The loss of weight in grams is the moisture content expressed in percentage.

\section{PERMEABILITY TEST}

A. Definition of Permeability

2. The term "permeability" as used in this test is that physical property of sand which permits the passage of gases. It is upon this property of permeability that the venting qualities of sand molds and cores depend. A sand of high permeability has good venting quality because of its "openress." The natural characteristics of the sand and its binders, the density with which these are packed, and the percentage of moisture used for tempering, are important factors in regulating the degree of permeability. The object of the permeability test is to determine that physical property of the sand called permeability. This is ascertained by measuring the rate of flow of air through a standard specimen of sand under a given pressure. It may have for its purpose the making of standardized tests or the routine control of sand mixtures in foundry operations.

\section{B. Apparatus Needed for Test}

3. Pedestal for specimen tube holder.-Permeability specimen tube holder. Rammer. Permeability air-flow apparatus.

\section{Description of Apparatus}

4. (a) Sand container or specimen tube.-The sand container ( $\mathrm{H}$, figs. 92 and 93 ) is a brass cylinder 5 inches $(12.70 \mathrm{~cm})$ high and 2 inches $(5.08 \mathrm{~cm})$ inside diameter.

5. (b) Sand container pedestal.-A pedestal is provided which the sand container ( $\mathrm{H}$, fig. 92) rests while the sand is being placed in it, and while the ramming operation is being performed, 
6. (c) Container auxiliary screen for testing dry sands and sands with very little bond.-If dry sand is to be tested, a special cap having a 60 -mesh brass screen bottom should be used, to slip into the lower end of the sand container. For ramming dry sand the pedestal must be placed upside down under the container. This supports the screen during ramming, and keeps the sand sample at proper height to use the tolerance marks (par. 8) as a guide.

stops is sufficient to permit a 2-inch $(5.08 \mathrm{~cm})$ movement of the weight. The total weight of the assembled rammer, including rammer head, rod, stops, and weight, is 17.5 pounds $(7,937.86 \mathrm{~g})$.

8. On top of the upper steel guide is attached a scale $(B)$ with three lines marked on it. If the upper end of the rammer rod is between the upper and lower of these lines after the third ram, it indicates that the sand sample is within the allowable limits of thick-

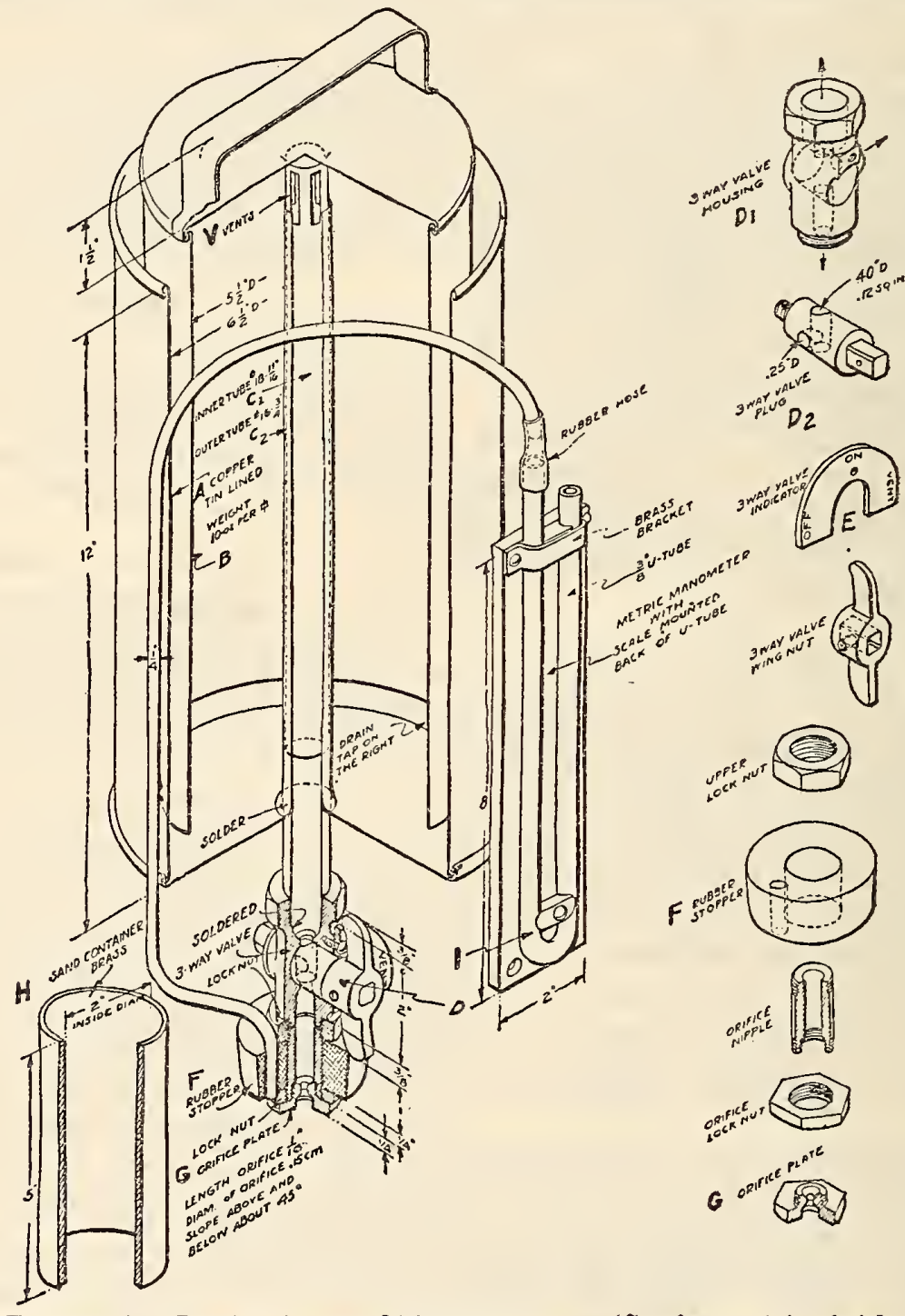

FIGURE 93.-Details of permeability apparatus. (See figure 92 for finished assembly view)

7. (d) Sand rammer.-The sand rammer ${ }^{17}$ is ness. The sample after ramming should be 2 inches shown in Figure 91 . It consists of a steel rod $(R)$ supported by two guides $\left(G, G^{\prime}\right)$. A steel rammer head $(D)$ is attached to the lower end of the rod $(R)$ and has a sliding fit in the sand container ( $H$, fig. 92). A castiron weight $(H)$ weighing 14 pounds $(6,350.29$ g) slides on the rod, its movement being regulated by two stops $\left(S, S^{\prime}\right)$. The distance between these

17 The committee investigations show that the character of the support on which the rammer sets has some influence on the test results and accordingly it recommends the use of a solid metal or concrete bench support for the rammer.

$(5.08 \mathrm{~cm})$ high, plus or minus $1 / 8$ inch $(0.3175 \mathrm{~cm})$.

9. (e) Permeability air flow apparalus.-The parts of the permeability apparatus (figs. 92 and 93) are as follows: A tank ( $A)$ made of copper (tin lined), or other suitable material, and provided with a vertical air outlet tube $(C)$ coming up through the bottom of the same. When in use this tank is partly filled with water as described in paragraph 16. A stopcock may be provided on the side of $\operatorname{tank}(A)$ at the bottom to drain off the water when the apparatus is not in use, or when adjusting the water level. 


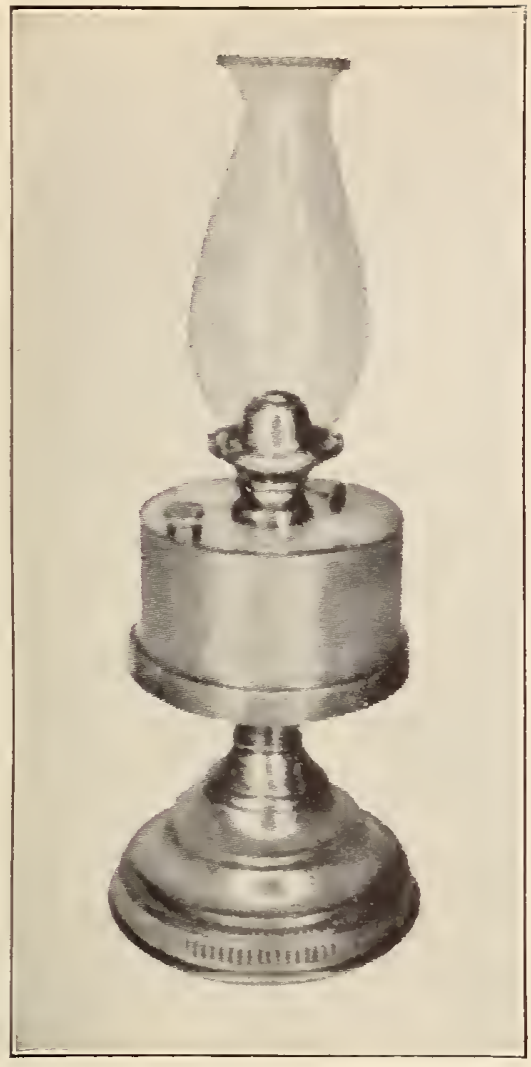

Figtre 42.-Test lamp and chimney

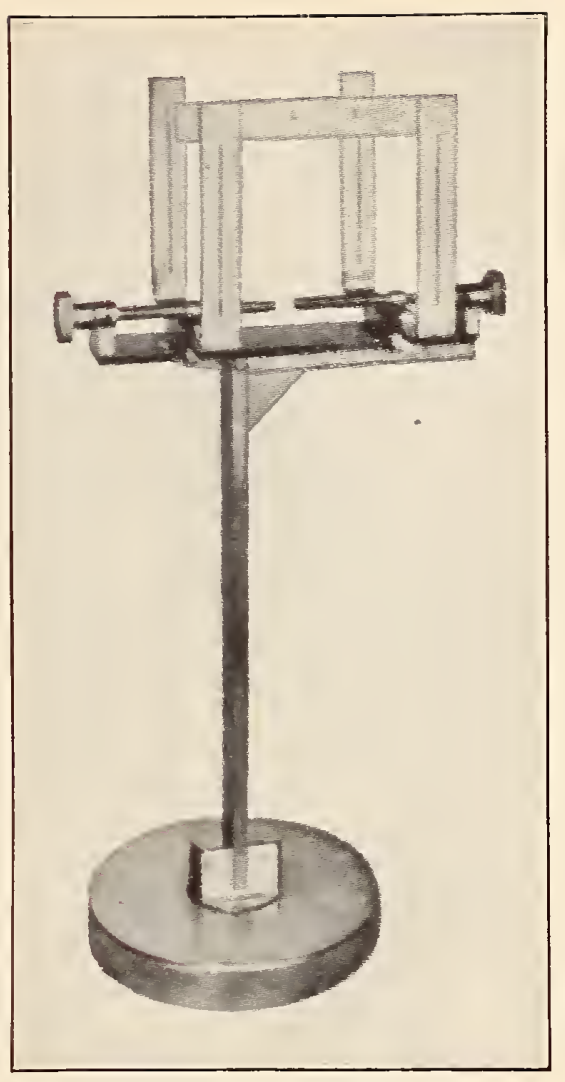

Figure 45.-Sight gage

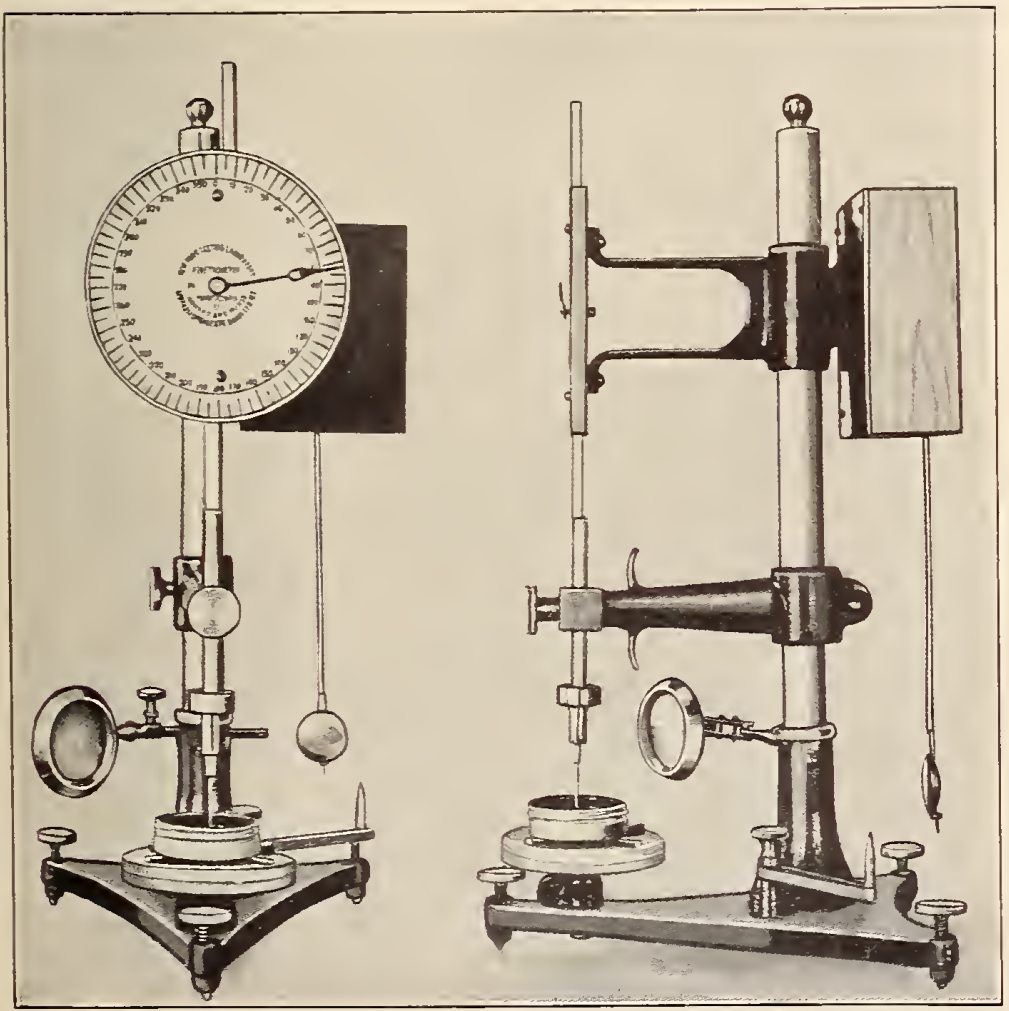

Figure 55.-Penetrometer

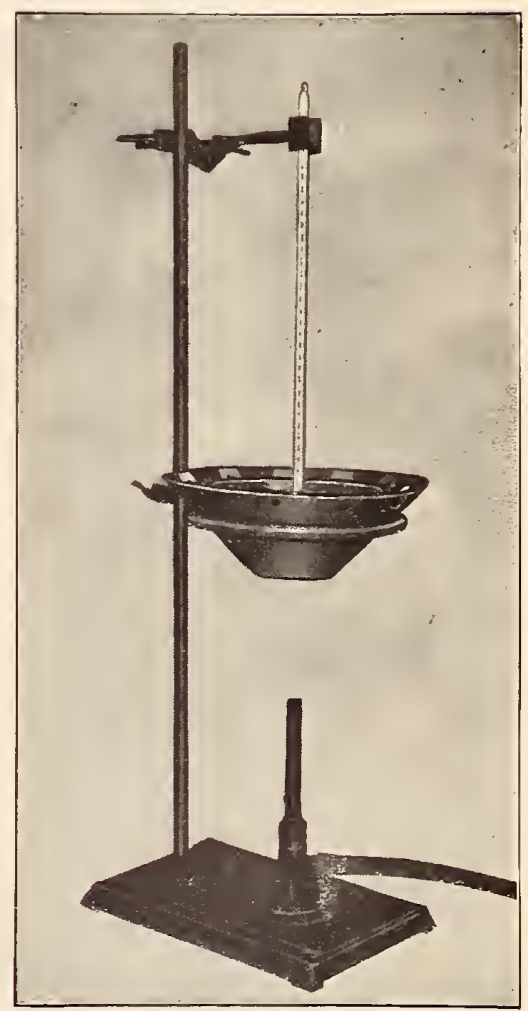

Figure 73.-Assembly of apparatus 


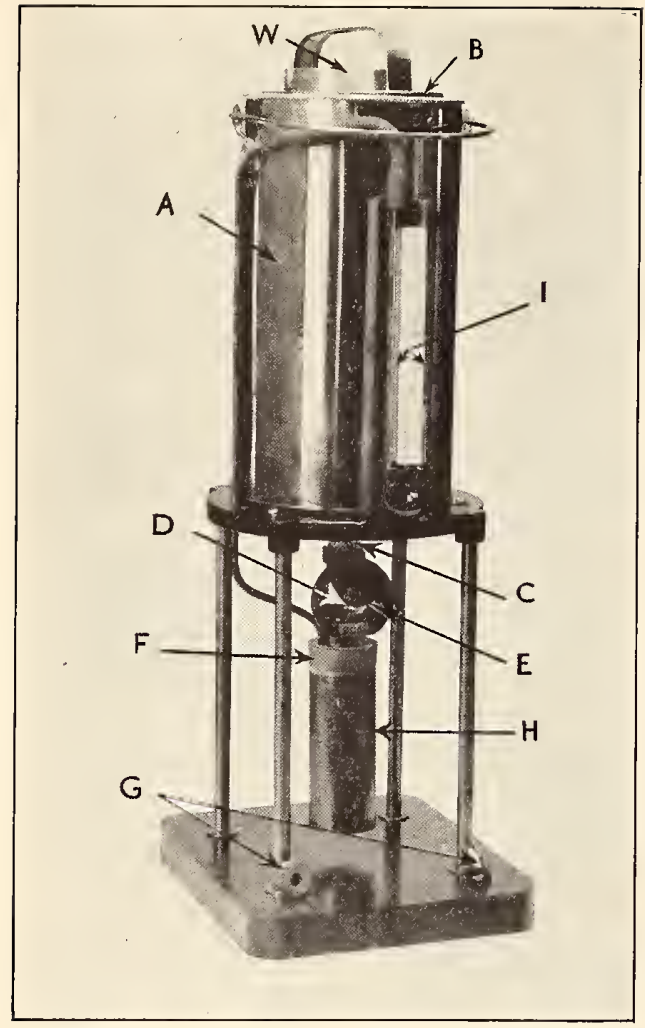

Figure 92.-Permeability testing apparatus See Figure 93 for details of construction.

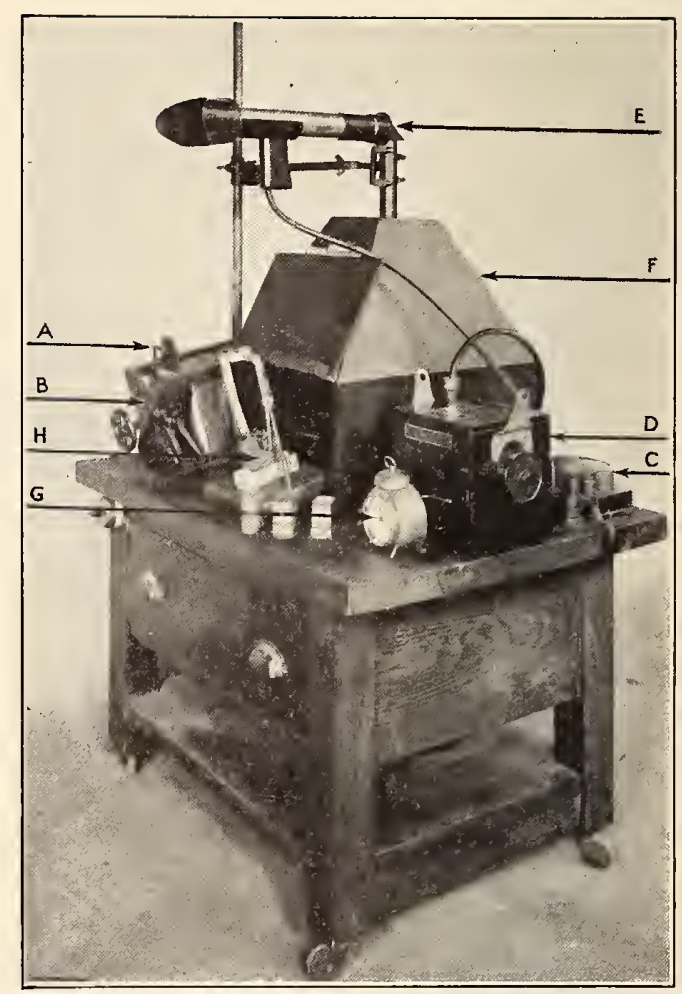

FIGURE 94.-Outfit assembled for testing sintering

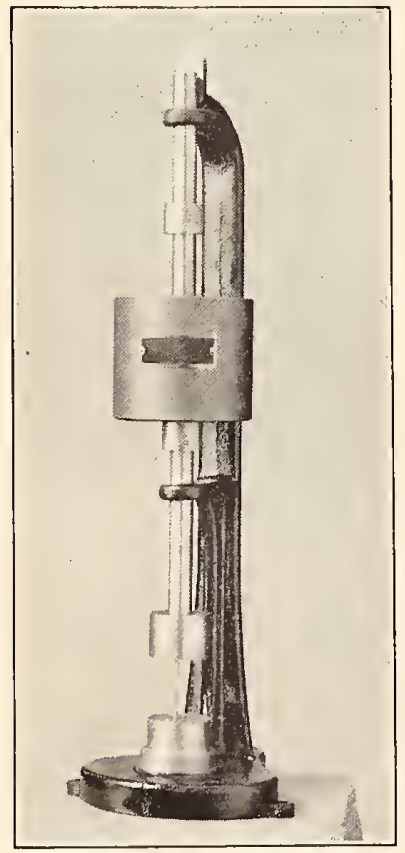

Figure 91.-Permeability rammer

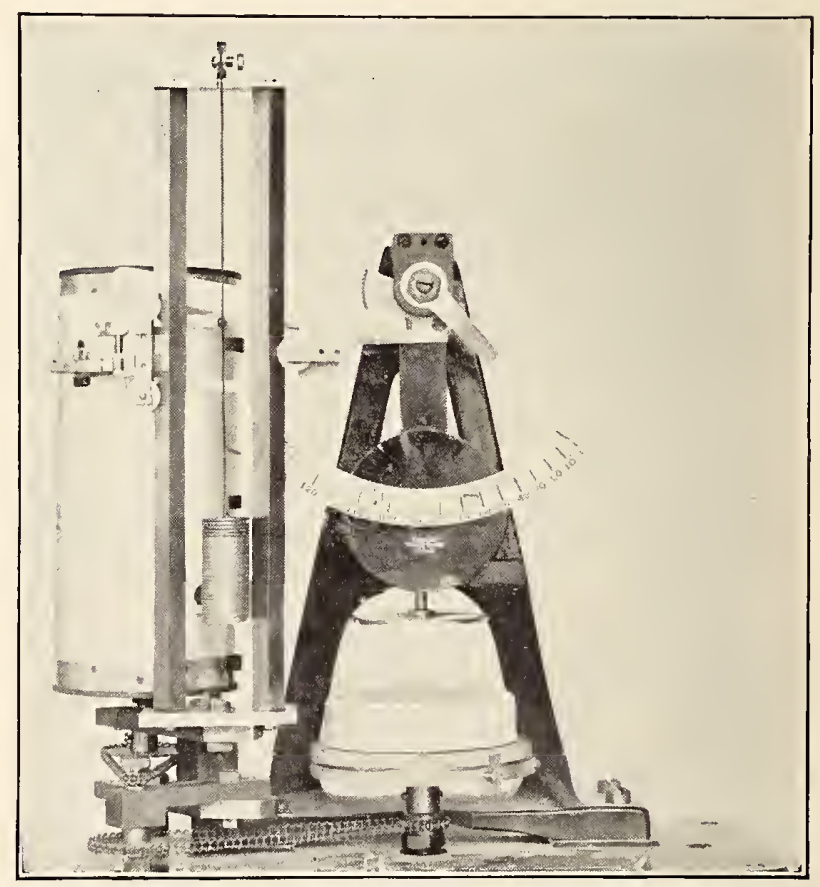

Figure 118.-Emley plasticimeter 
10. Bell $(B)$ has a vertical tube $(C 1$, fig. 93$)$ which slides inside the air outlet tube $(C 2)$ in tank $(A)$. Near the top of this tube are several vents $(V)$ to permit the air to be forced out of bell $(B)$. A 3-way valve $(D)$ is attached to the lower end of the outlet tube $(C)$ from tank $(A)$. The opening in the valve should not be too small, preferably not less than 0.03 square inch $\left(0.7742 \mathrm{~mm}^{2}\right)$ so as to permit the air to pass through freely. A valve indicator (E) is provided, marked "On," "Off," and "Vent," to show when the valve is in position to let air from bell $(B)$ through sand in sand container $(H)$, to shut off flow of air from bell, or to permit air to enter by-pass while raising the bell.

11. A nipple over which fits a rubber stopper ( $F)$, which is held in place by two locknuts, is attached to the lower side of the valve.

12. An orifice plate $(G)$ for use in rapid control work, is scrcwed into the lower end of the nipple below rubber stopper. The orifice plates $(G)$ can be seen on the shelf at base of tank. For a description of this rapid control method see paragraphs 45 to 58, inclusive.

13. The rubber stopper $(\vec{F})$ fits into a sand container $(H)$.

14. In front of the tank $(A)$ there is a manometer $(I)$, having a scale divided into centimeters and millimeters. This connects with a brass tube that passes down through the rubber stopper $(F)$.

15. All parts are of brass, except those described as being made of other materials, and except the stand or base.

16. Before using apparatus the tank $(A)$ is to bc filled with water to within $43 / 4$ inches $(12.065 \mathrm{~cm})$ of the top.

17. As the bell $(B)$ sinks into the water in tank (A) it forces air into the outlet pipe through the valve and through the sample of sand packed in the container $(H)$

18. The pressure of this air is read on the manometer ${ }^{18}(I)$. Since the pressure recorded on the manometer depends on the weight of the bell $(B)$, and on its cross-sectional arca, the weight should be madc such as to give a pressure of about, but not less than, $4.8 \mathrm{~g}$ per square centimctcr.

19. There should also be a weight $(W)$ provided, which can be placed on top of bell $(B)$, sufficient to increase the manometer pressure reading to 10 centimeters. ${ }^{19}$ This latter pressure is more convenicnt for testing very fine sands and for rapid work.

20. The bell $(B)$ has several lines marked on it, the lowest being " $X$," the second " $O$," the next "1,000" and the fourth "2,000." These indicate that the capacity of the bell between the " $O$ " and " 1,000 " marks is $1,000 \mathrm{cc}$ (61 cubic inches), and between " 0 " and " 2,000 " it is 2,000 cc ( 122 cubic inches).

21. The object of having the mark " $X$," which is about $3 / 4$ inches $(19 \mathrm{~mm})$ below the zero mark, is to insure raising the bell to the proper height, so that when it is necessary in a standard test to read the time required for the bell to sink from zero to 1,000 or 2,000 the zero mark will be clearly visible.

22 . To raise the bell, turn the indicator $(E)$ on valve plug to "vent." This allows air to enter through the by-pass instead of having to be drawn through the sand. Then turn indicator to "Off" and the bell will remain in its raised position. When

18 Expansion bulb manometer use permissible so permeability can be read directly.

19 If less than $5 \mathrm{~cm}$ pressure is recorded the bell sinks too slowly when a very fine sand is tested. The amount of pressure can be deter. mined by attaching the empty sand container to tbe apparatus,

plugging its lower end witb a rubber stopper, and opening the valve. ready to start the test, turn indicator to "On" and the bell will sink as air is forced through the valve.

\section{Preparation of Sample to be Tested}

23. (a) Selection.-The sample to be tested should be an average one, representative of the heap, floor, car, bank, or other source from which it is takcn.

24. In testing new sand for permeability it is absolutely necessary that the sand be properly sampled and uniformly tempered. For plant check or control tests upon facing or heap sands in daily use one may test the sand as tempered for molding.

25. (b) Preparing new sand for testing.- Since it is the object to determine the maximum permeability 20 under suitable foundry working conditions, in the examination of new sands, experiments should invariably be made with several water contents in order to ascertain that amount which develops the maximum degree of permeability.

26. It is advisable in most cascs to try percentages of water beginning with 4 per cent and increasing by stages of 2 per cent up to and including at least 8 per cent. Sometimes it will be found difficult to make a test with a water content of an exact predetermined pcrcentage.

27. The permissible extent of deviation from the predetermined amount should in no case be more than one-half per cent, and can be intelligently determined by the careful experimenter who observes critically the tendency of a sand to show widely differing permeability values as the water content is appreciably changed. A deviation not exceeding 0.2 per ccnt (for examplc, 5.8 per cent or 6.2 per cent in the casc of an attcmpt to get 6 per cent) can be considercd as entirely satisfactory for the determination of the permeability at the nearest fixed percentage.

28. The exact percentage of moisture, even if within 0.2 per cent, should be reported.

29. Supplementary tests with lower percentages of water than 4 per cent and higher percentages than 8 per cent should be made if and when the facts ascertained justify such tests. For example, when a strength value considered proper to report is obtained on a sand with a moisture content below 4 per cent or above 8 per cent and a permeability value on the sand is desircd, a test for permeability should be conducted with the sand tempered with the same amount of water as in the case of the strength test. In such cases, a sufficiently large sample of the sand should be tempered, to permit making both of these tests.

30. (c) Examination of new sands.-In the examination of new sands, procecd as follows: Dry at least $1,000 \mathrm{~g}$ of sand for one hour at a temperature not lower than $105^{\circ} \mathrm{C}$. nor above $110^{\circ} \mathrm{C}$. Carc should be exercised to spread the sand over a large area in a thin layer in order to expel all the moisture in a given time. This will make it possible to add the proper amount of water and give the sand the desired moisture content.

31. After the sand has cooled, measure out the desired quantity of water, adding sufficient extra water (usually from one-fourth to 1 per cent) to allow for evaporation during mixing. Thus if it is desired to add 4 per cent water and one-half per cent extra water is needed, one would add $47 \mathrm{cc}$ (since $1 \mathrm{cc}$ of

20 Optimum water content. Tbis term bas frequently been used in connection with the permeability and strengths of sands, and refers to that water content of the sand which gives the highest values for permeability or strength, respectively. As some misconceptions have arisen regarding tbe use of this term, the committee thinks it desirable to call attention to the fact that the optimum water content desirable to call attention to "best thm ered condition," or that tho does not necessarily nean the "'best tempered condition," or that the the sand is worked. 
water weighs $1 \mathrm{~g}$ ) to $1,000 \mathrm{~g}$, and secure a total weight of $1,047 \mathrm{~g}^{21}$

32 . For the teimpering operation, spread the sand on a smooth, flat, dry surface in a layer about 1 inch thick, sprinkle a small quantity of the required water evenly over the sand, and work the latter gradually. Again spread it into a thin layer and repeat the above operations, adding more water. Continue to do this until all of the water has been thoroughly distributed through the sand. There should be no dry lumps or other evidence of uneven tempering.

33. The sand should now be allowed to stand in order that the maximum temper may be developed. To secure this temper, place the sand in a humidor or air-tight receptacle 22 and allow it to stand for 24 hours. After this the sample is ready to be tested, as below.

34. Take the entire sample of sand from the humidor. Pass this entire sample twice through a coarse riddle and return the sand as quickly as possible to the humidor or receptacle. From this take sample to be tested for permeability; also sample to be tested for moisture content, and for strength if desired.

\section{E. Determination of Moisture Content}

35. Determine moisture as specified in paragraph 1.

\section{F. Ramming of Specimen}

36. Take a sufficient quantity (from 150 to $175 \mathrm{~g}$ ) of tempered sand to make a column 2 inches $(5.08$ cm) high, with a tolerance of 4 per cent. (Par. 8.) The sand should be carefully placed in the container ( $H$, fig. 92) and leveled off.

37. Place pedestal and container with sand in position beneath rammer.

38. Gently lower rammer rod with head into container until they are supported by the sand. Raise rammer head to the upper stop and let fall. Repeat twice, making a total of three rams. Note whether the upper end of the rod is within the tolerance marks. If not, discard the sample and put in another lot of tempered, sand of sufficient quantity to yield a column of the required height. This is usually accomplished on the second trial.

39. Lift rammer rod until disk at lower end of rod is free from the sand container, and take containcr off of pedestal. (A, fig. 91.)

\section{G. Measurement of Air Flow}

40. Before attaching sand container with specimen, open valve indicator ( $E$, fig. 92$)$ and raise bell (B) until mark " $X$ " appears. Then turn to "Vent" position. Attach sand container to rubber stopper ( $F$, fig. 92), moistening sides of stopper before applying, to prevent air leakage. Open valve to "On" position. Note scale on side of bell $(B)$, and as cup sinks, and zero mark on scale passes edge of tank (A), start stop watch.

41. Read pressure ${ }^{23}$ in manometer tube as soon as the pressure reading becomes steady. The instant the " 2,000 " mark on bell $(B)$ reaches upper edge of tank $(A)$, the stop watch should be stopped and time recorded. This represents the timc required to force $2,000 \mathrm{cc}$ of air through the sand. The time

21 Moisture content for all molding sand determinations and tests is to be expressed as tbe percentage of moisture in the damp sample of is to be expressed as tbe percentage of moisture in the damp sample of sand. It is not proper to calculate the amo

tionate to the weight only of the dry sand.
22 A 2 -quart preserve jar, with glass top and rubber ring, makes a ${ }^{22}$ A 2-quart

good humidor.

${ }^{23}$ In using a $U$ tube manometer, the pressure reading is obtained by taking the sum of the pressures in tbe two legs of tbe tube. If a single leg expansion bulb manometer is employed, only a single reading is, of course, necessary. and pressure obtained as above are to be used as described in paragraphs 42 to 44 , inclusive.

\section{Calculation of Permeability by Standard Method 24}

42. The degree of permeability as determined by this test is found by employing a formula. By its use, permeability is ascertained as the volume of air per minute, per gram per square centimeter pressure, per unit volume in specimen.

43. Permeability equals the number of cubic centimeters of air forced through the sand specimen, multiplied by the height of the sand specimen in centimeters; and this product divided by the product of the pressure in grams, the area of the sand specimen in square centimeters, and the time in minutes. Thus

$$
\text { Permeability }=\frac{\mathrm{cm}^{3} \text { of } \text { air } \times \mathrm{cm} \text { height of specimen }}{\text { grams pressure } \times \mathrm{cm}^{2} \text { a rea of specimen } \times \text { minutes }}
$$

44. The method of conducting the permeability test herein described calls for $2,000 \mathrm{cc}$ of air to be forced through the specimen; $5.08 \mathrm{~cm}$ ( 2 inches) to be the height of the specimen; and 20.268 square centimeters (3.1416 square inches, to be the area of the specimen. These fixed quantities are therefore substituted as constants in an equation as follows:

$2,000 \times 5.08$

$$
\text { Permeability }=\frac{2,000 \times 5.08}{20.268 \times \text { grams pressure } \times \text { minutes }}
$$

Reduced to its simplest terms this equation reads:

$$
\text { Permeability }=\frac{501.2}{\text { grams pressure } \times \text { minutes }}
$$

\section{Rapid Method for Using the Permeability Apparatus}

45. By making a slight change in the standard apparatus, which can be quickly readjusted for standardization purposes, greater rapidity of operation can be obtained at a slight sacrifice of accuracy.

46. This change consists only in inserting one of two standardized orifice plates ( $G$, fig. 92 ) in the lower end of nipple in stopper. These orifice plates must be lined with some material which does not corrode or wear rapidly. Hard gold is recommended for this purpose. ${ }^{25}$

47. (a) Orifices.-Two orifice plates of brass are used ( $G$, fig. 93). These plates are so designed as to be screwed into the lower end of the nipple in stopper ( $F$, fig. 92), which is threaded on the inner side and has the end faced square so that the orifice plate, which is provided with a gasket, can fit snugly against it and make an air-tight joint. Through the center of each orifice plate a hole is drilled, counterbored at both ends. The hole in each orifice plate is filled with a hard-gold plug, through which plug another hole is drilled. The holes constituting the two orifices should be drilled exactly $0.5 \mathrm{~mm}$ (0.0197 inch) and $1.5 \mathrm{~mm}$ (0.0590 inch), respectively. For the last-named hole drill No. 53 may be used.

48. The orifices are standardized ${ }^{26}$ as follows: The smaller orifice plate is screwed into its place in the nipple, but the sand container is not attached to the apparatus. The weight ( $W$, fig. 92$)$ is added to the top of the bell so as to give $10 \mathrm{~cm}$ pressure.

49. Raise the bell $(B)$ and allow it to descend, noting the time required for the cup to settlc from the " 0 " to the " 2,000 " mark. This gives the time

${ }_{24}$ For shop control, short-cut metbods are used as described under paragrapbs 45 to 58 .

${ }^{25}$ Tbe plugs through which the orifices are drilled are so small that it is not a serious item of expense to make tbem of gold. Jewelers have furnisbed tbese for $\$ 5$ a pair.

${ }_{26}$ Tbe permeability apparatus operated with the orifices employs the principle of tbe Saeger permeability apparatus (A. F. A. Transaction, 32. pt. 2, p. 134), and tbe relation between permeability and pressure on the sand is worked out exactly as for that apparatus. 
required to pass $2,000 \mathrm{cc}$ of air through the orifice, and should be 4.5 minutes. If the time required to accomplish this is greater than the above, the orifice is too small and should be carefully reamed out until it passes 2,000 ec of air in the proper time. If the time is less than 4.5 minutes the orifice must be compressed to a smaller size and rereamed until it is of the correct diameter.

50. The second orifice, with $1.5 \mathrm{~mm}$ opening, is standardized in the same manner, except that it should be made to pass $2,000 \mathrm{cc}$ of air in 0.5 minute.

51 . Both orifices should be calibrated to within 1 per cent. The smaller orifice is to be used with medium to fine grades of sand and the larger one with medium to coarse grades.

52. The orifice plate, which is adapted to the sand to be tested, is to bc screwed into its place in the nipple. The sand container with the sample of rammed sand is placed in position. Turn 3-way valve to "Vent" and raise bell $(B)$. Turn valve to "On" and allow bell $(B)$ to sink. Read pressure on manometer ( $I$, fig. 92) and close valve.

53. To test additional samples, proceed by placing them in position in the machine, turning valve to "On" each time and long enough to get a reading on the manometer. Bell $(B)$ need not be raised again until it has sunk to rest on the bottom of the tank.

54. Knowing the pressure obtained as above, the permeability of the sand can be obtained from the Table 1 given below. The columns headed "Pressure" give the readings on the manometer ranging from 0 to $10 \mathrm{~cm}$. The other columns give the permcability values for the two orifices corresponding to the pressures shown.

55. To obtain the permeability value of any sand from this table, note from it the pressure corresponding nearest to that indicated by the manometer. Then look on same line in column corresponding to orifice used and read the permeability value. ${ }^{27}$

56. (b) Selection of orifices.-The two sizes of orifices are provided so that there may be no difficulty in obtaining permeability values in testing sands of widely varying characteristics. As the experimenter acquires experience, he will be able to make the most discriminating selection of either orifice. It will be found that the orifice which has a diameter of $1.5 \mathrm{~mm}$ gives the most satisfactory results in permeability values from 36 up, as shown in Table 1 , and that the orifice having a diameter of $0.5 \mathrm{~mm}$ gives the most satisfactory results for permeability values from 49 down.

57. A neutral zone exists in which certain sands may conveniently be gaged as to permeability values, using either orifice, for the service ranges of the two orifices overlap to some extent. Within or close to the neutral zone the experimenter applying one orifice may obtain a value which does not afford a satisfactory comparison. In such cases, when a very slight difference in pressure gives a permeability value proportionately too great, the use of the other orifice should be resorted to. Experimental use of both orifices will readily determine the desirable one to employ, gaged by permeability values that are proportionate.

${ }^{27}$ For shop control work, the pressure reading is sometimes used without reference to the permeability chart.
TABLE 1.-Permeability test pressures and corresponding values, as obtained with orifice plates

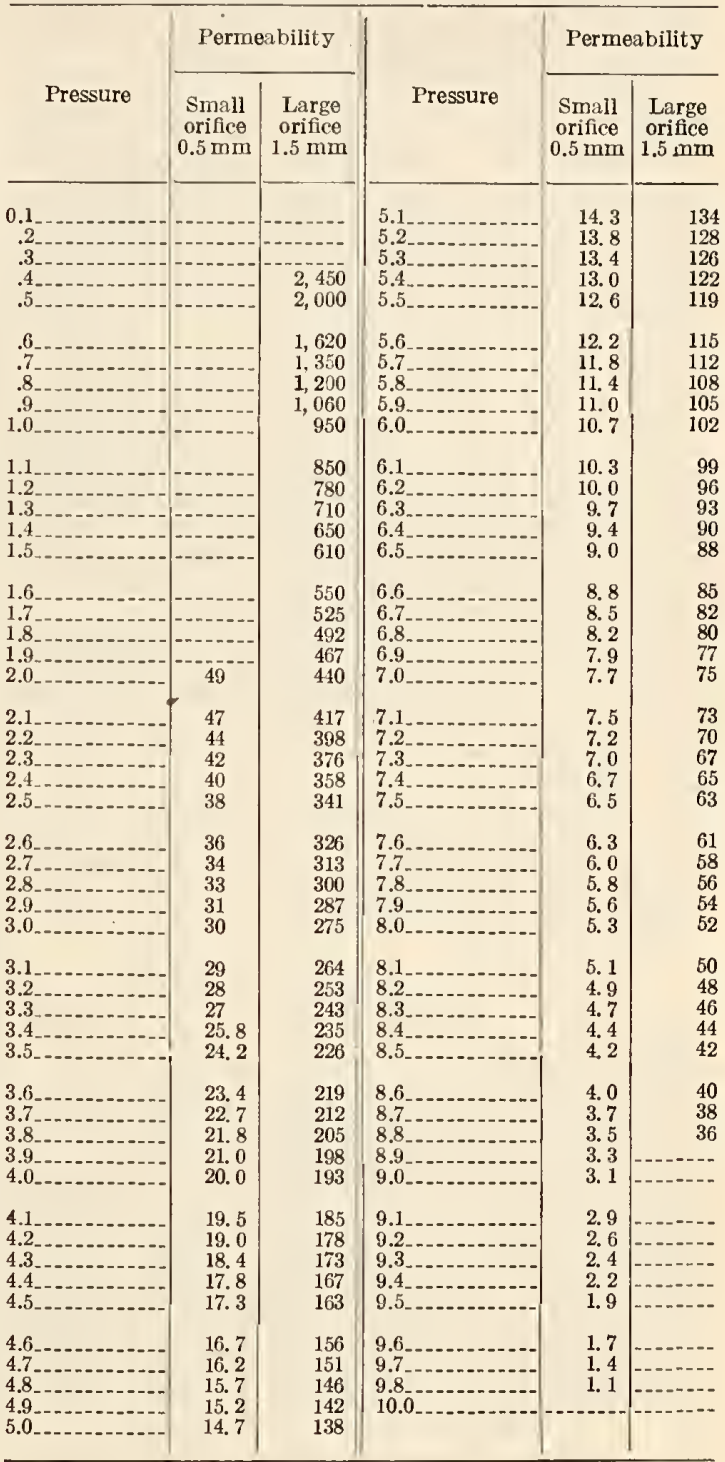

58. It is possible to make from Table 1 a directreading scale, which can be attached to the manometer, thus permitting the permeability value to be read without refcrence to the table.

\section{J. Testing Permeability of Dry Sand Specimens}

59. Where it is desired to determine the permeability of a dry sand containing little or no bond, this may be done by first placing on the bottom of the standard permeability test specimen holder an 
attachment consisting of a screen of 60 mesh. The required amount of sand is then placed in the specimen holder, rammed according to the method outlined in paragraphs 36 to 39 , inclusive, and the permeability determination made in the manner described in paragraphs 40 to 44 , inclusive.

\section{K. Precautions to be Observed in Using Permeability Apparatus}

60. While the permeability apparatus recommended by the subcommittee on tests has given almost universal satisfaction, occasional complaints have been received regarding it. Investigation of all of these complaints has shown that in nearly every case unsatisfactory results are due to lack of proper caution in handling the equipment.

61. Orifices. - These should be kept clean at all times. If any dirt collects in them it should be removed with a soft wooden point and never with a metal point or wire. This is because the orifices are carefully drilled to exact size and any scratch will vary the size of the opening. Care should be taken to prevent moisture from collecting in them, as even a film of water will change the calibration. In case of doubt regarding the presence of moisture, blow a current of dry air through the opening.

62. Accuracy.-Attention is called to the fact that the orifice method for determining permeability does not possess the accuracy of the standard method. In case there is any doubt as to which orifice to use, the determination can be checked against the standard method.

63. Leveling the machine.-In order to insure smooth descent of the bell, the machine should be set level and the weight that is placed on top of the bell should be set on the center of the latter. If the machine is used in the foundry it is desirable to have a small level on it.

64. Bell.-The bell should be raised slowly, and not with a jerk, to avoid splashing water into the air outlet tube and into the orifice with resultant stoppage of the latter.

65. Air outlet.-This should be kept dry. It is a good plan to wipe it out at frequent intervals with a rag fastened to the end of a stick to prevent moisture from condensing on the inner surface.

66. Checking up for leaks. - From time to time the air-flow system should be checked to see if leaks have developed. This can be done by placing a rubber stopper in the bottom of the permeability specimen tube holder, attaching the tube holder to the standard rubber stopper of the apparatus and opening the stopcock to the "On" position. If there are no leaks, the manometer tube should register a continuous pressure of $10 \mathrm{~cm}$.

\section{COMPRESSION STRENGTE (GREEN-SAND STRENGTE)}

\section{A. Equipment Needed}

67. Specimen tube (pcrmeability type) (par. 4). Rammer (permeability type) (fig. 91).

Stripping post (par. 71).

Compression apparatus (par. 74).

\section{B. Selection and Freparation of Sample Zor Testing}

68. The selection and preparation of the sample to be tested should be according to the procedure outlined for the selection and preparation of the sample to be tested for permeability. (See Sec. I, 2, pars. 23 to 35 .)

\section{Method of Procedure in Testing for Strength by Compression}

69. Take the entire sample of sand from the humidor. Pass this entire sample twice through a coarse riddle and return the sand as quickly as possible to the humidor or receptacle. From this take sample to be tested for strength and test as specified in paragraphs 71,72 , and 73 , which gives the controlling specifications as set up by the committee.

\section{Forming Test Specimen}

70. The test specimen used is the standard permeability specimen, made according to the specifications of Section I, 2, paragraphs 4, 36, 37, and 38 . The permeability test and moisture (Dietert method ${ }^{28}$ determination may be first made.

\section{E. Stripping Post}

71. The specimen is then removed from the permeability tube by the use of a stripping-post apparatus. A stripping post is a wooden or metal post $1 \frac{15}{16}$ inches in diameter and 5 inches long with a flange at the base $3 / 8$ inch wide and $1 / 4$ inch thick. In the center of the top of this post is a dowel projection about $1 / 8$ inch high and $3 / 16$ inch in diameter. On top of this post is set a metal disk (the bottom to take the dowel of this post for centering) which slides loosely into the sand container. The disk has a thickness of about $3 / 4$ inch with a slight outward taper from the bottom to the top. The metal disk on which the sand specimen is set in the "Adams" compression machine can be used for this purpose.

\section{F. Controlling Specifications in Making the Compression Test}

72. The compression test shall be made on the green-sand permeability specimen, after it has been removed from the permeability cylinder, by applying a load uniformly to the two plane surfaces of the specimen at a rate of 30 pounds per minute with a leeway of 10 pounds per minute in either direction, the load to be applied along the axis of the cylindrical specimen.

73. If the test result of one specimen varies more than 10 per cent from the average of all, this result must be discarded and another specimen tested. The compressive strength should be the average of three tests and is to be expressed as pounds per square inch of sand of cross-sectional area.

\section{G. Compression Test Apparatus}

74. Any apparatus in which the test can be performed following the specifications of paragraphs 72 and 73 may be used. Several different types of compression apparatus have been used.

Note.-The specification includes descriptions of two designs, (1) Saeger compression apparatus, and (2) Adams compression apparatus.

\section{FINENESS TEST}

\section{A. Foreword}

75. The fineness of a sand refers to the fineness of the grain. In general, the sand particles may be divided into two groups, the larger ones representing sand and the finer ones representing clay. Very fine sand particles are spoken of as silt. Sands may vary in their texture from coarse to fine, depending upon the size of the particles.

76. The texture of the sand affects the permeability, strength, amount of moisture required for mixing, and the surface of the casting.

77. The object of the fineness test is to determine the percentage of the differcnt sizes of grain which the sand contains.

\section{B. Apparatus Required}

78. Balance (sensitive to $1 / 100$ of a gram) with corresponding set of weights.

Set of standard half-heiglt sieves.

Washer or stirrer.

${ }_{28}^{8}$ Dietert, H. W., and Myler, W. M., jr., Molding Sand Control in the Foundry. Trans. A. F. A., 33, pp. 751-753. 
Four 1-quart preserve jars with rubber stoppers. Syphoning apparatus.

Buchner filter (3-inch).

Six 4 -inch watch glasses.

Filter pump.

Drying oven.

Camel's hair brush (1 inch broad).

Sieve shaking apparatus.

Time switch (desirable).

Nail brush.

Package black glazed paper.

Bottle of $\mathrm{NaOH}$ (sodium hrdroxide) solution.

Package $13 \mathrm{~cm}$ filter paper.

\section{Classes of Sands for Fineness Test}

79. Sands may be divided into two groups: Those containing no clay or bonding substance; those containing clay or bonding substance.

\section{Standard Sieves Osed}

80. The fineness test procedures for these two groups of sand vary, but the sieves used are the same for both groups.

81. Sizes of sieves used.-In testing for fineness the sieves used are National Bureau of Standards sieve numbers $6,12,20,30,40,50,70,100,140,200$, and 270. (See 500.2, p. 1.) The sieves are what are known as half-height and have a diameter of 8 inches.

82. In use, the coarsest sieve is placed on top and the finest at the bottom.

83. A pan fits onto the bottom of the set and a cover is placed on the sieves.

\section{E. Procedure for Sands Containing No Clay or Bonding Substance}

84. Fifty grams of dried sand are transferred to the first of the series of sieves, used for molding sands, and mentioned above, and placed in a testing sieve shaker.

85. The testing sieve shaker is then started and the specimens shaken on the sieves for a definite length of time as indicated for each type of sieve shaker as noted below:

Fifteen minutes, using Rotap (W. S. Tyler Co.); 15 minutes, using type HL, Coombs gyratory laboratory sifter (Great Western Manufacturing Co.); 10 minutes, using the Vibrote shaker (Traylor Vibrator Co.); 5 minutes, using the Adams shaker (Eoundry Supplies Manufacturing Co.).

86. The amount of sand remaining on each sieve is weighed ${ }^{2 \theta}$ and expressed in percentage of the original sample weight $(50 \mathrm{~g})$. The portion passing the No. 270 sieve is known as No. 270 (or pan material).

\section{F. Procedure for Sands Containing Clay or Bonding Substance}

87. Fifty grams of molding sand (dried as specified in paragraph 1) are put into a quart preserving jar, smooth on the inside, with no sharp shoulders in the neck, to permit the sand to be easily removed with a small stream of water.

88. Four hundred and seventy-five cubic centimeters of water and twent $v$-five cubic centimeters of a standard solution of sodium hydroxide (made by dissolving $10 \mathrm{~g}$ of sodium hydroxide, $\mathrm{NaOH}$, in 1,000 cc of water) ${ }^{30}$ are added, and the bottle or jar is covered and securely sealed.

29 For removing the sand from the sieres it is convenient to have a sheet of black glazed paper on which the sieve can be placed upside down. The sieve should be tapped to jar loose any grains adhering to the wire cloth, and it may be further cleaned by brushing the underside of the cloth with a brush while the sieve is held over the paper in an inverted position. The sand can then be slid from the paper in an inverted position. The sand can then be slid from the paper onto the scale pan of the balance,

${ }_{20}$ Distilled water preferable.
89. In using a preserving jar, instead of the usual rubber ring and glass cover, a rubber stopper may be employed, which fits into the mouth of the jar.

90. The receptacle is then placed in a shaking machine ${ }^{3 i}$ making about 60 revolutions per minute, in such a manner as to allow it to be up-ended at each revolution. At the end of one hour ${ }^{32}$ the receptacle is removed, the cover is unsealed, and the sand adhering to the cover is washed into the receptacle.

91. The receptacle is then filled with water, ${ }^{38}$ permitting the stream to stir up the contents, ${ }^{34}$ and allowed to stand for 10 minutes, when by means of a siphon extending to within 1 inch (approximately $2.5 \mathrm{~cm}$ ) of the bottom of the receptacle the water is siphoned off.

92. More water is added, filling the receptacle, and at the end of 10 minutes siphoned off. Water is added again, and at the end of five minutes siphoned off.

93. The process of five minutes' standing and siphoning is repeated until the water remains clear at the end of the 5-minute period. By this means the clav substance is separated from the grain.

94. The grain remaining in the bottle or jar is washed on to a filter paper, in a $9 \mathrm{~cm}$ Buchner's funnel drained by means of suction, and transferred, together with the filter paper, to a large watch glass, and dried for one-half an hour at a temperature which shall not be lower than $105^{\circ} \mathrm{C}$. nor higher than $110^{\circ} \mathrm{C}$.

95. The dried grain is weighed, and the difference between its weight and that of the original $50 \mathrm{~g}$ sample represents the clay substance. The grain is then treated as in paragraphs 84 to 86 , inclusive.

96. The method used for expressing the fineness 85 is explained under grading classification, Section III.

\section{CEEMICAL ANALYSIS OF FOUNDRY SAND}

\section{A. Preparation of Sample}

97. The sample shall be ground to pass through a 100-mesh sieve, be dry to the touch, and be representative of the whole material under test. All determinations, except the determination of water at $105^{\circ}$ to $110^{\circ} \mathrm{C}$., shall be made on the sample dried at said temperature and weighed out as follows: Dry, cool, and weigh the sample as below, quickly transfer the needed portion, restopper the bottle, and weigh again. No further sample should be taken from an opened weighing bottle until the contents have been redried. Distilled water shall be used throughout and concentrated acids are meant unless otherwise specified. All analyses shall be corrected by blank runs on the reagents carried along with the analyses.

1 The committee bas found that the use of an electric milk-shake stirrer of the type used at soda fountains is permissihle for mos sands and that fire minutes' stirring will accomplish the desired results. The vessel to contain the liquid should be supnlied with verticle baffics. For a few sands the electric stirrer does not function properly, and before the stirrer is used for any particular sand its performance should be checked up by the standard method.

32 A short-cut method for shop control work recommended by the committee is to weigh out $50 \mathrm{~g}$ of molding sand into a bottle, and add to 25 of to it 25 cc of a standard caustic solution (made hy dissolving 100 is the hottle is then sealed and minute for a Feriod of 15 minutes. The sands are then treated in the manner described in paragraphs 91 to 96 , inclusive.

33 It is absolutely necessary that the depth of water after filling the jar and the depth to which it is siphoned shall be standard. As quart reserving jars are slightly different in shape, the committee recom mends that the jar be flled with water to hring the liquid to a height of 6 inches from the bottom and that the siphon shall extend to exactly 1 inch from the bottom. In case the settled material is lifted by the siphon, a slight change in apparatus and procedure is necessary. A deeper vessel must he used and the length of the siphon must be such that it will lift out the water to a dcpth of 5 inches from the original surface.

34 If the stream of water does not stir the contents sufficiently ther means must be used.

35 Compound grains which are not broken apart in the fineness test manipulator shall be considered as unit grains. 


\section{B. Determination of Water at $105^{\circ}$ to $110^{\circ} \mathrm{C}$.}

98. Carefully air dry a portion of the sample and weigh $1 \mathrm{~g}$ portions in a tared, wide, low-form, flat-bottomed weighing bottle. Heat uncovered in a ventilated drying oven at a temperature not below $105^{\circ} \mathrm{C}$. nor above $110^{\circ} \mathrm{C}$. for one hour. Lightly stopper and cool in a desiccator. Lift the stopper momentarily just before weighing, restopper, and weigh. The use of a similar weighing bottle as a counterpoise carried through all of the operations is a desirable procedure. Calculate the percentage loss and report as " $\mathrm{H}_{2} \mathrm{O}$ at $105^{\circ}$ to $110^{\circ} \mathrm{C}$." This determination is not necessary in the summation of results, but is desirable for the study of different varieties of molding sands.

\section{Determination of Total Volatile Riatter (Loss on Ignition)}

99. Transfer $1 \mathrm{~g}$ of the sample, dried at a temperature not below $105^{\circ} \mathrm{C}$. nor above $110^{\circ} \mathrm{C}$., to a tared platinum crucible with cover, warm gently under oxidizing conditions until moisture has been expelled, then at dull redness until carbonaceous matter has been destroyed, and finally with cover on and at approximately $1,000^{\circ} \mathrm{C}$. to constant weight. Calculate the percentage loss and report as "loss on ignition." This determination represents water not driven off at $105^{\circ}$ to $110^{\circ} \mathrm{C}$. carbon dioxide and carbonaceous matter and is subject to any oxidation changes. A true water determination above $105^{\circ}$ to $110^{\circ} \mathrm{C}$. would require heating in a closed tube with absorption and weighing of the water.

\section{Determination of Silica}

100. Transfer $2 \mathrm{~g}$ of the sample, dried at $105^{\circ}$ to $110^{\circ} \mathrm{C}$. to a platinum crucible, mix intimately with $10 \mathrm{~g}$ of sodium carbonate, cover, and heat carefully until the cvolution of carbon dioxide occasioned by the reaction between silica and carbonate has ceased. Finally heat until the material is decomposed and the fusion is quiet. Cool, place the crucible cover in an evaporating dish, preferably of platinum to eliminate contamination, and for the sake of celerity in evaporation treat with a little dilute hydrochloric acid, scrub clean and remove. Cover the cooled melt with water and gently warm. Melts can usually be so loosened from crucibles with even sides. Transfer the loosened melt to the evaporating dish containing the faintly acid solution used to clean the cover and take care to avoid mechanical loss through effervescence. Wash out the crucible and remove any adhering matter by treatment with dilute $1: 1$ hydrochloric acid and scrubbing. Finally cover the evaporating dish and warm until the melt has been decomposed, hastening solution by breaking up resistant lumps with the flattened end of a glass rod. When effervescence has ceased, remove and wash the cover glass, and evaporate the contents of the dish to dryness, breaking up crusts from time to time with a platinum or glass stirring rod kept in the dish. When thoroughly dry cool and drench the residue with hydrochlolic acid and allow to stand for a few minutes. Add an equal volume of water, cover the dish and warm on the water bath for 10 minutes. Filter on a paper of suitable size, wash thoroughly with hot dilute hydrochloric acid (5:95) and finally with hot water. Reserve the residue.

101. Evaporate the filtrate to dryness, thoroughly dehydrate as before, and again treat with acid and filter, but using as little acid as possible and allowing but a few minutes' time for the digestion of the residue in acid. Filter the solution through a second and smaller paper, wash with a little cold dilute hydrochloric acid (1:99), and finally with a little hot water. No considerable washing of the small paper and silica recovery should be required. Reserve the filtrate and washings for the determination of iron and aluminum as below.

102. Transfer, wet or dry, the two papers containing the silica recoveries to a platinum crucible. Char carefully without allowing the paper to inflame, ignite until carbon has been destroyed, and finally with cover on at approximately $1,200^{\circ} \mathrm{C}$. to constant weight. Moisten the impure silica with a few drops of dilute sulphuric acid, carefully add sufficient hydrofluoric acid to decompose the silica, and slowly warm until all danger from effervescence is over. Finally evaporate to dryness, carefully ignite until sulphates are decomposed and then heat at approximately $1,000^{\circ} \mathrm{C}$. Cool, weigh, subtract this weight from the weight of the impure silica, add the weight of any recovery obtained in the determination of iron and aluminum, and calculate the percentage of silica.

\section{E. Determination of Total Iron, Aluminum, and Titanium Oxides}

103. Fuse the nonvolatile residue from the silica determination with as little sodium carbonate as possible, dissolve the cooled melt in dilute hydrochloric acid and add the solution to the filtrate reserved from the silica determination.

104. Add a few drops of nitric acid or bromine water and boil the solution until all trace of chlorine or bromide is gone. Then add hydrochloric acid, if not already present, sufficient to insure a total volume of 10 to $15 \mathrm{cc}$ of the acid. Add a few drops of methyl red solution, dilute to 200 to $250 \mathrm{cc}$ and heat the solution to boiling. Neutralize by the careful addition of dilute ammonia (1:1) (more dilute toward the end) until the color of the liquid just changes to a distinct yellow. Boil for one minute, allow to settle, filter, and wash the precipitate at once two or three times with hot 2 per cent ammonium chloride solution. Reserve the filtrate. Dissolve the precipitate on the paper in hot, dilute hydrochloric acid (1:1), catching the solution in the original beaker, and washing the paper thoroughly with hot water. Reserve the paper, as it may contain some undissolved aluminum hydroxide. Boil the solution, add a little macerated filter paper and reprecipitate with ammonia as above. Transfer the precipitate as completely as possible to a new filter by means of hot 2 per cent ammonium chloride solution. Finally remove any adhering hydroxide from the inside of the beaker by scrubbing with the reserved paper which is to be added and burned with the precipitate. Add the filtrate to the reserved filtrate and save for the determination of calcium as below.

105. Transfer the precipitate to a weighed platinum crucible and heat very gently until all danger of mechanical loss has passed, then at a higher temperature and under oxidizing conditions until carbon has been destroyed, and finally for 10 minutes with crucible covered over the blast lamp or its equivalent. Cool and weigh with the crucible tightly covered, as $\mathrm{Al}_{2} \mathrm{O}_{3}+\mathrm{Fe}_{2} \mathrm{O}_{3}+\mathrm{TiO}_{2}+\mathrm{SiO}_{2}$. Reserve the residue for the recovery of silica and determination of total ferric oxide and calculate the percentage of combined oxides.

\section{F. Recovery of Silica and Determination of Total Ferric Oxide}

106. (a) Recovery of silica.-Add 3 to $4 \mathrm{~g}$ of potassium or, preferably, sodium pyrosulphate to the combined oxides, snugly cover the crucible, and carefully heat until the salt is melted and in quiet fusion. Continue the heating at a very dull red until the oxides are in solution. This fusion can not be hurried and must be performed at the lowest temperature which will prevent the formation of a 
crust on the melt. Take up the cooled melt with so much dilute sulphuric acid that there shall be not less than $5 \mathrm{cc}$ of absolutely acid and enough water to effect solution.

107. Eraporate the solution and eventually heat until sulphuric acid fumes come off copiously. After cooling, dilute to approximately $100 \mathrm{cc}$, stir until salts are dissolved, and make sure that any small residue is silica and not unfused oxide, as shown by a light flocculent suspension as against a fine powder. Filter without delay, wash with hot water until free from soluble salts, and reserve the filtrate. Ignite the residue, weigh, correct with hydrofluoricacid treatment, and add the weight of silica recovered to that previously found and deduct from the gross weight of the combined oxides.

$10 \mathrm{~S}$. In case the ionvolatile residue is appreciable in amount, it must be recorered and added to the reserved filtrate by fusion with pyrosulphate and solution in sulphuric acid.

109. (b) Detcrmination of total ferric oxide.-Pass the hot reserved filtrate, preferably of 100 to $200 \mathrm{cc}$ volume and containing 5 per cent by rolume of sulphuric acid, through a Jones reductor using the customary precautions, and quiclly titrate the reduced solution with an approximately $0.03 N$ potassium perinanganate solution which has been standardized against the Bureau of Standards standard sodium oxalate. Reserve the titrate solution for the determination of titania, and after subtracting the permanganate required in a blank run calculate the percentage of $\mathrm{Fe}_{2} \mathrm{O}_{3}$. This value will be too high if titanium or vanadium are present. The latter need not be feared in this class of material; the effect of the former is overcome by subtracting the percentage of titania found below. (The $\mathrm{TiO}_{2}$ titer of a permanganate solution is the same as the $\mathrm{Fe}_{2} \mathrm{O}_{3}$ titer.) If a determination of ferrous oxide is made, the percentage found must be calculated to $\mathrm{Fe}_{2} \mathrm{O}_{3}$ and subtracted from the above value in order to get the percentage of ferric oxide in the sand.

110. The determination of iron under the above condition is not strictly accurate on account of the instability of the reduced titanium solution; the error is slight if the amount of titania is small.

\section{G. Determination of Titania}

111. Evaporate the titrated solution to a volume of approximately $75 \mathrm{cc}$, cool, transfer to a $100 \mathrm{cc}$ Nessler comparison tube, add $5 \mathrm{cc}$ of 3 per cent hydrogen peroxide, and dilute to the $100 \mathrm{cc}$ mark. In another tube mix the same amount of fusion salt, iron as ferric sulphate, and acid as are in the unknown, dilute to approximately $95 \mathrm{cc}$, and then add standard titanium sulphate solution until the colors match. Calculate the percentage of $\mathrm{TiO}_{2}$ and subtract this value from the calculated percentage of $\mathrm{Fe}_{2} \mathrm{O}_{3}$ obtained above.

\section{H. Determination of Alumina}

112. Subtract from the reight of $\mathrm{Al}_{2} \mathrm{O}_{3}+\mathrm{Fe}_{2} \mathrm{O}_{3}+$ $\mathrm{TiO}_{2}+\mathrm{SiO}_{2}$, (a) the weight of silica recovered from it, (b) the calculated weight of $\mathrm{Fe}_{2} \mathrm{O}_{3}$ obtained, and (c) the calculated weight of $\mathrm{TiO}_{2}$. Regard the remainder as $\mathrm{Al}_{2} \mathrm{O}_{3}$ and calculate its percentage.

\section{Determination of Lime}

113. (a) Gravimetric method.-Add a few drops of ammonia to the combined filtrates from the ammonia precipitation and bring the solution to boiling. To the boiling solution add $10 \mathrm{cc}$ of a saturated solution of ammonium oxalate and continue the boiling until the precipitated calcium oxalate assumes a granular form. Allow to settle for at least one hour, filter, and wash with boiling water, using no more than necessary on account of the solubility of the oxalate.
Reserve the filtrate and washing for the determination of magnesium. With the small amounts of calcium, magnesium, and aluminum involved, it is hardly necessary to dissolve the precipitate and attempt to recover the latter two. Ignite the precipitate to lime in a meighted platinum crucible with cover, at first uncovered until the carbon has been destroyed and then well covered to constant weight. Calculate the percentage of $\mathrm{CaO}$.

114. (b) Volumetric method.--Transfer the paper and precipitate obtained as in the grarimetric method to the well-mashed beaker in which the precipitation was made, spreading the paper out against the upper portion of the beaker. Wash the precipitates from the paper with a jet of hot mater, fold the paper, and leave it adhering to the upper portion of the beaker. Add to the contents of the beaker $50 \mathrm{cc}$ of dilute sulphuric acid $(1: 10)$, dilute to a volume of $250 \mathrm{cc}$ with hot water, and heat to a temperature of $80^{\circ}$ to $90^{\circ} \mathrm{C}$. Titrate with a standard approximately $0.03 N$ permanganate solution until the pink end point is obtained. Now drop the folded filter paper (which has been adhering to the side of the beaker) into the liquid, stir, and continue the titration until the pink color is again obtained. A Gooch crucible may be used instead of filter paper. From the total quantity of standard permanganate solution used calculate the percentage of $\mathrm{CaO}$.

\section{J. Determingtion of Magnesia}

115. Acidify the filtrate from the calcium precipitate with hydrochloric acid, and concentrate on the water bath to about $200 \mathrm{cc}$. Add $10 \mathrm{cc}$ of a saturated solution of microcosmic salt and then ammonia, dropwise, until a crystalline precipitate begins to form or the solution is alkaline. Finally add $10 \mathrm{cc}$ in excess and allow the solution to stand at room temperature for from 12 to 48 hours. Filter, rinse the beaker with a little 5 per cent by volume ammonia, wash the precipitate slightly, and discard the filtrate.

116. Dissolve the precipitate adhering to the beaker and on the paper in hot dilute hydrochloric acid, dilute to $100 \mathrm{cc}$, add $1 \mathrm{cc}$ of a saturated solution of microcosmic salt and ammoma, dropwise as before, and finally in 5 to 10 cc excess. Allow to stand for 6 to 24 hours, filter on paper, and wash with dilute 5 per cent by volume ammonia. Heat the wet or dry precipitate carefully (preferably in a muffle) until the paper chars without flaming, destroy the carbon under oxidizing conditions and at the lowest possible temperature, and finally ignite at approximately $1,000^{\circ} \mathrm{C}$. to constant weight. Calculate the percentage of $\mathrm{MgO}$ from the weight of $\mathrm{Mg}_{2} \mathrm{P}_{2} \mathrm{O}_{7}$ obtained.

\section{K. Determination of Cerbon Dioside}

117. Determine the carbon dioxide on a $10 \mathrm{~g}$ sample, dried at $105^{\circ} \mathrm{C}$. according to the method given in the United States Geological Survey Bulletin No. 700, pages $217-218$.

118. In brief, the method is as follows: Boil the meighed sample with dilute hydrochloric acid in a small Erlenmeyer flask attached to an upwardly inclined condenser, whence, after passing through a drying system-calcium chloride, anhydrous copper sulphate to retain hydrogen sulphide from decomposable sulphides and any hydrochloric acid that may pass over, then calcium chloride again-the carbon dioxide is caught by weighed absorption tubes filled with soda lime or with ascarite and calcium chloride. The drying system should employ, successively, calcium chloride, anhydrous copper sulphate, and calcium chloride again. The function of the anhydrous copper sulphate is to retain hydrogen sulphide from decomposable sulphides and any hydrochloric acid that may pass over. Of course, 
arrangement is made for a current of air free from carbon dioxide with which to sweep out the apparatus before and after the determination and for a slow current during its continuance. Calculate the percentage of carbon dioxide.

\section{Determination of Potassium and Sodium Oxides}

119. Grind a little more than $1 \mathrm{~g}$ of the sand in a small agate mortar with $0.5 \mathrm{~g}$ of ammonium chloride and thoroughly mix by grinding with the pestle. Next weigh out about $5 \mathrm{~g}$ calcium carbonate and use small portions as directed below. First transfer sufficient carbonate to form a thin layer on the bottom of an unweighed, deep form, platinum crucible, next thoroughly mix about $3 \mathrm{~g}$ of the carbonate with the already mixed sand and ammonium chloride, carefully transfer the mixture of the three to the platinum crucible and finally scrub the mortar and pestle with the remaining carbonate and pour on the mix as a cover.

120. Snugly cover the crucible and heat over a low flame for 10 minutes or until no more vapors are given off. Continue the heating with only the lower third of the crucible heated to dull red (best set in an asbestos shield) for three-quarters of an hour and allow to cool.

121. When cold, cover the sinter in the crucible with water, allow to slake and transfer the contents to a platinum dish. Dilute to $50 \mathrm{cc}$, rub up the mass with an agate pestle, rinse off the pestle and boil the solution for five minutes. Decant through a 9-cm filter into a 600-cc beaker. Repeat the rubbing and extraction treatment at least three times.

122. Treat the filtrate with a little ammonia, bring to boiling, add about $2 \mathrm{~g}$ of ammonium carbonate dissolved in $25 \mathrm{cc}$ of water, and continue the boiling for a minute or so. Allow the bulky precipitate to settle, filter into a 500-cc dish of porcelain or platinum, wash the residue with hot water, and evaporate the filtrate to dryness over a water bath.

123. Place the basin, covered with a glass, on a square of gauze over a low flame, heat very carefully until all danger of decrepitation is passed and then remove the cover and continue the heating until all ammonium salts have been expelled. Heat at as low a temperature as possible to avoid loss of alkali chlorides by volatilization. Cool the dish, add enough water to dissolve the chlorides, then a drop of barium chloride solution (or more if appreciable amounts of sulphates are indicated) and finally a few drops of ammonium carbonate solution to precipitate the excess of barium and the last traces of lime. Make sure that all soluble salts are in solution and again evaporate almost to dryness. Treat with 5-ce of water and filter through a $5.5-\mathrm{cm}$ filter placed in a $3.5-\mathrm{cm}$ funnel, into a previously weighed 35-ce crucible with cover. Wash the paper and residue with 3 to $4 \mathrm{ce}$ portions of hot water, add 1 drop of hydrochloric acid to the filtrate, and evaporate the solution to complete dryness on the water bath. When dry, cover, place on a triangle and very cautiously heat until danger of decrepitation is past. When vapors of ammonium chloride rise, raise the leat gently until all are driven off; finally raise the cover and heat it as well as the crucible gently till free from ammonium chloride. The heating of the cover should be done below red heat and the heating of the crucible should be gaged so that the chlorides suffer nor more than incipient fusion.

124. Cool the crucible and contents in a desiccator and weigh as $\mathrm{NaCl}+\mathrm{KCl}$. Test the mixed chlorides for purity by dissolving them in 5 to $10 \mathrm{ec}$ of water. If the solution is clear, proceed as below; if the solution is turbid, filter and wash as before and again evaporate to dryness and weigh.
125. (a) Determination of potassium oxides.Treat the solution of the mixed chlorides with suffcient chloroplatinic acid to combine with both sodium and potassium. (If the solution of chloroplatinic acid contains $0.05 \mathrm{~g}$ of platinum per cubic centimeter, this may be assured by adding as many cubic centimeters as 34 times the weight of the combined chlorides.) Place on the water bath, evaporate until the solution is sirupy, cool, and then half fill the crucible with alconol of 0.86 specific gravity (approximately 80 per cent by volume). Alicw to stand with occasional gentle stirring until sodium salt has dissolved and the residue is golden yellow. Filter through a weighed Gooch crucible, wash with the 80 per cent alcohol, dry at $130^{\circ} \mathrm{C}$. and weigh as $\mathrm{K}_{2} \mathrm{PtCl}_{6}$.

126. Subtract the weight obtained in the blank, multiply the corrected weight by 0.1938 to get the weight of $\mathrm{K}_{2} \mathrm{O}$ and calculate the percentage of $\mathrm{K}_{2} \mathrm{O}$.

127. (b) Determination of sodium oxide.-Multiply the weight of $\mathrm{K}_{2} \mathrm{PtCl}_{6}$ by 0.307 to reduce it to $\mathrm{KCl}$, subtract from the weight of the mixed chlorides and multiply the weight of sodium chloride so found by 0.5308 to reduce it to $\mathrm{Na}_{2} \mathrm{O}$. Correct this weight as indicated by the blank and calculate the percentage of $\mathrm{Na}_{2} \mathrm{O}$.

\section{Determination of Ferrous Oxide}

128. This determination is attended by several difficulties. Satisfactory results are impossible in the presence of carbonaceous matter. If the material is coarse and difficultly soluble, any grinding be most carefully done to avoid oxidation. The method to be described below is applicable in the absence of organic matter and does not require special apparatus.

129. Method.- Test a small portion of the sand by gently boiling for 20 minutes with hydrofluoric acid in a covered platinum crucible. If the above test shows that the sample leaves but little residue, take 0.5 to $1 \mathrm{~g}$ of the sample, dried at $105^{\circ} \mathrm{C}$. and place it directly in a platinum crucible of 80 to 100 cc capacity. In case the sample is not easily decomposed, transfer the weighed portion to an agate mortar and grind under absolute alcohol only long enough to yield a powder that will leave little or no residue on solution. Allow the alcohol to evaporate spontaneously and when the last trace has disappeared, transfer the powder to the platinum crucible and rinse the mortar and pestle with a jet of hot water.

130. In either procedure cover the powder with a little air-free water, and add $10 \mathrm{cc}$ of dilute sulphuric acid $(1: 3)$ carefully and with cover on in case efferveseence is noted. Add air free hot water until the crucible is half full and place the covered crucible on a triangle well down over a lamp turned low and protected from drafts. Rapidly displace the air in the crucible by a stream of carbon dioxide directed beneath the lid raised on one side. Before allowing the liquid to boil, discontinue the gas current and cover the crucible with the well-fitting lid. Crently boil, draw the lid a little to one side, quickly add $10 \mathrm{cc}$ of hydrofluoric acid, replace the lid, laise the temperature cautiously until steam comes off, and then lower until there is steady ebullition without danger of loss. Steam should issue continually for 20 minutes or as many minutes as may be deemed necessary or allowable. Transfer the crucibie, still covered, to the titration vessel, which may be of glass (containing a cold saturated solution of boric acid in freshly boiled water with an excess of the solid acid and $5 \mathrm{ce}$ of sulphurie acid) and which is already under the burette. Finally, titrate rapidly with a standard approximately $0.03 N$ permanganate solution until a first pink blush appears throughout the whole liquid. 
131. If an unattacked residue shows after titrating, allow it to settle completely, decant off the top liquid, wash once by decantation, and transfer to an agate mortar by the aid of a jet of water. Allow to settle, decant off most of the water, grind under the water that remains and wash back again into the large crucible. Repeat the treatment with hydrofluoric and sulphuric acids as before, in smaller amounts and for a shorter time, titrate, and, if necessary, repeat the operation a third time. Calculate the percentage of $\mathrm{FeO}$ from the total amount of permanganate required.

\section{TENTATIVE STANDARD TESTS}

\section{STRENGTH TISTS (GREEN SAND)}

132. Tentative standard methods are recommended for transverse, tensile, and shear strength tests. The compression strength test has been approved as the standard strength test. (See above, secs. 67 to 74 inclusive.) The publication points out that it is for the foundrrman to decide which test measures the kind of stress under which his sand is apt to fail.

133. For all strength tests, the selection and preparation of the sample to be tested should be according to the procedure outlined for the selection and preparation of the sample to be tested for permeability. (See above secs. 30 to 39 , inclusire.)

13t. The full text of the publication includes detailed descriptions and illustrations of apparatus and procedure for the tentative test methods to be used, which are as follows:

\section{BAR STRENGTH (TRANSTERSE) (DOTY METHOD) \\ 2. TEXSILE STREXGTH (AS DETELOPED BY A. A. GRUBB)}

A. This method has the following controlling specifications:

135. The tensile test shall he made by applying a load uniformly along the axis of the cylindrical test specimen 2 inches in diameter and 2 inches long \pm 36 inch (see par. 36 for forming test specimen) at a rate not to exceed 1 pound per minute.

136. If the test result of one specimen varies more than 10 per cent from the average of all, this result must be discarded and anothe specimen tested.

137. The tensile strength should be the arerage of three tests. The results for green sand strength should be expressed in pounds per square inch of sand of cross-sectional area.

\section{SHEAR STRENGTH TEST}

A. This method has the following controlling specifications:

13. The shear test shall be made on the green sand permeability specimen, after it has been remored from the permeahility cylinder. by applying a load uniformly to the two plane suriaces of the specimen, at a rate of 30 pounds per minute, with a leeway of 10 pounds per minute in either direction, the load to be applied along the axis of the cylindrical specimen.

139. If the result of one spccimen varies more than 10 per cent from the a verage of all, this resilt must be discarded and another specimen the average of all, this result must be discarded and another specimen is to be erpressed as pounds per square inch of sand of cross-sectional is to b.

\section{TESTIIG STRENGTH OF BAKED CORE SAND MIXTORES}

140. Two methods for testing the baked strength of core sand mixtures have been approved as tentative standards. One method $(a)$ uses the tensile test briquette, the other $(b)$ the transverse bar. The full text of the tentative standard presents descriptions and details of performing these methods, together with deseriptions and figures of the equipment used.

(a) Tensile Test (Briquette Method)

141. Measure of strength.-The tensile strength expressed in pounds per square inch shall be taken as a measure of the strength of dried or baked cores.

142. At least fire test pieces should be made and tested. Briquettes that are manifestly faulty or give strength differing more than 15 per cent from the average value of all the test cores made from the same sample and broken at the same period shall not be considered in determining the tensile strength. The average of the acceptable values will be reported as the tensile strength of the group.

\section{(b) Transverse Bar Method}

143. Measure of strength.-The transverse strength expressed in pounds per square inch shall be taken as a measure of strength of dried or baked cores when the transverse test bar is a 1 by 1 inch square bar 8 inches long, broken by applying a load at a rate of 24 pounds per minute, with a leeway of 10 pounds per minute in either direction, on the bar resting on supports 6 inches apart.

144. At least three specimens should be prepared and tested for each set of conditions being investigated.

\section{DYE ADSORPTION QUALITIES OF MOLDING SAND}

\section{A. Foreword}

145. Application.-The application of the dyeadsorption phenomenon to molding sand is solely for the purpose of ascertaining the nature of the clay substance present. Different sands possess midely different adsorption capacities, and this difference is due exelusively to the quantity and quality of colloidal material present.

146. The colloids in molding sands are mostly of an inorganic nature-hydrated aluminum silicate, hydrated iron oxide, hydrated silica acid, and other hydrated minerals. All of these constituents are of a gelatinous and sticky nature, and they impart to the sand the property of bond.

147. Strongly bonded molding sands commonly possess clay substance that is high in colloid content as measured by the dye-adsorption test.

148. The weaker bonded sands generally show a lower dye-adsorption figure corresponding to the smaller quantity of colloids present in the clay substance of those sands.

\section{B. Equipment and Materiais Needed}

149. Balance (sensitive to $1 / 100$ of a gram) and corresponding set of weights for weighing sand.

Drying oven (capable of maintaining a constant temperature of $105^{\circ}$ to $110^{\circ} \mathrm{C}$.).

Tide-mouth bottles 500 ce capacity

Washing apparatus.

Color comparison tube holder.

Pipette.

Balance (sensitive to $1 / 10 \mathrm{mg}$ ) is required for weighing out the dye.

Distilled water.

Ammonium hydrate.

Acetic acid.

Dye.-Du Pont erystal violet extra pure $\mathbf{B}$.

\section{Procedure}

150. Twenty-five grams of molding sand, dried for one hour at a temperature which shall not be lower than $105^{\circ} \mathrm{C}$., nor bigher than $110^{\circ} \mathrm{C}$., are weighed into a $500 \mathrm{cc}$ wide-mouth bottle fitted with a glass stopper, and $300 \mathrm{cc}$ of distilled water, plus $5 \mathrm{ec}$ of 10 per cent ammonium hydrate (made by adding $10 \mathrm{ce}$ ammonium hydrate of 0.90 specific gravity, to $90 \mathrm{cc}$ of water) are added.

151. The bottle is then stoppered, sealed with paraffin $\pi a x$, and rotated in a suitable machine for 30 minutes (any machine making approximately 60 revolutions per minute and up-ending the bottle with each revolution is satisfactory).

152. At the end of this period $90 \mathrm{cc}$ of distilled water are added, plus $5 \mathrm{cc}$ of 10 per cent acetic acid (made by adding $10 \mathrm{ce}$ acetic acid of 9912 per cent strength to $90 \mathrm{ec}$ of water). 
153. Crystal violet dye is then added in sufficient weight to allow for the adsorption by the colloidal matter and leave a slight excess. For molding sands of weak bond, $0.125 \mathrm{~g}$ of dye is a good amount to start with; while the stronger sands require an addition of 0.150 to $0.300 \mathrm{~g}$ or more of dye.

154. After adding the crystal violet the bottle is sealed again and rotated for another 30-minute period. If all the dye is taken up by the colloidal matter, more should be added, as it is necessary that an excess of dye be present over that required to satisfy the adsorption capacity of the colloids.

155. In order to determine the amount of dye adsorbed by the sand it becomes necessary to find the quantity unadsorbed or held in solution.

156. If the test is allowed to stand over night, suspended material settles out, leaving a clear solution of the dye, and the dye unadsorbed can be determined by color comparison. The standard color solution is made up by dissolving $0.500 \mathrm{~g}$ of crystal violet 30 in 500 ec distilled water. Twenty-five cubic centimeters of the clear dye solution are taken from the test by a pipette and run into one of a pair of "carbon" comparison tubes, such as are used in steel analysis, diluted to $50 \mathrm{cc}$ and thoroughly mixed. Forty cubic centimeters or more of distilled water are added to the second comparison tube, and the standard dye solution added from a burette until the color matches that of the test in question, taking care that the final volume is the same in both tubes.

157. If it required $2.5 \mathrm{cc}(0.0025 \mathrm{~g})$ in the standard tube to match the color in the test, then we have $0.0025 \mathrm{~g}$ of dye unadsorbed in $25 \mathrm{ec}$ or $0.040 \mathrm{~g}$ in $400 \mathrm{cc}$. This figure is subtracted from the amount of dye added to the test, multiplied by 4 , and the result expressed as milligrams of dye adsorbed per $100 \mathrm{~g}$ of sand.

\section{Fotes}

158. Electrouyte.-The presence of ammonium acetate in the test is helpful in that its presence tends to bring about rapidly the subsidence of the fine particles which otherwise would remain in suspension. It has no serious effect on crystal violet. The addition of ammonium hydrate acts as a partial deflocculator and thereby breaks up the agglomerations of clay or other bonding substances.

159. Dye.-Only the highest grade of crystal violet should be used. Impure dye gives low figures and is unstable. Standard dye solution should be kept in the dark, and a fresh quantity should be prepared frequently. For standard dye see footnote 36.

160. Used sands.- The dye adsorption test can not be relied upon for all grades of used sands. Impurities present in some heap sands, as, for example, sea coal, iron scale, organic binders, etc., seriously affect the adsorption results.

161. Dye concentration. - The final concentration of cyrstal violet should be not less than $0.024 \mathrm{~g}$, or more than $0.06 \mathrm{~g}$ in $400 \mathrm{cc}$ volume. A greater excess of dye gives high reading. After making a few tests it is a simple matter to judge the density of the clear dye solution, and it is essential that the proper concentration is obtained before the test settles overnight.

\section{SINTERING TEST OF MOLDING SAND}

\section{A. Foreword}

162. The true melting point of a molding sand, as observed by the full fusion of a cone or by other means, is not the only property upon which the practical "refractoriness" of the sand depends in

${ }_{36} \mathrm{Du}$ Pont crystal violet extra pure B obtained from E. I. du Pont de Nemours \& Co. (Ine), Wilmington, Del. foundry use. It is of more importance, to the foundrymen, to know the lower limit of the fusion range, that is, the temperature at which the sand sinters and "burns on" the casting, thus making cleaning difficult.

163. As a means of determining this lower limit or sintering point the following method, described below, has given satisfactory results:

\section{B. Preparation of Samples}

164. The sample to be tested should be an average one, representative of the heap, floor, car, bank, or other sources from which it is taken.

165. In carrying out the sintering test it is necessary that the sand be properly sampled and uniformly tempered. For plant check or control tests upon facing or heap sands in daily use, one may test the sand as tempered for molding.

166. The preparation of the sintering test specimen does not differ in any way from the preparation of the standard permeability specimens (see sections 23 to 34 , inclusive), except that the specimen is removed from the cylinder in which it is rammed and sufficient sand is cut away from the specimen along its length to leave a test piece 2 inches long, 2 inches high, and 1 inch thick. Specimens should be dried at least one hour at a temperature not lower than $105^{\circ} \mathrm{C}$., nor higher than $110^{\circ} \mathrm{C}$

\section{Sintering Apparatus}

167. The assembly of the sintering apparatus is shown in photograph, Figure 94. Sintering apparatus $(H)$ (see fig. 94): Carbon-plate resistance $(B)$ 1,000 watts; approximate range of resistance, 0.04 to 4 ohms; length, 18 inches; width, $6 \frac{1}{4}$ inches; height, 7 inches; ammeter $(C)$, range, 50 amperes; optical pyrometer meter $(D)$; optical pyrometer $(E)$; light-proof cabinet $(F)$ into which sintering apparatus is placed when conducting tests; interval timer $(G)$.

\section{Procedure of Sintering Test}

168. The sand specimen is placed in position in the sintering apparatus under the platinum ribbon heater $(H$, fig. 94). The specimen is centered between the platinum ribbon holder terminals and the platinum ribbon is placed in contact with the rounded surface of the sand specimen, allowing the weight of the platinum ribbon holder unit to be the applied load.

169. The optical pyrometer is sighted on the central point of the platinum ribbon and adjusted to read, the observed temperature starting with $923^{\circ} \mathrm{C}$., as shown in Table 2 .

\section{TABLE 2.-Temperature corrections for emissivity of} platinum

[Use a bsorption glass above 1,320 true]

\begin{tabular}{|c|c|c|c|}
\hline $\begin{array}{l}\text { Observed } \\
\text { tempera- } \\
\text { ture }\end{array}$ & $\begin{array}{l}\text { True } \\
\text { tempera- } \\
\text { ture }\end{array}$ & $\begin{array}{l}\text { Observed } \\
\text { tempera- } \\
\text { ture }\end{array}$ & $\begin{array}{l}\text { True } \\
\text { tempera- } \\
\text { ture }\end{array}$ \\
\hline $\begin{array}{c}{ }^{\circ} C . \\
923 \\
925 \\
967 \\
989 \\
1,011 \\
1,033 \\
1,055 \\
1,076 \\
1,098 \\
1,120 \\
1,141.5\end{array}$ & $\begin{array}{r}{ }^{\circ} C . \\
1,000 \\
25 \\
50 \\
75 \\
1,100 \\
25 \\
50 \\
75 \\
1,200 \\
25 \\
50\end{array}$ & $\begin{array}{l}{ }^{\circ} \mathrm{C} . \\
1,163 \\
1,184 \\
1,205.5 \\
1,227 \\
1,248 \\
1,269 \\
1,290.5 \\
1,312 \\
1,333 \\
1,354\end{array}$ & $\begin{array}{r}{ }^{\circ} C_{75} \\
75 \\
1,300 \\
25 \\
50 \\
75 \\
1,400 \\
25 \\
50 \\
75 \\
1,500\end{array}$ \\
\hline
\end{tabular}


170. Opposite the observed temperatures are the true temperatures secured by correcting the observed temperatures fur the emissivity of platinum. An electric curreut is passed through the platinum ribbon and by adjusting the earbon-plate resistance the brightmess of the platinum ribbon is made to match the biightness of the filament in the optical pyrometer which has been set at $923^{\circ} \mathrm{C}$. observed temperature, or $1,000^{\circ} \mathrm{C}$. true temperature. This accomplished, an interval timer or stop watch is started and the heated ribbon is allowed to remain in contact with the sand specimen for a period of four minutes. The electric current is then turned off and the platinum ribbon raised from the specimen, noting whether or not the ribbon has burned fast to the sand specimen. If the platinum ribbon has not burned fast, the specimen is moved forward about one-half an inch and the platinum ribbon again is placed in contact with the specimen. The above procedure is repeated, only this time increasing the true temperature by $25^{\circ}$. This corresponds to an observed temperature of $925^{\circ}$ or $1,025^{\circ}$ true temperature, as shown in the table. The above procedure is repeated each time, increasing the temperature by $25^{\circ}$ true temperature until the platinum ribbon has burned fast to the sand specimen. The true temperature at which this takes place is the sintering point.

\section{GRADING CLASSIFICATION}

\section{A. Foreword}

171. The subcommittee on grading of foundry sands has prepared and had approved tentative grading classifications for foundry sands. These classifications mere formulated as an aid in producing uniformity in a discussion of sand grades.

172. It is recommended that foundrymen grade their sands according to the system outlined below and that the A. F. A. grade numbers be used when practical; also that sand producers and sales organizations grade their products according to the same system and offer them under their A. F. A. grade numbers.

\section{B. Grain Fineness Number}

173. (a) Definition.-The grain fineness number of a sand is approximately the number of mesh per inch of that sieve which would just pass the sample if its grains mere averaged in size. It is approximately proportional to the surface area per unit weight of a sand, exclusive of clay.

174. (b) Method of determination-Make the standard A. F. A. fineness test (see I, 4, "Fineness test" p. 226) by removing the clay and sereening the grain. Multiply the weights or percentages of sand on the various sieves by the appropriate factors listed below. Add the products. Divide by the sum of the meights or percentages. The dividend is the A. F. A. grain fineness number. Below are listed the multipliers for the corresponding mesh numbers.

175. (c) Multipliers. - The weight or percentage of sand on each sieve is to be multiplied by the "approzimate mesh number" of the sieve through which it has passed. Inasmuch as mesh numbers differ among the testing sieve makers for sieves of approximately the same opening, the following "approximate mesh numbers" are listed as follows:

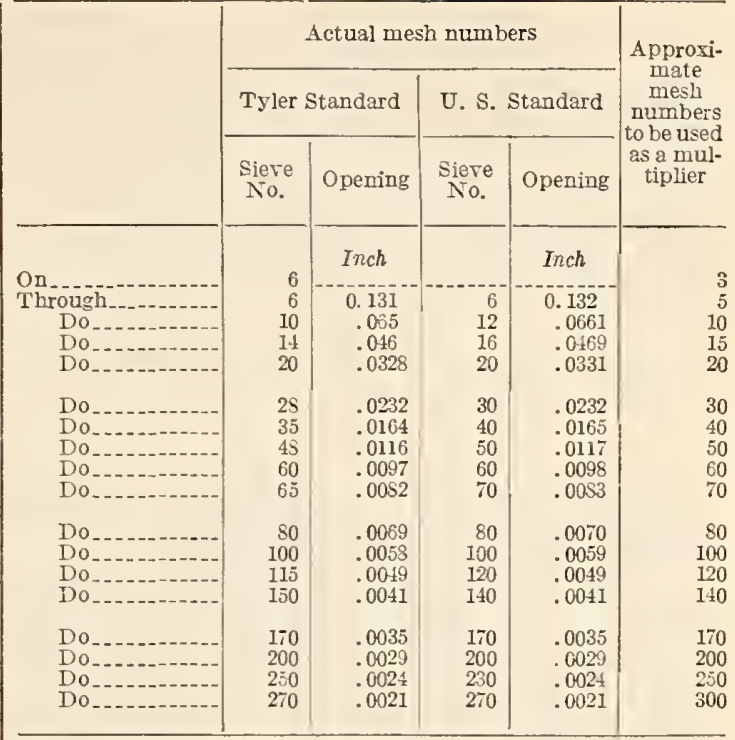

C. Grain Fineness Classification

\begin{tabular}{|c|c|}
\hline Grain class & Grain fineness zone \\
\hline $\begin{array}{l}\text { No. } 1 \\
\text { No. } 2 \\
\text { No. } 3 \\
\text { No. } 4 \\
\text { No. } 5\end{array}$ & $\begin{array}{l}200 \text { to } 300 \text {. } \\
140 \text { to but not including } 200 \text {. } \\
100 \text { to but not including } 140 \text {. } \\
70 \text { to but not including } 100 \text {. } \\
50 \text { to but not including } 70 \text {. }\end{array}$ \\
\hline 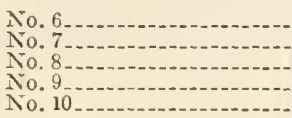 & $\begin{array}{l}40 \text { to but not including } 50 \text {. } \\
30 \text { to but not including } 40 \text {. } \\
20 \text { to but not including } 30 \text {. } \\
15 \text { to but not including } 20 \text {. } \\
10 \text { to but not including } 15 \text {. }\end{array}$ \\
\hline
\end{tabular}

A sand is in that grain class in which its grain fineness number falls according to the above grain fineness zones.

D. Clay Content Classification

Clay class

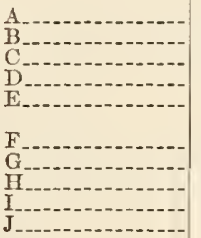

$\mathrm{A}$
$\mathrm{B}$
$\mathrm{C}$
$\mathrm{D}$
$\mathrm{D}$
$\mathrm{E}$
$\mathrm{H}$

A sand is in that clay content class in which its percentage of clay substance falls according to the above clay content zones.

176. The terms fine and coarse may be used to further define the grain fineness of sands; those sands whose fineness numbers lie in the upper part of a zone may be denoted as fine, while those in the lower part of a zone may be denoted as coarse. 
177. The terms angular, subangular, and rounded have been tentatively adopted for describing grain shape. It is to be recognized that some sands consist almost entirely of grains of one shape while others consist of mixtures of grains of widely varying shape.

American Railway Engineering Association, specifications for buildings for railway purposes.

\section{SAND FOR USE IN BRICKWORK}

See A. R. E. A. specification for brickwork, 518.83 , p. 475 .

American Society for Municipal Improvements, standard specification for sewers, 1922.

See 518.67 , p. 454 .

\section{SAND}

American Society for Testing Materials, standard specifications for gypsum plastering sand, serial designation C35-25, 1925.

1. Sand used for plastering in which gypsum is employed, shall consist of fine granular material, naturally or artificially produced by the disintegration of rock containing not less than 80 per cent by weight of silica, feldspar, dolomite, magnesite, or calcite, and shall be free from saline, alkaline, organic, or other deleterious substances.

2. It shall be graded from fine to coarse, and when dry not more than 6 per cent by weight shall be retained on a No. $8(2,380$-micron) sieve; not less than 80 per cent by weight shall be retained on a No. 50 (297-micron) sieve; and not more than 6 per cent by weight shall pass a No. 100 (149-micron) sieve. These sieves shall conform to the requirements of the Standard Specifications for Sieves for Testing Purposes (serial designation E11, see 500.2) of the American Society for Testing Materials.

American Society for Testing Materials, tentative specifications for sand for use in lime plaster, serial designation C66-27T, 1927.

1. Sand may be defined as follows: 37

Sand.-The fine granular material (usually less than one-fourth inch in diameter) resulting from the natural disintegration of rock, or from the crushing of friable sandstone rocks.

NoTw-- When used withouta qualifying adjective, the term "sand" is generally understood to mean the product of the natural disintegration of siliceous or calcareous rock. Sand should be distinguished from screenings, gravel, etc. The size of particle and other physical from screenings, gravel, etc. The size of particle and other physical material resulting from the crushing of blast-furnace slag is known as material r

2. Sand for lime plaster shall consist of hard, strong, durable, uncoated mineral or rock particles (as limited by the above definition), free from injurious amounts of saline, alkaline, organic, or other deleterious substances.

3. Sand for lime plaster shall be uniformly graded from fine to coarse within the following limits:

Per cent

Retained on a No. 8 (2,380-micron) sieve, not more than......Retained on a No. 30 (590-micron) sieve:

Not mora than

Not less than

Retained on a No, 50 (297-micron) sieve:

Not more than

Not less than

Petained on a No 100 (149-micron) sieve, not less than.--

Weight removed by decantation, not

4. The sieve analysis shall be made in accordance

with A. S. T. M. C41. (See 512.10 , p. 213.)

5. The decantation test shall be made in accordance with A. S. T. M. D136-28. (See 512.13, p. 240.)

6. Sand, when tested in accordance with A. S. T M. C40 (see 512.10 , p. 213) shall show a color not darker than the standard color, unless it is shown by adequatc tests that the impuritics causing the color are not larmful in plaster.

37 As defined by Committee E-8 on Nomenclature and Definition in A. S. T. M. C58-28T. (See 512,10, p. 213.)
American Society for Testing Materials, standard method of mechanical analysis of sand or other fine highway material, except fine aggregates used in cement concrete, serial designation D7-27, 1927.

1. A representative test sample of the aggregate weighing $50 \mathrm{~g}$ shall be taken.

2. The sample shall be dried to constant weight at a temperature not exceeding $110^{\circ} \mathrm{C} .\left(230^{\circ} \mathrm{F}\right.$.).

3. (a) The sample shall be passed through each of the standard sieves specified in Table 1.38

TABLE 1.-Requirements for sieve openings and wire diameter with permissible variations

\begin{tabular}{|c|c|c|c|c|c|c|c|c|}
\hline \multirow{2}{*}{$\begin{array}{l}\text { Mesh designa- } \\
\text { tion, United } \\
\text { States standard } \\
\text { sievo (series No.) }\end{array}$} & \multicolumn{2}{|c|}{$\begin{array}{l}\text { Sieve open- } \\
\text { ing }\end{array}$} & \multicolumn{2}{|c|}{$\begin{array}{l}\text { Wire diam- } \\
\text { eter }\end{array}$} & \multirow{2}{*}{$\begin{array}{l}\text { Toler- } \\
\text { ance } \\
\text { in } \\
\text { over- } \\
\text { age } \\
\text { open- } \\
\text { ing }\end{array}$} & \multicolumn{2}{|c|}{$\begin{array}{l}\text { Tolerance } \\
\text { on wire } \\
\text { diameter }\end{array}$} & \multirow{2}{*}{$\begin{array}{l}\text { Toler- } \\
\text { ance } \\
\text { in } \\
\text { maxi- } \\
\text { mum } \\
\text { open- } \\
\text { ing }\end{array}$} \\
\hline & $\begin{array}{l}\text { Milli- } \\
\text { meters }\end{array}$ & Inch & $\begin{array}{l}\text { Milli- } \\
\text { meter }\end{array}$ & Inch & & Under & Over & \\
\hline $\begin{array}{l}10 \\
20 \\
30 \\
40 \\
50 \\
80 \\
100 \\
200\end{array}$ & $\begin{array}{l}2.00 \\
.84 \\
.59 \\
.42 \\
.297 \\
.177 \\
.149 \\
.074\end{array}$ & $\begin{array}{r}0.0787 \\
.0331 \\
.0232 \\
.0165 \\
.0117 \\
.0070 \\
.0059 \\
.0029\end{array}$ & $\begin{array}{c}0.76 \\
.42 \\
.33 \\
.25 \\
.188 \\
.119 \\
.102 \\
.053\end{array}$ & $\begin{array}{r}0.0299 \\
.0165 \\
.0130 \\
.0098 \\
.0074 \\
.0047 \\
.0040 \\
.0021\end{array}$ & $\begin{array}{c}\text { Per } \\
\text { cent } \\
\pm 3 \\
\pm 5 \\
\pm 5 \\
\pm 5 \\
\pm 6 \\
\pm 6 \\
\pm 6 \\
\pm 8\end{array}$ & $\begin{array}{r}\text { Per } \\
\text { cent } \\
15 \\
15 \\
15 \\
15 \\
15 \\
15 \\
15 \\
15\end{array}$ & $\begin{array}{r}\text { Per } \\
\text { cent } \\
30 \\
30 \\
30 \\
30 \\
35 \\
35 \\
35 \\
35\end{array}$ & \begin{tabular}{|c} 
Per \\
cent \\
10 \\
25 \\
25 \\
25 \\
40 \\
40 \\
40 \\
60
\end{tabular} \\
\hline
\end{tabular}

Note.-The order in which the sieves are to be used in the process of sieving is immaterial and shall be left optional; but in reporting results the order in which the sieves have been used shall be stated.

(b) The percentage by weight retained on each sieve shall be determined and the sicving on each sieve shall be continued until less than 1 per cent of the weight retained on each sieve shall pass through the sieve during the last minute of sieving.

4. The percentages in mechanical analysis shall be reported in the following manner:

Passing the No, 200 sieve

Per cent

Passing the No. 100 sieve and retained on the No. 200 sieve.

Passing the No. 80 sieve and retained on the No. 100 sieve

Passing the No. 50 sieve and retained on the No. 80 sieve.--

Passing the No. 50 sieve and retained on the No. 80 sieve.-.

American Society for Testing Materials, standard specifications for materials for cement grout filler for brick and stone block pavements, serial designation D57-20, 1920.

See 512.16, page 253 .

American Society for Testing Materials, standard specifications for materials for cement mortar bed for brick, stone block, wood block, asphalt block, and other block pavements, serial designation D58-24, 1924.

\section{SAND FOR CEMENT MORTAR CUSHION}

See 518.21, page 368 .

American Society for Testing Materials, tentative specifications for sand for sheet asphalt and bituminous concrete pavements, serial designation D162-29, 1929 .

1. These specifications cover sand for use in the construction of the surface course of sheet asphalt pavements and those types of bituminous concrete pavements in which sand predominates.

2 . The grains of the sand shall be clean, tough, rough surfaced, and angular. The sand after drying shall be free from lumps or balls of clay or of clay and sand. Before being incorporated into

${ }_{38}$ The dimensions and tolerances for these sieves conform to the requirements of A.S. T. M. E11. (See 500.2, p. 1.) 
the paving mixturc it shall conform to the following requirements as to grading:

Passing No. 200 sieve

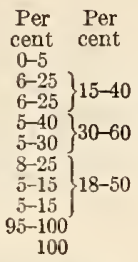

Passing No. 100 sieve, rctained on No. 200

Passing No. 80 sieve, retained on No. 100

Passing No. 50 sieve, retained on No. 80

Passing No. 40 sieve, rotained on No. 50

Passing No. 30 sieve, retained on No. 40

Passing No. 20 sieve, retained on No. 30

Passing No. 10 sieve,

Passing $1 / 3$-inch sereen

3. General characteristics.-The general characteristies shall be determined by examination under a microscope or a magnifying glass.

4. Mechanical analysis.-The tests for the mechanical analysis shall be made in accordance with A. S. T. M. D7 (given above).

5. The method of sampling shall conform to A. S. T. M. D75. (See 510, p. 184.)

Federal Specifications Board, specification No. 460, United States Government master specification for sands for use in sheet asphait or bituminous concrete pavements, January 25, 1927.

This specification is the same as A. S. T. M. D162 (given above), with the following additions:

Sand for binder course:

Passing No. 200 siere, not more tban

Per cent

Passing $1 / 4$-inch screen

6
-
-

Notes.-1. To meet conditions of service and available materials, the grading requirements may be changed by the purchasing department, provided that tbe maximum limits are not increased and tbe minimum limits are not decreased.

2. For binder course the sand shall consist of cloan, bard, durable grains.

Federal Specifications Board, specification No. 461, United States Government master specification for sand for cement grout filler, brick and stone block pavements, January 25, 1927.

The rcquirements of this specification are the same as those of the American Society for Testing Materials D57 (see 512.16, p. 253), except that the requirements for total passing No. 20 and No. 200 sieves are replaced by the requirements for passing No. 50 and No. 100 sieves as given in the recommended specifications of the National Paving Brick Manufacturers' Association.

Mechanical analysis of the sand shall be made in accordance with A. S. T. M. D7. (See 512.12, p. 234.)

Sampling shail be in accordance with A. S. T. M. D75-22. (See 510, p. 184.)

Federal Specifications Board, specification No. 462, United States Government master specification for sand for cement mortar bed for brick, stoneblock, or wood-block pavements, January 25,1927.

The requirements for this specification are the same as those of the American Society for Testing Materials standard specifications for materials for cement grout filler for brick and stone pavements, serial designation D57-20 (see 512.16, p. 253), except for the laboratory sieve requirement, which for this specification is as follows:

Passing $1 / 4$-inch screen

Mechanical analysis of the sand shall be made in accordance with A. S. T. M. D19. (See 512.10, p. 214.)

Sampling shall be in accordance with A. S. T. M.

D75. (See 510, p. 184.)

The Gypsum Institute, gypsum plasters.

Sand.-See 514.3, page 267.

National Paving Erick Manufacturers'Association, recommended specifications for sand filler.

Description. - Joints between the brick shall be filled with clean, dry sand of such sizes that all will pass a No. 12 sicve.

Filiing.-Sand shall be spread upon the surface of the roadway to a depth of not less than one-half inch. It shall then be swept into the joints completely filling them.

Sweeping.--Sand shall be swept into the joints by stiff brushes or by weighted brush drags drawn over the surface of the pavement. An excess of sand shall remain upon the surface.

Opening to traffic.-Immediately after the joints are filled, the roadway may be opened to traffic.

National Sand and Gravel Association, suggested commercial sizes of sand and gravel (tentative).

Sand

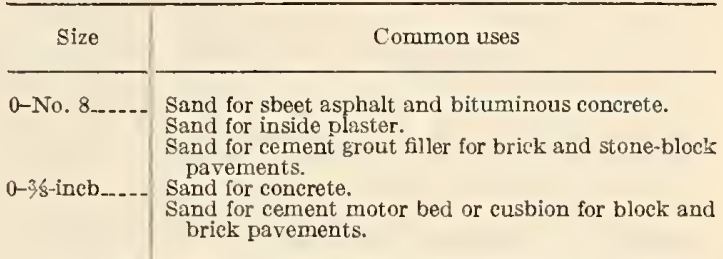

NoTE.-In connection witb sieve sizes, see A. S. T. M. E11, 500.2, p. 1

(For commercial sizes of gravel, see 512.11, p. 219). National Sand and Gravel Association, Bulletin 3, Representative Specifications for Different Uses of Sand and Gravel, November, 1928.

In this bulletin representative specifications for sand and gravel, many of which are presented in this volume, have been summarized.

\subsection{Fine Aggregate}

American Association of State Highway Officials, tentative specifications for highway materials, 1927.

\section{FINE AGGREGATE FOR PORTLAND CEMENT CONCRETE}

1. This specification covers two grades of fine aggregate for cement concrete, namely, grade $A$ and grade $B$. It also covers sand for mortar. The grade of fine aggregate required for the construction shall be as specified.

2. Sand.- Sand for fine aggregate shall consist of clean, hard, durable, uncoated particles, free from lumps of clay, soft or flaky material, loam, and organic matter. In no case shall fine aggregate containing lumps of frozen material be used.

Stone screenings.- Stone screenings, either alone or in comination with sand, shall not be used as fine aggregate except by written permission of the engineer.

Screenings, when used, shall consist of particles resulting from the crushing of clean, tough durable rock having a percentage of wear of not more than 6 per cent. In all others respects they shall conform to the requirements specified for grade A or grade B aggregate.

3. Fine aggregate shall be uniformly graded from coarse to fine, and when tested by means of laboratory sieves shall meet the following requirements:

Grade A

Per cent

Passing $3 / 8$ incb sieve $\ldots \ldots \ldots \ldots+\ldots$

Passing a No. 4 sieve, not less than (note 1)

Passing a No. - sieve (note 2)

Passing a No, 100 sieve ..... 5 Grade B:

Passing $3 / 8$ inch sieve

100

Passing $/ 8$ To. 4 sive

Passing a No - siove (note 2)

Passing No. 50 sieve (note 2) - ...

Passing a No. 100 sieve.

Sand for mortar:

Passing a No. 8 sieve

Passing a No. 50 sieve...

Passing a No, 100 sieve............ 0 
4. Grade A fine aggregate and sand for mortar shall contain not more than 3 per cent, and grade $B$ fine aggregate not more than 5 per cent, of clay and inorganic silt by actual dry weight.

5. All fine aggregate shall be free from injurious organic impurities. Aggregate subjected to the color test for organic impuritics and producing a color in the sodium hydroxide solution darker than the standard color shall be rejected unless subsequent mortar strength or concrete strength tests indicate them to be suitable for use.

6. Fine aggregate shall contain not less than per cent shale and other light weight material.

7. Grade A aggregate.-Grade A fine aggregate, when subjected to the mortar strength test, shall have a tensile or compressive strength, at the age of 7 and 28 days, of not less than 100 per cent of that developed by mortar of the same proportions and consistency, made of the same cement and standard Ottawa sand.

Grade $B$ aggregate and sand for mortar.-Grade B fine aggregate and sand for mortar shall develop a strength ratio, as described above, of not less than 85 per cent.

8. Fine aggregate passing the $3 / 8$-inch sieve, but otherwise failing to meet the requirements of paragraphs 5 or 7 may be used for concrete pavements, or failing to meet the requirements of paragraphs 4,5 , or 7 may be used for bridge structures, provided the proportions arc so adjusted that when tested in combination with the coarse aggregate to be used in the work, the crushing strength at the end of 7 and 28 days will at least equal that of concrete of the same consistency made with the same cement and coarse aggregate in combination with a fine aggregate meeting all of the requirements of paragraphs 3 to 7 , inclusive, and mixed in the proportions specified for the class of concrete under consideration.

9. Sampling and testing of fine aggregate shall be done in accordance with the Tentative Standard Methods of Sampling and Testing of the American Association of State Highway Officials.

NoTE 1.- -There shall be specified a percentage of 85 or more. Note 2.-It is understood that the graduation of the fine aggregate shall be further limited by the insertion of such requirements for intermediate mesh sieves as will insure that aggregates of satisfactory grading will be provided for the work in hand.

American Concrete Institute, tentative purchase specification for concrete aggregates, E-5A-29T, 1929.

\section{SCOPE OF SPECIFICATION}

The purpose of this specification is to provide a standard form for use in writing specifications to govern the purchase of concrete aggregates. It is presumed that the individual user will insert test limits that will meet his conditions as to projected use and characteristics of available materials.

The recommendations for test limits included in the specification are intended to govern the quality and size of aggregates proposed for use in Portland cement concrete for general purposes, and are based primarily upon considerations of strength. Where it is essential that the concrete possess additional characteristics, such as resistance to fire, action of sea water, unusual climatic conditions, etc., such additional requirements for the aggregates as may be found necessary should be inserted in the specifications.

In general, the test limits included show the range within which use is recommended. The individual user, in preparing his specifications, should include specific limits which best meet his local conditions, within the ranges herein recommended.

\section{QUALITY OF AGGREGATES}

It is recognized that for certain purposes satisfactory results may be obtained with materials not conforming to these specifications. In such cases the use of fine and coarse aggregates not conforming to these specifications should be authorized only under special provisions based upon laboratory studies of the possibility of designing a mixture of materials to be used on the job that will yield concrete equivalent to the specified mixture made with material complying with these specifications in all respects.

\section{FINE AGGRTGATE \\ GENERAI CEARACTERISTICS}

1. Fine aggregate shall consist of sand or other approved inert materials with similar characteristics, or a combination thereof, having hard, strong, durable particles, and shall conform to the requirements of these specifications.

\section{DELETERTOUS SUBSTANCES}

2. (a) The maximum percentages of deleterious substances shall not exceed the following values:

Per cent

by weight

Remored by decantation

Coal

Clay lumps

Other local deleterious substances (such as alkali, mica, coated grains, soft and flaky particles)

NoTE.--It is recognized that under certain conditions maximum percentages of deleterious substances less than those shown in the table should be specified.

(b) The sum of the percentages of shale, coal, clay lumps, soft fragments, and other deleterious substances shall not exceed 5 per cent by weight.

(c) All fine aggregate shall be free from injurious amounts of organic impurities. Aggregates subjected to the colorimetric test for organic impurities and producing a color darker than the standard shall be rejected unless they pass the mortar strength tcst as specified in section 4 .

\section{GRADING}

3. (a) Fine aggregate shall be well graded from coarse to fine and when tested by means of laboratory sieve shall conform to the following requirements:

Per cent

Passing a 3/8-inch sieve- 100
Passing a No. 4 sieve

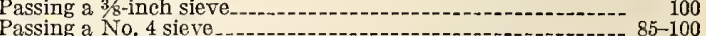
Passing a No. 4 sieve
Passing a No. 16 sieve.-Passing a No. 16 sieve_-_- $\begin{array}{r}45-80 \\ \text { Passing a No } 50 \text { sieve }\end{array}$

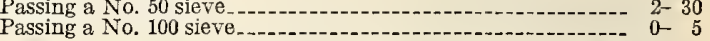

Note.-Figures in parentheses are suggested as limiting percentages but they may be altered within these limits to suit local conditions.

\section{MORTAR STRENGTH}

4. Fine aggregates, when subjected to the mortar strength test, shall have a tensile or compressive strength at the age of 7 and 28 days, equal to or greater than that developed by mortar of the same proportions and consistency made of the same cement and standard Ottawa sand.

\section{CONCRETE STRENGTH}

5. Fine aggregates passing the maximum size sieve rcquirement, but otherwise failing to meet the requirements herein provided for grading or mortar strength, may be used, if, when tested in combination with the coarse aggregate to be used in the work, in the proportion specified for the class of concrete under construction, the crushing or transverse strength of the concrete at the end of 7 and 28 days is at lcast equal to that of concrete of the same proportions and consistency made with the same cement and coarse aggregate in combination with a fine aggregate mecting all the requirements of these specifications. 


\section{COARSE ACGREGATE}

\section{GENERAI CHARACTERISTICS}

6. Coarse aggregate shall consist of crushed stone, gravel, blast-furnace slag, or other approved inert materials of similar characteristics, or combinations thereof, having hard, strong, durable pieces, free from adherent coatings and conforming to the requirements of these specifications.

\section{DELETERIOUS SUBSTANCES}

7. (a) The maximum percentages of deleterious substances shall not exceed the following values:

Per cent hy weight

Removed hy decantation.

Shales

Coal

Clay lumps.

Soft fragments

Other local deleterious suhstances (such as alkali, friahle, thin,

elongated, or laminated pieces)

Note.--It is recognized that under certain conditions maximum percentages of deleterious suhstances less than those shown in the table should he specified.

(b) The sum of the percentages of shale, coal, clay lumps, and soft fragments shall not exceed 5 per cent by weight.

\section{GRADING}

8. (a) Coarse aggregate shall be well graaed, between the limits specified, and shall conform to the following requirements:

Passing - inch sieve (maximum size), not less than--

Per cent by weight

Passing - inch sieve (intermediate size), not less than

(one-half maximum size), not more than

Passing - inch sieve (intermediate sizes), not less than.--.-.

Passing No. 4 sieve, not more than.

Tore - Where a range is shown the engineer should use range engineer should use an appropriate figure within the limits recommended. The figures in local conditions.

\section{CONCRETE STRENGTH}

(b) Coarse aggregate failing to meet the grading requirement may be used, if, when tested in combination with the fine aggregate to be used in the work, in the specified proportions, the crushing or transverse strength of the concrete at the end of 7 and 28 days is at least equal to that of concrete of the same proportions and consistency made with the same cement and fine aggregate in combination with a coarse aggregate meeting all the requirements of these specifications.

\section{WEIGHT OF SLAG}

9. Blast furnace slag that meets the grading requirements of these specifications shall conform to the following minimum weight requirements:

Lhs./ft.

General concrete 65

Concrete subject to abrasion

\section{DURABILITY}

10. Coarse aggregate shall pass a sodium sulphate accelerated-soundness test, except that aggregates failing in the accelerated-soundness test may be used if they pass a satisfactory freezing and thawing test

Note.-Many engineers feel that an abrasion test for coarse aggregato to be used in concrete suhject to ahrasion is important, hut no test limits are specified, due to the status of knowledge concerning suitahle specification limits for this test.

\section{METHODS OF SAMPLING AND TESTING} METHODS OF TESTING

11. The properties enumerated in these specifications shall be determined in accordance with the following methods of test:

(a) Sampling.-A. S. T. M. D75. (See 510, p. 184.) (b) Sieve analysis.-A. S. T. M. C41. (See 512.10 , p. 213.)

c) Decantation test.-A. S. T. M. D136. (See 512.13, p. 240.)

(d) Organic impurities.-A. S. T. M. C40. (See 512.10, p. 213.)

(e) Mortar strength.-U. S. D. A. Bulletin 1216, methods of making compression and tension tests of fine aggregate for concrete. (See 512.13, p. 242.)

(f) Compressive strength.-A. S. T. M. C39. (See 516.0, p. 284.)

(g) Soundness.-U. S. D. A. Bulletin 1216, method of test for soundness of coarse aggregate (sodium-sulphate soundness test). (See 512.14, p. 244.)

(h) Freezing and thawing.-A. S. T. M. C4, (C) Freezing and Thawing Tests of Drain Tile. (See 534.21, p. 610.)

(i) Shale and coal.-U. S. D. A. Bulletin 1216 , method of test for percentage of shale in aggregate. (See 512.14 , p. 243.)

(j) Soft fragments.-U. S. D. A. Bulletin 1216 , method of test for quantity of soft pebbles in gravel. (See 512.11 , p. 220 .)

(l) Weight of slag.-A. S. T. M. C29. (See 512.10 , p. 212.)

(l) Abrasion.-If abrasion tests are made the following methods of tests are recommended:

(1) Abrasion of gravel.-U. S. D. A. Bulletin 1216. (See 512.11, p. 219.)

(2) Abrasion of rock or crushed slag.-A. S. T. M. D2 (see 510, p. 182) except that for specific gravities lower than 2.2 , a 4,000 g sample shall be used.

American Railway Engineering Association, specifications for buildings for railway purposes, 1926 .

\section{FINE AGGREGATE}

See specification for concrete, 516.3, page 303 .

American Society for Municipal Improvements, 1922.

\section{FINE AGGREGATE}

(See A. S. M. I. specification for fine aggregate asphaltic concrete paving, 518.37 , p. 399.)

American Society for Testing Materials, tentative specifications for concrete aggregates, serial designation C33-28T, 1928 .

\section{QUALITY OF AGGREGATES}

It is recognized that for certain purposes satisfactory results may be obtained with materials not conforming to these specifications. In such cases the use of fine and coarse aggregates not conforming to these specifications should be authorized only under special provisions based upon laboratory studies of the possibility of designing a mixture of materials to be used on the job that will yield concrete equivalent to the specified mixture made with material complying with these specifications in all respects.

\section{FINE AGGREGATE}

1. Fine aggregate shall consist of sand or other approved inert materials with similar characteristics, or a combination thereof, having hard, strong, durable particles and shall conform to the requirements of these specifications.

2. (a) The maximum percentages of deleterious substances shall not exceed the following values:

Por cont by weight

Removed hy decantation

Shale

Clay lumps

Other local deleterious substances (such as alkali, mica, coated grains, soft and flaky particles)

Note.-It is recognized that under certain conditions maximum percentages of deleterious substances less than those shown in the tahle should he specified. 
(b) The sum of the percentages of shale, coal, clay lumps, soft fragments, and other deleterious substances shall not exceed 5 per cent by weight.

(c) All fine aggregate shall be free from injurious amounts of organic impurities. Aggregates subjected to the colorimetric test for organic impurities and producing a color darker than the standard shall be rejected unless they pass the mortar strength test as specified in section 4 .

3. (a) Fine aggregate shall be well graded from coarse to fine and when tested by means of laboratory sicves ${ }^{3 \theta}$ shall conform to the following requirements:

Passing a $3 / 8$-inch sieve

Passing a No. 4 sieve

Passing a No. 16 sieve

Passing a No. 50 sieve

Per cent

Passing a No. 100 sieve

NoTE-Figures in parentheses are suggested as limiting percentages, but they may be altered within these limits to suit local conditions.

(b) In case the concrete resulting from a mixture of aggregates approaching the extreme limits for gradation is not of a workable character, or when finished does not exhibit a proper surface, due to an excess of particles approximately one-eighth to one-half inch in size, either a fine aggregate having a sufficiently greater percentage of fine material, or a coarse aggregate having a sufficiently smaller percentage of fine naterial must be used.

4. Fine aggregates, when subjected to the mortar strength test, shall have a tensile or compressive strength at the age of 7 and 28 days of not less than (100) ${ }^{40}$ per cent of that developed by mortar of the same proportions and consistency made of the same cement and standard Ottawa sand.

\section{COARSE AGGREGATE}

5. Coarse aggregate shall consist of crushed stone, gravel, blast-furnace slag, or other approved inert materials of similar characteristics, or combinations thereof, having hard, strong, durable pieces, frce from adherent coatings and conforming to the requirements of these specifications.

6. (a) The maximum percentages of deleterious substances shall not exceed the following values:

Per cent by weight

Removed by decantation by weight

Coal.

Clay lumps

Soft fragments

delcterious substances (such as alkali, friable, thin --

ingated, or laminated pieces)

Note.-It is recognized that under certain conditions maximum percentages of deleterious substances less than those shown in the table should be specified.

(b) The sum of the percentages of shale, coal, clay lumps, and soft fragments shall not exceed 5 per cent by weight.

7. (a) Coarse aggregate shall be well graded, between the limits specified, and shall conform to the following requirements

\begin{tabular}{|c|c|c|}
\hline \multicolumn{3}{|c|}{ Per cent } \\
\hline size) & Not less than & 95 \\
\hline $\begin{array}{l}\text { ssing - meh sieve }{ }^{11} \text { or sereen: } \\
\text { Intermediate size }\end{array}$ & han & \\
\hline One-half maximum size & Not more than.- & \\
\hline $\begin{array}{l}\text { ssing - inch sieve or screen: } \\
\text { Intermediate sizes.-. }\end{array}$ & & \\
\hline $\begin{array}{l}\text { Intermediate sizes............ } \\
\text { As needed. }\end{array}$ & Not les & \\
\hline $\begin{array}{l}\text { As needed } \\
\text { ssing No. } 4 \text { sieve or a } 1 / 4 \text {-inch sercen }\end{array}$ & Not more than -- & \\
\hline
\end{tabular}

39 For detail requirements for these sieves, see A. S. T. M. E11, $500.2, \mathrm{p}, 1$.

500.2 Percentages in parentheses are recommended, but they may be ${ }^{40}$ Percentages in parentheses
altered to suit local conditions.

${ }_{41}$ The question whether round or square openings shall be used for testing aggregates is now being studied by the section on coarse screens of the Technical Committce on Size and Shape of Committee $\mathbf{E}_{-1}-1$ on Methods of 'Testing.
NoTE.-Where a range is shown the engineer should use an appropriate figure within the limits recommended. The percentages in parentheses are recommended but may need to be altered to suit local conditions.

(b) In case the concrete resulting from a mixture of aggregates approaching the extreme limits for gradation is not of a workable character, or when finished does not exhibit a proper surface, due to an excess of particles approximately one-eighth to onehalf inch in size, either a fine aggregate having a sufficiently greater percentage of fine material, or a coarse aggregate having a sufficiently smaller percentage of fine material, shall be used.

8. Blast-furnace slag that meets the grading requirements of these specifications shall conform to the following minimum weight requirements

Base concrete Lbs./tt. ${ }^{3}$ Surface conerete (subject to abrasion)

9. Coarse aggregate shall pass a sodium sulphate accelerated soundness test, except that aggregates failing in the accelerated soundness test may be used if they pass a satisfactory freezing and thawing test.

NoTE.-Many engineers feel that an abrasion test for coarse aggregate to be used in concrete suhject to abrasion is important, but no test limits are specified, due to the status of know ledge concerning suitable specification limits for this test.

\section{METHOD OF SAMPLING AND TESTING}

10. The properties enumerated in these specifications shall be determined in accordance with the following methods of test of the American Society for Testing Materials, except as specified in paragraph $(e)$.

(a) Sampling.-A. S. T. M. D75. (See 510, p. 184.

(b) Sieve analysis.-A. S. T. M. C41. (See 512.10, p. 213.$)$

(c) Decantation test.-A. S. T. M. D136. (See 512.13, p. 240.)

(d) Organic impurities.-A. S. T. M. C40. (See 512.10, p. 213.)

(e) Mortar strength.-Methods of making Compression and Tension Tests of Fine Aggregate for Concrete, United States Department of Agriculture, Bulletin 1216, see page 242 .

(f) Compressive strength.-A. S. T. M. C39. (See 516.0, p. 284 .)

(g) Soundness.-Proposed Method of Test for soundness of coarse aggregate (Sodium Sulphate Soundness Test). Appendix II to 1928 Report of A. S. T. M. Committee C-9 on Concrete and Concrete Aggregates.

(h) Freezing and thawing.-Method of freezing and thawing tests for drain tile as described in A. S. T. M. C4. (See 534.21, p. 610.)

(i) Shale and coal.-Proposed Method of Test for Percentage of Shale in Aggregate, Appendix III to 1928 report of Committee C 9 on Concrete and Concrete Aggregates. ${ }^{42}$

(j) Soft fragments.-Proposed Method of Test for Quantity of Soft Pebbles in Gravel, Appendix IV to 1928 Report of Committee C-9 on Concrete and Concrete Aggregates. ${ }^{42}$

(k) Coal and lignite.-Proposed Method of Test for Determination of Coal and Lignite in Sand, Appendix $V$ to 1928 Report of Committee C-9 on Concrete and Concrete Aggregates.12

(l) Moisture.-A. S. T. M. C70-28T. (See 512.13, p. 240.)

$(m)$ Consistency.-A. S. T. M. D138-26T. (See 516.0$, p. 286.$)$

(n) Weight of slag.-A. S. T. M. C29. (See 512.10, p. 212.)

(o) Abrasion.-If abrasion tests are made the following methods of test are recommended:

42 Proceedings, Am. Soc. Testing Mats., 28, p. 1; 1928. 
(1) Abrasion of gravel.-U. S. D. A. Bulletin 1216. (See 512.11, p. 219.)

(2) Abrasion of rock or crushed slag.-A. S. T. M. D2. (See 510, p. 183, (except that for specific gravities lower than 2.2 a $4000 \mathrm{~g}$ sample shall be used).)

American Society for Testing Materials, tentative method of test for field determination of approximate apparent specific gravity of fine aggregate, serial designation C68-28T, 1928.

1. This method of test is intended for use in the field for making approximate determinations of the apparent specific gravity of fine aggregate. The range of the apparatus is between specific gravities of 2.2 and 2.85 .

2. The apparatus shall consist of the following:

(a) Balance. - A balance, preferably of the torsion type, having a capacity of $2 \mathrm{~kg}$ or more and sensitive to $0.5 \mathrm{~g}$ or less.

(b) Flask.-A special graduated flast of the type, and conforming to the dimensions, shown in Figure 95.

3. A $1-\mathrm{kg}$ sample shall be selected which shall be as truly representative of the fine aggregate as possible. It shall be spread out on a flat surface and air dried until the surface moisture has evaporated and the sample is free flowing. The sample shall then be thoroughly mixed and $500 \mathrm{~g}$ accurately weighed out.

4. The graduated flask shall be filled to the 200-ce mark on the lower neck with water at room temperature. The 500-g sample of fine aggregate shall then be slowly poured into the flask, and the flask and contents agitated while introducing the agrregate to free any entrained air bubbles. The combined volume in cubic centimeters of the water and fine aggregate shall be read on the scale on the upper neck of the flask.

5. The approximate apparent specific gravity of the fine aggregate may be calculated from the formula:

$$
\text { A pprozimate apparent specific gravity }=\frac{500}{V-200}
$$

where $V=$ the combined volume in cubic centimeters of the water and fine aggregate in the flask.

6. Duplicate determinations should check to within 0.05 . Variations in the amount of water evaporated from the moist sample as described in section 3 beyond the point at which the surface moisture has disappeared and the sample is free flowing and apparently dry will cause variations in results of from 0.5 to 1 per cent, depending upon the absorption of the aggregate.

American Society for Testing Materials, tentative method of test for field determination of approximate percentage of voids in fine aggregate, serial designation C69-28T, 1928.

1. This method of test is intended for use in the field for making approximate determinations of the percentage of voids in fine aggregate when inundated with water.

2. The apparatus shall consist of the following:

(a) Balance.-A balance, preferably of the torsion type, having a capacity of $2 \mathrm{~kg}$ or more and sensitive to $0.5 \mathrm{~g}$ or less.

(b) Flask. - A special graduated flask of the type, and conforming to the dimensions, shown in Figure 95.

3. A sample weighing about $2 \mathrm{~kg}$ shall be selected which shall be as truly representative of the fine aggregate as possible. It shall be well mixed and spread out on a fiat surface and air dried until the surface moisture has evaporated and the sample is free flowing.

4. About $100 \mathrm{cc}$ of water shall be placed in the flask. Aggregate from the room-dry sample shall then be introduced slowly into the flask until the flask is filled to the 400-ce mark on the upper neck.
Water shall be added as required to keep the aggregate inundated as the filling proceeds. When the flask is filled to the 400-ce mark, both water and aggregate shall be at the same level. The aggregate in the flask shall not be agitated during or after the filling operation. The flask and contents shall be weighed to the nearest gram, and the weight of the flask shall be deducted, unless its weight has been counter balanced on the scale at the time of weighing.

5. (a) This method requires that the apparent specific gravity be known of the fine aggregate in a room-dry, free-flowing condition, but with the voids within the particles of the aggregate containing moisture. If the apparent specific gravity of the

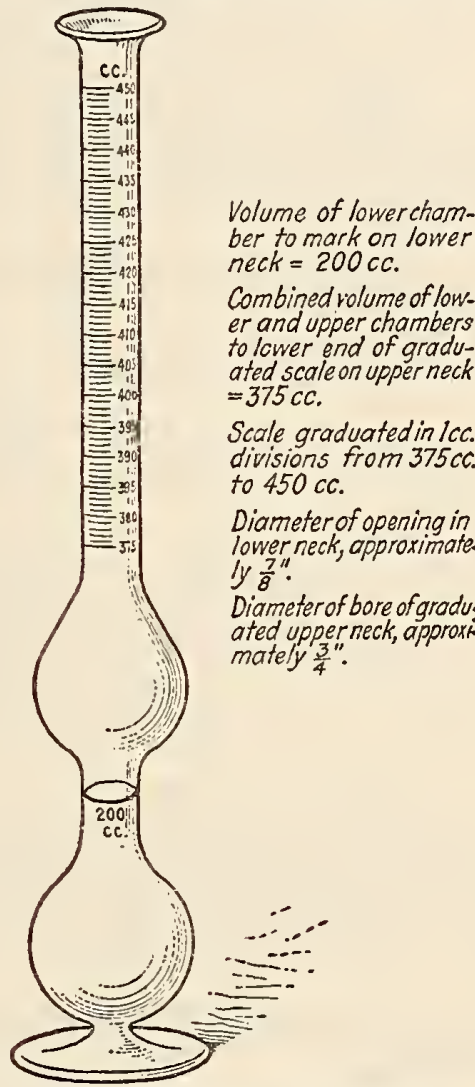

Figure 95.-Graduated flask for field testing of fine aggregate

aggregate in this condition is not known it shall be determined in accordance with A. S. T. M. C 68 , (above).

(b) The approximate percentage of voids in the inundated fine aggregate may be calculated from the formula:

$$
V=\frac{400 \times \text { sp. gr. }-W}{400(\text { sp.gr. }-1)} \times 100
$$

where

$V=$ the approximate percentage of voids sp. gr.=apparent specific gravity of the fine aggregate

$W=$ combined weight in grams of 400 ec of water and fine aggregate in the flask.

6. Duplicate determinations should check to within 1 per cent. Variations in the composition of the rock grains of the fine aggregate which result in variations of 0.05 in the approximate apparent specific gravity of the aggregate will cause an inac- 
curacy in results equal to from 2 to 3 per cent of voids.

American Society for Testing Materials, tentative method of test for field determination of surface moisture in fine aggregate, serial designation C70-28'T, 1928.

1. This method of test is intended for use in the field for making approximate determinations of the percentage of surface moisture in fine aggregate for use in computing the allowance to be made for water in the fine aggregate when proportioning concrete by the maximum water content method. The range of the apparatus is between the specific gravities of 2.2 for aggregate containing 10 per cent moisture and 2.85 for dry aggregate.

This method determines only surface moisture; that is, moisture on the outside of the particles. The moisture absorbed within the particles does not add to the volume of the particles and, therefore, does not make itself evident in this test.

2. The apparatus shall consist of the following:

(a) Balance.-A balance, preferably of the torsion type, having a capacity of $2 \mathrm{~kg}$ or more and sensitive to $0.5 \mathrm{~g}$ or less.

(b) Flask.-A special graduated flask of the type, and conforming to the dimensions, shown in Figure 95, page 239.

3. A sample weighing about $1 \mathrm{~kg}$ shall be selected which shall be as truly representative of the fine aggregate as possible. It shall be well mixed and spread out on a flat nonabsorbent surface. Five hundred grams shall be immediately weighed out, permitting moisture to evaporate as little as possible from the sample.

4. The graduated flask shall be filled to the 200 -cc mark on the lower neck with water at room temperature. The 500-g sample of damp aggregate shall then be slowly poured into the flask, and the contents of the flask agitated or stirred to free any entrained air bubbles. The combined volume, in cubic centimeters, of the water and fine aggregate shall be read on the scale on the upper neck of the flask.

5. (a) This method requires that the apparent specific gravity be known of the fine aggregate in a room-dry, free-flowing condition, but with the voids within the particles of the aggregate containing moisture. If the apparent specific gravity of the aggregate in this condition is not known it shall be determined in accordance with A. S. T. M. C68 (above).

(b) The percentage of surface moisture in the fine aggregate (air-dry basis) may be calculated from the formula:

$$
\text { Percentage of surface moisture }=\frac{V-\frac{500}{\text { sp. gr. }}-200}{200+500-V} \times 100
$$

where $V=$ the combined volume in cubic centimeters of the water and fine aggregate in the flask.

sp. gr.=Approximate apparent specific gravity on the fine aggregate.

6. Duplicate determinations should check to within 0.5 per cent. Variations in the composition of the rock grains of the fine aggregate which result in variations of 0.05 in the approximate apparent specific gravity of the aggregate will cause inaccuracy in results equal to from 1 to 1.5 per cent of moisture.

American Society for Testing Materials, standard method of decantation test for sand and other fine aggregates, serial designation D136-28, 1928.

1. This method of test covers the determination of the total quantity of silt, loam, clay, etc., in sand and other fine aggregates. ${ }^{43}$

43 This determination of the percentage of silt, clay, loam, etc., will include all water-soluble material present, the percentage of which may be determined separately if desired.
2. The pan or vessel to be used in the determination shall be approximately 9 inches $(230 \mathrm{~mm})$ in diameter and not less than 4 inches $(102 \mathrm{~mm})$ in depth.

3. The sample must contain sufficient moisture to prevent segregation and shall be thoroughly mixed. A representative portion of the sample sufficient to yield approximately $500 \mathrm{~g}$ of dried material, shall then be dried to a constant weight at a temperature not exceeding $110^{\circ} \mathrm{C}$. $\left(230^{\circ} \mathrm{F}\right.$.).

The dried material shall be placed in the pan, and sufficient water added to cover the sample (about $225 \mathrm{cc})$. The contents of the pan shall be agitated vigorously for 15 seconds, and then be allowed to settle for 15 seconds, after which the water shall be poured off, care being taken not to pour off any sand. This operation shall be repeated until the wash water is clear. As a precaution, the wash water shall be poured through a No. 200 sieve and any material retained thereon returned to the washed sample. The washed sand shall be dried to a constant weight at a temperature not exceeding $110^{\circ} \mathrm{C}$. $\left(230^{\circ} \mathrm{F}\right.$.) and weighed.

5. The results shall be calculated from the formula:

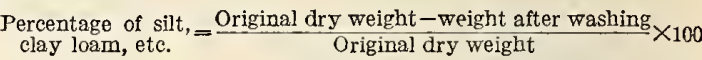

6. When check determinations are desired, the wash water shall be evaporated to dryness, the residue weighed, and the percentage calculated from the formula:

$$
\text { Percentage of silt, loam, clay, etc. }=\frac{\text { Weight of residue }}{\text { Original dry weight }} \times 100
$$

Federal Specifications Board, specification No. 455, United States Government master specification for broken stone, broken slag, or gravel for bituminous surface treatment, January 25, 1927.

\section{GENERAL REQUIREMENTS}

1. Broken stone shall consist of clean, tough, durable fragments, free from an excess of soft or disintegrated particles, dirt, or other objectionable matter.

2. Broken slag shall be air-cooled blast-furnace slag, and shall consist of angular fragments reasonably uniform in density and quality, and reasonably free from glassy pieces, dirt, or other objectionable matter.

3. Gravel shall consist of clean, hard, tough, durable stone particles, and shall be free from soft or disintegrated particles, dirt, or other objectionable matter.

\section{DETAIL REQUIREMENTS}

1. The broken stone, broken slag, or gravel, when tested by means of laboratory screens, shall meet the following requirements for size as designated:

(a) One-fourth to three-fourth inch size (broken stone or broken slag):

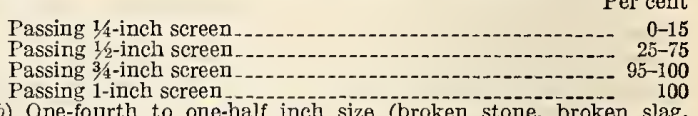
or gravel):

Passing 1/4-inch screen

Per cent

Passing 1/2-inch screen $0-15$

(100

Note.-The materials shall be supplied in the particular type(s) and size(s) ordered.

3. Sampling and testing.

Sampling. A. S. T. M. D75. (See 510, p. 184.)

Mechanical analysis.-A. S. T. M. D18. (See 512.15 , p. 250.) 
Federal Specifications Board, specification No. 464, United States Government master specification for fine aggregate for Portland cement concrete pavement or base, January 25, 1927.

\section{GRADES}

The materials covered by this specification shall be supplied in the particular grade or grades ordered by the purchasing department.

Grade A.-Fine aggregate for Portland cement concrete pavement or base.

Grade B.-Fine aggregate for Portland cement concrete base only.

\section{MATERIAL}

Fine aggregate shall consist of sand, or, subject to approval, other inert materials with similar characteristics, or combinations thereof.

\section{GENERAL REQUIREMENTS}

Fine aggregate shall consist of clean, hard, strong, durable uncoated grains, free from injurious amounts of dust, lumps, soft or flaky particles, shale, alkali, organic matter, loam, or other deleterious substances.

\section{DETAIL REQUIREMENTS}

1. Fine aggregate shall be well graded from coarse to fine, and when tested by means of laboratory screens and sieves, shall meet the following requirements:

Passing No. 100 sieve

Passing 1/4-inch screen

Ping $1 / 2$-inch screen.

2. Fine aggregate shall meet the following requirements for grades as designated:

\begin{tabular}{|c|c|c|}
\hline & $\left|\begin{array}{c}\text { Grade A, } \\
\text { parement } \\
\text { or base }\end{array}\right|$ & $\begin{array}{l}\text { Grade } \mathbf{B}, \\
\text { base only }\end{array}$ \\
\hline $\begin{array}{l}\text { Silt, loam, clas, etc., removed by decantation, } \\
\text { not more than. }\end{array}$ & $\begin{array}{r}\text { Per cent } \\
3.0\end{array}$ & $\begin{array}{r}\text { Per cent } \\
5.0\end{array}$ \\
\hline
\end{tabular}

3. Fine aggregate shall be of such quality that mortar briquettes made with 1 part by weight of Portland cement to 3 parts by weight of fine aggregate prepared and tested in accordance with the methods described in the standard specifications for Portland cement will show a tensile strength at the age of 7 and 28 days, as compared to the tensile strength developed in the same time by mortar of the same consistency made of 1 part of the same cement and 3 parts of Ottawa sand, for grades as designated:

\begin{tabular}{|c|c|}
\hline $\begin{array}{c}\text { Grade A, pavement or } \\
\text { base }\end{array}$ & Grade B, base only \\
\hline $\begin{array}{c}\text { Not less than (see Sec. } \\
\text { VI) - per cent. }\end{array}$ & $\begin{array}{c}\text { Not less than (see Sec. } \\
\text { VI) - per cent. }\end{array}$ \\
\hline
\end{tabular}

4. All fine aggregate shall be free from injurious organic impurities. Aggregates subjected to the color test for organic impurities and producing a color darker than the standard color shall be rejected unless subsequent mortar strength or concrete strength tests indicate them to be suitable for use.

\section{METHODS OF SAMPLING AND TESTING}

1. Methods of sampling shall be in accordance with A. S. T. M. D75. (See 510, p. 184.)

2. Tests of fine aggregate shall be made in accordance with the following methods: (a) Mechanical analysis.-A. S. T. M.: C41-24 (see 512.10, p. 213), using a $1 / 4$-inch screen instead of a No. 4 sieve as specified in A. S. T. M. method and United States standard sieves meeting the requirements of A. S. T. M. E11 (see 500.2, p. 1).

(b) Decantation test--A. S. T. M. D136-28 (Sce 512.13, p. 240.)

(c) Color-organic impurities.-A. S. T. M. C40-27. (See 512.10, p. 213.)

\section{NOTE}

The percentage referred to in Section IV, 3, should be inserted by the authorized representative of the department. The requirement will vary with the type of work and the characteristics of the available materials. It should preferably be 100 when the fine aggregate is to be used in the wearing course, and at least 85 when the fine aggregate is to be used in the base course. If fine aggregate meeting the suggested requirements for mortar strength can not be obtained, available material othervise complying with this specification may be used, provided the proportions can be so adjustcd that concrete of equal quality will be produced to that which would be secured by the use of a fine aggregate meeting the above recommcnded requirement for mortar strength. The moltar strength which will be developed by the fine aggregate available should be inserted in Section IV, 3, for the purpose of future control.

National Sand and Gravel Association, bulletin 3, representative specifications for different uses of sand and gravel, November, 1928.

In this bulletin representative specifications, many of which are presented in this volume, and including specifications for fine aggregate, have been suminarized.

United States Department of Agriculture, Bulletin 1216, method of mechanical analysis of extracted aggregates, 1928.

1 . The mineral aggregate left after extraction of the bituminous material shall be dried at not over $110^{\circ}$ C. $\left(230^{\circ}\right.$ F.) to a constant weight. It shall be separated by the use of a screen having openings one-quarter inch in diameter. The portion retained on the screen shall be examined in accordance with the method for mechanical analysis of broken stone, etc. The portion passing this screen shall bc examined in accordance with the method for the mechanical analysis of sand or other fine aggregate (see A. S. T. M. D7, 512.12, p. 234), except that the percentage of $200-$ mesh material shall be dctermined as follows: A 10-mesh sieve shall be superimposed on a 200-mesh sieve and the aggregate placed on the 10 -mesh sieve. The material is then immersed and gently oscillated in a bath of gasoline until all fine material has been washed off. The washed material is then dricd and weighed. The weight of material passing the 200-mesh sieve may be obtained as follows:

Weight of total mineral aggregate (including weight of mineral material recovered from extract) minus weight of material retained on sieve gives weight of mineral matter passing 200-mesh sieve.

2 . The mechanical analysis shall be recorded in the following manner:

Passing 200-mesh siove... Per cent

80 Passing 40 -mesh sieve and retained on 200-mesh sieve-.....

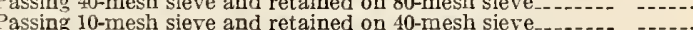
Passing $1 / 4$-inch screen and retained on 10 -mesh sieve Passing 1,5 -inch screen and retained on 1,4 -inch screen-.-.-Passing $3 / 4$-inch screon and retained on $1 / 2$-inch screen.......

3. In the sieve analysis of the sand fraction the following sizes of sieves shall be used: 10-mesh, 40-mesh, 80-mesh, and 200-mesh. 
United States Department of Agriculture, Bulletin 1216, methods of making compression and tension tests of fine aggregate for concrete, 1928.

1. Fine aggregate shall be tested for strength either by tension or compression in a mortar proportioned either by volume or by weight, using a 1:3 mix.

2. Mortars made of natural sand shall be compared to mortars made with the same cement and standard Ottawa sand mixed in the same proportion and of the same consistency.

\section{(A) TENSION TEST}

3. The briquets for the tension test shall be molded in the standard mold as used for testing Portland cement. Single or gang molds may be used. They shall be wiped with an oily cloth before using.

4. Immediately after mixing, the mortar shall be placed in the molds, pressed in firmly with the thumbs, and smoothed off with a trowel without ramming. Additional mortar shall be heaped above the mold and smoothed off with a trowel; the trowel shall be drawn over the mold in such a manner as to exert a moderate pressure on the material. The mold shall then be turned over and the operation of heaping, thumbing, and smoothing off repeated.

5. Tests shall be made with any standard machine. The briquets shall be tested as soon as they are removed from the water. The bearing surfaces of the clips and briquets shall be free from grains of sand or dirt. The briquets shall be carefully centered, and the load applied continuously at the rate of 600 pounds per minute.

6. Testing machines shall be calibrated frequently in order to determine their accuracy.

7. Briquets that are manifestly faulty, or which give strengths differing more than 15 per cent from the average value of all test pieces made from the sample and broken at the same period, shall not be considered in determining the tensile strength.

\section{(B) COMPRESSION TEST}

8. A cylindrical test piece 2 inches in diameter and 4 inches in length shall be used in making the compression test. The molds (single or gang molds) shall be made of noncorroding metal. The ends of the mold shall be parallel. They shall be oiled before using. During the molding of the test piece, the mold shall rest on a clean, plane surface (preferably a piece of plate glass which is allowed to remain in place until the mold is removed).

9. The mortar shall be placed in the molds in four layers about 1 inch in thickness, each layer being thoroughly compacted, but not rammed by a steel tamper. The steel tamper shall have a total length of about $6 \frac{1}{2}$ inches. It shall be 1 inch in diameter for $1 \frac{1 / 4}{4}$ inches with the handle $5 \frac{1}{4}$ inches long and five-eighths inch in diameter. It shall weigh approximately three-quarters of a pound. The surface of each layer shall be roughened before the addition of the next layer. In compacting the test pieces no mixing water shall be forced out of the mortar. In finishing the test piece, mortar shall be heaped above the mold and smoothed off with a trowel. As soon as the test pieces from one sample are molded, the top of each mold shall be covered with a piece of glass which shall be brought to a firm bearing on the fresh mortar. The cover glasses shall remain in place until the molds are removed.

10. Tests of mortar cylinders shall be made in any testing machine which is adapted to meet the specified requirements. The test pieces shall be broken as soon as they are removed from the water. The ends of the test cylinders shall be smooth, plane surfaces. The metal bearing plates of the testing machine shall be placed in direct contact with the ends of the test piece. During the test a spherical bearing block shall be used on top of the cylinder. In order to secure a uniform distribution of the load over the test cylinder, the spherical block must be accurately centered. The diameter of the spherical bearing block should be only a little greater than that of the test piece. The test piece shall be loaded continuously to failure. The moving head of the testing machine shall travel at the rate of not less than 0.05 or more than 0.10 inch per minute.

11. Testing machines should be frequently calibrated in order to determine their accuracy.

12. Cylinders that are manifestly faulty, or which give strengths differing more than 15 per cent from the average value of all test pieces tested at the same period and made from the same sample, shall not be considered in determining the compressive strength.

\section{(C) STORAGE OF TEST PIECES}

13. The moist closet may consist of a soapstone, slate, or concrete box, or a wooden box lined with metal. If a wooden box is used, the interior should be covered with felt or broad wicking kept wet. The bottom of the moist closet should be covered with water. The interior of the closet should be provided with nonabsorbent shelves on which to place the test pieces, the shelves being so arranged that they may be withdrawn readily.

14. Unless otherwise specified, all test pieces, immediately after molding, shall be placed in the moist closet for from 20 to 24 hours.

15. Cylinders or briquets shall be kept in molds on glass plates in the moist closet for at least 20 hours. After 24 hours in moist air, the briquets or cylinders shall be immersed in clean water in storage tanks of noncorroding material.

16. The air and water shall be maintained as nearly as practicable at a temperature of $21^{\circ} \mathrm{C} .\left(70^{\circ} \mathrm{F}.\right)$.

NoTE. - It is recommended that the laboratory method of proportioning fine aggregate, viz, by weight or by volume, for either the compression or tension test shall be the same as the method used in proportioning concrete in the field. When the mortar is proportioned by volume the required quantity of fine aggregate and cement shall be determined from the unit weight of the material.

Example:

Weight of sand, 100 pounds per cubic foot.

Weight of cement, 94 pounds per cubic foot.

Required, 75 cubic inches of mortar.

Quantity of sand, $\frac{75}{1728} \times 100 \times 453.6=1,969 \mathrm{~g}$.

Quantity of cement, $\frac{25}{172 \mathrm{~S}} \times 94 \times 453.6=617 \mathrm{~g}$.

\subsection{Coarse Aggregate.}

American Association of State Highway Officials, standard specifications for highway bridges and incidental structures, 1928.

\section{COARSE AGGREGATE}

\section{GENERAI}

Crushed stome and screened gravel.-Broken stone or gravel shall consist of uncoated particles of clean, hard, tough, durable rock. It shall contain no organic or other deleterious matter and shall be free from lumps of clay and soft pieces.

\section{SAMPLING AND TESTING}

Sampling and testing of coarse aggregate shall be done in accordance with the methods provided in the Tentative Standard Methods of Sampling and Testing of the American Association of State Highway Officials.

Sampling shall be done by the engineer sufficiently in advance of the required date for acceptance or rejection of the material to allow time for the necessary tests. Additional samples shall be 
sccured and tested at such times as the engineer may consider necessary. Samples shall weigh not less than 50 pounds.

\section{GRADING}

Coarse aggregate shall be uniformiy graded between the limits specified and shall meet the following requirements:

Per cent

Passing a - inch siere "4

95-100

Passing a - irch sieve :

Passing a No. 4 sieve

\section{SOUNDNESS}

Coarse aggregates shall pass the test for soundness. Samples showing disintegration shall be considered to have failed in this test.

American Association of State Highway Officials, standard specifications for highway bridges and incidental structures, 1928.

\section{RUBBLE OR CYCLOPEAN AGGREGATE}

One-man and derrick stone used in rubble or cyclopean concrete shall consist of tough, sound, and durable rock. The stone shall be frce from coatings, drys, seams, or flaws of any character. In general, the percentage of wear shall be not greater than 6 per cent as determined by A. S. T. M. D2. (See 510, p. 182.)

Preferably stone shall be angular in shape and shall have a rough surface such as will thoroughly bond with the surrounding mortar.

American Concrete Institute, tentative purchase specifications for concrete aggregates, E-5A-29T, 1929.

(See 512.13, p. 236.)

American Railway Engineering Association, specifications for buildings for railway purposes, 1926.

Coarse aggregate. (See A. R. E. A. specification for concrete, 516.3 , p. 303.)

American Society for Municipal Improvements, standard specifications for sewers, 1927.

Coarse aggregate. (See 518.67 , p. 453.)

American Society for Municipal Improvements, standard specifications for fine aggregate asphaltic concrete paring, 1922.

Coarse aggregate for use in paving. (See A. S. M. I. specification, 518.37 , p. 399 .)

American Society for Testing Materials, tentative specifications for concrete aggregates, serial designation C33-2ST, 1926.

Coarse aggregate. (See 512.13, p. 237.)

Federal Specifications Board, specification No. 454, United States Government master specification for coarse aggregate for Portland cement concrete pavement or base (stone, slag, or gravel).

The material covered by this specification shall be supplied in the particular size and type ordered, depending on the commercial sizes available.

\section{GENERAL REQUIREMENTS}

1. Broken stone shall consist of clean, tough, durable fragments, free from an excess of flat, elongated, soft, оr disintegrated pieccs, dirt, or other objectionable matter.

2. Broken slag sliall be air-cooled blast-furnace slag, and shall consist of angular fragments, reasonably uniform in density and quality, and reasonably free from thin, elongated, or glassy pieces, dirt, or other objectionable matter.

41 It is understood that the gradation of the coarse aggregate will be limited by the insertion of a marimum and intermediate size sieve which will insure that aggregates of satisfactory grading will be provided for the work in hasd. The maximum sizes of aggregstes for different classes of construction shall be as specifed under "Concrete masonry," Division III.
3. Gravel shall consist of clean, hard, tough, and durable particles of gravel, free from soft, thin, elongated, or laminated pieces, vegetable, or other extraneous deleterious matter.

\section{DETAIL REQUIPEMENTS}

(a) The broken stone shall have a percentage of wear of not more than 7.0 .

(This test value for quality of stone is suggested as the lowest safe requirement. With favorable conditions of available materials the maximum pcrcentage of wear permitted should be rcduced.)

(b) Broken slag of each size specified shall weigh not less than 70 pounds per cubic foot.

(c) The material shall be well graded between the limits specified, and when tested by means of laboratory screens and sieves shall meet the following requirements for size as designated in the following table:

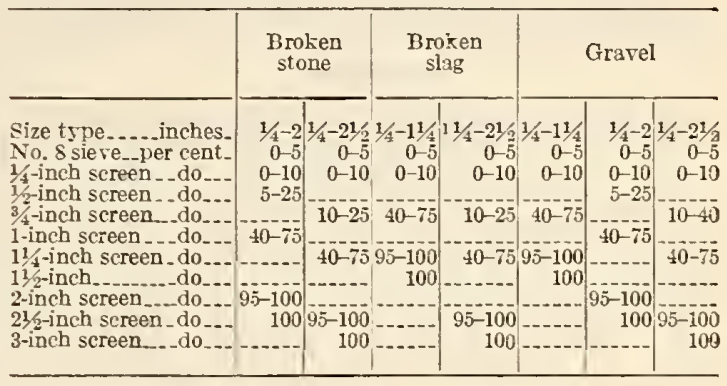

For use in base only.

\section{METHODS OF SAMPLING AND TESTING}

1. Methods of sampling shall be in accordance with A. S. T. M. standard method D75. (See 510., p. 184.)

2. Tests of coarse aggregate shall be made in accordance with the following methods:

(a) Percentage of wear: A. S. T. M. standard method D2. (See 510., p. 1S2.)

(b) Weight per cubic foot: A. S. T. M. standard method C9. (See 516.11, p. 2S7.)

(c) Mechanical analysis: A. S. T. M. Standard method D18 (see 512.15 , p. 250.), using a No. 8 sieve in addition to the screens specified in method D18. The No. $\delta$ sieve shall be a United States standard sieve meeting the requirements of A. S. T. M. standard specification E11, (see 500.2, p. 1).

National Sand and Gravel Association, bulletin 3 , representative specifications for different uses of sand and gravel, November, 1928.

(In this bulletin representative specifications, many of which are presented in this volume, and including specifications for coarse aggregate, have been summarized.)

United States Department of Agriculture, bulletin 1216, method of test for percentage of shale in aggregate, $192 \mathrm{~s}$.

It is suggested that for the separation of shale and other pieces having low specific gravity from concrete aggregate, a solution of zinc chloride $\left(\mathrm{ZnCl}_{z}\right)$ or some other satisfactory liquid having a specific gravity of approximately 1.95 be used. A sample of the pebbles should be first dried to constant weight at not over $110^{\circ} \mathrm{C}$., then placed in a container partially filled with the solution. Agitate for five minutes, skim off the lighter materials, and then pour the solution through a sieve which will retain the pebbles. Repeat the operation until the entire sample has been separated. Dry to constant weight, measure the volume of retained material, and compute the percentage of volume of shale or other soft material. 
NoTe.-Attention is called to the fact that this method of test is not applicable when the specific gravity of the shale exceeds the specific gravity of the solution. In cases where a solution of very high density is required, a mixture of bromoform and henzol may be employed.

United States Department of Agriculture, bulletin 1216 , method of test for soundness of coarse aggregate, 1928.

1. Immerse 10 small pieces (total weight about $1,000 \mathrm{~g}$.) of the rock in a saturated solution at $70^{\circ} \mathrm{F}$. of sodium sulphate $\left(\mathrm{Na}_{2} \mathrm{SO}_{4}\right)$ for 20 hours, after which place them for 4 hours in a drying oven maintained at $100^{\circ} \mathrm{C}$. Repeat the treatment - times. The operation of immersing, heating, reimmersing, etc., shall be continuous. Note the condition of the rock as to soundness at the end of the test.

2. Samples which exhibit marked disintegration shall be considered to have failed in this test..$^{45}$

\subsection{Broken Sione.}

American Association of State Highway Officials, tentative standard specifications for highway materials, 1927 .

\section{MATERIAL}

\section{NO. 1. STONE FOR TELFORD BASE COURSE AND RECON- STRUCTED BASE COURSE}

The material for this work shall consist of approved, sound, tough, durable stone, free from clay, loam, or other foreign substances. The pieces shall be approximately rectangular in section, having a depth equal to the thickness of the course, a width of from 2 to 6 inches, and a length of from 6 to 12 inches. The small stone for filling the voids in the large material shall consist of material at least equal in quality to that of the large stone.

\section{HO. 2. BROKEN STONE FOR WATER-BOUND BASE}

These specifications are substantially the same as A. S. T. M. D190. (See p. 250.)

\section{NO. 3. BROKEN SLAG FOR WATER-BOUND BASE}

1. This specification covers the quality and size of broken slag and slag sereenings for use in a waterbound base course which is to be covered with any type of surface.

2. The broken slag shall be air-cooled, blastfurnace slag and shall consist of angular fragments reasonably uniform in density and quality, and reasonably free from thin, elongated or glassy pieces, dirt or other objectionable matter.

3 . The slag shall conform to the following requirements:

(a) Per cent of wear, not more than

Pounds

(b) Wejght per cubic foot, not less tha

4. The coarse slag shall be of $(a)$ the $1^{1 / 4}$ to $2^{1 / 2}$ inch size, or $(b)$ the $2^{1 / 2}$ to $3^{1 / 2}$ inch size and shall be well graded between these limits. When tested by means of laboratory screens it shall conform to the following requirements:

(a) Passing 11/4-inch screen

Passing 21/2-inch screen

Passing 3-inch screen

(b) Passing 21/2-inch screen

Passing $31 / 2$-inch screen

Passing 4-inch screen

Per cent $0-15$ $0-15$
$95-100$

Either of the above sizes or a combination thereof may be specified as desired.

5. The sereenings shall be of either $(a)$ the 0 to $1 / 4$ inch size, or $(b)$ the 0 to $3 / 4$-inch size, well graded from coarse to fine and free from dirt or other foreign material. When tested by means of laboratory

48 A correct internretation of the results of the sodium-sulphate is $A$ correct crom accompanied by evidence of weathering of the exposed surface, may he accompanier by evidence of weathering of screens it shall conform to one of the following requirements:

(a) Passing 1/4-inch screen Per cent

Passing $1 / 4$-inch screen

Passing 1-ineh screen

Either of the above alternate sizes may be specfied as desired.

6. Sampling and testing methods.-(a) Wear.See. 510, page 187, United States Department of Agriculture bulletin 1216, method of test for abrasion of broken stone and broken slag, 1928.

(b) Weight. - See 512.10 , page 212 , A. S. T. M. method C29.

(c) Sampling.-Seè 510., page 184, A. S. T. M. method D75.

\section{NO. 4. BROKEN STONE FOR BITUMINOUS CONCRETE BASE}

Sections 2 and $7(a)$ of A. S. T. M. D190, (see p. 250) are part of this specification with the following additional:

1. This specification covers the quality and size of broken stone to be used in the construction of a bituminous concrete base.

2. The stone shall conform to the following requirements:

Percentage of wear, not more than Toughness, not less than ..........

3. The coarse aggregate shall consist either of (a) the $1 / 4$ to $1 \frac{1 / 4}{4}$ inch size or $(b)$ the $1 / 4$ to $2 \frac{1}{2}$ inch size as designated and shall be well graded between these limits. When tested by means of laboratory screens the size designated shall conform to the following requirements: $-0-5$ (30-60 een - 95-100

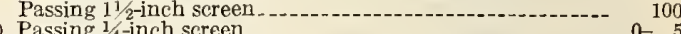

(b) Passing $1 / 4-1$ inch screen Passing $3 / 4$-inch screen Passing 11/4-inch screen

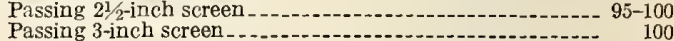

4. Test for toughness.-See 510. A. S. T. M. method D3.

\section{NO. 7. BROKEN STONE FOR PORTLAND CEMENT CONCRETE BASE COURSE}

Section $6(a)$ and $(c)$ of No. 3 and section 4 of No. 4 (above) are part of this specification, with the following additional:

1. This specification covers the quality and size of broken stone for use as coarse aggregate in a Portland cement concrete base course.

2. The broken stone shall consist of uncoated particles of clean, hard, tough, durable rock. It shall contain no organic or other deleterious matter and shall be free from lumps of clay and soft pieces.

3. At least - per cent of the stone shall come from ledges conforming to the following requirements: Per cent of wear, not more than Toughness, not less than

4. The broken stone shall show no evidence of disintegration when subjected to note 1 alternations in the sodium sulphate test for soundness.

5. Coarse aggregate shall be uniformly graded between the limits specified and shall meet the following requirements:

Passing $\frac{1 / 2}{1}$ inch screen (note 2 )

$\left\{\begin{array}{l}\text { Passing } 7 \text { inch screen (note 2) } \\ \text { Passing } 1 / 4 \text {-inch screen }\end{array}\right.$

NoTe 1-Five alternations are specified ordinarily. Attention, however, is called to the fact that a greatermumber may be specified if hower, is called to the fict that areater number may be specifed if, materials, or kind of service required make it advisable to do so. 
Note 2.- It is understood that the gradation of the coarse aggre ate sla! be further limited by the insertion of such requirements for intermediate size screens as will insure that aggregates of satisfactor $y$ grading will be prorided for the work in hand.

\section{NO. 8. BROKEN SLAG FOR PORTLAND CEMENT CONCRETE BASE COURSE}

Sections 2 and 6 of specification No. 3 and note 2 of specification No. 7 are part of this specification, with the following additional:

1. This specification covers the qualitiy and size of broken slag for use as coarse aggregate in a Portland cement concrete base course.

2 . The slag shall conform to the following requirements:

Per cent of wear, not more than

Weight per cubic foot, not less than

3. The broken slag shall be well graded between the limits specified. When tested by means of laboratory screens it shall conform to the following requirements:

Passing 21/2-inch screen

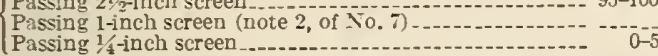

Per cent

TO. 9. BROKEN STONE FOR WATER-BOUND MACADAM SUR.

\section{FACE COURSE}

These specifications are substantially the same as A. S. T. M. D191. (See p. 250.)

\section{NO. 10. BROKEN SLAG FOR WATER-BOUND SURFACE COURSE}

This specification is the same as specification No. 3 (above), with the exception of sections 1 and 3 which for this specification are as follows:

1. This specification covers the quality and size of broken slag screenings for use in a water-bound surface course.

3. (a) Per cent of wear, not more than

(b) Weight per cubic foot

NO. 11. BROKEN STONE FOR BITUMINOUS MACADAM SURFACE COURSE

This specification is substantially the same as A. S. T. M. D192. (See p. 250.)

IO. 12. BROKEN SLAG FOR BITUMINOUS MACADAM SURFACE COURSE

This specification is substantially the same as A. S. T. M. D159 (see 512.2, p. 254), with the following requirement additional:

Per cent of mear, not more than.

15

NO. 13. BROKEN STONE FOR BITUMINOUS CONCRETE SUR-

This specification is substantially the same as A. S. T. M. D194 (see p. 251), omitting section 2, and substituting the following requirements in section 4 of the A. S. T. M. specification:

Passing $1 / 2$-inch screen

Per cent Passing $3 / 4$-inch screen

NO. 14. BROKEN SLAG FOR BITUMINOUS CONCRETE SURFACE

This specification is substantially the same as A. S. T. M. D160 (see 512.2, p. 251), with the following requirement additional.

Per cent of wear, not more than 15

NO. 15. BROKEN STONE FOR PORTLAND CEMENT CONCRETE SURFACE COURSE

Section $6(a)$ and $(c)$ of No. 3, section 4 of No. 4 and sections 2, 4, and 5 and notes 1 and 2 of No. 7 (above) are part of this specification with the following additional:

1. This specification covers the quality and size of broken stone for use as coarse aggregate in a Portland cement concrete surface course.
2. At least - per cent of the stone shall come from ledges conforming to the following requirements:

Per cent of wear, not more than Toughness, not less than

NO. 16. BROKEN SLAG FOR PORTLAND CEMENT CONCRETE SURFACE COURSE

Sections 2 and 6 of No. 3, Note 2 of No. 7 and section 3 of No. 8 (above) are part of this specification, with the following additional:

1. This specification covers the quality and size of broken slag for use as coarse aggregate in a Portland cement concrete surface course.

2 . The slag shall conform to the following requirements:

Per cent of wear, not more than Weight per cubic foot, not less than

American Railway Engineering Association, specification for stone ballast, 1925 .

(See 511.73, p. 210.)

American Society for Municipal Improvements, specifications for broken stone and gravel roads, 1922.

\section{PROPOSED SPECIFICATIONS FOR BROKEN STONE AND GRAVEL ROADS WITH AND WITHOUT BITUNIINOUS SURFACE TREATMENT}

1. General description.-The broken-stone road shall consist of three courses of broken stone, separately constructed, laid to conform to the required grades and cross sections and constructed as hereinafter specified.

\section{BROKEN STONE}

2. Quality of broken stone.-All broken stone shall be clean, rough surfaced, and sharp angled, of compact texture and uniform grain.

Tests for broken stone.-The broken stone shall be subjected to abrasion tests and toughness tests conducted by the engineer in accordance with methods adopted by the American Society for Testing Materials. (See 510, p. 182.) It shall show a "French coefficient of wear" of not less than 7.0 and its toughness shall be not less than 6.0.

3. Sizes.-The product of the crusher shall be passed over a rotary screen with sections having, respectively, circular openings of the following dimensions: First section, $5 / 8$-inch holes; second section, 11/4-inch holes; third section, 21/4-inch holes; fourth section, $3 \frac{1}{2}$-inch holes. If so directed the first section of the screen shall be fitted with a dust jacket having $1 / 4$-inch openings so placed as to separate the dust from the product passing through the first section. The screening plant shall also be fitted with a tailing chute so that no stone failing to pass the largest openings will fall into the bin for No. 4 size broken stone. The various sizes of broken stone shall be caught in separate bins and shall be designated as follows:

Dust, all passing through $1 / 4$-inch screen.

Screenings, all passing through $5 / 8$-inch screen.

No. 1 size, passing through $5 / 8$-inch screen and over 1/4-inch screen.

No. 2 size, passing over $5 / 8$-inch screen and through

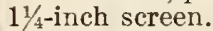

No. 3 size, passing over $1 \frac{1 / 4}{4}$-inch screen and through $2^{1 / 4}$-inch screen.

No. 4 size, passing over $2 \frac{1}{4}$-inch screen and through $3 \frac{1}{2}$-inch screen.

Tailings, passing over $3^{1 / 2-i n c h ~ s c r e e n . ~}$

Portable plants.- Portable crushing and screening plants shall be operated as directed.

Stationary plants.-If broken stone is to be supplied from stationary crushing and screening plants, the several sizes of broken stone shall not be used unless samples have been previously approved by the engineer. The various sizes of broken stone fur- 
nished shall be substantially the same as the samples approved.

\section{CONSTRUCTION}

4. First course.-After the subgrade course shall have been prepared as specified, a course of No. 4 broken stone shall be evenly spread so that it shall have after rolling the required thickness of $3 \frac{1}{2}$ inches. The depth of loose broken stone shall be gaged by the use of strings between iron stakes, as directed. The spreading of the broken stone must be from piles dumped on boards provided for the purpose or from piles dumped alongside the road, or as directed by the engineer. This course shall be thoroughly rolled with a 10 to 15 ton road roller. The rolling shall begin at the sides of the road and continue toward the center, and shall be kept up until there is no disturbance of the stone ahead of the roller. After the complction of the rolling, no teaming other than that necessary for bringing on the broken stone for the next course shall be allowed over the rolled broken stone. Should it be apparent after the rolling of the first course that the subgrade material shall have become churned up into or mixed with the broken stone of this course, whether by reason of the rolling, or by hauling over the broken stone or otherwise, the contractor shall at his own expense remove and replace such mixture of subgrade material and broken stone with clean broken stone of the proper size and shall roll the material to produce a uniform, firm, and even first course as required.

5. Second course.- On the completed first course shall be spread, in the manner specified in the preceding paragraph, No. 3 broken stone to form the second course. This broken stone shall be evenly spread to such a depth that it shall have, after rolling, the required thickness of $2 \frac{1}{2}$ inches. After the second course shall be compacted under the same provisions as prescribed for the first course, it shall be evenly covered with a thin layer of screenings. The quantity of screenings to be used shall be just sufficient to cover the larger stones, and care shall be exercised to avoid the use of an excess of the screenings. This covering shall then be rolled as heretofore provided. When the rolling shall have been completed the surface of the second course shall be firm, even, and true to the lines grades and cross sections.

6. Third course.-On the completed second course shall be spread in the manner above specified for the first course No. 3 broken stone to form the third course. This broken stone shall be evenly spread to such a depth that it well have after rolling the required thickness of $2 \frac{1}{2}$ inches. After the third course shall have been completed under the same provisions as prescribed for the first course, it shall be evenly covered with a thin layer of sereenings. The quantity of screenings to be used shall be just sufficient to cover the larger stones and care shall be exercised to avoid the use of an excess of the screenings. This covering shall then be rolled as heretofore provided, except that water shall be used in connection with the rolling as follows: After the screenings shall have been lightly rolled, water shall be sprinkled on the road surface just ahead of the roller in such quantity as will prevent the sticking to the wheels of the roller of the fine material on the surface, and the combined spreading of screenings, watering, and rolling shall be continued until the voids of the broken stone become so filled with the finel particles as to result in a wave of grout being pushed along the road surface by the front wheel of the roller. When the rolling shall have been completed the surface of the third course shall be firm, even, and true to the lines, grades, and cross sections. Aiter the third course has been compacted, puddled, and filled as above specified, it shall be evenly covered with a thin layer of screenings. Should at any time after its construction, and prior to the acceptance of the road, the larger stone be visible in the surface of the road, the contractor shall, without extra allowance, spread, sprinkle, and roll sufficient screenings to completely cover the same.

\section{PAYMENT}

7. Measurement and payment.-The quantity of broken-stone road to be paid for shall be the number of square yards, measured horizontally, satisfactorily completed in accordance with specifications. The price stipulated shall include the furnishing, crushing, and screening of the different sizes of broken stone, the placing, rolling, and watering of the broken stone, and all work and expenses incidental to the completion of the broken-stone road.

\section{PROPOSED SPECIFICATIONS FOR BROKEN-STONE ROAD WITH BITUMINOUS SURFACE}

8. General description.-The broken-stone road shall consist of three courses of broken stone, separately constructed, laid to conform to the required grades and cross sections and constructed as hereinafter specified.

\section{BROKEN STONE}

9. Quality of broken stone.-All broken stone shall be clean, rough surfaced, and sharp angled, of compact texture and uniform grain.

Test of broken stone.-The broken stone shall be subjected to abrasion tests and toughness tests conducted by the engineer in accordance with methods adopted by the American Society for Testing Materials. (See 510, p. 182.) The broken stone used for the construction of the first and second courses shall show a French coefficient of wear of not less than 7.0 and its toughness shall be not less than 6.0. The broken stone used for the construction of the third course and in connection with the bituminous surface shall show a French coefficient of wear of not less than 11.0 and its toughness shall be not less than 13.0.

10. Sizes. - The sizes shall be in accordance with the requirements as stated in the paragraph entitled "sizes" in the specifications for "Broken-stone road." (See above.)

\section{CONSTRUCTION}

11. Same as "construction" item of above specification with the following variations:

(1) The compacting and puddling process, applied only to the third course in the above specification, is applied for all three courses under this specification.

(2) A 12 to 15 ton roller is prescribed for this specification.

(3) Number 4 broken stone is prescribed for the second course of this specification and required thickness after rolling is $3 \frac{1}{2} 2$ inches.

(4) For this specification a surface sufficiently rough for bonding is required for the second and third courses.

(5) The following additional requirement is stipulated:

Each section of the broken-stone road shall be subjected to traffic for at least one month before the construction of the bituminous surface thereon.

12. This specification also contains specifications for high and low carbon tars for hot and cold surface treatments, which are practically identical with American Society for Testing Materials specification (see 505.31, p. 175), serial designations D104, D105, D108, and D109; also specifications for asphaltic oil for hot-surface treatment, practically identical with Federal Specifications Board No. 275. (See 505.22, p. 173.) 
13. Requirements relative to the application of these specified materials are as follows:

\section{SPECIFICATIONS FOR SURFACE TREATMENTS WITE HOT BITUMINOUS MATERIALS}

NotE.-The bituminous material under this designation may be NotE.-The bituminous material under this designation may bo be an asphaltic oil, the selection of the type and character of the materisl being optional with the engineer.

\section{HOT TARS}

14. Description of bituminous surface.-The bituminous surface shall consist of one application of refined tar covered with a top dressing of brokenstone chips, pea gravel, grit, or other mineral matter, constructed as hereinafter specified.

\section{BITUMINOUS MATERIAL}

15. Refined iar.- Refined tar used in the construction of the bituminous surface shall conform with either one of the specincations covering the chemical and physical properties of refined tar included under the item entitled "Refined tars for surface treatments."

16. Heating refined tar.-The refined tar shall be heated in kettles or tanks so designed as to admit of even heating of the entire mass, with an efficient and positive control of the heat at all times. It shall be heated as directed by the engineer to a temperature between $93^{\circ}$ C. $\left(200^{\circ}\right.$ F.) and $121^{\circ}$ C. $\left(250^{\circ}\right.$ F.) All refined tar heated beyond $121^{\circ} \mathrm{C}$. $\left(250^{\circ} \mathrm{F}\right.$.) shall be rejected. No tar shall be heated in kettles or takks containing any oil or asphalt ccment. Before changing from one type to another, kettles or tanks shall be scrupulously cleaned in order to avoid mixtures of the two. Any mixtures of different kinds of bituminous materials shall be rejected.

Thermometers furnished by contractors.-The contractor shall provide a sufficient number of accurate, efficient, stationary thermometers for determining the temperature of the refined tar in kettles or tanks.

\section{CONSTRUCTION}

17. Preparation of surface of road.-Prior to the application of the refined tar, the surface of the broken-stone road, when thoroughly dry, shall be swept clean of all dust, dirt, or other loose material with horse or power driven brooms of bass or other fine-fiber brooms, or with stiff fiber hand brooms of bass of other fine-fiber brooms, as directed by the engineer. When the cleaning is completed the upper surface of the No. 3 broken stone shall be exposed, forming a clean mosaic surface.

In addition to the sweepings above specified, the shoulders on each side of the road, if of earth, for a distance of 3 feet shall be thoroughly scraped with a blade grader in order to remove all dead material adjacent to the road, and thus prevent the formation of a weak and brittle crust at this point after the application of the bituminous material.

18. (a) Application of refined tar.-After the surface shall hare been cleaned to the satisfaction of the engineer, and when thoroughly dry, the refined tar shall be uniformly applied over the prepared surface of the road by means of a pressure distributor as hereinafter specified and in accordance with the directions of the engineer. The refined tar, when applied, shall have a temperature between $93^{\circ}$ C. $\left(200^{\circ} \mathrm{F}\right.$. $)$ and $121^{\circ}$ C. $\left(250^{\circ} \mathrm{F}\right.$.). The total amount of refined tar to be used in the construction of the bituminous surface shall be applied in one application and shall not be less than one-quarter nor more than one-half gallon per square yard, the precise quantity being determined by the engineer.

(b) Pressure distributor.-The pressure distributor employed shall be so designed and operated as to distribute the material specified uniformly under a pressure of not less than 20 pounds nor more than 75 pounds per square inch in the amount and between the limits of temperature specified. It shall be supplied with an accurate stationary thermometer in the tank containing the refined tar, and with an accurate pressure gauge so located as to be easily observed by the engineer while walking beside the distributor. It shall be so operated that at the termination of each run the refined tar will be at once shut off. It shall be so designed that tha normal width of application shall be not less than feet and so that it will be possible on either side of the machine to apply widths of not more than 2 feet. The distributor shall be provided with tirss of widths dependent upon the following relationship between the pressure per square inch of tire and tha diameter of the wheel; for a 2-foot diameter wheel 500 pounds shall be the maximum pressure per linear inch of width per wheel, an additional pressure of 30 pounds per inch being allowed for each additional 3 inches in diameter.

19. Application of top dressing.-Immediately after the completion of the application of the bituminous material there shall be uniformly applied to the road at the rate of from 20 to 30 pounds per square yard, broken-stone chips, pea gravel, grit or other mineral matter which will meet the following requirements: (The exact amount to be applied should be determined in advance by the engineer, depending on the rate of application of the bituminous material and character of the top dressing.)

The broken stone, or pea gravel, grit, or other mineral matter shall consist of clean, hard, tough durable material, free from soft or disintegrated particles, dirt, or other objectionable matter.

When tested by means of laboratory screens it shall meet the following requirements:

Per cent

Passing 3/4-inch screen, not less than. 85 Retained on 1/4-inch screen, not less than

NoTE.--If a comparatively soft stone is used, the mesh composition of the top dressing may be increased in size so that at least 95 per cent shall pass a 1 -inch screen and at least 85 per cent be retained on a 1 -inch screen.

20. Seasonal and weather limitations.-No refined tar shall be applied when the air temperature in the shade is below $10^{\circ} \mathrm{C}$. $\left(50^{\circ} \mathrm{F}\right.$.), except by the written permission of the engineer.

\section{PAYMENT}

21. Measurement and payment.-The quantity of broken-stone road with bituminous surface to be paid for under this item shall be the number of square yards, measured horizontally, satisfactorily completed in accordance with the specifications. The price stipulated in this item shall include the furnishing, crushing, and screening of the different sizes of broken stone, the placing, rolling, and watering of the broken stone, the heating and distributing of the refined tar, and all materials, work, and expenses incidental to the completion of the broken-stone road with bituminous surface except the furnishing of the refined tar, which will be included for payment under the item entitled "Refined tars for surface treatments."

\section{SPECIFICATIONS FOR REFINED TARS FOR HOT SURFACE TREATMENTS}

22. Previous service.-The contractor will be required to show to the satisfaction of the engineer that the company manufacturing the product he proposes to use under a given specification has for a period of at least two years manufactured the material in a thoroughly equipped plant; and that the product manufactured of bituminous material 
obtained from a similar source to that which he proposes to use shall have been in continuous and successful use in the surface treatment of brokenstone roads for a period of at least two years previous to the date of the letting in which his proposal was submitted.

23. Refined tar "A," optional with refined tar "B." (See A. S. T. M. D109-27T, 505.31, p. 175.)

24. Refined tar "B," optional with refined tar "A." (See A. S. T. M. D108-27T, 505.31, p. 175.)

25. Delivery. - The refined tar shall be delivered in suitable containers, far enough in advance of its use in the work to permit the necessary tests to be made. Each container shall be plainly labeled with the trade name, name of manufacturer, gross weight, and net weight. Each shipment and each carload shall be kept separate.

Bills of lading.-The contractor shall furnish the engineer on or before the arrival of each shipment at or near the site of the work, bills of lading, or correct copies thereof, which shall state the trade name, and the name and address of the company manufacturing and supplying it.

Samples.-Samples will be taken by the engineer from each carload when delivered at the work, unless satisfactory arrangements can be made for sampling before shipment. Such samples shall be analyzed by the engineer to assure the delivery of a product of the specified quality and to determine, for purpose of payment, the quantity of bitumen.

26. Work included under this item the contractor shall furnish and deliver on the work at such points as directed material which conforms with the specifications of either type specified.

27. Measurement and payment.-The quantity of bituminous material to be paid for under this item shall be the number of gallons placed on the highway in accordance with the specifications and requirements, or used as directed for other purposes. Bituminous material that is wasted by the contractor shall not be included in the measurement under this item. The price stipulated in this item shall include the cost of furnishing, hauling, and delivering the bituminous material on the work, and all expenses incidental thereto.

The quantity of bituminous material paid for will be computed on the basis of its volume at $60^{\circ} \mathrm{F}$.

\section{ASPHALTIC OIL FOR HOT SURFACE TREATMENT, OPTIONAL WITH RERINED HOT TARS A OR B}

(See Federal Specifications Board, No. 275, 505.22 , p. 173.)

28. The application of this product is subject to requirements similar to those listed under "Hot tars," in sections 14, 17, $18(b), 19,20,25,26$, and 27 (above).

Note.-In section 17 omit the last sentence in the first paragraph and substitute the following sentence: "Newly constructed broken stone and gravel roads shall be subjected to traffic for at least on month before the construction of the bituminous surface theron."

In scction $18(b)$ omit the reference to the stationary thermometer, which is not required in this specification.

The application of the product shall be as follows After the surfaces and shoulders have been cleaned, the bituminous material shall be applied to the road from a pressure distributor at a rate of between onethird and one-half gallon per square yard. The asphalt when applied shall have a temperature between $135^{\circ}$ C. $\left(275^{\circ} \mathrm{F}\right.$.) and $177^{\circ} \mathrm{C}$. $\left(350^{\circ} \mathrm{F}.\right)$; the exact amount to be applied per square yard to be determined by the engineer.

Measurement and payment.- The quantity of surface treatment to be paid for under this item shall be the number of square yards, measured horizontalJy, satisfactorily completed in accordance with the specifications. The price stipulated in this item shall include the furnishing and screening of the top dressing, the preparation of the roadway surface, the application of the bituminous material, and the spreading of the top dressing, and all materials, work and expenses incidental to the completion of the surface treatments, except the furnishing of the bituminous material, which will be included for payment under the item entitled "Asphaltic oil for for hot surface treatment."

\section{SPECIFICATIONS FOR SURFACE TREATMENTS WITH COLD BITUMINOUS MATERIALS}

Requirements listed under "Hot tars" (above) in sections $17,18(b), 19$, and 20 apply for these products.

NOTE.-In section 17 omit the last sentence in the first paragraph and substitute the following sentence: "Newly constructed broken stone aud gravel roads shall be subjected to traffic for at least one month before the construction of the bituminous surface thereon "

In section $18(b)$ omit the reference to the stationary thermometer, which is not required in this specification.

In section 19 omit the note referring to the mesh composition of the top dressing.

The following additional requirements are included in this specification:

29. Description of bituminous surface treatment.The bituminous surface treatment shall consist of one application of refined tar or cut-back asphalt cement covered with a thin layer of top dressing as hereinafter specified.

NoTE.-This specification provides for a surface treatment which may be used on thoroughly cleaned broken-stone or gravel roads in good condition; for the retreatment of tar surfaces or the maintenance of seal coats on tar-macedam or tar-conerete pavements, if refined tar is ual coats on tar-macadam or tar-concrete pavements, if refined tar macadam or asphaltic concrete pavements if cut-back asphalt cement macadam or asphaltic concrete pave

Refined tar and cut-back asphalt cement.-The refined tar or cut-back asphalt cement, hereinafter referred to as bituminous material, used in the construction of the surface treatments, shall conform with its respective specification covering the chemical and physical properties of bituminous materials included under the item entitled "Refined tar and cutback asphalt cement for cold surface treatment."

Application of bituminous material. - After the surface shall have been cleaned, and when thoroughly dry, the unheated bituminous material shall be uniformly applied to the prepared surface of the roadway by ineans of a pressure distributor as hereinafter specified, and in accordance with the directions of the engineer. The total amount of bituminous material to be used in the surface treatment shall be applied in one application and shall not be lcss than one-eighth nor more than one-half gallon per square yard, the precise quantity being determined by the engineer.

NотE.-The first treatment of broken-stone or gravel roads should consist of an application of between one-third and one-half gallon
per squarc yard. Generally, subsequent treatments on the above per square yard. Generally, subsequent treatments on the above
types of roads, and bituminous pavements, should be less than onetypes of roads, and bituminou
third gallon per square yard.

Measurement and payment.-The quantity of surface treatment to be paid for under this item shall be the number of square yards, measured horizontally, satisfactorily completed in accordance with the specifications. The price stipulated in this item shall include the furnishing and screening of the top dressing, the preparation of the roadway surface, the application of the bituminous material, and the spreading of the top dressing, and all materials, work, and expenses incidental to the completion of the surface treatments, except the furnishing of the bituminous material, which will be included for payment under the item entitled "Refined tar and cut-back asphalt cement for cold surface treatment."

30. Requirements listed under "Hot tars" (above) in sections $22,25,26$, and 27 apply for these products with the following additional 
High carbon tar, optional with low carbon tar, or cut-back asphalt cement. (See A. S. T. M. D104-27T, 505.31, p. 175.)

Low carbon tar, optional with high carbon tar, or cut-back asphalt cement. (See A. S. T. M. D105-27T, 505.31, p. 175.)

Cut-back asphalt cement, optional with low and high carbon tars.

The cut-back asphalt cement shall consist of a mixture of asphalt cement and naptha which will comply with the following specifications:

Its specific gravity of $25^{\circ} / 25^{\circ} \mathrm{C}$. $\left(77^{\circ} / 77^{\circ} \mathrm{F}\right.$.) shall be not less than 0.890 .

When $20 \mathrm{~g}$ of the material is maintained at a uniform temperature of $163^{\circ} \mathrm{C}$. $\left(325^{\circ} \mathrm{F}\right.$.) for five hours, in an open cylindrical tin dish $5 \frac{1}{2} \mathrm{~cm}$ (about $2 \frac{1}{4}$ inches) in diameter, with vertical sides measuring approximately $3 \frac{1}{2} \mathrm{~cm}$ (about $1 \frac{1}{2}$ inches) in depth, the loss in weight shall be not less than 30 nor more than 40 per cent.

When tested with a standard No. 2 needle under a load of $100 \mathrm{~g}$ for five seconds at $24^{\circ} \mathrm{C}$. $\left(77^{\circ} \mathrm{F}\right.$.) the penetration of the residue, expressed in hundredths of a centimeter, shall be between 50 and 85 .

iv hen tested by means of the Engler viscosimeter at $25^{\circ} \mathrm{C}$. $\left(77^{\circ} \mathrm{F}\right.$.) the specific viscosity of the first 50 ce passing the orifice of the viscosimeter shall be not less than 25 nor more than 35 .

When distilled according to the standard method of the American Society for Testing Materials (see 505.0, p. 182), the specific gravity of the distillate below $300^{\circ} \mathrm{C}$. $\left(572^{\circ} \mathrm{F}\right.$.) shall be not less than 0.730 nor more than 0.780 .

Its bitumen, as determined by its solubility in chemically pure carbon disulphide at room temperature, shall be not less than 99.5 per cent.

\section{TEMPORARY SUBSTITUTE FOR ROAD OIL}

31. Its specific gravity at $25^{\circ} / 25^{\circ} \mathrm{C}$. $\left(77^{\circ} / 77^{\circ} \mathrm{F}\right.$. $)$ shall not be less than 0.835 nor more than 0.970 .

It shall have a flash point of not more than $50^{\circ} \mathrm{C}$. $\left(122^{\circ} \mathrm{F}\right.$.) when tested in an open cup oil tester.

When tested by means of the Engler viscosimeter at $25^{\circ} \mathrm{C} .\left(77^{\circ} \mathrm{F}\right.$. $)$ the specific viscosity of the first 50 cc. passing the orifice of the viscosimeter shall be not less than 80 nor more than 120 .

When $20 \mathrm{~g}$. of the material are maintained at a uniform temperature of $163^{\circ} \mathrm{C}$. $\left(325^{\circ} \mathrm{F}\right.$.) for five hours, in an open cylindrical tin dish $5 \frac{1}{2} \mathrm{~cm}$. (about $2 \frac{1}{4}$ inches) in diametcr, with vertical sides measuring approximately $3 \frac{1}{2} \mathrm{~cm}$. (about $1 \frac{1}{2}$ inches) in depth, the loss in weight shall be not more than 30 per cent.

When the residue is tested by means of the New York Testing Laboratory float apparatus the float shall not sink in water maintained at $50^{\circ} \mathrm{C}$. $\left(122^{\circ} \mathrm{F}\right.$. $)$ in less than 90 seconds.

Its bitumen, as determined by its solubility in chemically pure carbon disulphide at room temperature, shall be not less than 99.5 per cent.

It shall be soluble in $86^{\circ}$ Baumé paraffin naphtha to the extent of not less than 94 per cent of its bitumen as determined by clause next preceding.

\section{PROPOSED SPECIFICATIONS FOR A GRAVEL ROAD}

32. General description.-The gravel road shall consist of three courses of mixtures of gravel, sand, and clay, separately constructed, laid to conform to the reqiured grades and cross sections and constructed as hereinafter specified. The transverse slope of the finished surface of the gravel roadway shall be 1 inch per foot.

Subgrade.-The subgrade for the gravel roadway shall consist of the natural earth roadbed prepared and rolled until firm, hard, and even, and shall con- form to the specified cross section. If sandy or other soil be encountered which will not compact readily under the roller, a small amount of clay, or other means satisfactory to the engineer, shall be used until a firm, even surface is obtained after rolling. Where the proposed grade allows the use of an old roadway for the subgrade, the roadway shall be shaped and rolled to the specified cross section and elevations and depressions removed so as to form an even surface before the construction of the first course. The roller used shall be a 10 to 15 ton road roller.

Shoulders.-After the roadbed has been graded, shoulders of firm carth or other suitable material 8 inches in depth after compacting shall be constructed on each side of the roadbed at such distances apart as may be required to retain the width of gravel specified. No material which contains wceds, sod, roots, or other perishable matter and which will not compact under the roller shall be placed in the shoulders. The shoulders shall extend to the side ditches or gutters with the same transverse slope as required for the finished roadway surface and shall be thoroughly rolled at the same time as the third or wearing course.

\section{MATERIALS}

Quality of gravel.-All gravel shall be hard and tough. Gravel which contains over 10 per cent of disintegrated stone shall not be used.

Sizes.-Two mixtures of gravel, sand, and clay shall be used, hereinafter designated in these specifications as No. 1 product and No. 2 product.

No. 1 product shall consist of a mixture of gravel, sand, and clay, with the proportions of the various sizes as follows: All to pass a $1 \frac{1 / 2}{2}$-inch screen and to have at least 60 and not more than 75 per cent retained on a $1 / 4$ inch screen; at least 25 and not more than 75 per cent of the total coarse aggregate (material over $1 / 4$-inch in size) to be retained on a $3 / 4$ inch screen; at least 65 and not more than 85 per cent of the total fine aggregate (material under $1 / 4$-inch in size) to be retained on a 200 -mesh sieve.

No. 2 product shall consist of a mixture of gravel, sand, and clay, with the proportions of the various sizes as follows: All to pass a $2 \frac{1}{2}$-inch screen and to have at least 60 and not more than 75 per cent retained on a 1/4-inch screen; at least 25 and not more than 75 per cent of the total coarse aggregate to be retained on a 1-inch screen; at least 65 and not more than 85 per cent of the total fine aggregate to be retained on a 200 -mesh sieve.

Test.- The mixture of gravel, sand, and clay of No. 1 product shall be subjected to a cementation test conducted by the engineer in accordance with the method recommended by the Special Committee on Materials for Road Construction of the American Society of Civil Engineers in January, 1916, and as described in the 1915 proceedings, page 2738 , except that the test shall be made on material which will pass a $1 / 4$-inch screen. Its coefficient of cementation shall not be less than 50 .

The method follows the "construction" item of proposed specifications for broken stone and gravel roads with and without bituminous surface treatment (above) with the following variations:

1. The compacting and puddling process, applied only to the third course in the above specification, is applied for all three courses under this specification.

2. First course shall consist of No. 2 product (as designated in this specification under "materials") laid to rolled thickness of 3 inches. The second course shall duplicate the first as to product and thickness and the third shall consist of No. 1 product (as designated in this specification under "materials") rolled to a thickness of 2 inches. 
3. The requirements referring to the use of water in the laying process, as given under section 6 (third course) above, should be included under requirements for the first course under this spceification. References to screenings, as included under section 6 (third course) in the construction requirements above, are omitted in this specification.

If necessary to satisfactorily bond the roadway surface, the third course shall be evenly covered with a thin layer of sand or sand and clay and rolled.

\section{PAYMENT}

33. Measurement and payment.-The quantity of gravel road to be paid for shall be the number of square yards, measured horizontally, satisfactorily completed in accordance with the specifications. The price stipulated shall include shaping and rolling of the subgrade, the furnishing of the different products of gravel, sand, and clay, the placing, rolling, and watering of the several courses, and all work and expenses incidental to the completion of the gravel road. (The quantity of shoulders shall be paid for under a separate item.)

Note.-The use of a bituminous surface treatment for gravel roads is not recommended under all conditions throughout the country. In certain sections gravel roads are constructed with local material carrying comparatively large percentages of clay, in which material carrying comparatively large percentages of clay, in which case it,

American Society for Testing Materials, standard method of mechanical analysis of broken stone or broken slag, except aggregates used in cement conerete, serial designation D18-16, 1916.

The method shall consist of (1) drying at not over $110^{\circ}$ C. $\left(230^{\circ}\right.$ F.) to a constant weight a sample weighing in pounds six times the diameter in inches of the largest holes required; (2) passing the sample through such of the following size screens having circular openings as are required or called for by the specifications, screens to be used in the order named: $8.89-\mathrm{cm}\left(3 \frac{1}{2}-\mathrm{inch}\right), 7.62-\mathrm{em}$ (3-inch), 6.35-cm (21/2inch), 5.08-cm (2-inch), 3.81-cm (1/1/2-inch), 3.18-cm (11/4-inch), 2.54-cm (1-inch), 1.90-cm (3/4-inch), 1.27$\mathrm{cm}(1 / 2-i n c h)$, and $0.64-\mathrm{cm}(1 / 4$-inch); (3) determining the percentage by weight retained on each screen; and (4) recording the mechanical analysis in the following manner:

Passing 0.64-em (1/4-inch) screen

Per cent

Passing 1.27-cm (1/2-inch) screen and retaincd on a 0.64

cm (1/4-inch) sercen.

Passing $1.90-\mathrm{cm}$ ( $3 / 2-$ inch) serecn and retained on a $1.27-\mathrm{cm}$

(1/2-inch) sereen.

Passing 2.54-cm ( $l$-inch) screen and retained on a $1.90-\mathrm{cm}$

$(3 / 4$-inch) screen $(l-i n c h)$ screen and retained on a $1.90-\mathrm{cm}$

100.00

American Society for Testing Materials, tentative specifications for commercial sizes of broken stone and broken slag for highway construction, serial designation D63-23T, 1923.

(See 511.71, p. 207.)

American Society for Testing Materials, tentative specifications for broken stone for water-bound base, serial designation D190-29T, 1929.

1. These specifications cover the quality and grading of broken stone and stone screenings for use in a water-bound broken stone base course which is to be covered with any type of surface.

2 . The broken stone shall consist of clean, tough, durable fragments, free from an excess of flat, elongated, soft, or disintegrated pieces.

3. Stone for screenings shall be of suitable binding quality.

4. The stone shall conform to the following requircment:

Percentage of wearNct more than 8

5. The coarse aggregate shall be of either $(a)$ the $1 \frac{1}{4}$ to $2 \frac{1}{2}$ inch size, (b) the $2 \frac{1}{2}$ to $3 \frac{1}{2}$ inch size, or $(c)$ the $2 \frac{1}{2}$ to $4 \frac{1}{2}$ inch size, and shall be well graded between the limits specified. When tested by means of laboratory screens, it shall conform to the following requirements:

Per cent

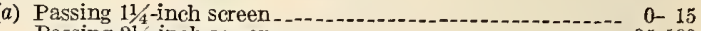

(b) Passing 21/2-inch sereen

Passing $31 / 2$-inch screen

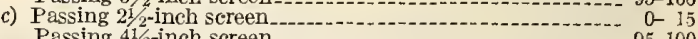

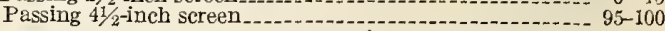

Noтe.-Any one of the above sizes or a comhination thereol may he specified as desired.

6. The screenings shall be of either (1) the 0 to $1 / 4$ inch size, or (2) the 0 to $3 / 4$ inch size, well graded from coarse to fine and free from dirt or other foreign material. When tested by means of laboratory screens they shall conform to one of the following requirements:

(a) Passing 1/4-inch screen

Per cent

Passing $3 / 4-i n c h$ screen

Note.-Either of the ahove alternate sizes may be specified as desired.

7. (a) The percentage of wear shall be determined in accordance with A. S. T. M. D2. (See 510 , p. 182.)

(b) Determinations of size shall be made in accordance with A. S. T. M. D18. (See 512.15, p. 250.)

8. The methods of sampling shall conform to A. S. T. M. D75. (See 510, p. 184.)

NOTE.-The test values fer quality of stone incorporated in these specifications are suggested as the lowest safe requirements. It is not expected, however, that the limits for size and quality will he applicable to all localities and conditions.

American Society for Testing Materials, standard specifications for broken stone for water-bound macadam surface course, serial designation D191-29, 1929.

Sections 2, 3, 5, ((a) and (b) sizes) 6, 7, and 8, and the footnote of A. S. T. M. D190 (given above) are part of this specification, with the following additional:

1. These specifications cover the quality and grading of broken stone and broken stone screenings for use in water-bound macadam surface course.

2. The stone shall conform to the following rcquirements:

Percentage of wear Not more than 6 . Toughness. Not less than 6.

3. The toughness shall be determined in accordance with A. S. T. M. D3. (Sec 510, p. 182.)

American Socieiy for Testing Materials, standard specifications for broken stone for bituminous macadam, serial designation D192-29, 1929.

Sections 2, 5, ((a) and (b) sizes) 7 , and 8, and the footnote of A. S. T. M. D150 (given above) and sections 2 and 3 of A. S. T. M. D191 (above) are part of this specification, with the following additional:

1. These specifications cover the quality and grading of broken stone for use in a bituminous macadam surface course or bituminous macadam base.

2. The broken stone shall be free from stone conted with dirt or other objectionable matter.

3 . The stone for filling the surface voids in the coarse aggregate shall be the $3 / 4$ to $1 \frac{1 / 4}{4}$ inch size, and when tested by means of laboratory screens shall conform to the following requirements:

Per cent

Passing $3 / 4$-inch screen $0-15$ Passing 1 -inch screen

4. Stone chips for use in seal coat shall be of the $1 / 4$ to $3 / 4$ inch size and when tested by means of the laboratory sereen shall conform to the following requirements: 
Per cent !

Passing $1 / 4$-inch screen

Passing $1 / 2$-inch screen 0-15

American Society for Testing Materials, tentative specifications for broken stone for bituminous concrete base, serial designation D193-29T, 1929.

Sections 2, 7, and 8 of A. S. T. M. D190 (given above) are part of this specification, with the following additional:

1. Thesc specifications cover the quality and grading of broken stone to be used in the construction of a closed mix and an open mix bituminous concrete base.

2. The stone shall conform to the following requirements:

Percentage of wear

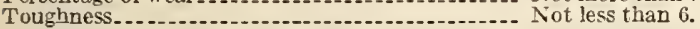

3. The coarse aggregate for open-mix bituminous concrete base shall consist of the $1 \frac{11 / 4}{4}$ to $2 \frac{1}{2}$ inch size and shall be well graded between these limits. When tested by means of laboratory screens it shall conform to the following requirements:

Per cent

Passing 11/4-inch screen

Passing $2 \frac{1}{2}$-inch screen

4. The coarse aggregate for closed-mix bituminous concrete base shall consist of the $1 / 4$ to $2 \frac{1}{2}$ inch size and shall be well graded between these limits. When tested by means of laboratory sereens it shall conform to the following requirements:

Per cent

Fassing 1/4-inch screen

0-5

Passing $1 \frac{1}{4}$-inch serecn

Passing 21,2-inch sereen.....--

5. The toughness shall be determined in accordance with A. S. T. M. D3. (See 510, p. 182.)

American Society for Testing Materials, standard specifications for broken stone for bituminous concrete surface, serial designation D 194-29, 1929.

Sections 2, 7, and 8 of A. S. T. M. D190 (given above), sections 2 and 3 of A. S. T. M. D191 (above), and scction 2 of A. S. T. M. D192 (above) are part of this specification with the following additional:

1. These specifications cover the quality and grading of broken stone to be used in the construction of fine-graded bituminous concrete pavements (modified Topeka or stone-filled sheet asphalt) and coarse-graded bituminous concrete, including asphaltic concrete binder.

2. Stone chips shall be of the $1 / 4$ to $1 / 2$ inch size. When tested by means of laboratory sereens they shall conform to the following requirements :

Passing $1 / 4$-inch screen

Per cent

Passing $1 / 2$-inch screen

3. The coarse aggregate for fine-grade bituminous concrete shall be of the $1 / 4$ to $3 / 4$ inch size and shall be well graded between these limits. When testcd by means of laboratory screens it shall conform to the following requirements.

Passing 1/1-inch screen

Per cent

Passing 1/2-inch screen

Passing $3 / 4$-inch screen.

4. The coarse agregate for coarse-graded bituminous concrete shall consist of the $1 / 4$ to $1 \frac{1}{4}$ inch size and shall be well graded between these limits. When tested by means of laboratory screens it shall conform to the following requirements:

Per cent

Passing $1 / 4$-inch screen

$0-10$

$25-75$

Passing $1 \frac{1}{4}$-inch screen

Federal Specifications Board, specification No. 456 , United States Government master specification for broken stone and broken slag for water bound base or wearing course, January 25, 1927.

\section{BROKEN STONE}

Sections 2, 3, 7, and 8 of A. S. T. M. D190 (given above) are part of this specification, with the following additional:

\section{DETAIT REQUIREMENTS}

(a) Stone for use as designated shall meet the following requirements:

\begin{tabular}{r|r|r}
\hline & $\begin{array}{c}\text { Base } \\
\text { course }\end{array}$ & $\begin{array}{l}\text { Surface } \\
\text { course }\end{array}$ \\
\hline Percentage of wear not more than_.......... & 10.0 & 6.0 \\
\hline
\end{tabular}

NOTE.-These test values for quality of stone are suggested as the lowest safe requirements. With favorable conditions of availahle materials, the maximum percentage of wear permitted should bo reduced.

(b) Broken stone shall be well graded between the limits specified and, when tested by means of laboratory screens, shall meet the following requirements for size as designated:

Note.-The particular size desired should be designated.

Coarse stone: (1) $2 \frac{1}{2}$ to $3 \frac{1}{2}$ inch size-

Per cent Passing 21/2-inch screen Passing 21/2-inch screen Passing 31/2-inch screen
Passing 4-inch screen

(2) $1 \frac{1}{4}$ to $2 \frac{1}{2}$ inch size-

Per cent Passing 11/4-inch screen.
Passing 2-inch screcn
Passing 21/2-inch screen. Passing 21/2-inch sereen

Screenings: (3) 0 to $\frac{3}{4}$ inch size-

Per cent

Passing 1/4-inch screen Passing $3 / 4$-inch screen Passing 1-inch screen --

NoтE-Either of these alternate sizes should be designated as desired. The 0 to $3 / 4$ inch size is preferred.

(4) 0 to $1 / 4$ inch size-

Pcr cent

Passing 1/4-inch screen 85-100

Passing 12 -inch screen

\section{METHOD OF TESTING}

Mechanical analysis, A. S. T. M. D18. (See 512.15, p. 250.)

\section{NOTES}

The materials covered by this specification are intended for use in the construction of water-bound base or surface courses, as follows:

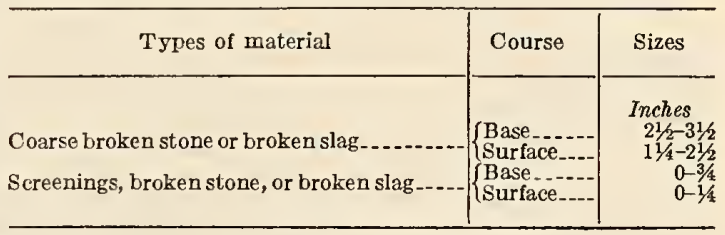

Federal Specifications Board, specification No. 457, United States Government master specification for broken stonc and broken slag for bituminous macadam base or surface course, January 25, 1927.

The requirements for broken stone under these specifications are substantially the same as for 
A. S. T. M. D191 (above), with the following variations:

Substitute the following for the A. S. T. M. requirement for screenings:

Chips, $1 / 4$ to $3 / 4$ inch size-

Passing 1/4-inch sereen.

assing 1/4-inch screen

Passing $1 / 2-$ inch screen

Passing $3 / 4$-inch screen

Passing 1-inch screen

Include the following requirement for filler stone:

Filler stone, $3 / 4$ to $1 \frac{1}{4}$ inch size-

Per cent

Passing 3/4-inch screen. $0-15$

Passing 1-inch screen

Passing 11/4-inch screen

Passing 1/2-inch screen

Note-For the part of this specification relating to slag, see A.S T.M. D159-27, page 254.

Federal Specifications Board, specification No. 458, United States Government master specification for broken stone and broken slag for bituminous concrete base or surface course, January 25, 1927.

\section{BROKEN STONE}

1. Broken stone shall consist of clean, tough, durable fragments, free from an excess of flat, elongated, soft, or disintegrated pieces, dirt, or other objectionable matter.

2. Detail requirements: (a) The stone shall meet the following requirements-

Note.-These test values for quality of stone are suggested as the lowest safe requirements. With favorable conditions of availahle materials the maximum percentage of wear permitted should he reduced and the minimum toughness permitted should be increased.

(1) Percentage of wear-_._._. (2) Toughness... Not less than 6.0 .

(b) Broken stone shall be well graded between the limits specified and, when tested by means of laboratory sereens, shall meet the following requirements for size as designated:

Note.-The particular size(s) desired should be designated.

(1) $1 \frac{1}{4}$ to $2 \frac{1}{2}$ inch size-

Per cent

Passing 11/4-inch screen.

Passing 2-inch screen

Passing $21 / 2$-inch screen

Passing $21 / 2$-inch seree

(2) $1 / 4$ to $2 \frac{1}{2}$ inch size-

Passing 1/4-inch screen

Passing 3/4-inch screen

Passing $1 \frac{1}{4}$-inch screen

Passing $21 / 2$-inch sereen

Passing 3-inch screen

(3) $1 / 4$ to $1 \frac{1}{4}$ inch size-

Passing 1/4-inch screen

Passing $3 / 1$-incb screen

Passing $1 \frac{1}{4}$-inch screen

Passing $1 \frac{1}{2}$-inch screen

(4) $1 / 4$ to $3 / 4$ inch size-

Passing 1/4-inch screen

Passing $1 / 2$-ineh screen

Passing 3/4-inch screen

Passing 1 -inch screen -

(5) $1 / 4$ to $1 / 2$ inch size-

Passing $1 / 4$-inch screen

Passing $1 / 2$-inch screen

Passing 3/4-inch screen

3. The materials covered by this specification are intended for use in the construction of bituminous concrete base or surface courses, as follows:

\begin{tabular}{|c|c|c|c|}
\hline Course & $\begin{array}{l}\text { Type of } \\
\text { material }\end{array}$ & Use of material & Size \\
\hline $\begin{array}{l}\text { Bituminous } \\
\text { concrete } \\
\text { hase. }\end{array}$ & $\begin{array}{l}\text { Broken stone } \\
\text { or broken } \\
\text { slag. } \\
\text { Broken stone } \\
\text { or hroken } \\
\text { slag. }\end{array}$ & $\begin{array}{l}\text { Coarse aggregate, open } \\
\text { mixture. } \\
\text { Coarse aggregate, closed } \\
\text { mixture. } \\
\text { Coarse aggregate, coarse- } \\
\text { graded hituminous con- } \\
\text { crete. } \\
\text { Coarse aggregate, fine- } \\
\text { graded hituminous con- } \\
\text { crete. }\end{array}$ & 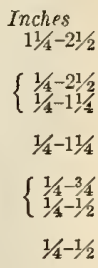 \\
\hline
\end{tabular}

4. Sampling and testing-

Sampling.-A. S. T. M. D75. (See 510, p. 184.)

Percentage of wear.-A. S. T. M. D2. (See 510, p. 182.)

Toughness.-A. S. T. M. D3. (See 510, p. 182.)

Mechanical analysis.-A. S. T. M. D18. (See p. 250.)

Federal Specifications Board, specification No. 459, United States Government master specification for broken stone and broken slag for binder course, sheet asphalt pavement, January 25, 1927.

\section{BROKEN STONE}

1. Broken stone shall consist of clean, tough, durable fragments, free from an excess of flat, elongated, soft or disintegrated pieces, dirt, or other objectionable matter.

2. Detail requirements: (a) Toughness, not less than 6 .

NотE.- This test value is suggested as the lowest safe requirement. With favorable conditions of available materials, the minimum toughness permitted should he increased.

(b) The broken stone, when tested by means of laboratory screens, shall meet the following requirements:

Passing $1 / 4$-inch screen -
Passing $3 / 4$-inch screen--
Passing $11 / 4$-inch screen

Passing $11 / 2$-inch screen

3. Sampling and testing-

Sampling: A. S. T. M. D75. (See 510, p. 184.)

Toughness: A. S. T. M. D3. (See 510, p. 182.)
Mechanical analysis: A. S. T. M. D18. (See p. 250.)

United States Department of Agriculture, Bulletin 1216 , method of test for apparent specific gravity and absorption of stone and other coarse materials, 1928.

1. The apparent specific gravity shall be obtained by weighing the water displaced by a sample of the material weighing approximately $1,000 \mathrm{~g}$, broken into pieces about $1 \frac{1}{4}$ inches in diameter. The vessel to be used consists of a galvanized-iron cylinder closed at one end and measuring 5 inches in diameter by 8 inches high. A brass spout one-half inch in diameter is soldered into the side of the cylinder 6 inches from the bottom. The spout is inclined at an angle of $2^{\circ}$ with the horizontal and is $2 \frac{1}{2}$ inches long. A notch is filed across its lower end to stop the drip from the displaced water. To determine the specific gravity and absorption, the dried and cooled sample shall be weighed to the nearest $0.5 \mathrm{~g}$ and immersed in water for 24 hours. The pieces shall then be surface dried individually with a towel, the sample reweighed and immediately placed in the cylinder, which has been previously filled to overflowing with water at room temperature.

2. The weight of water displaced by the sample shall be used to calculate its apparent specific 
gravity. The difference between the original weight of the sample and its weight after 24 hours shall be used to determine the absorption.

512.16 Fillers.

American Association of State Highway Officials, tentative standard specifications for highway materials, 1927.

\section{MINERAL FILLER}

1. This specification covers mineral filler for use in the construction of sheet asphalt or bituminous concrete pavements.

2. The mineral filler shall consist of Portland cement or approved dust prepared from crushed rock and shall be free from lumps or balls or any foreign material that may injure it for the purpose intended.

3. When tested by means of laboratory sieves, the mineral filler shall meet the following requirements:

Passing 100-mesh sieve, not less than

Per cent

Passing 200-mesh sieve, not less than

4. Sampling.-See A. S. T. M. D75, 510, p. 184

5. Testing-See A. S. T. M. D7, 512.12, p. 234.

American Society for Testing Materials, standard specifications for materials for cement grout filler for brick and stone-block pavements, serial designation D 57-20, 1920.

Approved as tentative American standard by the American Engineering Standards Committee, now the American Standards Association.

\section{SAND FOR CEMENT GROUT FILLER}

1. The sand shall consist of clean, hard, durable stone particles, preferably siliceous, free from lumps of clav and objectionable foreign matter.

2. (a) The sand shall be well graded from coarse to fine and when tested by means of laboratory sieves shall meet the following requirements:

Total passing No. 10 siere

Per cent

Total passing No. 10 siere

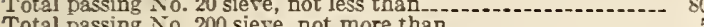

(b) This test shall be made in accordance with A. S. T. M. D7. (See 512.12, p. 234.)

3. (a) When the sand is mixed with Portland cement passing the requirements of the standard specifications and tests for Portland cement (serial designation C9, see 516.11, p. 287) of the American Society for Testing Materials, in the proportion of 1 part of cement to 3 parts of sand, by weight, according to the standard method of making $1: 3$ mortar briquets, the resulting mortar at the age of 7 and 28 days shall have a tensile strength of at least 75 per cent of that dereloped in the same time bv mortar of the same consistency, made of 1 part of the same cement and 3 parts of Ottawa sand.

(b) Preliminary acceptance samples shall be subjected to both 7 and 28 day mortar tests, and acceptance based thereon. Samples tested during the progress of the work will be accepted on the basis of the 7-dar test.

(c) The tests for mortar strength shall be made in accordance with the A. S. T. M. standard specifications and tests for Portland cement.

Note.-The Portland cement used in testing the proposed sand should preferably be that used or to be used in the work.

4. The sand shall be subject to inspection after delivery at the place of use, prior to and during the laving of the parement.

5 . The sand shall be sampled for testing at the source of supply, if required. Samples shall also be taken for testing from time to time, at the place of use prior to and during the laying of the pavement whenever desired by the engineer.

\section{PORTLAND CEMENT FOR CEMENT GROUT FILLER}

6. Portland cement for use in cement grout filler shall conform to the requirements of the A. S. T. M. standard specifications and tests for Portland cement.

\section{WATER FOR CEMENT GROUT FILLER}

7. Clean water free from harmful amounts of oil, acid, alkali, organic or vegetable matter shall be used.

Federal Specifications Board, specification No. 461, United States Government master specification for sand for cement grout filler, brick, and stoneblock pavements, January 25, 1927.

See par. 7 A.S. T. M. D57, above.

National Sand and Gravel Association, bulletin 3, representative specifications for different uses of sand and gravel, November, 1928.

(In this bulletin representative specifications, many of which are presented in this volume, and including specifications for "fillers and grouts, have been summarized.)

United States Department of Agriculture, Bulletin 1216, methods of sampling and testing premolded joint fillers, 1928 .

One section at least 1 foot long and the full width of the joint shall be submitted from each consignment and shall be tested in accordance with the following method:

(a) Absorption.-A sample 2 by 6 inches is cut from the joint material in such a manner that all edges are freshly cut and weighed. The specimen is then immersed in water for 24 hours, removed, and the surface water wiped off with a slightly dampened cloth. The specimen is then quickly weighed and the percentage of absorption computed.

(b) Brittleness. - A sample 2 by 6 inches is cut from the joint material parallel to the lay of the fiber and maintained at a temperature of from $4^{\circ}$ to $6^{\circ} \mathrm{C}$. in water for at least two hours prior to testing. It is then clamped between two boards so that the expansion joint cantilevers $3 \frac{1}{2}$ inches and is held in any suitable support. A cast-iron ball, weighing 0.95 pound and having a diameter of 1.875 inches, is suspended by a cord which is tied to an eyelet soldered to the ball. For samples having a thickness of nine-sixteenths inch or less the ball is suspended 1 foot above the center of the projected portion of the specimen. For samples over nine-sixteenths inch in thickness the ball is suspended 2 feet above the specimen. The ball is released by burning the string above the eyelet.

(c) Distortion.-A sample 2 by 6 inches, absolutely flat and straight, which has been cut parallel to the lay of the fiber, is clamped between two blocks so that the expansion joint cantilevers $3 \frac{1}{2}$ inches. The clamp with the expansion joint is then placed in an oven maintained at $125^{\circ} \mathrm{F}$. for two hours. The deflection from the horizontal is then measured.

United States Department of Agriculture, Bulletin 1216, method for determination of fineness of mineral filler, 1928.

1. The fineness test of mineral filler shall be made on a 200-mesh sieve which meets the requirements of the 200-mesh sieve specified for Portland cement.

2. The test shall be made with $50 \mathrm{~g}$ of filler. The sieve shall be thoroughly clean and dry. The filler shall be placed on the No. 200 sieve, with a pan and cover attached, if desired, and shall be held in one hand in a slightly inclined position so that the sample will be well distributed over the sieve, at the same time gently striking the side about 150 times per minute against the palm of the other hand on the upstroke. The sieve shall be turned every 
25 strokes about one-sixth of a revolution in the same direction. The operation shall continue until not more than $0.05 \mathrm{~g}$ passes through in one minute of continuous sieving. The fireness shall be determined from the weight of the residue on the sieve expressed as a percentage of the weight of the original sample.

3. Mechanical sieving devices may be used, but the filler shall not be rejected if it meets the fineness requirement when tested by the hand method deseribed in section 2 .

United States Department of Agriculture, Bulletin 1216, method of sampling mincral filler, 1928.

Mineral filler shall be sampled according to the method for sampling Portland cement received in carload lots. (See A. S. T. M. C9, 516.11, p. 287.)

\subsection{SIAG.}

American Association of State Highway Officials, standard specifications for highway materials, 1928.

This association has the following specifications for broken slag, which are presented under 512.15, page 244:

No. 3. For water-bound base.

No. 8. For Portland ccment concrete base course.

No. 10. For water-bound surface course.

No. 12. For bituminous macadam surface course.

No. 14. For bituminous concrete surface.

No. 16. For Portland cement concrete surface course.

American Society for Testing Materials, standard method of mechanical analysis of broken stone or broken slag, except aggregates used in cement concrete, serial designation D18-16, 1916.

(See 512.15, p. 250.)

American Society for Testing Materials, tentative specifications for commercial sizes of broken stone and broken slag for highway construction, serial designation D63-23T, 1923.

(See 511.71 , p. 207.)

American Society for Testing Materials, standard specifications for broken slag for water-bound base and wearing coursc, serial designation D65-23, 1923.

1. These specifications cover primarily slag screenings and coarse slag to be used in the construction of a water-bound base upon which some higher type of wearing course will be placed, preferably after the base has been firmly compacted by traffic. For a light traffic road it may also serve for both foundation and wearing course, provided the thickness of each is not less than 4 inches.

2 . The broken slag shall be air-cooled blast-furnace slag and shall consist of angular fragments reasonably uniform in density and quality, and reasonably free from thin, elongated or glassy pieces, dirt, or other objectionable matter.

3. The weight per cubic foot of each size specified shall be not less than 65 pounds.

4. The percentage of wear shall be not more than 12.0 per cent.

5. Screenings are that portion of the product of the crusher, including the dust of fracture, which, when tested by means of laboratory screens, shall meet the following requirements:

Passing $3 / 4$-inch screen, not less than

Per cent

Passing $3 / 4$-inch screen,
Passing $1 / 4$-inch screen 95

6. Coarse slag is that portion of the product of the crusher which, when tested by means of laboratory screens, shall meet the following requirements:

Passing 4-inch screen, not less than

Per cent

Passing 4-inch screen, not less than

7. (a) Weight per cubic foot.-The weight per cubic foot shall be determined as specified in A. S. T. M. C29. (See 512.10 p. 212.) (b) Percentage of wear.- The percentage of wear shall be determined as specified in A. S. T. M. D2 (see 510, p. 182), using, however, a volume of $4,000 \mathrm{cc}$ measured loose of the broken slag of the size specified in the standard test.

8. The method of sampling shall conform to A. S. T. M. D75. (See 510, p. 184.)

American Society for Testing Raterials, standard specifications for shovel-run or crusher-run broken slag for water-bound base, serial designation D66-23, 1923.

(Secs. 2, 3, 7, and 8 of A. S. T. M. D65, above, are part of this specification, with the following additional:)

1. These specifications cover primarily slag to be used in the construction of a water-bound base upon which some highcr type of wearing course will be placed, preferably af ter the base has been compacted by traffic.

2. The percentage of wear shall be not more than 15.0 per cent.

3. Shovel-run or crusher-run slag, when tested by means of laboratory screens, shall mect the following requirements:

Passing 4-inch screen, not less than

Per cent Passing $2 \frac{1}{2}$-inch screen.

25-75

American Society for Testing Materials, standard specifications for broken slag for bituminous macadam wearing course, serial designation D159-27, 1927.

(Sections 2, 7 (a), and 8 of A. S. T. M. D65 (above) are part of this specification with the following additional:)

1. These specifications cover $3 / 4$ to $1 / 4$ inch, $11 / 4$ to $3 / 4$ inch, and $2 \frac{1}{2}$ to $1 \frac{1}{4}$ inch or $3 \frac{1}{2}$ to $2 \frac{1}{2}$ inch size slag to be used in the construction of a bituminous macadam wearing course in which the bituminous material is applied by the penetration method. The maximum size of $3 \frac{1}{2}$ inches specified for the wearing course is on the basis of a course $2 \frac{1}{2}$ to $3 \frac{1}{2}$ inches in thickness after consolidation. The $1 \frac{1}{4}$ to $3 / 4$ inch size should be used to fill the surface voids after the first application of bituminous material and the $3 / 4$ to $1 / 4$ inch size after the application of the seal coat.

2. The weight per cubic foot of each size specified shall be not less than 70 pounds.

3 . The $3 / 4$ to $1 / 4$ inch size is that portion of the product of the crusher which, when tested by means of laboratory screens, shall meet the following requirements:

Passing 3/4-ingh sereen, not less than 95 Passing $1 / 4$-inch screen, not more than

4. The $1 \frac{1 / 4}{4}$ to $3 / 4$ inch size is that portion of the product of the crusher which, when tested by means of laboratory screens, shall meet the following requirements:

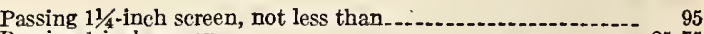
Per cent Passing 1 (1)

5. The $2 \frac{1}{2}$ to $1 \frac{1}{4}$ inch and $3 \frac{1}{2}$ to $2 \frac{1}{2}$ inch sizes are that portion of the product of the crusher which, when tested by means of laboratory screens, shall conform to one of the following requirements:

Passing 21/2-inch screen, not less than.

Per cent

-inch screen, not less than Total passing 11/4-inch screen, not more than............... 15 Potal $31 / 2$-inch screen, not less than.-.

NOTE.-One of the above alternate sizes for coarse slag should be specified to best suit the local and service conditions.

American Society for Testing Materials, standard specifications for broken slag for bituminous concrete (coarse-graded aggregate type), serial designation D160-27, 1927. 
(Sections 2, 7 (a), and 8 of A. S. T. M. D65 (above) and section 2 and 3 of A. S. T. M. D159 (above) are part of this specification with the following additional:

1. These specifications cover $3 / 4$ to $1 / 4$ inch and $1 \frac{1 / 4}{4}$ to $1 / 4$ inch size slag to be used in the construction of a coarse-graded bituminous concrete wearing course constructed by the mixing method. The $3 / 4$ to $1 / 4$ inch size should be used after the application of the seal coat when a seal coat is usea.

2. The $11 / 4$ to $1 / 4$ inch size is that portion of the product of the crusher which, when tested by means of laboratory scrcens, shall meet the following requirements:

Passing 11/1-inch screen Passing $3 / 1$-inch screen

American Society for Testing Materials, standard specifications for broken slag for bituminous concrete (fine-graded aggregate type), serial designation D161-27, 1927

(Sections 2, 7 (a), and 8 of A. S. T. M. D65 (above) are part of this specification with the following additional:)

1. These specifications cover slag aggregate to be used in the construction of a bituminous concrete wearing surface constructed by the mixing method.

2. The wcight per cubic foot shall be not less than 70 pounds.

3. The slag aggregate is that portion of the product of the crusher which, when tested by means of laboratory sereens, shall conform to the following requirements:

Passing $1 / 2$-inch screen

Per cent Passing $1 / 4$-inch screen 95-100 specifications for broken slag for bituminous macadan base, serial designation D195-27, 1927.

(Sections 2, 3, 7 (a), and 8 of A. S. T. M. D65 (above) are part of this specification with the following additional:)

1. These specifications cover $3 / 4$ to $1 \frac{1}{4}$ inch, $1 \frac{1 / 4}{4}$ to $2 \frac{1}{2}$ inch, and $2 \frac{1}{2}$ to $31 / 2$ inch size broken slag to be used in the construction of a bituminous macadam base in which the bituminous material is applied by the penetration method and which is to be covered with any type of surface course.

The $2 \frac{1}{2}$ to $3 \frac{1}{2}$ inch size is intended for use as a first course, which will be penetrated with bituminous material, when a base of over $3 \frac{1}{2}$ inches is desired.

The $1 \frac{1}{4}$ to $2 \frac{1}{2}$ inch size is intended for use as a second course which is to be penetrated with bituminous material.

The $3 / 4$ to $1 / 4$ inch size is intended to fill the surface voids when a sheet asphalt or a fine-graded bituminous concrete is to be placed directly upon it. This material can be omitted when a coarse-graded bituminous concrete or a binder course is to be laid directly upon the base.

2. The coarse slag when tested by means of laboratory screens shall conform to the following requirements:

(a) Passing 312-inch screen, not less than

Per cent

Total passing $21 / 2$-inch screen, not more than

(b) Passing 21 -inch screen, not less than

Total passing $1 \frac{1}{4}$-inch screen, not more than

3. The intermediate size slag when tested by means of laboratory screens shall conform to the following requirements:

Passing a 11/4-inch screen, not less than....................... 95 Per cent Passing $3 / 4$-inch screen, not more than.
American Society for Testing Materials, standard specifications for broken slag for bituminous concrete base, serial designation D196-27, 1927.

(Sections 2, 7(a), and 8 of A.S. T. M. D65 (above) are part of this specification with the following additional:)

1. These spccifications cover $1 / \frac{4}{4}$ to $1 \frac{1}{4}$ inch and $1 / 4$ to $21 / 2$ inch size broken slag, either of which is to be used in the construction of a bituminous concrete base prepared by the mixing method and upon which may be placed any type of surface course.

2. The weight per cubic foot of each size specified shall be not less than 70 pounds.

3. The slag when tested by means of laboratory screens shall conform to one of the following requirements:

Per cent

(a) Passing 11/4-inch screen, not less than ............................ Passing $3 / 4$-inch screen..........-.

Passing 1/4-inch screcn, not more than----.-- 5

Passing $21 / 1$-inch screen, not less than

Passing 1/1-inch screen, not more than -

Note,--One of the above alternate sizes for broken slag should be specified to best suit the local and service conditions.

Federal Specifications Board, specification No. 455, United States Government master specification for broken stone, broken slag or gravel for bituminous surface treatment, January 25, 1927.

(See 512.13, p. 240.)

Federal Specifications Board, specification No. 456, United States Government master specification for broken stone and broken slag for water-bound base or wearing course, January $25,1927$.

\section{BROYEN SLAG}

(Sections 2, 3, 7, and 8 of A. S. T. M. D65 (above) ale part of this specification with the following additional:)

Broken slag shall be that product of the crusher which, when tested by means of laboratory screens, shall meet the requirements for size as specified in the part of this specification devoted to "broken stone." (See 512.15 , p. 251 , sections 1 (b) and 2.)

\section{SHOVEL-RUN OR CRUSHER-RUN BROKEN SLAG FOR BASE}

The requirements for this product are the same as A. S. T. M. D66 (given above).

Federal Specifications Board, specification No. 457. United States Government master specification for broken slag for bituminous macadam base or surface course, January 25, 1927.

(The requirements for broken slag under these specifications are substantially the same as for A. S. T. M. D159 (above), with the following test method added:)

Mechanical analysis, A. S. T. M. D18. (See 512.15 , p. 250.)

Federal Specifications Board, specification No. 458, United States Government master specification for broken stone and broken slag for bituminous concrete base or surface course, January 25, 1927.

\section{BROKEN SLAG}

1. Broken slag shall be air-cooled blast-furnace slag, and shall consist of angular fragments reasonably uniform in density and quality, and reasonably free from thin, elongated, or glassy pieces, dirt, of other objectionable matter.

2. Detail requirements. - (a) Slag of each size specified shall meet the following requirement: Weight per cubic foot not less than 70 pounds. 
(b) The size requirements, uses, and methods of sampling and mechanical analysis in sections $2(b)$, 3 , and 4 of 512.15 , page 252, Federal Specifications Board, "broken stone", are part of this specification with the following test additional:

Weight per cubic foot, A. S. T. M. C29. (Sce 512.10 , p. 212.)

Federal Specifications Board, specification No. 459, United States Government master specification for broken stone and broken slag for binder course, sheet asphalt pavement, January 25, 1927.

\section{BROKEN SLAG}

1. Broken slag shall be air-cooled blast-furnace slag and shall consist of angular fragments reasonably uniform in density and quality, and reasonably free from thin, elongated, or glassy pieces, dirt, or other objectionable mattcr.

2. Detail requirements. - (a) Weight per cubic foot not less than 70 pounds.

(b) Size requirements and methods of sampling and testing are the same as for Federal Specifications Board "broken stone" (see 512.15, p. 251), with following test additional:

Weight per cubic foot, A. S. T. M. C29. (See 512.10 , p. 212.)

National Slag Association, recommended specifications for slag to be used in highways and highway structures, 1927.

\section{DEFINITION}

The term "slag" shall apply to the nonmetallic product, consisting essentially of silicates and alumino-silicates of lime, which is developed simultaneously with iron in a blast furnace.

\section{CLASSIFICATION}

The term "air-coolcd" shall apply to blastfurnace slag whose initial process of commercialization has included atmospheric cooling from molten to solid forms in pits or banks.

The term "granulated" shall apply to blastfurnace slag which has been cooled by pouring the slag while still molten into water.

\section{COMMERCIAL NAMES}

Commercial slag appears on the market under the following names:

(a) Coarse slag aggregate is broken air-cooled blast-furnace slag which usually passes a $4 \frac{1}{2}$-inch screen and is retained on a $1 / 4$ or $3 / 8$-inch screen. This product is subdivided by intermediate screens into sizes determined by the use to which it is to be put.

(b) Slag screenings is broken air-cooled blastfurnace slag which (according to the section in which it is produced) is furnished in sizes passing screens as follows: Upper limit, $1,3 / 4$, and $5 / 8$ inch; lower limit, $1 / 4,3 / 8$, or 0 inch.

(c) Slag sand is broken air-cooled blast-furnace slag, including dust of fracture, all of which usually passes a $1 / 4$-inch screen.

(d) Crusher-run slag is broken, but unscreened, air-cooled blast-furnace slag, sized approximately $4 \frac{1}{2}$ to 0 inch.

(e) Shovel-run slag is air-cooled blast-furnace slag marketed as an unprocessed material, sized approximately 6 to 0 inch.

$(f)$ Granulated slag is water-cooled blast-furnace slag, most of which will pass a $1 / 4$-inch screen.

\section{TESTS FOR BLAST-FURNACE SLAG}

WEIGHT-PER-CUBIC-FOOT DETERMINATION

Shall be made in accordance with A. S. T. M. C29. (See 512.10, p. 212.)

\section{METHOD OF SAMPLING}

Shall conform to A. S. T. M. D75. (See 512.10, p. 184.)

\section{SPECIFICATIONS 46}

Section (a).-The following specification covers the requirements (except for sizc) for slag to be used in the highway construction, highway maintenance, and highway structures listed below:

"The broken slag shall consist of roughly cubical fragments of air-cooled blast-furnace slag, reasonably uniform in density and quality and reasonably free from metallic iron, dirt, or other objectionable matter. The slag shall weigh not less than 70 pounds per cubic foot."

1. One-course plain or reinforced cement concrete highways.

2. Wearing course in 2-course plain or reinforced cement concrete highways.

3. Water-bound macadam wearing course.

4. Traffic-bound wearing course.

5. Bituminous macadam wearing course, penetration method.

6. Bituminous macadam wearing course, premixed method.

7. Rctread top bituminous construction.

8. Bituminous concrete, fine-graded aggregate type.

9. Bituminous concrete, coarse-graded aggregate type.

10. Single surface treatment.

11. Double surface trcatment.

12. Bituminous concrete binder course.

Section (b).-The following specification covers the requirements (with the exception of size) for slag to be used in the highway construction, highway maintenance, and highway structures listed below:

"The broken slag shall consist of roughly cubical fragments of air-cooled blast-furnace slag, reasonably uniform in density and quality and reasonably free from metallic iron, dirt, or other objectionable matter. The slag shall weigh not less than 65 pounds per cubic foot."

1. Plain or reinforced cement concrete base course.

2. Plain or reinforced cement concrete combination base and curb.

3. Plain or reinforced cement concrete highway structures. pipe.

5. Plain or reinforced cement concrete piling.

6. Drain tile, fence posts, markers, brick, and other cement concrete products.

7. Reconstructed rolled base course of slag or other matcrials.

8. Crusher-run slag base course.

9. Shovel-run slag base course.

10. Water-bound macadam base course.

11. Bituminous concrete "black base" course.

Section (c).- "Granulated slag for brick cushion and for granulated slag base course shall consist of water-cooled or steam-cooled blast-furnace slag,

40 These specifications agree substantially with those of the Federal Specifications Board, Committee E-5 of American Concretc Institute, and Committees C-9 and D-4 of American Society for Testing Materials, and with the recommendations made by $F . H$.Jackson and $J$ T. Pauls as the result of the Arlington Circular Track Tests of United States Bureau of Public Roads. 
substantially free from black cinder. For base course not less than 90 per cent shall pass threefourth inch, and for brick cushion not less than 95 per cent shall pass one-fourth inch."

United States Department of Agriculture, Bulletin 1216 , method of test for apparent specific gravity and absorption of stone and other coarse materials, 1928.

(See 512.15, p. 252)

\section{SAND-LIFE BRICK}

American Society for Testing Materials, tentative specifications for sand-lime building brick, serial designation C73-28T, 1928.

1. These specifications cover building brick made from sand and lime intended for use in brick masonry.

\section{PHYSICAL PROPERTIES AND TESTS}

2. (a) The bricks shall be classified as hard, medium, and soft on the basis of the following requirements: The classification of any lot of bricks shall be determined by the results of the tests for that requirement in which it is lowest.

\begin{tabular}{|c|c|c|c|c|}
\hline \multirow{2}{*}{ Classification } & \multicolumn{2}{|c|}{ Absorption limits } & \multicolumn{2}{|c|}{$\begin{array}{l}\text { Modulus of rupture } \\
\text { (brick flatwise) }\end{array}$} \\
\hline & $\begin{array}{l}\text { Mean of } 5 \\
\text { tests }\end{array}$ & $\begin{array}{l}\text { Individual, } \\
\text { maximum }\end{array}$ & $\begin{array}{c}\text { Mean of } 5 \\
\text { tests }\end{array}$ & $\begin{array}{l}\text { Indiridual, } \\
\text { minimum }\end{array}$ \\
\hline $\begin{array}{l}\text { Hard } \\
\text { Medium } \\
\text { Soft_. }\end{array}$ & $\begin{array}{c}\begin{array}{c}\text { Per cent } \\
9 \text { or less }\end{array} \\
9 \text { to } 15 \\
15 \text { or orer... }\end{array}$ & $\begin{array}{r}\text { Per cent } \\
12 \\
18 \\
\text { No limit. }\end{array}$ & $\begin{array}{l}\text { Lbs./in. } \\
600 \text { or over } \\
450 \text { or orer } \\
300 \text { or orer.- }\end{array}$ & $\begin{array}{r}\text { Lbs./in. }{ }^{2} \\
400 \\
300 \\
200\end{array}$ \\
\hline
\end{tabular}

(b) The tests shall be made in accordance with A. S. T. M. C67-28T. (See 534.10, p. 574.)

3 . For the purpose of tests, bricks representative of the commercial product shall be selected by a competent person appointed by the purchaser, the place or places of selection to be designated when the purchase order is placed. The manufacturer or seller shall furnish specimens for test without charge. All bricks shall be carefully examined and their condition noted before testing. For the purpose of tests, not less than 10 bricks shall be required. In general, two samples of 10 bricks each shall be tested for every 100,000 bricks contained in the lot under consideration, but where the total quantity exceeds 500,000 the number of samples tested per 100,000 bricks may be fewer, provided that they shall be distributed as uniformly as practicable over the entire lot. Additional representative samples may be taken at any time or place at the discretion of the purchaser.

\section{STANDARD SIZE}

4. The standard size of sand-lime brick shall be $2 \frac{1}{4}$ by $3 \frac{3}{4}$ by 8 inches with a permissible variation of plus or minus one-sixteenth inch in depth, oneeighth inch in width, and one-fourth inch in length.

\section{INSPECTION AND REJECTION}

5. All bricks shall conform to the following requirements for general properties under visual inspection:

(a) They shall be sound, of compact structure, reasonably uniform in shape, and free from cracks, warpage, large pebbles, balls of clay, or particles of lime that would affect their serviceability or strength. After being subjected to the absorption test they shall show no superficial disintegration.
6. If the visual inspection, section 5 , appears to indicate inferior strength, the bricks may be rejected unless the physical tests show the quality or grade to be as specified.

Federal Specifications Board, specification No. 505, United States Government master specification for brick, sand-lime, common, July 11, 1927.

This specification is applicable to common (not face) sand-lime brick of any of the following three classes: $H$, hard; $M$, medium; and $S$, soft.

\section{MATERIAL AND WORKMANSHIP}

Brick under this specification shall be of sand and lime hardened by treatment with high-pressure steam. They shall be sound, of compact structure, reasonably uniform in shape, and reasonably free from lime spots, large pebbles, and balls of clay.

\section{GENERAI REQUIREMENTS}

The standard size of brick shall be $2 \frac{1}{4}$ by $3 \frac{3}{4}$ by 8 inches with permissible variations of one-eighth inch in breadth or depth and one-fourth inch in length.

Bricks shall be delivered in good condition, with not more than 5 per cent broken.

At the completion of the absorption test the bricks shall show no evidence of a tendency to disintegrate.

\section{DETAIL REQUIREMENTS}

The brick shall meet the following absorption and strength requirements for its respective class. The standing of any set of bricks shall be determined by the requirements in which it is lowest. Unless otherwise specified in the invitation for bids, medium (M) or hard (H) brick shall be accepted in lieu of soft (S) brick and hard (H) brick in lieu of medium (M) brick.

\begin{tabular}{|c|c|c|c|c|}
\hline \multirow{2}{*}{ Class } & \multicolumn{2}{|c|}{ Absorption } & \multicolumn{2}{|c|}{$\begin{array}{c}\text { Transverse breaking load, } \\
\text { 7-inch span }\end{array}$} \\
\hline & Average of 5 & $\begin{array}{l}\text { Individual, } \\
\text { maximum }\end{array}$ & Average of 5 & $\begin{array}{l}\text { Individual, } \\
\text { minimum }\end{array}$ \\
\hline & $\begin{array}{c}\text { Per cent } \\
12 \text { or less.-.- } \\
12 \text { to } 20 .-1 \\
20 \text { or more.-- }\end{array}$ & $\begin{array}{r}\text { Per cent } \\
15 \\
24 \\
\text { No limit. }\end{array}$ & $\begin{array}{c}\text { Pounds } \\
1,080 \text { or more } \\
810 \text { or more } \\
540 \text { or more...- }\end{array}$ & $\begin{array}{r}\text { Pounds } \\
725 \\
540 \\
360\end{array}$ \\
\hline
\end{tabular}

\section{METHOD OF SAMPLING AND TESTS}

1. Sampling.-Ten bricks selected by the inspector, so as to be fairly representative of a quantity not exceeding 50,000 bricks, shall constitute a sample. If the bricks are delivered by car or boat, one sample shall be taken from each carload or boatload. If the bricks are delivered by truck or wagon, one or more samples shall be taken at the point of origin, covering all of the material from which shipments are to be made. Additional representative samples may be taken at any time or place at the discretion of the inspector.

2. Tests.-The sample shall be dried to constant weight at a temperature of $212^{\circ}$ to $220^{\circ} \mathrm{F}$.

(a) Absorption. - When cool, 5 of the 10 bricks in the sample shall be weighed separately on scales sensitive to within one-half of 1 per cent of the weight. They shall then be completely immersed in soft, distilled, or rain water at room temperature. The water shall be brought to a boil within one hour and boiling continued for five hours. The bricks shall be allowed to cool to room temperature in the water. They shall then be removed from the water and weighed, after wiping the surface 
with a damp cloth. This weight, minus the weight of the dry bricks, equals the weight of the water absorbed, which is calculated to per cent of the weight of the dry bricks.

Where means are not available for boiling the bricks, the absorption test may be made by immersing the dry bricks in soft distilled or rain water at ordinary temperature for five hours. When this method is used, the required absorption limits for the different classes shall be reduced one-fourth below the values given in the preceding section for both the average and the individual maximums.

In cases of disagreement as to the resulting classification, the absorption shall be determined by the boiling method and the full percentage absorption for the respective classes given in the preceding section applied. The same bricks, redried to constant weight, may be used in any such retest.

(b) Transverse strength. The other five bricks of the sample, previously dried, shall be tested laid flat wise on a span of 7 inches and with the load applied at the midpoint of the span with a standard testing machine or a calibrated portable or semiportable testing equipment. A steel bearing plate about $1 / 4$ inch thick by $1 \frac{1}{2}$ inches wide, shall be placed between the upper knife-edge and the brick. The knife-edges in contact with the brick shall be mounted so they will adjust themselves to the irregularities in the shape of the brick, and one or botll of the lower bearings shall be free to follow any movement of the brick during the test.

\section{NOTES}

1. Strength tests.-The acceptance procedure has been simplified to permit making the necessary tests at the building site or at the manufacturer's plant without the use of laboratory equipment, although the latter should be used where availablc. Responsibility for the accuracy, calibration, and general sufficiency of the equipment used necessarily devolves on the purchasing or inspecting officer concerned.

The Federal Specification Board specification contains specifications for equipment for conducting the transverse test, and also a list of manufacturers who have indicated willingness to supply equipment eomplying with the specifications.

2. Significance of the brick classification.-The elassification is based on strength and absorption values chosen so that gencrally bricks grading as medium or harder can be considered suitable for use in exterior walls. This should not be taken as a rigid distinction on account of the wide range in the character of materials used in brick manufacture, which makes it difficult to define weathering resistance in terms of properties that can be determined in acceptance tests. The purchasing officer should be guided in part by the experience with comparable bricks in the locality where those under test are to be used. In cases of doubt and where the time and equipment are available, acceptance in point of weathering resistance can be based on ability to withstand 100 alternations of freezing and thawing conducted according to generally accepted laboratory procedure. Failure is to be considered as reached when the samples are cracked or show superficial disintegration or spalling, with loss of weight of more than 5 per cent of the initial dry weight.

Where the wall is faced witl 3 inches or more of stone, terra cotta, brick, or other vencer, the weathering resistance of the material for the backing is without significance.

United States Department of Commerce, Bureau of Standards Simplified Practice Recommendation No. 38 , for sand-lime brick.
In accordance with unanimous action of a general conference of representatives of manufacturers, distributors, and users of sand-lime brick, the United States Department of Commerce, through the Bureau of Standards, recommends that one standard size of sand-lime brick be established as follows:

\begin{tabular}{c|c|c|c}
\hline Турө & Length & $\begin{array}{c}\text { Thick- } \\
\text { ness }\end{array}$ & Width \\
\hline Sand-lime brick_.................. & $\begin{array}{r}\text { Inches } \\
8\end{array}$ & $\begin{array}{r}\text { Inches } \\
21 / 4\end{array}$ & $\begin{array}{r}\text { Inches } \\
33 / 4\end{array}$ \\
\hline
\end{tabular}

\section{GYPSUM AND OTHER PLASTERS. 514.0 GENERAL ITEMS.}

American Society for Testing Materials, standard definition of terms relating to the gypsum industry, serial designation $\mathrm{C} 11-28,1928$.

Accelerator. - In the case of stucco, plaster, mortar, concrete, etc., a substance which will hasten the set.

Binder.- In the case of plaster and stucco, etc., a fibrous material which will increase their cohesiveness while they are in a plastic state.

Cement.-A material or a mixture of materials (without aggregate) which, when in a plastic state, possesses adhesive and cohesive properties, and which will harden in place.

NOTE.-The word "cement" is used without regard to the composition of the material.

Calcined gypsum.-Gypsum partially dehydrated by means of heat, liaving the approximate chemical formula $\mathrm{CaSO}_{4} \cdot 1 / 2 \mathrm{H}_{2} \mathrm{O}$.

Crushed gypsum.-Gypsun subjected to a primary crushing operation.

Gypsum.-Calcium sulphate, combined with two molecules of water, in crystallin form, having the approximate chemical formula $\mathrm{CaSO}_{4} \cdot 2 \mathrm{H}_{2} \mathrm{O}$.

Gypsum plastering sand.-The fine granular material naturally or artificially produced by the disintegration of rock, containing not less than 80 per cent by weight of silica, feldspar, dolomite, magnesite, or calcite.

Lath.-A material whose primary function is that of a base or background for the reception of plaster or stucco.

Mortar.-A material used in a plastic state, which can be troweled, and becomes hard in place, to bond units of masonry structures.

Note.-The word "mortar" is used without regard to the comnosition of the material, and is defined only with reference to its use as a bonding material as contrasted with the words "stucco" and "Plaster."

Plaster.-A material used in a plastic state, which can be troweled, to form a hard covering for the interior surfaces, walls and ceilings, etc., in any building or structure.

Noтe 1.-The word "plaster" is used without regard to the com. position of the material, and is defined only with reference to its use and location of use as contrasted with the words "stucco" and "mortar."

NOTE 2-This does not include decorative media ordinarily applied with a brush.

Rctarder.-In the case of stucco, plaster, mortar, concrete, etc., a substance which will retard the set

Sized gypsum.-Crushed gypsum of prescribed size of individual particles.

Stucco.-A material used in a plastic state, which can be troweled, to form a hard covering for the exterior walls or other exterior surfaces of any building or structure.

NoтE.-The word "stucco" is used without regard to the composition of the material, and is defined only with reference to it use and location of use as contrasted with the words "plaster" and "mortar," 
Wood fibcr.-A material produced by grinding or shredding wood.

Gypsum molding plaster.-A material consisting essentially of calcined gypsum for use in making interior embellishments, cornices, etc.

Gypsum pottcry plaster.-A material consisting essentially of calcined gypsum for use in making pottery, terra cotta, and other ceramic molds.

Keene's cement.- Anhydrous calcined gypsum, the set of which is accelerated by the addition of other materials.

American Society for Testing Materials, tentative definition of terms relating to the gypsum industry, serial designation C11-29T, 1929.

Aggregate.-Any inert material used as a filler in stucco, plaster, mortar, concrete, etc., without regard to its function as a binding material.

Gypsum plaster board.-A sheet composed of an incombustible core of gypsum, surfaced with paper or other fibrous material which is firmly bonded to the core, or with intermediate layers of such inaterial within the core, or an incombustible sheet of gypsum with not more than 15 per cent by weight of fiber intimately mixed and designed to be used as a lath for the reception of gypsum plaster.

Gypsum wall board.-A sheet composed of an incombustible core of gypsum, surfaced with paper or other fibrous material which is firmly bonded to the core, and designed to be used, without the addition of plaster, for walls, ceilings, or partitions and affording a surf ace suitable to receive decoration

Consistency. - A property of a material determined by the complete flow-force relation.

Plasticity. - A complex property of a material, involving a combination of the properties of mobility and of yield value.

Note.-A plastic material is distinct from a solid material in tbat it possesses mobility. It is distinct from a fuid material in that it requires a measurable force (yield value) to start llow.

Gypsum partition tile or block.-A gypsum building unit in form of tile or block for use in nonbearing construction in the interior of buildings and for the protection of columns, elevator shafts, etc., against fire.

Note 1.-Tbe words "tile" and "block" are synonymous and are used so as to be in accord with certain freight association nomenclature.

Not E 2.-Gypsum cored tile, 3 or 4 inches in thickness, may be split and used for furring purposes.

(NOTE.-These definitions, when adopted as standard, will be added to tbe present standard definitions of terms relating to tbe gypsum industry, C11-2 (given above).)

American Society for Testing Materials, standard methods of testing gypsum and gypsum products, serial designation $\mathrm{C} 26-27,1927$.

\section{DETERMINATION OF FREE WATER IN GYPSUM}

1. (a) Not less than 1 pound of the entire sample as received shall be weighed, spread out in a thin layer in suitable vessel, placed in a drying oven, and dried at $45^{\circ} \mathrm{C}$. for two hours. It shall then be cooled in an atmosphere free from moisture and weighed again. The loss of weight corresponds to the free water, and shall be calculated to percentage of sample as received.

(b) The dried sample shall be stored in an airtight container until used.

\section{DETERMINATION OF FINENESS}

2. Fineness shall be determined by sieving a known weight of the dried sample through sieves of the specified sizes and weighing the amount of material retained on each sieve. The material shall be shaken through the sieve with as little abrasion as possible.

The size of the sample to be used in this determination shall depend upon the size of grain of the sample. If the sample will all pass a 1/4-inch sieve, $100 \mathrm{~g}$ will be sufficient; if the largest particles are more than 1 inch in diameter, at least $1,000 \mathrm{~g}$ must be used. With these limitations the size of sample to be used is left to the discretion of the operator.

Note-The sizes of the sieves to be used are given in A. S , T. M. C22 (seo 514.1 p. 262), in A.S. T. M. C23 (see 514.2, p. 263), and in A. S. T. M. C2S (see 514.3 p. 265), covering the particular product examived.

\section{CHEMICAI ANALYSIS OF GYPSUM AND CAL- CINED GYPSUMI}

3. Dry sample as in section 1 , and reduce about $10 \mathrm{~g}$ until it all passes a No. 100 (149 micron) sieve, ${ }^{47}$ using extreme care not to unduly expose the material to the action of moisture or to overheating. The sample shall be kept in an air-tight container until ready for use.

4. Place $1 \mathrm{~g}$ of the sample in a covered crucible and dry at $215^{\circ}$ to $230^{\circ} \mathrm{C}$. to constant weight. Calculate the loss of weight to percentage of sample as received and report as combined water.

5. Place the residue obtained after drying, as described in section 4 , in a suitable flask and dissolve it in dilute $\mathrm{HCl}$ (not stronger than 1:4) in such a way that the gas evolved, after being freed from $\mathrm{H}_{2} \mathrm{O}$ vapor by calcium chloride or sulphuric acid, can be collected in either soda lime or caustic potash and weighed. The solution should be boiled for one minutc, and a current of $\mathrm{CO}_{2}$-free air kept passing through the apparatus for 30 minutes. The increase of veight of the soda lime or caustic potash corresponds to the weight of carbon dioxide, which is to be calculated to percentage of sample as received.

6 . Place $0.5 \mathrm{~g}$ of the sample prepared as described in section 3 in a porcelain casserole. Add about 25 cc of $1: 5 \mathrm{HCl}$, and evaporate to apparent dryness on a hot plate. Cool and add enough concentrated $\mathrm{HCl}$ to wet thoroughly. Add about $10 \mathrm{cc}$ of water, boil, filter, and wash. Put the filtrate back in the same casserole. Evaporate it to dryness and heat to about $120^{\circ} \mathrm{C}$. for one hour. Cool. Add enough concentrated $\mathrm{HCl}$ to wet thoroughly. Add about 25 cc of water, boil, filter, and wash. Transfer the two papers containing the two precipitates to the same crucible, ignite, and weigh. Calculate this weight to percentage of sample as received.

7. To the filtrate obtained as described in section 6, add a few drops of $\mathrm{HNO}_{3}$ and boil to insure oxidation of the iron. Add $2 \mathrm{~g}$ of $\mathrm{NH}_{4} \mathrm{Cl}$ previously dissolved in water. Make alkaline with $\mathrm{NH}_{4} \mathrm{OH}$. Digest hot for a few minutes until the precipitate coagulates. Filter, wash, ignite the precipitate, and weigh as $\mathrm{Fe}_{2} \mathrm{O}_{3}+\mathrm{Al}_{2} \mathrm{O}_{3}$. Calculate this weight to percentage of sample as received. This precipitate may be further treated to separate the two oxides, but this is generally unnecessary.

8. (a) To the filtrate obtained as described in section 7 , add $5 \mathrm{~g}$ of $\left(\mathrm{NH}_{4}\right)_{2} \mathrm{C}_{2} \mathrm{O}_{4}$ dissolved in water. Digest hot for one-half hour, making sure that the solution is always alkaline with $\mathrm{NH}_{4} \mathrm{OH}$. Filter, wash, and ignite in a platinum crucible over a strong blast to constant weight. Calculate this weight to percentage of sample as received.

(b) Alternative method.-To the filtrate obtained as described in section 7 , add $5 \mathrm{~g}$ of $\left(\mathrm{NH}_{4}\right)_{2} \mathrm{C}_{2} \mathrm{O}_{4}$ dissolved in water. Digest hot for one-half hour, making sure that the solution is always alkaline with $\mathrm{NH}_{4} \mathrm{OH}$. Filter and wash. Transfer the precipitate to a beaker, and wash the filter paper with hot dilute $\mathrm{H}_{2} \mathrm{SO}_{4}$, catching the washings in the same beaker. Heat gently to complete solution, adding more $\mathrm{H}_{2} \mathrm{SO}_{4}$ if necessary. While still warm, titrate with a solution of $\mathrm{KMnO}_{4}$ containing 5.6339 $\mathrm{g}$ per liter, until the pink color is permanent. The number of cubic centimeters of $\mathrm{KMnO}_{4}$ used gives

17 For detailed specifications for tbese sieves see serial designation E11 of A. S. T. M. 500.2 , see p. 1 . 
directly the percentage of lime in the dried sample. Recalculate to percentage of sample as received.

9. To the filtrate obtained as described in section $8(a)$ or $(b)$, add enough water to give a total volume of about $600 \mathrm{cc}$. Cool. Add $10 \mathrm{cc}$ of $\mathrm{NH}_{4} \mathrm{OH}$ and 5 g $\mathrm{NaNH}_{4} \mathrm{HPO}_{4}$ dissolved in water. Stir until precipitate begins to form. Let stand overnight. Filter, wash with a 2.5 per eent by weight solution of $\mathrm{NH}_{4} \mathrm{NO}_{3}$. Ignite and weigh. Multiply this weight by $40 / 111$ to find the weight of $\mathrm{MgO}$. Then calculate to percentage of sample as received.

10. Dissolve $0.5 \mathrm{~g}$ of the sample prepared as described in section 3 in $50 \mathrm{ec}$ of $1: 5 \mathrm{HCl}$. Boil. Add $100 \mathrm{cc}$ of boiling water, and continue boiling for five minutes. Filter immediately and wash thoroughly with hot water. Boil, and while boiling, add slowly $20 \mathrm{cc}$ of a boiling 10 per cent solution of $\mathrm{BaCl}_{2}$. Digest hot for one hour, or until precipitate settles. Filter and wash. Dry carefully. Ignite over Bunsen burner at lowest heat possible until filter paper is burned off. Ignite at bright red heat for 15 minutes, and weigh. Multiply this weight by $80 / 233$ to determine the weight of $\mathrm{SO}_{3}$. Then calculate to percentage of sample as received.

11. Dissolve in boiling water a $1 \mathrm{~g}$ sample (prepared as described in sec. 3 ), put on the filter, and wash with $250 \mathrm{cc}$ of boiling water then titrate the filtrate. Add two or three drops of potassium chromate solution and titrate with an $0.05 \mathrm{~N}$ solution of silver nitrate. Each cubic centimeter of silver nitrate solution $=0.002923 \mathrm{~g}$ of sodium chloride. Calculate to percentage of sample tested.

12. By the methods given above, the results are obtained and reported in the following form:

Free water

Combined water

Siliea and insoluble $\mathrm{SiO}_{2}$

Lime, $\mathrm{CaO}$

Magnesia, $\mathrm{MgO}$

Sulfur Trioxide, SO

Carbon Dioxide, $\mathrm{CO}$

Sodium Chloride, $\mathrm{NaCl}$

Per cent

100.001

Note.-Since it is frequently advisable to recalculate these results, that they may be nore enlightening, the following method is submitted for eonsideration:

(a) Multiply percentage of $\mathrm{MgO}$ by $84 / 40$, to find pereentage of

$\mathrm{MgCO}_{3}$

(b) Multiply the percentage of MgO by $44 / 40$ to find the percentage of $\mathrm{CO}_{2}$ as $\mathrm{MgCO}_{3}$.

(c) Deduct $\mathrm{CO}_{2}$ as $\mathrm{MgCO}_{3}$ from the $\mathrm{CO}_{2}$ determined.

(d) Multiply the $\mathrm{CO}_{2}$ remaining by $100 / 44$ to find percentage of $\mathrm{CaCO}_{3}$.

(e) Add together the pereentage of $\mathrm{SiO}_{2}, \mathrm{R}_{3} \mathrm{O}_{3}, \mathrm{MgCO}_{3}$, and $\mathrm{CaCO}_{3}$ and report in the aggregate.

(f) Multiply the percentage of $\mathrm{CaCO}_{3}$ by $56 / 100$ to find the pereen(f) Multiply the percent
tage of CaO as $\mathrm{CaCO}_{3}$.

tage of $\mathrm{CaO}$ as $\mathrm{CaCO}_{3}$.

(g) From the total percentage of $\mathrm{CaO}$, deduct the percentage

of $\mathrm{CaO}$ as CaCO3. The remainder may be ealled "available $\mathrm{CaO}$." Dctermine whieh (if either) is in excess.

(i) If the $\mathrm{CaO}$ is in excess, multiply the $\mathrm{SO}_{3}$ by $7 / 10$, and subtract the result from the "available $\mathrm{CaO}$." The remainder is reported as "excess CaO."

(j) If the $\mathrm{SO}_{3}$ is in excess, multiply the "available $\mathrm{CaO}$ " by $10 / 7$ and subtract the result from the $\mathrm{SO}_{3}$. The remainder is reported as "excess $\mathrm{SO}_{3}$."

(k) Add together the "available $\mathrm{CaO}$," and the $\mathrm{SO}_{3}$, and subtract the excess "CaO" or "exeess $\mathrm{SO}_{3} . "$ The remainder is $\mathrm{CaSO}_{4}$.

(l) If the $\mathrm{CaSO}_{4}$ is present as $\mathrm{CaSO}_{4} \cdot 1 / 2 \mathrm{H}_{2} \mathrm{O}$, the percentage of $\mathrm{CaSO}_{4}$ should bear to the percentage of combined water a ratio of $\mathrm{CaSO}_{4}$ should bear to the percentage of combined
136 to 9 . Determine which (if either) is in excess.

$(m)$ If the CaSO $\mathrm{C}_{4}$ is in excess some of it is present in the anhydrous form. Multiply the percentage of eombined water by $136 / 9$ to find the percentage of $\mathrm{CaSO}_{4}$ as $\mathrm{CaSO}_{4} \cdot 1 / 2 \mathrm{H}_{2} \mathrm{O}$. The difference between the total $\mathrm{CaSO}_{4}$ and the percentage of $\mathrm{CaSO}_{4}$ as $\mathrm{CaSO}_{4} \cdot 1 / 2 \mathrm{H}_{2} \mathrm{O}$, is the $\mathrm{CaSO}_{4}$ in the anhydrous form.

$(n)$ If the water is in excess, some of the $\mathrm{CaSO}_{4}$ is present as gypsum. Let $x=$ percentage of $\mathrm{CaSO}_{4} \cdot 1 / 2 \mathrm{H}_{2} \mathrm{O}$, and $y=$ percentage of CaSO $4 \cdot 2 \mathrm{H}_{2} \mathrm{O}$. Then $x+y=$ percentage of $\mathrm{CaSO}_{4}$ (as found in $k$ ) + percentage of water.

$$
\frac{9 x}{145}+\frac{36 y}{172}=\text { percentage of combined water. }
$$

Solve these equations for $x$ and $y$. Report $x$ as percentage of "calcined gypsum," CaSO $\mathrm{C}_{4} \cdot 1 / 2 \mathrm{H}_{2} \mathrm{O}$. Report $y$ as percentage of gypsum, $\mathrm{CaSO}_{4} \cdot 2 \mathrm{H}_{2} \mathrm{O}$.
Having made these calculations, the result may be reported as follows:

Per cent

Gypsum, $\mathrm{CaSO}_{4}+2 \mathrm{H}_{2} \mathrm{O}$

Calcined gypsum, $\mathrm{CaSO}_{4}+\frac{1}{2} \mathrm{H}_{2} \mathrm{O}$

Anhydrite CasO

Excess $\mathrm{CaO}$

Excess $\left.\mathrm{SO}_{3}\right\}$

Other ing, $\mathrm{NaCl}$

other ingredients.

$100.00 \pm$

The presence of the different forms of $\mathrm{CaSO}_{4}$ may be corroborated by a microscopic cxamination.

\section{PRECAUTIONS FOR PHYSICAL TESTS}

13. Gypsum products are peculiar in that their properties are very greatly affected by the small amounts of impurities which may be introduced by careless laboratory manipulation. In order to obtain concordant results, it is therefore absolutely essential to observe the following precautions:

(a) All apparatus shall be kept thoroughly clean. Especially shall all traces of set plaster be removed.

(b) Distilled water, free from chlorides and sulpliates, shall be used throughout.

(c) Standard Ottawa sand shall be used throughout. This shall be prepared for use by washing it once with 1:4 HCl and four times with distilled water, and then drying it.

\section{TESTING CONSISTENCY OF CALCINED GYPSUM}

14. In order that the results of testing samples of different plaster shall be directly comparable, it is necessary that all calcined gypsum be first brought to the same consistency, by the addition of the propcr amount of water. An accurate method for determining this consistency is the most important step in the standardizing of physical methods for testing cementing materials.

15. For the apparatus to be used for measuring consistency, reference is made to the Southard viscosimeter which has been used with satisfactory results.

The apparatus consists of a brass cylinder of 2-inch (5.08-cm) bore with a circular disk flange flush with its upper end. The screw actuating the piston is $5 / 8$ inch $(1.59 \mathrm{~cm})$ in outside diameter, $1 / 4$ inch $(0.635 \mathrm{~cm})$ pitch, right-hand square threads $1 / 16$ inch $(0.159 \mathrm{~cm})$ deep. The top of the brass disk flange is etched with concentric circles which vary in diameter from $6 \mathrm{~cm}$ up to $28 \mathrm{~cm}$ by increments of $2 \mathrm{~cm}$.

When in position for use the brass flange is to be maintained in a true horizontal position.

16. To prepare this viscosimeter for use see that the piston, cylinder walls, and top of plate are clean. Then by turning the crank, bring the top of the piston exactly flush with the top of the plate. Then by reverse cranking make 10 complete turns, which will lower the piston to a point $2 \frac{1}{2}$ inches $(6.35 \mathrm{~cm})$ below the top of the plate. Make a mixture of at least $300 \mathrm{~g}$ total of dry calcined gypsum and water. Shake the calcined gypsum into the water through a No. $8(2,380$-micron $)$ sieve ${ }^{48}$ allowing it to soak two minutes. Stir to an even fluidity for not to exceed 30 seconds. Pour this mixture immediately into the well in the center of the plate of the viscosimeter, filling the well just flush with the top of the plate. Then immediately turn the crank at the bottom of the viscosimeter 10 turns at the rate of 1 turn per second. The upward motion of the piston will cause the mixture to overflow into a circular pat, it being understood that the p. 1. 
top face of the circular disk of the instrument is to be adjusted and maintained in a true horizontal plane. Next take the average of the quadrant readings on the concentric lines on the top of the plate.

A neat mortar mixture is of testing consistency if with this operation, it gives a circular pat averaging $9.7 \mathrm{~cm}$ in diameter, and shall be expressed as the number of cubic centimeters of water required to be added to $100 \mathrm{~g}$ of the plaster.49

\section{WATER-CARRYING CAPACITY}

17. The water-carrying capacity is the amount of water, calculated as percentage by weight of the dry material, which is necessary to bring the mixture to testing consistency.

\section{DETERMINATION OF DRY BULK}

18. Place a No. 10 (2,000-mieron) sieve above a No. 20 (840-micron) sieve and lolding the two sieves together at a height of 12 inches $(30 \mathrm{~cm})$ above a perpendicular-sided receptacle of known volume and of about $400 \mathrm{cc}$ content.

Place the material under test on top of the No. 10 (2,000-micron) sieve and pass it through the No. 10 (2,000-mieron) sieve and No. 20 (840-micron) sieve filling the receptacle under the 12-inch head; then strike off and obtain the net weight of the contents in grams. Obtain the average of three tests according to this method and express the average result in cubic centimeters per $100 \mathrm{~g}$. Dry bulk is the volume in cubic centimcters occupied by $100 \mathrm{~g}$ of dry material. 50

Note.-All sieve numbers refer to United States Standard Sieve Series. ${ }^{s 1}$

\section{DETERMINATION OF WET BULK}

19. Wet bulk is the volume in cubic centimeters occupied by a mixture of $100 \mathrm{~g}$ of dry material mixed with water to testing consistency.

\section{DETERMINATION OF TIME OF SETTING}

20. Mix $200 \mathrm{~g}$ of the sample with enough water to make a paste of testing consistency. The directions for mixing are given under "Testing consistency." (See secs. 14, 15, and 16, above.)

Note.-If the sample is a sanded mixture use $300 \mathrm{~g}$ and mix to testing consisteucy for sanded mixture.

49 The following is recommended as a field method for the determination of testing consistency which will give fairly accurate resulis:

The apparatus shall consist of a piece of hrass tubing 2 inches $(5.08 \mathrm{~cm})$ in inside diameter and 4 inches $(10.15 \mathrm{~cm})$ long, and a piece of plate glass ahout 8 inches $(20.3 \mathrm{~cm})$ square. The brass tuhing shall he cut off true and square at the ends and to the exact length. Thoroughly wet the cylinder and glass plate hy immersion in clean water. Set the cylinder on end on top and in the center of the glass plate which is set and maintained in a true horizontal position.

Prepare a dry sample of calcined gypsum. Next make a mixture of calcined gypsum and water of sufficient quantity to slightly more than fll the cylinder. The mixture shall be made by adding the than fill the calcined gysura for two minutes. pour this mixture into the cylinder so as to completely fill it level pour this mixt

Immediately raise the cylinder from the glass with a quick, straight and upward motion so as to withdraw it from the mixture within. This act will allow the mixture to leave the cylinder, assuming a cone-shapcd circular pat upon the glass plate.

The testing consistency of a neat mortar is a mixture which will give a circular pat averaging $43 / 4$ inches ( $12 \mathrm{~cm}$ ) in diameter, and shall he expressed as the numher of cuhic centimeters of water required to be added to $100 \mathrm{~g}$ of the plaster.

The testing consistency of a sanded mortar is a mixture that, when placed in a 2 hy 4 inch (5.08 hy $10.15 \mathrm{~cm}$ ) cylinder immediately removed, the slump or settlement of the sanded mixture will he $1 / 2$ inch $(1.27 \mathrm{~cm})$.

${ }_{60}$ Dry hulk is expressed as the volume in cuhic centimeters occupied hy $100 \mathrm{~g}$ of dry material instead of "grams per $100 \mathrm{cc}$," since bulk is understood to mean volume per unit weight rather than weight per unit volume.

s1 See footnote 48 , p. 260.
Fill a rubber mold with the paste, and test for time of set with a Vicat needle. The specincations for the mold and needle are given in A. S. T. M. C9. (See 516.11, p. 287.)

When conducting the test the needle is allowed to sink into the paste at frequent intervals. After each penetration the needle must be wiped clean and the paste moved slightly so that the needle will not strike the same hole twice.

The frequency of the penetrations will depend upon the character of the material. If the sample is not retarded, it should be tested every two minutes until nearly set, and then every minute. If the sample is retarded, the intervals at first may be as long as one hour, but in any case they must be so selected that the time of sct is ascertained to the nearest minute.

Set is considered complete when the needle no longer penetrates to the bottom of the paste. The minutes elapsed from the time when the sample was first added to the water to the time when set is complete is recorded as the time of set of the sample.

Caution.-For test to determine the time of sct, it is ahsolutely essential that all dishes and u teusils he clean. Especially must they he free from all traces of set gypsum. Use distilled water.

\section{DETERMINATION OF TENSILE STRENGTH}

21. Mix $500 \mathrm{~g}$ of the sample to testing consistency. Cast into a 5-gang briquet mold, of the shape and size used for testing Portland cement. (See A. S. T. M. C9, 516.11, p. 287.) Do not cast each briquet successively, but move the containing vessel back and forth over the molds while pouring continuously. Work the briquet slightly with the point of the trowel to remove air bubbles, and level off the briquets. When sufficiently hard, remove and store in the room at a temperature of not less than $15.6^{\circ} \mathrm{C} .\left(60^{\circ} \mathrm{F}\right.$. $)$ nor more than $37.8^{\circ} \mathrm{C} .\left(100^{\circ} \mathrm{F}\right.$. $)$ for at least seven days. Weigh oncc a day. When the weight has become constant to within 0.1 per cent, proceed to test the specimens in a standard machine used for the determination of tensile strength.

Note.-When the material to he tested is a sanded mixture, use not less than $750 \mathrm{~g}$ of the sample and mix to testing consistency for sanded misture.

The average tensile strength shall be reported as the tensile strength of the material, except that if one or two briquets vary more than 15 per cent from the average of the five they shall be discarded and the tensile strength shall be reported as the average of the remaining specimens. In case three or more briquets vary more than 15 per cent from the average of the five, tests shall be continued upon three batches of five briquets and the average of the 15 briquets shall be reported. The tensile strength of all briquets shall be reported.

\section{DETERMINATION OF COMPRESSIVE STRENGTH}

22. Mix 2,000 g of the sample to testing consistency. Cast into five 2 by 4 inch split eylinders set plumb upon a metal or glass plate. Do not cast each cylinder successively, but move the containing vessel back and forth over the cylinders while pouring continuously. Work the cylinders slightly with the point of the trowel to remove air bubbles, and level off the top of the cylinders. When sufficiently hard, remove and store in the room at a temperature of not less than $15.6^{\circ} \mathrm{C}$. $\left(60^{\circ}\right.$ F.) nor more than $37.8^{\circ}$ C. $\left(100^{\circ}\right.$ F.) for at least seven days, and by weighing once a day, when the weight has become constant to within 0.1 per cent, proceed to test the specimens in a standard machine used for the determination of compressive strength.

Nore. - When the material to be tested is a sanded mixture, use not less than $3,000 \mathrm{~g}$ of the sample and mix to testing consistency for not less than 3,000 
The average compressive strength shall be reported as the compressive strength of the material, except that if one or twe of the cylinder specimens vary more than 15 per cent from the average the compressive strength shall be reported as the average of the remaining specimeins. The compressive strength of all the cylinders shall be reportcd.

\section{CONPRESSIVE STRENGTH OF GYPSUM PAETT- TION TIIE OR BIOCK}

23. (a) Not less than 10 fuil-size gypsum tile specimens shall be used.

(b) The test specimens shall be dried to constant weight at a temperature not exceeding $110^{\circ} \mathrm{F}$. $\left(43^{\circ}\right.$ C.), until two successive weighings of the same specimen do not show variation in excess of 0.5 per cent. At the option of the manufacturer or purchaser, strength tests may be conducted on samples that are dried at room temperature. However, in cases of controversy all test samples shall be dried to constant weight as herein prescribed.

(c) The tile or block shall be tested in the position in which they are designed to be used and shall bc bedded on and capped with a felt gasket not less than one-eighth inch nor more than one-fourth inch in thickness. At the option of the manufacturer or purchaser, or in cases of controversy, the test samples may be suitably bedded and capped with neat gypsum mortar, or the bearing surfaces of the tile may be planed or rubbed smooth and true. When neat sypsum mortar is used for bedding and capping, the test may be conducted after the mortar has set, but not sooner than 24 hours after the sample has been capped.

(d) The loading head shall completely cover the bearing area of the tile, and the applied load shall be transmitted through a spherical bearing block of proper design. The speed of the moving head of the testing machine shall not be more than 0.05 inch per minute.

\section{TRANSVERSE STRENGTH OF GYPSUM PARTITION TILE OR BLOCK}

24. (a) Not less than 10 full-size gypsum tile specimens shall be used.

(b) The test specimens shall be dried to constant weight as prescribed for the determination of the compressive strength in accordance with section $23(b)$

(c) The tile or block shall be supported on its face (flat) on fixed parallel bearings spaced 24 inches $(600$ mm) on centers, and shall be loaded through a similar bearing midway between the supports. All bearing and load suriaces shall be true, shall engage the full width of the test specimen, and shall be rounded to a radius of $1 / 2$ inch $(12.7 \mathrm{~mm})$.

(d) The speed of the moving head of the testing machine shall not be more than 0.05 inch per minute. The modulus of rupture shall be calculated in pounds per square inch from the formula:

$$
\text { Modulus of rupture }=\frac{3 w l}{2 b d^{2}}
$$

where $l=$ distance between supports in inches. $b=$ breadth (width) of tile in inches. $d=$ thickness of tile in inches. $w=$ load in pounds at failure of test specimen.

\section{ABSORPTION OF GYPSUM PARTITION TILE OR BLOCK}

25. (a) The absorption shall be determined upon a test specimen of partition tile or block consisting of approximately one-half of a full-size tile.

(b) The test specimen shall be dried to constant weight as prescribed for the determination of the compressive strength in succordance with section $23(b)$

(c) The dried test specimen shall be submerged in water until a constant saturated weight is attained, but in no event for a period of less than two hours, the water temperature beicg maintained at between $70^{\circ}$ and $80^{\circ} \mathrm{F} .\left(21^{\circ}\right.$ and $27^{\circ} \mathrm{C}$. $)$. The test specimen shall be removed, the surface wiped off with a damp cloth, and the specimen weirhed immediately. The percentage of absorption shall be calculated on the dry weight from the formula:

$$
\text { Percertage of absorition }=\frac{100(B-A)}{A}
$$

where $A=$ weight of dry test specimen.

$B=$ weight of saturated test specimen.

(d) The rate of absorption shall be determined by sealing upon the face of the test specimen, which shail first be dried to constant. weight as prescribed for determination of the compressive strength, a glass tube $1 \frac{1}{2}$ inches internal diameter and 12 inches in length, graduated in centimeters and located centrally over a core. Into this tube shall be poured $250 \mathrm{cc}$ of water at a temperature of between $70^{\circ}$ and $80^{\circ} \mathrm{F}$. $\left(21^{\circ}\right.$ and $27 \mathrm{C}$.). The rate of absorption shall be reported in cubic centimeters per minute.

\section{TRANSVERSE STRENGTH OF GYPSUN BOARDS}

26. (a) Test specimens shall be taken from not less than 10 gypsum boards.

(b) Strength test specimens shall be 12 inches $(300 \mathrm{~mm})$ in width and approximately 18 inches (460 $\mathrm{mm}$ ) in length. They shall be supported on fixed parallel bearings spaced 16 inches $(410 \mathrm{~mm})$ on centers and shall be loaded through a similar bearing midway between the supports. All bearing and load surfaces shall be true, shall engage the full width of the test specimen, and shall be rounded to a radius of $1 / 8$ inch $(3.2 \mathrm{~mm})$.

(c) Test loads shall be applied at a uniform rate of 60 pounds $(27.22 \mathrm{~kg})$ per minute with a permissible variation of \pm 10 per cent in the rate. Strength test results shall be reported when the load is applied across the fiber of the surfacing and also parallel to the fiber of the surfacing.

National Sand and Gravel Association, Bulletin 3, Representative Specifications for Different Uses of Sand and Gravel, November, 1928.

(In this bulletin representative specifications, many of which are presented in this volume, and including specifications for plaster sand, have been summarized.)

\subsection{GYPSUM.}

American Society for Testing Materials, standard specifications for gypsum, serial designation C22-25, 1925.

\section{MATERIALS AND STANDARDS}

1. The chemical and physical properties of gypsum shall be determined in accordance with A. S. T. M. C26 (given above).

2. (a) Pure gypsum is calcium sulphate combined in crystallin form with two molecules of water

(b) Its chemical formula is $\mathrm{CaSO}_{4} \cdot 2 \mathrm{H}_{2} \mathrm{O}$.

(c) No material may be considered gypsum within the meaning of these specifications which contains less than 64.5 per cent by weight of $\mathrm{CaSO}_{4} \cdot 2 \mathrm{H}_{2} \mathrm{O}$.

3. Gypsum may be in any of the following forms and sizes:

(a) Run-of-mine gypsum.-The form in which it comes from the mine or quarry.

(b) Crushed gypsum.-Run-oi-mine gypsum further reduced so that all of it will pass a 3 -inch $(76-\mathrm{mm})$ ring, and not more than 25 per cent shall pass a No. 100 sieve. 
(c) Sized gypsum.- Shall all pass a 11/2-inch (38-mm) ring, and not more than 10 per cent shall pass a 1 -inch sieve.

(d) Ground gypsum.-Gypsum further reduced for specific uses. Ground gypsum may be in any of the following standard forms:

No. 1.- This material shall all pass a No. 14 sieve, and not less than $\$ 5$ per cent of it shall pass a No. 100 sicve.

Note - For detailed specifications for these sieres, see A.S. T. M. L11, 500.2 (p. 1).

No. 2.-This material shall all pass a No. 14 sieve, and not less than 60 nor more than 85 per cent of it shall pass a No. 100 sieve.

No. 3.-This material shall all pass a No. S sieve, and not less than 40 nor more than 60 per cent of it shall pass a No. 100 sieve.

No. 4.- This material shall all pass a No. 100 sieve.

4. The chemical composition, within the limits prescribed by section 2 , shall be a matter of contract.

\section{SAMPIING}

5. When gypsum is shipped in bulk, samples of about 5 pounds $(2.25 \mathrm{~kg})$ each shall be taken at regular intervals during the complete loading or unloading of the carrier, in such a way as to accumulate at least 200 pounds $(90 \mathrm{~kg})$ of material, and to represent correctly the percentages of both the coarse particles and the fines in the shipment. This shall be broken so that it will all pass a 1 -inch (25- $\mathrm{mm})$ ring, thoroughly mixed, and reduced by quartcring to provide not less than a 1-pound $(0.5-\mathrm{kg})$ sample for the laboratory.

6. When gypsum is shipped in packages, at least 3 per cent of the packages shall be sampled. Samples shall be taken from both the surface and the center of the packages. These samples sliall then be broken, mixed, and quartered as directed above.

7. All laboratory samples shall be placed immediately in an air-tight container and shipped to the laboratory for test.

\section{PACKING AND MARKING}

8. (a) Gypsum may be shipped either in packages or in bulk.

(b) When shipped in packages for resale, the following information shall be legibly marked on each package or on a tag of suitable size attached thereto:

Name of manufacturer.

Form and size of material.

Chemical analysis of the material, giving the minimum percentages of $\mathrm{CaO}$ and $\mathrm{SO}_{3}$.

Net weight of package.

(c) When shipped in bulk, a card containing the required information shall be conspicuously placed in the carrier.

\section{INSPECTION AND REJECTION}

9. Inspection may be made either at the point of shipment or at the point of delivery. The inspector representing the purchaser shall have free access to the carriers being loaded for shipment to the purchaser. He sliall be afforded all reasonable facilities for inspection and sampling, which shall be so conducted as not to interfere unnecessarily with the loading of the carriers.

10. Any rejection shall be based upon the specific cause of failure to conform to the requirements of these specifications, and shall be reported within 10 working days from the receipt of the shipment by the consignee.
11. Claims for rehearing snall be valid only if made within 20 working days from receipt of notice of specific cause for rejection.

\subsection{CALCINED GYPSUM (PLASTER OF PARIS).}

American Society for Testing Materials, standard specifications for calcined gypsum, serial designation C23-22, 1922.

\section{MATERIALS AIED STANDARDS}

1. The chemical and physical properties of calcined gypsum shall be determined in accordance with A. S. T. M. C26. (See 514.0, p. 259.)

2. Calcined gypsum is the product resulting from the partial dehydration of gypsum by means of heat.

3. Calcincd gypsum may be marketed in either of the following sizes:

No. 1.- Material of this size shall all pass a No. 14 sieve, and not less than 75 per cent of it shall pass a No. 100 sieve.

No. 2.- Material of this size shall all pass a No. 14 sieve, and not less than 40 nor more than 75 per cent of it shall pass a No. 100 sicve.

Note.-A tolerance provision of 1 per cent on all siere determina. tions is permitted. All sieve numbers refer to United States stand. ard sieve series. (See A. S. T. M. E11, 500.2, p. 1.)

4. (a) The time of set of calcined gypsum shall be suitable for the purposes intended, and shall conform to the provisions prescribed for such in A. S. T. M. C28. (See 514.3, p. 265.)

(b) Calcined gypsum for molding or casting shall set in not less than 10 nor more than 40 minutes.

5. (a) Calcined gypsum shall have a tensile strength of not less than $200 \mathrm{lbs} . / \mathrm{in.}^{2}\left(14 \mathrm{~kg} / \mathrm{cm}^{2}\right)$.

(b) Calcined gypsum for molding or casting shall have a tensile streagth of not less than $200 \mathrm{lbs} . / \mathrm{in} .^{2}$ $\left(14 \mathrm{~kg} / \mathrm{cm}^{2}\right)$

6. (a) Calcined gypsum shall have a compressive strength of not less than $1,000 \mathrm{lbs} . /$ in..$^{2}\left(70 \mathrm{~kg} / \mathrm{cm}^{2}\right)$.

(b) Calcined gypsum for molding or casting shall have a compressive strength of not less than 1,000 lbs./in. ${ }^{2}\left(70 \mathrm{~kg} / \mathrm{cm}^{2}\right)$.

\section{SAMPLING}

7. At least 3 per ccnt of the packages shall be sampled, and sliall be so selected as to be representative of the contents of the shipment. Samples shall be taken from both the surface and the center of the packages. The material so obtained shall be thoroughly mixed and reduced by quartering to provide not less than a 15 -pound $(6.75-\mathrm{kg})$ sample for the laboratory.

8. All laboratory samples shall immediately be placed in an air-tight container and shipped to the laboratory for test.

\section{PACKING AND MARKING}

9. Calcined gypsum may be shipped in either packages or in bulk.

(a) When shipped for resale, the following information shall be legibly marked on each package or on a tag of suitable size attached thereto:

Name of manufacturer.

Brand.

Description.

Net weight of package.

(b) When shipped in bulk, a card containing the required information shall be conspicuously placed in the carrier. 


\section{INSPECTION AND REJECTION}

10. Inspection may be made either at the point of shipment or at the point of delivery. The inspector representing the purchaser shall have free access to the carriers being loaded for shipment to the purchaser. He shall be afforded all reasonable facilities for inspection and sampling, which shall be so eonducted as not to interfere unnecessarily with the loading of the carriers.

11. Any rejection shall be based upon the specific cause of failure to conform to the requirements of these specifications and shall be reported within ten working days from the receipt of the shipment by the consignee.

12. Claims for rehearing shall be valid only if made within 20 working days from receipt of notice of specific cause for rejection.

American Society for Testing Materials, tentative specifications for calcined gypsum for use in the preparation of dental plasters, serial designation C72-29T, 1929.

1. Calcined gypsums for use in the preparation of dental plasters are products of specifie properties with reference to time of set, fineness, and watercarrying capacity. These speeifications cover three grades, as follows:

Grade Q (quick setting); grade M (medium setting); and grade $\mathrm{S}$ (slow setting).

\section{REQUIREMENTS}

2. Calcined gypsum for use in the preparation of dental plasters shall contain not less than 93 per cent of calcined gypsum $\left(\mathrm{CaSO}_{4} \cdot 1 / 2 \mathrm{H}_{2} \mathrm{O}\right)$.

3. (a) Grade Q.- Shall set in not less than 2 nor more than 4 minutes.

(b) Grade $M$. - Shall set in not less than 6 nor more than 12 minutes.

(c) Grade S.- Shall set in not less than 20 nor more than 30 minutes.

4. The calcined gypsum, when tested in accordance with the requirements of section 9 , shall have a tensile strength of not less than $300 \mathrm{lbs}$. $/$ in. ${ }^{2}$.

5 . The calcined gypsum shall all pass a No. 30 sieve and not less than 95 per cent shall pass a No. 100 sieve.

6 . The calcined gypsum shall be dry and free from lumps.

\section{SAMPLING}

7. At least 3 per cent of the packages shall be sampled, and shall be so selected as to be representative of the contents of the shipment. Samples shall be taken from both the surface and the center of each package so selected. The material so obtained shall be thoroughly mixed and redueed by quartering to provide not less than a 15-pound $(6.75$-kg) sample for the laboratory.

8. All laboratory samples shall immediately be placed in an air-tight container and shipped to the laboratory for test.

\section{METHODS OF TESTING}

9. The chemical and physical properties of calcined gypsum for use in the preparation of dental plasters shall be determined in accordance with A. S. T. M. C26 (see 514.0 , p. 259 ), except that in the determinations of the time of set and the tensile strength the ealcined gypsum and water shall soak for 30 seconds in distilled water at $70^{\circ} \mathrm{F}$. and shall be mixed in the proportion of $100 \mathrm{~g}$ of ealcined gypsum to $00 \mathrm{cc}$ of water.

\section{PACKING AND MARIKING}

10. When shipped, the following information shall be legibly marked on each package or on a tag of suitable size attached thereto:

Name of manufacturer.

Brand.

Description.

Net weight of package.

\section{INSPECTION AND REJECTION}

11. Inspection may be made either at the point of shipment or at the point of delivery. The inspector representing the purchaser shall have free aecess to the carrier being loaded for shipment to the purchaser. He shall be afforded all reasonable facilities for inspection and sampling, which shall be so conducted as not to interfere unnecessarily with the loading of the carriers.

12. Any rejection shall be based upon the speeifie cause of failure to conform to the requirements of these specifications and shall be reported within 10 working days from the receipt of the shipment by the consignee.

13. Claim for rehearing shall be valid only if made within 20 working days from receipt of notice of specifie cause for rejection.

Federal Specifications Board, Specification No. 248, United States Government master speeification for calcined gypsum, November 5, 1924.

Calcined gypsum (plaster of Paris) sold under this specification may be of one of three classes: $\mathrm{C}$, easting; $\mathrm{G}$, general; and $\mathrm{F}$, finishing.

The calcined gypsum of any class may be either retarded or unretarded.

\section{MATERIAL AND WORKMANSHIP}

Calcined gypsum (plaster of Paris) sold under this specification is a fine white powder resulting from the partial dehydration of gypsum by means of heat.

\section{GENERAL REQUIREMENTS}

(a) Chemical composition-Calcined gypsum shall contain not less than 60.5 per cent by weight of $\mathrm{CaSO}_{4} \cdot 1 / 2 \mathrm{H}_{2} \mathrm{O}$.

(b) Tensile strength.-The tensile strength of ealcined gypsum shall be not less than 200 pounds per square ineh.

(c) Compressive strength. - The e o mpressive strength of ealcined gypsum shall be not less than 1,000 pounds per square inch.

\section{DETAIL REQUIREMENTS}

(a) Fineness.-Class $\mathrm{C}$ calcined gypsum shall all pass a No. 14 sieve, and not less than 75 per cent shall pass a No. 100 sieve.

Class G calcined gypsum shall all pass a No. 14 sieve, and not less than 40 nor more than 75 per cent shall pass a No. 100 sieve.

Class F calcined gypsum shall all pass a No. 14 sieve and not less than 60 per cent of it shall pass through a No. 100 sieve.

(b) Time of set.-Unretarded calcined gypsum shall set in not less than 10 nor more than 40 minutes.

Retarded caleined gypsum shall set in not less than 40 minutes nor more than 6 hours.

\section{METHODS OF INSPECTION AND TESTS}

(a) Sampling.-At least 3 per cent of the packages shall be sampled. Samples shall be taken from 
both the surface and the center of the packages. The material as obtained shall be thoroughly mixed and quartered to provide a 15-pound sample. All laboratory samples shall be placed immediately in an air tight container and sealed. Samples shall not be taken from broken packages.

(b) Chemical analysis.-For requirements of this specification under the captions "Preparation of sample," "Calcium oxide test," "Sulphur trioxide test," and "Combined water," see A. S. T. M. C26, 514.0 , page 259 .

NoтE.-The "Calcium oxide" test in this specification corresponds to the test captioned "Silica and insoluble" in the A. S.T. M. specification.

(c) Calculation.-Substitute the percentages found in the above tests in the equations $\mathrm{CaO}: \mathrm{SO}_{3}: \mathrm{H}_{2} \mathrm{O}=$ $56: 80: 9$. It will be found that one, and probably two, of the ingredients is present in excess of the amount required to satisfy these equations. Select that ingredient which is not in excess. (If more than one ingredient fulfills this condition either may be selected.) If this ingredient is $\mathrm{CaO}$, multiply the per cent $\mathrm{CaO}$ as found by 2.59 to find the per cent $\mathrm{CaSO}_{4} .1 / 2 \mathrm{H}_{2} \mathrm{O}$; if $\mathrm{SO}_{3}$, multiply per cent $\mathrm{SO}_{3}$ by 1.81; if $\mathrm{H}_{2} \mathrm{O}$, multiply per cent $\mathrm{H}_{2} \mathrm{O}$ by 16.11 .

(Additional methods under "Methods of inspection and tests" are as follows: $(d)$ Fineness, $(e)$ Testing consistency, $(f)$ Time of set, $(g)$ Tensile strength, and $(h)$ Compressive strength.)

(These methods are substantially the same as items under corresponding headings in A. S. T. M. serial designation C26, see 514.0, p. 259.)

\section{PACKING AND MARKING}

(a) Packing.-Calcined gypsum is shipped in packages.

(b) Marking.-Each package of calcined gypsum shall be legibly marked with a description of the contents, the name of the manufacturer, the brand (if any), and the net weight of the contents; also with some means of identification stating the particular contract on which the purchase and shipment were made.

\section{ADDITIONAI INFORMATION}

Class $\mathbf{C}$ calcined gypsum is designed to be used in casting ornamental work "on the bench," the casts to be later mounted in place. This class may also be used for running moldings, in place. It is known to the trade as "casting or molding plaster."

Class G calcined gypsum is intended for general structural purposes, such as preparing the setting mortar for gypsum block, pointing gypsum wall board, etc.

Class $\mathrm{F}$ calcined gypsum is intended for use as an addition to lime putty for the finishing coat of plaster.

The color of calcined gypsum of classes $\mathrm{C}$ and $\mathrm{F}$ is sometimes stressed, but it is believed that its importance is usually psychological rather than real.

Manufacturers can furnish calcined gypsum having any desired time of set within the limits specified.

NoTE.-Sieve numbers refer to United States standard sieve series. (See A. S. T. M. E11, 500.2, p. 1.)

\subsection{GYPSUM PLASTER.}

American Society for Testing Materials, standard specifications for gypsum plasters, serial designation C28-27, 1927.

1. The chemical and physical properties of gypsum plasters shall be determined in accordance with A. S. T. M. serial designation C26. (See 514.0, p. 259.)

\section{GYPSUM READY-SANDED PLASTER}

2. Gypsum ready-sanded plaster is a plastering material in which the predominating cementitious material is calcined gypsum, and which is mixed at the mill with all the constituent parts, including sand, in their proper proportion. It requires only the addition of water to make it ready for use.

3 . The following shall govern the composition of gypsum ready-sanded plaster for the desired coats:

(a) Scratch or first coat.-Gypsum ready-sanded plaster used for scratch or first coat shall contain not more than two-thirds by weight of sand. The other 33 per cent shall contain not less than 60.5 per cent by weight of calcined gypsum, $\mathrm{CaSO}_{4}$. $1 / 2 \mathrm{H}_{2} \mathrm{O}$, calculated from the $\mathrm{SO}_{3}$ content. The remainder may consist of materials to control the working quality, setting time, and the fibering.

(b) Browning or sccond coat.-Gypsum readysanded plaster used for browning or second coat shall contain not more than 75 per cent by weight of sand. The other 25 per cent shall contain not less than 60.5 per cent by weight of calcined gypsum, $\mathrm{CaSO}_{4} \cdot \frac{1}{2} \mathrm{H}_{2} \mathrm{O}$, calculated from the $\mathrm{SO}_{3}$ content. The remainder may consist of materials to control the working quality, setting time, and the fibering.

4. The time of set of gypsum ready-sanded plaster shall be as follows:

(a) Scratch or first coat.-This plaster shall set in not less than $1 \frac{1}{2}$ nor more than 7 hours.

(b) Browning or second coat.-This plaster shall set in not less than 2 nor more than 6 hours.

5. The tensile strength of gypsum ready-sanded plaster shall be as follows:

(a) Scratch or first coat.-This plaster shall have a tensile strength of not less than $75 \mathrm{lbs} . / \mathrm{in.}{ }^{2}\left(5 \frac{1}{4} \mathrm{~kg} /\right.$ $\mathrm{cm}^{2}$ ).

(b) Browning or second coat.-This plaster shall have a tensile strength of not less than $50 \mathrm{lbs} . / \mathrm{in.}{ }^{2}$ $\left(31 / 2 \mathrm{~kg} / \mathrm{cm}^{2}\right)$.

\section{GYPSUM NEAT PLASTER}

6. Gypsum neat plaster is a plastering material in which not less than 60.5 per cent of the cementitious material is calcined gypsum, $\mathrm{CaSO}_{4} \cdot 1 / 2 \mathrm{H}_{2} \mathrm{O}$, calculated from the $\mathrm{SO}_{3}$ content, and mixed at the mill with other materials.

7. Gypsum neat plaster shall contain not less than 60.5 per cent by weight of calcined gypsum, $\mathrm{CaSO}_{4}$. $1 / 2 \mathrm{H}_{2} \mathrm{O}$, calculated from the $\mathrm{SO}_{3}$ content. The remainder may consist of materials to control the working quality, setting time, and the fibering.

8. Gypsum neat plaster when mixed with three parts by weight of testing sand shall set in not less than 8 hours nor more than 32 hours.

9. Gypsum neat plaster shall have a tensile strength of not less than $150 \mathrm{lbs} . / \mathrm{in}^{2}\left(10 \frac{1}{2} \mathrm{~kg} / \mathrm{cm}^{2}\right)$.

\section{GYPSUM WOOD-FIBERED PLASTER}

10. Gypsum wood-fibered plaster is a gypsum plaster in which wood fiber is used as an aggregate.

11. Gypsum wood-fibered plaster shall contain not less than 60.5 per cent by weight of calcined gypsum, $\mathrm{CaSO}_{4}$. $1 / 2 \mathrm{H}_{2} \mathrm{O}$, calculated from the $\mathrm{SO}_{3}$ content, and not less than 1 per cent by weight of wood fiber made from a nonstaining wood. The remainder may consist of materials to control the working quality and setting time.

12. Gypsum wood-fibered plaster shall set in not less than $1 \frac{1}{2}$ nor more than 8 hours.

13. Gypsum wood-fibered plaster shall have a tensile strength of not less than $125 \mathrm{lbs} . / \mathrm{in.} .^{2}(8 \%$ $\left.\mathrm{kg} / \mathrm{cm}^{2}\right)$. 


\section{CAICINED GYPSUM FOR FINISHING COAT}

14. Calcined gypsum for finishing coat may, or may not, contain retarder. It may be classified in two grades, viz, "white" and "gray."

15. Calcined gypsum for finishing coat shall all pass a No. 14 sieve, and not less than 60 per cent of it shall pass through a No. 100 sieve.

Note.-All sieve numbers refer to United States standard sieve

16. Calcined gypsum for finishing coat when not retarded shall set in not less than 20 minutes nor more than 40 minutes, and when retarded shall set in not less than 40 minutes nor more than 6 hours.

17. Calcined gypsum for finishing coat shall have a tensile strength of not less than $200 \mathrm{lbs} . / \mathrm{in.} .^{2}(14 \mathrm{~kg} /$ $\mathrm{cm}^{2}$ ).

Note.-The water used for mixing gypsum plasters shall be fresh, pure, and clean. Trouble may occur from the presence of any excessive amount of mineral or organic substances in the water.

\section{PACKING AND MARKING}

Gypsum plaster shall be dry and free from lumps, and may be shipped either in packages or in bulk. Marking requirement is the same as in A. S. T. M. C23. (See 514.2, p. 263.)

(Requirements for sampling and inspection and rejection are the same as in A. S. T. M. C23.)

Anerican Society for Testing Materials, standard specifications for gypsum plastering sand, serial designation C35-25, 1925.

(See 512.12, p. 234.)

American Society for Testing Materials, tentative specifications for gypsum molding plaster, serial designation C59-29, 1929.

1. Gypsum molding plaster is a material employed in the making of interior embellishments, cornices, etc., and consists essentially of calcined gypsum.

\section{PROPERTIES}

2. Gypsum molding plaster shall contain not less than 80 per cent of calcined gypsum $\left(\mathrm{CaSO}_{4} \cdot{ }_{1}^{1} / 2 \mathrm{H}_{2} \mathrm{O}\right)$.

3. Gypsum molding plaster shall set in not less than 20 nor more than 40 minutes.

4. Gypsum molding plaster shall have a tensile strength of not less than $200 \mathrm{lbs} . / \mathrm{in} .^{2}\left(14.0 \mathrm{~kg} / \mathrm{cm}^{2}\right)$.

5. Gypsum molding plaster shall all pass a No. 30 sieve and not less than 90 per cent of it shall pass a No. 100 sieve.

\section{METHODS OF TESTING}

6. The chemical and physical properties of gypsum molding plaster shall be determined in accordance with A. S. T. M. C26. (See 514.0, p. 259.)

NotE.-The water used for mixing gypsum plasters shall be fresh, pure, and clean. Trouble may occur from the presence of any excessive amount of mineral or organic substances in the water.

\section{SAMPLING}

Same as A. S. T. M. C23. (See 514.2, p. 263.)

\section{PACKING AND MARKING}

Gypsum molding plaster shall be dry and free from lumps and may be shipped in 100-pound cloth sacks, 100-pound paper-lined cloth sacks, 80-pound paper sacks, or 250-pound (235-pound net) barrels. Marking requirement same as A. S. T. M. C23.

52 For detailed specifications for these sieves, see National Bureau of Standards, Letter Circular No, 74

\section{INSPECTION AND REJECTION}

Same as A. S. T. M. C23.

American Society for Testing Materials, tentative specifications for gypsum pottery plaster, serial designation $\mathrm{C} 60-29,1929$.

1. Gypsum pottery plaster is a material consisting essentially of calcined gypsum for use in making pottery molds.

\section{PROPERTIES}

2. Gypsum pottery plaster shall contain not less than 90 per cent of calcined gypsum $\left(\mathrm{CaSO}_{4} \cdot 1 / 2 \mathrm{H}_{2} \mathrm{O}\right)$.

3 . Gypsum pottery plaster, when mixed in accordance with the requirements of section six, shall set in not less than 20 minutes nor more than 40 minutes.

4. Gypsum pottery plaster, when mixed in accordance with requirements of section six, shall have a tensile strength of not less than $250 \mathrm{lbs} . / \mathrm{in} .{ }^{2}$ (17.5 $\mathrm{kg} / \mathrm{cm}^{2}$ ).

5. Gypsum pottery plaster shall all pass a No. 30 sieve and not less than 94 per cent of it shall pass a No. 100 sieve.

\section{IIETHODS OF TESTING}

6. The chemical and physical properties of gypsum pottery plaster shall be determined in accordance with A. S. T. M. C26 (see 514.0, p. 259), except that in the measurement of the setting time and tensile strength the plaster and water shall be mixed in the proportion of $100 \mathrm{~g}$ of plaster to $60 \mathrm{cc}$ of water.

Nore.-The water used for mixing gypsum plasters shall be fresh, pure, and clean. Trouble may oceur from the presence of any excessive amount of mineral or organic substances in the water.

Items "sampling," "packing and marking," and "inspection and rejection" are the same as for A. S. T. M. C59, (given above.)

Contracting Plasterers' International Association, standard specification for lath and plaster work, 1923.

(See 514.63, p. 280.)

(This specification includes requirements for gypsum plasters.)

Federal Specifications Board, specification No. 247, United States Government master specification for gypsum plaster, November 5, 1924.

\section{TYPES}

This specification covers four types of gypsum plaster: $W$, wood-fibered plaster; $N$, neat plaster; $B$, sanded brown coat plaster; and $S$, sanded scratch coat plaster.

The calcined gypsum to be used as an addition to lime putty for the finish coat of plaster is covered by the Federal Specifications Board specification No. 248. (See 514.2, p. 264.)

\section{MATERIAL AND WORKMANSHIP}

Gypsum plaster sold under this specification consists essentially of calcined gypsum to which has been added at the factory some of the materials which inust be mixed with it in the preparation of a wall plaster.

Wood-fibered plaster contains some wood fiber, retarded, and sometimes a material added to improve its working quality.

Neat plaster contains retarder and may or may not contain hair and some material added to improve its working quality.

Ready-sanded brown coat plaster contains all of the ingredients necessary, except water.

Ready-sanded scratch coat plaster contains all of the ingredients necessary, except water. 


\section{GENERAL REQUIREMENTS}

Specifications for calcined gypsum, F. S. B. No. 248 (see 514.2, p. 261 ), in effect at date of opening of bids, shall form part of this specification.

\section{DETAIL REQUIREMENTS}

The chemical composition and physical properties of gypsum plaster shall conform to the requirements given in Table 1.

TABLE 1.-Properties of gypsum plasters

\begin{tabular}{|c|c|c|c|c|}
\hline Property & $\begin{array}{c}\text { Wood } \\
\text { fibered } \\
W\end{array}$ & $\begin{array}{l}\text { Neat } \\
N\end{array}$ & $\begin{array}{c}\text { Sanded } \\
\text { brown } \\
\text { coat } \\
B\end{array}$ & $\begin{array}{l}\text { Sanded } \\
\text { scratch } \\
\text { coat } \\
S\end{array}$ \\
\hline $\begin{array}{l}\text { Content of } \mathrm{CaSO}_{4} \cdot 1 / 2 \mathrm{H}_{2} \mathrm{O} \text {, mini- } \\
\text { mum } \\
\text { Content of wood fiber, minimum }\end{array}$ & $\begin{array}{r}48.5 \\
1.0\end{array}$ & 51.5 & 11.5 & 15.0 \\
\hline $\begin{array}{l}\text { Content of sand, maximum } \\
\text { Time of set, minimuma } \\
\text { Time of set, maximum } \\
\text { Tensile strength, minimum. }\end{array}$ & $\begin{array}{r}1.5 \\
8.0 \\
125.0\end{array}$ & 150.0 & $\begin{array}{r}75.0 \\
2.0 \\
6.0 \\
50.0\end{array}$ & $\begin{array}{r}66.7 \\
1.5 \\
7.0 \\
75.0\end{array}$ \\
\hline
\end{tabular}

\section{METHOD OF INSPECTION AND TESTS}

(a) Sampling.-Same as F. S. B. No. 248, 514.2, page 264 .

(b) Chemical analysis.-

(1) Preparation of sample.-Dry about 1 pound of the material spread out in a thin layer in a suitable dish, in an oven at about $45^{\circ} \mathrm{C}$. for two hours. Cool in an atmosphere free from moisture. Scrcen $100 \mathrm{~g}$ of this material through a No. 14 sieve to remove any hair or wood fiber. (Probably some of the sand will also be retained.) Weigh the material passing through the sieve and record this weight as "per cent fines." Grind about $10 \mathrm{~g}$ of this material until it all passes a No. 100 sieve, using extrcme care that the material be not unduly exposed to moisture or overheating. Store in an air-tight container until used.

For additional requirements under "Chemical analysis," captioned as follow's: (2) Calciun oxide; (3) Sulphur trioxide; (4) combined water; and (5) calculation. (See A. S. T. M. C26, 514.0, p. 259.)

(c) Wood fiber.-Dry $100 \mathrm{~g}$ of the matcrial for two hours at $45^{\circ} \mathrm{C}$. Screen through a No. 14 sieve. Weigh the material retained on the sieve. Transfer it to a suitable dish and ignite to constant weight. The loss of weight is equal to the per cent wood fiber.

(d) Sand-Place $100 \mathrm{~g}$ of the material on a No. 100 sieve. Wash the material through the sieve by means of a stream of water from a faucet. Wash until the water comes through clear. Dry the material retained on the sieve and ignite to constant weight. This reight is equal to the per cent sand.

(e) Testing consistency. - To a known volume of water add a known weight of the sample. Sift the sample into the water by shaking it through a No. 4 sieve. Let soak for two minutes. Stir to an even fluidity and transfer immediately to a brass eylinder mold, 2 inches diameter by 4 inches liigh, mounted on a glass plate. Both the mold and the plate shall be clean and wet when the sample is introduced. Immediately raise the mold by lifting it vertically upward without distorting the specimen. The specimen, when thus released, will slump down on the glass plate. Measure its final height.

The mixture shall be considered of "testing consistency" when the final height is $3 \frac{1}{2}$ inches.
If the final height is greater or less than $3 \frac{1 / 2}{2}$ inches, repeat, using fresh materials throughout and varying the ratio of sample to water.

(f) Time of set.-Mix $200 \mathrm{~g}$ of the sample with enough water to make a paste of "testing consistency." (For quantity of water and directions for mixing see preceding section.) Test with Vicat needle. Set is considered complete when the needle no longer penetrates to the bottom of the specimen.

The frequency of the penetrations will depend upon the character of the material, and is therefore left to the discretion of the operator, provided that the time of set shall be determined to the nearest five minutes.

For this test it is absolutely essential that all ashes and utensils be clean. Especially must they be free from all traces of set gypsum. Distilled water must be used.

(g) Tensile strength.-Use $750 \mathrm{~g}$ of the sample. Additional procedure requirements are the same as F. S. B. No. 248. (See 514.2, p. 264.)

\section{PACKING AND MARKING}

Same as F. S. B. No. 248.

\section{ADDITIONAL INFORMATION}

Wood-fibered plaster may be used either with or without the addition of sand for either scratch or brown coat.

Neat plaster may be had either with or without hair. That with hair may be mised with 2 parts (by weight) of sand for scratch coat. Either the fibered or unfibered plaster may be mixed with 3 parts of sand for brown coat.

Sanded brown coat and seratch coat plasters contain all necessary ingredients for the respective coats, except the water. It is essential that no sand be added to these plasters.

Note.-Sieve numbers refer to United States standard sieve series. (See A. S.T. M. E11, 500.2, p. 1.)

The Gypsum Institute, gypsum plasters.

(The full text of these specifications includes instructions for superintendent and inspector, directions for application for all types of plasters specified, and the following specifications for materials:)

\section{WATER (FOR ALL PIASTERS)}

The water used for mixing must be clean, free from alkali and impurities. Do not wash tools in this water. Alum is used by some municipalities for purifying the drinking water. Alum in the water used for mixing will cause the plaster to set quickly. Water taken from stagnant pools or some wells contains organic or vegetable matter which will cause the plaster to set slowly. Water containing sulphur will also contribute to the slow setting of the plaster.

\section{SAND (FOR ALI PLASTERS)}

All sand used must be dry, clean, and sharp. If fine sand is used, the quantity must be reduced, since fine sand makes a wesker plaster. An increase of sand over the recommended proportions will cause the plaster to set more quickly and will give a weaker mixture. Quicksand should never be used, since plaster mixed with it will not harden. The use of quicksand can be detected by the mixture working short and having a watery appearance; also quicksand will settle to the bottom of the rixing 
box. A gypsum plastering sand should conform as closely as possible to the following specifications:

(a) Sand used for plastering purposes in which a gypsum plaster is employed shall be free from salt and from alkaline, organic, and other deleterious substances.

(b) It shall be graded from fine to coarse, and when dry not more than 10 per cent by weight shall be retained on a No. 8 sieve; not less than 80 per cent by weight shall be retained on a No. 50 sieve; and not more than 6 per cent by weight shall pass a No. 100 sieve. These sieves shall meet the specifications given in the Bureau of Standards' standard screen scale. (See 500.2, p. 1.)

NoтE.-While the foregoing specification covers what is necessary for an ideal plastering sand, good results are ohtained by the use of sands which approximate the specification.

\section{GYPSUM NEAT PLASTER}

\section{DESCRIPTION}

Gypsum neat plaster (sometimes called "hardwall," "cement," or "patent plaster") is the product of ground calcined gypsum which is carefully selected and tested as to its plastering value, strength, hardness, resistance to heat and moisture, and other important physical and chemical properties. The word "neat," in connection with the name of the plaster, implics that the product as shipped by the manufacturer does not contain sand; the sand necessary is added at the building site.

This plaster may or may not contain a small percentage of hair or other similar fiber. The fibered material is used for plastering on uneven surfaces and on wood and metal lath, while the unfibered is usually used for plastering on brick, tile, and similar solid surfaces. This plaster, with the addition of fine sand at the building site, is sometimes used for a sand-float finish in place of the usual lime putty.

\section{QUALITY}

The quality and composition of gypsum neat plaster shall be not less than that prescribed for such in A. S. T. M. C28. (See 514.3, p. 265.)

\section{GROUNDS}

Gypsum neat plaster shall be applied to a thickness of not less than is required for the kind of base or background, as follows:

Wood lath.-Three-fourths-inch grounds.

Gypsum plaster board, one-fourth inch thick.Three-fourths-inch grounds.

Gypsum plastcr board, three-eights inch thick.Seven-eighths-inch grounds.

Metal lath, expanded, sheet and wire-Threefourths-inch grounds.

Gypsum tile.-One-half-inch grounds.

Bricli and clay.-Five-eighths-inch grounds.

Note.-Following customary practice the grounds are of a thickness which includes the thickness of the lath. Where, of necessity, grounds are to he applied over (upon) gypsum plaster hoard, they shall he reduced in thickness an amount equal to the thickness of the plaster hoard and shall be secured by nailing through the plaster hoard into the supports.

\section{PROPORTIONS}

Gypsum neat plaster shall be mixed with sand not to exceed the proportions specified for the kind of base or background, as follows:

First or scratch coat-Wood lath.-To 1 part of the plaster (fibercd) add not morc than 2 parts by weight of dry sand.

Gypsum plaster board.-To 1 part of the plaster (fibered or unfibered) add not more than 2 parts by weight of dry sand.

Metal lath, expanded, sheet and wire.-To 1 part of the plaster (fibered) add not more than 2 parts by weight of dry sand.
Brick, gypsum and clay tile.-To 1 part of the plaster (unfibered) add not more than 3 parts by weight of dry sand.

Second or browning coat.-For the second or browning coat, on all bases or backgrounds, to 1 part of the plaster (unfibered) add not more than 3 parts by weight of dry sand.

\section{MIXING}

Raise one end of the mortar box about 4 inches. Place in the upper end of the box one layer of sand and on top of the sand a layer of plaster. Hoe the material dry from the upper end of the box down to the lower and back again until the dry mixture is of an even color. Put water into the empty or lower end of the box and hoe the plaster and sand into the water, mixing thoroughly to the proper consistence for application. Do not mix more material than will be used in about 1 hour. Do not retemper the mixture after it has started to set.

\section{COVERING CAPACITY}

The covering or spreading capacity of gypsum neat plaster depends to a large extent upon the quantity and quality of sand used, the plasticity of the mixture, the character of base or background to which it is applied, and the thickness of the plaster coats. Of minor importance in this respect might be included the weather (tempcrature) conditions and workmanship. Under average circumstances gypsum neat plaster, mixed and applied in accordance with the foregoing specifications, and to the grounds specified will spread or cover about as follows:

\section{One part of plaster to 2 parts of sand-}

On wood lath.-From 900 to 1,100 pounds per 100 square yards.

On gypsum plaster board.-From 800 to 900 pounds per 100 square yards.

On metal lath, expanded and wire.-From 1,700 to 2,000 pounds per 100 square yards.

On metal lath, sheet.-From 1,600 to 1,800 pounds per 100 square yards.

One part of plaster to 3 parts of sand-

On brick and clay tile.-From 1,400 to 1,700 pounds per 100 square yards.

On gypsum tile.-From 1,000 to 1,200 pounds per 100 square yards.

On first or scratch coats.-From 600 to 800 pounds per 100 square yards.

Nots.-Gypsum plaster is marketed full weight, 100 pounds in cloth sacks or 80 pounds in cloth or paper hags.

\section{GYPSUM WOOD-FIBERED PLASTER}

\section{DESCRIPTION}

Gypsum wood-fibered plaster is the product of ground calcined gypsum, which is carefully selected and tested as to its plastering value, strength, hardness, resistance to heat and moisture, and other important physical and chemical properties. The expression "wood-fibered," in connection with the name of the plaster, implies that the product as shipped by the manufacturer contains, intimately mixed, a suitable amount of nonstaining wood fiber made by the grinding or shredding of wood. When shipped this plaster docs not contain sand, and, in general, is intended to be used without the admixture of sand at the building site.

This plaster is extensively used where a high degree of fire resistance is necessary, for the improvement of acoustics, and in some instances where sand of the desired quality is not easily obtained. 
Note.-Gypsum wood-fibered plaster, with but 1 part by weight NOTE.-Gypsum wood-tibered plaster, with but 1 part by weight surfaces, and for the second (browning) coat upon gypsum plaster surfaces, and for the second (browning) coat upon gypsum plaster moard, wire, expanded or sheet metal lath. It is important to bear in mind that the addition of sand to gypsum wood-fibered plaster will hasten its time of setting. When it is intended to usesand, the manuplaster at the mill before shipment.

\section{QUALITY}

The quality and composition of gypsum woodfibered plaster shall be not less than that prescribed for such in A. S. T. M. C28.

\section{GROONDS}

Same as for neat plaster above.

\section{PROPORTIONS}

Gypsum wood-fibered plaster is manufactured to be used without the admixture of sand. It shall be mixed as specified for bases or backgrounds as follows:

First (scratch) and second (browning) coats on-

Wood lath.-Mix the plaster with water only. only.

Gypsum plaster board.-Mix the plaster with water

Metal lath, expanded, sheet and wire-Mix the plaster with water only.

Brick, gypsum and clay tile.-Mix the plaster with water only.

NoтE.--In certain work gypsum wood-fibered plaster, with but 1 part by weight of sand, will prove satisfactory on wood lath, brick, clay and gypsum tile surfaces, and for all second or browning coat work. The addition of sand to this plaster will hasten the time of set, and for this reason the manufacturer should be advised of such intention in order that the setting time of the material is sufficiently give good results when mixed with not more than 1 part by weight
of clean dry sand (which in some localities is common practice), these of clean dry sand (which in some localities is common practice), these specifications include instructions for mixing
ing or spreading capacity of such a mixture.

\section{MIXING}

With water only.-Raise the end of the mortar box about 4 inches. Place plaster in the raised end and water in the lower end of the mortar box. Hoe the material from the upper end of the box down into the water in the lower end, allowing the plaster to soak for a few minutes. After mixing to the proper consistence it is ready for application. Do not mix more material than will be used in about one hour. Do not retemper the mixture after it has started to set.

With not more than 1 part by weight of sand.Raise one end of the mortar box about 4 inches. Place in the upper end of the box one layer of sand and on top of this a layer of plaster. Hoe the material dry from the upper end of the box down to the lower and back again until the dry mixture is of even color. Put water into the empty or lower end of the box and hoe the plaster and sand into the water, allowing the mixture to soak for a few minutes. After mixing to the proper consistence it is ready for application. Do not mix more material than will be used in about one hour. Do not retemper the mixture after it has started to set.

\section{COVERING CAPACITY}

The covering or spreading capacity of gypsum wood-fibered plaster depends to a large extent upon the quantity and quality of wood fiber used, the plasticity of the mixture, the character of base or background to which it is applicd, and the thickness of the plaster coats. Of minor importance in this respect might be included the weather (temperature) conditions and workmanship. Under average circumstances gypsum wood-fibered plaster, mixed and applied in accordance with the foregoing specifications, and to the grounds specified, will spread or cover about as follows:
Plaster mixed with water only (no sand)-

On wood lath.-From 1,400 to 1,700 pounds per 100 square yards.

On gypsum plaster board.-From 1,300 to 1,600 pounds per 100 square yards.

On metal lath, expanded and wire.-From 2,200 to 2,700 pounds per 100 square yards.

On metal lath, sheet metal.-From 2,100 to 2,400 pounds per 100 square yards.

On brick and clay tile.-From 2,200 to 2,400 pounds per 100 square yards.

On gypsum tile.-From 1,800 to 2,000 pounds per 100 square yards.

One part of plaster to 1 part of sand-

On wood lath.-From 1,100 to 1,300 pounds per 100 square yards.

On brick and clay tile.-From 1,800 to 2,000 pounds per 100 square yards.

On gypsum tile.-From 1,400 to 1,600 pounds per 100 square yards.

On first or scratch coats.-From 1,000 to 1,300 pounds per 100 square yards.

NoтЕ.-Gypsum plaster is marketed full weight, 100 pounds in cloth sacks or $\$ 0$ pounds in cloth or paper bag.

\section{GYPSUM READY-SANDED PLASTER}

\section{DESCRIPTION}

Gypsum ready-sanded plaster (sometimes called "prepared" or "sanded" plaster) is the product of ground calcined gypsum which is carefully selected and tested as to its plastering value, strength, hardness, resistance to heat and moisture, and other important physical and chemical properties. The expression "ready-sanded," in connection with the name of the plaster, implies that the product, as shipped by the manufacturer, contains, intimately mixed, a suitable proportion of clean, sharp, dry sand, and that it is intended to be used without the admixture of additional sand at the building site.

In addition to the sand, this plaster may or may not contain a small percentage of hair or other similar fiber. The fibered material is used for plastering on uncven surfaces and on wood and metal lath, while the unfibered is usually used for plastering on brick, tile, and similar solid surfaces. Gypsum ready-sanded plaster, fibered or unfibered, is used for all of the purposes where a gypsum neat plaster with sand, fibered or unfibered, can be used. It is in demand in cases where a suitable grade of plastering sand can not economically be procured or during cold weather when local sand supplies are liable to contain frost.

\section{QUALITY}

The quality and composition of gypsum readysanded plaster shall be not less than that prescribed for such in A. S. T. M. C28.

\section{GROUNDS}

Same as for neat plaster, above.

\section{PROPORTIONS}

Gypsum ready-sanded plaster is manufactured to be used without the admixture of any additional sand at the building site. It shall be mixed as specified for bases or backgrounds as follows:

First (scratch) and second (browning) coats on-

Wood lath.-Mix the plaster (fibered) with water only.

Gypsum plaster board.-Mix the plaster (fibered or unfibered) with water only.

Metal lath, expanded, sheet and wire-Mix the plaster (fibered) with water only, 
Brick, gypsum, and clay tile.-Mix the plaster (unfibered) with water only.

Nore.-Gypsum ready-sauded plaster, for first (scratch) coat work, contains 1 part of gypsum and not more than 2 parts by weight of clean, sharp, dry sand. For the second (browning) coat work, the quantity of sand is increased to 3 parts by weight. Since the necessary proportions of gyspum and sand are made and controlled at the manufacturer's mill, it is necessary that in ordering gypurm readysanded plaster, statement is included as to whether the material is sanded plaster, statement is included as to whether the material is "fo be used ior first or second coat work, and whether it shall be coat work upon all open lath and uneven surfaces.

\section{MIXING}

With water only.-Put the plaster in one end and the water in the other end of the mortar box. Hoe the plaster into the water, mixing thoroughly to the proper consistency for application.

\section{COVERING CAPACITY}

The covering or spreading capacity of gypsum ready-sanded plaster depends to a large extent upon the quantity and quality of sand in the plaster, the plasticity of the mixture, the character of the base or background to which it is applied, and the thickness of the plaster coats. Of minor importance in this respect might be included the weather (temperature) conditions and workmanship. Under average circumstances gypsum ready-sanded plaster mixed and applied in accordance with the foregoing specifications, and to the grounds specified will spread or cover about as follows:

Plaster mixed with water only (no additional sand)-

On wood lath.-From 2,200 to 2,400 pounds per 100 square yards.

On gypsum plaster board.-From 2,000 to 2,200 pounds per 100 square yards.

On metal lath, expanded and wire.-From 4,500 to 5,000 pounds per 100 square yards.

On metal lath, sheet metal.-From 4,400 to 4,600 pounds per 100 square yards.

On brick and clay tile.-From 3,500 to 4,000 pounds per 100 square yards.

On gypsum tile.-From 2,500 to 2,800 pounds per 100 square yards.

On first or scratch coats.-From 1,900 to 2,200 pounds per 100 square yards.

Nore,-Gypsum plaster is markated full weight, 100 pounds in cloth sacks or 80 pounds in cloth or paper bags.

\section{GYPSUM PIASTERS ON COHCRETE SURFACES}

\section{GENERAL}

Plastering on concrete surfaces, regardless of the material used, presents unusual difficulties necessitating particluar care and precaution for this class of work. The dressed (smooth) lumber which is commonly used for form work, and also the use of metal forms, leaves a concrete surface so smooth that the necessary key or clinch for a proper bond for the plaster coat can not be obtaincd. Also, the common practice of oiling the forms simply aggravates these conditions. Concrete surfaces which are to be plastered should be rough. The character of surface desired may be procured by using rough forms or, better still, by hacking the surface to be plastered after the forms arc removed and before the concrete has become too hard.

The success and permanency of any plaster upon a concrete suriace (base or background) depends largely upon the condition of the concrete surface which is to receive and retain the plaster, the workmanship, and the character of plaster employed. Careful application of the plaster is important.

\section{QUALITY}

The quality and composition of the gypsum plaster used on concrete surfaces shall be not less than that prescribed for such in A. S. T. M. C28.

\section{PLASTER}

Gypsum plasters which are to be used for plastering upon concrete surfaces shall be either of the following:

(a) Gypsum plaster for concrete surfaces (specially prepared).

(b) Unsanded gypsum wood-fibered plaster.

\section{MIXIVG}

Raise one end of the mortar box about 4 inches. First put water in the lower end of the box, then in the raised end put the quantity of gypsum plaster to be used for one mixing. Hoe the plaster into the water, allowing it to soak for a few minutes. When soaked, work up the mixture in the usual manner, adding sufficient water, as you are mixing, to bring the plaster to the proper consistence for application. Do not mix more material than can be applied and straightened in about one and one-half hours. Do not retemper the mixture after it has started to set. Do not add sand to plasters that are to be applied to concrete surfaces.

Nore.-Gypsum wood-fibered plaster must be allowed to soak, after mixing, for about 15 minutes without further hoeing, before it is applied.

\section{GYPSUM FINISH PLASTERS-TROWEL AND SAND- FLOAT FINISHES}

\section{DESCRIPTION}

Prepared gypsum finish plasters, for trowel and sand-float finishes, are the product of ground calcined gypsum which is carefully selected with due consideration of the finished shade desired (from white trowel to light gray sand-float finishes), smooth working properties, strength, hardness, and other important physical and chemical properties.

\section{QUALITY}

The plaster used for trowel and sand-float finishes shall be of calcined gypsum of a quality not less than that prescribed in A. S. T. M. C28.

\section{PROPORTIONS}

Gypsum prepared finish plasters require the addition of water only and proper mixing to make them ready for application.

\section{MIXING}

Raise one end of the mortar box about 4 inches. Put water in the lower end of the box and plaster in the upper end, using (approximately) 1 part of water to 2 parts of plaster by volume. Allow the plaster to soak in the water, without hoeing, for at least 10 minutes. After all the material has soaked and there are no further signs of bubbles, mix thoroughly and, with particular care, break the material down to a smooth, even, creamy consistence. When properly mixed it will be too thin to carry in a hod but can be conveniently carried in a bucket.

\section{GYPSUM FINISHING PLASTER}

\section{DESCRIPTION}

Gypsum finishing plaster (sometimes termed gaging gypsum plaster) is a calcined gypsum product specially manufactured from ground calcined 
gypsum which is carefully selected with due consideration of purity and color. Since this plaster is manufactured to vield cool and smooth working properties it is extensively used in combination with lime putty for finish plaster coats. Gypsum finishing plaster is prepared with retarder, viz, "slow set," or without retarder, viz, "quick set." The principal purpose of this plaster is to harden the finish plaster coat and prevent check cracking of the surface.

\section{FROPORTIONS}

Use either with thoroughly slaked quicklime or "hydrated lime" in the proportion of about 1 measure of dry finishing plaster to 3 measures of lime putty. These proportions are about equal to 1 part of dry finishing plaster to 2 parts of dry "hydrated lime" by weight.

\section{MIXING}

Make a ring of thoroughly hydrated lime putty on the mixing board; put clean water in the center of the ring and sift the finishing plaster into the water, allowing it to soak for a few minutes. Mix to a creamy consistence, then cut the lime putty, together with the mixture, to a paste of uniform consistence.

Note.-Great care must be exercised to ascure that the lime used, whetker quicklime or hydrated, is thoroughly slaked.

\section{GYPSUM MOLDING AND CASTING PLASTER}

\section{DESCRIPTION}

Gypsum molding and casting plasters are specially manufactured for use in molds or castings of the most intricate forms, such as for shaping clay and terracotta forms. Also, it is used for staff work, plaster casts, relief, ornamental work, and cornices on walls and ceilings.

\section{MIING}

For casting and similar work mix thoroughly with water only. For moldings and work of like character, mix as directed for gypsum finishing plaster.

\section{COVERING CAPACITY OF FINISH PLASTERS}

The covering or spreading capacity of gypsum plasters used for, or in, the finishing coat depends upon the character and plasticity of the mixture, the condition of the surface or base coat of plaster to which the finishing coat is applied, and the thickness of the coat. Of minor importance in this respect might be included the weather (temperature) conditions and workmanship. Under average circumstances gypsum finish plasters, mixed and applied in accordance with the foregoing specifications, will spread or cover about as follows:

Gypsum trowel finish.- From 500 to 600 pounds per 100 square yards.

Gypsum sand float finish-From 700 to 800 pounds per 100 square yards.

Gypsum finishing (gaging).--From 400 to 500 pounds per 100 square yards.

Note.-Gypsum trowel, sand float, finishing, molding, and casting plasters are marketed full weight, 100 pounds in cloih sacks, 80 pounds in cloth or paper bags and 250 pounds in barrels, as desired.

United States Department of Commerce, division of simplified practice, recommendation No. 75, composition blackboard (including simplified list having gypsum core backing), April 1, 1928.

(See 545.9, p. 645.)

514.4. LIME PLASTER.

American Railway Engineering Association, specifications for buildings for railway purposes.

\section{IATHING AND PLASTERING (1926)}

(This specification includes requirements for the furnishing and setting of metal furring, metal lathing, wood lath, corner beads and in addition, the following requirements to be met in interior plastering and patching:)

The contractor shall provide all scaffolding, tools, labor, and materials, and everything necessary to complete the plastering and stucco work shown on the drawings or required to complete the building.

In general, all plastering on lathed surfaces shall consist of a scratch coat, a brown coat, and the finishing coat. On unfurred brick or tile surfaces the serateh coat will be omitted. Each coat shall be permitted to dry thoroughly before the next coat is applied. Before beginning his work the plasterer shall test and prove the lathing and grounds so that the finished plaster will be plumb, true, level, and waveless. Plastering shall run up behind all sill aprons, wainscoting, etc., and shall extend behind all bases.

Stone, brick, or terra cotta walls to be plastered and all wood lath shall be thoroughly drenched with water before applying the first coat of plaster.

In hot dry weather, especially if windy, close all openings in the building while plastering to prevent too rapid drying. In winter the temperature in the rooms being plastered shall be kept above the freezing point while plastering and until the plaster has hardened.

The scratch coat shall be well rubbed in and troweled against brick and tile and into lathed surfaces so as to form a perfect bond, and shall be scored and scratched in both directions to form a key for the brown coat.

The brown coat shall be applied to the seratch coat and brought flush with the grounds, with all surfaces straight, true, plumb, level, and waveless.

The finishing coat shall be applied to the brown coat and may be a sand float or white trowel finish as specifically designated. If a white trowel finish is called for, it shall be made of Keene's cement and lime putty troweled to a smooth hard finish free from trowel or brush marks.

The plasterer shall run all plaster molds, cornices, coves, etc., in accordance with models or full-sized profiles; all angles to be carefully and accurately mitered. Run work shall be carefully and accurately formed from templates to form continuous, unbroken, level lines. Ornamental enrichments shall be firmly secured in place with plaster of Paris, white lead and galvanized wire nails.

Unless otherwise permitted by the engineer, all plastering shall be done with "patent" or hard ivall plaster of a brand specifically approved by the engineer and mixed and applied in accordance with the manufacturer's directions. Plaster shall be delivered at the site in the original unbroken packages and stored in a dry place until used.

Lime putty used for plastering shall be made from first quality pure lump lime, screened and free from impurities, and shall be mixed at least two weeks before being used.

Sand for plastering shall be sharp and angular and free from dirt, oil, or impurities that will stain the plaster. It shall be sereened, washed and dried.

\section{PATCHING}

The plasterer shall do all necessary patching of plaster after the other mechanics have finished their work and shall leave same complete and perfect in every respect. 


\section{GENERAI, CONDITIONS}

The general conditions as given in 518.50 , page 416 shall be considered to apply to these specifications.

American Society for Testing Materials, tentative specifications for sand for use in lime plaster, serial designation: C66-27T, 1927.

See 512.12, page 234 .

Contracting Plasterers' International Association, standard specification for lath and plaster work, 1923.

See 514.63, page 280 .

(This specification includes requirements for lime plaster.)

National Iime Association, proposed specifications for lime plastering.

\section{PREFACE}

Sections 1, 2, and 3 are recommended practices. Sections 4 and 5 are the specifications for lime plastering.

1. Plastering failures may be caused by lack of rigidity in the building or by lack of preparation for the backing. A careful survey of the building should be made before plastering is started. Masonry walls and ceilings should be in true alignment and of sufficient rigidity, and should be clean and of rough texture. Frame structures should be well braced and properly reinforced against all concentrated loads. Any defects noted should be remedied or reported to the owner or his agent.

2. The finished plastering shall have true surfaces flush with the grounds or with the face where there are no grounds. All corners shall be straight and true. When dry, the surface shall not be marred by noticeable blotchies, pops, or joining lines.

3. Preparation of materials and backings.- $(a)$ Quicklime shall conform to, and be prepared for use in accordance with, A. S. T. M. C5. (See 517.2, p. 352.)

(b) Hydrated lime shall conform to A. S. T. M. C6 (see 517.2, p. 353), and shall be mixed with water to form a putty and stored, with reasonable care to prevent evapolation, for at least 24 hours prior to use.

(c) Sand shall conform to the proposed tentative specifications for sand for lime plaster of the American Society for Testing Materials.

(d) Hair or fiber shall be reasonably free from dust, grease, and balls or knots of hair or fiber. The individual hairs or fibers shall be from one-half to 2 inches long, averaging $1 \frac{1}{2}$ inches long; and the fiber employed shall become quickly pliable on wetting.

(e) Mixing water shall be sufficiently free from salts and organic material as to not produce deleterious effects on the finished plastering. In general, water which is potable will be satisfactory.

( $f$ ) Gaging material (calcined gypsum) Portland or Keene's cement, when used shall conform to the requirements of the American Society for Testing Materials, for the specific material employed.

(g) Wood lath, when used, shall be laid horizontally on walls and in one direction only on ceilings and shall be of such length that both ends of each lath will come over supports. They shall be nailed securely with fine blued wire lath nails, of at least $3 \mathrm{~d}$, at both ends and to every support they cross, the nails being staggercd along the length of the lath. Lath shall be spaced three-eighth inch apart. A space three-eighths inch shall be left between abutting ends. Joints shall be broken every sixth lath. The entire length of all angles and corners shall be reinforced with metal lath extending at least 6 inches on each surface. Wood lath shall be as damp as practicable when the plaster is applied. All warped, buckled, and unseasoned lath shall be replaced before plastering.

(h) Wire or metal lath when in sheets shall be in position with the longer dimension at right angles to the supports. The end of a sheet shall be located at least one support distant from an angle or corner, and the lath bent into or around the angle or oorner. Wherever possible the end joints between sheets shall come over supports. End joints over supports shall be lapped at least 1 inch; end joints not over supports shall be lapped 3 inches; side joints shall be lapped 1 inch. End joints when not over a support shall be tied with wire once every 6 inches; side joints on walls, once midway between each two supports, on ceilings, once every 6 inches. Each sheet shall be fastened to each support it crosses by fasteners spaced 6 inches apart across the sheet. Metal lath shall be fastened to wood supports by means of staples, or hooked head nails. Fasteners for metal lath to metal studs and furrings having no other provision for fastening shall be wire ties. Ties shall be at least equivalent to 18 gage annealed wire.

(i) Weights of expanded metal lath.-For walls where the spacing of supports is 16 inches on centers or less, the weight of expanded metal lath shall be not less than "heavy" (3.4 pounds per square yard) for the best grade of construction; "medium" (2.5 to 3 pounds per square yard) for the medium grade; and "light" (2.2 pounds per square yard) for the minimum grade. If the spacing of supports is 16 to 24 inches the lath shall be not less than "medium" for the minimum grade of construction; not less than "heavy" for the medium grade; and for the best grade, specially stiffened heavy expanded metal or wire lath or sheet lath shall be used. Supports shall not be spaced more than 24 inches on centers. For ceilings where the spacing of supports is 12 inches or less on centers, the weight of lath shall conform to the requirements given above for walls with supports spaced 16 inches, except that no "light" lath will be permitted. If the spacing of supports is 12 to 16 inches, then the weight of lath shall conform to the requirements given above for walls with supports spaced 16 to 24 inches. Ceiling supports shall not be spaced more than 16 inches on centers.

(j) Self-furring metal or wire lath may be used wherever metal furring strips and metal or wire lath are specified, provided that the lath proper, considered separately, meets the requirements of the grade of lath called for, and provided the design of the self-furring feature is such as to provide a space of not less than three-eighths inch between the back of the lath and the adjacent face of the wall, ceiling, or support. Self-furring lath shall be erected as specified for metal lath $3(g)$.

(k) Furring strips, if used, shall be rigidly attached to the structural support. They shall be of such a type as to provide a space of at least three-eighths inch between the structural support and the lath. Furring strips shall be used over wood supports wherever the face of the support is 4 inches or more in width.

(l) On masonry backings such as concrete, tile, stone, brick, etc., the surface should be thoroughly cleaned of all grease and loose particles before plastering. If the concrete surface is smooth or glazed it should be hacked or rougliened in some manner so as to offer suitable mechanical bond for the plaster. In order to thoroughly clean concrete walls they may be washed down with a dilute solution of muriatic acid and should in turn be rinsed with clear water. Masonry should be dampened, but not dripping wet when plaster is applied.

(m) Grourd strips or spot grounds shall be firmly attached to the backing. The thickness of the grounds shall conform to the required thickness of the plaster. 
(n) Protection during setting.-Both the scratch and brown coats shall be protected from drafts and frcezing during the settling period.

4. Proportions and application.-(a) Lime plaster on wood lath shall be a 3-coat work with not less than seven-eighths inch grounds. The finish coat may be either white or sanded, as specified.

(1) The scratch coat shall be composed of 1 volume of lime putty (made from either quicklime or hydrated lime) and 2 volumes of sand, with 1 bushel of hair or fiber per cubic yard of sand. The putty shall be prepared as directed in section 3 (a) (for quicklime), or 3 (b) (for hydrated lime). With hydrated lime, the ingredients may be mixed first dry and again wet. In either case, the wet mixture shall be stored as long as possible (at least 24 hours) without permitting it to dry out, and shall be retempered and the hair or fiber added just prior to use. The plaster shall be applied to the lath with sufficient pressure to force the plaster in between the laths so as to clinch over the back to form a good key. This coat shall cover the face of the lath about one-fourth inch. When nearly dry the surface of the coat shall be scratched with an appropriate tool, making scratches about 1/8 inch deep by $1 \frac{1}{2}$ inches apart. The brown coat shall be applied when the scratch coat has set sufficiently so that it takes considerable pressure to break down the edges of these scratches when rubbed with the thumb, and the application of the brown coat does not disturb the keys of the scratch coat.

NOTE.-If a more rapidly hardening plaster is desired, Portland or Keene's cement not excceding 25 per cent by volume of the lime may be suhstituted for an equal volume of lime.

(2) The brown coat shall be composed of 1 volume of lime putty (made from either quicklime or hydrated lime) and 3 volumes of sand. These shall be prepared and mixed as for the scratch coat; 4 (a) (1). This plaster shall be applied to the scratch coat with sufficient pressure to force it into the scratches. It shall be built out to the grounds, and rodded, darbied, and floated to a true, even surface with sharp, straight corners and angles. This coat shall be well worked. It shall be permitted to set until practically air dry.

(3) The white finish coat shall be composed of 3 volumes of lime putty (made from either quicklime or hydrated lime) and 1 volume of calcined gypsum for finish coat. The putty shall be prepared as directed in sections $3(a)$ (for quicklime) or $3(b)$ (for hydrated lime). A suitable amount of the putty should be circled out on a board, some water put into the circle, and calcined gypsum for finish coat equal to one-third the volume of the putty dusted into the water.

The whole is then mixed with a trowel, more water being added if necessary. Not more material than can be used in 30 minutes is to be mixed at one time. The mixture shall not be retempered, but each batch shall be started with clean board and tools. This plaster shall be applied in a thin, even layer over the brown coat. The workman shall watch carefully for the disappearance of the glaze, when the surface becomes slightly dull. When this occurs the coat shall be immediately trowelled down to a smooth, true finish using considerable pressure on the trowel, brushing the surface with water if necessary. This coat shall be as thin as possible without permitting the brown coat to show through. It shall be smooth, true, and free from trowel marks or blotches.

(4) The sand-finish coat shall be composed of 1 volume of lime putty (made from either quicklime or hydrated lime) and 2 volumes of sand. The putty shall be prepared as dirccted in section $3(a)$ (for quicklime) or $3(b)$ (for hydrated lime). The color of the sand shall be as approved by the pur chaser. Just before application, one-eighth volume of calcined gypsum for finish coat shall be mixed thoroughly with each batch of lime and sand, adding some more water if necessary. Not more material than can be used in 30 minutes shall be mixed at one time. This mixture shall not be retempered, but each batch shall be started with clean box and tools. This plaster shall be applied in a thin even layer over the brown coat. It shall be finished with a cork or felt float to an even, uniformly granular surface.

(b) Lime plaster on metal or wire lath shall be 3-coat work with not less than three-fourths inch grounds. The finish coat may be either white or sanded, as specified.

(1) The scratch coat shall be composed of 1 volume of lime putty (made from either quicklime or hydrated lime and 2 volumes of sand, with 1 bushel of hair or fiber per cubic yard of sand. The putty shall be prepared as directed in section $3(a)$ (for quicklime), or 3 (b) (for hydrated lime). With hydrated lime the ingredients may be mixed first dry and mixed again wet. In either case the wet mixture shall be stored as long as possible (at least 24 hours) without permitting it to dry out and shall be retempered and the hair or fiber added just prior to use. The plaster shall be applied to the lath with sufficient pressure to force the plaster in between the laths so as to clinch over the back to form a good key. This coat shall cover the face of the lath about one-fourth inch. When nearly dry the surface of the coat shall be scratched with an appropriate tool, making scratches about one-eighth inch deep by $1 \frac{1}{2}$ inches apart. The brown coat shall be applied when the scratch coat has set sufficiently so tlat it takes considerable pressure to break down the edges of these scratches when rubbed with the thumb, and the application of the brown coat does not disturb the keys of the scratch coat.

Note.-If a more rapidly hardening plaster is desired, Portland or Keene's cement not excecding 25 per cent by volume of the lime may be substituted for an equal volume of lime.

(2) The brown and finish coats shall be proportioned, mixed, and applied as specified in section 4 (a) (2), (3), and (4) above.

(c) Lime plaster on concrete ceilings shall be 2coat work with one-fourth inch grounds. The two coats shall be the scratch and finish coats, propor tioned, mixed, and applied as specified for the lime plaster on wood lath, 4 (a) (2), and (3) or (4), except that the scratch coat, instead of being scratched, is built out to grounds and rodded, darbied, and floated to a true, even surface with sharp, straight corners and angles. This shall be permitted to age until practically air-dry and the finish coat shall be applied directly to the surface of this scratch coat.

(d) Lime plaster on concrete or concrete block walls shall be 3 -coat work with three-fourths inch grounds. The three coats shall be the scratch, brown, and finish coats, proportioned, mixed, and applied as specified for lime plaster on wood lath, 4 (a) (1), (2), and (3) or (4).

(e) Lime plaster on brick or clay tile shall be 3-coat work with three-fourths inch grounds, proportioned, mixed, and applied as specified for lime plaster on wood lath, $4(a)(1),(2)$, and (3) or (4).

5. Miscellaneous.-(a) Doubled-up work shall be applied as follows: The scratch coat shall be proportioned, mixed, and applied as specified above, under $4(a)$. This scratch coat shall not be scratched nor permitted to harden, but shall be covered while still quite soft by application of the brown coat. The brown coat shall be built out to grounds, rodded, darbied, fioated, and permitted to set until practically air-dry, all as above cescribed. The finish coat shall be proportioned, mixed, and applied as specified for the finish coats, 4 (a) (3) or (4). 
(b) Lime plaster shall be applied to solid partitions only when the height does not exceed 12 feet and only when the studs are at least three-fourths inch steel channels or their equivalent, spaced not more than 16 inches on centers, and rigidly attached to the floors and ceilings. The metal lath shall be attached to the studs as specified in section $3(b)$. Thrce coats of lime plaster as specified in sections $4(b)$ (1), $4(a)(2)$, and $4(a)(3)$, or $4(a)$ (4) shall be applied to each side of the lath. The grounds shall be of such thickness that both faces of the lath and of the studs shall bc well covered by the brown coat. The over-all thickness of the finished partition shall be not lcss than 2 inches. The necessary changes in the thickness of the plaster shall be accomplished by changes in the thickness of the brown coat only. The sccond or scratch coat shall not be applied while the first is setting, but shall bc applied, either before the setting of the first starts, or not until after the setting of the first is complete.

(c) In certain instances where it is desired to apply the decorative finish directly to the brown coat specifications for scratch and brown coats, as regards composition and thickness as described in sections 4 (a) (1), and 4 (a) (2) for wood lath backings, and $4(b)(1)$ and $4(b)$ (2) for metal lath backing, shall be followed.

National Lime Association, proposed specifications for lime stucco.

\section{PREFACE}

All of Section I and Scction II, (1) and (2) and certain parts of Section III relating to ercetion details are recommended practices. The remaining sections are the specifications for lime stucco.

I. General. - A careful survey of the building shall be made before stuccoing is started. Masonry walls should be in true alignment and of sufficient rigidity, and should be clean and of rough texture. Frame structures should be well braced and properly rcinforced against all concentrated loads. Any defects noted should be remedied or reported to the owner or his agent.

The finished stucco shall have true surfaces flush with the grounds or with the face where there are no grounds. All corncrs shall be straight and true. When dry, the surfacc shall not be marred by noticcable blotches, pops, or joining lines.

The contractor shall furnish all necessary tools, scaffolds, and other appliances needed to fulfill the requircments of this contract, all scaffolding to be erected and maintained in accordance with the laws of the State or municipality having jurisdiction.

II. (1). Materials.-(a) Quicklime shall conform to and be prepared for use in accordance with A. S. T. M. C5. (See 517.2, p. 352.)

(b) Hydrated lime shall conform to A. S. T. M. C6 (see 517.2, p. 353), and shall bc mixed with water to form a putty and stored, with reasonablc care to prevent evaporation, for at least 24 hours prior to usc.

(c) Hair or fiber shall be reasonably free from dust, grease, and balls or knots of hair or fiber. The individual hairs or fibers shall be from one-half to 2 inches long averaging $1 \frac{1}{2}$ inches long, and the fiber empioyed shall become quickly pliable on wetting.

(d) Mixing water shall be sufficiently free from salts and organic matcrial as to not produce delcterious effects on the finished stucco. In general, water which is potable will be satisfactory.

(e) Portland cement shall conform to A. S. T. M. C9. (Sce 516.11 , p. 294.)

(f) Fine aggregate shall consist of natural sand or screenings from crushed stone and shall be well graded from fine to coarse within the limiting sizes. When dry, it shall all pass the No. 8 sieve and from 20 to 25 per cent shall pass the No. 50 sieve. It shall be free from loam, vegetable, or other dele- terious matter. (It may be tested by the colorimetric test as given by A. S. T. M. C40. (See 512.10, p. 213.)

2. Backings.-(a) Wood lath, when used, shall be No. 1 quality laid horizentally and shall be of such length that both ends of each lath shall come over supports. They shall be nailed securely at both ends and to every support they cross with fine blued or galvanized wire lath nails of at least $3 \mathrm{~d}$. Lath shall be spaced three-eighths inch apart and a space of three-eigh ths inch shall be left between abutting ends. Joints shall be broken every sixth lath. The cntire length of all angles and corners shall be reinforced with metal lath extending at least 6 inches on each surface. Wood lath shall be as damp as practicable when the stucco is applied. All warped, buckled, and unseasoned lath shall be replaced before plastering.

(b) Furring strips, if used, shall be rigidly attached to the masonry. They shall be of such a type as to provide a space of at lcast five-eighths inch between the masonry and the lath and shall not be over 2 inches in width. Furring strips shall be used over wood supports wherever the face of the support is 4 inches or more in width.

(c) Wire or metal lath when in sheets shall be in position with the longer dimension at right angles to the supports. The end of a sheet shall be located at least one support distant from an angle or corner, and the lath bent into or around the angle or corner. Wherever possible the end joints betwcen sheets shall come over supports. End joints over supports shall be lapped 1 inch; end joints not over supports shall be lapped 3 inches; side joints shall be lapped 1 inch. End joints when not over a support shall be tied with wire once every 6 inches and side joints once midway between each two supports. Each sheet shall be fastened to each support it crosses by fasteners spaced 6 inches apart across the sheet. Metal lath shall be fastcned to wood supports by means of staples, or hooked head nails. Fasteners for metal lath. to metal studs and furrings having no other provision for fastening shall be wire ties. Ties shall be at least equivalent to 18-gage annealed wire.

(d) Weights of expanded metal lath.-Supports shall not be spaced morc than 24 inches apart and the weight of the metal lath shall not be less than shown in the following table:

Weight in pounds per square yard of expanded metal lath

\begin{tabular}{l|r|r}
\hline \multirow{2}{*}{ Kind of constraction } & \multicolumn{2}{|c}{ Supports spaced } \\
\cline { 2 - 3 } & $\begin{array}{c}\text { 16 inches } \\
\text { or less }\end{array}$ & $\begin{array}{r}16 \text { to } 24 \\
\text { inches }\end{array}$ \\
\hline Best & $\begin{array}{r}3.4 \\
\text { Minimum }\end{array}$ & $\begin{array}{r}\text { Special. } \\
3.4\end{array}$ \\
\hline
\end{tabular}

(e) Self-furring metal or wire lath may be used wherever furring strips and metal or wire lath are specified, provided that the lath proper, considered separately, mects the requircments of the grade of lath called for, and provided the design of the selffurring feature is such as to provide a space of not less than five-cighths inch between the back of the lath and the adjacent face of the wall, or support. Self-furring lath shall be erected as specified for metal lath.

(f) On masonry backing, such as concrete, brick, stone, tile, etc., the surface should be thoroughly cleaned of all grease and loose particles before stuccoing. If the concrete surface is smooth or glazed, it should be hacked or roughened in some manner so as to offer suitable mechanical bond for the stucco. In order to thoroughly clean concrete 
walls they may be washed down with a dilute solution of muriatic acid, and should, in turn, be rinsed with clear water. Masonry should be dampened, but not dripping wet when stucco is applied.

(g) Ground strips or spot grounds shall be firmly attached to the backing of 4-foot centers. The width of a wooden ground strip or diameter of a wooden spot ground shall be not more than $1 \frac{1}{2}$ inches.

3. Proportions and mixing.-(a) The scratch coat shall be composed of 1 volume of quicklime putty or hydrated lime putty and 3 volumes of fine aggregate, with 1 bushel of hair or fiber per cubic yard of fine aggregate. The putty shall be prepared as directed in Section II $1(a)$ (for quicklime), or II 1 (b) (for hydrated lime). The ingredients of the mortar shall be thoroughly mixed with the minimum amount of water to obtain the required consistency. Mixing shall continue until the lime and fine aggregate are evenly distributed and the mass is uniform in color. With hydrated lime the ingredients may be mixed first dry and then mixed wet. In either case the wet mixture shall be stored as long as possible (at least 24 hours) without permitting it to dry out, and shall be retempered and the hair or fiber added just prior to use.

Note.-If a more rapidly hardening stucco is desired, Portland cement not excceding 33 per cent by volume of the lime may be substituted for an cqual volume of lime.

(b) The brown coat shall be composed of 1 volume of quicklime putty or hydrated lime putty, and 3 volumes of fine aggregate. These shall be prepared and mixed as for scratch coat II $3(a)$.

Note.-If a more rapidly hardening stucco is desired, Portland cement not excceding 25 per cent by volume of the lime may be substituted for an equal volume of lime.

(c) The finish coat shall be made with the same proportions as the brown coat, II $3(b)$, and shall be prepared and mixed as for the scratch coat II $3(a)$. If specified and provided for, a predetermined weight of finely ground mineral coloring matter shall be added to each batch of mortar prepared. In case hydrated lime is used, the coloring matter sliall be thoroughly mixed with it while dry, previous to the addition of any aggregate. In case quicklime is used, the coloring material shall be added to the fine aggregate while dry and the material thoroughly mixed. The ine aggregate shall be reduced by an amount equal to the volume of coloring material added.

4. A pplication and workmanship.-(a) Lime stucco, on wood, metal, or wire lath shall be 3-coat work.

(1) The scratch coat shall cover the face of the lath about one-fourth inch and shall be well troweled to insure a firm bond to the lath. When hard enough that the keys will not be disturbed, the surface of the coat shall be scratched with an appropriate tool making seratches about one-eighth inch deep and $11 / 2$ inches apart.

(2) The brown coat shall be applied, if possible, on the day following the application of the scratch coat. The scratch coat should be dampened if necessary, but not saturated before the brown coat is applied. The mortar shall be applied with sufficient pressure to force it into the scratches. It shall be approximately three-eighths to one-half inch thick and shall be brought to a true and even surface by screeding at"intervals not exceeding 5 feet and by constant use of the straightening rod. When the brown coat has stiffened sufficiently, it shall be dry floated with a wood float and lightly and evenily scored to form a good mechanical bond for the finish coat.

(3) The finishing coat should be approximately one-fourth inch thick and should be applied on the day following the application of the brown coat. The brown coat should be dampened if necessary, but not saturated before the finish coat is applied. The suiface finish shall be as specified by the owner or his agent.

(b) Lime stucco on brick, hollow tile, stone, concrete, or other masonry surfaces should preferably be 3-coat work, but the brown coat may be onitted if the surfaces of the masonry are very nearly true surfaces as usually obtained in mass concrete or brickwork.

(1) The scratch coat shall cover the base to a thickness of three-eighths inch measured from the normal masonry line. The mortar shall be applied to the backing with sufficient pressure to bond it to the surface. When hard enough that the bond will not be disturbed, the surface of the coat sliall be scratehed with an appropriate tool, making seratches about one-eighth inch deep and $1 \frac{1}{2}$ inches apart.

(2) Brown and finish coats shall be applied as specified for lime stucco on wood, metal, or wire lath, II $4(a)$ (2) and (3).

(c) If desired, the brown and finish coats may be doubled up; that is, as soon as the brown coat is stiff enough the finish coat may be applied. The scratch coat, however, should be allowed to set until quite firm before additional coats are applied.

III. Miscellaneous.-(a) Continuity of work.All stuccoing shall be carried on continuously from top to bottom. The coats thus applied shall proceed in one general direction without allowing the stucco to dry at the edge. If it is impossible to work the full length of the wall, the joining shall be at some chimney recess, projection, or other natural division in the surface. If this is impossible, the vertical joint shall be made behind a rain conductor or at a door or window opening. The stucco may be more smoothly blended at joints, if the edge to be joined is,first dampened.

(b) Protection of surfaces.-In rainy or threatening weather all fresh surfaces shall be protected against the direct washing effect of rain.

(c) Eaves, embedded trim, cornices, and sills should have large overhangs so detailed with barge boards, seat grooves, and drip grooves as to prevent any water from reaching the top or getting back of the stueco.

$(d)$ Flashings should be provided before starting stuccoing at the top and on the sides of openings wherever projecting wood trim occurs. They should be provided also across masonry walls under copings, cornices, and sills with mortar joints. The flashings should be made with material that will not oxidize and stain the stucco and should extend beyond and slightly over the face of the finished surfaces.

(e) Horizontal surfaces.-Stuccoed copings, cornices, and other exposed horizontal surfaces should be avoided whenever possible, All exposed stucco surfaces should shed water quickly.

(f) Finish above grade.- Stucco should terminate upon a base of stone, brick, or other suitable masonry not less than 18 inches above grade. A continuous damp coursing of slate or bituminous materials is recommended in masonry walls just below the starting line of any stucco, close to grade.

(g) It is recommended that finishes be rough to minimize the effect of any hair cracking that may occur.

(h) "Black" metal lath shall under no circumtances be used as a backing for lime stucco.

514.5. PORTLAND CEMENT PLASTER.

(No nationally recognized specifications available.)

\subsection{PLASTER MANUFACTURES.}

\subsection{Gypsum Plaster Board.}

American Society for Testing Materials, standard specifications for gypsum wall board, serial designation C36-25, 1925. 
1. Gypsum wall boards are sheets or slabs having an incombustible core of gypsum which, without the addition of plaster, furnish to interior walls, ceilings, or partitions a surface which may receive decoration.

2. Gypsum wall boards consist of an incombustible core of gypsum, with or without fiber, surfaced with paper or other fibrous material firmly bonded to the core.

3. Gypsum wall boards shall be nominally threeeighths inch $(9.5 \mathrm{~mm})$ in thickness with permissible variations in the average thickness of $\pm 1 / 64$ inch $(0.4$ $\mathrm{mm}$ ) and with permissible local variations of $\pm 1 / 32$ inch $(0.8 \mathrm{~mm})$ from the normal thickness.

Note. - Gypsum wall boards are manufactured in thicknesses from one-fourth inch $(6.3 \mathrm{~mm})$ to one-half inch $(12.7 \mathrm{~mm})$ with permissible local variations of $\pm 1 / 32$ inch $(0.8 \mathrm{~mm})$ from the nominal thickness.

4. (a) Gypsum wall boards that are to be laid with the joints butted shall have widths of 32 inches $(810 \mathrm{~mm}), 36$ inches $(910 \mathrm{~mm})$, or 48 inches $(1,220$ $\mathrm{mm})$, except that a variation of $3 / 32$ inch $(2 \mathrm{~mm})$ under the specified widths is permissible.

(b) Gypsum wall boards that are to be laid with the joints filled sball have widths one-fourth inch $(6.3 \mathrm{~mm})$ less than those specificd in paragraph $(a)$, except that the same variation is permissible.

(c) The lengths shall be from 4 feet $(1.22 \mathrm{~m})$ to 12 feet $(3.66 \mathrm{~m})$, inclusive, except that a tolcrance of thrce-eighths inch $(9.5 \mathrm{~mm})$ from the length of individual boards is permissible. Special boards may be cut to any desired length within the limits prescribed.

5 . The weight of gypsum wall board shall be not less than 1,400 pounds $(635 \mathrm{~kg})$ nor more than 2,100 pounds $(952.5 \mathrm{~kg})$ per 1,000 square feet.

6. (a) Strcngth test specimens shall be 12 inches $(300 \mathrm{~mm})$ in width and approximately 18 inches $(460 \mathrm{~mm})$ in length. When tested in accordance with the methods described in section 13 , specimens taken from the gypsum wall boards shall carry a load of not less than 60 pounds $(27.22 \mathrm{~kg})$ when the load is applicd across the fiber of the surfacing, and not less than 32 pounds ( $14.50 \mathrm{~kg}$ ) when the load is applied parallel to the fiber of the surfacing.

(b) With loads equal to or less than those specified in paragraph $(a)$ there shall be no breaking of the bond between the surfacing and the core.

7. That surface of the gypsum wall board designed to be exposed on erection shall be true and free from imperfections that would render the wall boards unfit for use with or without decorations. The edges and ends shall be straight and solid. Boards that are to be butted shall have corners square and opposite edges parallel to each other. With boards that are to have the joints filled, a negative variation of one-eighth inch $(3 \mathrm{~mm})$ from parallelism of opposite edges is permissible.

8. At least 1 per cent of the number of gypsum wall boards in a shipment, but not less than 10 boards shall be so selected as to be representative of the shipment. Twenty-five per cent of such selected boards, but not less than 10 individual boards, shall oonstitute a sample for test purposes. When a shipment consists of more than one car or carrier load, a sample shall be selected from each car or carrier.

9. Shipments of gypsum wall board shall conform to the following:

(a) They shall be shipped so as to be kept dry and free from moisture.

(b) When shipped for resale the following information shall be shown upon each board: Name of manufacturer, brand, and nominal thickness.

10. Inspection may be madc either at the point of shipment or at the point of delivery. The inspector representing the purchaser shall have free access to the carriers being loaded for shipment to the pur- chaser. He shall be afforded all reasonable facilities for inspection and sampling, which shall be so conducted as not to interfere unnecessarily with the loading of the carriers.

11. Any rejection shall be based upon failure to conform to the requirements of these specifications and shall be reported within 10 working days from the receipt of the shipment by the consignee. The notice of rejection shall contain a specific statement of the respects in which the boards have failed to meet the requirements of these specifications.

12. Claims for rehearing shall be valid only if made within 20 working days from the receipt of notice of specific cause for rejection.

\section{METHODS OF TESTING}

13. (a) Test specimens for the transverse strength test shall be taken from not less than ten gypsum boards.

(b) The test specimens shall be 12 inches $(300 \mathrm{~mm})$ in width and approximately 18 inches $(460 \mathrm{~mm})$ in length. They shall be supported on fixed parallel bearings spaced 16 inches $(410 \mathrm{~mm})$ on centers, and shall be loaded through a similar bearing midway between the supports. All bearing and load surfaces shall be true, shall engagc the full width of the test spccimen, and shall be rounded to a radius of oneeighth inch (3.2 $\mathrm{mm})$.

(c) Test loads shall be applied at a uniform rate of 60 pounds $(27.22 \mathrm{~kg})$ per minute with a permissible variation of \pm 10 per cent in the rate. Strength test results shall bc reported when the load is applied across the fiber of the surfacing and also parallel to the fiber of the surfacing.

American Society for Testing Materials, standard specifications for gypsum plaster board, serial designation: C37-25, 1925.

1. Gypsum plaster boards are sheets or slabs having an incombustible core of gypsum, and designed to be used as a lath or backing for gypsum plaster on the walls, ceilings, and partitions in the interior of buildings.

2. Gypsum plaster boards consist either of sheets or slabs of gypsum with not more than 15 per cent, by weight, of fiber intimately mixed; or an incombustible core of gypsum, with or without fiber, surfaced with paper or other fibrous material firmly bonded to the core, or with intermediate layers of such material within the core.

3. Gypsum plaster boards shall be one-fourth inch $(6.3 \mathrm{~mm})$, five-sixtcenths inch $(8.0 \mathrm{~mm})$, threeeighths inch $(9.5 \mathrm{~mm})$, or one-half inch $(12.7 \mathrm{~mm})$ thick, except that average thicknesses varying not more than one-sixteenth inch $(1.6 \mathrm{~mm})$ from the nominal thicknesses, and local variations of onesixteenth inch $(1.6 \mathrm{~mm})$ in thickness of the individual boards are permissible.

4. The nominal width of gypsum plaster boards shall be 32 inches $(810 \mathrm{~mm})$, and the nominal length 24 inches $(610 \mathrm{~mm}), 36$ inches $(910 \mathrm{~mm})$ or 48 inches $(1,220 \mathrm{~mm})$. A variation of one-fourth inch $(6.3 \mathrm{~mm})$ under specified width, and of three-eighths inch $(9.5 \mathrm{~mm})$ under or over the specified length is permissible.

5 . The weight of gypsum plaster board per 1,000 square feet shall conform to the following:

\begin{tabular}{|c|c|c|c|c|c|}
\hline \multicolumn{2}{|c|}{ Thickness } & \multicolumn{2}{|c|}{ Weight (minimum) } & \multicolumn{2}{|c|}{ Weight (maximum) } \\
\hline $\begin{array}{r}\text { Inch } \\
1 / 4 \\
5 / 16 \\
3 / 8 \\
1 / 2\end{array}$ & $\begin{array}{r}m m \\
6.3 \\
8 \\
9.5 \\
12.7\end{array}$ & $\begin{array}{c}\text { Pounds } \\
1,200 \\
1,250 \\
1,400 \\
2,000\end{array}$ & $\begin{array}{l}k g \\
544 \\
567 \\
635 \\
907\end{array}$ & $\begin{array}{c}\text { Pounds } \\
1,500 \\
1,650 \\
2,000 \\
3,000\end{array}$ & $\begin{array}{r}\mathrm{kg} \\
680 \\
750 \\
907 \\
\mathbf{1}, 361\end{array}$ \\
\hline
\end{tabular}


6. (a) Strength test specimens shall be 12 inches $(300 \mathrm{~mm})$ wide and approximately 18 inches (460 $\mathrm{mm}$ ) long. When tested in accordance with the methods described in section 13, specimens taken from the gypsum plaster boards shall carry not less than the following loads:

\begin{tabular}{|c|c|c|}
\hline \multirow[b]{2}{*}{$\begin{array}{l}\text { Board thick- } \\
\text { ness (incbes) }\end{array}$} & \multicolumn{2}{|c|}{ Load } \\
\hline & $\begin{array}{l}\text { Knife-edges } \\
\text { across fiber } \\
\text { of surfac- } \\
\text { ing }\end{array}$ & $\begin{array}{l}\text { Knife-edges } \\
\text { parallel to } \\
\text { fiber of } \\
\text { surfacing }\end{array}$ \\
\hline $\begin{array}{l}36 \\
516- \\
146\end{array}$ & $\begin{array}{c}\text { Pounds } \\
40 \\
35 \\
30\end{array}$ & $\begin{array}{c}\text { Pounds } \\
20 \\
17 \\
14\end{array}$ \\
\hline
\end{tabular}

(b) With loads equal to or less than those specified in paragraph $(a)$ there shall be no breaking of the bond between the surfacing and the core.

(c) Gypsum plaster boards that are one-half inch $(12.7 \mathrm{~mm})$ thick, or those that are not reinforced on the surfaces with a covering made from fibrous material, when tested as described in paragraph $(a)$, shall carry an ultimate center load of not less than 20 pounds $(9.07 \mathrm{~kg})$.

7. The surfaces shall be such that they will readily receive and retain gypsum plaster. The edges and ends shall be reasonably straight and solid. The corners shall be square with a permissible variation of one-fourth inch $(6 \mathrm{~mm})$ in the full width of the plaster boards. Gypsum plaster boards shall be free from cracks and imperfections that will render them unfit for use.

NoTE.-Gypsum plaster boards that bave their corners blurred or broken shall be considered acceptable, provided that tbe broken portion is not more than $11 / 2$ inches $(38 \mathrm{~mm})$ in length.

(See A. S. T. M. serial designation C36, sections 8 to 13 , inclusive (given above), for requisites for "sampling," "packing and marking," "inspection," "rejection," "rehearing," and "methods of testing.")

Federal Specifications Board specification No. 284, United States Government master specification for gypsum wall board, March 28, 1925.

This specification has the same essential requirements as that of the American Society for Testing Materials, serial designation C36 (given above) with the following variations:

\section{TYPES}

I. This specification covers two types of gypsum wall board: A, boards with square edges, designed for butted joints; B, boards with rounded edges, designed for filled joints.

\section{MATERIAL AND WORKMANSHIP}

Type $\mathrm{A}$ boards are 4 feet wide; type $\mathrm{B}$ boards are 3 feet $11 \frac{3 / 4}{4}$ inches wide.

\section{GENERAL REQUIREMENTS}

Weight.-Gypsum wall board shall weigh not less than 1,500 pounds nor more than 2,000 pounds per 1,000 square feet.

\section{METHOD OF INSPECTION AND TEST}

Sampling.-A strip 2 feet long by the width of the board shall be cut from one end of each of the 10 or more boards in the sample and forwarded to the laboratory for test.

\section{PACTING AND MARKING}

Gypsum wall board shall be packed in such a way as to be kept reasonably dry.

Gypsum wall board shall be stamped on each board with the name of the manufacturer and the brand, if any.

Federal Specifications Board specification No. 285, United States Government master specification for gypsum plaster board, March 28, 1925.

\section{TYPE}

One type of gypsum plaster board is covered by this specification.

\section{MATERIAL AND WORKMANSHIP}

Gypsum plaster board consists of set gypsum plaster, with or without fiber, reinforeed on the surfaces with sheets of fibrous material.

The nominal dimensions of a gypsum plaster board are 32 by 36 by $3 / s$ inches.

The surface of a gypsum plaster board shall be such that it will readily receive and retain gypsum plaster. The edges and ends shall be reasonably straight and solid and the corners square. They shall be free from cracks and imperfections which would render them unfit for use.

\section{GENERAL REQUIREMENTS}

\section{DTMENSION TOLERANCES}

(a) Thickness.-A tolerance of $\pm 1 / 16$ inch is permissible.

(b) Width.-A tolerance of $-1 / 4$ inch is permissible.

(c) Length.-A tolerance of $\pm 3 / 8$ inch is permissible.

\section{WEIGHT}

Gypsum plaster board shall weigh not less than 1,500 pounds nor more than 2,000 pounds per 1,000 square feet.

\section{STRENGTH}

When the load is applicd through a knife-edge parallel to the fibers of the surfacing material, the board shall withstand a pressure of at least 20 pounds; when the knife-edge is at right angles to the fibers, the breaking load shall be at least 40 pounds.

\section{BOND}

With loads equal to or less than those specified in Section III, 3, there shall be no breaking of the bond between the fibrous surfacing material and the plaster core.

\section{METHOD OF INSPECTION AND TEST}

See A. S. T. M. serial designation C36 (given above.)

\section{PACKING AND MARKING}

Gypsum plaster board shall be packed in such a way as to be kept reasonably dry.

Gypsum plaster board shall be stamped on each board with the name of the manufacturer and the brand, if any.

\section{ADDITIONAL INFORMATION}

Gypsum plaster boards one-fourth or one-half inch thick may be had for special purposes.

The Gypsum Institute, gypsum plaster board, specifications governing gypsum plaster board construction. 
Note. - The full text of these specifications includes four titles, as follows:

Title I. General uses.

Title II. Specifications, materials composition.

Title III. Specitications, erection. (a) On wood framing, (b) on metal supports.

Title IV. Advantages in the use of gypsum plaster board.

(The materials composition specifications, given under Title II, are as follows:)

1. Gypsum plaster boards shall consist either of sheets or slabs composed of one or more layers of hydrated calcined gypsum, with or without fiber, reinforced on the surfaces with a covering made from pulp, news, or felt stock, or of sheets or slabs composed of an intimate mixture of hydrated calcined gypsum and fibrous binding material.

\section{DIMENSIONS}

2. The thickness, length, and width of gypsum plaster boards shall conform to the following:

(a) Thickness.-Gypsum plaster boards shall not be less than $1 / 4$ inch nor more than 1 inch thick, except that average thicknesses varying not more than $1 / 16$ inch from the nominal thickness and local variations of $1 / 16$ inch in the thickncss of the individual boards are permissible. The thickness shall be taken as the average measurements, taken to the nearest $1 / 32$ inch, at the center of the plaster board. and at points on the diagonals, 2 inches from each of the four corners.

(b) Length and width.-The (nominal) width of gypsum plaster board shall be 32 inches, and the nominal length 24, 36, or 48 inches.

\section{STRENGTH}

3. The required strength of gypsum plaster boards shall be determined by tests which will be conducted as follows:

(a) Specimens for strength tests shall be 12 inches wide and approximately 22 inches long, and when tested shall be supported on parallel knife-cdge bearings spaced 20 inches apart, and shall be loaded through similar bearings placed 8 inches from either support.

(b) When tested as described, specimens taken from the gypsum plaster boards shall carry not less than the following loads:

\begin{tabular}{|c|r|r|}
\hline Board thickness (inch) & \multicolumn{2}{|c|}{ Knife-edges } \\
\hline $3 / 8$ & $\begin{array}{c}\text { Across } \\
\text { fiber of } \\
\text { surfacing }\end{array}$ & $\begin{array}{c}\text { Parallel } \\
\text { to fiber of } \\
\text { surfacing }\end{array}$ \\
\hline $5 / 16$ & Pounds & Pounds \\
$1 / 40$ & 35 & 20 \\
\hline
\end{tabular}

(c) With loads equal to or less than those specified under section $3(b)$ there shall be no breaking of the bond between the surfacing and the core.

(d) Gypsum plaster boards which are of a thickness greater than three-eighths inch, or which are not reinforced on the surfaces with a covering made from pulp, news, or felt stock, shall conform to all of the strength requirements of these specifications for a gypsum plaster board three-eighths inch in thickness.

(For requirements under headings "finish," "sampling," "packing and marking," "inspection," "rejection," and "rehearing," see A. S. T. M. specification C37 (given above).)

\subsection{Gypsum Tiles and Blocks.}

American Society for Testing Materials, standard specifications for gypsum partition tile or block, serial designation C52-27, 1927.
1. Gypsum partition tile or block is a building unit used for nonbearing construction in the interior of buildings.

Note.-The words "tile" and "block" are synonymous and are used so as to be in accord with certain freight association nomenclature.

2. (a) It is also used as a protection for columns, elevator shaits, etc., against fire.

(b) Gypsum cored tile, 3 or 4 inches in thickness, may be split and used for furring purposes.

\section{MANUFACTURE}

3. Gypsum tile consists of gypsum with or without an aggregate.

\section{DIMENSIONS AND PERMISSIBLE VARIATIONS}

4. Gypsum partition tile shall be rectangular in shape, with straight and square edges and true surfaces. They may also be of special shape, provided the requirements as otherwise mentioned herein are met. They may be solid or cored and shall preferably be 12 by 30 inches in face dimensions.

5. (a) The core spaces shall be symmetrically spaced.

(b) Shell thickness.-The minimum cross-sectional shell dimensions of gypsum partition tile with circular, elliptical, or rectangular core spaces shall conform to the following:

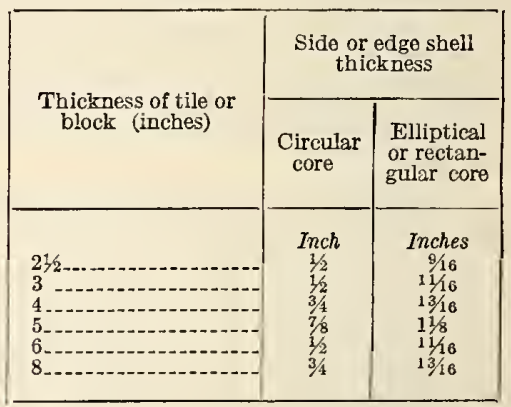

In tile or block having circular cores the sum of the widths of the two side shells plus the width of the center vertical web shall not be less than 2 inches in the case of 6 -inch tile and not less that $2 \frac{1}{2}$ inches in the case of 8-inch tile.

In tile or block having elliptical or rectangular cores the sum of the widths of the two side shells plus the width of the center vertical web shall be not less than $23 / 4$ inches in the case of 6 -inch tile and not less than $3 \frac{1}{4}$ inches in the case of 8 -inch tile.

6. (a) Gypsum tile may be of any convenient length and height, but in general they shall not exceed 30 inches in length and 12 inches in height. The thickness shall be uniform throughout and within the limits specified in paragraph $(b)$.

(b) Thickness.-Gypsum tile or block shall conform to the specified thicknesses with the following permissible variations:

\begin{tabular}{|c|c|c|}
\hline $\begin{array}{l}\text { Nominal size of tile } \\
\text { or block (inches) }\end{array}$ & $\begin{array}{l}\text { Minimum } \\
\text { thickness }\end{array}$ & $\begin{array}{l}\text { Maximum } \\
\text { thickness }\end{array}$ \\
\hline $\begin{array}{l}11 / 2 \text { (furring) } \\
2 \text { (furring) } \\
2 \\
21 / 2\end{array}$ & $\begin{array}{c}\text { Inches } \\
13 / 8 \\
17 / 8 \\
2 \\
23 / 8 \\
27 / 8\end{array}$ & $\begin{array}{c}\text { Inches } \\
15 / 8 \\
21 / 8 \\
21 / 4 \\
23 / 4 \\
31 / 4\end{array}$ \\
\hline $\begin{array}{l}4 \\
4 \\
6 \\
6 \\
8\end{array}$ & $\begin{array}{l}37 / 8 \\
47 / 8 \\
53 / 4 \\
73 / 4\end{array}$ & $\begin{array}{l}41 / 4 \\
51 / 4 \\
61 / 4 \\
81 / 4\end{array}$ \\
\hline
\end{tabular}


(c) Dimension determinations.-Over-all dimensions shall be measured from opposite face to opposite face of the tile or block, the greatest measurement observed being taken as the dimension.

Sectional dimensions of cored tile shall be taken 1 inch in from the ends of the tile or block, the least measurement observed being taken as the dimension.

Shell dimensions shall be measured from the surface of the core space to the normal surface of the tile or block inside the scoring.

(d) Dimension tolerances.-A variation of onefourth inch in the nominal width and three-eighths inch in the nominal length of the tile or block is permissible.

(e) Scoring.-The scoring upon gypsum tile or block shall be of such a nature as not to materially reduce the shell thickness.

\section{PHYSICAL PROPERTIES AND TESTS}

7. (a) When tested in accordance with the methods described in section 14, the compressive strength of gypsum tile or block shall be not less than 75 lbs./in. ${ }^{2}$ for the average of five specimens tested.

(b) When completely saturated the compressive strength shall be not less than $33 \frac{1}{3}$ per cent of the strength required for such tile or block when dried to constant weight.

(c) When tested in accordance with section 16 the rate of absorption of gypsum tile or block shall be not less than $8 \mathrm{cc}$ nor more than $30 \mathrm{cc}$ for the first minute, nor less than $4 \mathrm{cc}$ for each of the next five subsequent minutes.

8. In locations where fire resistance is an essential property gypsum tile or block shall meet the requirements of the specifications for fire tests of building construction and materials, (serial designation C19) of the American Society for Testing Materials (See 1928 book of A. S. T. M. tentative standards) as they apply for the construction in which the tile are used.

\section{SAMPLING}

9. At least 1 per cent of the number of gypsum tile or block in a shipment, but in no case less than 10 tile, shall be so selected as to be representative of the shipment. Twenty-five per cent of such selected tile, but in no case less than 10 individual tile, shall constitute a sample for test purposes. In case a shipment consists of more than one car or carrier load a sample shall be selected from each car or carrier. Samples for test shall be selected at thc factory or at the point of delivery, as specified in the sales contract.

\section{MARKING}

10. When gypsum tile or block are shipped for resale the manufacturer's "brand" name shall be shown upon each tile.

\section{INSPECTION AND REJECTION}

11. Inspection may be made either at the point of shipment or at the point of delivery. The inspector representing the purchaser shall have free access to the carriers being loaded for shipment to the purchaser. He shall be afforded all reasonable facilities for inspection and sampling, which shall be so conducted as not to interfere unnecessarily with the loading of the carriers.

12. Any rejection shall be based upon failure to conform to the requirements of these specifications and shall be reported within 10 working days from the receipt of the shipment by the consignee. The notice of rejection shall contain a specific statement of the respects in which the tile or block have failed to meet the requirements of these specifications.
13. Claims for rehearing shall be valid only if made within 20 working days from receipt of notice of specific cause for rejection.

\section{MLTHODS OF TESTING}

\section{COMPRESSIVE STRENGTH OF GYPSUT PARTITION TILE OR BLOCK}

14. (a) Not less than 10 full-size gypsum tile specimens shall be used.

(b) The test specimens shall be dried to constant weight at a temperature not exceeding $110^{\circ} \mathrm{F}$. $\left(43^{\circ} \mathrm{C}\right.$.) until two successive weighings of the same specimen do not show a variation in excess of 0.5 per cent. At the option of the manufacturer or purchaser, strength tests may be conducted on samples that are dried at room temperature. However, in cases of controversy all test samples shall be dried to constant weight as herein prescribed.

(c) The tile or block shall be tested in the position in which they are designed to be used and shall be bedded on and capped with a felt gasket not less than one-eighth inch nor more than one-fourth inch in thickness. At the option of the manufacturer or purchaser, or in cases of controversy, the test samples may be suitably bedded and capped with neat gypsum mortar or the bearing surfaces of the tile may be planed or rubbed smooth and true. When neat gypsum mortar is used for bedding and capping the test may be conducted after the mortar has set but not sooner than one hour after the sample has been capped.

(d) The loading head shall completely cover the bearing area of the tile and the applied load shall be transmitted through a spherical bearing block of propel design. The speed of the moving head of the testing machine shall not be more than 0.05 inch per minute.

\section{TRANSVERSE STRENGTH OF GYPSOM PARTITION TILE OR}

15. (a) Not less than 10 full-size gypsum tile specimens shall be used.

(b) The test specimens shall be dried to constant weight as prescribed for the determination of the compressive strength.

(c) The tile or block shall be supported on its face (flat) on fixed parallel bearings spaced 24 inches $(600 \mathrm{~mm})$ on centers, and shall be loaded through a similar bearing midway between the supports. All bearing and load surfaces shall be truc, shall engage the full width of the test specimen, and shall be rounded to a radius of one-half inch $(12.7 \mathrm{~mm})$.

(d) The speed of the moving head of the testing machine shall not be more than 0.05 inch per minute. The modulus of rupture shall be calculated in pounds per square inch from the formula:

where

$$
\text { Modulus of rupture }=\frac{3 w \ell}{2 b d^{2}}
$$

$l=$ distance between supports in inches

$b=$ breadth (widch) of tile in inches

$d=$ thickness of tile in inches

$w=$ load in pounds at failure of test specimen.

\section{ABSORPTION OF GYPSUM PARTITION TILE OR BLOCK.}

16. (a) The absorption shall be determined upon a test specimen of partition tile or block consisting of approximately one-half of a full-size tile.

(b) The test specimen shall be dried to constant weight as prescribed for the determination of the compressive strength.

(c) The dried test specimen shall be submerged in water until a constant saturated weight is attained, but in no event for a period of less than two hours, 
the water temperature being maintained at between $70^{\circ}$ and $80^{\circ} \mathrm{F}$. $\left(21^{\circ}\right.$ and $27^{\circ} \mathrm{C}$.). The test specimen shall be removed, the surfacc wiped off with a damp cloth and the specimen weighed immediately. The percentage of absorption shall be calculated on the dry weight from the formula:

$$
\begin{aligned}
& \text { Percentage of absorption }=\frac{100(B-A)}{A} \\
& \text { where } \\
& A=\text { weight of dry test specimen } \\
& B=\text { weight of saturated test specimen. }
\end{aligned}
$$

(d) The rate of absorption shall be determined by sealing upon the face of the test specimen, which shall first be dried to constant weight as prescribed for determination of the compressive strength, a glass tube $1 \frac{1 / 2}{}$ inches in diameter and 12 inches in length, graduated in centimeters and located centrally over a core. Into this tube shall be poured $250 \mathrm{cc}$ of water at a temperature of between $70^{\circ}$ and $80^{\circ} \mathrm{F}$. $\left(21^{\circ}\right.$ and $27^{\circ}$ C. $)$. The rate of absorption shall be reported in cubic centimeters per minute.

The Gypsum Institute, gypsum partition tile, 1928.

This publication includes A. S. T. M. standard specifications for gypsum partition tile or block, serial designation C52-27 (given above), and also specifications for the erection of gypsum partition tile. (See 518.53, p. 420.)

\subsection{Wall Plasters.}

American Society for Testing Materials, tentative specifications for Keene's cement, serial designation C61-29T, 1929.

Various manufacturers offer to the trade several grades of Keene's cement of different finenesses and setting times. These specifications are applicable to the grades manufactured for use in the base and finish coats of plastering. Grades intended for use in Scagliola, castings, and other special purposes, should conform to the requirements of these specifications in all respects except those for fineness and time of set, which will vary according to the particular use for which the grade is designed.

1. Keene's cement is anhydrous calcined gypsum, the set of which is accelerated by the addition of other materials.

\section{PROPERTIES}

2. Keene's cement shall not set in less than one hour nor more than four hours.

3. The cement shall have a tensile strength of not less than $450 \mathrm{lbs} . / \mathrm{in.}{ }^{2}\left(31.5 \mathrm{~kg} / \mathrm{cm}^{2}\right)$.

4. The cement shall all pass a No. 14 sieve, not less than 98 per cent shall pass a No. 40 sieve, and not less than 80 per cent shall pass a No. 100 sieve. (For sieve requirements, see 500.2, p. 1.)

5. The cement shall not show a combined water content of more than 2 per cent.

Requirements for items "Sampling," "Packing, and Marking," and "Inspection and Rejection," are the same as for A.S. T. M. C23. (See 514.2, p. 263.)

\section{METHODS OF TESTING}

-The chemical and physical properties of Keene's cement shall be determined in accordance with A. S. T. M. C26 (see 514.0, p. 259), except that in the determinations of the time of setting and tensile strength, the consistency used shall be such that a $350 \mathrm{~g}$ modificd Vicat needle (note) shall give a penetration of $15 \mathrm{~mm} \pm 2 \mathrm{~mm} 20$ seconds after dropping and the measurement being made 20 minutes after the addition of the Keene's cement to the gaging water. Furthermore, in the determination of testing consistency, 0.1 per cent of commercial retarder may be used.
Note.-In all essentials except the plunger tbe modified Vicat apparatus is the same as the Vicat apparatus described in A. S. T. M. $\mathrm{C} 26$. In the modified apparatus the plunger is made of aluminum tubing. The end that penetrates the mix is three-fourths incb in diameter and $13 / 4$ inches in lengtb, the stem is one-balf inch in diam. eter and 61/2 inches in length. The amount of penetration is determined by the drop of the plunger as read on the millimeter scale. The weigbt of the plunger may be varied by loading with shot.

Contracting Plasterers' International Association, standard specification for lath and plaster work, 1923.

Sand.-All sand to be clean, sharp sand free from loam.

Lime.-All lime to be fresh burned plasterers' lump lime or standard plasterers' hydrated lime.

Wood lath.-All wood lath to be No. 1 grade lath $1 \frac{1}{2}$ inches wide.

Nails.-To be three-penny fine, 16-gage wire nails.

Wire or metal lath. - All wire lath to be No. 18 gage 0.0475 three-eighth inch mesh painted wire lath, weighing 3.3 pounds per square yard, or No. 24 gage metal lath painted, weighing no less than 3.4 pounds per square yard.

Stucco.-All stucco to be fresh.

Hair to be well whipped cattle hair.

Fiber to be long vegetable fiber, well whipped.

Portland cement.- To be a brand that shall meet the requirements of the standard specifications for Portland cement of the American Society for Testing Materials as revised to date by said society. (See A. S. T. M. C9, 516.11, p. 287.)

Hard plaster.-To be an approved brand of one of the following types: A, Straight gypsum plaster; B, Gypsum and lime mixture type; C, Dark gypsum plaster.

Metal corner beads.-To be properly galvanized.

Lathing. - All wood lath to be nailed to each stud, joist, or bearing, with joints broken, not over seven lath to a break, no diagonal nor vertical lathing allowed, a full three-eighth inch key to be left for lime and mortar and not less than a full one-fourth inch for hard plaster.

Lime mortar.-To be composed of clean, coarse sand, fresh lump lime, and hair or fiber in proper proportions and to be well slaked and protected.

Putty.-Fresh-burned lump lime to be run off in a tight putty box, thoroughly slaked and screened through a fine putty screen. Hydrated lime, when used, to be soaked with water in a tight box for not less than 24 hours.

Hard finish.-To be composed of cold lime putty, plaster of Paris, and sand, to be well troweled to a smooth, even surface, free from blisters, checks, and other imperfections.

Sand finish.-All float sand finish to be composed of lime putty and sand to be water floated with a float to an even granular or sand surface.

Scratch coat.-All scratch coating to be well laid on and surface covered with a full coat which is to be well scratched, to be well undercut for the brown coat, all lime mortar scratch coating to be dry before applying the brown coat.

Brown coat.-All brown coating to be well applied, allowing only sufficient space for the finish coat, brown coat to be rodded and screeded with all angles straight and true, all hard plaster and patent stuccos to be mixed in accordance with the directions of the manufacturer, and no hard mortar to be floated with water, nor shall any "dead" material be retempered or used.

Wire or metal lath.-Shall be lapped at each joint or seam and shall be stapled every 6 inches with blued or galvanized staples.

Band iron furring.-As specified, to be $1 / 2,3 / 4$, or 1 inch crimped and painted band iron of No. 22 gage metal, such furring to be stapled to bearing and the wire or metal lath to be applied and stapled over such band iron furring. 
Suspended ceilings.-To be constructed of $1 \frac{1}{2}$ or 2 inch flat bar angles, tees, or channels, as may be called for; such principals shall be spaced not more than 4 feet 0 inch on centers hung with flat bar or 1/4-inch nild steel rod hangers, 4 feet 0 inch on centers, securely fastened with approved clips to the structural framing, or through the floor construction; in the event these hangers go through the floor construction they shall be provided with a 6-inch channel or flat bar anchor; no hanger shall be attached to or supported from the bottom flange of a tile arch. The flat bar, angle, tee, or channel runners shall be cross furred with $3 / 4$-inch steel chanels 12 inches on centers, with approved mild steel rod clips, or a double tie of not less than 14 gage annealed galvanized wire. The entire construction to be lathed with No. 18 gage wire lath or No. 24 gage metal lath of the quality and weight heretofore called for. Such lath to be placed with lapped joints and tied to cross furring every 6 inches with soft annealed No. 18 gage galvanized tie wire; all hangers, principals, and cross furrings to be coated with paint.

Furring.-All false beam or cornice furring to be constructed of $3 / 4$-inch channel or 1 -inch flat bar brackets not over 2 feet 0 inch apart, lined out with intermediate furring supports and anchored or toggle bolted into the construction to be made to conform to the design so as to allow for a minimum of plaster, such brackets to be covered with 18-gage wire or 24-gage metal painted lath secured with 18-gage galvanized wire, such furring to conform to the latest and best practice as to durability of construction.

Cornice work.-All molded beams and cornices will be screeded and run in place with molds, with true lines, and accurate miters.

Ornamental work.-All patterns to be turned out by skilled mechanics with true and accurate lines. All models for ornamental work shall be satisfactory to the architect.

Casts.-All casts to be well made, the contractor to supply a sufficient number to meet the requirements of the job; all casts to be made in line, well and truly undercut and free from warps and other irregularities supplying all necessary shrinkers and stretchers.

Stucco or roughcasting.-Where called for, lath the exterior with No. 18 gage galvanized wire lath, or No. 24 gage metal lath, galvanized after expansion, of the quality and weights herein called for, direct to the wood construction, or if called for, over one-half, three-fourths or 1 inch crimped band iron furring, stapled with blued or galvanized staples every 6 inches to the stud, joist, or bearings.

Scratch coat. - With mortar composed of 2 volumes of clean, coarse sand and 1 volume of Portland cement; to this mixture add hair or fiber; where lime is called for add not more than 5 per cent. Apply a scratch coat, thoroughly scratched and undercut, when this coat has set.

Brown coat with mortar composed of 3 volumes of clean, coarse sand and 1 volume of Portland cement, rod and straighten all surfaces; and when the brown coat has "set" roughcast with mortar compound of 2 volumes of Portland cement and 3 volumes of sharp sand, pebbles, or other aggregate specified, dashed on the surface with a scoop or paddle to an even artistic surface.

Cement stucco-On tile and brick.-Brush all masonry surfaces and coat with a seratch coat composed of 2 volumes of clean, coarse sand and 1 volume of Portland cement, scratched, when this coat has "set."

Brown coat with mortar composed of 3 volumes of clean, coarse sand and 1 volume of Portland cement, rod, straighten, and float to a uniform granular surface. Where third or finish coat is called for brown coat is to be scratched with wire scratcher.
Concrete walls and columns.-All work on concrete walls and columns shall have such concrete well brushed with steel brushes and such concrete shall then be covered with a light bond coat of cement as a bonding coat for the finish coat.

Concrete ceilings. - Shall be first washed with a solution of muriatic acid and water, such ceilings shall then be plastered as above.

Painted walls.-Walls that are to be coated with asphaltum waterproofing compounds should first be scratch coated, then browned or finished.

Patching of plaster.-All patching of plaster damaged by mechanies shall be paid for.

Scaffold.-The contractor shall supply all necessary tools, scaffold, and other appliances necessary to fulfill the requirements of the job; all scaffolding to be erected and maintained in accordance with laws of the State relating to scaffolds.

(The association also has a supplement to the above specification, containing recommended practice with regard to plaster measurements, selection of lath and grounds for various classes of work.)

515.

\section{MAGNESITE}

(No nationally recognized specifications available.)

\section{CEMENT AND CONCRETE}

\subsection{GENERAL ITEMS.}

American Association of State Highway Officials, standard specifications for highway bridges and incidental structures, 1928.

\section{WATER FOR USE WITH CEMENT}

Water for use with cement in mortar or concrete shall be subject to the approval of the engineer. It shall not be salt or brackish, and shall be reasonably clear and free from oil, acid, injurious alkali, or vegetable matter.

When required by the engineer, the quality of the mixing water shall be determined by the "tests for quality of water to be used in concrete" as provided in the United States Department of Agriculture Bulletin 1216, see page 287 .

In sampling water for testing, care shall be taken that the containers are clean and that the samples are lepresentative.

When comparative tests are made with a water of known satisfactory quality, any indication of unsoundness, marked change in time of setting, or a reduction of more than 10 per cent in mortar strength shall be sufficient cause for rejection of the water under test.

American Concrete Institute, standard specification of measurement of and estimating concrete, and standard methods for the measurement of concrete work, serial designation C-5A-26, 1926.

(The above specification and standard method gives separate modes of measurement for the following divisions of construction work:)

1. Plain and reinforced structural concrete.

2. Sidewalks and driveways.

3. Structural precast concrete.

4. Cast ornamental concrete.

5. Roads and pavements.

American Concrete Institute, tentative building regulations for reinforced concrete, serial designation E-1A-28T, 1928.

(These regulations have been adopted by the above organization for use as part of a general building code, to cover the use of reinforced concrete in any structure to be erected under the provisions of the code, to provide for the proper design and construction of structures of this material.) 
American Concrete Institute, standard definitions, serial designation $\mathrm{G}-4 \mathrm{~A}-23$, 1923.

(The institute has adopted 135 definitions of terms related to concrete and its uses.)

American Society for Testing Materials, standard method of making and storing specimens of concrete in the field, serial designation C31-27, 1927.

1. This method is intended to apply to the molding and storing of test specimens of concrete sampled from concrete being used in construction.

2 . The test specimens shall be cylindrical in form with the length twice the diameter. The standard shall be 6 by 12 inches cylinders where the coarse aggregate does not exceed 2 inches in size; for aggregates larger than 2 inches, 8 by 16 inch eylinders shall be used; 2 by 4 inch cylinders may be used for mixtures without coarse aggregate.

3. (a) The molds shall be cylindrical in form, made of nonabsorbent material, and shall be substantial enough to hold their form during the mold- work. All the concrete for each sample shall be taken from one place. A sufficient number of samples-each large enough to make one test specimen-shall be taken at different points so that the test specimens made from them will give a fair average of the concrete placed in that portion of the structure selected for tests. The location from which each sample is taken shall be noted clearly for future reference.

(b) In securing samples, the concrete shall be taken from the mass by a shovel or similar implement and placed in a large pail or other receptacle, for transporting to the place of molding. Care shall be taken to see that each test specimen represents the total mixture of the concrete from a given place. Different samples shall not be mixed together, but each sample shall make one specimen.

5. (a) The pails or other receptacles containing the samples of concrete shall be taken as quickly as possible to the place selected for molding test

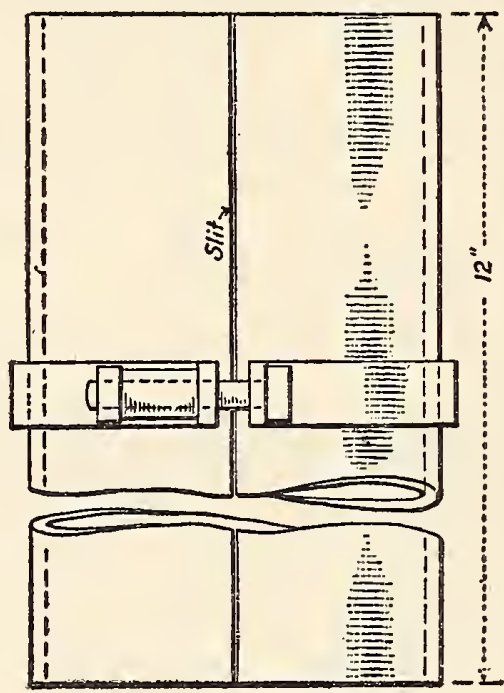

Side View.

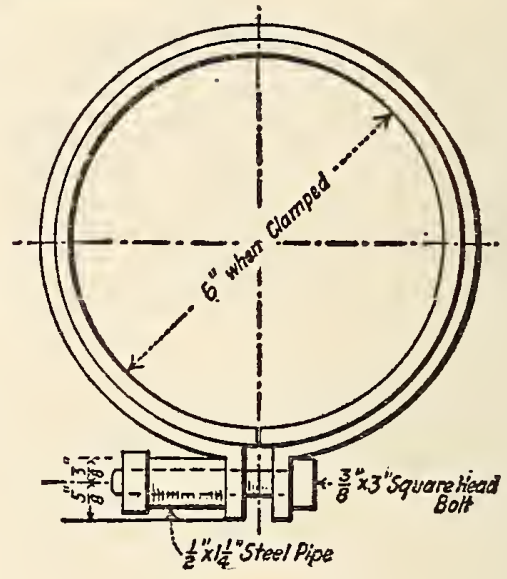

Top View.

Stock : 67"O. D. Cold-Drawn Seamless Steel Tubing; "Walls. Make Narrow Slit along one Element. May also Use 6"Stel Water-Pips, Machined Inside. Slit along one Element, so that when Closed will give 6 "Inside Diameter.

FIgure 96.-Cement mold

ing of the test specimens. They shall not vary in diameter more than $1 / 16$ inch in any direction, nor shall they vary in height morc than $1 / 16$ inch from the height required. They shall be substantially watertight so that there will be no leakage of water from the test specimen during molding.

Note,-The best type of mold consists of lengths of cold-drawn steel tubing, split along one element and closed by means of a circumsteel tubing, split along one element and closed by means of a circumferential band and bolt. Satisfactory of steel wator pipe machined on the inside, from rolled metal plates, from galvanized stcel, machined iron, or steel castings. Paraffined

(b) Each mold shall be provided with a base plate having a plane surface and made of nonabsorbent material. This plate shall be large enough in diameter to properly support the mold without leakage. Plate glass or planed metal is satisfactory for this purpose. A similar plate should be provided for covering the top surface of the test specimen when molded.

(c) Suggestions for suitable molds are shown in Figures 96,97 and 98 .

4. (a) Concrete for the test specimens shall be taken immediately after it has been placed in the specimens. To offset segregation of the concrete occurring during transportation, each sample shall be dumped into a nonabsorbent water-tight receptacle and, after slight mixing, immediately placed in the mold.

(b) The test specimens shall be molded by placing the concrete in the mold in layers 3 to 4 inches in thickness. Each layer shall be puddled with 25 strokes with a $5 / 8$-inch rod 24 inches in length, bullet pointed at the lower end. After puddling the top layer, the surface concrete shall be struck off with a trowel and covered with the top cover plate which may later be used in capping the test specimen.

6. Two to four hours after molding, the test specimens shall be capped with a thin layer of stiff neat-cement paste in order that the cylinder may present a smooth end for testing. The cap shall be formed by means of a piece of plate glass one-fourth inch in thickness or a machined metal plate one-half inch in thickness and of a diameter 2 or 3 inches larger than that of the mold. The plate shall be worked on the fresh cement paste until it rests on top of the mold. The cement for capping 
should be mixed to a stiff paste two to four hours before it is to be used in order to avoid the tendency of the cap to shrink. Adhesion of the concrete to
8. (a) The test specimens shall remain buried in damp sand until 10 days prior to the date of test. They shall then be well packed in damp sand or
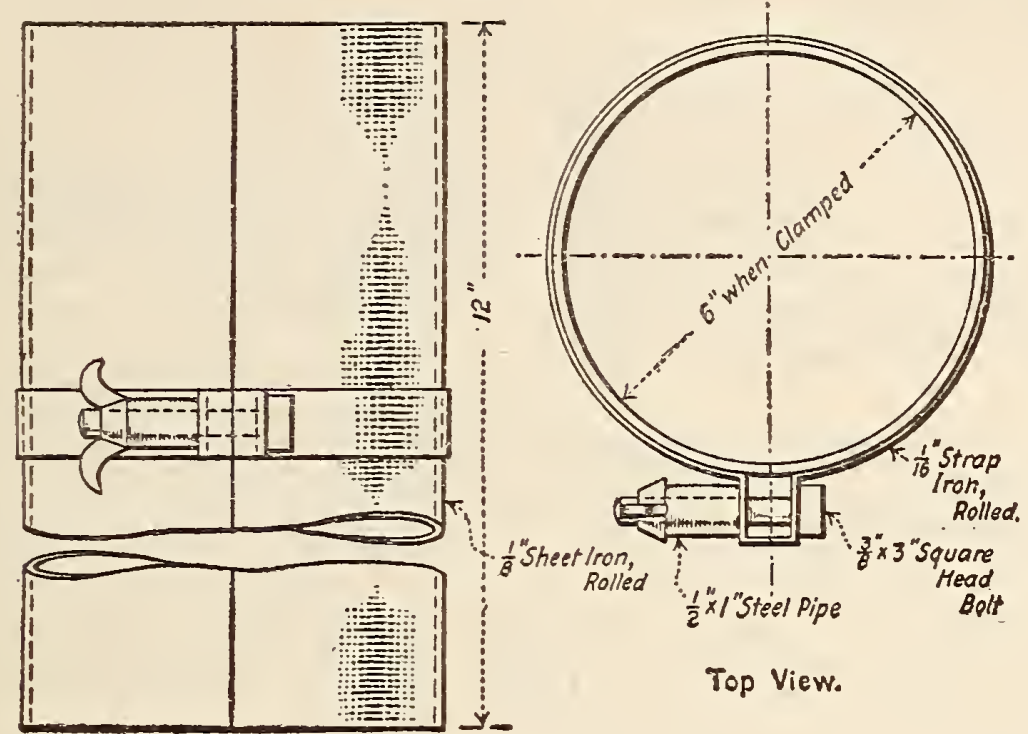

Top View.

\section{Side View}

\section{Figdre 97.-Cement mold}

the top and bottom plates may be avoided by oiling | wet shavings and shipped to the testing laboratory, the plates or by inserting a sheet of paraffined paper. where they shall be stored either in a moist room or

7. At the end of 48 hours the test specimens in damp sand until the date of test.
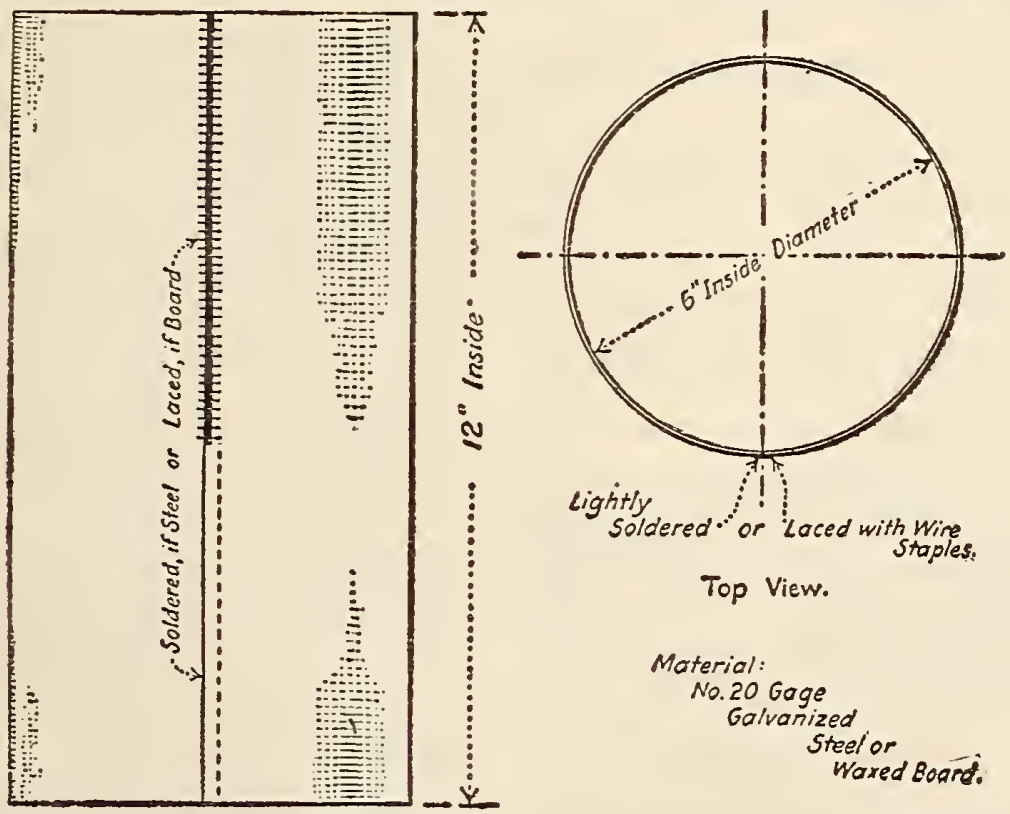

Top View.

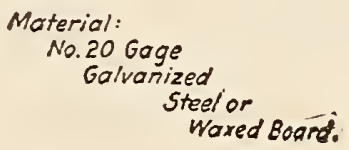

Side View.

Figure 98.-Cement mold

shall be removed from the molds and buried in damp sand except in case the molds shown in Figure 98 are used, when they may be buried in damp sand without removal of the mold, thus permitting shipping in the molds. (b) Should a 7-day test be required, the test specimens shall remain at the work as long as possible to harden and then shall be shipped so as to arrive at the laboratory in time for test on the required date. 
(c) Test specimens shall be protected from drying after removal from damp storage and before testing. American Society for Testing Materials, standard methods of making compression tests of concrete, serial designation C39-27, 1927.

1. These methods are intended to cover compression tests of concrete made in a laboratory where accurate control of quantities of materials and test conditions is possible. They are designed to apply primarily to hand-mixed concrete compression specimens, but may be used with slight modification in making tests of concrete for wearing resistance, bond between concrete and steel, impermeability, etc. The investigation of machine-mixed concrete will require certain obvious changes in the methods. For methods of conducting compression tests of concrete specimens made during the progress of construction work, see A. S. T. M. C31 (given above).

2. Materials shall be brought to room temperature ( $18^{\circ}$ to $24^{\circ} \mathrm{C}$., $65^{\circ}$ to $75^{\circ} \mathrm{F}$.) before beginning the tests. Cement shall be stored in a dry place, preferably in covered metal cans. The cement shall be thoroughly mixed in advance, in order that the sample may be uniform throughout the tests. It shall be sieved through a No. 16 (1,190 micron) sieve and all lumps rejected. Aggregates shall be in a room-dry condition when used in concrete tests. In general, aggregates should be separated on the No. 4 (4,760 micron), 3/8-inch and $1 \frac{1}{2}$ inch sieves and recombined to the average original sieve analysis for each batch. Fine aggregate should be separated into different sizes also, in cases where unusual gradings are being studied.

1 3. Representative samples of all concrete materials shall be secured for preliminary tests prior to the proportioning and mixing of the concrete. Cement test samples may be made up of a small quantity from each sack used in the concrete tests. Test samples of aggregates may be taken from larger lots by quartering.

4. Cement shall be tested in accordance with the methods described in A. S. T. M. C9. (See 516.11, p. 294.)

5. Fine aggregates (passing through a No. 4 (4,760 micron) sieve) shall be subjected, when required, to the following tests:

(a) Sieve analysis test made in accordance with A. S. T. M. C41. (See 512.10, p. 213.)

(b) Test for organic impurities made in accordance with A. S. T. M. C40. (See 512.10 , p. 213.)

(c) Test for quantity of silt, clay, and dust made in accordance with A. S. T. M. D136. (See 512.13, p. 240.)

(d) Test for unit weight made in accordance with A. S. T. M. C29. (See 512.10, p. 212.)

(e) Strength test of 1:3 mortar by weight at 7 and 28 days in comparison with standard sand mortar in accordance with A. S. T. M. C9, page 294.

6. Coarse aggregates (retained on a No. $4(4,760$ micron) sieve) shall be subjected when required to the following tests:

(a) Sieve analysis test as specified in section $5(a)$.

(b) Test for quantity of silt, clay, and dust made in accordance with A. S. T. M. D72. (See 512.11, p. 218.)

(c) Test for unit weight as specified in section $5(d)$.

7. The unit weight of mixed fine and coarse aggregates as used in concrete tests shall be determined in accordance with the method specified in section $5(d)$.

8. The quantities of each size of aggregate to be used in each batch shall be determined on the basis of the sieve analysis and the unit weight of the mixed aggregate. The exact quantities of cement and of each size of aggregate for each batch shall be determined by weight. The quantity of water for each batch shall be accurately measured. The quantities of materials may be expressed as (a) 1 volume of cement to .... volumes of total aggregate mixed as used, or $(b) 1$ volume of cement, .... volumes of fine aggregate, and . . . volumes of coarse aggregate, or (c) 1 part by weight of cement, .... parts by weight of fine aggregate, and .... parts by weight of coarse aggregate.

NotE.-It is impracticable to give a general method for proportioning concrete for experimental purposes; the details will necessarily vary widely with the purpose for which the tests are made. The following procedure is suggested for specific cases:

(a) Vary the cement content by 10 per cent intervals above and (a) Vary the cement co
below assumed quantity.

(b) Vary the proportions of fine to coarse aggregate, measured eparately, at intervals of 10 per cent

(c) Vary the quantity of mixing water by intervals of 10 per cent.

9. Compression tests of concrete shall be made on cylinders of diameter equal to one-half the length. The standard shall be 6 by 12 inch cylinders where the coarse aggregate does not exceed 2 inches in size; for aggregates larger than 2 inches, 8 by 16 inch cylinders shall be used; 2 by 4 inch cylinders may be used for mixtures without coarse aggregate.

10. (a) Concrete shall be mixed by hand in batches of such size as to leave a small quantity of concrete after molding a single test specimen. The batch shall preferably be mixed in a shallow metal pan with a 10-inch bricklayer's trowel which has been blunted by cutting off about $2 \frac{1}{2}$ inches of the point, as follows:

(b) The cement and fine aggregate shall be mixed dry until the mixture is homogeneous in color.

(c) The coarse aggregate shall be added and mixed dry.

(d) Sufficient water shall be added to produce concrete of the required workability.

Note.-Concrete tests should be made on plastic mixtures. It is of the utmost importance that a uniform degree of workability be secured in tests involving comparisons of different materials and methods.

(e) The mass shall be mixed thoroughly until the resulting concrete is homogeneous in appearance.

11. The workability or plasticity of each batch of concrete shall be measured immediately after mixing by one of the following methods:

(a) Slump test made in accordance with A. S. T. M. D138. (See p. 286.)

(b) Flow test made by placing a metal mold in the shape of a frustum of a cone $6 \frac{3}{4}$ inches in top diameter, 10 inches in bottom diameter, 5 inches in depth, on the table of the flow apparatus. The fresh concrete shall be placed in the mold in two layers. Each layer shall be puddled as described in section 13 . Immediately after molding, the surplus concrete shall be struck off with a trowel and the mold shall be removed by a steady upward pull; the table raised one-half inch and dropped 15 times in about 10 seconds by means of a suitable cam and crank. The spread of the fresh concrete, due to this treatment expressed as a percentage of the original bottom diameter of the cone is the "flow."

12. The molds shall preferably be of metal. Each mold shall be provided with a machined metal base plate, and shall be oiled with a heavy mineral oil before using. Particular care shall be taken to obtain tight molds so that the mixing water will not escape during molding.

Nоте.-The best type of mold consists of lengths of cold-drawn steel tubing, split along one element and closed by means of a circumferential band and bolt. Satisfactory molds can be made from lengths of steel water pipe machined on the inside, from rolled metal plates, from galvanized steel, machined iron or steel castings. Parafined cardboard molds will give good results under expert supervision.

13. Concrete test specimens shall be molded by placing the fresh concrete in the mold in layer's 3 to 4 inches in thickness. Each layer shall be puddled with 25 strokes with a $5 / 8$-inch rod 24 inches in length bullet-pointed at the lower end. After the top layer has been puddled the surplus concrete shall be 
struck off with a trowel and the mold covered with a piece of plate glass at least one-fourth inch in thickness or a machined metal plate which will be used later in capping the test specimen.

14. Two to four hours after molding the test specimens shall be capped with a thin layer of stiff neat-cement paste in order that the cylinder may present a smooth end for testing. The cap shall be formed by means of a piece of plate glass oncfourth inch in thickness or a machined metal plate one-half inch in thickness and of a diameter 2 or 3 inches larger than that of the mold. The plate shall be worked on the fresh cement paste until it rests on top of the mold. The cement for capping should be mixed to a stiff paste two to four hours before it is to be used in order to avoid the tendency of the cap to shrink. Adhesion of the concrete to the top and bottom plates may be avoided by oiling the plates or by inserting a sheet of paraffined paper.

15. Concrete test specimens shall be removed from the molds 20 to 48 hour's after molding, marked, weighed, and stored in damp sand, under damp cloths, or in a moist chamber until the date of test. The temperature of the curing room should not fall outside the range of $18^{\circ}$ to $24^{\circ} \mathrm{C}$. $\left(60^{\circ}\right.$ to $75^{\circ} \mathrm{F}$.)

16. Tests shall be made at the age of 7 and 28 days; ages of 3 months and 1 year are recommended if longer-time tests are required.

17. Three to five test specimens should be made on different days in investigations in which accurate comparisons are desired.

18. Compression tests shall be made immediately upon removal of the concrcte test specimens from the curing room; that is, the test specimens shall be loaded in a damp condition. The length and average diameter of the test specimen shall be measured in inches and hundredths; two diameters shall be measured at right angles near the mid length. The test spccimen shall be weighed immediately before testing.

19. In general, only the ultimate compressive strength of the cylinders need be observed. The metal bearing plates of the testing machine shall be placed in contact with the ends of the test specimen; cushioning materials shall not be used. An adjustable bearing block shall be used to transmit the load to the test specimen. The bearing block shall be used on top of the specimen in vertical testing machines. The diameter of the bcaring block shall be the same or slightly larger than that of the test specimen. The supper or lower section of the bearing block shall be kept in motion as the head of the testing machine is brought to a bearing on the test specimen.

20 . The load shall be applied uniformly and without shock. The moving head of the testing machine should travel at the rate of about 0.05 inch per minute when the machine is running idle.

21. The total load indicated by the testing machine at failure of the test specimen shall be rccorded and the unit compressive strength calculated in pounds per square inch, the area computed from the average diameter of the cylinder being used. The type of failure and appearance of the concrete shall be noted.

22. The weight of the concrete, in pounds per cubic foot, shall be determined from the weight of the specimens and their dimensions.

23. Density and yield of concrete when required shall be calculated from the unit volumes of the constituent materials and the volume of the fresh or set concrete as desired. Density is here understood to be the ratio of solids in the concrete to the total volume of the mass. Yield is the volume of con-. crete resulting from the volume of aggregate mixed as used.

24. The report shall include the following: (a) The kind and origin of concrete materials.

(b) Complete data on all tests of cement and aggregates.

(c) A description of methods of making and testing the concrete, where methods deviate from the proposed standards.

(d) The quantities of cement, aggregates, and water in each batch.

(e) The method of measuring workability or plasticity with "slump" or "flow" of concrete.

(f) The quantity of water expressed as a ratio to volume of cement.

(g) The age at test.

(h) The size of test specimens.

(i) The date of molding and testing each cylinder.

(j) The compressive strength in pounds per square inch of each test specimen and the average of tests in a set.

(k) A description of failure and appearance of concrete on each test specimen.

(l) The unit weight, density, and yield of the concrete.

American Society for Testing Materials, standard method of test for organic impurities in sands for concrete, serial designation $\mathrm{C} 40-27,1927$.

(See 512.10 , p. 213.)

American Society for Testing Materials, standard method of test for sieve analysis of aggregates for concrete, serial designation $\mathrm{C} 41-24,1924$.

(See 512.10, p. 213.)

American Society for Testing Materials, standard method of securing specimens of hardened concrete from the structure, serial designation C42-27, 1927.

1. These methods and precautions are intended to apply to sccuring test specimens from hardened concrete in the structure.

2. A specimen from hardened concrete to be tested for strength shall not be taken until the concrete has become hard enough so that the cutting of the specimen will not disturb the bond between the mortar and the coarse aggregate of the specimen.

3 . The specimen from the structure can best be secured by use of a core drill. For specimens taken perpendicular to a horizontal surface, a drill using chilled shot may be used; but when taken perpendicular to a vertical surface, a diamond drill should be used.

4. (a) The cord specimen taken shall be as nearly as possible a cylinder whose length is twice the diameter.

(b) In securing a specimen perpendicular to a horizontal surface care shall be taken to secure, if possible, a specimen whose beds shall be parallel to the horizontal bed of the concrete as originally placed.

(c) In securing a specimen perpendicular to a vcrtical surface, or to a surface with a batter, care shall be taken as to the place of securing the specimen. The lower portion of any one unit of depositing in mass concrete is more dense than is the upper portion of the unit, therefore a specimen shall be taken from near the middle of such unit of deposit.

5. A specimen to be taken from hardened concrete which has been removed from a structure shall be cut out by a drill or shall be cut into a symmetrical test piece by tooling or by sawing. The method of sawing can seldom be used to advantage in the field. In selecting this test specimen care shall be used to see that the concrete selected has not been injured or shattered by the method of its removal from the structure.

6. The specimen secured shall have ends, or beds, as nearly as possible perpendicular to its axis, and if the specimen has ends with uneven surfaces, these ends shall be made to parallel plane surfaces by capping with a thin layer of mortar 
richer than the mortar of the specimen or with a mixture of cement and calcined gypsum (plaster of Paris).

7. In case the dimensions of a specimen are necessarily appreciably different from the recommended shape which requires a height twice the diameter, allowance for the ratio of height to diameter must be made in determining the strength. When the height is more than twice the diameterthat is, when the ratio is greater than two-no correction need be made. For other ratios, corrections shall be made as indicated in the following

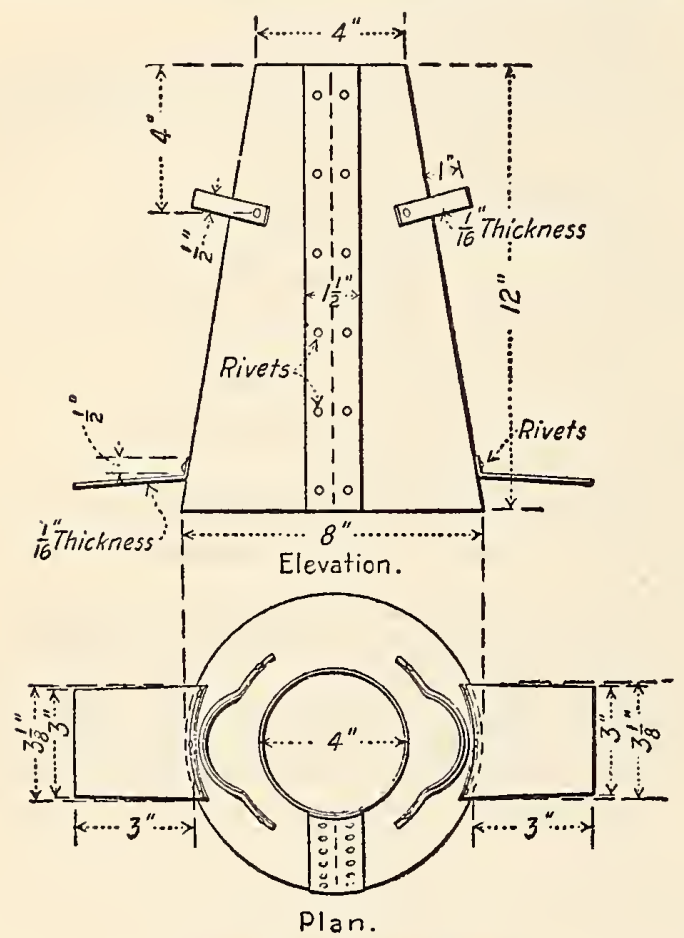

Figure 99.-Cement mold

table. Values not given in the table shall be determined by interpolation:

Ratio of height of cylinder to diameter $\frac{h}{d}$

1.75-Multiply crushing strength by 0.98 1.50-Multiply crushing strength by .95 . 1.25-Multiply crushing strength by .94 . 1.10-Multiply crushing strength by .90 .

1.00-Multiply crushing strength by .85 .

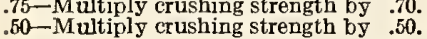

8. The direction of the application of the load in the compression test shall be stated with reference to the direction of the compaction of the mass of concrete in the structure, and where it is practicable the compression load shall be applied in the same direction. In order that the tests be made under uniform conditions as to moisture content, the test specimens shall be completely submerged in water for 48 hours and the compression test shall be made immediately thereafter.

American Society for Testing Materials, tentative rules for inspection of concrete and reinforced concrete work, serial designation C44-22T, 1922.

(In these rules is contained information relative to the status, responsibilities, and duties of an inspector of concrete work.)

American Society for Testing Materials, tentative method of test for consistency of Portland cement concrete, serial designation D138-26T, 1926.
1. This test covers the method to be used both in the laboratory and in the field for determining consistency of concrete.

NotE.-This test is not considered applicable when there is a considerable amount of coarse aggregate over 2 inches in size in the concrete.

2. The test specimen shall be formed in a mold of No. 16 gage galvanized metal in the form of the lateral surface of the frustum of a cone with the base 8 inches in diameter, the upper surface 4 inches in diameter, and the altitude 12 inches. The base and the top shall be open and parallel to each other and at right angles to the axis of the cone. The mold shall be provided with foot pieces and handles as shown in Figure 99.

3. When the test is made at the mixer, the sample shall be taken from the pile of concrete immediately after the entire batch has been discharged. When testing concrete that has been hauled from a central mixing plant, the sample shall be taken from the concrete immediately after it has been dumped on the subgrade.

4. The mold shall be placed on a flat, nonabsorbent surface, such as a smooth plank or a slab of concrete, and the operator shall hold the form firmly in place while it is being filled by standing on the foot pieces. The mold shall be filled to about onefourth of its height with the concrete, which shall then be puddled, using 25 strokes of a $5 / 8$-inch rod, 2 feet long, bullet pointed at the lower end. The filling shall be completed in successive layers similar to the first and the top struck off so that the mold is exactly filled. The mold shall then be removed by being raised vertically immediately after being filled. The molded concrete shall then be allowed to subside until quiescent and the heiglit of the specimen measured.

5. The consistency shall be recorded in terms of inches of subsidence of the specimen during the test, which shall be known as the slump.

Slump $=12$-inches of height after subsidence.

United States Department of Agriculture, Bulletin 1216, method of test for absorption of concrete, 1928.

1. Specimens for the absorption test shall be apparently sound, solid pieces of concrete and shall not show cracks or fissures. The specimens shall be so marked as to permit the identity of each one to be ascertained at any stage of the test.

2. Preparatory to the absorption test all specimens shall first be weighed and then dried in a drier or oven at a temperature of not less than $110^{\circ} \mathrm{C}$. $\left(230^{\circ}\right.$ F.) for not less than three hours. After removal from the drier, the specimens shall be allowed to cool to a temperature of $20^{\circ}$ to $25^{\circ} \mathrm{C}$. $\left(68^{\circ}\right.$ to $77^{\circ} \mathrm{F}$.) and reweighed. If the specimens were apparently dry when taken, and the second weight closely checks the first, the specimens shall be considered dry. If the specimens were known to be wet when taken, they shall be placed in the drier for a further drying treatment of two hours and reweighed. If the third weight checks the second the specimens shall be considered dry. In case of any doubt the specimens must be redried for 2-hour periods until check weights are obtained.

3 . The balance used shall be sensitive to $0.05 \mathrm{~g}$ when loaded with $1 \mathrm{~kg}$ and weighings shall be read at least to the nearest gram. When other than metric weights are used the same order of accuracy must be obtained. In reweighing after immersion the specimens shall be removed from the water, not allowed to drain more than one minute, the superficial water removed by towel or blotting paper, and the specimens at once put upon the balance.

4. Specimens after weighing shall be placed in a suitable receptacle, covered with distilled water 
or rain water, raised to the boiling point and boiled for five hours, and then cooled in water to a final temperature of $10^{\circ}$ to $15^{\circ} \mathrm{C}$. $\left(50^{\circ}\right.$ to $59^{\circ} \mathrm{F}$.).

5 . The test results shall be calculated as percentages of the initial dry weight carried to the nearcst-first decimal place. The results shall be reported separately for each individual specimen, together with the mean of the specimens comprising the sample.

United States Depariment of Agriculture, Bulletin 1216 , method of test for quality of water to be used in concrete, 1928.

1. Acidity and alkalinity.-The acidity or alkalinity shall be determined with standard solutions of one-tenth normal alkali or acid, respectively, using not less than $200 \mathrm{cc}$ of the water under examination. Phenolphthalein or methyl orange should be used as an indicator. Excessive acidity or alkalinity indicates the necessity for further tests.

2. Total solids and inorganic matter.-Five hundred cubic centimeters of the water shall be evaporated to dryness in a wighed dish. For this purpose a platinum dish of 100 to 200 cc eapacity is found most convenient. The dish shall be nearly filled with the water and placed on a water bath, additional portions of the sample of water being added from time to time until $500 \mathrm{cc}$ have been used. The contents of the dish shall be evaporated to dryness, and the dish and contents cooled in a desiccator and weighed. The weight of the residue in grams divided by 5 is the percentage of total solids in the water.

3. The total solids obtained as described may consist of organic matter, of inorganic matter, or of combinations of organic and inorganic matter. The platinum dish shall be ignited at low red heat, and the darkening of the residue during the early stage of the ignition usually indicates the presence of organic matter. The percentage loss on ignition at low red heat will usually be an indicator of the amount of organic matter, but it should be noted that some mineral salts tend to volatilize or partly decompose on heating.

4. The determination of the composition of the mineral matter in the water usually requires a complete chemical analysis of the total solids obtained by the evaporation of $500 \mathrm{cc}$ or more of the water and is not generally undertaken except when the percentage of total solids is large or the water appears to give abnormal tests in other respects.

5. A comparison of the given water with distilled water can be obtained by making standard soundness, time of setting, and 1:3 mortar-strength tests with standard sand, using the same cement of standard quality with each water. (Suggested limits for the last-named tests are as follows: Any indication of unsoundness, marked change in time of setting, or a variation of more than 10 per cent in strength from results obtained with mixtures containing the water of satisfactory quality shall be sufficient cause for rejection of the water under test.)

\subsection{CEMENT.}

\subsection{Portland Cement.}

American Association of State Highway Officials, standard specifications for highway bridges and incidental structures, 1928.

\section{PORTLAND CEMENT}

(This specification is the same as A. S. T. M. C9 (given below) as to quality, with the following provisions for sampling additional:)

Cement may be sampled either at the mill or at the site of the work as provided in the above speci- fication. The seals of cars containing cement which has been sampled shall not be broken except by the engineer; otherwise additional samples shall be taken from these cars.

The contractor shall notify the engineer of dates of delivery sufficiently in advance of such dates so that provision may be made for sampling the cement, either at the mill or upon delivery. If this is not done or if additional iests are necessary, the contractor may be required to rehandle the cement in the storehouse for the purpose of obtaining samples as directed.

American Association of State Highway Officials, tentative standard specifications for highway materials, 1927.

\section{PORTLAND CEMENT}

(This specification is identical with A. S. T. M. C9 (given below), sec. 1 to 15 inclusive.)

American Railway Engineering Association, specifications for Portland cement, 1927.

(Same as A. S. T. M. C9, see p. 294.)

American Society for Testing Materials, standard specifications and tests for Portland cement, serial designation $\mathrm{C} 9-26,1926$.

Approved as "Amcrican standard" by the American Engineering Standards Committee, now the American Standards Association.

1. Portland cement is the product obtained by finely pulverizing clinker produced by calcining to incipient fusion an intimate and properly proportioned mixture of argillaceous and calcareous materials, witl no additions subsequent to calcination excepting water and calcined or uncalcined gypsum.

\section{CHEMICAL PROPERTIES}

\section{The following limits shall not be exceeded:}

Per cont

Loss on ignition

Insoluble residue

Sulphuric anhydride (SO

Sulphuric anhydride $\left(\mathrm{SO}_{3}\right)$
Magnesia (MgO)

\section{PHYSICAL PROPERTIES}

3. The residue on a standard No. 200 sieve shall not exceed 22 per cent by weight.

4. A pat of neat cement shall remain firm and hard, and show no signs of distortion, cracking, checking, or disintegration in the steam test for soundness.

5. The cement shall not develop initial set in less than 45 minutes when the Vicat needle is used or 60 minutes when the Gillmore needle is used. Final set shall be attained within 10 hours.

6. The average tensile strength in pounds per square inch of not less than three standard mortar briquets (see sec. 47) composed of 1 part of cement and 3 parts of standard sand, by weight, shall be equal to or higher than the following:

\begin{tabular}{|c|c|c|}
\hline $\begin{array}{l}\text { Age at } \\
\text { test, } \\
\text { deys }\end{array}$ & Storage of briquets & $\begin{array}{c}\text { Tensile } \\
\text { strength }\end{array}$ \\
\hline 78 & $\begin{array}{l}1 \text { day in moist air, } 6 \text { days in water } \\
1 \text { day in moist air, } 27 \text { days in water. }\end{array}$ & $\begin{array}{r}\text { Los./in. }{ }^{2} \\
225 \\
325\end{array}$ \\
\hline
\end{tabular}

7. The average tensile strength of standard mortar at 28 days shall be higher than the strength at 7 days.

\section{PACKING, MARKING, AND STORAGE}

8. The cement shall be delivered in packages as specified with the brand and name of the manufacturer plainly marked thereon, unless shipped in 
bulk. When shipped in bulk, this information shall be contained in the shipping advices accompanying the shipment. A bag shall contain 94 pounds net. A barrel shall contain 376 pounds net. All packages shall be in good condition at the time of inspection.

9. The cement shall be stored in such a manner as to permit easy access for proper inspection and identification of each shipment, and in a suitable weather-tight building which will protect the cement from dampness.

\section{INSPECTION}

10. Every facility shall be provided the purchaser for careful sampling and inspection at either the mill or at the site of the work, as may be specified by the purchaser. At least 12 days from the time of sampling shall be allowed for the completion of the 7 -day test, and at least 33 days shall be allowed for the completion of the 28-day test. The cement shall be tested in accordance with the methods hereinafter prescribed. The 28-day test need not be made if waived by the purchaser.

\section{REJECTION}

11. The cement may be rejected if it fails to meet any of the requirements of these specifications.

12. Cement remaining in storage prior to shipment for a period greater than six months after test shall be retested and shall be rejected if it fails to meet any of the requirements of these specifications.

13. Cement shall not be rejected on account of failure to meet the fineness requirement if upon retest after drying at $100^{\circ} \mathrm{C}$. for one hour it meets this requirement.

14. Cement failing to meet the test for soundness in steam may be accepted if it passes a retest using a new sample at any time within 28 days thereafter. The provisional acceptance of the cement at the mill shall not deprive the purchaser of the right of rejection on a retest of soundness and time of setting at the time of delivery of cement to the purchaser.

15. Packages varying more than 5 per cent from the specified weight may be rejected; and if the average weight of packages in any shipment, as shown by weighing 50 packages taken at random, is less than that specified, the entire shipment may be rejected.

\section{TESTS}

\section{SAMPLING}

16. Tests may be made on individual or composite samples as may be ordered. Each test sample should weigh at least 4 pounds.

17. (a) Individual sample.-If sampled in cars. one test sample shall be taken from each 50 barrels or fraction thereof. If sampled in bins one sample shall represent each 200 barrels unless otherwise specified by the purchaser.

(b) Composite sample.-If sampled in cars, one sample shall be taken from 1 sack in each 40 sacks (or 1 barrel in each 10 barrels) and combined to form one test sample. If sampled in bins or warehouses one test sample shall represent not more than 200 barrels unless otherwise specified by the purchaser.

18. Cement may be sampled at the mill by any of the following methods that may be practicable, as specified:

(a) From the conveyor delivering to the bin.-At least 4 pounds of cement shall be taken from approximately each 100 barrels passing over the conveyor. This may be secured by taking the entire test sample at a single operation, known as the "grab method," or by combining several portions taken at regular intervals, known as the "composite method." (b) From filled bins by means of proper sampling tubes.-Tubes inserted vertically may be used for sampling cement to a maximum depth of 10 feet. Tubes inserted horizontally may be used where the construction of the bin permits. Samples shall be taken from points well distributed over the face of the bin.

(c) From filled bins at points of discharge.-Sufficient cement shall be drawn from the discharge openings to obtain samples representative of the cement contained in the bin, as determined by the appearance at the discharge openings of indicators placed on the surface of the cement directly above these openings before drawing of the cement is started.

19. The sampling shall be done by or under the direction of a responsible representative of the purchaser.

20. Samples preferably shall be shipped and stored in moisture-proof, air-tight containers. Samples shall be passed through a sieve having 20 meshes per linear inch in order to thoroughly mix the sample, break up lumps, and remove foreign materials.

\section{CHEMICAL ANALYSIS}

\section{IOSS ON IGNITION}

21. One gram of cement shall be heated in a weighed covered platinum crucible, of 20 to $25 \mathrm{cc}$ capacity, as follows, using either method $(a)$ or $(b)$ as specified:

Method (a).-The crucible shall be placed in a hole in an asbestos board, clamped horizontally so that about three-fifths of the crucible projects below, and blasted at a full red heat for 15 minutes with an inclined flame; the loss in weight shall be checked by a second blasting for 5 minutes. Care shall be taken to wipe off particles of asbestos that may adhere to the crucible when withdrawn from the hole in the board. Greater neatness and shortening of the time of heating are secured by making a hole to fit the crucible in a circular disk of sheet platinum and placing this disk over a somewhat larger hole in an asbestos board.

Method $(b)$.- The crucible shall be placed in a muffle at any temperature between $900^{\circ}$ and $1,000^{\circ} \mathrm{C}$. for 15 minutes and the loss in weight shall be checked by a second heating for 5 minutes.

22. A permissible variation of 0.25 will be allowed, and all results in excess of the specified limit but within this permissible variation shall be reported as 4 per cent.

\section{INSOLUBLE RESTDUE}

23. To a 1-g sample of cement shall be added 25 cc of water and 5 cc of concentrated hydrochloric acid (sp. gr. 1.19). Material shall be ground with the flattened end of a glass rod until it is evident that the decomposition of the cement is complete. The solution shall then be diluted to $50 \mathrm{cc}$ and digested on a steam bath for 15 minutes. The residue shall be filtered, washed with cold water and the filter paper, and contents digested in about 30 cc of a 5 per cent solution of sodium carbonate, the liquid being held at a temperature just short of boiling for 15 minutes. The remaining residue shall be filtered, washed with hot water, then with a few drops of hot hydrochloric acid (1:9) and finally with hot water, then ignited at a red heat and weighed as the insoluble residue.

24. A permissible variation of 0.15 will be allowed and all results in excess of the specified limit, but within this permissible variation shall be reported as 0.85 per cent.

\section{SULPEURIC ANEYDRIDE}

25. To a 1-g sample of cement shall be added 25 cc of water and 5 cc of concentrated hydrochloric 
acid (sp. gr. 1.19). Material shall be ground with the flattened end of a glass rod until it is evident that decomposition of the cement is complete. The solution shall be diluted to $50 \mathrm{cc}$ and digested on a steam bath for 15 minutes, filtered, and the residue washed thoroughly with hot water. The solution shall be diluted to $250 \mathrm{cc}$, hcated to boiling, and $10 \mathrm{cc}$ of a hot 10 per cent solution of barium chloride shall be added slowly drop by drop, from a pipette and the boiling continued until the precipitate is well formed. The solution shall then be digested on the steam bath at least three hours, preferably overnight. The precipitate shall be filtered, washed, and the paper and contents placed in a weighed platinum crucible and the paper slowly charred and consumed without flaming. The barium sulphate shall then be ignited and weighed. The weight obtained multiplied by 34.3 gives the percentage of sulphuric anhydride. The acid filtrate obtained in the determination of the insoluble residue may be used for the estimation of sulphuric anhydride instead of using a separate sample.

26. A permissible variation of 0.10 will be allowed, and all results in excess of the specified limit but within this permissible variation shall be reported as 2.00 per cent.

\section{MAGNESIA}

27. To $0.5 \mathrm{~g}$ of the cement in an evaporating dish shall be added $10 \mathrm{cc}$ of water to prevent lumping and then $10 \mathrm{cc}$ of concentrated hydrochloric acid (sp. gr. 1.19). The material shall be ground with the flattened end of a glass rod until attack is complete. The solution shall then be evaporated to complete dryness on a steam or water bath To hasten dehydration, the residue may be heated to $150^{\circ} \mathrm{C}$. or even $200^{\circ} \mathrm{C}$. for one-half to one hour. The residue shall be treated with $10 \mathrm{cc}$ of hydrochloric acid diluted with an equal amount of water. The dish shall be covered, and the solution digested for 10 minutes on a steam bath or water bath. The solution shall be diluted to $75 \mathrm{cc}$, filtered into a beaker, and the separated silica washed thoroughly with hot water (note 1). Five cubic centimeters of concentrated hydrochloric acid and two drops of methyl red indicator $(0.2$ per cent alcoholic solution) shall be added to the filtrate (about 250 cc) (note 2).

Notes.-1. Since this procedure does not involve the determination of silica, a second evaporation is unnecessary.

2. Manganese, if present, is distributed between the precipitates of iron and alumina, calcium and magnesium. In the amounts usually present in Portland cement, it may be neglected. If it is desired to determine the small amount of manganese that may be present in the magnesium pyrophosphate, this may be done colorimetrically as described in the United States Geological Survey Bulletin No $700, p .153$. If present in larger amounts, manganese Bulletin No. 700, p. 153. Ir present in larger amounts, manganese should be precipitated with the iron and alumina, preferably by the $700, \mathrm{p} .112$ ). If this method is used, more solution than in the method above described.

Dilute ammonium hydroxide shall be added drop wise until the color of the solution changes to a distinct yellow. The solution shall be boiled for one or two minutes and filtercd at once. The beaker and precipitate shall be washed slightly with a hot 2 per cent solution of ammonium chloride (or ammonium nitrate). Setting aside the filtrate, the precipitate shall be transferred by a jet of hot water to the precipitating vessel and dissolved in $10 \mathrm{cc}$ of hot hydrochloric acid. The paper shall then be extracted with acid, the solution and washings being added to the solution of the precipitate. The aluminum and iron shall then be reprecipitated at boiling heat by ammonium hydroxide as beforc in a volume of about $100 \mathrm{cc}$ and the second precipitate shall bc collected and washed with a hot 2 per cent solution of ammonium chloride (or ammonium nitrate) on the filter used in the first instance, if this is still intact. To the combined filtrates from the hydroxides of iron and aluminum, reduced in volume if need be, 1 ce of ammonium hydroxide shall be added, the solution brought to boiling, $25 \mathrm{cc}$ of a saturated solution of boiling ammonium oxalate added, and the boiling continued until the precipitated calcium oxalate has assumed a wcll-defined granular form. The precipitate after one hour shall be filtered and washed, and the filtrate set asidc. The filter shall be placed wet in a platinum crucible, and the paper burned off over a small flame of a Bunsen burner; after ignition, it shall be cautiously moistened with water, redissolved in hydrochloric acid, and the solution diluted to $100 \mathrm{cc}$; ammonia shall be added in slight excess, the liquid boiled, and filtered if a precipitate appears. The lime shall then be reprecipitated by ammonium oxalate, allowed to stand till settled, filtered, and washed. The combined filtrates from the calcium precipitates shall be acidified with hydrochloric acid, concentrated on the steam bath to about 150 cc and made slightly alkaline with ammonium hydroxide, boiled and filtered (to rcmove a little iron and aluminum, and perhaps calcium). When cool, the solution shall be acidified with hydrochloric acid, $10 \mathrm{cc}$ of saturated solution of sodium-ammonium-hydrogen phosphate added, and ammonia drop by drop, with constant stirring. When the crystalline ammonium-magnesium orthophosphate has formed, 5 cc excess ammonia shall be added. The solution shall be set aside for not less than four hours, preferably overnight, in a cool place, filtered, and washed with water containing 2.5 per cent $\mathrm{NH}_{3}$. The precipitate shall be dissolved in a small quantity of hot hydrochloric acid, the solution diluted to about $100 \mathrm{cc}, 1 \mathrm{cc}$ of a saturated solution of sodiumammonium-hydrogen phosphate added, and ammonia drop by drop, with constant stirring, until the precipitate is again formed as described and the ammonia is in moderate excess. The precipitate shall then be allowed to stand about two hours, filtered and washed as before. The paper and contents shall be placed in a weighed platinum crucible, the paper slowly charred, and the resulting carbon carefully burned ofi. The precipitate shall then be ignited to constant weight over a Meker burner, or a blast not strong enough to soften or melt the pyrophosphate. The weight of magnesium pyrophosphate obtained multiplied by 72.5 gives the percentage of magnesia. The precipitate so obtained always contains some calcium and usually small quantities of iron, aluminum, and manganese as phosphates.

28. A permissible variation of 0.4 will be allowed, and all results in excess of the specified limit but within this permissible variation shall be reported as 5.00 per cent.

\section{DETERMINATION OF FINENESS}

29. Wire cloth for standard sieves for cement shall be woven (not twilled) from brass, bronze, or other suitable wire, and mounted without distortion on frames about 2 inches below the top of the frame. The joint between the cloth and frame shall be smoothly filled with solder to prevent lodging of the cement. The sieve frames shall be circular, approximately 8 inches in diameter, and may be provided with a pan and cover.

30. A standard No. 200 sieve is one having nominally a 0.0029 -inch opening, certified by the National Bureau of Standards, and conforming to the specifications for this sieve in A. S. T. M. E11 (see 500.2, p. 1). The correction to the sieving value of the sieve shall be determined by sieving tests made in conformity with the standard specifications for these 
tests on a standardized cement which gives a residue of about 20 per cent on the No. 200 sieve.

31 . The test shall be made with $50 \mathrm{~g}$ of cement. The sieve shall be thoroughly clean and dry. The cement shall be placed on the No. 200 sieve, with pan and cover attached, if desired, and shall be held in one hand in a slightly inclined position so that the sample will be well distributed over the sieve, at the same time gently striking the side about one hundred and fifty times per minute against the palm of the other hand on the upstroke. The sieve shall be turned every 25 strokes about one-sixth of a revolution in the same direction. The operation shall continue until not more than $0.05 \mathrm{~g}$ passes through in one minute of continuous sieving (note 1 ). The fineness shall be determined from the weight of the residue on the sieve expressed as a percentage of the

\section{MIXING CEMENT PASTES AND MORTARS}

33. The quantities of dry materials to be mixed at one time shall be $500 \mathrm{~g}$ for neat cement mixtures and $1,000 \mathrm{~g}$ for mortar mixtures. The proportions of cement or cement and sand shall be stated by weight in grams of the dry materials; the quantity of water shall be expressed in cubic centimeters ( $1 \mathrm{cc}$ of water $=1 \mathrm{~g}$ ). The dry materials shall be weighed, placed upon a nonabsorbent surface, thoroughly mixed dry if sand is used, and a crater formed in the center, into which the proper percentage of clean water shall be poured; the material on the outer edge shall be turned into the crater by the aid of a trowel. After an interval of one-half minute for the absorption of the water the operation shall be completed by continous, vigorous mixing, squeezing and kneading with
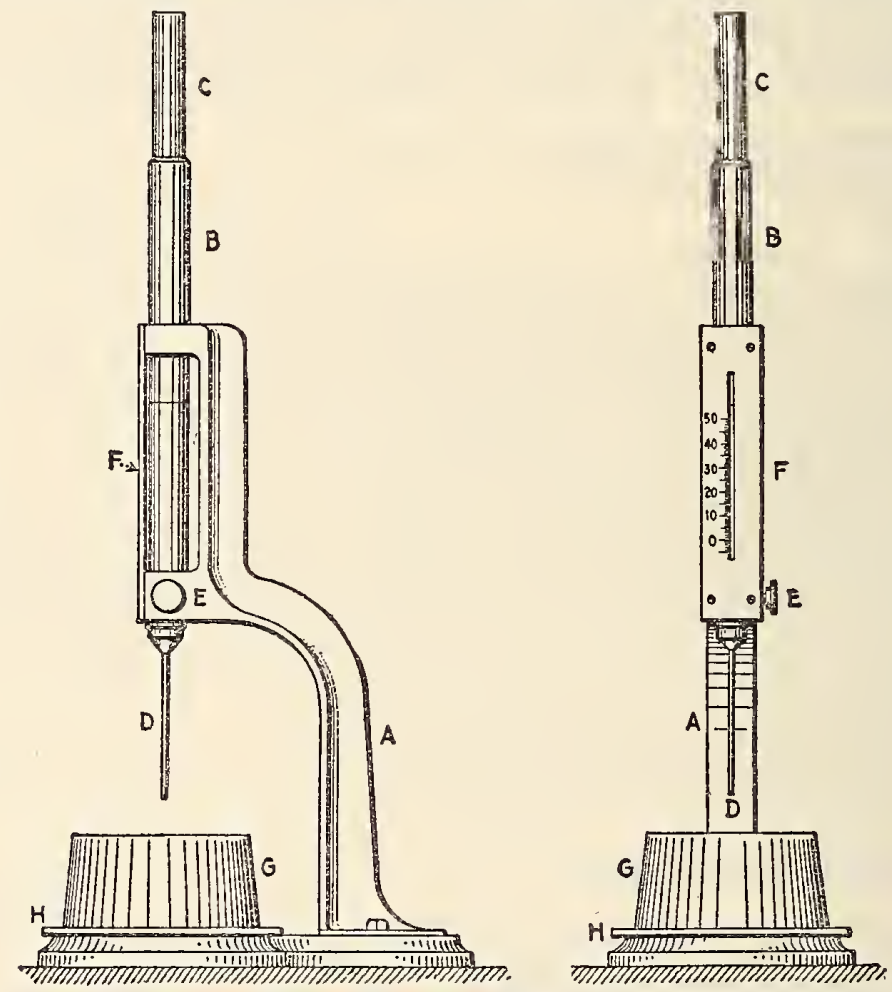

FIGURE 100.-Vicat apparatus

weight of the original sample, applying the sieve correction (note 2).

Nores.-1. The essential points in the sieving operation may he summarized as follows:

(1) Rotation of the sieve throughout the process.

(2) Guarding against loss of material Sieve over white paper and always tap the sieve gently.

(3) Use of a halance which will give results correct within $5 \mathrm{mg}$ and sufficiently sensitive so that the rest point will he deflected at least two divisions of the scale for an added load of $5 \mathrm{mg}$.

(4) Washers, shot, and slugs should never he used on the sieve.

(5) Excessive humidity interferes with good sieving. It tends to decrease the percentage of cement passing the sieve, and, in general to produce irregular results.

2. A plus $(+)$ sieve correction indicates the a mount to he added to and a minus (-) sieve correction the amount to he suhtracted from the per cent passing the sieve to ohtain the true fineness. The per cent passing is the amount ohtained hy suhtracting the per cent residue from 100 . The eorrections are un in the reverse order wen applied to the residues direct.

32. Mechanical sieving devices may be used, but the cement shall not be rejected if it meets the fineness requirement when tested by the hand method described in section 31 . the hands for at least one minute. During the operation of mixing, the hands shall be protected by rubber gloves.

NoTE-In order to secure uniformity in the results of tests for the time of setting and tensile strength the manner of mixing ahove descrihed shall he carefully followed. At least one minute is necessary to ohtain the desired plasticity which is not appreciahly affected oy continuing the mixing for several minutes. The exact time necessiry is dependent upon the personal equation of the operator. The error in mixing shall he on the side of overmixing.

34. The temperature of the room, the materials, the mixing water, the moist closet and storage-tank water shall be maintained as nearly as practicable at $21^{\circ}$ C. $\left(70^{\circ}\right.$ F.) and the mixing water, moist closet, and the water in the storage tank shall not vary from this temperature more than $3^{\circ} \mathrm{C}$. $\left(5^{\circ} \mathrm{F}\right.$.).

\section{NORMAL CONSTSTENCY}

35. The Vicat apparatus consists of a frame $A$ (fig. 100), bearing a movable rod, $B$, weighing $300 \mathrm{~g}$ 
one end $C$ being $1 \mathrm{~cm}$ in diameter for a distance of $6 \mathrm{~cm}$, the other having a removable needle $D, 1 \mathrm{~mm}$ in diameter, $6 \mathrm{~cm}$ long. The rod is reversible, and can be held in any desired position by a serew $E$, and has midway between the ends a mark $F$ which moves under a scale (graduated to millimeters) attached to the frame $A$. The paste is held in a rigid conical ring, resting on a glass plate about 10 $\mathrm{cm}$ square. The ring shall be made of a noncorroding, nonabsorbent material, and shall have an inside diameter of $7 \mathrm{~cm}$ at the base, $6 \mathrm{~cm}$ at the top, and a height of $4 \mathrm{~cm}$.

36. In making the determination, $500 \mathrm{~g}$ of cement, with a measured quantity of water, shall be kneaded ing percentages of water until the normal consistency is obtained. Each trial shall be made with fresh cement. The amount of water required shall be expressed in percentage by weight of the dry cement.

37. The consistency of standard mortar shall depend on the amount of water required to produce a paste of normal consistency from the same sample of cement. Having determined the normal consistency of the sample, the consistency of standard mortar made from the same sample shall be as indicated in Table 1 , the values being in percentage of the combined dry weights_of the cement and standard sand.

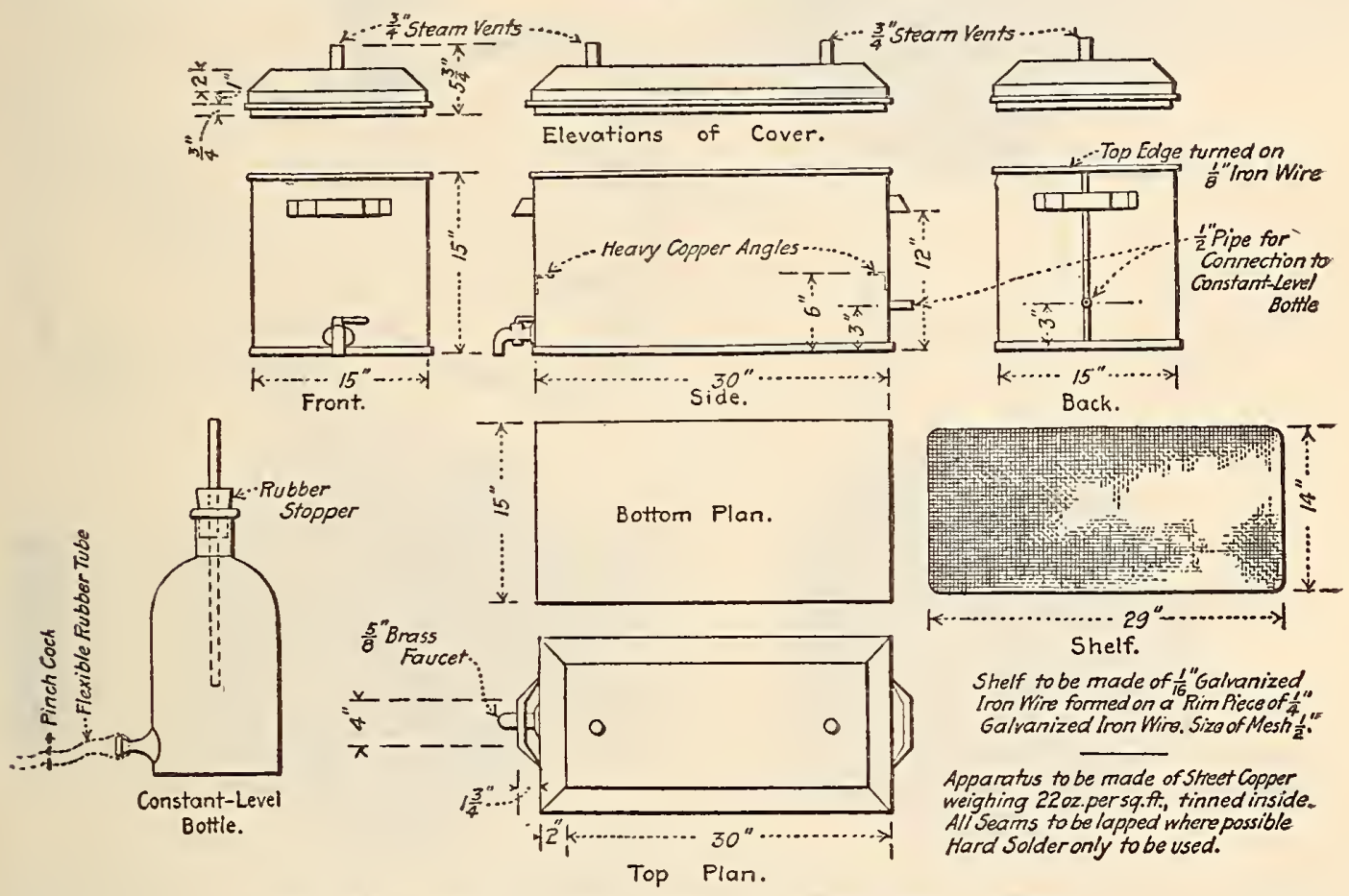

Figdre 101.-A pparatus for making soundness test of cement

into a paste, as described in section 33 , and quickly formed into a ball with the hands, completing the operation by tossing it six times from one hand to the other, maintained about 6 inches apart; the ball resting in the palm of one hand shall be pressed into the larger end of the conical ring held in the other hand, completely filling the ring with paste; the excess at the larger end shall then be removed by a single movement of the palm of the hand; the ring shall then be placed on its larger end on a glass plate and the excess paste at the smaller end sliced off at the top of the ring by a single oblique stroke of a trowel held at a slight angle with the top of the ring, and the top smoothed, if necessary, with a few light touches of the pointed end of the trowel. During these operations care shall be taken not to compress the paste. The paste confined in the ring, resting on the plate, shall be placed under the rod, the larger end of which shall be brought in contact with the surface of the paste; the scale shall then be read, and the rod quickly released. The paste shall be of normal consistency when the rod settles to a point $10 \mathrm{~mm}$ below the original surface in one-half minute after being released. The apparatus shall be free from all vibrations during the test. Trial pastes shall be made with vary-
TABLE 1.-Percentage of water jor standard mortars

\begin{tabular}{|c|c|c|c|}
\hline $\begin{array}{c}\text { Percentage } \\
\text { of water for } \\
\text { neat cement } \\
\text { paste of nor- } \\
\text { mal consist- } \\
\text { ency }\end{array}$ & $\begin{array}{c}\text { Percentage } \\
\text { of water for } \\
\text { one cement, } \\
\text { three stand- } \\
\text { ard Ottawa } \\
\text { sand }\end{array}$ & $\begin{array}{c}\text { Percentage } \\
\text { of water for } \\
\text { neat cement } \\
\text { paste of nor- } \\
\text { mal consist- } \\
\text { ency }\end{array}$ & $\begin{array}{c}\text { Percentage } \\
\text { of water for } \\
\text { one cement } \\
\text { three stand- } \\
\text { ard Ottawa } \\
\text { sand }\end{array}$ \\
\hline 15 & 9.0 & 23 & 10.3 \\
16 & 9.2 & 24 & 10.5 \\
17 & 9.3 & 25 & 10.7 \\
18 & 9.5 & 26 & 10.8 \\
19 & 9.7 & 27 & 11.0 \\
20 & 9.8 & 28 & 11.2 \\
21 & 10.0 & 29 & 11.3 \\
22 & 10.2 & 30 & 11.5 \\
\hline
\end{tabular}

\section{DETERMINATION OF SOUNDNESS}

Note.-Unsoundness is usually manifested by change in volume which causes distortion, cracking, checking, or disintegration. Pats improperly made or exposed to drying may develop what are known as shrinkage cracks within the first 24 hours and are not arr indication of unsoundness. These conditions are illustrated in Figure 102, facing page 292 .

The failure of the pats to remain on the glass or the cracking of the glass to which the pats are attached does not necessarily indicate unsoundness.

38. A steam apparatus, which can be maintained at a temperature between $98^{\circ}$ and $100^{\circ}$ C., or one 
similar to that shown in Figure 101, is recommended. The eapaeity of this apparatus may be inereased by using a raek for holding the pats in a vertical or inclined position.

39. A pat from eement paste of normal eonsisteney about 3 inches in diameter, $1 / 2$-inch thick at the center, and tapering to a thin edge, shall be made on flat clean glass plates about 4 inehes square, and stored in moist air for 24 hours. In molding the pat, the cement paste shall first be flattened on the glass and the pat then formed by drawing the trowel from the outer edge toward the eenter, then flattening the top. The pats used for the time of setting tests by the Gillmore method may be used for soundness tests.

40. The pat shall then be placed in an atmosphere of steam at a temperature between $98^{\circ}$ and

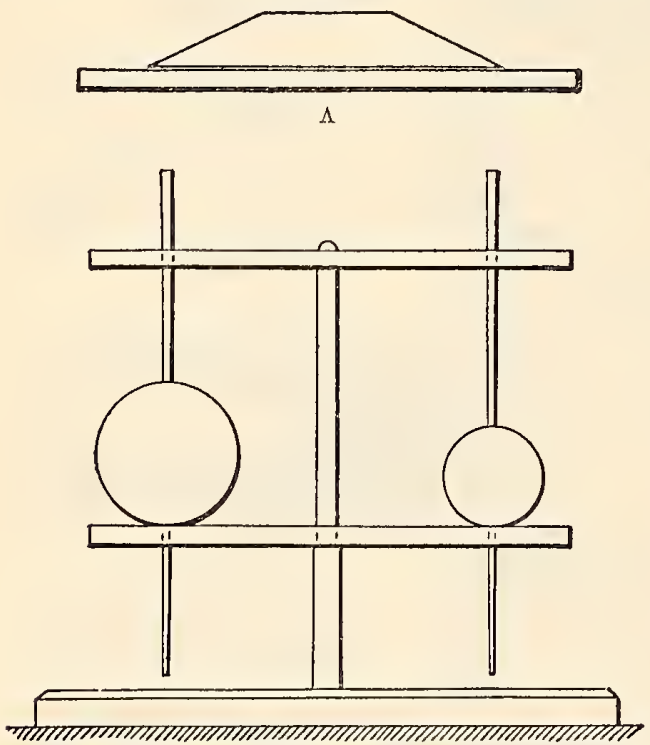

$\mathrm{B}$

Figure 103

a, Pat with top surface flattened for determining time of setting by Gillmore method.

$b$, Gillmore needles.

$100^{\circ} \mathrm{C}$., upon a suitable support 1 inch above boiling water for five hours.

NoтE.-It is important that the specimens be 24 hours old when placed in steam, since variations in their age will produce differences placed in the restalts of the steam tests. Particularly noticeable are the in the restes of steaming pats too soon, for many specimens steamed when only 10 or 12 hours old give apparently satisfactory results, while failure would be observed if they were not placed in steam until 24 hours old.

41. Should the pat leave the plate, distortion may be detected best with a straightedge applied to the surface which was in contact with the plate.

\section{DETERHINATION OF TIME OF SETTING}

42. The following are alternate methods, either of which may be used as specified.

43. The time of setting shall be determined with the Vicat apparatus described in section 35 . (See fig. 100.)

44. A paste of normal consistency shall be molded in the ring $G$ as deseribed in seetion 36 , and placed under the $\operatorname{rod} B$, the sinaller end of which shall then be carefully brought in contact with the surface of the paste, and the rod quickly releascd. The initial sct shall be said to have occurred when the needle ceases to pass a point $5 \mathrm{~mm}$, above the glass plate in one-half minute after being released; and the final set, when the needle does not sink visibly into the paste. The test pieces shall be kept in moist air during the test. This may be aecomplished by placing them on a rack over water contained in a pan and covered by a damp cloth, kept from contact with them by means of a wire screen; or they may be stored in a moist closet. Care shall be taken

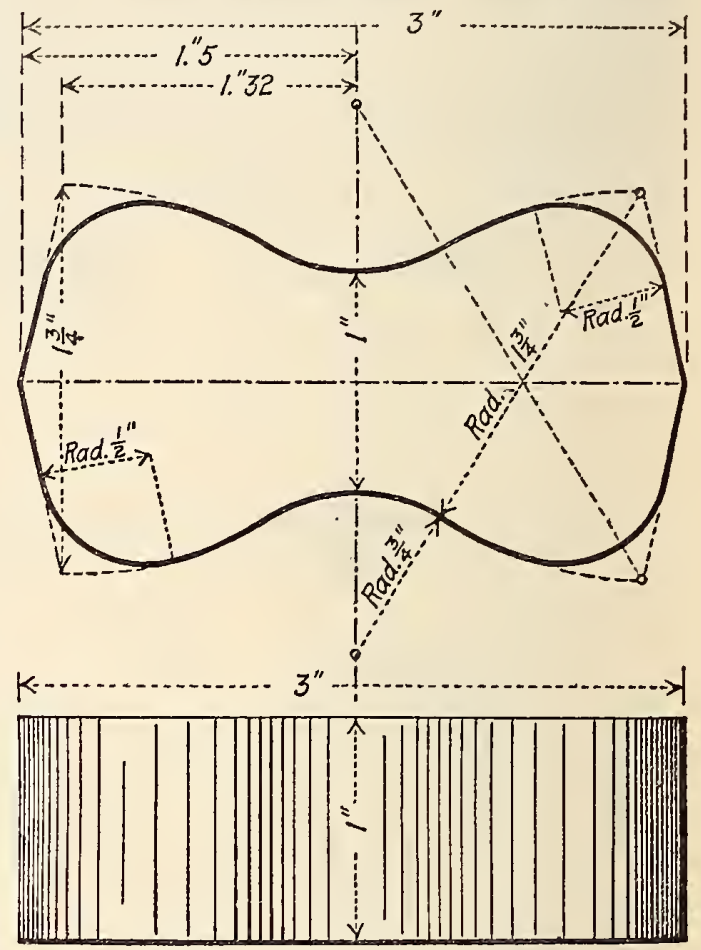

Figure 104.-Details for briquet

to keep the needle clean, as the colleetion of cement on the sides of the needle retards the penetration, while cement on the point may increase the penetration. The time of setting is affected not only by the percentage and temperature of the water used and the amount of kneading the paste received, but by the temperaturc and humidity of the air, and its determination is therefore only approximate.

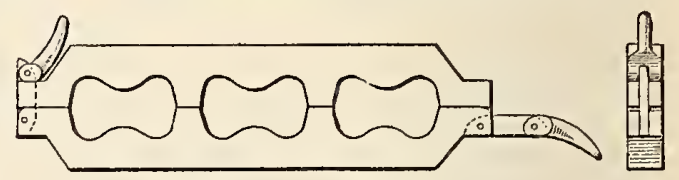

Figure 105.-Gang mold

45. The time of setting shall be determined by the Gillmore needles. The Gillmore needles should prefcrably be mounted as shown in Figure $103(b)$, and the needle ends shall be maintained in a clean condition and shall be plane and at right angles to the axis of the rod.

46. The time of setting shall be determined as follows: A pat of neat cement paste about 3 inches in diameter and one-half ineh in thickness with a flat top (fig. $103(a)$ ), mixed to a normal consistency, shall be kept in moist air at a temperature maintained as nearly as practieable at $21^{\circ} \mathrm{C}$. $\left(70^{\circ} \mathrm{F}\right.$.). The cement shall be considered to have acquired its initial set when the pat will bear, without appreciable 

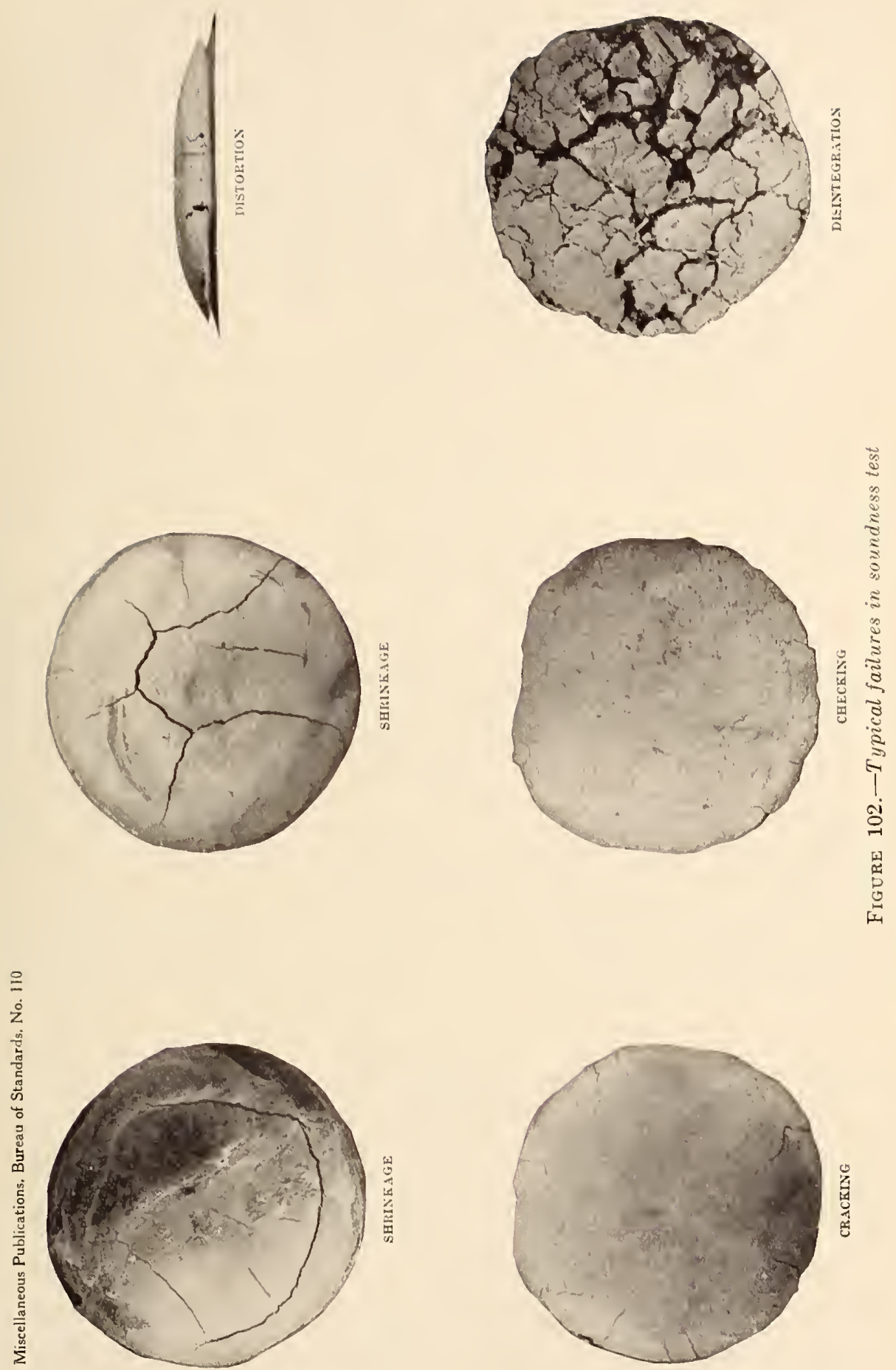

indentation, the Gillmore needle one-twelfth inch in diameter, loaded to weigh one-fourth pound. The final set has been acquired when the pat will bear, without appreciable indentation, the Gillmore needle one twenty-fourth inch in diameter, loaded to weigh 1 pound. In making the test, the needles shall be held in a vertical position and applied lightly to the surface of the pat.

\section{TENSION TESTS}

47. The form of test piece shown in Figure 104 shall be used. The molds shall be made of noncorroding metal and have sufficient material in the sides to prevent spreading during molding. Gang molds when used shall be of the type shown in Figure 105. Molds shall be oiled with a mineral oil.

48. The sand to be used shall be natural sand pounds, Each application of the thumbs shall be maintained not longer than sufficient to attain the specified pressure. Then the mortar shall be heaped above the mold and smoothed off with a trowel. The trowel shall be drawn over the mold in such a manner as to exert a pressure of not more than 4 pounds. The mold shall then be turned over upon a plane plate oiled with mineral oil, and the operation of heaping, thumbing and smoothing off repeated. No ramming or tamping shall be used, nor any troweling in excess of that required to smooth off the specimen.

52. The briquets shall be tested as soon as they are removed from the water. Tests may be made with any machine meeting the following requirements: The machine shall be capable of weighing the applied load within 0.5 per cent of the nominal value. The sensibility reciprocal (the weight re-

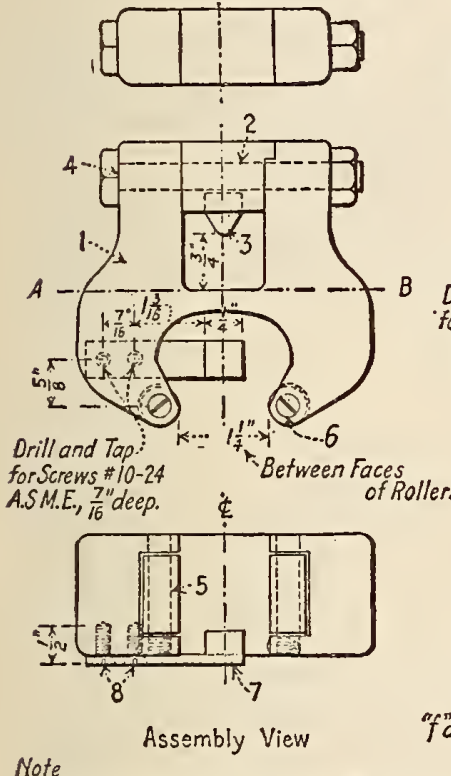

Note

Portion below Line A-B is Standard. As shown herein, Portion above Line A-B is Designed fos Use on One Type of Machine, and may be Varied to Adapt to other Machines.

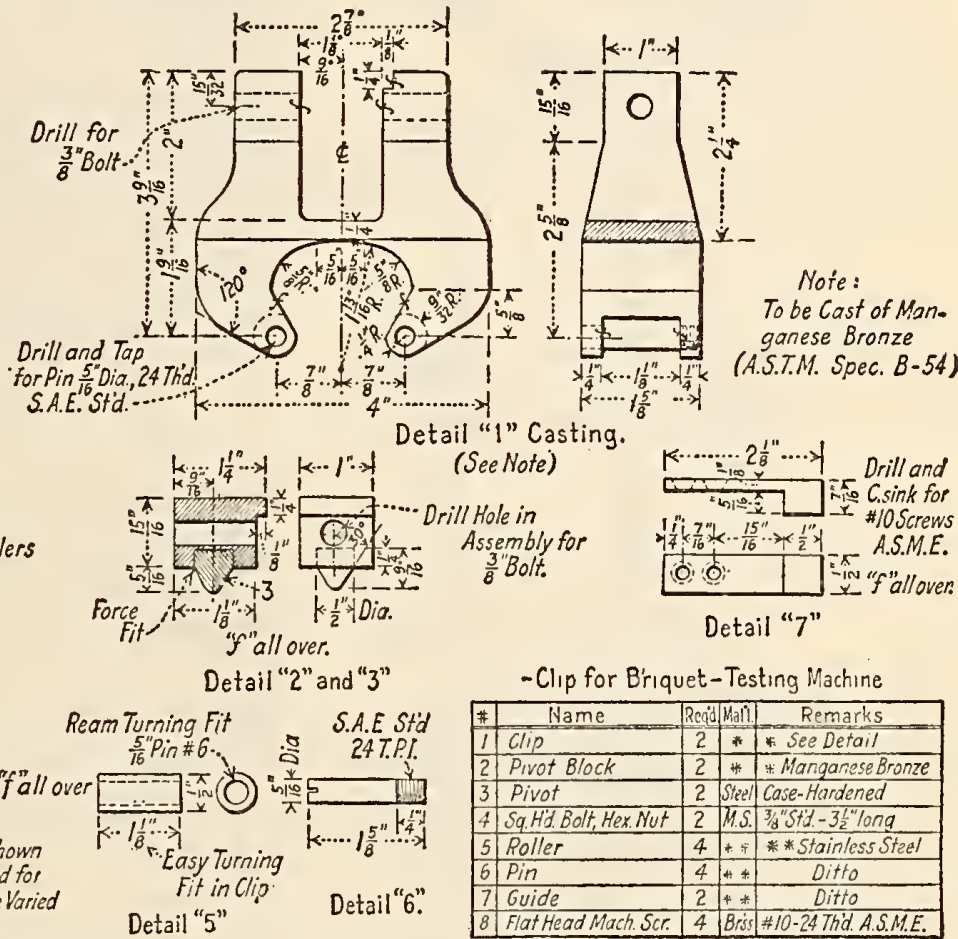

Detail " 5 "

FIgUre 106.-Clip for briquet-testing machine

from Ottawa, Ill., screened to pass a No. 20 sieve and retained on a No. 30 sieve. This sand may be obtained from the Ottawa Silica Co., Ottawa, Ill.

49. This sand shall be considered standard when not more than $5 \mathrm{~g}$ are retained on the No. 20 sieve, and not more than $5 \mathrm{~g}$ pass the No. 30 sieve, after one minute continuous sieving of a $100 \mathrm{~g}$ sample, in the manner specified for sieving cement on the No. 200 sieve. (See sec. 31 .)

50. The Nos. 20 and 30 sieves shall conform to the requirements for these sieves as given in $\mathrm{A}$. S. T. M. E11. (See 500.2, p. 1.)

51. Immediately after mixing the standard mortar in accordance with the methods for mixing cement pastes and mortars the molds shall be filled heaping full without compacting. Then the mortar shall be pressed in firmly with the thumbs, applying pressure twelve times to each briquet, at points to include the entire surface. The pressure shall be such that the simultaneous application of both thumbs will register a pressure of between 15 and 20 quired to be added to the load to move the beam from a horizontal position of equilibrium to a position of equilibrium at the top of the trig loop) shall not exceed 1 pound at the full capacity of the machine or at any lesser load. The clips for holding the tension-test specimens shall be in accordance with Figure 106. The bearing surfaces of the clips and briquets shall be free from sand or dirt, and the roller bearings shall be well oiled and maintained so as to insure freedom of turning. The briquets shall be carefully centered in the clips and the load applied continuously at the rate of 600 pounds per minute.

53. Testing machines shall be frequently calibrated in order to determine their accuracy.

54 . Briquets that are manifestly faulty, or which give strengths differing more than 15 per cent from the average value of all test specimens made from the same sample and tested at the same period, shall not be considered in determining the tensile strength. 


\section{STORAGE OF TEST SPECIMENS}

55. The moist closet may consist of a soapstone, slate or concrete box, or a wooden box lined with metal. The interior wall surfaces of all closets shall be covered with felt or broad wicking kept wet. The bottom of the moist closet shall bc covered with water to a depth of at least 2 inches.

56. All test specimens, immediately after molding, shall be kept in the molds on planc plates in the moist closet for írom 20 to 24 hours in such manner

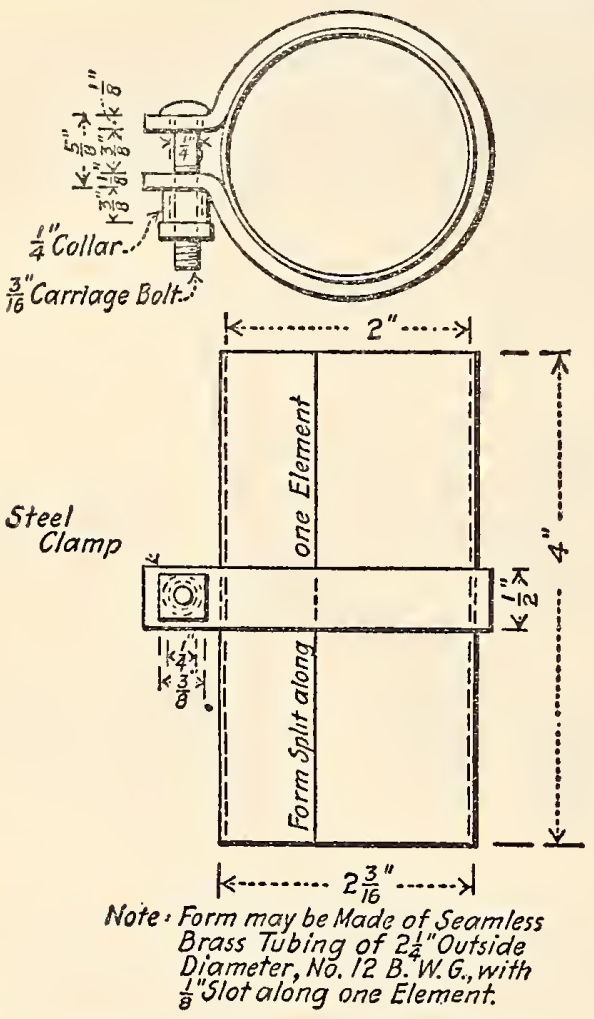

Figdre 107.-Details for $\mathscr{2}$ by 4 inch cylinder form

that the upper surfaces shall be exposed to the moist air.

57. The briquets shall then be removed from the molds and immersed in clean watcr in storage tanks constructed of noncorroding material.

American Society for Testing Materials, tentative specifications and tests for compressive strength of Portland-cement mortars, serial designation C9-16T 1916.

\section{SPECIFICATIONS}

1. The average compressive strength in pounds per square inch of not less than three standard mortar test pieces (see sec. 4) composed of 1 part cement and 3 parts standard sand, by weight, shall be equal to or higher than the following:

\begin{tabular}{c|c|c}
\hline $\begin{array}{c}\text { Age at } \\
\text { test, } \\
\text { days }\end{array}$ & Storage of test pieces & $\begin{array}{r}\text { Compres- } \\
\text { sive } \\
\text { strength }\end{array}$ \\
\hline & $\begin{array}{r}\text { 1 day in moist air, 6 days in water } \\
1 \text { day in moist air, 27 days in water... } \text { L }^{2} \\
1,200 \\
2,000\end{array}$ \\
\hline
\end{tabular}

2. The average compressivc strength of standard mortar at 28 days shall be higher than the strength at 7 days.

\section{TESTS}

3. The requirements governing the preparation of standard sand mortars for tension-test pieces shal! apply to compression test pieces. (See A. S. T. M/ . C9 above.)

4. A cylindrical test picce 2 inches in diameter and 4 inches in length is recommended for use in making compression tests of standard mortars. The molds shall be made of noncorroding metal. A satisfactory form of mold is shown in Figure 107. The ends of the mold shall be parallel. The tubing used in the molds shall be of sufficient thickness to prevent appreciable distortion. The molds shall be oiled beforc using. During the molding of the test piece, the mold shall rest on a clean, plane surface (preferably a piece of platc glass, which is allowed to remain in place until the mold is removed).

5. The mortar ${ }^{53}$ shall be placed in the mold in layers about 1 inch in thickness, cach layer being tamped by means of the steel tamper shown in Figurc 108. The weight of tamper shall be approximately three-fourths pound. In finishing the test piece, the mortar shall be heaped above the mold and smoothcd off with a trowel. As soon as the test pieces from one sample arc molded, the top of each test piece shall be covered with a piece of glass which is brought to a firm bearing on the fresh mortar. The cover glasses shall remain in place until the molds are removed.

6. The compression test pieces shall be stored in the same manner as the tension test pieces.

7. Tests of standard-mortar cylinders shall be made in any testing machine which is adapted to meet the specified requirements. The test pieces shall be tested as soon as removed from the water. The cnds of the test cylinders shall be smooth, plane surfaces. The metal bearing plates of the testing machine shall be placed in direct contact with the ends of the test picce. During the test a spherical bearing block shall be used on top of the cylinder. In order to secure a uniform distribution of the load over the test cylinder the spherical bearing block must be accurately centered. The diameter of the spherical bearing block should be only a little greater

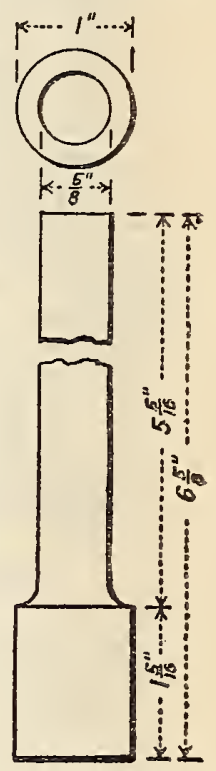
Details of steel tamper
Figdre 108.-

than that of the test piece. The test piece shall be loaded continuously to failure. The moving heat of the testing machine shall travel at the rate of not less than 0.05 or more than 0.10 inch per minute.

8. Testing machines should be frequently calibrated in order to determine their accuracy.

9. Cylinders that are manifestly faulty, or which give strengths differing more than 15 per ccnt from the average value of all test pieces tested at the same period and made from the same sample, shall not be considered in determining the compressive strength.

53 If sufficient mortar for six 2 by 4 inch cylinders is to be mixed in a single batch, approximately $3,000 \mathrm{~g}$ of material will be required. In this case the mixing shall be continued for one and one-half minutes. 
Federal Specifications Board, specification No. 1a, United States Government master specification for cement, Portland, June 10, 1927.

(This specification is identical with A. S. T. M. serial designation $\mathrm{C}$, given above, with the exception of the first sentence under the heading "rejection" (paragraph 11), this specification stipulating that the cement shall be rejected if it fails to meet any of the requirements of the specifications, whereas the A. S. T. M. specification stipulates merely that it may be rejected for failure to conform.)

\subsection{Natural Cement.}

American Society for Testing Materials, standard specifications for natural cement, serial designation C10-09, 1909.

1. Natural cement is the finely pulverized product resulting from the calcination of an argillaceous limestone at a temperature only sufficient to drive off the carbonic acid gas.

\section{PHYSICAL PROPERTIES}

2. The residue on a standard No. 100 sieve shall not exceed 10 per cent, and on a standard No. 200 sieve shall not exceed 30 per cent, by meight. For sieve descriptions, see A. S. T. M. E11, 500.2, p. 1 .

3. Pats of neat cement about 3 inches in diameter $1 / 2$ inch thick at center, tapering to a thin edge, shall be kept in moist air for a period of 24 hours.

(a) A pat shall then be kept in air at normal temperature.

(b) Another pat shall be kept in water maintained as near $70^{\circ} \mathbf{F}$. as practicable.

These pats shall be observed at intervals for at least 28 days, and, to satisfactorily pass the tests, shall remain firm and hard and show no signs of distortion, checking, cracking, or disintegrating.

4. The cement shall not develop initial set in less than 10 minutes, using the Vicat needle. Final set shall be attained in not less than 30 minutes nor more than 3 hours, using the Vicat needle.

5. The minimum requirements for tensile strength for briquets 1 square inch in cross section shall be as follows, and the cement shall show no retrogression in strength within the periods specified;

\section{Neat cement}

Age

Strength

24 hours in moist air (pounds)

7 days (1 day in moist air, 6 days in water)

28 days ( 1 day in moist air, 27 days in water)

One part cement, three parts standard Ottawa sand

Age (pounds)

7 days ( 1 day in moist air, 6 days in water)

28 days ( 1 day in moist air, 27 days in water)

\section{PACKAGES, MIARKING, AND STORAGE}

6. The cement shall be delivered in suitable bags or barrels with the brand and name of the manufacturer plainly marked thereon. A bag shall contain 94 pounds net. A barrel shall contain 282 pounds net.

7. The cement shall be stored in such a manner as to permit easy access for proper inspection and identification of each shipment, and in a suitable weathertight building which will protect the cement from dampness.

\section{INSPECTION}

8. (a) Every facility shall be provided the purchaser for careful sampling and inspection at either the mill or at the site of the work, as may be specified by the purchaser. At least 10 days from the time of sampling shall be allowed for the completion of the 7-day test, and at least 31 days shall be allowed for the completion of the 28-day test.

(b) The cement shall be tested in accordance with the provisions of A.S.T. M. C9. (See 516.11, p. 294.)

\section{RETECTION}

9. The cement may be rejected if it fails to meet any of the requirements of these specifications.

10. Cement failing to meet the 7 -day requirements may be held amaiting the results of the $28-d a y$ tests before rejection.

\subsection{Masonry Cement.}

Federal Specifications Board, specification No. 443, United States Government master specification for cement, masonry, October 28, 1926.

\section{TYPE AND GRADE}

This specification contains requirements for a material of one type and grade only, to be used in conjunction with sand in the making of mortar for laying units of masonry construction above grade.

\section{DETAIL REQUIREMENTS}

\section{PHYSICAL REQUIREMENTS}

(a) Fineness. - The residue on a standard No. 200 sieve shall not exceed 20 per cent by weight. Cement shall not be rejected for failure to meet this test if, after drying at $100^{\circ} \mathrm{C}$. for one hour and resieving, it meets this requirement.

(b) Time of selting. - The neat-cement paste, mixed to normal consistency, shall not develop initial set in less than 45 minutes as determined by the Vicat needle, or 60 minutes as determined by the Gillmore needle. Final set by either method shall be attained within 48 hours.

(c) Soundness.- Pats of neat cement, after being stored for 7 days, one in laboratory air and one in water, shall be firm and hard and show no signs of distortion, cracking, or disintegration under both conditions of storage. Mortar-strength specimens at the time of test shall show no signs of distortion, cracking, or disintegration.

(d) Strength.-The average compressive strength in pounds per square inch of not less than three 2-inch cubes, made of mortar composed of 1 part cement and 3 parts sand by weight, shall be not less than 175 at the are of 7 days. The average strength attained at 28 days shall not be less than that attained at 7 days.

\section{REJECTION}

The cement shall be rejected if it fails to meet any of the requirements of this specification. Cement remaining in storage prior to shipment for a period greater than six months after test shall be retested and shall be rejected if it fails to meet any of the requirements of this specification.

\section{METHODS OF INSPECTION, SAMPLING, AND TEST}

\section{TEMPERATURE}

The standard temperature shall be $70^{\circ} \mathrm{F}$. $\left(21^{\circ} \mathrm{C}\right.$.). The air of the laboratory, the materials, the mixing water, the moist closet, and storage tanks shall be maintained as near as practicable at this temperature and shall not vary from it more than plus or minus $5^{\circ} \mathrm{F}$. 


\section{FACILITIES FOR INSPECTION}

Every facility shall be provided by the vendor for the necessary sampling and inspection.

\section{STORAGE OF CEMENT}

The cement shall be stored in a suitable weathertight building which will protect it from dampness and in such manner as to permit safe and easy access for proper inspection and identification of each lot.

\section{SAMPLING}

Number and weight of samples.-Tests may be made on individual or composite samples as may be ordcred. Each test sample shall weigh at least 5 pounds and shall represent not more than 100 barrels.

\section{SHIPMENT OF SAMPLES}

Samples shall be shipped and stored in air-tight moisture-proof containers.

\section{TIME FOR TESTS}

At least 12 days from the time of sampling shall be allowed for the completion of the 7-day tests and 33 days shall be allowed for the completion of the 28-day tests.

\section{TREATMENT OF SAMPLES PRIOR TO TEST}

Samples shall be passed through a United States Standard No. 20 sieve, in order to mix the sample thoroughly, break up lumps, and remove foreign materials.

\section{PHYSICAI TESTS}

Physical tests used in this specification include $(a)$ determination of fineness, (b) mixing cement pastes and mortars, $(c)$ normal consistency, $(d)$ percentage of water for standard mortars, (e) determination of time of setting, $(f)$ determination of soundness, and (g) compression tests.

Items designated $(a),(b),(c)$, and $(e)$ are similar to methods described under corresponding descriptive headings in A. S. T. M. C9-26. (See 516.11, p. 294).

\section{(d) Percentage of water for standard mortars}

(1) Apparatus.-The flow-table apparatus consists of a rigid frame with a flat circular top, so mounted on a vertical shaft that it can be raised and dropped through a fixed height by means of a rotated cam. The top shall be of noncorrodible metal, 10 inches in diameter, and with the attached shaft shall weigh 9 pounds. The mold shall be of a noncorrodible material, 4 inches in inside diameter at the base, $23 / 4$ inches at the top, and 2 inches high.

(2) Method.-In making the determination the mortar shall be mixed in accordance with A. S. T. M. C9, paragraph 33, with a measured quantity of water. The table top shall be carefully wiped dry and the flow mold placed at the center and filled with mortar. In filling the mold the mortar shall not be rammed, but gently compacted to insure uniform filling. The mortar shall be smoothed off level with the top of the mold by aid of a trowel and the mold removed. Immediately the table shall be dropped through a height of one-eighth inch, 30 times at the rate of 60 drops per minute. The flow is the resulting increase in diameter of the mortar mass, expressed as the percentage of the original cliameter.

The mortar shall be of the standard consistency when the flow is 100 to 115 . Trial mortars shall be made with varying percentages of water until the standard consistency is obtained. Each trial shall be made with fresh mortar. The quantity of water shall be expressed as a percentage of the weight of the combined dry materials.

\section{(f) Determination of Soundness}

(1) Method.-Two pats from cement paste of normal consistency about 3 inches in diameter, one-half inch thick at the center, and tapering to a thin edge shall be made on clean, flat glass plates about 4 inches square, and stored in moist air for 24 hours. One of the pats shall then be stored in the air of the laboratory and the other shall be immersed in clean water in storage tanks of noncorrodible material.

Unsoundness is usually manifested by change in volume which causes distortion, cracking, checking, or disintegration. Pats improperly made or exposed to drying may develop what are known as shrinkage cracks within the first 24 hours.

The shrinkage cracks are not an indication of unsoundness. The failure of the pats to remain on the glass or the cracking of the glass to which the pats are attached docs not necessarily indicate unsoundness. Should the pat leave the plate, distortion may be detected best with a straightedge applied to the surface which was in contact with the plate, provided the glass plate was flat before test.

\section{(g) Compression Tests}

(1) Form of test piece.-The form of test piece used shall be a 2 -inch cube. The molds shall be made of a noncorrodible metal, and have sufficient material in the sides to prevent spreading during molding. Molds shall be oiled with a mineral oil before using.

(2) Molding of test pieces.-Immediately after mixing in accordance with the methods for mixing cement pastes and mortars, the standard mortar shall be placed in the cube molds which rest on plane nonabsorbent plates. The molds shall be filled heaping full without compacting. Then the mortar shall be gently worked into the molds with the thumbs, using only sufficient effort to ensure uniform filling of the molds. Additional mortar shall be heaped above the molds and smoothed off witl a trowel. The molds and plates shall then be turned over upon plane nonabsorbent plates oiled with mineral oil, the first plates removed, and the operation of heaping, thumbing, and smoothing off repeated. No ramming or tamping shall be used, nor any troweling in excess of that required to smooth off the specimen.

(3) Storage of test pieces-(a) Apparatus.-The moist closet may consist of a soapstone, slate, or conerete box, or a wooden box lined with metal. The interior wall surfaces of all closets shall be covered with felt or wicking, kept wet. The bottom of the moist closet shall be covered with water to a depth of at least 2 inches.

(b) Method.-All test pieces, immediately after molding, shall be kept in the molds on plane plates in the moist closet for from 20 to 24 hours in such a manner that the upper surfaces shall be exposed to the moist air. The cubes shall then be removed from the molds and placed in the air of the laboratory for six days, in such a manner as to allow free circulation of air around at least five faces of the specimens. At the age of 7 days the cubes for the 28-day tests shall be immersed in clean water in storage tanks of noncorrodible materials.

(4) Testing of cubes.-The tests may be made with any of the various types of machines acceptable to the office purchasing the cement. Those faces of the cube which have been in contact with the sides of the molds when molding, shall be placed in contact with the surfaces through which the compression is applied. A spherical seated bearing block shall be used between the lower face of the cube and the bed of the testing machine. The center of the sphere shall lie in that surface of the 
bearing block which is in contact with the cube, and the radius of the sphere shall be $1 \frac{1}{2}$ inches. The cube shall be carefully centered on the upper plate of the bearing block, and the two shall be slowly rotated as the head of the machine is brought to a bearing on the test piece. Cushioning materials shall not be used.

The load shall be applied uniformly and without shock. The moving head of the testing machine shall travel at the rate of approximately 0.05 inch per minute when the machine is running idle.

The 28-day specimens shall be tested as soon as they are removed from the water.

(5) Computation of strength. - The total load indicated by the testing machine at failure of the test piece shall be recorded and the unit compressive strength calculated in pounds per square inch of the cross-sectional area of the cube.

Cubes that are manifestly faulty, or which give strengths differing by more than 15 per cent from the average value of all test pieces made from the same sample and tested at the same period, shall not be considered in determining the compressive strength.

(6) Standard sand.-The sand to be used shall be natural sand, from Ottawa, Ill., screened to pass a No. 20 sieve and retained on a No. 30 sieve. This sand may be obtained from the Ottawa Silica Co., Ottawa, IIll.

This sand, having passed the No. 20 sieve, shall be considered standard when not more than 5 per cent passes the No. 30 sieve after one minute continuous sieving of a $100 \mathrm{~g}$ sample.

The sieves shall conform to the same requirements as for the No. 200 sieves, except that the average openings and wire diameters shall be as follows:

\begin{tabular}{|c|c|c|c|c|c|}
\hline Sieve No. & $\begin{array}{l}\text { Sieve } \\
\text { opening }\end{array}$ & $\begin{array}{c}\text { Wire } \\
\text { diameter }\end{array}$ & $\begin{array}{l}\text { Toler- } \\
\text { ance in } \\
\text { arerage } \\
\text { opening }\end{array}$ & $\begin{array}{l}\text { Tolerance } \\
\text { in wire } \\
\text { diameter }\end{array}$ & $\begin{array}{l}\text { Toler- } \\
\text { ance in } \\
\text { maxi- } \\
\text { mum } \\
\text { opening }\end{array}$ \\
\hline $30 \ldots$ & $\begin{array}{l}\text { Inch } \\
0.0331 \\
.0232\end{array}$ & $\begin{array}{l}\text { Inch } \\
0.0165 \\
.0130\end{array}$ & $\begin{array}{r}\text { Per cent } \\
\pm 5 \\
\pm 5\end{array}$ & $\begin{array}{c}\text { Per cent } \\
-15 \text { to }+30 \\
-15 \text { to }+30\end{array}$ & $\begin{array}{r}\text { Pet cent } \\
25 \\
25\end{array}$ \\
\hline
\end{tabular}

\section{PACKING AND MARKING}

The cement shall be delivered in suitable bags or barrels with the brand and name of the manufacturer and the net weight plainly marked thereon All packages shall be in good condition at the time of inspection.

Packages varying more than 5 per cent from the marked weight may be rejected; and if the average weight of packages in any shipment as shown by weighing 50 packages taken at random is less than that marked, the entire lot represented shall be rejected.

\subsection{Miscellaneous Cements.}

Federal Specifications Board, United States Government master specification No. 445 for cement, plastic magnesia, October 28, 1926.

(See 572 , p. 652.)

\subsection{STUCCO}

American Concrete Institute, standard recommended practice for Portland cement stucco, serial designation $\mathrm{C}-3 \mathrm{~A}-23,1923$.

The above recommendation contains methods for the use in building of Portland cement stucco, including general recommendations, design, construction, and the following specifications for materials:

\section{MATERIAIS}

Cement.-The cement should meet the requirements of the standard specifications for Portland cement of the American Society for Testing Materials, adopted by the American Concrete Institute, see A. S. T. M.: C9, 516.11, page 294

Fine aggregate.- Fine aggregate should consist of sand or screenings from crushed stone or crushed pebbles, graded from fine to coarse, passing when dry a No. 8 screen. Fine aggregate should preferably be of siliceous materials, clean, coarse, and free from loam, vegetable, or other deleterious matter.

Note.-The paragraphs relating to materials are sufficiently specific as to the quality of the stucco ingredients. However, reference may be made to the colorimetric test for detecting the presence of organic matter in sands, a descriotion of which is to be found in the report of Cormmittee C-9, American Society for Testing Materials, 1919.

Hydrated lime.-Hydrated lime should meet the requirements of the standard specifications for hydrated lime of the American Society for Testing Materials. (See A. S. T. M. C6, 517.2, p. 353.)

Note.-Hydrated lime should be specified to the exclusion of

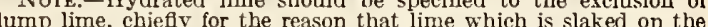
job can not as a rule be so thoroughly hydrated and so thoroughly mixed in the mortar as the mechanically hydrated product.

Hair or fiber.- There should be used only first quality long hair, free from foreign matter, or a long fiber well combed out.

Coloring matter.-Only mineral colors should be used which are not affected by lime, Portland cement, or other ingredients of the mortar or the weather.

Water.-Water should be clean, free from oil, acid, strong alkali, or vegetable matter.

Lath.-(a) Metal lath should be galvanized or painted expanded lath weighing not less than 3.4 pounds per square yard.

(b) Wire lath should be galvanized or painted woven wire lath, not lighter than 19 gage, $2 \frac{1}{2}$ meshes to the inch, with stiffeners at 8 inch centers.

(c) Wood lath should be standard quality, narrow plaster lath 4 feet long and not less than $3 / 8$-inch thick.

American Concrete Institute, tentative specifications, finish coat Portland cement stuceo, C-3C-29T, 1929.

\section{GENERAL}

1. The purpose of these specifications is to establish minimum requirements for finish coat Portland cement stucco.

2. The term "finish coat Portland cement stucco" shall be understood to mean a Portland cement mortar used to cover or decorate preceding coats of Portland cement stucco or other suitable bases on exterior walls and surfaces exposed to the elements.

3 . The minimum average compressive strength of finish coat Portland cement stucco at 28 days of age shall be $2,000 \mathrm{lbs} . / \mathrm{in}^{2}$ when molded and tested as 2 -inch cubes in the manner hereinafter specified.

4. Finish coat Portland cement stucco shall not absorb more than 10 per cent of water when tested as hereinafter specified.

5. Finish coat Portland eement stucco shall not contain more than 35 per cent by weight of the whole sample of material passing the 100-mesh sieve.

6. If pigments are used, they shall be pure mineral oxides guaranteed by the manufacturer to be of uniform quality and proof against action of lime and sun.

\section{METHOD OF IMAKING SPECIMENS}

7. Finish coat stucco to be used in making specimens for all tests shall be mixed to plastering consistency. The approximate amount of water 
required to mix any stucco to plastcring consistency shall be indicated by the manfacturer of that stucco. 8. In making specimens, molds shall be filled in two layers, each layer being lightly puddled with the finger. Stucco shall be left heaped on molds and be struck off at the end of 3 to 4 hours. Immediately after molding, specimens shall be covered with moist burlap for 24 hours, then removed from the molds.

9. After removal from molds specimens shall be immersed in water for 6 days and thereafter stored 21 days in dry air of the laboratory at approximately $70^{\circ} \mathrm{F}$. Specimens shall be tested at age of 28 days.

\section{METHOD OF TESTING}

10. Not less than 3 and preferably 5 specimens shall be required for each test. In the event of failure of the first set of specimens, the test shall be repeated on a second set of specimens.

11. Absorption tests shall be made on 2-inch cubes. After being cured, as provided in paragraph 9 , they shall be carefully weighed and then completely submerged in water at a temperature of between $60^{\circ}$ and $80^{\circ} \mathrm{F}$. for 24 hours. Specimens shall then be removed, the surface water wiped off with a damp cloth, and the specimens quickly weighed. The percentage of absorption is the difference in weight divided by the dry weight of the specimen and multiplied by 100 .

12. Specimens for compression test shall be tested in a vertical testing machine of not exceeding 50,000 pounds capacity. Load shall be applied through a spherical bearing block placed on top of the specimen. The dimensions of the bearing block shall be the same or slightly greater than those of the specimen.

13. Load shall be applied uniformly and without shock. The speed of the moving head of the testing machine shall be not more than 0.05 inch per minute when the machine is running idle.

14. Specimens shall be loaded to failure and unit compressive strength calculated in pounds per square inch. The type of failure and appearance of the specimen shall be noted.

American Railway Engineering Association, specifications for buildings for railway purposes.

\section{EXTERIOR STUCCO WORK (1926)}

The contractor shall provide all seaffolding, tools, labor, and materials, and everything necessary to complete the plastering and stuceo work shown on the drawings or required to complete the building.

The work required under this heading comprises the stuccoing of all exterior wall surfaces, as shown on the drawings and hereinafter described. Fresh stuceo shall be protected from the weather, and no stucco in which cracks, pits, streaks, discolorations, or other defects may oceur will be accepted. $\mathrm{Ce}-$ ment shall be Portland cement for the undercoats and white Portland cement for the finish coat in accordance with the specifications for Portland cement of the A. R. E. A., which are the same as A. S. T. M. C9. (See 516.11, p. 294.) Cement that has hardened or partially set shall not be used. Aggregate for the undercoats shall be thoroughly clean sand, graded from fine to coarse grains with the coarse grains predominating, and shall be free from loam, salt, vegetable, and other deleterious matter. Aggregate for the finish coat shall be thoroughly clean yellow gravel grit, marble, or granite screenings, as directed by the engineer. Hydrated lime and coloring compounds shall be first quality, of a brand acceptable to the engineer. Hair shall be first quality long cattle or goat hair.

Mortar for the first and second coats shall be composed of 1 part Portland cement, 3 parts sand, and one-tenth part of hydrated lime by volume, with sufficient hair added to bond the mortar to the lath.

Mortar for the finishing coat shall be composed of 1 part white Portland cement, 3 parts of aggregate and one-tenth part by volume of hydrated lime. This coat shall be brought to the tone selected by the addition of dry coloring compound not exceeding 10 per cent of the weight of the cement.

Mixing shall be done on a water-tight platform, the different constituents thoroughly mixed dry to a uniform color, water then added to obtain the proper consistency, and the whole turned over until the mass is uniform in color and consistency. No retempcred mortar shall be used and no more mortar shall be mixed than can be used in 30 minutes. The dry color in the finishing coat shall be carefully weighed or measured and thoroughly mixed with the sand. The cement and lime shall then be added and the entire mass thoroughly mixed by shoveling from one sidc of the platform to the other through a $1 / 4$-inch mesh sereen, and when the batch is of uniform color the water shall be added.

The stucco shall be applied in three coats, each coat not less than $1 / 4$ inch nor more then $3 / 8$ inch in thickness, the whole finishing $7 / 8$ inch thick beyond the normal masonry line or 1 inch thick over the furring strips. The plastering shall be carried on continuously in one general direction without allowing the mortar to dry at the edge. Where this is impossible the joints shall be made at a break, an opening, or other natural division of the surface. Stucco shall not be applied when the temperature is below fleezing. Masonry surfaces shall be cleaned and wet before the first coat is applied and brick walls shall have the joints raked out about $1 / 2$ inch. The first coat shall be applied under pressure so as to secure a perfect bond with the masonry wall or lathed surface. After the first coat has sct, but before it has dried, the second coat shall be applied and floated to a true plane. The undercoats shall be cross scratehed and scored before the initial set has taken place and shall be thoroughly wetted before the succeding coats are applied. The finishing coat shall be kept damp for at least four days, either by sprinkling after the mortar has hardened sufficiently to permit it or by hanging wet burlap over the surface.

After the second coat has set, but before it has dried, the finishing coat shall be applied and finished in accordance with one of the methods hereinafter specified as directed by the engineer.

Exposed aggregate (integral method).-The finishing coat shall be three-eighths inch thick and within 24 hours after it has been troweled to an even surface, shall be scrubbed with a stiff brush until the aggregate has been uniformly exposed. Should the cement be too hard to be readily removed by water, a solution of 1 part muriatic acid to 5 parts of water may be used, but as soon as the aggregate has been exposed particular care shall be taken to remove all trace of acid by spraying thoroughly with clean water from a hose.

Smooth troweled.-Finishing coat shall be smoothed with a metal trowel, with as little rubbing as possible.

Stippled.-Finishing coat shall be smoothed with a metal trowel, with as little rubbing as possible, and then shall be lightly patted with a brush of broom straw to give an even stippled surface.

Sand floated.-Finishing coat, after being brought to a smooth, even surface, shall be rubbed in a circular motion with a wood float. This floating shall be done when mortar has partially set.

Roughcast or spatter dash.-After the finishing coat has been brought to an even surface, and before attaining its final set, it shall be uniformly coated with a mixture of 1 part white cement to 2 parts white sand, thrown forcibly against the wall in 
such a manner as will produce a rough surface of uniform texture.

Pebble dash.-After the finishing coat has been brought to an even surface and before attaining its initial set, clean pebbles shall be forcibly thrown against the mortar and embedded therein. Pebbles shall vary in size from one-fourth inch to threeeighths inch, shall be well wetted before being cast, and shall he uniformly distributed over the surface. Ther may be pressed into the mortar with a clean wooden paddle, but the surface shall not be otherwise disturbed.

Note.-The above surface finishes are alternatires. Under no circumstances should the stuceo be worked after it has attained its initial set. Samples of the surface finish shall be laid up well in adrance of the work, and the approved sample shall be careully pre-

\section{GENERAL CONDITIONS}

All materials entering into the work and all methods used by the contractor shall be subject to the approval of the engineer and no part of the work will be considered as finally accepted until all of the work is completed and accepted.

The general conditions as given in 518.50 , page 416 , shall be considered to apply to these specifications.

National Sand and Gravel Association, Bulletin 3, representative specifications for different uses of sand and gravel, November, 1928.

In this bulletin representative specifications, many of which are presented in this volume, and including specifications for stucco sand, have been summarized.

Portland Cement Association, Portland cement stucco condensed specifications.

\section{GENERAI}

1. Preparation of surface.-All hangers, fasteners, trim, or other fixed supports or projections of any kind shall be in place previous to the application of stucco. In masonry backing the surface shall be cleaned thoroughly before stucco is applied and shail be sufficiently rough to provide a good mechanical bond for the first coat.

2. Flashing.-Flashing shall be in place previous to the application of stucco in the following locations: At the top and along sides of all openings wherever projecting trim occurs; across the wall and under coping, cornices, or brick sills with mortar joints, flashing to project beyond upper edge of stucco; under built-in gutters and around roof openings; at the intersection of walls and roofs; and at all other points whcre flashing would prevent water from getting behind the stucco.

3. Water protection.-All horizontal exposed surfaces which are of stucco, such as copings, cornices, belt courses, shall be given sufficient fall to prevent water from accumulating on such surfaces. In general, the construction shall protect the surface against excessive concentrated water flow, all horizontal projections being provided with overhanging drips and water-tight joints. Stucco wall surfaces shall be stopped 6 inches above grade line.

\section{MATERIALS}

4. Cement.-Portland cement shall conform to the current standard specifications of the American Society for Testing MIaterials. (See 516.11, p. 2S7.)

5. Fine aggregate.-Fine aggregate shall consist of clean sand, sereenings from crushed stone or pebbles, graded from fine to coarse, passing when dry a No. 4 screen, with not more than 20 per cent through a No. 50 screen, free from dust or other deleterious materials.

6. Water.- Water shall be clean, free from oil, acid, strong alkali, or vegetable matter.
7. Coloring materials.-Only permanent mineral oxides that are fully guaranteed by the manufacturer to be unaffected by lime, cement, or wcathering shall be used in coloring.

8. Hydrated lime.-Hydrated lime shall meet the requirements of the standard specifications of the American Society for Testing Materials, A. S. T. M. C6 (see 517.2, p. 353), and when used shall not exceed one-fifth the volume of the cement.

9. Reinforcement.-Reinforcement shall consist either of expanded metal cut from sheets not less than 20 gage in thickness with openings not less than $3 / 4$ by 2 inches nor greater than $1 \frac{1 / 2}{2}$ by 4 inches in size, the fabric to weigh not less than 1.8 pounds per square yard, or wire fabric composed of wires not smaller than 18 gage used with openings not less than $3 / 4$ inch square or with wires not smaller than 14 gage used with openings not greater than 2 inches square.

\section{CONSTRUCTION}

10. Proportions.-Mortar for both scratch and brown coats shall be mixed in the proportions of five sacks of Portland cement, one 50-pound sack of hrdrated lime, and 16 cubic feet of sand. The finish coat, if not a prepared Portland cement stucco, shall be of like proportions to those used in the previous coats, with such additions of mineral coloring pigments as necessary, but in quantities not to exceed 6 per cent of the volume of the cement used.

11. Mixing.-Dry mixing of ingredients shall be carried on until the color is uniform; wet mixing until the consistency is uniform. It is positively essential that a definite system be used which shall produce uniform mixes for scratch and brown coats. The quantity of water shall be determined by trial and thereafter used in the proper proportions.

12. Framing.-Spacing of studs shall not exceed 16 inches. Studding shall run from foundation to rafters without intervening horizontal members, tied together below second-floor joists with 1 by 4 inch boards let into the inner faces of the studs. In open construction without sheathing, the spacing of studs shall not exceed 12 inches. The corners of all walls shall be braced diagonally to secure the necessary rigidity of the structure. Bridging of studding with 2 by 4 inch braces shall occur at least once in each story height.

13. Sheathing. - Sheathing boards shall not be less than 6 inches nor more than 8 inches wide, dressed to a uniform thickness, laid horizontally, and fastened securely to each stud. Over the sheathing shall be laid, horizontally, beginning at the bottom, any standard asphalt saturated roofing felt weighing 15 pounds per square, the bottom layer lapping the baseboard and each strip lapping the strip below and all flashing at least 2 inches.

14. Application of reinforcement.-Reinforcement shall be placed horizontally, fastened with approved furring devices not more than 8 inches apart over the surface. Vertical laps shall occur at supports, horizontal joints being lapped and tightly laced with 18-gage annealed wire. The sheets shall be returned around corners at least 4 inches in sheathed construction and 16 inches in open construction. Corner beads shall not be used.

15. Furring. - All reinforeements shall be furred out from the studs, sheathing, or base three-eighth inch by any device which will not reduce the effective section of the scratch coat.

16. Half-ímbering. -Embedded trim or half-timbering shall be securely nailed directly upon sheathing or studs and shall have the insiae corners of vertical members grooved into which the mortar of the first coat shall be forced forming a water-tight 
joint. All joints on horizontal members shall be flashed.

17. Masonry walls.-Concrete, concrete block, brick, hollow tile, and similar walls shall be rigid and constructed upon solid footings, all units being set in Portland cement mortar. The surface on which stucco is to be applied shall be clean, free from all dust, dirt, or loose particles, preferably rough and of coarse texture. Wood lintels over wall openings shall not be used. Monolithic concrete walls shall be roughened by hacking, wire brushing, or other effective means. Concrete block, tile, or brick units shall have the joints cut back even with the surface. Clay tile shall be hard burned with dovetail or heavy, ragged scoring. Clay-brick walls shall be composed of rough, hard-burned clay brick, and if painted or waterproofed shall be covered with reinforcing fabric before overcoating with stucco.

18. Wetting the surface.-Immediately preceding the application of the stucco the surface of the wall shall be evenly wetted but not saturated. Water shall not be rapidly absorbed from the plaster nor remain standing on the surface.

19. Retempering.- Retempering by the addition of water shall not be permitted.

20. Consistency.-Only sufficient water to produce a workable consistency shall be used.

21. Application of stucco coats on frame construction.-The application shall be carried on continuously in one general direction without allowing the stucco to dry at the edges. If it is impossible to work the full width of the wall at one time the joining shall be at some natural division of the surface, such as a window or door. The scratch coat shall be shoved thoroughly through the metal reinforcement, forming a solid mass against the sheathing paper, thus completely encasing the metal. This coat shall be one-half inch thick, fully covering the face of the reinforcement, and shall have its surface heavily cross scratched to provide a strong mechanical key or bond. Allow this coat to become thoroughly dry. It shall be wet down, but not saturated before applying the second coat. The second or browning coat shall be at least one-half inch thick over the face of the first coat and shall be rodded straight and true in every direction or left untrue, giving a wavy effect, as the desired finish would suggest. If the finish is to be a float-type finish, the second coat shall be brought to a good even surface with wood floats. This coat shall be wet down for at least three days and allowed to become thoroughly dry before the finishing coat is applied. The finish coat shall be applied not less than one week after the application of the second coat and shall vary in thickness from one-eighth to one-fourth inch, depending upon the texture of the finish coat.

22. Scratch coat on masonry walls.-Mortar shall be troweled on to a thickness of approximately onehalf inch, heavily cross scratched, and allowed to become thoroughly dry before the browning coat is applied. (From this point on use specification covering "Application of stuceo coats on frame construction," par. 21.)

23. Freezing.-Stucco shall not be applied when the temperature is below $32^{\circ} \mathrm{F}$., unless protected with canvas and heat sufficient to prevent freezing for a period of at least 48 hours after application.

24. Curing.-Each coat shall be protected from drying rapidly from effects of intense sunlight or wind until it has sufficiently hardence to permit sprinkling. Each coat shall be kept moist by sprinkling for at least three days following its application.

25. Back-plastered construction.-In back-plastered construction the metal lath shall be attached directly to the face of the studs by an approved furring device and the mortar of the first or scratch coat applied with sufficient force to push it through the openings of the metal lath forming keys behind. The backplastering coat shall not be applied until the scratch coat has hardencd sufficiently to prevent injuring the keys of the scratch coat. The back-plastered coat shall not be less than one-half inch thick back of reinforcement, composed of the same proportions and materials as the scratch coat and shall be applied from side to side of the hollow space between studs. The application of the browning and finish coats on back-plastered construction is identical with other methods as previously given.

26. Open construction.-In open construction a standard 15-pound asphalt-saturated roofing felt shall be applied directly on the outside face of the studs being fastened by flat-headed roofing nails on 12-inch centers. Vertical laps shall be 12 inches and horizontal laps 6 inches over the lower course. Metal reinforcements shall be applied over the entire surface, held in place by approved furring devices, lapping at least 2 inches on all horizontal laps and at least 6 inches on all vertical laps. All horizontal laps between the studding shall have at least one tie with No. 18 annealed wire. All vertical laps shall occur on studding and shall be laced with No. 18 annealed wire. All metal reinforcement shall be returned around corners at least 16 inches. Corner beads will not be permitted.

27. Finish coat.-The finish coat shall be of the color and texture agreed upon by the owner and architect. If prepared Portland cement stucco is used it shall be applied according to the manufacturer's specifications. If a field mix is used for the finish coat it shall be applied in accordance with the practice outlined here. In all cases the architect will furnish samples of texture and color, which shall be inspected by all bidding contractors. A definite decision shall be arrived at as to color and texture before bids are taken, in order that bidding shall be done intelligently.

Portland Cement Association, suggested specifications for the application of Portland cement stucco on concrete block and tile walls.

(The above-entitled standard includes construction requirements and in addition the following:)

\section{MATERIALS}

\section{CEMERT}

Portland cement shall conform to the requirements of the latest standard specifications of the American Society for Testing Materials, A. S. T. M. C9 (see 516.11, p. 294).

\section{AGGREGATE}

Aggregate shall consist of clean sand or screenings from crushed stone, graded from fine to coarse. For the base coats (scratch and brown) all aggregates shall pass the No. 8 sieve. Fineness of the aggregate for the finish coat should be such as to produce the texture specified. (For the finish coat, aggregate may have to be finer than for the base coats, the degree of fineness depending upon the type of finish desired. For some finishes it is desirable that all of the material pass the No. 14 sieve, while for others up to 15 per cent may be retained on that sieve.) All aggregate must be free from loam, silt, and vegetable matter.

\section{HYDRATED LIME}

Hydrated lime shall meet the requirements of the standard specifications of the American Society for Testing Materials, A. S. T. M. C6 (see 517.2, p. 353), and shall be used in quantity not to exceed one-fifth the volume of cement. 


\section{WATER}

Only water that is fit to drink shall be used. It must be free from oil, acids, alkali, and vegetable matter.

\section{COLORING MATERIALS}

Only highest grade mineral pigments that are fully guaranteed by the manufacturer to be unaffected by lime, cement, or weather shall be used as coloring matter and in amounts not to exceed 6 per cent of the weight of the cement. In job-mixed stucco proper proportions to produce a desired shade shall be subject to the approval of the architect. A factoryprepared finish-coat stucco of the specified finish may be used upon the approval of the architect.

\section{SURFACE FINISHES}

It is recommended that the architect bring to his client's attention the possibilities in Portland cement stucco textures and colors. Samples of textures and colors should be submitted by competent stucco contractors and used as the basis for bids on the particular project under consideration. The following paragraph indicates briefly some of the more popular textures available, the number of finishes being limited only by the skill of the plasterer.

\section{COLONIAL}

The finishing coat, after being brought to a smooth, even surface and when slightly hardened should be rubbed with a circular motion of the float with the addition of a little sand to roughen the surface slightly.

\section{CALIFORNIA}

This texture consists of a rough cast surface partially smoothed by rubbing it with a piece of carpet or burlap before hardening has entirely taken place. The effect will then be similar to the walls of ancient adobe block covered with plaster, with the undulating surfaces revealing their contours.

\section{ITALIAN}

This is a rough-cast texture, spots of mortar being thrown onto the floated finished surface in various amounts, irrespective of placing. The steel trowel is then lightly run over the hillocks, troweling smoothly in spots the variations in the surface. This treatment gives the effect of depth and beauty, which may be further enhanced by the use of several colors for polychrome effect.

\section{ENGLISH COTTAGE}

Small dabs of mortar, placed on the steel trowel, are feathered off the edge of the trowel in short upward strokes, to the right or left, in indefinite direction and to avoid the appearance of a design. The irregular weaving can be varied by the amounts of mortar used on the trowel, the length of the strokes and the pressure applied, and constitute a very popular treatment adaptable to the smaller residence.

\subsection{CONCRETE AND MORTAR.}

American Association of State Highway Officials, standard specifications for bridges and incidental structures, 1928.

\section{CONCRETE MASONRY}

(The full text of the specification for concrete masonry includes material relative to methods of storing, measuring, mixing, depositing, and curing of concrete under varying conditions and for various types.)

\section{GENERAL}

Concrete masonry shall consist of an approved Portland cement, a fine aggregate, a coarse aggregate, and water, mixed in the proportions specified for the various classes of concrete hereinafter designated.

\section{CLASSIFICATION AND APPROXIMATE PROPORTIONS}

For the various concrete mixtures used in the work the proportions of cement, fine aggregate, and coarse aggregate shall be determined from the strength requirements for the particular class of work in question and from the particular limitations of the aggregate used and the consistency employed in the work.

As a basis for estimating and as a general indication of the mixture employed, the following approximate, classification of proportions is given:

\section{Class "A" concrete:}

1 part Portland cement.

2 parts fine aggregate.

4 parts coarsc aggregate passing a 11/2-inch opening. (For massive sections or those lightly reinforced $2 \frac{1}{2}$-inch coarse aggregate may be permitted. For concrete which is to be exposed to the action of sea water the maximum size of coarse aggregate shall be 1

Class "B" concrete:

1 part Portland cement.

$21 / 2$ parts fine aggregate.

5 parts coarse aggregate passing a $21 / 2$-inch opening.

Class " $\mathrm{C}$ " concrete:

1 part Portland cement.

3 parts fine aggregate.

6 parts coarse aggregate passing a 3 -inch opening.

Class "D" concrete (used for thin reinforced sections):

1 part Portland cement.

2 parts fine aggregate.

3 parts coarse aggregate passing a $3 / 4$-inch opening.

\section{STRENGTH OF CONCRETE}

The above specified approximate proportions shall be varied, as may be directed by the engineer, to secure from test cylinders the following minimum compressive strengths in pounds per square inch at the ages of 7 and 28 days:

\begin{tabular}{|c|c|c|}
\hline Class of concrete & 7 days & 28 days \\
\hline $\begin{array}{l}\mathrm{A} \\
\mathrm{B} \\
\mathrm{C}-1\end{array}$ & $\begin{array}{r}1,300 \\
1,000 \\
900 \\
1,700\end{array}$ & $\begin{array}{l}2,200 \\
1,700 \\
1,500 \\
2,800\end{array}$ \\
\hline
\end{tabular}

\section{LABORATORY AND FIELD CONTROL OF QUALITY}

Frequent tests, both in the laboratory and field, shall be made of the concrete mixtures, and the proportions of fine aggregate, coarse aggregate, and cement shall be varied in accordance with these tests in order to produce the above specified compressive strengths.

The laboratory tests made to determine the mortar and concrete making qualities of aggregates shall, in general, involve the use of these materials in a surface-dried condition, and in the records and reports of tests the quantities of aggregates shall be given for dry materials, either by weight or by volume or both.

Laboratory tests shall be made according to A. S. T. M. C39. (See 516.0, p. 284.) Field specimens shall be made according to A. S. T. M. C31. (See 516.0, p. 282.)

\section{ADJUDICATION OF COST OF CEMENT}

To form a basis for bidding and for payment ${ }^{-}$on concrete, the cement required for any structure shall be determined from the proportions as above specified for the different classes of concrete employed and the total yardage placed. For this purpose the following approximate quantities shall furnish a basis: 
Table of materials for 1 cubic yard of compacted concrete

\begin{tabular}{|c|c|c|c|}
\hline $\begin{array}{l}\text { Class of con- } \\
\text { crete }\end{array}$ & $\begin{array}{c}\text { Nominal } \\
\text { proportions }\end{array}$ & $\begin{array}{l}\text { Cement } \\
\text { sacks }\end{array}$ & $\begin{array}{c}\text { Weight of } \\
\text { ceinent }\end{array}$ \\
\hline $\begin{array}{l}\mathrm{A} \ldots \\
\mathrm{B} \\
\mathrm{C} \\
\mathrm{D}\end{array}$ & $\begin{array}{r}1: 2: 4 \\
1: 21 / 2: 5 \\
1: 3: 6 \\
1: 2: 3\end{array}$ & $\begin{array}{l}6.3 \\
5.2 \\
4.5 \\
7.3\end{array}$ & $\begin{array}{c}\text { Pounds } \\
592 \\
489 \\
423 \\
686\end{array}$ \\
\hline
\end{tabular}

If the contract price for concrete includes the cost of cement and if the proportions as adopted for the work vary from those specified above, necessitating a change in the quantity of cement, the difference in the cost of the work, based upon the actual cost of the cement delivered at the bridge site, shall be adjusted for or against the contractor, as the case may be.

Changes in the proportion of fine to coarse aggregate shall not be cause for any adjustment in compensation except as such changes affect the quantity of cement required. However, changes made at the contractor's request to permit the use of inferior aggregates shall not involve additional compensation for cement used.

\section{CONSISTENCY}

The quantity of mixing water to be used shall be determined in each case by the engineer, and no changes shall be made without his consent. In general, a mixture shall be used which contains the minimum amount of water consistent with the required workability.

In general, the consistency of concrete mixtures shall be such that:

1. The mortar clings to the coarse aggregate.

2 . The concrete is not sufficiently fluid to segregate when transported to the place of deposit.

3. The concrete, when dropped directly from the discharge chute of the mixer, shall flatten out at the center of the pile, but shall stand up and not flow at the edges.

4. The mortar shall show no free water when removed from the mixer.

5. The concrete shall settle into place when deposited in the forms and, when transported in metal chutes at an angle of $30^{\circ}$ with the horizontal, it shall slide and not flow into place.

6 . The upper layer of the set concrete shall show a cement film upon the surface, but shall be free from laitance.

\section{RUBDLE OR CYCLOPEAN CONCRETE}

Rubble or cyclopean concrete shall consist of either class $\mathrm{B}$ or class $\mathrm{C}$ concrete, as specified, in which is embedded large individual stones in accordance with the following requirements.

This class of concrete shall be used only in massive piers, gravity abutments, and heavy footings, and only with the approval of the engineer.

The stone shall be carefully placed (not dropped or cast) and the method of placing shall be such as to avoid injury to the forms or to the partially set adjacent inasonry. Stratified stone shall be placed upon its natural bed. All stone shall be thoroughly washed and saturated with water before being placed.

The total volume of the stone used shall not be greater than one-third of the total volume of the portion of the work in which it is placed.

For walls or piers greater than 2 feet in thickness 1 -man stone may be used. Each stone shall be completely surrounded by a layer of concrete not less than 6 inches in thickness, No stone shall extend above a point 1 foot below the top surface of any wall or pier nor shall it extend within less than 6 inches of any coping.

For walls or piers greater than 4 feet in thickness derrick stone may be used. Each stone shall be completely surrounded by a layer of concrete not less than 1 foot in thickness. No stone shall extend above a point 2 feet below the top surface of any wall or pier nor shall it extend within less than 8 inches of any coping.

\section{EXPANSION JOLNTS}

Expansion joints may be of the following types: Friction joints, open joints, filled compression joints, mortise joints, and special expansion joints.

Friction joints, when of metal, shall be composed of cast-iron or bronze plates, as noted on the plans. Sliding surfaces shall be planed true and smooth, the marks of the plane paralleling the movement of the joint. Expansion plates shall be well anchored. as shown on the plans. All sliding surfaces of expansion plates shall be thoroughly coated with graphite and grease just before being placed in position and special care taken to avoid placing concrete in such manner as to interfere with their free action.

Open joints shall be placed at locations designated on the plans and shall be formed by the insertion and subsequent removal of a templet of timber, metal, or other suitable material. The method of insertion and removal of joint templets shall be such as to avoid the possibility of chipping or breaking down at the edges, and the templets shall be so constructed that their removal may be readily accomplished without injury to the work.

Filled compression joints shall be made with an asphalt filler or premolded filler, the materials for which shall conform to "fillers," 505.15, American Association of State Highway Officials.

Mortise joints shall be as shown on the plans and, in general, shall consist of a tenon of concrete or metal sliding in a suitable concrete or metal socket or mortise. Concrete tenons in concrete mortise sockets, brass or bronze tenons sliding in concrete sockets, or metal tenons sliding in galvanized pipe sockets may be specified. In any case, the construction shall be such as to permit freedom of movement and such as to be, as far as possible, water-tight and rustproof.

Special types of expansion joints may be used when so specified on the plans or ordered in writing by the engineer.

Expansion joints through bridge floors preferably shall be constructed with a waterproof fiashing of copper or zinc to prevent the entrance of water into the joint. Such flashing shall be so arranged as to lead all drainage water to a point of discharge such as will avoid any staining of the exposed concrete surfaces.

\section{ORDINARY SURFACE FINISH}

The external surface of all concrete masonry shall be thoroughly worked during the operation of placing by means of a broad-tined fork or a concrete spade of an approved type. The working shall be such as to force all coarse aggregate from the surface and thoroughly work the mortar against the forms to produce a smooth finish free from water and air pockets or honeycomb.

As soon as the concrete has set sufficiently to permit, the forms shall be carefully removed and all depressions resulting from the removal of metal ties and all other holes and rough places shall be carefully pointed with a mortar of sand and cement in the proportion which has been employed for the particular class of concrete treated. The surface film of all such pointed surfaces shall be carefully removed before setting occurs. The surface shall then be given one of the following finishes: 
Class 1, surface finish.-As soon as the pointing has set sufficiently to permit it, the entire surface shall be thoroughly wet with a brush and rubbed with a No. 16 carborundum stone or an abrasive of equal quality, bringing the surface to a paste. The rubbing shall be continued sufficiently to remove all form marks and projections, producing a smooth dense surface without pits or irregularities.

The material which, in the above process has been ground to a paste, is now carefully spread or brushed uniformly over the entire surface and allowed to take a "reset." The final finish shall be obtained by a thorough rubbing with a No. 30 carborundum stone or an abrasive of equal quality. This rubbing shall continue until the entire surface is of a smooth texture and uniform in color.

After the final rubbing is completed the surface shall be thoroughly drenched and kept wet for a period of seren days, unless otherwise directed.

Railing balusters and other precast members which have been disfigured by the drip from the abrasive shall be thoroughly cleaned by means of a dilute solution of muriatic acid.

Class 2, surface finish.- After the pointing has set sufficiently to permit it, the entire surface shall be thoroughly wetted and rubbed with a No. 16 carborundum stone or an abrasive of equal quality to bring the surface to a smooth texture and remove all form marks. The paste formed by the rubbing, as above described, may be finished by carefully striping with a clean brush, or it may be spread uniformly over the surface and allowed to take a "reset," after which it may be finished by floating with a canvas, carpet-faced or cork float or rubbed down with dry burlap.

TREATMENT AND FLNISH FOR HORIZONTAL SURFACES ROT SUBJECTED TO WEAR

All upper horizontal surfaces, such as the tops of handrail posts and caps and the tops of parapets, copings, and bridge seats, shall be formed by placing an excess of material in the forms and removing or striking off such excess with a wooden templet, forcing the coarse aggregate below the mortar surface. The use of mortar topping for concrete railing caps and other surfaces falling under this classification shall in no case be permitted.

The final finish for caps and railings shall be obtained in one of the following ways, as specified:

Brush finish.- After the concrete has been struck off as above described, the surface shall be thoroughly worked and floated with a wooden, canvas, or cork float, the operation to be performed by skilled and experienced concrete finishers. Before this last finish has set, the surface shall be lightly striped with a fine brush to remove the surface cement film, leaving a fine-grained, smooth but sanded texture.

Float finish.-In lieu of the above, the surface may be finished with a rough carpet float or other suitable device, leaving the surface even, but distinctly sandy or pebbled in texture.

Ground or terrazzo finish.-When specified, the upper surfaces of rail caps, parapets, etc., may be finished by grinding with a carborundum stone or equally good abrasive to a smooth, dense, terrazzo finish. Finishing of this character shall be done as follows:

Using a No. 16 carborundum stone or an abrasive of equal quality, the surface shall be ground dry or in water until it is smooth and the individual pebbles and aggregate particles are cut and polished. The surface shall then be completely cleansed with water and the final rubbing done by means of a No. 30 stone. The finished surface shall present the texture of polished marble and shall show the various aggregate particles in polished outline.
Other types.- Special types of rail cap finish, such as tooled finish, sand-blasted finish, etc., shall be done in accordance with the general requirements governing "Special surface finishes," as hereinafter provided.

\section{SPECIAL SURFACE FINISHES}

The above specified surface finishes are to govern in ordinary concrete construction. When so specified, special surface finishes may be employed for ornamental panels, copings, and like construction. In general, the method and manner of performing this work will be fully provided for in the special provisions for the particular work in question.

In each case the contractor shall be required to prepare test or sample panels under the direction of the engineer, and the method and manner of finish, the choice, and selection of the aggregate, and other features affecting the work shall be approved before any further work is done.

Following are typical general requirements for the principal trpes of special surface finishes:

Tooled finish.- Finish of this character for panels and other like work may be secured by the use of a bushhammer, pick, crandall, or other approved tool. Air tools preferably shall be employed. No tooling shall be done until the concrete has set for at least 14 days and as much longer as may be necessary to prevent the aggregate particles from being "picked" out of the surface. The finished surface shall show a grouping of broken aggregate particles in a matrix of mortar, each aggregate particle being in slight relief.

Sand-blast finish.-The type of finish desired in this connection shall be similar to that above described for tooled finish but finer grained in texture. The sand blasting must be done by means of approved equipment and in such manner as to produce an eren, fine-grained surface in which the mortar has been cut away, leaving the aggregate particles exposed.

Wire brush or scrubbed finish.-This type of finish shall be produced by scrubbing the surface of a green concrete with stiff wire or fiber brushes, using a solution of muriatic acid in the proportion of 1 part acid to 4 parts water. As soon as the forms are removed and while the concrete is yet comparatively green the surface shall be thoroughly and evenly scrubbed as above described until the cement film or surface is completely removed and the aggregate particles are exposed, leaving an even pebbled texture presenting an appearance grading from that of fine granite to coarse conglomerate, depending upon the size and grading of aggregate used. Granite chips or colored aggregates may be used in this connection if desired. As soon as the scrubbing has progressed sufficiently to produce the texture desired the entire surface shall be thoroughly washed with water to which a small amount of ammonia has been added, to remove all traces of the acid.

American Railway Association, telegraph and telephone section, specification for communication underground conduit construction, No. 1-C-1, 1927.

(This specification contains requirements for concrete and materials used in concrete for underground work.)

(See 518.9 , p. 483.)

American Railway Engineering Association, specifications for Portland cement concrete, plain and reinforced, 1927.

\section{MATERIAIS} CEMENT

The cement shall meet the requirements of the A. R. E. A. specifications for Portland cement, 
which are the same as A. S. T. M. C9. (See 516.11, p. 294.)

\section{FINE AGGREGATE}

Fine aggregate shall consist of sand or other approved inert matelials with similar characteristics, or a combination thereof, having clean, hard, strong, durable, uncoated grains, and free from injurious amounts of dust, lumps, soft or flaky particles, shale, alkali, organic matter, loam or other delcterious substances.

\section{GRADING}

Fine aggregate shall range in size from fine to coarse within the limits indicated below, percentage by weight:

Passing through No. 4 sieve Per cent

Passing through No. 50 sieve, $\{$ not more than Passing through No. 100 sieve, when than -...than

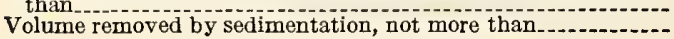

\section{ORGANIC IMPURITIES IN SAND}

Natural sand which shows a color darker than the standard color when tested in accordance with A. S. T. M. C40 (sce 512.10, p. 213) shall not be used unless the concrete made with the materials and in the proportions to be used on the work is shown by tests to be of the required strength.

\section{COARSE AGGREGATE}

\section{GENERAL}

Coarse aggregate shall consist of crushed stone, gravel, or other approved inert materials with similar characteristics, or combinations thereof, having clean, hard, strong, durable, uncoated particles free from injurious amounts of soft friable, thin, elongated, or laminated pieces, alkali, organic, or other deleterious matter.

\section{SLAG}

Provided the contract specifically pcrmits the usc of crushed slag as a coarse aggregate, it shall be air-cooled, blast-furnace slag, conforming to all the requirements for coarse aggregate specified in the above paragraph. The crushed slag shall weigh not less than 70 pounds per cubic foot and shall be obtained only from such banks as have the approval of the cngineer. All slag used shall have seasoned in the bank for a period of not less than one year, unless, in the opinion of the engineer a shorter period is sufficient.

\section{GRADING}

Coarse aggregate shall grade in size from fine to coarse, preferably within the following percentages, by weight:

Passing maximum size sieve not less than Per cent

Passing sieve one-half the maximum \{ not more than

Passing No. 4 sieve not more than

The maximum size of coarse aggregate shall be not more than 3 inches and shall be not more than four-fifths of the minimum clear space between reinforcement bar's or mesh.

\section{MIXING WATER}

Mixing water shall be free from oil, acid, and injurious amounts of vegetable matter, alkalies, or other salts.

\section{STORAGE OF CEMENT AND AGGREGATES}

\section{Storing Cement}

The cement shall be stored in a weather-tight structure with the floor raised not less than 1 foot from the ground, in such manner as to permit easy access for proper inspection and identification of each shipment. Cement that has hardened or partially set shall be removed from the site and not used in the work.

\section{Storing aggregates}

The fine and coarse aggregates shall be stored separately and in such manner as to avoid the inclusion of dirt and other foreign material in the concrete. Coarse aggregate shall be unloaded and piled in such manner as to maintain the grading of the sizes.

\section{STEEL REINFORCEMENT}

QUALITY

Steel rods or bars used for reinforcing shall conform to the requirements of the A. R. E. A. "specifications for billet-steel concrete reinforcement bars."

Structural steel shapes used for reinforcing shall conform to the requirements of the A. R. E. A. "Specifications for steel railway bridges."

\section{SIZE OF BARS}

Reinforcement bars shall conform to the areas and equivalent sizes shown on the following table:

\section{Sizes and areas of reinforcement bars}

\begin{tabular}{|c|c|c|}
\hline \multirow{2}{*}{ Size of bar (inches) } & \multicolumn{2}{|c|}{ Area (square inch) } \\
\hline & $\begin{array}{c}\text { Round } \\
\text { bar }\end{array}$ & $\begin{array}{c}\text { Square } \\
\text { bar }\end{array}$ \\
\hline $\begin{array}{l}3 / 8 \ldots \ldots \\
162 \ldots \\
5 / 8 \ldots \\
3 / 4 \ldots \\
7 / 8 \\
1 \\
1138 \\
11 / 4 \ldots\end{array}$ & $\begin{array}{r}0.110 \\
.196 \\
.306 \\
.441 \\
.601 \\
.785 \\
\end{array}$ & $\begin{array}{r}0.250 \\
1.000 \\
1.265 \\
1.562\end{array}$ \\
\hline
\end{tabular}

\section{DEFORMED BARS}

An approved deformed bar shall be one that will develop a bond at least 25 per cent greater than that of a plain round bar of equivalent cross-sectional area. The areas of deformed bars shall be determined by the minimum cross section thereof.

\section{WIRE}

Wire for concrete reinforcement shall be cold-drawn steel wire.

\section{SPIRAL}

Spirals for column reinforcement shall be colddrawn steel wire and consist of evenly spaced continuous spirals held firmly in place and true to line by at least three vertical spacer bars. At the ends of all spirals and at points of splice, the outside diameter shall be maintained.

\section{WORKMANSHIP}

\section{PROPORTIONING AND MIXING}

\section{General}

The unit of measure shall be the cubic foot. Ninety-four pounds (one sack or one-fourth barrel) of cement shall be assumed as 1 cubic foot.

\section{MEASURING INGREDIENTS}

The method of measurement shall be such as to secure the specified proportions in each batch. The 
aggregates shall be measured separately by volume or weight. In volume measurement the fine aggregate and the coarse aggregate shall be measured loose, as thrown into the measuring device, due allowance being made for bulking. The water shall be so measured as to insure the desired quantity in successive batches.

\section{PROPORTIONING}

\section{WATER-CEMENT RATIO}

The water-cement ratio is essentially equivalent to the void-cement ratio.

The proportioning of materials for the class of concretc specified or shown on the plans shall be based on the requirements for a plastic and workable mix containing not more than the amount of water per sack (94 pounds) of cement for each class of concrete as follows:

\begin{tabular}{|c|c|}
\hline $\begin{array}{c}\text { Class of con- } \\
\text { crete compres- } \\
\text { sion strength, } \\
\text { pounds per } \\
\text { square inch, } \\
\text { (28 days) }\end{array}$ & $\begin{array}{c}\text { Gallons of } \\
\text { water per } \\
\text { sack of } \\
\text { cement }\end{array}$ \\
\hline & \\
\hline 3,500 & 5.00 \\
3,000 & 5.50 \\
2,500 & 6.25 \\
2,000 & 7.00 \\
1,500 & 8.00 \\
\hline
\end{tabular}

These quantities of water must not be exceeded. Water in the aggregate must be included in the quantity specified and subtracted from the amount added to the mixture.

\section{Moisture in Aggregate}

Moisture in the aggregate shall be measured by methods satisfactory to the engineer, which will give results within 1 pound for each 100 pounds of aggregate.

\section{Workability}

The mixture shall be such as to produce concrete that can be worked readily into the corners and angles of the folm and around the reinforcement without excessive spading. The workability will be controlled by adding or deducting fine or coarse aggregate, but in no case shall the amount of coarse aggregate be such as to produce harshness in placing or honeycombing in the structure. ${ }^{54}$

\section{Slump}

The slump shall be within the following limits:

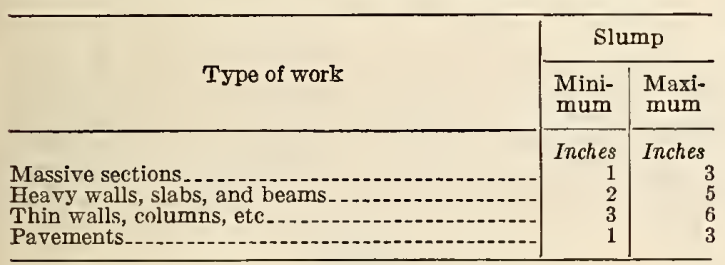

NOTE. - The slump test is a relative test and serves as a guide to gage tbe consistency to meet the workability adopted as satisfactory for special requirements. ${ }^{54}$ That combination of fine and coarse aggregate which will produce
the desired workability will vary with the grading of tbe aggregates. the desired workability will vary with the grading of tbe aggregates.

For three-fourths inch maximum size of aggregateNot more than $1^{2} 3$ parts of fine to 1 part of coarse, and Not less than two-thirds part of fine to 1 part of coarse.

For 1 incb and over maximum size of aggregate-

Not more tban 1 part of fine to 1 part of coarse, and

Not less than one-half part of fine to 1 part of coarse.

\section{TESTS}

Duplicate copies of all test data taken by the contractor for the control of concrete shall be filed with the engineer immediately after the data is obtained. The contractor shall furnish concrete for such test cylinders as the engineer may require.

Four test cylinders shall be made for each 500 cubic yards of concrete deposited. On no job shall less than four cylinders be made.

\section{COMPRESSION TESTS}

Specimens for compression tests shall be made and stored in accordance with A. S. T. M. C31. (See 516.0 , p. 282.) These specimens shall be tested in accordance with A. S. T. M. C39. (See 516.0, p. 284.)

\section{MIXING \\ Machine Mixing}

The mixing of concrete, unless otherwise authorized by the engineer, shall be done in a batch mixer of approved type which will insure a uniform distribution of the materials throughout the mass, so that the mixture is uniform in color and homogeneous. The mixer shall be equipped with a suitable charging hopper, water storage, and a water-measuring device controlled from a case which can be kept locked and so constructed that the water can be discharged only whilc the mixer is being charged. It shall also be equipped with an attachment for automatically locking the discharge lever until the batch has been mixed the required time after all materials are in the mixer. The entire contents of the drum shall be discharged before recharging. The mixer shall be cleaned at frequent intervals while in usc. The volume of the mixed material per batch shall not exceed the manufacturer's rated capacity of the mixer.

\section{Time of Mixing}

The mixing of each batch shall continue not less than one minute after all the materials are in the mixer, during which time the mixer shall rotate at a peripheral speed of about 200 feet per minute.

\section{Hand Mixing}

When it is permitted to mix by hand, the mixing shall be done on a water-tight platform of sufficient size to accommodate men and materials for the progressive and rapid inixing of at least two batches of concrete at the same time. The batches shall not exceed one-half cubic yard each. The materials shall be mixed dry until the mixture is of uniform color, the required amount of water added, and the mixing continued until the batch is of uniform consistency and character throughout. Hand mixing will not be permitted for concrete deposited under water.

\section{Retempering}

The retempering of concrete or mortar which has partially hardened - that is, remixing with or without additional cement, aggregate or water-will not be permitted.

\section{DEPOSITING CONCRETE}

\section{Cleaning Equipment}

Before beginning a run of concrete, hardened concrete and foreign materials shall be removed from the inner surfaces of the mixing and conveying equipment.

\section{General}

Before depositing any concrete, all débris shall be removed from the space to be occupied by the concrete, all steel reinforcing shall be secured in its 
proper location, all forms shall be thoroughly wetted, except in freezing weather, unless they have been previously oiled, and all form work and steel reinforcing shall be inspected and approved by the engineer.

\section{Handling and placing}

Concrete shall be handled from the mixer to the place of final deposit as rapidly as practicable by methods which shall prevent the separation or loss of the ingredients. It shall be deposited in the forms as nearly as practicable in its final position to avoid rehandling. It shall be so deposited as to maintain, until the completion of the unit, a plastic surface approxinately horizontal. Forms for walls or other thin sections of considerable height shall be provided with openings or other devices that will permit the concrete to be placed in a manner that will avoid accumulation of hardenerl concrete on the forms or metal reinforcement. Under no circumstances shall concrete that has partially hardened be deposited in the work.

\section{Chuting}

When concrete is conveyed by chuting, the plant shall be of such size and design as to insure a practically continuous flow in the chute. The angle of the chute with the horizontal shall be such as to allow the concrete to flow without separation of the ingredients. The delivery end of the chute shall be as close as possible to the point of deposit. When the operation is intermittent, the spout shall discharge into a hopper. The chute shall be thoroughly flushed with water before and after each run; the water used for this purpose shall be discharged outside the forms.

\section{Compacting}

Concrete, during and immediately after depositing, shall be thoroughly compacted by means of suitable tools. For thin walls or inaccessible portion of the forms, where rodding or forking is impracticable, the concrete shall be assisted into place by tapping or hammering the forms opposite the freshly deposited concrete. The concrete shall be thoroughly worked around the reinforcement, and around embedded fixtures and into the corners of the forms.

Removal of water

Water shall be removed from excavations before concrete is deposited, unless otherwise directed by the engineer. Any flow of water into the excavation shall be diverted through proper side drains to a sump, or be removed by other approved methods which will avoid washing the freshly deposited concrete. Water vent pipes and drains shall be filled by grouting or otherwise after the concrete has thoroughly hardened.

\section{Protection}

Exposed surfaces of concrete shall be protected from premature drying for a period of at least seven days after being deposited.

\section{Temperature}

Concrete when deposited shall have a temperature not below $50^{\circ} \mathrm{F}$ and not above $120^{\circ} \mathrm{F}$. In freezing weather suitable means shall be provided for maintaining the concrete at a temperature not lower than $50^{\circ} \mathrm{F}$. for not less than 72 hours after placing or until the concrete has thoroughly hardened. The methods of heating the materials and protecting the concrete shall be approved by the engineer. Salt, chemicals, or other foreign materials shall not be mixed with the concrete for the purpose of preventing freezing.

\section{Continuous depositing}

Concrete shall be deposited continuously and as rapidly as practicable until the unit of operation, approved by the engineer, is completed. Construction joints at points not provided for in the plans shall be made in accordance with the provisions in the section entitled "Construction joints" under the heading "Joints" (below).

\section{Depositing against other concrete}

Before depositing new concrete on or against concrete which has hardened, the forms shall be retightened, the surface of the hardened concrete shall be roughened as required by the engineer, thoroughly cleaned of foreign matter and laitance, and saturated with water. The new concrete placed in contact with hardened or partially hardened concrete shall contain an excess of mortar to insure bond. To insure this excess mortar at the juncture of the hardened and the newly deposited concrete, the cleaned and saturated surfaces of the hardened concrete, including vertical and inclined surfaces, shall first be slushed with a coating of neat-cement grout against which the new concrete shall be placed before the grout has attained its initial set.

Depositing concrete under water

\section{GENERAL}

When the depositing of concrete under water is authorized by the engineer the methods, equipment, and materials to be used shall be submitted to and approved by the engineer before the work is started. Concrete shall be deposited by a method that will prevent the washing of the cement from the mixture, minimize the formation of laitance, and avoid flow of water until the concrete has fully hardened. Concrete shall be placed so as to minimize segregation of materials. Concrete shall not be placed in water having a temperature below $35^{\circ} \mathrm{F}$.

\section{INCREASING AMOUNT OF CEMENT}

Concrete to be deposited under water shall contain one and three-fourths barrels (seven bags) or more of Portland cement per cubic yard of concrete in place.

\section{COFFERDAMS}

Cofferdams shall be sufficiently tight to prevent flow of water through the space in which the concrete is to be deposited. Pumping will not be permitted while concrete is being deposited nor before it has fully hardened.

\section{CONTINUOUS WORK}

When depositing concrete under water is permitted, concrete shall be deposited continuously, keeping the top surface as nearly level as possible, until it is brought above the water, or to the required height. The work shall be carried on with suflicient rapidity to prevent the formation of layers.

\section{METHOD}

One of the following methods of depositing concrete under water may be used:

(a) Tremie.-The tremie shall be water-tight and sufficiently large to permit a free flow of concrete. It shall be kept filled ${ }^{55}$ at all times during depositing.

55 The tremie may be filled by one of the following methods: (1) Place the lower end in a hox partly filled with concrete so as to sea the bottorn, then lower into position; (2) pluz the tremie with cloth the bottom, then loin in sacks or other material, which will be forced down as the pipe is flled with concrete; (3) plug the end of the tremie with cloth sacks filled 
The concrete shall be discharged and spread by raising the tremie in such manner as to maintain as nearly as practicable a uniform flow and avoid dropping the concrete through water. If the charge is lost during depositing, the tremie shall be withdrawn and refilled.

(b) Drop-bottom bucket.-The bucket shall be of a type that can not be dumped until it rests on the surface upon which the concrete is to be depositcd. The bottom doors when tripped shall open freely downward and outwald. The top of the bucket shall be open. The bucket shalk be completely filled, and slowly lowered to avoid backwash. Tihen discharged, the bucket shall be withdrawn slowly until well above the concrete.

(c) Bags.-Bags of jute or other coarse cloth shall be filled about two-thirds full of concrete and carefully placed by hand in a header-and-stretcher system so that the whole mass is interlocked.

\section{LATTANCE}

Great care shall be exercised to not disturb the concrete more than is unavoidable when it is being deposited under water in order to avoid the formation of laitance. On completing a section of concrete, the laitance shall be entirely removed before work is resumed.

\section{FORMS}

General

Forms shall conform to the shape, lines, and dimensions of the concrete as called for on the plans. Lumber used in forms for exposed surfaces shall be dressed to a uniform thickness, and shall be free from loose knots or other defects. Joints in forms shall be hoizontal or vertical. For unexposed surfaces and rough work, undressed lumber may be used. Lumber once used in forms shall have nails withdiawn, and surfaces to be in contact with " concrete thoroughly cleaned, before being used again.

\section{Design}

Forms shall be substantial and sufficiently tight to prevent leakage of mortal; they shall be properly braced or tied together so as to maintain position and shape. If adequate foundation for shores can not be secured, trussed supports shall be provided.

\section{Ties and Workmanship}

Bolts and rods shall preferably be used for internal ties; they shall be so arranged that when the forms arc removed no metal shall be within 1 inch of any surface. Wire ties will be permitted only on liglit and unimportant work; they shall not be used through surfaces where discoloration would be objectionable. Shores supporting successive stories shall be placed directly over those below, or so designed that the load will be transmitted directly to them. Forms shall be set to line and grade and so constructed and fastened as to produce true lines. Special care shall be used to prevent bulging.

\section{Molding}

Unless otherwise specified, suitable moldings or bevels shall be placed in the angles of forms to round oi bevel the edges of the concrete.

\section{Oiling Forms}

The inside of forms shall be coated with nonstaining inineral oil or other approved material or thoroughly wetted (except in freezing weather). Where oil is used, it shall be applied before the reinforcement is placed.

\section{Temporary Openings}

Temporary openings shall be provided at the base of column and wall forms, and at other points where necessary to facilitate cleaning and inspection immediately before depositing concrete.

\section{Remoral}

Forms shall not be disturbed until authorized by the engineer. Shoring shall not be removed until the member has acquired sufficient strength to safely support its weight and the load upon it. Members subject to additional loads during construction shall be adequately sholed to support both the member and construction loads in such a manner as will protect the meinber from damage by the loads; this shoring shall not be removed until the member has acquired sufficient strength to safely support its weight and the load upon it.

\section{METAL REINFORCEMENT}

\section{Cleaning}

Metal reinforcement, before being positioned, shall be thoroughly cleaned of mill and rust scale and of coatings that will destroy or reduce the bond. Reinforcement appreciably reduced in section shall be rejected. Where there is delay in depositing concrete, reinforcement shall be reinspected and, when necessary, cleaned.

\section{Bending}

Reinforcement shall be carefully formed to the dimensions indicated on the plans. Cold bends shall be made around a pin having a diameter of three or more times the least dimension of the reinforcement bars for steel of structural grade and six or more times that for steel of intermediate or hard grade.

\section{Straightening}

Metal reinforcement shall not be bent or straightcned in a manner that will injure the material. Bars with kinks or bends not shown on the plans shall not be used. Heating of reinforcement will be permitted only when the entire operation is approved by the engineer.

\section{Placing}

Metal reinforcement shall be accurately positioned, and secured against displacement by using annealed iron wire of not less than No. 18 gage or suitable clips at intersections, and shall be supported by concrete or metal chairs or spacers, or metal hangers, unless otherwise shown on the plans. The minimum clear distance between parallel bars shall be one and one-half times the diameter of round bars or one and one-half times the diagonal of square bars, and bars parallel to the face of any member shall be embedded a clear distance of not less than 1 inch from the face.

\section{Splicing}

Wherever it is necessary to splice the reinforcement otherwise than as shown on the plans, the character of the splice shall be decided by the engineer on the basis of safe bond stress and the stress in the reinforcement at the point of splice. Splices shall not be made at points of maximum stress nor shall adjacent bars be spliced at the same point.

Splices in columns, piers, and struts shall provide sufficient lap to transfer the stress by bond, 
Future Bonding

Exposed reinforcement bars intended for bonding with future extensions shall be protected from corrosion.

\section{Protective Concrete Covering}

\section{MOISTURE PROTECTION}

Metal reinforcement in wall footings and column footings shall have a minimum covering of 3 inches of concrete. At surfaces of concrete exposed to the weather, metal reinforcement shall be protected by not less than 2 inches of concrete.

\section{JOIN TS}

\section{General}

Instructions given on the plans, in the detailed specifications or schedule governing the work as to location and construction of joints, shall be strictly followed.

When the structures or portions of the structures are designed to be monolithic, they shall be cast integrally, except as hereinafter modified.

\section{Construction Joints}

(a) When necessary to provide construction joints not indicated or specified, such joints shall be located and formed so as not to impair the strength and to least impair the appearance of the structure. Where conditions require, the joints shall be reinforced as directed by the engineer in order to secure the necessary bond strength. Horizontal construction joints shall be prepared at the time the work is interrupted by thoroughly roughening the surface and providing keys or mortises, or by means of steel dowels set substantially at right angles to the plane of the joint.

(b) To prevent laitance in horizontal joints, excess water shall be removed from the surface forming the joint after depositing the concrete. Surfaces of contact shall be cleaned and wetted before depositing is resumed, and any laitance shall be removed.

(c) Where girders, beams, and slabs are designed to be monolithic with walls and columns they shall not be cast until four hours after completion of the walls or columns in order to permit of shrinkage or settlement. In case the columns are structural steel incased in concrete, the lapse of time to allow for shrinkage or settlement need not be observed.

\section{Joints in Columns}

Joints in columns shall be made at the underside of the floor. Haunches and column capitals shall be considered as part of and to act continuous with the floor. At least two hours must elapse after depositing concrete in the columns or walls before depositing in beams, girders, or slabs.

\section{Joints in Floors}

Construction joints in floors shall be located near the middle of spans of slabs, beams, or girders unless a beam intersects a girder at this point, in which case the joints in the girders shall be offset a distance equal to twice the width of the beam. Adequate provision shall be made for shear by use of inclined reinforcement.

\section{Expansion Joints}

At all expansion joints, the structure adjacent to the joint shall preferably be supported on separate columns or walls. Reinforcement shall not extend across an expansion joint; the break between the two sections shall be complete. Exposed edges of expansion joints in walls or abutments shall be rounded. Exposed expansion joints between two distinct concrete members shall be filled with an elastic joint filler of an approved quality.

\section{Sliding Joints}

The seat of sliding joints shall be finished to a smooth, plain surface and allowed to harden.

\section{Water-tight Construction Joints}

Where construction joints are required to be watertight the method of construction shall be as follows:

(a) Horizontal joints shall be constructed by forming a continuous keyway in the lower portion of concrete before the concrete has hardened. Before placing the superimposed concrete the joint shall be thoroughly cleaned of laitance or other foreign material, saturated with water and coated with neatcement grout. The superimposed concrete shall be placed in such a manner as will insure an excess of mortar over the entire surface of the joint.

(b) Vertical joints shall be made by a noncorrosive metal waterstop approved by the engineer.

\section{WATERPROOFING}

General

Concrete required to be water-tight shall be made with strict adherence to all provisions in these specifications regarding the choice of materials, proportions, consistency, mixing, placing, protection, and workmanship.

(a) Specification for membrane waterproofing on solid floor railway bridges may be found in the American Railway Engineering Association Manual, under Iron and Steel Structures.

(b) Integral compounds shall not be used for waterproofing unless specifically authorized by the engineer.

\section{CONCRETE IN SEA WATER}

Proportions

Plain concrete in sea water from 2 feet below low water to 2 feet above high water, or from a plane below to a plain above wave action, shall contain a minimum of $1 \frac{3}{4}$ barrels ( 7 bags) of Portland cement per cubic yard in place. Other plain concrete in sea water or exposed directly along the sea coast shall contain a minimum of $1 \frac{1}{2}$ barrels ( 6 bags) of Portland cement per cubic yard in place. Porous or weak aggregates shall not be used.

\section{Depositing in Sea Water}

Sea water shall not be allowed to come in contact with the concrete until it has hardened for at least four days. Concrete shall be placed in such a manner as to minimize the number of horizontal or inclined seams or work planes. The placing of concrete between tides shall be, so far as possible, a continuous operation. Concrete shall be deposited in sea water only when so directed by the engineer.

Protecting Concrete in Sea Water

In concrete exposed to sea water the metal reinforcement shall be placed not less than 3 inches from any plane or curved surface, and at corners it shall be not less than 4 inches from adjacent surfaces. Metal chair supports or ties shall not extend to the surface of the concrete. Where severe climatic conditions or severe abrasions are anticipated, the face of the concrete from 2 feet below low water to 2 feet above high water, or from a plane below to a plane above wave action shall be protected by stone of suitable quality, dense vitrified shale brick as designated on the plans or as required by the engineer, or in special cases the protection may be creosoted timber.

\section{CONCRETE IN ALKALI SOILS OR WATERS Proportions}

Concrete in alkali waters or below ground line of alkali soils shall contain a minimum of $13 / 4$ barrels ( 7 bags) of Portland cement per cubic yard in place. 
Plecing

Concrete in alkali soils or waters shall be placed in such a manner as to minimize the number of horizontal or inclined seams, or work planes.

\section{Protection}

Metal reinforcement or other corrodible metal shall not be placed closer than 2 inches to the surface of members exposed to alkali soils or waters. In foundations and in heary structures the metal reinforcement shall not be placed closer than 3 inches to the surface.

\section{SURFACE FINISH}

\section{General}

(a) The requirements in these specifications applying to forms, mixing, conveying, and depositing concrete shall be followed unless modified by the plans or by the direction of the engineer.

(b) The $\pi$ hole of an exposed surface between prescribed construction limits shall be cast in one continuous operation. Construction and expansion joints, when not shown on the plans, shall be made as directed by the engineer, and shall be true to line with sharp unbroken edges.

(c) The same brand of cement and the same kind and size of aggregate shall be used throughout the whole of any exposed surface.

(d) For exposed surfaces the forms shall be smooth and water-tight. If of rood, the boards shall be planed to a uniform thickness evenly matched with tongue and groove and tightly placed. They shall be so constructed that they can be removed without hammering or prying against the concrete. All offsets or mismatching of the boards shall be dressed to a smooth surface and all openings and cracks pointed flush with clay or plaster of Paris to prevent leakage and the formation of fins.

(e) The concrete for exposed surfaces shall be so mixed, placed, and worked with a spading tool that the aggregate is uniformly distributed and a full surface of mortar brought against the form, free from air pockets and roid spaces. If the finish is to be one that will expose the coarse aggregate, either by scrubbing, tooling, sand-blasting, or acid treatment, then after the full surface of mortar has been worked against the form, the coarse aggregate shall be spaded against the form to secure a uniform distribution at the face and a uniform texture of the exposed aggregate in the finished surface.

(f) The forms shall be removed from exposed surfaces as soon as the proper setting of the cement will permit, all fins and other projections carefully removed and offsets leveled; all voids and damaged places shall be immediately saturated with water, filled with a mixture of the same composition as the concrete and smoothed even with the surface with a wooden float or spatula. A steel trowel shall not be used for this purpose. Plastering and brush coating will not be permitted. The surface shall be finished free from streaks, discolorations, and other imperfections.

$(g)$ Whenever the forms are removed before the concrete has properly set, the surface shall be immediately wetted and kept wet for not less than three days.

Top Surfaces Not Subject to Wear

Top surfaces not subject to wear shall be smoothed with a mood float and be kept wet for at least seven days. Care shall be taken to avoid an excess of water in the concrete, and to drain or otherwise promptly remove any water that comes to the surface. Dry cement, or a dry mixture of cement and sand, shall not be sprinkled directly on the surface.

\section{Wearing Surface Finish}

\section{1-COURSE WORK}

Aggregates for the wearing surface in 1-course mork shall have a high resistance to abrasion, and shall be screened and when necessary thoroughly washed. The least quantity of mixing water that will produce a dense concrete shall be used. The mix shall not be leaner than 1 part of Portland cement and $2 \frac{1}{2}$ parts of aggregate. The surface shall be screeded even and finished with a wooden float. Excess water shall be promptly drained or otherwise removed. Overtroweling shall be avoided.

\section{2-COURSE TORK}

The rearing surface in 2-course work shall be placed within one-half hour after the base course. Where the mearing surface is required to be applied to a hardened base course, the latter shall be prepared by roughening with a pick or other effective tool. The roughened surface shall be thoroughly saturated with water and covered with a thin layer of neat cement paste immediately before the wearing surface is placed. The wearing course shall not be thinner than 1 inch.

\section{CURING}

Concrete rearing surfaces of roads and pavement made in accordance with the two previous paragraphs shall be kept wet for at least 21 days.

\section{Decorative Finishes}

RUBBED FINISH

Immediately after the forms are removed and all voids filled, the surface shall be thoroughly wetted and rubbed with carborundum brick, or other abrasive, to a smooth, even finish of uniform appearance without applying any cement or other coating.

\section{SCRUBBED FINISH}

The forms shall be removed while the concrete is still green and the surface scrubbed with stiff fiber or mire brushes, using water freely, until the aggregate is uniformly exposed to the desired extent. The whole surface shall then be thoroughly washed with clean water. If portions of the surface have become too hard to scrub to equal relief, or the film of cement is not removed from the surface of the exposed aggregate, diluted hydrochloric acid (1 part acid to 4 parts water) may be used to facilitate the scrubbing. All traces of the acid shall be thoroughly removed with clean water.

\section{SAND-BLAST FINISH}

In preparing the surface for a sand-blast finish, the concrete face shall be permitted to attain an intermediate degree of hardness; it shall then be air blasted with hard sand until the aggregate is in uniform relief.

\section{TOOLED FINISH}

The surface shall first be permitted to become dry and hard, and then dressed with tools, as called for, to a uniform texture and even face.

\section{SAND-FLOATED FINISH}

The form shall be removed before the surface has fully hardened; the surface shall be rubbed with a wooden float by a uniform circular motion, fine sand being rubbed into the surface until the resulting finish is even and uniform. 


\section{ACID-TREATED FINISH}

After the forms are removed and all voids filled, the surface shall be washed with commercial hydrochloric or nitric acid, diluted with water to a strength such that the bonding of the cement is not broken beyond the required depth. The solution shall be applied with stiff vegetable fiber brushes and the surface scrubbed until the aggregate is exposed to the desired extent. All traces of the acid shall then be quickly and completely washed off with water to prevent further action and permanent discoloration of the surface.

\section{COLORED PIGMENT FINISH}

Mineral pigment when used shall be thoroughly mixed dry with the cement and fine aggregate; care shall be taken to secure a uniform tint throughout.

\section{DESIGN}

\section{GENERAL ASSUMPTIONS}

The design of reinforced concrete members under these specifications shall be based on the following assumptions:

(a) Calculations are made with reference to working stresses and safe loads rather than with reference to ultimate strength and ultimate loads.

(b) A plane section before bending remains plane after bending, shearing distortions being neglected.

(c) The modulus of elasticity of concrete in compression is constant within the limits of working stresses and the distribution of compressive stress in beams is rectilinear.

(d) The moduli of elasticity of concrete in computations for the position of the neutral axis, for the resisting moment of beams, and for compression of concrete in columns, are as follows:

(1) One-fifteenth that of steel, when the compressive strength of the concrete at 28 days exceeds 1,500 and does not exceed 2,200 lbs./in.2.

(2) One-twelfth that of steel, when the compressive strength of the concrete at 28 days exceeds 2,200 and does not exceed 2,900 lbs./in. ${ }^{2}$.

(3) One-tenth that of steel, when the compressive strength of the concrete at 28 days is greater than 2,900 lbs./in.2.

(e) In calculating the moment of resistance of reinforced concrete beams and slabs the tensile resistance of the concrete is neglected.

( $f$ ) The bond between the concrete and the metal reinforcement remains unbroken throughout the range of working stresses. Under compression the two materials are therefore stressed in proportion to their moduli of elasticity.

(g) Initial stress in the reinforcement due to contraction or expansion of the concrete is neglected, except in the design of reinforced concrete columns.

American Railway Engineering Association, specifications for buildings for railway purposes, 1926 .

\section{MIORTAR FOR USE IN BRICKWORK}

(See A. R. E. A. specification for brickwork, 518.83$, p. 475.$)$

American Railway Engineering Association, specifications for buildings for railway purposes, 1926 .

\section{MORTAR FOR CEMENTING PIPE JOINTS}

(See specification for sewers and drainage, 518.67, p. 453.)

American Railway Engineering Association, specifications for buildings for railway purposes, 1926.

\section{MOKTAR FOR USE WITH CONCRETE ARCHITECTURAL STONE}

(See A. R. E. A. specification for concrete architectural stone, 516.4, p. 319 .)

American Railway Engineering Association, specifications for buildings for railway purposes, 1926 .

\section{HORTAR FOR USE IN STONE MASONRY}

(See A. R. E. A. specification for stone masonry and cut-stone work, 518.89, p. 478 .)

American Railway Engineering Association, specifications for buildings for railway purposes, 1926.

\section{MORTAR FOR USE WITH ARCHITECTURAL TERRA COTTA}

(See A. R. E. A. specification for architectural terra cotta, 534.24, p. 620.)

American Railway Engineering Association, specifications for buildings for railway purposes, 1926 .

\section{MORTAR FOR USE WITH CLAY HOLLOW TILE}

(See A. R. E. A. specincation for clay hollow tile, 534.22, p. 614 .)

American Society for Municipal Improvements, standard specifications for sewers, 1927.

\section{CONCRETE}

(See 518.67 , p. 453 )

American Society for Municipal Improvements, standard specifications for sewers, 1927.

\section{MORTAR}

(See 518.67, p. 453.)

Associated Tile Manufacturers, basic specification for tile work.

\section{CONCRETE AND MORTAR}

(See 534.20, p. 594.)

Common Brick Manufacturers' Association of America, specifications and general schedule of a brick house.

\section{MORTAR}

Lime mortar shall consist of 1 part slaked lump lime, or 1 part hydrated lime to $2 \frac{1 / 2}{2}$ parts of sand by volume.

Cement-lime mortar shall consist of 1 part lime as above and 1 part Portland cement to 6 parts sand by volume. Mix sand and cement together dry.

Cement mortar shall consist of 1 part Portland cement to 3 parts of sand by volume to which may be added lime in the proportion of 1 part of lime to 7 parts of Portland cement.

Grout for basement paving shall be mixed of the same material in the same proportion as specified for cement mortar mixed to a thin consistency so that it will run down to the bottom of the joints.

Natural cement when used instead of Portland cement in above mortars shall be properly proportioned in accordance with the directions of the manufactures.

All mortar containing cement shall be mixed immediately before it is required for use, and no such mortar shall be used after initial set has taken place.

Concrete Reinforcing Steel Institute, specifications for reinforced concrete in structures, 1928.

\section{Section 1. DEFINITION OF TERM}

The term "reinforced concrete" shall be understood to mean an approved concrete mixture in which steel bars of suitable area and shape are 
embedded for the purpose of acting with the concrete in resisting stress.

\section{Section 2. DRAWINGS}

The plans accompanying these specifications shall show typical details of all reinforced concrete construction. Detailed drawings showing number and location of bars, including complete bar lists and bending diagrams, shall be prepared by the contractor and submitted in duplicate form to the architect) for his approval. One copy of such approved drawings will be retained by him as a permanent record.

\section{Section 3. DEFINITIONS}

Aggregate.-Inert material which is mixed with Portland cement and water to produce concrete; in general, aggregate consists of sand, pebbles, gravel, crushed stone, or similar materials.

Anchorage. - The embedment in concrete of a portion of a reinforcement bar, either straight or with hooks, designed to prevent pulling out or slipping of the bar when subjected to stress.

Concrete.-A mixture of Portland cement, fine aggregate, coarse aggregate, and water. (See Mortar.)

Consistency. - A general term used to designate the relative plasticity of freshly mixed concrete or mortar.

Crushed stone.-Bedded rock or bowlders, which have been broken by mechanical means into fragments of varying shapes and sizes.

Deformed bar- Reinforcement bars with closely spaced shoulders, lugs, or projections formed integrally with the bar during rolling so as to firmly engage the surrounding concrete.

Gravel.-Rounded particles larger than sand resulting from the natural disintegration of rocks. (See Sand.)

Laitance.-Extremely fine material of little or no hardness which may collect on the surface of freshly deposited concrete or mortar, resulting from the use of excess mixing water, and usually recognized by its relatively light color.

Mixed aggregate. - A combination of sand and crushed stone, sand and pebbles, or sand and gravel. Mortar.-A mixture of Portland cement, fine aggregate, and water. (See Concrete.)

Plain concrete.-Concrete without metal reinforcement.

Portland cement.-The product obtained by finely pulverizing clinker produced by calcining to incipient fusion an intimate and properly proportioned mixture of argillaceous and calcareous materials, with no additions subsequent to calcination excepting water and calcined or uncalcined gypsum.

Sand.-Small grains resulting from the natural disintegration of rocks. (See Gravel.)

Screen.-A metal plate with closely spaced circular perforations. (See Sieve.)

Sieve.-Woven-wire cloth with square openings. (See Screen.)

\section{Section 4. CONCRETE MIXTURES}

Concrete in reinforced concrete construction when mixed in the following proportions, stated by volume, with aggregates which are clean and structurally sound, with water-cement ratios as indicated, shall be assumed to develop a laboratory cylinder compressive strength at an age of 28 days as follows:
Assumed strength of concrete mixture

PLASTIC CONCRETE

\begin{tabular}{|c|c|c|}
\hline $\begin{array}{c}\text { Water-cement } \\
\text { ratio United States } \\
\text { gallons per 94- } \\
\text { pound sack of } \\
\text { cement }\end{array}$ & $\begin{array}{l}\text { Approximate } \\
\text { mix volume of } \\
\text { Portland } \\
\text { cement to sum } \\
\text { of separate } \\
\text { volumes of fine } \\
\text { and coarse } \\
\text { aggregate as } \\
\text { measured dry }\end{array}$ & $\begin{array}{l}\text { Assumed } \\
\text { compressive } \\
\text { strength at } 28 \\
\text { days (in } \\
\text { pounds per } \\
\text { square inch) }\end{array}$ \\
\hline $\begin{array}{l}81 / 4 \ldots \ldots \\
71 / 2 \ldots \ldots \\
63 / 4 \ldots \\
6 \ldots \ldots-n\end{array}$ & $\begin{array}{l}1-7 \\
1-6 \\
1-51 / 1 \\
1-41 / 2\end{array}$ & $\begin{array}{l}1,500 \\
2,000 \\
2,500 \\
3,000\end{array}$ \\
\hline \multicolumn{3}{|c|}{ MODERATELY WET CONCRETE } \\
\hline $\begin{array}{l}81 / 4 \ldots \ldots \\
71 / 2-\ldots \\
630 \\
6 \\
6\end{array}$ & $\begin{array}{l}1-61 / 2 \\
1-51 / 2 \\
1-43 / 4 \\
1-4\end{array}$ & $\begin{array}{l}1,500 \\
2,000 \\
2,500 \\
3,000\end{array}$ \\
\hline
\end{tabular}

In interpreting the above table, water, or moisture contained in the aggregate must be included in the mixing water in computing the water-cement ratio.

(a) In no case shall concrete for any assumed strength be placed with a watel-cement ratio exceeding that shown. Where the aggregates are such that the mixes shown do not produce proper workability with the given water-cement ratio, the mixes shall be changed, but not the water-cement ratios.

(b) The graded sizes of the combined aggregate shall be such that when separated on a No. 4 standard square-mesh sieve, the amount retained sliall not be less than the amount passing, nor more than twice the amount passing.

(c) The structural plans show the proposed strength of concrete to be used, and the water-cement ratio necessary to produce that strength is indicated above. The water required in all cases includes that contained in the aggregates.

(d) When the above table is not used the strengtl of concrete must be predetermined. The strength used to carry out the design will be assumed to be equal to 80 per cent of the strength determined by tests conforming to the requirements of section 7 . These tests must be sufficient in number to satisfy the - as to their reliability. Tests must be continued throughout construction work. No concrete placing may proceed under this paragraph without an order in writing froin the approving the mixes to be used.

\section{Section 5. QUALITY OF MATERIALS}

All materials used in the work covered by this specification shall conform to the following requirements when tested. Such tests shall be made in accordance with the standard method of test, covering the particular material under consideration, of the American Society for Testing Materials in effect on the date of the adoption of these regulations by the and shall be made by competent persons.

(a) Steel.- New billet reinforcing steel is to be of intermediate grade, and shall conform to the specifications of the American Society for Testing Materials, serial designation A15-14. Material for spiral hooping shall have a yield point of not less than that required for intermediate grade steel.

(b) Cement.-Portland cement shall conform to the standard specifications and tests for Portland 
cement of the American Society for Testing Materials (serial designation C9-26).

(c) Sand.-All sand used for concrete aggregate shall be clean, sharp, and hard, containing not over 2 per cent of clay or loam and free from organic matter. It shall be graded from fine to coarse, but no material retained on a No. 4 standard squaremesh sieve shall be considered sand.

(d) Crushed stone or gravel.-All crushed stone or gravel for concrete aggregate shall be clean and hard, free from soft particles, and shall all be retained on a No. 4 standard square-mesh sieve. For beams, columns, solid slabs, flat slabs, and medium-sized sections, the maximum size of coarse aggregate shall consist of particles passing a 1-inch square-mesh sieve. For mass concrete, or for large reinforced concrete footings, the maximum size of coarse aggregate shall consist of particles passing a 2 -inch squaremesh sieve. For joists, thin slabs, thin or ornamental sections, or where fireproofing only is required, the maximum size of coarse aggregate shall consist of particles passing a one-half inch square-mesh sieve. In all cases where sizes of coarse aggregate are specified, there shall be a 5 per cent oversize allowed, larger than any specified maximum, and a 10 per cent undersized allowed, smaller than any specified minimum.

(e) Mixed aggregates.-All mixed aggregate used for concrete shall consist of a uniform mixture of sand and broken stone, sand and pebbles, or sand and gravel of such composition that when separated on a No. 4 standard square-mesh sieve the amount retained shall not be less than the amount passing, nor more than twice the amount passing. Material retained on a No. 4 standard square-mesh sieve shall conform to the specifications for "Crushed stone or gravel" and all passing to that for "Sand."

(f) Water.-Clean water, free from strong acids, alkalis, or organic materials shall be used in all concrete.

(g) Integral compounds.-Integral compounds to be used in concrete must first be approved by the

\section{Section 6. PROPORTION OF INGREDIENTS AND MIXING CONCRETE}

Concrete mixtures shall be designed according to the water-cement ratio theory to give the required strength as called for in the design and as required under section 4 .

(a) The builder superintendent shall keep proper sieves at the work and shall test materials whenever necessary or required, and shall be responsible at all times for the proper gradation of the aggregate and field control of the concrete.

(b) The methods of measuring materials shall be such that the proportions of concrete materials and water can be accurately controlled and easily checked during the progress of the work. The proportions of materials shall be such that they will not produce harshness in placing or honeycombing in the structure.

(c) The superintendent shall furnish a standard slump cone and will make frequent tests of the consistency of the concrete mixture as poured. The consistency shall be such as to produce a mixed concrete that can be puddled readily into the corners and angles of the forms and around reinforcement without excess of spading and without segregation or undue accumulation of water or laitance on the surface. This consistency shall be maintained at all times, but, while the mix must be workable, sufficient cement shall bc used to provide the strength for which the mixture was designed as specified in section 4 .

(d) No materials or liquids other than those conforming to the matcrial specifications herein incor- porated shall be used in concrete except when written permission is given by the

(e) Mixing concrete. - All mixing shall be done by machine if the total amount of concrete exceeds 50 cubic yards.

(f) The use of mechanical devices such as are approved by the ……_. to regulate automatically the water-cement ratio or to control the bulking of the aggregates is consistent with the provisions of these specifications.

(g) The use of central mixing plants is also consistent with the provisions of these specifications. Such provisions as apply to quality of materials, proportion of ingredients, mixing concrete, and placing concrete apply in their entirety to such plants. Where the interval between mixing and final placement in forms does not exceed 45 minutes, their use is not further restricted.

(h) The concrete shall be mixed until there is a uniform distribution of the materials, and the mass is uniform in color, and homogeneous. In machine mixing, only batch mixers of one-sack capacity or greater shall be used. Each batch shall be mixed at least one minute after all the materials are in the mixer and the mixer must be completely discharged before recharging.

(i) All concrete shall be placed immediately after mixing, and any concrete having a partial set before placing shall not be used in any portion of the work and shall not be returned to the mixer.

\section{Section 7. TESTS}

The contractor will be required to take test cylinders at the direction and under the supervision of the superintendent. These will be tested at the end of 7 days or 28 days, and the average ultimate unit compressive strength of the cylinders from each section of the structure must equal the strength for which the concrete was designed. All tests shall be made in accordance with the standard methods of making compression tests of concrete of the American Society for Testing Materials, serial designation C39. (See 516.0, p. 284.) In case of 7 -day tests the average strength must equal that given in the following table:

\begin{tabular}{|c|c|}
\hline $\begin{array}{c}\text { Assumed ulti- } \\
\text { mate compres- } \\
\text { sive strength } \\
\text { of concrete at } \\
\text { age of } 28 \text { days }\end{array}$ & $\begin{array}{c}\text { Assumed ulti- } \\
\text { mate compres- } \\
\text { sive strength } \\
\text { of concrete at } \\
\text { age of } 7 \text { days }\end{array}$ \\
\hline Lbs./in.2 & Lbs./in.2 \\
1,500 & 800 \\
2,000 & 1,100 \\
2,500 & 1,400 \\
3,000 & 1,800 \\
\hline
\end{tabular}

(a) In case of 7-day test, if the specimens fail to pass the above values, additional cylinders shall be retained for a test at the age of 28 days. If the additional tests indicate concrete of materially lower strength than that specified, the may require load tests of the structure itself.

(b) Samples shall be tested in the form of a cy]inder 6 inches in diameter by 12 inches long or 8 inches in diameter by 16 inches long.

\section{Section 8. CENTERING, FORMS AND CONDUITS}

All forms shall be built of sufficient strength and rigidity; and be thoroughly braced to safely support the loads which may come upon them during the course of construction without undue deflection. They shall have tight joints to prevent any appreciable part of the concrete mixture from escaping. Wooden forms, when used, shall be of dressed lumber. 
(a) In the case of column or wall forms, a suitable means shall be provided to permit the forms to be thoroughly cleaned, and immediately before the concrete is placed, all chips, ice, and other foreign material shall be removed. In the case of wooden forms, an opening shall be left in the bottom for cleaning the forms and adjusting the steel and these openings shall not be closed until the concrete is about to be placed.

(b) Side forms for beams, girders, walls, or columns shall not be removed within 6 days after placing concrete except in cases where such forms do not carry any vertical load, when they may be removed 24 hours after placing concrete.

(c) If ample shores are used to carry the full weight of the horizontal members, supporting member forms and removable steel tile may be removed as soon as the concrete has set hard, but in no case less than 24 hours after placing concrete.

(d) Before removing the shores under any horizontal member, the supporting member shall be stripped so that it may be examined on all sides.

(e) The minimum time which shall elapse before removing the shores or centering will vary with the design and conditions of the weather, but in no case shall be less than 15 days under ideal curing conditions.

(f) (See note.)

Note.-A clause should be inserted at this point specifying the actual dates between which this minimum time of removal of shores may be permitted. For the latitude of New York and Chicago, the dates of A pril I and November 15 are suggested. This clause should also ing the winter between the critical dates. A minimum of 25 days is suggested for the latitudes of New York and Chicago.

(g) During the period mentioned in subparagraph $(f)$ no forms may be removed in less than specified time unless permission is first obtained in writing from the to remove forms in less than the time specified herein. This permission will only be given in case conditions warrant such action, and provided that in the opinion of the

proper precautions are being taken, and that responsibility for accidents is assumed by the contractor. In no case, however, shall shores be removed in less than 10 days during the summer period above stated.

(h) The times given above are minimum times in all cases, and in no case shall shores be removed before the concrete is set hard and has sufficient strength to safely carry its own weight and all additional loads upon it or about to be put upon it.

(i) The builder, or his representative in charge of concrete construction, shall always be present during the removal of forms and shores, and shall personally be responsible for the safety of this operation at all times and under all conditions. During the winter months, no shores shall be removed until portions of the concrete have been chipped off, removed to a warm place, and tested for frost or ice.

(j) Electrical conduits or other pipes built in concrete construction shall not be of such character or so located as to reduce the strength of the construction. Conduits less than 1 inch in diameter will not be regarded as reducing the strength of concrete in compression, but when larger conduits are placed, special provisions must be made to distribute the stresses.

\section{Section 9. PLACING OF REINFORCEMENT}

Shop drawings, as specified in section 2, shall be submitted to the for approval before beginning fabrication of reinforcing materials. It is recommended that bars be shop fabricated. All reinforcing steel must be placed in accordance with the following requirements: (a) Bars shall be clean and sound-Metal reinforcement before being placed shall be free from loose rust scale, grease, clay, or other coatings or foreign substances that will destroy or reduce the bond. Steel bars shall not be bent or straightened in a manner that will injure the material. Bars with kinks or bends not shown on the plans shall not be used.

(b) Bars shall be formed to correct dimensions.Reinforcement shall be carefully formed to the dimensions indicated. Lengths, depths, and radii as shown on the bending details must be correctly reproduced.

(c) Bars shall be properly spaced.-The minimum center to center distance between parallel bars shall be two and one-half times the diameter for round bars or three times the side dimension for square bars. In no case shall the spacing between bars be less than 1 inch nor less than one and one-third times the maximum size of the coarse aggregate.

(d) Bars shall be continuous. - No splices of reinforcing bars shall be made without the approval of the architect or engineer, except where shown on the plans. Deformed bars shall be lapped a distance of 40 bar diameters, except that in columns and as longitudinal temperature reinforcing in walls or footings under walls they shall be lapped 24 bar diameters, but not less than 18 inches. In all splices the bars shall be spaced at the minimum distance specified in paragraph $(c)$.

(e) When required, bars shall be specially anchored.--Special anchorage shall consist of one of the following:

1. (a) In case the end of a beam or slab is not continuous, carrying the bottom reinforcement approximately 14 diameters beyond the face of support.

(b) In case the end of beam or slab is continuous, carrying the top reinforcement approximately 14 diameters beyond the one-fifth point of the adjoining clear span.

2. Bending the reinforcement around another bar or steel shape so placed that it will resist any longitudinal movement of the steel to be anchored.

3 . Bending the bar into a semicircular hook which has a radius large enough to insure proper bearing on the concrete.

(f) Bars shall be properly embedded.-All reinforcing steel shall have a protection of concrete not less than the following:

1. Three inches on bottoms of footings.

2. Two inches where concrete is exposed to action of weather or ground.

3 . One and one-half inches over all reinforcement in columns.

4. One and one-half inches on the bottom and sides of beams or girders.

5. Three-fourths inch on bottoms and sides of ribs and bottoms of all floor slabs.

6. Three-fourths inch on the bottoms of all flat slabs.

7. Three-fourths inch from the faces of all walls not exposed to action of weather or ground.

8. One diameter over all bars at the upper face of any member.

(g) Bars shall be accurately located and firmly held.-All reinforcing steel shall be accurately located in the forms and firmly held in place before and during the pouring of concrete, by means of metallic supports, spacer bars, wires, or other devices adequate to insure against displacement during the course of construction, and to keep the steel at the proper distance from the forms. Bar supports and spacers are to be sufficient in number, and sufficiently heavy to properly carry the steel 
they support. The number shall not be less than the following:

\section{Slabs}

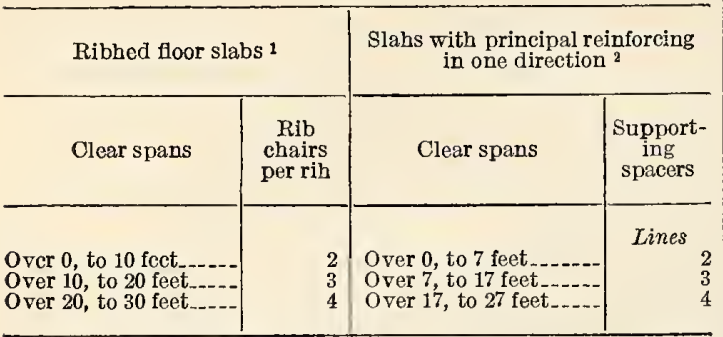

1 In addition to the above standard number of har supports and spacers it is recommended that the - specify the following: To support ends of hent hars in rihs, use har not less than $3 / 8$ inch diameter in each side of each beam crossed, supported ahove permanent or removable tile.

2 In addition to the ahove standard numher of bar supports and
nent or removable tile.

2 In addition to the ahove standard numher of bar supports and
spacers, it is recommended that the specify the following: spacers, it is recommended that the
To support cnds of hent hars in slahs, use har of not less than $1 / 2$ inch diameter on each side of each beam crossed, carried hy high chairs 3 feet apart.

The hars recommended to bc used in both footnotes will be found helpful in supporting the stirrups in the heams also. To he properly supported hy these hars, the stirrups should he bent into a $90^{\circ}$ bend instead of the usual hook. The supporting hars may then carry hoth the hent slab hars and the stirrups, and hold all steel rigidly in place. Care must he taken not to place these supporting bars too far from the edge of heam to allow them to operate for this dual purpose.

\section{Beams}

ORDINARY BEAMS (BARS 1 INCH SQUARE AND SMALLER)

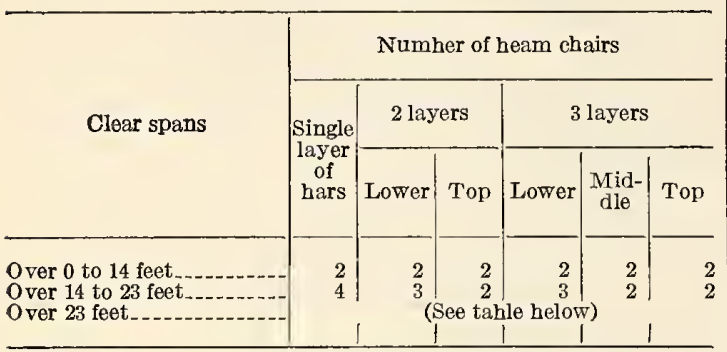

BEAMS OR SLABS (WITH LARGE NUMBER OF 11/8 OR $11 / 4$ INCH BARS)

\begin{tabular}{|c|c|c|c|c|c|c|}
\hline \multirow{3}{*}{ Clear spans } & \multicolumn{6}{|c|}{ Number of beam chairs } \\
\hline & \multirow{2}{*}{$\begin{array}{l}\text { Single } \\
\text { layer } \\
\text { of } \\
\text { hars }\end{array}$} & \multicolumn{2}{|c|}{2 layers } & \multicolumn{3}{|c|}{3 layers } \\
\hline & & Lower & Top & Lower & $\underset{\mathrm{dle}}{\mathrm{Mid}-}$ & Top \\
\hline $\begin{array}{l}\text { Over } 0 \text { to } 15 \text { feet.-. } \\
\text { Over } 15 \text { to } 30 \text { feet.- } \\
\text { Over } 30 \text { to } 45 \text { feet.- } \\
\text { Over } 45 \text { to } 60 \text { feet.- }\end{array}$ & $\begin{array}{l}2 \\
4 \\
6 \\
8\end{array}$ & $\begin{array}{l}3 \\
5 \\
7 \\
9\end{array}$ & $\begin{array}{l}2 \\
2 \\
3 \\
3\end{array}$ & $\begin{array}{r}3 \\
6 \\
9 \\
11\end{array}$ & \begin{tabular}{l|}
2 \\
2 \\
3 \\
3
\end{tabular} & 3 \\
\hline Spacing of & \multicolumn{6}{|c|}{ Beam chairs in spans other than ahove } \\
\hline All spans_-_. & $\begin{array}{cc}\text { Ft.in. } \\
8 & 0\end{array}$ & Ft. in. & $\begin{array}{ll}\text { Ft. in } \\
14 & 0\end{array}$ & Ft. in. & 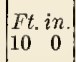 & Ft. in \\
\hline
\end{tabular}

Flat slabs

TWO AND FOUR WAY FLAT SLABS

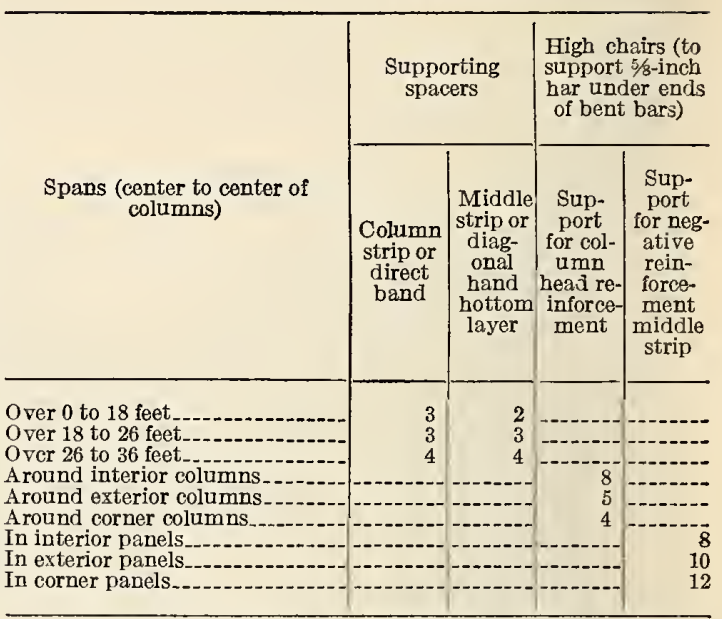

Note.-In roof slabs use one more supporting spacer under column trips, direct bands, and hottom layers of middle strips or diagonal bands, and one more $5 / 8$-inch chair har at column hesds than the number shown in the tahle ahove.

\section{Spirals}

\section{COLUMNS WITH SPIRAL HOOPING}

\begin{tabular}{|l|r|}
\hline Core diameter (outside to outside) & $\begin{array}{r}\text { Number } \\
\text { of spacers }\end{array}$ \\
\hline Over 0 to 24 inches._._. & 2 \\
Over 24 inches. & 3 \\
\hline
\end{tabular}

\section{Section 10. PLACTNG OF CONCRETE}

All concrete shall be so mixed that it will not contain any more water than is necessary to enable it to properly fill forms and surround reinforeing and no excess of water shall appear upon the surface without troweling.

(a) Before placing concrete each piece of reinforcement shall be in its proper position and be held until the placing is complete.

(b) Before placing concrete ail equipment for mixing and transporting the concrete shall be cleaned and all débris or ice shall be removed from the space to be occupied by the concrete; forms shall be thoroughly wetted (except in freezing weather) or oiled, and clay tile that will be in contact with concrete shall be well drenched (except in freezing weather).

(c) All excavations for placing of footings shall be drained of water before placing concrete unless otherwise directed in writing by the

(d) When concreting is once started it shall be carried on as a continuous operation until the section started is completed. If, for any reason, the concreting be stopped, care shall be taken to stop the work at such a point that the joint formed when the work is resumed will not weaken the member structurally. 
(e) Concrete shall be transported from the mixer to the place of final deposit as rapidly as is practicable by methods which will prevent the separation, segregation, or loss of the ingredients in transportation. It shall be deposited as nearly as practicable in its final position to avoid rehandling or flowing.

$(f)$ Collerete shall not be spouted directly into the forms but may be spouted into a movable bucket.

(g) Concrete shall be thoroughly compacted by puddling with suitable tools. In thin wall or inaccessible portions of the forms where rodding is impracticable the concrete shall be worked into place by tapping or hammering forms adjacent to the freshly deposited concrete.

(h) All vertical members shall be filled at least two hours ahead of the horizontal members to allow the concrete in the vertical members to take its initial settlement. The placing of vertical member concrete shall be in one continuous operation to the level of the bottom of the horizontal member supported by it.

(i) In placing vertical members the concrete shall be kept well puddled with a long pole or tamped to prevent voids and honeycombing. Filling the vertical members completely and puddling afterwards will not be allowed.

(j) When fresh concrete joins concrete that is set or partially set, the exposed surface of the old eoncrete shall be thoroughly cleaned, roughened, and have applied to it a coating of cement mortar, mixed in proportion of 1 part of cement to 1 part sand, before any more concrete is placed.

(k) All beam concrete shall be placed monolithic with the adjacent slab; that is, placed continuously from the bottom of the beam to the top of the slab.

(l) When a section of floor concrete is once placed, it shall be left entirely undisturbed until the concrete has thoroughly hardened.

$(m)$ Such surfaces as are to be finished must be brought to proper grade, struck off, and finished in a workmanlike manner. No honeycombing, rough spots, or protruding stones shall be left exposed. In case of floors, precautions must be taken to be sure that the thickness shown on the plans exists at all places.

(n) Where any deposits of laitance occur, proper provision must be made for their removal before finishing or before attempting to bond any further concrete to the surface so affected.

(o) The builder's superintendent in charge of concrete work shall mark in ink on the drawings the time and the date of the placing of concrete in the different members. Such drawings shall be kept on file at the job until the completion of the structure and shall be subject to the inspection of the at all times.

\section{Section 11. FREEZING WEATHER}

Concrete when deposited shall have a temperature of not less than $40^{\circ} \mathrm{F}$. and not more than $120^{\circ} \mathrm{F}$. In freezing weather suitable means shall be provided for maintaining the concrete at a temperature of at least $50^{\circ} \mathrm{F}$. for 72 hours or more after placing, or until the concrete has thoroughly hardened. The methods of heating the materials and protecting the eoncrete shall be approved by the - Salt, chemicals, or other foreign materials shall not be mixed with the concrete for the purpose of preventing freezing unless first approved in writing by the

(a) Manure shall not be used for protection when applied directly to concrete. All forms shall remain until assurance is had that all frost has left the conerete and it has developed sufficient strength to safely permit their removal. (b) No frozen material or materials containing ice shall be used.

(c) The use of chemicals or other materials to reduce the freezing point of materials used in concrete which, in the opinion of the - are injurious to the concrete, shall not be permitted.

(d) All methods and materials used for concreting in freezing weather shall be subject to the approval of the

\section{Section 12. HOT WEATHER}

Concrete laid during hot weather shall be thoroughly wet with clean water twice daily during the first week after placing.

\section{Section 13. CURING}

Exposed surfaces of concrete shall be kept moist for a period of at least seven days after being deposited, except when the has directed otherwise in writing.

\section{Section 14. SUPERINTENDENT}

All reinforced concrete construction shall be performed under the personal and constant supervision of a competent building superintendent experienced in this class of work, who must be approved by the - and may be removed for reasonable cause upon written notice.

\section{Section 15. EXPOSED CONCRETE}

Concrete which is exposed to weathering or comes in direct contact with water or chemicals shall be mixed and placed with special precautions so as to obtain the densest possible mass.

(a) Special precautions with regard to puddling the mixture must be observed so that no honeycomb pockets are left exposed.

(b) "Plastic mass concrete" is of relatively dry consistency and difficult to work into forms where considerable steel obstructs the flow of the material. If this consistency should be used, extreme precautions must be taken to insure a dense surface.

(c) Such surfaces as are so designated must in addition, be treated as indicated in sections 16 and 17.

\section{Section 16. WATERPROOFING}

Concrete required to be water-tight shall be made with the minimum amount of water consistent with proper work ability. It shall be made of materials which are themselves waterproof and shall be placed in position and puddled in a manner that will prevent segregation or the formation of voids. ${ }^{66}$

\section{Section 1\%. TREATMENT OF CONCRETE SURFACES}

Note.-Where special surface treatments are designated, they shall be made with reference to the standard specifications prepared by Committee $\mathrm{C}-3$ of the American Concrete Institute as revised from time to time. 0 ?

\section{Section 18. FLOORS AND CONCRETE FLOOR FINISH}

Note.-All floors and special floor finishes as designated shall be made with reference to the standard specifications prepared by Committee $\mathrm{C}-2$ of the American Concrete Institute as revised from time to time. ${ }^{59}$

56 Where the concrete is exposed to hydrostatic pressure, some recognized method of waterproofing shall be specified (such as mombranous or surface treatment waterproofing). Here insert specifcation for specific treatment desired.

37 Here insert specification for specific treatments desired.

68 Here insert specifications for floor and specific finishes desired 


\section{Section 19. SPECIAL PROVISIONS 50}

\section{Section 20. DISPUTES}

In case of discrepancies between the drawings and the specifications, the specifications shall govern; and in case of discrepancies between the scaled dimensions on the drawings and the figures written on them, the figures shall govern. Any questions regarding reinforced concrete not covered in these specifications or any questions as to any portions of these specifications shall be decided by reference to the Handbook on Reinforced Concrete published by the Concrete Reinforcing Steel Institute, Standard Building Regulations for Reinforced Concrete as developed jointly by the Concrete Reinforcing Steel Institute and the American Concrete Institute, such Committee Reports of the American Concrete Institute as are pertinent, and the Report of the Joint Committee on Standard Specifications for Concrete and Reinforced Concrete. These references shall be considered authoritative in the order in which they are mentioned.

\section{Section 21. CODE OF STANDARD PRACTICE}

These specifications are written in accordance with the Code of Standard Practice and the Specifications for Placing Reinforcement of the Concrete Reinforcing Steel Institute, published 1927, and all practices not herein specifically mentioned must conform to the provisions of said code and specifications.

Federal Specifications Board, specification No. 444a, United States Government master specification for integral waterproofing material, March 5, 1928.

\section{TYPES}

The materials covered by this specification are intended to be mixed with Portland cement mortar or concrete for the purpose of rendering them more completely impermeable and water repellent.

\section{MATERIALS AND WORKMANSHIP}

The materials shall be stable compounds not subject to disintegration either before or after incorporation with the mortar or concrete with which they are intended to be used.

\section{GENERAL REQUIREMENTS}

1. The material shall either mix readily with water or with dry Portland cement and shall become an insoluble integral part of the mortar or concrete.

2. The material when mixed with Portland cement mortar or concrete shall render such mortar or concrete more completely impervious to water and shall not impair their durability or reduce the compressive strength more than 10 per cent.

\section{DETAIL REQUIREMENTS}

The waterproofing properties shall be such that when combined in the manner, and in the proportions of waterproofing material to cement, specified by the manufacturers for use with Portland cement mortar and concrete, and tested as herein specified, the treated test pieces shall show an absorption of not more than 3 per cent and shall reduce the permeability (when tested under a pressure of 100 pounds) at least 95 per cent of test pieces made without the admixture of waterproofing materials, but in all other respects of identical materials, composition, and age.

\footnotetext{
${ }^{59}$ Here insert any special provisions desired.
}

\section{INSPECTION, SAMPLING, AND TESTS}

1. Test samples may be taken before shipment or after delivery at the discretion of the inspector. Representative samples shall be taken for at least every 500 pounds or 100 gallons of material. The samples when practicable shall be placed in a single container and sent to the laboratory as a composite sample representative of the lot under consideration.

2 . The appearance and chemical composition of the composite sample shall be recorded for comparison with those of samples from other shipments of materials of the same brand and make.

3 . The following tests shall be made for absorption, permeability, and compression.

4. Mortar for tests of waterproofing materials shall be made from Portland cement, and sand passing a No. 20 sieve and held on a No. 50 sieve, in the proportion of 1 volume of cement to 4 volumes of sand, with only sufficient water thoroughly worked to form a mixture which has a flow of 90 as measured by a cement laboratory flow table. All materials shall be mixed at, and the test pieces be stored in, a temperature of approximately $70^{\circ} \mathrm{F}$.

5. The waterproofing materials shall be incorporated in accordance with the methods and in the proportions to the amount of cement as specified by the manufacturers in their standard directions for the use of their materials.

6 . Test pieces shall be made at the same time and under the same conditions of both treated and untreatcd mortar. The permeability test pieces shall be made in the form of 1 inch thick disks in metal molds. The absorption test pieces shall be made in the form of 2 -inch cubes in metal molds. The molds shall be rcmoved after 24 hours.

7. The exposed surfaces of test pieces at the time of molding shall be struck off with a trowel, and 24 hours after molding all surfaces of the cubes and both faces of the disks shall be brushed with a wire brush to remove any neat cement brought to the surface in molding.

8. The test pieces shall be kept in a moist closet for 24 hours after molding, then be stored in a damp atmosphere for six days, after which period in the air of the laboratory.

9 . The tests shall be made when test pieces have aged 28 and 90 days. In each test each of the treated and untreated pieces shall be tested under identical conditions.

10. Pieces for the absorption tests shall be dried at $212^{\circ}$ to $230^{\circ} \mathrm{F}$. until no further loss in weight is shown. They shall then be allowed to cool in the air to room temperature. The pieces shall be completcly immersed in water for 24 hours, then be removed, the surfaces dried with a cloth, and weighed immediately. This process shall be repeated daily unil the pieces show less than one-tenth of 1 per ccnt daily increase in weight. The increased weight of the treated and the untreated pieces shall then be compared.

11. The impermeability tests shall be made by subjecting one side of the test picces to a water pressure of 100 pounds per square inch, using water free from any visible discoloration or matter in suspension. The test shall be continued 24 hours unless a measurable degree of permeability is shown in less time.

12. The degree of permeability shall be calculated in cubic centimeters of water passing through the piece per square centimeter of surface exposed to water pressure. The impermeability of the treated and the untreated pieces shall be then compared.

13. Compression tests shall be made of cubes that have been subjected to absorption tests. The cubes shall be tested to complete failure. The 
resistance to compression of the treated and the untreated pieces shall then be compared.

\section{PACKING, MARKING, AND SHIPMENT}

Materials for integral waterproofing shall be packed in suitable containers that will preserve the matelials in good condition. Each package shall be marked with the net weight or net liquid measure of the contents, and with the bland or trade name of the contents and the name of the manufacturer. Each order of the material shall be accompanied by printed directions for its use.

\section{NOTES}

1. The integral waterproofing materials should be mixed with the mortar or concrete with which it is to bs used in accordance with the printed directions of the manufacturer.

2. Treated mortar and concrete should be made with not more than 2 volumes of fine nor more than 4 volumes of coarse aggregate to each volume of cement. The aggregate should be so graded in size as to produce a mixture of maximum density. Only such a quantity of water should be used as necessary to make the completely mixed product sufficiently plastic for properly placing and finishing.

3 . In addition to various makes of integral waterproofing material, there are in the market brands of Portland cement with which a waterproofing material has been combined in its manufacture.

Joint Committee on Standard Specifications for Concrete and Reinforced Concrete.-This committee, consisting of affiliated committees of the American Society of Civil Engineers, American Society for Testing Materials, American Railway Engineering Association, American Concrete Institute and Portland Cement Association, submitted to constituent organizations August 14, 1924, a report on standard specifications for concrete and reinforced concrete.

The specifications are for the use of concrete in construction, including methods and materials under the chapter heads of (1) general instructions, (2) definitions, (3) quality of concrete, (4) materials, (5) proportioning and mixing concrete, (6) depositing concrete, (7) forms, ( 8 ) details of construction, (9) waterproofing and protective treatment, (10) surface finish, and (11) design.

The specifications given for materials include the following:

\section{PORTLAND CEMENT}

Portland cement shall conform to the standard specifications and tests for Portland cement of the American Society for Testing Materials, serial designation C9-26. (See 516.11, p. 294.)

\section{FINE AND COARSE AGGREGATE}

This specification is practically identical with American Society for Testing Materials, specification for aggregates, with the exception that in the A. S. T. M. specification for fine aggregate there is an added requirement that 100 per cent shall pass a $3 / 8$-inch sieve. Since publication of the joint committee specifications, the American Society for Testing Materials has revised its specification, C33-28T. (See 512.13, p. 237.)

\section{RUBBLE AND CYCLOPEAN AGGREGATE}

Rubble aggregate shall consist of clean, hard, durable stone or gravel larger than 3 inches and weighing not more than 100 pounds.

Cyclopean aggregate shall consist of clean, hard, durable stone or gravel weighing more than 100 pounds.
Aggregate shall be so stored as to avoid the inclusion of foreign materials. Frozen aggregate or aggregate containing lumps of frozen material shall be thawed before using.

Water for concrete shall be clean and free from injurious amounts of oil, acid, alkali, organic matter, or other deleterious substance.

National Sand and Gravel Association, Bulletin 3, representative specifications for different uses of sand and gravel, November, 1928.

(In this bulletin representative specifications, many of which are presented in this volume and including specifications for sand for brick mortar, have been summarized.)

Portland Cement Association, specifications for making, placing, and curing concrete in cold weather.

(This association has prepared the above-entitled specification to supplement regular concrete specifications and to be added thereto.)

United States Department of Agriculture, Bulletin 1216, Method of Proportioning Natural Sand and Gravel for Concrete Construction, 1928.

(See 512.10, p. 216.)

\subsection{CONCRETE BRICKS AND BLOCKS.}

American Association of State Highway Officials, standard specifications for highway bridges and incidental structures, 1928.

\section{CONCRETE CRIBBING}

(The full text of this specification includes construction methods and requirements for measurement and payment.)

\section{GENERAL}

The construction of concrete cribbing shall consist of the furnishing and installation of reinforced concrete crib members and the placing of the interior filling materials. Drift bolts shall be of wrought iron or galvanized steel not less than 1 inch in diameter and of the required length.

Casings for drift bolts shall be of galvanized steel or iron pipe not less than $1 \frac{1}{4}$ inches in diameter.

\section{GENERAL REQUIREMENTS}

The details of the crib members and their arrangement shall be as shown on the plans. If specific details for reinforcement are not shown on the plans, or if the contractor is permitted to purchase the crib members from manufacturers, he shall submit detailed specifications and plans for the approval of the engineer, and such plans must be approved before delivery of the material is begun.

All members shall be free from depressions and spalled, patched, or plastered surfaces or edges, or any other defect which may impair their strength or durability. Cracked or otherwise defective members will be rejected.

American Concrete Institute, standard specifications for concrete building block and concrete building tile, serial designation P-1A-29, 1929.

\section{GENERAI}

1. The purpose of these specifications is to define the requirements for concrete building block and concrete building tile to be used in construction.

2 . The word "concrete" shall be understood to mean Portland-cement concrete.

3. The average compressive strength of three or more concrete block or concrete building tile in pounds per square inch of gross cross-sectional area as laid in the wall shall not be less than 700 pounds, no one unit falling below 600 pounds 28 days after being manufactured or when shipped. 
Wherever concrete block or building tile are used to carry unusual heavy loads the average compressive strength of three or more of these units 28 days after being manufactured or when shipped in pounds per square inch of gross cross-sectional area as laid in the wall shall be at least ten times the figured superimposed load to be applied.

Nonload-bearing concrete block and concrete tile shall have sufficient strength necessary to prevent excessive breakage during delivery and handling.

4. The gross cross-sectional area of a 1-piece concrete block or tile shall be considered as the product of the length times the width of the unit as laid in the wall. No allowance shall be made for air spaces in hollow units. The gross cross-sectional area of each unit of a 2-piece block or tile shall be considered the product of the length of the unit times one-half the thickness of the wall for which the 2-piece block or tile is intended.

5. The compressive strength of the concrete in units of all classifications, except "nonload bearing block," shall be at least 1,000 lbs./in. ${ }^{2}$ when calculated on the minimum cross-sectional area in bearing.

6. Concrete building block and tile to be exposed to soil or weather in the finished work (without stucco, plaster, or other suitable protective covering) shall meet the requirements of the absorption test.

7. All concrete building block and tile not covered by paragraph 6 need not meet an absorption requirement.

8. Concrete block and tile shall not absorb more than 10 per cent of the dry weight of the unit when tested as hereinafter specified, except when it is made of concrete weighing less than $140 \mathrm{lbs} . / \mathrm{ft.}^{3}$ For block or tile made with concrete weighing Jess than $140 \mathrm{lbs} . / \mathrm{ft}^{3}$ the absorption in per cent by weight shall not be more than 10 multiplied by 140 and divided by the unit weight in pounds per cubic foot of the concrete under consideration.

9. Specimens for tests shall be representative of the commercial product of the plant.

10. Not less than three and preferably five specimens shall be required for each test.

11. The specimens used in the absorption test may be used for the strength test.

\section{METHODS OF TESTING}

Same as for A. C. I. P-1C-27T and P-1B-26T (given below) with the following additional:

The weight per cubic foot of the concrete in a block or tile is the weight of the unit in pounds divided by its volume in cubic feet. To obtain the volume of the unit fill a vessel with enough water to immerse the specimen. The greatest accuracy will be obtained with the smallest vessel in which the specimen can be immersed with its length vertical. Mark the level of the water, then immerse the saturated specimen and weigh the vessel. Draw the water down to its original level and weigh the vessel again. The difference between the two weights divided by 62.5 equals the volume of the specimen in cubic feet.

American Concrete Institute tentative specifications for cast stone, P-3A-29T, 1929.

\section{GENERAI}

1. The term "cast stone" shall be understood to mean a building stone manufactured from Portlandcement concrete, precast and set in place as trim or facing on or in buildings and other structures.

2. The minimum average compression strength of cast stone at the age of 28 days or less shall be 5,000 1bs./in. ${ }^{2}$ when tested as 2 by 2 inch cylinders or 2 by 2 inch cubes in the manner hereinafter specified.

3. At the age of 28 days or less the average absorption of cast stone shall be not less than 3 and not more than 7 per cent of water by dry weight of the specimen when tested as 2 by 2 inch cylinders or 2 by 2 inch cubes in the manner hereinafter specified.

4. All aggregate used in the manufacture of cast stone shall be of known durable quality.

\section{SELECTION OF SPECIMENS FOR TESTING}

5. Specimens for both compression and absorption tests shall be cut from stone as delivered on the job or from regular stock in the yard. Samples from which specimens will be cut shall be selected by the purchasel or his representative.

6. Specimens of faced cast stone for compression tests shall be cut in such a manner that the specimens are composed of approximately one-half of facing and one-half of backing material. Specimens shall be tested in the position in which the cast stone is laid in the wall.

\section{METHODS OF TESTING}

7. Not less than three and preferably five specimens shall be required for each test. In the event of failure to meet requirements in the first test, the test may be repeated on a second set of specimens.

8. Specimens for absorption test shall be dried at a temperature between $215^{\circ}$ and $225^{\circ} \mathrm{F}$. until the loss in weight is not more than 0.1 per cent in two hours of drying. They shall be weighed and then be submerged in water at a temperature of between $60^{\circ}$ and $80^{\circ} \mathrm{F}$. for 24 hours. The specimens shall then be removed from the water, the surface water wiped off with a damp cloth, and the specimens weighed. The percentage of absorption is the difference in weight divided by the dry weight of the specimen and multiplied by 100.

9. Specimens for strength test shall be dried at a temperature of between $215^{\circ}$ and $225^{\circ} \mathrm{F}$. until the loss in weight is not more than 0.1 per cent in two hours of drying.

10. If bealing surfaces of specimens for strength test are not smooth they shall be made so by grinding. If they can not be ground to a smooth surface they shall be capped with a mixture of one-half part Portland cement and one-half part plaster of Paris, which shall bu allowed to harden at least five hours before the test. The cap shall be formed by spreading the capping material upon a plate glass and pressing the specimen firmly on it, making the cap as thin as possible.

11. Load shall be applied through a spherical bearing block placed on top of the specimen in a vertical testing machine. The dimensions of the bearing block shall be the same, or slightly larger, than that of the test specimen.

12. Load shall be applied uniformly and without shock. The speed of the moving head of the machine shall be not more than 0.05 inch per minute when the machine is running idle.

13. Specimen shall be loaded to failure and the unit compressive strength calculated in pounds per square inch. The type of failure and appearance of specimen shall be noted.

American Concrete Institute tentative recommended practice for the manufacture of concrete building block, building tile and brick, serial designation P-6A-25T, 1925.

(These recommendations are intended to assist in the manufacture of concrete building block, building tile, and brick that will meet the requirements of the standard specifications of the American Concrete Institute. Particular attention is given to those features which will help to produce quality products economically.)

American Concrete Institute tentative specifications for concrete brick, serial designation $\mathrm{P}-1 \mathrm{~B}-26 \mathrm{~T}$, 1926. 


\section{GENERAI}

1. The purpose of these specifications is to definc the requirements for concrete brick to be used in construction.

2. The word "concrete" shall be understood to mean Portland-cement concrete.

3 . The average compressive strength of concrete brick 28 days after being manufactured or when shipped shall not be less than $1,500 \mathrm{lbs}$./in. ${ }^{2}$ of gross cross-sectional area as laid in the wall, and the compressive strength of any individual brick shall be not less than $1,000 \mathrm{lbs}$./in. ${ }^{2}$ of gross cross-sectional area as laid in the wall.

4. The gross cross-sectional area of a brick shall be considered as the product of the length times the width of the unit as laid in the wall.

5. Concrete brick shall not absorb more than 12 per cent of the dry weight of the brick when tested as hereinafter specified, except when they are made of concrete weighing less than $125 \mathrm{lbs} . / \mathrm{ft}^{3}{ }^{3}$ For brick made of concrete weighing less than $125 \mathrm{lbs} . / \mathrm{ft}^{3}$ the average absorption in per cent by weight shall not be more than 12 multiplied by 125 and divided by the unit weight in pounds per cubic foot of the concrete under consideration.

6. Specimens for tests shall be representative of the commercial product of the plant.

7. Five specimens shall be required for each test.

8. The specimens used in the absorption test may be used for the strength test.

\section{METHODS OF TESTING}

9. Absorption test.-The specimens shall be immersed in clean water at approximatcly $70^{\circ} \mathrm{F}$. for a period of 24 hours. They shall then be removed, the surface water wiped off, and the specimens weighed. Specimens shall be dried to a constant weight at a temperature of from $212^{\circ}$ to $250^{\circ} \mathrm{F}$. and reweighed. Absorption is the difference in weight divided by the weight of the dry specimens and multiplied by 100 .

10. Strength test. - Specimens for the strength test shall be dried to constant weight at a temperature of from $212^{\circ}$ to $250^{\circ} \mathrm{F}$.

11. The specimens to be tested shall be carefully measured for over-all dimensions of length, width, and thickness.

12. Bearing surfaces shall be made plane by capping with plaster of Paris, or a mixture of one-half Portland cement and one-half plaster of Paris, which shall be allowed to thoroughly harden (from three to six hours) before the test. No point on the surface shall deviate from the plane more than 0.003 inch. The cap shall not be thicker than one-eigh th inch. It shall be formed by means of an accurately machined metal plate or a heavy plate glass having a true surface.

13. Specimens shall be accurately centered in the testing machine.

14. The load shall be applied through a spherical bearing block placed on top of the specimen. The rate of loading after 50 per cent of the ultimate load has been applied shall not be greater than that which will produce a shortening of the specimen of 0.02 -inch per minute.

15. Metal plates of sufficient thickness to prevent appreciable bending shall be placed between the spherical bearing block and the specimen.

16. The specimen shall be loaded to failure.

17. The compressive strength in pounds per square inch of gross cross-sectional area is the total applied load in pounds divided by the gross crosssectional area in square inches.

American Concrete Institute tentative specifications for concrete sewer manhole and catch-basin block, serial designation P-1C-27T, 1927.

(See 518.65, p. 448.)
American Railway Engineering Association spccififications for buildings for railway purposes.

\section{CONCRETE ARCHITECTURAI STONE, 1926}

(The full text of the specification includes requirements for sctting, cleaning, and pointing, and protection.)

\section{GENERAI}

The contractor shall furnish all labor, tools, material, scaffolding, and equipment, except as otherwise noted, necessary to entirely complete any and all classes of concrete architectural stoncwork herein specified, according to the class of building and as shown on the drawings.

\section{COMPOSITION}

Concrete architectural stone shall be composed of Portland cement, meeting the requirements of the Specifications for Portland Cement of the American Railway Engineering Association, which are the same as A. S. T. M. C9 (see 516.11, p. 294), and sound, weather-resisting aggregates.

\section{TESTS}

Concrete architectural stone shall pass the following test requirements:

(a) Compression lest.-The ultimate compressive strength at 28 days must average not less than 1,500 lbs./in. ${ }^{2}$ of gross cross-sectional area of stone as used in the wall and must not fall below 1,000 lbs./in. ${ }^{2}$ gross area in any test. The gross area shall be taken as the minimum area in compression.

(b) Absorption.-The amount of water absorbed by the stone at 28 days shall not exceed 5 per cent by weight after 24 hours' immersion. (Per cent of absorption is obtaincd by dividing the weight of the water absorbed by the weight of the dry sample and multiplying the quotient by 100.)

At least three samples shall be tested for compression and three samples for absorption. Whenever practicable, tests shall be madc on full-sized samples, but if specimens are too large for testing in the ordinary machine or are in special shapes which can not be fitted in the machine, smaller or more regular spccimens may be used as directed, but thesc shall be made of the materials and proportions representing fully the manufactured product. The engineer may require further tests at any time, and unless tests are made under the immediate supervision of the engineer the contractor shall furnish a certificate of tests made by a laboratory of recognized standing showing a record of the compressive strength and absorption of the concrete stone.

\section{WORKING LOADS AND UNIT STRESSES}

Solid walls built of concrete architectural stone laid up in Portland-cement mortar may be loaded not to exceed $300 \mathrm{lbs}$./in. ${ }^{2}$ of actual bearing area. Lintels, mullions, and other parts carrying heavy loads shall be reinforced with steel sufficient to take the tensile and shearing stresses and shall be designed in accordance with recognized engineering practice. Where local ordinances do not conflict, the design shall be based on a unit tensile stress of $16,000 \mathrm{lbs}$./in. ${ }^{2}$ in reinforcement and 650 lbs./in. ${ }^{2}$ compression on the concrete in flexure.

\section{HANDLING}

Pieces weighing more than 300 pounds shall be provided with lewis holes or have hooks cast into the stone for hoisting purposes. Hooks shall be laid in flush or arranged to fit into depressions in adjoining stones. Hooks or lewis holes shall not 
be cast in the face of the stone or come within $2 \frac{1}{2}$ inches of the face of the stone, unless the stone is less than 5 inches thick.

\section{SURFACE FINISH AND CARVING}

A sample or samples of the architectural stone which the contractor proposes to furnish and erect shall be submitted to the engineer along with a diagram showing arrangement of joints, various sizes of units, and bonding to be employed.

If a surface finish of exposed special aggregate is specified this special facing must be not less than one-half inch thick on the exposed surface. Exposed surfaces shall be rubbed, tooled, scrubbed, or acid washed or otherwise treated to expose the aggregate and obtain the desired architectural results. All carving shall be done by skilled carvers from approved models, photographs, or drawings.

\section{MORTAR}

Portland cement shall satisfy the requirements of the A. R. E. A. specifications for Portland cement. (See A. S. T. M. C9, 516.11, p. 294.) Cement that has hardened or partiaily set shall not be used.

Sand and lime specifications as given in the A. R. E. A. specifications for brickwork, 518.83 (see p. 475), shall apply to sand and lime used for concrete architectural stone.

Mortar for concrete architectural stonework, except where otherwise specified, shall be composed of 1 part Portland cement and 3 parts sand, with the addition of sufficient lime putty to make the mortar work smoothly. The sand and cement shall be mixed dry, then wet to the proper consistency, and the lime putty added. Where colored mortar is to be used the mortar shall be composed of 1 part Portland cement and 2 parts sand, with lime putty added to make it work smoothly, and colored with approved mortar color. No more mortar shall be mixed at any one time than can be used within 30 minutes after mixing. Retempering cement mortar which has begun to set shall not be permitted. Mortar that has frozen shall not be used on the work.

\section{ANCHORS AND DOWELS}

Where anchors and dowels are required they shall be of the proper size and shape, thoroughly galvanized or painted with asphaltum paint.

\section{GUARANTY}

The contractor for concrete architectural stone shall guarantee the surface to be free from efflorescence, crazing, crumbling, or fading for a period of two years after completion of the building.

\section{GENERAL CONDITIONS}

All material entering into the work and all methods used by the contractor shall be subject to the approval of the engineer, and no part will be considered as finally accepted until all the work is completed and accepted.

The general conditions as given in 518.50, page 416, shall be considered to apply with equal force to this section of the specification.

American Railway Engineering Association specifications for buildings for railway purposes.

\section{CONCRETE ROOFING TILE, 1926}

(The full text of the specification includes requirements for laying the tile.)

\section{GENERAL CONDITIONS}

The contractor shall furnish all labor, materials, tools, and equipment needed to entirely complete the concrete tile roofing, including all valleys and flashing as specified or shown on drawings.

\section{TYPES}

Concrete roofing tile shall be divided into the following types:

1. Small tile supported directly on roofing paper and roof sheathing.

2. Large tile spanning between purlins.

Large tile shall be specified as:

(a) Interlocking tile for pitched roofs, and (b) flat tile.

\section{MATERIALS}

Concrete roofing tile shall be composed of Portland cement in accordance with specifications for Portland cement of the A. R. E. A. (which are the same as A. S. T. M. C9) (see 516.11, p. 294), and clean, hard, coarse sand or crushed rock. Large tile shall have suitable metal reinforcement. If colored tile are specified the color must be obtained by the use of pure mineral oxides, pure red oxide of iron for red, and chromium oxide for green. The coloring material shall be reground with the cement in a small ball mill or other closed grinder so that at least 78 per cent of the combined cement and coloring material will pass a 200 -mesh sieve as determined by samples.

\section{MANUFACTURE}

Small concrete roofing tile shall be made on approved cast-iron or strong steel pallets and upon an improved machine developing a pressure at the rollers or tampers of at least 100 lbs./in. ${ }^{2}$ Pallets shall be straight and free from warps. Large tile shall be made in smooth substantial molds to produce tile true to dimensions. Troweling bars shall have tapered channels which produce a compressive action.

The colored cement for colored tile shall be either mixed with sand and deposited as a facing in the molds or dusted dry onto the face of the tile and troweled in by special-shaped tools or rollers to give a dense, permanent, even-colored surface, or else applied in liquid form as the tile leaves the rollers.

As the tile are taken from the machine or molds the lower edge shall be painted with a mixture of colored cement and water applied with a brush, except where color is incorporated into or through the body of the tile when painting may be omitted. Tile shall stand for not less than three hours on cars or racks before placing in curing chambers, and shall be preferably cured in moist steam or under fog nozzles for 24 hours. In cold weather the temperature in the curing chamber shall be maintained not less than $75^{\circ} \mathrm{F}$. The tile shall then be taken from the pallet and stacked on end, kept under cover for 10 days and well wetted down at least twice a day. They shall then be stacked in the open air for 20 days more and sprinkled daily.

\section{DIMENSIONS}

Tile shall be true and of even thickness. The thickness of small concrete tile shall be not less than $3 / 16$ inch and edges and ends shall be smooth and clean. Tile shall be not warped more than $1 / 8$ inch and shall not vary more than $1 / 16$ inch in thickness. Starter tile shall be flanged and eave tile shall be made on special pallets that show solid edges.

Interlocking tile shall be -....-. by _...-.inches and one inch thick for the flat portion. The surface exposed to the weather shall be ......... by .......- allowing 4 -inch end laps. 
The tile must weigh not over $16 \mathrm{lbs} . / \mathrm{ft}^{2}$ and shall shall not vary from the specified thickness more than 1/16-inch nor be warped more than 1/8-inch.

Large flat tile shall be _....... by _...... - inch and $1 \frac{1}{2}$ inches thick. The ends of the tile shall be recessed so as to shoulder on the supports. The tile shall not weigh over $17 \mathrm{lbs}$./ft. ${ }^{2}$ and shall not vary in thickness more than $1 / 16$ inch nor be warped more than $1 / 8$ inch.

\section{TESTS}

All tests shall be made in a laboratory of recognized standing, and a certified copy of the tests shall be furnished by the manufacturer. Tests shall be on full-sized samples and at least six samples representing the ordinary commercial product shall be tested.

Concrete roofing tile shall not absorb more than 5 per cent of its own weight of water in 24 hours. Absorption from the face of the tile shall not exceed 2 per cent by weight in 24 hours with the face of the tile sample in contact with the water.

Tile shall be tested with meather face up. The tile shall be supported under the lugs near the ends of the tile, if the tile have lugs, but in no test shall the span be less than 13 inches for small tile nor less than _....... inches for large tile. The support under one end shall be rigid and the support under the other end shall rest on a roller bearing to allow for variation in the under surface of the tile. The load for small tile shall be applied in the center of the tile by placing a rigid bar having a semicircular bearing midway between the supports. From this crossbar shall be suspended a bucketlike receptacle which shall be loaded with shot, sand, or other suitable material until the tile breaks. The breaking load shall average not less than 150 pounds per tile when the load is applied in accordance with the method described above.

Large interlocking tile shall withstand a uniformly distributed load of $100 \mathrm{lbs} . / \mathrm{ft} .{ }^{2}$

Flat tile shall withstand a uniformly distributed load of $150 \mathrm{lbs} . / \mathrm{ft} .^{2}$ when supported on supports ..... feet apart. Lots of tile intended for building construction may be rejected, if more than 10 per cent of the samples tested break at loads less than 100 lbs./ft. ${ }^{2}$

\section{GUARANTY}

The roofing contractor shall furnish an unqualified guaranty, with good and sufficient bond, covering the maintenance of the roof in a water-tight condition for a period of ........- years from date of completion.

\section{GENERAL CONDITTONS}

All material entering into the work and all methods used by the contractor shall be subject to the approval of the engineer and no part of the work will be considered as finally accepted until all the work is completed and accepted.

The general conditions as given in 518.50 , page 416 , shall be considered to apply to this specification.

American Society for Municipal Improvements, standard specifications for sewers, 1927.

(This specification includes requirements for concrete sewers and segmental block sewers.)

See 518.67 , page 453 .

American Society for Testing Materials, tentative specifications for concrete building brick, serial designation C55-28T, 1928.

1. These specifications cover concrete building brick intended for use in brick masonry.

2. Concrete building brick shall be manufactured from a mixture of Portland cement and suitable aggregate.

\section{PHYSICAL PROPERTIES AND TESTS}

3. (a) Concrete building brick shall be classified as grades $\mathrm{B}$ and $\mathrm{C}$ on the basis of the following requirements. The classification of any lot of bricks shall be determined by the results of the tests for that requirement in which it is lowest, unless otherwise specified as provided in section 7 .

\begin{tabular}{|c|c|c|c|c|}
\hline \multirow{2}{*}{ Classification } & \multicolumn{2}{|c|}{$\begin{array}{l}\text { Compressive } \\
\text { strengtb (brick } \\
\text { flatwise) }\end{array}$} & \multicolumn{2}{|c|}{$\begin{array}{l}\text { Modulus of rupture } \\
\text { (brick flatwise) }\end{array}$} \\
\hline & $\begin{array}{c}\text { Mean of } \\
5 \text { tests }\end{array}$ & $\begin{array}{l}\text { Individ- } \\
\text { ual, min- } \\
\text { imum }\end{array}$ & $\begin{array}{c}\text { Mean of } \\
5 \text { tests }\end{array}$ & $\begin{array}{l}\text { Individ- } \\
\text { ual, min- } \\
\text { imum }\end{array}$ \\
\hline $\begin{array}{l}\text { Grade B } \\
\text { Grade C- }\end{array}$ & $\begin{array}{r}\text { Lbs./in.' } \\
2,250 \\
1,250\end{array}$ & $\begin{array}{r}\text { Lbs./in. }{ }^{2} \\
1,500 \\
1,000\end{array}$ & $\begin{array}{r}\text { Lbs./in.2 } \\
450 \\
300\end{array}$ & $\begin{array}{r}\text { Lbs./in.' } \\
300 \\
200\end{array}$ \\
\hline
\end{tabular}

NотE.-The above classifications are based on strength and do not necessarily measure weathering resistance.

(b) The tests shall be made in accordance with A. S. T. M. C67-28T. (See 534.10, p. 574.)

4. (a) For the purpose of tests, bricks representative of the commercial product shall be selected by a competent person appointed by the purchaser, the place or places of selection to be designated when the purchase order is placed. The manufacturer or seller shall furnish specimens for tests without charge. All bricks shall be carefully examined and their condition noted before testing. For the purpose of tests, not less than 10 bricks shall be required for each investigation. In general, two samples of 10 bricks each shall be tested for every 100,000 bricks contained in the lot under consideration; but where the total quantity exceeds 500,000 , the number of samples tested per 100,000 bricks may be fewer, provided that they shall be distrubuted as uniformly as practicable over the entire lot. Additional representative samples may be taken at any time or place at the discretion of the purchaser.

(b) Concrete building brick when sampled at the plant shall be at least 28 days old, unless otherwise mutually agreed upon between the manufacturer and the purchaser. Representative samples may be taken at any time by the purchaser from brick delivered on the job.

\section{STANDARD SIZE}

5. The standard size of concrete building brick shall be $2 \frac{1 / 4}{4}$ by $3 \frac{3}{4}$ by 8 inches with a permissible variation of plus or minus $1 / 16$ inch in depth, $1 / 8$ inch in width, and $1 / 4$ inch in length.

\section{VISUAL INSPECTION AND REJECTION}

6. Brick shall pass a visual inspection for freedom from cracks and irregularity. Cracked or misshaped brick shall be rejected.

7. At the option of the purchaser, acceptance may be based on the visual inspection requirements specified in section 6 and on the results of one of the strength tests (compressive or flexure) specified in section 3 .

Underwriters' Laboratories, standard for hollow concrete building units, February 21, 1929.

\section{GENERAI}

This standard covers hollow concrete building units which have been shown by fire tests to be eligible for a classification of $\mathbf{R}$ three hours under 
standard fire exposure conditions as specified in the American standard fire test specification.

This standard is based upon records of tests and field experience and is subject to revision as further experience and investigations may show to be necessary or desirable.

Devices or products which comply with this standard will not necessarily be acceptable if they have other features which, when examined and tested, are found to impair the results contemplated by this standard.

A device or product having materials or forms of construction differing from those detailed in this standard may be examined and tested according to the intent of the standard, and if found to be substantially equivalent may be given recognition.

\section{STRENGTH}

Hollow concrete building units shall bave an average strength in compression of $700 \mathrm{lbs} . / \mathrm{in}^{2}$ of gross cross-sectional area as laid in the wall when tested not more than 28 days after manufacture. Individual units shall have a minimum compressive strength of $600 \mathrm{lbs} / \mathrm{in}^{2}{ }^{2}$ gross cross-sectional area when tested not more than 28 days after manufacture.

Solid concrete building units shall have a minimum strength in compression of $1,000 \mathrm{lbs}$. $/ \mathrm{in}^{2}$ as laid in the wall when tested not more than 28 days after manufacture.

The procedure in making tests for compressive strength shall agree with the standard specifications of the American Concrete Institute.

\section{DIMENSTONS}

The height of hollow and solid units shall be not more than 8 inches (nominal).

The width (or thickness) of hollow and solid units shall be not less than 8 inches (nominal) as laid in the wall.

The length of hollow and solid units shall be not less than 12 inches nor more than 16 inches (nominal).

Note. - A tolerance of one-fourth inch plus or minus shall be recognized for the above dimensions, but actual dimensions shall be used in computing compressive strengtb values.

Face shells (side walls) of hollow units shall average not less than $1 \frac{3 / 4}{4}$ inches in thickness and shall be not less than $1 \frac{1}{2}$ inches thick at their thinnest point.

Internal and end webs of hollow units should not be less than 1 inch in thickness at their thinnest point.

Note.-Fnd webs of units adaptable for use in both 8 and 12 incb valls (laid all header courses in 12-inch walls) shall have an average thickness of $13 / 4$ inches and shan be not less than $1 \frac{1}{2}$ incbes tbick at their tbinnest point.

\section{DESIGN}

One-piece units with two or three rectangular or oval core holes and with plain or ornamental facing shall be considered as standard.

Other one-piece units may require fire endurance and other tests before they can be classed as standard.

\section{CEMENT}

Standard Portland cement (A. S. T. M. specification C9; see 516.11, p. 294) shall be used.

\section{AGGREGATE}

The aggregates used, both fine and coarse, including the sand, shall be secured from sources insuring uniformity in quality and kind.

Fine aggregate is defined as that passing a one-fourth inch screen and coarse aggregate as that retained on a one-fourth inch screen.
Coarse aggregates other than cinders shall not contain more than 65 per cent by weight of silicious material.

Aggregates containing silicious material in excess of this amount, require fire endurance and otber tests to determine their performance under standard fire exposure conditions.

Cinders when used as either fine or coarse aggregate or both, shall be of good quality, resulting from the combustion of bituminous coal or a mixture of bituminous and anthracite coal.

The combustible content of the mixed fine and coarse aggregate, when cinders are used, shall not exceed 20 per cent by weight of the dried mixed agglegates.

\section{PROPORTION}

The cement-aggregate proportion for hollow and solid units shall be $1: 3,1: 4,1: 5,1: 6,1: 7$, or any fraction therof not over $1: 7$.

NoтE.-Tbe above proportions are given in terms of volumes of cement to volumes of combined fine and coarse aggregates (after mixture).

No change shall be made in any particular cement-aggregate proportion specified above until strength and other tests have been made on specimens representative of the new proportion.

At least 25 per cent by volume of the mixed aggregate including cinders shall be coarse aggregate. It is recommended that the coarse aggregate pass a $5 / 8$-inch screen and that the maximum size of individual particles should not exceed one-half the thickness of the thinnest web or shell member of the unit in which they are used.

The percentage of coarse aggregate to total mixed aggregate shall not be changed from any specified value until strength and other tests have been made on specimens representative of the new proportion.

\section{CONSISTENCY}

The concrete used in the manufacture of hollow or solid units may be of a dry, damp, wet damp, or wet consistency.

No change in an established practice as to the consistency of the material shall be made until strength and other tests have been made on specimens representative of the new consistency.

\section{CURING METHODS}

Hollow and solid units may be air, water, or steam cured. Water or steam curing is preferred, due to the resultant uniformity of strength and other characteristics.

All units shall be held in storage for a period of not less than 28 days (including curing period) unless strength and other tests have indicated compliance with the standard of blocks shipped prior to that time. In cases where units are shipped before 28 days, steam or water curing is considered essential.

NoTE. - It is recommended that units be beld in storage for at least 10 days after manufacture.

\section{FIRE-ENDURANCE TESTS}

When fire-endurance tests are made on units not clearly conforming to all of the specifications of this standard, to determine their eligibility for classification and listing, the program of the American standard fire test specifications shall be followed, except that panels may be not more than 6 by 6 feet exposed areas and the fire hose stream test may be omitted if, in the judgement of the testing body, the data secured therefrom is not required to confirm or supplement that secured in the fireendurance test. Test performance in general 
corresponding to that recolded for panels $H, A$, G, B, D, and K, Underwriters' Laboratories' report $R-1555$ shall be the basis of judgement as to the outcome of such fire-endurance tests.

United States Department of Commerce, Bureau of Standards, Simplified Practice Recommendation No. 32, for concrete building units, June 1, 1925.

In accordance with a general conference of representatives of manufacturers, distributors, and users of concrete blocks, building tile, and bricks, the United States Department of Commerce, through the Bureau of Standards, recommends that the number of sizes of these units be reduced to the followving:

TABLE 1.-Concrete blocks

\begin{tabular}{|c|c|c|c|c|c|}
\hline $\begin{array}{l}\text { Meight } \\
\text { (inches) }\end{array}$ & $\begin{array}{l}\text { Toler- } \\
\text { ance }\end{array}$ & $\begin{array}{c}\text { Width } \\
\text { (inches) }\end{array}$ & $\begin{array}{l}\text { Toler- } \\
\text { ance }\end{array}$ & $\begin{array}{l}\text { Length } \\
\text { (inches) }\end{array}$ & $\begin{array}{l}\text { Toler- } \\
\text { ance }\end{array}$ \\
\hline 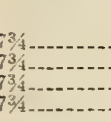 & $\begin{array}{r}\text { Inch } \\
-18 \\
-18 \\
-18 \\
-18 \\
-18\end{array}$ & $\begin{array}{l}6 \\
8 \\
8 \\
10 \\
12\end{array}$ & $\begin{array}{c}\text { Inch } \\
-1 / 4 \\
-1 / 4 \\
-1 / 4 \\
-1 / 4\end{array}$ & $\begin{array}{l}15^{3} 34 \\
15^{33} \\
15^{33} \\
15^{3} 4- \\
\end{array}$ & $\begin{array}{l}\text { Inch } \\
-1 / 8 \\
-1 / 8 \\
-1 / 8 \\
-1 / 8\end{array}$ \\
\hline
\end{tabular}

TABLE 2.-Concrete building tile 1

Poad bearing-.......................... \begin{tabular}{r|r|r|r}
\hline Inches & Inches & Inches \\
5 & $3^{3 / 4}$ & 12 \\
5 & 8 & 12 \\
5 & 12 & 12 \\
3 & 12 & 12 \\
4 & 12 & 12 \\
6 & 12 & 12 \\
8 & 12 & 12 \\
10 & 12 & 12 \\
12 & 12 & 12
\end{tabular}

1 Not more than 3 per cent permissible variation over or under for dimensions covering height, width, or length. The number of cells and weight per tile not considered at this conference.

TABLE 3.-Concrete brick

\begin{tabular}{c|r|r|r}
\hline Types & Height & Width & Length \\
\hline Face and common & Inches \\
$21 / 4$ & Inches \\
$33 / 4$ & Inches \\
8
\end{tabular}

\subsection{SIDEWALKS AND DRIVEWAYS.}

American Concrete Institute, standard specifications for Portland cement concrete sidewalks, C-2B-25, 1925.

(The full text of the specification includes requirements for construction in addition to the following specifications for materials.)

\section{USE}

These specifications cover both one and two course sidewalk construction. The specifications for materials apply to both one and two course types.

\section{MATERIALS}

Before delivery upon the job, and at such other times as the engineer deems necessary, the contractor shall furnish any required samples of the materials hereafter mentioned. Materials shall pass the following requirements.

(a) Cement.- The cement shall meet the requirements of the current standard specifications for Portland cement of the American Society for Testing Materials. (See C9, 516.11, p. 294.) (b) Fine aggregaie.-The fine aggregate shall consist of clean, hard, durable, uncoated particles of sand, stone, ol air-cooled blast-furnaee slag free from organic material. All of the fine aggregate shall pass a one-fourth-inch screen and 95 per cent shall be retained on a 100 -mesh screen. Not more than 25 per cent shall pass a 50 -mesh sereen. It shall be well graded from coarse to fine, and shall contain not more than 5 per cent by weight of clay or loam, none of which shall be in lumps. Blastfurnace slag used in the wearing course shall weigh not less than 70 pounds per cubic foot.

(c) Coarse aggregate.-Coar'se aggregate may be broken stone, gravel, or blast-furnace slag. All of the coarse aggregate shall pass a I-inch screen and at least 95 per cent shall be retained on a one-fourthinch screen, with all intermediate sizes retained.

The broken stone or gravel shall be clean, hard, durable, uncoated rock. It shall contain no vegetable or other deleterious matter and shall be practically free from soft, thin, elongated, or laminated pieces.

Slag shall be air-cooled blast-furnace slag weighing not less than 65 pounds per cubic foot when used in that part of the sidewalk not subject to abrasion and not less than 70 pounds per cubic foot when used in the wearing course.

(d) Water.-Water shall be clean and practically free from alkali, oils, or acid.

(e) Joint filler. - The joint filler shall be a suitable elastic waterproof compound that will not become soft and run out in hot weather, nor hard and brittle and chip out in cold weather, or prepared strips of fiber matrix and bitumen as approved by the engineer. The strips shall be one-half inch in thickness, their width shall at least equal the full thickness of the slab, and their length shall at least equal the width of the slab at the joint.

(f) Forms.-Forms shall be of lumber 2 inches thick, or of steel of equal strength, except on curves, where flexible strips may be used.

(g) Division plates.-Where division plates are used they shall be of $1 / 8$-inch steel as wide as the depth of the slab and as long as the width of the walk.

(h) Sub-base.-Only clean, durable material, such as coarse gravel ol steam-boiler cinders, free from ash or particles of unburned coal, shall be used in the sub-base.

Note.-Eliminate this clause if sub-base is not required.

\section{ONE-COURSE WORK}

The sidewalk shall consist of one 5-inch course of concrete in the proportion of 1 part of Portland cement, 2 parts of fine agglegate, and 3 parts of coarse aggregate.

The freshly-mixed concrete shall be placed promptly on the prepared subgrade. It shall then be struck off and tamped with a straightedge resting upon the side forms and advanced with a crosswise sawing motion. Collcrete may be delivered in trucks from a centrally located mixing plant provided the consistency of the concrete and the length of haul are such that the concrete can be delivered in a homogeneous and workable condition.

The concrete shall then be floated with a wooden float until the surface is true and the concrete thoroughly compacted.

\section{TWO-COURSE CONSTRUCTION}

Two-course sidewalks shall consist of a base 41/4-inches thick composed of concrete in the proportions of 1 part Portland cement, 3 parts of fine aggregate, and 5 parts coarse aggregate and a top coat three-fourths inch thick composed of mortar 
in the proportions 1 part Portland cement and 2 parts fine aggregate.

The base shall be deposited on the subgrade and thoroughly compacted by tamping. It shall then be struck off by a templet, which shall leave it nowhere less than three-fourths inch beiow the finished surface.

Within 45 minutes after the bottom course is laid and before the initial set has taken place, the material for the wearing surface shall be placed and brought to the established grade by means of a strike board.

After the wearing course has been brought to the established grade it shall be worked with a wood float in a manner which will thoroughly compact it and provide a surface free from depressions or irregularities of any kind.

American Society for Municipal Improvements, standard specifications for concrete sidewalks, 1927.

\section{GENERAL REQUIREMENTS}

\section{MATERIALS}

1. Cement.-The cement shall meet the requirements of the current standard specifications for Portland cement of the American Society for Testing Materials. (See A. S. T. M.: C9, 516.11, p. 294).

2. Aggregate.-Before delivery on the job, the contractor shall submit to the architect or engineer a 50-pound sample of each of the aggregates proposed for use. These samples shall be tested, and if found to pass the requirements of the specifications, similar material shall be considered as acceptable for the work. In no case shall aggregate containing frost or lumps of frozen material be used.

(a) Fine aggregate.-Fine aggregate shall consist of natural sand or screenings from hard, tough crushed rock or gravel consisting of quartz grains or other hard material, clean and free from any surface film or coating, and graded from fine, coarse particles predominating. Fine aggregate, when dry, shall pass a screen having 4 meshes to the linear inch; not more than 25 per cent shall pass a sieve having 50 meshes per linear inch, and not more than 5 per cent shall pass a sieve having 100 meshes per linear inch. Fine aggregate shall not contain injurious vegetable or other organic matter as determined by the colorimetric test nor more than 5 per cent by volume of clay or loam. Field tests may be made by the architect or engineer on fine aggregate as delivered at any time during progress of the work. If there is more than 7 per cent of clay or loam, by volume, in one hour's settlement after shaking in 100 per cent of water, the material represented by the sample shall be rejected.

Fine aggregate shall be of such quality that mortar composed of 1 part Portland cement and 3 parts fine aggregate, by weight, when made into briquets or cylinders, shall show a tensile or compressive strength, at 7 and 28 days, at least equal to the strength of briquets or cylinders composed of 1 part of the same cement and 3 parts standard Ottawa sand by weight. The percentage of water used in making the test specimens of cement and fine aggregate shall be such as to produce a mortar of the same consistency as that of the Ottawa sand test specimens of standard consistency. In other respects all briquets or cylinders shall be made in accordance with the methods of testing cement recommended by the American Society for Testing Materials. (See A. S. T. M.: C9, 516.11, p. 294.)

(b) Coarse aggregate.-Coarse aggregate shall consist of clean durable crushed rock, pebbles, or crushed blast-furnace slag, graded in size, free from vegetable or other organic matter, and shall contain no soft, flat, or elongated particles, The slag shall not contain more than $1 \frac{1}{2}$ per cent of sulphur as sulphides. The size of the coarse aggregate shall range from 1 inch down, not more than 5 per cent passing a screen having 4 meshes per linear inch, and no intermediate sizes shall be removed.

(c) No. 1 aggregate for wearing course.-No. 1 aggregate for the wearing course shall consist of clean, hard, tough, crushed rock or pebbles, free from vegetable or other organic matter and shall contain no soft, flat, or elongated particles. It shall pass, when dry, a screen having one-half inch square openings, and not more than 10 per cent shall pass a screen having 4 meshes per linear inch.

3. Mixed aggregate.-Crusher-run stone, bankrun gravel, or mixtures of fine and coarse aggregate prepared before delivery on the work shall not be used.

4. Subbase.-Only clean, durable material, such as coarse gravel or steam-boiler cinders free from ash or particles of unburned coal shall be used in the subbase.

Note.-Eliminate this clause unless subbase is required

5. Water.-Water shall be clean, free from acid, alkali, vegetable, or other organic matter.

6. Color.-If artificial coloring material is required, only those mineral colors shall be used which, in the amount hereinafter specified, will not appreciably impair the strength of the cement.

7. Reinforcement.-The reinforcing metal shall meet the requirements of the current standard specifications for steel reinforcement of the American Society for Testing Materials. It shall be free from excessive rust, scale, paint, or coating of any character which will tend to reduce or destroy the bond. The reinforcement shall have a weight of not less than 28 pounds per 100 square feet.

8. Joint filler.-The joint filler shall be of a suitable elastic waterproof compound that will not become soft and run out in hot weather, nor hard and brittle and chip out in cold weather, or prepared strips of fiber matrix and bitumen as approved by the architect or engineer. The strips shall be onehalf inch in thickness, their width shall at least equal the full thickness of the slab and their length shall at least equal the width of the slab at the joint.

\section{SOBGRADE}

9. Preparation.-All soft and spongy places shall be removed and all depressions filled with suitable material, which shall be thoroughly compacted in layers not exceeding 6 inches in thickness. The subgrade shall be thoroughly tamped until it is brought to a firm, unyielding surface. It shall have a slope of not more than one-half inch per foot.

When the concrete sidewalk is to be constructed over an old path composed of gravel or cinders, the old path shall be entirely loosened, the material spread for the full width of the subgrade and compacted as specified.

10. Deep fills.-All fills shall be made in the manner satisfactory to the architect or engineer. The use of muck, quicksand, soft clay, spongy, or perishable material is prohibited. The top of all fills shall extend beyond the walk on each side at least 1 foot, and the sides shall have a slope greater than 1 or $1 \frac{1}{2}$.

11. Drainage.-When required, a suitable drainage system shall be installed and connected with sewers or other drains indicated by the architect or engineer.

12. Depth.-The subgrade shall be not less than - inches below the finisbed surface of the walk.

Not E.-Subgrade to be at least 5 inches below the finished surface of the walk when the subbase is not required and at least 11 inches below when subbase is required. 


\section{SUBBASE}

(Omit these sections unless subbase is required) .

13. Thickness.-On the subgrade shall be spread a material as hereinbefore specified which shall be thoroughly rolled or tamped to a surface at least -. inches below the finished grade of the walk. On fills the subbase shall have the same slope as the sides of the fill.

14. Welting. - While compacting the subbase the material shall be kept thoroughly wet, and shall be wet when the concrete is deposited, but shall show no pools of water.

\section{FORMS}

15. Materials.-Forms shall be free from warp and of sufficient strength to resist springing out of shape.

16. Setting.-The forms shall be well staked or otherwise held to the established lines and grades and their upper edges shall conform to the established grade of the walk.

17. Division plates.-Suitable metal division plates shall be provided to completely separate adjacent slabs during construction unless otherwise permitted by the architect or engineer.

18. Treatment.-All wood forms shall be thoroughly wetted and metal forms oiled or coated with soft soap or whitewash before depositing any material against them. All mortar and dirt shall be removed from forms that have been previously used.

\section{CONSTRUCTION}

19. Size of slabs.-The slabs or independently divided blocks when not reinforced shall have an area of not more than 100 square fcet. Length of slab shall be equal to the width and all slabs of 100 square feet shall be reinforced.

20. Thickness of walk.-Minimum thickness to be 4 inches.

21. Joints.-A one-half inch joint shall be provided at least once every 50 feet in the length of the walk, which shall be filled with suitable material as specified under "Joint filler." A similar joint shall be provided at each intersection of sidewalk and street curb and at such other points as may be designated by the architect or engineer. Sidewalks in business districts shall be separated from abutting buildings by a one-half inch joint.

22. Protection of edges. - Where required by the architect or engineer, the edges of the slabs at the joints shall be protected by metal. Unless protected by metal, the upper edges of the slabs shall be rounded to a radius of one-half inch. The edges of all slabs abutting a business street which act as curbing must be protected by suitable metal angles or corner bars as approved by the architect or engineer

\section{MEASURING AND MIXING}

23. Measuring.-The method of measuring the materials for the concrete or mortar, including water, shall be one which will insure separate and uniform proportions of each of the materials at all times. A sack of Portland cement (94 pounds net) shall be considered 1 cubic foot.

24. Machine mixing.-All concrete shall be mixed by machine except when the architect or engineer shall otherwise permit under special conditions. A batch mixer of any approved type shall be used. The ingredients of the concrete or mortar shall be mixed to the specified consistency, and the mixing shall continue for at least one minute after all materials are in the drum. The drum shall be completely emptied before receiving material for the succeeding batch,
25. Hand mixing.-When it is necessary to mix by hand, the materials shall be mixed dry on a watcr-tight platform until the mixture is of uniform color, the required amount of water added, and the mixing continued until the mass is of proper consistency and homogeneous.

26. Retempering. - Retempering of mortar or concrete which has partially hardened-that is, remixing with or without additional materials or water-shall not be permitted.

\section{PROTECTION}

27. Treatment.-As soon as the finished work has hardened sufficiently to prevent damage, the surface of the walk shall be sprinkled with clean water or preferably covered with at least 1 inch of wet sand or earth and kept wet for at least seven days.

28. Protection.-The freshly finished work shall be protected from hot sun and drying winds until it can be sprinkled and covercd as above spccified. The concrete surface must not be damaged or pitted by rain drops, and the contractor shall provide and use, when necessary, sufficient tarpaulins to completely cover all sections that have been placed within the preceding 12 hours. The contractor shall erect and maintain suitable barricrs to protect the walk from traffic, and any section damaged from traffic or other causes occurring prior to its official acceptance shall be repaired or replaced by the contractor at his own expense in a manner satisfactory to the architect or engineer. Before the sidewalk is opencd to traffic the covering shall be removed and disposed of by the contractor. The walk shall not be opened to traffic until the architect or engineer so directs.

29. Temperature below $35^{\circ} F$.- If at any time during the progress of the work the temperature is, or in the opinion of the architect or engineer will, within 24 hours, drop to $35^{\circ} \mathrm{F}$, the water and aggregates shall be heated and precautions taken to protect the work from freezing for at least five days.

30. Name stamp.-The contractor shall mark in the walk at each end of every 150 feet of walk or fraction thereof, either by stamping or by inlaying an approved metal plate, his name and the year in which the walk is constructed. The stamped letters shall be 1 inch high and one-fourth inch deep. If a metal plate is used, the top of the plate shall be flush with the top of the walk.

\section{TWO-COURSE SIDEWALK}

For 2-course walks the following will apply in addition to the general requirements:

\section{CONCRETE BASE}

31. Proportions.-The concrete shall be mixed in the proportions by volume of 1 sack of Portland cement, 2 cubic feet of fine aggregate, and 4 feet of coarse aggregate.

32. Consistency.-The materials shall be mixed wet enough to produce a concrete of consistency that will flush readily under slight tamping, but which can be handled without causing a separation of the coarse aggregate from the mortar.

33. Placing.-After mixing, the concrete shall be handled rapidly, and the successive batches deposited in a continuous operation, completing individual sections to the required depth and width. Under no circumstances shall concrete that has partly hardened be used. The forms shall be filled and the concrete struck off and tamped to a surface the thickness of the wearing course below the established grade of the walk. The method of placing the various sections shall be such as to produce a straight clean- 
cut joint betreen them so as to make each section ari independent unit. If dirt, sand, or dust collects on the base it shall be removed before the wearing course is applied. Workmen shall not be permitted to walk on the freshly-laid concrete. Any concrete in excess of that needed to complete a section at the stopping of work shall not be used. In no case shall concrete be deposited upon a frozen subgrade or sub-base.

34. Reinforcing.-Slabs having an area of more than 100 square feet or having dimensions greater than 10 feet shall be reinforced with wire fabric or with plain or re-formed bars. The reinforcement shall be placed upon and slightly pressed into the concrete base immediately after the base is placed. It shall not cross joints and shall be lapped sufficiently to develop the full strength of the metal. The reinforcement shall not be less than 1 inch from the finished surface of the walk.

\section{WRARING COURSE}

35. Proportions for mixture No. 1.-The wearing course shall be mixed in the proportions of 1 sack of Portland cement and 2 cubic feet of fine aggregate. The minimum thickness shall be one-half inch.

NoTE.-Proportions and thickpess for residence districts or where traffic is light.

36. Proportions for mixture No. 2.-The wearing course shall be mixed in the proportions of 1 sack of Portland cement, 1 cubic foot of fine aggregate, and 1 cubic foot of No. 1 aggregate for wearing course. The minimum thickness shall be 1 inch.

NoTE.-Proportions and thickness for business districts or where traficic is heavy.

37. Consistency. - The mortar shall be the dryest consistency possible to work with a sawing motion of the strike board.

38. Placing.-The wearing course shall be placed immediately after mixing. It shall be deposited on the fresh concrete of the base before the latter has appreciably hardened and brought to the established grade with a strike board. In no case shall more than 45 minutes elapse between the time the concrete for the base is mixed and the wearing course is placed.

39. Finishing.-After the wearing course has been brought to the established grade by means of a strike board it shall be worked with a wood float in a manner which will thoroughly compact it and provide a surface free from depressions or irregularities of any kind. When required, the surface shall be steel troweled, but excessive working shall be avoided. In no case shall dry cement or a mixture of dry cement and sand be sprinkled on the suriace to absorb moisture or to hasten hardening. When division plates are not used the slab markings shall be made in the wearing course directly over the joints in the base with a tool which will completely separate the wearing course of adjacent slabs. Unless protected by metal, the surface edges of all slabs shall be rounded to a radius of one-half inch.

40. Coloring.-If artificial coloring is used it must be incorporated with the entire wearing course and shall be mixed dry with the cement and aggregate until the mixture is of uniform color. In no case shall the amount of coloring exceed 5 per cent of the weight of the cement.

\section{ONE-COURSE SIDEWAIK}

For 1-course walks the following will apply in addition to the general requirements:

41. Proportions. - The concrete shall be mixed in the proportions of 1 sack of Portland cement to not more than 2 cubic feet of fine aggregate and not more than 3 cubic feet of coarse aggregate, and in no case shall the volume of the fine aggregate be less than one-half the volume of the coarse aggregate.

A cubic yard of concrete in place shall contain not less than $68 / 10$ cubic feet of cement.

42. Consisicncy. - The materials shall be mixed with sufficient water to produce a concrete which will hold its shape when struck off with a strike board. The consistency shall not be such as to cause a separation of the coarse aggregate from the mortar in handling.

43. Placing.-After mixing, the concrete shall be handled rapidly and the successive batches deposited in a continuous operation, completing individual sections to the required depth and width. Under no circumstances shall concrete that has partly hardened be used. The forms shall be filled and the concrete brought by means of a strike board to surface one-quarter inch above the established grada (to allow for compacting by roller in finishing). The method of placing the various sections shall be such as to produce a straight, clean-cut joint between them so as to make each section an independent unit. Any concrete in excess of that needed to complete a section at the stopping of work shall not be used. Workmen shall not be permitted to walk on the freshly laid concrete. In no case shall concrete be deposited upon a frozen subgrade or subbase.

44. Reinforcing.-Slabs having an area of more than 100 square feet or having dimensions greater than 10 feet shall be reinforced with wire fabric or with plain or deformed bars. The reinforcement shall be placed 2 inches below the finished surface of the walk. The reinforcement shall not cross joints and shall be lapped sufficiertly to develop the full strength of the metal.

45. Finishing.-After the concrete has been brought by means of a strike board to a surface onequarter inch above the established grade it shall be rolled transversely with a metal roller having $a$ smooth even surface approximately 3 feet in length and from 6 to 8 inches in diameter and weighing not more than 50 pounds. The rolling must be done from the side or in such a manner that the surplus water is displaced from the surface of the concrete. The rolling must be continued at intervals of 15 to 40 minutes until all excess water is remover.

Unless protected by metal, the surface edges of all slabs shall be rounded to a radius of one-half inch.

(Cross out the following sections except for the surface desired:)

(a) Smooth surface.-Following the rolling above described, the surface of the concrete shall be troweled with a steel trowel to a smooth, even surface, free from depressions or irregularities of any kind. Excessive working of the surface with the trowel shall be avoided.

(b) Medium rough surface.-Following the rolling above described, the surface shall be floated with a wooden float only, producing an even, gritty finish. On wide sidewalks, after rolling, the finishing may be done with two applications of a canvas belt not less than 6 inches wide and about 2 feet longer than the width of the sidewalk. For the first application the belt shall be drawn across the surface with vigorous strokes at least 12 inches long and moved ahead vely slightly at each stroke. The second application shall be given immediately after the water glaze or sheen disappears, and the stroke of the belt shall be not more than 4 inches, while the longitudinal motion shall be greater than during the first application.

(c) Rough surface.-Following the rolling above described, the surface shall be floated with a wooden float, leveling all sags and producing an even surface. After being floated in this manner, the roughening shall be done by slapping the surface of the concrete with the face of the float, raising the float vertically from the concrete after each stroke. 


\subsection{MISCELLANEOUS SPECIFICATIONS} FOR CEMENT AND CONCRETE.

American Concrete Institute, standard specifications for concrete floors, serial designation $\mathrm{C}-2 \mathrm{~A}-24$, 1924.

(See 518.56, p. 423.)

American Concrete Institute, tentative recommended practice for treatment of exterior surfaces of industrial reinforced-concrete buildings, serial designation C-3B-25T, 1925.

(This recommended practice is designated to outline approved methods of treating the exterior surfaces of concrete factories, warehouses, and other industrial buildings in such manner as to produce pleasing and durable surfaces. The treatments are described in the recommendations under the following five general classes): (a) Pointing and patching; (b) correction of column and beam lines, fill joints, etc.; $(c)$ cement washes and proprietory paints; (d) rubbed finishes; and (e) tooled finishes.

American Concrete Institute, tentative specifications for concrete sewer manhole and catch-basin block, serial designation $\mathrm{P}-1 \mathrm{C}-27 \mathrm{~T}, 1927$.

(See 518.65 , p. 448.)

American Concrete Institute, sewer pipe and monolithic sewers.

1. Tentative specifications for reinforced-concrete sewer pipe, serial designation P-7C-25T, 1925. (See 518.67, p. 449 .)

2. Standard specifications for monolithic concrete sewers, serial designation S-3A-24, 1924. (See 518.67 , p. 451 .)

American Concrete Institute, cement concrete pavements.

(See 518.31 , p. 379 for the following standaids:)

1. Standard specifications for 1-course Portlandcement concrete pavements for highways, serial designation S-6A-28, 1928.

2. Standard specifications for 2-course Portlandcement concrete pavements for highways, serial designation S-6B-28, 1928 .

3. Standard specifications for 1-course Portlandcement concrete street pavement, serial designation S-6C-28, 1928.

4. Standard specifications for 2-course Portlandcement concrete street pavement, serial designation S-6D-28, 1928 .

American Concrete Institute, tentative specifications for concrete curb and concrete curb and gutter, serial designation S-6E-27T, 1927.

(See 518.63 , p. 441.)

American Electric Railway Association, specification for the manufacture of reinforced concrete poles (recommended), serial No. D108-26, 1926.

Note.-The iull text of this specification includes requirements for reinforcement, samples for tests, forms, assembling of reinforcement mixing and placing, curing, and regulations to be applied in tests, inspection, and rejection.

This specification includes the following requirements for materials and proportions:

\section{CEMENT}

Portland cement shall meet the requirements of the American standard specifications for Portland cement of the American Engineering Standards Committee. (See A. S. T. M. C9, 516.11, p. 294.) Cement shall be stored at the plant so that it wifl not be exposed to dampness, and if intended to be stored any length of time it should be placed in a weather-tight structure with floor raised not less than 1 foot from the ground. Cement that has hardened or partially set shall not be used.

\section{FINE AGGREGATES}

(a) General requirements.-Fine aggregate shal consist of natural sand or screenings from hard, tough, crushed rock, or gravel consisting of quartz grains, or other hard material, clean and free from any surface film or coating and graded from fine to coarse, with the coarse particles predominating.

(b) Grading.-Fine aggregate, when dry, shall pass a screen having 4 meshes to the linear inch; not more than 25 per cent shall pass a sieve having 50 meshes per linear inch and not more than 5 per cent shall pass a sieve having 100 meshes per linear inch.

(c) Impurities.-Fine aggregate shall not contain injurious vegetable or other organic matter as determined by the colorimetric test and not more than 5 per cent by volume of clay or loam. If more than 5 per cent of sediment appears above the fine aggregate in one hour's settlement after shaking a sample in a 100 per cent excess of water the material represented by the sample shall not be used in the work.

Colorimetric tests shall be made in accordance with A. S. T. M. C40. (See 512.10, p. 213.)

\section{COARSE AGGREGATE}

Coarse aggregate shall consist of clean, hard, tough, crushed rock or pebbles graded in size, free from vegetable or other organic matter, and shall contain no soft, flat, or elongated particles. The size of the coarse aggregate shall range from threefourths inch down, not more than 5 per cent passing a screen having four meshes per linear inch and no intermediate sizes shall be removed.

\section{WATER}

Water shall be clean, free from oil, acid, alkali, or vegetable matter.

\section{PROPORTIONS}

Using the separate fine and coarse aggregates and the consistency specified herein, the proportions shall be 1 part of Portland cement, $1 \frac{1 / 2}{2}$ parts fine aggregate, and 2 parts of coarse aggregate by volume. Under ordinary conditions this should produce a concrete having a compressive strength of $3,000 \mathrm{lbs}$. $/$ in. $^{2}$ at 28 days. The method of measuring the materials, including water, for the concrete should be one which will insure separate and uniform proportions of the materials at all times. As a rule, measurements by weight are more uniform and accurate than measurements by volume.

American Petroleum Institute, code of recommended field practice, standard rigs and derricks, July, 1928.

Spccifications for concrete derrick foundations.

(Prepared in cooperation with Portland Cement Association.)

The following is submitted as a type of specifications suitable for the construction of concrete rig and derrick foundations and other structures about an oil well where conditions are such that refinement of methods is practically impossible. This specification should not be used for other classes of work.

\section{MATERIALS}

Portland cement.-The cement shall be new and free from lumps. The foreman shall promptly reject any cement that has hardened or partially set, and shall protect all cement delivered on the site by storing it in a shed, if possible, or by covering it with tarpaulins until it is ready to be used. Cement shall not be piled on the ground, but shall be neatly stacked on a platform held at least 6 inches from the earth. Cement that has been rained upon or otherwise subjected to dampness shall not be used.

Aggregates.-The sand and stone shall be clean and free from lumps of earth or other foreign matter, 
and the particles shall not be coated with mud, oil, or any other injurious substances. The sand shall not be composed of uniform size grains, but shall contain both fine and coarse particles, the coarse predominating. The stone or gravel should be of different sizes, varying from one-fourth of an inch as the smallest to particles that will pass through a 2 -inch circular opening as the largest.

The foreman shall see that the stone and sand are stored in separate piles and protected from the addition of foreign substances.

Locally dug gravel or stone shall not be used except upon written permission of the oil company's representative.

Mixing water.-Mixing water shall be free from oil, acid, and injurious amounts of vegetable matter or salts. In general, if the water is unfit for drinking it is unfit for concrete. The foreman shall see that the best water available at the site is used.

\section{PROPORTIONING AND MIXING}

General.-The unit of measure shall be the cubic foot. One sack of cement (94 pounds) is assumed as 1 cubic foot. The foreman shall provide a wooden box whose inside dimensions shall be exactly 12 inches square and 12 inches deep, made with a bottom if concrete is mechanically mixed, and without a bottom if mixed by hand. The box shall have suitable handles for convenience in lifting.

If concrete is to be mixed by hand the foreman shall provide a water-tight mixing platform at least 7 feet wide and 12 feet long. He shall also provide a can of known gallon capacity for measuring the mixing water.

Measuring ingredients.-All ingredients shall be accurately measured to secure the specified proportions in each batch. The stone and sand must be measured scparately. The water shall be so measured as to insure the specified quantity in each batch.

Hand mixing.-In case concrete is hand mixed it shall be done in the following manner and order: The measured quantity of sand is to be spread out evenly on the platform. On this is evenly spread the required amount of cement. The cement and sand are then turned over thoroughly with square shovels enough times to produce a mass of uniform color. The measured quantity of broken stone or gravel is then spread in a layer on top of the cementsand mixture and all the materials again mixed by turning with shovels until the stone has been uniformly distributed throughout the mass. A depression or hollow is then formed in the center of the pile and the measured water added slowly while the materials are turned, this turning being continued until the whole mass is in a plastic or pasty jellylike consistency.

Machine mixing.-If a mechanical mixer is used the measured ingredients are to be placed in the mixer and mixed for not less than two minutes.

\section{PROPORTIONING}

The proportions of materials for concrete going into rig and derrick corner foundations shall be based on the requirements for a workable mix, containing not more than 7 gallons of water per sack of cement. In case the sand is very wet, as right after a rain, the water must be reduced to 6 gallons per sack of cement. If the sand is uniformly damp, $61 / 2$ gallons of water should be used per sack of cement. The other ingredients shall be proportioned as follows:

One sack of Portland cement.

Two cubic feet of sand.

Three and one-half cubic feet of gravel or stone.
The mixture shall be such as to produce concrete that can be worked readily into the corners and angles of the forms with ordinary spading. The workability will be controlled by adding or deducting sand or stone, but in no case shall the amount of stone be such as to produce difficulty in placing or honeycombing in the structure. Most important of all, the amount of water specified for each sack of cement shall in no case be exceeded.

Handling and placing.-Concrete shall be placed in the forms in such a manner as to prevent the separation or loss of ingredients. Each batch as placed shall be well spaded into the corners of the form so as to eliminate air pockets and prevent honeycombing. Concrete must be placed in the forms immediately after being mixed, and under no circumstances shall concrete that has partially hardened be deposited in the forms.

Admixtures.- Calcium chloride or other admixtures shall not be added to the concrete except upon written order of the oil company's representative, and then only in the proportions specified by him. If admixtures in liquid form are ordered the quantity used must be deducted from the mixing water.

American Railway Association, signal section, specification 11622, November, 1922, cement concrete battery box.

1. Purpose.-The purpose of this specification is to provide cement concrete battery box for housing primary and storage batteries.

2. Drawings.-Purchaser's drawings accompanying this specification and forming an essential part thereof are Figure 109, A. R. A. Sig. Sec. drawing 1597A and Figure 110, A. R. A. Sig. Sec. drawing 1598A

3. Material and workmanship.-Material and workmanship shall be first class in every respect.

4. Reinforcements.-(a) Reinforcing material shall be deformed one-fourth inch square steel rod or its equivalent.

(b) Reinforcement shall be placed not more than 1 inch nor less than one-half inch from inner surface of box.

(c) Bottom and walls of box, including the space above outlets, shall be completely reinforced with 0.068-inch steel mesh netting.

(d) Two reinforcing rods properly bent shall form two complete reinforcing bands around top of box above outlets; ends of each rod shall lap not less than 6 inches.

(e) One reinforcing rod of length 2 inches less than the height of wall shall be placed vertically 2 inches from inside corner in end walls and approximately 8 inches from inside corner in side walls.

(f) Bottom of box shall be reinforced lengthwise and crosswise with reinforcing rods of length 2 inches less than outside dimension of the box and spaced not more than 12 inches apart.

5. Cement.-The cement shall conform to the specifications of the purchaser.

6. Sand.-Sand shall be clean, sharp, and coarse. It shall be free from humus content and other foreign matter, but may contain a clay dust not to exceed 5 per cent.

7. Water.-Water shall be clean, fresh (not salt), and frec from sewage or other impurities detrimental to the concrete.

8. Stone or gravel.-(a) Stone or gravel shall be sound, hard, and durable and shall be clean and free from shale, dust, loam, clay, or any foreign substance.

(b) Stone or gravel shall be of such size as to pass over a 1/4-inch and through a $3 / 4$-inch screen.

9. Waterproofing. - A thin coat of mortar, or grout, shall be applied for a finishing coat upon which shall be placed a covering of suitable waterproofing. 

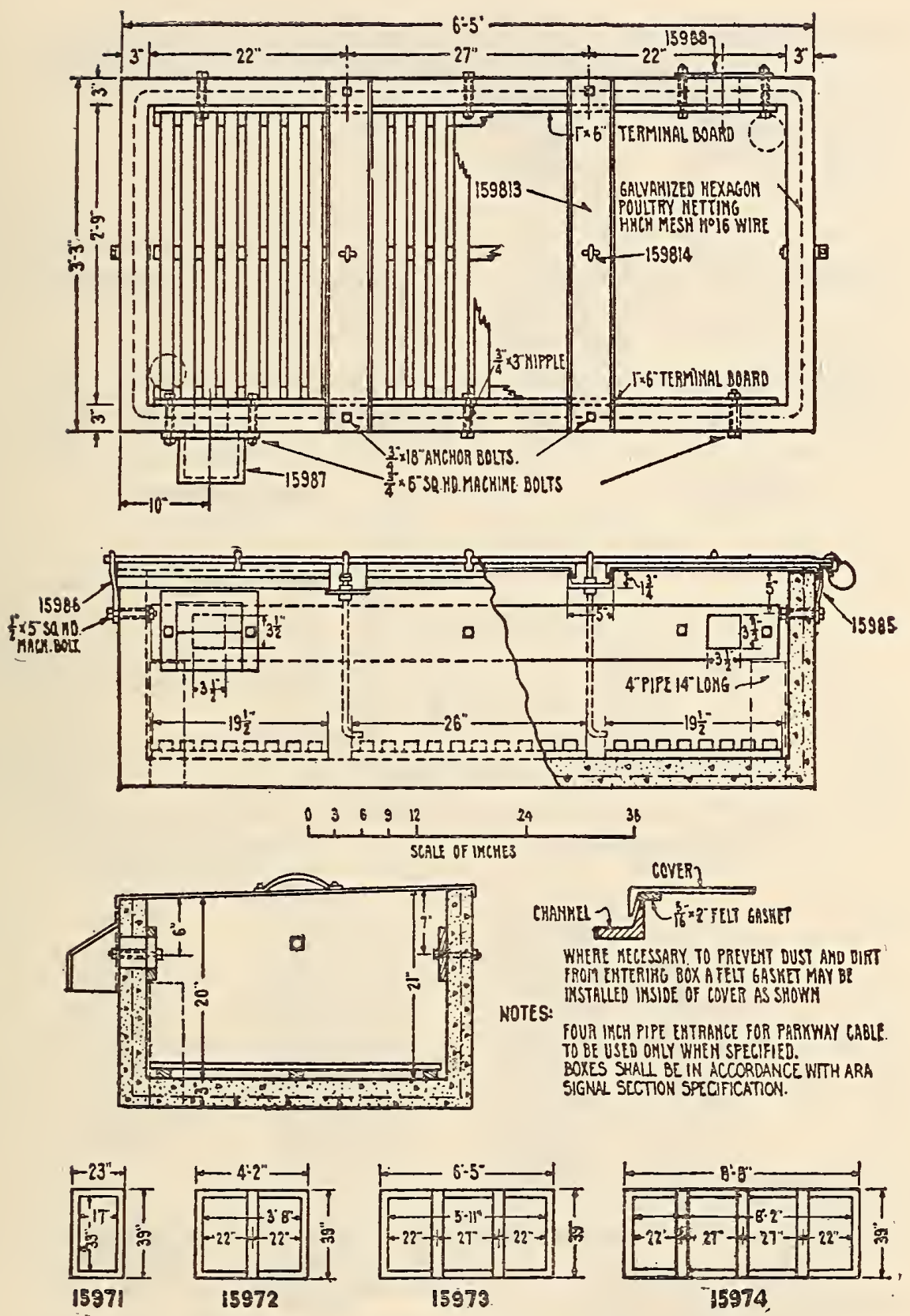

CONERETE OATTERY DQX

Figure 109.-A. R. A., S. S., drawing No. 1597 A

(For details see fig. 110) 


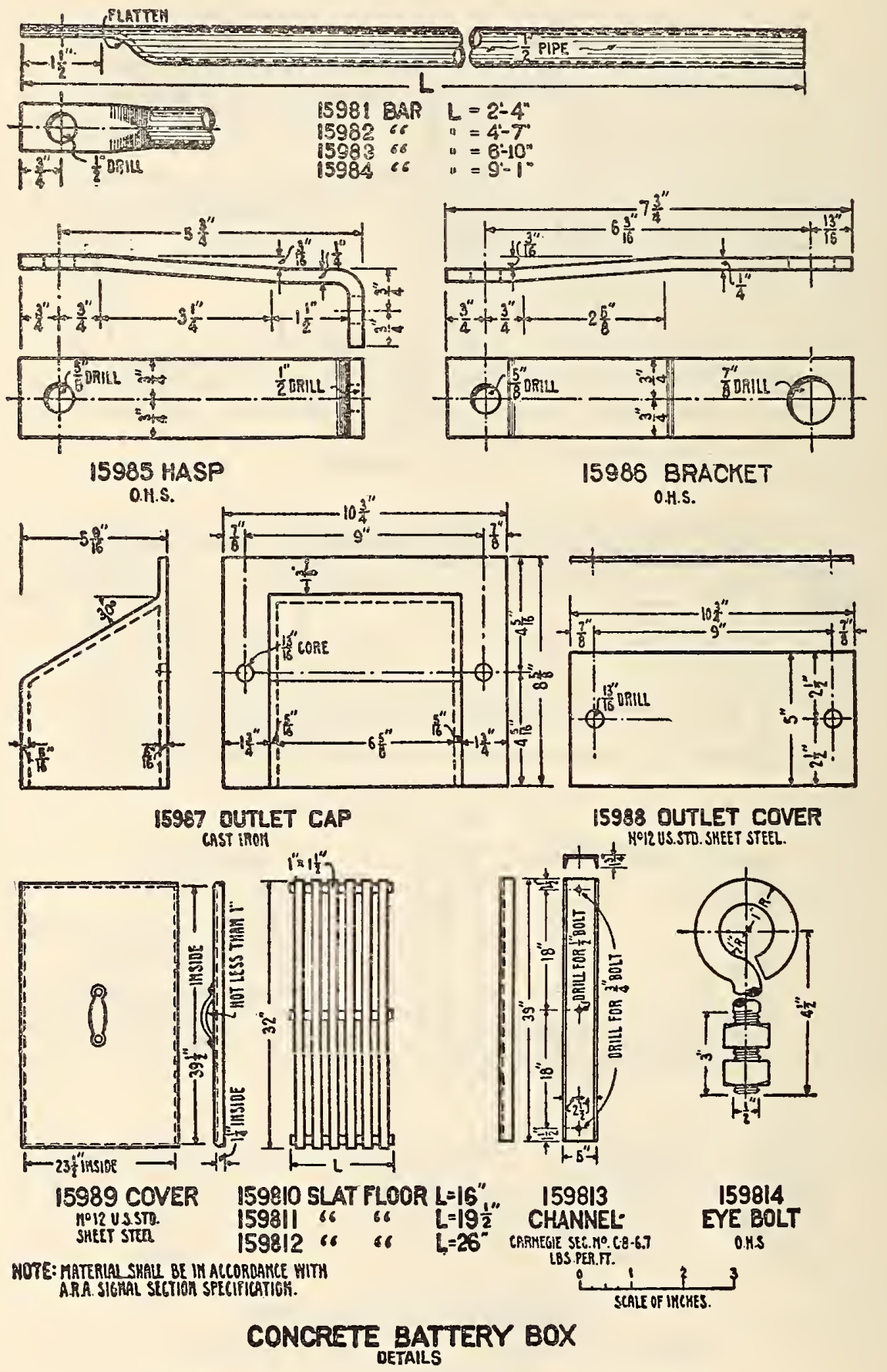

FIGURE 110.-A. R. A., S. S., drawing No. 1598A 
10. Proportions.-(a) The concrete shall be mixed in the proportion of 1 part of cement to not more than $1 \frac{1}{2}$ parts of sand, and 2 parts of stone or gravel, measured separately.

(b) The fine and coarse aggregates shall be used in such relative proportions as will insure maximum density.

11. Mixing.-(a) The mixing shall be done in a machine of the batch type.

(b) Each batch shall be mixed for not more than one and one-fourth minutes nor less than one minute.

12. Consistency.-The materials shall be wet mixed and of such consistency as with continual puddling, to barely flow into the form and about the metal reinforcement, without a separation of the aggregates while placing or while being conveyed from place of mixing to the forms.

13. Placing of concrete. - (a) The concrete shall be spaded along each surface of the mold as it is deposited to insure the smoothest possible finish. Every section shall be completely filled before any portion of the concrete has set.

(b) Concrete that has partially set shall not be used.

14. Temperature.-Concrete shall not be mised nor placed where and when the temperature is below $40^{\circ} \mathrm{F}$.

15. Forms.-The forms shall be substantially built and in such manner that the product will conform accurately to the measurements as specified on drawings. "The forms shall be water-tight and shall be coated with oil or grease to prevent concrete adharing to them. The oil or grease shall be of such character that it will not discolor the concrete.

16. Removing forms.-Forms shall not be removed until the concrete has set sufficiently to prevent its adhering to the forms when they are removed.

17. Outlets.- (a) Outlets shall be provided in accordance with Figure 109.

(b) One piece of 35-mesh copper screen, the size of the outlet cover, shall be provided for one outlet of each box for housing storage battery.

18. Cover.-(a) Cover shall be made of good quality, well-seasoned, fir lumber. The lumber shall be matched seven-eighths inch thick, surfaced both sides. Cleats shall be of $7 / 8$ by 4 inch lumber, surfaced four sides.

(b) Wooden cover shall be so constructed as to prevent rain entering the box. It shall be covered with galranized sheet iron. If two or more sheets of sheet iron are used, the joints shall be lap-turned. The sheet iron shall be fastened at front, back, and ends with No. 12 round-head wood screws, spaced 4 inches apart.

19. Hinges and hasps.-Hinges and hasps shall be fastened to cover beneath metal covering with 1 -inch No. 12 mood screws.

20. Eye rod.-An eye rod shall be provided at each end of box to support cover when raised, except as provided on A. R. A. Sig. Sec. 1597 A.

21. Ironwork.-There shall be no exposed ironwork inside of box; all bolts, screws, and nailheads shall be countersunk and protected with acid-proof compound.

22. Inside coating.-The entire inside surface and top edges of box for housing storage battery and all surfaces of wire board and cover shall be given two coats of asphaltum paint before installation of wire board.

23. Painting.-Exposed ironwork shall be thoroughly cleaned and given either a priming brush or dipping coat of red-oxide or graphite paint and two coats of light-gray paint.
24. Wire board and lining.-The wire board, lining, and frost board shall be of good quality, wellseasoned, cypress, fir, or white pine lumber.

25. Inspection.-Inspection requirements same as for A. R. A. Sig. Sec. specification 11328. (See 522, p. 491.)

26. Tests.-(a) Section 5 items $(a),(b),(c)$, and (d) of No. 11328 are part of the test requirements of this specification.

(b) The finished box shall not show visible defects, cracks, or leaks during or after completion of the following tests, which shall be made at the option of the purchaser on each separate box at the place of manufacture or on arrival at destination:

(1) The box shall be suspended continuously for five hours on a single rigid beam placed through the front outlet at one end and rear outlet at the other end, and supported at each end outside of the box. At the end of five hours the beam shall be changed to extend through the other two outlets and test continued for five hours more.

(2) The box shall be supported free from ground or floor on two beams of 2 inches surface placed directly beneath the box and ailowed to stand for 48 hours while filled with water to within 1 inch of the bottom of outlet opening.

(c) To determine humis content of sand, fill a wide-mouthed bottle two-thirds full of suspected material and add sufficient 4 per cent solution of sodium hydroxide to fill the bottle: shake occasionally, and if after 24 hours solution is not darker than a straw color, it is safe; if very dark, it shall not be used.

27. Curing.-Upon removal of the forms the finished product shall be protected from the direct rays of the sun and wind for a period of 48 hours and shall be kept wet by sprinkling for at least 7 days. The temperature of the air in which the curing is done shall be maintained above $40^{\circ} \mathrm{F}$., but artificial heat exceeding $110^{\circ} \mathrm{F}$. shall not be used.

28. Packing.-Material shall be so prepared as to permit convenient handling and to protect against loss or damage during shipment.

29. Marking.-(a) Purchaser's order, requisition and package number, name of consignor, and name and address of consignee shall be plainly marked on outside of package.

(b) Detail list of loose pieces, containers, and their contents shall be furnished for each shipment. Where carload shipments are made, routing and car identification shall be shown.

(c) There carload shipments are made, name and address of consigneee may be omitted.

30. Warranty. - $(\alpha)$ Contractor shall warrant the material covered by this specification to be free from defeets in material and workmanship under ordinary use and service, his obligation under this warranty being limited to making, at point of production, any part or parts to replace those which shall be found defective within one year after ship. ment to the purchaser. This warranty shall not apply to any apparatus which shall have been repaired or altered in any way by any one other than the manufacturer thereof, so as to affect, in the contractor's juagment, its proper functioning or reliability or which has been subject to misuse, negligence, or accident.

American Railway Association, signal section, specification 12422, November, 1922, concrete trunking, capping and supports.

(This specification is identical with A. R. A. Sig. Sec. No. 11622 (given a'oove) as to requirements under the following headings: Material and worḱinanship, cement, sand, water, stone, or gravel, proportions (except that this specification calls for 
2 parts of sand and 2 parts of stone or gravel to 1 of cement), consistency, placing of concrete, temperature, forms, removing forms, curing, inspection, packing, marking, and warranty. This specification has the following in addition):

Purpose.-The purpose of this specification is to provide for reinforced concrete trunking, capping, and supports to be used for the protection of rubberinsulated wire.

Drawings.-Purchaser's drawings accompanying this specification and forming an essential part thereof are Figure 111, A. R. A. Sig. Sec. drawing 1452, and Figure 112, A. R. A. Sig. Sec. drawing 1453.

Reinforcements.-Reinforcing material shall be deformed one-fourth inch square steel rod or its equivalent.

Mixing.-(a) The mixing shall be done in a machine of the batch type.

(b) Each batch shall be mixed for not less than one minute nor more than one and one-half minutes. Tests.-(Sec. 5, items $(a),(b),(c)$, and $(d)$ of A. R. A. Sig. Sec. specification 11328, are part of the test requirements of this specification, with the following additional:)

(a) Material shall be cured not less than 28 days before testing. Trunking shall be supported for a distance of 3 inches at each end. Where side walls are 5 inches high, outside dimensions, it shall support a weight of 500 pounds, concentrated within 12 inches in the center, without showing cracks. Where side walls are $6 \% 8$ inches high, outside dimensions, it shall support a weight of 800 pounds, concentrated within 12 inches in the center, without showing cracks.

(b) To determine humus content of sand, fill a wide-mouthed bottle two-thirds full of suspected material and add sufficient 4 per cent solution of sodium hydroxide to fill the bottle; shake occasionally, and if after 24 hours solution is not darker than a straw color, it is safe; if very dark, it shall not be used.

Finishing.-Before concrete is fully set all sharp edges shall be removed.

Outlets.-Outlets for branch leads, when specified, shall be provided when pouring concrete, using blocks of the size required to admit branch leads.

Finished product.-Trunking and capping shall be true to form and have a uniform thicknesss throughout not varying in thickness more than one-eighth inch from dimensions shown on drawings.

American Railway Association, telegraph and telephone section, reinforced concrete poles, specification $1-\mathrm{A}-8,1925$.

This specification includes the following requirements for materials and proportions:

\section{A. GENERAI}

This specification prescribes the general requirements for the manufacture of reinforced concrete telegraph and telephone poles.

\section{B. MATERIAL}

Cement.-An approved grade of Portland cement shall be used.

Sand.- Sand shall be clean, sharp, and coarse, free of humus content and other foreign materials, but may contain a clay dust not to exceed 5 per cent.

Water.-Water should be clean, fresh (not salt) and free from sewage or other impurities detrimental to the concrete.

Stone or gravel.-Stone or gravel shall be sound, hard, and durable, and shall be clean, and free from shale, dust, loam, clay, or any foreign substance. If necessary to meet these requirements, broken stone shall be screened and washed. The size of pieces shall range from a minimum of one-fourth inch to a maximum of three-fourths inch.

Steel reinforcing.- Steel reinforcing shall be deformed square rods of high grade, having an elastic limit of $55,000 \mathrm{lbs} . /$ in. $^{2}$

Proportions. - The concrete shall be mixed in the proportion of 1 part of cement to not more than 2 parts of sand and 2 parts of stone or gravel measured separately.

\section{PROCESS OF MANUFACTURE}

Method of casting. - The entire pole shall be cast at same time.

Method of reinforcing.-All steel reinforcement shall be of the cage type of construction, steel being placed in its designated position and maintained there throughout the process of manufacture. All chairs or other devices used to retain the reinforcement in position shall be of rustproof material, and if any portion of such chairs or devices is exposed at the surface it shall be confined to a point of contact with the form. Steel reinforcement shall be covered at all points by not less than three-fourths inch of concrete. No splicing will be permitted in the reinforcement rods unless made by such method that it will leave the splice equally as strong as the remainder of the rod, to the satisfaction of the purchaser. The surface of all steel reinforcement used shall be free from rust, oil, or grease, so that there will be a perfect and lasting bond between the concrete and the steel.

Mixing.-(a) The mixing shall be done in a machine of the batch type.

(b) Each batch to be mixed not less than one minute or more than one and one-half minutes.

Consistency. - The material shall be mixed by the wet process, the quantity of water employed being not less than 70 per cent by weight of the quantity of cement employed. The finished product shall be free from all air pockets or other voids, dense and waterproof.

Placing of concrete-(a) Every section shall be completely filled before any portion of the concrete has set.

(b) Concrete that has partially set or has been mixed over 30 minutes shall not be used.

(c) If a core is used it shall be so designed that no pressure shall be brought to bear on the concrete in removing same.

(d) In placing the concrete the larger aggregates shall be brought to the exterior surface of the finished poles, the space between the larger aggregates being completely filled with the smaller aggregates and the whole strongly bonded by the cement, the object being to produce a smooth surface. The mixture should be uniform over the entire exterior surface of the poles.

Dimensions.-Dimensions of poles shall be in accordance with Figure 113 A. R. A., T. \& T. No. 1293.

Temperature.-Concrete shall not be mixed nor placed when the temperature is below $40^{\circ} \mathrm{F}$. This applies to the place where manufactured.

Handling.-Forms shall not be removed until the concrete has attained a set sufficiently hard to prevent its adhering to them when they are removed.

Curing.-Upon removal of the forms the finished product shall be protected from the direct rays of the sun and wind for a period of 48 hours and shall be kept wet by spiinkling for at least 7 days. The temperature of the air in which the curing is done shall be maintained at above $40^{\circ} \mathrm{F}$., but artificial heat exceeding $110^{\circ} \mathrm{F}$. shall not be used, except when placed in a chamber containing unsaturated water vapor where temperatures up to $180^{\circ} \mathrm{F}$. are permissible. 


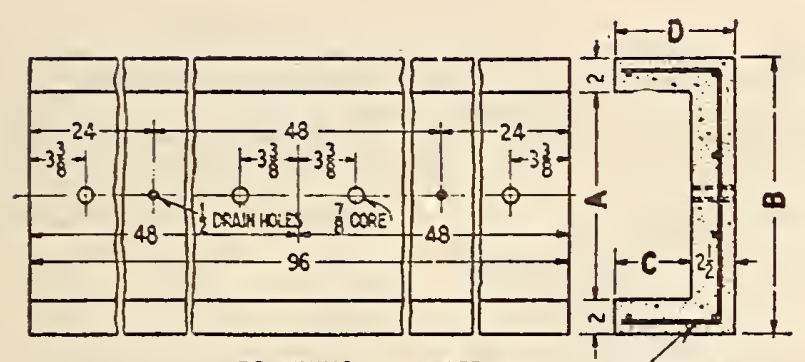

TRUKKING

NOTE: FOR DETAL OF RENFORCENIENT SEE DRAWI:G 1453.

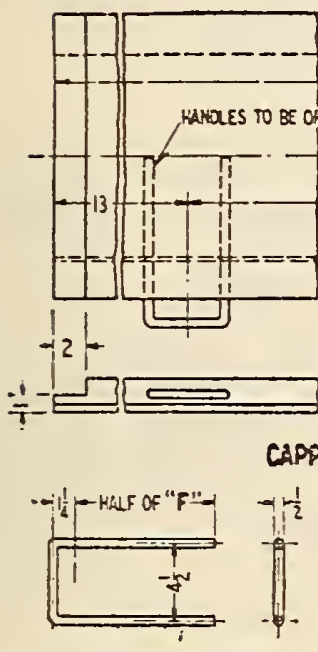

HANDLE POR CAPPING OH. STEEL

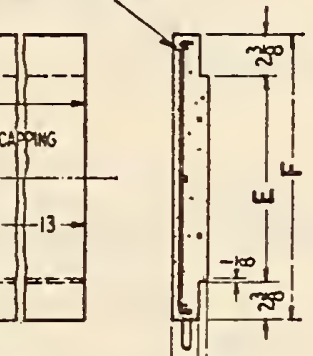

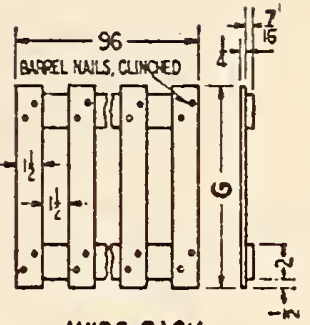

WIRE RACK

ROUGH YELLON PUE.
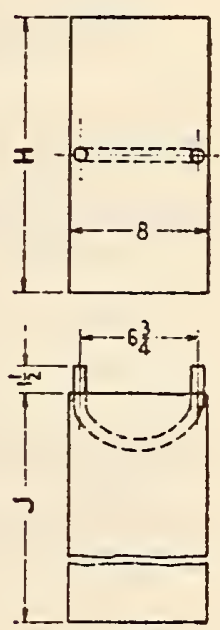

PIER

03399

SCUE ANO ALL DIMENSIONS GIVEN IN INEKES

\begin{tabular}{|c|c|c|c|c|c|c|c|c|c|c|c|c|c|c|c|c|c|}
\hline \multicolumn{6}{|c|}{ TRUAKIKG (8 FT. SECTION) } & \multicolumn{5}{|c|}{ CAPPIHG (4 FT. SECTIOHS) } & \multicolumn{3}{|c|}{ WHE RACK } & \multicolumn{4}{|c|}{ PIER } \\
\hline 4 & B & C & 0 & $\begin{array}{l}\text { LPPDOX: } \\
\text { WT. LRS }\end{array}$ & REE. NO & $E$ & $F$ & $\begin{array}{l}\text { APPRA } \\
\text { HT LAS }\end{array}$ & in & REF. NOO. & $G$ & EP'? & REF. MO & 4 & J & RE⿱宀㠯心 & REF: NO \\
\hline 2 & 61 & $2 \frac{1}{2}$ & 5 & 196 & 14521 & $1 \frac{3}{4}$ & $6 \frac{1}{2}$ & 42 & 2 & 14527 & & & & 6 & 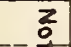 & 2 & 145217 \\
\hline 4 & 8 & " & ، & 240 & 14522 & $3 \frac{3}{4}$ & $8 \frac{1}{2}$ & 58 & 2 & 14528 & & & & 8 & 5 & 2 & 145218 \\
\hline 8 & 12 & 43 & 67 & 380 & 14523 & $7 \frac{3}{4}$ & $12 \frac{1}{2}$ & 82 & 2 & 11523 & $7 \frac{3}{4}$ & 1 & 145213 & 12 & ç & 2 & 5219 \\
\hline 12 & 16 & “ & “ & 475 & 14524 & $11 \frac{3}{4}$ & $6 \frac{1}{2}$ & 122 & 2 & 145210 & $11 \frac{3}{4}$ & 1 & 145214 & 16 & $\frac{1}{D}$ & 2 & 145220 \\
\hline 16 & 20 & " & 6 & 550 & 14525 & $15 \frac{3}{4}$ & $20 \frac{1}{2}$ & 154 & 2 & 211 & $15 \frac{3}{4}$ & 1 & 15 & 20 & & 2 & 145221 \\
\hline 20 & 24 & 6) & 6 & 625 & 16525 & $19 \frac{3}{4}$ & $24 \frac{1}{2}$ & 186 & 2 & 145212 & $19 \frac{3}{4}$ & 1 & 145216 & 24 & $\bar{z}$ & 21 & 145222 \\
\hline
\end{tabular}

\section{CONCRETE TRUAKMNG, CAPPIHG AND SUPPORT}

FIgURE 111.-A. R. A., S. S., drawing No. 1452 

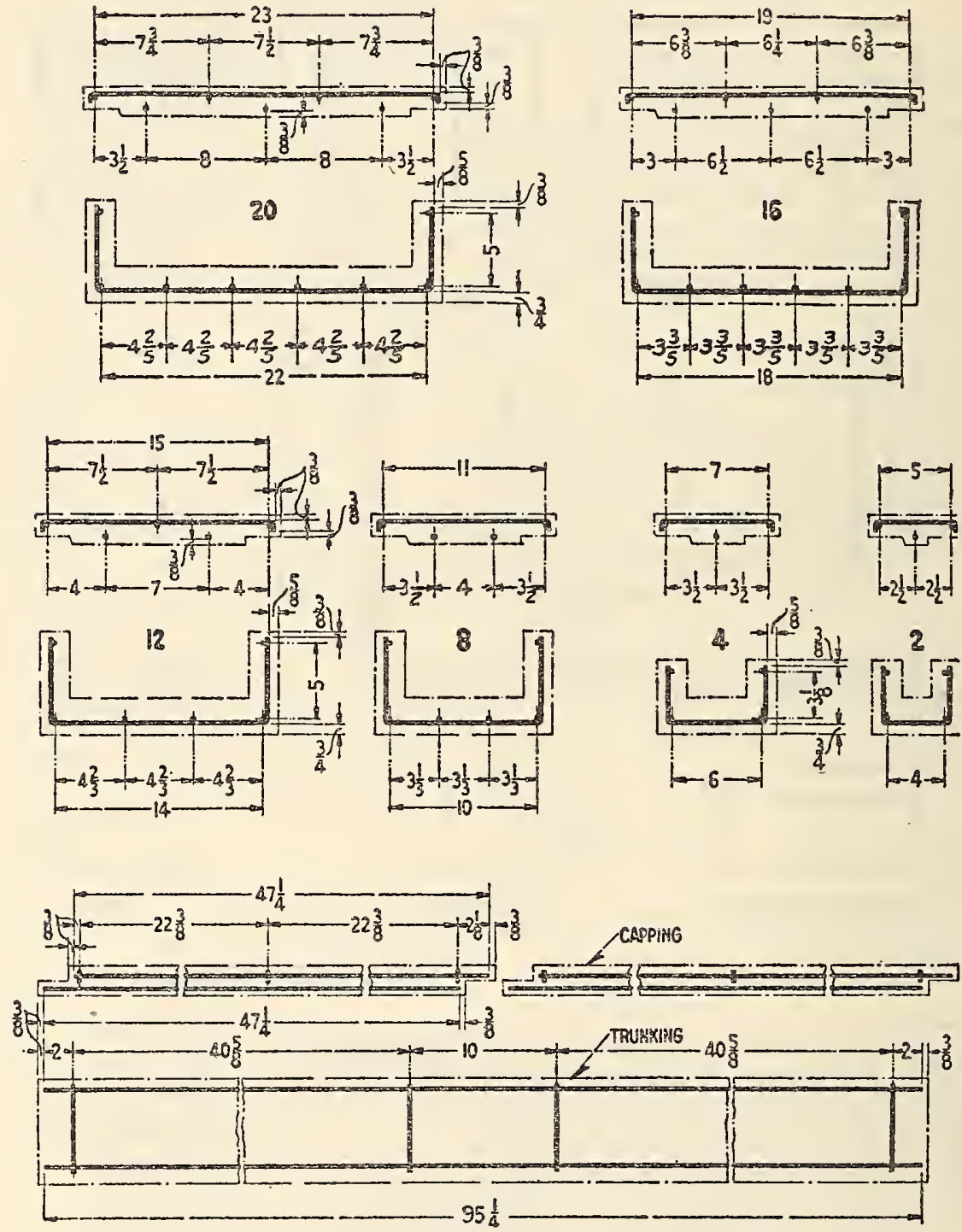

NOTE: PEIHFORCEMENT FOR TRLNWING AND CAPPIMG SHALL BE OF 1 SOISARE DEFORMEO STEEL RCOS OR THEIR EQUIVALENT, WIREO AT ALL POITTS OF COHTACT.

0369 is

FOR OTHEA DIMENSIONS AND FURTHER DETAILS

SEE CRAWNG 1452 AHD SPECIFICATIOH.

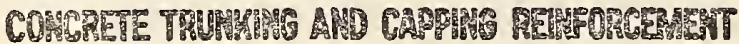

Figure 112.-A. R. A., S. S., drawing No. 1453 


\section{INSPECTION}

Purchaser may inspect the material at all stages of manufacture.

Purchaser may inspect the completed product to determine that the requirements of this specification have been met.

If the material has not been accepted at point of production, and if, upon arrival at destination, it does not meet the requirements of this specification, it may be rejected, and the contractor, upon request, shall advise the purehaser what disposition is to be made of the defective material. The contractor shall pay all freight charges.

If purchaser is to make inspection at point of production it shall be so stated.

\section{E. TESTS}

All poles furnished hereunder shall be sufficiently strong to withstand without any injury whatever, or permanent deformation, a stress of such force as
Test Table No. 2

\begin{tabular}{|c|c|}
\hline Length of pole & $\begin{array}{l}\text { Depth of } \\
\text { setting }\end{array}$ \\
\hline $\begin{array}{l}20 \\
30 \\
35 \\
45\end{array}$ & $\begin{array}{rl}\text { ft. } & \text { in. } \\
4 & 0 \\
5 & 0 \\
5 & 6 \\
6 & 0 \\
6 & 6 \\
7 & 0\end{array}$ \\
\hline
\end{tabular}

All poles furnished hereunder shall meet the above requirements as to required strength at any time subsequent to 30 days after they are cast.

Tests may be made at point of production or destination.

Contractor shall give the purchaser sufficient notice of date when material will be ready for testing.

\section{DIMENSIONS OF CIRCULAR CONCRETE POLES}

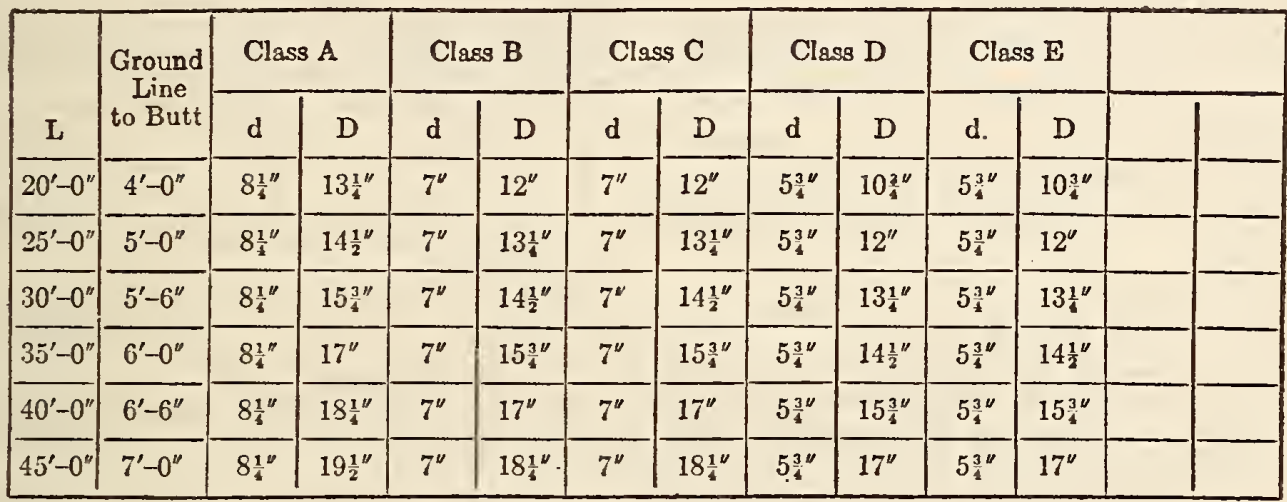

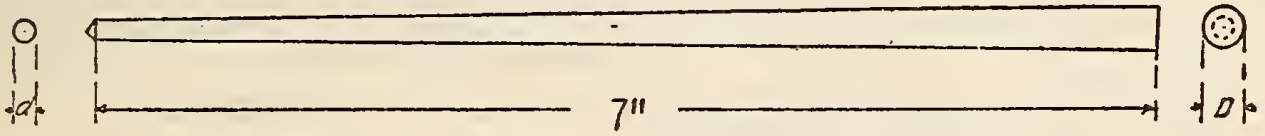

FigURE 113.-A. R. A., T. \& T. S., drawing No. 1293

specified in the following table, such stress being applied 2 feet from the top of the pole in any direction at right angles to the axis of the pole, the pole being supported only by its butt, which shall be set firmly in the ground or anchored to the testing apparatus. Maximum deflection shall not exceed 1 inch for each 2 feet of length above ground.

Test Table No. 1

\begin{tabular}{|c|c|}
\hline Class & Test load \\
\hline $\begin{array}{l}\mathrm{A} \\
\mathrm{B}_{1}-\ldots \\
\mathrm{C} \\
\mathrm{D} \\
\mathrm{E}\end{array}$ & $\begin{array}{c}\text { Pounds } \\
4,000 \\
3,000 \\
2,000 \\
1,500 \\
1,000\end{array}$ \\
\hline
\end{tabular}

Contractor shall provide at point of production apparatus and labor for making required tests under supervision of and without cost to the purchaser.

If tests are to be made at point of production, it shall be so stated. Purchaser will distinctly indicate which of the tests herein specified are to be made and what portion of the material shall be tested.

To test for humus deposits in sand, fill widemouth bottle two-thirds full of suspected material and add sufficient 4 per cent solution sodium hydroxide to fill bottle. Shake occasionally, and if after 24 hours solution is not darker than a straw color, it is safe; if very dark, it must not be used.

\section{F. LOADING ON CARS FOR TRANSPORTING}

All poles shall be carefully loaded on cars with proper supports in accordance with American Railway Association loading diagram. 


\section{G. MARKING}

Each pole shall be marked with a brand in the concrete at a point 8 feet from the butt end of the pole with the name of the manufacturer and the date manufactured, letters being not over 2 inches high or one-fourth inch deep.

\section{H. WARRANTY}

The $\left\{\begin{array}{l}\text { seller } \\ \text { contractor }\end{array}\right\}$ hereby expressly warrants that the material furnished under this specification shall be free from defects in design, material, and workmanship. The $\left\{\begin{array}{l}\text { seller's } \\ \text { contractor's }\end{array}\right\}$ obligation under this warranty shall not apply to any material which has been subject to misuse, negligence, or accident after acceptance.

The $\left\{\begin{array}{l}\text { seller } \\ \text { contractor }\end{array}\right\}$ hereby covenants and agrees to save harmless and indemnify the purchaser against all claims, suits, actions or proceedings, damages, costs, fees, and expenses by reason of alleged infringement of patents, or for patent royalties involved, in consequence of the purchase or the use of the material covered hereby.

Note-The full text of this specification includes items relating to the process of manufacture, inspection, tests, marking, and warranty.

American Railway Engineering Association, concrete fence posts, 1918.

1. Concrete fence posts are practical, economical, and a suitable substitute for wood.

2. Reinforcement should be placed as near to the surface of the post as practicable, say one-half inch from surface.

3. Posts should taper from base to top.

4. Square corner posts should be rounded off to radius of not less than 1 inch.

5 . Concrete should be made from clean, hard, aggregates, the percentage of the various sized grains being such as to produce a dense concrete, using screen analysis as a guide. The minimum size of the particles of gravel or crushed stone should not be less than one-fourth inch nor more than one-half inch. Concrete should be mixed in the proportion of 1 part cement to not more than 4 parts of mixed aggregate. Concrete should be of such a consistency that water can be brought to the surface by tamping; the use of an excess of water is detrimental. Concrete should be very thoroughly mixed in a batch, not a continuous mixer.

6. Reinforcing should be in the form of stiff, round or square rods, preferably deformed, made from steel with a high elastic limit. Crimped or stranded reinforcing that would be straightened out when brought into tension should not be used. Some method of positively holding the reinforcing in its proper place in the post throughout its entire length should be used.

7. Jogging or vibrating molds to compact the concrete in the post, or some other method that will accomplish the same purpose, should be employed.

8. Posts should be carefully made so as to secure a uniform strength in substantially all posts, and this strength should usually be such that the post will withstand a force of not less than 180 pounds at right angles to the axis of the post, the post acting as a cantilever beam supported at the ground line and the force being applied 60 inches above the ground line. It is not economical to make posts that will have the strength to resist a force of over 200 pounds when the post is tested in the manner above described.

9. Square, or nearly square, posts are more efficient than round posts in resisting the forces that ordinarily cause failure, but the difference is not very great and may in some cases be offset by an increased resistance to deterioration and better methods of manufacture.

10. Posts should not be made out of doors in freezing weather. They should not be exposed to the sun, and should be sprinkled with water the first 8 or 10 days after being made to aid curing.

11. Molds should be carefully oiled or soaked to prevent concrete sticking to them.

12. Posts should be cured for not less than 90 days, when cured naturally, before being set or shipped.

13. Posts should be carefully handled and packed in straw, sawdust, or other suitable material for shipment.

American Railway Engineering Association, specifications for constructing premolded concrete piles, 1921

See 518.42, page 411 .

American Railway Engineering Association, specifications for buildings for railway purposes, 1926 .

Sewers and drainage (reinforced concrete pipe).

See 518.67, page 453 .

American Society for Municipal Improvements, specifications for Portland cement, concrete pavements, February 1, 1926.

See 518.31 , page 389 .

American Society for Municipal Improvements, standard specifications for sewers, 1927.

(This specification includes requirements for cement concrete pipe, reinforced concrete pipe, concrete, and concrete sewers built in place.)

See 518.67, page 453 .

American Society for Municipal Improvements, standard specifications for curbs, 1927.

See 518.63, page 443 .

American Society for Testing Materials, standard specifications for cement concrete sewer pipe, serial designation C14-24, 1924.

See 518.67, page 464 .

Joint Concrete Culvert Pipe Committee, tentative standard specifications for reinforced concrete culvert pipe, 1928

(Constituent organizations of the above committee are: American Society for Testing Materials, Bureau of Public Roads of the United States Department of Agriculture, American Society of Civil Engineers, American Association of State Highway Officials, American Railway Engineering Association, American Concrete Institute, and American Concrete Pipe Association.)

\section{GENERAI}

1. These specifications apply to reinforced concrete pipe intended to be used for the construction of culverts.

2. Pipe, under these specifications, shall be of two classes known, respectively, as standard reinforced concrete culvert pipe, and extra strength reinforced concrete culvert pipe.

3 . The acceptability of pipe shall be determined by the results of the strength and absorption tests hereinafter specified, if and when required, and by inspection to determine whether the pipe comply with the specifications as to design, and freedom from defects.

\section{MATERIALS}

4. The reinforced concrete shall consist of Portland cement, mineral aggregate, and water in which steel has been embedded in such a manner that the steel and the concrete act together in resisting forces.

5. Portland cement shall meet the requirements of the current standard specifications and tests for 
Portland cement of the American Society for Testing Materials, A. S. T. M. C9. (See 516.11, p. 294.)

6. Reinforcement may consist of wire which meets the requirements of the current specifications for cold-drawn steel wire for concrete reinforcement of the American Society for Testing Materials, or of bars which meet the requirements of the current standard specifications for billet-steel concrete reinforcement bars of the American Society for Testing Materials.

7. (a) Fine aggregate shall consist of sand, stone screenings, or other inert materials with similar characteristics, or a combination thereof, having clean, hard, strong, durable, uncoated grains, and free from injurious amounts of dust, lumps, soft, or flaky particles, shale, alkali, organic matter, loam. or other deleterious substances. Fine aggregate shall be well graded and shall pass a $1 / 4$-inch screen.

(b) Coarse aggregate shall consist of crushed stone, gravel, slag, or other approved inert materials with similar characteristics, or combinations there of having clean, hard, strong, durable, uncoated particles, free from injurious amounts of soft, friable, thin, elongated or laminated pieces, alkali, organic, or other deleterious matter.

8. The aggregates shall be so graded and proportioned and thoroughly mixed with such a proportion of cement and water as will produce a homogeneous concrete mixture of such quality that the concrete will meet the test and design requirements herein specified.

9. The pipe shall be designed in accordance with the following assumptions:

(a) That the design load is equivalent to a vertical load uniformly distributed over the internal horizontal projection of the pipe, that the pipe is likewise uniformly supported and that no allowance is made for side pressure.

(b) The uniform load for the standard reinforced concrete culvert pipe shall be 2,000 pounds and for the extra strength reinforced concrete culvert pipe 4,000 pounds per square foot, respectively.

(c) The working stress per square inch in compression for the concrete shall not excced threeeighths of the strength of concrete upon which the design is based.

(d) The ratio $(n)$ of the modulus of elasticity of steel to that of concrete shall be 12 for concrete having an ultimate compressive strength at 28 days of $2,750 \mathrm{lbs}$./in. ${ }^{2}$ and 9 for concrete having an ultimate compressive strength of $4,000 \mathrm{lbs} . / \mathrm{in}^{2}$ or greater. Intermediate values of $n$ shall be proportional to the strength of concrete assumed in the design.

(e) The working stress for cold-drawn steel wire shall not exceed $27,500 \mathrm{lbs}$./in. ${ }^{2}$ For billet steel, intermediate and hard grades, the working stress shall not exceed $20,000 \mathrm{lbs}$./in. ${ }^{2}$; and for billet steel, structural grade, the working stress shall not exceed $18,000 \mathrm{lbs}$./in ${ }^{2}$

(f) The distance from the center of the reinforcement to the nearest or tension surface of the concrete shall not be less than three-fourth inch for pipe 12 inches or less in diameter, or less than 1 inch for pipe more than 12 inches in diameter.

$(g)$ The distance from the center of the tension reinforcemeni to the compression surface of the concrete and the area of the reinforcement shall not be less than that required by the formula

$$
\frac{w d}{16} \times \frac{d+t}{12}=j A t f_{\text {t }}
$$

in which

$w=$ uniform vertical load in pounds per square foot top and bottom of pipe.

$d=$ internal diameter of pipe in inches.

$t=$ distance from the center of the tension reinforcement to the compression surface of the concrete in inches.

$A=$ sectional area of tension reinforcement in square inches per lineal foot of the pipe.

$f_{s}=$ tensile stress in the reinforcement in pounds per square inch.

$j=$ ratio of the lever arm of the reinforcement to $t$ as determined by the usual formulas.

10. The shell thickness and the amount of circumferential reinforcement shall not be less than that given in the design tables for the classes and sizes of pipe and the strength of concrete therein specified.

11. Manufacturers may submit to the consumer or purchaser for approval designs based on strengths of concrete other than those given in the design tables. Such alternate designs shall comply with the design requirements given in Section III of these specifications. In no alternative design, however, shall the shell thicknesses be less than those given in Table 2, nor shall the strength of concrete be less than that given in Table 1 .

12. Pipe of the internal diameters listed in the design tables shall be considered standard sizes for culvert construction. In elliptical pipe the inside diameter at the minor axis shall be equal to the diameter of the corresponding size of circular pipe.

13. The ends of the pipe shall be of such design that the pipe when laid shall make a continuous conduit with a smooth and uniform interior surface.

14. When a single line of circular reinforcement is used in circular pipe it shall be placed at the center of the pipe shell. When two lines of reinforcement are used in circular pipe one shall be placed near the inner and onc near the outer surface of the pipe. The single line of elliptical reinforcement used in circular pipe, or the single line of circular reinforcement in elliptical pipe, shall be placed near the inner surface at the "top" and "bottom" of the pipe and near the outer surface at the sides. (Sec par. $20(d)$.)

15. Each line of circumferential reinforcement shall be assembled into a cage and have sufficient longitudinal bars or members extending through the barrel of the pipe to afford rigidity and maintain the reinforcemen $\mathrm{in}$ exact shape and correct posicion within the form.

16. The reinforcement shall be lapped not less than 30 diameters, or, if welded, the joints shall develop the full strength of the reinforcement. The spacing center to center of adjacent rings of circumferential reinforcement in a caga shall not exceed 4 inches up to and including pipe 48 inches in diameter, nor exceed the shell thickness for larger pipe, and shall in no case exceed 6 inches.

17. The bell shall have a circumferential reinforcement equal in unit area to that of a single line within the barrel of the pipe.

\section{WORKMANSHIP AND FINISH}

18. Pipe shall be substantially free from fractures, large or deep cracks, and surface roughness. The planes of the ends of the pipe shall be perpendicular to their longitudinal axes.

19. (a) Variations of the internal diameter shall not exceed $1 \frac{1}{2}$ per cent nor shall the shell thickness be less than that intended in the design by more than 5 per cent at any point. 
(b) Variation in the position of the reinforcement cages shall not exceed one-fourth inch from the position provided in the design nor shall the cover on the reinforcement be less than three-fourths inch at any point.

\section{MARTING}

20. The following shall be clearly stenciled on the pipe:

(a) The pipe class $\left\{\begin{array}{c}\text { by an " } \mathrm{S} \text { " for standard pipe, } \\ \text { and an "X" for extra } \\ \text { strength pipe. }\end{array}\right.$

(b) The date of manufacture.

(c) The name or trade-mark of manufacturer.

(d) Elliptical pipe with circular reinforcing and circular pipe with elliptical reinforcing shall have the words "Top or bottom" clearly stenciled on the inside of the pipe at the correct place to indicate the the proper position when laid.

\section{PHYSICAL TESTS}

21. Pipe may be tested for strength by either the 3 -edge or sand-bearing method:

(a) When the 3-edge bearing is used the lower bearing for the pipe shall consist of two wooden strips with vertical sides having their interior top corners rounded to a radius of approximately onehalf inch. The strips shall be straight and shall be securely fastened to a rigid block with the interior vertical sides spaced a distance apart not less than one-half inch nor more than 1 inch for each foot of diameter pipe. The upper bearing shall be a rigid wooden block, straight and true from end to end. The upper and lower bearings shall extend the full length of pipe, exclusive of bell. The pipe shall be placed symmetrically between the two bcarings, as illustrated in Figure 115. In testing pipe which is "out of line" the lines of the bearings chosen shall be from those which appear to give the most favorable conditions for fair test.

(b) When sand bcarings are used (see fig. 116) the ends of each specimen of pipe shall be accurately marked prior to the test in quarters of the circumference. Specimens shall be carefully bedded, above and below, in sand, for one-fourth the circumference of the pipe measured on the middle line of the barrel. The depth of bedding above and below the pipe at the thinnest points shall be one-half the radius of the middle line of the barrel.

The sand used shall be clean and moist and shall be such as will pass a 4,760-micron sieve (United States standard No. 4. (See 500.2, p. 1.)). The sand in the lower bearing shall be loose when the pipe is placed.

The top bearing frame shall not be allowed to come in contact with the pipe nor with the top bearing plate. The upper surface of the sand in the top bearing shall be struck level with a straightedge and shall be covered with a rigid top bearing plate, with lower surface a true plane, made of heavy timbers or other rigid material, capable of distributing the test load uniformly without appreciable bending. The test load shall be applied at the exact center of this top bearing plate, or in such manner as to produce uniform deflection throughout the full length of the pipe. For this purpose a spherical bearing is preferred, but two rollers at right angles may be used. The test may be made without the use of a testing machine by piling weights directly on a platform resting on the top bearing plate, provided, however, that the weights shall be piled symmetrically about a vertical line through the center of the pipe and that the platform shall not be allowed to touch the top bearing frame.

The frames of the top and bottom bearings shall be made of timber so heavy as to avoid appreciable bending by the side pressure of sand. The interior surfaces of the frames shall be dressed. No frame shall come in contact with the pipe during the test. A strip of cloth may, if desired, be attached to the inside of the upper frame on each side, along the lower edge, to prevent the escape of sand between the frame and the pipe.

(c) It is desirable that a machine shall be used which gives a uniform deflection throughout the full length of the pipe. Any mechanical or handpower device may be used in which the head that. applies the load moves at a speed of not more than 0.05 inch per minute while making the test. The testing machine shall be substantial and rigid throughout, so that the distribution of the load will not be affected appreciably by the deformation or yielding of any part. The load shall be applied eontinuously until the ultimate strength of the pipe is reached.

22. The ultimate load, as determined by one of the methods described in paragraph 21 , shall not be less than the ultimate load specified in Table 5 for the size and class of pipe that is being tested. When the test load reaches the cracking load specified in Table 5 for the size and class of pipe that is being tested there shall be in the barrel of the pipe no crack having a surface width of one one-hundredth inch ${ }^{60}$ or more, for a length of 1 foot or more. The ultimate load is reached when the pipe will sustain no greater load.

23. Elliptical pipe shall meet the test requirements for circular pipe having the same horizontal internal diameter.

24. Preliminary to placing an order, a consumer of pipe whose needs require shipments at intervals over extended periods of time shall be entitled to test not more than 10 pieces of pipe covering the size in which he is interested. The test specimens shall be selected in approximately equal numbers from the larger and smaller sizes of pipe. The acceptability of the larger sizes of pipe shall not be based on the results of tests in smaller sizes. After these preliminary tests, a consumer shall be entitled to additional tests in such numbers and at such times as he may deem necessary, provided that the total number of pipe tested shall not exceed 2 per cent of the total deliveries.

25. A purchaser who places occasional orders shall be entitled to test a number of pipe equal to 2 per cent of an order but not to exceed five pieces of any one size; otherwise the number of pipe desired for testing shall be included in the order.

26. All pipe for testing purposes shall be selected at random by the consumer or purchaser from the stock of the manufacturer and shall be pipe which would not otherwise be rejected under these specifications. The pipe shall be free from visible moisture when tested.

They shall not have been exposed to a temperature below $40^{\circ} \mathrm{F}$. for the 24 hours immediately preceding the test.

27. By agreement between the consumer and the manufacturer the continued acceptability of the pipe, after the preliminary pipe tests have been made, may be determined by tests of the quality of the concrete as placed in the pipe and examination of the quality, amount, and the accuracy of placement of the reinforcement. The quality of the concrete shall be determined on 6 by 12 inch test cylinders taken from the concrete used in making the pipe and manufactured and cured

${ }^{60}$ It is recommended that the width of the crack be measured hy means of a gage made from a leaf one one-hundredth inch thick from a set of standard machinists' gages, ground to a point one-sixtecnth inch wide, with corners rounded, and with a taper of onefourth inch per inch, as illustrated hy Figure 117. The crack shall he considered to he one one-hundredth inch wide when the point of the gage will just enter it at close intervals. 
under identical conditions with the pipe. When tested in accordance with the current standard methods prescribed by the American Society for Testing Materials these cylinders shall have a strength not less than that assumed in the design of the pipe.

28. Pipe shall be acceptable under the strength tests when all test specimens meet the test require- specimen that failed and the pipe shall be acceptable only when all of these retest specimens meet the test requirements. No further retests shall be permitted.

29. Absorption tests shall be made by the following method:

(a) The number of absorption specimens shall be equal to the number of pipe provided for testing.
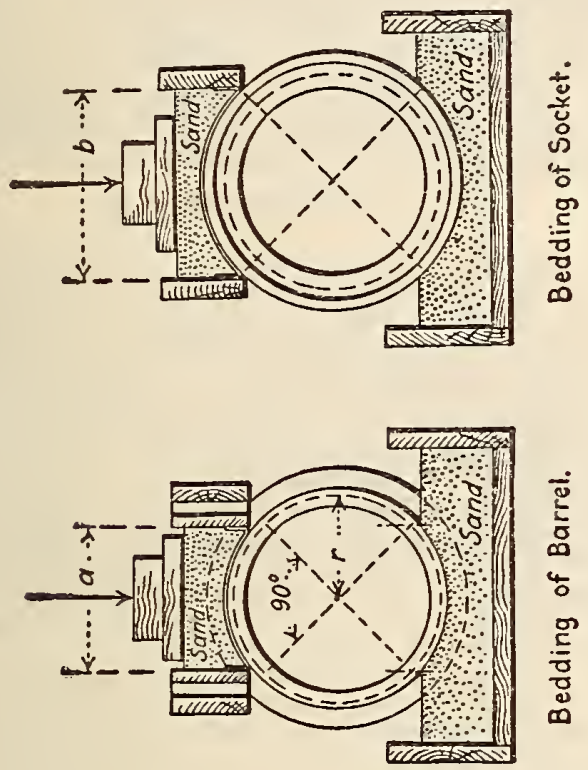

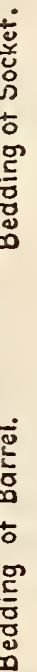
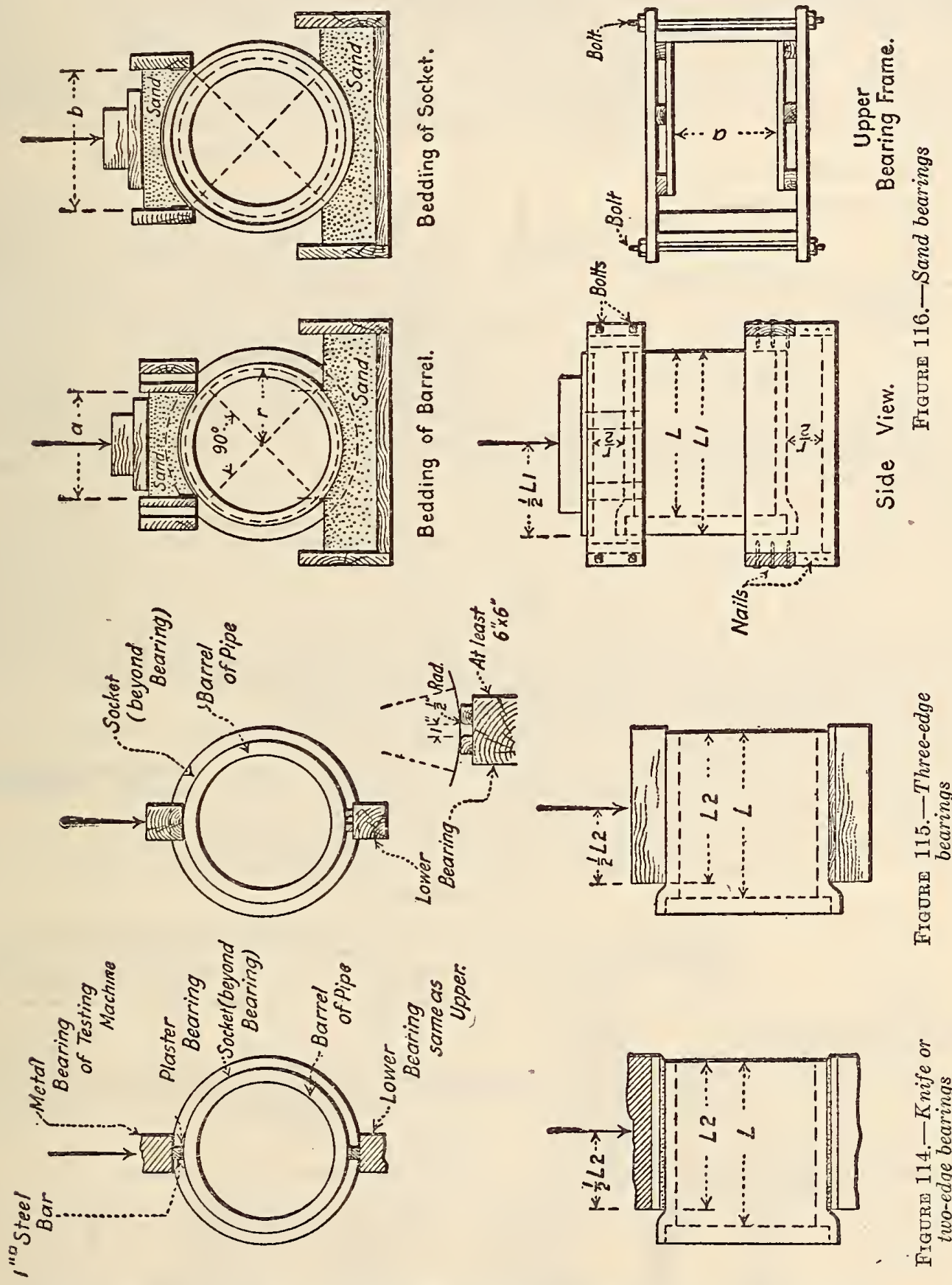

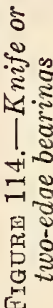

ments. Should less than 3 of the 10 preliminary test specimens or any one of the additional test specimens provided for in paragraph 24 or any one specimen provided for in paragraph 25 fail to meet the test requirements, then the manufacturer will be allowed a retest on 2 like specimens for each
The specimens shall be obtained from pipe that are acceptable as to strength and shall be taken from pipe used in making the strength test when that test is made. The specimens shall be marked with the number or identifying mark of the pipe from which they were taken. Each specimen shall have 
an area of 16 to 24 square inches and a thickness equal to the full depth of the pipe shell and shall be free from visible cracks.

(b) Specimens shall be dried at a temperature of approximately $110^{\circ}$ C. $\left(230^{\circ}\right.$ F.) until no loss of weight is shown by successive weighings at intervals of not less than four hours.

(c) The dried specimens shall be placed in a suitable receptacle, covered with distilled water or rain water, raised to the boiling point and boiled for five hours, and then cooled in water to a final temperature of from $15^{\circ}$ to $20^{\circ} \mathrm{C}$. $\left(59^{\circ}\right.$ to $68^{\circ} \mathrm{F}$.). When cool, the specimens shall be removed from the water, allowed to drain for not more than one minute, the

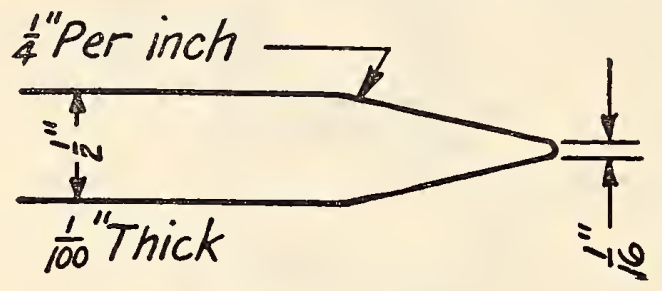

Figure 117.-Gage

superficial water removed by a towel or blotting paper, and the specimens immediately weighed.

(d) The balance used shall be sensitive to $0.5 \mathrm{~g}$ when loaded with $1 \mathrm{~kg}$ and weighings shall be read at least to the nearest gram. Where other than metric weights are used the same degree of accuracy must be obtained.

(e) The increase in weight of the boiled specimen over its dry weight shall be considered the absorption of the specimen and shall be calculated as a percentage of the dry weight. The results shall be reported separately for each specimen.

30 . The absorption shall not exceed 8 per cent for test specimens taken from pipe designed to be made of concrete having a compressive strength of 3,000 or more pounds per square inch, or 9 per cent for test specimens taken from pipe designed to be made of concrete having a compressive strength of less than 3,000 pounds per square inch. Pipe shall be considered to meet these specifications for absorption when not less than 80 per cent of the number of specimens tested, including any retested, meet the test requirements. When the initial absorption specimen from a pipe fails to meet these specifications the absorption test shall be made on another specimen from the same pipe and the results of the retest shall be substituted for the original test results.

31. Pipe will be considered ready for shipment when they meet the test requirements or when tests of 6 by 12 inch cylinders (sec. 27) show that the concrete has attained the strength assumed in the design of the pipe.

32. Every manufacturer furnishing pipe under these specifications shall furnish all facilities necessary to carry out the tests herein provided.

\section{INSPECTION}

33. All materials, processes of manufacture, and finished pipe shall be subject to inspection and approval by an inspector employed by the consumer or purchaser. The manufacturer, when so directed by the inspector, shall have holes cut in such sections of the finished pipe (not exceeding one hole in every 50 sections delivered) as desired, so that a proper inspection may be made of the quantity and placement of the reinforcement. If the pipes are tested for strength or absorption, inspection of the reinforcement shall be made on the pipe used for those tests, and in no case shall the total number of pipe cut open for inspection of reinforcement exceed the number to which the purchaser is entitled under the provisions of sections 24 or 25 .

34. Pipe shall be subject to rejection on account of failure to meet any of the specification requirements or on account of any of the following:

(a) Fractures or cracks passing through the shell, except that an end crack that does not exceed the depth of the joint, or a fracture that at its deepest point does not exceed the depth of the joint nor extend more than 10 per cent around the circumference, shall not be considered cause for rejection unless these defects exist in more than 5 per cent of the pipe inspected.

(b) Defects which indicate imperfect mixing and molding.

(c) Exposure of the reinforcement when such exposure would indicate that the reinforcement is misplaced.

TABLE 1.-Designs of standard reinforced concrete culvert pipe for uniform load of 2,000 pounds per square foot. Ultimate compressive strength of concrete, 2,750 pounds per square inch

$$
\left[f_{c}=1,030\right. \text { pounds] }
$$

\begin{tabular}{|c|c|c|c|c|c|c|c|}
\hline \multirow[b]{3}{*}{$\begin{array}{l}\text { Internal diameter } \\
\text { of pipe in inches } \\
\text { " } d \text { " }\end{array}$} & \multirow[b]{3}{*}{$\begin{array}{l}\text { Mini } \\
\text { thick } \\
\text { of } s .\end{array}$} & \multirow{2}{*}{\multicolumn{2}{|c|}{$\begin{array}{l}\text { Minimum distance cen- } \\
\text { ter reinforcement to } \\
\text { compressive surface } \\
\text { " } t \text { " }\end{array}$}} & \multicolumn{4}{|c|}{ Minimum area of circular reinforcement square inches per lineal foot of pipe " $A$ " " } \\
\hline & & & & \multicolumn{2}{|c|}{$\begin{array}{c}\text { Cold-drawn steel wire } f_{a}=27,500 \\
\text { lbs./in. }\end{array}$} & \multicolumn{2}{|c|}{$\begin{array}{l}\text { Billet steel, hard and intermediate } \\
\text { grades } f_{s}=20,000 \mathrm{lbs} . / \text { in }^{2}\end{array}$} \\
\hline & & $\begin{array}{l}\text { Circular } \\
\text { reinforce- } \\
\text { ment in } \\
\text { circular } \\
\text { pipe }\end{array}$ & $\begin{array}{l}\text { Elliptical } \\
\text { reinforce- } \\
\text { ment in } \\
\text { circular } \\
\text { pipe and } \\
\text { circular } \\
\text { reinforce- } \\
\text { ment in } \\
\text { elliptical } \\
\text { pipe }\end{array}$ & $\begin{array}{l}\text { Circular reinforce- } \\
\text { ment in circular pipe }\end{array}$ & $\begin{array}{l}\text { Elliptical rein- } \\
\text { forcement in } \\
\text { circular pipe and } \\
\text { circular reinforce- } \\
\text { ment in elliptical } \\
\text { pipe }\end{array}$ & $\begin{array}{l}\text { Circular reinforce- } \\
\text { ment in circular pipe }\end{array}$ & $\begin{array}{l}\text { Elliptical rein- } \\
\text { forcement in } \\
\text { circular pipe and } \\
\text { circular reinforce- } \\
\text { ment in elliptical- } \\
\text { pipe }\end{array}$ \\
\hline 18 & $\begin{array}{c}\text { Inches } \\
2 \\
21 / 4 \\
21 / 2 \\
3 \\
31 / 2\end{array}$ & $\begin{array}{l}\text { Inches } \\
1 \\
136 \\
-11 / 4 \\
11 / 2 \\
13 / 4\end{array}$ & $\begin{array}{l}\text { Inches } \\
11 / 4 \\
11 / 2 \\
2 \\
21 / 2\end{array}$ & 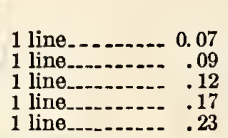 & 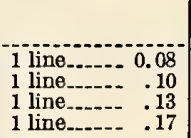 & 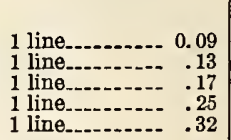 & 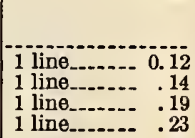 \\
\hline $\begin{array}{l}30 \\
36 \\
42\end{array}$ & $\begin{array}{l}31 / 2 \\
4 \\
41 / 2 \\
5\end{array}$ & $\begin{array}{l}21 / 2 \\
3 \\
31 / 2 \\
4\end{array}$ & $\begin{array}{l}21 / 2 \\
3 \\
31 / 2 \\
4\end{array}$ & $\begin{array}{ll}2 \text { lines, each.-. } & .17 \\
2 \text { lines, each.-- } & .20 \\
2 \text { lines, each--- } & .23 \\
2 \text { lines, each.-. } & .26\end{array}$ & 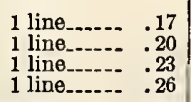 & $\begin{array}{ll}2 \text { lines, each... } & .23 \\
2 \text { lines, each.-- } & .28 \\
2 \text { lines, each } & .32 \\
2 \text { lines, each..- } & .37\end{array}$ & 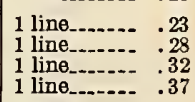 \\
\hline $\begin{array}{l}54 \ldots \ldots \\
60 \\
72 \ldots \\
84 \ldots\end{array}$ & $\begin{array}{l}51 / 2 \\
6 \\
7 \\
8\end{array}$ & $\begin{array}{l}41 / 2 \\
5 \\
6 \\
7\end{array}$ & $\begin{array}{l}41 / 2 \\
5 \\
6 \\
7\end{array}$ & $\begin{array}{ll}2 \text { lines, each.-- } & .30 \\
2 \text { lines, each--- } & .33 \\
2 \text { lines, each.-- } & .40 \\
2 \text { lines, each.-- } & .46\end{array}$ & 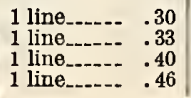 & $\begin{array}{ll}2 \text { lines, each... } & .42 \\
2 \text { lines, each-.- } & .46 \\
2 \text { lines, each.-- } & .56 \\
2 \text { lines, each..- } & .65\end{array}$ & 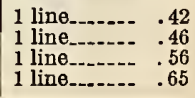 \\
\hline
\end{tabular}


TABLE 2.-Designs of standard reinforced concrete culvert pipe for uniform load of 2,000 pounds per square foot. Ultimate compressive strength of concrete, 4,000 pounds per square inch

$\left[f_{c}=1,500\right.$ pounds]

\begin{tabular}{|c|c|c|c|c|c|c|c|}
\hline \multirow[b]{3}{*}{$\begin{array}{l}\text { Internal diameter } \\
\text { of pipe in inches } \\
\text { " } d \text { " }\end{array}$} & \multirow[b]{3}{*}{$\begin{array}{c}\text { Minimum } \\
\text { thickness } \\
\text { of shell }\end{array}$} & \multirow{2}{*}{\multicolumn{2}{|c|}{$\begin{array}{l}\text { Minimum distance cen- } \\
\text { ter reinforcement to } \\
\text { compressive surface } \\
\text { " } t \text { " }\end{array}$}} & \multicolumn{4}{|c|}{ Minimum area of eircular reinforcement square inches per lineal foot of pipe " $a$ " } \\
\hline & & & & \multicolumn{2}{|c|}{$\begin{array}{c}\text { Cold-drawn steel wire } f_{s}=27,500 \\
\text { lbs./in. } .^{2}\end{array}$} & \multicolumn{2}{|c|}{$\begin{array}{l}\text { Billet steel, hard and intermediate } \\
\text { grades, } f_{s}=20,000 \text { lbs./in. }\end{array}$} \\
\hline & & $\begin{array}{l}\text { Circular } \\
\text { reinforce- } \\
\text { ment in } \\
\text { circular } \\
\text { pipe }\end{array}$ & $\begin{array}{l}\text { Elliptical } \\
\text { reinforce- } \\
\text { ment in } \\
\text { circular } \\
\text { pipe and } \\
\text { circular } \\
\text { reinforce- } \\
\text { ment in } \\
\text { elliptical } \\
\text { pipe }\end{array}$ & $\begin{array}{l}\text { Circular reinforce- } \\
\text { ment in circular pipe }\end{array}$ & $\begin{array}{c}\text { Elliptical rein- } \\
\text { forcement in } \\
\text { circular pipe and } \\
\text { circular reinforce- } \\
\text { ment in elliptical } \\
\text { pipe }\end{array}$ & $\begin{array}{l}\text { Circular reinforce- } \\
\text { ment in circular pipe }\end{array}$ & $\begin{array}{l}\text { Elliptical rein- } \\
\text { forcement in } \\
\text { circular pipe and } \\
\text { circular reinforce- } \\
\text { ment in elliptical } \\
\text { pipe }\end{array}$ \\
\hline 18 & \begin{tabular}{c|} 
Inches \\
$13 / 4$ \\
2 \\
$21 / 4$ \\
$25 / 8$ \\
3
\end{tabular} & $\begin{array}{r}\text { Inches } \\
78 \\
1 \\
136 \\
11 / 4 \\
11 / 2\end{array}$ & $\begin{array}{r}\text { Inches } \\
134 \\
158 \\
2\end{array}$ & 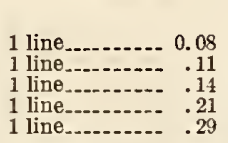 & 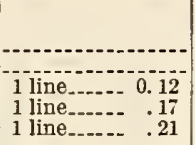 & 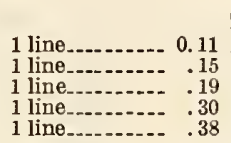 & $\begin{array}{ll}1 \text { line } & \\
1 \text { line } & \\
1 \text { line....... } & 0.17 \\
\end{array}$ \\
\hline $\begin{array}{l}30 \\
36 \\
48\end{array}$ & \begin{tabular}{l|}
3 \\
33 \\
33 \\
$41 / 4$ \\
$41 / 4$
\end{tabular} & $\begin{array}{l}2 \\
2^{3} / 8 \\
23 / 4 \\
31 / 4\end{array}$ & $\begin{array}{l}2 \\
236 \\
23 / 4 \\
31 / 4\end{array}$ & $\begin{array}{ll}2 \text { lines, each.-- } & .21 \\
2 \text { lines, each.-- } & .26 \\
2 \text { lines, each..- } & .30 \\
2 \text { lines, each.-- } & .34\end{array}$ & 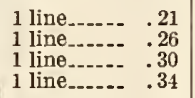 & $\begin{array}{ll}2 \text { lines, each--- } & .29 \\
2 \text { lines, each--- } & .36 \\
2 \text { lines, each--- } & .41 \\
2 \text { lines, each.-- } & .46\end{array}$ & 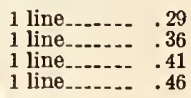 \\
\hline $\begin{array}{l}54 \\
60 \\
72 \\
84\end{array}$ & $\begin{array}{l}456 \\
5 \\
53 / 4 \\
658\end{array}$ & $\begin{array}{l}35 / 8 \\
4 \\
43 / 4 \\
558\end{array}$ & $\begin{array}{l}35 / 8 \\
4 \\
43 / 4 \\
558\end{array}$ & $\begin{array}{ll}2 \text { lines, each--- } & .38 \\
2 \text { lines, each--- } & .42 \\
2 \text { lines, each--- } & .51 \\
2 \text { lines, each.-- } & .60\end{array}$ & 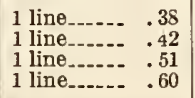 & $\begin{array}{ll}2 \text { lines, each_-.- } & .52 \\
2 \text { lines, each.-- } & .59 \\
2 \text { lines, each--- } & .71 \\
2 \text { lines, each--- } & .82\end{array}$ & 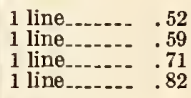 \\
\hline
\end{tabular}

TABLE 3.-Designs of extra strength reinforced concrete culvert pipe for uniform load of 4,000 pounds per square foot. Ultimate compressive strength of concrete, 2,750 pounds per square inch

[ $f_{\mathrm{c}}=1,030$ pounds]

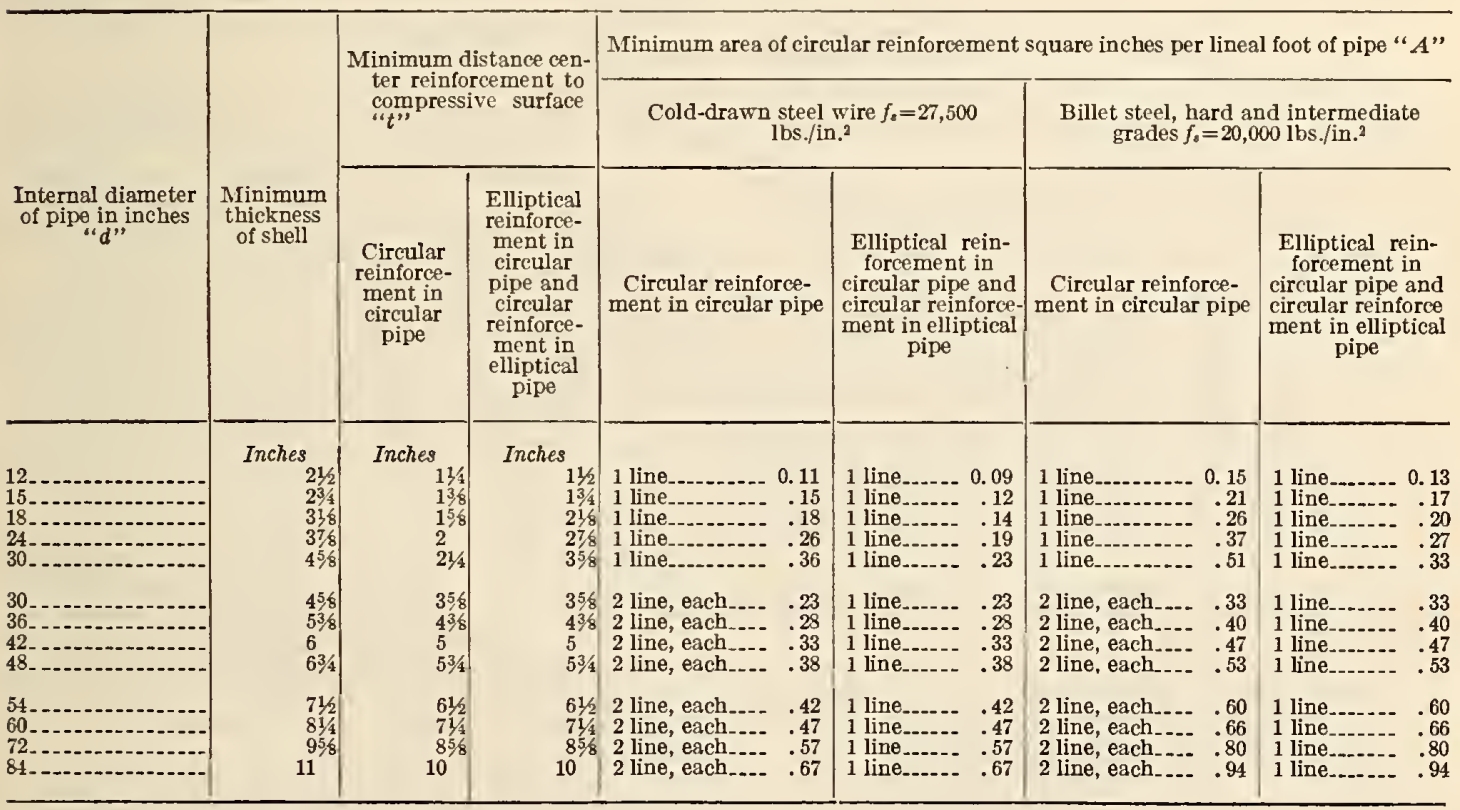


TABLE 4.-Designs of extra strength reinforced concrete culvert pipe for uniform load of 4,000 pounds per square foot. Ulimate compressive strength of concrete, 4,750 pounds per square inch

$\left[f_{\mathrm{c}}=1,780\right.$ pounds $]$

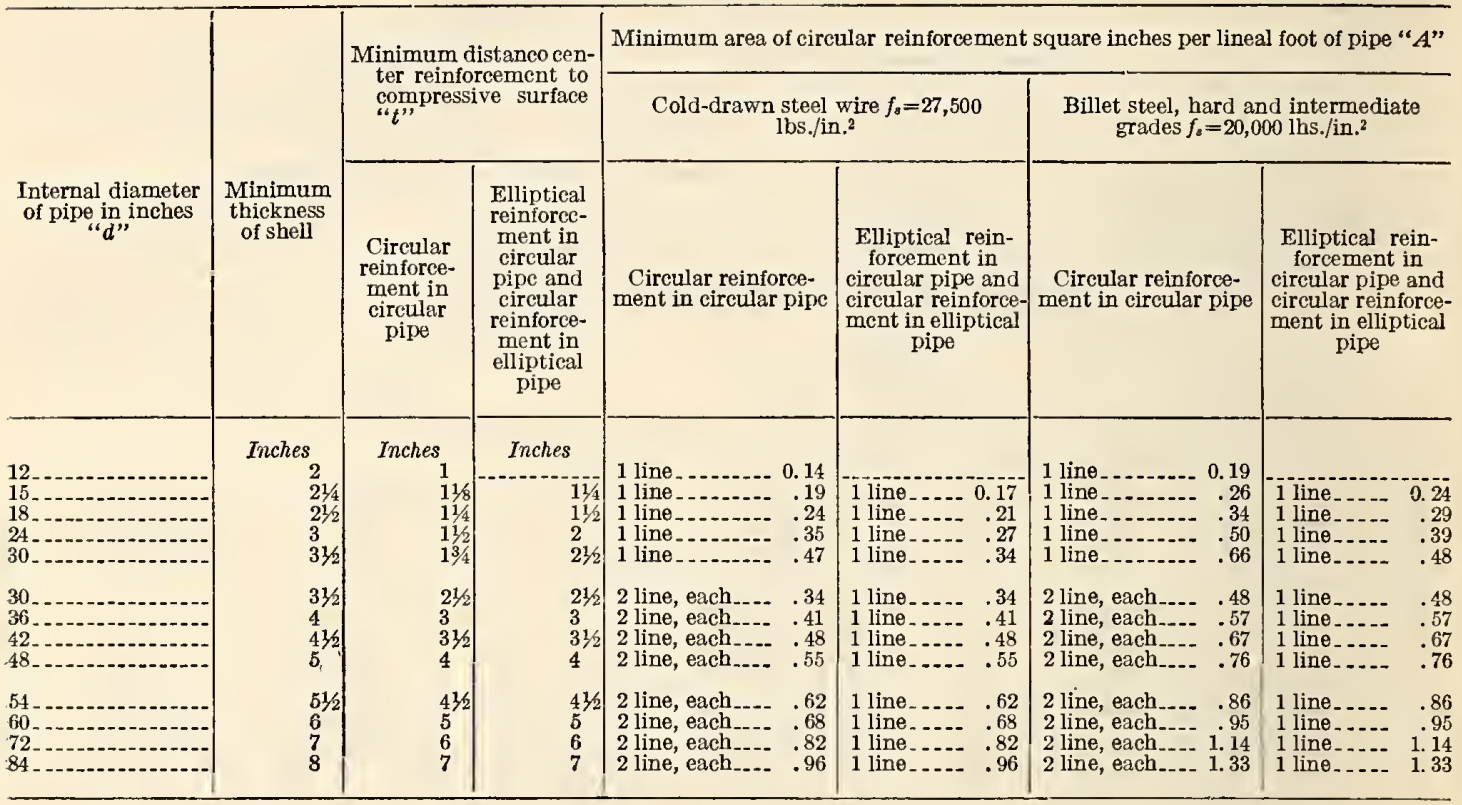

TABLE 5-Minimum strength of reinforced concrete culvert pipe

(Pipe in pounds per foot of laying length)

\begin{tabular}{|c|c|c|c|c|c|c|c|c|}
\hline \multirow{3}{*}{$\begin{array}{l}\text { Size of } \\
\text { pipe in } \\
\text { inches }\end{array}$} & \multicolumn{4}{|c|}{$\begin{array}{c}\text { Standard reinforced concrete } \\
\text { culvert pipe }\end{array}$} & \multicolumn{4}{|c|}{$\begin{array}{l}\text { Extra strength reinforced } \\
\text { concrete culvert pipe }\end{array}$} \\
\hline & \multicolumn{2}{|c|}{ 3edge hearing } & \multicolumn{2}{|c|}{ Sand bearing } & \multicolumn{2}{|c|}{ 3-edge hearing } & \multicolumn{2}{|c|}{ Sand hearing } \\
\hline & $\begin{array}{l}\text { Crack- } \\
\text { ing } \\
\text { load } 1\end{array}$ & $\begin{array}{l}\text { Ulti- } \\
\text { mate } \\
\text { load }\end{array}$ & $\begin{array}{c}\text { Crack- } \\
\text { ing } \\
\text { load } 1\end{array}$ & $\begin{array}{l}\text { Ulti- } \\
\text { mate } \\
\text { load }\end{array}$ & $\begin{array}{c}\text { Crack - } \\
\text { ing } \\
\text { load } 1\end{array}$ & $\begin{array}{l}\text { Ulti- } \\
\text { mate } \\
\text { load }\end{array}$ & $\begin{array}{l}\text { Crack- } \\
\text { ing } \\
\text { load } 1\end{array}$ & $\begin{array}{l}\text { Ulti- } \\
\text { mate } \\
\text { load }\end{array}$ \\
\hline 7 & $\begin{array}{l}1,600 \\
\mathbf{1}, 800 \\
2,000 \\
2,200 \\
2,500 \\
3,000 \\
3,500 \\
4,000 \\
4,500 \\
5,000 \\
6,000 \\
7,000\end{array}$ & $\begin{array}{r}2,000 \\
2,500 \\
3,000 \\
4,000 \\
5,000 \\
6,000 \\
7,000 \\
8,000 \\
9,000 \\
10,000 \\
12,000 \\
14,000\end{array}$ & $\begin{array}{r}2,400 \\
2,700 \\
3,000 \\
3,300 \\
3,750 \\
4,500 \\
5,250 \\
6,000 \\
6,750 \\
7,500 \\
9,000 \\
10,500\end{array}$ & $\begin{array}{r}3,000 \\
3,750 \\
4,500 \\
6,000 \\
7,500 \\
9,000 \\
10,500 \\
12,000 \\
13,500 \\
15,000 \\
18,000 \\
21,000\end{array}$ & $\begin{array}{r}3,200 \\
3,600 \\
4,000 \\
4.400 \\
5,000 \\
6,000 \\
7,000 \\
8,000 \\
9,000 \\
10,000 \\
12,000 \\
14,000\end{array}$ & $\begin{array}{r}4,000 \\
5,000 \\
6,000 \\
8,000 \\
10,000 \\
12,000 \\
14,000 \\
16,000 \\
18,000 \\
20,000 \\
24,000 \\
28,000\end{array}$ & $\begin{array}{r}4,800 \\
5,400 \\
6,000 \\
6,600 \\
7,500 \\
9,000 \\
10,500 \\
12,000 \\
13,500 \\
15,000 \\
18,000 \\
21,000\end{array}$ & $\begin{array}{r}6,000 \\
7,500 \\
9,000 \\
12,000 \\
15,000 \\
18,000 \\
21,500 \\
24,000 \\
27,000 \\
30,000 \\
36,000 \\
42,000\end{array}$ \\
\hline
\end{tabular}

I At the cracking load there shall he, in the harrel of the pipe, no crack having a surface width of 0.01 inch or more for a length of 1 foot or more.

National Paving Brick Manufacturers' Association recommended spccifications for cement grout filler for semimonolithic brick pavements.

Description. - Joints between the brick shall be filled completely with cement grout composed of 1 part Portland cement to 2 parts sand.

Cement and water.-Portland cement shall conform to the definition and meet the requirements of the standard specifications and tests for Portland cement of the American Society for Testing Materials, scrial designation C9. (See 516.11, p. 294.)

Water shall be clean, free from oils, acids, alkalies, or vegetablc matter.
Sand.-Sand for cement-grout filler shall consist of clean, sharp grains or of screenings from hard and durable rock or gravel.

(a) Sizes of grains shall be such that 100 per cent will pass a No. 12 sieve, not more than 40 per cent will pass a No. 50 sieve, and not more than 6 per cent pass a No. 100 sieve.

(b) Sand shall be frce from soft, friable materials, shale or slate, and vegetable or other organic matter. It shall not contain clay in excess of 5 per cent. Individual grains shall not be coated.

Sprinkling.- Sides and ends of the brick in the joints shall be thoroughly wet by gentle sprinkling immediately before the joints are filled.

Filling the joints.-Filler shall be mixed in and applied from a mechanical batch mixer of a type specially designed for the purpose. The mixer shall be self-contained and portable; it shall be of such a construction and operation that the surface of the pavement will not become splashed with oil or water.

The delivery of the mixer shall be such that the cement and sand will not separate in being applied to the joints.

Mixing.-A small batch of not more than 2 cubic feet of cement and sand combined shall be placed in the mixer and sufficient water shall be added while the mixer is in operation to produce the consistency of mortar. When this is thoroughly mixed more water shall be added slowly until the mixture reaches such a consistency that it will flow readily to the bottom of the joints without separation of the ingredients.

Applying.-Enough filler shall be applied to fill the joints. It shall be swept therein as applied, making certain that the joints are filied completely from top to bottom.

After the n̂rst application of filler has settled in the joints without having reached initial hardening a second application shall be made in the manner specified for the first, except that less water shall be used in mixing, producing a filler of thicker consistency. The filler shall immediately be worked into 
the joints by means of rubber-edged squeegees? which shall be operated at an angle with the joints.

Successive applications of filler shall be made if needed to insure completely filled joints.

Closing.- Whenever grouting shall cease or be interrupted the contractor shall provide metal strips one-sixteenth inch in thickness, 6 inches deep, and 3 feet in length, which shall be inserted in a transverse joint of the brick surface before discontinuing the grouting at any interval, in order that it shall end in a vertical joint. Strips shall remain in place until grouting reaches initial hardening.

Protecting the surface.-After the roadway has been inspected and sufficient time has elapsed to avoid injury to the filler a protective covering of sand or earth not less than 1 inch in depth shall be spread on the roadway. This shall be kept wet for not less than four days.

Closing to traffic. - The brick roadway shall be closed to traffic for not less than 15 days after grouting is completed.

National Paving Brick Manufacturers' Association, recommended specifications, concrete curb and header, 1924, also, integral concrete curb, 1924. (See 518.63 , p. 448.)

National Slag Association, specifications for slag to be used in highways and highway structures, 1927. (See 512.2, p. 256.)

(This specification covers the requirements, with the exception of size, for slag to be used in highway structures listed below.)

1. Plain or reinforced cement conerete highway structures.

2. Plain or reinforced cement concrete sewer pipe.

3. Plain or reinforced cement concrete piling.

4. Drain tile, fence posts, markers, brick, and other cement concrete products.

Portland Cement Association, suggested specifications for Portland-cement concrete sidewalks, 1927.

\section{ONE-COURSE CONSTRUCTION} GENERAL

1. Definition of terms. - The term engineer shall mean the city engineer or other person appointed by the municipality or the property owners to oversee sidewalk construction.

The term contractor shall mean the party or parties contracting to do any work described herein, or authorized representatives of such party or parties.

2. Sampling and testing materials.-Before delivery upon the job and at such other times as the engineer deems necessary the contractor shall furnish any required samples of the materials hereafter mentioned. Materials shall pass the following requirements:

3. Cement.-The cement shall meet the requirements of the current standard specifications for Portland cement of the American Society for Testing Materials. (See A. S. T. M. C9, 516.11, p. 294.) A sack containing 94 pounds of cement will be considered 1 cubic foot.

4. Fine aggregate.-The fine aggregate shall consist of clean, hard, durable, uncoated particles of sand or stone, free from all organic material. One hundred per cent shall pass a $1 / 4$-inch screen and 95 per cent shall be retained on a 100-mesh screen. Not more than 25 per cent shall pass a 50 -mesh screen. It shall be well graded from coarse to fine and shall not contain more than 3 per cent by weight of clay or loam, none of which shall be in lumps.

When the fine aggregate is mixed with Portland cement in the proportion of 1 part cement to 3 parts fine aggregate by weight, according to the standard method of making briquets, the resulting mortar at the age of 7 and 28 days shall have a tensile strength of at least equal to that developed in the same time by mortar of the same proportions and consistency made of the same cement and standard Ottawa sand.

5. Coarse aggregate.-Coarse aggregate may be broken stone, gravel, or blast-furnace slag.

The broken stone or gravel shall be clean, hard, durable, uncoated rock. It shall contain no vegetable or other deleterious matter, and shall be free from soft, thin, elongated or laminated pieces.

Slag shall be of a quality which has been successfully used in local concrete work. It shall be air cooled, nonglassy, and shall have been exposed to the weather at least one year.

One hundred per cent of the coarse aggregate shall pass a 1 -inch screen and at least 95 per cent shall be retained on a $1 / 4$-inch screen, with all intermediate sizes retained.

6. Water.-Water shall be clean and free from alkali, oils, or acid.

7. Joint filler.-Joint filler shall be premolded strips of asphalt or pitch, and fiber or mineral aggregate passing an 80-mesh screen, one-half inch thick, as wide as the thickness of the concrete slab and at least 2 feet long. It shall have such a consistency that it will not run in summer or chip out in winter.

8. Forms.-Forms shall be of lumber 2 inches thick, or of steel of equal strength, except on curves, where flexible strips may be used.

They shall be held rigidly in place by stakes or braces with top edges at true line and grade given by the engineer. Ends of adjoining forms shall be flush.

Where sidewalks fill the space between curb and building lines forms shall be set to give the walk a slope toward the curb of one-fourth inch per foot of width.

Where walks are bordered by lawns forms shall be set level and drainage of the walk secured by a crown of one-fourth inch for each foot from side to center.

9. Division plates.-Where division plates are used they shall be of $1 / 8$-inch steel, as wide as the depth of the slab and as long as the width of the walk.

Forms and division plates shall be thoroughly cleaned and oiled each time before they are used.

10. Subgrade. - That portion of the ground surface directly beneath the slab shall be called the subgrade.

All soft and spongy material in the subgrade shall be removed and replaced with suitable material. Fills shall be compacted in layers not exceeding 6 inches in thickness. The spots previously compacted by traffic shall be loosened to a depth of 6 inches. The whole subgrade shall be thoroughly and uniformly compacted to a firm surface having as nearly as possible a uniform bearing power.

A template, resting upon the side forms and having its lower edge at the exact elevation of the subgrade, shall be drawn along the forms before any concrete is laid. Any high places in the subgrade shall be removed so that the template will pass over without being raised off the side forms or being tipped at an angle to the sidewalk surface.

The subgrade shall be damp, but not muddy, when concrete is placed upon it.

11. Drains.- Where in the opinion of the engineer it is considered necessary to save the walk from damage by frost action, drains of 4 -inch concrete tile shall be laid on the lines and grades given by the engineer.

12. Subbase.- When drains are impracticable and the soil is poorly drained, colloidal clay, or a dobe, a 5-inch subbase shall be constructed of cinders, gravel, or other porous material approved by the 
enginecr. The subbase shall be thoroughly tamped until the surface is firm, and shall be drained into the street gutter.

13. Thickness and proportions.-The sidewalk shall consist of one 5-inch course of concrete in the proportions of 1 part of Portland cement, 2 parts of fine aggregate, 4 parts of coarse aggregate, and not over 7 gallons of water, including the free water in the aggregates. Less water may be used if desired in order to get the proper finish. The fine and coarse aggregates shall be proportioned on a dry, rodded basis in a manner approved by the engineer.

14. Mixing.-The ingredients of the concrete shall be thoroughly mixed until each particle of fine aggregate is coated with cement and each particle of coarse aggregate is coated with mortar.

Where a mechanical mixer is used it shall be of an approved batch type. Each batch shall be mixed at least one minute, from the time all the materials, including water, are in the drum until the beginning of the discharge.

The consistency of the mixed concrete shall be such that no separation of the ingredients takes place and some tamping is necessary to bring the mortar to the surface.

15. Placing and finishing.-The freshly mixed concrete shall be placed immediately on the prepared subgrade. It shall then be struck off and tamped with a straightedge or curved template having a crown of one-fourth inch per foot resting upon the side forms and advanced with a crosswise sawing motion.

It shall then be rolled with a light sheet-iron roller weighing approximately 1 pound per linear inch of surface contact and floated with a wooden float until the surface has a true contour and the concrete is thoroughly compacted. At the option of the engineer the surface may be brushed lightly with a broom.

The placing of concrete shall not be suspended for 45 minutes or longer except at the end of a slab.

No concrete shall be placed on a frozen subgrade or when the temperature is, or is liable to be within 24 hours below $35^{\circ}$ F., except with the written permission of the engineer.

16. Jointing. - The walk shall be cut into separate rectangular slabs. No plain concrete slab shall be longer than 6 feet on any one side.

Where division plates have been used they shall be removed after the concrete has hardened sufficiently to avoid breaking the edges or corners of the slabs.

Where division plates have not been used the partially set concrete shall be cut through to the subgrade. Care shall be taken to make the cut at right angles to the surface of the sidewalk.

The surface edges of each slab shall be rounded to a radius of about one-fourth inch. Markings shall be exactly at cuts between slabs.

17. Expansion joints.-Expansion joints shall extend from the surface to the subgrade, be truly at right angles to the sidewalk surface and be made by putting the specified joint filler in place before placing the concrete. They shall be placed as follows, and supported during construction by a board header:

At or near all places where the sidewalk line intersects a curb line or other sidewalk a 1-inch expansion joint shall be made at right angles to the center line of the walk.

Wherever the sidewalk abuts a curb or building, a $1 / 2$-inch joint shall be placed between the curb and the sidewalk and between the sidewalk and the building.

A 1/2-inch expansion joint shall be made across the walk at approximately 50 -foot intervals.

18. Curing. - As soon as the concrete has set sufficiently it shall be sprinkled and kept moist until covered. As soon as it can be done without damage to the walk it shall be covered with 2 inches of earth or sand which shall be kept wet seven days. The walk shall then be cleaned and opened to traffic.

The contractor shall protect the concrete from damage by rain, pedestrians, and animals with suitable covers and barricades, and by red lights at night.

\section{TWO-COURSE CONSTRUCTION}

The specifications for 1-course sidewalks concerning terms, materials, forms, subgrade, drainage, subbase, mixing, placing, finishing, jointing, and curing shall govern the construction of 2-course sidewalks except as noted below.

19. Thickness and proportions.-Two-course sidewalks shall consist of a base $4 \frac{1}{4}$ inches thick composed of concrete in the proportions of 1 part Portland cement, 2 parts of fine aggregate, 4 parts of coarse aggregate, and not over 7 gallons of water, including the free water in the aggregates. The top coat shall be three-fourths of an inch thick and composed of mortar in the proportions of 1 part Portland cement, 3 parts of fine aggregate, and $6 \frac{1}{2}$ gallons of water, including the free water in the aggregates. The fine and coarse aggregate shall be proportioned on a dry, rodded basis in a manner approved by the engineer.

20. Laying base.- The base shall be deposited on the subgrade and thoroughly compacting by tamping. It shall then be struck off by a template which shall leave it nowhere less than three-quarters of an inch below the finished surface.

21. Laying wearing surface.-Within 45 minutes after the bottom course is laid and before the initial set has taken place it shall be covered by the mortar for the top coat.

Portland Cement Association, specifications for plain and reinforced concrete (water-ccment ratio method).

(These specifications are based on the proposed standard building regulations submitted by Committee $\mathbf{E}-1$ of the American Concrete Institute, 1928.)

\section{MATERIALS}

1. Portland cement.-Portland cement shall conform to A. S. T. M. C9. (See 516.11, p. 294.)

2. Concrete aggregates. - (a) Concrete aggregates shall consist of natural sands and gravels, crushed rock, crushed air-cooled blast furnace slag, or other inert material having clean, uncoated grains of strong and durable minerals. Aggregates containing soft, friable, thin, flaky, elongated, or laminated particles totaling more than 3 per cent, or containing shale in excess of $1 \frac{1}{2}$ per cent, or silt and crusher dust finer than the No. 100 standard sieve in excess of 2 per cent shall not be used. These percentages shall be based on the weight of the combined aggregates as used in the concrete. When all three groups of these deleterious substances are present in the aggregates, the combined amounts shall not exceed 5 per cent by weight of the combined aggregate.

(b) Aggregates shall not contain strong alkali or organic material which gives a color darker than the standard color when tested in accordance with A. S. T. M. C40. (See 512.10, p. 213.)

(c) The maximum size of the aggregate shall be not larger than one-fifth of the narrowest dimension between forms of the member for which the concretc is to be used nor larger than three-fourths of the minimum clear spacing between reinforcing bars.

01 The construction of 1-course concrete sidewalks is recommended in preference to 2-course. 
By maximum size of aggregate is meant the clear space between the sides of the smallest square opening through which 95 per cent by weight of the material can be passed.

3. Water.- (a) Water used in mixing concrete shall be clean, and free from strong acids, alkalies, oil, or organic materials.

4. Metal reinforcement.-(a) Metal reinforcement shall conform to the requirements of the Standard Specifications for Billet-Steel Concrete Reinforcement Bars of intermediate grade (serial designation A15-14), or for Rail-Steel Concrete Reinforcement Bars (serial designation A16-14) of the American Society for Testing Materials. The provision in these specifications for machining deformed bars before testing shall be eliminated.

(b) Wire for concrete reinforcement shall conform to the requirements of the Tentative Specifications for Cold-Drawn Steel Wire for Concrete Reinforcement (serial designation A82-27) of the American Society for Testing Materials.

(c) Structural steel shall conform to the requirements of the Standard Specifications for Structural Steel for Buildings (serial designation A9-24) of the American Society for Testing Materials.

(d) Cast-iron sections for composite combination columns shall conform to Standard Specifications for Cast-Iron Pipe and Special Castings (serial designation A44-04) of the Amcrican Society for Testing Materials.

5. Storage of materials.-(a) Cement and aggregates shall be stored at the work in a manner to prevent deterioration or the intrusion of foreign matter. Any material which has deteriorated or has been damaged shall be immediately and completely removed from the work.

\section{CONCRETE QUALITY AND PROPORTIONING}

6. Concrete quality. - (a) The working stresses for the design of this structure are based upon the minimum ultimate 28-day strength of the concrete to be used. The strength of concrete for which all parts of the structure were designed is shown on the several plans. The strength of concrete shall be fixed in terms of the water-cement ratio in accordance with one of the following methods: (1) By established results for average materials, as provided in section 7 ; (2) by specific test of materials for the structure, as provided in section 8 .

(b) By the water-cement ratio is meant the total quantity of water entering the mixture, including the surface water carried by the aggregate, expressed in terms of the quantity of cement. The watercement ratio shall be expressed in United States gallons per sack (94 pounds net) of cement.

7. Water-cement ratio for average materials.-(a) Where no preliminary tests of the materials to be used are made, the watcr-cement ratio shall not exceed the values in the following table:

\section{Assumed strength of concrete}

\begin{tabular}{|c|c|}
\hline $\begin{array}{c}\text { Water-cement } \\
\text { ratio 1 United } \\
\text { States gallons } \\
\text { per sack of } \\
\text { cement (94 }\end{array}$ & $\begin{array}{c}\text { Assumed } \\
\text { compressive } \\
\text { strength 2 at } \\
\text { 25 days in } \\
\text { pounds per } \\
\text { pounds net) } \\
\text { square inch }\end{array}$ \\
\hline 4 & 5,000 \\
5 & 3,750 \\
6 & 3,000 \\
$63 / 4$ & 2,500 \\
$71 / 2$ & 2,000 \\
$81 / 4$ & 1,500 \\
\hline
\end{tabular}

1 Surface water contained in the aggregate must be included as part of the mixing water in computing the water-cement ratio.

2 The strengths indicated are for average materials, thorough mixed, and concrete moisture-cured at normal temperature of $70^{\circ} \mathrm{F}$ tested damp. (b) During the progress of the work a reasonable number of compression tests shall be made as may be required by the architect, but at least one specimen shall be tested for each 100 cubic yards of concrete being placed. The tests shall be made in accordance with the provisions for making field tests of concrete, as provided in section 9 .

(c) Should the average 28-day strength of the contiol cylinders cured moist at $70^{\circ} \mathrm{F}$. fall below the minimum ultimate strength called for on the plans, the architcct shall have the right to change the watercement ratio. In the event that the architect finds it necessary to change the water-cement ratios from those specified, adjustment, covering amount of cement and aggregates affected, will be made as an extra or a credit under the provisions of the contract.

(d) Should the average strengths shown by the cylinders cured on the job and tested subsequent to 28 davs fall below the required strength, the architect shall have the right to require conditions of temperature and moisture necessary to secure the required strength.

8. Water-cement ratio by tests of materials.-(a) Where the water-cement ratios for the various strengths of concrete are to be established by test, these tests shall be made in advance of the beginning of operations using the materials proposed and consistencies suitable for the work in accordance with A. S. T. M. C39 (see 516.0, p 284.), including the provisions for curing in a moist room at $70^{\circ} \mathrm{F}$. and testing wet. A curve representing the relation between the average 28-day strength of the concrete and water-ccment ratio shall be established for a range of values, including all of the strengths called for in the plans. The tests shall include at least four different water-cement ratios and at least four specimens for each water-cement ratio, each specimen made from a separate batch. The water-cement ratio to be used in the structure shall be that corresponding to a point on the curve, established by these tests, representing a strength of concrete 15 per cent higher than the minimum ultimate strength called for on the plans, and satisfactory evidence shall be submitted to show that these water-cement ratios are not exceeded during the construction of the building. No substitution shall be made in the materials being used on the work without additional tests in accordance herewith, to show the new water-cement ratios to be used.

(b) During the progress of the work, a reasonable number of additional 28-day compression tests may be required, but at least one specimen shall be tested for each 50 cubic yards of concrete of any one strength, and not less than two specimens of each strength of concrete for any one day's operation. Such tests shall be made in accordance with the provisions in section 9 for making field tests of concrete. Should the average strengths of the control cylinders cured moist at $70^{\circ} \mathrm{F}$. shown by these tests for any portion of the structure fall below the minimum ultimate 28-day strengths called for on the plans, the architect shall have the right to order a change in the mix or the watercement ratios for the remaining portion of the structure, but no extra claim shall be allowed for such changes. Should the average strengths shown by the cylinders cured on the job and tested subsequent to 28 days fall below the required strength, the architect shall have the right to require conditions of temperature and moisture necessary to secure the required strength.

9. Field tests of concrete.-(a) Field tests of concrete, when required, shall be made in accordance with A. S. T. M. C39, with the following exceptions:

(1) Two sets of samples of concrete for test specimens shall be taken as the concrete is being 
delivered at the point of deposit, care being taken to obtain a sample representative of the entire batch.

(2) One set designated as control cylinders shall be placed under moist curing conditions at approximately $70^{\circ} \mathrm{F}$. within 24 hours after molding and maintained therein until tested.

(3) The second set, designated as job cylinders, shall be kept as near to the point of sampling as possible and yet receive the same protection from the elements as is given to the portions of the structure being placed. Specimens shall be kept from injury while on the work. They shall be sent to the laboratory not more than seven days prior to the time of test and while in the laboratory shall be kept in the ordinary air at a temperature of approximately $70^{\circ} \mathrm{F}$.

(b) All specimens and tests shall be made by a properly qualified person or testing laboratory, who shall furnish the architect with a report, certified in the presence of a notary public, showing the results of tests and stating that they were made in accordance with the provisions of this specification.

10. Concrete proportions and consistency.- $-(a)$ The proportions of aggregates to cement for concrete of any water-cement ratio shall be such as to produce concrete that will work readily into the corners and angles of the form and around the reinforcement without excessive puddling or spading and without permitting the materials to segregate or free water to collect on the surface. The combined aggregate shall be of such composition of sizes that when separated by the No. 4 standard sieve, the weight retained on the sieve shall not be less than one-third nor more than two-thirds of the total, nor shall the amount of coarse material be such as to produce harshness in placing or honeycombing in the structure. When forms are removed, the faces and corners of the members shall show smooth and sound throughout.

(b) The methods of measuring concrete material shall be such that the proportion of water to cement ean be accurately controlled during the progress of the work and easily checked at any time by the architect or his authorized representative.

\section{MIXING AND PLACIJG CONCRETE}

11. Removal of water from excavation.-(a) Water shall be removed from excavations before concrete is deposited, unless otherwise directed by the architect. Any flow of water into the excavation shall be diverted through proper side drains to a sump or be removed by other approved methods which will avoid washing the freshly deposited concrete. Water vent pipes and drains shall be filled by grouting, or otherwise, after the concrete has thoroughly hardened.

12. Cleaning forms and equipment.-(a) Before placing concrete all equipment for mixing and transporting the concrete shall be cleaned, all debris and ice shall be removed from the places to be occupied by the concrete, forms shall be thoroughly wetted (except in freezing weather) or oiled, and clay or cement tile that will be in contact with concrete shall be well drenched (except in freezing weather). Reinforcement shall be thoroughly cleaned of ice or other coatings.

13. Inspection.-(a) Concrete shall not be placed until the forms and reinforcement have been inspected by the architect or engineer responsible for the design or his authorized representative.

14. Mixing.-(a) The concrete shall be mixed until there is a uniform distribution of the materials and the mass is uniform in color and homogeneous. The mixer shall be of such type as to insure the maintaining of the correct proportion of the ingreeients.
The mixing shall continue for at least one minute after all the ingredients are in the mixer.

15. Transporting.--(a) Concrete shall be handled from the mixer to the place of final deposit as rapidly as practicable by methods which will prevent the separation or loss of the ingredients. It sliall be deposited as nearly as practicable in its final position to avoid rehandling or flowing. Under no circumstances shall concrete that has partially hardened be deposited in the work.

(b) When concrete is conveyed by chuting, the plant shall be of such size and design as to insure a practically continuous flow in the chute. The siope of the chute shall be such as to allow the concrete to flow without separation of the ingredients. The delivery end of the chute shall be as close as possible to the point of deposit. When the operation is intermittent, the spout shall discharge into a hopper. The chute shall be thoroughly flushed with water before and after each run; and water used for this purpose shall be discharged outside the forms.

16. Placing--(a) When concreting is once started it shall be carried on as a continuous operation until the placing of the section or panel is completed.

(b) Concrete shall be thoroughly compacted by puddling with suitable tools during the operation of placing, and thoroughly worked around the reinforcement, around embedded fixtures, and into the corners of the forms.

(c) Where conditions make puddling difficult, or where the reinforcement is congested, batches of mortar containing the same proportion of cement to sand used in the concrete shall first be deposited in the forms and the operation of filling with the regularly specified mix be carried on at such a rate that the mix is at all times plastic and flows readily into the spaces between the bars.

(d) A record shall be kept on the work of the time and date of placing the concrete in each portion of the structure. Such record shall be kept until the completion of the structure and shall be open to the inspection of the architect.

Note.-Specifications covering finishing of concrete surfaces should be added. Methods of finishing are so varied they can not be given in a general specification.

17. Curing.-(a) Exposed surfaces of concrete shall be kept moist for a period of at least seven days after being deposited. In hot weather, exposed concrete shall be thoroughly wetted twice daily during the first $\pi c e k$.

18. Depositing in cold weather.-(a) When depositing concrete at freezing or near freezing temperatures, the concrete shall have a temperature of at lest $50^{\circ} \mathrm{F}$. but not more than $120^{\circ} \mathrm{F}$. The concrete shall be maintained at a temperature of at least $50^{\circ} \mathrm{F}$. for not less than 72 hours after placing or until the concrete has thoroughly hardened. When necessary, concrete materials shall be heated before mixing. Dependence shall not be placed on salt or other chemicals for the prevention of freezing. No frozen materials or materials containing ice shall be used. Manure shall not be applied directly to concrete when used for protection.

\section{GORP AND DETAILS OF CONSTRUCTION}

19. Design of forms.-(a) Forms shall conform to the shape, lines, and dimensions of the member as called for on the plans. They shall be substantial and sufficiently tight to prevent leakage of inortar; they shall be properly braced or tied together so as to maintain position and shape and insure safety to workmen and passers-by. Temporary openings shall be provided where necessary, to facilitate cleaning and inspection immediately before depositing concrete, 
20. Removal of forms.-(a) The removal of forms shall be carried out in such a manner as to insure the complete safety of the structure. Where the structure as a whole is supported on shores, removable floor folms, beams, and girder sides, column and similar vertical forms may be removed within 24 hours, providing the concrete has hardened sufficiently that it is not injured. In no case shall the supporting forms be disturbed until the concrete has hardened sufficiently to permit their removal with safety. Shoring shall not be removed until the member has acquired sufficient strength to support safely its weight and the load upon it.

21. Cleaning and bending reinforcement.(a) Metal reinforcement, before being placed, shail be free from rust scale or other coatings that will destroy or reduce the bond. Reinforcement shall be formed to the dimensions indicated on the plans. Cold bends shall be made around a pin having a diameter of four or more times the least dimension of the bar

(b) Metal reinforcement shall not be bent or straightened in a manner that will injure the material. Bars with links or bends not shown on the plans shall not be used. Heating of reinforcement for bending will not be permitted.

22. Placing reinforcement.-(a) Metal reinforcement shall be accurately placed and secured and shall be supported by concrete or metal chairs or spacers or metal hangers.

23. Splices and offsets in reinforcement.-(a) In slabs, beams, and girders splices of reinforcement shall not be made at points of maximum stress without the approval of the architect. Splices, where permitted, shall provide sufficient lap to transfer the stress between bars by bond and sheer.

(b) Splices in column bars shall provide a lap of 24 diameters for deformed bars and 30 diameters for plain bars.

24. Construction joints.-(a) Joints not indicated on the plans shall be so made and located as to least impair the strength of the structure. Where a horizontal joint is to be made, any excess water and laitance shall be removed from the surface after concrete is deposited. Before depositing of concrete is resumed, the hardened surface shall be cleaned and roughened and all weak concrete removed.

(b) At least two hours must elapse after depositing concrete in the columns or walls before depositing in beams, girders, or slabs supported thereon. Beams, girders, brackets, column capitals, and haunches shall be considered as part of the floor system and shall be placed monolithically therewith.

(c) Construction joints in floors shall be located near the middle of spans of slabs, beams, or girders unless a beam intersects a girder at this point, in which case the joints in the girders shall be offset a distance equal to twice the width of the beam. In this last case provision shall be made for shear by use of inclined reinforcement.

Simplex Concrete Pile Association, specifications for standard simplex concrete piles.

(See 518.42, p. 413.)

\section{7.}

\section{LIME}

\subsection{GENERAL ITEMS.}

American Society for Testing Materials, standard methods of sampling, inspection, packing, and marking of quicklime and lime products, serial designation C50-27, 1927.

\section{A. GENERAI}

1. (a) Samples of quicklime and lime products shall be taken at one of the following places, as may be agreed upon by the contracting parties:
At point of manufacture, or destination.-From conveyors delivering to bins or piles, from filled bins by means of sampling tubes, from filled bins at the point of discharge, from piles by means of sampling tubes ol shovels, or from loaded cars.

(b) All samples shall be taken in triplicate and immediately sealed in air-tight mositure-proof containers.

(c) One sample shall be immediately delivered to the consignee; one sample shall be delivered to the consignor, if requested, within 10 days from the date of sampling; the third sample shall be retained with seal unbroken until such time as the material has been accepted or a referee called for.

(d.) Samples shall be of an equal weight of not less than 5 pounds each when the quantity sampled equals 30 tons or more. For smaller quantities, the weights of the samples may be reduced proportionately, provided that no sample shall weigh less than 2 pounds, except by special agreement of the contracting parties.

(e) If the samples are taken at any place other than the place of manufacture of the material being sampled, such samples shall be taken within 24 hours of the receipt of the material.

(f) Sampling shall be conducted as expeditiously as possible in order to avoid undue exposure of the material to the air.

(g) Samples shall not be taken from the broken packages.

\section{B. LUMP OR GRANUIAR MIATERAI}

2. (a) In bulk (piles or cars).-Material shall be selected so that it wili represent an average of all parts of the pile or car and shall not contain a disproportionate share of the top or bottom layers. This material shall comprise at least 10 shovelsful taken from different parts of the pile or car. The total material so taken shall weigh not less than 100 pounds for a 30 -ton unit, with a proportionate quantity of material for multiples of this unit, provided that at no time shall the total amount of material so taken weight less than 50 pounds. It shall be broken if necessary, to pass a 1 -inch ring, thoroughly mixed, and ouartered, and the triplicate samples for testing shall be taken from the material so drawn and prepared.

(b) In bulk (at conveyors to or from bins, piles, or cars).-Material shall be taken at regular intervals during the entire time of movement of the mass being sampled. The quantity to be taken at each interval and the number of such intervals shall be so proportioned that the final quantity of material taken shall conform to the requirements of paagraph $(a)$.

3. In packages.-At least 2 per cent of the packages shall be sampled, the packages to be taken from various parts of the material being sampled, and at no time shall less than five packages be sampled. The contents of each of the packages so taken shall be put in a pile, broken if necessary, to pass a I-inch ring, thoroughly mixed, and quartered, and the triplicate samples for testing shall be taken from the material so drawn and prepared.

\section{POWDERED MATERIAL}

4. (a) In bulk (bins, piles, or cars).-A sampling tube shall be used which takes a core not less than 1 inch in diameter, and which is of sufficient length to permit the taking of a core from the top to the bottom of the mass being sampled. Cores shall be taken from at least 10 separate parts of the mass. The cores so taken shall be thoroughly mixed and quartered and the triplicate samples for testing shall be taken from the material so drawn and prepared. 
A minimum quantity of 100 pounds shall be drawn for a unit of 30 tons of material with a proportionate quantity of material for multiples of this unit, provided that at no time shall less than 50 pounds be drawn as the original portion.

(b) In bulk (at conveyors to or from bins, piles, or cars, at packing machines, etc.).- Sampling at conveyors, packing machines, etc., shall conform to the provisions of section $2(b)$.

5. In packages.-A sampling tube shall be used which takes a core not less than one-half inch in diameter. The tube shall be so inserted into the package that it will take a core of the material being sampled from substantially the entire length of the package. Material thus taken from individual packages shall be thoroughly mixed and quartered, and the triplicate samples for testing shall be taken from the material so drawn and prepared. Individual packages shall be taken from various parts of the unit being sampled. At least 1.5 per cent of the packages shall be sampled, and in no case shall less than five packages be sampled.

\section{TREATMENT OF SAMPLE AT LABORATORY}

6. The sample as received at the laboratory shall be thoroughly mixed, and reduced in quantity by the usual methods of quartering to give a minimum portion of $75 \mathrm{~g}$. This portion shall be ground, either by a suitable mechanical device or by hand in an agate mortar, until it all passes a No. 100 (149 micron) sieve. (See A. S. T. M.: E 11, 500.2, p. 1 , for sieve requirements.) It shall then be thoroughly mixed, placed in an air-tight container, and used in the determination of the chemical composition.

7. Samples to be used for such special tests as may be lequired by individual specifications shall be obtained by mixing and quartering the material remaining after removal of the $75-\mathrm{g}$ portion for chemical analysis. The quantity of sample and the screen size of the particles will depend upon the requirements of the individual specifications.

\section{INSPECTION}

8. When inspection and sampling are to be conducted at the point of manufacture, the manufacturer shall afford the inspector repiesenting the purchaser all reasonable facilities for inspection and sampling, which shall be so conducted as not to interfere unnecessalily with the operation of the works. The duties of the inspector or sampler shall be confined exclusively to sampling and inspection of the finished material as ready for shipment, and shall not under any circumstances be construed to permit investigation of methods or processes of manufacture.

\section{REJECTION}

9. (a) Rejection of material based on failure to pass the tests prescribed in the specifications shall be reported wichin 10 days from the taking of samples, and the cause for rejection shall be stated.

(b) The samples which represent rejected material shall be kept in air-tight, moisture-ploof containers for at least five days from the date of the original test report.

\section{RETESTING}

10. (a) Either of the contracting parties may make claim for a retest within five days of the date of the original test report. The expense of the retest shall be borne by the party demanding such retest.

(b) Should the contracting parties be unable to reach a mutually satisfactory agreement based upon the results of the original test, the third sample of material shall be delivered unopened to a referee laboratory for test and the results of this referee iest shall be binding on both parties.

\section{PACKING}

11. Lump lime-Lump lime may be shipped in bulk in carload lots, in wooden barrels, or steel containers holding 180 or 280 pounds net each, or in pasteboard cartons holding 90 pounds net each.

12. Pulverized lime.-Pulverized lime may be shipped in bulk, in carload lots, or in cloth or paper bags.

13. Hydrated lime.-Hydrated lime shall be shipped in cloth bags holding 100 pounds net each, or in paper bags holding $\mathbf{5 0}$ pounds net each.

\section{MARKING}

14. Each shipment of material shall carry with it some means of identification, stating the particular contract on which the purchase and shipment were made.

15. Each package shall have legibly marked thereon, the net weight of its contents, the name of the manufacturer, the place of manufacture, and the brand name, if any. This marking shall comply with all requirements of the Federal lime barrel act, entitled "An act to standardize lime barrels (Public No. 228-Congress-S. 5425)," and with such other Federal or State laws as may be applicable to the shipment in question.

16. In addition to the above information, the following guarantee may be marked on each package or shipment: "Guaranteed by (name of manufacturer) to meet the specifications of the American Society for Testing Materials" for (name of material).

American Society for Testing Materials, standard definitions of terms relating to lime, serial designation C51-28, 1928.

Quicklime.-A calcined material, the major part of which is calcium oxide or calcium oxide in natural association with a lesser amount of magnesium oxide, capable of slaking with water.

Hydrated lime.-A dry powder obtained by treating quicklime with water enough to satisfy its chemical affinity under the conditions of its hydration.

NoTE.-It consists essentially of calcium hydroxide or a mixture of calcium hydroxide and magnesium oxide and magnesium hydroxide.

Lump lime.-Quicklime as it comes from the kilns.

Lump lime screened.-Lump lime after forking or screening to remove the finer portion.

Note.-The portion which is removed is usually that which will pass a $1 / 2$-inch sieve.

Pulverized lime--Quicklime which will pass a fine sieve of specified size.

NOTE.-The size of the sieve is usually one-fourth inch.

\subsection{IIME.}

American Railway Engineering Association, specifications for buildings for railway purposes.

\section{IIME FOR USE IN BRICKWORK}

(See A. R. E. A. specification for brickwork, 518.83, p. 475 .)

Common Brick Manufacturers' Association of America, specifications and general schedule of a brick house.

(See 516.3, p. 310.) MORTAR

National Lime Association, basic specification for lime stuceo, 1922. (See 514.4, p. 274).

National Lime Association, standard specifications for lime plaster. (See 514.4, p. 272.) 
National Lime Association, uses of lime.

(The association has prepared recommendations covering the use of lime in various chemical processes, including general requirements for the lime to be used in the manufacture of paper, strawboard, glass, leather, insecticides, sugar, bleaching powder, soda, sodium bicarbonate and soda ash, sand-lime brick, silica brick, pigments, water paints, dyestuffs and intermediates, calcium carbide and cyanamide, rubber, lubricating greases, animal glue and gelatin, varnish and textiles; also requirements for lime to be used in water softening, fluxing, neutralization and purification processes, trade wastes, causticization, and other processes.)

\subsection{HYDRATED IIME OR QUICKLIME.}

American Ceramic Society, standard specifications for limestone, quicklime, and hydrated lime, 1928.

\section{(A) GENERAI}

\section{(FOR USE IN THE MANUFACTURE OF GLASS)}

1. Use of lime.-Most common glasses are fused mixtures of alkali (usually soda), alkaline earth (usually lime), and silica. Freedom from color is important in determining the quality of glass, which, in turn, depends upon the absence of coloring oxides (usually iron) from the raw material.

Limestone, quicklime, or hydrated lime may be used for making glass. The decision as to which to use is generally based on availability, cost, and present practice.

2. Definition of limestone, quicklime, and hydrated lime.-Limestone consists essentially of calcium carbonate, or of calcium and magnesium carbonates where the amount of the latter does not exceed 45.5 per cent. Quicklime is the product resulting from the calcination of limestone, and consists essentially of calcium oxide, or of calcium and magnesium oxides. It will slake when water is added to it, and this slaking is accompanied by an evolution of heat and an increase in volume. Hydrated lime is a dry powder which is made by treating quicklime with enough water to satisfy its chemical affinity under the conditions of manufacture. It consists essentially of calcium hydroxide, or of a mixture of calcium hydroxide and magnesium oxide or hydroxide.

3. Packing.-Limestone is shipped in bulk in carload lots, or in cloth or paper bags. Quicklime is shipped either in bulk, in carload lots, or barrels holding 180 pounds net or 280 pounds net each. Hydrated lime is shipped in paper bags holding 50 pounds net each.

\section{(B) REQUIREMENTS}

1. Quality.-Except for the constituents enumerated below, the composition of a limestone, lime, or hydrated lime may vary within wide limits and still be satisfactory to the glass manufacturer. Either high calcium or high magnesium material may be used. It is essential, however, that the composition be reasonably uniform from day to day. Otherwise the difficulty of controlling the process of manufacture would become too great.

2. Composition.-Quicklime shall not contain more than 3 per cent $\mathrm{CO}_{2}$ as shipped. Hydrated lime shall not contain more than 5 per cent $\mathrm{CO}_{2}$ as shipped, and shall contain sufficient water to meet the chemical requirements of the calcium oxide.

Limestones, quicklimes, and hydrated limes may be divided into three classes, depending upon the character of glass for which they are suitable. To meet these specifications, the constituents listed below shall not exceed the maximum percentages or fall below the minimum percentages given in the following table:

TABLE 1.-Composition of the nonvolatile portion of limestone, quicklime, or hydrated lime

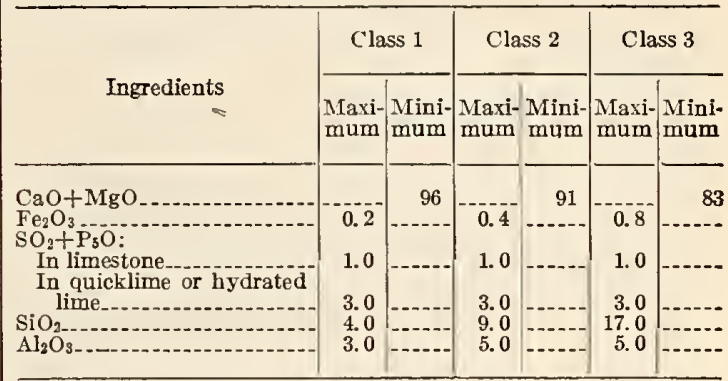

The sum of the calcium and magnesium oxide is specified in theabove table. In order to prevent undue variation in the relative proportions of these two ingredients the per cent calcium oxide shall be established by contract, and it is specified that the per cent calcium oxide shall not vary more than 2 per cent either way from the contract figure.

An approximate figure for the content of alumina should also be included in the contract. This figure must come within the limits set in the above table, and it is specified that the amount of alumina in the material delivered shall not vary more than 1 per cent either way from the contract figure.

3. Fineness.-Unless otherwise specified, limestone, quicklime, and hydrated lime shall be crushed so that all will pass a No. 16 sieve.

Note.-A No. 16 sieve has an opening of $1.19 \mathrm{~mm}$ and a wire diameter of $0.54 \mathrm{~mm}$ with a permissible tolerance of 2 per cent in the opening and 10 per cent in the wire diameter.

4 Marking.-Each carload of material, or fraction thereof, shall be legibly marked with the names of the consignor and consignee, and with some means of identifying the particular contract on which the shipment is made.

This information is in addition to that required by the Federal lime-barrel law.

5. Retesting.-Notice of the rejection of a shipment based on these specifications must be in the hands of the consignor within 10 days after the receipt of the shipment at the point of destination. If the consignor desires a retest, he shall notify the consignee within five days of receipt of the notice of rejection. The consignee shall provide all reasonable facilities to permit the consignor to resample the material. This retest shall be at the expense of the consignor.

\section{(C) SAMPLING AND TESTING}

1. Sampling.-The purchaser will bear all expense of sampling and testing. When limestone or quicklime is shipped in bulk, the sample shall be so taken that it will represent an average of all parts of the shipment from top to bottom, and shall not contain a disproportionate share of the top and bottom layers, which are most subject to changes. The sample shall consist of 1 shovelful for each 3 tons of material, but not less than 10 shovelfuls, taken from different parts of the shipment. The total sample taken shall weigh at least 100 pounds, shall be mixed thoroughly, and "quartered" to provide a 15-pound sample for the laboratory. In case a shipment consists of more than one car, a separate sample shall be taken from each car.

When quicklime is shipped in barrels, at least 3 per cent of the number of barrels shall be sampled. They shall be taken from various parts of the ship- 
ment, dumped, mixed, and sampled as specified in the above paragraph.

In the case of hydrated lime the sample shall be a fair average of the shipment. Three per cent of the packages shall be sampled. The sample shall be taken from the surface to the center of the package. The material so obtained shall be thoroughly mixed and quartered to provide a 2-pound sample for the laboratory.

When sampling quicklime or hydrated lime, it is essential that the operation be conducted as expeditiously as possible, in order to avoid undue exposure of the material to the air. The sample to be sent to the laboratory shall immediately be placed in an air-tight container in which the unused portion shall be stored until the shipment has been finally accepted or rejected by the purchaser.

The sample may be taken either at the point of shipment or at the point of destination, as agreed upon by the contracting parties. If it is desired to enforce the requirements as to carbon dioxide, the sample must be taken at the point of shipment.

2. Testing.-The following directions are a brief summary of the analytical methods which are recommended: Blast $0.5 \mathrm{~g}$ of the sample for 15 minutes in a platinum crucible. Cool and transfer to an evaporating dish. Mix to a slurry with distilled water. Add 5 to $10 \mathrm{cc}$ concentrated $\mathrm{HCl}$. Heat gently until solution is complete, breaking up lumps if necessary. Evaporate to dryness on water bath. Add 5 to $10 \mathrm{cc}$ concentrated $\mathrm{HCl}$ and dilute with an equal volume of distilled water. Digest on water bath for 10 minutes. Filter and wash with hot water. Evaporate the filtrate to dryness. Dissolve in acid and water as before. Filter and wash with hot water. Ignite the two precipitates together and weigh as silica and insoluble matter.

Dilute the above filtrate to $250 \mathrm{cc}$; add $\mathrm{HCl}$ if necessary to insure a total volume of 10 to $15 \mathrm{cc}$; make alkaline with $\mathrm{NH}_{4} \mathrm{OH}$; boil until odor of $\mathrm{NH}_{3}$ is barely noticeable. Filter, and wash slightly with hot water. Dissolve the precipitate with hot dilute $\mathrm{HCl}$ and repeat the precipitation as before. Filter, and wash thoroughly with hot water. Ignite and weigh as oxides of iron, aluminum, and phosphorus.

To the filtrates from the above add a few drops of $\mathrm{NH}_{4} \mathrm{OH}$ and bring to a boil. Add $25 \mathrm{ce}$ of a saturated solution of $\left(\mathrm{NH}_{4}\right)_{2} \mathrm{C}_{2} \mathrm{O}_{4}$. Continue boiling until the precipitate becomes granular. Let stand until precipitate settles clear. Filter and wash with boiling water. Ignite the precipitate, dissolve in dilute $\mathrm{HCl}$, and dilute to $100 \mathrm{cc}$. Add excess of $\mathrm{NH}_{4} \mathrm{OH}$, and boil. Filter out any insoluble matter; ignite and weigh; add its weight to the oxides of iron and aluminum found previously. To this filtrate add $\left(\mathrm{NH}_{4}\right)_{2} \mathrm{C}_{2} \mathrm{O}_{4}$, proceeding as before. Filter, and wash with boiling water. Ignite and blast to constant weight as calcium oxide.

Acidify the filtrates from the above with $\mathrm{HCl}$. Evaporate to $150 \mathrm{cc}$. Add $10 \mathrm{cc}$ of a saturated solution of $\mathrm{NaNH}_{4} \mathrm{HPO}_{4}$ and boil; cool; add $\mathrm{NH}_{4} \mathrm{OH}$ drop by drop, with constant stirring, until the precipitate starts to form. Then add moderate excess of $\mathrm{NH}_{4} \mathrm{OH}$. Stir for several minutes. Let stand overnight. Filter and dissolve the precipitate in hot dilute $\mathrm{HCl}$. Dilute to $100 \mathrm{cc}$, add $1 \mathrm{cc}$ of saturated solution of $\mathrm{NaNH}_{4} \mathrm{HPO}_{4}$, and precipitate as before. Filter, and wash with an alkaline solution made by diluting $\mathrm{NH}_{4} \mathrm{OH}$ until it contains about 2.5 per cent $\mathrm{NH}_{3}$ and then add a few drops of $\mathrm{HNO}_{3}$; ignite and weigh as $\mathrm{Mg}_{2} \mathrm{P}_{2} \mathrm{O}_{7}$; multiply this weight by 0.3621 to find the weight of $\mathrm{MgO}$.

Place $5 \mathrm{~g}$ of the sample of quicklime or hydrated lime, or $0.5 \mathrm{~g}$ of limestone, in a small Erlenmeyer flask and cover with hot distilled water. Connect this flask into a carbondioxide train, set up as follows: Next to the flask is a reflux condenser, to to which is connected a calcium-chloride drying tube, followed by a tube containing anhydrous $\mathrm{CuSO}_{4}$, then another tube of $\mathrm{CaCl}_{2}$, then by two tubes filled with soda lime, and finally by another tube of $\mathrm{CaCl}_{2}$. The entire train must be so arranged that a stream of $\mathrm{CO}_{2}$ free air can be kept passing through it. Start this stream of air. Weigh the tubes containing soda lime and replace them in the train. Add to the sample in the flask about $25 \mathrm{cc}$ of $1: 1 \mathrm{HCl}$, being careful that no gas is lost and that the effervescence is not too violent during the operation. When the effer vescence diminishes, heat the flask, bringing the liquid gradually to boiling. Boil for 10 minutes. Remove the flame and allow the flask to cool for 15 minutes, while the stream of air is still flowing. Disconnect and weigh the soda-lime tubes. Their increase in weight is recorded as carbon dioxide.

Dissolve $5 \mathrm{~g}$ of the sample in $\mathrm{HCl}$ and evaporate to dryness. Dissolve the residue in $\mathrm{HCl}$; filter and wash with hot water. Fuse the residue with $\mathrm{Na}_{2} \mathrm{CO}_{3}$. Treat the filtrate with $\mathrm{NaOH}$ in boiling solution; filter and wash with hot water. Dissolve both this precipitate and the fused residue in dilute $\mathrm{H}_{2} \mathrm{SO}_{4}$. Reduce with $\mathrm{Zn}$ and titrate with $N / 20$ $\mathrm{KMnO}_{4}$. Calculate the result to per cent $\mathrm{Fe}_{2} \mathrm{O}_{3}$.

Dissolve $2 \mathrm{~g}$ of the sample in $10 \mathrm{cc}$ water and 15 cc $1: 1 \mathrm{HCl}$. Filter out insoluble matter. Dilute filtrate to $250 \mathrm{cc}$. Heat to boiling and add $10 \mathrm{cc}$ of a boiling 10 per cent solution of $\mathrm{BaCl}_{2}$, drop by drop, with constant stirring. Let stand overnight; filter; wash with hot water; ignite and weigh as $\mathrm{BaSO}_{4}$. Calculate to per cent $\mathrm{SO}_{3}$.

Dissolve $10 \mathrm{~g}$ of the sample in 80 cc of $1: 1 \mathrm{HNO}_{3}$; filter; wash; fuse the residue $\mathrm{Na}_{2} \mathrm{CO}_{3}$; dissolve the melt in $\mathrm{HNO}_{3}$ and add this solution to the filtrate. Boil the filtrate with $10 \mathrm{cc}$ of 1.5 per cent $\mathrm{KMnO}_{4}$ solution until $\mathrm{MnO}_{2}$ is precipitated. Add enough $\mathrm{H}_{2} \mathrm{SO}_{3}$ to dissolve the $\mathrm{MnO}_{2}$. Neutralize with $\mathrm{NH}_{4} \mathrm{OH}$. Add 1 ce of concentrated $\mathrm{HNO}_{3}$ for every $100 \mathrm{cc}$ of solution. Bring to $40^{\circ}$ C. and precipitate with ammonium molybdate. Shake for 10 minutes and let stand at $40^{\circ} \mathrm{C}$. for 12 hours. Filter and wash with 1 per cent $\mathrm{KNO}_{3}$. Dissolve the precipitate in a known volume of $N / 10 \mathrm{NaOH}$ and $\mathrm{CO}_{2}$ free water. Titrate the excess $\mathrm{NaOH}$, using phenolphthalein as indicator. Calculate the per cent $\mathrm{P}_{2} \mathrm{O}_{5}$, using the proportion, $\mathrm{P}: \mathrm{NaOH}:: 1: 23$.

American Railway Engineering Association, specifications for quicklime to be used in water treatment, 1922.

\section{DEFINITION}

1. Quicklime for use in water treatment is a material the major part of which is calcium oxide, which will slake on the addition of water.

\section{GRADES}

2. Quicklime is divided into two grades:

(a) Selected.-Shall be well burned, picked free from ashes, coke, clinker, or other foreign material.

(b) Run-of-kiln. - Shall be well burned, without selection. It shall be free from large lumps of unburned or foreign material.

\section{FORMS}

3. Quicklime is shipped in two forms:

(a) Lump. - Shall be kiln size.

(b) Pulverized. - Shall be reduced in size to pass a $1 / 4$-inch screen. 


\section{CIASSES}

4. Quicklime for water treatment is divided into two classes:

(a) High-calcium.

(b) Calcium.

\section{BASIS OF PURCHASE}

5. The particular grade, form, and class of quicklime desired shall be specified in advance by the purchaser. Unless otherwise specified high-calcium quicklime shall be furnished.

\section{CHEMICAL PROPERTIES AND TESTS}

\section{(A) SAIIPLING}

Lime in Barrels

6. Quicklime shall be shipped in barrels, or other containers of similar size. At least 3 per cent of the number of barrels shall be sampled. They shall be taken from various parts of the shipment, dumped, mixed, and sampled. The samples shall comprise at least 10 shovelfuls taken from different parts of the shipment. The total sample taken shall weigh at least 100 pounds and shall be crushed to pass a 1 -inch ring, and quartered to provide a 15-pound sample for the laboratory.

Laboratory Samples

7. All samples to be sent to the laboratory shall be immediately transferred to an air-tight container in which the unused portion shall be stored until the quicklime shall finally be accepted or rejccted by the purchaser.

Chcck tests on samples taken from car at destination will occasionally be made, and should agree within reasonable limits with initial sample.

\section{(B) CHEMICAL TESTS}

\section{Chemical Properties}

8. (a) The classes and chemical properties of quicklime shall be determined by standard methods of chemical analysis.

(b) Samples shall be taken as specified in sections 6 and 7.

(c) Quichlime shall conform to the following requirements as to chemical composition:

\section{Chemical composition}

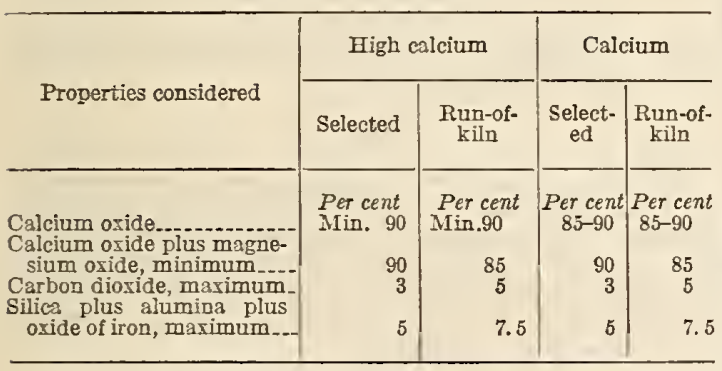

\section{INSPECTION, PENAIIZATION, AND REJECTION}

\section{INSPECTION}

9. (a) All quicklime shall be subject to inspection.

(b) The quicklime may be inspected either at the place of manufacture or the point of delivery as arranged at the time of purchase.

(c) The inspector representing the purchaser shall have free entry at all times, while work on the con- tract of the purchaser is being performed, to all parts of the manufacturer's works which concern the manufacture of the quicklime ordered. The manufacturer shall afford the inspector all reasonable facilities for inspection and sampling, which shall be so conducted as not to interfere unnecessarily with the operation of the works.

(d) The purchaser may make the tests to govern the acceptance or rejection of the quicklime in his own laboratory or elsewhere. Such tests, however, shall be made at the expense of the purchaser.

\section{PENALIZATION}

All prices shall be based upon minimum percentage of calcium oxide. If a lower grade is furnished than the one specified it may, at the option of the purchaser, be accepted upon making a rebate cqual to the percentage of calcium oxide below the minimum designated.

\section{REJECTION}

11. (a) Unless otherwise specified, any rejection based on failure to pass thc tests prescribed in these specifications shall be reported within 10 working days from the taking or receipt of samples.

(b) Rejected quicklime shall be returned to the shipper or as he may direct. All freight charges in both directions to be paid by the shipper.

\section{REHEARING}

12. Samples which represent rejected quicklime shall be preserved in air-tight containers for 10 working days from the date of test report. In case of dissatisfaction with the results of the tests, the manufacturel may make claim for a lchcaring within that time.

American Railway Engineering Association, specification for hydratcd lime to be used in water treatment, 1922.

\section{DEFINITION}

1. Hydrated lime is a dry flocculent powder resulting from the hydration of quicklime.

\section{CLASSES}

2. Hydrated lime for water treatment is commercially divided into two classes: (a) High-calcium, (b) calcium.

\section{BASIS OF PURCHASE}

3. The particular class of hydrated lime desired shall be specified in advance by the purchaser. Unless otherwise specified high-calcium lime shall be furnished.

\section{CHEMICAL PROPERTIES AND TESTS SAMIIING}

4. The sample shall be a fair average of the shipment. Three per cent of the packages shall be sampled. The sample shall be taken from the surface to the center of the package. A 2-pound sample to be sent to the laboratory shall immediately be transferred to an air-tight retainer, in which the unused portion shall be stored until the hydrated lime has been finally accepted or rejected by the purchaser. Check tests on samples taken from cars at their destination will occasionally be made, and should agree within reasonable limits with initial sample.

\section{CHEMICAL PROPERTIES}

5. (a) The classes and chemical properties of hydrated lime shall be determined by standard methods of chemical analysis. 
(b) The hydrated lime shall conform to the following requirements as to chemical composition:

High-calcium, not less than 90 per cent calcium hydroxide $\left(\mathrm{Oa}(\mathrm{OH})_{2}\right)$.

Calcium, not less than 85 per cent calcium hydroxide $\left(\mathrm{Ca}(\mathrm{OH})_{2}\right)$.

\section{PHYSICAI PROPERTIES AND TESTS FTNEN ESS}

6. A $100 \mathrm{~g}$ sample shall leave by weight a residue of not over 2 per cent on a standard 100-mesh sieve and not over 0.5 per cent on a standard 20 -mesh sieve.

\section{PACKING AND MARKING}

\section{PACKING}

7. Hydrated lime shall be packed either in cloth or in papcr bags of not more than 50 pounds net weight.

\section{MARKING}

8. The weight shall be plainly marked on each package, together with the name of the manufacturer.

\section{INSPECTION, PENALIZATION, AND REJEC- TION}

\section{INSPECTION}

9. (a) All hydrated lime shall be subject to inspection.

(b) The hydrated lime may be inspected either at the place of manufacture or the point of delivery, as arranged at the time of purchase.

(c) The inspector representing the purchaser shall have frce entry at all times while work on the contract of the purchaser is being performed, to all parts of the manufacturer's works which concern the manufacture of the hydrated lime ordered. The manufacturcr shall afford the inspector all reasonable facilities for inspection and sampling, which shall be so conducted as not to interfere unnecessarily with the opcration of the works.

(d) The purchaser may make the tests to govern the acceptance or rejection of the hydrated lime in his own laboratory or elsewhere. Such tests, however, shall be made at the expense of the purchaser.

\section{PEN ALIZATION}

10. All prices shall be based upon the minimum percentage of calcium hydroxide. If a lower grade is furnished than the one specified, it may at the option of the purchaser be accepted upon making a rebate equal to the percentage of calcium hydroxide below the minimum designated.

\section{REJECTION}

11. (a) Unless otherwise specified, any rejection based on failure to pass tests prescribed in these specifications shall be reported within 10 working days from the taking or receipt of samples.

(b) Rejected hydrated lime shall be returned to the shipper or as he may direct. All freight charges to be paid by the shipper in both directions.

\section{REHEARING}

12. Samples which represent rejected hydrated lime shall be preserved in air-tight containers for 10 working days from the date of the test report. In case of dissatisfaction with the rcsults of the tests the manufacturer may make claim for a rehearing within that time.

American Society for Municipal Improvements, standard specifications for sewers, 1927.

(This specification includes requirements for hydrated lime for use in sewer construction.)

(See 518.67, p. 453.)
American Society for Testing Materials, standard specifications for quicklime for structural purposes, serial designation C5-26, 1926.

\section{REQUIREMENTS}

1. The quicklime shali conform to the foilowing requirements as to chemical composition, calculated to the nonvolatile basis:

\begin{tabular}{|c|c|c|}
\hline$=$ & $\begin{array}{l}\text { Cal- } \\
\text { cium } \\
\text { lime }\end{array}$ & $\begin{array}{l}\text { Mag- } \\
\text { nesium } \\
\text { lime }\end{array}$ \\
\hline Calcium oxide, minimum & $\left|\begin{array}{c}\text { Per cent } \\
75\end{array}\right|$ & Per cent \\
\hline Magnesium oxide, minimum & & 20 \\
\hline Calcium and magnesium oxides, minimum & 95 & 95 \\
\hline Silica, alumina, and oxide of iron, maximum & 5 & \\
\hline Carbon dioxide, maximum: & & \\
\hline If sample is taken at the kiln & 3 & 3 \\
\hline If sample is taken at any other place... & 10 & 10 \\
\hline
\end{tabular}

2. When tested in accordance with the method described in section 4, quicklime shall contain not more than 15 per cent by weight of residue.

\section{METHODS OF TEST}

3. The chemical analysis of the lime shall be made in accordance with A. S. T. M. C25. (See p. 354.)

4. An average $21 \frac{1}{\mathrm{~kg}}$ (5-pound) sample shall be put in a box of wood or of some material of similarly low thermal conductivity and slaked by an experienced operator with sufficient water at $70^{\circ}$ to $80^{\circ} \mathrm{F}$. $\left(21^{\circ}\right.$ to $27^{\circ} \mathrm{C}$.) to produce the maximum quantity of lime putty, care being taken to avoid "burning" or 'drowning" the lime. It shall be allowed to stand for one hour and then washed through a No. 20 sieve by a stream of water having a moderate pressure. No material shall be rubbed through the sieve. The washing shall be continued until the residue on the screen appears to consist wholly of coarse particles, but in no case shall washing be continued more than 30 minutes. The residue shall be dried to constant weight at a temperature of $212^{\circ}$ to $225^{\circ} \mathrm{F}$. $\left(100^{\circ}\right.$ to $107^{\circ}$ C.). The sample of lump lime taken for this test shall be broken so as to all pass a 1-inch ring and be retained on a $1 / 4$-inch sieve. Pulverized lime shall be tested as received.

\section{SAMPLING, RETESTING, PACKING, AND MARKING}

5. The sampling, retesting, packing, and marking shall be conducted in accordance with A. S. T. M. C50. (See 517.0, p. 347.)

\section{APPENDIX}

Quicklime can never be used as such for structural purposes; it must always be slaked first. Since the method of slaking is an important factor in determining the quality of the finished product, the following directions are given, not as a part of the specifications, but as information for the further protection of the purchaser.

\section{PREPARATION OF LIME PUTTY}

1. (a) Introduction.-Different kinds of lime vary considerably in the way in which they behave with water. A little supervision over the operation of slaking will amply pay for itself by insuring the production of the greatest possible quantity and the best possible quality of putty. To find out how to slake a new lot of lime, it is safest to try a little of it and see how it works. Since different lots of the 
same brand of lime vary somewhat, and since the weather conditions at the time have a decided influence, it is wise to try a sample from each lot used, whether familiar with the brand or not.

(b) Classification of limes.-In a bucket, put two or three lumps of lime about the size of one's fist, or, in the case of granular lime, an equivalent amount. Add enough water to just barely cover the lime, and note how long it takes for slaking to begin. Slaking has begun when pieces split off from the lumps or when the lumps crumble. Water of the same temperature should be used for test and field practice.

If slaking begins in less than 5 minutes, the lime is quick slaking; from 5 to 30 minutes, medium slaking; over 30 minutes, slow slaking.

(c) Directions for slaking.-For quick-slaking lime, always add the lime to the water, not the water to the lime. Have enough water at first to cover all the lime completely. Have a plentiful supply of water available for immediate use - a hose throwing a good stream, if possible. Watch the lime constantly. At the slightest appearance of escaping steam, hoe thoroughly and quickly, and add enough water to stop the steaming. Do not be afraid of using too much water with this kind of lime.

For medium-slaking lime, add the water to the lime. Add enough water so that the lime is about half submerged. Hoe occasionally if steam starts to escape. Add a little water now and then if necessary to prevent the putty from becoming dry and crumbly. Be careful not to add any more water than required and not too much at a time.

For slow-slaking lime, add enough water to the lime to moisten it thoroughly. Let it stand until the reaction has started. Cautiously add more water, a little at a time, taking care that the mass is not cooled by the fresh water. Do not hoe until the slaking is practically complete. If the weather is very cold, it is preferable to use hot water; but if this it not available, the mortar box may be covered in some way to keep the heat in.

2. (a) White coat.-After the action has ceased run off the putty through a No. 10 sieve and store for a minimum of two weeks.

(b) Base coats.- After the action has ceased run off the putty through a No. 8 sieve. Add sand up to equal parts by weight, all of the hair required, and store for a minimum of two weeks.

(c) Mason's mortar.-After the action has ceased add part or all of the sand required and store for a minimum of 24 hours.

American Society for Testing Materials, standard specifications for hydiated lime for structural purposes, serial designation C6-24, 1924.

1. These specifications cover two classes of hydrated lime, namely, masons' hydrate and finishing hydrate.

2. (a) Masons' hydrated lime may be used for scratch or brown coat of plaster, for stucco, for mortar, and for addition to Portland-cement concrete.

(b) Finishing hydrated lime may be used for any of the purposes enumerated in paragraph $(a)$ for masons' hydrated lime, and, in addition, it may be used as an ingredient in the final or white coat of plaster.

\section{REQUIREMENTS}

3. Masons' hydrated lime shall conform to the chemical and physical requirements herein specified except that for plasticity, specified in seciion 8 .

4. Finishing hydrated lime shall conform to all chemical and physical requirements herein specified, including that for plasticity, specified in section 8 .

5. (a) Carbon dioxide.-Carbon dioxide in the sample shall not exceed 5 per cent if the sample is taken at the place of manufacture, or 7 per cent if the sample is taken at any other place. The percentages in both cases are based on the nonvolatile portion. (See sec. 9.)

(b) Calcium and magnesium oxides.-Calcium and magnesium oxides shall constitute not less than 95 per cent of the nonvolatile portion. (See sec. 9.)

6 . The sample shall leave a residue of not more than 0.5 per cent on a No. 30 sieve, and not more than 15 per cent on a No. 200 sieve. (See sec. 10.)

7 . If the steam has no visible effect on the pat (see sec. 11), the sample shall be reported as being "sound." If the pat disintegrates, the sample shall be reported as being unsound, and the shipment rejected. If the pat cracks, pops, or shows other minor defects, it shall not be reported as either sound or unsound, but its behavior shall be noted.

8. Finishing hydrated lime shall have a plasticity figure of not less than 200. (See sec. 13.)

\section{METHODS OF TEST}

9. The chemical analysis of the hydrated lime shall be made in accordance with serial designation C25 of the American Society for Testing Materials (given below).

10. Fineness of hydrated lime shall be determined as follows:

One hundred grams of the sample as received shall be placed on a No. 30 sieve, which shall be nested above a No. 200 sieve. The material shall be washed by means of a stream of water from a faucet. The washing shall be continued until the water coming through the sieve is clear, but in no case should the washing be continued for more than 30 minutes. The residues on both sieves shall be dried to constant weight in an atmosphere free from carbon dioxide at a iemperature between $100^{\circ}$ and $120^{\circ}$ C. The weights shall be calculated to pcrcentages of the original sample, the weight of the material retained on the No. 30 sieve being added to the wcight of the material retained on the No. 200 sieve to obtain the correct weight of the material retained on the No. 200 sieve.

The sieves used shall meet the requirements given in the United States Standard Sieve Series. (See A. S. T. M. E $11,500.2$, p. 1.)

A piece of rubber tubing attached to a water faucet is to be used for the washing. The velocity of the water may be increased by pinching the tubing, but should not be sufficient to cause any splashing of the sample over the sides of the sieve.

Care must be taken not to let water accumulate on the No. 200 sieve, else the openings will become clogged and the operation can not be completed in 30 minutes.

11. Constancy of volume shall be determined as follows:

To $20 \mathrm{~g}$ of the sample, $100 \mathrm{~g}$ of standard Ottawa sand shall be added, mixed thoroughly and enough water added to the mixture to make a good plastic mortar of rather dry consistency. This shall be spread on a clean glass plate to form a layer about $1 / 4$ inch thick by 4 inches square. The pat shall be of even thickness throughout, and not tapering at the edges. If the mortar is too dry to work well, more water may be added. The pat shall be placed in a closet for 24 hours. The temperature in the closet shall be between $65^{\circ}$ and $75^{\circ} \mathrm{F}$. $\left(18^{\circ}\right.$ and $24^{\circ}$ C.), and there shall be free circulation of air, but no direct draft striking the pat. The pat shall then be soaked in water until a film of water stands unabsorbed on the surface of the pat. If the pat has cracked, the consistency of the mortar was too wet and a new pat must be made. Two or three pats made at the same time from mortars of slightly different consistencies will avoid delays, 
Twenty grams of the sample shall be mixed with enough water to form a thick cream. This shall be spread in a thin layer on the surface of the pat. It shall be allowed to stand for 15 minutes to permit air bubbles to form. It shall be troweled to an even surface, making this skim coat as thin as possible without allowing the sand to show through. The pat shall be put in the closet, under the conditions specified above, for 24 hours. It shall be examined carefully to see that there are no cracks or pops. The pat shall be suspended in a vessel partially filled with cold water, in such a way that the water can boil without touching the pat. The water shall be brought gradually to boiling and kept boiling gently for five hours, the pat being surrounded by steam during this time. The water shall then be permitted to cool for at least 12 hours. The pat shall then be removed and examined.

12. Consistency of lime putty shall be measured as follows:

The lime shall be made into a stiff putty with water and permitted to soak overnight. It shall be molded in a ring such as is used with a Vicat needle, resting the specimen on a glass plate.

The needle used is a modified form of Vicat needle, $12.5 \mathrm{~mm}$ in diameter and weighing $30 \mathrm{~g}$. This may conveniently be made from a suitable piece of aluminum tubing. The lower end should be closed without shoulders or curvature, and the tube loaded with shot to the specified weight. It is mounted in the Vicat needle stand.

The initial reading shall be taken with the bottom of the needle in contact with the surface of the sample. The final reading shall be taken 30 seconds after the plunger is released. A penetration of 20 Inm shall be considered standard.

If the penetration is less than standard, the sample may be removed from the mold, mixed with more water, and retested. If the penetration is more than standard, the sample shall be discarded and a new one prepared.

The ring and the Vicat needle stand are described in the Standard Specifications and Tests for Portland Cement (serial designation C9) of the American Society for Testing Materials, 516.11, page 294.

13. Plasticity shall be determined by means of the apparatus shown in Figure 118, facing page 223, as follows: Three hundred grams of the sample shall be mixed with enough water to form a thick putty. After aging in a vessel covered with a damp cloth for not less than 16 nor more than 24 hours this putty shall be adjusted to standard consistency, as defined in section 12 , with a permissible variation of $\pm 5 \mathrm{~mm}$.

A ring such as is referred to in section 12 shall be lubricated with a thin film of water, placed on a porcelain base plate, filled with the paste and struck off level. The mold shall be removed by raising it vertically without distorting the paste. The base plate and paste shall be placed in the instrument and the carriage turned up by hand until the surface of the paste is in contact with the disk and the distance between the disk and the top of the base plate is $1 \frac{1}{4}$ inches.

The carriage shall be thrown into gear and the motor started. It is essential that the motor shall be started exactly 120 seconds after the first portion of the paste has been put in the mold. The time when the first portion of paste is put in the mold shall be recorded as zero time-the motor is therefore started at two minutes.

Care shall be taken to protect the specimen from draits during the test.

The scale reading shall be recorded every minute until the test is completed.

The test shall be considered complete when $(a)$ the scale reading reaches $100,(b)$ any reading is less than the one before, or (c) the scale reading remains constant for three consecutive readings (two minutes) and the specimen has visibly ruptured or broken loose from the base plate.

The time and the scale reading at the end of the experiment shall be noted. The plasticity figure shall be calculated from the formula $P=\sqrt{F^{2}+(10 T)^{2}}$, in which $P$ is the plasticity figure, $F$ is the scale reading at the end of the experiment, and $T$ is the time in minutes from when the first portion of paste was put in the mold to the end of the experiment.

After each test the porcelain base plate shall be washed with hot water, treated with dilute hydrochloric acid to remove any lime from the surface pores, washed again with water to remove the acid, dried by heating above $100^{\circ} \mathrm{C}$., and cooled to room temperature before it is used again.

\section{SAMPLING, INSPECTION, REJECTION, ETC.}

14. The sampling, retesting, packing, and marking shall be conducted in accordance with serial designation C50 of the American Society for Testing Materials. (See 517.0, p. 347.)

American Society for Testing Materials, standard methods of chemical analysis of limestone, quicklime, and hydrated lime, serial designation C25-29, 1929.

Nore.-Bulletin 700, United States Geological Survey, should be used as a reference hook in connection with these methods, especially when very exact and detailed analyses are desired. Blank determinations should he made on all reagents, and corrections applied when called for.

\section{TPEATMENT OF SANPLE}

The sample, taken and prepared in accordance with the requirements of the particular specifications of the society applicable to the material shall be treated as follows:

Weigh out approximately $0.5 \mathrm{~g}$ of the substance (note 1) and, if a limestone or hydrated lime, ignite in a covered platinum crucible in an electric muffie (note 2) for 15 minutes, or longer if the heat is not powerful enough to effect complete decomposition within that time. Transfer to an evaporating dish, preferably of platinum (note 3 ) for the sake of celerity in evaporation, mix to a thin slurry with distilled water, add 5 to $10 \mathrm{cc}$ of $\mathrm{HCl}$ (sp. gr. 1.20) and digest with aid of gentle heat and agitation until solution is complete. Solution may be aided and the time shortened by light pressure wioh the flattened end of a glass rod upon resistant lumps. Then evaporate the solution to dryness, so far as this may be possible on the water bath.

\section{DETERHINATION OF SIIICA AND INSOLUBLT MATTER, INCLUDING SIIICA}

When dry, or nearly so, place the dish and its contents in an air bath or (covered) on a platinum triangle resting on a hot plate and, if it is a high calcium limestone that is under treatment, heat for one hour at $200^{\circ} \mathrm{C}$.; if a high magnesian stone is under treatment the temperature should not exceed $120^{\circ} \mathrm{C}$. Drench the cooled mass with $\mathrm{HCl}$ (sp. gr. 1.20) and allow to stand for a few minutes. Add an equal volume of water, cover the dish and place on the watcr bath for 10 minutes (note 4). Separate the silica by filtration on a filter of suitable size. Wash thoroughly with dilute $\mathrm{HCl}$ and then wash twice with cold water.

Evaporate the filtrate to dryness. Extract the residue with $\mathrm{HCl}$ as before, but allowing only a few minutes time. Filter this solution through a second and smaller paper.

Transfer, wet, the papers containing the separated residue to a weighed platinum crucible. Char 
carefully without allowing the paper to inflame, and ignite to constant weight in an electric muffle, and reigh (note 2). The increase in weight represents the insoluble and siliceous matter and is reported as insoluble matter, including silica.

Silica.-To determine the amount of silica $\left(\mathrm{SiO}_{2}\right)$, treat the "insoluble matter, including silica," in the crucible with $5 \mathrm{cc}$ of water, $5 \mathrm{cc}$ of $\mathrm{HF}$ and one or two drops of $\mathrm{H}_{2} \mathrm{SO}_{4},{ }^{62}$ and evaporate to dryness. Heat the residue for two or three minutes in an electric muffle and again weigh. The difference between this weight and that previously obtained gives the weigh' of "silica" $\left(\mathrm{SiO}_{2}\right)$ (note 5).

If the silica is determined by rolatilization with $\mathrm{HF}$, the residue in the crucible is fused with a little $\mathrm{Na}_{2} \mathrm{CO}_{3}$ (note 6), and the cooled melt is dissolved in diluted $\mathrm{HCl}$ and the solution added to the filtrate from the second evaporation for "insoluble matter, including silica." If the "insoluble matter, including silica," is reported as such and no HF treatment is given, the "insoluble matter, including silica," is not fused or further treated.

Insoluble matter, excluding silica.-The difference between the weight of "insoluble matter, including silica," and "silica" represents the "insoluble matter, exclusive of silica."

\section{DETERMINATION OF TOTAL IRON AND ALU- MINUM}

To the $\mathrm{HCl}$ solution from the determination of silica add a few drops of bromine water or $\mathrm{HNO}_{3}$ and boil the solution until all trace of bromine or chlorine is gone. Then add $\mathrm{HCl}$, if not already present, sufficient to insure a total volume of 10 to $15 \mathrm{cc}$ of $\mathrm{HCl}$ (sp. gr. 1.20).68 Add a fen drops of methyl red solution and after diluting to 200 to 250 cc heat the liquid to boiling. Neutralize by means of $\mathrm{NH}_{4} \mathrm{OH}$ (dilute toward the end) until the color of the liquid changes to a distinct yellow. Boil for one or two minutes, allow to settle, filter and wash the precipitate at once two or three times with hot $\mathrm{NH}_{4} \mathrm{Cl}$ (2 per cent) solution and suck dry. ${ }^{04}$

Set the filtrate aside. Dissolve any precipitate from the paper in hot, dilute $\mathrm{HCl}$, the solution passing into the beaker in which the precipitation was made and wash the paper thoroughly with hot water. Boil the solution to expel any trace of chlorine and again precipitate the hydroxides with $\mathrm{NH}_{4} \mathrm{OH}$ as described above. Ignite the precipitate, moist (note 2), in a weighed platinum crucible, cool and weigh as $\mathrm{Al}_{2} \mathrm{O}_{3}$ and $\mathrm{Fe}_{2} \mathrm{O}_{3}$ (note 7 ).

\section{DETERMINATION OF TOTAL IRON}

Fuse the combined iron and aluminum oxides (note 8 ) in a platinum crucible at a very low temperature with 3 to $4 \mathrm{~g}$ of $\mathrm{K}_{2} \mathrm{~S}_{2} \mathrm{O}_{7}$ or better $\mathrm{Na}_{2} \mathrm{~S}_{2} \mathrm{O}_{7}$ (note 9). Take up the melt with so much dilute $\mathrm{H}_{2} \mathrm{SO}_{4}$ that there shall be not less than $5 \mathrm{~g}$ absolute acid and enough water to effect solution on heating. Evaporate the solution and eventually heat until acid fumes come off copiously. After cooling and redissolving in water, filter out the small amount of silica, wash, ignite, weigh, and correct by $\mathrm{HF}$ and $\mathrm{H}_{2} \mathrm{SO}_{4}$ (note 10). Add the weight so corrected to the welght of silica previously found and deduct from the gross weight of the oxides of iron and aluminum.

Reduce the filtrate by zinc (note 11 ). Titrate with $\mathrm{KMnO}_{4}$ using a $N / 20$ solution.

62 See Bulletin 700, United States Geologicol Survey, p. 103.

63 See Bulletin 700, U. S. Geological Surtey, p. 258.

a See Bulletin 700, U. S. Geological Survey, p. 107.

\section{DETERMINATION OF ALUMINUM}

Subtract the calculated weight of $\mathrm{Fe}_{2} \mathrm{O}_{3}$ obtained by the method described above from the weight of $\mathrm{Fe}_{2} \mathrm{O}_{3}+\mathrm{Al}_{2} \mathrm{O}_{3}$. Report the remainder as $\mathrm{Al}_{2} \mathrm{O}_{3}$. In case phosphorus is determined, calculate it to $\mathrm{P}_{2} \mathrm{O}_{5}$. and deduct also.

\section{DETERMINATION OF TOTAL IRON}

\section{ALTERNATIVE METHOD (Note 8)}

Dissolve 2 to $5 \mathrm{~g}$ (depending upon the amount of iron present) of the properly prepared sample in $\mathrm{HCl}$ and evaporate rapidly to dryness. Treat the residue with water and $\mathrm{HCl}$, filter off the silica and wash several times with hot water. Precipitate the iron in a boiling solution with fixed alkali, allow to settle, filter, and wash free of chlorides, using hot water. Dissolve in dilute $\mathrm{H}_{2} \mathrm{SO}_{4}$.

Ignite the insoluble matter from the evaporated hydrochloric acid solution in a platinum crucible. Treat with $\mathrm{H}_{2} \mathrm{SO}_{4}$ and $\mathrm{HF}$ and heat until fumes of $\mathrm{H}_{2} \mathrm{SO}_{4}$ appear. Bring the residue into solution with. the addition of a few drops more of $\mathrm{H}_{2} \mathrm{SO}_{4}$ and combine the solution with that of the bulk of the iron. Reduce this solution by zinc and titrate the iron with $\mathrm{KMnO}_{4}$ in the usual manner.

\section{DETERMINATION OF CALCIUM}

(a) GRAVIMETRIC METHOD (Note 12)

Add a few drops of $\mathrm{NH}_{4} \mathrm{OH}$ to the combined filtrate from the $\mathrm{R}_{2} \mathrm{O}_{3}$ precipitate, and bring the solution to boiling. To the boiling solution add $35 \mathrm{co}$ of a saturated solution of $\left(\mathrm{NH}_{4}\right)_{2} \mathrm{C}_{2} \mathrm{O}_{4}$, and continue the boiling until the precipitated $\mathrm{CaC}_{2} \mathrm{O}_{4}$ assumes a granular form; then allow it to stand 20 minutes, or until the precipitate has settled and the supernatent liquid is clear, filter and wash thoroughly with boiling water (note 13). Place the wet filter and precipitate in a platinum crucible, and burn off the paper over the small flame of a Bunsen burner, or in a muffle. Ignite the residue, dissolve in hot dilutc $\mathrm{HCl}$, and make up the volume of the solution to $100 \mathrm{cc}$ with water. Add $\mathrm{NH}_{4} \mathrm{OH}$ in slight excess and boil the liquid. If a small amount of $\mathrm{Al}_{2}(\mathrm{OH})_{e}$ separates, filter it out, wash with $\mathrm{NH}_{4} \mathrm{Cl}$ (2 per cent solution), ignite, weigh, and add this weight to that found in the first determination. Then reprecipitate the lime by $\left(\mathrm{NH}_{4}\right)_{2} \mathrm{C}_{2} \mathrm{O}_{4}$, allow to stand until settled, filter, wash thoroughly with boiling water (note 13), and weigh as calcium oxide after ignition to constant weight in a covered platinum crucible.

\section{(b) VOLUMETRIC METHOD (Note 14)}

Make the filtrate from the iron and alumina precipitate alkaline with $\mathrm{NH}_{4} \mathrm{OH}$, boil, and add $35 \mathrm{cc}$ of a boiling saturated solution of $\left(\mathrm{NH}_{4}\right)_{2} \mathrm{C}_{2} \mathrm{O}_{4}$. Stir vigorously and allow to stand until the precipitate has settled, filter on an $11 \mathrm{~cm}$ paper, and wash ten times with hot water (note 15). Transfer the paper and precipitate to the beaker in which the precipitation was made, spreading the paper out against the upper portion of the beaker. Wash the precipitate from the paper with a jet of hot water, fold the paper, and leave it adhering to the upper portion of the beaker. Add to the contents of the beaker $50 \mathrm{cc}$ of dilute $\mathrm{H}_{2} \mathrm{SO}_{4}(1: 10)$, dilute to a volume of $250 \mathrm{cc}$ with hot water and heat to a temperature of $80^{\circ}$ to $90^{\circ} \mathrm{C}$. Titrate with the standard $\mathrm{KMnO}_{4}$ solution ${ }^{65}$ until the pink end point is obtained. Now drop the folded filter paper

66 See directions for preparing this solution. 
(which has been adhering to the side of the beaker) into the liquid; the pink color of the latter will be discharged. Finish the titration by adding $\mathrm{KMnO}_{4}$, a drop at a time, until the pink end point is again obtained. A Gooch crucible may be used instead of filter paper. From the total quantity of standard $\mathrm{KMnO}_{4}$ solution used, calculate the percentage of calcium oxide.

\section{DETERMINATION OF STRONTIUM}

Transfer the weighed oxides obtained in the gravimetric determination of calcium to a small flask of 20 ce capacity and dissolve in $\mathrm{HNO}_{3}$. Evaporate to dryness and heat at $150^{\circ}$ to $160^{\circ} \mathrm{C}$. Treat the thoroughly dried nitrates with as little (rarely over $2 \mathrm{cc}$ ) of a mixture of equal parts of absolute alcohol and ether as may be needed to dissolve the calcium salt, solution being hasiened by occasional gentle agitation. After standing over night in the colked flask collect the insoluble matter on the smallest

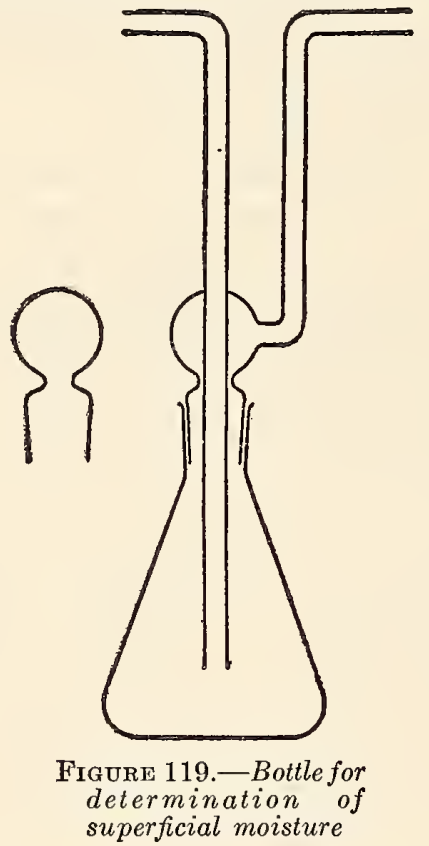

possible filter and wash with more of the above mixture of alcohol and ether. After drying, pass a few cubic centimeters of hot water through the filter, on which may remain a few tenths of a milligram of residue, which does not usually contain any lime or other alkaline earth and whose wcight is therefore to be deducted from that of the lime, unless it can be shown that it is derived from the glass of the little flask in which the nitrates of calcium and strontium were evaporated. To the solution of strontium nitrate in a small beaker add a few drops of $\mathrm{H}_{2} \mathrm{SO}_{4}$ and then its volume of alcohol, whereby the strontium is precipitated as sulphate. After 12 hours, filter on a small filter paper and wash the residue with 50 per cent alcohol. Ignite at a low temperature, moisten with dilute $\mathrm{H}_{2} \mathrm{SO}_{4}$, evaporate again ignite and weigh as strontium sulphate. Test the sulphate spectroscopically as to treedom from calcium and barium.

Calculate the weight of strontium oxide and deduct from the weight of calcium oxide obtained as above.

\section{DETERMINATION OF MAGNESIUM}

Acidify the combined filtrates from the calcium precipitates with $\mathrm{HCl}$ and concentrate on the water bath to about $150 \mathrm{cc}$, and heat to boiling. To the boiling solution add $10 \mathrm{cc}$ of a saturated solution $\mathrm{Na}\left(\mathrm{NH}_{4}\right) \mathrm{HPO}_{4}$ and continue the boiling for several minutes. Then cool to room temperature, and add $\mathrm{NH}_{4} \mathrm{OH}$ drop by drop with constant stirring until the crystallin ammonium-magnesium orthophosphate begins to form and then in moderate excess, the stirring being continued for several minutes. Allow the liquid to stand in a cool atmosphere for from 12 to 48 hours (note 16) and filter.

Dissolve the precipate in hot dilute $\mathrm{HCl}$, make up the solution with water to about $100 \mathrm{cc}$ add $1 \mathrm{cc}$ of the saturated solution of $\mathrm{Na}\left(\mathrm{NH}_{4}\right) \mathrm{HPO}_{4}$ and $\mathrm{NH}_{4} \mathrm{OH}$ drop by drop with constant stirring until the precipitate is again formed as above dcscribed and the ammonia is present in moderate excess. Then allow it to stand in a cool atmosphere for from 12 to 48 hours (note 16) filter on paper or a Gooch cruciblc, wash with dilute $\mathrm{NH}_{4} \mathrm{OH}$ containing $\mathrm{NH}_{4} \mathrm{NO}_{3}$ (note 17), ignite (note 2), cool, and weigh as $\mathrm{Mg}_{2} \mathrm{P}_{2} \mathrm{O}_{7}$ (note 18).

\section{DETERMINATION OF TOTAL VOLATILE MATTER}

\section{(LOSS ON IGNITION)}

Place $1 \mathrm{~g}$ of the properly prepared sample (note 19 ) in a weighed platinum crucible, cover with the lid and heat gently for five minutes (note 2), gradually increasing the temperature to the maximum of the muffle, and maintain at this temperature until constant weight is obtained (usually about 15 minutes).

\section{DETERIMINATION OF MECHANICAL MOISTURE}

Limestone.-Weigh $1 \mathrm{~g}$ of the properly prepared sample in a tared, wide, low-form, flat-bottomed weighing bottle. Heat, uncovered, in a ventilated drying oven at $120^{\circ} \mathrm{C}$. for two hours. Quickly stopper and cool in a desiccator. Lift the stopper momentarily just before weighing and weigh. The use of a similar weighing bottle as a counterpoise carried through all of the operations is a desirable procedure. The loss in weight represents "mechanical moisture" or "hydroscopic water" at $120^{\circ} \mathrm{C}$.

Hydrated lime.-Mechanical moisture in hydrated limes is to be determined by aspirating over the sample in a closed container at $120^{\circ} \mathrm{C}$. a slow stream of dry, $\mathrm{CO}_{2}$-free air. The container for the sample is to be equipped with two interchangeable stoppers, one to be used during weighings and the other to be used during the drying. The second stopper is equipped with two entry tubes for conducting the air stream over the sample. (Note 20.) (See fig. 119.)

The container is connected in a train, having before it a soda-lime tower, a limewater bottle, a sulphuric-acid bottle, and a phosphoric anhydride bulb, in the order named, and after it another protective phosphoric anhydride bulb. The lime-water bottle is useful for giving indication of the exhaustion of the soda lime. The train is placed in position, with connection for the sample bottle, in a drying oven at $120^{\circ} \mathrm{C}$.

The manipulations are as follows: Place 2.5 to $3 \mathrm{~g}$ of the properly prepared sample into the previously weighed bottle and immediately restopper it. A glazed paper funnel will aid rapid manipulation. Insert the bottle in the train by quickly exchanging the stoppers and draw a slow current of dry, $\mathrm{CO}_{2-}$ free air through the apparatus for two hours. Remove the sample bottle from the oven with another 
quick exchange of stoppers and set in a desiccator to cool. When cool, remove it to the balance case for several minutes before weighing it, and just before weighing lift the stopper slightly for an instant to relieve any vacuum that may exist in the bottle. The loss in weight of the sample represents "mechanical moisture" or "hydroscopic water" at $120^{\circ} \mathrm{C}$.

Use a bottle similar to the one containing the sample as a counterpoise in all weighings.

\section{DETERMINATION OF CARBON DIOXIDE}

Carbon dioxide is to be determined upon the properly prepared sample according to the method given in United States Geological Survey Bulletin No. 700, page 217. An illustration showing the arrangement of the component parts of the necessary apparatus is shown on page 218 of that bulletin.

With limestone, use a $0.5 \mathrm{~g}$ sample; with burned lime, hydrated lime, etc., use a $5 \mathrm{~g}$ sample, in the former case, at once in the latter, light the burner under the flask and start the flow of water through the condenser. Keep the flame low, so as to secure steady but quiet ebullition, and do not interrupt the air current although it should be reduced to a slow rate. With much carbon dioxide the rate of absorption is very readily noted by holding the hand to the soda-lime tubes, which become hot or warm when absorption is taking place. Sufficient time having elapsed (note 21), extinguish the flame and increase the air current. When cool, disconnect the soda-lime tubes from the apparatus and allow to stand in the balance case until two weights taken 30 minutes apart agree within $0.5 \mathrm{mg}$.

The soda lime for use in this determination must be porous, not hard and unabsorptive like that sometimes used for the combustion of nitrogenous organic substances.

Alternative method.-The apparatus illustrated in Figure 120, which is a modification of the usual

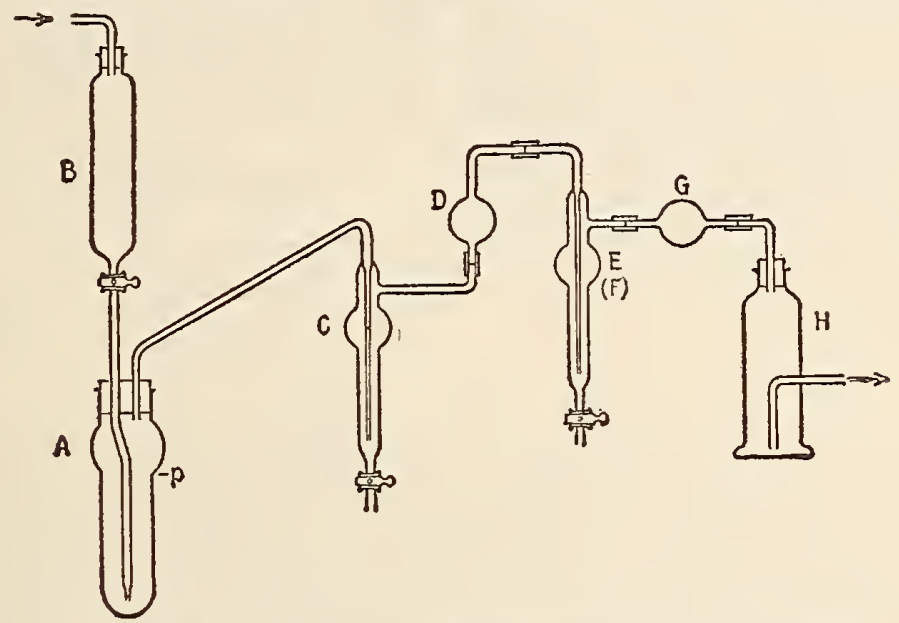

FigdRe 120.-A pparatus for determination of carbon residue

Boil the weighed sample with dilute $\mathrm{HCl}$ in a small Erlenmeyer flask attached to an upwardly inclined condenser, whence, after passing through a drying system-calcium chloride, anhydrous copper sulphate to retain hydrogen sulphide from decomposable sulphides and any $\mathrm{HCl}$ that may pass over, then calcium chloride again-the carbon dioxide is caught by absorption tubes filled with soda lime followed by calcium chloride. Of course, arrangement is made for a current of air free from $\mathrm{CO}_{2}$ with which to sweep out the apparatus before and after the experiment and for a slow current during its continuance.

The results are very accurate and the determination can be quickly carried out.

The manipulations are as follows: Pour hot water upon the powder in the flask fitted with a separatory funnel and delivery tube; attach this to the condenser and force a current of air free from $\mathrm{CO}_{2}$ through the whole system, except the weighed absorption tubes, until the original air has been displaced, the observation bulbs (containing $\mathrm{H}_{2} \mathrm{SO}_{4}$ to show the rate of gas flow) being attached directly to the drying system. Then close the stopcock in the separatory funnel, half fill the latter with $\mathrm{HCl}(1: 1)$, replace the rubber stopper of the funnel, insert the absorption tubes between the drying system and the observation bulbs, and allow the acid to flow into the flask, slowly if there is much carbon dioxide, rapidly if there is but little. When effervescence diminishes form, may be used, if desired. The principle of its operation is the same as that of the procedure described above.

The apparatus consists of the following:

A tube, $A$, of decomposition of the sample.

A tube, $B$, to contain acid. A soda-lime tower is placed ahead of tube $B$ so that air drawn through the apparatus is free of $\mathrm{CO}_{2}$.

A "microdrier," $C$, containing water, to act as a condenser and to retain most of the acid volatilized from tube $A$.

A bulb, $D$, containing mossy zinc to remove the last traces of $\mathrm{HCl}$.

A "microdrier," $E$, containing $\mathrm{H}_{2} \mathrm{SO}_{4}$ to dry gases. If the sample evolves $\mathrm{H}_{2} \mathrm{~S}$, another microdrier, $F$, containing cadmium chloride solution, is inserted between bulb $D$ and microdrier $E$.

A bulb, $G$, containing $\mathrm{P}_{2} \mathrm{O}_{5}$ to complete the drying of the gases.

A midvale bulb, $\mathrm{H}$, for absorption of $\mathrm{CO}_{2}$. It is filled as follows: A layer of glass wool extending above the end of the outlet tube is placed in the bottom and on this a layer of $\mathrm{P}_{2} \mathrm{O}_{5}$, about threeeighths inch thick. Immediately on this is placed another layer of glass wool, and the rest of the bulb is filled with "ascarite," except for a final layer of glass wool on the top.

The manipulations are as follows: Sweep out tubes $C, D, E, F$ and $G$, with a current of $\mathrm{CO}_{2}$-free air. Place in tube $B$ sufficient $\mathrm{HCl}$ (sp. gr. 1.1) to completely decompose the sample, but so regulated in 
volume that the liquid level in tube $A$ shall not be above the point $P$. Connect a soda-lime tower to the top of tube $B$.

With limestone, use an $0.5 \mathrm{~g}$ sample; with burned lime, hydrated lime, etc., use a 3 to $5 \mathrm{~g}$ sample.

Introduce rapidly into tube $A$ a weighed amount (note 1) of properly prepared sample and immediately cover it with $\mathrm{CO}_{2}$-frce water and attach to the apparatus. Then attach the weighed midvale bulb. Allow the acid from tube $B$ to flow into tube $A$, the rate being governed by the rate of cvolution of gas. Then gently heat tube $A$ and boil to expel all $\mathrm{CO}_{2}$ (note 21), while drawing a slow current of $\mathrm{CO}_{2}$-free air through the apparatus. Continue the air current for three minutes after removing the flame. Disconnect the midvale bulb and weigh it after it has come to equilibrium in the balance case. Use a second midvale bulb as counterpoise in all weighings.

\section{DETERMINATION OF SULFURIC ANHYDRIDE}

Place $2 \mathrm{~g}$ of the properly preparcd sample in a small, dry beaker and stir it up with 10 cc of cold water until all lumps are broken up and the lighter particles are in suspension. Add $15 \mathrm{cc}$ of dilute $\mathrm{HCl}(1: 1)$ and heat until solution is complete. Filter through a small paper and wash the residue thoroughly with hot water. Dilute the filtrate to $250 \mathrm{cc}$, heat to boiling, and add $10 \mathrm{cc}$ of a boiling 10 per cent solution of barium chloride drop by drop with constant stirring. Stir well and allow to stand overnight. Filter, wash with boiling water, ignite, and weigh as $\mathrm{BaSO}_{4}$.

\section{DETERMINATION OF TOTAL SULPHUR}

Weigh $1 \mathrm{~g}$ of a properly prepared sample. Add approximately $0.5 \mathrm{~g}$ of sodium carbonate. Mix thoroughly in a porcelain crucible and heat gently until sintered. Then ignite for 15 minutes at a temperature of approximately $1,000^{\circ}$ C., taking care to allow access of air to the contents of the crucible. Cool and place the crucible in a $250 \mathrm{cc}$ beaker and cover with hot water. Add $10 \mathrm{cc}$ of bromine water, then $30 \mathrm{cc}$ of hydrochloric acid $(1: 1)$ and boil until solution is complete and all bromine has been expelled. Remove the crucible, washing it with water. Add a few drops of methyl red and render the solution alkaline with ammonium hydroxide $(1: 1)$. Boil the solution for one or two minutes, filter, and wash with hot water. To the filtrate add 5 ce of hydrochloric acid (1:1), adjust the volume to about $200 \mathrm{cc}$, bring the solution to boiling, and while boiling add $10 \mathrm{cc}$ of hot 10 per cent barium chloride solution. Allow to stand overnight. Filter, wash with hot water, ignite, and weigh as $\mathrm{BaSO}_{4}$.

Nore.-It is usually desirable to make a fusion in a platinum crucible. However, it has been found that with limestones very high in impurities, when the fusion is made in a muffle, the damage to porcelain crucibles introduces no appreciable error.

\section{DETERMINATION OF PHOSPHORUS}

Dissolve $10 \mathrm{~g}$ of the sample in 80 ce of dilute $\mathrm{HNO}_{3}(1: 1)$, filter, and wash the residue with hot watcr. Ignite and fuse the residue with a little $\mathrm{Na}_{2} \mathrm{CO}_{3}$, cool the melt and add its nitric-acid solution to the main filtrate. (Note 22.)

In case the rock is rather argillaceous, mix the powder with half its weight of $\mathrm{Na}_{2} \mathrm{CO}_{3}$ and blast strongly. Dissolve the cooled melt in $\mathrm{HNO}_{3}$ and evaporate to dryn:ss. Take up the dry residue with dilute $\mathrm{HNO}_{3}(1: 2)$, filter, and wash the residue with hot water. Ignite the residue in platinum and evaporate with $\mathrm{HF}$ and $\mathrm{HNO}_{3}$. Finally, evaporate twice with $\mathrm{HNO}_{3}$ alone to expcl all fluorine, dissolve the residue in $\mathrm{HNO}_{3}$ and add the solution to the main filtracc. (Note 22.)

Oxidize the nitric-acid solution by boiling with 10 cc of 1.5 per cent $\mathrm{KMnO}_{4}$ solution until manganese dioxide is precipitated. Dissolve the oxide by addition of $\mathrm{H}_{2} \mathrm{SO}_{3}$ or a sulphite free from phosphorus and boil to expel the oxides of nitrogen and sulphur. Neutralize the $\mathrm{HNO}_{3}$ with $\mathrm{NH}_{4} \mathrm{OH}$ and then add $1 \mathrm{cc}$ of $\mathrm{HNO}_{3}$ (sp. gr. 1.42) for every $100 \mathrm{cc}$ of solution. Bring the solution to a temperature of $40^{\circ} \mathrm{C}$., add ammonium molybdate solution ${ }^{86}$ and shake for 10 minutes. Allow to stand at a temperature of not over $40^{\circ} \mathrm{C}$. for 1 to 12 hours. Filter and wash ten times with a 1 per cent $\mathrm{KNO}_{3}$ solution. Return the precipitate to the precipitating vessel, add a measured excess of $0.1 \mathrm{~N} \mathrm{NaOH}$ and $25 \mathrm{cc}$ of water free from $\mathrm{CO}_{2}$ and shake or stir until the precipitate is dissolved. Dilute to 100 to $200 \mathrm{cc}$ with water free from $\mathrm{CO}_{2}$, add three drops of 0.2 per cent phenolphthalein solution, and discharge the pink eolor with standard acid. Finish the titration by adding standard alkali until the reappearance of the pink color. The alkali solution should be free from earbonate and be standardized against the Bureau of Standards standard benzoic acid. ${ }^{67}$ The ratio of phosphorus to $\mathrm{NaOH}$ should be considered as 1:23 in calculating results. (Note 23.)

\section{DETERMINATION OF MANGANESE}

Dissolve $10 \mathrm{~g}$ of the sample in 100 ce of dilute $\mathrm{HNO}_{3}$ (1:1), filter, and wash the residue with hot water. Ignite the residue in platinum, fuse with a little $\mathrm{Na}_{2} \mathrm{CO}_{3}$, and add the nitric-acid solution of the melt to the main filtrate.

Dilute the solution to $150 \mathrm{cc}$ and add $0.5 \mathrm{~g}$ of sodium bismuthate. (Note 24.) Heai for a few minutes, or until the pink color has disappeared and dioxide has precipitated. If manganese dioxide does not precipitate add more bismuthate. Clear the solucion by adding a few drops of a saturated solution of sodium bisulphite or other suitable reducing agent free trom ehlorides and boil to expel all oxides of nitrogen and sulphur. Cool to $15^{\circ} \mathrm{C}$., add an excess of sodium bismuthate, agitate, and let stand for a few minutes. Add 50 ee of 3 per cent $\mathrm{HNO}_{3}$ and filter through asbestos. Wash with 3 per cent $\mathrm{HNO}_{3}$ until the washings run through colorless. Add a measured excess of a standard $\mathrm{FeSO}_{4}$ solution and titrate back with a scandard $\mathrm{KMnO}_{4}$ solucion of which the strength has been determined by means of the Bureau of Standards sodium oxalatc. (Note 25.)

\section{DETERMINATION OF FERROUS IRON SOLUBLE} IN SULPHURIC ACID

In limestones the presence of carbonaceous matter renders the exact or even approximate determination of ferrous iron often impossible. Nevertheless, even in its presence aceeptable results are sometimes obtainable if there is not much of such matter and if it does not give with acid a colored solution. Occasionally limestones show films of manganese peroxide, which likewise interferes with the determination.

(a) In absence of carbonaceous matter.-The powder, one to several grams, is introduced into a stout flask of about 200 to 250 cc capacity and boiled with a little water till all air is expelled. While still boiling, dilute $\mathrm{H}_{2} \mathrm{SO}_{4}$ is added, a little at a time, till effervescence ceases, and then a further amount. Calcium sulphate precipitates, but the iron will remain in solution. The flame is then removed and

66 See Blair, The Chemical Analysis of Iron, 8th Ed. p. 92.

ө See J. Am. Chem. Soc., 34, p. 1027; 1912; 35, p.1309; 1913. 
a stopper tightly inserted, through which passes a small stopcock funnel. When cool, or nearly so, cold water is poured into the funnel, the cock cautiously opened, and the water drawn into the flask, more water being poured into the funnel as fast as it empties till the solution amounts to 100 to $150 \mathrm{cc}$. Such precaution to exclude air is hardly necessary in most cases, however, for in presence of $\mathrm{H}_{2} \mathrm{SO}_{4}$ the oxidation of ferrous iron is exceedingly slow. It is ordinarily quite sufficient to equalize the internal and external pressures by opening the cock, removing the stopper, and pouring in cold water. The flask is then brought under a burette containing dilute $\mathrm{KMnO}_{4}$ solution and the iron titrated without delay. With a carbonate which is wholly decomposable without the aid of heat solution may be accomplished in a flask filled with $\mathrm{CO}_{2}$, using cold acid, whereby the danger of attack of silicates is lessened if these are present. If the preference is for the $\mathrm{K}_{2} \mathrm{Cr}_{2} \mathrm{O}_{7}$ method of titration, $\mathrm{HCl}$ may be used instead of $\mathrm{H}_{2} \mathrm{SO}_{4}$ in absence of manganese peroxide. In this case there is, of course, no separation of an insoluble calcium salt, a fact which renders easier the subsequent determination of the iron in any insoluble residue the rock may yield. The ferrous iron thus found is mostly, if not altogether, that existing as carbonate. ${ }^{88}$

(b) In presence of carbonaceous matter.-Decomposition is effected in a flask by dilute $\mathrm{H}_{2} \mathrm{SO}_{4}$ in an atmosphere of $\mathrm{CO}_{2}$. With limestones and active agitation no heat need be used, but with dolomites it will be necessary. The solution is then quickly filtered through asbestos (in an atmosphere of $\mathrm{CO}_{2}$ if much iron is present), the residue and filter washed a few times with water, and the filtrate titrated at once with permanganate. If it is colored by organic matter the result may be in error. As above, $\mathrm{HCl}$ and titration by $\mathrm{K}_{2} \mathrm{Cr}_{2} \mathrm{O}_{7}$ may be used in absence of manganese pcroxide. In either case it is important to allow the acid to act no longer than is necessary and to filter quickly. A determination of the ferrous iron in the insoluble matter is usually not worth attempting because of the admixed organic matter.

\section{DETERMINATION OF AVAILABLE LIME IN QUICK- LIME AND HYDRATED IIME BY THE MODIFIED SCAIFE METHOD}

"Available lime" in quicklime or hydrated lime is that (or those) constituent (or constituents) which enters (or enter) into the reaction under the conditions of the specified method or process.

The interpretation of results obtained by the following method shall be restricted by the above definition.

Place $1.4 \mathrm{~g}$ of the carefully prepared and finely ground (passing a No. 100 sieve) lime in a $400 \mathrm{cc}$ beaker, add 200 ec of hot water, cover, heat carefully, and then boil for three minutes.

Cool, wash down cover, add 2 drops of phenolphthalein, and titrate with normal hydrochloric acid, adding the acid dropwise as rapidly as possible and stirring vigorously to avoid local excess of acid. When the pink color disappears in strealss retard the rate of addition of acid somewhat, but continue until the pink color disappears entirely and does not reappear for one or two seconds. Note the reading and ignore the return of color.

Repeat the test, substituting for the $400 \mathrm{cc}$ beaker a 1-liter graduated flask, carrying a 1-hole stopper fitted with a short glass tube drawn out to a point. Cool and add dropwise and with vigorous stirring $5 \mathrm{cc}$ less acid than before. Call the number of cubic centimeters used $A$. Grind up any small lumps with a glass rod flattened at

${ }^{68}$ If a determination of ferrous iron in the insoluble residue is desired, see U. S. Geological Survey Bulletin 700, p. 265.
d. one end, dilute to the mark with freshly boiled distilled water, close the flask with a solid stopper, mix thoroughly for 4 or 5 minutes, and let settle for 30 minutes.

Pipette a 200 ce portion, add phenolphthalein and titrate slowly with $0.5 \mathrm{~N}$ hydrochloric acid until the solution remains colorless on standing one minute. Call this addiiional number of cubic centimeters $B$. Then the percentage of available $\mathrm{CaO}=2 A+5 B$.

Notes.-To secure accurate and concordant results it is essential that this method be followed in minute detail.

For very accurate work $0.1 \mathrm{~N}$ acid may te used in place of the 0.5 For very accurate work $0.1 \mathrm{~N}$ acid may be used in place of the $\mathbf{0 . 5}$
$N$ acid prescribed. The calculation then becomes: Available $\mathrm{CaO}=2 A+B$

Standard acid solutions must be standardized by the National Bureau of Standards benzoic acid No. $48-\mathrm{A}$ through a sodiumhydroxidesolution.

\section{PREPARATION OF STANDARD SOLUTION OF POTASSIUM PERMINGANATE}

Prepare a solution of potassium permanganate containing $5.64 \mathrm{~g}$ of the pure crystallized salt in each liter. Such a solution will be of such strength that $1 \mathrm{cc}$ will approximately equal $0.005 \mathrm{~g}$ of $\mathrm{CaO}$. (Note 26.)

The best and simplest way to prepare such a solution is to weigh out the required quantity of potassium permanganate and place in a bottle containing the proper volume of water a week or 10 days before the solution is to be standardized. The bottle and its contents must be kept in a dark place and shaken occasionally for the first three or four days. When ready for standardization siphon off the solution through a glass siphon into another bottle, leaving a deptl of about 1 inch of solution undisturbed in the original bottle. (Note 27.) Thoroughly mix the solution in the second bottle by shaking and standardize as follows (note 28):

Dissolve $0.5 \mathrm{~g}$ of $\mathrm{Na}_{2} \mathrm{C}_{2} \mathrm{O}_{4}$ (note 29) in a $400 \mathrm{cc}$ beaker with 200 to $250 \mathrm{cc}$ of hot water $\left(80^{\circ}\right.$ to $90^{\circ} \mathrm{C}$.) and add $10 \mathrm{cc}$ of dilute $\mathrm{H}_{2} \mathrm{SO}_{4}(1: 1)$ to the solution. Titrate at once with the permanganate solution being standardized, stirring vigously and continuously. The permanganate solution must not be added more rapidly than 10 to $15 \mathrm{ec}$ per minute, and the last 0.5 to 1 ce must be added dropwise, with particular care to allow each drop to be fully decolorized before the next is introduced. The excess of permanganate solution necessary to give the end-point color is determined by running a blank determination in another beaker containing the same volume of acid and water present in the original beaker and heated to the same temperature. (Note 30.) The value of the permanganate solution in grams of $\mathrm{CaO}$ per cubic centimeter of solution may be calculated from the following equations: $5 \mathrm{CaC}_{2} \mathrm{O}_{4}+2 \mathrm{KMnO}_{4}+8 \mathrm{H}_{2} \mathrm{SO}_{4}=$ $\mathrm{K}_{2} \mathrm{SO}_{4}+2 \mathrm{MnSO}_{4}+10 \mathrm{CO}_{3}+8 \mathrm{H}_{2} \mathrm{O}$. $5 \mathrm{CaSO}_{4}+\mathrm{K}_{2} \mathrm{SO}_{4}+2 \mathrm{MnSO}_{4}+10 \mathrm{CO}_{2}+8 \mathrm{H}_{2} \mathrm{O}$. Notes. - 1 . Due to the rapidity with which quicklime and hy.
drated limes become contaminated by absorption of water and carbon dioxide from the air, samples must be protected in tightly stopped contaimers at all times. Samples for analysis are to be samples being determined by the difference in wcight of the bottles before and after their removal

2. Ignitions in electric muffles arc far superior to flame ignitions. If an electric muffle is not available, flame ignition and the blast lamp may be used.

3. If a platinum dish is not avalable, porcelain may be used. A glass container positively must not be used.

4. Instead of strong acid, that of half strength may be used and the heating begun at once.

5. For ordinary control work in the plant laboratory this correction may, perhaps, be neglected; the dcuble evaporation must never be neglected.

6. Fusion with pyrosulphate is to be avoided on account of the subsequent introduction of sulfates into the solution.

7. This precipitate may also contain $\mathrm{TiO}_{2}, \mathrm{P}_{2} \mathrm{O}_{6}$, and $\mathrm{Mn}_{3} \mathrm{O}_{4}$. 
8. Where the iron is present in small quantities it is rather unsatisfaetory to determine it in the ignited oxides from the $0.5 \mathrm{~g}$ sample. Under these conditions the alternative method may be used.

9 . The pyrosulphates are less troublesome and more eficetive than the bisulphates.

10. This eorreetion for impurities should not be made when the 10. This eorreetion for impurities should not be made when the hydrofluoric aeid eorreetion of the main siliea determination has been
omitted. After two evaporations, 1 to $2 \mathrm{mg}$ of siliea are still to be omitted. After two evaporations,

11. Zine will also reduee titanium. If this metal is to be determined and dedueted from $\mathrm{Al}_{2} \mathrm{O}_{3}$, then $\mathrm{H}_{2}, \mathrm{~S}$ must be used for reduction (boiling out the $\mathrm{H}_{2} \mathrm{~S}$ in a stream of $\mathrm{CO}_{2}$ before titration). If titanium is not to be determined, the s'ight error introdueed by its reduction with zine is so small as to be negligible, except in the most exaet and detailed analyses.

12. The gravimetric method must be employed when a reeovery of aluminum is desired or when a determination of strontium is contemplated.

13. Care must be exeercised in this washing, as 1,000 ce of boiling water will dissolve over $1 \mathrm{cg}$ of $\mathrm{CaC}_{2} \mathrm{O}_{4}$.

water will dissolve over 14 cg of $\mathrm{CaC}_{2} \mathrm{O}_{4}$. in the plant laboratory. It must be borne in mind that in this method all strontium oxide is reported as calcium oxide.

15. Use not more than 125 ee for this washing.

15. Use not more than 125 ee for this washing.
16. The less the amount of magnesium present the longer the precipitate must be allowed to settle.

17. Made by diluting $\mathrm{NH}_{4} \mathrm{OH}$ with distilled water until the solution eontains 21,2 per cent $\mathrm{NH}_{3}$ by weight. Three or four drops of $\mathrm{HNO}_{3}$ (sp. gr. 1.42) are then added.

18. When manganese is present in the limestone it will be caught in large part with the magnesium precipitate. (See United States Geolo rieal Survey Bulletin 700, p. 134.) In case manganese was not eliminate $\mathrm{l}$ before this preeipitation, the weighed pyrophosphate must be dissolved in $\mathrm{INO}_{3}$, tested for manganese with hismuthate, the manganese determined and deducted as $\mathrm{Mn}_{2} \mathrm{P}_{2} \mathrm{O}_{7}$

manganese determined and deducted as $\mathrm{Mn}_{2} \mathrm{P}_{2} \mathrm{O}_{7}$. 19. This determination may be made, if desired, upon the $0.5 \mathrm{~g}$ sample isnted for solution in HCl.

under "Treatment of sample."

20. A little bottle of 20 to 30 ce capacity with glass stoppers, as descrihed, ground to fit, is a convenient form of apparatus. (See fig. 119.)

21. Boiling for one minute is usually sufficient

22. In limestones very low in phosphorus it may be deemed desirable to concentrate the phosphorus from a considerahle weight of sample. In this case proceed as direeted by F. Hinden (Zeitschr. Anal. Chemie, 54, p. 214; 1915,) as follows: To the nitrie aeid solution of the large sample add a few drops of $\mathrm{Fe}_{2} \mathrm{Cl}_{0}$ solution and then $\mathrm{NH}_{4} \mathrm{OH}$ until the liquid beeomes slightly turhid. Then add $0.5 \mathrm{~g}$ of pure preeipitated $\mathrm{CaCO}_{3}$ and boil for five minutes. Filter, dissolve of pure preeipitated $\mathrm{CaCO}_{3}$ and boil for five minutes.

the preeipitate in dilute $\mathrm{HNO}_{3}$, and proeeed as above. 23. Titanium and vanadium, whieh interfere in this determination, should not

material.

Instead of the speeified alkalimetrie titration method, the phosphorus in the phosphomolybdate may be determined as magnesium pyrophosphate (United States Geologieal Survey Bulletin 700, pp. 178-179), or by the reductor method (A. A. Blair, Chemical Analysis of Iron, 8th ed. pp. 91-92)

24. The persulphate method may be employed if desired.

25. The separation of manganese with the $\mathrm{R}_{2} \mathrm{O}_{3}$ precipitate by the use of hromine is not recommended on aeeount of the incompleteness of the preeipitation. Separation of manganese by means of ammonium sulphide after the ammonia preeipitation is also undesirahle nium sulphide after the ammonia preeipitation is also undesirahle of aceount of the int

(See also note 18 coneerning the contamination of magnesium pyro(See also note 18 coneerning the contamination of magnesium pyro-
phosphate by manganese pyrophosphate and the need of eorrective measures when manganese is present in the material under analysis.) 26. With high-ealeium limes more than $50 \mathrm{cc}$ of this solution will probahly be required to titratc the liherated oxalie acid. In sueh cases the use of a larger burette is to he preferred to increasing the strength of the permanganate solution.

27. The siphon should not extend nearer than 1 inch from the bottom of the original bottle.

28. This solution should be standardized at least once every week. It will be found more convenient to make a solution as above deseribed and standardize it evcry week than to attempt, by boiling and filtering to make a solution whieh will not change.

and filtering to make a solution whieh will not change. 29. Sodium oxalate for standardization of potassium permanganate
solution should be purchased from the National Bureau of Standards, Washington, D. C. This material is sold by the Bureau of Standards as sample No. $40 \mathrm{a}$

30 . When the end point is reaehed the temperature of the solution should not be below $60^{\circ} \mathrm{C}$.

American Society for Testing Materials, standard specifications for quicklime for use in the manufacture of sulphite pulp, serial designation $\mathrm{C} 46-27$, 1927.

1. Lime is used in the "milk of lime" or "tank system" of sulphite-pulp manufacture for making the cooking liquor. The milk of lime is held in solution or suspension in a series of tanks equipped with suitable agitators. The sulphur dioxide is forced or drawn through these tanks successively. In some cases the tanks are built on top of each other in the form of a tower. The contents of the first tank are drawn off when the liquor has reached a certain strength $\left(3.5\right.$ to 6 per cent total $\mathrm{SO}_{2}$ ) and the contents of the second and third tanks progress to the first and second tanks, respectively. The third tank is again charged with fresh milk of lime. There are other systems of absorption which provide for continuous instead of intermittent operation. The function of the lime is to furnish the base for the formation of the bisulphites of calcium and magnesium.

\section{REQUIREMENTS}

2. (a) Either calcium or magnesium lime may be used, but owing to the greater solubility and reactivity of magnesium bisulphite, high-magnesium lime gives the best results. The ratio $\mathrm{MgO}$ to $\mathrm{CaO}$ shall be reasonably constant and the lime shall be moderately free from impurities.

(b) The quicklime shall conform to the following requirements as to chemical composition, all percentages to be calculated on the basis of the weight of the sample taken at the point of manufacture: ${ }^{69}$

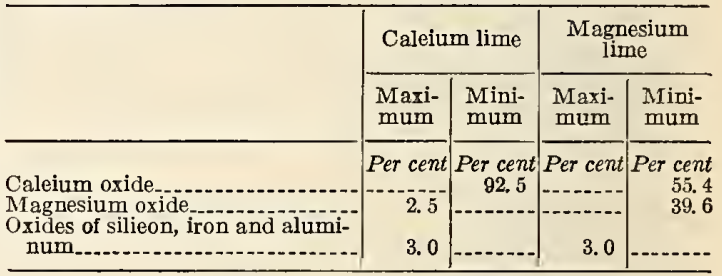

\section{METHODS OF TEST}

3. The chemical analysis of the lime shall be made in accordance with A. S. T. M. C25-27T (given above).

\section{SAMPLING, INSPECTION, REJECTION, ETC.}

4. The sampling, inspection, rejection, retesting, packing, and marking shall be conducted in accordance with A. S. T. M. C50. (See 517.0, p. 347).

American Society for Testing Materials, standard specifications for hydrated lime for the manufacture of varnish, serial designation C47-27, 1927.

1. Hydrated lime is used in the manufacture of oleo-resinous varnishes to harden and partially neutralize the rosin. About 8 pounds of hydrated lime are gradually added to 100 pounds of the melted rosin, and heated for a short period.

\section{REQUIREMENTS}

2. (a) The hydrated lime shall be of such a color that no pronounced darkening of the finished varnish will result. It shall be of such a texture and of such a fineness that all portions of it will rapidly come into intimate contact with the resins so as to enter into the chemical reactions with rapidity.

(b) The lime shall be completely hydrated, 70 since a high degree of hydration is important.

${ }^{62}$ If $i t$ is desired to use the nonvolatile basis the figures given above should be changed to the following:

\begin{tabular}{|c|c|c|c|c|}
\hline & \multicolumn{2}{|c|}{ Caleium lime } & \multicolumn{2}{|c|}{$\begin{array}{l}\text { Magnesium } \\
\text { lime }\end{array}$} \\
\hline & $\begin{array}{l}\text { Maxi- } \\
\text { mum }\end{array}$ & $\begin{array}{l}\text { Mini- } \\
\text { mum }\end{array}$ & $\underset{\text { mumi- }}{\text { Maxi- }}$ & $\begin{array}{l}\text { Mini- } \\
\text { mum }\end{array}$ \\
\hline $\begin{array}{l}\text { Caleium oxide } \\
\text { Magnesium oxide } \\
\text { Oxides of silicon, iron, and } \\
\text { aluminum }\end{array}$ & $\left|\begin{array}{r}\text { Per cent } \\
2.6 \\
3.1\end{array}\right|$ & $\begin{array}{c}\text { Per cent } \\
94.3\end{array}$ & $\mid \begin{array}{r}\text { Per cent } \\
-3.1\end{array}$ & $\begin{array}{r}\text { Per cent } \\
56.5 \\
40.4\end{array}$ \\
\hline
\end{tabular}

$70 \mathrm{~A}$ vcry high ealeium lime is preferred. It is espeeially important that the iron eontent be low in order to avoid undesirable color
effects. 
(c) Fineness.-Not more than 10 per cent of the hydrated lime shall be retained on a No. 230 (62 micron) sieve.

(d) Reactivity. -The hydrated lime shall conform to the requirements of the tung-oil heat test as indicated in section 5 .

(e) The hydrated lime shall conform to the following requirements as to chemical composition, calculated to the nonvolatile basis:

Per cent

Calcium oxide $(\mathrm{CaO})$ minimum

Magnesium oxide (M I

Iron 0 ide, alumina 0 ide, and insoluhio matter, maxima.....

Iron oxide $\left(\mathrm{Fe}_{2} \mathrm{O}_{3}\right)$, maximum

Carhon dioxide $\left(\mathrm{CO}_{2}\right)$, maximum:

If sample is taken at place of manufacture.

If sample is taken at other than place of manufacture....-

\section{METHODS OF TEST}

3. The chemical analysis of the lime shall be made in accordance with A. S. T. M. C25, p. 354.

4. One hundred grams of the sample as received shall be placed on a No. 230 (62 micron) sieve. (See A. S. T. M. E11, 500.2, p. 1.) The material shall be washed by means of a stream of water from a faucet (note 1). Washing shall be continued until the water coming through the sieve is clear. The residue upon the No. 230 (62 micron) sieve shall be dried to constant weight in an atmosphere free from $\mathrm{CO}_{2}$ in a drying oven whose temperature is maintained between $100^{\circ}$ and $120^{\circ} \mathrm{C}$. $\left(212^{\circ}\right.$ and $248^{\circ}$ F.). The weight of this residue shall be calculated as percentage of the original sample.

Note 1.-A small piece of ruhher tuhing attached to a water faucet will he found convenient. The velocity of the stream of water may he increased hy pinching the tuhe, hut the velocity should not he sufficient to cause any danger of splashing the sample over the sides of the sieve.

5. Fifty grams of raw tung oil ${ }^{71}$ shall be placed in a $100 \mathrm{cc}$ beaker and $1 \mathrm{~g}$ of hydrated lime stirred in. A cloudy mass will result. Heat shall then be applied. High-quality hydrated lime will usually start to react at approximately $80^{\circ} \mathrm{C}$. $\left(176^{\circ} \mathrm{F}.\right)$, small bubbles rising to the surface. As heating is continued, large flakes of lime tungate will form and rise to the surface to form a thick white scum and the oil below will gradually become fairly clear. These changes will usually be completed when a temperature of $120^{\circ} \mathrm{C}$. $\left(248^{\circ} \mathrm{F}\right.$.) is reached. The heating shall be continued until the mass reaches a temperature of $140^{\circ} \mathrm{C}$. $\left(284^{\circ} \mathrm{F}\right.$.). It shall then be allowed to cool and the formation of the thick scum on the surface noted which will, when freed of air, gradually drop to the bottom of the beaker. This scum consists of tung-oil soaps of lime which are quite insoluble in the oil. Addition of rosin will readily effect solution of these soaps.

A poor grade of lime will fail to react, the absence of bubbles or flakes of lime tungate being noticeable. The oil will remain quiescent and cloudy. Only small amounts of soaps will form on the surface.

\section{SAMPLING, INSPECTION, REJECTION, ETC.}

6. The sampling, inspection, rejection, retesting, packing, and marking shall be conducted in accordance with A. S. T. M. C50. (See 517.0, p. 347.)

American Society for Testing Materials, standard specifications for quicklime and hydrated lime for use in the cooking of rags for the manufacture of paper, serial designation $\mathrm{C} 45-25,1925$.

1. Rags are cooked for the manufacture of paper in a digester under steam pressure with lime or with lime and soda-ash. They are then washed to eliminate as much of the noncellulose material as possible.

71 Tung oil for this test should conform to the requirements of the tentative specifications for raw tung oil (serial designation D12) of the American Society for Testing Materials,

\section{REQUIREMENTS}

2. (a) Either quicklime or hydrated lime may be used. It shall be clean and free from gritty substances.

(b) The standard quicklime for use in the cooking of rags for the manufacture of paper shall contain 90 per cent of available lime calculated on the basis of the weight of the sample taken at the point of manufacture (note).

(c) The standard hydrated lime for use in the cooking of rags for the manufacture of paper shall contain 64.3 per cent of available lime ${ }^{72}$ calculated on the basis of the weight of the sample taken at the point of manufacture (note).

\section{METHODS OF TEST}

3. The chemical analysis of the lime shall be made in accordance with A. S. T. M. C25 (given above).

\section{SAMPIING, INSPECTION, REJECTION, ETC.}

4. Sampling, inspection, rejection, retesting, packing, and marking shall be conducted in accordance with A. S. T. M. C50. (See 517.0, p. 347.)

NoTE.-A standard composition withoutrejection limits is specified for the reason that lime of either higher or lower total oxides, a vailable lime, calcium oxide or calcium hydroxide, than the standard may safely he used under suitable conditions for the purpose herein specified, depending primarily upon purely economic considerations. In the present state of the art it is believed that the more serviceable type of specification for the product herein specified is that which defines a reasonahle standard rather than one that fixes actual rejection limits. It is generally recognized that, other things heing equal, lime meeting this standard is preferable to lime not meeting it, and lime surpassing the standard should be considered at a premium.

American Society for Testing Materials, standard specifications for quicklime and hydrated lime for use in the textile industry, serial designation C48-24, 1924.

1. Lime is used in the textile industry for the following operations: (a) Liming the kiers, $(b)$ boiling out or scouring, $(c)$ bleaching, $(d)$ mercerizing, and (e) water softening.

These specifications apply only to the first three operations in connection with the treatment of the vegetable fibers-cotton, linen, and jute. (For specifications for lime for water softening, see A. S. T. M. C53, p. 362, and A. S. T. M. C54, p. 362.)

2. (a) Kier liming consists of applying a coating of whitewash to the inside of the kier and heating with steam. These two operations are repeated until a coat of the desired thickness is obtained. The lime serves to prevent the fibers from being stained by the iron kier.

(b) The boiling-out process consists of soaking the fibers in a milk of lime, passing them through rollers into a kier, covering the fibers with water and boiling with steam either at atmospheric or higher pressures. The fibers then pass through a series of washings and treatments with acid and soda ash. The function of the lime is to saponify, hydrolyze and neutralize whereby it makes possible, in following operations, the removal of fatty and greasy matters and pectin bodies. The fibers then consist of practically pure cellulose, the impurities being coloring matter not decomposed by alkalis.

(c) Lime is used in the bleaching operation for making the "chemic" solution of hypochlorite bath. This bath may be made from bleaching powder or from lime and liquid chlorine. These specifications are limited to the process involving the use of lime and liquid chlorine. The operation consists of making a milk of lime and introducing chlorine under carefully controlled conditions until the mixture is nearly neutral. The sludge is allowed to settle and the clear hypochlorite solution is drawn off. After

72 Availahle lime of 64.3 per cent is equivalent to 85 per cent of available calcium hydroxide; 
bleaching, the fibers are scoured and washed, and then passed on to the soda-ash boil. Lime serves as a carrier of chlorine under conditions whereby the liberation of nascent oxygen and the resulting oxidation can be controlled in accordance with the special requirements of textile fibers. Bleaching decolorizes the fibers by oxidation of the coloring matter. It probably performs several other functions also about which there is not general agreement.

\section{REQUIREMENTS}

3. (a) Either quicklime or hydrated lime of fairly high calcium content may be used. ${ }^{73}$ The lime shall be fresh and substantially free from ash, dirt, and core, and shall be very reactive chemically.

(b) The quicklime or hydrated lime shall conform to the following requirements as to chemical composition, calculated to the nonvolatile basis:

Calcium oxide $(\mathrm{CaO})$, minimum

Per cent

Magnesium oxide (MgO), maximum

( Carbon dioxide $\left(\mathrm{CO}_{2}\right)$, maximum:

If sample is taken at place of manufacture-

$$
\text { Quicklime }
$$

If sample is taken at other than place of manufacture

Quicklime

Hydrated lime

\section{METHODS OF TEST}

4. The chemical analysis of the lime shall be made in accordance with A. S. T. M. C25. (See p. 354.)

\section{SAMPLING, INSPECTION, RRJECTION, ETC.}

5. The sampling, inspection, rejection, retesting, packing, and marking shall be conducted in accordance with A. S. T. M. C50. (See 517.0, p. 347.)

American Society for Testing Materials, standard specifications for quicklime and hydrated lime for the manufacture of silica brick, serial designation C49-24, 1924.

1. In the manufacture of silica brick, silica, preferably quartzite, is ground in a wet pan until the particles are less than $1 / 4$ inch in size. Milk of lime is then added in quantities varying from 1.5 to 4 per cent $\mathrm{CaO}$ and the shapes are molded and dried. When bone-dry they are burned in down-draft kilns until most of the quartzite has been converted into tridymite and christobalite.

\section{REQUIREMENTS}

2. Either quicklime or hydrated lime may be used. It shall be substantially free from ash, dirt, and core. Fineness of hydrate and rapidity of slaking of quicklime are desirable qualities.

(b) The quicklime or hydrated lime shall conform to the following requirements as to chemical composition, calculated to the nonvolatile basis:

Calcium oxide $(\mathrm{CaO})$, minimum

Per cent

Magnesium oxide (MgO), maximum

maximum

Silica $\left(\mathrm{SiO}_{2}\right)$ and insoluble matter, maximum

Carbon dioxide $\left(\mathrm{CO}_{2}\right)$, maximum:

If sample is taken at place of manufacture

If sample is taken at other than place of manufacture-.--

Therc is a tendency to prefer quicklime for the boiling out and kier process, and hydrated lime for the mordanting process. Quicklime or hydrated lime which contains iron in a readily soluble form should be avoided as should also hydrated lime which contains a considerable amount of unhydrated magnesia.

\section{METHODS OF TEST}

3. The chemical analysis of the lime shall be made in accordance with A. S. T. M. C25. (See p. 354.)

\section{SAMPLING, INSPECTION, REJECTION, ETC.}

4. The sampling, inspection, rejection, retesting, packing, and marking shall be conducted in accordance with A. S. T. M. C50. (See 517.0, p. 347.)

American Society for Testing Materials, standard specifications for quicklime for use in water treatment, serial designation C53-27, 1927.

1. (a) In the treatment of water for public supplies, lime is used alone or with iron sulphate or aluminum sulphate to produce a precipitate which assists in the clarification of the water and in the removal of the bacteria by filtration. An excess of lime is sometimes used to remove part of the hardness of the water.

(b) Lime and soda ash are used together for softening water.

(c) The lime serves as a chemical reagent in water treatment.

The only useful constituent of the lime is the calcium oxide capable of reacting with the other chemicals which are in the water or which are added to it. Inert material, besides reducing the value in proportion to its amount, also makes more sludge to be disposed of for a given amount of chemical action and thus reduces the capacity of the equipment in which it is used.

\section{REQUIREMENTS}

2. (a) The lime shall be substantially free of core, ash, and dirt, and shall be capable of disintegrating, in water, into a suspension of finely divided material.

(b) The standard quicklime for use in water treatment shall contain 90 per cent of available lime, calculated on the basis of the weight of the sample taken at the point of manufacture (note).

\section{METHODS OF TEST}

3. The chemical analysis for available lime shall be made in accordance with A. S. T. M. C25. (See p. 354.)

\section{SAMPLING, INSPECTION, REJECTION, ETC.}

4. The sampling, inspection, rejection, retesting, packing, and marking shall be conducted in accordance with A. S. T. M. C50. (See 517.0, p. 347.)

Note.-A standard composition without rejcction limits is specified for the reason that lime of either higher or lower total oxides, available lime, calcium oxide, or calcium bydroxide, than the standard may safely be used under suitable conditions for the purpose herein specified depending primarily upon purely economic considerations. In the present state of the art it is believed that the more serviceable type of specification for the product herein specified is that which defines a reasonable standard rather than one that fixes actual rejection limits. It is generally recognized that, other things being equal lime meting this standard is preferable to lime not meeting it and lime surpassing the standard should be considered at a premium.

American Society for Testing Materials, standard specifications for hydrated lime for use in water treatment, serial designation C54-27, 1927.

1. (a) In the treatment of water for public supplies, lime is used alone or with iron sulphate or aluminum sulphate to produce a precipitate which assists in the clarification of the water and in the removal of bacteria by filtration. An excess of lime is sometimes used to remove part of the hardness of the water.

(b) Lime and soda ash are used for softening water. 
(c) Hrdrated lime serves as a ehemical reagent in water treatment. The only useful constituent of the hydrated lime is the calcium hydroxide capable of reacting with the other chemicals which are in the water or which are added to it. Inert material, besides redueing the value in proportion to its amount, also makes more sludge to be disposed of for a given amount of ehemical action and thus reduces the capacity of the equipment in whieh it is used.

\section{REQUIREMENTS}

2. The standard hydrated lime for use in water treatment shall contain 68.1 per eent of available lime ${ }^{i t}$ calculated on the basis of the weight of the sample taken at the point of manufacture (note).

\section{METHODS OF TESTS}

3. The chemical analysis for available lime shall be made in aecordance with A. S. T. M. C25. (See p. 354.)

\section{SAMPLING, INSPECTION, REJECTION, ETC.}

4. The sampling, inspection, rejeetion, retesting, packing, and marking shall be conducted in accordance with A. S. T. M. C50. (See 517.0, p. 347.)

Note.-Same as for_C53 (given above).

Federal Specifications Board, specification No. 249, United States Government master specification for hydrated lime for struetural purposes, November 5,1924

\section{TYPES}

1. Hydrated lime sold under this specifieation may be of either of two types: MI, masons, and $F$, finishing.

\section{MATERIAL AND WORKMANSHIP}

2. Hydrated lime sold under this specification is a fine white powder produced by the aetion of water on quicklime. It consists essentially of calcium hydroxide naturally associated with more or less magnesium oxide or hydroxide.

\section{REQUIREMENTS}

3. Same as A. S. T. M. serial designation C6 517.2, page 353 .

\section{METHOD OF INSPECTION AND TESTS}

\section{A. SAMPLING}

4. One and one-half per cent of the number of paekages (but not less than five packáges) shall be sampled. Samples shall be taken by means of a sampling tube not less than one-half ineh in diameter, inserted longitudinally in the paekage and extending its entire length. The material so collected shall be mixed and quartered to 15 pounds. This shall be divided into three parts of 5 pounds eaeh, whieh shall immediately be sealed in air-tight containers. One of these shall be sent to the consignor, one to the consignce, and the third held for retest in case of dispute.

Sampling shall be eonducted as expcditiously as possible in order to avoid undue exposure to the air.

Samples shall not be taken from broken packages.

\section{B. CHEMICAI ANAIYSIS}

1. Calcium oxide.-Weigh out $0.5 \mathrm{~g}$ of the material and transfer to a platinum or porcelain evaporating dish, mix to a thin slurry with distilled water, add 5

7 Available lime of 68.1 per cent is equivalent to 90 per cent of to $10 \mathrm{cc}$ of $\mathrm{HCl}$ (sp. gr. 1.20) and digest with gentle heat and agitate until solution is complete. Evaporate this to dryness so far as this may be possible on the water bath. Heat the dish and its contents on a hot plate for one hour at $120^{\circ} \mathrm{C}$. Dreneh the cooled mass with $\mathrm{HCl}$ (sp. gr. 1.20) and allow to stand for a few minutes. Add an equal volume of water and heat on water bath for 10 minutes. Filter. Wash thoroughly with dilute $\mathrm{HCl}$ and then trice with cold water. Evaporate the filtrate to dryness. Extract with $\mathrm{HCl}$ as before bui allowing only a few minutes time. Filter through a seeond paper. Ignite both precipitates to constant weight in a platinum crueible. Add $5 \mathrm{cc} \mathrm{HF}$ and two drops $\mathrm{H}_{2} \mathrm{SO}_{4}$. Evaporate to dryness and ignite for two or three minutes. Fuse tle residue with a little $\mathrm{Na}_{2} \mathrm{CO}_{3}$. Dissolve the cooled melt in $\mathrm{HCl}$ and add the solution to the filtrate obtained above. Add a few drops of bromine water ol nitric acid and boil until all trace of $\mathrm{Br}$ or $\mathrm{Cl}$ is gone. Then add $\mathrm{HCl}$ if necessary to insure the presence of $10 \mathrm{cc}$ of concentrated aeid. Add a few drops of methyl red solution, dilute to $200 \mathrm{cc}$ and boil. Neutralize with $\mathrm{NH}_{4} \mathrm{OH}$ (dilute toward the end) until the color of the liquid changes to a distinet yellow. Boil for one or two minutes, allow to settle, filter, and wash two or three times with a hot 2 per cent solution of $\mathrm{NH}_{4} \mathrm{Cl}$. Suck dry. Dissolve the preeipitate by letting hot dilute $\mathrm{HCl}$ run through the paper into the beaker in which the precipitation was made. Wash thoroughly with hot water. Boil to expel any trace of $\mathrm{Cl}$, and repreeipitate with $\mathrm{NH}_{4} \mathrm{OH}$ as described above. Filter, wash two or three times with a hot 2 pcr cent solution of $\mathrm{NH}_{4} \mathrm{Cl}$, and combine this filtrate with the one obtained above. Add a few drops of $\mathrm{NH}_{4} \mathrm{OH}$ and boil. Add $35 \mathrm{cc}$ of a saturated solution of $\left(\mathrm{NH}_{4}\right)_{2} \mathrm{C}_{2} \mathrm{O}_{4}$ and continue the boiling until the preeipitate $\mathrm{CaC}_{2} \mathrm{O}_{4}$ assumes a granular form. Allow to stand 20 minutes or until the supernatant liquid is elcar. Filter and wash thoroughly with hot water. Ignite the precipitate in a platinum crueible. Dissolve in hot dilute $\mathrm{HCl}$ and make up to $100 \mathrm{ec}$ with water. Add a slight exeess of $\mathrm{NH}_{4} \mathrm{OH}$ and boil. If any $\mathrm{Al}_{2}(\mathrm{OH})_{6}$ separates out, filtcr, wash with 2 per cent $\mathrm{NH}_{4} \mathrm{Cl}$ and discard the preeipitate. Treat the filtrate with $\left(\mathrm{NH}_{4}\right)_{2} \mathrm{C}_{2} \mathrm{O}_{4}$ and proeeed as before. Ignite the preeipitate to constant weight in a platinum crucible, and record as caleium oxide.

Additional items under "Chemical analysis," including tests for magnesium, carbon dioxide and nonvolatile matter, are in substantial conformance with tests under corresponding headings in $\mathrm{A}$. S. T. M. serial designation C25. (See p. 354.) This specification contains additional methods of test for the material ineluding tests for 1, fineness; 2 , soundness; 3 , standard consistency; and 4 , plasticity. (The proeedure followed under these tests is the same as that under corresponding test headings in A. S. T. M. serial designation C6, p. 353.)

\section{PACKING AND MARKING}

\section{A. PACKING}

5. Hydrated lime is shipped in cloth bags holding 100 pounds each or paper bags holding 50 pounds each.

\section{B. NLARBLTE}

Eaeh bag shall have legibly marked thereon the net weight of its contents, the name of the manufacturer, the place of manufacture, the brand (if any) and some means of identifying the particular contract on which the purehase and shipment were made.

\section{ADDITIONAL INFORMATION}

6. There are two kinds of hydrated lime, known to the trade as "masons" and "finishing." Masons 
hydrate is intended to be used for any structural purpose except the finishing coat of plaster. Finishing hydrate is intended for use in the finishing coat of plaster but may be used for any stiuctural purpose.

Federal Specifications Board, specification No. 250, United States Government master specification for quicklime for structural purposes, November 5 , 1924

\section{TYPES}

1. Quicklime sold under this specification may be of either of two types: C, calcium; M, magnesium.

\section{MATERIAL AND WORKMANSHIP}

2. Quicklime sold under this specification shall be well burned from a good quality of limestone, dolomite, or marble.

\section{REQUIREMENTS}

3. Requirements agree with those for A. S. T. M. C5 (p. 352), except that for this specification the quicklime, when tested in accordance with the methods given, shall contain not more than 3 per cent, by weight, of waste.

\section{METHOD OF INSPECTION, TESTS, ETC.}

\section{A. SAMPLING}

4. In sampling bulk lime, 10 shovelfuls (not less than 100 pounds) shall be selected by an inspector from different parts of the car. This shall be broken if necessary so that it will all pass a 1 -inch ring. It shall be mixed and quartered to obtain a final sample of 15 pounds. This shall be divided into three equal parts and each part shall be sealed in an air-tight container.

In sampling lime in barrels 2 per cent (but not less than 5) of the barrels shall be opened and their contents dumped in a pile. This pile shall then be sampled as under "bulk lime."

Sampling shall be done as expeditiously as possible in order to avoid undue exposure of the material to the air.

One of the triplicate samples shall be sent to the consignor, one to the consignee, and the third shall be held for retest in case of dispute.

Samples shall not be taken from broken packages.

\section{B. GHEMICAI ANALYSIS}

Test methods and procedure under this heading are in substantial conformance with tests given in serial designation C25, American Society for Testing Materials. (See p. 354). The tests called for under this specification are as follows:

I. Silica.

II. Iron and aluminum oxides.

III. Calcium oxide.

IV. Magnesium oxide.

V. Carbon dioxide.

VI. Nonvolatile matter.

In No. IV (magnesium oxide), to find the weight of the magnesium oxide, multiply the weight of the $\mathrm{Mg}_{2} \mathrm{P}_{2} \mathrm{O}_{7}$ by 0.3621 .

Calculations.-The weights in grams of the oxides, as found above, are multiplied by 200 to reduce them to percentages. The weight of the carbon dioxide in grams is multiplied by 20 . All of these figures must then be divided by the weight of the nonvolatile matter, as found in the preceding paragraph, to reduce them to the nonvolatile basis called for in the table of requirements.

\section{WASTE}

After the sample for chemical analysis has been set aside, all of the rest of the sample as received shall be screened on a No. 3 sieve. Weigh the lime retained on the sieve and transfer it to a slaking box. This lime is then slaked by an experienced operator to produce the maximum quantity of putty, care being taken to avoid "burning" or "drowning" the lime. Cover the box to prevent evaporation and let stand 24 hours. Transfer the putty to a No. 20 sieve, and wash it through the sieve with a stream of water from a hose. This operation must be completed within 30 minutes. The sieve and the residue contained thereon are then dried to constant weight in a current of $\mathrm{CO}_{2}$-free air at a temperature of $110^{\circ}$ to $120^{\circ} \mathrm{C}$. The dry weight of this residue is divided by the weight of the lime slaked, multiplied by 100 , and the result reported as "per cent waste."

\section{PACKING AND MARKING}

\section{A. PACKING}

5. Quicklime may be delivered in bulk if in carload lots; otherwise it shall be delivered in barrels holding 180 or 280 pounds each.

\section{B. MARKING}

Each barrel of lump lime shall be legibly marked with a description of the contents, the name of the manufacturer, the place of manufacture, the brand (if any), the net weight of the contents, and some means of identification of the particular contract on which purchase and shipment were made.

Each carload of bulk lime shall carry the above information on a card conspicuously posted inside the car.

Most of the above marking is required by the Federal lime barrel act, Public No. 228, Sixty-fourth Congress.

\section{8.}

\section{CONSTRUCTION WORK}

\subsection{GENERAL ITEMS.}

American Society for Municipal Improvements, standard municipal contract form, 1927.

(The above-entitled standard is intended for use as a guide both as to general order of the subject matter and as to the general intent of its principal clauses. The three major items within the standard fall under the headings: (1) Agreement, (2) approximate quantities and unit prices, and (3) general conditions.

For use in any particular municipality the standard contract form should be checked over and revised to conform with local and State laws, and in many instances its general conditions may properly be modified in order to cover more completely the outstanding details of the particular project.)

American Society for Testing Materials, standard method of mechanical analysis of sand or other fine highway material, except fine aggregates used in cement concrete, serial designation D7-27, 1927.

(See 512.12, p. 234.)

National Sand and Gravel Association, Bulletin 3 , representative specifications for different uses of sand and gravel, November, 1928.

(In this bulletin, representative specifications, many of which are presented in this volume, and including specifications for sand and gravel for use in construction work, have been summarized.)

United States Department of Agriculture, department bulletin No. 1216, 1928, tentative standard methods of sampling and testing highway materials. (Adopted by the American Association of State Highway Officials and approved by the Secretary of Agriculture, for use in connection with Federal-aid road construction.)

(The above-mentioned bulletin contains 70 methods, of which number 58 deal with nonmetallic 
minerals. American Society for Testing Materials standard and tentative standard methods have been incorporated into the bulletin wherever applicable, in some cases with slight alteration of the A. S. T. M. presentation. Those of the methods given in the bulletin which are not duplicated by standards and methods of other organizations are given in this volume, under decimal classifications corresponding with the subject matter of the individual methods.)

\subsection{GRADING FOR ROADS.}

\subsection{General Items.}

American Electric Railway Association, classification and bearing power of soils, serial No. W201-15, 1915.

\section{Classification}

\begin{tabular}{|c|c|c|c|c|c|}
\hline \multirow{2}{*}{ Key } & \multirow{2}{*}{ Material } & \multicolumn{2}{|c|}{ Size } & \multicolumn{2}{|c|}{ Screens } \\
\hline & & From- & To- & $\begin{array}{c}\text { Pass } \\
\text { through- }\end{array}$ & $\begin{array}{c}\text { Retain } \\
\text { on- }\end{array}$ \\
\hline $\begin{array}{l}1 \\
2 \\
3 \\
4 \\
5 \\
6 \\
7\end{array}$ & $\begin{array}{l}\text { Fine gravel } \\
\text { Coarse sand } \\
\text { Medium sand } \\
\text { Fine sand. } \\
\text { Very fine sand } \\
\text { Silt } \\
\text { Clay. }\end{array}$ & $\begin{array}{l}m m \\
2 \\
1 \\
.5 \\
.25 \\
.10 \\
.05 \\
.005\end{array}$ & $\begin{array}{l}1^{m m} \\
.5 \\
.25 \\
.10 \\
.05 \\
.005 \\
.0000\end{array}$ & $\begin{array}{l}\text { No. } 10 \\
\text { No. } 18 \\
\text { No. } 32 \\
\text { No. } 70 \\
\text { No. } 160 \\
\end{array}$ & $\begin{array}{l}\text { No. } 18 . \\
\text { No. } 32 . \\
\text { No. } 70 . \\
\text { No. } 160 . \\
\text { No. } 230 . \\
\end{array}$ \\
\hline
\end{tabular}

By the number of sieve is meant the number of meshes per lineal inch of wire cloth, woven from brass wire, having the following diameters for

Diameter (inches)

Sieves Nos. 10 and 18 0.016

Sieve No. 32

Sieve No. 70

Sieves Nos, 160 and 230

The mesh should be regular in spacing, and the cloth should be mounted on the frame without twisting.

\section{Bearing power}

\begin{tabular}{|c|c|}
\hline Material & $\begin{array}{l}\text { Safe load } \\
\text { in tons per } \\
\text { square foot }\end{array}$ \\
\hline $\begin{array}{l}\text { Quick sands and wet soil } \\
\text { Dry earth according to depth below surface } \\
\text { Moderately dry clay, confined } \\
\text { Dry, stiff clay } \\
\text { Sand, confined } \\
\text { Sand, compact and cemented } \\
\text { Gravel, cemented. } \\
\text { Rock }\end{array}$ & $\begin{array}{r}0.1-1.0 \\
1.0-3.0 \\
2.0-4.0 \\
4.0-6.0 \\
2.0-6.0 \\
4.0-8.0 \\
8.0-10.0 \\
25.0-200.0\end{array}$ \\
\hline
\end{tabular}

American Society for Testing Materials, standard methods of sampling stone, slag, gravel, sand, and stone block for use as highway materials, including some material survey methods, serial designation D75-22, 1922.

(See 510, p. 184.)

\subsection{Roadway.}

American Association of State Highway Officials, tentative standard specifications for highway materials, 1927

(See 512.15 , p. 244.)

American Electric Railway Association, specification for street-railway track construction, 1923.

(Prepared jointly with American Society for Municipal Improvements.)
(The above specification, in addition to requirements for excavation, drainage, rails, rail joints, bonds, rail fastenings, and ties, includes the following:)

\section{TYPIS OF FOUNDATION}

\section{(A) PLAIN BALLAST CONSTRUCTION}

Where the track is of the "plain ballasted type" such ballast shall preferably consist of either clean, hard, durable crushed stone, crushed slag, clean gravel, cinders, or other suitable material. Where the character of the subsoil is suitable the use of the natural ballast material encountered in the trench excavation may be permitted for this purpose.

Distribution and rolling.-(a) Ballast shall be evenly distributed under the ties so that after the track has been surfaced and the ballast tamped and compacted it shall have a minimum depth of inches. Where practical to do so the ballast should be thoroughly rolled with a roller weighing not less than 10 tons before the ties are placed in the trench.

(b) The best results will be obtained from the use of a ballast which will provide a maximum density after tamping, such as the run of the crusher with dust removed.

\section{(B) CONCRETE SLAB SUBBALIAST CONSTRUCTION}

Where a "concrete slab subballast construction" is used such concrete shall be laid upon the subgrade to a depth of - inches. The concrete shall conform to the A. S. M. I. specifications for concrete for pavement foundation.

Track shall be laid and surfaced on this concrete slab; the ballast used shall consist of clean, hard, durable, crushed stone, gravel, slag, or other suitable material, and shall be thoroughly tamped under the ties. The size of the ballast should preferably be run of the crusher up to 1 inch with dust removed.

\section{(C) SOLID CONCRETE CONSTRUCTION}

The placing of the concrete shall follow immediately after the surfacing and lining of the track, and the concrete shall be brought to a height of - inches below the top of the rail and shall coincide with the grade established for the top surface of the pavement base. Concrete below and around ties and rails shall be thoroughly rammed and tamped. Care shall be taken in tamping concrete to see that the surface and line of the track is not disturbed. In no case shall the ends of the ties be covered by a board while placing concrete. The concrete shall conform to the A. S. M. I. specifications for concrete for pavement foundations.

\section{PAVEMENT BASE}

\section{A. S. M. I. SPECIFICATIONS TO CONTROL}

Except in the case of solid concrete track construction, when the track has been properly lined and surfaced, the paving base, where type of pavement requires same, shall be laid. It shall be of concrete which shall conform to the requirements of the American Society for Municipal Improvements for concrete pavement foundation.

\section{DEPTH DEPENDS ON PAVENGETT}

The depth of the concrete paving base shall depend on the type of paving to be laid, and in no case shall it extend below 1 inch above the bottom of the tie.

\section{RAIL PLASTER}

Immediately preceding the laying of the pavement the space along both sides of girder-rail webs between the head and underside of the lip and the base shall 
be filled with a plaster composed of 1 part Portland cement to about 3 parts sand or other suitable material in order to properly support the paving laid next to the rails. A mastic may be substituted for this filler, consisting of asphaltic concrete binder.

\section{PAVEMENT}

The pavement shall be laid in accordance with the specifications of the American Society for Municipal Improvements for the type of pavement to be installed. Where practicable to do so, vehicular traffic should be kept off the track until the pavingjoint filler has had sufficient time to set thoroughly.

American Society for Municipal Improvemen's, specifications for broken stone and gravel roads, 1922. (See 512.15, p. 245.)

American Society for Municipal Improvements, specifications for street-railway track, 1923. (Prepared jointly with American Electric Railway Engineering Association.)

(Given above.)

National Paving Brick Manufacturers Association, recommended specifications for grading.

Clearing and grubbing.-All objectionable brush, stumps, fences, boulders, stone or other material within the limits of or overhanging the highway shall be removed. All trees, stumps, roots, and brush shall be cleared and grubbed from the area to be improved in such a manner that in case of a fill no part of them shall be within 18 inches of the finished surface of the subgrade; or, in the case of an excavation, no part of them shall be within 3 inches of the finished surface of the subgrade. All stones that are less than 3 inches below the finished surface of the subgrade shall also be removed.

Grading.-The roadway and approaches to be improved shall be graded to such lines and cross sections that when rolled the finished surface of the subgrade shall conform to that shown in the plans.

Excavation. - All vegetable matter and soft, spongy earth, muck, or quicksand which will not compact by rolling shall be removed and replaced with material that can be compacted.

Embankment.-Earth in embankment shall be applied in layers not exceeding 12 inches in depth and each layer shall be thoroughly rolled and compacted before successive layers are applied. All vegetable matter and soft, spongy earth, muck, or quicksand which will not compact by rolling shall be excluded from embankment as provided for in excavation.

Rolling.-The roller used in compacting the subgrade shall be of the self-propelling. 3-wheel type, weighing not to exceed 10 tons. Rolling shall continue until the subgrade is thoroughly compacted. Any depressions in the surface thereof created by 1'olling shall be brought to grade by the addition of suitable material. Rolling shall then be repeated until the subgrade is of uniform density, with a smooth and even surface.

Protection of materials. - No earth from excavation or embankment shall be deposited against or upon any material, brick or stone, sand or gravel to be used in the pavement. Such materials shall be so placed and protected that they will be uninjured by travel or by grading operations.

Testing.-Immediately before the artificial foundation is laid the surface of the subgrade shall be tested, and if not found as required it shall be corrected to conform to the true grade shown in plans.

\subsection{Excavation and Embankment.}

American Society for Testing Miaterials, standard method of test for the determination of moisture equivalent of subgrade soils in the field, serial designation D220-29, 1929.
1. This method covers the determination of the moisture equivalent of subgrade soils by means of a field test.

2. The apparatus shall consist of a bowl, a spatula, and a suitable device for adding water dropwise.

3. A sample weighing approximately $500 \mathrm{~g}$ shall be taken from the field, air dried, and the lumps reduced in size to approximately one-eighth inch, all rock above this size being removed.

4. (a) The soil sample (prepared as described in sec. 3) shall be placed in a bowl and water slowly added from a burette, the water and soil being mixed by means of a spatula until the soil reaches the consistency of putty, and so the soil may be compacted with the spatula without any free water remaining on the surface. The soil shall then be struck of smooth and a drop of water allowed to fall on the surface. If this drop is immediately absorbed by the soil it indicates that the moisture equivalent has not been reached A small increment of water shall then be added and thoroughly mixed with the soil. The surface shall again be struck off smooth and an additional drop allowed to fall thereon. This process is repeated until, upon the addition of a drop of water, the surface remains moist and has a shiny appearance.

By adding small increments of water and by watching the surface of the soil carefully the critical point may readily be determined.

(b) The sample shall then be dried out at a temperature not exceeding $100^{\circ} \mathrm{C}$. and the percentage of moisture determined on the basis of the dry weight of the sample.

Note.-This test is inaccurate when applied to soils whose moisture equivalent is below 20.

The Asphalt Association, specification C-1, for preparation of subgrade (tentative), September 1, 1920.

Section 1. General description.-(a) Preparation of subgrade shall include all clearing and grubbing, excavations and embankment, installation of underdrainage and replacements, grading, shaping, and compaction of subgrade materials to produce a subgrade which shall conform to the grades and cross sections shown on the plans.

(b) All materials shall conform to the requirements of these specifications and to the plans.

SEc. 2. Clearing and grubbing.-The ground shall be cleared of all dead trees, stumps, brush, weeds, roots, and other objectionable material within the limits of the highway, and all trees shall be removed from within the limits of the roadway and from slopes as directed before the grading is started. Timber of any value which it may be necessary to cut shall be piled neatly on the abutting property unless otherwise directed. All branches of trees extending within the roadway limits shall be trimmed as directed by the engineer, and branches which hang within 12 feet of the roadway surface shall be removed, all of which shall be done carefully. All trees, stumps, etc., removed in the excavation shall be cut off, excavated, and removed to a depth of not less than 9 inches below the subgrade surface. Where an embankment is to be made not more than $2 \frac{1}{2}$ feet in depth, trees, stumps, roots, etc., shall be eut off flush with the existing ground surface. Where embankments exceeding $2 \frac{1}{2}$ feet are to be made, trees, stumps, roots, etc., shall be cut off to within 6 inches of the ground surface. The contractor shall burn or otherwise dispose of all such trees, stumps, brush, weeds, roots, etc., and all sod taken from the excavation in a satisfactory manner, and shall remove all rubbish and refuse to such point or points beyond the limits of the roanway as may be directed. All excavations made below the subgrade suríace by the removal of trees, stumps, ete., shall be filled with suitable material, which 
shall be compacted thoroughly so as to make the bearing power of the surface at these points conform to the surrounding subgrade. All slopes of cuts, embankments, ditches, and waterways and all culverts, whether old or new, shall be cleaned and cleared of obstructions in a satisfactory manner and shall be left in a neat and trimmed condition.

SEC. 3. Roadway excavation.-Roadway excavation shall include the removal and satisfactory disposal of all materials taken from within the limits of the work contracted for necessary for the construction and preparation of the roadbed, embankment, subgrade, shoulders, slopes, slide ditches, trenches, waterways, under sections, approaches, etc., as indicated on the plans. All suitable materials removed from the excavation shall be used as far as practicable in the formation of embankment, shoulders, and for replacements as directed by the engineer. All drainage and slides shall be removed and disposed of as directed by the engineer. Ditches and waterways shall be excavated to the depth and width shown on the plans.

SEc. 4. Embankments.-Embankments shall be formed of suitable subgrade material placed in successive layers of not more than 12 inches in depth for the full width of the cross section and each layer shall be rolled thoroughly with a 3 -wheel powerdriven roller weighing not less than 10 tons. Stumps, trees, rubbish, sod, or any other unsuitable materials or substances shall not be placed in the embankment. When embankments are to be made on a hillside the slope of the original ground on which the embankment is to be placed shall be plowed deeply or cut into steps before the filling is commenced. All such embankments shall be tamped thoroughly where it is impracticable to use a roller.

SEc. 5. Installation of underdrainage.-Underdrainage shall be provided as shown on the plans and as directed by the engineer and shall be completed and approved by the engineer prior to laying the base.

SEc. 6. Replacements.-All soft and yielding material and other portions of the subgrade which will not compact readily when rolled or tamped shall be removed as directed by the engineer, and shall be filled with suitable material.

SEc. 7. Grading and shaping.-The subgrade material shall be graded and shaped by suitable means so that after compaction it will conform to the grades and cross sections shown on the plans.

SEC. 8. Compacting.-After having been shaped the subgrade shall be thoroughly rolled with a 3wheel power-driven roller weighing not less than 10 tons until uniformly compacted to a firm even surface. All portions of the subgrade which are not accessible to the roller shall be compacted thoroughly with hand tampers weighing not less than 50 pounds, the face of which shall not exceed 100 square inches in area. All soft and yielding material which will not compact readily when rolled or tamped shall be replaced as specified under section 6 .

SEc. 9. Protection of subgrade.-All ditches and drains shall be completed to drain the highway effectively before the placing of any base-course material. After the subgrade has been rolled it shall be protected from traffic in so far as practicable and any irregularities resulting from traffic or other causes which develop before the base-course material is laid shall be remedied by reshaping and rolling the subgrade material at such places. At all times the subgrade surface shall be kept in such condition that it will drain readily and correctly.

\subsection{Slopes and Shoulders.}

American Association of State Highway Officials, standards of practice 1928.

\section{SHOULDERS}

Wherever practicable shoulders along the edge of pavements shall have a standard width of not less than 8 feet.

American Society for Municipal Improvements, specifications for broken stone and gravel roads, 1922. (See sec. 32.)

See 512.15, page 249 .

Ashpalt Association, specification C-3, for shoulders, headers, curbs and gutters (tentative), September 1, 1920.

See 518.63 , page 446 .

\subsection{Subbases for Roads.}

American Society for Municipal Improvements, specifications for broken stone and gravel roads, 1922. (See sec. 32.)

See 512.15 , page 249 .

Asphalt Association, specification C-2, for subbase (tentative), September 1, 1920.

(The full text of this specification includes methods of construction and the following specifications for materials.)

Section 1. General description.-(a) The sub-base shall consist of mineral aggregate placed and compacted in excavations made by the removal of unsuitable subgrade materials as shown on the plans and as directed by the engineer, and shall conform to the grades and cross sections as shown on the plans.

(b) All materials shall conform to the requirements of these specifications.

\section{MATERIALS}

Section 2. Mineral aggregate.-The mineral aggregate shall consist of approved stone, mine tailings, slag, or gravel composed of reasonably hard durable fragments not more than 5 inches in their largest dimension and free from excess of clay. If the product does not contain sufficient fine material to fill the voids between the coarser fragments when compacted, similar material in suitable size and quantity for this purpose shall also be furnished by the contractor.

National Sand and Gravel Association, Bulletin 3 , representative specifications for different uses of sand and gravel, November, 1928.

(In this bulletin representative specifications, many of which are presented in this volume, and including specifications for material for subgrade treatment, have been summarized.)

\subsection{FOUNDATION FOR ROADS.}

\subsection{General Items.}

American Association of State Highway Officials, tentative standard specifications for highway materials, 1927.

See 512.15, page 244 .

American Electric Railway Association, design of proper foundation for tracks in paved streets (recommended), serial No. W102-23, 1923.

For most soils, except very heavy clays, heavy traffic and heaviest cars up to 35 tons.

Note.-The ties, rails, and paving foundation are shown merely to complete the drawing and should not be considered a part of this recommended design of track foundation.

American Society for Testing Materials, tentative specifications for commercial sizes of broken stone and broken slag for highway construction, serial designation D63-23T, 1923.

See 511.71 , page 207 .

Federal Specifications Board, specification No. 465, United States Government master specification. 
for materials for cushion course, brick, stone block, or wood block pavement. (Sand, slag, limestone screenings, etc.) January 25, 1927.

\section{TYPES}

The materials covered by this specification shall be supplied in the particular type or types ordered by the purchasing department.

Sand.

\section{TYPE OF MATERIAI}

Granulated slag.

Slag screenings.

Limestone screenings.

\section{MATERIAL}

The materials shall be (a) sand free from lumps of clay, (b) granulated slag, (c) slag screenings, or $(d)$ limestone screenings.

\section{DETAIL REQUIREMENTS}

1. The material shall be well graded from coarse to fine and, when tested by means of laboratory
2. (a) The sand shall be well graded from coarse to fine and when tested for gradation as to size shall meet the following requirements:

Total passing 1/4-inch screen.

Per cent

Total passing No. 10 sieve, not less than

(b) These tests shall be made in accordance with A. S. T. M. D7 (see 512.12, p. 234) with the exception that a $1 / 4$-inch screen conforming to the requirements given in A. S. T. M. D19 (see 512.10, p. 214) shall be used in addition to the others specified in method D7.

3. (a) The sand shall be passed through a No. 10 sieve and that portion retained by the No. 10 sieve shall be discarded. The portion passing a No. 10 sieve shall be mixed with Portland cement conforming to the requirements of A. S. T. M. C9 (see 516.11, p. 294) in the proportion of 1 part cement to 3 parts of sand, by weight, according to the standard method of making 1:3 mortar briquets. The resulting mortar at the age of 7 and 28 days shall have a tensile strength of at least 75 per cent of that developed in the same time by mortar of the same consistency, made of 1 part of the same cement and 3 parts of Ottawa sand.

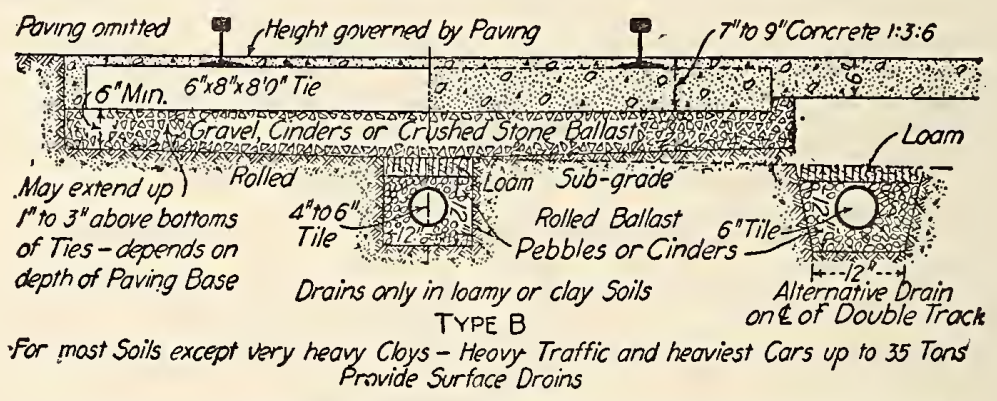

FigURE 121.-Design of proper foundation for tracks in paved streets

screens and sieves, shall meet the following requirements:

Passing No. 20 sieve

Per cent

Passing 1/4-inch screen

2. It shall meet the following requirement:

Per cent

Silt, loam, clay, etc., removed by decantation, not more than 10.0

\section{METHODS OF SAMPLING AND TESTING}

1. Methods of sampling shall be in accordance with A. S. T. M. standard method D75. (See 510, p. 184.)

2. Tests of the material shall be made in accordance with the following methods:

(a) Mechanical analysis, A. S. T. M. D19 (see 512.10 , p. 214), using United States standard sieves meeting the requirements of A. S. T. M. E11 (see 500.2 , p. 1.)

(b) Decantation test A. S. T. M. tentative method D136. (See 512.13, p. 240.)

\subsection{Cement Concrete Foundation.}

American Society for Testing Materials, standard specifications for materials for cement mortar bed for brick, stone block, wood block, asphalt block, and other block pavements, serial designation D58-24, 1924.

\section{SAND FOR CEMENT MORTAR CUSHION}

1. The sand shall consist of clean, hard, durable stone particles, preferably siliceous, free from lumps of clay and any objectionable foreign matter. (b) Preliminary acceptance samples shall be subjected to both 7 and 28 day mortar tests, and acceptance based thereon. Samples tested during the progress of the work will be accepted on the basis of the 7-day test.

(c) The tests for mortar strength shall be made in accordance with A. S. T. M. C9.

Note.-The Portland cement used in testing the proposed sand should preferably be that used or to be used in the work.

4. The sand shall be subject to inspection after delivery at the place of use, prior to and during the laying of the pavement.

5. The sand shall be sampled for testing at the source of supply, if required. Samples shall also be taken for testing from time to time at the place of use prior to and during the laying of the pavement, whenever desired by the engineer.

\section{PORTLAND CEMENT FOR CEMENT MORTAR BED}

6. Portland cement for use in the cement mortar cushion shall conform to the requirements of A. S. T. M. C9.

Asphalt Association, specification B-5, for Portland cement concrete base (tentative), September $1,1920$.

(The full text of the specification includes methods of construction and the following specifications for materials.)

Section 1. General description.-(a) The Portland cement concrete base shall consist of a mixture of coarse aggregate, fine aggregate, and Portland cement having a finished thickness of 6 inches, except where otherwise indicated on the plans, and shall conform to the grades and cross sections shown 
on the plans. The base shall include integral curbs or binders if so shown on the plans, and of the dimensions indicated on the plans.

(b) The materials shall conform to the requirements of these specifications.

\section{MATERIALS}

Same as section 4 of specification $\mathrm{C}-3$ (see 518.63 p. 446) with exception of $(b)$, which for this specification is as follows:

(b) When tested by means of laboratory screens the coarse aggregate shall meet the following requirements:

Per cent

Passing 236-inch screen, not less than. 95
$25-75$ Passing 11/4-inch screen

Passing 1/4-inch screen, not more than

Sec. 3. Fine aggregate-Same as section 5 of specification $\mathrm{C}-3$.

SEc. 4. Portland cement.-Requirements same as section 6 of specification $\mathrm{C}-3$.

SEC. 5. Water.-Same as section 7, specification $\mathrm{C}-3$.

SEC. 6. Sources of supply.-Same as section 8, specification $\mathrm{C}-3$.

SEc. 7. Methods of testing.-Same as section 9, specification $\mathrm{C}-3$.

National Paving Brick Manufacturers' Association, recommended specifications, reconstructed base course.

\section{WORN CONCRETE PAVEMENTS OR OLD CON. CRETE BASES}

\section{DESCRIPTION}

Worn concrete pavements or old concrete base courses used as the base for brick wearing surfaces shall be reconstructed, if required, under the following conditions:

(a) Where existing concrete remaining is inadequate to meet the requirements of depth as shown on the plans, such portions shall be removed and replaced with new concrete to the depth required.

(b) Such portions of existing pavement as have been destroyed or damaged so as to prevent uniform support for the wearing surface shall be replaced with new concrete.

(c) Cracks, or other minor imperfections of the surface which might impair its value for foundation purposes and make impossible an even bedding course, shall be cleaned and filled with bituminous material, cement mortar, or finely crushed slag or stone thoroughly wetted and compacted.

\section{MATERIALS AND MTXING}

Materials and mixing for concrete required for replacements shall conform to the specifications for concrete base (see above) except that, where the quantity of concrete to be replaced does not make machine mixing economical, concrete may be mixed and placed by hand.

The surface of the reconstructed pavement as prepared for laying the brick thereon shall be reasonably smooth and even.

\section{WORN MACADAM OR GRAVEL ROADS}

\section{DESCRIPTION}

Worn macadam or gravel wearing surface used as the base for brick pavements shall be reconstructed by shaping, adding new material, where required, and by rolling to form a uniformly dense and thoroughly compacted base.

\section{MATERIALS}

Broken stone, crushed slag, or gravel used in reshaping the base shall conform to the specifications under rolled bases.

The existing road surface shall be thoroughly cleaned of all dust, earth, or refuse. It shall be brought to required grade, cross section, and condition for laying the brick thereon as follows:

Where existing surface is broken stone, crushed slag, or gravel, it shall be scarified, if needed, to obtain a uniformly dense surface for the entire width of the pavement and to a depth sufficient to remove all depressions and irregularities and to permit of uniform reshaping. The existing road must not be disturbed or scarified below the newly established grade line.

After loosening, the material shall be shaped to conform to grade and cross section as shown in the plans by the addition of new material if required.

\section{ROILING}

The reconstructed base shall then be compacted by rolling to uniform and thorough density and the addition of screenings or sand not exceeding onefourth inch in maximum size as a binder. During this operation the road shall be thoroughly wetted to assist in the compaction of the material.

The base shall be brought to the required uniformity and density by a self-propelling roller weighing not to exceed 10 tons. Rolling shall begin at the shoulders and continue backward and forward until the center of the roadway is reached. Depressions developing during rolling shall be brought to the required surface by the addition of material and rerolling. Rolling shall continue until the base is firmly consolidated and shows no tendency to creep in front of the roller. During the rolling the surface of the base shall be swept with stiff wire or rattan brooms so that all fine material shall enter the interstices of the base.

\section{TESTING}

Where the existing surface overlies a Telford or other large stone base, projecting stones, if any, shall be napped off to a uniform elevation. All depressions or irregularities shall be brought to the required grade and cross section by the addition of new material, if required, and by rolling.

National Paving Brick Manufacturers'Association, recommended specifications, artificial bases, cement concrete.

\section{MATERIALS}

Concrete shall be composed of Portland cement and of fine and coarse aggregate in the form of:

(a) Fine aggregate- - Sand, stone, or slag screenings.

(b) Coarse aggregate.-Broken stone, gravel, or crushed slag.

(c) Combined aggregate-Bank-run gravel or crusher-run stone.

\section{PORTLAND CEMENT}

Portland cement shall conform to the definition and meet the requirements of A. S. T. M. C9. (See 516.11, p. 294.)

\section{FIND AGGREGATE}

Fine aggregate shall consist of clean sand or screenings from hard durable rock, gravel, or slag, all of which shall be uniformly graded and which will pass through a 1/4-inch sieve.

Fine aggregate shall be free from soft friable material, shale, or slate, vegetable or other organic matter. It shall not contain clay or silt in excess of 5 per cent by weight. 


\section{COARSE AGGREGATE}

Coarse aggregate shall consist of broken stone, gravel, or crushed slag.

(a) Broken stone.-Broken stone used as the coarse aggregate shall be reasonably sound. It shall be uniformly graded in sizcs between a maximum of what will pass a $1 \frac{1}{2}$-incl and a minimum that will be retained on a $1 / 4$-inch circular opening in a revolving screen. It shall be reasonably clean and free from dust.

(b) Gravel.-Gravel used as the coarsc aggregate shall be composed of reasonably sound stone uniformly graded in sizes between a maximum of what will pass through $1 \frac{1}{2}$-inch and a minimum that will be retained on a $1 / 4$-inch circular opening in a revolving screen. It shall be clcan and free from soft, friable material, vegetable or other organic mattcr.

(c) Crushed slag.-Crushed slag used as the coarse aggregate shall be hard and sound. It shall weigh not less than 1,800 pounds per cubic yard. It shall meet the requirements of stone and gravel for sizes. Slag shall be reasonably clean and free from dust.

\section{COMBINED AGGREGATE}

Combined aggregate shall be of a character and quality that the separate ingredients shall meet the requirements for coarse and fine aggregate as above specified when separated, provided:

(a) That run-of-bank gravel may be used containing not more than 70 nor less than 50 per cent by volume of fine aggregatc.

(b) That run-of-crusher stone may be used containing not more than 70 nor less than 50 per cent by volume of fine aggregate.

\section{WATER}

Water shall be clean, free from oils, acids, alkalies, and vegetable matter.

\section{PROPORTIONS}

Cement, fine and coarse aggregate, shall be mixed in the proportions of 1 part cement, 3 parts of fine aggregate, and 6 parts coarse aggregate. If combined aggregate is used then the proportions shall be 1 part ccment to 7 parts combined aggregate.

The full text of the specification includes requirements for mixing, depth, forms, depositing concretc, retempering, and protection.

National Slag Association, recommended specifications for slag to be used in highways and highway structures, 1927.

See 512.2, page 256 .

\subsection{Telford Foundation.}

American Association of State Highway Officials, tentative standard specifications for highway materials, 1927.

No. 1 stone for Telford base course and reconstructed base course.

See 512.15, page 24.4 .

Asplalt Association, specification B-4, for Telford base (tentative), September 1, 1920.

(The full text of the specification includes methods of construction and the following specifications for materials:)

Section 1. General description.-(a) The Telford base shall consist of a single course of large stones wedged in place by the addition of small stones, constructed upon the prepared subgrade and having the dimensions and cross sections shown on the plans.

(b) The materials shall be in accordance with these specifications.

\section{RATERIALS}

SEc. 2. Stones.- (a) The stones shall consist of approved, sound, tough, durable rock, free from clay, loam, or other foreign substance.

(b) Large stone shall be approximately rectangular in section and have a depth of about 8 inches after napping, a width of from 2 to 6 inches and a length of from 6 to 12 inches.

(c) Sinall stone shall be of suitable sizc for wedging between the coarse stone as laid.

\subsection{Fiacadam Foundation.}

American Society for Municipal Improvements, standard specifications for subgrade and foundations for pavements, 1928.

(This specification includes directions for laying a new macadam foundation and directions for utilizing an old macadam foundation.)

See 518.24, page 371 .

Asphalt Association, specification B-2, for macadam base (tentative), September 1, 1920.

(The full text of the specification includes methods of construction and the following specifications for materials.)

Section 1. General description.-- (a) The macadam base shall consist of two courses of mineral aggregate, each course composed of coarse aggregate and screenings and having a compacted thickness of not less than 3 inches. The two courses shall form a foundation having a compacted thickness of not less than 6 inches and shall conform to the grades and cross sections shown on the plans.

(b) The materials shall conform to the requirements of these specifications.

\section{MATERIALS}

SEc. 2. Mineral aggregate-(a) The mineral aggregate shall consist of broken stone, mine tailings, or slag. It shall be of reasonably uniform quality throughout and free from an excess of flat or elongated pieces. Rock or mine tailings from which it is produced shall have a per cent of wear of not more than 8. Slag from which it is produced shall have a per cent of wear of not more than 12 and for each size specified shall weigh not less than 65 pounds per cubic foot.

(b) When tested by means of laboratory screens the coarse aggregate and screenings shall meet, respectively, the following requirements and be uniformly graded between the limits given:

\begin{tabular}{|c|c|c|c|c|c|c|}
\hline \multirow{2}{*}{$\begin{array}{l}\text { Size (in } \\
\text { inches) }\end{array}$} & \multirow{2}{*}{ Name } & \multicolumn{5}{|c|}{ Per cent passing screen } \\
\hline & & $\begin{array}{c}31 / 2 \\
\text { inches }\end{array}$ & $\begin{array}{c}21 / 2 \\
\text { inches }\end{array}$ & s/4inch & $1 / 2$ inch & $1 / 4$ inch \\
\hline $21 / 2$ to $31 / 2$ & $\begin{array}{c}\text { Coarse aggre- } \\
\text { gate. }\end{array}$ & $95-100$ & $0-15$ & & & \\
\hline $\begin{array}{l}\text { or } \\
0 \text { to } 1 / 2 \text {. }\end{array}$ & Screenings & & & $95-100$ & $95-100$ & $\begin{array}{l}40-80 \\
40-80\end{array}$ \\
\hline
\end{tabular}

SEc. 3. Sources of supply.-Approval of sources of supply of mineral aggregate under these specifications shall be obtained from the engineer prior to delivery of material, and samples shall be submitted as directed by the engineer.

SEc. 4. Methods of testing.-Methods of testing shall be as follows:

(a) Per cent of wear, A. S. T. M. D2. (See 510, p. 182.) When testing slag 4,000 cc loose measure of the size specified in the standard test shall be used.

(b) Weight per cubic foot of broken slag, A. S. T. M. standard method C20. (See 534.10, p. 572.) 
(c) Screen tests, A. S. T. M. standard method D1S. (See 512.15, p. 250.)

Asphalt Association, specification B-3, for reconstruction of old macadam to serve as base course (tentative), September 1, 1920.

(The full text of the specification includes methods of construction and the following specifications for materials.)

Section 1. General description.-(a) The base course shall consist of an existing stone or slag road to which, after scarifying, there shall be added a mineral aggregate composed of coarse aggregate and screenings compacted so as to forin a base course having a total thickness of not less than 6 inches, and conforming to the grades and cross sections shown on the plans.

(b) The materials and methods of construction shall conform to the requirements of these specifications.

\section{MATERIALS}

Same as specification B-2 (above).

Asphalt Association, specification B-7, for asphalt macadam base (penetration method), May 1, 1927.

See 518.27 , page 374 .

National Paving Brick Manufacturers' Association, recommended specifications, reconstructed base course.

\section{WORI MACADAM OR GRAVEL ROADS}

See 518.21, page 369

National Slag Association, recommended specifications for slag to be used in highways and highway structures, 1927.

See 512.2, page 256 .

(Includes specification for'slag for water-bound macadam base course)

\subsection{Broken Stone Foundation.}

American Association of State Highway Officials, tentative standard specifications for highway inaterials, 1927.

See 512.15 , page 244 .

American Society for Municipal Improvements, specifications for broken stone and gravel roads, 1922.

See 512.15, page 245 .

American Society for Municipal Improvements, standard specifications for subgrades and foundations for pavements, 1928.

\section{BROKEN STONE FOUNDATIONS FOR PAVEMENTS}

Subgrade.- In the case of the construction of new broken stone foundations for pavements the subgrade shall be prepared by cutting or filling as may be required to produce a surface parallel with the finished grade of the wearing surface and a depth below the finished grade to the specified thickness of the foundation and paving material.

After rough grading has been completed, the surface of the subgrade shall be brought to the required grade and cross section and rolled with a power roller weighing not less than 12 tons, until the surface thereof is unyielding. If required by the engineer, the subgrade shall be drenched with water before rolling. Depressions or ruts occuring from any cause shall be brought to grade with good earth and thoroughly rolled as above specified. Any portions of the subgrade which are inaccessible to the roller shall be thoroughly tamped with tampers weighing not less than 40 pounds, and having a face which shall not exceed 80 square inches in area. All soft, spongy, and unyielding spots and all perishable matter shall be entirely removed and the space filled with suitable matter.
Whenever clay, adobe, or soils of similar character are encountered, they shall be excavated to a depth of at least 2 inches below the established grade of the subbase. There shall then be provided a layer of coarse gravel, sharp sand, or other suitable material which will afford proper drainage. This material will be brought to the established subgrade and properly shaped and rolled.

Subdrainage. - When, in the opinion of the engineer, the soil is of such a character that it retains an excessive amount of moisture, such as clay subject to swelling or heaving under the action of frost, or sands similar to quicksand that do not afford a ready natural drainage, subdrains shall be provided and constructed as directed by the engineer.

New macadam foundation.-After the subgrade has been properly prepared, there shall be spread a layer of clean, broken trap rock or other suitable stone, which shall pass a 3 -inch ring and be retained on a 2-inch ring, said stone to be spread in a uniform layer and shall have a depth, after final compression, of 6 inches.

On this course of stone there shall be spread in uniform layers about 1 inch in thickness, sereenings or sand, or a mixture thereof, acceptable to the engineer, which shall be swept into the interstices, and this operation shall continue until said interstices are completely filled. During the spreading of this binder the rolling shall continue until the foundation is thoroughly filled and solidly compacted to the satisfaction of the engineer.

This binder shall not be applied in such quantity as to cover or form a coating over the top of the stone foundation. Any surplus binder which shall remain over the surface of this foundation shall be removed before the surface pavement or any second course of stone is laid.

Bank gravel or sand may be used for filling the interstices when same is approved by the engineer.

Old macadam foundation. - The surface shall be thoroughly swept and cleaned of all fine material that may be caked upon the surface of the stone or lying loose as dust, thereby exposing the clean, coarse stone for the reception of bituminous concrete.

If the old macadam does not present the desired coarse, grainy surface, or is not at proper and satisfactory grade after cleaning, it shall be spiked up and redressed to the desired crown and grade, the coarse stone being brought to the top by harrowing or otherwise, or new stone added when, in the opinion of the engineer in charge, it is needed to give the necessary thickness or character of surface. It shall then be thoroughly rolled with the use of water as may be required.

In the case of bituminous pavements a layer about one stone deep of new, clean stone, averaging $1^{1 / 2}$ inches in size, shall be spread over the surface aiter redressing and rolling the old macadam and finally rolled until firmly set in place without the application of water. This is intended to form a coarse, grainy surface of fresh, clean stone which will bond with the bituminous paving material.

\section{SPECIFICATION FOR ASPHALTIC BASE}

\section{ASPHALTIC CONCRETE TYPE}

(Same as Asphalt Association, specification B-8, for asphalt concrete base (see 518.27, p. 374), with the following variations:)

1. Rock or mine tailings from winich the coarse aggregate is produced shall have a per cent of wear of not more than 5 .

2. Laboratory screens for the coarse aggregate shall be $2 \frac{1}{2}$ inch, $11 / 4$ inch, and $1 / 4$ inch. The screentest percentages are identical with $\mathrm{B}-8$. 
3. Asphalt cement, in addition to the requirements of B-8, shall have a specific gravity at $25^{\circ} \mathrm{C} .25^{\circ} \mathrm{C}$. of not less than 1.000 .

\section{SPECIFICATION FOR TAR-BOUND BASE, HOT PENETRATION TYPE}

Drainage.-The roadbed shall be perfectly drained. Side drains, cross drains, and $V$ drains shall be provided as shown on engineer's plan, where required by local conditions.

Roller.-The roller used shall be a power roller of a type suitable for macadam road construction and shall weigh 10 to 15 tons.

Subgrade.-In the case of the construction of new broken-stone foundations for pavcments the subgrade shall be prepared by cutting or filling as may be required to produce a surface parallel with the finished grade of the wearing surface and a depth below the finished grade equal to the specified thickness of the foundation and paving material.

Stone.-The stone shall be of best quality crushed rock (or other approved road material) free from dust, loam, or clay.

Base course.-The lower course shall consist of crushed stone which shall pass a $3 \frac{1}{2}$-inch ring and be retained on a $2 \frac{1}{4}$-inch ring, spread to a finished depth of . . . . . inches, but _. . . _ inches below the finished grade. The course shall be filled thoroughly with clean sand, gravel, stone sereenings, or similar filler approved by the engincer, and rolled until smooth and firm. No filler shall be left on the surface.

Waterproofing course.-The waterproofing course shall be placed on the base and shall consist of a layer of crushed stone which shall pass a $2 \frac{1}{4}-$-inch

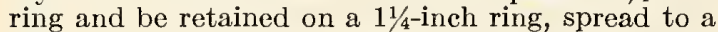
finished depth of not less than $2 \frac{1}{2}$ inches. (See note.) This course shall not be filled, but shall be keyed thoroughly together by rolling. The surface shall be left smooth and of even texture, clean, and free from dirt, clay, stone dust, or other material which will prevent the easy penetration of the refined tar.

Nore.-This size stone is best for trap rock or hard rock which does not break under the roller. If limestone or other soft rock is used, a larger size stone may be used to advantage.

First coat of refined tar.--Refined tar, as hereinafter specified, heated to a temperature of not less than $200^{\circ} \mathrm{F}$. and not more than $275^{\circ} \mathrm{F}$., shall be spread uniformly over the surface when dry to the amount of not less than one and seven-tenths gallons, nor more than one and eight-tenths gallons to the square yard.

Three-quarter-inch stone.-T $\mathrm{h}$ r e e-qu ar t e r-i $\mathrm{c}$ ch crushed stone without dust, that is, stone which shall pass a $1 \frac{1}{1 / 4}$-inch ring and be retained on a 1/2-inch ring, shall be spread over the surface, filling the voids but leaving none on the surface. This course shall then be rolled until firm. The road sliall be swept free from any particles of stone not held by the refined tar.

If the wearing course is to be a block pavement, a sand-tar cushion as hereinafter specified shall be laid over the base as completed in the above section.

If the wearing course is to be sheet asphalt or bituminous conerete the waterproofing course shall be finished as follows:

Second coat of refined tar.- Not less than $1 / 2$ nor more than $3 / 4$ of a gallon of refined tar to the square yard, heated to a temperature of not less than $200^{\circ} \mathrm{F}$. nor more than $275^{\circ} \mathbf{F}$., shall be spread over the surface of the waterproofing course, and covered with stone screenings or sharp sand. The road shall then be rolled until compacted. All loose screenings or sand shall be swept off, and the wearing course shall be laid directly on the completed waterproofing course.
Sand-tar cushion.-Upon the completed foundation course shall be spread a course three-fourths inch thick, of sand, mixed with refined tar for surface treatments, in the proportion of 90 per cent sand and 10 pcr cent refined tar, by volume. The sand shall be clean and dry and shall be mixed with the refined tar until uniform, homogeneous mixture is obtained. It shall then be spread on the foundation course and shaped to a true surface parallel to the surface of the finished pavement.

1. Refined iar "G."-The refined tar shall be homogeneous, free from watcr, and shall not foam when heated to $150^{\circ} \mathrm{C}$. $\left(302^{\circ} \mathrm{F}\right.$.).

2. Its specific gravity at a temperature of $25^{\circ} \mathrm{C}$., $\left(77^{\circ}\right.$ F.) shall be not less then 1.200 nor more then 1.300 .

3. When tested by means of the New York Testing Laboratory float apparatus the float shall not sink in water maintained at $50^{\circ} \mathrm{C}$. $\left(122^{\circ} \mathrm{F}\right.$.) in less than 140 seconds nor more than 170 scconds.

4. Its bitumen as determined by its solubility in chemically pure carbon disulphide at room temperature shall not be less than 75.0 per cent nor more than 90.0 per cent, and it shall not show more than 0.2 per cent ash upon ignition of the material soluble in carbon disulphide.

5. When distilled according to the tentative method recommended by Committee D-4 of the American Society for Testing Materials (see A. S. T. M. D20, 505.0, p. 120), it shall yield no distillate at a temperature lower than $170^{\circ} \mathrm{C} .\left(338^{\circ} \mathrm{F}\right.$.) ; not more than 10.0 per cent by weight shall distill below $270^{\circ}$ C. $\left(518^{\circ}\right.$ F.) and not more than 20.0 per cent by weight shall distill below $300^{\circ} \mathrm{C}$. $\left(572^{\circ} \mathrm{F}\right.$.).

6 . The total distillate from the test made in accordance with clause 5 shall have specific gravity at a temperature of $25^{\circ} \mathrm{C}$. $\left(77^{\circ} \mathrm{F}\right.$.) of not less than 1.030 .

7. The melting point, as determined in water by the cube method, of the pitch residue remaining after distillation to $300^{\circ}$ C. $\left(572^{\circ}\right.$ F.) in accordance with the test described in clause 5 shall not be more than $75^{\circ}$ C. $\left(167^{\circ}\right.$ F.).

Refined tar for sand tar cushion.-The refined tar for cushion shall conform to the requirements for "Refined tar for surface treatment," A. S. M. I. specifications for 1918, page 365 of the proceedings for 1918.

Its specific gravity at $25^{\circ} / 25^{\circ}$ C. $\left(77^{\circ} / 77^{\circ} \mathrm{F}\right.$.) shall not be less than 1.100 nor more than 1.180 .

When tested by means of the Engler viscosimeter at $40^{\circ} \mathrm{C} .\left(104^{\circ} \mathrm{F}\right.$.) the specific viscosity of the first 50 cubic centimeters passing the orifice of the viscosimeter shall be not less than 10 nor more than 35 , with a maximum range not exceeding 5 .

NotE.-Low specific viscosities should be used for work in the Northern States and high specific viscosities for work in the Southern States.

Its bitumen, as determined by its solubility in chemically pure carbon disulphide at room temperature, shall be not less than 90 per cent.

When distilled according to the American Society for Testing Materials standard method it shall yield not more than 5 per cent of distillate at a temperature lower than $170^{\circ} \mathrm{C}$. $\left(338^{\circ} \mathrm{F}\right.$.) ; not more than 30 per cent shall distill below $270^{\circ} \mathrm{C} .\left(518^{\circ} \mathrm{F}\right.$.); and not more than 40 per cent shall distill below $300^{\circ} \mathrm{C}$. $\left(572^{\circ}\right.$ F.).

\section{SPECIFICATIONS FOR TAR-BOUND BASE, COLD PENETRATION TYPE}

Requirements for 1 , drainage; 2 , rollers; 3 , subgrade; 4, stone; and 5 , base course, same as above specification, except that for this specification the lower course crushed stone shall pass a $3 \frac{1}{2} 2$-inch ring and be retained on a $2 \frac{1}{2}$-inch ring. 
If a deep foundation course is necessary, the stone shall be laid and rolled in separate layers not over 4 inches in thickness. When local conditions require, Telford may be substituted for part of the brokenstone foundation.

Waterproofing course.-The waterproofing course shall be placed over the base thus prepared and consist of a layer of stone which will pass a $2 \frac{1}{4}$-inch ring, and be retained on a $11 / 4$-inch ring, spread to a finished depth of not over 3 inches. This stone shall be rolled dry until it is thoroughly locked together and shows no movement under the roller and shall be even and true to the lines, grades, and cross sections.

Filling waterproofing course.-The filler shall be broken-stone or broken-slag screenings, or sand, and be rolled into the stone by spreading in light even applications over the surface, keeping the roller passing back and forth and adding more filler as it rattles into the voids, and continuing these operations until the filler is flush with the surface of the stone; to be done without water. When the voids are filled, water shall be used and more filler added as needed to bring it flush with the surface of the stone. If sand is used for the filler, slag or stone screenings may be used with the water to finish up. Sufficient filler and water to form the usual grout or to cover the stone shall not be used. Traffic shall be kept off the road while it is drying out and any excess of filler on the surface shall be swept off, so that the stone shall be exposed.

Applying refined tar.-When the road is dry, 1 gallon of refined tar, as hereinafter specified, per square yard, or more if the road will absorb it, shall be applied in two or more uniform applications. Each application shall be allowed to penetrate until there is no excess bitumen on the surface of the road before making additional application.

Covering and rolling.-The covering material shall be either clean, dry sand or granulated slag. The covering shall be spread within two to foul hours after the last application of refined tar and sufficient used to prevent the roller from picking up the binder. The foundation shall then be thoroughly rolled. At the conclusion of the rolling the refined tar sliall just show through the cover.

If the wearing course is to be sheet asphalt it shall be laid on this surface. If the wearing course is to be a block pavement, it shall be laid on a sand-tar cushion.

Sand-tar cushion.-Upon the completed foundation course shall be spread a course three-fourth inch thick of sand, mixed with refined tar as hereinafter specified, in the proportion of 90 per cent sand and 10 per cent refined tar, by volume. The sand shall be clean and dry and shall be mixed with the refined tar until a uniform, homogeneous mixture is obtained. It shall then be spread on the base course and shaped to a true surface parallel to the surface of the finished pavement.

Refined tar for base and cushion.-The refined tar for base and cushion shall conform to the requirements for "Refined tar for surface treatments," A. S. M. I. specifications for 1918 , page 365 of the proceedings for 1918 .

Its specific gravity at $25^{\circ} / 25^{\circ} \mathrm{C}$. $\left(77^{\circ} / 77^{\circ} \mathrm{F}\right.$. $)$ shall not be less than 1.100 nor more than 1.180 .

When tested by means of the Engler viscosimeter at $40^{\circ} \mathrm{C}$. $\left(104^{\circ} \mathrm{F}\right.$.) the specific viscosity of the first $50 \mathrm{cc}$ passing the orifice of the viscosimeter shall be not less than 10 nor more than 35 with a maximum range not exceeding 5 .

NotE.-Low specific viscosities should be used for work in the Northern States and high specific viscosities for work in the Southern States.
Its bitumen, as determined by its solubility in chemically pure carbon disulphide at room temperature, shall not be less than 90 per cent.

When distilled according to A. S. T. M. D20, (see 505.0, p. 120 ), it shall yield not more than 5 per cent of distillate at a temperature lower than $170^{\circ} \mathrm{C}$. $\left(338^{\circ} \mathrm{F}.\right)$, nor more than 30 per cent shall distill below $270^{\circ} \mathrm{C}$. $\left(518^{\circ} \mathrm{F}\right.$.); and not more than 40 per cent shall distill below $300^{\circ} \mathrm{C}$. $\left(572^{\circ} \mathrm{F}\right.$.).

American Society for Testing Materials, standard form of specifications for certain commercial grades of broken stone, serial designation D35-18, 1918 .

See 511.70 , page 206 .

American Society for Testing Materials, tentative specifications for commercial sizes of broken stone and broken slag for highway construction, serial designation D63-23T, 1923.

See 511.71, page 207.

American Society for Testing Materials, tentative specifications for broken stone for water-bound base, serial designation D190-29T, 1924.

See 512.15 , page 250 .

Federal Specifications Board, specification No. 456 United States Government master specification for broken stone and broken slag for water-bound base or wearing course, January 25, 1927.

See 512.15, page 251 (for broken stone) and 512.2, page 255 (for broken slag)

National Paving Brick Manufacturers' Association, recommended specifications for artificial bases, 1924 .

Rolled bases of gravel, stone, or slag, with or without shoulders or bituminous binding.

See 518.28, page 376 .

\subsection{Bituminous Macadam Foundation.}

American Society for Testing Materials, tentative specifications for broken stone for bituminous macadam, serial designation D192-29, 1924.

See 512.15, page 250.

American Society for Testing Materials, standard specifications for broken slag for bituminous macadam base, serial designation D195-27, 1927.

See 512.2 , page 255 .

Federal Specifications Board, specification No. 457, United States Government master specification for broken stone and broken slag for bituminous macadam base or surface course, January 25, 1927.

See 512.15, page 251 (for broken stone), and 512.2, page 255 (for broken slag).

\subsection{Bituminous Concrete Foundation.}

American Society for Testing Materials, standard specifications for broken slag for bituminous concrete (coarse-graded aggregate type), serial designation D160-27, 1927.

See 512.2 , page 254 .

American Society for Testing Materials, standard specifications for broken slag for bituminous concrete (fine-graded aggregate type), serial designation D161-27, 1927.

See 512.2 , page 255 .

American Society for Testing Materials, tentative specifications for broken stone for bituminous concrete base, serial designation D193-29T, 1924.

See 512.15 , page 251 .

American Society for Testing Materials, standard specifications for broken slag for bituminous concrete base, serial designation D196-27, 1927.

See 512.2 , page 255 .

American Society for Testing Materials, tentative specifications for gravel for bituminous concrete base, serial designation $\mathrm{D} 309-29 \mathrm{~T}, 1929$.

See 512.11 , page 218 .

Federal Specifications Board, specification No. 458, United States Government master specification 
for broken stone and broken slag for bituminous concrete base or surface course, January $25,1927$.

See 512.15, page 251 (for broken stone), and 512.2 page 255 (for broken slag).

National Slag Association, recommended specifcations for slag to be used in highways and highway structures, 1927.

See 512.2, page 256 .

\subsection{Asphaltic Foundation.}

American Society for IIunicipal Improvements, standard specifications for subgrades and foundations for pavements, 1928.

(This specification includes requirements for asphaltic base, asphaltic concrete type.)

See 518.24 , page 371 .

Asphalt Association, specification B-7, for asphalt macadam base (penetration method), April 10, 1929.

(The full text of the specification includes methods of construction and the following specifications for materials).

Section 1. General description.-(a) The asphalt macadam base shall consist of two courses of mineral aggregate and asphalt cement combined as hereinafter specified and having a total compacted thickness of not less than 6 inches. The bottom course shall have a compacted thickness of not less than $3 \frac{1}{2}$ inches and the second course shall have a compacted thickness of not less than $2 \frac{1}{2}$ inches. The method of construction shall proceed substantially as follows:

I. A layer of No. 1 coarse aggregate rolled to the thickness of the bottom course shall be treated with hot asphalt cement as hereinafter specified.

II. A layer of No. 2 coarse aggregate shall then be laid, rolled to the thickness of the second course, and treated with hot asphalt cement as hereinaiter specified.

III. If a sheet asphalt of fine graded aggregate asphaltic concrete surface course is to be laid directly upon the asphalt macadam base, the surface voids in the base course shall be filled by applying and rolling in sufficient intermediate aggregate. The use of intermediate aggregate shall be omitted if an asphalt macadam, coarse graded aggregate asphaltic concrete or binder course is to be laid directly upon the asphalt macadam base.

(b) All materials shall conform to the requirements of these specifications.

\section{MATERIALS}

SEc. 2. Mineral aggregate.-(a) The mineral aggregate shall consist of broken stone, mine tailings, or slag. It shall be of reasonably uniform quality throughout and shall be clean and free from dust and an excess of flat or elongated pieces. Rock or mine tailings from which it is produced shall have a per cent of wear of not more than 8 . Slag from which it is produced, shall weigh not less than 70 pounds per cubic foot for each size specified.

(b) When tested by means of laboratory screens the coarse and intermediate aggregates shall meet, respectively, the following requirements and be uniformly graded between the limits given:

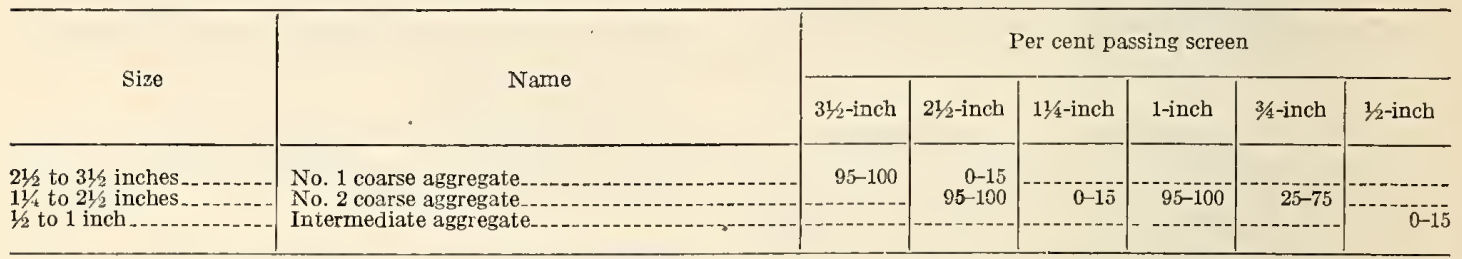

Sec. 3. Asphalt cement.-The asphalt cement shall be homogeneous and free from water and shall not foam when heated to $175^{\circ} \mathrm{C}$. $\left(347^{\circ} \mathrm{F}\right.$.). It shall meet the following requirements for penetration:

Penetration at $25^{\circ} \mathrm{C}$. ( $77^{\circ} \mathrm{F}$.) $100 \mathrm{~g}, 5$ seconds.......... $60-150$ (Between these limits the enginecr shall specify as desired: 60 to 70 , 85 to 100,100 to 120 , or 120 to 150 .)

SEc. 4. Sources of supply.-(a) Approval of sources of supply of mineral aggregate under these specifications shall be obtained from the engineer prior to delivery of material and samples of each size specified shall be submitted as directed by the engineer.

(b) A 1-pound sample accompanied by an analysis of the aspinalt cement that the contractor proposes to use in his work must be submitted with his bid. No asphalt cement, other than that submitted with bid, shall be used by any contractor except with the written consent of the engineer.

SEc. 5. Methods of testing.- Same as for specification B-8, omitting A. S. T. M. D7.

Notes.-The above specifications are recommended by the Asphalt Association for use under what may be termed average conditions. It is realized, however, that no single standard specification will satisfactorily cover all variations in local conditions which may prevail for individual jobs. Before adopting these specifications verbatim the engineer should, therefore, give particular consideration to the items listed below, and, if necessary, make such changes as are suggested.

I. Thickness. - These specifications call for a total finished thickness of base course of 6 inches. If a different thickness is desired one of the following combinations is suggested:

(a) Total thickness, 7 inches.

SECTION 1. (a) First sentenco, change 6 to 7 ; second sentence, change $31 / 2$ to 4 ; and $2 \frac{1}{2}$ to 3 .
SECTION 1. (a) II. Insert "second" after first word, and strike out No. 2.

SEc. 2. (b) Table, strike out second line of requirements in connection with No. 2 coarse aggregate.

SEc. 8 . Strike out No. 2.

(b) Total thickness, 8 inches.

Section 1. (a) First senteuce, change 6 to 8 . Strike out second sentence and insert: "Each course shall have a compacted thiskness of not less than 4 inches."

Make all other changes as suggested above for total thickness of 7 inches.

iI Che

11. Coarse aggregate.-If only a very soft product with a per cent of wear greater than 8 is available, it is advisable to increase the thickness of the base to 7 or 8 inches and to make the chauges recommended under "I. Thickness." In addition, the following changes should be made.

SEC. 2. (a) Strike out third and fourth sentences.

SEC. 5. (a) and (b) Strike out and change $(c)$ and $(d)$ to $(a)$ and $(b)$, respectively.

III. Use of crushed gravel.-If it is desired to allow for the use of gravel in the construction of the asphalt macadam base, the following sentence should bo inserted after the first sentence in section $2(a)$ : If produced by crushing gravel, only that portion of the gravel which has been retained upon a screon with $31 / 2$-inch or larger openings shall be used in its manufacture.

Asphalt Association, specification B-8, for asphaltic concrete base (commonly known as "black base"), April 10, 1929.

(The full text of the specification includes methods of construction and the following specifications for materials:)

SECTION 1. General description.-(a) The asphaltic concrete base shall consist of a mineral aggregate composed of coarse aggregate and fine aggregate, uniformly mixed with asphalt cement and shall have a compacted thickness of 3 inches.

(b) All materials shall conform to the requirements of these specifications. 


\section{MATERIAIS}

SEc. 2. Coarse aggregate.-(a) The coarse aggregate for asphaltic conerete base mixture shall consist of broken stone, broken slag, mine tailings, or gravel. It shall be of reasonably uniform quality and shall be free from dust and an excess of flat or elongated pieces. Rock or mine tailings from which it is produced shall have a per cent of wear of not more than 8. Slag from which it is produced shall, for each commercial size used, weigh not less than 70 pounds per cubic foot. If gravel is used, it shall be composed of sound, hard, durable pebbles, free from clay or coatings of any character.

(b) When tested by means of laboratory screens the coarse aggregate, which may consist of one or more commercial products, shall mect the following requirements:

Passing 11/2-inch screen not less than

Per cent

Passing $3 / 4$-inch screen

Passing $1 / 2-$ inch screen.

Material which passes the $1 / 4$-inch screen in such commercial products shall in laboratory tests and for the purpose of proportioning the mixture be considered as fine aggregate.

SEc. 3. Fine aggregate.-The fine aggregate for asphaltic concrete base mixture shall consist of sand composed of clean, hard, durable grains, free from clay, loam, and other foreign matter, together with particles which pass a 1/4-inch laboratory screen which may be present in the coarse aggregate material. When tested by means of laboratory screcns and sieves the sand or total fine aggregate shall meet the following requirements:

\begin{tabular}{|c|c|c|}
\hline Passing- & Retained on- & Per cent \\
\hline 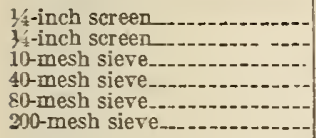 & $\begin{array}{l}\text { 10-mesh sieve } \\
\text { 40-mesh sieve. } \\
\text { 200-mesh sieve }\end{array}$ & $\begin{array}{r}100 \\
0-20 \\
15-50 \\
22-65 \\
7-10 \\
0-6\end{array}$ \\
\hline
\end{tabular}

SEc. 4. Asphalt cement.-The asphalt cement shall be homogeneous, and free from water and shall not foam when heated to $175^{\circ} \mathrm{C}$. $\left(347^{\circ} \mathrm{F}\right.$.). It shall meet the following requirements for penetration:

Penetration at $25^{\circ} \mathrm{C} .\left(77^{\circ} \mathrm{F}.\right), 100 \mathrm{~g}, 5 \mathrm{sec}$ $40-70$ (Between these limits the engineer shall specify as desired, 40 to 50 , 50 to 60 , or 60 to 70 .)

SEc. 5. Sources of supply.-(a) Approval of sources of supply of material under these specifications shall be obtained from the engineer prior to delivery of material, and samples of each shall be submitted as directed by the engineer.

(b) A 1-pound sample accompanied by an analysis of the asphalt cement that the contractor proposes to use in his work must be submitted with his bid. If the contractor proposes to prepare the asphalt cement at the paving plant then in lieu of the above, a 1-pound sample accompanied by an analysis each of flux and refined asphalt must be submitted with his bid, together with a statement of the proportions in which they will be combined to produce the asphalt cement which he proposes to use. No asphalt cement, flux, or refined asphalt ther than that specified in his bid shall be used by any contractor except with the written consent of the engineer.

SEC. 6. Methods of testing.-Methods of testing shall be as follows:

(a) Per cent of Trear of rock or mine tailings, A. S. T. M. D2. (See 510, p. 182.)

(b) Weight per cubic foot of broken slag, A. S. T. M. C29. (See 512.10 , p. 212.) (c) Screen test of coarse aggregate, A. S. T. M. D18. (See 512.15, p. 250.)

(d) Sieve test of fine aggregate, A. S. T. M. D7. (See 512.12, p. 234.)

(e) Penetration of asphalt cement, A. S. T. M. D5, 505.0, p. 118 .

\section{PREPARATION AND COMPOSITION OF MIXTURE}

SEc. 7. Preparation of asphalt cement.-(a) The asphalt cement shall be melted in kettles or tanks designed to secure uniform heating of the entire contents and shall be brought to a temperature of $250^{\circ}$ to $350^{\circ} \mathrm{F}$.

(b) When refined asphalt is to be combined with a flux the mixture shall be thoroughly agitated until a homogeneous asphalt cement of the required penetration is produced. The penetration of the asphalt cement shall be tested at suitable intervals to insure that it is maintained at a uniform consistency throughout the period of use.

SEc. 8. Preparation of mineral aggregate.-(a) The aggregate shall be dried and heated at the paving plant in suitably designed revolving driers, and when delivered to the mixer shall be at a temperature of $225^{\circ}$ to $350^{\circ} \mathrm{F}$. The aggregates may be fed simultaneously into the same drier, but in such case, immediately after heating, they shall be screened into coarse and fine aggregate and stored in separate bins, except in plants where the aggregates are proportioned and dried by the batch method.

(b) A registering pyrometer shall be installed at a suitable point at the discharge end of the drier with the registering device so located as clearly to indicate to the drum firemen the temperature of the mineral aggregate when discharged.

SEc. 9. Preparation and composition of asphaltic concrete base mixture.-(a) The coarse and fine aggregate for asphaltic concrete base mixture shall be measured separately and accurately either by weight or volume for each batch to be mixed. The required quantity of hot asphalt cement for each batch shall be measured by actual weighing with scales attached to the asphalt cement bucket. The mixture shall be made in an approved twin pug or batch mixer first by charging it with coarse aggregate and fine aggregate. After these have been thoroughly mixed for a period of not less than 15 seconds the asphalt cement shall be added and the mixing continued for a period of at least 30 seconds or longer if necessary to produce a homogeneous mixture in which all particles of the mineral aggregate are uniformly coated.

(b) The constituents of the asphaltic concrete base mixture shall be combined in such proportions as to produce a mixture conforming to the following composition limits by weight:

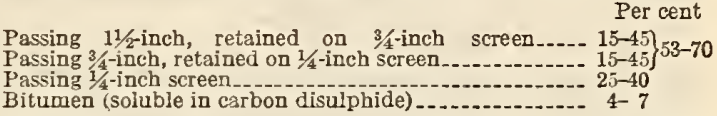

SEc. 10. Paving-plant inspection.-For the verification of weights or proportions and character of materials and determination of temperatures used in the preparation of the mixture the engineer or his authorized representatives shall have access at any time to all parts of the paving plant.

SEC. 11. Transportation of mixture.-The mixture shall be transported from the paving plant to the work in tight vehicles previously cleaned of all foreign materials, and when directed by the engineer each load shall be covered with canvas or other suitable material of sufficient size to protect it from weather conditions. No loads shall be sent out so late in the day as to interfere with spreading and compacting the mixture during the daylight unless 
artificial light satisfactory to the engineer is provided.

Note.-The above specifications are recommended by the Asphalt Association for use under what may be termed average conditions. It is realizcd, however, that no single standard specification will cover satisfactorily all variations in local conditions which may prevail for individual projects. Before adopting these specifications verbatim the engineer should, therefore, give particular consideration to the items listed below and if necessary make such changes as are suggested.

I. Subgrade.-Experience of the past few years has emphasized earlier knowledge, but omission in practice, of the absolute necessity of properly draining the subgrade, irrespective of what type or thickness of foundation or pavement surface is used. This is especially important in connection with country-road construction.

Under modern traffic conditions any type of road will fail sooner or later if subjected to traficic while the subgrade is more or less saturated with water at certain seasons of the year no matter whether the base or foundation course is a rigid slab of Portlandcement concrete or of flexible asphaltic concrete or compressed stone.

No foundation will satisfactorily overcome poor drainage.

It is assumed, therefore, that before using this or any other specifications that the engineer will provide for appropriate and sufficient drainage for the subgrade.

II. Thickness of base.-Experience covering the past 10 years and a very large mileage on the Pacific Coast and in many Western States has proved that pavements consisting of dense asphaltic concrete base and a stable asphaltic surface of a combined thickness of 5 inches is ample for heavy modern traffic when laid directly upon a properly drained and compacted subgrade.

This specification, therefore, provides for a finished thickness of asphaltic concrete base of 3 inches. In some instances it may be desirable to reduce the thickness to $2 \frac{1}{2}$ inches and in others to increase it to 4 inches. In such cases the following change should be made:

SEc. 1. (a) Last line, change 3 to $3 \frac{1}{2}$ or 4 as desired.

If, in the opinion of the engineer, all or any portion of the subgrade or existing road is not of sufficient strength to serve as a subbase it should be reinforced by the addition of new crushed stone, slag, gravel, or other equivalent available material, as for reasons of economy this is generally preferable to increasing the thickness of the asphaltic concrete base.

In connection with any type of foundation, areas of greater or less extent are liable to develop during construction which have an unanticipated weak subsoil. To provide for such necessarily unforeseen conditions it is recommended that unit prices be provided in bids for each class of material as may be required for such emergencies.

III. Coarse aggregate.- If it is desired to use a larger size of coarse aggregate than that specified the following changes should be made:

SEc. $2(b)$. Third line, change $1 \frac{1}{2}$ to $2 \frac{1}{2}$.

Sec. $2(b)$. Fourth line, change $3 / 4$ to $1 \frac{1}{4}$.

SEc. $9(b)$. Third line, change $1 \frac{1}{2}$ to $2 \frac{1}{2}$ and change $3 / 4$ to $1 \frac{1}{4}$.

Sec. 9 (b). Fourth line, change $3 / 4$ to $1 \frac{1}{4}$.

IV. Asphalt cement.-The penetration limits for asphalt cement may be made to conform to those which are selected for the asphalt cement for surface course.

V. Sheet asphalt surface course.-When a sheet asphalt surface course is to be laid in conjunction with an asphaltic concrete base the usual binder course may be eliminated.
Federal Specifications Board, specification No. 276a, United States Government master specification for asphalt for use in road and pavement construction, May 8, 1925.

See 505.11 , page 145 .

United States Department of Commerce, Bureau of Standards, Simplified Practice Recommendation No. 4, for asphalt (penetration limits), January 1, 1924.

\section{See 505.0, page 145 .}

\subsection{Slag Foundation.}

American Association of State Highway Officials, tentative standard specifications for highway materials, 1927.

See 512.15, page 244 .

American Society for Testing Materials, standard specifications for broken slag for water-bound base and wearing course, serial designation D65-23, 1923.

See 512.2 , page 254 .

American Society for Testing Materials, standard specifications for shovel-run or crusher-run broken slag for water-bound base, serial designation D66-23, 1923.

See 512.2, page 254 .

Federal Specifications Board, specification No. 456, United States Government master specification for broken stone and broken siag for waterbound base or wearing course, January 25, 1927.

See 512.15, page 251 (for broken stone) and 512.2, page 255 (for broken slag).

National Paving Brick Manufacturers' Association, recommended specifications for artificial bases, 1924 .

Rolled bases of gravel, stone, or slag, with or without shoulders or bituminous binding.

Material and size.-Broken stone or crushed slag as called for on the plans and in the bids for artificial base course shall be run-of-crusher material of good grade and reasonably sound, that will all pass 3-inch circular openings in a revolving screen and be retained on 1/4-inch openings. Gravel shall be composed of durable particles, together with clay or other suitable binding material. One hundred per cent shall pass through a 3-inch screen, not more than 75 per cent shall pass through a 3/4-inch screen, and not more than 30 per cent shall pass through a No. 10 revolving screen. The gravel shall contain not less than 10 nor more than 20 per cent by weight of clay or other suitable binding material.

Depth.-The broken stone, crushed slag, or gravel shall be uniformily distributed on the prepared subgrade to a depth approximately 30 per cent greater than the finished depth of ..... inches, called for on the plans, and thoroughly rolled in one course until the base is of uniform and thorough density throughout, conforming to the given grade and the cross section.

Rolling.-The roller used for this purpose shall be self-propelling tandem or 3-wheel type, weighing not more than 10 tons nor less than 5 tons. The rolling shall commence at the edges of the pavement and work toward the center and shall be continued until the base is thoroughly consolidated and shoms no tendency to creep in front of the roller. During this operation the surface of the base shall be swept with stiff brooms so that all fine material shall enter the interstices of the base.

Binder course.- With broken stone or crushed slag the base shall be bound with screenings of the same material that will all pass a $1 / 4$-inch sieve opening. The screenings shall be spread over the surface of the base and swept and rolled into the voids.

Stone shoulder.-On pavements where the plans call for rolled shoulder and where curbs are not provided 
for on the plans for the project the rolled base course of stone, slag, or gravel shall extend at least 12 inches beyond the edges of the brick wearing surface as shown on the plans. On completion of the base course 2 by 4 inch wooden forms shall be set on edge upon the prepared base and securely staked to act as guides for placing the brick wearing surface. The guide forms shall remain in place when the pavement is completed where shown in the plans.

After the completion of the brick wearing surface, a shoulder of broken stone or crushed slag shall be placed. The width thereof shall be not less than 12 inches and the depth as built up upon the extended base shall equal the combined depth of the brick surface and the bedding course as shown on the plans. The shoulder shall be thoroughly compacted by rolling, using the same roller, if practicable, as specified for the brick surface.

Bituminous binding. - Where a bituminous-bound base or shoulder is indicated on the plans the surface of the rolled base or shoulder shall receive a bituminous penetration not exceeding $1 \frac{1}{2}$ gallons per square yard.

Immediately after bituminous material is applied the surface shall be covered with a light application of screenings.

National Slag Association, recommended specifications for slag to be used in highways and highway structures, 1927.

See 512.2 , p. 374 .

\subsection{Miscellaneous Specifications for Foun- dation for Roads.}

Asphalt Association, specification B-1, for gravel base (tentative), September 1, 1920.

(The full text of the specification includes methods of construction and the following specifications for materials:

Section 1. General description.-(a) The gravel base shall consist of two courses of gravel, each course having a compacted thickness of not less than 4 inches. The two courses shall form a foundation having a compacted thickness of not less than 8 inches and shall conform to the grades and cross sections shown on the plans.

(b) All material shall conform to the requirements of these specifications.

\section{MATERIAI}

SEc. 2. Gravel.-The gravel shall be composed of hard durable rock, together with sand and clay or other binding material, and shall be free from an excess of thin or elongated pieces. When tested by means of laboratory screens it shall meet the following requirements:

Total retained on $1 / 4$-inch screen

The material retained on the $1 / 4$-inch screen shall meet the following requirements:

Retained on 11/2-inch screen

Per cent

SEc. 3. Sources of supply.-Approval of sources of supply of gravel under these specifications shall be obtained from the engineer prior to delivery of material, and samples shall be submitted as directed by the engineer.

SEC. 4. Method of testing.--Screen tests of gravel shall be made according to A. S. T. M. D18. (See 512.15 , p. 250 .)

Asphalt Association, specification B-6, for truing up old pavements to serve as base course (tentative), September 1,1920

(The full text of the specificarion includes methods of construction and the following specifications for materials.)
Section 1. General description.-(a) The old pavement of macadam, brick, block, or Portlandcement concrete shall where shown on the plans be made to serve as a base course by filling all depressions having a depth of three-fourths inch or more with asphaltic concrete compacted so as to produce a surface conforming as closely as possible to the grades and cross sections shown on the plans.

(b) All materials and methods shall conform to the requirements of these specifications.

\section{ASPHALT CONCRETE FOR FILLING DEPRESSIONS}

SEc. 2. Sheet-asphalt surface course.-If sheetasphalt binder and surface courses are to be laid on the old pavement, the asphaltic concrete used for truing up the old surface shall be composed of the same materials combined in the same proportions and in the same manner as specified for binder course in the "Specifications for sheet asphalt binder and surface courses," 518.37, page 406 .

SEc. 3. Asphaltic concrete surface course.-(a) If an asphaltic concrete surface course of the coarsegraded aggregate type is to be laid on the old pavement, the asphaltic concrete used for truing up the old surface shall be composed of the same materials combined in the same proportions and in the same manner as specified in the "Specifications for asphaltic concrete surface course," 518.37, page 405.

(b) If an asphaltic concrete surface course of the fine-graded aggregate type is to be laid on the old pavement, the asphaltic concrete used for truing up the old surface shall consist of coarse aggregate and sand, uniformly mixed with asphalt cement, the sand and asphalt cement conforming with specifications for sand and asphalt cement in the specifications for "Asphaltic concrete surface course." The coarse aggregate shall consist of broken stone produced from tough durable rock, of reasonably uniform quality throughout, and shall be clean and fre 2 from dust and an excess of flat or elongated pieces. When tested by means of laboratory screens it shall meet the following requirements and be uniformly graded between the limits given:

Per cent

Passing 11/1-inch screen, not less than Passing 1 1 -inch screen, not more than

SEc. 4. Asphalt-macadam surface course.-If an asphalt-macadam surface course is to be laid on the old pavement, the asphaltic concrete used for truing up the old surface shall consist of a mineral aggregate of broken stone, mine tailings, or slag, uniformly mixed and coated with from 4 to 6 per cent by weight of asphalt cement as directed by the engineer. The mineral aggregate shall conform to the specifications for intermediate aggregate, and the asphalt cement shall conform to the specifications for asphalt cement in the "Specifications for asphalt-macadam surface course," 518.37, page 403. Immediately before mixing the mineral aggregate shall be heated to a temperature of $150^{\circ}$ to $300^{\circ} \mathrm{F}$. and the asphalt cement shall be heated to a temperature of from $250^{\circ}$ to $350^{\circ} \mathrm{F}$. The mixture shall be made in a mechanical mixer or with hot shovels on a clean mixing board and the proportions shall be varied within the limits designated as directed by the engineer.

National ?aving Brick Manufacturers' Association, recommended specifications for bedding course, 1924.

Sand, granulated slag, slag screenings, or limestone screenings.

Description.-Upon the prepared base after it has been thoroughly cleaned of all dirt and débris shall be spread a bedding of sand, granulated slag, slag screenings, or limestone screenings, as called for in the plans, not more than 1 nor less than $1 / 2$ inch in finished depth. 
Sizes.-Bedding material shall not exceed 1/4 inch in maximum grain size. It may contain fine material passing a No. 20 standard mesh sieve, not exceeding 15 per cent by weight. In the case of sand, this fine material may be loam or clay.

Shaping.-Bedding shall be shaped to a true surface parallel with the surface of the finished roadway by means of a template extending the entire width of the roadway, drawn forward upon the curbs or other guide rails as provided. When the width of the roadway precludes the use of a template spanning the entire distance the bedding shall be shaped in sections, using scantling laid upon the base as guide rails. The bedding course shall be struck off at least twice with the template. Any depressions developing shall be filled in and the bed again struck off with the template. This operation shall be continued until perfect alignment is presented.

Rolling.-If directed by the engineer, in addition to shaping with a template, the bedding course shall also be compacted with a hand roller. The roller shall be not less than 36 inches in diameter, 24 inches in width, and weigh not less than 10 pounds per inch of width.

The bedding course shall be struck off with the template and then rolled. Any depression existing shall be filled in, struck off with the template, and again rolled. This operation shall be repeated until perfect alignment is presented.

Hand finishing.- When the use of the template and guide rails is impracticable in finishing the bedding surface it shall be shaped to the surface required by hand lutes.

No disturbance.-The bedding shall not be disturbed after final shaping prior to laying the brick.

National Paving Brick Manufacturers' Association, recommended specifications for natural base, 1924.

Clearing and grubbing.-All objectionable brush, stumps, fences, boulders, stones, or other material within the limits of or overhanging the highway shall be removed. All trees, stumps, roots, and brush shall be cleared and grubbed from the area to be improved in such a manner that in case of a fill no part of them shall be within 18 inches of the finished surface of the subgrade, or in the case of an excavation no part of them shall be within 6 inches of the finished surface of the subgrade. All stones that are less than 6 inches below the finished surface of the base also shall be removed.

Grading.-The roadway and approaches to be improved shall be graded to such lines and cross sections that when rolled the finished surface of the base shall conform to that shown in the plans.

Excavation.-All vegetable matter and soft, spongy earth, muck, or quicksand which will not compact by rolling shall be removed and replaced with material that can be compacted.

Embankment.-Earth in embankment shall be applied in layers not exceeding 12 inches in depth and each layer shall be thoroughly rolled and compacted before successive layers are applied. All vegetable matter and soft, spongy earth, muck, or quicksand which will not compact by rolling shall be excluded from embankment as provided for earth in excavation.

Rolling.-The roller used in compacting the base shall be of the self-propelling 3-wheel type weighing not to exceed 10 tons. Rolling shall continue until the base course is thoroughly compacted. Prior to and during the rolling the natural base shall be thoroughly wetted to assist in and insure thorough and uniform compaction. Any depressions in the surface thereof created by rolling shall be brought to grade by the addition of suitable material. Rolling and wetting then shall be repeated until the base course is of uniform density with a smooth and even surface.

Protection of materials.-No earth from excavation or embankment shall be deposited against or upon any material to be used in the pavement. Such materials shall be placed and protected so that they will be uninjured by travel or by grading operations.

Testing.-The surface of the natural base shall be brought to the crown shown on the plans and before completion be tested with the template. Any variation from the true grade and cross section shown on the plans shall be corrected.

National Paving Brick Manufacturers' Association, recommended specifications for cement-sand bed for semimonolithic brick pavements, 1924.

Description.- Upon the base as prepared shall be added a cement-sand bedding which shall become an integral part of the base and which shall be included in and constitute a part of the full depth of base as shown on the plans. Bedding shall be composed of 1 part Portland cement and 4 parts sand. When finally finished the depth of bedding shall not exceed 1 inch nor be less than $1 / 2$ inch.

Cement.-Portland cement shall conform to the definition and meet the requirements of A. S. T. M. C-9. (See 516.11, p. 294.)

Sand.- Sand for the bedding shall be clean, all of which shall be uniformly graded and which will pass a $1 / 4$-inch sieve.

Sand shall be free from soft, friable material, shale or slate, vegetable, or other organic matter. It shall not contain clay or silt in excess of 5 per cent by weight.

Premixing.-Cement and sand for the bedding shall be premixed in the proportions specified, by hand or by mechanical batch mixer. Mixing shall continue for each batch until the mass is of uniform shade.

Shaping.-Bedding shall be shaped to a true surface parallel with the surface of the finished roadway by means of a template extending the entire width of the roadway, drawn forward upon the curbs or guide rails. When the width of the roadway precludes the use of a template spanning the entire distance the bedding shall be shaped in sections, using scantling laid upon the base as guide rails. The bedding course shall be struck off at least twice with the template. Any depressions developing shall be filled in and the bed again struck off with the template. This operation shall be continued until perfect alignment is presented.

Rolling.-If directed by the engineer, in addition to shaping with a template, the bedding course shall also be compacted with a hand roller. The roller shall be not less than 36 inches in diameter, 24 inches in width, and weigh not less than 10 pounds per inch of width.

The bedding course shall be struck off with the template and then rolled. Any depressions existing shall be filled in, struck off with the template, and again rolled. This operation shall be repeated until perfiect alignment is presented.

Hand finishing.- When the use of the template and guide rails is impracticable in finishing the bedding surface it shall be shaped to the surface required by hand lutes.

No disturbance.-The bedding shall not be disturbed after final shaping prior to laying the brick.

Naticnal Sand and Gravel Association, Bulletin 3, representative specifications for different uses of sand and gravel, November, 1928.

(In this bulletin representative specineations, many of which are presented in this volume, and including specifications for sand for cushion course and sand for cement-mortar bed, have been summarized.) 
518.3 PAVING SURFACES.

\subsection{General Items.}

American Association of State Highway Officials, tentative standard specifications for highway materials, 1927.

See 512.15, page 244 .

American Association of State Highway Officials, tentative standard specifications for highway materials, 1927.

No. 18. Oif asphalt filler (type A).

No. 19. Asphalt filler (type B).

No. 20. Asphalt cement (prepared from petroleum), penetration grades 30 to 40,40 to 50,50 to 60,60 to 70 .

No. 21. Asphalt cement (prepared from petroleum), penetration grades 85 to 100,100 to 120 , 120 to 150 .

No. 22. Asphalt cement (prepared from native asphalt), penetration grades 30 to 40,40 to 50 , 50 to 60,60 to 70 .

No. 23. Asphalt cement (prepared from native asphalt), penetration grades 85 to 100,100 to 120 , 120 to 150 .

(For properties and methods of sampling and testing, see 505.13, p. 146.)

American Society for Testing Materials, standard definitions of terms relating to materials for roads and pavements, serial designation D8-18, 1918.

See 510, page 182 .

American Society for Testing Materials, tentative specifications for commercial sizes of broken stonc and broken slag for highway construction, serial designation D63-23T, 1923.

See 511.71 , page 207 .

American Society for Testing Materials, standard methods of sampling stone, slag, gravel, sand, and stone block for use as highway materials, including some material survey methods, serial designation D75-22, 1922 .

See 510, page 184 .

\subsection{Cement Concrete Pavements.}

American Association of State Highway Officials, standards of practice, 1928.

\section{HIGH EARLY STRENGTH CONCRETE}

In the construction of concrete pavements, provision should be made for requiring high early strength concrete on special sections of the road when the requirements of traffic justify early opening of the pavement.

\section{LONGITUDINAL JOINTS}

In constructing concrete pavements some suitable form of construction joint shall be used or method of construction adopted that will divide the pavement longitudinally into sections corresponding in width with the traffic lanes; and that dowel bars shall be installed across the joint between the sections.

\section{WIDTH OF TRAFFIC LANES}

On pavements 10 feet shall be considered as the standard width for each trafic lane.

\section{CROWN}

The crown of a 2-lane concrete pavement shall be 1 inch

\section{THICKNESS}

No part of a concrete pavement shall have a thickness of less than 6 inches and all unsupported edges shall be strengthened.

American Concrete Institute, standard specifications for 1-course Portland cement concrete pave- ments for highways, serial designation $\mathrm{S}-6 \mathrm{~A}-28$ 1928.

\section{GENERAL}

1. It is the intent of these specifications to cover the requirements for materials and construction of Portland cement concrete pavement, wherein the concrete is of uniform proportions from top to bottom of the slab.

\section{MATERIALS}

\section{(A) CEMENT}

2. Cement shall be a standard Portland cement which, at the time it is incorporated in the pavement mixture, shall conform to A. S. T. M. C9. (See 516.11, p. 294.)

\section{(B) AGGREGATES}

3. Prior to placing any orders for aggregates the contractor shall advise the engineer of the proposed source or sources of supply of aggregates. The engineer may require the contractor to submit 50 -pound samples of all aggregates proposed for use. If the engineer finds such samples fulfill the requirements of these specifications for aggregates, similar material shall be considered as acceptable. Acceptance of samples shall not be construed as a guarantee of acceptance of all materials from the same source, and it shall be understood that any aggregates which do not meet with the requirements of tliese specifications will be rejected. Upon receiving notification of the proposed source or sources of aggregate supply, the engineer may elect to investigate and test the aggregate supply at the source; in which case he shall notify the contractor as to acceptability or nonacceptability of the proposed aggregates. The engineer shall notify the contractor, after agreement upon a source or sources of aggregate supply, whether routine tests of aggregates during construction will be made at the source of supply or at the point of receipt.

4. (a) Fine aggregate.-Fine aggregate shall consist of natural sand, stone screenings, slag sand, tailings, chatts, or other inert materials with similar characteristics, or a combination thereof, having clean, hard, strong, durable, uncoated grains. When incorporated in the pavement mixture, fine aggregate shall be free from frost, frozen lumps, injurious amounts of dust, mica, soft or flaky particles, shale, alkali, organic matter, loam, or other deleterious substances. Ninetyive per cent of the fine aggregate, when dry, shall pass a 1/4-inch screen; not more than 25 per cent shall pass a 50-mesh sieve, and not more than 5 per cent by weight shall pass a 100 -mesh sieve. In no case shall fine aggregate be accepted containing more than 3 per cent, by dry weight, nor more than 5 per cent by dry volume, nor more than 7 per cent by wet volume, of clay, loam, or silt. If any sample of fine aggregate shows more than 7 per cent of clay, loam, or silt in one hour's settlement after shaking in an excess of water, the material represented by the sample will be rejected. Fine aggregate shall be of such a quality that mortar composed of Portland cement and the fine aggregate when made into 2 by 4 inch cylinders, in the same proportions as will be used in the concrete mixture for the pavement, shall show compressive strength at 7 and 28 days equal to or greater than the compressive strength of cylinders composed of mortar of the same proportions of Portland cement and standard Ottawa sand. For proportioning test cylinders, Portland cement and fine aggregate and standard Ottawa sand shall be measured by weight, and the same Portland cement shall be used with 
the Ottawa sand as with the fine aggregate to be tested.

5. (b) Coarse aggregate.-Coarse aggregate shall consist of one of the following materials, or a combination thereof: Crushed rock, pebbles (gravel), air-cooled blast-furnace slag, chatts, or tailings. The particles of coarse aggregate shall be of clean, hard, tough, durable material, free from vegetable or other deleterious substances, and shall contain no soft, flat, or elongated pieces. Coarse aggregate, excepting air-cooled furnace slag, shall show not more than 6 per cent loss in the wear test.

Note.-In many cases, it will be necessary for the engineer to specify the sizes, grading, and quality of coarse aggregate in accordance with local conditions. In every case, the engineer should provide specifications which will reauire the use of the best coarse aggrcgate whieh is economically available. The following specifications covering size and grading of coarse argregate will be found applicable in most sections of the country, and are intended for use with the $1: 2: 31 / 2,1: 2: 3$, or $1: 1 \frac{1}{2}: 3$ mixture.

6. The size of the coarse aggregate shall be such as to pass a 3 -inch round opening. Coarse aggregate shall be uniformly graded within the limits shown in the following table, and any material which does not come within the limits specified shall be rejected.

\begin{tabular}{|c|c|c|}
\hline & Minimum & Maximum \\
\hline Passing 3-inch round opening & Per cent & Per cent \\
\hline Passing 2 -inch round opening & 82 & $\overline{95}$ \\
\hline Passing $1 / 2$-inch round opening & 15 & 25 \\
\hline Passing $1 / 4$-inch sieve. & -- & 5 \\
\hline
\end{tabular}

7. Crushed rock shall consist of particles of rock produced by quarrying and crushing ledge rock, field boulders, or pebbles, from which, after crushing, all dust and pieces below $1 / 4$-inch size have been screened out. Crushed rock shall conform in quality to the specifications under "Coarse aggregate."

8. Pebbles (gravel) shall consist of loose material containing only particles retained upon a $1 / 4$-inch screen, resulting from the natural crushing and erosion of rocks. Pebbles must have wearing qualities at least equal to crushed stone. Pebbles shall conform in quality to the specifications under "Coarse aggregate."

9. Air-cooled blast-furnace slag.-The broken slag shall consist of roughly cubical fragments of aircooled blast-furnace slag, reasonably uniform in density and quality and reasonably free from metallic iron, containing no dirt or other objectionable matter. The slag shall weigh not less than 70 pounds per cubic foot.

10. Chatts, or tailings, are terms locally applied to by-products, or waste products, of certain mining and industrial operations. When used as coarse aggregate for concrete pavements, such materials shall substantially conform to the specifications under "Coarse aggı'egate."

11. Mixed aggregaie.-Mixed aggregate shall consist of a combination of fine and coarse aggregates. That portion of mixed aggregate passing a 1/4-inch screen shall conform to the requirements for fine aggregate; and that portion of mixed aggregate retained on a $1 / 4$-inch screen shall conform to the requirements for coarse aggregate.

\section{(C) WATER}

12. Water shall be clean, free from oil, acid, alkali, or vegetable matter.

\section{(D) REIN FORCEMENT}

13. Reinforcement shall consist of steel fabric, or of steel bars, or a combination of both and shall have an effective weight exclusive of dowel bars at joints and of circumferential bars of at least .... pounds per 100 square feet.

14. (a) Steel fabric.-Steel fabric shall be manufactured from cold-drawn wire and shall comply with tentative standards of the American Society for Testing Materials, serial designation A82-27.

15. The spacing of primary members shall be not more than _..... inches, and of secondary members not more than ...- inches.

16. Steel-bar reinforcement.-This style of reinforcement shall consist of steel bars of the size, shape, and spacing shown on the plans, and shall be properly formed into mats. All intersections of longitudinal and transverse bars along the exterior edges of the mat and evcry other intersection of the longitudinal and transverse bars in the interior of the mat shall be securely wired or clipped together to resist displacement during handling and concreting operations. The materials shall have an effective weight of not less than ...... pounds per 100 square feet exclusive of laps, ties, clamps, chairs, and such portions of the bars as are not in the plane of the mat for their full lengths.

17. Steel bars shall comply with the standard requirements for concrete reinforcement bars, structural and intermediate grades, of the American Society for Testing Materials, serial designation A15-14. All bar reinforcement when placed in the pavement shall be free from excess rust, scale, or other substance which prevents the bonding of the concrete to the reinforcement. When in storage on the work, bars shall be protected from corrosion by placing them on a dry platform under a weatherproof cover.

\section{(E) JOINT FILLER}

Joint fillers shall consist of prepared strips of fiber matrix and bitumen, or a uniform mixture of fiber and bitumen, or a combination of both, containing not more than 25 per cent by weight of inert material having thicliness of _. inches, and width equal to -inches greater than the thickness of the pavement at any point. The bitumen used in the manufacture of the joint filler may be either tar or asphalt of a grade that will not become soft enough to flow in hot weather, nor brittle in cold weather. The prepared strips shall be cut to conform to the cross section of the pavement and in lengths equal to the width of the pavement, except that strips equal in length to half the width of the pavement may be used when laced or clipped together at the center in a workmanlike and effective manner.

\section{(F) SHOULDERS}

(Any special materials for the construction of shoulders should be here described as desired by the engineer.)

\section{SUBGRADE}

18. Subgrade will be considered as that portion of the highway upon which the pavement is to be placed.

\section{(A) FINE GRADING}

19. Fine grading will include the finished excavation and embankment which may be necessary to bring the subgrade to the required elevation, alignment, and cross section. All suitable materials removed from the excavation in fine grading shall be used as far as practicable in the formation of the embankment, as may be required. Such material not used in embankment may be deposited on the shoulders as directed by the engineer. When the amount of the embankment exceeds the amount of the material available from excavation, suitable material shall be obtained byl the contractor from 
borrow pits located bevond the limits of the shoulders or embankment slopes. Such borrow pits shall be left in neat condition, such as will drain completely. Ditch sections and back slopes of cuts must conform to the plans, and be left with neat and uniform appearance.

\section{(B) PREPARATION AND MIINTENAICE}

20. The subgrade shall be constructed to have, as nearly as practicable, a uniform density throughout its entire width. Wherever the subgrade extends beyond the lateral limits of an old roadway, or wherever an old gravel, macadam, or other hard compacted crust comes within 6 inches of the elevation of the finished subgrade, such old roadway or crust shall be ploughed, loosened or scarified to a depth of at least 6 inches and the loosened material redistributed across the full width of the subgrade, adding suitable material, when necessary, so that when compacted to the required elcvation, alignment, and cross section, the subgrade will approach as nearly as possible, a condition of uniform density. Compression of the subgrade material shall be accomplished with a self-propelled roller weighing not less than 3 tons. Hand-tamping portions of the subgrade may be dirccted by the engineer when necessary. There shall not be left on the subgrade or shoulders. bcams, or ridges of earth or other material that will interfere with the immediate discharge of water from the subgrade to the side ditches, and the subgrade shall be maintained free from ruts so that it will, at all times, drain properly.

21. All depressions developing under traffic on the subgrade, or in conncetion with rolling, shall be filled with suitable material. Rolling shall bc continued until the subgrade is uniformly compacted, properly shaped, and true to grade and alignment. It is not intended that the rolling shall be continued beyond this point, as the purpose of rolling is not to produce a subgrade that can not be further compacted, but to produce a uniformly compacted subgrade. All hauling shall be distributed over the width of the subgrade so far as practicable, so as to leave it in a uniformly compacted condition.

22 . After being prepared in the above manner, the subgrade shall be so maintained until the concrete pavement has been placed thereon.

\section{(C) SUBGRADE-CHECKING AND ACCEPTANCE}

23. Immediately prior to placing concrete pavement on the subgrade, it shall be checked by means of an approved scratch template, resting on the side forms, having the scratch points placed not less than 8 inches apart, and to the cxact elevation and cross section for the subgrade surface. The scratch template shall be drawn along the forms so that the plane of the points will be at a right angle to the grade line, and the long axis of the template at a right angle to the center line of the pavement. All high places indicated by the scratch points shall be removed to true grade, and any low places back filled with suitable material and rolled or hand tamped until smooth and firm. The subgrade shall be checked and completed in accordance with these requirements for a distance of not less than 100 feet in advance of the concrete. If hauling over the subgrade after it has been finished and checked as above specified, rcsults in ruts or other objectionable irregularities, the contractor shall reroll or handtamp the subgrade and place it in smooth and satisfactory condition before the pavement is deposited upon it. If the condition of the subgrade is such that it can not be placed in satisfactory condition to receive the pavement by the above methods, placing pavement may be stopped by the engineer unless the contractor can provide and haul over suitable trackways or use other satisfactory means for the protection and maintenance of the subgrade.

\section{(D) SPECIAL TREATMENT}

24. (Special treatment may be specified for certain subgrades such as sand, gumbo, adobe, and other materials, which can not be satisfactorily prepared for pavement by the methods specified in the foregoing paragraphs.)

\section{FORMS}

\section{(A) MATERIALS}

25. Wooden forms shall bc dressed to 3-inch thickness, and equal in depth to the thickness of the pavement at the sides. Forms shall rest upon stakes driven into the ground within 1 foot of each end of each scparate piece, and at intervals not greater than 5 feet elsewhere. Forms shall be held in place by stakes driven into the ground along the outside cdgc at intervals of not more than 6 feet, two stakes being placed at each joint. The forms shall be firmly nailed to the side stakes, and firmly braced at any point where necessary to resist the pressure of the concrete or the impact of the tamper. Forms shall be capped along the inside upper edge with 2-inch angle irons.

26. Metal forms shall be of shaped stecl sections not less than 10 feet in length, for tangents and for curves having radii of 150 fcet and over. For curves of less radii, sections 5 feet long may be used. Forms must have a depth equal to the side thickness of the pavement. Forms shall be made of steel plate of approved scction. At least three bracing pins or stakes shall be used to cach 10 feet of form, and the bracing and support must be ample to resist the pressure of the concrete and the impact of the tamper without springing.

\section{(B) SETTING}

27. Forms shall set to exact grade and alignment at least 500 feet in advance of the point of depositing concrete. Before setting, the sections must be thoroughly cleancd. After setting, they shall be thoroughly oiled before concrete is placed against them. Forms in place will be subject to check and correction of line or grade at any time.

\section{PAVEMENT SECTION}

28. Width, thickness, and crown of concrete pavement shall be as shown on the plans for the improvement.

\section{JOINTS}

29. The joints to be formed shall be transverse or longitudinal. They shall be tested during and after finishing with a 10-foot straight-edge, and any irregularities in the surface shall be immediately corrected. Expansion joints shall be formed between the pavement under construction and all other rigid types of pavement or structures to which it may be adjacent. All joints shall be edged to a radius of one-eighth inch. Joints shall be made as follows:

\section{(A) TRANSVERSE EXPANSION JOINTS}

30. Transverse expansion joints shall be -.-inches wide, spaced .... feet apart. A bulkhead cut to the exact cross section of the pavement, shall be securely staked in place at right angles to the center line and surface of the pavement. The premolded joint filler shall be placed against the bulkhead and held in position by pins on which there is an outstanding lug. Concrete shall be deposited on both sides of the bulkhead before it is removed. 
After the concrete has been struck off, the bulkhead shall be removed by lifting it slowly from one end and replacing it with concrete as it is lifted, so that the joint filler will be left in the correct position.

31. When expansion joints are made at the end of the day's work they shall be formed by finishing the concrete to the bulkhead, placed as before specified. When work is resumed the joint filler shall be placed against the hardened concrete and held in position by pins until fresh concrete is placed against it.

32. In pavements with integral curb the joint shall be continuous in a straight line through pavement and curb.

33. Joints shall be opened on the edges for their entire depth, upon removal of the forms.

34. Before the pavement is opened to traffic the joint filler shall be trimmed off to a uniform height of one-fourth inch above the surface of the pavement.

\section{(B) LONGITUDINAL EXPANSION JOINTS}

35. Longitudinal expansion joints shall be formed by placing the filler against the form, bulkhead, curb, or adjacent structure and placing the concrete against it. The filler shall extend the full depth of the pavement, and be flush with the pavement surface.

\section{(C) TRANSVERSE CONSTRUCTION JOINTS}

36. Transverse construction joints shall be formed whenever it is necessary to stop concreting for 30 minutes or longer, except at expansion joints, by staking in place a bulkhead, as specified for transverse expansion joints, and finishing the concrete to the bulkhead. An edging tool shall be used along the bulkhead to make the construction joint a regular and well-defined line. When the plans require steel dowels across transverse joints in this bulkhead there shall be holes spaced 3 feet, center to center, 3 inches below the surface of the finished pavement, through which $3 / 4$-inch plain round steel rods 4 feet long shall be inserted with 2 feet projecting. At leas.t one-half length of each bar shall be encased in heavy paper or coated with paint or oil in such a manner as to prevent a bond between the steel and the concrete.

37. When work is resumed the plank shall be removed, care being taken not to disturb the rods or the concrete. The fresh concrete shall be placed directly against the face of the concrete previously laid and carefully worked around the rods.

38. If concreting must be stopped within 10 feet of a previously made transverse joint the concrete shall be removed to this joint.

\section{(D) LONGITUDINAL CONSTRUCTION JOINTS}

39. Longitudinal construction joints shall be formed where required and must be straight and vertical. When so indicated on the plans, steel dowels shall be used as provided in the preceding section.

\section{WATER SUPPLY}

\section{(A) EQUIPMENT}

40. Where necessary for the supply of water for all operations described in these specifications, duplicate pumps, connected to an adequate pipe line along the improvement, shall be provided by the contractor. The pipe line must be fitted with drains at the low points, and air relief valves at the high points, and with convenient outlets for all paving operations. Where the concrete mixer operates on the subgrade, the pipe line shall have a minimum diameter of 2 inches. For supplying a mixer using more than four sacks of cement per batch, 60 per cent of the pipe line shall have a minimum diameter of 3 inches, and the remaining 40 per cent shall have a minimum diameter of 2 inches. The large diameter pipe shall lead from the pump.

\section{(B) PRIORITY TO WATER SUPPLY}

41. The concrete pavement in place, for 10 days after laying, and the subgrade preparation, shall have prior rights to the water supply. If it should develop there is not sufficient water for all purposes, the concrete mixer shall be shut down until the water needs of the curing and subgrading operations have been cared for.

\section{PROPORTIONING AND MIXING CONCRETE}

\section{(A) PROPORTIONING}

42. (a) Measuring materials.-The method of measuring the materials for the concrete, including water, shall be such as to insure the required proportions of each of the materials as directed by these specifications. One sack of Portland cement (94 pounds net) shall be considered 1 cubic foot.

43. (b) Proportions.-The concrete shall be proportioned 1 sack of Portland cement, not more than ...- cubic feet of fine aggregate, and not more than -..- cubic feet of coarse aggregate. A cubic yard of concrete in place, measured between neat lines, must contain . ... barrels of Portland cement. The engineer shall compare the calculated amount of cement required by these specifications and tables with the amounts actually used in each section of concrete _... feet long, or between successive transverse joints. If the amount of cement actually used in the pavement varies from the specified amount by more than 3 per cent for any section, the engineer may require the proportions of the concrete to be adjusted so as to use the specified amount of cement. If it is found that the amount of cement used in any section is $921 / 2$ per cent or less of the specified quantity, the contractor shall be required to remove such section or sections and replace them with concrete made in accordance with these specifications. Such removal and replacement shall be done at the expense of the contractor.

\section{(B) MIXING}

44. (a) Operation of mixer.-The concrete shall be mixed in a batch mixer with the "boom and bucket" type of delivery. The capacity of the drum shall be such that only whole bags of cement are used in each batch. Mixing shall continue for at least 1 minute after all materials, including water, are placed in the drum, and before any part of the batch is discharged. The drum shall be revolved not less than 14 nor more than 18 revolutions per minute. The drum shall be completely emptied before receiving materials for the succeeding batch. The volume of the mixed material in each batch shall not exceed the mixer manufacturer's rated capacity of the drum.

45. The mixer shall be provided with a watermeasuring tank into which mixing water shall be discharged, having a visible gage so that the amount of water for each batch may be separately and accurately measured. The mixer shall be provided with an approved batch-timing device which will automatically lock the batch-discharging device during the full mixing time and release it at the end of the mixing period. The timer device shall have a bell which will automatically ring at the end of the mixing period. This device shall be subject to inspection and adjustment by the engineer at any time.

46. (b) Retempering.-Mortar or concrete which has partially set shall not be retempered by being mixed with additional materials or water. 
47. (c) Central mixing plants.-The use of central mixing plants and the transportation of mixed concrete is permitted under these specifications, provided there is no segregation of the mixed concrete when it is delivered at the point where it is to be deposited in the pavement. The period between mixing and placing in the pavement shall not exceed 40 minutes, and this period may be reduced at the direction of the engineer. The concrete must be of workable consistency when placed on the subgrade.

48. (d) Consistency. - The concrete mixture shall contain no more water than is necessary to produce a workable mass which can be brought to a satisfactory finish in the pavement. The amount of water used shall not exceed $6 \frac{1}{4}$ gallons per sack of cement, when the aggregates are dry.

\section{PLACING CONCRETE AND REINFORCEMENT}

\section{(A) INSPECTION OF SUBGRADE}

49. (a) Rechecking subgrade.-Immediately before placing concrete, or any type of reinforcement, the subgrade shall be rechecked by means of a scratch template as provided in paragraph 23 of these specifications, and any inequalities corrected as therein provided.

50. (b) Condition of subgrade.-Concrete shall be placed only on a moist subgrade, but there shall be no pools of standing water. If the subgrade is dry, it shall be sprinkled with as much water as it will absorb readily. The engineer may direct that the subgrade may be sprinkled or thoroughly wet down from 12 to 36 hours in advance of placing concrete, where such procedure may be deemed necessary.

\section{(B) PLACLNG REINFORCEMENT}

51. Steel fabric reinforcement of the size and weight shown on the plans, shall be placed 2 inches below and parallel to the finished surface of the pavement unless otherwise indicated. Fabric shall extend to within 2 inches of sides and ends of slabs. All laps of fabric sections shall be not less than threefourths of the spacing of members in the direction lapped. Steel bar reinforcement shall be placed 3 inches below the finished surface of the pavement unless otherwise indicated on the plans. Transverse bars shall extend to within 2 inches of the margins of the pavement. Bar reinforcement shall be placed and securely supported in correct position before any concrete is laid. All intersections of longitudinal and transverse bars shall be securely wired or clipped together to resist displacement during concrete operations.

\section{(C) PLACING CONCRETE}

52. The mixed concrete shall be deposited rapidly on the subgrade to the required depth and for the entire width of the pavement section, in successive batches and in a continuous operation without the use of intermediate forms or bulkheads between joints. While being placed, the concrete shall be vigorously sliced and spaded with suitable tools to prevent formation of voids or honeycomb pockets. The concrete shall be especially well spaded and tamped against the forms. When the concrete is placed in two horizontal layers to permit use of steel reinforcement, the first layer shall be roughly struck off with? a template or screed, riding on the side forms at the correct elevation to permit placing the reinforcement in specified position. The concrete above the reinforcement shall be placed within 15 minutes after the first layer has been placed. Any dust, dirt, or foreign matter which collects on the first layer shall be careifully removed before the upper layer is placed,
53. In case of a breakdown of the mixer, involving stopping operations for more than 40 minutes, a transverse joint shall be formed at the point directed by the engineer, to close the section. Any concrete in excess of that needed to complete a section, when work is stopped for more than 40 minutes, shall not be used in the pavement.

\section{(D) FINISHING}

54. (a) General.-Experienced and skillful workmen must be employed at all times for preparing the surface of the pavement. The concrete shall be brought to the specified contour by means of a heavy screed or template, fitted with handles, weighing not less than 15 pounds per linear foot. This screed or template may be of steel, or of wood shod with steel. It shall be shaped to the cross section of the pavement, and have sufficicnt strength to retain its shape under all working conditions. The template or screed shall rest on the side forms and shall be drawn ahead with a sawing motion. At transverse joints the template shall be drawn not closer than 3 fect toward the joint, and shall then be lifted and set down at the joint and drawn backwards away therefrom. Surplus concrete shall then be taken up with shovels and thrown ahead of the joint.

55. (b) Belting. -The concrete shall be finished by using a belt of wood, canvas, or rubber, not less than 6 nor more than 12 inches wide, and at least 2 inches longer than the width of the pavement. The belt shall be applied with a combined crosswise and longitudinal motion. For the first application vigorous strokes at least 12 inches long shall be used, and the longitudinal movement along the pavement shall be very slight. The second application of the belt shall be immediately after the water sheen disappears, and the stroke of the belt shall be not more than 4 inches and the longitudinal movement shall be greater than for the first belting.

56. (c) Machine finishing.-When a finishing machine is used it shall be so designed and operated as to strike off and consolidate the concrete, eliminating ridges and producing a true and even surface. The operation of the machine shall be so controlled as to keep the coarse aggregate near the finished surface of the pavement. Repeated operation of the machine over a given area is to be avoided.

57. A hand tamping template and belt must be kept for use in case the tamping machine breaks down.

58. (d) Longitudinal floating.-Immediately after the screeding specified under IX (D), $54(a)$ has been completed, the surface should be inspected for high or low spots and any needed corrections made by adding or removing concrete. Rough spots should be gone over with a long-handled float and worked to proper contour and grade. The entire surface shall then be floated longitudinally, with a float board not less than 16 feet long and 8 inches wide. This float board shall have convenient plow handles at each end. It shall be operated by two men, one at each end, each man standing on' a bridge spanning the pavement. The lower surface of the float board shall be placed upon the surface of the concrete with the long dimension parallel to the center line of the pavement. The float shall then be drawn back and forth in slow strokes about 2 feet long, and advancing slowly from one side of the pavement to the other. The purpose of this operation is to produce a uniform even surface on the concrete, free from transverse waves. The two bridges on which the workmen stand should be placed about 18 feet apart when the length of the float is $\mathbf{1 6}$ feet. When the entire width of the pavement has been floated in this manner from one position of the bridges, they shall be moved ahead about 12 feet so that the next 
section to be floated shall overlap the one previously so floated from 3 to 4 feet. After this floating has been completed, and all transverse waves eliminated, the surface shall be finished by the belting process specified in paragraph $\mathbf{5 5}$.

59. (e) Finishing at joints and tooling.-The contractor shall provide a suitable split float or split roller, having a slot to fit over expansion joints. This device shall be so arranged as to float the surface for a width of at least 3 feet on each side of the joint simultaneously. This device shall be used in such manner as to produce a true surface across the joint. Edges of the pavement, at joints and side shall be tooled for a width of 2 inches, the corners rounded to a radius of $1 / 4$ inch.

60. (f) Trueness of surface.-The finished surface of the pavement must conform to the grade, alignment, and contour shown on the plans. Just prior to the final finishing operation, the surface shall be tested with a light straight-edge, 10 feet in length, laid parallel to the center line of the pavement. Any deviation shall be immediately corrected.

61. The contractor shall be held responsible for the trueness of surface of the pavement, and shall be required to make good any deviation from the alignment, grade, and contour shown on the plans in excess of the tolerance stipulated in this section.

\section{CURING AND PROTECTION}

\section{(A) BURLAP COVER}

62. The contractor shall provide a sufficient amount of burlap or canvas for every mixer on the job, to cover all of the pavement laid in any one day's maximum run. Burlap or canvas cover shall be made up in sheets 12 feet wide, and 4 feet longer than the width of the pavement. Burlap or canvas cover shall be placed on the concrete immediately after the final belting, and shall then be sprayed with water in such a manner that the surface of the pavement will not be damaged. Burlap or canvas cover shall be kept continuously moist by spraying until the concrete has taken final set.

\section{(B) WET EARTH COVER}

63. As soon as it can be done without damaging the concrete, the surface of the pavement shall be covered with not less than 2 inches of earth or 6 inches of hay or straw. This cover shall be kept continuously wet by spraying for 10 days after the concrete is laid.

\section{(C) SPRINKIING OR PONDING}

64. The sprinkling system of curing may be used if approved. The sprinkling equipment shall be placed carefully, and without injuring the concrete surface. The sprinkling system shall be so arranged, and supplied with sufficient water at ample pressure, to keep every portion of the pavement surface continuously wet (both night and day) for 10 days after laying the concrete. Dikes shall be constructed along both edges of the pavement, with cross dikes where necessary, and the water flowing off the surface of the pavement shall be collected and led to the ditches or culverts as directed by the engineer. The contractor shall be held responsible for any damage to the roadway, shoulders, or adjacent property by reason of escaping water.

65 . The ponding system of curing may be used at the option of the contractor. Dikes shall be built along both edges of the pavement, with cross dikes at sufficiently frequent intervals, and the pavement flooded with sufficient water within the dikes to keep all portions of the pavement surface continuously covered with water for 10 days after the concrete is laid.

\section{(D) CLEANING}

66. After 14 days the earth or other cover may be removed. After 30 days the contractor may use a mormon or a fresno scraper to remove the cover, except that scrapers shall not be used within 1 foot of expansion joints. Cover within 1 foot of expansion joints must be removed by hand. Road machines, or blade graders of the 2 or 4 wheel type shall not be used for removing the cover.

67. After the cover has been removed, or ponds emptied and dikes removed, the entire surface of the pavement shall be swept clean and free from dirt and debris. Horse or motor drawn sweepers shall not be operated on the pavement till 30 days have elapsed after the concrete is placed.

\section{(E) COLD WEATHER PRCTECTION}

68. Concrete shall not be mixed nor deposited when the temperature is below freezing, except under such conditions as the engineer may direct in writing. If at any time during the progress of the work the temperature is, or in the opinion of the engineer will, within 24 hours, drop to $38^{\circ} \mathrm{F}$., the water and aggregates shall be heated and precautions taken to protect the concrete from freezing until it is at least 10 days old. In no case shall concrete be deposited upon a frozen subgrade, nor shall frozen materials be used in the concrete.

\section{PROHIBITION OF TRAFFIC}

\section{(A) BARRICADES}

69. The contractor shall provide and maintain substantial barricades across the pavement, with suitable warning signs by day and by night, to prevent traffic of any kind upon the pavement before it is 21 days old, or before the cover has been removed. The contractor shall provide and maintain watchmen at each mixer, whenever the paving crew is not at work, who shall prevent destruction or removal of barricades and keep traffic off the pavement.

70. No section of pavement shall be opened to traffic until written instructions have been given by the engineer.

\section{(B) CROSSINGS}

71. At public highway and private crossings the contractor shall provide suitable structures to carry the traffic across the pavement without injury to the concrete. All such structures shall be subject to the approval of the engineer, and he may direct their improvement, or repair, as conditions may require.

\section{CONDITION BEFORE ACCEPTANCE}

72. Before the road will be considered completed in accordance with these specifications, and acceptable to the engineer, the pavement, shoulders, ditches, back slopes, and structures shall be placed in a neat and orderly condition, conforming to the plans and specifications in all respects. Equipment, surplus materials, and construction débris of every description shall be removed from the right of way.

American Concrete Institute, standard specifications for two-course Portland cement concrete pavements for highways, serial designation S-6B-28, 1928.

\section{GENERAL}

1. It is the intent of these specifications to cover the requirements for materials and construction of Portland cement concrete pavement composed of two layers of concrete made with unlike coarse aggregates but of the same proportions. 


\section{MATERIALS}

2. The requirements for -
(A) Cement,
(B) Aggregates,

(a) Fine aggregate,

shall be as specified in Section II, paragraphs (A), (B), and $(a)$ of A. C. I., serial designation S $-6 \mathrm{~A}-28$ (given above).

3. (b) Coarse aggregate for bottom course.-Structurally sound material considered too soft for a pavement surface may be used as the coarse aggregate in the bottom course. It shall consist of crushed rock, pebbles (gravel), air-cooled blast-furnace slag, chatts, or tailings. The particles of coarse aggregate shall be of clean, durable material, free from vegetable or other deleterious substances and shall contain no flat or elongated pieces.

Note.-In many cases it will be necessary for the engineer to specify the sizes, grading, and quality of coarse aggregate in accordance with local conditions. In every case the engineer should provide specifica. tions which will require the use of the best coarse aggregate which is economically arailable. The following specifications covering size and grading of coarse aggregate will be found applicable in most sections of the country and are intended for use with proportions from $1: 2: 4$ to $1: 1-1 / 2: 3$.

4. The size of the coarse aggregate shall be such as to pass a 3 -inch round opening. Coarse aggregate shall be uniformly graded within the limits shown in the following table, and any material which does not come within the limits specified shall be rejected.

\begin{tabular}{l|r|rr}
\hline & Minimum & Maximum \\
\cline { 1 - 2 } & & \\
\hline Passing 3-inch round opening cent & Per cent \\
Passing 2-inch round opening & 100 & 82 & 95 \\
Passing 3/2-inch round opening & 15 & 25 \\
\hline
\end{tabular}

5. (c) Coarse aggregate for top course.- Shall consist of crushed rock, pebbles (gravel), air-cooled blast-furnace slag, chatts, or tailings. The particles of coarse aggregate shall be of clean, hard, tough durable material, free from vegetable or other deleterious substances, and shall contain no soft or elongated pieces. The crushed rock shall wear not more than 6 per cent. when subjected to the standard Deval abrasion test. When subjected to the abrasion test described in United States Department of Agriculture Bulletin 1216 (see 510, p. 187) pebbles shall show a loss of not more than 12 per cent.

6. The size of the particles shall be such that at least 95 per cent shall pass a 1 -inch round opening and not more than 5 per cent shall pass a $1 / 4$-inch sieve, with all the intermediate sizes retained.

7. The requirements for crushed rock, pebbles (gravel), air-cooled blast-furnace slag and chatts or tailings shall be as specified in Section II (B) (b) of A. C. I. specification, S-6A-28 (given above).

8. The requirements for-

(d) Mixed aggregate.

(C) Water,

(D) Reinforcement.

(E) Joint filler,

(F) Shoulders,

shall be as specified in Section II, (B), (C), (D), (E), (F) of A. C. I. serial designation S-6A-28.

\section{SUBGRADE}

9. The requirements for subgrade, including-

(A) Fine grading,

(B) Preparation and maintenance of subgrade,

(C) Checking and acceptance of subgrade,

(D) Special treatment, shall be as specified in Section III, (A), (B), (C), (D) of A. C. I. serial designation $\mathrm{S}-6 \mathrm{~A}-28$.

\section{FORMS}

10. The requirements for-
(A) Materials,

(B) Setting,

shall be as specified in Section IV, (A) and (B) of A. C. I., S $-6 \mathrm{~A}-28$.

\section{PAVEMENT SECTION}

11. Width and thickness of concrete pavement and the depth of the top and bottom courses shall be as shown on the plans for the improvement.

\section{JOINTS}

12. The requirements for joints, including -

(A) Transverse expansion joints,

(B) Longitudinal expansion joints,

(C) Transverse construction joints,

(D) Longitudinal construction joints,

shall be as specified in Section VI, (A), (B), (C), (D) of A. C. I., S-6A-28.

\section{WATER SUPPLY}

13. The requirements for -

(A) Equipment,

(B) Priority to water supply,

shall be as specified in Section VII, (A) and (B) of A. C. I., S-6A-28.

\section{PROPORTIONING AND MIXING CONCRETE}

\section{(A) PROPORTIONING}

14. (a) Measuring materials.-The method of measuring the materials for the concrete, including water, shall be such as to insure the required proportions of each of the materials as directed by these specifications. One sack of Portland cement (94 pounds net) shall be considered 1 cubic foot.

15. (b) Proportions. - The concrete in both the top and bottom course shall be proportioned 1 sack of Portland cement, not more than - - cubic feet of fine aggregate, and not more than - - cubic feet of coarse aggregate. A cubic yard of concrete in place, measured between neat lines, must contain -- barrels of Portland cement. The engineer shall compare the calculated amount of cement required by these specifications and tables with the amounts actually used in each section of concrete -- feet long, or between successive transverse joints. If the amount of cement actually used in the pavement varies from the specified amount by more than 3 per cent for any section, the engineer may require the proportions of the concrete to be adjusted so as to use the specified amount of cement. If it is found that the amount of cement used in any section is $92 \frac{1}{2}$ per cent or less, of the specified quantity, the contractor shall be required to remove such section or sections, and replace them with concrete made in accordance with these specifications. Such removal and replacement shall be done at the expense of the contractor.

16. The contractor may, at his option, construct the top course of mortar composed of cement and fine aggregate mixed in the proportion of 1 sack of cement to _- cubic feet of fine aggregate.

17. The requirements for-

\section{(B) MIXING}

(a) Operation of mixer,

(b) Retempering,

(c) Central mixing plants,

(d) Consistency, 
shall be as specified in Section VIII, paragraphs (B), $(a),(b),(c),(d)$ of A. C. I., S-6A-28.

\section{PLACING CONCRETE AND REINFORCEMENT}

18. The requirements for-

\section{(A) INSPECTION OF SUBGRADE}

shall be as specified in Section IX, (A) A. C. I., S-6A-28.

\section{(B) PLACING REINFORCEMENT}

19. Steel fabric reinforcement of the size and weight shown on the plans shall be placed between the bottom and top courses, unless otherwise indicated. Fabric shall extend to within 2 inches of sides and ends of slabs. All laps of fabric sections shall be not less than the spacing of members in the direction lapped. Steel bar reinforcement shall be placed between the top and bottom courses unless otherwise indicated on the plans. Transverse bars shall extend to within 3 inches of the margins of the pavement. Bar reinforcement shall be placed and securely supported in correct position before any concrete is laid. All intersections of longitudinal and transverse bars shall be seeurely wired or clipped together to resist displacement during concreting operations.

\section{(C) PLACING CONCRETE}

20. The mixed concrete shall be deposited rapidly on the subgrade to the required depth and for the entire width between longitudinal joints, without the use of intermediate forms or bulkheads between joints. While being placed the concrete shall be vigoroulsy sliced and spaded with suitable tools, to eliminate voids or honeycomb pockets. The concrete shall be especially well spaded and tamped adjacent to forms, bulkheads, and curbs. The bottom course shall be struck off at the correct elevation with a template, or screed riding on the side forms. The top course shall be placed within 15 minutes after the bottom course was placed. Any dust, dirt, or foreign matter which collects on the surface of the bottom course shall be carefully removed before the top course is placed.

21. Whenever, because of a breakdown or for any other reason operations will be stopped for more than 40 minutes, a transverse joint shall be formed at the point directed by the engineer, to elose the section. Both the top and bottom courses shall be completed to this joint. Any concrete in excess of that needed to complete a section, when work is stopped for more than 40 minutes, shall not be used in the pavement.

22. The requirements for-

\section{(D) FINISHLTG}

(a) General,

(b) Belting,

(c) Machine finishing,

(d) Longitudinal floating,

(e) Finishing at joints and tooling,

(f) Trueness of surface,

shall be as specified in Section IX, $(D)(a),(b),(c)$, $(d),(e),(f)$ of A. C. I., S-6A-28.

\section{CURING AND PROTECTION}

23. The requirements for-

(A) Burlap cover,

(B) Wet earth

(C) Sprinkling or ponding,

(D) Cleaning,

(E) Cold weathel protection, shall be as specified in Section X, (A), (B), (C), (D), (E) of A. C. I., S-6A-28.

\section{PROHIBITION OF TRAFFIC}

24. The requirements for-
(A) Barricades,

(B) Crossings,

shall be as specified in Section XI, (A) and (B) of A. C. I., S-6A-28.

\section{CONDITION BEFORE ACCEPTANCE}

25. The condition before acceptance shall be as specined in Section XII, of A. C. I., S-6A-28.

American Concrete Institute, standard specifications for one-course Portland cement concrete street pavement, serial designation S-6C-28, 1928.

\section{GENERAL}

1. It is the intent of these specifications to cover the requirements for the materials and construction of Portland cement concrete street pavement wherein the concrete is of uniform proportion from top to bottom of slab.

\section{MATERIALS}

2. Materials shall meet the requirements given in A. C. I. serial designation S-6A-28 (given above), under Section II in paragraphs-
(A) Cement,
(B) Aggregates,
(C) Water,
(D) Reinforcement,
(E) Joint filler.

\section{SUBGRADE}

3. Subgrade will be considered as that portion of the highway upon which the pavement is to be placed.

\section{(A) PREPARATION AND MAINTENANCE}

4. Preparation and maintenance shall meet the requirements given in III subgrade, paragraph (B) of A. C. I., S-6A-28.

\section{(B) CHECKING AND ACCEPTANCE}

5. Just before the concrete pavement is placed the subgrade shall be checked by means of an approved scratch template when the crown is secured by screeding or with stakes or T's when the crown is obtained by luting. All high places shall be removed to true grade and any low places filled with suitable material and rolled or hand tamped until smooth and firm. The subgrade shall be checked and completed in accordance with these requirements for a distance of not less than 100 feet in advance of the concrete. If hauling over the subgrade after it has been finished and checked as above specified results in ruts or other objectionable irregularities, the contractor shall reroll or hand tamp the subgrade and place it in smooth and satisfactory condition before the pavement is deposited upon it. If the condition of the subgrade is such that it can not be placed in satisfactory condition to receive the pavement, placing pavement may be stopped by the engineer unless the contractor can provide and haul over suitable trackways or use other means for the protection and maintenance of the subgrade.

\section{(C) AGGREGATES ON THE SUBGRADE}

6. Neither fine nor coarse aggregates shall be deposited upon the subgrade before the subgrade has been shaped and brought to the true finish. 


\section{(D) SPECIAL TREATMENT}

7. (Special treatment may be specified for certain subgrades, such as sand, gumbo, adobe, and other materials, which can not be satisfactorily prepared by the methods specified in the foregoing paragraphs.)

\section{ADJUSTING STREET STRUCTURES}

8. Manhole and catch basin covers, valve boxes, and similar existing structures within the area to be paved shall be adjusted by the contractor to come flush with the parement surface.

\section{FORMS}

\section{(A) MATERIAIS}

10. (a) Wooden forms shall be straight, dressed on at least one side, not less than 2 inches in thichness, and equal in width to the depth of the concrete which is to be placed against them. Wooden forms shall be held in place by stakes driven into the ground along the outside edge at intervals of not more than 6 feet, two stakes being placed at each joint. The forms shall be firmly nailed to the side stakes and braced to resist the pressure of the concrete or the impact of tamping.

11. (b) Metal forms shall be of shaped steel sections. They shall be straight, have a depth equal to the depth of the concrete to be placed against them, and sufficient strength to resist without springing the working strains to which they are subjected.

\section{(B) SETTING}

12. Forms shail be set to exact grade and alignment at least 200 feet in advance of the point where concrete is being deposited. Forms shall be thoroughly cleaned and oiled before concrete is placed against them. Forms in place will be subject to check and correction of line or grade at any time.

\section{JOINTS}

\section{(A) GENERAL}

13. The joints to be formed shall be transverse or longitudinal. Ther shall be tested during and after finishing with a 10-foot straightedge and any irregularities in the surface shall be immediately corrected.

14. All joints shall extend through the entire thickness of the pavement, shall be perpendicular to the surface of the pavement, and be edged to a radius of one-fourth inch.

\section{(B) TRANSVERSE EXPANSION JOINTS}

15. Transverse expansion joints shall be placed across the pavement perpendicular to the center line. They shall be three-eighths inch wide and spaced 35 feet apart between intersections. A bulkhead, cut to the exact cross section of the pavement, shall be securely staked in place at right angles to the center line and surface of the pavement. The premolded joint filler shall be placed against the bulkhead and held in position by pins on which there is an outstanding lug. Before the bulkhead is removed, concrete shall be deposited on both sides of it. After the concrete has been brought to the proper crown, the bulkhead shall be removed by lifting it slowly from one end and replacing it with concrete as it is lifted so that the joint filler will be left in the correct position.

16. When expansion joints are made at the end of the day's work they shall be formed by finishing the concrete to the bulkhead, placed as before speci- fied. When work is resumed the joint filler shall be placed against the hardened concrete and held in position by pins until fresh concrete is placed against it.

17. In parements with integral curb the joints shall be continuous in a straight line through pavement and curb.

18. Expansion joints in intersections shall be located as shown on the plans or as directed by the engineer.

19. Before the pavement is opened to traffic the joint filler shall be trimmed off to a uniform height of one-fourth inch above the surface of the pavement.

\section{(C) LONGITODINAL EXPANSION JOINTS}

20. The requirements for longitudinal expansion joints shall be as specified in VI, joints, paragraph (B) of A. C. I., S $6 \mathrm{~A}-28$.

\section{(D) TRANSVEESE CONSTRDCTION JOINTS}

21. The requirements for transverse construction joints shall be as specified in VI, joints, paragraph (C), of A. C. I., S-6A-28.

\section{(E) IONGITUDINAL CONSTRUCTION JOINTS}

22. These joints, located as shown on the plans, shall divide all pavements 30 feet or more in width.

When the whole width of the pavement is placed in one operation longitudinal joints shall be formed by embedding in the pavement a strip of galvanized or painted 18 gage, corrugated sheet metal 1 inch less in depth than the depth of the pavement at the joint. The metal shall be accurately staked to line and grade by means of pins driven vertically through holes provided for that purpose at not more than 4 foot intervals. The pins shall be of mild steel onefourth inch in diameter and at least 15 inches long, and shall be left in place. The metal strips may be of any length. Punched or cut holes shall be provided in the strips at 5 -foot intervals to receive $1 / 2$-inch round tie bars. The metal shall be carefully placed in the proper location and be vertical when the concrete is deposited about it.

23. When the pavement is built in successive longitudinal strips, longitudinal joints shall be formed by painting the edge of sections first built with bituminous paint and depositing the concrete in adjacent section against the painted edge.

24. Deformed bars having a sectional area of not less than 0.196 square inch and 4 feet long shall be placed at 5-foot intervals across all longitudinal joints extending 2 feet into the concrete on either side of the joint.

\section{(F) MANHOLE AND CATCH-BASIN COVERS}

25. Manhole and catch-basin covers and all other fixed objects in the pavement shall be separated from the concrete by joint filler.

\section{WATER SUPPLY}

\section{(A) FROM CITY MAINS}

26. Water taken from the city mains shall be paid for by the contractor at the rate of ...... per thousand gallons.

\section{(B) PRIORITY TO WATER SUPPLY}

27. The concrete pavement in place, for 10 days after laying, and subgrade preparation, shall have prior rights to the water supply. If it should develop that there is not sufficient water for all purposes the concrete mixer shall be shut down until the water needs of the curing and subgrading operations have been cared for. 


\section{PROPORTIONING AND MIXING CONCRETE}

28. The requirements for -

(A) Proportioning.

(B) Mixing.

(a) Measuring materials.

(b) Proportions.

(a) Operation of mixer,

(b) Retempering

(c) Central mixing plants,

(d) Consistency,

shall be as specified under VIII, Proportioning and Mixing Concrete, in A. C. I., S-6A-28.

\section{PLACING CONCRETE AND REINFORCEMENT}

\section{(A) INSPECTION OF SUBGRADE}

29. (a) Rechecking subgrade-Immediately before placing concrete, or any type of reinforcement, the subgrade shall be rechecked by the means provided in Section III (B) of these specifications.

30 . The requirements for-

(b) Condition of subgrade,

\section{(B) PLACING REINFORCEMENT}

shall be as specified in Section IX, (b) (B), A. C. I., $\mathrm{S}-6 \mathrm{~A}-28$.

\section{(C) PLACING CONCRETE}

Substantially the same as section IX, (C), in A. C. I., S-6A-28.

\section{FINISHING}

Same as IX (D) (a), A. C. I., S-6A-28, with the following in addition:

35. After the concrete has been struck off the template shall be used as a tamp. In this operation one end of the template shall rest on the side support while the other is lifted and dropped, advancing at such a rate that the whole pavement is struck at least once. The opposite end shall then be lifted and dropped and advanced in the same manner. In no case shall either end be advanced more than 1 foot ahead of the other.

36 . Or the correct pavement contour may be secured by the use of a lute. in that case steel grade stakes provided with lugs shall be driven into the subgrade with the top of the lugs accurately set at the elevation for the finished pavement. These stakes shall be set at intervals of 10 feet along the subgrade and in a straight line perpendicular to the center line of the pavement, one at the center line and one at at each quarter point and at as many additional points as the engineer may direct. The concrete shall then be spread to the elevation indicated by the stakes.

37. Cement mortar gathered from the surface of the concrete already placed shall not be used in filling boot tracks or stony areas, but such imperfections shall be dug out and refilled with concrete to the depth of the reinforcing and worked smooth. No workmen shall then be allowed to walk over the area so completed.

38. The requirements for-
(B) Belting,
(C) Finishing at joints and tooling,
(D) Longitudinal floating,
(E) Trueness of surface,

shall be as specified in Section IX, paragraphs (D), $(b),(d),(e),(f)$, of A. C. I., S-6A-28.

\section{CURING AND PROTECTION}

39. The requirements for-
(A) Burlap cover,
(B) Wet earth,
(C) Sprinkling,
(D) Ponding,

shall be as specified in Section $\mathrm{X},(\mathrm{A}),(\mathrm{B})$, and (C), of A. C. I., S-6A-28.

\section{(E) CLEANING}

42. The requirements for cleaning and for-

(F) COLD WEATHER WORK

shall be as specified in Section X, (D), and (E) of S $-6 \mathrm{~A}-28$.

\section{PROHIBITION OF TRAFFIC}

\section{(A) BARRICADES}

44. The requirements for barricades shall be as specified in Section XI, (A), of S-6A-28.

\section{CONDITION FOR ACCEPTANCE}

Substantialy the same as XII of A. C. I., S-6A-28.

American Concrete Institute, standard specifications for two-course Portland cement concrete street pavement, serial designation S-6D-28, 1928.

\section{GENERAL}

1. It is the intent of these specifications to cover the requirements for the materials and construction of Portland cement concrete street pavements composed of two layers of concrete made with unlike coarse aggregates but of the same proportions.

\section{MATERIALS}

2. The requirements for-
(A) Cement
(B) Aggregates,

(a) Fine aggregates,

shall be as specified in Section II, (A), (B), and (a) of A. C. I., serial designation 'S-6A'-28 (given above).

3. The requirements for-

(b) Coarse aggregate for bottom course,

(c) Coarse aggregate for top course,

shall be as specified in Section II, (b) and (c) of (B) in A. C. I., serial designation S-6B-28 (given above).

4. The requirements for-

Crushed rock,

Pebbles (gravel),

Air-cooled blast-furnace slag,

Chatts or tailings, and for-

(d) Mixed aggregates,

(C) Water,

(D) Reinforcement,

(E) Joint filler,

shall be as specified in Section II, of A. C. I., S-6A-28.

\section{SUBGRADE}

5. The requirements for subgrade, including-

(A) Preparations and maintenance of subgrade,

(B) Checking and acceptance of subgrade,

(C) Aggregates on subgrade,

(D) Special treatment,

shall be as specified in Section III, (A), (B), (C), (D), of A. C. I., S-6A-28. 


\section{ADJUSTING STREET STRUCTURES}

6. Manhole and catch-basin covers, valve boxes, and similar existing structures within the area to be paved shall be adjusted by the contractor to come flush with the parement surface.

\section{FORMS}

7. The requirements for-
(A) Materials,
(a) Wooden forms,
(b) Metal forms,

(B) Setting

shall be as specified in Section V, of A. C. I., S-6C-28 (given above).

\section{JOINTS}

8. The requirements for-

(A) General,

(B) Transverse expansion joints,

(C) Longitudinal expansion joints,

(D) Longitudinal construction joints,

(E) Manhole and catch-basin covers, shail be as specified in Section VI, (A), (B), (C), (E), and $(\mathrm{F})$, of A. C. I., S-6C-28.

\section{WATER SUPPLY}

9. The requirements for-

(A) Water taken from the city mains,

(B) Priority to water supply, shall be as specified in Section VII, of A. C. I., S $-6 \mathrm{C}-28$.

\section{PROPORTIONING AND MIXING CONCRETE}

10. The requirements for-

(A) Measuring materials,

(B) Proportions,

(C) Mixing

(a) Proportioning concrete with mixed aggregates,

(D) Retempering,

(E) Central mixing plants,

(F) Consistency,

shall be as specified in Section VIII, of A. C. I., S-6B-28.

\section{PLACING CONCRETE AND REINFORCEMENT}

11. The requirements for-

(A) Inspection of subgrade,

(B) Condition of subgrade,

C) Placing reinforcement,

shall be as specified in Section IX, (A), (B) of A. C. I., S-6B-28.

\section{(D) PLACING CONCRETE}

Substantially the same as IX (C), A. C. I., S-6A-28.

\section{FINISHING}

14. The requirements for-

(A) Leveling surface,

shall be as specified in Section $\mathrm{X}$, (A) of A. C. I., S-6C-28.

The requirements for-

(B) Belting

(C) Longitudinal floating,

(D) Finishing at joints and tooling,

(E) Trueness of surface,

shall be as specified in Section IX, paragraphs $(\mathrm{D}),(b),(d),(e),(f)$, of A. C. I., S-6A-28.

\section{CURING AND PROTECTION}

15. The requirements for-

(A) Burlap cover

(B) Wet earth,

shall be as specified in Section $X,(A)$, and (B) of A. C. I., S-6A-28.

16. The requirements for-

(C) Sprinkling,

(D) Ponding,

(E) Cleaning,

(F) Cold weather work,

shall be as specified in Section $\mathrm{X},(\mathrm{C}),(\mathrm{D}),(\mathrm{E})$ of A. C. I., S-6A-28.

\section{PROHIBITION OF TRAFFIC}

17. The requirements for-

(A) Barricades,

shall be as specified in Section XI, of A. C. I., S-6A-28.

\section{XUI. CONDITION FOR ACCEPTANCE}

18. The requirements are substantially the same as in Section XII, A. C. I., S-6A-28.

American Society for Municipal Improvements, specifications for Portland cement concrete pavements, February 1, 1926.

Introduction.- These specifications are intended to cover average conditions for concrete and reinforced concrete pavements. Throughout the specifications certain minimum requirements, which have been known to give satisfactory results for average conditions, are used. For other conditions of traffic and for unusual conditions of subgrade and drainage it will be necessary for the engineer to make provisions for such conditions by increasing the requirements for the thickness of pavement and the quantity of reinforcement.

\section{RECOMMENDATIONS ON DESIGN}

The principles of the design of concrete pavements as outlined herein have been evolved gradually from independent sources by observation, experiment, and theoretical considerations, and are assembled in this section for the convenience and guidance of the engineer. While the equations are based on sound physical principles, they are in reality only reasonably safe approximations when applied to concrete slabs.

1. Pavement cross section.-It is recommended that the thickness of all pavement slabs for a distance of at least 2 feet from any unsupported edge over which maximum wheel loads may pass be not less than that determined by the following equation:

$$
t=\sqrt{\frac{3 P}{f}}
$$

in which $t=$ thickness of slab in inches.

$P=$ maximum wheel load in pounds.

$f=$ allowable fiber stress of concrete in tension, pounds per square inch.

The maximum wheel load to be provided for is one-third of the total weight of the loaded truck, on the assumption that two-thirds of the total load is distributed over the rear axle and that the tread of each rear wheel bears one-half of the rear axle load. The allowable fiber stress of the conerete should not be greater than one-half of the modulus of rupture. The modulus of rupture should be determined on test specimens of the concrete as mixed for the work. 
For concrete complying with the minimum requirements of these specifications (Sec. V, pars. 16 to 19), a modulus of rupture not greater than $500 \mathrm{lbs} . / \mathrm{in.}{ }^{2}$ may be assumed, so that $f$ would be $250 \mathrm{lbs} . / \mathrm{in} .^{2}$ The thickness of the slab at a distance of 4 feet or more from an unsupported edge may be reduced to seven-tenths of $t$, or the thickness may be represented by a double parabolic curve decreasing from $t$ at the unsupported edge to seven-tenths of $t$ at the center.

2. Width and length of pavement slabs.-The width of the pavement is necessarily determined by traffic and economic conditions. It is lecommended that when conditions do not justify the paving of the whole area of the street between curbs the minimum width of pavement shall be 20 feet. It is recommended that the maximum size of slabs of plain concrete shall be 20 feet in width by 25 feet in length, and of reinforced concrete 20 feet in width by 60 feet in length.

3. Reinforcement.-It is recognized that one of the functions of steel in pavement slabs is to prevent the widening of contraction cracks, and it is believed that the steel may also aid in the prevention or diminution of cracks formed during the period of curing. The relief of the first condition may be accomplished by using sufficient steel, so that when contraction of the slab takes place the steel across a crack or joint, located midway between the edges or joints, will not be over stressed because of the friction developed between the pavement and subgrade. It is recommended, therefore, that the area of both transverse and longitudinal steel be proportional in accordance with the following equation,

$$
A s=\frac{0.5 l p c}{f s}
$$

in which $A s=$ effective cross-sectional area of steel in square inches per foot of length or width of section.

$l=$ distance between transverse joints when computing longitudinal steel or the distance between longitudinal joints when computing transverse steel.

$p=$ weight of concrete slab per square foot.

$f s=$ allowable stress of steel used.

$c=$ the coefficient of friction of concrete on the subgrade soil (a coefficient of 2.0 is recommended). (See Goldbeck, "Friction Tests of Concrete on Various Sub-bases," Highway Engineer and Contractor, August, 1924, p. 29.)

If a longitudinal joint with bonded dowel bars is used, $l$ should be taken as the full width of the pavement or the width between longitudinal joints having no such bonded dowels. In other words, $l$, in any case, should be taken as the distance between margins where free contraction may occur. It is recommended that the allowable working stresses shall not cxceed $25,000 \mathrm{lbs} . / \mathrm{in} .^{2}$ for wire fabric or $18,000 \mathrm{lbs}$./in. ${ }^{2}$ for deformed bars.

(a) Fabric reinforcement.-Parallel members of the fabric shall be spaced not less than 4 nor more than 12 inches. No member shall have an effective area of less than 0.028 of a square inch. Adjacent sheets shall be lapped not less than one-half of the width of the spacing, provided that, in all cases, the edge members of each sheet parallel to the lap shall overlap at least 1 inch.

(b) Bar mats. - No member shall have an effective cross-sectional area of less than 0.11 of a square inch. Members shall be spaced not more than 18 inches apart in either direction. Bars shall be lapped not less than 30 diameters.

\section{MATERIALS}

4. Cement.-See A. S. T. M., C9, 516.11, page 294.

5. Concrete aggregates.-See A. S. T. M., C33-28T, 512.13, page 237. Crushed stone shall have a per cent of wear of not more than 6.5 as determined by A. S. T. M., D2. (See 510, p. 182.)

6. Water.-Water for concrete shall be clean and frce from injurious amounts of oils, acids, alkalis, or other deleterious substances. Test specimens of mortar made from the materials and the water to be used in the work shall develop a tensile or compressive strength at seven days not less than that developed by the same materials and distilled water.

7. Reinforcement.-Reinforcing materials shall be either cold-drawn steel wire fabric or deformed steel bars, structural grade.

(a) Wire fabric shall consist of sheets or strips manufactured for the purpose. The steel wire shall comply with the standard specifications for cold-drawn steel wire for concrete reinforcement, serial designation A82 of the American Society for Testing Materials. Intersecting members shall be rigidly welded at right angles in such a manner as to develop the full tensile strength across the weld.

(b) Deformed bars shall be made by either the Bessemer or the open-hearth process, and shall be rolled from new billets. No rerolled material will be accepted. The chemical properties, tensile properties, and bend-test requirements shall conform to the standard specifications for billet-steel concrete reinforcement bars, serial designation A15-14, structural grade, of the American Society for Testing Materials.

8. Joint fillers shall be either semisolid bituminous compounds which shall be heated and poured into the joints or premolded bituminous composition strips. Poured fillers.-(a) Oil asphalt.-Similar to A. S. T. M. D241-26T, 505.15, page 150 .

(b) Asphalt grout shall consist of an intimate mixture of asphalt and fine sand. "The asphalt shall comply with paragraph $8(d)$ of these specifications. The sand shall pass a 10-mesh sieve. The materials shall be heated separately to between $300^{\circ}$ and $400^{\circ} \mathrm{F}$. The hot sand shall be run into a measured volume of hot asphalt. The volume of sand shall not exceed the volume of asphalt, and, in general, volume proportions of 3 parts of asphalt to 2 parts of sand will will be found satisfactory. The contents of the mixing receptacle shall be thoroughly mixed with a rake or hoe.

(c) Asphalt mastic shall be composed of an intimate mixture of from 30 to 50 per cent of limestone dust and from 50 to 70 per cent of asphalt by weight. The asphalt shall comply with paragraph $8(d)$ of these specifications. The limestone dust shall be free from lumps or balls or any foreign material; not less than 85 per cent shall pass a 100 -mesh sieve and not less than 65 per cent shall pass a 200-mesh sieve. The asphalt mastic may be either prepared on the job or shipped ready for use. When it is prepared on the job the ashalt shall be heated to between to $350^{\circ}$ and $400^{\circ} \mathrm{F}$., and the proper amount of limestone dust then added cold. The mixture shall then be mixed by agitation with $a$ spade or hoe.

(d) Asphalt for grout or mastic shall be homogeneous and free from water. It shall meet the following requirements for physical or chemical properties:

I. Penetration at $25^{\circ}$ C. $\left(77^{\circ}\right.$ F.) $100 \mathrm{~g}$, five seconds, 85-100. Material delivered under one contract shall not vary more than 10 points either way from the penetration called for by the engineer.

II. Flash point (open-cup), not less than $177^{\circ} \mathrm{C}$. $\left(350^{\circ}\right.$ F.).

III. Loss at $163^{\circ}$ C. $\left(325^{\circ}\right.$ F.) in five hours, not more than 3 per cent. Reduction in penetration of 
residue at $25^{\circ} \mathrm{C} .\left(77^{\circ} \mathrm{F}.\right), 100 \mathrm{~g}$, five seconds, not more than 50 per cent of the original penetration.

IV. Ductility at $25^{\circ} \mathrm{C}$. $\left(77^{\circ} \mathrm{F}\right.$.), not less than $30 \mathrm{~cm}$.

V. Proportion of bitumen soluble in carbon tetrachloride, not less than 99 per cent.

Premolded joint filler.-(e) The strips of filler shall correspond in thickness to the width of joint specified; the width of the strips shall be at least 1 inch greater than the depth of the pavement and the length shall be not less than 4 feet. The joint strips shall be of such character that they will not be deformed by ordinary handling during the hot summer months or become hard and brittle in cold weather. Thin strips of stiffener will be allowed. The bitumen shall be uniformly impregnated with suitable filler, to reduce the brittleness at low temperatures to a minimum. The premolded joint filler shall comply with the requirements under the heading "Physical properties" of American Association of State Highway Officials specification for premolded expansion joints. (See 505.15, p. 150.)

\section{PAVEMENT SECTION}

10. Width, thickness, and crown of the pavement shall be as shown on the plans for the improvement. In no case shall the thickness of concrete pavement be less than 6 inches.

\section{FORIS}

11. General.-Steel forms shall be used when practicable. Steel or wooden forms shall be free from warp and of sufficient strength to resist springing out of shape. If wooden forms are used they shall be capped with a metal strip. Forms once used shall be thoroughly cleaned before being used again.

12. Setting.-Forms shall be well staked on hubs placed not more than 4 feet on centers and driven well into the ground so that the forms shall hold firmly to established line and grade. The inside of forms shall be coated with mineral oil or thoroughly wetted before depositing concrete. Special precautions shall be taken to prevent the leakage of mortar through or under the side forms.

13. Removal.-Forms shall not be disturbed until the concrete is adequately hardened, and in no case shall the forms be removed in less than 24 hours after the concrete has been deposited.

\section{PROPORTIONS}

14. Cement and aggregates.-The proportions shall be $1 \mathrm{bag}$ of cement to - parts by volume of fine aggregate to - parts by rolume of coarse aggregate as specified by the engineer.

15. The quality of the concrete shall be measured by its workability as determined by the slump test or other accepted tests for workability, and by its compressive strength at 28 davs as determined by compression tests of the concrete to be used, or by other metliods of test which may be adopted by the American Society for Testing Materials for the determination of quality of concrete in road slabs.

16. The proportions shall not be less than 1 part by volume of cement to 2 parts by volume of fine aggregate to 4 parts by volume of coarse aggregate.

17. The compressive strength of the concrete mixed in the proportion $1: 2: 4$ shall be not less than 2,500 lbs./in. ${ }^{2}$ at 28 days. The test specimens shall be made in accordance with A. S. T. M. C31. (See 516.0, p. 282.)

18. For concrete designed and mixed in proportions other than $1: 2: 4$ the compressive strength shall be specified by the engineer in charge of the work.

19. Water.-The proportion of water to be used shall be determined by the slump test, in A. S. T. M.
D138. (See 516.0, p. 286.) The quantity of water used shall be such tliat the maximum slump shall not exceed:

Hand finished pavement

Inches Machine finished pavement

\section{IHIXING CONCRETE}

20. In handling the measuring materials for the concrete, including water, methods shall be used which will insure complete separation of the materials at all times until the materials are brought together in the mixer. Cement shall be either emptied directly from bags into the charging skip or conveyed to the mixer in compartments separated from the aggregate and provided with a water-tight cover. Aggregates shall not be piled on the prepared subgrade. The unit of measure shall be the cubic foot of 94 pounds of cement (1 bag or onefourth barrel shall be considered as 1 cubic foot). The methods of handling and measuring shall be such as to insure uniform proportions throughout the work.

21. Mixing.-The mixing of concrete shall be done in a batch mixer of approved type which will insure a uniform distribution of the materials throughout the mass so that the mixture is uniform in color and homogeneous. The mixer shall be eouipped with suitable charging hopper, waterstorage tank, and a water-measuring device controlled from a case which can be kept locked and so constructed that the water can be discharged only while the mixer is being charged. It shall also be equipped with an attachment for automatically locking the discharge lever until the batch has been mixed the required time after all materials are in the mixer. The entire contents of the drum shall be discharged before recharging The mixer shall be cleaned at frequent intervals while in use. The volume of the mixed material per batch shall not exceed the manufacturers' rated capacity of the mixer. The mixing of each batch shall continue not less than one minute after all the materials are in the mixer.

22. Retempering.-Retempering of mortar or concrete which has partially hardened-that is, remixing with or without additional materials or water-will not be permitted.

\section{DEPOSITING CONCRETE}

23. General.-Immediately prior to placing the concrete, the subgrade shall be brought to an even surface and checked with an approved templet before any concrete is laid. The subgrade shall be thorcughly wetted from 6 to 12 hours before placing concrete to prevent absorption of water from the concrete, but shall show no pools of water where the concrete is being laid.

24. Placing reinforcement. ${ }^{75}$-If steel reinforcement is used it shall be of the effective area and spacing shown on the plans for the improvement. The reinforcement shall be so placed that it shall be 2 inches below and parallel to the finished surface of the pavement and shall extend to within 2 inches of the sides and ends of the pavement slabs.

(a) Fabric reinforcement shall be placed on a first layer of conerete which has been struck off at the correct elevation to permit the location of the fabric in the position specified. Adjacent sheets shall be lapped not less than one-half of the width of the spacing, provided that, in all cases, the edge members of each sheet parallel to the lap shall overlap at least 1 inch.

75 The use of reinforcement is optional with the engineer. If reinforcement is not specified the sections between brackets should be omitted. 
(b) Bar mats shall be made up in sections of the proper length and width and shall be placed before any concrete is deposited. The bars shall be free from scale, oil, excessive rust, or other deleterious substances. In the construction of the mat, accuracy of bending is essential Members shall be fastened together at each intersection by means of a spring steel clip or other device which will not loosen or permit the bar to rotate. Bars shall be rigidly supported on approved chairs, bends, or stirrups. Bars shall be lapped not less than 30 diameters.

25. Depositing. - The mixed concrete shall be deposited rapidly on the subgrade to the required depth and for the entire width of the pavement section, in successive batches and in a continuous operation, without the use of intermediate forms or bulkheads between joints. While being placed the concrete shall be vigorously sliced and spaded with suitable tools to prevent the occurrence of voids or honeycomb pockets. The concrete shall be especially well spaded and tamped against the forms When the concrete is placed in two horizontal layers to permit use of steel fabric, the first layer shall be roughly struck off with a templet or screed, riding on the side forms, at the correct elevation to permit placing the reinforcement in specified position. The concrete above the reinforcement shall be placed within 15 minutes after the first layer has been placed. Any dust, dirt, or foreign matter which collects on the first layer shall be carefully removed before the upper layer is placed.

26 . In case of a breakdown of the mixer, involving stopping operations for more than 40 minutes, a transverse joint shall be formed at the point directed by the engineer. Any concrete in excess of that needed to form this joint shall not be used in the pavement.

27. Freezing.-In no case shall concrete be placed upon a frozen subgrade nor shall it be mixed and placed when the atmospheric temperature is below ireezing. If at any time the air temperature is likely to drop to or is already $38^{\circ} \mathbf{F}$., the concrete shall not be mixed or deposited unless the mix can be brought to a temperature of not less than $50^{\circ} \mathrm{F}$. nor more than $100^{\circ} \mathrm{F}$. Concrete shall be maintained at a temperature of not less than $50^{\circ} \mathrm{F}$. for a period of not less than 72 hours after placing.

28. Transverse expansion joints.-Transverse joints shall be formed perpendicular to the surface of the pavement and at right angles to the axis of the pavement, at the end of any run, and at such points as may be indicated on the plans, provided that no section shall be less than $\mathbf{1 5}$ feet in length. The joints shall be not less than one-half inch and not more than three-fourths inch in width and shall extend the full width and depth of the slab. The joints shall be filled either with poured asphaltic filler or with premolded composition strips set in place.

(a) Poured filler shall be applied after the concrete has acquired its final set. The joint opening must be free from all foreign material, open to the full depth, and the faces shall be clean and dry when the hot filler is applied. The poured filler shall have a temperature of between $325^{\circ}$ and $400^{\circ} \mathrm{F}$. when poured into the joint. As the filler cools and subsides in the joint additional material shall be added in order that the joint will be flush full when the poured filler has cooled to the prevailing atmospheric temperature.

(b) Premolded joint strips shall be set in place before the concrete is deposited. They shall be held against a rigid form so that the concrete can be worked against them without displacement and the form shall be such that it can be removed without lifting the joint from the subgrade.

29. Longitudinal expansion joints.-Longitudinal joints shall be formed perpendicular to the surface and parallel to the axis of the pavement. The width of the joints and the method of joint filling shall be the same as specified for transverse expansion joints.

30. Construction joints-that is, joints built in the pavement for any other purpose than that of providing for the simple forces of expansion and contraction-shall be butt joints, dowel joints, or metal strip tongue-and-groove joints, as shown on the plans for the improvement.

\section{FINISHING CONCRETE}

31. Finishing, general.-The concrete pavement shall be finished either (a) by screening and belting, (b) by a finishing machine, or (c) by luting and belting or floating.

$\left(a_{1}\right)$ Screeding. - The concrete shall be brought to the specified contour by means of a heavy screed or templet, fitted with handles, weighing not less than 15 pounds per linear foot. This screed or templet may be of steel or of wood shod with steel. It shall be shaped to the cross section of the pavement and have sufficient strength to retain its shape under all working conditions. The templet or screed shall rest on the forms and shall be drawn ahead with a sawing motion to within not less than 3 feet of the next transverse joint, when it shall be lifted, set down at the joint, and drawn backward away therefrom. Surplus concrete shall then be taken up with shovels and thrown ahead of the joint.

$\left(a_{2}\right)$ Belting.-The concrete shall be finished by using a belt of wood, canvas, or rubber, not less than 6 nor more than 12 inches wide and at least 2 feet longer than the width of the pavement. The belt shall be applied with a combined crosswise and longitudinal motion. For the first application vigorous strokes at least 12 inches long shall be used, and the longitudinal movement along the pavement shall be very slight. The second application of the belt shall be immediately after the water sheen disappears; the stroke of the belt shall not be more than 4 inches and the longitudinal movement shall be greater than for the first belting.

(b) Machine finishing.-When a finishing machine is used it shall be so designed and operated as to strike off and consolidate the concrete, eliminating ridges and producing a true and even surface. Tamping that will produce a mortar top will not be permitted. A heavy screed or templet and belt must be kept for use in case the tamping machine breaks down.

(c) Luting.-In case the pavement is of such width that a screed can not be used, the concrete may be placed by means of a lute or a solid rake, stakes being set not more than 5 feet apart with their tops at proper elevation. The stakes shall be removed after the surface has been properly formed and the holes filed with concrete and tamped. After luting, the pavement shall be finished either by belting, as described in paragraph $31\left(a_{2}\right)$, or by floating.

32. During the operation of screeding or luting the concrete adjacent to the side forms shall be spaded with a heavy fork or a special spade with 2-inch round holes in the blade to push large aggregate a way from the forms and to work the mortar into contact with the side forms, thus preventing the occurrence of porous concrete on the sides of the section.

33. Surface testing.-After the final finishing operation the surface shall be tested with a 10-foot straightedge, laid parallel to the center line of the pavement. Any irregularity in the surface exceeding one-fourth inch shall be corrected immediately.

34. Brooming.-The surface of the pavement shall be broomed at right angles to the axis of the pavement if it is so directed by the engineer. An ordinary house broom may be used. The brooming 
shall commence when the concrete has acquired such consistency as to retain the impression of the broom marks, which shall be from one-sixteenth to oneeighth of an inch deep. During the brooming the brooms shall be kept free from adherent mortar by frequent rinsing in clean water.

\section{CURING AND PROTECTION}

35. Curing.-(a) Immediately after the final finishing and checking of the surface the entire surface of the pavement shall be covered with wetted burlap which shall be kept wet until replaced by at least 2 inches of earth or 6 inches of hay, marsh grass, or straw, which shall be kept wet for at least 10 days. Material containing manure shall not be used.

(b) Ponding.- The system of ponding under water for 10 days may be used as a method of curing, if local conditions warrant the specification of this method by the engineer.

(c) Sprinkling.-Automatic sprinkling by means of pipe lines under pressure and lawn sprinklers throwing a fine spray, properly spaced to cover the pavement, may be used as a method of curing, provided the pavement be kept moist for a period of at least 10 days.

36. Rain.-During rainy weather green concrete shall be protected by canvas supported above the surface until the concrete has hardened.

37. Duration of curing and hardening.-In hot weather the pavement shall be closed to traffic for at least 14 days and in cool weather for such additional time as may be considered necessary by the engineer.

38. Protection.-The contractor shall erect and maintain suitable barriers to protect the pavement from all traffic, and any part of the pavement damaged from traffic or other causes occurring prior to its official acceptance shall be repaired or replaced by the contractor at his own expense. Before the pavement is opened to traffic the covering shall be remored and disposed of as directed by the engineer.

(The full tcxt of this specification includes appendixes containing specifications for subgrade-preparation and maintenance.)

National Slag Association, recommended specifications for slag to be used in highways and highway structures, 1927.

See 512.2, page 256 .

\subsection{Gravel Pavements.}

American Society for Municipal Improvements, specifications for broken stone and gravel roads, 1922. See 512.15 , page 245 .

American Society for Testing Materials, tentative specifications for commercial sizes of sand and gravel for highway construction, serial designation D64-20 T, 1920.

See 512.10 , page 215 .

American Society for Testing Materials, standard method of test for quantity of clay and silt in gravel for highway construction, serial designation D72-21, 1921.

See 512.11 , page 218.

Federal Specifications Board, specification No. 453, United States Government master specification for gravel for gravel roads, January 25, 1927.

See 512.11, page 218 .

\subsection{Macadam Pavements.}

American Society for Municipal Improvements, standard specifications for asphalt macadam pavement, 1927 .

See 518.37, page 402 .

Asphalt Association, specification A-1, for asphalt macadam surface course (penetration method), June 24, 1925. E

See 518.37, page 403.
National Slag Association, recommended specifications for slag to be used in highways and highway structures, 1927.

See 512.2 , page 256 .

\subsection{Bituminous Macadam and Concrete Pavements. (See also .505.34.)}

American Society for Testing Materials, standard specifications for broken slag for bituminous concrete (coarse-graded aggregate type), serial designation D160-27, 1927.

See 512.2 , page 254 .

American Society for Testing Materials, standard specifications for broken slag for bituminous concrete (fine-graded aggregate type), serial designation D161-27, 1927.

See 512.2, pagc 255.

American Society for Testing Materials, tentative specifications for broken stone for bituminous macadam, serial designation D192-29T, 1924.

See 512.15 , page 250 .

American Society for Testing Materials, tentative specifications for broken stone for bituminous concrete surface, serial designation D194-29, 1924.

See 512.15 , page 251 .

Federal Specifications Board, specification No. 457, United States Government master spccification for broken stone and broken slag for bituninous macadam basc or surface course, January 25, 1927.

See 512.15, page 251 (for broken stone), and 512.2 , page 255 (for broken slag)

Federal Specifications Board, specification No. 458, United States Government master specification for broken stone and broken slag for bituminous concrete base or surface course, January 25, 1927.

See 512.15, page 251 (for broken stone), and 512.2, page 255 (for broken slag).

National Slag Association, recommended specifications for slag to be used in highways and highway structures, 1927

See 512.2 , page 256 .

\subsection{Brick Pavements. (See also 534.1.)}

American Society for Municipal Improvements, specifications for brick pavements, 1921.

(The requirements for paving brick and the methods of test included in this specification are similar to A.S.

T. M. serial designation C7-29T, 534.11, p. 577.)

American Society for Testing Materials, standard specification for paving brick, serial designation C7-29T, 1929.

See 534.11, page 577 .

American Society for Testing Materials, standard specifications.for cement grout filler for brick and stone block pavements, serial designation D57-20, 1920.

See 512.16, page 253 .

Federal Specifications Board, specification No. 505, United Statcs Government master specification for brick, sand-lime, common, July 11, 1927.

See 513 , page 257 .

National Paving Brick Manufacturers Association, recommended specifications, vitrified paving brick.

See 534.11, page 585 .

United States Department of Commerce, Bureau of Standards, Simplified Practice Recommendation No. 38 , for sand-lime brick.

See 513, page 258 .

\subsection{Stone Block Pavements.}

American Society for Municipal Improvements, specifications for stone block pavements, 1927.

\section{STANDARD SPECIFICATIONS FOR STONE BLOCK PAVING}

1. Description.-This pavement shall consist of stone blocks and sand cushion, or cement-sand bed 
paved on the previously constructed concrete base course. The type of block and character of joint filler and material for the cushion or bed shall be as designated in the proposal.

\section{NEW GRANITE BLOCKS}

2. Quality of granite.-The blocks shall be of granite of medium-size grain, showing an even distribution of constituent minerals. They shall be of uniform quality and texture throughout and free from seams or disintegrated materials.

The quality of granite shall conform to the requirements for class A or class B, for extra-heavy or heavy traffic, respectively, as designated in the proposal:

Class $A$ :

French coefficient

(Percentage of wear Class $B$ :

rench coefficient

(Percentage of wear

Toughress. Not less than 11. Not less than 9 .

Not less than 8 Not more than 5.0 ).

The average of three tests shall be used for determining both the perccntage of wear and toughness.

3. Size and dressing of blocks. - The blocks shall be of the following dimensions: Not less than 8 nor more than 12 inches long on top, not less than $43 / 4$ nor more than $5 \frac{1}{4}$ inches deep, and the width on the top shall be either $3 \frac{1}{2}$ to $4^{1 / 2}$ inches or $4^{1 / 2}$ to $5^{1 / 2}$ inches.

The blocks shall be so dressed that the faces will be approximately rectangular in shape and the ends and sides sufficicntly smooth to permit the blocks to be laid with joints not exceeding one-half inch in width at the top and for 1 inch downward therefrom, and not exceeding 1 inch in width at any other part of the joint. The top surface of the block shall be so cut that there will be no depressions measuring more than three-eighths of an inch from a straight edge laid in any direction on the top and parallel to the general surface thereof.

\section{RESURFACING BLOCKS}

4. Size and character.-Resurfacing blocks for use on old concrete foundations where less than the standard depth of blocks must be used shall be 7 to 11 inches long, $3 \frac{3 / 4}{4}$ to $4 \frac{1}{4}$ inches wide, and $3 \frac{1}{2}$ to 4 inches deep, and shall in all other respects meet the requirements as to quality and dressing for new granite paving blocks.

\section{NEW SAN DSTONE BLOCRS}

5. Quality, size, and dressing.-The paving blocks shall be of sound, hard sandstone free from clay, seams, or defects which would injure them for paving purposes, of uniform quality and texture.

They shall be quarried from fine-grained live rock, showing a straight and even fracture and shall be made by splitting and breaking from large quarried blocks and not by redressing old paving blocks.

The blocks shall be of the following dimensions: Not less than 8 nor more than 10 inches long; not less than $3 \frac{1}{2}$ nor more than 5 inches wide on top; not less than 5 nor more than $5 \frac{1}{2}$ inches dcep.

These blocks shall conform in character of dressing to the requirements for granite blocks in section 3 .

\section{DURAX PAVING BLOCKS}

6. Quality, size, and dressing.-Durax paving blocks shall be eut from granite complying with the requircments specified herein for new granite paving blocks.

The blocks shall be cubes of granite with six approximately square surfaces, the edges of which measure not more than 4 nor less than 3 inches in length. They shall be dressed so as to conform with the requirements specificd for new granite blocks except as to form and dimensions.

7. Method of paving.-They shall be laid as specified for ncw granite blocks except that the courses shall be laid in circular concentric ares with the largest blocks at the center of the arc and the smaller ones at the springing line. The radii of the ares may be varied from 3 to 7 feet.

\section{SAMPLING AND INSPECTION OF BLOCKS}

8. Preliminary samples and certificates.-Contractors shall file with the engineer at or before the time of bidding, a certificate showing the name and location of the quarry or quarries from which it is proposed to obtain the blocks, together, in the case of granite blocks, with a copy of a report or reports from an approved laboratory showing results of tests for toughness and per cent of wear. The contractor shall further file with the engineer, prior to the letting, six specification blocks representing the stone proposed for use, except that such samples will not be required where a previous sample of the same blocks has been filed and approved.

9. Sampling and inspection of shipments.-Samples selected from shipments for physical tests by the engineer or his representative shall consist of not less than six blocks so chosen as to fairly represent actual deliveries. No samples shall include blocks that would be rejected by a visual examination, and the bedding plane shall be marked on at least two of the blocks selected.

All deliveries shall be subjected to further inspcction at the place of use prior to and during laying, and blocks which fail to conform to the requirements of these specifications shall be rejected.

10. Materials for cement sand bed.-Cement-sand bed shall consist of 1 part Portland cement and 4 parts by volume of clean, coarse sand, well graded from a maximum size of $1 / 4$-inch particles.

11. Materials for grout filler.-Grout joint filler shall consist of 1 part Portland cement and 1 part by volume of clcan, sharp, well-graded, fine sand free from particles of such size as to obstruct the ready flow of the grout to the bottom of the joints.

12. Materials for bituminous mastic filler.-Bituminous mastic filler shall consist of a combination of approximately equal parts by volume of sand with one of the bituminous materials hereinafter specified.

A. Sand.-Sand for bituminous mastic filler shall consist of elean durable grains of which all shall pass a standard 10 -mesh sieve and 85 per cent by weight shall pass a 20 -mesh sieve.

B. Tar pitch filler.-Similar to A. S. T. M. D11227T. (See 505.31, p. 175.) Tests are to be made on the sample as rcceived, but results reported on a dry basis.

C. Asphalt cement filler.-The asphalt cement shall be homogeneous and free from water. It shall conform to the following requirements:

(a) Penetration at $25^{\circ} \mathrm{C} .\left(77^{\circ} \mathrm{F}\right.$. $), 100 \mathrm{~g}, 5$ seconds, 60 to 70

(b) Flash point (open cup), not less than $175^{\circ} \mathrm{C}$. (347 ${ }^{\circ} \mathrm{F}$.)

(c) Loss on heating at $163^{\circ}$ C. $\left(325^{\circ}\right.$ F. $), 50 \mathrm{~g}, 5$ hours, not more than 2 per cent.

Penetration at $25^{\circ}$ C. $\left(77^{\circ}\right.$ F.), $100 \mathrm{~g}, 5$ seconds, of residue after heating at $163^{\circ} \mathrm{C}$. $\left(325^{\circ} \mathrm{F}\right.$. $)$ as compared with penetration of asphalt cement before heating, not less than 60 per cent.

(d) Ductility at $25^{\circ} \mathrm{C}$. $\left(77^{\circ} \mathrm{F}\right.$.), not less than $30 \mathrm{~cm}$.

(e) Proportion of bitumen soluble in carbon tetrachloride, not less than 99 per cent.

D. Tar and asphalt filler.-This material for use in bituminous mastic filler shall be a mixture of coaltar pitch and refined asphalt, consisting of 100 parts of coal tar and 20 parts of refined asphalt. These products shall comply, respectively, with the requirements of sections $12 \mathrm{~B}$ and $12 \mathrm{C}$, except that the penetration of the asphalt at $77^{\circ} \mathrm{F}$. shall be not less than 30 nor more than 40 . 
(The full text of the specification includes requirements for construction.)

American Society for Testing Materials, standard specifications for cement grout filler for brick and stone block pavements, serial designation D57-20, 1920.

See 512.16 , page 253 .

American Society for Testing Materials, standard specifications for block for granite pavements, serial designations D59-26, D131-23, D132-23.

See 511.71 , page 208

Federal Specifications Board, specification No. 510, United States Government master specification for block for granite pavements.

See 511.71, page 208 .

\subsection{Asphalt Pavements.}

American Association of State Highway Officials, standard specifications for highway bridges and incidental structures, 1928.

\section{PAVING BLOCKS (ASPHALT)}

\section{GENERAI}

Asphalt blocks shall be composed of asphaltic cement, mineral aggregate, and inorganic dust, as herein specified, thoroughly mixed while hot in such proportions, depending on their character, that the finished blocks shall conform to the specified requirements.

The ingredients of the blocks shall be tested in accordance with the methods provided in the tentative standard methods of sampling and testing of the American Association of State Highway Officials.

Each bidder shall submit with his bid a specimen block of the size and quality described in these specifications, labeled with the name of the bidder, the name of the manufacturer, and the place of manufacture. Bids not accompanied by specimen blocks will not be accepted.

\section{ASPHALTIC CEMENT}

The asphaltic cement shall conform to the following requirements:

1. It shall be homogeneous and free from water.

2. Specific gravity $25^{\circ} / 25^{\circ} \mathrm{C}$. $\left(77^{\circ} / 77^{\circ} \mathrm{F}\right.$.) not less than 1.00

3. Flash point -Not less than $190^{\circ} \mathrm{C}\left(374^{\circ} \mathrm{F}\right)$

4. The penetration at $25^{\circ} \mathrm{C}$. $\left(77^{\circ} \mathrm{F}\right.$ ), $100 \mathrm{~g}, 5$ seconds, shall be varied to suit climatic and traffic conditions in accordance with the following table:

\begin{tabular}{|c|c|c|c|}
\hline \multirow{2}{*}{ Traffic } & \multicolumn{3}{|c|}{ Temperature } \\
\hline & Low & Moderate & High \\
\hline 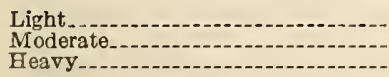 & $\begin{array}{l}15-25 \\
15-25 \\
15-20\end{array}$ & $\begin{array}{l}15-25 \\
15-20 \\
10-20\end{array}$ & $\begin{array}{r}10-15 \\
10-15 \\
5-15\end{array}$ \\
\hline
\end{tabular}

5. Loss on heating. - At $163^{\circ} \mathrm{C} .\left(325^{\circ} \mathrm{F}\right.$.), $50 \mathrm{~g}, 5$ hours, not more than

6. Penetration of residue after heating - At $25^{\circ} \mathrm{C},\left(77^{\circ} \mathrm{F}\right), 100 \mathrm{~g}, 5$ seconds, as compared to penetration before heating, not less

than 50 per cent
7. Ductility. At $25^{\circ}$ C. $\left(77^{\circ}\right.$ F.), 5 to 10

8. Proportion of bitumen soluble in carbon tetrachloride.-Not less than 99.0 per cent.

\section{MINERAL AGGREGATE}

The mineral aggregate shall be clean, hard, unweathered trap rock, dolomite, limestone, copper conglomerate, or other suitable material which shall show a percentage of wear of not more than 5 percent. Its grading shall be such as to produce, with the inorganic dust, the mesh composition hereinafter specified.

\section{INORGANIC DUST}

The inorganic dust, or filler, shall be produced from sound limestone or other approved material and shall be powdered to such a fineness that 95 per cent shall pass a 30 -mesh sieve and not less than 50 per cent shall pass a 200 -mesh sieve. Sufficient inorganic dust shall be used to give the mesh composition hereinafter specified.

\section{MANUFACTURE OF BLOCKS}

Before being mixed the asphaltic cement and crushed mineral aggregate shall be heated separately. In no case shall either be heated to a higher temperature than $350^{\circ} \mathrm{F}$., but the temperature of each shall be so regulated that the temperature of the block mixture as delivered to the press molds shall be not less than $225^{\circ} \mathrm{F}$.

The mineral aggregate, inorganic dust, and asphaltic cement in the proper proportions shall be thoroughly mixed to produce a homogeneous mass in which all particles are thoroughly coated with asphaltic cement.

The blocks shall receive a compression in the molds of not less than 4,000 lbs./in.2, applied on the 5 by 12 inch surface. After presssing they shall be cooled by passing through water or by other suitable means.

\section{CHARACTERISTICS OF FINISHED BLOCKS}

Size and shape.-The blocks shall be 5 inches in width by 12 inches in length unless the depth required is less than 2 inches, in which case they shall be 4 inches in width by 8 inches in length. The depth shall be as shown on the plans. A variation either way from these dimensions of one-fourth inch in length or one-eighth inch in width or depth will be sufficient cause for rejection of any block

Composition.-The composition of the blocks, as delivered on the work, shall conform to the following requircments:

Retained by $1 / 4$-inch screen (circular openings), not more than Passing 1/4-inch screen and retained by 20 mesh sieve than- 10 Passing 20-mesh sieve and retained by 100-mesh sieve Passing 200-m Passing 100-mesh sieve (including all tones) - - $200-$ mesh sieve, not less than Bitumen content sieve, not less than $61 / 2-9$

Specific gravity. - The blocks shall have a specific gravity of not less than 2.35 at $77^{\circ} \mathrm{F}$.

Absorption test.-The blocks to be tested shall be cleaned of loose particles, carefully weighed, placed in a pressure vesssel, and subjected to a vacuum of 22 inches for one hour at room temperature. While still maintaining the vacuum, water shall then be admitted to the vessel until the blocks are completely immersed, after which the vacuum shall be relieved and water pressure carried up to $100 \mathrm{lbs}$./in. ${ }^{2}$ and there maintained for one hour. The blocks shail then be removed from the vessel and after mopping off all surface water shall be carefully weighed. The average of a set of four blocks tested in this manner shall show absorption not exeeding 0.5 per cent by weight.

American Association of State Highway Officials, tentative standard specifications for highway materials, 1927.

See 505.13, page 146 .

American Society for Municipal Improvements, standard specifications for sheet asphalt paving, 1927.

(This specification includes methods of construction and requirements for maintenance and the following specifications for materials:)

1. General description.-The sheet-asphalt pavement shall consist of a binder course and a wearing course, laid upon a foundation constructed in accord- 
ance with the specifications for foundation. The binder course shall be laid upon the prepared foundation to a finished thickness of $1 \frac{1}{2}$ inches and shall be composed of broken stone, sand, and asphalt cement. The surface course shall be laid upon the compacted binder course to a finished thickness of $1 \frac{1}{2}$ inches and shall be composed of sand, mineral filler, and asphalt cement. All materials and methods of construction shall conform to the requirements of these specifications.

Note.- When this pavement is to be laid on a Portland cement conerete foundation it is recommended that tbe foundation mix be not richer than $1: 3: 6$ or its equivalent. In cases where weak subnot richer than $1: 3: 6$ or its equivalent. In cases wisere weak subgrade is encountered, the engineer must exercise his own judgment as to tbickening the concrete foundation, enriching the

adding reinforcement, or otherwise providing for safety.
Other types of foundation than conerete sbould not be ignored, Other types of foundation than concrete sbould not be ignored,
and it is recommended tbat old gravel or macadam be taken into consideration when, in the judgment of the engineer, conditions warrant thoir use for foundation purposes. The engineer must, bowever, see that the old gravel or macadam foundations be carcfully trued and brought to uniform surface and otberwise be of proper strength before the wearing surface is placed.

\section{MATERIALS}

2. Sources of supply.-(a) Approval of sources of supply of binder stone, sand, and mineral filler shall be obtained from the engineer prior to the delivery of materials, and samples of each shall be submitted as directed by the engineer.

(b) A 1-pound sample of the asphalt cement that the contractor proposes to use must be submitted to the engineer before the use of such material in the work will be permitted. If the contractor proposes to prepare the asphalt cement at the paving plant, then, in lieu of the above, a 1-pound sample each of flux and refined asphalt must be submitted, together with a statement of the proportions in which they will be combined to produce the asphalt cement which he proposes to use. Every refined asphalt and asphalt cement admitted under these specifications shall be equal in quality to the recognized standard for its particular kind or type of asphalt.

(c) Unless otherwise permitted by the engineer, all samples shall be submitted at least thrce weeks prior to use of the materials in the work and in no case shall they be used until they have been examined and approved by him. Whenever during the course of the work new deliveries of paving materials are received by the contractor, samples of these shall at once be submitted to the engineer and their use in the work will not be permitted until they have been examined and approved by him.

3. Methods of testing.--Unless otherwise specified, all tests shall be conducted in accordance with the latest standards or tentative standards of the American Society for Testing Materials.

4. Stone for binder course shall be produced from tough durable rock having a per cent of wcar of not more than 6 . It shall be freshly crushed, of reasonably uniform quality throughout, and shall be clean and free from dust and an excess of flat or elongated pieces. As delivered to the mixer it shall comply with the following requirements: (a) The particles of stone shall be free from adhering dust; (b) it shall have a mesh composition within the following limits:

Per cent

Passing 10-mesh, not more tban Passing 1/4-inch screen, retained on 10-mesh sieve............... 10-25 Passing 1/2-inch screen, retained on 1/4-inch screen........

5. Sand for binder course shall consist of clean, tough, angular grains, free from clay, loam, or other foreign matter, and all of it shall pass a $1 / 4$-inch laboratory screen. As delivered to the mixer it shall be frce from clayey lumps or loosely bonded aggregations of finer particles.
6. Sand for surface course shall consist of clean tough, rough-surfaced and angular grains, free from clay, loam, and other foreign matter. As delivered to the mixer it shall comply with the following requirements: (a) The particles shall be free from adhering dust; $(b)$ it shall be free from clayey lumps or loosely bonded aggregations of finer particles; (c) it shall have a mesh composition within the following limits:

\begin{tabular}{|c|c|c|}
\hline Passing- & Retained on- & Per cent \\
\hline $\begin{array}{l}\text { 200-mesh } \\
\text { 100-mesh } \\
\text { 80-mesb } \\
\text { 40-mesh } \\
\text { 30-mesh } \\
20 \text {-mesh } \\
10 \text {-mesh } \\
10-\text { mesh }\end{array}$ & $\begin{array}{l}\text { 200-mesh } \\
\text { 100-mesh } \\
\text { 50-mesb } \\
\text { 40-mesh } \\
\text { 30-mesh }\end{array}$ & $\left.\begin{array}{l}0-5 \\
6-25 \\
6-25 \\
5-40 \\
5-30 \\
8-25 \\
5-15 \\
5-15 \\
98-100\end{array}\right\} 30-60$ \\
\hline
\end{tabular}

The engineer shall select or approve from available sources of supply a sand coming within the above limits and which, in his judgment, will be suitable for the climatic and traffic conditions. All deliveries of this sand to the plant must conform within reasonable limits to the sample which has been approved.

7. Mineral filler shall consist of thoroughly dry limestone dust, slate dust, or Portland cement which shall meet the following requirements:

Passing 200-mesh sieve, not less tban.

Per cent Passing 100-mesh sieve, not less than 65
85

8. Asphalt cement shall be homogeneous, free from water, and shall not foam when heated to $175^{\circ}$ C. $\left(347^{\circ} \mathrm{F}.\right)$. It shall meet the following requirements for physical and chemical properties:

I. Penetration at $25^{\circ} \mathrm{C}$. ( $\left(77^{\circ} \mathrm{F}\right.$ ) $100 \mathrm{~g}, 5$ seconds (Between these limits the exact penetration desired, 35,45 , or 55 , shall be specified by the engineer. An asphalt cement baving a penetration 5 points above or below that specificd shall be considered acceptable for use.)

II. Ductility at $25^{\circ} \mathrm{C}$. $\left(77^{\circ} \mathrm{F}\right.$.), not less tban $\ldots \ldots . . . .-30 \mathrm{~cm}$ III. Loss on heating at $163^{\circ} \mathrm{C}$. $\left(325^{\circ} \mathrm{F}\right.$.) $, 50 \mathrm{~g}, 5$ bours, not more than of residue at $25^{\circ}$ C. $\left(77^{\circ}\right.$ F.), $100 \mathrm{~g}, 5$ Penctration of residue at $25^{\circ} \mathrm{C}$. ( $\left(77^{\circ} \mathrm{F}.\right), 100 \mathrm{~g}, 5$
seconds, as per cent of original penetration, not less than IV. Flash point (open cup) not less tban $175^{\circ} \mathrm{C}$.
$\left(347^{\circ} \mathrm{F}.\right)$. Proportion of bitumen soluble in carbon tetra. chloride, not less than..... 99 per cent

Note.-When less than 99 per cent of the asphalt cement is soluble in carbon tetrachloride the percentage of bitumen (solubility in carbon disulphide) shall be reported.

\section{PAVING PLANT}

9. Plant essentials.-(a) The plant used in manufacturing the pavement must be of the batch type and capable of mixing in the manner herein specified not less than 80 tons of surface mixture per day of eight hours and must be provided with separate chambers for heating and mixing the ingredients. The stone and sand shall be heated in an approved type of revolving drier. The mixing shall be done in a twin-shaft mixer of the pug mill type capable of holding and properly mixing not less than a 750-pound batch of surface mixture or in a rotary mixer in which the asphalt is sprayed into and incorporated with the mineral aggregate under pressure. Each plant must be provided with asphalt kettles of such capacity that one filling of them will be sufficient for a day's run and so designed as to secure uniform heating of the entire contents. There shall also be provided a bin for the storage of hot aggregate having a storage capacity of not less than 1 ton and provided with at least two compartments 
each having a separate gate. It shall have an overhead rotary screen not less than 6 feet long for removing oversize binder stone and separating the coarse and fine aggregates and delivering them to the separate compartments; bins and screens to be provided with overflow pipes. The hopper from which the hot aggregate is drawn from the bin must rest upon scales so arranged that a definite weight of material can be weighed out of each bin and delivered to the mixer.

(b) The asphalt bucket used to measure and apply the asphalt must be balanced upon scales and so arranged that the asphalt can be easily and quickly weighed and be applied in a thin sheet the full width of the mixer.

(c) The plant shall be provided with an asphalt thermometer for the melting kettles, an electric pyrometer at the discharge chute of the dricr, a platform scale, and at least two inspector's armored thermometers.

Plant-testing equipment.-The contractor sliall provide and maintain in good order at the plant the following testing equipment, which may be used by the engineer or an inspector designated by him to determine the penetration of asphalt and asphalt cement, the grading of the mineral aggregate, and such other tests as are deemed necessary:

1 penetration machine, with needles, glass dish, and thermometer. 1 set of standard sieves, $200,-100,80,50,40,30,20$, and 10 mesh. 1 laboratory stone scale.

1 laboratory sand scale.

1 set of standard screens, $1 / 4,1 / 2,8 / 4$. and $11 / 4$ inch circular openings. 1 roll of manila paper for making pat tests.

All of the above-mentioned apparatus shall be of standard type and approved by the engineer and shall be properly housed by the contractor in a separate building or room not less than 10 by 10 feet and provided with a workbench. This room shall be used exclusively for testing purposes by the contractor and the engineer or his inspector.

\section{PAVING-PIANT OPERATION}

11. Sand and stone storage.-The different kinds of sand and stone used shall be kept separate and such provisions as are deemed necessary by the cngineer to keep them from becoming mixed shall bc made by the contractor.

12. Preparation of asphalt cement.-(a) The asphalt cement shall be melted at the paving plant in kettles or tanks and shall be brought to a temperature of $250^{\circ}$ to $350^{\circ} \mathrm{F}$.

(b) When refined asphalt is to be combined with a flux or when the asphalt cement contains over 5 per cent mineral matter the mixture shall be thoroughly agitated until a homogeneous asphalt cement of the required penetration is produced, after which only sufficient agitation shall be provided to keep the asphalt cement homogeneous. The penetration of the asphalt cement shall be tested at suitable intervals to insure that it is maintained at a uniform consistency throughout the period of use.

13. Preparation of mineral aggregates.-(a) The stone and sand for binder course shall be dried and heated in the revolving drier and when delivered to the mixer shall be at a temperature between $250^{\circ}$ and $325^{\circ} \mathrm{F}$. The temperature between these limits shall be regulated according to the temperature of the atmosphere and the working of the mixture. Both aggregates may be simultaneously fed into the same drier, but in such case they should previously be mixed in substantially the desired proportion and must immediately after heating be screened into coarse and fine aggregate and stored in separate bins.

(b) The sand for surface course shall be dried and heated in the revolving drier and when delivered to the mixer shall be at a temperature between $275^{\circ}$ and $400^{\circ} \mathrm{F}$. The temperature between these limits shall be regulated according to the temperature of the atmosphere and the working of the mixture. When a mixture of two or more sands is required in order to produce the grading specified in section 6 such combination shall be made before the sand is fed into the drier by proportioning the sands from separate storage piles and mixing them by means of shovels.

14. Preparation and composition of binder mixture.-(a) After the ingredients have been separately and accurately weighed in accordance with the approved formula each batch of mixture shall be made by first charging the mixer with the mineral aggregate and then adding the asphalt cement. Mixing shall then be continued for a period of at least 30 seconds, or longer if necessary, to produce a homogeneous mixture in which all particles of the mineral aggregate are uniformly coated.

(b) The binder mixture shall conform to the following composition limits by weight:

\begin{tabular}{|c|c|c|}
\hline & Per cent & $\begin{array}{c}\text { Toler- } \\
\text { ances, } \\
\text { above or } \\
\text { below }\end{array}$ \\
\hline $\begin{array}{l}\text { Bitumen soluble in carbon disulphide } \\
\text { Sand and material passing 10-mesh sieve. } \\
\text { Coarse aggregate retained on } 10 \text {-mesh sieve... }\end{array}$ & $\begin{array}{r}4-6 \\
15-30 \\
65-80\end{array}$ & $\begin{array}{r}\text { Peт cent } \\
0.5 \\
2.0 \\
2.0\end{array}$ \\
\hline
\end{tabular}

The engineer shall specify or approve a mixture coming within the above limits which, in his judgment, will be suitable for the traffic and climatic condition.

In no case shall these permissible variations from the approved formula be construed as authorizing a mixture which does not come within the broad limits set for the composition of the binder mixture.

15. Preparation and composition of surface mixture.-(a) After the ingredients have been separately and accurately weighed in accordance with the approved formula each batch of mixture shall be made by first charging the mixer with the sand and mineral filler. After the two have been thoroughly mixed the asphalt cement shall be added and the mixing continued for a period of at least one minute, or longer if necessary, to produce a homogeneous mixture in which all particles of the mineral aggregate are uniformly coated.

(b) The surface mixture shall conform to the following composition limits by weight:

\begin{tabular}{|c|c|c|c|}
\hline Passing- & Retained on- & Per cent & $\begin{array}{l}\text { Toler- } \\
\text { ances, } \\
\text { above or } \\
\text { below }\end{array}$ \\
\hline $\begin{array}{l}\text { Bitumen soluble in carbon di- } \\
\text { sulphide. } \\
\text { 200-mesh } \\
80-\text { mesh } \\
\text { 10-mesh }\end{array}$ & $\begin{array}{l}200 \text {-mesh } \\
80 \text {-mesh... } \\
40 \text {-mesh.... }\end{array}$ & $\begin{array}{r}9.5-12 \\
10.0-20 \\
14.0-32 \\
22.0-45 \\
12.0-40\end{array}$ & $\begin{array}{r}\text { Per cent } \\
0.5 \\
1.5 \\
3.0 \\
8.0 \\
4.0\end{array}$ \\
\hline
\end{tabular}

The engineer shall specify or approve a mixture coming within the above limits which, in his judgment, will be suitable for the traffic and climatic conditions.

In no case shall these permissible variations from the approved formula be construed as authorizing a mixture which does not come within the broad limits set for the composition of the surface mixture.

16. Paving-plant inspection.-The engineer or his authorized representatives shall have access at any 
time to all parts of the paving plant in order to insure the manufacture of the pavement in strict accordance with these specifications.

\section{NOTES}

The accompanying specifications are recommended for use under what may be termed average conditions. It is realized, however, that no singlestandard specification will satisfactorily cover all variations in local conditions which may prevail for individual jobs. Before adopting these specifications verbatim the engineer should, therefore, give particular attention to the items listed below and if necessary make such changes as are suggested. In the matter of cross section a crown of one-fourth inch to the foot is recommended.

I. Thickness.-These specifications call for a total finished thickness or depth of 3 inches. If it is desired to make the depth $2 \frac{1}{2}$ inches the requirement for thickness of the binder course should be changed from $1 / 2$ inches to 1 inch in sections 1 and 20.

II. Use of broken slag in binder course.-If it is desired to permit the use of broken slag in the binder course the following changes should be made:

Sec. 3. Add the following sentence: "When determining the per cent of wear of slag $4,000 \mathrm{ec}$ loose measure of the size specified in the standard test shall be used."

Sec. 4. End of first sentence insert "or of broken slag having a per cent of wear of not more than 10 and weighing not less than 70 pounds per cubic foot for the size specified."

SEc. 14. (b). Change requirement for per cent of bitumen soluble in carbon disulphide from 4 to 6 to " 4 to 7 ."

III. Use of gravel in binder course.-While gravel is not recommended for use in binder course, when it is the only readily available material for coarse aggregate it may be permitted, in which case the following change should be made:

SEc. 4. End of first sentence insert "or of gravel composed of hard, durable rock particles free from clay or coatings of any character."

IV. Sand for surface course.-Under heavy concentrated traffic conditions the sand for the surface course should preferably approach as closely as possible the following typical grading. It is impracticable to specify any exact grading, but under the conditions mentioned where it is possible to approximate this grading with a single sand or a combination of two or more available sands the engineer should change the limits specified in section 6 so as to more closely define the grading required without making the specification so rigid as to be impracticable for his work. If this is not done he should at least require the contractor to work to the typical grading as closely as practicable within the specification limits.

\section{Typical sand grading for surface mixture}

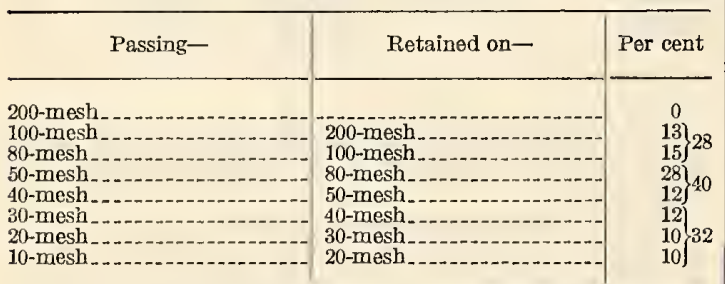

V. Asphalt cement.-The most desirable consistency or "penetration" for asphalt cement will vary somewhat with climatic and traffic conditions.
In section 8 , I, limits of penetration of from " 30 to 60 " are specified with a statement that between these limits the exact penetration desired shall be specified by the engineer. The ranges of penetration suggested for various combinations of climate and traffic are shown in the following table:

\begin{tabular}{|c|c|c|c|}
\hline \multirow{2}{*}{ Traffic } & \multicolumn{3}{|c|}{ Temperature } \\
\hline & Low & Moderate & High \\
\hline $\begin{array}{l}\text { Light } \\
\text { Moderate_... } \\
\text { Heavy }\end{array}$ & $\begin{array}{l}50-60 \\
50-60 \\
40-50\end{array}$ & $\begin{array}{l}50-60 \\
50-60 \\
40-50\end{array}$ & $\begin{array}{l}40-50 \\
40-50 \\
30-40\end{array}$ \\
\hline
\end{tabular}

VI. Composition of binder and surface mixtures.The limits given in the specifications are intended to cover a wide range of traffic and climatic conditions and locally available supplies of material. The variations permitted are too great to be applied to any one contract. After approving the materials to be used, the engineer should set or approve formulas which will produce binder and surface mixtures suitable for the traffic and climatic conditions and falling within the broad composition limits given in the specifications.

A reasonably efficient mixing crew should be able to produce binder and surface mixtures complying with the formula requirements within the following limits:

Bitumen Filler (200-mesh derived therefrom) -- 1.5 per cent above or below. Stone Sand

Occasional variations slightly in excess of those given above may be permitted by the engineer, but the average composition of the mixtures turned out at the plant should fall within the limits given. The wide limits appearing in the specifications are not intended to apply to any one contract.

VII. Samples.-It is recommended that the following procedure be adopted in connection with sampling materials for test:

Samples should be put in clean, dry containers, perferably tin boxes or cans. The following amounts of the different materials are required for test:

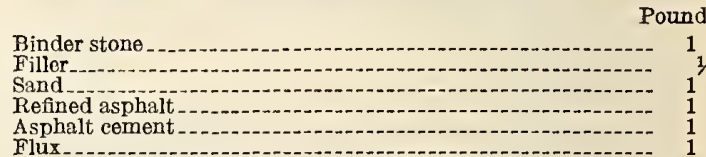

Method of sampling.-Extreme care should be taken in every case to obtain a sample which is truly representative of the material to be examined. These samples are for the use of the testing laboratory only and should not be used for testing at the plant before submitting them to the laboratory. The particular precautions to be observed in each case are given below:

Binder stone.-A sufficient number of 5-pound samples to be taken from different parts of the pile. These should be thoroughly mixed together and be reduced by quartering to the desired size.

Filler.-Samples should be taken from several bags and mixed.

Sand.-Samples should be taken from the interior of the pile where the sand is damp, a sufficient number of 1-pound samples to be taken from different parts of the pile. These should be thoroughly mixed together and reduced by quartering to the desired size.

Refined asphalt and asphalt cement.-

(a) In barrels. - At least one sample should be taken from each batch. It should be taken at sufficient 
depth below the surface to insure obtaining representative material free from all dirt or other extraneous matter and at a point not less than 4 inches distant from the top and sides of the barrel.

(b) In tank cars. - The contents of the tank should be heated until completely liquid throughout. It should then be agitated and thoroughly mixed by means of air or steam, after which the sample shall be taken from the dome in such a manner as to obtain the asphalt from a point at least 3 feet below the surface.

(c) In kettles.-The contents of the kettles must be completely liquid and thoroughly agitated previous to and during sampling. The sample may be taken from the pipe through which the material is delivered to the mixer by means of a clean dipper.

Flux.-The directions given for sampling refined asphalt and asphalt cement apply to this material except that under ordinary conditions it is not necessary to agitate the contents of the tank car.

Surface and binder mixtures.-Samples may be taken at the plant or on the street.

If taken at the plant they should preferably be obtained from the wagons or trucks, care being observed to obtain an average representative sample which is not contaminated with dust, sand, or oil from the wagon bodies and shows no evidence of separation having taken place after mixing and dumping. The first or last portions of a batch as it comes from the mixer should not be included in the sample.

If obtained on the street the sample should be taken after the mixture has been shoveled and raked, care being observed to obtain an average sample which shows no signs of segregation in transit or excessive richness due to accumulation of bitumen at the bottom or top of the load or oil from the wagon bodies.

Samples of surface mixture should be pressed between a sheet of paper and trimmed while hot to a convenient size.

VIII. Methods of testing binder and surface mixtures.- It is recommended that the following laboratory methods be used to determine conformity of binder and surface mixtures with specification requirements:

Determination of bitumen content and mesh composition of binder mixture.- Weigh out from 350 to $500 \mathrm{~g}$ of the binder mixture and extract the bitumen from it in a centrifugal extractor or suitable continuous hot extractor, using chemically pure carbon disulphide as a solvent for the bitumen. Follow the same general method for the drying and sifting of the mineral aggregate as described in the method for analyzing surface mixtures. The screens and sieves to be used are as follows: 11/4-inch, 1/2-inch, and 1/4-inch circular opening, and 10-mesh.

Remarks.-The limits of accuracy of this test are as follows: For bitumen content, 0.3 per cent above or below the result obtained. For mesh composition, 10 per cent of the result obtained (above or below).

Determination of bitumen content and mesh composition of surface mixtures.-The sample of surface mixture should be heated to about $300^{\circ} \mathrm{F}$. until soft and 10 to $20 \mathrm{~g}$ of it weighed onto a tared S. \& S. filter paper No. 595, $11 \mathrm{~cm}$ in diameter. The filter paper and contents should be placed in a funnel and washed with chemically pure carbon disulphide until the washings run through practically colorless. Dry the filter paper and residue at $250^{\circ} \mathrm{F}$. for onehalf hour. Open the filter paper carefully and remove the mineral aggregate. Scrape off the dust adhering to the paper as thoroughly as possible with a blunt palette knife and add it to the mineral aggregate. Evaporate the filtrate containing the bitumen, burn the bitumen, add the filter paper to it, and burn to an ash. Add the ash to the mineral aggregate previously removed from the filter paper and weigh. The difference between the weight of surface mixture originally taken and the combined weight of the ash and residue is considered as the weight of bitumen in the sample. This combined ash and residue is then sifted through the following sieves (in the order named) and the percentages of the various sized particles calculated: $200,100,80$, $50,40,30,20,10$.

Sifting shall be continued on each sieve until less than 1 per cent passes through the sieve during the last minute of sifting.

If desired, the surface mixture may be extracted in a centrifuge or in any suitable form of extractor with hot, chemically pure carbon disulphide and the combined ash from the extracted bitumen and the mineral aggregate sifted as above.

Remarks. - The limits of accuracy of this test are as follows: For bitumen content, 0.3 per cent above or below the result obtained. For mesh composition, 10 per cent of the result obtained (above or below).

American Society for Municipal Improvements, standard specifications for fine aggregate asphaltic concrete paving, 1927.

(The full text of the specification includes methods of construction and the following specifications for materials.)

1. General description.-The fine aggregate asphaltic concrete pavement shall consist of a mixture of broken stone, or broken stone and sand, mineral filler, and asphalt cement. It shall be laid to a finished thickness of 2 inches upon a foundation constructed in accordance with the specifications for foundation and shall be finished with a seal coat of hot asphalt cement and stone chips or fine gravel. All materials shall conform to the requirements of these specifications.

Note. - When this parement is to be laid on a Portland cement concrete foundation it is recommended that the foundation mix be not richer than $1: 3: 6$ or its equivalent. In cases where weak subgrade is encountered the engineer must exercise his own judgment as to thickening the concrete foundation, enriching the mixture, adding reinforcement, or otherwise providing for safety

Other types of foundation than concrete should not he ignored, and it is recommended that old gravel or macadam be taken into consideration when, in the jud oment of the en rineer, conditions warrant thcir use for foundation pures. The enineer must, however see that the or the old gravel or macadam foundations be carefully trued and brought wearing surface is placed.

\section{MATERIALS}

2. Sources of supply.-(a) Approval of sources of supply of stone, sand, and mineral filler shall be obtained from the engineer prior to the delivery of materials, and samples of each shall be submitted as directed by the engineer.

(b) A 1-pound sample of the asphalt cement that the contractor proposes to use must be submitted to the engineer before the use of such material in the work will be permitted. If the contractor proposes to prepare the asphalt cement at the paving plant, in lieu of the above, a 1 -pound sample each of flux and refined asphalt must be submitted together with a statement of the proportions in which they will be combined to produce the asphalt cement which he ртороses to use. Every refined asphalt and asphalt cement admitted under these specifications shall be equal in quality to the recognized standard for its particular kind or type of asphalt.

(c) Unless otherwise permitted by the engineer, all samples shall be submitted at least three weeks prior to use of the materials in the work, and in no case shall they be used until they have been examined and approved by him. Whenever, during the course of the work, new deliveries of paving materials are received by the contractor, samples of these shall at 
once be submitted to the engineer, and their use in the work will not be permitted until they have been examined and approved by him.

3. Methods of testing.- Unless otherwise specified, all tests shall be conducted in accordance with the latest standards or tentative standards of the American Society for Testing Materials.

4. Coarse aggregate.-The coarse aggregate shall consist of broken stone produced from tough, durable rock having a per cent of wear of not more than 6. It shall be freshly crushed, of reasonably uniform quality throughout, and shall be clean and free from dust and an excess of flat or elongated pieces, and must not contain over 5 per cent of weathered or partly decomposed rock. It must be free from particles of sandstone, feldspar, slate, shale, and stone having a per cent of wear of over 6 . As delivered to the mixer, it shall comply with the following requirements:

(a) The particles of stone shall be free from adhering dust.

(b) It shall have a mesh composition within the following limits:

Per cent

Passing 10-mesh, not more than

Passing 1/4neh sereen, retained on 10-mesh sieve-..-.

Passing 1/2-inch screen, retained on $1 / 1$-inch screen

5. Fine aggregate. - This shall be composed of sand or stone screenings, or a mixture of both.

The sand shall consist of clean, tough, roughsurfaced and angular grains, free from clay, loam, or other foreign matter.

The stone screenings must be frcshly crushed from rock of the character specified under "Coarse aggrcgate."

As delivered to the mixer, the fine aggregate shall comply with the following requirements: dust.

(a) The particles shall be free from adhering

(b) It shall be free from claycy lumps or loosely bonded aggregations of finer particles.

(c) It shall have a mesh composition within the following limits:

\begin{tabular}{|c|c|c|}
\hline Passing- & Retained on- & Per cent \\
\hline $\begin{array}{l}\text { 200-mesh } \\
80 \text {-niesh } \\
40-\text { mesh } \\
10-\text { mesh } \\
1 / 4-\text { inch screen }\end{array}$ & $\begin{array}{l}200 \text {-mesh } \\
40 \text {-mesh } \\
10-\text { mesh }\end{array}$ & $\begin{array}{r}0-5 \\
15-40 \\
30-60 \\
18-50 \\
0-3\end{array}$ \\
\hline
\end{tabular}

The engineer shall select or approve from available sources of supply a fine aggregate coming within the above limits and which, in his judgment, will be suitable for the climatic and traffic conditions. All deliveries to the plant must conform within reasonable limits to the sample which has been approved.

6. Stone chips or pea gravel for seal coat shall be of reasonably uniform quality, free from dust, and shall meet the following requirements:

Passing 1/4-inch screen, not more than

Per cent Passing 1/2-inch screen, not less than

7. Mineral filler shall consist of thoroughly dry limestone dust, slate dust, or Portland cement which shall meet the following requirements:

Per cent

Passing 200-mesh sieve, not less than

Passing 100-mesh sieve, not less than

Per 65

8. Asphalt cement.-Practically indentical with Asphalt Association, B-8, section 4. (See 518.27, p. 374.)

NoтE.- Where less than 99 per cent of the asphalt cement is soluhle in carbon tetrachloride, the percentage of hitumen (soluhility in carhon disulphide) shall be reported.

\section{PAVING PIANT}

9. Plant essentials.-(a) The plant used in manufacturing the pavement must be of the batch type and capable of mixing in the manner herein specified not less than 80 tons of surface mixture per day of eight hours and must be provided with separate chambers for heating and mixing the ingredients. The stone and sand shall be heated in an approved type of revolving drier. The mixing shall be done in a twin-shaft mixer of the pug-mill type capable of holding and properly mixing not less than a 750 -pound batch of surface mixture or in a rotary mixer in which the asphalt is sprayed into and incorporated with the mineral aggregate under pressure. Each plant must be provided with asphalt kettles of such capacity that one filling of them will be sufficient for a day's run and so designed as to secure uniform heating of the entire contents. There shall also be provided a bin for the storage of hot aggregate having a storage capacity of not less than 1 ton and provided with at least two compartments, each having a separate gate. It shall have an overhead rotary scleen not less than 6 feet long for removing oversize stone and separating the coarse and fine aggregate and delivering them to the separate compartments; bins and screen to be provided with overflow pipes. The hopper from wlich the hot aggregate is drawn from the bin must rest upon scales so arranged that a definite weight of material can be weighed out of each bin and delivered to the mixer.

(b) The asphalt bucket used to measure and apply the asphalt must be balanced upon scales and so arranged that the asphalt can be easily and quickly weighed and be applied in a thin sheet the iull width of the mixer.

(c) The plant shall be provided with an asphalt thermometer for the melting kettles, an electric pyrometer at the discharge chute of the drier, a platform scale, and at least two inspectors' armored thermometers.

10. Plant testing equipment.-The contractor shall provide and maintain in good order at the plant the following testing equipment, which may be used by the engineer or an inspector designated by him to determine the penetration of asphalt and asphalt cement, the grading of the mineral aggregatc, and sucl other tests as are deemed necessary:

1 penetration machine with needles, glass dish, and thermometer. 1 set of standard sieves, $200,100,80,50,40,30,20$, and 10 mesh. 1 lahoratory stone scale.

1 lahoratory sand scale. $11,1 / 2,3 / 4$, and $11 / 4$ inch circular openings.

1 set of standard screens, $1 / 4,1 / 2,3 / 4$, and $11 / 4$

All of the above-mentioned apparatus shall be of standing type and approved by the engineer and shall be properly housed by the contractor in a separate building or room not less than 10 by 10 feet and provided with a workbench. This room shall be used exclusively for testing purposes by the contractor and the enginecr or his inspector.

\section{PAVING-PLANT OPERATION}

11. Sand and stone storage.-The different kinds of sand and stone used shall be kept separate, and such provisions as are deemed necessary by the engineer to keep them from becoming mixed shall be made by the contractor.

12. Preparation of asphalt cement.-(a) The asphalt cement shall be melted at the paving plant in kettles or tanks and shall be brought to a temperature of $250^{\circ}$ to $350^{\circ} \mathrm{F}$.

(b) When refined asphalt is to be combined with a flux or when the asphalt cement contains over 5 per cent mineral matter, the mixture shall be thoroughly agitated until a homogeneous asphalt cement of the required penetration is produced, after 
which only sufficient agitation shall be provided to keep the asphalt cement homogeneous. The penetration of the asphalt cement shall be tested at suitable intervals to insure that it is maintained at a uniform consistency throughout the period of use.

13. Preparation of mineral aggregates.-The coarse and fine aggregates shall be dried and heated in the revolving drier, and when delivered to the mixer shall be at a temperature between $275^{\circ}$ and $350^{\circ} \mathrm{F}$. The temperature between these limits shall be regulated according to the temperature of the atmosphere and the working of the mixture. Both coarse and fine aggregates may be simultaneously fed into the same drier, but in such case they should previously be mixed in substantially the desired proportions, and must immediately after heating be screened into coarse and fine aggregate and stored in separate bins. The screen shall have openings of such size that the fine aggregate will all pass a 10 -mesh sieve and the coarse aggregate will all pass a $3 / 4$-inch screen and be retained on a 10-mesh sieve.

14. Preparation and composition of asphaltic concrete mixture.-(a) This shall be composed of asphalt cement, fille1, coarse and fine aggregates in the following proportions by weight:

\begin{tabular}{|c|c|c|}
\hline & Per cent & $\begin{array}{l}\text { Toler- } \\
\text { ances } \\
\text { above or } \\
\text { below }\end{array}$ \\
\hline $\begin{array}{l}\text { Asphalt cement (99 per cent+bitumen) } \\
\text { Filler } \\
\text { Fine aggregate } \\
\text { Coarse aggregate }\end{array}$ & $\begin{array}{r}6.5-9.0 \\
5.0-15.0 \\
30.0-40.0 \\
45.0-55.0\end{array}$ & $\begin{array}{r}\text { Per cent } \\
0.5 \\
1.5 \\
2.0 \\
2.0\end{array}$ \\
\hline
\end{tabular}

The engineer shall specify or approve a formula coming within the above limits which, in his judgment, will produce a pavement which will be suitable for the traffic and climatic conditions.

In no case shall these permissible variations from the approved formula be construed as authorizing a mixture which does not come within the board composition limits as set forth in the last portion of this section

(b) After the ingredients for each batch have been separately and accurately weighed in accordance with the approved formula, the coarse and fine aggregates and the mineral filler shall be charged into the mixer. After these have been thoroughly mixed the asphalt cement shall be added and the mixing continued for a period of at least one minute, or longer if necessary, to produce a homogeneous mixture in which all particles of the mineral aggregate are uniformly coated.

(c) The asphaltic concrete mixture shall conform to the following composition limits by weight:

\begin{tabular}{|c|c|c|c|}
\hline Passing- & Retained on- & Per cent & $\begin{array}{l}\text { Toler- } \\
\text { ance, } \\
\text { above or } \\
\text { below }\end{array}$ \\
\hline $\begin{array}{l}\text { Bitumen soluble in car- } \\
\text { bon disulphide. } \\
200 \text {-mesh sieve } \\
80 \text {-mesh sieve } \\
\text { 40-mesh sieve } \\
10 \text {-mesh sieve } \\
1 / 4 \text {-inch screen } \\
1 / 2 \text {-inch screen }\end{array}$ & $\begin{array}{l}\text { 200-mesh sieve } \\
\text { 40-mesh sieve } \\
10 \text {-mesh sieve } \\
1 / 1 \text {-inch screen } \\
1 / 2 \text {-inch screen. }\end{array}$ & $\begin{array}{r}6.5-9.0 \\
4.0-10.0 \\
4.0-15.0 \\
7.0-22.0 \\
5.0-20.0 \\
10.0-25.0 \\
15.0-25.0 \\
5.0-20.0\end{array}$ & $\begin{array}{r}\text { Per cent } \\
0.5 \\
\\
1.5 \\
3.0 \\
3.0 \\
3.0 \\
3.0 \\
3.0 \\
3.0\end{array}$ \\
\hline
\end{tabular}

The engineer shall specify or approve a mixture coming within the above limits which, in his judg- ment, will be suitable for the traffic and climatic conditions.

15. Paving-plant inspection.-The engineer or his authorized representatives shall have access at any time to all parts of the paving plant in order to insure the manufacture of the pavement in strict accordance with these specifications.

\section{NOTES}

The accompanying specifications are recommended for use under what may be termed "average conditions." It is realized, however, that no single standard specification will satisfactorily cover all variations in local conditions which may prevail for individual jobs. Before adopting these specifications verbatim the engineer should, therefore, give particular attention to the items listed below and, if necessary, make such changes as are suggested. In the matter of cross section a crown of one-fourth inch to the foot is recommended.

I. Thiclcness.- These specifications call for a finished thickness or depth of 2 inches for surface course mixture. If it is desired to make the depth $2 \frac{1}{2}$ inches, the following changes should be made in the specifications:

Section 1 , second sentence, change " 2 " to " $21 / 2$."

Section 21 , second sentence, change " 2 " to " $21 \%$."

Section 21 , second sentence, change " $1 \frac{13}{4}$ " to " $2 \frac{1}{4}$."

Section 21 , second sentence, change " $2 \frac{1 / 2}{2}$ " to " 3 ."

II. Asphalt cement. - The most desirable consistency or penetration for asphalt cement will vary somewhat with climatic and traffic conditions. In section 8, I, limits of penetration of from 40 to 70 are specified with a statement that between these limits the exact penetration within a 10-point range shall be as directed by the engineer. The ranges of penetration suggested for various combinations of climate and traffic are shown in the following table:

\begin{tabular}{|c|c|c|c|}
\hline \multirow{2}{*}{ Traffic } & \multicolumn{3}{|c|}{ Temperature } \\
\hline & Low & Moderate & High \\
\hline $\begin{array}{l}\text { Iight } \\
\text { Moderate.. } \\
\text { Heavy.... }\end{array}$ & $\begin{array}{l}60-70 \\
50-60 \\
50-60\end{array}$ & $\begin{array}{l}50-60 \\
50-60 \\
50-60\end{array}$ & $\begin{array}{l}50-60 \\
50-60 \\
40-50\end{array}$ \\
\hline
\end{tabular}

III. Cut-back asphalt cement for seal coat.-In case it is desired to use a cut-back asphalt cement for seal coat, the following changes should be made in the specifications:

Section 1, last sentence, strike out "hot" and substitute "cut-back."

Section 2, insert the following between paragraphs (b) and (c):

'(c) A 1-pound sample of the cut-back asphalt cement that the contractor proposes to use in his work, together with a statement as to its source and character, must be submitted with his bid. No cutback asphalt cement, other than that specified in his bid, shall be used by any contractor except with the written consent of the engineer and provided that the cut-back asphalt cement shall comply in all respects with the requirements of these specifications.'

Change designation of former paragraph " $(c)$ " to " $(d)$."

Insert after section 8 the following new section:

"SEc. 9. See Asphalt Association specification A-2 (p. 404), 'Notes'-III, section 7, Cut-back asphalt cement."

Renumber sections 9 to 24 .

V. Samples.-It is recommended that the following procedure be adopted in connection with sampling materials for test. 
Samples should be put in clean containers, preferably tin boxes or cans. The following amounts of the different materials are required for test:

Broken stone _... Filler-10.

Sand

Refined asphalt

Asphalt cement

Flux

Method of sampling-Fxtreme care should be taken in every case to obtain a sample which is truly representative of the material to be examincd. These samples are for the use of the testing laboratory only and should not be used for testing at the plant before submitting them to the laboratory. The particular precautions to be observed in each case are given below:

Broken stone.-A sufficient number of 5-pound samples to be taken from different parts of the pile. These should be thoroughly mixed together and be reduced by quartering to the desired size.

Filler.-Samples should be taken from several bags and mixed.

Sand.-Samples should be taken from the interior of the pile where the sand is damp; a sufficient number of 1-pound samples to be taken from different parts of the pile. These should be thoroughly mixed together and reduced by quartering to the desired size.

Refined asphalt and asphalt cement-(a) In barrcls. - At least one sample should be taken from each batch. It should be taken at sufficient depth below the surface to insure obtaining representative material free from all dirt or other extraneous matter, and at a point not less than 4 inches distant from the top and sides of the barrel.

(b) In tank cars. - The contents of the tank should be heated until completely liquid throughout. It should then be agitated and thoroughly mixed by means of air or steam, after which the sample shall be taken from the dome in such a manner as to obtain the asphalt from a point at least 3 feet below the surface.

(c) In kettles.- The contents of the kettles must be completely liquid and thoroughly agitated previous to and during sampling. The sample may be taken from the pipe through which the material is delivered to the mixer by means of a clean dipper.

Flux.-The directions given for sampling refined asphalt and asphalt cement apply to this material except that under ordinary conditions it is not necessary to agitate the contents of the tank car.

Surface mixture.-Samples may be taken at the plant or on the street.

If taken at the plant, they should preferably be obtained from the wagons or trucks, extreme care being observed to obtain an average representative sample which is not contaminated with dust, sand, or oil from the wagon bodies, and shows no evidence of separation having taken place after mixing and dumping. The first or last portions of a batch as it comes from the mixer should not be included in the sample.

If obtained on the street, the sample should be taken after the mixture has been shoveled and raked, care being observed to obtain an average sample which shows no signs of segregation in transit or excessive richness due to accumulation of bitumen at the bottom or top of the load or oil from the wagon bodies.

Samples of surface mixture should weigh not less than 2 pounds and should be placed in a box or suitable container.

VI. Method of testing asphaltic concrete surface mixtures.-It is recommended that the following laboratory method be used to determine conformity of surface mixtures with specification requirements.
Determination of bitumen content and mesh composition of asphaltic concrete surface mixture.Weigh out from 350 to $500 \mathrm{~g}$ of the mixture and extract the bitumen from it in a centrifugal extractor or suitable continuous hot extractor, using chemically pure carbon disulphide as a solvent for the bitumen. Remove the mineral aggregate from the extractor after the bitumen has been completely extracted and dry it at $250^{\circ} \mathrm{F}$. for one-half hour. Evaporate the filtrate containing the bitumen, burn the bitumen, add the filter paper to it (if filter paper is used), and burn to an ash. Add the ash to the dried mineral aggregate and weigh. The difference between the weight of surface mixture originally taken and the combined weight of the ash and dried mineral aggregate is considered as the weight of the bitumen in the sample. The combined ash and mineral aggregate is then sifted through the following sieves and screens (in the order named) and the percentages of the various. sized particles calculated: $200,100,80,50,40,30$, 20, 10, 1/4-1nch, 1/2-inch, and 3/4-inch. Sifting shall be continued on each sieve until less than 1 per cent passes through the sieve during the last minute of sifting.

Remarks.-The limits of accuracy of this test are as follows:

For bitumen content, 0.3 per cent above or below the result obtained. For mesh composition, 10 per cent of the result obtained (above or below).

American Society for Municipal Improvements, standard specifications for asphalt macadam pavement, 1927.

1. General description.-The asphalt macadam pavement shall consist of coarse, broken stone or slag consolidated by rolling while in place on the roadbed, the interstices between the coarse particles being subsequently filled with hot asphalt cement. and finer particles of crushed stone or slag. It shall be laid to a finished thickness of 3 inches upon a foundation constructed in accordance with the specifications for foundation and shall be finished with a seal coat of hot asphalt cement and stone or slag chips or fine gravel. All materials shall conform to the requirements of these specifications.

Note.- When this pavement is to be laid on a Portland cement concrete foundation it is recommended that the foundation mix be not richer than $1: 3: 6$ or its equivalent. In cases where weak subre is encountered the engineer unust exercise his own judpment as to thickening the concrete foundation, enriching the mixture, adding reinforcement, or otherwise providing for safety.

Other types of foundation than providing for safety. and it is recommended that old gravel or macadam be taken into consideration when in the judgment of the engineer, conditions warrant their use for foundation purposes. The engineer must, however, see that the old gravel or macadam foundations be carefully trued and brought to uniform surface and otherwise be of proper strength before the wearing surface is placed.

\section{MATERIAIS}

2. Sources of supply.-(a) Approval of sources of supply of mineral aggregate shall be obtained from the engineer prior to the delivery of materials, and samples of each shall be submitted as directed by the engineer.

(b) A 1-pound sample of the asphalt cement that the contractor proposes to use must be submitted to the engineer before the use of such material in the work will be permitted. Every refined asphalt and asphalt cement admitted under these specifications shall be equal in quality to the recognized standard for its particular kind or type of asphalt.

(c) Unless otherwise permitted by the engineer, all samples shall be submitted at least three weeks prior to use of the materials in the performance of the work and in no case shall they be used until they have been examined and approved by him. Whenever during the course of the work new deliv- 
eries of paving materials are received by the contractor samples of these shail at once be submitted to the engineer and their use in the work will not be permitted until they have been examined and approved by him.

3. Methods of testing.-Unless otherwise specified, all tests shall be conducted in accordance with the latest standards or tentative standards of the American Society for Testing Materials.

4. Mineral aggregate.-Requirements same as for Asphalt Association specification A-1. (See 518.37.) 5. Asphalt cement.-Requirements same as for Asphalt Association specification B-7. (See p. 374.)

Nors.- Where less than 99 per cent of the asphalt cement is soluble in carbon tetrachloride the percentage of bitumen (solubiliry in carbon disulphide) shall be reported.

\section{NOTES ON SPECIFICATIONS FOR ASPHAIT MAC- ADAM PAVEMENT}

The accompanying specifications are recommended for use under what may be termed average conditions. It is realized, however, that no single standard specification will satisfactorily cover all variations in local conditions which may prevail for individual jobs. Before adopting these specifications verbatim the engineer should, therefore, give particular consideration to the items listed below and if necessary make such changes as are suggested. In the matter of cross section a cromn of three-eighth inch to the foot is recommended.

I. Thickness.-These specifications call for a finished thickness of 3 inches. If it is desired to make the thickness $2 \frac{1}{2}$ inches the following changes should be made:

Section 1 , sixth line, change 3 to $3 \frac{1}{2}$.

II. Mineral aggregate. - When it is necessary to use rock or mine tailings with a per cent of wear of more than 6 a maximum of 8 should be fixed if practicable, in which case in section $4(a)$, fifth line, change 6 to 8 .

If only a very soft product with a per cent of wear greater than 8 is available it is advisable to increase the thickness of the surface course to 315 inches and to make the following changes:

Section 1, sixth line, change 3 to 31

Section $4(a)$, strike out third and fourth sentences and insert the necessary description of the material to be used.

Section 4 (b) table, first column, change $2 \frac{1}{2} 2$ to 3 inches; third column, change $2 \frac{1}{2}$ to 3 inches.

Section 5, I, change 85 to 150 and 120 to 150 and strike out the sentence in brackets. This change should be made without reference to the recommendation under IV, Asphalt cement.

III. Use of crushed gravel.-If it is desired to allow for the use of gravel in the construction of the asphalt macadam the following sentence should be inserted after the first sentence in section $4(a)$. If produced by crushing gravel only that portion of the gravel which has been retained upon a screen with $31 / 2$ inch or larger openings shall be used in its manufacture.

IT. Asphalt cement.-The most desirable consistency or "penetration" for asphalt cement may vary somewhat with climate and traffic conditions. In section 5 , I, limits for penetration of from " 85 to 150 " are specified with a statement that between these limits the exact penetration limits desired ( 85 to 100,100 to 120 , or 120 to 150 ) shall be specified by the engineer. The ranges of penetration suggested for various combinations of climate and traffic are shown in the following table:

\begin{tabular}{|c|c|c|c|}
\hline \multirow{2}{*}{ Traffic } & \multicolumn{3}{|c|}{ Temperature } \\
\hline & Low & Moderate & High \\
\hline 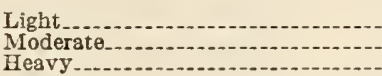 & $\begin{array}{r}120-150 \\
100-120 \\
85-100\end{array}$ & $\begin{array}{r}100-120 \\
100-120 \\
85-100\end{array}$ & $\begin{array}{l}85-100 \\
85-100 \\
85-100\end{array}$ \\
\hline
\end{tabular}

American Society for Testing Materials, asphalt cement.

(See 505.13, p. 147.)

Asphalt Association, specification A-1, for asphaltmacadam surface course (penetration method), April 10, 1929 .

(The full text of the specification includes methods of construction and the following specifications for materials:)

Section 1. General description.-(a) The asphaltmacadam surface course shall consist of mineral aggregate and asphalt cement combined as hereinafter specified and shall be laid upon the prepared base to a finished thickness of not less than $2^{1 / 2}$ inches. The method of construction shall proceed substantially as follows:

I. A layer of coarse aggregate rolled to the approximate thickness of the finished surface course shall be treated with hot asphalt cement applied as hereinafter specified.

II. The surface voids shall then be filled by applying and rolling in sufficient intermediate aggregate.

III. A seal coat of hot asphalt cement shall then be applied and covered with fine aggregate.

(b) All material shall conform to the requirements of these specifications.

\section{MATERIALS}

SEc. 2. Mineral aggregate-(a) The mineral aggregate shall consist of broken stone, mine tailings, or slag. It shall be of reasonably uniform quality throughout and shall be clean and free from dust and from an excess of flat or elongated pieces. Rock or mine tailings from which it is produced shall have a per cent of wear of not more than 6. Slag from which it is produced shall weigh not less than 70 pounds per cubic foot for each size specified.

(b) When tested by means of laboratory screens the coarse, intermediate, and fine aggregate shall meet, respectively, the following requirements and be uniformly graded between the limits given:

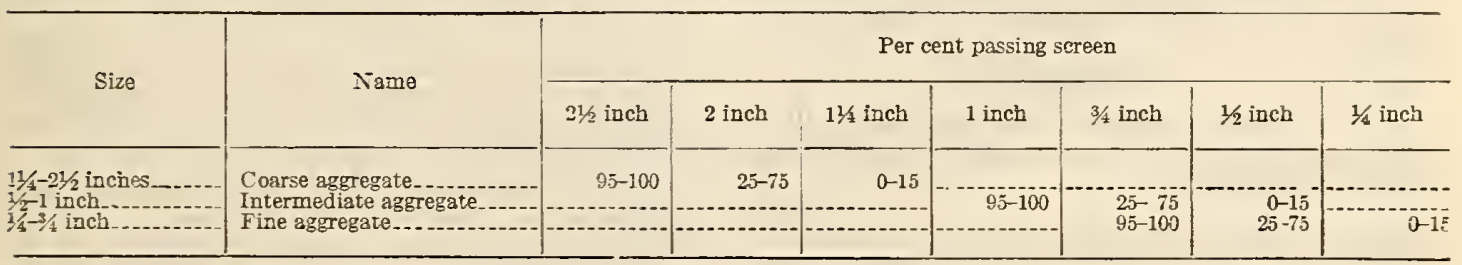


Sec. 3. Asphalt cement.-The asphalt cement shall be homogeneous, free from water, and shall not foam when heated to $175^{\circ}$ C. $\left(347^{\circ} \mathrm{F}\right.$.). It shall meet the following requirements for penetration:

Penetration at $25^{\circ} \mathrm{C}$. ( $\left.77^{\circ} \mathrm{F}.\right) 100 \mathrm{~g}, 5$ seconds, 60 to 150 . Between these limits the cngineer shall specify as desired (60 to 70,85 to 100 , 100 to 120 , or 120 to 150 .)

Sec. 4. Sources of supply.--Requirements same as for specification $\mathrm{B}-7$.

SEc. 5. Methods of testing.-Test methods same as for specification B-8, omitting A. S. T. M. D7.

The accompanying specifications for asphaltmacadam surface course are recommended by the Asphalt Association for use under what may be telmed average conditions. It is realized, however, that no single standard specification will cover satisfactorily all variations in local conditions which may prevail for individual jobs. Before adopting these specifications verbatim the engineer should, thereforc, give particular consideration to the items listed below and if neccssary make such changes as are suggested. In the inatter of cross section a crown of three-eighths inch to the foot is recommended.

I. Thickness. - These specifications call for a finished thickness of $2 \frac{1}{2}$ inches, which is the minimum recommended. If it is desired to make the thickness 3 inches the following change should be made in the specifications.

Secrion 1. (a) Third line, change $2 \frac{1}{2}$ to 3.

II. Coarse aggregate.-When it is necessary to use rock or mine tailings with a per cent of wear of more than 6 a maximum of 8 should be fixed, if practicable, in which case in section $2(a)$, fourth line, change 6 to 8 .

If only a very soft product with a per cent of wear greater than $\delta$ is available it is advisable to increase the thickness of the surface course to 3 inches and to make the changes recommended under I. Thickness. In addition the following changes should be made:

Section $2(a)$, strike out third and fourth sentences.

Section $2(b)$, table, first column, change $2 \frac{1}{2}$ to 3 ; third column, change $2 \frac{1}{2}$ to 3 .

III. Use of crushed gravel. - If it is desired to allow for the use of gravcl in the construction of the asphalt macadam the following sentence should be inserted after the first sentence in section $2(a)$ : "If produced by crushing gravel only that portion of the gravel which, has been retained upon a screen with $3 \frac{1}{2}$-inch or larger openings shall be used in its manufacture."

Asphalt Association, specification A-2, for asphaltic concrete surface course, April 10, 1929 (coarse graded aggregate type).

(The full text of the specification includes methods of construction and the following specifications for materials:)

Section 1. (a) General description.-The asphaltic concrete surface course shall consist of a mineral aggregate composed of coarse aggregate, fine aggregate, and mineral filler, uniformly mixed with asphalt cement, and shall be laid upon the prepared base to a finished thickness of 2 inches. A seal coat of hot asphalt cement shall then be applied and covered with intermediate aggregate.

(b) All materials and methods of preparation shall conform to the requirements of these specifications.

\section{MATERIALS}

SEc. 2. Coarse aggregate.--Requirements same as for specification $\mathrm{B}-8,518.27$, page 374 , with the following exceptions:

Percentage of wear, not more than

Per cent Passing $1 \frac{1}{4}$-inch screen (instead of $1 \frac{1}{2}$-inch)

Add.- When only one commercial product is used to furnish coarse aggregate not more than 10 per cent of this product shall pass a $1 / 4$-inch laboratory screen.
SEC. 3. Fine aggregate.-Requirements same as for specification B-8.

SEc. 4. Mineral filler.-The mineral filler shall consist of thoroughly dry limestone dust or other mineral dust approved by the engineer which, when tested by means of laboratory sieves, shall meet the following requirements:

Passing 30-mesh sieve.

Per cent

Per 100

SEc. 5. Intermediate aggregate. - The intermediate aggregate for seal coat shall consist of broken stone, broken slag, mine tailings, or pea gravel. It shall be of reasonably uniform quality and shall be free from dust. When tested by means of laboratory screens it shall meet the following requirements:

Passing 1/2-inch screen, not less than.

Per cent

Passing 1/4-inch screen, not more than......

SEc. 6. Asphalt cement.-The asphalt cement shall be homogeneous, free from water, and shall not foam when heated to $175^{\circ} \mathrm{C}$. $\left(347^{\circ} \mathrm{F}\right.$.). It shall meet the following requirements for penetration.

Penetration at $25^{\circ} \mathrm{C}$. ( $\left.77^{\circ} \mathrm{F}.\right) 100 \mathrm{~g}, 5$ seconds 40 to 70

Between these limits the engineer shall specify as desired: 40 to 50 , 50 to 60 , or 60 to 70 .

SEc. 7. Sources of supply.-Practically the same as for specification B-8.

Stc. 8. Methods of testing.--Same as for specification B-8.

\section{PREPARATION AND COMPOSITION OF MIXTURE}

SEc. 9. Preparation of asphalt cement.-Same as for specification B-8.

SEc. 10. Preparation of mineral aggregate.-Same as for specification B-8.

Sec. 11. Preparation and composition of surface course mixture.-(a) The coarse and fine aggregate and mineral filler for surface course mixture shall be measured separately and accurately either by weight or volume for each batch to be mixed. The required quantity of hot asphalt cement for each batch shall be measured by actual weighing with scales attached to the asphalt-cement bucket. The mixture shall be made in an approved twin pug or batch mixer by first charging it with the coarse aggregate, fine aggregate, and mineral filler. When the inixture is prepared in a twin pug mixer the volume of mineral aggregate and asphalt cement shall not be so great as to extend above the tips of the mixer blades when these blades are in a vertical position. After the total mineral aggregate has been thoroughly mixed for a period of not less than 15 seconds the asphalt cement shall be added and the mixing continued for a period of at least 30 seconds or longer if necessary to produce an homogeneous mixture in which all particles of the aggregate are uniformly coated.

(b) The constituents of the surface course mixture shall be combined in such proportions as to produce a mixture conforming to the following. composition limits by weight:

Passing 11/4-inch, retained on $3 / 4$-inch screen Per cent

Passing $3 / 4$-inch, retained on $1 / 4$-inch screen

Passing $1 / 4$-inch, retained on

Bitumen (soluble in carbon disulphide)

The proportions shall be varied within the limits designated as directed by the engineer.

SEc. 12. Paving-plant inspection.-Same as for specification B-8.

SEC. 13. Transportation of mixture.-Same as for speciñcation $\mathrm{B}-8$. 


\section{NOTES}

These specifications are recommended for use under what may be termed average conditions. It is realized, however, that no single standard specification will cover sarisfactorily all variations in local conditions which may prevail for individual jobs. Before adopting thesc specifications verbatim the engineer should, therefore, give particular consideration to the items listed below and, if necessary. make such changes as are suggested. In the matter of cross section a crown of one-fourth inch to the foot is recommended.

I. Stone, slag, or mine tailings.-In section 2 at least 95 per cent of the broken stone, broken slag, or mine tailings is required to pass a $1 / 2$-inch screen. If desired, a slightly larger maximum size may, however, be used, in which case the following change should be made:

Section 2, change one-half inch to three-fourths inch and change 95 per cent to 100 per cent.

II. Use of gravel in surface course. - If it is desired to allow for the use of gravel in the construction of the surface course, insert "Gravel" in sections $1,2,6$, 7,9 , and 10 where needed.

Asphalt Association, specification A-4, for sheetasphalt binder and surface courses, April 10, 1929.

(The full text of the specification includes methods of construction and the following specifications for materials:)

Section 1. General description.-(a) The binder course shall consist of coarse aggregate and sand, uniformly mixed with asphalt cement, and shall be laid upon the prepared base to a finished thickness of $1 \frac{1}{2}$ inches.

(b) The surface course shall consist of sand and mineral filler, uniformly mixed with asphalt cement and shall be laid upon the compacted binder course to a finished thickness of $1 \frac{1}{2}$ inches.

(c) All materials and methods of preparation shall conform to the requirements of these specifications.

\section{MATERIALS}

SEC. 2. Coarse aggregate.-The coarse aggregate shall consist of broken stone produced from tough, durable rock having a per cent of wear not more than 6. It shall be of reasonably uniform quality throughout and shall be clean and free from dust and an excess of flat or elongated pieccs. When tested by means of laboratory screens and sieves it shall meet the following requirements and be uniformly graded between the limits given:

Passing 11/4-inch screen, not less than

Per cent

Passing

Passing 10-mesh sieve, not more than

SEc. 3. Sand for binder course.-The sand for binder course shall consist of clean, hard, durable grains, free from clay, loam, or other foreign matter, and when tested all shall pass a 1/4-inch laboratory sereen.

Sec 4. Sand for surface course-See specification A-3, Section 3, Sand (a) (given above).

Sec. 5. Mineral filler.-Same as for specification A-2 (above).

SEc. 6. Asphalt cement.-Same as for specification $\mathrm{A}-3$.

Src. 7 Sources of supply.-Same as for specification $\mathrm{B}-8,518.27$, page 374 .

Sec. 8. Methods of tcsting.-Same as for specification $\mathrm{B}-8$.

PREPARATYON AND COMPOSTTION OF MIXTURE

Sec. 9. Preparation of asphalt cement.-Same as for specification B-8.
SEc. 10. Preparation of mineral aggregates.-(a) The coarse aggregate and sand for binder course shall be dried and heated at the paving plant in suitably designed revolving driers. They shall be heated to a temperature of $225^{\circ}$ to $350^{\circ} \mathbf{F}$., as determined on the mixing platform. Both may be fed simultaneously into the same drier, but, in such case, immediately after heating they shall be screened into coarse and fine aggregate and stored in separate bins.

(b) The sand for surface course aggregate shall be dried, heated, and stored separately at the paving plant as described in the preceding paragraph, and, when delivered at the mixer, shall be at a temperature of $275^{\circ}$ to $400^{\circ} \mathrm{F}$. When a mixture of two or more sands is required in order to produce a material conforming to the requirements of section 4, such combination shall be made either before the sand is fed into the drier by proportioning the sands from separate storage piles and mixing them by means of shovels or suitable mechanical device.

(c) A registering pyrometer shall be installed at a suitable point at the discharge end of the drier with the registering device so located as clearly to indicate to the drum freman the temperature of the mineral aggregate when discharged.

SEc. 11. Preparation and composition of binder course mixture.-(a) The hot coarse aggregate and sand for binder course shall be measured separately and accurately either by weight or volume for each batch to be mixed. The required quantity of hot asphalt cement for each batch shall be measured by actual weighing with scales attached to the asphalt cement bucket. The mixture shall be made in an approved twin pug mixer or a rotary mixer in which the asphalt is sprayed into and incorporated with the mineral aggregate under pressure by first charging it with the mineral aggregate. After these have been thoroughly mixed for a period of not less than 15 seconds the asplialt cement shall. be added and the mixing continued for a period of at least 30 seconds, or longer if necessary, to produce a homogeneous mixture in which all particles of the mineral aggregate are uniformly coated.

(b) The constituents of the binder course shall be combined in such proportions as to produce a mixture conforming to the following compesition limits, by weight:

Coarse aggregate retained on 10-mesh sieve............... 60 -80 Sand and material passing 10-mesh sieve................ Bitumen (soluble in carbon disulphide) .................... 4-6

The engineer shall specify or approve a mixture coming witlin the above limits and the maximum permissible variations from the mixture so specified are given below:

Bitumen Sagd .

SEc. 12. Preparation and composition of surface coursc mixture.-(a) The hot sand and the inineral filler shall be measured separately and accurately by weight or volume for each batch to be mixed. The hot asphalt cement for each batch shall be measured by actual weighing with scales attached to the asphalt cement bucket. The mixture shall be made in an approved twin pug mixer, or a rotary mixer in which the asphalt is sprayed into and incorporated with the mineral aggregate under pressure, by charging it first with the sand and mineral filler. When the mixture is prepared in a twin pug mixer the volume of mineral aggrcgate and asphalt cement shall not be so great as to extend above the tips of the mixer blades when these blades are in a vertical position. After the sand and mineral filler have been thoroughly mixed for a 
period of 15 to 30 seconds as directed by the engineer, the asphalt cement shall be added and the mixing continued for a period of at least 30 seconds, or longer, if necessary, to produce a homogeneous mixture, in which all particles of the mineral aggregate are coated uniformly.

(b) The constituents of the surface course shall be combined in such proportions as to produce a mixture conforming to the following composition limits, by weight, and having a density when thoroughly compressed of not less than 95 per cent of the calculated maximum density of a voidless mixture composed of the same materials in like proportions:

Passing 10-mesh, retained on 40-mesh sieve

Per cent Passing 40-mesh retained on 80-mesh sieve

Passing 80-mesh, retained on 200 -mesh sieve

Passing 200-mesh sicve.

$10-40$

on 200-mesh sieve

The engineer shall specify or approve a mixture coming within the above limits which, in his judgment, will be suitable and the maximum permissible variations from the mixture as specified are given below:

Passing 10-mesh

Passing 40-mes:

Passing 80-mesh

Passing 200-mesh_._._._._._._._. 1.5 per cent above or below.

Bitumen 0.5 per cent above or below.

SEc. 13. Paving plant inspection.-Same as for specification $\mathrm{B}-8$.

SEc. 14. Transportation of mixiures.-Same as for specification $\mathrm{B}-8$.

\section{NOTES}

The above specifications are recommended by the Asphalt Association for use under what may be termed average conditions. It is realized, however, that no single standard specification satisfactorily will cover all variations in local conditions which may prevail for individual jobs. Before adopting these specifications verbatim the engineer, therefore, should give particular attention to the items listed below and, if necessary, make such changes as are suggested. In the matter of cross section a crown of one-fourth inch to the foot is recommended.

I. Thickness.- These specifications call for a total finished thickness or depth of 3 inches. If it is desired to make the depth $2 \frac{1}{2}$ inches, the following changes should be made in the specifications:

Section $1(b)$, last line, change $1 \frac{1 / 2}{2}$ to 1 .

II. Use of broken slag in binder course.-If it is desired to permit the use of broken slag in the binder course the following changes should be made:

Section 2, end of first sentence, insert " or of broken slag weighing not less than 70 pounds per cubic foot for the size specified."

III. Use of gravel in binder course.-If it is desired to permit the use of gravel in the binder course the following change should be made:

Section 2, end of first sentence, insert "or of gravel composed of hard, durable rock particles free from clay or coatings of any character."

IV. High filler mixtures.-Investigations which have been conducted indicate definitely that where exceptionally high stability is required, as in the case of very heavy traffic pavements, this may be secured by an increase in the 200-mesh filler particles in the mixture above the maximum of 20 per cent allowed by these specifications. When such increase is made in filler content there should be a corresponding increase in maximum temperature requirements to permit of proper raking of the hot mix.

Asphalt Association, specification $\mathrm{A}-5$, for asphaltic concrete binder and surface courses. April 10 , 1929.
(The full text of the specification includes material relative to construction and the following specification for materials:)

Section 1. General description.-(a) The binder course shall consist of coarse aggregate and sand, uniformly mixed with asphalt cement, and shall be laid upon the prepared base to a finished thickness of $1 \frac{1}{2}$ inches.

(b) The asphaltic concrete surface course shall consist of a mineral aggregate composed of broken stone, broken slag, or mine tailings, sand, and mineral filler, uniformly mixed with asphalt cement, and shall be laid upon the compacted binder course to a finished thickness of $1 \frac{1 / 2}{2}$ inches.

(c) All materials and methods of preparation shall conform to the requirements of these specifications.

\section{MATERIALS}

Sec. 2. Coarse aggregate for binder course.-The coarse aggregate shall consist of broken stone produced from tough, durable rock having a per cent of wear of not more than 6 , or of broken slag weighing not less than 70 pounds per cubic foot for the size specified. It shall be of reasonably uniform quality throughout and shall be clean and free from dust and an excess of flat or elongated pieces. When tested by means of laboratory screens and sieves it shall meet the following requirements and be uniformly graded between the limits given:

Passing 11/4-inch screen, not less than

Per cent

Passing $1 / 2$-inch, retained on $1 / 4$-inch screen

Passing 1/4-inch screcn, retained on 10-mesh sieve-

Passing 10-mesh sieve, not more than

SEc. 3. Sand for binder course.-Same as A-4, 518.37 (above).

SEc. 4. Stone, slag, or mine tailings for wearing course.-Same as A-3 (above).

SEc. 5. Sand for wearing course.-Same as A-3.

Sec. 6. Mineral filler.-Same as A-2 (above).

SEc. 7. A sphalt cement.-The asphalt cement shall be homogeneous and free from water and shall not foam when heated to $175^{\circ} \mathrm{C}$. $\left(347^{\circ} \mathrm{F}\right.$.). It shall meet the following requirements for penetration:

Penetration at $25^{\circ} \mathrm{C}$. ( $\left(77^{\circ} \mathrm{F}.\right) 100 \mathrm{~g}, 5$ seconds, 25 to 60 . (Between these limits the engincer shall specify as desired, 25 to 30,30 to 40,40 to 50 , or 50 to 60 .)

SEc. 8. Sources of supply.-Same as Asphalt Association, specification B-8, 518.27, page 374

SEC. 9. Methods of testing.-Same as B-8.

\section{PREPARATION AND COMPOSITION OF MIXTURES} $\mathrm{B}-8$.

SEc. 11. Preparation of mineral aggregates.-Same as for specification B-8, with the following addition: The broken stone, broken slag, or mine tailings and sand shall be dried and heated at the paving plant in suitably designed revolving driers. They shall be heated to a temperature of $275^{\circ}$ to $375^{\circ} \mathrm{F}$. as determined on the mixing platform. Both may be simultaneously fed into the same drier in proper porportions or mixed in such proportions prior to being heated.

SEc. 12. Preparation and composition of binder course mixture.-Same as A-4.

Sec. 13. Preparaition and composition of surface course mixture.-The hot broken stone, broken slag, or mine tailings and sand shall be measured accurately by weight or volume, either as a single, correctly proportioned combination or separately, for each batch to be mixed. The mineral filler shall be measured in like manner. The hot asphalt cement for each batch shall be measured by actual weighing with scales attached to the asphalt cement bucket. 
The mixture shall be made in an approved twin pug mixer, or a rotary mixer in which the asphalt is sprayed into and incorporated with the mineral aggregate under pressure, by first charging it with the broken stone, slag or mine tailings, sand and mineral filler. When the mixture is prepared in a twin pug mixer the volume of mineral aggregate and the asphalt cement shall not be so great as to extend above the tips of the mixer blades when these blades are in a vertical position. After the total mineral aggregate has been thoroughly mixed for a period of 15 to 30 seconds as directed by the engineer, the asphalt cement shall be added and the mixing continued for a period of at least 30 seconds or longer, if necessary, to produce a homogeneous mixture in which all particles of the mineral aggregate are coated uniformly.

(b) The constituents of the surface course shall be combined in such proportions as to produce a mixture conforming to the following composition limits, by weight: Passing 200-mesh sieve

Passing 80-mesh, retained on 200-mesh sieve. Passing 40-mesh, retained on 80-mesh sieve...... 11 Passing 10-mesh, retained on 40-mesh sieve $\ldots . . . . . . .7 \quad 7$ Passing 1/2-inch screen, retained on 10-mesh sieve....... $20-35$

The engineer shall specify or approve a mixture coming within the above limits and the maximum permissible variations from the mixture so specified are given below:

Bitumen.

Sand

aber or below

SEc. 14. Paving plant inspection.-Same as B-8.

SEc. 15. Transportation of mixtures.-Same as B-8.

Federal Specifications Board, specification No.

276a, United States Government master specification for asphalt for use in road and pavement construction, May 8, 1925.

See 505.11, page 145 .

Federal Specifications Board, specification No. 459, United States Government master specification for broken stone and broken slag for binder course, sheet asphalt pavement, January 25, 1927.

See 512.15 , page 251 (for broken stone), and 512.2 , page 255 (for broken slag).

United States Department of Commerce, Bureau of Standards, Simplified Practice Recommendation No.

4, for asphalt (penetration limits), January 1, 1924. See 505.0, page 145 .

\subsection{Sand Clay or Topsoil Pavements.}

American Society for Testing Materials, tentative specifications for commercial sizes of sand and gravel for highway construction, serial designation D64-20T, 1920.

See 512.10, page 215 .

American Society for Testing Materials, tentative specifications for natural or artificial sand-clay mixtures for road surfacing, serial designation D67-23T, 1923.

1. The sand-clay shall be composed of either a naturally occurring or artificially prepared mixture of hard, durable, preferably angular, fragments of sand, together with silt and clay with or without gravcl, and shall be free from an excess of feldspar or mica.

2. (a) When tested by means of laboratory sieves and screens the matcrial shall conform to the following requirements as to grading:

Passing 2-inch screen
Passing 1/4-inch screen

Per cent

(b) The material, if any, retained on the $1 / 4$-inch screen shall be uniformly graded from the maximum size present to $1 / 4$ inch. (c) The material passing the 1/4-inch screen shall conform to the following requirements:

Per cent

Total sand Sand retained on No. 60 sieve. Silt The tests for the mechanical analysis of the

3. The tests for the mechanical analysis of the sand, clay, natural sand-clay or topsoil mixtures shall be made in accordance with sections 4 to 7 , except that the determination of the percentage of suspension clay may be omitted and the percentage of total clay may be calculated by difference as follows:

$100-$ (percentage of sand + percentage of silt $)=$ percentage of clay.

4. The sample as received shall be dried in an oven at a temperature not to exceed $100^{\circ} \mathrm{C} .\left(212^{\circ} \mathrm{F}\right.$.), and shall then be broken up in a mortar by means of a rubber-covered pestle, care being taken not to break any fragments of rock or sand. The sample shall then be passed through a $1 / 4$-inch screen, and the material passing this screen further pulverized by rolling out in a thin layer on a rubber pad, using a rubber-covered roller, after which the sample shall be passed through a No. 10 sieve.

5. The apparatus shall consist of a wide-mouthed cylindrical glass jar of at least 10 liters capacity; a soil centrifuge with a 4 or 8 tube head of such diameter that a speed of $1,500 \mathrm{r}$. p. m. will exert a centrifugal force approximately 500 times the force of gravity; 4 centrifuge tubes of 100 cc capacity; and a chemical balance sensitive to $0.001 \mathrm{~g}$.

6 . By the method of quartering, a sample weighing approximately $25 \mathrm{~g}$ and passing the No. 10 sieve shall be selected and placed in a beaker with approximately $500 \mathrm{cc}$ of distilled water. This mixture shall be gradually brought up to the boiling point during a period of one hour and then be allowed to simmer for an additional hour. After standing until cool, the material in the beaker shall be thoroughly brushed and dispersed with a stiff brush for 2 or 3 minutes and then allowed to stand for 8 minutes. The supernatant liquid shall then be decanted to a depth of $8 \mathrm{~cm}$ from the surface of the liquid into a vessel having a capacity of about 10 liters. About $500 \mathrm{cc}$ of ammoniated water (concentration $1: 500$ ) shall be added to the material remaining in the beaker and the brushing repeated as before. After again standing for 8 minutes, the supernatant liquid shall be decanted into the large vessel. This process shall be repeated until the supernatant liquid becomcs clear after 8 minutes sedimentation. The sand and silt have now been separated from the clay and suspension clay, which are contained in the large vessel. The material in the beaker shall then be transferred to an evaporating dish, dried to constant weight at a temperature not to exceed $100^{\circ} \mathrm{C}$. $\left(212^{\circ}\right.$ F.), cooled, and a mechanical analysis made, using No. 20, No. 60, No. 100, and No. 200 sieves. Material retained on each sieve shall be recorded as percentage of the original sample, and that which passes the No. 200 sieve shall be recorded as the percentage of silt in the original sample.

The volume of liquid in the large vessel shall now be brought up to at least 10 liters by the addition of ammoniated water, and the vessel thoroughly shaken until all material is in suspension. Seventy cubic centimeters of the liquid shall then be siphoned from this vessel at a depth of about one-half the total depth of the liquid into each of 4 centrifuge tubes. The tubes shall then be mounted in the centrifuge and run for 30 minutes at a speed which for the diameter of the head used will exert a centrifugal force approximately 500 times the force of gravity. The material which has been thrown out of the liquid by centrifuging shall be classified as clay and that which remains in suspension as suspension clay. 
The solution containing the suspension clay shall then be decanted, evaporated to dryness, and the weight of the suspension clay determined. The clay remaining in the tube shall also be dried and weighed.

The weight of suspension clay is found froin the liquid in four tubes, or $280 \mathrm{cc}$; consequently this weight must be multiplied by the factor obtained by dividing the total volume used by $280 \mathrm{cc}$ in order to obtain the total suspension clay in the original sample. The weight of clay is determined from the clay in one tube, or $70 \mathrm{cc}$; therefore, the total volume divided by 70 cc gives the factor by which this weight should be multiplied to give the total clay in the original sample. The total weights of clay and suspension clay thus obtained are converted into percentages of the original $25 \mathrm{~g}$ sample. The determination of the percentage of suspension clay by this method includes any water-soluble material present which may be determined separately if desired.

7. In case it is not considered necessary to make a determination of the percentage of suspension clay, the following procedure for determination of total clay (clay plus suspension clay) may be followed:

An aliquot portion of the total volume of water used in washing the sample shall be taken and evaporated to dryness and the weights of clay plus suspension clay obtained, which shall be expressed as the percentage of total clay in the original $25 \mathrm{~g}$ sample.

American Society for Testing Materials, tentative method of test for quantity of clay in sand-clay, topsoil, and semigravel for highway construction, serial designation D73-20T, 1920.

1. This test covers the determination of the quantity of clay in that portion of sand-clay, topsoil, and semigravel for highway construction which passes a No. 10 sieve. ${ }^{76}$

2. Five hundred grams of material shall be dried to constant weight at a temperature below $176.6^{\circ} \mathrm{C}$. $\left(350^{\circ} \mathrm{F}\right.$.). The material shall be gently pulverized to break down soft clods or masses, but not to grind or break hard material. It shall be passed through a No. 10 sieve and the coarse residue weighed and recorded as "coarse material." The material passing the No. 10 sieve shall be used as a starting point of a percentage analysis as follows:

3. Two samples of $50 \mathrm{~g}$ of this material shall be weighed out for duplicate analyses. Each shall be placed in a tared wide-mouth bottle $(5$ to $6 \mathrm{~cm}$ in diameter and 12 to $15 \mathrm{~cm}$ high). Five cubic centimeters of dilute ammonia water and about $200 \mathrm{cc}$ of water shall be added. The bottle shall be closed with a cork or glass stopper and shaken vigorously for 20 minutes. The sample shall be allowed to settle eight minutes and decanted carefully, or the supernatant liquid siphoned off to a depth of $8 \mathrm{~cm}$ below the surface of the liquid. (The depth of the liquid in the bottle should be sufficient to leave about $4 \mathrm{~cm}$ below the point of siphoning.) The bottle shall again be filled with water, shaken for 3 minutes, allowed to settle and siphoned off as before. This process shall be repeated until the supernatant liquid is clear. Care should be taken to wash the stopper and neck of the bottle free from coarse material before decanting. The bottle and washed material shall be dried to constant weight at a temperature between $100^{\circ}$ and $110^{\circ} \mathrm{C}$. $\left(212^{\circ}\right.$ to $230^{\circ} \mathrm{F}$.) weighed, and the net weight of washed material determined.

4. The percentage of clay shall be calculated from the formula:

Percentage of clay $=\frac{\text { Original weight-weight after washing }}{\text { Origin }} \times 100$

70 For specifications for sieves, see A. S. T. M. E 11, 500.2, p. 1.
5. As a check the washings drawn off shall be collected and evaporated to dryness for direct recovery of the fine sediment classed as clay:

$$
\text { Percentage of clay }=\frac{\text { Weight of residue }}{\text { Original weight }} \times 100
$$

6. The determinations of percentage of clay on the two samples shall check within \pm 1 percentage of clay to be acceptable.

Federal Specifications Board, specification No. 452, United States Government master specification for materials for topsoil or sand-clay road surfaces, January 25, 1927.

\section{GRADES}

The materials covered by this specification shall be supplied in the particular grade or grades ordered (see Note 2), grade A, grade B, and grade C.

\section{GENERAL REQUIREMENTS}

Materials for topsoil or sand-clay road surfaces shall be composed of naturally occurring or artificially prepared mixtures of hard, durable fragments of sand, together with silt and clay of suitable binding properties, and may carry a considerable amount of gravel. The material shall be free from an excess of feldspar or mica.

\section{DETAIL REQUIREMENTS}

1. Materials for topsoil or sand-clay road surfaces, when tested by means of laboratory screens, shall meet the following requirements:

Passing $1 / 4$-inch screen

Per cent Passing 2-inch screcn 70-100

2. Material, if any, retained on the $1 / 4$-inch screen shall be uniformly graded from the maximum size present to $1 / 4$ inch.

3. The material passing the 1/4-inch screen shall conform to the following requirements for the respective grades as designated:

\begin{tabular}{|c|c|c|c|}
\hline & \multicolumn{3}{|c|}{ Grade } \\
\hline & $\mathbf{A}$ & B & $\mathrm{C}$ \\
\hline $\begin{array}{l}\text { Clay } \\
\text { Silt } \\
\text { Total sand } \\
\text { Sand retained on No. } 60 \text { sieve }\end{array}$ & $\begin{array}{r}\text { Per cent } \\
10-20 \\
5-15 \\
65-80 \\
45-60\end{array}$ & $\begin{array}{r}\text { Per cent } \\
10-25 \\
5-20 \\
60-80 \\
30-60\end{array}$ & $\begin{array}{r}\text { Per cent } \\
10-25 \\
5-20 \\
55-80 \\
30-60\end{array}$ \\
\hline
\end{tabular}

\section{METHODS OF SAMPLING AND TESTING}

The topsoil or sand-clay materials shall be tested in accordance with the following methods:

1. Preparation of sample.-Former A. S. T. M. tentative method D137-24T, paragraph 2, proceedings A. S. T. M., 1924, Part I, page 922.

2. Mechanical analysis of total sample.-A.S. T. M. D18. (See 512.15, p. 250.)

3. Mechanical analysis of material passing 1/4inch screen.-Former A. S. T. M. tentative method D137-24T, proceedings A. S. T. M., 1924, Part I, page 922 , using U. S. Standard sieves meeting the requirements of A. S. T. M. standard specification E 11. (See 500.2 , p. 1.)

The determination of "suspension clay" shall be omitted, and the percentage of clay may be calculated by difference as follows:

Percentage ofcray $=100$ - percentage of sand-percentage of silt 


\section{NOTES}

1. In economical construction of topsoil and sand-clay roads, it is necessary to use the best available local materials. In promulgating this master specification, the Federal Specifications Board does not intend, therefore, that the use of its requirements shall be strictly mandatory; where material conforming to the requirements is not economically available, the master specification should serve as a guide in the selection of material.

2 . The material designated as grade $\mathrm{A}$ is suitable for use on roads carrying an average traffic of not more than 400 vehicles per day. The material designated as grade $B$ is of intermediate value, and that designated as grade $\mathbf{C}$ is suitable for secondary roads carrying only very light traffic.

National Sand and Gravel Association, Bulletin 3, Representative Specifications for Different Uses of Sand and Gravel, November, 1928.

(In this bulletin, representative specifications, many of which are presented in this volume, and including specifications for sand-clay road surfaces, have been summarized.)

\subsection{Iiscellaneous Pavement Specifica- tions.}

American Association of State Highway Officials, tentative standard specifications for highway materials, 1927.

See 512.15, page 244 .

American Society for Testing Materials, standard specifications for broken slag for bituminous wearing course, serial designation D159-27, 1927.

See 512.2 , page 254 .

American Society for Testing Materials, tentative specifications for broken stone for water-bound macadam surface course, serial designation D19129,1924 .

See 512.15, page 250 .

National Slag Association, recommended specifications for slag to be used in highways and highway structures, 1927.

See 512.2 , page 256 .

National Slate Association, specification for slate floors, terraces, and walks, 1925.

See 518.56 , page 425 .

\subsection{BRIDGE CONSTRUCTION.}

\subsection{General Items.}

American Association of State Highway Officials, standard specifications for bridges and incidental structures, 1928.

This organization has prepared specifications relating to bridge construction, including five divisions, captioned (1) Gencral provisions, (2) materials, (3) general construction, (4) special construction, and (5) design. Specifications contained in the volume relating to nonmetallic minerals have been placed in this compilation under their proper commodity headings, as follows:

Portland cement, 516.11, page 287.

Water for use with cement, 516.0, page 281.

Fine aggregate. (See A. A. S. H. O., highway materials, fine aggregate, 512.13, p. 235.)

Carse aggregate, 512.14 , page 242 .

Rubble or cyclopean aggregate, 512.14, page 243.

Stone for masonry, 511.9 , page 212 .

Brick, 534.10 , page 559.

Bituminous materials:

Fillers, 505.15, page 149 .

Bituminous carpets, 505.31, page 174.

Bituminous carpets, 505.31 , page 174 .

Asphalt for bituminous carpets, 505.13, pase

Waterproofing materials, 505.16
Paving blocks, 518.37, page 395 .

Paving blocks, 518.37, page 395
Bearing piles, 518.42 , page 411 .

Bearing piles, 518.42 , page 411.
Concrete masonry, 516.3 , page 301

Concrete masonry, 516.3 , page 301

Mortar rubble masonry, 518.82, page 474

Dry rubble masonry, 518.81 , page 473 .

Brick masonry, 518.83 , page 475 .
Riprap, 511.72, page 208.

Concre te cribbing, 516.4, page 317 .

Waterproofing, 505.16, page 151 .

Foundations and substructures, (given below).

Monolithic concrete culverts, 518.61, page 441.

Reinforced concrete slab and girder bridges, 518.44, page 413.

Concrete viaducts, 518.72, page 471 .

Arches, 518.46, page 414.

Bridge floors, 518.45, page 414.

Wearing surfaces for bridge floors, 518.45 , page 413 .

Railings and parapets, 518.43, page 413, and 516.3, page 301.

Control of materials, 518.40 , see below.

Excavation and fill, 518.49, page 415 .

American Association of State Highway Officials, standard specifications for highway bridges and incidental structures, 1928.

\section{CONTROL OF MATERIAIS}

\section{SOURCE OF SUPPLY AND QUALTTY}

These specifications contemplate the use of firstclass materials throughout, used in such a manner as to produce completed construction which is workmanlike and acceptable in every detail. Only materials conforming to the requirements of these specifications and approved by the engineer shall be used in the work. The source of supply of materials shall be approved by the engineer before shipment is made.

\section{TESTS OF MATERIAIS}

All tests of materials shall be made by the engineer in accordance with approved methods as described and designated in these specifications.

When tests of materials are necessary, such tests shall be made by and at the expense of the commission unless otherwise provided. The contractor shall afford such facilities as the engineer may require for collecting and forwarding samples, and shall hold the materials represented by the samples until tests have been made and the materials found equal to the requirements of the specifications or to approved samples. The contractor in all cases shall furnish the required samples without charge.

\section{STORAGE OF MATERIAIS}

Materials shall be stored so as to insure the preservation of their quality and fitness for the work. Stored materials shall be located so as to facilitate prompt inspection. That portion of the right of way not required for public travel may be used for storage purposes and for the placing of the contractor's plant and equipment, and any additional space required, unless otherwise stipulated, shall be provided by the contractor at his expense.

\section{DEFECTIVE MATERIALS}

All materials which do not meet the requirements of these specifications will be rejected, and shall be removed immediately from the work unless permitted by the engineer to remain on the work.

American Association of State Highway Officials, standard specifications for highway bridges and incidental structures, 1928.

\section{FOUNDATIONS AND SUBSTRUCTURES}

(This specification consists largely of construction requirements, with the following specifications for classes of concrete.)

All concrete for mass footings, except concrete placed under water, shall be of class $C$ concrete unless otherwise specified. All massive pier shafts, gravity walls and other mass construction shall be of class B concrete unless otherwise specified. All reinforced work such as cantilever footings, walls, pier diaphragms, and other like construction, shall be of class A concrete.

Note.-For definitions of the various classes of concrete mentioned abovc, see 516.3, page 301, American Association of State Highway Officials, specification for "Concrete masonry." 
518.41 Bridges.

American Association of State Highway Officials, standard specifications for bridges and incidental structures, 1928.

See 518.40 , page 410 .

American Railway Engineering Association, specifications for stone masonry.

\section{BRIDGE AND RETAINING WALI MASONRY}

See 518.89 , page 479 .

\subsection{Piling.}

American Association of State Highway Officials, standard specifications for highway bridges and incidental structures, 1928.

\section{BEARING PILES, CONCRETE}

(The full text of this specification includes material relative to form work, methods of casting, storing, setting, measuring, and payment, also specifications for timber piles, and the following additional requirements.)

\section{MANUFACTURE OF PRECAST CONCRETE PILES}

Size and shape.-Precast concrete piles shall be of approved size and shape. If a square section is employed, the corners shall be chamfered at least 1 inch. Piles perferably shall be cast with a driving point and for hard driving preferably shall be shod with a metal shoe of approved pattern. Piling may be either of uniform section or tapered. In general, tapered piling shall not be used for trestle construction except for that portion of the pile which lies below the ground line; nor shall tapered piles be used in any location where the piles are to act as columns. In general, concrete piles shall have a crosis-sectional area, measured above the taper, of not less than 140 square inches and when they are to be used in salt water they shall have a cross-sectional area of not less than 220 square inches.

Class of concrete. - Class D concrete shall be used for precast concrete piles. (See 516.3, p. 301, American Highway Officials specification for "Concrete masonry.")

Reinforcement.-Reinforcement for precast concrete piles shall consist of longitudinal bars in combination with lateral reinforcement in the form of hoops or spirals. The longitudinal reinforcement shall be not less than 1 per cent and preferably not less than $1 \frac{1}{2}$ per cent of the total cross section of the pile. The reinforcement shall be placed at a clear distance from the face of the pile of not less than 2 inches and when the piles are for use in salt water or alkali soils this clear distance shall be not less than 3 inches. The driving point and also the top of the pile shall be protected against impact by means of special spiral winding or bands designed for this purpose. The reinforcing system preferably shall be of the unit type, rigidly wired or fastened at all intersections and held to true position in the forms by means of concrete blocks or other suitable device. When piles exceed 55 feet in length, additional longitudinal reinforcement shall be added throughout the central one-third of the length. Piling under retaining walls, arch footings, abutments, etc., shall be designed to withstand the lateral stresses induced.

\section{MANUFACTURE OF CAST-IN-PLACE CONCRETE PILES}

Description.-Cast-in-place concrete piles shall be cast in strong metal shells which shall remain -permanently in place.

Metal shells.- The metal shall be of a sufficient thickness and reinforced to such an extent that it will hold its original form and show no signs of distortion after the core has been withdrawn. The design of the shell shall be submitted to and approved by the engineer before any driving is done.

Inspection of shells.- After the shell has been driven and the core withdrawn, the shell shall be inspected and approved before any concrete is placed. No payment will be made for any shell which has been improperly driven, is broken, or otherwise defective and, if necessary, any such shell shall be removed and replaced.

Class of concrete.-Class D concrete shall be used for cast-in-place concrete piles. (See 516.3 p. 301. American Association of State Highway Officials specification for concrete masonry.)

Reinforcement.-Reinforcement for cast-in-place piles shall be of the unit type, rigidly fastened together and lowered into the shell before concrete is placed. No loose bars will be permitted. The reinforcement shall be secured in such a manner as to insure its proper location in the finished pile.

Placing concrete. - No concrete shall be placed until all driving within a radius of 15 feet has been completed, or until all the shells for any one bent have been completely driven. If this can not be done, all driving within the above limits shall be discontinued until the concrete in the last pile cast has set at least seven days.

Concrete shall be placed continuously in each pile, care being used to fill every part of the shell and to work concrete around the reinforcement without displacing it. No concrete shall be placed in shells containing an accumulation of water.

\section{EXTENSIONS OR "BUILD UPS"}

Extensions, splices or "build ups" on concrete piles shall be avoided, but when necessary they shall be made as follows:

After the driving is completed, the concrete at the end of the pile shall be cut away, leaving the reinforcing steel exposed for a length of 40 diameters. The final cut of the concrete shall be perpendicular to the axis of the pile. Reinforcement similar to that used in the pile shall be securely fastened to the projecting steel and the necessary form work shall be placed, care being taken to prevent leakage along the pile. The concrete shall be of the same quality as that used originally in the pile. Just prior to placing concrete the top of the pile shall be thoroughly wetted and covered with a thin coating of neat cement, retempered mortar, ol other suitable bonding material. The forms shall remain in place not less than seven days and shall then be carefully removed and the entire exposed surface of the pile finished as above specified.

American Railway Engineering Association, specifícations for constructing premolded concrete piles, 1927.

1. Piles shall be made in accordance with the dimensions shown on the drawings.

2. The workmanship and materials shall be in accordance with the specifications for concrete, plain and reinforced, of the American Railway Engineering Association, (see 516.3, p. 303) with the modifications as shown on the following page.

\section{AGGREGATES}

3. The coarse aggregate shall consist of material such as crushed stone or gravel varying in size from one-fourth inch to three-fourths inch.

\section{PROPORTIONS}

4. Concrete shall be of such mixture as to give a strength of $3,000 \mathrm{lbs} . /$ in. $^{2}$ at 28 days. 


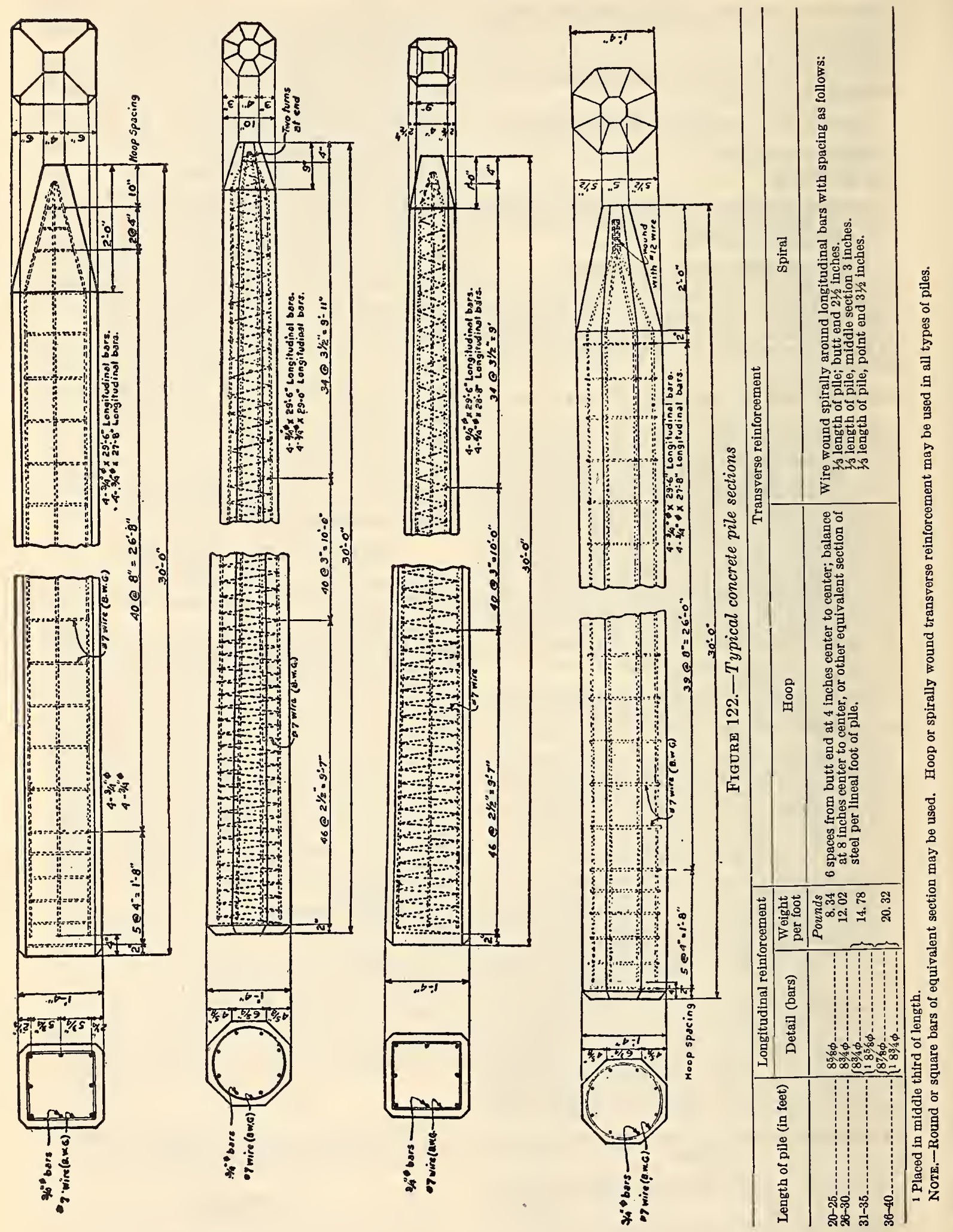


5. Where ordinary method of sheltered curing is employed, piles shall be seasoned for a period of not less than 3 days before being moved and not less than 28 days before handling and moving to the site of driving. No method of accelerated seasoning shall be used until approved by the engineer.

6. Each pile shall be stamped or marked with the date of its manufacture.

\section{HANDLING}

7. Piles shall be handled carefully, avoiding any dropping or heavy jarring while in horizontal positions.

Simplex Concrete Pile Association, specifications for standard simplex concrete piles.

General.-Piles shall be made of concrete, cast-inplace, and shall be of the standard simplex type. Materials and workmanship shall in all respects comply with the requirements of the best engineering practice for such work. The contractor for piling shall furnish and install complete all piling shown on the drawings and called for in the specifications, and shall at all times cooperate with the other contractors doing work on the same project.

Concrete.-The concrete shall be mixed thoroughly and to proper consistency in the proportions as specified under general specifications for the work to be done and shall be placed in such a manner as not to disturb the proper distribution of the mixture.

Method.-Standard simplex concrete piles are cast in place by driving to proper penetration a cylindrical steel driving form fitted at its lower end with a detachable cast-iron or pressed-steel base. The driving form is then filled with concrete to the necessary height and withdrawn, leaving the castiron or pressed-steel base in the ground with a column of concrete on top of it completely filling the hole to its compacted walls.

NotE.-The full text of the specification includes requirements for driving forms, base, capacity, driving, basis of contract, length and spacing of piles, and reinforcement.

\subsection{Parapets and Railings.}

American Association of State Highway Officials standard specifications for highway bridges and incidental structures, 1928.

\section{RAILINGS AND PARAPETS}

This specification covers masonry and brick parapets and railings, concrete railings, and pipe railings. The specification in full gives construction requirements.

For concrete railings, the material specified is class "D" concrete. (See 516.3, p. 301, American Association of State Highway Officials, "Concrete masonry.")

\subsection{Slabs and Girders.}

American Association of State Highway Officials, standard specifications for highway bridges and incidental structures, 1928.

\section{REINFORCED CONCRETE SLAB AND GIRDER BRIDGES}

(This specification consists largely of construction requirements, with the following specifications for classes of conorete:)

In concrete slab and girder bridges, class "A" concrete shall be used throughout except for railings. Class " $D$ " concrete shall be used for railings, lamp-posts and other ornamental parts of the structure above the curbs.

NoтE.-For definitions of the various classes of concrete mentioned above, see 516.3, page 301, American Association of State Highway Officials, specification for "Concrete masonry."

\subsection{Floors and Wearing Surfaces.}

American Association of State Highway Officials, standard specifications for highway bridges and incidental structures, 1928.

\section{WEARING SURFACES FOR BRIDGE FLOORS}

CONCRETE WEARING SURFACE

Concrete wearing surfaces for concrete floors may consist either of an additional thickness of concrete placed monolithically with the floor proper or of a separately placed wearing surface of concrete.

For monolithic construction the additional floor thickness shall be not less than three-fourths inch and the entire floor slab shall be of class $D$ concrete. (See 516.3, p. 301, American Association of State Highway Officials, "Concrcte masonry.") Separately placed concrete wearing surfaces shall be not less than 4 inches thick at the center and $2 \frac{1}{2}$ inches thick at the edges and shall be of class " $D$ " concrete.

\section{BRICK WEARING SURFACE}

Brick wearing surfaces for concrete floors shall be placed only after the concrete floor has been thoroughly cured.

\section{Materials}

All materials used in the construction of brick wearing surfaces shall conform to 534.11 , page 577 , American Association of State Highway Officials, "Paving brick." Sand for the sand-cement bedding course shall conform to the requirements for grade B fine aggregate, see 512.13, American Association of State Highway Officials, "Fine aggregate."

\section{SAND-CEMENT BED}

The sand-cement bedding course shall consist of sand and Portland cement, in the proportion of 1 part cement and 4 parts sand, mixed dry until the mass is of a uniform color. The mixing may be done in an approved batch mixer or by hand on a clean, tight surface.

\section{ASPHALT FILIER}

All joints shall be filled with hot asphalt filler and a surface dressing applied on the day of laying the brick.

\section{SORFACE DRESSING}

Immediately after the joints have been filled, and while the filler is still soft and pliable, the pavement shall be covered with a thin layer of dry sand, stone, or slag screenings, or granulated slag. This top dressing shall be of such sizes that all will pass a $1 / 4$-inch sieve.

\section{ASPHALT BLOCK WEARING SURFACE}

Asphalt block wearing surfaces for concrete floors shall be placed only after the concrete floor has been thoroughly cured.

\section{Materials}

All materials used in the construction of asphalt block wearing surfaces shall conform to 518.37, page 395, American Association of State Highway Officials, "Asphalt paving block." Sand for the mortar bed shall conform to the requirements for grade B fine aggregate. (See 512.13, American Association of State Highway Officials, "Fine aggregatc."

\section{MORTAR BED}

The mortar bed shall consist of sand and Portland cement, in the proportion of 1 part cement to 4 parts sand, mixed with sufficient water to thoroughly moisten the ingredients and to make a mortar of such consistency that it can easily be spread upon 
the foundation and struck with a templet to a smooth and even surface. It must not, however, be so soft as to allow the blocks to sink into the mortar when they are placed thereon.

\section{BITUMINOUS CARPETS}

\section{Materials}

Bituminous materials.-The bituminous materials used in the construction of bituminous carpets shall conform to the requirements of American Association of State Highway Officials, 505.13, page 147 , "Asphalt for bituminous carpets," and 505.31, page 174, "Bituminous carpets."

Aggregate.-The aggregate shall consist of gravel, stone chips, or slag which shall be clean, sound, and hard, and thoroughly dry when applied. Material containing soft or partially disintegrated particles of stone or shale will not be accepted. The aggregate shall be washed and screened to such size that all of it will pass a sieve having $1 / 2$-inch square openings, and not more than 15 per cent shall pass a $1 / 4$-inch sieve.

NotE-The full text of the specification includes material regarding construction of the types given above, also specifications for creasoted wood block wearing surface.

American Association of State Highway Officials, standard specifications for highway bridges and incidental structures, 1928.

\section{BRIDGE FLOORS (CONCRETE)}

The concrete used for bridge floors shall be class "A" concrete unless otherwise specified. (See 516.3, p. 301, American Association of State Highway Officials, "Concrete masonry.")

Expansion joints shall be constructed as shown on the plans and in accordance with the requirements specified in American Association of State Highway Officials, "Concrete masonry."

Note.-The full text of the specification includes directions for construction and curing, also specifications for plank and laminated or strip floors.

American Association of State Highway Officials, standard specifications for highway bridges and incidental structures, 1928.

\section{BITUMINOUS MATERIALS (ASPHALT FOR BITU- MINOUS CARPETS)}

See 505.13, page 147 .

\subsection{Arches}

American Association of State Highway Officials, standard specifications for bridges and incidental structures, 1928.

\section{ARCHES}

(This specification includes material relative to construction and the following additional:)

\section{CONCRETE ARCEES}

\section{Class of Concrete}

Unless otherwise specified, mass footings shall be of class $\mathrm{C}$ concrete; mass piers and abutments of class $\mathrm{B}$ concrete; and arch rings, ribs and roadway floor slabs of class A concrete. Other portions of the work shall be of class A concrete if reinforced and of class B concrete if unreinforeed.

\section{EXPANSION JOINTS}

Expansion joints, where required, shall be constructed as shown on the plans and in accordance with the requirements specified in 516.3, page 301, American Association of State Highway Officials, specifications for concrete masonry.

\section{SURFACE FINISH}

Unless otherwise specified, all arch ribs or rings, rails, copings, and exposed faces of spandrel and wing walls shall be finished in accordance with the requirements specified for class 1 surface finish. The exposed surfaces of piers and abutments below the spring line of the arches shall be finished in accordance with the requirements for class 2 surface finish.

Particular care shall be exercised in order to produce a pleasing and uniform surface finish throughout the entire portion of the structure visible above the ground.

On filled spandrel arches the extrados of the rings shall be screeded to correct contour and finished with a wooden float unless extrados forms are used, and the extrados and the inside faces of the spandrel walls shall be left smooth to receive the waterproofing.

\section{ASHLAR MASONRY ARCHES}

Ashlar masonry arches shall be constructed in accordance with the requirements of "Ashlar masonry" (see 518.82 p. 473) with the following modifications:

\section{Arch Rings}

The number of courses and the depth of voussoirs shall be as shown on the plans. Voussoirs shall be placed in the order indicated; shall be full size throughout, dressed true to templet, and shall have bond not less than the thickness of the stone. Beds and joints shall be fine finished and mortar joints shall not exceed three-eighths inch in thickness. Exposed surfaces of the intrados and arch ring shall be given the surface finish indicated on the plans.

\section{Backing}

Backing may consist of class B concrete or large stone, shaped to fit the arch, bonded to the spandrels, and laid in full beds of mortar. The extrados and inside faces of the spandrel walls shall be given a finishing coat of 1 to $2 \frac{1}{2}$ cement mortar which shall be troweled smooth to receive the waterproofing.

\section{RUBBLE MASONRY ARCHES}

Rubble masonry arches shall be constructed in accordance with the requirements of "Mortar rubble masonry" (see 518.82, p. 474, American Association of State Highway Officials), with the following modifications:

Arch Rings

The number of courses and the depth of voussoirs shall be as shown on the plans. Voussoirs shall be placed in the order indicated; shall be full size throughout and shall have bond not less than their thickness. Beds shall be roughly pointed to bring them to radial planes. Radial joints shall be in planes parallel to the transverse axis of the arch and, when measured at the intrados, shall not exceed three-fourths inch in thickness. Joints perpendicular to the arch axis shall not exceed 1 inch in thickness when measured at the intrados. The intrados face shall be dressed sufficiently to permit the stone to rest properly upon the centering. Exposed faces of the arch ring shall be rock faced with edges pitched to true lines.

The work shall be carried up symmetrically about the crown, the stone being laid in full mortar beds and the joints grouted where necessary. No spalls will be permitted.

\section{Backing}

Backing may consist of class B concrete or large stone shaped to fit the arch, bonded to the spandrels, and laid in full beds of mortar. The extrados and 
inside faces of the spandrel walls shall be given a finishing coat of 1 to $2 \frac{1}{2}$ cement mortar which shall be troweled smooth to receive the waterproofing.

(For definitions of the various classes of concrete and surface finish mentioned above, see 516.3 , p. 301 , American Association of State Highway Officials, specifications for "Concrete masonry.")

\subsection{Miscellaneous Items under Bridge Construction.}

American Association of State Highway Oficials, standard specifications for highway bridges and incidental structures, 1928.

\section{EXCAVATION AND FILL}

\section{GENERAL}

Foundation excavation shall include the removal of all material of whatever nature necessary for the construction of foundations and substructures in accordance with the plans or as directed by the engineer. It shall include the furnishing of all necessary equipment and the construction of all cribs, cofferdams, caissons, etc., which may be necessary for the execution of the work. It shall also include the subsequent removal of cofferdams and cribs and the placement of all necessary back fill as hereinafter specified.

\section{CLASSIFICATION OF EXCAVATION}

Classification of excavation, if any, will be made on the basis of the material to be removed between certain elevations. Such classification will be indicated on the plans and set forth in the proposal.

\section{DEPTH OF FOOTINGS}

The elevations of the bottoms of footings, as shown on the plans, shall be considered as approximate only, and the engineer may order, in writing, such changes in dimensions or elevations of footings as may be necessary to secure a satisfactory foundation.

\section{TREATMENT OF FOONDATION MATERIALS}

Solid rock.-All rock or other hard foundation material shall be freed from all loose material, cleaned and cut to a firm surface, either level, stepped, or serrated, as may be directed by the engineer. All seams shall be cleaned out and filled with concrete, mortar, or grout.

Other materials. - When masonry is to rest on an excavated surface other than rock, special care shall be taken not to disturb the bottom of the excavation and the final removal of the foundation material to grade shall not be made until just before the masonry is to be placed.

\section{COFFERDAMS}

Construction.-Cofferdams or cribs for foundation construction shall, in general, be carried well below the bottom of the footings and shall be well braced and as water-tight as practicable. In general, the interior dimensions of cofferdams shall be such as to give sufficient clearance for the construction of forms and the inspection of their exteriors, and to permit pumping nutside of the forms. Cofferdams or cribs which are tilted or moved laterally during the process of sinking shall be righted or enlarged so as to provide the necessary clearance and this shall be at the sole expense of the contractor.

When conditions are encountered which, in the opinion of the engineer, render it impracticable to unwater the foundation before placing masonry, he may require the construction of a concrete foun- dation seal of such dimensions as may he necessary. The foundation shall then be pumped out and the balance of the masonry placed in the dry. When weighted cribs are employed and the weight utilized to partially overcome the hydrostatic pressure acting against the bottom of the foundation seal, special anchorage, such as dowels or keys, shall be provided to transfer the entire weight of the crib into the foundation seal. When a foundation seal is placed under water, the cofferdam shall be vented or ported at low-water level.

Cofferdams shall be constructed so as to protect green concrete against damage from a sudden rising of the stream and to prevent damage to the foundation by erosion. No timber or bracing shall be left in cofferdams or cribs in such a way as to extend into the substructure masonry, without written permission from the engineer.

For substructure work, the contractor shall submit, upon request, drawings showing his proposed method of cofferdam construction and other details left open to his choice or not fully shown on the engineer's drawings. Such drawings shall be approved by the engineer before construction is started.

Removal.-Unless otherwise provided, cofferdams or cribs with all sheeting and bracing shall be removed by the contractor after the completion of the substructure. The removal shall be effected in such a manner as not to disturb or mar the finished masonry.

\section{PUMPING}

Pumping from the interior of any foundation inclosure shall be done in such manner as to preclude the possibility of any portion of the concrete materials being carried away. No pumping will be permitted during the placing of concrete or for a period of at least 24 hours thereafter, unless it be done from a suitable sump separated from the concrete work by a water-tight wall.

Pumping to unwater a sealed cofferdam shall not commence until the seal has set sufficiently to withstand the hydrostatic pressure.

\section{INSPECTION}

After each excavation is completed, the contractor shall notify the engineer, and no masonry shall be placed until after the engineer has approved the depth of the excavation and the character of the foundation material.

\section{BACK FLLING}

All material used for back fill shall be of a quality acceptable to the engineer and shall be free from large or frozen lumps, wood, or other extraneous material.

All spaces excavated and not occupied by abutments, piers, or other permanent work shall be refilled with earth up to the surface of the surrounding ground, with a sufficient allowance for settlement. All back fill shall be thoroughly compacted and, in general, its top surface shall be neatly graded.

The filling behind abutments and wing walls of all bridge structures shall be deposited in horizontal layers and well compacted. Special precautions shall be taken to prevent any wedging action against the masonry and the slope bounding the excavation for abutments and wing walls shall be destroyed by stepping or serrating to prevent wedge action.

Fill placed around culverts and piers shall be deposited on both sides to approximately the same elevation at the same time.

Adequate provision shall be made for the thorough drainage of all back filling. French drains shall be placed at weep holes.

For foundations through hard material exposed to erosion, the back fill around piers and in front of 
abutments and wings may be ordered by the engineer to be of stone or of lean concrete, in which case, unless otherwise provided, this back fill shall be paid for as extra work.

No back filling shall be placed against any masonry abutment, wing, wall or culvert until permission shall have been given by the engineer and preferably not until the masonry has been in place 21 days.

\section{APPROACH EMBANKMENT}

When the contract for any bridge structure involves the placement of approach embankments, these shall be constructed and paid for in accordance with the highway specifications governing this class of work in effect for the particular locality involved.

\section{MEASUREMENT AND PAYMENT}

Payment for foundation excavation shall include the cost of all labor, material, and equipment, and other items that may be necessary or convenient to the successful completion of the excavation to the elevation shown for the bottom of the footings. It shall also include the cost of removing cofferdams and any surplus material which may have been thrown up during the process of excavation, and shall include the cost of back filling an amount of material equal to the amount of excavation. Any back fill required in excess of the amount excavated shall be paid for as extra work unless a price is asked for and tendered on extra back fill.

The yardage of excavation paid for will be that actually removed, except that no payment will be made for material removed outside of an area which is bounded by vertical planes 1 foot outside of the footings and parallel thereto.

When it is necessary to carry footings deeper than the elevation shown on the plans, payment will be made on the basis of the prices tendered for such excavation or, in case no such prices are tendered, it may be paid for as extra work.

\subsection{BUILDING CONSTRUCTION AND MA- TERIALS.}

\subsection{General Items. 'See also 505.16 and 505.36.)}

American Railway Engineering Association, specifications for buildings for railway purposes.

These specifications contain various items for construction of, and materials used in, buildings for railway purposes. Items appearing elsewhere in this volume are as follows:

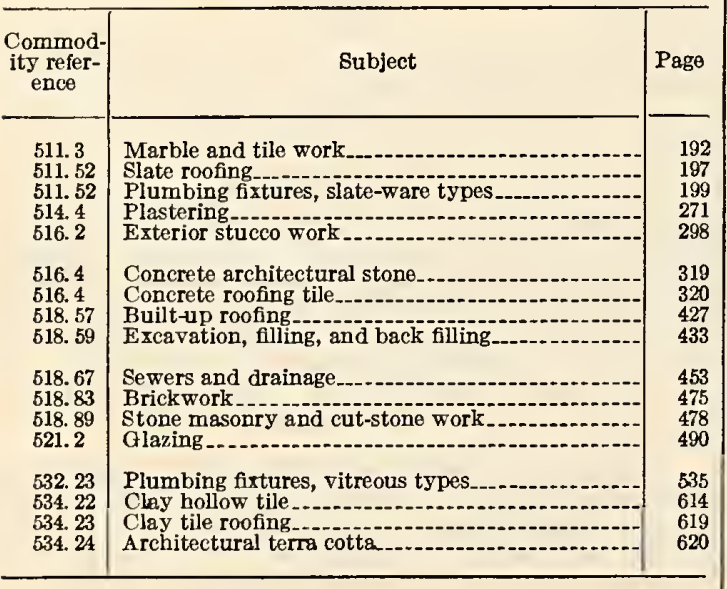

General conditions relating to these specifications are given below:

\section{GENERAL CONDITIONS (1927)}

\section{GENERAL}

These general conditions are intended to be used in connection with the American Railway Engineering Association construction contract form as published in the manual, and shall apply to all sections of these specifications with equal force.

\section{COMPANY, ENGINEER, AND CONTRACTOR DEFINED}

As used in these specifications, the term company shall be understood to mean the railroad or railway company, the term engineer shall be understood to mean the chief engineer of the company or his duly authorized representative, and the term contractor shall be understood to mean the person, firm, or corporation agreeing to perform the work covered by these specifications.

\section{DRAWINGS FURNISHED BY THE COMPANY}

The drawings furnished by the company shall be considered as part of and illustrating these specifications. These specifications are intended to supplement the drawings, the two being considered cooperative. Drawings and specifications will be part of the contract and are equally binding. They are the property of the company and shall be returned when work is completed.

The drawings show the general character of detail work, but the company reserves the right to furnish proper scale details of such portion as may, in the judgment of the engineer, require them. In preparing such details slight modifications may be made if necessary without in any way invalidating the contract. The contractor shall not execute any work requiring such details until these have boen furnished, and all work shall conform with these details when executed.

Figures on drawings shall take precedence over measurements by scale, detail drawings over smallscale drawings, and full-size details over all other drawings. The decision of the engineer shall be final as to the interpretation of drawings and specifications.

\section{ERRORS OR DISCREPANCIES}

If the contractor in course of the work finds any discrepancy between the plans and the physical conditions of the locality, or any errors in the plans, or in the points given for the construction of the work, it shall be his duty to immediately inform the engineer in writing, and the company will promptly verify and, if necessary, correct such errors. Any work performed before these discrepancies or errors have been corrected will be done at the contractor's risk.

\section{WORKING DRAWINGS}

The contractor shall submit to the engineer in triplicate copies of all working drawings and erection diagrams of all parts of the work, including both structure and equipment.

Said drawings shall include list of all parts of equipment with pattern numbers or other necessary designation in order that repair parts may be readily ordered in the future.

These drawings shall measure _....... outside and ........ inside, ........ border lines. All such drawings must be approved by the engineer before the work involved is started. The approval of said working drawings by the engineer shall not imply any change in the specifications or relieve the contractor from the responsibility of any errors thereon. No change shall be made on any approved drawing without the written consent of the engineer. 
The contractor shall supply additional copies of working drawings and erection diagrams on request.

Upon completion of the work the contractor shall furnish a complete set of corrected ink tracings on cloth, or Van Dyke prints on cloth made from ink tracings, of all working drawings and erection diagrams and this set $\approx$ hall be a true record of the work as constructed.

\section{IAYTNG OUT WORE}

Necessary lines, corners, and elevations will be established on the site of the building by the engineer. The contractor shall erect permanent batter boards and protect the points so established until the work is completed and accepted. Using the points established by the engineer, the contractor shall lay out his own work and be responsible for its accuracy.

\section{PROSECDTION OF THE WORK}

When the work of a subcontractor engages with the work of any other subcontractor, he must cooperate with the other subcontractor and exercise extraordinary care to prevent injury to any work or material. Each subcontractor shall do all necessary cutting, fitting, and patching of his work where it engages the work of another contractor or the company.

\section{MATERIALS}

All materials shall be new and of the grade specified, and shall be the best of their respcctive kinds for the uses intended.

Priced materials. - Where the quality or kind of material can not be definitely specified, the amount of money the contractor is to pay for same is given in these specifications. The sum so given is intended to cover the purchase price of the materials and freight charges, but this sum shall not include any cost of hauling, cartage, supervision, preparatory work, profit, or the cost of erection; it being intended that the contractor shall include such foregoing items in his contract price. The engineer will select such materials and notify the contractor of his selection and the price agrecd upon, but the contractor shall contract for the material and supervise its delivery and erection as fully as other parts of the work.

If the required payment for such priced material should be more than the sum herein specified, the difference is to be paid by the company, and if it should be less, the difference is to be deducted from the sum to be paid the contractor under the contract.

"Approved" materials.-The term "approved" in this specification signifies that the engineer must be consulted as to the source from which the material is to be purchased as well as its general quality and construction, but such approval does not mean the acceptance of the material actually furnished if it should be defective.

Special materials.-Special brands of materials or devices mentioned in these specifications or shown on the drawings are named for the purpose of establishing a standard or criterion of quality and character desired. Other materials of equal quality and adaptability to the purpose for which they are intended may be substituted, but only with the written approval of the engineer. If the contractor desires to substitute some other brand of material for that called for, he must submit a statement with his proposal, clearly and fully describing such substitutions as he desires to make. Where a specific make or kind of apparatus is called for and furnished by the contractor, the furnishings of such apparatus does not relieve the contractor of liability until he shall make such apparatus or appliance operative so that it will successfully perform the function for which it is intended. The contractor shall protect the com- pany against claims on account of patented devices or parts proposed by him.

\section{EQUIPMENT}

The contractor shall provide all equipment required for the execution and completion of the work, including all staging, scafiolding, apparatus, tools, etc., which are necessary All equipment must meet with the approval of the company and the engineer may require the removal of any portion of equipment which is defective or unsuitable for the proper prosecution of the work and the contractor will be required to substitute therefor satisfactory equipment without delay.

\section{PERMITS, IAWS, AND ORDINANCES}

All work shall conform with the State or municipal laws, ordinances, or regulations governing such work. The contractor shall give all requisite notices in connection with his work to the proper authorities, and shall procure at his own expense all permits, licenses, etc., of every description, necessary for the construction and completion of the work, and shall deliver to the company all certificates of inspection for plumbing, electric wiring, or any other branch of the work for which such certificates may be required in connection with this contract.

Whenever these specifications or any document which they supplement conflict with the building code of the city or town in which the work is executed the contractor shall submit the question as to which to follow to the engineer and abide by his decision.

\section{TEMPORARY TOILET FACHLITLES}

The contractor shall establish and maintain in a location approved by the engineer temporary toilet facilities for the accommodation of his employees.

\section{TEMPORARY OFFICE}

The contractor shall provide in a suitable location on the site, for the exclusive use of the engineer, a temporary office, which shall be weatherproof and have a door and window.

\section{TEMPORARY HEAT}

Where temporary heat or heat during the construction of the building is required for drying plaster or paint, for the prevention of damage to materials by freezing, or for any other reason, such heat shall be provided by the contractor at his own expense unless otherwise specified hereinafter.

\section{FORCE-ACCOONT WORK}

Whenever any work is done or material furnished on a force-account basis - that is, for a price based upon the actual cost of labor and materials plus an added percentage to cover overhead expenses, superintendence, profit, use of tools and equipment, and contractor's risk and liability - the actual cost shall not exceed the fair market value of the labor and material furnished. Where work is done on this basis the time of all employes shall be entered by the contractor on forms supplied him for that purpose and checked and signed in duplicate daily by the contractor and the engineer, and no labor not so entered and checked will be allowed.

\section{ACCOUNTING REQUIREMIENTS}

At the completion of the work the contractor shall furnish a complete list of all quantities in accordance with the company's classification for all work underground for each item or structure, and shall furnish 
in lump-sum form the cost of the superstructure for each item or building, this cost to include the proportionate part of the contractor's overhead and profit.

Where the work is of such nature that existing facilities are removed or remodeled by the contractor he shall furnish the company with a statement showing in detail the cost of such work, the materials removed, and the disposition of the materials. The above information shall be furnished in order to comply with Interstate Commerce Commission accounting requirements.

American Society for Testing Materials, tentative specifications for fire tests of building construction and materials, serial designation C19-26T, 1926.

See 534.10 , page 569 .

American Society for Testing Materials, building brick.

1. Tentative specifications for building brick (made from clay or shale) serial designation C62$29,1929$.

See 534.11 , page 583 .

2. Tentative specifications for sand-lime building brick, serial designation C73-28T, 1928.

See 513, page 257.

Asphalt Shingle and Roofing Association (now Institute), tests for granular surfacing materials, 1922.

See 510, page 186.

Asphalt Shingle and Roofing Association (now Institute), tests for powdered surfacing materials (recommended), 1923.

See 510, page 186.

Asaociated Tile Manufacturers, basic specification for tile work, 1924.

See 534.20 , page 594 .

National Sand and Gravel Association, Bulletin 3, representative specifications for different uses of sand and gravel, November, 1928.

(In this bulletin representative specifications, many of which are presented in this volume, and including specifications for sand for use in building construction, have been summarized.)

\subsection{Buildings and Dwellings.}

American Railway Engineering Association, specifications for buildings for railway purposes.

See 518.50 , page 416 .

Common Brick Manufacturers' Association of America, specifications and general schedule of a brick house.

The above standard includes in the general schedule estimating sheets, specification and contract forms, and specifications for construction. Items included in this volume are as follows:

Mortar. (See 516.3, p. 310.)

Ideal Construction. (See 518.53, p. 419.)

Chimneys and fireplaces. (See 518.55, p. 422.)

Bricklaying. (See 518.83, p. 477.)

Nationai Association of Marble Dealers, standard specifications for interior marble.

See 511.3 , page 194 .

National Building Granite Quarries Association, granite specificatious.

See 511.1 , page 187 .

National Fire Protection Association, specifications for standard industrial buildings more than one story in height, 1929.

(This standard consists of specifications for the construction of industrial buildings more than one story in height, with separate sections on reinforced concrete construction, heavy timber (mill) construction, and protected steel construction. The general provisions common to all three types of construction are covered in a section on general requirements. Explanatory and descriptive material is included in an appendix.)

\subsection{Foundations for Building Construc. tion.}

American Railway Engineering Association, specifications for constructing premolded concrete piles, 1921.

See 518.42 , page 411 .

Associated Factory Mutual Fire Insurance Companies, inspection department, specifications for gravity tank foundations.

(The Associated Factory Mutual Fire Insurance Companies and the National Board of Fire Underwriters have the same specifications for gravity tank foundations, the latter being contained in "Regulations for the Construction and Installation of Gravity and Pressure Tanks, Towers, etc.," "Foundations in the Ground," given below.)

National Board of Fire Underwriters, regulations for the construction and installation of tanks, gravity and pressure, towers, etc.

\section{FOUNDATIONS IN THE GROUND}

\section{MIATERIAI}

Foundations shall be built of concrete of $1: 3: 5$ mixture if not reinforced or $1: 2: 4$ mixture if rcinforced. The aggregate shall be clean, and coarse aggregate shall consist of stone or gravel not larger than 3 inches for $1: 3: 5$ concrete, or $1 \frac{1}{2}$ inches for reinforced concrete.

\section{FORM}

The tops of foundations shall be level and at least 12 inches above grade. The tops of foundation piers shall be located accurately at the correct elevations. The bottom of foundations shall be located bclow the frost line, and in the case of piers at least 4 feet below grade, and shall rest on thoroughly tamped soil or rock.

Piers shall ordinarily be made pyramidical in form with smooth or stepped sides. If supporting a tower their center of gravity should preferably lie in the continued center of gravity line of the tower column. The height of piers sball be not less than the mean width. The top surface shall extend at least 3 inches beyond the bearing plates on all sides and shall be chamfered at the edge.

\section{DESIGN}

The details of design shall conform with the current Report of the Joint Committee on Standard Specifications for Concrete and Reinforced Concrete. (See 516.3, p. 317.)

\section{ANCHORAGE}

The weight of foundations and design of anchorage shall in all cases be sufficient to resist the maximum net uplift occurring with the tank empty and wind loads on the structure, as specified in previous sections.

The weight of a foundation pier shall be not less than the maximum net uplift when the pier is buried to a depth of not less than two-thirds of its height and one and one-half times the uplift when buried to a less amount.

Anchor bolts shall be arranged to securely engage a weight sufficient to develop their full working strength. Their lower ends should preferably be hooked or fitted with anchor plates.

Anchor bolts shall be accurately located with sufficient free length of thread to fully engage their nuts. Expansion bolts are not acceptable. The minimum size of anchor bolts shall be $1 \frac{14}{4}$ inches when not replaceable.

\section{GROUTING AND FLASHING}

Bearing or base plates shall be laid on cement grout to secure a uniform and complete bearing sur- 
face on piers. Flat-bottom tanks not supported by beams shall be placed on a layer of unset cement thicker than any projecting metal. Joints between cement and steel plates and anchor bolts shall be pointed up with cement mortar and flashed with asphalt.

\section{BEARING ON FOUNDATIONS}

The pressure in pounds per square inch of column base plates or steel footings on foundations shall not exceed the following in pounds per square inch:

Portland-cement concrete $1: 2: 4$ 500

Portland-cement concrete 1:3:5

\section{SOIL-BEARING PRESSURES}

The soil shall be carefully inspected after excavation for foundations has been made. If the pressure on the soil is to be more than 2 tons per square foot, or if there is any indication of poor soil conditions, tests for bearing shall be made and the foundation redesigned, if necessary, to carry the maximum loads without excessive settlement. The soil pressure in tons per square foot shall not exceed the following: Ordinary clay or sand.

Compact dry clay or coarse sand

Compact sand and gravel.

Shale rock

Hard rock $-$

Although the foregoing pressures are reasonably conservative, in present good practice bearing pressures higher than $1 \frac{1}{2}$ tons per square foot are seldom used.

Simplex Concrete Pile Association, specifications for standard simplex concrete piles.

See 518.42, page 413 .

\subsection{Walls and Partitions.}

American Railway Engineering Association, specifications for buildings for railway purposes.

\section{BRICKWORK}

See 518.83 , page 475 .

\section{CLAY HOLLOW TILE, 1926}

See 534.22 , page 614 .

American Society for Testing Materials, standard specifications for gypsum, serial designation C22-25, 1925.

See 514.1, page 262 .

American Society for Testing Materials, standard specifications for calcined gypsum, serial designation C23-22, 1922 .

See 514.2, page 263 .

American Society for Testing Materials, standard specifications and tests for hollow burned-clay loadbearing wall tile, serial designation C34-27, 1927.

See 534.22, page 616 .

American Society for Testing Materials, standard specifications for gypsum wall board, serial designation C36-25, 1925.

See 514.61 , page 275 .

American Society for Testing Materials, standard specifications for gypsum plaster board, serial designation C37-25, 1925.

See 514.61, page 276 .

American Society for Testing Materials, standard specifications for gypsum partition tile or block, serial designation C52-27, 1927.

See 514.62, page 278 .

American Society for Testing Materials, tentative specifications for hollow burned clay fireproofing, partition and furring tile, serial designation C56-28T, 1928.

See 534.22 , page 618 .

Common Brick Manufacturers' Association of America, specifications and general schedule for a brick house.

\section{IDEAL CONSTRUCTION}

The specifications for ordinary brickwork (see 518.83, p. 477) shall apply to brick walls built hollow, known as ideal brickwork, and the materials of which it is constructed, except that the arrangement of the bricks in the wall shall be as specified herein, and excepting also the provisions of paragraphs 2 and 4 of "Bricklaying." (See 518.83, p. 477.) All flues

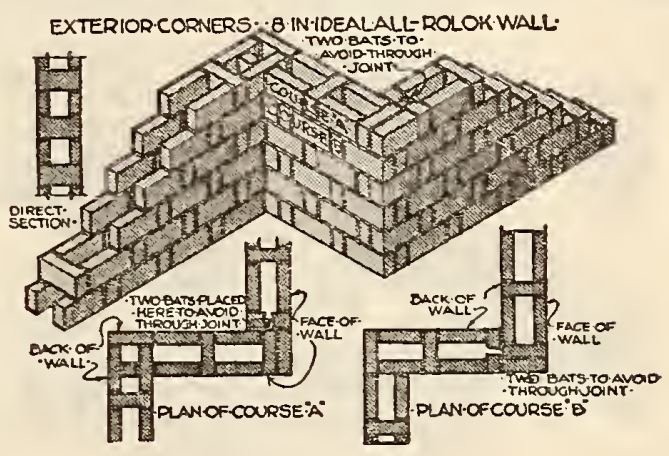

Figdre 123

shall be constructed with a minimum thickness of 4 inches of solid brickwork.

All ideal brickwork shall be laid in cement-lime mortar with joints thoroughly filled.

All ideal walls shall have at least the following number of vertical webs running continuously in the direction of the wall with full mortar joints, and each web shall have a thickness of at least $2 \frac{1}{4}$ inches of solid material, namely, 8-inch walls, two webs; 12 inch walls, three webs; 16-inch walls, four webs. All walls of ideal all-rolok construction shall be built entirely of brick on edge to form a hollow wall. All ideal all-rolok walls 8 inches thick shall have each course laid in Flemish bond with headers and stretch-

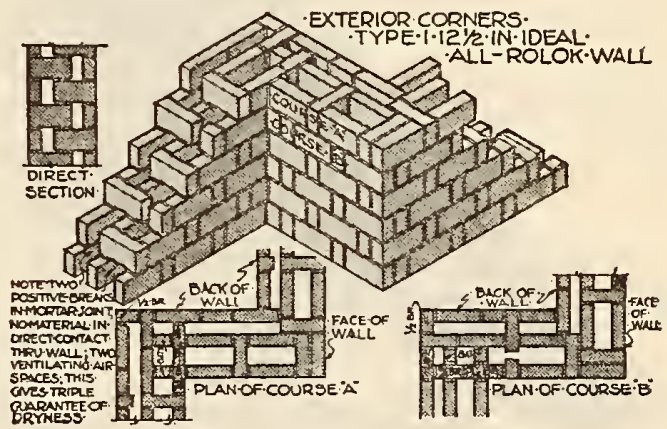

Figure 124

ers alternating as shown in Figure 123, and all headers shall be through headers. The exposed face of ideal all-rolok walls 12 or more inches in thickness shall be laid in Flemish bond, and the wall shall be bonded together in accordance with Figure 124 or Figure 125 at the option of the contractor.

All walls of ideal rolok-bak construction shall be built with the exposed face $3 \frac{3}{4}$ inches thick of brick laid flat. Backing shall be brick on edge. The exposed face of ideal rolok-bak walls 8 inches thick shall be laid with not exceeding five stretcher courses of brick laid flat between courses containing headers. Each course containing headers shall be composed of one header and one through stretcher alternating the full length of the course. Ideal rolok-bak walls 12 inches or more in thickness shall have the facing 
bonded to the backing as described for walls 8 inches thick, and the backing shall be bonded together as provided for ideal all-rolok walls.

In laying ideal walls, reasonable care shall be taken to prevent mortar dropping into the cavities.

Joists, rafters, and wall plates shall be supported upon a continuous course of headers as shown in Figure 126, excepting where there are no openings in the wall below, and in the latter case joist support may be obtained by laying two or three courses of

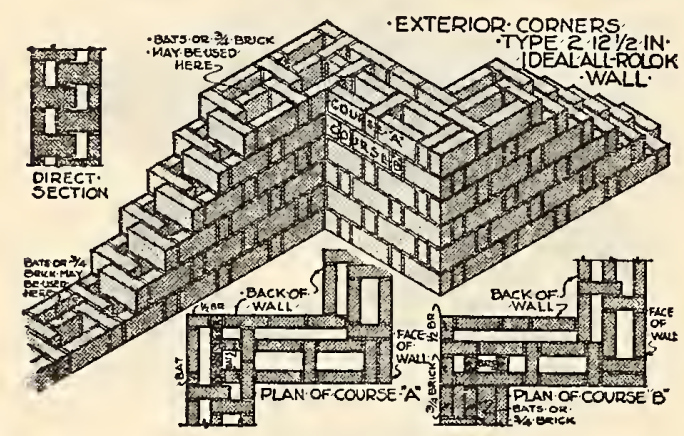

Figure 125

4-inch brickwork under the joists as shown in Figure 127.

The bearing of structural members transmitting to the wall greater loads than those from ordinary joists or rafters shall be increased in proportion to the load to be carried.

When a continuous header course is provided as joist support, the proper number of vertical joints in that course shall be of colored mortar to match the brick for the purpose of making this course appear as

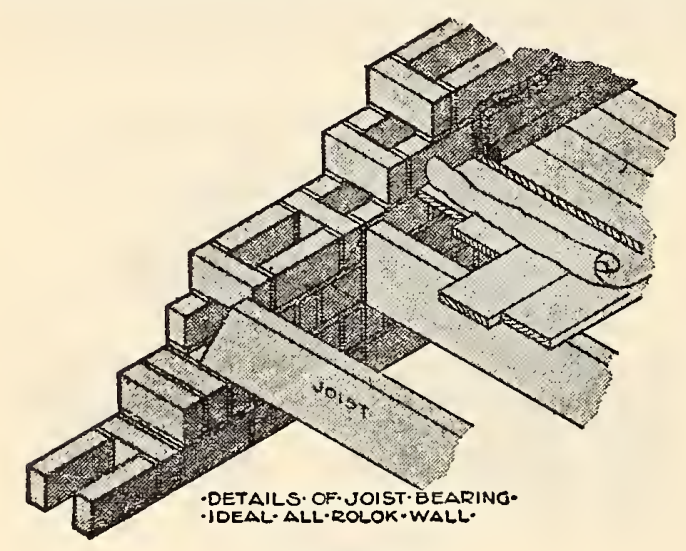

Figure 126

a course of alternate headers and stretchers, as shown in Figure 127.

Contracting Plasterers' International Association, standard specification for lath and plaster work, 1923.

See 514.63 , page 280 .

Federal Specifications Board, specification No. 54, United States Government master specification for asbestos millboard, February 1, 1923.

See 545.2 , page 639 .

Federal Specifications Board, specification No. 248, United States Government master specification for calcined gypsum, November, 5, 1924.

See 514.2 , page 264 .
Federal Specifications Board, specification No. 284, United States Government master specification for gypsum wall board, March 28, 1925.

See 514.61, page 277 .

Federal Specicfiations Board, specification No. 285, United States Government master specification for gypsum plaster board, March 28, 1925.

See 514.61, page 277.

Federal Specifications Board, United States Government master specifications for hollow clay tile, July 11, 1927.

(1) Specification No. 507, load-bearing wall tile (see 534.22, p. 619). (2) Specification No. 508, fireproofing, partition, and furring tile (see 534.22, p. 619).

The Gypsum Institute, gypsum partition tile, 1928.

(This publication includes A. S. T. M. standard specifications for gypsum partition tile or block, scrial designation C52-27 (see 514.62, p. 278) and the following specifications for the erection of gypsum partition tile:)

1. Gencral.-All permanent partitions and furring shall be started on the rough floor. All partitions of a temporary nature or that may be moved from time to time shall be started on the finished floor Tile courses must not be started upon frozen concrete nor upon cinder concrete when such is used for the purpose of fill only.

2. Partitions.-All partitions shall be laid in horizontal superimposed courses with vertical joints broken. Such partitions shall extend from floor to

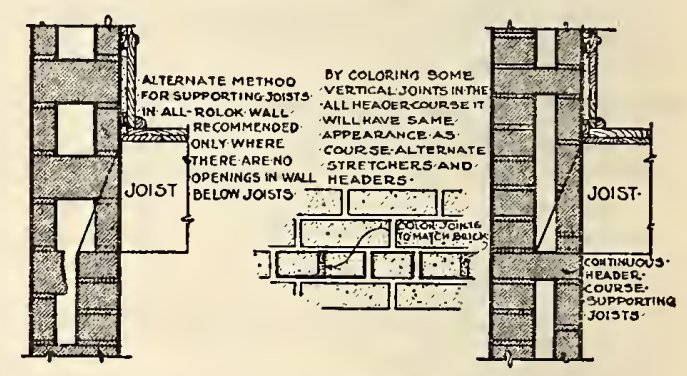

FIgURE 127

ceiling, and shall be set plumb, straight, and true, and shall be wedged at the ceiling, and the joints slushed with gypsum mortar.

3. Anchors.- (To be placed in masons' specifications.) Partitions intersecting brick, or other masonry walls, shall be sccurely anchored to such walls by means of corrugated or other approved anchors furnished and set by the masonry contractor approximately $121 / 4$ inches on centers so that they will project in the joints of the partition not less than 5 inches.

4. Corners and intersections.-Partitions shall be bonded at the corners and intersections by overlapping alternate courses back and forth (log cabin fashion). Overlapping tile shall cover the courses below to the full thickness of the tile.

5. Furring.-Gypsum tile furring shall be laid as is herein required for partitions. All close standing furring and that in contact with the construction shall be securely anchored to the masonry by means of tenpenny steel cut nails driven into the masonry joints at intervals not greater than every 2 feet horizontally and vertically, or shall be securely anchored by metal ties or other approved methods for securing furrring to masonry. Free standing furring shall be of not less thickness, nor of greater height, than shown in the table below.

6. Height.- The clear height of nonbearing partitions of gypsum tile shall not exceed the following: 


\begin{tabular}{|c|c|}
\hline Nominal thickness of tile & $\underset{\text { height }}{\text { Maximum }}$ \\
\hline $\begin{array}{l}\text { 2-inch solid partition or inclosure } \\
\text { 3-inch solid partition or inclosure } \\
\text { 3-inch hollow partition or inclosure } \\
\text { 4-inch hollow partition or inclosure } \\
\text { 5-inch hollow partition or inclosure } \\
\text { 6-inch hollow partition or inclosure } \\
\text { 8-inch hollow partition or inclosure. }\end{array}$ & Feet 10 \\
\hline
\end{tabular}

7. Bucks.-(To be placed in carpenters' specifications.) (a) General. - In partition construction, regardless of the material employed, the frequency of openings and the size and height of such are factors that should receive consideration when designing, since all such openings weaken the construction. The best constructions involve the use of door bucks which extend from floor to ceiling. Bucks of this character should be employed for large openings or in cases where such openings are of frequent occurrence.

(b) Wood.-Where wood bucks are used they shall be of not less than 2-inch lumber and of a width equal to the total thickness of the tile and plaster construction. They may be rabbeted to receive the ends of the tile, or they may be of a width equal to the thickness of the tile, only with $1 / 2$-inch grounds nailed to the sides and projecting beyond the buck not less than three-fourths inch forming a rabbet to receive the ends of the partition tile. All wood bucks shall be secured to the ends of the tile partition with corrugated or other approved anchors nailed to the buck and extending at least 5 inches into every horizontal joint.

(c) Metal.-Where metal bucks are used they should be of sufficient width to receive the ends of the tile. When the metal bucks are of a width equal to the thickness of the tile construction, furring strips of wood shall be secured to the buck as a stop for the plaster coats and for a nailing base for trim. Where the metal bucks are of a width to include the total thickness of the tile and plaster construction, the trim shall be secured direct to the metal bucks or to wood nailing blocks bolted to the face of the bucks for such purpose.

8. Lintels.-(a) Built-up.-Openings in partitions of gypsum tile which are not more than 22 inches in width may be spanned by a gypsum tile which shall have a bearing at each end upon the jamb of not less than 4 inches. When such openings are more than 22 inches, but not more than 4 feet in width the gypsum tile over the openings shall be laid in the form of a jack arch. Skewbacks shall be cut in the tile used over the jambs, intermediate tile shall be beveled to fit the skewbacks, and a key tile to fit shall be set in the center of the jack arch. Bevel cuts shall not be less than 4 inches to the foot. The skewback tile shall have a bearing on the jambs of not less than 12 inches, nor shall they be set so as to project beyond the face of the jambs to a distance greater than one-half of the bearing.

(b) Reinforced and cast.-Openings in partitions of gypsum tile which are more than 4 feet, but not over 6 feet in width may be spanned by lintels of reinforced gypsum of monolithic design.

All reinforced gypsum lintels shall have a bearing upon each jamb of not less than 8 inches.

Mat reinforcement.-Mat reinforced lintels shall be 12 inches in height and shall be reinforced with a galvanized welded wire mat on each side (face) of the lintel. The mat shall not be lighter than No. 12 gage longitudinal wires 5 inches on centers, and No. 12 gage cross wires 9 inches on centers.

Rod reinforcement.-Rod reinforced lintels shall be 12 inches in height and shall be reinforced on each side (face) of the lintel with not less than two $1 / 4$-inch rods for the full length of the lintel. One of the required rods shall be straight and the other shall be bent up for shear. The horizontal part of the reinforcement, at the bottom, shall be 1 inch from the lower face of the lintel and both ends of both rods shall, at their extreme ends, be bent so as to form an anchor within the material.

(c) Metal.-Openings in partitions of gypsum tile which are more than 6 feet in width shall be spanned by metal lintels of approved design. They shall be of such form as to provide a distributed and uniform bearing upon the supporting construction and shall provide a bearing upon each jamb of not less than 8 inches. Metal lintels shall be furnished by the steel contractor but set by the partition contractor. (This same sentence must be inserted in the steel specifications.)

9. Mortar.-All gypsum tile construction shall be laid up with a gypsum mortar composed of 1 part of unfibered gypsum neat plaster to not more than 3 parts of clean, sharp sand. Gypsum mortar shall not be retempered.

10. Plastering.- (To be placed in plasterers' specifications.) All plastering on gypsum tile shall be done with gypsum plasters applied in accordance with the specifications for such of The Gypsum Institute, or other specifications approved by the architect.

All joints between gypsum tile and other masonry construction shall, before the application of the plaster, be covered with a strip of metal lathing not less than 12 inches in width and weighing not less than 2.2 pounds per square yard.

When bucks extend from floor to ceiling that portion of the buck above the opening shall before the application of the plaster be covered with a strip of metal lathing weighing not less than 2.2 pounds per square yard and not less than 16 inches in width.

When bucks do not extend from floor to ceiling there shall be attached a strip of metal lathing 12 inches wide and not less than 2 feet long weighing not less than 2.2 pounds per square yard, to each side of the partition on both sides of the opening. This strip should run diagonally across the head of the opening over the buck.

11. Conduits. - Where conduits occur in walls they shall be so placed as to allow the full thickness of the plaster being applied. A strip of metal lathing weighing not less than 2.2 pounds per square yard shall be applied over all pipe chases occurring in partitions.

NoTE. - When conduits in partitions are placed within 12 inches of door hucks proper hearing of lintols or jack arches can not he obtained. It is therefore recom specification requiring conduits to be kept at least 12 inches from the door bucks.

12. Trim.-(To be placed in carpenters' specifications.) All wood or metal trim shall be of such design as to completely cover any junction between metal bucks or furring and the plaster coats.

(a) Baseboards and similar trim shall be secured to grounds or to nailing blocks or shall be attached by other approved means. Nailing blocks shall be furnished and placed by the carpenter contractor. They shall be not less than seven-eighth inch thick, shall be nailed directly to the end of the gypsum tile and shall be of such other dimensions as to completely cover the end of the tile. When nailing blocks are used they shall be spaced not to exceed 30 inches.

Note.-Conditions on the job vary this practice, as sometimes the nailing hlocks are furnished hy the carpenter and set hy the mason.

(b) Chair rail, picture molding, and plaster grounds shall be secured as is herein required for baseboards.

(c) Blackboards, toilet, and heavy fixtures shall be secured by bolting through the tile construction with 
galvanized iron bolts, or shall be nailed to nailing blocks not less than $1 \frac{1}{1} / 2$ inches thick of the character required for other trim, and spaced not to exceed 15 inches.

United States Department of Commerce, division of simplified practice, recommendation No. 12, hollow building tile, March 1, 1926. (Simplification of sizes of tile.)

See 534.22 , page 619 .

United States Department of Commerce, division of simplified practice, recommendation No. 19 (revised), asbestos paper and asbestos millboard, March 1, 1927. (Sizes and weights.)

See 545.2 , page 640 .

\subsection{Inclosures.}

American Railway Engineering Association, specifications for buildings for railway purposes.

\section{PLUMBING (SLATE TYPE CLOSET STALIS)}

See 511.52, page 199 .

\subsection{Chimneys.}

Common Brick Manufacturers' Association of America, specifications and general schedule of a brick house.

\section{CHIMNEYS AND FIREPLACES}

Brickwork in chimneys shall consist of a minimum thickness of 4 inches of solid brickwork laid with full mortar joints. All flues shall be lined with fireclay flue lining from below smoke entrance to 4 inches above chimney top, with joints filled and pointed tight with mortar. Put 2-inch cement wash on top of all chimney caps. Provide and set angle-iron support if fireplace opening is not arched, also iron ash dump, clean-out door to ash pit, and fire-clay or terra-cotta thimbles of proper size for furnace and range.

Facing and lining of fireplace and hearth shall be laid with common brick, from which those selected for color, markings, or other characteristics desired shall be faced out and the remainder used for backing up and for all other brickwork. All exposed exterior brickwork shall be hard burned brick. If exposed brickwork back of kitchen range is shown on drawings, the same shall be of similar brick.

Eastern Clay Products Association, specification for brick chimneys with fire-clay flue linings (recommended).

This specification is written for one or more chimneys constructed of brick either singly or in connection with walls, fireplaces, etc., in any building. Where materials other than brick are to be used, it will serve as a basis on which such changes as are necessary may be made.

1. Brick.-(a) All brick shall be good, sound, hard, well-burned common brick. Brick shall be well wetted just before laying, if laid in dry, warm weather, or shall be perfectly dry if laid in cold weather.

(b) (If face brick or fire brick are to be used for any exposed work, stipulate the kind here, or elscwhere.)

2. Mortar.-(a) Mortar for all freestanding chimneys (and for fireplaces, breasts, or other portions thereof) and for all chimneys above roofs shall be proportioned as follows: Two bags, 200 pounds, of Poriland ccment and one bag, 50 pounds, of dry hydrated lime thoroughly mixed dry, three times this volume of clean, sharp sand, and sufficient water. All shall be well tempered to an even color.

(b) Mortar for chimneys forming parts of walls (and for breasts, fireplaces, hearths, or other portions thereof) is to be as elsewhere specified for brickwork of walls.
3. Fire-clay flue linings.-(a) Fire-clay flue linings shall be manufactured from suitable refractory clay, either natural or compounded, which has a softening point not lower than $1,994^{\circ} \mathrm{F}$. (Segar Cone 03) and shall be adapted to withstand high temperatures and flue gases.

(b) All flue linings shall be of the nearest stock size commercially obtainable to the sizes shown or marked on the drawings unless the same are stated to be made of special size to order for the particular installation shown.

NoTE: Care should be taken that only standard sizes are shown or used, as this reduces the cost and facilitates prompt delivery.

(c) A certificate shall, if required (or upon request), be furnished by the manufacturer stating that the flue lining supplied is in accordance with these specifications.

4. Construction.-(a) Build all chimneys (chimney breasts, fireplaces, hearths, etc.) to dimensions, forms, sizes, heights, and thicknesses shown on drawings.

(b) Bricks shall be laid in the best and most workmanlike manner with full beds and full slushed joints, leaving no voids or empty spaces in the walls, and all shall be built perfectly straight and true to line on all faces.

(c) All flues shall be lined their entire height with fire clay flue linings starting at bottom of each flue above clean-out space (and at top of each smoke chamber over fireplaces) and extending 4 inches above the top course of brick. No broken flue linings shall be used. Any change in direction shall be accomplished by carefully chipping off the ends of linings.

(d) All flues shall be independent of one another, but where the plans require that flues be built without a "withe" or dividing walls, joints of the linings shall be staggered at least 7 inches.

(e) Leave openings for all stovepipe holes and any registers where indicated or required and furnish and neatly build in fire clay flue rings of the proper sizes for all pipes entering flues.

(f) Clean-out openings shall be provided and set at bases of all flues (except for fireplaces), each to be full width of flue. For each furnish and set a cast-iron frame, well anchored, fitted with a tight closing cast-iron door.

Nore.-If fireplaces are a part of the building for which this specification is used and metal frames or fire-clay flues for ash drops are desired this will serve as a reminder that they should be called for.

(g) Each length of lining shall be set in place and the brick laid around it and under no circumstances shall more than three courses of the brick chimney wall be laid before the flue lining is inserted.

$(h)$ All linings shall be laid with absolutely full joints of the mortar before specified, carefully pointed, and smoothed on inside. The space between flue linings and inclosing walls shall be slushed full with mortar as each course of brick is laid.

(i) In the case of any flue which is built with an offset, a bag of burlap or similar material attached to a rope and filled with excelsior or similar material tightly fitting the flue shall be inserted below the first offset and drawn up as each few sections of flue lining are installed.

(j) A wash of rich cement mortar having a slightly concave surface shall be laid on top of the chimney tapering off from 2 inches thick against the flue lining.

(k) Build in all flashings as shown and elsewhere specified, carefully construct or set any caps shown, thoroughly clean down any exposed brickwork and leave everything finished and complete.

5. Smoke test.-(a) Before the scaffolding is removed from around any chimneys and before any chimney walls or breasts are plastered, but not until after the mortar has seasoned, each flue shall be 
given a separate and thorough smoke test by the mason contractor. The tests shall be made in the presence of the mason contractor, the heating contractor and the architect, or their representatives.

(b) Leaks into adjacent flues shall be especially looked for and not more than one flue shall be tested at one time.

(c) Any leaks which may develop shall be promptly made tight before the chimney work will be accepted as satisfactory.

\subsection{Floors.}

American Concrete Institute, standard specifications for concrete floors, serial designation $\mathrm{C}-2 \mathrm{~A}-2^{4}$ 1924.

\section{APPLICATION OF SPECIFICATIONS}

These specifications apply to floors in buildings, whether subjected to moderate or heavy traffic, and cover the laying and finishing of the floor, also its protection during early hardening.

\section{USE OF SPECIFICATIONS}

For architects, engineers, and others desiring to embody these specifications in their general specifications covering a particular piece of work, the following outline of the paragraphs necessary to meet different conditions will prove convenient:

Floors laid on ground.-Moderate or light traffic:

Two-course.-Paragraphs 1 to 19 (except 8), 34 to 52,54 to 57 .

One-course.-Paragraphs 1 to 19 (except 8), 34 to 47,58 to 62 .

Floors laid on ground.-Heavy traffic:

Two-course.-Paragraphs 1 to 19,34 to 51,53 to 57 .

Reinforced-concrete floors.-Moderate or light traffic, paragraphs 1 to 26 (except 8), 28 to 33 .

Reinforced-concrete floors.-Heavy traffic: Paragraphs, 1 to 25,27 to 33 .

\section{MATERIALS}

\section{A. PORTLAND CEMENT}

1. Portland cement shall meet the requirements of the current standard specifications for Portland cement adopted by the American Society for Testing Materials, C9, 516.11 , page 287.

\section{B. AGGREGATES}

2. Before delivery on the job, the contractor shall submit to the architect or engineer a 50-pound sample of each of the aggregates proposed for use. These samples shall be tested, and if found to pass the requirements of the specifications, similar material shall be considered as acceptable for the work. In no case shall aggregate containing frost or lumps of frozen material be used.

Crusher run stone, bank run gravel or mixtures of fine and coarse aggregate prepared before delivery on the work shall not be used.

\section{FIVE AGGREGATE}

3. Fine aggregate shall consist of natural sand or screenings from hard, tough, crushed rock, or gravel consisting of quartz grains or other hard material, clean and free from any surface film or coating and graded from fine to coarse, with the coarse particles predominating.

4. Fine aggregate, when dry, shall pass a screen having 4 meshes to the linear inch; not more than 25 per cent shall pass a sieve having 50 meshes per linear inch and not more than 5 per cent shall pass a sieve having 100 meshes per linear inch.

5. Fine aggregate shall not contain injurious vegetable or other organic matter as determined by the colorimetric test nor more than 5 per cent by volume of clay or loam. Field tests may be made by the architect or engineer on fine aggregate as delivered at any time during the progress of the work. If there is more than 7 per cent of clay or loam by volume in one hour's settlement after shaking in 100 per cent excess of water, the material represented by the sample shall be rejected.

6. Fine aggregate shall be of such quality that mortar composed of 1 part Portland cement and 3 parts fine aggregate, by weight, when made into briquets, shall show a tensile strength at 7 and 28 days at least equal to the strength of briquets composed of 1 part of the same cement and 3 parts standard Ottawa sand, by weight. The percentage of water used in making the briquets of cement and fine aggregate shall be such as to produce a mortar of the same consistency as that of the Ottawa sand briquets of standard consistency. In other respects, all briquets shall be made in accordance with the methods of testing cement recommended by the American Society for Testing Materials, C9, 516.11, page 287.

\section{COARSE AGGREGATE}

7. Coarse aggregate shall consist of clean, hard, tough, crushed rock, pebbles, or air-cooled blastfurnace slag, graded in size, free from vegetable or other organic matter, and shall contain no soft, flat, or elongated particles. The size of the coarse aggregate shall range from $1 \frac{1}{2}$ inches down, not more than 5 per cent passing a screen having 4 meshes per linear inch, and no intermediate sizes shall be removed. The weight per cubic foot of blast furnace slag shall not be less than 65 pounds per cubic foot in that portion of the slab not subject to abrasion, and not less than 70 pounds per cubic foot for use in the,wearing surface.

Note.-The above paragraph is printed as revised, and is a tentative standard under A. C.I. procedure.

8. No. 1 aggregate for the wearing course shall consist of clean, hard, tough, crushed rock or pebbles, free from vegetable or other organic matter, and shall contain no soft, flat, or elongated particles. It shall pass when dry a screen having $3 / 8$-inch openings and not more than 10 per cent shall pass a screen having four meshes per linear inch.

\section{E. WATER}

9. Water shall be clean, free from oil, acid, alkali, or vegetable matter.

\section{F. COLOR}

10. If artificial coloring matter is required, only those mineral colors shall be used which, in the amount hereinafter specified, will not appreciably impair the strength of the cement.

\section{G. REINFCRCEMENT}

11. The reinforcing metal shall meet the requirements of the current standard specifications for steel reinforcement of the American Society for Testing Materials. It shall be free from excessive rust, scale, paint, or coatings of any character which will tend to reduce or destroy the bond.

\section{H. JOIN T FILLER}

12. The joint filler shall be a suitable compound that will not become soft and run out in hot weather, nor hard and brittle and chip out in cold weather; or, prepared strips of fiber matrix and bitumen as approved by the architect or engineer. The strips shall be one-half inch in thickness and their width shall at least equal the full thickness of the slab. 


\section{CONSTRUCTION}

\section{A. PROPORTIONING}

13. The method of measuring the materials for the concrete or mortar, including water, shall be one which will insure separate and uniform proportions of each of the materials at all times. A sack of Portland cement ( 94 pounds net) shall be considered as 1 cubic foot.

\section{B. MIXING}

14. All concrete shall be mixed by machine except when the architect or engineer shall otherwise permit under special conditions. A batch mixer of an approved type shall be used. The ingredients of the concrete or mortar shall be mixed to the specified consistency, and the mixing shall continue for at least one minute after all the materials are in the drum. Raw materials shall not be permitted to enter the drum until all the material of the preceding batch has been discharged.

15. When it is necessary to mix by hand, the materials shall be mixed dry on a water-tight platform until the mixture is of uniform color, the required amount of water added, and the mixing continued until the mass is of uniform consistency and homogeneous.

16. Retempering of mortar or concrete which has partially hardened, that is, mixing with or without additional materials or water, shall not be permitted.

\section{CURING}

17. As soon as the finished floor has hardened sufficiently to prevent damage thereby, the floor shall be covered with at least 1 inch of wet sand, or 2 inches of sawdust, which shall be kept wet by sprinkling with water for at least 10 days.

18. The freshly finished floor shall be protected from hot sun and drying winds until it can be sprinkled and covered as above specified. The concrete surface must not be damaged or pitted by raindrops and the contractor shall provide and use when necessary sufficient tarpaulins to completely cover all sections that have been placed within the preceding 12 hours.

\section{TEMPERATURES BELOW $40^{\circ} \mathrm{F}$.}

19. If at any time during the progress of the work the temperature is, or in the opinion of the architect or engineer will, within 24 hours, drop to $40^{\circ} \mathrm{F}$., the water and aggregates shall be heated and precautions taken to protect the work from freezing for at least five days.

\section{IV., REINFORCED-CONCRETE FLOORS}

For reinforced-concrete floors the following will apply in addition to paragraphs 1 to 19, inclusive.

\section{A. FORMS}

20. The forms shall be substantial, unyielding and so constructed that the concrete will conform to the designed dimensions and contours, and shall also be tight to prevent the leakage of mortar. The supports for floors shall not be removed until the concrete has hardened sufficiently and then only with the consent of the engineer or architect in charge. Permanent shores shall be placed in such a manner as to assure safety of the floors after temporary supports are removed.

\section{B. REINFORCEMENT}

21. Reinforcing metal shall be provided as called for on the plans. It shall be placed as indicatcd and mechanically held in position so that it will not become disarranged during the depositing of the concrete. Whenever it is necessary to splice tension reinforcement the character of the splice shall be such as will develop its full strength. Splices at points of maximum stress shall be avoided. Splicing by lapping bars without contact and with space between bars along the overlap equal to twice the thickness of the bars is preferable to mechanical splices or clamps.

\section{CONCRETE SLAB}

22. The concrete shall be mixed in the proportions by volume of 1 sack of Portland cement, 2 cubic feet of fine aggregate, and 4 cubic feet of coarse aggregate.

23. Only sufficient water shall be used to produce a workable plastic mix which will flow sluggishly into the forms and around the reinforcement and which can be conveyed from the mixer to the forms without the separation of the coarse aggregate from the mortar.

24. The concrete shall be placed in a manner to insure a smooth ceiling and thoroughly worked around the reinforcement and into the recesses of the forms. Concrete shall be deposited in its final position as soon as possible after mixing. It shall be struck off to a surface at least 1 inch below the established grade of the finished surface of the floor. Workmen shall not be permitted to walk in freshly-laid concrete, and if sand or dust collects on the base it shall be carfully removed before the wearing course is applied.

25. When it is necessary to make a joint in a flcor slab its location shall be designated by the architect or engineer; joints to be vertical.

\section{WEARING COURSE}

26. Mixture No. 1.-The mortar shall be mixed in the proportions of 1 sack of Portland cement and 2 cubic feet of fine aggregate. The minimum thickness shall be $3 / 4$ inch.

27. Mixture No. 2.-The mortar shall be mixed in the proportions of 1 sack of Portland cement, 1 eubic foot of fine aggregate, and 1 cubic foot of No. 1 aggregate for wearing course. The minimum thickness shall be $3 / 4$ inch.

28. The mortar shall be of the dryest consistency possible to work with a sawing motion of the strike board.

29. The wearing course shall be placed immediately after mixing. It shall be deposited on the fresh concrete of the base before the latter has appreciably hardened and brought to the established grade with a strike board.

Nore.-When placing the wearing course after the concrete slab has hardened eliminate paragraph 29 and substitue paragraphs 30 and 31 .

30. The surface of the slab shall be thoroughly roughened by picking or other means and cleaned of all dirt and débris.

31. The slab shall be thoroughly moist but free from pools of water when the grout and mortar for wearing course is placed. A neat-cement grout shall be brushed on the surface of the slab, the wearing course immediately applied and brought to the established grade with a strike board. Grout and mortar shall be used within 45 minutes after mixing with water.

32. After the wearing course has been brought to the established grade by means of a strike board it shall be worked with a wood float in a manner which will thoroughly compact it and provide a surface free from depressions or irregularities of any kind. When required, the surface shall be steel troweled, but excessive working shall be avoided. A mixture of dry cement, sand, and No. 1 aggregate may be 
applied to the fresh concrete of the base for a wearing course, but in no case shall dry cement or a mixture of dry cement and sand be sprinkled on the surface of the wearing course to absorb moisture or to hasten the hardening. Special methods not conflicting with these specifications may be used.

33. If artificial coloring is used it must be incorporated with the entire wearing course and shall be mixed dry with the cement and aggregate until the mixture is of uniform color. In no case shall the amount of coloring exceed 5 per cent of the weight of the cement.

\section{PLAIN CONCRETE FLOORS}

For plain concrete floors the following will apply in addition to paragraphs 1 to 29 , inclusive:

\section{A. SUBGRADE}

34. All soft and spongy places shall be removed and all depressions filled with suitable material which shall be thoroughly compacted in layers not exceeding 6 inches in thickness. The subgrade shall be thoroughly tamped until it is brought to a firm, unyielding surface.

35. All fills shall be made in a manner satisfactory to the architect or engineer. The use of muck, quicksand, soft clay, spongy, or perishable materia is prohibited.

36 . When required, a suitable drainage system shall be installed and connected with sewers or other drains indicated by the engineer.

37. The subgrade shall not be less than - inch below the finished surface of the floor.

Note. - Subgrade is to be - inch below the finished surface of the floor when sub-base is not required, and at least - inch below when sub-base is required.

\section{B. SUB-BASE}

(Omit these sections when sub-base is not required.)

38. Only clean, hard material, such as coarse gravel or steam-boiler cinders free from ash or particles of unburned coal, shall be used in the subbase.

39. The material as specified shall be spread on the subgrade thoroughly rolled or tamped to a surface at least - inch below the finished grade of the floor. On fills, the sub-base shall extend the full width of the fill.

40. While compacting the sub-base the material shall be kept thorouglily wet and shall be in that condition when the concrete is deposited.

\section{FORMS}

41. Forms shall be free from warp and of sufficient strength to resist springing out of shape.

42. The forms shall be well staked or otherwise held to the established lines and grades and their upper edges shall conform to the established grade of the floor.

43. All wood forms shall be thoroughly wetted and metal forms oiled or coated with soft soap or whitewash before depositing any material against them. All mortar and dirt shall be removed from the forms that have been previously used.

American Society for Testing liaterials, standard specifications and tests for hollow burned clay floor tile, serial designation C57-27, 1927.

See 534.22 , page 616 .

Associated Tile Manufacturers, specification for tiled bathrooms and tiled floors or wainscots, 1920 . See 534.20 , page 594 .

Federal Specifications Board, specification No. 445, United States Government master specification for cement, plastic magnesia (magnesia- oxychloride) used as flooring, bases, wainscots, etc. October 28, 1926

See 572 ., page 652 .

Federal Specifications Board, specification No. 506, United States Government master specification for tile, hollow clay, floor, July 1, 1927.

See 534.22 , page 618 .

National Slate Association, specification for slate floors, terraces, and walks, 1925.

A natural split or "quarry cleft" surface is desirable for ordinary outdoor purposes and for distinctive interior lobbies, aisles, display rooms, etc., although the standard rubbed surface can be furnished when desired.

Slate for these purposes is seldom required to be of an exact thickness, the run of stock being entirely suitable. The thickness will depend largely upon the method of laying and location. In sand or soil with grass it should be from $7 / 8$ to $11 / 4$ inches thick, but when properly laid in cement a thickness of $1 / 2$ inch will be satisfactory and economical. The size and shape of the pieces will be detcrmined by the arrangement and pattern desired. Seven of the many styles of cutting and laying which are in common use are illustrated in Figure 128.

For estimating weight of slate the quarries usually figure from 16 to 20 pounds per square foot on a basis of 1 -inch thickness.

(Suggested paragraphs suitable for rewriting into an architect's specification. Paragraphs not applying to the type of walk, floor, or terrace desired may be omitted without destroying the effectiveness of the whole.)

\section{SLATE}

The contractor shall furnish and lay all slate for walks, floors, and terraces listed below. Slate shall be (here specify color, thickness, surface, size when necessary, shape of pieces, style of edges, and width of joints).

\section{TYPE OF INSTALLATION}

Note.-Specification writer may copy here only those paragraphs covering type of installation desired.

\section{(A) ON SOIL WITH GRASS JOINTS (SOIL OR TURF SUB-BEN)}

Mark out location of all walks and terraces to be laid with grass joints. If ground is not well settled, wet and tamp thoroughly. Fill any holes or depressions with thoroughly tamped soil. Lay slate directly on soil in (here specify arrangement and pattern). Each piece of slate shall rest on a solid, even bed with the top surface level.

\section{(B) ON SOIL WITH GRASS JOINTS (CINDER SUB-BED)}

Mark out location of all walks and terraces to be laid with slate with grass joints and excavate to a depth of 8 to 10 inches. Fill excavations with cinders from 6 to 8 inches deep, well rolled and tamped to an even surface. On top of cinder bed place a 2-inch layer of soil and on soil lay slate in (here specify arrangement and pattern). Fill all joints with soil to level of surface of slate and sow grass seed.

\section{(C) LAID ON CONCRETE ON EARTH}

Note.-If experience in a given locality warrants greater depth specification writer can change figures accordingly.

(a) Mark out location of all walks and terraces to be laid with slate in concrete and excavate to a depth of 10 inches. Fill excavation with cinders well rolled and tamped to within 5 inches of level desired.

(b) On cinder bed place a course of cinder concrete 3 inches deep. Follow at once with the setting mixture (3 parts sand with 1 part of cement). As 
soon as this setting bed is leveled sprinkle it with pure Portland cement to make perfect bond between slate and setting bed and lay slate in (here specify arrangement and pattern). Thoroughly wet the pieces of slate as set and bed to desired level. Point all joints with concrete mixed 2 parts well-sieved sand and 1 part cement.
(E) ON MASONRY SLAB LAID IN CONCRETE WITHOUT WATER. PROOFING MEMBRAN E

(a) The surface of the masonry shall be carefully swept and all unnecessary obstructions removed.

(b) Place from 1 to 2 inches (depending on final level) of setting mixture (3 parts sand with 1 part of cement). As soon as this setting bed is leveled
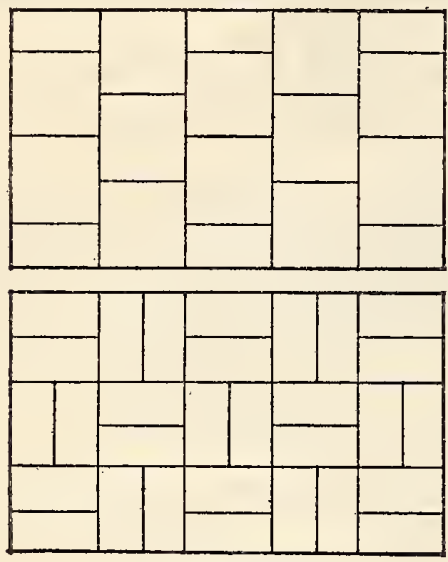

No. 1 and No. 1-A

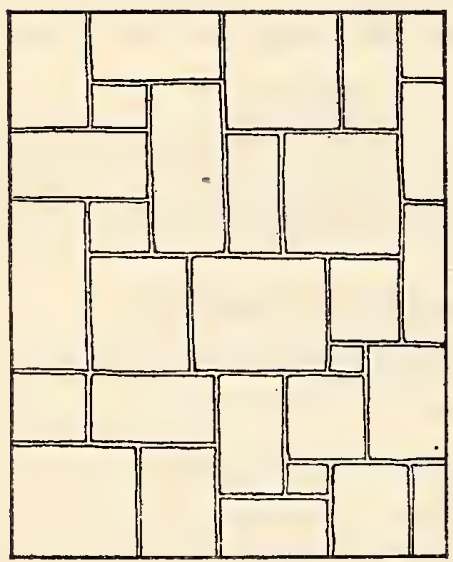

No. 4

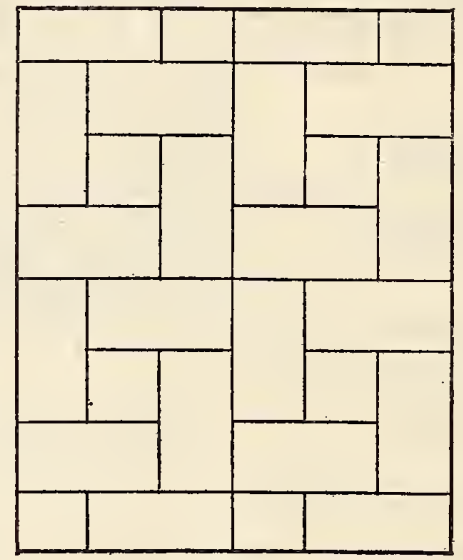

No. 2

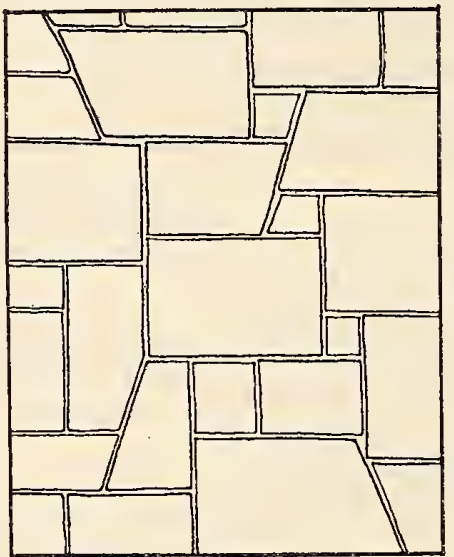

No. 5

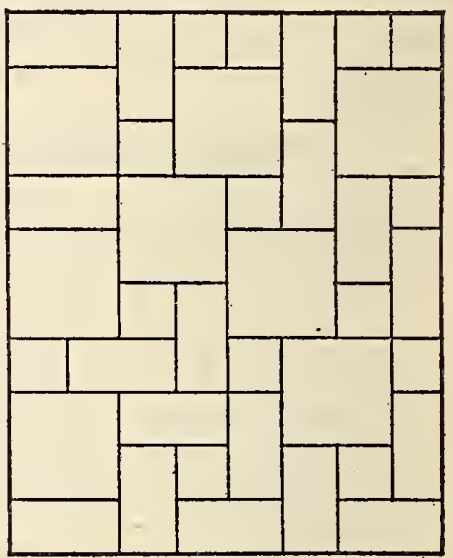

No. 3

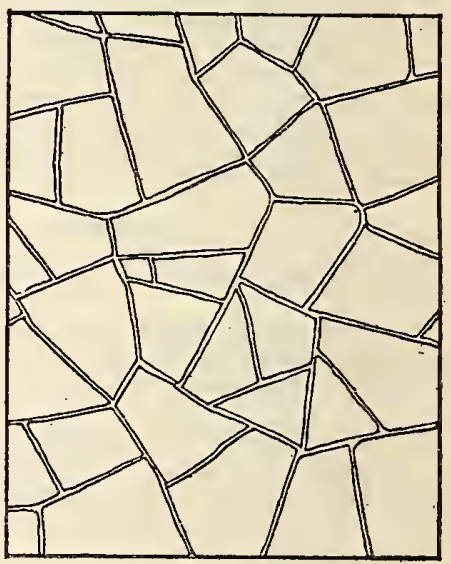

No. 6

FIgURE 128.-Patterns for slate floors, terraces and walks

Nos. 1 and 1A, units of one size; No. 2, units of two sizes; No. 3, units of three sizes; No. 4, random rectangular; No. 5 , semiirregular No. 6, irregular.

(D) ON MASONRY SLAB IAID IN CONCRETE WTTH WATERPROOFING MEMBRANE

NorE.-Only required where it is necessary to prevent wate damage underneath or where there are occupied quarters below.

(a) (Here specify method of waterproofing. Method should be that found by the designer to be most satisfactory in accordance with locality.)

(b) On top of waterproofing place a concrete setting bed from 2 to $2 \frac{1}{2}$ inches thick (depending on level desired). Suitable expansion joints extending the full depth of the setting bed shall be provided at intervals of 15 feet in each direction and shall be filled with caulking or plastic compound stiff enough not to ooze out under pressure and not soft enough to run.

(c) On this bed place the setting mixture. When leveled, sprinkle with pure Portland cement and firmly embed the slate in (here specify arrangement and pattern), pointing each joint with concrete (2 parts well-sieved sand and 1 part cement) to a level with the surface of the slate. Thoroughly wet each slate before bedding it. sprinkle with pure Portland cement to perfect bond between bed and slate and lay slate in (here specify arrangement and pattern). Thoroughly wet pieces of slate and firmly embed to desired level. Point all joints with concrete mixed 2 parts well-sieved sand and 1 part cement.

\section{(F) ON MASONRY SLAB IN EMBEDDING COMPOUND}

(a) On top of the (waterproofing) (masonry slab) spread the embedding compound. This compound shall be (several methods and brands of compounds and plastics have been found successful in various localitites and under certain conditions. It would be well to investigate those experience has proven most satisfactory in a given locality before specifying the method to be followed), using not less than - pounds per 100 square feet.

(b) In this compound firmly embed the slate with joints butted and pointed flush with the same compound. All shall be perfectly dry when set. 


\section{(G) IN PLACE OF EXISTING WOOD FLOORS}

(a) Remove the present wood floor.

(b) Wherever so shown, or practicable, the floor joists shall be lowered to the depth required for the construction of slate floors. On top of such joists lay a false wood floor of not less than $13 / 16$ inch thick by not over 6 -inch-wide boards. Place $1 / 4$ inch apart and securely nail to each joist.

(b1) Where floor joists are not lowered their tops shall be beveled off to a sharp edge. To the sides of the joists at a distance below the finished floor required for the construction of the slate floors securely nail not less than 1 by $2 \frac{1}{2}$ inch cleats. In between the joists cut and set 1 inch by not over 6-inch-wide boards laid about $1 / 4$ inch apart and securely nailed to the cleats. Where joists are chamfered the point of the joist shall be at least $3 / 4$ inch below the top of the concrete setting bed.

(c) Cover this new false floor with two layers of asphalt-saturated rag felt of not less than commercial 14-pound weight (allowable tolerance 1 pound more or less). Nail only as necessary to hold in place.

(d) On the felt place a setting bed of concrete ( 3 parts sand to 1 part cement), from 1 to 2 inches thick, depending on desired level. After this setting bed is leveled follow immediately with from $1 / 2$ to 2 inches (depending on level desired) of setting mixture ( 3 parts sand to 1 part cement). After this mixture is leveled sprinkle with pure Portland cement and bed slate thereon in (here specify general arrangement or pattern). Thoroughly wet each piece of slate before bedding. Point all joints with concrete mixed 2 parts well-sieved sand and 1 part cement.

(e) At any openings where level of new slate floor is above that of adjoining floors install slate saddle of required dimensions and cut off all doors, etc., to allow proper clearance for use with new floor levels.

\section{(H) OVER EXISTING WOOD FLOORS}

(a) Cover existing floor with two layers asphaltsaturated rag felt of not less than commercial 14-pound weight (allowable tolerance 1 pound more or less). Each sheet of felt shall lap over preceding one and shall be nailed only as is necessary to hold it in place.

(b) Lay wire mesh or metal floor lath on the felt and cover with from $1 / 2$ to 2 inches (depending on level desired) of setting mixture (3 parts sand, 1 part cement). After this bed is leveled sprinkle with pure Portland cement and bed slate thereon in (here specify general arrangement or pattern). Thoroughly wet each piece of slate before bedding. Point all joints with concrete mixed 2 parts wellsieved sand and 1 part cement.

(c) Cut off all doors, etc., to allow proper clearance for use with raised level of new floor. At all openings install slate saddle of required dimensions.

\section{CLEANING SLATE}

Note.-Only required for slate floors with concrete mixture joints.

Sponge all slate as set on exposed surface with clean water to remove mortar stains, and if necessary upon completion, rub down with a solution 3 parts coal oil and 1 of linseed oil. (If light or weak solution of muriatic acid is needed and used for cleaning, care should be taken to wipe only exposed slate surfaces to avoid acid eating out cement joints.)

\section{COMPLETION}

Upon completion, all slate shall be sound, whole, and clean, and the work shall be left smooth and in every respect a neat example of craftsmanship.
518.57 Ceilings and Roofing. (See also 505.16 and 505.36.)

American Railway Engineering Association, specifications for buildings for railway purposes.

\section{BUILT-UP ROOFING, 1926}

\section{MATERIALS}

1. General

The contractor shall furnish all labor, material, tools, and equipment necessary to entirely complete the roofing, to make the work water-tight, and shall leave it in a neat and finished condition.

\section{Materials}

Pitch.-Pitch shall be the best quality, straightrun American coal-tar pitch, distilled direct from coal tar, and, free from water and ammonia. It shall contain not less uhan 15 per cent nor more than 35 per cent of free carbon. The melting point shall be not below $140^{\circ} \mathrm{F}$. nor above $150^{\circ} \mathrm{F}$. (A. S. T. M. D61, cube in water method; see 505.0, p. 123), and it shall show a flash point of not less than $240^{\circ} \mathrm{F}$.

Asphalt.-Asphalt shall be a combination of natural asphalt prepared and combined properly in which inherent mineral matter will be permitted, but to which no mineral matter shall be added. The nuelting point of asphalt shall be between $140^{\circ}$ and $180^{\circ} \mathrm{F}$. The penetration at $77^{\circ} \mathrm{F}$. under a load of $100 \mathrm{~g}$ for 5 seconds shall be not less than 20 nor more than 30 . The ductility at $77^{\circ} \mathrm{F}$. when a briquette of the material having a minimum cross section of $1 \mathrm{~cm}$ is pulled apart at the rate of $5 \mathrm{~cm}$ per minute shall be not less than $10 \mathrm{~cm}$ for asphalt having a melting point under $165^{\circ} \mathrm{F}$. nor less than $3 \mathrm{~cm}$ for asphalt having a melting point between $165^{\circ}$ and $180^{\circ} \mathrm{F}$. The loss of a $50 \mathrm{~g}$ sample at $325^{\circ} \mathrm{F}$. for five hours shall not exceed 1 per cent. The penetration of the residue at $77^{\circ} \mathrm{F}$. under a load of $100 \mathrm{~g}$ for five seconds shall be not less than 10 nor more than 15 . The methods of making tests shall be in accordance with the standard of the A. S. T. M. and penetrations are in hundredths of a centimeter.

Asphalt primer.-Asphalt primer for concrete and gypsum decks shall weigh not less than 7 pounds per gallon and shall be made from asphalt of the quality specified under "Asphalt," flushed to the proper consistency. The melting point of the asphalt primer shall be such as will permit the subsequent application of the hot asphalt to amalgamate immediately with it.

Roofing felts.-Impregnated felts shall be 32 or 36 inches wide, and shall be saturated thoroughly. They shall have sufficient pliability to meet the requirements of application, and samples taken 5 feet from the outside ends of rolls shall bend flat without cracking through $180^{\circ}$ at a temperature of $60^{\circ} \mathrm{F}$. A variation of 7 per cent from the weights specified will be allowed. The impregnating compounds shall be of the qualities specified and shall be of the proper consistency for saturating the felts.

Tarred and asphalted felts shall be rag felts, and for built-up roofings shall weigh not less than 14 pounds per 108 square feet.

Unimpregnated felt or rosin-sized building paper shall weigh not less than 5 pounds per 108 square feet.

Manufactured roofing.-Manufactured roofing material shall be delivered at the site in the original sealed packages of the imanufacturer, and each package shall be wrapped and labeled properly in order that it may be identified easily.

Gravel and slag.-Gravel shall be hard, durable, free from clay, loam, and other foreign substances, and shall range from $1 / 4$ to $5 / 8$ inch. 
Slag shall be granulated furnace slag, free from sand, dirt, and other foreign substances, and shall range from $1 / 4$ to $5 / 8$ inch.

\section{Application}

The roof decks shall be clean, smooth, thoroughly dry, and free from projections which would injure the roof coverings. All roofing shall be laid smoothly, without wrinkles or buckles, and finished surfaces shall be free from cracks and bubbles.

Before applying the roofing over wood decks all loose knots and other flaws shall be removed and knotholes and large cracks shall then be covered with tin or other sheet metal nailed in place.

For gypsum or concrete decks the joints and surfaces shall be made tight and flush and all loose and uneven surfaces shall be leveled up before the roofing is applied.

\section{TYPE A-1}

\section{Pitch and Gravel (or Slag) over Wood or Precast Gypsum Slabs}

Roofing constructed of five layers of tarred felt, pitch, and gravel (or slag) and a minimum of 150 pounds of pitch per 100 square feet.

1. If on wood, lay one thickness of rosin-sized building paper or unimpregnated felt weighing not less than 5 pounds per 108 square feet, lapping the sheets at least 1 inch; if on precast gypsum, this shall be omitted.

2. Over the entire surface lay two layers of tarred felt weighing not less than 14 pounds per 108 square feet per layer, lapping each sheet of each layer 17 inches over the preceding sheet, end laps not less than 4 inches. Sheets shall be nailed as often as necessary to hold them in placc until the remaining felt is laid. These layers shall be turned up vertical, surfaces not less than 6 inches, and securely fastened.

3 . Coat the entire surface uniformly with hot pitch, using not less than 25 pounds per 100 square feet.

4. Over the entire surface lay three layers of tarred felt, lapping each sheet of each layer 22 inches over the preceding sheet, with end laps not less than 6 inches. Hot pitch shall be mopped the full 22 inches on each sheet so that in no place shall felt touch felt, using not less than 25 pounds of pitch per 100 square feet between successive layers. Any nailing necessary shall be done in such a manner that all nails will be covered by not less than two layers of felt. Nails shall be galvanized and shall be driven through flat tin or zinc caps. These layers shall be turned up vertical, surfaces not less than 6 inches, and securely fastened.

5. Coat the entire surface uniformly with hot pitch poured from a dipper, using not less than 75 pounds per 100 square feet, into which, while hot, embed not less than 400 pounds of gravel (or 300 pounds of slag).

\section{TYPE A-2}

\section{Pitch and Gravel (or Slag) over Concrete or Poured Gypsum}

Roofing constructed of one coating hot pitch and five layers of tarred felt, pitch, and gravel (or slag), and a minimum of 215 pounds of pitch per 100 square feet.

1. The entire surface shall be coated uniformly with hot pitch, using not less than 40 pounds per 100 square feet.

2. Over this lay two layers of tarred felt weighing not less than 14 pounds per 108 square feet per layer, lapping each sheet of each layer 17 inches over the preceding sheet, end laps not less than 4 inches. Hot pitch shall be mopped the full 17 inches on each sheet so that in no place will felt touch felt, using not less than 25 pounds of pitch per 100 square feet between successive layers. These layers shall be turned up vertical, surfaces not less than 6 inches, and securely fastened.

3. Coat the entire surface uniformly with hot pitch, using not less than 25 pounds per 100 square feet.

4. Over the entire surface lay three layers of tarred rag felt, lapping each sheet of each layer 22 inches over the preceding sheet, end laps not less than 6 inches. Hot pitch shall be mopped the full 22 inches on each sheet so that in no place will felt touch felt, using not less than 25 pounds of pitch per 100 square feet between succeeding layers.

If roof decks are of gypsum, in addition to the above, the upper edge of each course of the third layer of felt shall be nailed to the roof deck with galvanized nails driven through flat tin or zinc caps. These layers shall be turned up vertical, surfaces not less than 6 inches, and securely fastened.

5. Coat the entire surface uniformly with hot pitch poured from a dipper, using not less than 75 pounds per 100 square feet, into which while hot not less than 400 pounds of gravel (or 300 pounds of slag) shall be embedded.

\section{TYPE B-I}

\section{Asphalt and Gravel (or Slag) over Wood or Precast Gypsum Slabs}

Roofing constructed of five layers of asphalted felt, asphalt, and gravel (or slag), and a minimum of 150 pounds of asphalt per 100 square feet.

1. If on wood, lay one thickness of rosin-sized building paper or unimpregnated felt weighing not less than 5 pounds per 100 square feet, lapping the sheet at least 1 inch; if on precast gypsum, this shall be omitted.

2 . Over the entire surface lay two layers of asphalted felt weighing not less than 14 pounds per 108 square feet per layer, lapping each sheet of each layer 17 inches over the preceding sheet, end laps not less than 4 inches. Sheets shall be nailed as often as necessary to hold them in place until the remaining felt is laid. These layers shall be turned up vertical, surfaces not less than 6 inches, and securely fastened.

3 . Coat the entire surface uniformly with hot asphalt, using not less than 25 pounds per 100 square feet.

4. Over the entire surface lay three layers of asphalted felt, lapping each sheet of each layer 22 inches over the preceding sheet, end laps not less than 6 inches. Hot asphalt shall be mopped the full 22 inches on each sheet so that in no place will felt touch felt, using not less than 25 pounds of asphalt per 100 square feet between successive layers.

Any nailing necessary shall be done in such manner that all nails will be covered by not less than two layers of felt. Nails shall be galvanized and shall be driven through flat tin or zinc caps. These layers shall be cut off at vertical, surfaces not less than 6 inches, and securely fastened.

5. Coat the entire surface uniformly with hot asphalt poured from a dipper, using not less than 75 pounds per 100 square feet into which while hot embed not less than 400 pounds of gravel (or 300 pounds of slag).

\section{TYPE B-2}

\section{Asphalt and Gravel (or Slag) over Concrete or Poured Gypsum}

Roofing constructed of five layers asphalted felt, asphalt, and gravel (or slag), and a minimum (not including primer) of 205 pounds of asphalt per 100 square feet.

1. The entire surface, if of concrete, shall be coated uniformly with asphalt primer, using not less than 10 pounds per 100 square feet, and allowed to dry. 
If of gypsum, the surface shall be given two such coats. Upon this there shall be applied a uniform coat of hot asphalt, using not less than 30 pounds per 100 square feet.

2. Over this lay two layers of asphalted rag felt weighing not less than 14 pounds per 108 square feet per layer, lapping each sheet of each layer 17 inches over the preceding sheet, end laps not less than 4 inches, and securely fastened. Hot asphalt shall be mopped the full 17 inches on each sheet so that in no place will felt touch felt, using not less than 25 pounds of asphalt per 100 square feet between successive layers. These layers shall be turned up vertical, surfaces not less than 6 inches, and securely fastened.

3. Coat the entire surface uniformly with hot asphalt, using not less than 25 pounds per 100 square feet.

4. Over the entire surface lay three layers of asphalted rag felt, lapping each sheet of each layer 22 inches over the preceding sheet, end laps not less than 6 inches. Hot asphalt shall be mopped the full 22 inches on each sheet so that in no place will felt touch felt, using not less than 25 pounds of asphalt per 100 square fcet between successive layers.

If roof decks are of gypsum, in addition to the above, the upper edge of each course of the third layer of felt shall be nailed to the roof deck with galvanized nails driven through flat tin or zine caps. These layers shall be turned up vertical, surfaces not less than 6 inches, and securely fastened.

5. Coat the entire surface uniformly with hot asphalt poured from a dipper, using not less than 75 pounds per 100 square feet, into which while hot embed not less than 400 pounds of gravel (or 300 pounds of slag).

\section{TYPE C-1, CLASSES A AND B}

Asphalt Impregnated Asbestos Felt and Asphalt Cement Over Wood

Roofing composed of one layer approximately 60 pounds, two layers for class $\mathrm{A}$, one layer for class $\mathrm{B}$, approximately 14 pounds, asphalt impregnated asbestos felt (the latter lapped 17 inches for class $A$ and 3 inches for class B), and a minimum of 85 pounds for class A and 55 pounds for class B, asphalt roofing cement, per 100 square feet.

1. Lay sheets of asphalt impregnated asbestos felt weighing approximately 60 pounds per 108 square foot dircctly over the sheathing. Lap the joints 3 inches and seal them with asphalt cement. Nail these sheets along the lap with barbed nails driven through flat tin caps, spaced 6 inches apart, and in parallel lines approximately 10 inches apart and approximately 10 inches from the laps, these nails to be spaced 18 inches apart and staggered. This 60-pound felt shall be laid parallel to pitch of roof and turned up 5 inches above level of roof on all vertical surfaces but not cemented to vertical surfaces.

Over the 60-pound felt edging strips composed of the fiashing material shall be applied at eaves and gable overhang, extending 4 inches on roof, cemented and nailed, and turned down over and secured to facia and projected one-half inch beyond to form a drip edge.

2. Mop the entire surface of the 60-pound felt with the asphalt cement, heated to flow freely, and while the cement is hot embed in it sheets of asphalt impregnated asbestos felt weighing approximately 14 pounds per 108 square feet in 2-ply construction for class $\mathrm{A}$ and in single-ply construction for class B roofing, this 14-pound felt to be run at right angles to the 60-pound felt, lapped 17 inches for class $A$ and 3 inches for class B, and turned up 5 inches for both classes, above level of roof on all vertical surfaces.
Start at low point of roof with a one-half width sheet of the 14-pound felt, then a full width sheet of the same felt laid flush with and entirely covering the one-half width sheet. For class A then lay full width sheets, setting the first so as to overlap the one-half width, starting sheet 2 inches, and then exposing 15 inches of each succeeding shcet to the weather. For class B lay full-width sheets, overlapping the preceding ones 3 inches, with balance exposed to the weather.

Mop the entire surface between plies with the asphalt cement, heated to flow freely, and roll the felts closely behind the mop so that no missing of asphalt can possibly take place. Approximately 30-pounds of asphalt shall be used per square for each mopping.

Nail the back edge of each sheet with the barbed nails, driven through flat tin caps, spaced 9 inches apart, the center line of nail heads to be approximately three-fourths inch below back edge of sheet.

3 . Walls and all other elevations above roof surface shall be carried vertically at least 12 inches to provide for proper flashing. Roofing material shall be carried up 5 inches above level of roof on all vertical surfaces.

All flashings, except those around ventilators, standpipes, exhausts, etc., shall be composed of base flashing of special asbestos flashing material approximately $10 \frac{1}{2}$ inches wide, cemented and nailed to vertical surface. Such flashings shall be counterflashed with approved fibrous mineral and bituminous cement, and applied with a reinforcement of impregnated asbestos felt.

4. When the roofing is otherwise complete cover the entire surface with a mopping of the asphalt cement, to be applied hot, using approximately 25 pounds to the square.

\section{TYPE C-2, CLASSES $\triangle$ AND B}

Asphalt Impregnated Asbestos Felt and Asphalt Cement Orer Dry Concrete or Gypsum

Roofing composed of 1 coat asphalt primer, 1 coat asphalt cement over primer, 1 layer, approximately 60 pounds, and 2 layers for class A, 1 layer for class $\mathrm{B}$, approximately 14 pounds, asphalt impregnated asbestos felt (the latter lapped 17 inches for class A and 3 inches for class B), and a minimum of 85 pounds for class A, 55 pounds for class $B$, asphalt roofing cement per 100 square feet, not including primer or first coat over primer.

1 . Coat the concrete or gypsum with cold asphalt primer, using approximately 1 gallon over concrete and 1 to 2 gallons over gypsum per 100 square feet of roof surface to provide a proper bond between roof deck and asphalt. Allow the primer to dry.

2. Mop the entire surface thus primed with asphalt cement, heated to flow freely, and while the cement is hot embed into it sheets of asplaalt impregnated asbestos felt, weighing approximately 60 pounds per 108 square feet. Lap the joints 3 inches and seal them with the hot asphalt cement. The 60-pound felt shall be laid parallel to pitch of roof and turned up 5 inches above level of roof on all vertical surfaces (for class B, not cemented to vertical surfaces).

Over the 60-pound felt, edging strips composed of flashing materials shall be applied at eaves and gable overhang, extending 4 inches on roof, cemented, and turned down over and secured to facia and projected one-half inch beyond to form a drip edge.

3 . Mop the entire surface of the 60-pound felt with the hot asphalt cement, and while the cement is hot embed into it sheets of asphalt impregnated asbestos felt, weighing approximately 14 pounds per 108 square feet in 2-ply construction for class A, single-ply construction for class B; this 14-pound felt to be run at right angles to the 60-pound felt, lapped 17 inches and turned up 5 inches for class A, 
and lapped 3 inches and turned up 5 inches for class $B$, above level of roof on all vertical surfaces.

Start at low point of roof with a $1 / 2$-width sheet of the 14-pound felt, then with a full width shect of the same felt laid flush with and entirely covering the $1 / 2$-width sheet. For class A then lay full-width sheets, setting the first so as to overlap the one-half width, starting sheet 2 inches and then exposing 15 inches of each succeeding sheet to the weather. For class B lay full-width sheets, overlapping the preceding ones 3 inches, with balance exposed to the weather.

Mop the entire surface between plies with asphalt cement, heated to flow freely, and roll the felts closely behind the mop so that no missing of asphalt can possibly take place. Approximately 30 pounds of asphalt shall be used per square for each mopping.

4. On gypsum and other types of roof decks that permit of nailing, the back edges of all felts shall be securely nailed to roof slab with cut nails driven through flat tin caps in addition to mopping.

5. Walls and all other elevations above roof surface shall be carried vertically at least 12 inches to provide for proper flashing.

Roofing material shall be carried up 5 inches above level of roof on vertical surfaces. All flashings except those around ventilators, stand-pipes, exhausts, etc., shall be composed of base flashing of special asbestos flashing material approximately $10 \frac{1}{2}$ inches wide, cemented, and nailed to vertical surface. Such flashings shall be counterflashed with approved fibrous mineral and bituminous cement, and applied with a reinforcement of impregnated asbestos felt.

When the roofing is otherwise complete, cover the entire surface with a mopping of the asphalt cement, to be applicd hot, using approximately 25 pounds to the square.

Note.-On concrete surfaces maximum pitch upon which this roofing may heapplied without nailing is 3 inches to the foot, except on short runs, as in saw-tooth construction. If wood nailing strips are inserted in concrete, the pitch may he increased.

Provision for expansion should he made in covering over expansion joints in concrete slah.

\section{GUARANTY}

The roofing contractor shall furnish an unqualified guaranty, with good and sufficient bond, covering the maintenance of the roof in a water-tight condition for a period of years from date of completion.

\section{GENERAL CONDITIONS}

All materials entering into the work and all methods used by the contractor shall be subject to the approval of the engineer, and no part of the work will be considered as finally accepted until all of the work is completed and accepted.

The general conditions as given in 518.50 , page 416 , shall be considered to apply to the above specifications.
American Railway Engineering Association, specifications for buildings for railway purposes (clay tile roofing, 1926).

See 534.23, page 619 .

American Railway Engineering Association, specifications for buildings for railway purposes, section $10-\mathrm{C}$, slate roofing, 1927.

See 511.52, page 197.

Asphalt Shingle and Roofing Association (now Institute), standards for the laying of roofing.

This association has adopted detailed methods for the laying of roofing, as follows:

1. Directions for laying roll roofings, adopted December 8, 1920 .

2. Directions for laying shingles, adopted March $9,1921$.

Asphalt Shingle and Roofing Association (now Institute), tests for powdered surfacing materials (to be used in connection with roofings), 1923.

See 510, page 186.

Asphalt Shingle and Roofing Association (now Institute), mill analysis of bituminous roofings (recommended), 1922.

See 505.0, page 139 .

Contracting Plasterers' International Association, standard specifications for lath and plaster work, 1923.

See 514.63 , page 280 .

Federal Specifications Board, specification No. 380, United States Government master specification for asphalt plastic cement. For use in roofing.)

See 505.13, page 148 .

National Slate Association, slate roofs, January, 1926.

(a) The following specifications for "standard," "textural," and "graduated" slate roofs give in detail the procedure to be followed in the laying of each type of roof on any type of structure from the smallest bungalow to the largest mansion.

(b) In each specification it is assumed that sheathing boards, crickets, cant strips, or other underroof surface will be specified elsewhere under the proper trade.

(c) The notes, under the various items in the specifications, are informative and indicate optional changes which may be made in the specifications.

(d) The full text of the specifications as published by the National Slate Association includes for each specification a prefatoly "short form" to be written into the architect's specification.

(e) The association has a separate specification for sheet-metal work.

$(f)$ The association has specificational data relating to flat slate roofs, but has no specification for this type.

\section{NOTES FOR "STANDARD," "TEXTURAL" AND} "GRADUATED" ROOF SPECIFICATIONS

1. The specifications used as here given will provide for a complete slate roof as follows:

\begin{tabular}{|c|c|c|c|}
\hline & Standard & Textural & Graduated \\
\hline Felt, saturated asphalt_....- & 14 pounds per square... & Commercially known as 30 pounds & 30 pounds per square. \\
\hline Flashings... & 16-ounce copper & $\begin{array}{l}\text { per square. } \\
\text { 16-ounce copper }\end{array}$ & 16-ounce copper. \\
\hline Hips & Saddle.............. & Saddle_............... & Saddle. \\
\hline Valleys.... & Open & Closed & \\
\hline Ridges & Saddle & Saddle & Do. \\
\hline Cement................ & Elastic waterproof & Elastic waterproof & Elastic waterproof. \\
\hline Slate & $\begin{array}{l}\text { Commercial standard (approxi- } \\
\text { mately } 3 / 16 \text { inch). }\end{array}$ & $\begin{array}{l}3 / 16 \text { to } 8 / 8 \text { inch, random width, ap- } \\
\text { proved lengths. }\end{array}$ & $\begin{array}{l}3 / 4 \text { to } 1 / 4 \text { inch thick; } 24 \text { to } 12 \text { inches } \\
\text { long. }\end{array}$ \\
\hline Nails. & Copper & Copper & Copper. \\
\hline
\end{tabular}


STANDARD SPECIFICATION FOR A STANDARD SLATE ROOF

(Standard specification "S," covering in detail all materials and labor in connection with a standard slate roof.)

\section{GENERAL AND GUARANTY}

(a) The general conditions of the contract are hereby made a part of the contract, and this contractor shall examine these general conditions and thoroughly acquaint himself with all the requirements therein contained.

(b) This contractor shall furnish all materials and labor in accordance with the drawings and these specifications.

(c) This contractor shall inspect all surfaces prepared for slating by other trades, point out to the proper authority all defects, and shall not proceed with the laying of felt, flashings, or slate until the necessary corrections have been made.

(d) Roofing shall be applied by workmen experienced in the applying of slate.

(e) The roofing contractor shall furnish his own scaffold or rigging, or arrange with the general contractor for the use of scaffolds furnished by others.

(f) A written guaranty shall be furnished that the materials used are in strict accordance with these specifications, and that any and all repairs required on the roof due to defective materials or workmanship furnished under this contract shall be made without cost to the owner for a period of one year.

\section{ROOFING FELT}

(a) On all boarding to be covered with slate, furnish and lay asphalt-saturated rag felt, not less in weight than that commercially known as "14pound" felt. In this weight, per 100 square feet, a tolerance of 1 pound plus or minus will be allowed.

(b) Felt shall be laid in horizontal layers with joints lapped toward eaves and at ends at least 2 inches and well secured along laps and at ends as necessary to properly hold the felt in place and protect the structure until covered by the slate. All felt shall be preserved unbroken, tight, and whole.

(c) The felt shall lap over all hips and ridges.

(d) Felt shall be lapped 2 inches over the metal of any valleys or built-in gutters.

\section{SLATE}

(a) Slate shall be of commercial standard quality and thickness.

Note.-Commercial standard slate is approximately $3 / 16$-inch in thickness. If any other thickness is desired, so state and omit "commercial."

(b) Size of slate shall be widths of ..... length(s).

Note.-For table of standard sizes, see Department of Commerce Simplified Practice Recommendation No. 14. (See 511.52, p. 203.) A definite size must be stated to place all estimates on an equal basis.

To obtain a roof at a minimum cost, confer with local roofers as to the size or sizes economically available. Standard roofs are usually laid with slate of one size on any one arca. If random widths are desired, so state.

(c) Color of slate shall be

Note.-For list of slate colors see Simplified Practice Recommendation No. 14, 511.52, p. 203.

If weathering slate are desired, so state, otherwise unfading colors will he furnished.

If a particular quarry product is desired, so state.

(d) All slate shall be hard, dense, sound rock, machine punched for two nails each. All exposed corners shall be practically full. No broken corners on covered ends which sacrifice nailing strength or the laying of a water-tight roof will be allowed. No broken or cracked slates shall be used.
NotE.-." Machine punched" does not exclude hand punching by means of the punch and mawl or hand punching with the hammer, es the slate is laid, to properly locate holes for fitting hips, etc or obtaining suitable nailing to the roof boards.

\section{HIPS}

(a) All hips shall be laid to form "saddle" hips without metal underneath.

Note.-If any other typc of hip is desired, so state.

\section{RIDGES}

(a) All ridges shall be laid to form "saddle" ridges. The nails of the combing slate shall pass through the joints of the slates below.

Note.-If combed ridges, ridge rolls, or cresting are desired, so state.

\section{VALLEYS}

(a) All valleys shall be laid to form "open" valleys Note.-If "closed" valleys are desired, so state.

\section{ELASTIC CEMENT}

(a) Cement shall be an approved brand of waterproof elastic slaters' cement colored to match as nearly as possible the general color of the slate.

\section{NAIIS}

(a) All slate shall be fastened with two large-head slaters' solid copper nails. Use threepenny (1/4-inch) nails for slates 18 inches or less in length, fourpenny $(11 / 2-i n c h)$ for 20 inches or longer, and sixpenny (2-inch) for slates on hips and ridges.

\section{SLATING}

(a) The entire surface of all main and porch roofs, the roofs and sides of any dormer windows, if shown, and all other surfaces so indicated on the drawings, shall be covered with slate in a proper and water-tight manner.

(b) The slate shall project 2 inches at the eaves and 1 inch at all gable ends, and shall be laid in horizontal courses with the standard 3-inch head lap, and each course shall break joints with the preceding one. Slates at the eaves or cornice line shall be doubled and canted one-fourth inch by a wooden cant strip.

(c) Slates overlapping sheet-metal work shall have the nails so placed as to avoid puncturing the sheet metal. Exposed nails shall be permissible only in top courses where unavoidable.

(d) Neatly fit slate around any pipes, ventilators, etc.

(e) Nails shall not be driven in so far as to produce a strain on the slate.

(f) Cover all exposed nailheads with elastic cement. Hip slates and ridge slates shall be laid in elastic cement spread thickly over unexposed surface of under courses of slate, nailed securely in place, and pointed with elastic cement.

(g) Build in and place all flashing pieces furnished by the sheet-metal contractor and cooperate with him in doing the work of flashing.

(h) On completion, all slate must be sound, whole, and clean, and the roof shall be left in every respect tight and a neat example of workmanship.

Nore.--Vertical surfaces may be laid with 1-inch head lap.

Specify cant strips of proper thickness under carpentry.

Where cant strips occur above gutters, they should be placed before the metal.

\section{STANDARD SPECIFICATIONS FOR A TEXTURAL SLATE ROOF}

(Standard specification "T," covering in detail all materials and labor in connection with a textural slate roof.) 


\section{GENERAL AND GUARANTY}

(Same as for standard slate roof (above) with following additional:)

(c) The roofing contractor shall furnish samples of the slate he proposes to use. If required, a layout shall be prepared by the producer and submitted to the architect for approval.

\section{ROOFING FELT}

(a) On all boarding to be covered with slate, furnish and lay asphalt-saturated rag felt, not less in weight than that commercially known as 30pound felt.

(Items $(b),(c)$, and $(d)$, section 2 of specification for a standard slate roof, are part of this specification.)

\section{SLATE}

(a) Slate shall be in combinations of thicknesses from $3 / 8$ to $3 / 16$ inch thick.

- Note.-If a percentage of thicker slates is desired, state the thickness and percentage of each.

(b) Slates shall be of random widthe and of such lengths as approved by the architect.

Note.-If a layout is required, state "in accordance with approved layout."

If definite sizes must be furnished, so state. To obtain a roof at minimum cost, confer with local roofers as to sizes economically available.

(c) The predominating color of the slates shall be

Note.-For list of slate colors, see Simplified Practice Recommendation No. 14, 511.52, page 203.

Colors will be of "weathering" class unless otherwise noted.

(d) Same as section 3 (d) of specification for a standard slate roof (above).

Sections 4 (hips), 5 (ridges), 7 (elastic cement), and 9 (slating) of specification for a standard slate roof are part of this specification.

\section{VALIEYS}

(a) All valleys shall be laid to form "closed" valleys.

NotE.-If open valleys are desired, so state. "Round" or "cance" valleys may be used if desired, but will add to the cost of the roof and are more frequently used with graduated roofs.

\section{NAIIS}

(a) All slate shall be fastened with large-head slaters' copper nails of sufficient lengths to adequately penetrate roof boarding. Care shall be taken to avoid exposing the nails on cornice, soffits, overhanging eaves, etc.

\section{STANDARD SPECIFICATION FOR A GRADUATED SLATE ROOF}

(Standard specification " $\mathrm{G}$," covering in detail all materials and labor in connection with a graduated slate roof.)

\section{GENRRAL AND GUARANTY}

Same as for standard slate roof (above) with following additional:

(b) The roofing contractor shall furnish samples of the slate he proposes to use. A layout showing graduations, courses, and color distribution shall be prepared by the producer and submitted to the architect for approval.

\section{ROOFING FELT}

Same as for textural slate roof (above).

\section{SLATE}

(a) Slate shall be in combination of thicknesses from $3 / 4$ to $1 / 4$ inch.

Note.-Any desired range may be used, inserting the desired thickness and lengths.

(b) Slates shall be random widths from 24 to 12 inches in length.

(c) Color of slate shall be -.......

NoTE.-State color or color mixture, permanent or weathering, and percentages of each desired.

(d) All slate shall be hard, dense, sound rock, machine punched for two holes. Slates $3 / 4$ inch and thicker, when 20 inches or more in length, shall have four holes. All exposed corners shall be practically full and no broken corners or covered ends which sacrifice nailing strength or the laying of a watertight roof will be allowed. No broken or cracked slates shall be used.

NoтE.- "Machine punched" does not exclude hand punching by means of the punch and mawl or hand punching with the hammer, as slate is laid, to properly locate holes for fitting hips, etc., or obtaining suitable nailing to the roof boards.

Sections 4 (hips), 5 (ridges), and 7 (elastic cement) of specification for a standard slate roof are part of this specification.

\section{VALIEYS}

(a) All valleys shall be

Note.-Insert necessary following paragraphs:

1. Laid to form "open" valleys.

1. Laid to torm "open "valleys.

2. Laid to form "closed" valeys. securely nailed, and shall be 4 inches longer than the slates used in the corresponding courses on the roof and laid to allow the tail coursings, to line up. Proper copper flashing under each course.

\section{NAIIS}

(a) All slate shall be fastened with large flat-head slaters' copper nails of sufficient length to adequately penetrate nailing surface. Use four nails for slates $3 / 4$ inch and thicker when 20 inches or more in length. Care should be taken to avoid exposing the nails on cornice, soffits, overhanging eaves, etc.

(b) Nails securing slate must in no instance be driven through flashing.

\section{SLATING}

(a) The entire surface of all main and porch roofs, the roofs and sides of any dormer windows, if shown, and all other surfaces so indicated on the drawings, shall be covered with slate in combinations of colors, sizes, and thicknesses shown on approved layout drawing furnished by slate producer.

(b) The slate shall project at the eaves and at all gable ends as directed, and shall be laid in horizontal courses with the standard 3-inch head lap, and each course shall break joints with the preceding one. Slates at the eaves or cornice line shall be doubled and canted so that the succeeding course will have flat contact.

Note.-If other construction at eaves and gables is desired, this should be noted here.

Items in section 9 (slating), subsections $(c),(d)$, $(e),(f),(g),(h)$, and note following, of specification for a standard roof, are part of this specification.

Underwriters Laboratories, specifications for Class $\mathrm{C}$ asphalt rag-felt sheet roofing and shingles, March, 1929.

See 505.16, page 169 .

United States Department of Commerce, Bureau of Standards, Simplified Practice Recommendation No. 14, for roofing slate, July, 1928.

See 511.52, page 203. 


\subsection{Miscellaneous Specifications for Building Construction and Mate- rials.}

American Concrete Institute, standard recommended practice for Portland cement stucco, serial designation C-3A-23, 1923.

See 516.2, page 297 .

American Concrete Institute, tentative specifications for concrete building block and concrete building tile, serial designation $\mathrm{P}-1 \mathrm{~A}-28 \mathrm{~T}, 1928$.

See 516.4 , page 317 .

American Concrete Institute, standard specifications and building regulations for concrete staves, serial designation $\mathrm{P}-4 \mathrm{~A}-26,1926$.

\section{GENERAL}

1. Concrete staves meeting the requirements of the following specifications may be used in the construction of silos, coal pockets, corncribs, grain bins, and other structures for which these units are suitable.

\section{TESTS}

2. Concrete staves must be subjected to transverse and absorption tests. All official tests must be made in a testing laboratory of recognized standing. Six samples representing the ordinary commercial product selected at random from stock must be provided for the purpose of testing.

\section{TRANSVERSE STRENGTH}

3. The ultimate transverse strength of the test staves at 28 days after being manufactured or when shipped must average not less than 90 pounds with no test falling below 75 pounds for each inch of width of the stave.

4. The absorption at 28 days after being manufactured or when shipped must not in any case exceed 6 per cent.

\section{TRANSVERSE TEST}

5. The transverse test shall be made as follows: The sample to be tested shall be placed flatwise in the testing machine and supported at one end on a ${ }_{3}^{3} /$-inch round rod and at the other end by a spherical bearing block using a steel plate 2 inches wide and of sufficient stiffness to properly distribute the load between concrete and load point. The distance between points of support shall be exactly 24 inches. The load shall be applied at midspan through a spherical bearing block.

\section{ABSORPTION}

6. In the absorption tests the samples shall be first thoroughly dried to a constant weight at a temperature not to exceed $230^{\circ} \mathrm{F}$. After drying, the sample shall be completely submerged in clean water at a temperature of between $60^{\circ}$ and $80^{\circ} \mathrm{F}$. for a period of 48 hours. The specimen shall then be removed, the surface water wiped off, and the sample reweighed. The percentage absorption is the weight of the water absorbed divided by the weight of the dry specimen and the quotient multiplied by 100 .

\section{VERTICAL LOADING}

9. The load on any concrete stave wall, including the superimposed weight of the wall, shall not exceed 200 lbs./in. ${ }^{2}$.

\section{LATERAL IOADING}

10. Silos, grain tanks, coal pockets, corn cribs, etc., constructed of concrete staves shall be hooped with steel rods or bands of such cross-sectional area that the steel will not be stressed to exceed 16,000 lbs./in., ${ }^{2}$ and the hoops shall be placed at such intervals that the staves will not be loaded to exceed 25 per cent of their average transverse strength.

American Concrete Institute, tentative specifications for concrete brick, serial designation $\mathrm{P}-1 \mathrm{~B}-$ $26 \mathrm{~T}, 1926$.

See 516.4 , page 318 .

American Railway Engineering Association, specifications for buildings for railway purposes.

\section{EXCAVATION, FILLING, AND BACKFILLING (1926)}

\section{GENERAL}

The contractor shall furnish all labor, material, tools, and equipment cxcept as otherwise noted, necessary to entirely complete all excavations for foundation walls, piers, footings, pits, ducts, tunnels, basements, and any other excavation which may be implied or shown on the drawings to receive the subsequent work.

Any excavation paid for or deducted on a unitprice basis shall be for the actual measured yardage.

No allowance shall be made on account of slope to the sides of excavation, but measurements for quantities of excavation shall be taken to outside of shceting.

The unit price paid or deducted shall include the whole value of the sheeting, bracing, or any other material actually used in connection with the work, either as a form for concrete foundations, as a protection against eaving during the process of excavating, or as a cofferdam, and shall also include any pumping or bailing which may be necessary.

\section{CLASSIFICATION}

All material excavated shall be classified as rock excavation, wet excavation, and common excavation.

\section{ROCK EXCAVATION}

Rock excavation shall comprise rock in solid beds or masses in its original position which, in the judgment of the engineer, may best be removed by blasting and detached rock or bowlders, measuring 1 cubic yard or more.

\section{WET EXCAVATION}

Wet excavation shall comprise that material, not included under rock excavation, which requires pumping or sheet piling to overcome seepage and overflow.

\section{COMMON EXCAVATION}

Common excavation shall include all materials that do not come under the classifications of rock or wet excavation.

\section{SOIL TEST}

Before any foundation work is placed the contractor shall satisfy himself that the soil encountered is of such a nature that it will support the structure as designed; in case of doubt he must notify the engineer, and no foundations shall be laid until a proper investigation is made.

\section{BEDS FOP FOOTTINGS}

The beds for footings shall be leveled and free of all loose material before any foundations are put in place. No footings shall rest on filled ground except where absolutely necessary, and all filling under such footings shall be sand or other approved filling, puddled and tamped in place. No such footings shall be put in place by the contractor without first obtaining permission from the engineer, 


\section{QUICKSAND POCKETS}

If any quicksand pockets or other soft spots are encountered beneath foundation walls, piers, or footings, the same shall be excavated and filled with concrete, the extra work being paid for on the basis of unit prices provided in contract.

\section{PUMPING AND BAILING}

The contractor shall perform all pumping and bailing necessary to keep all excavation entirely free from water during the progress of the work under all circumstances and contingencies which may arise, using such means as may be best adapted to conditions. The cost of pumping and bailing shall be included in the contractor's bid for excavation.

\section{BLASTING}

The contractor shall do all blasting necessary in connection with the excavation as shown on the drawings. All drilling, placing of charges, and shooting, together with the covering of blasts, shall be done in an approved manner. All work in connection with blasting shall be done in strict accordance with any laws or ordinance in effect where the work is located.

\section{DISPOSAL OF EXCAVATED MATERIAL}

Excavated material shall be used for back filling around all underground work. After forms of such work have been removed and the work has been inspected by the engineer, the contractor shall fill up to the finished grade as shown on the drawings.

Only material suitable for back filling shall be so used. Large frozen lumps, bowlders, etc., shall not be used. Back filling must be placed in layers not to exceed 6 inches, each layer being thoroughly tamped and puddled.

The contractor, when so required, shall haul and place surplus excavated material within a distance not to exceed 300 feet from the building as directed by the engineer.

Any surplus excavated material which can not be disposed of within 300 feet of the building shall be disposed of by the contractor, unless otherwise released by the company.

\section{FILIING}

Sand or cinder filling where called for on the drawings, shall be thoroughly tamped, rolled, and compacted in place by the contractor. Where floors are on fill, the fill shall be placed in layers and thoroughly puddled, tamped, and rolled or flooded. Wherever such fill occurs it shall be included in the lump-sum price for the structure in which it occurs. Sand fill shall be clean sand, free from sticks or other foreign matter. When cinder fill is used, cinders will be furnished by the company, in cars as near as practicable to the site of the work, but must be unloaded and placed by the contractor.

No filling or back filling shall be done at a time when there is danger of frost entering the material, except at the discretion of the engineer.

\section{GRADING AND FINAL CLEANING}

All grading that may be necessary around the buildings, as shown by the drawings, shall be done by the contractor. Cinders, sand, or clean dirt shall be used for the work as called for by the drawings.

At the completion of the work the contractor shall thoroughly clean up and remove any rubbish, dirt, or excavated material from site as called for under disposal of excavated material, and leave the site clean and graded to finish grades as shown by the drawings.

\section{PIEE FOUNDATIONS}

Where timber foundation piles are required or shown on the drawings these shall be furnished and driven by the contractor, unless otherwise provided for in the contract. Contractors shall quote in their bids a unit price per linear foot penetration below cut-off, for furnishing, driving, and cutting of piles.

Timber piles shall be furnished in accordance with the American Railway Engineering Association specifications covering piles for trestles, except that where piles are to be untreated they shall not be peeled. Piles that will be cut off below permanent moisture will not be treated. Piles which will extend above the line of permanent moisture shall be treated with creosote oil, full-cell process, in accordance with the A. R. E. A. specification for treated timber piles. Pile shall be driven by a steam or drop hammer to refusal, or until the penetration per blow under the last blows of a 2,000-pound hammer, falling 20 feet, does not exceed 1 inch. They shall be driven in location shown on the drawings without variation of more than 1 foot in any direction. The contractor shall excavate around and cut off the piles at the elevations shown and properly prepare the piles to receive the masonry or other parts of the structure. Piles shall be cut off in a horizontal plane unless otherwise shown on the drawings. Where the contract provides that the piles shall be furnished and driven by the railway company, the contractor shall provide in his bid for excavating around and cutting off the piles as described above.

The lengths of piles necessary shall be fixed by the engineer after test piles have been driven. The cost of driving test piles will be paid for by the railway company.

\section{UNDERGROUND AND OVERHEAD STRUCTURES}

All gas, water, and drainage pipes, sewers, or conduits, shall be supported in place by the contractor, and all expense attending their renewal shall be borne by him. All telegraph, electric light, or telephone wires, signals, etc., which, in the judgment of the engineer, interfere with the progress of the work shall be removed without expense to the contractor. During construction the contractor shall maintain in safety, permanent poles, wires, sewers, pipes, or conduits affecting his work or with which it may interfere. If damaged through his negligence, all expenses attending repairs thereto shall be borne by him.

\section{GENERAL CONDITIONS}

All materials entering into the work and all methods used by the contractor shall be subject to the approval of the engineer, and no part of the work shall be considered as finally accepted until all the work is completed and accepted.

The general conditions as given in 518.50 shall be considered to apply to these specifications.

American Society for Testing Materials, standard specifications for quicklime for structural purposes, serial designation C5-26, 1926.

See 517.2, page 352 .

American Society for Testing Materials, standard specifications for hydrated lime for structural purposes, serial designation C $6-24,1924$.

See 517.2 , page 353 .

American Society for Testing Materials, tentative specifications for concrete building brick, serial designation C55-28T, 1928.

See 516.4 , page 321 . 
Common Brick Manufacturers' Association of America, specifications and general schedule of a brick house.

\section{MORTAR}

See 516.3 , page 310 .

Concrete Reinforcing Steel Institute, specifications for reinforced concrete in structures, 1928 .

See 516.3 , page 310 .

Federal Specifications Board, specification No. 249, United States Government master specification for hydrated lime for structural purposes, November 5, 1924.

See 517.2 , page 363 .

Federal Specifications Board, specification No. 230, United States Government master specification for quicklime for structural purposes,

November 5, 1924.

See 517.2 , page 364 .

Federal Specifications Board, specification No. 505 , United States Government master specification for brick, sand-lime, common, July 11, 1927.

See 513, page . 257

National Fire Protection Association, vaults.

Specifications for ground supported vaults, adopted 1926 .

\section{FIRE RESISTANCE CLASSIFICATIONS}

Ground supported vaults are those supported directly on the ground and independent of the building in which they are located. They are intended to afford full protection to their contents even in the event of complete destruction of the building.

\section{SIX-HOJR VAULTS}

This classification is recommended where the construction and occupancy are such that a degree of fire resistance equivalent to six hours of the standard test is deemed necessary.

\section{FOOR-HOOR VAOLTS}

This classification is recommended where the construction and occupancy are such that a degree of fire resistance equivalent to four hours of the standard test is deemed necessary.

\section{TWO-HOJR VAULTS}

This classification is recommended where the construction and occupancy are such that a degree of fire resistance equivalent to two hours of the standard test is deemed necessary.

\section{SIX-HOUR VAULTS}

\section{FOUNDATIONS}

Foundations shall be of concrete, stone, or brick masonry of ample size to take the entire load of the vault structure and its contents.

\section{WALIS}

(a) Materials.-Walls shall be built solid of reinforced concrete, or brick well bedded in mortar, or of protected steel or reinforced concrete framework with panels of reinforced concrete, plain concrete, or brick. Stone and gravel aggregates for concrete shall be selected with regard to their fire-resistive properties. Siliceous gravel shall not be used for the coarse aggregate, unless adequately reinforced against spalling as by wire mesh near the surface, as this material is particularly subject to disintegration under heat. There shall be a covering of at least 2 inches of concrete over all reinforcement.

Nore.-The concrete and reinforcement shall comply with the recommendations of the current report of the Joint Committee on Concrete and Reinforced Concrete. (See 516.3, p. 317.) Brick shall con to the Society for Testing Materials. (See A. S. T. M. C62-29, 534.11, p. 583. .)

Where a structural steel framework is used, the steel shall be protected with at least 4 inches of concrete, brick, or its equivalent tied with steel ties or wire mesh equivalent to No. 7 (A. S. W. gage, 0.177 inch diameter) wire on 8-inch pitch. Brick protection if used, shall be filled solidly to the steel with concrete. At the joints between columns, beams, and panels, the panels shall be well bonded to the columns by notching or rabbeting into the concrete to a depth and width of at least 1 inch. Temperature reinforcement for concrete shall consist of steel rods at least one-half inch in diameter spaced 4 inches on centers and running at right angles in both directions. Rods shall be securely wired at intersections not over 12 inches apart in both directions and be installed centrally in each panel wall. Any equivalent form of temperature reinforcement may be used.

(b) Thickness.-Walls shall be at least of the thickness specified in Table 1 , the variations depending on the materials used, the type of building construction, and the number of stories. However, in the lower story of buildings over two stories in height, the minimum thickness shall in no case be less than 12 inches for reinforced concrete nor 16 inches for brick. These minimum thicknesses apply to the vault construction only.

TABLE 1.-Minimum wall thicknesses, ground supported vaults

A. SIX-HOUR VAULTS

\begin{tabular}{|c|c|c|c|c|}
\hline \multirow{3}{*}{$\begin{array}{l}\text { Number of stories } \\
\text { counting from } \\
\text { top down }\end{array}$} & \multicolumn{4}{|c|}{ Kind of wall construction } \\
\hline & \multirow{2}{*}{$\begin{array}{l}\text { Reinforced } \\
\text { concrete }\end{array}$} & \multirow{2}{*}{$\begin{array}{l}\text { Brick or } \\
\text { plain } \\
\text { concrete }\end{array}$} & \multicolumn{2}{|c|}{$\begin{array}{l}\text { Protected steel or rein. } \\
\text { forced concrete frames }\end{array}$} \\
\hline & & & $\begin{array}{l}\text { Reinforced } \\
\text { concrete } \\
\text { panels }\end{array}$ & $\begin{array}{l}\text { Brick or } \\
\text { plain con- } \\
\text { crcte panels }\end{array}$ \\
\hline 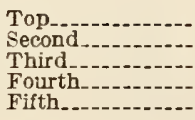 & $\begin{array}{l}10 \\
10 \\
10 \\
12 \\
12\end{array}$ & $\begin{array}{l}12 \\
12 \\
12 \\
16 \\
16\end{array}$ & $\begin{array}{l}10 \\
10 \\
10 \\
10 \\
12\end{array}$ & $\begin{array}{l}12 \\
12 \\
12 \\
12 \\
16\end{array}$ \\
\hline $\begin{array}{l}\text { Sixth } \\
\text { Seventh } \\
\text { Eighth } \\
\text { Ninth } \\
\text { Tenth }\end{array}$ & $\begin{array}{r}12 \\
\\
\end{array}$ & $\begin{array}{r}20 \\
0 \\
0\end{array}$ & $\begin{array}{l}12 \\
12 \\
12 \\
12 \\
14\end{array}$ & $\begin{array}{l}16 \\
16 \\
16 \\
16 \\
16\end{array}$ \\
\hline
\end{tabular}

B. FOUR-HOUR VAULTS

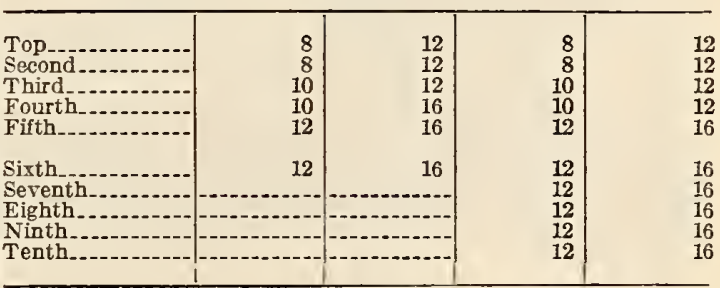


TABLE 1.-Minimum wall thicknesses, ground supported vaults-Continued

\section{TWO-HOUR VAULTS}

\begin{tabular}{|c|c|c|c|c|c|c|}
\hline \multirow{3}{*}{$\begin{array}{l}\text { Number of } \\
\text { stories counting } \\
\text { from top down }\end{array}$} & \multicolumn{6}{|c|}{ Kind of wall construction } \\
\hline & \multirow[b]{2}{*}{$\begin{array}{l}\text { Rein- } \\
\text { forced } \\
\text { con- } \\
\text { crete }\end{array}$} & \multirow[b]{2}{*}{ Brick } & \multirow{2}{*}{$\begin{array}{c}\text { Hollow } \\
\text { clay tile } \\
\text { or con- } \\
\text { crete } \\
\text { block }\end{array}$} & \multicolumn{3}{|c|}{$\begin{array}{l}\text { Protected steel or rein- } \\
\text { forced concrete frames }\end{array}$} \\
\hline & & & & $\begin{array}{l}\text { Rein- } \\
\text { forced } \\
\text { con- } \\
\text { crete } \\
\text { panels }\end{array}$ & $\begin{array}{c}\text { Brick } \\
\text { or plain } \\
\text { con- } \\
\text { crete } \\
\text { panels }\end{array}$ & $\begin{array}{l}\text { Hollow } \\
\text { clay tile } \\
\text { or con- } \\
\text { crete } \\
\text { block } \\
\text { panels }\end{array}$ \\
\hline Top. & 6 & 8 & 12 & 6 & 8 & 12 \\
\hline Second. & 8 & 12 & 12 & 8 & 12 & 12 \\
\hline Third & 10 & 12 & 16 & 10 & 12 & 16 \\
\hline Fourth & 10 & 16 & 20 & 10 & 12 & 16 \\
\hline Fifth & 12 & 16 & & 12 & 16 & 20 \\
\hline Sixth.. & 12 & 16 & -1 & 12 & 16 & 20 \\
\hline Seventh & - & 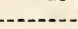 & $1-2-2-1$ & 12 & 16 & 20 \\
\hline Eighth..- & ------- & -2 & $-2-2-2$ & 12 & 16 & 20 \\
\hline Ninth & & & -- & 12 & 16 & 20 \\
\hline Tenth & & & 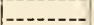 & 12 & 16 & 20 \\
\hline
\end{tabular}

(c) Independence.-Vault walls shall be structurally independent of the building wherever possible. If connceted in any manner, the connection shall be so made that in event of the collapsc of the building the building members may move or fall without affecting the stability or fire-resistive qualities of the vault.

In fire-resistive construction provision shall be made for expansion of the interior building members, as otherwise severe thrusts may be exerted on the vault structure.

Note.-Under moderately severe conditions of heating an expansion in the concrete or steel equivalent to one-sixteenth inch per foot is possible.

Where building members join those of the vault they shall project into the vault not more than 4 inches, and in no case shall the wall thickness be less than 8 inches at these points. All beams or bearing members adjoining the vault shall be designed to release freely in case of failure. Where the outside walls of a building are used to serve as a portion of the vault walls, the latter shall be effectively bonded to the building walls.

NoTE.-Attention is called to the fact that the method of building a vault wall against the outer wall of building, and omitting bonding will not insure the integrity of the vault, and that vault walls erected after the building are likely to settle and break connections with building walls. Also falling building walls may tear away wall used jointly for building and vaults and cause failure of vault.

\section{ROOF}

(a) Materials.-Roof construction shall be of reinforced concrete or protected structural steel with reinforced concrete slabs or fillers of adequatc strength and fire resistance.

(b) Thickness.-Roof shall be unpierced and shall be at least 8 inches in thickness. Greater thickness may be necessary to provide strength for loads and impacts as specified below.

(c) Independence. - Vault roofs shall be entirely independent of floors, roofs, or ceilings of buildings. Vaults should preferably be built to extend to the top floor of the building and the top of the vault located as close to the underside of the building roof as possible.

(d) Bonding.-The roof and walls of the vault shall be thoroughly bonded together. If construction is of reinforced concrete throughout, the rein- forcing steel in the roof shall be carried into the walls and the wall reinforcement into the roof. If there are steel beams in the roof, these sha!l be sccurely fastened to structural members embedded in the walls. If walls are of brick suitable anchors shall be provided.

(e) Provision against impact.- Where the roof is more than 12 inches below the roof of the building the vault walls should be parapeted at least 12 inches above the vault roof and the space thus formed filled with sand, gypsum, or similar material, to act as a cushion against impact from falling materials and also to serve as further insulation against accumulation of burning débris on the vault roof. Adequate drainage shall be provided for this space above the roof.

(f) Design.- Roof shall be designed for a live load of at least $350 \mathrm{lbs} . / \mathrm{ft} .^{2}$ to take care of impact loading. Where local conditions are especially severe, such as near masonry walls or large tanks, loads of from 500 to $1,000 \mathrm{lbs}$./ft. ${ }^{2}$ should be assumed and maximum spans in at least one direction should not exceed 10 feet.

Note. - It is not deemed practicable to design the roof entirely to prevent possibility of damage from a heavy safe or machine falling through a considerable distance. Such equipment should be so located as not to endanger vault structures below.

(g) Interior supports.-Where long spans are needed, the introduction of interior columns, girders, or division walls may be necessary. All interior stcel work and reinforcing shall be protected with a covering having a fire-resistance classification of not less than three hours.

\section{FLOORS}

(a) Materials and thickness.-Floors shall be of noncombustible material of a construction having a fire resistance classification not less than two hours. Floors shall be unpierced, not less than 6 inches thick, and greater if neccssary to support the full load of floor and contents.

(b) Flooring.-No wood or other combustible material shall be used in the floor or surfacing.

(c) Independence.-Floors shall be thoroughly bonded to the vault walls and shall be independent of floors of the building.

\section{DOORS}

Shall conform to specifications as given in the section on vault doors and shall be of the same fireresistance classification as the vault in which instalicd.

\section{WATER-TIGHTNESS}

(a) Walls, roofs, and floors shall be effectively waterproofed, preferably using a mixture of concrete of proper grading, mixture, and placing for the purpose. No combustible membrane or coating shall be employed except on a roof exposed to the weather.

(b) Provision shall preferably be made to prevent entrance of water at door openings. Raised or sloping sills and large drains in building floors outside of vaults are suggested.

\section{VENTILATION}

Ventilation of interior shall be only through door openings. Walls, floors, and roofs shall not be picrced.

\section{INSPECTION}

The construction of the vault shall be under the immediate supervision of a competent engineer or architect to insure that it is built in accordance with the above rccommendations and that careful workmanship is obtained throughout. 


\section{FOUR-HOUR VAULTS}

\section{FOUNDATIONS}

Same as for 6-hour vaults.

\section{WALLS}

(a) Materials.-Same as for 6-hour vaults.

(b) Thickness.--Shall be as specified for 4-hour classification in Table 1 . In the lower story, however, the minimum thickness shall in no case be less than 12 inches for ruinforced concrete nor 16 inches for brick. These minimum thicknesses apply to the vault construction only.

(c) Independence.-Same as for 6-hour vaults.

\section{ROOF}

Same as for 6-hour vaults.

\section{FLOORS}

Same as for 6-hour vaults.

\section{DOORS}

Shall conform to specifications for vault doors as given in the section on vault doors and shall be of the same fire-resistance classification as the vault in which installed.

\section{WATER-TIGHTNESS}

Same as for 6-hour vaults.

\section{VENTILATION}

Same as for 6-hour vaults.

\section{INSPECTION}

Same as for 6-hour vaults.

\section{TWO-HOUR VAULTS}

FOUNDATIONS

Same as for 6-hour vaults.

\section{WALIS}

(a) Materials.-Walls shall be built of reinforced concrete, brick well bedded in mortar, load-bearing hollow-clay tile, hollow concrete blocks, or of protected steel, or reinforced concrete framework with panels of these materials.

The hollow concrete building block shall have cement proportions from $1: 3$ to $1: 7$ and either air or steam cured, and mixed with either dry, damp, or wet consistencies and of fine and coarse aggregates of crushed limestone, of crushed slag, or crushed cinders or of sand and calcareous pebbles when assembled into walls one unit thick.

The hollow-clay tile shall be not less than 2-cell for 8-inch and not less than 3-cell for 12-inch, conforming with the current specifications of the A. S. T. M. for load-bearing wall tile. (See A. S. T. M. C34-27, 534.22, p. 616.)

Hollow walls shall be plastered on both sides with at least five-eighths inch of gypsum or Portland cement plaster. Where a structural steel framework is used the steel framework shall have protection having a fire-resistance classification of not less than two hours. At the joints, between columns, beams, and panels, the latter shall be well bonded to the columns and beams, the panels to be notched or rabbeted into the concrete of the columns for a depth of at least 1 inch, but in no case shall the construction be such that the fire-resistance classification of any portion is less than two hours.

(b) Thickness. - Walls and wall panels shall be not less than the minimum thickness specified in Table 1 for 2-hour vaults. (c) Independence-To conform with requirements for 6-hour vaults, except that when concrete block or hollow clay tile are used for walls without frames, such walls shall serve as bearing members for the vault only.

\section{ROOF}

Same as for 6-hour vaults.

\section{FLOORS}

\section{Same as for 6-hour vaults.}

\section{DOORS}

Shall conform to specifications given in the section on vault doors, and shall be of the same fire-resistance classification as the vault on which installed.

\section{WATER-TIGHTNESS}

Same as for 6-hour vaults.

\section{VENTILATION}

Same as for 6-hour vaults.

\section{INSPECTION}

Same as for 6-hour vaults.

Specifications for structure supported vaults, adopted 1927.

\section{FIRE-RESISTANCE CLASSIFICATIONS}

Structure-supported vaults are thosc supported by the framework of buildings of fire-resistive construction. These vaults may be located individually on any floor of such a building and are designed to afford full protection to their contents, assuming the integrity of the supporting structure.

\section{SIX-HODR VADLTS}

This classification is recommended where the construction and occupancy are such that a degree of fire resistance equivalent to six hours of the standard test is deemed necessary.

\section{FOUR-HOUR VAULTS}

This classification is recommended where the construction and occupancy are such that a degree of fire resistance equivalent to four hours of the standard test is deemed necessary.

\section{TWO-HOUR VAULTS}

This classification is recommended where the construction and occupancy are such that a degree of fire resistance equivalent to two hours of the standard test is deemed necessary.

\section{GENERAL SPECIFICATIONS}

\section{SUPPORTING STRUCTURE}

(a) Strength.-The structure supporting the vault shall be of adequate strength to carry the full building loads as well as the entire weight of the vault structure and contents.

(b) Fire resistance.-There shall be no combustible material in any portion of the supporting members of the structure. All structural members of the building shall have a degree of fire resistance equivalent to at least the same number of hours of the standard test as that for which the vault is rated.

\section{WALIS}

(a) Location and arrangement.-The walls of the vault shall follow the panels of the building whenever possible and shall extend from floor to ceiling 
of the building in the stories where the vault is located.

If vaults are located on more than one story, they shall preferably be placed one above the other in the several stories.

(b) Materials.-Walls of vaults of the various classifications shall be built of the materials and in the manner specified for ground supported vaults of equivalent classifications.

(c) Thickness.- The thickness of vault walls of the various classifications shall be not less than the minimum thicknesses for the various materials specified for the top stories of ground-supported vaults as given in Table 1 except that the minimun thickness of walls of hollow clay tile or concrete block for a 2-hour vault may be 8 inches.

(d) Bonding.-Vault walls shall be effectively bonded at the top and bottom to the floor or roof of the building in the stories where the vault is located. Suitable bonding shall also be secured between the walls and adjoining building columns as well as between vault walls and outside walls of the building where the latter are used to serve as a portion of the vault walls.

\section{ROOF AND FLOOR}

(a) The building floors or roof of the building shall serve for the roof and floors of the vault. The roof or floor shall be unpierced.

(b) No wood or other combustible material shall be used in the flooring or surfacing.

\section{INTERIOR SUPPORTS}

Where there are interior supporting columns in a vault they shall have a degree of fire resistance equivalent to not less than three hours of the standard fire test.

\section{DOORS}

Shall conform to the specifications as given in the section on vault doors and shall be of the same fireresistance classification as the vault on which installed.

\section{WATER-TIGBTNESS}

Shall conform to the specifications for groundsupported vaults.

\section{VENTILATION}

Ventilation of interior shall be only through door openings. Walls, floors, and roofs shall not be pierced.

\section{INSPECTION}

The construction of the vault shall be under the immediate supervision of a competent engineer or architect to insure that it is built in accordance with the above recommendations and that careful workmanship is obtained throughout.

\section{SPECIFICATIONS FOR VAULT DOORS}

\section{[CLASSIFICATION}

Vault doors are classified as six-hour, four-hour, or two-hour when the construction is such that they are capable of withstanding an exposure equivalent to six hours, four hours, or two hours of the standard test.

\section{GENERAL DESIGN}

Doors may be single or mounted in pairs, but in all cases to have insulation mounted between the inner and outer face plates.

Note.-It is believed that inner uninsulated doors bave some value in keeping combustihle material well inside the vault chamber value in keeping combustihle material
and they may be installed if desired.

\section{STRENGTH}

The door structure shall have adequate strength to withstand the wear and tear of normal operation, and such rough usage as may be reasonably anticipated for a device of its class.

\section{INSULATING MATERIAL}

Doors shall be insulated with nonflammable heat insulating material.

Note.-Experience with present insulating materials and metbods of construction indicates that desired results can he obtained with insulation baving thicknesses as sbown in the following tahle:

\begin{tabular}{|c|c|}
\hline Classification & Tbickness of insulation \\
\hline $\begin{array}{l}\text { 6-hour } \\
\text { 4-hour } \\
\text { 2-hour }\end{array}$ & $\begin{array}{l}\text { Not less tban } 5 \text { incbes. } \\
\text { Not less than } 4 \text { inches. } \\
\text { Not less tban } 3 \text { inches. }\end{array}$ \\
\hline
\end{tabular}

DETATIS OF CONSTRUCTION

(a) Doors shall be mounted in a vestibule with or without inner doors creating a dead air space and shall have a total thickness sufficient to provide for the specified thickness of insulation and sufficient additional room for locking mechanism. All four edges of the door shall be so formed as to provide tongued-and-grooved joints. A hinged metal bridge shall be provided over the sill to protect tongues and grooves against mechanical injury.

(b) Vestibule shall consist of an open steel box carrying on its inner face the insulated head, sill, and jamb structures formed to engage all four edges of the door. Edges of the box shall be provided with flanges overlapping the outer and inner surfaces of the wall at least 3 inches on the outside and 2 inches on the inside. Sides of the vestibule shall be at least $1 / 16$ inch thick, and the entire structure shall be braced and stiffened in such a manner as to provide a rigid support for the doors.

(c) When in the closed position doors shall be secured to the frame at intervals not exceeding 2 feet on all four sides.

Note.-Hinges are considered as fastenings, but must be reinforced with bolts if tbespacing does not come within the specifica tions given.

(d) Inner doors, if used, shall be of steel plate, at least 1/16 inch thick, reinforced to give stability, closing against stops and provided with suitable hardware.

\section{UNINSULATED STEEL VADLT DOORS}

Since the uninsulated vault door is widely used, it is felt that it should be given a classification at this point for reference purposes, although this classification is outside the group recommended for use in connection with vaults. On the basis of such furnace tests as have been made, a classification of one-half hour is given to the type of uninsulated door described in connection with record rooms.

\section{VAULT INTERIOR EQUIPMENT}

(Under this heading are grouped a number of points which relate to the proper equipment and maintenance of a vault.)

\section{(Adopted 1927)}

\section{FILING EQUIPMENT}

Filing equipment should be noncombustible throughout. Containers should be entirely inclosed if possible; but if contents are such as to make complete inclosure impracticable, containcrs having only the front end open are advisable. Cubical contents of individual containers should be kept as small as possible.

\section{LIGH THNG}

The lighting should be electric, so arranged that both wires of the circuit are cut off when the doors are closed. Common methods of accomplishing this are given below in order of merit.

(a) Interior wiring in conduit with as many fixed lamps as are needed for adequate illumination; 
this interior system to be supplied by a short extension cord through doorway to live receptacle outside vault. Cord may be protected by flexible armor or by short length of rigid steel conduit with bushings and taped in place, on the cord at the point of door closure.

(b) Interior wiring controlled by door switch, switch opening both sides of the circuit.

(c) Interior wiring controlled by outside switch with red pilot light.

Tiring should be in conduit preferably exposed, and there should be no pendant or extension cord within the vault.

Care should be taken to make vault lighting adequate. Otherwise matches or other hazardous forms of illumination are likely to be used.

\section{HEATING}

Ordinarily vaults require no heating. Where steam coils are used, a hazard is invariably introduced. Open-flame heaters, electrical heaters, etc. should not be employed.

\section{VENTILATION}

Many examples of hazards introduced into vaults by ventilating systems are found. Openings are cut through walls to permit of ventilation. Fans are installed, usually supplied by makeshift wiring.

It is possible to install mechanical rentilation, either drawing through or discharging through doorway, and not necessitating additional wall openings. Increase in height of door will be desirable when this is done, and any such system should be so arranged that electric power supply to any apparatus within the vaults will be cut off when doors are closed.

Ducts passing through walls, even though protected by doors or shutters, create an additional hazard.

\section{HOOSEREEPIVG}

General cleanliness should be of the highest type. Toreign materials should not be kept in vaults. This has been emphasized by finding in vaults such things as gasoline, kerosene, lubricating oils, oily rags, nitrocellulose moving-picture and X-ray films, and the film mounts used by dentists, turpentine, reserve supply of matches, etc

Smoking inside raults should be positively forbidden.

No refinishing of any sort should be done inside vaults, since this means the presence of common and special hazards incidental to refinishing work. Special emphasis should be laid upon the undesirability of any refinishing by means of spraying now so commonly used.

\section{TELEPHONE AND ALARH}

Where possible telephone connections should be installed inside the vault or alarm bells should be arranged so that a person locked inside the vault can ring an alarm on the outside.

Alarm bells have also been successfully arranged that ring inside when the door is about to be closed, a second switch on the outside door or the other half of a double inside door shutting off the bell.

Underwriters' Iaboratories, supplementary standard for hollow concrete building units, 1929.

See 510.4, page 321 .

United States Department of Commerce, Bureau of Standards, Simplified Practice Recommendation No. 32, for concrete building units, June 1, 1925.

See 516.4, page 323

United States Department of Commerce, Bureau of Standards, Simplified Practice Recommendation No. 38 , for sand-lime brick.

See 513 , page 258 .
518.6 DRAINAGE STRUCTURES. (See also 531 and 534.21.)

\subsection{General Items.}

American Society for Testing Materials, standard definitions of terms relating to sewer pipe, serial designation: C8-24, 1924.

\section{FORCES ACTING UPON SEWER PIPE}

External forces.-Forces resulting from pressures or impact acting from the outside upon the pipe.

Internal forces.-Forces resulting from interior fluid pressure.

Abrasion.- Wearing away of surface material by friction.

\section{RAW MATERIALS}

Physical properties.-Those sensible properties of raw materials, which in their combinations affect the manufacture and use of the product.

Chemical properties.-Those properties resulting from combinations of elements in the raw materials which in their composition affect the manufacture and use of the product.

Clay.-An earthy or stony mineral aggregate consisting essentially of hydrous silicates of alumina, plastic when sufficiently pulverized and wetted, rigid when dry, and vitreous when burned at a sufficiently high temperature.

Surface clay.-An unconsolidated, unstratified clay, occurring on the surface.

Fire clay.-A sedimentary clay of low-flux content.

NoTE. -It is usually associated with coal measures.

Shale.-A thinly stratified, consolidated sedimentary clay with well-marked cleavage parallel to the bedding.

\section{PIPE}

(a) Sewer pipe--Separate pipe suitable for the conveyance of domestic and industrial sewage and storm water and capable of being properly joined to form a conduit.

Clay pipe.-Made from red burning plastic clay devoid of fissile structure. Maturing temperature about $1,170^{\circ} \mathrm{C}$. Vitrification not ordinarily produced, and salt glazing not always effective.

Fire-clay pipe.-Made from buff, gray, or reddish burning fire clay showing conchoidal structure. Maturing temperature about $1,250^{\circ} \mathrm{C}$. Complete stony vitrification may be produced, but an absorption lower than 3 per cent is not general, not desirable for maximum strength.

Shale pipe.-Made from red burning hard clay with a distinct fissile structure. Vitrification at from $1,050^{\circ}$ to $1,250^{\circ} \mathrm{C}$., and salt glazing successful only at highest temperature.

Cement-concrete pipe.-Pipe formed by consolidating in a mold a mixture of Portland cement, water, sand, stone, or other hard material, and permitting it to harden by natural process prior to handling and use.

Special.-A pipe other than a straight pipe.

Branch.-A pipe attached to and diverging from the barrel of another pipe, such as $Y$ branches, $T$ branches, etc.

(b) Demands upon sewer pipes.-Requirements of qualities which are desirable and attainable under conditions of actual practice.

\section{PARTS OF SEWER PIPE}

Barrel or shell.-Main body of a pipe, exclusive of differently formed ends.

Ends.-Those parts of a pipe which terminate it and are so formed as to permit of making a proper joint.

Beveled end.-End surfaces of pipes inclined at an angle with the pipe axis so formed that the end of 
one pipe can enter the end of the adjoining pipe with a close fit.

$H u b$ or bell. - That end of a pipe which is sufficiently enlarged for a short distance to receive and inclose the spigot end of the adjoining pipe, to form a joint.

Spigot.-That end of a pipe which enters and is formed to fit the hub or bell of the adjoining pipe; it is sometimes scored or has a head.

Butt.-Plain end of a pipe sometimes scored on the outside, to close up against a similar end of an adjoining pipe for the purpose of forming a joint, the two ends being surrounded and covered by a collar.

Collar.-A flat band to surround and cover a butt joint.

Base, form of.- Shape of that part of a pipe which rests upon a foundation.

\section{JOINTS}

Beveled.-Formed by joining pipes with beveled ends and applying the jointing material.

$H u b$ and spigot.-Formed by inserting the spigot end of one pipe into the hub of another and applying the jointing material.

Butt and collar.-Formed by abutting the butt ends of two adjacent pipes and, after applying the jointing material, surrounding the joint with a collar.

Joiniing materials.-The materials which are inserted between the ends of adjoining pipes for the purpose of forming a continuous closed conduit.

Elasticity of joints.-Ability of jointed pipes to resist strains caused by bending and returning to original position.

\section{FINISHED PRODUCT}

(A) MATERIAL

Durability.-Resistance to disintegration or deterioration.

Serviceability.-Ability to readily and effectively render satisfactory service.

Thickness.-Distance between outside and inside surfaces.

Strength.-Ability to resist physical forces imposed during handling and after pipe has been put in use.

Toughness.-Relative degree of resistance to impact without fracture as opposed to brittleness.

Hardness.-Intensity of molecular cohesion as measured by resistance to penetration by a standard tool.

Smoothness.-Evenness of surface without projections or irregularities.

Vitrification. - The consolidation of material under high heat.

Shrinkage-Diminution of dimensions and mass of the material.

Porosity.-Ratio of the volume of interstices of the material to the volume of its mass.

Percolaition.-Passage of water through the interstices of the material.

\section{(B) COVERING}

Coating.-A covering of other materials applied in liquid form.

Glazing.-Hard glassy surface covering.

Sall glazing.-Glazing produced by application of salt during vitrification.

Lining.-A covering of other material applied in solid form to inside surfaces.

Waterproofing.-Materials resistant to penetration by water.

\section{(C) DEFECTS}

Warp.-Twisted out of normal shape.

Fracture.-Rupture of the material by a break through its entire thickness.
Crack.-Fissure or open seam not necessarily extending through body of material.

Fire crack.-A crack resulting from lack of uniformity in shrinkage after the application of excessive heat.

Hair crack.-Irregularly running, fine, narrow crevice or fissure at the surface of a pipe not penetrating deeply and caused by a shrinkage of material during manufacture.

Blister.-Convex hollow prominence formed by separation and raising of a film in the process of burning.

Leakage.-Passage of water contrary to intention.

Lamination.-Division of material into thin layers or sheets.

\section{IDENTIFICATION}

Marking and imprints.--Impressions made upon pipe at the place and time of manufacture for the purpose of identification.

American Society for Testing Materials, tentative specifications for required safe crushing strengths of sewer pipe to carry loads from ditch filling, serial designation $\mathrm{C} 15-17 \mathrm{~T}, 1917$.

The following table gives required safe crushing strengths per linear foot of pipe, to carry loads from ditch-filling materials only when pipe is laid in accordance with Recommended Practice for Laying Sewer Pipe, A. S. T. M. D12 (see 1927 book of standards, Part II, p. 210), for sand and for thoroughly wet clay ditch-filling materials. In the preparation of this table a safety factor of $1 \frac{1}{2}$ has been used, which has been found necessary to prevent cracking from the loads of ditch filling. ${ }^{77}$

Ordinary pipe laying is pipe laying in accordance with customary good practice in pipe-sewer construction, whereby the underside of the pipe is well bedded on soil for $60^{\circ}$ to $90^{\circ}$ of the circumference.

First-class pipe laying is pipe laying in accordance with the best customary practice in pipe-sewer construction, whereby the entire underside of the pipe is very thoroughly bedded on soil and the entire pipe is surrounded by well-compacted soil, under the direction of an inspector constantly present on the work.

Safe crushing strengths of sewer pipe to carry loads. from ditch filling for ordinary sand and for thoroughly wet clay ditch-filling materials (for ordinary pipe-laying methods)

[Strength in pounds per linear foot]

\begin{tabular}{|c|c|c|c|c|c|c|c|c|c|c|}
\hline \multirow{4}{*}{$\begin{array}{l}\text { Height of } \\
\text { fill above } \\
\text { top of pipe, } \\
\text { in feet }\end{array}$} & \multicolumn{10}{|c|}{ Breadth of ditch a little below top of pipe } \\
\hline & \multicolumn{2}{|c|}{1 foot } & \multicolumn{2}{|c|}{2 feet } & \multicolumn{2}{|c|}{3 feet } & \multicolumn{2}{|c|}{4 feet } & \multicolumn{2}{|c|}{5 feet } \\
\hline & \multicolumn{10}{|c|}{ Ditch-filling material } \\
\hline & 总 & 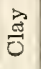 & 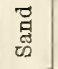 & 悹 & 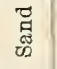 & $\frac{\vec{g}}{0}$ & $\begin{array}{l}\text { D् } \\
\text { G } \\
\text { W }\end{array}$ & 焉 & 司 & $\stackrel{\Xi}{\sigma}$ \\
\hline $\begin{array}{l}4=- \\
4 \\
6 \\
8 \\
10 \\
10-\end{array}$ & $\begin{array}{l}265 \\
409 \\
470 \\
505 \\
525\end{array}$ & $\begin{array}{l}280 \\
450 \\
545 \\
605 \\
640\end{array}$ & $\begin{array}{r}615 \\
1,055 \\
1,370 \\
1,600 \\
1,765\end{array}$ & $\begin{array}{r}635 \\
1,125 \\
1,500 \\
1,790 \\
2,015\end{array}$ & $\begin{array}{r}970 \\
1,745 \\
2,370 \\
2,875 \\
3,275\end{array}$ & $\begin{array}{r}990 \\
1,825 \\
2,525 \\
3,115 \\
3,610\end{array}$ & $\begin{array}{l}1,330 \\
2,455 \\
3,405 \\
4,215 \\
4,900\end{array}$ & $\begin{array}{l}1,350 \\
2,535 \\
3,575 \\
4,495 \\
5,295\end{array}$ & $\begin{array}{l}1,690 \\
3,165 \\
4,460 \\
5,595 \\
6,590\end{array}$ & $\begin{array}{l}1,710 \\
3,250 \\
4,640 \\
5,890 \\
7,020\end{array}$ \\
\hline $\begin{array}{l}12 \\
14 \\
16 . \\
18 \\
20\end{array}$ & $\begin{array}{l}535 \\
540 \\
545 \\
545 \\
545\end{array}$ & $\begin{array}{l}660 \\
675 \\
680 \\
685 \\
690\end{array}$ & $\begin{array}{l}1, y 00 \\
2,025 \\
2,070 \\
2,100\end{array}$ & $\begin{array}{l}2,320 \\
2,425 \\
2,505\end{array}$ & $\begin{array}{l}3,600 \\
3,855 \\
4,065 \\
4,230 \\
4,365\end{array}$ & $\begin{array}{l}4,030 \\
4,380 \\
4,675 \\
4,920 \\
5,130\end{array}$ & $\begin{array}{l}5,485 \\
5,975 \\
6,395 \\
6,750 \\
7,050\end{array}$ & $\begin{array}{l}6,000 \\
6,620 \\
7,165 \\
7,630 \\
8,060\end{array}$ & $\begin{array}{l}7,460 \\
8,225 \\
8,890 \\
9,480 \\
9,995\end{array}$ & $\begin{array}{r}8,035 \\
8,950 \\
9.775 \\
10,520 \\
11,190\end{array}$ \\
\hline $\begin{array}{l}22 \\
21 \\
28 \\
30 \\
\text { Very great }\end{array}$ & $\begin{array}{l}545 \\
545 \\
545 \\
545 \\
545 \\
545\end{array}$ & $\begin{array}{l}690 \\
690 \\
690 \\
690 \\
690 \\
690\end{array}$ & $\begin{array}{l}2,125 \\
2,140 \\
2,150 \\
2,160 \\
2,165\end{array}$ & $\begin{array}{l}2,610 \\
2,645 \\
2,675 \\
2,695 \\
2,715\end{array}$ & $\begin{array}{l}4,470 \\
4,560 \\
4,630 \\
4,685 \\
4,725\end{array}$ & $\begin{array}{l}5,305 \\
5,455 \\
5,575 \\
5,680 \\
5,765\end{array}$ & $\begin{array}{l}7,305 \\
7,525 \\
7,705 \\
7,860 \\
7,990 \\
8\end{array}$ & $\begin{array}{r}8,425 \\
8,750 \\
9,035 \\
9,280 \\
9,500 \\
11,075\end{array}$ & $\begin{array}{l}10,445 \\
10,840 \\
11,185 \\
11,490 \\
11,755 \\
13,635\end{array}$ & $\begin{array}{l}11,795 \\
12,340 \\
12,830 \\
13,270 \\
13,670 \\
17,305\end{array}$ \\
\hline
\end{tabular}

77 Prepared from the Standard Specifications for Drain Tiles A. S.'T. M. C4. (See 534.21, p. 608.) 
When pipes are laid in a concrete or other permanent masonry cradle, strong enough to carry the entire load to the subbase without breaking and large enough to prevent material settlement, the standard strengths for all dimensions of ditches and all filling materials shall be those specified for standard semer pipe.

National Paving Brick Manufacturers' Association, recommended specifications, underdrainage and elimination of water, 1924 .

Localion.-Tile and broken stone drains shall be provided of such size and in such location as shown on the plans for the improvement accompanying these specifications.

Quality.-The drain tile or vitrified pipe as called for on the plans shall be hard burned to insure against disintegration or crumbling, reasonably smooth on the inside and free from cracks, warps, and imperfections that would appreciably decrease its strength or service.

Size.- The minimum length of such pipe shall be 12 inches and minimum diameter 4 inches.

Tile or pipe drains. - The tile or pipe shall be laid true to line and grade with close joints and shall rest on a solid bed throughout its length. The tile or pipe shall be covered as laid with gravel, broken stone, or crushed slag, placed around and above it to a height of at least 1 foot above the tile or pipe. The back fill above the stone, grarel, or slag filling shall be of suitable earth tamped in place in layers not exceeding 4 inches in thickness.

Lateral and suriace connections shall be made with suitable specials. The drains shall have suitable and frequent inlets and outlets desimned and protected as shomn on plans.

Stone drains. - In stone drains the trench shall be excavated to the dimensions shomn on the plans, finished to the given line and grade and be uniform in surface and tamped if necessarr. The bottom course, from $S$ inches to 10 inches in depth, shall be of sound, hard, and durable stone, gravel, or slag varying in size from that which will pass through a revolving screen having holes $2 \frac{1}{2}$ inches in diameter to that which will pass through a revolving screen having holes 5 inches in diameter. It shall be placed so as to give maximum roids.

The top course of stone, slag, or gravel, all of which will pass through a screen having holes from $3 / 4$ inch to $21 / 2$ inches in diameter, shall then be placed over the bottom course to fill the trench to a total depth of at least 12 inches.

Above this the earth filling shall be placed as heretofore specified and suitable outlets provided.

Sione underbedding or subbase. - There the plans call for a layer or course of broken stone, gravel, or slag to be spread under the entire area of the road for acceleration of drainage and to prevent water entering the upper structure of the road, the stone or other material shall be sufficiently large and uneven to contain air volume that will prevent capillary movement of moisture. Sufficient fine material shall be orerlaid to prevent filtration of any superstructure material, and artificially placed over it. This layer shall be thoroughly compacted before an superstructure material is placed upon it.

\subsection{Culverts. (See also 518.4.)}

American Association of State Highway Oficials, standard specifications for highway bridges and incidental structures, 1928 .

\section{MONOLITEIC CONCRETE CULVERTS}

(This specification consists largely of construction requirements, with the following specifications for classes of concrete:)

Unless otherwise specified, all reinforced concrete culverts shall be of class A concrete except the foot- ings, which may be of either class A or class B concrete as specified.

Gravity sections used for wings and culvert barrel walls shall be of either class A or class B concrete as specified.

Nоте.-For definitions of the various classes of concrete mentioned abore, see 516.3, page 301, American Association of State Highway Officisis, specification for "Concrete Masonry."

American Railway Engineering Association, specifications for stone masonry.

See 518.89, page 478 .

\subsection{Drains.}

American Concrete Institute, standard specifications for concrete drain tile, serial designation P- $7 \mathrm{~B}-25,1925$.

See 534.21, page 606 .

American Railway Engineering Association, specifications for buildings for railway purposes.

\section{SEWERS AND DRAINAGE, 1926}

See 518.67 , page 453 .

American Society for Testing Materials, standard specifications for drain tile, serial designation C4-24, 1924.

See 531.21 , page 608 .

\subsection{Curbs.}

American Concrete Institute, tentative specifications for concrete curb and concrete curb and gutter, serial designation S-6E-27T, 1927.

\section{GENERAL}

The curb, or combined curb and gutter shall be constructed of Portland cement concrete in accordance with these srecifications and shall comply with the requirements and dimensions shown on the plans.

Preparaiion. - The excavation for curbs shall be of sufficient width and depth as to permit the proper placing of forms, tile where necessary, and the required thickness of foundation.

All soft and spongy materials shall be removed and all depressions fllled with suitable material which shall be thoroughly compacted in layers not exceeding 4 inches in thickness. While compacting the subbase, the material shall be kept thoroughly net and shall be in that condition when the concrete is deposited.

Drainage. - Then required, a suitable drainage system shall be installed and connected with sewers or other drains indicated by the engineer. The size and requirements for drain tile shall be as shown on the plans or as specified by the engineer.

\section{MATERIAIS}

The cement used shall be a standard brand of Portland cement, and shall meet the requirements of the American Society for Testing Materials, C9. (See 516.11, p. 294.)

Fine aggregate.-Fine aggregate shall consist of natural sand, or when the engineer so directs, stone screenings, slag, sand, tailings, chatts, or other inert materials with similar characteristics, or a combination thereof, having clean, hard, strong, durable, uncoated grains. Then incorporated in the mixture, fine aggregate shall be free from frost, frozen lumps, injurious amounts of dust, mica, soft or flaky particles, shale, alkali, organic matter, loam or other deleterious substances. It shall be uniformly graded between the $1 / 4$ inch and 100 -mesh sieves. Not more than 25 per cent of the material shall pass a 50 -mesh sieve, and not more than 3 per cent of dry material, by weight, shall pass a 100mesh sieve.

Coarse aggregate.-Coarse aggregate shall consist of crushed rock, pebbles (gravel), chatts or tailings, 
or a combination of these materials. The particles shall be clean, hard, tough, durable material, free from vegetable or other deleterious substances, and shall contain no soft, flat, or elongated pieces. The coarse aggregate shall be uniformly graded from fine to coarse with 100 per cent passing the $1 \frac{11}{2}$-inch sieve and not more than 5 per cent passing the $1 / 4$-inch sieve.

If blast-furnace slag is used for coarse aggregate, it shall be air-cooled and exposed to the weather for a period of at least six months prior to use. It shall contain not more than 2 per cent of sulphur and dried slag when shaken to refusal shall have a weight
Forms.-All forms shall be of metal, straight, free from warp, and of sufficient strength to remain in alignment and sustain the pressure of the concrete. Forms must be kept clean, oiled or greased, and in good condition.

Division plates shall be of $1 / 8$-inch steel, oiled and cleancd each time prior to use.

\section{CONSTRUCTION}

Separate curb dimensions.-The cross section for a separate curb shall be similar to that shown in Figure 129.

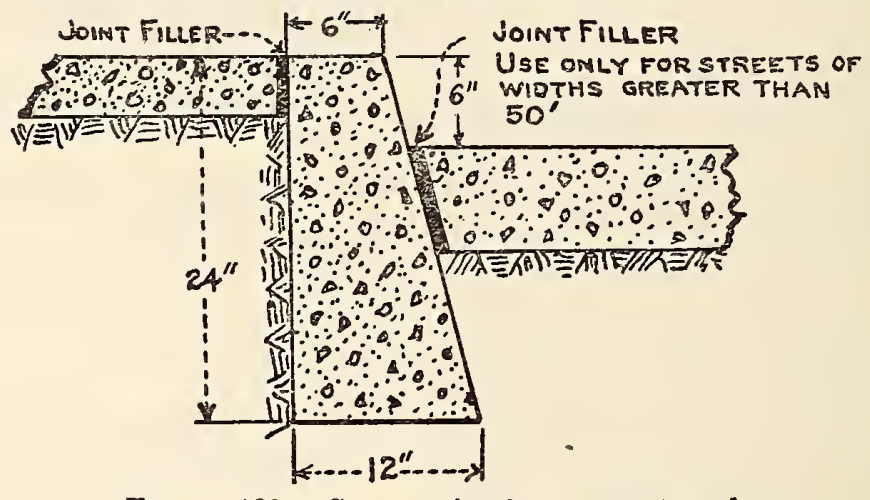

Figdre 129.-Cross section for a separate curb

of not less than $70 \mathrm{lbs} . / \mathrm{ft}^{3}$ In "general, slag shall, conform to the specifications for "coarse aggregate." (See 512.13, p. 237.)

Natural mixed aggregatcs. - Natural mixed, or pit-run aggregates shall not be used as they come from the pit, but shall be screened and remixed to agree with the proportions specified. In any case, the quality and grading of the fine and coarse agoregates separated by screening, shall be at least equivalent to that specified in the foregoing paragraphs for fine and coarse aggregates. ${ }^{78}$

Mixing water.-The mixing water used shall be clean and free from injurious amounts of oil, acid, alkali, or vegetable matter. Ordinarily any potable water will mect these requirements.
The back shall be straight and perpendicular and the street side shall have a batter of approximately 1 to 4 . The thickness at the base shall be not less than 12 inches and not more than 6 inches at the top.

Combined curb and gutter dimensions.-The cross section of a combined curb and gutter shall be similar to that shown in Figure 130.

The top of the curb shall be 6 inches above the gutter line, but this design may be varied according to the locality and drainage facilities.

The combined curb and gutter shall be divided into scctions not more than 10 feet long. The construction joint must insure complete separation of the sections.

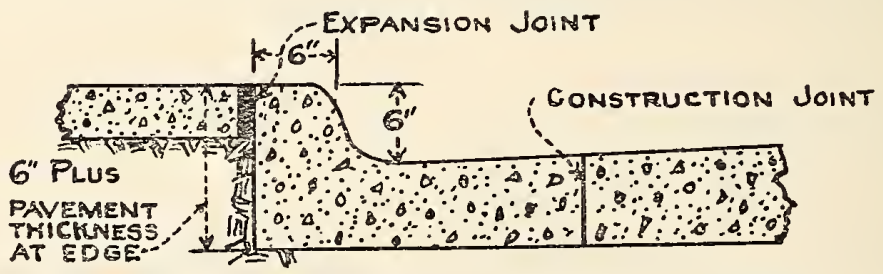

FIGURE 130.-Cross section of a combined curb and gutter

Coloring.-If artificial coloring is used in the concrete curb it shall consist only of permanent mineral oxides that are fully guaranteed by the manufacturer to be unaffected by lime, cement, or weathering.

Joint filler.-The material for filling joints in concretc curbs and gutters shall be a premolded bituminous mastic or suitable waterproof compound that will not become soft and run out in hot weather nor become hard and brittle and chip out in cold weather.

if In many cases it will be nccessary for the engincer to specify the sizes, grading, and quality of coarse ageresate in accordance with sizes, grading, and quality of coarse aggiegate in accordance with cations which will insure the use of the best coarse agregate which is economically available.
At street intersections where two curbs meet the corner shall be rounded to a radius of not less than 20 feet.

Expansion joints.-Expansion joints shall be required at each curb return, and at such intervais as defined by the engineer or as shown on the plans. Unless otherwise designated on the plans the expansion joint shall be one-half inch in thickness and of a material as defined in these specifications under materials, joint filler.

Proportions. - The proportions of aggregate to cement shall be such as to produce concrete that can be puddled readily into the corners and angles of the form without excessive spading and without undue accumulation of water or laitance on the surface, The proportion of fine and coarse aggregates 
shall be such that the ratio of the coarse to the fine shall not be less than 1 nor more than 2, nor shall the amount of coarse material be such as to produce harshness in placing or honeycombing. Proportions approximating a 1:2:3 mix are recommended.

Measuring materials. - The method of measuring materials, including water, shall be one which will insure separate accurate proportions at all times. A bag of Portland cement (94 pounds net) shall be considered as I cubic foot.

Machine mixing. - The concrete shall be mixed in a batch mixer of such capacity that only whole bags of cement are used in each batch. It shall be a type approved by the engineer.

Mixing shall continue for at least 1 minute, but preferably $1 \frac{1}{2}$ minutes after all materials, including water, are placed in the drum, and before any part of the batch is discharged.

Hand mixing.- Mixing by hand shall only be permitted on jobs that are too small to justify the use of a machine mixer. When it is necessary to mix by hand, the mixing shall be done on a water-tight platform and the materials shall be turned at least three times and until the mass is a uniform color and all the particles coated with a cement mortar.

Central mixing.- The use of central mixing plants, and the transportation of mixed concrete is permitted under these specifications, provided there is no segregation of the mixed concrete when it is delivered at the point where it is to be deposited. The concrete must be of a workable consistency when placed. Under no circumstances shall concrete, mixed at a central plant, be deposited directly into the forms, but shall be rehandled from a platform, sled, trough, or other suitable device which can travel alongside the curb forms.

Relempering.-Concrete which has partially set shall not be retempered by being mixed with additional materials or water.

Consistency. - The concrete mixture shall contain no more water than is necessary to produce a workable mass which can be brought to a satisfactory finish. The amount of water used shall not exceed $61 / 4$ gallons per sack of cement.

Placing.--After mixing, the concrete shall be placed in the forms in separate layers 4 or 5 inches thick until the forms are completely filled. Each layer shall be thoroughly tamped and spaded before the next is added. Particular attention shall be given to spading the concrete close to the forms in order to prevent honeycombing and so that the exposed surface may have a smooth uniform appearance.

When the forms are completely filled the concrete shall be struck off with a templet cut to the desired curb design. The concrete shall then be finished with a wooden float, in a manner which will thoroughly compact the mass and leave a true even surface. When required, the surface may be troweled smooth, but excessive working with a steel trowel should be avoided. The upper edges of the curb shall be rounded with an edging tool.

The forms shall be removed within 24 hours after the concrete has been placed and minor defects filled with mortar composed of 1 part of Portland cement and 2 parts of fine aggregate. The front or face forms shall be removed within 12 hours after concrete has been placed, and the top and face of the curbing, from the top to 8 inches below shall be finished while the concrete is still green by frequently wetting a soft brick or a wood block and rubbing the surface until it is smooth. Within 24 hours the curbing shall be rubbed once more with a block and water as before to produce a smooth surface. Brush finishing or plastering shall not be permitted on the top or the face of the curbing. All separator joints shall be opened from top to bottom, the edges adjacent to the joint being sharp and clean cut. Curing and protection. - The concrete curb shall be kept wet for seven days after placing. It may be covered with wet earth, or burlap, hay or straw, and kept moist by intermittent sprinkling.

During the curing period the curb shall be protected from injury by proper warning signs and barricades.

Concrete shall not be mixed nor deposited when the temperature is below freezing, except under such conditions as the engineer may direct in writing. If, at any time during the progress of the work, the temperature is, or in the opinion of the engineer will, within 24 hours, drop to $38^{\circ} \mathrm{F}$. the water and aggregates shall be heated, and precautions taken to protect the concrete from freezing until at least 10 days old. In no case shall concrete be deposited upon a frozen subgrade, nor shall frozen materials be used in the concrete.

American Society for Irunicipal Improvements, standard specifications for curbs, 1927.

\section{THE TEST FOR QUALIFICATIONS OF CURBSTONE}

If a test is desired it shall be provided for in the local specifications.

1. Abrasion.-The loss by abrasion shall be determined by the "rattler test." (See A. S. T. M. C7-29T, 534.11$, p. 577.$)$

The rattler, abrasive charge, the test and record shall be as described therein. Four blocks of the material to be tested shall be axed or hammered to about 5 by 6 by 10 inches and dried (these four blocks will weigh from 110 to 120 pounds, approximating the weight of 10 brick).

For the present a loss of 12 per cent by abrasion will be permissible.

If made through the "Deval abrasion test" as deseribed in A. S. T. M. D2 (see 510 p. 182) a coefficient of wear of 7 is permissible.

2. Absorption. - The dry blocks shall be weighed, then immersed in water for 48 hours, drained off for five minutes and then weighed. The increase in weight is the absorption and should not exceed $2 \frac{1}{2}$ per cent.

\section{SETTING CURB}

3. Grading.-Curb shall not be set until the rough grade has been prepared and rolled, if the latter is specified.

4. Temperature.-Curb shall not be laid in concrete when there is indication of freezing. If overtaken by temperature falling to $35^{\circ} \mathrm{F}$., the concrete in place shall be covered with dirt from the street or otherwise protected as the engineer directs.

\section{FOR SETTING CURB IN CONCRETE}

5. Curb trench.-The curb trench should be 18 inches wide, 6 inches in front of the curb, and 12 inches back from face of curb. It should be at least 22 inches deep. If the back of the trench suggests caving in, it should be held with planks, so as to insure the full width of concrete throughout its depth, with square shoulder at the top.

6. Concrete.- Shall be made with Portland cement, aggregates, and water, conforming to those specified for paving base, except that the coarse aggregate should pass a 1-inch mesh. These are mixed in the proportion of 1 part of cement to 3 of fine and 7 of the coarse aggregate.

Should a gravel carrying adequate sand be used, the engineer shall specify the more exact proportions according to run of material and the details for mixing. 
The concrete shall be spread in the bed not more than 10 feet in advance of placing the curb.

As soon as the curb is bedded to its true line and grade, the trench should be filled with the concrete in front of the curb to the bottom of the paving base and back to the curb to within 6 inches of the top of the curb. A light tamping or spading is advisable, but the curb must not be disturbed. The remainder of the trench back of the curb shall be filled with dirt from the street or with a specified material.

7. Drain tile.-If drain tile is required, the bottom of the trench should be 36 inches below the top of the curb, and at least 6 inches wide.

The tile should be a commercial size, not less than 3 or 4 inches inside diameter. It shall be laid to line and grade and connected by vitrified tile, closed joint, to sewers or eatch basins. If outlet is available it is best to make these connections about 300 feet apart. The tile shall be covered with sereened gravel or broken stone, none of which shall pass a $1 / 2$-inch sereen (the coarse aggregate for concrete may be used). This covering shall be carried up to the bottom of the curb trench, thus it will be 14 inches deep.

8. If not set in concrete. - If the curb is not set in concrete, the trench and drain tile should be as above.

9. Drain tile not used.-If, however, drain tile is not required, the curb trench may be of such depth and may be drained off as local conditions suggest.

NотE.-In the trench described above about 1.3 to 1.5 cubic feet of concrete will be required for 1 linear foot of curb.

\section{DRESSING OR CUTTING}

10. The face shall be dressed to vary not more than $3 / 8$ inch from a plane surface, for a depth of 12 inches from the top. The remainder of the face shall be free from projections of more than $1 / 2$ inch.

The back shall likewise be dressed parallel to the face for a depth of 1 inch from the top. The remainder of the back shall be free from projections of more than 1 inch.

The top shall likewise be dressed having a bevel ol rise of $1 \frac{1}{4}$ inch from face to back of eurb.

The ends shall likewise be dressed at right angles to the face and top to vary not more than $1 / 8$ inch from a plane surface for a depth of 12 inches from the top.

11. Circular curb for round corners and closures should meet the above except as to length.

12. Bull nose or round edge curb are regarded as special features and entail extra cutting to meet local practice.

13. Headers or protection curb shall be dressed to a plane surface on top, ends cut at right angles to the face and top to a $3 / 8$-inch joint for a depth of 8 inches.

Note.-Except for special purposes the standard methods of dressing or cutting as employed for the several kinds of stone are dressing or cutting as employed for the several kinds of stone are a particular method.

\section{GRANITE CURB}

The curb shall be straight split, medium grained, of uniform texture and color, free from seams, excess of mica and disintegration.

14. If set in concrete the following dimensions are recommended:

The granite splits more readily into shapes where the depth is three times the width:

Length not less than 4 feet.

Top width, 5 inches or as required, having in mind the quarrying proportion of one-third the depth.

Bottom width should be not less than 3 inches rough squared.
Depth.-For not less than three-fourths of the length, the depth should be 16 inches for a curb 5 inches wide.

15. If not set in concrete a special depth up to 20 or 26 inches may be required.

\section{HARD SAINDSTONE}

16. The curb shall be of best quality hard sandstone, uniform color, free from seams and laminations.

17. If set in concrete the following dimensions are recommended:

Length not less than 36 inches nor more than 42 inches.

Top width, 4,5 , or 6 inches, as required.

Bottom width should be not less than one-half the width specified for the top.

Depth. - For not less than three-fourths of the length, the depth should be 16 or 18 inches for the 5 or 6 inch width, respectively.

18. If not set in concrete a special depth up to 20 or 25 inches may be required.

\section{BLTESTONE AND LIMESTONE}

19. Same as for hard sandstone, except that the length may be from 36 to 48 inches.

\section{SOFT SANDSTONE AID IIMESTONE}

20. Soft sandsione and limestone.-As the softer stone are of ten machine cut or sawed, the dimensions need not be limited by strata or natural cleavage, but for handling and laying they should, if set in concrete, have a length not less than 4 feet nor more than 6 feet. Top width 5 or 6 inches. Bottom width not less than top.

Top width, 5 or 6 inches.

Bottom width, same as top.

Depth.-For a length not less than three-fourths of the top length, the depth should be 16 or 18 inches for a 5 or 6 inch width, respectively.

If not set in concrete a depth up to 24 inches may be required.

21. Header or protection curb should have a depth of 12 to 14 inches, a width not less than 4 inches, and length not less than 24 inches.

It shall be set in a concrete bed 6 inches deep and 18 inches wide and backed with concrete to within 8 inches of its top on the paving side and flush with the top on the unpaved or exposed side.

\section{SPECIFICATIONS FOR CONCRETE CURB}

22. Dimensions.-Standard cross section for straight curb shall be not less than 6 inches at top, 8 inches at bottom, and 18 inches in depth. The top corner on face of curb shall be rounded to a radius of 1 inch.

23. Proportions. - The curb shall be made of Portland cement concrete in either ${ }_{\mathrm{k}}^{\mathrm{s}}$ of the following proportions: 1 part (by volume) of Portland cement to 3 parts (by volume) of mixed aggregate, or 1 part Portland cement to 2 parts fine aggregate to 4 parts coarse aggregate.

\section{MATERIAIS}

24. The cement shall meet the requirements of the current standard specifications for Portland cement of the American Society for Testing Materials. (See 516.11, p. 287.)

25. Mixed aggregate shall consist of natural sand, gravel, or screenings from hard, tough, crushed rock or gravel, consisting of quartz grains or other hard material, clean and free from any surface film or coating and graded from fime to coarse, the latter 
particles predominating. Mixed aggregate, when dry, shall pass a screen having $3 / 4$-inch circular openings; not more than 20 per cent shall pass a sieve having 50 meshes per linear inch, and not more than 5 per cent shall pass a sieve having 100 meshes per linear inch. It shall not contain injurious vegetable or other organic matter as determined by the colorimetric test nor more than 5 per cent, by volume, of clay or loam.

That portion of the mixed aggregate which passes the 10-mesh-per-linear-inch screen shall be of such quality that the mortar composed of 1 part Portland cement and 3 parts aggregate to be tested, by weight, when made into briquets or cylinders, shall show tensile and compressive strength, at 7 and 28 davs, at least equal to the strength of briquets or cylinders composed of 1 part of the same cement and 3 parts standard Ottawa sand by weight. The percentage of water used in making the test specimens of cement and aggregate to be tested shall be such as to produce a mortar of the same consistency as that of the Ottawa sand test specimens of standard consistency. In other respects all briquets or cylinders shall be made in accordance with the methods of testing cement recommended by the American Society for Testing Miaterials. (See C9, 516.11,p. 294.)

26. Fine aggregate shall consist of natural sand or screenings from hard, tough, crushed rock or gravel consisting of quartz grains or other hard materials, clean and free from any surface film or coating and grading from fine to coarse, the latter particles predominating. Fine aggregate shall meet all the requirements for mixed aggregate, except as to size, which are as follows: All of it shall pass a screen having four meshes to the linear inch; not more than 25 per cent shall pass a sieve having 50 meshes per linear inch and not more than 5 per cent shall pass a sieve having 100 meshes per linear inch.

27. Coarse aggregate shall consist of clean, durable, crushed rock or pebbles, graded in size, free from vegetable or other organic matter, and shall contain no soft, flat, or elongated particles. The size of the aggregate shall range from that passing through a $3 / 4$-inch circular opening to not more than 5 per cent passing a screen having four meshes per linear inch, and no intermediate sizes shall be removed.

\section{IFEASURING AND MIYING}

28. Requirements same as for concrete sidewalks (see 516.5 , p. 324 ) with following additional:

29. Consistency.-The materials shall be mixed wet enough to produce a concrete of consistency that will flush readily under slight tamping, but which can be handled without causing a separation of the coarse aggregate from the mortar.

\section{FORMS}

30. Forms for concrete curb shall be made of substantial materials, preferably steel forms. They shall be braced or staked so as to insure perfect alignment and grade. If the curb is precast; that is, not molded in place, the forms, preferably steel, shall be so constructed that they will permit uniform spacing and finish as herein specified.

\section{FINISE}

31. After pouring concrete into the forms it shall be puddled and spaded so as to insure a thorough mixture, eliminate air pockets, and create uniform and smooth sides.

Before the concrete has thoroughly set, and while the concrete is still green, the forms shall be removed and the front and top sides shall be finished with a float or steel trowel to make a uniform finished surface.

\section{ROUNDING CORNERS}

32. Wherever corners are to be rounded, special steel trowels shall be used while the concrete is workable and the corners constructed to the dimensions herein specified.

33. For combined curb and gutter the top and side of curb and gutter may be finished by the use of a special-shaped trowel or curb-and-gutter machine which will shape the entire upper suriaces in aceordance with the dimensions and shape specified. This trowel shall be used immediately upon removing the front form of the curb and while the concrete is still workable but firm enough to stand up.

\section{PROTECTIOT}

Requirements same as sections 27,28 , and 29 of specifications for concretc sidewalks. (See 516.5, p. 32 t.)

\section{JOINTS}

34. All curb shall be cut clear through so it will consist of sections about 6 feet long. These shall be cut by the use of steel templates where the curb is cast in place.

35. Expansion joints.- Shall be placed between sections of curb at intervals of 50 feet. These joints shall be filled with premolded bituminous material which will meet the requirements of A. S. M. I. specifications for coal-tar pitch filler or asphalt filler. These joints shall be one-half inch in thickness.

At street intersections, where the straight curb meets the curbed section of curb, similar expansion joints shall be placed.

\section{COMBINED CURB AND GUTTER}

36. Dimensions. - The standard cross section of combined curb and gutter shall be as follows: Total width at base, 24 inches; thickness of gutter slab, 6 inches; thickness at top of curb, 5 inches; thickness of curb at gutter line, 6 inches; height of curb above gutter, 6 inches. The front face of the curb will have a batter of 1 inch from bottom to top and the top corner of curb face will be roughened to 1-inch radius, while the intersection of curb and gutter shall be rounded to $1 \frac{1}{2}$-inch radius. The top of the gutter slab at the street edge shall be 5 inches below the top of the curb.

37. Proportion, materials, forms, measurements, mixing, consistency, finish, construction, protection, joints, expansion joints, etc., shall be in accordance with the specification herein given for concrete curb.

\section{PREMOLDED REINFORCED-CONCRETE CURB}

38. Reinforced concrete curb, premolded, of the materials and dimensions shown on the plans, shall be manufactured in standard lengths of 6 feet by the method hereinafter specified, or some method equally good.

39. Forms.-Collapsible forms shall be used which may be designed to cast a series of units. Ends must be square with top and sides.

Of the two faces which are to be exposed after laying, one or both shall be cast against a steel form. If only one is cast against a steel form the other shall be trowel dressed.

The above-mentioned forms shall have fastened to them by means of bolts not more than 3 feet apart, a metal corner bar. This bar must be correctly placed so that its rounded portion makes a smooth connection with the top and face surfaces.

40. Concrete for curb. - The concrete in curb manufacture shall contain for each sack of cement 2 cubic feet of dry sand and 3 cubic feet of washed gravel or crushed stone, screened so that all pieces shall pass 
a $3 / 4$ inch ring and 95 per cent of the gravel or stone shall be retained on a $1 / 4$-inch ring.

It shall be machine-mixed by batches, each batch being revolved for at least three minutes after the last ingredient has been placed in the drum.

41. Consistency.-Concrete shall be of such consistency that it may be placed without excessive ramming, but the use of excess water in order to secure a flowing concrete will not be permitted. The consistency required is that generally described as creamy. The amount of water to be used in mixing will be determined from time to time by the engineer, and when once determined shall be used for each batch until further notice. This uniformity shall be secured by measuring the water in the water tank on the mixer, which tank shall be equipped with a gauge glass graduated to gallons and quarters.

42. Withdrawal of a mixed batch.-Under no circumstances will a mixed batch or any part thereof be removed from the drum during the process of placing the succeeding batch in the drum.

43. Finish.-The concrete shall be poured and manipulated in such a manner that the top and face of the curb shall have plane surfaces, showing no coarse aggregate or pockets. Care must be taken not to to disturb the corner bar.

As the forms are removed, and while the concrete is green, the top and 10 inches of the face shall be rubbed down with a plane wood block.

44. Protection.-While curing, the curb shall be protected from direct rays of the sun and shall be kept moist for at least 10 days before placing in curb trench.

45. Closures.-Special: Cutting of concrete curbstones will in no case be permitted. Short lengths for final closures, which are to permit of special locations of drop curbs, shall be made to dimension for the particular purpose, either separately, as in the case of standard lengths, or may be cast in place in the street after the other curb has been set and concreted.

Asphalt Association, specification C-3, for shoulders, headers, curbs, and gutters (tentative), September 1, 1920.

(The full text of the specification includes methods of construction and the following specifications for materials:)

Section 1. General description.-(a) The surface course shall be protected at the sides by shoulders, headers, curbs, or gutters of the type shown on the plans and covered by these specifications and shall conform to the dimensions and grades shown on the plans.

(b) All materials shall conform to the requirements of these specifications and to the plans.

\section{MATERIALS}

Sec. 2. Gravel for shoulders.-Gravel shall be composed of hard, durable rock, together with sand and clay or other binding material, and shall be free from an excess of thin or elongated pieces. When tested by means of laboratory screens it shall meet the following requirements:

Passing 21/2-inch screen, not less than

Per cent Total retained on $1 / 4$-inch screen meet the following requirements:

Retained on 1-inch screen.

Per cent

SEc. 3. Broken stone, mine tailings, or broken slag for shoulders.-Broken stone, mine tailings, or broken slag for shoulders shall be composed of sound, durable particles. It shall be of reasonably uniform quality throughout and free from an excess of flat or elongated pieces. It shall consist of coarse aggregate, and fine aggregate or screenings as required in sections 11 or 12 as the case may be; which when tested by means of laboratory screens shall meet, respectively, the following requirements and be uniformly graded between the limits given:

\begin{tabular}{|c|c|c|c|c|c|c|}
\hline \multirow[b]{2}{*}{ Size } & \multirow[b]{2}{*}{ Name } & \multicolumn{5}{|c|}{ Per cent passing screen } \\
\hline & & 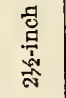 & 题 & 열 & 舟 & 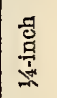 \\
\hline $\begin{array}{l}11 / 4 \text { to } 21 / 2 \text { inches. } \\
1 / 2 \text { to } 3 / 4 \text { inch.... } \\
0 \text { to } 3 / 1 / 1 \text { inch. }\end{array}$ & $\begin{array}{l}\text { Coarse aggregate } \\
\text { Fine aggregate } \\
\text { Screenings }\end{array}$ & 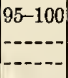 & $\mid \begin{array}{c}0-15 \\
-\cdots\end{array}$ & $\mid$\begin{tabular}{|}
$95-100$ \\
$95-100$
\end{tabular} & $\mid \begin{array}{l}25-75 \\
-\end{array}$ & $\mid \begin{array}{r}-75 \\
0-15 \\
40-80\end{array}$ \\
\hline
\end{tabular}

SEc. 4. Coarse aggregate for concrete curbs, headers, and gutters.- $(a)$ The coarse aggregate shall consist of broken stone, mine tailings, slag, or gravel. It shall be of reasonably uniform quality throughout and shall be free from dust and an excess of flat or elongated pieces. Rock or mine tailings from which it is produced shall have a per cent of wear of not more than 8. Slag from which it is produced shall have a per cent of not more than 12 , and shall weigh not less than 65 pounds per cubic foot for the size specified in section $4(b)$. If gravel is used it shall be composed of sound, hard, durable pebbles, free from clay or coatings of any character.

(b) When tested by means of laboratory screens the coarse aggregate shall meet the following requirements:

Per cent

Passing 13/2-inch screen, not less than Passing $3 / 4$-inch screen $-1 .-15$

SEc. 5. Fine aggregate for concrete curbs, headers, and gutters.- $(a)$ The fine aggregate shall consist of sand or a mixture of sand and stone screenings containing not more than 50 per cent by volume of screenings, which shall conform to the following requirements:

(b) Sand shall be composed of clean, hard, durable grains and shall contain not more than 5 per cent of clay and silt. It shall be free from other foreign material, well graded from coarse to fine, and when tested by means of laboratory screens and sieves it shall meet the following requirements:

Per cent

Passing 1/4-inch screen, not less than. 95 Passing 200-mesh sieve, not more than

Mortar composed of 1 part by weight of Portland cement and 3 parts by weight of the sand, shall have a tensile strength at the age of 7 and 28 days of not less than 75 per cent of that developed by mortar of the same proportions, made of the same cement, and standard Ottawa sand.

(c) Stone screenings shall consist of material produced by crushing clean, tough, durable rock and shall contain not more than 3 per cent of clay and silt. It shall be free from other foreign material, well graded from coarse to fine, and when tested by means of laboratory screens and sieves shall meet the following requirements:

Per cent

Passing 1/2-inch screen, not less than

Passing 200-mesh sieve, not more than

95

At the time of use stone screenings shall be free from cakes or crusts of hardened materials. For purposes of proportioning on the work and in laboratory tests only that portion of the entire product passing a $1 / 4$-inch laboratory screen shall be regarded as fine aggregate.

$(d)$ Combinations of sand and stone screenings shall be well graded from coarse to fine, and when tested by means of laboratory sieves that portion 
which passes a $1 / 4$-inch laboratory screen shall meet the following requirements:

Passing a 50-mesh sieve, not more than

Per cent Passing a 100-mesh sieve, not more than

Mortar composed of 1 part by weight of Portland cement and 3 parts by weight of the combination of sand and stone screenings, which have been passed through a $1 / 4$-inch laboratory screen, shall have a tensile strength at the agc of 7 and 28 days of not less than 90 per cent of that developed by mortar of the same proportions, made of the same cement, and standard Ottawa sand.

SEc. 6. Portland cement for concrete curbs, headers and gutters.- (a) Portland cement shall be the product obtained by finely pulverizing clinker produced by calcining to incipient fusion an intimate and proper proportioned mixture of argillacious and calcareous materials, with no additions subsequent to calcination excepting water and calcined or uncalcined gypsum.

I. The following limits of composition shall not be exceeded:

Per cent

Loss on ignition

Sulphuric anhydride (SO)

Magnesia (MgO)

II. Its specific gravity shall be not less than 3.10 . Should the test of cement as received fall below this requirement a second test may be made upon an ignited sample. The specific gravity test will not be made unless specifically ordered.

III. The residue on a standard No. 200 sieve shall not exceed 22 per cent by weight.

IV. A pot of neat cement shall remain firm and hard, and show no signs of distortion, cracking, checking, or disintegration in the strain test for soundness.

V. The cement shall not develop initial set in less than 45 minutes when the Vicat needle is used or 60 minutes when the Gillinous needle is used. Final set shall be attained within 10 hours.

VI. The average tensile strength in pounds per square inch of not less than three standard mortar briquettes composed of 1 part cement and 3 parts standard sand by weight shall be equal to or higher than the following:

\begin{tabular}{c|c|r}
\hline $\begin{array}{c}\text { Age at } \\
\text { test } \\
\text { days }\end{array}$ & Storage of briquettes & $\begin{array}{r}\text { Tensile } \\
\text { strength }\end{array}$ \\
\hline & & Lbs./in.2 \\
200 \\
300
\end{tabular}

VII. The average tensile strength of standard mortar at 28 days shall be higher than the strength at 7 days.

(b) The cement shall be delivered in suitable bags or barrels with the brand and name of the manufacturer plainly marked thereon, unless shipped in bulk. A bag shall contain 94 pounds net, a barrel shall contain 376 pounds net. The cement shall be stored in such manner as to permit easy access for proper inspection and identification of each shipment, and in a suitable weather-tight building which will protect the cement from dampness.

(c) Every facility shall be provided the purchaser for careful sampling and inspection at either the mill or at the site of the work, as may be specified by the purchaser. At least 10 days from the time of sampling shall be allowed for the completion of the 7-day test, and at least 31 days shall be allowed for the completion of the 28-day test. The cement shall be tested in accordance with the method hereinafter prescribed. The 28-day test shall be waived only when specifically so ordered.

(d) The cement may be rejected if it fails to meet any of the requirements of these specifications. Cement shall not be rejected on account of failure to meet the fineness requirement if upon retest after drying at $100^{\circ} \mathrm{C}$. for one hour it meets this requirement. Cement failing to meet the test for soundness in strain may be accepted if it passes a retest, using a new sample at any time within 28 days thereafter. Packages varying more than 5 per cent from the specified weight may be rejected; and if the average weight of packages in any shipment, as shown by weighing 50 packages taken at random, is less than that specified, the entire shipment may be rejected.

SEc. 7. Water for concrete.-The water used in mixing the Portland cement concrete shall be reasonably clear, free from harmful amounts of oil, acid, alkali, or vegetable substance, and neither brackish, or salty.

SEc. 8. Sources of supply.-Approval of sources of supply of gravel, broken stone, mine tailings, broken slag, coarse aggregate, fine aggregate, and Portland cement under these specifications shall be obtained from the engineer prior to delivery of materials, and samples of each shall be submitted as directed by the engineer.

SEc. 9. Methods of testing.-Methods of testing shall be as follows:

(a) Per cent of wear of rock or slag, standard test D2 American Society for Testing Materials. (See 510, p. 182.) When testing slag 4,000 cc loose measure of the size specified in the standard test shall be used.

(b) Weight per cubic foot of broken slag, standard method C29, American Society for Testing Materials. (See 512.10 , p. 212.)

(c) Screen tests of gravel, broken stone, mine tailings, broken slag, coarse aggregate, and fine aggregate, standard method D18, American Society for Testing Materials. (See 512.15, p. 250.)

(d) Sieve test of fine aggregate, standard method D7, American Society for Testing Materials. (See 512.12 , p. 234 .)

(c) Mortar tensile strength test of fine aggregate, A. S. T. M. C9 (see 516.11, p. 294) (tension tests). Preliminary acceptance samples shall be subjected to both 7 and 28 day tests and acceptance based thereon. Samples tested during progress of the work will be accepted on the basis of the 7-day test. (f) All tests for Portland cement, A. S. T. M. C9. National Paving Brick Manufacturers Association, recommended specifications for integral brick curb, 1924.

Material.-Vitrified paving brick used for integral brick curb shall be of the same quality as those used in the balance of the pavement.

Mortar in which the brick are set and which is used to fill the joints shall be made of 1 part Portland cement to 1 part fine aggregate, conforming to the requirements for these materials outlined under artificial bases, cement concrete. (See 518.21, p. 369.)

Constructing curb.-As the concrete base is constructed, and before the same has been allowed to set, a layer of 1 to 1 mortar shall be spread over the base a sufficient distance from the edge of the base course to provide a mortar bed in which the brick are immediately set and the joints promptly filled with mortar.

The brick shall be laid with its 4 -inch face as its depth, and unless otherwise specified they shall be laid crosswise of the pavement as headers.

When the pavement proper is to be constructed of brick 3 inches in depth, no appreciable amount of 
mortar is to be put under the brick to avoid making the bedding course immediately adjacent to the brick curb more than 1 inch in depth.

When the pavement proper is to be constructed of brick $3 \frac{1}{2}$ or 4 inches in depth, the thickness of the mortar bed in which the brick are set should be such as to provide a bedding course at least one-half inch in depth immediately adjacent to the brick curb.

The surface of the brick pavement proper when constructed is brought even with the surface of the brick curb.

Protection.-The same protection must be afforded the integral brick curb as is provided for concrete base afier its construction.

National Paving Brick Manufacturers Association, recommended specifications, concrete curb and header, 1924.

(This specification includes the following requirements for materials:)

Materials and mixing.-The concrete for the curb shall be composed of materials and be mixed as specified for concrete base course (see 518.21, p. 369) except that the coarse aggregate shall be of such maximum size that all will pass $3 / 4$-inch circular openings in a revolving screen. It shall be thoroughly mixed in the proportions of 1 part cement, 2 parts fine aggregate, and 4 parts coarse aggregate. Concrete shall be deposited in the forms, thoroughly spaded and settled to insure a uniform contact of mortar with the forms, with a nilm of mortar flushed to the surface. The upper face of the curb shall then be finished smooth and even. The upper inside edge of the curb shall be finished to a radius of $1 \frac{1}{2}$ inches. Upon removing the forms, the exposed portion of the curb shall be finished to make smooth any roughened condition.

Ileaders. - Where concrete headers are used at the ends of the pavement and at intersecting roadways, they shall be of the width and depth shown on the plans and specifications. Concrete shall be in the proportions of 1 part cement, 3 parts fine aggregate, and 6 parts coarse aggregate.

National Paving Brick Manufacturers Association, recommended specifications, integral concrete curb, 1924 .

Material, size, and mixing.-Integral concrete curb shall be built at the same time, and of the same materials, mixed in similar proportions, as the concrete base, and shall not exceed 6 inches in width on the surface.

Cement, coarse aggregate, fine aggregate, water and mixing thereof, shall conform to the specifications for concrete base.

Forms.-Forms used to retain the concrete curb shall be of wood or metal. They shall be straight. and free from warp or bending. Forms shall be held securely in position, true to line and grade, by stakes and clamps, with the ends closely joining.

Forms shall not defiect from position more than $1 / 4$ inch.

They shall be cleaned thoroughly and oiled with mineral oil immediately before placing conerete against them. They shall be staked and clamped not less than 50 feet in advance of placing concrete. Forms shall remain in place not less than 48 hours.

Placing concrete.-Concrete for the curb shall be placed so that curb and base proceed simultaneously. Concrete shall be thoroughly consolidated within the forms for the entire depth of the curb. The exposed top surface of the integral curb shall be given a smooth finish.

Protection.-The same protection must be afforded the integral concrete curb as is provided for concrete base after its construction. Integral curb shall be protected so that it shall not be chipped or injured in any way prior to laying the brick.
Hational Paving Brick Manufacturers Association, recommended specifications, stone curb and header, 1924.

Quality, size, and finish.-The curbing shall be of the best quality sandstone, limestone, or granite as indicated on the plan and bidding form, homogeneous and free from cracks, seams, and other defects, and shall not be more than 8 feet or less than 4 feet in length. The stone shall be - inches in width on the top after dressing, not less than inches in depth, and not less than the same width on the base as is specified for the top.

The stone shall be dressed at least 10 inches down on the face and ends and 3 inches down on the back.

Radial curb shall be not less than 5 feet in length, conforming to the dimensions and dressed as for straight curb to the radius shown on the plans, and with the ends cut radially.

The joints shall not exceed $1 / 4$ inch for a distance of 12 inches from the top, but may be wider from this point to the bottom.

Setting.- The curbing shall be set on edge to the line and grade given, prior to the completion of the grading. Both the bed and the backing shall be thoroughly tamped with heavy tampers to the full depth of the stone.

Headers.-Headers when shown on the plans at the ends of the pavement or at intersections with unpaved streets, or at intersections with streets paved with a dissimilar material, shall be set to the grade of the pavement surface. Stone for this purpose shall have a minimum width of 4 inches, a deptli sufficient to extend to the bottom of the base course, and shall be not less than 3 feet in length.

\subsection{Gutters.}

American Concrete Institute, tentative specifications for concrete curb and concrete curb and gutter, serial designation S-6E-27T, 1927.

See 518.63 , p. 441 .

Asphalt Association, specification $\mathrm{C}-3$, for shoulders, headers, curbs, and gutters (tentative), September 1, 1920.

See 518.63 , p. 446.

518.65 Catch Basins, Iniets, Ifanholes, Etc.

American Concrete Institute, standard specifications for concrete sewer, manhole, and catch-basin block, serial designation P-1C-29, 1929.

\section{GENERA亡}

1. The purpose of these specifications is to define the requirements for solid concrete manhole and catch-basin block.

2. The word "concrete" shall be understood to to mean Portland cement concrete.

3. The ultimate compressive strength of solid concrete manhole and catch-basin block at 28 days of age or when delivered upon construction work shall average not less then $1,500 \mathrm{lbs} . / \mathrm{in} .^{2}$ of crosssectional area of the unit as laid in the wall. No individual unit shall test less than 1,200 lbs./in. ${ }^{2}$ of cross-sectional area under the same conditions.

4. Concrete manhole and catch-basin block shall not absorb more than 8 per cent of the dry weight of the unit when tested as hereinafter specified. No individual unit shall absorb more than 10 per cent of the dry weight of the unit.

5. Specimens for tests shall be representative of the commercial product of the plant.

Not less than three and preferably five specimens shall be provided for each test.

6. Specimens used in the absorption test may be used for the strength test. 


\section{METHODS OF TESTING}

(Methods of testing for this specification are similar to those given in A. C. I. P-1B-26T (given above), with the following additions:)

1. In the absorption test, the average of the tests of not less than three specimens shall be taken as the absorption.

2 . In the strength test, when testing other than rectangular block or tile care must be taken to see that the load is applied through the center of gravity of the specimen.

3. In the strength test, in no case shall the distance between the edge of the spherical bearing block and the end of the bearing plate be greater than twice the thickness of the plate. Where a number of thin plates are used, in no case shall the plates be less than 1 inch thick nor shall any plate extend beyond the one immediately above it a greater distance than twice the thickness of the plate.

4. The average of the tests of strength of not less than three specimens shall be taken as the comparative strength.

American Society for Municipal Improvements, standard specifications for sewers, 1927.

\section{MATHOLES AND INLETS}

See 518.67 , page 464 .

\subsection{Retaining and Head Wails.}

American Association of State Highway Officials, standard specifications for bridges and incidental structures, 1928.

\section{RIPRAP}

See 511.72 , page 208

American Railway Engineering Association, specifications for stone masonry.

\section{BRIDGE AND RETAINING WALL MASONRY}

See 518.89 , page 479 .

\subsection{Sewers and Sewer Pipe. (See also 531.5.)}

American Concrete Institute, tentative specifications for plain concrete.sewer pipe, serial designation $\mathrm{P}-7 \mathrm{~A}-24 \mathrm{~T}, 1924$.

(This specification is practically identical with serial ciesignation C14, American Society for Testing Materials.) (See 518.67, p. 464. )

American Concrete Institute, tentative specifications for reinforced-concrete sewer pipe, serial designation P-7C-25T, 1925.

\section{GENERAL}

1. These specifications apply to reinforced-concrete pipe intended to be used for the conveyancy of sewage, industrial wastes and storm water. Pipe furnished under these specifications shall be of a single class as to strength.

Pipe may be made in either of two types $(a)$ fieldmade cast-concrete pipe or (b) shop-made mechanically-compacted pipe as hereinafter described.

2. The acceptability of pipe shall be determined by the purchaser; by the results of the load tests hereinafter specified, if and when required, and by inspection, to determine whether the pipe comply with the specincations as to dimensions, shape, and freedom from external and internal defects.

\section{IRATERIAIS}

3. Pipe shall be manufactured from concrete in which steel has been imbedded in such manner that the steel and concrete shall assist each other in taking stress.

$\mathrm{By}$ concrele is meant a suitable mixture of Portland cement, mineral aggregates, and water, hardened by hydraulic chemical action.

4. Portland cement shall meet the requirements of the standard specifications and tests for Portland cement (serial designation, C9) of the American Society for Testing Materials. (See 516.11, p. 294.)

5. Reinforeement may consist of wire or fabric which will meet the requirements of the tentative specifications for cold-drawn steel wire for concrete reinforcement (serial designation A82-21T) of the American Society for Testing Materials, or of rods and bars which meet the requirements of the standard specifications, for billet steel concrete reinforcement bars (serial designation A-15-15).

6. Fine aggregate shall consist of sand, stone screenings, or other inert materials with similar characteristics, or a combination thereof, having clean, hard, strong, durable uncoated grains and free from injurious amounts of dust, lumps, soft or fiaky particles, shale, alkali, organic matter, loam, or other deleterious substances. Fine aggregate shall be well graded and shall pass a 1/4-inch screen.

Coarse aggregate shall consist of crushed stone, gravel, slag, or other approved inert materials with similar characteristics or combinations thereof, having clean, hard, strong, durable, uncoated particles, free from injurious amounts of soft, friable, thin, elongated or laminated pieces, alkali, organic or other deleterious matter.

Where the shell thickness is 5 inches or less, coar'se aggregate shall be used which will pass a screen having 1 -inch openings. On $5^{1 / 2}$ to 9 inch shells, the stone may pass a screen having openings of $1 \frac{1}{2}$ inches.

7. The mix shall be such as to produce a pipe that will meet the physical test requirements hereinafter provided, but under no circumstances shall the mixture consist of less than 1 part Portland cement to 2 parts fine and 4 parts coarse aggregate with a minimum amount of water to produce a workable mix.

\section{TABLE 1.-Minimum dimensions of field-cast} reinjorced concrete pipe

\begin{tabular}{|c|c|c|}
\hline $\begin{array}{l}\text { Interior diameter, } \\
\text { in inclues }\end{array}$ & $\begin{array}{l}\text { Shell } \\
\text { thick- } \\
\text { ness }\end{array}$ & $\begin{array}{l}\text { Total minimum cross-sectional area of } \\
\text { steel, square inch per linear foot of } \\
\text { pipe }\end{array}$ \\
\hline $\begin{array}{l}24-\cdots \\
27-\cdots \\
30--- \\
33-- \\
36-- \\
42-\cdots\end{array}$ & $\begin{array}{c}\text { Inches } \\
3 \\
3 \\
31 / 2 \\
33 / 4 \\
4 \\
412\end{array}$ & $\begin{array}{ll}1 \text { line } & \\
1 & \text { line } \\
1 & \text { line }\end{array}$ \\
\hline $\begin{array}{l}48-- \\
54-- \\
60-- \\
66-- \\
72--\end{array}$ & $\begin{array}{l}5 \\
51 / 2 \\
6 \\
61 / 2 \\
7\end{array}$ & 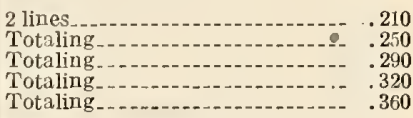 \\
\hline
\end{tabular}

\section{DESIGN}

8. Field-made cast concrete pipe shall be made with concrete having a consisteney which will permit the concrete to be placed in the forms and will require spading to properly work it around the reinforcement. The pipe may be made with wall thicknesses and steel quantities shown in Table 1 provided they meet all the strength and other requirements of these specifications. It is clearly understood that the wall thicknesses and quantities of steel given in this table are not specified wall thicknesses and steel quantities, but are minimums, 
and that manufacturers shall use greater shell thicknesses or greater steel quantities or both if necessary to meet all other requirements.

9. Shop-made mechanically compacted pipe must be manufactured in a facti:y and by a process which will automatically compact the concrete into the forms and around the reinforcement in such a manner as to uniformly obtain the maximum quality and strength for the lesser amount of concrete allowed.

The pipe may be madc with wall thicknesses and steel quantities shown in Table 2 provided they meet all the strength and other requirements of these specifications. It is clearly understood that the wall thicknesses and quantities of steel given in this table are not specified wall thicknesses and steel quantities, but are minimums and the manufacturers shall use greater shell thicknesses or greater steel quantities, or both, if necessary to meet all other requirements.

It is clearly understood that these requirements constitute factory conditions and, therefore, pipe shall not be considered as meeting these specifications unless made at a plant established for permanent operation.

TABLE 2.-Minimum dimensions of shop-made mechanically-compacted reinforced-concrete pipe

\begin{tabular}{|c|c|c|}
\hline $\begin{array}{l}\text { Interior diameter, } \\
\text { in inches }\end{array}$ & $\begin{array}{l}\text { Shell } \\
\text { thick- } \\
\text { ness }\end{array}$ & $\begin{array}{l}\text { Total minimum cross-sectional area of } \\
\text { steel, square inch per linear foot of } \\
\text { pipe }\end{array}$ \\
\hline 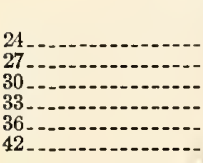 & $\begin{array}{r}\text { Inches } \\
21 / 2 \\
25 / 8 \\
23 / 4 \\
23 / 4 \\
3 \\
33 / 8\end{array}$ & 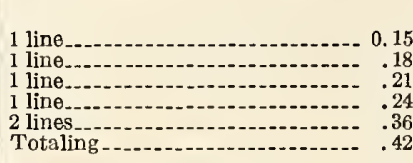 \\
\hline $\begin{array}{l}48 \\
54-\cdots \\
60 \\
66-\cdots \\
72 \\
72-\cdots\end{array}$ & $\begin{array}{l}33 / 4 \\
41 / 8 \\
41 / 2 \\
43 / 4 \\
5\end{array}$ & 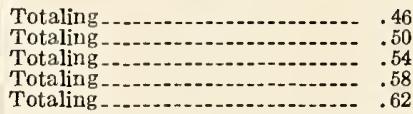 \\
\hline
\end{tabular}

10. The reinforcement shall extend throughout the barrel of the pipe. It shall be assembled into units so designed that they may be readily placed and maintained of true, exact shape and proper position within the pipe form during the manufacturing process.

11. The ends of reinforced-concrete pipe shall be so formed that when the pipes are laid together and the joints cemented, they shall make a continuous and uniform line of pipe with a smooth and regular interior surface. The joints shall be of such a design that when cemented they will prevent leakage and infiltration as well as appreciable irregularities in the flow line of the sewer.

12. Reinforcement shall bc placed not less than three-fourth inch nor more than $1 \frac{1 / 4}{4}$ inches from the inner surface of the pipe shcll when one line of steel is used and from either surface when two lines are used.

The reinforcement may be of clliptical form and placed in pipe near the inner surface on the vertical axis and near the outer surface on the horizontal axis of the pipe, providing all other requirements given in Tables 1 and 2 are complied with.

\section{WORKMANSHIP AND FINISH}

13. Pipe shall be substantially free from fractures, large or deep cracks, and surface roughness. The ends of pipe shall be square with their longitudinal axes.

14. Variations of the internal diameter shall not exceed 1.5 per cent. The shell thickness shall not be less than that intended in the design by more than 5 per cent at any point.

\section{MARKING}

15. When shipment of pipe is made in any manner other than direct from manufacturer to user, pipe shall bc so marked that the manufacturer of the pipe can be identificd. The date of manufacture shall be plainly marked on the pipe in all cases.

\section{IOAD TEST}

16. The test specimens shall be full-size pipe which will in every respect pass the inspection requirements hereinafter provided.

17. The specimens to be tested shall be selected by the purchaser or his representative at the point or points designated by him when placing the order. The manufacturer shall furnish, for testing purposes and at his own expense, one pipe of each size included in the order; the purchaser bearing all expense of testing such pipe. Sliould additional tests be made upon the demand of the purchaser or manufacturer, as hereinafter provided, then cost of such additional test specimens and the expense of testing shall be borne by the party making such demand.

18. Should the test specimens furnished by the manufacturer meet the test requirements, then all pipe represented by such specimens shall be accepted; provided, howcver, that the purchaser shall have the right to require an additional test in any size or sizes of pipc.

Should any of the specimens first tested fail to meet the test requirements, then the manufacturer shall have the right to submit an additional test of the size or sizes of pipe which have failed.

In either case, when an additional test is made in any size of pipe, such test shall consist of three pipes or multiple thereof, including the pipe first tested; if two-thirds of the pipe so tested shall meet the test requirements, then all pipe represented by such test shall be accepted, otherwise they shall be rejected.

\section{TEST METHODS}

The sand bearing and 3-edge bearing tests are used in this specification, and are substantially the same as given in sections 18 and 20, A. S. T. M. C-4. (See 534.21, p. 608.)

The test specimens shall show an ultimate strength not less than that given in Table 3 for the various sizes and methods of test stated and shall show no clearly visible crack, caused by the application of the load, extending the full length of pipe when tested to one-half the ultimate load designated in Table 3. Ultimate load is the point beyond which no additional load can be sustained.

TABLE 3.-Ultimate strength iest requirements of reinforced concrete sewer pipe at not less than 28 days

\begin{tabular}{|c|c|c|}
\hline \multirow{2}{*}{ Internal diameter of pipe, in inches } & \multicolumn{2}{|c|}{$\begin{array}{l}\text { Ultimate load in pound } \\
\text { per linear feet of pipe }\end{array}$} \\
\hline & $\begin{array}{c}\text { 3-edge bear- } \\
\text { ing }\end{array}$ & $\begin{array}{c}\text { Sand bear- } \\
\text { ing }\end{array}$ \\
\hline $\begin{array}{l}24 \\
27 \\
336 \\
42\end{array}$ & $\begin{array}{l}3,000 \\
3,300 \\
3,600 \\
3,900 \\
4,200 \\
4,700\end{array}$ & $\begin{array}{l}4,500 \\
4,950 \\
5,400 \\
5,850 \\
6,300 \\
7,050\end{array}$ \\
\hline $60^{4}$ & $\begin{array}{l}5,100 \\
5,500 \\
5,800 \\
6,000 \\
6,200\end{array}$ & $\begin{array}{l}7,650 \\
8,250 \\
8,700 \\
9,000 \\
9,300\end{array}$ \\
\hline
\end{tabular}

Note. - The load per foot of pipe shall be determined by dividing the total test load by the net inside length of the barrel of the pipe, measuring from the bottom of the socket to the end of the spigot. 


\section{INSPECTION}

26. All pipe shall be subject to inspection at the factory, trench, or other point of delivery by a competent inspector employed by the consumer or purchaser. The purpose of the inspection shall be to cull and reject pipe which, independent of the phrsical tests herein specified, fail to meet the requirements of these specifications.

27. Pipe shall be subject to rejection on account of any of the following:

(a) Variations in any dimensions exceeding the permissible variations given in section 13 .

(b) Fractures or cracks passing through the shell, except that cracks at either end of the pipe not extending beyond the joint nor fracture not extending beyond the joint nor extending more than 10 per cent around the circumference, will not be considered cause for rejection.

(c) Defects which indicate imperfect mixing and molding.

(d) Exposure of the reinforcement when such exposure mould indicate that the reinforcement is misplaced.

28. All rejected pipe shall be plainly marked by the inspector and shall be replaced by the manufacturer or seller with pipe which meets the requirements of these specifications, without additional cost to the consumer or purchaser.

American Concrete Institute, standard specifications for monolithic concrete sewers, serial designation S-3A-24, 1924.

iThe above specification includes the following requirements for materials.)

\section{CEMENT}

Secriox 1. All cement shall conform to the current specifications for Portland cement of the American Society for Testing Materials, and shall be tested in accordance with the methods of testing described in the specifications of that society. (See C9, 516.11, p. 294.)

\section{FINE $\triangle G G R E G A T E$}

SEC. 2. Fine aggregate shall consist of sand graded from fine to coarse and passing, when dry, a sereen having holes $1 / 4$ inch in diameter. It shall be clean, coarse, free from dirt, vegetable loam, or other deleterious matter. Not more than 6 per cent shall pass a sieve having 100 meshes per linear inch. ${ }^{79}$

SEc 3. Fine aggregate shall be of such quality that mortars composed of the proportions of cement and fine aggregate hereinafter specified for the various classes of concrete shall show a compressive strength after 14 days at least equal to the strength of mortar made of Portland cement and standard Ottawa sand in corresponding proportions and of the same consistency. ${ }^{80}$

\section{COARSE AGGREGATE}

SEc. 4. The coarse aggregate shall consist of crushed stone, gravel, or blast-furnace slag which is retained on a screen having 1/4-inch diameter holes and graded from the smallest to the largest parti-

it Crushed stone screenings may be permitted for use as fine aggregate provided that they shall comply with all the specifications of sections 2 and 3 , and further that they shall be produced from stone having a French coefficient of wear of not less than 8 , as described in A. S. T. M. D2. (See 510. p, 182.)

A It is recommended that, if possible, available fine aggregates be tested before awarding contracts. It if appears necessary to use an aggregate of poorer quality than above specified the proportion of cement in the various classes of concrete should he increased in order that the strength of the mortar actually used shall not be less than that with the Ottawa sand. If, however, the strength of the resulting mortars is less than 70 per cent of those with Ottawa sand, fine aggregate should be rejected entirely. cles. It shall be clean, hard, durable, free from all deleterious matter and soft, flat, or elongated particles. Crusher dust in sufficient quantity to weaken the concrete will not be permitted. For reinforcedconcrete arches or for plain concrete arches less than 6 inches in thickness, the maximum size of particles shall be such as will pass a screen having 1 -inch diameter holes. For inverts and plain concrete arches over 6 inches in thickness, the maximum size of particles shall be such as will pass a screen having $1 \frac{1}{2}$ inch diameter holes.

SEC. 5. Where crushed stone is used it shall have a French coefficient of wear of not less than 8, as described in A. S. T. M. D2. (See 510. p. 182.)

(The above paragraph is for use in locations where limestone or sandstone of a questionable value are common. If all available stone is suitable, the paragraph may be omitted.)

SEc. 6. Samples of not less than one-half cubic foot of fine aggregate and not less than 1 cubic foot of coarse aggregate shall be delivered in suitable boxes or containers. All samples shall be plainly labeled with the places where taken, where to be used, the date, and the name of the collector.

Sec. 7. For the purpose of determining proportions of materials for concrete, each bag of cement containing 94 pounds shall be considered as containing 1 cubic foot. Sand and coarse aggregate shall be measured loose in approved boxes or hoppers.

\section{WATER}

SEc. 8. Water used for concrete shall be free from oil, acid, alkalies, or organic matter.

\section{CONCRETE REINFORCEMENT BARS}

SEc. 9. All steel reinforcement shall consist of cold-drawn steel wire fabric having an elastic limit of not less than 55,000 lbs./in..$^{2}$; or of expanded metal having an elastic limit of not less than 55,000 lbs./in. ${ }^{2}$, and expanded cold from steel sheets; or of reinforcing bars.

SEc. 9. (a) Steel bars for reinforced-concrete sewers shall conform to the current specifications of the American Society for Testing Materials for (A) Billet Steel or (B) Rail Steel, except that rail steel bars may be used in sizes of 1 inch and under only, and hot-twisted bars will not be permitted.

SEc. 10. Dimensions of bars given on the plans are based on square sections. The net area and weights of bars shall not be less than 95 per cent of the values for square bars as indicated. In computing the weights of steel, 1 cubic inch of steel shall be regarded as 0.283 pounds.

SEC. 11. The quantity of metal to be paid for shall be the number of pounds actually placed, as shown on the drawings as ordered. It shall not include any waste metal due either to the nature of the construction or to the fact that the lengths supplied are too long or too short for their purpose.

The quantity paid for shall, however, include extra metal in laps, where authorized, due to the fact that a single bar would be unreasonably long.

All bars shall be of the length ordered and shall be in one piece where required up to 30 feet in length.

The compensations shall cover the cost of furnishing and delivering metal, including any royalty, the cutting, bending, placing, fastening in position, coating with cement, and all other work and materials connected therewith.

\section{CASTINGS}

SEc. 12. Circular cast-iron frames and covers for manholes and catch basins and any other iron castings shown on the drawings, or specified herein, 
necessary to complete the work, shall be furnished and placed.

SEC. 13. All castings shall be of tough, closegrained, gray iron, free from blowholes, shrinkage, and coldshuts. They shall be sound, smooth, clean, and free from blisters and all defects.

SEC. 14. All castings shall be made accurately to dimensions to be furnished and shall be planed where marked or where otherwise necessary to secure perfectly flat and true surfaces. Allowances shall be made in the patterns so that the thickness shall not be reduced. Manhole covers shall be true and shall seat at all points.

SEC. 15. All castings shall be thoroughly cleaned and painted before rusting begins, and before leaving the shop, with two coats of high-grade asphaltum or other suitable varnish that the engineer may direct. After the castings have been placed in a satisfactory manner, all foreign adhering substances shall be removed and the castings given two additional coats of asphaltum or other varnish as directed by the engineer.

SEc. 16. No casting shall be accepted the weight of which shall be less than that computed to its dimensions by more than 5 per cent.

\section{MATERIAL FOR IINING INVERTS}

SEc. 17. All vitrified brick shall be uniform in size, and be not less than 8 by 4 by 2 inches, nor more than 10 by $41 / 2$ by $21 / 2$ inches in length, width or thickness, respectively. The brick shall be free from lime or other impurities, uniformly vitrified and annealed, and shall have one edge face such that if the brick is laid on a horizontal plane on that face no portion thereof shall be more than one-eighth inch from the planc.

SEC. 18. Concrete block for sewer lining shall be uniform in size, not more than 18 by 12 inches in surface area and not less than 3 inches in thickness. They shall be made of class "A" or better concrete, as hereinafter specified, in satisfactory molds, and thoroughly cured. They shall have an ultimate compressive strength at 28 days of not less than $2,000 \mathrm{lbs}$. /in. ${ }^{2}$

SEC. 19. Tile liners for inverts shall not be more than 8 by 12 inches in surface area, and not less than 2 inches in thickness. The back of the tile shall be roughened and equipped with lugs or projections for bedding in mortar. They shall be manufactured under the general requirements covering vitrified sewer pipe and shall comply with the standard tests of the American Society for Testing Materials for clay sewor pipe in so far as applicable.

\section{Part II. CONCRETE FOR MONOLITHIC CONCRETE SEWERS}

SEc. 20. Concrete shall consist of a mixture of cement, fine and coarse aggregate, and water of the qualities hereinbefore specified.

Concrete shall be of three classes, proportioned as follows:

\begin{tabular}{r|r|r|r}
\hline Class & Cement & $\begin{array}{c}\text { Fine } \\
\text { aggregate }\end{array}$ & $\begin{array}{c}\text { Coarse } \\
\text { aggregate }\end{array}$ \\
\hline & Sack & Cubic feet & Cubic feet \\
\hline A & 1 & 2 & 4 \\
$2 \frac{1}{2}$ & 5 \\
\hline
\end{tabular}

\section{NIXING}

The relative proportions of fine and coarse aggregates may be modified at the direction of the engineer, provided that the proportions of cement to the total of the aggregates measured separately shall not be changed.

SEc. 21. Concrete shall be machine mixed. The concrete mixer shall be designed to take one completed batch of materials (using whole bags of cement) and to mix that batch thoroughly before any portion of it is withdrawn or any portion of the succeeding batch is introduced. The mixer shall be equipped with a tank so designed that when once set it will automatically supply to the mixer the amount of water so determined. The mixer shall be equipped with an instrument for measuring the time of mix.

Sec. 22. Concrete shall be mixed at least one minute after all the ingredients, including water, have been discharged into the mixer. Where the character of the work will permit, concrete shall be mixed in batches of one-half to 1 cubic yard, and the mixer speed shall not be less than 12 nor more than 19 revolutions per minute. Where small mixers are used, the spced shall not exceed 22 revolutions per minute.

SEc. 23. No concrete shall be hand mixed except relatively small quantities, and then only by special permission of the engineer.

SEc. 24. Where concrete is mixed by hand, the cement and fine aggregate shall be mixed dry on a properly constructed wooden or steel platform built for the purpose until it shall have obtained an even and uniform color throughout. The mixture shall then be spread to make a bed of uniform thickness, on which shall be spread the coarse aggregate, and the whole wet with the required amount of watel and turned with square-pointed shovels at least three times or until a uniform mixture is secured, water being added from time to time, if necessary.

\section{RUBBLE OR STONE IN CONCRETE}

Sec. 25. In all plain concrete, where the thickness is 15 inches or more, there may be embedded broken pieces of sound stone, the greatest dimension of which does not exceed 6 inches, and the least dimension of which is not less than three-quarters of the greatest dimension. These stones shall be set in the concrete as layers are being rammed, in a satisfactory manner, and so placed that each stone is completely and perfectly embedded. In general, there shall be a space of 4 inches between the stones and no stone shall come within 4 inches of any exposed face. The stone shall be thoroughly cleaned and wet before placing.

\section{CONSISTENCY}

Sec. 26. In mixing concrete, it is advisable to use the least possible amount of water required to obtain a workable mix, and when the aggiegate is dry, 6 gallons of water to a sack of Portland cement is the maximum which should be used. (For slag aggregate this may be somewhat increased.) Where comparatively dry mix is to be used, as in inverts, and near the crown of the arches, the concrete must be thoroughly tamped until the water flushes to the surface.

\section{WORI IN FREEZING WEATHER}

SEc. 27. Concrete shall not be mixed nor deposited in the work in freezing weather except as directed. If the work on concrete structures is prosecuted in cold weather, proper precautions shall be taken for removing ice and frost from the materials, including heating the water and aggregates; for protecting the newly laid masonry from freezing, and for securing work satisiactory in all respects. Satisfactory covering for the newly laid concrete and such additional appliances and materials as may be required therefor, including steam pipes for keeping the air warm beneath the said covering shall be provided. 
American Railway Engineering Association, specifications for buildings for railway purposes.

\section{SETERS AND DRAINAGE, 1926}

(The full text of this specification contains requirements for construction, and includes the following general conditions and requirements for materials:)

\section{GRNERAL}

The contractor shall furnish all materials and labor required to make the excavation and construct the sewers, manholes, catch basins, sewer connections to existing manholes, etc., as called for on the drawings.

\section{VITRIFIED SEWER PIPE}

The pipes and specials shall be of standard length and of the best quality of salt glazed vitrified double strength sewer pipe of the "hub and spigot pattern." The pipe shall be smooth, dense, and sound, thoroughly burned, impervious to moisture, free from laminations, cracks, flaws, blisters, or other imperfections. The interior surface shall be smooth and well glazed, and straight pipe shall be true cylinder and the interior diameter shall be the full specified dimension, the inner and outer surfaces shall be concentric. No pipe less than 6 inches shall be used except for downspout connections, or unless otherwise shown on the plans.

The pipe shall be subject to inspection and approval or rejection by the engineer.

\section{MORTAR}

Mortar for cementing the pipe joints shall be neat Portland cement or a mixture of equal parts of Portland cement and sand, as directed by the engineer, with only enough water added to give it the proper consistency. Mortar shall be mixed only as needed for use. The retempering of mortar that has become partly set will not be permitted.

Mortar for brickwork shall consist of Portland cement thoroughly mixed with sand, in the proportion by volume of 1 part loose cement and 3 parts sand.

CEMENT, SAND, STONE

Cement, sand, and stone shall be of the quality as specified in the A. R. E. A. specifications for conerete work. (See 516.3, p. 303.)

\section{BRICX}

The brick used shall be of the best quality, sound, and hard burned, uniform and free from lime and cracks and shall not absorb more than 15 per cent in weight after being thoroughly dried, when immersed in water for 24 hours, and samples must be approved by the engineer.

\section{MANHOLES AND CATCH BASINS}

Manholes and catch basins shall be built at the places shown on the plans or as otherwise directed by the engineer and shall be of the form and dimensions shown on the detailed drawings.

\section{CAST-IRON COVERS}

All covers shall be of tough gray iron, free from defects which would tend to weaken them, such as cold shuts or blowholes, and shall be absolutely clean and have a workmanlike finish. They shall conform to the standards as shown on the plans.

\section{CAST-IRON AND REINFORCED CONCRETE PIPE}

Cast-iron pipes and special castings shall be used where shown on the plans or as directed by the engi- neer. They shall be the bell and spigot type manufactured in accordance with the "Standard specifications for cast-iron pipe and special castings" of the American Water Works Association for class "A" pipe. The joints between cast-iron pipe and special castings shall be made in the usual manner. Reinforced concrete pipe of a design acceptable to the engineer shall be used if called for by the plans. Joints shall be made as specified for vitrified pipe.

\section{FOUNDATION DRAINS}

When the ground is wet or of a swampy nature drain or farm tile not less than 4 inches in diameter shall be placed along all foundations on a very slight grade at approximately the level of the footings. These drains shall be placed in a layer of clean, coarse gravel or broken stone not less than 1 foot thick, and shall be connected with the main drainage system.

\section{SPECIAL FITTINGS}

In case vitrified sewer pipe is to be paid for on a unit-price basis all "specials" including wyes, tees, bends, etc., will be figured as two lengths of straight pipe.

\section{GENIRAL CONDITIONS}

All materials entering into the work and all methods used by the contractor shall be subject to the approval of the engineer and no part of the work will be considered as finally accepted until all of the work is completed and accepted.

The general conditions as given in 518.50 , page 416 , shall be considered to apply to these specifications.

American Society for Municipal Improvements, standard specifications for sewers, 1927.

(This specification includes vitrified sewer pipe, cement concrete pipe, reinforced concrete pipe, concrete sewers built in place, brick sewers, segmental block sewers, and pipe sewers.)

\section{MATERIALS}

\section{GENERAL NOTE}

All materials shall be subject to the inspection of the engineer and to tests herein outlined. Any material failing to pass the required tests shall at once be removed from the work. Failure to condemn material on preliminary inspection shall not be grounds for aceeptance if future defects are found.

\section{CEMENT}

All cement shall conform to the current specifications for Portland cement of the American Society for Testing Materials, and shall be tested in accordance with the methods of testing described in the specifications of that society. (See C9, 516.11, p. 294.)

The engineer is to be notified of the location of wharves, yards, or sheds where the cement is to be stored. No cement will be tested in cars, in course of transportation, or on the street. All cement not satisfactory to the engineer is to be branded and immediately removed and all cement accepted by him to be so marked and kept in a weather-tight storehouse, having a tight floor a proper distance above the ground. A sufficient quantity shall be kept in storage to allow for 7-day tests, and if required, 28day tests. Cement kept in storage may be subjected to repeated tests. Cement in bags shall not be piled to a height exceeding 7 feet.

\section{HYDRATED LIME 81}

Hydrated lime shall conform to the current specifications for hydrated lime of the American Society

81 Hydrated lime is commercially divided into four classes, highcalcium, calcium, magnesian, and high-magnesian, and the particular class of lime desired should be specified in each case. 
for Testing Materials and shall be tested in accordance with the methods of testing described in the specifications of that society. (See C6, 517.2, p. 353.)

\section{SAND}

Sand shall be of the best quality locally obtained and shall be clean, coarse, free from dirt, vegetable loam, organic acids, or other deleterious matter. It shall contain not more than 4 per cent by volume of clay and no clay shall be artificially added.

Grading for mortar. - Sand for mortar shall be graded from fine to coarse and passing when dry a screen having 10 meshes per linear inch and not less than 94 per cent retained on a sieve having 100 meshes per linear inch.

Grading for fine aggregate for concrete.-Sand for fine aggregate for concrete shall be graded from fine to coarse and passing when dry a screen having holes $1 / 4$ inch in diameter and not less than 94 per cent retained on a sieve having 100 meshes per linear inch.

Tests.--Sand shall be of such quality that mortars composed of the proportions of cement and sand hereinafter specified for the various classes of concrete shall show a compressive strength after 14 days at least equal to the strength of mortar made of Portland cement and standard Ottawa sand in corresponding proportions and of the same consistency.

Samples.- Samples of not less than $1 / 2$ cubic foot of fine aggregate shall be delivered in suitable boxes or containers. All samples shall be plainly labeled with the places where taken, where to be used, the date, and the name of the collector.

\section{COARSE AGGREGATE}

Quality.-Coarse aggregate shall consist of crushed stone, gravel, or blast-furnace slag, which is retained on a screen having holes $1 / 4$ inch in diameter and graded from the smallest to the largest particles. It shall be sound, hard, and durable; be free from loam, clay, organic matter, and all deleterious material, and shall not contain any soft, flat, or elongated particles. Crushed dust in sufficient quantity to weaken the conerete will not be permitted.

Maximum sizes.-For plain arches less than 6 inches in thickness and reinforced arches where steel spacing is 2 inches or less from face, the maximum size of particles shall be such as will pass a screen having holes 1 inch in diameter. For inverts, plain arches over 6 inches in thickness, and reinforced arches where spacing of steel is greater than 2 inches, the maximum size of particles shall be such as will pass a screen having holes $1 \frac{1}{2}$ inches in diameter.

Samples. - Samples of not less than 1 cubic foot of coarse aggregate shall be delivered in suitable boxes or containers. All samples shall be plainly labeled with the place where taken, where to be used, the date, and name of the collector.

Tests.-Where crushed rock is used it shall have a specific gravity of not less than 2.6 and shall have a French coefficient of wear of not less than 8 . (See A. S. T. M. D2, 510, p. 182.)

\section{COMIMON BRICK}

Quality.-Common brick shall be sound, burnt hard entirely through, of compact texture, with true, even faces and rectangular edges. At least one edge face of each brick shall be such that if laid on a horizontal plane, no portion of that face shall be more than $1 / 8$ inch from that plane. Brick shall be regular in size and shape and for use on any one contract shall vary not more than $1 / 8$ inch in dimension.

Samples, tests, inspection.-Each shipment of between 12,000 and 20,000 brick shall be sampled. Each sample, at the discretion of the engineer, may be subjected to cross breaking and crushing tests under the three conditions given below, the brick to be tested fiat and any unevenness in the surface to be leveled up with a plaster of Paris bed:

(a) After having been thoroughly dried.

(b) After having been immersed in water for 24 hours.

(c) After being subjected to 20 freezings, alternating with 20 thawings.

The following figures fix minimum and average values to be met by the brick after the above treatment:

\begin{tabular}{|c|c|c|c|c|}
\hline & \multicolumn{2}{|c|}{$\begin{array}{l}\text { Modulus of rup- } \\
\text { ture, pounds } \\
\text { per square inch }\end{array}$} & \multicolumn{2}{|c|}{$\begin{array}{l}\text { Crushing } \\
\text { strength, } \\
\text { pounds per } \\
\text { square inch }\end{array}$} \\
\hline & $\begin{array}{l}\text { Mini- } \\
\text { mum }\end{array}$ & $\begin{array}{l}\text { Aver- } \\
\text { age }\end{array}$ & $\begin{array}{l}\text { Mini- } \\
\text { mum }\end{array}$ & $\begin{array}{l}\text { A ver- } \\
\text { age }\end{array}$ \\
\hline (a) Dried & $\begin{array}{l}350 \\
300\end{array}$ & 500 & 3,000 & 4,000 \\
\hline (c) Frozen and thawed & 225 & 275 & $\begin{array}{l}2,000 \\
2,000\end{array}$ & $\begin{array}{l}0,000 \\
2,500\end{array}$ \\
\hline
\end{tabular}

The absorption of the brick after immersion in water for 24 hours shall not exceed 15 per cent of the weight of the dry brick.

All brick must be satisfactory in every respect to the engineer and subject to his acceptance or rejection.

\section{VITRIFIED BRICK}

Quality.-Vitrified brick shall be sound, uniformly vitrified and annealed, be free from lime or other impurities with true, even faces and rectangular edges. At least one edge face shall be such that if laid on a horizontal plane, no portion of that face shall be more than $1 / 8$ inch from that plane. Brick shall be regular in size and shape and for use on any one contract shall vary not more than $1 / 8$ inch in dimensions.

Inspection.-All brick shall be satisfactory to the engineer and subject to his acceptance or rejection.

Samples.-Brick shall be sampled and submitted to a standard rattler test and shall not show a loss of more than 28 per cent. This test shall be made according to the method used in A. S. T. M. C 7 . (See 534.11, p. 577.)

Tests.-Brick, after having been thoroughly dried and then immersed in water for 24 hours, shall not absorb more than 4 per cent of their weight of water.

\section{VITRIFIED SEWER PIPE}

Quality.-Sewer pipe shall be of the best quality of standard hard burned, salt glazed, vitrified clay or shale pipe, shall be free from blisters, cracks, and pronounced laminations and must give a metallic ring when struck with a hammer- All pipes shall be socket pipes with sockets true, circular, and concentric with the base of the pipe and the plane of the ends at right angles to the axis of the pipe. The inner surface of the socket and the outer surface of the spigot end shall be scorcd by triangular shaped or semicircular rings about $1 / 8$ inch deep.

Sizes and dimensions.- (Same as A. S. T. M. serial designation $\mathrm{C} 14$, see 518.67 , p. 464 , for the sizes specified, which for this specification are sizes with internal diameters of $6,8,10,12,15,18,21,24$, $27,30,33$, and 36 inches.)

Specials.-(Substantially the same as A. S. T. M. C14, section 31 "Specials.")

Inspection.-(Substantially the same as A. S. T. M. C13. See 531.5, p. 517.)

Tests.-(Same as A. S. T. M. C14.)

\section{CEMENT CONCRETE PIPE}

Quality.-Cement concrete pipe shall be made of a suitable mixture of Portland cement, mineral aggregates, and water, and the materials used shall 
meet the requirements of these specifications. Pipe shall be free from fractures, large or deep cracks and blisters, laminations, and surface roughness. All pipe shall be socket pipe with sockets true, circular, and concentric with the base of the pipe.

(The requirements for this material under (1) Sizes and dimensions, (2) Specials, (3) Inspection (omitting requirements regarding glaze), and (4) Tests, are the same as for Vitrified sewer pipe (above).)

NOTE.-Sizes above 24 inches are not recommended.

\section{REINFORCED CONCRETE PIPE}

Size and shape.-Reinforced concrete pipe shall be made circular in cross section, and in sizes from 24 inches to 96 inches inside diameter. Diameters shall be as specified with a permissible variation of not more than three-fourths of 1 per cent. They shall be in sections not less than 3 feet in length and ends so formed that when laid together and cemented they shall make a continuous and uniform line of pipe.

Materials.-Pipe shall be made of class A concrete and reinforcement. All materials used shall meet the requirements for the different classes of materials used as prescribed in these specifications.

Forms. - All pipe shall be made in forms composed of sheet steel cores and casings, and cast-iron bottom and top rings which form the joint. The forms shall be rigidly held together and the core and casing so placed as to insure a uniform wall thickness.

Air curing.- The top rings, cores, and casings shall not be removed from the pipe until the concrete has obtained its final set. Pipe shall not be lifted from the bottom rings until the concrete is from 60 to $\mathbf{7 2}$ hours old. After the cores and casings have been removed form the pipe, they shall be kept constantly and thoroughly wetted by sprinkling with water at least three times a day until they are removed from the bases and yarded. After being placed in the yard the pipe shall be sprinkled thoroughly at least three times a day until they are six days old. Air-cured pipe are not to be laid until at least 14 days old.

Steam curing.- Pipe may be cured by the use of wet steam in the following manner. After the pipes have been cast they shall be covered with canvas or other material known as steaming jackets and wet steam be turned into these jackets for one day after casting. Then the casings and cores may be removed and steam again applied in the same manner for one day. After this has been done the pipes may be removed from the bases and yarded, no other curing being necessary. Steam-cured pipes may be laid when they are six days old.

Samples for testing.-Any or all of the following tests may be applied to samples selected by the engineer for the pipe delivered on the work. For the purpose of making such tests, the contractor shall furnish and deliver, when directed, five lengths of each size pipe used in the work.

Crushing tests.- When supported at the bottom upon a knife-edge 1 inch in width, in such a manner that an even bearing is provided throughout the whole length, exclusive of the bell, and load is applied at the crown uniformly through a similar knife-edge, the various sizes of pipe shall withstand, without fracture, the loads shown in the following table:

\begin{tabular}{|c|c|}
\hline $\begin{array}{l}\text { Diameter, in } \\
\text { inches }\end{array}$ & $\begin{array}{l}\text { Load per } \\
\text { linear foot }\end{array}$ \\
\hline $\begin{array}{l}24 \\
27 \\
30 \\
33 \\
36 \\
39 \\
42\end{array}$ & $\begin{array}{r}\text { Pounds } \\
2,149 \\
2,369 \\
2,583 \\
2,830 \\
3,080 \\
3,300 \\
3,521\end{array}$ \\
\hline
\end{tabular}

When supported on a sand saddle which extends the full length of the pipe, exclusive of the bell, and whose upper surface fits accurately the outer curved surface of the pipe, and whose width is equal to an arc of $15^{\circ}$, in such a manner that an even bearing is provided throughout the whole length, and the load is applied at the crown uniformly through a knifeedge 1 inch in width, the various sizes of pipe with diameters greater than 42 inclies shall withstand the following loads:

\begin{tabular}{|r|r|}
\hline $\begin{array}{c}\text { Diameter, in } \\
\text { inches }\end{array}$ & $\begin{array}{c}\text { Load per } \\
\text { linear foot }\end{array}$ \\
\hline & $\begin{array}{r}\text { Pounds } \\
5,700 \\
48 \\
54\end{array}$ \\
60 & 6,600 \\
66 & 7,500 \\
72 & 8,250 \\
78 & 9,000 \\
84 & 9,750 \\
90 & 10,500 \\
96 & 11,250 \\
& 12,000 \\
\hline
\end{tabular}

Absorption test.-The specimens for absorption test shall be sound pieces with all edges broken and may be from pipes broken in the crushing test. One specimen shall be taken from each pipe broken in the crushing test or it may be taken from other pipes. They shall be from 12 to 20 square inches in area and shall be as nearly square as they can be readily prepared. They shall be free from observable cracks, fissures, laminations or shattered edges. These specimens after being dried at a temperature of between $300^{\circ}$ and $325^{\circ} \mathrm{F}$. until there is no further loss of weight, cooled in a desiccator and then immersed in water for 25 hours shall not absorb more than 10 per cent of their weight in water.

\section{STEEL REINFORCEMENT}

Material.-All steel reinforcement shall consist of cold-drawn steel-wire fabric, conforming to the current specifications of the American Society for Testing Materials for "cold-drawn steel-wire for concrete reinforcement," or of expanded metal having an elastic limit of not less than $55,000 \mathrm{lbs}$./in.2 and expanded cold from sheets; or of reinforcing bars.

Bars.-Steel bars for reinforcing shall conform to the current specifications of the American Society for Testing Materials for (A) Billet Medium Steel, or (B) Rail Steel, except that rail steel bars may be used in sizes of 1 inch and under only, and hot twisted bars will not be permitted.

Weights.-Dimensions of bars given on plans are based on square sections. The area and weight of bars shall not be less than 95 per cent of the values for square bars as indicated. In computing the weights of steel, 1 cubic inch of steel shall be regarded as 0.283 of a pound.

\section{TWO-RING SEGMENTAL THE BLOCKS}

Quality.-All segmental blocks shall be of the best quality of vitrified clay ware, thoroughly burned, and true to form. They shall be made in two rings so that the blocks of one ring will interlock with those of the adjoining ring and the longitudinal joints of one ring will break joints with those of the other ring. The inner or wearing surface shall be evenly salt glazed and free from checks or fire cracks. All surfaces which will come in contact with the mortar in joints shall be scarified or grooved in order to secure a satisfactory bond.

The least allowable total thickness of material from the inside face of the sewer to the nearest opening in the block shall be $1 \frac{1}{2}$ inches for sewers 30 to 
42 inches in diameter, $13 / 4$ inches for sewers 45 to 54 inches in diameter and 2 inches for sewers 57 to 84 inches in diameter. The lengths of the blocks to be used on tangents shall not exceed 24 inches and on curves 12 inches. The thickness of the shell shall not be less than indicated in the following table:

\begin{tabular}{|c|c|}
\hline $\begin{array}{l}\text { Size, inside } \\
\text { diameter, in } \\
\text { inches }\end{array}$ & $\begin{array}{c}\text { Thickness } \\
\text { of shell }\end{array}$ \\
\hline $\begin{array}{l}30 \\
33 \\
36 \\
39 \\
42 \\
45 \\
48 \\
51 \\
54 \\
57 \\
60 \\
63 \\
66 \\
69 \\
72 \\
75 \\
78 \\
81 \\
84\end{array}$ & $\begin{array}{c}\text { Inches } \\
434 \\
434 \\
434 \\
51 / 2 \\
51 / 2 \\
51 / 2 \\
6 \\
6 \\
61 / 8 \\
618 \\
71 / 4 \\
71 / 4 \\
71 / 4 \\
71 / 4 \\
71 / 2 \\
71 / 2 \\
73 / 4 \\
73 / 4 \\
81 / 4\end{array}$ \\
\hline
\end{tabular}

Junctions.-Junctions for connections on segmental block sewers shall be made with $\mathrm{T}$ or $\mathrm{Y}$ branches. Where the size of the branch is such as to permit, it shall be burned on the block as an integral part thereof. Where the size if such that this can not be done, openings of the necessary size shall be left in the block, into which the necessary pipe or slant shall be inserted and tightly cemented in.

Tests.- If the design or material of the block is such as to cause the engineer to doubt its strength for the work, the contractor, when so directed, shall be required to make a loading test on a full-size section before it is used in the work. The sections so tested must bear without fracture the maximum load to which the finished sewer will be subjected.

\section{ONE-RING SEGMENTAL TILE BLOCKS}

Quality.-All blocks shall be of the best quality of vitrified clay ware, thoroughly burned and true to form and shape and shall be jointed with abutting webs or surfaces to form a backing for mortar so as to insure tight joints. The inner or wearing surface shall be evenly salt glazed and free from checks or fire eracks. The sides shall be of such design as to insure tight joints.

Thickness, weights, and lengths.-The maximum length of block shall be 24 inches, shorter lengths to be furnished where required for curves and specials. Blocks furnished on any one contract shall not vary more than 1/4-inch in width. The thickness of the shell shall not be less than indicated in the following table:

\begin{tabular}{|c|c|}
\hline $\begin{array}{l}\text { Size, inside } \\
\text { diameter, } \\
\text { in inches }\end{array}$ & $\begin{array}{c}\text { Thickness } \\
\text { of shell }\end{array}$ \\
\hline & Inches \\
\hline $30 \ldots \ldots$ & $\begin{array}{l}43 / 4 \\
43\end{array}$ \\
\hline $36 \ldots$ & $43 /$ \\
\hline $39 \ldots$ & $51 / 2$ \\
\hline $42 \ldots$ & $51 / 2$ \\
\hline $45 \ldots$ & $51 / 2$ \\
\hline $48-\ldots$ & 6 \\
\hline $51 \ldots$ & 6 \\
\hline $54 \ldots$ & $\begin{array}{l}6 \\
6\end{array}$ \\
\hline 60 & $6^{3} / 4$ \\
\hline $63 \ldots$ & $\begin{array}{l}63 \% \\
6^{3}\end{array}$ \\
\hline $69 \ldots$ & 7 \\
\hline & 7 \\
\hline 78 - & 8 \\
\hline & 8 \\
\hline
\end{tabular}

Junctions, inspection, lests.-The provisions of the paragraphs titled junctions, inspection, tests of these specifications for 2-ring segmental tile blocks shall apply similarly to 1-ring blocks.

\section{CASTINGS}

Quality.-All castings for manhole heads, covers, and other purposes must be of tough, gray iron free from cracks, holes, swells, and cold shuts. The quality shall be such that a blow from a hammer will produce an indentation on a rectangular edge of the casting without flaking the metal.

Workmanship.-All castings shall be made accurately to dimensions to be furnished and shall be planed where marked or where otherwise necessary to secure perfectly flat and true surfaces. Allowance shall be made in the patterns so that the thickness shall not be reduced.

No plugging, burning in, or filling will be allowed. Covers must fit the frames in any position. All castings shall be carefully coated, both inside and out, with coal-tar-pitch varnish. The varnish shall be made from a good cuality of coal tar, with sufficient oil added to make a smooth coating, tough and tenacious when cold, and not brittle nor with any tendency to scale off. The castings shall be evenly heated to a temperature of $300^{\circ} \mathrm{F}$. immediately before being dipped and shall possess not less then this temperature when put in the vat; they shall remain in the vat five minutes unless otherwise directed by the engineer. The varnish shall be heated to a, temperature of $300^{\circ} \mathrm{F}$., unless otherwise directed by the engineer, and shall be maintained at this temperature during the time the casting is immersed. Fresh pitch and oil shall be added when necessary to keep the mixture at the proper consistency, and the vat shall be emptied of its contents and refilled with fresh material when considered necessary by the engineer. After being coated the casting shall be carefully drained of all surplus varnish. No casting shall be allowed to become wet before being dipped. Any casting that is to be recoated shall first be thoroughly scraped and cleaned.

\section{WROUGHT IRON}

Quality.-All wrought iron to be used on the work shall be of the class known as "Merchant iron, grade A," and must be free from cinder spots, injurious flaws, buckels, blisters, or cracks. Wrought iron shall be made by the puddling process or rolled from fagots or piles made from wrought-iron scrap, alone or with muck bar added. All iron shall have a minimum tensile strength of $50,000 \mathrm{lbs}$./in. ${ }^{2}$ an elastic limit of at least $25,000 \mathrm{lbs}$./in. ${ }^{2}$. A specimen cut from the iron when nicked on one side and bent back through an angle of $180^{\circ}$ shall show a long, clean, silky fiber, free from slag, dirt, or coarse crystalline spots.

Finishing painting.-Wrought iron before leaving the factory shall be thoroughly cleaned and dipped in a preparation of asphalt or coal tar and oil as described under "Castings."

\section{LUMBER}

Lumber shall be straight and sound, free from shakes, cracks, large or loose knots, and other defects impairing its strength or durability. It shall be squared to the required dimensions throughout its entire length. Matched and grooved sheeting shall have one planed side.

\section{WOOD PILES}

All piles shall be composed of straight, sound, live timber, free from cracks, shakes, or rotten knots, cut from mixed oaks, maple, elm, birch, 
Norway pine, yellow pine, tamarack, cypress, Douglas fir, spruce, or other timber satisfactory to the engineer. At no point shall the pile deviate more than one-half of its diameter from a straight line drawn between its ends.

Each pile must show an even and gradual taper between the ends and must be free from sudden and excessive enlargement at the butt. Branches and knots must be neatly trimmed.

The diameters at the butt and small ends for different length piles must not be less than shown on the following table. All diameter dimensions are to be measured exclusive of the bark.

\begin{tabular}{|c|c|c|}
\hline Length below cut-off, in feet & $\begin{array}{l}\text { Minimum } \\
\text { diamcter } \\
\text { at point }\end{array}$ & $\begin{array}{l}\text { Mini- } \\
\text { mum } \\
\text { diameter } \\
\text { at butt }\end{array}$ \\
\hline $\begin{array}{l}\text { Less than } 20 \\
20 \text { to } 25 \\
26 \text { to } 35 \\
36 \text { to } 45 \\
46 \text { and } \text { orer. }\end{array}$ & $\begin{array}{r}\text { Inches } \\
6 \\
6 \\
8 \\
8 \\
8\end{array}$ & $\begin{array}{r}\text { Inches } \\
10 \\
11 \\
12 \\
13 \\
11\end{array}$ \\
\hline
\end{tabular}

\section{WATER}

Water used for concrete or mortar shall be clean and fresh and be free from oil, acid, alkalies, or organic matter.

\section{JOINING COMPOONDS}

Joining compounds shall have preferably a bituminous base, shall adhere firmly to the glazed surfaces of the pipes, shall melt and run freely at a temperature as low as $250^{\circ} \mathrm{F}$., and when set shall be sufficiently elastic to permit of a slight morement of the pipes without injury to the joints or breaking the adhesion of the compound to the pipes. The compound shall not deteriorate when submerged in fresh or salt water or normal domestic sewage. It shall show no deterioration of any kind when immersed for a period of five days in a 1 per cent solution of hydrochloric acid or a 5 per cent solution of caustic potash.

\section{Part II. METHODS OF CONSTRUCTION}

\section{EXCAVATTON}

Classification.-All exeavation shall consist of three classifications, namely, class A, class B, and class C.

Class A excavation defined-Class A excavation shall comprise solid rock in the original bed or welldefined ledges and which can only be removed by continuous blasting and all boulders or detached pieces of rock 8 cubic feet or more in content. ${ }^{62}$

Class $B$ excavation defined.--Class $B$ excavation shall comprise all shale, soapstone, slate, hardpan, fire clay, cemented gravel, macadam, or telford parements, brick masonry, except in pavements, and detached pieces of rock less than 8 cubic feet or more than 1 cubic foot in content.

Class $C$ excavation defined.-Class $\mathrm{C}$ excavation shall consist of all materials not mentioned in class

${ }^{82}$ In localities where limestones, which might vary by imperceptible degrees from shales and other stones, oceur, the Chicago definition of rock is considered valuable. "Rock shall mean any material geologically in place, cemented into a mass by natural causes, and of geologically in place, cemented into a mass by natural causes, and of cr greater in the scale of mineral hardness. Frozen material shall not be classified as rock."
$A$ and class $B$ excavation or in the paragraph entitled "Removing and replacing parements." 83 A. Work included in excavation.-Under the headings class $A$, class $B$, and class $C$ excavation is included the work of removing all obstructions on the surface of streets, alleys, rights of way, and public places; of making all necessary excavations not provided for in the paragraph headed "Removing and replacing pavements"; of furnishing and putting in place all shoring and bracing necessary to excarate and protect the work; of doing all pumping and bailing necessary to keep the trenches free from water; of providing for the uninterrupted flow of sewers and surface water and the temporary disposal of water from all other sources during the progress of the work; of protecting all pipes, conduits, culrerts, street-car and railroad tracks, poles, wires, fences, buildings, and other public and private property along the field of operation; of removing after completion of the work all shoring and bracing not necessary to support the sides of the trenches; of hauling away all surplus excavation and material; of doing all back filling; of restoring all property injury to which is contemplated in this contract; and of restoring the surface and curbing of all streets, alleys, and rights of way to as good condition as they were prior to the beginning of excavation, excepting where such work is included under the item "Removing and replacing pavements."

5B. Work included in excavation. - Subject to the provisions of section $6 \mathrm{~B}$, the prices named under all items requiring excavation are to include all the work specified in $5 \mathrm{~A}$.

6A. Payment.-Payment for class A, class B, and class $C$ excavation shall be made at the respective prices bid per cubic yard, and the amount shall be full payment for all riork done in connection with class A, class B, and class $C$ excavation as described in section $5 \mathrm{~A}$.

6B. Payment for class $A$ and class $B$ excavation.Payment for class A and class $B$ excavation shall be made at the respective prices bid per cubic vard, which prices shall be in addition to the amounts paid per linear foot of completed sewer.

7. Open cut and tunnels.-In general, excavation shall be made in open cut from the surface and the contractor shall not be allowed to do any tunneling without obtaining permission from the engineer, and then only according to methods approved by him. This permission will only be given where a line is to be laid behind the curb, across a paved street, under street-car or railroad tracks, or where, in the opinion of the engineer, it is necessary to tunnel short sections on account of proximity of adjacent walls or structures. Such excavation then can be made in alternate sections of open cut and tunnel, the length of the tunnel sections to be specified by the engineer, and the head room in them to be not less than 3 feet measured from the top of the sewer. These tunnel sections shall be cut underneath to a wedge with its edge horizontally across the sewer and back filled tightly by tamping from each end.

It is the intention of the A.S. M. I. committee in the above class ification of excavation to include all classes of exeavation which there is a reasonable possibility of encountering. In localities where quicksand is known to occur and can be defined its inclusion as a definito item in the contract is recommended. Quicksand may be defined as a material the satisfactory excavation of which requires the use of close-sheeting driven well below sewer re or the use of well points. close-sheeting driven we we In this instance the reuld to include the cast ef leaving in seme up to the and the furnishing and operation of well points, if used. If quicksend classfication is inserted it
be mentioned in subsequent clause with classifications listed. 
NorE.-The above paragraph is only intended for short, isolated tunnels and should not be used for a regular tunneling specification. It was not considered feasible to write a general tunnel specification because of the large variation of local practice and of materials encountered. In localities where tunnel practice has not been well developed it is recommended that experts in tunneling be retained to advise in the preparation of tunnel plans and specifications.

8. Excavation above spring line of sewers built in place. - All excavations above spring line of sewers built in place will be estimated and paid for as a trench with vertical sides, the distance between which shall be 1 foot greater than the outside horizontal dimensions of the masonry at the spring line. ${ }^{84}$

9. Class $B$ and class $C$ excavation below spring line of sewers built in place.-Class B and class C excavation below spring line of sewers built in place shall be taken out and will be computed to the shape and outside dimensions of the masonry, except in cases of special sections where the width of masonry is greater below than at spring line. In this case the trench line described in the preceding paragraph shall apply until it coincides with the outside of the masonry.

10. Class $A$ excavation and concrete refill below spring line of sewers built in place.-Class A excavation below spring line of sewers built in place shall be made so as to conform as nearly as possible to the outside shape of the sewer wall, and a smooth bed made for the masonry by filling the uneven surface of the rock with class $\mathrm{C}$ concrete or rubble masonry, this refill being carried up to the spring line whether above the rock surface or not. With monolithic concrete sewers, concrete of the same class as the sewer walls may, at the option of the engineer, be substituted for the class $\mathrm{C}$ in the concrete refill. The amount of class A excavation to be paid for below the spring line will be the area of a segment of a circle with its chord at the surface of the rock and a radius $41 / 2$ inches larger than the radius of the outside of the sewer section. The amount of the concrete refill to be paid for will be the difference in area of two semicircles whose radii are, respectively, equal to and $41 / 2$ inches greater than the outside of the sewer section. The outside of the sewer section for the monolithic concrete section shall be considered as the outside of a regular concrete section; of the brick sewer as the outside of a 1-ring brick sewer; of the 1-ring segmental block as the outside of a full block section; and of the 2-ring segmental block as a semicircle having a radius $21 / 2$ inches larger than the inside radius of the sewer, the outer block being omitted in the latter type of sewer and the excess space filled with mortar. ${ }^{85}$

11A. Class $B$ and class $C$ excavation for vitrified and premolded pipe.-Class $B$ and class $C$ excavation for vitrified tile and premolded concrete or cement pipe will be estimated as a trench with vertical sides, a length equal to the distance from $\mathrm{c}$ to $\mathrm{c}$ of manholes and a width 12 inches greater than the inside diameter of the pipe for pipe up to and including 18 inches; 15 inches greater than inside diameter for pipes 21 to 27 , inclusive, and 18 inches greater for pipes 30 inches larger, provided that no trench shall have a width of less than 30 inches. The depth of trench shall include the thickness of the pipe. Where rock is encountered in the trench the width for class B and class $C$ overlying excavation shall be the same as in section 12 .

11B. Same as $11 \mathrm{~A}$, omitting all reference to class $\mathrm{C}$ excavation.

12. Class $A$ excavation for vitrified and premolded pipe.-Class A excavation for vitrified tile and premolded concrete or cement pipe will be estimated as described in the preceding paragraph, except that

${ }^{84}$ For firm soils not requiring bracing or for rock easily shot to line the width may be made equal to the width of masonry. For section of irregular shape pay lines should be shown on the plans.

88 For shaves of inverts other than circular pay lines for excavation should be shown on plans. the width and depth of trenches in rock excavation hall be increased 4 inches.

13. Excavation for manholes.-Excavation for manholes will be considered as being included in the excavation for the sewer, unless the maximum outside width of manhole masonry is greater than the pay-line width of trench, in which case the excavation necessary outside the pay lines will be estimated and paid for.

14. Underdrains.-Pipes for underdrains are to be laid below the sewer where directed by the engineer. They are to be laid with open joints, the joints wrapped in one thickness of muslin, the pipes bedded in gravel or broken stone and the refilling material placed solidly about them. T's for plugging the underdrains are to be turned up in the invert at such intervals as may be directed and the underdrains carefully plugged upon completion of the work. Excavation shall be made to the depth and width necessary and its area of cross section computed as a rectangular cross section. The trench shall be refilled with material specified by the engineer, which material shall be computed and paid for as the difference in area of the cross section of excavation and the outside cross section of the drain pipe. Pipe will be paid for by the linear foot at the price bid.

15. Unusual ground conditions.-Where saturated ground is encountered and when it is the opinion of the engineer that such ground can not be controlled by the ordinary methods of bracing, the engineer will instruct that other forms of bracing or sheeting be used. The excess in cost of such bracing or sheeting used over ordinary methods of bracing or shoring will be estimated and paid to the contractor, this amount to be the actual cost as determined by the engineer plus 15 per cent. ${ }^{86}$

16. Excavation below grade.-If, in the opinion of the engineer, the material at or below grade line is of improper nature for a foundation it is to be removed to such depths and widths and in such a manner as he may direct. Such excavation shall be refilled to grade with such materials as the engineer may direct and confined in place by poling boards or vertical sheeting in a manner satisfactory to the engineer. Such excavation and materials used for refill will be estimated and paid for at the respective prices bid.

17. Disposal of material.-Excavated material shall be so placed as not to unreasonably interfere with travel on the streets by the occupants of adjoining property or with access to fire hydrants. Such surplus excavation as is suitable shall be hauled and spread as directed on such property as is noted on the plans. Where directed, macadam, telford, gravel, street surfacing, surface loam, sod, etc., shall be kept separate from the remainder of the excavated material and replaced in its original position after back filling of the trench. All surplus excavation not disposed of as stated above shall be removed from the site of the work by the contractor, but none shall be deposited on private property until written consent of the owner or owners thereof has been filed with the engineer. As before stated, all costs of disposal of surplus excavated material shall be included in prices bid requiring excavation.

18. Fences and lights. - The contractor shall provide suitable fences around trenches and street obstructions and shall provide red cautionary lights at night and watchmen when necessary.

19. Ownership of excavated material.-Underground structures removed, such as brick, sewer

${ }^{86}$ In making plans for any sewer job the engineer should, if he anticipates encountering quicksand, saturated ground, etc., show on his plans and in his specifications exactly how he proposes to take care of it and cover the cost of the work by the necessary bid items. The above clause is only intended to apply in case such conditions are encountered unexpectedly during the progress of the work. 
pipe, etc., shall become the property of the contractor unless otherwise noted on the plans. Where noted on the plans such structures are to be carefully removed, separated into convenient units for handling and conveniently placed for removal by the city.

20. Bracing and shoring.-The sides of all trenches shall be securely held by bracing and sheathing which, if not ordered left in, may be removed in units when the lerel of the back fill has reached their elevation. When, in the opinion of the engineer, sheeting or shoring can not be safely removed it shall be left in place, or, if he so desires, the sheeting shall be cut and the upper part withdrawn. The contractor shall be paid for the lumber so left in place or withdrawn at the prices bid, but no payment will be made for sheeting cut off and remored in excess of 8 feet in length of which not more than 2 fect shall be above the surface. Timber left in place shall be cut off at least 2 feet below the surface. Paymert for matched and grooved sheeting will be made in each case for the length of trench occupied by such sheeting, with no allowance for wastage due to tongues and splines, respectively. In the case of timber no thickness of sheeting over 2 inches and no other lumber exceeding in size that customarily used shall be paid for unless the use of such large sizes shall have been ordered in writing by the engineer.

21. Temporary bridges, crossings.-The contractor shall, where ordered, construct bridges, crossings, etc., the surfaces of the bridges being flush with the street. The bridges shall be capable of supporting at any point a wagonload of 3 tons on each wheel or its equivalent without inducing fiber stresses in beams greater than 1,000 pounds for wood and 16,000 for steel per square inch. Bridges or crossings so ordered will be paid for by the square foot of floor area, according to the dimensions ordered put in by the engineer.

22. Restoration of surface of ground.-During the construction of the sewer all work of leeping alleys and rights of way in a passable condition by refilling sunken ditches, laying temporary paving over trenches, removing surplus material, etc., shall be done in a manner satisfactory to the engineer. If any such work is not done within five days after written notice given by the engineer the work may be done by the city and the expense thereof be paid by the contractor or deducted from sums due the contractor under this contract.

23. Amount of trench.- Without the written permission of the engineer, not more than 200 feet of trench shall be opened in advance of the completed sewer and in rock work not less than 30 feet in advance of the completed sewer.

\section{REMOVING AND REPLACING PAVEMENTS}

24. Work included.-Under the heading "Removing and replacing pavement" is included the work of tearing up such areas of pavement other than macadam or telford as may be required for the construction of sewers therein, of placing a temporary pavement to carry traffic until such time as the permanent pavement may be laid, and of permanently repaving these areas after the back fill has settled.

25. Temporary pavement.-In placing the temporary parement the back fill shall be compacted or flushed as directed up to the level of the pavement subgrade, after which the remainder shall be filled with crushed stone or gravel bonded with limestone screenings and compacted as far as possible without requiring the use of a roller. This temporary pavement may be crowned, at the discretion of the engineer, but in no case shall this crown be more than $3 / 4$ inch in height for each foot of width of trench. The contractor shall maintain this temporary pavement in condition adequate for its usual traffic until such time as it is replaced by the permanent pavement and shall pay any claims or damages arising from his neglect to maintain the temporary roadway.

26. Permanent pavement.-The permanent pavement shall be laid at such time as the engineer may permit, but in no case in less than six months after the trench was back filled nor more than one year after the temporary parement was laid. The contractor shall replace the permanent pavement during the above-stated period upon receipt of notice from the engineer. Such replacement must be made under city inspection and in accordance with the city standard specifications for the kind of pavement to be laid.

27. Payment. - The contractor will be paid for work done under this paragraph by the square yard at the price bid for removing and replacing pavements, and the amount to be paid for shall be determined by the length of pavement actually disturbed with a width 2 feet greater than the pay-line width of the trench. If a greater width of pavement shall have become displaced, the contractor shall satisfactorily restore such at his own expense.

\section{PIIING}

28. Driving.-Piles of the quality specified under Part I of these specifications are to be furnished and driven by the contractor as shown on plans or as directed by the engineer. They are to be replaced by the contractor at his own expense where broken in driving or where driven off line. Piles are not to be driven until the trench has been excavated to the grade line.

They must be properly pointed before being driven and the butt protected by an iron ring to prevent brooming. They must be driven to sufficient depth to insure proper bearing power, the necessary penetration to be determined by the engineer.

29. Cut-off.-Each pile is to be cut off by the contractor exactly level, so that the cap or masonry which rests on it will have a firm and even bearing.

30. Caps.-Pile caps, if used, are to be of the dimensions shown on the plans or as directed by the engineer, and are to be securcly fastened to the piles by oak-tree nails or by bolts and washers.

31. Payment.-In determining the length of new pile to be paid for the part sawed off is not to be deducted unless it exceeds 2 feet in length, in which case all but 2 feet of the part sawed off is to be deducted. Caps, if used, will be paid for by the board foot at the price bid, which shall include the cost of fastenings.

\section{CONCRETE}

32. Definition.-Whenever the term concrete is used herein it is understood to mean an intimate and homogeneous mixture of cement, sand, and coarse aggregate, with sufficient water.

33. Proportions.-Concrete shall be of four classes, proportioned as follows:

\begin{tabular}{|c|c|c|c|}
\hline Class & $\begin{array}{l}\text { Cement } \\
\text { sacks }\end{array}$ & Sand & $\begin{array}{c}\text { Coarse } \\
\text { aggregate }\end{array}$ \\
\hline $\begin{array}{l}\mathrm{A} \\
\mathrm{B} \\
\mathrm{B}\end{array}$ & $\begin{array}{l}1 \\
1 \\
1 \\
i\end{array}$ & $\begin{array}{r}\text { Cubic feet } \\
2 \\
21 / 2 \\
3 \\
41 / 2\end{array}$ & $\begin{array}{r}\text { Cubic feet } \\
4 \\
5 \\
6 \\
9\end{array}$ \\
\hline
\end{tabular}

In the case of concrete used in sufficiently large masses and of such consistency as to allow of packing without settlement bowlders not exceeding 
$1 / 2$ cubic foot in volume may, at the option of the engineer, be placed therein by hand in such manner that each stone is surrounded by not less than 4 inches of concrete. Bowlders so used will be paid for by volume, 2,000 pounds of bowlders being considered as being equivalent to $1 / 2$ cubic yard. The volume of bowlders shall be deducted from the total volume of concrete, the remainder being the amount of concrete which will be paid for ${ }^{87}$

34. Mixing by machine.-The concrete mixer shall be designed to take one complete batch of materials (using whole bags of cement) and to mix that batch thoroughly before any portion of it is withdrawn or any proportion of the succeeding batch is introduced. The mixer shall be equipped with a tank so designed that when once set it will automatically supply to the mixer the amount of water so determined. The mixer shall be equipped with an instrument for measuring the time of the mix. Concrete shall be mixed at least one minute after all the ingredients, including water, have been discharged into the mixer. Where the character of the work will permit concrete shall be mixed in batches of $1 / 2$ to 1 cubic yard and the mixer speed shall be not less than 12 nor more than 19 revolutions per ininute. Where small mixers are used the speed shall not exceed 22 revolutions per minute.

35. Hand mixing.-Where concrete is mixed by hand the cement and sand shall be mixed dry on a properly constructed wooden or steel platiorm until it shall have an even and uniform color throughout. This mixture shall then be spread to make a bed of uniform thickness, on which shall be spread the coarse aggregaie and the whole wet with the required amount of water and turned with square-pointed shovels until a uniform mixture is secured, water being added from time to time if necessary.

36. Consistency.- In mixing concrete it is advisable to use the least amount of water reouired to obtain a workable mix, and when the aggregate is dry 6 gallons of water to a sack of Portland cement is the maximum which should be used. Where a comparatively dry mix is to be used, as in inverts and near the crown of arches, the concrete must be thoroughly tamped until the water flushes to the suriface.

37. Work in freezing weather. - When the temperature falls below $32^{\circ}$ F. all the materials which enter into the construction of masonry of any kind are to be thoroughly heated and used while hot. For brick masonry and pipe joining this is to be done by heating the brick and sand and the water with which the mortar is used. For concrete masonry it is to be done by heating the aggregates and water with which the concrete is mixed. As soon as the mortar or masonry is placed it is to be protected from frost, either by back filling or by covering with hay, straw, manure, or some similar substance to a depth of at least 1 foot. A covering of canvas or boards will not be considered sufficient, but where steam is available and it is practicable to cover all exposed masonry with canvas and introduce steam underneath the canvas it will be deemed a sufficient protection to do so. When the temperature falls to or below $20^{\circ} \mathrm{F}$. no mason work of any kind is to be done except by special permission of the engineer and strictly according to his directions, provided that masonry may be laid in trenches 12 feet or more in depth and width not exceeding one-third the depth.

${ }_{87}$ For concrete sewer projects of considerable magnitude, where foilities arc available for making compression tests of concrete the A S J I comited recommends a study of the Report of the Joint Committee on Concrete and Reinforced Concrete. (See 516.3, p.317.)

\section{CONCRETE SEWERS BUIT IN PLACE}

38. Line and grade.-Concrete sewers shall be constructed to the alignment, grade, and dimensions indicated on the plans or as designated by the engineer.

Unless otherwise specified or indicated on the plans, they shall be constructed with class A concrete.

39. Machine and hand mixing.-Concrete shall be machine mixed, except in emergencies or on isolated small jobs, when liand mixing may be allowed upon express permission from the engineer. Both machine and hand mixing shall be done as specified under concrete.

40. Transporting concrete-Provision shall be made for quickly transporting the concrete from the mixer to the work with as little shaking as possible, so that the tendency of water to rise to the top may be reduced to a minimum. Any concrete which may have been compacted during transportation shall be mixed before being placed in the work. Any concrete delayed more than one-half hour in transit shall not be used in the work.

41. Placing concrete.-Concrete shall be deposited so as to maintain a nearly level surface and avoid flowing along the forms. It shall be continuously and sufficiently worked to expel air and to force the aggregate away from the forms. In special cases where concrete is to be deposited on slopes a comparatively dry mix may be used, but great care shall be exercised to spread such concrete evenly in layers not more than 6 inches in thickness and to ram it thoroughly. In general, the methods used shall be such as to give a compact, dense, and impervious concrete with a smooth surface.

42. Construction joints. - No horizontal construction joints shall be made except at the places shown on the plans. Joints between succecding sections of the sewer shail be made vertically. All joints shall be provided with dovetails or keyways for bonding with next section.

43. Joining new work to old.- When new work is joined to that already in place the contact surfaces of the old concrete shall be thoroughly cleaned, using a stiff wire brush, and, if necessary, a stream of water, and shall be clean and wet at the moment the fresh concrete is placed. A thick wash of rich mortar shall be run over the contact surface of the old conciete. When consicered necessary by the engineer, the first 1 or 2 inches of the abutting concrete shall be picked oft so as to remove the laitance or the old cement shall be washed ofi with acid or alkali and then with water to remove all traces of them.

44. Finish of concrete surfaces.-Special care shall be taken that all inside concrete surfaces shall be smooth and free from indentations or projections. All surfaces shall be free from voids, exposed stones, and other imperfections. If such imperfections are found upon removing the forms the fault shall be corrected at the contractor's expense by immediately filling with mortar or otherwise as directed.

45. Plastering of concrete surfaces. - No plastering of any concrete surface shall be done unless expressly permitted, and if so permitted shall be made in strict accordance with directions. No payment will be made for plastering done to correct defective work.

46. Masonry in water.-No concrete or other masonry shall be deposited under water without permission, and then only in accordance with directions. Water shall not be permitted to rise on any masonry unitil it shall have set at least 12 hours.

47. Drying concreie.-Precautions shall be taken to prevent concrete from drying until there is no danger of cracking from lack of moisture. Con- 
crete shall be kept moist for at least one week, unless sooner covered with earth. This may be done by a covering of wet sand, burlap, continuous sprinkling, or by other methods approved by the engineer.

45. Forms.-There shall be provided suitable collapsible centers or forms with smooth surfaces of ample strength and rigidly braced. The bracing shall be adequate to prevent deviations from the correct hines. All steel forms shali be reatly and accurately made with all similar parts in each longitudinal section of form interchangeable with other sections. Bent plates required to fit shall be rolled and fabricated to the correct eurves before assembling. Suitable forms shall be provided for bends in the semer. Steel filler plates shall be furnished. All wooden forms shall be built of clean, sound lumber, reasonably free from knots, dressed on all sides, and neatly ñted. Tongued-and-groove material shall be used where required. The form surface shall be water-tight, securely fastened to the ribs or supports. No forms built up in the trench or ribs mith separate pieces of mooden lagging will be allowed except for specials or eurves. No center or form shall be used which is not clean and of proper shape and strength and in every way suitable. Before placing concrete or reinforcement the forms shall be coated with lubricants to prevent adherence of concrete. ${ }^{85}$

49. Removal of arch forms.-Arch forms shall not be slackened until the back filling has been carried to a height of at least 1 foot above the top of the arch and tamped. The contractor shall be responsible for all damage caused by premature removal of forms.

50. Back flling.-Back fill over and around arch sewers shall be placed as soon as possible after concrete has set. The filling up to the plane of the top of arch shall be made from the best earth and shall not contain a sufficient amount of stones to allow the pieces to become wedged. This part of the fill shall be made in layers of not over 6 inches and carefully tamped. If the remainder of the back fill is dumped from buckets the contents of the buckets shall not be allowed to fall directly on the sewer but shail be deposited on existing back fill and allowed to slide into the trench unless the impact is broken by timber grillage. Bracing should generally be removed only when the trench below it has become substantially filied, and every precaution shall be taken to prevent any large slips of earth from the side of the trench onto or against the green arch.

51. Reinforcement.-Steel bars of the dimensions shown for the reinforcement of concrete semers shall be put in place as shown on plans. Bars shall be bent without beating to the shapes shown and shall be placed in exact positions and with the spacings shown. They shall be securely fastened so as to prevent displacement during the placing of concrete. The engineer reserves the right to order the use of special fastenings in order to hold the reinforcement rigidly in place. All bars where spliced are to have a lap of at least forty times their diameter.

52. Construction of inverts. - On all sections having a comparatively flat invert the complete invert shall be built first, while on all circular sections a center strip of not less than one-fourth the circumference shall be built. The invert or center strip shall be placed in sections of not orer 16 feet where the surface is to be finished with end guides and a longitudinal straight edge and not more than 20 feet where a separate lining of vitrified brick or tile is to be used.

ss Ordinary crude petroleum oil makes a satisiaciory lubricant for forms and is the one incst commonly used.
53. Finish of unlined inverts.-The concrete for the invert is to be laid in the trench as one operation and the invert surface formed by the use of end guides and straightedges and floated and troweled to true lines and cross section. Within two hours after the concrete is laid a dry mix of 2 parts of Portland cement and 1 part of sand, free from crusher dust and particles larger than $1 / 8$ inch, shall be sprinkled over it, floated, and troweled. This treatment shall be repeated at least once and as often as necessary to obtain a smooth impervious finish.

51. Vitirified brick lining.-The concrete bottom shall be shaped up to a hine $1 / 2$ inch below the bottom of the brick hining and allowed to set before bricklaying commences. Brick shall be laid by skilled bricklayers, using mortar of the quality as hereinafter speciffed under the heading "Mortar." Brick shall be laid on edge as stretchers, to lines stretched between end guides for each three courses of brick and so that end joints are broken by a lap of at least 2 inches. Mortar joints shall not exceed $1 / 4$ inch in thickness and shall be carefully struck as soon as each length of section is completed, and all loose mortar shall be carefully removed. No brick shall be laid loose and subsequently grouted.

55. Vitrificd tile invert lining for inverts.- Vitrified tile for lining inverts shall be of the same quality as specified for segmental blocks. They shall be not more than 12 by 18 inches in surface area and not less than 1 inch in thickness. The back of the tile shall be roughened and equipped with lugs or projections for bedding the mortar. The tile shall be carefully bedded in wet mortar, the mortar bed to be approximately one-half inch in thickness. Blocks shall be laid in limes stretched between end guides for each course of block to be laid. Each block must have an even bearing over its entire surface and the finished surface shall be smooth and even and conform accurately with the shape of the invert as shown on the plans. ${ }^{89}$

56. Hydrated lime.-Hydrated lime may be added to concrete inix to increase its workability, at the option of the engineer, and in the amounts ordered by him. Hydrated lime so used will be estimated and paid for by the ton, which price shall include the entire cost of furnishing, mixing, and placing, and shall be in addition to the payment for concrete or concrete semers. 80

$57 \mathrm{~A}$. Payment.-Concrete shall be estimated in place and paid for by the cubic yard at the price bid, which price shall be full payment for all the cost of furnishing, mixing, placing, protecting, and pointing of all concrete, and of furnishing, placing, and removing all centering and form work necessary.

57B. Payment.-Concrete sewers will be paid for by the linear foot of completed sewer, this price to include all labor material and all ordinary excavation necessary to build the sewer in accordance with the plans and specifications. In making the classification the depth of the sewer shall be taken as the distance from the surface of the ground to the invert.

58A. Steed bars for reinforcement will be estimated and paid for by the pound at the respective prices bid for reinforcing steel bars, which prices shall be full payment for furnishing, protecting, bending, and placing all steel bars, and of furnishing and placing all wires, clips, chairs, or other devices to be used for securing the bars. Payment will be made for all laps used for splices on transverse bars as shown on the plans. On longitudinal bars the contractor will be paid for laps according to length of sections ordered put in.

89 The above three methods of finishing invert are given complete. Any one may be used as preferred.

$80^{\circ}$ Hydrated lime may be used to advantage in increasing the workability of concrete. Published tests indicate that it slightly reduces strength. 
59A. Invert finishing and invert lining will be estimated and paid for by the square foot at the respective prices bid, which prices shall be full payment for the finished invert or finished lining complete. These prices shall be in addition to the amount paid for concrete in place contained in the invert.

\section{MORTAR}

60. Composition.-Mortar for brick or scgmental block masonry shall be made of 1 part Portland cement and 3 parts of sand. Mortar for pipc joints shall be made of 1 part of Portland cement and 1 part sand. The sand and ccment shall be of the quality specificd in Part I, and shall bc measured by volume, 94 pounds of cement to be used as 1 cubic foot.

61. Manner of preparation.-For either class of mortar the sand and cement shall be thoroughly mixed dry in proper boxes and such quantity of clcan water added as will produce a stiff mortar of the proper consistency. The whole mass shall be thoroughly worked with proper tools.

62. Hydrated lime.-Hydrated lime may be added to the mortar mix to increase its workability and paid for in the manncr hereinbcfore specified under concrete.

\section{BRICK IRASONRT}

63. Quality.-All brick masonry, whether in sewcrs, manholes, inlets, or other structures, shall be made of brick and mortar of the quality hereinbefore specified and laid in a careful and workmanlike manner by skilled bricklayers.

64. Wetting.-All common brick shall be thoroughly wettcd before bcing laid.

65. Freezing weather.-The provisions of the paragraph entitled "Work in freezing weather," under the heading Concrete, shall apply to brick masonry.

66A. Measurement and payment.-All brick masonry will be measured and paid for by the cubic foot at the price bid, which price shall be full payment for masonry in placc, including plaster coat where specified. Wliere dimensions are not shown on plans onc ring will be estimated as $4 \frac{1}{2}$ inches, two rings or wall masoniy one brick length in thickness as 9 inches, thrce rings or wall masonry $1 \frac{1}{2}$ brick lengths in thickness as 13 inches, four rings or wall masonry two brick lengths in thickness as 18 inches.

\section{BRICK SEWERS}

67. Line and grade-Brick scwers shall be so constructed of the form and dimensions indicated on the plans and shall be built to the lines and grades given by the engineer

68. Laying brick.-Brick shall be laid by placing sufficient mortar on the bed and forcing the brick into it at one operation. All bricks in each course shall be strctchers and shall break joints with those in adjoining courscs, except at spring line, where corners may be bonded with headers. No brick shall be laid loose and subsequently grouted.

69. Racking courses.-All unfinished brickwork must be racked back in courses and the mortar removed from the exposed surface of the bricks before it has had time to harden. When new work is joined to old the surface of the bricks where joints are to be made must be washed and cleaned.

70. Brick laterals.-When two brick sewcrs intersect the junction shall be constructed as a plain intersection of the two barrcls and no bell-mouth arch built unless specifically shown on the plans. Great carc shall be taken to obtain first-class brickwork on the two arches near the intersection and to trowel the inside surface wherever clipped brick have been used.

71. Pipe laterals and slants--Pipe laterals and slants shall be built into the brickwork wherever shown on the plans or designated by the engineer. In all cases the laterals shall enter the sewer at an angle of at least $45^{\circ}$. Where slants can not be used the pipe shall be neatly clipped to conform to the inside face of the sewer and the edges neatly plastered with cement mortar. In no case will jagged edges be allowed to project into the barrel of the sewer.

72A. Payment. - Brick masonry will be estimated and paid for as specified in section $66 \mathrm{~A}$, no deductions being made for manhole openings or gaps in the wall for junctions and slants.

72B. Payment.-Brick sewers will be paid for by the linear foot of completed sewer, which price shall include all labor and material amd all ordinary earth excavation necessary to build the sewer in accordance with the plans and specifications. In making the classifications the depth of the sewer shall be taken as the distance from the surfacc of the ground to the invert, the length as the distance from center to center of manholes.

\section{CONSTRDCTION OF INVERTS OF BRICK SEWERS}

73. Profiles.-In building inverts a profile of the same dimensions as the brickwork shall be securely fixed at the forward end of the section and a line stretched between nails spaced around its inner surface and corresponding points on the completed invert for cvery three courses.

74. Joints. - The mortar joints on the inside ring shall not exceed $1 / 4$ inch in thickness. In the outer ring, a thickness of $1 / 4$ to $1 / 2$ inch will be permitted.

75. Striking joints and cleaning.-All joints on the inside of the sewer shall be carefully struck as soon as the section of invert is completed and the loose mortar removed from the sewer.

76. Water in trench.-Water which may collect in the trcnch ahead of the completed invert shall not be bailed up and thrown on the brickwork nor allowed to overflow onto it until at least eight hours after laying.

\section{CONSTROCTION OF ARCHES OF BRICK SEWERS}

77. Centers. - The arch shall be built on strongly built centers, the working face of which shall be of the samc dimensions as the arch to be constructed. The lagging shall be planed on one face and two edges, laid on the templates in a tight and workmanlike manner,

78. Cleaning at spring line.-Before the centers are placed all earth or loose mortar shall be carefully scraped from the spring line of the completed invert, and if at any time before the arch is started loose material falls from the side walls it shall be thoroughly cleaned off before brick are laid.

79. Joints. - Where centers are to be pulled while the mortar is still green the longitudinal joints shall bc made as thin as it is practicable to make a full joint, and in no case greater than one-fourth inch in thickness.

80. Plastering.-The exterior surface of the arch shall be covered with a coating of mortar not less than three-eighths inch thick.

81. Drawing centers. - When a section of arch is completed the entire space between the curved masonry surface and the sides of the trench shall be filled with carth placed in 6-inch layers, each thoroughly rammed to a height level with the extrados of the brick arch. When this has been done to the satisfaction of the engineer, and when the spring line of the invert ahead has been cleaned as provided in the paragraph styled "Cleaning at spring line," the center may bc loosened and drawn ahead. They shall be scraped clean immediately upon drawing.

82. Striling joints and cleaning.-As soon as the centcr has been drawn before bricklaying is recommenced the inside joints of the arch just completed 
shall be carefully rubbed full and struck to give a smooth interior surface, and the loose mortar from this work, together with any other rubbish, immediately removed from the sewer.

\section{SEGMENTAI BLOCE SEWERS}

83. Line and grade.-Segmental block sewers shall be constructed of the form and dimensions shown and shall be built to the line and grade given by the engineer. ${ }^{01}$

84. Joints.- Segmental blocks shall be laid so that all joints are staggered, using short blocks where necessary. Care shall be taken that all joints are filled with mortar, those on the inside of the sewer not to exceed one-fourth inch in thickness. On the 2-ring blocks the joints between the inner and outer rings shall be formed by placing the last ring to be laid on a bed of mortar spread on the ring in place and tamping or squeezing the block into place. In order to insure that all space between blocks is filled, it will be necessary to spread an excess amount of mortar, the excess being squeezed out as the block is pushed into place. With 1-ring blocks the same procedure shall be taken in closing the ship-lap joint between blocks. With either kind of block the blocks shall be so bedded that there are no offsets between blocks and that the inside face of the sewer shall present a smooth straight surface.

85. New and old work.-When joining new work to that already in place the surface of the block where joints are to be made must be washed and cleaned.

86. Mortar.-Mortar for segmental block scwers shall be of the quality as specified under the heading "Mortar."

87. $T$ and $Y$ junctions shall be installed as shown on plans or as designated by the engineer, and when not to be immediately used shall be closed with earthenware covers held lightly in place by mortar. Payment will be made at the price bid, which shall be in addition to the payment for sewers to which these specials are attached, and shall include the cost of covers.

88. Reducers.-Gradual reducers of a length of at least 10 feet shall be built wherever the size is changed. These reducers shall be built of brick masonry or concrete, according to the specifications for those classes of sewers, and will be paid for according to the prices bid for the items used.

89A. Measurement and payment.-Segmental block sewers will be paid for by the linear foot of completed sewer, and the length of the particular size to be paid for shall be the actual invert length of that size laid, which price shall be full payment for segmental block sewers in place complete. On 2-ring work, where the outer ring is omitted in the invert, the sewer will be paid for at threefourths the price bid for full section.

89B. Measurement and payment.-Segmental block sewers will be paid for by the linear foot of completed sewer, which price sha!l include all labor and material and all ordinary earth excavation necessary to build the sewer in accordance with the plans and specifications. In making the classification the depth of the sewer shall be taken as the distance from the surface of the ground to the invert, the length as the distance from center to center of manholes.

CONSTRUCTION OF INVERTS OF SEGMEITAL BLOCK SEWERS

90. Templets.-In building inverts a templet of the same dimensions as the section of the sewer

91 Segmental tile is a comparatively new material for sewer construction. It can usually be used to advantage in competition with other sewer materials. Several types are availahle. The engineer considering the use of this material should carefully investigate its applicability to the particular conditions as well as the various types, their safe bearing load, and facility for construction as compared to other standard materials. shall be securely fixed at the forward end of the section and a line stretched between rails spaced around its inner surface and corresponding points on the completed invert for each course of block to be laid.

91. Bearing.-In laying the outer ring of the invert care should be taken to obtain a solid and even bearing for each block and concrete or mortar used when necessary to smooth up the bed.

92. Inverts in rock.-On 2-ring work, where solid rock is encountered in the invert, the outer block may be omitted and the inner block with mortar and concrete backing used. The class $\mathrm{C}$ concrete backing shall be accurately brought to a line $2 \frac{1}{2}$ inches from the inside face of the block. Mortar of the proper consistency shall then be spread on this concrete backing and the block brought to line by pressing into the mortar bed.

93. Striking joints and cleaning.-All joints on the inside of the sewer shall be rubbed full and jointed as soon as a section of the invert is completed and all loose mortar shall be removed from the sewer.

94. Water in trench.-Water which may collect in the trench ahead of the completed invert shall not be bailed up and thrown on the completed invert nor allowed to overflow onto it until at least eight hours after laying.

\section{CONSTRUCTION OF ARCHES OF SEGMENTAL BLOCK SEWERS}

95. The provisions of the paragraph titled "Centers, cleaning at spring line, drawing centers, and striking joints and cleaning," under the heading "Construction of arches of brick sewers," shall apply similarly to the construction of arches of segmental block sewers. ${ }^{22}$

96. Exterior joints.-The exterior joints of the arch shall be filled with mortar before any back filling is done.

\section{PIPE SEWERS}

97. Grade.-The grade as shown on the profile is that of the inner side of the invert and to which the work must conform. A variation of one-half inch from this will be deemed sufficient reason to cause the work to be rejected and rebuilt. The engineer will set all stakes for lines and grades, which the contractor must maintain and keep uncovered, so that they can be examined at any time. The grade shall be kept by line or ranging rods, as the engineer shall direct. Such ranging rods, poles, levels, straightedges, etc., as are required for the work will be furnished by the contractor.

98. Shaping trench bottom.-The bottom of the trench shall be shaped so as to conform as nearly as possible to the outside of the pipe, particular care being taken to recess the bottom of the trench in such manner as to relieve the bell of all load. In rock the excess excavation shall be refilled to subgrade with $3 / 4$ inch gravel or class D concrete, thoroughly tamped and shaped to fit the bottom of the pipe so that at least the lower third of the pipe is evenly supported throughout its entire length. Gravel or class D concrete used for this purpose will be paid for at the prices bid, the amount to be paid for being the same as the amount of class A excavation below subgrade.

99. Laying.-Each pipe shall be laid to the line and grade given by the engineer and in such a manner as to form a close concentric joint with the adjoining pipe and prevent sudden offsets of flow line. All pipes shall be laid with bells uphill. The subgrade must be kept free from water while pipes

92 For loadings which might exceed the strength of segmental block arches the strength can be materially and cheaply incressed by partially filling the haunch with concrete instead of earth. 
are being laid. Joints shall be made in the manner specified in the following two paragraphs.

100. Cement joints.-A closely twisted hemp or oakum gasket of such diameter as required to support the spigot of the pipe at the proper flow-line grade and make truly concentric joints, and in one piece of sufficient length to pass around the pipe and lap at the top, shall be thoroughly saturated in coment mortar. This gasket shall be laid in the bell for the lower third of the circumference of the joint and covered with mortar specified for pipe joining. The spigot of the pipe shall then be inserted and carefully driven home, after which a small amount of mortar shall be inserted in the annular space around the entire circumference of the pipe. The ends of the gasket shall then be wrapped around the pipe and solidly rammed into the joint with a calking tool, the mortar previously placed being driven ahead of the gasket. The remainder of the joint shall then be completely filled with mortar and beveled off to an angle of $45^{\circ}$ with the outside of the pipe. On pipes of 18 inches or larger in diameter, the joints shall be pointed and smoothed from the inside.

101. Hoi poured joints.-Joints shall be carefully centered and callked as specified in the preceding paragraph. A suitable runner then shall be placed and the compound, heated to a temperature of approximately $400^{\circ} \mathrm{F}$., shall be poured into it in such a manner that the annular space shall be completely filled. When bituminous joints are used it will be permissible to make alternate joints before lowering pipe into trenches. ${ }^{95}$

102. Cleaning. - The interior of the sewer shall, as the work progresses, be cleared of all dirt, cement, and superfluous materials of every description. On small sewers, where cleaning after laying may be difficult, a swab or drag shall be kept in the pipe line and pulled forward past each joint immediately after its completion.

103. Back filling.-Before the usual back filling is commenced, ine earth shall be tamped around and above the pipe to a depth of 1 foot above the extrados. No walking over the pipe will be allowed until 1 foot of back fill is complete. Care shall be taken to prevent any disturbance to the pipe or damage to newly made joints.

104. Junctions. - T or $\mathrm{Y}$ junctions shall be installed at the places shown on plans or designated by the engineer, and when not to be immediately used shall be closed with earthenware covers held tightly in place by mortar. Payment will be made at the price bid, which shall be in addition to amount paid per linear foot of sewer, and shall include the cost of covers.

105A. Measurement and payment.-Pipe sewers will be paid for by the linear foot of completed sewer, the length of the particular size to be paid for shall be the actual length of that size laid. This price shall be full payment for furnishing pipe and joining materials and incorporating them into a completed sewer as specified above.

105E. Measurement and payment.-Pipe sewers will be paid for by the linear foot of completed sewer, which price shall include all labor and materials and all ordinary earth excavation necessary to build the sewer in accordance with the plans and specifications. In making the classification the depth of the sewer shall be taken as the distance from the surface of the ground to the invert, the length as the distance from center to center of manholes.

63 For combined sewers and sanitary sewers in dry ground, cement joints are considered satisfaetory. For sanitary sewers below the ground water table, bituminous joints are preferred. The use of a concrete cradle in the latter ease may sometimes be advisable.

\section{MANEOIES AND INLETS}

106. Materials.-Manholes and inlets may be built of either concrete or brick masonry.

107. Concrete.-Concrete manholes and inlets are to be constructed of class A concrete, using tight, smooth forms, and shall be of the same quality of workmanship and materials as specified under "Concrete and concrete sewers."

108. Brick.-Brick manholes and inlets shall be built of brick masonry of the quality as specified under "Brick masonry." Every fifth course of brick shall be laid as stretchers, the remainder being laid as headers. Every brick shall have full mortar joints on the bottom and sides, which shall be formed at one operation by placing sufficient mortar on the bed and forcing the brick into it. Horizontal joints shall not exceed $3 / 3$ inch, and the vertical joints on the inside of the manholes shall not exceed $1 / 4$ inch. Joints on the inside face are to be carefully rubbed full and struck as the manhole is built up. A single rowlock shall be turned over all incoming and outgoing pipes.

109. Steps.-During the construction of each manhole, steps of wrought iron or cast iron shall be set in place on the inside of the manhole, beginning 2 feet above the bottom and placed not more than 18 inches apart. These steps are to be of the dimensions shown on the plans and shall be firmly built in the wall, allowing them to project $3 \frac{1}{2}$ inches from the inside of the manhole.

110. Frames and covers.- When the manhole or inlet masonry is completed, frames or covers of the dimensions and materials as shown on the plans are to be set in place to the line and grade given by the engineer.

111A. Payment.-Masonry in manholes and inlets will be estimated to the dimensions shown on the plans and paid for at the price bid per cubic foot of masonry of the class used. Frames and covers will be paid for by the piece at the price bid. In all cases the amount paid shall be full payment for all work in place complete.

111B. Payment.-Manholes and inlets will be paid for at a lump-sum price for each manhole and inlet respectively complete, including ordinary earth excavation, covers, steps, and masonry. In making the classification the depth of the manhole shall be taken as the distance from the surface of the ground to the flow line grade of the manhole.

American Society for Testing Materials, standard specifications for clay sewer pipe, serial designation C13-24, 1924.

See 531.5, page 517.

American Society for Testing Materials, standard specifications for cement concrete sewer pipe, serial designation C14-24, 1924.

1. These specifications cover cement-concrete products intended to be used for the conveyance of sewage, industrial wastes and storm water.

2. Pipes furnished under these specifications shall be of a single class to be designated "A.S.T. M., Cement-Concrete Sewer Pipe."

\section{MATERIAT AND MANUSACTURE}

3. (a) Cement-concrete pipes shall be manufactured from Portland-cement concrete.

(b) Concrete.-By concrete is meant a suitable mixture of Portland cement, mineral aggregates, and water, hardened by hydraulic chemical action.

(c) Cement.-Portland cement shall meet the requirements of A.S.T.M. C9. (See 516.11, p. 294.)

(d) The materials shall possess such physical and chemical properties that when molded into pipes and properly cured the product will be strong, 
durable, and serviceable, free from objectionable defects, and in compliance with these specifications and tests.

\section{CHEMICAL TESTS AND REQUIREMENTS}

4. The consumer or purchaser may prescribe in advance special chemical requirements in cases where sewage, industrial wastes, or ground waters have marked acid or alkaline character, or are of abnormaliy high temperatures. He may make use of chemical analysis of the pipe materials to ascertain whether these special requirements are met. The presence of deleterious materials causing slaking or disintegration shall be cause for rejection.

\section{PHYSICAL TESTS}

5. The physical tests of pipes shall include crushing test, hydrostatic pressure test, and absorption test.

6 . The specimens to be tested shall be selected by the purchaser or his representative at the point or points designated by him when placing the order. The manufacturer or seller shall furnish specimens for test, without separate charge, up to 1 per cent of the number of pipes to be delivered or furnished in each size of pipe, except that in no case shall the number of specimens furnished be less than five.

7. (a) Failure of 20 per cent of the specimens to meet the requirements of any of the tests imposed, shall result in rejection of all the pipe in the shipment or delivery, corresponding to the sizes thus failing to comply; except that in the event of 20 per cent of the specimens in any size failing to meet the lequirements, the manufacturer or seller may, with the consent of the consumer or purchaser, furnish for test, without charge, additional specimens from the same shipment to be selected as specified in section 6 . In case more than 80 per cent of the specimens tested, including those first tested, shall show substantial compiance for each of the various tests performed, then the entire shipment or delivery for this size shall be accepted; otherwise it shall be rejected.

(b) In addition to the foregoing requirements, failure of individual specimens to develop 75 per cent of the average crushing strength requirements shall be cause for rejection of the shipment, but the seller may cull the pipe and submit the balance of the shipment for retest, and, if the shipment then passes ail of the requirements of these specifications, it shall be accepted.

TABLE 1.-Dimensions, etc., of cement-concrete sewer pipe furnished as test specimens

\begin{tabular}{|c|c|c|c|c|c|c|c|c|c|c|c|c|c|c|c|c|c|c|c|c|c|c|c|}
\hline \multirow{3}{*}{ 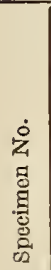 } & \multirow[b]{3}{*}{ 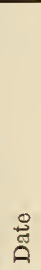 } & \multirow[b]{3}{*}{$\begin{array}{l}\dot{0} \\
\dot{z} \\
\circ \\
\stackrel{0}{\circ}\end{array}$} & \multirow[b]{3}{*}{ 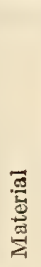 } & \multirow{3}{*}{ 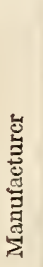 } & \multirow{3}{*}{ 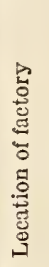 } & \multirow{3}{*}{ 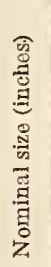 } & \multirow{3}{*}{ 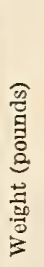 } & \multirow{3}{*}{ 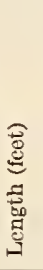 } & \multirow{2}{*}{\multicolumn{4}{|c|}{$\begin{array}{c}\text { Thickness of } \\
\text { spigot (inches) }\end{array}$}} & \multirow{2}{*}{\multicolumn{4}{|c|}{$\begin{array}{l}\text { Thickness of } \\
\text { socket (inches) }\end{array}$}} & \multicolumn{6}{|c|}{ Internal diameter (inches) } & \multirow{3}{*}{ Remarks } \\
\hline & & & & & & & & & & & & & & & & & \multicolumn{2}{|c|}{ Spigot } & \multicolumn{2}{|c|}{$\begin{array}{l}\text { Back of } \\
\text { socket }\end{array}$} & \multicolumn{2}{|c|}{ Socket } & \\
\hline & & & & & & & & & $\begin{array}{l}\vec{L} \\
\text { 离 }\end{array}$ & : & : & : & 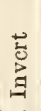 & ह & : & $\frac{0}{\mathscr{2}}$ & 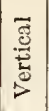 & 竎 & 胥 & 它 & 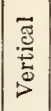 & 突 & \\
\hline & & & & & & & & & & & & & & & & & & & & & & & \\
\hline & & & & & & & & & & & & & & & & & & & & & & & \\
\hline & & & & & & & & & & & & & & & & & & & & & & & \\
\hline & & & & & & & & & & & & & & & & & & & & & & & \\
\hline & & & & & & & & & & & & & & & & & & & & & & & \\
\hline & & & & & & & & & & & & & & & & & & & & & & & \\
\hline & & & & & & & & & & & & & & & & & & & & & & & \\
\hline & & & & & & & & & & & & & & & & & & & & & & & \\
\hline & & & & & & & & & & & & & & & & & & & & & & & \\
\hline & & & & & & & & & & & & & & & & & & & & & & & \\
\hline & & & & & & & & & & & & & & & & & & & & & & & \\
\hline & & & & & & & & & & & & & & & & & & & & & & & \\
\hline & & & & & & & & & & & & & & & & & & & & & & & \\
\hline & & & & & & & & & & & & & & & & & & & & & & & \\
\hline & & & & & & & & & & & & & & & & & & & & & & & \\
\hline & & & & & & & & & & & & & & & & & & & & & & 1 & \\
\hline
\end{tabular}

8. The specimens of pipes shall be sound, full-size pipe. They shall first be freed from all visible moisture. When dry, each specimen shall be weighed, measured, and inspected. The results of these observations shall be recorded and preserved as shown in Table 1.

9. Specimens which, when placed in a vertical position, do not give a metallic ring when struck with a hammer, or are observed to have cracks or other defects in form or dimensions in excess of the limits permitted in these specifications, shall be discarded and replaced with additional specimens from the shipment.

\section{(A) CRUSHING TEST}

10. (a) Any prime mover or hand power which will apply the load at a uniform rate of about 2,000 pounds per minute, or in increments of not more than 100 pounds at the same rate, may be used in making the test.

(b) The pipe shall not be allowed to stand under load longer than is required to apply the load and to observe and record it.

(c) The testing machine shall be substantial and rigid throughout, so that the distribution of the load will not be affected appreciably by the deformation or yielding of any part. 
(d) The bearings and the specimen shall be accurately centered so as to secure a symmetrical distribution of the loading on each side of the center of the pipe in every direction.

(e) The load shall be applied until the pipe yields by eracks passing through the shell.

11. Two edge bearing test.-(Sce A. S. T. M. C4-24, 534.21 , p. 608 , section 19.)

The crushing strength shall be calculated by dividing the total load required to break each pipe by the net inside length of the barrel of the pipe, measuring from the bottom of the socket to the end of the spigot.

12. Three edge and sand bearings.-(See A. S. T. M. C4-24, 534.21, p. 608, sections 18 and 20.)

\section{(B) HYDROSTATIC TEST}

13. Sound full-size pipe not exceeding about 25 per cent of the specimens received for test in each size of pipe, shall be tested for leakage under internal hydrostatic pressure.

The ends of the pipe shall be tightly closed by wooden or metallic bulkheads or covers faced with rubber or leather so that no leakage shall occur through the covers at the test pressure. One cover shall be provided with a $3 / 4$-inch wrought-iron nipple passing through the cover, and held securely in place and made water-tight by means of lock nuts and washers or gaskets. The outer end of the nipple shall be connected with a pump discharge or water service line.

Water pressures, as measured by a standardized gauge attached to the delivery pipe close to the specimen, shall be internally applied to the specimen as follows:

5 lbs./in. ${ }^{2}$ for 5 minutes $10 \mathrm{lbs}$./in. ${ }^{2}$ for 10 minutes 15 lbs./in. ${ }^{2}$ for 15 minutes

The specimens shall show no leakage under these pressures. Moisture appearing on the surface of the pipe in the form of patches or beads, adhering to the surface, shall not be considered leakagc.

\section{(C) ABSORPTION TEST}

(The requirements for this test are the same as for the absorption test in A. S. T. M. C4, see 534.21, p. 608, with the exception of the following items peculiar to the type of commodity in this spccification.)
14. (a) The specimens shall be sound pieces with all edges broken, and may be from pipes broken in the crushing or other tests. They shall be from 12 to 20 square inches in area, and shall be as nearly square as they can be readily prepared. They shall be free from observable cracks, fissures, laminations, or shattered edges.

(b) Each specimen shall be marked so that it may be identified with the pipe used in the crushing test from which the specimen was taken. The marking shall be applied so that the pigment used shall not cover more than 1 per cent of the total superficial area of the specimen.

TABLE 2.-Physical test requirements of cementconcrete sewer pipe

\begin{tabular}{|c|c|c|c|}
\hline \multirow{2}{*}{ Internal diameter (inches) } & \multicolumn{2}{|c|}{$\begin{array}{l}\text { Average crushing } \\
\text { strength (pounds } \\
\text { per linear foot) }\end{array}$} & \multirow{2}{*}{$\begin{array}{l}\text { Maximum } \\
\text { absorption }\end{array}$} \\
\hline & $\begin{array}{c}\text { Knife-edge } \\
\text { and } 3-e d g e^{2} \\
\text { bearings }\end{array}$ & $\begin{array}{l}\text { Sand bear- } \\
\text { ings }{ }^{3}\end{array}$ & \\
\hline $\begin{array}{l}4 \\
6 \\
8 \\
10\end{array}$ & $\begin{array}{l}1,000 \\
1,000 \\
1,000 \\
1,100 \\
1,200\end{array}$ & $\begin{array}{l}1,430 \\
1,430 \\
1,430 \\
1,570 \\
1,710\end{array}$ & $\begin{array}{r}\text { Per cent } \\
8 \\
8 \\
8 \\
8 \\
8\end{array}$ \\
\hline $\begin{array}{l}15 \\
18 \\
21 \\
24\end{array}$ & $\begin{array}{l}1,370 \\
1,540 \\
1,810 \\
2,150 \\
2,360\end{array}$ & $\begin{array}{l}1,960 \\
2,200 \\
2,590 \\
3,070 \\
3,370\end{array}$ & $\begin{array}{l}8 \\
8 \\
8 \\
8 \\
8\end{array}$ \\
\hline $\begin{array}{l}39 \\
33 \\
36 \\
39 \\
42\end{array}$ & $\begin{array}{l}2,580 \\
2,750 \\
3,080 \\
3,300 \\
3,520\end{array}$ & $\begin{array}{l}3,600 \\
3,930 \\
4,400 \\
4,710 \\
5,030\end{array}$ & $\begin{array}{l}8 \\
8 \\
8 \\
8 \\
8\end{array}$ \\
\hline
\end{tabular}

1 See sec. 11. 2 See sec. $12 . \quad$ 3 See sec. 13.

\section{PHYSICAL TEST REQUIREMENTS}

15. The test requirements of cement-concrete pipe shall be as given in Table 2 . The individual results of the various tests for each size of pipc and for each shipment class and mill shall be tabulated separately so as to show the percentage which fails to comply with the requirements of each test.

TABLE 3.-Dimensions of cement-concrete sewer pipe

\begin{tabular}{|c|c|c|c|c|c|c|}
\hline $\begin{array}{l}\text { Internal diameter (D), } \\
\text { inches }\end{array}$ & Laying length (L) & $\begin{array}{c}\text { Inside } \\
\text { diameter } \\
\text { at mouth } \\
\text { of socket } \\
\left(\mathrm{D}_{2}\right)^{1}\end{array}$ & $\left\{\begin{array}{c}\text { Depth of } \\
\text { socket } \\
\left(\mathrm{L}_{\mathrm{l}}\right)\end{array}\right.$ & $\begin{array}{l}\text { Mini- } \\
\text { mum } \\
\text { taper of } \\
\text { socket } \\
\text { (H) }\end{array}$ & $\begin{array}{l}\text { Thick: } \\
\text { ness of } \\
\text { barrel ('T) }\end{array}$ & Thickness of socket $\left(T_{3}\right)$ \\
\hline $\begin{array}{l}6 \\
6 \\
6 \\
10 \\
12\end{array}$ & 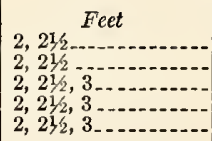 & $\begin{array}{c}\text { Inches } \\
6 \\
81 / 4 \\
1034 \\
13 \\
151 / 4\end{array}$ & $\begin{array}{r}\text { Inches } \\
11 / 2 \\
2 \\
21 / 2 \\
21 / 2 \\
21 / 2\end{array}$ & $\begin{array}{l}1: 20 \\
1: 20 \\
1: 20 \\
1: 20 \\
1: 20\end{array}$ & $\begin{array}{c}\text { Inches } \\
9 / 6 \\
5 / 8 \\
3 / 4 \\
3 / 8 \\
1 \\
1\end{array}$ & $\begin{array}{l}\text { The thickness of the socket } 1 / 4 \text { incb from its outer } \\
\text { end shall be not less tban three-fourtbs of tbe } \\
\text { tbickness of tbe barrel of the pipe. }\end{array}$ \\
\hline 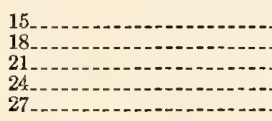 & $\begin{array}{l}2.21 / 2,3 \ldots \\
2,21 / 2,3 . \\
2,21 / 2,3 . \\
2,21 / 2,3 . \\
21 / 2,3\end{array}$ & $\begin{array}{l}183 / 4 \\
2234 \\
26 \\
291 / 2 \\
331 / 4\end{array}$ & \begin{tabular}{l|l}
213 \\
23 \\
23 \\
3 \\
314 \\
314
\end{tabular} & $\begin{array}{l}1: 20 \\
1: 20 \\
1: 20 \\
1: 20 \\
1: 20\end{array}$ & $\begin{array}{l}11 / 4 \\
11 / 2 \\
13 / 4 \\
21 / 8 \\
21 / 4\end{array}$ & \\
\hline 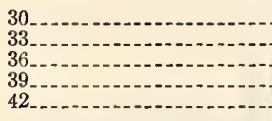 & $\begin{array}{l}21 / 2,3 \\
21 / 2,3 \\
21 / 2,3 \\
21 / 2,3 \\
21 / 2,3\end{array}$ & $\begin{array}{l}37 \\
4014 \\
44 \\
471 / 4 \\
51\end{array}$ & $\begin{array}{l}31 / 2 \\
4 \\
4 \\
4 \\
4\end{array}$ & $\begin{array}{l}1: 20 \\
1: 20 \\
1: 20 \\
1: 20 \\
1: 20\end{array}$ & $\begin{array}{l}21 / 2 \\
23 / 4 \\
3 \\
31 / 4 \\
31 / 2\end{array}$ & \\
\hline
\end{tabular}

${ }^{1}$ When pipes are furnished baving an increase in tbickness over that giveu in last column, tben tbe diameter of socket shall be increased by an amount equal to twice the increase of thickness of barrel. 


\section{A. S. T. M. SIZES AND DIMENSIONS}

16. Pipes shall be furnished of the sizes, internal diameter, and with the dimensions give in Tables 3 and 4 . Where several lengths are mentioned in the table the consumer or purchaser shall indicate, at the time of purchase, which lengths shall be furnished; and unless so indicated the manufacturer shall furnish such lengths as he may elect.

17. The permissible variation from the dimensions given in Table 3 shall not exceed those stated in Table 4. Where the thickness of barrel is increased beyond that given in Table 3 in order to meet the specified requirements of strength, the diameter at inside of socket shall be increased by double the increase in thickness of barrel. Pipes intended to be straight shall not have variation in alignment of more than $1 / 8$ inch per foot length.

\section{WORKMANSHIP AND FINISH}

18. Pipes shall be substantially free from fractures, large or deep cracks and blisters, laminations and surface roughness.

19. The ends of the pipes shall be square with their longitudinal axis.

20. (a) Special shapes shall have a plain spigot end and a socket end corresponding in all respects with the dimensions specified for pipes of the corresponding internal diameter. Branches shall be furnished to lay the same lengths as straight pipe. All specials shall conform in finish to the workmanship and finish specifications for pipes.

TABLE 4.-Permissible variations in dimensions of cement-concrete sewer pipe

\begin{tabular}{|c|c|c|c|c|c|}
\hline \multirow{3}{*}{$\begin{array}{l}\text { Normal size, } \\
\text { in inches }\end{array}$} & \multicolumn{5}{|c|}{ Limits of permissible variation in- } \\
\hline & \multirow{2}{*}{$\begin{array}{l}\text { Length } \\
\text { per foot } \\
(-)\end{array}$} & \multicolumn{2}{|c|}{ Internal diameter } & \multirow{2}{*}{$\begin{array}{l}\text { Depth of } \\
\text { socket } \\
(-)\end{array}$} & \multirow{2}{*}{$\begin{array}{l}\text { Thick- } \\
\text { ness of } \\
\text { barrel } \\
(-)\end{array}$} \\
\hline & & $\underset{( \pm)}{\text { Spizot }}$ & $\begin{array}{l}\text { Socket } \\
( \pm)\end{array}$ & & \\
\hline $\begin{array}{l}4 . \ldots . \\
6 \\
80 \\
12 \\
12\end{array}$ & Inch \begin{tabular}{r|}
$1 / 4$ \\
$1 / 4$ \\
$1 / 4$ \\
$1 / 4$ \\
$1 / 4$ \\
$1 / 4$
\end{tabular} & $\begin{array}{l}\text { Inch } \\
16 \\
3 / 6 \\
3 / 6 \\
14 \\
14 \\
1 / 4\end{array}$ & $\begin{array}{c}\text { Inch } \\
118 \\
3 / 6 \\
1 / 4 \\
1 / 4 \\
1 / 4 \\
1 / 4\end{array}$ & Inch 1. & $\begin{aligned} & \text { Inch } \\
& 3 / 16 \\
& 1116 \\
& 1 / 16 \\
& 1 / 16 \\
& 1 / 16\end{aligned}$ \\
\hline $\begin{array}{l}15 \\
18 \\
21 \\
24 \\
27\end{array}$ & $\begin{array}{l}1 / 4 \\
1 / 4 \\
134 \\
3,8 \\
3 / 8\end{array}$ & $\begin{array}{l}1 / 4 \\
1 / 4 \\
518 \\
5 / 16 \\
5 / 16\end{array}$ & $\begin{array}{l}1 / 4 \\
1 / 4 \\
5 / 10 \\
5 / 10 \\
5 / 10\end{array}$ & $\begin{array}{l}3 / 4 \\
1 / 4 \\
1 / 4 \\
1 / 4 \\
1 / 4\end{array}$ & $\begin{array}{l}3 / 32 \\
3 / 32 \\
138 \\
1,8 \\
1 / 8\end{array}$ \\
\hline 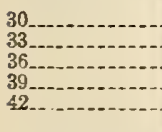 & $\begin{array}{l}38 \\
38 \\
38 \\
38 \\
38\end{array}$ & $\begin{array}{l}3 / 8 \\
3 / 8 \\
1 / 2 \\
1 / 2 \\
1 / 2\end{array}$ & $\begin{array}{l}3 / 8 \\
38 \\
1 / 2 \\
1 / 2 \\
1 / 2\end{array}$ & $\begin{array}{l}1 / 4 \\
1 / 4 \\
1 / 4 \\
1 / 4 \\
1 / 4\end{array}$ & $\begin{array}{l}3 / 8 \\
3 / 16 \\
3316 \\
316 \\
3 / 16\end{array}$ \\
\hline
\end{tabular}

Note.-The minus sign ( - ) alone indicates that the plus variation is not limited; the plus and minus sign $( \pm)$ indicates variation in both excess and deficiency in dimension.

(b) Slants shall have their spigot ends cut at an angle of approximately $45^{\circ}$ with the longitudinal axis.

(c) Curves shall be at angles of $90^{\circ}, 45^{\circ}, 221^{\circ}$, as required. They shall conform substantially to the curvature specified.

(d) Branches shall be furnished with the connection or connections of the size or sizes specified, securely and completely fastened in the process of manufacture to the barrel of the pipe. T-branches and double $\mathrm{T}$-branches shall have their axes perpendicular to the longitudinal axis of the pipe. Y-branches, double Y-branches, and V-branches shall have their axes approximately $45^{\circ}$ from the longitudinal axis of the pipe measured from the socket end. All branches shall terminate in sockets, and the barrel of the branch shall be of sufficient length to permit making a proper joint when the connecting pipe is inserted in the branch socket.

(e) Channel or split pipe, curves, and branches shall be accurate half sections of the corresponding size of pipe or other specials.

\section{MARKINGS}

21. Pipes shall bear the initials or name of the person, company, or corporation by whom they are manufactured, and the location of the mill. The markings shall be indented or stenciled on the exterior or interior of the barrel near the socket and shall be plainly legible for purpose of identification.

\section{INSPECTION}

22. All pipes shall be subject to inspection at the factory, trench, or other point of delivery by a competent inspector imployed by the consumer or purchaser. The purposes of the inspection shall be to cull and reject pipes which, independent of the physical tests herein specified, fail to meet the requirements of these specifications.

23. Pipes shall be subject to rejection on account of the following:

(a) Variations in any dimension exceeding the permissible variations given in Table 4.

(b) Fracture or cracks passing through the shell or socket, except that a single crack at either end of a pipe not exceeding 2 inches in length or a single fracture in the socket not exceeding 3 inches in width nor 2 inches in length will not be deemed cause for rejection unless these defects exist in more than 5 per cent of the entire shipment or delivery.

(c) Blisters where the surface is broken or which project more than 1/8 inch above the surface.

(d) Defects which indicate imperfect mixing and molding.

(e) Cracks sufficient to impair the strength, durability or serviceability of the pipe.

(f) Variation of more than 1/8 inch per linear foot in aligument of a pipe intended to be straight.

(g) Failure to give a clear ringing sound when placed on end and dry-tapped with a light hammer.

(h) Insecure attachment of branches on spurs.

24. All rejected pipes shall be plainly marked by the inspector and shall be replaced by the manufacturer or seller with pipes which meet the requirements of these specifications, without additional cost to the consumer or purchaser.

American Society for Testing Materials, standard specifications for clay sewer brick, serial designation C32-24, 1924.

See 534.11, page 581 .

Federal Specifications Board, specification No. 495, United States Government master specification for pipe, concrete, plain, June 10, 1927

(This specification is based on American Society for Testing Materials specification C14 (see p. 464), and is practically identical with that specification. This specification does not include requirements for the use of sand bearings under the crushing test, which are part of the crushing test requirements in the A. S. T. M. specification, and makes no provision for chemical tests and requirements, which are included in the A. S. T. M. specification.)

\subsection{RESERVOIRS, TAINKS, POOIS, ETC.}

\subsection{Fuel Storage Tanks.}

National Board of Fire Underwriters, regulations for the installation of containers for storing and handling flammable liquids, 1927. 


\section{DESIGN AND CONSTRUCTION OF CONCRETE FUEL OIL STORAGE TANKS}

\section{ACCEPTABILITY OF CONCRETE TANKS}

Concrete tanks may be used for the storage of fuel oil when the liquid is under $35^{\circ} \mathrm{B}$.

\section{SIZE AND LOCATION}

The size of the tank and its location also, whether it shall be underground, above ground, or inside a building shall conform in all respects with the current Regulations of the National Fire Protection Association for the Construction and Installation of Oil-Burning Equipments. flanged sections with a space of about $11 / 2$ inches left between the flange and the concrete on each side of the wall. This space shall be calked later with litharge and glycerin or other satisfactory oil-proof material. It is also advisable to provide a ring projecting about 2 inches around the pipe sleeve to engage the concrete.

\section{SUPERVISION: OF DESIGN AND CONSTRUCTION}

A competent, experienced designing and constructing engineer satisfactory to or representing the owners shall be responsible for the design of the tank. Detailed plans shall be submitted for approval to the inspection department having jurisdiction before the installation is started.
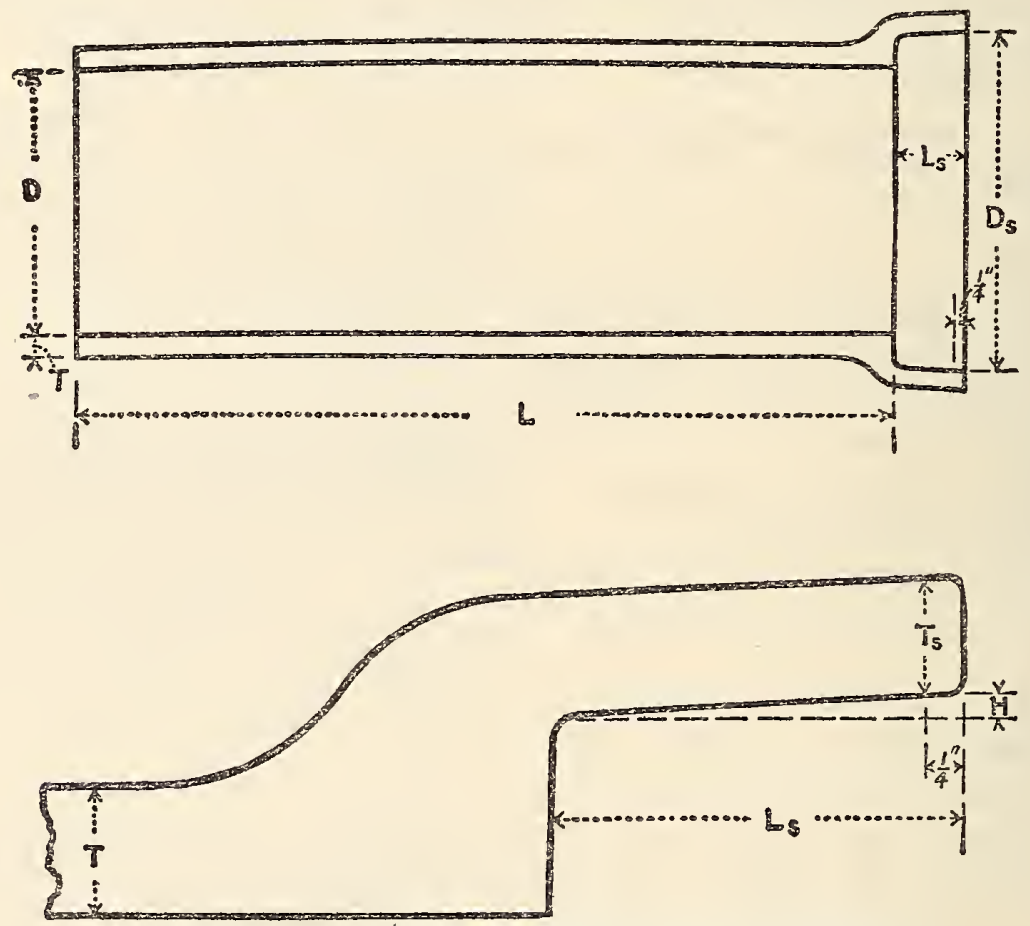

Figure 131.-A pplication of dimensions given in Table 3

ROOF

All tanks shall be built with a concrete roof. Roof shall be made gas tight at junction with walls and shall be constructed without unprotected openings.

\section{FOJNDATIONS}

The tanks shall be set on firm well tamped earth, rock, or other suitable foundation to guard against settlement. The foundations shall be constructed to provide sull bearing for the tank bottom.

\section{PIPE CONNECTIONS, FITTINGS, AHD AUXILIARY EOUIPMENT}

(a) The installation and arrangement of all pipe connections and fittings, including vent pipe, filling pipe, manhole covers, oil level indicating device, leaters and heating pipe, oil pipes, and embankments or dikes shall conform in all respects with the current Regulations of the National Fire Protection Association for the Construction and Installation of Oil Burning Equipments.

(b) All pipes shall pass through the roof if possible. Where pipes pass through the tank there shall be
The work of construction shall be under the supervision of a competent engineer who shall superintend and inspect the placing of the concrete.

\section{DESIGN OF CONCRETE TANK}

\section{Loads}

(a) Tanks shall be designed to provide for all internal and external loads. These include:

1. Full hydrostatic pressure of contents upon floor and walls.

2. External hydrostatic pressure upon floors and walls.

3. Earth pressure on walls.

4. Live and dead loads on roof.

(b) Column loads shall be distributed over a proper area by providing adequate footings and floor reinforcement.

(c) The hydrostatic pressure in the soil shall be considered equivalent to a fluid pressure of $621 / 2$ pounds per cubic foot for the full head of water.

NoTE.-While the hydrostatic pressure in soil is generally about 50 per cent of the full hydrostatic pressure, the greater value is recommended as a factor of safety for unforeseen conditions. 
Temperature Reinforcement

Temperature reinforcement shall be placed in the walls and floor independent of other reinforcement, the steel ratio being not less than one-third of 1 per cent. The minimum range in temperature should be predetermined as a basis of calculation.

\section{Thickness of Walls}

The minimum thickness of walls shall be 8 inches at the top and 10 inches at the bottom in order to give sufficient space for spading.

\section{Unit Stresses}

(a) Steel in tension in the walls and floor shall be designed for a safe working stress of $10,000 \mathrm{lbs} . / \mathrm{in} .{ }^{2}$, whether in circumferential tension or in tension due to bending moments.

(b) In continuous or restrained members positive and negative bending moments shall be given equal consideration.

(c) In circumferential walls the thickness shall be based upon a maximum tensile strength in the concrete of $150 \mathrm{lbs}$. $/ \mathrm{in.}^{2}$, but in no case shall be less than the minimum thickness given above. (Par. 9.)

(d) Working stresses in the concrete and steel for all cases not covered in (a) and (b) shall confor'm with the recommendations of the current Report of the Joint Committee on Concrete and Reinforced Concrete.

\section{CONSTRUCTION}

\section{Materials}

(a) The cement shall meet the requirements of the current standard specifications for Portland cement adopted by the American Society for Testing Materials and the American Concrete Institute. (See 516.11, p. 287.) It shall be stored in a weather-tight structure with the floor raised not less than 1 foot from the ground. Cement that has hardened or partially set shall not be used.

(b) Before delivery on the job, the contractor shall submit to the engineer in charge a 50-pound sample of each of the aggregates proposed for use. These samples shall be tested and if found to pass the requirements of the specifications, similar material shall be considered as acceptable for the work.

(c) Fine aggregate shall consist of natural sand or screenings from hard, tough, crushed rock or pebbles, clean and free from any suriace film or coating and graded from fine to coarse particles passing when dry, a sieve having four meshes per lineal inch. Fine aggregate shall not contain injurious vegetable or other organic matter as indicated by the colorimetric test, nor more than 7 per cent by volume of clay or loam. Field tests may be made by the engineer on fine aggregate as delivered at any time during the progress of the work. If there is more than 7 per cent of clay or loam by volume in one hour's settlement after shaking in an excess of water the material represented by the sample shall be rejected.

Nот:.-The colorimetric test may be applied in the field as follows: Fill a 12-ounce graduated prescription bottle to the $41 / 2$-ounce mark with the sand to be tested. Add a 3 per cent solution of sodium hydroxide until the volume of sand and solution, after shalcing, amounts to 7 ounces. Shake thoroughly and let stand for 24 hours. The sample should then show a practically colorless solution or at most a soiution not darker than straw color.

(d) Coarse aggregate shall consist of clean, hard, tough, crushed rock, or pebbles graded in size, free from vegetable or other organic matter and should contain no soft, flat, or elongated particles. The size of the coarse aggregate shall range from 1 inch down, not more than 5 per cent passing a screen having four meshes per linear inch and no intermediate sizes shall be removed. (e) Crusher-run stone, bank-run gravel, or mixtures of fine and coarse aggregates prepared before delivery on the work shall not be used because the ratio of fine to coarse material varies so widely as to lead to concrete mixtures of greatly varying proportions.

$(f)$ The water shall be free from oil, acid, and injurious amounts of vegetable matter, alkali, or other salts.

(g) The reinforcing metal shall meet the requirements of the current standard specifications for billet steel reinforcement of the American Society for Testing Materials excepting that twisted square bars shall not be employed in the construction. Reinforcing shall be free from excessive rust, scale, paint, or coatings of any character which will tend to reduce or destroy the bond.

\section{Proportions of Concrete Mixture}

(a) The unit of measure shall be the cubic foot. Ninety-four pounds (one sack or one-fourth barrel) of Portland cement shall be assumed as 1 cubic foot.

(b) The concrete shall be mixed in a proportion by volume not leaner than 1 sack of Portland cement, $1 \frac{1}{2}$ cubic feet of fine aggregate and 3 cubic feet of coarse aggregate.

(c) The method of measuring the materials for the concrete, including water, shall be one which will insure separate and uniform proportions of each of the materials at all times.

\section{NIethod of Mixing}

(a) All concrete shall be mixed by machine in a batch mixer of an approved type equipped with suitable charging hopper, water storage, and a water measuring device which can be locked. The mixing plant shall be of sufficient capacity and power to carry out each prearranged operation without danger of delay during the process.

(b) The ingredients of the concrete shall be mixed to the required consistency and the mixing continued not less than one and one-half minutes after all materials are in the mixer and before any part of the batch is discharged. The mixer shall be emptied before receiving materials for the succeeding batch. The volume of the mixed material used per batch shall not exceed the manufacturer's rated capacity of the drum.

(c) In no case shall aggregates containing frost or lumps of frozen material be used.

(d) The quantity of water used in mixing shall be the least that will produce a plastic or workable mixture which can be worked into the forms and around the reinforcement. Under no circumstances shall the consistency of the concrete be such as to permit a separation of the coarse aggregate from the mortar in handling. An excess of water shall not be permitted as it seriously affects the strength of the concrete and any batch containing such an excess shall be rejected.

(e) The retempering of mortar or concrete which has partially hardened; that is, remixing with or without additional materials or water shall not be permitted.

\section{Depositing of Concrete}

(a) Before beginning a run of concrete, all hardened concrete or foreign material shall be completely removed from the inner surfaces of all conveying equipments.

(b) Before depositing any concrete, all débris shall be removed from the space to be occupied by the concrete, all steel reinforcing shall be secured in its proper location, all forms shall be thoroughly wetted except in freezing weather unless they have been previously oiled and all form work and steel 
reinforcing shall be inspceted and approved by the engineer.

(c) Concrete shall be handled from the mixer to the place of final deposit as rapidly as possible and by methods of transporting which will prevent the separation of the ingredients. Chutes shall not be used. The concrete shall be deposited directly into the forms as nearly as possible in its final position so as to avoid rehandling. The piling up of concrete material in the forms in such manner as to permit the escape of mortar from the coarser aggregate shall not be allowed. Under no circumstances shall concrete that has partially set be depositcd in the work.

(d) Where continuous placing of concrete in floor and walls is impracticable, the operations shall be in the following order:

1. The concrete of all footings and floor, which shall be placed monolithically.

2 . The concrete of walls.

3. The concrete of columns, if any.

4. The concrete of the roof.

(e) No break in time of over 45 minutes shall occur during any one operation. Between placing of columns and supported roof slabs an interval of at least six hours shall elapse to permit the settlement of concrete in the columns. In placing concrete in floors, it shall not be allowed to set up on exposed vertical faces where work is temporarily discontinued

(f) In walls, the concrete shall be placed in layers of not over 12 inches for the entire wall so that a monolithic structure will result. The concrete shall be thoroughly worked around the reinforcing material so as to completely surround and embed the samc.

(g) If the placing of concrete is unavoidably interrupted by accident or otherwise, the previous surface shall be roughened and washed clean with a hose, a mixture of $1: 1$ mortar slushed on uniformly before further concreting is done and the new concrete deposited immediately thereafter.

(h) When deposited in the forms, concretc shall be thoroughly spaded against the inner and outer faces of the forms so that it will densely compact and force out the trapped air and work back the coarser particles from the face of the forms.

Note.-More and better work can be accomplished by using light wooden sticks 1 by 2 inches, planed smooth with sheet steel blade at lower end rather than with heavy spades. Enough laborer should be employed, spading continuously, to obtain satisfactory results.

(i) During freezing weather, the stone, sand, or water or all three materials shall be heated so that the concrete mixture will have a temperature of at least $60^{\circ} \mathrm{F}$. After the concrete is deposited, precaution shall be taken to prevent freezing for at least 48 hours. Concreting shall not be begun when the temperature is below $15^{\circ} \mathrm{F}$.

\section{Forms}

(a) The forms shall be of a good grade of lumber, planed to a uniform thickness and width, tongued and grooved for walls, strongly made, and located or held in place either by exterior bracing or, on the outside of circular walls, by circumferential bands, so that no distortion allowing displacement of concrete is possible.

(b) Joints in forms sliall be tight so that no mortar will escape. If forms are to be reused, they shall be thoroughly cleaned. (A slush mixture of one-half petrolatum and one-half kerosene makes a good mixture for oiling forms.) The use of bolts or wires through the concrete shall be prohibited. All spreaders shall be removed.

(c) The forms shall not be removed until the concrete has sufficiently hardened so that no deflec- tion or damage will result. in warm weather column and wall forms shall remain undisturbed for at least 48 hours and roof forms at least 7 days. In cold weather no predetermined rules can be made.

(d) Contractors equipped to handle the work with sliding forms mav be permitted to do so, provided the forms are left at one level until the concrete which will be exposed on raising them has hardened sufficiently to sustain the weight of the concrete abovc.

\section{Details of Construction}

(a) Reinforcing steel shall be cleaned of all mill and rust scales before being placed in the forms. All reinforcement shall be bent or curved true to templates, placed in its proper position as required by the plans and securely wired or fastened in place well in advance of the concreting. Reinforcement shall be inspected and approved by the engineer before any concrete is deposited.

(b) Wherever it is necessary to splice the reinforcement, no lap splice shall be less than 40 diameters. No two laps of adjacent rods shall be directly opposite each other in circular walls.

(c) All reinforcement shall be interconnected and grounded by an approved method as a protection against lightning. Any pipe passing through the tank shall be properly bonded to the reinforcement.

(d) If walls and floors are not deposited in one operation a suitable joint or dam shall be provided between the floor and walls. This can be made as follows: (1) Provide a recess in the floor to engage the wall and insert a galvanized iron strip at least 10 inches wide with joints riveted and soldered so as to form a continuous band on one side of the recess, or (2) place a 10-inch strip of deformed sheet metal 1 inch back from the inside form and engaging floor and wall equally. After wall form is removed the 1 inch recess is to be plastered with a $1: 11 / 2$ mortar to make a 6-inch covered base.

(e) Unless the roof is insulated against temperature changes by sufficient earth cover or the reinforcing in walls and roof is designed to take care of temperature stresses likely to occur, an expansion joint shall be provided between the tops of walls and the bottom of roof slabs so that any expansion of the roof due to tempcrature will not transmit bending moment into the walls.

(f) In roof slabs where tcmporary stops are necessary they shall be made on the planes of least shear; that is, at the middle of beams or slabs.

(g) When tanks are being built in such locations that they may be subjected to hydraulic pressure through the soil, suitable provision shall be made to keep this pressure from acting on the fresh concrete until the latter has attained sufficicnt strength. If sheet piling or shores are used they shall be so placed that they will not pass through the walls.

\section{Finishing}

(a) The floors and roof shall be brought to grade with a straight-edge or strike board, finished with a wood float and troweled to a smooth surface as soon as possible after the concrete is deposited. As soon as the forms are removed, voids in walls, if any, shall be filled with a $1: 1^{1 / 2}$ mortar.

(b) The interior of the tank shall be oil proofed. This work shall be done only by persons familiar with the process. A bond guaranteeing the work for a term of years shall be furnished.

NotE.-Oil proofing is deemed essential owing to the possibility of using the tank as a container for oils of various character.

(c) The tanks shall be tested as soon as practicable in the opinion of the engineer by filling with water, and shall show no signs of leakage during a period of seven days. 
(d) Back filling shall not be placed around the walls nor deposited on the roof until after the water test has been made, nor until a time when, in the opinion of the engineer in charge, it can be safely done.

(The full text of this specification includes requirements for pipe connections, fittings and auxiliary equipment, and supervision of design and construction.)

\subsection{Viaducts.}

American Association of State Highway Officials, standard specifications for highway bridges and incidental structures, 1928.

\section{CONCRETE VIADUCTS}

\section{DESCRIPTION}

Concrete viaduct construction shall consist of a series of reinforced concrete slab or girder spans supported on bents or towers composed of concrete columns suitably braced by means of longitudinal and transverse struts and sway braces. The superstructure shall be constructed in accordance with the requirements governing the construction of concrete slab and girder structures. The following provisions relate to the construction of the column substructure.

\section{CLASS OF CONCRETE}

All columns, caps, braces, and reinforced footings shall be of class "A" concrete. Mass footings shall be of class "B" concrete unless otherwise specified.

\section{COLUMN FORMS}

Column forms shall be composed of material not less than $11 / 4$ inches in thickness after being dressed, and shall be effectively braced. When possible, forms shall be daylighted at intervals not greater than 10 feet vertically, the openings being sufficient to permit of free access to the forms for the purpose of inspecting, working and spading the concrete.

\section{PLACING CONCRETE}

Concrete in columns shall be placed in one continuous operation, unless otherwise directed. The columns shall be allowed to set at least 12 hours before the caps are placed.

Unless otherwise permitted by the engineer, no concrete shall be placed in the superstructure until the column forms have been stripped sufficiently to determine the character of the concrete in the columns. The load of the superstructure shall not be allowed to come upon the bents until they have been in place at least 21 days, unless otherwise permitted by the engineer.

\section{SURFACE FTNISH}

Unless otherwise specified, exposed surfaces of all columns, struts and bracing shall be given a finish in accordance with class 1 , surface finish.

Note.-For definitions of the various classes of concrete mentioned above. (See 516.3, p. 301, American Association of State Highway Officials, specifications for "Concrete masonry.")

\subsection{Swimming Pools.}

Associated Tile Manufacturers, basic specification for tile work, 1924

(This specification contains provisions governing requirements for construction of various structures of tile, including swimming pools.)

For specifications for materials. (See 534.20, p. 594 .)

Portland Cement Association, condensed specifications for concrete swimming-pool construction.

\section{GENERAL}

\section{GENERAL CONDITIONS}

The plain and reinforced concrete work shall be governed by the contract, these specifications and the plans.

\section{MATERIALS}

\section{CEMENT}

The cement shall meet the requirements of the current standard specifications for Portland cement of the American Society for Testing Materials. (See 516.11, p. 294.)

\section{FINE AGGREGATE}

Fine aggregate shall consist of sand or screenings from crushed rock or pebbles, well graded from fine to coarse, passing, when dry, a sereen having four meshes to the linear inch. Fine aggregate shall not contain injurious amounts of vegetable or other organic matter, nor more than 7 per cent by volume of clay, dust, or loam.

\section{COARSE AGGREGATE}

Coarse aggregate shall consist of hard crushed rock or pebbles, free from vegetable or other organic matter, and shall be practically free from soft, flat, or elongated particles. It shall be well graded from one-quarter inch to 1 inch in size, not more than 5 per cent passing a screen having four meshes per linear inch.

\section{MIXED AGGRIGATE}

Crusher-run stone, bank-run gravel, or mixtures of fine and coarse aggregate prepared before delivery on the work shall not be used, unless same are tested beforehand and proportioned so as to insure obtaining a concrete of the required quality, density, and strength.

\section{WATER}

Water shall be clean and free from oil, acid, alkali, organic matter, or other deleterious substances. It should be pure enough to drink.

\section{REIN FORCEMENT}

Rcinforcing metal shall meet the requirements of the current standard specifications for steel reinforcement of the American Society for Testing Materials. It shall be free from excessive rust, scale, paint, or coatings of any character which would tend to reduce or destroy the bond. Either plain or deformed steel bars or wire mesh shall be used.

\section{JOINT FLLLER}

The joint filler shall be an elastic waterproof material that will not become soft and run out in hot weather nor hard and brittle in cold weather.

\section{CONSTRUCTION}

\section{EXCAVATION}

Excavation shall be made to the proper dimensions and elevations, allowing for exterior forms and line of drain tile, and for sand fill under floor if required. All refuse material encountered at grade shall be removed and all soft or spongy spots excavated and holes refilled with well tamped sand or gravel.

\section{SUB-BASE}

After the walls have been erected and the forms removed, a 4-inch sub-base of sand shall be provided to insure a firm foundation for the floor. The sand should be wet down and well compacted by rolling 
and should be wet, but with no standing water, when the concrete for the floor is placed.

Note.-The sub-base may be omitted if pool is built in porous sandy soil or gravel.

\section{DRAIN AGE}

A line of 6-inch drain tile extending completely around the pool shall be placed around the outer lower edge of the wall footings and also under the floor of the pool. The lowest point of the drain should be connected with another drain leading to a suitable outlet.

\section{PLOMBING}

The necessary piping for filling and emptying the pool, as well as for an overflow system, should be provided and placed as required.

\section{MEASURII!G}

The method of measuring each of the constituent materials shall be such as to insure the specified proportions in each batch. A sack of Portland cement (94 pounds net) shall be considered as 1 cubic foot or one-fourth of a barrel.

\section{CONCRETE MIXING}

All concrete shall be mixed in an approved type of batch mixer (except when under special conditions the engineer otherwise permits). The ingredients of the concrete shall be mixed to the required consistency for at least one and one-half minutes after all materials are in the mixer before any part of the batch is discharged from the drum. The mixer should be equipped with a water-measuring device which can be locked, also with a suitable charging hopper.

\section{RETEISPERING}

Retempering of concrete or mortar which has partially hardened-that is, remixing with or without additional materials or water-shall not be permitted.

\section{FORMS}

Forms shall be smooth, substantial, unyielding, and so constructed that the concrete will conform to the designed shape and dimensions.

\section{REINFORCEMENT}

Reinforcement shall be placed in the exact location shown on the plans and wired at intersections so that it will not become disarranged during the depositing of concrete. If the bars must be spliced, not less than forty times the diameter of the bar shall be allowed for the splice, and bars shall be separated at least $1 \frac{1}{2}$ diameters of the larger bar. In splicing wire mesh or fabric at least 4 inches shall be allowed for the lap. Splices should be avoided at points of maximum stress.

\section{PROPORTIONS}

The concrecie should be mixed in the proportions of 1 sack of Portland cement, 2 cubic feet of fine aggregate, and 3 cubic feet of coarse aggregate.

\section{CONSISTENCY}

In mixing the concrete the least amount of water should be used which it is possible to employ and still obtain a workable plastic mixture which can be worked into the forms and around the reinforcement. The proper consistency is, perhaps, best described as "quaky." An excess of water must be avoided, as it seriously affects the strength and impermeability of the concrete, and any batch of concrete containing such an excess should be rejected.

\section{PLACING}

The concrete shall be placed in its final position as soon as possible after mixing and must be in place within 30 minutes after the water has been added to the dry materials. It should be placed in horizontal layers in the walls not more than 9 inches deep in as continuous an operation as possible so as to avoid construction joints. Concrete should not be placed in water. If underground springs are encountered they should be drained to a sump outside the forms and pumped out until the concrete is above the water line. The concrete should be well spaded and if the forms are rapped with mallets while placing it will help settle the concrete $\mathrm{n}$ place and eliminate air bubbles and stone pockets.

\section{JOINTS}

The surface of the footings covered by the floor slab shall be coated with tar or asphalt to prevent a bond between footings and slab, thus allowing the floor to act independently of the footings.

\section{SURFACE FINISH}

Immediately after the forms have been removed all projections and irregularities on the exposed surfaces shall be carefully removed and all cavities neatly filled with mortar. Enough 1:2 Portland cement and sand mortar should be used to fill cavities, but no plastering should be permitted. If desired, the interior surface of the walls may be more smoothly finished by wetting and thoroughly rubbing with a concrete brick or carborundum stone to obtain a still denser surface. The top of the walls should be left gritty to prevent slipping.

\section{GENERAL}

All exposed surfaces should be finished with a smooth and neat appearance.

\section{PROTECTION}

Exposed surfaces should be kept wet for a period of at least 10 days after placing in dry, hot weather.

\section{TEMPERATURE BELOW $35^{\circ} \mathrm{F}$,}

If at any time during the progress of the work the temperature reaches or in the opinion of the engineer may within 24 hours drop to $35^{\circ} \mathrm{F}$., the water and aggregate should be heated and precautions taken to protect the work from freezing for at least five days.

\section{REMOVAL OF FORMS}

The wall forms should remain in place at least 36 hours after the concrete has been placed or until the concrete has hardened sufficiently to be self-sustaining. When freezing weather occurs, forms and bracing should remain in place an additional time equal to the time the structure has been exposed to freezing. No outside pressure should be placed on the concrete before the removal of all forms.

\subsection{Watering Troughs.}

(No nationally recognized specifications available.)

\subsection{Reservoirs.}

(No nationally recognized specifications available.)

\subsection{Spillways.}

(No nationally recognized specifications available.)

\subsection{Cofferdams.}

(No nationally recognized specifications available.)

\subsection{MASONRY.}




\subsection{General Items.}

American Association of State Highway Officials standard specifications for bridges and incidental structures, 1928.

\section{STONE FOR MASONRY}

See 511.9 , page 212 .

American Railway Engineering Association, masonry specifications, 1902 .

It is recommended that railway companies prepare and use specifications complete in themselves for all kinds of masonry in such form that they may be attached to and form part of specifications and contracts for other railway construction when desirable.

\subsection{Dry Rubble Masonry.}

American Association of State Highway Officials, standard specifications for highway bridges and incidental structures, 1928.

\section{DRY RUBBLE MASONRY}

(The full text of this specification includes material relative to construction methods.)

\section{DESCRIPTION}

Dry rubble masonry as here specified shall include the classes commonly known as coursed, random, and random range work and shall consist of roughly squared and dressed stone laid without mortar.

\section{MATERIALS}

Stone for this class of masonry shall conform to the requirements of "Stone for masonry." (See 511.9 , p. 212, American Association of State Highway Officials).

\section{SIZE OF STONE}

The stones shall conform in size to the requirements specified for "Mortar rubble masonry." (See 518.82, p. 474, American Association of State Highway Officials.)

\section{HEADERS}

Headers shall conform to the requirements specified for "Mortar rubble masonry."

\section{SHAPING STONE}

The stones shall be roughly squared on joints, beds, and faces. Selected stone, roughly squared and pitched to line, shall be used at all angles and ends of walls.

\section{COPIIGS, BRDGGE SEATS, AND BACK WALIS}

Copings, bridge seats, and back walls, when used in connection with dry rubble masonry, shall conform to the requirements specified for "Mortar rubble masonry."

\section{MEASUREMENT AND PAYMENT}

The quantity of stone masonry to be paid for under this item shall be the number of cubic yards measured in the completed work, and the limiting dimensions shall not exceed those shown upon the plans or fixed by the engineer. The contract price shall include all labor, tools, materials, and other expense incidental to the satisfactory completion of the work.

Concrete used in connection with rubble masonry shall be paid for as in the case of other concrete construction.

American Association of State Highway Officials standard specifications for bridges and incidental structures, 1928 .

\section{RIPRAP}

See 511.72, page 208 .

American Railway Engineering Association, specifications for stone masonry.

See 518.89, page 478 .

\subsection{Cement Rubble Masonry.}

American Association of State Highway Officials, standard specifications for highway bridges and incidental structures, 1928.

\section{ASHLAR MASONRY}

(The full text of this specification includes material relative to construction methods and the following specifications for materials:)

Ashlar masonry shall consist of first-class cut stone masonry laid in regular courses and shall include all work in which, as distinguished from rubble masonry, the individual stones are dressed or tooled to exact dimensions.

\section{STONE}

The stone for this class of masonry shall conform to the requirements of "Stone for masonry" (see 511.9 , p. 212 , American Association of State Highway Officials), and shall be kept free from dirt, oil, or any other injurious material which may prevent the proper adhesion of the mortar.

\section{MORTAR}

Mortar for laying the stone and pointing shall be composed of 1 part of Portland cement and 2 parts of sand, unless otherwise provided.

The individual stones shall be large and well proportioned. They shall not be less than 12 nor more than 30 inches in thickness. The thickness of courses, if varied, shall diminish regularly from bottom to top of wall.

For the purpose of this specification the surface finishes of a stone are defined as follows:

Smooth finished.-Having a surface in which the variations from the pitch line do not exceed $1 / 16$ inch. Fine finished.- Having a surface in which the variations from the pitch line do not exceed $1 / 4$ inch. Rough finished.- Having a surface in which the variations from the pitch line do not exceed $1 / 2$ inch. Scabbled.-Having a surface in which the variations from the pitch line do not exceed $3 / 4$ inch.

Rock faced.-Having an irregular projecting face without indications of tool mark. The projections beyond the pitch line shall not exceed 3 inches, and no part of the face shall recede back of the pitch line.

Stones shall be dressed to exact sizes and shape before being laid and shall be cut to lie on their natural beds with top and bottom truly parallel. Hollow beds will not be permitted. The bottom bed shall be the full size of the stone; and no stone shall have an overhanging top.

Beds of face stone shall be fine finished for a depth of not less than 12 inches.

Vertical joints of face stone shall be fine finished and full to the square for a depth of not less than 9 inches.

Exposed surfaces of the face stone shall be given the surface finish indicated on the plans, with edges pitched to true lines and exact batter. Chisel drafts $1 \frac{1}{2}$ inches wide shall be cut at all exterior corners. Face stone forming the starling or nosing of piers shall be rough finished unless otherwise specified.

Holes for stone hooks shall not be permitted to show in exposed surfaces.

Stretchers shall have a width of bed of not less than one and one-half times their thickness. They 
shall have a length not less than twice their thickness, nor less than 3 feet, and not more than three and one-half times their thickness.

Headers shall be placed in each course and shall have a width of not less than one and one-half times their thickness. In walls having a thickness of 4 feet or less, the headers shall extend entirely through the wall. In walls of greater thickness, the length of headers shall be not less than two and one-half times their thickness when the course is 18 inches or less in height and not less than 4 feet in courses of greater height. Headers shall bond with the core or backing not less than 12 inches. Headers shall hold in the heart of the wall the same size shown in the face and shall be spaced not farther apart than 8 feet center to center. There shall be at least one header to every two stretchers.

Corcs and backing shall consist either of roughly bedded and jointed headers and stretchers, as specified above, or of class B concrete (see 516.3, page 301, American Association of State Highway Officials, specification for "concrete masonry"), as may be specified.

When stone is used for cores or backing, at least half of the stone shall be of the same size and character as the face stone and with parallel ends. No course shall be less than 8 inches thick.

Concrete used for cores and backing shall conform to the requirements specified for "Concrete masonry." When the wall is 3 feet or less in thickness, the face stone shall pass entirely through the wall and backing will not be allowed.

The mortar shall be hand or machine mixed as may be required by the engineer. In the preparation of hand-mixed mortar, the sand and cement shall be thoroughly mixed together in a clean, tight mortar box until the mixture is of uniform color, after which clean water shall be added in such quantity as to form a stiff paste. Machine-mixed mortar shall be preparcd in an approved mixer and shall be mixed not less than $1 \frac{1}{2}$ minutes. Mortar shall be used within 45 minutes after mixing. Retempering of mortar will not be permitted.

Stones for copings of walls, piers, and abutment bridge seats shall be carefully selected and fully ciimensioned stones. On picrs not more than two stones shall be used to make up the entire width of coping, and the copings of abutment bridge seats shall be of sufficient width to extend at least 4 inches under the back wall. Each step forming the coping of a wing wall shall be formed by a single stone which shall overlap the stone forming the step immediately below it at least 12 inches.

Tops of copings shall be given a bevel cut at least 2 inches wide, and beds, bevel cuts, and tops shall be fine finished. The vertical joints shall be smooth fillished and the copings shall be laid with joints not more than $1 / 4$ inch in thickness. The undersides of projecting copings preferably shall have a drip bead.

Joints in copings shall be located so as to provide not less than a 12-inch bond with the stones of the under course and so that no joint will come directly under the superstructure masonry plates.

Pointing shall not be done in freezing weather or when the stonc contains frost.

Joints not pointed at the time the stone is laid shall be thoroughly wet with clean water and filled with mortar. The mortar shall be well driven into the joints and finished with an approved pointing tool. The wall shall be kept wet while pointing is being done and in hot or dry weather the pointed masonry shall be protected from the sun and kept wet for a period of at lcast three days after completion.

Aiter the pointing is completed and the mortar set, the wall shall be thoroughly cleaned and left in a neat and workmanlike condition.
The quantity of stone masonry to be paid for under this item shall be the number of cubic yards measured in the completed work and the limiting dimensions shall not exceed those shown upon the plans or fixed by the engineer. The contract price shall include all labor, tools, materials, and other expense incidental to the satisfactory completion of the work.

American Association of State Highway Officials, standard specifications for highway bridges and incidental structures, 1928.

\section{MORTAR RUBBLE MASONRY}

(The full text of this specification includes material relative to construction methods, and the following specifications for materials:)

Mortar rubble masonry as here specified, shall include the classes commonly known as coursed, random, and random range work, and shall consist of roughly squared and dressed stone laid in cement mortar.

\section{STONE}

Stone for mortar rubble masonry shall conform to "Stone for masonry" (see 511.9, p. 212, American Association of State Highway Officials), and shall be kept free from dirt, oil or any other injurious material which may prevent the proper adhcsion of the mortar.

The mortar used shall conform, as regards materials, proportions, and mixing, to the mortar specified for "Ashlar masonry" (given above).

Individual stones shall have a thickness of not less than 8 inches and a width not less than one and onehalf times the thickness. No stones, except headers, shall have a length less than one and one-half times their width. Stones shall decrease in thickness from bottom to top of wall.

Headers shall hold in the heart of the wall the same size shown in the face and shall extend not less than 12 inches into the core or backing. They shall occupy not less than one-fifth of the face area of the wall and shall be evenly distributed. Headers in walls 2 feet or less in thickness shall extend entirely through the wall.

The stones shall be roughly squared on joints, beds, and faces. Selected stone, roughly squared and pitched to line, shall be used at all angles and ends of walls. If specified, all corners or angles in exterior surfaces shall be finished with a chisel draft.

All shaping or drcssing of stone shall be done before the stone is laid in the wall and no dressing or
hammering which will loosen the stone will be permitted after it is placed.

Copings, bridge seats, and back walls shall be of the materials shown on the plans and when not otherwise specified shall be of class "A" concrete which shall conform to the requirements for "Concrete masonry."

Concretc copings shall be made in sections extending the full width of the wall, not less than 12 inches in thickness, and from 5 to 10 feet long. The sections may be cast in place or precast and set in place in full mortar beds.

The quantity of stone masonry to be paid for under this item shall be the number of cubic yards measured in the completed work and the limiting dimensions shall not exceed those shown upon the plans or fixed by the engineer. The contract price shall include all labor, tools, materials, and other expense incidental to the satisfactory completion of the work.

Concrete used in connection with rubble masonry shall be paid for as in the case of other concrete construction. 
American Association of State Highway Officials, standard specifications for bridges and incidental structures, 1928.

\section{CONCRETE MASONRY}

See 516.3 , page 301

Federal Specifications Board, specification No. 443, United States Government master specification for cement, masonry, October 28, 1926.

See 516.11, page 295 .

\subsection{Brick Masonry.}

American Association of State Highway Officials, standard specifications for highway bridges and incidental structures, 1928.

\section{BRICK MASONRY}

(The full text of this specification includes construction methods.)

\section{DESCRIPTION}

Brick masonry shall consist of brick laid in cement mortar, and shall include such construction with building brick or ornamental brick as may be specified. Brick pavements are not included under this designation.

\section{MATERIALS}

Brick

Brick used for this elass of work shall conform to the requirements specified in A. S. T. M. C62-29. (See 534.11, p. 583.)

\section{Mortar}

The mortar used shall conform, as regards materials, proportions and mixing, to the mortar specified for "Ashlar masonry," see 518.82, American Association of State Highway Officials.

\section{COPINGS}

The top of all exposed brickwork shall be provided with a coping which shall project at least 1 inch beyond the face of the masonry.

This coping shall be provided with a drip bead or shall be beveled so as to cause water to drip clear of the wall. Copings shall be of class "A" concrete (see 516.3, p. 301, American Association of State Highway Officials, specification for "Concrete masonry"), or of the same mortar as that with which the brick are laid and shall be not less than 4 inches thick and cast in sections not less than 3 feet nor more than 5 feet long.

\section{MEASUREMENT AND PAYMENT}

The quantity of brickwork to be paid for under this item shall be the number of cubic yards of brick masonry actually placed in the structure in accordance with the plans or as modified by written instructions from the engineer. This price sliall include all labor, materials, and other expense incidental to the satisfactory completion of the work. Filling material for the interior of the wall, when not of brick, and concrete or mortar copings, shall be paid for on the basis of the number of cubic yards actually placed.

American Face Brick Association, specifications for face brickwork, 1923.

(This association has prepared a standard to be used in writing specifications for face brickwork, with requirements stipulated for the laying, mortar, joining, bonding and cleaning, and pointing.)

American Railway Engineering Association, specifications for buildings for railway purposes.

\section{BRICKWORK, 1926}

\section{GENERAL}

The contractor shall furnish all labor, materials, tools, scaffolding, and equipment, except as otherwise noted, necessary to entirely complete any or all classes of brickwork herein specified according to the class of building and as shown or implied on the accompanying drawings, including all backing, covering of iron and steel, all piers, walls, chimneys, and other special work shown, specified, or otherwise implied.

\section{CLASSIFICATION OF BRICKWORK}

Brickwork shall be classified as either common brickwork or face brickwork. The class of brickwork to be used shall be determined by the class of the building or by notations on the accompanying drawings. Unless otherwise specified common brickwork shall be used on all buildings of mechanical terminals, shops, storehouses, isolated freight houses, and similar buildings. In general, face brick shall be used for passenger stations and auxiliary buildings, combination passenger and freight stations, and freight houses built in conjunction with passenger stations.

\section{COMMON BRICEWORK}

All common brickwork shall be laid even and true to line, plumb, level, and with all joints accurately kept. All brickwork shall be laid with joints not more than three-eighths inch thick and bonded together with full headers every sixth course. All brick shall be good, hard, well-burned brick, free from cracks and uniform in size, shape, and quality, and shall not absorb more than 10 per cent of their weight in water. They shall be laid in a full bed of mortar with shoved joints neatly struck on all exposed walls. The bricks used on the face of the wall shall be selected whole bricks of a uniform size and with true rectangular face.

Porous or salmon brick shall be thoroughly wetted either by immersion or sprinkling before being laid, except in freezing weather.

\section{FACE BRICKWORY}

The exterior face brickwork shall be laid up with a selected and approved pressed face brick. The contractor as a basis for his proposal shall figure on a face brick to cost $\$$........ per thousand f. o. b. building site or company's lines, as provided in the letter of invitation, and any variation from this price, more or less, will be adjusted according to the actual cost of the brick. Face brickwork shall be laid with all stretchers unless otherwise shown and shall be bonded either by blind headers or an approved metal wall tie every sixth course.

All pressed brickwork shall be laid true to line, plumb, level, and with all joints accurately kept. All work shall be laid so that four courses shall not exceed 11 inches in height, and joints shall be raked out to a depth of at least $3 / 8$ inch unless otherwise shown on drawings or ordered by engineer. Where rough face brick is used, instead of raking the horizontal joints, strips shall be placed in those joints as the brick is laid up and after the mortar has hardened properly these strips shall be removed. The vertical joints shall be raked in the usual manner.

The company reserves the right to deviate from the type of joint specified above so as to conform with the type of brick selected. All brick courses shall be so proportioned that they will work out evenly with height of windows and doors. No split or fractional courses will be permitted. All 
backing up of pressed face brick shall be as specified under common brickwork.

Porous or salmon brick shall be thoroughly wetted either by immersion or sprinkling before being laid, except in freezing weather.

\section{DETAII OF BRICKWORK}

All brickwork details, such as lintels, belt courses, and other trim, shall be laid up according to details shown on accompanying drawings and as specified under either common brickwork or face brickwork.

\section{SAMPLES}

The contraetor will furnish samples of all brick to be used, together with prices for the various kinds of face brick submitted for approval of the engineer. The engineer also shall have the option of obtaining samples and prices for face brick. The samples selected and approved will be filed with the engineer and taken as a standard of material to be furnished, and all material used in the work must be equal in all respects to the approved samples.

\section{CEMENT}

The cement shall meet the requirements of the American Railway Engineering Association's specifications, which are identical with those of the American Society for Testing Materials, see A. S. T. M. C9, 516.11, page 294.

\section{SAND}

Sand shall be clean, sharp, coarse, and of grains varying in size. It shall be free from sticks or other foreign matter, but it may contain clay or loam not to exceed 2 per cent.

Where so required for pointing, face work sand shall be clean, sharp, white sand of the very best quality.

\section{IIIME}

Lime usea snall be of good quality, in large lumps, free from cinders, or clinkers, must contain less than 10 per cent impurities and must slake readily in water, making a very soft paste, free from core. Before being used all lime shall be thoroughly slaked with water. No air-slaked lime shall be used. The use of hydrated lime of an approved brand will be permitted at the discretion of the engineer.

\section{MORTAR COLOR}

A mortar color of an approved brand shall be used to color mortar for face brickwork, color and mixture shall be as approved by the engineer. The contractor shall upon request of the engineer lay up samples of face brickwork with different shades of mortar in order that the engineer may decide by comparison the proper shade of mortar to use. These samples shall be of a size not to exceed 6 square feet in area, and the contractor shall, if requested, build not to exceed six such samples. In general, unless otherwise specified, or ordered by the engineer, the mortar shall be colored slightly darker than the face brick used.

\section{MORTAR}

Mortar for all common brickwork except where otherwise specified shall be composed of 1 part Portland cement and 4 parts sand thoroughly mixed and tempered with lime paste to make it work smoothly. Where directed, the mortar is to be as above specified, omitting lime paste.

Mortar for all pressed brickwork shall be of either of the following mixtures, as directed by the engineer: 1 part Portland cement, 1 part lime putty, and 2 parts sand, the sand and cement to be mixed dry, then wet to proper consistency and lime putty added. If pressed face brick is to be laid with colored mortar, the following mixture to be used: 1 part Portland cement to 2 parts sand with lime paste added to make it work smoothly and colored with approved mortar color as directed by engineer. No more mortar containing cement shall be mixed at any one time than can be used within 30 minutes after mixing. Retempering cement mortar which has begun to set will not be permitted. No mortar which has frozen shall be used on the work.

In lieu of cement mortar, the contractor may, when permitted by the engineer, use a patent cement or a natural cement of a brand acceptable to the engineer, in which case 1 part of the patent cement shall be used with $2 \frac{1}{2}$ to 3 parts of sand. Such mortar shall be mixed and used strictly in accordance with the manufacturer's instructions and these specifications. No more mortar than is required for the current day's work shall be mixed.

\section{WATER}

The water shall be clean, reasonably clear, and free from oil, acid, and injurious amounts of vegetable matter, alkalies, or other salts. The contractor shall arrange for his own water supply and shall pay for same.

\section{WOOD CENTERINGS}

The contractor shall provide wood centers for all openings wherever necessary. Centers shall be strongly constructed, made to fit accurately to the work, be well supported and rigidly braced so as to carry all loads until the brickwork has set. At the completion of the work all centering shall be removed from the premises.

\section{SCAFFOLDING, PROTECTION, ETC.}

The contractor shall provide all scaffolding, staging, ladders, etc., necessary for the work. All walls or other parts shall be securely braced and protected against damage by wind and storm during construction.

\section{AITCHORS, STEEL, ETC.}

The contractor shall provide chases for all pipes, set bearing plates for beams, etc., and build into the brickwork all anchors, bolts, ties, nailing blocks, etc., as indicated on drawings and will be responsible for accurate location of same.

\section{BACRING}

Where so shown iron, steel, and other material shall be backed up with brickwork in a manner indicated on details.

\section{FLUE LININGS}

Brick chimneys or flues which are not of such dimensions that fire brick lining is required or called for on detailed plans, shall be provided with a terra cotta flue lining from a point 2 feet below the lowest smoke pipe entering same to base of chimney cap. All joints in this lining must be completely filled with cement mortar and carefully pointed up. No lime mortar shall be used in laying up tile linings or brick flues.

\section{FIRE-BRICB LININGS}

Brick linings for circular concrete stacks shall be laid up with radial fire brick in cement mortar. The interior surface shall be true, plumb, perfectly smooth and without taper, with bed joints not more than one-eighth inch thick. This lining shall be entirely independent and separate from the stack proper. 


\section{VITRIFIED TILE WALL COPING}

Vitrified tile wall coping shall be provided where indicated on the accompanying drawings. It shall be best hard-burned, salt-glazed tile, laid in full bed of mortar of 1 part cement to 3 parts sand, omitting all lime.

\section{CAST CONCRETE COPING}

All walls where so indicated on the drawings shall be coped with cast concrete coping. This is to be of the section as detailed and made in lengths of approximately 6 feet.

\section{CAST CONCRETE SILLS, LINTELS, ETC.}

Where so indicated on drawings, window and door sills, lintels, chimney caps, etc., shall be of cast concrete according to details shown for same.

\section{REQUIREMENTS FOR CAST CONCRETE}

Concrete for cast copings, lintels, sills, caps, etc., shall be composed of 1 part Portland cement, 2 parts sand, and 3 parts crushed stone or gravel of a size to pass a 13 -inch ring. Exposed surfaces shall be troweled smooth, and edges shall be smooth and unbroken. Cast concrete copings, sills, lintels, caps, etc., shall be set true, level, and plumb and carefully pointed out. No cast concrete member shall be set until the concrete is sufficiently hard to prevent damage. Copings, sills, and caps shall be provided with drips.

\section{NEW MASONRY JOINING TO OLD}

The contractor shall use special precaution where new masonry nork joints up with old masonry work, to see that the old work is sufficiently roughed up, anchors provided, and work keyed so that an absolutely tight and neat bond is assured between old and new work.

The contractor shall do all work in connection with cutting out old brickwork, stonework, or concrete where required. Care shall be exercised to see that only such portion of the masonry is disturbed as is necessary.

\section{PROTECTION AND POINTING UP}

The contractor must keep his work covered and protected from the action of the weather or frost. He shall also protect by boxing all dressed or ornamental work liable to damage. At the completion of the work or at any time when so ordered he shall do all patching in a most satisfactory manner, clean down and point up all brickwork, etc., removing all surplus mortar and stains. All window and door frames shall be carefully caulked with oakum and pointed up after they have been inspected and before staff bead is applied.

\section{GENERAL CONDITIONS}

All materials entering into the work and all methods used by the contractor shall be subject to the approval of the engineer, and no part of the work will be considered as finally accepted until all of the work is completed and accepted.

The general conditions as given in 518.50 , page 416 , shall be considered to apply these specifications.

American Society for Municipal Improvements, standard specifications for sewers, 1927.

\section{BRICK MASONRY (SEWER CONSTRUCTION)}

(See 518.67, p. 453. )

Common Brick Manufacturers' Association of America, specifications and general schedule for a brick house.

\section{BRICKLAYING}

1. All brickwork shall be built level, plumb, square, and true to dimensions shown and to approximate thickness indicated on the plans. Leave openings, chases, and build corbels, offsets, pilaster and relieving arches shown or required. Solidly bed all sills or plates resting on masonry. All floor joists shall rest directly on the brick and no bond timbers or wood plates shall be inserted in the brick wall for leveling the joists. Corbel out brick walls and do all necessary beam filling to form fire stops at each floor level as shown on drawings. Build in any and all nailing blocks required.

2. Build in plasterers lath laid with broken joints for the purpose of attaching furring strips.

3. All porous or absorbent brick shall be laid wet, except in freezing weather.

4. All brick shall be laid on a full bed of mortar. Outside 4-inch thickness of exterior walls, entire thickness of any parapet walls or other brickwork exposed to the weather on more than one side of the wall or pier, and walls of flues 4 inches thick shall be laid with full joints. All other brick shall be laid with dry vertical joints with bricks touching end to end.

5. All header courses and all arches and heads of openings, all footings, sills, chimney and parapet caps or copings shall be laid with full mortar joints.

6. All projections of footings shall be formed of headers.

7. Lay all window sills of brick on edge with a slope of 2 inches to the foot to conform to standard slope of wood window sills except where otherwise shown.

8. Lay any brick door sills and steps so shown with brick on edge sloping three-eighths inch to the foot.

9. Porch and terrace floors where shown to be paved with brick shall be laid on a $1: 8$ concrete bed 3 inches thick composed of 1 part Portland cement to 8 parts sand, gravel, crushed stone, or cinders, graded to proper size. Brick shall be laid on a setting bed one-half inch thick of cement mortar. Vertical joints shall be thoroughly filled and struck flush.

10. Basement floors paved with brick, shall be laid on edge on a well-tamped bed of sand 2 inches thick. Joints shall be completely filled with grout swept in with a broom.

11. If basement is to have a cement floor, same shall be of $1: 8$ concrete 3 inches thick, troweled or floated to a smooth finish.

12. Unless otherwise indicated on the drawings or agreed upon, all brickwork shall be laid up in common bond with not exceeding five stretcher courses between courses containing headers. Each course containing headers shall be composed of one header and one stretcher alternating the full length of the course.

13. Footings, sills, chimneys, and parapet caps, brick steps, cheek walls to steps, brick porch and terrace floors shall be laid in cement mortar.

14. All exterior walls below first floor level and brickwork below first floor level except footings shall be laid in cement-lime mortar.

15. All brick walls above first story level and other brickwork not otherwise mentioned may be laid in lime mortar.

16. All joints shall be approximately one-half inch thick.

17. The joints of all exterior exposed brickwork fireplace, and any brickwork showing behind range, shall be neatly struck. All other joints shall be plain cut, unless otherwise indicated. 
18. Wherever brick arches are indicated, the radius of same shall be made up in the joints and not in the brick.

19. Furnish and set any steel angles over openings indicated or noted on drawings, also any steel beams indicated on drawings. All steel angles and steel beams shall be at least 8 inches longer than the clear span.

\section{BRICKLAYING IN FREEZING WEATHER}

20. When brickwork is laid in freezing weather in localities where alternate freezing and thawing may be expected, cement mortar shall be used.

21. In localities where a continuous cold spell may be expected, lime mortar may be used if the brickwork is laid a sufficient length of time before the usual date of the spring thaw to allow the mortar to set.

22. Precautions shall be taken to prevent the mortar freezing before it is placed in the wall and the next course of brick placed upon it. Brick laid in freezing weather shall not be wet.

\subsection{Arches.}

American Railway Engineering Association, specifications for stone masonry.

See 518.89, below.

American Society for Municipal Improvements, standard specifications for sewers, 1927.

(This specification includes requirements for the construction of arches of segmental block sewers.) See 518.67 , page 453 .

\subsection{Monuments.}

(No nationally recognized specifications available.)

\subsection{Miscellaneous Specifications for Ma- sonry.}

American Railway Engineering Association, specifications for buildings for railway purposes.

\section{STONE MASONRY AND CUT STONE WORK, 1926}

(In addition to the material given below, the full text of this specification includes requirements for setting, protection, and pointing.)

\section{GENERAI}

The contractor shail furnish all labor, materials, tools, scaffolding, and equipment, except as otherwise noted, necessary to entirely complete any or all classes of stone masonry and cut stone work herein specified, according to the class of building and as shown on the drawings.

\section{DESCRIPTION OF STONE}

All stone specified or shown on drawings shall be sound, durable, well seasoned, from quarries approved by the engineer, and each stone shall be laid on its natural bed. When required by the engineer sample shall be furnished, which shall be about 4 by 7 by 1 inch thick produced so that the large faces shall show the grain or rift of the stone, the finish specified to be indicated on the large faces and at least two of the edges shall be rock face. Similar samples shall be provided when select stock is required for certain positions in the building. Samples submitted are to be typical of the extremes which the contractor proposes to furnish.

\section{CUTTING AND SETTING DRAWINGS}

The cut-stone contractor shall prepare and submit to the engineer for his approval, when required, complete cutting and setting drawings (in triplicate) for all the cut-stone work in this contract. Such drawings shall show in detail sizes and dimensions of stones, the arrangements of joints and bondings, anchoring, and other necessary details.

\section{CARVING}

All carving shall be done by skilled carvers from approved models.

\section{CUTTING AND FINISH}

All exposed faces shall be cut true. The stone shall be cut full to the square with joints as required. The arrises shall be full and true. Beds, ends, and tops shall be dressed straight and at right angles to the face unless otherwise shown. No patching or hiding of defects will be permitted, and lewis holes shall not be cut on exposed surfaces.

Washes shall be as steep as practical and drips of sufficient depth to shed water shall be provided on all projecting stones and courses.

Specially graded stone acceptable as to hardness and color, as per samples to be submitted, shall be employed for grade course steps and all other positions exposed to direct wear. Steps shall be cut with a slight pitch to the front.

The finish on exposed surfaces, except rock-face work, shall be hand-tooled work not less than 10 cuts to the inch.

Where molded work is tooled, the tooling shall run in the direction of the molding and not across.

Rock-face work shall have no projection exceeding 2 inches. Edges shall be pitched to a straight line.

\section{IEWIS HOLES}

Lewis holes shall be cut in all stones weighing more than 300 pounds. No lewis or other holes shall come within $2 \frac{1}{2}$ inches of the exposed face of the stone, unless the stone is less than 5 inches thick.

\section{MORTAR}

Mortar for all stonework, except where otherwise specified, shall be composed of 1 part Portland cement, 1 part lime putty, and 2 parts sand, the sand and cement to be mixed dry, then wet to proper consistency, and the lime putty added. If stonework is to be laid with colored mortar the following mixture shall be used: 1 part Portland cement, 2 parts sand, with lime paste added to make it work smoothly and colored with approved mortar color. No more mortar containing cement shall be mixed at any one time than can be used within 30 minutes after mixing. Retempering cement mortar which has begun to set will not be permitted. No mortar which has frozen shall be used on the work.

The cement shall meet the requirements of the American Railway Engineering Association (which are the same as for A.S.T. M. C9; see 516.11, p. 294). Cement that has hardened or partially set shall not be used.

Sand and lime specifications as given in A. R. E. A. specification for brickwork (518.83, see p. 475) apply to sand and lime for stonework.

\section{ANCHORS AND DOWELS}

Proper provision shall be made for anchoring and doweling the stone.

Anchors and dowels shall be of the proper size and shape and thoroughly galvanized or coated with asphaltum paint.

\section{BROKEN COURSED ASHLAR}

The face stones shall be rock-faced edges pitched to a straight line, shall have parallel beds and rectangular joints.

The beds and joints for 6 inches back from face shall be dressed to lay not over $1 / 4$-inch joint. 
The stones need not be laid up in a regular course, but shall be laid level on their natural beds, shall be well-bonded, having at least one header, extending entirely through the wall, for every 10 square feet of face surface. Headers shall be placed so that they come halfway between the header of the course above and below. No stone shall be less than 6 inches thick, and no stone shall measure in its least horizontal dimensions less than 9 inches.

\section{BOWLDER MASONRY}

Where shown on the drawings, foundation walls shall be composed of stone of proper size and thickness. They shall be of fair shape, and spalled so that they will lay with good even bearings on the wall. All stones shall be laid in full beds of mortar, all interstices filled, and all exposed faces neatly pointed. All work must be thoroughly done and well bonded. A header, extending entirely through the wall, shall be built in every 8 square feet of surface. Each header shall break joint with the headers in the courses above and below.

\section{GENERAL CONDITIONS}

All material entering into the work and all methods used by the contractor shall be subject to the approval of the engineer and no part of the work will be considered as finally accepted until all the work is completed and accepted.

The general conditions as given in 518.50, page 416 shall be considered to apply to these specifications.

American Railway Engineering Association, specification for stone masonry.

\section{GENERAL}

\section{STANDARD SPECIFICATIONS}

1. The requirements for cement and concrete shall be those adopted by the American Railway Engineering Association.

\section{GENERAL REQUIREMENTS}

\section{STONE}

2. Stone shall be (state kind), and shall be sound, hard, and durable, of approved quality and shape, free from holes, seams, drys, or other imperfections.

\section{MORTAR}

3. Mortar shall be mixed in a suitable box, or in a machine mixer, preferably of the batch type, and shall be kept free from foreign matter. The size of the batch and the proportions and the consistency shall be as directed by the engineer. When mixed by hand the sand and cement shall be mixed dry, the requisite amount of water then added and the mixing continued until the cement is uniformly distributed and the mass is uniform in color and homogeneous.

\section{IAYING}

4. The arrangement of courses and bond shall be as indicated on the drawings, or as directed by the engineer. Stone shall be laid to exact lines and levels, to give the required bond and thickness of mortar in beds and joints.

5. Stone shall be cleansed and dampened before laying.

6. Stone shall be well bonded, laid on its natural bed and solidly settled into place in a full bed of mortar.

7. Stones shall not be dropped on or dragged over the wall, but shall be placed without jarring stones already laid.
8. Heavy hammering shall not be allowed on the wall after a course is laid.

9. Stone becoming loose after the mortar is set shall be relaid with fresh mortar.

10. Stones shall not be laid in freezing weather unless authorized by the engineer. If laid, the stone shall be first freed from ice, snow, or frost by warming. The sand and water used in the mortar shall be heated.

\section{POINTING}

11. Before the mortar has set in beds and joints, it shall be removed to a depth of not less than $1 \frac{1}{2}$ inches. Pointing shall not be done until the wall is complete and mortar set; nor when frost is in the stone.

12. Mortar for pointing shall consist of equal parts of sand, sieved to meet the requirements, and Portland cement. In pointing, the joints shall be wet, and filled with mortar, pounded in with a "set-in" or calking tool and finished with a beading tool the width of a joint, used with a straightedge.

\section{BRIDGE AND RETAINING WALL MASONRY, ASHLAR STONE}

13. The stone shall be large and well proportioned. The thickness of the courses shall diminish regularly from bottom to top. The maximum thickness shall not be more than twice the minimum thickness and no course shall be less than 12 inches thick.

\section{DRESSING}

14. Beds and builds shall be square with each other and dressed true and out of wind. They shall be fine pointed, so that the mortar layer shall not be more than three-eighths of an inch thick when the stone is laid. Hollow beds shall not be permitted.

15. Joints in face stone shall be full to the square for a depth equal to at least one-half the height of the course, but in no case less than 10 inches. The backs shall be roughly scabbled to avoid overhang.

\section{FACE OR SURFACE}

16. Exposed surfaces of the face stone where not otherwise specified shall be rock faced with edges pitched to the true lines and exact batter. The face shall not project more than 3 inches beyond the pitch line.

17. Chisel drafts, not less than $1 \frac{1}{2}$ inches wide, shall be cut at exterior edges and shall be neat and accurate.

18. Holes for stone hooks shall not be permitted to show in exposed dressed surfaces. Such stone shall be handled with clamps, keys, lewis, or dowels.

\section{STRETCEERS}

19. Stretchers shall not be less in length than two and one-half times their thickness with an average width of bed at least one and a quarter times their thickness.

\section{HEADERS}

20. Headers shall not be less in length than two and one-half times their thickness, shall occupy one-fifth of the face of the wall, and shall not be less in width than one and one-fourth times their thickness.

21. Headers shall hold in heart of wall the same size shown in face, so arranged that a header in a superior course shall not be laid over a joint, and a joint shall not occur over a header; the same disposition shall occur in back of wall.

22. Headers in face and back of wall shall interlock when thickness of wall will admit, 
23. Where the wall is 3 feet thick or less, the face stone shall pass entirely through. Backing shall not be permitted.

\section{BACKING}

24a. At least one-half of the backing stone shall be of the same size as the face stone and be roughly squared; the remainder of backing stone shall be large, well shaped, and roughly bedded and jointed. Bed joints shall not exceed 1 inch. Vertical joints in back of wall shall not exceed 2 inches. The interior vertical joints shall not exceed 4 inches.

Voids shall be thoroughly filled with (1) concrete, or (2) spalls, fully bedded in cement mortar.

$24 b$. Backing shall be of (1) concrete, or (2) headers and stretchers, as specified in paragraphs 19 and 20 , and heart of wall filled with concrete.

(a) Paragraphs $24(a)$ and $24(b)$ are so arranged that either may be eliminated according to requirements.

25 . Where the wall will not admit of such arrangement, stone not less than 4 feet long shall be placed transversely in heart of wall to bond the opposite sides.

26. Where stone is backed with two courses, neither course shall be less than 9 inches thick.

\section{BOND}

27. Bond of stone in face, back, and heart of wall shall be at least one-half the thickness, but not less than 10 inches. Backing stones shall be laid to break joints with the face stone and with one another.

\section{COPING}

28. Coping stone shall be full size throughout, of dimensions indicated on the drawings.

29. Beds, joints, and top shall be fine pointed.

30. Location of joints shall be determined by the position of the bed plates, as indicated on the drawings.

\section{IOCKS}

31. Where required, coping stones, stones in steps of abutment wings, and stones on piers and ice breakers shall be fastened together with iron cramps or dowels in the position indicated on the drawings.

\section{ICE BREAKERS}

32. In large piers with ice breakers, the face of the stones forming the ice breakers shall have a 2-inch tooled margin draft all around, and shall be dressed off between to a uniform surface with a point or pick. The beds and vertical joints shall be pick dressed the full width of the stones, so as not to exceed $1 / 4$ of an inch in width. The backs of the stones shall be scabbled off so as to form square and vertical joints with the backing. The courses shall be arranged as shown in detail plans.

\section{TRESTLE PIERS}

33. Small piers carrying columns shall have backing of precisely the same thickness as the face stones, with beds dressed with the same care. The vertical joints shall be square, and shall not exceed $1 / 2$ inch in width. Such piers shall be built wholly of dimension stones, and the courses shall be arranged as shown in detailed plans. The footings shall in all cases be of concrete, reinforced, if necessary.

\section{BRIDGE AND RETAINING WALL MASONRY- BLOCK RUBBLE}

34. The stone shall be roughly squared, and may be laid in irregular courses. Beds shall be parallel, roughly dressed, and the stone laid horizontal to the wall. Face joints shall not be more than 1 inch thick. Bottom stone shall be large, selected, flat stone.

35. The wall shall be compactly laid, having at least one-fifth the surface of back and face headers arranged to interlock, having all voids in the heart of the wall thoroughly filled with concrete or suitable stones and spalls, fully bedded in cement mortar.

\section{ARCH MASONRY, ASHLAR STONE}

\section{FALSE WORK}

36. The contractor shall provide and erect false work for the temporary support of arch centers. This may be of any preferred design, subject to approval, but shall be of ample strength and stiffness to safely, and without undue deformation, carry the whole load of completed arch. The contractor shall be entirely responsible for the stability of the false work, and for any damage that may result from overloading, wind, flood, backwater, logs, ice, fire, or other cause. Should piling be necessary, the company may, at its option, drive the piles at the cost of the contractor.

\section{CENTERS}

37. The contractor shall provide and erect centers strongly framed and braced longitudinally and transversely, the upper surface conforming accurately to the curve of the intrados of the arch, after making proper allowance for settlement under load. The lagging shall consist of 2 by 3 inch dressed plank laid transversely along the joints of the voussoir. Arch centers may be of any preferred design (subject to approval). At the ends, and in large arches at the intermediate posts, the centers shall be supported on sills or plates, provided with sand boxes, folding oak or other hardwood wedges, or both. Centers shall not be unequally or eccentrically loaded, and great care shall be taken in striking the arch centers to insure a slow and even subsidence, and to avoid unequal stresses. They shall not be struck without the express permission of the engineer.

38. Voussoirs or ring stones shall be full size throughout and dressed true to templet, and shall have bond not less than width on intrados.

\section{DRESSING}

39. Joints of voussoirs and intrados shall be fine pointed. Mortar joints shall not exceed one-fourth inch.

\section{FACE OR SURFACE}

4.0. Exposed surface of the ring stone shall be (1) fine pointed, or (2) rock faced, with a marginal draft.

41. Number of courses and depth of voussoirs shall be indicated on the drawings.

42. Voussoirs shall be placed in the order indicated on the drawings.

\section{BACKING}

43. Backing shall consist of (1) concrete, or (2) large stone, shaped to fit the arch bonded to the spandrel and laid in full bed of mortar.

44. Extrados shall be grouted and finished with $11 / 2$-inch coat of mortar applied evenly for a finishing coat, upon which, when required, shall be placed a covering of approved waterproofing material.

BENCH WALLS, PIERS, SPANDREIS, ETC.

45. Bench walls, piers, spandrels, parapets, wing walls, and copings shall be built under the specifications for Bridge and Retaining Wall Masonry, Ashlar Stone, as far as applicable. 
ARCH MASONRY-BLOCK RUBBLE

\section{DRESSING}

46. Voussoirs or ring stones shall be full size throughout and shall have bond not less than width on intrados.

47. Beds shall be roughly dressed to bring them to radial planes.

48. Mortar joints shall not exceed 1 inch.

\section{FACE OR SURFACE}

49. Exposed surfaces of ring stones shall be rock faced, and edges pitched to true lines.

50 . Voussoirs shall be placed in the order indicated on the drawings.

\section{BACRING}

51. Backing shall consist of (1) concrete, or (2) large stone, shaped to fit the arch, bonded to the spandrel, and laid in full bed of mortar.

52. Extrados shall be grouted and finished with 11/2-inch coat of mortar applied evenly for a finishing coat, upon which, when required, shall be placed a covering of approved waterproofing material.

BENCH WALIS, PIERS, SPANDKELS, ETC.

53. Bench walls, piers, spandrels, parapets, wing walls, and copings shall be built under the specifications for Bridge and Retaining Wall Masonry, Block Rubble, as far as applicable.

\section{CULVERT MASONRY}

54. Culvert masonry shall be laid in cement mortar. Character of stone and quality of work shal be the same as specified for Bridge and Retaining Wall Masonry, Block Rubble.

\section{SIDE WALLS}

55. One-half the top stones of the side walls shall extend entirely across the wall.

\section{COVER STONES}

56. Cover stones shall be sound and strong and of thickness indicated in drawings.

\section{END WALLS, COPING}

57. End walls shall be covered with suitable coping, as indicated on the drawings.

\section{DRY MASONRY}

58. Dry masonry shall include dry retaining walls and slope walls.

\section{RETAIIIIG WAILS}

59. Retaining walls and dry masonry shall include all walls in which stone laid without mortar is used for retaining embankments or for similar purposes. Flat stones at least one and one-half times as wide as thick shall be used.

60 . Bed and joint shall be roughly squared.

61. Beds and face joints shall not exceed 1 inch. Vertical joints at back 2 inches and interior joints 3 iriches.

62. Stone of different sizes shall be evenly distributed over entire face of wall, generally keeping the larger stone in lower nart of wall.

63. The work shall be well bonded, and shall present a reasonably true and even surface, free from holes or projections.

\section{SIOPE WALLS}

64. Slope walls shall be built of such thickness and slope as indicated on plans or directed by the engineer. Stone shall be placed at right angles to the slopes.

\subsection{MISCELLANEOUS SPECIFICATIONS FOR CONSTRUCTION WORK.}

American Electric Railway Association, specification and form of contract for electrical conduit construction, serial No. D200-26, 1926.

(This specification includes items for proposal sheet, contract, general condition of contract, specification for materials, and construction. Appendixes containing specifications for tile duct and fiber duct are also given. Below are given specifications for materials and tile duct.)

\section{SPECIFICATION FOR MATERIALS QUAITY}

All materials used throughout shall be the best of their respective kinds, unless specified to the contrary and samples of same shall be submitted whenever requested; also all models, patterns, and templates required, and same shall be subject to the inspection and approval of the engineer before any work is done or materials ordered.

\section{OPTION}

When more than one kind of manufacture of a material is specified the option shall be with the contractor. When one kind or "approved as equal" is specified the choice shall be made by the engineer, who may select an "equal" if requested by the contractor.

\section{DUCT}

All duct used for conduit shall be in accordance with the standard specification of the A. E. R. A., as given below. ${ }^{94}$

\section{CEMENT}

Cement used for conduit construction shall be a moderately quick-setting, high-grade brand of Portland cement, which shall fulfill the requirements of the standard specifications and tests for Portland cement of the American Society for Testing Materials. (See 516.11, p. 294.) When the cement is furnished by the contractor he shall provide a sufficient quantity of cement and a suitable building for storage of same on the site at least two weeks before the cement is required on the work to enable the en gineer to make 7-day tests. The contractor shall keep records of the dates and quantities of the various lots of cements received and of the cement used, and said records shall be accessible to the engineer at all times.

\section{SAND}

Sand used for conduit construction shall be considered to be all the fine portion of the aggregate which shall pass through a $1 / 4$-inch diameter hole. It shall be a clean siliceous material, free from vegetable loam and other organic matter with not more than 6 per cent passing a 100 -mesh sieve. It shall have sizes of grain varying between the largest and smallest sizes and shall not interfere with the normal set of the cement. Comparison tests on the supplied sand, with standard sand, if required by the engineer shall be made by making a briquet of 1 part of cement and 3 parts sand, and these briquets shall develop an average strength at seven days of at least 70 per cent of the standard briquet.

94 The specification for the tile duct is not a part of the conduit pecification, as it will usually be found preferable for the company specication, as it will usually be found preferable for the company to purchase the thle duct and deliver same to the conduit contractor. If, however, it is desirable to have the conduit contractor furnish the tile duct, this paragraph should be changed to correspond with
the specification for tile duct, as given below. 


\section{STONE}

Stone used for tile conduit construction shall include all the aggregate, too large to pass through a $1 / 4$-inch diameter hole, and that which will pass through a 1-inch diameter ring. ${ }^{95}$ It shall be clean, hard, and durable and shall be graded in sizes from the largest to the smallest. Clean gravel, if approved by the engineer, may be substituted for stone.

\section{WATER}

Water used for mixing concrete shall be fresh, free from acid, alkaline, or organic matter which would injure the concrete or brickwork or would interfere with the normal set of the cement.

\section{CONCRETE}

Concrete, unless otherwise ordered by the engineer, shall be composed of 1 part cement, - parts of sand, and - parts of stone. These proportions,
If the temperature should fall below the freezing point, after the concrete has been deposited, it shall be so thoroughly protected that the cement can attain its final set before frost reaches it. If the temperature is near the freezing point when the concrete is mixed, the material shall be warmed throughout before being placed in the machine. With this precaution it will be allowable to work with the thermometer as low as $20^{\circ} \mathrm{F}$. if the indications point to a rising temperature. If there is a probability of a drop in temperature, however, concreting shall not be started with the temperature below $40^{\circ} \mathrm{F}$. Work shall not be started at any time if the engineer does not approve, although the responsibility for injury by frost shall be solely upon the contractor.

\section{MORTAR}

Mortar shall be composed of 1 part cement and 3 parts of sand, both as previously specified. Mortar that has been mixed over one-half hour or that

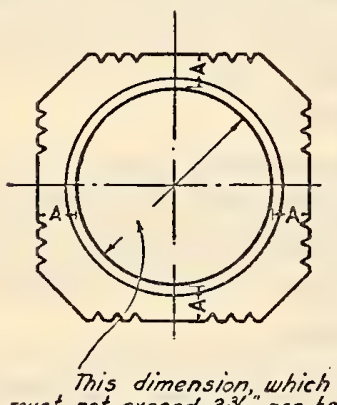
must not exceed 3 is, nor be less than. $3 \%$; must be unitorm in alf the duct of any ane onder.

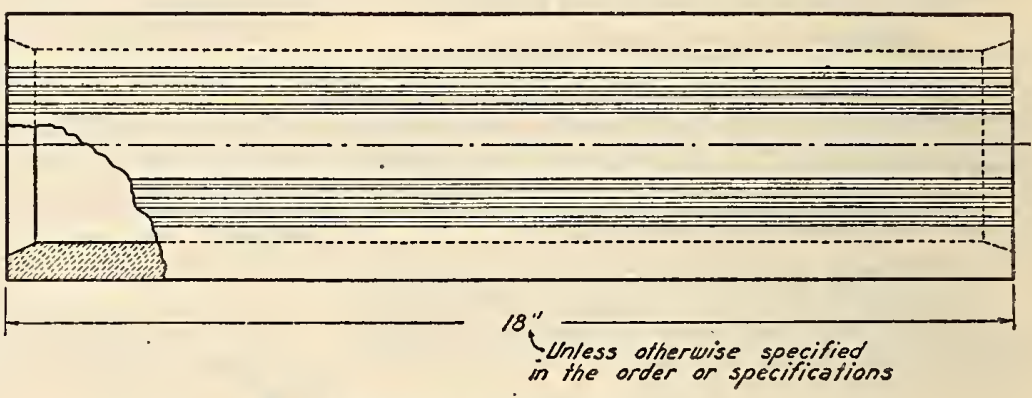

To be furnished in accordance with Company's Standard Specifications

A $\left\{\begin{array}{l}\text { Hinimum Thickness } \% 16^{4} \\ \text { Average Thickness } 5 \% 8\end{array}\right.$

FIGURE 132.-Three and one-half inch square tile duct for conduit

however, may be varied at the discretion of the engineer.96 One bag of cement containing 94 pounds net shall be considered equivalent to 1 cubic foot.

The concrete shall be machine mixed, or if approved by the engineer, hand mixed, in which case the mixing shall be done on a water-tight surface.

In either case, the materials shall be placed dry and turned long enough to be well mixed before the water is added.

The whole shall then be turned until the mixture shall be such that it shall show a mass uniform in color and homogeneous.

All materials shall be mixed wet enough to form a concrete of such consistency as may be rammed readily into the forms and about the conduit and which at the same time can be conveyed from the mixer to the forms without separation of the coarse aggregate from the mortar.

After interruption of the mixing for any length of time, the mix for the first two batches shall be enriched by using more cement and sand for the purpose of feeding the mixer; the proportion to be determined by the engineer.

No retempering of mortar or concrete will be allowed.

05 Fiber conduit laid with 1 -inch spacing requires a finer stone. The so-called "pea stone" which will pass through a $1 / 2$-inch hole is The so-called "pea stone" whe

commonly used in such case. The usual proportions found desirab
cement, 3 parts sand, and 5 parts stone. has commenced to set shall not be used. The mortar shall be as dry as practicable to obtain adhesion.

\section{SPECIFICATION FOR TILE DUCT}

\section{DEFINITION OF TERMS}

\section{Terminology}

The terminology used is the standard of the American Electric Railway Association.

\section{Parties to Contract}

The word "company" where occurring in this specification shall mean the purchaser of the material and labor hereinafter referred to, or its duly authorized representative.

The word "manufacturer" where occurring in this specification shall mean the party accepting the order to furnish the material and labor hereinafter referred to, or its duly authorized representative.

\section{PHYSICAL PROPERTLES}

\section{Mechanical}

\section{REQUIRED STRENGTH}

The completed duct must have the necessary mechanical strength and toughness to prevent chipping at the ends and breakage in ordinary handling. 


\section{DRAWINGS OF DIMENSIONS}

\section{Compliance with Drawings}

Each piece of duct must conform in shape and allowable dimensions to the company's drawing for the kind of duct ordered, but the dimensions must be uniform in all duct furnished.

\section{Bore Test}

A straight mandrel, 18 inches long, having the same shape in cross section as the duct bore as shown on the company drawing, and having dimensions equal to the minimum bore permitted by the drawing, shall be pushed through each piece of duct. (See figs. 132 and 133.)

\section{Short Pieces}

In each shipment there shall be included two pieces each of $4,6,8,9$, and 12 inch lengths for every thousand feet of duct.

\section{WORK NANSEIP AND FLNISE}

Workmanship and Materials

All workmanship and materials shall be the best of their respective kinds and shall be in full accord with the best modern engineering practice.

\section{SPECIFICATIONS FOR CONCRETE DERRICK FOUNDATIONS}

(Prepared in cooperation with Portland Cement Association.)

See 516.9 , page 327 .

American Railway Association, telegraph and telephone section, specification for communication underground conduit construction, No. 1-C-1, 1927.

(This specification includes the following specifications for materials:)

\section{MANHOLES AND HANDHOLES}

Manholes and handholes shall be constructed of either concrete or hard-burned brick. In any case the material should be used that will provide the most satisfactory and economical construction.

\section{MATERIAL ENTERING INTO CONCRETE AND MORTAR}

Concrete consists of certain proportions of cement, sand, broken stone or gravel, and water. The quality of each of these materials used in connection with the conduit system shall be as outlined below:

(a) Portland cement shall be used.

(b) Sand shall be clean, sharp, coarse, and free from clay, loam, dirt, and any vegetable matter.

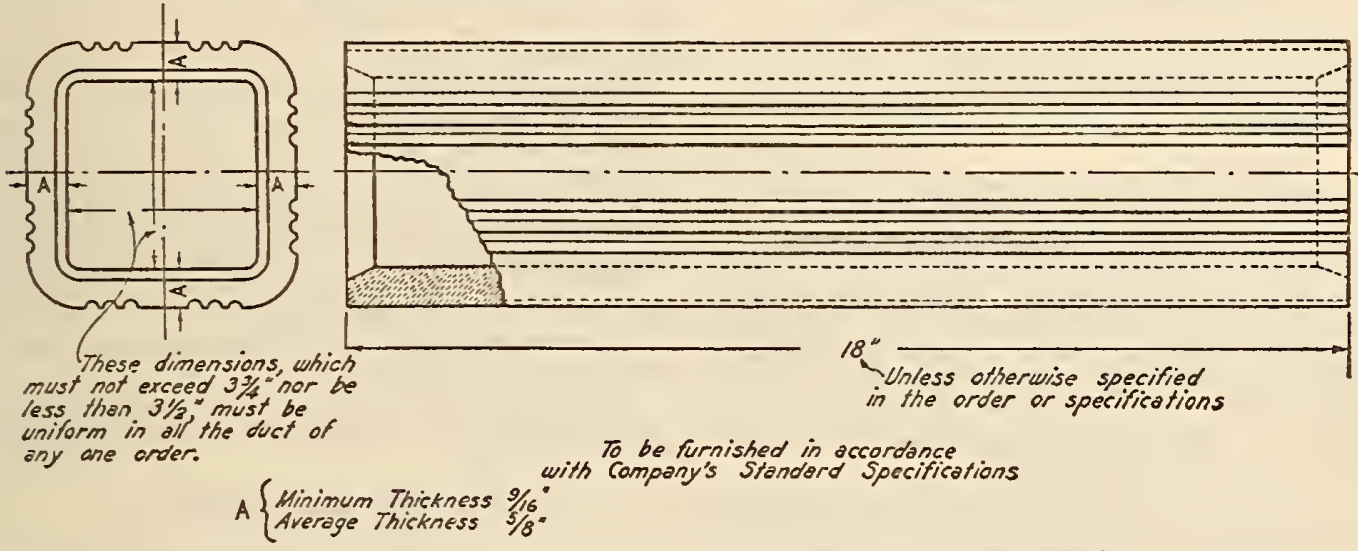

FIGURE 133.-Three and one-half inch round tile duct for conduit

\section{Finish}

Each piece of duct must consist of whole, sound, thoroughly vitrified and glazed tile, straight from end to end, with smooth interior surface, free from blisters, sharp corners, and obstructions.

The bore must be symmetrical with, and the ends must be cut off square with, the longitudinal axis of the duct. The inner edge of the ends of each piece of duct must be chamfered so that sharp edges will not be encountered when cable is drawn in.

The outer surface of all the ducts must be scored approximately as shown on the company's standard drawing and in such manner as to give cement a hold on the surface of the duct.

\section{INSPECTION AND REJECTION}

All duct will be subject to inspection at the factory or on delivery, or both, as desired by the company. The inspection will cover quality, checking up with drawings, and breakage.

Only such duct as passes the inspection will be accepted.

American Petroleum Institute, code of recommended field practice, standard rigs and derricks, July, 1928. (c) The broken stone shall be hard, free from all impurities and dust, and shall be of such size that it will pass through a $3 / 4$-inch mesh and be retained on a $1 / 4$-inch mesh.

Coarse gravel may be substituted for the broken stone if the latter is not available. The gravel shall be clean, hard, durable pebbles, which shall range in size from $1 / 4$ to $3 / 4$ inch. If necessary, it shall be screened.

(d) The water shall be clean, fresh, and free from sewage, oils, or other detrimental impurities.

\section{PROPORTIONS}

The proportions of the ingredients entering into concrete for trench work and manhole floors shall be no weaker than the following mixture: 1 part cement, 3 parts sand, and 6 parts broken stone or gravel.

The proportions of the ingredients entering into concrete for manhole or handhole walls and roofs shall be no weaker than the following mixture: 1 part cement, $2 \frac{1}{2}$ parts sand, and 5 parts brokenstone.

The proportion of the ingredients entering into mortar shall be no weaker than the following mixture: 1 part cement and 3 parts sand. 
520.

\section{GENERAI ITEMS}

American Ceramic Society, tentative methods of analysis of glass, 1928.

\section{(A) PREPARATION OF SAMPIE}

The sample is washed with distilled water (if necessary) and dried thoroughly with a clean cloth.

The cleaned and dried glass is chipped into an agate mortar and ground to pass 200 mesh. All of the sample is ground if the glass does not appear homogeneous. The finely ground glass is then placed in a clean bottle and tightly stoppered, as it is very hygroscopic.

Note.-The steel mortar was discarded by the committee, due to its possibilities of contamination.

The sample may be sieved if the sieve is used for glass only and well cleaned by brushing and air.

\section{(B) FUSION OF THE SAMPIE}

If the sample is fused immediately no ignition is necessary. Otherwise the ignition must be run and the analysis calculated back to a moisture-free basis. Example:

$$
\frac{\mathrm{SiO}_{2} \times 100}{100-\text { moisture }}=\mathrm{SiO}_{2} \text { in moisture-free glass. }
$$

A $1 \mathrm{~g}$ portion of the prepared sample is carefully and thoroughly mixed with $5 \mathrm{~g} \mathrm{Na} \mathrm{CO}_{3}$ in a platinum crucible of 30 to 40 cc capacity. At first the crucible (covered) is heated over a small flame. The heat is gradually increased until a quiet and perfectly clear fusion is obtained. At no time should there be a violent reaction. The flame should be directed against the bottom and sides at an angle and should not envelop the whole crucible

When fusion is complete, the flame is removed and the cake allowed to solidify in a thin layer around the sides and bottom of the crucible. This effect may be obtained by seizing the crucible at the top with a pair of tongs and using a gentle gyratory motion.

When cool, the cake is loosened by gentle pressure on the sides of the crucible or by applying a small flame to melt a thin layer noxt to the crucible when the cake may be easily removed.

NotE.-According to Dittrich, potassium salts are more prone to pass into the precipates than sodium and a fusion with the double salts is not as advisable as one with $\mathrm{Na}_{2} \mathrm{CO}_{3}$. The high melting point of $\mathrm{Na}_{2} \mathrm{CO}_{3}$ is also an aid to complete fusion.

The fusion niay he made in an electric muffle hut is not as quick as the Bunsen burner, as the heat is slower of regulation.

\section{(C) SIIICA}

The fusion is transferred to a platinum dish, porcelain casserole, or Pyrex beaker of 150 to $400 \mathrm{cc}$ capacity with hot $\mathrm{H}_{2} \mathrm{O}$, making sure that the transfer is complete. This may be done with 75 to $100 \mathrm{cc}$ $\mathrm{H}_{2} \mathrm{O}$. Cover with a watch glass and place on the steam bath till distintegration is complete. This may be aided with a blunt glass rod, breaking up all the large lumps. After disintegration is complete $\mathrm{HCl}$ is added in excess, being careful that the reaction is not too violent and that the dish is tightly covered. When the reaction is complete remove the cover and wash into the dish with $\mathrm{H}_{2} \mathrm{O}$. Wash down the sides of the dish and place uncovered on the steam bath and evaporate to apparent dryness.
Notes.-A platinurn dish is preferred, since porcelain and Pyrez are attacked by the silicic acid of the solution.

Water is added to the melt before adding $\mathrm{KCl}$, as it affects the precipitate in such a way as to make it hreak up easily and facilitates better washing, as it is less likely to contain occluded soda.

There is no need of elaborate haking after the first evaporation as the baking affects the $\mathrm{SiO}_{2}$ and the bulk of this is already taken down anyway. Refnement comes after the second ovaporation.

Moisten the dry mass with $\mathrm{HCl}(1: 1)$ and add 50 to $100 \mathrm{cc}$ hot $\mathrm{H}_{2} \mathrm{O}$. Digest on the steam bath for 10 minutes and decant through the filter. Add $50 \mathrm{cc}$ hot 1 per cent $\mathrm{HCl}$, break up any large lumps with a blunt stirring rod, and decant again. Transfer the precipitate to the filter, police the beaker with hot 1 per cent $\mathrm{HCl}$, and wash the precipitate four times with hot $\mathrm{H}_{2} \mathrm{O}$.

Evaporate the filtrate to apparent dryness on the steam bath and bake at not more than $110^{\circ} \mathrm{C}$. for one hour to dehydrate the silica. Moisten the dry mass with hot $\mathrm{HCl} 1$ per cent and add $50 \mathrm{cc}$ hot $\mathrm{H}_{2} \mathrm{O}$. Digest on the steam bath for 10 minutes and filter into a $400 \mathrm{cc}$ beaker. Police the beaker with hot $\mathrm{HCl}, 1$ per cent, and wash the precipitate four times with hot $\mathrm{H}_{2} \mathrm{O}$.

Place the combined precipitates in a platinum crucible, dry thoroughly, and burn off the papers without allowing to burst into flame. Blast for 15 minutes at $1,200^{\circ} \mathrm{C}$. Weigh and blast again at $1,200^{\circ} \mathrm{C}$. to constant weight. Moisten the contents of the crucible with $\mathrm{H}_{2} \mathrm{O}$, add 3 drops $\mathrm{H}_{2} \mathrm{SO}_{4}$ and fill the crucible with $\mathrm{HF}$. Evaporate to dryness and blast for a few minutes at $1,200^{\circ} \mathrm{C}$. The loss in weight is $\mathrm{SiO}_{2}$. Report as $\mathrm{SiO}_{2}$.

Fuse the residue left in the crucible with $\mathrm{Na}_{2} \mathrm{CO}_{3}$ and add to the filtrate.

Note.-The time and temperature of baking was selected by the committee in order to prevent the loss of silica by formation of soluble magnesium silicates.

\section{(D) IRON AND ALUMTNA}

The combined filtrates from the silica are adjusted to a volume of approximately 200 cc. One drop of $\mathrm{HNO}_{3}$ is added as an oxidizing agent. The solution is then brought to a boil and a few drops of methy? red indicator added. Ammonium hydroxide is now added drop by drop till the solution turns distinctly yellow. The solution is then boiled for three or four minutes and the precipitate allowed to settle. A small paper, $7 \mathrm{~cm}$, is used for the filtration.

Transfer the precipitate to the filter with hot $\mathrm{NH}_{4} \mathrm{NO}_{3} 2$ per cent. Poiice the beaker and wash the precipitate with hot $\mathrm{NH}_{4} \mathrm{NO}_{3} 2$ per cent till free from chlorides. The precipitate is ignited while moist in a platinum crucible at $1,200^{\circ} \mathrm{C}$. to constant weight and weighed as the combined oxide of iron and alumina.

If it is desired to separate the two, the ignited residue is fused with $2 \mathrm{~g}$ of fused $\mathrm{KHSO}_{4}$. The fusion cake is dissolved in hot $\mathrm{H}_{2} \mathrm{O}, 5$ cc concentrated $\mathrm{H}_{2} \mathrm{SO}_{4}$ added and the iron reduced with $\mathrm{H}_{2} \mathrm{~S}$ and titrated with standard permanganate solution.

If the electrometric method is used, the fusion cake is dissolved in 1:1 $\mathrm{HCl}$. Enough $1: 1 \mathrm{HCl}$ is added to bring the solution up to $150 \mathrm{cc}$, brought to a boil, reduced with stannous chloride, and titrated in the usual manner electrometrically.

The oxide of alumina is obtained by difference. In the ordinary analysis this separation is not carried out. 
$\mathrm{TiO}_{2}$ may be determined before or after the $\mathrm{Fe}_{2} \mathrm{O}_{3}$ determination by the colorimetric method and also subtracted from the alumina.

Nore.-The oxides of iron and alumina are slightly soluhle in $\mathrm{H}_{2} \mathrm{O}$. Methyl red is preferred to methyl orange, as its $p_{\text {H }}$ value is hetter suited to this work.

\section{(E) LIME}

The filtrate from the iron and alumina determination is made slightly ammoniacal. The solution is brought to a boil and $25 \mathrm{cc}$ saturated solution of ammonium oxalate added with vigorous stirring. Boil for 10 or 15 minutes and allow the precipitate to settle. Filter through a small filter paper. Police the beaker and wash the precipitate five times with $\left(\mathrm{NH}_{4}\right)_{2} \mathrm{C}_{2} \mathrm{O}_{4} 1.5$ per cent. Wash once with hot $\mathrm{H}_{2} \mathrm{O}$ to remove the excess $\left(\mathrm{NH}_{4}\right)_{2} \mathrm{C}_{2} \mathrm{O}_{4}$. The precipitate is then washed into a beaker with hot $\mathrm{H}_{2} \mathrm{O}$ through the punctured filter paper and dissolved in an excess of 25 per cent $\mathrm{H}_{2} \mathrm{SO}_{4}$. The solution is made up to $75 \mathrm{cc}$ with hot $\mathrm{H}_{2} \mathrm{O}$ and is titrated with standard potassium permanganate to the first permanent faint pink color. The filter paper is placed on the inside of the beaker near the top and added to the solution near the end point. The calcium is calculated to $\mathrm{CaO}$.

NotE.-Calcium oralate is appreciahly soluhle in hot $\mathrm{H}_{2} \mathrm{O}$.

If the filter paper is added directly to the solution, high results will he ohtajned.

\section{(F) MAGNESIA}

The filtrate from the calcia is made slightly acid with $\mathrm{HCl}$ and brought to a boil. The volume may range upward to $600 \mathrm{cc}$. One-half gram microcosmic salt is added and the solution stirred thoroughly. One-ninth of the total volume of ammonium hydroxide is added, the solution stirred vigorously for 15 minutes and allowed to stand 6 hours, or overnight before filtering. Frequent stirring and cooling will hasten the precipitation. The precipitate is filtered through a Gooch filter. Police the beaker and wash the precipitate five timcs with 5 per cent $\mathrm{NH}_{4} \mathrm{OH}$. Dry in a drying oven or over a small flame before igniting. Ignite to constant weight at $1,200^{\circ} \mathrm{C}$. Calculate to $\mathrm{MgO}$ by using the factor 0.3621 .

For greater accuracy the magnesia may be filtered through a filter paper, dissolved, and reprecipitated.

One precipitation is generally accurate enough for the routine method.

\section{(G) ALKALIES}

In most routine analyses the alkalies are determined by difference. This throws all the error on the alkalies.

Example: $\quad 100-\left(\mathrm{SiO}_{2}+\mathrm{Al}_{2} \mathrm{O}_{3}+\mathrm{Fe}_{2} \mathrm{O}_{3}+\mathrm{CaO}+\right.$ $\mathrm{MgO})=$ alkalies.

If a more accurate method is desired, the $\mathrm{J}$. Lawrence Smith fusion method is recommended. National Sand and Gravel Association, Bulletin 3 Representative Specifications for Different Uses of Sand and Gravel, November, 1928.

(In this bulletin, representative specifications, many of which are presented in this volume, and including specifications for glass sands, have beel summarized.)

521.

\section{FLAT GLASS}

\subsection{GENERAL ITEMS.}

Federal Specifications Board, specification No. 448, United States Government master specification for plumbing fixtures, November 22, 1926.

(The specification is published in full as Bureau of Standards Circular No. 310 , and gives general and detail requirements for 22 plumbing fixture items, including marble and glass for use in plumbing, general requirements for which are as follows:)

Quality of these materials and finish of same shall be described in the specification for the work.

\subsection{PLATE GLASS.}

American Marine Standards Committee, glass for air ports and fixed lights, standard $\mathrm{H}$ No. 1, 1925.

(This committee has indorsed for marine use Federal Specifications Board specification No. 123 (given below) and glass furnished under this standard is to be of the kind and grade classified as "polished plate glass glazing quality.")

\section{[ALL DTMENSIONS IN INCERS]}

\begin{tabular}{|c|c|c|c|c|}
\hline \multirow{2}{*}{$\begin{array}{l}\text { Port or light size clear } \\
\text { glass diameter }\end{array}$} & \multicolumn{2}{|c|}{$\begin{array}{l}\text { Standard dimen- } \\
\text { sions of finished } \\
\text { glass }\end{array}$} & \multicolumn{2}{|c|}{$\begin{array}{l}\text { Variation toler- } \\
\text { ances over or } \\
\text { under }\end{array}$} \\
\hline & $\begin{array}{l}\text { (A) Di- } \\
\text { ameter }\end{array}$ & $\begin{array}{l}\text { (B) } \\
\text { Thick- } \\
\text { ness }\end{array}$ & $\underset{\text { eter }}{\text { Diam- }}$ & $\begin{array}{l}\text { Thick- } \\
\text { ness }\end{array}$ \\
\hline $\begin{array}{l}8 \\
10 \\
12 \\
16 \\
18\end{array}$ & $\begin{array}{l}81 / 2 \\
101 / 2 \\
121 / 2 \\
141 / 2 \\
161 / 2 \\
181 / 2\end{array}$ & 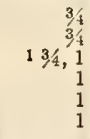 & $\begin{array}{l}1 / 16 \\
1 / 16 \\
1 / 16 \\
1 / 10 \\
1 / 16 \\
1 / 16\end{array}$ & $\begin{array}{l}1 / 32 \\
1,32 \\
1 / 32 \\
1 / 32 \\
1,32 \\
1 / 32\end{array}$ \\
\hline
\end{tabular}

1 Three-fourths inch thickness for 12 -inch size is for internal lights only.

1. The thickness of any piece shall be practically uniform and shall not vary more than $1 / 64$ inch along its whole periphery.

2. Edge of glass shall be cut or ground evenly with corners slightly chamfered.

3 . Where location or service requirements demand greater thickness of either plain or laminated (nonshatterable) glass, the standard thickness (as tabulated above) shall be increased by quarter inches.

Federal Specifications Board, specification No. 123, United States Government master specification for flat glass for glazing purposes, April 1, 1924.

\section{CLASSIFICATION}

Polished plate glass...... $\left\{\begin{array}{l}\text { Second silvering quality. } \\ \text { Glazing quality. }\end{array}\right.$

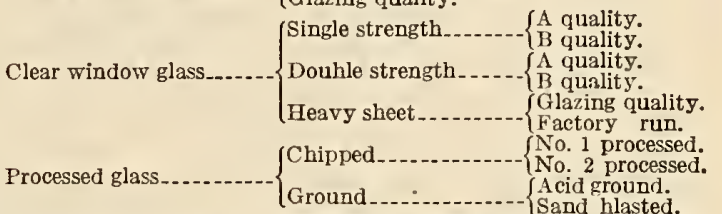

Rolled figured sheet....-_Figured sheet (large variety of patterns) Colored figured sheet.

Polished wire.

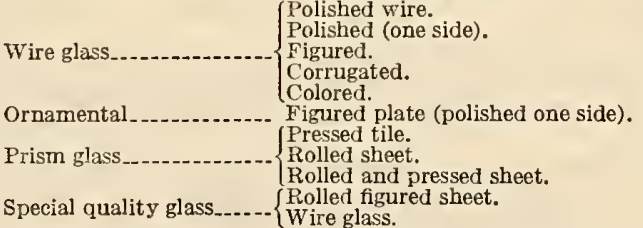

\section{DEFINITIONS OF THE GENERAI CLASSES OF} PLATE, CLEAR WINDOW, AND ROLIED SHEET GLASS

Plaie glass.-Transparent, flat, relatively thin glass having plane polished surfaces and showing no distortion of vision when viewing objects through it at any angle.

Plate glass is made at present by casting and rolling large sheets periodically or by rolling a continuous sheet. The sheets are then ground and polished. 
Clear window glass.-Transparent, relatively thin, flat glass having glossy, fire finished, apparently plane and smooth surfaces, but having a characteristic waviness of surface which is visible when viewed at an acute angle or in reflected light.

Clear window glass is made at present by hand blowing or by machine blowing and drawing into cylinders and flattening, or by drawing directly into a sheet, the surface finish being that obtained during the drawing process.

Processed glass. - There are three kinds of processed glass either in plate or window glass, viz, ground glass, chipped one process, and chipped two processes.

The ground glass is made by either sand blasting or acid etching of one surface. The chipped glass is made by applying either one or two coatings of glue to the ground surface.

Rolled figured glass.-A flat glass in which the vision is more or less obscured either by the roughened surface produced in rolling or by the impression of a large variety of decorative designs on one surface of the sheet.

Wire glass.-Rolled flat glass having a layer of meshed wire incorporated approximately in the center of the sheet.

This glass is produced with polished or figured surfaces.

Ornamental plate.-A figured plate glass made by rolling or rolling and pressing and having the plain surface ground and polished.

Prism glass.-A flat glass having prism-shaped parallel ribs designed for deflecting light.

This is made as a rolled plate or as a pressed plate, of which one side may be ground and polished, or as a pressed tile.

\section{DETAILED SPECIFICATIONS OF VARIOUS TYPES OF GLAZING GLASS}

\section{GENERAL PRINCIPLES IN OLVID IN GRADING GLASS}

All flat glass contains some imperfections and the principle employed in grading is to exclude all defects that would be objectionable in a given grade. This is difficult to do, since there are no sharp lines of demarcation between grades, and experienced inspectors will differ in judgment as the quality of the glass approaches the limits of the grades. Small lights must be quite free from imperfections as compared with larger ones, and the center of any sheet should be clear, whereas the edges may contain more pronounced defects.

Method of examination.- The method of examination is described in these specifications in order to make the results more uniform, and defines the condition under which glass should be examined because the distance from the glass, the angle between the glass and the line of sight, and the intensity of light all affect the visibility of imperfections.

These specifications should be interpreted by examining the glass in the following manner, with reference to the definitions of defects listed in the glossary.

The glass should be examined when placed in a position similar to that of a glazed light with the observer's eye on a level with the center of the sheet, and looking through the glass from a distance of about 36 inches into the light from a clear sky without any sun or any close background.

The visibility of waves, lines, or cords depends chiefly upon the angle of observation, and the intensity of these defects can be classified on this basis. The values given for angles are the angles the line of sight makes with the sheet of glass when in a vertical position. Slight movement of the head horizontally through an angle of $2^{\circ}$ or $3^{\circ}$ will make waves or lines more perceptible.
Acceptance or rejection.-Acceptance or rejection of a shipment or delivery shall be based on an examination of the following quantities:

For orders of 100 lights or less all shall be examined.

For orders of 101 to 500 lights at least 50 per cent shall be examined.

For orders of 501 or more lights at least 25 per cent shall be examined.

Boxes shall be selected from the shipment at random.

If not more than 10 per cent of the lights examined are below quality, the shipment shall be accepted provided the lights below the specified grade are not distinctly below the upper limit of the next lower grade.

If, however, an entire shipment of 500 lights or more is examined, not more than 5 per cent may be below quality.

\section{SPECIFICATIONS FOR POLISHED PLATE GLASS}

Sizes and thickness.-The standard thicknesses of plate glass shall be $1 / 8,3 / 16,1 / 4,3 / 8,1 / 2,5 / 8,3 / 4,1,11 / 4$, and $1 \frac{1}{2}$ inches. Sheets are available $1 / 4$ inch thick in sizes having a maximum area of 250 square feet. Glass of $1 / 4$ inch thickness may be furnished having almost any desired dimension under the following maximums: 120 by 280 inches, 144 by 260 inches, 160 by 240 inches.

The standard stock thickness for glazing purposes is $1 / 4$ inch, but this may vary between $7 / 32$ and $5 / 16$ inch, although $1 / 8$ and $3 / 16$ inch glass are carried in stock in the larger cities.

Tolerances in thickness.-The maximum and minimum thicknesses allowed shall not be more than the given thickness plus or minus one-half the difference between the standard thicknesses, although for $1 / 4$ inch glass occasional plates as thick as $5 / 16$ inch are supplied. The general variation in thickness should not be more than $1 / 32$ inch for individual lights under 10 square feet, in thicknesses up to $1 / 4$ inch. The variation in lights over $1 / 4$ inch in thickness should not exceed one-half the total tolerance for that thickness.

Tolerances in dimensions. - Variation from dimensions ordered shall not be more than $1 / 32$ inch per $1 / 8$ inch of thickness.

Grades.-Plate glass shall be furnished for glazing purposes in one of two grades as specified. These grades will be known as second silvering and glazing qualities.

Second silvering quality is invariably used where the highest standard of glazing is required and imperfections are discoverable only on close inspection. This quality is rarely sold for glazing purposes in sizes over 20 square feet. Glazing quality represents the usual selection of plate glass supplied when quality is not otherwise definitely specified.

As allowable tolerances in quality must vary considerably with size of sheet required, different specifications will apply in each of the following four divisions according to size: Division I, sheets up to and including 10 square feet in area; division II, sheets having an area greater than 10 square feet, but not greater than 25 square feet; division III, sheets having an area greater than 25 square feet, but not greater than 75 square feet; division IV, sheets having an area greater than 75 square feet.

\section{DIVISION I}

\section{SIZES UP TO AND INCLUDING 10 SQUARE FEET}

Second silvering quality.-This glass shall not contain any major defects. The central area of this glass may contain only well-scattered seeds. Ream, skim, short finish, and scratches which can 
not be removed by buffing are not permissible. The edges may contain coarse seeds, but none shall be larger than $1 / 32$ inch in diameter.

Glazing quality.-The central area of this quality may contain numerous scattered seed, including an occasional coarse seed, but no heavy seed. Small bubbles may occur on the edge. Stones, large bubbles, skim, ream, or long or heavy scratches are not permissible. Faint strings in the corners or upper edge of the light are permissible. The polish shall not show areas of short finish.

\section{DIVISION II}

\section{SIZES FROM 10 TO 25 SQUARE FEET, INCLUSIVE}

Second silvering quality. - The central area of this quality may contain more numerous fine seed than the small sizes and all occasional coarse seed. The edges may contain occasional small bubbles and fine strings. No heavy defects or sciatches which can not be removed by buffing are permissible. The polish must be good and free from visible short finish.

Glazing quality.-The central area may contain small bubbles and fine strings or ream which does not give visible distortion when looking straight through the glass, but no long or heavy scratches. The edges may contain bubbles over $3 / 32$ inch, visible scratches shorter than 10 inches, small areas of ream, strings, and small stones not larger than $1 / 32$ inch, but these defects should not be grouped nor interfere with the vision. The polish over the central area should be good, but patches of light short finish may be present about the edges.

General.- None of the above grades or sizes may contain any heavy or long lines, streaks of ream, any bubbles larger than $1 / 8$ inch, visible poor polish, open bubbles, areas of skim, or stones over $1 / 32$ inch in diameter.

\section{DIVISION III}

\section{SIZES FROM 25 TO 75 SQUARE FEET}

Glazing quality.-Lights of this size may contain numerous visible and larger imperfections not allowed in the smaller lights. But these must not be grouped or so prominent that they noticeably interfere with the vision. The central area of the plate shall be frce from these larger defects.

The sheets may contain seed of any size, but not heavy seed, except in relatively small patches on the outer border of the sheet, occasional bubbles up to $1 / 8$ inch in the center and up to $3 / 16$ inch on the borders, strings, ream, and skim in very limited areas if not causing a deformation of objects viewed through the plate, occasional scratches, and small stones under $1 / 16$ inch.

Heavy ream, heavy cords, bubbles larger than $3 / 16$ inch in diameter, stones larger than $1 / 16$ inch in diameter, large fire cracks, areas of unpolished glass, easily visible poor polish, large open bubbles, or sand holes are not permitted. The large defects should be confined to the upper edge and upper corners of the sheet, the lower and central areas to be relatively free from major defects.

\section{DIVISION IV}

\section{SIZES GREATER THAN 75 SQUARE FEET}

Sheets larger than 75 square feet may contain defects of almost any kind, except that they must not show large areas of heavy seed or bubbles, nor have any defects which will cause spontaneous breakage, such as skim or large stones (1/8 inch diameter) or show any areas of unpolished glass.

\section{SPECIFICATIONS FOR CLEAR WINDOW GLASS FOR GLAZING}

Clear window glass for glazing is made in several different qualities and in the varying thickncsses shown in Table 1 below.

Single strength and double strength window glass is regularly supplied in two standard qualities, known as A quality and B quality. A limited amount of this glass, known as A quality, which is especially free from defects, is sometimes selected for special purposes and may be specified if desired. It must be borne in mind, however, that the total amount of AA glass produced by the manufacturers does not exceed 3 per cent of the total amount of window glass produced.

There is also a limited amount of singlc-strength and double-strength glass produced in a quality inferior to B quality and is known as fourth quality. The amount of glass produced in this quality also represents a very small percentage of the total window glass produced.

A quality of single-strength glass inferior to fourth quality is also produced in very limited quantities and is known as C quality. This is the lowest grade of glass that is packed and marketed in this country.

Window glass is also produced in thicknesses hcavier than double strength, and according to its thickness it is classified as (1) 26-ounce glass; (2) 29-ounce glass; (3) 34-ounce glass; and (4) 39 ounce or $3 / 16$-inch glass.

Twenty-sixth-ounce and twenty-nine-ounce glass are produced only in A and B qualities.

Thirty-four-ounce and thirty-nine-ounce, or $3 / 10^{-}$ inch glass, are produced in glazing and factory-run quality.

TABLE 1-Tolerances in thickness and average weight of clear window glass

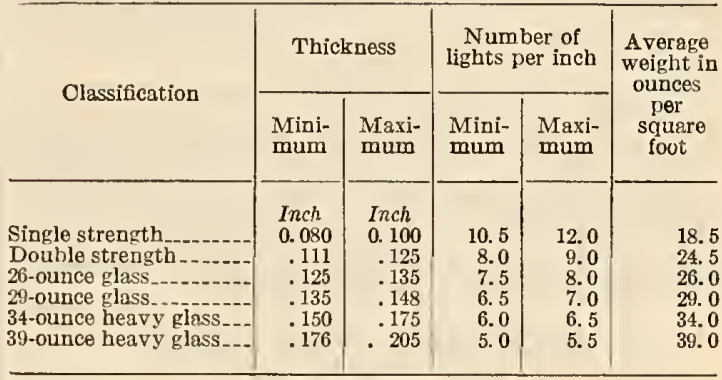

Sizes obtainable-The maximum dimensions recommended are:

\begin{tabular}{|c|c|c|}
\hline & Width & Length \\
\hline $\begin{array}{l}\text { For single strength } \\
\text { For double strength } \\
\text { For heavy sheet }\end{array}$ & $\begin{array}{c}\text { Inches } \\
40 \\
60 \\
66\end{array}$ & \begin{tabular}{|r} 
Inches \\
50 \\
80 \\
90
\end{tabular} \\
\hline
\end{tabular}

TOLERANCES AND THICKNESS

Thickness of individual sheets shall not vary more than one-half of the total variation allowed for that particular strength of glass as shown in Table 1 above for sizes up to 50 united inches. For larger sizes variations in thickness may be equal to the tolerance allowed for that class.

\section{FLATNESS}

All clear window glass shall be relatively flat. Slight curvature, provided it is regular, will be 
allowed, but the maximum deformation or bow shall not make an arc higher than 0.5 per cent of the length of the sheet. Reverse curve or crooked glass is not allowable.

\section{GLAZING}

Window glass should always be glazed with the convex side out.

\section{DIMENSIONS}

Glass must be cut to dimensions ordered with an allowable tolerance of $1 / 32$ inch per $1 / 8$ inch of thickness.

\section{A QUALITY}

The defects permitted in this quality are faint strings or lines, slight burn, small seeds, small blisters, and light scratches.

No light shall contain all of these defects, and those present may not be grouped when in the central area of the sheet.

Strings, lines, or burn spccks shall not be of such intensity that they are visible when observing the sheet at an angle greater than $30^{\circ}$ between the line of sight and the glass.

Waves shall not be visible at an angle greater than $20^{\circ}$ with the glass.

Blisters shall not exceed one-fourth inch in length unless they occur near the edge of the sheet.

In general, the central area of the light shall be practically free from defects, and the appearance of the light as a whole shall be such that there is no perceptible interference with the vision as long as one is not looking through the glass at an acute angle.

\section{B QUALITY}

This quality admits of the same kind of defects as A quality, but they may be larger, heavier, and more numerous.

Occasional scattered blisters not more than onehalf inch long may occur over the central area of the sheet. Larger blisters up to 1 inch in length may occur about the bordering areas.

Waves should not be of such intensity that they are visible when observing the sheet at an angle greater than $45^{\circ}$ with the glass, unless on the border.

Burn spots may be visible when looking directly through the glass, but they must not cause any appreciable depression and the speckled appearance must not be so great as to interfere with vision when examining the glass in the specified position.

\section{HEAVY SHEET WINDOW GLASS}

\section{GLAZING QUALITY}

The same specifications for selecting provided for A quality single-strength and double-strength glass shall apply.

\section{FACTORY-RUN QUALITY}

This quality is the run of glass as produced by the factory. It may contain glass of very good quality and some glass of verv ordinary quality. However, the glass that contains heavy cords, lines, or strings over the entire surface, raised blisters, cap strings, stoncs, or batch particles causing a rough surfacc, or depression, or having its surface covered with heavy burn, wrinkles, deep scratches, or stone, shall not be included in this quality.

\section{SPECIFICATIONS FOR ROLIED FIGURED SHEET} GLASS

This type of glass is supplied in but one quality for glazing purposes. It is made in a wide variety of surface finishes which serve to obscure the vision as well as provide a decorative finish and also special patterns for increasing illumination by diffusion or deflection.

Figured sheet glass is made in the following thicknesses: $1 / 8,3 / 16,1 / 4$, and $3 / 8$ inch, and can be obtained in sizes up to 48 inches wide and 130 inches long.

\section{SPECIFICATIONS FOR WIRE GLASS}

This type of glass is supplied in but one quality for glazing purposes. It is made polished and in a wide variety of surface finishes which serve to obscure the vision as well as provide a decorative finish and also special patterns for increasing illumination by diffusion or deflection.

Wirc glass can be made in the following thicknesses: $1 / 8,1 / 4,5 / 16,3 / 8,1 / 2$, and $3 / 4$ inch. The standard type of wire used has a mesh about $1 \frac{1 / 4}{4}$ by $7 / 8$ inch, and is not lighter than 24 B. \& S. gage, except for $1 / 3-i n c h$, in which $26 \mathrm{~B}$. \& S. gage wire is used. Wire glass can be obtained in sizes up to 60 inches wide and 144 inches long.

Corrugated wire glass is made in one thickness, which is about $5 / 16$ inch. Deep angle sheets are $2 \frac{1}{2}$ inches $\mathrm{C}$ to $\mathrm{C}$ of corrugations and mesh with corrugated asbestos. Shallow angle sheets are $211 / 16$ inches $\mathrm{C}$ to $\mathrm{C}$ and mesh with corrugated iron.

\section{SPECIFICATIONS FOR SPECIAL QUALITY GLASS FOR ABSORBING AND INTERCEPTING ULTRA- VIOLET AND INERA-RED RAYS}

This glass may be made as rolled figured sheet or wire glass in flat or corrugated sheets. It shall be of such composition that not less than 80 per cent of the ultra-violet rays ${ }^{1}$ and not less than 50 per cent of the infra-red rays are excluded.

\section{SPECIFICATIONS FOR POLISHED FIGURED OR ORNAMENTAL PLATE GLASS}

Ornamental plate glass is a figured sheet glass, the smooth surface of which has been polished. It is made $1 / 4$ inch thick in sizes up to 50 inches wide by 100 inches long and in a variety of patterns. Chipped plate glass, single and double processed, is also sold as polished figured plate glass.

\section{SPECIFICATIONS FOR PRISM GLASS}

This glass is made as rolled sheet glass with or without wire and as a pressed tile. It is made with one side polished, sold as prism plate glass or as a rough rolled prism glass, and can be furnished in sizes up to 42 by 138 inches, $1 / 4$ inch thick, and in sizes between 42 and 60 inches by 138 inches, $5 / 16$ inch thick. The prism ribs are furnished running horizontally with the length of the sheet. Prism wire glass is made in sizes up to 42 by 138 inches, with a thickness of $3 / 8$ inch.

Pressed prism tile are made either 4 by 4 inches square or 5 by 5 inches square.

\section{GENERAL SPECIFICATIONS FOR ROLLED FIGURED SHEET GLASS, WIRE GLASS, ORNAIIENTAL PLATE GLASS, AND PRISM GLASS}

Quality.-The glass shall contain no visible stones which may cause spontaneous breakage of the sheet, no fire cracks or exposed wire. The pattern shall be uniform over the surface without any easily visible blemishes.

Tolerance for thickness.-The total variation in thickness of this glass shall not be more than $3 / 64$ inch for any single sheet; the maximum variation allowed

1 In order to obtain this value, the ultra-violet transmission, including rellection, should not exceed the following values: At 0.405 Tr. $=30$ to 40 per cent; at 0.3625 , Tr. $=12$ to 15 per cent; and at 0.313 $\mathrm{Tr} .=0.5$ to 1.0 per cont. 
for anr specified thickness may not be greater than $3 / 64$ inch over or under that thickness.

Dimensions.-Glass must be cut to dimensions ordered with an allowable tolerance of $1 / 32$ inch per $1 / 8$ inch of thickness.

\section{GLOSSARY OF TERMS USED IN THESE SPECIFI- CATIONS}

The various terms used in describing imperfections appearing in flat glass are the following: Seeds, boil bubbles, blisters, open bubbles, cords, strings, ream, knots, scratches, light grazes, cullet cuts, sand lash, sleck, short finish, sand holes, feathers, skim, stones, wares, lines, burns, burn spots, and fire cracks.

Many of these terms may be grouped under one general term and a number are not necessary in preparing specifications, although they are of ten used in a factory as identifying certain imperfections.

The following terms shall be used in specifications:

\section{Plate glass}

Seeds.

Short finish.

Skim.

Strings.

Scratches.

Bubbles.

Open bubbles.

Ream.

Stones.

Fire cracks.

Sand holes.

\section{Clear window glass}

Seeds.

Blisters.

Scratches.

Lines.

Strings.

Burns.

Cords.

Stones.

Definitions, causes, and brief description of these various imperfections are given herewith.

Bubbles. - Gas inclusions in any rolled glass. These inclusions are practically always spherical and brilliant in appearance. The term applies to all such inclusions larger than $1 / 32$ inch in diameter. The term small bubbles (commonly known as boil) refers to sizes between $1 / 32$ and $3 / 32$ inch.

Seeds. - Minute bubbles less than $1 / 32$ inch in diameter. Fine seeds are visible only on close inspection, usually appearing as small specks and are an inherent defect in the best quality of plate glass. Seed about $1 / 64$ to $1 / 32$ inch in diameter are usually considered as coarse seed.

Heavy seed.-Refers to a condition when the fine and coarse seed are very numberous, such as 25 or more to the square inch.

Scattered seed.-Indicates a condition of few and occasional easily visible coarse seed. Two or three may be spaced 1 or 2 inches from each other, but one here and there at much greater distances apart is the usual intention of the term.

Open bubbles.-Bubbles which have been broken into by grinding, leaving a hemispherical hole in the glass surface.

Blisters.-Elongated bubbles or seeds, elliptical in shape. This form of bubble is generally peculiar to window glass, but may be found in plate glass manufactured by recently improved methods. In both cases the method of manufacture draws out practically all bubbles in one direction.

Shim.-Streaks of dense seed, with accompanying small bubbles.

Strings.-Wavy, transparent lines appearing as though a thread of glass had been incorporated into the sheet.

Cords.-Heavy strings incorporated in the sheet, occurring without any regularity of direction, and appearing to be of considerable thickness rather than on the surface.
Ream.-An area of unhomogeneous glass incorporated in the sheet, producing a wavy appearance. Scratches.-Any marking or tearing of the surface appearing as though it had been done by either a sharp or rough instrument. Scratches occur on sheet glass in all degrees from various accidental causes.

Short finish.-Insufficient polish or lack of brilliancy; improperly finished surface which has the appearance of being slightly pitted and wavy when the surface is viewed in reflected light. These indentations, which are slight, have a polished rather than a ground surface, but the general effect is a slight dulling of the surface. Poor polish is usually caused by improper grinding.

Stones.-Any opaque or partially melted particle of rock, clay, or batch ingredient embedded in the glass.

Lines.-Waves which extend continuously across the sheet so that the reflection from the surface appears as a line or series of lines extending either the full width or a considerable distance across the sheet.

Burns.-Small projections or indentations on the surface, appearing as an area of small specks, together with some destruction of the surface polish. An imperfection occurring during the flattening of window glass caused by flattening furnaces becoming too hot.

Fire cracks.-Small cracks penetrating the surface of the sheet. Usually in the shape of short hooked crescents. Caused by sudden heating or chilling of the surface.

Sand holes.-Rough spots on the polished surface produced during coarse grinding which fine grinding did not later remove; due, to some extent, to coarse grains of grinding sand becoming mixed with finer grades.

Central area of sheet.-This term is used with slightly different interpretation with reference to plate or window glass. In plate glass the central area is considered to form an oval or circle centered on the sheet whose axes or diameters do not exceed 80 per cent of the over-all dimension. This allows a fairly large area at the corners which may have imperfections not allowed in the central area.

In window glass the central area is considered as being a circle having a diameter equal to half the width of the sheet, or an ellipse having one diameter equal to half the length of the sheet and the other diameter equal to half the width of the sheet.

Plate Glass Manufacturers of America, specifications for polished plate glass, revised to May 23, 1929.

(This specification is the same as Federal Specifications Board specification No. 123, "Specifications for Polished Plate Glass," see above, with the following variations):

Sizes and thickness, paragraph 2 reads:

The standard stock thickness for glazing purposes is $1 / 4$ inch, but this may vary between $5 / 32$ and $9 / 32$ inch.

Tolerance in thickness. - When a certain thickness is specified for a specific purpose, the tolerance in thickness shall be half the difference allowed on standard thickness, although $1 / 4$ inch glass for glazing purposes is accepted within the latitude of $5 / 32$ to $\% 32$ inch. The general variation in thickness should not be more than $1 / 32$ inch for individual lights under 10 square feet in thickness up to $1 / 4$ inch. The variation in lights over $1 / 4$ inch in thickness should not exceed one-half the total tolerance for that thickness.

Polished plate glass $1 / 8$ inch and $8 / 10$ inch thick is carried in stock in the larger cities.

Society of Automotive Fngineers, 1928, recommended practice. 


\section{PLATE GLASS}

The glass producer shall be furnished with a templet of the finished glass.

Only polished plate glass shall be used in windshields and front-quarter door windows.

Polished plate glass for windshields and passenger-car windows shall be of two grades: "Selected glazing" and "Glazing."

"Selected glazing" plate glass shall contain practically no visible imperfections under specified conditions of inspection. Very fine scattered sceds are permissible.

"Glazing" plate glass shall contain no other visible imperfections than a few scattered seeds and occasional faint strings or faint short finish marks.

The thickness of plate glass shall be $7 / 32$ inch, with tolerances of plus and minus $1 / 16$ inch, with variations in individual plates of not more than 1/64 inch.

For definitions of terms used in the above specifcations see F. S. B. No. 123, page 485.

\subsection{CLEAR WINDOW GIASS.}

American Railway Engineering Association, specifications for buildings for railway purposes.

\section{GLAZING (1926)}

The contractor shall furnish all labor, tools, equipment, and everything necessary to complete the glazing required, and shall take proper precautions to protect the public and employees of the company from any and all damage from his operations.

The contractor shall furnish and set all glass of every description for window, door, transom, and ceiling sash, including metal sash and all glass in skylights and marquises. Sash must be primed and thoroughly dry before any glass is set.

All putty, excepting for metal sash, shall be firstquality white-lead putty mixed with pure linseed oil.

Unless otherwise marked on the drawings, glass for wood sash shall be American, double strength, class A.

Glass for main-entrance doors shall be polished 1/4-inch plate glass.

Glass for skylights, marques, metal sash, and metal doors, unless otherwise specified or called for on the drawings, shall be $1 / 4$-inch factory ribbed wire glass.

Where opaque or figured glass is called fo: on the drawings this shall be Florentine, Maze, or other "approved" figured glass.

Samples of all glass to be used shall be submitted to the engineer for approval, and all glass to be used in the work must conform strictly in quality with the approved samples.

Glazing in metal sash.-Glass in metal sash and doors shall be bedded in litharge putty, then clips or stops applied, back puttied and neatly face puttied; finished surface of putty shall show absolutely smooth, true, and free from sags or wrinkles.

Glazing in wood sash.-Glass in wood sash shall be back puttied, securely fastened with glazier's points and neatly face puttied.

Glazing in doors.-Glass in wood doors shall be fastened in place with removable wood stops.

Glazing skylights and marquises.-Glass in skylights and marquises shall be secured in place by the use of copper strips and copper screws and shall be made water-tight.
FIN AI CLEANING

Before the building is tendered for final acceptance all broken glass shall be replaced and all glass of every description shall be thoroughly cleaned.

\section{GENERAL CONDITIONS}

All materials entering into the work and all methods used by the contractor shall be subject to the approval of the engineer, and no part of the work will be considered as finally accepted until all of the work is completed and accepted.

The general conditions as given in 518.50 , page 416 , shall be considered to apply to these specifications.

Federal Specifications Board, specification No. 123, United States Government master specification for flat glass for glazing purposes, April 1, 1924.

\section{CLEAR WINDOW GLASS}

See 521.1, page 485 .

\subsection{WIRE GLASS.}

Federal Specifications Board, specification No. 123, United States Government master specification for flat glass for glazing purposes, April 1, 1924.

\section{WIRE GLASS}

See 521.1, page 485 .

\subsection{ROLLED FIGURED SHEET GLASS.}

Federal Specifications Board, specification No. 123, United States Government master specification for flat glass for glazing purposes, April 1, 1924.

\section{ROLLED FIGURED SHEET GLASS}

See 521.1, page 485.

\subsection{PRISM GLASS.}

Federal Specifications Board, specification No. 123, United States Government master specification for flat glass for glazing purposes, April 1, 1924.

\section{PRISM GIASS}

See 521.1, page 485 .

\subsection{SPECIAI QUALITY GLASS.}

Federal Specifications Board, specification No. 123, United States Government master specification for flat glass for glazing purposes, April 1, 1924.

\section{SPECIAI QUALITY GLASS}

See 521.1, page 485 .

\subsection{SIDEWALK AND SKYLIGHT GLASS.}

American Railway Engineering Association, specifications for buildings for railway purposes.

\section{GIAZING (1926) (SKYLIGHT GLASS)}

See 521.2, above.

United States Department of Commerce, division of simplified practice, recommendation No. 49, sidewalk, floor, and roof lights, March, 1926.

In accordance with the unanimous action of a general conference of representatives of manufacturers, distributors, and users of sidewalk, floor, and roof lights, the United States Department of Commerce, through the Bureau of Standards, recommends that simplified sizes, shapes, and styles be established, as follows: 
TABLE 1.-Sidewalk lights (stock sizes and shapes) ROUND GLASS

\begin{tabular}{l|r|r|r}
\hline Diameter (in inches) & $\begin{array}{c}\text { Thickness } \\
\text { at support- } \\
\text { ing edge }\end{array}$ & $\begin{array}{c}\text { Diameter } \\
\text { of shield }\end{array}$ & $\begin{array}{c}\text { Distance } \\
\text { on centers }\end{array}$ \\
\hline & Inch & Inches & Inches \\
$31 / 4$ & $31 / 4$ \\
31 & $31 / 4$ & $41 / 4$ \\
\hline
\end{tabular}

SQUARE GLASS

\begin{tabular}{c|c|c|c}
\hline Size (in inches) & $\begin{array}{c}\text { Thickness } \\
\text { at support- } \\
\text { ing edge }\end{array}$ & $\begin{array}{c}\text { Size of } \\
\text { shield }\end{array}$ & $\begin{array}{c}\text { Distance } \\
\text { on centers }\end{array}$ \\
\hline $31 / 2$ by $31 / 2$ & Inch & Inches & Inches \\
\hline
\end{tabular}

1 Inclosed in a cast-iron shield. The space between the glass and shield should be filled with plastic compound when protection against the weather or traffic is necessary.

TABLE 2.-Floor lights ${ }^{1}$ (stock sizes)

SQUARE GLASS

\begin{tabular}{|c|c|c|c|}
\hline Size (in inches) & $\begin{array}{l}\text { Thickness } \\
\text { at support- } \\
\text { ing edge }\end{array}$ & $\begin{array}{l}\text { Size of } \\
\text { shield }\end{array}$ & $\begin{array}{l}\text { Distance } \\
\text { on centers }\end{array}$ \\
\hline $\begin{array}{l}4 \text { by } 4{ }^{2} \\
61 / 8 \text { by } 61 / 8^{2} \\
31 / 2 \text { by } 31 / 23\end{array}$ & $\begin{array}{cc}\text { Inch } & \\
& \\
& \\
& \\
& 3 / 4\end{array}$ & $\begin{array}{l}\text { Inches } \\
\text { No shield. } \\
\text { No shield. } \\
4\end{array}$ & $\begin{array}{c}\text { Inches } \\
\begin{array}{c}5 \\
71 / 2 \\
5\end{array}\end{array}$ \\
\hline
\end{tabular}

1 Where neither weather conditions nor heavy traffic have to be considered, the cast-iron shield may be omitted at the option of the architect or owner.

2 Without cast-iron shields.

${ }^{3}$ Inclosed in a cast-iron shield. The space between the glass and shield should be filled with plastic compound.

TABLE 3.-Roof lights $^{1}$ (stock sizes)

SQUARE GLASS

\begin{tabular}{|c|c|c|c|}
\hline Size (in inches) & $\begin{array}{l}\text { Thickness } \\
\text { at support- } \\
\text { ing edge }\end{array}$ & $\begin{array}{l}\text { Size of } \\
\text { shield }\end{array}$ & $\begin{array}{c}\text { Distance } \\
\text { on centers }\end{array}$ \\
\hline $81 / 4$ by $81 / 4-$ & Inch $\quad 3 / 2$ & $\begin{array}{c}\text { Inches } \\
83 / 4 \text { by } 83 / 4\end{array}$ & Inches \\
\hline
\end{tabular}

1 Glass used in this construction to be wire glass, and in all cases set in a metal shield.

522.

\section{GLASS CONTAINERS}

American Railway Association, signal section, specification 11328, July, 1928, battery jar.

\section{PURPOSE}

(a) The purpose of this specification is to provide primary battery jar for signaling.

\section{DRAWINGS}

(a) Figure 134, A. R. A., S. S., drawing 1053B, or Figure 135, A. R. A., S. S. drawing 1419, forms an essential part hereof.

\section{MATERIAI AND WORKMANSHIP}

(a) Material and workmanship shall be first class in every respect. (b) Manufacturer's identification mark shall be blown on side of each jar.

(c) Variations shall be within the limits specified in Figure 134 or 135.

\section{INSPECTION}

(a) Purchaser may inspect the material at all stages of manufacture.

(b) Purchaser may inspect the completed product to determine that the requirements of this specification have been met.

(c) If the material has not been accepted at point of production and if, upon arrival at destination, it does not meet the requirements of this specification, it may be rejected, and the contractor, upon request, shall advise the purchaser what disposition is to be made of the defective material. The contractor shall pay all freight charges.

(d) If purchaser is to make inspection at point of production it shall be so stated.

\section{TESTS}

(a) Tests may be made at point of production, or on samples submitted, and may also be made at destination.

(b) Contractor shall give the purchaser sufficient notice of time when material will be ready for testing.

(c) Contractor shall provide, at point of production, apparatus and labor for making the required tests under supervision of the purchaser.

(d) If tests are to be made at point of production it shall be so stated. Purchaser will distinctly indicate which of the tests herein specified are to be made and what portion of the material shall be tested.

(e) Purchaser shall select from the proposed shipment either 4 or 20 jars for the heating and chilling test and 1 for the solubility test.

$(f)$ Breakage for each test shall not exceed 1 when 4 jars are tested or 2 when 20 jars are tested.

\section{SOLUBILITY}

(a) Expose 110 square inches of inside of jar surface by filling to the required level with a solution consisting of $20 \mathrm{~g} \mathrm{NaOH}$ (caustic soda) by titration in $100 \mathrm{cc}$ of distilled water, then place in oven maintained at $120^{\circ} \mathrm{F}$. for 100 hours. The jar shall not show a loss of weight in excess of $0.5 \mathrm{~g}$.

\section{HEATING TEST}

(a) Jars having a specified wall thickness of threesixteenths inch, placed one-third their depth in a mixture of crushed ice or snow and salt at $5^{\circ} \mathrm{F}$. for 10 minutes shall not check or crack due to being half filled with paraffin at $375^{\circ} \mathrm{F}$. Temperature shall be read with bulb of thermometer in ice and against bottom edge of jar. Jars having a specified wall thickness of one-fourth inch shall withstand a similar test with a paraffin temperature of $340^{\circ} \mathrm{F}$.

\section{CHILLING TEST}

(a) Jars having a specified wall thickness of threesixteenths inch maintained at a temperature of $225^{\circ}$ F. for 30 minutes, either in an oven or in a boiling saturated salt solution, shall not check or crack due to being thrust bottom downward in a tub of ice water at $40^{\circ} \mathrm{F}$. and then tipped over and allowed to fill. Jars having a specified wall thickness of one-fourth inch shall withstand a similar test at a temperature of $212^{\circ} \mathrm{F}$. 


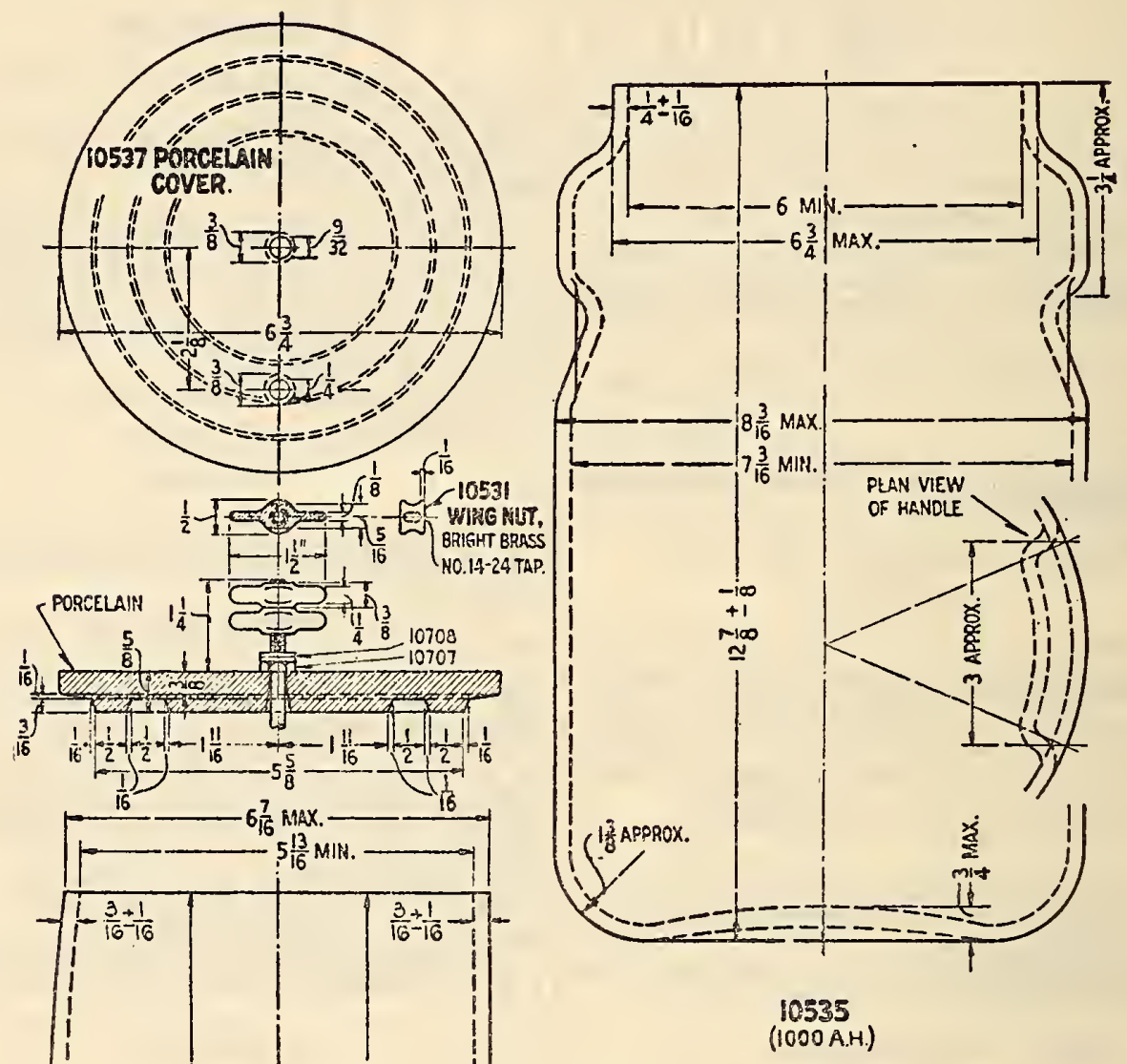

HOTZS: JARS SHALL EE OF HEAT RESISTING GLASS ANO SO GROUND THAT COVER SHALL KAVE A TRUE BEARING. PORCELAIN COVER SHALL BE GLAZED ON TOP AND EDGE.

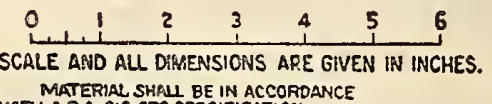

10532 MARREL SMAPE.

10533 STRALGHT SIDES.

10534

(500 A.H)

DOUND JARS AMD COVER

(PRIMARY BATTERY)

FIGURe 134.-A. R. A., S. S., drawing No. 1053B 


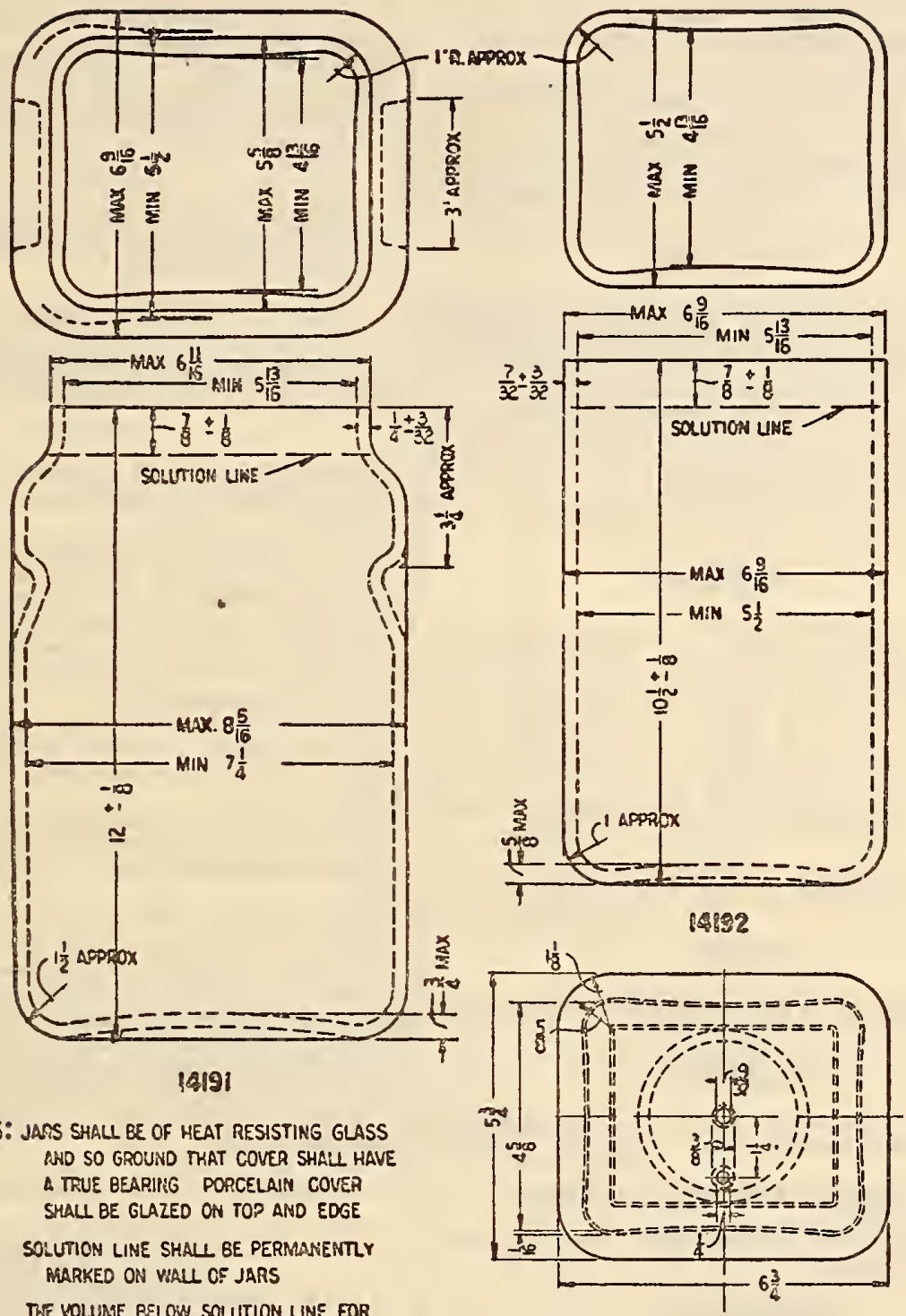

THE VOLUME BELOW SOLUTION LINE FOR JAR 14152 SHALL EE NOT LESS THAN $\triangle A O O C C$

\section{$0 \div 235$}

SCALE AND ALL DIMENSIONS ARE GIVEN IN INCHES

\section{REGTAMGULAR JAS ARO COVER}

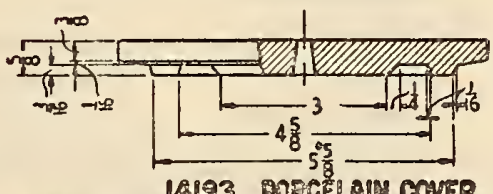

(PPIMARY BATERY)

Figdre 135.-A, R. A., S. S., drawing No. 1419 


\section{PACKING}

(a) Material shall be so prepared as to permit convenient handling and to protect against loss or damage during shipment.

(b) Jars shall be furnished in multiples of the contents of a standard package. When not so ordered the manufacturer may correct the order to the nearest standard-package quantity.

(c) Standard package for jars in accordance with Figure 134, details 10532, 10533, and 10534, and Figure 135, detail 14192 , shall hold 16 jars.

(d) Standard package for jars as shown on Figure 134, detail 10535, and Figure 135, detail 14191, shall hold 8 jars.

\section{MARKING}

(a) Purchaser's order, requisition, and package number, name of consignor, and name and address of consignee shall be plainly marked on outside of package.

(b) Detail list of loose pieces, containers, and their contents shall be furnished for each shipment. Where carload shipments are made routing and car identification shall be shown.

(c) Where carload shipments are made name and address of consignee may be omitted.

\section{WARRANTY}

(a) Contractor shall warrant the material covered by this specification to be free from defects in material and workmanship under ordinary use and service, his obligation under this warranty being limited to making, at point of production, any part or parts to replace those which shall be found defective after shipment to the purchaser. This warranty shall not apply to any apparatus which has been subjected to misuse, negligence, or accident.

American Railway Association, signal section, specification 11728, July, 1928, battery jar.

\section{PURPOSE}

(a) The purpose of this specification is to provide storage-battery jar for signaling.

\section{DRAWINGS}

(a) Figure 136, A. R. A., S. S., drawing 1224B, forms an essential part hereof.

\section{MATERIAL AND WORKMANSHIP}

(a) Material and workmanship shall be first class in every respect.

\section{VARIATIONS}

(a) Variations shall be within the limits specified in Figure 136.

\section{INSPECTION}

(a) Inspection requirements same as for A. R. A. signal section, specification 11328 (given above).

\section{TESTS}

Section 5, items $(a),(b),(c)$, and $(d)$ of specification 11328 are part of the test requirements of this specification.

(e) In checking the sizes of jars a mandrel one thirty-second inch larger than the maximum outside dimensions and one thirty-second inch smaller than the minimum inside dimensions specified in Figure 136 shall be used. Mandrel shall move freely into the inside of the jar for a distance of five-sixths of the depth of the jar and over the outside for a distance of 1 inch from top of the jar.

$(f)$ Jar shall be filled with water at a temperature of $50^{\circ} \mathrm{F}$, after which live steam shall be injected at such a rate as will raise the temperature to $200^{\circ} \mathrm{F}$. in one and one-half minutes. Jar shall then be allowed to stand for one minute, after which cold water shall be injected at such a rate that jar will be cooled to a temperature of $50^{\circ} \mathrm{F}$. in one and onehalf minutes.

\section{PACKING}

(a) Material shall be so prepared as to permit convenient handling and to protect against loss or damage during shipment.

\section{MARKING}

(Sec. 10 of specification 11328 covers the marking requirements of this specification.)

\section{Warranty}

(Same as sec. 11, specification 11328.)

Glass Container Association of America, types of finish.

(This association has adopted dimensional standards for 42 types of finishes, or closure structures, for glass containers, in cooperation with the cap and closure and glass manufacturers.)

Interstate Commerce Commission, regulations for transportation of explosives and specifications for shipping containers.

(The specifications of the commission, including glass containers, may be obtained upon request from the Interstate Commerce Commission, Washington, D. C.)

United States Department of Commerce, division of simplified practice, recommendation No. 10 , for milk and cream bottles and bottle caps, March, 1927.

In accordance with unanimous action of manufacturers, distributors, and users of milk bottles, the United States Department of Commerce, through the Bureau of Standards, recommends that in respect to bottles used in the sale of milk and cream the quarter-pint size be eliminated and that the recognized sizes, capacities, and dimensions of the quart, pint, and 1/2-pint bottles, and the sizes of caps for such bottles, be in conformity with the following tables:

TABLE. 1-Sizes of milk and cream bottles

\begin{tabular}{|c|c|c|c|c|c|c|c|}
\hline Capacity & Weight & $\begin{array}{l}\text { Over- } \\
\text { all } \\
\text { height }\end{array}$ & $\begin{array}{c}\text { Out- } \\
\text { side } \\
\text { diam- } \\
\text { eter } \\
\text { of } \\
\text { body }\end{array}$ & $\begin{array}{l}\text { Diam- } \\
\text { eter of } \\
\text { cap } \\
\text { seat } \\
\text { (maxi- } \\
\text { mum) }\end{array}$ & $\begin{array}{c}\text { Out- } \\
\text { side } \\
\text { diam- } \\
\text { eter } \\
\text { of neck }\end{array}$ & $\begin{array}{l}\text { Out- } \\
\text { side } \\
\text { diam- } \\
\text { eter } \\
\text { of roll1 }\end{array}$ & $\begin{array}{l}\text { Depth } \\
\text { of roll }\end{array}$ \\
\hline & Ounces & Inches & Inches & Inches & Inches & Inches & Inch \\
\hline Quart. & $251 / 2$ & $\left\{\begin{array}{l}914 \\
916\end{array}\right.$ & $3^{13} / 16$ & $1^{2} 1 / 32$ & $12 \% / 32$ & $21 / 4$ & $11 / 16$ \\
\hline $\begin{array}{l}\text { Pint } \\
\text { Half- } I\end{array}$ & $\begin{array}{l}151 / 2 \\
10\end{array}$ & $\begin{array}{l}71 / 4 \\
53 / 8\end{array}$ & $\begin{array}{l}31 / 16 \\
21 / 2\end{array}$ & $\begin{array}{l}121 / 32 \\
1^{21 / 32}\end{array}$ & $\begin{array}{l}113 / 16 \\
13 / 4\end{array}$ & $\begin{array}{r}21 / 4 \\
23 / 16\end{array}$ & $\begin{array}{r}11 / 16 \\
2 / 16\end{array}$ \\
\hline
\end{tabular}

1 For maximum and minimum limits, see table in Figure 138.

TABLE 2.- Size of milk and cream bottle caps ${ }^{1}$

\begin{tabular}{c|c|c}
\hline Size number & Die & $\begin{array}{r}\text { Thickness of } \\
\text { paper board }\end{array}$ \\
\hline No. 2 & $\begin{array}{r}\text { Inches } \\
121 / 32\end{array}$ & $\begin{array}{c}\text { Inch } \\
0.035-0.040\end{array}$ \\
\hline
\end{tabular}

1 One size of cap will meet the requirements for the four varieties of bottles shown in Table 1. 


\begin{tabular}{|c|c|c|c|c|c|c|c|c|c|c|c|c|c|c|c|c|}
\hline \multirow{3}{*}{ AH. } & \multicolumn{8}{|c|}{ BATTERY JARS } & \multicolumn{3}{|c|}{ SAND TRAYS } & \multicolumn{3}{|c|}{ CEL COVERS } & \multicolumn{2}{|c|}{ HOLD DOWhS } \\
\hline & \multirow{2}{*}{ REF. NO. } & \multicolumn{2}{|c|}{$A$} & \multicolumn{2}{|c|}{$B$} & \multirow{2}{*}{ C } & 0 & \multirow{2}{*}{ EII } & \multirow[b]{2}{*}{ REF NO. } & \multirow[b]{2}{*}{$F$} & \multirow[b]{2}{*}{ G } & \multirow[b]{2}{*}{ REF. NO. } & \multirow[b]{2}{*}{ H } & \multirow[b]{2}{*}{$J$} & \multirow[b]{2}{*}{ REF. NO. } & \multirow[b]{2}{*}{$\mathbf{K}$} \\
\hline & & sio. & max & sio & gax & & MiN. & & & & & & & & & \\
\hline 40 & 12241 & $77^{3.1}$ & 75 & $44^{3}$ & $5^{\circ}$ & $11^{\circ}$ & $68^{\circ}$ & $4 \frac{10}{8}$ & 122401 & $9 ! 8^{\prime \prime}$ & $6 \frac{1}{2}^{10}$ & 122411 & $4^{\circ}$ & $6 \frac{11}{2}$ & 122421 & $3 \frac{7}{8}$ \\
\hline 80 & 12242 & $9 \frac{1}{4}$ & $9 !_{2}^{\circ}$ & $5 \frac{1}{2}$ & $5_{4}^{30}$ & $12 \frac{3}{4}$ & $8 \frac{1}{2}$ & $4 \frac{3}{4}^{\circ}$ & 122402 & $11^{\circ}$ & $7 \frac{14}{4}$ & 122412 & $5^{3} \frac{3}{4}^{\circ}$ & $7 \frac{11}{4}$ & 66 & $3 \frac{71}{8}$ \\
\hline 120 & 12243 & $9 \frac{1}{4}$ & $9 \frac{1}{2}$ & 7 & $7 \frac{10}{4}$ & $123^{3}$ & $81^{10}$ & $6 \frac{1}{4}$ & 122403 & $11^{\circ}$ & $8 \frac{3}{4}$ & 122413 & $5 \frac{34^{3}}{4}$ & $8 \frac{3}{4}^{30}$ & 122422 & $5 \frac{1}{2}$ \\
\hline 240 & 12245 & $12 \frac{30}{8}$ & $12 \frac{5}{8}$ & 78 & $78^{\circ}$ & $17^{\prime}$ & $11 \frac{11}{2}$ & $68^{\circ}$ & 122405 & $14 \frac{1}{8}$ & $9 \frac{1}{8}$ & 122415 & $8 \frac{1}{2}$ & $9_{8}^{10}$ & 122424 & $62^{\circ}$ \\
\hline 400 & 12246 & $128^{\circ}$ & 125 & $10 \frac{5}{8}$ & 11 & 174 & $1 \frac{10}{2}$ & 970 & 122406 & $148^{\prime 2}$ & $12 \frac{3}{8}$ & 122416 & $8 \frac{10}{2}$ & $12_{8}^{3 n}$ & 122423 & $9^{\circ}$ \\
\hline
\end{tabular}

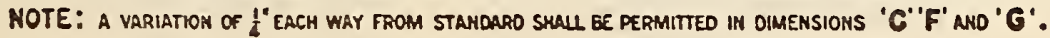

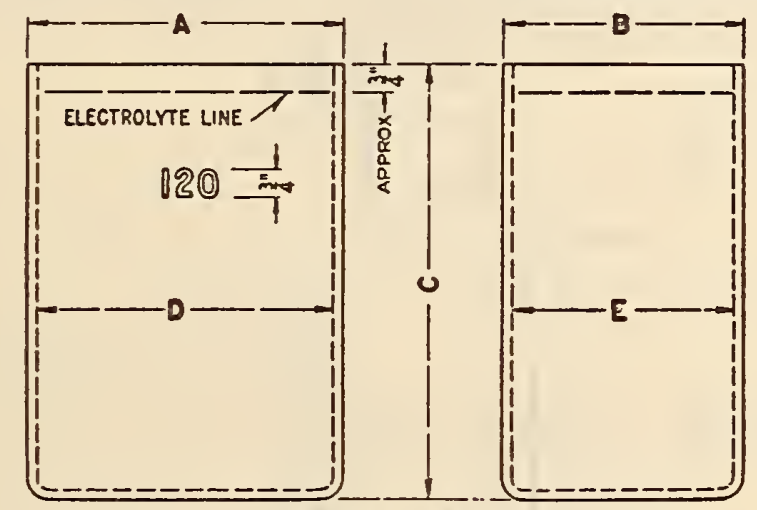

BATTERY JAR.

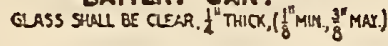

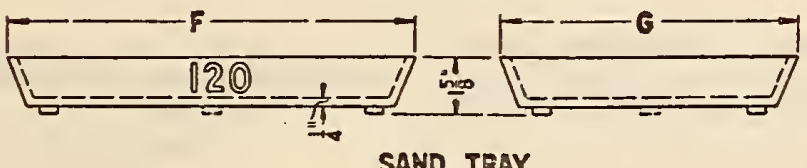

SAND TRAY.

SAND TRAYS FOR 2 40 AHAND 400 AH JARS

SHALL HAVE MINE (9) INSULATOR FEET.

HOTES: CAPACITY OF JAR SHALL BE MOULDED OR ETCHED IN GLASS OF JAR AND SAND TRAY.

CELL COVERS AND HOLD-DOWNS SHALL HAVE ROUNDEO EDGES AND CORNERS.

MATERIRL SHALL BEIN ACCORDANCE WITH AR.A SIG. SEC. SFECIFICATION.

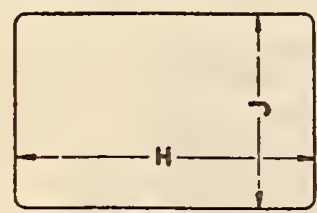

CELL COVER.

SHEET GLASS 3"TO I" THICK. CORRUGATED ON ONE SIOE PARALLEL TO DIMENSION ' $J$ '.

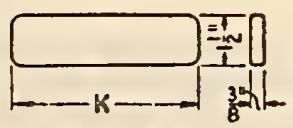

HOLD-DOWN.

\section{AEQ'D FOR 240 AH.AND} 400 A.H. CELLS.

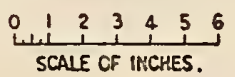

\section{STOHAGE BRTERY JARS, COVERS, HOLD-DOWHS AMD SAND TRAYS.}

\section{STATIONARY TYPE.}

Figure 136.-A. R. A., S. S., drawing No. $1224 B$ 

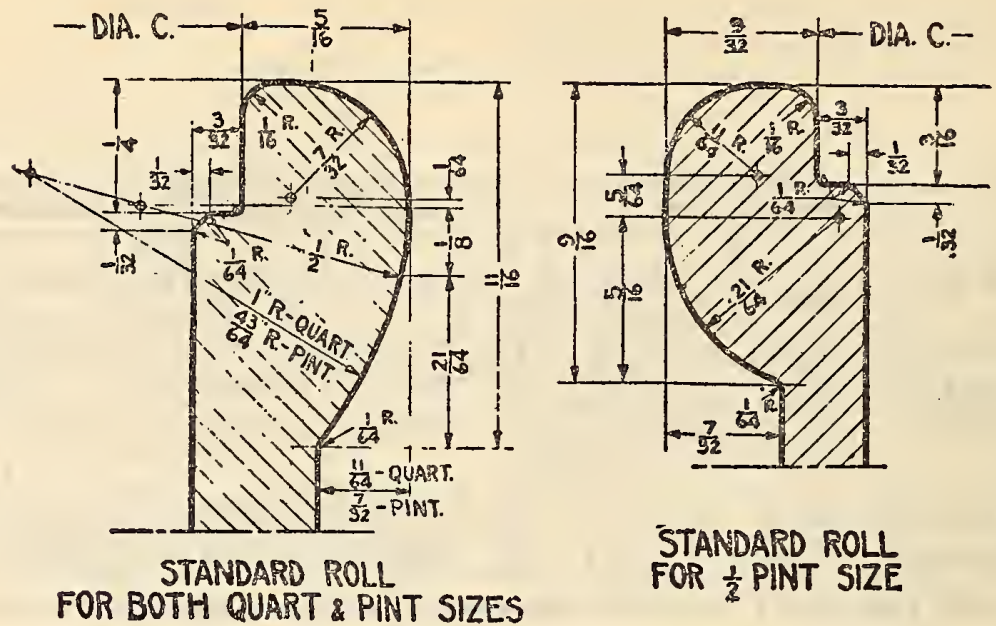

\section{STANDARD ROLL FOR $\frac{1}{3}$ PINT SIZE}

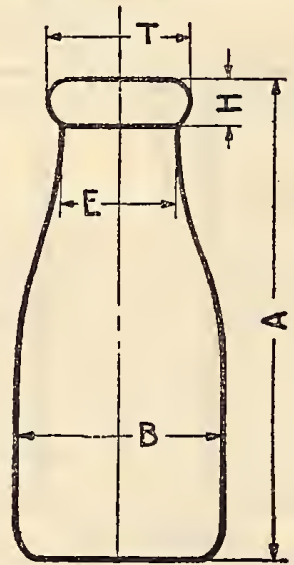

Figure 137

Onited States Department of Commerce, division of simplified practice, recommendation No. 91, glass containers for preserves, jellies, and apple butter, January, 1929.

\section{MILK BOTTLE GLASS FINISH}

\begin{tabular}{|c|c|c|c|c|c|c|c|c|}
\hline ADOR & A & B & C & $E$ & 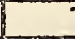 & $\Gamma$ & $H$ & VEI \\
\hline ZZE & & & MAX. & & MAX. & MIN. & & \\
\hline$\frac{1}{2}$ PINT & $5 \frac{3}{8}$ & 2.500 & 1.656 & 1.750 & 2.197 & 2.167 & $\frac{9}{16}$ & $10 \mathrm{oz}$ \\
\hline PINT & $7 \frac{1}{4}$ & 3.062 & 1.656 & 1.812 & 2.260 & 2.230 & $\frac{11}{16}$ & $15 \frac{1}{2} 02$ \\
\hline QUART & $9 \frac{1}{4}$ & 3.812 & 1.656 & 1.906 & 2.260 & 2.230 & $\frac{11}{16}$ & $25 \frac{1}{2} 0 z$ \\
\hline CHART & $9 \frac{1}{2}$ & 3.812 & 1.656 & 1.906 & 2.260 & 2.230 & $\frac{11}{16}$ & $25 \frac{1}{2}$ \\
\hline
\end{tabular}

\section{NOTE}

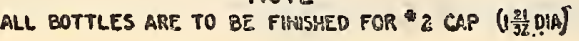

Figure 138

A general conference of representative manufacturers, distributors, and users unanimously recommends to the industry, through the Bureau of Standards of the Department of Commerce, the fol- lowing simplified list of stock sizes based on the avoirdupoise weight of the food content.

\begin{tabular}{|c|c|c|}
\hline Preserves & Jellies & $\begin{array}{l}\text { Apple } \\
\text { butter }\end{array}$ \\
\hline $\begin{array}{c}\text { Ounces } \\
2 \\
4 \\
8 \\
12 \\
16 \\
32\end{array}$ & $\begin{array}{c}\text { Ounces } \\
2 \\
4 \\
6 \\
8 \\
10 \\
12\end{array}$ & $\begin{array}{c}\text { Ounces } \\
7 \\
14 \\
28 \\
38\end{array}$ \\
\hline $\begin{array}{l}43 \\
64\end{array}$ & & $\cdots-$ \\
\hline
\end{tabular}

523.

\section{TABLE GLASSWARE}

523.1 BOTTLES (VINEGAR).

American IMarine Standards Committee, kinds and sizes of glassware for ship equipment, standard $\mathrm{O}$ No. 8-1926, (Bottles).

See 523.9 , page 497 .

Federal Specifications Board, specification No. 121a, United States Government master specification for tableware, glass, September 25, 1926.

\section{TYPES AND ITEMS}

1. This specification includes two types of glass tableware: A, blown or pressed ware and $\mathrm{B}$, cut ware. 
2. The following items are included: Tumbler, vinegar bottle, sirup pitcher, salt shaker, and pepper shaker.

\section{MATERIAI}

1. Blown or pressed ware.-Shall be made from either a lime or a lead flint glass, which may be melted in a pot or tank and may be either hand or machine made.

2. Cut ware.-Shall be made from a lead silica alkali glass commonly known as lead crystal. It shall contain not less than 30 per cent of lead oxide and have a specific gravity not less than 3.0.

\section{GENERAL RIQUIREMENTS}

1. Glass shall be of such composition and so well annealed that articles will pass the shock test and boiling test without showing any indications of corrosion, chipping, scumming, or cracking.

2. Design. - Ware shall be furnished conforming to figures shown on drawings of ware adopted for Government use or as otherwise indicated in the order, measurements and weights to be within the following tolerances:

Dimensions

Per cent

Capacity

Weight

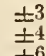

\section{DETAIL REQUIREMENTS}

1. All tableware shall be free from heavy cords, stones, and fine cracks. It shall be straight and true so that when rotated about its vertical axis the top edges shall show an eccentricity of less than one-eighth of an inch and the bottoms must be sufficiently plane so that the ware will not rock on a smooth surface. All edges must be properly finished.

2. Pressed and blown ware may show faint color and have a few scattered bubbles (not excceding five one-hundredths inch in diameter) providing the glass is of what is generally considered as good color and the defects do not damage the appearance of the ware. Surfaces shall be fire polished enough to eliminate dullness.

3. Cut ware. - IVare shall be cut on a wheel from smooth blanks and all of the exterior surface shall be smoothed and polished, including scallops and notches. The interior of the blanks must be protected from acid. during manufacture.

\section{METHOD OF SAMPLING AND TESTING}

1. Sampling.-In shipments of 10 dozen or less of any item, 2 articles of that item shall be selected at random for test. For shipments of over 10 dozen, 2 samples for each 10 dozen or fraction thereof shall be selected for test.

\section{METHODS OF TESTING}

(a) Boiling test.-Articles shall be completely immersed in distilled water in a closed vessel having a small steam vent and boiled continuously for six hours, boiling water being occasionally added to compensatc for evaporation losses. The article shall be suspended in the water so that it does not come in contact with the containing vessel. Any article which shows corrosion, scumming, chipping, or cracking as a result of the boiling shall be considered as having failed to pass the test.

(b) Shock test for tableware.-Articles shall be immersed in tap water at $18.5^{\circ} \mathrm{C}$. $\pm 21 / 2^{\circ}\left(65^{\circ} \mathrm{F}\right.$. $+5^{\circ}$ ) allowed to remain 10 minutes, then transferred suddenly into boiling water. This operation shall be repeated five times. Cracking of the article during the test shall be considered as a failure.

(c) Determination of specific gravity.-Article shall be dried at room temperature and weighed. It shall then be weighed suspended in water, completely immersed. Very light cord or thread shall be used for suspending the articlc, depending upon its weight. Specine gravity may be calculated from the formula:

$$
\frac{\text { Dry weight }}{\text { Dry weight-suspended weight }}=\text { spccific gravity }
$$

The entire article or broken portions of it may be used for this test. Weighing shall be done accurately to at least one-tenth of 1 per cent of the total weight.

(d) Failure of any item to pass any of the requirements shall be considered as a basis of rejection of the article.

(e) If all samples taken pass the requirements, the order shall be accepted; if any one item, such as tumblers or vinegar bottles, pass the requirements, but another item fails to pass, that portion of the order which passes shall be accepted, and that portion failing to pass shall be rejected.

\section{PACKING}

Shall be in accordance with commercial practice unless otherwise specified.

\subsection{PITCHERS.}

American Marine Standards Committee, kinds and sizes of glassware for ship equipment, standard $\mathrm{O}$ No. 8-1926. (Pitchers.)

See 523.9, below.

Federal Specifications Board, specification No. 121a, United States Govcrnment master specification for tableware, glass; September 25, 1926. (Sirup pitcher, glass.)

Sec 523.1 (above).

\subsection{SHAKERS.}

American Marine Standards Committee, kinds and sizes of glassware for ship equipment, standard $O$ No. 8-1926. (Shakers.)

Sce 523.9, belowv.

Federal Specifications Board, spccification No. 121a, United States Government master specification for tableware, glass; September 25, 1926. (Salt and pepper shakers, glass.)

See 523.1 (above).

\subsection{TURBLERS.}

American Marine Standards Committee, kinds and sizes of glassware for ship equipment, standard $\mathrm{O}$ No. 8-1926. (Tumblers.)

See 523.9 , below.

Federal Specifications Board, specification No. 121a, United States Government master specification for tableware, glass; September 25, 1926. (Glass tumblers.)

See 523.1 (above).

\subsection{MISCELLANEOUS TABLE GLASS. WARE.}

American Marine Standards Committee, kinds and sizes of glassware for ship equipment, standard $\mathrm{O}$ No. 8-1926.

\section{BLOWN WARE}

All for passenger service and/or officers' mess.

Tumblers:

$2 \frac{1}{2}$-ounce beverage.

5-ounce tall, grape or orange juice.

7-ounce tall, ginger ale.

10-ounce squat, saloon or stateroom. 
10-ounce tall, soda or lemonade.

12-ounce tall, soda or ice tea.

14-ounce tall, ice tea.

Stemware:

1-ounce stemmed glass.

3 -ounce stemmed glass.

$31 / 2$-ounce stemmed glass.

5 -ounce parfait.

5 -ounce, low stem sherbert.

6-ounce, high.

Miscellaneous:

54-ounce jug.

5 -inch ice cream dish.

41/2-ounce handled sherbert cup.

Liners for silver pots for horseradish or mustard.

(For replacements.)

Water bottle, standard size.

\section{PRESSED WARE}

For passenger service and/or officers' mess.

Bottle, wide mouth, ground stopper. (For separate use for oil, vingear, etc.)

Bottle, ground stopper, for oil, vinegar, etc. (For silver frame caster.)

Dish, preserve, 7-inch oval.

Dish, grapefruit or baked apple.

Punch bowl, 16-inch.

Shakers, salt and pepper, metal or glass top.

Shakers, salt, pepper, and paprika, silver-plated tops. (For individual caster.)

Shakers, glass only. (For replacements.)

3-pint hallboy jug.

Tray, celery, 11-inch.

Tumblers, 3-ounce oyster cocktail.

For crew and/or third-class service.

Dish, $91 / 2$-inch.

Pitcher, molasses or sirup, metal top.

Shakers, salt and pepper, metal or glass top.

Shakers, pepper and salt, large, metal top.

Tumbler, 10-ounce.

\section{IIGHTING GLOBES AND SHADES}

American Marine Standards Committee, fixed lights for ships, standards $\mathrm{H}$ No. $3, \mathrm{H}$ No. 4, and H No. 5, 1925.

(These standards are for fixed ship lights of type A, pressed-steel frame; type B, cast-brass frame; and type $\mathrm{C}$, cast-brass finished frame. The standards include the recommended glass sizes of American Marine Standards Committee, standard H No. 1, 1925. (See 521.1, p. 485.))

American Railway Association, signal section, specification 5927, July, 1927, hand lantern globes.

\section{GENERAI DESCRIPTION}

(a) This specification covers all clear, red, yellow, green, and blue globes.

\section{KIND OF GLASS}

(a) Globes shall be made of low expansion heatresisting glass.

(b) Colored globes shall be made of solid color glass; all globes shall be clear and free from opalescence, affecting the light transmitted.

\section{FURNISHING SAMPLES}

(a) Samples shall be furnished free of cost and shall be in excess of the number of globes ordered.

(b) The manufacturer must submit samples of globes, showing the extreme limits of colors which it is proposed to furnish. These shall bear labels showing the photometric values, and, if approved, will be kept as the standards.

\section{PERMISSIVE VARIATIONS}

Globes shall conform to A. R. A. Sig. Sec. 1610A or 1611B. (Figs. 139 and 140.)

\section{MARKING}

(a) Lettering specified in the order shall have the marking blown, etched, or sand-blasted upon the glass.

(b) Glnbes shall have the manufacturer's trademark marked not more than $1 \frac{1}{2}$ inches below the top of the globe.

\section{WORKMANSHIP}

(a) Globes shall be clear in color, of smooth surface, and have both ends finished without sharp edges.

\section{TESTS}

(a) Manufacturer shall give the purchaser sufficient notice of time when material will be ready for testing.

(b) Manufacturer shall provide, at point of production, apparatus and material for making required tests.

\section{BOILING WATER TEST}

(a) Globes shall stand immersion in boiling distilled water for two hours without showing any disintegration.

\section{PHOTOMETRIC TESTS}

(a) Globes in the respective colors tested on a photometer, shall be in accordance with the scale used in selecting the limits of photometric values in signal section specification 6918 .

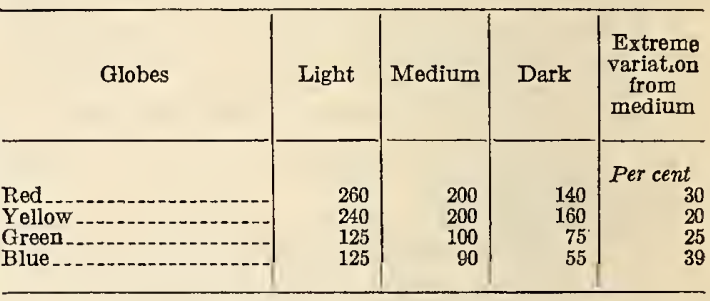

(b) The photometric value shall be the average of three readings taken, $90^{\circ}$ apart, around the circumference of the test globes, on a level with the flame. No readings shall be taken through the lettering.

(c) If any of the samples fail to pass the photometric test, the purchaser may require any or all of the colored globes to be tested photometrically in his presence, and all globes failing to pass within the limits shown shall be rejected.

\section{CHILIING TEST}

(a) Clear, red, and yellow globes.--In making this test the globe is placed in a hand-lantern frame, suitable for the globe tested. The fount shall contain signal oil or kerosene. Suitable burners and wicks for the lantern involved shall be used. The wick shall be turned up as far as possible without causing the lantern to smoke. The lantern shall then be tilted to an angle of $45^{\circ}$, keeping the flat edge of the flame in the same plane as the flat edge of the wick and with globe so placed that the flame is directed against the side opposite the lettering; it shall be allowed to remain in this position for five minutes, during which period the flame shall be regulated, if necessary, so that not more than 1 


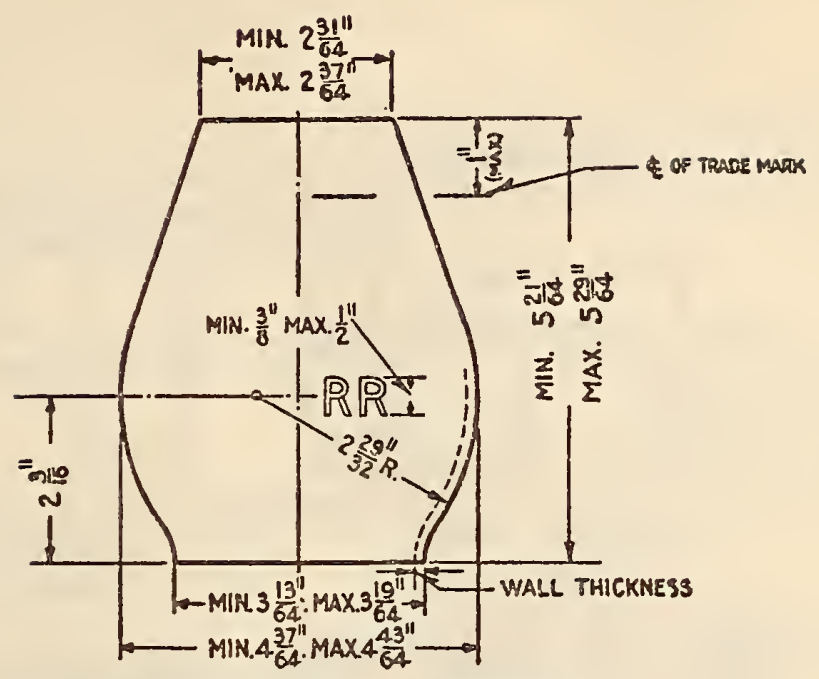

\begin{tabular}{|c|c|c|c|c|c|}
\hline GLOBE & COLOR & $\frac{\text { WALI }}{\text { MIM! }}$ & $\frac{1 \text { MCKNESS }}{M A X}$ & $\frac{\text { WEIG }}{M \text { MWN }}$ & $\frac{\pi S-a x^{2}}{M A x}$ \\
\hline 16101 & CLEAR & $3 / 32$ & $7 / 32^{10}$ & 10 & 14 \\
\hline 16102 & RED & II & 11 & $\pi$ & 16 \\
\hline 16103 & YELLOW & 11 & II & II & 16 \\
\hline 15109 & BLUE & $5 / 64$ & $1 / 4^{\prime \prime}$ & 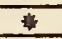 & + \\
\hline 15105 & GREEN & II & 11 & $\$$ & 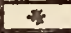 \\
\hline
\end{tabular}

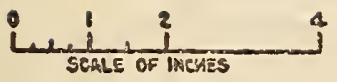

NOTE:- WABES OH ACCOUT OF MAMIL-

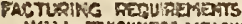
s

WALL THICKXES5 MESUEED AT EADS CKLY.

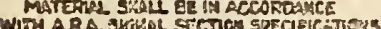

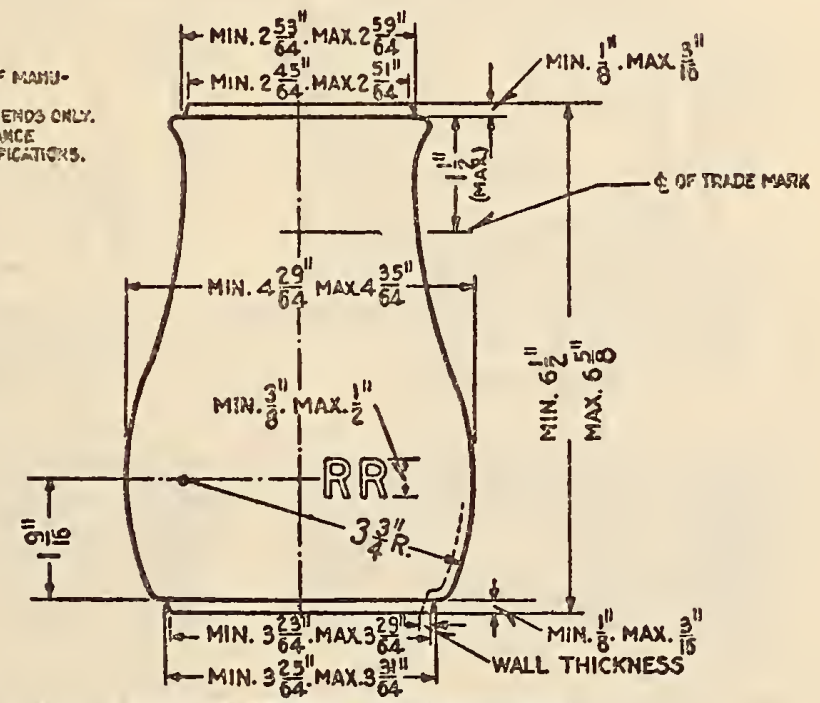

\begin{tabular}{|c|c|c|c|c|c|}
\hline GLOBE & COLOR & $\frac{\text { WALLT }}{\text { MIN. }}$ & MACANS & $\begin{array}{l}\text { WEIG } \\
\text { MIN: }\end{array}$ & $\frac{5-O Z}{M A X}$ \\
\hline 16106 & CLEAR & $3 / 32^{11}$ & $7 / 32$ & 10 & 10 \\
\hline 16107 & FED & II & 11 & 11 & 17.5 \\
\hline 16108 & YELLOW & II & 11 & II & 17.5 \\
\hline 10109 & BLUE & $5 / 64^{11}$ & $1 / 4^{11}$ & 澏 & 表 \\
\hline 16010 & GREEN & "I & II & 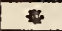 & 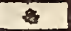 \\
\hline
\end{tabular}

HAND LAMTERR GLOBES

Figure 139.-A. R. A., S. S., drawing No. 1610A 


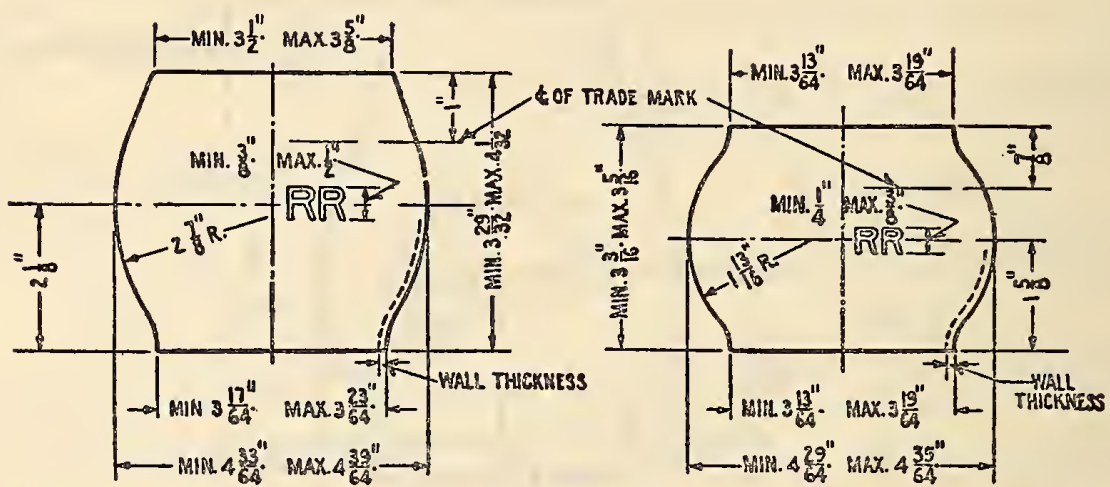

\begin{tabular}{|c|c|c|c|c|c|}
\hline GLOB & CDLOR & \multicolumn{4}{|c|}{ 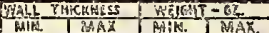 } \\
\hline $6 y^{2}$ & CLEAR & $5 / 64$ & $3 / 15^{11}$ & 6 & 11 \\
\hline 1612 & RED & II & II & 7 & $i 2$ \\
\hline Songens & VELCOW & it & 11: & 7 & 12 \\
\hline 76149 & 8LUE & $\bar{n}$ & $7 / \sqrt{6}$ & 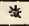 & 亦 \\
\hline 18. & 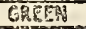 & B & it & \% & $*$ \\
\hline
\end{tabular}

\begin{tabular}{|c|c|c|c|c|c|}
\hline GLOBE & COLOR & \multicolumn{4}{|c|}{ 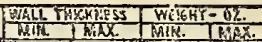 } \\
\hline 616 & CLEAP & $3 / 32^{4}$ & $7 / 32$ & 50 & 9.0 \\
\hline 1458 & RED & to & $\|$ & 5.5 & 10.5 \\
\hline 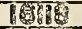 & YELEGY & 71 & i1 & 5.5 & 10.5 \\
\hline 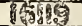 & Sप1 & $5 ; 66^{\circ}$ & 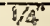 & * & * \\
\hline 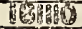 & GREES & t & 62 & th & * \\
\hline
\end{tabular}
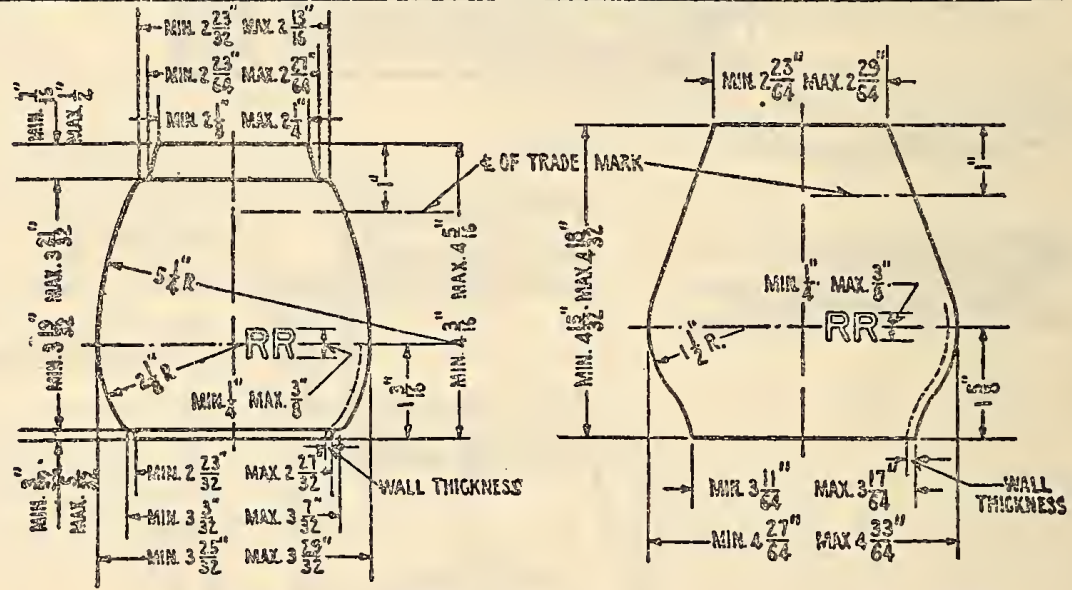

\begin{tabular}{|c|c|c|c|c|c|}
\hline GLOEE & 50 & \multicolumn{2}{|c|}{ WALL THICKAESS } & \multirow{2}{*}{\multicolumn{2}{|c|}{ WLIGHT-02 }} \\
\hline & & MIN. & MAX: & MH & \\
\hline 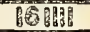 & CLEAR & $3 / 32$ & $7 / 32$ & 5.0 & 9.9 \\
\hline 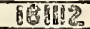 & RED & " & 11 & 5.5 & 10.5 \\
\hline 8 & YELLOW & "1 & $" 1$ & 5.5 & 10.5 \\
\hline 85. & BtuE & $5 / 54^{\prime \prime}$ & $1 / 8^{n}$ & te & * \\
\hline 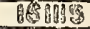 & Gereps & 11 & 11 & * & $\%$ \\
\hline
\end{tabular}

\begin{tabular}{|c|c|c|c|c|c|}
\hline GLOBE & COres & \multicolumn{4}{|c|}{ 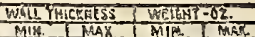 } \\
\hline 16838 & CLEAR & $3 / 32$ & $3 / 16$ & 5.5 & 9.0 \\
\hline 66418 & RED & 4 & $\because$ & 5.5 & 31.9 \\
\hline 16968 & VELLQY & 18 & 10 & 55 & 11.0 \\
\hline 6utBS & GLLE & 0 & 11 & 4 & 4 \\
\hline 86909 & GREEY & $\because 0$ & $\because 1$ & 徣 & $\%$ \\
\hline
\end{tabular}

$\underbrace{\frac{1}{1}}_{\text {SCALE OF IRCHES }}$

WOTE:- WAVEO OH RCCOUNT OF MANUFACTURIHG

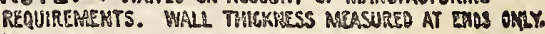
BATERIAL SHALL BE IH RCCORDANGE WITH

A.RA. SIG SEC SPCLIBRTQA.

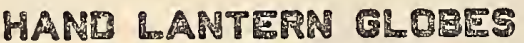

Figure 140.-A. R. A., S. S., drawing No. 1611B 
square inch of the globe surface is smoked. The globe shall then be removed from the frame while in a vertical position with the large end downward, dropped immediately into a tub of ice water, maintained at a temperature of between $43^{\circ}$ and $50^{\circ} \mathrm{F}$., care being taken that the globe does not strike the ice on being dropped into the water.

(b) Green and blue globes.-In making this test, the globe is placed in a hand-lantern frame, standing vertically, and with the wick so regulated that the flame is not more than 1 inch high, or just below the smoking point, and allowed to remain for three minutes. The globe is then dropped in ice water as described in section $(a)$.

(c) Percentage of failures.-Globes of the various colors, tested as described in sections $(a)$ and $(b)$, shall not show a breakage in excess of the percentage given below:

$\begin{array}{ccccc}\text { Clear } & \text { Red } & \text { Yellow } & \text { Blue } & \text { Green } \\ 1236 & 25 & 25 & 30 & 30\end{array}$

(d) Additional chilling tests.-If samples fail to pass the chilling test, an equal number of additional samples will be taken for a second test, and if the average of both lots meets the tests the entire quantity shall be accepted, otherwise the entire quantity shall be rejected.

\section{NUMBER OF GLOBES TESTED}

(a) Samples shall be selected at random for photometric and chilling tests, as follows:

1. On orders of any one color aggregating-

100 dozen

50 to 99 dozen

26 to 49 dozen

2 per cent of total.

1 to 25 dozen

4 per cent of total.

(b) At option of the purchaser, three additional

globes from each lot nay be required to make boiling tests.

(c) Inspection and tests may be waived on orders for less than 25 dozen globes.

\section{REJECTION}

(a) Globes which do not conform to the requirements of this specification shall be rejected.

\section{REHEARING}

(a) When tests are made at the purchaser's laboratory, samples of rejected material shall be held for one month from date of test report. In case the manufacturer is dissatisfied with results of tests, he may claim for rehearing within that time. Failure to raise a question within one month will be construed as evidence of satisfaction with results of tests. The samples shall be scrapped and claim for a rehearing will not be considered.

\section{INSPECTION}

Inspection requirements same as for A. R. A. signal section, No. 11328, see 522, page 491 .

\section{NUMBER OF PACKAGES EXAMINED}

(a) Not less than 10 per cent of all packages of globes shall be examined for conformity to the requirements in sections covering "Permissive variations," "Marking," and "Workmanship," of this specification.

\section{FREIGHT CHARGES}

(a) Rejected material shall be returned to the contractor, who shall pay freight charges both ways.

\section{ORDERING}

(a) Globes shall be ordered in 5 dozen lots, as far as possible.

\section{PACKING}

(a) Globes shall be carefully packed in barrels or boxes, marked to show style of globe, color, number of globes, weight, order number, requisition number, and the purchaser's private mark. Not more than 5 dozen globes shall be packed in each container.

\section{SHIPPING}

(a) Globes shall be held at place of manufacture until inspected, sampled, and tested, and, if accepted, manufacturer shall load and ship them as required by the purchaser.

Federal Specifications Board, specification No. 122, United States Government master specification for glass lantern globes and lamp chimneys, April $1,1924$.

\section{DESIGNATION}

Articles covered in these specifications are clear and colored lantern glcbes used for hand lanterns, railway signal lanterns, and similar uses, and for clear lamp chimneys as used for interior lighting.

\section{MATERIAI}

Lantern globes.-These shall be made from a glass which may be classed as a heat-resisting glass because of its having a low coefficient of expansion or some other property producing the ability to withstand sudden heating and cooling without rupture.

The glass may be either pot or tank melted and the articles may be either hand or machine made.

Lamp chimneys. - These may be made of any type of glass provided it passes the rcquirements. The glass may be either pot or tank melted and the articles may be either hand or machine made.

\section{REQUIREMENTS}

Lantern globes.-Clear glass may show faint pink or green color, but shall have what is generally considered a good color. A few scattered bubbles are permissible providing they do not interfere with the performance of the globe. Surfaces shall be polished enough to eliminate dullness.

All colored globes shall be of solid-color glass and pass photometric requirements specified in the order.

Glass shall be of such composition and so well annealed that globes will pass the shock test for lantern globes and the boiling test without showing any cracking, chipping, scumming, or corrosion.

Lamp chimneys. - Glass may show faint pink or green color, but should have what is generally considered a good color.

It may contain a few scattered bubbles (not exceeding $5 / 100$ inch in diameter) providing such defects do not damage the appearance of the ware. Surfaces of chimneys shall be well fire polished.

Glass shall be of such composition that it will pass the shock test for lamp chimneys and the boiling test without showing any cracking, chipping, scumming, or corrosion.

Lantern globes and lamp chimneys shall be free from heavy cords, stones, and fine cracks. Ware shall be straight and true so that when rotated about its vertical axis, the top edges shall show an eccentricity of less than $1 / 8$ inch, and the bottoms must be sufficiently plain so that the ware will not rock on a smooth surface. Edges shall be either molded or ground to a smooth surface. 
Design.-Ware shall be furnished conforming to figures shown on drawings or as otherwise indicated in the order, dimensions and weights to be within the tolerances specified.

\section{METHOD OF TESTING}

Shock test for lantern globes.-Same as "chilling test," American Railway Association, signal section, specification 5927, see 524 (above), with the exception of the percentage limits for failures, which for this specification are as follows:

$$
\begin{array}{ccccc}
\text { Clear } & \text { Ruby } & \text { Yellow } & \text { Green } & \text { Blue } \\
121 / 2 & 331 / 3 & 33 \frac{1}{3} & 50 & 75
\end{array}
$$

Shock test for lamp chimneys. - Chimneys shall be heated in an oven to $120^{\circ} \mathrm{C}$. $\left(250^{\circ} \mathrm{F}\right.$.) for at least 20 minutes. The chimney shall then be rapidly removed and sprayed on one side with a finely divided spray of water at temperatures between $60^{\circ}$ F. $\left(16^{\circ}\right.$ C. $)$ and $68^{\circ}$ F. $\left(20^{\circ}\right.$ C. $)$. Chimneys which crack as a result of this test shall be considered as having failed.

Boiling test. - Articles shall be completely immersed in distilled water in a closed vessel having a small steam vent and boiled continuously for six hours, boiling water being occasionally added to compensate for evaporation losses.

The article shall be suspended in the water so that it does not come in contact with the walls of the containing vessel.

Any article which shows corrosion, scumming, chipping, or cracking as a result of the boiling shall be considered as having failed to pass the test.

\section{SAMPLING}

Same as $\Lambda$. R. A. specification 5927 (above).

\section{ACCEPTANCE}

Failure of any type of article to pass any of the requirements shall be considered as a basis of rejection of the article.

If all samples taken pass the requirements, the order shall be accepted. If any one type of article passes, but another type fails to pass, that portion of the order which passes shall be accepted and that portion which fails to pass shall be rejected.

525.

\section{LENSES}

\subsection{LIGHTHOUSE IENSES.}

(No nationally recognized specifications available.) 525.2 REFLECTORS. able.)

(No nationally recognized specifications avail-

\subsection{SIGNAL IENSES.}

American Electric Railway Association, sizes of lenses for use in light signals (recommended), serial No. S105-25, 1925.

For light signals employed in electric railway signaling, the diameters of the lenses should be as follows: $5 \frac{3}{8}, 6 \frac{3}{8}$, or $8 \frac{3}{8}$ inches.

American Railway Association, signal section, specification 6918, signal roundels, lenses, and glass slides.

\section{GENERAL}

(a) Material.-1. Glass shall be of a composition which is durable on prolonged exposure to the atmosphere. Colored glass shall be of a uniform solid color. Flashed glass will not be accepted.

2. Glass shall have a refraction index of not less than 1.50 and a specific gravity of not less than 2.50.

(b) Workmanship.-Workmanship shall be of the best; glasses shall be true to size and form and practically free from chips, bubbles, streaks, and wrinkles and shall have the name or trade-mark of manufacturer pressed on the outer zone.

(c) Color.-Red, green, yellow, blue, purple, and lunar white glasses will be purchased.

(d) Wrapping.-Each glass shall be wrapped in paper of corresponding color.

(e) Tests.-1. Manufacturer shall give the purchaser sufficient notice of time when material will be ready for testing.

2. Manufacturer shall provide at point of production apparatus and labor for making required tests under the supervision of the purchaser.

3. Manufacturer shall test each glass, placing thereon a label showing that the photometric value falls within the dark and light limits shown in section (b) under "Roundels," and shall submit all red glass to the sodium test.

4. Purchaser reserves the right to make repetition of the above tests.

(f) Samples.-Manufacturer shall submit samples of glasses, showing the extreme limits of colors which it is proposed to furnish. These shall bear labels showing the photometric values, and, if approved, will be kept in the office of the signal engineer, as standard.

(g) Inspection.-(Inspection requirements same as for A. R. A., signal section, specification 11328, see 522 , p. 491.)

(h) Rehearing.-1. When tests are made at the purchaser's laboratory, samples of the rejected material will be held for one month from date of test report. In case manufacturer is dissatisfied with result of tests, he may make claim for rehearing within that time. Failure to raise a question within one month will be construed as evidence of satisfaction with result of tests. The samples will be scrapped and claim for rehearing will not be considered.

\section{ROUNDELS}

(a) Design.-1. Roundels shall either be of the convex or flat type, as specified, and of high transmission glass, and be between twenty-one hundredths of an inch and twenty-nine hundredths of an inch in thickness.

2. New designs shall be confined to $8 \% \frac{1}{8}$ inches in diameter for high signals and $5 \% 3$ inches in diameter for low signals.

(b) Photometric values.-1. Roundels will be subjected to spectrophotometric analysis. The following table gives an analysis of roundels of the various colors of medium intensity, the first row of figures, marked "Wave length," being the wave length of the light in different parts of the spectrum measured in thousandths of a millimeter, and the other rows of figures are the percentages of light of the wave length given in the first row, which the different roundels transmit. Roundels of medium intensity should transmit light as nearly as possible of this composition.

\begin{tabular}{|c|c|c|c|c|c|c|}
\hline $\begin{array}{l}\text { Wave } \\
\text { length }\end{array}$ & Red & Yellow & Green & Blue & Purple & $\begin{array}{l}\text { Lunar } \\
\text { white }\end{array}$ \\
\hline $\begin{array}{r}0.41 \\
.43 \\
.45 \\
.47\end{array}$ & $\begin{array}{l}0 \\
0 \\
0 \\
0\end{array}$ & $\begin{array}{l}0 \\
0 \\
0 \\
0\end{array}$ & $\begin{array}{l}40 \\
53 \\
62 \\
68\end{array}$ & $\begin{array}{l}80 \\
73 \\
68 \\
54\end{array}$ & $\begin{array}{l}90 \\
82 \\
74 \\
59\end{array}$ & $\begin{array}{l}90 \\
80 \\
69 \\
65\end{array}$ \\
\hline $\begin{array}{l}.49 \\
.51 \\
.53 \\
.55\end{array}$ & $\begin{array}{l}0 \\
0 \\
0 \\
0\end{array}$ & $\begin{array}{r}0 \\
2 \\
8 \\
18\end{array}$ & $\begin{array}{l}70 \\
64 \\
48 \\
30\end{array}$ & $\begin{array}{r}27 \\
10 \\
2 \\
2\end{array}$ & $\begin{array}{r}18 \\
5 \\
1 \\
.5\end{array}$ & $\begin{array}{l}54 \\
38 \\
22 \\
23\end{array}$ \\
\hline $\begin{array}{l}.57 \\
.59 \\
.61 \\
.63\end{array}$ & $\begin{array}{r}0 \\
0 \\
0 \\
47\end{array}$ & $\begin{array}{l}20 \\
42 \\
50 \\
54\end{array}$ & $\begin{array}{r}16 \\
6 \\
3 \\
1\end{array}$ & $\begin{array}{l}1 \\
0 \\
0 \\
0\end{array}$ & $\begin{array}{r}5 \\
0 \\
0 \\
0\end{array}$ & $\begin{array}{r}26 \\
11 \\
12 \\
11.5\end{array}$ \\
\hline $\begin{array}{l}.65 \\
.67 \\
.69 \\
.71\end{array}$ & $\begin{array}{l}74 \\
78 \\
75 \\
74\end{array}$ & $\begin{array}{l}57 \\
57 \\
55 \\
52\end{array}$ & $\begin{array}{l}0 \\
0 \\
0 \\
0\end{array}$ & $\begin{array}{l}0 \\
0 \\
1 \\
2\end{array}$ & $\begin{array}{r}0 \\
.2 \\
12 \\
52\end{array}$ & $\begin{array}{r}10.5 \\
23 \\
51 \\
79\end{array}$ \\
\hline
\end{tabular}


2. Comparison of old and new R. S. A. (A. R. A. signal section) photometric scales. The R. S. A. (A. R. A. signal section) in 1918 adopted certain medium photometric standards for the respective signal colors, designating such medium values as 100 (that is, 100 per cent). The present high transmission colors corresponding to the table of wave lengths above permit an increase of the medium photometric values as shown in the following table. Manufacturing conditions require a reasonable variation from the medium as shown by the light and dark limits, respectively.

\begin{tabular}{|c|c|c|c|c|}
\hline Globes & $\begin{array}{l}\text { Light } \\
\text { limit }\end{array}$ & $\begin{array}{l}\text { Medium } \\
\text { intensity }\end{array}$ & $\begin{array}{l}\text { Dark } \\
\text { limit }\end{array}$ & $\begin{array}{l}\text { Extreme } \\
\text { variation } \\
\text { from } \\
\text { medium }\end{array}$ \\
\hline $\begin{array}{l}\text { Red } \\
\text { Green } \\
\text { Yellow } \\
\text { Blue } \\
\text { Purple } \\
\text { Lunar white }\end{array}$ & $\begin{array}{l}160 \\
175 \\
140 \\
125 \\
125 \\
120\end{array}$ & $\begin{array}{l}130 \\
150 \\
120 \\
100 \\
100 \\
100\end{array}$ & $\begin{array}{r}100 \\
125 \\
100 \\
75 \\
75 \\
80\end{array}$ & $\begin{array}{c}\text { Per cent } \\
23 \\
16^{2} / 3 \\
162 / 3 \\
25 \\
25 \\
20\end{array}$ \\
\hline
\end{tabular}

3. Red.- Shall be of such quality that all yellow rays of light emitted by the sodium flame are absorbed, the spectrum being either red or red and orange.

4. Green.-Shall be of the color known as admiralty green, having a slightly bluish tint as seen in daylight. The spectrum shall show most of the blue and green, a slight amount of yellow, and not more than a trace of orange and red. (d) Spreadlite.-1. Spreadlite lenses may be either of the optical or inverted type with cylindrical segments on the smooth face.

(e) Yard (wide angle).

(f) Fresnel.

(g) Multifresnel.

2. Lenses $6 \frac{3}{8}$ inches and over in diameter, except the yard (wide angle) type, shall have at least five zones, and smaller lenses four zones.

3. New designs shall be confined to lenses of the diameter shown in sections $3(a)$ and $3(c)$.

(b) Color.-1. Colored lenses shall have the same photometric and spectrophotometric value as roundels of the same color.

(c) Focal length.-1. The focal length of each lens shall be pressed on the outer zone, and shall be in accordance with following table:

\begin{tabular}{|c|c|}
\hline Diameter, in inches & $\begin{array}{l}\text { Focal } \\
\text { length }\end{array}$ \\
\hline $\begin{array}{l}4 \\
4152- \\
5 \\
538 \\
638 \\
836 \\
10\end{array}$ & $\begin{array}{l}\text { Inches } \\
23,4 \\
131 / 2 \\
31 / 2 \\
31 / 2 \\
33,4 \\
11 / 2 \text { or } 5 \\
6\end{array}$ \\
\hline
\end{tabular}

1 When used in lamp with 5-inch lens.

\section{GLASS SLIDES}

(a) Material.-1. Thickness of slide shall be not less than ninety-five thousandths of an inch, and not more than fifteen hundredths of an inch.

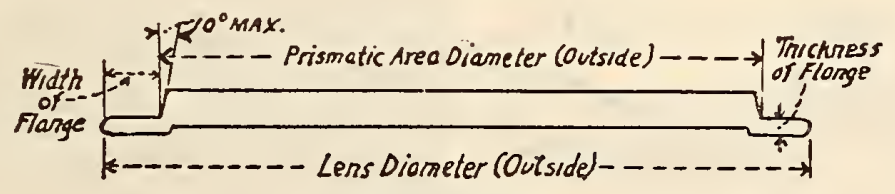

Figure 141

5. Yellow.- Shall give a spectrum sñowing most of the red, all of the yellow, and part of the green, but no blue.

6. Blue.-Shall give a spectrum showing most of the blue, some green, and almost no red.

7. Purple.-Shall give a spectrum showing most of the blue, some green, and a narrow band of the extreme red.

8. Lunar white.-Shall show blue and green, with about 10 per cent of yellow and orange and some red. It shall appear a light blue when viewed by daylight, and when placed in front of a yellow kerosene flame it shall make this flame appear white.

\section{IENSES}

(a) Design.-1. Lenses shall be of the polyzonal design and one of the following types or the equivalent according to requirements:

(a) Optical.-1. Optical lenses shall have a smooth outer face.

(b) Inverted.-1. Inverted lenses shall a have a smooth inner face.

(c) Optical and inverted.-1. Optical and inverted lenses must focus to an area not exceeding $3 / 8$-inch in diameter, for all lenses up to the 6-inch size, nor exceeding $1 / 2$-inch in diameter for the larger sizes. When observed along the axis from a distance of 20 to 40 feet, a small oil flame being placed in the focus, the whole lens shall appear equally illuminated, the risers of the steps showing only as narrow dark rings. (b) Design.-1. Slides shall be $\left\{\begin{array}{c}\text { cut } \\ \text { pressed }\end{array}\right\}$ to size ordered.

(c) Color.-1. Slides shall have the same photometric value as roundels of the same color.

\section{ILLUMINATION}

(a) The R. S. A. (A. R. A. signal section) standard color light signal is based on the use of the ordinary yellow kerosene flame as a source of illumination.

\section{MARKING}

(a) Purchaser's order and requisition number, name of consignor, and name and address of consignee shall be plainly marked on outside of package.

(b) Detail list of loose pieces, containers, and their contents shall be furnished for each shipment. Where carload shipments are made, show routing and car identification.

\section{PACKING}

(a) Material shall be packed so as to permit convenient handling and to prevent loss or damage during shipment.

\subsection{LAMP LENSES.}

Society of Automotive Engineers, lamp lenses (standard, 1929). 
Head-lamp lenses and prismatic-area diameters

\begin{tabular}{|c|c|c|c|}
\hline \multicolumn{2}{|c|}{ Outside diameter } & \multicolumn{2}{|c|}{ Diameter of prismatic area } \\
\hline $\begin{array}{l}\text { Wide flange } \\
\text { (approxi- } \\
\text { mately 1/2 } \\
\text { inch) }\end{array}$ & $\begin{array}{c}\text { Narrow } \\
\text { flange } \\
\text { (approxi- } \\
\text { mately 14 } \\
\text { inch) }\end{array}$ & Maximum & Minimum \\
\hline $\begin{array}{l}8.00 \\
8.25 \\
8.50 \\
8.75 \\
9.00\end{array}$ & $\begin{array}{l}7.50 \\
7.75 \\
8.00 \\
8.25 \\
8.50\end{array}$ & $\begin{array}{l}6.980 \\
7.230 \\
7.480 \\
7.730 \\
7.980\end{array}$ & $\begin{array}{l}6.955 \\
7.205 \\
7.455 \\
7.705 \\
9.955\end{array}$ \\
\hline $\begin{array}{r}9.25 \\
9.50 \\
9.75 \\
10.00\end{array}$ & $\begin{array}{l}8.75 \\
9.00 \\
9.25 \\
9.50\end{array}$ & $\begin{array}{l}8.230 \\
8.480 \\
8.730 \\
8.980\end{array}$ & $\begin{array}{l}8.205 \\
8.455 \\
8.705 \\
8.955\end{array}$ \\
\hline $\begin{array}{l}10.25 \\
10.50 \\
10.75 \\
11.00\end{array}$ & $\begin{array}{r}9.75 \\
10.00 \\
10.25 \\
10.50\end{array}$ & $\begin{array}{l}9.230 \\
9.480 \\
9.730 \\
9.980\end{array}$ & $\begin{array}{l}9.205 \\
9.455 \\
9.705 \\
9.955\end{array}$ \\
\hline $\begin{array}{l}11.25 \\
11.50 \\
11.75 \\
12.00\end{array}$ & $\begin{array}{l}10.75 \\
11.00 \\
11.25 \\
11.50\end{array}$ & $\begin{array}{l}10.230 \\
10.480 \\
10.730 \\
10.980\end{array}$ & $\begin{array}{l}10.205 \\
10.455 \\
10.705 \\
10.955\end{array}$ \\
\hline $\begin{array}{l}12.25 \\
12.50 \\
12.75 \\
13.00\end{array}$ & $\begin{array}{l}11.75 \\
12.00 \\
12.25 \\
12.50\end{array}$ & $\begin{array}{l}11.230 \\
11.480 \\
11.730 \\
11.980\end{array}$ & $\begin{array}{l}11.205 \\
11.455 \\
11.705 \\
11.955\end{array}$ \\
\hline
\end{tabular}

The thickness of the lens flange for all sizes of head-lamp lenses shown in the light-face type shall be $5 / 32$ of an inch, plus $1 / 64$, minus 0 , at the edge of

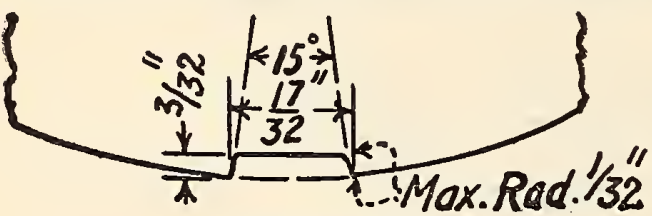

Figdre 142

the prismatic area, and shall be not less than $1 / 8$ of an inch at the edge of a lens. For sizes shown in boldface type the thickness of the flange shall be $3 / 16$ of an inch, plus $1 / 64$, minus 0 , and shall be not less than $1 / 8$ of an inch at the edge of a lens.

The rear surface of the lens flange shall be plane.

This standard applies only to motor-vehicle electric head lamps.

Locating notch.-A lens-locatirg notch as illustrated shall be located at the bottom of all lenses except those having no prismatic structure.

The center of the locating notch shall not be more than one sixty-fourth of an inch to either side of the vertical center line of the lens.

\section{TAIL-LAMP LENS DIAMETER}

The lens diameter used for tail lamps shall be 3 inches, with tolerances of plus 0 and minus $1 / 32$ inch. The minimum light opening shall be $2 \frac{1}{2}$ inches.

\section{SPOT-LAMP IENS DIAMETERS}

The lens diameter used for spot-lamp lenses shall be $41 / 2$ and 6 inches, with tolerances of plus and minus $1 / 32$ inch. The minimum light openings in the lamp door shall be $3 \frac{1}{2}$ and 5 inches respectively.

\section{SIDE-LAMP LENS DIAMETERS}

The lens diameters used in side lamps shall be as specified in the following table:
Side-lamp lens diameters and light openings

\begin{tabular}{|c|r|r|r|}
\hline \multicolumn{2}{|c|}{ Outside diameter } & \multicolumn{2}{|c|}{$\begin{array}{c}\text { Minimum light } \\
\text { opening in door }\end{array}$} \\
\cline { 1 - 3 } Nominal & Tolerances & Nominal & Tolerances \\
\hline 2 & $+0,-1 / 32$ & 156 & $+0,-132$ \\
$21 / 2$ & $+0,-1 / 32$ & $21 / 8$ & $+0,-1 / 32$ \\
3 & $+0,-1 / 32$ & $21 / 2$ & $+0,-1 / 32$ \\
5 & $\pm 1 / 32$ & 4 & $\pm 1 / 32$ \\
6 & $\pm 1 / 32$ & 5 & $\pm 1 / 32$ \\
7 & $\pm 1 / 32$ & 6 & $\pm 1 / 32$ \\
\hline
\end{tabular}

\section{MOTOR CYCLE HEAD-LAMP LENSES}

Motor cycle head-lamp lenses shall have a $61 / 8$-inch outside diameter. The diameter of their prism area shall be $5 \% \frac{3}{8}$ inches. The tolerances for these diameters shall be $\pm 1 / 32$ inch.

The thickness of the bevel edge shall be $5 / 32$ inch at the edge of the prism area and $1 / 8$ inch at the edge of the lens, with tolerances of plus $1 / 64$ inch and minus 0 . Four locking notches shall be used in other than plain lenses.

\section{CHEMICAL AND OTHER GLASSWARE.}

\subsection{INSULATORS.}

(No nationally recognized specifications available.)

\subsection{CHEMICAL GLASSWARE.}

American Chemical Society, standard sizes and shapes of apparatus, 1927.

(Standard sizes and shapes for laboratory apparatus are recommended in a report of the committee on standard apparatus of the American Chemical Society. The report lists standard sizes for a number of items made of glass.)

American Drug Manufacturers Association, standardized tablet bottles, 1923.

This association has adopted a series of Blake bottles for tablets which has been adopted by a number of the association members and is in use.

The bottle numbers, capacity (to base of neck), corkage numbers, and weights of the Blake tablet series are as indicated in the following table:

\begin{tabular}{|c|c|c|c|c|c|c|c|}
\hline No. & $\begin{array}{c}\text { Capac- } \\
\text { ity }\end{array}$ & $\begin{array}{c}\text { Cork- } \\
\text { age } \\
\text { No. }\end{array}$ & Weight & No. & $\begin{array}{c}\text { Capac- } \\
\text { ity }\end{array}$ & $\begin{array}{l}\text { Cork- } \\
\text { age } \\
\text { No. }\end{array}$ & Weight \\
\hline $\begin{array}{l}1882 \\
1900 \\
1901 \\
1883 \\
1884\end{array}$ & $\begin{array}{c}\text { Ounces } \\
16 \\
1 / 4 \\
5 / 16 \\
1 / 3 \\
1 / 2\end{array}$ & $\begin{array}{l}2 \\
2 \\
2 \\
2 \\
2\end{array}$ & $\begin{array}{c}\text { Ounces } \\
1 / 2 \\
5 / 8 \\
11 / 16 \\
3 / 4 \\
1^{3 / 4}\end{array}$ & $\begin{array}{l}1905 \\
1906 \\
1907 \\
1908 \\
1909\end{array}$ & $\begin{array}{r}\text { Ounces } \\
5 \\
6 \\
7 \\
8 \\
10\end{array}$ & $\begin{array}{r}9 \\
10 \\
10 \\
10 \\
10\end{array}$ & $\begin{array}{c}\text { Ounces } \\
51 / 2 \\
61 / 4 \\
7 \\
71 / 2 \\
91 / 4\end{array}$ \\
\hline $\begin{array}{l}1885 \ldots \\
1886 \\
1887 \\
1888 \\
1902\end{array}$ & $\begin{array}{l}5 / 8 \\
3 / 4 \\
11 / 4 \\
11 / 2\end{array}$ & $\begin{array}{l}4 \\
4 \\
4 \\
4 \\
5\end{array}$ & $\begin{array}{l}11 / 4 \\
11 / 2 \\
13 / 4 \\
\mathbf{2} \\
21 / 2\end{array}$ & $\begin{array}{l}1910 \\
1911 \\
1912 \\
1913\end{array}$ & $\begin{array}{l}12 \\
14 \\
15 \\
16\end{array}$ & $\begin{array}{l}14 \\
14 \\
14 \\
14\end{array}$ & $\begin{array}{l}11 \\
121 / 2 \\
13 \\
131 / 2\end{array}$ \\
\hline $\begin{array}{l}1889 \\
1890 \\
1891 . \\
1903 . \\
1904\end{array}$ & $\begin{array}{l}2 \\
21 / 2 \\
3 \\
31 / 2 \\
4\end{array}$ & $\begin{array}{l}6 \\
7 \\
7 \\
7 \\
7\end{array}$ & $\begin{array}{l}23 / 4 \\
31 / 4 \\
31 / 2 \\
4 \\
41 / 2\end{array}$ & $\begin{array}{l}1914 \\
1915 \\
1916 \\
1917\end{array}$ & $\begin{array}{l}18 \\
20 \\
24 \\
28\end{array}$ & $\begin{array}{l}14 \\
16 \\
16 \\
18\end{array}$ & $\begin{array}{l}151 / 2 \\
17 \\
19 \\
21\end{array}$ \\
\hline
\end{tabular}

\subsection{COVER GLASSES.}

(No nationally recognized specifications available.)

\subsection{TUBES AND TUBING.}

(No nationally recognized specifications available.) 
526.6 WATER GAOGES.

American Railway Association, mechanical division, specifications for water gauge and lubricator glasses, 1925.

1. Scope.-These specifications cover glasses for water gauges and lubricators for use on locomotive and stationary boilers classified as follows:

Class A. Reflex type water-gauge glasses.

Class B. Tubular type water-gauge glasses.

Class C. "Bull's-eye" type water-gauge glasses.

Class D. "Bull's-eye" type lubricator gauge glasses.

\section{PHYSICAL PROPERTIES AND TESTS}

2. Optical test-Class A.-The test glass placed reflex side down upon a dead black surface shall show under sky or diffused artificial light not to exceed 25 per cent dark or nonreflective area of the reflex surfaces.

3. Dipping test-(a) Classes A, B, and C.-The test glass shall be suspended for two minutes in glycerin at a temperature of $200^{\circ} \mathrm{F}$., after which it shall be withdrawn and immediately plunged into water having a temperature of from $35^{\circ}$ to $40^{\circ} \mathrm{F}$. This procedure shall be repeated with the temperature of the glycerin raised $25^{\circ}$ each time until the glass fails. So tested, glasses shall meet the requirements of the respective classes shown below, viz:

Class $A$ and $C$.- Not spawl at or below $275^{\circ} \mathrm{F}$. nor shatter nor break at or below $300^{\circ} \mathrm{F}$.

Class B.-Not spawl at or below $350^{\circ}$ F. nor shatter nor break at or below $375^{\circ} \mathrm{F}$.

(b) Class D.-The test glass shall be heated in boiling water $\left(212^{\circ}\right.$ F.) for a period of two minutes, quickly removed therefrom and then immediately plunged into water having a temperature of from $35^{\circ}$ to $40^{\circ} \mathrm{F}$. The test glass shall withstand nine heatings and dippings without spalling or breaking.

4. Solubility test-Classes $A, B, C$, and D.-The test glass shall be placed in a digester and subjected to steam of 50 pounds pressure (with an allowable variation of 5 pounds either way) for 48 hours. Under this treatment the glass shall not crack, become permanently cloudy or otherwise show any change which would impair its value as a gauge glass.

5. Solubility test (optional)-(a) Class A.-The test glass shall be mounted in a standard reflex water gauge holder and live steam at a pressure of 125 lbs./in. ${ }^{2}$ (with an allowable variation of 5 pounds either way) allowed to blow through for 72 hours. Under this treatment the glass shall not crack, become permanently cloudy, or otherwise show any change which would impair its value as a gauge glass.

(b) Class B.-The test glass shall be mounted in a standard tubular water-gauge holder and live steam at a pressure of $90 \mathrm{lbs} . /$ in. $^{2}$ (with an allowable variation of 5 pounds either way) allowed to blow through for 72 hours. Under this treatment the glass shall show practically no cutting, corrosion or permanent clouding, due to the action of the steam or other change which would impair its value as a gauge glass.

Note.-Either the "Solubility test" or the "Solubility test (optional)" given above or both may, at the option of the purchaser, be made of the respective classes of glasses to which they are applicable.

6. Pressure test-Class B.-The test glass when placed in a suitable holder shall stand without bursting a hydraulic pressure of $1,000 \mathrm{lbs} . / \mathrm{in} .^{2}$ maintained for one minute.

7. Samples.-Two samples each of classes A, C, and $\mathrm{D}$ and three samples of class $\mathrm{B}$ shall be taken at random from each lot of 10 dozen glasses. Samples shall be furnished free of charge by the manufacturer and shall be in excess of the number of glasses ordered.
8. Place of making test.-The purchaser may make the tests to govern the acceptance or rejection of the material in his own laboratory or elsewhere. Such tests shall be made at the expense of the purchaser.

\section{PERMISSIBLE VARIATIONS}

9. Permissible variations-(a) Class A.-Glasses shall conform to the limiting dimensions shown on the purchaser's drawing within the following permissible variations:

Length

Width-0 inch plus, $1 / 16$ inch minus. inch plus, $1 / 16$ inch minus.

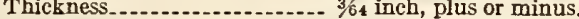

The variation in width shall not exceed $1 / 32$ inch, and the variation in thickness between the gasket faces shall not exceed $1 / 64$ inch for individual glasses. Glasses shall not show convex or concave gasket seat surfaces in excess of $1 / 64$ inch.

(b) Class B.-Glasses shall conform to the limiting dimensions shown on the purchaser's drawing within the following permissible variations:

Length.-Not less, nor more than $1 / 8$ inch greater, than that specified on order.

Diameter. - Not more than 0.02 inch over nor 0.04 inch under that specified on order.

Out-of-round.-Not more than 0.01 ineh.

Thickness of walls.-Be equal to $1 / 8$ of the nominal outside diameter with permissible variations of $1 / 64$ inch under and $3 / 64$ inch over.

(c) Classes C and D.-Glasses shall conform to the dimensions shown on the purchaser's drawing.

\section{WORKMANSHIP AND FINISH}

10. Workmanship and finish.-All glasses shall be properly annealed, clear, and free from flaws and defects.

Class A.-The reflex and front surfaces of glasses shall be highly polished. The ends of the reflexes shall be brought down and terminated in an arc across the ends of the glass. The gasket seat on the reflex side shall be ground to a smooth flat surface.

Class B.-Glasses shall be straight, of uniform size and section, have smoothly finished ends, and be clear and free from flaws.

Classes $C$ and $D$.- Glasses shall be highly polished on both inside and outside surfaces and be free from fins and sharp edges.

\section{WRAPPING AND LABELING}

11. Wrapping.-(a) All glasses shall be carefully wrapped to prevent scoring and breaking.

(b) Labeling-Classes $A$ and B.-Each individual glass shall be indelibly labeled with the name or trade-mark of the manufacturer.

(c) Class B.-Glasses shall be put up in lots of one dozen pieces, and each package labeled to show the name of the manufacturer and number and size of glasses contained therein.

(d) Classes $C$ and $D$.- Packages shall be labeled to show the name of the manufacturer and the number and kind of glasses contained therein.

\section{INSPECTION AND REJECTION}

12. Inspection.-The inspector representing the purchaser shall have free entry at all times while work on the contract of the purchaser is being performed to all parts of the manufacturer's works which concern the manufacture of the material ordered. The manufacturer shall afford the inspector free of charge all reasonable facilities to satisfy him that the material is being furnished in accordance with these specifications. 
13. Rejection.-Material represented by samples which fail to conform to the requirements of these specifications will be rejected.

14. Rehearing.- Samples tested in accordance with these specifications which represent rejected material shall be preserved for 14 days from date of test report.

\subsection{MISCELLANEOUS SPECIFICATIONS FOR GLASS.}

Federal Specifications Board, United States Government master specification No. 368, for sponge and pin cups, March 24, 1926.

\section{TYPES}

There shall be but one size and type of cup to serve the purpose of both sponge and pin cup.

\section{MATERIAL}

The material shall be glass.

\section{DETAIL REQUIREMENTS}

The glass used in the cups shall be clear. The general dimensions shall be approximately as follows: Over-all diameter, $3 \frac{1 / 4}{4}$ inches; height $1 \frac{5}{8}$ inches. The cups shall have slightly internally concave side walls and all edges shall be rounded.

\section{METHOD OF INSPECTION AND TESTS}

The glass shall be of such quality and strength that when the cup is dropped edgewise from the height of a flat-top desk (approximately 30 inches) to a wooden floor three times, it will not break.

\section{PACKING AND MARKING}

Goods purchased under this specification shall be packed and marked as required by the purchaser.

527.

\section{MIRRORS}

(No nationally recognized specifications available.) 
531.

\section{CLAY}

\subsection{GENERAL ITEMS.}

American Ceramic Society, test for apparent porosity, 1928.

The apparent porosity shall be calculated by means of the following formula:

$$
P=\frac{S y-W f}{V f} \times 100
$$

in which $P=$ the per cent apparent porosity.

$S f=$ weight of the saturated fired test piece in grams.

$W f=$ weight of the fired test piece in grams.

$V f=$ volume of the fired test piece in cubic centimeters.

American Ceramic Society, standard method for sampling ceramic materials as delivered, 1922.

\section{(A) SHIPPED AND STORED IN AN ONGROOND CONDITION}

"Unground condition" shall mean material as mined which has not been crushed, ground, or intentionally mixed.

\section{TIME OF SAMPLING}

The material shall be sampled when it is being placed into or taken from railroad cars, ships, barges, wagons, casks, bags, etc. Samples can usually be taken adrantageously as the material passes through the crusher. Samples collected from the surface of piles, bins, cars, etc., are generally unreliable. If it is necessary, however, to sample under such conditions, one-half the sample shall be taken from the lower third and one-half from the upper two-thirds.

\section{COLLECTION OF THE GROSS SAMPLE}

To collect samples, a shovel or specially designed tool or mechanical means shall be used for taking equal portions or increments. The increments shall be regularly and systematically collected so that the entire quantity of material sampled will be represented proportionately in the gross sample, and with such frequency that the gross sample of the required amount shall be collected.

\section{SIZE}

The standard gross sample shall not be less than 1 per cent of the total shipment.

(B) SHIPPED AND STORED IN A CPUSHED OR PULVERIZED CONDITION

\section{TIMS OF SAMPLTTG}

If the material is shipped loose, the sampling shall be done during loading or unloading as described under section A1.

If the material is in containers, all containers of each shipment shall be so marked, tagged, or labeled that they can be distinguished from all other shipments, and that ready reference can be had to the shipper's name and the date of delivery.

\section{JETEOD OF COLLECTION}

If the material is loose, the gross sample shall be taken as described under paragraph 2 above. If in containers, an opening shall be made in the sack or barrel and a portion of the contents shall be withdrawn. This sample should not be taken from the top alone, but should represent all portions of the contained material. This can be done with the aid of an open pipe, spoon-shaped rod, or other suitable apparatus. If there is any reason to doubt the uniformity of the shipment, it is best to test separately a larger sample from each container.

\section{SIZE}

The standard gross sample shall not be less than 0.2 per cent of the total lot sampled.

\section{(C) TREATMENT OF THE GROSS SAMPLE}

\section{CROSHING}

After the gross sample has been collected in the case of unground material, it shall be systematically crushed, mixed, and reduced in quantity by quartering to convenient size for testing. The largest sizes of pieces allowable with various weights of samples are shown in the following table:

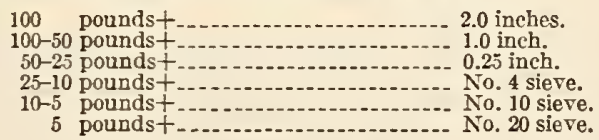

\section{QUARTERING}

The sample crushed to the required size shall be thoroughly mixed by coning and reconing on a clean surface. The cone shall be flattened and then marked into quarters by two lines which intersect at right angles directly under a point corresponding to the apex of the original cone. The diagonally opposite quarters shall then be removed and the space that they occupied brushed clean. The material remaining shall be successively crushed, mixed, coned, and quartered until two opposite corners shall equal the size necessary for testing.

The final portion shall be placed in a container suitable for storage or shipment and carefully marked.

American Ceramic Society, tentative method for sampling clay deposits, 1928.

\section{PRELIMINARY SAMPLING}

A face of the body of clay to be sampled shall be carefully stripped of loose or foreign materials, and a series of parallel trenches cut, each a straight line, so as to make as nearly as may be a vertical section entirely across the outcrop. In the case of stratified or bedded deposits which are inclined, the direction of these trenches shall be, preferably, across the dip, so that a proportionate amount of clay will be obtained from each individual layer. Each trench shall be about 12 inches wide, and deep enough to produce at least 100 pounds of material. Where natural outcrops are not available for a sampling, preliminary trials may be made with a hand auger, and test pits dug to the necessary depth to expose a section (or face) of the clay.

As this may yield more material than is necessary for even a complete series of tests, the clay from the different trenches shall be reduced to lumps not exceeding 2 inches in diameter, mixed together, and reduced by quartering to about 100 pounds, which is 
to be sent to the testing laboratory. The quartering shall be done on a heavy sailcloth at least 8 feet square.

If the deposit shows distinct difference in regard to structure, color, or texture, each bed showing these individual differences shall be sampled separately, provided they are sufficiently thick to be mined by themselves or can be thrown out if undesirable.

Where the clay is stored in stock piles the sample may be taken from these, provided they are representative. At least one-half of the sanuple shall be taken from the lower third of the pile.

In the case of those clays which are being purified a sample of both the crude material and the clay as prepared for the market shall be taken.

The samples collected as outlined above shall be placed in clean tight-weave strong sacks and carefully labeled by means of two tags each bearing the proper identification marks. One folded tag shall be placed within the sack and the other securely attached to the outside.

\section{EXTENDED SAMPLING}

After a clay proves satisfactory in the preliminary testing, the surrounding tract must be surveyed and systematically sampled. A topographical survey of the tract shall be made. Holes shall be drilled or dug through the deposit not more than 100 feet apart. A record shall be kept of the thickness of each stratum encountered. By plotting these results to scale the shape, size, and dip of the strata can be estimated.

A few larger holes may be dug at the extremities of the property or at any points of doubtful value in order to get samples large enough for a complete series of tests. These should be taken under the direction of "Preliminary sampling."

American Ceramic Society, standard method for drying shrinkage, 1928.

\section{SIZE AND SHAPE}

The test pieces shall bc made approximately 30 by 20 by $45 \mathrm{~mm}$ ( $1 \frac{1}{8}$ by $1 \frac{1}{8}$ by $1 \frac{7}{8}$ inches). It is apparent that the dry dimensions will vary with different clays.

\section{PREPARATION}

The clay shall be thoroughly dried at a temperature above $64^{\circ} \mathrm{C}$. but under $76^{\circ} \mathrm{C}$., and crushed to pass a standard No. 20 sieve. (Tentative Standard Scales for Testing Sieves.) It shall then be made up with water to a soft plastic consistency and thoroughly wedged and kneaded by hand. ${ }^{1}$

\section{MAKING TEST PIECES}

The test pieces shall be made in a suitable metal mold measuring approximately 30 by $30 \mathrm{~mm}$ ( $1 \frac{1 / 8}{b y} 1 \frac{1}{8}$ inches) by any desired length. After making, the pieces shall be eut into $45-\mathrm{mm}(17 / 8$-inch) lengths. The mold shall be thinly and evenly oiled with kerosene or a light machine oil only as frequently as is necessary to keep the clay from sticking. The test piece shall be formed by taking in the hand a lump of clay, somewhat larger than required to fill the mold, and kneading it into a roll approximately the length of the mold. It shall then be placed in the mold andforced into the corners by blows with the cheek of the fist. The excess shall then be struck off with a wire and the piece slicked with a spatula and appropriately marked or numbered for identification. Care should be taken by the operator to keep his nands free from oil. The

1 This consistency will permit of the careful handling of the pieco immediately after forming. test piece shall be removed from the mold immediately and transferred to a smooth, straight pallet, care being taken not to distort it.

If preferred, the test piece may be made in a piston plunger press fitted with a die $30 \mathrm{~mm}$ ( $1 \frac{1 / 8}{8}$ inches) square. In either event the corners of the test piece shall be slightly rounded.

\section{PLASTIC VOLUME}

The plastic volume shall be determined immediately in a volumeter of the Seger type. Kerosene with s specific gravity of about 0.8 shall be used as the measuring fluid. The volume shall be read to the nearest $0.1 \mathrm{ec}$.

\section{DRYING}

After the plastic volume is determined the surfaces of the test pieces shall be dried lightly with a soft cloth to remove the film of kerosene, and allowed to dry at room temperature until air dry. They shall then be dried at a temperature between $64^{\circ}$ and $76^{\circ}$ C. for at least five hours and finally at $110^{\circ} \mathrm{C}$. to approximately constant weight. They shall not be allowed to cool to room temperature between these two drying operations unless they be placed in a desiccator. After the final drying treatment they shall be placed in a desiccator to cool and remain there until the test is continued.

\section{DRY VOLUME}

The dry test pieces shall then be allowed to soak in kerosene of the same specific gravity as that used in the volumeter for at least 12 hours, after which the volume shall be determined in the same manner as specified for the plastic volume in paragraph 4 . The volume shrinkage shall be calculated by the following formula:

in which

$$
b=\frac{V p-V d}{V d} \times 100
$$

\footnotetext{
$b=$ per cent volume shrinkage.

$V p=$ the plastic volume.

$V d=$ the dry volume.
}

\section{LINEAR SHRINKAGE}

This value shall be calculated from the per cent volume shrinkage by the formula:

in which

$$
a=100\left[\sqrt[3]{1+\frac{b}{100}}-1\right]
$$

$a=$ per cent linear shrinkage.

\section{REPORT}

In reporting drying shrinkage, the following information shall be given, and these values shall be the means of results obtained on two test pieces: (a) Volume shrinkage in percentage of the dry volume, and $(b)$ linear shrinkage in percentage of the dry length.

American Ceramic Society, standard method for behavior in firing, 1928.

\section{(A) PREFACE}

\section{DATA REQUIRED}

In order to determine the behavior of a clay in firing, the following data must be obtained: (a) Progressive change in porosity and (b) progressive change in volume.

\section{ADDITIONAL USEFOL DATA}

Knowledge of the following properties will always be useful, checking, in a measure, the accuracy of 
the above determinations: Changes in (c) color, $(d)$ hardness, $(e)$ absorption, $(f)$ mechanical strength, and $(g)$ apparent specific gravity.

\section{(B) METHOD}

\section{TEST PIECES}

The clay shall be prepared and the test pieces formed as defined in the Method for drying shrinkage (given above) sections 1,2 , and 3 .

They shall then be dried and the dry volume determined as defined in sections 5 and 6 of the same method.

\section{FIRING}

The test pieces shall be placed in a refractory muffle or sagger as thin-walled as possible, which is set in the test kiln. The test pieces shall not be stacked together too compactly. The test kiln shall be of such size that the effective space available is not less than $1 \frac{1}{2}$ cubic feet. The heating may be accomplished by any convenient means, but the temperature distribution should be thoroughly explored during the first few firings. A set of pyrometric cones of the Seger formulas as made by Edward Orton, jr., shall be placed as closely to the stacked-up test pieces as possible; but temperature control by means of a platinum platinum-rhodium thermocouple is required also. The temperature, therefore, shall be reported in terms of cones and the pyrometric readings, in which correction has been made for the cold-junction temperature.

The range of cones used shall cover the firing range of the clay, which must be judged by the operator. Generally, the cones to be placed in the kiln are 014 to 3 for impure, common brick clays, $012 \frac{1}{2}$ to 5 for shales and the better types of ferruginous clay, and 02 to 15 for clays of the fire-clay type. Oxidizing conditions must be maintained during firing.

The heating shall preferably be at the rate of $45^{\circ} \mathrm{C}$. per hour from the start of the firing until a heat treatment is reached, corresponding to the softening point of the third consecutive cone below that at which the first trial is to be drawn; and $20^{\circ} \mathrm{C}$. per hour from that point until the end of the firing. In no case shall the rate of heating be greater than $60^{\circ} \mathrm{C}$. per hour or less than $30^{\circ} \mathrm{C}$. per hour for the first period, or greater than $25^{\circ} \mathrm{C}$. per hour or less than $15^{\circ} \mathrm{C}$. per hour for the last period.

\section{DRAWING THE TEST PIECES}

According to the nature of the clay, test pieces are first drawn at cone 010 for impure, common brick clays; cone 08 for shales and the better grades of ferruginous clay; cone 2 for clays of the fire-clay type. A test piece is then drawn from the kiln at each interval of two cones, and immediately before drawing the temperature indicated by the pyrometer shall be recorded. The removal of the test piece from the kiln shall be done as quickly as possible to avoid cooling down the kiln too much. It will be necessary'as a rule to increase the rate of application of heat a little after each draw.

Upon removal from the test kiln the test piees shall be $(a)$ immediately covered with hot sand or (b) placed in a small supplementary furnace kept at a dark-red heat and large enough to hold all of the test pieces to be drawn. Upon completion of the firing, the auxiliary furnace shall be allowed to cool at the natural rate. (While it is recognized that furnace control by means of a thermocouple is more definite and accurate than with pyrometric cones, especially for testing purposes, trials have demonstrated that the use of cones minimizes the variations between one firing and another, due to slight differences in rate and uniformity of heating.)

\section{WETGHIVG}

When cool enough to handle the test pieces shall be placed in a desiccator containing concentrated sulphuric acid until they have cooled to room temperature. They shall then be carefully inspected and any adhering particles of sand or other material, picked up during firing, earefully removed. They shall then be weighed on a balance to an accuracy of 0.1 gram.

\section{SATURATION}

The weighed test pieces shall be placed in distilled water in a suitable vessel and boiled for two hours, then allowed to cool to room temperature, while still immersed in water. During boiling the test pieces shall not be in contact with the heated bottom of the container.

\section{SATURATED WEIGHT}

When cooled to as near $20^{\circ} \mathrm{C}$. as possible in the room atmosphere each test piece shall be dried lightly with a damp towel to remove the excess water and weighed in air to an accuracy of $0.1 \mathrm{~g}$.

\section{FIRED VOLUME}

The fired volume shall be determined in a volumeter of approved type. 928.

American Ceramic Society, sag tests (tentative),

\section{(A) PREFACE}

The purpose of such a test as here defined is to determine the temperature and rate of softening under fixed conditions of load and heat treatment as evideneed by transverse bending. For grogged mixtures that must support transversely applied load it supplies a direct test.

"Fine grog bodies" are understood as those containing grog finer than that which will pass a No. 20 sieve, and "coarse grog mixtures" those containing a grog of a coarser size in any percentage.

\section{(B) METHOD FOR CLAY AND FINE GROG BODIES}

\section{SHAPE AND SIZE}

The test pieces shall be bars measuring $12 \mathrm{~mm}$ ( $1 / 2$ inch) thick by $25 \mathrm{~mm}$ (1 inch) wide by $230 \mathrm{~mm}$ ( 9 inches) long when thoroughly dry.

\section{MAKING}

The bars shall be made in plaster molds by casting the clayslip to such thickness that when dry it will be somewhat thicker than $12 \mathrm{~mm}$ (1/2 inch). This shall be allowed to dry slowly at room temperature until leather hard and then cut into strips somewhat wider than $25 \mathrm{~mm}$ (1 inch) and longer than $230 \mathrm{~mm}$ (9 inches). The oversize necessary will depend on the drying shrinkage of the clay. When thoroughly dry the bars shall be sized exactly by rubbing or seraping.

\section{SETTING}

The bars shall be set with the $25 \mathrm{~mm}$ ( 1 inch) dimension, horizontal, in covered saggers. They shall rest on two fire-clay knife edges spaced $177 \mathrm{~mm}$ (7 inches) apart.

\section{KNIFE-EDGES}

It is recommended that the knife-edges be triangular in cross section and $45 \mathrm{~mm}$ ( $13 / 4$ inches) high.

\section{HEAT TREATMENT}

The test kiln shall be of such size that the effective space available is not less than $1 \frac{1}{2}$ cubic feet. The 
heating may be accomplished by any convenient means, but the temperature distribution should be thoroughly explored during the first few firings. A set of pyrometric cones of the Seger formulas as made by Edward Orton, jr., shall be placed as closely to the test pieces as possible, but temperature control by means of a platinum platinum-rhodium thermocouple is required also. It is obvious that the kiln must be fired separately for each heat treatment.

The heating shall preferably be at the rate of $45^{\circ}$ C. per hour from the start of the firing until a temperature of $800^{\circ} \mathrm{C}$. is reached and $20^{\circ} \mathrm{C}$. from that point until the end of the firing. In no case shall the rate of heating be greater than $60^{\circ} \mathrm{C}$. per hour or less than $30^{\circ} \mathrm{C}$. per hour for the first period, or greater than $25^{\circ} \mathrm{C}$. per hour or less than $15^{\circ} \mathrm{C}$. per hour for the last period.

\section{NUMBER OF BARS}

Two bars of the same clay shall be set for each heat treatment. The number of heat treatments is optional, but for data upon which to base conclusions on rate of softening, at least five heat treatments at two cone intervals shall be made.

\section{WARPAGE RATIO}

The amount of warpage shall be indicated by the ratio, to the second decimal place, of the amount of deflection in tenths of a millimeter to the span of $177 \mathrm{~mm}$ ( 7 inches), and shall be the average of two bars.

\section{VARIATION}

If one bar shows a deformation more than 20 per cent greater than the other, the test shall be considered faulty and the results discarded.

\section{(C) METHOD FOR COARSE GROG MIXTURES}

\section{SHAPE AND SIZE}

The test piece shall be $65 \mathrm{~mm}$ (21/2 inches) square and $330 \mathrm{~mm}$ (13 inches) long when fired. A variation of $\pm 3 \mathrm{~mm}$ ( $1 / \mathrm{sinch}$ ) is allowable in the crosssectional dimensions.

\section{MAKING}

The bars shall be made in a suitable mold. The mixture shall be made up to soft plastic consistency and thrown forcibly into the mold, so as to completely fill out the lower corners. The first lump shall be thrown in the center and then the ends filled in a similiar manner. The excess shall then be struck off with a wire and the top of the bar slicked off with a spatula and appropriately marked. The bars shall then be thoroughly dried and fired to at least one cone higher than that at which warpage is to be determined. Such care shall be taken in setting that no warpage or sagging occurs in this preliminary firing.

\section{SETTING}

The bars shall be set on flat fire-clay supports with a span of $255 \mathrm{~mm}$ (10 inches) and loaded in the center with a fire-clay stirrup having a knifeedge support. The load shall be $4.5 \mathrm{~kg}$ (10 pounds) for bars of exactly $65 \mathrm{~mm}$ (21/2 inches) square cross section. For bars within $\pm 3 \mathrm{~mm}$ ( $1 / 8$ inch) of these dimensions the load shall be calculated as follows:

\section{where}

$P=0.0185 b d^{2}$

$P=$ load in kilograms.

$b=$ breadth of bar in centimeters.

$d=$ depth of bar in centimeters.

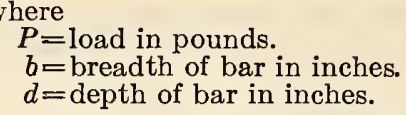

\section{HEAT TREATMENT}

The heat treatment shall be the same as specified above for clay and fine grog bodies.

\section{NUMBER OF BARS}

The number of bars shall be the same as specified above for clay and fine grog bodies.

\section{WARPAGE RATIO}

The warpage ratio shall be calculated as already specified for clay and fine grog bodies.

\section{VARIATION}

Allowable variation shall be the same as specified above for clay and fine grog bodies.

American Ceramic Society, standard method for shrinkage and pore water, 1928.

\section{(A) PREFACE}

"Shrinkage water" is defined as that portion of the water of plasticity which is driven off up to the point where shrinkage ceases. "Pore water" is defined as that portion of the water of plasticity which is driven off from the point where shrinkage ceases until the clay piece has reached approximately constant weight at $110^{\circ} \mathrm{C}$. Thus shrinkage water plus pore water equals the water of plasticity.

\section{(B) METHOD}

\section{DATA}

The data necessary for the calculations here involved are the plastic volume and the dry volume, as determined in the Method for Drying Shrinkage, and the dry weight and water of plasticity as determined in the Method for Water of Plasticity.

\section{SHRINKAGE WATER}

The following formula shall be used:

in which

$$
t_{1}=\frac{V p-V d}{W d} \times 100
$$

$t_{1}=$ the per cent shrinkage water.

$V p=$ the plastic volume in cubic centimeters.

$V d=$ the dry volume in cubic centimeters.

$W d=$ the dry weight in grams.

\section{PORE WATER}

The following formula shall be used:

in which

$$
t_{2}=T-t_{1}
$$

$t_{2}=$ the per cent pore water.

$T=$ the per cent water of plasticity.

American Ceramic Society, standard method for slaking test, 1928.

\section{(A) PREFACE}

The method here defined differs but little from the practice which is generally followed. The principal amendments are in the closer specifications on the size of screen and temperature of water. The latter particularly has a marked effect upon the slalking values obtained. 


\section{(B) METHOD}

\section{TEST PIECES}

The test pieces shall be cubes made of a mixture of 50 per cent by weight of ground flint and 50 per cent of the clay to be tested which has been ground to pass a No. 30 sieve. (Tentative Standard Series of Testing Sieves.) The flint shall be of the grade and fineness ordinarily termed "potters' flint" and shall all pass a No. 100 sieve. (Tentative Standard Series of Testing Sieves.) The cubes shall be 2.5 $\mathrm{cm}$ ( 1 inch) on an edge in the wet state.

\section{MAKING THE TEST PIECES}

The test pieces shall be made by pugging or working the mixture by hand to its best plastic consistency, batting out into a slab $2.5 \mathrm{~cm}$ (1 inch) thick, cutting from this a strip $2.5 \mathrm{~cm}$ (1 inch) wide, and subdividing this strip into $2.5 \mathrm{~cm}$ (1-inch cubes).

\section{DRYING}

The drying shall be accomplished in three stages, (a) at a room temperature until air dry, (b) at a temperature between $64^{\circ}$ and $76^{\circ} \mathrm{C}$. for at least five hours, and finally, (c) without allowing to cool to room temperature, at $110^{\circ} \mathrm{C}$. until approximately constant in weight. The test pieces shall then be cooled to room temperature in a desiccator.

\section{THE SCREEN}

The slaking shall be done on screens corresponding to a No. $2 \frac{1}{2}$ sieve. (Tentative Standard Series of Testing Sieves.)

\section{WATER}

The temperature of the water shall be held at $25^{\circ} \mathrm{C} . \pm 1^{\circ} \mathrm{C}$. , or $77^{\circ} \mathrm{F} . \pm 2^{\circ} \mathrm{F}$.

\section{SLAKING}

After the samples are thoroughly dried and cooled (par. 1 above) they shall be placed on a screen and carefully immersed in water, care being taken to prevent agitation of the water during the slaking operation. The water shall be at least $2.5 \mathrm{~cm}(1$ inch) deep under the test piece and the top of the piece shall be immersed in the water to a depth of not less than $1.2 \mathrm{~cm}^{*}(1 / 2$ inch) and not more tlian $2.5 \mathrm{~cm}$ (1 inch)

\section{REPORT}

The only datum to be obtained is the time required in minutes for the whole of the test piece to slake and settle through the screen. The time reported shall be the average of two determinations.

American Ceramic Society, test for apparent specific gravity, 1928.

The apparent specific gravity shall be determined by the formula

$$
G=\frac{W f}{V f-(S f-W f)}
$$

in which

$G=$ the apparent specific gravity.

\section{BULK SPECIFIC GRAVITY}

The bulk specific gravity shall be calculated by the formula

in which

$$
G b=\frac{W f}{l f f}
$$

$G b=$ the bulk specific gravity.

American Ceramic Society, standard method for true specific gravity, 1928.

\section{SAMPLE}

For raw ceramic materials, the sample shall be taken with the same care and precision as for chemical analysis. For fired materials, the sample shall consist of at least two portions of the test piece or ware, from different positions, and the ratio of original surface to volume shall not be greater than in the whole piece.

The sample shall be crushed between hardened steel surfaces, when crushing is necessary, and then ground in an agate mortar, to pass a No. 100 sieve (Tentative Standard Scale for Testing Sieves). In fired samples or those not likely to contain magnetic material as an original constituent, any magnetic material present after crushing shall be removed with a magnet. About $10 \mathrm{~g}$ shall be prepared in this manner, dried to constant weight at $110^{\circ} \mathrm{C}$., and placed in a glass-stoppered weighing bottle.

\section{THE PYCNOMETER}

The pycnometer shall be of good quality and workmanship with the mouth ground flat so that there shall be no rounded recess between stopper and bottle when the stopper is in place. The stopper with a capillary tube shall be used.

\section{WEIGHING}

All weighings shall be made on an accurate chemical balance to the third decimal place in grams, with material, and in an atmosphere at a temperature of $20^{\circ} \mathrm{C} . \pm 1^{\circ} \mathrm{C}$. The weight of the bottle and stopper dried at $110^{\circ} \mathrm{C}$. shall be recorded as $p$ and the weight of the bottle filled with distilled water with the stopper in place as $w_{1}$.

\section{INTRODUCING THE SAMPLE}

About $2 \mathrm{~g}$. of the sample shall be placed in the pycnometer (which has been dried at $110^{\circ}$ C.) and weighed with the stopper, the weight being recorded as $w$. The bottle shall then be filled to approximately one-third its capacity with distilled water, the stopper put in place with a piece of paper between stopper and bottle to prevent sticking, and boiled with care for 15 minutes. The bottle shall then be filled with distilled water to the base of the neck and allowed to cool to $20^{\circ} \mathrm{C} . \pm 1^{\circ} \mathrm{C}$. When cool and the sample settled, the filling shall be completed and the bottle wiped dry with a soft cloth.

\section{FINAL WEIGHING}

The pycnometer shall then be weighed and the weight recorded as $w_{2}$.

\section{CALCULATION}

The true specific gravity shall be obtained by the formula:

in which

$$
G t=\frac{w-p}{(w-p)-\left(w_{2}-w_{1}\right)}
$$

$G t=$ the true specific gravity.

$w=$ weight of the stoppered pycnometer and sample.

$p=$ weight of the stoppered pycnometer.

$w_{1}=$ weight of the stoppered pycnometer filled with water.

$w_{2}=$ the weight of the stoppered pycnometer sample and water.

\section{CHECKS}

Two determinations shall be made and reported and they shall check to within 0.005 .

American Ceramic Society, tentative method for transverse strength, 1928. 


\section{METHOD}

\section{PREPARATION}

The clay shall be thoroughly dried at $64^{\circ} \mathrm{C}$. or above, but under $76^{\circ} \mathrm{C}$., crushed and screened to pass a No. 20 sieve. It shall then be thoroughly mixed dry with an equal amount by weight of standard silica sand that passes a No. 20 sieve (Tentative Standard Series of Testing Sieves), and is retained on a No. 30 sieve. The mixture shall then be made up to soft plastic consistency with water and thoroughly pugged by hand.

\section{SHAPE}

The test piece shall be made in a suitable metal or wooden mold. It shall be $17.5 \mathrm{~cm}$ (7 inches) long and have a $2.5 \mathrm{~cm}$ (1 inch) square cross section in the plastic state.

\section{FORMING}

The mold shall be evenly and thinly oiled with kerosene and placed on a firm, smooth surface. A lump of the clay mixture, somewhat larger than is required to fill the mold, shall be thrown forcibly into the mold so as to completely fill out the lower corners. The excess shall then be cut off with a spatula, and the top slicked off and appropriately marked. The marking should be near the ends, so as not to deform the center of the bar.

\section{DRYING}

The test pieces shall be allowed to dry under a cloth at room temperature for two days, then exposed at room temperature until air dry. During this period they shall be turned every 12 hours to make the drying more uniform. They shall then be placed in a drier operating between $64^{\circ}$ and $76^{\circ} \mathrm{C}$. for at least five hours, and from there transferred to a drying oven, operating at $110^{\circ} \mathrm{C}$., where they shall remain until approximately constant in weight.

\section{BREA EING}

The test pieces shall be cooled in a desiccator and then broken in a suitable machine, having knife edges with a $6 \mathrm{~mm}$ (1/4 inch) radius and $12.5 \mathrm{~cm}$ ( 5 inches) apart. The machine should have an automatic shut off for the shot, and the rate of loading should be about $45 \mathrm{~kg}$ (100 pounds) per minute. The depth and breadth of the bar shall be taken at the break, and each shall be the average of three measurements taken to the nearest 0.25 $\mathrm{mm}(0.01$ inch).

\section{MODULUS OF RUPTURE}

The modulus of rupture shall be calculated by the formula

where

$$
M=\frac{3 P l}{2 b d^{2}}
$$

$M=$ modulus of rupture in $\mathrm{kg}$ per square centimeter.

$P=$ breaking load in $\mathrm{kg}$ (read to the nearest 0.1 $\mathrm{kg}$ ).

$l=$ distance between knife edges in $\mathrm{cm}$.

$b=$ breadth of bar in $\mathrm{cm}$.

$d=$ depth of bar in $\mathrm{cm}$.

or, if the English system is used, the units will be pounds and inches, and the modulus will be obtained in pounds per square inch by the same formula.

Ten bars shall be broken and the average modulus of rupture reported. Two faulty test pieces are permissible in the case of which the average of the eight or nine remaining shall be reported.

\section{VARIATION}

A variation of plus or minus 15 per cent from the average modulus of rupture is permissible. Test pieces showing a greater variation shall be considered faulty and discarded; but as indicated in 6 preceding, if more than two are thus discarded, the test shall be repeated.

American Ceramic Society, test for volume change, 1928.

The volume change shall be determined by the relation

in which

$$
b_{1}=\frac{V d-V f}{V d} \times 100
$$

$b_{1}=$ per cent volume change.

$V d=$ volume of the dry test piece in cubic centimeters.

American Ceramic Society, standard method for water of plasticity, 1928 .

\section{TEST PIECES}

The test pieces shall be of the same size and shape and made in the same manner as defined in the Method for Drying Shrinkage (see p. 508), sections 1, 2 , and 3 .

\section{PLASTIC WEIGHT}

The edges and corners of three test pieces shall be rubbed lightly with the finger to prevent loss in handling. They shall then be weighed on a balance to an accuracy of $0.1 \mathrm{~g}$.

\section{DRYING}

After the plastic weight is obtained the test pieces shall be allowed to dry at room temperature until air dry. They shall then be dried at between $64^{\circ}$ an $76^{\circ} \dot{\mathrm{C}}$. for at least five hours and finally at $110^{\circ} \mathrm{C}$. until approximately constant in weight.

\section{DRY WEIGHT}

The dried test pieces shall be cooled to room temperature in a desiccator and then weighed with the same accuracy as before (par. 2).

\section{CALCULATION}

The water of plasticity shall be calculated as a percentage of the weight of the dry clay bar by the following formula:

in which

$$
T=\frac{W p-W d}{W d} \times 100
$$

$T=$ per cent water of plasticity.

$W p=$ weight of the plastic test piece.

$W d=$ weight of the dry test piece.

\section{REPORT}

The average of the three values obtained shall be reported as the per cent water of plasticity.

\subsection{BALI CLAY.}

(No nationally recognized specifications available.)

\subsection{CHINA CLAY.}

(No nationally recognized specifications available.)

\subsection{FIRE CLAY.}

Eastern Clay Products Association, specification for brick chimneys with fire-clay flue linings (recommended)

(See 518.55, p. 422.)

Eastern Clay Products Association, standards, fire-clay flue linings and fittings. 
(The association has prepared dimensional standards for the following items: Rectangular fire-clay lining, round fire-clay flue lining, and fire-clay flue rings.)

Federal Specifications Board, specification No. $334 a$, United States Government master specification for clay, fire, August 7, 1926.

\section{CLASSES}

Fire clay shall be of the following classes: F, fine, and $\mathrm{C}$, commercial.

\section{MATERIAL}

This specification covers a heat-resistant material composed entirely, or principally, of fire clay and which is used in laying up fire-clay refractories. It shall be sufficiently free from grit and of such quality and plasticity that it will spread satisfactorily with a trowel when tempered with water.

\section{DETAIL REQUIREMENTS}

1. Class $F$.-The material as furnished shall be air dry and ground to the following fineness: Not more than 4 per cent shall be retained on a United States standard sieve No. 20.

2. The material shall show satisfactory bonding power.

3. The softening point shall be not more than 3 cones (approximately $60^{\circ} \mathrm{C}$., or $108^{\circ} \mathrm{F}$.) lower than that of the fire-clay brick or shape with which it is to be used.

4. When specified, the material shall pass the Navy simulated service test.

5. Class C.-The material as furnished shall be air dry and ground to the following fineness: Not more than 10 per cent shall be retained on a United States standard sieve No. 20.

6 . The material shall show satisfactory bonding power.

7. The softening point shall be not more than 3 cones (approximately $60^{\circ} \mathrm{C}$., or $108^{\circ} \mathrm{F}$.) lower than that of the fire-clay brick or shape with which it is to be used.

\section{METHOD OF INSPECTION AND TESTS}

1. Sampling.-(a) When shipped in containers a sample shall be taken from one of every 100 containers or less. The sample shall be taken by dumping the container and quartering the material to provide a 5 -pound sample for the laboratory.

(b) When shipped in bulk the sample shall be so taken that it will represent an average of all parts of the shipment from top to bottom and shall not contain a disproportionate share of the top and bottom layers. The sample shall consist of 1 shovelful for each 3 tons of material, but not less than 10 shovelfuls taken from different parts of the shipment. The total sample taken shall weigh at least 100 pounds and shall be mixed thoroughly and "quartered" to provide a 5-pound sample for the laboratory. In case a shipment consists of more than one car a sample shall be taken from each car.

2. Fineness test.-One hundred grams of the sample as received shall be placed on a No. 20 sieve and shall be washed by means of a stream of water from a faucet. The washing shall be continued until the water coming through the sieve is clear, but in no case should the washing be continued for more than 30 minutes. The residue on the sieve shall be dried to constant weight at a temperature between $110^{\circ}$ and $150^{\circ} \mathrm{C}$. The sieve carrying the dried residue shall then be held in one hand in a slightly inclined position so that the sample will be well distributed over the sieve, at the same time gently striking the side about 150 times per minute against the palm of the other hand on the upstroke. The sieve shall be turned every 25 strokes about one-sixth of a revolution in the same direction. The operation shall continue until not more than $0.05 \mathrm{~g}$ passes through in one minute of continuous sieving. The fineness shall be determined from the weight of the original sample.

$\AA$ piece of rubber tubing attached to a water faucet is to be used for the washing. The velocity of the water may be increased by pinching the tubing but should not be sufficient to cause any splashing of the sample over the sides of the sieve.

Mechanical sieving devices may be used, but the material shall not be rejected if it meets the fineness requirements when tested by the hand method described.

3. Bond test.- The material shall be tested for bonding power by mixing one portion with water to a creamy consistency and another portion to a good troweling consistency. These mixtures shall then be used in laying up a pier of three of the fire-clay brick, laid flat, with which the clay is to be used in service. The faces of the brick to be bonded shall be dipped in the mixture of creamy consistency, a thin layer of the other mixture added, and the brick then firmly rubbed into place. In no case shall the mortar "joint" exceed one-eighth inch.

When the pier is air-dry it is to be fired uniformly in a suitable furnace to a tempe-ature $300^{\circ} \mathrm{C}$., or $540^{\circ} \mathrm{F}$. lower than the minimum allowable softening point of the fire-clay brick as defined in the Federal Specifications Board specification for fire-clay brick No. 268a. (See 534.12, p. 591.) The pier shall be allowed to cool in the furnace and when cold shall be sufficiently well bonded to remain intact when lifted by grasping the top brick.

4. Softening-point test.-The softening point is to be determined according to A. S. T. M. C24. (See 534.10 , p. 573.)

5. Navy simulated service tests.-(a) Tests are conducted in small oil-fired furnaces, the dimensions and method of construction of which are shown in Figure 143. For comparative purposes one side wall of the combustion chamber is built up of brick and fire clay of approved brands and the other side wall of brick and fire clay of the samples under examination. Both walls are backed uniformly with 3 inches of insulation. An air-atomizing fuel-oil burner is used. The flame sweeps the length of the furnace, curves upward and returns to the front, then up the stack from which it escapes horizontally toward the rear of the furnace.

(b) The test consists of two runs, each of 24 hours' duration, at furnace temperatures of $1,590^{\circ}$ and $1,650^{\circ} \mathrm{C}$. (approximately $2,895^{\circ}$ and $3,000^{\circ} \mathrm{F}$.), respectively.

(c) During each run the following temperature determinations are made:

(1) Furnace temperatures.

(2) Temperatures of outer face of brickwork of each side wall at front and rear of furnace.

(d) Refractory furnace face temperatures are recorded at 30 -second intervals by means of a recording radiation pyrometer sighted into a closedend refractory tube placed in the rear wall of the furnace. The radiation pyrometer is checked at quarter-hourly intervals with an optical pyrometer sighted on the refractory walls through the front of the furnace above the burner. Control of the furnace is ordinarily carried out from observations of the recording radiation pyrometer.

(e) Temperatures of the outer face of the brickwork of each side wall are determined at half-hourly intervals with an optical pyrometer sighting on the brickwork through sillimanite tubes, the ends of which are placed flush with the wall. The tubes are 
carefully lagged and plugged to prevent radiation losses.

(f) A spalling test is conducted at the conclusion of each run by injecting cold air at high velocity into the furnace immediately after shutting off the oil supply to the burner. The injection continues until the walls are cool.

(g) The comparative heat-insulating properties, together with the relative conditions of the side walls, determine whether or not the material under test is acceptable for use in service. rial, the contract number, and the name of the contractor.

\section{NOTES}

Fire clay of class $\mathbf{F}$ is intended particularly for use by the Navy.

Notice of the rejection of a shipment based on this specification must be in the hands of the consignor, unless otherwise specified, within 10 days after the receipt of the shipment at the point of destination. If the consignor desires a retest, he

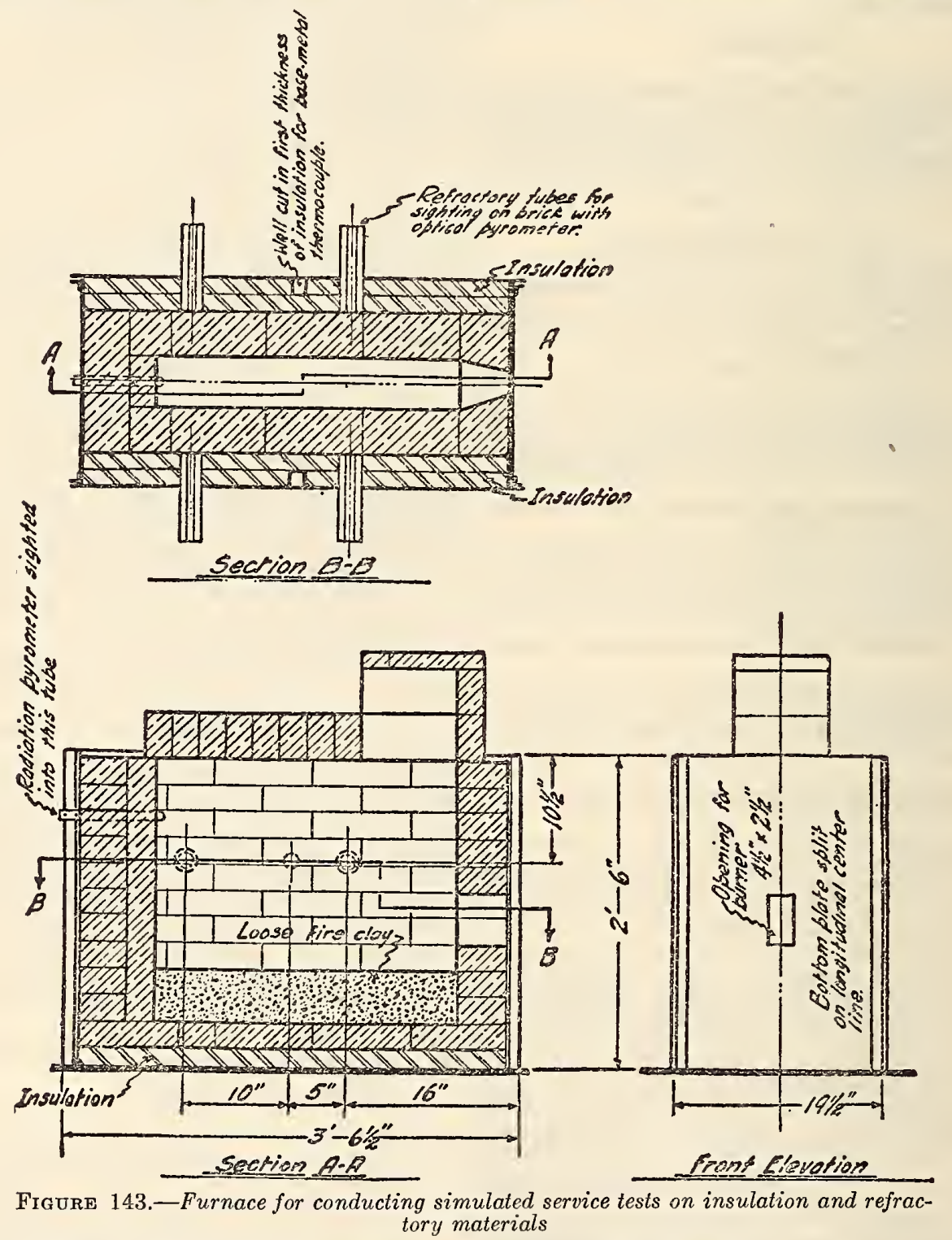

6. The combined results of fineness, bonding power, and softening point shall be considered as a suitability test, but (at the discretion of the purchaser) the simulated service test may replace all other tests included in the suitability test.

7. Fineness and softening point shall be considered as a control test.

\section{PACKING AND MARKING}

When delivered in containers each container shall be plainly marked with the trade name of the mate- shall notify the consignee within five days of receipt of said notice.

The cones referred to in this specification are known as the Orton pyrometric cones.

The sieve used shall meet the requirements given in the United States Standard Sieve Series. (See A. S. T. M. E11, 500.2, p. 1.)

\subsection{KAOLIN.}

(No nationally recognized specifications available.) 


\subsection{CLAY PRODUCTS.}

American Railway Association, signal section, specification 2412 , vitrified clay conduit.

(This specification is practically identical with American Railway Association, telegraph and telephone section, specification $1-\mathrm{C}-9$, given below.) Emerican Railway Asscciation, telegraph and telephone section, specification for vitrified clay conduit, No. 1-C-9, 1920.

\section{B. DRAWING}

1. Purchaser's drawing accompanying this specification and forming an essential part thereof, is Figure 144, A. R. A., T. \& T. drawing No. 1024.

\section{MATERIAL}

1. All conduit shall be made of finely divided compact clay, thoroughly vitrified, and shall be free from stones and pebbles.

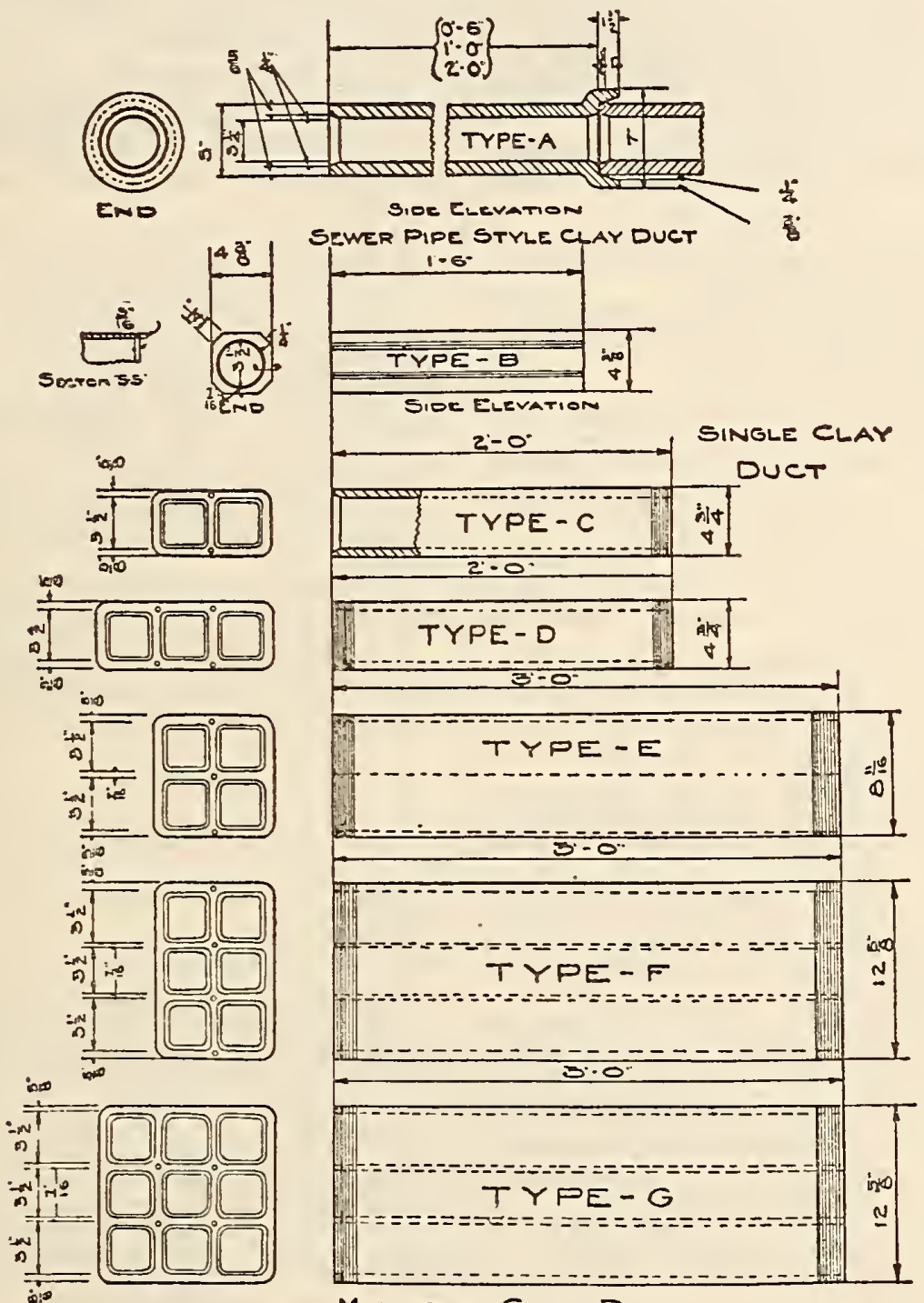

\section{VITREFIED CLAY DUCT}

Figure 144.-A. R. A., T. \& T'. S., drawing No. 1024

\section{A. PURPOSE}

1. The purpose of this specification is to provide for the furnishing of sewer pipe, style round or square bore, single duct and square bore $2,3,4$, 6 , or 9 duct multiple clay conduit for wires and cables.

\section{WORKMANSHIP}

1. The shape of the conduit and arrangement of the ducts in the multiple conduit shall be as shown on the drawing referred to. All conduit shall be as symmetrical throughout as is consistent with the best commercial practice. The ends of 
each piece shall be perpendicular to its sides and shall be practically smooth and free from projections. In the case of multiple duct the interior eorners of all duct holes and the exterior edges of the conduit, excluding the ends, shall be slightly rounded. All duct holes shall be beveled at their ends.

2. The holes in single duct shall be well centered.

3. Single and multiple conduits may contain eracks within the limits as to size and location specified below, provided they are not of such a nature as to appreciably weaken the conduit. The presence of cracks which appreciably weaken the conduit shall be determined by sounding each piece with a steel hammer or its approved equivalent, the piece of conduit to be rejected if, when so sounded, it fails to yield a clear metallic ring.

4. Single conduit may contain through cracks at the end not exceeding 2 inches in length or $1 / 8$ inch in width.

5. The inner walls of multiple conduit may contain through cracks at the ends, not exceeding 6 inches in length or $1 / 8$ inch in width.

6 . The outside walls of multiple conduit may contain through cracks at the ends, not exceeding 3 inches in length or $1 / 8$ inch in width.

7. At least two dowel pin holes in each end of each piece of conduit shall be free from through cracks exceeding 3 inches in length or $1 / 16$ inch in width.

8. No conduit shall eontain transverse through cracks in its inner walls, which exceed $1 / 16$ inch in width or which extend perceptibly into its outer walls.

9. The outer walls of all conduits shall be free from transverse through cracks or other transverse eracks, which appreciably weaken the conduit, as shown by sounding test.

10. All conduit shall be free from cracks, whose edges protrude into the duct holes or more than $3 / 16$ inch beyond the outer surface of the conduit.

11. All conduit shall be free from air checks, as shown by the sounding test.

12. Conduit having clipped ends may be accepted under this specification, provided the fracture does not extend farther into the conduit than the beveling of the ends.

13. The interior walls of single conduit shall be free from rough or sharp unbroken blisters, or other projections and from smooth, rounded, unbroken blisters, which project more than $1 / 10$ inch above the surface of the wall.

14. The interior walls of multiple duct may contain unbroken blisters or other projections, provided they do not project more than $1 / 8$ inch above the surface of the wall, and are so located in the corners of the duct holes that there are at least $2 \frac{1}{2}$ inches of sinooth, flat surface on all interior walls, or provided they are smooth and round and do not project more than $1 / 16$ inch above the surface of the wall.

15. Unbroken blisters or other projections on the outer surfaces of single or multiple duct shall not project more than $3 / 16$ inch above the surface of the wall.

16. Smooth salt drip which does not project more than $1 / 8$ of an inch below the surface is not classed as an objectionable form of unbroken blister.

17. Recesses in the walls of single or multiple conduit caused by broken blisters or other defects shall not decrease the thickness of the walls by more than $3 / 16$ of an inch. When on the interior walls the edges of the recesses shall be smooth so far as to offer no projecting edges.

18. The outer walls of single conduit shall be eounbed with two sets of three combings each, running lengthwise on the conduit and placed adjacent to the corners.
19. The outer walls of multiple conduit shall be scarified or otherwise suitably roughened for at least 2 inches at each end.

20. All inside wall of at least 75 per cent of the pieces in any lot of conduit shall be thoroughly glazed. All inside walls not thoroughly glazed shall be smooth and the conduit shall be thoroughly vitrified.

\section{E. DIMENSIONS}

1. The dimensions of all pieces of conduit in any lot shall as uniformly conform to the required dimensions as is consistent with the best commercial practice.

2. The desired lengths of the standard pieces in any lot of conduit are shown in the following table. The average length of the standard pieces in any lot shall not be less than the figures shown in this table, nor shall any piece be more than 1 inch shorter than these figures:

(1) Sewer pipe style conduit, 24 inches.

(2) Single-duct conduit, 18 inches.

(3) Two-duct conduit 24 inches.

(4) Three-duct conduit, 24 inches.

(5) Four-duct conduit, 36 inches.

(6) Six-duct conduit, 36 inches.

(7) Nine-duct conduit, 36 inches.

3. Short lengths shall be approximately 6,9 , and 12 inches long and orders shall state the per cent of each length of short pieces desired.

4. All duct holes shall be not more than $3 \frac{1}{2}$ inches nor less than 31/4 inches in diameter. Measurements of square duct holes shall be made between opposite surfaces.

5 . The minimum thickness of the walls of single and multiple conduit shall be shown in the following table. Variations from these thicknesses shall be in excess of them and shall not be greater than is consistent with the best commercial practice.

\begin{tabular}{|c|c|c|}
\hline \multirow{2}{*}{ Type of conduit } & \multicolumn{2}{|c|}{ Wall thickness } \\
\hline & Outer & Inner \\
\hline $\begin{array}{l}\text { Sewer-pipe style } \\
\text { Single-duct conduit } \\
\text { Multiple-duct conduit }\end{array}$ & $\begin{array}{c}\text { Inch } \\
78 \\
7 / 6 \\
5 / 8\end{array}$ & \begin{tabular}{c} 
Inch \\
\hdashline $7 / 16$
\end{tabular} \\
\hline
\end{tabular}

6. All multiple conduit shall be provided with at least two dowel-pin holes at each end. The dowelpin holes shall be not more than $15 / 32$ of an inch nor less than $11 / 32$ of an inch in diameter, and shall be not less than $1 \frac{3}{4}$ inches deep. They shall be located in the center of the intersections of the partition walls, and, when necessary, in the center of the intersections of the partition walls and the outside walls, so that when two sections of conduit are assembled on the pins, one piece being fixed and the other placed in turn in all symmetrical positions, a mandrel 12 inches long and 3 inches square may be drawn through the assembled sections.

\section{F. EQUIPMENT}

1. At least two dowel pins shall be furnished for each multiple conduit. They shall be of a good grade of wrought iron or mild steel, not less than $3 \frac{1}{4}$ inches long. A suitable circular flanged collar shall be securely fastened to the middle of the pin to prevent it from slipping too far into the dowel-pin holes of either section of conduit. Both ends of the dowel pin shall be rounded so that they will readily enter the holes of the conduit. The pins shall fit properly into the dowel-pin holes 
of the duct. In no case shall their diameter be less than five-sixteenths inch.

\section{G. INSPECTION}

1. Purchaser may inspect the material at all stages of manufacture.

2. Purchaser may inspect the completed product to determine that the requirements of this specification have been met.

3 . If the material has not been accepted at point of production and if, upon arrival at destination, it does not meet the requirements of this specification it may be rejected, and the contractor, upon request, shall advise the purchaser what disposition is to be made of the defective material. The contractor shall pay all freight charges.

4. If purchaser is to make inspection at point of production it shall be so stated in the order.

\section{H. TESTS}

1. Material offered for inspection under this specification shall be factory run, from which no conduit of superior quality has been removed.

2. A straightedge laid lengthwise on the concave side or sides of a 3 -foot section of conduit may show on one side an off-set of not more than threeeighths of an inch; provided that if the section contains another concave side the off-set of the second concave shall not be greater than oneeighth of an inch. The allowable off-set in conduit shorter than 3 feet in length shall be proportional to the limits allowed for 3 -foot lengths. Not more than 30 per cent of the number of pieces of conduit accepted from any lot offered for inspection shall contain noticeable bends within the limits specified above.

3. A section of the finished conduit weighing from 3 to 4 pounds broken, so as to have all edges unglazed and in the case of multiple conduit, so as to be free from scarification, after being thoroughly dried and then completely immersed for 24 hours in water having a temperature of from $60^{\circ}$ to $80^{\circ} \mathrm{F}$. shall show an absorption of water of not more than 5 per cent of its weight.

4. The finished conduit shall be free from soluble alkalies, as determined by testing with red litmus paper.

5. The presence of cracks, which appreciably weaken the conduit, shall be determined by sounding each piece with a steel hammer or its approved equivalent, the piece of conduit to be rejected if, when sounded, it fails to yield a clear metallic ring.

6 . Tests may be made at point of production or on samples submitted and may also be made at destination.

7. Contractor shall give the purchaser sufficient notice of time when material will be ready for testing and cooperate to set a time convenient to the purchaser for inspection.

8. Contractor shall provide at point of production apparatus and labor for making the required tests under supervision of the purchaser.

\section{J. PACKING}

1. Multiple duct conduit, when shipped in cars, shall be so packed that the ends and each layer shall be separated. The ends of all conduit at the center of the car shall be firmly braced to prevent movement of the conduit during shipment.

\section{B. MARKING}

1. Conduit shipped in less than carload lots shal have the name of the contractor, the purchaser's order, inspection number, and the shipping address plainly marked on a tag securely fastened to 10 per cent of the pieces in the shipment.

\section{WARRANTY}

1. Contractor shall warrant the material covered by this specification to be free from defects in material and workmanship under ordinary use and service, his obligation under this warranty being limited to making at point of production any part or parts to replace those which shall be found defective within one year after shipment to purchaser. This warranty shall not apply to any material which has been subject to misuse, negligence, or accident.

American Railway Engineering Association, specifications for burnt clay ballast, 1921.

See 511.73, page 209 .

American Railway Engineering Association, specifications for buildings for railway purposes.

\section{VITRIFIED SEWER PIPE}

See specification for sewers and drainage, 518.67, page 453 .

American Society for Municipal Improvements, standard specifications for sewers, 1927.

\section{VITRIFIED SEWER PIPE}

See 518.67, page 453 .

American Society for Testing Materials, standard specifications for clay sewer pipe, serial designation, C13-24, 1924.

1. These specifications cover clay products intended to be used for the conveyance of sewage, industrial wastes, and storm water.

2. Sewer pipes furnished under these specifications shall be of a single class to be designated "A. S. T. M. Clay Sewer Pipe."

\section{MATERIAL AND MANUFACTURE}

3. (a) Clay pipes shall be manufactured from surface clay, fire clay, or shale, or a combination of these materials.

(b) Surface clay is an unconsolidated, unstratified clay, ${ }^{2}$ occurring on the surface.

(c) Fire clay is a sedimentary clay ${ }^{2}$ of low flux content.

Note.-It is usually associated with coal measures.

(d) Shale is a thinly stratified, consolidated sedimentary clay ${ }^{2}$ with well-marked cleavage parallel to the bedding.

$(e)$ The materials shall possess such physical and chemical properties that when molded into pipes and subjected to a suitable temperature the product will be strong, durable, and serviceable, free from objectionable defects, and in compliance with these specifications and tests.

\section{CHEMICAL TESTS AND REQUIREMENTS}

Same as A. S. T. M. C14; see 518.67, page 464. PHYSICAL TESTS

Same as A. S. T. M. C14.

\section{PHYSICAI TEST REQUIREMENTS}

Same as A. S. T. M. C14.

2 The definitions for the terms "surface clay," "fire clay," and "shale" are based upon the following definition for the term "clay". "Clay. An earthy or stony mineral ageregate conisting essentially

Clay.-An earthy or stony mineral aggregate consisting essentially of hydrous silicates of alumina, plastic when suficiently pulverized and wetted, rigid when dry, and vitreous when burned at a sufficiently nigh temperature. 


\section{SIZES AND DIMENSIONS}

Same as A. S. T. M. C14 as to sections 27 and 28 and Table 2.

Variances occur in Tables 3 and 4, which for this specification are as follows:

TABLE 3.-Dimensions of clay sewer pipe

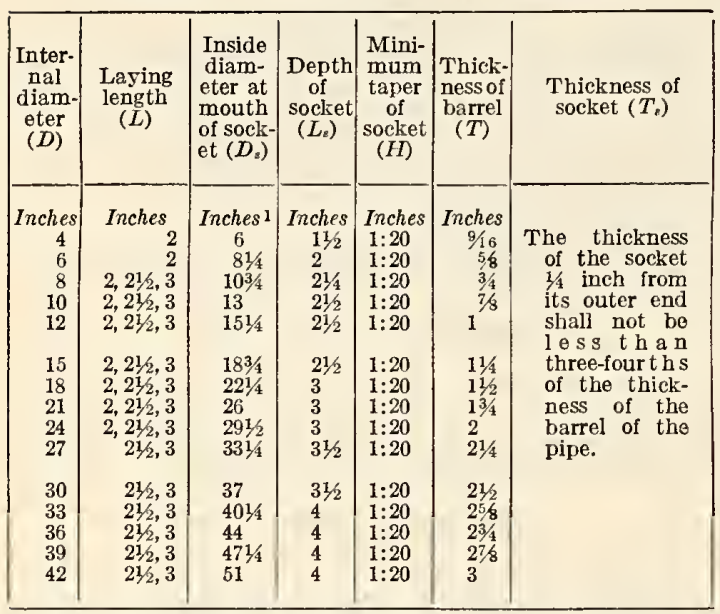

1 When pipes are furnished having an increase in thickness over that given in last column, the diameter of socket shall be increased by an amount equal to twice the increase of thickness of barrel.

TABLe 4.-Permissible variations in dimensions of clay sewer pipe

\begin{tabular}{|c|c|c|c|c|c|c|}
\hline \multirow{3}{*}{$\begin{array}{l}\text { Nor- } \\
\text { mal } \\
\text { size }\end{array}$} & \multicolumn{6}{|c|}{ Limits of permissible variation in- } \\
\hline & \multirow{2}{*}{ Length } & \multirow{2}{*}{$\begin{array}{c}\text { Lengths } \\
\text { of two } \\
\text { opposite } \\
\text { sides }\end{array}$} & \multicolumn{2}{|c|}{$\begin{array}{c}\text { Internal diam- } \\
\text { eter }\end{array}$} & \multirow{2}{*}{$\begin{array}{l}\text { Depth } \\
\text { of } \\
\text { socket }\end{array}$} & \multirow{2}{*}{$\begin{array}{l}\text { Thick- } \\
\text { ness of } \\
\text { barrel }\end{array}$} \\
\hline & & & Spigot & Socket & & \\
\hline $\begin{array}{c}\text { Inches } \\
4 \\
6 \\
8 \\
10 \\
12\end{array}$ & $\begin{array}{c}\text { In. per } \\
\text { foot } \\
-1 / 4 \\
-14 \\
-1 / 4 \\
-1 / 4 \\
-1 / 4\end{array}$ & $\begin{array}{r}\text { Inch } \\
1 / 8 \\
1 / 8 \\
1 / 8 \\
1 / 8 \\
1 / 8\end{array}$ & $\begin{array}{l}\text { Inch } \\
\pm 18 \\
\pm 3 / 16 \\
\pm 1 / 4 \\
\pm 1 / 4 \\
\pm 5 / 16\end{array}$ & $\begin{array}{r}\text { Inch } \\
\pm 3 / 16 \\
\pm 1 / 4 \\
\pm 5 / 16 \\
\pm 5 / 16 \\
\pm 3 / 8\end{array}$ & $\begin{array}{l}\text { Inch } \\
-1 / 8 \\
-1 / 4 \\
-1 / 4 \\
-1 / 4 \\
-1 / 4\end{array}$ & $\begin{array}{l}\text { Inch } \\
-1 / 16 \\
-1 / 16 \\
-1 / 16 \\
-1 / 16 \\
-1 / 16\end{array}$ \\
\hline $\begin{array}{l}15 \\
18 \\
21 \\
24 \\
27\end{array}$ & $\begin{array}{l}-1 / 4 \\
-1 / 4 \\
-1 / 4 \\
-3 / 8 \\
-3 / 8\end{array}$ & $\begin{array}{r}1 / 6 \\
3 / 16 \\
3 / 16 \\
1 / 4 \\
1 / 4\end{array}$ & $\begin{array}{r} \pm 5 / 16 \\
\pm 33 \\
\pm 7 / 6 \\
\pm 1 / 2 \\
\pm 58\end{array}$ & $\begin{array}{r} \pm 5 / 8 \\
\pm 7 / 16 \\
\pm 1 / 2 \\
\pm 9 / 16 \\
\pm 11 / 16\end{array}$ & $\begin{array}{l}-1 / 4 \\
-1 / 4 \\
-1 / 4 \\
-1 / 4 \\
-1 / 4\end{array}$ & $\begin{array}{r}-3 / 32 \\
-3 / 32 \\
-1 / 8 \\
-1 / 8 \\
-1 / 8\end{array}$ \\
\hline $\begin{array}{l}30 \\
33 \\
36 \\
39 \\
42\end{array}$ & $\begin{array}{l}-3 / 8 \\
-3 / 6 \\
-3 / 8 \\
-3 / 8 \\
-3 / 8\end{array}$ & $\begin{array}{l}1 / 4 \\
3 / 8 \\
3 / 8 \\
3 / 8 \\
3 / 8\end{array}$ & $\begin{array}{l} \pm 58 \\
\pm 3 / 4 \\
\pm 3 / 4 \\
\pm 3 / 4 \\
\pm 3 / 4\end{array}$ & $\begin{array}{l} \pm^{11 / 16} \\
\pm^{13} 16 \\
\pm^{131} 16 \\
\pm^{131} 16 \\
\pm^{13} / 16\end{array}$ & $\begin{array}{l}-1 / 4 \\
-1 / 4 \\
-1 / 4 \\
-1 / 4 \\
-1 / 4\end{array}$ & $\begin{array}{l}-1 / 6 \\
-3 / 16 \\
-3 / 16 \\
-3 / 16 \\
-3 / 16\end{array}$ \\
\hline
\end{tabular}

Note.-The minus sign (-) alone indicates that the plus variation is not limited; the plus and minus sign $( \pm)$ indicates variation in both excess and defieiency in dimension.

\section{WORIMANSHIP AND FINISH}

Same as A. S. T. M. C14, with the following additional:

The inner surface of the socket and the outer surface of the spigot end shall be scored by triangular shaped or semicircular shaped rings about $1 / 8$ inch deep.

The number of scorings shall be as follows:

For pipes 4 to 6 inches in internal diameter, inclusive...

For pipes 8 to 10 inches in internal diameter, inclusive

For pipes 12 to 21 inches in internal diameter, inclusive

For pipes 12 to 21 inches in internal diameter, inclusive......

For pipes 33 to 42 inches in internal diameter, inclusive......
The glaze shall consist of a continuous layer of bright or semibright glass substantially free from coarse blisters and pimples. If present, none of these shall project more than $1 / 8$ inch above the surrounding surface. Not more than 10 per cent of the inner surface of any pipe barrel shall be bare of glaze except the socket, where it may be entirely absent. Glazing will not be required on the outer surface of the barrel at the spigot end for a distance from the end equal to two-thirds the specified depth of socket for the corresponding size of pipe. Where glazing is required there shall be absence of any well-defined network of crazing lines or hair cracks. All glazing shall be equal to that produced by the best salt-glazing process.

\section{MARKINGS}

Same as A. S. T. M. C14.

\section{INSPECTION}

Same as A. S. T. M. C14 with the following exceptions:

Substitute: "Laminations which indicate extended voids in the pipe material" for item $(d)$ in C14, "Defects which indicate imperfect mixing and molding."

Add (i) "Glaze which does not fully cover and protect all parts of the shell and ends except those exempted; also glaze which is not equal to the best salt glaze."

Eastern Clay Products Association, standards of manufacture, 1925.

This association has prepared dimensional standards for the following items:

Salt glazed vitrified sewer pipe, single strength.

Salt glazed vitrified sewer pipe, double strength.

Channel pipe.

Salt glazed vitrified sewer pipe fittings, including $T$ branches, $Y$ branches, double $T$ branches, double $Y$ branches, curves, elbows, cut curves, cut elbows, reducers and increasers, slants, $P$ traps, $R$ traps, $\mathrm{HH}$ traps, center HH traps, bowls (closet or slop), stoppers and strainers, and grease taps.

Rectangular fire-clay flue lining.

Round fire-clay flue lining.

Fire-clay flue rings.

Straight stove pipe.

Stove pipe fittings (bottoms), openings, double openings, drop bottoms, chimney bases, and anchor bonnets.

Chimney tops (crown top, octagon hooded top, and cone top).

Wall coping, double-slant style (straight sections, ends, starters, corners, right and left).

Wall coping, camel back style, (straight sections, ends, starters corners, right and left).

Interstate Commerce Commission, regulations for transportation of explosives, and specifications for shipping containers.

(The specifications of the commission, including earthenware containers, may be obtained upon request from the Interstate Commerce Commission, Washington, D. C.)

\section{CHINA AND PORCELAIN WARE}

\subsection{GENERAL ITEMS.}

American Chemical Society, standard sizes and shapes of apparatus, 1927.

(Standard sizes and shapes for laboratory apparatus are recommended in a report of the committee on standard apparatus, of the American Chemical Society. The report lists standard sizes for a number of items made of porcelain.) 


\subsection{TABLE CHINAWARE.}

Federal Specifications Board, specification No. 243a, United States Government master specification for chinaware, vitrified, September 25, 1926.

\section{TYPES AND CLASSES}

The material covered in this specification is vitrified china of three weights known as thick china, hotel (or rolled edge) china, and medium-weight china.

\section{MATERIAL AND WORKMANSHIP}

Vitrified china is glazed chinaware having a white body and burned to such a temperature that a dense, practically nonabsorbent body is produced. Fracture shows a dense structure, which may appear granular. Generally, the ware will not be translucent in the thicknesses specified.

\section{GENERAL REQUIREMENTS}

1. Thick ware.-The only articles of this ware described in this specification are: A cup, unhandled, and saucer; a C. P. O. cup, handled, and saucer; a sauce boat; a bowl, and an individual vegetable dish. The thickness of these articles varies from five-sixteenths to three-eighths inch, and is the heavy type of ware generally used for lunch-counter service.

2. Hotel-weight (or rolled edge) ware.-This ware has a thickness varying from five thirty-seconds to one-fourth inch, depending upon the size of the piece, and in flatware is characterized by a "roll" or bead under the outer edge. This ware is a type used for general restaurant and hotel service.

3. Medium-weight ware.-This ware is somewhat lighter than rolled-edge ware, and the outer edge is not rolled or beaded. It is a type generally used for hospital as well as for restaurant and hotel service.

\section{DETAIL REQUIREMENTS}

1. Design.-All ware furnished shall conform to the shapes adopted for Government use. Measurements and weights shall be within the tolerances indicated in lists 1 to 3 . All flatware shall have a glazed foot.

2. Grade.- Ware for enlisted men's service shall be run of kiln, except thirds. All other china shall be of a grade known as firsts. Ware shall be free from bad warping and imperfections in decoration.

3. Absorption. - The average absorption of all hotel and medium weight ware having a maximum dimension of less than 10 inches shall not exceed 0.3 per cent. The average absorption of all thick ware shall not exceed 0.5 per cent. The average absorption of all ware having a maximum dimension of 10 inches or more shall not exceed 1.5 per cent.

4. Impact.-Impact tests shall be made only on 8,7 , and 5 inch plates, saucers, cups, bowls, platters, and bakers. The ware shall give at least the following average values in the impact test:

ENLISTED MEN'S SERVICE

\begin{tabular}{|c|c|}
\hline Article & $\begin{array}{l}\text { A verage of } \\
\text { impact } \\
\text { values on } \\
\text { all samples } \\
\text { tested }\end{array}$ \\
\hline $\begin{array}{l}\text { Cup } \\
\text { Coffee saucer } \\
\text { 14-inch plate dish, oval platter } \\
\text { 10-inch haker. } \\
30 \text { 's bowl }\end{array}$ & $\begin{array}{r}\text { Ft. }-16 . \\
0.250 \\
.200 \\
.200 \\
.450 \\
.350 \\
.300\end{array}$ \\
\hline
\end{tabular}

HOTEL CHINA (ROLLED EDGE)

\begin{tabular}{|c|c|}
\hline Article & $\begin{array}{l}\text { A verage of } \\
\text { impact } \\
\text { ralues on } \\
\text { all samples } \\
\text { tested }\end{array}$ \\
\hline $\begin{array}{l}8, \pi \text {, and } 5 \text { inch plates } \\
\text { Saucer, coffee or tea } \\
14 \text {-inch dish, oval platter } \\
\text { 10-inch dish, oval platter } \\
\text { 8-inch dish, oval platter- } \\
8 \text { or } 10 \text { inch haker } \\
\text { Teacup } \\
24 \text { 's howl }\end{array}$ & $\begin{array}{r}F t .-l b . \\
0.200 \\
.200 \\
.450 \\
.350 \\
.250 \\
.350 \\
.060 \\
.300\end{array}$ \\
\hline
\end{tabular}

HOSPITAL SERVICE (MEDIUM WEIGHT)

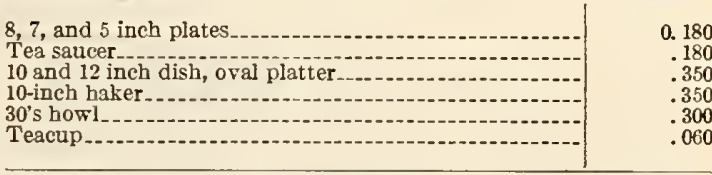

5. Chipping.-Chipping tests shall be made only on 8,7 , and 5 inch plates and on saucers. The ware shall give at least the following average value in the chipping test:

\begin{tabular}{|c|c|}
\hline Service & $\begin{array}{l}\text { A verage of } \\
\text { chipping } \\
\text { values on } \\
\text { all samples } \\
\text { tested }\end{array}$ \\
\hline $\begin{array}{l}\text { Enlisted men's service. } \\
\text { Hotel china, rolled edge } \\
\text { Hospital service, medium weight }\end{array}$ & $\begin{array}{r}F t .-l b . \\
0.150 \\
.150 \\
.075\end{array}$ \\
\hline
\end{tabular}

\section{METHOD OF INSPECTION AND TESTS}

\section{SAMPLING}

(a) Articles sampled.-Samples for test will usually include only plates, cups, saucers, platters, bowls, and bakers, but may, at the option of the purchaser, include other articles which are usually ordered in small quantities. Such articles will be tested for absorption only.

(b) Number of samples required.-Samples of each shape shall be taken at random from shipments of each 100 dozen or fraction thereof for tests as follows:

\begin{tabular}{|c|c|c|}
\hline \multirow{2}{*}{ Articles } & \multicolumn{2}{|c|}{ Test } \\
\hline & Chipping & Impact \\
\hline $\begin{array}{l}\text { Plates_- } \\
\text { Saucers } \\
\text { Cups.-. }\end{array}$ & 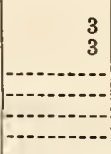 & $\begin{array}{l}6 \\
6 \\
6 \\
3 \\
3 \\
6\end{array}$ \\
\hline
\end{tabular}

Other articles (for ahsorption only)

(c) Marking samples.- Separate samples shall be used for the chipping and impact tests. Additional samples for the absorption test are not required when chipping and impact tests are specified, but six samples of the odd shapes will be required for absorption tests when specified by the purchaser. Samples for test should be so marked that the part of the shipment they represent can be identified. (See "Method of inspections and tests," 3, (a), (b), and $(c)$. 


\section{METHODS OF TESTING1}

(a) Chipping test.-This test shall be made using the apparatus and 6-ounce hammer shown in Figure $145 .^{3}$

The plate or saucer resting on its foot is placed between two cast-iron blocks forming a $90^{\circ} \mathrm{V}$, so adjusted that when the hammer is hanging vertically the center of the impact face of the chipping hammer touches the edge of the test piece in the plane of the bisector of the angle of the V.

The sample is repeatedly struck on the edge by the hammer with blows of increasing force until failure occurs, beginning with the initial blow and
A plate, saucer, platter, or baker is supported against the three equally spaced 1/8-inch steel balls, so that when the hammer is hanging vertically the impact point of the hammer touches the center of the bottom of the test piece.

A cup or bowl resting on its foot is placed between two cast-iron blocks forming a $90^{\circ} \mathrm{V}$, whose sides are sufficiently high to support the upper edge of the cup or bowl, and is so adjusted that when the hammer is hanging vertically its impact point touches the cup at its upper edge and in the plane of the bisector of the angle of the $\mathrm{V}$.

The samples are repeatedly struck, until failure occurs, with blows of increasing force, beginning

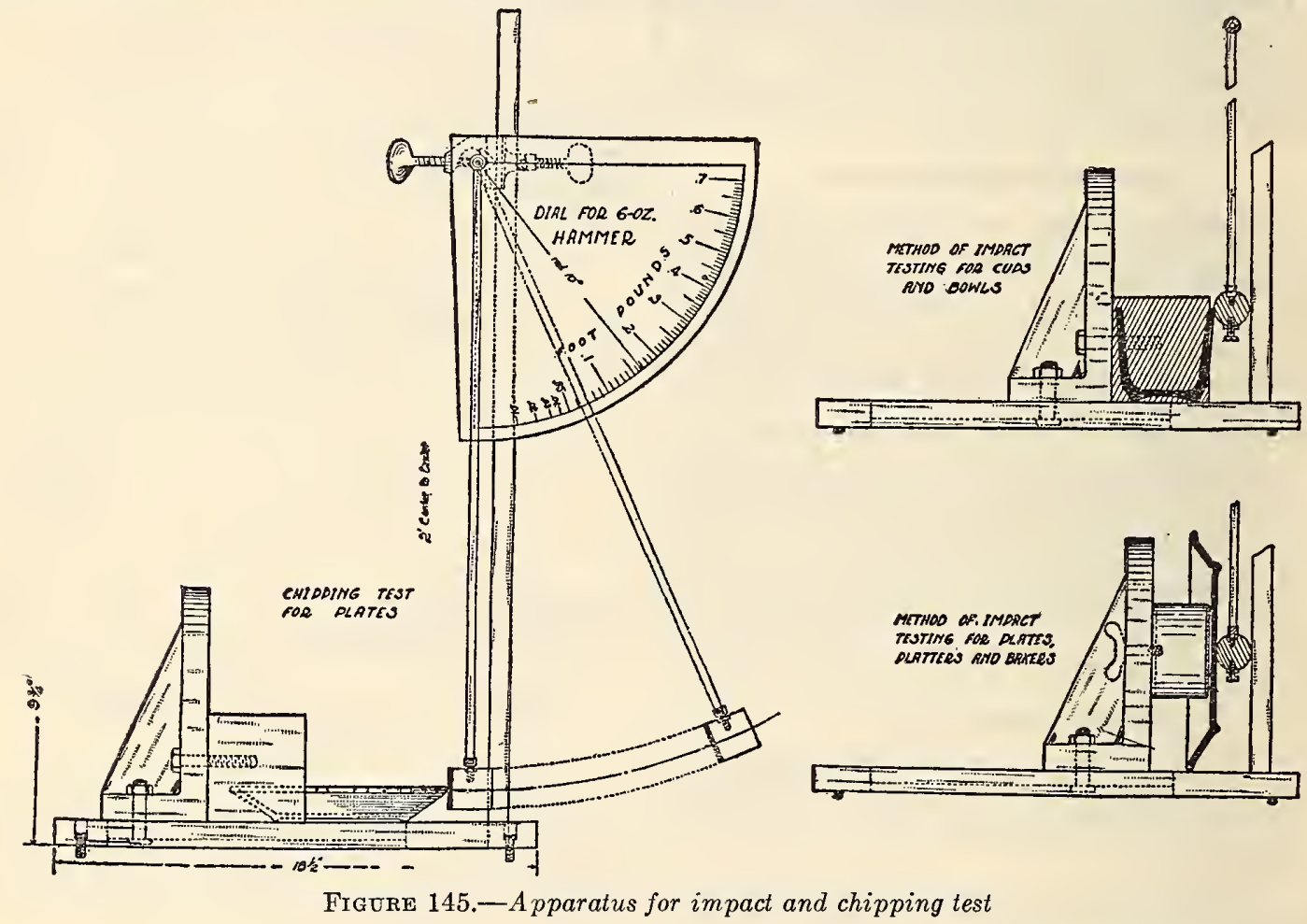

increasing the force of each succeeding blow in the increment given below.

\begin{tabular}{|c|c|c|c|}
\hline Service or type oi ware & $\begin{array}{c}\text { Weight } \\
\text { of } \\
\text { hammer }\end{array}$ & $\begin{array}{l}\text { Initial } \\
\text { blow }\end{array}$ & $\begin{array}{l}\text { Incre- } \\
\text { ment }\end{array}$ \\
\hline $\begin{array}{l}\text { Enlisted men's service and hotel } \\
\text { Ware } \\
\text { Hospitalware- }\end{array}$ & $\begin{array}{r}\text { Ounces } \\
6 \\
6\end{array}$ & $\begin{array}{l}F t .-l b . \\
0.10 \\
.02\end{array}$ & $\begin{aligned} F t .-l b . \\
0.02 \\
.01\end{aligned}$ \\
\hline
\end{tabular}

Failure consists in chipping the edge so that fragments of glaze and body are removed. The article shall be tested at three equally spaced points on its periphery and the average value for the three points shall be the value for the individual article.

(b) Impact test.-This test shall be made using the apparatus and 4-ounce hammer as shown in Figure 145.

${ }^{3}$ For graduating a dial in divisions representing $0.01 \mathrm{ft}$. $\mathrm{ll}$. for use with the 6-ounce hammer, project points 0.133 inch apart, measured along the vertical axis, to the circumference of a circle whose radius is 10 inches, and for the 4 -ounce hammer project points 0.2 inch apart in a similar manner. with the initial blow and increasing the force of each succeeding blow in the increments given in the following table:

\begin{tabular}{|c|c|c|c|}
\hline Article & $\begin{array}{l}\text { Weight of } \\
\text { hammer }\end{array}$ & $\begin{array}{l}\text { Initial } \\
\text { blow }\end{array}$ & $\begin{array}{l}\text { Incre- } \\
\text { ment }\end{array}$ \\
\hline $\begin{array}{l}\text { Plate, saucer, platter, baker, bowl } \\
\text { Enlisted men's service cup } \\
\text { Teacup }\end{array}$ & $\begin{array}{r}\text { Ounces } \\
4 \\
4 \\
4\end{array}$ & $\begin{array}{r}F t .-l b . \\
0.10 \\
.10 \\
.02\end{array}$ & $\begin{array}{r}F t .-l b . \\
0.02 \\
.02 \\
.01\end{array}$ \\
\hline
\end{tabular}

Failure of plates, saucers, platters, and bakers is defined as the development of a rupture which appears as a hole through the body or as a body crack extending through the rim of the article. Failure of cups and bowls is defined as the development of a body crack extending into any portion of the foot or as the breaking away of a portion of the body.

(c) Absorption test.-Absorption shall be made on glazed pieces having an area of approximately $2 \frac{1}{2}$ square inches, broken from three different samples used in the chipping or impact tests, The samples, 
after being dried to constant weight at $110^{\circ} \mathrm{C}$., shall be completely immersed and vigorously boiled in water for five hours and then allowed to remain in the water for 20 hours. The samples are then removed, wiped with a damp cloth to remove excess moisture, and immediately weighed to the nearest $0.01 \mathrm{~g}$. The percentage of absorption is computed from the formula:

$$
\text { Percentage absorption }=\frac{\text { Wet weight }- \text { dry weight }}{\text { Dry weight }} \times 100
$$

\section{ACCEPTANCE AND REJECTION}

(a) Failure of one type only.-Failure of any type of article to conform to any of tie requirements of this specification shall cause rejection of that type. If any one type of article, such as plates or saucers, meets the requirements, but another type, such as cups, fails to comply with these requirements, that portion of the shipment which conforms shall be accepted and the portion failing to conform shall be rejected.

(b) Failure of portion of samples of same type.Also, if the sample representing one part of a shipment of ware of the same type of article fail to meet the requirements, and samples representing other parts of the same shipment conform to the requirements of the specification, only that part of the shipment represented by the samples that fail to meet the requirements of the specifications shall be rejected.

(c) Retest.-If the first set of samples does not meet the requirements of this specification, the manufacturer shall, within 10 days after notification, demand a retest, which must be made on a second set of samples taken from the shipment in question. If the test on the second set of samples is satisfactory, the material shall be accepted.

\section{METHOD OF MARKING}

All ware shall be marked on the bottom in underglaze figures showing the manufacturer's name, trade-mark, or other legend characteristic of said manufacturer's ware. The contract year, as indicated on the order, shall also be placed on the bottom in underglaze figures when so specified by the purchaser.

\section{NOTES}

Further detail requirements for dimensions, with proper tolerances, of any article of chinaware used by the Navy Department may be added to the requirements of this specification in order that such articles may be obtained in sizes that will fit in the compartments of the baskets used in their dishwashing machines.

List 1.-Hotel weight china for dining room service ${ }^{1}$

\begin{tabular}{|c|c|c|c|c|c|}
\hline Article & Trade size & Actual size & Tolerance & \multicolumn{2}{|c|}{$\underset{\text { weight }}{\text { Maximum }}$} \\
\hline & 7 inches, RI & 9 inches. & $1 / 8$ inch.- & Lbs. & 02. \\
\hline lates...... & 4 inches, RY & 614 inches.-. & 18 inch... & & 9 \\
\hline Do...... & 5 inches, $\mathrm{RE}$ & 718 inches & 18 inch & & 12312 \\
\hline Do-..... & 7 inches, $\mathrm{RE}$ & 9 inches...... & 18 inch & & 24 \\
\hline $\begin{array}{l}\text { Do } \\
\text { Soup, rim, deep.-...... }\end{array}$ & 8 inches, $\mathrm{RE}$ & 95,8 inches & $1 / 8$ inch & & 27 \\
\hline Soup, rim, deep-.... & 5 inches, $\mathrm{RE}$ & 7 inches... & 18 inch... & & 12 \\
\hline Tea saucer, wide foot------ & $R E_{-\ldots}$ & 618 inches. & 18 inch & & $93 / 2$ \\
\hline Coffee saucer (after-dinner) ----- & $\mathrm{RE}_{-}$ & 478 inches. & 38 inch-.. & & $51 / 2$ \\
\hline xon (after-dinner & & $31 \%$ ounces & $1 / 4$ ounce... & & 5 \\
\hline Tesc & & $71 / 2$ ounces-. & $1 / 2$ ounce & & 7 \\
\hline Bouillon cup, Boston & No. $2, \mathrm{BH} . .$. & $71 / 2$ ounces_-. & $3 / 2$ ounce-- & & $1^{71 / 2}$ \\
\hline $\begin{array}{l}\text { Egg cup, wheat, } \\
\text { Baker }\end{array}$ & 8 inches, $\mathrm{RE}$ & $\begin{array}{l}61 / 2 \text { ounces } \\
10 \text { inches.-. }\end{array}$ & $1 / 4$ inch... & & 29 \\
\hline Do & 10 inches, $\mathrm{RE}$ & 1178 inches & 38 inch.- & 3 & 0 \\
\hline Do & 5 inches, $\mathrm{RE}$ - & 714 inches & 18 inch & & $121 / 2$ \\
\hline Do...- & 3 inches, $\mathrm{RE}$. & $5 \frac{3}{4}$ inches $\ldots$ & 18 inch... & & 7 \\
\hline Salad bowl & No. $3, R_{E}$ & $91 / 2$ inches ... & $1 / 4$ inch... & 2 & 8 \\
\hline Do & No. $6, \mathrm{RE}$ - & 6 inches...- & $1 / 4$ inch.-. & & 15 \\
\hline Sauce boat & Large, RE. & 11 ounces.- & 1 ounce... & & 18 \\
\hline pickle) ...... & No. 3 & $71 / 2$ inches. & $1 / 8$ inch & & $81 / 2$ \\
\hline Pin tray-- & No 2 & $\begin{array}{l}51 / 4 \text { inches } \\
11 / 2\end{array}$ & $\begin{array}{l}1 / 8 \text { inch } \\
1 / 4 \text { ounce }\end{array}$ & & 5 \\
\hline Cream jug, handled, medium & No. 1,54 's & $41 / 2$ ounces_- & $1 / 2$ ounce--- & & 6 \\
\hline Sugar, individual, covered, round, unhandle & & 5 ounces & $1 / 4$ ounce & & 8 \\
\hline Sugar.. & No. $1, \mathrm{RE}_{\mathrm{B}}$ & 17 ounces & $11 / 2$ ounces & & 28 \\
\hline Butter, indiv & 232 inches, $\mathrm{RE}$. & $31 / 2$ inches... & $1 / 8$ inch... & & 3 \\
\hline unhandled.. & No. 1 ..-.......... & 4 ounces..... & $1 / 2$ ounce... & & 11 \\
\hline Cake c & & $61 / 4$ inches & 18 inch-- & & \\
\hline Jug, he & $24 \mathrm{~s}_{-}$ & $23 / 4$ pints & $1 / 2$ pint.- & 2 & 12 \\
\hline Jug ... & 6 'S RE & 6 pints.. & 34 pint & 5 & 0 \\
\hline Do & 12 's, RE. & $43 / 2$ pints.- & 12 pint... & 4 & 0 \\
\hline Do & 24 's, $\mathrm{RE}$ E. & 31 pints... & $3 / 8$ pint... & 2 & 8 \\
\hline Do & 36 's, RE & $11 / 4$ pints... & $1 / 4$ pint... & & 22 \\
\hline Do. & 42 's, RE & $3 / 4$ pint $\ldots$ & 38 pint... & & 17 \\
\hline Do.- & 48 's, $\mathrm{RE}_{-}$ & 512 ounces.-. & $3 / 4$ ounce-. & & 9 \\
\hline Dish, oval, platter..... & 4 inches, $\mathrm{RE}$ & 7 inches & 18 inch-- & & $91 / 2$ \\
\hline 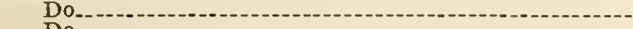 & 6 inches, RE- & 918 inches... & is inch.- & & 16 \\
\hline Do & 8 inches, $\mathrm{RE}$ & $113 / 4$ inches... & 14 inch-- & & 27 \\
\hline - & 10 inches, $\mathrm{RE}$ & 1314 inches... & $1 / 4$ inch & 2 & 10 \\
\hline$M_{1}$ & 14 inches, $\mathrm{RE}$ & $171 / 2$ inches...... & $1 / 2$ inch......- & 6 & 0 \\
\hline Casserole, plain & 8 inches, $R D$ & 234 pints & 14 pint & 2 & 8 \\
\hline Match stands, round, hooded..- & & $61 / 2$ inches. & $1 / 2$ inch.- & & 12 \\
\hline Ice tub.- & & $91 / 4$ pints. & 1 pint & 8 & \\
\hline Fruit & 4 inche & 538 inches. & $1 / 8$ inch-. & & $61 / 2$ \\
\hline Bow & $24{ }^{\prime} \mathrm{s}_{-}$ & 112 pints.- & & & 26 \\
\hline 7ered, removable dr & & $53 / 4$ inches & $1 / 8$ inch & & 27 \\
\hline
\end{tabular}

1 The ware described in this list is, in general, that specified for the Public Health Service, the Veterans' Bureau, Navy officers' mess, and the General Supply Committee. 
List 2.-Thick china for enlisted men's service ${ }^{1}$

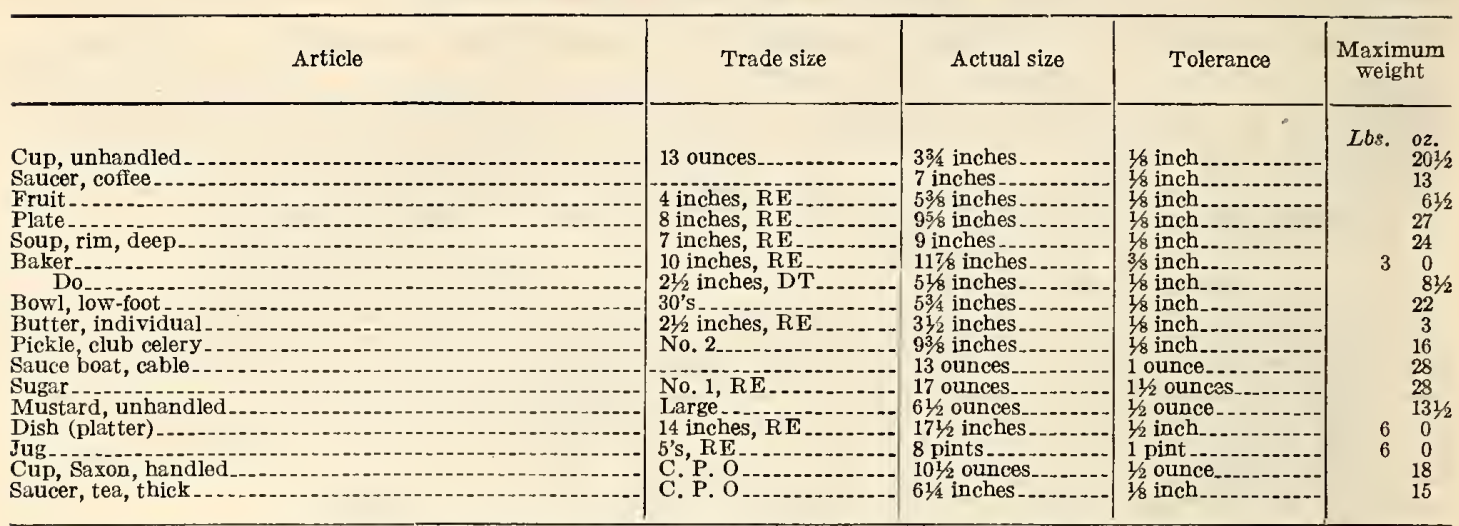

1 The ware described in this list is, in general, that specified for the Army, Navy, and General Supply Committee.

List 3.-Medium-weight vitrified china for hospital service ${ }^{1}$

\begin{tabular}{|c|c|c|c|c|c|}
\hline Article & Trade size & Actual size & Tolerance & \multicolumn{2}{|c|}{$\begin{array}{c}\text { Maximum } \\
\text { weight }\end{array}$} \\
\hline Plate, dinner ... & & 9 inches...- & & $L b 8$. & $0 z$. \\
\hline Plate, bread and butter & 4 inches... & 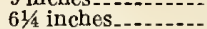 & $1 / 8$ inch..... & & 21 \\
\hline Plate, pie & 5 inches.-- & $71 / 8$ inches.... & 18 inch..... & & 11 \\
\hline Dishes, platter & 10 inches. & $131 / 4$ inches.- & $1 / 4$ inch... & 2 & 9 \\
\hline Do & 12 inches. & 1518 inches. & $1 / 4$ inch.. & 3 & 8 \\
\hline Bowl, low-foot & 30 's & 534 inches & $18 \mathrm{inch}$... & & 19 \\
\hline Celerv, pickle & No. $3 \ldots$ & $71 / 2$ inches & 18 inch... & & $81 / 2$ \\
\hline Cup, tea, Boston, bhk., hd & - n..... & $71 / 2$ ounces... & $1 / 2$ ounce & & 7 \\
\hline Saucer, tea, reinforced well hole & -......- & $5^{3} 4$ inches & $1 / 8$ inch.... & & $71 / 2$ \\
\hline Bakers, open vegetable dish & 8 inches & $101 / 8$ inches. & $1 / 4$ inch.-. & 2 & 0 \\
\hline 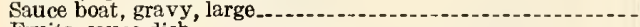 & ----- & 11 ounces & 1 ounce-.- & & 18 \\
\hline Fruits, sauce dish & 4 inches... & $53 / 8$ inches.. & $1 / 8$ inch... & & 6 \\
\hline Mustard, Vienna, unhandled... & No. 1..... & 4 ounces & $1 / 2$ ounce.... & & 11 \\
\hline Cream, Vienna, unhandled & -----1 & $13 / 4$ ounces.......-- & $1 / 8$ ounce $\ldots .$. & & $51 / 2$ \\
\hline $\begin{array}{l}\text { Jug, pitcher } \\
\text { Jug, handled }\end{array}$ & 5's, RE. - & 8 pints...$--1 .-1$ & 1 pint & 6 & 0 \\
\hline $\begin{array}{l}\text { Jug, handled } \\
\text { Do }\end{array}$ & 54 's $-\cdots-$. & $\begin{array}{l}41 / 2 \text { ounces.- } \\
11 / 4 \text { pints }-. .\end{array}$ & $\begin{array}{l}1 / 2 \text { ounce } \ldots \ldots \\
1 / 4 \text { piat } \ldots \ldots \ldots\end{array}$ & & $\begin{array}{r}6 \\
18\end{array}$ \\
\hline Sugar bowl, individual, round, covered, unhan & & 5 ounces.... & $1 / 4$ ounce.-- & & 8 \\
\hline Egg cup, double & & 4 ounces_. & $1 / 4$ ounce-....- & & 7 \\
\hline Bakers, individual vegetable & 3 inches. & $5 \% \frac{3}{4}$ inches.- & 18 inch........ & & $71 / 2$ \\
\hline Bowl, oatmeal, cereal & & 6 inches & $1 / 8$ inch $\ldots . . . . .$. & & 9 \\
\hline $\begin{array}{l}\text { Sugar bowl } \\
\text { Cofiee pot, individual }\end{array}$ & No. $1, \mathrm{RE}_{\text {. }}$ & 17 ounces $\ldots . . . .$. & $11 / 2$ ounces & & 28 \\
\hline Cofiee pot, individual.- & & 12 ounces.. & 1 ounce & & 15 \\
\hline
\end{tabular}

1 The ware described in this list is, in general, that specified for the Medical Corps.

United States Department of Commerce, division of simplified practice.

In accordance with unanimous action of conferences of representatives of manufacturers, distributors, and users of chinaware, the United States Department of Commerce, through the Bureau of Standards, has prepared the following simplified practice recommendations for standard types and sizes of chinaware:

No. 5, hotel chinaware, January 1, 1924.

No. 33, cafeteria and restaurant chinaware, January 1, 1924.

No. 39, dining-car chinaware, July 1, 1925.

No. 40, hospital chinaware, July 1, 1925 
Recommended list of sizes, types, and capacities of chinaware for hotel, restaurant, cafeteria, hospital, dining car, and Government service

\begin{tabular}{|c|c|c|c|c|}
\hline To. & Items & Trade size & Actual size & Tolerance \\
\hline $\begin{array}{l}1 \\
2 \\
3 \\
4 \\
5\end{array}$ & 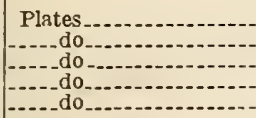 & $\begin{array}{l}3 \text { inche } \\
4 \text { inche } \\
41 / 2 \text { inch } \\
5 \text { inche } \\
6 \text { inche }\end{array}$ & $\begin{array}{l}51 / 2 \text { inches -- } \\
61 / 4 \text { inches -- } \\
658 \text { inches -- } \\
71 / 8 \text { inches -- } \\
818 \text { inches -- }\end{array}$ & $\begin{array}{l}16 \text { inch. } \\
1 / 8 \text { inch. } \\
1 / 8 \text { inch. } \\
18 \text { inch. } \\
1 / 8 \text { inch. }\end{array}$ \\
\hline 6 & 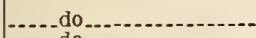 & $\because:$ & 9 inch & Is inch. \\
\hline $\begin{array}{l}7 \\
8\end{array}$ & do do & & 958 inches .- & inch. \\
\hline $\begin{array}{l}8 \\
9\end{array}$ & Plates, lunch, or grille & 6 ins & $97 / 8$ inches - - & $1 / 4$ iuch. \\
\hline & $\begin{array}{l}\text { Plates, soup, coupé } \\
\text { deep. }\end{array}$ & 6 inc & $71 / 2$ incl & 1/8 inch. \\
\hline 10 & Plates, soup, rim deep & 5 inc & 7 inches.... & $1 / 8 \mathrm{inch}$. \\
\hline 11 & . & & 9 inches.... & $1 / 8 \mathrm{inch}$. \\
\hline 12 & Butters, i & 2 inc & 3 inches .... & \\
\hline 13 & $\begin{array}{l}\text { Butters, individual, } \\
\text { thick. }\end{array}$ & & 3 inches.... & $1 / 8 \mathrm{inch}$. \\
\hline 14 & Butters, individual & $21,2 \mathrm{i}$ & $31 / 2$ inches .. & $1 / 8$ inch. \\
\hline 15 & $\begin{array}{l}\text { Ice cream ha } \\
\text { shell. }\end{array}$ & & & \\
\hline 16 & do & No & es.- & $1 / 8$ in \\
\hline 17 & Dishes, ova & & --- & \\
\hline & $\cdots$ & & es - - & \\
\hline 20 & -..do & 10 & 1314 inch... & $1 / 1$ inch. \\
\hline 21 & . & & aes. & $1 / 4$ \\
\hline 22 & $\mathrm{do}_{-}$ & & hes. & \\
\hline 23 & Bakers, douhle & 21 & 51,8 inc & $1 / 8$ inch. \\
\hline 25 & -...do_. & & es_. & \\
\hline 25 & -....do do & & 5 L/ inches.. & 1/s inch. \\
\hline 25 & ...do. & & $S \ldots$ & 78 \\
\hline 27 &.$--40 \ldots$ & & & \\
\hline 28 & -_do do..... & 10 & inches_. & $3 / 8$ inch. \\
\hline 29 & $\begin{array}{l}\text { Bakers, open vegetahle } \\
\text { dish. }\end{array}$ & & $101 / 8$ inches. & $1 / 4$ inch. \\
\hline 30 & Sauce boats . . & Lar & 11 ounces_.- & 1 ounce. \\
\hline 31 & - do do. & $\mathrm{Me}$ & -...- & $7 / 2$ \\
\hline 32 & ...-do (cahle) & & s... & 10 \\
\hline 33 & (-..-- do $\mathrm{do}_{-2}$ & & 3 & ce. \\
\hline 34 & Bowls, low & $30^{\prime}$ & $5^{3 / 4}$ inches & 18 \\
\hline 35 & Bowls, foc & $30^{\prime}$ & hes.... & $1 / 4$ inch. \\
\hline 36 & Bow & & & \\
\hline $\begin{array}{l}37 \\
38\end{array}$ & Bowls, sug & & es... & $11 / 2$ \\
\hline $\begin{array}{l}38 \\
39\end{array}$ & -_-do do & & es_-- & 1 ou \\
\hline 39 & $\begin{array}{l}\text { Bowl, sugar, covered } \\
\text { tankard shape, maxi- } \\
\text { mun weight. }\end{array}$ & & es _.. & 10 \\
\hline 40 & $\begin{array}{l}\text { Bowls, sugar, individ- } \\
\text { ual, covered, un- } \\
\text { handled. }\end{array}$ & & s.... & $1 / 4$ ounce. \\
\hline 41 & $\begin{array}{l}\text { Sugar, open oval } \\
\text { rihbed. }\end{array}$ & In & 5 & $1 / 2$ ounce. \\
\hline 42 & Salad howls ... & No & es. & $1 / 4$ \\
\hline 43 & _._. _ do do & & les.. & $1 / 4$ inch. \\
\hline $\begin{array}{l}44 \\
45\end{array}$ & - - do do & & es.- & $1 / 4$ inch. \\
\hline & $---\alpha$ & & & \\
\hline 46 & -...--do_--- & & & $1 / 4$ \\
\hline 47 & $\begin{array}{l}\text { Comport, footed, sa- } \\
\text { lad, Illinois shape. }\end{array}$ & & & \\
\hline 48 & 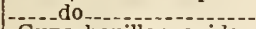 & No. 3 & hes_. & 3/8 inch. \\
\hline 49 & $\begin{array}{l}\text { Cups, houillon, ovide, } \\
\text { heavyweight. }\end{array}$ & & & \\
\hline 50 & $\begin{array}{l}\text { Cups, houillon, ovide, } \\
\text { medium weight, } 2 \\
\text { hlock handle. }\end{array}$ & & $71 / 2$ ounces. & $1 / 2$ ounce. \\
\hline 51 & $\begin{array}{l}\text { Liner, houillon, un- } \\
\text { handled, fa ncy } \\
\text { shape. }\end{array}$ & & Tou & $1 / 2$ ounce \\
\hline 52 & Cover, houilion. & $\mathrm{T}$ & & $1 / 6$ \\
\hline 53 & $\begin{array}{l}\text { Cups, cofiee, ovide, } \\
\text { heavyweight. }\end{array}$ & & & \\
\hline 54 & $\begin{array}{l}\text { Cups, tea, ovide, } \\
\text { heavyweight. }\end{array}$ & & 71 & 1/2́ ounce \\
\hline 55 & $\begin{array}{l}\text { Cups, tea, ovide, me- } \\
\text { dium weight. }\end{array}$ & & 71 & $1 / 2$ ounce \\
\hline 56 & vide_. & & & $1 / 2$ \\
\hline 57 & $\begin{array}{l}\text { Cups, extra tea, ovide, } \\
\text { medium weight. }\end{array}$ & & es_- & $1 / 2$ ounce \\
\hline 58 & $\begin{array}{l}\text { Cups, coffee, heary, } \\
\text { straight side. }\end{array}$ & & 90 & $1 / 2$ ounce \\
\hline 59 & $\begin{array}{l}\text { Cups, coffee, heavy, } \\
\text { ovide. }\end{array}$ & & & $1 / 2$ ounce. \\
\hline 60 & $\begin{array}{l}\text { Cups, coffee, Saxon } \\
\text { (after dinner). }\end{array}$ & & $3^{1}$ & \\
\hline
\end{tabular}

Recommended list of sizes, types, and capacities of chinaware for hotel, restaurant, cafeteria, hospital, dining car, and Government service-Continued

\begin{tabular}{|c|c|c|c|c|}
\hline No. & Items & Trade size & Actual size & Tolerance \\
\hline 61 & Cups, unhandled.- & 13 ounce & $33 / 4$. Inches & 18 inch. \\
\hline 62 & Cups, egg, douhle. & & 4 ounces... & $1 / 4$ ounce. \\
\hline 63 & $\begin{array}{l}\text { Cups, cgg, wheat, un- } \\
\text { handled. }\end{array}$ & - & $61 / 2$ ounces & $1 / 2$ ounce. \\
\hline 64 & $\begin{array}{l}\text { Cups, egg, footed, } \\
\text { single. }\end{array}$ & 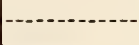 & $11 / 2$ ounces. & $1 / 4$ ounce. \\
\hline 65 & $\begin{array}{l}\text { Cups, egg, footed, } \\
\text { Boston. }\end{array}$ & No. 1 & $61 / 2$ ounces. & $1 / 2$ ounce. \\
\hline 66 & $\begin{array}{l}\text { Cups, egg, covered, } \\
\text { Boston. }\end{array}$ & & $41 / 4$ inches & 1/8 inch. \\
\hline 67 & Saucers, tea, medium & & 578 inches. & $1 / 8$ inch. \\
\hline 68 & Saucers, tea, wide foot & & 616 inches & 18 inch. \\
\hline 69 & $\begin{array}{l}\text { Saucers, tea, reinforced } \\
\text { well hole. }\end{array}$ & & $53 / 4$ inches & 38 inch. \\
\hline 70 & Saucers, coffee.- & & 656 inches & 36 inch. \\
\hline 71 & - do $_{-} \ldots$ & & 7 inches & $1 / 8$ inch. \\
\hline 72 & $\begin{array}{l}\text { Saucers, coffee (after } \\
\text { dinner). }\end{array}$ & & $47 / 8$ inches. & $1 / 8$ inch. \\
\hline 73 & Pot, coffee, individual & & 12 ounces... & 1 ounce. \\
\hline$\underline{74}$ & Cover, cake, plain. & & 614 inches.. & $1 / 8$ inch. \\
\hline 75 & Trays, celery, oval & No. & 12 inches... & 14 inch. \\
\hline 76 & -_-_do_. & No. 2 & 10 inches & $1 / 4$ inch. \\
\hline 77 & ... do & No. 3 & $71 / 2$ inches & 38 inch. \\
\hline 78 & - & Fancy & 11 inches & $1 / 4$ inch. \\
\hline 79 & $\begin{array}{l}\text { Creams, Vienna, han- } \\
\text { dled or unhandled. }\end{array}$ & No. 1. & $21 / 4$ ounces & $1 / 4$ ounce. \\
\hline 80 & -...-do do & No & $11 / 2$ ounces. & $1 / 4$ ounce. \\
\hline 81 & $\begin{array}{l}\text { Creams, Vienna, un- } \\
\text { handled. }\end{array}$ & & 134 ounces. & 1/s ounce. \\
\hline 82 & Creams, unhandled & & 1 ounce.... & 38 cunce. \\
\hline $\begin{array}{l}83 \\
84\end{array}$ & Jugs, cream, handled & 54 's & $41 / 2$ ounces. & $1 / 2$ ounce. \\
\hline 84 & $\begin{array}{l}\text { Dishes, fruit, coupé } \\
\text { shape. }\end{array}$ & 3 inche & $41 / 8$ inches _. & 1/8 inch. \\
\hline 85 & di & $31 / 2$ & $43 / 4$ inches. & $1 / 8$ inch. \\
\hline 86 & ..... do & 4 in & $53 / 8$ inches & 1/8 inch. \\
\hline 87 & -...... do & $41 / 2$ & $5^{3} / 4$ inches & 1/8 inch. \\
\hline 88 & Dishes, ice cream & 4 inches & $41 / 6$ inches & 16 inch. \\
\hline $\begin{array}{l}89 \\
90\end{array}$ & Dishes, grape fruit.- & 41 & $\begin{array}{l}5 \text { inches... } \\
61 / 4 \text { inches. }\end{array}$ & $\begin{array}{l}1 / 8 \text { inch. } \\
1 / 8 \text { inch. }\end{array}$ \\
\hline 91 & Dishes, pickle, oval & & $81 / 2$ inches & I/4 inch. \\
\hline 92 & Dishes... & 5 in & $81 / 4$ inches & $1 / 8$ inch. \\
\hline 93 & $\ldots \mathrm{do}_{\ldots} .$. & $7 \mathrm{in}$ & $101 / 2$ inches. & 1/s inch. \\
\hline 94 & Ice tuhs.-. & & $91 / 4$ pints... & 1 pint. \\
\hline 95 & Jugs......... & 6's.. & 6 pints..... & $3 / 4$ pint. \\
\hline 96 & .... do & 12 & $41 / 4$ pints & $1 / 2$ pint. \\
\hline 98 & . do & $24^{\prime}$ & $S_{-\ldots}$ & 38 pint. \\
\hline 98 & -..... do & $36^{\prime}$ & $11 / 4$ pints... & $1 / 4$ pint. \\
\hline 100 & Jug R/E............... & $46^{\prime} \mathrm{s}$ & 8 ounces.... & $\begin{array}{l}18 \text { pint. } \\
1 / 2 \text { ounce. }\end{array}$ \\
\hline 101 & Jugs & $48^{\prime}$ & aces_. & $3 / 4$ ounce. \\
\hline 102 & Jugs, hall ho & & ats... & $1 / 2$ pint. \\
\hline 103 & Jugs, pitcher_.... & No & 8 pints $\ldots$ & 1 pint. \\
\hline 10 & $\begin{array}{l}\text { Match stands, round } \\
\text { hooded. }\end{array}$ & & $61 / 2$ inches & $1 / 2$ inch. \\
\hline 105 & Mugs, coffee & 24 & 91/2 ounces. & $-1 / 2$ ounce. \\
\hline 105 & ._do do...... & & $71 / 2$ ounces & $1 / 2$ ounce. \\
\hline 107 & $\begin{array}{l}\text { Mustard, unhandled, } \\
\text { large. }\end{array}$ & & & $1 / 2$ ounce. \\
\hline 108 & Mustard pots, Vienna, & $\mathrm{N}$ & 4 ounce & $1 / 2$ ounce. \\
\hline 109 & Nappies (howls) & 3 in & 5 in & $1 / 8$ inch. \\
\hline 110 & & 4 inche & $53 / 4$ inches & $1 / 8$ inch. \\
\hline 111 & Nappies & & $57 / 16$ ounces & $1 / 4$ ounce. \\
\hline 112 & Nappi & & 578 ounces. & $1 / 1$ ounce. \\
\hline 113 & Pickle, cluh celery & No. 2 & $93 / 8$ inches & $1 / 8$ inch. \\
\hline 11 & Trays & & $51 / 4$ inches.. & 18 inch. \\
\hline & Casserole, plain & 8 in & $23 / 4$ pints & 1/4 pint. \\
\hline
\end{tabular}

It is further recommended that the recognized items be made in three weights only, as covered by the trade names "rolled edge," "medium weight," and "lightweight," respectively.

532.2 PORCELAIN.

532.21 Crucibles.

Manufacturing Chemists' Association of the United States, standard specifications for laboratory apparatus, 1922 


\section{LABORATORY PORCELAIN WARE}

Porcelain ware, to be acceptable, shall be:

(a) Uniform in size and true shape, in accordance with given dimensions.

(b) Free from cracks in body, glaze, or similar imperfections.

(c) Marked with manufacturer's name.

(d) Able to meet specified tests for that article as hereafter described.

(e) Shall be uniformly white and free from specks. Allowable tolerances-Capacity.-Plus or minus 5 per cent of specified capacity when filled to brim. Specification tests.-1. The article shall withstand, without cracking of glaze or body, heating to $225^{\circ} \mathrm{C}$. filled with high-flash eylinder oil and cooling by floating on water at $25^{\circ} \mathrm{C}$.

2. The article shall withstand, without cracking of glaze or body, being placed cold in the full flame of a Meker burner, heated for three minutes, and removed with cold platinum-tipped tongs 10 seconds after flame has been removed.

3. The article shall withstand treatment for six hours with a 10 per cent sodium-hydroxide solution on closed steam plate without appreciable dulling of surface.

4. The article shall show no evidence of pores or channels in the body after soaking overnight in an aqueous solution of methyl violet, rinsing quickly, and wiping dry.

5. Softening of the glaze shall not take place when two articles are taken, one placed inside the other so that the glazed surfaces are in contact, heated rapidly to $1,000^{\circ} \mathrm{C}$. and then at the rate of $200^{\circ} \mathrm{C}$. per hour to $1,125^{\circ} \mathrm{C}$., removed, and cooled on a fire-clay slab. After cooling the pieces shall not adhere.

\section{ANTRALING CUP}

Standard size.-

\begin{tabular}{c|c|c|c|c}
\hline Capacity & $\begin{array}{c}\text { Diameter } \\
\text { of rim }\end{array}$ & $\begin{array}{c}\text { Diameter } \\
\text { of bottom }\end{array}$ & Height \\
\hline${ }^{2} l$ & $m m$ & $m m$ & $m m_{25}$ \\
\hline
\end{tabular}

To be glazed inside and outside with exception of outside bottom surface.

Shall meet tests Nos. 1, 2, 3, 4, and 5 .

Standard sizes.-

BOATS

\begin{tabular}{|c|c|c|c|}
\hline & Length & Width & Height \\
\hline$A$ & $m_{60}$ & $\mathrm{~mm}_{10}$ & $m m$ \\
\hline B- & $\begin{array}{r}88 \\
100\end{array}$ & $\begin{array}{l}12 \\
20\end{array}$ & $\begin{array}{r}8 \\
13\end{array}$ \\
\hline
\end{tabular}

To be glazed inside and outside with exception of outside bottom surface.

Shall meet tests, Nos. 1, 2, 3, 4, and 5 . CASSEROLES

Standard sizes.-

\begin{tabular}{|c|c|c|c|c|}
\hline & Capacity & $\begin{array}{c}\text { Diameter, } \\
\text { outside }\end{array}$ & $\begin{array}{c}\text { Diameter, } \\
\text { inside }\end{array}$ & Height \\
\hline $\begin{array}{l}\mathrm{A}-1 \\
\mathrm{~B} \\
\mathrm{~B} \\
\mathrm{C} \\
\mathrm{D} \\
\mathrm{E} \\
\mathrm{F} \\
\mathrm{F}\end{array}$ & $\begin{array}{r}m l \\
75 \\
150 \\
210 \\
375 \\
500 \\
750 \\
1,250\end{array}$ & $\begin{array}{r}m m \\
70 \\
85 \\
95 \\
110 \\
133 \\
140 \\
165\end{array}$ & $\begin{array}{r}m m \\
66 \\
80 \\
90 \\
105 \\
130 \\
130 \\
160\end{array}$ & $\begin{array}{l}m m \\
35 \\
45 \\
50 \\
62 \\
68 \\
88 \\
95\end{array}$ \\
\hline
\end{tabular}

To be glazed inside and out with exception of rim and end of handle, with lip and flat porcelain handle.

Shall meet tests Nos. 1, 2, 3, 4, and 5 .

\section{COMBUSTION CAPSULE}

Standard size.-

\begin{tabular}{|c|c|c|c|c|}
\hline & Capacity & $\begin{array}{l}\text { Diam- } \\
\text { eter at } \\
\text { top }\end{array}$ & $\begin{array}{l}\text { Diam- } \\
\text { eter at } \\
\text { bottom }\end{array}$ & Height \\
\hline A & ${ }^{m l}{ }_{25}$ & $m m_{42}$ & $m m_{20}$ & ${ }_{25}^{m m}$ \\
\hline
\end{tabular}

To be glazed entirely.

Shall meet tests Nos. 1, 2, 3, 4, and 5 .

\section{CRUCIBLES, TYPE FOR TALI FORM}

Standard sizes.-

\begin{tabular}{r|r|r|r|r}
\hline & Capacity & $\begin{array}{r}\text { Diam- } \\
\text { eter at } \\
\text { rim }\end{array}$ & $\begin{array}{r}\text { Diam- } \\
\text { eter at } \\
\text { bottom }\end{array}$ & Height \\
\hline & $m l$ & $m m$ & $m m$ & $m m$ \\
\hline B. & 15 & 35 & 17 & 27 \\
\hline
\end{tabular}

Glazed inside and outside with exception of outside bottom surface.

Shall meet tests Nos. 1, 2, 3, 4, and 5 .

CRUCIBLE COVERS, TYPE FOR HIGH-FORM CRUCIBLES

Standard sizes.-

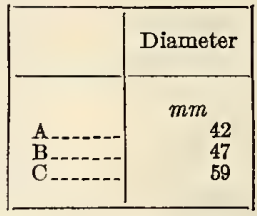

To be glazed entirely except rim.

Shall meet tests Nos. 1, 2, 3, 4, and 5 .

CRUCIBLES, TYPE FOR LOW FORM

Standard sizes.-

\begin{tabular}{r|r|r|r|r}
\hline & Capacity & $\begin{array}{r}\text { Diameter, } \\
\text { rim }\end{array}$ & $\begin{array}{l}\text { Diameter, } \\
\text { bottom }\end{array}$ & Height \\
\hline & $m l$ & $m m$ & $m m$ & $m m$ \\
\hline B & 12 & 37 & 15 & 21 \\
\hline & 30 & 46 & 18 & 29 \\
23 & 44 \\
\hline
\end{tabular}

To be glazed inside and outside with exception of outside bottom surface.

Shall meet tests Nos. 1, 2, 3, 4, and 5 .

CRUCIBLE_COVERS, TYPE FOR LOW-FORM CRUCIBLES

Standard sizes.-

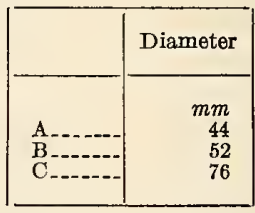

To be glazed entirely inside and outside, except rim.

Shall meet tests Nos. 1, 2, 3, 4, and 5 . 
DISHES, DEEP FORM

\section{Standard sizes.-}

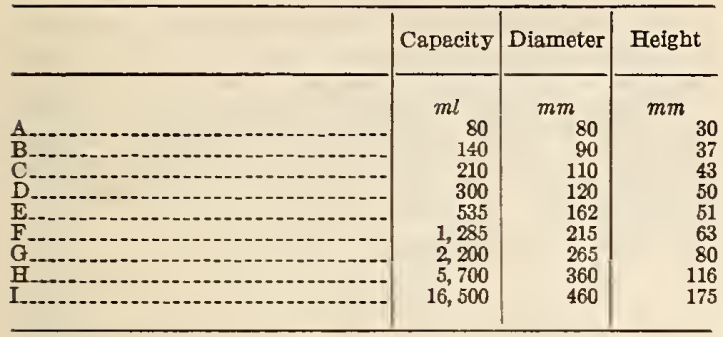

To be glazed outside and inside up to and including dish of 300 cc capacity. Larger sizes glazed inside and for a short distance below the rim on the outside, remaining outside surface being unglazed.

Shall meet tests Nos. 1, 2, 3, 4, and 5 .

\section{FUNNELS, BÜCHNER}

\section{Standard sizes.-}

\begin{tabular}{|c|c|c|c|c|c|c|}
\hline & \multirow{2}{*}{$\begin{array}{c}\text { Outside } \\
\text { diam- } \\
\text { eter }\end{array}$} & \multicolumn{3}{|c|}{ Diameter } & \multirow[b]{2}{*}{$\begin{array}{l}\text { Length } \\
\text { of stem }\end{array}$} & \multirow{2}{*}{$\begin{array}{l}\text { Depth } \\
\text { of rim } \\
\text { to per- } \\
\text { forated } \\
\text { plate }\end{array}$} \\
\hline & & $\begin{array}{l}\text { Inside } \\
\text { diam- } \\
\text { eter }\end{array}$ & $\begin{array}{l}\text { Perfo- } \\
\text { rated } \\
\text { area }\end{array}$ & $\begin{array}{l}\text { Diam- } \\
\text { eter } \\
\text { perfo- } \\
\text { rations }\end{array}$ & & \\
\hline 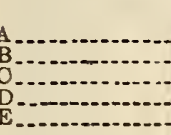 & $\begin{array}{r}m m \\
66 \\
102 \\
135 \\
163 \\
200\end{array}$ & $\begin{array}{r}m m \\
60 \\
95 \\
130 \\
155 \\
190\end{array}$ & $\begin{array}{r}m m \\
45 \\
80 \\
115 \\
140 \\
175\end{array}$ & $\begin{array}{r}m m \\
1 \\
1 \\
1 \\
1 \\
1\end{array}$ & $\begin{array}{r}m m \\
50 \\
80 \\
100 \\
110 \\
130\end{array}$ & $\begin{array}{l}m m \\
27 \\
46 \\
61 \\
66 \\
75\end{array}$ \\
\hline
\end{tabular}

To be glazed inside and outside. There shall be not more than $5 \mathrm{~mm}$ between the centers of the perforations, and the perforated plate shall be flat.

Shall meet tests Nos. 3 and 4 .

\section{GOOCH CROCIBLES, LOW FORM}

Standard size.-

\begin{tabular}{l|c|c|c|c}
\hline & $\begin{array}{c}\text { Diameter } \\
\text { at top }\end{array}$ & $\begin{array}{c}\text { Diameter } \\
\text { at bottom }\end{array}$ & Height & $\begin{array}{c}\text { Diameter } \\
\text { perfora- } \\
\text { tions }\end{array}$ \\
\hline & $m m$ & $m m_{35}$ & $m m_{24}$ & $m m$ \\
\hline
\end{tabular}

To be glazed inside and outside excepting outside bottom surface. There shall be not more than $5 \mathrm{~mm}$ between the centers of perforations.

Shall meet tests Nos. 2, 3, and 5 .

\section{GOOCH CROCIBLES, TELL FORM}

Standard sizes.-

\begin{tabular}{r|r|r|r|r|r|}
\hline Capacity & $\begin{array}{c}\text { Diameter } \\
\text { at top }\end{array}$ & $\begin{array}{r}\text { Diameter } \\
\text { at bottom }\end{array}$ & Height & $\begin{array}{r}\text { Diameter } \\
\text { perfora- } \\
\text { tions }\end{array}$ \\
\hline$m l$ & 10 & $m m$ & $m m$ & $m m$ & $m m$ \\
\hline & 25 & 35 & 22 & 30 & 1 \\
\hline \\
\hline
\end{tabular}

To be glazed inside and outside except outside bottom surface.

Shall meet tests Nos. 2, 3, and 5.

\section{GOOCH CRUCIBLE COVERS-TYPE FOR TALL FORM}

Standard sizes.-

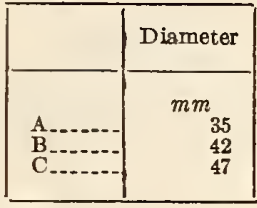

To be glazed entirely.

Shall meet tests Nos. 2, 3, and 5.

\section{PERFORATED PLATES FOR FUNNEL USE}

Standard sizes.-

\begin{tabular}{l|r|r|r}
\hline & Diameter & $\begin{array}{c}\text { Thick- } \\
\text { ness }\end{array}$ & $\begin{array}{c}\text { Diameter } \\
\text { perfora- } \\
\text { tions }\end{array}$ \\
\hline & $m m$ & $\begin{array}{r}m m \\
\text { A }\end{array}$ & $m m$ \\
\hline & 38 & 4.5 & 1 \\
4.0 & & 1 \\
\hline
\end{tabular}

Edge to be beveled to $60^{\circ}$ with top surface

To be glazed only on top surface.

Shall meet tests Nos. 3 and 4.

\section{PLATES FOR COLOR REACTIONS}

Standard size.-

\begin{tabular}{r|r|r|r|r|r}
\hline & $\begin{array}{c}\text { Dimen- } \\
\text { sions }\end{array}$ & $\begin{array}{c}\text { Number } \\
\text { of depres- } \\
\text { sions }\end{array}$ & $\begin{array}{c}\text { Thick- } \\
\text { ness }\end{array}$ & $\begin{array}{c}\text { Diameter } \\
\text { of depres- } \\
\text { sions }\end{array}$ & $\begin{array}{c}\text { Depth of } \\
\text { depres- } \\
\text { sion }\end{array}$ \\
\hline $\begin{array}{c}m m \\
110 \text { by } 90\end{array}$ & 12 & $m m$ & $m m$ & $m m$ \\
\hline
\end{tabular}

To be glazed entirely with exception of bottom surface. Surface with depressions to be uniformly white and free from colored specks.

Shall meet test No. 4.

PORCELAIN SPATULA, TYPE FOR LONG SPATULA AT ONE END, KNOB AT OTHER

Standard sizes.-

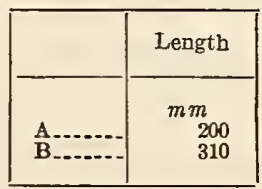

To be glazed entirely.

Shall meet test No. 4.

\subsection{Porcelain for Electrical Purposes.}

American Electric Railway Association, standard design of cap and cone insulators, serial No. D7-13, 1913.

(Standard dimensions for this product are given under this specification, without reference to material.)

American Electric Railway Association, specification for overhead line material for 750-volt d. c., direct suspension, serial No. D102-21, 1921 . 
(These specifications cover iron and steel fittings, wood cross arms, bronze castings, 7-strand steel cable, switch boxes, tree and cable guards, wood insulator pins and brackets, wood break-strain insulators, and the following specifications for porcelain strain and feeder insulators:)

\section{PORCELAIN}

\section{GENERAL}

Porcelain or its equivalent shall be used for angles and other points of unusual strain in feeder work and may be used for strain and feeder insulators.

\section{CHARACTER}

Porcelain for insulators shall be high grade, fine grained and dense, true to shape, free from bubbles and other imperfections, and smooth. An approximately cubical specimen weighing not

\section{FEEDER INSULATORS-SMALL}

(See fig. 147)

Feeder insulators for cables of diameter less than 1 inch shall be approximately 4 inches diameter at base by 4 inches high, top and side grooves swept out on radius of $5 / 8$ inch, and having a depth of at least $1 / 2$ inch, and jaws at top having thickness at point $1 / 2$ inch above bottom of groove of not less than $5 / 8$ inch. The thread shall be 2 inches long, starting from point $3 / 4$ inch above bottom of insulator.

\section{FEEDER INSULATORS-LARGE}

(Seg fig. 147)

Feeder insulators for cables of diameter of 1 inch or more shall be approximately 5 inches rliameter at base by 5 inches high, top and side grooves swept out on radius of 1 inch and having a depth

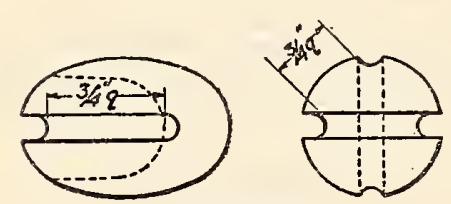

Type 1

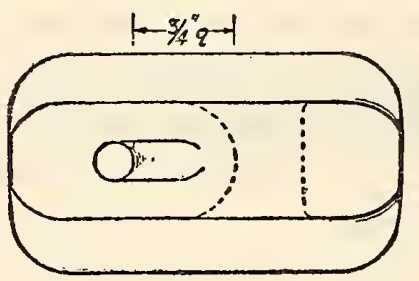

Type 4

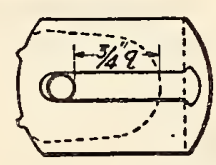

Type 2

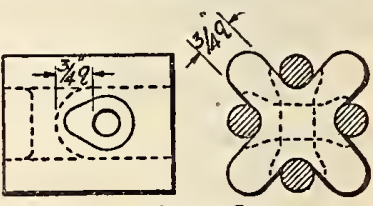

Type 3

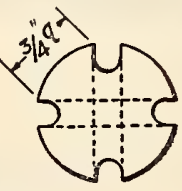

2

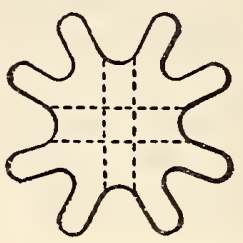

\begin{tabular}{|l|l|c|}
\hline \multicolumn{3}{|c|}{ PERMISSIBLE VARIATION } \\
\hline & OVER & UNDER \\
\hline$q$ & NOLIMIT & $0^{\circ}$ \\
\hline
\end{tabular}

TYPICAL- PORCELAIN STRAIN INSULATORS

Figdre 146

less than 1 ounce, freed from all glaze, after thorough drying, immersion for 48 hours under 12 inches of water and the removal, by wiping off, of any surface water, shall not show an increase in weight of over one-tenth of 1 per cent.

The glaze shall be smooth, firmly adherent, thoroughly vitrified, free from bubbles, cracks, crazing, or undissolved particles.

\section{STRAIN INSEIATORS}

(See fig. 146)

Strain insulators of porcelain shall be of type in which the connections will securely interlock in case of failure of the porcelain. If of egg or similar type the passages for the strands shall be of full size, smooth, of easy curves so that the strands shall have large area of contact without abrupt bends and of depth sufficient to insure that entire strand is below the surface of the insulator body throughout the space in which the two parts of strand overlap, with at least three-fourths inch porcelain between the two strands at the closest point.

\section{FEEDER IISULATORS-GENERAL}

Feeder insulators shall fit the standard pin with diameter of 1 inch at top. of at least $1 / 2$ inch, and jaws at top having thickness at point $1 / 2$ inch above bottom of groove of not less than $5 / 8$ inch. The thread shall be 2 inches long, starting from a point 1 inch above bottom of insulator.

American Railway Association, signal section, specification 14428, July, 1928, porcelain insulation.

\section{PURPOSE}

(a) The purpose of this specification is to provide dry process porcelain insulation for terminal blocks and for mounting lightning arresters, switches, fuses, and similar apparatus used in signal work, where apparatus is installed in housings and voltages do not exceed 660 .

\section{DRAWINGS}

Contractor shall furnish with his tender, drawings forming an essential part thereof.

\section{TENDER}

(a) The tender shall bc for apparatus meeting the requirements of this specification. If the contractor wishes to vary from the specification, a tender may be submitted covering the apparatus 
he desires to furnish. This tender shall be accompanied by full information showing wherein the requirements of the specification are varied from.

\section{MATERIAL AND WORKMANSHIP}

(a) The material shall be of the best commercial grade so as to provide a homogeneous body or mass, which shall be clean white color, hard, tough, dense, and uniform throughout.

(b) Parts shall be true to shape and free from sharp edges, projections, checks, flaws, cracks, chips, holes, laminations, metallic substances, or other irregularities or defects due to improper or impcrfect mechanical treatment, faulty drying or firing, inconsistent with the best commercial manufacture.

(c) Surfaces of parts shall be glazed where specified on drawings. The glaze shall be smooth, bright, firmly adherent, thoroughly vitrified and

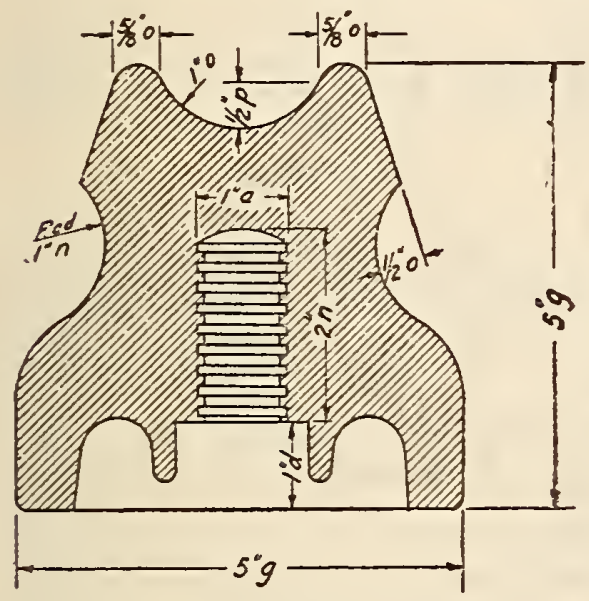

LARGE FEEDER INSULATOR (d) If purchaser is to make inspection at point of production it shall be so stated.

\section{TESTS}

(a) Tests may be made at point of production, or on samples submitted, and may also be made at destination.

(b) Contractor shall give the purchaser sufficient notice of time when material will be ready for testing.

(c) Contractor shall provide, at point of production, apparatus and labor for making the required tests under supervision of the purchaser.

(d) If tests are to be made at point of production it shall be so stated. Purchaser will distinctly indicate which of the tests herein specified are to be made and what portion of the material shall be tested.

(e) Absorption.-1. Samples of finished parts, broken for the test into pieces of approximately 1 cubic inch in volume and with at least 50 per cent

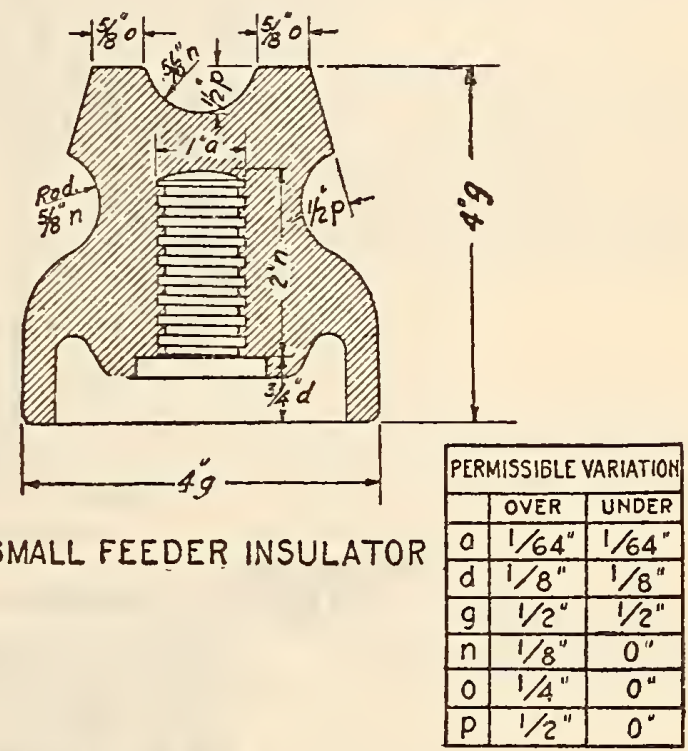

FIGURE 147.-Feeder insulators

free from bubbles, cracks, checks, crazing, or undissolved particles, and shall be continuous over the specified portion of the surface.

(d) In the compounding of the glaze, lead oxide or lead salts, injurious to the glaze, shall not be used and the mixture shall have as nearly as possible the same coefficient of expansion as the porcelain.

(e) The finished parts shall conform to dimensions and tolerances shown on drawings.

(f) A minimum tolerance will be allowed of 1.5 per cent plus or minus.

\section{INSPECTION}

(a) Purchaser may inspect the material at all stages of manufacture.

(b) Purchaser may inspect the completed product to determine that the requirements of this specification have been met.

(c) If the material has not been accepted at point of production and if, upon arrival at destination, it does not meet the requirements of this specification, it may be rejected, and the contractor, upon request, shall advise the purchaser what disposition is to be made of the defective material. The contractor shall pay all freight charges, of surface unglazed, shall be dried at a temperature of at least $100^{\circ} \mathrm{C}$. to a constant weight.

2. Samples shall then be boiled in pure water for at least 2 hours and allowed to cool in this water to room temperature.

3 . Upon being removed from the water the surface shall be wiped dry and samples weighed.

4. Absorption shall not exceed 0.5 per cent of the constant dry weight.

\section{PACKING}

(a) Material shall be so prepared as to permit convenient handling and to protect against loss or damage during shipment.

\section{MARKING}

(a) Purchaser's order, requisition, and package number, name of consignor, and name and address of consignee shall be plainly marked on outside of package.

(b) Detail list of loose pieces, containers, and their contents shall be furnished for each shipment. Where carload shipments are made, routing and car identification shall be shown. 
(c) Where carload shipments are made, name and address of consignee may be omitted.

\section{WARRANTY}

(a) Contractor shall warrant the material covered by this specification to be free from defects in material and workmanship under ordinary use and service, his obligation under this warranty being limited to making, at point of production, any

\section{B. DRAWINGS}

Drawing included and referred to: Figure 148, A. R. A., T. \& T. 1285, porcelain filler button.

\section{GENERAI}

1. The quality of the material used and the methods of manufacture shall be such as to insure for the completed filler button the properties called for in this specification.

\section{TOLERANCE-}

\section{OLNE GREEN "F"}

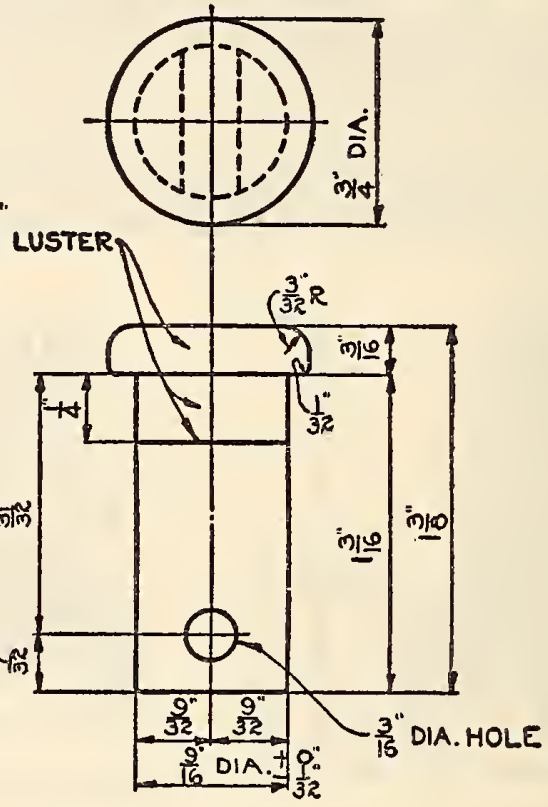

CLEAR WHITE PORCELAIN

$\pm \frac{1}{32}$ FOR ALL DIMENSIONS UNLESS OTHERWISE SPECIFIED

GLAZE SHALL BE FREE'FROM LUMPS, BURNS, CRACKS AND OTHER DEFECTS. FREE FROM WARPAGE, IRREGULARITIES CHIPS AND FLAWS.

WHEN BUTTON IS BROKEN UP AND IMMERSED FOR 48 CONSECUTIVE HOURS IN WATER AT $40^{\circ} \mathrm{TO} 100^{\circ} \mathrm{F}$ IT SHALL NOT ABSORB MORE THAN $1 \%$ OF ITS ONN WEIGHT.

\section{PORCELAIA FILLER BUTTON ARA-3-A}

Figure 148.-A. R. A., T. \& T. S., drawing No. 1285

part or parts to replace those which shall be found defective after shipment to the purchaser. This warranty shall not apply to any apparatus which has been subjected to misuse, negligence, or accident.

American Railway Association, telegraph and telephone section, porcelain filler button, ARA-3-A, No. 2-G-14, 1924 .

\section{A. PURPOSE}

The purpose of this specification is to guide the seller/contractor in the manufacture of porcelain filler buttons to be used in connection with porcelain jack panel (given below).
2. The specification and drawing are intended to cooperate with and supplement each other so that any detail indicated in one and not in the other shall be executed the same as if indicated in both, unless exceptions are specifically mentioned within the body of this specification.

3. The seller/contractor must make sure that all material and work is in accordance with the specification before the filler buttons are offered.

\section{MATERIAL AND WORKMANSHIP}

1. All material and workmanship shall be of the best commercial grade and all parts shall be made 
in accordance with the dimensions and within the tolerances shown on the drawing.

2. The filler button shall be made of vitrified homogeneous clear white porcelain. It shall not absorb water to a greater extent than 1 per cent of its weight, when immersed for 48 consecutive hours in water at $40^{\circ}$ to $100^{\circ} \mathrm{F}$.

3. The completed porcelain filler button shall have no chips, flaws, warpage, or other irregularities inconsistent with the best commercial manufacture.

\section{E. DIMENSIONS}

All dimensions shall be within the tolerances shown on the drawing included in this specification. In all cases the cumulative effect of all variations shall be such as not to exceed the given limits for any aggregate dimensions.

\section{F. FINISH}

1. The knob and part of the shank as shown on the drawing shall be smoothly and evenly colored with a permanent olive green glazing, which shall be without luster. Sample showing the desired color and finish to be furnished upon request.

\section{G. INSPECTION}

1. The purchaser shall have the right to make such inspcction and tests as he may desire of the porcelain filler button at any stage of manufacture. Such inspection not to include the process of manufacture. The inspector of the purchaser shall have the right to reject any material or finished porcelain filler buttons which are defective in any respect. Inspection shall not however, relieve the seller/ contractor from the obligation of furnishing perfect material and sound reliable work.

2. Any imperfect material or unfaithful work that may bc discovered bcfore the final acceptance of the completed porcelain filler button shall be corrected immediately, upon the requirement of the purchaser, notwithstanding that it may have been overlooked by the purchaser. If the requirements of this specification are not fulfilled when the porcelain filler buttons are offered for final acceptance, the purchaser shall have the right to reject them.

\section{H. TESTS}

1. Tests may be made at point of production or on samples submitted and may also be made at destination.

2 . The seller/contractor shall give the purchaser sufficient notice of time when the filler buttons will be ready for testing.

3. The seller/contractor shall provide at point of production apparatus and labor for nlaking the required tests under supervision of the purchaser.

4. One porcelain filler button shall be selected at random from each lot of 50 and broken into not less than three pieces. The broken button, after having been immersed in water at a temperature between $40^{\circ}$ and $100^{\circ} \mathrm{F}$. for 48 consecutive hours, shall not have increased more than 1 per cent in weight.

\section{J. PACKING}

1. Each porcelain filler button shall be wrapped in tissue paper, and 10 buttons packed in a substantial pasteboard carton.

2. Each carton shall bear on one end an inscription in bold type reading as follows:

10 Porcelain Filler Buttons ARA-3-A

Name of seller/contractor
The seller's/contractor's name shall appear in the space provided for same.

3. The cartons containing the procelain filler buttons shall be packed in a strong suitable case to prevent loss or damage to shipment during transit.

\section{K. MARKING}

1. The quantity of porcelain filler buttons, purchaser's order and requisition number, name of consignor, and name and address of consignee shall be plainly marked on the outside of each case.

2 . Detail list of loose species, containers, and their contents shall be furnished for each shipment.

\section{WARRANTY}

1. The seller/contractor hereby expressly warrants that the material furnished under this specification shall be free from defects in design, material and workmanship. The seller's/contractor's obligation under this warranty shall not apply to any material which has been subject to misuse, negligence, or accident after acceptance.

2. The seller/contractor hereby covenants and agrees to save harmless and indemnify the purchaser against all claims, suits, actions, or proceedings, damages, costs, and fees and expenses by reason of alleged infringement of patents or for patent royalties involved in consequence of the purchase or the use of the material covered hereby.

American Railway Association, telegraph and telephone section, porcelain jack panel, ARA-4-B, No. 2-G-9, 1924.

\section{A. PURPOSE}

The purpose of this specification is to guide the seller/contractor in the manufacture of a poicelain panel to be used in mounting jacks on switchboard frame works, test panel units, or other apparatus when specified.

\section{B. DRAWINGS}

Drawings included and referred to:

Figure 149, A. R. A., T. \& T. 1193, porcelain jack panel ARA-4-B assembly; and Figure 150, A. R. A., T. \& T. 1194, porcelain jack panel ARA4-B method of testing.

\section{GENERAL}

1. The quality of the material used and the methods of manufacture shall be such as to insure for the completed panel the properties called for in this specification.

2. The specification and drawings are intended to cooperate with and supplement each other so that any detail indicated in one and not in the other shall be executed the same as if indicated in both unless exceptions are specifically indicated within the body of this specification.

3 . The seller/contractor must make sure that all material and work is in accordance with the specification before the panels are offered.

\section{MATERIAL AND FINISH}

1. The panel shall be of vitrified homogeneous clear white porcelain, and shall not be warped to a greater extent than allowed in the drawing or have any irregularities, chips, or flaws.

2. The face and four sides shall be smoothly and evenly colored with a hard, fired, olive green glazing, which shall be without luster. (Sample showing the desired color and finish will be furnished upon request.) 
3. Each panel shall have its name and code num- Isch broken piece shall not absorb water to a greater ber "Jack Panel ARA-4-B" molded on the back as shown in Figure 149.

\section{E. ELECTRICAL TEST}

1. Two per cent, but not less than three jack panels selected at random from each lot shall be extent than 1 per cent of its weight when immersed for 48 consecutive hours

\section{G. INSPECTION}

1. The seller/contractor shall submit two sample jack panels for approval before proceeding with the

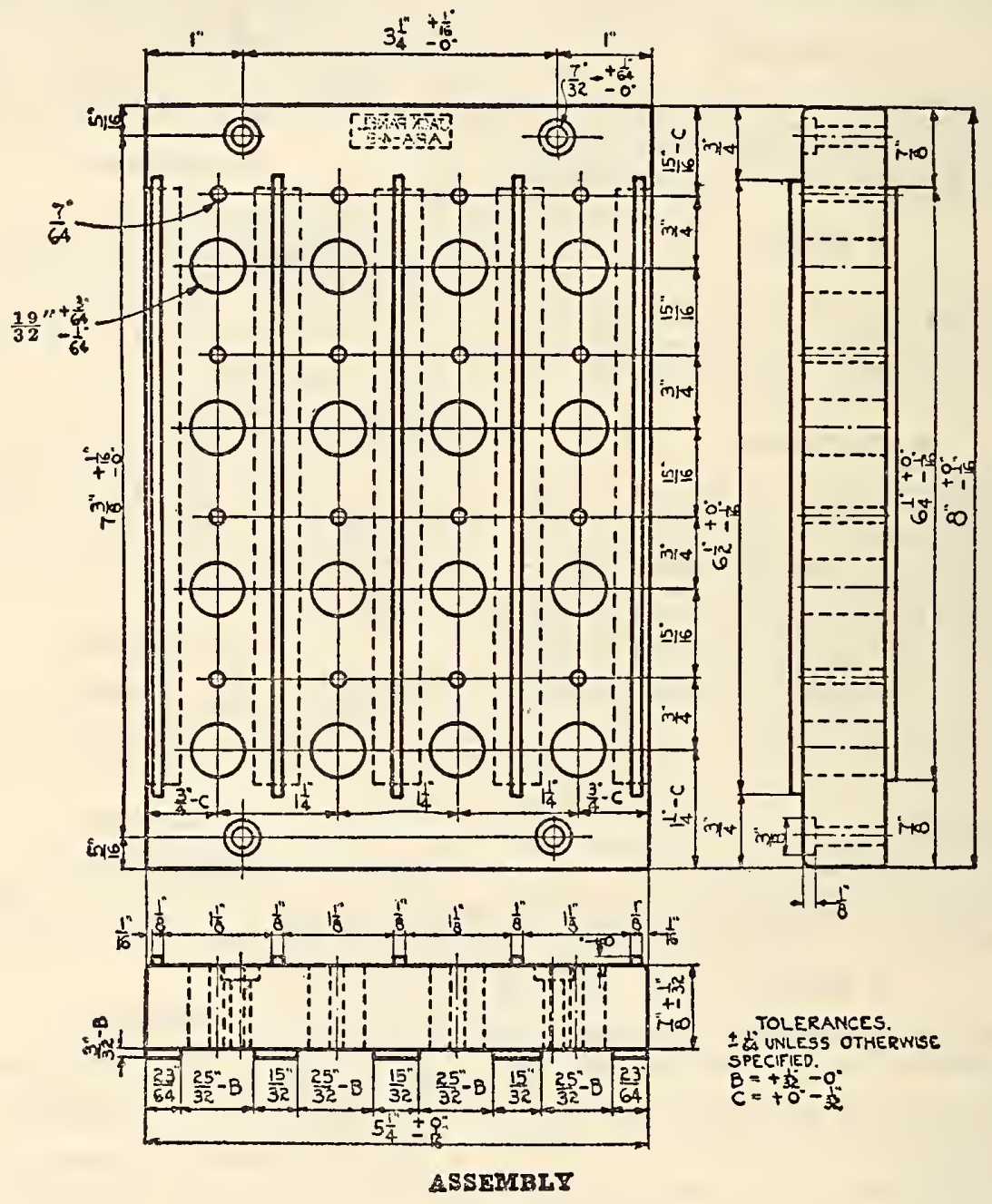

\section{PORCELAIN JACK PANEL ARA-G-B}

Figdre 149.-A. R. A., T. \& T. S., drawing No. 1193

tested for insulation resistance according to the following method:

2. Each panel shall be equipped with 16 jacks connected together in two groups of eight jacks each as shown in Figure 149, and when so tested shall have an insulation resistance of not less than 100 megohms between the two groups of jacks.

\section{F. ABSORPTION TEST}

One jack panel shall be selected at random from each lot and broken into several pieces. Any manufacture of the entire order; one of these samples if approved shall be returned to the seller/rontractor and may be retained either as a permanent record or applied on the order as desired. The other will be retained by the purchaser.

2. A one hundred per cent inspection shall be made of the following features:

Finish; warps; width and length; size of $19 / 32^{-}$ inch and $7 / 64$-inch holes; distance between $7 / 32$-inch holes.

3. If any individual panel fails for any of the above features it shall be reported as not conforming. 
4. If no defects or a few slight defects not detrimental to the serviceability of the material are found, the entire lot under consideration shall be reported as conforming.

5 . If the defects are general, affecting the serviceability of the material the entire lot shall be reported as not conforming.

6. If the panels have not been inspected at the seller's/contractor's establishment and if upon arrival at destination they do not meet the require-
2. Each carton shall bear on one end an inscription in bold type reading as follows:

\section{Porcelain Jack Panel ARA-4-B \\ Name of seller/contractor}

The seller's/contractor's name shall appear in the space provided therefor.

3. The cartons containing the jack panel shall be packed in strong wooden cases in order to prevent breakage or injury to shipment during transit.

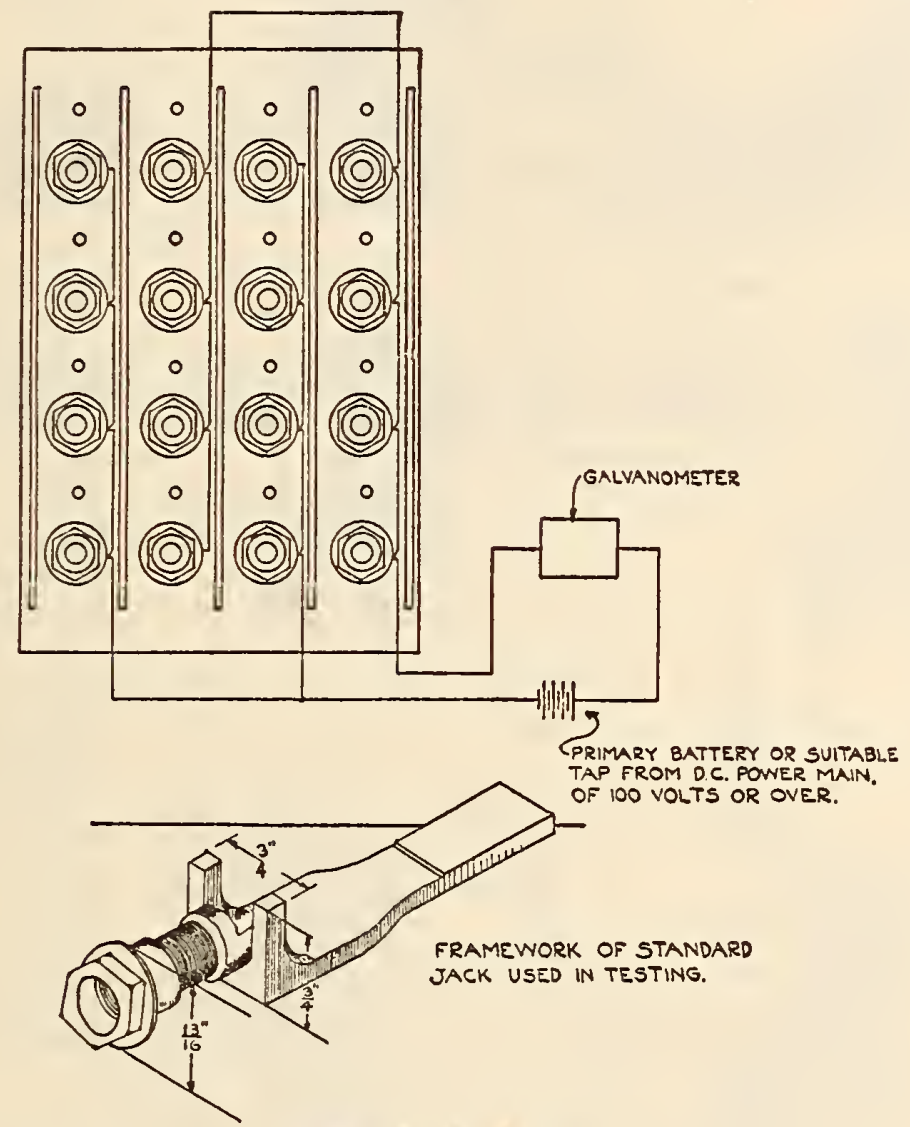

METHOD OF TESTIM

\section{PORCELAIN JACK PANEL ARA-4-B}

Figure 150.-A. R. A., T. \& T. S., drawing No. 1194

ments of this specification, they may be rejected and the seller/contractor upon request, shall advise the purchaser what disposition is to be made of the defective panels. The seller/contractor shall pay all transportation charges on such rejected material.

\section{H. PACKING}

1. Each completed jack panel shall be packed in a corrugated strawboard carton strong enough to permit individual shipment and sufficient packing material shall be included to prevent any independent motion of the jack panel in the carton and avert breaking or chipping.

\section{J. MARKING}

1. Quantity of jack panels, purchaser's order and requisition number, name of consignor, and name and address of consignee shall be plainly marked on outside of package.

2. Detail list shall be furnished for each shipment.

\section{K. WARRANTY}

Same as for procelain filler button (given above). American Society for Testing Materials, tentative methods of testing electrical porcelain, serial designation D116-29T, 1929. 
(These tests are intended to apply to porcelain which is to be used for electrical insulating purposes. Included are tests for tensile strength, compressive (crushing) strength, transverse strength, resistance to impact, dielectric strength, resistance to thermal change, and porosity.)

United States Department of Commerce, division of simplified practice, recommendation No. 73, one-piece porcelain insulators, October 1, 1927.
In accordance with the unanimous action of a general conference of representative manufacturers and users of 1-piece porcelain insulators, the United States Department of Commerce, through the Bureau of Standards, recommends that stock varieties of this product be limited to those listed in the following tables.

It is further recommended that stock varieties be specified wherever practicable.

\section{Legend}

[See also Figures 151 to 166 , inclusive]

Cook

Findlay

Franklin -

Gen. Porc

Hartford. Porc.
Cook Porcelain Insulator Corporation. Findlay Electric Porcelain Co. Findlay Electric Porcelain

Franklin Porcelain Co

Hartford Faience Co.

Knox Electric Porcelain Co.

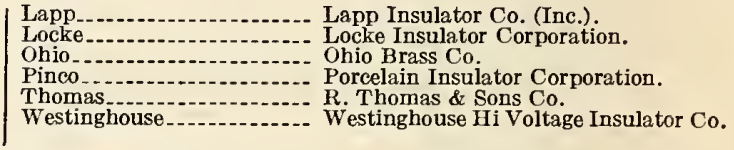

\begin{tabular}{|c|c|c|c|c|c|c|c|c|c|c|}
\hline \multirow[b]{2}{*}{ Manufacturers } & \multicolumn{2}{|c|}{ Figure 151} & \multicolumn{2}{|c|}{ Figure 152} & \multicolumn{2}{|c|}{ Figure 153} & \multicolumn{2}{|c|}{ Figure 154} & \multicolumn{2}{|c|}{ Figure 155} \\
\hline & $\begin{array}{c}\text { Stock } \\
\text { catalogue } \\
\text { No. }\end{array}$ & $\begin{array}{l}\text { Cata- } \\
\text { logue No. } \\
\text { to be dis- } \\
\text { couraged }\end{array}$ & $\begin{array}{l}\text { Stock } \\
\text { catalogue } \\
\text { No. }\end{array}$ & $\begin{array}{l}\text { Cata- } \\
\text { logue No. } \\
\text { to be dis- } \\
\text { couraged }\end{array}$ & $\begin{array}{c}\text { Stock } \\
\text { catalogue } \\
\text { No. }\end{array}$ & $\begin{array}{l}\text { Cata- } \\
\text { logue No. } \\
\text { to be dis- } \\
\text { couraged }\end{array}$ & $\begin{array}{c}\text { Stock } \\
\text { catalogue } \\
\text { No. }\end{array}$ & $\begin{array}{l}\text { Cata- } \\
\text { logue No. } \\
\text { to be dis- } \\
\text { couraged }\end{array}$ & $\begin{array}{c}\text { Stock } \\
\text { catalogue } \\
\text { No. }\end{array}$ & $\begin{array}{l}\text { Cata- } \\
\text { logue No. } \\
\text { to be dis- } \\
\text { couraged }\end{array}$ \\
\hline Cook .. & $\begin{array}{l}10 \\
15\end{array}$ & & $\begin{array}{r}20 \\
129\end{array}$ & & 155 & & 85 & & 30 & 90 \\
\hline Findlay.- & & & $\begin{array}{l}186 \\
192\end{array}$ & & 182 & 180 & 188 & & 194 & \\
\hline Franklin_ & & & & & 1000 & & 1033 & 1032 & 1011 & \\
\hline Gen. Porc & $\begin{array}{l}109 \\
112\end{array}$ & & $\begin{array}{l}129 \\
136\end{array}$ & & $\begin{array}{l}133 \\
134\end{array}$ & 155 & 2822 & $6-\mathrm{C}$ & 2904 & \\
\hline Hartford. & & & & & $A-105$ & & $A-106$ & & $A-107$ & \\
\hline Ill. Porc-- & $\begin{array}{l}109 \\
112\end{array}$ & $\begin{array}{l}107 \\
108\end{array}$ & $\begin{array}{l}222 \\
224\end{array}$ & & 235 & 237 & 253 & & 247 & \\
\hline Knox & $\begin{array}{l}\mathbf{K}-5 \\
\mathbf{K}-6\end{array}$ & & $\mathbf{K}-8$ & & $\mathbf{K}-10$ & & $\mathbf{K}-40$ & & $\mathbf{K}-30$ & \\
\hline Lapp... & 593 & & & & $591-A$ & & 588 & & 592 & \\
\hline Locke_- & & & & & 44 & -- & 8881 & 12 & 2 & \\
\hline Ohio & $\begin{array}{r}9400 \\
10565\end{array}$ & & & & 10387 & $\left\{\begin{array}{r}29207 \\
9990\end{array}\right.$ & 12847 & & 9404 & \\
\hline Pinco . - & & & $\begin{array}{l}499 \\
799\end{array}$ & & & $\begin{array}{r}325 \\
359-\mathrm{L}\end{array}$ & $401-\mathrm{L}$ & 61 & 223 & $\begin{array}{l}674-\mathrm{L} \\
623-\mathrm{L}\end{array}$ \\
\hline Thomas. & $\begin{array}{l}1011 \\
1012\end{array}$ & & 1094 & & 1111 & $\begin{array}{l}1101 \\
1159\end{array}$ & 1009 & & 1010 & 1049 \\
\hline Westinghouse & $\begin{array}{l}100 \\
110\end{array}$ & & & & 104 & & 146 & & 136 & \\
\hline & Figures 15 & 56 and 157 & Figu & re 158 & Figu & re 159 & Figur & re 160 & Figur & re 161 \\
\hline Cook & 615 & & 50 & & 55 & & 100 & 95 & & \\
\hline Findlay... & 202 & & 195 & & 196 & 200 & --- & & $\begin{array}{l}1 \\
2198 \\
2199\end{array}$ & \\
\hline Franklin_. & 1024 & 1001 & & & 1002 & $\cdots$ & $\begin{array}{l}1012 \\
1013\end{array}$ & & $\begin{array}{l}1029 \\
1031\end{array}$ & $--\cdot$ \\
\hline Gen. Porc & 145 & $3-C$ & $\begin{array}{l}1151 \\
2151\end{array}$ & & $41 / 2-C$ & $\begin{array}{l}131 \\
140\end{array}$ & $\begin{array}{l}12905 \\
22905\end{array}$ & & - & -- \\
\hline Hartford & $A-130$ & & $A-108$ & & $A-109$ & & & & $\begin{array}{l}A-110 \\
A-111\end{array}$ & $-\cdots$ \\
\hline III. Porc. & 256 & & $\begin{array}{l}257 \\
261\end{array}$ & & 361 & & 259 & & $\begin{array}{l}1366 \\
2366\end{array}$ & 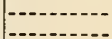 \\
\hline Knox.... & $\mathrm{K}-46$ & & $\mathbf{K}-50$ & & $\mathrm{~K}-55$ & & $\mathbf{K}-49$ & & $\mathrm{~K}-55$ & $-\cdots$ \\
\hline Lapp_. _. & -- & & 586 & & 584 & - & $\begin{array}{r}587 \\
1590\end{array}$ & 2589 & $\begin{array}{l}1582 \\
2583\end{array}$ & (---n \\
\hline Locke. & 3749 & & $\begin{array}{r}396 \\
3693\end{array}$ & & $\begin{array}{l}14451 \\
24451\end{array}$ & & $\begin{array}{r}5-\mathbf{A} \\
55\end{array}$ & & $\begin{array}{l}6510 \\
6511\end{array}$ & - \\
\hline Ohio & 9953 & & $\begin{array}{l}12848 \\
10636\end{array}$ & & 10044 & & $\begin{array}{r}9890 \\
11913\end{array}$ & & 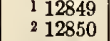 & -15237 \\
\hline Pinco_- & 264 & $\left\{\begin{array}{l}645-\mathrm{A} \\
645-\mathrm{L}\end{array}\right.$ & & & 366 & $\left\{\begin{array}{r}300 \\
622-\mathrm{L}\end{array}\right.$ & $\begin{array}{r}645 \\
645-A\end{array}$ & ---- & $\begin{array}{r}164 \\
264-A\end{array}$ & 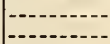 \\
\hline Thomas. & 1108 & & $\begin{array}{ll} & 1067 \\
1 & 1177 \\
2 & 1196\end{array}$ & 1164 & 1153 & $\ldots$ & 1022 & & $\begin{array}{l}1151 \\
1152\end{array}$ & - \\
\hline Westinghouse_ & 106 & & $\begin{array}{l}1166 \\
2176\end{array}$ & & 1311 & & 156 & & $\begin{array}{ll}1 & 1013 \\
2 & 1113\end{array}$ & (1) \\
\hline
\end{tabular}




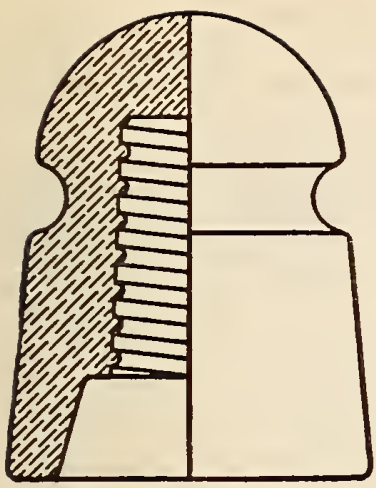

FigdRE 151

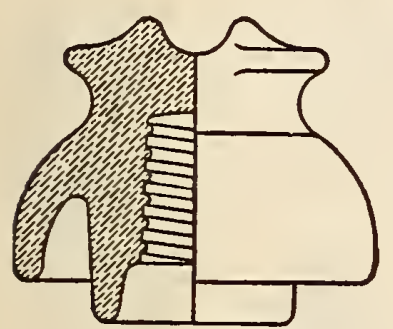

Figdre 154

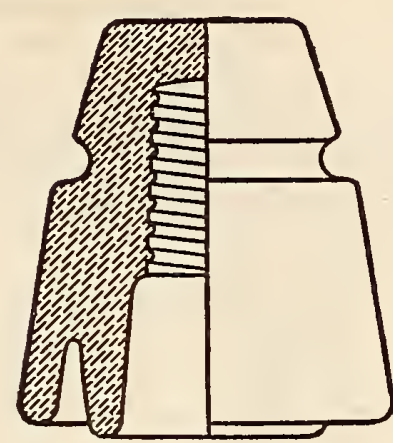

Figdre 152

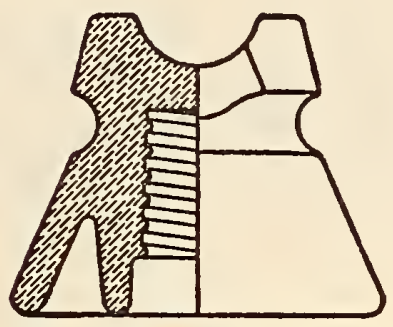

FIgURe 155

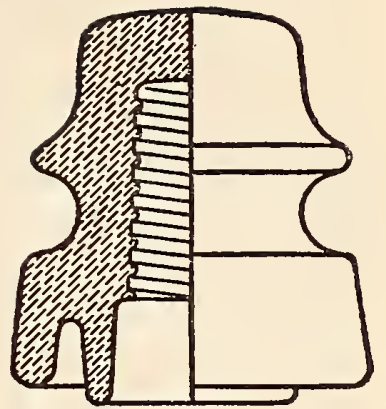

Figure 153

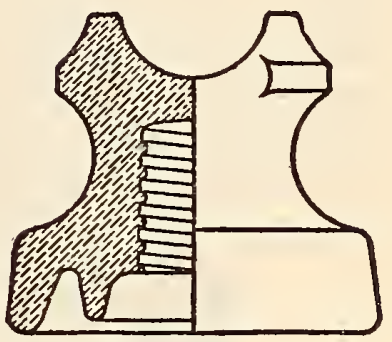

Figure 156

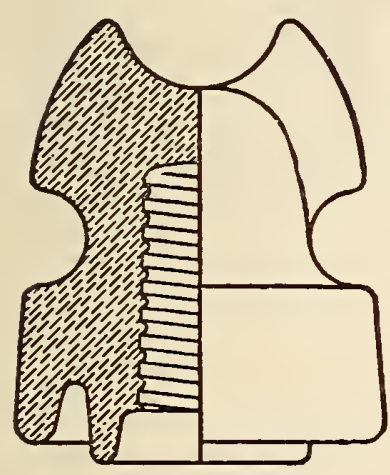

Figure 157

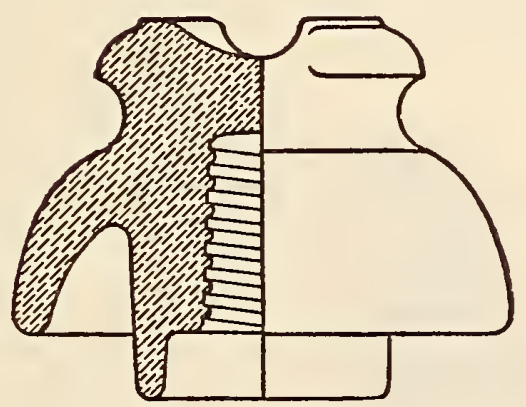

FIGURE 158

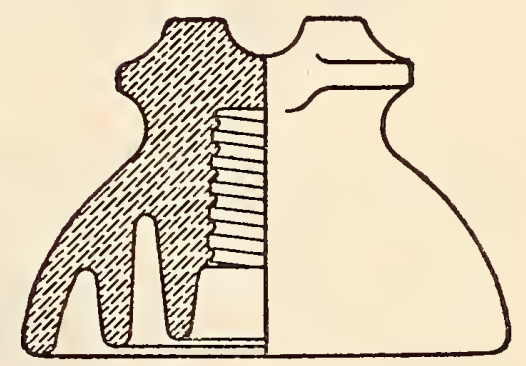

Figure 159
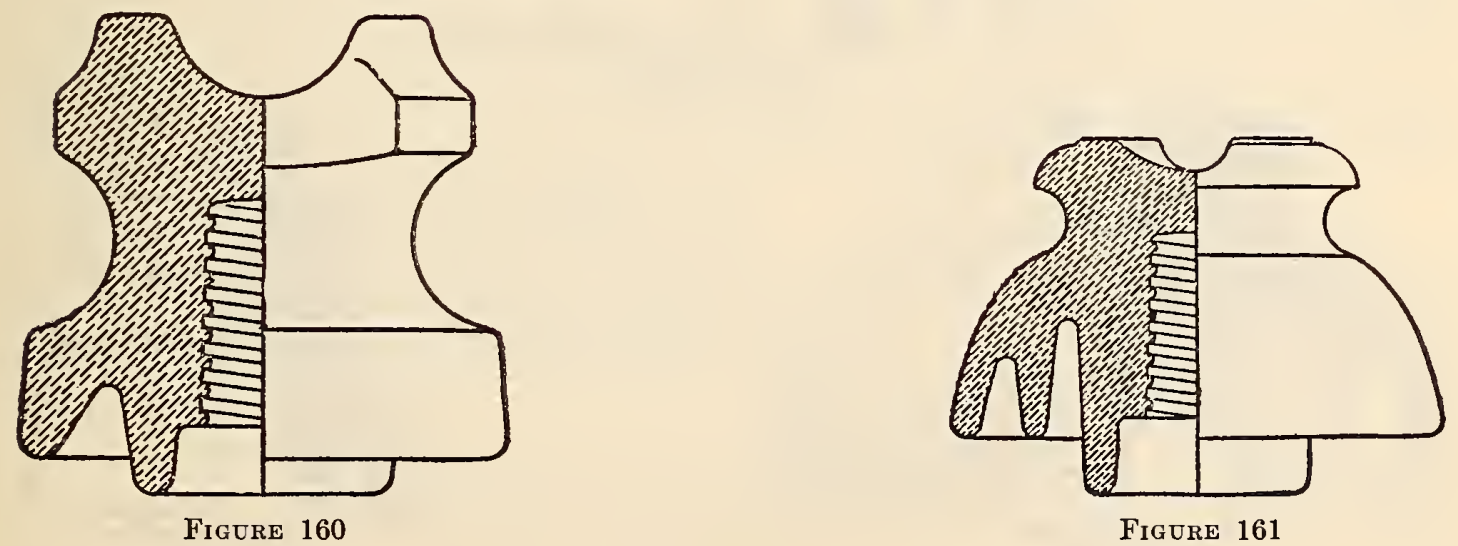
Legend

\begin{tabular}{|c|c|c|c|c|c|c|c|c|c|c|}
\hline \multirow[b]{2}{*}{ Manufacturers } & \multicolumn{2}{|c|}{ Figure 162} & \multicolumn{2}{|c|}{ Figure 163} & \multicolumn{2}{|c|}{ Figure 164} & \multicolumn{2}{|c|}{ Figure 165} & \multicolumn{2}{|c|}{ Figure 166} \\
\hline & $\begin{array}{c}\text { Stock } \\
\text { catalogue } \\
\text { No. }\end{array}$ & $\begin{array}{l}\text { Cata- } \\
\text { logue No. } \\
\text { to be dis- } \\
\text { couraged }\end{array}$ & $\begin{array}{c}\text { Stock } \\
\text { catalogue } \\
\text { No. }\end{array}$ & $\begin{array}{l}\text { Cata- } \\
\text { logue No. } \\
\text { to be dis- } \\
\text { couraged }\end{array}$ & $\begin{array}{c}\text { Stock } \\
\text { catalogue } \\
\text { No. }\end{array}$ & $\begin{array}{l}\text { Cata- } \\
\text { logue No. } \\
\text { to be dis- } \\
\text { couraged }\end{array}$ & $\begin{array}{c}\text { Stock } \\
\text { catalogue } \\
\text { No. }\end{array}$ & $\begin{array}{c}\text { Cata- } \\
\text { logue No. } \\
\text { to be dis- } \\
\text { couraged }\end{array}$ & $\begin{array}{c}\text { Stock } \\
\text { catalogue } \\
\text { No. }\end{array}$ & $\begin{array}{l}\text { Cata- } \\
\text { logue No. } \\
\text { to be dis- } \\
\text { couraged }\end{array}$ \\
\hline $\begin{array}{l}\text { Cook } \\
\text { Findlay }\end{array}$ & $\begin{array}{r}60 \\
196-A\end{array}$ & & 910 & & 65 & & 70 & & 114 & \\
\hline Franklin... & & & & & & & $\begin{array}{l}1008 \\
1123\end{array}$ & & 1014 & \\
\hline Gen. Porc & $\begin{array}{r}168 \\
12907\end{array}$ & & $\begin{cases}1 & 3045 \\
2 & 3045\end{cases}$ & & $\begin{array}{l}11-\mathrm{C} \\
21-\mathrm{C}\end{array}$ & & $\begin{array}{ll}1 & 3050 \\
2 & 3050\end{array}$ & & 118 & 3060 \\
\hline Hartford. & - & & 1 & & & & A-112 & & $A-147$ & \\
\hline 기. Porc. & $\begin{array}{l}1266 \\
22266\end{array}$ & 265 & $\begin{array}{l}1270 \\
2270\end{array}$ & & $\begin{array}{l}1370 \\
2370\end{array}$ & & $\begin{array}{l}1380 \\
2380\end{array}$ & & 212 & \\
\hline Knox _- & $\mathbf{K}-60$ & & & & $\mathrm{~K}-65$ & & $\begin{array}{ll}1 & \mathrm{~K}-70 \\
2 & \mathrm{~K}-70\end{array}$ & & & \\
\hline Lapp .... & 585 & $-\cdots$ & $-\cdots-$ & & & & 2063 & & $588-\mathrm{A}$ & \\
\hline Locke....... & 298 & 6512 & ${ }^{2} 303$ & & & & $\begin{array}{l}6508 \\
6509\end{array}$ & & 6507 & \\
\hline Obio_ & & & & & & & $\begin{array}{l}12851 \\
12852\end{array}$ & 25118 & 10747 & \\
\hline Pinco. & & & & & $\begin{array}{r}165 \\
265-A\end{array}$ & & $\begin{array}{r}1367 \\
2367-A\end{array}$ & & $420-\mathrm{L}$ & 717-L \\
\hline Thomas. & $\begin{array}{l}11188 \\
21190\end{array}$ & 1128 & $\begin{array}{l}1178 \\
1179\end{array}$ & & & & $\begin{array}{l}1157 \\
1158\end{array}$ & & 1185 & 1140 \\
\hline Westinghouse. - & $\begin{array}{l}11111 \\
21211\end{array}$ & $\begin{array}{l}11012 \\
21112\end{array}$ & $\begin{array}{l}11213 \\
21313\end{array}$ & & & & $\begin{array}{l}11014 \\
21114\end{array}$ & & 120 & \\
\hline
\end{tabular}

$213 / 8$-inch pinhole.

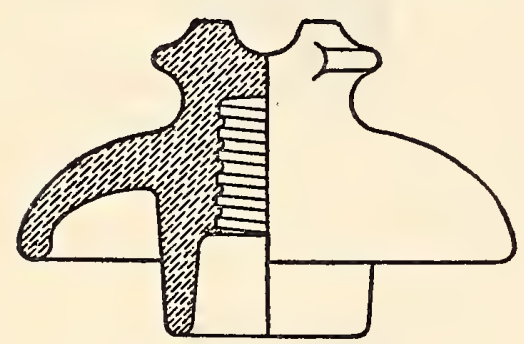

Fradre 162

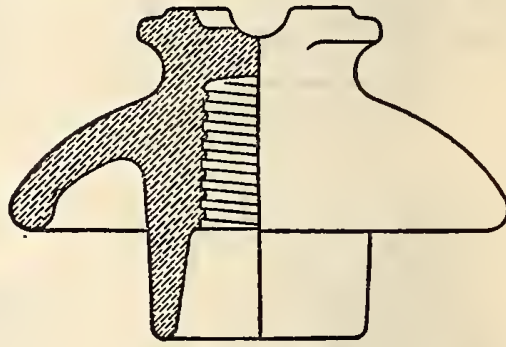

Frgdre 163

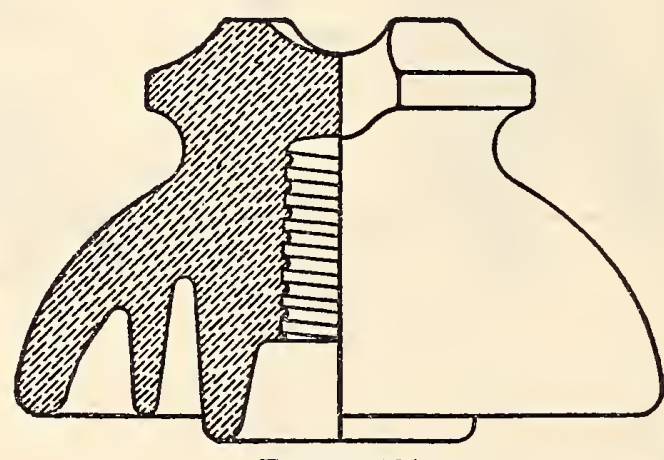

Frgure 164

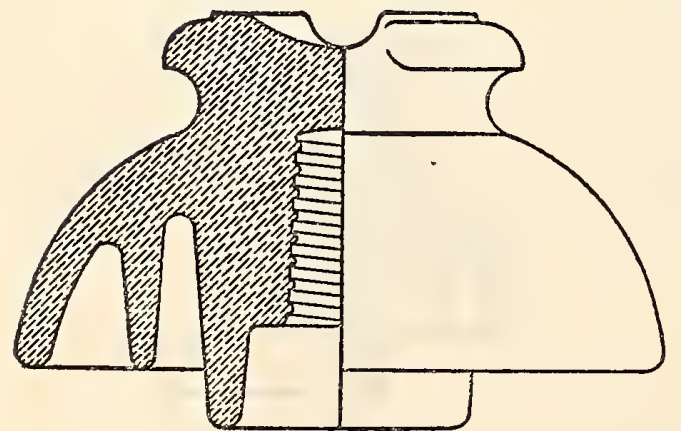

FIGURE 165

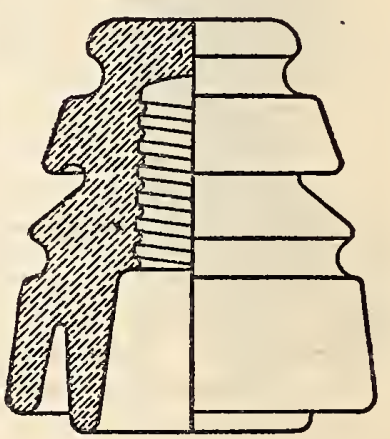

FIGURE 166 
532.23 Tubs, Sinks, and Lavatories.

American Railway Engineering Association, specifications for buildings for railway purposes.

\section{PLUMBING (1926)}

This specification includes requirements for the installation of plumbing fixtures, and in addition, typical types for guidance in use of plumbing specifications, as shown in Figures 167 to 175 , inclusive.

Federal Specifications Board, specification No. 448, United States Government master specification for plumbing fixtures, November 22, 1926. The specification is published in full as Bureau of Standards Circular No. 310, and gives general and detail requirements for 22 plumbing fixture items, including vitreous ware for use in plumbing, general requirements for which are as follows:

\section{VITREOUS WARE}

(a) Quality of vitreous ware.-Vitreous fixtures shall be best quality vitreous china, thoroughly fused and vitrified, producing a material white in color, which when fractured shall show a homogeneous mass, close grain, and free from pores. All surfaces coming in contact with walls, floors, or surfaces or other fixtures shall be ground true. Closet bowl base with extended horn is not required to be ground.

(b) Dimensions and variations.-The bowls of water-closets, urinals, lavatories, slop sinks, and other vitreous fixtures shall be not less than $1 / 2$ inch thick at any point, except flushing rims and part forming overflows, which must be of substantial thickness. The dimensions shown on the figures shall be followed where given, but a variation of not more than 5 per cent plus or minus will be allowed. A warpage of not more than $1 / 4$ inch per foot will be allowed, but in no case shall the total warpage exceed $1 / 2$ inch.

(c) Glazing. - Shall be white, thoroughly fused, and united to body without discoloration, chips, or flaws on the glazed surfaces, and shall be absolutely free from craze. All surfaces shall be glazed except those coming in contact with walls or floors, and portion of rear of aprons on lavatories set off from wall required to be left unglazed for supporting fixture in kiln.

(d) Stamp.-The manufacturer's name or trademark and Government designation shall be in ink under glaze on each fixture. The Government designation shall be placed within a shield at the point indicated and shall bc a guaranty that ware of that makc. will stand the following prescribed test. Government designation is given under description of fixture. Shield shall be as indicated in Figure 176 .

(e) Test.-A fractured piece of material taken from any part of a vitreous china plumbing fixture, after being immersed in red aniline ink of good color strength for one hour, shall not show any discoloration through the glaze and shall not show absorption when broken, to a depth greater than $1 / 8$ inch below surface of fracture at any point.

Federal Specifications Board, specification No. 448, United States Government master specification for plumbing fixtures, November 22, 1926. The specification is published in full as Bureau of Standards Circular No. 310, and gives general and detail requirements for 22 plumbing fixture items, including porcelain ware for use in plumbing, general requirements for which are as follows:

\section{PORCELAIN WARE}

(a) Quality of porcelain ware.-Porcelain fixtures shall be best quality regular selection. The ware shall be made with a glaze over a heavy vitreous coating covering the fire-clay body, producing an exterior white in color. All exposed surfaces shall be glazed. All surfaces coming in contact with walls, floors, or surfaces of other fixtures shall be ground true.

(b) Dimensions and variations.-All fixtures shall be heavy pattern, and all parts shall be of substantial thickncss. The dimensions as given shall be followed but a variation of not more than 5 per cent plus or minus will be allowed.

(c) Glazing.- All surfaces cxposed after fixtures are set shall be glazed; backs and undersides of urinals and laundry trays will not be required to be glazed. Glazing shall be throughly fused and united to body of fixture. Crow feet or cracks will not be permitted in water-flushed surfaces. Crow feet in glaze, except on water-flushed surfaces, shall not exceed $1 / 2$ inch in diameter and shall be semiglazed. Cracks in glaze, except on waterflushed surfaces, shall not exceed 1 inch in length nor $1 / 32$ inch in width. Discolored spots or bumps under the glaze shall not exceed 1 inch in diameter.

(d) Stamp.-The manufacturer's namc or trademark shall be stamped in ware on each fixture.

United States Department of Commerce, trade standards unit, Commercial Standard CS4-29, staple porcelain (all-clay) plumbing fixtures, 1929.

(Adopted at a conference of representative manufacturers, distributors, and users, and later accepted and approved by the industry for promulgation by the Department of Commerce.)

\section{GENERAI}

The nomenclature, definitions, grading rules, types, sizes, dimensions, and general practices given herein are recommended as standard by the manufacturers, distributors, and users of porcclain (all-clay) plumbing fixtures.

Types and sizes of urinals, sinks, laundry trays, lavatories, receptors, and baths not specifically mentioned are considered as special.

\section{GRADING RULES}

Noteworthy advances recently have been made in the structure, glaze, and appearance of porcelain (all-clay) plumbing fixtures as a result of continual effort to attain perfection. In common with all other industries, certain tolerances or unimportant variations occuring in the finished product are recognized, which here take the form of unavoidable minor blemishes, invisible to the untrained eye, and which result from traces of ccrtain impurities, which are sometimes segregated, in the refined clays and earths of which the ware is made. Within duly prescribed limitations such minor blemishes have no effect on the utility or value of the fixture. However, they are employed as the simplest commercial basis for grading the ware.

All of the described ware which grades below regular selection on any one blemish is classified as "culls."

\section{NOMENCLATURE AND DEFINITIONS}

Break.-A damage resembling a dunt, but resulting from accident or handling after shipment from factory.

Chipping.-Unglazed parts along ground edges extending more than $1 / 4(0.25)$ inch from edge.

Craze.-Fine cracks in the glaze.

Culls.-Serviceable ware which grades below "regular selection."

Discoloration. - A single colored spot over $1 / 4(0.25)$ inch in diameter or a sufficient number of "spots" 
to give the effect of a change in color. Faint green spots are not classified as discoloration.

Dull or eggshell finish.-Dead or flat finish. Undeveloped glaze. Slightly matted. A semiglazed finish with appearance of numerous very fine pinholes. Not glossy.

Dunt.- A hair-line fracture extending through the body caused by strains set up in the process of manufacture.

Finish--Texture and condition of surface other than color.

Fire check.-Fine shallow crack in the body, not covered with glaze. No pieces with open fire checks shall be marketed. (When sufficiently
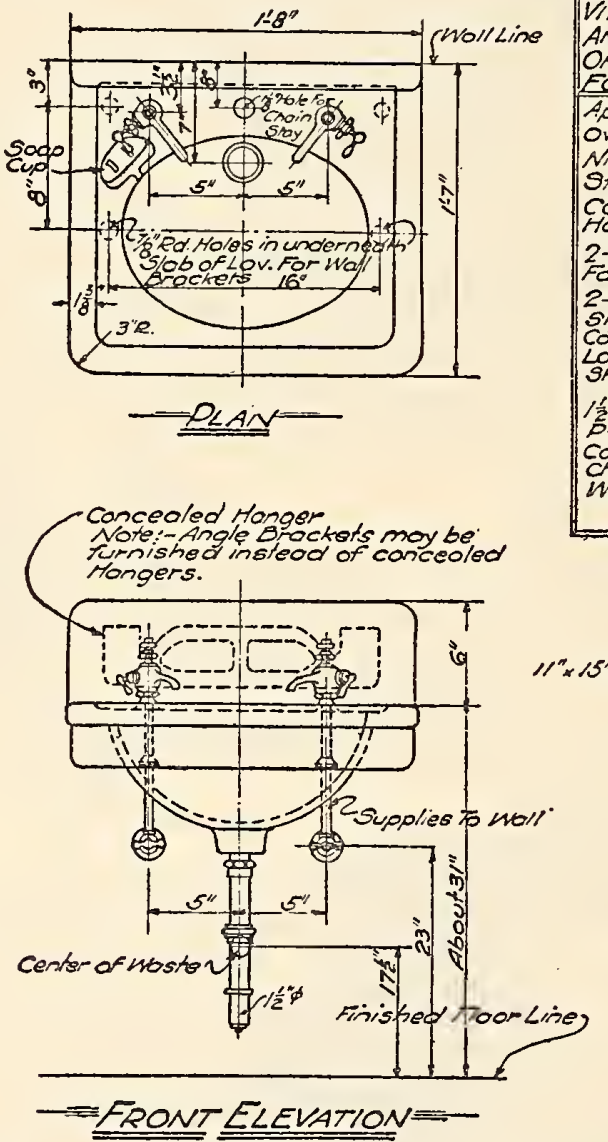

Pinhole.-Unglazed portion of body, or small hole under $1 / 16(0.063)$ inch in diameter.

Polishing mark.-A spot where some minor blemish has been ground off and surface polished and with an area not greater than that of a $3 / 8$ (0.375) inch circle.

Pottery square.-A square 2 inches on a side. For grading purposes it may be a 2-inch square hole cut in a small sheet of any flexible material, such as rubber or paper, for convenience in sliding over irregular surfaces to determine segregations.

Projection.-A raised portion of the surface $1 / 32(0.031)$ inch to $1 / 4(0.25)$ inch in maximum dimension.

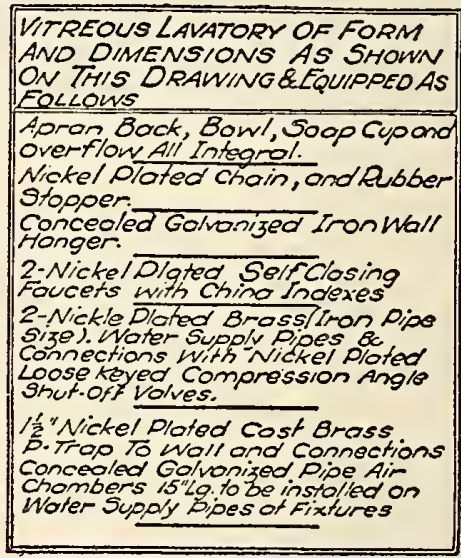

For use in Importont Stations and Office Buldings.

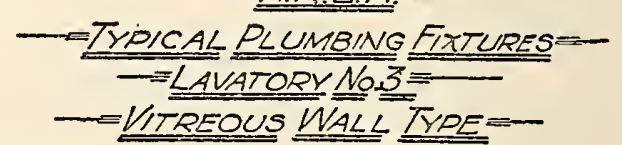

Figure 167

covered with glaze as to be easily cleaned, it is not detrimental.)

Fire check stopped.-Fire check not over 1/8 $(0.125)$ inch wide which has been filled with cement or other satisfactory repair material forming a clean and complete repair.

Green spot.-Dark centered green spots are classified as discoloration.

Kiln support marks.-Large unglazed spots resulting from blocks necessary to support the ware while firing and allowable on the back or one end of fixtures. They are regularly covered with white enamel paint.
Large projection.-A raised uncolored portion of the surface greater than $1 / 4(0.25)$ inch in maximum dimension.

Regular selection.-First-class ware in conformity with the limitations of the grading rules.

Roughing-in measurement.-Dimension from finished wall or floor to center of waste or supply opening.

Segregation.-More than 4 spots, 4 projections, or 8 pinholes in any possible "pottery square."

Spot.-Colored portion of the surface $1 / 32$ (0.031) inch and less than $1 / 8(0.125)$ inch in maximum dimension. 


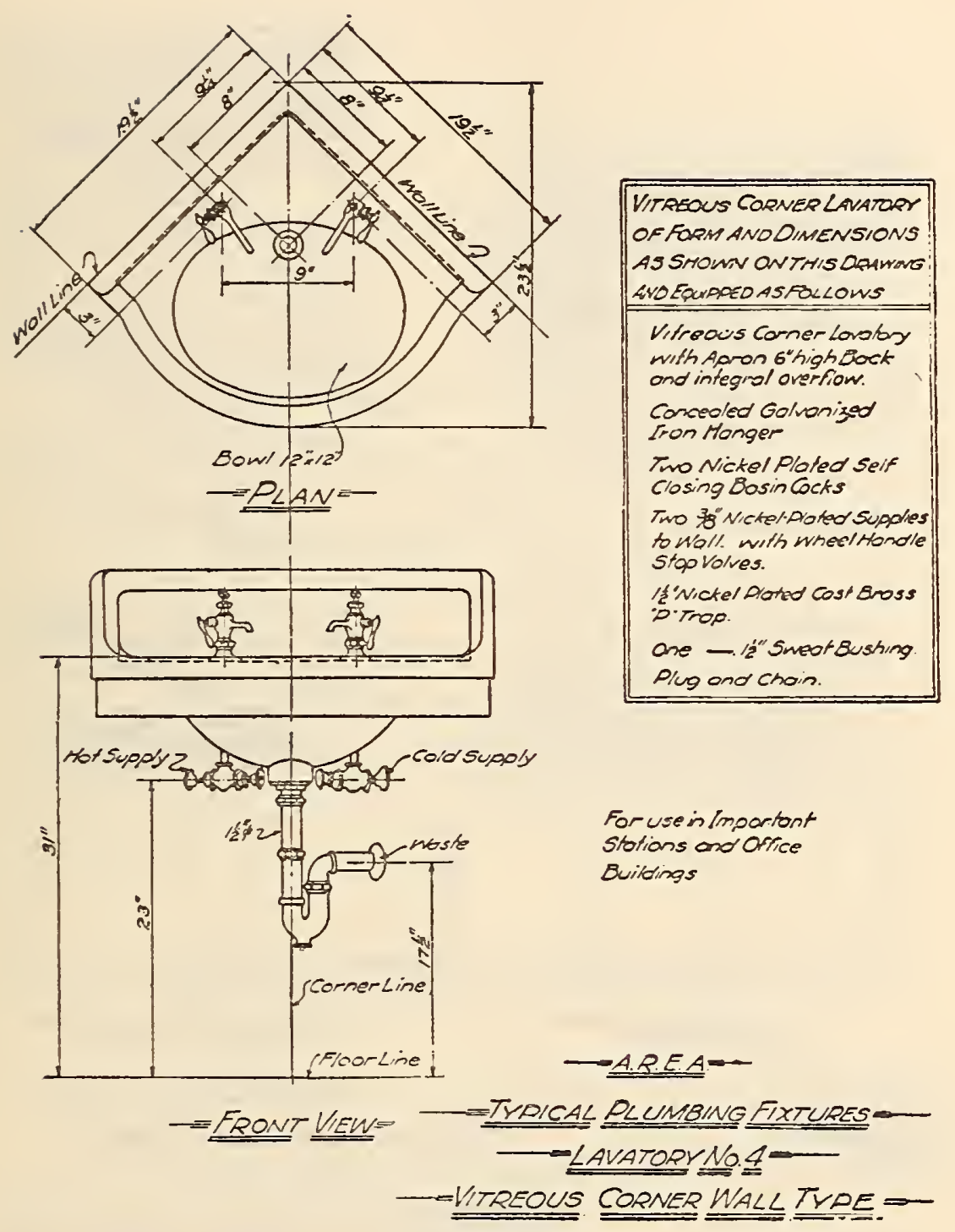

FIGURE 168 


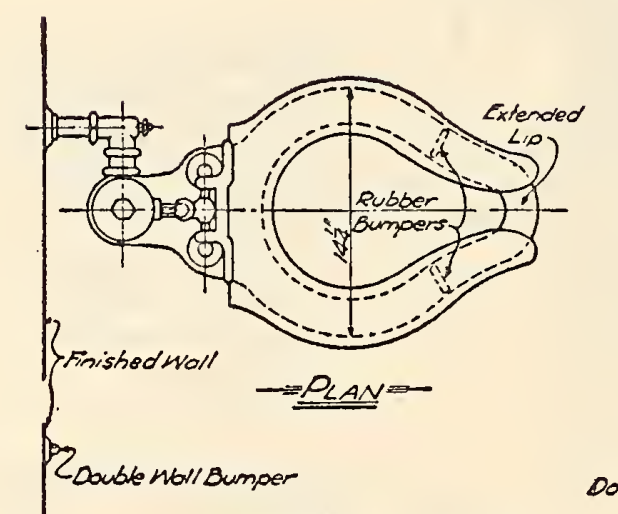

\begin{tabular}{|c|}
\hline 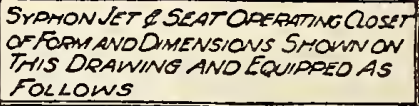 \\
\hline $\begin{array}{l}\text { Vitheow Whe Automatic Sypton Jet } \\
\text { Bowl. With Extanded Lip. }\end{array}$ \\
\hline Seot Qperating Flush \\
\hline $\begin{array}{l}\text { Whe With N.P Pigid Connections. } \\
\text { With N.P.Ange Stop And Connoctions } \\
\text { To woll }\end{array}$ \\
\hline 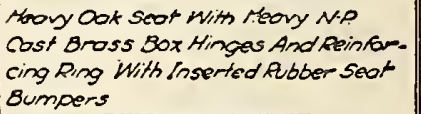 \\
\hline S-Nickel Doted Floor Bolts \\
\hline $\begin{array}{l}\text { 1- Double Wall Bumper-Seanel } \\
\text { Fostened to Wall. }\end{array}$ \\
\hline
\end{tabular}

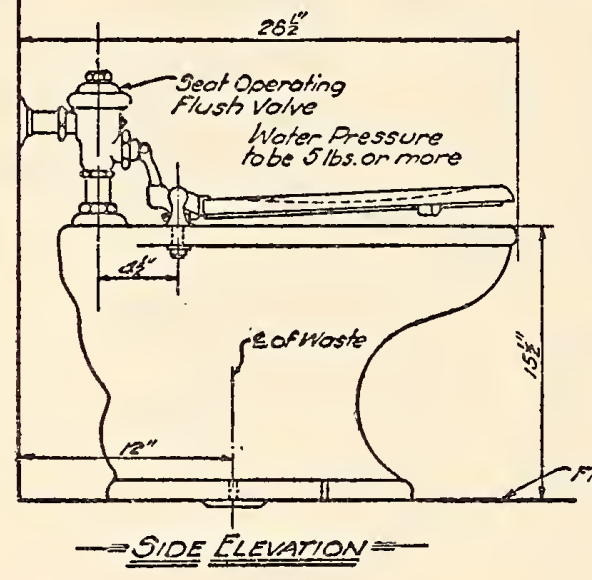

oublentiloumper

Fer use in Office

Buildrings and

importont stitions

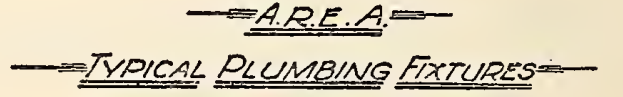

$\Longrightarrow$ CLOSET No 1

- SYPHON JET SEAT OPERATING FLUSHVAVE TYPE

Figure 169 


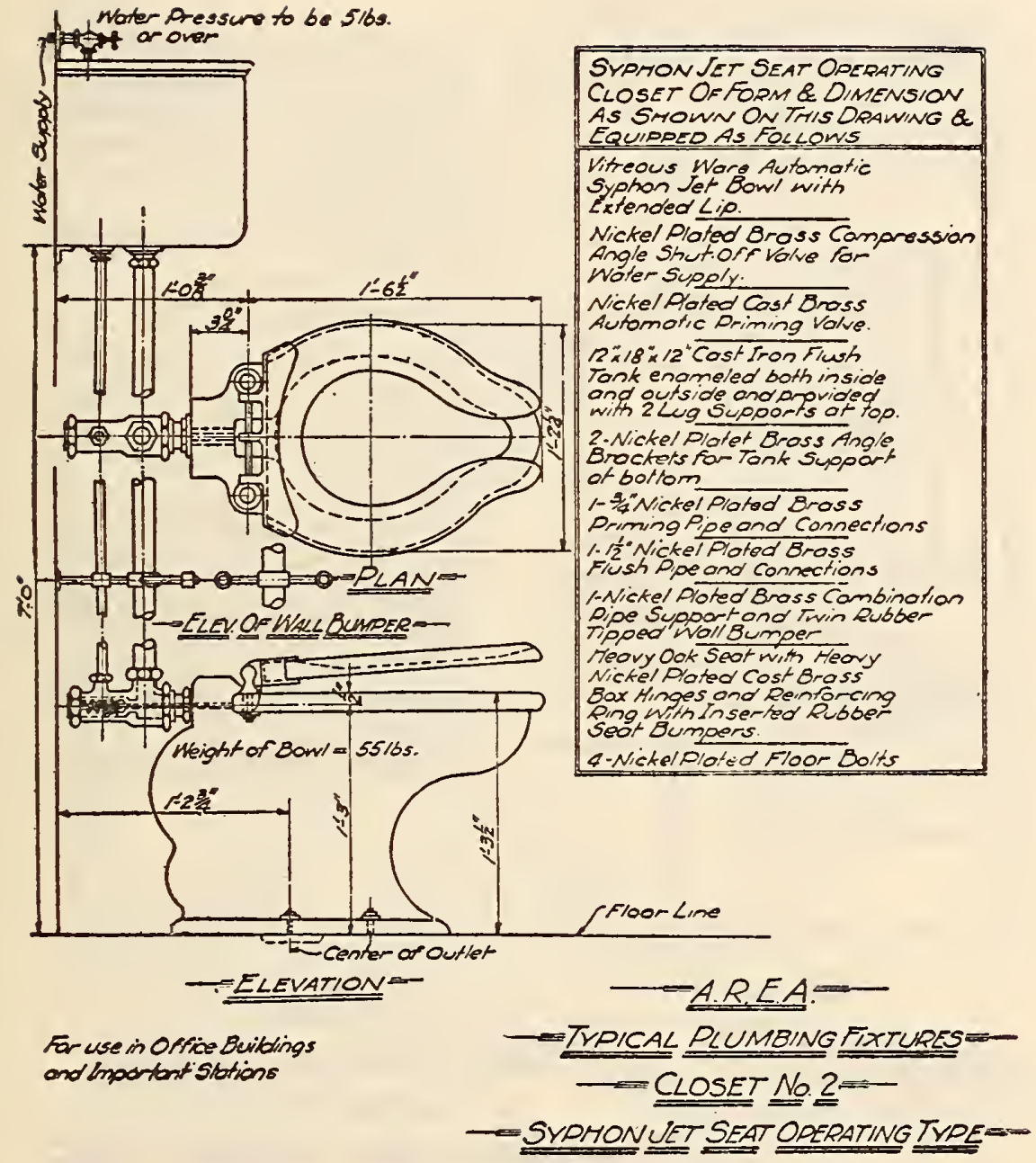

FIGURE 170 


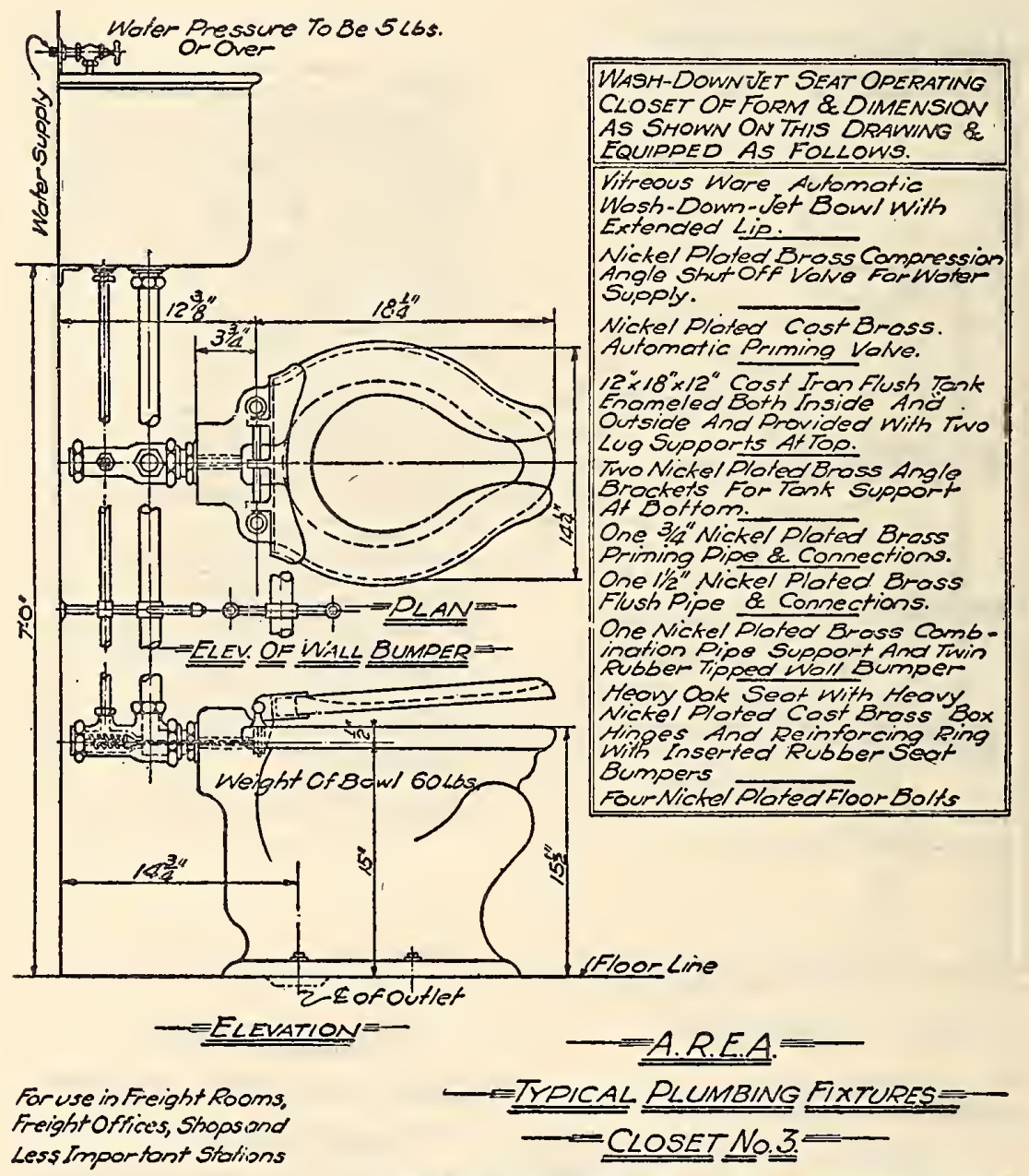

WNASH DOWN DET SEAT OPERATINO TYPES

FigdRe 171 


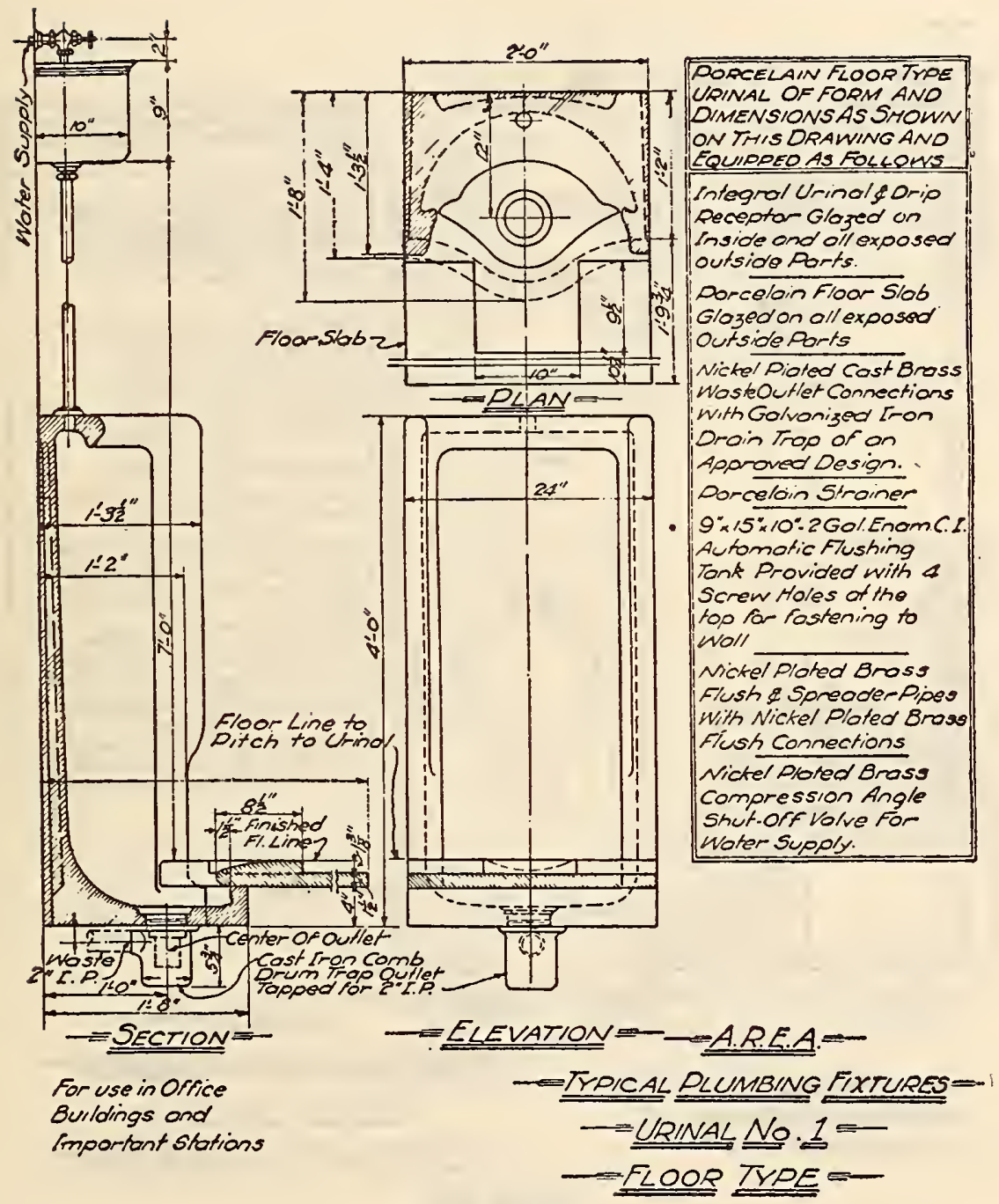

Figdre 172 


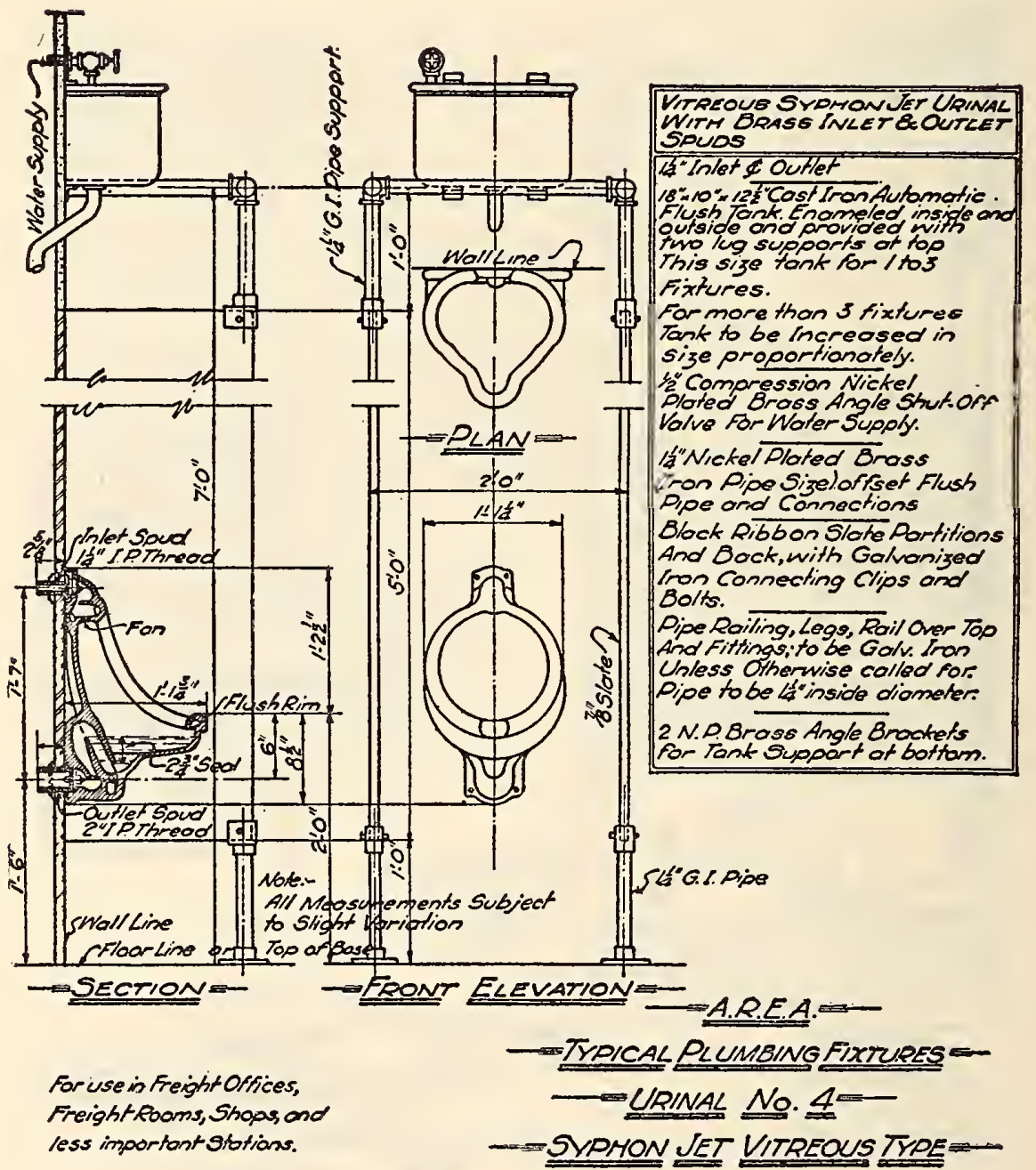

Figure 173 


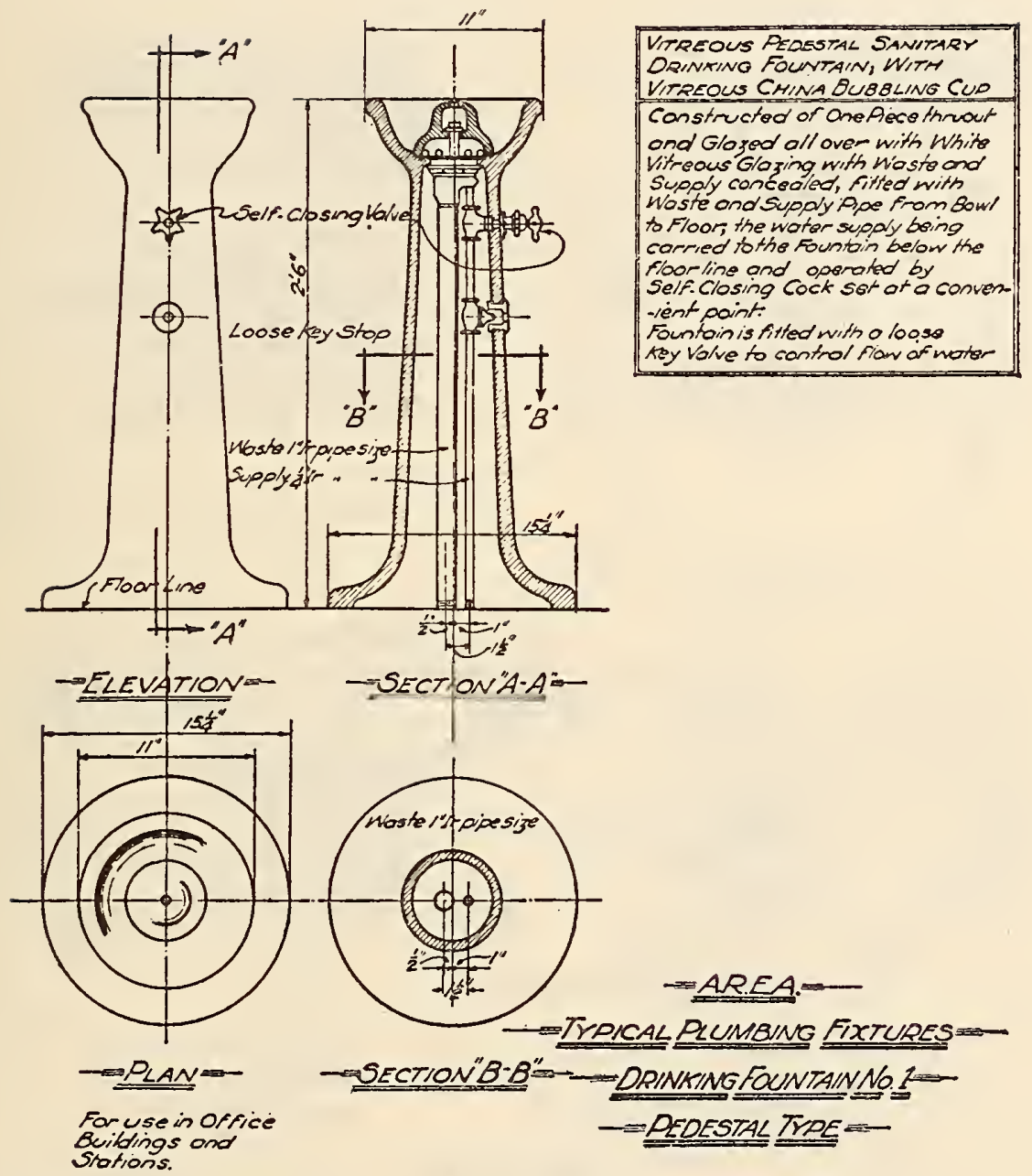

Figure 174 


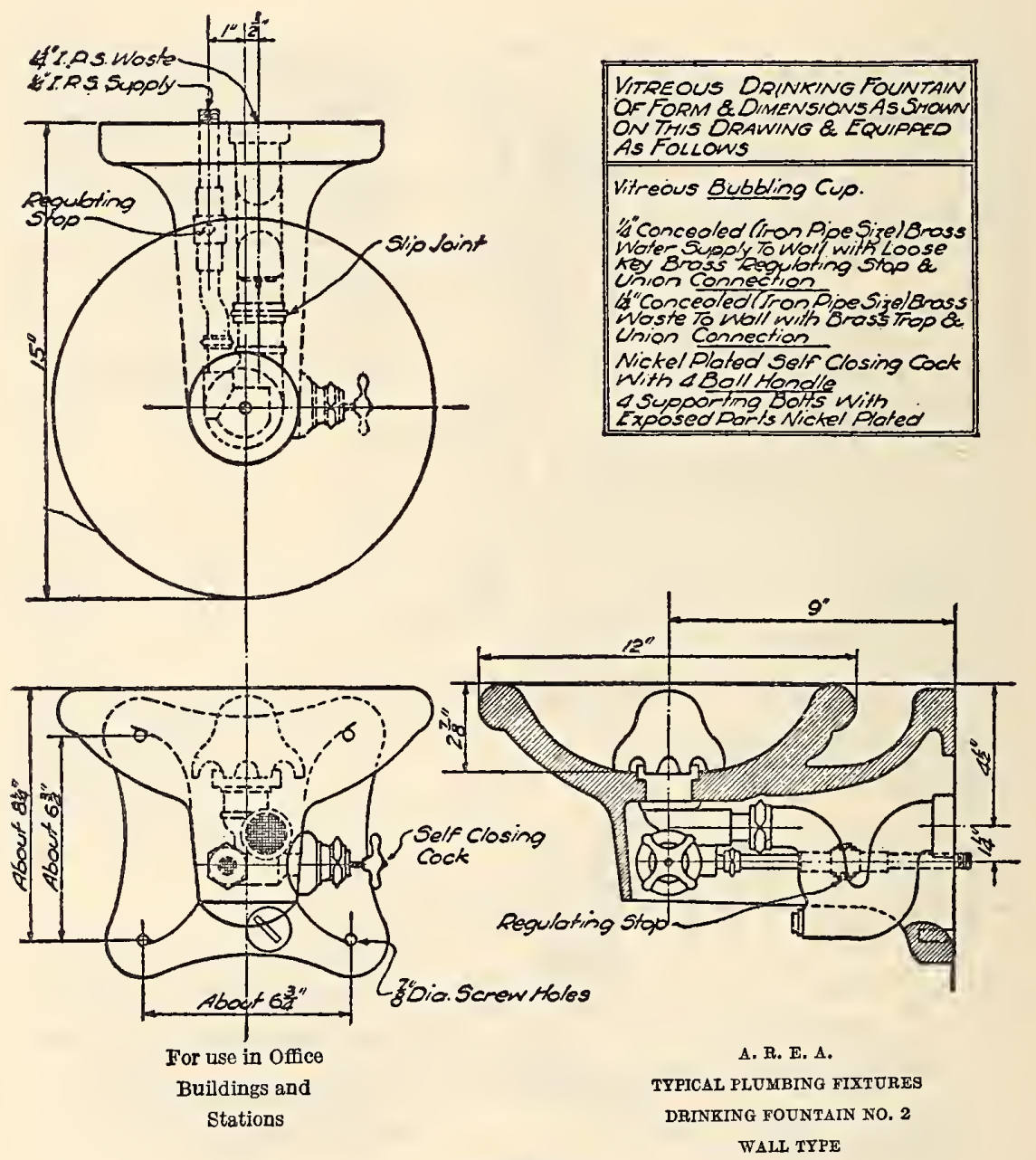

FigdRE 175 
Large spot.-A eolored portion $1 / 8(0.125)$ inch to $1 / 4(0.25)$ ineh in maximum dimension.

Streak.-A slight defect in the finish, giving an appearance similar to paintbrush marks.

Visible surface.-The surface other than wet surface, readily visible after installation of the fixture, by an observer in normal standing position.

Warpage.-The maximum deviation from a straightedge laid as nearly parallel to the edge of the piece as possible.

Wavy finish.-A defeet in the finish having the appearanee of numerous runs in the glaze; irregular or mottled.

Wet surface.-The surface which may be wet during the normal use or operation of the fixture.

\section{METHOD OF GRADING STALI URINALS}

Examine elosely the wet surface, which ineludes the top of the extended lip, for exposed body, fire eheeks, pinholes, projections, and spots. Fire eheeks behind the splash rim or lip are considered to be on unseen surfaces, exeept those near the bottom of the urinal. Examine remainder of piece for dunts or other defeets.

Sinee most stall urinals are set up in batteries spaeed only 6 inehes apart or with joints filled and covered, defeets oeeurring on the sides are not eonsidered to be as serious as on other parts of the fixtures.

Stall urinals are graded aecording to the maximum blemishes listed in Table 1.

Note.-It is not intended that the inspector shall measure or count any blemishes except in case of doubt, since with practice dimensional limits and numbers can readily be gaged by eye.

\section{TABLE 1.—Stall urinals}

\begin{tabular}{|c|c|c|}
\hline Location & Blemish or defect & Regular selection \\
\hline $\begin{array}{l}\text { Entire glazed } \\
\text { surface. }\end{array}$ & $\left\{\begin{array}{l}\text { Discolorations } \\
\text { Projections or large } \\
\text { spots. } \\
\text { Warpage.............. } \\
\text { Wavy finish. }\end{array}\right.$ & $\begin{array}{l}\text { Not more than } 3 \text {. } \\
\text { Not more than } 5 \text {. } \\
\text { Not warped more than } 1 / 2 \\
\text { inch at sides or front. } \\
\text { Not over } 10 \text { square inches. }\end{array}$ \\
\hline Wet surface. & $\left\{\begin{array}{l}\text { Spots and pinholes-- } \\
\text { Stopped fire check -- }\end{array}\right.$ & $\begin{array}{l}\text { A total of not more than } 15 \text {; } \\
\text { no segregation. } \\
\text { Not more than 1; not over } \\
1 / 2 \text { inch. }\end{array}$ \\
\hline $\begin{array}{l}\text { Visible surface } \\
\text { other than } \\
\text { sides. }\end{array}$ & $\left\{\begin{array}{l}\text { Spots and pinholes }-- \\
\text { Stopped fire check -- }\end{array}\right.$ & $\begin{array}{l}\text { A total of not more than 15; } \\
\text { no segregation. } \\
\text { Not more than 1; not over } \\
1 \text { inch long. }\end{array}$ \\
\hline Sides.... & $\left\{\begin{array}{l}\text { Other permissible } \\
\text { blemishes. }\end{array}\right.$ & $\begin{array}{l}\text { Not over } 2 \text { inches long; not } \\
\text { more than } 2 \text {. } \\
\text { Not more than twice the } \\
\text { number permissible on the } \\
\text { "visible surface other than } \\
\text { sides." }\end{array}$ \\
\hline
\end{tabular}

\section{METHOD OF GRADING SINKS, ROLL-RIM LAUN. DRY TRAYS, COMBINATION SINK AND LAUN- DRY TRAYS, AND SIOP SINKS}

Examine elosely the wet surface, which ineludes the baek and top of rim, for exposed body, fire ehecks, pinholes, projections, and spots. Examination should be made with the eyes of observer about 2 feet from the surface observed. Examine the remainder of the piece for dunts or other defects.

Sinks, roll-rim laundry trays, eombination sink and laundry trays, and slop sinks are graded aeeording to the maximum blemishes listed in Table 2.
Legs are graded on the same basis as the fixture.

TABLE 2.-Sinks, roll-rim laundry trays, combination sink and laundry trays, and slop sinks

\begin{tabular}{|c|c|c|}
\hline Location & Blemish or defect & Regular selection \\
\hline \multirow{3}{*}{$\begin{array}{l}\text { Entire glazed } \\
\text { surface. }\end{array}$} & $\left\{\begin{array}{l}\text { Discolorations } \\
\text { Projections or large }\end{array}\right.$ & \multirow{4}{*}{$\begin{array}{l}\text { Not more than } 3 \text {. } \\
\text { Not more than } 5 \text {. } \\
\text { Not warped inward more } \\
\text { than } 1 / 2 \text { inch. } \\
\text { Not over } 10 \text { square inches. } \\
\text { A total of not more than } \\
20 \text {; no segregation. } \\
\text { Not over } 1 / 2 \text { inch long; not } \\
\text { more than } 2 \text {. }\end{array}$} \\
\hline & warpage & \\
\hline & (Wavy finish... & \\
\hline Wet surface. & $\left\{\begin{array}{l}\text { Spots and pinholes.- } \\
\text { Stopped fire check.. }\end{array}\right.$ & \\
\hline \multirow{2}{*}{ Visible surface } & [Spots and pinholes.- & \multirow{2}{*}{$\begin{array}{l}\text { A total of not more than } 20 \text {; } \\
\text { no segregation. } \\
\text { Not over } 2 \text { inches long; not } \\
\text { more than } 1 \text {. }\end{array}$} \\
\hline & Stopped fire & \\
\hline
\end{tabular}

\section{METHOD OF GRADING BATHS AND SHOWER RECEPTORS}

Examine elosely the wet surface, which ineludes top of rim, for exposed body, fire eheeks, diseoloration, pinholes, projections, and spots. Examiner tion should be made with the eyes of observaabout 2 feet from the surfaee observed. Examine the remainder of the piece for dunts or other defects.

Baths and shower reeeptors are graded aeeording to the maximum blemishes listed in Table 3.

\section{TABLE 3.-Bath and shower receptors}

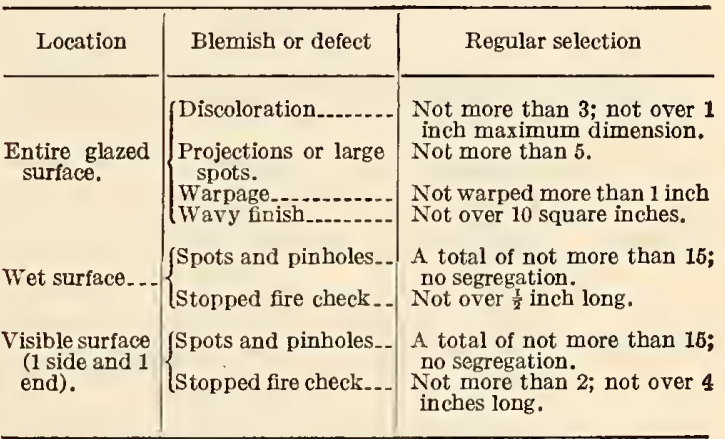

\section{METHOD OF GRADING LAVATORIES}

Lavatories should be examined with the eyes of the observer about 2 feet from the surfaee observed and are graded according to the maximum blemishes listed in Table 4 .

Blemishes or defects on other than visible surfaces are not counted.

The top of slab, front apron, inside of bowl, and faee of baek of lavatories with baek are most important. Sides should not be subjected to the same rigid inspeetion.

Warpage tests are made at faetory

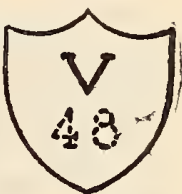

Figure 176 by use of horizontal plane, this being a level table upon which lavatory is allowed to rest, face down, and tested with thickness gages placed between lavatory and table.

Pedestals and legs are graded on the same basis as the lavatory.

Pedestals and legs are not to be warped out of perpendieular line more than one-half inch. 
TABLE 4.-Lavatories

\begin{tabular}{|c|c|c|}
\hline Location & Blemish or defect & Regular selection \\
\hline $\begin{array}{l}\text { Entire glazed sur- } \\
\text { face. }\end{array}$ & $\left\{\begin{array}{l}\text { Discolorations } \\
\text { Projections or large } \\
\text { spots. } \\
\text { Warpage }\end{array}\right.$ & $\begin{array}{l}\text { Not more than } 1 \text {. } \\
\text { Not more than } 3^{\circ} \\
\text { On } 24 \text { hy } 20 \text { inch lava- } \\
\text { tories and larger,warp- } \\
\text { age of slah out of hori- } \\
\text { zontal plane not to } \\
\text { exceed } 3 / 8 \text { inch. }\end{array}$ \\
\hline $\begin{array}{l}\text { Service space, top of } \\
\text { slah, inside of howl, } \\
\text { and front of apron. }\end{array}$ & $\left\{\begin{array}{l}\text { Polishing mark } \\
\text { Spots and pinholes.-- } \\
\text { Stopped fire check.-- }\end{array}\right.$ & $\begin{array}{l}\text { Not more than } 2 \text {. } \\
\text { A total of not more } \\
\text { than } 8 \text {; no segrega- } \\
\text { tion. } \\
\text { None allowed. }\end{array}$ \\
\hline $\begin{array}{l}\text { Face of integral back } \\
\text { and side. }\end{array}$ & Spots and pinholes.- & $\begin{array}{l}\text { Not more than } 5 \text { on } \\
\text { either side or hack; } \\
\text { no segregation; a } \\
\text { total of not more } \\
\text { than 15. }\end{array}$ \\
\hline
\end{tabular}

\section{METHOD OF GRADING FLAT-RIM LAUNDRY TRAYS}

Examine closely the wet surface, which includes only the inside of tray, for exposed body, fire checks, pinholes, projections, and spots. Examination should be made with the eyes of the observer about 2 feet from the surface observed. Examine the remainder of the piece for dunts or other defects.

Flat-rim laundry trays are graded according to the maximum blemishes listed in Table 5 .

Legs are graded on the same basis as the fixture.

\section{TABLE 5.-Flat-rim laundry trays}

(Top of rims are unglazed)

\begin{tabular}{|c|c|c|}
\hline Iocertion & Blemish or defect & Regular selection \\
\hline $\begin{array}{l}\text { Entire glazed } \\
\text { surface. }\end{array}$ & $\left\{\begin{array}{l}\text { Discolorations } \\
\text { Projections or large } \\
\text { spots. } \\
\text { Warpage............. } \\
\text { Wavy finish }\end{array}\right.$ & $\begin{array}{l}\text { Not more than } 3 \text { per com- } \\
\text { partment. } \\
\text { Not more than } 5 \text { per com- } \\
\text { partment. } \\
\text { Not warped more than } 8 / 4 \\
\text { inch. } \\
\text { Not over } 10 \text { square inches. }\end{array}$ \\
\hline Wet surface... & $\left\{\begin{array}{l}\text { Spots and pinholes.. } \\
\text { Stopped fire check .- }\end{array}\right.$ & $\begin{array}{l}\text { A total of not more than } 20 \text {; } \\
\text { no segregation. } \\
\text { Not more than 2; not over } \\
1 \text { inch long. }\end{array}$ \\
\hline Visible surface & $\left\{\begin{array}{l}\text { Spots and pinholes.- } \\
\text { Stopped fire check .- }\end{array}\right.$ & $\begin{array}{l}\text { A total of not more than } 20 \text {; } \\
\text { no segregation. } \\
\text { Not more than } 2 \text {; not over } \\
2 \text { inches long. }\end{array}$ \\
\hline
\end{tabular}

\section{MARKING AND LABELING}

Porcelain (all-clay) plumbing fixtures shall bear the trade-mark or name of the actual manufacturer and the words "Made in U. S. A." applied in such manner as to be permanent.

It is recommended that no name, brand, or label other than that of the actual manufacturer be used on the ware.

"Regular selection" labels shall be used only on such ware as conforms to the requirements for "regular selection" as set forth in the grading rules. No label shall be used on ware which grades lower than "regular selection." Labels shall be applied only at the factory.

The following wording shall be used on labels for "regular selection" ware:

\section{PORCELAIN (ALL-CLAY) REGULAR SELECTION}

We certify that this porcelain (all-clay) plumbing fixture conforms to regular selection in accordance with grading rules incorporated in Commercial Standard CS4-29 adopted by the industry in cooperation with the Bureau of Standards of the Department of Commerce.

\section{CULLS}

Manufacturers shall mark all culls with two small dots cut through the glaze and filled with red ink or enamel at the locations specified below: Fixture Location

Urinals............... On the top behind the inlet.

Lavatories...-..-..... Underneath on the right side of howl.

Sinks and trays.......- On front just ahove the right leg.

Receptors..............- On front at the right side.

All crates containing "culls" shall be marked with two splashes of red on one end of the crate so as to be visible without tearing down stacks.

\section{DIMENSIONAL STANDARDS}

The clearance between fixture and wall for all fixtures set away from wall shall be 2 inches for regular installation.

Where not otherwise specified, a variation of 5 per cent will be permitted from all dimensions indicated herein.

Underlined dimensions are identical for all sizes and types of similar items.

Illustrations 177 to 200, inclusive, indicate types and sizes but do not represent standard designs.

\section{STANDARD TYPES AND SIZES}

The following types, items, sizes, and dimensions are recommended as standard for the industry. Other types, items, sizes, and dimensions are considered special.

\section{STALI URINALS}

When stall urinals are set in mortar, plaster, cement, or concrete at least one-half inch should be allowed beneath for "rocking in" with dry sand. This will properly allow for the natural contraction and expansion of walls, partitions, concrete, etc.

Stall urinals ground on the sides to butt together for battery installation without partitions shall be eliminated for the reason that the type of installation is impracticable and unsanitary.

Eliminate 27-inch interlocking stall urinals.

\section{RESULTS}

The foregoing recommendations contemplate a reduction of variety considered as staple as indicated by the following table:

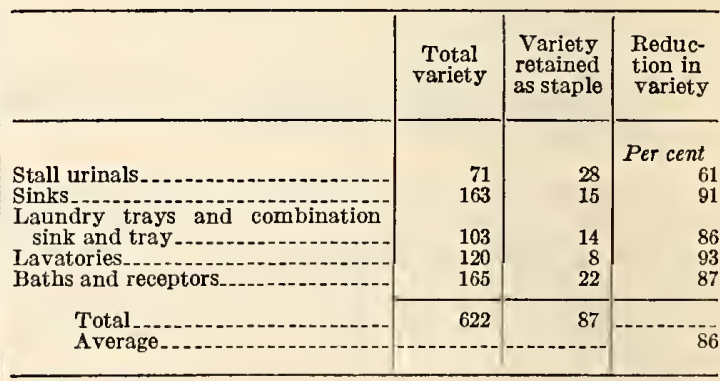

United States Department of Commerce, division of simplified practice, Simplified Practice Recommendation No. 52, staple vitreous china plumbing fixtures, October 1, 1926. 

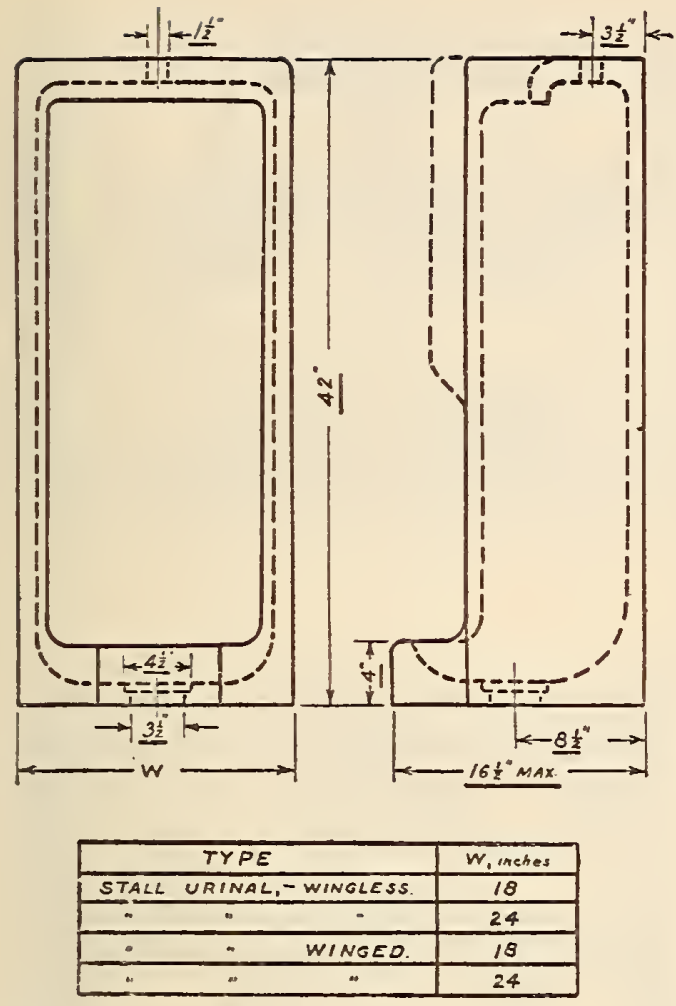

FIGURE 177.- Winged and wingless stall urinals
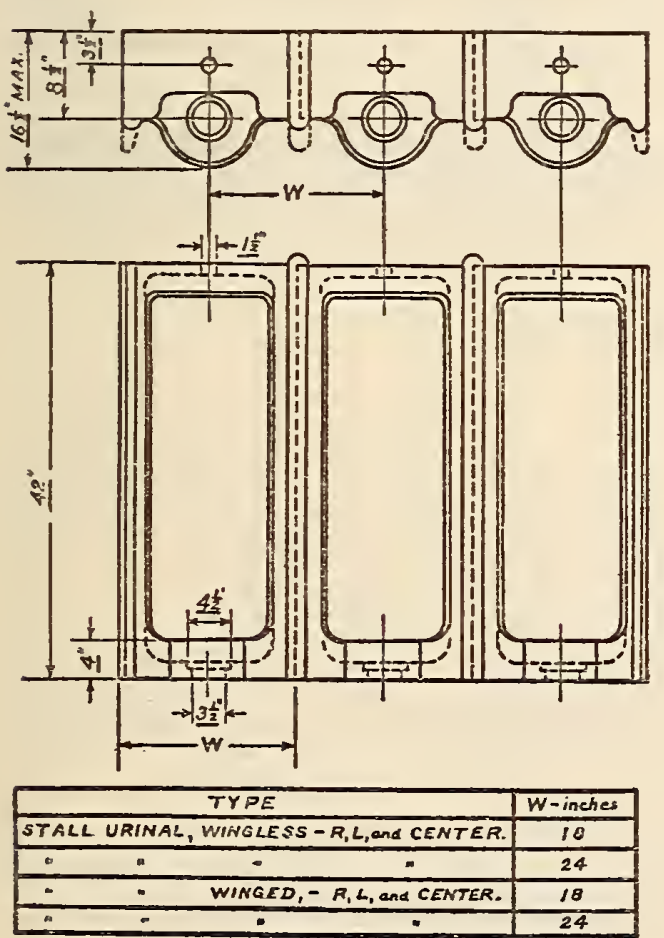

FIGURE 178.-Integral interlocking stall urinals, winged and wingless
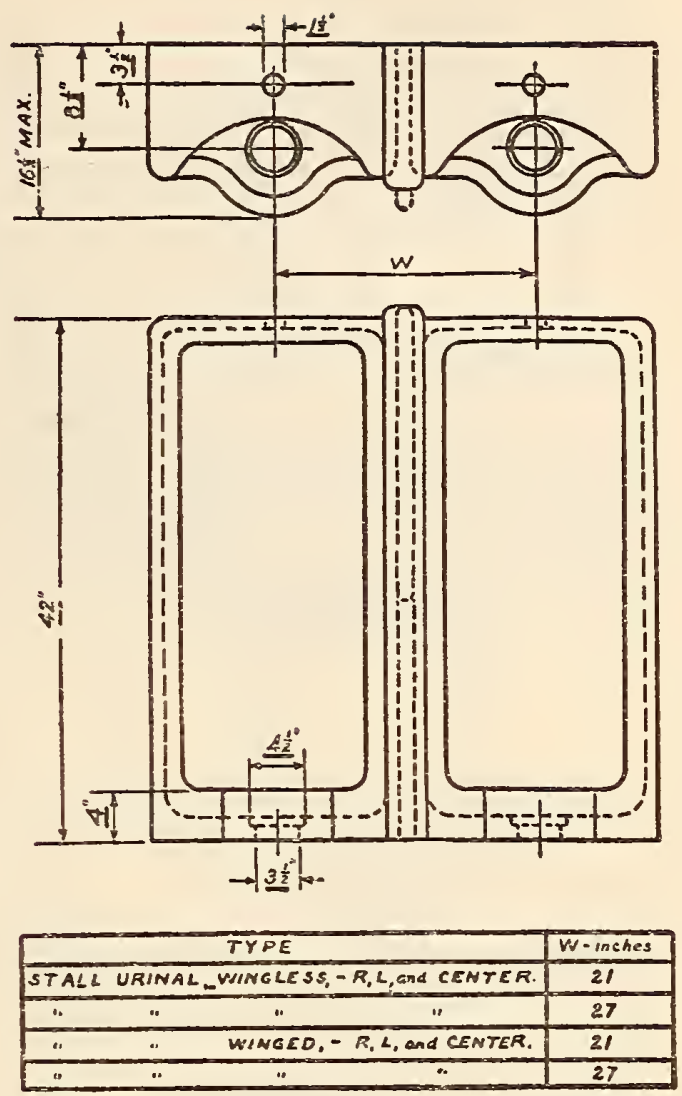

FIGURE 179.-Stall urinals in battery with partition, winged and wingless
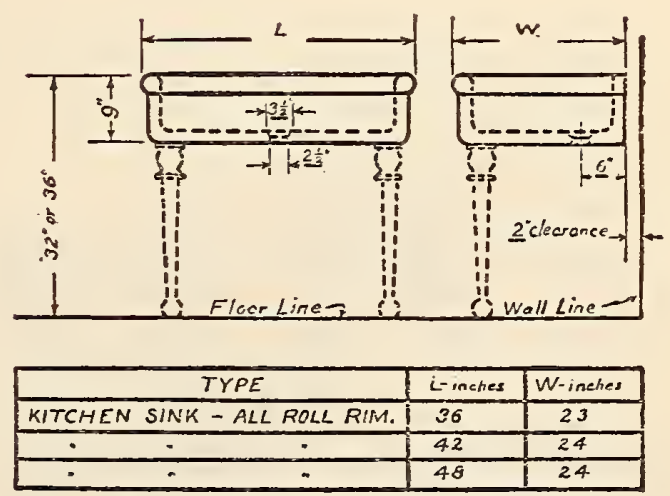

FIGURE 180.-Kitchen sinks, all roll rim, with or without two legs 

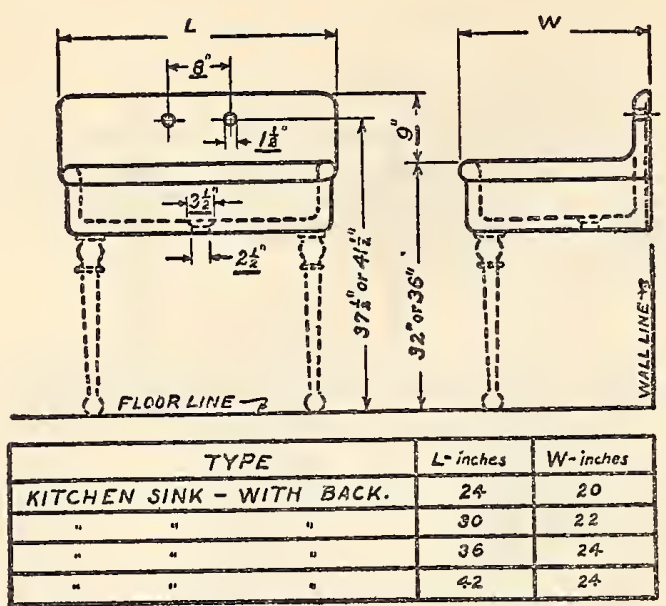

FIgURE 181.-Kitchen sinks with back, with or without two legs

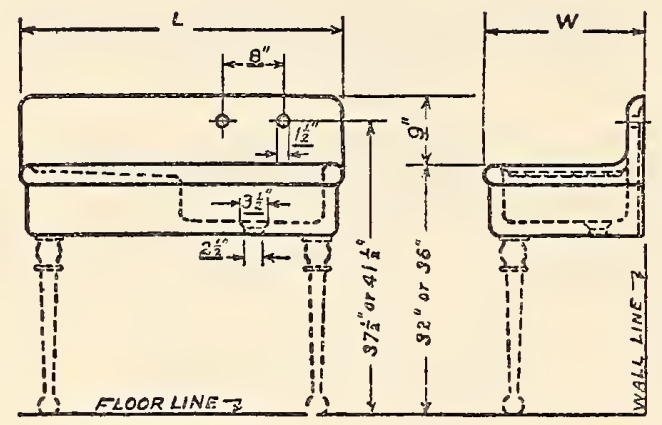

\begin{tabular}{|c|c|c|}
\hline TYPE & L-inches & W-inches \\
\hline KITCHEN SINK, - INTEGRAL BACK & 42 & 21 \\
\hline AND DRAINBOARD. R.PL & 52 & 24 \\
\hline
\end{tabular}

Figure 182.-Kitchen sinks, integral back and drainboard, with or without two legs (R.\& L.)

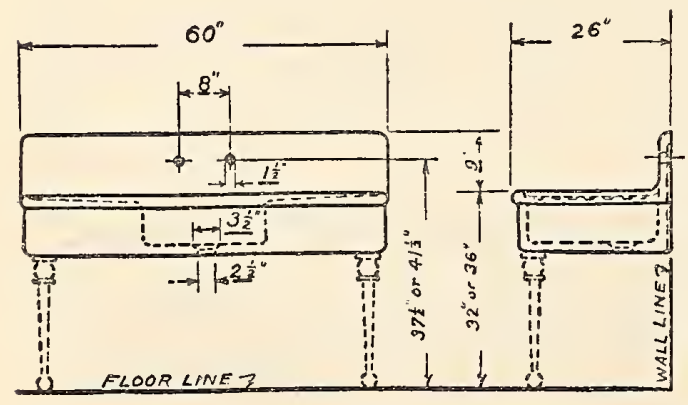

KITCHEN SINK - BACK AND DOUBLE ORAIN SHELF.

FIGURE 183.-Kitchen sinks, back and double drain shelf, with or without two legs

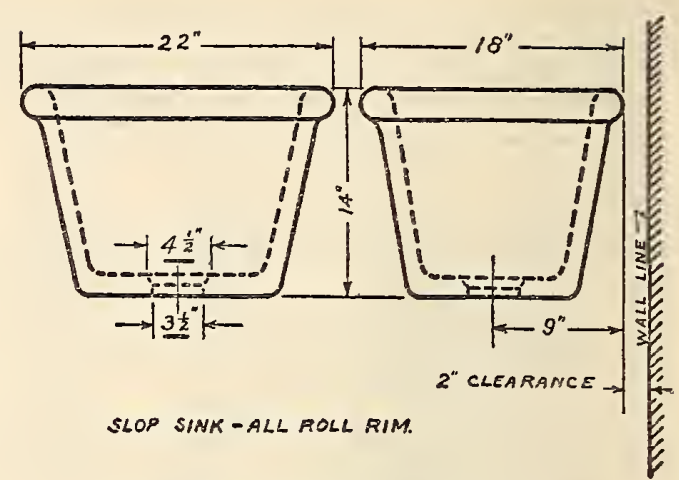

Figure 184.--Slop sinks, all roll rim
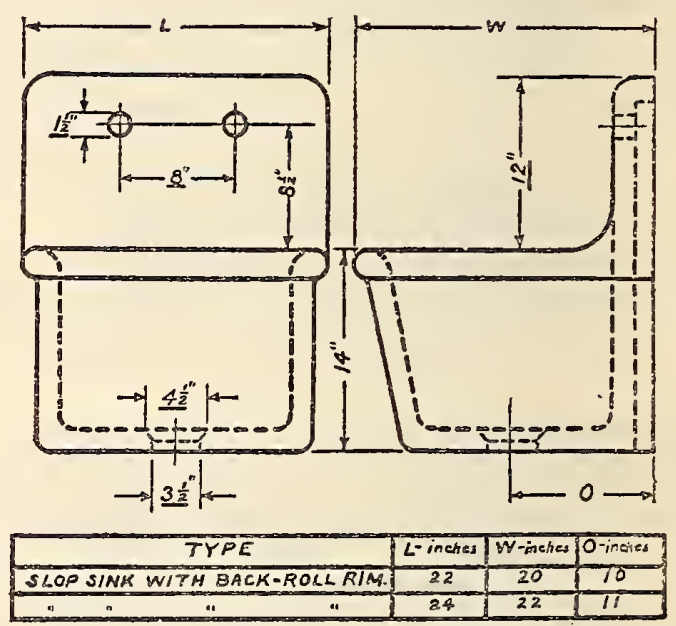

FIgdRE 185.-Slop sinks with back, roll rim

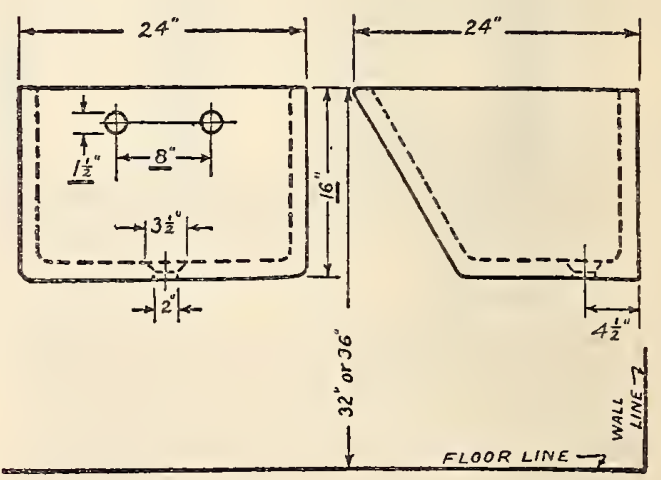

LAUNORY TRAY - FLAT RIM.

FigdRE 186.-Laundry trays, flat rim 

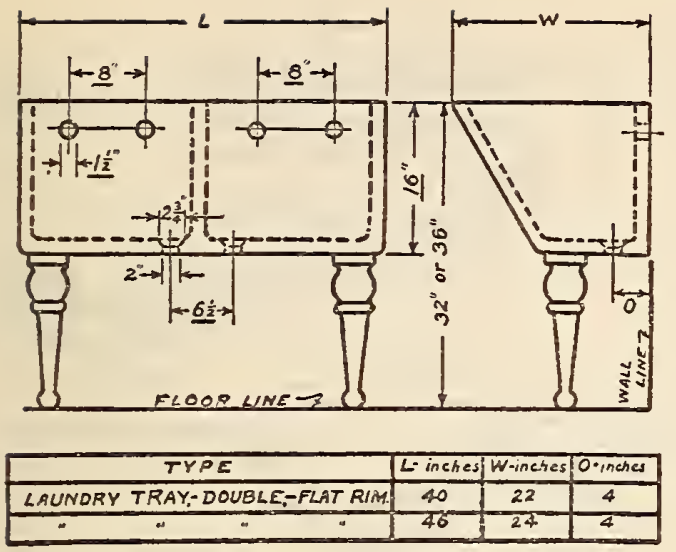

FIGURE 187.-Double laundry trays, flat rim
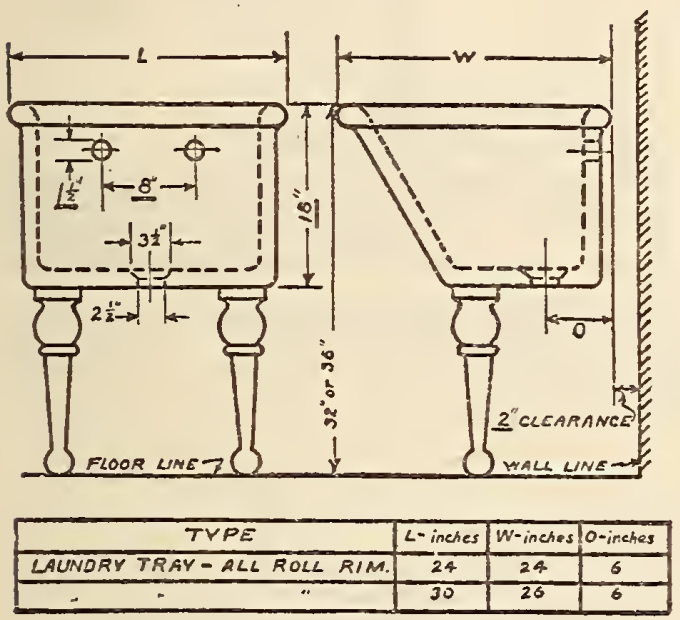

FIGURe 188.-Laundry trays, all roll rim
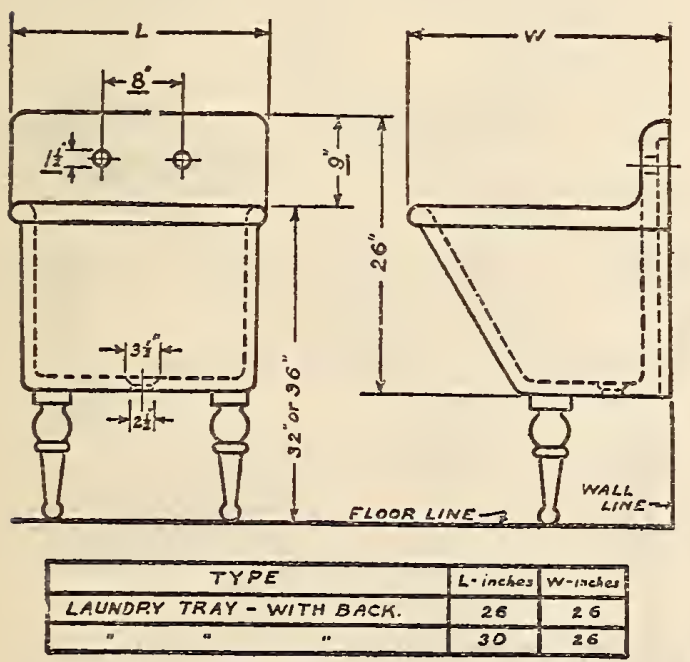

FIGURE 189.-Laundry trays with back

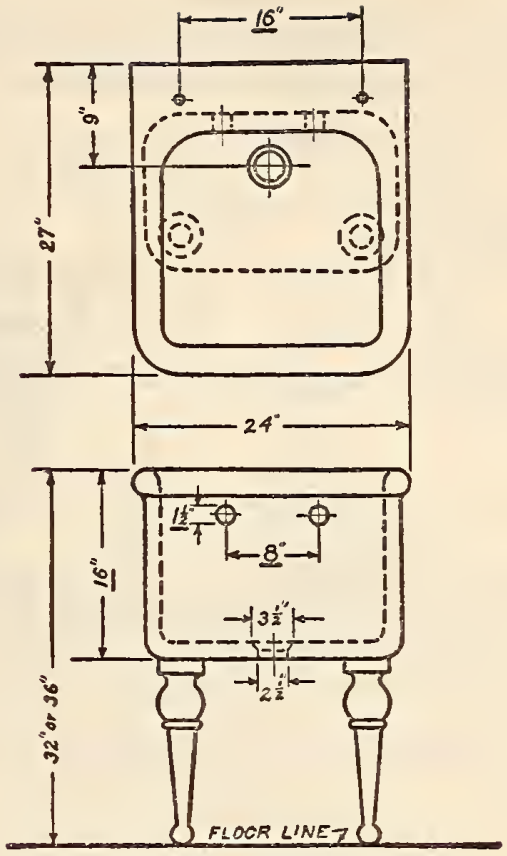

FIGURE 190.-Laundry trays with extended ledge
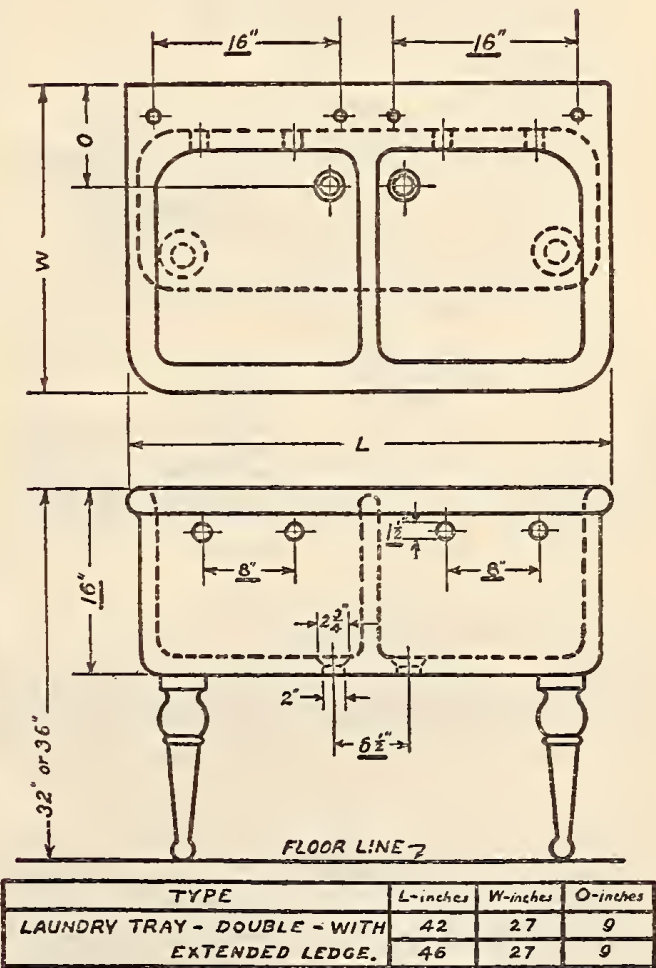

FIGURE 191.-Double laundry trays with extended ledge 
In accordance with unanimous action of a general conference of representatives of manufacturers, distributors, and users the Department of Commerce, through the Bureau of Standards, recom-

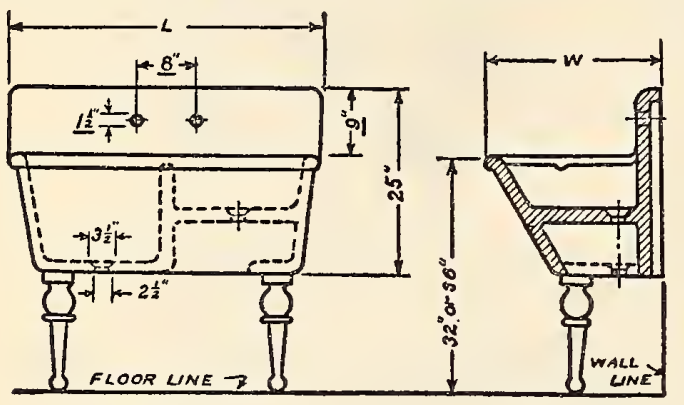

\begin{tabular}{|c|c|c|}
\hline TYPE & $L$-inches & W-inches \\
\hline SINK AND TRAY-COMBINATION & 42 & 24 \\
WITH BACK.- $R$ and $L$. & 50 & 24 \\
\hline
\end{tabular}

FIGURE 192.-Combination sink and tray with back mends the establishment of the following standards for staple vitreous china plumbing fixtures:

\section{GENERAL}

1. The nomenclature, definitions, grading rules, types, sizes, dimensions, and general practices given herein are recommended as standard.

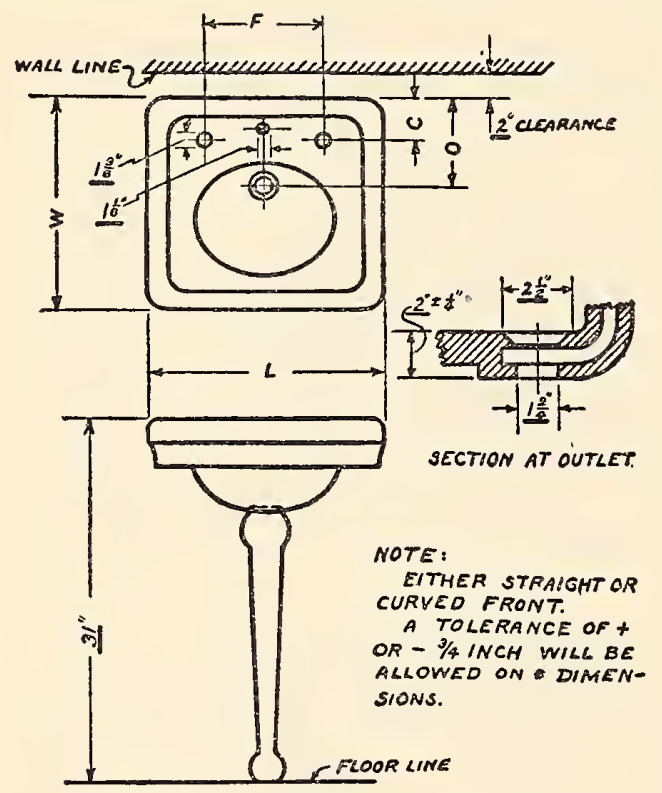

\begin{tabular}{|c|c|c|c|c|c|c|}
\hline TYPE (Dimemsrons in inches) & 6 & $W$ & $F$ & 0 & $C$ \\
\hline RECTANGULAR LAVATORY WITH LEG & 20 & 18 & 10 & $7 \%$ & $3 \%$ \\
\hline$"$ & $"$ & 24 & 20 & 12 & $7 \%$ & $4 / 2$ \\
\hline
\end{tabular}

FigURE 193.-Reclangular lavatories with leg

2. Types and sizes of water-closet bowls, tanks, and lavatories not specifically mentioned shall be considered as special.

3. Catalogues displaying vitreous china plumbing fixtures should, as near as possible, conform to recommended standard items and where specials are exhibited they should be so indicated.

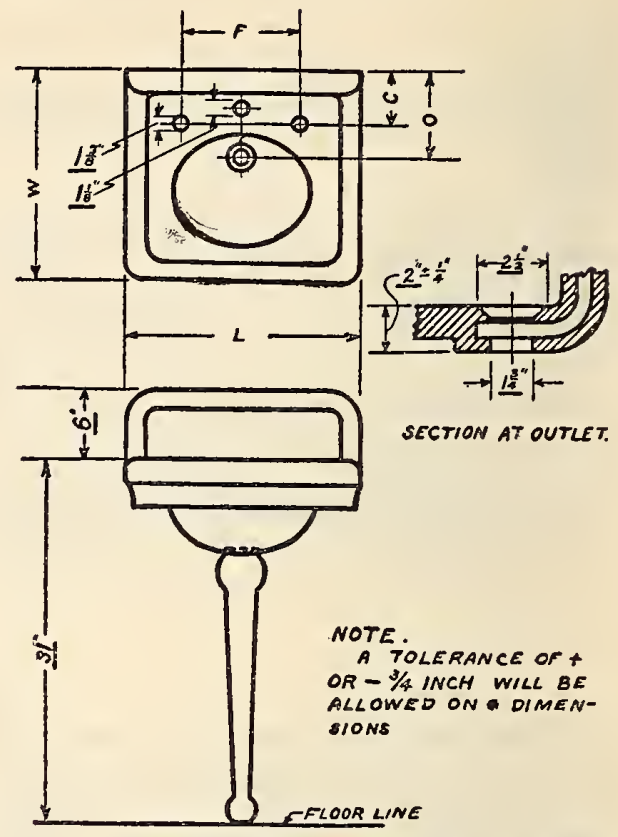

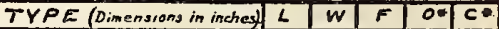
\begin{tabular}{|l|l|l|l|l|l|}
\hline RECTANGULAR LAVATORY WITH LEG & 20 & 18 & 10 & $7 \%$ & $4 \%$ \\
\hline
\end{tabular} AND BACK.

Figdre 194.-Rectangular lavatories with back and leg

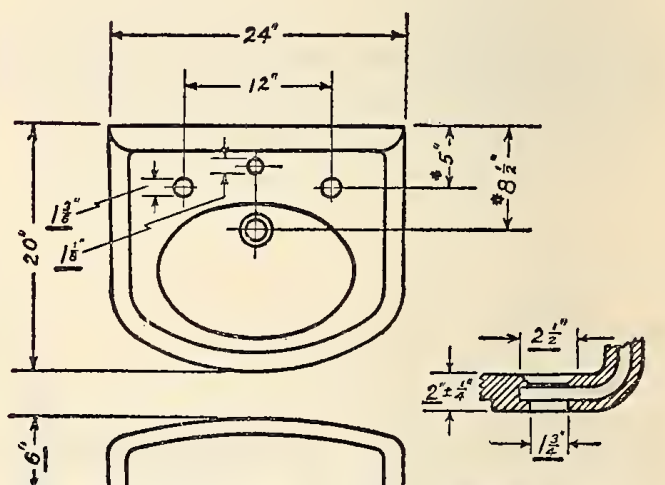

SECTION AT OUTLET.

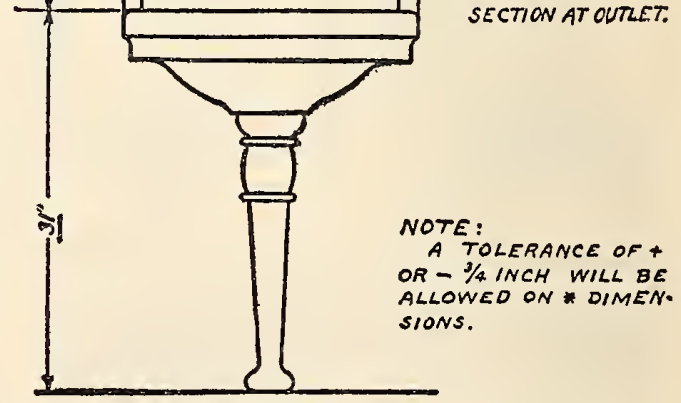

FIGURE 195.-Elliptical front lavatories with back and leg 


\section{GRADING ROLES}

4. Vitreous china plumbing fixtures shall be graded in accordance with the grading rules as given herein.
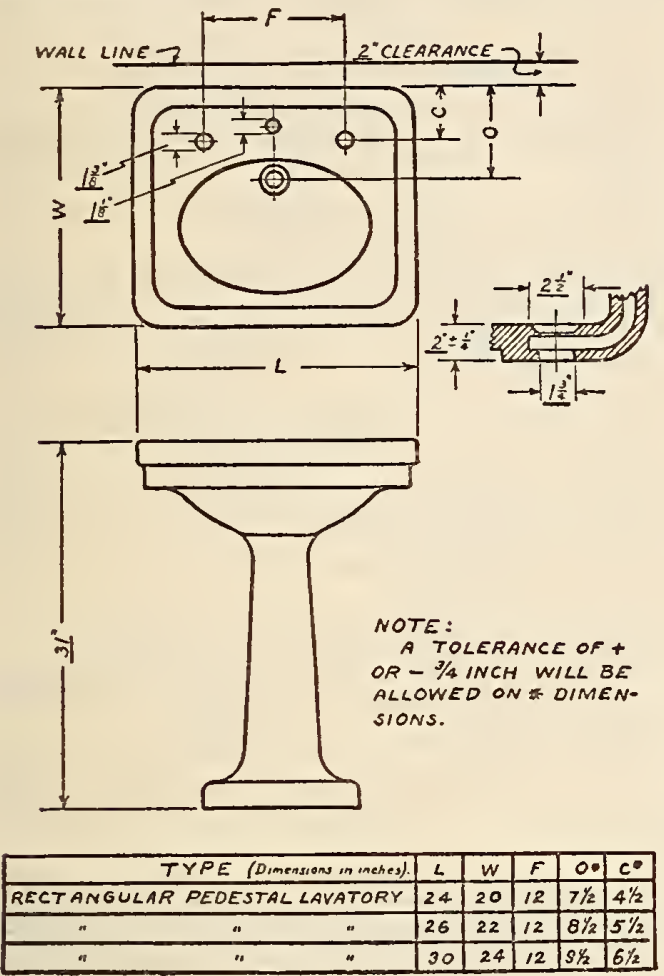

FigURE 196.-Rectangular pedestal lavatories

5. Vitreous china plumbing fixtures are made of materials mined from the earth, containing metallic elements and foreign matter which can not be
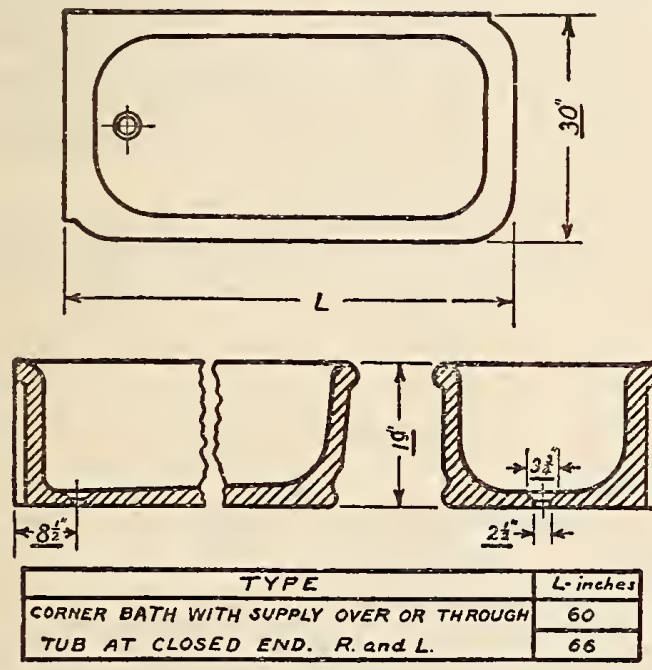

FigURE 197.-Corner baihs with supply over or through tub at closed end

entirely eliminated in practice. As they are made and finished by hand, and subjected to a high degree of heat, it is an accepted fact that they can not be regularly produced free from unimportant variations and minor blemishes.

6. Careful inspection is employed in each department of manufacture and each of the imperfections
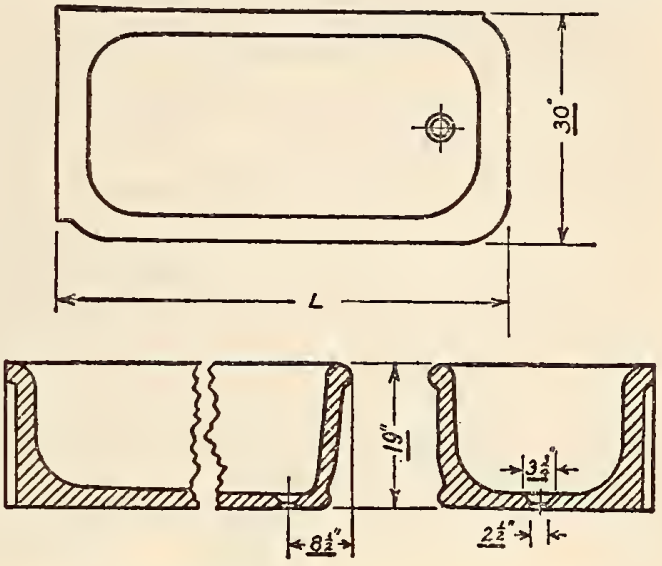

\begin{tabular}{|l|l|}
\hline TYPE & L-inches \\
\hline CORNER BATH WITH SUPPLY THROUGH TUB & 60 \\
\cline { 2 - 2 } & 66 \\
\hline
\end{tabular}

Figure 198.-Corner baths with supply through tub at open end

listed herein as acceptable under the "regular selection" grading is caused by some unavoidable condition in the manufacturing process. Perfection is not guaranteed nor commercially possible.

7. The blemishes permitted under the grading rules do not affect the utility or value of the fixture.
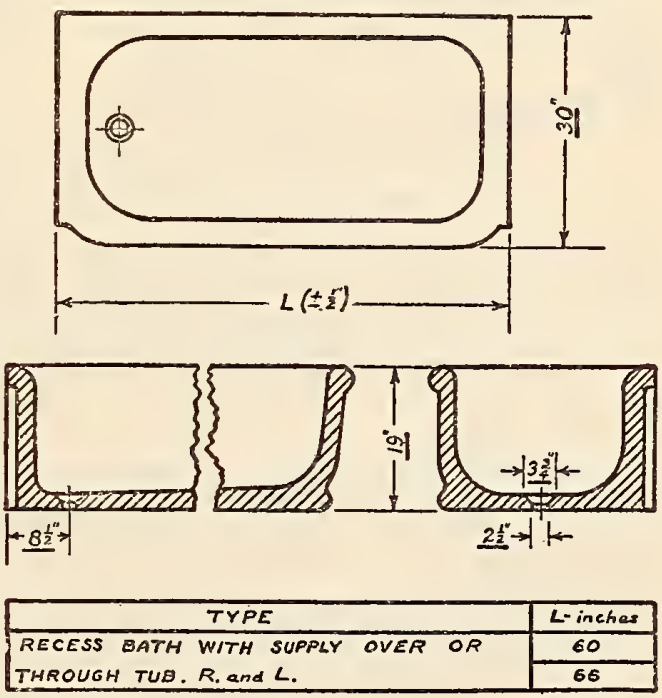

Figure 199.-Recess baths with supply over or through tub (R.\& L.)

8. The terms "regular selection" and "culls" shall be used to replace the terms "grade A" and "grade B" for grading vitreous china plumbing fixtures, as it is recognized that the terms "grade A" and "grade B" are confusing to the trade and to the consumer.

9. Ware which grades below "regular selection" shall be classified as "culls." 


\section{NOMENCLATURE AND DEFINITIONS}

Blister.-A raised uncolored portion of the surface, $1 / 32(0.031)$ inch and less than $1 / 8(0.125)$ inch in maximum dimension.

Large blister.-A raised uncolored portion of the surface $1 / 8(0.125)$ inch to $1 / 4$ ( 0.25 inch), inclusive, in maximum dimension.

Bubble.-An uncolored raised portion of the surface, or a sand speck smaller than $1 / 32(0.031)$ inch in maximum dimension.

Craze.-Fine cracks in the glaze.

Culls.-Ware which grades below "regular selection."

Discoloration.-A colored spot over 1/4 (0.25)

inch in maximum dimension, or a sufficient number of specks or spots to give the effect of a change in color.

Dull or eggshell finish.-Dead or flat finish. Undeveloped glaze. A semiglazed finish with

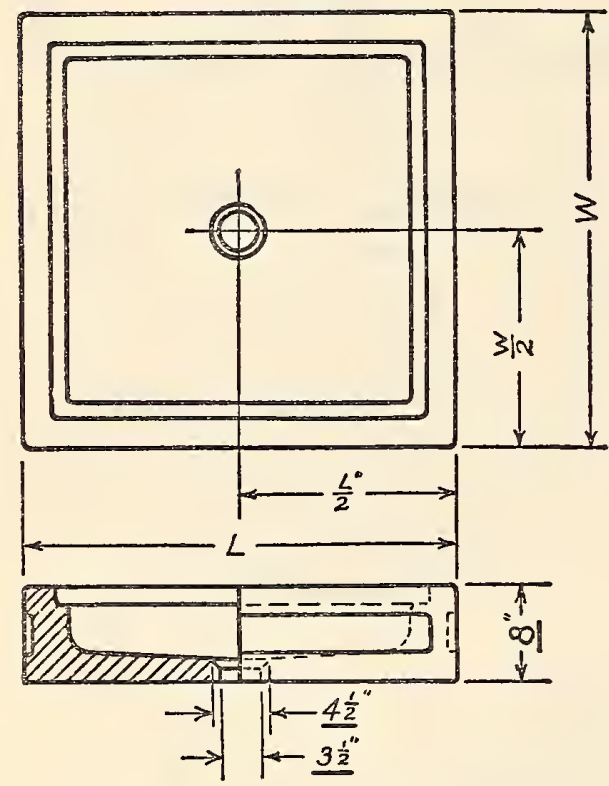

\begin{tabular}{|c|c|c|}
\hline TYPE & L-INCMES & W-INCHES \\
\hline RECEPTOR, UNIVERSAL & 36 & 36 \\
\hline " U & 42 & 42 \\
\hline
\end{tabular}

FIGURE 200.-Universal type shower receptors

numerous very fine pinholes or slightly matted in appearance. Not glossy.

Dunt.-A hair-line fracture extending through the body, and due to strains set up in the process of manufacture.

Exposed body.-Unglazed portion $1 / 16(0.063)$ inch in maximum dimension or over.

Finish.-Texture and condition of surface other than color.

Fire check. - Fine shallow crack in the body not covered with glaze. (When sufficiently covered with glaze as to be easily cleaned, it is not detrimental.)

Flushing surface.-The surface which may be wet during the operation of the fixture.

Pinhole.-Unglazed portion of body, or small liole under $1 / 16$ (0.063) inch in maximum dimension.

Polishing mark. - A spot not larger than $3 / 8(0.375)$

inch in maximum dimension where some minor blemish has been ground off and the surface polished.
Pottery square.-A square 2 inches on each side. For grading purposes may be a 2-inch square hole cut in a small sheet of any flexible material, such as rubber or paper, for convenience in sliding over irregular surfaces to determine segregation.

Projection.-A raised uncolored portion of the surface over $1 / 4(0.25)$ inch in maximum dimension.

Regular selection.-First-class ware in conformity with the limitations of the grading rules.

Roughing in measurement.-Dimension from finished wall or floor to center of waste or supply opening.

Segregation.-More than four spots, blisters, or pinholes in any "pottery square."

Speck.-A colored portion less than 1/32 (0.031) inch in maximum dimension. Specks less than $1 / 100$ (0.01) inch in maximum dimension, unless in sufficient number to form a discoloration, are not counted.

Spot.-A colored portion of the surface $1 / 32$ $(0.031)$ inch and less than $1 / 8(0.125)$ inch in maximum dimension.

Large spot.-A colored portion $18(0.125)$ inch to $1 / 4(0.25)$ inch, inclusive, in maximum dimension.

Spud.-Threaded brass connection inserted in the vitreous chinaware.

Streak.-A slight defect in the finish giving an appearance similar to painters' brush marks.

$V$ isible surface.-The surface readily visible after installation of the fixture by an observer in normal standing position.

Vitreous china plumbing fixtures.-The term "vitreous china" shall be applied only to such plumbing fixtures as will pass the following red-ink test:

A fractured piece of material taken from any part of a vitreous china plumbing fixture, after being immersed in red aniline ink of good color strength for one hour, shall not show any discoloration through the glaze and shall not show absorption when broken to a depth greater than one-eighth inch below the surface of fracture at any point.

Water-closet bowl.-The term "water-closet bowl" is the accepted general term applicable to such fixtures.

Reverse trap.-The term "reverse trap" shall be applied only to water-closet bowls having back supply; integral flushing rim; a minimum water seal of $21 / 2$ inches; a minimum water area of $83 / 4$ by $71 / 2$ inches; a siphon trap way at the rear of closet which shall pass a $11 / 2$-inch diameter solid ball; and a minimum weight of 38 pounds.

Reverse trap, with jet.-Same as reverse trap, with jet added.

Siphon jet.-The term "siphon jet" shall be applied only to watercloset howls having top supply, ivtegral flushing rim and jet: a minimum water area of 12 by 10 inches. 2 minimum depth of seal of 3 in ball; minimum horizontal over-all dimensions or 14 by 23 inches; and a minimum weight of 48 pound

Wash down.-The term "wash down" shall be applied only to water-closet howls having back supply; integral flushing rim; a minimum water area of 8 by 7 inches, a minimum water seal of $21 / 2$ inches; a siphon trap way at the front of closet which shall pass a $11 / 2$-inch diameter solid hall; and a minimum weight of 34 pounds.

Wash down with jet.-Same as wash down, with jet added.

Water area.-Elliptical area of the still water in the water-closet bowl, when filled to the top of the dam.

Wavy finish.-A defect in the finish having the appearance of numerous runs in the glaze; irregular or mottled.

10. Examine the well hole closely for excess glaze, spots, blisters, pinholes, etc. With eyes about 2 feet directly above rim, rock the bowl first to one side and then the other to an angle of about $45^{\circ}$, then tilt backward at the same angle, noting only the defects which can be observed in those positions. Minor blemishes which are not observed in this operation are assumed to be on unseen surfaces. Examine the remainder of the bowl for dunts, craze, or other serious defects.

Note.-It is not intended that inspectors shall measure or count any blemishes except in case of douht, since with practice dimensional limits and numbers can be readily gaged by eye. 
11. Tater-closet botrls are graded in accordance with the maximum blemishes listed. (See Table 1.)

12. Bowls having more than the maximum grading limit permissible for "regular selection" on any one bleinish shall be classified as "culls."

TABLE 1.- Tater-closet bowls

\begin{tabular}{|c|c|c|}
\hline Location & Blemish or defect & Regular selection \\
\hline Genera].... & 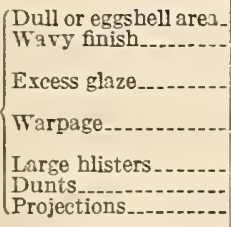 & $\begin{array}{l}\text { Not over } 4 \text { square inches. } \\
\text { Not more than } 4 \text { square } \\
\text { inches. } \\
\text { Not more than } 18 \text { inch thick } \\
\text { in well. } \\
\text { Not noticeahly warped when } \\
\text { seat is attached. } \\
\text { Not more than } 2 . \\
\text { None allowed. } \\
\text { Do. }\end{array}$ \\
\hline $\begin{array}{l}\text { Flushing sur- } \\
\text { face. }\end{array}$ & $\left\{\begin{array}{l}\text { Exposed bods } \\
\text { Unglazed fire check } \\
\text { Spots, hlisters, or } \\
\text { pinholes. } \\
\text { Buhbles or specks_.. }\end{array}\right.$ & $\begin{array}{l}\text { Do. } \\
\text { Do. } \\
\text { No segregation; a total of not } \\
\text { orer } 10 . \\
\text { Not orer } 10 \text { in } 1 \text { "pottery } \\
\text { square"; a total of not orer } \\
25 .\end{array}$ \\
\hline Tisible surface. & $\left\{\begin{array}{l}\text { Englazed fire check } \\
\text { Spots, hlisters, or } \\
\text { pinholes. } \\
\text { Bubhles or specks -- }\end{array}\right.$ & $\begin{array}{l}\text { Not orer } 1 / 4(0.25) \text { inch on } \\
\text { foot; not orer } 18(0.125) \\
\text { inch on more prominent } \\
\text { surfaces. } \\
\text { Not orer } 1 / 2 \text { inch long. } \\
\text { No segregation; a total of not } \\
\text { orer } 10 . \\
\text { Not orer } 5 \text { in } 1 \text { "pottery } \\
\text { square"; a total of not over } \\
25 \text {. }\end{array}$ \\
\hline
\end{tabular}

13. Low tanks are installed at a level where blemishes are more readily visible than on watercloset bowls and, although less likely to become soiled, are graded about as closely for appearance. Tanks and covers are graded separately on an equal basis for segregation of blemishes. The covers shall be limited to about one-half the total blemishes permitted for tanks. No blemishes on the inside surface are counted. Minor blemishes on the outside surface, hidden by the cover, are not counted. Examination should be made with the eves of the observer about 2 feet from the surface observed.

14. Low tanks are graded in accordance with the maximum blemishes listed. (See Table 2.)

15. Low tanks having more than the maximum grading limit permissible for "regular selection" on any one blemish shall be classified as "culls."

TABLE 2.-Low tanks

\begin{tabular}{|c|c|c|}
\hline Location & Blemish or deiect & Regular selection \\
\hline General.. & $\left\{\begin{array}{l}\text { Warpage....... } \\
\text { Dunts........ }\end{array}\right.$ & $\begin{array}{l}\text { Not noticeably warped. } \\
\text { None allowed. }\end{array}$ \\
\hline & $\left\{\begin{array}{l}\text { Dull or eggshell area } \\
\text { Wary finish...-... }\end{array}\right.$ & $\begin{array}{l}\text { Not orer } 2 \text { square inches. } \\
\text { Not more than } 4 \text { square } \\
\text { inches. }\end{array}$ \\
\hline Visible surface. & $\left\{\begin{array}{l}\text { Exposed hody } \\
\text { Unglazed fire check } \\
\text { Spots, hlisters, or } \\
\text { pinholes. } \\
\text { Buhhles or specks... }\end{array}\right.$ & $\begin{array}{l}\text { Not orer } \$(0.125) \text { inch. } \\
\text { Not orer } 1 / 4 \text { inch long. } \\
\text { No segregation; a rotal of not } \\
\text { over } 10 \text {. } \\
\text { Not orer } 5 \text { in } 1 \text { "pottery } \\
\text { souare"; a total of not over } \\
25 \text {. }\end{array}$ \\
\hline
\end{tabular}

Noт:.-Corers showing more than 50 per cent of the allowahle numher of hlemishes listed for low tanks shall he classified as "culls."

\section{IIETHOD OF GRADING VITREOUS CHIYA LAVA- TORIES, PEDESTAIS, AND LEGS}

16. Since lavatories are installed at a level where blemishes are more readily noticeable than on water- closet bowls and tanks, they should be graded more closely than any other vitreous china fixture.

17. Lavatories should be examined with the eyes of observer about 2 feet from the surface observed.

18. The top of slab, front apron, inside of bowl, and face of back of lavatories with back are most important. Sides should not be subjected to the same rigid inspection.

19. IVarpage tests are made at factory by use of horizontal plane, this being a level table upon which lavatory is allowed to rest, face down, and tested with thickness gages placed between lavatory and table.

20. Pedestals and legs are graded the same as water-closet bowls.

21. Pedestals and legs are not to be warped out of perpendicular line more than one-half inch. To be free from rough projections. No exposed body over one-half inch.

22. Vitreous china lavatories are graded in accordance with the maximum blemishes listed in Table 3; any vitreous china lavatory having more than the maximum grading limit permissible for " regular selection" on any one blemish shall be classified as a "cull."

TABLE 3.-Vitreous china lavatories

\begin{tabular}{|c|c|c|}
\hline Location & Blemish or defect & Regular selection \\
\hline \multirow[t]{2}{*}{ General... } & 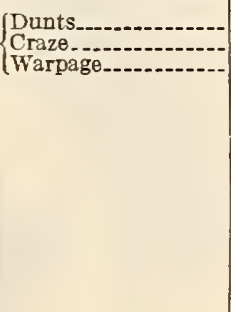 & $\begin{array}{l}\text { None allowed. } \\
\text { Do. } \\
\text { On } 24 \text { hy } 20 \text { inch laratories } \\
\text { and larger, warpage of } \\
\text { slah out of horizontal } \\
\text { plane not to exceed } 3 / 8 \\
\text { inch. On smaller than } \\
24 \text {-inch lavatories, warp- } \\
\text { age of slah out of hori- } \\
\text { zontal plane not to } \\
\text { exceed } 1 / 4 \text { incll. (The } \\
\text { same deviation to apply } \\
\text { on lavatories with back, } \\
\text { when attached to wall.) }\end{array}$ \\
\hline & Dull or eggshell & One allowed; not over \\
\hline \multirow[t]{4}{*}{$\begin{array}{l}\text { Serrice space, top } \\
\text { of slah, inside } \\
\text { of howl, and } \\
\text { front of apron. }\end{array}$} & $\begin{array}{l}\text { Exposed hody } \\
\text { Unglazed fire check- } \\
\text { Spots, hlisters, and } \\
\text { pinholes. } \\
\text { Buhhles or specks... } \\
\text { Polishing mark_._-- }\end{array}$ & $\begin{array}{l}\text { None allowed. } \\
\text { Do. } \\
\text { No segregation; a total } \\
\text { of not more than } 8 \text {. } \\
\text { Not more than } 3 \text {, in } 1 \\
\text { "pottery square"; a to- } \\
\text { tal of not more than } 8 \text {. } \\
\text { No segregation; not more } \\
\text { than } 2 \text { allowed. }\end{array}$ \\
\hline & (Dull or eggshell. & One allowed; not over \\
\hline & Exposed body....... & None orer $1 / 2$ inch; not \\
\hline & Large blisters... & $\begin{array}{l}\text { Not more than } 2 \text { on either } \\
\text { side or hack; a total of } \\
\text { not more than } 6 \text {. }\end{array}$ \\
\hline \multirow{2}{*}{$\begin{array}{l}\text { Face of integral } \\
\text { hack and sides. }\end{array}$} & Unglazed fire check & None on hack; not more \\
\hline & $\begin{array}{l}\text { Spots, hlisters, and } \\
\text { pinholes. } \\
\text { Buhhles or specks_-- }\end{array}$ & $\begin{array}{l}\text { No segregation; not more } \\
\text { than } 5 \text { on either side or } \\
\text { hack; a total of not more } \\
\text { than } 15 \text {. } \\
\text { Not more than } 4 \text { in } 1 \\
\text { "pottery square"; a } \\
\text { total of not more than } \\
12 \text {. }\end{array}$ \\
\hline
\end{tabular}

\section{GRADING RULES FOR OTHER VITREOUS-CHINA PLUMBING FIXTURES}

23. The grading rules for water-closet bowls shall apply to slop sinks, clinic sinks, and bidets.

24. The grading rules for, and method of grading lavatories, pedestals, and legs shall apply to drinking fountains, manicure tables, and toilet tables.

25. All vitreous-china plumbing fixtures not specifically mentioned in the foregoing shall take the grading rules for water-closet bowls, 


\section{IMARKING AND LABELING}

26. Water-closet bowls, tanks, tank covers, lavatories, and all other vitreous-china plumbing fixtures shall bear the trade-mark or name of the actual manufacturer and the words "Made in U. S. A.," applied in such manner as to be permanent and visible after installation. It is recommended that no name, brand, or label other than that of the actual manuiacturer be used on the ware.

27. "Regular selection" labels shall be used only on such ware as conforms to the requirements for "regular selection," as set forth in the grading rules. No label shall be used on ware which grades lower than "regular selection." Labels shall be applied only at the factory.

28. The following wording shall be used on labels for "regular selection" ware:

This is a high-class and valuable piece of vitreous china and should be handled as such. This piece has been classified as "regular selection" after a thorough inspection by competent and experienced
32. Soap-dish depressions shall be used on all lavatories to and including the 24 -inch size, but not on larger sizes.

33. Wherever soap-dish depressions are used on slabs of lavatories, they shall be located on the lefthand side as one faces the lavatory and shall be approximately $3 \frac{1}{4}$ inches long, by $2 \frac{1}{4}$ inches wide, by $1 / 8$ inch deep at the deepest point, and shall drain into bowl.

34. Where not otherwise specified, a variation of 5 per cent from the dimensions indicated herein will be permitted.

35. Underlined dimensions in Figures 202 to 209, inclusive, are identical for all sizes and types of similar items.

36 . The total thickness of lavatories at faucet or valve holes shall be not less than one-half inch nor more than $1 \frac{1}{4}$ inches.

37. The total thickness of lavatories at waste outlets shall be not less than $1 \frac{1}{4}$ inches nor more than $17 \% 8$ inches.

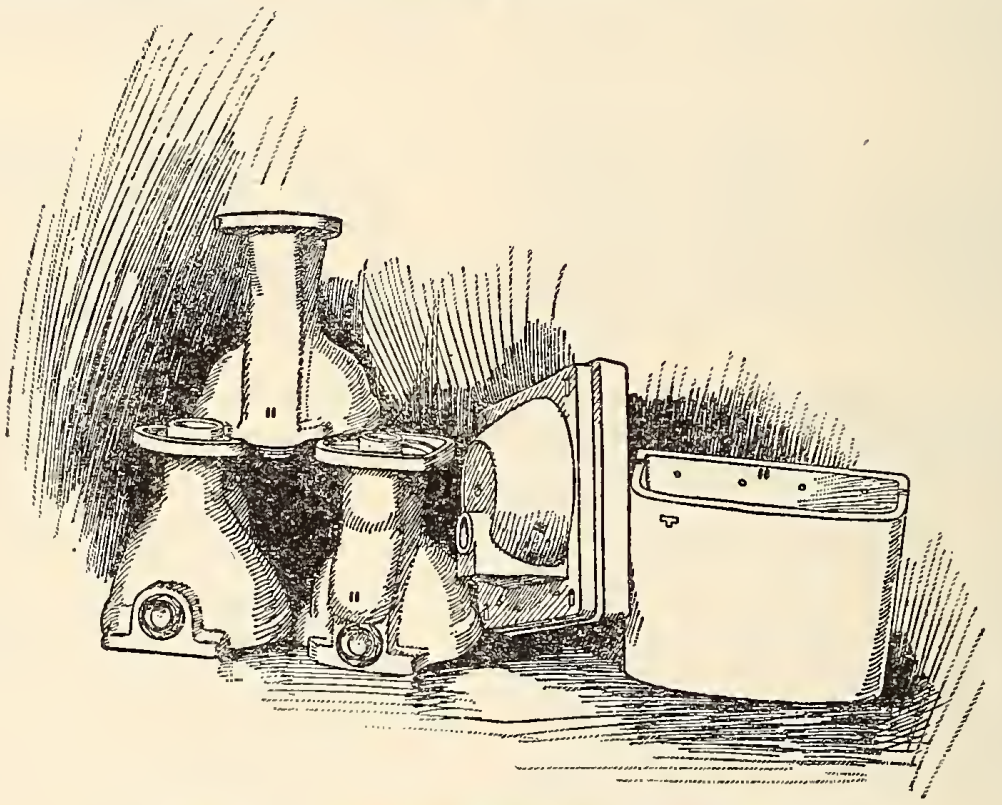

FIGURE 201.-Location and character of marking for culls

men. The term "regular selection" does not mean that this article is without blemish. It is impossible to make vitrcous china plumbing fixtures without flaws of some kind and these have not been overlooked in the grading of this article.

This piece has been graded in accordance with uniform grading rules adopted by the Sanitary Potteries in conjunction with the United States Bureau of Standards of the Department of Commerce.

29. Culls shall be marked by the maker with two parallel lines cut through the glaze into the body of the ware at the location (see fig. 201) recommended by the Manufacturers' Advisory Committee on Vitreous China Plumbing Fixtures. These cuts shall be filled with a bright red varnish or enamel which is resistant to the action of hot water.

30. All crates containing "culls" shall be marked with two splashes of red on one end of the crate so as to be visible without tearing down stacks.

\section{DIMENSIONAL STANDAEDS}

31. The standard size for spuds on all watercloset bowls shall be as follows: 2 inches for all closet bowls operated under low-down tanks; $11 / 2$ inches for all other installations.
38. Supply pipes to floor for standard washdown combinations are furnished 19 inches long.

\section{STANDARD TYPES AND SIZES}

39. The following items are recommended as standard for the industry and constitute a reduction to 58 from the previous 441 items.

\section{WATER-CLOSET BOWLS AND TANKS}

Staple floor outlet siphon jet closet with silencing chamber:

Top supply, regular bowl.

Extended top supply, regular bowl.

Top supply, extended lip.

Extended top supply, extended lip.

Staple floor outlet siphon jet closet:

Top supply, regular bowl (regular height).

Extended top supply, regular bowl (regular height).

Top supply, extended lip (regular height).

Extended top supply, extended lip (regular height).

Top supply, extended lip (juvenile height). 


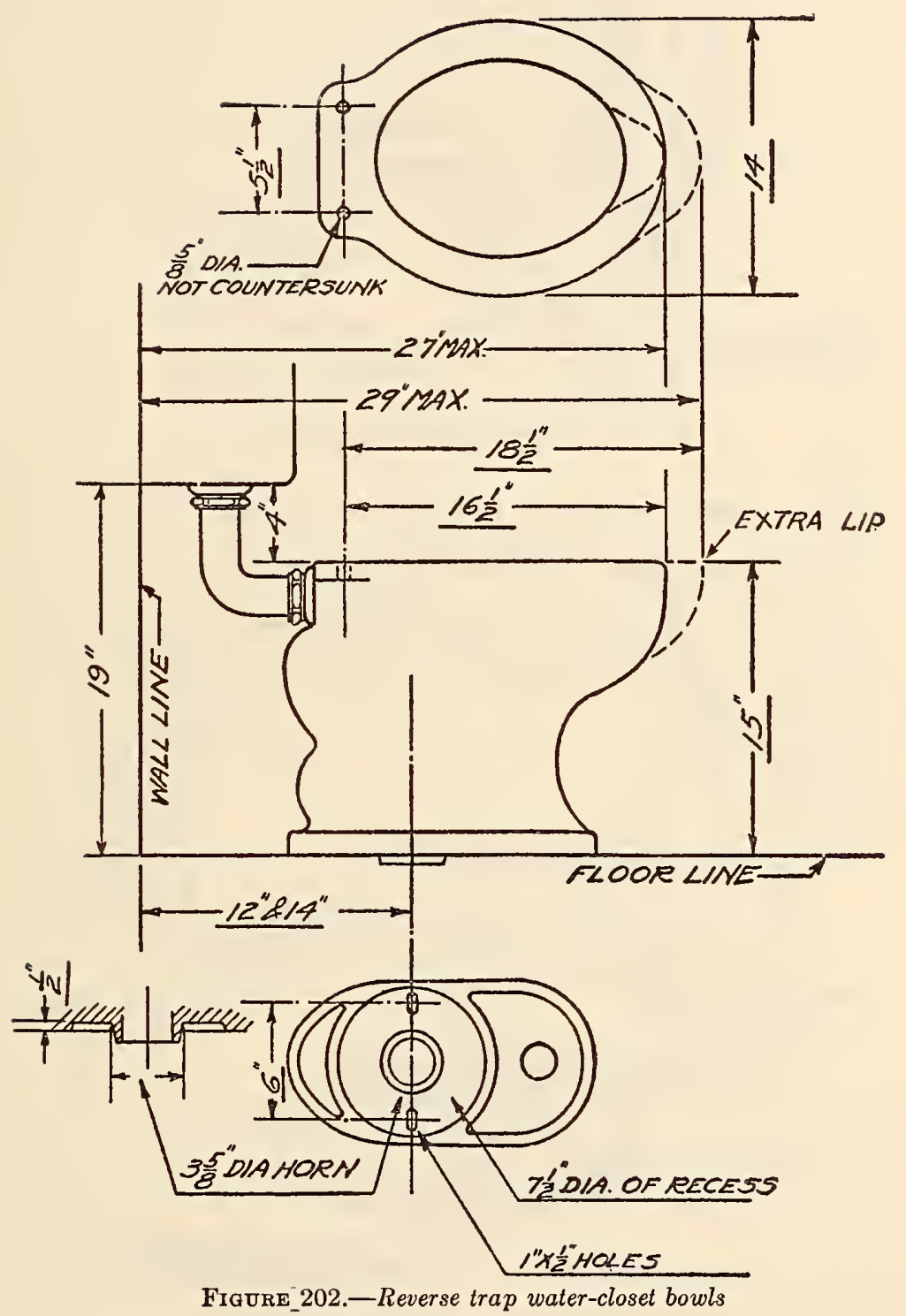




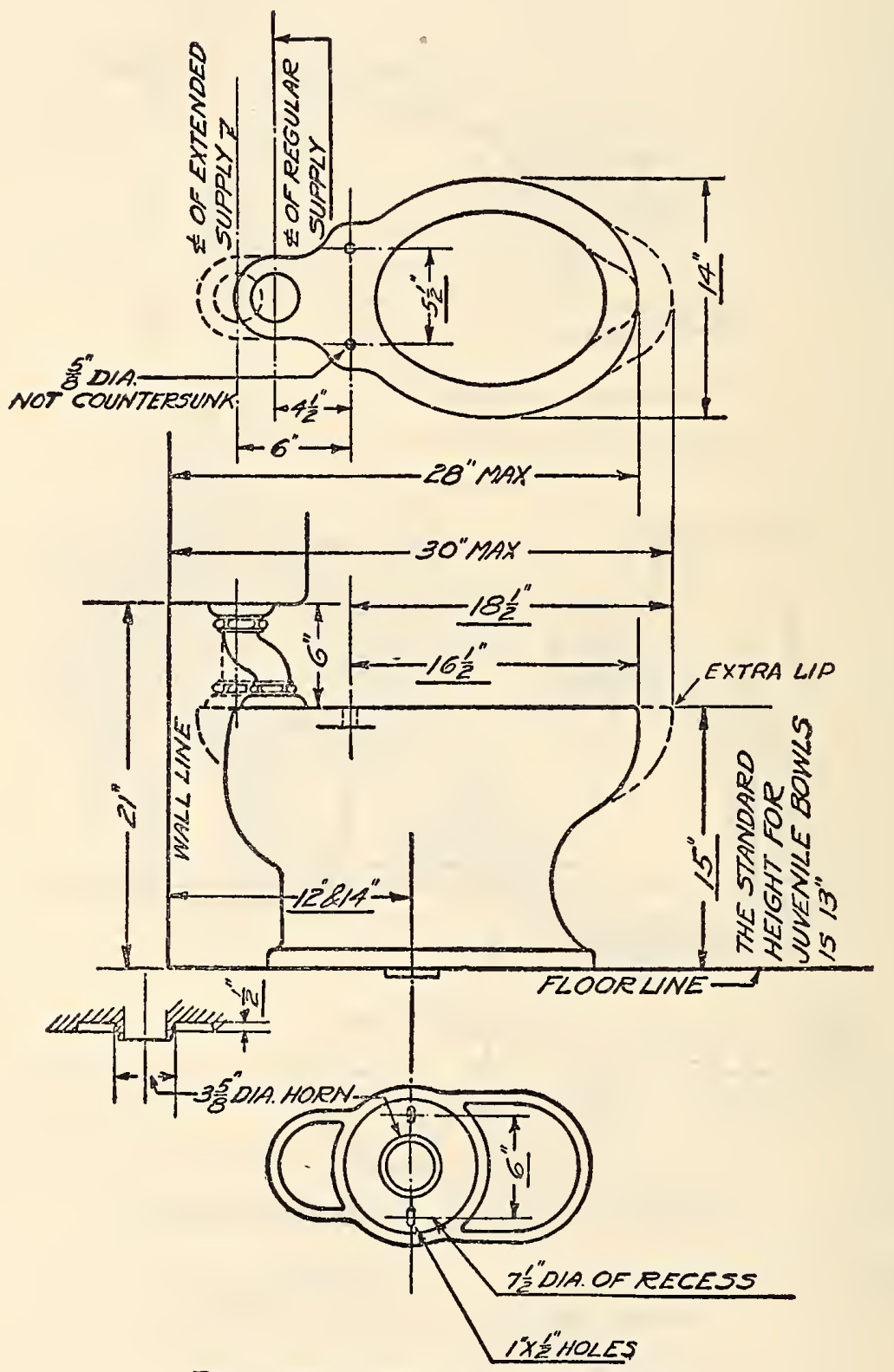

FIGURe 203.-Siphon jet water-closet bovols 


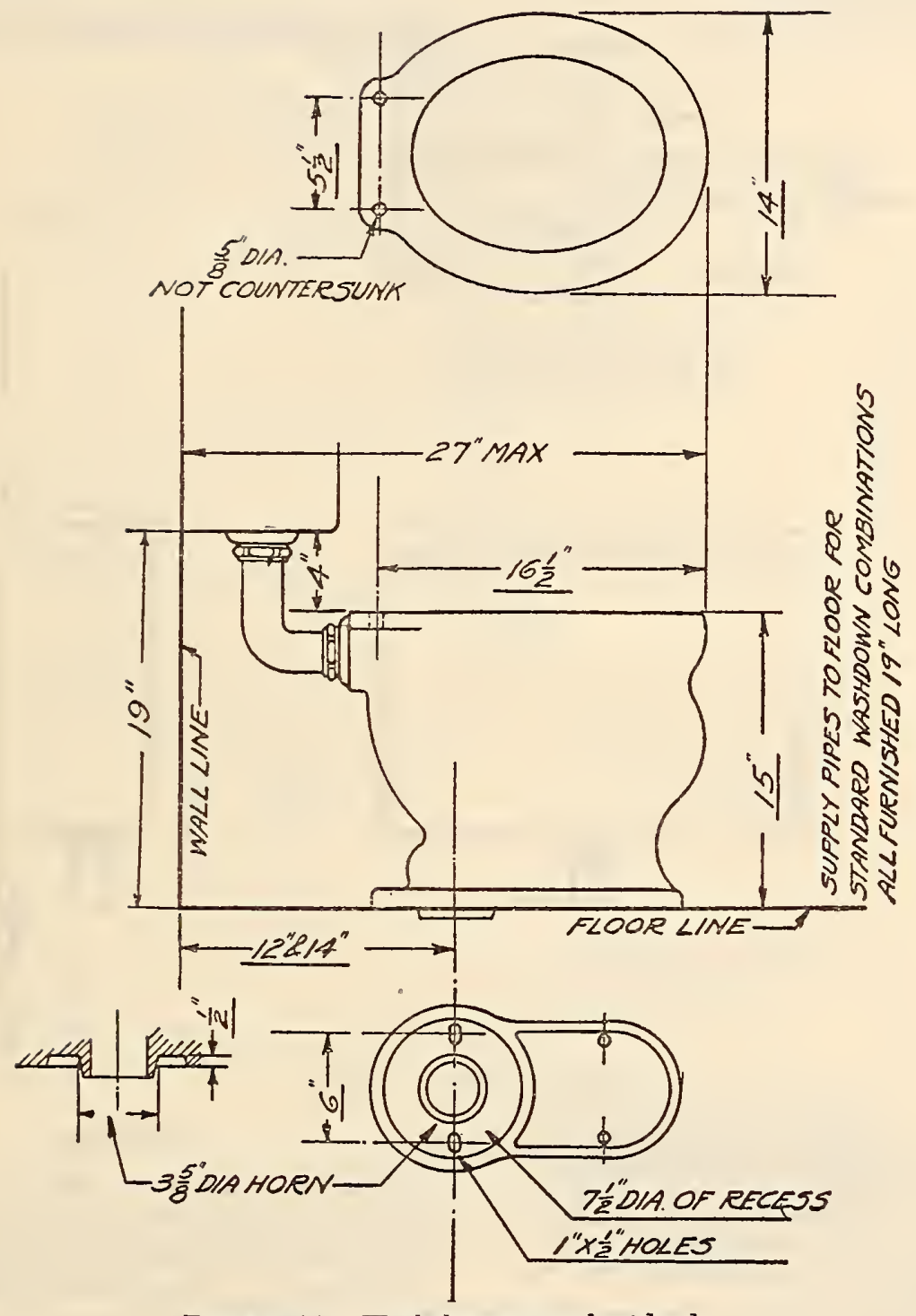

Figure 204.-Wash-down water-closet bowls 
Wall hanging siphon jet closet:

Top supply, extended lip.

Back supply, extended lip.

Side supply, extended lip.

Staple floor outlet siphon jet with raised rear vent:

Top supply, extended lip (regular height).

Top supply, extended lip (juvenile height).

Wall hanging siphon jet closet with raised rear vent:

Top supply, extended lip.

Back supply, extended lip.

Side supply, extended lip.

Staple floor onitlet reverse trap closet:

Back supply, regular bowl (regular height).

Wall hanging blow-out closet with raised rear vent:

Back supply, extended lip.

Side supply, extended lip.

Low-down tank with upper left-hand lever:

Small (approximately 6-gallon capacity).

Large (approximately 8-gallon capacity).

High-up tanks, with center outlet and over top supply. Where used, lever shall be on left side.

Small (approximately 3-gallon capacity).

Large (approximately 6-gallon capacity).

NotE.-The above action eliminates all side supply water-closet bowls of the floor type and all wash-down water-eloset bowls with extended lip.

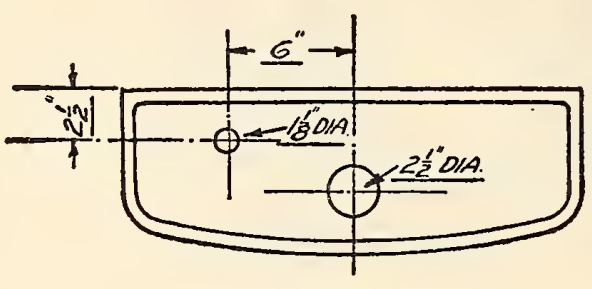

TOP VIEW OF TANK

WITH COVER REMOVED
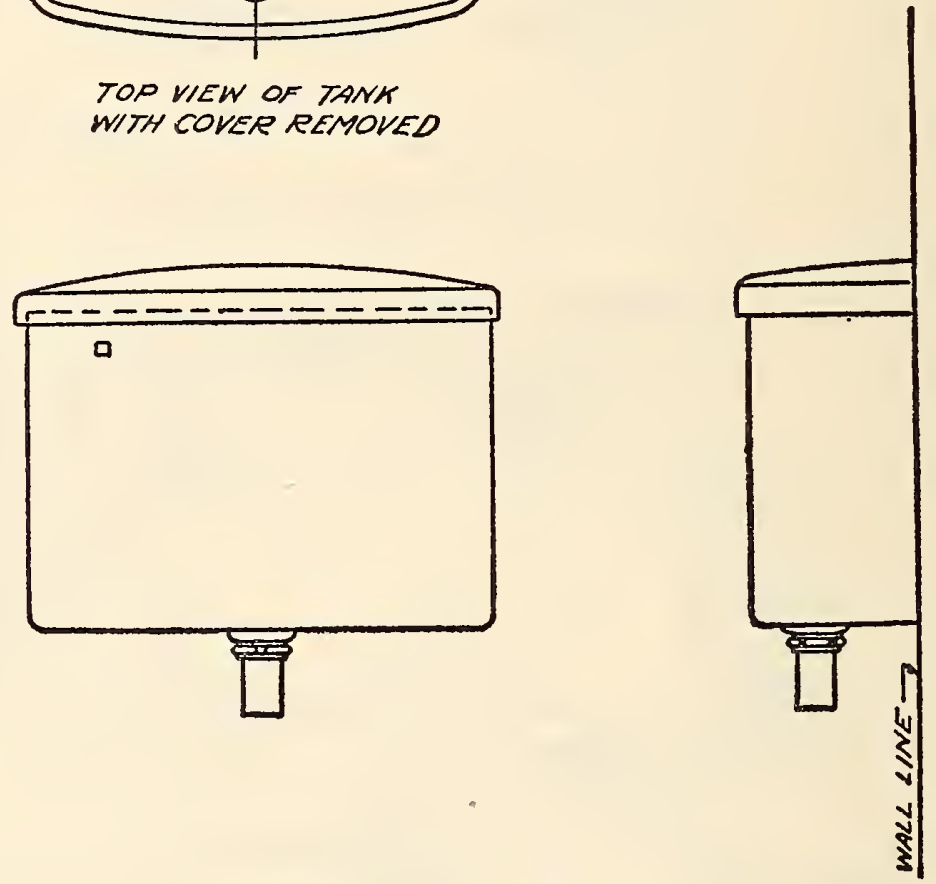

Figure 205.-Low tanks

Back supply, extended lip (regular height).

Staple floor outlet reverse trap closet with jet:

Back supply, regular bowl (regular height).

Back supply, extended lip (regular height).

Staple floor onitlet siphon wash down:

Back supply, regular bowl, 12-inch roughing (regular height).

Back supply, regular bowl, 14-inch roughing (regular height).

Back supply, regular, integral seat, 12-inch roughing (regular height).

Back supply, regular, integral seat, 14-inch roughing (regular height).

Staple floor outlet siphon wash down with jet:

Back supply, regular bowl (regular height).

Wall hanging blow-ont closet:

Back supply, extended lip.

Side supply, extended lip.

Back supply, extended lip, integral seat.

Side supply, extended lip, integral seat.

\section{LAVATORIES}

40. It is recommended that all lavatories be made with an overflow; that standardized supply and waste punchings be limited $(a)$ for two lavatory faucets and pop-up waste or chain-stay or combination fittings; $(b)$ for combination fitting for integral nozzle and pop-up waste.

Straight front lavatory:

18 by 20 inches, with back.

18 by 20 inches, without back.

20 by 24 inches, with back.

20 by 24 inches, without back.

22 by 27 inches, without back.

24 by 30 inches, without back.

Round front lavatory:

18 by 20 inches, with back.

18 by 20 inches, without back.

Round front corner lavatory:

17 by 17 inches, with back. 
533. EARTHENWARE AND STONEWARE

\subsection{BOWLS.}

(No nationally recognized specifications available.)

\subsection{CROCKERY.}

(No nationally recognized specifications available.)

\section{BRICK}

A structural unit in the form of a rectangular prism (usually solid and 8 by $33 / 4$ by $2 \frac{1}{4}$ inches in size).

NOTE-In the present state of the art the term "brick" when used without a qualifying adjective is generally understood to mean a structural unit of clay or shale formed while plastic and subse-

quently fired.
When substances other than clay or shale are employed, such as When substances other than clay or shale are employed, such as
lime and sand, cement and sand, fire clay, adobe, etc., the term "brick" should be suitably qualified.

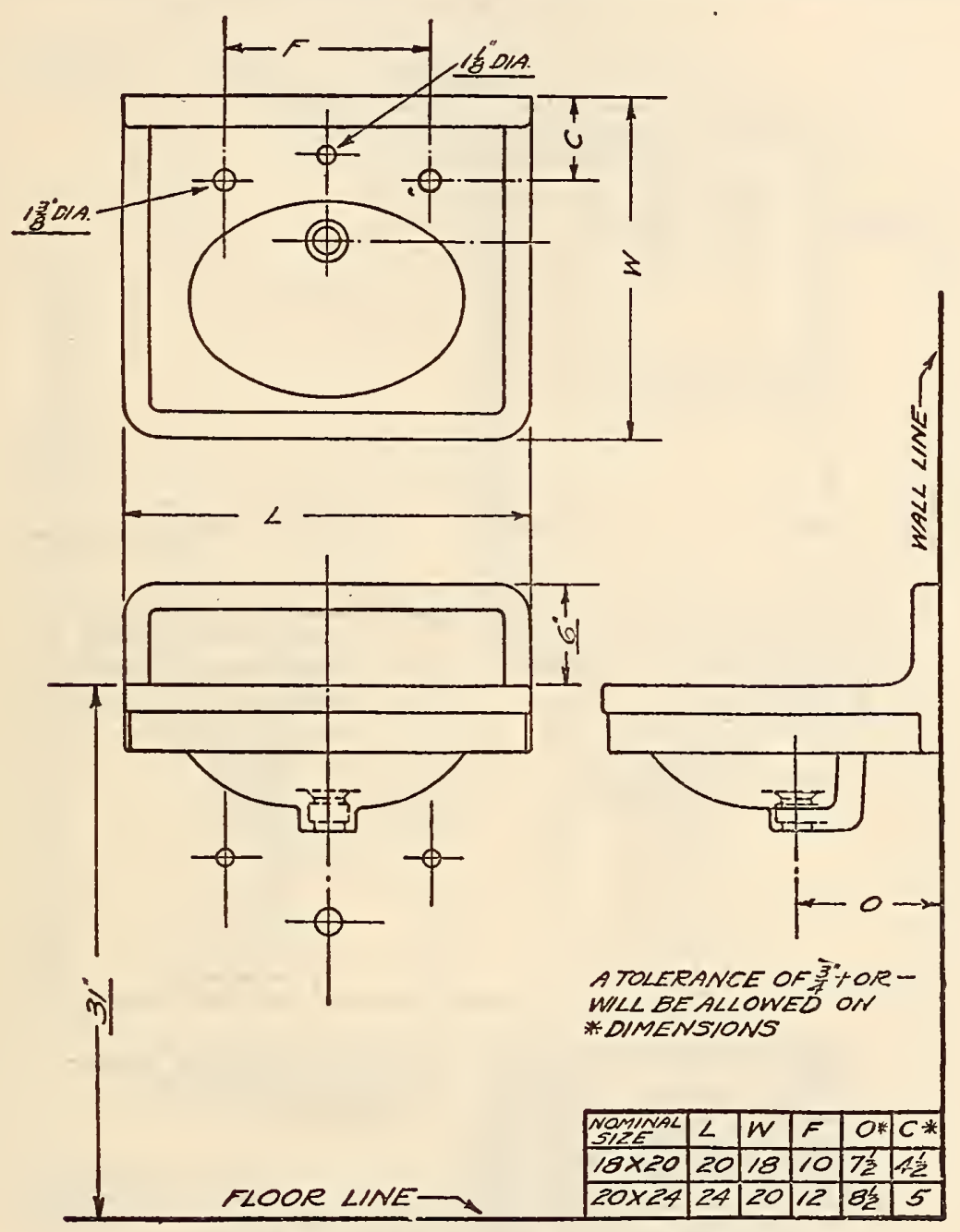

FIgURE 206.-Straight front lavatories, with back

533.3 POTS.

(No nationally recognized specifications available.)

534. BRICKS AND TILES

\subsection{BRICKS.}

534.10 General Items.

American Ceramic Society, tentative definitions, 1928.

\section{APPARENT SPECIFIC GRAVITY}

Apparent specific gravity is the specific gravity of the water-impermeable portion of the specimen; that is, solid material plus sealed pores or cavities. Apparent specific gravity is then the weight per unit of volume of water-impermeable portion of the specimen.

\section{BULK SPECIFIC GRAVITY}

Bulk specific gravity is the specific gravity of the composite bulk; that is, solid material plus 
sealed pores or cavities, and water-permeable pores. Bulk specific gravity is therefore the weight per unit of exterior volume.

\section{TRUE SPECIFIC GRAVITY}

True specific gravity is the weight per volume of the solid material in which all sealed pores or cavities and all water-permeable pores have been obliterated by fine grinding. at high temperatures, serial designation C16-20, 1920.

1. The object of this test is to determine the resistance of the specimen to deformation at a specified temperature for a specified time, when subjected to a compressive load of $25 \mathrm{lbs} . / \mathrm{in.}^{2}$ $\left(1.765 \mathrm{~kg} / \mathrm{cm}^{2}\right)$.

2. The apparatus consists essentially of a furnace and loading device. It shall be constructed in accordance with Figures 210 and 211 ,

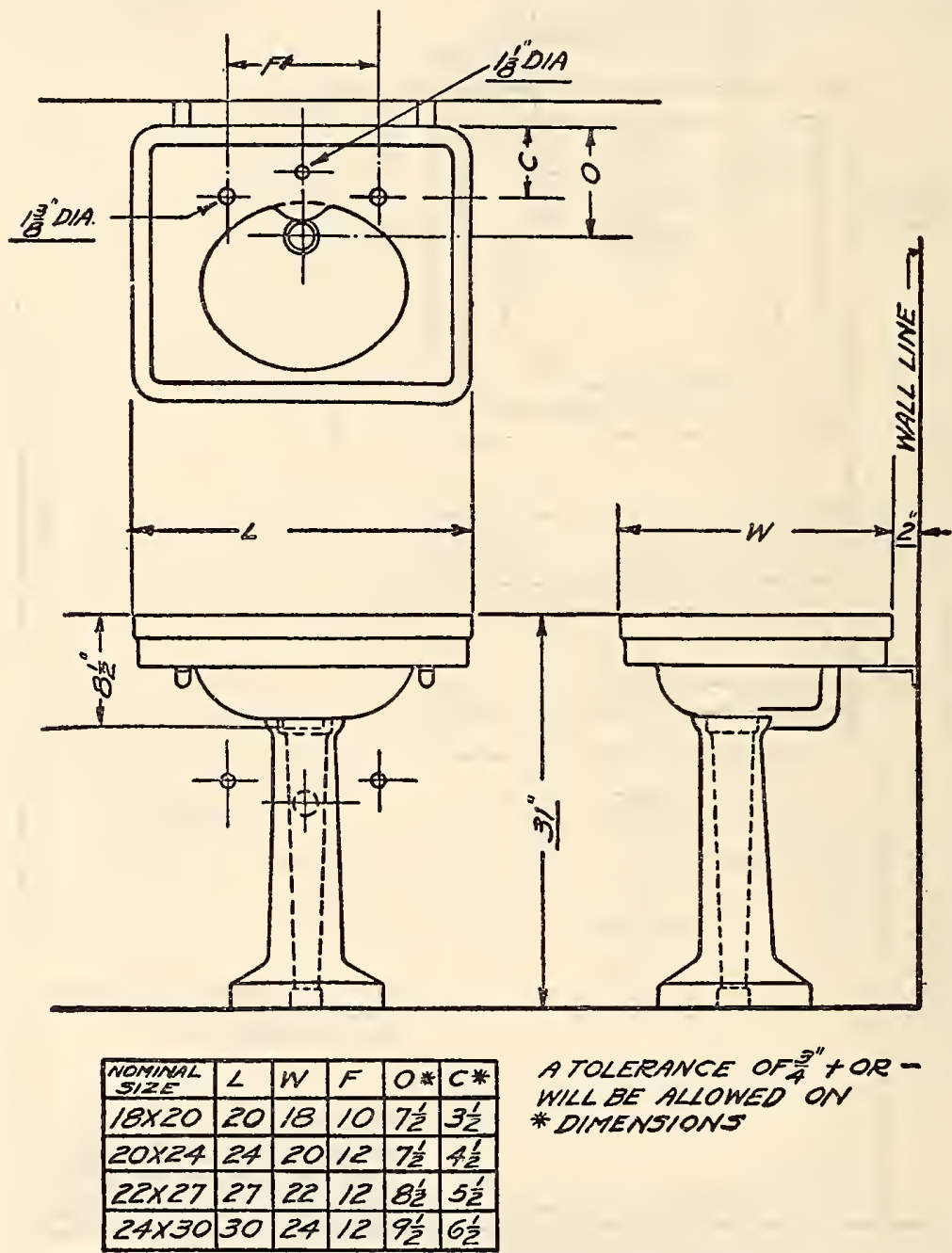

FIGURe 207.-Straight front lavatories, without back

American Ceramic Society, standard method of testing for pyrometric cone equivalent, 1928.

(This specification is similar to American Society for Testing Materials, C24, see p. 573, with the following variations:)

1. Test cones to measure $7 \mathrm{~mm}$ along the edge of the base and $30 \mathrm{~mm}$ high.

2 . The base of the test pieces and pyrometric cones shall be embedded not more than $2 \mathrm{~mm}$ in the plaque and their troweled faces shall make an angle $82^{\circ}$ of with the plaque.

3. Two or more tests at the proper temperature shall be made.

American Society for Testing Materials, standard method of test for refractory materials under load (a) The furnace shall be cylindrical in form, 18 inches $(457 \mathrm{~mm})$ in internal diameter, as shown in Figure 210.

(b) The heating shall be done with gaseous or oil fuel and compressed air, using not less than two burners located tangentially and so arranged that no flame can impinge upon the test specimen. The burners shall be such as will insure a uniform temperature in all parts of the furnace and be under complete control.

(c) The method of loading shown in Figure 210 shall be used, and the details shall be such as will insure accuracy in the applied load and freedom from eccentric loading, both in the original application and during the testing. It is advantageous 
to make the crossbeams as light as possible, so that | ice. A recording form of indicator is recommended the greater portion of the load may be concentrated where possible.

in the weights.

(d) The temperature may be measured either with a calibrated platinum-rhodium thermocouple, encased in a double protecting tube with the junc- approximately 9 by $4 \frac{1}{2}$ by $2 \frac{1}{2}$ inches (228 by 114

3. The test specimen shall consist, whenever possible, of a standard 9-inch brick placed vertically on end. In the case of blocks or shapes, sections

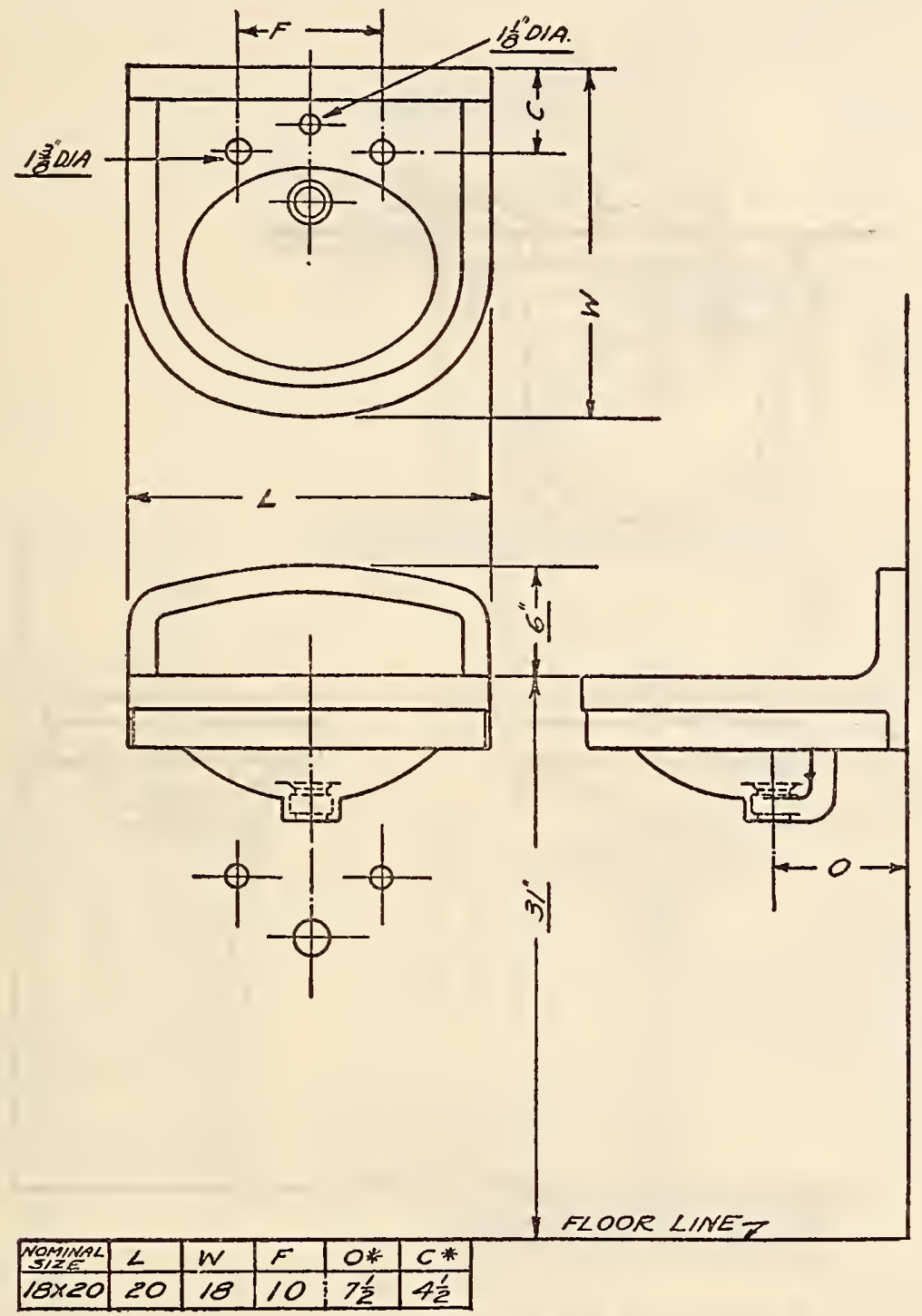

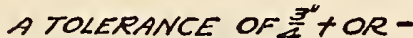 $W / L L$ BE ALLOWED ON * DIMENSIONS}

Figure 208.-Round front lavatories, with back

tion not more than 1 inch $(25 \mathrm{~mm})$ from the side or edge of the specimen and approximately opposite the center; or with some form of optical pyrometer that has been calibrated against a thermocouple in the furnace. If the thermocouple is used, the coldend temperature should be kept constant in melted by $64 \mathrm{~mm}$ ) shall be cut, utilizing as far as possible existing plane surfaces. The ends of the specimen shall be either groundsso that they are parallel and perpendicular to the vertical axis, or, if this is impossible, shall be bedded in a neutral cement, so that the specimen is perpendicular to the base of the furnace. 

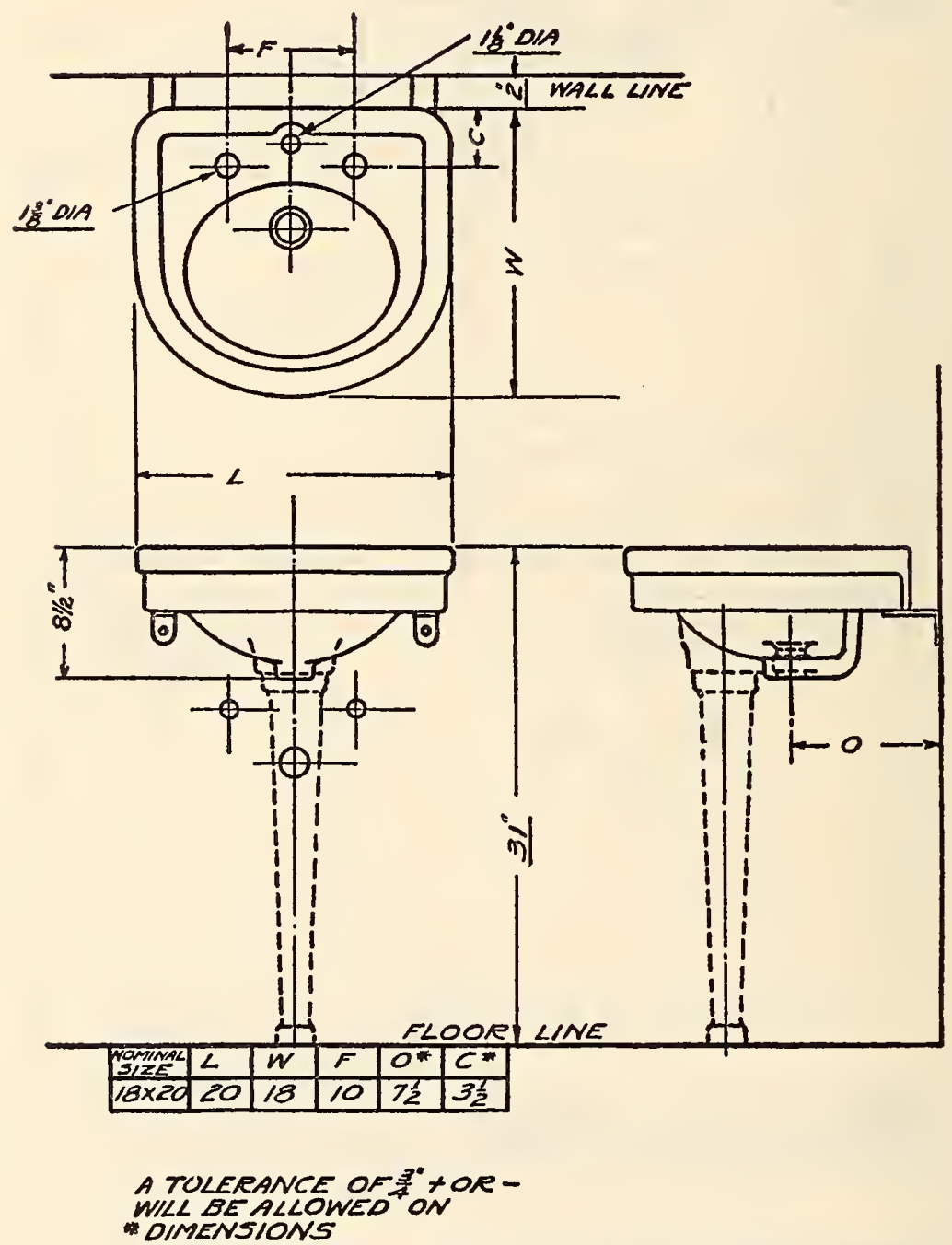

FIgURe 209.--Round front lavatories, without back 

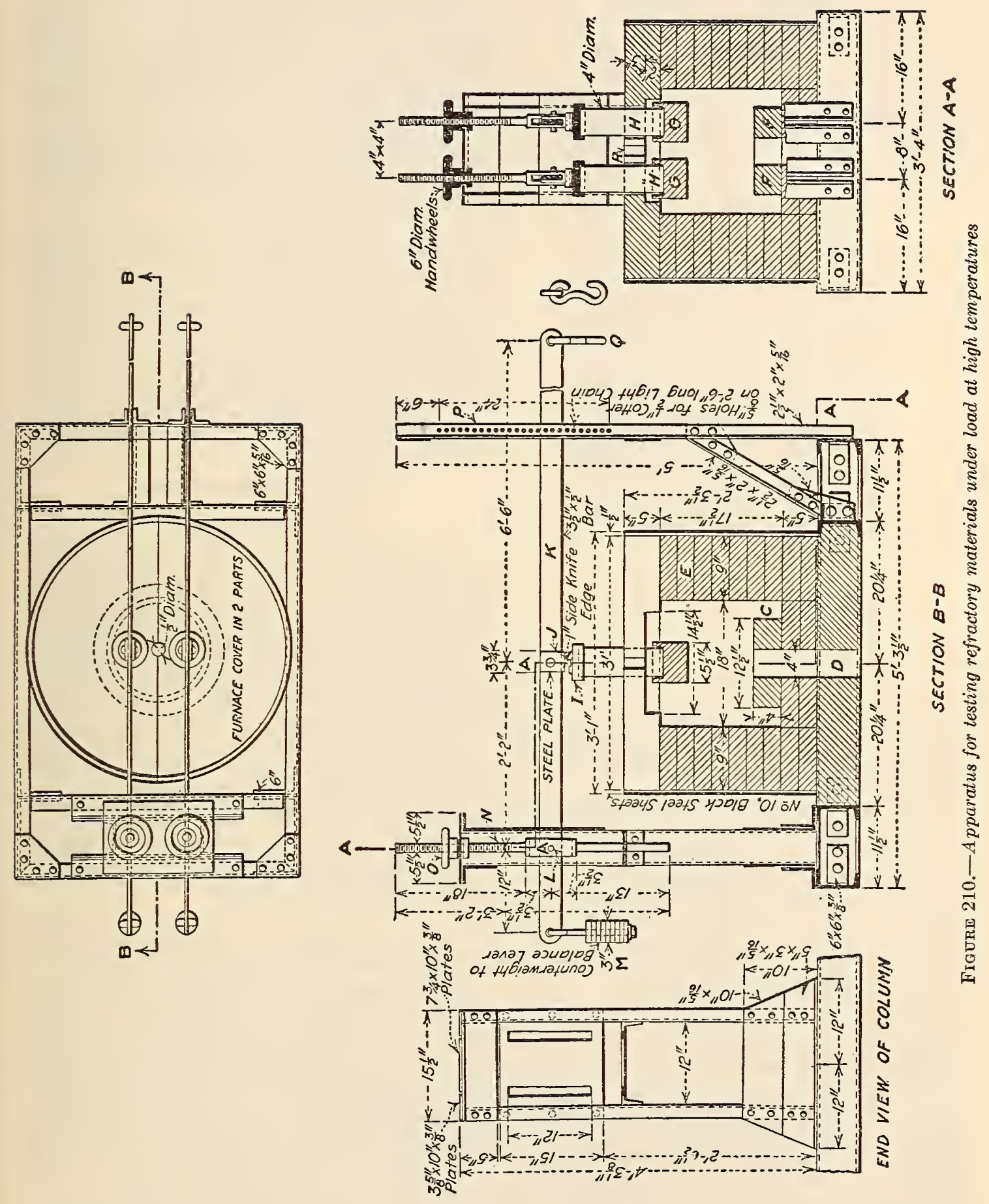
TABLE 1.-Temperature to be attained at time specified

[AII temperatures in degrees centigrade]

\begin{tabular}{|c|c|c|c|c|}
\hline \multirow[b]{2}{*}{ Time } & \multirow[b]{2}{*}{ Silica } & \multicolumn{3}{|c|}{ Fire clay } \\
\hline & & $\begin{array}{l}\text { Heavy } \\
\text { duty }\end{array}$ & $\begin{array}{l}\text { Moder- } \\
\text { ate duty }\end{array}$ & $\begin{array}{l}\text { Light } \\
\text { duty }\end{array}$ \\
\hline \multicolumn{5}{|l|}{$H$} \\
\hline $\begin{array}{rr}0 & 15 \\
& 30 \\
& 45\end{array}$ & $\begin{array}{r}40 \\
80 \\
140\end{array}$ & $\begin{array}{l}160 \\
280 \\
400\end{array}$ & $\begin{array}{l}160 \\
280 \\
400\end{array}$ & $\begin{array}{l}160 \\
280 \\
400\end{array}$ \\
\hline \multirow{3}{*}{$\begin{array}{r}11 \\
1 \\
3 \\
4\end{array}$} & 200 & 500 & 500 & 500 \\
\hline & 260 & 620 & 595 & 570 \\
\hline & 290 & 720 & 685 & 640 \\
\hline 45 & 300 & 815 & 770 & 700 \\
\hline \multirow{3}{*}{$\begin{array}{rr}2 & 1 \\
& \\
& \\
& 4\end{array}$} & 310 & 900 & 850 & 755 \\
\hline & 320 & 980 & 920 & 810 \\
\hline & $\begin{array}{l}385 \\
490\end{array}$ & $\begin{array}{l}1,045 \\
1,100\end{array}$ & $\begin{array}{r}990 \\
1,050\end{array}$ & $\begin{array}{l}860 \\
905\end{array}$ \\
\hline \multirow{4}{*}{$\begin{array}{ll}3 & \\
& 1 \\
& \\
& 4\end{array}$} & 590 & 1,150 & 1,100 & 950 \\
\hline & 695 & 1,195 & 1,145 & 985 \\
\hline & 800 & 1,235 & 1,185 & 1,020 \\
\hline & 900 & 1,270 & 1,220 & 1,050 \\
\hline 40 & 1,000 & 1,300 & 1,250 & 1,075 \\
\hline $\begin{array}{l}15 \\
30\end{array}$ & $\begin{array}{l}1,100 \\
1000\end{array}$ & $\begin{array}{l}1,330 \\
1,350\end{array}$ & 1,275 & 1,090 \\
\hline 45 & 1,250 & 1,350 & 1,300 & 1,100 \\
\hline $\begin{array}{ll}5 & 0\end{array}$ & 1,300 & 1,350 & 1,300 & 1,100 \\
\hline 15 & 1,350 & 1,350 & 1,300 & 1,100 \\
\hline 30 & 1,380 & 1,350 & 1,300 & 1,100 \\
\hline 45 & 1,410 & 1,350 & 1,300 & 1,100 \\
\hline $\begin{array}{ll}6 & 0\end{array}$ & 1,440 & 1,350 & 1,300 & 1,100 \\
\hline $\begin{array}{l}15 \\
30\end{array}$ & $\begin{array}{l}1,470 \\
1,500\end{array}$ & (end) & (end) & (end) \\
\hline 45 & 1,500 & & & \\
\hline \multirow{3}{*}{$\begin{array}{ll}7 & \\
& 15 \\
30\end{array}$} & 1,500 & & & \\
\hline & 1,500 & & & \\
\hline & $\begin{array}{l}1,500 \\
1,500\end{array}$ & & & \\
\hline \multirow[t]{2}{*}{80} & 1,500 & & & \\
\hline & (end) & & & \\
\hline
\end{tabular}

The test specimen shall be measured before testing, making not less than five observations in each direction to within \pm 0.02 inch $(0.5 \mathrm{~mm})$. The average dimensions shall be reported and the cross section calculated.

4. The test specimen shall occupy approximately the center of the furnace and should rest on a block of some highly refractory material, having a minimum expansion or contraction. A siliconcarbide brick has been found satisfactory. At the top of the test specimen a block of similar highly refractory material should be placed, extending through the furnace top to receive the load.

Note.-Gross errors which may more than double the contraction will result if the specimen is not set perpendicular to the base of the support or if the load is eccentrically applicd.

5. The rate of heating shall be in accordance with the requirements of Table 1 and the timetemperature curves of Figure 212 , which give the rate and time of heating suggested for different grades of material.

6. (a) The load is calculated from the average cross section as determined on the untested specimen and the requirement of the test. It is recommended that for general purposes, 25 lbs./in. ${ }^{2}$ $\left(1.765 \mathrm{~kg} / \mathrm{cm}^{2}\right)$ be used.

(b) The additional masses required to give the desired loading should be equally distributed on each side of the beam.

7. (a) At the expiration of the time of heating, the supply of heat shall be stopped and the furnace allowed to cool, during not less than five hours before removing the load and examining the test specimen.

Note.-The specimen shall be examined, immediately after the heating is stopped, for evidences of cracking and spalling, as such defects may develop later due to the rapid cooling of the furnace.

(b) After the test specimen has cooled to the room temperature it shall be remeasured as before described, and the change in length recorded and reported as percentage of the original length.

Noте.-It is recommended that a photograph be made of the specimen beforc and after testing, as yielding valuable information at a minimum of time and expense.

American Society for Testing Materials, standard methods of ultimate chemical analysis of refractory materials, including chrome ores and chrome brick, serial designation C18-21, 1921.

It will be understood that the making of a complete silicate analysis is a difficult procedure requiring a wide knowledge of the chemistry involved in the operations and a thorough training in carrying out the work. A skilled analyst of good training is therefore required to do the work. The descriptions here given cover the vital points of procedure, but frequent reference in regard to the details of the various manipulations must be made to Bulletin No. 422, United States Geological Survey on Analysis of Silicate and Carbonate Rocks, by W. F. Hillebrand; also to Treatise on the Ceramic Industries, volume 1 (1913), by J. W. Mellor; and to similar publications.

\section{GENERAI REFRACTORIES}

\section{GENERAL CONSIDERATIONS}

1. The sample shall be crushed in a hardened tool-steel mortar, using a pestle of the same sort. Fine grinding shall be done in an agate mortar, either by hand or by a mechanical sample grinder of the MeKenna, Carling, or similar type, so constructed as to prevent the introduction of impurity.

2. Moisture shall be determined in the sample in its ordinary air-dried condition, and all other percentage compositions shall be calculated to a moisture-free basis. Whenever a sample is weighed out for any determination, a moisture determination shall also be made. If preferred, the sample may be dried in a weighing bottle, from which the required samples shall be weighed out.

3. In all cases, check determinations shall be made, and the results shall be redetermined if satisfactory checks are not obtained. It shall be considered satisfactory if the differences between check determinations do not exceed the following limits:

Per cent

For silica or other constituent amounting to 30 per cent or over- 0.3 For alumina or other constituent amounting to 10 to 30 per cent For alumina or other constituent amounting to 10 to 30 per cent $\quad: 2$

These figures are stated in terms of the whole sample as 100 per cent.

\section{SOLUTIONS REQUIRED}

4. The acids referred to as concentrated shall be of approximately the following specific gravities:

Hydrochloric acid (HCl) _._._._._._. 19 Sulfuric acid $\left(\mathrm{H}_{2} \mathrm{SO}_{4}\right)$ Nitric acid $\left(\mathrm{HNO}_{3}\right)$

5. Dissolve $1.5 \mathrm{~g}$ of ammonium carbonate in 50 ec of cold water.

6. Dissolve $107 \mathrm{~g}$ of $\mathrm{NH}_{4} \mathrm{Cl}$ in $1,000 \mathrm{cc}$ of warm water.

7. Neutralize 20 cc of concentrated $\mathrm{HNO}_{3}$ with $\mathrm{NH}_{4} \mathrm{OH}$ and dilute to $1,000 \mathrm{cc}$. Test with litmus; the solution shall not be acid. 
8. Dissolve $1 \mathrm{~g}$ of ammonium oxalate in $50 \mathrm{cc}$ of water, heating gently.

9. The strength of the final standard titania solution shall be $1 \mathrm{cc}=0.0001 \mathrm{~g} \mathrm{TiO}_{2}$. To prepare the solution, weigh out in a platinum crucible an amount of potassium titanium fluoride $\left(\mathrm{K}_{2} \mathrm{TiF}_{6}\right)$ sufficient to make from $1 / 2$ to 1 liter of "stock solution" in which $1 \mathrm{cc}=0.0001 \mathrm{~g} \mathrm{TiO}_{2}$. Evaporate several times with $\mathrm{H}_{2} \mathrm{SO}_{4}$ without taking to dryness, thus driving out all fluorine. Take up the residue with water containing enough $\mathrm{H}_{2} \mathrm{SO}_{4}$ to make at least 5 per cent of the solution, when finally diluted to the standard solution strength. To standardize the stock solution take out two 50-cc portions,

\section{METHODS}

MOISTURE

11. To determine moisture, heat approximately $1 \mathrm{~g}$ of the sample at a temperature not under $105^{\circ}$ nor over $110^{\circ} \mathrm{C}$., to constant weight.

\section{LOSS ON IGNITION}

12. To determine loss on ignition, heat about $1 \mathrm{~g}$ of the sample over a blast lamp or in an electric furnace at $900^{\circ}$ to $1,000^{\circ} \mathrm{C}$., to constant weight. Ignition loss may be determined in the sample from which the moisture has been removed. The per-
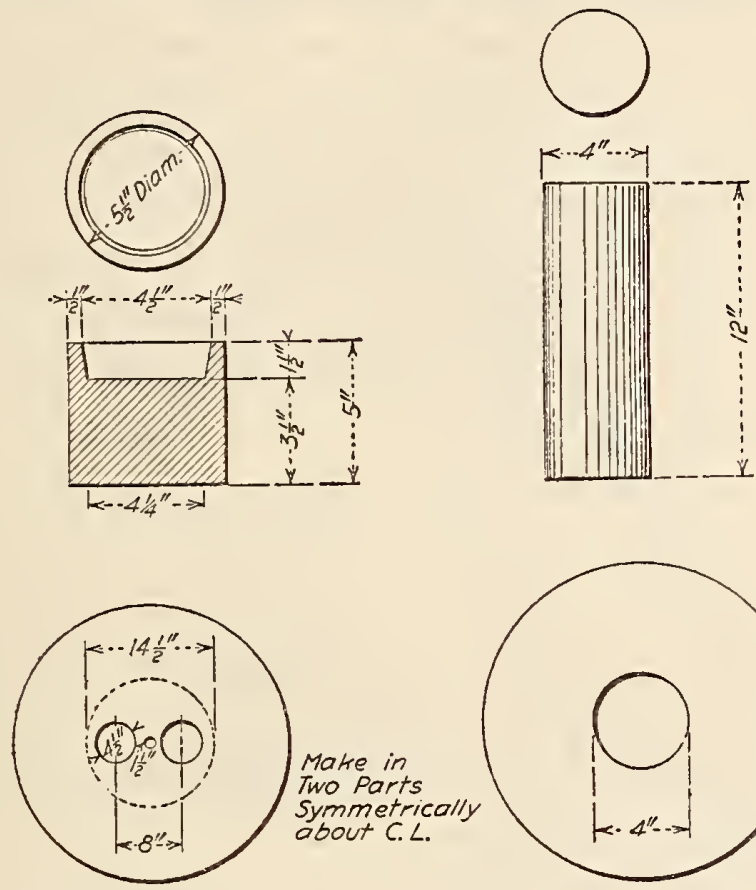

Matre in wo parts symmetricall

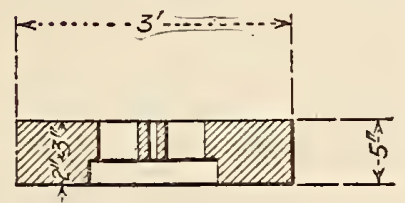

A

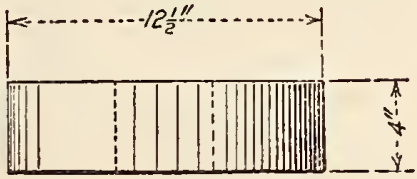

B

FigdRE 211.-Special shapes required for furnace

dilute, boil, and precipitate with ammonia. Filter, wash with hot water until free from alkali, ignite, blast, and weigh. The duplicate should check very closely. From the weight of titania thus determined calculate the strength of the stock solution. The standard solution to be used is obtained from the stock solution by diluting until $1 \mathrm{cc}=0.0001 \mathrm{~g} \mathrm{TiO}_{2}$.

10. Dissolve about $0.4 \mathrm{~g}$ (a little less) of $\mathrm{KMnO}_{4}$ in 1 liter of water and check against an iron solution of known purity and strength. Dilute the solution until $1 \mathrm{cc}=0.001 \mathrm{~g} \mathrm{Fe}_{2} \mathrm{O}_{3}$.

The ordinary chemical iron wire is not sufficiently pure for this use. If preferred, the solution may be standardized against sodium oxalate, a pure form of which is prepared expecially for such work by the National Bureau of Standards, Washington, D. C. centage of ignition loss is calculated to a moisturefree basis.

\section{SIIICA}

13. To determine silica, weigh out approximately $0.5 \mathrm{~g}$ of the sample and mix with $5 \mathrm{~g}$ of $\mathrm{Na}_{2} \mathrm{CO}_{3}$. Put a little $\mathrm{Na}_{2} \mathrm{CO}_{3}$ in the bottom of the crucible before introducing the mixture, and then cover the mixture with a little more $\mathrm{Na}_{2} \mathrm{CO}_{3}$. Fuse over a Meker burner or blast lamp until complete solution is obtained. Cool the fusion, and as it solidifies rotate the crucible to spread the mass up the side walls. Dissolve the fusion in about $100 \mathrm{cc}$ of water in a platinum or porcelain evaporating dish. Add about $20 \mathrm{cc}$ of concentrated $\mathrm{HCl}$, introducing it slowly by means of a pipette, keeping the dish covered with a watch glass, Evaporate to dryness 
and heat until the fumes of $\mathrm{HCl}$ are gone. Add about $5 \mathrm{cc}$ of concentrated $\mathrm{HCl}$ and 30 to $40 \mathrm{cc}$ of water. Warm on a water bath for 10 to 15 minutes and break up the lumps. Decant the clear liquid onto a filter paper and collect the filtrate in a 400-cc beaker. Add more $\mathrm{HCl}$ and water to the evaporating dish, warm again, and decant. Repeat this a third time.

Finally, transfer the contents of the dish to the filter paper. Wash with cold water until silver nitrate shows no chlorides to be left. Transfer the filtrate to the original evaporating dish, evaporate again to dryness, take up with a little $\mathrm{HCl}$ and water, transfer to a new filter paper, and wash as before, Transfer both silica precipitates to a plati-

\section{ALUMINA}

14. Regarding the determination of alumina, reference should be made to Scientific Paper No. 286 of the National Bureau of Standards, by Doctor Blum on Determination of Alumina as Oxide.

To determine alumina, to the filtrate from the silica determination, add about $10 \mathrm{cc}$ of $\mathrm{NH}_{4} \mathrm{Cl}$ solution and heat to boiling. Then add $\mathrm{NH}_{4} \mathrm{OH}$ very slowly and with constant stirring until there is a slight excess. The $\mathrm{NH}_{4} \mathrm{OH}$ must be free from $\mathrm{CO}_{2}$. Filter hot and rapidly, and wash four times by decantation with hot solution of ammonium nitrate. Carefully wash the precipitate from the filter paper into a beaker, using hot water.

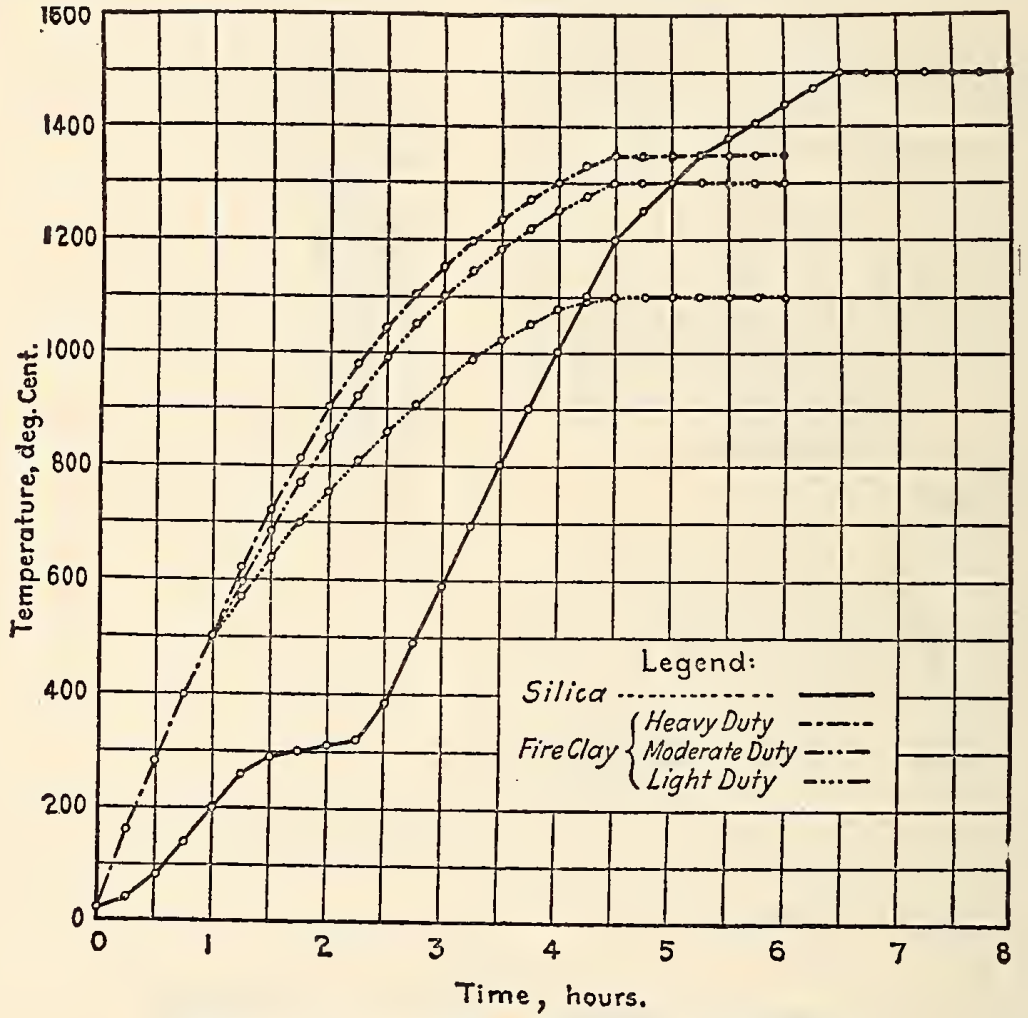

Figure 212.-Time-temperature curve for load test

num crucible. Ignite carefully over a Bunsen flame until the filter paper is burned off, then blast for about 30 minutes, cool, and weigh. Repeat blasting for five minutes, wcigh again, and repeat until constant weight is obtained.

To the residue in the crucible add about $5 \mathrm{cc}$ of water and five drops of $\mathrm{H}_{2} \mathrm{SO}_{4}$. Add $\mathrm{HF}$ drop by drop at first, and then slowly until the crucible is almost one-half filled. Warm on a hot plate until almost dry, add 2 or 3 ce more of HF and evaporate to dryness. Hcat the crucible to bright redness and then blast for five minutes. Cool and weigh and repeat blasting to constant weight. The loss in weight from the original silica residue represents the actual silica content $\left(\mathrm{SiO}_{2}\right)$, except for that part of silica which is later recovered from alumina, etc. The residue from the $\mathrm{HCl}$ evaporation is left in the crucible and the total precipitate of alumina, etc., is added to this same crucible in which it is ignited and weighed.
Dissolve the precipitate in hot dilute $\mathrm{HCl}$. Repeat the precipitation with $\mathrm{NH}_{4} \mathrm{Cl}$ and $\mathrm{NH}_{4} \mathrm{OH}$. Decant four times as before, using hot ammonium nitrate. Transier to the filter paper and wash with hot ammonium nitrate until the washings are free from chlorides when tested by silver nitrate. Evaporate the filtrate nearly to dryness, add a little $\mathrm{NH}_{4} \mathrm{OH}$ and continue the evaporation. Keep the solution alkaline to coagulate any iron and aluminum hydroxides. Transfer the precipitate to a filter paper and wash thoroughly. Transfer the moist filter paper to the platinum crucible containing the residue from the silica. Burn off the filter paper and blast the precipitate. Repcat blasting and weighing to a constant weight.

\section{IRON OXIDI}

15. To detcrmine iron oxidc, fuse the ignited alumina precipitate with about six times its weight 
of potassium pyrosulphate or potassium bisulphate. Avoid sputtering. Heat carefully to redness and continue heating until the residue is all dissolved. Cool, dissolve in warm water, and add about $10 \mathrm{cc}$ of dilute $\mathrm{H}_{2} \mathrm{SO}_{4} \quad(1: 5)$. Evaporate to a small volume; then heat to a higher temperature until copious fumes of $\mathrm{H}_{2} \mathrm{SO}_{4}$ are evolved. Sufficient $\mathrm{H}_{2} \mathrm{SO}_{4}$ should be present to form a pasty mass when cooled. Take up with water, filter off, wash, ignite, and weigh the silica as before and evaporate with $\mathrm{HF}$ as before. The loss in weight is extra silica to be added to the original silica determination and subtracted from the alumina. Fuse the residue with a little potassium pyrosulphate or potassium bisulphate and add the solution of this cake to the main solution. Transfer the total filtrate to a $250 \mathrm{cc}$ graduated flask and dilute to $250 \mathrm{cc}$, mixing thoroughly. Draw off 50 cc of this solution and reserve for titania determination. Pass the remaining $200 \mathrm{cc}$ through a Jones reductor or otherwise reduce the solution with pure zinc and $\mathrm{H}_{2} \mathrm{SO}_{4}$. To the solution add $3 \mathrm{cc}$ of 10 per cent $\mathrm{CuSO}_{4}$ solution and titrate with standard $\mathrm{KMnO}_{\text {t }}$ solution (1 ce equivalent to $0.001 \mathrm{~g}$ $\mathrm{Fe}_{2} \mathrm{O}_{3}$ ), until a faint pink tinge is seen. If Jones reductor or similar apparatus is used, subtract the $\mathrm{KMnO}_{4}$ equivalent, which has previously been determined on blank samples run through the apparatus. From the result calculate the iron as $\mathrm{Fe}_{2} \mathrm{O}_{3}$.

\section{TTTANIA}

16. To determine titania, place in a small Nessler tube the $50 \mathrm{cc}$ of filtrate previously set aside for titania determination, and fill the tube up to the graduation mark. Add $1 \mathrm{cc}$ of $\mathrm{H}_{2} \mathrm{O}_{2}$ (free from fluorine) and shake well. The color of this solution is now compared in any standard color comparator with the color of a known standard solution, preferably of such strength that $1 \mathrm{cc}$ equals $0.0001 \mathrm{~g}$ of titanium dioxide $\left(\mathrm{TiO}_{2}\right)$. To make the comparison, put $10 \mathrm{cc}$ of this standard solution into a second small Nessler tube and dilute with water from a burette until the color is matched. Note the amount of water added and calculate the percentage of $\mathrm{TiO}_{2}$ in the sample.

\section{LIME}

17. To determine lime, evaporate the filtrate from the alumina precipitation to about $250 \mathrm{cc}$. While still boiling add about 5 ce of acetic acid. Meanwhile dissolve about $1 \mathrm{~g}$ of oxalic acid in a little hot water and add to the solution. In about five minutes add a slight excess of $\mathrm{NH}_{4} \mathrm{OH}$ and boil until precipitation is complete. Cool thoroughly and filter. Wash three times by decantation with dilute $\mathrm{NH}_{4} \mathrm{OH}(1: 10)$ or a 1 per cent solution of ammonium oxalate. Dissolve the precipitate by adding about $50 \mathrm{cc}$ of dilute $\mathrm{HNO}_{3}(1: 5)$. Again add a slight excess of $\mathrm{NH}_{4} \mathrm{OH}$ and a few drops of oxalic-acid solution. Boil well and let stand a couple of hours to cool. Filter and wash as before. Transfer the precipitate to a platinum crucible, carefully burn off the paper and ignite over a blast lamp for about 10 minutes. Cool and weigh quickly. Repeat the blasting to constant weight. The increase in weight of crucible is calcium oxide.

\section{IIAGNESIA}

18. To determine magnesia, add to the filtrate from lime precipitation about $2 \mathrm{~g}$ of sodium-ammonium-phosphate dissolved in $15 \mathrm{cc}$ of water. Stir vigorously, and while stirring add drop by drop about one-third of the volume of the solution of $\mathrm{NH}_{4} \mathrm{OH}$. Let stand 12 to 24 hours. Filter and wash the precipitate with dilute $\mathrm{NH}_{4} \mathrm{OH}(1: 10)$. Discard the filtrate. Wash the precipitate until the washings show no discoloration when tested with silver nitrate. Redissolve the precipitate in warm dilute $\mathrm{HNO}_{3}(1: 5)$, using about $50 \mathrm{cc}$, and collect in a beaker. Precipitate and wash as before. Collect the precipitate on a Gooch asbestos crucible. Dry slowly and then heat over a Meker burner for about 10 minutes; cool and weigh. Reignite to constant weight.

Tbe $\mathrm{Mg}_{2} \mathrm{P}_{2} \mathrm{O}_{7}$ is never pure, being contaminated by small amounts of calcium, barium, aluminum, iron manganese, etc but in the analysis of refractories this error is negligible, and correction for tbese impurities is not required unless previously agreed upon.

\section{ALKALIES}

19. To determine alkalies, the J. Lawrence Smith method shall be used. Weigh out about $0.5 \mathrm{~g}$ of the sample and mix well with $0.5 \mathrm{~g}$ of $\mathrm{NH}_{4} \mathrm{Cl}$ and $3 \mathrm{~g}$ of $\mathrm{CaCO}_{3}$. Transfer to a platinum crucible, placing about $0.5 \mathrm{~g}$ of $\mathrm{CaCO}_{3}$ in the bottom of the crucible and a similar amount over the top of the mixture. Heat gently over a low flame for about 15 minutes to volatilize $\mathrm{NH}_{4} \mathrm{Cl}$. Then raise the temperature until the lower three-quarters of the crucible is dull red. Hold this temperature for about one hour. Cool, take up with about $50 \mathrm{ce}$ of water and heat over a water bath, adding water to replace that lost by evaporation. Break up any lumps with a small pestle. Decant the clear liquid through a filter paper and wash four times by decantation. Then transfer the residue to the filter. Wash until silver nitrate shows only a very faint turbidity. To the filtrate add $\mathrm{NH}_{4} \mathrm{OH}$ and ammonium carbonate and heat to boiling. Filter and again digest the precipitate with $\mathrm{NH}_{4} \mathrm{OH}$ and ammonium carbonate. Filter and allow the filtrate to collect with the previous filtrate, then evaporate to dryness in a platinum or porcelain dish. Remove ammonium salts by gentle ignition in a moving flame. Treat with water and remove the last trace of lime by adding ammonium oxalate to the boiling solution and let stand over night. Filter, evaporate to dryness, ignite gently, and allow to cool. Then moisten the residue with $\mathrm{HCl}$, evaporate again to dryness, ignite gently, and weigh. Dissolve in water. Ignite and weigh any insoluble residue, deducting this from the alkali chlorides.

Dilute the solution of the mixed chlorides and add hydrochloroplatinic acid in amount equal to $0.3 \mathrm{cc}$ more than would be necessary if all chlorides were present as $\mathrm{NaCl}$. Evaporate to a sirupy consistency. Cool and treat with a few cubic centimeters of 80 per cent ethyl alcohol. Stir and decant through a weighed Gooch crucible. Treat again with 80 per cent alcohol and decant. Repeat washing and decantation until the alcohol leaving the Gooch crucible is colorless and the precipitate appears golden yellow, not orange. Transfer the precipitate to the Gooch crucible, wash with 80 per cent alcohol about six times, and dry at $130^{\circ} \mathrm{C}$. to constant weight. This residue is potassium chloroplatinate.

The oxides are calculated as follows:

Weigbt of potassium chloroplatinate $\times 0.3068=\mathrm{KCl}$. Weigbt of potsssium chloroplatinate $\times 0.1941=\mathrm{K}_{2} \mathrm{O}$ Total chlorides $-\mathrm{KCl}=\mathrm{NaCl}$.

$\mathrm{NaCl} \times 0.5303=\mathrm{Na}_{2} \mathrm{O}$

\section{CHROME ORES AND CHROME BRICF}

20. The sample shall be secured after the manner of any of the approved methods of sampling. If from ores or chrome-bearing refractories, it shall not be less than 10 pounds, in small nut size particles when sent to the laboratory. If from chrome brick the sample shall consist of a small portion from the interior of from 15 to 25 bricks. This $10-$ pound sample shall be crushed to pass a No, 20 
sieve ${ }^{4}$ and quartered until a sample of $100 \mathrm{~g}$ is secured, which shall be ground to pass a No. 100 sieve. From this portion a smaller working sample shall be quartered and ground in an agate mortar to a very nearly gritless powder. In the case of hard-burned brick or hard lump chrome ore the very fine grinding assists materially in solution of the sample.

21. Dry the fine, ground powder at $110^{\circ} \mathrm{C}$. for not less than one hour. Cool in desiccator. Fuse $0.5 \mathrm{~g}$ of the thoroughly dried sample in approximately $10 \mathrm{~g}$ of fused potassium bisulphate in a platinum crucible. The usual cautions of a bisulphate fusion should be observed; that is, the flame turned very low so that the bisulphate just melts, increasing the flame so that the bisulphate remains in a liquid condition, very gradually increasing until the crucible reaches a dull-red heat. The object of the slow heating is to retain the sulphuric acid, which is the effective part of the flux. The most refractory ores should be thoroughly fused in one and one-half to two hours. Remove the crucible from the burner and allow to cool in the air. The bisulphate melt will shrink from the crucible and may be removed easily in one cake. Dissolve the fusion in $200 \mathrm{cc}$ hot $\mathrm{HCl}(1: 1)$, boiling until the solution acquires a clear, bright green color. Transfer the solution to a porcelain evaporating dish and evaporate to dryness on a water bath. Bake at exactly $120^{\circ}$ C. until free from hydrochloric acid fumes. Moisten with hot $\mathrm{HCl}$ (1:1), evaporate on water bath and bake a second time at $120^{\circ} \mathrm{C}$. Dissolve in $200 \mathrm{cc}$ hot dilute $\mathrm{HCl}(1: 1)$ and filter out the silica. Ignite and weigh the silica in a platinum crucible. Add two drops concentrated $\mathrm{H}_{2} \mathrm{SO}_{4}$ and sufficient $\mathrm{HF}$ to remove all silica. Evaporate, ignite, and weigh. The difference gives the amount of silica present in the sample. Should there be any appreciable residue left in the crucible, it should be fused with a small smount of fused $\mathrm{KHSO}_{4}$ and added to the filtrate from the silica.

Dilute the filtrate from the silica to about 500 cc and precipitate iron, aluminum, chromium, and titanium, if present, with $\mathrm{NH}_{4} \mathrm{OH}$, using care to have not more than two drops of $\mathrm{NH}_{4} \mathrm{OH}$ in excess in order to avoid solution of chromium and aluminum hydroxide in the alkaline solution. On account of the relatively large amount of magnesium that is usually present in chrome ores and chrome brick it is advisable to dissolve the precipitate in hot dilute $\mathrm{HCl}$ and precipitate a second time. Filter out the combined hydroxides and dissolve them on the filter paper with $\mathrm{HNO}_{3}$, catching the solution in a porcelain casserole. Add a few crystals of $\mathrm{KClO}_{3}$ and evaporate the nitric solution, adding a crystal of $\mathrm{KClO}_{3}$ from time to time until the chromium is completely oxidized, which is indicated by a change from a dull green to a brilliant orange color. Dilute this solution with hot water and again precipitate with $\mathrm{NH}_{4} \mathrm{OH}$, filtering as before. The filtrate contains a large percentage of the chromium, while the residue on the filter consists of iron and aluminum hydroxides and titanium, if present. Repeat the oxidation two or three times until the filtrate from the iron and aluminum hydroxide precipitation shows no further traces of chromium. Concentrate and combine the filtrates containing chromium to approximatcly 500 cc. Add $\mathrm{H}_{2} \mathrm{SO}_{3}$ until the liquid changes from a bright orange to a brilliant green color and heat to boiling. Add $\mathrm{NH}_{4} \mathrm{OH}$ to precipitate chromium as hydroxide, having not more than two drops in excess, as stated above. Filter, wash well with hot water, ignite,

4 For sieve requirements, see A. S. T. M. E11,500.2, p. 1 . and weigh as $\mathrm{Cr}_{2} \mathrm{O}_{3}$. The iron and aluminum and titanium hydroxides are ignited and weighed in a platinum crucible. Fuse the combined oxides with a small amount of $\mathrm{KHSO}_{4}$, following the same precautions as mentioned above. Dissolve the fusion in dilute $\mathrm{H}_{2} \mathrm{SO}_{4}$, reduce with $\mathrm{H}_{2} \mathrm{~S}$ and titrate the iron with standard permanganate solution. Determine the titanium present by the colorimetric method, as described in section 16 . Subtract the combined amounts of $\mathrm{Fe}_{2} \mathrm{O}_{3}$ and $\mathrm{TiO}_{2}$ from the total weight of the three oxides and thus determine $\mathrm{Al}_{2} \mathrm{O}_{3}$. If titanium is absent, reduce the iron with metallic zinc, and titrate witll $\mathrm{K}_{2} \mathrm{MnO}_{4}$, which is a little quicker method than the $\mathrm{H}_{2} \mathrm{~S}$ reduction.

To the combined filtrates from the precipitations of iron, aluminum, chromium, and titanium hydroxides add bromine, allow to stand for a few minutes and then add sufficient $\mathrm{NH}_{4} \mathrm{OH}$ to precipitate the manganese hydroxide. Boil to concentrate the manganese hydroxide if any be present. Filter, ignite, and weigh as $\mathrm{Mn}_{3} \mathrm{O}_{4}$. In the filtrate from the manganese add $\mathrm{NH}_{4} \mathrm{OH}$ and $\left(\mathrm{NH}_{4}\right)_{2} \mathrm{C}_{2} \mathrm{O}_{4}$. Heat to boiling, and when the calcium oxide has settled filter with the usual precautions. The calcium oxide may be determined by ignition or by the volumetric method. (Refer to the determination of calcium oxide as described in sec. 17.)

Evaporate the filtrate from the calcium oxalate to dryness with $\mathrm{HNO}_{3}$ to remove the excess of ammonium salts. Dissolve the residue in hot water, filter, allow to cool, and determine $\mathrm{MgO}$ as described in section 18.

Determine alkalies by the J. Lawrence Smith method, as described in section 19 .

\section{RAPID ROUTINE METHOD FOR THE ANALYSIS OF CHROME
BRICK AND CHROME ORE}

22. The sample shall be secured after the manner of any of the approved methods of sampling. If from ores or chrome-bearing refractories, it shall not be less than 10 pounds in small nut-sized particles when sent to the laboratory. If from chrome brick, the sample shall consist of a small portion from the interior of from 15 or 25 bricks. This $10-$ pound sample shall be crushed to pass a No. 20 sieve, and quartered, until a 4-ounce sample is secured, which shall be ground to pass a No. 100 sieve (above). From this portion a smaller working sample shall be quartered and ground in an agate mortar to a very nearly gritless powder. In the case of hard-burned brick or hard-lump chrome ore the very fine grinding assists materially in solution of the sample. Dry the fine, ground powder at $110^{\circ} \mathrm{C}$. for not less than one hour. Cool in desiccator.

23. Fuse $0.5 \mathrm{~g}$ of the sample in 8 to $10 \mathrm{~g}$ of sodium peroxide in a nickel or porcelain crucible over a Bunsen burner, being careful not to heat too strongly, When fused leach out with cold water and boil 15 minutes to decompose any sodium peroxide. Cool and add $100 \mathrm{cc}$ of $\mathrm{H}_{2} \mathrm{SO}_{4}(1: 1)$. Cool to tap-water temperature and titrate with ferrous ammonium sulphate and potassium permanganate. Time 30 to 45 minutes.

\section{SILICA AND IRON}

24. Fuse $0.5 \mathrm{~g}$ of the sample in a nickel crucible over a Bunsen burner. Leach out with cold water, make acid with $\mathrm{HCl}$, and evaporate to dryness. Take up with $200 \mathrm{cc}$ water and add $10 \mathrm{cc} \mathrm{HCl}$. Filter off silica and wash well with hot water and hot dilute $\mathrm{HCl}$. For high silica a second evaporation is necessary. The filtrate or combined filtrate is made alkaline with sodium hydroxide and about 5 cc of bromine added. The mass is allowed to digest for one-lialf hour on a steam bath. This 
oxidizes the iron and chromium, precipitates the iron, and dissolves the chromium and aluminum. After precipitating the iron twice it is filtered off on an asbestos plug and washed well with hot water. This precipitate of iron may be dissolved off the plug with hot $\mathrm{HCl}(1: 1)$ and determined by the stannous chloride reduction and permanganate method.

A blank should be run in conjunction with this method and deductions made when necessary to determine the purity of the chemicals used.

American Society for Testing Materials, tentative specifications for fire tests of building construction and materials, serial designation: C19-26T, 1926.

Approved as "Tentative American Standard" by the American Engineering Standards Committee, now the American Standards Association.

The performance of walls, columns, floors, and other building members uncer fire exposure conditions is anitem of major importance in securing constructions which are safe and which are not a menace to neighboring structures nor to the public. Recognition of this is registered in the codes of many authorities, municipal and other. It is important to secure balance of the many units and also to promote uniformity in requirements of rarious authorities throughout the country. To do this it is necessary that the fire-resistire propthe country. To do this it is necessary that the fire-resistire properties of materials and assemblies be measured and specified according to a common standard expressed in terms which are applicable
alike to a wide variety of materials, situations, and conditions of exposure.

Such a standard is found in the specifications which follow. They prescribe a standard exposing fire of controlled estent and severity. Periormance is defined as the period of resistance to standard exposure elapsing before the first critical point in behavior is observed. Results are reported in units in which ficld exposures can be judged and expressed.

The specifications may be cited as the Standard "Fire Test Specification" and the performance or exposure shall be expressed as "two bour," "six hour," "one-half hour," etc.

When a factor of safety exceeding that inherent in the test condi. tions is desired, a proportional increase should be made in the specitions is desired, a proportional in
fed time-classification period.

Classifications of assemblies involving combustibles in sufficient quantity or so arranged as to continue burning after the derived time period shall be designated by the term "combustible" after the period assigned.

1. (a) The test methods are applicable to assemblies of masonry units and to composite assemblies of structural materials for buildings, including bearing and other walls and partitions, columns, girders, beams, and slabs and composite slab and beam assemblies for floors and roofs. They are also applicable to other assemblies and structural units which constitute permanent integral parts of a finished building.

(b) It is the intent that classifications shall register performance during the period of exposure and shall not be construed as having determined suitability for use after fire exposure.

\section{CONTROL OF FIRE TESTS}

2. The conduct of fire tests of materials and construction shall be controlled by the standard time-temperature curve shown in Fig. 213. The points on the curve which determine its character are:

$1,000^{\circ} \mathrm{F}$. (approximately $538^{\circ} \mathrm{C}$.) $1,300^{\circ} \mathrm{F}$. (approximately $704^{\circ}$ C.) $1,550^{\circ} \mathrm{F}$. (approximately $843^{\circ}$ C.) $1,700^{\circ} \mathrm{F}$. (approximately $927^{\circ} \mathrm{C}$. $1,850^{\circ} \mathrm{F}$. (approximately $1,010^{\circ} \mathrm{C}$ ) $2,000^{\circ} \mathrm{F}$. (approximately $1,093^{\circ} \mathrm{C}$.) $2,300^{\circ} \mathrm{F}$. (approximately $1,260^{\circ} \mathrm{C}$.)

3. (a) The temperature fixed by the curve shall be deemed to be the average temperature obtained from the readings of several thermocouples (not less than three) symmetrically disposed and distributed to show the temperature near all parts of the sample, the thermocouples being inclosed in sealed porcelain tubes $3 / 4$ inch in outside diameter and of $1 / 8$ inch wall thickness. The exposed length of the porcelain tube and couple in the furnace chamber shall be not less than 12 inches. Other types of protecting tubes or pyrometers may be used that under test conditions give the same indications as the above standard within the limit of accuracy that applies for furnace-temperature measurements. For greater difference of design and size the time-temperature curve followed shall be modified to give an exposure equal to that sobtained by using the standard pyrometer and curve above described.

(b) The temperatures shall be read at intervals not exceeding five minutes during the first hour, and thereafter the intervals may be increased to not more than 15 minutes.

(c) The accuracy of the furnace control shall be such that the area under the time-temperature curve, obtained by averaging the results from the pyrometer readings, is within 15 per cent of the corresponding area under the standard time-tem. perature curve shown in Figure 213 for fire tests of one hour or less duration, within 10 per cent for those over one hour and not more than two hours, and within 5 per cent for tests exceeding two hours in duration.

4. (a) Temperatures at unexposed surfaces shall be measured with thermocouples or thermometers ${ }^{5}$ placed under oven dry asbestos fire felt pads 6 inches square, 0.4 inch thick and weighing not less than 1.0 lb./ft. ${ }^{2}$ nor more than $1.4 \mathrm{lbs}$. $/ \mathrm{ft}^{2}$ The wire leads of the thermocouple or the stem of the thermometer shall have an immersion under the pad and be in contact with the unexposed surface for not less than $3 \frac{1}{2}$ inches. The hot junction of the thermocouple or the bulb of the thermometer shall be placed approximately. under the center of the pad. The outside diameter of protecting tubes of glass, clay, or porcelain, and of thermometer stems shall not be more than $5 / 16$ inch. The pad shall be held firmly against the surface, and shall fit closely about the thermocouples or thermometer stems. Thermometers shall be of the partial-immersion type, with a length of stem between the end of the bulb and the immersion mark of 3 inches. The wires for the thermocouple in the length covered by the pad shall not be heavier than No. 18 B. \& S. gage ( 004 inch) and shall be electrically insulated with heat and moisture resistant coatings.

(b) The temperature readings shall be taken at not less than five points on the surface, one of which shall be approximately at the center and four at approximately the centers of the quarter sections. If additional points are used, they shall be symmetrically disposed about the center, with no location nearer than one and one-half times the thickness of the construction or nearer than 12 inches to the edges. None shall be located opposite or on top of beams, girders, pilasters, or other structural members.

(c) Temperature readings shall be taken at intervals not exceeding 15 minutes until a reading exceding $212^{\circ} \mathrm{F}$. $\left(100^{\circ} \mathrm{C}\right.$.) has been obtained at any one one point. Thereafter the readings may be taken more frequently at the discretion of the testing body, but the intervals need not be less than five minutes.

(d) Where the conditions of acceptance place a limitation on the rise of temperature of the unexposed surface, the temperature end point of the fire-endurance period shall be determined by the average of the measurements taken at individual points; excepting that if a temperature rise 30 per cent in excess of the specified limit occurs at any one of these points, the remainder shall be ignored and the fire-endurance period judged as ended.

\section{CLASSIFICATION AS DETERMINED BY TEST}

5. Results shall be reported in accordance with the performance in the tests prescribed in these

$\checkmark$ Under certain conditions it may be unsafe or impracticable to use thermometers. 
specifications. They shall be expressed in time periods of resistance, as for example four hour, onehalf hour, etc.

\section{TEST STRUCTURES}

6. (a) The test structure may be located at any place where all the necessary facilities for properly conducting the test are provided.

(b) Entire freedom is left to each investigator in the design of his test structure and the nature and use of fuel, provided the test requirements are met.

\section{TEST SAMPLES}

7. The test sample shall be truly representative of the construction for which classification is desired and shall be built under conditions representative of those obtaining as practically applied in building construction. The physical properties of the materials and/or ingredients used in the test sample shall be determined and recorded.
TABLE 1

\begin{tabular}{|c|c|c|c|}
\hline $\begin{array}{l}\text { Parts of } \\
\text { structure }\end{array}$ & Resistance period & $\begin{array}{c}\text { Water } \\
\text { pres- } \\
\text { sure at } \\
\text { nozzle }\end{array}$ & $\begin{array}{l}\text { Dura- } \\
\text { tion of } \\
\text { appli- } \\
\text { cation } \\
\text { per } 100 \\
\text { square } \\
\text { feet ex- } \\
\text { posed } \\
\text { area }\end{array}$ \\
\hline $\begin{array}{l}\text { Floors and } \\
\text { roofs. }\end{array}$ & $\left\{\begin{array}{l}8 \text { hours and over } \\
4 \text { hours and over if less than } 8 \text { hours } \\
2 \text { hours and over if less than } 4 \text { hours. } \\
1 \text { hour and over if less than } 2 \text { hours. } \\
\text { Less than } 1 \text { hour }\end{array}\right.$ & $\begin{array}{r}\text { Lb./in. }{ }^{2} \\
45 \\
45 \\
45 \\
30 \\
30\end{array}$ & $\begin{array}{c}\text { Min- } \\
\text { utes } \\
6 \\
5 \\
23 \\
11 / 2 \\
1\end{array}$ \\
\hline $\begin{array}{l}\text { Walls and } \\
\text { partitions. }\end{array}$ & $\left\{\begin{array}{l}8 \text { hours and over } \\
4 \text { hours and over if less than } 8 \text { hours } \\
2 \text { hours and over if less than } 4 \text { hours } \\
1 \text { hour and over if less than } 2 \text { hours.- } \\
\text { Less than } 1 \text { hour }\end{array}\right.$ & $\begin{array}{l}45 \\
45 \\
30 \\
30 \\
30\end{array}$ & $\begin{array}{l}6 \\
5 \\
21 / 2 \\
11 / 2 \\
1\end{array}$ \\
\hline
\end{tabular}

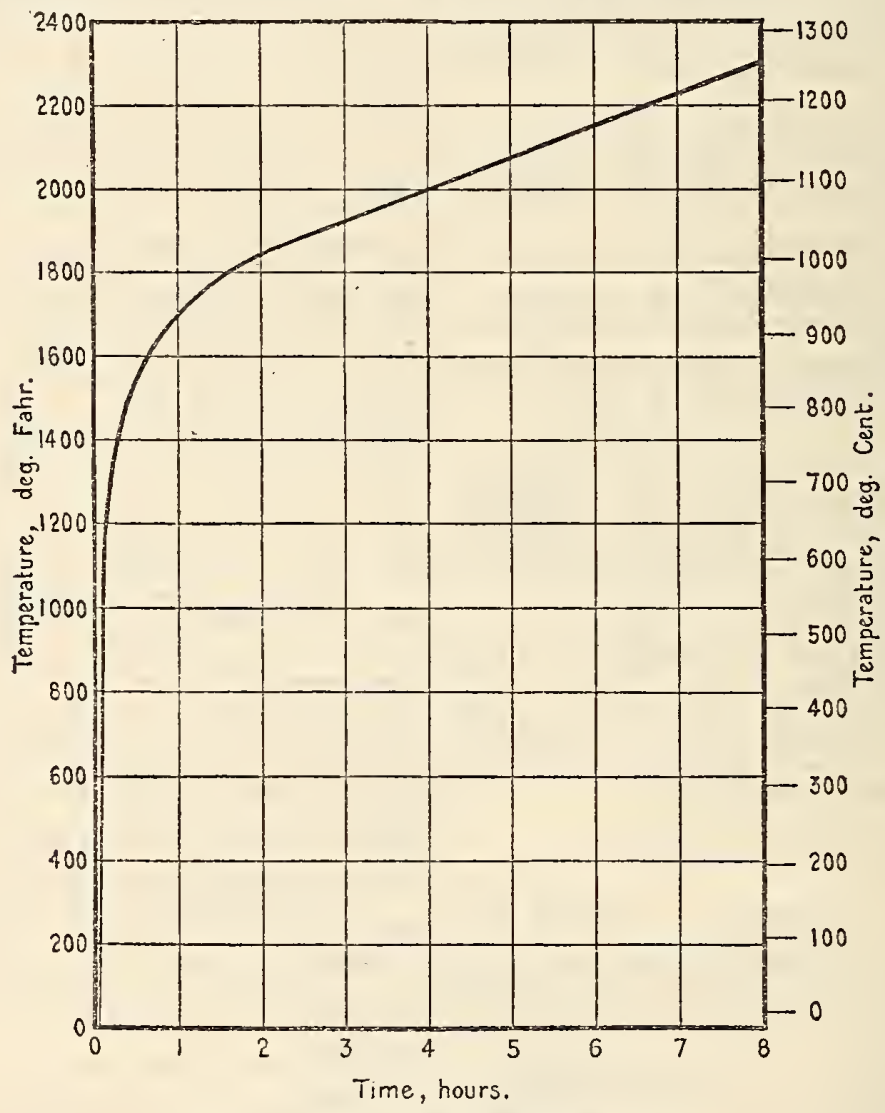

FIgURE 213.-Time-temperature curve

\section{CONDUCT OA FIRE TESTS}

The fire-endurance test on the sample with its applied load, if any, shall be continued until failure occurs, or until it has withstood the test conditions for a period equal to that herein specified in the conditions of acceptance for the given type of construction,
9. (a) Where required by the conditions of acceptance, a duplicate sample shall be subjected to a fire-exposure test for a period equal to one-half of that indicated as the resistance period in the fire-endurance test, but not for more than one hour, immediately after which the sample shall be subjected to the impact, erosion, and cooling effects of a fire-hose stream directed first at the middle 
and then at all parts of the exposed face, changes in direction being made slowly.

(b) The fire-hose stream test shall not be required in the case of constructions having a resistance period, indicated in the fire-endurance test, of one-half hour or less.

(c) The submitter may elect, with the advice and consent of the testing bociy, to have the fire-hose stream test made on the sample subjected to the fire-endurance test and immediately following the expiration of the fire-endurance test, provided that the testing body reserves the right to decide that a fire-hose stream test should be made on a duplicate sample as specified in paragraph $(a)$.

(d) The stream shall be delivered through 21/2inch hose, discharging through a National Standard clay pipe of corresponding size equipped with a 11/8-inch discharge tip of the standard-taper smoothbore pattern without shoulder at the orifice. The water pressure and duration of application shall be as specified in Table 1 .

(e) The nozzle orifice shall be 20 feet from the center of the exposed surface of the test sample if the nozzle is so located that when directed at the center its axis is normal to the surface of the test sample. If otherwise located, its distance from the center shall be less than 20 feet by an amount equal to 1 foot for each $10^{\circ}$ of deviation from the normal.

10. The material or construction shall not be tested until a large proportion of its final strength has been attained, and, if it contains free water, until the excess has been given off; this will usually require about 30 days' time under favorable drying conditions. Where this can not be attained within 40 days, artificial drying with air temperature not exceeding $100^{\circ} \mathrm{F}$. ( $38^{\circ} \mathrm{C}$.) shall be used.

\section{TESTS OF BEARING WALLS AND PARTITIONS}

11. The area exposed to fire shall be not less than 100 square feet, with neither dimension less than 9 feet. The test specimen shall not be restrained on its vertical edges.

12. During the fire-endurance and fire and firestream tests the construction shall be loaded in a manner calculated to develop theoretically as nearly as practicable the working stresses contemplated by the design.

13. The test shall not be regarded as successful unless the following conditions are met:

(a) The wall or partition shall have sustained the applied load during the fire-endurance test without passage of flame or gases hot enough to ignite cotton waste for a period equal to that for which classification is desired.

(b) The wall or partition shall have sustained the applied load during the fire and fire-stream test as specified in section 9, without passage of flame, stream, or gases hot enough to ignite cotton waste, and after cooling, but within 72 hours after its completion, shall sustain a total load equal to the dead load plus twice the superimposed load specified above.

(c) The fire stopping, if any, shall have functioned to prevent passage of fire for a period equal to that for which classification is desired.

(d) Transmission of heat through the wall or partition during the fire-endurance test shall not have been such as to raise the temperature on its unexposed surface more than $250^{\circ} \mathrm{F}$. $\left(139^{\circ} \mathrm{C}\right.$. $)$ above its initial temperature.

VII. TESTS OF NONBEARING WAILS AND PARTITIONS

14. The area exposed to fire shall be not less than 100 square feet, with neither dimension less than 9 feet. The test specimen shall be restrained on all four edges.

15. The tost shall not be regarded as successful unless the following conditions are met:

(a) The wall or partition shall have withstood the fire-endurance test without passage of flame or gases hot enough to ignite cotton waste for a period equal to that for which classification is desired.

(b) The wall or partition shall have withstood the fire and fire-stream test as specified in section 9 , without passage of flame, stream, or gases hot enough to ignite cotton waste.

(c) The fire stopping, if any, shall have functioned to prevent passage of fire for a period equal to that for which classification is desired.

(d) Transmission of heat through the wall or partition during the fire-endurance test shall not have been such as to raise the temperature on its unexposed surface more than $250^{\circ} \mathrm{F}$. $\left(139^{\circ} \mathrm{C}\right.$.) above its initial temperature.

\section{TESTS OF COLUMNS}

16. The length of the column exposed to fire shall, when practicable, approximate the maximum clear length contemplated by the design, and for building columns shall not be less than 9 feet. The contemplated details of connections, and their protection, if any, shall be applied according to the methods of acceptable field practice.

17. (a) During the fire-endurance test the column shall be loaded in a manner calculated to develop theoretically as nearly as practicable the working stresses contemplated by the design. Provision shall be made for transmitting the load to the exposed portion of the column without unduly increasing the effective column length.

(b) If the submitter and the testing body jointly so decide, the column may be subjected to one and three-fourths times its designed working load before the fire-endurance test is undertaken. The fact that such a test has been made shall not be construed as having had a deleterious effect on the fireendurance test performance.

18. The test shall not be regarded as successful unless the column shall have sustained the applied load during the fire-endurance test for a period equal to that for which classification is desired.

\section{TESTS OF FLOORS AND ROOFS}

(The following contemplates application of fire exposure to the underside of constructions and omission from the upper surface of all units which are not essential to the constructions. Specifications and test procedure with fire applied to the upper side have not been developed.)

19. The area exposed to fire shall be not less than 180 square feet with neither dimension less than 12 feet. Beams or girders, if a part of the construction under test, shall lie within the combustion chamber and have a clearance of not less than 8 inches from its walls.

20. During the fire-endurance and fire and firestream tests the construction shall be loaded in a manner calculated to develop theoretically as nearly as practicable the working stresses in each member contemplated by the design.

21. The test shall not be regarded as successful unless the following conditions are met:

(a) The construction shall have sustained the applied load during the fire-endurance test without passage of flame or gases hot enough to ignite cotton waste for a period equal to that for which classification is desired.

(b) The construction shall have sustained the applied load during the fire and fire-stream test as specified in section 9, without passage of flame, 
stream or gases hot enough to ignite cotton waste, and after cooling but within 72 hours after its completion shall sustain a total load equal to the dead load plus twice the superimposed load specified above.

(c) Transmission of heat through the construction during the fire-endurance test shall not have been such as to raise the temperature on its unexposed surface more than $250^{\circ} \mathrm{F}$. $\left(139^{\circ} \mathrm{C}\right.$.) above its initial temperature.

\section{TESTS OF FINISH FOR WALIS, PARTITIONS, AND CEILINGS}

22. The test panel shall be a wall, partition, or floor.

(a) Test panels carrying interior wall and partition finish shall be finished on both faces with the finish which is the subject of the test; excepting that with the advice or consent of the testing body this provision may be waived with respect to panels of solid or masonry construction.

(b) Test panels carrying exterior wall finish on the exposed face shall be finished on the unexposed face with an interior wall finish judged by the testing body to be suitable for purposes of the test, excepting that with the advice or consent of the testing body this provision may be waived with respect to panels of solid or masonry construction.

(c) Test panels carrying cciling finish shall be finished on the upper face with a flooring judged by the testing body to be suitable for purposes of the test, excepting that with the advice or consent of the testing body this provision may be waived with respect to panels of solid or masonry construction.

23. The area exposed to fire shall be for tests of wall and partition finish, not less than 100 square feet, with neither dimension less than 9 feet; for tests of ceiling finish, not less than 180 square feet, with neither dimension less than 12 feet.

24. The test shall not be regarded as successful unless the following conditions are met:

(a) The finish shall have withstood the fireendurance test without developing openings capable of passing flame or hot gases for a period equal to that for which classification is desired.

(b) The finish shall have withstood the fire and fire-stream test as prescribed, respectively, for floors, walls, and partitions as specified in section 9, without developing openings capable of passing flame, hot gases, or stream.

(c) Transmission of heat through the finish during the fire-endurance test shall not have been such as to raise the temperatures at its contact with the structural members of the test panel or elsewhere on its unexposed surface more than $250^{\circ} \mathrm{F}$. $\left(139^{\circ}\right.$ C. $)$ above the initial temperatures at these points.

American Society for Testing Materials, standard method of test for porosity and permanent volume changes in refractory materials; serial designation C20-20, 1920.

1. The object of this test is to determine the porosity and permanent volume changes in refractory materials when heated to series of specified temperatures.

2. (a) The sample shall consist of at least seven standard-size brick.

(b) Test specimens measuring $2 \frac{1}{2}$ by $2 \frac{1}{2}$ by $11 / 4$ inches shall be cut so as to remove the original surfaces of the bricks; for this a "cut-off" grinding wheel is recommended. There should be five test specimens for each of the seven heat treatments specified in section 4 , or 35 test specimens for each kind of brick. The test specimens shall be brushed or washed frec from all adhering dust and marked serially with a refractory stain, for which 5 per cent cobalt-kaolin mixture is suggested.

3. After the test specimens have been cut and cleaned, they shall be dried and the volumes and porosity of each obtained as described in sections 5 and 6 . They shall be heated as specified in section 4 , and the changes in volume and porosity determined.

4. (a) Dry the test pieces prior to placing in the kiln.

(b) Raise the temperature as rapidly as is consistent with even heat distribution to $1,200^{\circ} \mathrm{C}$. From $1,200^{\circ} \mathrm{C}$. raise the temperature at the rate of $30^{\circ}$ per hour, drawing samples at each $50^{\circ}$ interval from $1,200^{\circ}$ to $1,500^{\circ} \mathrm{C}$.

(c) If it is possible, and the number of brands being tested warrant, it is best that separate burns to each temperature be made and the kiln sealed and allowed to cool by radiation. In case separate burns can not be made, the five test specimens from each temperature increment should be covered with hot sand immediately on being drawn, or placed in a supplementary furnace and kept at about $500^{\circ} \mathrm{C}$. until all drawings are completed, and then cooled with the furnace sealed to cool wholly by radiation.

5. (a) The test specimens shall be cleaned from adhering or loosely attached pieces and particles, care being taken not to alter the exterior volume as originally prepared for this test.

(b) The test specimens shall be heated if necessary to $110^{\circ} \mathrm{C}$. to remove moisture, and their dry weight $(D)$ obtained to $0.10 \mathrm{~g}$.

(c) The test specimens shali be placed in kerosene of known density $(\delta)$ under a vacuum of 24 inches for four hours at $25^{\circ} \mathrm{C}$. and cooled to room temperature while yet immersed.

(d) When cool, each test specimen shall be weighed suspended in kerosene at $25^{\circ} \mathrm{C}$. to determine its suspended weight $(S)$, in grams.

$(e)$ The saturated weight $(W)$, in grams, of each test specimen shall be obtained immediately after obtaining the suspended weight by drying lightly with a kerosene-moistened towel to remove the excess kerosene and then weighing in air.

$(f)$ The exterior volume $(V)$, in cubic centimeters, of each test specimen is obtained by subtracting the suspended weight $(S)$ from the saturated weight $(W)$, and dividing by the density $(\delta)$ of the kerosene. Thus

$$
V=\frac{W-S}{\delta}
$$

(g) The actual volume of open pores $\left(V_{1}\right)$, in cubic centimeters, is obtained by subtracting the dry weight $(D)$ from the saturated weight $(W)$, and dividing by the density $(\delta)$ of the kerosene. Thus

$$
V_{1}=\frac{W-D}{\delta}
$$

(h) The apparent specific gravity $\left(T_{1}\right)$ of that portion of the test specimen which is impervious to liquid is obtained by dividing the dry weight by the difference between the dry and suspended weights, and multiplying by the density of the kerosene. Thus

$$
T_{1}=\frac{D}{D-S} \delta
$$

(i) The true specific gravity $(T)$ of the wholly solid or burned clay portion is obtained by crushing a portion of the dried test specimen to powder passing a No. 120 sieve and determining the displacement at $25^{\circ} \mathrm{C}$. under 24-inch vacuum, of a $20 \mathrm{~g}$ sample in a $50 \mathrm{cc}$ straight-wall pyknometer using kerosene, and correcting for density of the kerosene.

(j) The volume of sealed pores $\left(V_{2}\right)$, in cubic centimeters, is obtained by subtracting the quo- 
tient of dry weight $(D)$ divided by true specific gravity $(T)$ from the volume of the impervious portion of the test specimen; or

$$
V_{2}=\frac{D-S}{\delta}-\frac{D}{T}
$$

(k) The volume shrinkage is obtained by subtracting the volumes; that is, the values of $\frac{W-S \text {, }}{\delta}$ before and after the heat treatment.

6. To show progressive changes in the several volumes, refer all volumes back to the original exterior volume of the test specimen as 100. This is done by multiplying all volumes by the factor $100 / V$, in which $V$ is the exterior volume of the test specimen prior to the subjection to heat treatment.

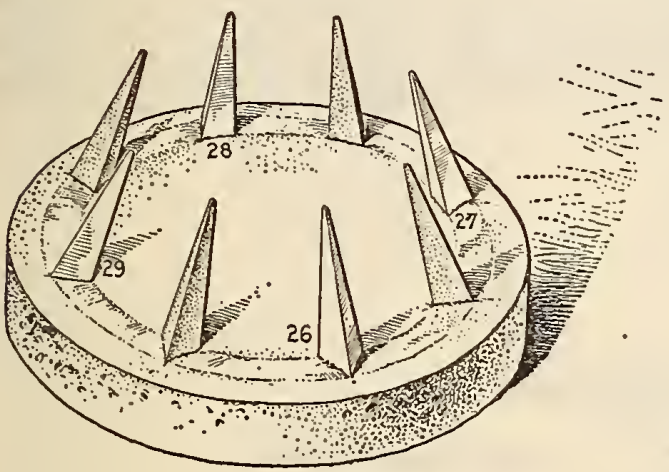

Figure 214

The volume data should be determined for each test specimen and multiplied by the above factor to reduce all volumes for each test specimen to terms of 100 original exterior volumes of that test specimen before the average of the five for each heat treatment is calculated.

American Society for Testing Materials, standard method of test for softening point of fire-clay brick, serial designation $\mathrm{C} 24-28,1928$.

1. The object of this test is to determine the softening point of fire-clay brick by comparison of test cones with standard Orton pyrometric cones heated in a suitable furnace.

2. A $1 \mathrm{~kg}$ (2-pound) sample shall be taken by chipping off approximately equal portions from the corners of the brick. These fragments shall be reduced in size by means of rolls or a jaw crusher adjusted to pass a lump $6 \mathrm{~mm}$ ( $1 / 4$ inch) in diameter. They shall be mixed thoroughly, and the amount of material reduced to about $250 \mathrm{~g}$ ( 5 pounds) by quartering. A magnet shall be repeatedly passed through the crushed material until all particles of metallic iron are removed. This portion shall be ground in a porcelain or agate mortar to pass a No. 60 sieve of the United States Standard Sieve Series. ${ }^{\circ}$ In order to avoid excessive reduction of the fines, they shall be removed frequently during the process of reduction by throwing the sample on the sieve and continuing the grinding of the coarser particles until all the sample will pass through the sieve.

3. (a) The sample thus prepared shall be thoroughly mixed and after the addition of sufficient dextrin or glue and water shall be formed into test cones in a metal mold in the shape of tetrahedrons measuring $5 \mathrm{~mm}(3 / 16$ inch) on the sides at the base and $25 \mathrm{~mm}$ (1 inch) high.

(b) When dry the test cones may be subjected to a preliminary burn at a temperature not exceeding

- For detailed specifications for this sieve, see A. S. T. M. E11, $500.2, \mathrm{p}, 1$. $1,300^{\circ} \mathrm{C} .\left(2,372^{\circ} \mathrm{F}\right.$. $)$ for the purpose of sintering them into a firm condition to permit handling.

4. The test cones shall be mounted on plaques of refractory material of such a composition as will not affect the fusibility of the cones. ${ }^{7}$ They shall be mounted with the base embedded approximately $1 \mathrm{~mm}(0.04$ inch) in the plaque and the face of one side inclined at an angle of $82^{\circ}$ with the horizontal. The arrangement with respect to the Orton cones shall be substantially as shown in Figure 214; that is, alternating with the Orton cones in such a way that Orton cones of successive numbers will be placed opposite each other. The plaque may be any convenient size and shape and may be biscuited before using, if desired.

5. (a) The heating shall be carried out in a suitable furnace at a rate to conform to the following requirements:

To reach cone 20

Minutes

Time interval to cone:

23 .

26 -

27

29

30

31.

32

35

Note.-At $1,000^{\circ} \mathrm{C}$. the old cone pat may be removed and a new one put in without cooling the furnace down below red heat.

(b) That type of furnace in which a neutral or oxidizing atmosphere may be maintained is to be preferred. Excessive reducing conditions should be avoided. Care should be taken that the flame does not strike directly against the cone or cone plaque. The furnace should be tested at intervals for the determination of the uniformity of the distribution of the heat.

6. (a) The softening of the cone will be indicated by the top bending over and assuming the position shown in Figure 215. The bloating, squatting, or unequal fusion of small constituent particles should always be reported. The softening point shall be reported in terms of Orton cones and shall be that cone which most nearly corresponds in time of softening with the test cone. If the test cone

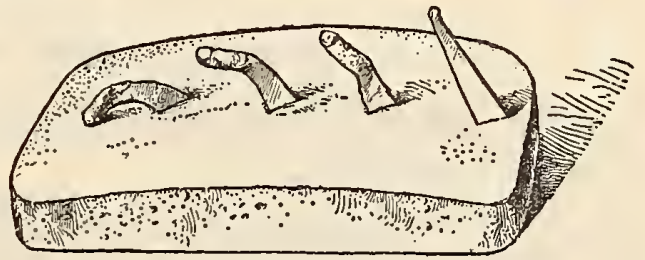

FIGURE 215

softens later than one Orton cone but earlier than the next Orton cone and approximately midway between, the softening point shall be reported thus: Cone No. 31-32.

(b) If the test cone starts bending at an early cone but is not down until a later cone, the fact should be reported.

American Society for Testing Materials, tentative method of test for resistance of fire-clay brick to thermal spalling action, serial designation C38-27T, 1927.

1. The object of this test is to determine the resistance of high-duty fire-clay brick to spalling

7 A mixture of equal parts of a highly refractory clay, such as a good grade of china clay, and fused alumina which will pass a No. 100 good grade of china clay, and fused alumina which will pass a No. 100 sievo (U.tory. 
action by subjecting them to repeated rapid temperature changes.

2. (a) The sample shall consist of at least five standard 9-inch brick or of five shapes, which may be cut to standard size with a grinding wheel.

(b) Test specimens of shapes such as boiler arch brick shall be cut to the standard 9-inch size. For this purpose a "cut-off" wheel is recommended.

3. (a) The specimens shall be weighed and placed in a door of a furnace operated at $1,350^{\circ} \mathrm{C}$. $\left(2,462^{\circ}\right.$ F.), care being taken to prevent the direct heating of more than the $2 \frac{1}{2}$ by $4 \frac{1}{2}$-inch end. After one hour the specimen shall be withdrawn from the furnace and stood on end in a tank of flowing cold water from $10^{\circ}$ to $21^{\circ} \mathrm{C}$. $\left(50^{\circ}\right.$ to $70^{\circ} \mathrm{F}$.), so that the hot end of the brick will be immersed to a depth of 2 inches. After three minutes the sample shall be withdrawn from the water, allowed to steam five minutes, and then returned to the furnace. The door of the furnace shall be closed while the bricks are out to prevent the lowering of the temperature.

\section{COMPRESSION TEST \\ APPARATUS}

2. Any form of standard testing machine may be used for the compression test together with a spherical bearing block large enough entirely to cover the specimen.

\section{TEST SPECIMEN}

3. The test specimen shall consist of a half brick. Any method which will produce a specimen with approximately plane and parallel ends, without shattering the specimen, may be used.

NoTE.-This permits the use of a suitable half brick from the flexure test.

4. A half brick to be used for the compression test shall be prepared as follows: The two surfaces intended to receive the load from the testing machine shall receive a thin coating of shellac and be allowed to dry thoroughly. One of the shellacked bearing surfaces shall then receive a thin coat of a neat

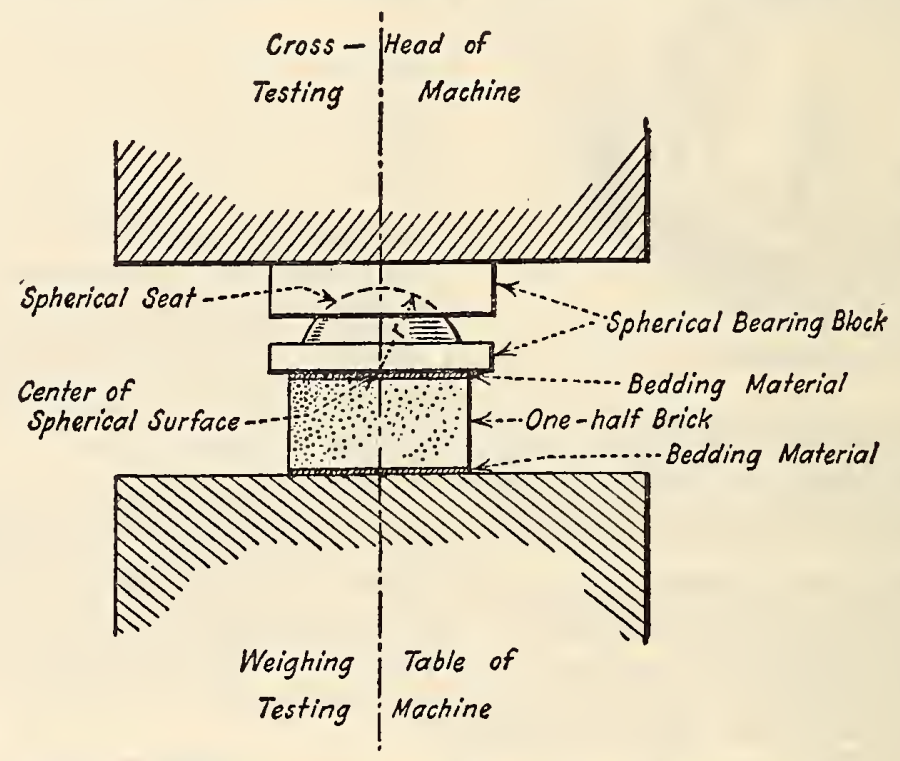

Figure 216.-Special bearing block for use in testing brick

The alternate heating and cooling shall be continued in hourly cycles until a loss of 20 per cent by weight has occured in each brick.

(b) When pieces begin to fall off, the sample is laid on a 4.5 by 9.0 -inch asbestos board, divided into 100 equal squares, and the approximate percentage of loss estimated after each removal from the water. After the completion of the test, the brick is again weighed and the percentage of loss determined.

4. The report shall show for each brick the number of dips before loss started the percentage of loss after each cooling and the total number of dips until a loss of 20 per cent by weight has occurred.

American Society for Testing Materials, standard specifications for quicklime and hydrated lime for the manufacture of silica brick, serial designation C49-24, 1924.

See 517.2 , page 364 .

American Society for Testing Materials, tentative methods of testing brick (compression, flexure, and absorption), serial designation C67-28T, 1928.

1. These methods cover the following tests for brick: The compression test, the flexure or crossbending test, and the absorption test. mortar of calcined gypsum (plaster of Paris). This mortared face of the specimen shall then be pressed on plate glass or a machined metal plate, previously oiled or covered with waxed paper, and the bedding material allowed to harden. This procedure shall be repeated with the other shellacked surface. Care shall be taken that the opposite faces so coated with bedding material shall be parallel. Depressions of recessed or paneled bricks shall be filled with neat Portland cement mortar which shall stand at least 24 hours before testing.

5. Compression tests shall be made on at least five half-brick test specimens from different bıicks.

\section{PROCEDURE}

6. (a) The half-brick test specimen shall be tested, placed flat.

(b) The bealing block shall be used on top of the test specimen in vertical testing machines. The center of the sphere of the bearing block shall be used in contact with the top bearing surface of the specimen and in the vertical axis of the specimen. (Fig. 216.) The spherical bearing block shall 
be kept thoroughly lubricated to insure accurate adjustment, which should be made by hand under a small initial load.

(c) The speed of the moving head of the testing machine, during the application of the load, shall 110 t be more than 0.05 inch per minute.

(d) During the test the beam of the testing machine shall be kept constantly in a floating position.

7. The compressive strength in pounds per square inch shall be reported as the total maximum load $W$, in pounds, indicated by the testing machine, divided

\section{PROCEDURE}

11. (a) A test-specimen brick shall be placed flatwise on the knife-edges with a span of 7 inches and the load applied at mid span.

(b) A steel bearing piece one-fourth inch in thickness, one-half inch in width, and of a length equal to the full width of the specimen shall be placed between the upper knife-edge and the specimen.

(c) The speed of travel of the head of the testing machine, during the application of the load, shall not be more than 0.05 inch per minute.

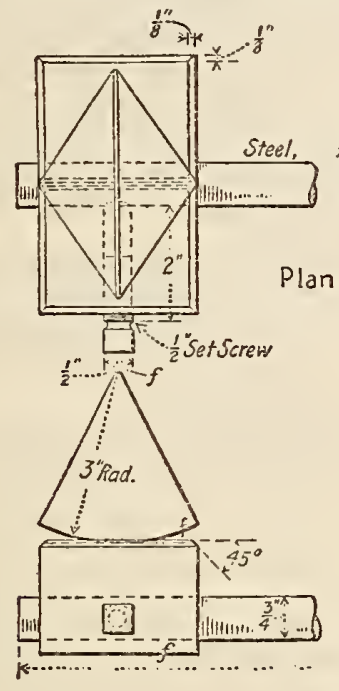

Side Elevation.

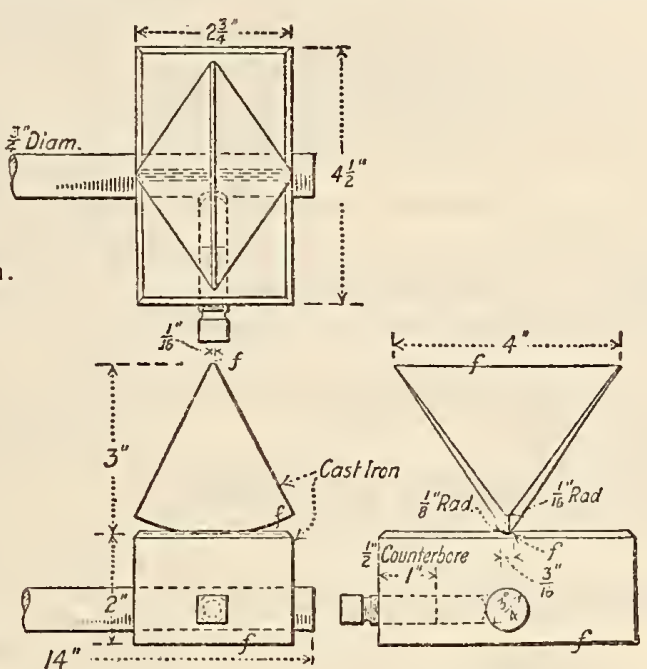

End Views.

FigdRE 217

by the area $A$, in square inches, of the minimum section of the specimen perpendicular to the line of application of the load.

The compressive strength in pounds per square inch shall be calculated from the formula:

$$
\text { Compressive strength }=\frac{W}{A}
$$

\section{FLEXURE TEST}

\section{APPARATUS}

8. (a) Any form of standard testing machine with knife-edges as described in paragraph $(b)$ may be used for the flexure test.

NotE.-Portable apparatus properly calibrated may be used.

(b) Knife-edges shall be straight and have full contact with the specimen or bearing plate and they shall have a length at least equal to the width of the $b_{i}$ ick to be tested. Their bases shall be so curved or rounded as to permit free movement of the working edges, both longitudinally and transversely. The use of knife-edges of the type shown in Figure 217 is recommended, although other types may be used which will provide free movement and at the same time insure full and even bearing at all times during the test.

\section{TEST SPECIMEN}

9. The test specimen shall be a whole brick.

10. Flexure tests shall be made on at least five whole bricks.
12. The modulus of rupture $R$, in pounds per square inch, shall be reported as calculated from the following formula:

$$
R=\frac{3 W l}{2 b d^{2}}
$$

where

$W=$ the total load in pounds at which the brick failed.

$l=$ the distance between the supports in inches. $b=$ the width of the brick in inches.

$d=$ the depth of the brick in inches.

\section{ABSORPTION TEST}

\section{TEST SPECIMEN}

13. The test specimen shall be a whole brick.

14. The absorption test shall be made on at last five whole bricks.

\section{PROCEDURE}

15. The sample shall be dried to constant weight at a temperature of $100^{\circ}$ to $105^{\circ} \mathrm{C}$. $\left(212^{\circ}\right.$ to $221^{\circ} \mathrm{F}$.).

16 . When cool the bricks shall be weighed separately on scales sensitive to within 0.5 per cent.

17. The dry bricks shall be totally immersed in soft, distilled, or rain water at a temperature of $15.5^{\circ}$ to $30.0^{\circ} \mathrm{C}$. $\left(60^{\circ}\right.$ to $86^{\circ} \mathrm{F}$.) for five hours. The bricks shall then be removed, the surface water wiped off with a damp cloth and the bricks quickly weighed. 
18. The percentage of absorption shall be calculated on the dry weight from the formula:

$$
\text { Percentage of absorption }=\frac{100(b-a)}{a}
$$

Where $a=$ weight of $\operatorname{dry}$ bricks and $b=$ weight of saturated bricks.

American Society for Testing Materials, standard definitions of terms relating to refractories, serial designation C71-28, 1928.

Fire elay.-A sedimentary $\operatorname{clay}^{8}$ of low flux content.

Note.-It is usually associated with coal measures.

Plastic or bond fire clay.-A fire clay of sufficient natural plasticity to bond nonplastic materials.

Flint fire clay. - A hard or flint-like fire clay occurring as an unstratified massive rock, practically devoid of natural plasticity and showing a concoidal fracture.

Diaspore clay.-A rock consisting essentially of diaspore bonded by fire clay.

Nodular fire clay.-A rock containing aluminous or ferruginous nodules or both bonded by fire clay.

NoTE.-In some districts such clays are called "burly" or "burley flint" clay.

American Society for Testing Materials, tentative definitions of terms relating to refractories, serial designation C71-28T, 1928.

Spalling.-Breaking or cracking of refractories to such an extent that fragments are separated, presenting newly exposed surfaces of the residual mass.

NoтE.-The causes of spalling may be classified under three main headings-namely, thermal, mechanical, and structural-as follows:

\section{THERMAL}

(a) Factors related to service-Rapidity and range of thermal fluctuation; contamination by slags and fluxes; tightness of joints; previous vitrification.

(b) Factors related to refractories.-Degree and uniformity of reversible thermal expansion; heat transfer; elasticity; plastic flow.

\section{IECHANICAL}

(a) Factors related to service.-Rapid heating of wet brick; abuse in removing clinker and slag; unequal and excessive stresses; pinching; no provision for expansion; thin joints.

(b) Factors related to refractories.-Mechanical strength; toughness; accuracy of shape.

\section{STRUCTURAL}

(a) Factors related to service.-Slags and fluxes; character of the material in joints; insulation of refractories.

(b) Factors related to refractories.-Vitrification; shrinkage; nature of bond; structure; degree of burning.

Pyrometric cone equivalent (P.C.E.).-An index to the degree of fusion resulting in a cone of the material bending until the tip touches the plaque as a result of a definite heat treatment, in accordance with A. S. T. M. C24. (See p. 573.)

Noтe.-The terms "fusion point," "softening point," "deformation point," and "melting point" have heretofore bcen loosely used for "pyrometric cone equivalent."

8 The definition of the term "fire-clay" is based upon the following definition of the term "clay":

Clay.-An earthy or stony mineral aggregate consisting essentially of hydrous silicates of alumina, plastic when sufficiently pulverized and wetted, rigid when dry, and vitreous when burned at a sufficiently
high temperature.
Common Brick Manuiacturers' Association of America, specifications and general schedule of a brick house.

(The above standard includes, in the general schedule, estimating sheets, specification and contract forms, and specifications for construction. Items included in this volume are as follows: (1) Mortar (see 516.3, p. 310) (2) ideal construction (see 518.53, p. 319), (3) chimneys and fireplaces (see 518.55, p. 422), and 4) bricklaying (see 518.38, p. 477).)

United States Department of Agriculture, Bulletin 1216, method of sampling paving brick, 1928 .

1. Place of sampling.- Where a standard rattler has been installed and is kept in an approved condition, and acceptable facilities for testing are furnished by the manufacturer, samples will be taken and tested at the plant by an inspector while cars are being loaded, provided daily shipments are sufficient to warrant it. Under all other conditions, samples will be taken from the cars upon arrival at their destination.

2. Method of selecting samples.-A single sample will be taken to represent each 10,000 to 15,000 brick according to conditions described later. No brick are to be included in the sample which are cracked or so deformed as to be unfit for laying under the specifications.

3. (a) Samples from kilns.- When samples are taken directly from the kiln, the first set will usually be chosen as soon as enough brick have been removed from the front of the kiln to permit of obtaining a sample representative of both the full width and height of the kiln. Later samples are to be taken with the idea of representing three degrees of burning in the kiln, one sample to be selected from localities (usually the top and sides) to represent the hardest-burned brick, one sample usually selected from the more central portion of any cross section of the kiln to represent mediumburned brick, and one sample, usually from the bottom layers and away from the sides, to represent the least-burned brick. Each of these samples should represent approximately 15,000 brick.

(b) Samples taken from piles. - When the appearance of the pile of brick shows clearly that there is a considerable range in the degree of burning, samples to represent each of three degrees should be selected, as in the case of samples taken from kilns, each sample to represent about 10,000 brick. When the surface appearance of the brick does not furnish any indication of their uniformity samples are to be taken at random representing approximately 10,000 brick.

(c) Samples from cars.-When a sample is taken from a carload, it shall be selected in the same manner as previously described to represent three approximate degrees of burning.

4. Shipment of samples.- Samples which must be transported long distances by freight or express shall be carefully put up in packages holding not more than 12 brick each. When more than 6 brick are shipped in one package, it shall be so arranged as to carry two parallel rows of brick side by side and these rows shall be separated by a partition. In event of some of the brick being cracked or broken in transit, the sample shall be disqualified if there are not remaining 10 sound, undamaged brick.

5. Storage and care of samples.-Samples shall be carefully handled to avoid breakage or injury. They shall be kept in the dry so far as practicable. If wet when received, or known to have been immersed or subjected to recent prolonged wetting, they shall be dried for at least six hours in a temperature of $100^{\circ} \mathrm{F}$, before testing. 
534.11 Face, Common, and Vitrified Brick.

American Association of State Highway Officials, standard specifications for bridges and incidental structures, 1928.

\section{BRICK (PAVING)}

Same as A. S. T. M. C7 (see below) except that sampling shall conform to the requirements of United States Department of Agriculture, Bulletin 1216, method of sampling paving brick. (See 534.10, p. 576.)

The brick used shall be one of the varieties included in United States Department of Commerce, division of simplified practice, recommendation No. 1. (See p. 586.)

\section{BRICK FOR MASONRY}

Brick for masonry construction shall conform to the requirements of A. S. T. M. C62-28T. (See p. 583 .)

American Railway Engineering Association, specifications for buildings for railway purposes.

\section{FACE AND COMMON BRICK}

See A. R. E. A. specification for brickmork, 518.83 , page 475 .

American Railway Engineering Association, specifications for buildings for railway purposes.

\section{BRICK FOR USE IN SEWERS}

See specification for sewers and drainage, 518.67, page 453

American Society for Municipal Improvements, standard specifications for sewers, 1927.

\section{COMMON BRICK}

See 518.67, page 453 .

\section{VITRIFIED BRICK}

See 518.67, page 453 .

American Society for Testing Materials, tentative specifications for paving brick, serial designation C7-29T, 1929.

1. These specifications cover vitrified brick used in the construction of pavements.

2. The consumer or purchaser shall specify the size or sizes and variety or varieties of brick which are to be supplied.

Note.-The sizes and varieties recognized by the Permanent Committee on Simplification of Varieties and Standards for Vitrified Paring Brick of tbe United States Department of Commerce given bclow are recommended for use wherever practicable:

\begin{tabular}{|c|c|c|c|}
\hline & Depth & Width & Length \\
\hline $\begin{array}{l}\text { Plain wire-cut (vertical fiber lugless as } \\
\text { usually laid) } \\
\text { Wire-cut lug (Dunn) } \\
\text { Repressed lug-- }\end{array}$ & $\begin{array}{l}\text { Inches } \\
\left\{\begin{array}{c}21 / 2 \\
3 \\
3 \\
3 \\
4 \\
4\end{array}\right.\end{array}$ & $\begin{array}{c}\text { Inches } \\
4 \\
4 \\
4 \\
31 / 2 \\
31 / 2 \\
31 / 2\end{array}$ & $\begin{array}{r}\text { Inches } \\
81 / 2 \\
81 / 2 \\
81 / 2 \\
81 / 2 \\
81 \\
81\end{array}$ \\
\hline
\end{tabular}

The above sizes and varieties are those recognized by tbe permanent committee for the year 1929, and are subject to cbange from year to year.

\section{MANUFACTURE}

3. Paving brick shall be manufactured from fire clay, semifire clay or shale, or combinations thereof. The manufacturing process shall be such that the brick are thoroughly annealed, tough, durable, and evenly burned so that, when broken, they show a uniformly dense structure free from lime, air pockets, and marked laininations.

4. Lug brick shall be provided with not less than two lugs on one side of the brick. The lugs shall project not less than $1 / 8$ inch nor more than $1 / 4$ inch from the side of the brick and the total area of contact of the lugs shall not exceed 30 per cent of the area of the side of the brick.

5. Beveled-end brick shall be double beveled not more than $1 / 8$ inch on each end.

6 . The edges of repressed brick shall be rounded to a radius of not more than $3 / 16$ inch.

7. Paving brick shall conform to the following nominal sizes. The dimensions of the brick shall be construed as applying to the body of the brick only, exclusive of lugs or beveled ends:

Transverse dimensions, inclusive

Length, inches $21 / 6 \mathrm{~b} 4$ 3 by $31 / 2$ by $4-$ $31 / 2$ by 4 these specifications, the individual bricks shall not vary from the nominal size requirements specified in section 7 by more than $\pm 1 / 8$ inch in either transverse dimension, or by more than $\pm 1 / 4$ inch in length.

\section{PHYSICAL PROPERTIES AND TESTS}

9. The quality and acceptability of paving brick shall be determined by the rattler test as described in sections 17 to 29 , inclusive, and by visual inspection, as described in section 16 . The acceptance of paving brick as satisfactorily meeting either of these tests shall not be construed as in any way waiving the other.

10. The percentage of loss in the rattler test of the respective sizes of paving brick specified shall conform to the following maximum requirements:

\begin{tabular}{|c|c|c|}
\hline \multicolumn{2}{|c|}{ Size of brick } & $\begin{array}{c}\text { Loss in } \\
\text { rattler } \\
\text { test }\end{array}$ \\
\hline $\begin{array}{c}\text { Transverse } \\
\text { dimensions }\end{array}$ & Length & \\
\hline Inches & Inches & Per cent \\
$21 / 2$ by 4 & $81 / 2$ & 26 \\
3 by $31 / 2$ & $81 / 2$ & 26 \\
3 by 4 & $81 \frac{2}{2}$ & 24 \\
$31 / 2$ by 4 & $81 / 2$ & 22 \\
\hline
\end{tabular}

\section{SAMPLING}

11. The standard sample for the rattler test shall consist of 10 whole undamaged brick which conform individually to the visual inspection requirements specified in section 16 .

12. (a) Samples shall preferably be selected at the place of manufacture and tested prior to shipment. However, in special cases where less than 100,000 brick are involved, samples, when required, may be selected subsequent to delivery at the destination.

(b) In general, one standard sample shall be selected for each 15,000 brick or fraction thereof; provided, however, that when the sampling is done at the place of manufacture prior to shipment and the total number of brick involved is more than 100,000 brick, the number of brick for which one standard sample is considered representative may be increased at the option of the purchaser to not more than 50,600 brick.

13. Acceptance of any lot of paving brick shall be based on the results of the rattler test on the sample representing that lot, subject only to rejection at 
any time of individually imperfect or unsatisfactory brick, in accordance with the requirements under visual inspection as described in section 16 .

14. (a) If the percentage of loss on any sample exceeds by 2 per cent or less the maximum permissible rattler loss specified in section 10 for the size specified, the lot shall not be rejected, but two additional tests shall be made, and if the average of the three tests equals or is less than the maximum permissible rattler loss the entire lot shall be accepted, provided no test used in computing the average of the three tests exceeds the maximum permissible rattler loss by more than 2 per cent.

(b) Rejection.- Where a lot of paving brick is rejected for failure to pass the rattler test, the purchaser may, at his option, permit the seller to regrade the rejected brick and again offer the brick for test and acceptance. The regraded brick shall be resampled and retested, and if it again fails the purchaser may definitely and finally reject the lot for which the test is representative.

15. Unless otherwise specified, the seller shall furnish at the place of manufacture, without charge to the purchaser, the use of an approved rattler, in efficient operating condition, conforming to the requirements specified in sections 17 to 25 , inclusive. Payment of the cost of testing shall be made by the purchaser, except that the cost of testing additional samples in accordance with section 14, made necessary by the failure of any lot or lots, or of testing supplemental samples to determine the admissibility of a portion of any lot or lots, shall be paid by the seller. Provided, however, that if the supplemental tests show the brick under examination to be acceptable, then the cost of such tests shall be paid by the purchaser.

\section{VISUAL INSPECTION}

16. Paving brick shall be subject to visual inspection prior to shipment, after delivery, or during laying and culling out and rejection subject to the exception specified in paragraph $(b)$, of individual imperfect bricks or unsatisfactory brick for the following causes:

(a) Variation in size.-Brick which vay from the nominal sizes specified in section 7 by more than $\pm 1 / 8$ inch in either transverse dimension, or more than $\pm 1 / 4$ inch in length.

(b) Broken or chipped brick.-Brick which are chipped or broken so that neither wearing surface remains substantially intact, or so that the lower, or bearing surface, is reduced in area more than 15 per cent, provided brick so rejected, but otherwise acceptable, may be used for half brick for beginning courses and for batting.

(c) Cracked brick.-Brick which are cracked to such a degree, either from shocks received in shipping and handling, or from defcctive manufacture, as to produce defects described in paragraph $(b)$, unless such cracks are plainly superficial and insufficient to weaken the brick under the conditions of use.

(d) Warped or kiln-marked brick-Brick which are misshaped, bent or twisted, or kiln marked more than $3 / 16$ inch so that they will not form a proper surface, or align with other brick in conformance with the specifications for laying brick in the pavement.

(e) Underburned brick.-Brick which are obviously too soft and too poorly vitrified to endure traffic wear. If disagreement on this requirement arises between the purchaser and the seller, it shall be the right of the purchaser to make one or more rattler tests of the brick he wishes to exclude, and if in any or all tests the brick fail to conform to the requirements of these specifications then all brick having the same objectionable appearance may be excluded.

\section{RATTLER TEST}

\section{APPARATUS}

17. The machine used in the rattler test shall be of good mechanical construction, self-contained, and shall conform to the following details of material and dimensions, and shall consist of barrel, frame, and driving mechanism as described in sections 18 to 22. In Figure 218 is illustrated a rattler which will meet the requirements specified, and to which reference should be made.

18. The barrel of the machine shall consist of the heads, head liners, staves, and stave liners conforming to the following requirements:

(a) Heads. - The heads may be cast in one piece with the trunnions which shall be $27 / 16$ inches in diameter, and shall have a bearing 6 inches in length; or they may be cast with heavy hubs, which shali be bored out for $2 \% 16$-inch shafts and shall be key seated for two keys, each $1 / 2$ by $3 / 8$ inch and spaced $90^{\circ}$ apart. The shaft shall be a snug fit and when keyed shall be entirely free from lost motion. The distance from the end of the shaft or trunnion to the inside face of the head shall be $15 \%$ inches in the head for the driving end of the rattler and $113 / 8$ inches for the other head, and the distance from the face of the hubs to the inside face of the heads shall be $5 \frac{1}{8}$ inches.

The heads shall not be less than three-fourths nor more than seven-eighths inch in thickness. In outline, each head shall be a regular 14 -sided polygon inscribed in a circle 283/8 inches in diameter. Each head shall be provided with flanges not less than $3 / 4$ inch in thickness and extending outward $2 \frac{1}{2}$ inches from the inside face of the head to afford a means of fastening the staves. The surface of the flanges of the head shall be smooth and give a true and uniform bearing for the staves. To secure the desired true and uniform bearing, the surfaces of the flanges of the head shall be either ground or machined. The flanges shall be slotted on the outer edge, so as to provide for two $3 / 4$-inch bolts at each end of each stave, the slots to be $13 / 16$ inch in width and $23 / 4$ inches center to center. Each slot shall be provided with a recess for the bolt head, which shall act to prevent the turning of the same. Between each two slots there shall be a brace three-eighth inch in thickness extending down the outward side of the head not less than 2 inches.

(b) Hcad liners.-There shall be for each head a cast-iron head liner, 1 inch in thickness and conforming to the outline of the head, but inscribed in a circle $28 \frac{1}{8}$ inches in diameter. This head liner shall be fastened to the head by seven $5 / 8$-inch cap screws through the head from the outside. Whenever these head liners become worn down $1 / 2$ inch below thcir initial surface level, at any point of their surface, they shall be replaced with new ones. The metal of these liead liners shall be hard machinery iron and should contain not less than 1 per cent of combined carbon.

(c) Staves.-The staves shall be made of 6 -inch medium carbon-steel structural channels $271 / 4$ inches in length and weighing 15.5 pounds per lineal foot. The staves shall have two holes $13 / 16$ inch in diameter, drilled in each end, the ccnter line of the holes being

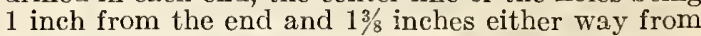
the longitudinal center line. The spaces between the staves shall be as uniform as practicable, but shall not exceed $5 / 16$ inch.

(d) Stave liners.-The interior, or flat side, of each stave shall be protected by a liner three- 

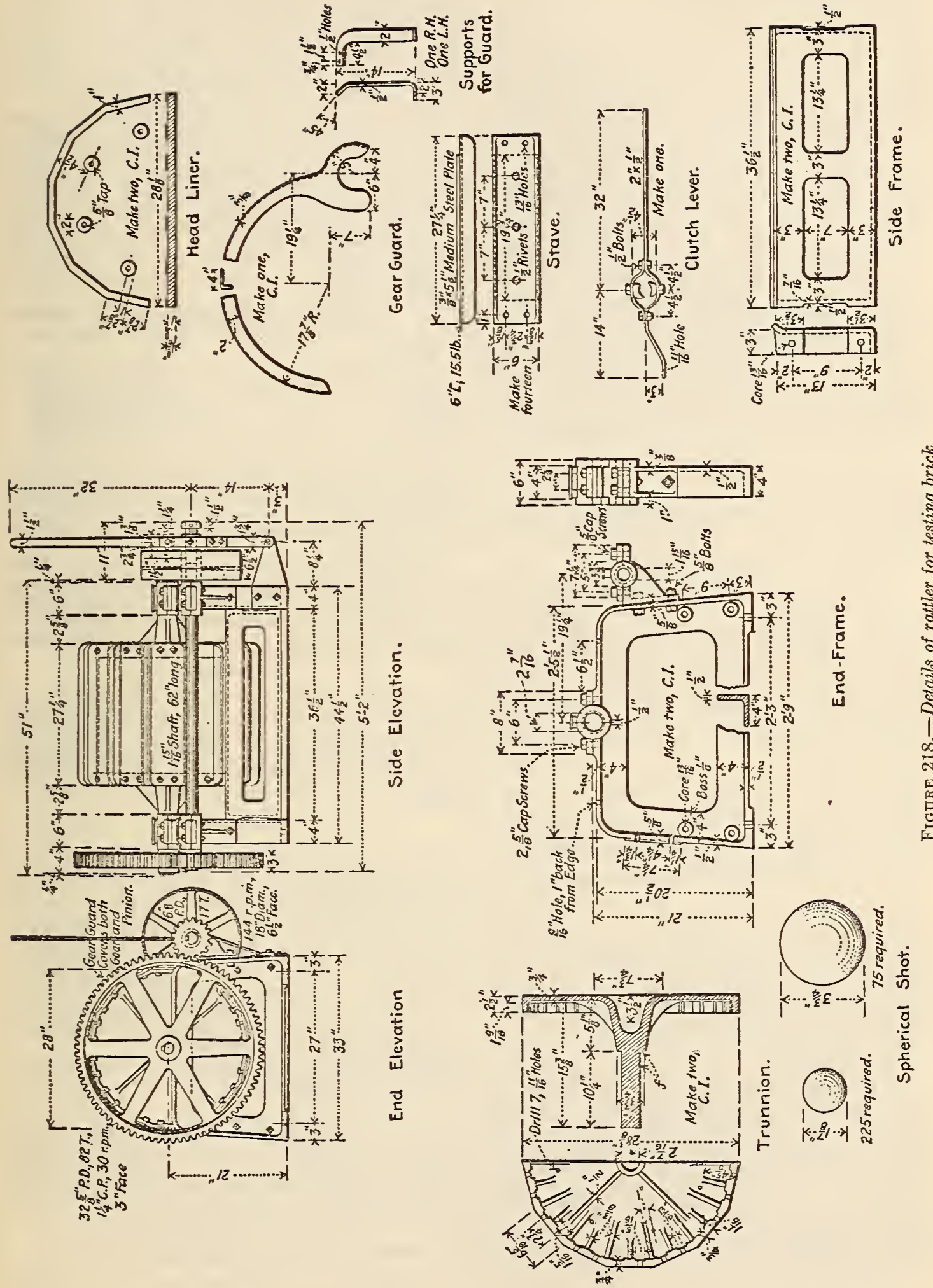
eighths inch thick by $5 \frac{1}{2}$ inches wide by $19 \% 4$ inches long. The liner shall consist of medium carbon steel plate, and shall be riveted to the channel by three $1 / 2$-inch rivets, one of which shall be on the center line both ways and the other two on the longitudinal center line and spaced 7 inches from the center each way. The rivet holes shall be countersunk on the face of the liner and the rivets shall be driven hot and chipped off flush with the surface of the liners, These liners shall be inspected from time to time. and if found loose shall be at once reriveted.

19. When a new rattler, in which a complete set of new staves is furnished, is first put into operation, it shall be charged with 400 pounds of shot of the same sizes, and in the same proportion as provided in section 23 , and shall then be run for 18,000 revolutions at the rate of speed specified in section $27(a)$. The shot shall then be rcmoved and a standard shot charge inserted after which the rattler may be charged with brick for a test.

20 . No stave shall be used for more than 70 3onsecutive tests without renewing its lining. Two of the 14 staves shall be removed and relined at a time in such a way that of each pair, one falls upon one side of the barrel and the other upon the opposite side, and also so that the staves changed shall be consecutive, but not contiguous; for example 1 and 8,3 and 10,5 and 12,7 and 14,2 and 9,4 and 11,6 and 13, etc., to the end that the interior of the barrel at all times shall present the same relative conditions of repair. The changes in the staves should be made at the time when the shot charges are being corrected, and the record must show the number of charges run since the last pair of new lined staves was placed in position.

The staves when bolted to the heads shall form a barrel 20 inches in length, inside measurement, between head liners. The liners of the staves shall be so placed as to drop between the head liners. The staves shall be bolted tightly to the heads by four $3 / 4$-inch bolts, and each bolt shall be provided with a locknut and shall be inspected at not less frequent intervals than every fifth test and all nuts kept tight. A record shall be made after each inspection showing in what condition the bolts were found.

21. The barrel shall be mounted on a cast-iron frame of sufficient strength and rigidity to support it without undue vibration. It shall rest on a rigid foundation with or without the interposition of wooden plates and shall be fastened thereto by bolts at not less than four points.

22 . The barrel shall be driven by gearing whose ratio of driver to driven is not less than 1 to 4 . The countershaft upon which the driving pinion is mounted shall not be less than $1^{15} / 16$ inches in diameter, with bearing not less than 6 inches in length. If a belt drive is used, the pulley shall be not less than 18 inches in diameter and $6 \frac{1}{2}$ inches in face. $A$ belt at least 6 inches in width, properly adjusted to avoid unnecessary slipping, should be used.

23. The abrasive charge shall consist of cast-iron spheres of two sizes.

(a) Large spheres.-When new, the larger spheres shall be 3.75 inches in diameter and shall weigh approximately 7.5 pounds $(3.40 \mathrm{~kg})$ each. Ten spheres of this size shall be used. These shall be weighed separately after each 10 tests, and if the weight of any large sphere falls to 7 pounds $(3.175$ $\mathrm{kg})$ it shall be discarded and a new one substituted; provided, however, that all of the large spheres shall not be discarded and substituted by new ones at any single time, and that in so far as possible the large spheres shall compose a graduated series in various stages of wear.

(b) Small spheres.-When new, the smaller spheres shall be 1.875 inches in diameter and shall weigh approximately 0.95 pound $(0.43 \mathrm{~kg})$ each. In general, the number of small spheres in a charge shall not fall below 245 nor exceed 260 . The collective weight of the large and small spheres shall be as nearly 300 pounds as possible. No small sphere shall be retained in use after it has been worn down so that it will pass a circular hole 1.75 inches in diameter, drilled in an iron plate $1 / 4$ inch in thickness, or weigh less than 0.75 pound $(0.34 \mathrm{~kg})$.

Further, the small spheres shall be tested by passing them over the plate mentioned or by weighing, after every 10 tests, and any which pass through or fall below the specified weight, shall be replaced by new spheres, provided, further, that all of the small spheres shall not be rejected and replaced by new ones at any one time, and that so far as possible the small spheres shall compose a graduated series in various stages of wear. At any time that any sphere is found to be broken or defective it shall at once be replaced.

24. The cast-iron abrasive spheres shall conform to the following requirements as to chemical composition:

Combined carbon

Graphitic carbon

Silicon.

Manganese

Phosphorus

Not under 2.50 per cent: Not over 0.25 per cent. Not over 1.00 per cent. Not over 0.50 per cent Not over 0.25 per cent. Not over 0.08 per cent.

For each new batch of spheres used, the chemical analysis shall be furnished by the maker or be obtained by the user, before introducing the spheres into the charge, and unless the analysis conforms to the requirements specified, the batch of spheres shall be rejected.

25. The scales used shall be placed on a solid level foundation and shall be sufficiently sensitive to determine the initial weight of 10 bricks to the nearest 0.1 pound. The scale shall be tested by a standard test weight at intervals of not more tban every 10 tests.

\section{TEST SPECIMEN}

26. The number of brick per test shall be 10 whole bricks for all weights of brick, and the brick shall be weighed to the nearest 0.1 pound. No brick should be selected as part of a regular test that would be rejected by any other requirements of the specifications under which the purchase is madc.

\section{PROCEDURE}

27. (a) The test shall consist of 1,800 revolutions of the rattler at the rate of not less than 29.5 nor more than 30.5 revolutions per minute. A counting machine shall be attached to the rattler for counting the revolutions. A margin not to exceed 10 revolutions will be allowed for starting and stopping. Only one start and stop per test is, in general, acceptable. If, from accidental causes, the rattler is stopped and started more than once during a test, and the percentage loss exceeds the maximum permissible under the specifications, the test shall be disqualified and another made.

(b) Any test, at the expiration of which a stave liner is found detached from the stave or seriously out of position, shall be rejected.

28 . The loss shall be calculated as a percentage of the original weight of the brick composing the charge, by weighing the brick remaining in the rattler at the end of the test, deducting this weight from the original weight of the brick, dividing this difference by the original weight of the brick and multiplying by 100 .

Percentage of loss $=\frac{\text { Original weight }- \text { weight after testing }}{\text { Original weight }} \times 100$

In weighing the rattled brick, any piece weighing less than 1 pound shall be rejected. 


\section{RECORD}

29. A complete and continuous record shall be kept of the operation of all rattlers working under the rattler test. This record shall contain the following data concerning each test made:

1. The name of the person, or corporation furnishing each sample tested.

2. The name of the maker of the brick represented in each sample tested.

3. The name of the street or contract which the sample represented.

4. The brands or marks upon the bricks by which they were identified.

5. The number of bricks furnished.

6 . The date on which they were received for test.

7. The date on which they were tested.

8. The drying treatment given before testing, if any.

9. The length, width, and thickness of the bricks.

10. The collective weight of the 10 large spherical shot used in making the test at the time of their last standardization.

11. The number and collective weight of the small spherical shot used in making the test at the time of their last standardization.

12. The total reight of the shot charge after its last standardization.

13. Certificate of the operator that he examined the condition of the machine as to staves, liners, and any other parts affecting the barrel and found them to conform to the requirements at the beginning of the test.

14. Certificate of the operator of the number of charges tested since the last standardization of shot charge and last renewals of stave liners.

15. The time of the beginning and ending of each test and the number of revolutions made by the barrel during the test as shown by the counting machine.

16. Certificate of the operator as to number of stops and starts made in each test.

17. The initial collective weight of the 10 bricks composing the charge and their collective weight after rattling.

18. The loss calculated as a percentage of the initial weight and the calculation itself, the correction factor, and the corrected percentage of loss.

19. The number of broken bricks and remarks upon the portions which were included in the final weighing.

20. General remarks upon the test and any irregularities occurring in its execution.

21 . The certificate of the operator that the test was made in accordance with the requirements of the American Society for Testing Materials and that the record is a true record.

22. The signature of the operator or person responsible for the test.

23. The location of the laboratory where the test was made and name of the owner.

24 . The serial number of the test.

In the event of more than one copy of the record of any test being required, they may be furnished on separate sheets and marked duplicate, but the original record shall always be preserved intact and complete.

American Society for Testing Materials, standard specifications for clay sewer brick, serial designation C32-24, 1024.

1. These specifications cover four classes of clay bricks intended to be used in drainage structures for the conveyance of sewage, industrial waste, and storm water, as follows, the purposes for which they are intended to be especially suitable being indicated:
Class $A$ vitrified brick, for the interior of structures where resistance to the abrasive action of sewrage having velocities up to 18 feet per second is required.

Class $B$ vitrified brick, for the interior of structures where resistance to the abrasive action of sewage having velocities up to 12 feet per second is required.

Hard brick, for structures requiring imperviousness to internal and external pressures and resistance to the abrasive action of sewage having velocities up to 8 feet per second.

Medium brick, for storm-water conduits, where a high degree of imperviousness is not rcquired and where abrasive action is due to sewage having low velocities only.

For definitions of the above classes, see Table 1.

2 . The consumer or purchaser shall specify the class or classes of brick to be supplied.

3. (a) The acceptability of brick shall be determined (1) by the results of the chemical and physical tests hel einafter specified, and (2) by visual inspection to determine whether the bricks comply with the specifications as to dimenslons, shape, and freedom from visible defects.

(b) The acceptance of brick as satisfactorily meeting one of these two general requirements shall not be construed as in any way waiving the other.

\section{MATERIALS}

4. (a) Clay bricks shall be manufactured from surface clay, fire clay, or shale or a combination of these materials.

(b) Surface clay is an unconsolidated, unstratified clay 9 of low flux content.

Note. - It is usually associated with coal measures.

(d) Shale is a thinly stratified, consolidated sedimentary clay with well-marked cleavage parallel to the bedding.

(e) The material shall posses such physical and chemical properties that when molded into bricks and subjected to a suitable temperature the product will be strong, durable, and serviceable, free from objectionable defects, and in compliance with these specifications and tests.

\section{SIZE}

5. Sewer brick shall be not less than $2 \frac{1}{4}$ by $3 \frac{3}{4}$ by 8 inches nor more than $2 \frac{1}{2}$ by 4 by $8 \frac{1}{2}$ inches, except that No. 1 vitrified brick may be 3 by 4 by $8 \frac{1}{2}$ inches, but not more than $3 \frac{1}{2}$ by 4 by $8 \frac{1}{2}$ inches.

\section{SAMPLING}

6. For the purpose of tests, bricks shall be selected by a competent person appointed by the purchaser, the point or points of selection to be designated when the order is placed. The manufacturer or seller shall furnish specimens for tests without separate charge. All bricks shall be carefully examined and their condition noted before being subjected to any test. No sample which fails to meet the requirements of the visual inspection as given below shall be subjected to test. For the purpose of the tests not less than 15 bricks shall be required for each delivery; they shall be dried to constant weight in a suitable oven at a temperature of from $225^{\circ}$ to $250^{\circ} \mathrm{F}$. ( $107^{\circ}$ to $121^{\circ} \mathrm{C}$.).

\footnotetext{
"The definitions for the terms "surface clay," "fire clay," and "shale" are based upon the following definitions for the term "clay" Clay.-An earthy or stony mineral aggregate consisting essentially of hyarous silicates of alumina, plastic when sufficiently pulverized and wetted, rigid when dry, and vitreous when burned at a suffic iently high temperature.
} 


\section{CHEMICAL TESTS AND REQUIREMENTS}

7. The consumer or purchaser may preseribe in advance special chemical requirements in cases where sewage, industrial wastes, or ground waters have a marked acid or alkaline character or are of abnormally high temperatures. He may make use of chemical analyses of the brick or brick materials to detect deleterious materials, such as caustic lime, iron pyrites, or any other materials which are known to cause slaking or disintegration. Such deleterious matter contained in the brick material shall be cause for rejection.

\section{PHYSICAL TESTS}

8. (a) The brick shall be subjected to absorption, compression and transverse tests, and shall meet testing machine the edge surface shall be bedded in a thin coat of calcined gypsum (plaster of Paris) spread upon plate glass previously coated with a film of oil. Before applying the calcined gypsum (plaster of Paris) the bearing surface of the brick shall receive a coating of shellac. The brick shall be pressed firmly upon the surface, making the layer as thin as possible, and be permitted to remain undisturbed until set.

(b) The machine used for the compression tests shall be equipped with a spherical bearing block kept thoroughly lubricated to insure accurate adjustment, which should be made by hand under a small initial load. The radius of the spherical bearing surface should be such that the center of the sphere falls in the adjacent end surface of the specimen. During the test the beam of the testing

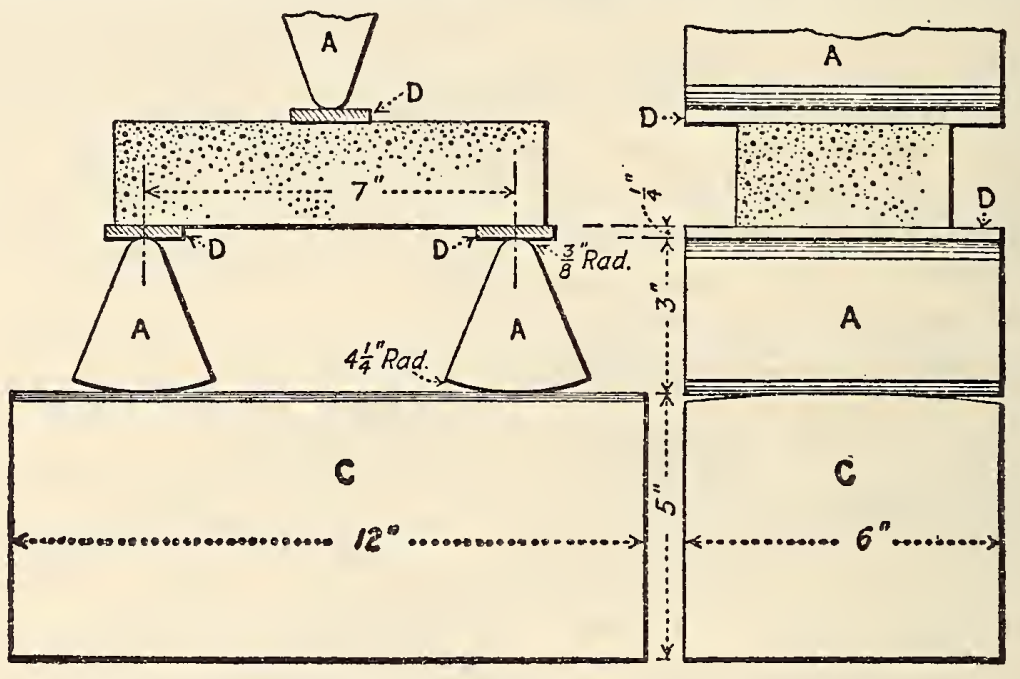

$A=$ Cast-Iron Wedges; $C=$ Sofr Wood Block, $D=$ Cold-Rolled Steel Plates

FIGURE 219

the requirements specified in Table 1 for such tests.

(b) The standing of any set of bricks shall be determined by that one of the three requirements in which it is the lowest.

9. (a) At least five bricks after being dried to constant weight shall be completely submerged in water at a temperature of between $60^{\circ}$ and $80^{\circ} \mathrm{F}$. $\left(15^{\circ}\right.$ and $27^{\circ}$ C.). The water shall be heated to boiling within one hour, boiled continuously for five hours, and then allowed to cool to a temperature between $60^{\circ}$ and $80^{\circ} \mathrm{F}$. $\left(15^{\circ}\right.$ and $27^{\circ} \mathrm{C}$.). The brick shall then be removed, the surface water wiped off with a damp cloth, and the bricks quickly weighed.

(b) The percentage of absorption shall be calculated on the dry weight from the formula:

$$
\text { Percentage-of absorption }=\frac{100(b-a)}{a}
$$

where $a$ equals weight of dry bricks and $b$ equals weight of saturated bricks.

10. (a) Compression tests shall be made on at least five half bricks, previously dried, each taken from a different brick. The half brick shall be prepared either by sawing or cutting upon a yielding bed with a sharp mason's chisel which shall be the full width of the brick. The specimens shall be tested on_edge, To secure a uniform bearing in the machine shall be kept constantly in a floating position.

(c) The breaking load shall be divided by the area in compression and the results reported in pounds per square inch.

11. (a) At least five bricks, previously dried, shall be tested, laid flatwise on knife-edges with a span of 7 inches and with the load applied at mid span. Steel bearing plates about $1 / 4$ inch thick by $1 \frac{1}{2}$-inch wide may be placed between the knifeedges and the brick. The use of a wooden base block, slightly rounded transversely across the top, upon which to rest the lower knife-edges (see fig. 219), or the form of lower knife-edge shown in Figure 220 is recommended. If the knife-edges shown in Figure 219 are used they should rest upon smooth plane blocks of wood at least 2 inches thick.

(b) The modulus of rupture shall be calculated in pounds per square inch by the following formula:

$$
R=\frac{3 W l}{2 b d^{2}}
$$

in which $l$ equals the distance between supports in inches, $b$ equals the breadth, and $d$ equals depth of the brick in inches, and $W$ equals the load in pounds at which the brick failed. The measurement $d$ is taken at the fractured surface after the specimen is broken. 
$T_{A B L E}$ 1.-Physical test requirements for different classes of brick

\begin{tabular}{|c|c|c|c|c|c|c|}
\hline \multirow[b]{2}{*}{ Class } & \multicolumn{2}{|c|}{ Absorption limits } & \multicolumn{2}{|c|}{ Compressive strength (on edge) } & \multicolumn{2}{|c|}{ Modulus of rupture } \\
\hline & Mean of 5 tests & $\begin{array}{l}\text { Individual, } \\
\text { maximum }\end{array}$ & Mean of 5 tests & $\begin{array}{l}\text { Individual, } \\
\text { minimum }\end{array}$ & Mean of 5 tests & $\begin{array}{l}\text { Individual, } \\
\text { minimum }\end{array}$ \\
\hline $\begin{array}{l}\text { Class A, vitrified } \\
\text { Class B, vitrified........ } \\
\text { Hard } \\
\text { Medium }\end{array}$ & $\begin{array}{l}\text { Per cent } \\
3 \text { or less } \\
5 \text { or less } \\
5 \text { to } 10 \\
10 \text { to } 15\end{array}$ & $\begin{array}{r}\text { Per cent } \\
6.0 \\
12.0 \\
17.0\end{array}$ & $\begin{array}{c}\text { Lbs./in. } \\
5,000 \text { or over } \\
3,500 \text { or over } \\
2,000 \text { or over }\end{array}$ & \begin{tabular}{r|} 
Lbs./in. ${ }^{2}$ \\
4,000 \\
2,500 \\
1,500
\end{tabular} & $\begin{array}{c}\text { Lbs./in.2 } \\
1,200 \text { or over }\end{array}$ & $\begin{array}{r}\text { Lbs./in. } \\
\begin{array}{r}800 \\
400 \\
300\end{array}\end{array}$ \\
\hline
\end{tabular}

\section{VISUAL INSPECTION}

12. (a) All brick shall be given a thorough visual inspection at the sewer trench by a competent inspector employed by the purchaser to determine whether the brick, independently of the chemical and physical requirements, comply with the specifications of general properties as follows:

(b) Bricks shall be of rectangular cross section, full size, and of uniform dimensions throughout. Recessed bricks shall not be accepted.

(c) Bricks shall have straight, parallel edges and square corners. The ends and at least one edge
1. These specifications cover brick made from clay or shale and properly burned, intended for use in brick masonry.

\section{PHYSICAL PROPERTIES AND TESTS}

2. (a) The bricks shall be classified as grades $\mathrm{A}, \mathrm{B}$, and $\mathrm{C}$ on the basis of the following requirements: The classification of any lot of bricks shall be determined by the results of the tests for that requirement in which it is lowest, unless otherwise specified as provided in section 8

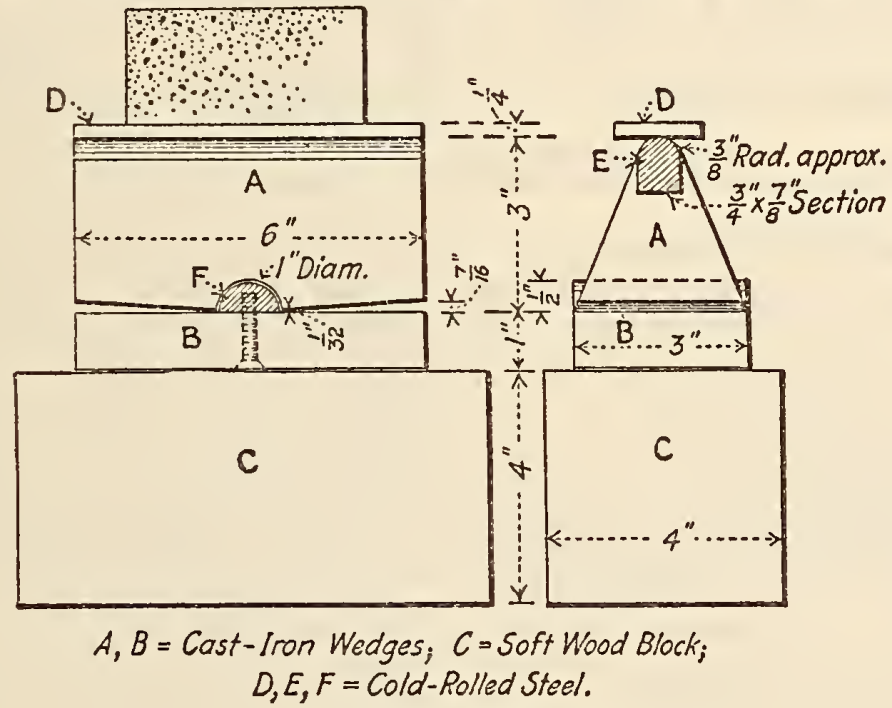

FIGURE 220

shall have a smooth plane surface; kiln marks not exceeding one-sixteenth inch in depth shall be permitted on the opposite edge.

(d) Bricks shall be thoroughly and uniformly burned and when broken shall show a fracture of uniform and compact texture.

(e) The color of the bricks shall be considered to indicate their burning, providing the meaning of the variation in color be first determined from an investigation of the materials and the process of manufacture.

(f) Bricks shall be free from checks or cracks extending into the body of the brick in a manner that would affect their serviceability or strength. Bricks shall be free from stones, pebbles, and visible grains or masses of lime.

(g) Bricks when struck together or when struck with a trowel shall give a clear ringing sound.

American Society for Testing Materials, tentative specifications for building brick (made from clay or shale), serial designation C62-29, 1929.

\begin{tabular}{|c|c|c|c|c|}
\hline \multirow[b]{2}{*}{ Name of grade } & \multicolumn{2}{|c|}{$\begin{array}{l}\text { Compressive strength } \\
\text { (bricks flatwise) }\end{array}$} & \multicolumn{2}{|c|}{$\begin{array}{l}\text { Modulus of rupturs } \\
\text { (bricks flatwise) }\end{array}$} \\
\hline & Mean of 5 tests & $\begin{array}{l}\text { Indi- } \\
\text { vidual } \\
\text { mini- } \\
\text { mum }\end{array}$ & $\begin{array}{l}\text { Mean of } 5 \\
\text { tests }\end{array}$ & $\begin{array}{l}\text { Indi- } \\
\text { vidual } \\
\text { mini- } \\
\text { mum }\end{array}$ \\
\hline $\begin{array}{l}\text { Grade A } \\
\text { Grade B } \\
\text { Grade C. }\end{array}$ & $\begin{array}{c}\text { Lbs./in. } \\
4,500 \text { or over } \\
2,500 \text { to } 4,500 .- \\
1,250 \text { to } 2,500-.\end{array}$ & $\begin{array}{c}\text { Lbs./in. } 2 \\
3,500 \\
2,000 \\
1,000\end{array}$ & $\begin{array}{l}\text { Lbs./in. } \\
600 \text { or over.- } \\
450 \text { or over-- } \\
300 \text { or over.- }\end{array}$ & $\begin{array}{r}\text { Lbs./in. }{ }^{2} \\
400 \\
300 \\
200\end{array}$ \\
\hline
\end{tabular}

Note. - The above classifications are based on strength and do not necessarily measure weather resistance.

(b) The tests shall be conducted in accordance with A. S. T. M. C67-28T. (See 534.10, p. 574.)

3. For the purpose of tests, bricks representative of the commercial product shall be selected by a competent person appointed by the purchaser, the 
place or places of selection to be designated when the purchase order is placed. The manufacturer or seller shall furnish specimens for tests without charge. All bricks shall be carefully examined and their condition noted before testing. For the purpose of tests, not less than 10 bricks shall be required for each investigation. In general, two samples of 10 bricks each shall be tested for every 100,000 bricks contained in the lot under consideration, but where the total quantity exceeds 500,000 the number of samples tested may be fewer, provided that they shall be distributed as uniformly as practicable over the entire lot. Additional representative samples may be taken at any time or place, at the discretion of the purchaser.

\section{STANDARD SIZES}

4. The standard sizes shall conform to the following dimensions, with a permissible variation, plus or minus, of $1 / 16$ inch in depth, $1 / 8$ inch in width, and $1 / 4$ inch in length:

\begin{tabular}{|c|c|c|c|}
\hline Type & Depth & Width & Length \\
\hline $\begin{array}{l}\text { Common brick } \\
\text { Rough-face brick } \\
\text { Smooth-face brick }\end{array}$ & $\begin{array}{r}\text { Inches } \\
21 / 4 \\
21 / 4 \\
21 / 4\end{array}$ & $\begin{array}{r}\text { Inches } \\
33 / 4 \\
33 / 4 \\
37 / 8\end{array}$ & $\begin{array}{r}\text { Inches } \\
8 \\
8 \\
8\end{array}$ \\
\hline
\end{tabular}

\section{WORKMANSHIP AND FINISH}

5. When any particular surface appearance or uniformity is desired, it shall be as mutually agreed upon.

\section{INSPECTION AND REJECTION}

6. All brick shall comply with the following requirements for general properties under visual inspection:

(a) They shall be free from cracks, warpage, stones, pebbles, or particles of lime that would affect their serviceability or strength.

(b) Bricks of Grades A or B when struck together shall give a ringing sound.

7. If the visual inspection (sec. 6) appears to indicate inferior strength, the brick may be rejected unless the physical tests show the quality or grade to be as specified.

8. At the option of the purchaser, acceptance may be based on the visual inspection requirements specified in section 6 and the results of one of the strength tests (compression or flexure) specified in scction 2. While the compressive strength is considered as generally giving the better basis for classification purposes, the flexure test gives important information on the strength and proper manufacturing details.

Federal Specifications Board, specification No. 504, United States Government master specification for brick, clay, common, July 11, 1927.

\section{CIASSES}

This specification is applicable to common solid or hollow (not face) clay brick of any of the following four classes: $V$, vitrified; $H$, hard; $M$, medium; and $\mathrm{S}$, soft.

\section{MATERIAL AND WORKIMANSHIP}

Brick under this specification shall be of clay or shalc, be sound, of compact structure, reasonably uniform in shape, free from stones and pebbles that would affect their serviceability or strength, and without excessive laminations or warpings.

\section{GENERAL REQUIREMENTS}

The standard size of brick shall be $23 / 4$ by $3 \frac{3}{4}$ by 8 inches, with permissible variations of $1 / 8$ inch in breadth or depth and $1 / 4$ inch in length.

Bricks shall be delivered in good condition, with not more than 5 per cent broken.

At the completion of the absorption test the bricks shall show no evidence of disintegration.

\section{DETAIL REQUIREMENTS}

The brick shall meet the following absorption and strength requirements for its respective class. The standing of any set of bricks shall be determined by the requirements in which it is lowest. Unless otherwise specified in the invitation for bids, medium (M) or hard $(\mathrm{H})$ brick shall be accepted in lieu of soft $(\mathrm{S})$ brick, and hard $(\mathrm{H})$ brick in lieu of medium (M) brick.

Physical requirements

\begin{tabular}{|c|c|c|c|c|}
\hline \multirow{2}{*}{ Class } & \multicolumn{2}{|c|}{ Absorption } & \multicolumn{2}{|c|}{$\begin{array}{c}\text { Transverse breaking load, } \\
7 \text {-inch span }\end{array}$} \\
\hline & Average of 5 & $\begin{array}{l}\text { Individual } \\
\text { maximum }\end{array}$ & Average of 5 & $\begin{array}{l}\text { Individual } \\
\text { minimum }\end{array}$ \\
\hline $\begin{array}{l}\mathrm{V}_{-} \\
\mathrm{H}_{-} \\
\mathrm{M}_{-} \\
\mathrm{S}_{-}\end{array}$ & $\begin{array}{l}\quad \text { Per cent } \\
5 \text { or less } \\
5 \text { to } 12 \\
12 \text { to } 24 \\
24 \text { or more }\end{array}$ & $\begin{array}{r}\text { Per cent } \\
6 \\
15 \\
28 \\
\text { No limit. }\end{array}$ & $\begin{array}{l}\text { Pounds } \\
2,170 \text { or more... } \\
1,080 \text { or more... } \\
810 \text { or more } \\
540 \text { or more...-. }\end{array}$ & $\begin{array}{r}\text { Pounds } \\
1,450 \\
725 \\
540 \\
360\end{array}$ \\
\hline
\end{tabular}

\section{METHOD OF SAMPIING AND TESTS}

1. Sampling.-Ten bricks selected by the inspector, so as to be fairly representative of a quantity not exceeding 50,000 bricks, shall constitute a sample. If the bricks are delivered by car or boat, one sample shall be taken from each carload or boatload. If the bricks are delivered by truck or wagon, one or more samples shall be taken at the point of origin, covering all of the material from which shipments are to be made. Additional representative samples may be taken at any time or place, at the discretion of the inspector.

2. Tests.-The sample shall be dried to constant weight at a temperature of $212^{\circ}$ to $220^{\circ} \mathrm{F}$.

(a) Absorption.- When cool, 5 of the 10 bricks in the sample shall be weighed separately on scales sensitive to within one-half of 1 per cent of the weight. They shall then be completely immersed in sof $t$, distilled, or rain water at room temperature. The water shall be brought to a boil within one hour and boiling continued for five hours. The bricks shall be allowed to cool to room temperature in the water. They shall then be removed from the water and weighed, after wiping the surface with a damp cloth. This weight minus the weight of the dry bricks equals the weight of the water absorbed, which is calculated to per cent of the weight of the dry bricks.

Where means are not available for boiling the bricks, the absorption test may be made by immersing the dry bricks in sof $t$, distilled, or rain water at ordinary temperature for five hours. When this method is used, the absorption limits for the different classes shall be reduced one-fourth below the values given in Section $V$ for both the average and the individual maximums.

In cases of disagreement as to the resulting classification, the absorption shall be determined by the boiling method and the full percentage absorption for the respective classes given in Section $V$ applied. The same bricks, redried to constant weight, may be used in any such retest. 
(b) Transierse strength.-The other five bricks of the sample, previously dried, shall be tested laid flatwise on a span of 7 inches and with the load applied at the mid-point of the span with a standard testing machine or a calibrated portable or semiportable testing equipment. A steel bearing plate about $1 / 4$ inch thick by $1 \frac{1}{2}$ inches wide shall be placed between the upper knife-edge and the brick. The knife-edges in contact with the brick shall be mounted so they will adjust themselves to the irregularities in the shape of the brick, and one or both of the lower bearings shall be free to follow any movement of the brick during the test.

\section{NOTES}

1. Strength tests.-The acceptance procedure has been simplified to permit making the necessary tests at the building site or at the manufacturer's plant without the use of laboratory equipment, although the latter should be used where available. Responsibility for the accuracy, calibration, and general sufficiency of the equipment used necessarily devolves on the purchasing or inspecting officer concerned. The following specification is suggested for portable or semiportable equipment for conducting the transverse test:

Portable or semiportable equipment for conducting transverse tests of building brick shall be capable of applying a center load of not less than 4,000 pounds on bricks laid flatwise on a span of 7 inches. A steel bearing plate about $1 / 4$ inch thick by $1 \frac{1}{2}$ inches wide shall be placed between the upper knife-edge and the brick. The knife-edges in contact with the brick shall be mounted so they will adjust themselves to the irregularities in the shape of the brick, and one or both of the lower bearings shall be free to follow any movement of the brick during the test.

The equipment shall be constructed so it can be calibrated in a standard testing machine and shall accommodate bricks having $1 / 2$ inch variation above or below the standard $2 \frac{1}{4}$ inch thickness and $33 / 4$ inch width. It shall be constructed so the load can be applied by hand with a maximum force of 50 pounds. The parts shall be designed so as not to be reacilily damaged or displaced by shipment or handling and a suitable carrying case with one handle for the portable and two handles for the semiportable equipment shall be provided.

The weight of the portable equipment, inclusive of carrying case, shall be not more than 55 pounds, and the accuracy and sensitiveness shall be within 30 pounds up to 2,000 pounds applied load and within 60 pounds for higher loads.

The weight of the semiportable equipment, inclusive of carrying case, shall be not more thar 110 pounds and the accuracy and sensitiveness shall be within 10 pounds up to 2,000 pounds applied load and within 20 pounds for higher loads.

Bidders shall submit description of apparatus it is proposed to supply and the general limits of sensitiveness and accuracy obtainable with it.

2. Significance of the brick classification.-The classification is based on strength and absorption values chosen so that bricks grading as medium or harder can be considered suitable for use in exterior walls. This should not be taken as a rigid distinction on account of the wide range in the character of clays and processes used in brick manufacture, which makes it difficult to define weathering resistance in terms of properties that can be determined in acceptance tests. The purchasing officer should be guided in part by the experience with comparable bricks in the locality where those under test are to be used. In cases of doubt and where the time and equipment are available acceptance in point of weathering resistance can be based on ability to withstand 100 alternations of freezing and thawing conducted according to generally accepted laboratory procedure. Failure is to be considered as reached when the samples are cracked or show superficial disintegration or spalling, with loss of weight of more than 5 per cent of the initial dry weight.

Where the wall is faced with 3 inches or more of stone, terra cotta, brick, or other veneer the weathering resistance of the material for the backing is without significance.

National Paving Brick Manufacturers' Association, recommended specifications, vitrified paving brick, 1924.

(The full text of the specification includes directions for laying the brick.)

\section{QUAIITY}

Vitrified paving brick shall be evenly burned and thoroughly annealed. They shall be hard and tough. The texture and structure of the brick shall appear uniform and shall disclose freedom from open or marked lamination.

\section{SIZES}

The transverse dimensions of vitrified paving brick, as shown in the plans and designated in the bids, shall be 3 by 4 inches or $3 \frac{1}{2}$ by 4 inches, and variation from these dimensions shall not exceed $1 / 8$ of an inch. The length of the brick shall be $81 / 2$ inches and variation from this dimension shall not exceed $1 / 2$ of an inch.

\section{STYLE OF BRICK AND DEPTH OF WEARING SURFACE}

Wire-cut brick shall be used for wearing surface as indicated on the plans for the project, $3,3 \frac{1}{2}$ or 4 inches in depth; repressed brick for wearing surface, 4 inches in depth.

\section{SHAPE}

Ends of the brick may be double-beveled not more than $1 / 8$ of an inch. Lugs, if any, shall be not less than two nor more than four in number, extending from the body of the brick not more than onefourth of an inch. Special brick for hillside grades, exceeding from 5 to 8 per cent, shall have one or more longitudinal edges or faces chamfered or grooved not more than three-fourths of an inch.

\section{INSPECTION AND TESTS}

Shipments in excess of 100,000 brick to one or more projects under the jurisdiction of the engineer shall be inspected and tested at the place of manufacture. Shipments of less than 100,000 brick may, at the option of the cngineer, be tested at the plant or on the cars or street after delivery. Brick shall not lose of their weight more than _..... per cent when tested in the standard rattler of, and in accordance with, the standard specifications for paving brick of the American Society for Testing Materials, serial designation C7-29T. (See 534.11, p.577.) The forementioned standard specifications shall likewise govern visual inspection and are hereby made a part of these specifications.

\section{DELIVERY AND PROTECTION OF BRICK}

Brick shall be handled carefully and piled neatly outside the roadway before grading is completed in sufficient quantity to complete the brick surface, provided that they may be brought upon the work immediately prior to laying if delivery can be accomplished with no injury or delay to work in 
progress. Brick shall be so piled and protected that they will be kept clean. They shall be clean when they are laid in the pavement.

United States Department of Commerce, division of simplified practice.

In accordance with unanimous action of joint conferences of representatives of manufacturers, distributors, and users, the United States Department of Commerce recommends that recognized dimensions of brick be reduced to the following lists:

\section{RECOMMENDATION NO. 1, MARCH 31,1927}

\section{Varieties}

[Sizes in inches] ${ }^{1}$

\begin{tabular}{|c|c|c|c|c|c|}
\hline \multicolumn{3}{|c|}{$\begin{array}{l}\text { Plain wire-cut brick } \\
\text { (vertical fiber lugless) } \\
\text { as usually laid }\end{array}$} & \multicolumn{3}{|c|}{$\begin{array}{l}\text { Wire-cut lug brick } \\
\text { (Dunn) }\end{array}$} \\
\hline Width & Depth & Length & Width & Depth & Length \\
\hline $\begin{array}{l}4 \\
4 \\
4\end{array}$ & $\begin{array}{l}21 / 2 \\
3 \\
31 / 2\end{array}$ & $\begin{array}{l}81 / 2 \\
81 / 2 \\
81 / 2\end{array}$ & $31 / 2$ & 4 & $8 \frac{1 / 2}{2}$ \\
\hline \multicolumn{6}{|c|}{ Repressed lug brick } \\
\hline \multicolumn{2}{|c|}{ Width } & \multicolumn{2}{|c|}{ Depth } & \multicolumn{2}{|c|}{ Length } \\
\hline \multicolumn{2}{|c|}{$31 / 2$} & \multicolumn{2}{|c|}{4} & \multicolumn{2}{|c|}{$81 / 2$} \\
\hline
\end{tabular}

1 Tolerance of $1 / 8$ inch in width and depth and of $1 / 2$ inch in length allowable.

\section{RECOMMENDATION NO. 7, JUNE 21, 1923}

\section{Approximate dimensions}

[Expressed in inches]

\begin{tabular}{|c|r|r|r|}
\hline Types & Length & $\begin{array}{c}\text { Thick- } \\
\text { ness }\end{array}$ & Width \\
\hline Common brick & 8 & $21 / 4$ & 33 \\
\hline Rough face brick & 8 & 214 & 334 \\
Smooth face brick ----- & 8 & $21 / 4$ & $37 / 8$ \\
\hline
\end{tabular}

\subsection{Fire Brick and Fire-Clay Brick.}

American Marine Standards Committee, fire-clay refractories for marine service, Standard $\mathrm{E}$ No. $13,1927$.

\section{(A) MATERIALS}

1. Fire-clay brick.-This term covers brick of standard or special shape composed of heat-resisting clay or clays which has been burned to produce the desired strength and structure. The brick or shape shall be compact, of homogeneous structure, free from checks, cracks, voids, or soft centers. They shall be free from such swells, warps, twists or distortions as would prevent ready and accurate laying up with a maximum joint of $1 / 8$ inch. All corners shall be sufficiently solid and strong to prevent excessive crumbling or chipping when handled.

All brick of the standard 9-inch series shall not vary from specified dimensions more than $1 / 8$ inch in width and thickness, and $3 / 16$ inch in length. For special shapes no dimension shall vary more than 2 per cent from the dimension specified, unless greater variation is allowed by contract, but in no case shall a variation of less than $1 / 8$ inch be specified.
2. Fire clay shall be heat resistant and shall be of essentially the same composition as that of the brick with which it is to be used. It shall be sufficiently free from grit and of such quality and plasticity that it will spread satisfactorily with a trowel when tempered with water.

3. Plastic refractory.-The material covered by this specification is essentially a mixture of grog, raw clay, and sufficient water to render the mass plastic and of a stiff mud consistency. The grog is composed principally or entirely of calcined clay, and the raw clay is a plastic grade used to supply the necessary working properties and bond.

\section{(B) GRADES}

1. Fire-clay bricks shall be furnished in either of the four following grades as required:

SH75.-Intended for use under most severe conditions of boiler practice where installations are operated at an average rating of not less than 175 . Material of this class should have high resistance to slagging, spalling, and severe temperatures.

H75.- Intended for use under conditions such as are common in general boiler practice, where resistance to slagging, spalling, and high temperature is important.

M73.-Intended for use at moderate temperatures such as are encountered in hand-fired boilers operating at a rating not exceeding 125 and where resistance to spalling and slagging is important under these conditions of temperature.

H25.- Intended for service in which resistance to slagging and spalling is not of particular importance, but in which the refractory is expected to resist deformation under load as well as under high temperature such as are encountered in spring arches.

2. Fire clay shall be furnished in one grade only as described in A2.

3. Plastic refractory shall be furnished in one grade only as described in A3.

\section{(C) DETAIL REQUIREMENTS}

1. Fire clay brick-Grade SH75.-(a) The material shall contain not more than 65 per cent total silica $\left(\mathrm{SiO}_{2}\right)$ as determined by chemical analysis.

(b) The softening point shall be not less than that of standard pyrometric cone No. 31 (approximately, $1,680^{\circ} \mathrm{C}$., or $3,056^{\circ} \mathrm{F}$.).

(c) The product shall withstand 15 quenchings without failure.

Grade H75.- (a) The softening point shall be not less than that of standard pyrometric cone No. 31 (approximately, $1,680^{\circ} \mathrm{C}$., or $3,056^{\circ} \mathrm{F}$.).

(b) The product shall withstand 12 quenchings without failure.

Grade M73.-(a) The softening point shall not be less than that of standard pyrometric cone No. 29 (approximately, $1,640^{\circ} \mathrm{C}$., or $2,984^{\circ} \mathrm{F}$.).

(b) The product shall withstand 2 quenchings without failure.

Grade H25.- (a) Refractories of this grade shall contain 65 per cent or more total silica $\left(\mathrm{SiO}_{2}\right)$.

(b) The softening point shall be not less than that of standard pyrometric cone No. 28 (approximately, $1,615^{\circ} \mathrm{C}$., or $2,939^{\circ} \mathrm{F}$.) .

(c) The product shall withstand 6 quenchings without failure.

(d) The deformation under load shall not exceed 3 per cent.

2. Fire clay.-(a) The softening point shall not be more than three cones (approximately $60^{\circ} \mathrm{C}$., or $108^{\circ} \mathrm{F}$.) lower than that of the fire brick with which is to be used.

(b) Material shall be furnished air dry and ground to such fineness that not less than 90 per cent shall 
pass through a United States standard sieve No. 20. (See A.S. T. M. E11, 500.2, p. 1.)

(c) The material shall show satisfactory bonding power, as defined in D2.

(d) It shall contain not more than 65 per cent total silica $\left(\mathrm{SiO}_{2}\right)$ as determined by chemical analysis.

3. Plastic refractory.-(a) The moisture content when delivered shall not exceed 15 per cent (mechanical water).

(b) The minimum softening temperature shall not be less than that of standard pyrometric cone No. 31 (approximately, $1,680^{\circ} \mathrm{C}$., or $3,055^{\circ} \mathrm{F}$.).

(c) The total linear dry and burned shrinkage shall not exceed 4 per cent of the plastic length.

(d) The material shall contain not more than 65 per cent total silica $\left(\mathrm{SiO}_{2}\right)$ calculated on the dry weight.

\section{(D) TESTS}

1. Fineness.-(a) One hundred grams of the sample as received shall be placed on a No. 20 sieve and shall be washed by means of a stream of water from a faucet. The mashing shall be continued until the water coming through the sieve is clear, but in no case should the washing be continued for more than 30 minutes. The residue on the sieve shall be dried to constant weight at a temperature between $110^{\circ}$ and $150^{\circ} \mathrm{C}$. The sieve carrying the dried residue shall then be held in one hand in a slightly inclined position, so that the sample will be well distributed over the sieve, at the same time gently striking the side about 150 times per minute against the palm of the other hand on the upstroke. The sieve shall be turned every 25 strokes about one-sixth of a revolution in the same direction. The operation shall continue until not more than $0.5 \mathrm{~g}$ passes through in olle minute of continuous sieving. The fineness shall be determined from the weight of the residue on the sieve expressed as a percentage of the weight of the original sample.

(b) A piece of rubber tubing attached to a water faucet is to be used for the washing. The velocity of the water may be increased by pinching the tubing, but should not be sufficient to cause any splashing of the sample over the sides of the sieve.

(c) Mechanical sieving devices may be used, but the material shall be accepted if it meets the fineness requirement when tested by the hand method described.

2. Bond.- (a) The material shall be tested for bonding power by mixing one portion with water to a creamy consistency and another portion to a good troweling consistency. These mixtures shall then be used in laying up a pier of three of the fireclay brick, laid flat, with which the clay is to be to be used in service. The faces of the brick to be bonded shall be dipped in the mixture of creamy consistency, a thin layer of the other mixture added, and the brick then firmly rubbed into place. In no case shall the mortar "joint" exceed one-eighth inch.

(b) When the pier is air dry, it is to be fired uniformly in a suitable furnace to a temperature of $300^{\circ} \mathrm{C}$ :, or $540^{\circ} \mathrm{F}$., lower than the minimum allowable softening point of the fire clay brick (as defined herein) with which it is to be used in service. The pier shall be allorred to cool in the furnace and when cold shall be sufficiently well bonded to remain intact when lifted by grasping the top brick.

3. Softening point.-This is to be determined according to A. S. T. M. method C21. (See 534.10, p. 573.)

4. Composition.-The content of total silica shall be determined by A. S. T. M. method C18-21. (See 534.10 p. 564 .)

5. Quenching.-(a) This test shall be conducted on standard 9-inch straight brick which have been brought uniformly, under no load, to a temperature of $1,400^{\circ} \mathrm{C} .\left(2,552^{\circ} \mathrm{F}\right.$.) in not less than five hours, held for five hours, and allowed to cool in the kiln, without induced draft, to room temperature.

(b) The quenching shall be conducted in the following manner: The brick shall be heated by placing it in the door of a suitable furnace which is being held at a temperature of $850^{\circ} \mathrm{C} .\left(1,562^{\circ} \mathrm{F}\right.$.). The heated end of the brick should be flush with the inner face of the furnace and the outer end should be exposed to the free circulation of air.

(c) At hourly intervals the hot end of the brick shall be immersed in running water for three minutes and to a depth of 4 inches. The brick shall then be removed, allowed to steam in the air for five minutes, and returned to the furnace door. This cycle is repcated 15 or less times, according to grade requirements as specified in $\mathrm{Cl}$, unless the specimen has failed previously.

(d) The brick is considered to have failed when the entire plane surface of the heated end has completely spalled away, or when the structure of the brick has become so weakened that the end can be easily removed with the fingers.

(e) The results for any one brand shall be reported as the average of five specimens.

6. Load test. - The load test shall be conducted according to A. S. T. M. method C16-20. (See 534.10 , p. 560 .)

7. Shrinkage, plastic refractory.-The total linear dry and burned shrinkage shall be conducted on brick which have been prepared of the material (as delivered) by hand ramming with a wooden mallet in suitable wooden molds of inside dimensions approximately $2 \frac{1}{2}$ by $4 \frac{1}{2}$ by 9 inches. The brick shall be removed immediately from the mold and set on edge on a suitable pallet and marked on the 9 by $4 \frac{1}{2}$ inch faces with gage marks approximately 8 inches apart. The sample shall then be carefully dried, then burned uniformly, under no load, to $1,400^{\circ}$ C. $\left(2,552^{\circ} \mathrm{F}\right.$.) in not less than five hours, and held at this temperature for five hours and allowed to cool in the kiln and without induced draft to room temperature.

The distance between gage marks shall be measured to the nearest thirty-second of an inch and the shrinkage calculated in accordance with the following formula:

$$
\text { Per cent shrinkage }=\frac{L_{w}-L_{b}}{L_{w}} \times 100
$$

where

$L_{w}=$ length of the specimen in plastic state.

$L_{b}=$ length of specimen after burning at $1,400^{\circ} \mathrm{C}$.

The shrinkage of any one specimen shall be reported as the average of the distance between the two sets of gage marks and results of any one brand shall be reported as the average of five specimens.

NotE.-The cones referred to in this specification are known as the Orton pyrometric cones.

\section{(ए) PJRCHASE CONDITIONS AS TO TESTS}

(a) The tests herein specified define the characteristics of the materials for the guidance of manufacturers and dealers. The purchaser shall have the right to test materials in accordance with these specifications before accepting them, but before exercising such right he shall invite the manufacturer to make a joint selection of the materials to be tested so as to avoid any question as to fairness of selection.

(b) In current purchases, based on these specifications, of materials for immediate use, without opportunity to the purchaser to make tests, it will be assumed that the materials supplied by the soller conform to the requirements, and in such cases, or whenever tests by the purchaser are waived, the seller shall, upon request, furnish satisfactory evidence that the materials supplied were 
successfully tested in an approved laboratory according to the methods prescribed in this specification, or shall give other satisfactory guaranty that they possess the requisite characteristies to meet the requirements under the specified tests.

American Society for Testing Materials, standard definitions for clay refractories, serial designation C27-28, 1928.

1. The following definitions relating to the resistance to heat of clay refractories are recommended for the purpose of classification:

2 . The softening point referred to in the following definitions shall be determined in accordance with A. S. T. M. C24. (See 534.10, p. 573.)

\section{HIGH HEAT DUTY BRICK}

\section{(A) CLAY FIRE BRICK}

(Silica content less than 70 per cent)

3. The softening point of clay fire brick for high heat duty shall not be lower than that of standard cone No. 31 (about $1,680^{\circ} \mathrm{C}$., or $3,055^{\circ} \mathrm{F}$.).

4 . When a brick of this type softens at a temperature not below the softening point of standard cone No. 29 (about $1,640^{\circ}$ C., or $2,985^{\circ} \mathrm{F}$.) it may be tested according to classification (B) for silicious clay fire brick without losing in standing if it passes the tests.

\section{(B) SILICIOUS CLAY FIRE BRICK}

(Silica content 70 per cent or over)

5. The softening point of silicious clay fire brick for high heat duty shall not be lower than that of standard cone No. 28 (about $1,615^{\circ} \mathrm{C}$., or $2,940^{\circ} \mathrm{F}$.).

6. All silicious clay fire brick for high heat duty shall be subjecied io a load test in accordance with A. S. T. M. C16. (See 534.10, p. 560.) The pressure to be applied upon the brick (placed on end) shall be 25 lbs./in.2 and the maximum furnace temperature $1,350^{\circ} \mathrm{C}$. $\left(2,460^{\circ} \mathrm{F}\right.$.). The brick shall not show a contraction of more than 4 per cent of the original length nor an expansion of more than 1 per cent.

\section{INTERMEDIATE HEAT DUTY BRICK}

7. The softening point of brick for intermediate heat duty shall not be lower than that of standard cone No. 28 (about $1,615^{\circ} \mathrm{C}$., or $2,940^{\circ} \mathrm{F}$.).

\section{MODERATE HEAT DUTY BRICK}

8. The softening point of brick for moderate heat duty shall not be lower than that of standard cone No. 26 (about $1,595^{\circ}$ C., or $2,905^{\circ}$ F.).

\section{LOW HEAT DUTY BRICK}

9. The softening point of brick for low heat duly shall not be lower than that of standard cone No.19 (about $1,520^{\circ} \mathrm{C}$., or $2,770^{\circ} \mathrm{F}$.).

American Society for Testing Materials, standard specifications for clay fire brick for malleable furnaces with removable bungs and for annealing ovens, serial designation C63-28, 1928.

(Paragraphs designated $(b)$ and $(f)$-under the heading "Purpose and utility of specifications," and also sections 1 and 2 of A.S.T. M. C64, see below, are part of this specification.)

Purpose and utility of specifications.-The purpose of these specifcations is the selection of clay fire brick that are suitable for service in malleable furnaces under general conditions of operation and furnace design.

Factors directly or indirectly affecting the life of the brick are as follows:

A. Factors dependent upon fuel or firing:

Character and kind of fuel.

Character of ash or fluxing ingredients.
Quantity of ash or fluxing ingredients.

Method of firing.

Character of blast and length of flame.

Pressure of blast.

Direction of flame and impinging of flame upon brickwork.

B. Factors dependent upon furnace design or construction:

Rise and span of arch.

Type of bung frame.

Care taken and method followed in laying brick and building bungs.

Tightness with which brick are clamped in bung frames.

Kind of clay used in laying brick.

Thickness of walls.

C. Factors dependent upon character of charge:

Kind and amount of scrap used.

Amount of foreign material and rust on scrap

Percentage of pig in charge.

Percentage of pig in charge.
D. Factors dependent upon operating conditions

Method of charging.

operating temperature.

Duration of melting period.

Size of heats and number of heats per day.

Care taken and method followed in handling bungs.

Positions of brick in furnace and practice followed in shifting bungs.

These specifications do not include a requirement for slag resistance, although evidence indicates that the brick, and especially the side-wall brick, should be resistant to the corrosive action of slag. In the light of present knowledge, however, none of the slag tests that have been proposed can be regarded as satisfactory for malleable that have been

The bungs and side walls must withstand high-operating temperaThe bungs and side walls must withstand high-operating tempera-
tures, This is insured by the softening-point requirement. They tures. This is insured by the softening-point requirement. They must be resistant to rapid temperature changes; hence the spalling-
test requirement. The spalling requirements for side walls have been made less stringent than for bungs, as the spalling conditions are less severe. Side-wall brick must also withstand slag action at the slag line, and the brick which are most resistant to slagging do not usually withstand spalling to the highest degree. Practical experience has shown that in bungs and side-wall brick the silica content should not be excessively high. This is cared for by imposing a maximum limit on the percentage of silica A minimum ing a maximum limit on the perccntage of silca. A minimum limit is placed upon the cold breaking strength, merely to insure that the bung brick shall be strong enough to withstand clamping in the bung frames and to withstand shipping and handling with-
out excessive scuffing. It is recognized that high strength is not out excessive scuffing.

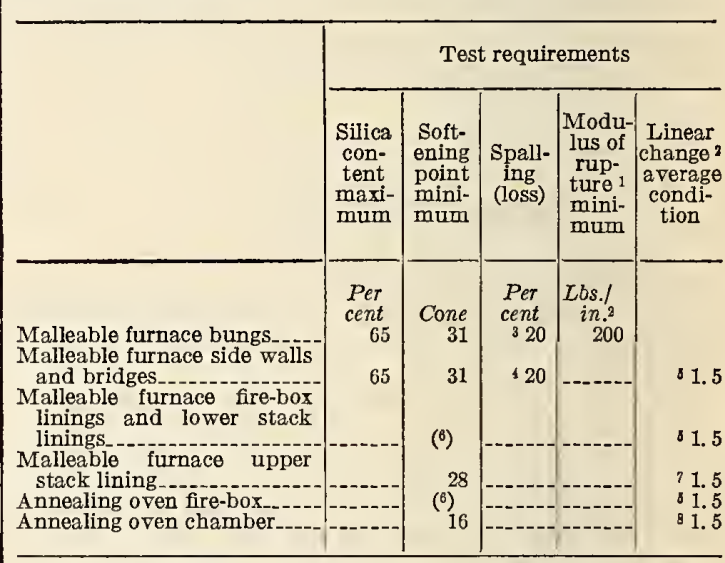

1 Tested cold.

2 When duplicate samples are heated uniformly and maintained at the temperatures as designated below for five hours and cooled. 18 dips.

4 dips.

- The softening point shall not be below cone 31 in the case of the nonsiliceous grade and not below cone 28 in the case of the siliceous grade. Brick are considered as being of nonsiliceous grade when the silica content is 65 per cent or less and of siliceous grade when the silica content is greater than 65 per cent.

$7,350^{\circ} \mathrm{C}$.

$81,150^{\circ} \mathrm{C}$.

The test methods for this specification are the same as for A. S. T. M. C64, below. The spalling test shall be made on standard 9-inch straight brick or standard 9 or 13 inch bung brick. The explanatory note at the conclusion of $\mathrm{C} 64$ is also part of this specification.

American Society for Testing Materials, standard specifications for clay fire brick for stationary boiler service, serial designation C64-28, 1928. 


\section{GENERAL}

Purpose and utility of specifications.-(a) The purpose of these specifications is the selection of the clay fire hrick for general hoiler service. They may not apply to the special conditions sometime encountered in high-power utility hoilers. In these plants gencral conditions may not apply and the selection of the refractories often becomes a special prohlem for the particular plant involved.

(b) Use of these specifications will not relieve the huyer of all responsihility in the selection of the refractories hest suited to his particular practice of the hrands which meet these requirements particular practice. Or the hrands which moth some may give highy satistactory servico in one installion and fal to do so in another. Certain hrands may give best service in one plant, other hrands in another plant. The reason for this variahility in service lies in the almost countless combinations of conditions which may exist in hollcr-furnace operation. Some of the factors which directly or indirectly affect the life of the brick are the following:

A. Factors dependent upon design or construction of the setting: Furnace volume.

Thickness and height of walls.

Use of relieving arches or sectional wall supports.

Degree of insulation.

Provision for air or water cooling of refractories.

Type of stoker arch.

Type of stoker arch.

Height of stoker arch above grates or stoker.

Provision for expansion of the hrick laying hrick.

Care taken and method followed in laying

Character of mortar used in laying hrick.

Hand or mechanical firing

Type of grate, stoker, or hurner.

Character and kind of fuel.

Direction of flame and impingement on hrickwork

Character of ash or fluxing ingredients.

Quantity of ash or fluxing ingredients,

Fusihility and viscosity of ash.

C. Factors dependent upon operation:

C. Factors dependent up

Air leaks in furnace walls.

Temperature of operation.

Care and promptness in

Character of furnace atmosphere.

Violence of temperature fluctuations.

Rate of operation.

Segregation of different sizes of coal on chain grates.

Pressure or draft conditions in furnace.

Care and promptness of making minor repairs.

Method of removing adhering clinker.

Ahrasion of hrickwork.

(c) In these specifications hrick are divided into siliceous and non(c) In these specifications hrick are dividement is stipulated as to siliceous grades, hut no other denship hetween analysis and service has analysis, as no definite relationship hetween analysis and service has heen demonstrated. The softening-point requirements are well ahove the temp

boiler practice. action. The spalling test specified will select hrick of satisfactory resistance to thermal shock for general hoiler practice. In those installations where exceptionally severe spalling seems to occur the failure of the hrick may in many cases he the result of factors which can not he measured in the spalling test. Such conditions call for study by a competent engineer.

(e) Brick which are suhjected to severe slagging conditions in (e) Brick which are suhjected to severe slagging conditions in service should not be required to meet a severe spalling test requirement. Although slagging is one of the major causes of failure of the refractories, no test for slag resistance is included in these specincations for the reason that in the light of present knowledge none of
the methods of making slag tests that have been proposed can he regarded as satisfactory.

(f) Revision of the requirements is to be expected as methods of testing are further perfected and as the effect of operating conditions upon the life of the hrick hecomes hetter understood. However, clay fire hrick which fail to meet the requirements of these specifications may he regarded as unsatisfactory for general service.

1. These specifications are intended to cover only burned products made from fire clay by the usual processes of manufacture and containing not more than 55 per cent alumina or 85 per cent silica. Even within this range of composition it is not intended to include products other than those regularly sold as clay fire brick.

\section{SIZE TOLERANCE}

2. Variations (plus or minus) of 2 per cent from specified dimensions covering both shrinkage and warpage shall be allowed on dimensions of 4 inches or over. On dimensions under 4 inches 3 per cent variation shall be allowed.

\section{TEST REQUIREMENTS}

\section{MODERATE HEAT DUTY BOILER SETTINGS}

3. The requirements for brick for moderate high duty boiler settings are intended to apply to boiler furnaces in which the temperatures are not over $2,400^{\circ}$ F. $\left(1,315^{\circ}\right.$ C. $)$.

A. Zone of highest temperatures (side walls, arches, doors, and jamhs)

4. (a) Softening point.-The softening point shall not be below cone 29 in the case of the nonsiliceous grade ${ }^{10}$ and not below cone 27 in the case of the siliceous grade.

(b) Linear change.- When duplicate samples are heated uniformly to a temperature of $1,350^{\circ} \mathrm{C}$., maintained at this temperature for five hours and cooled, they shall not show an average contraction of more than 1.5 per cent of the original length.

B. Zone of lower temperatures (hacking-up brick for highest temperature zone and walls beyond first tuhe hank)

5. (a) Softening point.-The softening point shall not be below cone 18 .

(b) Modulus of rupture.-The modulus of rupture of the brick tested cold shall not be under 500 lbs./in. ${ }^{2}$

\section{HIGE HEAT DUTY BOLLR SETTINGS}

A. Zone of highest temperatures (side walls, hridge walls, arches, doors, and jamhs, and first pass side walls)

6. The requirements for brick for zone of highest temperatures in high heat duty boiler settings are intended to apply to boiler furnaces in which the temperature of the combustion chamber is continuously or a significant portion of the time above $2,400^{\circ}$ F. $\left(1,315^{\circ}\right.$ C. $)$ under norml conditions of operation. The refractoriness of the nonsiliceous grade is assured by the softening point, but for this requirement in the case of the siliceous grade it is necessary to incorporate both the softcning point and load test.

7. (a) Softening point.-The softening point shall not be below cone 31 in the case of the nonsiliceous grade and not below cone 28 in the case of the siliceous grade.

(b) Linear change.-When duplicate samples are heated uniformly to a temperature of $1,400^{\circ} \mathrm{C}$. maintained at this temperature for five hours, and cooled, they shall not show an average contraction of the original length of more than 1.5 pcr cent in the case of the nonsiliceous grade and 0.75 per cent in the case of the siliceous grade.

(c) Spalling. - When subjected to the spalling test, brick shall withstand an average of five dips in the case of the nonsiliceous grade and three dips in the case of the siliceous grade.

(d) Deformation.-Deformation in the standard load test at $1,350^{\circ}$ C. under a load of $25 \mathrm{lbs}$./in. ${ }^{2}$ shall not be over 6 per cent in the case of the siliceous grade.

8. Service condition No. 1.-For division walls heatcd on both sides the following requirements shall apply:

(a) Softening point.-The softening point shall not be bclow cone 31 in the case of the nonsiliceous grade and not below cone 28 in the case of the siliceous grade.

(b) Deformation.-Deformation in the standard load test at $1,350^{\circ} \mathrm{C}$. under a load of $25 \mathrm{lbs} . / \mathrm{in}^{2}$ shall not be over 6 per cent in the case of the nonsiliceous grade and not over 4 per cent in case of the siliceous grade.

NoTE.-It is not practical to include a spalling test for hrick subject to rigid load test requirements.

9. Service condition No. 2.-Under severe conditions of spalling, the following requirements shall apply:

(a) Softening point.-The softening point shall not be below cone 31 in the case of the nonsili-

10 Brick are considered as heing of siliceous grade when the silica content is greater than 65 per cent and of nonsiliceous grade when the silica content is 65 per cent or less. 
ceous grade and not below cone 28 in the case of the siliceous grade.

(b) Linear change.-When duplicate samples are heated uniformly to a temperature of $1,400^{\circ} \mathrm{C}$., maintained at this temperature for five hours and cooled, they shall not show an average contraction of the original length of more than 2 per cent in the case of the nonsiliceous grade and 1 per cent in the case of the siliceous grade.

(c) Spalling.-When subjected to the spalling test, brick shall withstand an average of nine dips in the case of the nonsiliceous grade and four dips in the case of the siliceous grade.

Note.- It is not practical to include a limit for deformation under load since fire-clay brick which arc so made as to give the highest resistance to spalling are relatively weak under load.

10. Service condition No. 3.-For service where slagging conditions are severe the following requirements shall apply:

(a) Softening point.-The softening point shall not be below cone 31 in the case of the nonsiliceous grade and not below cone 28 in the case of the siliceous grade.

(b) Linear change.- When duplicate samples are heated uniformly to a temperature of $1,400^{\circ} \mathrm{C}$., maintained at this temperature for five hours, and cooled, they shall not show an average contraction of the original length of more than 1 per cent in the case of the nonsiliceous grade and 0.5 per cent in the case of the siliceous grade.

(c) Water absorption. - Water absorption shall not be over 10 per cent in the case of the nonsiliceous grade and 18 per cent in the case of the siliceous grade.

Note.-Tbese tests are specified for conditions of severe slag erosion to insure good refractoriness and high density, as these properties are known to he important. No slag test has been devised wbicb gives quantitative results that can te used in specifications.

11. When any one of the three special service conditions described in sections 8,9 , and 10 is to be met, it shall be expressly stipulated by the purchaser.

B. Zone of lower temperatures (backing-up brick for highest

temperature zone, and walls of gecond and third passes).

12. The service to which brick in the zone of lower temperatures are subjected varies with the size and type of boiler, thickness of walls, degree of insulation, draft conditions, and position in the furnace. Leaky baflles may sometimes cause the brick beyond the first tube bank to be subjected to unexpectedly high temperatures. Depending upon the particular conditions and upon the margin of safety desired, the brick used may be of the same grade as used in the zone of highest temperatures as described in section 7 or of one of the following grades:

\begin{tabular}{|c|c|c|c|}
\hline & Grade $A$ & Grade B & Grade C \\
\hline Softening paint, not under cone & 28 & 26 & 18 \\
\hline $\begin{array}{l}\text { Modulus of rupture, lbs./in. }{ }^{2} \text {, not } \\
\text { under }\end{array}$ & 450 & 500 & 500 \\
\hline
\end{tabular}

\section{METHODS OF TESTING}

13. The determination of silica content shall be made in accordance with A. S. T. M. serial designation C18. (See 534.10 , p. 564.)

14. (a) The softening point (fusion point) shall be determined in accordance with A. S. T. M. C24. (See 534.10, p. 573.)

(b) In the event that the material fails to pass the specified requirements by one cone or less, two additional softening point determinations shall be made as checks on the accuracy of the test and the final results judged on the basis of the three determinations. This procedure is necessary because of variations that have been found to exist in this test even where the standard methods are followed.

15. (a) The spalling test shall be conducted in accordance with A. S. T. M. C38-27'T (note 1). (See 534.10, p. 573.) The test shall be continued until each brick shall have lost 20 per cent by weight or until the total number of immersions necessary to give the required average has been obtained. Individual samples shall not be continued in the test after a loss of 20 per cent has been sustained. The brick shall be dried and weighed before and after testing and the loss computed as a percentage of the original dry weight.

(b) The results in the spalling test for any one brand shall be reported as the average loss of 10 specimens sclected at random. If desired, the test may be made on only 5 specimens, but when this is done an additional 5 specimens shall be tested in the event that the first 5 do not meet the requirements, and the results reported as the average of the entire 10 specimens.

16. (a) The modulus of rupture shall be determined in accordance with the method of test for modulus of rupture of building brick, as described in sections 8 to 12 of the Tentative Methods of Testing Brick (A. S. T. M. C67-28T). (See 534.10, p. 574.$)$

(b) The results in the modulus of rupture test for any one brand shall be reported as the average of 10 specimens selected at random. If desired, the test may be made on only 5 specimens, but when this is done an additional 5 specimens shall be tested in the event that the first 5 do not meet the requirements, and the results reported as the average of the entire 10 specimens.

17. The load test shall be made in accordance with A. S. T. M. C16. (See 534.10, p. 560.)

\section{IXPLANATORY NOTE}

Note.-It will be noted tbat tbe present Tentative Metbod of Test for Resistance of Fire-Clay Brick to Thermal Spalling Action (C38-27T) of the American Society for Testing Materials is tbe one (C) referred to in thcse specifications. It is recognized that tbere are water-dip spalling test devoloped at the Bureau of Stanciards; the air spalling test used by a large contracting company; and possibly
others. It seems very probable, however, in the light of past work and that now going on, that the ultimate spalling test may be different from any of these. Every thing considered, it seems best to use metbod C38 for the present.

American Society for Testing Materials, standard specifications for clay fire brick for marine boiler service, serial designation C65-28, 1928.

(This specification is identical in many respects with C64-28, given above. Variations in the specifications are indicated in the following presentation of C65-28:)

\section{CENERAT}

Purpose and utility of specifications.-(a) Tbe purpose of tbese specifications is the selection of clay fire brick for marine boiler service.

(b) Idcntical with paragrapb (b) C64, omitting the following factors under "A" in C64: (1) Use of relieving arches or sectional wall supports, and (2) provision for expansion of the brickworl, and substituting under " $\mathrm{C}$ " the factor "vihration and shocks" for factor listed in $\mathrm{C} 64$, "segregation of different sizes of coal on chain grates,"

(c) The most important causes of failure are softening (fusion), spalling, slag action, and shrinkage. The softening point requirepants, slag all ahove the temperatures actually attained by tbo bricl ark in marine boiler practice. The spalling test specified will sclect brick of satisfactory resistance to thermal shock for this class sclect brick of satisfactory resistance to thermal shock for this class of service. In those installations where exceptionally sovere spalling
seems to occur the failure of the brick may in many cases be the result seems to occur the failure of the brick may in many cases be the result
of factors which can not be measured in the spalling test. Such conditions call for study by a competent engineer.

(Pars. $(d)$ and $(e)$ identical witb pars. $(e)$ and $(f)$ of C64.)

These specifications are intended to cover only burned products made from fire clay by the usual processes of manufacture and containing not more than 55 per cent alumina or 85 per cent silica. Even 
within this range of composition it is not intended to include products other than those regularly sold as clay fire brick.

\section{SIZE TOLERANCE}

Same as for $\mathrm{C} 64$

\section{TEST REQUIREMENTS}

MODERATE HEAT DUTY BOILER SETTINGS

Same as section $4(a)$ and $(b)$, under "Test requirements, moderate heat duty boiler settings," C64.

\section{HIGH FEAT DUTY BOIIER SETTINGS}

Same as section 7 , items $(a),(b),(c)$, and $(d)$, under "High heat duty boiler settings," of C64, with the following note added:

NotE.-The reiractoriness of the nonsilicious grade is assured by the softening point, but for this requirement in the case of the silicious grade, it is necsssary to incorporate both the softening point and the load test.

\section{METHODS OF TESTING}

Same as for C64.

Federal Specifications Board, specification No. 268a, United States Government master specification for brick, fire clay, January 22, 1925.

(This specification is similar in certain particulars to American Marine Standards Committee, fireclay refractories for marine service, given above, this specification including the four classes of clay brick given in the A. M. S. C. specification, with identical requirements and methods of test for softening point, composition, and quenching, for the four classes. To avoid unnecessary duplication the items mentioned as identical are omitted in the presentation of this specification.)

\section{CLASSES}

Fire-clay brick shall be of the following classes: SH75, H75, H57, M73, H25, and M7.

For derivation of the class nomenclature used see Section VI of this specification.

\section{MATERIAI AND WORKMANSHIP}

See (A), Materials; 1. Fire-clay brick, A. M. S. C. specification.

\section{DETAIL REQUIREMENTS}

For requirements for classes SH75, H75, M73, and H25, see A. M. S. C. specification.

\section{CLASS H57}

1. The softening point shall be not less than that of pyrometric cone No. 31 (approximately $1,680^{\circ} \mathrm{C}$. or $3,056^{\circ} \mathrm{F}$.).

2. The brick shall withstand five quenchings without failure.

3. The absorption after reheating shall be not less than 6 per cent nor more than 16 per cent.

\section{CLASS M7}

1. Silicious brick.-The $\mathrm{SiO}_{2}$ content shall be more than 65 per cent.
2. The softening point shail be not less than that of pyrometric cone No. 28 (approximately $1,615^{\circ} \mathrm{C}$., or $2,939^{\circ} \mathrm{F}$.).

3. The brick shall withstand three quenchings without failure.

4. The deformation under load shall not exceed 4 per cent.

Note.-When specified, brick of class SB75 shall pass the Navy simulated service test.

\section{METHODS OF INSPECTION AND TESTS}

Sampling.-Ten 9-inch bricks shall be taken at random from every shipment of one carload or less and submitted for test. These bricks shall constitute a sample. Five bricks of the sample shall be tested as required in this specification. If they fail to meet the requirements of a test (or tests) it shall constitute rejection. If the consignor desires a retest (see Sec. VIII, 1, Notice of rejection) the test (or tests) in which the material failed shall be repeated, using the necessary number of bricks from the balance of the sample.

Softening point, composition, and quenching tests same as A. M. S. C. specification.

The absorption shall be determined for brick which have been brought uniformly under no load to $1,400^{\circ} \mathrm{C}$. in not less than five hours and held for five hours and allowed to cool in the kiln and without induced draft to room temperature.

The test shall be conducted on specimens not less than $100 \mathrm{~g}$ in weight, one specimen to be taken from each of five bricks of any one brand and the average result reported.

The per cent absorption shall be determined according to the following formula:

$$
\text { Per cent absorption }=\frac{W-D}{D} \times 100
$$

$W=$ weight of specimen after having been boiled in water for two hours and allowed to cool in the water.

$D=$ weight of specimen after having been dried to constant weight at $110^{\circ} \mathrm{C}$.

The load test shall be conducted according to A. S. T. M. C16-20. (See 534.10, p. 560.)

The Navy simulated service tests. (See F. S. B. No. 334 a, 531.3, p. 513.)

The combined results of workmanship, chemical analyses, softening point, and absorption and load tests, where required, shall be considered as a suitability test, but (at the discretion of the purchaser) the simulative service test may replace all other tests included in the suitability test.

Workmanship and softening-point determination shall be considered as a control test.

\section{MARIING}

In each brick shall be molded the trade name, or the name of the manufacturer, or such a mark as will serve to identify the material.

\section{NOTES}

1. The consignor shall be notified of the rejection of a shipment based on this specification, unless otherwise specified, within 10 days after receipt of a shipment at the point of destination. If the 
consignor desires a retest, he shall notify the consignee within 5 days of receipt of said notice.

2 . The cones referred to in this specification are known as the Orton pyrometric cone.
3. The class nomenclature used in Section II of this specification is based on the following scheme devised by Committee C-8 on Refractories of the American Society for Testing Materials:

\begin{tabular}{|c|c|c|c|c|c|c|c|c|c|c|}
\hline \multirow{2}{*}{\multicolumn{2}{|c|}{$\begin{array}{l}\text { Temperature indicated by prefixing proper letter to number: } \\
\qquad \begin{array}{c}H=\text { High temperature } \\
M I=\text { Moderate temperature } \\
L=\text { Low temperature }\end{array}\end{array}$}} & \multicolumn{3}{|c|}{ Load unimportant } & \multicolumn{3}{|c|}{ Load moderate } & \multicolumn{3}{|c|}{ Load important } \\
\hline & & $\begin{array}{l}\text { Abra- } \\
\text { sion, } \\
\text { unim- } \\
\text { por- } \\
\text { tant }\end{array}$ & $\begin{array}{l}\text { Abra- } \\
\text { sion, } \\
\text { moder- } \\
\text { ate }\end{array}$ & $\begin{array}{l}\text { Abra- } \\
\text { sion, } \\
\text { impor- } \\
\text { tant }\end{array}$ & $\begin{array}{l}\text { Abra- } \\
\text { sion, } \\
\text { unim- } \\
\text { por- } \\
\text { tant }\end{array}$ & $\begin{array}{l}\text { Abra- } \\
\text { sion, } \\
\text { moder- } \\
\text { ate }\end{array}$ & $\begin{array}{l}\text { Abra- } \\
\text { sion, } \\
\text { impor- } \\
\text { tant }\end{array}$ & $\begin{array}{l}\text { Abra- } \\
\text { sion, } \\
\text { unim- } \\
\text { por- } \\
\text { tant }\end{array}$ & $\begin{array}{l}\text { Abra- } \\
\text { sion, } \\
\text { moder- } \\
\text { ate }\end{array}$ & $\begin{array}{l}\text { Abra- } \\
\text { sion, } \\
\text { impor- } \\
\text { tant }\end{array}$ \\
\hline $\begin{array}{l}\text { Slag action, unimpor- } \\
\text { tant. } \\
\text { Slag action, moderate. - } \\
\text { Slag action, important - }\end{array}$ & $\begin{array}{l}\text { Spalling, unimportant } \\
\text { Spalling, moderate } \\
\text { Spalling, important } \\
\text { Spalling, unimportant } \\
\text { Spalling, moderate } \\
\text { Spalling, important } \\
\text { Spalling, unimportant } \\
\text { Spalling, moderate } \\
\text { Spalling, important }\end{array}$ & $\begin{array}{r}1 \\
10 \\
19 \\
28 \\
37 \\
46 \\
55 \\
64 \\
73\end{array}$ & $\begin{array}{r}2 \\
11 \\
20 \\
29 \\
38 \\
47 \\
56 \\
65 \\
74\end{array}$ & $\begin{array}{r}3 \\
12 \\
21 \\
30 \\
39 \\
48 \\
57 \\
66 \\
75\end{array}$ & $\begin{array}{r}4 \\
13 \\
22 \\
31 \\
40 \\
49 \\
58 \\
67 \\
76\end{array}$ & $\begin{array}{r}5 \\
14 \\
23 \\
32 \\
41 \\
50 \\
59 \\
68 \\
77\end{array}$ & $\begin{array}{r}6 \\
15 \\
24 \\
33 \\
42 \\
51 \\
60 \\
69 \\
78\end{array}$ & $\begin{array}{r}7 \\
16 \\
25 \\
34 \\
43 \\
52 \\
61 \\
70 \\
79\end{array}$ & $\begin{array}{r}8 \\
17 \\
26 \\
35 \\
44 \\
53 \\
62 \\
71 \\
80\end{array}$ & $\begin{array}{r}9 \\
18 \\
27 \\
36 \\
45 \\
54 \\
63 \\
72 \\
81\end{array}$ \\
\hline
\end{tabular}

Note.-Class SH75 (special high temperature) is so designated because it is meant to apply to especially severe boiler practice.

4. Definition of classes-Class SH75.-Brick of this class are intended for use under the most severe conditions of boiler practice, such as marine boilers used by the Navy and in plant installations designed to operate at an average rating of not less than 175 . Material of this class should have high resistance to slagging, spalling, and severe temperatures.

In the United States Navy service brick of class SH75 are used in oil-fired boilers operated at greater than 500 per cent rating, and where severe vibrations and rapid changes in temperature occur. In this service the brick are secured by anchor bolts.

Class H75.-Brick of this class are intended for use under conditions such as are encountered in general boiler practice. For this class, resistance to slagging, spalling, and high temperature is important.

Class H57.-Brick of this class are intended for use under conditions where resistance to spalling is not of great importance and where resistance to slagging and high temperature is important. In general boiler practice they may be used in the side walls, but, if the refractories used are limited to one brand, material of class H75 is recommended.

Class M73.--Brick of this class are intended for use at moderate temperatures such as are encountered in hand-fired boilers operated at average rating not exceeding 125 . Resistance to spalling and slagging is important under these conditions of temperature.

Class H25.-This class is intended primarily for brick of silicious nature and for scrvice in which resistance to slagging and spalling is not of particular importance, but in which the refractory is expected to rcsist deformation under load at relatively high temperatures.

Brick of class H25 are particularly adapted for service under conditions where resistance to deformation under load, with soaking heats at relatively high temperatures, is important, but where there is no marked fluctuation of temperature bclow approximately $650^{\circ} \mathrm{C}$. $\left(1,202^{\circ} \mathrm{F}\right.$.).

NOTE-Brick of class H75 which withstand the load test satisfactorily may be included in this class.

Class $M \%$.-This class is intended primarily for brick of silicious nature, for service at moderate temperatures, and under the conditions where resistance to spalling and slagging is not important, but where resistance to deformation under load is important.

Brick of this class are particularly adapted for service under conditions where resistance to defor- mation under load, with soaking heats at moderate temperatures, is important, but where there is no marked fluctuation of temperature below approximately $650^{\circ}$ C. $\left(1,202^{\circ} \mathrm{F}\right.$.).

Note.-Brick of class M73 which withstand the load test satisfac. torily may be included in this class.

Federal Specifications Board, specification No. 335a, United States Government master specification for refractories, fire clay, plastic, October 1 , 1925.

\section{GRADE}

The material furnished under this specification shall be of one grade only.

\section{MATERIAL AND WORKMANSHIP}

The material covered by this specification is essentially a mixture of grog, raw clay, and sufficient water to render the mass plastic and of a stiff mud consistency. The grog is composed principally or entirely of calcined clay, and the raw clay is a plastic grade used to supply the necessary working properties and bond.

\section{DETAIL REQUIREMENTS}

1. The material shall contain not more than 65 per cent total silica $\left(\mathrm{SiO}_{2}\right)$ calculated on the dry weight.

2. The softening point shall be not less than that of standard pyrometric cone No. 31 (approximately $1,680^{\circ} \mathrm{C}$. or $3,056^{\circ} \mathrm{F}$.) .

3. The material as delivered shall contain not more than 15 per cent water calculated on the plastic weight.

4. The total linear dry and burned shrinkage shall not exceed 4 per cent of the plastic length.

5 . When specified, the material shall pass the Navy simulated service test.

\section{METHOD OF INSPECTION AND TESTS}

1. Sampling.-A sample of not less then 50 pounds shall be taken from one container, taken at random, for every shipment of one carload or less and shall be so taken as to be truly representative of the material in the container.

2. Testing.-_a (a) The content of total silica shall be determined by analytical methods described under A. S. T. M. C18, 534.10, page 564 .

(b) The softening point shall be determined according to A. S. T. M. C24, 534.10, page 573 .

(c) The water content shall be determined by drying a specimen of not less than $200 \mathrm{~g}$ (approxi- 
mately 7 ounces) to constant weight at a temperature of not less than $110^{\circ} \mathrm{C}$. and not more than $150^{\circ}$ C. The per cent water shall then be determined in accordance with the following formula:

$$
\text { Per cent water }=\frac{W-D}{W} \times 100
$$

where

W = the weight of the material as received.

$\mathrm{D}=$ the weight of the material after having been dried to constant weight.

(d) The total linear dry and burned shrinkage shall be conducted as in section D (7) of American Marine Standards Committee, standard E, No. 13 (given above).

(e) Navy simulated service test. (See F. S. B. No. $334 a, 531.3$ p. 513 .)

(f) The combined results of chemical analyses, softening point, water content, and shrinkage (burned) shall be considered as a suitability test, but (at the discretion of the purchaser) the Navy simulated service test may replace all other tests included in the suitability test.

\section{PACKING AND MARKING}

The material shall be packed in containers so constructed as to insure delivery of the material in satisfactory condition. The size and type of the container shall be as specified by the purchaser.

Each container shall be plainly marked with the trade name of the material, the contract number, and the name of the contractor.

\section{NOTES}

1. The consignor shall be notified of the rejection of a shipment based on this specification, unless otherwise specified, within 10 days after receipt of a shipment at the point of destination. If the consignor desires a retest, he shall notify the consignee within five days of receipt of said notice.

2 . The cones referred to in this specification are known as the Orton pyrometric cone.

3. The material covered by this specification is particularly adapted to those portions of installations where special fire-clay shapes have been, or are being, used. It is recommended also for the construction of small furnaces such as are used in domestic heating plants, in baking ovens, and in laboratory apparatus.

United States Department of Commerce, division of simplified practice, recommendation No. 79 , malleable foundry refractories, February 1, 1928.

In accordance with the unanimous action of a general conference of representative manufacturers, distributors, users, and interested associations, the industry has adopted, and approved for promulgation by the United States Department of Commerce, a simplified practice recommendation limiting the stock shapes and sizes of brick and tile for malleable foundry refractories to the following:

Section I. Door opening tile.

5 by 9 by 24 inches.

5 by $131 / 2$ by 24 inches.

Section II. Tap-out blocks.

Outside dimensions $71 / 2$ by 736 by 9 inches, with suitable flare from the hole to the face to allow room for the tapping rod and plugging

one block to have a $3 / 4$ inch diameter hole for foundries using hand ladles.

One block to have a 1-inch diameter hole for furnaces served with bull ladles.
Section III. Shapes for side walls, bridge walls, etc.

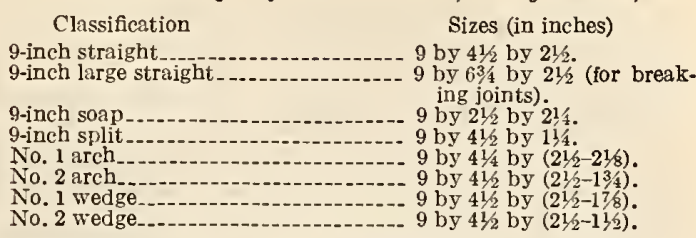

Section IV. Shapes for bungs and roofs.

Classification

Sizes (in inches)

No. 101 square bung

$131 / 2$ by $41 / 2$ by 3 .

No. 102 angle bung

No. 103 arch bung

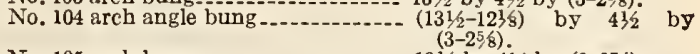

No. 105 arch bung-

Section $V$. Shapes for round stacks.

Circle brick

24-inch circle

Sizes (in inches)

36 -inch circle 9 by $41 / 2$ by $21 / 2$

48-inch circle

60-inch circle

72-inch circle

No. 1 wedge

No. 1 wedge

Section VI.

Upon depletion of present stocks the eliminated items shall be considered as specials, and extended delivery dates are to be anticipated.

Section VII.

It is recommended that in the issuance of new catalogues or lists only standard items be shown. If special items are also exhibited, some designating mark should be used to indicate the standards.

\subsection{Miscellaneous Specifications for Brick.}

Federal Specifications Board, specification No. 505, United States Government master specification for brick, sand lime, common, July 11, 1927.

See 513 , page 257 .

National Paving Brick Manufacturers' Association, recommended specifications for integral brick curb, 1924.

See 518.63, page 447 .

United States Department of Commerce, Bureau of Standards, Simplified Practice Recommendation No. 38, for sand-lime brick.

See 513, page 258 .

\subsection{TILES.}

\subsection{General Items.}

American Concrete Institute, standard specifications for concrete building block and concrete building tile, serial designation P-1A-29, 1929.

See 516.4, page 317 .

American Society for Testing Materials, standard definitions of terms relating to hollow tile, serial designation C43-24, 1924.

\section{TILE}

1. Hollow tile.-Hollow building units with parallel cells.

Note.-In the present state of the art the term "hollow tile," if used without a qualifying adjective, is understood to mean clay hollow tile.

The term "terra cotta," which is applied to ornamental building units of burned clay, should not be used to designate hollow tile. 
2. Load-bearing wall tile.-Hollow tile for use as a load-bearing structural unit in walls.

(a) Hollow floor tile.-Hollow tile for use as a load-bearing structural unit in floors.

(b) Foundation tile.-Hollow tile for use as a load-bearing structural unit in foundations.

(c) Side-construction tile.-Hollow tile designed to receive its principal stress at right angles to the direction of its cells.

(d) End-construction tile-Hollow tile designed to receive its principal stress parallel to the direction of its cells.

(e) Book tile.-Hollow tile with tongue-andgrove edges resembling a book in shape.

(f) Salt-glazed tile.-Clay hollow tile with a vitreous glaze on its surfaces produced by burning salt in the kiln at the temperature used in finishing the burning.

3. IIollow tile fireproofing.-Hollow tile for use as a protection for structural members against fire.

(a) Split tile.-Hollow tile which has been knifed parallel with its cells in the process of manufacture for the purpose of separation into two equal units.

(b) Partition tile.-Hollow tile for use in building interior partitions, subdividing areas into rooms or inclosing stairways or shaft's, and carrying no superimposed load.

(c), Furring tile.-Tile of suitable design for lining the inside of walls and carrying no superimposed load.

(d) Porous hollow tile.-Clay hollow tile in which the natural porosity of the clay has been increased by the admixture of other ingredients.

\section{RAW MATERIALS}

4. Shale.-A thinly stratified, consolidated sedimentary clay ${ }^{11}$ with well-marked cleavage parallel to the bedding.

5. Fire clay.-A sedimentary clay of low flux content.

Note.-It is usually associated with coal measures.

6. Surface clay.-An unconsolidated, unstratified, clay, occurring on the surface.

\section{DESIGNATION OF DIMENSIONS}

7. Length.- In the case of hollow tile, that dimension measured between its cut ends.

8. Thickness.-In the case of hollow tile, that dimension designed to lie at right angles to the face of the wall, floor, or other member in which it is used.

9. Width.-In the case of hollow tile, that dimension measured at right angles to the direction of its thickness and length.

Note.--In practice, the first dimension given represents thickness; the second, width; the third, length.

\section{PARTS, OPENINGS, AND SURFACE FEATURES}

10. Shell.- In the case of hollow tile, the outer walls.

11. Webs.- In the case of hollow tile, the partitions dividing it into cells.

12. Cells.- In the case of hollow tile, the openings parallel with its shell and webs.

13. Scoring.- In the case of hollow tile, the grooves formed in the exterior faces of the shell to increase the bond of mortar, plaster, or stucco.

11 The definitions for the terms "surface clay," "fire clay," and "shale" are based upon the following definition for the term "clay:" Clay.-An earthy or stony mineral aggregate consisting essentiali of hydrous silicates of alumina, plastic when sufficiently pulverized and wetted, rigid when dry and vitreous when burned at a sufficiently high temperature.
Associated Tile Manufacturers, basic specification for tile work, 1924.

\section{EXTENT OF WORK}

1. The following specification shall be known and hereafter identified as Basic Specification for Tile Work, Second Edition, 1924, as issued by the Associated Tile Manufacturers. It specifies how the work shall be done, beginning at the bottom of the concrete setting beds and at the back of the scratch coats, carrying each through to and including the finished tile surfaces.

2 . The location and extent of tile work, together with the kinds, grades, sizes, colors, and designs of the tiles, shall be governed by the architect's drawings, specifications, and schedules.

3. All materials and labor necessary for the completion of the tile work shall be furnished in accordance with this basic specification except as modified, if at all, by the architect's drawings, specifications, and schedules.

4. Any such modifications, and the general conditions, and other provisions of the architect's specification, shall be accepted as additions to this basic specification, and where conflicting, shall take precedence over it.

\section{TILES}

\section{SAIIPLES}

5. If required in the architect's specification, typical samples of each kind and grade of tiles as specified and proposed to be used, and shop or setting drawings, or rubbings shall be submitted to the architect for approval.

6 . Each sample shall be marked with the name of the manufacturer and the grade of the tile. Approved samples shall be retained by both the architect and the tile contractor.

\section{GRADES AND CERTIFICATE}

7. Before setting any tiles, the tile contractor shall furnish to the architect a certificate of grade, properly filled in on the form of grade certificate of the Associated Tile Manufacturers. This certificate shall be signed by the manufacturer of the tiles; shall state the grade, kind, and full quantities of tiles; and give identification marks for all packages of tiles furnished under this contract. Packages shall be branded with corresponding shipping marks and shall be subject to inspection by the architect or his representative before being opened.

\section{CEMENTS, LIME, AND AGGREGATES}

8. All cement and hydrated lime shall be delivered in the original containers bearing the brand and maker's name.

\section{PORTLAND CEMENT}

9. All Portland cement (including white) shall comply with the Standard Specifications and Test for Portland Cement of the American Society for Testing Materials, serial designation C9. (See 516.11 , p. 294 .)

10. All white cement shall be white Portland of an acceptable brand of American manufacture.

\section{LIME}

11. All hydrated lime shall comply with A. S. T. M. C6. (See 517.2, p. 353.)

\section{SAND}

12. All sand shall pass an 8-mesh sieve and shall be free from organic matter, salt, or alkali, and if 
it contains more than 5 per cent by volume of material passing a 100 -mesh sieve shall be well washed. All sand for concrete shall be graded from fine to coarse.

\section{CRUSHED STONE OR GRAVEL}

13. Crushed stone or gravel shall be hard and well graded from $1 / 4$ to 1 inch ring size. If loam or clay coat the particles, or are present to a greater extent than 5 per cent by volume, the stone or gravel shall be washed till not more than that amount is contained.

\section{SLAG}

14. Slag shall be clean, dense, crushed blastfurnace sla,g, weighing not less than 70 pounds per cubic foot when loosely placed in the measure, and containing not more than 1.3 per cent of sulphur as sulphides.

\section{CINDERS}

15. Cinders shall be thoroughly vitrified, and shall be free from ashes, unburned coal, or coke. Large lumps shall be broken up. All cinders shall be screened free from particles smaller than one-fourth inch and shall be well washed. House ashes and cinders containing sulphur in any form shall not be used. (See par. 17.)

NotE.- Where cinders or cinder concrete are used it is recommended that metal plumbing and heating pipes be wrapped.

\section{CONCRETE AND MORTAR}

\section{CONCRETE}

16. Concrete shall consist of 1 volume of Portland cement, $2 \frac{1}{2}$ volumes of sand, and 5 volumes of gravel, crushed stone, or slag, or shall consist of 1 part Portland cement and 5 parts of well-graded coarse sand. At the option of the contractor, 5 pounds of hydrated lime may be added for each bag of Portland cement.

17. Cinders may be used in place of gravel, crushed stone, or slag, unless prohibited by the architect's specification.

18. Thoroughly mix all cement and aggregates until the concrete is of uniform color and consistency throughout, using a minimum quantity of clean, fresh water. Mixing may be by hand or by acceptable batch machine mixers.

19. Concrete shall be spread promptly after mixing, and shall be well compacted to uniformly rough surfaces at proper level to receive the setting mortar.

\section{MORTAR}

20. Sand, cement, and lime shall be thoroughly mixed in the proportions hereinafter specified under setting, paragraph 57, until of uniform color and required consistency. Mortar shall not be retempered, and tile shall not be set in mortar that has reached its initial set.

\section{METAL IATH, SHRINKAGE MESH, AND PAPER}

\section{METAL LATH}

21. Metal lath shall be coated expanded metal, coated perforated metal, or coated wire lath.

22. Lath on vertical surfaces where supports are not over 16 inches on centers and lath for ceilings shall be metal lath weighing not less than 3.4 Ibs./yd. ${ }^{2}$ or wire lath not less than No. 18 gage (0.047 inch diameter), two and one-half mesh to the inch.

23. Lath on vertical surfaces, where supports are placed not over 12 inches on centers shall be metal lath weighing not less than $3 \mathrm{lbs} . / \mathrm{yd} .^{2}$, or wire lath not less than No. 20 gage (0.035 inch diameter), wo and one-half mesh to the inch.

24. If the distance between the centers of the structural supports of any lath is more than 16 inches, the lath behind the tilework shall be provided with stifieners not over 12 inches on centers.

25. Lath shall be stretched tight and shall be secured at all bearings with fastenings not more than 6 inches apart, as follows: To woodwork with not less than one 1-inch bright staple, or one $6 \mathrm{~d}$. bright wire nail; to metal furring with hammered prongs or twisted loops of wire; and to gypsum blocks and to masonry with specially hardened steel nails or self-clinching nails. In driving staples or nails the lath shall not be flattened or damaged.

26. Vertical joints in lath shall not occur except at structural supports and shall there be lapped one full mesh. All horizontal joints of lath shall be butted and laced or tied with wire at least once between supports.

\section{SERINKAGE MESH}

27. Shrinkage mesh where specified herein for use in concrete setting beds shall be No. 16 gage, unpainted expanded metal of from 2 to 3 inches mesh, or galvanized wire fabric of one of the following sizes: Rectangular mesh-No. 8 gage $(0.162$ inch diameter) wire at 3 -inch centers crossed by No. 10 (0.135 inch diameter) wire at 8-inch centers, or triangular mesh, No. 8 wire at 4 -inch centers, diagonally laced with No. 14 wire at 4 -inch centers; or 4 by 7 inch hexagonal mesh of No. 10 gage wire.

28. Shrinkage mesh shall be placed well within the mass of the concrete setting bed and where more than one width or length is required shall be lapped not less than one-third of a mesh. The mesh shall butt against walls and partitions and shall not be turned up.

\section{BUITDIRG PAPER}

29. Building paper shall be tar or asphalt saturated paper weighing not less than 11 pounds per 100 square feet. It shall be lapped at least 2 inches and, in connection with floors, shall be turned up at the walls at least 2 inches.

\section{SETTING OF TILES}

30. General.-All tiles shall be firmly secured in place. Joints shall be well filled, all lines shall be kept straight and true, and all finished surfaces brought to true and level planes. The completed work shall be free from cracked or broken tiles.

31. Borders and patterns. - Where borders, lines, patterns, panels, or other effects are a part of the work, the tiles shall be properly spaced, and shall accurately reproduce designs shown on the drawings or effects described in the specification of the architect.

32. Edges.-All intersections and returns shall be perfectly formed. All cutting and drilling of tiles shall be neatly done without marring the surface. The cut edges of tiles against any trim, finish, builtin fixtures, etc. shall be carefully ground and jointed. Around electric outlets, plumbing pipes, or fixtures and fittings, the tile shall fit close, so that the regular plates, collars, or coverings will overlap the tile.

33. Laying out.-All tilework shall be so laid out on floors and lengthwise on walls that, wherever possible, no tiles less than half full size shall occur. For heights stated in feet and inches, unless tilework is intended to exactly fill vertical spaces, courses shall be maintained full to produce nearest attainable height within a variation above or below 
equivalent to less than one-half course to avoid cutting of tiles which would otherwise be necessary. 34. Tile packages kept dry.-All tiles shall be kept dry while in packages and shall not be allowed to lie in or upon wet sawdust or similar materials.

35. Soaking before laying.-All tiles, except vitreous tiles and ceramic mosaic, shall be soaked in clean water before being set.

36. In freezing weather.-In freezing weather concrete setting beds or scratch coats or tiles shall not be placed in unheated portions of the structure.

Note.-For Floating Method and Buttering Method of setting tiles, see paragraphs 95,97 , and 98 .

\section{TILED FLOORS AND HORIZONTAI SURFACES}

\section{(Other than ceilings, soffits, and sills)}

\section{CONCRETE SETTING BEDS}

37. General.-Concrete settings beds of stone, gravel, or slag, or sand concrete, shall be 2 inches thick, except that directly on earth or fills referred to in paragraph 53 they shall be 3 inches thick.

38. Concrete setting beds of cinder concrete (par. 17) shall be $2 \frac{1}{2}$ inches thick, except that directly on earth or fills referred to in paragraph 53 they shall be 3 inches thick.

39. Where joists are chamfered, the point of the joist shall be at least $3 / 4$ inch below the top of the concrete setting bed.

40. The upper flanges of any steel beams projecting into concrete setting beds shall be covered with loose sheet metal or building paper to prevent adhesion of the concrete setting beds.

41. All floor arches or slabs, and all wood floors shall be swept free of loose particles before placing any concrete setting beds.

42. The contractor shall not install any tile work in floors of shower baths, except when located in basements, until a pan or safing of lead or other metal has beeen placed, turned up on all sides, and made water-tight by other contractors. The finished surface of the tile floor shall be sloped to drain properly into the outlet.

43. Concrete setting beds on new wood construction. Unless otherwise provided for in the architect's specifications, a layer of building paper, lapped and turned up, shall be placed over wood floors as described in paragraph 29.

44. Concrete setting beds shall be reinforced with shrinkage mesh as described in paragraphs 27 and 28.

45. Concrete setting beds on old wood construciion.-Where tile floors are to be laid over existing wood floors, the tile contractor shall cover the existing wood upper floor, or under floor, as the case may be, with building paper in accordance with the provisions of paragraph 29.

46. On the surface thus prepared a concrete setting bed, 1 inch thick, composed of sand and cement as described in paragraph 16 , with shrinkage mesh as described in paragraphs 27 and 28 , shall be spread. This concrete setting bed shall finish at such thresholds as are provided by the architect's specification, the thresholds being of sufficient thickness to receive a $1 / 2$-inch layer of setting mortar plus the thickness of the tiles. Where joists are chamfered the point of the joist shall be at least $3 / 4$-inch below the top of the concrete setting bed.

47. Concrete setting beds on pressed-steel construction.-In connection with pressed-steel joist systems of floor construction, the metal reinforcement and concrete slabs will be furnished and placed by other contractors as a part of such systems. Mortar setting beds only shall be provided by the tile contractor, and shall be placed directly on the concrete slab, unless joists have been set low as mentioned in paragraph 48 ,
48. If steel joists are shown or specified lowered for the passage of pipes, concrete setting beds of thickness and as specified in paragraphs 49 and 50 shall be provided.

49. Concrete setting beds on concrete slabs or other masonry construction.-Concrete setting beds shall not be placed until the exposed surfaces of masonry floor slabs, arches, or other structural work shall have been brought to the required level or surface for the concrete setting beds by other contractors, and not until such surfaces are free from mortar droppings, projecting joists, etc., and so they shall present comparatively smooth and even surfaces for concrete setting beds without any depressions, cracks, holes, or open joints.

50 . On the surfaces thus prepared spread the concrete setting beds in accordance with paragraphs 37 or 38.

51. Concrete setting beds on other concrete over earth or fills-Interior.-Where concrete setting beds for tile work are to be laid on concrete which has been placed over earth or fills, the setting beds shall not be laid until the lower work is suitably prepared by others as specified for concrete slabs and other masonry construction, paragraph 49. On the surface thus prepared place concrete setting beds to the thickness specified in paragraphs 37 or 38 .

52. Exterior.-Follow specifications for interior concrete setting beds, paragraph 51, except that cinders shall not be used in the concrete.

53. Concrete setting beds directly on earth or fills-Interior.-Where concrete setting beds for tile work are to be laid on earth or on fills of cinders, gravel, or other materials (sand cushions separately specified) without intervening slabs of concrete, the concrete setting beds shall be 3 inches thick, of stone, gravel, slag, or sand concrete, and shrinkage mesh shall be placed in these setting beds, in accordance with paragraphs 27 and 28 . Cinders may be used in place of other aggregates unless prohibited by architect's specification.

54. Exterior.-Follow specifications for interior concrete setting beds (par. 53), except that cinders shall not be used.

\section{CLEAVAGE PLANES}

55. Sand cushions.--Where sand cushions are provided for in the architect's specification as a means of absorbing vibration or to afford a cleavage plane, a layer of clean, sharp sand, not less than one-half inch thick, shall be spread and thoroughty compacted to a smooth and level surface. Over sand cushions shall be laid a layer of building paper as described in paragraph 29 , ready to receive the concrete setting beds.

56. Building paper.-Where so provided in the architect's specification, spread a layer of building paper in accordance with paragraph 29, before placing concrete setting beds, to form a cleavage plane between concrete setting beds and surfaces underlying them.

\section{MORTAR SETTING BEDS}

57. Mortar for setting beds shall consist of 1 part Portland cement and 3 parts sand and shall not be less than one-half inch thick.

58. Unless the mortar setting bed is spread the same day or the day after the concrete setting bed has been placed, the concrete setting bed shall be thoroughly saturated with clean, fresh water, and its surface uniformly hand dusted with Portland cement immediately before spreading the mortar of the setting bed.

59. The mortar shall be spread until the surface of the mortar setting bed is absolutely true and even in plane, either level or uniformly sloped for drainage, as the case may be, For all surfaces 
orer 100 square feet in area, screed strips shall be set as temporary guides to assure these results.

60 . As large an area as can be covered with tile before the mortar has reached its initial set shall be placed at one operation. When more setting mortar has been spread than can thus be covered, the unfinished portion shall be removed and cut back to a clean, beveled edge.

\section{SETTING OF TILES}

61. Portland cement shall be uniformly hand dusted over the surfaces of the mortar setting beds immediately preceding the setting of the tile The tiles shall be placed upon and firmly pressed and tamped into the mortar until exactly true and even with the finished floor lines.

62. In the case of ceramic mosaic, the joint between the sheets shall be kept the same width as those between the mounted tile and set without showing where the sections join.

63. Where the area of any floor is over approximately 100 square feet, the tiles or ceramic mosaic sheets shall be laid to a straightedge at regular interrals.

64. Wherever borders or defined lines occur, they shall be laid before the field or bodies of the floors or spaces to be tiled, and the rile shall be set as before specified. The inner edges of all borders against fields or bodies shall be kept straight and any cutting of tiles for irregularities in wall lines or rertical planes shall be done along the outer edges.

65. Thresholds, if specified to be included, shall be set in a similar manner to borders; and if not included, the tile work of floors shall be brought to true lines, level with adjoining floors, and stopped under doors, unless shown or specified to be continued into closets or other contiguous spaces.

66. Hearths, unless otherwise detailed or specified, shall be set flush with the surface of adjoining floors. In connection with hearths and fireplaces, supply and set any ash drops, features, fittings, or other materials specified or scheduled by the architect.

67. Stair treads, floors of shower baths, swimming pools, special hospital floors, and other surfaces not intended to be level shall be sloped as detailed or directed for purposes of draining.

68. Tiled nosings, coves, curbings, gutters, or other molded or shaped features shall be thoroughly backed or built up with mortar or concrete. They shall be rigidly placed, reinfolced or otherwise made firm and secure. Tiled window sills and jambs, partitions, copings, or other similar features shall be set as described herein under "Tiled $W$ alls and Vertical Surfaces.'

69. Where bathtubs are of built-in types, or extend to floors, the concrete setting beds over wood construction shall be continuous under them, and shail form close junetion with all surrounding vertical planes. Tiling shall be omitted under such tubs, but under free standing tubs the tiling of floors shall be continuous to the surrounding tiling of vertical planes.

70. As soon as the cement mortar beds have sufficiently set, the tiles on floors or other horizontal surfaces shall be well washed with clean mater, and the joints between the tile grouted or jointed as mentioned under paragraphs 71 to 75 , inclusive.

\section{GROUTING AND JOINTING}

71. The joints between all units of ceramic mosaic and between the abutting sheets as laid shall maintain the standard mounting width approximating one-sisteenth inch.

72. Width of floor joints.-Unless otherwise shown specified, or stipulated, the joints of other tiles shall approximate the following width, with uniform variance therefrom, either more or less, as may be desirable to cause the units, in the hands of skilled workers, to accommodate themselves to given spaces:

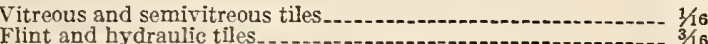
Flint and hydraulic tiles Quarry tiles

Joints in the fields of walls and floors should not run straight through with the joints in the base and top moldings, but should break in order to emphasize the different function of the molding as distinguished from the field.

73. All ceramic mosaic and other tiles sct with close joints shall be grouted with Portland cement mixed with water to the consistency of thick cream. The grout shall be forced into the joints, sprinkled with dry cement, and finished flush and true. All surplus grouting shall be removed and the faces of tiles left clean. In cases where acid solutions are required to elean the face of the finished tile work of surplus grouting or other particles of foreign matter, all hardware, such as hinges, cupboard trim, etc., shall be covered first by a coating of vaseline to protect the metal from the possible effect of acid fumes.

74. All joints not grouted shall be completely filled with mortar consisting of 1 volume of Portland cement and 2 volumes of sand, as before specified and, at the option of the tile contractor, tempered with hydrated lime to extent of not more than 10 per cent of the volume of cement and sand. All surplus mortar shall be miped off, and the faces of tiles left clean. White Portland cement, white sand, or mortar colors, in joints of tiles on horizontal surfaces-or firc clay for fireplace backs and jambsshall be used only when and where so provided in the architect's specification or schedule, and shall then replace the Portland cement or plain sand as here specified.

75. After being cleaned as just described, floors or horizontal surfaces in each room or portion as completed shall be closed to traffic or work until the tiles are firmly set. (Par. 118.) After this, all completed tile work shall be finally turned over in clean condition as described in paragraphs 119,120 , and 121.

\section{TILED WALIS AND VERTICAL SURFACES}

\section{PREPARATION FOR THE SCRATCH COAT}

76. General.-Scratch coats shall not be applied until substantial grounds, plugs, and other provisions have been installed by respective contractors to receive plumbing fixtures, electric outlets, radiator brackets, or any other fixtures or fittings to be secured against tiled surfaces.

77. In new construction work, when scratch coats are to be applied directly to concrete, brick, hollow building tile, stone, or other masonry, without furring, the masonry surfaces shall be thoroughly moistened but not saturated

78. In existing structures, where scratch coats are to be applied directly to masonry without furring, when such masonry has been previously coated with cement mortar or cement plaster, the mortar or plaster shall be removed, or hacked and wire brushed in such a manner as to restore satisfactory suction for complete bonding of the scratch coat.

79. Where such existing masonry has been coated with mortar or plaster containing gypsum or lime, the mortar or plaster, or any painted surfaces, shall be entirely removed and the masonry hacked and wire brushed as just described. In 
lieu of removing or hacking and brushing, such existing surfaces, if conformable to architect's details or if approved, may be metal lathed to comply with requirements of paragraphs 86 and 88 .

80. Scratch coats for tile work shall not be applied to new unfurred surfaces of concrete or other masonry construction until such surfaces shall have been brought to the required plane, plumb, reasonably straight and true, by other contractors, with the faces free of fins, excessive voids, or projecting joints, and left fairly rough, ready for the scrubbing specified in paragraph 82 .

81. Old surfaces of concrete, brickwork, or stone, if smooth, shall be hacked, roughened, or raked by the contractor applying scratch coats as may be necessary to provide satisfactory bonding for the scratch coats.

82. Unfurred concrete to be tiled, whether new or old, shall be thoroughly scrubbed to clean the surface and give it additional roughness. At the option of the tile contractor, this may be done by the use of wire brushes or by using muriatic acid diluted with 6 to 10 parts of water, scrubbed on until the aggregate is exposed, after which all traces of acid shall be removed by thorough rinsing.

83. On wood studding or wood furring.-On wood studding or wood furring to be tiled, furnish and attach building paper and metal lath as described in paragraphs 21 to 26 , inclusive, and paragraph 29 . This shall apply in the case of either old or new structures, as any existing lime or gypsum plaster shall invariably be removed by whatever contractor is specificd in such cases; old wood lath to remain but shall be covered with metal lath.

84. Where tiles form architraves of doors, windows, or medicine cabinets, trims of openings, or other features and bases, furnish and secure strips of metal lath as described in paragraphs 21 to 26 , inclusive.

85. On metal studding or metal furring.-Where pressed-steel studs, metal channels, tees, or other metal supports are used for partitions or furring, the metal lath in connection with same will be furnished and applied by other contractors.

86. On gypsum blocks. - Where tiles are to be set against gypsum blocks, furnish and place a layer of building paper; this is to be overlaid with stiffened expanded metal or stiffened wire lath as described in paragraph 26 , the lath to be secured in place by special anchorage or lacing, as conditions require.

87. Alternate.- At the option of the tile contractor, one heavy coat of asphaltum paint or other equal damp-proofing coat may be substituted for the building paper back of the metal lath before specified for use on gypsum blocks.

88. On cork or other insulation.-Where tiles are to be set against cork or other insulation, furnish and attach metal lath as described in paragraphs 21 to 26 , inclusive. In such installations the scratch coat shall contain suitable proportions of an established brand of waterproofing compound mixed in accordance with manufacturer's directions.

\section{SCRATCH COAT}

89. A scratch coat shall be placed back of all tiles. The scratch coat on unfurred masonry shall be applied in conformity with paragraphs 76 to 82 , inclusive. In all other cases the scratch coat shall be applied to metal lath.

90. Where bathtubs of built-in types are set against walls or partitions of masonry or gypsum construction, the scratch coat behind tubs shall be omitted below the undersides of rims. Against supports involving metal lath on wood, or steel studs, or furring, the scratch coat shall continue to and form close junction with concrete setting beds of floors. The tiling on all vertical surfaces in contact with built-in tubs shall abut the top of the rim, separated therefrom by a full-width joint.

91. The mortar for scratch coat shall consist of 1 volume of Portland cement and 3 volumes of sand. At the option of the contractor, hydrated lime may be added not to exceed 10 per cent of the volume of ccment and sand, and plasterer's fiber or hair may be incorporated in the scratch coat.

92. The scratch coat shall be $1 / 2$ inch thick, or more if necessary, to make an even and true surface at the proper distance from the face of the tiles, allowing for the thickness of the tile and for a 1/4-inch bed of setting mortar for floating work and a 3 i-inch bed of setting mortar for buttered work. The scratch coat, at the option of the contractor, may be applied in two coats instead of one.

93. The scratch coat shall be applied not less than 24 hours nor more than 48 hours before commencing to set the tiles. While still plastic the scratch coat shall be deeply scored or scratched, horizontally, approximately 1 inch apart.

\section{SETTING OF TILES}

94. Immediately before setting tiles the scratch coat shall be thoroughly moistened with water, but not saturated.

95. On the surface thus prepared the mortar for setting the tiles shall be applied in accordance with either of the methods described in paragraphs 97 and 98. In the absence of stipulations to the contrary, tiles may be set by either the floating or buttering method.

96. Setting mortar shall consist of 3 volumes of sand and 1 volume of Portland cement, to which hydrated lime not to exceed one-tenth the volume of the cement and sand combined shall be added.

97. Floating method.-Screeds or temporary guide strips shall be mortared plumb and true onto the scratch coat, to accurately indicate the surface plane of the mortar setting bed, which shall be rodded and floated flush with the guide strips. A skim of neat Portland cement shall be applied to the mortar setting bed or the back of each tile as laid. Each tile shall be beaten into place and brought flush in plane with the other tiles.

98. Buttering method.-The scratch coat shall be spotted with small pieces of tile mortared plumb and true to accurately indicate the plane of the tiled wall when finished. Each tile shall be buttered and tamped in place and brought to a plumb and true suriace, flush with the spots and with the other tiles. The back of each tile shall be completely covered with the mortar, bed at back of tiles shall be full and even, and all corners and crevices filled.

99. Fireplaces.-Tiles of fireplace facings or mantel breasts shall be set by either the floating or buttering method, but tiles of backs and jambs shall be set by the floating method unless shown or specified to be set flat, when they shall be tightly bedded against the surrounding masonry and set with full joists pointed on the face. Hearths to be as specified in paragraph 66.

100. Inserts. - In the absence of stipulations to the contrary, any tile inserts indicated or specified may be set by either the buttering or floating method.-

101. Trimmers.-All caps, bases, coves, moldings, or other trim tiles shall be backed full with mortar and tamped into place. The reference by the architect in his specification or schedule to any cap, base, quarter-round, bead, cove, combination tiles with cove corner or angles, or any molded tile shall be taken to carry with it the inclusion of all requisite returns, stops, angles, corners, or other "trimmers" which are an established standard of the Associated Tile Manufacturers, incidental to the trimmers 
mentioned, and they shall be furnished and set by the tile contractor, as is especially called for.

\section{GRODTING AND JOINTING}

102. Vertical units and joints shall be maintained plumb and even, and all caps, bases, moldings, and horizontal units or joints shall be maintained level and even. Joints in the tile work of shower-bath partitions, windows jambs, alcoves, or in returns to contiguous spaces shall be maintained level and true with the joints of main fields. Every fourth course of tile shall be brought to a level and straight line. Wooden wedges, if used for this purpose, shall be removed before grouting is done.

103. As soon as the mortar setting bed has sufficiently hardened, the tiles on walls or other vertical surfaces shall be well washed with clean, fresh water, and joints between tiles shall be grouted or jointed as mentioned under paragraphs 104,105 , and 106, unlesss otherwise stipulated. With the exception of the white Portland cement specified for joints of white tiles in paragraph 104 , white cement, white sand, or mortar colors shall not be used in grouting or jointing except when expressly so provided as mentioned in paragraph 74 .

104. Joints in white bright glazed tiles shall be maintained at the customary size, approximately $3 / 64-$ inch, and shall be filled with white Portland cement mixed with water to the consistency of thick cream. All traces of this grouting shall be wiped from faces of tiles before hardening, and all joints shall be left full and smooth.

105. Width of wall joints.-Unless otherwise shown, specified, or stipulated, the joints of other tiles shall approximate the following widths with uniform variance therefrom, either more or less, as shall be desirable to cause the units in the hands of skilled workers to accommodate themselves to given spaces.

Inch

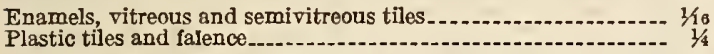

106. Where other kinds of tiles, including ceramic mosaic, are set on vertical surfaces, they shall be grouted or pointed and left clean in accordance with paragraphs 71 to 74 'nclusive, and 119 to 121 , inclusive.

\section{TILED CEILINGS, SOFFITS, AND SILLS}

\section{PREPARATION FOR THE SCRATCH COAT}

107. On hollow building tile.-When ceilings, soffits, or sills are to be tiled on unfurred hollow building tile, the provisions contained in paragraphs 76 and 77 shall be complied with before applying the scratch coat, which shall contain suitable proportions of an established brand of waterproofing compound, mixed in accordance with manufacturer's directions.

108. On concrete.-The scratch coat shall not be placed on an unfurred concrete ceiling or soffit, but shall be applied to metal lath furnished and attached by another contractor.

109. On wood joists or wood stripping.-To the underside of new wood joists or new wood stripping furnish and attach expanded metal or wire lath as described in paragraphs 21 to 26 , inclusive. For existing wood joists or stripping paragraph 83 shall be followed.

110. On suspended ceilings with metal supports.Supports and metal lath for such types of construcstruction will be furnished and applied by other contractors.

111. On gypsum or on cork or other insulation.Preparation for scratch coat against gypsum, or against cork, or other insulation shall be made in accordance with paragraphs 86,87 , and 88 .

\section{SCRATCH COAT}

112. A scratch coat shall be placed back of all tiles and be applied either directly to the hollow building tile as mentioned in paragraph 107 or applied to expanded metal or wire lath, either furnished and attached by the tile contractor as described in paragraphs 109 and 111 or by other contractors, in accordance with the type of support, as mentioned in paragraph 110 .

113. The mortar for scratch coat shall be as described in paragraph 91 , and shall be applied to conform to paragraphs, 92 and 93 , providing a $1 / 4$-inch mortar setting bed instead of $1 / 2$-inch.

\section{SETTING OF TILES}

114. All tiles shall be set in mortar and in such manner as to comply with the descriptions in paragraphs 94 to 98 , inclusive, and with paragraphs 100 to 106 , inclusive.

\section{SWIMMING POOLS}

115. The tile work of swimming pools, or of Turkish baths, steam rooms, etc., shall be prepared for and set in accordance with the requirements of all other portions of the basic specification, and, in addition, the mortar setting beds and joints shall contain suitable proportions of an established brand of waterproofing compound mixed in accordance with manufacturer's dircetions. When the walls and floors of swimming pools are not waterproofed, the scratch coats and concrete setting beds shall be waterproofed as specified for the mortar setting beds.

116. Life rails shall be reinforced with properly shaped expanded metal and the high point shall be brought to an exact level on all sides of the pool. Scum gutters shall be formed in accordance with details and drained to the outlets with a high point between each pair of outlets.

\section{ACCESSORIES}

117. The furnishing and setting of accessories, such as andirons, grates, medicine cabinets, mirrors, recessed heaters, paper holders, soap cups, towel racks, shelves, etc., are not included, unless specified or scheduled as a part of the tile contract and listed in detail. When included, they shall be furnished, delivered, and set by the tile contractor.

\section{FINAL CLEANING AND RESPONSIBILITY}

118. The contractor shall post suitable notices or make other provisions to the effect that no one shall pound about freshly tiled walls or ceilings, or walk upon freshly tiled floors, for several days after the tiles are set.

\section{CLEANING}

119. Upon completion of the various portions of his work, the tile contractor shall remove all unused materials, rubbish, etc., in connection with this contract and shall give the tile work one thorough cleaning at the time of its completion. (See par. 73.)

120. After completion and cleaning in accordance with paragraph 119 the obligation of the tile contractor shall cease as to any damage or injury which may be done to the tile work by others, and as to any further cleaning of the tile work upon final completion of the building as a whole, which additional cleaning shall be done only if so required by the architect's specification.

\section{RESPONSIBILTTY}

121. Neither the final certificate nor payment nor any provision in the basic specification shall relieve the contractor of responsibility for faulty 
materials or workmanship, and he shall remedy any defects due thereto which shall appear within a period of one year from the date of final completion of the structure, unless a longer date is stipulated in the architect's general conditions or in his modifications to this basic specification.

\section{DEFINITIONS OF CERTAIN MATERIALS AND METHODS REFERRED TO IN THE BASIC SPECI- FICATION \\ BUTTERING}

122. A method of setting tiles in which the back of each tile is "buttered" with mortar and then set in place. Floating is the alternate method. (See "Buttering method" and "Floating method" in basic specification.)

\section{CLEAVAGE PLANE}

123. A layer of material such as sand or building paper, used under certain conditions to separate the concrete setting bed from the under slab or other structural support. This is intended to allow for possible independent movement of the two planes.

\section{(See setting beds.) \\ FLOATING}

\section{CONCRETE SETTING BED}

124. A method of setting tiles by beating tiles onto the fresh surface of the mortar setting bed. It is the method used for setting mounted sheets of tile, and is the alternative of the buttering method for setting unmounted tiles. (See "Floating method" and Buttering method" in basic specification.)

\section{GROUTING}

125. The method of finishing the joints of tile work in floors, walls, etc., by filling with a mixture of cement and water of the consistency of thick cream. This mixture is called a." grout."

\section{HYDRATED LIME}

126. Powdered lime resulting from the factory hydration of quicklime. It is delivered in bags as distinguished from quicklime, which latter-in lumps or granular form-requires hand slaking. A standard paper bag of hydrated lime weighs 50 pounds and in volume is equal to about $11 / 4$ cubic feet.

\section{INSERTS}

127. In the basic specification this term refers to isolated or grouped tiles set in a finished surface of other material such as brick, stone, stucco, concrete, or cement whether on interior or exterior walls or floors. It includes bands and borders as well as panels and accentuation spots.

\section{MORTAR SETTING BED}

See setting beds.

\section{MOUNTED TILES}

128. For accuracy in setting as well as convenience in handling certain small tiles are mounted on paper pasted to the face of the tiles. These mounted sheets are set as units by the floating method. The paper is soaked and removed after setting.

\section{SAND CUSHION}

129. A layer of sand sometimes placed under the concrete setting bed of a tiled floor to insulate it from the structure, thereby tending to prevent vibration due to machinery in motion, wind stresses, etc. from being transmitted to the tiled surface, as well as to minimize the possibility of structural cracks appearing on the surface.

\section{SCRATCH COAT}

130. The Portland cement plaster forming the backing for the setting mortar of tiled walls and ceilings. It is scratched, scored, or grooved, to give key for the mortar setting bed.

\section{SCREEDS OR SCREED STRIPS}

131. A wooden strip, or a strip of mortar, laid on a floor or wall at intervals, to gage the thickness of setting beds, or to indicate the finished tiled surface.

\section{SETTING BED, CONCRETE}

132. The term "concrete setting bed," as used in the basic specification, refers to a layer or bed of concrete under a tiled floor or other horizontal tiled surface. This does not come in contact with the tiles, but serves as a foundation on which to apply the setting mortar. It is sometimes known as the underfill or base.

\section{SETTING BED, MORTAR}

133. Wherever tiles are to be set, a layer of mortar is applied, known as the setting mortar or the mortar setting bed. Into this the tiles are beaten when set by the floating method.

\section{SHRINKAGE MESH}

134. A layer of expanded metal or wire fabric, of a large mesh. Its use tends to prevent cracking of setting beds due to contraction and expansion when affected by temperature changes or due to cracks or settlements in structural supports from other causes.

\section{TRIMMERS}

135. The terms "trim tile" and "trimmers" are used interchangeably to designate bases, caps, corners, angles, architraves, and other tile moldings and shapes, as made and standardized by the Associated Tile Manufacturers to suit the various kinds, grades, and sizes of tiles.

United States Department of Commerce, division of simplified practice, recommendation No. 61 , white glazed tile and unglazed ceramic mosaic, January 1, 1927.

In accordance with the unanimous action of a general conference of representatives of manufacturers, dealers, contractors, architects, and users, the Department of Commerce, through the Bureau of Standards, recommends the establishment of the following standards for white glazed tile and unglazed ceramic mosaic:

\section{GENERAL}

1. The types, shapes, patterns, sizes, dimensions, grade nomenclature, grade marks, grade specifications, and certifications of grades given herein are recommended as standards.

2. Catalogues displaying white glazed tile and unglazed ceramic mosaic should, as near as possible, conform to recommended standard items, and where specials are exhibited they should be so indicated.

3. Replacements playing a very important part in the use of tile and ceramic mosaic, manufacturers should exercise discretion in the retention or destruction of molds which become obsolete through the operation of these recommendations.

4. Various names are now used by architects, manufacturers, and contractors without definition, and new designations are from time to time applied to established grades or to mixed grades of tiles, which are indefinite or confusing, and tend to encourage misrepresentation and substitution.

5. Plain grade marking - that is, practicable in application, and which even the layman purchaser 
can understand-mould avoid the possibility of tampering, discourage the shipping of mixed grades in the same container, allay undue suspicion when the identity of the grade is concealed, properly place responsibility, and serve as the consumer's guaranty.

It would also serve as a medium to build public confidence in tile and would encourage the consumer to place his order or contract for tile work with the assurance of receiving what he requires at the lowest cost.

Grade marking is considered fundamental to an understanding of tile grades, to the proper and satisfactory use of tiles, to the consumer's good will, and to the fullest development of the tile industry.

6. Warpage and manufacturing blemishes on the surface of tile are factors which must be considered in grading. As all grades of tile are made from the same raw material and by the same processes, there is a dire necessity for some uniform practice on the part of producers in the sorting and grading of tile. As grade names have little significance until their relative meanings are clearly defined and the terms which apply to the definitions explained, some uniformity which can be used as measures of relative values must be secured.

7. The establishment and use of standardized minimum grade specifications would be greatly facilitated by the adoption of a uniform method for certification of grade. Based on definite grade specifications, the certification of grades would be more effective than the method of certification by grade name without definition.

The adoption of certificates to cover this condition will be of real value to the industry.

\section{DIMENSIONAI SIMPLIFICATION}

TaBLE 1.-White glazed tiles

\begin{tabular}{|l|l|l|}
\hline \multicolumn{3}{|c|}{ Inches } \\
\hline 6 by 6 & 6 by 3 & $41 / 4$ by 41/4 \\
\hline
\end{tabular}

TABLe 2.-Ceramic mosaic

\begin{tabular}{|c|c|}
\hline & Inches \\
\hline $\begin{array}{l}\text { Square } \\
\text { Oblong.-- } \\
\text { Hexagon.- }\end{array}$ & $\begin{array}{l}11 / 32,15 / 32,3 / 4,11 / 6,23 / 16 . \\
1 / 2 \text { by } 11 / 16,3 / 4 \text { by } 19 / 16,1 / 16 \text { by } 23 / 16 . \\
1,11 / 4 .\end{array}$ \\
\hline
\end{tabular}

TABLE 3.-Mounting sheets and paper sizes

\begin{tabular}{|c|c|c|}
\hline Size of tile & $\begin{array}{l}\text { Number of } \\
\text { rows-width, } \\
\text { length }\end{array}$ & Size of paper \\
\hline $\begin{array}{l}15 / 32 \text { inch square } \\
3 / 4 \text { inch square } \\
11 / 16 \text { inches square } \\
2316 \text { inches square } \\
11 / 16 \text { by } 1 / 2 \text { inch oblong } \\
11 / 16 \text { by } 1 / 2 \text { inch herringhone diagonal } \\
19116 \text { by } 3 / 4 \text { inch ohlong } \\
1916 \text { by } 3 / 4 \text { inch herringbone diagonal } \\
2316 \text { by } 11 / 16 \text { inches oblong } \\
2316 \text { by } 11 / 16 \text { inches herringbone } \\
\text { diagonal. } \\
1 \text { inch hexagon } \\
11 / 4 \text { inches hexagon }\end{array}$ & $\begin{array}{l}24 \text { by } 48 \text { rows } \\
16 \text { by } 32 \text { rows } \\
12 \text { hy } 24{ }^{1}- \\
6 \text { by } 12{ }^{2} \\
24 \text { by } 24^{3} \\
16 \text { by } 32 \\
16 \text { by } 16 \\
11 \text { hy } 22 \\
12 \text { by } 12 \\
8 \text { by } 16 \\
12 \text { by } 24 \\
12 \text { by } 18\end{array}$ & $\begin{array}{l}\text { Inches } \\
1318 \text { by } 261 / 4 \\
121 / 2 \text { by } 251 / 4 \\
1318 \text { by } 261 / 4 \\
1318 \text { by } 261 / 4 \\
1318 \text { by } 261 / 4 \\
121 / 2 \text { by } 251 / 4 \\
1216 \text { by } 251 / 4 \\
121 / 4 \text { by } 241 / 2 \\
1318 \text { by } 261 / 4 \\
1134 \text { by } 233 / 4 \\
101 / 4 \text { by } 241 / 2 \\
121 / 2 \text { by } 223 / 8\end{array}$ \\
\hline
\end{tabular}

1 Mounted by itself or in combination with $11 / 16$ by $1 / 2$ and $15 / 32$ squares.

Mounted by itself or in combination with $23 / 16$ by $11 / 16,11 / 16$ by $11 / 16,11 / 16$ by $1 / 2$, and $15 / 32$ squares.

Mounted by itself or in combination with $15 / 32$ squares.

1 Mounted by itself or in comhination with $3 / 4$ squaresand $11 / 32$ dot.

5 Mounted hy itself or in comhination with $11 / 16$ by $11 / 16,136$ by $1 / 2$, and $15 / 32$ squares.
TABLE 4.-Container for shipping flat white glazed tiles

\begin{tabular}{|c|c|}
\hline Type of container & Capacity 1 \\
\hline Barrel__......-. & $\begin{array}{c}\text { Square feet } \\
62.5\end{array}$ \\
\hline
\end{tabular}

1 A barrel of designated capacity will contain 250 pieces of 6 by 6 tiles or 500 pieces of 6 by 3 or $41 / 4$ by $41 / 4$ tiles.

Figures 221, 222, 223, and 224 show the recommended styles, patterns, etc., for trimmers in white glazed tiles.

\section{UNIFORM GRADE NOMENCLATURE}

(See fig. 225)

White glazed tiles.-Selected, standara, commercial.

Ceramic mosaic.-Selected, commercial.

\section{GRADE MARKS AND SEALS \\ WHITE GLAZED TILES}

To consist of strips of paper in distinctive color, on which the grade name is printed in plain type.

These strips of paper are to be pasted firmly across the joints of the cants of the barrel top and bottom with waterproof paste, thereby sealing the containers.

All white glazed tiles to be shipped in sealed packages with grade of contents indicated by "grade seal."

Color and grade imprint seals to be used on all containers to plainly designate contents. Color of paper for grade seals to be: Pink for selected grade, blue for standard grade, and yellow for commercial grade.

\section{CERAMIC MOSAIC}

The paper used for mounting ceramic mosaic provides the practical medium on which the grade of the tile is to be indicated.

Ceramic mosaic of selected grade to oe mounted on pink mounting paper, imprinted with the grade name "selected grade."

Ceramic mosaic of commercial grade to be mounted on yellow mounting paper, imprinted with the grade name "commercial grade."

\section{GENERAL}

Grade marks and seals in the manner and color prescribed may be used by any manufacturer or producer of tiles.

The words "Made in Accordance with Simplified Practice Recommendation No. 61 of the United States Department of Commerce" may be printed upon grade labels and seals in addition to the grade name.

The above is predicated upon the provision that the product is in conformity with the standards as adopted by the industry and published in Simolified Practice Recommendation No. 61.

\section{MINIMUM GRADE SPECIFICATIONS}

Warpage and manufacturing blemishes on the surface of tiles being primary factors incident to grading, the following minimum grade specifications are hereby established:

\section{WHITE GLAZED TILES}

Selected grade.-The tiles of this grade are as perfect as it is possible to manufacture; they are free from warpage exceeding two-tenths of 1 per cent ( $=12 / 1000$ of an inch for a 6 -inch length), 


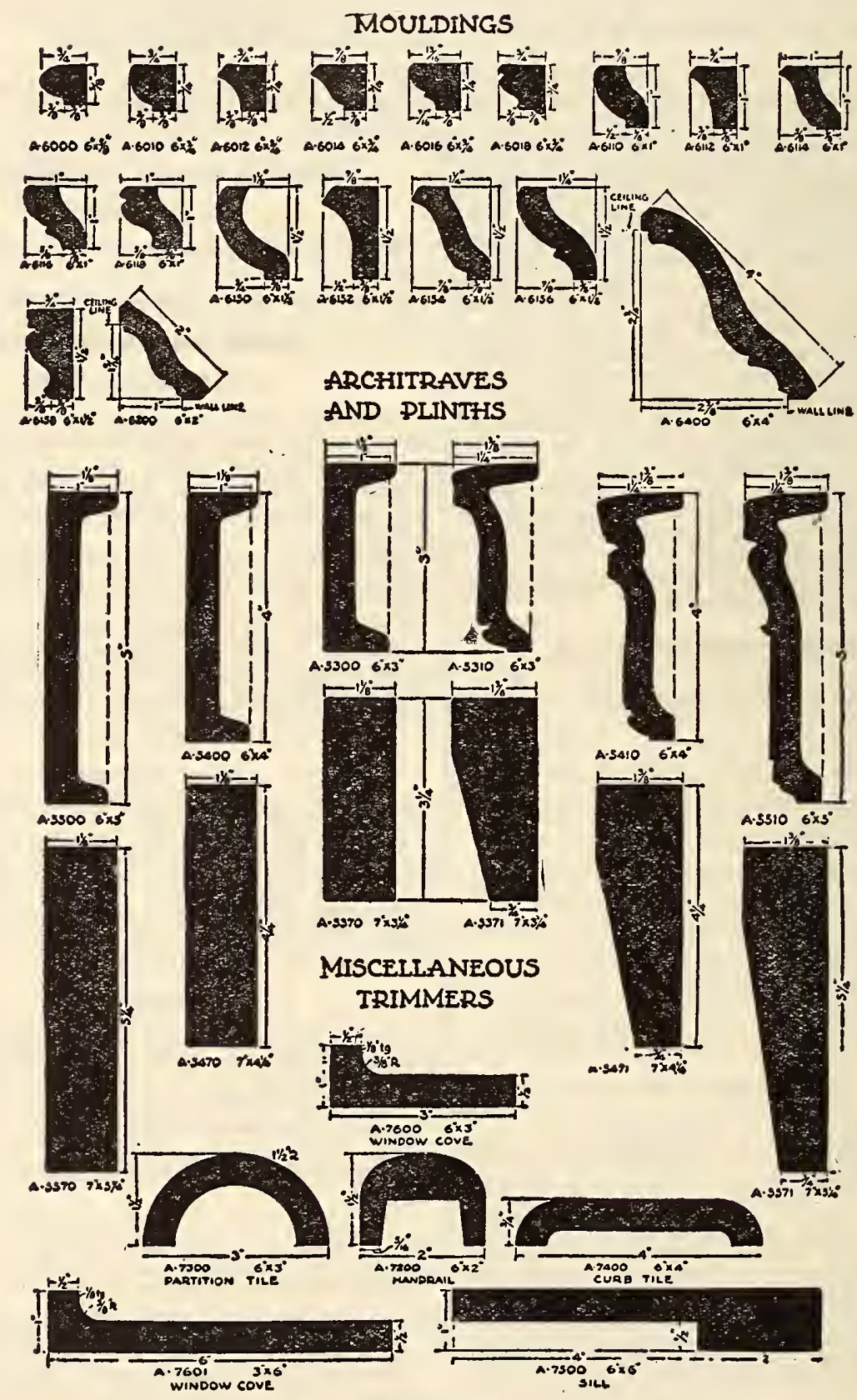

Figuae 221 


\section{$6^{\circ} \times 6^{\prime \prime}$ AND $6^{\circ} \times 3^{\prime \prime}$ RADIUS TILES}
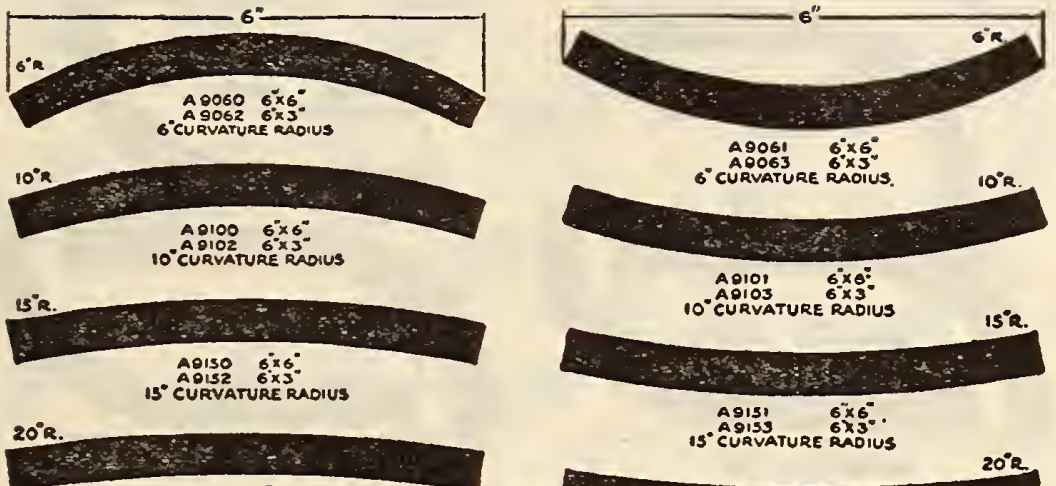

- Árvature ráius

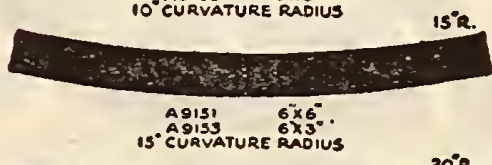

$1920066^{\circ} \times 5^{\circ}$

$00^{\circ}$ AURVATURE RAOIUS
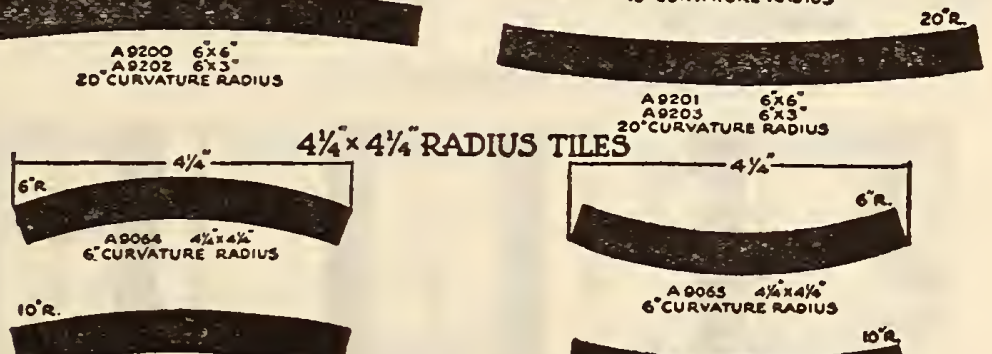

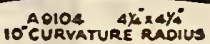

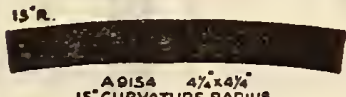

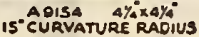
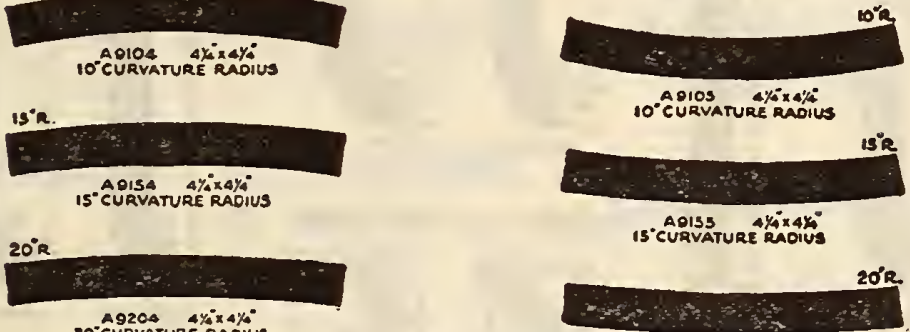

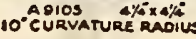

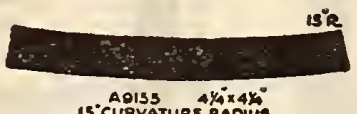

$15^{\circ}$ AOISS 4 144 $\times 4 x_{0}^{\circ}$

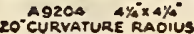

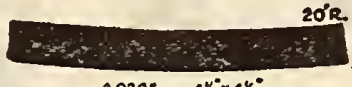

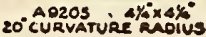

$6^{\circ} \times 3^{\prime}$ RADIUS TULE COMBINATIONS
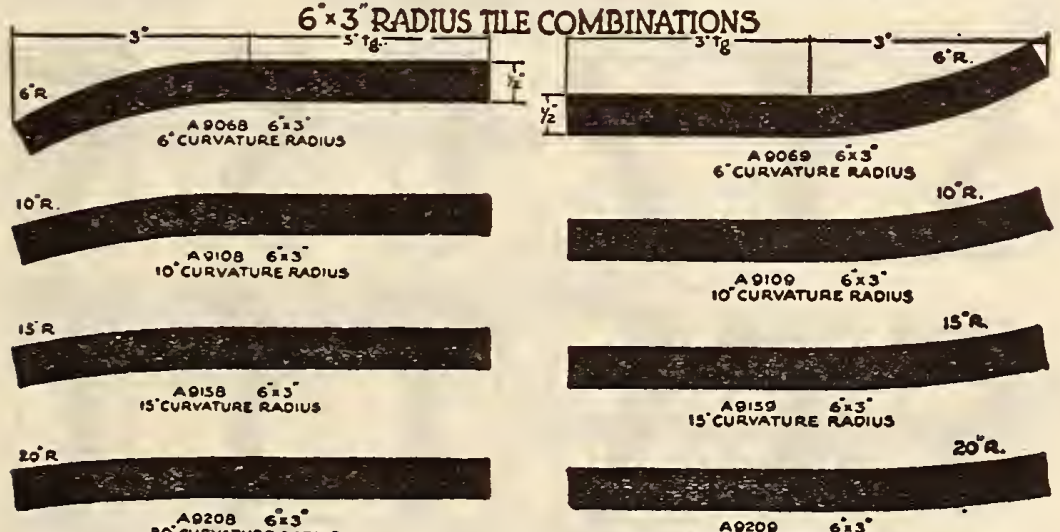

20. APRTER $6^{\circ} 3^{\circ}$

20. APQRVR

Figure 222 
LARGE RADIUS COMBINATIONS
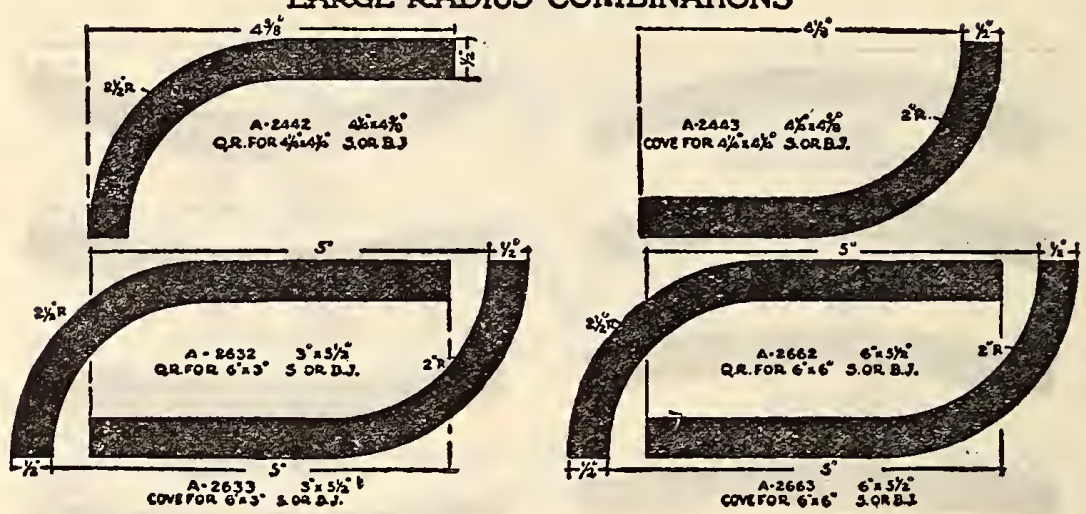

BASES
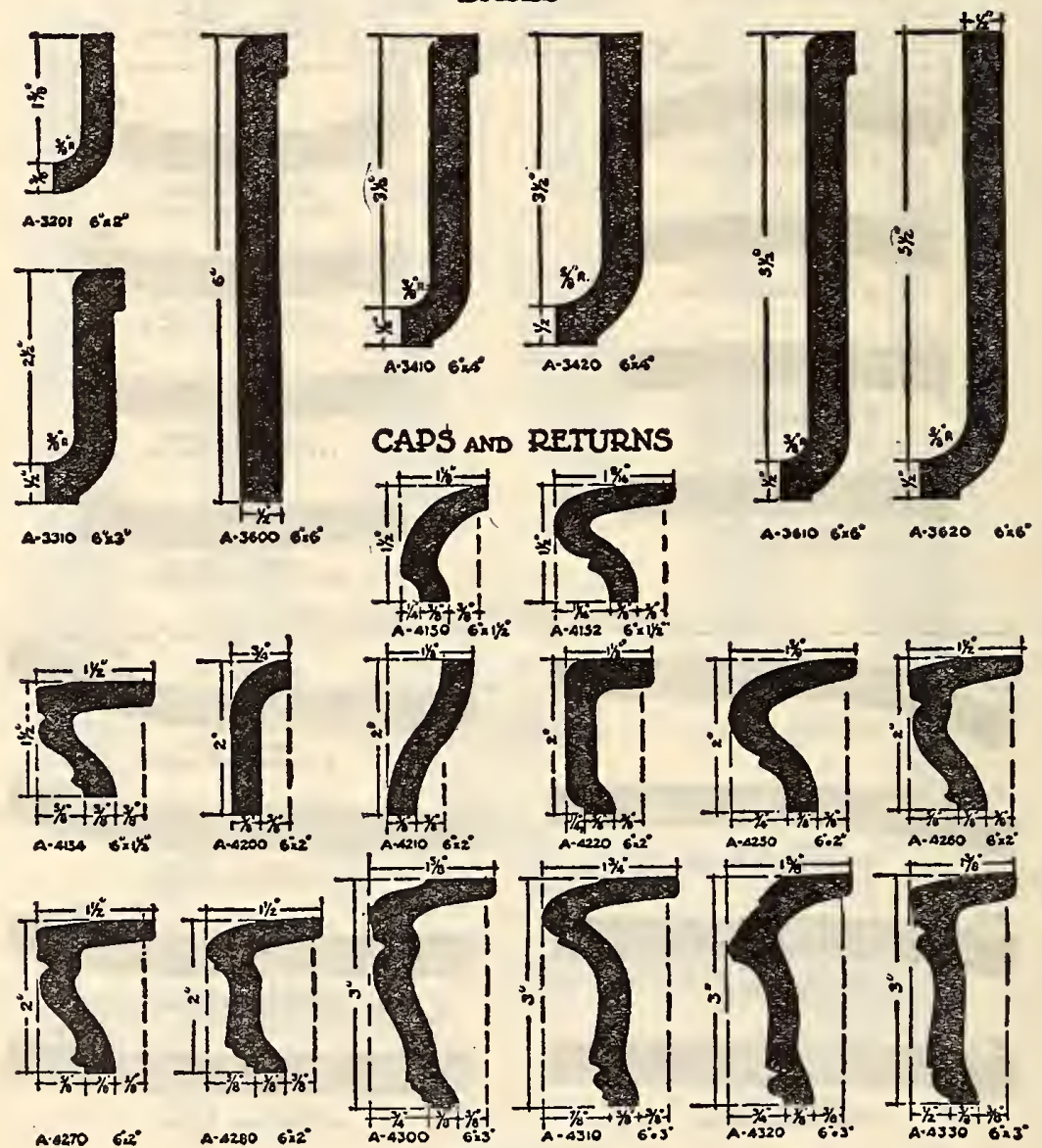

Figure 223 
QUARTER ROUNDS AND COVES

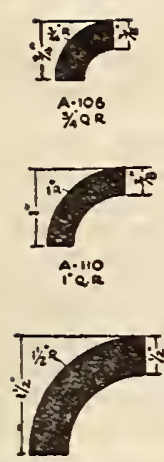

A.150
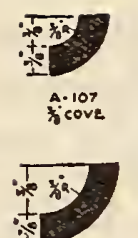

Ficove
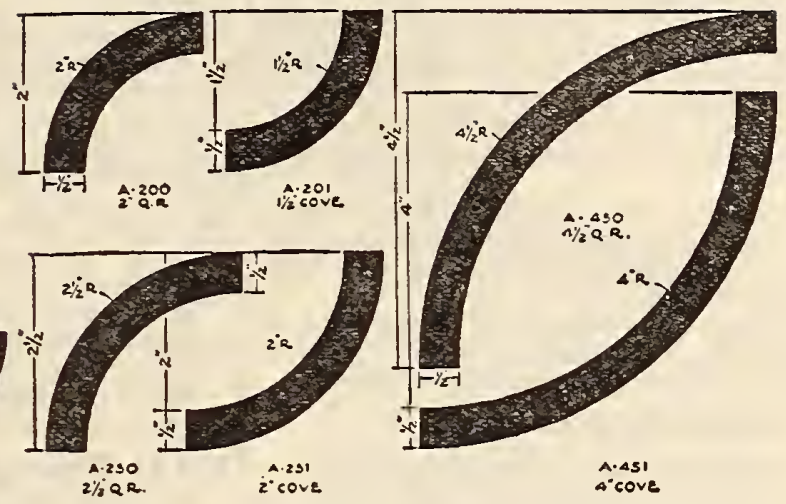

SMALL RADIUS COMBINATIONS
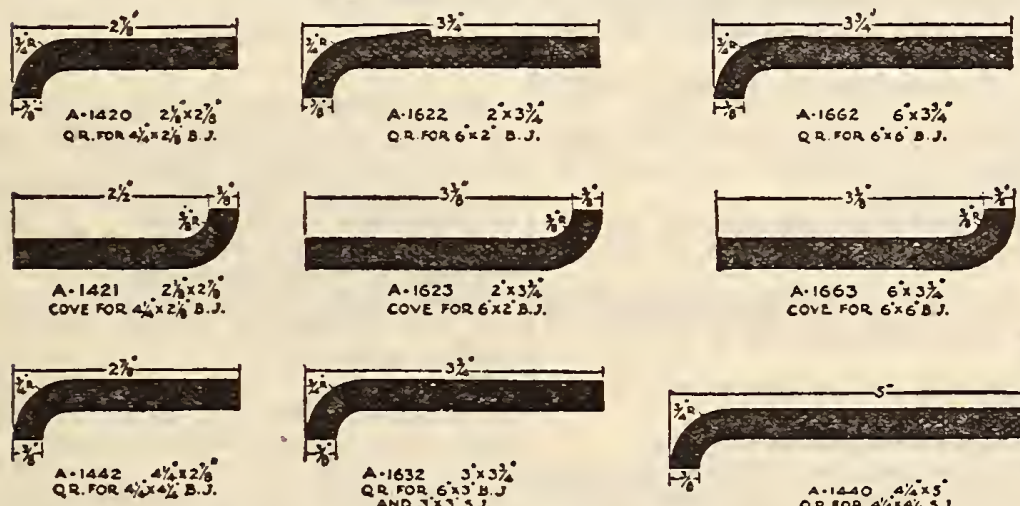

AR FOR $1632 \times 3 \times 33^{\circ}$
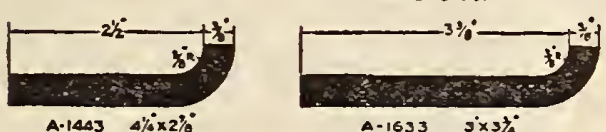

A.14A3 $4 \% \times 2 \%$,

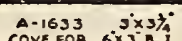

COVE FOR GXYZJ

$6 \%$

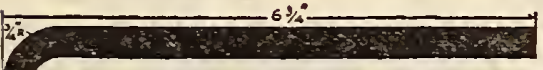

A.1630
C.R.FOR $6 \times 3 \times 3 \times 1 \%$
5.3

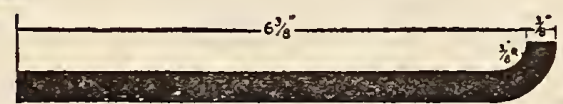

$63 \%$

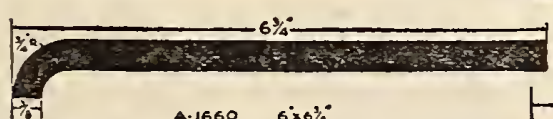

A-1631 $3 \times 6 \%$

A. 1660
RR.FOR $6 \times 6 \times 5 \%$

COVE FOR $6 \times 3^{2} \times 5 \%$

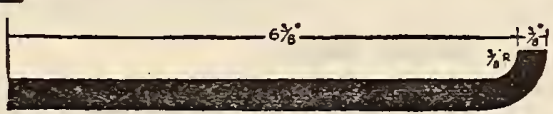

A- 1661
COVE FOR $6^{\circ} \times 6^{\circ} \times 64^{\circ}$
S.J

B. $J=$ Broken Joint

S.J $=$ Straight Joint

Figure 224 
wedging and crooked edge exceeding three-tenths of 1 per cent, and free from specks, spots, blots, pulls, biscuit cracks, biscuit chips, shivered edges, welts, dry spots, scum, sand, or stickers.

Standard grade.-The tiles of this grade are reasonably straight and true in shape and without objectionable surface blemishes. They are free from warpage exceeding four-tenths of 1 per cent, ( $=24 / 1000$ of an inch for a 6 -inch length), wedging or crooked edge exceeding five-tenths of 1 per cent and free from spots, blots, biscuit cracks, biscuit chips, shivered edges, welts, dry spots, scum, or stickers. The grade permits of certain minor surface blemishes such as occasional specks, sand, and pulls.

Commercial grade.-The tiles of this grade permit of all of the blemishes and defects which are not permissible in the higher grades but are free from blots and biscuit cracks.

Grade tolerances.-A tolerance of 5 per cent of standard grade tile in selected and of 5 per cent of commercial grade tile in standard is permissible.

\section{EXPLANATION OF TERMS}

(Used in grade specifications for flat white bright glazed tiles)

Warpage.-A surface curvature, either convex or concave, measured on the face of the tiles along the edges. The degree of warpage is the variation from the plane expressed in percentage of the tile length.

Crooked sides. - A curvature of the sides, either convex or concave, measured along the sides. The degree of crooking is the departure from a straight line drawn between two corners, expressed in percentage of the tile length.

Wedging.-A difference in the lengths of two opposite sides, expressed in percentage of the tile length.

Specks.-Any dark dot on the face less than 1/64 inch in diameter and noticeable at a distance of more than 3 feet.

Spots.-Any dark dot on the face more than 1/64 inch in diameter.

Blots.-Green marks or stains on the face.

Pulls.-Small depressions or scratches in the body noticeable through the glaze at a distance of more than 3 feet.

Biscuit cracks.-Any fracture in the body of the tile visible both on face and back.

Biscuit chips.-A glazed-over chip on the edge or corner of the body.

Shivered edge.-A minute fracture of the glaze running along the edge which appears as a fine silvery thread when struck by light from an angle.

Welts.-An unusually heavy accumulation of glaze in the form of a ridge along the edge.

Dry spots.-Small areas on the face which have been insufficiently glazed.

Scum.-Lack of gloss, crystalline or frosted in effect, appearing on the face of the tile.

Sand.-Grains of sand embedded in the glaze.

Stickers.-Small rough or raised spots in the glaze.

\section{CERAMIC MOSAIC}

Selected grade.-Tiles of this grade are as perfect as it is possible to inanufacture. They are harmonious in color, easily cleaned, uniformly mounted, and of uniform surface and texture. The vitreous colors are fired to vitrification. The semivitreous colors are fired to as high a degree of vitrification as the particular color permits.

Commercial grade. This grade of ceramic mosaic does not represent the same degree of perfection as the selected grade. Variations and irregularities in either textures, surfaces, edges, firing, or combinations of these variations are in evidence. They are, however, satisfactory for some installations.

\section{CERTIFICATION OF GRADES}

The adoption of minimum grade specifications will be greatly facilitated by the use of a uniform method for certification of grades. Based upon definite grade specifications, the certification of grades will be more effective than the present method of certification by grade name without definition.

Certification shall consist of: (a) Package grade certificate and (b) master grade certificate. Minimum grade specification and the grade of tile are to be given on both forms.

The package grade certificate to be in the same distinctive colors for each grade as the "grade seal"; namely, pink for selected grade, blue for standard grade, and yellow for commercial grade.

Grade certificates to be placed on container before closing and affixation of grade seal.

Master grade certificate to be issued to tile contractors on request only, and to be used for certifying shipments consisting of one or more grades.

Both certificates to give adequate information for identification of the containers to which they apply.

534.21 Drainage Tiles. (See also 518.62.)

American Concrete Institute, standard specifications for concrete drain tile, serial designation: P-7B-25, 1925.

\section{GENERAL}

These specifications apply to concrete drain tile consisting of two classes, namely, standard drain tile and extra-quality drain tile.

The purposes for which these classes are intended to be suitable are as follows:

Standard drain tile, for ordinary district land drainage at moderate depths.

Extra-quality drain tile, for district land drainage, at considerable depths and where an extra quality is desired.

The purchaser shall specify the class of tile to be supplied. Standard drain tile shall be supplied where no advance selection is stated.

The acceptability of drain tile shall be determined by the results of the chemical and physical tests hereinafter specified, any or all of which may be required by the purchaser; and by inspection, to determine whether the tile comply with the specifications as to dimensions, shape, and freedom from external and internal defects.

\section{MATERIAL}

Concrete drain tile shall be manufactured from Portland cement concrete. By concrete is meant a suitable mixture of Portland cement, mineral aggregates, and water, hardened by hydraulic chemical action.

Portland cement shall meet the requirements of A. S. T. M. C9. (See 516.11, p. 294.)

The materials shall possess such physical and chemical properties that when molded into tile and properly cured the product will be strong, durable, and serviceable, free from objectionable defects, and in compliance with these specifications and tests.

\section{SIZES AND DIMENSIONS}

All drain tile shall be of circular cross section, except when otherwise specified in advance. They shall be approximately straight, except in the case of special connections. The ends shall be so regular and smooth as readily to admit of making close joints by pressing together adjoining tile.

The sizes of drain tile shall be designated by their internal diameters. 
Drain tile smaller than 12 inches in diameter shall have a minimum length of 12 inches. Tile from 12 to 30 inches in diameter, inclusive, shall have lengths not less than the diameters. Tile larger than 30 inches in diameter shall have a miniruum length of 30 inches.

Permissible variations as given in Table 1 shall in no case be exceeded. as to decrease the strength appreciably. Tile shall not be chipped or broken in such a manner as to decrease their strength materially or to admit earth into the drain.

\section{MARKING}

When shipment of tile is made in any manner other than direct from manufacturer to user, tile

\section{RECORMENHEO METHOOS OF

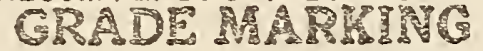

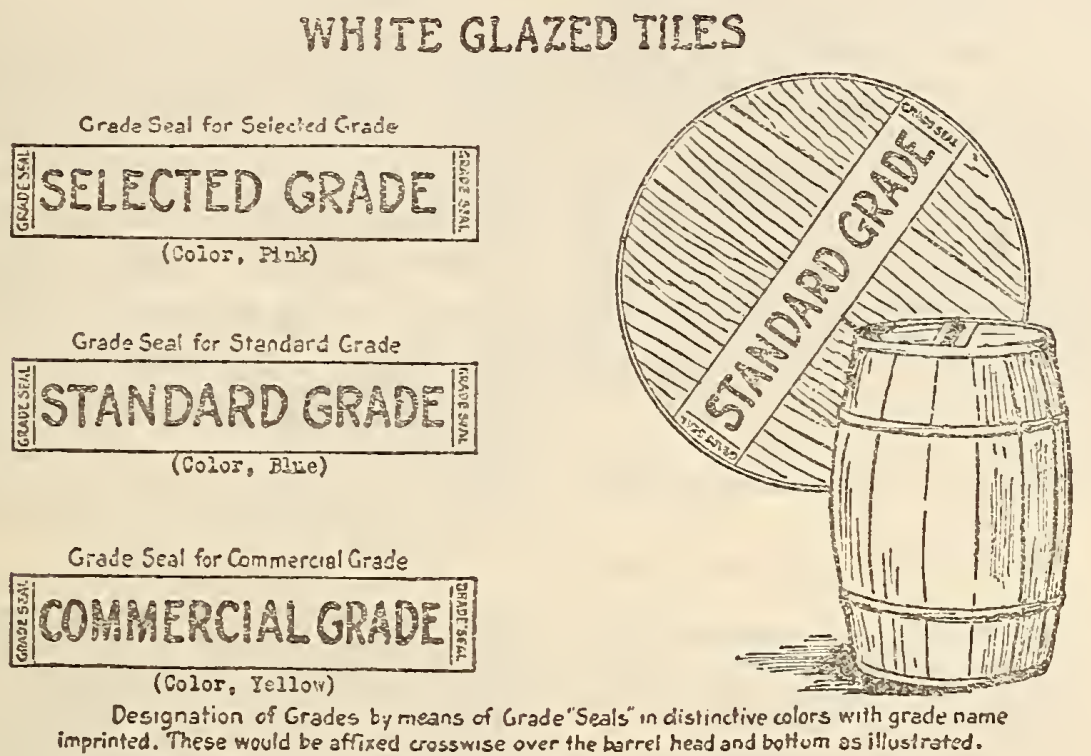

imprinted. These would be affized crosswise over the barrel head and bottum as illustrated.

\section{CERAMIC MOSAIC}
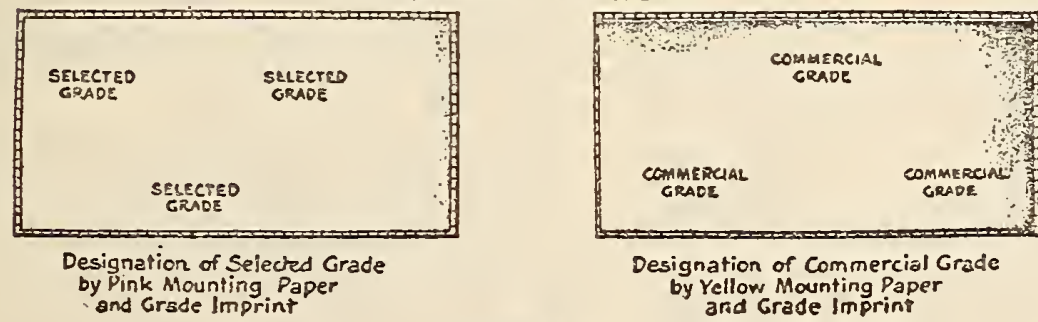

Designation of Commercial Grade by Yellow Mounting Paper

Figure 225

TABLE 1.-Permissible variations in drain tile

\begin{tabular}{|c|c|c|}
\hline Physical properties specified & $\begin{array}{l}\text { Standard } \\
\text { drain tile }\end{array}$ & $\begin{array}{c}\text { Extra- } \\
\text { quality } \\
\text { drain tile }\end{array}$ \\
\hline $\begin{array}{l}\text { Allowable variation of arerage diameter of any } \\
\text { tile above or below specified diameter }\end{array}$ & \multirow[b]{2}{*}{$\begin{array}{l}3 \\
2\end{array}$} & \multirow[b]{2}{*}{$\begin{array}{l}3 \\
2\end{array}$} \\
\hline Allowable variation from straightness & & \\
\hline
\end{tabular}

\section{WORKMAISHIP AND FINISH}

Drain tile shall be substantially uniform in structure throughout, and shall be reasonably smooth on the inside.

Drain tile shall be free from cracks and checks extending into the body of the tile in such a manner shall be so marked that the manufacturer of the tile can be identified.

\section{CHEIICAL TEST AND REQUIREMENTS}

Same as A. S. T. M. C4 (given below), section 6, paragraph 1.

\section{PHYSICAL TESTS}

The physical tests included in this specification are substantially the same as for A. S. T. M. C4-24 (see 534.21, p. 608), omitting sections 15,16 , and 17 and the 2-edge bearing test (sec. 19) of the A. S. T.M. specification.

\section{PHYSICAL TEST REQUIREMENTS}

The average test load for all of the tile constituting a standard sample shall not be less than given 
in Table 2, and no individual tile shall show a strength of less than 75 per cent of such requirement. The average absorption for all the specimens constituting a standard sample shall not be greater than that given in Table 2 , and the average test of an individual tile shall in no case show an absorption of more than 125 per cent of such requirement.

In the freezing and thawing test, at least 95 per cent of all the tile tested shall meet the requirements.

In the event that a standard sample of tile fails to meet the requirements of the absorption test, the manufacturer or other seller may demand recourse to the freezing and thawing test, to be made at his expense. In such recourse, the number of tiles tested shall be four times the number represented by the standard sample. If the material passes the freezing and thawing test satisfactorily, it shall not be rejected on account of its failure to meet the absorption requirements.

In the event of the failure of a standard sample to meet the above requirements, the manufacturer or other seller may thoroughly cull the material and submit a portion for retest at his own expense, and for such retest the number of tiles per sample shall be 10 for the strength and absorption tests and 20 for the freezing and thawing test. In the event of the failures of the material after culling to pass the requirements, it shall be rejected without further test.

TABLE 2.-Physical test requirements, drain tile

\begin{tabular}{|c|c|c|c|c|c|c|}
\hline \multirow{3}{*}{$\begin{array}{l}\text { Internal diam- } \\
\text { eter of tile, } \\
\text { in inches }\end{array}$} & \multicolumn{3}{|c|}{ Standard drain tile } & \multicolumn{3}{|c|}{ Extra-quality drain tile } \\
\hline & \multicolumn{2}{|c|}{ Average load } & \multirow{2}{*}{$\begin{array}{c}\text { A ver- } \\
\text { age ab- } \\
\text { sorp- } \\
\text { tion }\end{array}$} & \multicolumn{2}{|c|}{ A verage load } & \multirow{2}{*}{$\begin{array}{l}\text { Aver- } \\
\text { age ab- } \\
\text { sorp- } \\
\text { tion }\end{array}$} \\
\hline & $\begin{array}{c}\text { Sand } \\
\text { bearing }\end{array}$ & $\begin{array}{l}\text { 3-edge } \\
\text { bearing }\end{array}$ & & $\begin{array}{c}\text { Sand } \\
\text { bearing }\end{array}$ & $\begin{array}{l}\text { 3-edge } \\
\text { bearing }\end{array}$ & \\
\hline $12=$. & $\begin{array}{c}\text { Lbs./ } \\
\text { lin. ft. } \\
1,200 \\
1,200 \\
1,200 \\
1,200 \\
1,200\end{array}$ & \begin{tabular}{|r|} 
Lbs./ \\
lin. ft. \\
800 \\
800 \\
800 \\
800 \\
800
\end{tabular} & \begin{tabular}{|c|} 
Per cent \\
10 \\
10 \\
10 \\
10 \\
10
\end{tabular} & $\begin{array}{r}\text { Lbs./ } \\
\text { lin.ft. } \\
1,600 \\
1,600 \\
1,600 \\
1,600 \\
1,600\end{array}$ & $\begin{array}{c}\text { Lbs./ } \\
\text { lin.ft. } \\
1,067 \\
1,067 \\
1,067 \\
1,067 \\
1,067\end{array}$ & $\begin{array}{r}\text { Per cent } \\
9 \\
9 \\
9 \\
9 \\
9\end{array}$ \\
\hline 20 & $\begin{array}{l}1,200 \\
1,300 \\
1,400 \\
1,500 \\
1,600\end{array}$ & $\begin{array}{r}800 \\
867 \\
933 \\
1,000 \\
1,067\end{array}$ & $\begin{array}{l}10 \\
10 \\
10 \\
10 \\
10\end{array}$ & $\begin{array}{l}1,600 \\
1,600 \\
1,800 \\
2,000 \\
2,200\end{array}$ & $\begin{array}{l}1,067 \\
1,067 \\
1,200 \\
1,333 \\
1,467\end{array}$ & $\begin{array}{l}9 \\
9 \\
9 \\
9 \\
9\end{array}$ \\
\hline $\begin{array}{l}24 . \\
26 . \\
28 \\
30 .\end{array}$ & $\begin{array}{l}1,700 \\
1,800 \\
1,900 \\
2,000 \\
2,100\end{array}$ & $\begin{array}{l}1,133 \\
1,200 \\
1,267 \\
1,333 \\
1,400\end{array}$ & $\begin{array}{l}10 \\
10 \\
10 \\
10 \\
10\end{array}$ & $\begin{array}{l}2,400 \\
2,600 \\
2,800 \\
3,000 \\
3,200\end{array}$ & $\begin{array}{l}1,600 \\
1,733 \\
1,867 \\
2,000 \\
2,133\end{array}$ & $\begin{array}{l}9 \\
9 \\
9 \\
9 \\
9\end{array}$ \\
\hline 40. & $\begin{array}{l}2,200 \\
2,300 \\
2,400 \\
2,500 \\
2,600\end{array}$ & $\begin{array}{l}1,467 \\
1,533 \\
1,600 \\
1,667 \\
1,733\end{array}$ & $\begin{array}{l}10 \\
10 \\
10 \\
10 \\
10\end{array}$ & $\begin{array}{l}3,400 \\
3,600 \\
3,800 \\
4,000 \\
4,200\end{array}$ & $\begin{array}{l}2,267 \\
2,400 \\
2,533 \\
2,667 \\
2,800\end{array}$ & $\begin{array}{l}9 \\
9 \\
0\end{array}$ \\
\hline
\end{tabular}

Note. - When the freezing and thawing test is made as provided herein, the number of freezings and thawings to be endured shal be as follows: For standard drain tile, 36; for extra-quality drain tile, 48 .

\section{INSPECTION}

All tile shall be subject to inspection at the factory, trench, or other point of delivery by a competent inspector employed by the consumer or purchaser. The purposes of the inspection shall be to cull and reject tile which, independent of the physical tests herein specified, fail to meet the requirements of these specifications. ${ }^{12}$

2 When the point of inspection is not designated in the contract or order, it shall be understood as being at the point of manufacture.
Tile shall be subject to rejection on account of any of the following:

(a) Variations in any dimensions exceeding the permissible variations given in Table 1 .

(b) Cracks, fractures, or defects in excess of those allowed in IV, workmanship and finish.

(c) Failure to give a clear ringing sound when a dry tile placed on end is tapped with a light hammer.

All rejected tile shall be plainly marked by the inspector and shall be replaced by the manufacturer or seller with tile which meets the requirements of these specifications, without additional cost to the consumer or purchaser.

The manufacturer or other seller may appeal from decisions of the inspector on questions of strength or structure when such decisions are based on visual inspection alone, in which case the point at issue shall be determined by standard physical tests, the cost of which shall be paid by the appellant, if the inspector was right, or by the purchaser if his inspector was in error.

American Society for Municipal Improvements, standard specifications for sewers, 1927.

(This specification includes requirements for one and two ring segmental tile blocks, vitrified clay ware, vitrified tile lining for inverts and specifications for segmental block sewers.)

See 518.67, page 453 .

American Society for Testing Materials, standard specifications for drain tile, serial designation $\mathrm{C} 4-24$, 1924.

Approved as "American standard," by the American Engineering Standards Committee, now the American Standards Association.

1. (a) These specifications cover three classes of drain tile, namely, farm drain tile, standard drain tile, and extra-quality drain tile.

(b) The purposes for which these classes are intended to be suitable are as follows:

Farm drain tile, for ordinary private drainage work on farms, for moderate sizes and depths.

Standard drain tile, for ordinary district land drainage at moderate depths.

Extra-quality drain tile, for district land drainage, for considerable depths, and where an extra quality is desired.

2 . The purchaser shall specify the class or classes of tile to be supplied, whether farm drain tile, standard drain tile, or extra-quality drain tile. Standard drain tile shall be supplied where no other advance selection is stated.

3. (a) The acceptability of drain tile shall be determined (1) by the results of the chemical and physical tests hereinafter specified, and (2) by visual inspection to determine whether the tiles comply with the specifications as to dimensions, shape, and freedom from visible external and internal defects.

(b) The acceptance of drain tile as satisfactorily meeting one of these two general requirements shall not be construed as in any way waiving the other.

\section{MATERTALS AND MANUFACTURE}

4. (a) These specifications shall apply to drain tile made of shale, fire clays, or surface clays and to drain tile made of concrete.

(b) Shale is a thinly stratified, consolidated sedimentary clay 13 with well-marked cleavage parallel to the bedding.

(c) Fire clay is a sedimentary clay of low fux content.

Note.-It is usually associated with coal measures.

13 The definitions for the terms "surface clay," "fire clay," and "shale" are based upon the following definition for the term "clay"; Clay.-An earthy or stony mineral aggregate consisting essentially of hydrous silicates of alumina, plastic when sufficiently pulverized and wetted, rigid when dry, and ritreous when burned at a sufficiently high temperature. 
(d) Surface clay is an unconsolidated, unstratified clay occurring on the surface.

(e) $\mathrm{By}$ concrete is meant a suitable mixture of Portland cement, mineral aggregates, and water hardened by hydraulic chemical reaction.

$(f)$ If the purchaser desires to exclude any of these materials he shall so specify in advance. All materials used shall be first class of their kind and suitable for the purpose.

5. The method of manufacture shall be such as to insure excellence of product and uniformity in quality.

\section{CHEMICAI TESTS AND REQUIREMENTS}

6. The purchaser may specify special chemical requirements as to resistance of the tile to chemical action in cases where soils or drainage waters have marked acid or alkaline character, or are of abnormally high temperature, and may prescribe chemical tests of the tile to ascertain whether these special requirements are met. Without a special agreement in advance, no drain tile shall be rejected by reason of its composition as determined by ultimate chemical analysis.

The presence in drain tile of visible grains or masses of caustic lime, iron pyrites, or any other minerals which are known to cause slaking or disintegration of the tile, shall be construed as a valid ground for rejection, unless satisfactory proof be submitted that the tiles are permanent and durable, and that the objectionable minerals are not present in quantity or condition to work damage.

\section{PHYSICAL TESTS}

7. The physical tests of drain tile shall include (A) strength tests and (B) absorption test, and may include (C) freezing and thawing tests, when specified by the purchaser in advance or when called for by the manufacturer or other seller as provided in sections $34,35,47$, and 52 .

8. The specimens of tile shall all be selected at the factory or at the shipping destination, or at the trench, at the option of the purchaser. The selection shall be made by a competent inspector employed by the purchaser. The inspector shall divide the tile into subclasses if lack of uniformity in any important particular warrants such division, and shall select enough representative specimens of tile from each subclass for a complete set of standard physical tests.

9. A standard physical test shall comprise tests of five individual tiles. Specimens of tile may be selected by the inspector in such number as he judges necessary to determine fairly the quality of all the tile. The manufacturer or other seller shall furnish specimens of tile without separate charge up to 0.5 per cent of the whole number of tile, and the purchaser shall pay for all in excess of that percentage at the same rate as for other tile.

\section{(A) STRENGTH TESTS OF DRAIN TILE}

10. The specimens of tile shall be unbroken, full-size tile.

11. The walls of the tile shall, at the time of testing, be as thoroughly wet as will result from completely covering with hay, cloth, or similar absorbent material, and keeping the covering wet for not less than 12 hours.

12. No specimen of tile shall be exposed to water or air temperatures lower than $40^{\circ} \mathrm{F}$. from the beginning of wetting until tested. Frozen tile shall be completely thawed before the wetting begins.

13. Each specimen of tile shall be weighed on reliable scales just prior to testing, and the weights shall be reported.
14. Any machine or hand method which will apply the load continuously, or in increments not esceeding 5 per cent of the estimated total breaking load, may be used in making the test. The tile shall not be allowed to stand under load longer than is required for observing and recording the loads. All solid parts of the bearing frames and bearing blocks shall be so rigid that the distribution of the load will not be affected appreciably by the deformation of any part. All bearings and the specimens of tile shall be so accurately centered as to secure a symmetrical distribution of the loading on each side of the center of the tile in every direction.

15. The purchaser shall choose (1) sand bearings, (2) two-edge bearings, or (3) three-edge bearings, for use in making strength tests of drain tile. (See secs. 18,19 , and 20 .)

16. The test results shall be calculated and reported, in pounds per linear foot of tile, in terms of the "ordinary supporting strength." 14

The ordinary supporting strength shall be calculated by multiplying the test breaking loads by the following factors: For sand bearings, 1.00; for 2-edge bearings, 1.50 ; for 3 -edge bearings, 1.50 .

The results of the strength tests shall be reported separately for each of the five individual specimens of tile constituting a standard test, together with the average.

17. The modulus of rupture may or may not be calculated and reported, at the option of the purchaser. When reported it shall be calculated by the equations. ${ }^{15}$

$$
\begin{gathered}
\mathrm{M}=0.20 r \frac{W}{12} \\
f=\frac{6 \mathrm{M}}{t^{2}}
\end{gathered}
$$

where-

$M=$ maximum bending moment in wall in poundinches per inch of length,

$r=$ radius of middle line of tile wall in inches,

$W=$ ordinary supporting strength, calculated as prescribed in section 16, in pounds per linear foot of tile,

$f=$ modulus of rupture in pounds per square inch, and

$t=$ thickness of tile wall in inches.

Five-eighths of the weight of the tile per linear foot for sand bearings, or three-fourths for 2-edge or 3 -edge bearings, shall be added to $W$ in computing the maximum bending moment $M$, when such addition exceeds 5 per cent of $W$. The value of $t$ used shall be the average thickness of the wall at the top of the tile or that at the bottom, selecting the lesser of the two.

18. When sand bearings are used, the ends of each specimen of tile shall be accurately marked in quarters of the circumference prior to the test. (See fig. 116, p. 339.) Specimens shall be carefully bedded, above and below, in sand, for one-fourth the circumference of the tile measured on the middle line of the wall. The depth of bedding above and below the tile at the thinnest points shall be one-half the radius of the middle line of the wall.

The sand used shall be clean, and shall be such as will pass a No. 4 screen.

The top bearing frame shall not be allowed to come in contact with the tile nor with the top bearing plate. The upper surface of the sand in the top bearing shall be struck level with a straight-edge, and shall be covered with a rigid top bearing plate, with lower surface a true plane, made of heavy timbers or other rigid material, capable of distributing the test load

14 The "or dinary supporting strength," when calculated as specified in section 16 , is approximately equal to the actual supporting strength of a tile when laid in a ditch by the "ordinary" method. (See note under Table 2.)

is The coefficient of 0.20 in equation (1) approximates the value found by theoretical analysis and also that determined by extended tests. 
uniformly without appreciable bending. The test load shall be applied at the exact center of this top bearing plate, in such a manner as to permit free motion of the plate in all directions. For this purpose a spherical bearing is preferred, but two rollers at right angles may be used. The test may be made without the use of a testing machine, by piling weights directly on a platform resting on the top bearing plate; provided, however, that the weight shall be piled symmetrically about a vertical line through the centcr of the tilc, and that the platform shall not be allowed to touch the top bearing frame.

The frames of the top and bottom bearings shall be made of timbers so heavy as to avoid appreciable bending by the side pressure of the sand. The interior surfaces of the frames shall be dressed. No frame shall come in contact with the tile during the test. A strip of cloth may, if desired, be attached to the inside of the upper frame on each sidc, along the lower edge, to prevent the escape of sand between the frame and the tile.

19. When 2-edge bearings are used, the ends of each specimen of tile shall be accurately marked in halves of the circumference prior to the test. (See fig. 114, p. 339.)

The ile to be tested shall be supported by a, metallic knife bearing 1 inch wide and extending the length of the tile. Before the tile is placed, a fillet of plaster of Paris and sand 1 inch wide, and thick enough to compensate for all the inequalities of the tile barrel, shall be cast on the surface of the knifeedge bearing. The tile shall be placed upon the fillet while the plaster of Paris is still somewhat plastic. The load shall be applied through an upper knife bearing of the same size and length as the lower bearing. A plaster of Paris fillet 1 inch wide shall be cast along the length of the crown of the tile to equalize the lower bearing before the upper one is brought into contact.

Both of the bearings shall be sufficiently rigid to transmit and receive uniform loads throughout their lengths without deflection, and shall be so attached to the machine as to transmit and receive the maximum scresses produced by the test without lost motion, vibration, or sudden shock.

20. When 3-edge bearings are used the ends of each specimen of tile shall be accurately marked in halves of the circumference prior to the test. (See fig. 115 , p. 339 .)

The lower bearings shall consist of two wooden strips with vertical sides, each strip having its interior top corner rounded to a radius of approximately one-half inch. They shall be straight and shall be securely fastened to a rigid block with their interior vertical sides 1 inch apart.

The upper bearing shall be a wooden block, straight and true from and to end.

The test load shall be applied through the upper bearing block in such a way as to leave the bearing free to move in a vertical plane passing midway between the lower bearings.

In testing a tile which is "out of straight" the lines of the bearings chosen shall be from those which appear to give most favorablc conditions for fair bearings.

\section{(B) ABSORPTION TESTS OF DRAIN 'TLE}

21. Not less than three separaic test specimens from each five separate tiles shall be taken as a "standard sample" for the absorption test. Of the three specimens from each tile, one shall be taken from one end, another from the opposite end, and the third shall be taken from the middle portion of the tile. Each specimen shall be of from 12 to 20 square inches in area, measured upon the exterior or convex side, and shall bo as nearly square as the nature of the material will readily permit. The speimens shall be obtained by breaking the tile and shall be apparently sound, solid pieces of the wall of the tile, and shall not show cracks or fissures or shattered edges due to the shock of breaking or cutting. The specimens may be obtained from the broken pisces of the tiles used in the strength test if the restrictions as to the size and location of the specimens can be duly observed. The specimens shall be so marked as to permit the identity of each one to be ascertained at any stage of the test.

22. Preparatory to the absorption test all specimens shall be first weighed and then dried in a drier or oven at a temperature of not less than $110^{\circ} \mathrm{C}$. $\left(230^{\circ} \mathrm{F}\right.$.) for not less than three hours. After removal from the drier the specimens shall be allowed to cool to a temperature of $20^{\circ}$ to $25^{\circ} \mathrm{C} .\left(68^{\circ}\right.$ to $77^{\circ} \mathrm{F}$.) and reweighed. If the specimens were apparently dry when taken and the second weight closely checks the first the specimens shall be considered dry. If the spccimens were known to be wet when taken they shall be placed in the drier for a further drying treatment of two hours and reweighed. If the third weight checks the second the specimens shall be considered dry. In case of any doubt the specimens must be redried for 2-hour periods until check weights are obtained.

23. The balance used shall be sensitive to $0.5 \mathrm{~g}$ when loaded with $1 \mathrm{~kg}$ and weighings shall be read at least to the nearest gram. Where other than metric weights are used the same order of accuracy must be obtained.

In reweighing after immersion the specimens shall be removed from the water, not allowed to drain for more than one minute, the superficial water removed by towel or blotting paper, and the specimens at once put upon the balance.

24. Specimens after weighing shall be placed in a suitable woven-wire receptacle, packed tightly enough to prevent jostling, covered with distilled water or rain water, raised to the boiling point and boiled for five hours, and then cooled in water to a final temperature of $10^{\circ}$ to $15^{\circ} \mathrm{C}$. $\left(50^{\circ}\right.$ to $59^{\circ} \mathrm{F}$.).

25. The test results shall be calculated as percentages of the initial dry weight, carried to the nearest first decimal place. The results shall be reported separately for each individual specimen, together with the mean of the $\mathbf{1 5}$ or more specimens comprising the standard sample, the maximum and the minimum single observations entering into the mean, and the variation between the maximum and the minimum of the three specimens of each tile represented in the standard sample.

\section{(C) FREEZING AND THAWING TESTS OF DRAIN TILE}

26. The test specimens employed in making the freezing and thawing test shall be another set selected as specified in section 21 .

27. The specimens shall be dried as specified in section 22 .

28. The same scales and weights as are specified in section 23 for the absorption test or others of equivalent sensitiveness and accuracy shall be employed for the weighings required in the freezing and thawing test. The same procedure in weighings and reweighing as specified in section 23 shall be used.

29. The specimens shall be immersed for 72 hours in water having a temperature of $18^{\circ}$ to $24^{\circ}$ C. $\left(65^{\circ}\right.$ to $75^{\circ}$ F.) and then weighed as specified in section 23.

30 . When the specimens (either from the absorption test or from a specially prepared series) have been weighed after saturation with water they shall be returned to the water and kept immersed till the freezing test is begun. For freezing, they shall be placed with their concave faces upward in watertight metal trays, suitably mounted in a rigid metal 
crate, ${ }^{18}$ and immersed in ice water until the specimens have attained substantially the temperature of the water, after which the water shall be drawn down to a depth of one-half inch in each tray. The crate shall then be lifted as a whole, without disturbing the specimens, and placed in the freezing apparatus.

Freezing shall be performed in a quiet atmosphere, free from perceptible natural or artificial until the water in the trays is frozen solid. Exposure to freezing conditions in excess of this requirement shall be considered as without significance.

At the conclusion of freezing under the specified conditions the crate of specimens shall be withdirawn and at once immersed in water at a temperature of $18^{\circ}$ to $24^{\circ} \mathrm{C}$. $\left(65^{\circ}\right.$ to $75^{\circ} \mathrm{F}$.) in a special receptacle of proper size. Heating shall be continued so that the water will regain the required

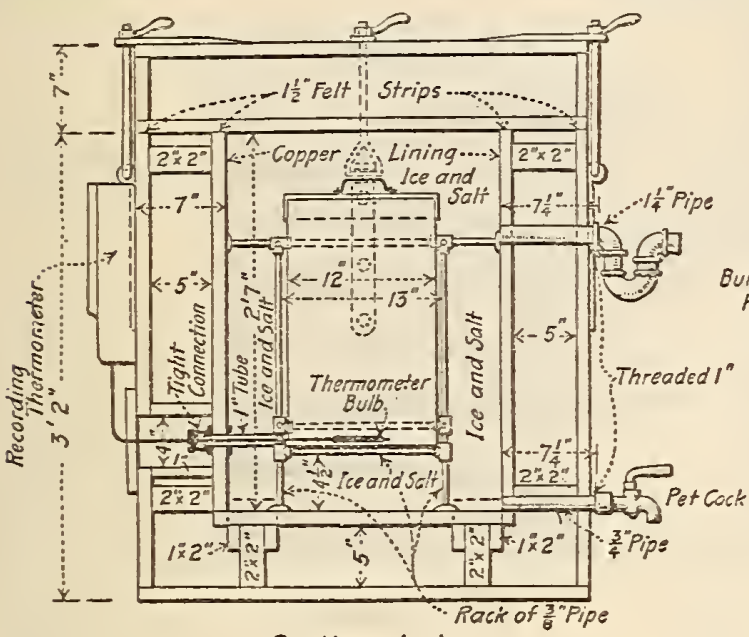

Section $A-A$.

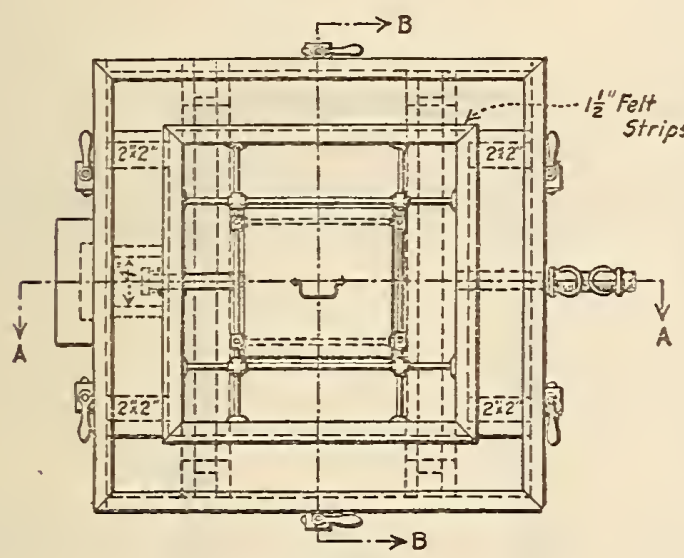

Plan View,

Top Removed.

Note: Box to be Constructed of Seasoned White Pire, free from Dafacts, or other Suitable Timber, $1 " x 6$; inless otherwise Specified.

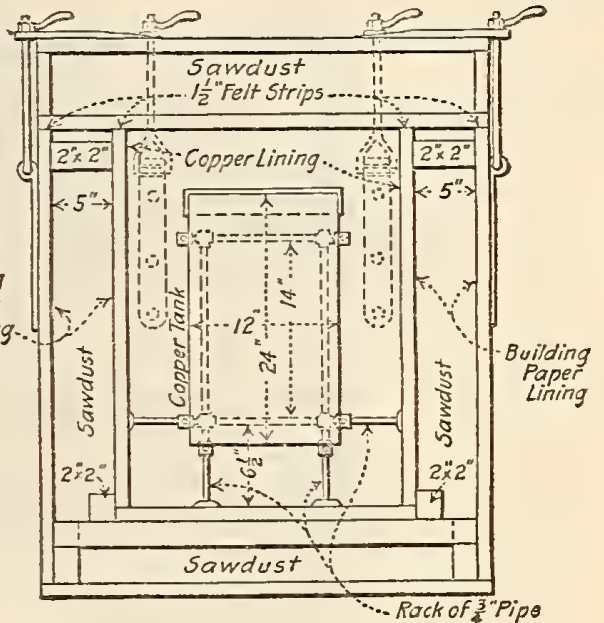

Section $B-B$

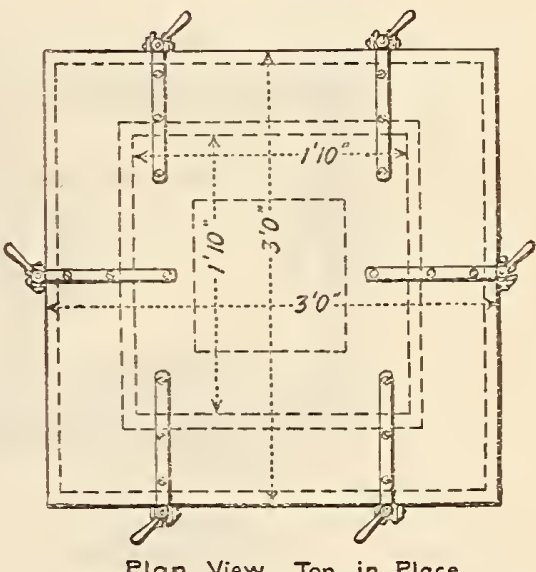

SUGGESTED PLANS

FOR

FREEZING BOX

USING SALT AND ICE TO FREEZE

FigUre 226

currents. If artificial freezing apparatus is em-l temperature as soon as practicable after the speciployed ${ }^{17}$ the apparatus shall have sufficient heatabsorbent capacity to enable the temperature of the freezing chamber to be brought to $-10^{\circ} \mathrm{C}$. $\left(14^{\circ} \mathrm{F}\right.$ ) or below within 30 minutes after the introduction of the specimens. The temperature in the freezing apparatus shall not fall lower than $-20^{\circ} \mathrm{C}$. $\left(-4^{\circ} \mathrm{F}\right.$.). The freezing shall be continued

13 Fig. 225 shows a crate and trays suitable for use in the box for artificial freezing illustrated in fig. 224.

17 Artificial freezing will generally be necessary. It may be conducted in a commercial zero (F.) refrigerating room or in an artificial freezing box similar to the one shown in Figure 226 , in which zero
(F.) temperatures can readily be produced by the use of salt and ice. mens are immersed. A temperature of $18^{\circ}$ to $24^{\circ}$ C. $\left(65^{\circ}\right.$ to $75^{\circ} \mathrm{F}$.) shail then be maintained for not less than two hours. At the conclusion of the thawing treatment the crate of specimens shall be inspected and the condition of each sample after each thawing shall be noted in the records.

31. Failure under the freezing and thawing treatment shall be considered to be reached when (a) The specimens show superficial disintegration or spalling with loss of weight of more than 5 per cent of the initial dry weight, or $(b)$ the specimens are badly cracked in other than lamination planes; 
or $(c)$ the specimens show evident serious loss of structural strength.

\section{PHYSICAL-TEST REQUIREMENTS}

32. The physical-test requirements for the diferent classes of drain tile shall be as given in Table 1.

33. Drain tile made of mixtures of surface clays with other clays shall conform to the absorption number represented by the standard sample (sec. 21 . If the material passes the freezing and thawing test satisfactorily it shall not be rejected on account of its failure to meet the absorption requirements specified in Table 1 , but the average percentage of absorption of the specimens used in the freezing and thawing test shall be adopted as the maximum allowable mean absorption for the contract in question.

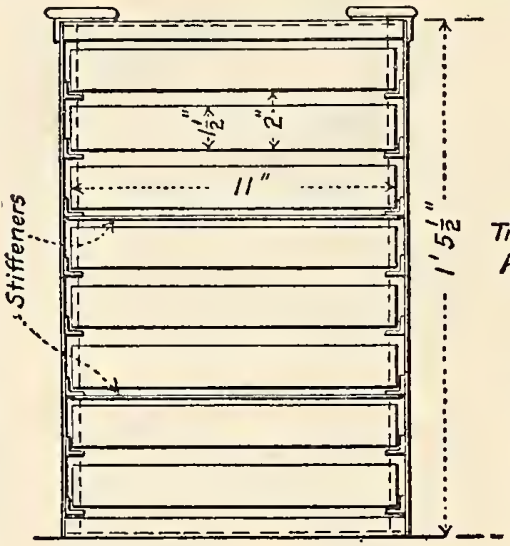

Front Elevation.

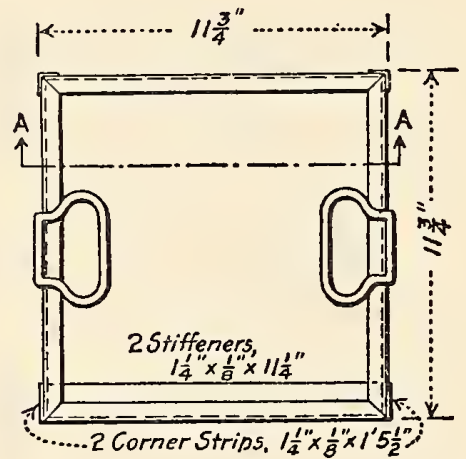

Top View, Trays Removed.

Notes:

Rack Constructed of $\frac{5^{\prime \prime}}{8} \times \frac{5^{\prime \prime}}{8} \times \frac{1}{8}$ Galvanized Angles, except as Noted. All Connections Riveted or Soldered.

Trays, $\|\ddot{x}\| " x / \frac{1}{2} "$ outside, Made of No. 17 Galvanized Steel.

Figure 227.-Suggested plans for freezing crate and trays

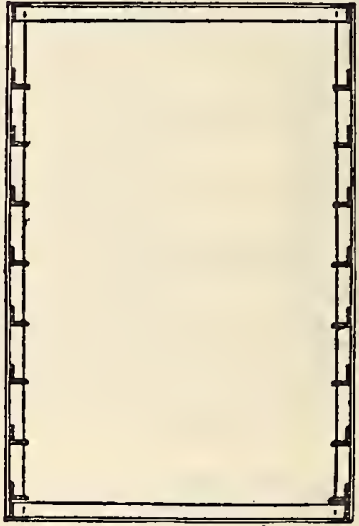

Section A-A, Trays Removed. requirements for surface-clay tile in Table 1, when the proportion of surface clay is 75 per cent or more and to the requirements for shale and fire-clay tile for all other proportions.

34. In the event that a standard sample (sec. 21) of tile fails to meet the requirements of the absorption test the manufacturer or other seller may demand recourse to the freezing and thawing test, to be made at his expense. In such recourse the number of tiles tested shall be four times the
35. In the strength tests individual tiles of a standard test whose mean strength is satisfactory may fall 25 per cent below the requirement for the average without causing rejection. In the absorption test the absorption of individual tiles of a standard sample (sec. 21) which gives a satisfactory mean absorption percentage may exceed the average by 25 per cent without causing rejection. In the freezing and thawing test at least 95 per cent of all the tiles tested shall meet the requirement. 
In the event of the failure of a standard sample (secs. 9, 21, and 26) to meet the above requirements the manufacturer or other seller may thoroughly cull the material and submit a portion for retest at his own expense, and for such retest the number of tiles per sample shall be 10 for the strength and absorption tests and 20 for the freezing and thawing test. In the event of the failure of the material after culling to pass the requirements it shall be rejected without further test.

\section{TABLE 1.-Physical-test requirements for different classes of drain tile}

[Caution.-Drain tile meeting these requirements are not necessarily safe against cracking in ditches. For safe requirements as against cracking see Table 2]

\begin{tabular}{|c|c|c|c|c|c|c|c|c|c|c|c|c|}
\hline \multirow{3}{*}{$\begin{array}{c}\text { Internal diameter of tile in } \\
\text { inches }\end{array}$} & \multicolumn{4}{|c|}{ Farm-drain tile } & \multicolumn{4}{|c|}{ Standard drain tile } & \multicolumn{4}{|c|}{ Extra-quality drain tile } \\
\hline & \multirow{2}{*}{$\begin{array}{l}\text { Mini- } \\
\text { mum } \\
\text { average } \\
\text { ordinary } \\
\text { support- } \\
\text { ing } \\
\text { strength }\end{array}$} & \multicolumn{3}{|c|}{$\begin{array}{l}\text { Marimum average ab- } \\
\text { sorption by standard } \\
\text { boiling test }\end{array}$} & \multirow{2}{*}{$\begin{array}{l}\text { Mini- } \\
\text { mum } \\
\text { average } \\
\text { ordinary } \\
\text { support- } \\
\text { ing } \\
\text { strength }\end{array}$} & \multicolumn{3}{|c|}{$\begin{array}{l}\text { Maximum arerage ab- } \\
\text { sorption by standard } \\
\text { boiling test }\end{array}$} & \multirow{2}{*}{$\begin{array}{l}\text { Mini- } \\
\text { mum } \\
\text { average } \\
\text { ordinary } \\
\text { support- } \\
\text { ing } \\
\text { strength }\end{array}$} & \multicolumn{3}{|c|}{$\begin{array}{l}\text { Maximum average ab- } \\
\text { sorption by standard } \\
\text { boiling test }\end{array}$} \\
\hline & & $\begin{array}{l}\text { Shale } \\
\text { and } \\
\text { fire-clay } \\
\text { tile }\end{array}$ & $\begin{array}{l}\text { Surface- } \\
\text { clay tile }\end{array}$ & $\begin{array}{l}\text { Con- } \\
\text { crete } \\
\text { tile }\end{array}$ & & $\begin{array}{l}\text { Shale } \\
\text { and } \\
\text { fire-clay } \\
\text { tile }\end{array}$ & $\begin{array}{l}\text { Surface- } \\
\text { clay tile }\end{array}$ & $\begin{array}{l}\text { Con- } \\
\text { crete } \\
\text { tile }\end{array}$ & & $\begin{array}{c}\text { Shale } \\
\text { and } \\
\text { fire-clay } \\
\text { tile }\end{array}$ & $\begin{array}{l}\text { Surface- } \\
\text { clay tile }\end{array}$ & $\begin{array}{l}\text { Con- } \\
\text { crete } \\
\text { tile }\end{array}$ \\
\hline $\begin{array}{l}4 \\
6 \\
8\end{array}$ & $\begin{array}{l}\text { Lbs. per } \\
\text { lin. ft. } \\
800 \\
800 \\
800 \\
800\end{array}$ & $\begin{array}{r}\text { Per cent } \\
11 \\
11 \\
11 \\
11\end{array}$ & $\begin{array}{r}\text { Per cent } \\
14 \\
14 \\
14 \\
14\end{array}$ & $\begin{array}{r}\text { Per cent } \\
12 \\
12 \\
12 \\
12\end{array}$ & $\begin{array}{r}\text { Lbs. per } \\
\text { lin. }, t . \\
1,200 \\
1,200 \\
1,200 \\
1,200\end{array}$ & $\begin{array}{r}\text { Per cent } \\
9 \\
9 \\
9 \\
9\end{array}$ & $\begin{array}{r}\text { Per cent } \\
13 \\
13 \\
13 \\
13\end{array}$ & $\begin{array}{r}\text { Per cent } \\
10 \\
10 \\
10 \\
10\end{array}$ & $\begin{array}{r}\text { Lbs. per } \\
\text { lin. ft. } \\
\text { I, } 600 \\
1,600 \\
1,600 \\
1,600\end{array}$ & $\begin{array}{r}\text { Per cent } \\
7 \\
7 \\
7 \\
7\end{array}$ & $\mid \begin{array}{r}\text { Per cent } \\
11 \\
11 \\
11 \\
11\end{array}$ & $\begin{array}{r}\text { Per cent } \\
9 \\
9 \\
9 \\
9\end{array}$ \\
\hline $\begin{array}{l}10 \\
12 \\
15 \\
18\end{array}$ & $\begin{array}{r}800 \\
800 \\
1,000\end{array}$ & $\begin{array}{l}11 \\
11 \\
11\end{array}$ & $\begin{array}{l}14 \\
14 \\
14\end{array}$ & $\begin{array}{l}12 \\
12 \\
12\end{array}$ & $\begin{array}{l}1,200 \\
1,200 \\
1,300 \\
1,400\end{array}$ & $\begin{array}{l}9 \\
9 \\
9 \\
9\end{array}$ & $\begin{array}{l}13 \\
13 \\
13 \\
13\end{array}$ & $\begin{array}{l}10 \\
10 \\
10 \\
10\end{array}$ & $\begin{array}{l}1,600 \\
1,600 \\
1,600 \\
1,800\end{array}$ & $\begin{array}{l}7 \\
7 \\
7 \\
7\end{array}$ & $\begin{array}{l}11 \\
11 \\
11 \\
11\end{array}$ & $\begin{array}{l}9 \\
9 \\
9 \\
9\end{array}$ \\
\hline $\begin{array}{l}21 \\
24 \\
27 \\
30\end{array}$ & & Not per & rmitted & & $\begin{array}{l}1,550 \\
1,700 \\
1,850 \\
2,000\end{array}$ & $\begin{array}{l}9 \\
9 \\
9 \\
9\end{array}$ & $\begin{array}{l}13 \\
13 \\
13 \\
13\end{array}$ & $\begin{array}{l}10 \\
10 \\
10 \\
10\end{array}$ & $\begin{array}{l}2,100 \\
2,400 \\
2,700 \\
3,000\end{array}$ & $\begin{array}{l}7 \\
7 \\
7 \\
7\end{array}$ & $\begin{array}{l}11 \\
11 \\
11 \\
11\end{array}$ & $\begin{array}{l}9 \\
9 \\
9 \\
9\end{array}$ \\
\hline 33. & & & & & $\begin{array}{l}2,150 \\
2,300 \\
2,450 \\
2,600\end{array}$ & $\begin{array}{l}9 \\
9 \\
9 \\
9\end{array}$ & $\begin{array}{l}13 \\
13 \\
13 \\
13\end{array}$ & $\begin{array}{l}10 \\
10 \\
10 \\
10\end{array}$ & $\begin{array}{l}3,300 \\
3,600 \\
3,900 \\
4,200\end{array}$ & $\begin{array}{l}7 \\
7 \\
7 \\
7\end{array}$ & $\begin{array}{l}11 \\
11 \\
11 \\
11\end{array}$ & $\begin{array}{l}9 \\
9 \\
9 \\
9\end{array}$ \\
\hline
\end{tabular}

1 Larger sizes recommended as standard are as follows: 45, 48, 54, and 60 inches.

NoTE. - When the freezing and thawing test is made as provided in section 7 , the number of freezings and thawings to be endured shall be as follows: For farm-drain tile, 24; for standard drain tile, 36; for extra-quality drain tile, 48.

TABLE 2.-Standard ordinary supporting strengths of drain tile for ordinary sand and for thoroughly wet clay ditch filling materials

[Strengths in pounds per linear foot]

\begin{tabular}{|c|c|c|c|c|c|c|c|c|c|c|c|c|c|c|c|c|c|c|c|c|}
\hline \multirow{6}{*}{$\begin{array}{c}\text { Height of fill } \\
\text { above top of } \\
\text { tile, in feet }\end{array}$} & \multicolumn{20}{|c|}{ Breadth of ditch at top of tile } \\
\hline & \multicolumn{4}{|c|}{1 foot } & \multicolumn{4}{|c|}{2 feet } & \multicolumn{4}{|c|}{3 feet } & \multicolumn{4}{|c|}{4 feet } & \multicolumn{4}{|c|}{5 feet } \\
\hline & \multicolumn{20}{|c|}{ Method of laying pipe } \\
\hline & \multicolumn{2}{|c|}{ Ordinary } & \multicolumn{2}{|c|}{ First class } & \multicolumn{2}{|c|}{ Ordinary } & \multicolumn{2}{|c|}{ First class } & \multicolumn{2}{|c|}{ Ordinary } & \multicolumn{2}{|c|}{ First class } & \multicolumn{2}{|c|}{ Ordinary } & \multicolumn{2}{|c|}{ First class } & \multicolumn{2}{|c|}{ Ordinary } & \multicolumn{2}{|c|}{ First class } \\
\hline & \multicolumn{20}{|c|}{ Ditch-filling material } \\
\hline & Sand & $\mathrm{Clas}$ & Sand & Clay & Sand & Clay & Sand & Clay & Sand & Clay & Sand & Clay & 'Sand & Clay & Sand & Clay & Sand & Clay & Sand & Clay \\
\hline & $\begin{array}{l}265 \\
400 \\
470 \\
505\end{array}$ & $\begin{array}{l}280 \\
450 \\
545 \\
605\end{array}$ & $\begin{array}{l}220 \\
335 \\
390 \\
420\end{array}$ & $\begin{array}{l}235 \\
375 \\
455 \\
505\end{array}$ & $\begin{array}{r}615 \\
1,050 \\
1,370 \\
1,600\end{array}$ & $\begin{array}{r}635 \\
1,120 \\
1,500 \\
1,790\end{array}$ & $\begin{array}{r}510 \\
880 \\
1,140 \\
1,330\end{array}$ & $\begin{array}{r}530 \\
935 \\
1,250 \\
1,490\end{array}$ & $\begin{array}{r}970 \\
1,750 \\
2,370 \\
2,870\end{array}$ & $\begin{array}{r}990 \\
1,820 \\
2,530 \\
3,110\end{array}$ & $\begin{array}{r}810 \\
1,460 \\
1,980 \\
2,390\end{array}$ & $\begin{array}{r}830 \\
1,520 \\
2,110 \\
2,590\end{array}$ & $\begin{array}{l}1,330 \\
2,450 \\
3,410 \\
4,220\end{array}$ & $\begin{array}{l}1,350 \\
2,540 \\
3,580 \\
4,490\end{array}$ & $\begin{array}{l}1,110 \\
2,040 \\
2,840 \\
3,510\end{array}$ & $\begin{array}{l}1,130 \\
2,110 \\
2,980 \\
3,740\end{array}$ & $\begin{array}{l}1,690 \\
3,160 \\
4,460 \\
5,590\end{array}$ & $\begin{array}{l}1,710 \\
3,250 \\
4,640 \\
5,890\end{array}$ & $\begin{array}{l}1,410 \\
2,640 \\
3,720 \\
4,660\end{array}$ & $\begin{array}{l}1,430 \\
2,710 \\
3,870 \\
4,910\end{array}$ \\
\hline $\begin{array}{l}10 \ldots \\
12 \ldots \\
14 \ldots \\
16 \ldots\end{array}$ & $\begin{array}{l}525 \\
535 \\
540 \\
545\end{array}$ & $\begin{array}{l}640 \\
660 \\
675 \\
680\end{array}$ & $\begin{array}{l}440 \\
445 \\
450 \\
455\end{array}$ & $\begin{array}{l}535 \\
550 \\
560 \\
565\end{array}$ & $\begin{array}{l}1,760 \\
1,850 \\
1,960 \\
2,030\end{array}$ & $\begin{array}{l}2,010 \\
2,190 \\
2,320 \\
2,420\end{array}$ & $\begin{array}{l}1,470 \\
1,570 \\
1,640 \\
1,690\end{array}$ & $\begin{array}{l}1,680 \\
1,820 \\
1,930 \\
2,020\end{array}$ & $\begin{array}{l}3,270 \\
3,600 \\
3,850 \\
4,060\end{array}$ & $\begin{array}{l}3,610 \\
4,030 \\
4,380 \\
4,670\end{array}$ & $\begin{array}{l}2,730 \\
3,000 \\
3,210 \\
3,390\end{array}$ & $\begin{array}{l}3,010 \\
3,355 \\
3,650 \\
3,890\end{array}$ & $\begin{array}{l}4,900 \\
5,480 \\
5,980 \\
6,400\end{array}$ & $\begin{array}{l}5,290 \\
6,000 \\
6,620 \\
7,160\end{array}$ & $\begin{array}{l}4,080 \\
4,570 \\
4,980 \\
5,330\end{array}$ & $\begin{array}{l}4,410 \\
5,000 \\
5,520 \\
5,970\end{array}$ & $\begin{array}{l}6,590 \\
7,460 \\
8,230 \\
8,890\end{array}$ & $\begin{array}{l}7.020 \\
8,030 \\
8,950 \\
9,770\end{array}$ & $\begin{array}{l}5,490 \\
6,220 \\
6,850 \\
7,410\end{array}$ & $\begin{array}{l}5,850 \\
6,690 \\
7,460 \\
8,150\end{array}$ \\
\hline $\begin{array}{l}18 \\
20 \\
22 \\
24\end{array}$ & $\begin{array}{l}545 \\
545 \\
545 \\
545\end{array}$ & $\begin{array}{l}685 \\
690 \\
690 \\
690\end{array}$ & $\begin{array}{l}455 \\
455 \\
455 \\
455\end{array}$ & $\begin{array}{l}570 \\
575 \\
575 \\
575\end{array}$ & $\begin{array}{l}2,070 \\
2,100 \\
2,120 \\
2,140\end{array}$ & $\begin{array}{l}2,500 \\
2,560 \\
2,610 \\
2,650\end{array}$ & $\begin{array}{l}1,720 \\
1,750 \\
1,770 \\
1,780\end{array}$ & $\begin{array}{l}2,090 \\
2,140 \\
2,180 \\
2,210\end{array}$ & $\begin{array}{l}4,230 \\
4,370 \\
4,470 \\
4,560\end{array}$ & $\begin{array}{l}4,920 \\
5,130 \\
5,310 \\
5,450\end{array}$ & $\begin{array}{l}3,530 \\
3,640 \\
3,730 \\
3,800\end{array}$ & $\begin{array}{l}4,100 \\
4,280 \\
4,420 \\
4,540\end{array}$ & $\begin{array}{l}6,750 \\
7,050 \\
7,310 \\
7,520\end{array}$ & $\begin{array}{l}7,630 \\
8,060 \\
8,430 \\
8,750\end{array}$ & $\begin{array}{l}5,630 \\
5,880 \\
6,090 \\
6,270\end{array}$ & $\begin{array}{l}6,360 \\
6,720 \\
7,020 \\
7,290\end{array}$ & $\begin{array}{r}9,4801 \\
9,9901 \\
10,4001 \\
10,8001\end{array}$ & $\begin{array}{l}10,520 \\
11,200 \\
11,800 \\
12,300\end{array}$ & $\begin{array}{l}7,900 \\
8,33 \\
8,700 \\
9,030\end{array}$ & $\begin{array}{r}8,770 \\
9,330 \\
9,830 \\
10,300\end{array}$ \\
\hline $\begin{array}{l}26 \\
28 \\
30 \\
\text { Very gre }\end{array}$ & $\begin{array}{l}545 \\
545 \\
545 \\
545\end{array}$ & $\begin{array}{l}690 \\
690 \\
690 \\
690\end{array}$ & $\begin{array}{l}455 \\
455 \\
455 \\
455\end{array}$ & $\begin{array}{l}575 \\
575 \\
575 \\
575\end{array}$ & $\begin{array}{l}2,150 \\
2,160 \\
2,170 \\
2,180\end{array}$ & $\begin{array}{l}2,670 \\
2,700 \\
2,710 \\
2,770\end{array}$ & $\begin{array}{l}1,790 \\
1,800 \\
1,810 \\
1,820\end{array}$ & $\begin{array}{l}2,230 \\
2,250 \\
2,260 \\
2,310\end{array}$ & $\begin{array}{l}4,630 \\
4,680 \\
4,730 \\
4,910\end{array}$ & $\begin{array}{l}5,580 \\
5,680 \\
5,770 \\
6,230\end{array}$ & $\begin{array}{l}3,850 \\
3,900 \\
3,940 \\
4,090\end{array}$ & $\begin{array}{l}4,640 \\
4,740 \\
4,810 \\
5,190\end{array}$ & $\begin{array}{l}7,700 \\
7,860 \\
7,990 \\
8,730\end{array}$ & $\begin{array}{r}9,030 \\
9,280 \\
9,500 \\
11,100\end{array}$ & $\begin{array}{l}6,420 \\
6,550 \\
6,660 \\
7,270\end{array}$ & $\begin{array}{l}7,530 \\
7,740 \\
7,920 \\
9,230\end{array}$ & $\begin{array}{l}11,200 \\
11,500 \\
11,800 \\
13,600\end{array}$ & $\begin{array}{l}12,800 \\
13,300 \\
13,700 \\
17,300\end{array}$ & $\begin{array}{r}9,320 \\
9,570 \\
9,800 \\
11,400\end{array}$ & $\begin{array}{l}10,700 \\
11,100 \\
11,400 \\
14,400\end{array}$ \\
\hline
\end{tabular}

Note.-Ordinary pipe laying is pipe laying in accordance with customary good practice in tile-drain construction whereby the under

side of the pipe is well bedded on soil for $60^{\circ}$ to $90^{\circ}$ of the circumference.
First-class pipe laying is pipe laying in accordance with the best customary practice in pipe-sewer construction whereby the entire under side of the pipe is very thoroughly bedded on soil and the entire pipe is surrounded by well-compacted soil under the direction of an inspector constantly present on the work.

When pipe is laid in a concrete or other permanent masonry cradle strong enough to carry the entire load to the subbase without breaking and large enough to prevent material settlement the standard strengths for all dimensions of ditches and all filling materials shall be those specified for standard drain tile in Table 1. 
36. The manufacturer or other seller shall not be held responsible for cracking of drain tile in ditches unless by special agreement in advance, and in any event his obligation shall be held to be discharged by the delivery of drain tile having the minimum ordinary supporting strengths specified in Table 2 ; and, if it is not otherwise specified in advance by the purchaser, tile shall be supplied of the strengths specified for clay ditch filling, for "ordinary" pipe laying, and for widths of ditch at the level of the top of the tile equal to 0.5 foot greater than the outside diameters of the tile. The purchaser shall furnish to the manufacturer or other seller complete information, in advance of receiving bids, as to the number of linear feet of drain tile of each diameter required for each different depth of ditch, measured to the nearest foot from the surface of the ground to the top of the tile.

\section{VISUAJ INSPECTION}

37. All drain tile shall be given a thorough visual inspection at the trench by a competent inspector employed by the purchaser. The purposes of the visual inspection shall be (1) to cull and reject imperfect individual tiles, and (2) to determine whether the tiles, independently of meeting the chemical and the physical test requirements, comply with the spccifications of general properties, especially as stated hereinafter.

38. All drain tile shall be of approximately circular cross section, except when otherwise specified in advance. They shall be approximately straight, except in the case of special connections. The ends shall be so regular and smooth as readily to admit of making close joints by turning and pressing together adjoining tile.

39. The sizes of drain tile shall be designated by their interior diameters.

40. Drain tile smaller than 12 inches in diameter shall have a minimum length of 12 inches. Tile of from 12 to 30 inches in diameter, inclusive, shall have lengths not less than the diameters. Tile larger than 30 inches in diameter shall have a minimum length of 30 inches.

41. Drain tile shall be substantially uniform in structure throughout, and the inspector shall investigate this property by examining fractured surfaces.

42. Drain tile shall give a clear ring when stood on end and while dry tapped with a light hammer.

43. The inspector may use the color of drain tilc as a general guide in sorting and inspecting, but he shall first so familiarize himself with the raw materials and the processes used in the manufacture of the particular tile in question as to be competent to interpret the true meaning of variations in their color.

44. Drain tile shall be reasonably smooth on the inside.

45. Drain tile shall be free from cracks and checks extending into the body of the tile in such a manner as to decrease the strength appreciably. Tile shall not be chipped or broken in such a manner as to decrease their strength materially or to admit earth into the drain.

46. All drain tile shall be sufinciently "vitrified" or "hard burned" to afford the degree of supporting strength, percentage of absorption, and resistance to freezing and thawing specified in the physical-test requirements prescribed in Table 1 .

47. The manufacturer or other seller may appeal from decisions of the inspector on questions of strength or structure when such decisions are based on visual inspection alone, in which case the point at issue shall be determined by standard physical tests, the cost of which shall be paid by the appel- lant if the inspector was right or by the purchaser if his inspector was in error.

TABLE 3.-Distinctive general physical properties of different classes of drain tile

\begin{tabular}{|c|c|c|c|}
\hline Physical properties specified & $\begin{array}{l}\text { Farm } \\
\text { drain } \\
\text { tile }\end{array}$ & $\begin{array}{l}\text { Stand- } \\
\text { ard } \\
\text { drain } \\
\text { tile }\end{array}$ & $\begin{array}{l}\text { Extra- } \\
\text { quality } \\
\text { drain } \\
\text { tile }\end{array}$ \\
\hline $\begin{array}{l}\text { Allowable variation of average diameter } \\
\text { below specified diameter....-per cent } \\
\text { Allowable variation between maximum and } \\
\text { minimum diameters of same tile, or aver- } \\
\text { age diameters of adjoining tile }\end{array}$ & 5 & 3 & 3 \\
\hline Allowable variation from straightness & 85 & 75 & 65 \\
\hline $\begin{array}{l}\text { Allowable thickness of exterior blisters, } \\
\text { lumps and flakes which do not weaken tile } \\
\text { and are few in number }\end{array}$ & 5 & 3 & 3 \\
\hline $\begin{array}{l}\text { Allowable diameters of above blisters, } \\
\text { lumps and flakes }\end{array}$ & 25 & 20 & 15 \\
\hline General inspection & $(1)$ & $\left({ }^{2}\right)^{15}$ & (3) 10 \\
\hline
\end{tabular}

1 Careful. 2 Rigid. Very rigid.

48. Drain tile of the different classes shall, in addition to all requirements heretofore specified, have the distinctive physical characteristic prescribed in Table III.

\section{TESTING, INSPECTION, AND REJECTION}

49. All tests shall be made by experts employed by the purchaser. Full reports of all tests shall be furnished the manufacturer or other seller on his request. Tests shall be made and reported promptly.

50. The purchaser shall pay the expense of making all tests except as otherwise specified in sections $9,34,35,47$, and 52 .

51. The number of standard tests to be made shall be determined by the purchaser.

52. In all contracts for 10 or more carloads of tile preliminary general tests and inspection shall be made at the factory by the purchaser upon demand of the manufacturer or other seller. The expense of such tests and inspection shall be paid by the manufacturer or other seller.

53. The inspector shall be employed by the purchaser.

54. The manufacturer or other seller of the drain tile shall afford the inspeetor all reasonable facilities for his work, both as to the selection of specimens for test and as to visual inspection. Inspection shall be completed promptly.

55. The inspector shall plainly mark all drain tile which he rejects, and such rejected tile shall be removed promptly by the manufacturer or other seller. Upon the request of the purchaser, the manvfacturer or other seller shall givefull account of the removal of rejected tile.

\subsection{Hollow Building Tile.}

American Railway Engineering Association, specifications for builaings for railway purposes.

\section{CLAY HOLLOW TILE, 1926}

\section{GENERAI}

The contractor shall furnish all labor, materials, tools, scaffolding, and equipment, except as otherwise noted, necessary to entirely complete any or all classes of clay hollow-tile work herein specified, according to the class of building and as shown or implied on the drawings. 
The contractor shall provide and erect all hollowtile exterior and interior bearing walls of hard-burned hollow tile, true and regular in size.

Tile to which plastering is to be applied shall have all faces scored with special dovetail scoring to furnish a good bond.

All subdividing, nonbearing partitions where shown on plans shall be of hollow partition tile.

\section{HOLLOW-TLE MATERIAL}

All hollow building tile shall be straight uniform, free from objectionable cracks, and burned to such a degree of hardness that it will pass the following requirements:

(a) Standard load-bearing tile shall have an absorption not to exceed 12 per cent and be capable of sustaining a load of at least $700 \mathrm{lbs}$./in. ${ }^{2}$ of gross area when designed to be laid with the cells horizontal and tested in that position, and $1,200 \mathrm{lbs} . /$ in. $^{2}$ of gross area when designed to be laid with the cells vertical and when tested in that position.

(b) Finished face tile shall have an absorption not to exceed 10 per cent and be capable of sustaining a load of at least $700 \mathrm{lbs} /$ in. $^{2}$ of gross area when designed to be laid with the cells horizontal and when tested in that position and 1,200 lbs./in. ${ }^{2}$ of gross area when designed to be laid with the cells vertical and when tested in that position.

(c) Vitrified foundation tile shall have an absorption not exceeding 8 per cent and be capable of sustaining a load of at last 1,200 lbs./in. ${ }^{2}$ over the gross area when tested in the same position as when laid in the wall.

LAYING

Tile in the exterior walls and interior bearing walls shall be load-bearing tile laid to develop their full strength. No vertical or head joints shall be mortared through the wall, but a generous air space shall be left in the center of the walls by buttering the two edges of each tile either before or after it is set in the wall. All tile must be wet before concrete or mortar is placed.

\section{MORTAR}

All mortar used for laying up the hollow tile shall consist of Portland cement and clean, sharp sand in the proportion of 1 part cement to 3 parts sand, well mixed to a smooth, moderately stiff mortar. Cold lime putty not to exceed 10 per cent of cement by volume will be allowed in the mortar.

\section{FODNDATION TILES}

Where so indicated on the drawings the foundation walls from top of footings to the underside of first-floor beams shall be constructed of hard-burned foundation tile. Where columns or piers supporting heavy loads rest on the foundation walls same shall be filled with concrete from footing to top of walls.

\section{SUBDIVIDIMG PARTITIONS}

All partitions shall be started on the structural floor and be wedged against the floor above.

\section{JAMB TILE}

The contractor shall provide for all door and window openings, where indicated, jamb tile with rabbitted openings to receive the door or window frame box. The space between the tile and the frame box shall be well filled with mortar to within 1 inch of stop bead and calked to stop bead with roofer's cement or oakum to prevent the passage of air or moisture.

\section{IINTELS}

Openings not exceeding 5 feet in clear span may be spanned with arch lintel tile or with regular tile reinforced with proper steel rods in the lower cells and filled solidly with stone concrete.

Openings over 5 feet in clear span shall be spanned with reinforced concrete or with steel lintels faced with tile, as shown on drawings.

\section{SILLS}

Where called for by the drawings, sills of special hollow-sill tile shall be used. Special care must be taken to fill all joints so as to prevent moisture working through them; wood sill of window frame shall be set in heavy bed of roofer's cement.

\section{ARCH OPENINGS}

All arch openings shown on drawings shall be spanned with 2-course rowlock hollow brick header arches, carefully laid on substantial centers. Arches shall spring from the hollow tile and must be well bedded on them.

\section{COLUMNS AND PIERS}

Columns and piers, so indicated, shall be constructed of hollow tile to sizes shown. Where column finish is round they shall be built of circular hollow tile column covering filled with concrete where the second-story walls are supported by them. Square columns shall be built of the proper size hollow tile laid as indicated under "laying."

\section{FLOOR JOIST BEARINGS}

Tile slabs 1 inch thick shall be provided and set under all floor joists as bearing plates for end construction tile.

\section{WALI PLATES}

In the upper courses of wall at intervals of 5 feet $3 / 4$-inch bolts projecting 6 inches above the top shall be embedded in cement grout to allow the plate being fastened down with washers and nuts.

\section{FLOORS}

Floors shall be segmental arch or flat arch of hollow-tile construction, as indicated on the drawings.

\section{DEPTH OF TILE}

Depth of hollow tile shall be regulated by span and load to be carried and as indicated on the drawings.

\section{CENTERS}

Centers must be of such size as to insure against deflecting, and must not be removed before the floor has properly set, and under long spans a center line of supports must be maintained for at least three weeks after the floor has been completed.

\section{CLEANING, ETC.}

Upon completion of this work the contractor shall repair all damaged tile, clear away all rubbish of every description, leaving this part of the work clean and in perfectly good condition.

\section{GENERAL CONDIMIONS}

All materials entering into the work and all methods used by the contractor shall be subject to the approval of the engineer, and no part of the work will be considered as finally accepted until all the work is completed and accepted. 
The general conditions as given in 518.50 , page 416 shall be considered to apply to this specification.

American Society for Testing Materials, standard specifications and tests for hollow burned clay tile, 1927.

C34-27, load-bearing wall tile.

C57-27, floor tile.

1. The tile shall be made from surface clay, shale, fire clay or admixtures thereof.

2. (a) According to the results of physical tests, tile shall be classified as hard, medium, and soft on the basis of the following strength and absorption requirements, both of which must be met for a given class:

Note.-As different types of clay are used in the manufacture of tile, color can not be taken as indicative of classification.

\section{Absorption}

\begin{tabular}{|c|c|c|}
\hline Class & Mean of 5 tests & $\begin{array}{l}\text { Individual, } \\
\text { maximum }\end{array}$ \\
\hline Hard.--... & $\begin{array}{l}\text { Per cent } \\
12 \text { or less... }\end{array}$ & $\begin{array}{l}\text { Per cent } \\
15\end{array}$ \\
\hline $\begin{array}{l}\text { Medium... } \\
\text { Soft_........ }\end{array}$ & $\begin{array}{l}16 \text { or less. } \\
25 \text { or less........ }\end{array}$ & $\begin{array}{l}19 \\
28\end{array}$ \\
\hline
\end{tabular}

Compressive strength

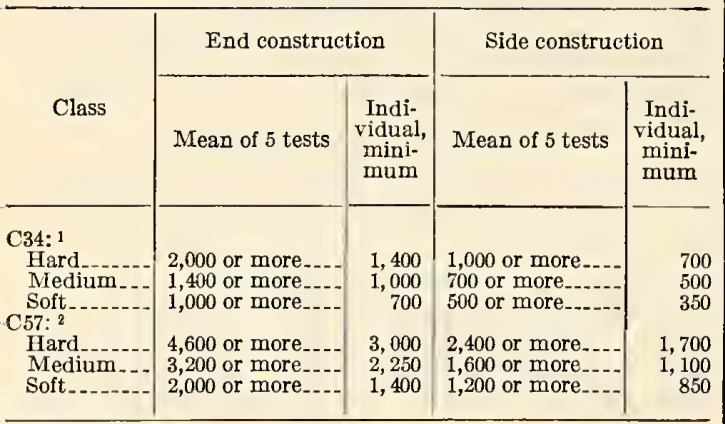

1 Based on gross area (lbs./in.2). Gross area shall be taken as the total area inclosed hy the outside dimensions of the unit taken in a direction perpendicular to that in which the load is carried.

2 Based on net area (lbs./in. ${ }^{2}$ ).

(b) Where end-conscruction tiles are used on the side they shall meet the requirements of that construction, and vice versa.

\section{WEIGHTS}

3. (a) The tile shall have the following dry weights determined as hereinafter specified:

$$
C-34
$$

\begin{tabular}{|c|c|c|}
\hline Size of unit, in inches & $\begin{array}{l}\text { Num- } \\
\text { her of } \\
\text { cells }\end{array}$ & $\begin{array}{l}\text { Stand- } \\
\text { ard } \\
\text { weight }\end{array}$ \\
\hline $\begin{array}{l}33 / 4 \text { by } 12 \text { by } 12 \\
6 \text { by } 12 \text { by } 12 \\
8 \text { by } 12 \text { by } 12 \\
12 \text { by } 12 \text { by } 12 \text { by } 12\end{array}$ & $\begin{array}{l}3 \\
6 \\
6 \\
6 \\
6\end{array}$ & $\begin{array}{r}\text { Pounds } \\
20 \\
30 \\
36 \\
42 \\
48\end{array}$ \\
\hline $\begin{array}{l}3 \text { Do by } 5 \text { hy } 12 . \\
8 \text { by } 5 \text { by } 12 \\
\text { Do by } 5 \text { by } 12 \text { (L-shaped) }\end{array}$ & $\begin{array}{l}9 \\
1 \\
2 \\
3\end{array}$ & $\begin{array}{r}52 \\
9 \\
16 \\
16 \\
16\end{array}$ \\
\hline $\begin{array}{l}8 \text { by } 61 / 2 \text { by } 12 \text { (T-shaped) } \\
8 \text { hy } 73 / 4 \text { by } 12 \text { (square) } \\
8 \text { hy } 101 / 4 \text { hy } 12 \text { (H-shaped) } \\
8 \text { by } 8 \text { hy } 8 \text { (cube) }\end{array}$ & $\begin{array}{l}4 \\
6 \\
7 \\
9\end{array}$ & $\begin{array}{l}16 \\
24 \\
32 \\
18\end{array}$ \\
\hline
\end{tabular}

$C-57$

Flat arch

\begin{tabular}{|c|c|}
$\begin{array}{c}\text { Depth of } \\
\text { arch, in } \\
\text { inches }\end{array}$ & $\begin{array}{c}\text { Average } \\
\text { weight per } \\
\text { square foot }\end{array}$ \\
\hline & Pounds \\
6 & 26 \\
7 & 29 \\
8 & 32 \\
9 & 35 \\
10 & 38 \\
12 & 42 \\
15 & 50 \\
\hline
\end{tabular}

Segmental arch

\begin{tabular}{|c|c|}
$\begin{array}{c}\text { Depth of } \\
\text { arch, in } \\
\text { inches }\end{array}$ & $\begin{array}{c}\text { Average } \\
\text { weight } \\
\text { per square } \\
\text { foot of floor }\end{array}$ \\
\hline & $\begin{array}{c}\text { Pounds } \\
6\end{array}$ \\
8 & 36 \\
10 & 40 \\
\hline
\end{tabular}

Tile for use in combination hollow tile and concrete construction

\begin{tabular}{|c|c|c|}
\hline Size of unit, in inches & $\begin{array}{l}\text { Mini- } \\
\text { mum } \\
\text { number } \\
\text { of cells }\end{array}$ & $\begin{array}{l}\text { Standard } \\
\text { weight }\end{array}$ \\
\hline $\begin{array}{l}4 \text { by } 12 \text { by } 12 \\
6 \text { by } 12 \text { by } 12 \\
8 \text { hy } 12 \text { by } 12 \\
10 \text { hy } 12 \text { by } 12 \\
12 \text { by } 12 \text { by } 12 .\end{array}$ & $\begin{array}{l}3 \\
3 \\
4 \\
4 \\
4 \\
4\end{array}$ & $\begin{array}{r}\text { Pounds } \\
16 \\
22 \\
25 \\
30 \\
35 \\
40\end{array}$ \\
\hline
\end{tabular}

(b) A tolerance of 5 per cent will be allowed on the above standard weights.

\section{DIMENSIONS}

4. No dimension shall vary more than 3 per cent from the standard dimensions for any form of tile.

\section{WORKMANSHIP AND FINISH}

5. All tile shall be well burned, reasonably free from laminations, and from such cracks, blisters, surface roughness, and other defects as would interfere with the proper setting of the tile or impair the strength or permanence of the construction.

The exterior suiface of all the tile intended for plaster or stucco shall be scored in such a manner as to give good adhesion.

\section{MARKING}

6. All tile shall bear the initials, name, or trademark of the manufacturer. Tile supplied in filling specification C34 shall bear the words "Load bearing " and hollow tile intended for use in partitions under specification C57 shall be marked "Partition." These marks shall be indented on the exterior of the tile and shall be plainly legible.

\section{INSPECTION AND REJECTION}

7. Proper facilities shall be provided the purchaser for sampling and inspection, either at the factory or at the site of the work, as may be specified 
in the contract. At least 10 days from the time of sampling shall be allowed for the completion of the test. The inspection shall be based on the requirements specified above. All tests shall be made in accordance with the methods hereinafter prescribed.

8. Individual tile shall be rejected for failure to meet the weight, size, or workmanship and finish requirements. In case of failure to meet the absorption and strength requirements for the class specified the seller may sort the shipment, and new samples shall be selected by the purchaser from the retained lot and tested at the expense of the seller. In case the second set of samples fails to meet the test requirements the entire lot shall be rejected.

9. Except as specified in the above paragraph and unless otherwise agreed, the expense of inspection and testing shall be borne by the purchaser.

\section{TESTS}

\section{SAMPIING}

10. Samples of tile for testing shall be selected by the purchaser or by a competent representative authorized by him to do his work. In all cases the samples shall be representative of the whole lot of tile from which they are selected. Full-size tile shall be taken in all cases.

11. For the strength, absorption, and weight determinations, five individual tile shall be selected from each kiln or from each 100 -ton lot. Where tile of several designs are used, enough samples shall be weighed to determine the true average weight in pounds per square foot for the construction. In no case shall less than five tile be taken. For the fire test the size of the test panel will govern the number of tile required. Samples for the freezing tests shall be taken from tile that have not been subjected to strength or fire tests.

12. Samples for the strength and absorption tests shall be selected at the factory or at the site of the work, as specified in the contract. If the fire or freezing tests are to be made, it shall be so specified at the time of placing the order, samples being selected at the factory at least 45 days in advance of the time of filling the order, and 90 days in advance for freezing tests.

13. All tile selected for test shall be plainly and permanently marked for reference by the testing operator.

\section{WEIGHT DETERMINATIONS}

14. The five tile, if not in kiln-dry condition, shall be dried to constant weight at a temperature of not less than $100^{\circ} \mathrm{C}$. $\left(212^{\circ} \mathrm{F}\right.$.) and be weighed separately. The scale shall be sensitive to within 0.5 per cent of the weight of the smallest unit.

\section{STRENGTH TESTS}

15. Five full-size dry tile shall be used.

16. The speed of the moving head of the testing machine shall not be more than 0.05 inch per minute.

17. A spherical bearing block of proper design shall be placed on top of the test sample.

18. (a) Bearing surfaces of the test samples and portions adjoining them which are liable to absorb water from the plastic capping shall be coated with shellac and allowed to dry. A quantity of plastic mortar made of a mixture of 3 parts (by volume) of Portland cement and 1 part of unretarded gypsum (plaster of Paris) mixed with sufficient water to spread evenly shall be placed on a plain surface which has been coated with oil, and allowed to harden sufficiently to bear the weight of the tile. The surface to be capped shall be placed on this mortar, and while holding the specimen so that its axis is at right angles to the capping surface it shall be given a single firm pressure.

(b) The average thickness of the cap after the extruded plaster has been removed and the edges trued shall not be more than one-eighth inch. Patching of caps after setting shall not be permitted. Imperfect caps shall be removed and replaced with new ones.

(c) Where time is not available for aging the cement-gypsum cap, a cap of neat gypsum may be used, although the resulting tile strength will generally be lower than with the cement-gypsum cap. If the tile so capped fail to pass specification requirements on the score of strength, they shall be retested with Portland-cement-gypsum caps aged not less than three days.

19. When the cement-gypsum cap is used it shall age at least three days before the tile is tested. Where the neat gypsum cap is used the tile may be tested as soon as the plaster has been well set, but not sooner than one hour after the sample has been capped.

20. All tile shall be tested in a position such that the load is applied in the same direction as in service.

\section{ABSORPTION TESTS}

21. The samples shall consist of five tile or three representative pieces from each of these five tile. If small pieces are used two shall be taken from the shell and one from an interior web, the weight of each piece to be not less than $227 \mathrm{~g}$ ( $1 / 2$ pound). The samples shall have had their rough edges or loose particles ground off and be free from cracks from the failure of the tile in compression, where taken from tile which have been subjected to strength tests.

22. Each piece shall be marked so that it may be identified at any time with the tile from which it was taken. Markings which do not cover more than 5 per cent of the total superficial area of the piece shall be used.

23. Preparatory to the absorption tests all samples shall be dried to constant weight in a drier or oven at a temperature of not less than $100^{\circ} \mathrm{C}$. $\left(212^{\circ} \mathrm{F}\right.$.).

24. The balance used shall be sensitive to within 0.2 per cent of the weight of the smallest unit or piece tested.

25. After obtaining the dry weight of the samples they shall be immersed in soft, distilled, or rain water, raised to the boiling point and boiled continuously for one hour, and then allowed to cool in water to room temperature.

26. After saturation, the sample shall be removed from the water and allowed to drain for not more than one minute. The superficial water shall be removed with a damp cloth, after which they shall be weighed immediately.

27. The test results shall be calculated as percentages of the initial dry weight, carried to the nearest first decimal place. The results shall be reported separately for each tile, with the average for the five tile.

\section{FREEZING TESTS}

(This test is used in connection with Specification C-34 only.)

28. Where the freezing test is to be made, five separate representative tile shall be selected.

29. If not possible to use the whole tile, a piece consisting of a cell not less than 4 inches long shall be sawed from the tile. These pieces shall be saturated by immersion in cold water for at least 72 hours prior to starting the freezing.

30. Any practical method of obtaining alternate freezings and thawings may be used, the freezings to be always made with fully saturated samples 
and the time and temperature to be such as to insure full freezing and thawing throughout the specimen. The initial weighing and all weighings for loss shall be made on dry specimens.

31. The balance used shall be sensitive to within 0.1 per cent of the weight of the smallest unit or pieoe tested.

32. Failure under the freezing and thawing treatment shall be considered to be reached when-

(o) The samples show superficial disintegration or spalling with loss of weight of more than 5 per cent of the initial weight; or $(b)$ the samples are badly cracked; or $(c)$ the samples show evident loss of structural strength.

\section{ADDITIONAI}

In addition to the foregoing, the following requirements apply individually to the specifications as designated:

\section{SPECIFICATION C34-27}

All tile shail be so designed that substantially the same masonry strength will be developed in all wall thicknesses for which they are to be used.

\section{WEATHERING RESISTANCE}

All tile used in exterior work subjected to weathering conditions shall be able to withstand 100 alternate freezings and thawings. Tile classed as hard or medium by these specifications may be considered as meeting the weathering requirement, provided they are burned to the normal maturity for the given clay. Tile classed as soft shall be accepted as meeting the weathering requirement only on the basis of freezing tests.

\section{FIRE RESISTANTE}

The tile shall meet the following requirements as tested according to A. S. T. M. C19-26T (see 534.10, p. 569), as the tests apply for bearing walls and partitions, and to be acceptable shall develop the following resistance periods as tested unplastered:

\begin{tabular}{|c|c|c|c|}
\hline $\begin{array}{l}\text { Thickness } \\
\text { of wall }\end{array}$ & $\begin{array}{c}\text { Number of } \\
\text { units in } \\
\text { wall thick- } \\
\text { ness }\end{array}$ & $\begin{array}{c}\text { Number of } \\
\text { cells in } \\
\text { wall }\end{array}$ & $\begin{array}{c}\text { Pesistanco } \\
\text { period I }\end{array}$ \\
\hline $\begin{array}{c}\text { Inches } \\
8 \\
8 \\
12 \\
12 \\
12 \\
16 \\
16\end{array}$ & $\begin{array}{r}1 \\
1 \\
1 \\
1 \\
\\
2 \\
2 \text { or } 3 \\
2 \text { or } 3\end{array}$ & $\begin{array}{l}2 \\
3 \\
3 \\
3 \\
4 \\
4 \\
6\end{array}$ & $\begin{array}{c}\text { Hours } \\
11 / 2 \\
2 \\
3 \\
4 \\
5 \\
6 \\
8\end{array}$ \\
\hline
\end{tabular}

These are near the minimum values developed in tests. The average results will gcnerally be higher. Plaster coatings $3 / 4$ inch thick applied on both sides and remaining in place throughout the thick applied on both sides and by 1 to 2 hours.

\section{SPECIFICATION C57-27}

\section{FIRT PESISTANCE}

In cases where the fire resistance is an essential property the purchaser shall specify the degree of fire resistance (n̂re-resistance period) required, and the manufacturer shall supply such available information on the fire test performance of the given or closely similar product as will aid the purchaser in deciding whether the requirements are inet. Further tests in accordance with A. S. T. M. C19-26T. (See 534.10, p. 569.)

By agreement, acceptance may be based on dry wreight of the units, percentage of absorption, fire resistance, and the workmanship and finish.
American Society for Testing Materials, tentative specifications and tests for hollow burned-clay fireproofing, partition and furring tile, serial designation C56-28T, 1928.

(This specification is practically the same as A. S. T. M. C57, given above, with the exception of the absorption and weight requirements, which for this specification are as follows:)

1. According to the results of the physical tests, tile shall be classified as hard, medium, and soft on the basis of strength and absorption requirements, the class of any lot being determined by the requirement which gives it the lowest rating.

\section{Absorption}

\begin{tabular}{l|r|r|r}
\hline C56 & $\begin{array}{c}\text { Mean of } \\
5 \text { tests }\end{array}$ & $\begin{array}{c}\text { Individ- } \\
\text { ual maxi- } \\
\text { mum }\end{array}$ & $\begin{array}{c}\text { Individ- } \\
\text { ual mini- } \\
\text { mum }\end{array}$ \\
\hline Hard & $6-12$ & 15 & 5 \\
Medium & $12-16$ & 19 & 5 \\
Soft_... & $16-25$ & 25 & 5 \\
\hline
\end{tabular}

Weights-Standard partition tile

\begin{tabular}{l|r|r|r}
\hline \multicolumn{1}{c|}{ Dimensions, in inches } & $\begin{array}{c}\text { Mini- } \\
\text { mum } \\
\text { number } \\
\text { of cells }\end{array}$ & $\begin{array}{c}\text { Standard } \\
\text { weight }\end{array}$ \\
\hline & & & Pounds \\
3 by 12 by 12 & 3 & 15 \\
4 by 12 by $12 \ldots$ & 16 \\
6 by 12 by 12 & 3 & 22 \\
Do & & 4 & 25 \\
10 by 12 by 12 & & 4 & 30 \\
12 by 12 by 12 & & 4 & 35 \\
\end{tabular}

Federal Specifications Board, specification No. 506, United States Government master specification for tile, hollow clay, floor, July 11, 1927.

(This specification is substantially the same as for the medium and soft grades of tile in A. S. T. M. C57, given above, with the exception of rcquirements for compressive strength, method of sampling, and basis of acceptance, which for this specification arê as follows:)

\begin{tabular}{|c|c|c|c|c|}
\hline \multirow{3}{*}{ Class } & \multicolumn{4}{|c|}{ Compressive strength (pounds per square inch of net area) } \\
\hline & \multicolumn{2}{|c|}{ End construction } & \multicolumn{2}{|c|}{ Side construction } \\
\hline & Average of 5 & $\begin{array}{c}\text { Individ- } \\
\text { ual, } \\
\text { minimum }\end{array}$ & Average of 5 & $\begin{array}{l}\text { Individ- } \\
\text { ual, } \\
\text { minimum }\end{array}$ \\
\hline $\begin{array}{l}M_{-} \\
S_{-. .}\end{array}$ & $\begin{array}{l}3,000 \text { or more } \\
2,000 \text { or more }\end{array}$ & $\begin{array}{l}2,000 \\
1,400\end{array}$ & $\begin{array}{l}1,500 \text { or more } \\
1,000 \text { or more }\end{array}$ & $\begin{array}{r}1,000 \\
700\end{array}$ \\
\hline
\end{tabular}

A set of five representative tiles for test shall be taken at random from shipments not excecding 100 tons, and additional sets of five for each 100 tons for larger shipments. Where a shipment contains tile from several sources, separate sets of samples shall be taken of the tile from each. Where sampled at the plant, one set shall be taken from each kiln or from each 100-ton lot for continuous kilns. Separate samples shall be taken of each size and design of tile included. 


\section{BASIS OF ACCEPTANCE}

The requirements have been simplified to permit acceptance based on weight, absorption, material, and workmanship in cases where the tile is used under loads considerably below working load values, which is frequently the case. These determinations can be made if necessary at the site of the work or at the plant with equipment that can be provided without much difficulty. For more exacting use the compression tests should be made and acceptance based on their results in addition to those from the weight and absorption determinations, the standing of any set of samples being determined by the requirement which gives it the lowest classification.

Federal Specifications Board, specification No. 507, United States Government master specification for tile, hollow clay, load-bearing wall, July 11 , 1927.

(This specification is substantially the same as A. S. T. M. C34 (given above) with the exception of requirements for sampling and basis of acceptance, which are given above under F. S. B. 506, and weathering resistance requirements, which for this specification are as follows:)

\section{WEATHERING RESISTANCE}

The classification is based on requirements chosen so that hollow tile grading as medium (M) or hard (H) can be considered as suitable for use in exterior walls. This should not be taken as a rigid distinction on account of the wide range in the character of clays and processes used in tile manufacture, which makes it difficult to define weathering resistance in terms of properties that can be determined in acceptance tests. The purchasing officer should be guided in part by the experience with comparable material in the locality where those under test are to be used. In cases of doubt and where the time and equipment are available, acceptance in point of weathering resistance can be based on ability to withstand 100 alternations of freezing and thawing conducted according to A. S. T. M. C34. Failure is to be considered as reached when the samples are cracked or show superficial disintegration or spalling with loss of weight of more than 5 per cent of the initial dry weight.

Where the wall is faced with 3 inches or more of stone, terra cotta, brick, or other veneer, the weathering resistance of the material in the backing is without significance.

Federal Specifications Board, specification No. 508, United States Governnent master specification for tile, hollow clay, fireproofing, partition and furring, July 11, 1927 .

(This specification is substantially the same as for the medium and soft grades of tile in A. S. T. M., C56, given above, with the exception of requirements for compressive strength and sampling, which are given above under F. S. B. No. 506, and basis of acceptance, which for this specification is as follows:)

\section{BASES OF ACCEPTANCE}

Acceptance of hollow tile under this specification can generally be based on weight, absorption, and workmanship. The strength tests should be required only where the other determinations do not appear to fully define the product. In any case, the standing of a set of samples is determined by the requirement which gives it the lowest classification.

(This specification has the following additional to A. S. T. M. C56:)
Furring tile are made in sizes $11 / 2$ by 12 by 12 and 2 by 12 by 12 , and are split from 3 by 12 by 12 and 4 by 12 by 12 tile which should weigh 15 and 16 pounds, respectively.

United States Department of Commerce, division of simplified practice, recommendation No. 12, hollow building tile, March 1, 1926.

In accordance with unanimous action of a general conference of representatives of manufacturers, distributors, and users, the United States Department of Commerce, through the Bureau of Standards, recommends that the number of sizes of hollow building tile be reduced to the following:

TABLE 1

STANDARD LOAD BEARING WALL TILE

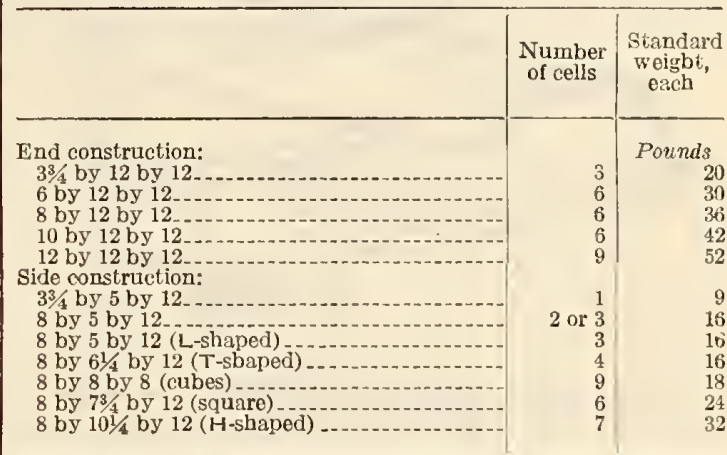

STANDARD PARTITION TILE

\begin{tabular}{|l|l|l}
\hline 3 by 12 by 12 & 3 & 15 \\
4 by 12 by 12 & 3 & 16 \\
6 by 12 by 12 & 3 & 22 \\
8 by 12 by 12 & 4 & 30 \\
10 by 12 by 12 & 4 & 36 \\
12 by 12 by 12 & 4 & 40 \\
\hline
\end{tabular}

STANDARD SPLIT FURRING TILE

2 by 12 by $12 \ldots$

STANDARD BOOK TILE

\begin{tabular}{|l|r|r}
\hline 3 by 12 by 18 to 24 & & Lbs./ft. ${ }^{2}$ \\
\end{tabular}

Not more than 5 per cent tolerance under allow. able for weights and 3 per cent over or under for dimensions eovering thickness, width, and length.

\subsection{Roof Tiles.}

American Railway Engineering Association, specifications for buildings for railway purposes.

\section{CLAY ROOFING TILE, 1926}

\section{GENERAL}

The contractor shall furnish all labor, material, tools, scaffolding, and equipment, unless otherwise noted, necessary to complete any or all classes of clay roofing tile, herein specified, according to the class of building and as shown or implied on the drawings.

\section{MATERIALS}

All pitched roofs so indicated on the drawings shall be covered with ...... pattern clay tile as made by _......-, or approved equal, with stock fittings, suitable for each pattern. 
Clay tile shall be hard-burned, true in shape, and of uniform natural deep color, in accordance with samples submitted to and approved by the engineer. The contractor shall submit with his bid representative samples of the clay tile to be used, and the tiles subsequently furnished must be equal in quality to the samples.

\section{FLASHINGS}

All raglets or special flashing tiles of every description, in connection with this roof, shall be furnished

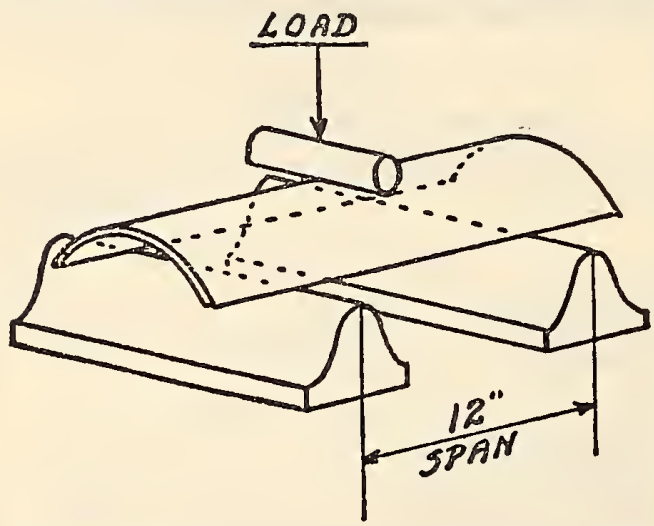

FiguRe 228.-Method recommended for use in testing all curved tiles

and placed by this contractor. Metal flashings will be furnished and placed by the sheet-metal contractor.

\section{ROOFING FELT}

The roofer shall cover the roof sheathing with one thickness of approved roofing felt weighing not less than ...... pounds to the square, laying same with a 3-inch lap and securing in place with capped nails not over 10 inches apart. The felt should be laid parallel with the eaves and lapped over all valleys about 4 inches and laid under all hips and ridge flashings about 6 inches.

\section{LAYING TILE}

Tiles shall be fastened with copper nails, and sh.ill be well locked together and laid smoothly, and no attempt shall be made to stretch the courses.

The tiles must be laid so that the vertical lines are parallel with each other and at right angles to the eaves. The tiles that verge along the hips shall be cut close against the hip boards, and a water-tight joint made by cementing cut hip joint to hip board with best quality elastic cement. Each piece of hip roll shall then be nailed to the hip board and the hip rolls cemented where they lap each other. No broken or cracked tiles shall be used or left in the roof when complete.

Gable rakes shall be furnished with special detached gable rake fittings.

\section{GUARANTY}

The roofing contractor shall furnish an unqualined guaranty, with good and sufficient bond, covering the maintenance of the roof in a watertight condition for a period of ........ years from date of completion.

\section{GENERAL CONDITIONS}

All material entering into the work and all methods used by the contractor shall be subject to the approval of the engineer and no part of the work will be considered as finally accepted until all the work is completed and accepted.

The general conditions as given in 518.50, page 416 , shall be considered to apply to these specifications.

Clay Products Institute of California, strength tests for roofing tiles.

The Clay Products Institute recommends that any curved roofing tile when tested in the manner shown in Figures 228 and 229 should safely withstand a caref ully applied load of 400 pounds without cracking or failure. This test may be performed on a job by laying two pieces of pipe on the ground forming four contact points of support for the tile and another picce of pipe laid across the tile at the middle and then a 1 by 10 inch board 3 or 4 feet long may be laid over the pipe, being held at either end, and then four sacks of cement carefully laid on the board, thus approximately providing the required 400 pounds load on the tile, as shown in Figure 229. Spanish (S) tile may be tested in the same manner.

To be safe, roofing tiles should be attached in some substantial manner to the roof construction by means of wires, nails, lugs, interlocking, or the like.

A paragraph similar to the following is recommended for specifications:

The roofing tile shall not ahsorh more than 15 per cent of the constant dry weight of the tile immediately after immersed in water for 48 hours. The tiles shall he capahle of sustaining without rupture or crack a load of at least 400 pounds carefully applied at the center point of the tile when supported on four points of hearing 6 inches each side of the center point of the tile, making a 12-inch span. A test result shall consist of the average of five tiles tested. Tiles shall he secured to the roof construction in the following manner:

(Insert desired provisions.)

\subsection{Terra Cotta.}

American Railway Engineering Association, specifications for buildings for railway purposes.

\section{ARCHITECTURAI TERRA COTTA, 1926}

\section{GENERAL}

The contractor shall furnish all labor, materials, tools, scaffolding, and equipment, except as other-

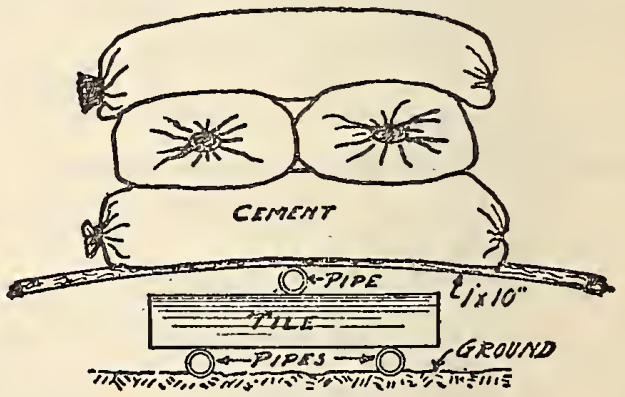

FIGURE 229

wise noted, necessary to entirely complete any or all classes of architectural terra-cotta work herein specified, according to the class of buildings and as shown or implied on the drawings.

\section{QUAIITY OF MATERIAI}

Material for all architectural terra cotta shall be carefully selected clay, to be in perfect condition after burning, of uniform fracture hard enough to resist scratching by knife. 
Architectural terra cotta shall develop an average ultimate compressive strength of not less than $5,000 \mathrm{lbs} . /$ in. $^{2}$

Coloring shall be as indicated and must be uniform.

\section{DEFECTIVE WORK}

All work shall be carefully modeled by skilled workmen in strict accordance with detail drawings. All pieces shall be perfect when set in place and any work damaged after installation, before acceptance, shall be replaced.

\section{DRAWINGS}

The architectural terra-cotta contractor shall prepare and submit to the engineer, for his approval, complete detail and setting drawings (in triplicatc) for all terra-cotta work covered by this contract. Such drawings shall show, in detail, jointing, bonding, anchoring, and other construction features. All blocks shall be numbered serially.

\section{MODELS}

If desired by the engineer, full-sized plaster models prepared by experts shall be submitted for his approval. Price for models shall be agreed upon.

\section{MOLDING AND FITTING}

Templates for molded work shall be made according to details and models. Carving and molding must be sharp, straight, true, and well undercut. Blocks must be straight, true, and out of wind. A reasonable number of additional blocks must be provided to prevent delay from defective materials or injury. So far as possible all grinding of joints and fitting of matcrial shall be done at the factory. Provide washes and drips for all projecting courses. Wherever flashing will occur provide raglets. Proper provision shall be made for anchors, tie rods, etc.

\section{MORTAR}

Mortar for terra-cotta work, unless otherwise specified, shall be composed of 1 part Portland cement, 3 parts sand, with the addition of sufficient lime putty to make the mortar work smoothly. The sand and cement shall be mixed dry, then wet to the proper consistency and the lime putty added. Where terra cotta is to be laid with colored mortar, the following mixture shall be used: 1 part Portland cement, 2 parts sand, with lime paste added to make it work smoothly and colored with approved mortar color. No more mortar containing cement shall be mixed at any one time than can be used within 30 minutes after mixing. Retempering cement mortar which has begun to set will not be permitted. No mortar which has frozen shall be used on the work.

Portland cement shall be in accordance with the A. R. E. A. specifications, which are the same as A. S. T. M. C9. (See 516.11, p. 294.)

Sand and lime specifications, as given in A. R. E. A. specification for brickwork, 518.83 (see p. 475), shall apply to sand and lime for terra cotta.

\section{SETTING AND ANCHORING}

All blocks must be cleaned and wetted before setting.

Mortar shall be kept one-half inch from the face of the terra cotta to allow for pointing. Splashing exposed faces of the terra cotta with mortar shall be avoided.

All beds and vertical joints shall be of maximum width of three-eighths inch unless otherwise indicated. The terra cotta shall be set accurately, true to line, and level. Face blocks shall be set on thoroughly wetted wooden wedges, which are not to be removed until the building is cleaned and pointed.

All terra-cotta work shall be thoroughly bonded to masonry backing. Cornices, column caps, and blocks with greater projection than bed shall be thoroughly anchored.

Anchors and dowels, rods, and hooks shall be of the proper size and shape and thoroughly galvanized or coated with asphaltum paint.

This contractor shall do all cutting and fitting of terra cotta to accommodate other trades.

\section{PROTECTION}

Wherever necessary, all projecting courses or individual blocks shall be protected against injury during the sctting process by wooden covering, which shall be maintained in good and substantial condition until removed for the purpose of clcaning down the work.

\section{CLEANING AND POINTING}

The face of the terra-cotta work under this contract shall be thoroughly cleaned upon completion, such cleaning to be done with soap powder boiled in clean water and applied vigorously with stiff fiber brushes. If necessary, clean, sharp, finc white sand may be added to the soap and water mixture. The use of wire brushes or acids will not be permitted for cleaning terra-cotta work.

All face joints shall bc brushed out one-half inch in depth and pointed flush with mortar consisting of 1 part stainless cement, 2 parts clean white sand and sufficient cold lime putty to make a mixture as stiff as can be worked. All joints shall be wetted before pointing.

\section{GENERAL CONDITIONS}

All material entering into the work and all methods used by this contractor shall be subject to the approval of the engincer, and no part of the work will be considered as finally accepted until all the work is completed and accepted.

The general conditions as given in 518.50 , page 416 , shall be considered to apply to thcse specifications.

National Terra Cotta Society, standard specification for the manufacture, furnishing, and sctting of terra cotta, 1923 .

(This specification includes provisions under heads of A, General information; B, Material; C, Design and structure; D, Transportation, storage, and protection; and E, Erection. The standard specification is included in a publication of the society entitled "Terra Cotta, Standard Construction," which also includes 67 plates of illustrative architectural forms of assumed proportions, and their proper constructional features showing uses of terra cotta. The society has, in addition to the standard specification, a short form spccification for incorporation in the architect's specifications. The following glossary of terms relating to terra cotta is included in the standard.)

Surface finish designates the texture of the surface of the clay body prior to application of the ceramic finish.

It may be-

(a) Smooth.

(b) Tooled or drove

(b1) Eight lines to the inch.

(b2) Six lines to the inch.

(c) Light irregular drag or combing.

(d) Heavy irregular drag or combing.

(e) Special.

A special surface finish like "bush-hammered," "pitted," "vermiculated," etc., involves extra expense and, if required, should be clearly specified. 
Surface finish for unglazed surfaces may be smooth or may be tooled with a light or heavy drag. Flat surfaces of sufficient width may be tooled, while the curved surfaces of mouldings may be left smooth.

Surface finish for glazed ceramic finish (whether lustrous or mat) is usually made sinooth.

Granite colors, if unglazed, may be made smooth or with irregular drag, or pitted. A bush-hammered or special surface involves extra expense, and, if required, should be clearly specified. If glazed ceramic finish is used for granite colors the surface treatment is usually smooth.

Ceramic finish designates the surface and color applied by the ceramic processes of coating, glazing, burning, etc.

1. Unglazed terra cotta.-Terra cotta with a ceramic finish producing an unglazed finish made in various shades of buff, gray, salmon, red, and brown. Most colors thus made are vitreous.

2. Glazed or enameled terra colta.-Terra cotta having an impervious ceramic finish of a glassy texture which may be either lustrous or mat (sometimes designated as full or dull glazes or enamels) made in various colors.

3. Granite color terra cotta.-(a) Unglazed granite color:-A mottled ceramic finish similar to unpolished granite.

(b) Glazed or enameled granite color.-A mottled ceramic finish similar to polished granite, made either lustrous or mat.

4. Polychrome terra cotta or faïence.-Terra cotta baving two or more colors on the same piece. (a) Polychrome, unglazed.-Unglazed terra cotta having two or more colors on the same piece.

(b) Polychrome, glazed.-Glazed terra cotta having two or more colors on the same piece.

(c) Polychrome, blended colors.-Made only in glazed terra cotta. If, in polychrome glazed work, the colors are not to be separated by definite lines or contours of ornaments, but are to be blended together by brush treatment or the like, the term "polychrome, blended colors" shall be used. The character of work expected should be explicitly described.

Note.-For polychrome work always clearly specify the work to be done and the number of colors on a single piece.

5. Special.-There are a number of ceramic finishes used by individual manufacturers, the processes for which are patented or the names copyrighted which are not included in this glossary.

6. Semiglaze.-An ambiguous term which should never be used.

7. Fire-gilding.-A coating of gold glaze, either mat or lustrous, fixed by an additional burning. (The area of surface to be gilded should be clearly described.)

\subsection{Miscellaneous Specifications for Tile.}

American Electric Railway Association, specification for tile duct, 1926.

(This specification is given in full under American Electric Railway Association specification and form of contract for electrical conduit construction, serial No. D200-26, (See 518.9, p. 481.) 
541.

\section{ABRASIVES, NATURAL AND ARTIFICIAL}

\subsection{GENERAL ITEMS.}

Grinding Wheel Manufacturers Association of the United States and Canada, safety code for the use, care, and protection of abrasive wheels, 1926.

(This association, in cooperation with the International Association of Industrial Accident Boards and Commissions, prepared the above code. The code has been approved by the American Engineering Standards Committee, now the American Standards Association, and has been issued as an American standard.)

National Sand and Gravel Association, Bulletin 3. representative specifications for different uses of sand and gravel, November, 1928.

(In this bulletin, representative specifications, many of which are presented in this volume, and including specifications for sand for polishing, grinding, and abrasives, have been summarized.)

\subsection{FLINT.} able.)

(No nationally recognized specifications avail-

\subsection{HONES, WHETSTONES, AND OIL-} STONES. able.)

(No nationally recognized specifications avail-

\subsection{ABRASIVE WHEELS.}

Grinding Wheel Manufacturers Association of the United States and Canada, standard types of grinding wheels.

(This association originated the movement for simplification of sizes and types of grinding wheels resulting in Simplified Practice Recommendation No. 45, of the United States Department of Commerce (given below), the recommendation incorporating, with only slight additional simplification of sizes, the previously adopted standards of the association.)

Society of Automotive Engineers, crank-shaft grinding wheels, recommended practice, 1920.

\begin{tabular}{c|c}
\hline Nominal width, in incbes & Radii of edges. ? \\
$\begin{array}{c}11 / 2,15 / 8,134,17 / 8,2,21 / 8,21 / 4,2 \frac{1}{3}, 21 / 2,23 / 4,3 \\
31 / 4,31 / 2,33 / 4,4\end{array}$ & $3 / 32$ incb, marimum.
\end{tabular}

1 Wheels are to be ordered by the nominal width. Wheel manufacturers allow 0.029 inch additional to the nominal widths for truing. 2 Radii of edges are specified as maximum to permit dressing wbeels to desired crank-sbaft pin radii.

This specification applies only to unfinished wheels for grinding crank shafts.

United States Department of Commerce, division of simplified practice, recommendation No. 45 , grinding wheels, Januarý, 1929.

In accordance with unanimous action by representative manufacturers, distributors, and users of grinding wheels, the United States Department of Commerce, through the Bureau of Standards, recommends that stock sizes of the commodity conform to those shown in the attached tables.

TABLE 1.-Types of grinding wheels

\begin{tabular}{|c|c|c|c|}
\hline $\begin{array}{l}\text { Type } \\
\text { No. }\end{array}$ & Cross section & $\begin{array}{l}\text { Type } \\
\text { No. }\end{array}$ & Cross section \\
\hline- & $\begin{array}{l}\text { Straight. } \\
\text { Cylinder. } \\
\text { Eliminated. } \\
\text { Tapered } 2 \text { sides. } \\
\text { Recessed } 1 \text { side. } \\
\text { Straigbt cup. } \\
\text { Recessed } 2 \text { sides. }\end{array}$ & $\begin{array}{l}8 \\
9 \\
10 \\
11 \\
13 \\
14\end{array}$ & $\begin{array}{l}\text { Eliminated. } \\
\text { Flaring cup. } \\
\text { Disb. } \\
\text { (Saw gummer) saucer. } \\
\text { Eliminated. }\end{array}$ \\
\hline
\end{tabular}

1 Wheels of types 6 and $11 \mathrm{can}$ be furnisbed witb beveled face.

The committee proposes for elimination three cross sections, as follows:

Type No. 3.- Tapered one side.

Type No. 8.-Countersunk dovetail.

Type No. 10.-Raised dovetail.

The nine types retained are representative of practically all grinding wheels used on the standard makes of grinding machines. This classification of grinding wheels will greatly simplify the stocking of wheels wherever a quantity is kept on hand, and it will also enable the user to accurately order a grinding wheel by giving the type number and the complete dimensions (see Table 2) necessary to construct such a wheel, as designated by the cross section of that type.

TABLE 2.-Key to letter dimensions

A, flat spot of beveled wall.

$\mathrm{D}$, diameter (over all).

E, center or back thickness.

$F$, deptb of recess. (See type 5 , fig. 233.)

G, depth of recess." (See types 5 and 7 , figs. 233 and 235 .)

$\mathrm{H}$, arbor hole.

$J$, diameter of flat or small diameter.
$\mathrm{K}$, diameter of flat inside. $\mathrm{M}$, large diameter of bevel. $\mathrm{P}$, diameter of recess. $\mathbf{R}$, radius.

$\mathbf{T}$, tbickness (over all).

T, widtb of face.

$\mathrm{U}$, widtb of face.

W, tbickness of wall. 
TABLE 3.-Type No. 1-Straight wheels-Vitrified and silicate

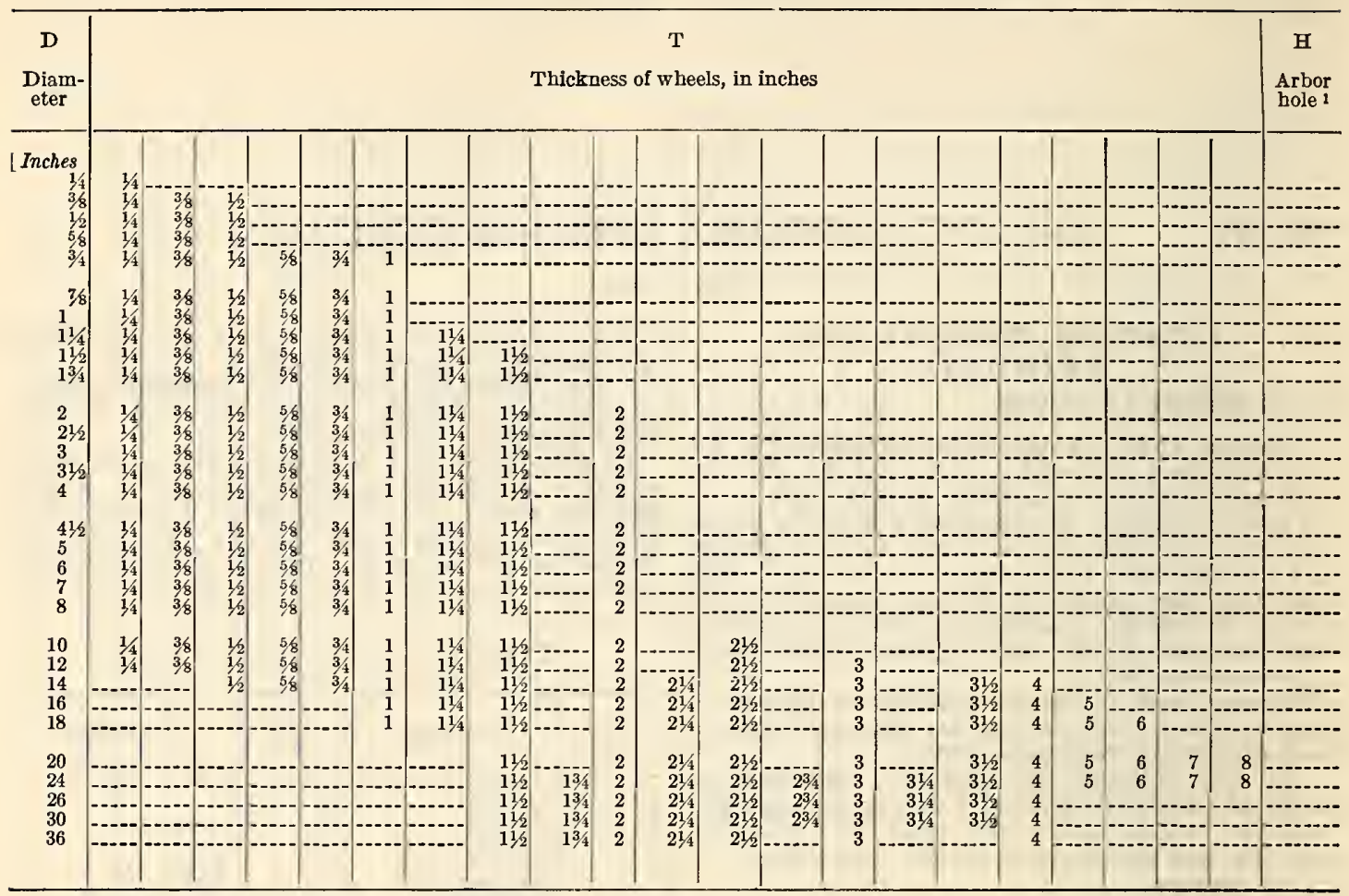

1 As ordered. Refer to Table 14 for minimum arbor holes. Also see Tables 16, 17, and 21.

TABLE 4.-Type No. 1-Wheels (organic bonds)

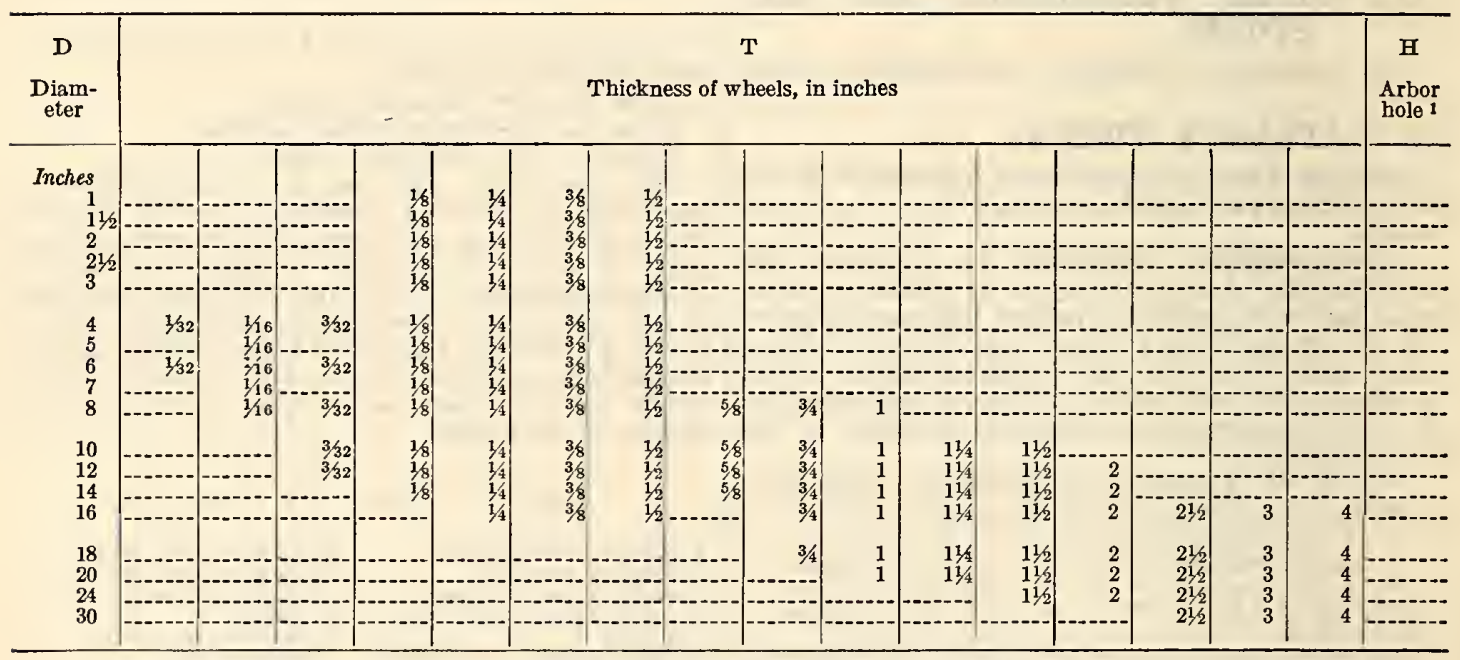

1 As ordered. Refer to Table 14 for minimum arbor holes. Also see Tables 16, 17, 21, and 22. 
ABRASIVE MATERIALS, ASBESTOS, AND CHALK

TABLE 5.-Type No. 2-Cylinder wheels

\begin{tabular}{|c|c|c|}
\hline $\begin{array}{c}\text { D } \\
\text { Diameter }\end{array}$ & $\begin{array}{c}\mathbf{T} \\
\text { Thickness }\end{array}$ & $\begin{array}{c}\text { W } \\
\text { Thickness } \\
\text { of wall }\end{array}$ \\
\hline $\begin{array}{c}\text { Inches } \\
8 \\
10 \\
10 \\
10 \\
12 \\
12\end{array}$ & $\begin{array}{c}\text { Inches } \\
4 \\
4 \\
4 \\
4 \\
4 \\
4\end{array}$ & $\begin{array}{l}\text { Inches } \\
1 \\
1 \\
11 / 4 \\
11 / 2 \\
11 / 1 \\
11 / 2\end{array}$ \\
\hline $\begin{array}{l}14 \\
14 \\
14 \\
16 \\
16\end{array}$ & $\begin{array}{l}4 \\
4 \\
5 \\
4 \\
4\end{array}$ & $\begin{array}{l}11 / 4 \\
11 / 2 \\
11 / 2 \\
11 / 2 \\
2\end{array}$ \\
\hline $\begin{array}{l}16 \\
16 \\
16 \\
16 \\
16\end{array}$ & $\begin{array}{l}4 \\
5 \\
5 \\
6 \\
6\end{array}$ & $\begin{array}{l}3 \\
11 / / 4 \\
11 / 2 \\
2 \\
3\end{array}$ \\
\hline $\begin{array}{l}16 \\
18 \\
18 \\
18 \\
18\end{array}$ & $\begin{array}{l}8 \\
4 \\
4 \\
5 \\
5\end{array}$ & $\begin{array}{l}2 \\
2 \\
4 \\
11 / 4 \\
11 / 2\end{array}$ \\
\hline $\begin{array}{l}18 \\
18 \\
18 \\
20 \\
20\end{array}$ & $\begin{array}{l}5 \\
6 \\
6 \\
4 \\
6\end{array}$ & $\begin{array}{l}4 \\
2 \\
4 \\
4 \\
2\end{array}$ \\
\hline $\begin{array}{l}20 \\
20 \\
24 \\
30 \\
30\end{array}$ & $\begin{array}{l}6 \\
6 \\
6 \\
6 \\
6 \\
6\end{array}$ & $\begin{array}{l}21 / 2 \\
4 \\
21 / 2 \\
2 \\
3\end{array}$ \\
\hline
\end{tabular}

TABLE 6.-Type No. 4-Wheels (tapered two sides)

\begin{tabular}{|c|c|c|c|}
\hline D & $\begin{array}{l}\text { Taper } 3 / 4 \\
\text { inch } \\
\text { per foot }\end{array}$ & $\begin{array}{c}\text { Taper 1/2 } \\
\text { inch } \\
\text { per foot }\end{array}$ & ㅍ \\
\hline Diameter & $\begin{array}{c}\mathrm{U} \\
\begin{array}{l}\text { Width of } \\
\text { face }\end{array}\end{array}$ & $\begin{array}{c}\mathrm{U} \\
\text { Width of } \\
\text { face }\end{array}$ & $\begin{array}{l}\text { Arbor } \\
\text { hole } 1\end{array}$ \\
\hline Inches & Inches & Inches & \\
\hline $\begin{array}{l}12 \\
12 \\
12 \\
14\end{array}$ & $\begin{array}{l}13 / 4 \\
21 / 4 \\
1\end{array}$ & -2 & \\
\hline $\begin{array}{l}14 \\
14 \\
16 \\
16 \\
16\end{array}$ & $\begin{array}{l}11 / 2 \\
2 \\
11 / 2 \\
2\end{array}$ & 3 & \\
\hline $\begin{array}{l}18 \\
18 \\
18 \\
20 \\
20\end{array}$ & $\begin{array}{l}2 \\
21 / 2 \\
3 \\
2 \\
21 / 2\end{array}$ & $\begin{array}{l}2 \\
21 / 2 \\
3 \\
2 \\
21 / 2\end{array}$ & \\
\hline $\begin{array}{l}20 \\
20 \\
24 \\
24 \\
24\end{array}$ & \begin{tabular}{l}
3 \\
\hdashline 2 \\
$21 / 2$ \\
3
\end{tabular} & $\begin{array}{l}3 \\
31 / 2 \\
2 \\
21 / 2 \\
3\end{array}$ & \\
\hline $\begin{array}{l}24 \\
24 \\
26 \\
26\end{array}$ & $\begin{array}{l}31 / 2 \\
4 \\
3 \\
4\end{array}$ & $\begin{array}{r}31 / 2 \\
4\end{array}$ & \\
\hline $\begin{array}{l}30 \\
30 \\
30 \\
30\end{array}$ & $\begin{array}{l}21 / 2 \\
3 \\
-4\end{array}$ & $\begin{array}{l}21 / 2 \\
3 \\
31 / 2 \\
4\end{array}$ & \\
\hline
\end{tabular}

1 As ordered. Refer to Table 14 for minimum arbor holes.
TABLE 7.-Type No.5-Wheels (recessed one side)

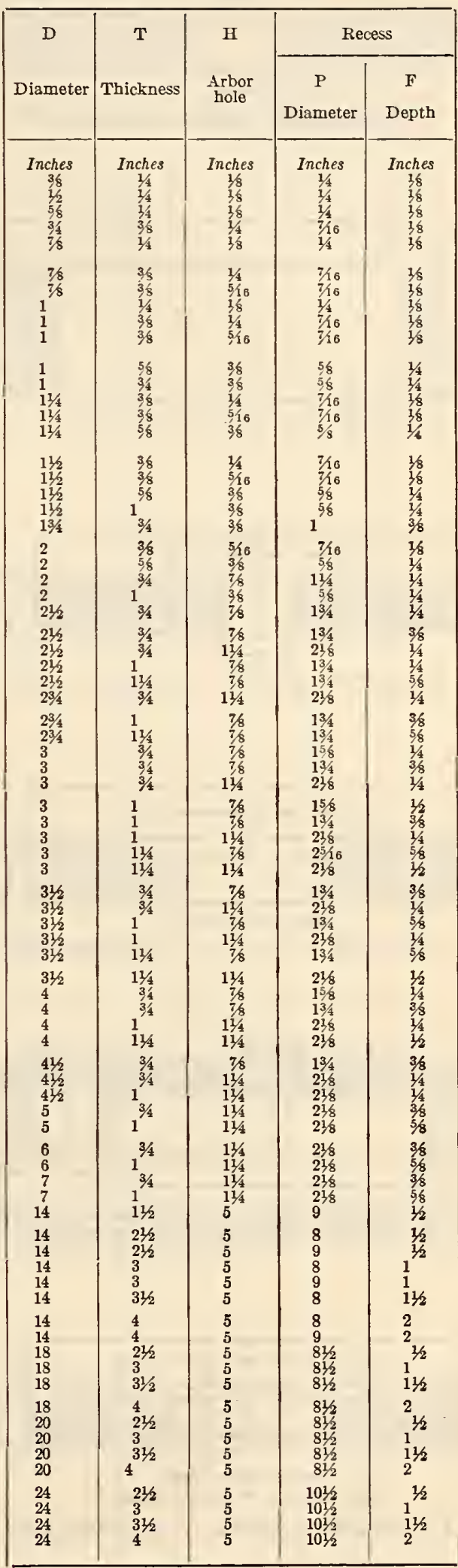




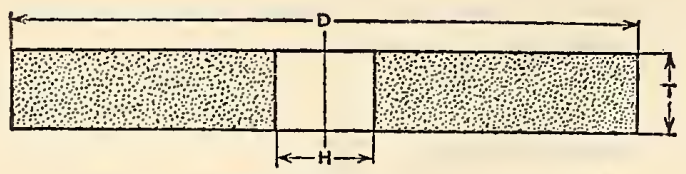

FigURe 230.-Type No. 1, straight

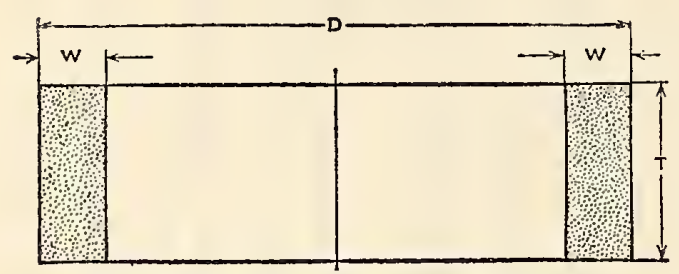

Figure 231.-Type No. 2, cylinder

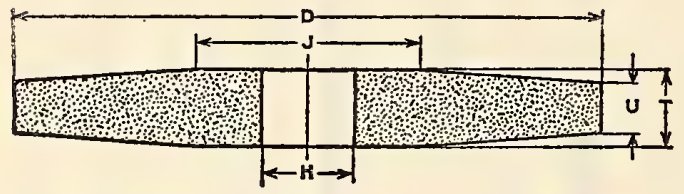

FigURe 232.-Type No. 4, tapered two sides

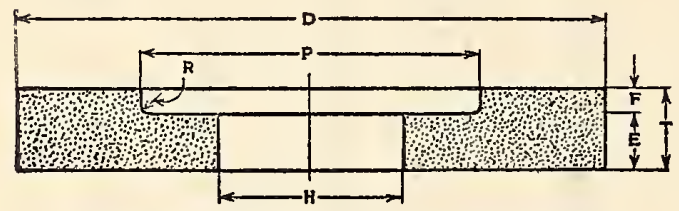

FigdRe 233.-Type No. 5, recessed one side

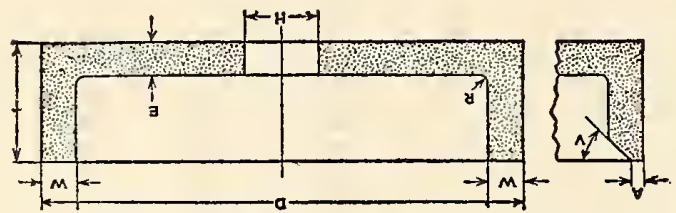

FIgURE 234.-Type No. 6, straight cup (wheels of this type can be furnished with beveled face

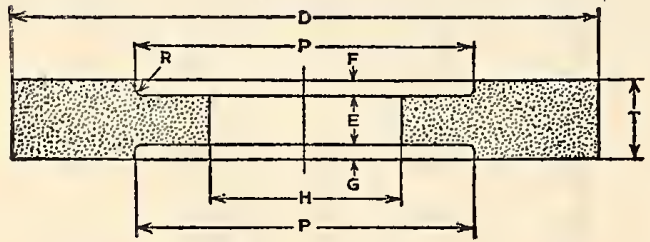

Figdre 235.-Type No, 7, recessed two sides
TABLE 8.-Type No. 6-Straight-cup wheels

\begin{tabular}{|c|c|c|c|c|c|c|c|}
\hline \multicolumn{6}{|c|}{ Straight face } & \multicolumn{2}{|c|}{ Bevel face } \\
\hline$\underset{\text { ter }}{\text { Diame- }}$ & $\begin{array}{c}\mathrm{T} \\
\text { Thick- } \\
\text { ness }\end{array}$ & $\begin{array}{c}\text { H } \\
\text { Arbor } \\
\text { hole } 1\end{array}$ & $\begin{array}{c}\text { W } \\
\text { Thick- } \\
\text { ness of } \\
\text { wall }\end{array}$ & $\begin{array}{c}E \\
\text { Center } \\
\text { or back } \\
\text { thick- } \\
\text { ness }\end{array}$ & Radius & $\begin{array}{c}\text { A } \\
\text { Flat } \\
\text { spot of } \\
\text { beveled } \\
\text { wall }\end{array}$ & $\begin{array}{c}\mathrm{V} \\
\text { Angle } \\
\text { of bevel }\end{array}$ \\
\hline \begin{tabular}{|c|} 
Inches \\
$21 / 2$ \\
3 \\
$31 / 2$ \\
4 \\
5
\end{tabular} & $\begin{array}{r}\text { Inches } \\
11 / 2 \\
11 / 2 \\
13 / 4 \\
11 / 2 \\
11 / 2\end{array}$ & Inches & $\begin{array}{r}\text { Inches } \\
3 / 8 \\
3 / 8 \\
3 / 8 \\
3 / 8 \\
3 / 8\end{array}$ & $\begin{array}{r}\text { Inches } \\
3 / 8 \\
3 / 8 \\
3 / 8 \\
3 / 3 \\
3 / 8\end{array}$ & \begin{tabular}{r|} 
Inch \\
$1 / 16$ \\
$1 / 16$ \\
$1 / 16$ \\
116 \\
$1 / 16$
\end{tabular} & $\begin{array}{r}\text { Inch } \\
1 / 8 \\
1 / 8 \\
1 / 8 \\
1 / 1 \\
1 / 8\end{array}$ & $\begin{array}{l}\cdot \\
30 \\
30 \\
30 \\
30 \\
30\end{array}$ \\
\hline $\begin{array}{r}8 \\
8 \\
8 \\
10 \\
10\end{array}$ & $\begin{array}{l}4 \\
5 \\
6 \\
4 \\
4\end{array}$ & & $\begin{array}{l}1 \\
1 \\
1 \\
1 \\
11 / 4\end{array}$ & $\begin{array}{l}1 \\
1 \\
1 \\
1 \\
11 / 4\end{array}$ & & & -- \\
\hline $\begin{array}{l}10 \\
12 \\
12 \\
12 \\
12\end{array}$ & $\begin{array}{l}4 \\
4 \\
4 \\
5 \\
6\end{array}$ & - & $\begin{array}{l}11 / 2 \\
11 / 4 \\
11 / 2 \\
11 / 2 \\
13 / 4\end{array}$ & $\begin{array}{l}11 / 2 \\
134 \\
11 / 2 \\
115 \\
13 / 4\end{array}$ & & & $\ldots$ \\
\hline $\begin{array}{l}14 \\
14 \\
14 \\
14 \\
16\end{array}$ & $\begin{array}{l}4 \\
4 \\
5 \\
6 \\
5\end{array}$ & $\begin{array}{l}--- \\
--- \\
--- \\
---\end{array}$ & $\begin{array}{l}11 / 4 \\
11 / 2 \\
11 / 4 \\
11 / 2 \\
2\end{array}$ & $\begin{array}{l}11 / 4 \\
11 / 2 \\
11 / 4 \\
11 / 2 \\
2\end{array}$ & & & $\begin{array}{l}--- \\
-- \\
-\cdots\end{array}$ \\
\hline $\begin{array}{l}20 \\
24 \\
24 \\
30\end{array}$ & $\begin{array}{l}6 \\
4 \\
6 \\
8\end{array}$ & -- & $\begin{array}{l}2 \\
13 / 4 \\
21 / 2 \\
21 / 2\end{array}$ & $\begin{array}{l}2 \\
13 / 4 \\
21 / 2 \\
21 / 2\end{array}$ & & & $-\ldots$ \\
\hline
\end{tabular}

1 As ordered.

TABLE 9.-Type No. 7-Wheels (Recessed two sides)

\begin{tabular}{|c|c|c|c|c|c|}
\hline \multirow{2}{*}{$\begin{array}{c}\text { D } \\
\text { Diam- } \\
\text { eter }\end{array}$} & \multirow{2}{*}{$\begin{array}{c}\text { T } \\
\text { Thick- } \\
\text { ness }\end{array}$} & \multirow{2}{*}{$\begin{array}{c}\text { H } \\
\underset{\substack{\text { Arbor } \\
\text { hole }}}{ }\end{array}$} & \multicolumn{3}{|c|}{ Recess } \\
\hline & & & $\begin{array}{c}\mathbf{P} \\
\text { Diam- } \\
\text { eter }\end{array}$ & $\begin{array}{c}\text { F } \\
\text { Depth }\end{array}$ & $\begin{array}{c}G \\
\text { Depth }\end{array}$ \\
\hline $\begin{array}{c}\text { Inches } \\
12 \\
12 \\
14 \\
14 \\
14\end{array}$ & $\begin{array}{c}\text { Inches } \\
11 / 2 \\
11 / 2 \\
11 / 2 \\
2 \\
2\end{array}$ & $\begin{array}{c}\text { Inches } \\
5 \\
5 \\
5 \\
5 \\
5\end{array}$ & $\begin{array}{c}\text { Inches } \\
71 / 2 \\
71 / 2 \\
71 / 2 \\
71 / 2 \\
71 / 2\end{array}$ & $\begin{array}{c}\text { Inches } \\
3 / 16 \\
1 / 2 \\
3 / 16 \\
3 / 16 \\
3 / 16\end{array}$ & $\begin{array}{c}\text { Inches } \\
7 / 16 \\
112 \\
7 / 16 \\
7 / 16 \\
15 / 16\end{array}$ \\
\hline $\begin{array}{l}14 \\
14 \\
14 \\
18 \\
18\end{array}$ & $\begin{array}{l}4 \\
5 \\
6 \\
2 \\
21 / 2\end{array}$ & $\begin{array}{l}5 \\
5 \\
5 \\
8 \\
8\end{array}$ & $\begin{array}{r}8 \\
8 \\
8 \\
11 \\
11\end{array}$ & $\begin{array}{l}1^{1 / 2} \\
1 / 2 \\
\frac{1}{3 / 8}\end{array}$ & $\begin{array}{l}1^{1 / 2} \\
2 \\
3 / 8 \\
5 / 8\end{array}$ \\
\hline $\begin{array}{l}18 \\
24 \\
24 \\
24 \\
24\end{array}$ & $\begin{array}{l}3 \\
11 / 4 \\
13 / 8 \\
11 / 2 \\
15 / 8\end{array}$ & $\begin{array}{r}8 \\
12 \\
12 \\
12 \\
12\end{array}$ & $\begin{array}{l}11 \\
151 / 4 \\
151 / 4 \\
151 / 4 \\
151 / 4\end{array}$ & $\begin{array}{l}1 / 2 \\
5 / 16 \\
5 / 16 \\
3 / 8 \\
3 / 8\end{array}$ & $\begin{array}{l}3 / 4 \\
5 / 16 \\
5 / 16 \\
3 / 8 \\
3 / 8\end{array}$ \\
\hline $\begin{array}{l}24 \\
24 \\
24 \\
24 \\
24\end{array}$ & $\begin{array}{l}13 / 4 \\
17 / 8 \\
2 \\
2 \\
21 / 4\end{array}$ & $\begin{array}{l}12 \\
12 \\
12 \\
12 \\
12\end{array}$ & $\begin{array}{l}1514 \\
151 / 4 \\
151 / 4 \\
1414 \\
151 / 4\end{array}$ & $\begin{array}{l}7 / 16 \\
7 / 16 \\
7 / 16 \\
9 / 16 \\
1 / 2\end{array}$ & $\begin{array}{l}7 / 16 \\
7 / 16 \\
7 / 16 \\
9 / 16 \\
1 / 2\end{array}$ \\
\hline $\begin{array}{l}24 \\
24 \\
24 \\
24 \\
24\end{array}$ & $\begin{array}{l}21 / 4 \\
21 / 4 \\
21 / 2 \\
23 / 4 \\
23 / 4\end{array}$ & $\begin{array}{l}12 \\
12 \\
12 \\
12 \\
12\end{array}$ & $\begin{array}{l}141 / 4 \\
151 / 4 \\
141 / 4 \\
151 / 4 \\
141 / 4\end{array}$ & $\begin{array}{l}9 / 16 \\
6 / 8 \\
916 \\
5 / 8 \\
9 / 16\end{array}$ & $\begin{array}{l}9 / 16 \\
916 \\
9 / 16 \\
5 / 8 \\
9116\end{array}$ \\
\hline $\begin{array}{l}24 \\
24 \\
24 \\
24 \\
24\end{array}$ & $\begin{array}{l}3 \\
3 \\
31 / 4 \\
31 / 2 \\
4\end{array}$ & $\begin{array}{l}12 \\
12 \\
12 \\
12 \\
12\end{array}$ & $\begin{array}{l}151 / 4 \\
141 / 4 \\
141 / 4 \\
141 / 4 \\
141 / 4\end{array}$ & $\begin{array}{l}11 / 16 \\
13 / 16 \\
13 / 16 \\
13 / 16 \\
13 / 16\end{array}$ & $\begin{array}{l}11 / 16 \\
13 / 16 \\
13 / 16 \\
13 / 16 \\
13 / 16\end{array}$ \\
\hline $\begin{array}{l}26 \\
20 \\
26 \\
26\end{array}$ & $\begin{array}{l}21 / 4 \\
21 / 4 \\
21 / 2 \\
21 / 2\end{array}$ & $\begin{array}{l}12 \\
12 \\
12 \\
12\end{array}$ & $\begin{array}{l}151 / 4 \\
141 / 4 \\
151 / 4 \\
141 / 4\end{array}$ & $\begin{array}{l}5 / 8 \\
9 / 16 \\
5 / 8 \\
9 / 16\end{array}$ & $\begin{array}{r}1 / 8 \\
9 / 6 \\
8 / 8 \\
9 / 16\end{array}$ \\
\hline $\begin{array}{l}26 \\
26 \\
26 \\
26\end{array}$ & $\begin{array}{l}23 / 4 \\
23 / 4 \\
3 \\
3\end{array}$ & $\begin{array}{l}12 \\
12 \\
12 \\
12\end{array}$ & $\begin{array}{l}151 / 4 \\
141 / 4 \\
151 / 4 \\
141 / 4\end{array}$ & $\begin{array}{c}5 / 8 \\
9 / 16 \\
5 / 8 \\
13 / 16\end{array}$ & $\begin{array}{c}5 / 8 \\
9 / 16 \\
7 / 8 \\
13 / 16\end{array}$ \\
\hline
\end{tabular}


TABLE 10.-Type No. 11-Flaring-cup wheels

\begin{tabular}{|c|c|c|c|c|c|c|c|c|c|}
\hline \multicolumn{8}{|c|}{ Straight face } & \multicolumn{2}{|c|}{ Bevel face } \\
\hline $\begin{array}{c}\text { Diam- } \\
\text { eter }\end{array}$ & $\begin{array}{c}\text { Thick- } \\
\text { ness }\end{array}$ & $\begin{array}{c}\text { H } \\
\text { Ar. } \\
\text { bor } \\
\text { bole' }\end{array}$ & $\begin{array}{c}\text { W } \\
\text { Thick- } \\
\text { ness } \\
\text { of wall }\end{array}$ & $\begin{array}{c}\text { E } \\
\text { Cen- } \\
\text { ter or } \\
\text { back } \\
\text { thick } \\
\text { ness }\end{array}$ & $\begin{array}{l}\text { J } \\
\text { Diam- } \\
\text { ter of } \\
\text { flat or } \\
\text { small } \\
\text { diam- } \\
\text { eter }\end{array}$ & $\begin{array}{c}\mathbf{K} \\
\text { Diam- } \\
\text { eter of } \\
\text { flat in } \\
\text { side }\end{array}$ & $\begin{array}{c}\text { Radi- } \\
\text { us }\end{array}$ & $\begin{array}{l}\text { A } \\
\text { Flat } \\
\text { spot } \\
\text { of } \\
\text { bev- } \\
\text { eled } \\
\text { wall }\end{array}$ & $\begin{array}{c}\mathrm{V} \\
\text { Angle } \\
\text { of } \\
\text { bevel }\end{array}$ \\
\hline $\begin{array}{l}\text { ches } \\
3 \\
314 \\
331 \\
4 \\
416 \\
5 \\
6 \\
7\end{array}$ & $\begin{array}{c}\text { Inches } \\
1114 \\
11 / 4 \\
11 / 2 \\
11 / 2 \\
2 \\
13 / 4 \\
2 \\
2\end{array}$ & 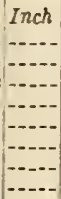 & \begin{tabular}{r|} 
Inch \\
$1 / 4$ \\
$1 / 2$ \\
$1 / 4$ \\
$1 / 4$ \\
$1 / 1$ \\
$1 / 4$ \\
3.8
\end{tabular} & $\begin{array}{r}\text { Inch } \\
36 \\
38 \\
38 \\
36 \\
1 / 2 \\
1 / 2 \\
1 / 2 \\
1 / 2\end{array}$ & \begin{tabular}{|c|} 
Inches \\
211 \\
218 \\
218 \\
3 \\
$33 / 4$ \\
334 \\
$41 / 2$ \\
5
\end{tabular} & \begin{tabular}{r|} 
Inches \\
136 \\
$11 / 2$ \\
112 \\
258 \\
318 \\
318 \\
334 \\
334
\end{tabular} & $\begin{array}{r}\text { Inch } \\
110 \\
110 \\
110 \\
110 \\
110 \\
110 \\
116\end{array}$ & $\begin{array}{r}\text { Inch } \\
16 \\
16 \\
1 / 8 \\
16 \\
16\end{array}$ & 30 \\
\hline
\end{tabular}

1 As ordered.

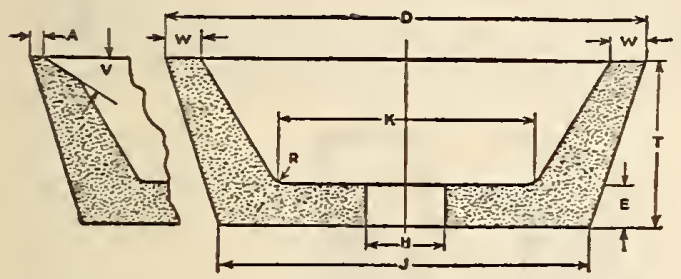

Figdre 236.-Type No. 11, flaring cup (wheels of this type can be furnished with beveled face)

TABLE 11.-Type No. 12-Dish wheels

\begin{tabular}{|c|c|c|c|c|c|c|}
\hline $\begin{array}{c}\text { Dism- } \\
\text { eter }\end{array}$ & $\begin{array}{c}\text { Thick- } \\
\text { ness }\end{array}$ & $\begin{array}{l}\text { Arbor } \\
\text { hole } 1\end{array}$ & $\begin{array}{c}\mathrm{E} \\
\text { Center } \\
\text { or back } \\
\text { thick- } \\
\text { ness }\end{array}$ & $\begin{array}{l}\mathrm{K} \text { and J } \\
\text { Diameter } \\
\text { of flat in- } \\
\text { side and } \\
\text { diameter } \\
\text { of flat or } \\
\text { small } \\
\text { diameter }\end{array}$ & $\begin{array}{c}\text { U } \\
\text { Width } \\
\text { of face }\end{array}$ & $\begin{array}{c}\text { M } \\
\text { Large } \\
\text { diame- } \\
\text { ter of } \\
\text { bevel }\end{array}$ \\
\hline $\begin{array}{c}\text { Inches } \\
3 \\
31 / 2 \\
4 \\
41 / 2 \\
5 \\
6 \\
6 \\
8 \\
8\end{array}$ & $\begin{array}{c}\text { Inch } \\
1 / 2 \\
1 / 2 \\
1 / 2 \\
1 / 2 \\
1 / 2 \\
3 / 2 \\
3 / 4 \\
3 / 4 \\
1\end{array}$ & & $\begin{array}{l}\text { Inch } \\
5 / 16 \\
5 / 16 \\
5 / 16 \\
5 / 16 \\
5 / 16 \\
5 / 16 \\
36 \\
1 / 2 \\
1 / 2\end{array}$ & $\begin{array}{c}\text { Inches } \\
11 / 2 \\
13 / 4 \\
2 \\
21 / 4 \\
21 / 2 \\
3 \\
3 \\
4 \\
4\end{array}$ & $\begin{array}{l}\text { Inch } \\
116 \\
332 \\
332 \\
332 \\
16 \\
16 \\
16 \\
16 \\
16 \\
16 \\
16\end{array}$ & $\begin{array}{c}\text { Inches } \\
23 / 4 \\
31 / 8 \\
33 / 8 \\
4 \\
41 / 2 \\
51 / 4 \\
51 / 4 \\
7 \\
7\end{array}$ \\
\hline
\end{tabular}

1 As ordered.

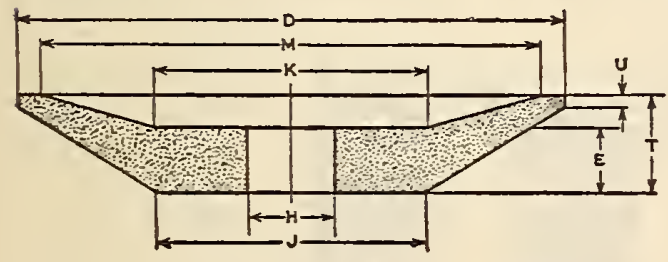

Figdre 237,-Type No. 12, dish
TABLE 12.-Type No.13-Wheels (saw gummer), saucer

\begin{tabular}{|c|c|c|c|c|}
\hline Diameter & $\begin{array}{c}\text { T } \\
\text { Thick- } \\
\text { ness }\end{array}$ & $\begin{array}{c}\text { H } \\
\text { Arbor } \\
\text { hole } 1\end{array}$ & $\begin{array}{c}\mathrm{E} \text { and } \mathrm{U} \\
\text { Center or } \\
\text { back thick- } \\
\text { ness and } \\
\text { width of } \\
\text { face }\end{array}$ & \begin{tabular}{|c|}
$\mathrm{K}$ \\
$\begin{array}{c}\text { Diameter } \\
\text { of flat } \\
\text { inside }\end{array}$ \\
\end{tabular} \\
\hline $\begin{array}{c}\text { Inches } \\
6 \\
6 \\
6 \\
6 \\
6\end{array}$ & $\begin{array}{c}\text { Inches } \\
1 / 2 \\
96 \\
3 / 8 \\
1 / 8 \\
1\end{array}$ & & $\begin{array}{l}\text { Inches } \\
14 \\
13 \\
1 / 8 \\
12 \\
58 \\
3 / 4\end{array}$ & $\begin{array}{c}\text { Inches } \\
3 \\
3 \\
3 \\
3 \\
3 \\
3\end{array}$ \\
\hline $\begin{array}{l}6 \\
6 \\
6 \\
6 \\
8 \\
8\end{array}$ & $\begin{array}{l}11 / 8 \\
114 \\
13 \\
11 / 2 \\
1 / 2 \\
1 / 2\end{array}$ & & $\begin{array}{l}3 / 8 \\
1 / 8 \\
11 / 8 \\
1314 \\
1 / 4\end{array}$ & $\begin{array}{l}3 \\
3 \\
3 \\
3 \\
3 \\
3\end{array}$ \\
\hline $\begin{array}{l}8 \\
8 \\
8 \\
8 \\
8\end{array}$ & $\begin{array}{l}9 / 8 \\
3 / 4 \\
3 / 8 \\
11 / 8 \\
11 / 8\end{array}$ & & $\begin{array}{l}3 / 8 \\
1 / 8 \\
5 / 8 \\
3 / 4 \\
7 / 8\end{array}$ & $\begin{array}{l}3 \\
3 \\
3 \\
3 \\
3 \\
3\end{array}$ \\
\hline $\begin{array}{r}8 \\
8 \\
8 \\
10 \\
10\end{array}$ & $\begin{array}{r}114 \\
13 / 2 \\
115 \\
112 \\
5 \%\end{array}$ & & $\begin{array}{c}1 \\
11 / 3 \\
114 \\
1 / 4 \\
3 / 8\end{array}$ & $\begin{array}{l}3 \\
3 \\
3 \\
31 / 2 \\
31 / 2 \\
31 / 2\end{array}$ \\
\hline $\begin{array}{l}10 \\
10 \\
10 \\
10 \\
10\end{array}$ & $\begin{array}{l}3 / 4 \\
1 / 8 \\
11 / 8 \\
11 / 4\end{array}$ & & $\begin{array}{r}1 / 2 \\
0^{6 / 8} \\
3 / 4 \\
1^{1 / 8}\end{array}$ & $\begin{array}{l}31 / 2 \\
31 / 2 \\
31 / 2 \\
3132 \\
31 / 2\end{array}$ \\
\hline $\begin{array}{l}10 \\
10 \\
12 \\
12 \\
12\end{array}$ & $\begin{array}{r}13 / 8 \\
132 \\
132 \\
5 \% \\
34\end{array}$ & & $\begin{array}{l}11 / 8 \\
11 / 4 \\
1 / 4 \\
13 \\
13\end{array}$ & $\begin{array}{l}31 / 2 \\
31 / 2 \\
4 \\
4 \\
4\end{array}$ \\
\hline $\begin{array}{l}12 \\
12 \\
12 \\
12\end{array}$ & $\begin{array}{l}1^{7 / 8} \\
11 / 8 \\
11 / 4\end{array}$ & & $\begin{array}{r}3 / 8 \\
3^{3 / 8} \\
1^{1 / 8}\end{array}$ & $\begin{array}{l}4 \\
4 \\
4 \\
4\end{array}$ \\
\hline $\begin{array}{l}12 \\
12 \\
12 \\
12\end{array}$ & $\begin{array}{l}13 / 8 \\
11 / 2 \\
108 \\
13 / 4\end{array}$ & & $\begin{array}{l}11 / 8 \\
114 \\
118 \\
11 / 2\end{array}$ & $\begin{array}{l}4 \\
4 \\
4 \\
4\end{array}$ \\
\hline
\end{tabular}

1 As ordered.

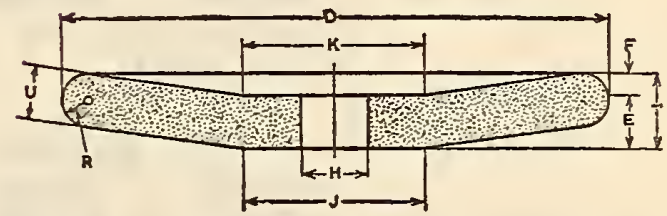

FIGURE 238.-Type No.-19 (saw gummer), saucer

No wheel should be operated which has smaller arbor hole than shown in following table for varying diameters and thicknesses: 
TABLE 14.-Minimum sizes of arbor holes given in inches for various diameters and thickness of grinding wheels

\begin{tabular}{|c|c|c|c|c|c|c|c|c|c|c|c|c|c|c|c|c|c|c|c|}
\hline Diam- \\
eter
\end{tabular}
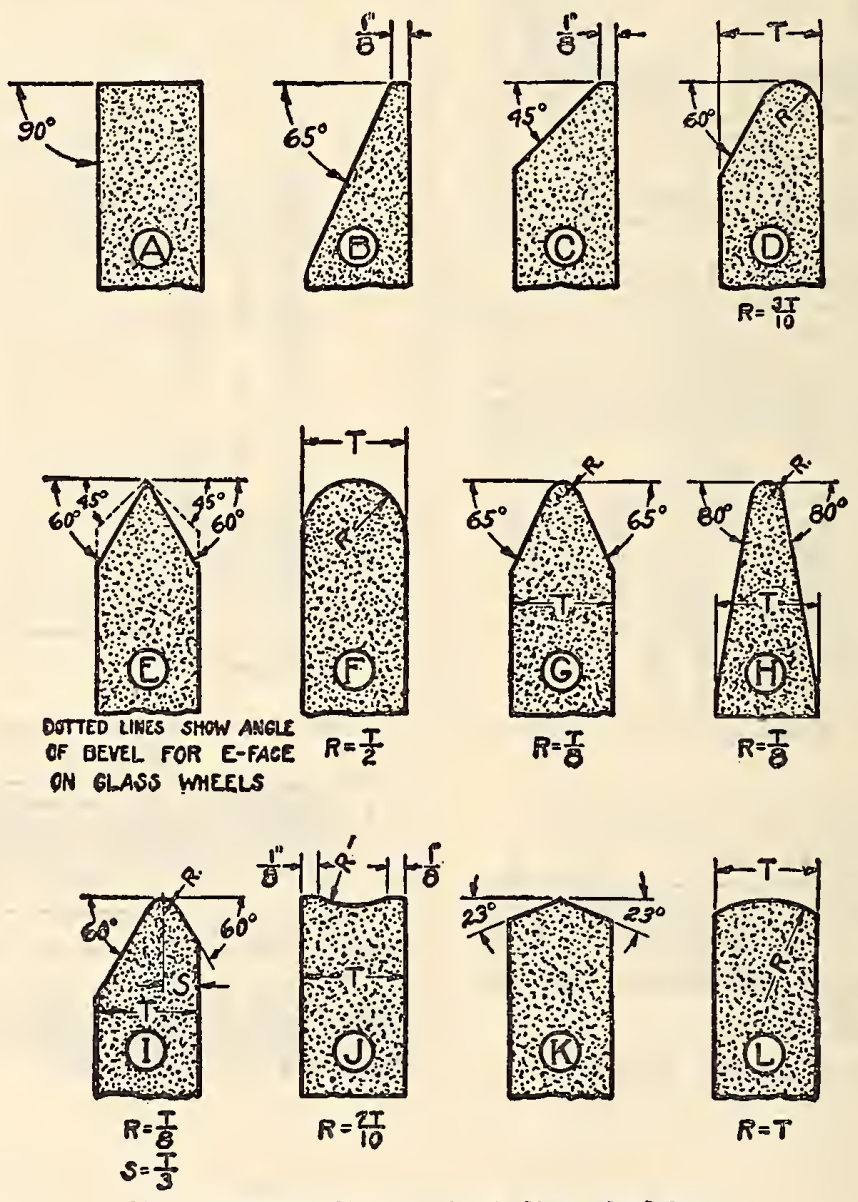

FigURE 239.-Shapes of grinding wheel faces 
TABLE 15.-Type No. 5-Wheels for internal grinding (recessed one side)

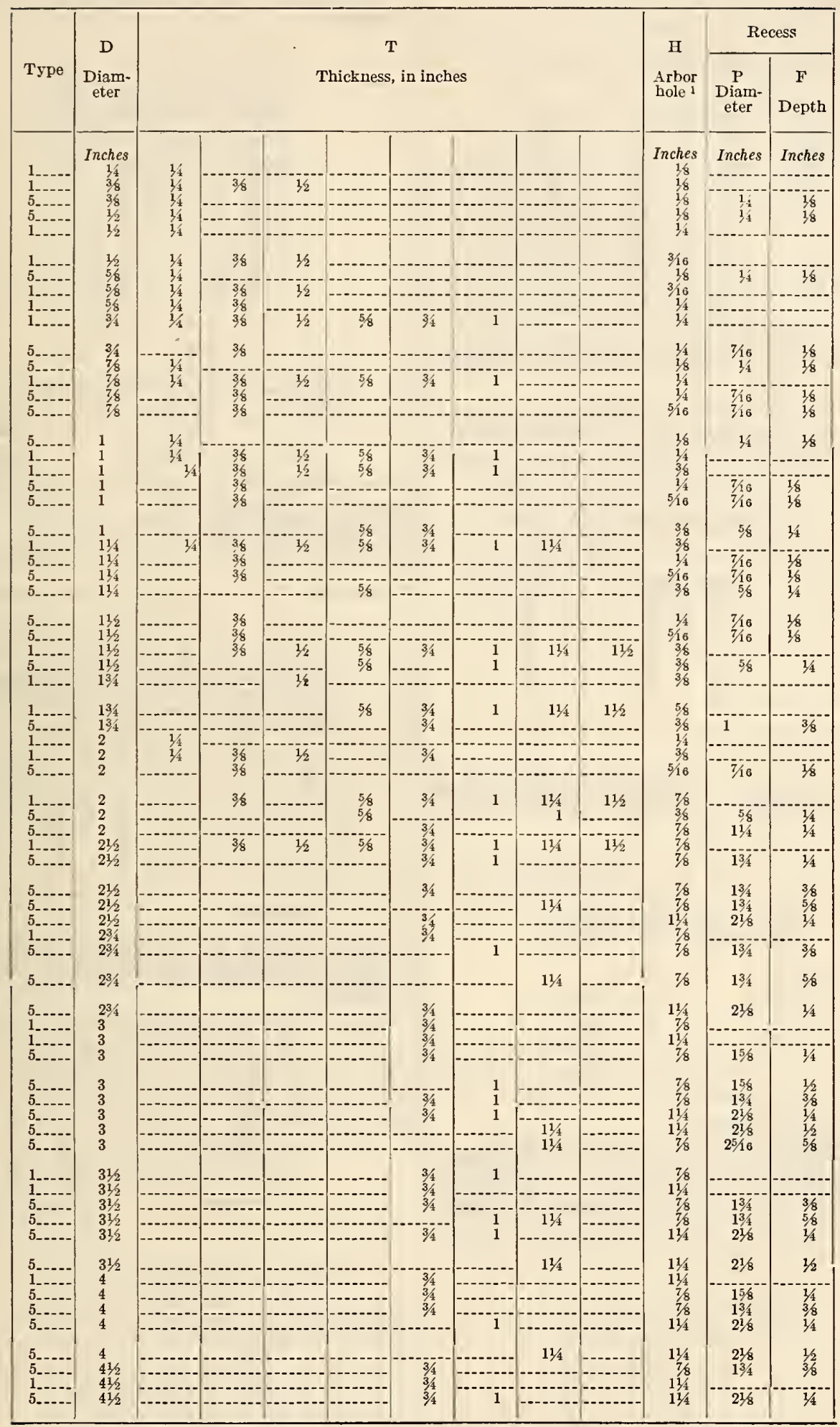

I Arbors are sizes now in use. 
TABLE 16.-Type No. 1-Internal grinding wheels recommended for new machine design

\begin{tabular}{|c|c|c|c|}
\hline $\begin{array}{l}\text { Wheel } \\
\text { diameter }\end{array}$ & Arbor & $\begin{array}{l}\text { Wheel } \\
\text { diameter }\end{array}$ & Arbor \\
\hline $\begin{array}{c}\text { Inches } \\
1 / 4 \\
3 / 8 \\
1 / 2 \\
5 / 8 \\
5 / 8 \\
3 / 4 \\
7 / 8 \\
1 \\
1 \\
11 / 4 \\
11 / 2 \\
13 / 4 \\
2\end{array}$ & $\begin{array}{c}\text { Inches } \\
1 / 8 \\
1 / 8 \\
3 / 16 \\
3 / 16 \\
1 / 4 \\
1 / 4 \\
1 / 4 \\
114 \\
3 / 8 \\
3 / 8 \\
8 / 8 \\
112 \\
3 / 8\end{array}$ & $\begin{array}{l}\text { Inches } \\
2 \\
2 \\
2 \\
21 / 2 \\
21 / 2 \\
3 \\
3 \\
31 / 2 \\
31 / 2 \\
4 \\
4 \\
4\end{array}$ & $\begin{array}{c}\text { Inches } \\
1 / 2 \\
5 / 8 \\
7 / 8 \\
5 / 8 \\
7 / 8 \\
5 / 8 \\
7 / 8 \\
5 / 8 \\
7 / 8 \\
5 / 8 \\
7 / 8 \\
11 / 4\end{array}$ \\
\hline
\end{tabular}

Note.-Thickness (in inches), 1/4, $8 / 8,1 / 2,5 / 8,3 / 4,7 / 8,1,114,136,134$, 2 , and $21 / 2$.

TABLE 17.-Type No. 1-Straight cylindrical grinding wheels recommended for new machine design

\begin{tabular}{|c|c|c|c|c|c|}
\hline $\begin{array}{c}\text { D } \\
\text { Diam- } \\
\text { eter }\end{array}$ & $\begin{array}{c}T \\
\text { Thick- } \\
\text { ness } 1\end{array}$ & $\begin{array}{c}\mathrm{H} \\
\text { Arbor } \\
\text { hole }\end{array}$ & $\begin{array}{c}\text { D } \\
\text { Diam- } \\
\text { eter }\end{array}$ & $\begin{array}{c}\text { T } \\
\text { Thick- } \\
\text { ness } 1\end{array}$ & $\begin{array}{c}\mathrm{H} \\
\text { Arbor } \\
\text { hole }\end{array}$ \\
\hline $\begin{array}{c}\text { Inches } \\
10 \\
12 \\
14 \\
16 \\
18 \\
18 \\
20 \\
20\end{array}$ & $\mid$\begin{tabular}{c}
$-1---1$ \\
\hdashline-1
\end{tabular} & \begin{tabular}{|c|} 
Inches \\
5 \\
5 \\
5 \\
5 \\
5 \\
8 \\
5 \\
8
\end{tabular} & $\begin{array}{c}\text { Inches } \\
20 \\
24 \\
24 \\
24 \\
26 \\
30 \\
36\end{array}$ & & $\begin{array}{c}\text { Inches } \\
12 \\
5 \\
8 \\
12 \\
12 \\
12 \\
12\end{array}$ \\
\hline
\end{tabular}

1 As ordered.

TABLE 18.-Type No.5-Cylindrical grinding wheels (recessed one side) recommended for new machine design

\begin{tabular}{|c|c|c|c|}
\hline $\begin{array}{c}\text { D } \\
\text { Diam- } \\
\text { eter }\end{array}$ & $\begin{array}{c}\mathrm{T} \\
\text { Thick- } \\
\text { ness }{ }^{1}\end{array}$ & $\begin{array}{c}\text { H } \\
\text { Arbor } \\
\text { hole }\end{array}$ & $\begin{array}{c}\text { P } \\
\text { Diam- } \\
\text { eter of } \\
\text { recess }\end{array}$ \\
\hline $\begin{array}{c}\text { Inches } \\
14 \\
18 \\
20 \\
24\end{array}$ & & $\begin{array}{c}\text { Inches } \\
5 \\
5 \\
5 \\
5\end{array}$ & $\begin{array}{c}\text { Inches } \\
8 \\
81 / 2 \\
81 / 2 \\
101 / 2\end{array}$ \\
\hline
\end{tabular}

1 As ordered.

Depth of recess for type No. 5 wheels.-

$\mathrm{W}$ ith $\mathrm{T} 2$ inches and less.

With $\mathrm{T} 21 / 2$ inches

$=0$.

With $\mathrm{T} 3$ inches

$\mathbf{F}=1 / 2$ inch

With $\mathrm{T} 31 / 2$ inches

$\mathrm{F}=1$ inch.

With $\mathrm{T} 4$ inches.

$\mathrm{F}=2$ inches.
TABLE 19.-Type No. 7-Cylindrical grinding wheels (recessed two sides) recommended for new machine design

\begin{tabular}{|c|c|c|c|}
\hline $\begin{array}{c}\text { D } \\
\text { Diam- } \\
\text { eter }\end{array}$ & $\begin{array}{c}\mathrm{T} \\
\begin{array}{c}\text { Thick- } \\
\text { ness }\end{array}\end{array}$ & $\begin{array}{c}\text { H } \\
\text { Arbor } \\
\text { hole }\end{array}$ & $\begin{array}{c}\text { P } \\
\begin{array}{c}\text { Diam- } \\
\text { eter } \\
\text { of recess }\end{array}\end{array}$ \\
\hline $\begin{array}{c}\text { Inches } \\
14 \\
16 \\
18 \\
20 \\
20 \\
24 \\
24 \\
26 \\
30\end{array}$ & & $\begin{array}{c}\text { Inches } \\
5 \\
5 \\
8 \\
8 \\
12 \\
8 \\
12 \\
12 \\
12\end{array}$ & $\begin{array}{c}\text { Inches } \\
8 \\
8 \\
11 \\
11 \\
141 / 4 \\
11 \\
141 / 4 \\
141 / 4 \\
1414\end{array}$ \\
\hline
\end{tabular}

1 As ordered:

Depth of recess for 8 and 12 inch hole

\begin{tabular}{|c|c|c|}
\hline \multirow{2}{*}{ Thickness (in inches) } & \multicolumn{2}{|c|}{ Depth of recess } \\
\hline & $F$ & $\mathrm{G}$ \\
\hline $\begin{array}{l}\text { With } \mathrm{T} \text { less than } 2 \text { inches. } \\
\text { With } \mathrm{T} \text { from } 2 \text { inches up to } 2^{3} 1 / 32 \text {, inclusive. } \\
\text { With } \mathrm{T} 3 \text { inches and larger }\end{array}$ & $\begin{array}{c}\text { Inch } \\
0 \\
9 / 16 \\
1316\end{array}$ & 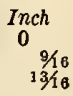 \\
\hline
\end{tabular}

TABLE 20.-Type No. 1-Straight cutting-off and slotting wheels

\begin{tabular}{|c|c|c|c|c|c|}
\hline $\begin{array}{c}\text { D } \\
\text { Diam- } \\
\text { eter }\end{array}$ & $\begin{array}{c}\text { T } \\
\text { Thick- } \\
\text { ness- }\end{array}$ & $\begin{array}{c}\mathrm{H} \\
\text { Arbor } \\
\text { holel }\end{array}$ & $\begin{array}{c}\text { D } \\
\text { Diam- } \\
\text { eter }\end{array}$ & $\begin{array}{c}\text { T } \\
\text { Thick- } \\
\text { ness- }\end{array}$ & $\begin{array}{c}\text { H } \\
\text { Arbor } \\
\text { hole }\end{array}$ \\
\hline $\begin{array}{c}\text { Inches } \\
4 \\
4 \\
4 \\
4 \\
5 \\
5 \\
6 \\
6 \\
6 \\
6\end{array}$ & $\begin{array}{c}\text { Inch } \\
132 \\
132 \\
1 / 16 \\
3 / 32 \\
168 \\
1 / 16 \\
168 \\
1 / 32 \\
1 / 16 \\
3 / 32 \\
1 / 6\end{array}$ & 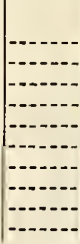 & $\begin{array}{c}\text { Inches } \\
7 \\
7 \\
8 \\
8 \\
8 \\
10 \\
10 \\
12 \\
12\end{array}$ & $\begin{array}{c}\text { Inch } \\
1 / 16 \\
1 / 8 \\
1 / 16 \\
3 / 32 \\
168 \\
3 / 32 \\
168 \\
3 / 32 \\
168\end{array}$ & \\
\hline
\end{tabular}

1 As ordered.

TABLE 21.-Type No. 1-Straight wheels with large holes for tool grinding recommended for new machine design

\begin{tabular}{|c|c|c|}
\hline $\begin{array}{c}\text { D } \\
\text { Diameter }\end{array}$ & $\begin{array}{c}\text { T } \\
\text { Thickness } 1\end{array}$ & $\begin{array}{c}\text { H } \\
\text { Arbor hole }\end{array}$ \\
\hline $\begin{array}{c}\text { Inches } \\
14 \\
20 \\
24 \\
26 \\
36\end{array}$ & $\mid \begin{array}{l}- \\
- \\
-\end{array}$ & $\begin{array}{c}\text { Inches } \\
5 \\
9 \\
10 \\
12 \\
24\end{array}$ \\
\hline
\end{tabular}

1 As ordered. 
TABLE 22.-Type No. 1-Straight wheel sizes recommended for new design high speed swing frame and floor stand grinding machines (organic bonded wheels only)

\begin{tabular}{|c|c|c|}
\hline $\begin{array}{c}\text { D } \\
\text { Diameter }\end{array}$ & $\begin{array}{c}\mathrm{T} \\
\text { Thickness }\end{array}$ & $\begin{array}{c}\mathrm{H} \\
\text { Arbor hole }\end{array}$ \\
\cline { 1 - 2 } Inches & Inches & Inches \\
16 & $2,21 / 2,3$ & 6 \\
18 & $\mathbf{2}, 21 / 2,3$ & 8 \\
20 & $2,21,2,3$ & 8 \\
24 & $2,21,3,3,4$ & 12 \\
30 & $3,31,2,4$ & 12 \\
36 & $3,312,4$ & 12 \\
\hline
\end{tabular}

TABLE 23.-Type No. 6-Straight cup wheels for tool-room grinding

\begin{tabular}{|c|c|c|c|c|c|c|c|}
\hline \multicolumn{6}{|c|}{ Straight face } & \multicolumn{2}{|c|}{ Bevel face } \\
\hline $\begin{array}{c}\mathrm{D} \\
\text { Diam- } \\
\text { eter }\end{array}$ & $\begin{array}{c}\text { T } \\
\text { Thick- } \\
\text { ness }\end{array}$ & $\begin{array}{c}\mathrm{H} \\
\text { Arbor } \\
\text { hole } 1\end{array}$ & $\begin{array}{c}\text { Wr } \\
\text { Thick- } \\
\text { ness of } \\
\text { wall }\end{array}$ & $\begin{array}{c}\text { E } \\
\text { Center } \\
\text { or back } \\
\text { thick- } \\
\text { ness }\end{array}$ & Radius & $\begin{array}{c}\text { A } \\
\text { Flat } \\
\text { spot of } \\
\text { beveled } \\
\text { wall }\end{array}$ & $\begin{array}{c}\text { V } \\
\text { Angle } \\
\text { of bevel }\end{array}$ \\
\hline $\begin{array}{c}\text { Inches } \\
21 / 2 \\
3 \\
31 / 2 \\
4 \\
5\end{array}$ & $\begin{array}{r}\text { Inches } \\
11 / 2 \\
11 / 2 \\
13 / 4 \\
11 / 2 \\
11 / 2\end{array}$ & 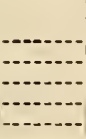 & \begin{tabular}{r|} 
Inch \\
$3 / 8$ \\
38 \\
38 \\
38 \\
38 \\
$3 / 8$
\end{tabular} & \begin{tabular}{|r|} 
Inch \\
$3 / 8$ \\
$3 / 8$ \\
$3 / 8$ \\
38 \\
36 \\
36
\end{tabular} & $\begin{array}{r}\text { Inch } \\
1 / 16 \\
116 \\
1 / 16 \\
1 / 16 \\
1 / 10\end{array}$ & $\begin{array}{r}\text { Inch } \\
1 / 8 \\
1 / 8 \\
18 \\
1 / 8 \\
1 / 8 \\
18\end{array}$ & $\begin{array}{r}\cdot \\
30 \\
30 \\
30 \\
30 \\
30\end{array}$ \\
\hline
\end{tabular}

1 As ordered.

This recommendation in no way seeks to embarrass users who possess grinding machines which may have been designed to accommodate certain of the eliminated sizes of grinding wheels. In the course of time new wheels and new grinders will be made to conform to the simplified list. Meanwhile all current equipment will be serviced. There is no need to scrap or discard grinding machines or any parts thereof, for that would be wasteful and diametrically opposed to the benefits that rest potentially within this recommendation for all interests.

NOTE.-The full test of this standard as published includes a supplement of standard nomenclature for various classes of work found in the grinding industry.

\subsection{ABRASIVE CLOTHS AND PAPERS.}

Abrasive Paper and Cloth Manufacturers' Exchange, standards and specifications for abrasive coated products, 1929 .

(The above-entitled publication includes standard gradings for grits, including standard sands for flint, emery, garnet, aluminum oxide, and silicon carbide; specifications and tests for backings (cloth, paper, combination cloth and paper, and fiber); types of coatings (open type, closed type); standards for packing of abrasives, and finished abrasive products standards, as follows:)

No. 1 , flint.

No. 2 , emery cloth.

Nos. 3, 3a, garnet.

No. 4 , chalk flint.

Nos. 5, 5a, aluminum oxide (for metalwork).

Nos. 6, 6a, aluminum oxide (for woodwork).

Nos. 7, 7a, 7b, silicon carbide.

Federal Specifications Board, specification No. 385a, United States Government master specification for paper, flint, October 1, 1928.

\section{TYPES AND CLASSES}

The product shall be of one type and of two classes, as follows: Class A, ream; and class B, roll.

\section{MATERIAL AND WORKMANSHIP}

The product shall be made under approved methods, with high-grade backing abrasive and adhesive materials, and shall be free from any defects which may affect the serviceability of the material.

\section{GENERAL REQUIREMENTS}

1. (a) The product shall be furnished in the following commercial grain sizes, as specified: $4 / 0$ $3 / 0,2 / 0,0,1 / 2,1,1 \frac{1}{2}, 2,2 \frac{1}{2}, 3$, and $3 \frac{1}{2}$.

(b) The designations given above represent in the order given the finest to the coarsest grain covered by this specification.

(c) The size of the grain for the respective designating number shall conform to the size of grain on the corresponding standard sample.

2. All flint abrasive shall be of good quality, crushed from clean, hard quartz, and shall be free from any extraneous material.

\section{DETAIL REQUIREMENTS}

1. Class A, ream, shall be furnished in sheets 9 by 11 inches, unless otherwise specified.

2. Class B, roll, shall be furnished in rolls 50 yards long and in widths as specified.

3. The backing on ream paper shall be of good quality of Kraft paper.

4. The backing on roll paper shall be of a good quality of Kraft or rope paper.

5. The weight of the paper backing shall be within the following limits:

\begin{tabular}{l|c}
\hline & $\begin{array}{c}\text { Nomi- } \\
\text { nal paper } \\
\text { weight }\end{array}$ \\
\hline Ream flint, Nos. $4 / 0$ to 1 , inclusive & $\begin{array}{c}\text { Pounds } \\
60-70 \\
70-90 \\
125-135\end{array}$ \\
Ream flint, Nos. $11 / 2$ to $31 / 2$, inclusive flint, all numbers
\end{tabular}

These weights are based on 24 by 36 inch sheets, 480 sheets per ream.

6. The average tensile strength of flint paper per inch of width shall be not less than the following:

\begin{tabular}{l|r|r}
\hline & $\begin{array}{r}\text { Stronger } \\
\text { direction }\end{array}$ & $\begin{array}{r}\text { Weaker } \\
\text { direction }\end{array}$ \\
\hline & Pounds & Pounds \\
Ream flint, Nos. $4 / 0$ to 1 , inclusive & 42 & 22 \\
Ream flint, Nos. $11 / 2$ to $31 / 2$, inclusive & 49 & 45 \\
Roll flint, all numbers........... & 85 & 45 \\
\hline
\end{tabular}

7. Ream flint paper shall be packed in quires containing 24 sheets each. Either 5 or 10 of these l-quire packages shall be tied in a bale with string or wrapped in strong paper. The bales shall be packed in bundles having wood top and bottom boards, and bound securely with wire. Standard bundles shall contain the following quantities:

\begin{tabular}{|r|c|}
\hline Grain number & $\begin{array}{c}\text { Reams per } \\
\text { bundle }\end{array}$ \\
\hline $4 / 0,3 / 0,2 / 0,0$ & 5 \\
$1 / 2$ & 4 \\
$11 / 2$ & $31 / 2$ \\
$11 / 2$ & 3 \\
$2,1 / 2$ & 2 \\
3 & $11 / 2$ \\
$31 / 2$ & 1 \\
\hline
\end{tabular}


8. Roll paper shall be carefully wrapped in strong paper.

9. Each sheet shall be marked with the manufacturer's name and the grain number. When shipment is made in paper packages, the manufacturer's name, the grain number, and the quantity contained shall appear on the wrapper. The manufacturer's name, the grain number, and the quantity contained shall also appear on the end boards of the shipping bundles.

10. Roll flint paper shall be plainly marked, at least every 3 feet, with the manufacturer's name and the grain number, and the outside wrapper shall also be marked.

\section{METHOD OF INSPECTION AND TESTS}

1. Samples taken from each shipment shall be subjected to test by rubbing against the standard samples. The specimens tested shall be equal to the standard sample with respect to the quality and the adhesion of the grain, as evidenced by the comparative condition of the surfaces in rubbing contact.

2. Strength test pieces 1 inch in width shall be cut, both longitudinally and transversely, from samples taken from each delivery. At least five such test pieces from each direction shall be placed in a tensile testing machine with the jaws 3 inches apart, and pulled to rupture at the rate of 12 inches per minute. The required strength is not specified for the longitudinal and transverse directions, respectively, as the sheets are not always cut in the same direction of the paper.

\section{PACKING AND MARKING OF SHIPMENTS}

Each shipment should be marked with the name of the material, name of the contractor, and the number of the contract or order under which shipment is made.

Federal Specifications Board, specification No. 386a, United States Government master specification for paper, garnet, October 1, 1928.

(This specification is identical as to certain items with Federal Specifications Board No. 385a (given above), the items in common being so designated below:)

\section{TYPES AND CLASSES}

(Same as 385a.)

\section{MATERIAI AND WORKMANSHIP}

(Same as 385a.)

\section{GENERAL REQUIREMENTS}

(Subsection 1 of No. 385a is part of this specification, with the following additional:)

All garnet abrasive shall be of good quality, equal in all respects to the best American product, and shall be free from any extraneous material.

\section{DETAIL REQUIREMENTS}

(Subsections 1, 2, 8, 9, and 10 of No. 385a are part of these requirements, with the following additional:)

The backing on ream garnet shall be of good quality kraft or rope paper.

The backing on roll garnet shall be of a good quality of rope paper.

The weights of the paper backing shall be within the following limits:

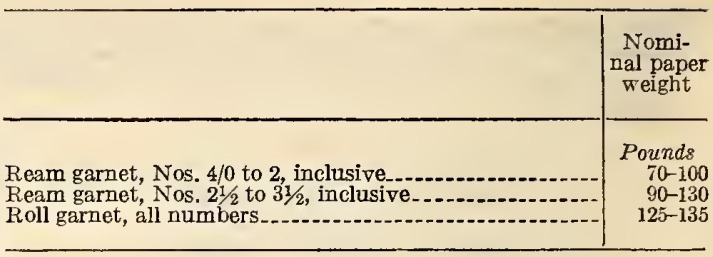

These weights are based on 24 by 36 inch sheets, 480 sheets per ream.

The average tensile strength of garnet paper per inch of width shall be not less than the following:

\begin{tabular}{l|r|r}
\hline & $\begin{array}{r}\text { Stronger } \\
\text { direction }\end{array}$ & $\begin{array}{r}\text { Weaker } \\
\text { direction }\end{array}$ \\
\hline & & \\
Ream garnet, Nos. 4/0 to 2, inclusive & 49 & Pounds \\
Ream garnet, Nos. 21/2 to 31/2, inclusive.... & 65 \\
Roll garnet, all numbers._. & 32 \\
& 85 & 45 \\
\hline
\end{tabular}

Ream garnet paper shall be shipped in quires containing 24 sheets each. Either 5 or 10 of these 1-quire packages shall be tied in a bale with string or wrapped in strong paper. The bales shall be packed in bundles having wood top and bottom boards, and bound securely with wire. Standard bundles shall contain the following quantities:

\begin{tabular}{|r|r||c|c|}
\hline Grain No. & $\begin{array}{c}\text { Reams per } \\
\text { bundle }\end{array}$ & Crain No. & $\begin{array}{c}\text { Reams per } \\
\text { bundle }\end{array}$ \\
\hline $4 / 0,3 / 0$ & 5 & 1 & $21 / 2$ \\
$2 / 0$ & 4 & $11 / 2$ & 2 \\
0 & $31 / 2$ & 2 & $11 / 2$ \\
$1 / 2$ & 3 & $21 / 2,3,31 / 2$ & 1 \\
\hline
\end{tabular}

\section{METHOD OF INSPECTION AND TESTS}

(Same as in 385a.)

\section{PACKING AND MARKING OF SHIPMENTS}

(Same as in 385a.)

Federal Specifications Board, specification No. $387 a$ United States Government master specification for cloth, abrasive, aluminum oxide, October $1,1928$.

\section{TYPE}

Aluminum oxide abrasive cloth shall be of but one type.

\section{MATERIAL AND WORKMANSHIP}

The product shall be made under approved methods, with high-grade backing, abrasive, and adhesive materials, and shall be free from any defects which may affect the serviceability of the material.

\section{DETAIL REQUIREMENTS}

1. The product shall be furnished in sheets 9 by 11 inches, unless otherwise specified.

2. The backing shall be of jean or drill of good quality. The cloth backing shall have a minimum weight of 4 ounces per yard, 30 inches wide. The cloth shall be properly dyed and back filled preparatory to coating. 
3. Aluminum oxide abrasive shall be of best quality fused aluminum oxide, free from extraneous material.

4. Aluminum oxide abrasive cloth shall be ordered in accordance with the commercial grading numbers given in Section VI of this specification. The size of aluminum oxide grain for the respective designating number shall conform to the size of grain on the corresponding standard samples of aluminum oxide cloth.

5. The average tensile strength of the abrasive cloth per inch of width shall be not less than the following: Longitudinal, 90 pounds; and transverse, 45 pounds.

6. Aluminum oxide abrasive cloth shall be shipped in quires containing 24 sheets each. Either 5 or 10 of these 1-quire packages shall be tied in a bale with string or wrapped in strong paper. The bales shall be packed in bundles having wood top and bottom boards, and bound securely with wire. Standard bundles shall contain the following quantities:

\begin{tabular}{|r|r|}
\hline Grain No. & $\begin{array}{c}\text { Reams per } \\
\text { bundle }\end{array}$ \\
\hline $320,240,180,150,120,100$ & $21 / 2$ \\
80,60 & 2 \\
50,40 & $11 / 2$ \\
$36,30,24$ & 1 \\
& \\
\hline
\end{tabular}

7. Each sheet shall be marked with the manufacturer's name and the grain number. When shipment is made in paper packages the manufacturer's name, the grain number, and the quantity contained shall also appear on the wrapper. The manufacturer's name, the grain number, and the quantity contained shall also appear on the end boards of the shipping bundles.

\section{METHOD OF INSPECTION AND TESTS}

1. Samples taken from each shipment shall be tested by rubbing against the standard samples. The specimens tested shall be equal to the standard sample with respect to the quality and the adhesion of the grain, as evidenced by the comparative condition of the surfaces in rubbing contact.

2. Strength test pieces 1 inch in width shall be cut, both longitudinally and transversely, from samples taken from each delivery. At least five such test pieces from each direction shall be placed in a tensile testing machine with the jaws 3 inches apart and pulled to rupture at the rate of 12 inches per minute.

\section{PACKING AND MARKING OF SHIPMENTS}

Each shipment should be marked with the name of the material, name of the contractor, and the number of the contract or order under which the shipment is made.

\section{NOTES}

1. The size of the aluminum-oxide grain should be specified in accordance with the commercial designation numbers: $320,240,180,150,120,100$, $80,60,50,40,36,30$, and 24 .

2. The numbers range in order from the finest to the coarsest grain required under this specification. The relation between the grain designations of aluminum-oxide cloth and emery cloth is as follows, corresponding grade designations appearing opposite each other:

\begin{tabular}{|c|c|c|c|}
\hline $\begin{array}{l}\text { Aluminum } \\
\text { oxide } \\
\text { grades }\end{array}$ & $\begin{array}{l}\text { Correspond- } \\
\text { ing Emery } \\
\text { cloth grades }\end{array}$ & $\begin{array}{l}\text { Aluminum } \\
\text { oxide } \\
\text { grades }\end{array}$ & $\begin{array}{l}\text { Correspond- } \\
\text { ing Emery } \\
\text { cloth grades }\end{array}$ \\
\hline $\begin{array}{r}320 \\
240 \\
180 \\
150 \\
120 \\
100 \\
80\end{array}$ & $\begin{array}{l}\mathrm{FF} \\
\mathrm{F} \\
3 / 0 \\
2 / 0 \\
0 \\
1 / 2 \\
1 / 2\end{array}$ & $\begin{array}{l}50 \\
-40 \\
36 \\
30\end{array}$ & $\begin{array}{c}2 \\
-21 / 2 \\
-3\end{array}$ \\
\hline & 1 & 24 & \\
\hline
\end{tabular}

Federal Specifications Board, specification No. 388a, United States Government master specification for cloth, emery, October 1, 1928.

(The requirements of this specification conform in general with F. S. B. No. 387 a (given above) with the exception of items numbered III (3), (4), and (6), which for this specification are as follows:)

3. Emery abrasive shall be of best quality Turkish emery or equal, free from extraneous material. ${ }^{1}$

4. Emery cloth shall be furnished in the following commercial grain sizes, as specified: $\mathrm{FF}, \mathrm{F}, 3 / 0$, $2 / 0,0,1 / 2,11 / 2,2,21 / 2,3$, and $31 / 2$.

These designations, in the order given, indicate the finest to the coarsest grain covered by this specification.

The size of emery grain for the respective designating number shall conform to the size of grain on the corresponding standard sample of emery cloth.

6. Emery cloth shall be packed in quires containing 24 sheets each. Either 5 or 10 of these 1-quire packages shall be tied in a bale with string or nrapped in strong paper. The bales shall be packed in bundles having wood top and bottom boards and be bound securely with wire, Standard bundles shall contain the following quantities:

\begin{tabular}{|c|c|}
\hline Grain No. & $\begin{array}{c}\text { Reams per } \\
\text { bundle }\end{array}$ \\
\hline FF $, F, 3 / 0,2 / 0,0,1 / 2 \ldots$ & $21 / 2$ \\
$1,11 \frac{1}{2}, 2 \ldots$ & $21 / 2$ \\
$21 / 2$ & 1 \\
\hline $3,31 / 2 \ldots$ & 1 \\
\hline
\end{tabular}

Federal Specifications Board, specification No. 582, United States Government master specification for paper, abrasive, artifical, waterproof, October 1, 1928.

(Section II (Material and Workmanship), Subsections IV (9) and V (2), and Section VI (Packing and Marking) of Federal Specifications Board specification No. 385a (given above) are part of this specification, with the following additional:)

\section{TYPE}

Waterproof artificial abrasive paper shall be of but one type.

\section{DETAIL REQUIREMENTS}

1. Size.-Paper shall be furnished in sheets 9 by 11 inches, unless otherwise specified.

2. Backing.-(a) Backing of artifical waterproof abrasive paper shall be of good quality kraft or rope paper.

1 Artificial aluminum-oxide abrasive, as covered by F. S. B. No. 387 , in effect on date of invitation for bids, will be considered acceptable in lieu of natural emery. Contractors furnishing aluminumoxide cloth in place of emery cloth will mark on the outside wrapper oxide cloth in place of emery cloth will mark on the outside wrapper and the nearest coresponding grade of emery cloth. 
(b) Weight of the paper backing shall be within the following limits:

\begin{tabular}{|c|c|}
\hline Grain No. & Nominal paper weights \\
\hline $150,120,100,80$, and $60 \ldots-1$ & 90 -pound kraft. \\
$180,-140,280,320$, and 400 & $\begin{array}{l}70 \text {-pound kraft. } \\
20 \text {-pound or } 40 \text {-pound } \\
\text { krait or } 40 \text {-pound } \\
\text { rope. }\end{array}$ \\
\hline
\end{tabular}

(c) There shall be printed on the backs of sheets of abrasive paper, dependent upon the weight of the paper, one of the following designations:

\begin{tabular}{|c|c|}
\hline Weight & $\begin{array}{c}\text { Designe. } \\
\text { tion }\end{array}$ \\
\hline $\begin{array}{c}\text { 90-pound paper } \\
\text { 70-pound paper }\end{array}$ & $\mathrm{D}$ \\
40-pound paper...... & $\mathrm{C}$ \\
\hline
\end{tabular}

(d) The paper weiglits are based on 480,24 by 36 inch sheets per ream.

(e) A variation of plus or minus 5 per cent in the nominal paper weights will be allowed.

$(f)$ The paper backing shall be so treated or waterproofed that it will withstand the softening action of water when used wet and stand up during the usual friction or rubbing to which it is subjected in ordinary use.

3. Artificial abrasive.-(a) Ouality-All artificial abrasive shall be either silicon carbide or aluminum oxide, of good quality, equal in all respects to the best American product, and shall be free from any extraneous material.

(b) Grain size.-Waterproof artificial abrasive paper shall be ordered in accordance with the commercial designation numbers given in Section IV of this specification. The size of the artificial grain for the respective designating numbers shall conform with the size of grain in the corresponding standard samples of waterproof artificial abrasive paper.

4. Adhesive.- The adhesive shall be made of good quality drying oils, varnishes, or other adhesive materials and be suitable for this special type of product.

5. Strength.-The average tensile strength of waterproof artificial abrasive paper per inch of width, when dry, shall be not less than the following:

\begin{tabular}{|c|c|c|}
\hline Grain No. & $\begin{array}{l}\text { Stronger } \\
\text { direction }\end{array}$ & $\begin{array}{c}\text { Weaker } \\
\text { direction }\end{array}$ \\
\hline $\begin{array}{l}150 \text { to } 60 \text {, marked " } \mathrm{D} \text { ", } \\
180 \text {, marked " } \mathrm{C} \text { " } \\
220 \text { to } 400 \text {, marked " } \mathrm{G} \text { ", } \\
220 \text { to } 400 \text {, marked " } \mathrm{A} \text { ", }\end{array}$ & $\begin{array}{r}\text { Pounds } \\
65 \\
49 \\
49 \\
15\end{array}$ & $\begin{array}{r}\text { Pounds } \\
32 \\
25 \\
\\
10\end{array}$ \\
\hline
\end{tabular}

6. Waterproof artificial abrasive paper shall be shipped in quires containing 24 sheets each. Ten of these 1-quire packages shall be tied together in a bale. The bales shall be packed in bundles, having wooden top and bottom boards, and securely bound with wire. Standard bundles shall contain the following quantities of different grain numbers:

\begin{tabular}{l|r|}
\hline Grain No. & $\begin{array}{l}\text { Reams } \\
\text { per } \\
\text { bundle }\end{array}$ \\
\hline 80 and 60 & 2 \\
150,120, and 100 & 3 \\
220 and 180 & 4 \\
$400,320,280$, and 240 & 5 \\
\hline
\end{tabular}

\section{METHOD OF INSPECTION AND TESTS}

1. Samples taken from each shipment shall be tested by rubbing against the standard samples. Both the specimen to be tested and the standard sample shall be dipped in water just before making this rubbing test. The specimens tested shall be equal to the standard samples with respect to the quality and the adhesion of the grain, as evidenced by the comparative condition of the surface in rubbing contact.

\section{NOTES}

The size of the artificial grain should be specified in accordance with the commercial designating numbers: $400,320,280,240,220,180,150,120$, 100,80 , and 60 .

The numbers range in order from the finest to the coarsest grain

Federal Specifications Board, specification No. 583, United States Government master specification for paper, garnet, waterproof.

(Section II (Material and Workmanship), SubSections IV, (9), and V, (2), and Section VI (Packing and Marking) of F. S. B. No. 385a, are part of this specification.)

(Subsections II, (2), (d), (e), and $(f)$; II, (4), and III (1), of F. S. B. No. 582 (above) are part of this specification.)

(The following is additional to the above references:)

\section{TYPE}

Waterproof garnet paper shall be of but one type.

\section{DETAIL REQUIREMENTS}

1. Size.-Paper shall be furnished in sheets 9 by 11 inches unless otherwise specified.

2. Backing.-(a) Backing shall be of good quality kraft paper.

(b) Weight of paper backing shall be as follows:

\begin{tabular}{c|r}
\hline Grain No. & $\begin{array}{c}\text { Nominal } \\
\text { paper } \\
\text { weight }\end{array}$ \\
\hline $4 / 0,3 / 0,2 / 0$, and $0 \ldots$ & $\begin{array}{r}\text { Pounds } \\
70 \\
5 / 0,6 / 0, \text { and } 7 / 0\end{array}$ \\
\hline
\end{tabular}

3. Garnet abrasive-(a) Quality.-All garnet shall be of good quality, equal in all respects to the best American product, and shall be otherwise free from extraneous material.

(b) Grain sizes. - Waterproof garnet paper shall be ordered in accordance with the commercial designating numbers given in Section III of this specification. The size of the garnet grain for the respective designating numbers shall conform with the size of the grain on the corresponding standard samples of waterproof garnet paper.

4. Strength.-The average tensile strength of waterproof garnet paper per inch of width when dry shall be not less than the following:

\begin{tabular}{c|r|r}
\hline Grain No. & $\begin{array}{r}\text { Stronger } \\
\text { direction }\end{array}$ & $\begin{array}{r}\text { Weaker } \\
\text { direction }\end{array}$ \\
\hline $4 / 0,3 / 0,2 / 0$, and 0 & $\begin{array}{r}\text { Pounds } \\
49\end{array}$ & $\begin{array}{r}\text { Pounds } \\
25 \\
5 / 0,6 / 0, \text { and } 7 / 0\end{array}$ \\
\hline
\end{tabular}


5. Traterproof garnet paper shall be shipped in quires containing 24 sheets each. Ten of these 1-quire packages shall be tied together in a bale. These bales shall be packed in bundles having wooden top and bottom boards and securely bound with wire. Standard bundles shall contain the following quantities of the different grain numbers:

\begin{tabular}{|c|c|}
\hline Grain No. & $\begin{array}{l}\text { Reams } \\
\text { per } \\
\text { bundle }\end{array}$ \\
\hline $\begin{array}{l}0,2 / 0, \\
3 / 0.4 / 0,0, \\
5 / 0,6 / 0, \text { and } 1 / 0\end{array}$ & $\begin{array}{l}3 \\
4 \\
5\end{array}$ \\
\hline
\end{tabular}

\section{NOTES}

The size of the garnet grain desired should be specified in accordance with the following commercial designating numbers: $7 / 0,6 / 0,5 / 0,4 / 0,3 / 0$, $2^{\prime} 0$, and 0 .

The numbers range in order from the finest to the coarsest grain.

United States Department of Commerce, division of simplified practice, recommendation No. 89, coated abrasive products, September 1, 1928 .

This simplified-practice recommendation was drafted at a joint conference of representative producers, distributors, and users. The recommendation, which limits the list of stock sizes and rarieties to those listed, is subject to annual revision by a conference of the standing committee of the industry.

\section{SIMPLIFIED LIST OF COATED ABRASIVE PROD. UCTS}

EXPLATATION OF SYMECLS AND ABBREVIATIONS USED IN THIS TABOLATION

" 1 ," used in the main body of the tabulation, stanc's for "class 1 " or standard items of manu- facture. Class 1 items would be regularly made by some or all manufacturers and carried in stock by them at their factories and where required at their branch warehouses.

" 2 ," used in the main body of the tabulation, stands for "class 2" or nonstandard items which are of limited sale for specific purposes, which will not be catalogued for general sale, which may be discontinued when the special demand for them changes, and which will be distinguished by being left unprinted except for class name and grit number.

Under "Class of goods" a "ream" is 480 sheets; a standard "roll" is 50 yards long; "combination" backing is paper reinforced with cloth.

Under "Backing" A indicates 40-pound, or light paper; C, 70-pound; D, 100-pound; E, 130-pound, or heary paper, $J$, a jeans cloth; $\mathbf{X}$, drill cloth; $\mathrm{H}$, heary twill weave cloth.

Under "Coating," "R Wt." metal cloth disks indicates regular or standard coating for surface grinding disks. "DC" metal cloth disks indicates double coating or extra heavy weight for surface grinding disks.

\section{DEFINITIONS}

Closed coating.-One in which the abrasive is put in on such quantity as entirely to cover the paper or cloth backing.

Open coating.-One in which approximately 50 per cent of the backing is covered, thus leaving open spaces between the grains of abrasive.

Grade numbers.-The upper of the two rows of figures, indicating grade numbers, is an arbitrary nomenclature established by the industry a hundred years ago. The figures in the lower row, constituting a more descriptive system of nomenclature, indicate the mesh markings or the approximate size of the screen through which the abrasive was passed. Thus, No. 80 means that the control screen is composed of 80 strands of silk to the square inch, the strands running in two directions and set perpendicular to each other. These systems are used alternately or coincidently according to the custom of the individual manufacturer and the kind or character of the product so marked.

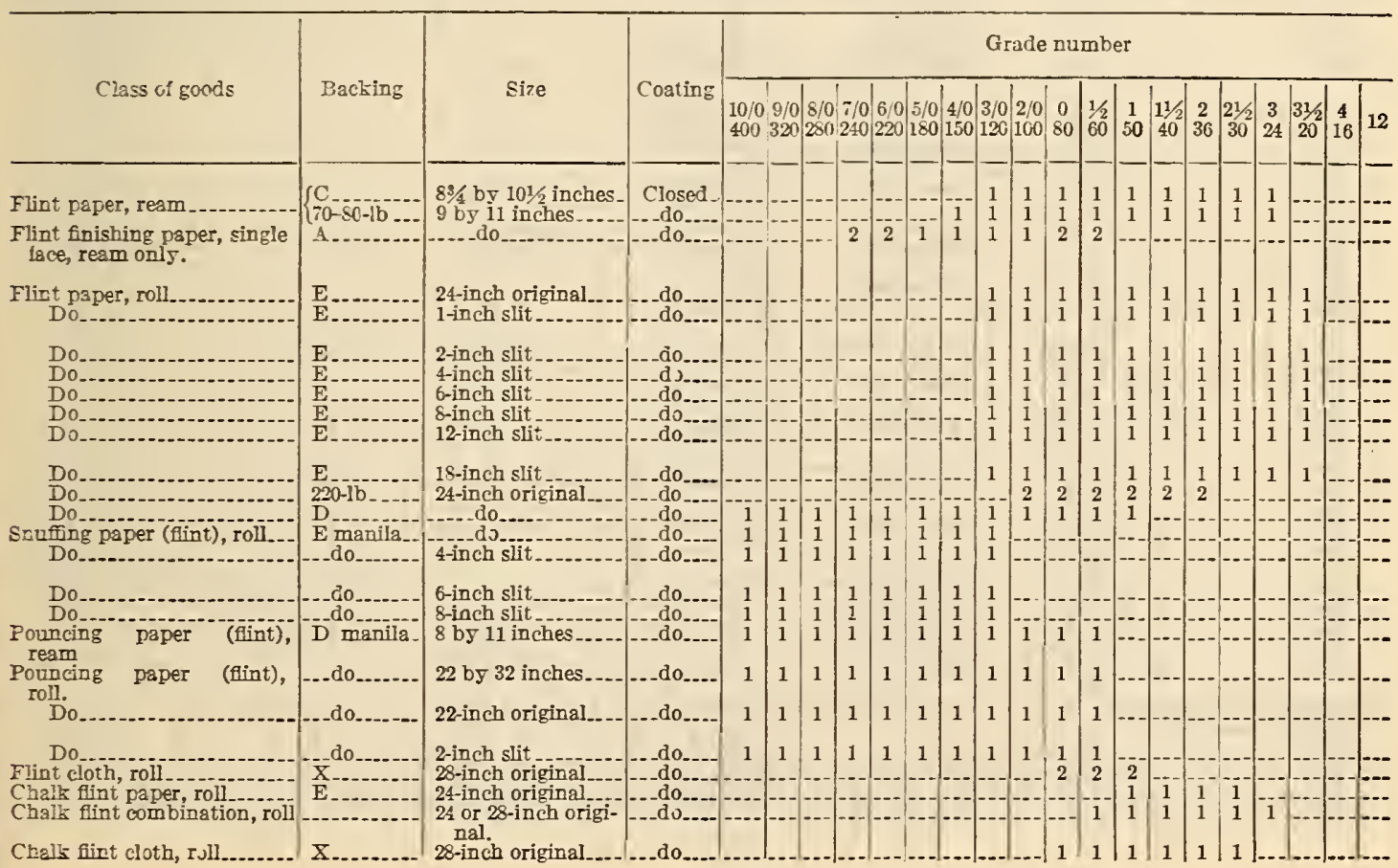




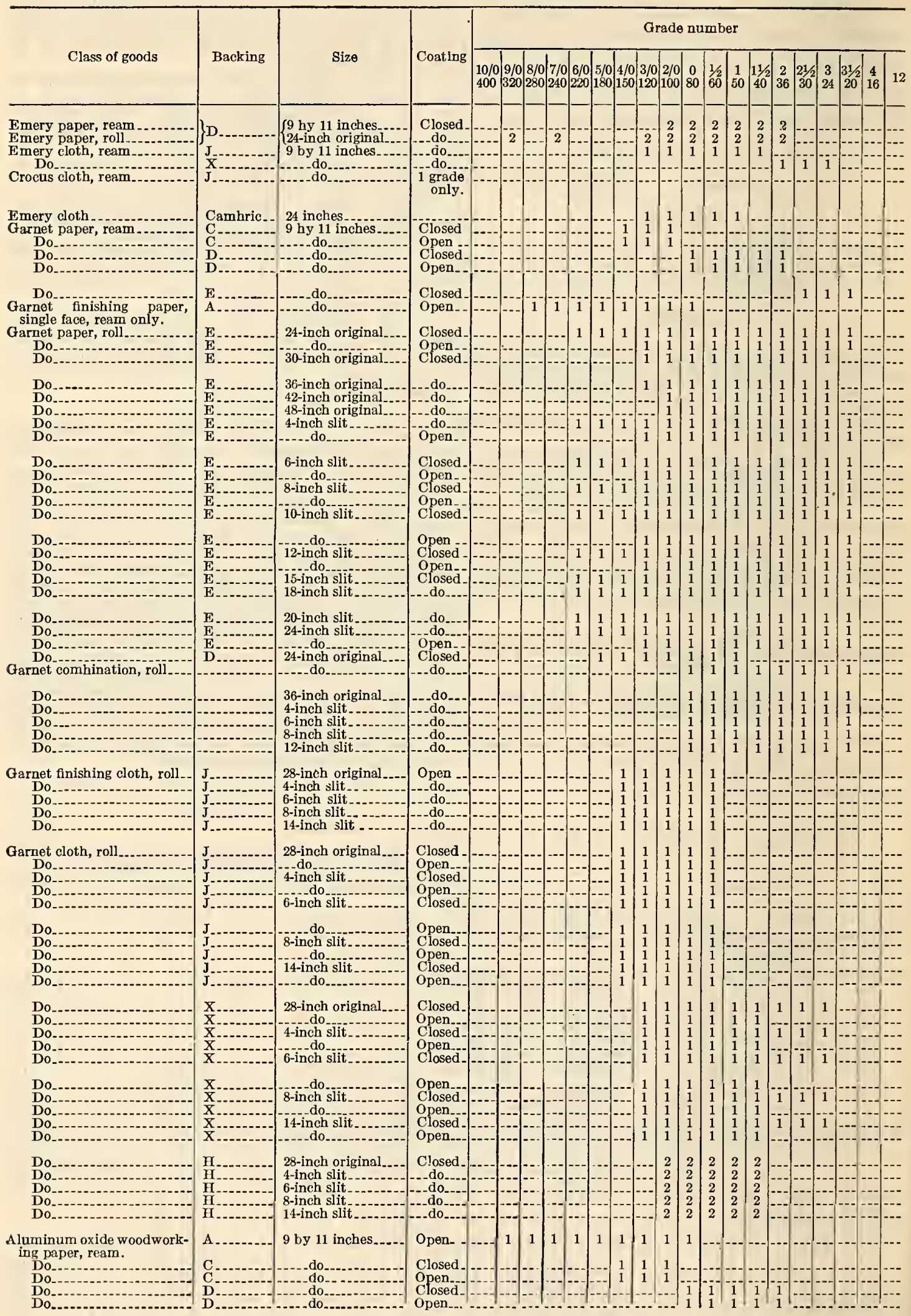




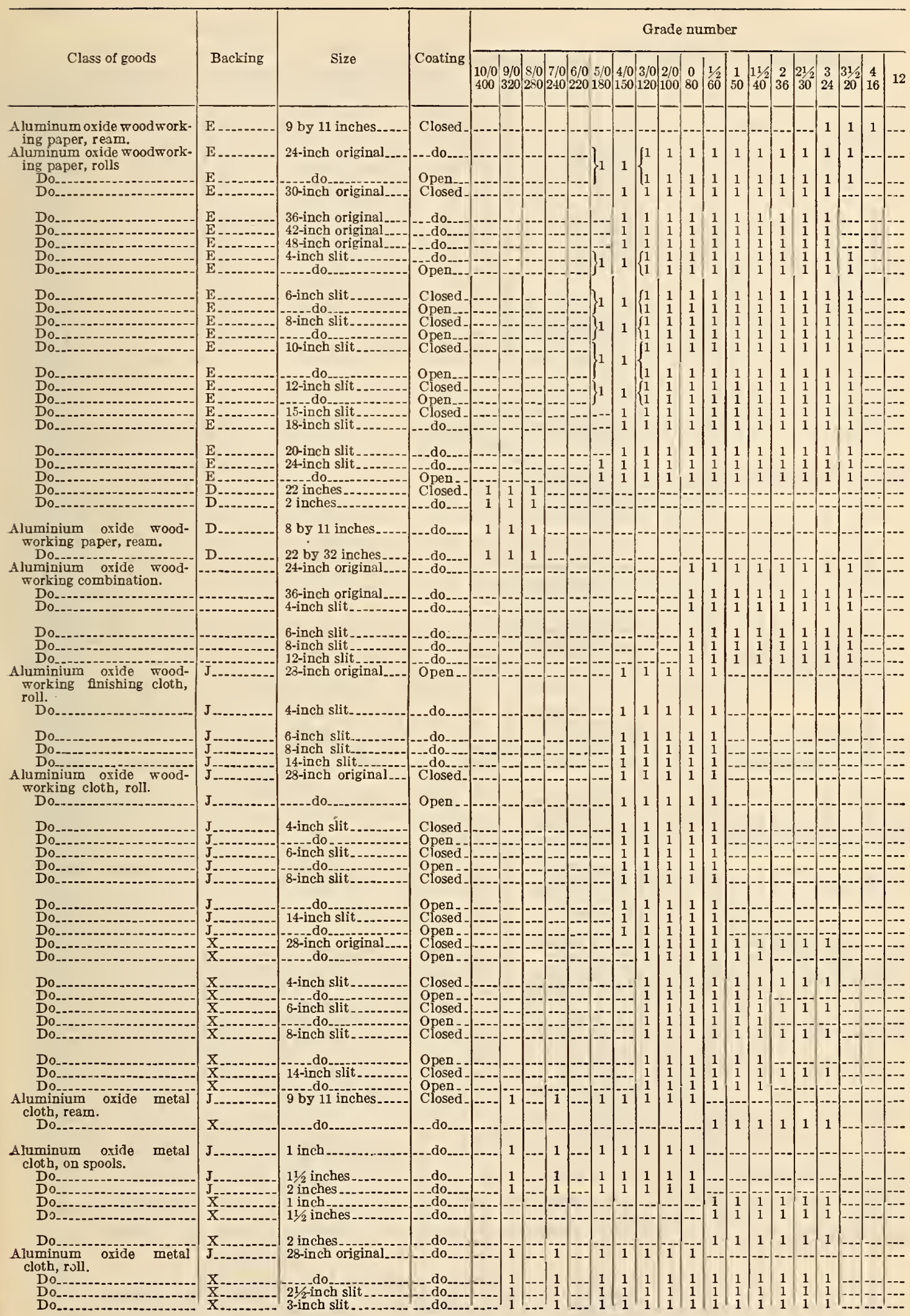




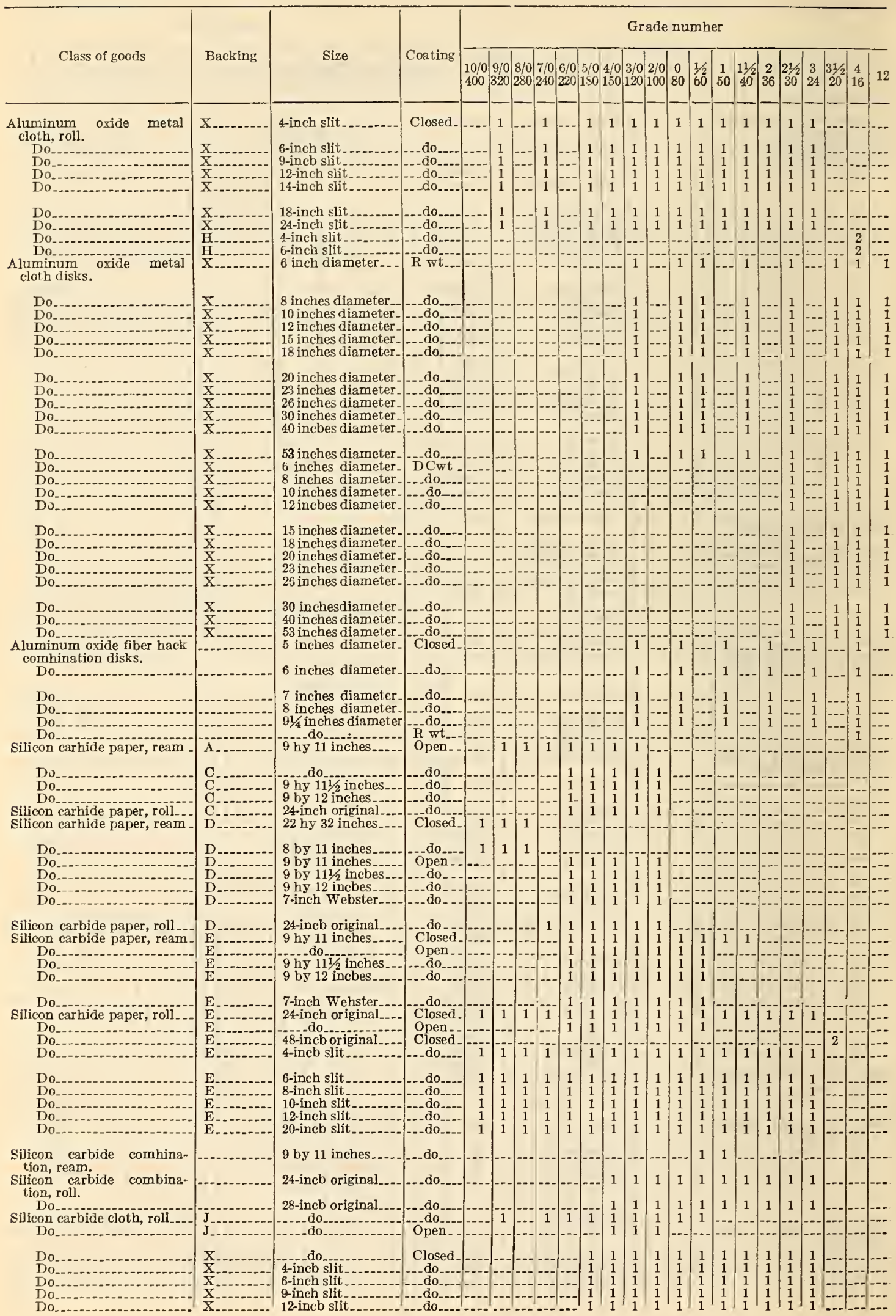




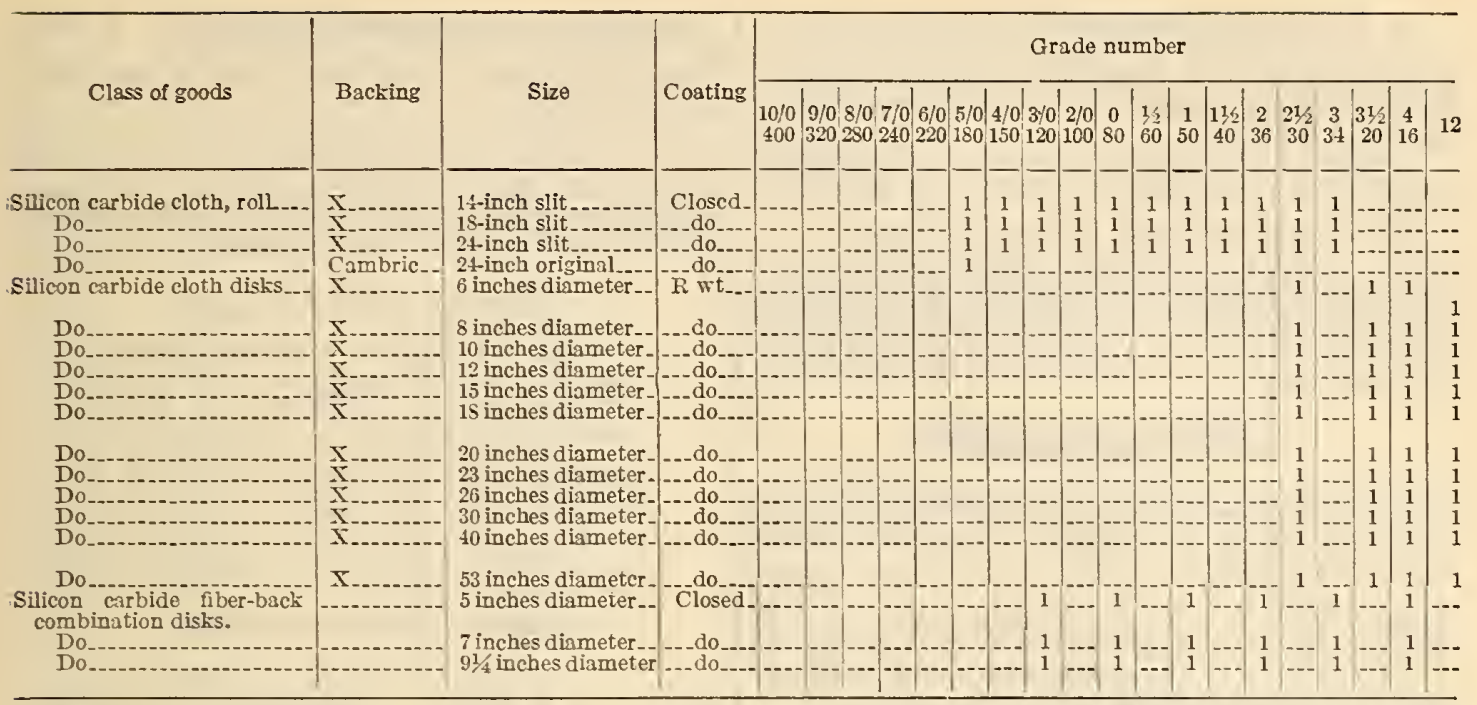

Waterproof flint paper, waterproof garnet paper, waterproof aluminum oxide paper, and waterproof silicon carbide paper are all made in a rariety of grit numbers and backings which arc determined and controlled by a patent license agreement.

Converted shapes and sizes that can be cut from the products listed:

Shoe trade shapes.-Heel scouring rolls will be made in the loug-established and most-used shapes FS, CT, A, B, C, OG102, and OG104.

Heel breasters will be made in the six well-established standard shapes (300 to 305 ) and in a few semistandard shapes.

Pin wheels. (See disk list).

Pads will be made in the six well-established shapes.

Sit rolls (in intermediate sizes).- Slit rolls, although considered as special items, can be supplied in intermediate widths.

Nonstandard length rolls can be supplied on special order only in lengths of 25 yards or greater.

Joined belts can be supplied in the materials and grades listed, at the widths listed, or at intermediate widths and in lengths as ordered, except-

Combination and paper belts will be made only in lengths up to 37 inches.

Standard belt joints may be "butt reinforced" either straight or diagonal, "dovetailed" either straight or diagonal, or "skived" either straight or single diagonal.

Cones and sleeves (joined) can be supplied on special order.

Nonstandard size sheets can be supplied on special order in sizes up to a maximum area of 400 square inches with a maximum dimension of 35 inches.

Disks of standard material (other than $R$ weight grinding disks) can be furnished in the following diameters: $1,2,3,3 \frac{1}{2}, 4,6,8,10,12,15,18$, and 20 inches, or in intermediate sizes.

Assorted sheet packages.-Flint paper, $4^{3 / 3}$ by $5^{1 / 4}$ inches, 20 sheets (fine, medium, and coarse) ream flint paper.

Emery paper, $4 \frac{1}{2}$ by $5 \frac{1}{2}$ inches, 10 sheets (fine, medium, and coarse) ream emery paper.

Emery cloth, $4 \frac{1}{2}$ by $5 \frac{1}{2}$ inches, 6 sheets (fine, medium, and coarse) ream emery cloth.

\subsection{PUMICE.}

(No nationally recognized specifications available.) 545. ASBESTOS

545.1 ASBESTOS, UNMANUFACTURED.

(No nationally recognized specifications available.)

\subsection{ASBESTOS PAPER, MILIBOARD, AND ROLI BOARD.}

American Marine Standards Committee, asbestos millboard, standard E No. 17, 1928.

(The above specification is identical mith Federal Specifications Board, No. 54 (given below), with the exception that in this specification the $3 / 8$-inch nominal thickness grade is omitted.)

Federal Specifications Board, specification No. 54 , United States Government master specification for asbestos millboard, February 1, 1923.

\section{GRADES}

1. Asbestos millboard shall be supplied in the following grades as required: Medium and hard.

\section{MATERIAL}

2. (a) Material covered by these specifications shall be boards of uniform thickness, composed of asbestos fiber, with a small amount of binding material.

(b) Vegetable matter shall not exceed 3 per cent by weight of the composition of the millboard and the same shall only be used as sizing.

\section{GENERAL REQUIREMENTS}

3. (a) Asbestos millboard shall be supplied in standard sheets 42 by 48 inches, unless otherwise specified. The thickness of the millboard referred to hereafter in these specifications shall be the mean of the four thicknesses determined by measurements taken at the middle of each side.

(b) The thickness of each board shall be within the limits given in Table $\mathrm{A}$.

(c) The average thickness of any 10 boards, taken at random, shall be within the limits given in Table B.

Thickness

\begin{tabular}{c|r|r|r|r}
\hline \multirow{2}{*}{$\begin{array}{c}\text { Nominal thick- } \\
\text { ness (inch) }\end{array}$} & \multicolumn{2}{|c|}{ Table A } & \multicolumn{2}{c}{ Table B } \\
\cline { 2 - 5 } & Minimum & Maximum & Minimum & Maximum \\
\hline & Inch & Inch & Inch & Inch \\
$1 / 8$ & 0.11 & 0.14 & 0.12 & 0.13 \\
$1 / 4 / 8$ & .29 & .24 & .26 \\
$3 / 2.22$ & .41 & .36 & .39 \\
\hline
\end{tabular}


(d) For a special thickness, the minimum or maximum variation shall be that permitted for the next larger thickness. If over $1 / 2$ inch nominal thickness, the same percentage of tolerance shall be permitted as for $1 / 2$ inch thick.

(e) Millboard shall show no change of texture and no softening when heated indefinitely in a dry heat at $400^{\circ} \mathrm{F}$.

( $f$ ) The loss of weight of millboard after it has has been dried to a constant weight at a temperature not exceeding $220^{\circ} \mathrm{F}$. shall not exceed 20 per cent when a $2 \mathrm{~g}$ sample is cut up and heated to a bright cherry-red heat in an open crucible in a gas muffie for 30 minutes.

\section{DETAIL REQUIREMENTS}

4. (o) The maximum acceptable weights for millboard reduced to the basis of 1 square foot area, 1 inch thick, shall be.

Pounds

Medium millboar

6.00

Hard miliboard.

The thickness of the sample sheet shall be determined as in paragraph $(a)$.

(b) Samples of millboard within 0.01 inch of the nominal thickness shall not show signs of cracking when submitted to the following weights nor deflections less than noted in the following table:

\begin{tabular}{|c|c|c|c|}
\hline Thickness (incb) & Hard load & $\begin{array}{l}\text { Medium } \\
\text { load }\end{array}$ & $\begin{array}{l}\text { Deflection } \\
\text { medium } \\
\text { minimum }\end{array}$ \\
\hline $\begin{array}{l}1 / 8-1 \\
1 / 4 \\
3 / 8 \\
1 / 2\end{array}$ & \begin{tabular}{r|} 
Pounds \\
4.5 \\
18.0 \\
36.0 \\
54.0
\end{tabular} & $\begin{array}{r}\text { Pounds } \\
1.3 \\
6.0 \\
12.0 \\
19.0\end{array}$ & $\begin{array}{l}\text { Inch } \\
0.40 \\
.35 \\
.25 \\
.20\end{array}$ \\
\hline
\end{tabular}

TESTS

5. (a) The test pieces for the deflection test shall be 4 inches wide and 12 inches long, cut with the direction of the fiber. Fach sample shall be tested by placing it upon two knife-edges, the tops of which are approximately $1 / 8$-inch radius, placed parallel 10 inches apart. The load specified in paragraph $5(b)$ for the size being tested shall be applied entirely across the test specimen at a point equidistant from the supporting knife-edges. The knife-edge supporting this centrally applied load shall have a face with a radius of approximately $1 / 8$ inch. At least one minute shall be allowed for the specimen to take its final set.

(b) Tests shall be conducted to determine the composition as provided for in paragraph $3(e)$ and $(f)$.

\section{PACKING AND MARKING}

6. Deliveries shall be made in substantial crates marked with the grade, thickness, and number of sheets contained, the contract, requisition, or other number and the name of the contractor.

Federal Specifications Board, specification No. 211, United States government master specification for magnesia asbestos plaster, September 30, 1924.

(See 572, page 650.)
United States Department of Commerce, division of simplified practice recommendation No. 19 (revised), asbestos paper and asbestos millboard, March 1, 1927.

The Department of Commerce, as the result of unaminous action of a conference of manufacturers, distributors, and users, recommends that sizes, weights, and thickness of asbestos paper and asbestos millboard be redueed to the following list:

TABLE 1.-Asbestos paper

\begin{tabular}{|c|c|c|c|}
\hline $\begin{array}{l}\text { Weight } \\
\text { of rolls }\end{array}$ & $\begin{array}{l}\text { Width of } \\
\text { rolls }\end{array}$ & $\begin{array}{l}\text { Weight per } 100 \\
\text { square feet }\end{array}$ & $\begin{array}{c}\text { Thick- } \\
\text { ness }\end{array}$ \\
\hline $\begin{array}{c}\text { Pounds } \\
100 \\
50 \\
50\end{array}$ & $\begin{array}{c}\text { Inches } \\
36 \\
24 \\
18\end{array}$ & \begin{tabular}{lllll}
\multicolumn{5}{c}{ Pounds } \\
8 & 10 & 12 & 14 & 16 \\
8 & 10 & 12 & 14 & 16 \\
8 & 10 & 12 & 14 & 16
\end{tabular} & \begin{tabular}{l}
\multicolumn{2}{c}{ Inch } \\
$1 / 16 \quad 1 / 8$ \\
$1 / 16$ \\
$1 / 16$
\end{tabular} \\
\hline
\end{tabular}

TABLE 2.-Asbestos millboard

\begin{tabular}{|c|c|}
\hline $\begin{array}{l}\text { Size of } \\
\text { sheet }\end{array}$ & $\begin{array}{l}\text { Thickness of } \\
\text { sheet }\end{array}$ \\
\hline $\begin{array}{l}\text { Inches } \\
42 \text { by } 48\end{array}$ & $1 / 16 \quad \begin{array}{l}\text { Inch } \\
1 / 8\end{array} 1 / 4 \quad 1 / 2$ \\
\hline
\end{tabular}

\subsection{ASBESTOS PIPE COVERING AND CEMENT.} able.)

\subsection{TEXTILES, YARNS, AND PACKING.}

American Society for Testing Materials, tentative specifications for tolerances and test methods for asbestos yarns, serial designation D299-28T, 1928.

1. These specifications cover the tolerances and methods of testing for plain asbestos yarn and metallic asbestos yarn.

\section{DEFINITIONS}

2. (a) Asbestos yarn.-Asbestos yarn is yarn consisting of (1) asbestos fiber, or (2) asbestos and cotton fibers, or (3) asbestos and cotton fibers and wire.

(b) Plain asbestos yarn.-Plain asbestos yarn is yarn consisting of (1) asbestos fiber, or (2) asbestos. and cotton fibers.

(c) Metallic asbestos yarn.-Metallic asbestos yarn is yarn consisting of plain asbestos yarn twisted with brass, copper or other fine wire.

(d) Cut.-The term "cut" indicates the size of asbestos yarn. The word "cut" preceded by a number indicates, in multiples of 100 yards, the yardage per pound of single-ply yarn. For example "5-cut" indicates that a pound of single-ply yarn so designated measures approximately 500 yards, "10-cut" indicates a yarn that measures approximately 1,000 yards per pound.

(e) Ply.-The term "ply," applied to asbestos yarn, indicates the number of strands of single yarn twisted together to form a heavier yarn. For example, "2-ply 10-cut" indicates that each of the two strands forming the 2-ply shall be 10 -cut. 


\section{TOLERANCES}

3. The yardage of plain, single-ply asbestos yarn shall conform to the following requirements:

\begin{tabular}{|c|c|c|}
\hline Size designation & $\begin{array}{l}\text { Nominal } \\
\text { yardage } \\
\text { per } \\
\text { pound }\end{array}$ & $\begin{array}{l}\text { Permissible } \\
\text { range of } \\
\text { variation in } \\
\text { yardage }\end{array}$ \\
\hline $\begin{array}{l}\text { 5-cut } \\
\text { 6-cut } \\
\text { 7-cut } \\
\text { 8-cut } \\
\text { 9-cut }\end{array}$ & $\begin{array}{l}500 \\
600 \\
700 \\
800 \\
900\end{array}$ & $\begin{array}{ll}450- & 549 \\
550- & 649 \\
650- & 749 \\
750- & 849 \\
850- & 949\end{array}$ \\
\hline $\begin{array}{l}\text { 10-cut } \\
12 \text {-cut } \\
14-c u t \\
16-c u t \\
18-c u t\end{array}$ & $\begin{array}{l}1,000 \\
1,200 \\
1,400 \\
1,600 \\
1,800\end{array}$ & $\begin{array}{r}950-1,049 \\
1,150-1,249 \\
1,300-1,499 \\
1,500-1,699 \\
1,700-1,899\end{array}$ \\
\hline $\begin{array}{l}20 \text {-cut } \\
24 \text {-cut } \\
26 \text {-cut } \\
28-\text { cut } \\
\text { 30-cut }\end{array}$ & $\begin{array}{l}2,000 \\
2,400 \\
2,600 \\
2,800 \\
3,000\end{array}$ & $\begin{array}{l}1,900-2,099 \\
2,300-2,499 \\
2,500-2,699 \\
2,700-2,899 \\
2,900-3,099\end{array}$ \\
\hline
\end{tabular}

4. The diameter of the wire used in metallic asbestos yarn shall not rary from the size specified more than plus or minus 0.001 inch.

5. (a) The cotton content of both plain asbestos yarn and of metallic asbestos yarn shall conform to the following requirements:

Grade A

Per cent

Grade A A

Grade AAA.

$15-20$

(15

(b) The cotton content of metallic asbestos yarn shall be determined after all the wire has been removed from the test specimen.

\section{METHODS OF TESTING}

6. The asbestos yarn shall be washed thoroughly with chloroform or ether to remove all grease, fats, and oily matter and shall be dried to constant weight at from $100^{\circ}$ to $105^{\circ} \mathrm{C}$.

A test specimen of about $1 \mathrm{~g}$ of the extracted and dried yarn shall be weighed and placed in a combustion boat and the boat placed in a combustion furnace. The drying and absorption train shall be attached and a gentle current of thoroughly dried and purified oxygen shall be started through the apparatus. Heat shall then be applied until the combustion tube becomes dull red and the tube shall be subjected to this temperature for 15 or 20 minutes. The flow of oxygen shall then be continued for an additional 15 minutes to remove the products of combustion from the apparatus. The water from the products of combustion shall be removed by passing them through bulbs containing sulphuric acid, and then through tubes containing calcium chloride. The carbon dioxide shall be removed by absorption by passing through caustic potash ( $\mathrm{KOH}$ ) in a Vanier or other absorption bulb. The weight of the bulb being known, any increase in weight indicates the amount of carbon dioxide absorbed.

7. The percentage of cellulose shall be calculated from the following formula:

Percentage of cellulose $\left(\mathrm{C}_{6} \mathrm{H}_{30} \mathrm{O}_{5}\right)=\frac{\text { Weight of } \mathrm{CO}_{2} \times 0.6137}{\text { Weight of sample }} \times 100$

Federal Specifications Board, specification No. $94 \mathrm{~b}$, United States Government master specification for packing, asbestos metallic cloth sheet, May 12, 1927.

\section{GRADES}

Packing shall be furnished in the following grades as required: Grade I, not less than 90 per cent asbestos; Grade II, not less than 75 per cent asbestos.

\section{MATERIAL AND WORKMANSHIP}

Asbestos metallic cloth sheet packing shall be made of woven asbestos wire insertion cloth treated with a rubber compound. The workmanship shall be first class in every respect.

\section{GENERAL REQUIREMENTS}

1. Sheet packing one-sixteenth inch in thickness shall be one ply of cloth. Sheet packing one-eighth, three-sixteenths, and one-fourth inch in thickness shall be made of two, three, and four plies, respectively, of $1 / 16$-inch cloth cemented together.

2. A plus tolerance of one sixty-fourth inch will be permitted. No minus tolerance in thickness shall be allowed.

3. The finished packing shall not crack nor show any signs of injury when bent flat on itself in any direction.

The brass or copper wire shall be 0.007 inch in diameter, plus or minus 0.001 inch.

\section{DETAIL REQUIREMENTS}

(a) The cloth shall be woven from yarn containing not less than 90 per cent for Grade I and 75 per cent for Grade II, by weight, long-fiber asbestos of not less than 12 per cent, by weight, water of composition.

(b) The weave of the cloth shall consist of not less than 20 for I and 16 for II strands in the warp and 10 for I and 8 for II strands in the filler per linear-inch.

(c) For packing one-sixteenth inch in thickness, each strand of the warp and filler shall consist of two plies of asbestos yarn and one for I and not more than two for II brass or copper wires twisted together.

(d) The weight per square yard of one-sixteenth-inch asbestos metal cloth before application of the rubber compound shall be not less than 2.75 pounds per square yard.

(e) The rubber compound shall be not more than 50 for I and 55 for II per cent by weight of the finished packing.

(f) Packing shall show no signs of flowing, cracking, material hardening or disintegration of the rubber compound when bolted between iron plates and subjected to the action of saturated steam at 275 for I and 200 for II pounds per square inch for a total of 24 hours.

Weight of finished products

\begin{tabular}{|c|c|c|}
\hline \multirow{2}{*}{$\begin{array}{l}\text { Thickmess } \\
\text { (inch) }\end{array}$} & \multicolumn{2}{|c|}{ Weight per square yard } \\
\hline & Minimum & Maximum \\
\hline $\begin{array}{l}1 / 16- \\
1 / 8-\cdots \\
3 / 16- \\
1 / 4 \ldots\end{array}$ & $\begin{array}{r}\text { Pounds } \\
5.50 \\
10.50 \\
16.00 \\
21.50\end{array}$ & $\begin{array}{r}\text { Pounds } \\
6.25 \\
11.75 \\
17.75 \\
24.00\end{array}$ \\
\hline
\end{tabular}




\section{METHOD OF INSPECTION AND TESTS}

A sample of finished packing 12 inches square and of untreated fabric 12 inches square shall be taken at random by the inspector from each lot of 2,000 pounds of packing or less and forwarded to the testing laboratory.

\section{PACKING AND MARKING OF SHIPMENTS}

1. Packing shall be supplied in rolls weighing approximately 100 pounds or 250 pounds unless otherwise ordered. Each roll shall be wrapped in waterproof paper.

2. The packing shall be marked with the maker's name and brand neatly stenciled upon each linear yard. The date of manufacture shall be marked on each end of each roll.

\section{NOTES}

1. Grade 1 packing is suitable for steam pressure up to $300 \mathrm{lbs}$./in. ${ }^{2}$ and temperature up to $700^{\circ} \mathrm{F}$.

2. Grade II packing is suitable for steam pressure up to $200 \mathrm{lbs}$. $/ \mathrm{in.}^{2}$.

3. Requirements for test and analyses which are applicable to this specification are contained in Federal Specifications Board specification 59a, general specifications for rubber goods.

Federal Specifications Board, specification No. 95, United States Government specification for asbestos valve-stem packing, December 29, 1923.

\section{GENERAI}

(a) This specification covers the requirements for asbestos valve-stem packing to be used under steam pressures up to $300 \mathrm{lbs}$./in. ${ }^{2}$ and a maximum temperature of $700^{\circ} \mathrm{F}$.

\section{CONSTRUCTION AND MATERIAI}

(a) This packing shall be furnished in any of the following types as required and consist of-

1. Braided asbestos, lubricated with satisfactory oils.

2. Braided asbestos, frictioned with vulcanizable gums.

3. Twisted asbestos; lubricated with satisfactory oils.

4. Twisted asbestos.

(b) The finished packing, except type 4, shall be dipped in pure flake graphite.

(c) The yarn shall contain not less than 90 per cent of long-fiber asbestos of not less than 12 per cent water of composition.

(d) The weight of the single ply of asbestos yarn shall not be more than 1 pound per 1,000 yards.

(e) The lubricant and graphite in types 1 and 3 shall not exceed 33 per cent by weight of the completed packing. Types 2 and 4 shall contain no lubricants.

Nore.-Requirements for construction, materials, sampling, and testing which are applicable to this specification are contained in Foderal Specifieations Board specification No. 59a, general specifications for rubber goods.

Federal Specifications Board, specification No. 96c, United States Government master specification for packing, asbestos sheet, compressed, May 22, 1928.

\section{GRADES}

Packing shall be furnished in two grades, as required-grade A and grade B. (See Section VII.)

\section{MATERIAL AND WORKMANSHIP}

Packing shall be made of asbestos fiber, treated with a rubber compound and felted into a sheet of compact and uniform texture, with smooth surfaces and free from imperfections. The finished packing shall be free from gasoline or other solvents used in the process of manufacture.

\section{GENERAL REQUIREMENTS}

1. The fiber shall be chrysotile asbestos of not less than 12 per cent water of composition.

2. The rubber shall be the best quality new, wild, or plantation rubber.

3. Packing shall be furnished in the dimensions ordered. A tolerance in thickness, plus or minus, will be permitted as follows: For thickness three thirty-second inch or less, 10 per cent; for greater thickness, 5 per cent.

4. The hardness, measured in depth of indentation produced by a spherical surface $3.2 \mathrm{~mm}(0.125$ inch) in diameter under a dead-weight pressure of $1 \mathrm{~kg}$ (2.2 pounds) applied for one minute, shall be not less than $0.15 \mathrm{~mm}$ nor more than $0.25 \mathrm{~mm}$. At least five impressions shall be made on both faces and the average of all readings reported as the hardness.

\section{DETAIL REQUIREMENTS}

CHEMICAL COMPOSITION

\begin{tabular}{|c|c|c|}
\hline & Grade A & Grade B \\
\hline Asbestos fiber, minimum. & $\begin{array}{r}\text { Per cent } \\
\text { by weight } \\
75\end{array}$ & $\begin{array}{l}\text { Per cent by } \\
\text { weight }\end{array}$ \\
\hline Rubber & $9-16$ & 9 \\
\hline $\begin{array}{l}\text { Sulphur, maximum } \\
\text { Mineral fillers }\end{array}$ & $0.50-1$ & Remain \\
\hline
\end{tabular}

Weights per square yard

\begin{tabular}{|c|c|c|c|c|}
\hline \multirow{2}{*}{$\begin{array}{l}\text { Size of packing } \\
\text { (inch) }\end{array}$} & \multicolumn{2}{|c|}{ Grade A } & \multicolumn{2}{|c|}{ Grade B } \\
\hline & Maximum & Minimum & Maximum & Minimum \\
\hline $\begin{array}{l}1 / 64 \ldots \\
1 / 32-\cdots \\
1 / 16 \\
1 / 8 \\
3 / 16 \\
1 / 4 \\
1 / 4\end{array}$ & $\begin{array}{r}\text { Pounds } \\
1.3 \\
2.7 \\
5.4 \\
10.8 \\
16.2 \\
21.6\end{array}$ & $\begin{array}{r}\text { Pounds } \\
1.0 \\
2.2 \\
4.4 \\
8.8 \\
13.2 \\
17.6\end{array}$ & $\begin{array}{r}\text { Pounds } \\
1.5 \\
3.0 \\
5.9 \\
11.8 \\
17.7 \\
23.6\end{array}$ & $\begin{array}{r}\text { Pounds } \\
1.2 \\
2.4 \\
4.8 \\
9.6 \\
14.4 \\
19.2\end{array}$ \\
\hline
\end{tabular}

\section{GRADE A PACKING}

The asbestos fiber shall be thoroughly carded and not less than 90 per cent shall remain on the $1 / 2$-inch screen and not more than 6 per cent shall pass through the $1 / 4$-inch screen when 1 pound of fiber is subjected to a 2-minute test in a standard asbestos-shaking machine. The tensile strength shall be not less than $4,000 \mathrm{lbs}$./in. ${ }^{2}$.

\section{GRADE B PACKING}

The tensile strength of cross-laminated sheets shall be not less than $3,000 \mathrm{lbs} . /$ in. $^{2}$ in either the longitudinal or transverse directions. Single-ply sheets having a minimum tensile strength of 1,800 lbs./in. ${ }^{2}$ will be acceptable if the average tensile strength in the longitudinal and transverse directions is not less than 3,000 lbs./in. ${ }^{2}$.

\section{METHODS OF INSPECTION AND TESTS}

1. Two samples of finished packing 12 inches square shall be selected by the inspector from each lot of 2,000 pounds of packing or less of each thickness and forwarded to the testing laboratory for 
such tests as may be necessary to determine compliance with this specification.

2. Accurate records of each box or mix of material shall be maintained by the manufacturer and be open for examination by the inspector, who shall at intervals be permitted to check the mixing operation.

3. The inspector may make a screen analysis of the asbestos fiber during the process of manufacture to determine compliance with Section IV(Grade A Packing, above.)

4. The tension test piece shall have a constricted portion 2 inches long and one-half inch wide. Three tension tests shall be made in each direction from one sample of each lot, the other sample being used for the hardness test.

\section{PACKING AND MARKING OF SHIPMENTS}

Shall be in accordance with the best commercial practice unless otherwise specified.

\section{NOTES}

1. Grade A packing is for use in steam joints under a maximum pressure of $350 \mathrm{lbs} . / \mathrm{in.}^{2}$ and a maximum temperature of $700^{\circ} \mathrm{F}$, and for joints of internal-combustion engines.

2. Grade B packing is for use in steam joints under a maximum pressure of $300 \mathrm{lbs}$. in. $^{2}$ and a maximum temperature of $600^{\circ} \mathrm{F}$., and for internalcombustion engines.

3. By using a number of rings of $1 / 16$-inch compressed asbestos sheet packing, sufficient to fill the packing space in boiler blow-off valves, the individual rings vulcanize into one solid ring and serve the same purpose as the special molded rings previously covered by Federal Specifications Board specification No. 109, which has been canceled.

(Requirements for tests and analyses which are applicable to this specification are contained in Federal Specifications Board specification 59a, general specifications for rubber goods.)

Federal Specifications Board, specification No. $97 \mathrm{~b}$, United States Government master specification for gaskets, asbestos metallic cloth, December $29,1923$.

\section{TYPES AND GRADES}

Gaskets shall be furnished in the following types and grades as required: Type A, seamless; type B, jointed.

Grade I.-Not less than 90 per cent asbestos.

Grade $I I$.- Not less than 75 per cent asbestos.

\section{MATERIAL AND WORKMANSHIP}

All gaskets shall be made from woven asbestos wire insertion cloth treated with a rubber compound. All layers of the gasket shall be uniformly impregnated with the rubber compound. The workmanship shall be first class in every respect.

\section{GENERAL REQUIREMENTS}

1. The order shall specify the type, grade, shape, and size desired, and the gaskets shall be so finished as to retain their shape and size under the ordinary conditions of handling before and during installation. (See "Notes," 3.)

2. All gaskets one-eighth inch in thickness and greater shall be folded and contain not less than 3 plies of cloth. No gaskets less than one-eighth inch thick shall be ordered under this specification. (See "Notes," 3 and 5.)

3. No minus tolerance will be permitted. Plus tolerance will be permitted as follows: $1 / 32$ inch in thickness on all gaskets, $1 / 32$ inch in all other dimen- sions of handhole gaskets, and $1 / 16$ inch on all other dimensions of all other gaskets.

4. A gasket shall not crack nor show any signs of injury when bent fiat on itself.

5 . The brass or copper wire shall be 0.007 inch in diameter, plus or minus 0.001 inch.

6. Seamless gaskets shall be composed of an integral piece of the fabric in which the filler threads run continuously around the gasket, while the warp threads run transversely. The fabric shall be folded on itself to form the desired thickness, the fold forming that edge of the gasket which is exposed to steam.

7. Jointed gaskets shall be composed of cloth cut on the bias and folded into the required shape and thickness after the ends have been securely joined together. The fold shall form that edge of the gasket which is exposed to steam.

\section{DETAIL REQUIREMENTS}

(Items $(a),(b),(e)$, and $(f)$ of "Detail requirements," F. S. B. No. 94b (given above) apply to Grades I and II as designated in this specification.)

Cloth for 1/8-inch, 3-ply gaskets shall be constructed as follows: Each strand of the warp shall consist of two plies of asbestos yarn and one for Grade I and not more than two for Grade II brass. or copper wires twisted together; each strand of the filler shall be composed of one or more plies of asbestos yarn and one for I and not more than two for II brass or copper wires twisted together. In construction of Grade II not more than one wire shall be used in the filler when only one ply of yarn is used.

Cloth for 3/16-inch 3-ply and 1/4-inch 4-ply gaskets shall be constructed as follows: Each strand of the warp and filler shall consist of two plies of asbestos yarn for I and not more than two for II brass or copper wires twisted together.

The weight of the asbestos metallic cloth before application of the rubber compound shall be not less than 2.25 pounds per square yard for $1 / 8$-inch, 3 -ply gaskets, and 2.75 pounds per square yard for $3 / 16$-inch 3-ply and 1/4-inch 4-ply gaskets.

\section{METHOD OF INSPECTION AND TEST}

One sample gasket and 1 square foot of untreated fabric shall be taken at random from each lot of 5,000 or less handhole gaskets, 1,000 or less muddrum and pipe-flange gaskets, or 500 or less manhole gaskets and forwarded to the testing laboratory.

\section{PACKING AND MAREING OF SHIPMENTS}

Packing and marking shall be in accordance with the usual commercial practice unless otherwise specified in the proposal.

\section{NOTES}

1. Grade I gaskets are suitable for steam pressure up to $300 \mathrm{lbs} . /$ in. $^{2}$ and temperature up to $700^{\circ} \mathrm{F}$.

2. Grade II gaskets are suitable for steam pressure up to $200 \mathrm{lbs}$. $/ \mathrm{in}^{2}$

3. This specification includes all folded gaskets for boiler manholes, handholes, and flanged joints. Handhole and tube-plate gaskets may be either type A or type B; all other gaskets should be type B.

4. When ordered, gasket tape shall conform to the requirements of jointed gaskets, Grade I and II, as required.

5. Stamped asbestos gaskets should be made from material described in Federal Specifications No. 94 and 96 (given above).

(Requirements for tests and analyses which are applicable to this specification are contained in 
Federal Specifications Board, specification No. 59a, general specifications for rubber goods.)

Federal Specifications Board specification No. 103a, United States Government master specification for asbestos high-pressure rod packing, June 15, 1925.

Packing shall be supplied in one grade only.

\section{MATERIAL AND WORKMANSHIP}

1. Packing shall be made from woven asbestos cloth, treated with a protective coating of rubber compound, wrapped tightly upon itself, in layers. No wire insertion will be permitted.

2. It shall be square and shall be made plain, with a central rubber core, or with a rectangular rubber spring back as specified and shall be sufficiently pliable to move or flow toward the rod as wear takes place. Packing less than three-eighths inch in size shall not contain a rubber core or spring back.

3. When the packing is made with a rubber spring back the outside cover stock shall consist of not less than two complete layers of cloth.

4. Rubber friction shall not be used on the outside of the packing where it will come ip contact with the rod.

\section{REQUIREMENTS}

1. The asbestos cloth shall be made as follows: Each strand of the warp and filling shall consist of two asbestos yarns, and the weave shall run not less than 20 strands of warp and not less than 10 strands of filling per linear inch.

2 . The yarn shall contain not less than 90 per cent of long-fiber asbestos of not less than 12 per cent water of composition.

3. New, wild, or plantation rubber of best quality shall be used in the spring back, central core, and friction.

4. The compound used for the spring back or central core shall contain not less than 60 per cent by volume of rubber. The total sulphur, exclusive of that contained in barytes, shall not exceed 8 per cent of the weight of rubber as compounded. The rubber compounds shall be free from all substances which might injuriously affect the quality.

5. The packing shall be coiled into helices and vulcanized. The inside diameter of the helix shall be not less than four nor more than six times the thickness of the packing.

6. Packing with core or spring back shall contain not less than 40 nor more than 60 per cent by weight of rubber compound, including the core or spring back, prior to lubrication. Packing without core or spring back shall contain not less than 30 nor more than 50 per cent by weight of rubber compound prior to lubrication.

7. The cross-sectional area of the rubber core or spring back shall not exceed 12 per cent of the nominal cross-sectional area of the packing.

8. The lubrication shall not exceed 20 per cent by weight of the finished packing. Lubrication may be either oil or glycerin.

9. The finished packing shall be dipped in pure flake graphite.

10. When subjected to the action of a saturated steam at $250 \mathrm{lbs}$./in. ${ }^{2}$ gauge pressure for eight hours the finished packing shall not harden nor soften materially, and the friction compound shall retain most of its elasticity and strength as indicated by the "tooth" when the plies are separated.

\section{METHODS OF INSPECTION AND TEST}

1. A sample of untreated eloth 12 inches square shall be taken from each lot.
2. A sample 12 inches long of each size of packing as finished and also without lubrication or graphite shall be taken from each 1,000 pounds or less.

\section{PACKING AND MARKING}

Each helix of packing shall be wrapped in waxed paper.

\section{ADDITIONAL INFORMATION}

1. This packing is suitable for use as a piston-rod, valve-stem, or slip-joint packing under steam pressures up to $300 \mathrm{lbs} . /$ in. $^{2}$ and with a maximum temperature of $700^{\circ} \mathrm{F}$.

2. The packing as furnished shall weigh within 5 per cent, plus or minus, of the proposed weights as submitted with the proposals.

(Requirements for tests and analyses which are applicable to this specification are contained in Federal Specifications Board specification 59a, general specifications for rubber goods.)

Federal Specifications Board, United States Government master specification No. 487 , for asbestos wick and rope packings, May 12, 1927.

\section{GENERAL SPRCIFICATIONS}

Unless otherwise specified herein, tests and analyses shall be made in accordance with the methods described in United States Government master specification No. 59a, general specifications for rubber goods (methods of physical tests and chemical analysis), in effect on date of invitation for bids.

\section{GRADE AND TYPES}

Wick and rope packing shall be made in one grade only, as follows: Wick packing, one-fourth inch only. Rope packing, all sizes, three-eighths inch and larger.

\section{MATERIAI AND WORKMANSHIP}

Wick and rope packing shall be made from longfiber carded asbestos spun into yarns and laid up into the general form of a common lamp wick or a loosely twisted cord which can be separated and rewound into grommets or braided into small gaskets. If a core is employed it shall be of the same material and construction.

\section{DETAIL REQUTREMENTS}

1. Packing shail be made from yarn containing not less than 85 per cent by weight of asbestos of at least 12 per cent water of composition.

2. No wire insertion, sizing, or lubrication shall be permitted.

3. The weight per linear yard shall not be less than stated in the following table:

\begin{tabular}{|c|c|}
\hline $\begin{array}{c}\text { Nominal } \\
\text { size }\end{array}$ & $\begin{array}{c}\text { Minimum } \\
\text { Weight per } \\
\text { linear yard }\end{array}$ \\
\cline { 1 - 2 } Inches & Pounds \\
$1 / 4$ & 0.045 \\
$3 / 8$ & .12 \\
$1 / 2$ & .20 \\
$5 / 8$ & .30 \\
$3 / 4$ & .40 \\
$7 / 8$ & .50 \\
$11 / 4$ & 1.00 \\
135 & 1.00 \\
\hline
\end{tabular}

\section{METHOD OF INSPECTION AND TEST}

1. Three balls or reels from each lot of 25 or less of the same size under inspection shall be selected by 
the inspector, measured, and weighed to obtain the average weight per linear yard.

2. A test sample weighing not less than 6 ounces shall be selected from each lot of 100 pounds or less of each size.

3 . Tests shall be made for (a) hygroscopic moisture, $(b)$ asbestos content, and (c) water of composition.

\section{PACKING OF SHIPMENTS}

Packing shall be furnished as follows unless otherwise specified: $1 / 4$-inch wick packing in 1-pound balls; $3 / 3$ to $1 \frac{1}{4}$ inch rope packing in 25-pound reels.

\subsection{MISCELIANEOUS OF ASBESTOS.}

Society of Automotive Engineers, brake lining (standard, 1926).

(This standard has been incorporated in its entirety into Simplified Practice Recommendation 66, of the United States Department of Commerce, given below.)

United States Department of Commerce, Division of Simplified Practice, recommendation No. 66, automobile brake lining, December 1, 1927.

The Department of Commerce, as a result of unanimous action of a conference of manufacturers, distributors, and users, recommends that stock sizes of automobile-brake lining be limited to the sizes in the following table:

\begin{tabular}{|c|c|c|c|c|}
\hline Thickness (inches) & $\begin{array}{r}5 \% 2 \\
+0.000 \\
-.020\end{array}$ & $\begin{array}{c}3 / 16 \\
+0.000 \\
-.020\end{array}$ & $\begin{array}{c}1 / 4 \\
+0.000 \\
-.031\end{array}$ & $\begin{array}{r}5 / 16 \\
+0.000 \\
-.031\end{array}$ \\
\hline Widths (inches $\pm 1 / 16$ ) & $\begin{array}{c}116 \\
11 / 4 \\
115 \\
153 \\
13 / 4 \\
178 \\
2 \\
21 / 2 \\
\end{array}$ & $\begin{array}{r}11 / 8 \\
11 / 4 \\
11 / 2 \\
15 / 8 \\
13 / 4 \\
2 \\
21 / 4 \\
21 / 2 \\
23 / 4 \\
3 \\
31 / 4 \\
31 / 2 \\
-\end{array}$ & $\begin{array}{c}13 / 4 \\
2 \\
21 / 4 \\
21 / 5 \\
23 / 4 \\
3 \\
31 / 4 \\
3162 \\
334 \\
4 \\
41 / 2 \\
5 \\
6\end{array}$ & \begin{tabular}{|c|} 
\\
\\
\hdashline
\end{tabular} \\
\hline
\end{tabular}

United States Department of Commerce, Division of Simplified Practice, recommendation No. 75, composition blackboard, April 1, 1928.

In accordance with unanimous action of a general conference of representative manufacturers, wholesalers, and users of composition blackboard, the United States Department of Commerce, through the Bureau of Standards, recommends that the following table constitute the accepted simplified list of stock items:

\section{TABLE 1.-Cement asbestos backing} TYPE 1

\begin{tabular}{|c|c|c|}
\hline Color & Widths (feet) & Lengths (feet) \\
\cline { 1 - 3 } Black _......- & $31 / 2$ and 4....... & 6,7 , and 8. \\
\hline
\end{tabular}

TABLE 2,-Wood pulp backing

TYPE 2

\begin{tabular}{|c|c|c|}
\hline Color & Widths (feet) & Lengths (feet) \\
\hline 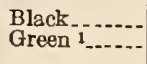 & $\begin{array}{l}31 / 2 \text { and } 4 \\
31 / 2 \text { and } 4 \ldots\end{array}$ & $\begin{array}{l}6,7,8,10 \text {, and } 12 . \\
6,7,8,10 \text {, and } 12 .\end{array}$ \\
\hline
\end{tabular}

OBSOLESCENT

\begin{tabular}{|c|c|c|}
\hline All.......... & $3^{2}-\ldots$ & 9 and 11. \\
\hline
\end{tabular}

1 The present volume in green board is sufficiently large to wartant its retention. The volume of demand at the expiration of the period of this recommendation will determine the subsequent action.

2 The present volume in the 3 -foot width is sufficiently large to warrant its retention. The volume of demand at the expiration of the period of operation of this recommendation will determine the subsequent action.

TABLE 3.-Gypsum core backing

TYPE 3

\begin{tabular}{|c|l|l|}
\hline Color & Widths (feet) & Lengths (feet) \\
\hline Black_...... & $31 / 2$ and 4.-..-. & 6,7 , and 8. \\
\hline
\end{tabular}

OBSOLESCENT

\begin{tabular}{|c|}
\hline All... \\
\hline
\end{tabular}

1 The present volume in the 3 -foot widch is sufficiently large to warrant its retention. The volume of demand at the expiration of the period of operation of this recommendation will determine the subsequent action.

\section{$546 . \quad$ CHALK}

American Ceramic Society, standard specifications for ceramic whiting, 1924 .

\section{(A) GENERAI}

1. Definition.-Ceramic whiting is a finely ground white powder composed of nearly pure calcium carbonate or calcium carbonate and magnesium carbonate obtained from pulverizing and sizing chalk, marble, or limestone, or as a chemically precipitated product.

2. Use in ceramic products.-Whiting is used to furnish the calcium oxide component of glazes, enamels, and fluxed ceramic bodies. It is an active fluxing agent, rarely used in large quantities in body mixtures. It may be used either as a raw ingredient in glazes or fritted or smelted with other glaze materials before application.

3. Packing.-Whiting is packed and shipped in barrels which do not hold more than 325 pounds or bags which do not hold more than 125 pounds. The package should be labeled, stating whether the content is natural rock whiting or chemically precipitated whiting.

\section{(B) REQUIREMENTS}

1. Quality.-Whiting shall be uniform in quality (from shipment to shipment), both as to fineness of grain and composition. The calcium, magnesium, or total carbonates shall not vary more than \pm 1 per cent and the silica not more than tone-half of 1 per cent of a figure set by contract within the limits 
of the composition shown in class 1 or class 2 . It should be manufactured from the purest rock available and should be free from particles of pyrites, iron-bearing silicates, metallic iron, and gypsum.

2. Composition.-Whitings shall be divided into two classes, No. 1 being practically a pure calcium carbonate and No. 2 containing calcium carbonate with a considerable percentage of magnesium carbonate within the limits of the composition given. This does not indicate that one class is inferior in quality to the other, but indicates that numerous users prefer the magnesium whiting to the pure calcium whiting.

OEASS 1

\begin{tabular}{l|c|c|c|c|c|c}
\hline Material & $\begin{array}{c}\text { Total } \\
\text { carbon- } \\
\text { ates }\end{array}$ & $\mathrm{CaCO}_{3}$ & $\mathrm{MgCO}_{3}$ & $\mathrm{Fe}_{2} \mathrm{O}_{3}$ & $\mathrm{SiO}_{2}$ & $\mathrm{SO}_{3}$ \\
\hline Maximum & Per cent & Per cent & Per cent & Per cent \\
0.25 & $\begin{array}{r}\text { Per cent } \\
2.0\end{array}$ & Per cent \\
0.1
\end{tabular}

CLASS 2

\begin{tabular}{l|r|r|r|r|r}
\hline Maximum & & 8 & 0.25 & 2.0 & 0.1 \\
Minimum & & & & \\
\hline
\end{tabular}

3. Fineness.-Screening samples by washing for 10 minutes with a stream of water practically without pressure shall not leave a residue of more than 1 per cent on a No. 140 screen (or more than 2 per cent on a No. 200 screen), and at least 98 per cent of the material shall pass a No. 200 screen. It shall also be so fine that a separation made by a Pearson $^{2}$ air separator will show at least 85 per cent of the material finer than $0.02 \mathrm{~mm}$ and at least 48 per cent finer than $0.01 \mathrm{~mm}$.

As an alternative to the Pearson air separator method the following (not so desirable) may be used: $2 \mathrm{~g}$ of the material shall be shaken for 10 minutes in a 250-cc cylindrical graduate with 250 ce of distilled water. On allowing to settle it shall require not less than 20 minutes for visible settling to cease when the cylinder is viewed in reflected light, using clear, north sky light for illumination.

4. Marking.-Each shipment of material shall be legibly marked with the names of consignor and consignee and with some means of identifying the particular contract on which the shipment is made.

5. Retesting.-Notice of the rejection of a shipment based on these specifications must be in the hands of the consignor within $\mathbf{1 0}$ days after the receipt of the shipment at the point of destination. If the consignor desires a retest, he shall notify the consignee within five days of receipt of said notice.

\section{(C) SAMPLING AND TESTING}

1. Sampling.-Twenty-five per cent of the number of packages shall be selected for sampling. A core representing the contents of each of these packages shall be taken with a sampling tube. The total material so collected shall weigh not less than 5 pounds. This shall be thoroughly mixed and quartered to provide a 1/2-pound sample for the laboratory.

2. Testing.-The following directions are a brief summary of the analytical methods which are recom-

2 The Pearson air separator developed at the National Bureau of Standards by J. C. Pearson and F. A. Hitchcock gives excellent separations of fine, dry powders and is the only machine which can be used for this purpose for particles as small as $0.01 \mathrm{~mm}$. Its use is therefore recommended for manufacturers and users of fine powder such as whiting, flint, feldspar, paint pigments, etc. mended. ${ }^{3}$ Blast $0.5 \mathrm{~g}$ of the sample for 15 minutes in a platinum crucible. Cool and transfer to an evaporating dish. Mix to a slurry with distilled water. Add 5 to $10 \mathrm{cc}$ concentrated $\mathrm{HCl}$. Heat gently until solution is complete, breaking up lumps: if necessary. Evaporate to dryness on water bath. Add $15 \mathrm{cc}$ concentrated $\mathrm{HCl}$ and dilute with an equal volume of distilled water. Digest on water bath for 10 minutes. Filter and wash with hot water. Evaporate the filtrate to dryness. Dissolve in acid and water as before. Filter and wash with hot water. Ignite the two precipitates. together and weigh as silica and insoluble matter.

Dilute the above filtiate to $250 \mathrm{cc}$. Make alkaline with $\mathrm{NH}_{4} \mathrm{OH}$. Boil until odor of $\mathrm{NH}_{3}$ is barely noticeable. Filter and wash slightly with hot water. Dissolve the precipitate with hot dilute $\mathrm{HCl}$ and repeat the precipitation as before. Filter and wash thoroughly with hot water. Ignite and weigh as oxides of iron, aluminum, and phosphorus.

To the filtrates from the above add a few drops of $\mathrm{NH}_{4} \mathrm{OH}$ and bring to a boil. Add $25 \mathrm{cc}$ of a saturated solution of $\left(\mathrm{NH}_{4}\right)_{2} \mathrm{C}_{2} \mathrm{O}_{4}$. Continue boiling until the precipitate becomes granular. Let stand one hour. Filter and wash with hot water. Ignite the precipitate, dissolve in dilute $\mathrm{HCl}$, and dilute to 100 cc. Add excess of $\mathrm{NH}_{4} \mathrm{OH}$ and boil. Filter out any insoluble matter, ignite, and weigh; add its weight to the oxides of iron and aluminum found previously. To this filtrate add $\left(\mathrm{NH}_{4}\right)_{2} \mathrm{C}_{2} \mathrm{O}_{4}$, proceeding as before. Filter, and wash with boiling water. Ignite, and blast to constant weight as calcium oxide.

Acidify the filtrates from the above with $\mathrm{HCl}$. Add 10 ce of a saturated solution of $\mathrm{NaNH}_{4} \mathrm{HPO}_{4}$ and evaporate to $150 \mathrm{cc}$; cool; add $\mathrm{NH}_{4} \mathrm{OH}$ drop by drop with constant stirring until the precipitate starts to form. Then add moderate excess of $\mathrm{NH}_{4} \mathrm{OH}$. Stir for several minutes. Let stand overnight. Filter and dissolve the precipitate in hot dilute $\mathrm{HCl}$

Dilute to $100 \mathrm{cc}$, add $1 \mathrm{cc}$ of saturated solution of $\mathrm{NaNH}_{4} \mathrm{HPO}_{4}$, and precipitate as before. Filter and wash with dilute $\mathrm{NH}_{4} \mathrm{OH}$ (25 per cent $\mathrm{NH}_{3}$ containing a few drops of $\mathrm{HNO}_{3}$ ); ignite and weigh as $\mathrm{Mg}_{2} \mathrm{P}_{2} \mathrm{O}$; multiply this weight by 0.3621 to find the weight of $\mathrm{MgO}$.

Place $0.5 \mathrm{~g}$ of the sample in a small Erlenmeyer flask and cover with hot distilled water. Connect this flask into a carbon-dioxide train, set up as follows: Next to the flask is a reflux condenser, to which is connected a calcium-chloride drying tube followed by a tube containing anhydrous $\mathrm{CuSO}_{4}$, then another tube of $\mathrm{CaCl}_{2}$, then by two tubes filled with soda lime, and finally by another tube of $\mathrm{CaCl}_{2}$. The entire train must be so arranged that a stream of $\mathrm{CO}_{2}$-free air can be kept passing through it. Start this stream of air. Weigh the tubes containing soda lime and replace them in the train. Add to the sample in the flask about $25 \mathrm{cc}$ of $1: 1 \mathrm{HCl}$, being careful that no gas is lost and that the effervescence is not too violent during the operation. When the effervescence diminishes, heat the flask, bring the liquid gradually to boiling. Boil for 10 minutes. Remove the flame and allow the flask to cool, while the stream of air is still flowing, for 15 minutes. Disconnect and weigh the soda lime tubes. Their increase in weight is recorded as carbon dioxide.

To $5 \mathrm{~g}$ of the sample in a covered vessel, add 25 cc of water, and then cautiously $10 \mathrm{cc}$ of concentrated hydrochloric acid. Break up any lumps with the flattened end of a glass rod and boil for two or three minutes. Reduce the hot solution

3 For more complete information on this subject reference should be made to The Analysis of Silicate and Carbonate Rocks, by W. F. Hillebrand, U. S. Geol. Surv. Bull. No, 700, 
with a few drops of stannous chloride solution, cool, barium is absent, the residue may be rejected; if dilute to $100 \mathrm{cc}$, add $10 \mathrm{cc}$ of mercuric chloride solution, and titrate with standard potassium dichromate solution $(N / 20)$, using potassium ferricyanide as outside indicator. Calculate the results to $\mathrm{Fe}_{2} \mathrm{O}_{3}$. A blank determination is desirable for comparison. The permanganate method for determination of total iron, as given by Blair in The Chemical Analysis of Iron, may also be used.

Fuse $2.5 \mathrm{~g}$ of the sample with about $10 \mathrm{~g}$ of sodium carbonate, and dissolve the melt in 10 per cent hydrochloric acid. (Any insoluble residue should be filtered off and tested by flame test for barium.

present, the residue should be again fused with an excess of sodium carbonate, and the water extract tested for $\mathrm{SO}_{3}$ ) Dilute the clear solution to $250 \mathrm{cc}$, heat to boiling, and add slowly $10 \mathrm{ce}$ of hot 10 per cent solution of barium chloride. Formation of a white precipitate indicates the presence of sulphur. A blank test should be made to insure the purity of reagents used.

For extremely accurate work any insoluble matter may be filtered off at this point, ignited, fused with sodium carbonate, and the melt dissolved in 10 per cent hydrochloric acid and added to the filtrate. 


\section{MICA, RARE MINERALS}

551. CRUDE MICA

552. MANUFACTURED MICA

(No nationally recognized specifications avail- $\begin{gathered}\text { (No nationally recognized specifications avail- } \\ \text { able.) }\end{gathered}$ able.) 
560.

\section{GENERAL ITEMS}

National Jewelers Board of Trade, recommended practice regarding classification of precious stones (in advertising and selling), 1928.

For trade purposes and in order to simplify the consideration of stones, it is urged that they be considered under five general headings; that is, 1 , genuine; 2 , reconstructed; 3 , synthetic; 4 , doublet; 5 , imitation. The definitions and limitations of each group are set forth in the following:

\section{GENUINE}

All stones produced by nature, in other words, natural stones, would continue to be called by their name or names without adjective modification other than that referring to color or quality.

Example.-A pink beryl might be so termed or sold as a "morganite," or reddish agate sold as "carnelian," or white zircon as "jargoon," etc.

We recommend that agate, regardless of the fact that over 90 per cent of it is dyed or treated before sale, continue to be sold as agate or under the various headings which now designate the various colors of dyed agate.

Example.-Agate which has been subjected to blackening be sold as "black onys," etc.

\section{RECONSTRUCTED}

All stones produced by fusing or amalgamation of natural stones or parts thereof without the introduction of extraneous elements.

Note.-This type of stone is to-day practically nonexistent and the committee knows of no such stones for sale commercially. In times past, however, the natural ruby was successfully simulated by fusing small pieces of genuine rubies. In order to provide for further discoveries along this line, classification No. 2 is herewith recommended.

\section{SYNTHETIC}

Produced by artificial means but having approximately the exact characteristics of the genuine stones, such as:

(a) Same hardness with a tolerance of 5 per cent over or under the extremes of the genuine.

(b) Same dichroism.

(c) Same specific gravity or density with a tolerance of 5 per cent over or under the extremes of the genuine.

(d) Same chemical constituents with reasonable tolerance in the proportion of the constituents.

\section{DOUBLET}

Stones produced by the cementing together of two or more parts, one of which must be of genuine stone.

\section{IMITATION}

All other productions made to simulate genuine stones. It is recommended that in the discussion of stones they be at all times referred to as "precious stones" and not as "precious stones" and "semiprecious stones."

\section{DIAMONDS AND PEARIS}

National Jewelers Board of Trade, recommended practice regarding the description of diamonds (in advertising and selling), 1928.

(The above-entitled recommended practice includes data relative to the following trade terms or factors relating to diamonds: (1) Perfection, (2) color, (3) cutting (or make), (4) weight, (5) price, and (6) imitations. The recommendations set forth what should not be done in trade practices, and do not lay down any hard, fast rules as to grading the various qualities.)

National Jewelers Board of Trade, recommended. practice regarding the description of pearls and imitation pearls (in advertising and selling), 1928.

\section{GENOINE PEARLS}

Genuine pearls should be advertised and sold as "pearls." Where the term "oriental pearl" is used it shall be limited to pearls found in salt water.

\section{IMITATION PEARLS}

In the advertising of imitation pearls, if the word "pearl" is used, the word "imitation" should always be used in conjunction in clearly legible type-immediately before, following, or directly underneath.

"Indestructible," or its equivalent, as applied to imitation pearls is inaccurate and should not be used.

\section{CULTURE PEARLS}

Culture pearls should be advertised and sold as "culture pearls."

562.

\section{SAPPHIRES}

(No nationally recognized specifications available.) 
571.

\section{SULPHUR}

(No nationally recognized specifications available.)

572.

\section{MAGNESIA}

American Marine Standards Committee, specification for magnesia asbestos plaster, standard E No. 16, 1928.

(This specification is substantially identical with Federal Specifications Board No. 211, given below.)

American Marine Standards Committee, specification for magnesia molded pipe covering and blocks, standard E No. 15, 1928.

(This specification is substantially identical with that issued by the Federal Specifications Board for the same materials, given below.)

Federal Specifications Board, specification No. 211, United States Government master specification for magnesia asbestos plaster, September 30, 1924.

\section{GRADE}

1. Magnesia asbestos plaster shall be furnished in but one grade.

\section{MATERIAL AND WORKMANSHIP}

2. Magnesia asbestos plaster shall contain not less than 85 per cent pure hydrated magnesium carbonate $\left(4 \mathrm{MgCO}_{3} \cdot \mathrm{Mg}(\mathrm{OH})_{2} \cdot 5 \mathrm{H}_{2} \mathrm{O}\right)$, and not less than 10 per cent asbestos fiber.

\section{DETAIL REQUIREMENTS}

3. (a) The plaster shall be dry, the magnesia content shall be finely ground, and ingredients shall be uniformly mixed.

(b) When properly mixed with water, the plaster shall be such that it can be laid on with a trowel or by hand.

(c) The plaster upon drying shall form a firm, light, incombustible, porous heat insulating covering for pipes and other surfaces.

\section{METHOD-OF INSPECTION, TESTS, ETC.}

4. (a) From each delivery samples shall be taken at random. The amount taken shall vary with the amount of the delivery. Sufficient shall be taken to thoroughly represent the material presented for inspection. The samples taken shall be thorougbly mixed, quartered, and at least $25 \mathrm{~g}$ forwarded to the Government laboratory for analysis.

(b) A sample of the material which has been molded in a form and dried shall be subjected to furnace heat without baking hard, burning, or producing a flame.

(c) The weight of the material after molding and drying shall not exceed 20 pounds per cubic foot.

(d) Umpire method for the analysis of 85 per cent magnesia products.-I. The preparation of the samples taken for analysis is important. From each lot at least 10 small fragments are taken.
II. With a sharp knife each fragment is trimmed to a smooth surface, care being taken that no fibers project from any surface.

III. The entire sample is thoroughly crushed and, if necessary, is quartered down to a $25-\mathrm{g}$ sample, which is stored in a glass bottle provided with a rubber stopper.

IV. Hygroscopic moisture.-A 2-g portion of the sample is placed on a weighed watch glass and dried at $100^{\circ} \mathrm{C}$. to constant weight. The loss in weight is taken as hygroscopic moisture.

It is considered advisable to make a moisture determination on a small sample and that the other determinations be made on the undried material. A less desirable procedure is to dry the crushed sample and preserve it in a desiccator.

V. Fiber.-A 5-g sample is taken for the chemical analysis, $100 \mathrm{cc}$ of water and 2 drops of methyl orange are added, and $N \mathrm{HCl}$ is slowly added with stirring until a permanent pink color appears. (This will require about $125 \mathrm{cc}$.) The asbestos is collected by filtering through a weighed Gooch crucible and is thoroughly washed with cold water. The crucible and its contents are dried at $110^{\circ} \mathrm{C}$. and weighed. ('The weight of the fiber so obtained may be corrected by a blank determination on the asbestos used in the product.)

It is well known that the fiber used in this product loses weight on being washed with the distilled water and that a very considerable percentage of its original weight is lost by washing with hydrochloric acid. It is apparent, therefore, that determination of fiber in 85 per cent magnesia should be investigated before a standard method of analysis can be prepared.

VI. The combined filtrate and washings obtained above are made up to 1 liter in a standardized flask; $100 \mathrm{cc}$ of this solution are employed for further analyses.

VII. Iron and alumina.-A 100-ce portion of the above solution is diluted to $200 \mathrm{cc} ; 10 \mathrm{cc}$ of concentrated hydrochloric acid are added and the solution is brought to the boiling temperature. Dilute $\mathrm{NH}_{4} \mathrm{OH}$ is slowly added to slight excess; the solution is boiled for a minute or two to coagulate the precipitate and to expel the excess of ammonia. The precipitate, which consists of the hydroxides of aluminum and iron, is filtered off, washed with hot water, and, if desired, is ignited and weighed as $\mathrm{Fe}_{2} \mathrm{O}_{3}+\mathrm{Al}_{2} \mathrm{O}_{3}$.

VIII. Lime. - The combined filtrate and washings obtained above are brought to the boiling temperature and made slightly acid with $\mathrm{HCl}$. About $10 \mathrm{cc}$ of a saturated solution of ammonium oxalate are then added, and the solution is made slightly alkaline with $\mathrm{NH}_{4} \mathrm{OH}$. After boiling for five minutes, the solution is allowed to cool, and when thoroughly cold the bulk of the solution is decanted through a filter and the precipitate in the beaker is washed two or three times by decantation with cold water, using about 50 ce of water each time. The precipitate on the filter is dissolved in dilute $\mathrm{HCl}$ and is returned to the beaker in which the precipitation was made. Sufficient $\mathrm{HCl}$ is added to redissolve the entire precipitate, and the solution is brought to boiling, a few cubic centimeters of ammonium oxalate solution are 
added and then dilute $\mathrm{NH}_{4} \mathrm{OH}$ in slight excess. When cold, the precipitate of calcium oxalate is collected on a filter, washed with cold water, ignited, and weighed as $\mathrm{CaO}$. It is especially important that the double precipitation of lime be carried out in order that the occlusion of magnesia with the lime shall be avoided.

1X. Magnesia.-The combined filtrates, but not the washings from the last lime precipitation, are acidified with hydrochloric acid and evaporated on a hot plate to about $500 \mathrm{cc}$. A sufficient quantity of a saturated solution of sodium ammonium phosphate to precipitate all of the magnesia is added, and a considerable excess of ammonia. The solution is thoroughly stirred until precipitation is complete and is allowed to stand at least 12 hours in the cold. The bulk of the liquid is then decanted through the filter; the precipitate on the filter is washed back into the beaker and dissolved in the least possible amount of dilute hydrochloric acid. A few drops of phosphate solution are added and then ammonia in slight excess, with stirring, as before. After standing at least six hours, the precipitate is filtered off and washed with dilute ammonia $(1: 10)$. It is then dried, detached from the paper, and the latter burned on a platinum wire above the crucible containing the bulk of the precipitate and the ash added to the contents of the crucible. The covered crucible is first heated gently and, when ammonia is no longer evolved, the heating is increased to a full blast until constant weight is obtained. The residue is magnesium prro-phosphate $\left(\mathrm{Mg}_{2} \mathrm{P}_{2} \mathrm{O}_{7}\right)$. The following formula may be used to determine the weight of the basic magnesium carbonate:

$$
\mathrm{MIg}: \mathrm{P}: \mathrm{O}_{7} \times 0.8723=4 \mathrm{MrgCO} \cdot \mathrm{Mrg}(\mathrm{OH})_{3} \cdot 5 \mathrm{H}_{2} \mathrm{O}
$$

$$
\text { (In place of } 0.8723 \text {, the fraction } \frac{0.349}{0.4} \text { may be used.) }
$$

In redissolving the precipitates of calcium oxalate and of magnesium ammonium phosphate, it is the practice at the Mellon Institute to use dilute nitric acid. It is believed that these precipitates are less soluble in ammonium nitrate than in ammonium chloride. If desired nitric acid may be used in place of hydrochloric acid all through the analysis.

$\mathrm{X}$. Loss on ignition.-About $2 \mathrm{~g}$ of the sample is placed in a weighed, covered crucible. This is heated slowly to redness and then at the full temperature of the blast for 15 minutes. After weighing, blast for 5 minutes more to insure constant weight. The loss in weight includes water and $\mathrm{CO}_{2}$ from the magnesium carbonate and loss due to heating the asbestos.

The loss on ignition is a simple test which is believed to be of value as indicating the extent to which "calcination" has taken place during service.

XI. Carbon dioxide.-About $1 \mathrm{~g}$ of the sample is introduced into a weighed Schroedter alkalimeter. Dilute hydrochloric acid $(1: 1)$ is allowed to run in on the sample, and when the main reaction is over the solution is boiled gently for two minutes. When cold, air is drawn through to displace the carbon dioxide and the apparatus is weighed. The loss in weight is $\mathrm{CO}_{2}$. This method is subject to errors of precision of as much as 0.5 per cent.

The determination of carbon dioxide will, in all probability, rarely be made. It is given here, however, in order that methods for the complete analysis may be recorded.

\section{PACKING AND MARKING}

5. (a) The material shall be delivered in substantial cloth or burlap sacks; net weight of each container shall be about 60 pounds. (b) Each container shall be marked with the name of the contents manufacturer's name and purchase order number.

\section{ADDITIONAL INFORMATION}

6. The material is suitable for insulating surfaces, the temperatures of which do not exceed $600^{\circ} \mathrm{F}$., and is intended for use in connection with magnesia molded pipe covering and blocks or where it is not practicable to use the material delivered in forms or blocks.

Federal Specifications Board, specification No. 212, United States Government master specification for magnesia molded pipe covering and blocks, September 30, 1924.

\section{GRADE}

1. But one grade of material shall be furnished under these specifications.

\section{MATERIAI}

2. Magnesia insulating covering or lagging material shall contain not less than 85 per cent of pure hydrated magnesium carbonate $\left(4 \mathrm{MgCO}_{3} \cdot \mathrm{Mg}(\mathrm{OH})_{2}\right.$. $5 \mathrm{H}_{2} \mathrm{O}$ ) and not less than 10 per cent long fiber asbestos.

\section{GENERAI REQUIREMENTS}

3. (a) The weight per cubic foot of the finished material shall not exceed 18 pounds.

(b) The mixture of the magnesia and the asbestos shall be homogeneous throughout.

\section{DETAIL REQUIREMENTS}

4. (a) Standard thickness pipe covering for sizes from one-half inch up to and including 10 inches shall be furnished in sections 3 feet long, split in half lengthwise. Covering for sizes larger than 10 inches shall be furnished in segments.

(b) In double-standard thickness pipe covering the inner layer shall be furnished in sections for pipe sizes up to and including 10 inches, and in curved blocks for larger sizes. The outer layer shall be furnished in sections for pipe sizes up to and including 8 inches, and in curved blocks for larger sizes.

(c) The thicknesses shall not be less than those given

\begin{tabular}{|c|c|c|}
\hline $\begin{array}{l}\text { Nominal size of } \\
\text { pipe (inches) }\end{array}$ & $\begin{array}{l}\text { Standard } \\
\text { thickness } \\
\text { of cover- } \\
\text { ing }\end{array}$ & $\begin{array}{c}\text { Double } \\
\text { standard } \\
\text { thickness }\end{array}$ \\
\hline 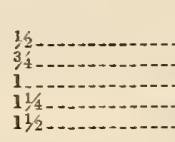 & $\begin{array}{c}\text { Inches } \\
7 / 3 \\
7 / 8 \\
7 / 8 \\
7 / 3 \\
7 / 8\end{array}$ & $\begin{array}{c}\text { Inches } \\
13 / 4 \\
13 / 4 \\
13 / 4 \\
13 / 4 \\
13 / 4\end{array}$ \\
\hline $\begin{array}{l}2 \\
21 / 2-1 \\
3 \\
3132 \\
4\end{array}$ & $\begin{array}{l}11 / 32 \\
11 / 32 \\
11 / 32 \\
11 / 32 \\
11 / 8\end{array}$ & $\begin{array}{l}21 / 16 \\
21 / 16 \\
21 / 16 \\
2116 \\
21 / 4\end{array}$ \\
\hline $\begin{array}{l}41 / 2-\ldots \\
5 \\
6 \\
7\end{array}$ & $\begin{array}{l}11 / 5 \\
11 / 3 \\
11 / 8 \\
11 / 4\end{array}$ & $\begin{array}{l}21 / 4 \\
21 / 4 \\
21 / 4 \\
21 / 2\end{array}$ \\
\hline 10 and above 1 & $\begin{array}{l}11 / 4 \\
11 / 4 \\
11 / 4 \\
11 / 2\end{array}$ & $\begin{array}{l}21 / 2 \\
21 / 2 \\
21 / 2 \\
3\end{array}$ \\
\hline
\end{tabular}
for the various sizes in the following table:

1 Covering for 12-inch pipe may be furnished in sections or segments. 
(d) A tolerance of one-fourth inch over the thickness given will be permitted.

(e) Sectional pipe covering shall have an attached canvas jacket weighing not less than 3.80 ounces per linear yard, unless heavier canvas is required by the purchase order. Segmental block covering shall not be supplied with canvas.

(f) Unless solid brass bands, lacquered, are specifically required by the contract or order, steel bands, black japanned, may be furnished. All bands shall be at least three-fourths inch wide for covering for pipe sizes up to and including 5 inches and at least 1 inch wide for pipe sizes larger than 5 inches. The thickness of the brass bands shall be not less than 0.01 inch and the thickness of the steel bands shall be not less than 0.007 inch. Bands shall be furnished at the rate of 5 for 2 sections.

(g) Rectangular flat blocks shall be 6 inches wide, 18 inches or 3 feet long, as required, and of thicknesses as required.

\section{METHOD OF INSPECTION, TESTS, ETC.}

5. (a) From each lot of 100 pieces or less presented for inspection samples shall be selected for test. The amount taken for chemical analysis shall vary with the size of the delivery. Sufficient shall be taken to thoroughly represent the material presented for inspection. After crushing the samples taken and thoroughly mixing, a sample not less than $25 \mathrm{~g}$ shall be secured by quartering and the analysis carried out by an approved method.

(b) From each lot submitted for inspection several lengths or blocks shall be selected, weighed, and measured and the weight per cubic foot determined.

(c) Umpire method for the analysis of 85 per cent magnesia products.- Same as (d) Method of Inspection, Tests, etc., of F. S. B. No. 211 (given above).

\section{PACKING AND MARKING}

6. The material shall be packed in substantial boxes in a manner to insure against breakage in transit. Each box shall be marked with the name of the material, size, amount contained, name of contractor or manufacturer, purchase order number, and the item number under which delivery is made.

\section{ADDITIONAL INFORMATION}

7. (a) Purchase orders for pipe covering shall specifiy standard or double thickness and the type of brands required.

(b) If canvas jacket heavier than 3.80 ounces per linear yard is required the weight of canvas desired shall be stated in the proposal.

(c) This material is suitable for pipe covering where the temperature does not exceed $500^{\circ} \mathrm{F}$.

Federal Specifications Board, specification No. 445, United States Government master specification for cement, plastic magnesia (magnesia-oxychloride) used as flooring, bases, wainscots, etc., October 28, 1926.

\section{TYPE}

Plastic magnesia cement is used largely for making the so-called resilient types of flooring. The quality depends upon the proportion of the various aggregatcs and cement. Hence, as a floor of a certain quality may be desired it is not essential that the plastic magnesia cement should be made according to any required formula, but the manufacturer is allowed to use any combination of ingredients that will give the specified physical properties.

\section{MATERIAL AND WORKMANSHIP}

Plastic magnesia cement is a dry intimate mixture composed of calcined caustic magnesia, fibrous materials, finely ground inert fillers, and colors (if desired) which on gaging with a solution of magnesium chloride sets to a resilient product.

\section{GENERAL REQUIREMENTS}

1. If the chloride solution fails to meet the requirements for concentration, no further tests shall be made with it nor any tests made with the dry mixture until the concentration has been corrected.

2 . The principle of the tests being the determination of the quality of the finished product, the consistency used shall be that given by the vendor furnishing the dry mixture and chloride as that which will be used on the work. Hence there shall be furnished by the contractor a statement as to the weight of chloride solution which will be used per unit weight of the dry mixture on the work.

\section{DETAIL REQUIREMENTS}

\section{TENSLE STRENGTH}

The average tensile strength of not less than three briquets, when made and stored according to the "Method of tests," shall not be less than-

200 pounds per square inch at the end of 24 hours for the first coat.

300 pounds per square inch at the end of 7 days for the first coat. 250 pounds per square inch at the end of 24 hours for the finish coat.

350 pounds per square inch at the end of 7 days for the finish coat.

\section{TRANSVERSE STRENGTH}

The average transverse strength of not less than three bars ( $1 / 2$ by 2 by 12 inches) when made, stored, and tested according to the "Method of tests," shall not be less than-

400 pounds per square inch at the end of 24 hours for the first coat.

700 pounds per square inch at the end of 7 days for the first coat. 600 pounds per square inch at the end of 24 hours for the finish coat.

900 pounds per square inch at the end of 7 days for the finish coat.

\section{EXPANSION}

The coefficient of expansion as determined on test pieces, prepared and stored according to the "Method of tests," shall lie within the following limits:

Between final set and 24 hours after final set: -0.10 per cent, +0.15 per cent.

Between final set and seven days after final set: -0.10 per cent, +0.18 per cent.

Specimens which crack or warp or separate from the scratch coat or concrete base shall be considered as having given results beyond the above limits.

\section{TIME OF SET}

A pat of the dry material when gaged with a solution of the chloride, as described in the "Methods of tests," shall develop on initial set in not less than one hour and a final set in not more than eight hours when determined by the Gilmore needle.

\section{FINEN ESS}

The residue on a standard 100-mesh sieve shall not exceed 30 per cent by weight for the finish coat.

\section{SOUNDNESS}

A pat of the dry mixture when gaged with the solution of chloride according to the "Method of tests," shall not distort, crack, check, or disintegrate in the steam test for soundness.

\section{CHEMICAL LIMITS FOR MAGN ESIOM CHLORIDE}

The solid chloride or the salts in the liquid chloride shall meet the following requirements:

$\mathrm{MgCl}_{2}, 6 \mathrm{H}_{2} \mathrm{O}$, not less than 97 per cent. $\mathrm{CaO}$ (as $\mathrm{CaCl}_{2}$ ), not more than 2 per cent. $\mathrm{SO}_{3}$ (as $\mathrm{MgSO}_{4}$ ), not more than 2 per cent. $\mathrm{NaCl}+\mathrm{KCl}$, not more than 3 per cent.

The concentration of the solution of chloride shall not be less than $21^{\circ}$ Baumé. 


\section{METHOD OF INSPECTION AND TESTS}

\section{INSPECTION AND STORAGE}

Such facilities shall be furnished the purchaser for careful inspection and sampling at the mill, or at the site of the work, as may be called for in the contract by the purchaser. The material shall be stored in a suitable weather-tight building which will protect it from dampness, in such manner as to permit of ready inspection and sampling. Sampling must be done within 72 hours after delivery of the materials to the site of the operation.

\section{TIME FOR TESTING}

Tests shall be completed within 10 days after sampling.

\section{SAMPLING}

(a) Tests.-Tests may be made on individual or composite samples as may be required by the contract. Each test sample of the dry mixture shall weigh at least 15 pounds; each test sample of dry chloride shall weigh at least 4 pounds, or if a solution is used the test sample shall weigh 15 pounds.

(b) Individual samples.-If the dry mixture is delivered in bags, one test sample shall be taken from each 50 bags; if in barrels, one from each 10 barrels. If the solid chloride is used, one test sample shall be taken from each 5 drums or 15 bags; if a solution is used, one test sample shall be taken from each 10 drums.

(c) Composite samples.-If the dry mixture is delivered in bags, one sample shall be taken from each 10 bags and every five samples so taken combined to form one composite test sample. If in barrels, one sample shall be taken from each 2 barrels and every five samples so taken combined to form a composite test sample. If the solid chloride is used, one sample shall be taken from each 5 drums or 15 bags and every three samples so taken combined to form a composite test sample. If a solution is used, one sample shall be taken from each 4 drums and every three samples so taken combined to form a composite test sample.

(d) If the shipment represents amounts greater than 500 bags, or 150 barrels, of dry mixture, and greater than 25 drums, or 75 bags, of chloride or 50 drums of solution, it should be divided into two groups. Each group should be sampled and tested as a single shipment. Larger shipments should be divided into groups in the same ratio.

(e) Samples shall be placed, shipped, and kept during test in air-tight containers.

\section{ANALYSIS OF COMMERCIAL MAGNESIUM CHLORIDE}

(a) Preliminary procedure. Place about $10 \mathrm{~g}$ of the sample in a weighing bottle, stopper, and weigh accurately. Transfer the sample to a short-stem funnel placed in the neck of a $500 \mathrm{cc}$ graduated flask. Replace stopper in weighing bottle and again weigh accurately to determine the weight of sample used. Dissolve the sample in distilled water (that has been well boiled to free it from carbon dioxide,) allowing the solution to run into the flask; wash off funnel and stem and remove. Dilute the solution in the flask to the mark with water, stopper, and mix thoroughly.

(b) Total alkalinity (expressed as $\mathrm{Mg}(\mathrm{OH})_{2}$ ).Titrate a $100 \mathrm{cc}$ aliquot of the solution with $0.1 \mathrm{~N}$ $\mathrm{HCl}$ solution (see Reagents), using methyl orange as an indicator. Calculate the total alkalinity to percentage of magnesium hydroxide, $\mathbf{M g}(\mathrm{OH})_{2}$. (1 cc $0.1 \mathrm{~N} \mathrm{HCl}=0.002916 \mathrm{~g} \mathrm{Mg}(\mathrm{OH})_{2}$.)

(c) Calcium (expressed as $\mathrm{CaCl}$ ). - Transfer a 50 cc aliquot of the original solution to a beaker, add about $50 \mathrm{cc}$ of freshly boiled water, about $5 \mathrm{~g}$ of $\mathrm{NH}_{4} \mathrm{Cl}$ and a few drops of $\mathrm{NH}_{4} \mathrm{OH}$. Heat the solution to boiling, add slowly with stirring $20 \mathrm{cc}$ of a hot saturated solution of ammonium oxalate and continue the boiling for five minutes. Let stand from one to two hours. Filter through a 9 $\mathrm{cm}$ filter paper, catching the filtrate in a $250 \mathrm{cc}$ graduated flask, and wash the beaker and filter two times with boiling hot water. Set the filtrate aside, place the beaker under the funnel, dissolve the precipitate by pouring about $10 \mathrm{cc}$ of hot $\mathrm{HCl}(1: 4)$ through the filter, and wash the filter several times with hot water. Add $1 \mathrm{~g}$ of $\mathrm{NH}_{4} \mathrm{Cl}$ to the solution in the beaker, render just alkaline with $\mathrm{NH}_{4} \mathrm{OH}$, heat to boiling, and add $5 \mathrm{cc}$ of a hot saturated solution of ammonium oxalate. Boil about five minutes, let stand for two hours, and filter through the original paper, catching the filtrate in the flask used in the previous filtration. Wash the beaker and filter with boiling hot water until $10 \mathrm{cc}$ of the washings are not decolorized in two to three minutes after adding $0.5 \mathrm{cc}$ of $\mathrm{H}_{2} \mathrm{SO}_{4}$, heating to about $70^{\circ} \mathrm{C}$, and adding two drops of $0.1 N \mathrm{KMnO}_{4}$ solution. Set the filtrate aside, place the beaker in which precipitation was made under the funnel, pierce the apex of the filter paper with a stirring rod and wash the precipitate into the beaker with a jet of hot water; pour $30 \mathrm{cc}$ of warm dilute $\mathrm{H}_{2} \mathrm{SO}_{4}(1: 4)$ through the filter so that the acid comes in contact with all of the paper, and wash the funnel and filter thoroughly with hot water. Dilute to about $250 \mathrm{cc}$ with water, heat to about $90^{\circ} \mathrm{C}$, and titrate at once with $0.1 \mathrm{~N}$ $\mathrm{KMnO}_{4}$ solution (see Reagents) to a faint pink color not disappearing in two to three minutes (the temperature of the solution should not be below $60^{\circ} \mathrm{C}$. when the end point is reached). Calculate the total calcium to percentage of $\mathrm{CaCl}_{2}$ (1 cc of $N \mathrm{KMnO}_{4}=0.00555 \mathrm{~g} \mathrm{CaCl}_{2}$ )

(d) Magnesium (expressed as $\mathrm{MgCl}_{2} \cdot 6 \mathrm{H}_{2} \mathrm{O}$ ).Dilute the combined filtrates (from the calcium oxalate precipitations) to the mark with water, stopper, and mix thoroughly. Transfer a $100 \mathrm{cc}$ aliquot of this solution to a beaker, acidify with $\mathrm{HCl}$, add $10 \mathrm{cc}$ of a cold saturated solution of diammonium hydrogen phosphate $\left(\left(\mathrm{NH}_{4}\right)_{2} \mathrm{HPO}_{4}\right)$, boil for five minutes, cool, add $\mathrm{NH}_{4} \mathrm{OH}$ drop by drop, with constant stirring, until the solution is alkaline and the crystallin ammonium magnesium orthophosphate has formed. Then add $5 \mathrm{cc}$ of $\mathrm{NH}_{4} \mathrm{OH}$ (sp. gr. 0.90), stir to mix, and let stand in a cool place at least four hours, preferably overnight. Filter on an $11 \mathrm{~cm}$ paper and wash four or five times with dilute $\mathrm{NH}_{4} \mathrm{OH}(1: 19)$. Dissolve the precipitate in a small quantity of hot dilute $\mathrm{HCl}$ $(1: 1)$, catching the solution in the beaker used for the previous precipitation, wash the paper with hot water, and dilute the solution to about $100 \mathrm{cc}$ with water. Add $1 \mathrm{cc}$ of a cold saturated solution of $\left(\mathrm{NH}_{4}\right)_{2} \mathrm{HPO}_{4}$, and $\mathrm{NH}_{4} \mathrm{OH}$ drop by drop, with constant stirring, until the precipitate is again formed as described; then add 5 cc of $\mathrm{NH}_{4} \mathrm{OH}$ (sp. gr. 0.90 ), stir to mix, and let stand in a cool place for not less than two hours. Filter on a weighed Gooch crucible, wash with dilute $\mathrm{NH}_{4} \mathrm{OH}(1: 19)$, ignite gently at first, and finally to constant weight over a Meker burner or blast or in a muffle at a temperature (about $1,000^{\circ} \mathrm{C}$.) that will not soften or melt the pyrophosphate. Cool and weigh as $\mathrm{Mg}_{2} \mathrm{P}_{2} \mathrm{O}_{7}$. Calculate the $\mathrm{Mg}_{2} \mathrm{P}_{2} \mathrm{O}_{7}$ to percentage of $\mathrm{MgCl}_{2} \cdot 6 \mathrm{H}_{2} \mathrm{O}$. Then calculate the percentage of $\mathrm{Mg}(\mathrm{OH})_{2}$ found above to $\mathrm{MgCl}_{2} \cdot 6 \mathrm{H}_{2} \mathrm{O}$ and the percentage of $\mathrm{MgSO}_{4}$, as determined below, to $\mathrm{MgCl}_{2}$. $6 \mathrm{H}_{2} \mathrm{O}$. Subtract the sum of these two results from the $\mathrm{MgCl}_{2} \cdot 6 \mathrm{H}_{2} \mathrm{O}$ calculated from the $\mathrm{Mg}_{2} \mathrm{P}_{2} \mathrm{O}_{7}$ and report the difference as the percentage of $\mathrm{MgCl}_{2} \cdot 6 \mathrm{H}_{2} \mathrm{O}$ in sample.

$\mathrm{Mg}_{2} \mathrm{P}_{2} \mathrm{O}_{7} \times 1.8261=\mathrm{MgCl}_{2} \cdot 6 \mathrm{H}_{2} \mathrm{O}$ $\mathrm{Mg}(\mathrm{OH})_{2} \times 3.4855=\mathrm{MgCl}_{2} \cdot 6 \mathrm{H}_{2} \mathrm{O}$ $\mathrm{MgSO}_{4} \times 1.6890=\mathrm{MgCl}_{2} \cdot 6 \mathrm{H}_{2} \mathrm{O}$ 
(e) Sulphate (expressed as $\mathrm{MgSO}_{4}$ ).-Transfer a $100 \mathrm{cc}$ aliquot of the original solution to a beaker, acidify with $\mathrm{HCl}$ and add $1 \mathrm{cc}$ more of this acid, dilute to $250 \mathrm{cc}$ with water, heat to boiling, filter if necessary, add drop by drop from a pipette $10 \mathrm{cc}$ of a hot 10 per cent solution of barium chloride, and continue the boiling until the precipitate is well formed. Let stand on the steam bath for two hours, filter on paper, and wash with hot water until $5 \mathrm{cc}$ of the washings give no test for chloride with 10 per cent $\mathrm{AgNO}_{3}$ solution. Place the paper and contents in a crucible, heat so that the paper is slowly charred and consumed without flaming, ignite, cool, and weigh the $\mathrm{BaSO}_{4}$. Calculate to percentage of $\mathrm{MgSO}_{4}\left(\mathrm{BaSO}_{4} \times 0.5157=\mathrm{MgSO}_{4}\right)$.

(f) Chlorine.-Transfer a 25 cc aliquot of the solution prepared in the preliminary procedure to a 400 cc beaker, acidify with $\mathrm{HNO}_{3}$ (see Reagents), and then add 5 cc more of this acid. Dilute with water to about $150 \mathrm{cc}$ and add from a burette or pipette an excess of $0.1 \mathrm{~N} \mathrm{AgNO}_{3}$ (see Reagents), noting the volume added $\left(1 \mathrm{~g}\right.$ of pure $\mathrm{MgCl}_{2} \cdot 6 \mathrm{H}_{2} \mathrm{O}$ requires about $100 \mathrm{cc}$ of $0.1 \mathrm{~N} \mathrm{AgNO}_{3}$ ); stir thoroughly to coagulate the precipitate, heat until hot to the touch, let settle, filter on a filter paper or on a Büchner funnel, and wash with water until the washings give no test for $\mathrm{AgNO}_{3}$. To the combined filtrate and washings from the $\mathrm{AgCl}$, add $5 \mathrm{cc}$ of ferric alum indicator. (See Reagents.) Upon addition of the ferric indicator no color should develop; if a reddish or yellowish color results, more of the $\mathrm{HNO}_{3}$ is required to destroy this. The amount of $\mathrm{HNO}_{3}$ when within reasonable limits does not affect the results. Now titrate the excess of silver with $0.1 \mathrm{~N} \mathrm{NH}_{4} \mathrm{CNS}$ or KCNS (see Reagents), stirring thoroughly until a permanent reddish-brown color is obtained when viewed against a white background. From the volume of $0.1 \mathrm{~N} \mathrm{AgNO}_{3}$ added, subtract the $\mathrm{AgNO}_{3}$ equivalent of the sulphocyanate used and calculate the result obtained to percentage of chlorine. The percentage of chlorine multiplied by the factor 2.8673 should give a result not smaller than that reported for $\mathrm{MgCl}_{2} \cdot 6 \mathrm{H}_{2} \mathrm{O}$. A lower result indicates that the sample contains compounds of magnesium other than the sulphate and chloride; a higher result indicates the presence of other chlorides, such as calcium, sodium, or potassium. (g) Sodium and potassium (expressed as $\mathrm{Na}_{2} \mathrm{SO}_{4}+$ $\mathrm{K}_{2} \mathrm{SO}_{4}$ ).-Transfer a 50 ce aliquot of the solution prepared in the preliminary procedure to a beaker, add $50 \mathrm{cc}$ of absolute ethyl alcohol (or sufficient 95 per cent alcohol to give a solution containing 50 per cent by volume of absolute alcohol), and then add $50 \mathrm{cc}$ of the alcoholic solution of ammonium carbonate. (See Reagents). Stir for five minutes, allow the mixture to stand at least 20 minutes, filter on asbestos in a Gooch crucible and wash several times with the precipitant (total volume of washings should be about 50 to $60 \mathrm{cc}$ ). Evaporate the filtrate to dryness in a weighed platinum dish on the steam bath, drive off the ammonium salts by gentle heating, cool, add about $1 \mathrm{cc}$ of water and $1 \mathrm{cc}$ of dilute $\mathrm{H}_{2} \mathrm{SO}_{4}(1: 4)$; again evaporate to dryness, heat gently at first and finally by means of a frec flame to dull redness until fumes of sulphuric anhydride cease to come off; let cool, add about $1 \mathrm{~g}$ of solid ammonium carbonate, ignite as before, cool, and weigh. Repeat the ignition with solid ammonium carbonate until a constant weight is obtained. From the weight found calculate the percentage of alkali sulphates $\left(\mathrm{Na}_{2} \mathrm{SO}_{4}+\mathrm{K}_{2} \mathrm{SO}_{4}\right)$.

Reagents-(1) Tenth normal hydrochloric acid solution.-To pure concentrated $\mathrm{HCl}$ (sp. gr. 1.19) add distilled water until the specific gravity of the solution is about 1.020 . Transfer 100 ce of this solution to a graduated liter flask, dilute to the mark with water and mix thoroughly. Transfer $100 \mathrm{cc}$ of this latter solution to a beaker, dilute with water to
300 cc, add a few drops of $\mathrm{HNO}_{3}$ and then (gradually and with constant stirring to coagulate the precipitate) a 5 per cent solution of $\mathrm{AgNO}_{3}$, until no further precipitation takes place. Heat the liquid to boiling and set the whole aside (in a dark place) to cool. When completely settled filter off the $\mathrm{AgCl}$ on asbestos in a Gooch crucible (previously weighed), wash thoroughly with water containing a few drops of $\mathrm{HNO}_{3}$ to the liter and then with a little pure water. Dry first at $100^{\circ} \mathrm{C}$. and then at $130^{\circ}$ to $150^{\circ}$ C., cool, and weigh. From the weight of $\mathrm{AgCl}$ found, calculate the normality of the solution. Instead of the above procedure, the solution may be standardized against standard $\mathrm{NaOH}$ or $\mathrm{KOH}$ solution that has been standardized against Bureau of Standards benzoic acid.

(2) Methyl orange solution.-Dissolve $0.1 \mathrm{~g}$ of pure methyl orange in $100 \mathrm{cc}$ of hot water, let cool, and filter off any deposited matter.

(3) Potassium permanganate solution.-Dissolve $3.2 \mathrm{~g}$ of pure potassium permanganate in a liter of distilled water, let stand 8 to 14 days, siphon off the clear solution (or filter through an asbestos filter), and standardize as follows: In a 400 ce beaker dissolve 0.40 to $0.50 \mathrm{~g}$ of Bureau of Standards' sodium oxalate in $250 \mathrm{cc}$ of hot water $\left(80^{\circ}\right.$ to $90^{\circ} \mathrm{C}$.) and add $15 \mathrm{ce}$ of dilute sulfuric acid (1:1). Titrate at once with the potassium permanganate solution, stirring the liquid vigorously and continuously. The permanganate must not be added more rapidly than 10 to 15 ce per minute, and the last 0.5 to 1 cc must be added dropwise with particular care to allow each drop to be fully decolorized before the next is introduced. The solution should not be below $60^{\circ} \mathrm{C}$. by the time the end point is reached. (Too rapid cooling may be prevented by allowing the beaker to stand on a small asbestos-covered hot plate during the titration. The use of a small thermometer as a stirring rod is most convenient.) The weight of sodium oxalate used multiplied by 0.8334 gives its iron equivalent. The permanganate solution should be kept in a glass stoppered bottle painted black to keep out light. The Fe value of the $\mathrm{KMnO}_{4}$ multiplied by 0.995 gives the $\mathrm{CaCl}_{2}$ value. The weight of sodium oxalate used multiplied by 0.8283 gives its $\mathrm{CaCl}_{2}$ equivalent.

(4) Tenth normal silver nitrate solution.-Dissolve $17.1 \mathrm{~g}$ of pure $\mathrm{AgNO}_{3}$ in distilled water, dilute to 1 liter, and mix. Standardize against pure $\mathrm{NaCl}$ and adjust to decinormal strength, or calculate the normality and use the solution as it is.

(5) Tenth normal sulphocyanate solution.-Dissolve about $8 \mathrm{~g}$ of $\mathrm{NH}_{4} \mathrm{CNS}$ or $10 \mathrm{~g}$ of $\mathrm{KCNS}$ in water, dilute to 1 liter, and mix. To standardize, transfer $20 \mathrm{cc}$ of the $0.1 \mathrm{~N} \mathrm{AgNO}_{3}$ solution to a beaker, add $50 \mathrm{cc}$ of water and $2 \mathrm{cc}$ of the ferric alum indicator. Then add the sulphocyanate solu. tion from a burette, with constant stirring, until a permanent reddish-brown color is obtained. Adjust so that $1 \mathrm{cc}$ of the solution will be equivalent to $1 \mathrm{ec}$ of the $\mathrm{AgNO}_{3}$ solution; or, record its value in terms of the $\mathrm{AgNO}_{3}$ solution and use it as it is.

(6) Pure nitric acid.-Dilute pure concentrated $\mathrm{HNO}_{3}$ (sp. gr. 1.42) with water until a solution is obtained containing about 50 per cent of $\mathrm{HNO}_{3}$, boil until perfectly colorless, and keep in the dark.

(7) Ferric alum indicator.-To a cold, saturated solution of ferric ammonium sulphate add sufficient pure, colorless $\mathrm{HNO}_{3}$ to cause the disappearance of the brown color.

(8) Alcoholic solution of ammonium carbonateMix $18 \mathrm{cc} \mathrm{NH}_{4} \mathrm{OH}$ (sp. gr. 0.90), $80 \mathrm{cc}$ of water, and 90 ce of absolute ethyl alcohol (or sufficient 95 per cent alcohol to give a solution containing 50 per cent by volume of absolute alcohol). Shake the solution with powdered ammonium carbonate until saturated, let stand several hours, and filter out the excess of the latter. 


\section{DENSITY OF SOLUTION}

(a) Apparatus.-A picnometer, Westphal balance or hydrometer may be used. As in the great majority of cases a hydrometer will be used, it should conform to the following specifications: Overall length, 81/2 inches (variance of one-fourth inch either way permissible); shot bulb, three-fourths inch in diameter; air chamber, $3 \% 16$ inches long and thirteen-sixteenths inch in diameter; take-up bulb approximately thirteen-sixteenths inch in diameter.

\section{FINENESS}

(a) Apparatus.-A United States standard sieve series No. 100 sieve shall be used. In this sieve the wire cloth should conform to the following requirements:

Siere opening $0.149 \mathrm{~mm}$ or 0.0059 inch.

Wire diameter $0.102 \mathrm{~mm}$ or 0.0040 inch.

Tolerance in average opening, 6 per cent.

Tolerance in maximum opening, 40 per cent.

Tolerance in wire diameter -15 per cent to +35 per cent.

The sieve frame should be circular, about $20 \mathrm{~cm}$ (about 8 inches) in diameter and about $5 \mathrm{~cm}$ (about 2 inches) between top of the frame and the cloth.

(b) Methods.-The test shall be made with $50 \mathrm{~g}$ of the dry mixture. This shall be placed upon the clean dry sieve pan and cover attached, and held in one hand in a slightly inclined position, so that the sample shall be well distributed over the sieve, and struck about one hundred and fifty times per minute against the palm of the other hand on the upstroke. The sieve shall be turned in the same direction every 25 strokes about one-sixth of a revolution. The operation shall be continued until not more than 0.05 of a gram passes through in one minute of continuous sieving. The fineness shall be determined from the weight of the residue on the sieve, expressed as a percentage of weight of the original sample. Mechanical sieving devices may be used, but rejection shall be based only upon the results of hand sieving.

\section{CONSISTENCY}

The principle of the tests being the determination of the quality of the finished product, the consistency used shall be that given by the vendor furnishing the dry mixture and chloride as that which will be used on the work. Hence there shall be furnished by the contractor a statement as to the weight of chloride solution which will be used per unit weight of the dry mixture on the work. This weight converted and expressed in cubic centimeters per thousand grams of dry mixture shall be used in the testing laboratory throughout all tests.

\section{MUXING DRY MIXTURE AND CHLORIDE}

The quantity of dry mixture to be mixed with chloride at one time shall not exceed $2,000 \mathrm{~g}$, nor be less than $300 \mathrm{~g}$. The quantity of chloride solution shall be expressed in cubic centimeters. The dry material shall be weighed, placed on a nonabsorbent surface, a crater formed in the center, and the chloride solution added. The outer edge of the dry mixture shall then be turned into the crater with a trowel. After an interval of one-half minute to allow for absorption, the operation shall be completed by a continuous mixing and kneading with the hands for one minute. Duling the operation the hands shall be covered with rubber gloves. The air of the laboratory during this and all other tests shall be maintained as near as practicable at $21^{\circ} \mathrm{C} .\left(70^{\circ} \mathrm{F}\right.$.).

\section{DETERMINATION OF SOUNDNESS}

(a) Apparatus.-An apparatus similar to that shown in Figure 101, page 291, or one which will fulfill the same requirements, is recommended.

(b) Method.-A pat of the plastic magnesia cement mixed as above shall be made on a clean glass plate (about 4 inches square) and stored in moist air for 24 hours. The pat shall be about 3 inches in diameter, one-half inch thick at the center and tapering to a thin edge. In molding the pat the plastic magnesia cement shall be first flattened on the glass and the pat formed by drawing the trowel from the outer edge to the center. At the end of 24 hours storage it shall be placed in a loosely covered vessel in an atmosphere of steam at a temperature of not less than $98^{\circ} \mathrm{C}$. for about five hours.

\section{DETERMTNATION OF THE TIME OF SET}

(a) Apparatus.-The time of set shall be determined by the Gilmo e needles (see fig. 103, p. 292).

(b) Method. - The pat which has been prepared for the determination of soundness may be used, provided its surface has been flattened for an area of about $1 \frac{1}{2}$ inches in diameter. The plastic magnesia cement shall be considered to have acquired its initial set when the pat will bear without any indentrtion the Gilmore needle one-twelfth inch in diameter, loaded to weigh one-fourth pound. Final set has been acquired when the pat will bear without any indentation the Gilmore needle one-twentyfourth inch in diameter loaded to weigh 1 pound. Care should be taken to apply the needles in a vertical position on the flattened surface of the pat.

\section{TENSILE STRENGTE}

(a) Form of test piece.-The standard form of test piece is that used in testing Portland cement.

(b) Making the test piece.-Immediately after mixing, as described in Section V, 8 above, the plastic magnesia cement shall be placed in the molds, pressed in firmly with the thumbs and smoothed off with the trowel without any ramming. The mold shall then be turned over and placed upon a heavy glass plate and more plastic magnesia cement added, and the operation repeated as before.

(c) Storage of test pieces.-During the first 24 hours the filled molds shall be tightly covered with a glass plate. At the end of this period the specimen shall be removed and stored in the air until broken. During the air storage the specimens shall be so placed that the air may have free access to them.

(d) Testing.-Tests may be made with any standard briquette machine acceptable to the purchaser of the cement. The bearing surface of the clips of the machine and the briquettes should be free from grains of sand or dirt. The briquettes should be carefully centered and the load applied continuously at the rate of 600 pounds per minute. The testing machines should be frequently calibrated in order to determine their accuracy.

(e) Faulty briquettes.-Briquettes that are manifestly faulty, or which give strengths differing more than 15 per cent from the average of test pieces made from the same sample and broken at the same time, shall not be considered in determining the tensile strength.

\section{TRANSVERSE STRENGTH}

(a) Form of test piece.-Any mold which will permit of making a test piece $1 / 2$ by 2 by 12 inches. is satisfactory. Such a mold is shown in Figure 240. 
(b) Making the test piece.-The plastic magnesia cement after mixing as described in Section $V$, 8 should be spread along the length of the mold and worked by the trowel into the corners. Somewhat more than that needed to fill the molds should be used, and the excess struck off by not more than three strokes of the trowel forward and three backward over the full length of the mold. Further troweling should not be done, as excessive troweling materially changes the surface of the test piece.

(c) Storage of test piece.-The test piece shall be stored in the air of the laboratory for the 24 hours following making and should be removed from the
After breaking the test pieces should be carefully measured for thickness to the nearest 0.02 inch. The transverse strength (modulus of rupture) shall be calculated from the formula

wherein

$$
M R=\frac{3 W L}{2 B D^{2}}
$$

$W=$ the breaking load,

$L=$ the length between supports $=10$ inches,

$B=$ the width of the bar $=2$ inches,

$D=$ the thickness of the bar, measured to the nearest 0.02 inch.
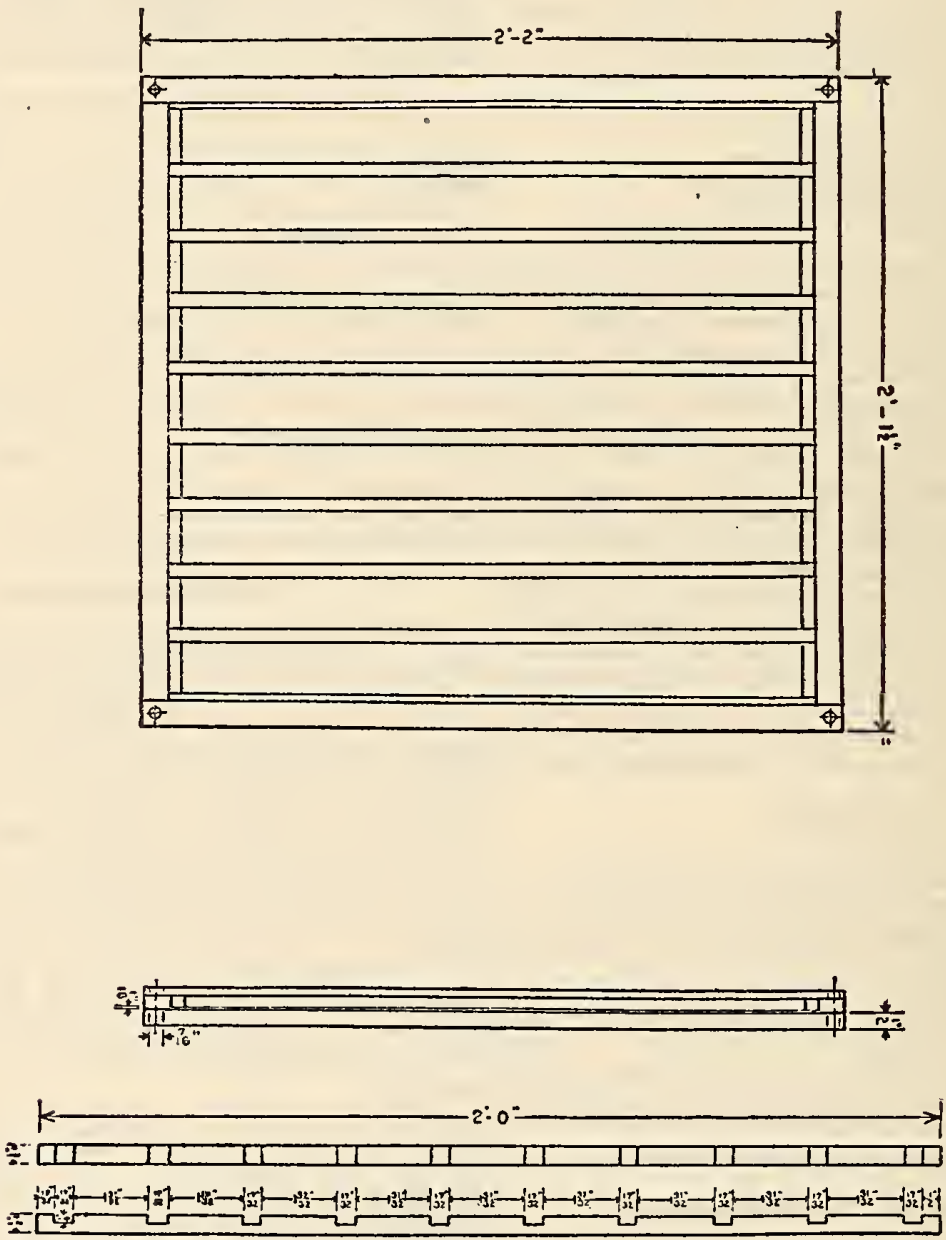

FrgURE 240.-Mold for transverse strength test pieces

mold at the end oi this period and stored on edge supported at two points only. Each test piece should be at least 1 inch from the neighboring test pieces.

(d) Testing.-A suitable testing machine for this purpose is not yet produced by testing-machine manufacturers. Figure 241 shows a homemade type which can readily be made in any shop and will serve the purpose. Figure 242 shows a more elaborate but far more satisfactory machine.

In testing, the test piece should be supported on roller bearings 10 inches apart. The load shall be applied midway between these bearings through a knife-edge upon that surface which was lowermost in the mold.
Hence for a length of 10 inches and a breadth of 2 inches the transverse strength is equal to seven and one-half times the breaking load, divided by the square of the thickness. The need of careful measurement of the thickness is hence evident.

\section{COEFFICIENT OF EXPANSION}

(a) Apparatus.-Any suitable device for measuring lengths of approximately 10 inches which may be read to 0.001 inch can be used. A suitable type of instrument is the Berry gage or a similar type as shown in Figure 243.

(b) Test piece.-Plastic magnesia flooring during hardening frequently shows apparently excessive changes in volume. However, it has been noted 
that the same plastic magnesia cement used in covering old wooden floors or concrete does not show any eracking, buckling, or noticeable volume changes. Hence the following procedure is given for preparing the test piece for coefficient of expansion:

Bars approximately 1 by 2 by 12 inches should be made of 1:3 Portland cement sand mortar with apart.

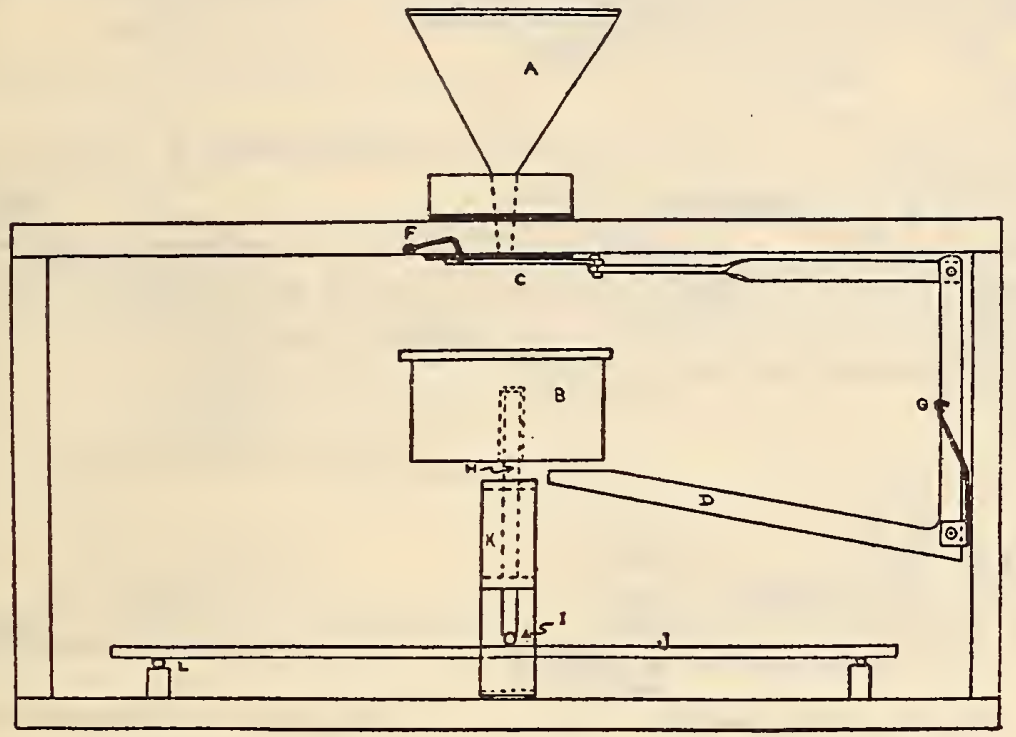

FIGURE 241.-A readily made type of transverse testing machine

a roughened top surface, such as may be obtained by brushing before set with a broom. These to permit of easy handling should be reinforced lengthwise, midway between top and bottom, with about three No. 16-gauge iron wires. After storage for two weeks in a damp atmosphere they should be stored in the air for six weeks before use. Upon coat work the plastic magnesia cement should be applied in a thickness of one-half inch. The surface should be smoothed with not more than three forward and three backward passes of the trowel, after which gauge marks of the type suggested in Figure 243 should be placed approximately 10 inches

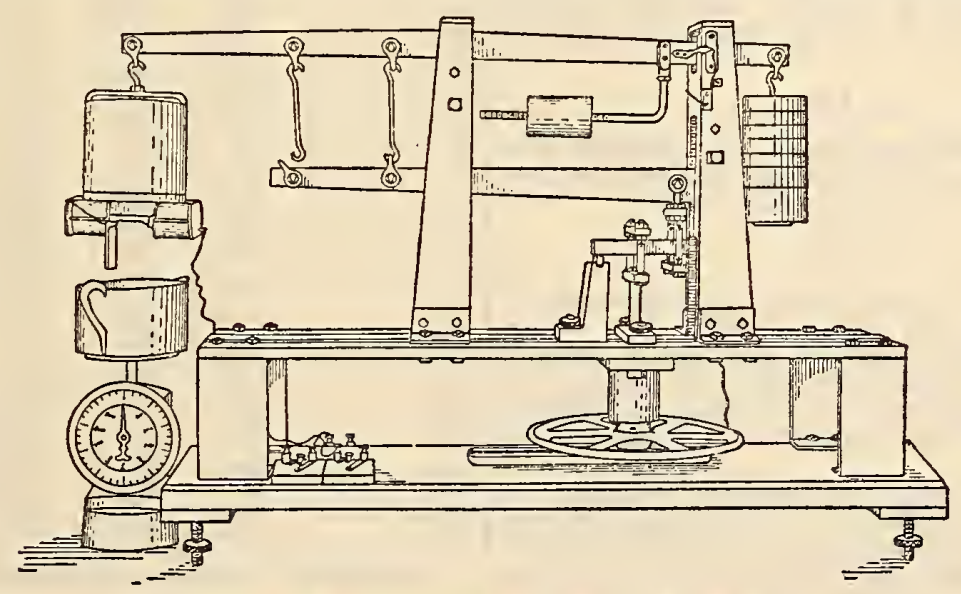

FIGURE 242.-Transverse testing machine

the rough surface of the bars after such aging should be placed the plastic magnesia cement, using as a binding material a cream of magnesite and chloride. The plastic magnesia cement should be prepared as given in Section V, 8 and should be applied in a thickness of about one-fourth inch for each coat of two-coat work. The ninish coat should be applied to the first coat 24 hours after the first has been applied. If intended for one- (c) Making measurements.-The initial measurements with the gauge should be made at the time of final set, as determined by the Gilmore needle on the expansion test piece. Several readings should be made on each bar and should check each other to within one-half of the smallest division of the dial. The second measurement should be made 

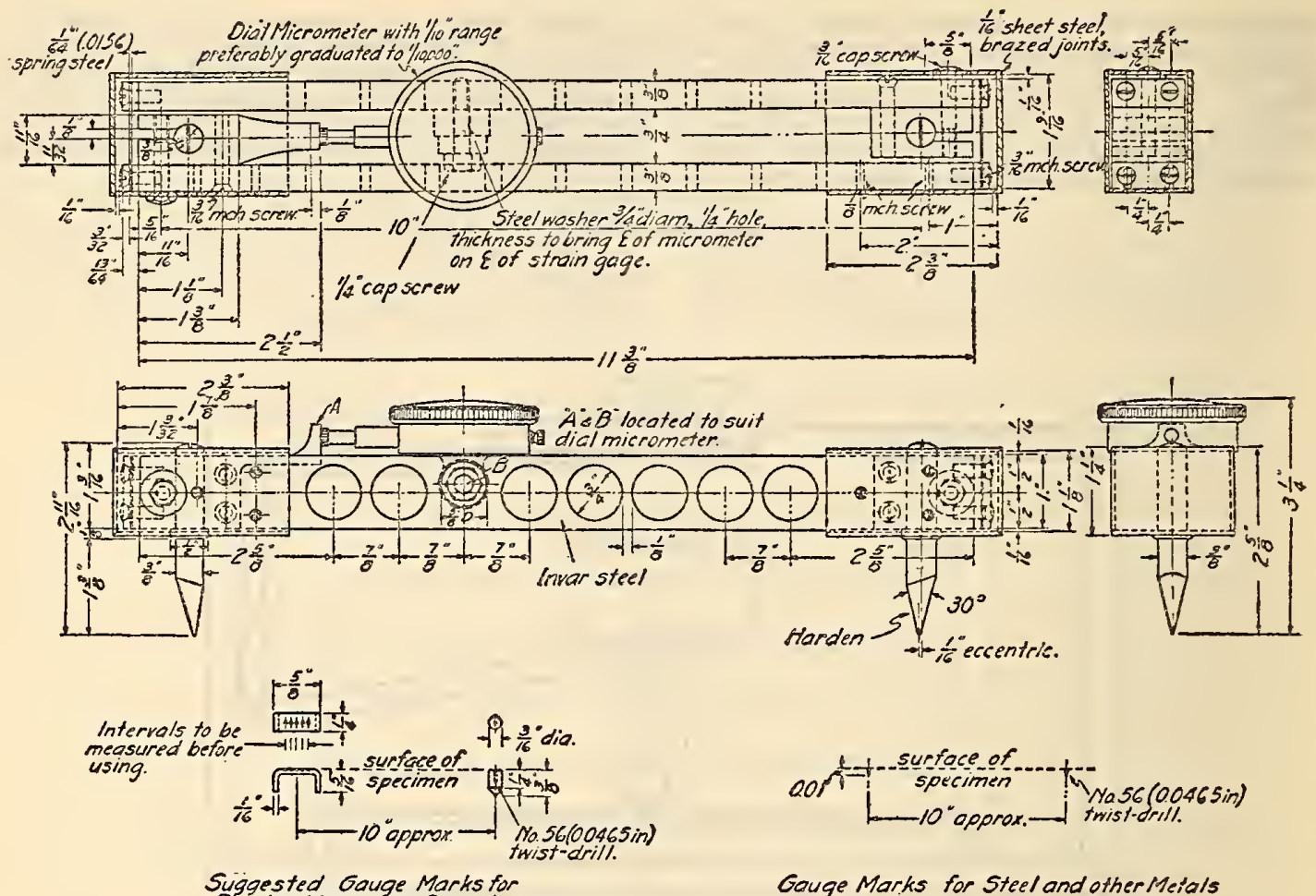

Suggested Gauge Marks for
Plastic Magnesia Cement

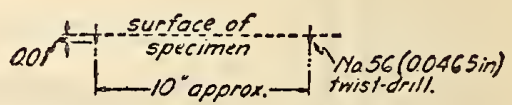

FIGURE 243.-An instrument for measuring length change

\section{PACKING AND MARKING}

The dry mixture shall be delivered in suitable containers with brand or manufacturer's name plainly marked thereon. The chloride, if in the solid form, may be delivered in original containers. If delivered in the liquid form it should be in such containers as can be readily delivered without spilling, and permit of sealing after sampling and during the testing.

\section{SAIT}

Federal Specifications Board, specification No. 364 , United States Government master specification for condiments, December 24, 1925.

\section{SALT}

Quality.-Shall be of the best quality, fine-grained, crystalline, table salt. Shall contain on a waterfree basis not more than 1.4 per cent of calcium sulphate $\left(\mathrm{CaSO}_{4}\right)$, nor more than 0.5 per cent of calcium and magnesium chloride $\left(\mathrm{CaCl}_{2}\right.$ and $\left.\mathrm{MgCl}_{2}\right)$ nor more than 0.1 per cent of matter insoluble in water.

Method of analysis.-Chemical analysis, if required by the purchaser in the examination and testing of samples and deliveries under this specification, shall be made by the methods of the Association of Official Agricultural Chemists in effect on date of proposal.

\section{GRAPHITE AND MANUFAC- TURES THEREOF}

The Plumbago Crucible Association, 1923.

The association has adopted the following standard sizes of plumbago crucible, which have been approved by the American Engineering Standards Committee, now the American Standards Association.

\begin{tabular}{|c|c|c|c|c|c|}
\hline No. & Height & Top & Bilge & Bottom & $\begin{array}{l}\text { A pproxi- } \\
\text { mate ca- } \\
\text { pacity in } \\
\text { pounds } \\
\text { water } 1\end{array}$ \\
\hline $\begin{array}{l}10 \\
12 \\
14 \\
16 \\
18\end{array}$ & $\begin{array}{l}81 / 16 \\
81 / 2 \\
87 / 8 \\
91 / 4 \\
913 / 26\end{array}$ & $\begin{array}{l}61 / 16 \\
6^{3} / 8 \\
6^{11} 16 \\
6^{15} / 16 \\
75 / 16\end{array}$ & $\begin{array}{l}69 / 16 \\
67 / 8 \\
73 / 16 \\
712 \\
715 / 16\end{array}$ & $\begin{array}{l}413 / 16 \\
51 / 16 \\
51 / 4 \\
51 / 2 \\
5^{13 / 16}\end{array}$ & $\begin{array}{l}4.8 \\
5.6 \\
6.4 \\
7.2 \\
8.6\end{array}$ \\
\hline $\begin{array}{l}20 \\
25 \\
30 \\
35 \\
40\end{array}$ & $\begin{array}{l}105 / 16 \\
10^{15 / 16} \\
111 / 2 \\
12 \\
121 / 2\end{array}$ & $\begin{array}{l}711 / 16 \\
83 / 16 \\
85 / 8 \\
9 \\
93 / 8\end{array}$ & $\begin{array}{c}83 / 8 \\
87 / 8 \\
95 / 16 \\
93 / 4 \\
101 / 8\end{array}$ & $\begin{array}{l}61 / 8 \\
61 / 2 \\
613 / 16 \\
71 / 8 \\
7716\end{array}$ & $\begin{array}{l}10 \\
12 \\
14 \\
16 \\
18\end{array}$ \\
\hline $\begin{array}{l}45 \\
50 \\
60 \\
70\end{array}$ & $\begin{array}{l}133 / 16 \\
133 / 4 \\
147 / 16 \\
151 / 16\end{array}$ & $\begin{array}{l}97 / 8 \\
10^{1 / 4} \\
10^{13} / 16 \\
111 / 4\end{array}$ & $\begin{array}{l}10^{1} 1 / 6 \\
11^{1} / 8 \\
11^{1} 1 / 16 \\
12^{3} / 16\end{array}$ & $\begin{array}{l}713 / 16 \\
81 / 8 \\
89 / 16 \\
815 / 16\end{array}$ & $\begin{array}{l}21 \\
24 \\
28 \\
32\end{array}$ \\
\hline $\begin{array}{l}80 \\
90 \\
100 \\
125\end{array}$ & $\begin{array}{l}155 / 8 \\
16^{3} / 16 \\
16^{1} 1 / 16 \\
173 \% 8\end{array}$ & $\begin{array}{l}11^{11} / 16 \\
121 / 8 \\
121 / 2 \\
13\end{array}$ & $\begin{array}{l}12^{11 / 16} \\
131 / 8 \\
131 / 2 \\
141 / 16\end{array}$ & $\begin{array}{c}91 / 4 \\
99 / 16 \\
97 / 8 \\
105 / 16\end{array}$ & $\begin{array}{l}36 \\
40 \\
44 \\
50\end{array}$ \\
\hline $\begin{array}{l}150 \ldots \ldots \\
175 \\
200 \\
225\end{array}$ & $\begin{array}{l}183 / 8 \\
191 / 4 \\
20 \\
203 / 4\end{array}$ & $\begin{array}{l}133 / 4 \\
143 / 8 \\
15 \\
151 / 2\end{array}$ & $\begin{array}{l}147 / 8 \\
159 / 16 \\
16^{1 / 4} / 4 \\
16^{13} / 16\end{array}$ & $\begin{array}{l}107 / 8 \\
113 / 8 \\
117 / 8 \\
125 / 16\end{array}$ & $\begin{array}{l}60 \\
70 \\
80 \\
90\end{array}$ \\
\hline $\begin{array}{l}250 \ldots \ldots \\
275 \\
300 \\
400\end{array}$ & $\begin{array}{l}213 / 8 \\
22 \\
221 / 2 \\
245 / 16\end{array}$ & $\begin{array}{l}16 \\
167 / 16 \\
167 / 8 \\
183 / 16\end{array}$ & $\begin{array}{l}175 / 16 \\
1713 / 16 \\
181 / 4 \\
19^{1} 1 / 6\end{array}$ & $\begin{array}{l}12^{1} / 16 \\
13 \\
13^{3} / 8 \\
14^{7} / 16\end{array}$ & $\begin{array}{l}100 \\
110 \\
120 \\
160\end{array}$ \\
\hline
\end{tabular}

11 pound water $=0.96$ pints, or 27.7 cubic inches.

Dimensions given are outside demensions in inches.

575. CARBONS AND ELECTRODES

575.1 CARBON, POWDERED.

(No nationally recognized specifications available.)

575.2 CARBON ELECTRODES.

(No nationally recognized specifications available.) 


\section{FLUORSPAR AND CRYOIITE} able.)

(No nationally recognized specifications avail592.

\section{FEIDSPAR} able.)

(No nationally recognized specifications avail593.

\section{SIIICA} able.)

\section{MISCELIANEOUS NONMETAIIIC IMIN ERALS}

American Institute of Mining and Metallurgical Engineers, recommended American practice for rock dusting coal mines, 1925.

(Approved by the American Engineering Standards Committee, now the American Standards Association.)

(This recommended practice includes sections related to definitions, mines to be rock dusted, parts of mine to be dusted, sampling dust and record of sampling, and the following requirements for the dust to be used:)

\section{KIND OF DUST TO BE USED}

The kind of dust to be used shall be as specified by United States Bureau of Mines, and subject to the approval of the State mine inspection service. It shall not contain more than 5 per cent of combustible matter, nor more than 25 per cent of quartz or free silica particles, nor absorb moisture from the air to such an extent as to cake and destroy its effectiveness as a dry dust. It may be made from limestone, dolomite, gypsum, anhydrite, shale, talc, adobe, or other inert material which meets the foregoing specifications. The lighter colored dusts are to be preferred.

\section{SIZE OF DUST TO BE USED}

The dust to be used shall be pulverized so that 100 per cent will pass through a sieve having 20 meshes per linear inch, and 50 per cent or more will pass through a sieve having 200 meshes per linear inch (40,000 perforations per square inch).

\section{AYIOUNT OF DUST TO BE USED}

In all places where rock dust is distributcd, enough shall be used so that the percentage of incombustible material in the samples of dust collected in tha places shall be maintained at lcast 55 per cent. Along room entries or gangways where methane gas is found in the ventilating current, the amount of incombustible material above specified shall be raised 10 per cent for each 1 per cent of gas. Where rock dust barriers are installed, the amount of dust used shall be at least $100 \mathrm{lbs} . / \mathrm{ft}^{2}$ of average cross section of entry, at the barrier zone. 


\section{TECHNICAL SOCIETIES, TRADE ASSOCIATIONS, AND OTHER ORGANIZATIONS ISSUING STANDARDS AND SPECIFICATIONS DEALING WITH NONMETALLIC MINERALS}

There are given below the names and addresses of organizations the standards and specifications of which are reproduced herein either in full or in substance by means of abstracts and cross references. Under the procedure of many of these organizations provision is made for periodical revision of standards on an annual, biannual or similar basis. Persons interested in obtaining information concerning the status or revisions of standards should communicate with the organizations at the offices as listed below:

Abrasive Paper and Cloth Manufacturers Exchange, W. H. Chafe, chairman, Standardization Committee, care of American Glue Co., 121 Beverly Street, Boston, Mass.

Amcrican Association of State Highway Officials, W. C. Markham, secretary, National Press Building, Washington, D. C.

American Ceramic Society, Ross C. Purdy, secretary, 2525 North High Street, Columbus, Ohio.

American Chemical Society, Charles L. Parsons, secretary, Mills Building, Washington, D. C.

American Concrete Institute, Harvey Whipple, secretary, 2970 West Grand Boulevard, Detroit, Mich.

American Concrete Pipe Association, M. W. Loving, sccretary, 33 West Grand Avenue, Chicago, Ill.

American Drug Manufacturers Association, Carson P. Frailey, secretary, Albee Building, Washington, D. C.

American Electric Railway Association,G. C. Hecker, gencral secretary, 292 Madison Avenue, New York, N. Y.

American Face Brick Association, G. S. Eaton, secretary, 130 North Wells Street, Chicago, Ill.

American Foundrymen's Association, R. E. Kennedy, technical secretary, 222 West Adams Street, Chicago, Ill.

American Gas Association (Inc.), Alexander Forwär managing director, 420 Lexington Avenue, New York, N. Y.

American Institute of Mining and Metallurgical Engineers, H. Foster Bain, secretary, 29 West Thirty-ninth Street, New York, N. Y.

American Marine Standards Committee, A. V. Bouillon, secretary, Department of Commerce Building, Washington, D. C.

American Mining Congress, J. F. Callbreath, secretary, Munsey Building, Washington, D. C.

American Oil Burner Association (Inc.), Harry F. Tapp, executive secretary, 342 Madison Avenue, New York, N. Y.

American Petroleum Institute, C. A. Young, director of division of standardization, 1508 Kirby Building, Dallas, Tex.

American Railway Association, Mechanical Division, V. R. Hawthorne, secretary, 431 South Dearborn Street, Chicago, Ill.

American Railway Association, Signal Section, H. S. Balliet, secretary, 30 Vesey Street, New York, N. Y.

American Railway Association, Telegraph and Telephone Section, W. A. Fairbanks, secretary, 30 Vesey Street, New York, N. Y.

American Railway Engineering Association, E. H. Fritch, secretary, 431 South Dearborn Street, Chicago, Ill.
American Society of Civil Engineers, George T. Seabury, secretary, 33 West Thirty-ninth Street, New York, N. Y.

American Society of Mechanical Engineers, Calvin W. Rice, secretary, 29 West Thirty-ninth Street, New York, N. Y.

American Society for Municipal Improvements, C. IV. S. Sammelman, secretary, 4359 Lindell Boulevard, St. Louis, Mo.

American Society for Testing Materials, C. L. Warwick, secretary-treasurer, 1315 Spruce Street, Philadelphia, $\mathrm{Pa}$.

American Standards Association (formerly American Engineering Standards Committee), P. G. Agnew, secretary, 29 West Thirty-ninth Street, New York, N. Y.

American Wood-Preservers' Association, H. L. Dawson, secretary, 228 North La Salle Street, Chicago, Ill.

Asphalt Association, J. E. Pennybacker, secretary, 441 Lexington Avenue, New York, N. Y

Asphalt Shingle and Roofing Institute, J. S. Bryant, manager, 2 West Forty-fifth Street, New York, N. Y

Associated Factory Mutual Fire Insurance Companies, Inspection Department, H. O. Lacount, consulting engineer, 184 High Street, Boston (9), Mass.

Associated Tile Manufacturers, M. A. Illing, secretary, 420 Lexington Avenue, New York, N. Y.

Clay Products Institute of California, Seward C. Simons, secretary-manager, 611 Architects Building, Fifth and Figueroa Streets, Los Angeles, Calif.

Common Brick Manufacturers' Association of America, Ralph P. Stoddard, secretary, Guarantee Title Building, Cleveland, Ohio.

Concrete Reinforcing Steel Institute, M. A. Beeman, secretary, Tribune Tower, Chicago, Ill.

Contracting Plasterers' International Association, Edward McDonnell, secretary-treasurer, 605 Lincoln Building, Detroit, Mich.

Eastern Clay Products Association, Henry T. Shelley, secretary, Colonial Building, Philadelphia $\mathrm{Pa}$.

Federal Specifications Board, Major Robert W. Voeth, vice chairman and technical secretary, National Bureau of Standards, Washington, D. C. Glass Container Association of America, W. L. Davis, secretary of the standardization committee, 19 West Forty-fourth Street, New York, N. Y.

Grinding Wheel Manufacturers Association of the United States and Canada, F. R. Henry, secretary, Summit Street and Negley Place, Dayton, Ohio. Gypsum Institute, W. J. Fitzgerald, secretary, 110 West Fortieth Street, New, York, N. Y.

Independent Oil Men of America, Jay Trexler, 205 West Wacker Drive, Chicago, Ill.

Indiana Limestone Institute, H. S. Brightly, secretary, Bedford, Ind.

International Association of Industrial Accident Boards and Commissions, Ethelbert Stewart, secretary, U. S. Bureau of Labor Statistics, Washington, D. C. 
TECHNICAL SOCIETIES, TRADE ASSOCIATIONS, ETC.

Interstate Commerce Commission, Washington, D. C.

Joint Committee on Standard Specifications for Concrete and Reinforced Concrete, Duff A. Abrams, secretary-treasurer, care of International Cement Corporation, 342 Madison Avenue, New York, N. Y.

Joint Concrete Culvert Pipe Committee, M. W. Loving, secretary, 33 West Grand Avenue, Chicago, Ill.

Manufacturing Chemists' Association of the United States (office of the secretary), 921 Woodward Building, Fifteenth and H Streets NW., Washington, D. C.

National Association of Marble Dealers, Victor Mosel, secretary, Rockefeller Building, Cleveland, Ohio.

National Association of Purchasing Agents (Inc.), G. A. Renard, secretary-treasurer, 11 Park Place, New York, N. Y.

National Board of Fire Underwriters, W. E. Mallalieu, general manager, 85 John Street, New York, N. Y.

National Building Granite Quarries Association (Inc.), H. H. Sherman, secretary, 31 State Street, Boston, Mass.

National Fire Protection Association, Franklin H. Wentworth, managing director, 60 Batterymarch Street, Boston, Mass.

National Jewelers Board of Trade, B. L. Shinn, secretary, 22 West Forty-eighth Street, New York, N.'Y.

National Lime Association, N. G. Hough, general manager, 927 Fifteenth Street, NW., Washington, D. C.

National Paving Brick Manufacturers Association, G. F. Schlesinger, managing director and chief engineer. National Press Building, Washington, D. C.
National Sand and Gravel Association, Stanton Walker, director, engineering and research division, Munsey Building, Washington, D. C.

National Slag Association, H. J. Love, secretary, 937 Leader Building, Cleveland, Ohio.

National Slate Association, W. S. Hays, sceretary, Drexel Building, Philadelphia, Pa.

National Terra Cotta Society, W. F. Lockhardt, secretary, 230 Park Avenue, New York, N. Y.

Natural Gasoline Association of America, Ray E. Milleı, secretary, Tulsa Building, Tulsa, Okla.

New York Produce Exchange, W. C. Rossman, secretary, 2 Broadway, New York, N. Y.

Pennsylvania Slate Institute, R. S. Tibbals, secretary, Pen Argyl, Pa.

Plate Glass Manufacturers of America, P. A. Hughes, secretary, First National Bank Building, Pittsburgh, Pa.

Plumbago Crucible Association, C. H. Rohrbach, secretary, 90 West Street, New York, N. Y.

Portland Cement Association, W. M. Kinney, general manager, 33 West Grand Avenue, Chicago, Ill. Simplex Concrete Pile Association (Inc.), W. M. B. Freeman, 38 Chauncey Street, Boston, Mass.

Society of Automotive Engineers (Inc.), C. F. Clarkson, sccretary and general manager, 29 West Thirty-ninth Street, New York, N. Y.

Underwriters' Laboratories, Dana Pierce, president, 207 East Ohio Street, Chicago, Ill.

United States Department of Agriculture, Bureau of Public Roads, Washington, D. C.

United States Department of Commerce, Bureau of Mines, Washington, D. C.

United States Department of Commerce, National Bureau of Standards, commercial standards unit, Washington, D. C.

United States Department of Commerce, National Bureau of Standards, division of simplified practice, Washington, D. C. 



\section{IN DEX}

A. P. I. gravity, definition of

Abrasion, broken stone and broken slag, method of test for.. gravel, method of test for rock, method of test for

Abrasire cloth, aluminum oxido, specification for $--{ }^{-}$

Abrasive products, coated, recommendation for

A brasive paper, artificial, waterproof, specification for

Absorption, concrete, method of test for. sand, gravel, stone or other nonbituminous highway materials, method of test for stone and other coarse materials, method of test for --.-water, of slate, method of test for

Absorption test, brick

drain tile...

作

sewer pipe

A cidity in gasoline, test for

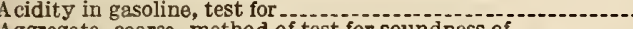

Aggregate, coarse, method of test for soundness of - ............. Portland cement concrete pavement or base, specification for

specification for

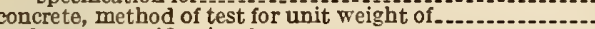

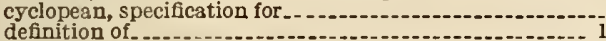

fine, concrete, method of making compression and tension tests of

method of test for determination of voids in

method of test for field determination of approximate

method of test for field determination of approsimate

percentage of voids in . ................................

method of test for field determination of surface mois

ture in

Portland cement concrete pavement or base, specifi-

cation for

method of test for percentage of shale in.-...-...-...-...

tile work

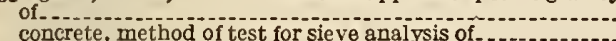

specification for

extracted, method of mechanical analysis of

fine, method of decantation test for

Air-flow apparatus for permeability test of foundry sands...-.

A ir ports, glass for

Aluminum oxide abrasive cloth, specification for

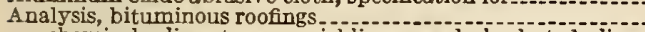

chemical, limestone, quicklime, and hydrated lime,

method of refractory materials, including chrome ores and chrome
brick coal and coke, method of glass, method for grease

mechanical, broken stone or broken slag, except aggre-

gates used in cement concrete, method of

mixtures of sind or other fine material with brol-stone or broken slag, except aggregates used in
cement concrete

sand or other fine highway material, except fine aggre-

gates used in cement concrete, method of .......... sand, method for A nnealing cup, porcelain.

Annealing orens, specification for clay fire brick for Anthracite, method of test for size of. Apparatus, laboratory, specification for (laboratory porcelain

Apparent porosity, test for

Apparcnt specific gravity, coarse aggregates, method of test for fine aggregate, approximate, mothod of test for field determination of

sand, gravel, stone or other nonbituminous highway materials, method of test for.

materials, method of test for
sand, stone, and slag screenings and other fine nonbitumi-

nous highway materials, method of test for - nonbitumi stone and other coarse materials, method of test for

pplication of Portland cement stucco on concrete block and tile walls, spccification for.

Arch masonry, ashlar stone

block rubble.

Arches, specification for

Architectural stone, concrete, specification for

Architectural terra cot ta, specification for

Artificial abrasive paper, waterproof, specification for

Artificial bases, cement concrete, specification for

specification for . .
Page Artificial sand-clay mixtures for road surfacing, specification for roofs, specification for Asbestos high-pressure rod packing, specification for Asbestos metallic cloth sheet packing, specification for Asbestos millboard, specification for

Astos millboard, spillberd

Asbestos plaster, magnesia, specification for

Asbestos prepared roofing, slate-surfaced, specification for

Asbestos prepared roofing, slate-surfaced, specification for

Asbestos valve-stem packing, specification for

Asbestos wick and rope packings, specification for

Asbestos yarns, specification for tolerances and test method for

Ash determination for coal and coke

Ashlar masonry, specification for

Ashlar stone, specification for

Asphalt, bituminous carpet, specification for mineral surfaced roofing, specification for

penetration limits

unsurfaced built-up roofing specification for

unaterproofing and daup profing, specification for----.--

Asphalt block pavements, specification for materials for cement

mortar bed for

Asphalt block wearing surface for bridge floors.................. 413

Asphalt cement, speciflcation for

Asphalt cements, method of test for specific gravity of

Asphalt fibrous roof coating, specification for ..............

Asphalt filler, brick pavement, specification for (blown typo) oil, specification for ( $t$ ype $A$ )

Asphalt for use in constructing built-up roof coverings, specification for

235 Asphalt for use in damp proofing and waterproofing below

and above ground level, specification for

Asphalt for use in road and pavement construction, specifica-

tion for

Asphalt macadam base, specification for

Asphalt macadam pavement, specification for

A sphalt macadam surface course, specification for

Asphalt mastic, acid-resisting, specification for waterproofing, spccification for

Asphalt pavement, sheet, specification for broken slag for binder course for

specification for broken stone for binder course for ...... specification for mineral filler for

Asphalt paving sheet, speciflcation for -----

A sphalt paving blocks, specification for

Asphalt penetrometer

Asphalt plastic cement, specification for

Asphalt prepared roofing, slate surfaced, specification for

Asphalt prepared roofing and shingles, slate surfaced, specifcation for.

primer for roofing and waterprooling, specification for Asphalt roll roofing, smooth-surfaced, method of testing....surfaced with granular talc, specification for surfaced with powdered tilc, specification for

Asphalt roll roofing and shingles, heavyweight slate surfaced, pecification for

late surfaced, method of testing -...-.

specification for
Asphalt saturated asbestos felt for use in constructing built-up

roofs, specification for

Asphalt saturated rag felt, flashings, specification for roofing and waterproofing, specification for Asphalt saturated roofing felt for use in waterproofing and in Asphalt saturated woven cotton fabric for waterproofing, specification for Asphalt varnish, specification for

239 Asphaltic base

Asphaltic compounds, method of test for loss on heating of Asphaltic concrete base, specification for Asphaltic concrete binder and surface courses, specification Asphaltic concrete binder and surface courses, specification Asphaltic concrete paving, fine aggregate, specification for Asphaltic concrete surface course, coarse-graded aggregato
type, specification for fine-graded aggregate type, specification for

300
480
Asphaltic oil for hot surface treatment

480 Asphalts, definition of

14 Asphalts sufficiently solid to be hander to be handled in fragments, method Asphaltum, petroleum, specification for

Autogenous ignition temperatures, method of test for the

determination of
A utomobile brake lining, recommendation for
408

\section{8}

643 
Back filling, specification for

Ballast, burned clay, specification for pit-run gravel, specification for stone, specification for

washed gravel, specification for

Barometric pressure, Sydney Young equation for correction

table of approximate corrections for

Base, asphalt macadam, specification for

asphaltic concrete, specification for

bituminous concrete specification for broken slag for specification for broken stone for specification for broken sto
specification for gravel for

bituminous specification for broken stone for specification for broken stone for

gravel, specification for --

macadam, specification for

natural, specification for

ortland cement concrete, specification for

specification for coarse aggregate for (stone, slag, or gravel) tar-bound

Telford, specification for

specification for hroken stono

siecification for shovel-run or crusher-run broken slag

Base course, reconstructed, specification for

specification for reconstruction of old macadam to serveas.

Bases, artificial, cement concrete, specification for ........... specification for

Baths, method of grading

Battery box, ccment concrete, specification for

Battery jar, specification for

Beaker sampling of petroleum product

Bearing piles, concrete, specification for

Bearing power of soils

aring test, sand, for

Bedding coge, for pipe--.-.-.-.

Behavior in firing, method for

Berry gage for coefficient of expansion

Binder course, asphaltic concrete,

sheet-asphalt, specification for specification for broken stone for

Bitumen, insoluble in carhon tetrachloride, method of test for

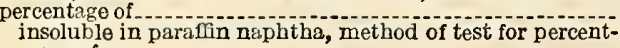

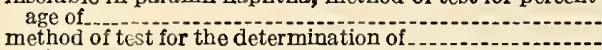
meluble, test for soluble in carhon disulphide, method of test for percentsoluble in carbon tetrachloride, method of test for the determination of proportion of

Bitumens, definition of

Bituminous built-up roofing, specification for installation of metal flashings with

specification for installation of plastic flashings with

specification for surfacing materials for -...-..............-.

Bituminous carpets, specincation for

specification for asphalt for
Bituminous carpets for hridge $100 r s-$

Bituminous coal, crushed, method of test for cubic-foot wcight

of - - method of test for sieve analysis of --..--

method of test for sieve analysis of
Bituminous concrete, coarse-graded aggregate type, specificaion for hroken slag for

finc-graded aggregate type, specification for hroken slag for.

Bituminous concrete base, specification for broken slag for.-.--

specification for hroken stone for
specification for gravel for

Bituminous concrete base or surface course, specification for broken slag for

specification for broken stone for-

Bituminous concrete pavements, spccification for mineral filler for specification for sand for.

Bituminous concrete surface, spccification for broken stone for.

Bituminous emulsions, method of testing

Bituminous grout for use in waterproofing below and above ground level, specification for

Bituminous macadarn, specification for hroken stone for -.-.-.-.

Bituminous macadam base, specification for broken slag for -

Bituminous macadam base or surface course, specification for hroken slag for

specification for hroken stone for

Bituminous macadam wearing course, specification for broken slag for -

Bituminous mastics, grouts, and liko mixtures, method of testing

Bituminous materials, definition of

method of float test for.

method of sampling - -

method of test for coarso particles in, hy means of elutriation . of test for coarso particles in, by meass of elutia-
Page

(3)
Bituminous materials, method of test for ductility of

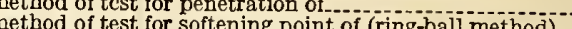

method of test for specific gravity of (ring-ball method) -.-

method of test for water in..................................... specification for-

asphalt for bituminous carpets

hituminous carpets

fillers

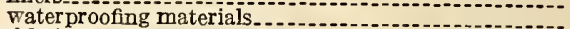

suitable for road treatment, method of test for distillation

Bituminous mixtures, examination of

method of sampling ......................

Bituminous protective coatings, method of test for steam disti]on of

ituminous roofings, analysis of -

ituminous sand, specification fo

Black hase, specification for

Backboard, composition, recommendation for

Blackboard slate, recommendation for

Blake bottles for tablets.

durax-granite pavement, specification for -

granite hlock pavement specification for --

gypsum partition, specification for

recut granite hlock pavement, specification for

Block pavements, granite, specification for block for-

recut granite, specification for block for

specification for materials for cement mortar bed for

stone, specification for sand for cement grout filler for

tone or wood, specification for sand for cement mortar bed

Blocks, magnesia molded, specification for

paving, asphalt, specification for

specification for coal-tar oil for

segmental tilo

Boiler service, marine, specification for clay fire brick for...... stationary, specification for clay fire hrick for ............ Borings sampling of petroleum products Bottle caps, recommendation for

Bottle sampling of petroleum products. tlles, milk and cream, recommendation for tahlet, standardized

Brake lining, automohile, recommendation for $---z_{-2}$ building, concrete, specification for

specification for

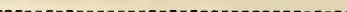

common clay, specification for

-

作

concrete, specification for

fire clay, malleahle furnace and annealing oven, specifica-

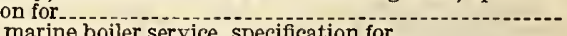
marine boiler service, specification for method of test for resistance of, to thermal spalling action.

method of test for softening point of specification for

stationary boiler service, specification for

method of testing

aving method 0 specification for

rough and smooth face and common, recommendation for send ime, recommendation for--

sand lime, recolding, specification

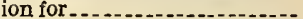

scwer, clay, specification for
silica, specification for quicklime and hydrated lime for the

manufacture of -.--

vitrified, for sewers

Brick chimneys with fire clay flue linings, specification for Brick curb, integral, specification for

Brick bouse, specification and general schedulo for-bricklaying

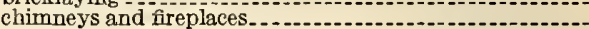

ideal construetion

mortar-

Brick masonry, specification for --

Brick masonry for sewers

Brick pavements, scmimonolitbic, specification for cement grout filler for ................................................ specification for cement sand bed for

specification for asphalt fller for (blown type)

specification for materials for cement mortar hed for......-

specification for materials for cushion course of

specification for sand for cement grout filler for

specification for sand for cement mortar bed for

Brick sewers..

wearing surface for bridge foors

Bricklaying, specification for

Bridge liools, concrete, specification for

specification for wearing surfaces for

Bridge masonry, ashlar stone

Block ruhble..........-.-.-.-.-
Bridges, highway, specification for-

arches.

ashlar masonry 
Bridges, bigbway, specifications for-Continued.

asphalt paving blocks.

asphalt for bituminous carpets.

bituminous carpets

fillers.

waterproofing materials

brick masonry

coarse aggregate.-

concrete bearing piles

concrete bridge floors

concrete cribhing.

concrete masonry

concrete viaducts

contrcl of materials

dry rubble masonry

excavation and fil

foundations and substructure

monolithic concrete culverts.

mortar rubble mas

railings and parapets

riprap

rubble or cyclopean aggregate

water for use witb cement.

wearing surfaces for bridge floors

reinforced concrete slab and girder, specification for

Broken slag, binder course, sheet aspbalt pavement, specifica-

tion for

bituminous concrete, coarse-graded aggregate typo, speci-

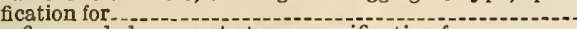

fine-graded aggregate type, specification for

bituminous concrete base, specification for

bituminous concrete base or surface course, specification

for.

bituminous concrete surface.

bituminous macadam base, specification for

bituminous macadam base or surface course, specification for

bituminous macadam surface course

bituminous macadam wearing course, specification for

bituminous surfaco treatment, specification for

bituminous surface treatment, specification for ---

mighway construction, specificat ion for commercial sizes of -

method of mechanical analysis of mixtures of sand or otber

fine material witb, except aggregates used in cement concrete

method of test for abrasion of

Portland cement concrete base course

Portland cement concrete surface course

sbovel-run or crusber-run, for water-bound base, specifica. tion for

water-bound base

water-bound base and wearing course, specification for.- 254

Water-bound surface course

Broken stone, binder course, sbeot aspbalt pavement, specifica-

bituminous conerete base, specification for

bituminous concrete base or surface course, specification

for bituminous concrete surface, specification for

bitumirous macadam, specification for

bituminous macadam base or surface course, specification

bituminous macadam surface course

bituminous surface treatment, specification for

form of specification for certain commercial grades of

highway construction, specification for commercial sizes of

method of mechanical analysis of, except aggregates used in

cement concrete

metbod of mecbanical analysis of mixtures of sand or other

fine material witb, except aggregates used in cemcnt concrete

metbod of test for abrasion of

Portland cement concrete base course

Portland cement concrete suriace cours

water-bound base, specification for

water-bound base or wearing course, specification for -

Broken stone foundations for pavements

Broken stone roads, specification for

Building block and tile, concrete, specification for

sand lime, specification for

specification for

Building construction and materials, specification for fire tests

of -

Building regulations for concrete staves

Building tile, hollow, recommendation for

Building units, concrete, recommendation for

hollow concrete, standard for

Buildings for railway purposes, specification for-

architectural terra cotta.

brickwork.

built-up roofing

clay bollow tile-

clay roofing tile..

concrete arcbitectural ston

concrete roofing tile

excavation, falling, and back filling

exterior stucco wor

Buildings for railway purposes, specifications for-Contd. glazing

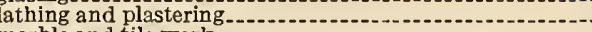

marble and tile work

plumhing

sewers and drainage.

slate roofing

stone masonry and cut stone work

Built-up roofing, bituminous, specification for installation of specification for installation of plastic fiasbings witb...

specification for

Built-up roofs, specification for coal-tar saturated roofing felt

for use in constructing

surfaced witb slag or gravel, specification for high-carbon

and high-bitumen coal-tar pitches for use in constructing

Burlap saturated witb bituminous substances for use in water-

proofing, specification for

Burned clay ballast, specification for

Burned clay fireproofing, partition and furring tile, bollow, specification and test for

Burned clay floor tile, bollow, specification and test for-..-.-

Burned clay load-bearing wall tile, hollow, specification and test for-

$$
\begin{aligned}
& \text { urner, Dressel "D" or No. } \\
& \text { Miller No. } 2 \text { sun hinge. }
\end{aligned}
$$

Burning quality, kerosene oils, method of test for

long-time burning oil for railway use, metbod of test for--mineral seal oil, method of test for........................-

Cafeteria cbinaware, recommendation for

Calcined gypsum, specification for

Calcined gypsum for use in tbe preparation of dental plasters,

specification for
Caicium cbloride for dust provention, specification for

Calcinm chloride metbod for determination of percentage of

water in bituminous emulsions

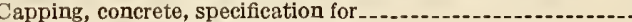

Caps, bottle, recommendation for

Capsules, combustion, porcelain

Carbon disulphide, metbod of test for percentage of bitumen

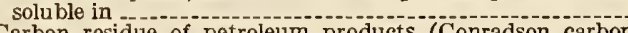

Carbon residue of petroleum products (Conradson carbon
residue), method of test for

Carbon tetracbloride, metbod of test for percentage of bitumen

insoluble in specification for

specification for asphalt for

Casseroles, porcelain -..---.-

Catch-basin block, conerete, specification for

Cell space of lump coke, method of test for volume of

ment, asphalt, specification for

cbemical analysis of

consistency of

dotermination of fineness of

determination of soundness of

Keene's, speciócation for

masonry, specinication for

natural, specification for

plastic magnesia, specification for

Portland, cement grout filler -

specification and test for

tar, bigb and low carbon, specification for specification for -

tension tests of

tile work

time of setting of -

Cement concrete artificial bases, specification for.--

Cement concrete battery box, specification for

Cement concrete pipe- pipe, specification for
Cement concrete sewer pavements, speci-

fication for materials for and stone block pavements, specisemimonolithic brick, pavements, specification for

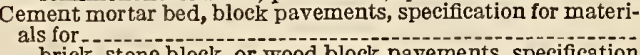

brick, stone block, or wood block pavements, specification for sand for

Cement sand bed for semimonolitbic brick pavements, specification for

Cements, asphalt, metbod of test for specific gravity of tar, bigh and low carbon, for use cold in repair work (cut-
back product), specification for Centrifugal method for examination of bituminous mixtures.-

Centrifuge tubes

Ceramic materials, method for sampling -

Ceramic whiting specification for

Cbarcoal adsorption test method for testing natural gas for

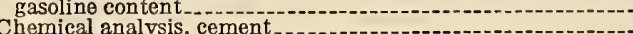

chrome ores and ehrome brick ${ }_{\text {foundry sands } . . . .-}$ 
Chemical analysis, limestone, quicklime, and hydrated lime, method of brick, method of

Chimney, Keystone No. 24 Macbeth-Evans No. 514 pearl top

Chimneys, brick, with fire clay flue linings, specification for-lamp, glass, specification for ..................... specification for

Chinaware, cafeteria and restaurant, recommendation for dining car, recommendation for hospital, recommendation for

hotel, recommendation for

vitrifed, specification for -

Chrome ores and chrome

analysis of

Classification, Indiana limestone. .

precious stones.

soils

solid fuels.

Clay, fire, specification for

Clay ballast, burned, specification for

Clay brick, common, specification for

Clay conduit, vitrified, specification for
Clay deposits, method for sampling

Clay deposits, method for sampling y for specification for marine boiler service, specification for

clay fireproofing, partition and furring tile, hollow, specification for

Clay floor tile, hollow, specification for -

Clay hollow tile, specification for

Clay in gravel for highway construction, method of test for quantity of -

clay in sand-clay, topsoil, and semigravel for highway construction, metbod of test for quantity of
clay load-bearing wall tile, hollow, specification for $\ldots . . . .$.

Clay load-bearing wall tile, hollow, specification for

Clay roofing tile, specification for

Clay sewer brick, specification for

Clay sewer pipe, specification for

Cleveland open cup Cloth, abrasive, aluminum oxide, specification for emer'y, specification for

Cloud point of petroleum products, method of test for

Coal, calorimetric determination for

crushed bitumiucus, method of test for cuhic-foot weight ofmethod of test for sieve analysis of

definition of terms relating to

Kjeldahl-Gunning method for determination of nitrogen method of lahoratory sampling and analysis of

method of sampling

Coal-ash fusion furnace

Coal mines, rock dusting in.

Coal-tar oil for flooring and paving blocks, specification for

Coal-tar pitch, roofing, spccificatiou for stone-block filler, specification for

Coal-tar pitches, high-hitumen, for use in damp proofing and

and waterproofing below and above ground lerel, specification for.

high-carhon, for use in damp proofing and waterproofing below and ahove ground level, specification for--.-.--
high-carbon and high-bitumen, for use in constructing high-carbon and high-bitumen, for use in constructing
built-up roofs surfaced with slag or gravel, specification for

Coql-tar saturated rag felt for roofing and waterproofing, specification for

Coal-tar saturated roofing felt for use in waterproofing and in constructing built-up roofs, specification for

Coarse aggregate, method of test for apparent specific gravity of..

method of test for soundness of
Portland cement concrete pavement or base, specification

Portland cement concrete pavement or base, specification for

Coarse particles in bituminous materinls by means of elutriation, method of test for

Coated abrasive products, recommendation for

Coatings, bituminous protective, method of test for stcam disillation of

test for discoloration of

Code for testing natural gas for gasoline content

Coke, calorimetric determination for

definition of terms relating to.

Kjeldahl-Gunning method for determination of nitrogen

in

lump, method of test for volume of cell space of

method of lahorator $y$ sampling and analysis of

method of shatter test for

method of test for cuhic-foot weight of -

method of test for sieve analysis of -

method of tumbler test for

Coking coais, specification for

Color, lubricating oils, by means of union colorimeter, method

of test for...
Page

Color,

trum, by means of union colorimeter, method of refined petrolcum oil, by means of Saybolt chromometer, method of test for

160

Color standards of union colorimeter.

Combustion capsules, porcelain.

ommercial grades of broken stone, form of specification for ommercial sizes, broken stone and broken slag for highway construction, specification for gravel

sand.

sand and gravel for highway construction, specification for

recommendation for

sand lime, specification for

sewer

Communication underground conduit construction, specifica-

tion for

Composition blackhoard, rccommendation for. method for

Compounded cutting oil, requirements for

Compressed asbestos sheet packing, specification for

Compression strength test for foundry sands

ompression and cooling test method for testing natural gas

for gasoline content.-.

concrete, method of making

concrete, method of making agregate for concrete, method of making

Compressive strength of Portland cement mortars, specifica-

tion and test for

oncrete, bituminous, coarse-graded aggregate type, specification for broken slag for

fine-graded aggregate type, specification for broken slag for hardened, method of securing specimens of, from the method of making compression and tension tests of fine method of making compression tests of

method of test for-

absorption of

determination of voids in fine aggregate for

organic impurities in sands for

sieve analysis of aggregates for

unit weight of aggrcgate for..

water to be used in

plain and reinforced, specification for (water-cement ratio method)

Portland cement, method of test for consistency of plain and reinforced, specification for............... spccification for fine aggregate for specification for gravel for

reinforced, in structures, specification for

sewer.

tile work

Concrete aggregates, spccification for

Concrete and reinformed concrete, specification for

Concrete architectural stonc, specification for

crete base, asphaltic, snccification for

bituminous, specification for broken slag for specifcation for broken stone for

specification for gravel for

Portland cement, specification for

Concrete base or surface course, bituminous, specification for broken slag for. specifican

Concrete bearing prorifin for Concrete block and tile walls, specification for application of Portland cement stucco on

Concrete brick, specification for

Concrete bridge floors, specification for

Concrete building brick, specification for

Concrete building units, hollow, standard for

recommendation for

Concrete construction, method of proportioning natural sand

and gravel for

Concrete cribhing, specification for -

Concrete culvert pipe, reinforced, specification for

Concrete culverts, monolithic, specification for

Concrete curb, in tegral, specification for Concrete curb and concrete curb and gutter, specification forConcrete curb and header, specification for Concrete drain tile, specification for -

Concrete fence posts, specification for

Concrete floors, specification for
Concrete fuel oil storage tanks, specification for design and construction of

Concrete masonry, specification for

Concrete pavement or base, Portland cement, specification for coarse aggregate for (stone, slag, or gravel)

specification for fine aggregate for

Conerete pavements, bituminous, specification for mineral filler for.

specification for sand for

one-course Portland cement, for highways, specification for.

Portland cement, specification for

two-course Portland cement, for highways, specification

for.-.................. Concrete paving, fine aggregate asphaltic, specification for.-.- 
Concrete sewer, manhole, and catch-basin block, specification

for-..........................

Concrete sewers, built in place

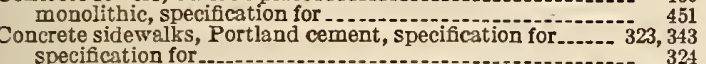

Concrete specimens, metbod of making and storing in the field

Concrete staves, specification and building regulations for...

concrete street pavement, 1-course Portland cement, specification for -

two-course Portland cement, specification for.

Concrete surface, bituminous, specification for broken ston for-

Concrete surface course, asphaltic, coarse-graded aggregate

type, speciñcation for

fine-graded aggregate type, specification for

Concrete swimming-pool construction, specification for

Concrete trunking, capping, and supports, specification for.--

Concrete viaducts, specification for

Concrete viaducts, specification for
Concrete wearing surface for bridge floors

Concrete wearing surface for bridge

Conduit, vitrified clay, specification for-

Conduit construction, communication underground, specification for

electrical, specification and form of contract for

Conradson carbon residue test (method of test for carbon

residue of petroleum products)

Consistency, cement

Portland cement concrete, metbod of test for Construction, built-up roofing, specification for
concrete, method of proportioning natural sand and gravel for..

concrete fuel-oil storage tanks, specification for. concrete strimming pools, specification for

highway, method of test for quantity of clay and silt in gravel for specification for commercial sizes of sand and gravel
for

specification for refined tar for

Containers, glass, for preserves, jellies, and apple butter, recommendation for

Containers for storing and handling flammable liquds, rezu-

lations for tbe installation of (design and construction of concrete fuel oil storage tanks)

Continuous sampling of petroleum products

Corrosion test for lubricants and liquid fuels

Corrosion test for lubricants and liquid fuels

Corrosive sulphur compounds in gasoline, method of test for

detection of

specification for

Cotton fabrics, woven, saturated with bituminous substances

for use in waterproofing, specification for

Crank-case lubricating-oil viscosity numbers...-..-..--

Crank-pin grease, requirement for

Crank-shaft grinding wheels

Cream bottles, recommendation for

Creosote oil for priming coat with coal-tar pitch in damp proof-

ing and waterproofing below and above ground level, speci-

ing and waterproofing below and above ground level, speci-

Cribbing, concrete, specification for

Crucibles, Gooch

plumbago, sizes of

Crude petroleum, definition of

method of test for distillation of

Crude shale oil, definition of

Crushed

method of test for sieve analysis of

Crushed stone for roofing, requirement for

Crusher-run broken slag for water-bound base, specification

for

Crushing strengths of sewer pipe to carry loads from ditch

filling, specification for

Crushing test of sewer pipe

Cubic-foot weight, coke, method of test for

crushed bituminous coal, method of test for.

Culvert masonry

Culverts, monolithic concrete, specification for

Cups, sponge and pin, specification for -

Curb, concrete, specinication for

integral brick, specification for

integral concrete, specification for

stone, specification for

Curb and gutter, concrete, specification for

Curbs, specification for

specificetion for materials for

Cutting oils, specification for

Cyclopean aggregate, specification for

Damp proofing, specification for asphalt for

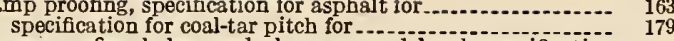

Damp proofing below and above ground level, specification

specification for creosote oil for priming coat with coal-tar pitch in

specification for high-bitumen coal-tar pitches for use in -.

specification for high-carbon coal-tar pitches for use in.--

specification for primer for use with aspbalt in

Decantation test for sand and other fine aggregates, method of

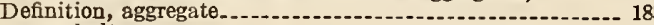

asphalts...

bitumens...-

brick.

clay refractories

methods of sampling petroleum products ..................

nonbituminous materials for road and pavement making. penetration

petroleum.

pitches

sand.

screen

sieve

slag -

slate

terms relating to-

coal and coke

hollow tile_-_.-

materials for roads and pavements.-1

petroleum.

refractories

sewer pipe

iscosity

Demulsibility tests

218 Dental plasters, specification for calcined gypsum for use in the preparation of

Description of pearls end imitation pearls

Description of pearis and imitation pearis.

Design or concrete fuel oil storage tanks, specincation for

Detection of free sulphur and corrosive sulphur compounds in gasoline, method of test for.

67

Determination, approximate apparent specific gravity of fine aggregate, method of test for

approximate percentage of voids in fine aggregate, method of test for.

autogenous ignition temperatures, method of test for

bitumen, method of test for

fineness of mineral filler, metbod for

moisture equivalent of subgrade soils in the field, method

percentage of water in bituminous emulsions, calcium

chloride method for in bituminous emulsions, calcium

proportion of bitumen soluble in carbon tetrachloride, metbod of test for

surface moisture in fine aggregate, metbod of test for

voids in fine aggregate for concrete, metbod of test for

Determining insulating qualities of slate, method of test for-.particles, metbod of

Dining-car chinaware, recommendation for

Dipper sampling of petroleum products........-.

Discoloration of coatings

Displacement methods for specific gravity of bituminous

materials

Distillation, bituminous materials suitable for road treatment, method of test for

gasoline, naphtha, kerosene, and similar petroleum prod-

ucts, method of test for.

natural gas gasoline, method of test for Distilling flask, Saybolt

Doctor test for lubricants and liquid fuels

Domestic fuel oils

rain tile, concrete, specification for

specification for

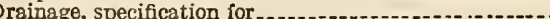

Dressel "D" bumer

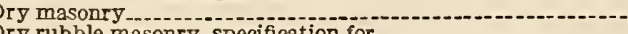

Drying shrinkage, method for

Duct, tile, specification for

Ductility of bituminous materials, method of test for

Durax granite pavements, specification for block for

Dust prevention, specification for calcium chloride for

E

Elasticity, modulus of, of slate, determination of -

Electrical conduit construction, specification and form of contract for

195 
Electrical insulating oils, method of testing

Elimination of water, specification for

Elutriation method of test for coarse particles in bituminous materials

Emery cloth specifion

Emiey plasticim

Emulsion test

Emulsion test for soluble cutting oils.

Emulsions, bituminous, method of testing

Engine distillate, definition of

Engler flask

Eschka method for determination of sulphur in coal and coke.-

Ether, petroleum, specification for.

Examination of bituminous mixtures.

Excavation, sewer.-

Excavation and fill, specification for

Expansion joints, premolded, specification for

Expressible oil and moisture in paraffin waxes, method of test

for - Exterior stucco work, specification for

Exterior stucco work, specification for
Extracted aggregates, method of mechanical analysis of...--

\section{F}

Fabrics, felted and woven, saturated with bituminous substances, for use in waterproofing and roofing, method of testing

woven cotton, saturated with bituminous substances, for use in waterproofing, specification for

Face brick, rough and smooth, recommendation for Feeder insulators

Felt, asphalt-saturated asbestos, for use in constructing builtup roofs, specification for

asphalt-saturated rag, flashings, specification for roofing and waterproofing, specification for

asphalt-saturated roofing, for use in waterproofing and in constructing built-up roofs, specification for

coal-tar saturated rag, for roofing and waterproofing, specification for

coal-tar saturated roofing, for use in waterproofing and in constructing built-up roofs, specification for

Felted fabrics saturated with bituminous substances for use in

waterproofing and roofing, method of testing ............

Fence posts, concrete, specification for

Fibrous roof coating, asphalt, specification for

Field determination, approximate apparent specific gravity of fine aggregate, method of test for

approximate percentage of voids in fine aggregate, method of test for.

surface moisture in fine aggregate, method of test for

Figured sheet glass

Filer, asplualt, brick pavement, specification for (blown type)specification for (type B)

cement grout, brick and stone-block pavement, specification for materials for specification for sand for

semimolithic brick pavement, specification for

mineral, method for determination of fineness of sheet asphalt and bituminous concrete pavement, specification fo

specification for

oil asphalt, specification for (type A)

sand, specification for

stone-block, specification for coal-tar pitch for-

Filler button, procelain, specification for

Fillers, premolded joint, method of sampling and testing specification for

Fine aggregate, concrete, method of making compression and

tension tests of method of test for determination of voids in.

method of decantation test for method of test for field determination of approximate pcrmethod of test for field determination of approximate percentage of voids in

method of test for field determination of surface mois-

ture in Portland cement concrete, specification for

Portland ceinent concrete pavement or base, spccification for

Fine aggregate asphaltic concrete paving, specification for

Fineness, cement, determination of

mineral filler, method for determination of

powdered coal, method of test for

Fincness test for foundry sands

Finish coat Portland cement stucco, specification for

Fire clay, specification for

ire clay brick, furnace and annealing oven, spccification for marine boiler service, specification for

method of test for resistance of, to thermal spalling action

method of test for softening point of ..

specification for

stationary boiler service, specification for

Fire clay flue linings, specification for brick chimneys with.

Fire clay refractories, marine service, specification for

plastic, specification for

Page

Fire point by mears of open cup, method of test for

ire tests of building construction and materials, specification

Fireplaces, specification for.-.

Fireproofing tile, hollow burned clay, specification and test for. hollow clay, specifications for iring hellow clay, specilications

Firing, method for behavior in $\overrightarrow{\text {. }}$, for coal and coke Fixed lights, glass for Fixed lights, glass for
Fixtures, plumbing, specification for-porcelain ware vitreous ware

vitreous china, recommendation for

Flammable liquids, containers for storing and lations for the installation of (design and construction of concrete fuel-oil storage tanks)

volatile, method of test for flash point of

Flash point by means of open cup, method of test for

Flash point by means of the Pensky-Martens closed tester, method of test for

lashings, metal, specification for installation of, with bituminous built-u, roofing plastic, specification for installation of, with bituminous built-up roofing-

specification for asphalt saturated rag felt for

Flat glass for glazing purposes, specification for

130

167

158

160

160

156

160

179

130

239

239

240
488

415

146

253

235
342

253

150

146

146
177
17

528
253
149

242

213
240

239
239

240

235

\begin{tabular}{l}
241 \\
399 \\
\hline
\end{tabular}

289

253
6
226

226
513

588

590

573
573

573
591

588
422
586

592

Flexure testing of slate, method of (determination of modulus of rupture and modulus of elasticity)

Flint paper, specification for

Float test for bituminous materials, method of

lock test, kerosene

mineral seal oil

loor lights, recommendation for - -

Floor tile, hollow burned clay, specification and test for. hollow clay, specification for

Flooring, specification for coal-tar oil for

Floors, bridge, concrete, specification for specification for wearing surfaces for

concrete, specification for

slate, specification for

tiled

Form of specification for certain commercial grades of broken

stone-_-
Forty-five-degrees-below-zero-Fahrenheit lubricating oil, speci-

fication for

Foundation for tracks in paved streets, design of

Foundations, bridge, specification for pavement, specification for

Foundry, coke, specification for

Foundry refractories, malleable, recommendation for

Foundry sands, method of testing and grading-...-

bituminous highway materials, method of test for.

Free sulphur in gasoline, method of test for detection of.-.-.--

Freezing and thawing tests of drain tile.

Fuel oil, definition of domestic and industrial

method of test for thermal value of

specification for

Fuel-oil storage tanks, concrete, specification for design and

construction of

fuel oils

gasoline.-.-

illuminating oil

methods of test

motor, method of test for sulphur in (lamp method) -..... telassification of

Funnels, Büchner, porcelain

Furnace, coal-ash fusion

urnaces, malleable, specification for clay firs brick for

Furnishing of terra cotta, specification for

burned clay, specification and test for

Fusibility of ash, determination of, for coal and coke...........

\section{G}

Garnet paper, specification for

waterproor, specification for

Gas, natural, code for testing, for gasoline content.

Gas coals, specification for ....................

Gas oil, definition of

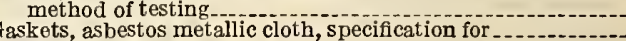

Gasoline, asbestos metallic cloth, specifcation for -............ method of test for detection of free sulphur and corrosive sulphur compounds in.................... method of test for distillation of motor, specification for

natural, spccification and test method for

natural gas, method of test for distillation of

rules regulating the petroleum trade for

specification for 
Page

Gasoline content, code for testing natural gas for.

General conditions for railway building

General scbedule for a brick bouso-bricklaying ideal construdion mortar.

Gillmore needles

Glacial gravels, metbod of determining quality of, by lithological count of particles

Glass, airport and fixed light

flat, for glazing purposes, specification for metbod of analysis of

plate, recommended practice for

polisbed plate, specification for

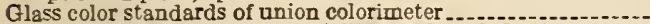

Glass containers for preserves, jellies, and apple butter, recommendation for

Glass for use in plumbing, requirement for

Glass lantern globes and lamp chimneys, specification for.....

Glass slides, specification for

Glass tableware, specification for.

Glasses, water gauge and lubricator, specification fo

Glassware for ship equipment, kinds and sizes of

Glazing, specification for

Globes, band lantern, specification for lantern, glass, specification for

Gooch crucibles

Grab sampling of petroleum products

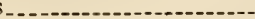

Grades or broken stone, for

Grading, specilication for -

Grading metbod for found

Granite, specification for

Granite block pavements, recut, specification for block for.---

Granite pavements, durax, specif

Granular specifications for slates

Granular tale, specification for aspbalt roll-roofing surfaced with.

Grapbite lubricating grease, requirement for

Gravel, bituminous concrete base, specification for bituminous surface treatment, specification for

commercial sizes of

gravel roads, specification for

highway construction, method of test for quantity of clay ing silt in

and silt in specification for commercial sizes of method of sampling . . . method of test for abrasion of metbod of test for apparent specific gravity and absorption

or free moisture content for
metbod of test for quantity of soft pebbles in.
metbod of test for toughness of

natnral, for concrete construction, method of proportion-

Portland cement concrete, specification for

Portland cement concrete pavement or base, specification$$
\text { roofing, requirement for }
$$

Gravel ballast, pit run, specificetion for

washed, specification for

Gravel base, specification for

Gravel roads, specification for

Gravels, glacial, method of determining quality of, by litho-

logical count of particles

Gravity, A. P. I., definition of petroleum and petroleum products, metbod of test for, by means of tbe bydromete

specific, bituminous materials, method of test for definition of

Gravity tanks, regulations for construction and installation of

Grease, crank-pin, requirement for grapbite lubricating, requirement for metbod of mineral lubricating, requirement for

Greases, metbod of test for penetration of specification for

Green-sand strengtb test for foundry sands

Grinding wheels, crank-shaft recommendation - for

Ground supported vaults, specification for

Grout, bituminous, for use in waterproofing below and above

ground level, specification for

Grouts, bituminous, method of testing.

Gutters, specification for

Gypsum, calcined, for use in tbe preparation of dental plasters, specifieation for

$$
\begin{aligned}
& \text { specification } \mathrm{f} \\
& \text { pecification for }
\end{aligned}
$$

specification for

Gypsum finisbing plaster

Gypsum finisbing plaster-

Gypsum industry, definition of terms

Gypsum molding plaster, specification for

Gypsum neat plaster

Gypsum partition tile, specification for

Gypsum partition tile or block, specifications for

Gypsum plaster, specification for

G jpsum plaster board, specification for

Gypsum plastering sand, specification for

Gypsum plasters, concrete surfaces.

specification for
Gypsum pottery plaster, specification for

Gypsum wall board, specification for--------

Gypsum wo

\section{$\mathrm{H}$}

Iand lantern globes, specification for

eader, concrete, specification for

stone, specification for

Headers, specification for

Heavy-weight slate-surfaced asphalt roll-roofing and shingles, specification for

Hempel distilling fiask.

Higb-bitumen coal-tar pitcb for use in constructing built-up

roofs surfaced witb slag or gravel, specification for

waterproofing below and above ground level, specification for

High-carbon coal-tar pitch for use in constructing built-up roofs surfaced witb slag or gravel, specification for - - - -.---ligh-carbon coal-tar pitcb for lise in damp proofing and waterproofing below and above ground level, specification for -..Higb-carbon tar cement for use cold in repair work (cut-back product), specification for

Higb-carbon tar for surface treatment, cold a pplication, s pecification for

hot application, specification for

High-pressure rod packing, asbestos, specification for

Higbway bridges, specification for -

arches

ashlar masonry

bituminous minteriols, aspholt for bituminous

bituminous carpets

fillers

Waterproofing materials

brick masonry.

coarse aggregate--

concrete bridge floors

concrcte cribbing

concrete masonry

concrete viaducts

control of materials.

dry rubble masonry

excavation and fill

foundations and substructures

mortar rubble masonry

paving blocks, as pbalt

railings and parapets

rainfors and parapets

riprap-1e or cyclopean aggregate

stone for masonry

water for use with cement

Higbway construction, method of test for quantity of clay and

silt in gravel for

specification for commercial sizes of broken stone and bro-

sen slag for

Higbway material, fine, method of mechanical analysis of .-.-

Higbway materials, nozbituminous, metbod of test for apparent specific gravity of method of test for apparent specific gravity and $\mathrm{ab}-$ specification for -

asphalt fillers and cements

bituminous sand

fine aggregate for Portland cement concrete

fine aggregate for Portland cement concrete...-.......

gravel for

mineral filler

premolded expansion joints.

Higbway structures, specification for slag to be

Highways, specification for 1-course Portland cement concrete pavements for

specification for slag to be used in

Hollow building tile, recommendation for

Hollow burned clay fireproofing, partition and furring tile,

specification and test for

Hollow burned clay load-bearing wall tile, specification and test for

Hollow clay fireproofing, partition and furring tile, specifica-

tion for

Hollow clay load-bearing wall tile, specification for

Hollow concrete building units, standard for

ollow tile, clay, specification for

definition of terms relating to

Hospital chinaware, recommendation for

Hot extraction metbod for examination of bituminous miz-

Hotel chinaware, recommendation for

House, brick, specification and general schedule of

Hydrated lime, method of cbemical analysis of

specification for

498
448
44
44
15
3
17
17
17
17
178
17
17
17
64 
Hydrated lime for-

structural purposes, specification for

362

the manufacture of varnish, specification for

use in the cooking of rags for the manufacture of paper, specification for

use in the textile industry, specification for

use in water treatment, specification for
Bydrometer method for specific gravity of bituminous mate-

rials.

Hydrostatic test of sewer pipe.

\section{I}

Ideal construction of a brick house, speciflcation for

Ignition temperatures, autogenous, method of test for the determination of nluminating oils, method of tets for sulphur in (lamp method)specification for

Imitation pearls, description of

Impedance bonds, specification for petrolatum for use in Impurities, organic, in sands for concrete, method of tests for Indiana limestone, classification of

Industrial fuel oils

nlets for sewcrs

Inspection methods for quicklime and lime products

Installation, containers for storing and handling flammahle liquids, regulations for (design and construction of concrete fuel oil storage tanks)

metal lashings with bituminous huilt-up roofing, specimetal lashings with bituminous huilt-up roofing, specification for
plastic flashings with bituminous built-up roofing, specification for
tanks, gravity and pressure, towers, etc., regulations for tanks, gravity and pressure, towers, etc., regulations for

Insulating oils, electrical, method of testing.

Insulating qualities of slate, method of test for determining the Insulation, porcelain, specification for porcelain, 1-piece, recommendation for

porcelain

Integral hrick curh, specification for

Integral concrete curh, specification for

Integral waterproofing material, specification for.-.-.-...-..-.

Interior marble, specification for

\section{$\mathrm{J}$}

Jack panel, porcelain, specification for

Jackson test for apparent specific gravit

Jar, hattery, specification for

Joint fillers, premolded, method of sampling and testing

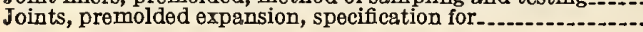

\section{$\mathbf{K}$}

Keene's cement, specification for

Kerosene, definition of flock test for

method of test for distillation of

Kerosene oils, method of test for burning quality of Keystone No. 24 chimney.

Kinds of glassware for ship equipment

Kjeldahl-Gunaing method for determination of nitrogen in

\section{L}

Lahoratory apparatus, specification for (lahoratory porcelain

ware) -
Lamp, standard brass Sayholt test

Lamp chimneys, glass, specification for

Lamp lenses

Lantern glohes, glass, specification for

hand, specification for.

Lard, oil, requirement for

Lath, specification for $---m e t h$

Laundry trays, flat rim, method of grading roll, rim, method of grading

Lavatories, method of grading

vitreous china, method of grading

Le Chatelier test for apparent specific gravity

Lenses, lamp

light signal, sizes of

specification for

ights, fixed, glass for sidewalk, floor and roof, recommendation for

Lime, definition of terms relating to

hydrated, for structural purposes, specification for

for the manufacture of silica hrick, specification for -

for the manufacture of varnish, specification for

for use in the cooking of rags for the manufacture of paper, specification for

for use in water treatment, specification for

method of chemical analysis of specification for.

Lime plaster, specification for sand for use in
Lime plastering, specification for

Lime products, method of sampling, inspection, packing, and Lime stucco, specification for.

Limestone, Indiana, classification of method of chemical analysis of. specification for

Le material, overhead, for 750 -volt direct current, direct suspension, specification for

iquid fuels, specification for-

fuel oils

gasoline method of oist

Liquids, flammahle, containers for storing and handling, regulations for the installation of (design and construction of concrete fuel oil storage tanks)
Load-hearing wall tile, hollow hurned clay, specification and

test for

Long-time burning oil for railway use, method of test for hurning quality of

Loss on heating of oil and asphaltic compounds, method of test for

Low-carhon tar cement, specification for

Low-carhon tar cement for use cold in repair work (cut-back product), specification for

Low-carhon tar for surface treatment, cold application, specifcation for

ubricants, method of test for neutralization numher of method of test for viscosity of specification for-

cutting oils

greases

lubricating oils

Luhricating grease, graphite, requirement for mineral, requirement for

Luhricating oil, forty-five-degrees-helow-zero-Fahrenheit, specification for

zero Fahrenheit, specification for

Lubricating oil vis, sity number

Luhricating oils, method of test for color of, by means of union colorimeter

method of test for precipitation number of

method of test for steam emulsion of specification for

transmission, specification fo

Lubricator glass, specification fo

Lump coke, method of test for volume of cell space of
494

150

Macadam, hituminous, specification for broken stone for old, reconstruction of, to serve as hase course, specification for

Macadam base, asphait, specification for hituminous, specification for broken slag for .......... specification for

Macadam hase or surface course, bituminous, specification for

broken slag for specification for hroken stone for

Macadam pavement, asphalt, specification for

Macadam surface course, water-hound, specification for broken stone for.

Macadam wearing course, bituminous, specification for hroken slag for.

Macheth-Evans No.514 pearl top chimney-a

Magnesia asbestos plaster, specification for

Magnesia cement, plastic, specification for

Magnesia molded pipe covering and hlocks, specification for Magnesia oxychloride

Making specimens of concrete in the field, method of

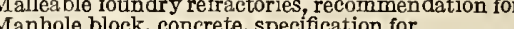

anhole block, concrete

Manufacture of reinforced cometo poles, specification

Manufacture of terra cotta, specification for pluming

plumbing, requirement for

beation for - service,

Marine boiler service, specification for clay fire brick for

$$
\text { hlock rubble }
$$

ashlar, specification for hlock retainin

concrete, specification for culvert speciflcation for

dry ruhhle, specification for

mortar ruhhle, specification for stone, specification for

Masonry cement, specification for

Mastic, asphalt, acid resisting, specification for........ waterproofing, specification for.........

Mastics, bituminous, method of testing
Tan 


\section{Page}

Materials, bituminous, method of test for specific gravity of.. specification for-

asphalt for bituminous carpets fillers. waterproofing materials

cement grout filler, for brick and stone block parements,

cement mortar bed, for block parements, specification for.control of, for highway bridges, specifiestion for

cushion course, brick, stone block or wood block pavement,

specification for

granular surfacing, test for

high

bituminous sand.

fine ageregate for Portland cement concrete--

gravel for Portland cement concrete.

mineral filler

premolded expansion joints

nonbituminous highway, method of test for apparent

specific gravity, and absorption or free moisture content

for

powdered surfacing, test for

road and parement, definition of terms relating to

surfacing, for bituminous built-up roofing, specification for topsoil or sand-clay road surface, specification for

waterproofing, specification for.

Techanical

gates used in cement concrete, method of

extracted aggregates, method of

extracted aggregates, method of . .

mixtures of sand or other fine material with broken stone or

broken slag, except aggregates used in cement concrete.

sand or other fine highway material, except fine aggregates used in cement concrete.

Melting point, paraffin max, method of test for..... petroleum, method of test for

Metal flashings, specification for the installation of, with bituminous built-up roofing -

Metal lath for tile work

Ietallic cloth gaskets, asbestos, specification for

Metallic cloth gasets, asbestos, specifation for

Milk bottles, recommendation for

Milk bottles, recommendation for

Millboard, asbestos, specificatior

Mineral filler, method for determination of uncress of sheet as phalt and bituminous concrete pavement, specification for

specification for.

Mineral lubrica ting grease, requircment for

Nineral seal oil, flock test for method of test for burning quality of

Mineral surfaced roofing, specification for asphalt for

Mines, coal, rock dusting in

Mirtures, bituminous, exsmination of method of sampling

sand or other fine material with broken stone or broken

slag, method of mechanical analysis of, except aggregates used in cement concrete

Modulus of elasticity of slate, determination of

Modulus of rupture of slate, determination of

Moisture, expressible, in paraffin mases, method of test for.-surface, in fine aggregate, method of test for field determination of

Moisture content, free, for sand, grarel, stone, or other non-

Moisture determination materials _. foundry sands

Moisture equifalent of subgrade soils in the feld, method of

test for determination of

Molding plaster, g5psum, specification for

Monolithic concrete culverts, specification for

Monolithic concrete sewers, specification for

Mortar, sewer masonry tile work.

Mortar bed, cement, block parements, specification for materials for

brick, stone block, or wood block pavements, specification for send for

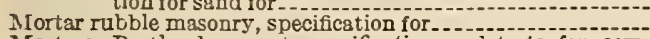

Mortars, Portland cement, specification and tests for com-

pressive strength of

Mosaic, unglazed ceramic, recommendation for

Motor fuel $V$, specification for

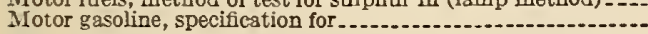

\section{$\mathbf{N}$}

Naphtha, method of test for distillation of petroleum, definition of

rules regulating the petroleum trade for

Naphthas, method of test for sulphur in (lamp method)

National Standard Petroleum Oil Tables, abridgment of ....-

Natural base, specification for

Natural cement, specification for

Totural gas, code for testing for gasoling content

Notur los,

Natural gasoline, specification and test method for..........-.

Natural sand and gravel for concrete construction, method of proportioning Neutralization number of petroleum products and lubricants, method of test for

Nonbituminous highway materials, method of test for apparent specific gravity and absorption or free moisture content for

Tonbituminous materials for road and pavement making,

Oil, asphaltic, for hot surface treatment

coal-tar, for flooring and paving blocks, specification for... creasote, for priming coat with coal-tar pitch in damp proofing and Waterproofing below and above ground

expressible, in paraffin waxes, method of test for fuel, method of test for thermal value of.

illuminating, specification for

long-time burning, for railway use, method of test for burning quality of

lubricating, 45-degrees-below-zero Fahronheit, specification for. zero Fabrenheit, specification for method of test for loss on heating of mineral seal, flock test for ............... method of test for burning quality of refined petroleum, method of test for color of, by means of Saybolt chromometer.

road, for hot application, specification for

transformer, specification for

Oil absorption test method for testing natural gas for gasoline content.

il asphalt filler, specification for (type A) -

il shale, definition of

electrical insulating, method of testing

fuel, domestic and industrial testing specification for

gas, method of testing luminating, method of test for sulphur in (lamp method)-

specification for
kerosene, method of test for burning quality of
lubricating, nethod of test for color of, by means of Union colorimeter

method of test for precipitation number of method of test for steam emulsion of specification for

petroleum, abridged volume correction table for heavier than illuminating oil, method of test for sul-

road, method of test for specific gravity of

transmission lubricating, specification for Old pavements, truing up, to serve as base course, specifica-

One-course Portland cement concrete pavements for highways, specification for
One-course Portland cement concrete street pavoment, specification for.

One-piece porcelain insulators, recommendation for

open cup, method of test for flash and fire points by means of Organic impurities in sands for concrete, method of test for Ornamental glass

Orens, annealing, specification for clay fire brick for
Orerhesd line material for 750 -volt direct current direct susverhesd line material for 750 -volt direct current direct sus-
pension, specification for........

Packing, asbestos high-pressure rod, specification for-

asbestos metallic cloth sheet, specincation for

compressed asbestos steet, specification for

Packing methods for quicklime and lime products

aper, abrasire, artificial, waterproof, specification for-...... asbestos, recommendation for

flint, specification for

garnet, specification for

pecification for quicklime and hydrated lime for use in

the cooking of rags for the manufacture of tile work -

Paraffin naptha, method of test for percentage of bitumen insoluble in.

method of test for melting point of Paraffin wases, method of test ior expressible oil and moisture Parapets, for bridges, specincation for

Partition tile, gypsum, specification for hollow burned-clay, specification and test for-

hollow clay, specification for

Partition tile or block, gypsum, specification for

Pared streets, design of proper foundation for tracks in...-.-

644 
Page

Pavement, aspbalt macadam, specification for

brick, stone block, or wood block, specification for mate-

rials for cushion course of for (stone, slag, or gravel)

specification for fine aggregate for

sheet asphalt, specification for broken slag for binder

course for
specification for broken stone for binder course for...-

street, 1-course Portland cement concrete, specification for two-course Portland cement concrete, specification for

Pavement construction, specification for asphalt for use in

Pavements, bituminous concrete, specification for mineral filler for

block, specification for materials for cement mortar hcd for brick, specification for asphalt filler for (blown type) -.-brick and stone block, specification for sand for cement

brick, stone block, or wood block, specification for sand for cement mortar bed for

definition of terms relating to materials for

durax granite, specification for block for

granite hlock, specification for block for

one-course Portland cement concrete, for highways, specification for

Portland coment concrete, specification for

recut granite block, specifcation for block tor for

specification for cemcrt sand bed for sheet asphalt and bituminous concrete, specification for sand for

specification for subgrades and foundations for stone block, specification for

two-course Portland cement concrete, for higbways, speci fication for

Pavements to serve as base course, specification for truing up Paving, fine ageregate asphaltic concrete, specification forsheet asphalt, specification for

sheet asphalt, specification for
Paving blocks, asphait, specification for specification for cos]-tar oil for

Paving brick, method of campling

specification for

vitrified, specification for

Pearls, desciption of

Pehhles, soft, in gravel, method of test for quantity of

Penetration, bituminous matcrials, metbod of test for

definition of

greases and petrolatum, method of test for

Penetrometer, asphalt

Pensky-Martens closed tester

Percentage of bitumen insoluble in carbon tetracbloride

method of test for

Percentage of bitumen insoluble in parafing naphtha, method

of test for
Pcrcentage of bitumen soluhle in carhon disulpbide, method of

test for

Percentage of shale in aggregate, metbod of test for

Percentage of $\nabla$ oids in ine aggregate, approximate, metbod of

tcst for field determination of

Percentage of water in bituminous cmulsions, calcium cbloride method for determination of

Permanenis volume cbanges in refractory materials, metbod of

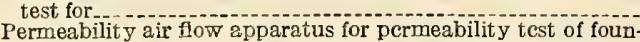
dry sands

Permeability test for foundry sands

Permeahility test pressures, table of

Petrolatam, method of test for color of, by means of the Union colorimeter.

method of test for melitng point of

metbod of test for penetration of.-

Pctrolatum for use in impedance bonds, specification for Petroleum, crude, definition of

method of test for distillation of

method of tcst lor distillation of definition of

definition of terms relating to

refined, rules regulating the petroleum trade for

Petroleum and petroleum products, method of sampling method of test for, by means of the hydrometer

Petroleum asphaltum, specification for

Pctroleum ether, specification for

Petroleum lubricating grease, dcfinition of

Petroleum oil, refined, I

Petroleum oil tahlos

Petroleum oils, abridged volume correction table for

beavier than illuminating oil, method of test for sulphur in.

Petroleum products, method of test for carhon residue of

(Conradson carbon residue)

method of test for-

cloud and pour points of

neutralization

viscosity of -..---water and

Petroleum spirits, definition of

specification for

Petrolcum trade, rules regulating the-crude petroleum

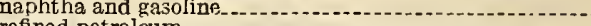
refined petrolcum

Phosphorus in ash, determination of, for coal and coke.-...

iles, bearing concrete, specificetion for premolded concrete, specification for constructing.-...... simplex concrete, specification for

Pin cups, specifiention for

Pipe, cement cication

, cilvert, reinforcod concrete, s. reinforced concrcte. er, ccment concrete, specification for

clay, specification for

definition of terms rclating to

reinforced conerete, snecification for

required safe crushing strengths of, to carry loads from ditch $f$

Pipe covering and blocks, magnesia molded, snecification for

Pipe scwers.

Pit run gravel ballast, specification for

tch, coal tar, roofing, specification for
stone hlock filler, specification for

Pitches, definition of

high-bitumen coal-tar, for use in damp proofing and waterproofing below and above ground level, specification for high-carbon and high-bitumen coal-tar, for use in constructing built-up roofs surfaced with slag or gravel, specification for

bigh-carbon coal-tar, for use in damp proofing and waterproofing below and abore ground level, specification for. soft tar, metbod of test for specific gravity of

tar, sufficiently solid to be bandled in fragments, method

of test for snecific gravity of . . ......................

Plain and reinforced concrete (water-cement ratio method), specification for

aster, gypsum, specification for

gypsum finishing

gypsum molding and casting.

gypsum neat.

gypsum pottery, specification for

gypsum ready sanded

lime, specification for sand for use in.

magnesia asbestos, specification for ...

Plister board, gypsum specification for

Plaster work, specification for

Plastering lime, specification for

specification for

Plastering sand, gypsum, specification for

Plesters, dental, specification for calcined gypsum for use in tbe preparation of

gypsum, concrete surface specification for

lastic cement, asphalt, specification for

Plastic firc clay refractories, specification for

Plastic flashings, specification for installation of, with bitu-

minous built-up roofing

Plastic magnesia cement, specification for

Plasticimetcr, Emley

Plasticity, water of, metbod for

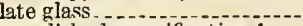

polished, specification for

Plumbago crucible, sizes o:

Plumbing, specification for

Plumbing fixtures, specification for-

marble and glass

porcelain ware.

slate.

soapstone.

vitreous ware

staple porcelain (all-clay)

vitreous china, recommendaticn for -1

Polished plate glass, specification for

Porcelain filler button, specification for

Porelain incerion,

Porcelon insulation, specification for

Porcelain jack panel, specification for

Porcelain plumbing fixtures.

rcelain ware, lahoratory, specification for specificatiou for

Pore water, method for

refra, apparent, test for

Portland ccment, cement grout filler specification and test for

Port land cement concrete, method of test for consistency of plain and reinforced, spccification for plain and reinforced, spccification for

specification for gravel for

Portland cement concrete base, specification for

Portland cement concrete pavement or base, spccification fo coarse aggrcgate for (stone, slag, or $\mathrm{B}$

Portland cement concrcte pavements, 1-course, for highways, specification for -

specification for

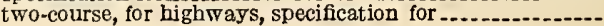


Page

Portland coment concrete sidewalks, specification for

Portland cement concrete street pavement, 1-course, specifitwo-course, specification for

Portland cement mortars, specification and test for compressive strengtb of

Portland cement stucco, application of, on concrete block and tile walls, specification for

finis b coat, specification for

recommended practice for

specification for

Posts, fence, concrete, specification for

Pottery plaster, gypsum, specification for

Pour point of petroleum products, method of

Powdered coal, method of test for fineness of

Powdered surfacing materials, test for

Powdered talc, specification for asphelt roll roofing surfaced

witb.

Precious stones, classification of

Precipitation number of lubricating oils, metbod of test for

Premolded concrete pipes, specification for constructing.

Premolded expansion joints, specification for

Premolded joint fillers, metbod of sampling and testing.....

Preparation of subgrade

Prepared roofing, asphalt, specification for

asphalt and asbestos, slate surfaced, specification for

Pressure tanks, regulations for the construction and installa-

tion of

Primer, asphait, for roofing and waterproofing, specification for-

Primer for use witb asphalt in damp proofing and watcrproof-

Priming coat, specification for creosote oil for

Prism glass

Processed glass.

Promenade tile, requirement for

Proportion of bitumen soluble in carbon tetrachloride, method of test for tbe determination of

Proportioning natural sand and gravel for concrete construction, metbod of

Protection test for lubricants and liquid fuels

Protective coatings, bituminous, metbod of test for steam

distillation of

Pulp, sulphite, specification for quicklime for use in tbe manu

furchaso specification for concrete aggregates.

Pycnometer metbod for specific gravity of bituminous materials

Pyrometric cone equivalcnt, method of testing for ............

\section{$Q$}

Quality, glacial gravels by lithological count of particles, method of determining -

water to be used in concrete, method of test for

Quantity, clay and silt in gravel for highway construction,

method of test for

clay in sand day, topsoil, and semigravel for bigbway construction, metbod of test for construction, metbod of test for

Quicklime, method of chemical analysis of method of sampling, inspection, packing and marking of specification
uicklime for-

structural purposes, specification for tbe manufacture of silica brick, specification for use in the cooking of rags for the manufacture of paper, specification for

use in the manufacture of sulphite pulp, specification for -use in t be textile industry, specification for

use in water treatment, specification for -.

\section{$\mathbf{R}$}

Rag felt, asphalt saturated, flashings, specification for roofing and w aterproofing, specification for coal-tar saturated, for roofing and waterproofing, specifcation for

Rag felt sbect roofing and shingles, asphalt, specification for.Railings for bridges, specification for

Rammer for permeability test of foundry sands

Rattler for testing paving brick

Reaction test for lubricants and liquid fuels

Reclaimed oil, specification for

Recommendation for-

asbestos paper and asbestos millboard

automobile brake lining

Cing

blackboard slate

cafeteria and restaurant chinaware

coated abrasive products

composition black board

dining car cbinaware-..-

glass containers for preserves, jellies, and apple butter.-. grinding wheels

bollow building tile

hospital chinaw are

botel chinaware

malleable foundry refractories

milk and cream bottles and bottle

one-piece porcelain insulators

roofing slate

rough and smooth face and common brick.

sand lime brick
Recommendation for-Continued.

staple vitreous chine plumbing fixtures

structural slate for plumbing and sanitary purposes..... vitrified paving brick

(tice ande

Recommended practice for Portland cement stucco-..-.-.

Reconstructed base course, specification for

Reconstruction of old macadam to serve as base course, specification for

Recuperator grease, requirement for

Recut granite pavements, specification for block for

Refined petroleum oil, metbod o I test for color of, by means o Saybolt chromometer

Refined tar, construction, specification for hot application, specification for hot application, specincation for

Refractories, clay, definition o

definition of terms relating to

fire clay, marine service, specification for malleable foundry, recommendation for

Refractory materials, including cbrome ores and chrome brick method of ultimate chemical analysis of

method of test for porosity and permanent volume changes in

Rofractory materials under load at high temperatures, metbod of test for

Regulations, construction and installation of tanks, gravity

and pressure, towers, etc...

mable liquids (design and construction of concrete fueloil storage

Reinforced concrete, specification for

structural, specification for

Reinforced concrete culvert pipe, spccification for .......... 336

Reinforced concrete pipe- Reinforced concrets poles, specification for

Reinforced concrete sew $\mathrm{cr}$ pipe, specification for

Reinforced concrete slab and girder bridges, specification for

Repair work, specification for higb and low carbon tar cements for use in (cut-back product)

specification for tar for use in
Residuo, desired penetration, metbod of test for perccntago of. specified penctration, method of test for Resistance of fire-clay brick to thermal spalling action, metbod
of test for

Restaurant chinaware, recommendation for Retaining wall masonry, asblar stone block rubble

Rigs and derricks, standard

Ring-ball method of test for softening point of bituminous matcrials.

Riprap, specification for

Road construction, specification for asphalt for use in

Road oil for hot application, specification for

Road oil substitute

Road oils, metbod of test for specific gravity of

Road surfaces, topsoil or sand clay, specification for materials

for - surfacing, specification for natural or artificial sand clay mixtures for

Road tars, method of test for specific gravity of

Roads, broken stone and gravel, spccification for definition of terms relating to materials for gravcl, specification for gravel for

Rock, method of test for abrasion of method of test for toughness of -

Rod packing, asbestos hicb pressure, specification for

Roll roofing, asphalt, smooth surfaced, metbod of testing. surfaced $w^{*}$ ith granular talc, specification for surfaced with granular talc, specification for-

beavy weight slate surfaced asphalt, specification for slate surfaced asphalt, metbod of testing................. specification for

Rolled figured sheet, glass

Roof coating, aspbalt frbrous, spccification for

Roof covcrings, built-up, specification for asphalt for use in constructing -

Roof lights, recommendation for

Roofing, asphalt prepared, specification for aspbalt rag felt shcet, specification for aspbalt roll, surfaced witb granular talc, specification for surfaced with powdered talc, specification for

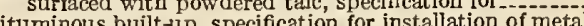
flashings witb.

llashings witb -
specification for installation of plastic flashings witb.-

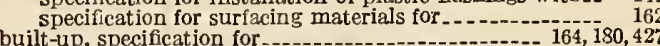
built-up, specification for

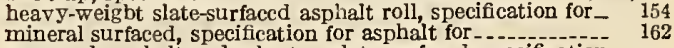
prepared, asphalt and asbestos, slate surfaced, specification slate, specification for

late-surfaced aspbalt prepared, specification for -...-...-. slate-surfaced aspbalt roll, method of testing specification for

smootb-surfaced aspbait roll, method of testing

specification for asphalt primer for

specification for asphalt paturated rag felt for

specification for coal-tar pitch for

specification for coal-tar saturated rag felt for-

unsurfaced built-up, specification for asphalt for ........... 


\section{Page}

Roofing felt, asphalt saturated, for use in waterproofing and in constructing built-up roofs, specification for - -..-..coal-tar saturated, for use in waterproofing and in con-
structing built-up roofs, specification for Roofing gravel, requirement for ...-...Roofing materials

Roofing slag, requirement for

Roofing slate, recommendation for standard for

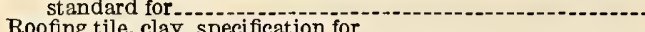
concrete, specification for strengtb test for.

Roofings, bituminous, analysis of

Roofs, built-up, specification for asphait-saturated asbestos felt for use in constructing

specification for asphalt-saturated roofing felt for use in constructing

specification for coal-tar saturated roofing felt for use in constructing

constructing surfaced with slag or gravel, specification for highcarbon and higb-bitumen coal-tar pitches for use in constructing

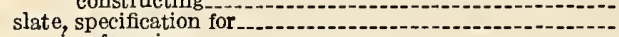

Rope, wire, for mines

Rope packings, asbestos, specification for

Rough-face brick, recommendation for

Roundels, signal, specification for--
Rubble, definition of

specification for-

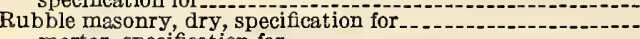
mortar, specification for

Rubblestone, specification for ...

Rules regulating the petroleum trado-

crude petroleum

refined petrolcum

Rupture, modulus of, of slate, determination of

\section{$\mathrm{S}$}

Sag tests

Salt, specification for

Sampling metbod for--

bituminous materials.

bituminous mixtures.

ceramic materials

clay

coal

coal and coke-

petroleum and petroleum products.

powdered coal

premolded joint fillers

quicklime and lime products.

stone, slag, gravel, sand, and stone block for use as highway materials

Sand, bituminons, specification for-cement grout filler

cement grout filler brick and stone block pavement, specification for

cement mortar bed for brick, stone block, or wood block

pavements, specification for

commercial sizes of - -

concrete, method of test for organic impurities in.-.-.-.-.-

definition of tbe term
foundry, metbod of testing and grading

gypsum plaster

gypsum plastering, specification for
highway construction, specification for commercial sizes of

himway construction, specification for comatarcial sizes or.

ime plaster, specification for--

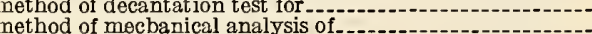

method of sampling

method of test for apparent specific gravity and absorption

or free moisture content for

natural, for concrete construction, metbod of proportioning

sbeet asphalt and bituminous concrete pavement, speci-

fication for

Sand analysis, method for

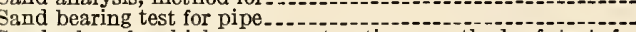

Sand clay for highway construction, method of test for

quantity of clay in specification for

Sand clay road surfaces, specification for materials for--..---.

Sand filler, specification for Sand lime brick, common, specification for.--10
recommendation for

Sand lime building brick, specification for

Sand or other finc matcrial witb broken stone or broken slag,

method of mechanical analysis of mixtures of, except aggre-

Saponification number, method of test for

Saybolt chromometer

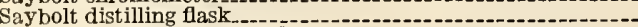

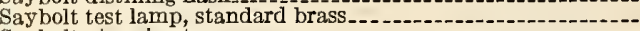

Saybolt viscosimeters

Screening, slag, method of test for apparent specific gravity of.

Securing specimens of hardened concrete from the structure,

method of.
Sediment in lubricants and liquid fuels, test for
Sediment in petroleum products, method of test for, by means
of centrifuge of centrifuge Segmental block sewers

62 Segmental tile blocks

54 Semigravel for highway construction, method of test for quan-

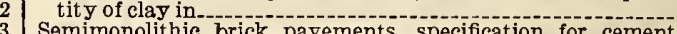
Semimonolithic brick pavements, specification for cement grout filler for

specification for cement sand bed for

Setting of cement, determination of time of

Setting of terra cotta, specification for-

Sewer block, concrete, specification for Sewer brick, clay, specification for clay, specification for

definition of terms relating to

reinforced concrete, specification for

equired safe crushing strengths of, to carry loads from ditch filling, specification for vitrified.

Sewers, concrete, monolithic, specification for---.--.--.---.-. Shale, percentage of, in aggregate, metbod of test for.-.-.-.-.-. Sinatter test for coke, method of

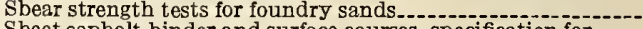

Sheet asphalt binder and surface courses, specification for----

Sheet asphalt pavement, specification for broken slag for binder

specification for broken stone for binder course for
specification for mineral filler for specification for mineral fill
specification for sand for

Sheet aspbalt paving, specification for

Sheet packing, asbestos metallic cloth, specification for

Sheet roofing and shingles, asphalt rag felt, specification for hingles, aspbalt rag felt sheet, specification for heavyweight slate surfaced asphalt, specification for----. slate surfaced asphalt, metbod of testing

slate surfaced aspbalt prepared, specification for--.--.-.-.

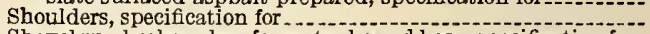

Sbovel run broken slag, for water-bound base, specification forSbower receptors, method of grading--.--_Sbrinkage, drying, method for

Shrinkage mesh for tile work
Shrinkage water, method for

Shrinkage water, method for

Sidewalk lights, reommendation for Sidewalks, concrete, specification for

Portland cement concrete, specification for

Sieve, definition of the term
Sieve analysis, aggregates for concrete, method of test for

coke, method of test for crushed bituminous coal, method of test for-.-.-.-.-.-

Sieve openings, requirement for

Sieves, method of testing

Sieves for testing purposes, specification for

Sight gage

for thick, specification for quickllme and hydrated lime Sills, tiled

Silt, quantity of, in gravel for highway construction, method of test for

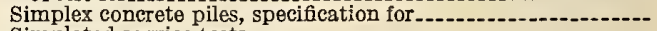
Simulated service tests.

ss, method of grading

Sintering test of molding sand broken stone and broken slag for highway construction,

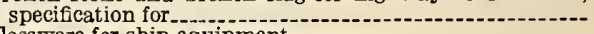

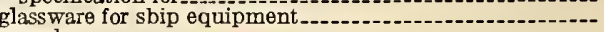
gravel

lenses for use in light signals

plumbago crucibles

sand and gravel for highway construetion, specification for.

Slag, broken, binder course, shcet asphalt pavement, specifcation for

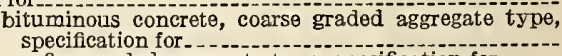

fine graded aggregate type, specification for --..cation for

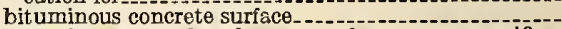

bituminous macadam base or surface course, specification for

bituminous macadam surface course
bituminous macadam wearing course, specification forbituminous surface treatment, specification for...... highway construction, specification for commercial sizes of -

metbod of mechanical analysis of, except aggregates used in cement concrete

method of mecbanical analysis of mixtures of sand or other fine material with, except aggregates used in

method of test for abrasion of

Portland ccment concrete base course

Portland cement concrete surface course

shovel run or crusher run, for water-bound base, specification for - . - -

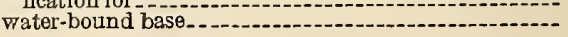


Page

Slag, water-bound base or wearing course, specification for definition of...

method of sampling Port roofing, requirement for

Sla screenings method of test for apparent specific gravity of Slag to be used in higbways and highway structures, specification for

slaking test, method for

Slaking test, method for--.-1 ate, hlack board, recommendation for

definition of the term metbod of flexure testing of (determination of modulus of rupture and modulus of elasticity)
method of test for determining the insulating qualities of

method of test for water absorption of plumbing, requirement for

roofing, recommendation for structural, for plumbing and sanitary purposes, recommendation for

Slate floors, terraces, and walks, specification for

Slate roofing, specification for

Slate roofs, specification for

Slate surfaced asphalt and asbestos prepared roofing, specifica-

tion for
Slate surfaced esphalt prepared roofing and shingles, specifica-

tion for

slate surfaced aspbalt roll roofing and sbingles, heavy weight,

specification for

method of testing

specification for

Slates, granular specification for

Slides, glass, specification for -

slop sinks, method of grading

Smooth-face brick, recommendation for

Smooth-surfoced asphalt roll roofing metbod of testing--

Soapstone for use in plumhing, specification for

Soffits, tiled

Soft pebbles in gravel, metbod of test for quantity of

Soft tar pitcbes, method of test for specific gravity of .........

Softening point, bituminous materials, method of test for (ring-ball metbod)

fre-clay brick, metbod of test for

tar products, method of test for (cuhe in water method) -

Soils, clsssification and bearing power of

subgrade, metbod of test for the determination of moisture equivalent of, in the field

Solid fuels, classification of

test code for
Solids in flue gases, quantitative test for

Soluhle bitumed, test for
Soluble cutting oil, requirement for

Soluble cutting oil, requirement for

Spalling action, tbermal, metbod of test for resistance of fineclay brick to

Spatula, porcelain

Specific gravity, apparent, approximate, of fine aggregate,

method of test for field determination of

coarse aggregates, method of test for -............

sand, gravel, stone, or other nonbituminous higbway

materials, metbod of test for

sand, stone, and slag screenings and other fine non-

hituminous highmay materials, metbod of test for stone and other coarse materials, method of test for.... test for .

asphalts and tar pitches sufficiently solid to be handled in fragments, method of test for

bituminous materials, metbod of test for definition of

lump coke, determination of

road oils, road tars, asphalt cements, and soft tar pitches, true, method for

Specific viscosity, method of test for

Specifications for

acid resisting aspbalt mastic

ond

application of Portland cement stucco on concrete hlock and tile walls

arches.

architectural terra cotta.

artificial bases

as bestos higb-pressure rod packing

asbestos metallic cloth gaskets.

asbestos metallic cloth sheet packing

asbestos millboard

asbestos valve stem packing

asbastos wick and rope packings

ashlar masonry

asphait bituminous carpet.-.

mineral surfaced roofing

unsurlaced huilt-up roofing

asphalt cement

aspholt flller, t5........

asphalt filler, type B

aspbalt filler for brick pavements (blown type)

asphalt for nse in damp proofing and waterproofing helow

and above ground level

Specifications for-Continued.

asphalt for use in rogd and pavement construction_-... 145

aspbalt macadam base

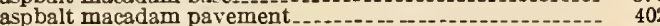

asphalt macadam surface course

asphalt mastic for use in waterproofing

aspbalt paving blocks....

asphalt plastic cement._._...

asphalt prepared roofing -

ssphalt rag felt sheet roofing and shingles...---.---.--

aspbalt roll roofing, surfaced with granular talc........... 154

surfaced with powderer talc....................... 154

asphalt saturated asbestos felt for use in constructing built.

asphalt saturated rag felt, flashings

roofing and waterproofing ..... in waterproofing and in constructing built-up roofs

asphalt saturated woven cotton fahric for waterproofing.asphalt varnish

aspbaltic concrete base.

asphaltic concrete binder and surface courses

asphaltic concrete surface course, coarse graded aggregate

ype

fine graded aggregate type_...

battery jar......

bedding course

hituminous carpets

hituminous grout for use in waterproofing below and ahove

bituminous materials - asphalt for bituminous carpets.

bituminous carpets.

fillers.

waterproofing materials

bituminous sand

block, durax granite pavement

granite block pavement

recut granite block pavement

brick chimneys witb fire clay fue linings

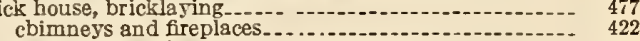

ideal construction

mortar

brick masonry-...

bricklaying.-

brickwork

broken slag, binder course, sheet aspbalt parement...... bituminous concrete, coarse graded aggregate type.fine graded aggregate type

bituminous concrete base or wearing course

bituminous conaca

bituminous macadam wearing course.

bituminous macadam wearing course.-bound base or wearing course

hituminous concrete base or surface course

bituminous concrete surface.

bituminous

hituminous macadam base or surface course......-. 251

broken slag, or gravel for bituminous surface treatment.

water-bound base or wearing course.

water-bound macadam surface course

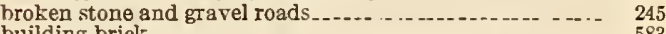

building brick

uildings for railway purposes, architectural terra cotta... $\quad 620$

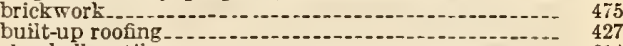

clay bollow tile

clay roofing tile.-. 619

concrete architectural stone

concrete roofing tile._.

excavation, filling, and back filling

exterior stucco work

latbing and plastering.

marble and tile work

plumbing

plumbing

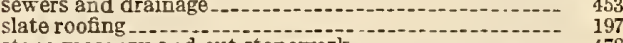

$\begin{array}{lll}\text { stone masonry and cut stonework. } & 478\end{array}$

burlap saturated with bituminous substances for use in

waterproofing

burned clay baliast_- - - .

calcined gypsum . . .

calcined gypsum for use in the preparation of dental

plasters

cement concrete artificial bases.

cement concrete battery box.

cement concrete sewer pipe

cement concrete sewer pipe-..--

cement sand bed for semimonolithic hrick pavements..

ceramic whiting -... . ..................

chimneys and fireplaces

clay fire brick, furnaces and annealing ovens marine boiler service.

stationary boiler service

clay hollow tile.

clay roofing tile- 
Specifications for-Con tinued.

clay sewer pipe

coal-tar oil for flooring and paving blocks............................

coal-tar pitch, roofing

stone block filler

waterproofing and damp proofing

coal-tar saturated rag felt for roofing and waterproofing

coal-tar saturated roofing felt for use in waterproofing and

in constructing built-up roofs

coarse aggregate

Portland cement concrete pavement or base (stone, slag, or gravel)

commereial grades of broken stone form of commercial sizes, brokcn stone and broken slag for highway

sand and gravel for highway construetion.

common clay brick

common sand lime brick

communication underground conduit construction.

compressed asbestos sheet packing - -

compressive strength of Portland cement mortars

concrete aggregates

concrete and reinforced concret

concrete architectural stone

concrete bearing piles

concrete brick

concrete bridge floors

concrete building block and concrete huilding tile

concrete huilding brick

concrete crihhing

concrete curb and concrete curh and gut ter

concrete curh and header.

concrete drain tile

concrete fence posts.

concrete floors

concrete masonry

concrete roofing tile

concrete

concrete sidcwalks .

concretes star

concrete swimming pool construction

conts

condiments (salt)

constructing premolded concrete piles

construction of built-up roofing

control of materials for highway hridgc

creosote oil for priming coat with coal-tar pitch in damp

proofing and waterproofing below and above ground level.

curbs

design and construction of concrete fuel-oil storage tanks.

drain tile

drain tile

dry ruhble masonry.

electrical conduit construction

emery cloth

excavation, filling, and back filling

excavation and fill

exterior

fine aggregate, Portland cement concrete

Portland cement concrete pavement or base

fine aggregate asphaltic concrete.

finish coat

fire clay brick

fire clay refractorics for marine service

fire tests of building construction and materials............

fiat glass for glazing purposes

flint paper

forty-five-degrecs-below-zero-Fahrenheit lubricating oil.

foundations and substructures

foundry coke.

fuel oils

garnet paper.

gas and coking coals

gasoline

glass, airport, and fixed light.

glass lantern glohes and lamp chimney

glass tableware.

glazing.

grading

granite

granular slates

gravel, bituminous concrete base

glavel road

gravel hase.

greases

ground supported vaults

gypsum.

gypsum molding plaster

gypsum partition tile or block

gypsum plaster

gypsum plaster board

gypsum plastering sand

gypsum pottery plaster

gypsum wall board

heavy weight slate surfaced asphalt roll roofing and heavy

weight slate surfaced asphalt shingles...................
Page | Specifications for-Continued.

high and low carbon tar cements ......................... 178 high and low carbon tar cements for use cold in repair work

high and low carbon tars for surface treatment, cold application

high hitumen coal-tar pitches for use in darmp proofing and water proofing below and above ground level

high-carbon and high-bitumen coal tar pitches for use in constructing built-up roofs surfaced with slag or gravel

high-carbon coal-tar pitches for use in damp proofing and waterproofing below and above ground level............ hot application

highway bridges and incidental structures, arches.......

ashlar masonry

bituminous matcrials, asphalt for bituminous carpets.

bituminous carpets phalt for bituminous carpets.

fillers.

waterproofing materials

brick masonry.

coarse aggregate

concrete bridge floors

concrete cribbing

concrete masonry

concrete viaducts

control of materials

dry rubble masonr

foundations and substructures...

monolithic concrete culverts.

mortar rubhle masonry

Portland cement - -

railings and parapets

reinforced concrete slab and girder bridges

rubble or cy clopean a ggregate

stone for masonean

Water for use with

wearing surfaces for bridge floors.

bituminous sand

fine aggregate for Portland cement concrete

gravel for Portland cement concrcte.

material

mineral filler

premolded expansion joints

tars and $t$ ar cements
hollow burned clay fireproofing, partition and furring tile.

hollow burned clay floor tile

hollow burned clay load bearing wall tile--

hollow clay fireproofing, partition and furring tile........

hollow clay foor tile.

hollow clay foor tile.

hollow clay load bearing wall tile

hydrated lime for structural purposes

hydrated lime for the manufactire of varnish_...........- 360

ideal construction of a brick house

illuminating oils

installation of metal flashings with bituminous built-up

roofing

installation of plastic flashings with bituminous built-up roofing

integral brick curb-

integral concrete curb

nterral waterproofing

interior marble

Keene's cement ..-...

labaratory apparatus (laboratory porcelain ware)

lath and plaster work

lathing and plastering

limestucco

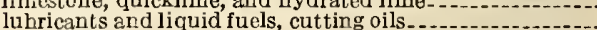

fucl oils

gasoline

greases

illuminating oils

lubricating oils.

methods of test

macadam base

magnesia asbestos plaster

molded pipe covering and blocks.

manufacture, furnishing and setting of

manufacture of rein forced concrete poles.

marble and tile work

masonry cement

pavements.

cement mortar bed for block pavements

cushion coursc, brick, stone block, or wood block pave-

ment

topsoil or sand clay road surface . . . mineral filler

shect asphalt and bitumionus concrete pavement.-.-. monolit hic concrete culverts

monolit hic concrete culverts_-_-_-_-

mortar rubble masonry

motor fuel

natural base... 
Specifications for-Continued. natural cement

natural or artificial sand clay mixtures for road surfacing.oil asphalt filler (type A)

one-course Portland cement concrete pavements for high-

waJS - course Portland cement concrete street pavement

overhead line material for 750-bolt direct current, direct

suspension.

paving brick .

petrolatum for use in impedance bonds...................

petroleum asphaltum

petroleun asphabtu

petroleum spirits (mineral spirits)

pit run gravel ballast . .

plain and reinforced concrete (water-cement ratio method).

plastic fire-clay refractories.

plastic magnesia ccment.

plumbing.

plumbing fistures, marble and glass

porcelain ware

slate

soapstone

vitreous ware

polished plate glass...

porcelain filler button

porcelain insulation

porcelain jack panel.

porcelain ware.

Portland cement

Portland cement concrete, plain and reinforced

Portland cement concrete base

Portland cement concrete pavements

Portland cement concrete sidewalks.

Portland ccment stucco

premolded expansion joints

primer for use with asphalt in damp proofing and water-

proofing below and above ground level.

proofing below and above ground level
quicklime and hydrated lime for the manuacture of silica

quicklime and hydrated lime for the manufacture of silica

quicklime and hydrated lime for use in the cooking of rags

for the manufacture of paper

quicklime for structural purposes.-.

quicklime for use in the manufacture of sulphite pulp.

railings and parapets.

reclaimed oil.

reconstructed base course

reconstruction of old macadam to serve as base

refined tar for construction

refined tar for hot application.

reinforced concrete, in structures

reinforced concrete culvert pipe.

reinforced concrete poles

reinforced concrete sewer pipe

reinforced concrete slab and girder bridges

required safe crushing strengths of sewer pipe to carry loads

from ditcb filling.

riprap

road oil for hot application.

roof coating, asphalt fibrous .

salt

sand, cement grout filler, brick and stono block pavcments

cement mortar bed for brick, stone block, or wood

block pavement

sand fillcr

sand-lime building brick .

sewer

sewers and drainago

sheet asphalt binder and surface courses

sheet asphalt paving

shoulders

shoulders, headers, curbs, and gutters

shovel rus or crusher run broken slag for water-bound base

sieves for testing purposes.

sieves for testing purposes,
signal roundels, lenses, and glass slides.

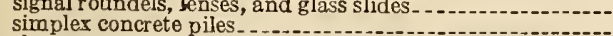

slag to be used in highways and highway structures...-.

slate floors, terraces, and walks . ...

slate roofing

slate roofs

slate suriaced aspbalt and asbestos prepared roofing

slate surfaced asphalt prepared roofing and shingies

slatesurfaced asphalt roll roofing and slate surfaced asphalt shingles

soapstone for use in plumbing

stone ballast.

stone block pavements

stone curb and header.

stone masonry

stone masony and cut stonowor.

street ralway track constrene wor

structure supported vaults

subbase

subgrades and foundations for pavements

surfacing materials for bituminous built-up roofing

tar cement.
Page

295

408

146

379
386

525
577

113

113

38
71

209
344

344
592

652
, 535

535

196

206
535

489

528

526
529

23

pecifications for-Continued.

tar for surface treatment (cold and hot application) ........ 174

tar for use in repair work joints in concrete roads............. tars for cold application

Telford base

tbermometers for tests.

tile duct.

tile work-

tolerances for asbestos yains

transformer oil.

transmission lubricating oils

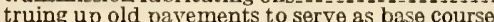

two-course Portland cement concrete pavements for highways

two-course Portland cement concrete strect pavement...-

underdrainage and elimination of water

vitreous ware.

vitrified cbinaware

vitrified clay conduit

vitrified paving brick

washed gravel ballast.

water for use witb cement

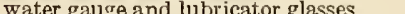

waterproof artificial abrasive paper.

waterproof garnet paper

waterproofing materials

wearing surfaces for bridge floors

woven cotton fabries saturated with bituminous sub-

stances for use in waterproofing

zero Fahrenheit lubricating oil

Specimens of hardened concrete from the structure, method of securing mineral, specification for

Sponge cups, specification for

Stall urinals, method of grading -

Standard for roofing slate

Standardized tablet bottles

Standards of practice, cement concrete pavements............ shoulders.

Staplo porcclain (all-clay) plumbing fixtures

Staple vitreous china plumbing fixtures, recommendation for.

Stationary boiler service, specification for clay fire brick for--

Staves, concrete, specification and building regulations for --

team distillation of bituminous protective coatings, method

Stone, broken, binder course, sheet asphalt pavement, speci-

fication for

bituminous concrete base or surface course, specifica-

tion for _. .

bituminous concrete surface, specification for

bituminous macadam, specification for.---
bituminous macadam base or surface course, specification for

bituminous macadam surface course

bituminous surface trcatment, specification for

form of specification for certain commercial grades of

higbway construction, specification for commercial sizes of

method of mecbanical analysis of, cxcept aggregates used in ccmcnt concrete method of mechanical analysis of mixtures of sand or other fino material with, except aggregates used in cement concrete.

metbod of test for abrasion of

Portland cement concrete base course

Portland cement concrete surface course

water-bound base or wearing course, specification

water-bound macadam surface course, speciñcation

cast, specification for

concrete, arcbitectural, specification for

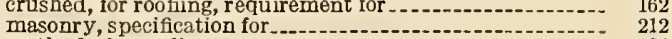

method of sainpling -

method of test for apparent specific gravity and absorption

or free moisture content for
Portland cement concrete pavement or base, specification

for ford base course and reconstructed base course Stone ballast, specification for

Stone block, method of sampling

Stone block filler, specification for coal-tar pitcb for

one block pavements, specification for

specification for materials for cement mortar bed for

specification for materials for cushion course of

specification for sand for cement grout filler for

specification for sand for cement mortar bed for

Stone curb and header, specification for

Stone masonry, specification for

$\begin{array}{ll}\text { Storing specimens of concrete in the field, method of } & 649 \\ & \end{array}$

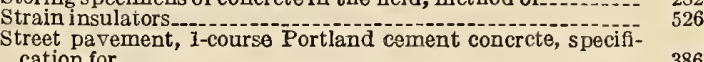

two-course Portland cement concrete, specification for

treet railway track construction, specificarion for 
Page

Strength test, drain tile
roofing tiles.

roofing tiles

Structural slate, for plumbing and sanitary purposes, recommendation for

Structure supported vaults, specification for

Structures, higbway, spccification for slag to be used in
Stucco, finish coat Portland cement, specification for lime, specification for

Portland cement, application of, on concrete block and tile walls, spccification for

Stucco work, exterior, specification for

Stucco work, exterior, specification for

Subbase, specification for

Subgrade, preparation of

Subgrades for pavements, specification for

Substructures for bridges, spccification for

Sulphite pulp, specification for quicklime for use in tbe manu-

facture of

Sulphur, dctermination of, for coal and coke.

Sulphur compounds, corrosive, in gasoline, method of test for

Sulphur in gasoline, free, method of test for detection of

Sulpbur in motor fuels, naphthas, and illuminating oils (lamp method), method of test for

Sulphur in petroleum oils heavier tban illuminating oil, metbod of test for

Supports, concrete, specification for

Surface, bituminous concrete, specification for broken stone for

for -

aspbaltic concrete, coarse graded aggregate type, specification for

fane graded aggregate type, specification for

bituminous concrete, specification for broken stone for

bituminous macadam, specification for broken slag for.-specification for broken stone for

sheet asphalt, specification for -

specification for broken slag for

water-bound macadam, specification for broken stone for

Surface moisture in fine aggregate, metbod of test for field

determination of

Surface treatment, specification for higb and low carbon tars

for -

Surface treatments witb cold bituminous materials..........

Surfacing materials, bituminous built-up roofing, specification for

granular, test for

powdered, test for

Swimming pool construction, specification for

Swimming pools, tiled

$\mathrm{T}$

Table of permeability test pressures.

Tahle of volume corrections for petroleum oils

Tahlet bottles, standardized.

'Tablcware, glass, specification for

Talc, granular, specification for asphalt roll roofing surfaced wowdered, specification for asphalt roll roofing surfaced witb

Tanks, fuel-oil storage, concrete, specification for design and construction of

gravity and pressure, regulations for the construction and installation of

vitreous cbina, method of grading

Tar, definition of

refined, for construction, specification for refined, for hot application, specification for

Tar-bound base

Tar cement, high and low carbon, for use cold in repair work (cut-hack product), specification for

bigh and low carbon, specification for

cation for.

Tar for use in repair work, specification for

Tar for use in the maintenance of cracks and for sealing joints in

concrcte roads, specification for

Tar pitches sufficiently solid to be handled in fragments, method of test for specific gravity of

Tar products, method of test for softcning point of (cube in water method)

Tars, definition of

high and low carhon, for surface treatment, hot applica-

refined, for bot surface treatments

road, method of test for specific gravity of

Tars for cold application, specification for-

Telford base, specification for

Tensile strength test for foundry sands.

Tensile test of baked core sand mixtures

Tension test, cernent.

fine aggregate for concrete, method of making

Terms relating to cypum industry, definition of --

Terms rclating to gypsum industry, defnition or-.-.-.-.-...-

Terms relating to lime, definition of

Terms relating to lime, definition of ----

Terms relating to petroleum, definition of

Terms relating to scwer pipe, definition of

Terra cotta, architectural, specification for specification for the manufacture, furnishing, and setting Terraces, slate, specification for Tests and test methods-

abrasion, broken stone, and broken slag gravel.-.-. stone, rock

absorption of concrete

apparent porosity.

apparent specific gravity coarse aggregates.

sand, stone, and slag screenings and other fine non-

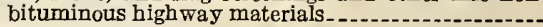
apparent specific gravity and absorption of stone and other coarse materials. content for sand, gravel, stone, or other nonbituminous

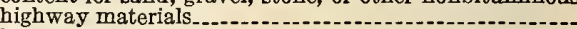
ashestos yarns

bituminous emulsions.--

bituminous masties, grouts, and like mixtures

brick.

burning quality, kerosene oils

long-time burning oil for railway use carbon residue of petroleum products (Conradson carbon residue) -... petroum residue) coarse particles in bituminous materials by means of elutriation

color of luhricating oils by means of union colorimeter.-.

color of petrolatum by means of the union colorimeter

color of refined petroleum oil by means of Saybolt chromometer :-

compression and tension, of fine aggregate for concrete, metbods of making

metbods of making

consistency of Portland cement concrete

cubic-foot weight, coke. crushed bituminous coal.

decantation fest for sand and other fime detection of free sulphur and corrosive sulphur compounds

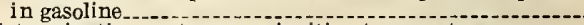
determination, autogenous ignition temperatures........ bitumen. equivalent of subgrade soils in the field proportion of bitumen soluble in carbon tetrachloride. voids in fine aggregate for concrcte

determining the insulating qualities of slate. distillation, bituminous materials suitable for road treatment.

crude petroleum

gasoline, naptha, kerosene, and similar petroleum products . natural gas gasoline ductility of bituminous materials electrical insulating oils

expressible oil and moisture in paraffin waxes............. stances ior use in waterproofing and roofing field determination, apparent specific gravity of fine aggreapproximate percentage of voids in fine aggregate.....-

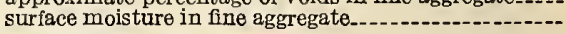
fineness of powdered coal.

flash and fire points by means of open cup flasb point by means of the Pensky-Martens closed tester.flash point of volatile flammable liquids. flexure testing of slate (determination of modulus of rup-

ture and modulus of elasticity). ture and modulus of elasticity)

float test for bituminous materials.
foundry sands foundry

gas oils gravity of petroleum and petroleum products by means of hydrometer.

gypsum and gypsum products hollow burned clay floor tile

hollow burned clay load-bearing wall tile

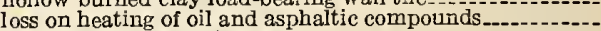
lubsicants and liquid fuels

clting point, paraffin wax petrolatum.

natural gas for gasoline content. neutralization number of petroleum products and lubricants.

organic impurities in sands for concretepenetration, bituminous materials grcases and petrolatum

percentage of bitumen insoluhle in carbon tetracbloride.percentage of bitumen insoluhle in paraffin naphtha....-percentage of bitumen soluhle in carhon disulpbide...-.percentage of residue of desired penetration..............

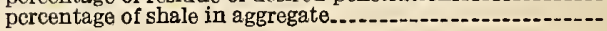


Tests and test methods-Continued.

porosity and permanent volume changes in refractory materials c...ent portland cement...

powdered surfacing materials.

precipitation number of lubricating oils

premolded joint fillers.

pyrometric cone equivalent

quality of water to be used in concrete

quantity of clay and silt in gravel for highway construction.-

quantity of clay in sand clay, topsoil, and semigravel for

quantity of clay in sand clay, topsoil, and semigravel for highway construction $---1 .-$
quantity of soft pebbles in gravel

quantity of sort peboles materials under load at bigh temperatures....

refractory materials under load at bigh temperatures....-

residue of specified penetration
resistance of fire clay brick to thermal spalling action.... roofing tiles

saponification number

shatter, for coke, method of

sieve analysis, aggregates for concrete

$$
\text { coke }
$$

sieves.

size of anthracit

smooth surfaced asphalt roll roofing, slate surfaced asphalt

roll roofing and slate surfaced asphalt shingles.

softening point, bituminous materials (ring-ball method) fire clay brick

tar products (cube in water method)

solid fuels.

soluble bitumen

soundness of coarse aggregate

specific gravity, asphalts and tar pitches sufficiently solid

to be handled in fragments.

bituminous materials

road oils, road tars, asphalt cements, and soft tar pitches.

specific viscosity.

steam distillation of bituminous protective coatings

steam emulsion of lubricating oils

sulpbur in motor fuels, naphthas, and illuminating oils

(lamp method)

sulphur in petroleum oils heavier than illuminating oil.-. thermal value of fuel oil

toughness, gravel

rock

tumbler, for coke, method of

unit weight of aggregate for concrete

vapor pressure.

viscosity of petroleum products and lubrients

volume

volume of cell space of lump coke.

water absorption of slate

water absorption of slate

water in petroleum products and otber bituminous materials

Textile industry, specification for quicklime and bydrated

Thermal spalling action, method of test for resistance of fire

Thermal spalli

Thermal value of fuel oil, metbod of test for.

Thermometers for tests, specifications for

Thief sampling of petroleum products.

Three edge bearing tests for pipe.

Tile, clay roofing, specification for

concrete building, specification for

concrete building, specification for

drain, concrete, specification for

fleproofing, partition and furring bolow burmed

scecification and test for furring, hollow burned clay,

floor, hollow clay, specification for

gypsum partition, specification for

hollow, definition of terms relating to

hollow clay, freproofing, partition and furring, specifica-

tion for

method of setting

promenade, requirement for

roofing, strength test for

(1)

Wall, hollow clay load bearing, specification for

Thite glazed, recommendation for

Tile duct, specification fo

Tile work, specification for

Tiled ceilings, soffits and sills

Tiled floors

Tolerances for asbestos yarns, specification for

Topped crude petroleum, definition of

Topsoil for highway construction, method of test for quantity of clay in.

Topsoil road surfaces, specification for materials for

Toughness, gravel, metbod of test for

rock, method of test for-

Towers, regulations for the construction and installation of -

Track construction, street railway, specification for

Tracks in paved streets, design of proper foundation for.....

Transformer oil, specification for

Transmission lubricating oils, specification for
Page

572

287

90

903
560
257

287

218

409

220

135
Transverse bar test of baked core sand mixtures--

Transverse strength test for foundry sands.

True specific gravity, method for

Truing up old pavements to serve as base course, specifica-

tion for---.--

Tumbler test for coke, metbod of

Two-course Portland cement concrete pavements for higbways, specification for

Two-course Portland cemont concrete street pavement, speci-

fication for

\section{U}

Ultinate cbemical analysis of refractory materials, including cbrome ores and chrome brick, method of Underdrainage, specification for

Underground conduit construction, specification for

Unglazed ceramic mosaic, recommendation for

Union colorimeter

Unit weight of aggregate for concrete, method of tests for

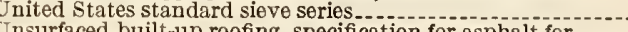

Unsurfaced built-up roofing, specification for asphalt for

\section{V}

Valve stem packing, asbestos, specification for...............

Vapor pressure testing method

specification for hydrated lime for tbo manufacture of

Vaults, ground supported, specification

structure supported, specification for

Viaducts, concrete, specification for.-

Vicat apparatus

Viscosimeters, Saybolt

scosit $y$, definition of

petroleum products and lubricants, method of test for.... specific, metbod of test for.

iscosity numbers, crank case lubricating oil

Vitreous cbina plumbing fixtures, recommendation for.-......

itreous ware, specification for

$\checkmark$ itrified brick for sewers

Vitrified chinaware, specification for

Vitrified clay conduit, specification for

Vitrified paving brick, specification for -

Voids in fine aggregate, concrete, metbod of test for determination of method of test for field determination of approximate percentage of

Volatile fammable liquids, metbod of test for flash point of.

Volatile matter, determination of, for coal and coke..........

Volume change, test for

Volume changes in refiactory materials, mothod of test for....

Volume correction table for petroleum oils, abridged.......-

Volume of cell space of lump coke, method of test for

W

Walks, slate, specification for

Wall board, gypsum, specification for

all tile, bollow clay load bearing, specification for

Walls, concrete block and tile, specification for application of tiled

Tashed gravel ballast, specification for

Water, shrinkage and pore, method for

Water absorption of slate, method of test for

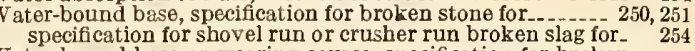

Water-bound base or wearing course, specification for broken

slag for..

Water-bound macadam surface course, specification for broken

stone for.

Water-closet bowls, vitreous cbina, method of grading

Water for cement grout filler..........

Water for gypsum plaster

Water for use with cement, specification for

Water-gauge glass, specification for

Water in bituminous emulsions, calcium chloride method for

determination of percentage of

Water in lubricants and liquid fuels by centrifuge, test for

Water in petroleum products, method of test for, by means of

Water in petroleum products and ot ber bituminous materials,

metbod of test for.

Water of plasticity, mothod for

$\begin{array}{ll}\text { Water to be used in concrete, method of test for quality of }-.- & 512 \\ \end{array}$

Water treatment, specification for hydrated lime for us in $_{--}-\overline{3} 51,362$ specification for quicklime for use in -

Waterproof artificial abrasive paper, specification for....$---6 \quad 633$

Waterproof garnet paper, specification for _.....- 634

specification for -

asphalt mastic for use in

asphalt primer for

152

164 
asphalt saturated roofing felt for use in

asphalt saturated woven cotton fabric for

coal-tar pitch for

coal-tar saturated rag felt for

coal-tar saturated roofing felt for use in

woven cotton fabrics saturated with bituminous substances for use in

Waterproofing below and above ground level, specification for

asphalt for use in.-

bituminous grout for use in

bituminous grout for use in

with coaltar pitch in

high-bitumen coal-tar pitches for use in

high-carbon coal-tar pitches for use i

primer for use with asphalt in

Waterproofing material, integral, specification for

Wax, paraffin

method of test for expressible oil and moisture in method of test for melting point of

Wearing course, bituminous macadam, specification for broken slag for.

specification for broken slag for specification for broken stone for

water bound, specification for broken slag for

Wearing surfaces for bridge floors, specification for

Wearing surfaces for bridge thoors, specincation

Weight, unit, of aggregate for concrete, method of test for

Wheels, grinding, crank shaft recommendation for

White glazed tile, recommendation for

Whiting, ceramic, specification for-_._.

Wick feed test_.._._-_._-_._- 57

$\begin{array}{ll}\text { Window glass. } & \\ \text { Wind } & \end{array}$

Wire diameter, requirement for

Wire glass

Wire rope for mines.....

Wood block pavement, specification for materials for cement mortar bed for specification for materials for cushion course of specification for sand for cement mortar bed for

Woven cotton fabric, asphalt saturated, for waterproofing,

specification for - .

woven cotton fabrics saturated with bituminous substances

Woven fabrics saturated with bituminous substances for use in

waterproofing and roofing, method of testing

Yarns, asbestos, specification for tolerances and test methods for Young, Sydney, equation for correction for barometric pressure

\section{Z}

Zero Fahrenheit lubricating oil, specification for 





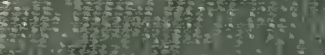

H.

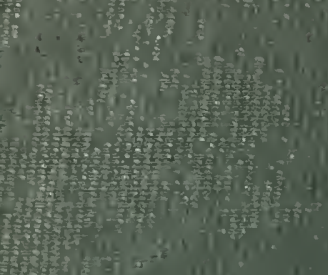

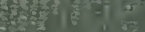

a.

ing in

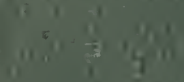

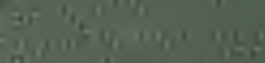

in

11

\section{givinge}

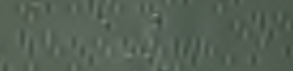

